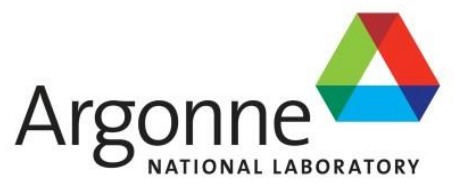

ANL-NSE-2

\title{
Experimental Breeder Reactor II (EBR-II) Level 1 Probabilistic Risk Assessment
}

Nuclear Science and Engineering Division

June 30, 1991 (Final Draft)

August 1, 2018 (Publication) 


\begin{abstract}
About Argonne National Laboratory
Argonne is a U.S. Department of Energy laboratory managed by UChicago Argonne, LLC under contract DE-AC02-06CH11357. The Laboratory's main facility is outside Chicago, at 9700 South Cass Avenue, Argonne, Illinois 60439. For information about Argonne and its pioneering science and technology programs, see www.anl.gov.
\end{abstract}

\title{
DOCUMENT AVAILABILITY
}

Online Access: U.S. Department of Energy (DOE) reports produced after 1991 and a growing number of pre-1991 documents are available free via DOE's SciTech Connect (http://www.osti.gov/scitech/)

Reports not in digital format may be purchased by the public from the National Technical Information Service (NTIS):

U.S. Department of Commerce

National Technical Information Service

5301 Shawnee Rd

Alexandria, VA 22312

www.ntis.gov

Phone: (800) 553-NTIS (6847) or (703) 605-6000

Fax: (703) 605-6900

Email: orders@ntis.gov

Reports not in digital format are available to DOE and DOE contractors from the Office of Scientific and Technical Information (OSTI):

U.S. Department of Energy

Office of Scientific and Technical Information

P.O. Box 62

Oak Ridge, TN 37831-0062

www.osti.gov

Phone: (865) 576-8401

Fax: (865) 576-5728

Email: reports@osti.gov 


\title{
Experimental Breeder Reactor II (EBR-II) Level 1 Probabilistic Risk Assessment
}

\author{
D. J. Hill ${ }^{1}$, W. A. Ragland ${ }^{1}$, and J. Roglans-Ribas ${ }^{2}$
}

Technical Editor: J. B. Heineman ${ }^{1}$

Contributors: Y. W. Chang ${ }^{1}$, G. H. Chisholm ${ }^{3}$, R. M. Fryer ${ }^{3}$, J. P. Herzog ${ }^{2}$, A. A. Kamal', A. E. Knox , K. J. Miles ${ }^{2}$, J. A. Morman ${ }^{1}$, P. A. Pfeiffer ${ }^{1}$, R. W. Schaefer ${ }^{5}$, D. Schurman ${ }^{5}$, J. Trainer ${ }^{7}$, and C. Wang ${ }^{1}$

${ }^{1}$ Reactor Engineering Division, Argonne National Laboratory

${ }^{2}$ Reactor Analysis Division, Argonne National Laboratory

${ }^{3}$ EBR-II Division, Argonne National Laboratory

${ }^{4}$ Technology Division, Argonne National Laboratory

${ }^{5}$ Engineering Division-AW, Argonne National Laboratory

${ }^{6}$ Science Applications International Corporation

${ }^{7}$ TENERA

June 30, 1991 (Final Draft)

August 1, 2018 (Publication)

Acknowledgment: The EBR-II PRA report owes much to individuals not included above particularly the unselfish assistance provided by EBR-II Division staff, W. K. Lehto, G. L. Lentz, W. H. Radtke, W. S. Barak, R. W. King, and E. M. Dean; members of the EBR-II Experimental Safety Review Group (ESRG) and Plant Safety Review Group (PDRG); P. $R$. Davis of PRD Consulting and L. Koch for their helpful reviews of earlier versions of this report; L. W. Deitrich for his leadership during the early stages of this project and N. A. Hanan for his assistance and advice on PRA methodology. 


\title{
TABLE OF CONTENTS
}

\author{
PART 1 of 3
}

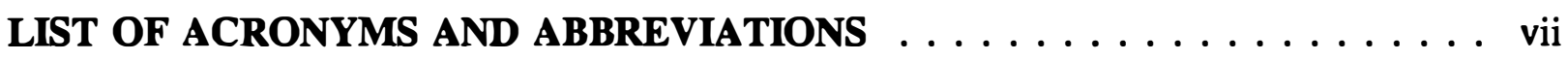

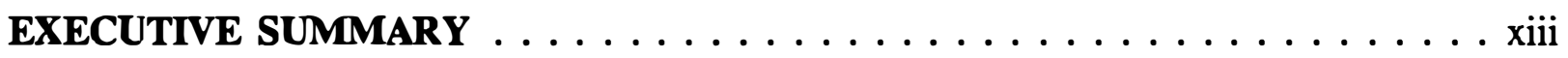

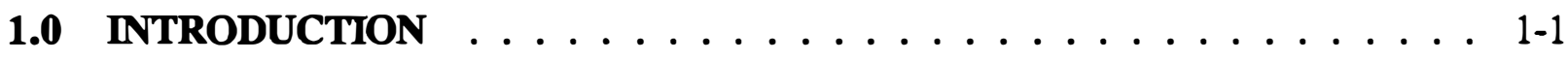

1.1 Project Motivation and Initiation ................. 1-1

1.2 Project Scope ....................... . . . . . . . . . . .

1.3 Topical Summaries of PRA Report Sections . . . . . . . . . . 1-2

1.4 Document Chronology .................. . . . . . . . . . . . . . . .

Section 1.0 References ..................... . . 1-7

2.0 PROJECT PLANS AND METHODS ASSESSMENT . . . . . . . . . 2-1

2.1 Project Organization ................... 2-1

2.2 Objectives and Scope .................. 2-2

2.3 Methodology .................... 2-7

2.4 Quality Assurance ................... 2-24

Section 2.0 References . . . . . . . . . . . . . . . . . 2-25

3.0 OVERVIEW OF EBR-II . . . . . . . . . . . . . . . 3-1

3.1 Site Location ......................... 3-1

3.2 Function and History of EBR-II ............... . . 3-5

3.3 Plant Description .................... 3-11

3.4 Reactor Shutdown System ................... 3-31

3.5 Decay Heat Removal .................... 3-33

3.6 Unique Features of EBR-II . . . . . . . . . . . . . . 3-38

3.7 Whole Plant Experiments in EBR-II . . . . . . . . . . . . . 3-41

Section 3.0 References . . . . . . . . . . . . . . . . . . . . . 3-48

4.0 SPECIAL ISSUES FOR THE EBR-II PRA . . . . . . . . . . . 4-1

4.1 Passive Safety Features .................. 4-1

4.2 Natural Circulation .................... . . 4-4

4.3 Reactivity Feedbacks .................... 4-7

Section 4.0 References ...................... . . 4-10 


\section{TABLE OF CONTENTS (Cont'd)}

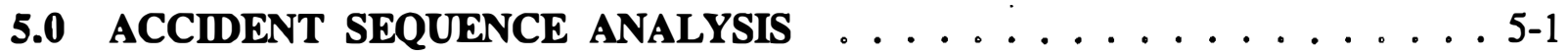

5.1 Initiating Event Identification .............. . . .

5.2 Initiating Event Quantification ............... 5-43

5.3 Event Tree Development ................ . . 5-69

5.4 Core Damage Analysis ................. . . 5-152

5.5 Uncertainity Analysis For Reactivity Feedbacks . . . . . . . 5-186

Section 5.0 References . . . . . . . . . . . . . . . . 5-208

6.0 SYSTEMS ANALYSIS ...................... . . . .

6.1 Systems Analysis Procedures ................ 6-1

6.2 Analysis of Frontline Systems .............. 6-13

6.3 Analysis of Support Systems ............... 6-49

Section 6.0 References .................. 6 . . . . . . . . . . . . .

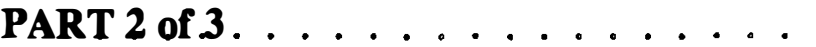

7.0 DATA BASE DEVELOPMENT

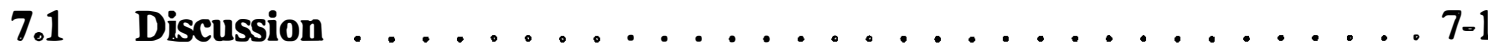

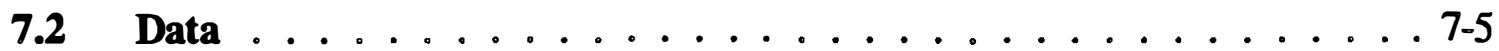

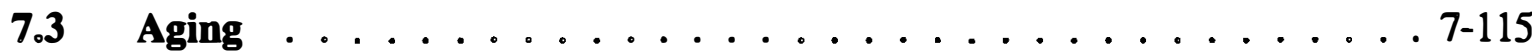

7.4 Dependency Analysis ................... 7-128

Section 7.0 References . . . . . . . . . . . . . . 7-148

8.0 HUMAN RELIABLITY ANALYSIS . . . . . . . . . . . . . 8-1

8.1 General Evaluation of EBR-II Human Factors . . . . . . . . 8-1

8.2 Human Reliability Assessment . . . . . . . . . . . . . . 8-3

8.3 Insufficient Subcriticality Margin during Refueling . . . . . . 8-45

8.4 Other Human Errors During Operation . . . . . . . . . . 8-58

8.5 Human Errors During Recovery .............. . 8-66

8.6 Summary ........................... 8-76

Section 8.0 References . . . . . . . . . . . . . . 8-77

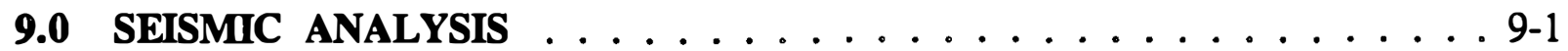

10.0 EXTERNAL EVENTS .................... . . . . . .

10.1 Introduction . . . . . . . . . . . . . . . . . 10-1

10.2 Sodium Fires ........................ 10-6

10.3 Fires ....................... 10-27 
TABLE OF CONTENTS (Cont'd)

10.4 Tornadoes and High Winds . . . . . . . . . . . . . . 10-30

10.5 Volcanism . . . . . . . . . . . . . . . . . . . . . 10-34

10.6 External Floods . . . . . . . . . . . . . . . . . . 10-36

10.7 Lightning . . . . . . . . . . . . . . . . . . 10-37

10.8 Aircraft Impact . . . . . . . . . . . . . . . . . . . . 10-38

10.9 Accidents at Other INEL Facilities (Industrial and Military) . . . 10-40

10.10 Turbine Missiles . . . . . . . . . . . . . . . . . . . 10-40

10.11 Internal Floods . . . . . . . . . . . . . . . . . . . . . . 10-42

10.12 Summary $\ldots \ldots \ldots \ldots$. . . . . . . . . . . . . . . 10-43

Section 10.0 References $\ldots \ldots \ldots \ldots$. . . . . . . . . . 10-46

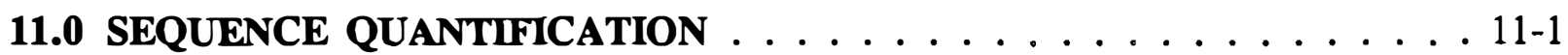

11.1 Introduction . . . . . . . . . . . . . . . . . . . 11-1

11.2 Sequence Quantification Methodology . . . . . . . . . . . . . 11-2

11.3 Sequence Quantification Results . . . . . . . . . . . . . . 11-10

12.0 UNCERTAINTY, SENSITIVITY, AND IMPORTANCE ANALYSES . . . 12-1

12.1 Introduction . . . . . . . . . . . . . . . . 12-1

12.2 Uncertainty Analysis . . . . . . . . . . . . . . . . 12-1

12.3 Sensitivity Analysis . . . . . . . . . . . . . . . . . 12-8

12.4 Importance Calculations . . . . . . . . . . . . . . . . 12-16

13.0 INTERPRETATION OF RESULTS . . . . . . . . . . . . 13-1

13.1 Internal Events . . . . . . . . . . . . . . . . . . 13-1

13.2 Seismic Event . . . . . . . . . . . . . . . . . . . . . . 13-14

13.3 External Events (non-seismic) . . . . . . . . . . . . . . . 13-14

13.4 Summary . . . . . . . . . . . . . . . . . . . . 13-14

13.0 References . . . . . . . . . . . . . . . . . . 13-17

14.0 SUMMARY AND CONCLUSIONS . . . . . . . . . . . . . . . . . 14-1

14.1 Summary of Key Results . . . . . . . . . . . . . . . . . . . 14-1

14.2 Conclusions . . . . . . . . . . . . . . . . 14-3

PART 3 of 3

APPENDIX A: AN ASSESSMENT OF RISK DURING CORE REFUELING AT EBR-II APPENDIX B: FAULT TREES 


\section{ACRONYMS AND ABBREVIATIONS}

I. General

$\begin{array}{ll}\text { ACRDS } & \text { Automatic Control Rod Drive System } \\ \text { ACS } & \text { Argon Cooling System } \\ \text { ANL } & \text { Argonne National Laboratory } \\ \text { ANL-E(ast) } & \text { Argonne National Laboratory, Illinois Site } \\ \text { ANL-W(est) } & \text { Argonne National Laboratory, Idaho Site } \\ \text { ANSI } & \text { American National Standards Institute } \\ \text { ASME } & \text { American Society of Mechanical Engineers } \\ \text { ATR } & \text { Advanced Test Reactor } \\ \text { ATWS } & \text { Anticipated Transient Without Scram } \\ & \\ \text { BFTF } & \text { Breached Fuel Test Facility } \\ \text { BOP } & \text { Balance-of-Plant } \\ \text { BWR } & \text { Boiling Water Reactor } \\ & \\ \text { CCF } & \text { Common Cause Failure } \\ \text { CDF(cdf) } & \text { Cumulative Distribution Function } \\ \text { CDFR } & \text { Commercial Demonstration Fast Reactor } \\ \text { CRBR(P) } & \text { Clinch River Breeder Reactor (Plant) } \\ \text { CRDM } & \text { Control Rod Drive Mechanism } \\ \text { CREDO } & \text { Centralized Reliability Data Organization } \\ \text { CP } & \text { Control Power (Relays) } \\ \text { CPS } & \text { Constant (Continuous) Power System } \\ \text { DAS } & \text { Data (Digital) Acquisition System } \\ \text { DBW } & \text { Design Basis Wind } \\ \text { DCS } & \text { Digital Control Station } \\ \text { DHR } & \text { Decay Heat Removal } \\ \text { DN } & \text { Delayed Neutron } \\ \text { DSNP } & \text { Dynamic Simulator for Nuclear Power Plants } \\ \text { EBR-II } & \text { Experimental Breeder Reactor II } \\ \text { ECCS } & \text { Emergency Core Cooling System } \\ \text { EP } & \text { Emergency Procedures } \\ \text { EP } & \text { Engineering Physics Division (ANL) } \\ \text { EPRI } & \text { Electric Power Research Institute } \\ \text { ESF } & \text { Engineered Safety Feature } \\ \text { ESRP } & \text { Eastern (portion) of the Snake River Plain } \\ & \\ \text { FAA } & \text { Federal Aviation Agency } \\ \text { FCF } & \text { Fuel Cycle Facility (ANL) } \\ \text { FERD } & \text { Failure Modes and Effects Tree Analysis Program } \\ \text { FFTF } & \\ \text { FMEA } & \\ \text { FTAP } & \\ & \\ & \end{array}$




\section{ACRONYMS AND ABBREVIATIONS (Cont'd)}

GLASS

GSCS .

HEP

HEPA

HFBR

HFEF

HFIR

HMLD

HPP

IDCOR

IE

IEEE

IFR

IFR

IHX

INEL

IOT

IREP

LCDL

LCRA

LLNL

LMFBR

LMR

LOCA

LOCP

LOF(WS)

LOHS(WS)

LONP

LOS(A)

LOPI

LPP

LWR

MG (M/G)

MSL

MV/I

NAS

NCPS

NESC

NITF

NQA-1

OC

PDF(pdf)

PICT

P\&ID

PFR
Germanium-Lithium Argon Scanning System

Graphite and Shield Cooling System

Human Error Probability

High-Efficiency Particulate-Air

High Flux Beam Reactor

Hot Fuel Examination Facility (ANL)

High Flux Isotope Reactor

Hydrogen Meter Leak Detector

High Pressure Plenum

Industry Degraded Core Rulemaking Group

Initiating Event

Instititute of Electrical and Electronic Engineers

Integral Fast Reactor

Instrument Flight Rules

Intermediate Heat Exchanger

Idaho National Engineering Laboratory

Inherent Operability Tests

Interim Reliability Evaluation Program

Delayed Loss of Circulation

Rapid Loss of Circulation

Lawrence Livermore National Laboratory

Liquid Metal Fast Breeder Reactor

Liquid Metal Reactor

Loss of Coolant Accident

Loss of Constant Power

Loss of Flow (without Scram)

Loss of Heat Sink (without Scram)

Loss of Normal Power

Loss of Sodium (Accident)

Loss of Piping Integrity

Low Pressure Plenum

Light Water Reactor

Motor-Generator

Mean Sea Level

Millivolt to Current Converter

National Academy of Sciences

Numerically Controlled Positioning System

National Energy Software Center

Nuclear Instrument Test Facility

Quality Assurance Program Requirements for Nuclear Facilities (ANSI/ASME Standard)

OverCooling

Probability Density Function

Plant Inherent Control Test

Piping and Instrumentation Diagram

Prototype Fast Reactor 


\section{ACRONYMS AND ABBREVIATIONS (Cont'd)}

$\begin{array}{ll}\text { PRA } & \text { Probabilistic Risk Assessment } \\ \text { PRD } & \text { Power Reactivity Decrement } \\ \text { PTCGSSS } & \text { Primary Tank Cover Gas Sampling Supply System } \\ \text { PWR } & \text { Pressurized Water Reactor } \\ \text { QA } & \text { Quality Assurance } \\ \text { QAE } & \text { Quality Assurance Element } \\ \text { QAPD } & \text { Quality Assurance Program Description } \\ & \\ \text { RA } & \text { Reactor Analysis Division (ANL) } \\ \text { RAS } & \text { Reactor Analysis and Safety Division (ANL) } \\ \text { RAW } & \text { Risk Achievement Worth } \\ \text { RBCB } & \text { Run-Beyond-Clad-Breach } \\ \text { RDF } & \text { Rod Drop Feedback Reactivity } \\ \text { RE } & \text { Reactor Engineering Division (ANL) } \\ \text { RIFL } & \text { Large Fast Reactivity Insertion } \\ \text { RRW } & \text { Risk Reduction Worth } \\ \text { RSS } & \text { Reactor Shutdown System } \\ & \\ \text { SA (S/A) } & \text { Subassembly } \\ \text { SAP6 } & \text { Structural Analysis Program - Version 6 } \\ \text { SASSYS } & \text { Safety Analysis Code for Plant Transient Analysis } \\ \text { SAS4A } & \text { Safety Analysis Code for Severe Accident Analysis } \\ \text { SDD } & \text { System Design Description } \\ \text { SGTR } & \text { Steam Generator Tube Rupture } \\ \text { SHRT } & \text { Shutdown Heat Removal Test } \\ \text { SOT } & \text { Subassembly Outlet Temperature } \\ \text { SRP } & \text { Savannah River Plant } \\ \text { SRP } & \text { Standard Review Plan } \\ \text { TAN } & \text { Test Area North } \\ \text { THTB } & \text { Transient Heat Transfer Code - Version B } \\ \text { TMI-2 } & \text { Three Mile Island - Unit 2 } \\ \text { TOP } & \text { Transient OverPower } \\ \text { TSOF } & \text { Total Sodium Flow } \\ & \\ \text { UOR } & \text { Unusual Occurrence Report } \\ \text { WRC } & \text { Reactor Upper Plenum Flow - Channel B } \\ \text { UPFB } & \text { Uninterruptible Power Supply } \\ \text { UPS } & \text { United States Department of Energy } \\ \text { USDOE (DOE) } & \text { United States Nuclear Regulatory Commission } \\ \text { USNRC (NRC) } & \\ & \\ \text { VFR } & \\ & \\ \text { WR } & \\ & \end{array}$


ACRONYMS AND ABBREVIATIONS (Cont'd)

II. Accident Sequence Analysis

1. Initiating Events

$\begin{array}{ll}\text { ANTC } & \text { Anticipatory Shutdown } \\ \text { DKHR } & \text { Decay Heat Removal Sub-tree } \\ \text { EVTR } & \text { Evaporator Tube Rupture } \\ \text { I } & \text { Initiating Event - Reloading Errors } \\ \text { IDAM } & \text { Fuel Damage Sub-tree } \\ \text { IE } & \text { Initiating Event } \\ \text { LCDL } & \text { Loss of Circulation - Delayed } \\ \text { LCRA } & \text { Loss of Circulation - Rapid } \\ \text { LF1A } & \text { Single Pump Loss of Flow - Group A } \\ \text { LF1B } & \text { Single Pump Loss of Flow - Group B } \\ \text { LF1C } & \text { Single Pump Loss of Flow - Group C } \\ \text { LF1D } & \text { Single Pump Loss of Flow - Group D } \\ \text { LF2A } & \text { Double Pump Loss of Flow - Group A } \\ \text { LF2B } & \text { Double Pump Loss of Flow - Group B } \\ \text { LF2C } & \text { Double Pump Loss of Flow - Group C } \\ \text { LF2D } & \text { Double Pump Loss of Flow - Group D } \\ \text { LF2E } & \text { Double Pump Loss of Flow - Group E } \\ \text { LF2F } & \text { Double Pump Loss of Flow - Group F } \\ \text { LMFR } & \text { Liquid Metal Fire } \\ \text { LOCP } & \text { Loss of Constant Power } \\ \text { LONP } & \text { Loss of Normal Power } \\ \text { LOPI } & \text { Loss of Piping Integrity } \\ \text { MDLF } & \text { Local Faults: Metal Driver Breach Initiator } \\ \text { MELF } & \text { Local Faults: Metal Experiment Breach Initiator } \\ \text { OCPP } & \text { Overcooling by Primary Pump Overspeed } \\ \text { OCSL } & \text { Overcooling by Major Steam Leak } \\ \text { OCSP } & \text { Overcooling by Secondary Pump Overspeed } \\ \text { OELF } & \text { Local Faults: Oxide Experiment Breach Initiator } \\ \text { RIFL } & \text { Fast Reactivity Insertion ( > 1.00 \$ ) } \\ \text { RIFS } & \text { Fast Reactivity Insertion ( < 0.35 \$ ) } \\ \text { RISA } & \text { Slow Reactivity Insertion - Group A ( < 0.20 \$) } \\ \text { RISB } & \text { Slow Reactivity Insertion - Group B ( 0.20-0.65 \$) } \\ \text { RISC } & \text { Slow Reactivity Insertion - Group C ( 0.65-1.10 \$) } \\ \text { RISD } & \text { Slow Reactivity Insertion - Group D ( > 1.10 \$) } \\ \text { SCRM } & \text { Scram } \\ \text { SDFR } & \text { Shutdown Cooler NaK Fire in Containment } \\ \text { SFLE } & \text { Subassembly Local Fault from Loading Error } \\ \text { SFPB } & \text { Subassembly Local Fault from Partial Blockage } \\ \text { SHDL } & \text { Long Shutdown } \\ \text { SHDS } & \text { Short Shutdown } \\ \text { SPTR } & \text { Superheater Tube Failure } \\ \text { SSFR } & \text { Secondary Sodium Fire in Containment } \\ \text { TAIR } & \text { Transient - Loss of Instrument Air } \\ \text { TBOP } & \text { Loss of Steam Plant Heat Removal } \\ & \end{array}$




\section{ACRONYMS AND ABBREVIATIONS (Cont'd)}

TNDO Tornado

TSDC Transient - Unrecoverable Loss of a Shutdown Cooler

TSEC Loss of Secondary System Heat Removal

TSHC Transient - Unrecoverable Loss of Shield Cooling

2. Event Tree TOPs

$\begin{array}{ll}\text { BPHR } & \text { Balance of Plant Decay Heat Removal } \\ \text { DETP } & \text { Detect in Time to Shutdown and Prevent Propagation } \\ \text { DETS } & \text { Detect in Time to Shutdown and Prevent Propagation } \\ \text { DHR } & \text { Decay Heat Removal } \\ \text { DHRL } & \text { Decay Heat Removal Long Term } \\ \text { DHRS } & \text { Decay Heat Removal Short Term } \\ \text { DRLA } & \text { Decay Heat Removal Long Term (1 sd cooler + shield cooling) } \\ \text { DRLB } & \text { Decay Heat Removal Long Term (2 shutdown coolers) } \\ \text { DRSA } & \text { Decay Heat Removal Short Term (1 sd cooler + shield cooling) } \\ \text { DRSC } & \text { Decay Heat Removal Short Term (1 sd cooler) } \\ \text { DSRC } & \text { Decay Heat Removal Short Term } \\ \text { DUME } & \text { Water and Sodium Dump before Leak Escalation } \\ \text { DUMS } & \text { Water and Sodium Dump before Leak Escalation } \\ \text { FROD } & \text { Sufficient Control or Safety Rods Drop during LOF Event } \\ \text { FSDG } & \text { Less than 2 DEG Failures within First 0.2 Seconds } \\ \text { FSIG } & \text { Scram Signal during LOF Event } \\ \text { LKDT } & \text { Leak Detection by HMLDs } \\ \text { LLRF } & \text { Limited Leak } \\ \text { LMFD } & \text { Liquid Metal Fire Detection } \\ \text { LROD } & \text { Sufficient Control or Safety Rods Drop during LOF Event } \\ \text { MODS } & \text { Leak Escalates within First Minute } \\ \text { MRSS } & \text { Manual SCRAM Under LOHS Conditions } \\ \text { NBFM } & \text { No Boiling or Fuel Melting in Average Core Assembly } \\ \text { NPFB } & \text { No Pin Failure or Boiling in T.S. Limit Pins } \\ \text { NPPB } & \text { No Pin-to-Pin Propagation } \\ \text { NPPD } & \text { No Pin-to-Pin Propagation } \\ \text { NPPE } & \text { No Pin-to-Pin Propagation } \\ \text { NPPO } & \text { No Pin-to-Pin Propagation } \\ \text { NPPP } & \text { No Pin-to-Pin Propagation } \\ \text { NSSP } & \text { No Subassembly-to-Subassembly Propagation } \\ \text { NSSS } & \text { No Subassembly-to-Subassembly Propagation } \\ \text { PKRU } & \text { Pumps Keep Running for 1 Hour } \\ \text { PRRE } & \text { Pressure Relief by Secondary Boundary Failure } \\ \text { PRRS } & \text { Pressure Relief by Secondary Boundary Failure } \\ \text { PRUN } & \text { Primary Pumps keep Running } \\ \text { PS } & \text { Protective Surveillance } \\ \text { RFIR } & \text { Reactor Shutdown } \\ \text { RIHX } & \text { Reactor Shutdown before IHX Failure } \\ \text { RROD } & \text { Sufficient Control or Safety Rods Drop during TOP Event } \\ \text { RSIG } & \text { Scram Signal during TOP Event } \\ & \end{array}$




\section{ACRONYMS AND ABBREVIATIONS (Cont'd)}

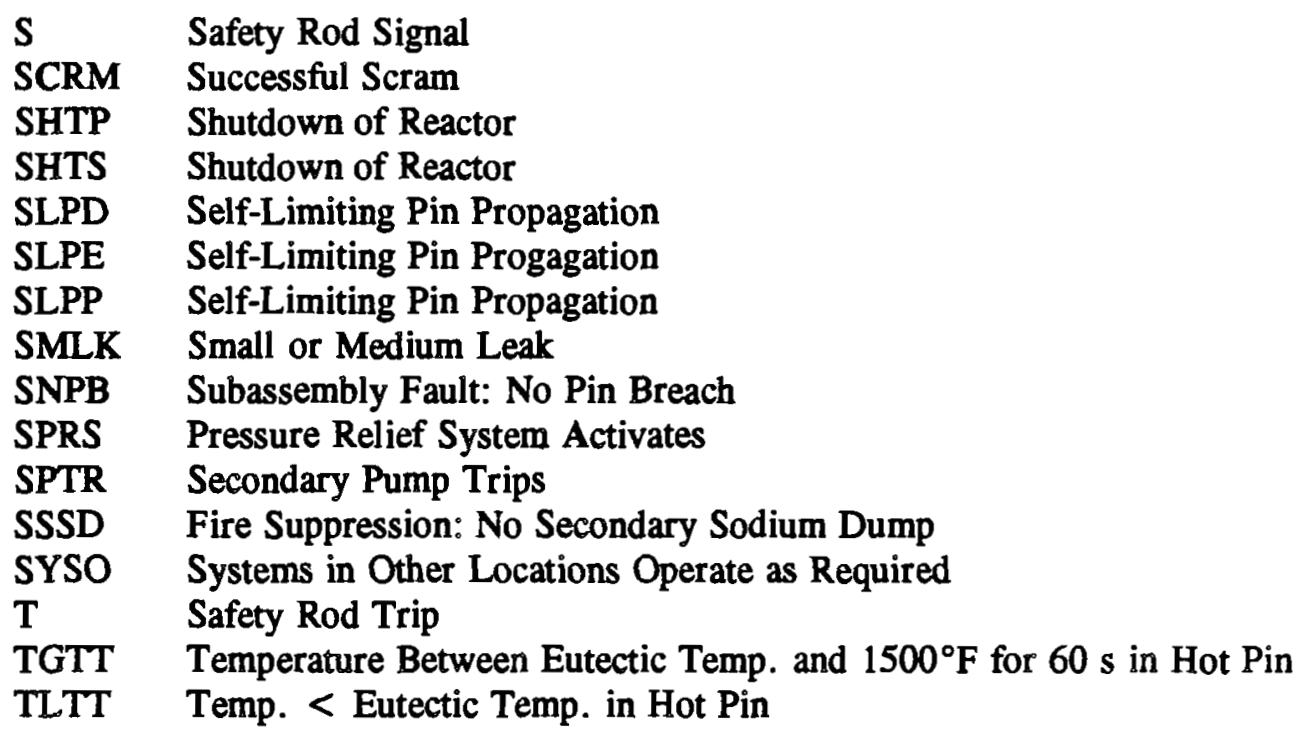

3. Fuel Damage Categories (End States)

$\begin{array}{ll}\text { CD } & \text { Core Damage } \\ \text { CSD } & \text { Core Structural Damage } \\ \text { MCD } & \text { Minor Core Damage } \\ \text { ND } & \text { No Damage } \\ \text { PED } & \text { Potential Experiment Damage }\end{array}$




\section{EXECUTIVE SUMMARY \\ EXPERIMENTAL BREEDER REACTOR-II LEVEL 1 PRA}

\subsection{BACKGROUND}

The National Academy of Sciences Study of the USDOE Category A Reactors recommended that a Probabilistic Risk Assessment (PRA) should be performed on the EBR-II Reactor. In January 1989, a Level 1 PRA was initiated. This report documents work performed in completing the Level 1 PRA except for seismic analysis; it describes the methodology used, assumptions made, the analysis performed, and the final results. The seismic risk assessment is in progress. It will be separately documented.

Probabilistic Risk Assessments are categorized at three levels. The product of a Level 1 PRA is the identification of the dominant component failure combinations that may lead to fuel damage and their frequencies. In a Level 2 PRA the physical processes occurring during an accident are analyzed and the response of the confinement system determined. The time and mode of confinement failure as well as the inventories of radionuclides released to the environment are predicted. A Level 3 PRA analyzes the transport of radionuclides through the environment and can assess the public health or economic consequences of an accident.

\subsection{OBJECTIVES}

The EBR-II Level 1 PRA successfully fulfilled four primary objectives:

- Identification of the plant features that are the most important contributors to the EBR-II fuel-damage frequency.

- Development of plant logic models for use in analyzing safety and reliability issues. 
- Identification of dominant EBR-II accident sequences and their frequencies.

- Establishment of an Argonne National Laboratory team with demonstrated expertise in performing risk and reliability analyses.

\subsection{SCOPE}

The EBR-II Level 1 PRA examined internal events such as equipment failures, operator and maintenance errors, seismic events and internal fire and flooding. Initiating events were identified through a review of plant event reports and a functional analysis of the plant. An examination of plant records provided a plant-specific data base. Where required by lack of sitespecific data, generic data bases were employed with careful consideration of EBR-II unique features and conditions. A thorough consideration of common-cause events was included, particularly in the reactor shutdown system.

\subsection{VALIDITY AND REVIEW}

To ensure a high degree of validity of the EBR-II models, there was extensive involvement of EBR-II operations, engineering analysis, and safety staff personnel in performing the study. This close cooperation of the EBR-II staff in the initial analyses will facilitate downstream use of the PRA for risk related design or operational decisions.

In addition, review cycles of the program were implemented. These included:

- Review and approval of the system descriptions, success criteria, and fault trees by the plant system and nuclear safety engineers.

- External (non-ANL) peer review of the program methodology and results by PRA consultants.

- Independent review of the methodology and results by EBR-II division, ANLWest. 
- Independent review of the scope and methodology by a senior management review panel (ANL).

\subsection{RESULTS}

An important result of a Level 1 PRA is the estimation of the frequency of events resulting in damage. The EBR-II Level 1 PRA calculated a total accident sequence frequency from internal events of slightly less than $210^{-6}$ per year. Figure 1 compares the results of this study with the results from a recent study of commercial LWRs. It should be emphasized that the EBR-II is not directly comparable to LWRs as EBR-II has many unique design features and mitigating safety systems which act to limit the progression of reactor fuel damage. Also the definition of damage for the EBR-II PRA is cast in such a way as to include accidents that may lead only to minor or no core damage under specific circumstances, while Level 1 PRAs for commercial LWRs often assume that incipient core melt will always progress to full core melt. Therefore caution should be used in comparing the EBR-II results with any specific LWR. However, as shown in Fig. 1, the EBR-II risk profile is lower then commercial LWRs.

The risk from external events is also low. The dominant nonseismic external event is a liquid metal fire under the deck plates which is judged to lead to damage with an annual frequency of $3.610^{-6}$ per year. Table 1 compares the results of this study with other USDOE Category A reactors. Again it can be seen that EBR-II appears to represent a low risk when compared to these reactors.

The conclusions reached by the Level 1 study are:

- The frequency of damage at EBR-II is generally lower than that reported for commercial LWRs and the USDOE Category A reactors.

- There is no single dominant source or cause of the various accident sequences.

- The passive decay heat removal function and the ability of reactivity feedbacks to limit the temperature excursion in a transient lead to low risk. 
- The passive decay heat removal function and ability of reactivity feedbacks to limit temperature excursion in a transient also lead to a sparse set of requirements for active safety systems or operator intervention.

- EBR-II is relatively insensitive to human error and only a few important human actions were identified.

- EBR-II has little or no requirement for electrical power to prevent fuel damage. 


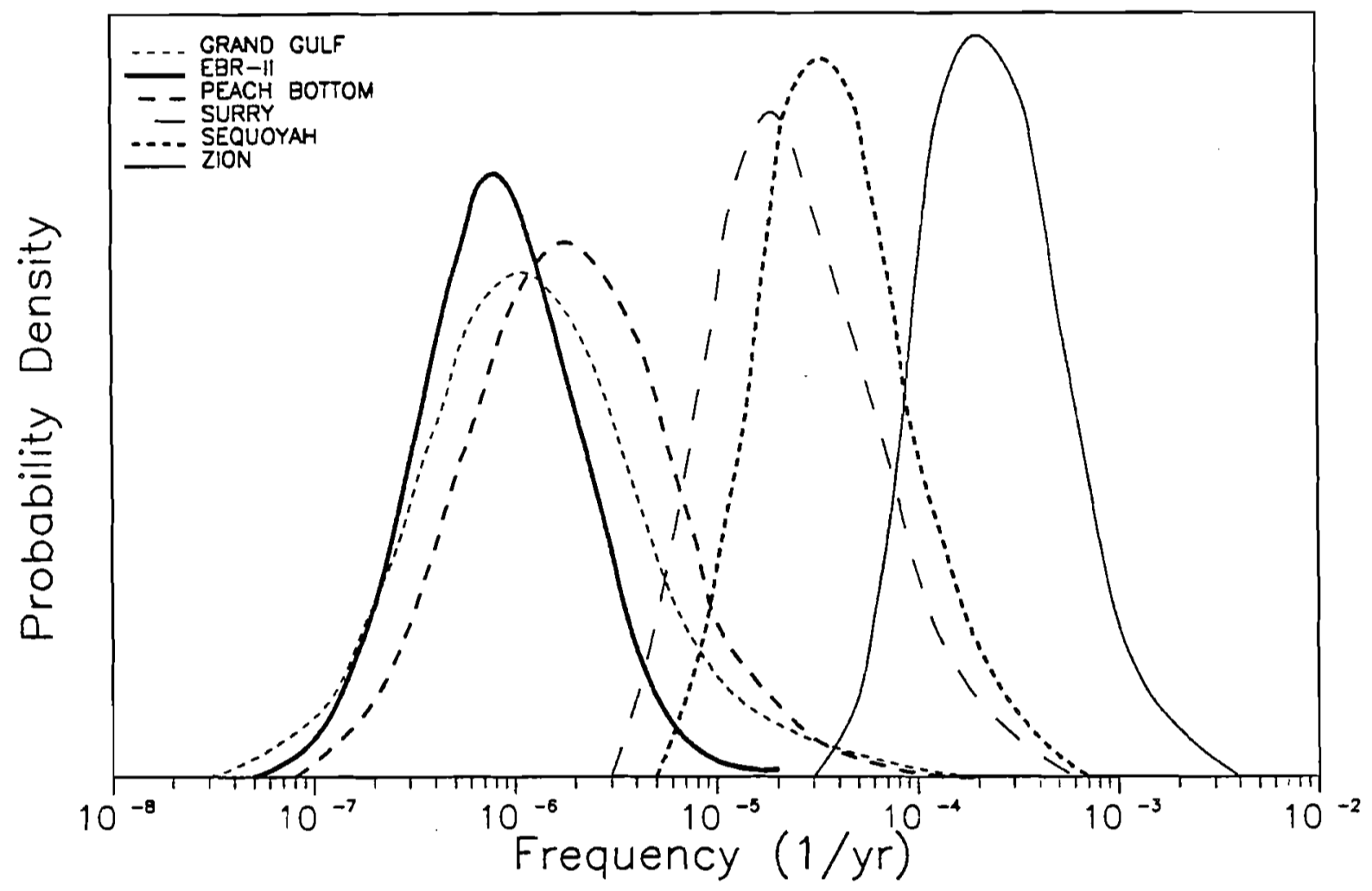

Fig. 1. Comparison of EBR-II Damage Frequency with Core Damage at Commercial LWRs (LWR data from NUREG-1150) 
Table 1. Mean Core Damage Frequencies for USDOE Facilities

\begin{tabular}{||l|l|c|c|c|c|c|c||}
\hline & & \multicolumn{2}{|c|}{ Mean Core Damage Frequencies (Yr. $\left.{ }^{-1}\right)$} & \multicolumn{2}{||}{} \\
\hline & & $\begin{array}{c}\text { Total } \\
\text { Internal }\end{array}$ & Seismic & Fire & $\begin{array}{c}\text { Wind/ } \\
\text { Tornado }\end{array}$ & $\begin{array}{c}\text { Total } \\
\text { External }\end{array}$ & Total \\
\hline \hline & EBR-II & $1.610^{-6}$ & & $3.610^{-6}$ & - & & \\
\hline$[13.4]$ & ATR & $1.810^{-4}$ & - & - & - & - & - \\
\hline$[13.5]$ & HFBR & $3.510^{-4}$ & - & - & - & - & \\
\hline$[13.3]$ & HFIR & $3.110^{-4}$ & $1.210^{-4}$ & $1.910^{-5}$ & $2.910^{-4}$ & $4.310^{-4}$ & $7.410^{-4}$ \\
\hline$[13.6]$ & $\begin{array}{l}\text { N- } \\
\text { Reactor }\end{array}$ & $6.710^{-5}$ & $1.710^{-4}$ & $1.710^{-5}$ & - & $1.910^{-4}$ & $2.510^{-4}$ \\
\hline $\begin{array}{l}{[13.7]} \\
{[13.8]}\end{array}$ & $\begin{array}{l}\text { SRP K- } \\
\text { Reactor }\end{array}$ & $2.110^{-4}$ & $1.210^{-4}$ & $1.410^{-7}$ & - & $2.210^{-4}$ & $4.310^{-4}$ \\
\hline \hline
\end{tabular}


1.0 INTRODUCTION 


\section{Table of Contents}

Page

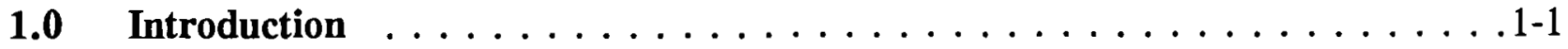

1.1 Project Motivation and Initiation $\ldots \ldots \ldots \ldots \ldots \ldots \ldots \ldots \ldots \ldots \ldots \ldots$

1.2 Project Scope . . . . . . . . . . . . . . . . . . .1-2

1.3 Topical Summaries of PRA Report Sections . . . . . . . . . . . 1-2

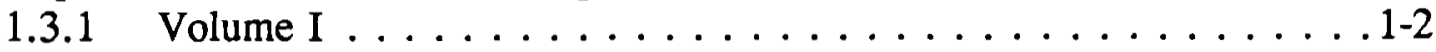

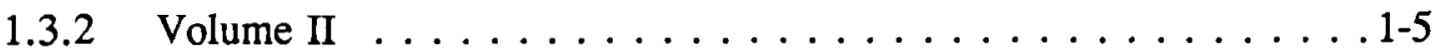

1.3 .3 Volume III ...................... . . .

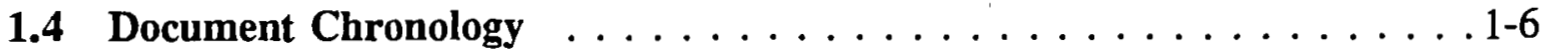

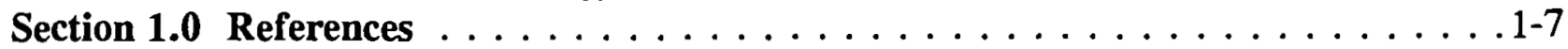




\section{List of Tables}

Page

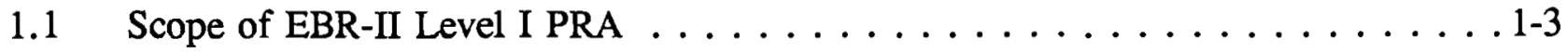




\subsection{INTRODUCTION}

\subsection{Project Motivation and Initiation}

The Committee to Assess Safety and Technical Issues at DOE Reactors of the National Academy of Sciences issued a report entitled "Safety Issues at the DOE Test and Research Reactors" in August 1988 [1.1]. Among the recommendations in this report is: "All of the DOE Class A reactors should have Level I PRAs. The need for Level II and Level III PRAs should be given careful consideration...". Specifically with respect to EBR-II, "The Committee recommends that a Level I PRA be performed for the EBR-II plant and that refueling accidents and the reliability of the reactor protection system and containment isolation be carefully examined in the context of the PRA." In response to this recommendation, a Level I Probabilistic Risk Assessment (PRA) for EBR-II which addresses identification and quantification of the frequencies of sequences which might lead to core damage was initiated.

A major benefit of performing a PRA for EBR-II is to explicitly evaluate the risks of the EBR-II plant by bringing together modern systems analysis and the existing analytical and experimental results pertaining to EBR-II safety in the integrated PRA framework. The PRA results include an integrated model of the plant that delineates the interfaces and relationships among the inherent characteristics of the reactor, the equipment, procedures, and human activities, and quantifies the contributions of these components to safety and to risk. The PRA models also allow assessment of the impacts of plant modifications, aging of plant equipment, procedure changes, and technical specifications changes on risk. In addition, the PRA models can be used to assess risks associated with both irradiation and plant experiments, and allow evaluation of experimental limits and controls intended to limit risk. Finally, the PRA provides a basis for operator training in identifying and responding to risk dominant accident sequences.

Realization of all of the potential benefits of an EBR-II PRA requires that there be an ongoing process of development and improvement of PRA models of the plant, the data base supporting quantification of the models, the evaluations of accident sequences, and applications 
of the PRA in decision making processes. Thus, the EBR-II PRA should not be regarded as a closed-ended study, but as an on-going process of model development, analysis, and applications in performance of the EBR-II mission.

Upon completion and review of the Level I study, which is the subject of this document, effort will be applied to consideration of a limited scope containment study.

\subsection{Project Scope}

The scope of the EBR-II PRA is summarized in Table 1.1.

\subsection{Topical Summaries of PRA Report Sections}

\subsubsection{Volume I}

Section 2.0 of this report describes the formulation of EBR-II PRA project plans and the assessment and selection of PRA methodologies applicable to this study.

Section 3.0 provides a descriptive overview of the EBR-II plant, including an historical perspective of operations and some of the major plant improvements over 25 years. This section also provides an insight into the unique features of EBR-II and some of the integral plant experiments performed to demonstrate the relatively benign behavior of the reactor when subjected to severe loss-of-flow and loss-of-heat-sink transients.

Section 4.0 presents a discussion of special features of EBR-II, including the passive safety features of the reactor with respect to inherent shutdown from full power.

Section 5.0 provides a description of the accident sequence selections pertinent to EBR-II operations and the concomitant analyses that could lead to several categories of core damage. 
Table 1.1. Scope of EBR-II Level I PRA

Sources of Radioactivity Considered

- Accumulated fission products and actinides in core and blanket subassemblies.

- Induced activity in structural materials and in sodium coolant.

- Accumulated fission products in subassemblies in the storage basket or undergoing fuel transfer between core and basket.

Plant States Considered

- Plant configuration is as of October 1,1989 (except as noted).

- Core configuration is considered to be mostly driver fuel, with irradiation experiments operating within Technical Specification (Tech. Spec.) limits.

- Initial operating conditions are considered to be within Tech. Spec. limits, with allowable conditions assumed to occur.

Initiating Events. Considered

- Generic initiating event categories usually examined for LMRs such as Loss-of-Flow (LOF), Transient Over Power (TOP), Loss-of-Heat-Sink (LOHS), Over Cooling (OC) and Local Faults.

- Initiating events with LWR analogues such as Loss-of-Coolant-Accident (LOCA) - for EBR-II, Loss-of-Sodium Accident.

- General Transients.

- Criticality (fuel handling).

- External events, particularly earthquakes and fires. 
Section 6.0 summarizes all of the detailed EBR-II systems analyses contained in Appendix $\mathrm{C}$ of this document. The summary includes schematic system diagrams, a discussion of failure data and the system failure rates obtained from fault tree analysis.

Section 7.0 describes the criteria for selection of failure rates, aging effects, and event probabilities from generic PRA literature, LMR plant data, and EBR-II plant-specific data.

Section 8.0 provides the rationale and data selection for human factors analysis as applied to EBR-II operations and procedures.

Section 9.0 describes the basis for seismic analysis of the EBR-II site and plant components. An earthquake is considered to be the major external event that could affect plant integrity.

Section 10.0 considers the PRA effects of other external events, specifically fires, internal floods, and tomadoes.

Section 11.0 presents the process and results of sequence quantification, which combines the accident sequence event trees with the results of fault tree analyses of EBR-II systems to produce the core damage frequencies and the percentage contribution of each sequence to each core damage state.

Section 12.0 discusses the importance of each core damage contributor and the attendant uncertainties and sensitivities of the data input.

Section 13.0 presents an interpretation of all the qualitative and quantitive results of the EBR-II PRA.

Section 14.0 presents the conclusions and recommendations resulting from the PRA effort. 
Appendix A describes the risk analysis of core refueling operations.

Appendix B contains all fault trees and input data used in the quantification of the EBR-II PRA.

\subsubsection{Volume II (System Notebooks)}

Appendix C contains the details of systems analyses for the EBR-II front-line and support systems including system descriptions, data selection, fault tree analyses, and quantification leading to system failure rates. This Appendix contains twelve system notebooks as follows:

- C.1 Reactor Shutdown System

- C.2 Primary Pumps

- C.3 Primary Pumps Motor/Generator Sets

- C.4 Shutdown Coolers

- C.5 Shield Cooling

- C.6 Thimble Cooling

- $\quad$ C.7 Stack Exhaust System

- C.8 Secondary Sodium Systems/Balance-of-Plant Systems

- C.9 Electrical Systems

- C.10 Instrument and Plant Air Systems

- C.11 Cooling Water Systems

- C.12 Argon Systems

\subsubsection{Volume III}

Appendix D contains documents written specifically to support the EBR-II PRA with respect to PRA methodology, passive safety features of EBR-II, local fuel element faults, lossof-sodium accidents and seismic analysis. 


\subsection{Document Chronology}

The first version of the system notebooks, Appendix C, was released as Revision 0 in February 1990. The main body of the text was issued for ANL internal review in October and November 1990 and the updated system notebooks in January and February 1991 and labelled

Revision 1. After resolution of all the review comments, the entire document was released as Revision 2 in June 1991. 
Section 1.0 References

1.1 Committee to Assess Safety and Technical Issues at DOE Reactors, "Safety Issues at the DOE Test and Research Reactors," National Academy Press, (1988). 
2.0 PROJECT PLANS

AND METHODS ASSESSMENT 


\section{Table of Contents}

Page

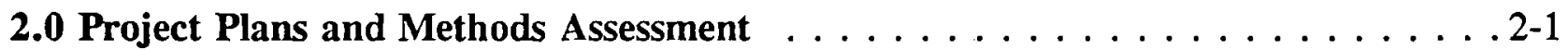

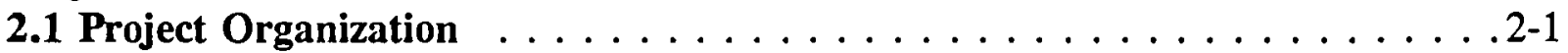

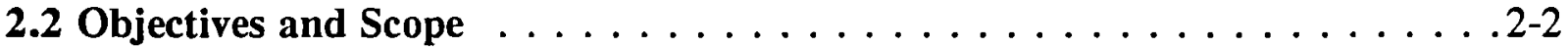

2.2 .1 Objectives . . . . . . . . . . . . . . . . .2-2

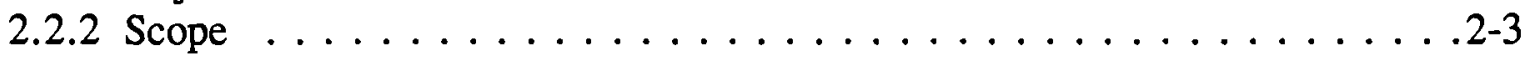

2.3 Methodology . . . . . . . . . . . . . . . . . . . 2-7

2.3.1 Plant Familiarization . . . . . . . . . . . . . . . . 2-11

2.3.2 Accident Sequence Analysis . . . . . . . . . . . . . . 2-12

2.3.2.1 Internal Initiating Events . . . . . . . . . . 2-12

2.3.2.2 External Initiating Events $\ldots \ldots \ldots \ldots \ldots \ldots . \ldots . \ldots . \ldots .13$

2.3.2.2.1Earthquakes . . . . . . . . . . . . 2-14

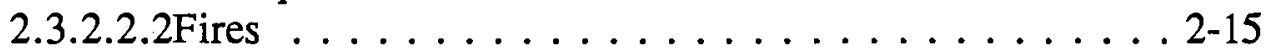

2.3.2.2.3Other External Events $\ldots \ldots \ldots \ldots \ldots \ldots . \ldots 2-15$

2.3.2.3 Event Trees . . . . . . . . . . . . . . . 2-15

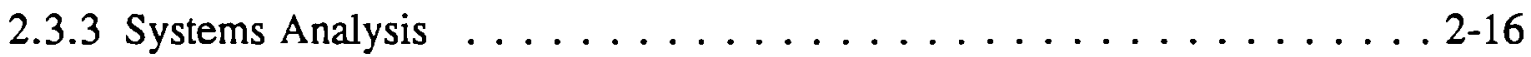

2.3.3.1 System Modeling . . . . . . . . . . . . 2-17

2.3.3.2 Human Reliability . . . . . . . . . . . . . . . 2-17

2.3.3.3 Common Cause . . . . . . . . . . . . . . 2-18

2.3.3.4 Data Base . . . . . . . . . . . . . . . . 2-18

2.3.4 Accident Sequence Quantification . . . . . . . . . . . 2-20

2.3.4.1 Core Damage . . . . . . . . . . . . . . . . . . 2-20

2.3.4.2 Uncertainty and Importance in Accident Sequences . . . . . 2-21

2.3.5 Mathematical and Computing Methods . . . . . . . . . . . 2-21

2.3.5.1 Event Trees . . . . . . . . . . . . . . 2-22

2.3.5.2 Fault Trees . . . . . . . . . . . . . . . . 2-22

2.3.5.3 Uncertainty and Importance . . . . . . . . . . . . 2-23

2.3.5.4 Sequence Evaluation ................. 2-23

2.3.6 Interpretation and Application of Results . . . . . . . . 2-23

2.4 Quality Assurance . . . . . . . . . . . . . . . . . 2-24

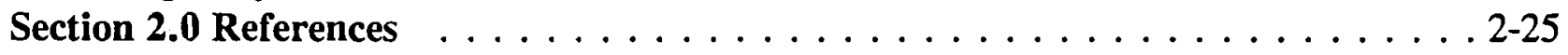




\section{$\underline{\text { List of Figures }}$}

\section{$\underline{\text { Page }}$}

2.1 Task Flow Diagram . . . . . . . . . . . . . . . . . . . 2-10

2.2 Data Flow in the EBR-II PRA . . . . . . . . . . . . . . . 2-19 


\section{List of Tables}

\section{Page}

2.1 General and Specific PRA Objectives . . . . . . . . . . . . . . 2-4

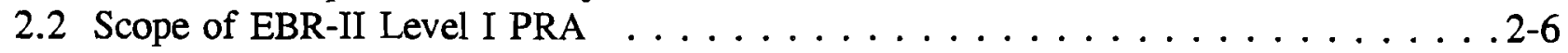

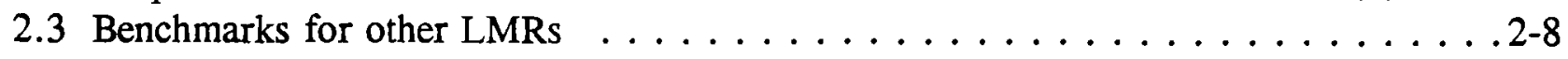




\subsection{PROJECT PLANS AND METHODS ASSESSMENT}

\subsection{Project Organization}

In response to the recommendation in the report entitled "Safety Issues at the DOE Test and Research Reactors" [2.1] in August 1988, an effort was begun in mid-1989 at ANL to organize a project dedicated to the production of a Level I Probabilistic Risk Assessment (PRA) for EBR-II. At the direction of the Laboratory Director and the Associate Laboratory Director for Engineering Research, the principal responsibility for planning and performing the EBR-II PRA was vested in the Reactor Analysis and Safety Division (RAS) at ANL. Within the project duration, a reorganization of the Engineering Research divisions resulted in the dissolution of RAS and the subsequent creation of two new divisions, Reactor Analysis (RA) and Reactor Engineering (RE). The principal EBR-II PRA responsibility currently is delegated to RA, with staff support from RE, Engineering Physics (EP), and EBR-II. Because of the matrix management structure of the project, the project organization has remained intact (see Section 2.4, "Quality Assurance" and the details of the QA Program [2.2]).

The principal individual responsibility for production of the PRA is vested in the EBR-II PRA Project manager, who reports to RA division management along with periodic reports to the Senior Review Panel which consists of senior ANL technical management who are also responsible for review of the PRA final draft (internal review). The Project Manager is responsible for coordination of the technical tasks and advisory reviews performed by the Systems Analysts in conjunction with the EBR-II Technical Support Group. $\mathrm{He}$ is also responsible for ensuring that the methodology adopted is state-of-the-art; to this end, the USNRC PRA procedures guide [2.3] is used for initial guidance, complemented by the inclusion of current expertise from outside contractors in the areas of PRA methodology, Human Reliability, and Seismic Analysis. 
The EBR-II PRA was initiated with a one week EBR-II familiarization course held at ANL-W, Idaho Falls, in January 1989. It was planned that a first draft of the final report be delivered to ANL management, the EBR-II Division, and the EBR-II PRA Senior Review Panel in October 1990, with submission of the report for DOE review in 1991.

\subsection{Objectives and Scope}

\subsubsection{Objectives}

Probabilistic risk assessments can be used to achieve many different objectives, ranging from evaluation of a plant's performance in comparison to a probabilistic safety goal, through evaluation of the effects of technical specification modifications, to support of an operational risk tracking system. The details of the PRA modeling required depend on the objectives. The objectives of the EBR-II PRA are summarized as follows:

1. To quantitatively assess the level of safety of EBR-II and the uncertainties in the assessment.

2. To identify changes in design or operations which could significantly improve safety.

Initially, these objectives are addressed by completion of a Level I PRA analysis focused on assessment of core damage frequency. The need for extension of the analysis to Level II and Level III will be evaluated when Level I results for core damage frequency are available.

3. To develop a method for continuing use by EBR-II in assessing effects of plant modifications, experiments, and operational changes.

This objective will be addressed by making the initial PRA models available in convenient form for use in on-going analysis of EBR-II. A continuing process of upgrading and extending the initial PRA models, adding new models, and improving the data base supporting the models is visualized. It is intended that the PRA will become a part of the EBR-II evaluation methods for 
use as needed in the decision-making process. In order to fully implement the PRA in its intended role, a final objective is:

4. To develop staff expertise in performance and application of risk analysis.

Development of expertise at ANL in performance and application of PRA techniques, and in interpretation of results is essential to the continuing development and applications of the PRA. Table 2.1 presents the specific objectives of the PRA in relationship to the general objectives stated above.

It is noted that general objectives 1 and 2 and the specific objectives 1 through 7 represent the first order of business for the EBR-II PRA Project, and is addressed in this report. A quantitative assessment of the safety of EBR-II in PRA terms is considered to be the most important near term goal of the project. In the longer term, integration of the PRA into the overall battery of analysis methods available to EBR-II, and continuing utilization of the PRA as a decision-making tool is perhaps more important to the continued safe and efficient operation of the plant.

\subsubsection{Scope}

The EBR-II PRA Project has concentrated on completing a Level I PRA for the reactor system, which focuses on producing a comprehensive list of accident scenarios for EBR-II together with a measure of expected occurrence frequencies and associated uncertainties and an assessment in terms of core damage alone. At the conclusion of the Level I study, a limited study will be conducted to assess the need for more detailed analysis of containment loads, containment performance, and radiological consequences of releases from containment. The scope of the Level I analysis of the reactor system is summarized in Table 2.2. 


\section{General Objectives}

1. To quantitatively assess the level of safety of EBR-II and the uncertainties in the assessment.

2. To identify changes in design or operations which could significantly improve safety.

\section{Related Specific Objectives}

1. To identify those accident sequences which are the dominant contributions to core damage frequency.

2. To identify and model those systems and components which are important to accident sequence initiation and/or. mitigation.

3. To identify those human actions which are important to accident sequence initiation and/or mitigation.

4. To assess the effects of external events (in particular, seismic events) on core damage frequency.

5. To assess the effects of EBR-II passive safety features in reducing both the frequency and consequences of the accident sequences.

6. To assess important dependencies.

7. To develop a database for use in quantifying system and component failure rates and external events frequencies.

8. To develop a computerized PRA database for continuing use in analysis of EBR-II. continuing use in assessment of plant modifications, experiments, and operational changes in EBRII. 
Table 2.1 General and Specific PRA Objectives (Cont'd)

General Objectives

4. To develop staff expertise in performance and application of risk analysis.
Related Specific Objectives

9. To develop a computerized method for updating the database as additional experience is accumulated.

10. To extend existing models and add new models as needed.

11. To familiarize EBR-II analysis, engineering, and operations personnel with PRA concepts and their utilization.

12. To establish in-house expertise in all major areas of PRA analysis, to facilitate continuing development of the PRA and it's application in decision-making. 


\section{Table 2.2. Scope of EBR-II Level I PRA}

\section{Sources of Radioactivity Considered}

- Accumulated fission products and actinides in core and blanket subassemblies.

- Induced activity in structural materials and in sodium coolant.

- Accumulated fission products in subassemblies in the storage basket or undergoing fuel transfer between core and basket.

\section{$\underline{\text { Plant States Considered }}$}

- Plant configuration is as of October 1, 1989 (except as noted)

- Core configuration is considered to be mostly driver fuel, with irradiation experiments operating within Technical Specification (Tech. Spec.) limits.

- Initial operating conditions are considered to be within Tech. Spec. limits, with allowable conditions assumed to occur.

\section{Initiating Events Considered}

- Generic initiating event categories usually examined for LMRs such as Loss-of-Flow (LOF), Transient Over Power (TOP), Loss-of-Heat-Sink (LOHS), Over Cooling (OC) and Local Faults.

- General Transients.

- Criticality (fuel handling).

- External events, particularly earthquakes and fires. 
Not included in the scope are reactor start-up events, partial power operation, plant experiments performed under special procedures, such as the automatic control rod drive system in high-speed mode, and human error-acts of commission. None of these categories is considered to introduce significant effects on the level of risk associated with EBR-II operations.

In order to assess the comparative level of risk associated with operation of EBR-II the core damage frequencies derived are compared to those for commercial LWRs such as given in NUREG-1150 [2.4], any relevant USDOE goals, the results of other PRAs being performed on USDOE Class A Facilities and the results for other LMRs. These benchmarks are summarized in Table 2.3.

\section{$2.3 \quad$ Methodology}

The purpose of this description is to provide an introduction to, and an overview of, the basic risk analysis methodology used in this study. The various review bodies, whose comments on DOE class A facilities provided the impetus for this study, have emphasized state-of-the-art methodology [2.1]. The individual components of the methodology will be developed in later sections.

The USNRC PRA procedures guide [2.3] has been used to define the scope and nature of the present work. This risk analysis therefore fits within a previously defined formal structure for such activities. However for each specific PRA there are still many decisions on methodology which must be made. In the first instance, it is intended that a comprehensive Level I [2.3] PRA be performed. Therefore, the first task of this risk analysis was to produce a comprehensive list of accident scenarios for EBR-II together with a measure of expected occurrence frequencies and associated uncertainties, and an assessment of the consequences in terms of core damage. 
Table 2.3. Benchmarks for Comparative Risk

- Commercial LWRs (NUREG-1150) [2.4]

- USDOE goals [2.5]

- $\quad$ PRAs for other USDOE Category A facilities [2.1]

- $\quad$ PRAs for oṭher LMRs $[2.9,2.11]$ 
The accidents which are identified are intended to encompass all potential accident scenarios which might possibly lead to core damage in EBR-II. In particular this includes external events, with emphasis on fire, internal floods, and earthquake. At the initiation of the present analysis, it was anticipated that these three external events would contribute significantly to the core damage frequency. It should be noted that, in view of the life extension studies at EBR-II, careful note will be taken of scenarios which threaten availability as well as safety.

In this risk analysis, the event trees have been developed to the level of detail necessary to clearly define the accident sequence but with front-line and support systems treated using fault trees. That is, we have adopted the "small event tree-linked fault tree" approach to the analysis. In the quantification of the trees, uncertainty is built in from the outset, using standard methodology [2.3].

Figure 2.1 is a diagrammatic representation of the process which was followed in performing the Level I EBR-II PRA. The various activities are discussed in detail in later sections. However, it is important to recognize the first, and key, task of plant familiarization. Obviously a meaningful PRA cannot result unless those charged with performing the PRA become thoroughly familiar with the design and operation of EBR-II. This was attained by plant walkthroughs and by accessing available sources of information on these subjects. The acquisition of the basic information is crucial to the success of the project.

Two features of EBR-II introduce different considerations into this PRA. The first of these is the ability of EBR-II passive safety characteristics, such as reactivity feedbacks and decay heat removal, to mitigate the effects of several accident sequences. Indeed, an unprotected Loss-of-Flow (LOF) and an unprotected Loss-of-Heat-Sink (LOHS) have been performed as plant experiments in EBR-II [2.6]. One of the challenges in performing this PRA is to take account of these characteristics of EBR-II in an appropriate manner. Second, it is important to consider the mission of EBR-II as a research facility, which conducts experimental irradiations and plant transient experiments, within the PRA. These factors must be considered in characterizing the reactor and in assessing accident sequence initiation and outcome, so that 


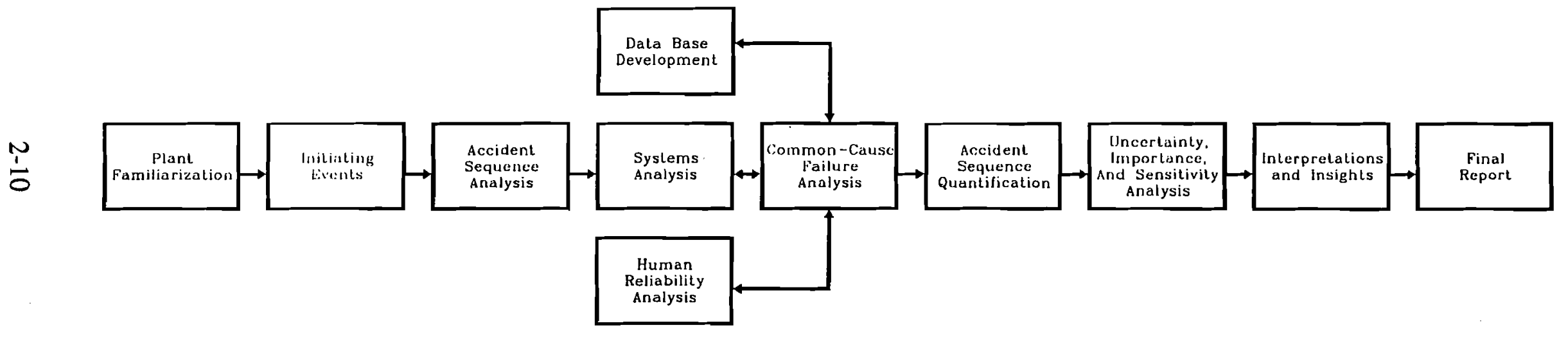

Fig. 2.1. Task Flow Diagram 
the risk assessment adequately considers the impact of experiments on facility risk. In this way, the PRA can be used as a factor in decisions on experiment acceptability.

\subsubsection{Plant Familiarization}

In Fig. 2.1, the first activity identified is plant familiarization. As noted above, this activity is crucial to the successful performance of the entire PRA. Plant and system familiarizations were continuous activities which took place throughout the project in order that the PRA analysts were fully aware of how the plant is built and operated.

The assembly of the necessary information for analysis of EBR-II was a prodigious task. The first step in the process was a short course on EBR-II given by EBR-II staff to the PRA staff with the dual objective of providing a background for consideration of EBR-II and to open lines of communication between the largely Illinois-based PRA staff and the Idaho-based engineers whose understanding of EBR-II needed to be transferred. To further enhance this activity a list of principal engineering, operations, and maintenance contacts for each system, and subsystem, within EBR-II was established.

An integral part of the familiarization process is the plant walkthrough. A first general walkthrough formed part of the short course on EBR-II, thereafter individual analysts undertook system specific inspections with the cognizant EBR-II engineer(s). A great deal of emphasis was placed upon these personal contacts because, for a plant having the extensive operating experience of EBR-II, the personal insights of an experienced staff are of high value to systems analysts.

The systems analyses of the EBR-II systems operating characteristics are documented in a series of system notebooks (Appendix $C$ of this document and a summary in Section 6.0). The system notebooks are an important product of the PRA, independently of the risk analysis implications, because they represent a consolidation of EBR-II documentation and operating data and experience which heretofore was not available. E.g., one important feature of the systems analyses is to identify and document all the systems interfaces and dependencies. 
Documents which are important to the systems analysis procedure are the System Design Descriptions (SDDs), Operating Instructions and Maintenance Procedures, Piping and Instrumentation Diagrams (P\&IDs) and detailed drawings. EBR-II documents used for developing the initiating events and event trees include existing safety documentation, the Unusual Occurrence Reports (UORs), and the Emergency Procedures. Excellent background material is found in the training manuals and the Life Extension study [2.7].

\subsubsection{Accident Sequence Analysis}

The first formal step in the risk analysis is to produce the list of initiating events and to layout the corresponding event trees. This is discussed further below.

\subsubsection{Internal Initiating Events}

The safety of LMRs with respect to large radiological releases has been traditionally discussed in terms of three generic whole-core accident sequences, broadly categorized as TOP (Transient $\underline{\text { Over }}$ Power), LOF (Loss-of-Flow) and LOHS (Loss of Heat Sink) [2.8], together with Local Faults. For PRA application, this categorization must be broadened to list all possible scenarios. Use of the methods in reference 2.3, shows that, beyond these three categories above, a number of other possibilities must be considered, including plant transients and a LOCA (Loss-of-Coolant Accident). The classic primary system LOCA is inappropriate for a pool-type LMR (i.e., EBR-II); for the EBR-II PRA, the appropriate analogue is a Loss-of-Sodium Accident, so designated throughout this document.

Having established the broad categories of accident scenarios, a list of initiating events was compiled. This process enumerated all possible events which could (conceptually) lead to core damage and or radionuclide release. This list of initiating events was generated by:

(1) Determining how each of the categories from reference 2.3 can occur. 
(2) Reviewing generic lists of accident initiating events from other LMR studies ([2.9], [2.10]], [2.11]).

(3) Reviewing the EBR-II UORs (Unusual Occurrence Reports) for actual events which have occurred at EBR-II.

(4)

Considering events for which emergency procedures are included in the EBRII Emergency Procedures manual [2.12].

There are some special issues at EBR-II which are incorporated into the analysis. The National Academy of Sciences (NAS) review drew attention to refueling accidents [2.1] and these are the subject of a separate study within the PRA. Similarly, the NAS review identified containment isolation as requiring particular attention. Containment isolation will be discussed in follow-on studies.

Once the provisional list of initiating events was in place it was reviewed for completeness. This review process, which involved EBR-II operations staff, EBR-II management and PRA specialists, was essential to establishing the base list of initiating events which underpins the analysis. The initiating events are grouped in terms of the expected system response to reduce the number of event models. Finally, the frequency for each initiating event was derived from plant specific data, fault tree analysis or expert elicitation.

\subsubsection{External Initiating Events}

There is a special class of initiating event which are typically considered separately because of the unique effects members of this class can have on the behavior of the system. These are the external events, of which three were expected to be particularly important for EBR-II, fires, internal floods, and earthquakes. Other potential external events were screened for inclusion. 


\subsection{Earthquakes}

Following guidelines laid down in earlier PRA studies, the seismic analysis involved:

Seismic Hazard: This is a determination of how frequently ground motions occur at the INEL site and what accelerations are associated with them. There was some information on site characterization already available from a study of DOE sites conducted by LLNL [2.13], and from actual earthquakes [2.14]. However, it was decided that a new hazard curve for the ANL-West site was needed to extend the frequency range and uncertainty bands and to remove any undue conservatism for PRA applications. In addition, seismological estimates of earthquake magnitude are needed to validate assumptions regarding vertical and horizontal motion.

(2) Fragility: This requires the determination of the responses of the structures and plant equipment to any given earthquake.

(3) Plant Logic: This is the exercise of determining the effects of the combinations of system failures on the plant behavior.

These three features are then assembled to provide a description of plant damage states and the associated probabilities.

The basic methodology for the seismic risk assessment is that referred to in the NRC procedures guides as the Zion Method, first used in the Oyster Creek Seismic Risk Assessment [2.15] and later improved and used in the Zion Probabilistic Safety Study [2.16]. In this method, the various fragilities are expressed in terms of a ground motion parameter (peak ground acceleration). Dynamic analyses of the structures or the equipment are required to determine the response of that system to an earthquake. For EBR-II, a limited amount of analysis was available which provided some of the required information. Additional dynamic 
analyses were performed at ANL. Clearly it is not practical to perform a dynamic analysis of every part of EBR-II, so a priority list was developed on the basis of potential hazards of seismic failures. In particular, priority was given to systems in which failure could lead directly to core damage or which could disable passive safety functions.

\subsubsection{2 $\quad \underline{\text { Fires }}$}

The fire analysis follows the steps outlined in the NRC procedures guide [2.3]. A screening analysis was performed to identify critical fire impact locations and effort concentrated upon these locations. Event tree and fault tree logic models were constructed to identify any routes that lead from fires to potential core damage.

\subsection{Other External Events}

The usual lists of potential external events were reviewed for applicability to EBR-II [2.3]. For those external events which warranted further consideration, a two-tiered screening analysis was performed to identify the routes to potential core damage. If a particular external event was identified which has a significant impact on plant risk then that event was the subject of more detailed analysis. External events are discussed in detail in Section 10.

\subsubsection{Event Trees}

The process of event tree development was to some degree iterative, in that only when a complete understanding of the EBR-II accident responses was acquired could the definitive set of headings be produced, and yet this process provided the frame upon which the other activities are based.

For the EBR-II PRA, the top events and their success criteria were chosen to lead to the spectrum of possible accident sequences and their associated probabilities given a specific initiating event. It is not possible to immediately step from this result to estimates of core 
damage, since the EBR-II core varies in composition from run to run. Any particular core is composed of assemblies of some or all of the following types, Mk II driver fuel (U-5Fs, 316SS), Mk III (U-10Zr, D9), Mk IIIA (U-10Zr, 316SS), metal fuel experiments (U-10Zr, U/Pu/Zr, HT-9, D9, 316SS) and oxide fuel experiments $\left(\mathrm{UO}_{2}, \mathrm{PuO}_{2}, 316 \mathrm{SS}, \mathrm{D} 9, \mathrm{HT}-9\right)$. There are also other types of fuel (e.g., UN) which conceivably could be irradiated in the future. Irradiation experiments are likely to be of high burnup and to be run-to-clad-breach (RTCB) in order to find burnup limits for a particular fuel type/clad combination, or even run-beyond-clad-breach (RBCB). These experiments are typical of those being used to qualify a new driver fuel for use in EBR-II. The essential point is that the probability of a given sequence can be derived from general consideration of the plant, assuming that the particular core configuration does not affect

the plant system and passive response, whereas the extent of core damage in any particular sequence is heavily dependent on the core configuration. Therefore, the event trees are constructed in a manner which defines accident sequences, but these are not automatically combined into core damage, or not core damage, bins. The response of the reactor core and individual types of subassemblies to particular accident sequences is described in a separate event tree and the conditional probability of damage given an accident sequence is evaluated.

At the conclusion of the Accident Sequence Analysis task there was available a complete list of initiating events, internal and external, each of which was screened for its contribution to core damage frequency and grouped according to their system response. Event trees have been developed for each initiating event or initiating event group. The top events in the event trees provide the focus for systems analysis in terms of definitive success and failure criteria for those system responses. The event trees themselves define sets of accident sequences which can be modelled using coupled neutronic/thermal-hydraulic codes such as SASSYS [2.17].

\subsubsection{Systems Analysis}

Once the event trees are complete the Level I analysis requires systems analysis input to proceed to quantification. This task is lengthy and complex, requiring a full analysis of each front-line and support system to establish the probability of success for each safety function and associated uncertainties, together with the initiating event frequencies, in order to 
provide the probabilities of each sequence. The probabilities associated with each top event can be different from sequence to sequence if there is dependency between system performance and the initiating event.

\subsubsection{System Modeling}

The systems analysis used standard fault tree methodology to describe the failure modes of each system analyzed. The design of EBR-II is such that there are very few mitigative functions, apart from scram and natural convection decay heat removal, required to protect the core from damage and therefore much of the emphasis in the systems analysis was upon identifying dependencies and searching for potential initiating events.

A system notebook was produced for each system analyzed. This is discussed further in Section 6; the notebooks constitute Appendix C.

\subsubsection{Human Reliability}

In a system such as EBR-II, humans are relied upon to perform many tasks in the event of off-normal occurrences at the plant, e.g., manual scram, manual activation of safety rods, etc. As a result, human reliability will be a major consideration in the possible causes of system failure. The importance of human factors is shown, for example, by the fact that, in the accident at TMI-2, a major contributing cause was operator error prior to and throughout the accident. Other areas where human error can be implicated in accident progression are inadequate procedures, training or accident response planning. The human reliability analysis involved screening the PRA model to identify human factors related initiating events and then investigating the role of the operator in accident sequences, particularly through recovery actions. 


\subsubsection{Common Cause}

Common causes for failures can lead to unexpectedly high probabilities of redundant system failures where they are found to exist. In general terms, the more reliable a system from the viewpoint of random independent failure, the more likely it is that common cause will dominate the total failure frequency.

In the analysis the $\beta$ factor method was used to treat common cause. The potential for common cause failure was assessed using a methodology similar to that expounded in a recent joint USNRC/EPRI report [2.18].

\subsubsection{Data Base}

Twenty-five years of operating history at EBR-II provides a potentially large data base for the evaluation of system reliability. This is particularly so for the more recent operating history where detailed records have been kept. However, these facility records were not kept with a PRA in mind. Nevertheless, the raw data from maintenance, testing, and operation of EBR-II was applied where possible. This plant specific data was augmented by using generic industry data. In addition, the EBR-II division has been contributing data to the Oak Ridge LMR data base, CREDO [2.19]. Maximum use was made of all the data held in CREDO, EBR-II, Fast Flux Test Facility (FFTF), and Japanese (JOYO) databases, to establish EBR-II or LMR specific data. Also, generic data sources for non-LMR specific components were utilized where appropriate (e.g. electronics). Figure 2.2 gives an outline of the total information flow required to establish an EBR-II data base and the corresponding PRA data base. It should be noted that all data relevant to the production of the PRA, including source data, event trees, and fault trees has been centralized in a single database, created using the relational database ORACLE [2.20]. 


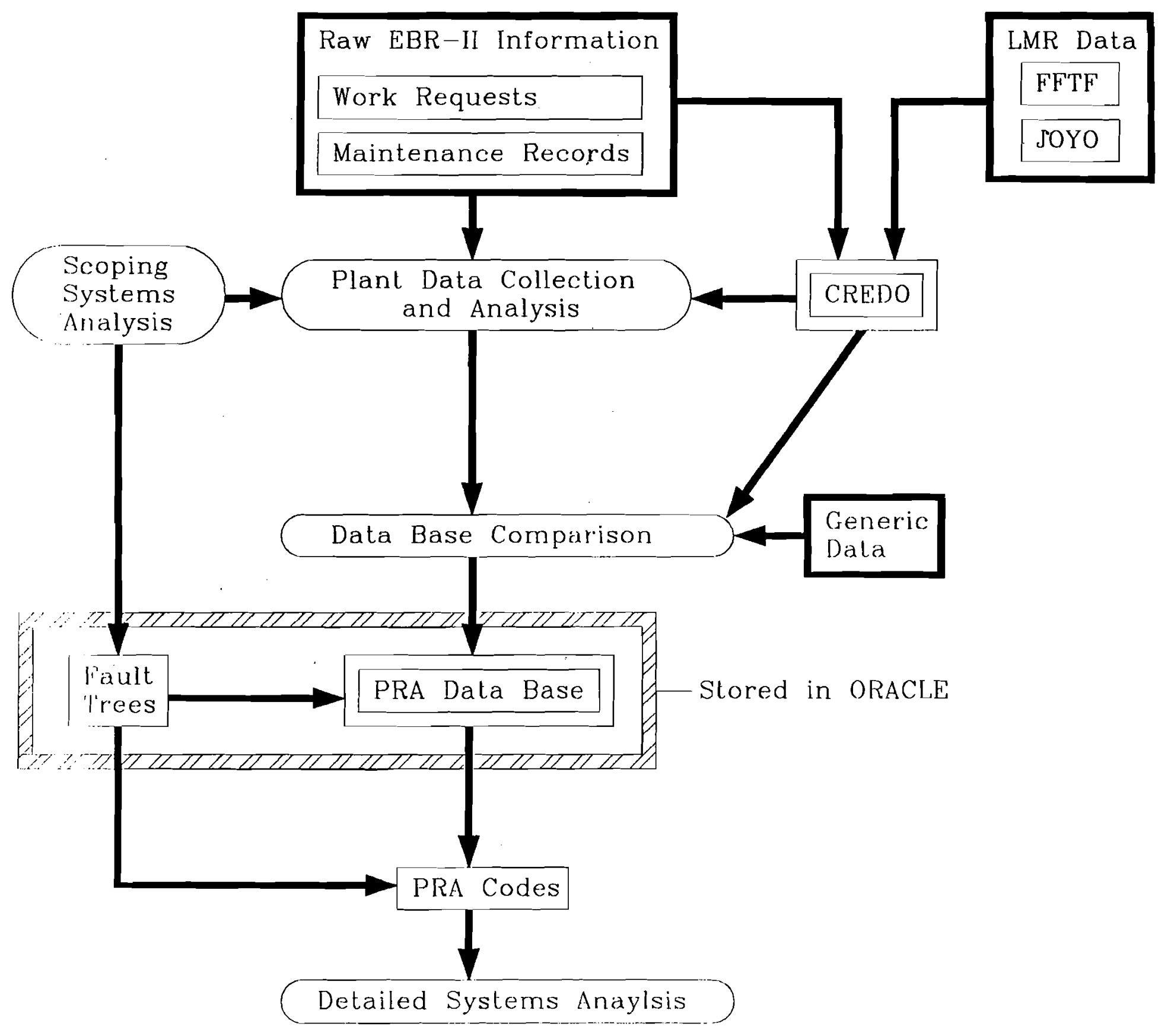

Fig. 2.2. Data Flow in the EBR-II PRA 


\subsubsection{Accident Sequence Ouantification}

Accident sequence quantification is the process by which the results from the separate tasks are synthesized to give overall frequencies for a given accident sequence. For each initiating event, the various sequences are defined by linking the appropriate fault trees and this, combined with the initiating event frequency, leads to the overall frequency for any specific sequence. In order to provide a link for future risk estimates, some estimate of core damage for a given sequence is required. Core damage is not arbitrarily assumed. The technique applied is to perform thermal hydraulic analyses of the specific sequences to determine the level of damage to driver fuel and various experiments. This was done using the SASSYS code [2.17].

\subsubsection{Core Damage}

The definition of what constitutes core damage is a special issue in the EBR-II PRA for two reasons, firstly because the core varies in composition from run to run and secondly because EBR-II is a research reactor modified to routinely accommodate fuel experiments with breached cladding. As discussed earlier a particular core might contain metallic driver fuel and both oxide and metal fueled experimental assemblies as well as more exotic fuel types which might be irradiated as experiments. The top events were defined in terms of temperature limits upon driver fuel. Driver fuel in this context is anything which satisfies the current or anticipated Technical Specification limits in power and flow conditions and burnup. Therefore, it can be evaluated whether a particular sequence leads to damage in driver fuel and an assessment could be made as to the degree of damage from each experiment. It is highly time-consuming to assess the damage for each assembly on a run by run basis and continually update the assessment, (note that burnup affects the potential damage); therefore, for the purposes of damage assessment, the damage is defined solely in terms of driver fuel, but a special damage category for experiments is included. 


\subsubsection{Uncertainty and Importance in Accident Sequences}

Uncertainty analysis is carried out in order to determine the uncertainty in the results for various identifiable uncertainties. These include model uncertainties, statistical uncertainties, and the uncertainty associated with completeness. In general, uncertainty becomes more important when accident sequence frequencies are low.

A related topic is that of importance. Importance analysis seeks to identify those systems whose existence is most important in maintaining the current level of risk and those systems whose improvement would most reduce the existing risk. There are various possible measures of importance, the more common measures of importance are Risk Achievement Worth (RAW), Risk Reduction Worth (RRW) and Fussell-Vesely. All three of these measures have been applied.

Sensitivity studies seek to determine if variation of model parameters and assumptions significantly affect the results of the PRA. This is carried out in a less systematic manner than the statistical treatment of uncertainty but is important in providing insight into the results of a PRA.

\subsubsection{Mathematical and Computing Methods}

Over the last fifteen years many computer codes have been written which are designed to facilitate the performance of PRAs. The selection of appropriate codes for application in this PRA depends as much upon availability as their appropriateness for the task. The final product of this PRA consists of the elements of a risk based management system, which EBR-II operations can use routinely to assess the effect of plant modifications upon risk. Therefore, it was a requirement of the code system adopted that it be portable, i.e. easily transferred from ANL-East to ANL-West. Ideally the code system would be self contained, i.e. it would not rely on any particular commercial product, and that ANL would control the source code so that the configuration could be monitored without being dependent upon any external organization. 
Several commercial codes, USNRC or EPRI sponsored, were evaluated and adapted to EBR-II PRA requirements to perform cross-check calculations as verification of the ANL chosen codes and also to demonstrate that the code package chosen had been implemented successfully.

The chosen code suite (EBR2PRA) has been implemented for use on the SUN computer systems at ANL.

\subsubsection{Event Trees}

The event trees used for the Level I PRA are analyzed by resolving the accident sequences. Sequences are analyzed by an in-house program that uses some of the subroutines of MODULE [2.21] for quantification.

\subsubsection{2 $\quad \underline{\text { Fault Trees }}$}

Since the preferred methodology used in this study involves the study of large fault trees, the most important modeling decision to be made was the selection of the computer code (or suite of codes) used to handle the complex problems which arise from large fault trees.

Once the qualitative understanding of the system failure is acquired, the fault tree analysis code can be used to generate sequence probabilities, primarily based upon the minimal cutsets and the system data base. A version of a Korean-authored fault tree code MODULE [2.21], was installed upon the SUN workstation and used in this project. Several sample problems were run and compared to better known codes such as FTAP to provide verification for the code. MODULE was combined with other special purpose codes and TREDRA [2.22] to create a package termed EBR2PRA. The applicability and precision of this package has been benchtested against the PRA results from a commercial code using EBR-II input data. 


\subsubsection{Uncertainty and Importance}

Uncertainty in both fault and event trees is handled within the codes, or code suites chosen. For fault trees it was a functional requirement of the code selected that it handle uncertainty and importance readily. MODULE has an integrated treatment of uncertainty and importance. This capability was extended into EBR2PRA.

\subsubsection{Sequence Evaluation}

Accident sequences in LMRs have received a great deal of attention and a number of codes have been written to follow the course of accidents up to and including fuel damage. The best example of this capability is the ANL-authored fast reactor accident analysis code SAS4A/SASSYS [2.17]. In the case of EBR-II, the EBR-II Division of ANL has two plant specific models which are used for routine thermohydraulic and safety analysis, NATDEMO [2.23] and DSNP [2.24]. Neither of the latter two codes treats fuel failure or events beyond fuel failure. However, any of the three can be used to estimate whether a given plant state will likely lead to core damage using thermal criteria. This allows cross-check calculations to be performed.

\subsubsection{Interpretation and Application of Results}

When the quantification portion of the PRA is complete the process of interpretation of the numerical results determines whether the final product meets the stated objectives. Identification of the risk dominant accident sequences at the end of the Level I PRA provides insight into those potential plant vulnerabilities that lead to core damage. Each dominant sequence is examined to determine the factors that are the major contributions to its frequency. The potential plant vulnerabilities thus identified, are then examined to determine whether improvements can be identified that might significantly reduce the likelihood of core damage. 


\subsection{Quality Assurance}

The EBR-II PRA Project Quality Assurance Staff has established a Quality Assurance Program (QAPD) [2.2] in accordance with the ANL-East Quality Assurance Manual [2.25]. Specifically the QAPD was evolved by applying the standards of NQA-1 [2.26] to the various activities involved in the production of the EBR-II PRA. Of the eighteen (18) Quality Assurance Elements (QAEs) continued in NQA-1, ten (10) are applicable to the EBR-II PRA Project. Most important of these are QAE 3.0 - Review of Technical Work and QAE 6.0 - Document Control. 


\section{Section 2.0 References}

2.1 Committee to Assess Safety and Technical Issues at DOE Reactors, "Safety Issues at the DOE Test and Research Reactors," National Academy Press, (1988).

2.2 Argonne National Laboratory, unpublished information (1990).

U.S.N.R.C., "PRA Procedures Guide," NUREG/CR-2300, USNRC (1983).

2.3

U.S.N.R.C., "Severe Accident Risks: An Assessment for Five U.S. Nuclear Power

2.4 Plants, " NUREG-1150, Vols. 1 and 2, (1990).

USDOE, Draft of "Policy Statement on Nuclear Safety Objectives for DOE 2.5 Facilities" (1988).

EBR-II Division, ANL, "The Experimental Breeder Reactor-II Inherent Safety

2.6 Demonstration," Nuclear Engineering and Design 101, pp. 1-90 ( 1987).

R. W. King, E. C. Filewicz, J. Poloncsik and W. H. Radtke, "Extending the Operating

2.7 Lifetime of EBR-II to 30 Years and Beyond," ANL/EBR-127 (LD), (1985).

R. D. Smith, "Design and Development of Systems and Components for Safety," Proc.

2.8 of the International Meeting on Fast Reactor Safety Technology, Seattle, WA, p. 576 (1979).

"CRBR Safety Study: An Assessment of Accident Risks in the CRBRP," CRBRP-1

2.9 Volumes 1 and 2 (1977).

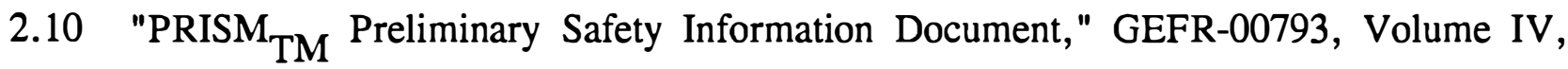
Appendix A (1986). 
2.11 "Clinch River Breeder Plant Probabilistic Risk Assessment" CRBRP-4, 1984.

2.12 Argonne National Laboratory, unpublished information.

2.13 D. W. Coats and R. C. Murray, "Natural Phenomena Hazards Modeling Project: Seismic Hazard Models for Department of Energy Sites," UCRL-53582, Lawrence Livermore National Laboratory (1984).

2.14 J. G. Gale and W. K. Lehto, "Seismic Response of the EBR-II to the Mt. Borah Earthquake," Proc. of the International Topical Meeting on Fast Reactor Safety, Knoxville, Tennessee, pp. $709-714$ (1985).

2.15 R. P. Kennedy and M. K. Ravindra, "Seismic Fragilities for Nuclear Power Plant Risk Studies," Nuclear Engineering and Design 79, pp. 47-68 (1984).

2.16 Commonwealth Edison Company, Zion Probabilistic Safety Study (1981).

2.17 A. M. Tentner et al., "THE SAS4A LMFBR Whole Core Accident Analysis Code," Proc. of the International Topical Meeting on Fast Reactor Safety, pp. 989-990, Knoxville, Tennessee, (1985), and F. E. Dunn et al., "The SASSYS-1 LMFBR Systems Analysis Code," ibid, pp. 999-1006.

2.18 A. Mosleh et al., "Procedures for Treating Common Cause Failures in Safety and Reliability Studies," EPRI NP-5613, NUREG/CR-4780 (1988).

2.19 J. J. Manning, et al., "A Guide for Completing Input Forms for CREDO - A Centralized Reliability Availability and Maintainability Analysis Center for Liquid-Metal-Cooled Reactor and Test Facility Components," ORNL/TM-9892 (1986).

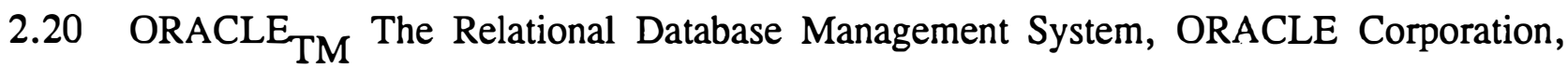
Belmont, California. 
2.21 S. H. Han, T. W. Kim and K. J. Yoo, "Development of an Integrated Fault Tree Analysis Computer Code MODULE by Modularization Technique", Reliability Engineering \& System Safety, Vol. 21, 145-154 (1988)

2.22 L. S. Baker et al., "TREDRA, A Computer Program to Draft Fault Trees," JBF Associates Inc., NESC No. 1021 (1978).

2.23 D. Mohr and L. Chang, "Perturbations of Reactor Flow and Inlet Temperature for EBRII Reactivity Feedback Validation," Proc. of the International Topical Meeting on Fast Reactor Safety, pp. 617-628, Knoxville, Tennessee (1985).

2.24 E. M. Dean and J. F. Koenig, "Simulation of LMFBR Pump Transients and Comparison to LOF that Occurred at EBR-II," Proc. of the International Topical Meeting on Fast Reactor Safety, pp. 629-634, Knoxville, Tennessee (1985).

2.25 Argonne National Laboratory, unpublished information (1989).

2.26 "Quality Assurance Program Requirements for Nuclear Facilities," ASME/ANSI NQA-1 (American Society of Mechanical Engineers/American National Standards Institute), 1989 Edition. 
3.0 OVERVIEW OF EBR-II 


\section{Table of Contents}

Page

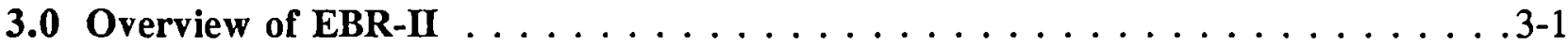

3.1 Site Location . . . . . . . . . . . . . . . . . . .

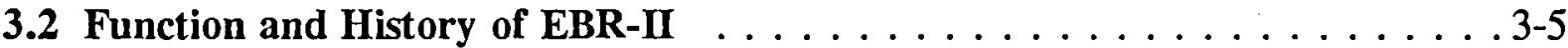

3.3 Plant Description . . . . . . . . . . . . . . . . 3-11

3.3.1 Major Structures $\ldots \ldots \ldots \ldots \ldots \ldots \ldots \ldots \ldots$. . . . . . . . . . . . .

3.3.2 Reactor System . . . . . . . . . . . . . . . . . . 3-12

3.3.3 Primary Sodium System . . . . . . . . . . . . . . . . . . 3-20

3.3.4 Secondary Sodium and Balance of Plant . . . . . . . . . . . . 3-29

3.4 Reactor Shutdown System . . . . . . . . . . . . . . . . 3-31

3.5 Decay Heat Removal . . . . . . . . . . . . . . . . . . . . . 3-33

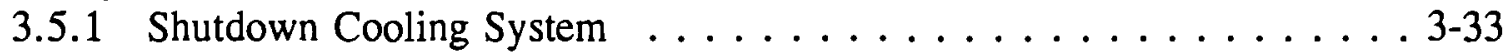

3.5.2 Shield Cooling System . . . . . . . . . . . . . . . . 3-35

3.6 Unique Features of EBR-II . . . . . . . . . . . . . . . . 3-38

3.7 Whole Plant Experiments in EBR-II . . . . . . . . . . . . . . . 3-41

3.7.1 LOF/Scram to Natural Circulation . . . . . . . . . . . . . . . 3-43

3.7.2 Scram with Delayed LOF to Natural Circulation . . . . . . . . . . 3-43

3.7.3 Reactivity Feedback Characterization . . . . . . . . . . . . . 3-43

3.7.4 Loss of Flow without Reactor Scram . . . . . . . . . . . . . . 3-44

3.7.5 Balance of Plant Transients . . . . . . . . . . . . . . . . 3-45

3.7.5.1 Dynamic Frequency Response Tests . . . . . . . . . . . . . 3-45

3.7.5.2 Loss of Heat Sink Without Scram . . . . . . . . . . 3-45

3.7.5.3 Steam Drum Pressure Reduction . . . . . . . . . . . 3-46

3.7.6 Overcooling Transients . . . . . . . . . . . . . . 3-46

3.7 .7 Summary . . . . . . . . . . . . . . . . . . . 3-46

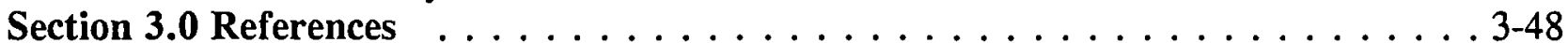




\section{List of Figures}

Page

3.1 Location of INEL in Relation to Surrounding States . . . . . . . . . . . . 3-2

3.2 Location of Primary INEL Facilities and Relationship to INEL Site Boundary . . . 3-3

3.3 ANL-W Site Plan . . . . . . . . . . . . . . . . . . . . . 3-4

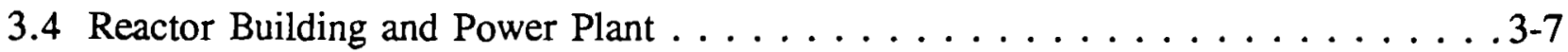

3.5 EBR-II Operating Floor $\ldots \ldots \ldots \ldots \ldots \ldots \ldots \ldots \ldots . . \ldots \ldots$

3.6 EBR-II Primary-system Components $\ldots \ldots \ldots \ldots \ldots \ldots \ldots \ldots \ldots . . \ldots \ldots$

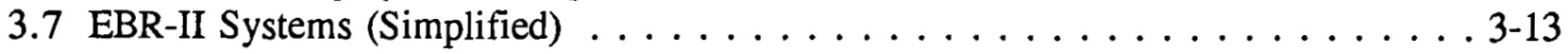

3.8 Power Plant - Location of Components . . . . . . . . . . . . . . . . . 3-14

3.9 Location of Secondary System Components in Sodium Boiler Building . . . . . . 3-15

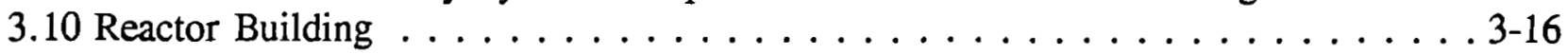

3.11 EBR-II Core . . . . . . . . . . . . . . . . . . . . 3-17

3.12 Primary Cooling Systems . . . . . . . . . . . . . . . . . . . 3-21

3.13 Cutaway View of a Primary-coolant Pump . . . . . . . . . . . . . . 3-23

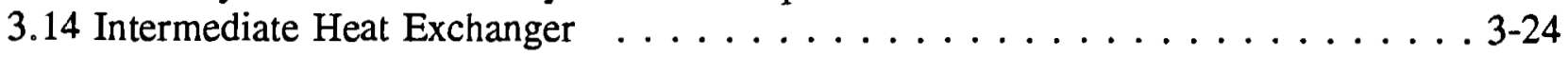

3.15 Primary-tank Assembly . . . . . . . . . . . . . . . . . . . 3-26

3.16 Roller Support System . . . . . . . . . . . . . . . . . . . . . . . . 3-27

3.17 Primary-tank Cover . . . . . . . . . . . . . . . . . . . . . . 3-28

3.18 Reactor Shutdown Circuits - Strings A and B . . . . . . . . . 3-32

3.19 Shutdown Cooling System . . . . . . . . . . . . . . . . 3-34

3.20 Shield Cooling System $\ldots \ldots \ldots \ldots$. . . . . . . . . . . . . . . . . . . 


\subsection{OVERVIEW OF EBR-II}

\subsection{Site Location}

The Experimental Breeder Reactor II (EBR-II) is located in Bingham County, Idaho, at latitude $43^{\circ} 35^{\prime} 10^{\prime \prime} \mathrm{N}$, longitude $112^{\circ} 40^{\prime} 10^{\prime \prime} \mathrm{W}$. The average altitude is $5125 \mathrm{ft}(1562 \mathrm{~m})$ above sea level. The EBR-II is approximately in the center of the Argonne National LaboratoryWest (ANL-W) portion of the Idaho National Engineering Laboratory (INEL).

The INEL, (Fig. 3.1), in east-central Idaho, has been a nuclear research site since 1949. This large site is a federal government complex of facilities which was initially called the National Reactor Testing Station, but was renamed the INEL in 1974. The reservation is a 572,000 -acre $(231,500 \mathrm{ha})$ government-owned reservation under the control of the U. S. Department of Energy (DOE). The INEL is established on a large high-desert, sagebrush covered and treeless plain in the north Rocky Mountains of the United States that has few significant terrain features. All activities at the INEL are limited to those permitted by DOE, and the establishment of permanent private residence is prohibited. The INEL reservation covers parts of four Idaho counties, with the 810-acre (328 ha) area administered by ANL-W being entirely in Bingham County. Underneath a large portion of the INEL is the large Snake River Plain Aquifer.

ANL-W encompasses an area in the southeastern corner of INEL (Fig. 3.2), and contains a number of nuclear facilities (Fig. 3.3) including HFEF, FCF, and EBR-II. EBR-II lies 10 miles $(16 \mathrm{~km})$ from the eastern boundary of INEL, and 3 miles $(5 \mathrm{~km})$ north of east-west U. S. Highway 20.

In the vicinity of ANL-W, the area is mostly undeveloped, the climate semiarid, and the population density low. The Snake River and the city of Idaho Falls, both approximately 30 miles $(48 \mathrm{~km})$ to the east are, respectively, the most significant natural and manmade features 


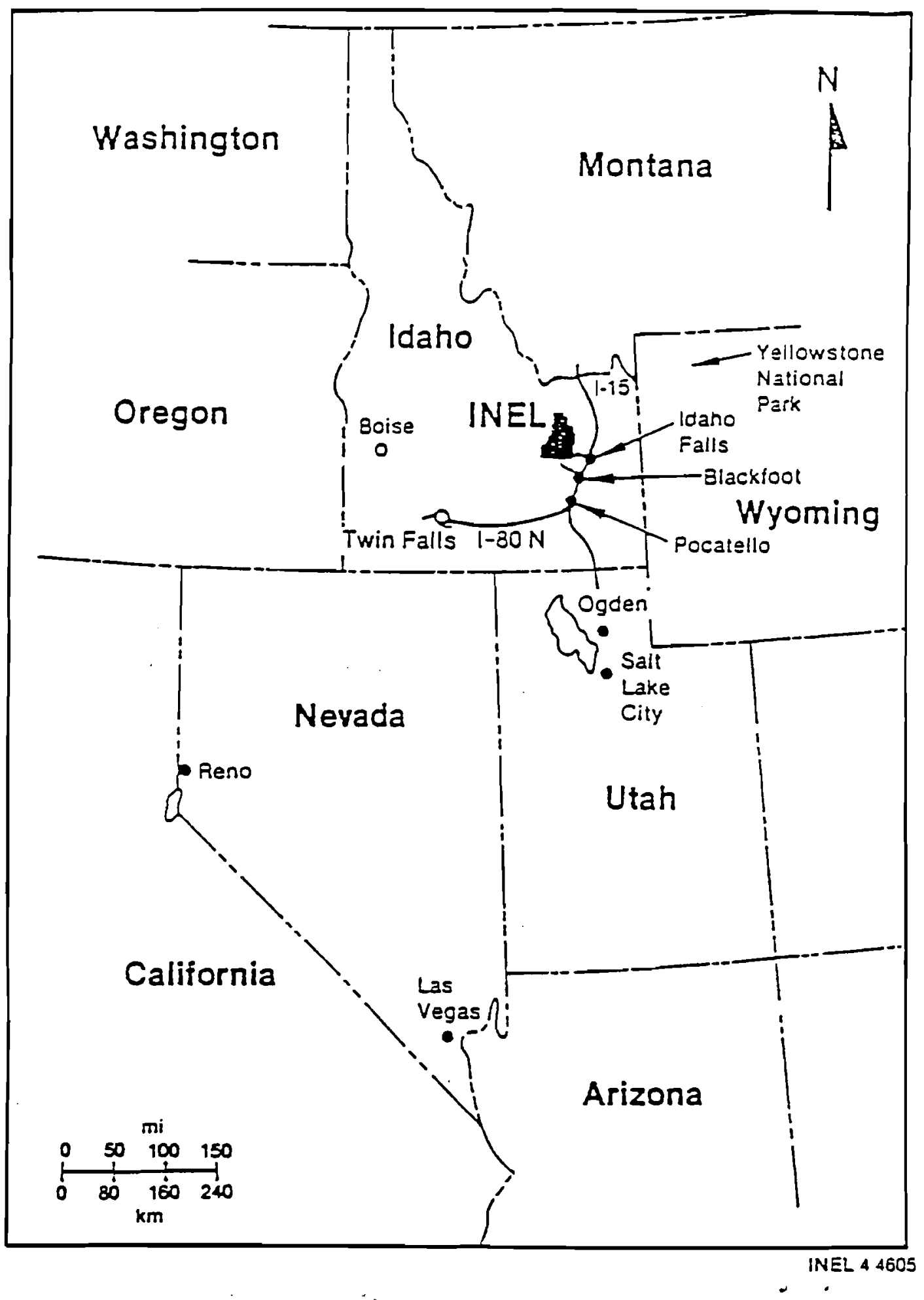

Fig. 3.1. Location of INEL in Relation to Surrounding States 


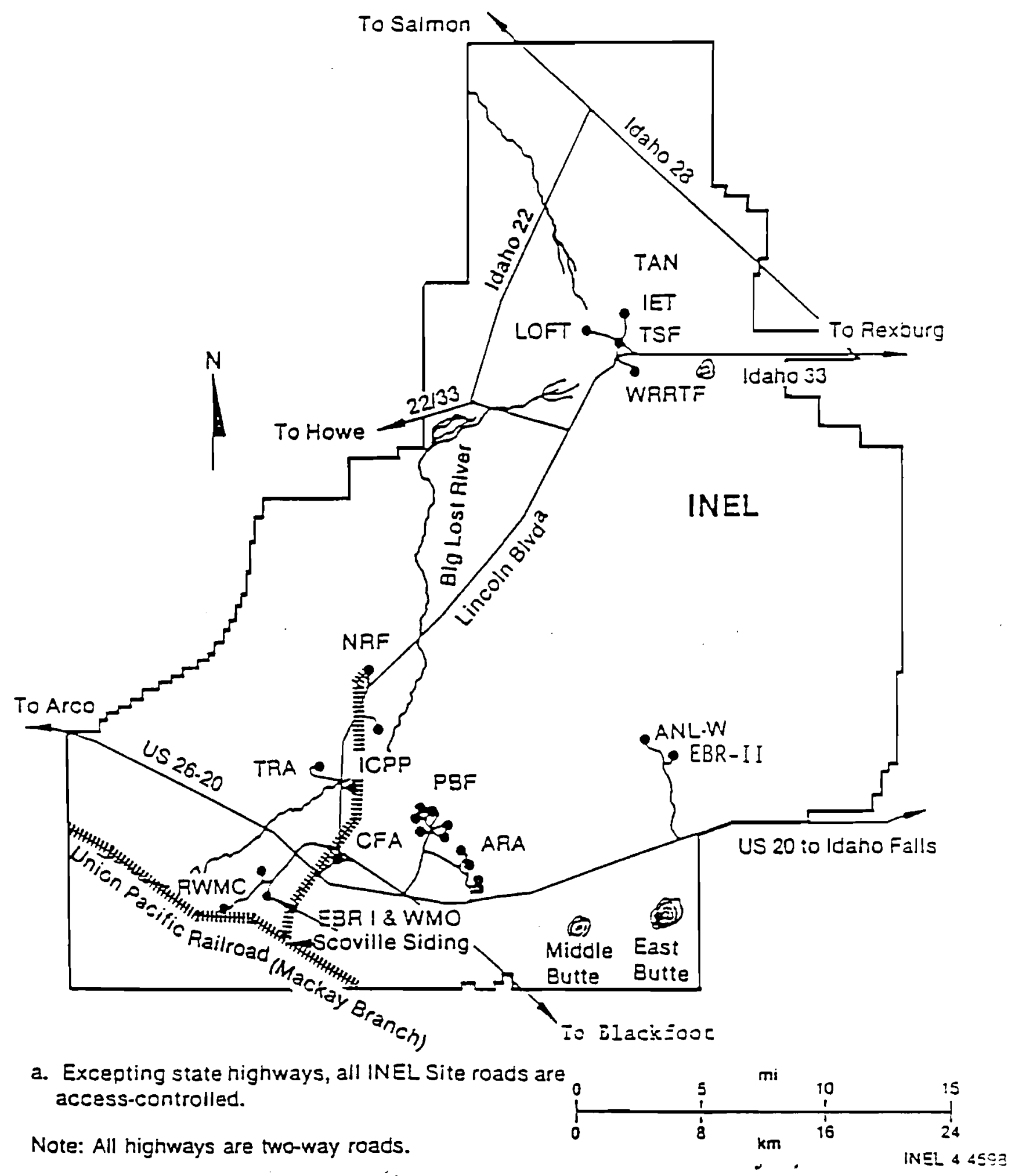

Fig. 3.2. Location of Primary INEL Facilities and Relationship to INEL Site Boundary 


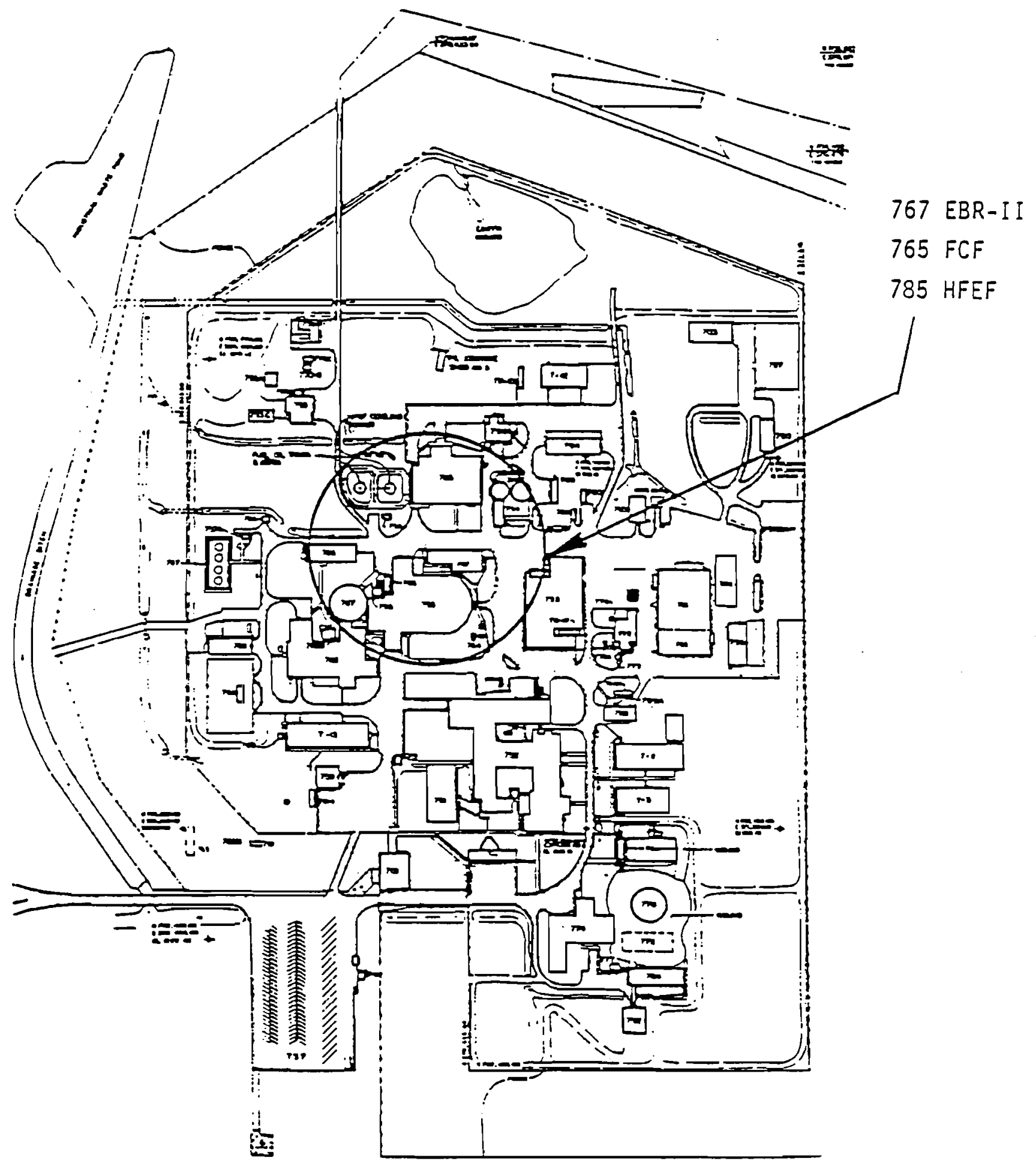

Fig. 3.3. ANL-W Site Plan 
of the area. Other prominent natural features are several nearby volcanic-origin buttes to the south and southwest, and mountains to the west and north which are visible on a clear day. Sagebrush and similar dry-country vegetation covers much of the INEL, and irrigated farms are adjacent on lands not within the INEL reservation.

The terrain of the government-owned reservation is characteristic of a cool high-elevation desert plain, and has climatological conditions typical of such regions. The climate is dry, with moderately hot summers and cold winters. Moderate winds from the southwest are frequent. The predominant native vegetative species are mainly sagebrush, bitterbrush, buffalo grass and similar desert plants which provide a sparsely distributed groundcover.

The overall topography of the ANL-W site is that of a shallow, slightly concave ground depression in the low-rolling rises and depressions that generally characterize the terrain of this area of the INEL. The ground surface consists generally of unvegetated composite layers of interbedded volcanic rock and water-permeable sedimentary materials. The latter include alluvium, alluvial-fan deposits, lake-bed (lacustrine) and playa deposits, and wind-blown (aeolian) deposits. This soil material is only a few meters deep, covering the volcanic bedrock below. The voids and cracks in and between the layers of basalt and sedimentary material are readily traversed by precipitated and ground-source waters. The sandy soils are characterized as having high ion-exchange characteristics. Beneath ANL-W, the uppermost portion of the ground water layer of the Snake River Plain aquifer is between 600 and $700 \mathrm{ft}$ (183 and $214 \mathrm{~m}$ ) below the surface. The principal sources of groundwater recharge of the aquifer are from the streams and canals of the Birch Creek Valley and the Big and Little Lost River Valleys to the north and northwest, and from the impoundments and irrigation canals from the Mud Lake area to the northeast. The level terrain, absorbent soil characteristics and low annual precipitation preclude significant soil erosion.

\subsection{Function and History of EBR-II}

Experimental Breeder Reactor II (EBR-II) is a complete power-producing liquid metal fast breeder reactor, with rated thermal power of $62.5 \mathrm{MW}$ and electrical output of 
approximately $20 \mathrm{MW}$ to on-site and utility loads. It includes a pool-type sodium-cooled reactor, sodium-cooled secondary-heat-transport system, and conventional steam turbine-generator for power conversion. In addition, a fuel reprocessing facility was originally available to complete the closed fuel-cycle operation. After the fuel-cycle concept was demonstrated in 1965-1968, the fuel reprocessing was replaced by fuel-evaluation programs. The reactor building and power plant are shown in Fig. 3.4 and the reactor operating floor is shown in Fig. 3.5.

The primary system is a submerged pool-type design. The entire reactor and primary coolant system are contained in a single tank (primary tank) (Fig. 3.6). There is no outside piping containing primary sodium, except for small-diameter piping connections to the primary purification cell, the radioactive sodium chemistry loop, and the fuel element rupture detector.

About $90,000 \mathrm{gal}\left(341 \mathrm{~m}^{3}\right)$ of sodium are contained in the primary tank, and it has a normal operating temperature of $700^{\circ} \mathrm{F}\left(371^{\circ} \mathrm{C}\right)$. The sodium is pumped through the reactor at $9000 \mathrm{gpm}\left(0.568 \mathrm{~m}^{3} / \mathrm{s}\right)$ and is heated to $883^{\circ} \mathrm{F}\left(473^{\circ} \mathrm{C}\right)$ at $62.5 \mathrm{MWt}$. The primary sodium, which is radioactive, is confined to the primary tank and is never mixed with the secondary system.

The secondary system is an intermediate closed loop between the primary system and the steam system. About $14,000 \mathrm{gal}\left(53.0 \mathrm{~m}^{3}\right)$ of sodium are contained in the secondary sodium loop. The secondary sodium is circulated through the tube side of the intermediate heat exchanger at a rate of $5580 \mathrm{gpm}\left(0.352 \mathrm{~m}^{3} / \mathrm{s}\right)$ at $62.5 \mathrm{MWt}$ and is heated to $865^{\circ} \mathrm{F}\left(463^{\circ} \mathrm{C}\right)$. The heated sodium flows through the shell side of the superheaters and evaporators and is cooled to about $587^{\circ} \mathrm{F}\left(308^{\circ} \mathrm{C}\right)$.

The steam system consists essentially of a steam generator, turbine, condenser, feedwater heaters, and pumps. Feedwater at a temperature of $550^{\circ} \mathrm{F}\left(288^{\circ} \mathrm{C}\right)$ flows to the steam drum and then through the tubes of seven parallel-connected evaporators and is heated to a water-steam mixture at about $580^{\circ} \mathrm{F}\left(304^{\circ} \mathrm{C}\right)$. After the moisture is removed from the steam in the steam drum, the saturated steam flows through the tubes of two parallel-connected superheaters where 


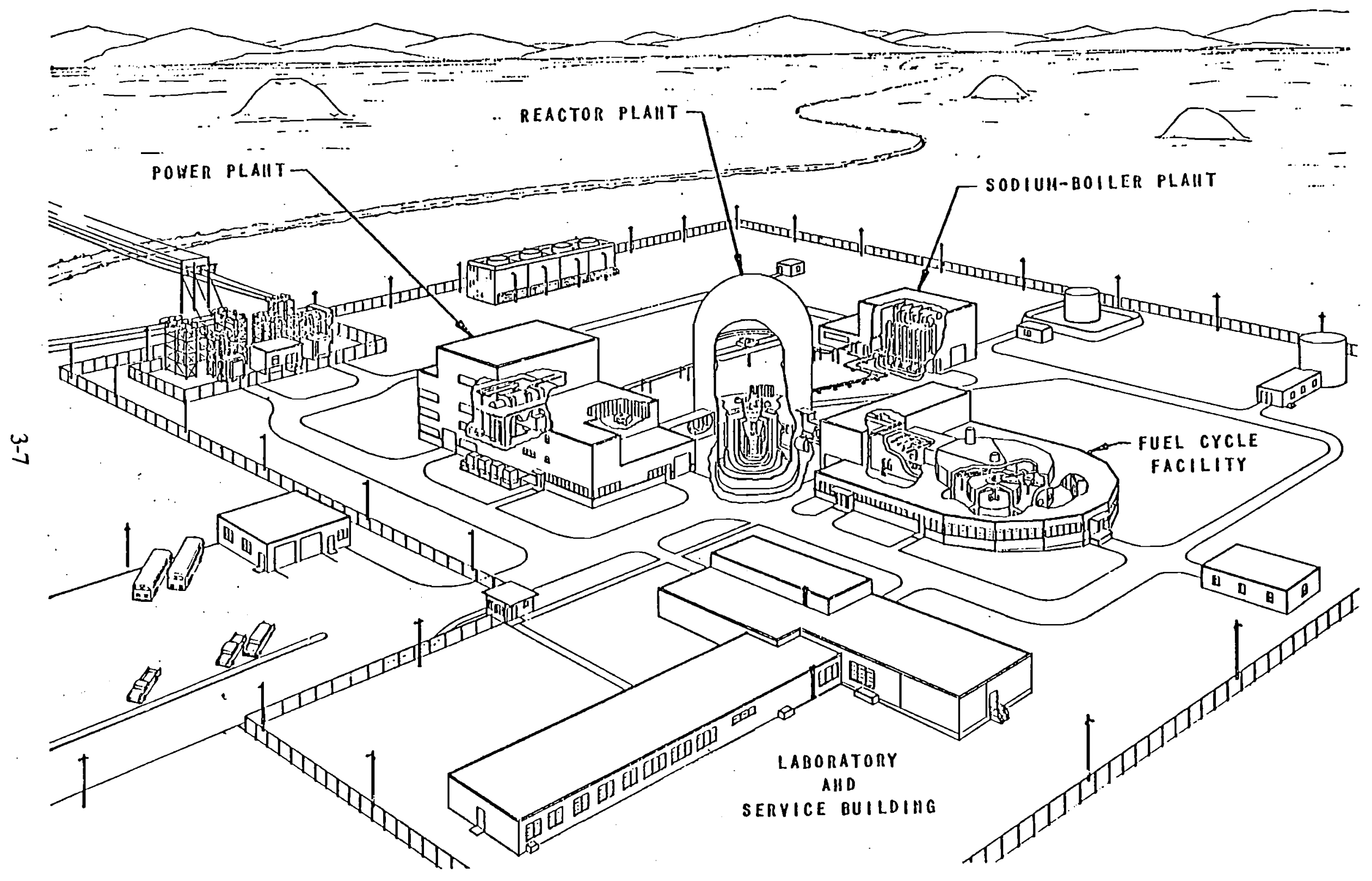

Fig. 3.4. Reactor Building and Power Plant 


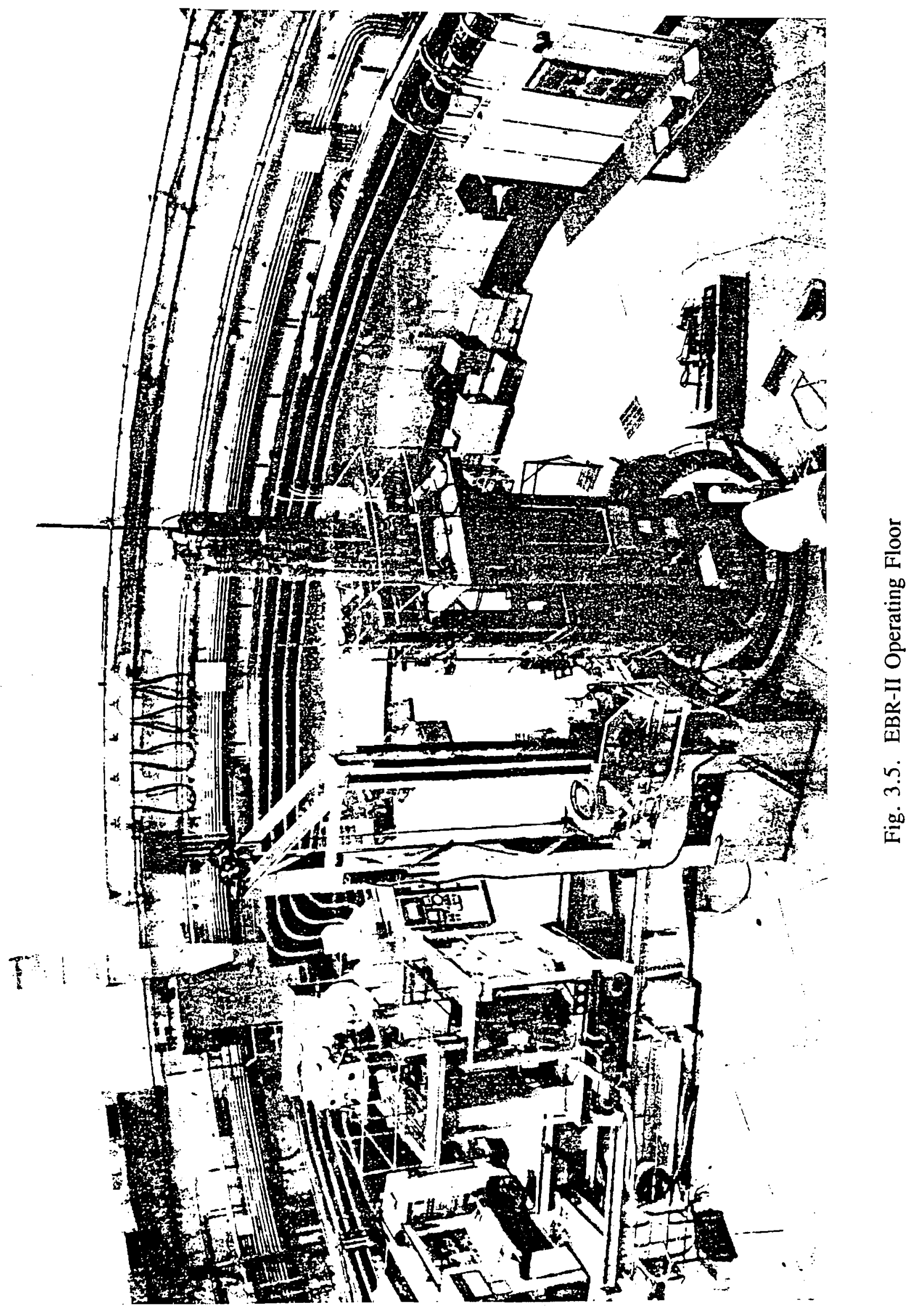




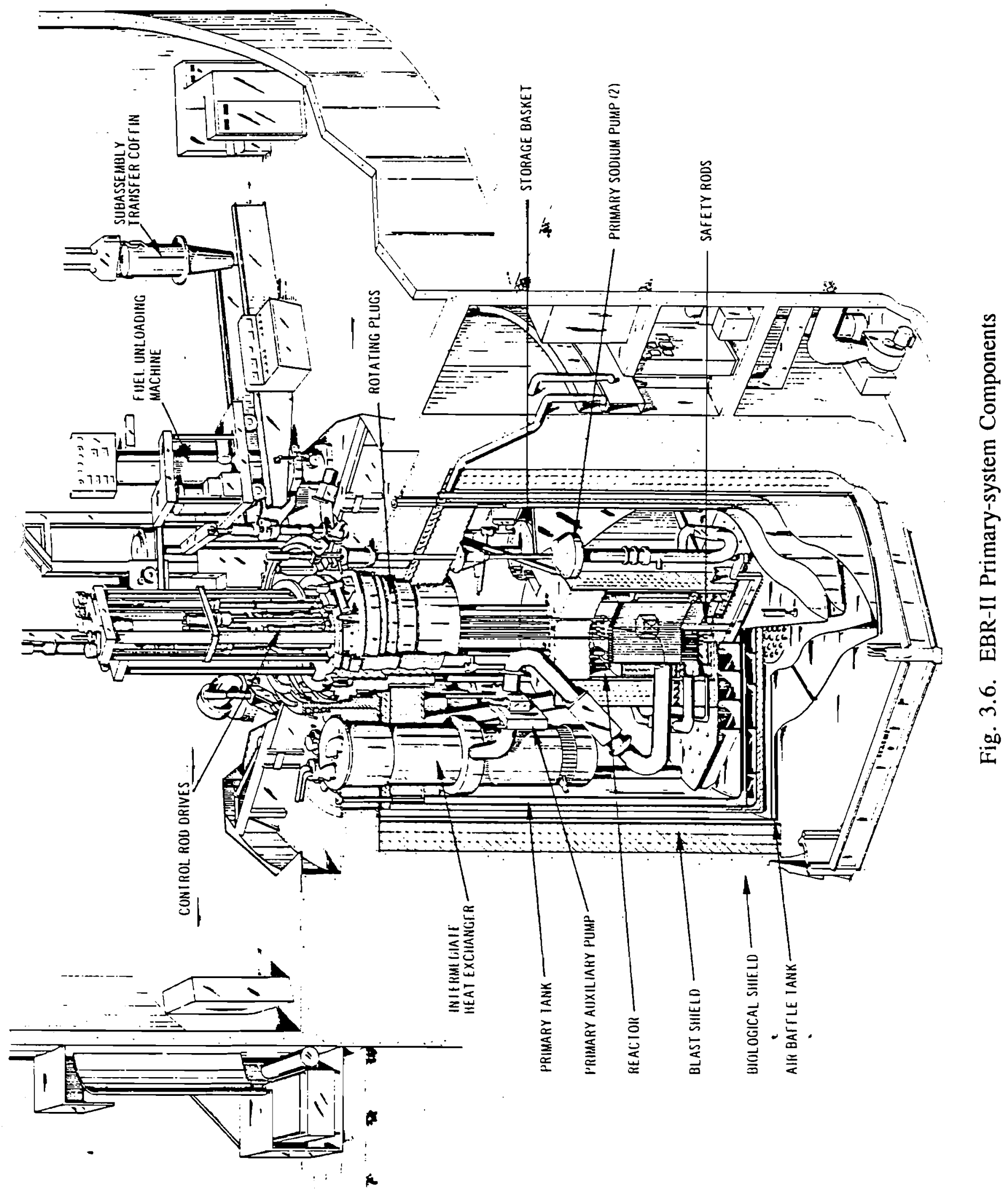


it is heated to $820^{\circ} \mathrm{F}\left(438^{\circ} \mathrm{C}\right)$. The superheated steam then flows either to the turbine or directly to the condenser.

EBR-II completed its 25th year of operation in August 1989. Since the early 1960s, the facility has been compiling an outstanding operating record and a growing list of accomplishments. A review of EBR-II's operating history reveals a plant that has demonstrated high availability, stable and safe operating characteristics, and excellent performance of its sodium components. The record of high capacity factor documents the reliability and maintainability that can be achieved in a pool-type sodium-cooled fast reactor.

A major reason for the high capacity factor of recent years and the reliable operation of EBR-II has been the ongoing simplification of the plant. What started as a relatively complex plant to operate has become an increasingly simple, safe, and reliable plant today, and efforts are constantly under way to further improve and simplify its operation. A case in point is the simplification of the reactor shutdown system. The number of input trip signals to the shutdown system and the number of startup interlocks have been reduced from the original design. This was possible because of the inherent safety of the design and the increasing evidence of the benign behavior of EBR-II event under upset conditions.

EBR-II has proved to be a very versatile facility in which to perform various programs reliably and safely. Its mission has changed several times to meet the needs of the LMFBR base program. In its initial operation, EBR-II successfully demonstrated the feasibility of a sodiumcooled fast breeder reactor operating as an integrated reactor, power plant, and fuel-processing facility. In 1965, the role of EBR-II was changed to that of a steady-state irradiation facility. Since that time, over 11,000 individual experiments have been irradiated. The experiments have consisted of various fuel types (oxides, carbides, nitrides, and metal), cladding and structural materials, and reactor control materials. Peak burnups have reached nearly 20 at. $\%$ for mixedoxide and mixed-carbide fuels, and nearly 19 at. \% for metal fuels.

Since early 1978, the reactor has been operating for extended periods with failed (breached) fuel elements as part of a national program to determine the lifetime and survivability 
of failed fuel in fast reactors, and the effects of such fuel on reactor operation. In preparation for this mode of operation, a cover-gas cleanup system (CGCS) was installed in 1977 to remove fission gases from the primary cover gas by cryogenic distillation.

The performance of the reactor and its primary cooling system has been very good. Throughout its operating history, the reactor has been highly stable and readily controllable. An ever-present and predictable negative power coefficient ensures kinetics stability under all foreseeable operating conditions.

Mechanical stability of the core components has been excellent. No indication of vibration or coolant-flow blockage has been observed, nor has any indication of gas entrainment been detected in the coolant. Post-irradiation examinations conducted on discharged core components (subassemblies, control- and safety-rod thimbles, etc.) have uncovered no evidence of excessive wear, fretting, ratcheting, vibration, or abrasion.

In 1976, a program of annual nondestructive inspection was begun on the most critical areas of the secondary-sodium and high-pressure-steam systems. Items examined included pipe welds, sections of steam and secondary piping, hangers, and valve-body casings.

The programs for shutdown-heat-removal testing and use of EBR-II as a test bed for components such as steam generators and pumps, a man-machine-interface program, demonstration of automatic control of the reactor, and the initiative for an Integral Fast Reactor are all examples of the various research missions of EBR-II.

\subsection{Plant Description}

\subsubsection{Major Structures}

A plot plan of the ANL-W site is given in Fig. 3.3. The buildings of direct relevance are 767 , EBR-II reactor building, 768 , the power plant, and 769 , the sodium boiler building. Also worthy of note are 757 , the cooling tower, 758 , the electrical substation and 765 , 
originally called the fuel cycle facility, changed to Hot Fuels Examination Facility/South (HFEF/S) and recently renamed Fuel Cycle Facility (FCF).

The EBR-II systems are shown in simplified form in Fig. 3.7. The reactor, intermediate heat exchanger, control rods and safety rods all reside in the reactor building. Secondary sodium is piped from the IHX to the sodium-boiler plant through the yard piping. The sodiumboiler plant houses the secondary electromagnetic (EM) pump, seven evaporators, two superheaters, surge tank, steam drum etc. The power plant adjoins the reactor building and houses the primary pump motor generator sets, feedwater pumps and feedwater heaters, the turbine generator, the reactor control room, electrical supply cabinets and all other equipment. In addition there are a number of offices in the power plant. Figures 3.8 and 3.9 give detailed layouts of the power plant and the sodium boiler building. Both these buildings are steel framed and not designed to contain or confine any energy release. In fact the north and east walls of the sodium-boiler plant are designed with blowout panels in the event of a large energy release in the sodium boiler plant.

Access to the reactor building is gained from the power plant via a personnel air lock and an emergency air lock. There is also an equipment air lock for transfer of fuel between FCF and EBR-II, as well as large pieces of equipment. The reactor containment building is a steel shell, one inch thick with 12 inches of concrete on the inside surface. There is a polar crane used for transferring fuel. The primary tank, containing the reactor, 90,000 gallons of liquid sodium and the pumps and heat exchanger, lies below grade as shown in Fig. 3.10. The control rod drives are situated on the reactor floor and enter the core through the small rotating plug.

\subsubsection{Reactor System}

The EBR-II reactor core (see Fig. 3.11) consists of a 7-row hexagonal central core containing enriched uranium which is completely surrounded by 3 rows of stainless steel reflections and a 6-row radial blanket of depleted uranium. The blanket regions contain either depleted uranium or stainless steel and nickel. The core- and blanket-region materials are 


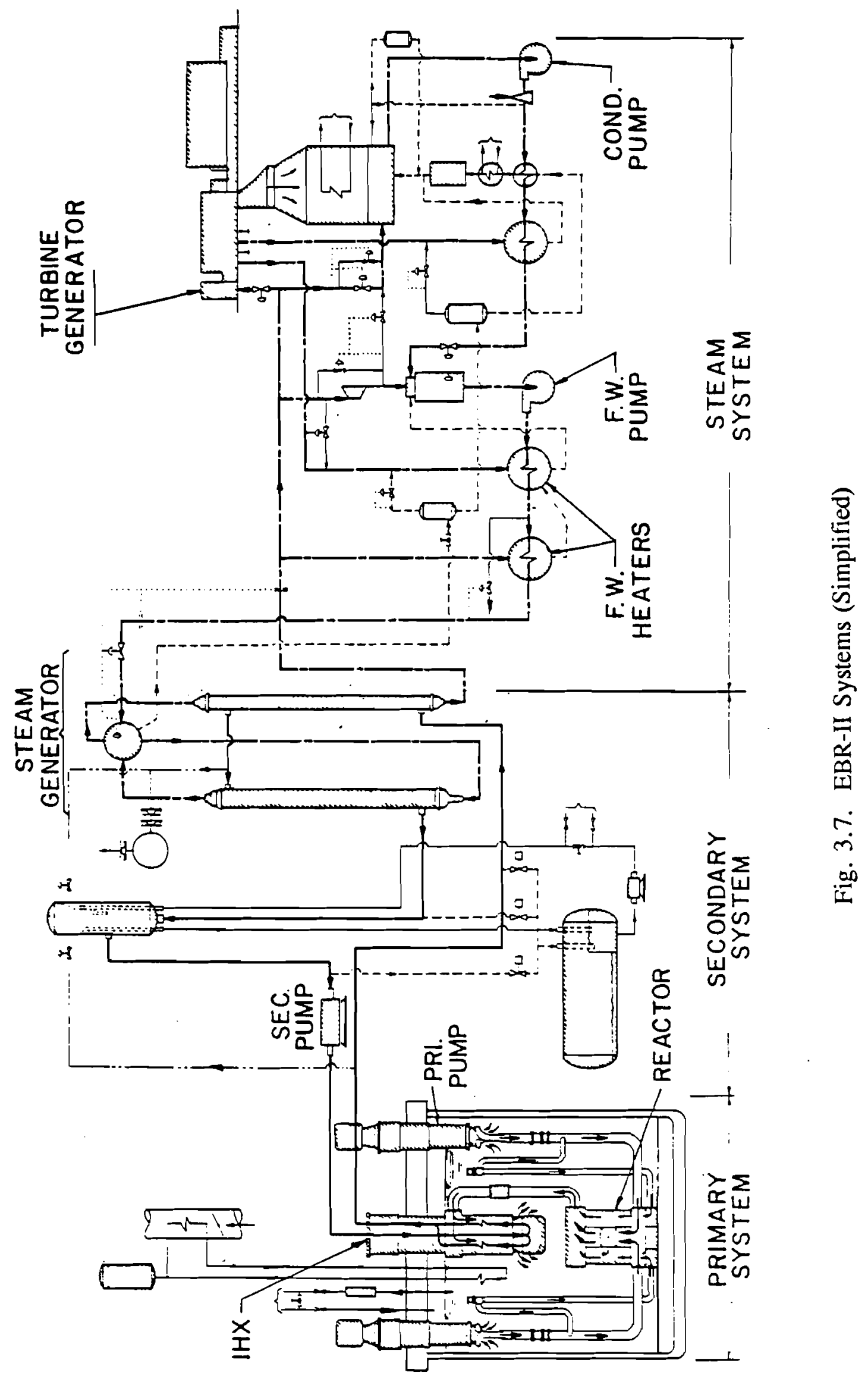




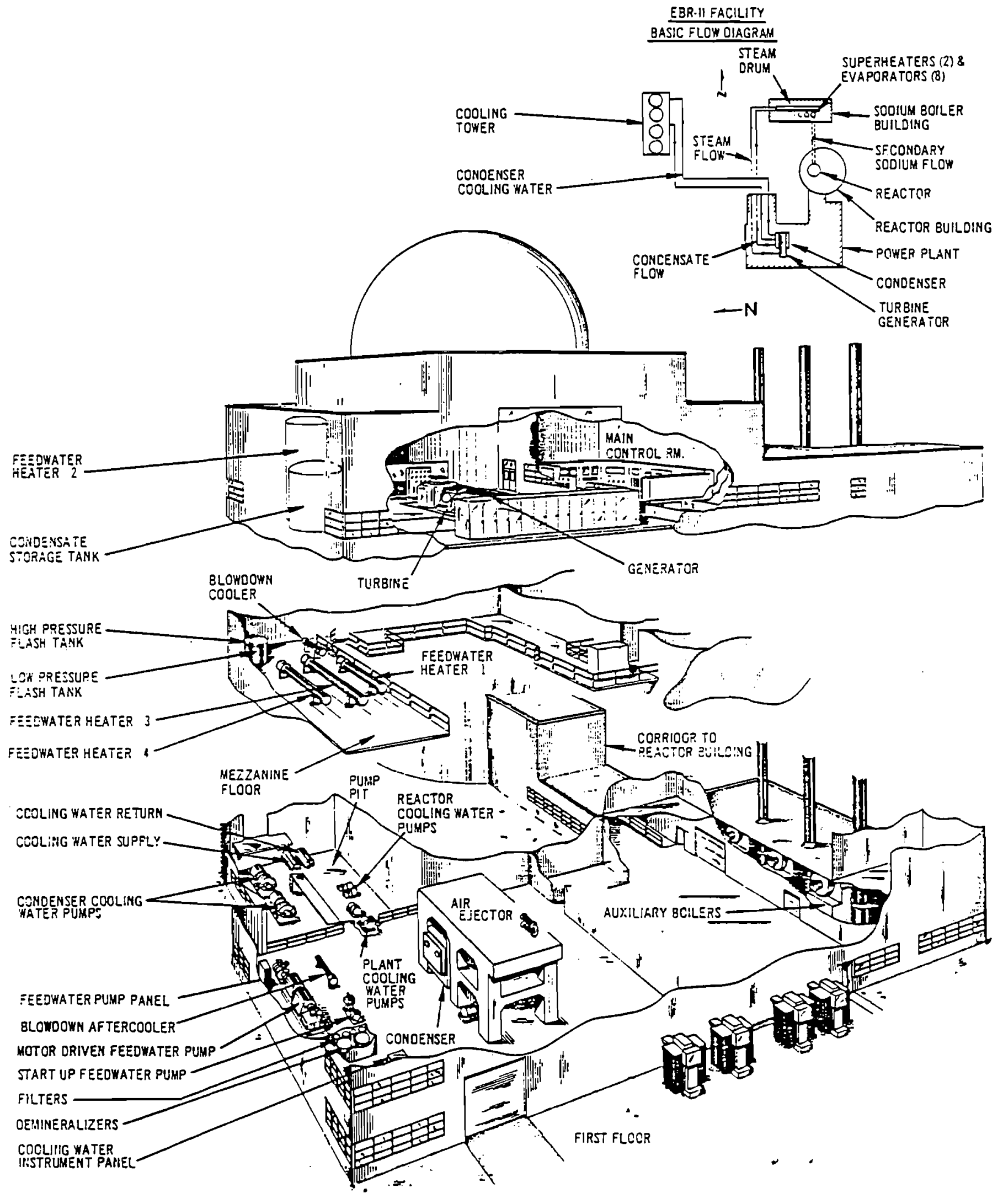

Fig. 3.8. Power Plant - Location of Components 


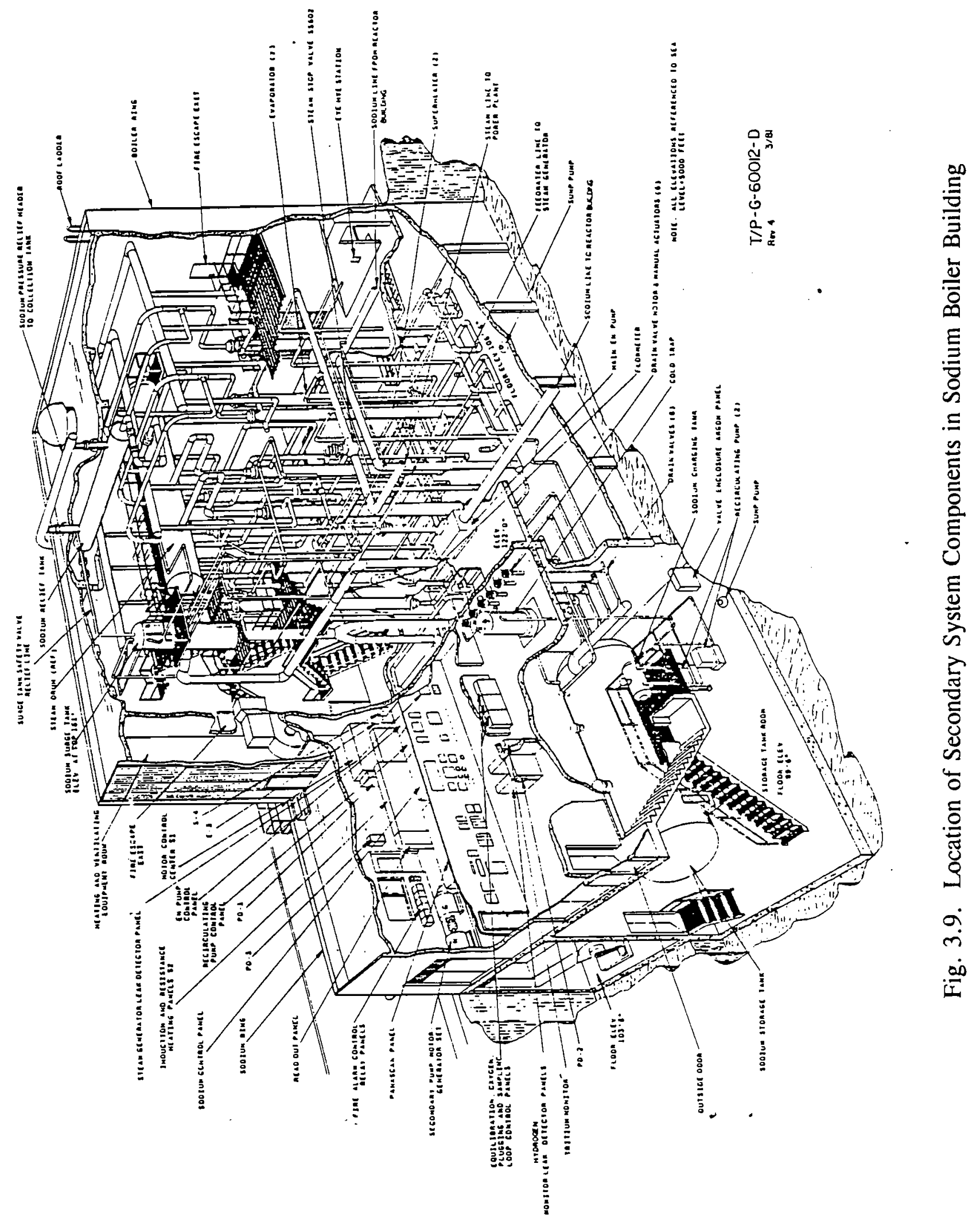




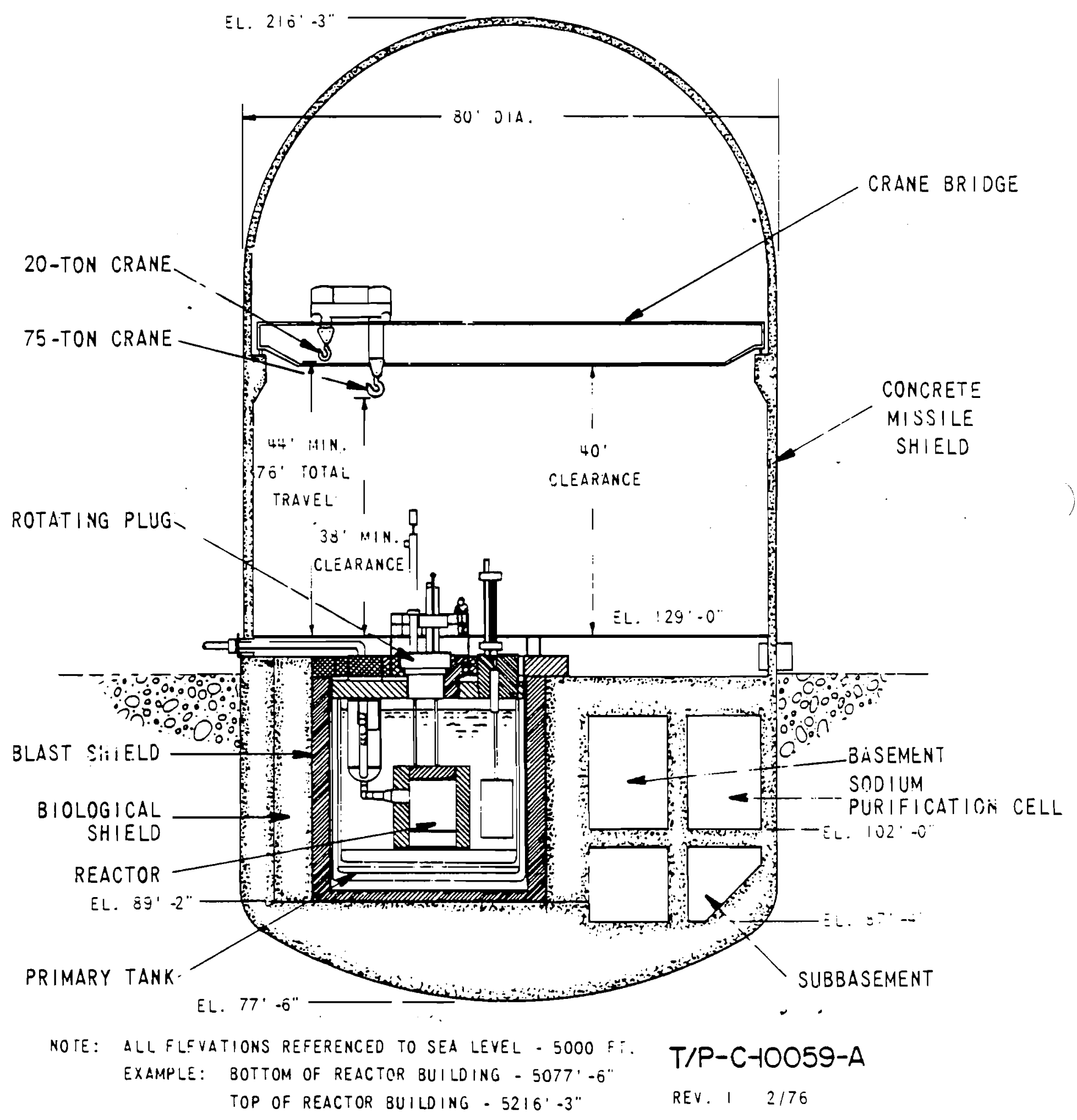

Fig. 3.10. Reactor Building 


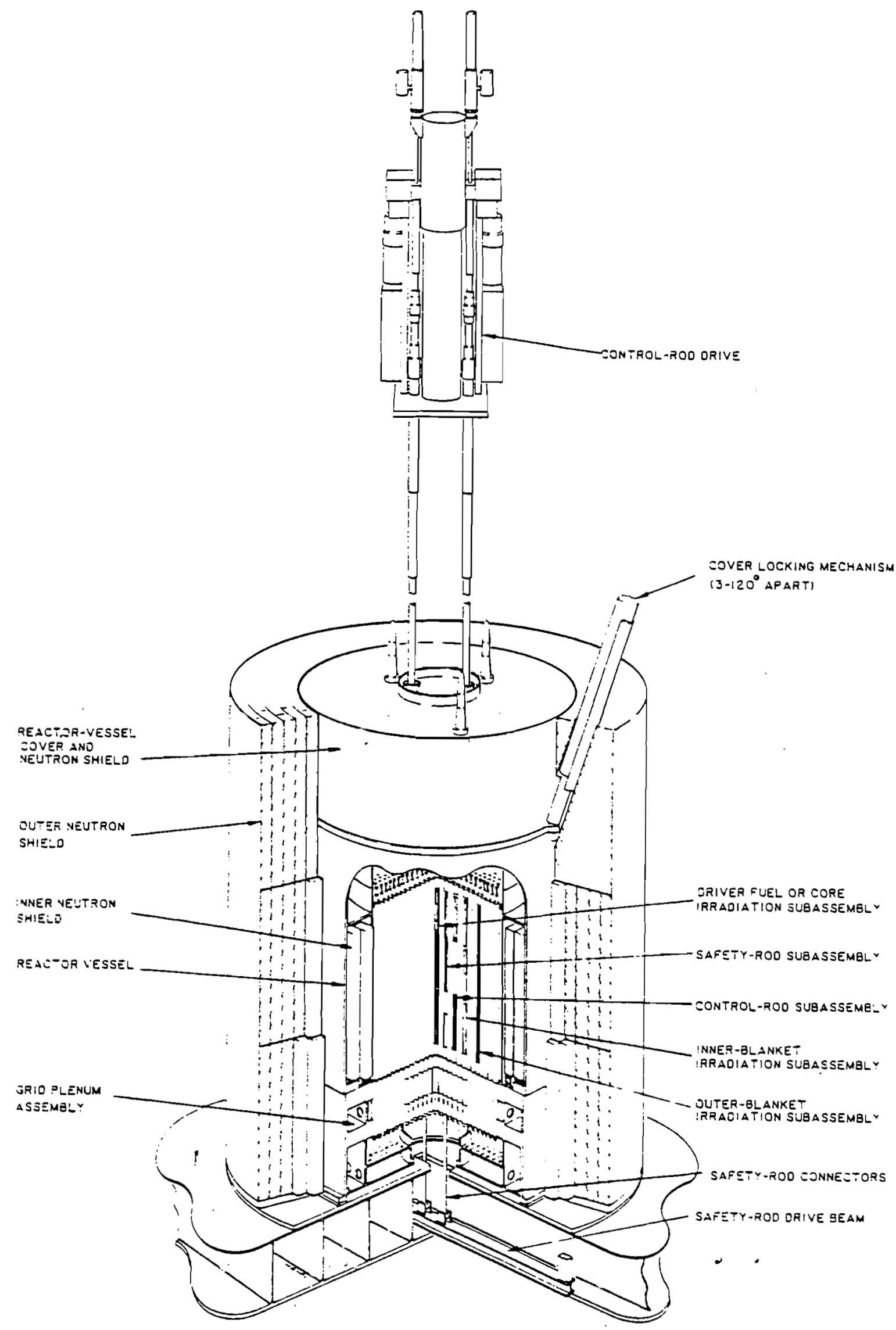

Fig. 3.11. EBR-II Core 
contained in 637 subassemblies, which are contained in and supported by the reactor vessel. The reactor vessel, consisting of a grid plenum assembly, inner and outer reactor-vessel shells, and a reactor-vessel cover, is surrounded by a radial neutron shield and is submerged beneath approximately $10 \mathrm{ft}$ of sodium. The reactor-vessel cover, which also contains neutron shielding, raises to permit fuel handling. It contains penetrations for control-rod drive shafts to provide reactor control and for the holddown and gripper shafts used for fuel handling.

The reactor vessel is constructed of an outer shell, an inner shell, and thermal baffles, all of Type 304 stainless steel. It is well shielded by the inner radial neutron shield and well protected thermally. The neutron shield is composed of a seven-layer radial shield and an axial shield in the reactor-vessel cover. Two layers of the seven-layer radial shield are between the two reactor-vessel shells. The other five layers cover the outside of the reactor vessel from below the grid plenum to the top of the vessel cover. The shield is composed of plain graphite blocks and borated graphite blocks, in stainless steel cans, that moderate and absorb neutrons passing through the reactor blanket. The blanket subassemblies also act as neutron and gamma shields to reduce radiation damage to the reactor vessel and the components in or directly outside the reactor vessel.

The grid plenum assembly, which supports the hexagonal subassemblies in the reactor vessel, has 637 apertures arranged to position the subassemblies in a tightly packed triangular lattice. The subassemblies are all outwardly identical: each has an upper adapter to permit handling (removal and insertion) by the same gripper and transfer devices; each has a hexagonal support body that contains the fuel or blanket material; and each has a lower adapter. The lower adapters have different bottom configurations to prevent positioning in the wrong region and have different coolant orifices to ensure the appropriate amount of sodium flow.

Operation of the reactor is controlled by vertical movement of fueled control rods with $\mathrm{B}_{4} \mathrm{C}$ followers in thimbles in row 5 of the core region. Control-rod drive shafts, operating through penetrations in the reactor-vessel cover, are connected to the control rods. The controlrod drive shafts are connected to independently operated electromechanical drives mounted above 
the primary-tank assembly on the small rotating plug. Twelve rods were originally in the core; currently two original control-rod positions contain test facilities. The control-rod drives are operated through 3 in. of travel or less at least once every 24 hours to check for the possibility of control-rod binding.

Because control rods are made of fuel with a poison follower the core is made critical by raising the control rods into the core, and scram is effected by dropping the control rods out of the core.

Safety rods located in thimbles in row 3 of the core region are connected to safety-rod connectors that extend up through the reactor-vessel grid plenum assembly. The connectors are connected through a safety-rod drive beam and safety-rod shafts outside the reactor vessel (but within the primary tank) to the safety-rod drive mechanism at the top of the primary-tank cover. The safety-rod drive system is a part of the reactivity control system; it provides negative reactivity when the reactor is operating, or when it is shutdown and the control rods are disconnected. In addition, use of the safety rods as shim rods, operating in conjunction with the control rods, has recently been implemented.

Sodium coolant, which surrounds the reactor, is pumped into the high-pressure and lowpressure chambers in the grid plenum of the reactor vessel (two inlets for each chamber, on opposite sides of the reactor vessel) from the two centrifugal main primary-sodium pumps, which operate in parallel. The high-pressure-coolant plenum is the source of coolant for the core and inner-blanket-region subassemblies. The low-pressure-coolant plenum is the source of coolant for the outer-blanket-region subassemblies.

The reactor's nuclear instrumentation consists of three wide-range nuclear instrument channels (A, B, and C) and two linear channels (7 and 7A). Channels $\mathrm{A}, \mathrm{B}$, and $\mathrm{C}$ each provides continuous neutron-flux monitoring, alarm functions, and automatic protective functions in the form of a reactor scram for short periods and over power conditions. Channels 7 and $7 \mathrm{~A}$ each provide linear power indication for startup of the reactor and indication for reactor control 
in the power range. The detectors for all the channels are in the reactor's $\mathrm{J}$ thimbles (J1, J3, and J4).

\subsubsection{Primarv Sodium System}

The main function of the primary cooling system is to remove the heat produced in the reactor core by nuclear fission and transport it to the intermediate heat exchanger (IHX), where it can be transferred to the secondary cooling system. To perform this function, liquid sodium is pumped from the primary tank by two centrifugal pumps, through high pressure and low-pressure (throttled) piping to the reactor, and then to the shell side of the shell-and-tube IHX, from which it is returned to the primary tank. The entire primary cooling system, and the reactor vessel itself, are contained within the primary tank. Operation with the reactor vessel and other primary-system components completely submerged in the liquid sodium coolant ensures a high degree of reliability and safety of the primary system.

The primary cooling system, shown in Fig. 3.12, consists of the following major components: two primary-coolant pumps, a primary auxiliary pump, the IHX (primary side), reactor supply and discharge piping, and instrumentation associated with the system.

When the reactor is operating at the full design power of $62.5 \mathrm{MWt}$, the primary-system flow is $1064 \mathrm{lb} / \mathrm{s}(484 \mathrm{Kg} / \mathrm{s})$ through the reactor vessel, the reactor inlet sodium temperature is $700^{\circ} \mathrm{F}\left(371^{\circ} \mathrm{C}\right)$, and the mixed mean outlet temperature is $883^{\circ} \mathrm{F}\left(473^{\circ} \mathrm{C}\right)$. Certain leakage paths from the cooling system to the primary tank have been designed into the system. These leakages occur: at the ball-joint disconnects of the primary pumps; at the low-pressure throttle valves; at the reactor inlet plenums; from the reactor-vessel cover, rod-drive penetrations, etc.; at the outlet plenum; and at the IHX inlet.

The primary-cooling-system instrumentation originally included 10 flowmeters, both electromagnetic (EM) and head type, on the high-pressure and low-pressure legs of each primary pump and on the reactor outlet piping to the IHX. Of these flowmeters, only three remain operable. Similarly, the original system had 12 pressure transmitters, two in each of the 


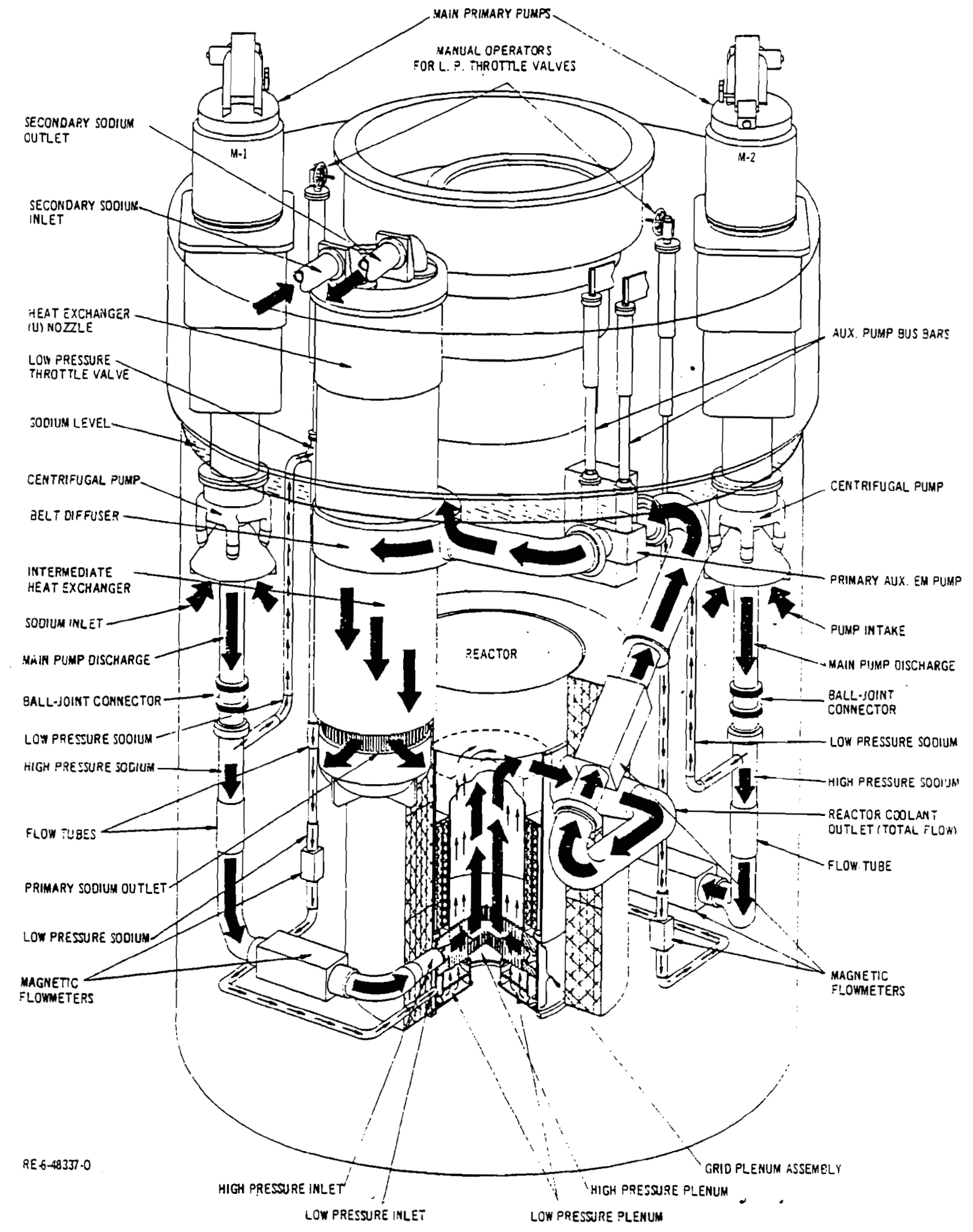

Fig. 3.12. Primary Cooling System 
primary-pump discharge pipes, one in each of the high- and low-pressure piping to the reactor, one in the high-pressure plenum, one in the low-pressure plenum, one in the upper plenum, and one in the reactor discharge pipe. Of these transmitters, only three remain operable. Of the many thermocouples and resistance temperature detectors installed throughout the system, most are still operable. The loss of instruments, particularly flowmeters, is a concern for EBR-II. Currently the number available is sufficient for plant protection needs, however, loss of more flowmeters would cause difficulties. Strategies to alleviate the problem include using pressure transmitters as flowmeters and adding pump speed sensors.

The EBR-II primary-coolant pumps are a matched pair of vertically mounted centrifugal pumps designed to pump liquid sodium from the primary tank, through in-tank piping, and through the reactor core. Each pump is mounted on a penetration nozzle in the primary-tank cover, and the pump structures are inside the primary tank. Figure 3.13 is a cutaway view of a primary pump.

The pumps take their suction directly from the $700^{\circ} \mathrm{F}\left(371^{\circ} \mathrm{C}\right)$ bulk sodium in the primary tank and discharge to a 12 -in. $(0.3 \mathrm{~m})$ pipe nozzle that is integral with the pump assembly. Each pump has its own motor-generator (MG) set located outside the reactor building, in the power plant. Pump speed is controlled by varying the generated frequency to the pump drive motor through use of a magnetic slip clutch between the motor and generator of the MG set.

Heat from the radioactive primary sodium is transferred to the nonradioactive secondarysodium system by the intermediate heat exchanger (IHX). The IHX is a shell and counterflow, single-tube-wall unit which operates completely submerged in the reactor sodium coolant. It is suspended from above by a shield plug and positioned within a well casing which is an integral extension of its primary-tank nozzle. The IHX and shield plug assembly, shown in Fig. 3.14, is approximately 5 - $\mathrm{ft}(1.52 \mathrm{~m})$ dia and $24-\mathrm{ft}(2.32 \mathrm{~m})$ long and weighs about $69,000 \mathrm{lb}(31,364$ $\mathrm{Kg}$ ) when flooded with sodium. The total heat transfer surface is about $4800 \mathrm{sq} \mathrm{ft}\left(446 \mathrm{~m}^{2}\right)$. 


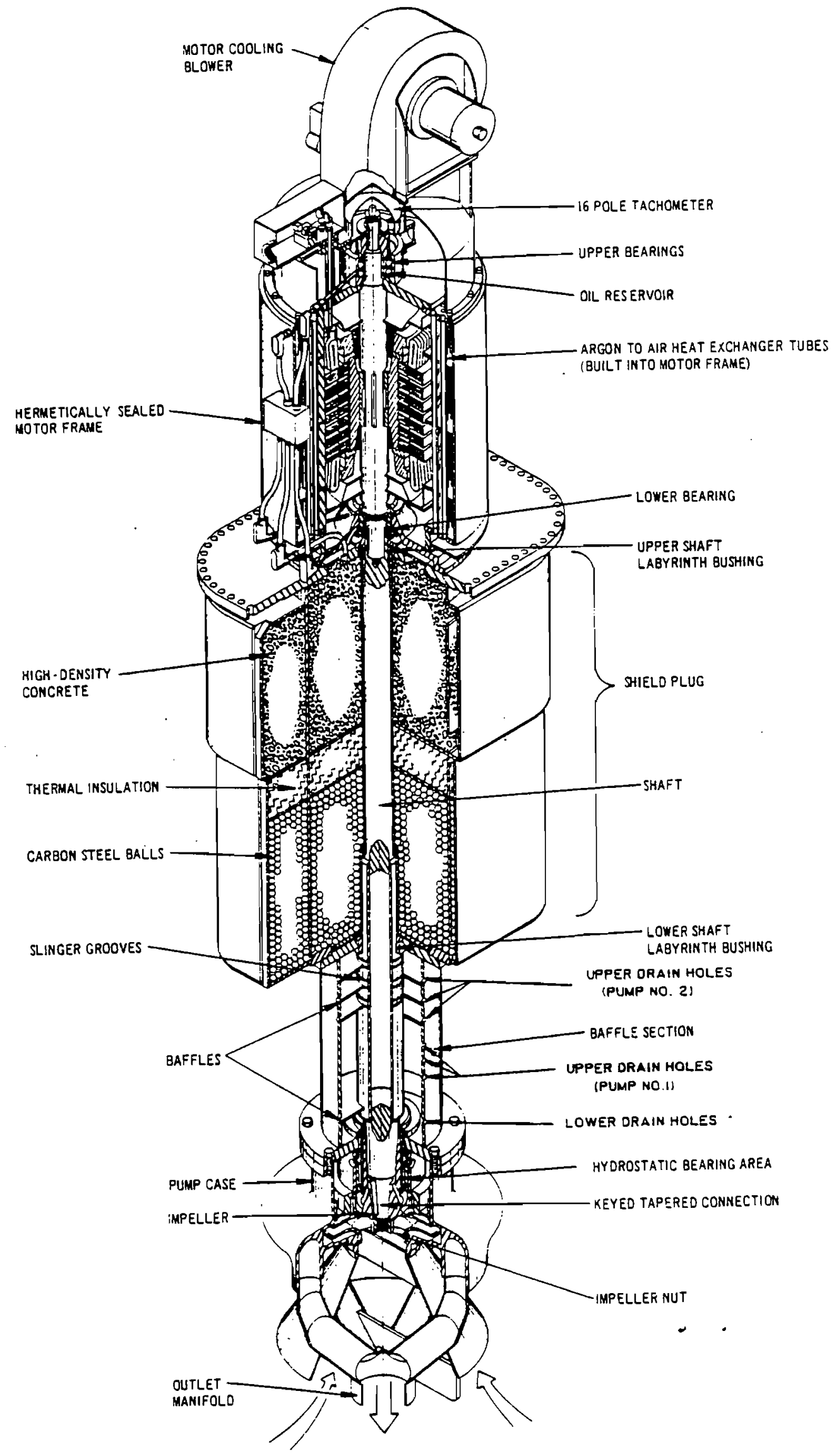

Fig. 3.13. Cutaway View of a Primary-coolant Pump 


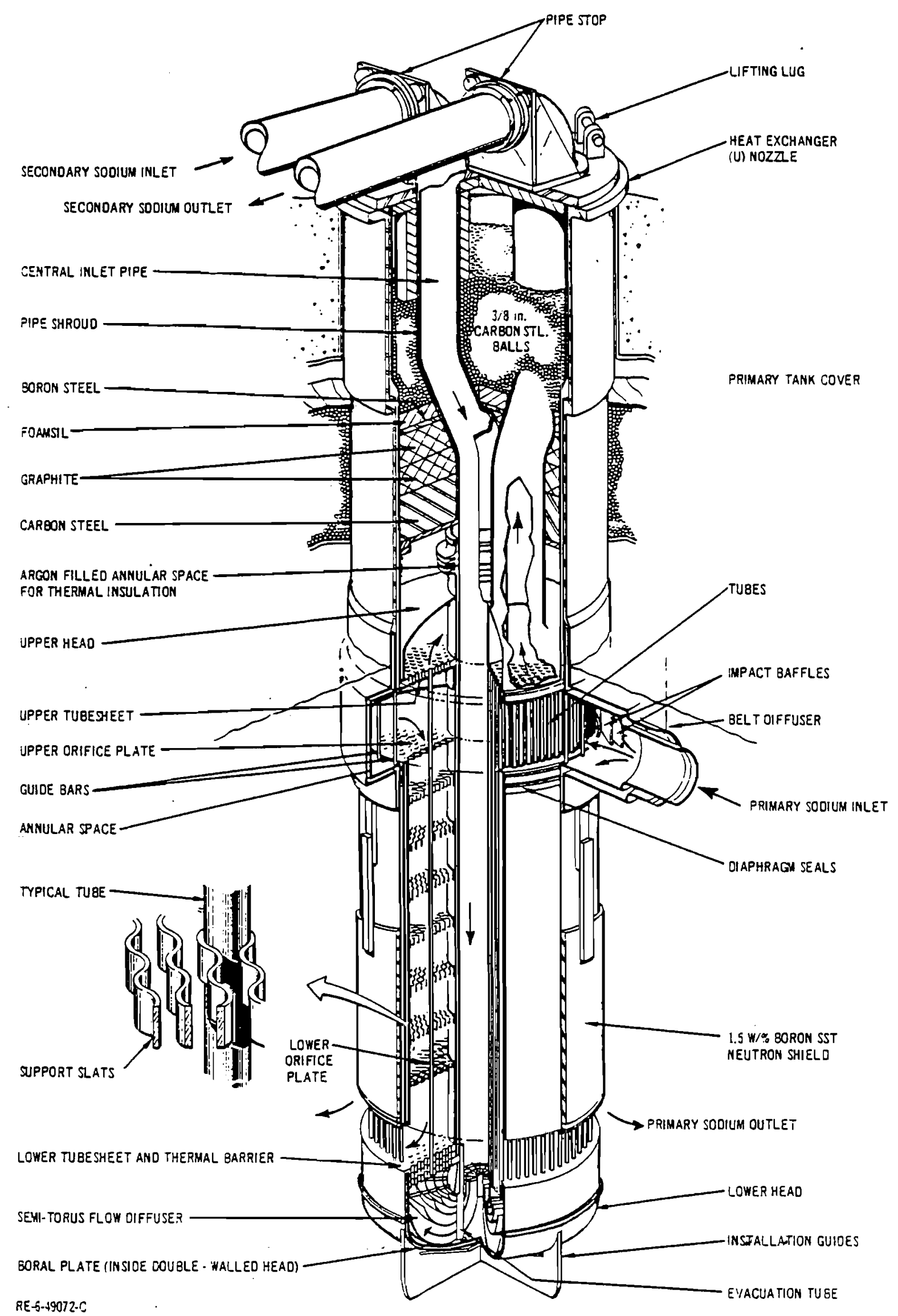

Fig. 3.14. Intermediate Heat Exchanger 
The primary tank is a double-walled cylindrical tank about $27 \mathrm{ft}(8.23 \mathrm{~m})$ in diameter by $27-\mathrm{ft}(8.23 \mathrm{~m})$ high. It contains the nuclear reactor, the entire primary cooling system, and portions of the fuel handling system, all submerged in $700^{\circ} \mathrm{F}\left(371^{\circ} \mathrm{C}\right)$ sodium. The primarytank assembly (see Fig. 3.15) consists of the primary tank and cover, biological and blast shields, an air baffle tank, and a primary-tank support structure. All penetrations into the tank are through the primary-tank cover, above the bulk sodium.

The primary tank and the support structure are completely independent of each other except at the.top. The primary tank, containing the bulk sodium, and those components connected to the primary-tank cover are supported by six hangers, which are welded to each of the radial beams in the tank cover and bear on six high-strength steel rollers supported by the top support structure. This roller support system (see Fig. 3.16) is necessary to avoid high stresses caused by the differential expansion between the primary tank and the top support structure. As shown in Fig. 3.15, the basic top support structure consists of six radial beams, spaced at $60^{\circ}$ intervals; and a large central ring. The basic structure of the primary-tank cover, shown in Fig. 3.17, is essentially the same as the basic top support structure, and the six radial beams of the support structure and six similar beams within the tank cover are coincident.

Containment of the 90,000 gals $\left(341 \mathrm{~m}^{3}\right)$ of radioactive primary sodium by the primary tank system is essential for the continued, safe, operation of EBR-II. The primary tank is of double-wall construction to provide maximum reliability, The tank consists of two vessels completely independent of each other, except for their common attachment to the primary-tank cover, from which they are suspended. Both tanks and the cover are constructed entirely of Type 304 stainless steel and of welded construction. There are no penetrations in the tanks except for the cover.

Because of their unique construction, the uniform temperature maintained on both tanks, and the common cover, mechanical and thermal stresses are very low. An argon atmosphere is maintained in the annulus between the tanks and thermocouples and a sodium leak detector probe is also provided in the annulus. Corrosion of stainless steel when exposed to argon or $700^{\circ} \mathrm{C}\left(371^{\circ} \mathrm{C}\right)$ sodium is nil. If a small leak in the main tank developed, sodium would slowly 


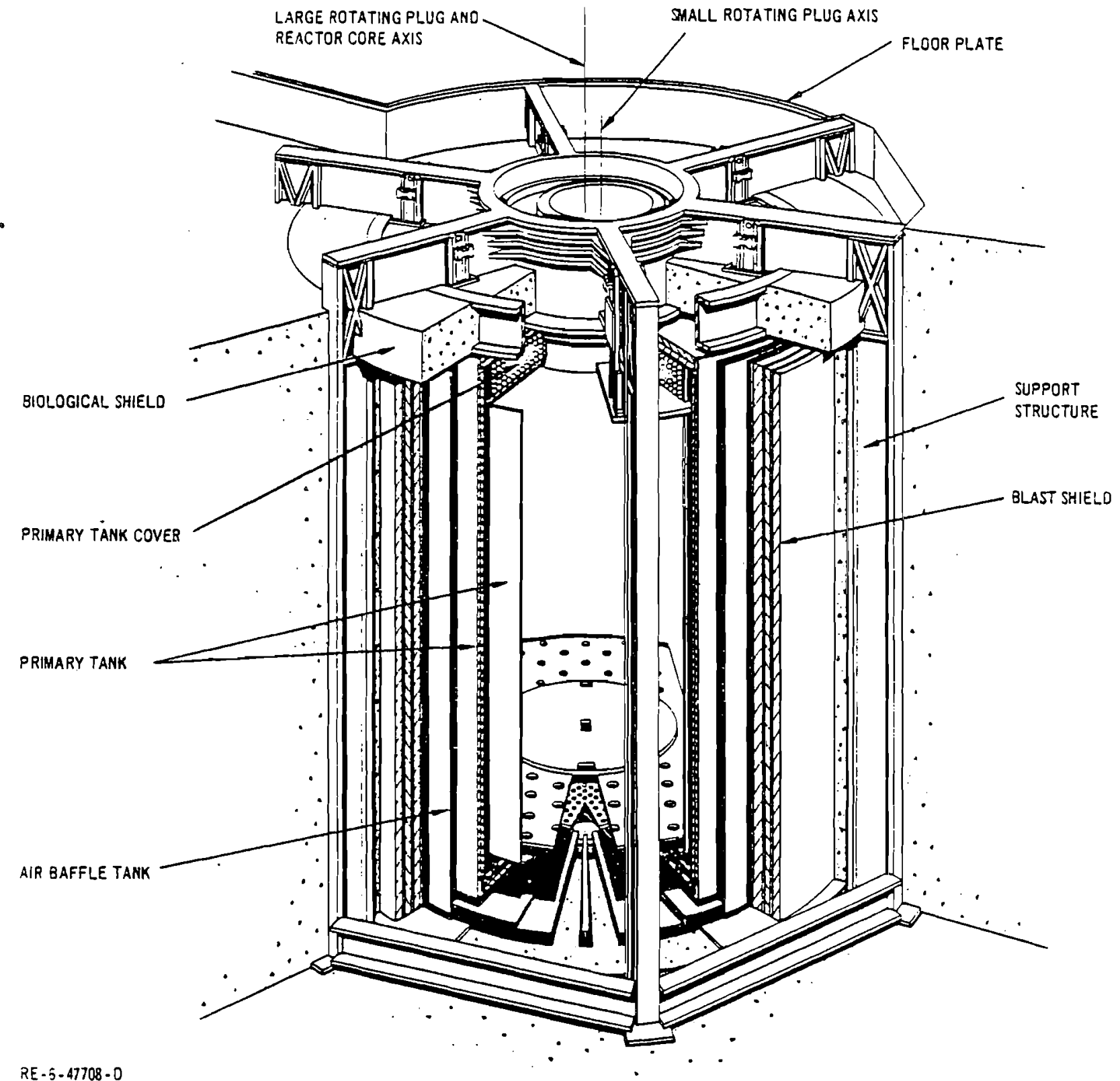

Fig. 3.15. Primary-tank Assembly 


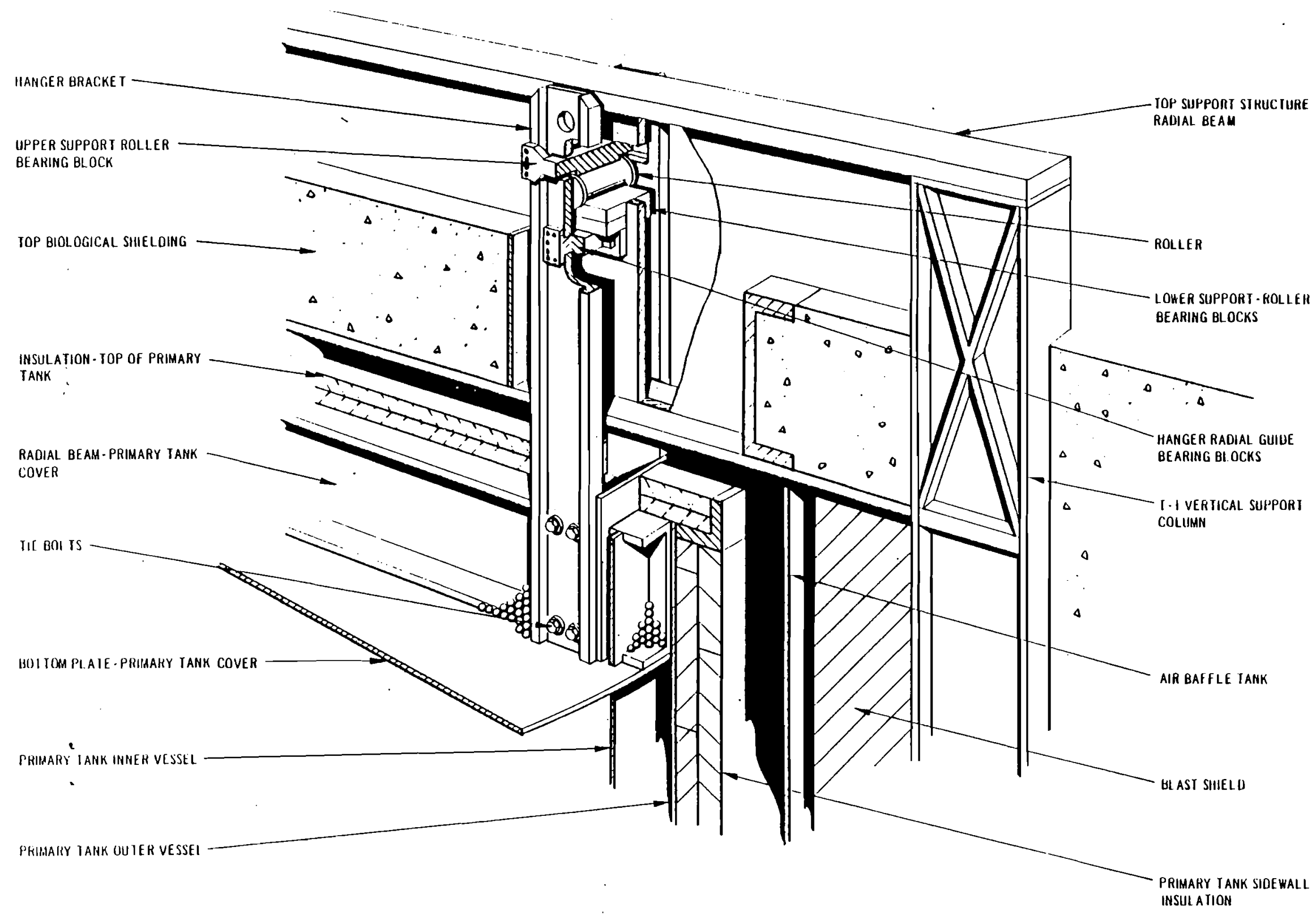

Fig. 3.16. Roller Support System 


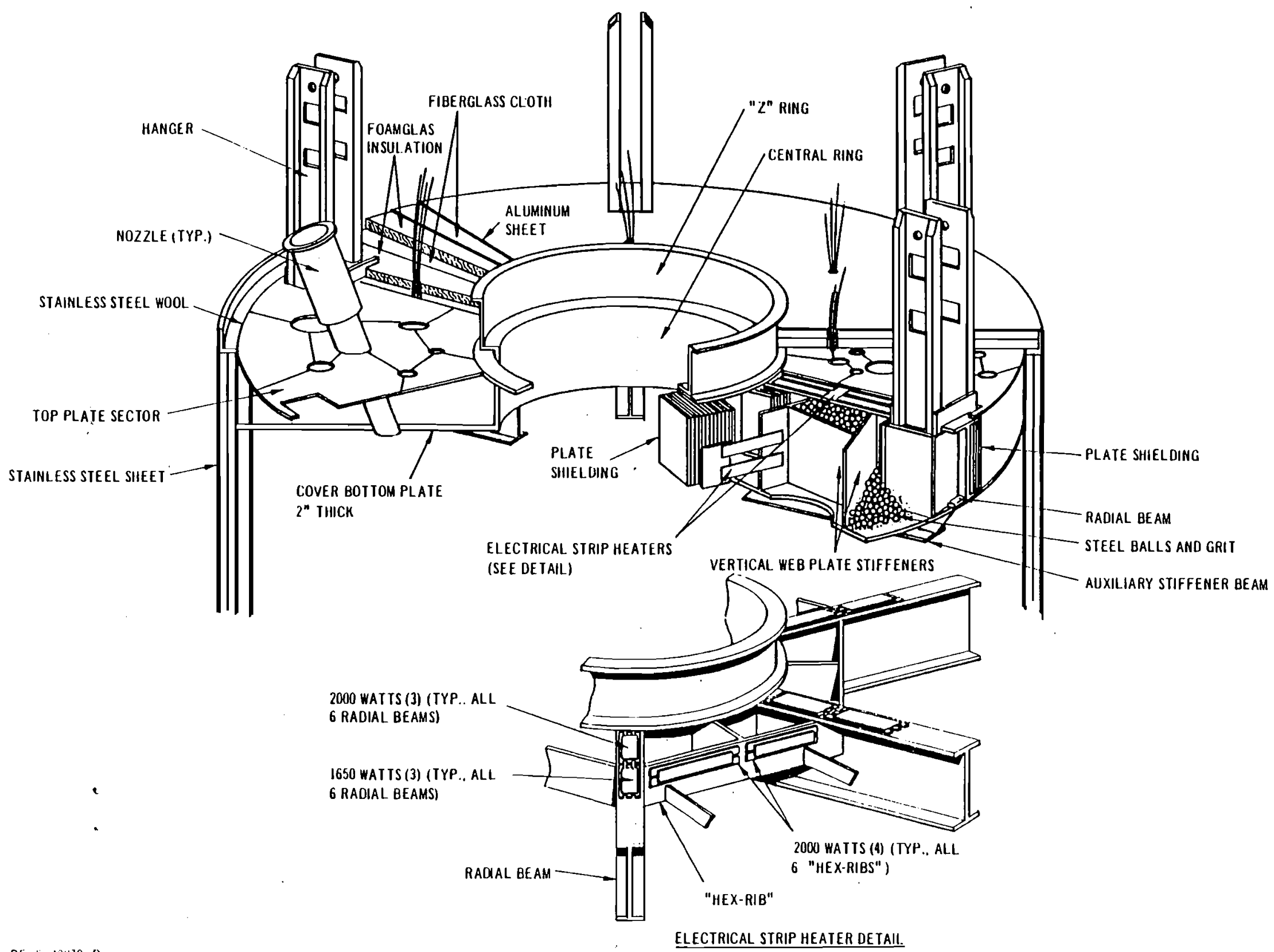

RE - 6 - 491170 - D

Fig. 3.17. Primary-tank Cover 
fill the annulus and be contained by the outer tank. The volume of the annulus is approximately $990 \mathrm{ft}^{3}$ or $7400 \mathrm{gal}\left(28.0 \mathrm{~m}^{3}\right)$. If a leak in the inner tank filled the annulus, it would drop the primary tank bulk sodium level about $22 \mathrm{in}$. $(0.56 \mathrm{~m})$, which would still maintain the reactor core under sodium. If a slight leak developed, there is a reasonable chance that it could be repaired or its effects minimized to permit continued reactor operation.

For shielding, the cover is filled to a depth of about 26 in. $(0.66 \mathrm{~m})$ with 3/8-in. $(9.5$ $\mathrm{mm}$ ) -dia carbon steel balls. The entire tank is insulated to minimize heat losses from the bulk sodium. Numerous thermocouples are located throughout the tank to monitor temperatures of the sodium, the inert-cover-gas blanket above the sodium, the shells of the inner and outer vessels, and the cover.

\subsubsection{Secondary-Sodium System and Balance of Plant}

The secondary-sodium system removes heat from the primary-sodium system, via the intermediate heat exchanger (IHX), and transports it to the steam generator where it is used to produce steam. The secondary-sodium system consists of the main secondary-sodium loop, the secondary-sodium recirculation system and the secondary-sodium auxiliary and subsystems.

The function of the main secondary-sodium loop is to transport reactor-generated heat from the primary cooling system to the steam generator, where high-pressure, high-temperature steam is produced. At the full design reactor power of $62.5 \mathrm{MWt}$, the nonradioactive secondary sodium is circulated at about $5580 \mathrm{gpm}\left(0.352 \mathrm{~m}^{3} / \mathrm{s}\right)(93 \%$ flow) through the tube side of the intermediate heat exchanger (IHX), where heat is transferred from the primary to the secondary sodium. The secondary sodium leaves the IHX at about $865^{\circ} \mathrm{F}\left(463^{\circ} \mathrm{C}\right)$ and flows through mainsecondary-sodium-loop piping to the sodium boiler building, where it enters the shell side of the superheaters. It then flows to the evaporators, where it enters the shell side at about $800^{\circ} \mathrm{F}$ $\left(427^{\circ} \mathrm{C}\right)$, to the surge tank at about $587^{\circ} \mathrm{F}\left(308^{\circ} \mathrm{C}\right)$, the main EM pump, and back to the IHX in the reactor building. 
For reliability, the main secondary-sodium loop contains no valves. Unlike the primary system, most secondary-system components, including instrumentation, are accessible for maintenance or replacement.

The main secondary loop is fabricated of mainly two materials -- type 304 stainless steel, and $21 / 4 \% \mathrm{Cr}-1 \%$ Mo ferritic steel. Generally, the stainless steel is used in the reactor building and yard piping, and the chrome-moly steel is used in the sodium boiler building.

During plant standby, the thermal driving head caused by heating in the IHX and cooling in the steam generator can produce a natural-convection flow of about $6 \%$. In this condition the secondary sodium pump is operated by reversing the pump current. This technique is used to reduce, but not actually reverse, secondary flow during a shutdown so that only the decay heat is removed.

The total sodium volume contained in the secondary-sodium system, which includes the storage tank, dump piping and recirculating system, as well as the main secondary loop, is about $14,000 \mathrm{gal}\left(57.9 \mathrm{~m}^{3}\right)$ at $\left.250^{\circ} \mathrm{F}\left(121^{\circ} \mathrm{C}\right)\right)$. The sodium volume in the secondary system has diminished by about $1800 \mathrm{gal}\left(6.8 \mathrm{~m}^{3}\right)$ over the years because of maintenance, repair, and sampling activities.

Sodium is circulated through the main secondary loop by an AC linear induction electromagnetic (EM) pump. This pump forms an integral part of the secondary piping system and circulates sodium through the IHX to the superheaters and evaporators where heat is transferred to water and/or steam. A conventional steam system, balance-of-plant converts the steam to electricity using a single turbine-generator. Steam is also used for site heating and feedwater heating in four separate heaters. 


\subsection{Reactor Shutdown System}

The reactor shutdown system (RSS) is designed to automatically shut down the reactor in the event of equipment or operator malfunction or an environmental problem that could damage the reactor, injure personnel, or release contamination to the environment.

The RSS will cause the control rods (and, in some cases, the safety rods) to automatically scram to their least reactive position, to shut down reactor operation, if any of the selected plant parameters exceed established limits. During fuel handling, the safety rods will scram to their least reactive position if the fuel-handling shutdown circuit opens.

For redundancy, two shutdown circuits are provided. One is called string $A$, and the other string B. The purpose of string B is to provide reactor shutdown if string A fails to do so. String $B$ is only used during reactor operation; it has no function during fuel handling.

String A provides two modes of operation: (1) reactor operation, and (2) unrestricted fuel handling.

Basically, the shutdown strings will provide plant shutdown in the event of two major problem conditions: (1) overpower, and (2) undercooling. Overpower conditions are indicated by high neutron-flux levels and high temperatures. Undercooling conditions are indicated by high temperatures and low coolant flow rates. Both strings also provide protection from other problem conditions, such as earthquakes or malfunctions of other essential plant equipment.

The reactor shutdown circuits consist of relay coils and contacts and keyswitch contacts, connected in series and series-parallel, to form a circuit between the two electrical buses, RCL-1 and RCL-2. Figure 3.18 is a simplified schematic diagram of string A and string B. The actual path of the electrical circuit depends on the mode of operation and the reactor power level. When all shutdown-string parameters are satisfied and their associated contăcts are closed, string A control-power relays CPA, CPC-1, CP-1, and CP-2 will be energized, as will string-B relays CPB-1, CPB-2, and CPB-3. 


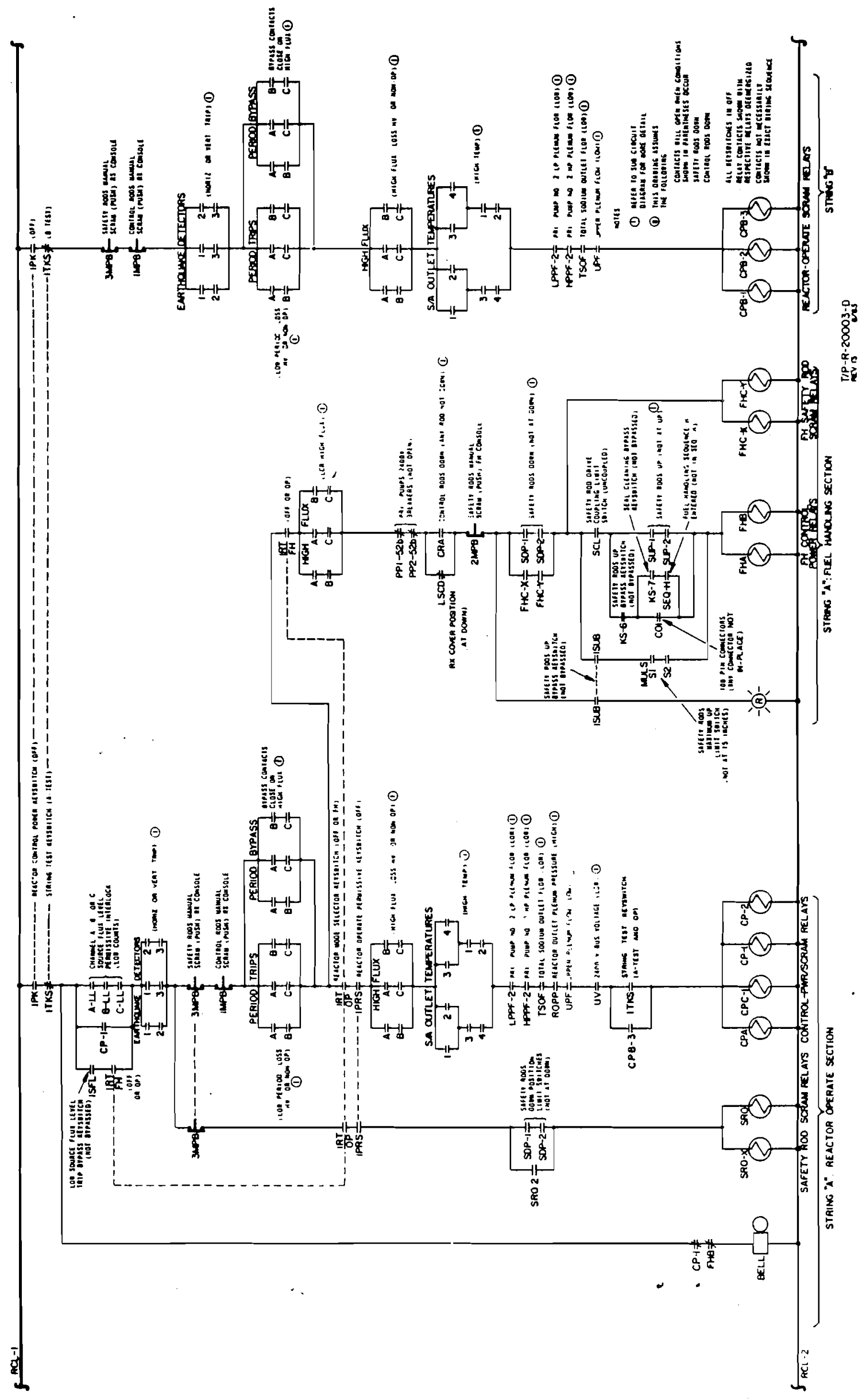

Fig. 3.18. Reactor Shutdown Circuits - Strings A and B 


\subsection{Decay Heat Removal}

\subsubsection{Shutdown Cooling System}

Fission-product decay heat generated in the reactor during shutdown is normally removed from the primary system by the secondary-system sodium. However, if the secondary-system sodium is not flowing through the intermediate heat exchanger in the primary tank, or if the reactor cover is raised, the decay heat will be transferred to the primary bulk sodium. Although the bulk sodium has a large thermal capacity, an undesirable temperature rise would occur if decay heat continued to be transferred to the bulk sodium over an extended time period. Under these conditions the shutdown cooling system would transfer the excess heat from the primary system to the air outside the reactor building and would keep the primary bulk sodium within the acceptable operating temperature limit of $700^{\circ} \mathrm{F}+10^{\circ} \mathrm{F}$.

The shutdown cooling system is one of the EBR-II sensitive systems and removes a total of $360 \mathrm{~kW}$ of heat when the temperature of the bulk sodium in the primary tank is $700^{\circ} \mathrm{F}$. The decay heat produced in the reactor after shutdown decreases continually with time in a nonlinear manner. For this reason, a nominal value for decay-heat removal could not be selected as a reference value in the design of the shutdown coolers. Instead, the total integrated amount of decay heat produced in the reactor from shutdown to various times after shutdown was considered in the shutdown-cooler design.

Two identical cooling loops comprise the shutdown cooling system (see Fig. 3.19). Each consists of a shutdown-cooler plug, shutdown-cooler box assembly, and associated piping equipment. The system provides heat transfer through the natural convection of the NaK eutectic alloy ( $22 \% \mathrm{Na}$ and $78 \% \mathrm{~K})$. In each closed loop, flow occurs between a heat-exchanger plug, located in the primary tank and mounted in the L-1 or L-2 nozzle and heat-exchanger core in a box assembly on the outside wall of the reactor 

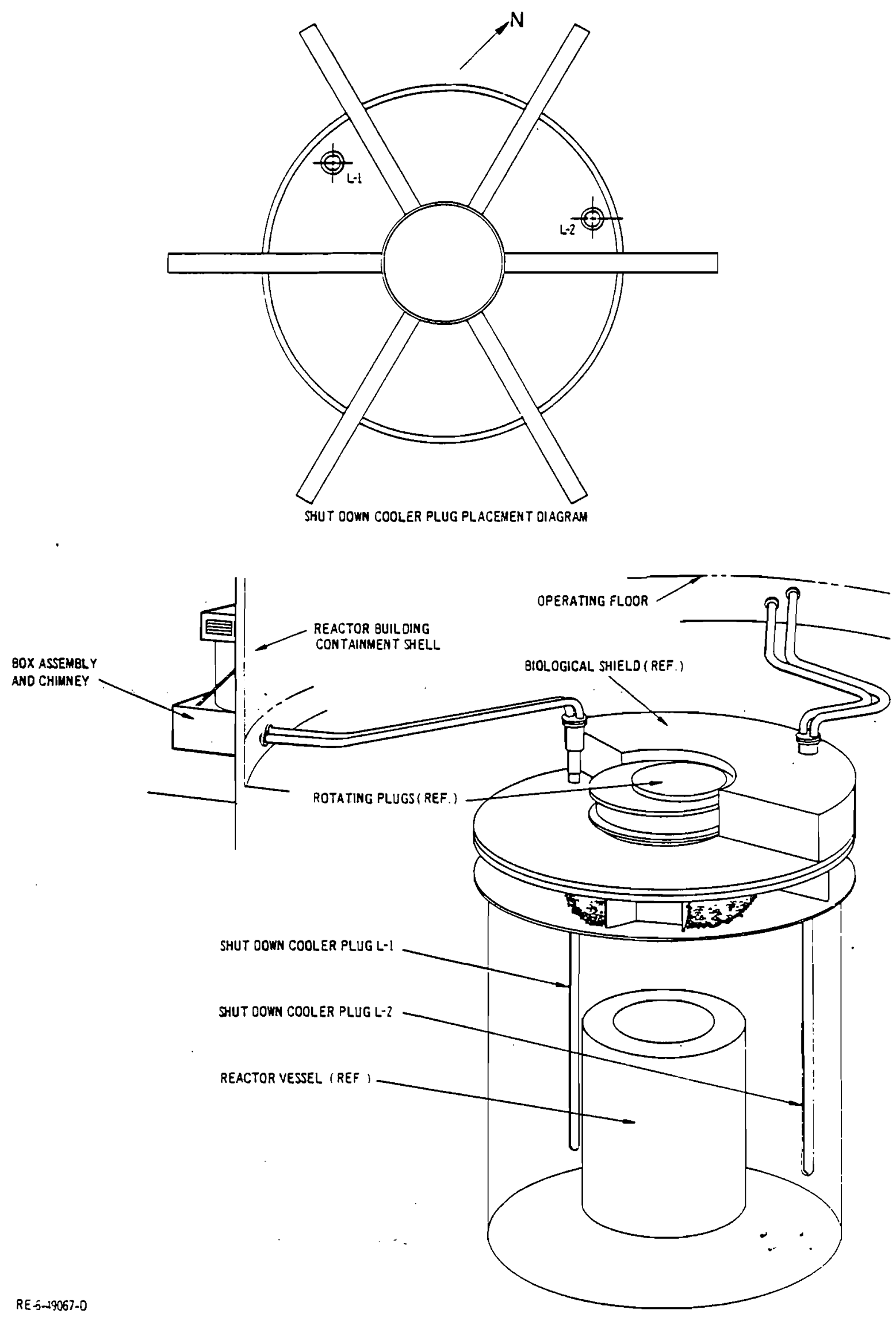

Fig. 3.19. Shutdown Cooling System 
building. NaK flow varies from 2 to $10 \mathrm{gpm}(0.0076$ to $0.038 \mathrm{~m} / \mathrm{min})$, when the air dampers (described below) are closed and the bulk sodium is at $700^{\circ} \mathrm{F}\left(371^{\circ} \mathrm{C}\right)$, to 40 to $70 \mathrm{gpm}(0.15$ to $0.76 \mathrm{~m}^{3} / \mathrm{min}$ ) when the bulk-sodium temperature increases to $710^{\circ} \mathrm{F}$ and the air dampers are open.

The natural-convection flow of $\mathrm{NaK}$ in the closed-loop system is completely independent of any power source and functions whenever the bulk sodium in the primary tank is hotter than the outside air. A small surge tank, which is part of the box assembly and is blanketed with argon gas, permits thermal expansion in the system. Decay heat is transferred from the shutdown-cooler $\mathrm{NaK}$ to the atmosphere by the natural convection of air tube is controlled by dampers above and below the heat exchanger and by a sheet-metal chimney above the upper damper. The NaK cooling system, external to the primary tank, is instrumented with thermocouples, electromagnetic flowmeters, and level indicators.

The shutdown cooling system has been in operation since 1963 and has provided very reliable operation. Only minor maintenance has been required, mainly on the air dampers and actuators.

\subsubsection{Shield Cooling System}

The shield cooling system provides forced-air cooling of the biological shield, the blast shield, and the rotating plugs. It circulates air between these components and cooling and filtering components through ductwork embedded in concrete or exposed. As shown in Fig.3.20, there are actually two separate but identical cooling and filtering systems available for use. One is operating while the other is on standby. These systems are operated alternately to equalize fan operating times and to allow maintenance activity during standby periods. 


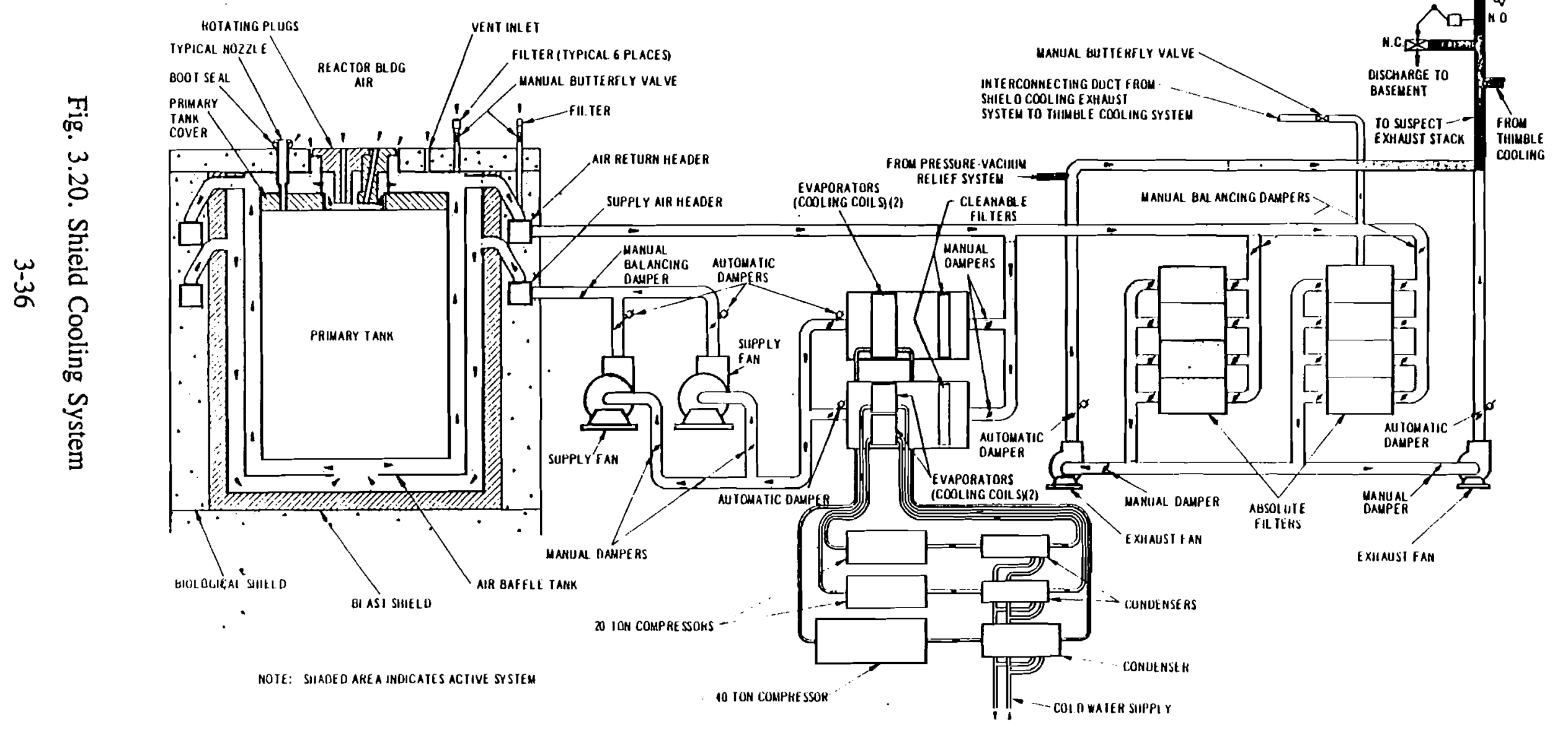


The shield cooling system is part of the reactor building ventilation system. It operates at a pressure slightly less than that of the building atmosphere. This permits a certain amount of in-leakage and simplifies the cooling of certain areas in the shield that cannot be connected to a closed system. The top structure of the primary-tank support system, the rotating shield plugs, and the top surface of the primary-tank cover are cooled primarily by air drawn from the building atmosphere. This air is drawn into the shield cooling system through ducting in the rotating plugs, the annuli between some of the primary-tank nozzles and the biological-shield sleeves, and around the top cover of the primary tank through air ducts in the top biological shield. It is then joined by air which has circulated through the air baffle tank, an annular tank surrounding the primary tank. The blast shield, the radial and lower biological shield, and the primary tank are cooled by the recirculated, cooled air passing through the air baffle tank. The air flow is then split into two paths, one to the exhaust stack and the other through coolers and then into the shield cooling system.

The system recirculates $15,000 \mathrm{cfm}\left(7.08 \mathrm{~m}^{3} / \mathrm{s}\right)$ of air and exhausts $5000 \mathrm{cfm}\left(2.36 \mathrm{~m}^{3} / \mathrm{s}\right)$ from the reactor building. An amount equal to the exhaust, $5000 \mathrm{cfm}(7.08 \mathrm{~m} / \mathrm{s})$, is drawn into the system from the reactor building. The total heat load in the system is about $506,000 \mathrm{Btu} / \mathrm{hr}$ $(148 \mathrm{~kW})$.

The recirculated air is filtered by cleanable filters before it enters the cooling coils. The exhausted air passes through high-efficiency particulate-air (HEPA) filters, referred to as "absolute" filters, before it is discharged to the suspect exhaust stack. The exhaust from the shield cooling system combines with the exhaust from the thimble cooling system and the combined exhaust is discharged from the reactor-building containment through a penetration. One valve outside the reactor building and two mechanically interlocked valves inside the building are controlled by the reactor-building isolation system. If the two valves in the exhaust duct to the suspect exhaust stack are closed under isolation conditions, the third valve opens to discharge the air back to the basement of the building.

Thermocouples in the rotating plugs monitor temperatures to avoid causing distortion of the plugs, which could cause binding of the rod drives or other equipment that penetrate the 
plugs. Dampers adjust the amount of air flowing through the rotating plugs. Thermocouples are also in the blast and biological shields and in the cooling-air stream. Some of these thermocouples are connected to high-temperature alarms. Low air flow in the operating system also will cause an alarm.

Under normal operating conditions, power for the shield cooling system is supplied by the $480-\mathrm{V}$ distribution system. Under emergency conditions, the $100-\mathrm{kW}$ diesel generator supplies power for the exhaust-air portion of the system, and the $400-\mathrm{kW}$ diesel generator supplies power for the recirculating portion of the systems, including the power for the refrigeration system that provides the cooling.

The shield cooling system is comprised of the following subsystems, each with its own components. The components of the regular (40-ton) refrigeration system are a compressor, a water-cooled condenser, a cleanable-filter bank, and an evaporator (cooling coil). The standby (20-ton) refrigeration system has two compressors, two water-cooled condensers, two evaporators, and cleanable-filter bank. The recirculating air system has two automatic dampers, three manual dampers, and a supply fan with a capacity of $14,420 \mathrm{cfm}$ at $58^{\circ} \mathrm{F}$ and 12.3 psia. The fan is powered by a 20 -hp electric motor. These components are duplicated in the standby recirculating-air system. The exhaust air system has a HEPA filter bank, two manual dampers, one automatic damper, and a fan with a capacity of $7730 \mathrm{cfm}$ at $96^{\circ} \mathrm{F}$ and $14-\mathrm{in} .-\mathrm{H}_{2} \mathrm{O}$ static pressure. A 30-hp motor drives the fan. These components are duplicated in the standby exhaust air system.

\subsection{Unique Features of EBR-II}

EBR-II has a variety of unique features that directly enhance reactor safety through relatively benign responses to accident initiating events. These features can all be grouped under the umbrella phrases "passive safety" or "inherent safety" and refer to the system's ability to survive potential accident sequences without core damage via natural features of the materials and design, rather than through the action of any active mitigating function. These attributes are not just theoretical concepts. EBR-II has carried out an extensive program of plant testing 
which demonstrated the performance of these passive safety features under a variety of accident conditions. The Shutdown Heat Removal Test series (SHRT) culminated in the Inherent Safety Demonstration Tests performed in April 1986 during which an Unprotected Loss-of-Normal Power and an Unprotected Loss-of-Heat Sink were performed without incurring any damage and demonstrating passive neutronic shutdown capability.

The essential characteristics which make this behavior possible are use of sodium coolant with its good heat transfer capability, metallic uranium fuel with its high thermal diffusivity and the pool design which makes available a large heat sink and inherent negative reactivity feedbacks.

Specifically, the features of EBR-II which lead to this benign response to various accident conditions are as follows:

\section{Large Excess Heat Capacity}

The design of EBR-II is such that in the primary tank, sodium and structure there is a large heat capacity with the result that, in the event of loss of all decay heat removal capability, it takes several days at decay heat levels to increase temperatures to fuel or structural damage levels.

\section{Large Heat Removal Capability}

The heat removal capability of the balance of plant exceeds the heat generated following a scram and the plant would cool down if measures were not taken to prevent this happening. A unique operational consideration at EBR-II involves overcooling the system rather than overheating. This cooldown is driven by natural circulation of the secondary sodium, and the cooldown rate is easily controlled by running the secondary pump backwards in order to retard the natural circulation flow. Cooldown is undesirable because of the time required to return to temperature and possibly stressing of components. There is no safety concern associated with cooldown following scram. 


\section{Natural Circulation Decay Heat Removal}

Removal of the decay heat from the fuel does not require that primary pumps be operating. The ability to remove the decay heat using natural circulation of the primary coolant has been demonstrated experimentally in the Shutdown Heat Removal Test (SHRT) series.

\section{Reactivity Feedbacks}

The reactivity feedbacks in EBR-II are such that any heating leads to power reduction. Many different feedbacks contribute but because of the small core size the dominant feedback is the sodium density effect. Various unprotected accidents have been run as experiments in EBR-II to explicitly demonstrate this effect.

\section{Metal Fuel}

EBR-II uses metallic uranium fuel enclosed in stainless steel clad. Metal fuel has a high thermal diffusivity which means that it responds faster under accident conditions than oxide fuel.

In addition, because EBR-II was designed in the 1950's as a research reactor, throughout the design of EBR-II there are conservative features which were included because of lack of knowledge concerning possible accidents. For example the steam generator tubes are doublewalled with a double walled tube-sheet thus requiring two failures to bring sodium and water into contact. Furthermore there is a detection system to monitor for such leaks.

One interesting feature of EBR-II is the leakage paths between primary system pipes and plena and the bulk sodium in the primary tank. These allow for natural circulation flow to remove fission product decay heat from the core even if the main coolant circuits were to be disabled for some reason. 


\subsection{Whole Plant Experiments in EBR-II}

The thermal hydraulic testing program at EBR-II was initially conducted in support of the continued safe and reliable operation of EBR-II and evolved into an experimental program contributing to the design assessment of advanced LMRs. The goals of the experiments have been to understand the detailed response of EBR-II to a wide variety of upset conditions both with and, ultimately without scram. The full scope of the testing program is described in detail in ref. 3.1. The following is a brief description of the experiments and their significance, both in general and more specifically to the Probabilistic Risk Assessment.

Early testing concerned natural circulation phenomena at either decay power or fission power levels, [3.2]. The next area addressed was the transition from forced flow to natural circulation from a wide variety of conditions with different sequences of scram, primary pump trip, and secondary pump trip times.

Loss-of-forced-flow transients were conducted from full primary flow at hot-standby conditions, from auxiliary pump flow at decay power levels, and from various reduced levels of fission power and primary pump flow. The analysis of these test results clearly demonstrated that transients initiated from conditions in which relatively normal temperature gradients existed in the system (i.e., power-to-flow ratios close to unity) lead to a smooth and benign transition to natural circulation [3.3]. However, as predicted in the original Hazards Summary Report [3.10], when the transient imposed resulted in a significant degradation of the thermal head in the primary circuit prior to the loss of forced flow, a substantial and temporarily sustained total reversal of flow occurred in the lower power regions of EBR-II (i.e., the radial blanket and reflector regions) [3.4]. No damage to the blankets was predicted or observed.

The SHRT Program was developed jointly by thermal-hydraulic specialists from EBR-II and Department of Energy contractor organizations, largely in support of the design of LMR plants in the U.S. The objectives of this program were to: 
1. demonstrate passive removal of decay heat following reactor shutdown by natural circulation of primary sodium coolant;

2. demonstrate passive reactor shutdown following a loss of forced circulation without scram (LOFWS);

3. demonstrate passive reactor shutdown following a loss of balance-of-plant (BOP) heat sink without scram (LOHSWS); and

4. provide test data for validating computer codes used in the design, licensing, and operation of LMRs.

Each of the total of 58 tests in the SHRT Program was one of the five following types:

- LOF/scram to natural circulation

- Scram with delayed LOF to natural circulation

- Reactivity feedback characterization

- LOFWS

- Balance of plant transients

- dynamic frequency response test

- LOHSWS

- steám drum pressure reduction 


\subsubsection{LOF/Scram to Natural Circulation}

The essential parameters that were studied in this sequence are the initial power and primary flowrate and the trip/non-trip of the secondary pump. The first test, which duplicates one that was conducted earlier was initiated from $36 \%$ of full power and $40 \%$ of full flow by tripping the primary pumps. In addition, several different combinations of primary pump rundown times and delays in the secondary pump trip were studied. In all cases, the final state of the plant is natural circulation in both the primary and secondary systems. The subsequent test initial conditions were power and primary flow levels of $75 \% / 100 \%, 75 \% / 75 \%$, and $100 \% / 100 \%$, respectively. For each of these test conditions (with the auxiliary pump turned off), the secondary flow was in the first case slowly reduced to about $20 \%$ or in the second case permitted to rapidly coastdown to natural circulation. Thus, the final test in this group directly simulated a total loss of pumping power (normal and emergency) to the plant while operating. at full power and flow.

\subsubsection{Scram with Delayed LOF to Natural Circulation}

Most of these tests were initiated from normal full power and flow where the first step was to scram the reactor while maintaining either full primary flow or auxiliary pump ( $~ 5 \%$ ) flow. After a prescribed delay during which time the primary system approached isothermal conditions, the primary pumps (or the auxiliary pump) were tripped and the flow coasted down to natural circulation. The effect of the level and rate of coastdown of secondary flow was also studied. During these transients, the coolant flow in the radial blanket-reflector region either temporarily stagnated or reversed due to the adverse thermal conditions delaying the transition to upward natural convection.

\subsubsection{Reactivity Feedback Characterization}

These tests were designed to provide measurements of the dynamic plant response to flow and inlet temperature perturbations in order to permit validation of reactivity feedback models. A series of thermal-hydraulic disturbances was imposed upon the core, 
maintaining fixed (or precisely changed) control rod positions, in order to measure the resulting power changes and thereby to compute the effective reactivity feedbacks through an inverse kinetics calculation. The first type of disturbance was a change in primary flowrate, holding the reactor inlet temperature and control rod positions fixed. This causes the reactor $\Delta \mathrm{T}$ to change initially, affecting the reactivity balance, which drives a power change. Initial power levels of $25 \%, 50 \%$, and $70 \%$ were used.

The second type of disturbance was a change (either decrease or increase) in the reactor inlet temperature (caused by changes in the secondary flowrate), while the primary flowrate and control rod positions were fixed. Initial power levels of $25 \%, 50 \%$, and $70 \%$ were used. A variation of the two types of disturbances employed was a flowrate or inlet temperature disturbance followed by a change in control rod position sufficient to return the power to its initial value. The results of some of these feedback tests are described in [3.5].

\subsubsection{Loss of Flow without Reactor Scram}

This sequence of tests involved coolant pump trips without permitting a reactor scram. The initial tests used different combinations of rate of primary pump coastdown, trip or non-trip of the secondary pump, and operation of the auxiliary pump on emergency power, battery power, or not operated at all. These tests were initiated from various power and flowrates, culminating in $100 \%$ power and flow. The mildest transients, in terms of maximum core temperatures, were conducted first, with the severity gradually increased. Included among these tests were an unprotected LOF to natural circulation from full power as well as an unprotected station blackout (loss of normal and emergency a-c electric power without scram), [3.6]. 


\subsubsection{Balance of Plant Transients}

\subsubsection{Dynamic Frequency Response Tests}

These tests were designed to generate data over a wide disturbance frequency range that can be used to validate whole plant dynamic simulation models. There were types of cyclic tests in which a relatively small periodic disturbance was added to either control-rod-inserted reactivity or secondary loop electromagnetic pump voltage. This periodic disturbance consisted of a fundamental frequency and a number of harmonics. The propagation and attenuation of these frequencies in the form of power, temperature, flow, and pressure disturbances was measured throughout the plant. Spectral analysis was applied to both the measured output and the simulation results, providing transfer functions for each. A comparison of the two sets of results (measured vs. calculated) is a direct measurement of the efficacy of the calculational models.

\subsubsection{Loss of Heat Sink Without Scram}

This test had as its objective the demonstration that the reactor will passively shut itself down following a total loss of heat sink, including the emergency decay heat removal system. Experimentally, this was accomplished by essentially stopping the secondary flow (including natural circulation) without permitting the reactor to scram, and maintaining full primary flow.

These particular conditions were chosen to umbrella all possible loss of normal heat sink transients. In this case, the loss of heat removal from the primary system caused the reactor inlet temperature to increase and thus the reactor power to decrease due to the negative reactivity feedbacks. Although the inlet temperature rose substantially, the resulting power reduction was sufficiently large and rapid to cause a reduction in the outlet temperature so that the core $\Delta \mathrm{T}$ actually decreased to almost zero and the entire primary system approáchéd an isothermal condition [3.6]. 


\subsubsection{Steam Drum Pressure Reduction}

Two tests were run which involved a rapid opening of the steam bypass valve to the condenser and causing a rapid steam depressurization. The primary and secondary flowrates were held constant and reactor power and temperatures were allowed to freely respond. The decrease in the steam drum pressure and in its water saturation temperature reduced the secondary sodium temperature exiting the evaporators. This reduced secondary sodium temperature overcooled the primary sodium in the IHX, thus reducing the reactor inlet temperature after a time delay. Due to the negative reactivity feedbacks, the reactor power slowly increased to meet the increased steam power demand. The reactor and secondary loop $\Delta$ Ts were fixed by the reactor and secondary flowrates and the power demand [3.7]. These tests represent ways in which Balance-of-Plant faults could lead to reactivity insertion and hence an overpower transient.

\subsubsection{Overcooling Transients}

One potential source of reactor overpower and fuel overheating arises if the primary system is overcooled. If there is no scram, the negative inlet temperature coefficient combined with the falling inlet temperature leads to increasing power. This condition was investigated in the PICT and IOT experiments [3.8, 3.9]. It was demonstrated that variations in the primary flow rate were accommodated by power level changes so that the power-to-flow ratio remained at less than 1 . Overcooling by faults on the secondary side, such as the increase of secondary flow to the maximum possible, will lead to a power-to-flow ratio of greater than 1. In this case the transient is limited by the capacity of the secondary pump to remove heat and the accident terminates without any damage to fuel.

\subsubsection{Summary}

Throughout its long and successful operating history the response of EBR-II to all conceivable forms of off-normal conditions has been analyzed and wherever practical this behavior confirmed by experiment. The implication for the EBR-II PRA is that the outcome of 
system transients can be predicted with a high degree of confidence, allowing for a finer discrimination between degrees of damage than might normally be applied. 


\section{Section 3.0 References}

3.1 H. P. Planchon et al., "The Experimental Breeder Reactor II Inherent Shutdown and Heat Removal Tests - Results and Analysis," Nucl. Engrg. Des. 91 (1986) pp. 287-296.

3.2 R. M. Singer et. al., "Decay Heat Removal and Dynamic Plant Testing at EBR-II", Second Specialists' Meeting on Decay Heat Removal and Natural Convection in LMFBRs, Brookhaven National Laboratory, April 17-19, 1985.

3.3 D. Mohr and E. E. Feldman, "A Dynamic Simulation of the EBR-II Plant During Natural Convection with the NATDEMO Code", Decay Heat Removal and Natural Convection in Fast Breeder Reactors, Hemisphere Publ. Corp., New York, 1981.

3.4 R. M. Singer, D. Mohr and J. L. Gillette, "Transition from Forced to Natural Convective Flow in an LMFBR Under Adverse Thermal Conditions," Proc. of the Seventh International Heat Transfer Conference, Munchen, Germany (1982).

3.5 D. Mohr and L. K. Chang, Perturbation of Reactor Flow and Inlet Temperature for EBR-II Reactivity Feedback Validation", Intl. Topical Meeting on Fast Reactor Safety, Knoxville, Tennessee, April 21-25, 1985.

3.6 D. Mohr et al., "Loss-of-Primary-Flow-Without-Scram Tests: Pretest Predictions and Preliminary Results", Nucl. Engrg. Des. 101 (1987) pp. 45-56 and E. E. Feldman et al., "EBR-II Unprotected Loss-of-Heat-Sink Predictions and Preliminary Test Results, Nucl". Engrg. Des. 101 (1987) pp. 57-66.

3.7 E. E. Feldman et al., "Prediction and Measured Response of EBR-II Plant to Large Pressure Changes", Topical Meeting on Anticipated and Abnormal Transients in Nuclear Power Plants, Atlanta, GA, April 12-15, 1987. 
3.8 L. K. Chang et al., "Plant Inherent Control Testing in EBR-II", Topical Meeting on Anticipated and Abnormal Transients in Nuclear Plants, Atlanta, GA, April 12-15, 1987.

3.9 W. K. Lehto et al., "Reactor Safety Implications of Pump Run-Up Tests in EBR-II," Nucl. Engrg. Des., 110, p. 47 (1988). 


\subsection{SPECIAL FEATURES OF EBR-II}




\section{Table of Contents}

\section{Page}

4.0 Special Issues for the EBR-II PRA $\ldots \ldots \ldots \ldots \ldots \ldots \ldots$. . . . . . . . . .

4.1 Passive Safety Features . . . . . . . . . . . . . . . . 4-1

4.2 Natural Circulation . . . . . . . . . . . . . . . . .4-4

4.3 Reactivity Feedbacks . . . . . . . . . . . . . . . . 4-7

Section 4.0 References $\ldots \ldots$. . . . . . . . . . . . . . . . . . . . . . . . . 4-10 


\section{List of Figures}

Page

4.1 Design Flow Leakage Paths in EBR-II . . . . . . . . . . . . . . . . . 4-6 


\section{List of Tables}

Page

4.1 EBR-II Design Features Contributing to Passive Safety . . . . . . . . . . . 4-3 


\subsection{SPECIAL FEATURES OF EBR-II}

\subsection{Passive Safety Features}

The term passively safe or inherently safe has been used to characterize various reactor designs in recent years. The particular features which lend themselves to this description have no mechanical moving parts and rely upon natural phenomena to provide the mitigating functions rather than active systems which are asked to perform, on demand, in response to a given stimulus.

EBR-II has many design features which contribute to the safety of the reactor which can be termed 'passive' or 'inherent'. The role that these features play are reflected in the PRA. In an upset condition, some of these design features provide times for operator recovery on the order of eight hours. One example of this type of feature is the large heat capacity of the system. Other contributors to passive safety are the inherent physical limits to system performance which appear as constraints to the severity of the associated initiating event, e.g., a physical limit on pump over speed. The final type is the use of natural phenomena to mitigate accidents, e.g., natural circulation cooling of the core and reactivity feedbacks limiting the temperature excursion in unscrammed transients.

The classic functional approach to reactor safety and accident sequence mitigation utilizes a fundamental safety philosophy which can be described as follows: first, scram the reactor to ensure neutronic shutdown and second, provide means of removing decay heat. In LWRs, the front-line systems designed to prevent core damage are active, on-demand systems; if these systems fail, there is a high probability of the occurrence of core damage. In pool-type LMRs, if all active systems fail, passive systems provide sufficient shutdown and heat removal such that there is a low probability of even minor core damage.

EBR-II is designed with the same fundamental safety philosophy as LWRs but with a different architecture. Just as in LWRs, a failsafe scram system is provided in EBR-II to shut 
off the nuclear chain reaction; if the mechanical scram system fails, neutronic shutdown is effected by reactivity feedbacks. Thereafter the decay heat removal can be carried out by passive means, utilizing natural circulation. In the primary tank, if the primary pumps are not operating, natural circulation transfers heat from the core to the bulk sodium. Heat can be extracted from the tank sodium in several ways. The preferred heat transport path is through the secondary sodium loop. In fact, if the secondary sodium is flowing under natural circulation conditions, the rate of extraction of heat is too large and the system will cool too rapidly. This is prevented by retarding the natural circulation flow by reversing the field of the secondary electromagnetic pump. Under certain conditions heat is not removed through the secondary system and the decay heat is then removed by two shutdown coolers which are passive systems.

Table 4.1 lists the various design features which can be considered as part of the passive safety position for EBR-II and how they are reflected in the PRA. A number of these design features serve to reduce the frequency of initiating events by removing or reducing the consequences of a single failure. An example of this is the passive accommodation of sodium after a primary vessel leak by the guard vessel which therefore requires a coincident leak or breach of the guard vessel before a core threatening sequence can occur.

In EBR-II the sodium coolant is at a low pressure and therefore the stresses in the coolant piping are very low and the probability of crack-initiated piping failure is much less than a high pressure system such as an LWR. Furthermore any pipe break results in a loss-of-flow rather than any loss-of-sodium because of the submerged primary system. The result is that the only events with the potential for loss of a significant amount of sodium are very low probability events such as rupture of the primary tank and guard vessel. This is discussed further in Section 5 and Appendix D.1.

Other design features ensure that for anticipated events, e.g., a fault on a single coolant pump, the core will not be threatened even in the event of total failure of the safety systems. This is ensured by an appropriate design of the pumps, with a suitably long coast down time. In the event of a fault affecting both coolant pumps (such as loss of normal power) the coastdown is sufficiently long to preclude core damage even if all protective systems fail. 
Table 4.1. EBR-II Design Features Contributing to Passive Safety

Design Features

Low pressure system

Guard vessel

Submerged primary system

Inherent limits on pumps

Pump coastdown time

Large heat capacity and heat removal capability

Double-walled tubes

Natural circulation

Reactivity feedbacks

\section{Effect on EBR-II PRA}

Low initiating event frequency

Limits severity of initiating event

Limits severity of initiating event

Limits severity of initiating event

Characteristic of initiating event

Success criteria for decay heat removal

Low initiating event frequency

Sequence analysis

Sequence analysis 
Similarly the capacity of the pumps has been chosen such that the maximum overspeed is not sufficient to lead to damaging transients.

The large heat capacity of the primary tank sodium and associated structure ensures that, in the event of some form of loss of heat removal capability, there are long times available to act and there are several passive systems capable of removing the heat.

Use of double-walled tubes in the steam generators ensure that the probability of any sodium-water reaction is reduced to a minimum. This contrasts with designs in place at commercial and prototype LMRs which have less conservative designs.

Two natural phenomena are relied upon in the design of EBR-II, and to a certain extent in all LMRs, to achieve passive safety. Natural circulation of the sodium ensures passive accomplishment of the decay heat removal function and negative reactivity feedbacks ensure passive shutdown in a wide variety of anticipated transients, including Loss-of-Flow, Loss-ofHeat-Sink and reactivity addition events. These phenomena are discussed in detail below.

\subsection{Natural Circulation}

The passive safety features that lead to the benign response to protected transients are natural circulation heat removal from the core and the large heat capacity of the primary tank, sodium and associated structures.

Normal forced cooling of the core is carried out by two centrifugal pumps pumping liquid sodium through the core. If the pumps lose power, or for any other reason cease to operate, the cooling of the core is carried out by natural circulation. Hot sodium generated in the core region rises through the flow paths available to the tank while cold sodium, at tank temperature,

replaces it in the core. By this means the core heat is transferred to the bulk sodium in the reactor tank. The large thermal capacity in the tank ensures a very slow rate of temperature rise in the tank and very long times for heat removal by the secondary system, shutdown coolers, shield cooling and heat losses. 
It is necessary to ask how natural circulation might be defeated. Firstly, normal natural circulation cannot function if critical flow paths are blocked or if there is excessive flow resistance. Secondly, natural circulation cannot be sustained if the necessary thermal conditions are not preserved. No arbitrary unspecified means of defeating natural circulation has been hypothesized and it has not proved possible to establish any mechanism that can defeat natural circulation, other than massive structural failure which alters the geometry totally, disrupting the flow paths.

As discussed in Section 5, blockage of the large inlet and outlet pipes are considered to be very low frequency events, as there is no source of debris large enough to completely block pipes apart from massive structural failure. The auxiliary pump is on the outlet Z-pipe and conceivably could retard natural circulation if the head were reversed. The pump is tested at reactor startup for each run and no mechanism for reversing polarity has been identified. There are no valves on the main inlet and outlet flow paths which could be misaligned. The throttle valve on the low pressure piping is locked fully open and never manipulated. Gas bubbles could conceivably form by disentrainment, for example, in the Z-pipe but this is not possible at the flow velocities found under forced flow conditions. Furthermore EBR-II is designed with a variety of flow leakages which bypass the main natural circulation path (see Fig. 4.1). These leakages are sufficient to allow cooling of the core under decay heat conditions.

Extensive experimentation has been performed in EBR-II demonstrating the ability of natural circulation to remove the decay (shutdown) heat. These are the Shutdown Heat Removal Tests (SHRT) summarized in reference [4.2].

In summary, no credible means of defeating natural circulation in EBR-II has been identified. Therefore the existence of natural circulation will be assumed in sequence calculations. The thermal effects of the transition to natural circulation will be included in the evaluation of accident response. 


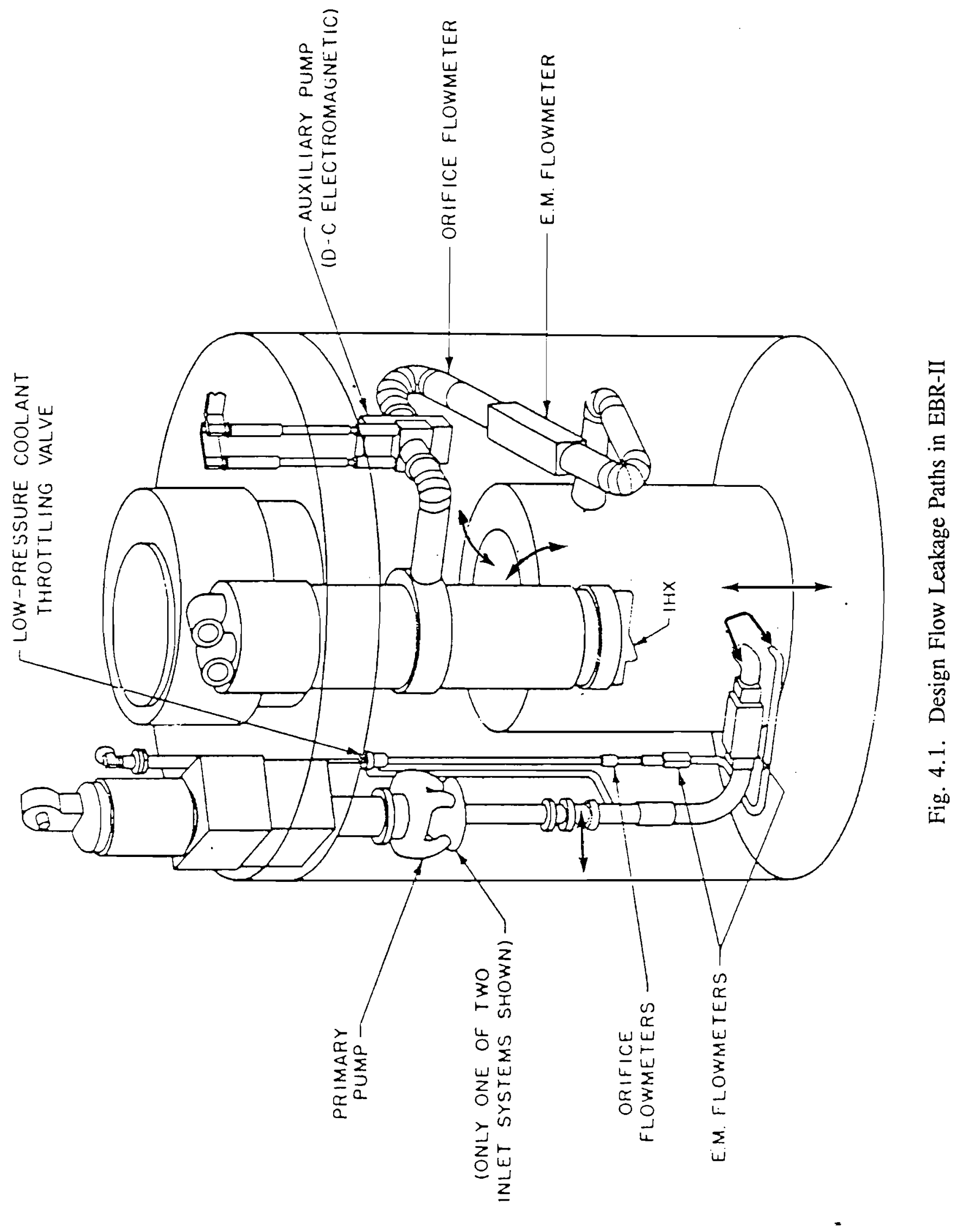




\subsection{Reactivity Feedbacks}

The passive safety features that control the response to Anticipated Transient Without Scram (ATWS) events are the reactivity feedbacks. Although they are sometimes referred to as a shutdown capability, reactivity feedbacks alone cannot drive the reactor subcritical. They do, however, tend to reestablish a stable state (which may be essentially zero fission power) where heat production and removal are in balance.

The passive feedbacks are temperature-driven reactivity changes - expansion, bowing and Doppler broadening. They can be divided into three categories of importance $[4.3,4.4]$. Sodium expansion reactivity is by far the strongest feedback. Because of the small core size, the sodium expansion reactivity has a large leakage component and is negative everywhere. The second category consists of fuel axial expansion, control driveline expansion, steel expansion and radial expansion/bowing. Doppler broadening is the least important of the feedbacks, accounting for only a few percent of the total; its smallness is due to three factors: the hard neutron spectrum, the high fuel enrichment and the small temperature gradient across the metal fuel. All these feedbacks are always negative except for bowing, which can be positive or negative, depending on the particular core loading. Since bowing reactivity never dominates, the net feedback reactivity is always negative. Thus if an accident initiator makes the core temperature rise, the resulting feedback reactivity tends to reduce the reactor power and thereby reduce the fuel temperatures.

The reactivity feedbacks of EBR-II have proven to be stable over the years, despite the differences in core loadings dictated by its role as an irradiation facility. Since 1977 the measured prompt power coefficient has varied $21 \%$ and the measured average power coefficient has varied $36 \%$. For four widely different loadings, the calculated average power coefficient varied by only $10 \%$ while the fraction of fueled subassemblies that were non-metal experiments varied from 0 to $19 \%$ [4.4]. This stability occurs because the coolant volume fraction is nearly constant, independent of core subassembly type, and the coolant expansion dominates the passive feedbacks. As a consequence of the stability, it is reasonable to use a generic metal-fueled 
loading in the sequence analyses. The influence of experimental subassemblies on EBR-II feedbacks can be treated as one source of feedback uncertainty.

The reactivity feedbacks are simply a manifestation of the laws of physics. For example, it is inevitable that the sodium will expand as its temperature rises. This will necessarily be a negative reactivity effect. The only way the feedback influence could be negated is if an accident initiator significantly modifies the core geometry. Only a severe earthquake appears capable of this. Thus, except in the presence of such severe external initiators, reactivity feedbacks are assumed to be present when performing sequence analyses.

Reactivity feedbacks will fail to prevent damage if they are not strong enough to keep temperature rises caused by the accident initiator within safe bounds. The failure analysis for passive safety consists of predicting the effect of feedbacks on the transient outcome and. evaluating the reliability of the prediction. The predictions for EBR-II should be more reliable than predictions made for most LMR designs, since there is a wealth of experimental data related to the feedbacks, including the Inherent Safety Demonstration Tests. Data from these tests have been used to test calculational predictions. This is discussed further in Section 5.5.

The results of calculations presented in Sections 5.4 and 5.5 and supported by a large number of plant experiments, including direct demonstration of many transients, is summarized below.

- Loss of Heat Sink - In the event of a Loss of Heat Sink the reactor temperatures reduce uniformly as directly demonstrated by the SHRT B302R experiment [4.7].

- Pump Overspeed - The power-to-flow ratio does not exceed unity and the temperature does not increase. This result has also been directly demonstrated in EBR-II [4.8].

- Loss of Flow - The SHRT plant experiments directly demonstrated that no damage occurs if the flow coastdown is long enough [4.7]. However, 
each initiating event must be considered on its own merits because different coastdown times can occur.

- Loss of Normal Power - This transient is a particular example of a loss of flow with long coastdown time combined with a Loss of Primary Heat Sink simulated by the SHRT 45 experiment [4.7]. In this case, the peak coolant temperatures are close enough to boiling to require detailed analysis of the effect of uncertainties in the feedback, but no damage occurs with a nominal prediction.

- Reactivity Insertion - For the case of slow reactivity insertions, feedbacks limit the rate of rise of power, and damage will result only if the total reactivity insertion is large enough. Therefore, the reactivity insertion events are classed according to the total reactivity inserted. 


\section{Section 4.0 References}

4.1 H. P. Planchon et al., "The Experimental Breeder Reactor II Inherent Shutdown and Heat Removal Tests - Results and Analysis", Nucl. Engrg. Des. 91 (1986) pp. 287-296.

4.2 D. Mohr et al., "Loss-of-Primary-Flow-Without-Scram Tests: Pretest Predictions and Preliminary Results", Nucl. Engrg. Des. 101 (1987) pp. 45-56.

4.3 D. Mohr and L. K. Chang, "Perturbations of Reactor Flow and Inlet Temperature for EBR-II Reactivity-Feedback Validation," Proc. Int. Topical Meeting on Fast Reactor Safety, Knoxville, TN, April 21-25, 1986, CONF-850410, Vol. II, p. 617 (1985).

4.4 D. Meneghetti and D. A. Kucera, "Comparisons of PRD Components for Various EBRII Configurations," Ann. Nucl. Energy, Vol. 14, No. 9, p. 473 (1987).

4.5 D. C. Wade and Y.I. Chang, "The Integral Fast Reactor (IFR) Concept: Physics of Operation and Safety," Proc. Int. Topl. Mtg. on Advances in Reactor Physics, Mathematics and Computation, Paris, France, April 27-30, 1987.

4.6 D. Meneghetti and D. A. Kucera, "Delineations of Power, Power-to-Flow and InletTemperatures Feedbacks of EBR-II," Ann. Nucl. Energy, Vol. 17, No. 1, p. 19 (1990).

4.7 H. P. Planchon et al., "Implications of the EBR-II Inherent Safety Demonstration Test," Nucl. Engrg. Des., 101, p. 75 (1987).

4.8 W. K. Lehto et al., "Reactor Safety Implications of Pump Run-Up Tests in EBR-II," Nucl. Engrg. Des., 110, p. 47 (1988). 


\subsection{ACCIDENT SEQUENCE ANALYSIS}


Table of Contents

$\underline{\text { Page }}$

5.0 Accident Sequence Analysis $\ldots \ldots \ldots \ldots \ldots \ldots \ldots \ldots \ldots \ldots$. . . . . . . . .

5.1 Initiating Event Identification $\ldots \ldots \ldots \ldots \ldots \ldots \ldots \ldots \ldots \ldots \ldots \ldots \ldots$

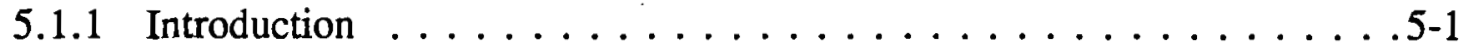

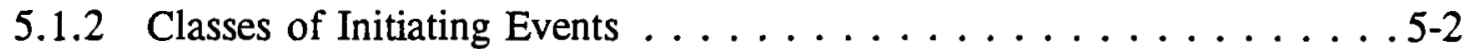

5.1 .3 Loss-of-Flow . . . . . . . . . . . . . . . . 5-7

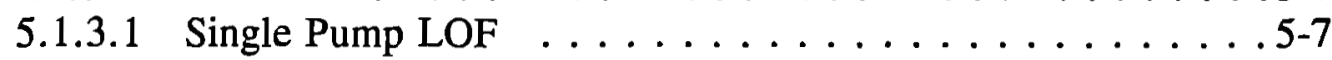

5.1.3.1.1 Electrical Faults . . . . . . . . . 5-7

5.2.3.1.2 Mechanical Faults . . . . . . . . . 5-12

5.1.3.2 Double Pump LOF - General . . . . . . . . . . . . . 5-14

5.1.3.3 Double Pump LOF - Loss of Vital Electrical Power . . . . 5-15

5.1.3.3.1 Loss of Normal Power (LONP) . . . . . . . . 5-15

5.1.3.3.2 Double Pump LOF - Loss of Constant Power . 5-16

5.1.3.4 Loss of Piping Integrity (LOPI) . . . . . . . . . 5-17

5.1.3.4.1 Pipe Break . . . . . . . . . . . . . 5 5-17

5.1.3.4.2 Ball-Joint Bellows Failure . . . . . . . . 5-18

5.1.3.5 Other Primary System LOF Considerations . . . . . . . . 5 5-19

5.1.3.5.1 Auxiliary Pump . . . . . . . . . . . 5 5-19

5.1.3.5.2 Primary Flow Path Blockage . . . . . . . . 5-19

5.1 .4 Reactivity Insertion . . . . . . . . . . . . . . . . . . 5-19

5.1 .5 Loss of Primary Heat Sink $\ldots \ldots \ldots \ldots \ldots \ldots \ldots \ldots$. . . . . . . . . .

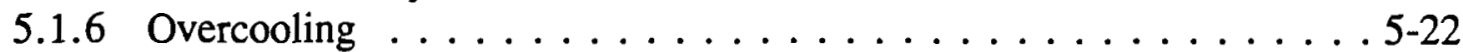

5.1 .6 .1 Steam Leak . . . . . . . . . . . . . . 5-22

5.1.6.2 Primary Pump Run-up . . . . . . . . . . . . . 5-22

5.1.6.3 Secondary Pump Run-up . . . . . . . . . . . . . . 5-23

5.1.6.4 Shutdown Coolers Inadvertent Operation . . . . . . . . . 5-23

5.1 .7 Steam-Generator Failures . . . . . . . . . . . . . . . 5-23

5.1.8 Loss of Primary Sodium Accidents (LOSA) . . . . . . . . . 5-24

5.1.8.1 Primary Vessel Rupture . . . . . . . . . . . . . . . 5-26

5.1.8.2 Primary and Guard Vessel Rupture . . . . . . . . . . 5-27

5.1.8.3 Breached Fuel Test Facility (BFTF) Failure . . . . . . . . 5-27

5.1.8.4 Primary Na Purification System Leak . . . . . . . . 5-28

5.1 .8 .5 IHX Tube Failure . . . . . . . . . . . . . 5-28

5.1 .9 Support System Failures . . . . . . . . . . . . . . . . . . . . . 5-29

5.1.9.1 Instrument and Plant Air . . . . . . . . . . . . . . . 5-29

5.1.9.2 Reactor Auxiliaries and Plant Cooling Water Systems . . . 5-31

5.1 .9 .3 Electrical Systems $\ldots \ldots \ldots \ldots \ldots \ldots \ldots \ldots$. . . . . . . . . . . . . . .

5.1 .9 .4 Shutdown Coolers . . . . . . . . . . . . . . 5-34

5.1.9.5 Shield and Thimble Cooling . . . . . . . . . 5-35

5.1.10 Shutdown and Scram . . . . . . . . . . . . . . 5-36 
5.1 .11 Local Faults . . . . . . . . . . . . . . . . . 5-37

5.1 .12 Initiating Event Categories $\ldots \ldots \ldots \ldots \ldots \ldots \ldots \ldots . . \ldots \ldots$

5.2 Initiating Event Quantification $\ldots \ldots \ldots \ldots \ldots \ldots \ldots \ldots \ldots$.43 $\ldots \ldots \ldots$

5.2.1 Flow Transients (Single Pump) $\ldots \ldots \ldots \ldots \ldots \ldots \ldots$. . . . . . . . . .

5.2.2 Flow Transients (Double Pump) . . . . . . . . . . . . . 5-49

5.2.2.1 Double Pump LOF Fault Tree . . . . . . . . . . . . 5 5-49

5.2.2.2 Pipe Breaks . . . . . . . . . . . . . 5-52

5.2 .3 Loss of Vital Electrical Power . . . . . . . . . . . . 5-54

5.2.3.1 Loss of Normal Power (LONP) . . . . . . . . . 5-54

5.2.3.2 Loss of Constant Power (LOCP) . . . . . . . . . 5-54

5.2 .4 Reactivity Insertion . . . . . . . . . . . . . . . . . . 5-54

5.2 .5 Loss of Primary Heat Sink $\ldots \ldots \ldots \ldots \ldots \ldots \ldots \ldots .56$

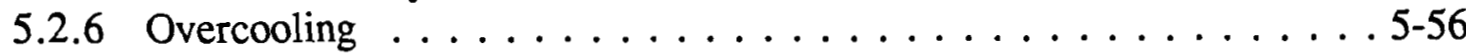

5.2 .7 Steam Generator Tube Rupture $\ldots \ldots \ldots \ldots \ldots \ldots \ldots$. . . . .57

5.2 .8 Rapid Loss of Circulation . . . . . . . . . . . . . . . . 5-57

5.2 .9 Delayed Loss of Circulation (LCDL) . . . . . . . . . . . 5-60

5.2.10 Support System Failures . . . . . . . . . . . . . . . 5-61

5.2 .11 Shutdowns and Scrams $\ldots \ldots \ldots \ldots \ldots \ldots \ldots \ldots . \ldots \ldots 2$

5.2 .12 Local Faults . . . . . . . . . . . . . . . . . . .5-62

5.2.13 Final Initiating Event Frequencies . . . . . . . . . . 5-64

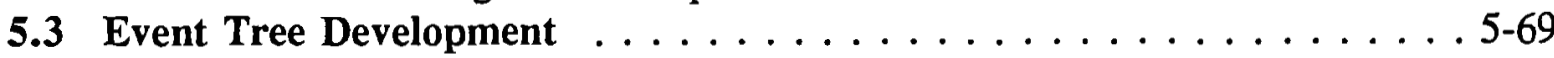

5.3 .1 Introduction $\ldots \ldots \ldots \ldots \ldots \ldots \ldots \ldots \ldots \ldots \ldots \ldots$

5.3 .2 Safety Functions . . . . . . . . . . . . . . . . . 5-69

5.3.3 Damage Definitions . . . . . . . . . . . . . 5-86

5.3.4 EBR-II Event Trees . . . . . . . . . . . . . . . . 5-93

5.3.4.1 Review of Event Trees in Other Relevant PRAs . . . . . 5 5-93

5.3.4.2 Event Tree Construction . . . . . . . . . . . . . 5-96

5.3.5 Event Trees for Flow Transients . . . . . . . . . . . . . . . 5-99

5.3.5.1 Initiating Event Description . . . . . . . . . . . . . 5-99

5.3.5.2 Normal Plant Response and Success Criteria . . . . . . . 5-99

5.3.5.3 Event Trees and Accident Sequences . . . . . . . . . . 5 5-99

5.3.6 Event Trees for Loss of Vital Electrical Power . . . . . . . . . . 5-110

5.3.6.1 Initiating Event Description . . . . . . . . . . . 5-110

5.3.6.2 Normal Plant Response and Success Criteria . . . . . . . 5-110

5.3.6.3 Event Trees and Accident Sequences . . . . . . . . 5-110

5.3.7 Event Trees for Slow Reactivity Insertion . . . . . . . . . . 5-113

5.3.7.1 Initiating Event Description . . . . . . . . . . 5-113

5.3.7.2 Normal Plan Response and Success Criteria . . . . . . . 5 5-113

5.3.7.3 Event Trees and Accident Sequences . . . . . . . . . 5-113 
5.3.8 Event Trees for Fast Reactivity Insertions . . . . . . . . . 5-119

5.3.8.1 Initiating Event Definition . . . . . . . . . . . . 5 5-119

5.3.8.2 Normal Plant Response and Success Criteria . . . . . . . 5-119

5.3.8.3 Event Tree and Accident Sequences . . . . . . . . . . 5-119

5.3.9 Event Trees for Loss of Primary Heat Sink . . . . . . . . . . . 5-122

5.3.9.1 Initiating Event Description . . . . . . . . . . . 5-122

5.3.9.2 Normal Plant Response and Success Criteria . . . . . . 5 5-122

5.3.9.3 Event Trees and Accident Sequences . . . . . . . . . . 5-122

5.3.10 Event Trees for Overcooling . . . . . . . . . . . . . 5-125

5.3.10.1 Initiating Event Description . . . . . . . . . . 5-125

5.3.10.2 Normal Plant Response and Success Criteria . . . . . . . 5-125

5.3.10.3 Event Trees and Accident Sequences . . . . . . . . . 5-125

5.3.11 Event Trees for Steam Generator Tube Rupture . . . . . . . . . 5-129

5.3.11.1 Initiating Event Description . . . . . . . . . . . . 5-129

5.3.11.2 Normal Plant Response and Success Criteria . . . . . . 5-129

5.3.11.3 Event Trees and Accident Sequences . . . . . . . . . 5-130

5.3.12 Event Tree for Rapid Loss of Circulation . . . . . . . . . . . 5-133

5.3.12.1 Initiating Event Definition . . . . . . . . . . . . . 5-133

5.3.12.2 Normal Plant Response and Success Criteria . . . . . . 5 5-133

5.3.12.3 Event Tree and Accident Sequences . . . . . . . . . . 5-133

5.3.13 Event Trees for Support System Failures . . . . . . . . . 5-135

5.3.13.1 Initiating Event Description . . . . . . . . . . . 5-135

5.3.13.2 Normal Plant Response and Success Criteria ... . . . 5 5-135

5.3.13.3 Event Trees and Accident Sequences . . . . . . . . . 5-135

5.3.14 Event Trees Shutdowns . . . . . . . . . . . . . . . . . . 5-139

5.3.14.1 Initiating Event Description . . . . . . . . . . . . . 5-139

5.3.14.2 Normal Plant Response and Accident Sequences . . . . . 5-139

5.3.14.3 Event Trees and Accident Sequences . . . . . . . . . . 5-139

5.3.15 Event Trees for Local Faults . . . . . . . . . . . . . . . 5-144

5.3.15.1 Initiating Event Description . . . . . . . . . . . 5-144

5.3.15.2 Normal Plan Response and Success Criteria . . . . . . . . 5-144

5.3.15.3 Event Trees and Accident Sequences . . . . . . . . . 5- 5-144

5.4 Core Damage Analysis . . . . . . . . . . . . . . . . . . . 5-152

5.4 .1 SASSYS Model of EBR-II $\ldots \ldots \ldots \ldots \ldots \ldots \ldots \ldots$ 5-152

5.4 .2 Accident Calculations . . . . . . . . . . . . . 5-154

5.4.2.1 Loss-of-Flow Events . . . . . . . . . . . . . . . 5-154

5.4.2.2 Loss of Normal Power . . . . . . . . . . . . 5-165

5.4.2.3 Loss of Constant Power . . . . . . . . . . . . . . 5-169

5.4.2.4 Reactivity Insertion Events . . . . . . . . . . . . . . 5-170 
5.4.2.5 Loss of Primary Heat Sink ... . . . . . . . . . 5-181

5.4.2.6 Loss-of-Sodium Events . . . . . . . . . . . . 5 5-181

5.5 Uncertainity Analysis For Reactivity Feedbacks . . . . . . . . . 5-186

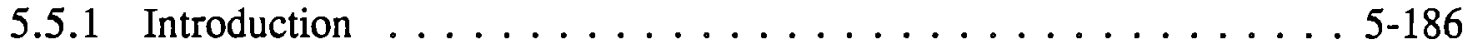

5.5 .2 Analysis Method and Results ............... 5-188

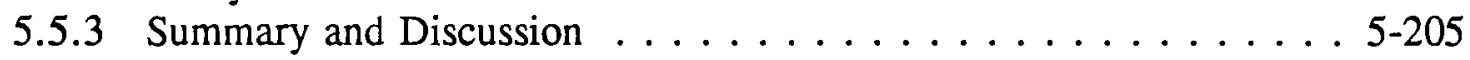

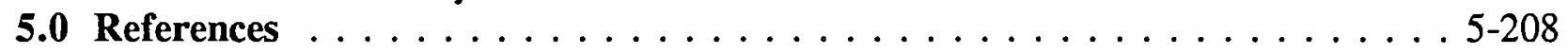




\section{List of Figures}

Page

5.1 Schematic of Pump and Motor Generator Set $\ldots \ldots \ldots \ldots \ldots \ldots$. . . . . . . . .

5.2 Coastdowns for Four Types . . . . . . . . . . . . . . . . . . . . 5-9

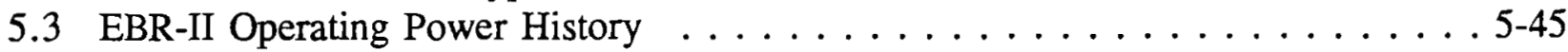

5.4 Probability Density Function for Single-CR Scram Reactivity . . . . . . . . . . 5 5-72

5.5 Exact Probability Density Functions for Scram Worth . . . . . . . . . . . . 5-76

5.6 EBR-II Decay Heat . . . . . . . . . . . . . . . . . . . . . 5 5-81

5.7 Core Response Tree for Fuel Damage Definition . . . . . . . . . . . . . 5-91

5.8 Functional Event Tree for EBR-II . . . . . . . . . . . . . . . . . . . . . . . 5-94

5.9 Transient Event Tree for N-Reactor with EBR-II Analogues (Local Effects Removed) . . . . . . . . . . . . . . . . . . . . . . 5-95

5.10 Core-Damage Event Tree for Internal Initiators in the CRBR PRA . . . . . . . . 5-97

5.11 Event Tree for Single Pump Fault - Group A . . . . . . . . . . . . . . . . 5-100

5.12 Event Tree for Single Pump Fault - Group B . . . . . . . . . . . . . . . . 5-101

5.13 Event Tree for Single Pump Fault - Group C . . . . . . . . . . . . . . . 5 5-102

5.14 Event Tree for Single Pump Fault - Group D . . . . . . . . . . . . . . . . 5-103

5.15 Event Tree for Double Pump Fault - Group A . . . . . . . . . . . . . 5-104

5.16 Event Tree for Double Pump Fault - Group B . . . . . . . . . . . . . . 5-105

5.17 Event Tree for Double Pump Fault - Group C . . . . . . . . . . . . . 5 5-106

5.18 Event Tree for Double Pump Fault - Group D . . . . . . . . . . . . . . . 5 5-107

5.19 Event Tree for Double Pump Fault - Group E . . . . . . . . . . . . . . 5 5-108

5.20 Event Tree for Double Pump Fault - Group F . . . . . . . . . . . . . . . . 5-109

5.21 Event Tree for Loss of Normal Electric Power . . . . . . . . . . . . . . 5 5-111

5.22 Event Tree for Loss of Constant Power . . . . . . . . . . . . . . . . . 5-112

5.23 Event Tree for Reactivity Insertion - RISA . . . . . . . . . . . . 5-115

5.24 Event Tree for Reactivity Insertion - RISB . . . . . . . . . . . . . 5-116

5.25 Event Tree for Reactivity Insertion - RISC . . . . . . . . . . . . . . 5-117

5.26 Event Tree for Reactivity Insertion - RISD . . . . . . . . . . . . . . . 5-118

5.27 Event Tree for Small Reactivity Insertion . . . . . . . . . . . . . . 5-120

5.28 Event Tree for Large Reactivity Insertion . . . . . . . . . . . . . . . 5-121

5.29 Event Tree for Loss of Primary Heat Sink (TSEC) . . . . . . . . . . . . . . . . 5-123

5.30 Event Tree for Loss of Primary Heat Sink (TBOP) . . . . . . . . . . . . . . 5-124

5.31 Event Tree for Overcooling (Steam Leak) . . . . . . . . . . . . . . 5-126

5.32 Event Tree for Overcooling (Primary Pump Overspeed) . . . . . . . . . . . 5 5-127

5.33 Event Tree for Overcooling (Secondary Pump Overspeed) . . . . . . . . . . . 5-128

5.34 Event Tree for A Steam Tube Rupture in A Superheater Module . . . . . . . . . 5-131

5.35 Event Tree for A Steam Tube Rupture in An Evaporator Module . . . . . . . 5-132

5.36 Event Tree for Delayed Loss of Circulation . . . . . . . . . . . . . . . . . 5-134

5.37 Event Tree for Transient Loss of Shield Cooling . . . . . . . . . . . . . . . 5 5-136

5.38 Event Tree for Transient Loss of Shutdown Coolers . . . . . . . . . . . . . . 5-137 
5.39 Event Tree for Transient Loss of Instrument Air . . . . . . . . . . . . . . 5-138

5.40 Event Tree for Scram . . . . . . . . . . . . . . . . . . . . . . . 5-140

5.41 Event Tree for Anticipatory Shutdown $\ldots \ldots \ldots \ldots \ldots \ldots \ldots \ldots$ 5-141

5.42 Event Tree for Shutdown Short . . . . . . . . . . . . . . . . 5-142

5.43 Event Tree for Shutdown Long $\ldots \ldots \ldots \ldots \ldots \ldots \ldots . \ldots \ldots$ 5-143 $\ldots \ldots \ldots$

5.44 Event Tree for Local Fault - Driver Pin Failure . . . . . . . . . . . . . . . 5-147

5.45 Event Tree for Local Fault - Metal Experiment Pin Failure . . . . . . . . . . . 5 5-148

5.46 Event Tree for Local Fault - Oxide Experiment Pin Failure . . . . . . . . . . 5 5-149

5.47 Event Tree for Local Fault - Partial Subassembly Blockage . . . . . . . . . . 5-150

5.48 Event Tree for Local Fault - Loading Error . . . . . . . . . . . . . . . . 5-151

5.49 Pump Coastdowns in EBR-II . . . . . . . . . . . . . . . . . . . 5-155

5.50 Hot Pin Coolant Temperature during Protected Single Pump Faults . . . . . . 5 5-159

5.51 Hot Pin Coolant Temperature during Unprotected Single Pump Faults . . . . . . 5-161

5.52 Hot Pin Coolant Temperature during Protected Double Pump Faults . . . . . . . . 5-164

5.53 Hot Pin Coolant Temperature during Unprotected Double Pump Faults . . . . . 5-167

5.54 Hot Pin Coolant Temperature during an Unprotected

Loss-of-Normal-Power Event . . . . . . . . . . . . . . . . . . . . . . . . 5 5-168

5.55 Hot Pin Temperatures during a Safety Rod Insertion at $0.0029 \$ / \mathrm{s}$. . . . . . . 5-172

5.56 Reactivity Response during a Safety Rod Insertion at $0.0029 \$ / \mathrm{s}$. . . . . . . . . 5-173

5.57 Hot Pin Temperatures during a Control Rod Insertion at $0.0042 \$ / \mathrm{s}$. . . . . . . 5-174

5.58 Hot Pin Cladding Temperature during an Instantaneous Reactivity Insertion . . . 5-177

5.59 Hot Pin Peak Fuel Temperature during an Instantaneous Reactivity Insertion . . 5 5-178

5.60 Time to Reach Core Damage Conditions during Instantaneous TOP Accidents . . 5-179

5.61 SHRT B302R Top of Core Thermocouple and Reactor Inlet Temperatures . . . 5-182

5.62 Hot Pin Coolant Temperature and Flowrate for a Protected Rapid LOS . . . . 5 5-184

5.63 LONP-4 Outcome Distribution For Potential Experiment Damage . . . . . . . 5-200

5.64 LONP-4 Outcome Distribution for Minor Core Damage . . . . . . . . . . 5 5-201

5.65 LONP-4 Outcome Distribution for Core Damage . . . . . . . . . . . . . . 5-202 
List of Tables

Page

5.1 Generic Classes of LMR Initiating Events . . . . . . . . . . . 5-3

5.2 EBR-II Potential Initiating Events Listed by Event Category . . . . . . . . . . . . 5-4

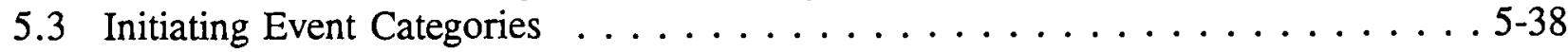

5.4 Comparison of EBR-II Initiating Events with Risk Significant

Accident Initiators in the Reference LMFBR of Reference $5.5 \ldots \ldots$. . . . 5-41

5.5 Annual Initiating. Frequencies for Single Pump LOFs by Cause . . . . . . . . . 5-46

5.6 Annual Initiating Frequencies for Single Pump LOFs by Group . . . . . . . . . . . 5-47

5.7 Annual Initiating Frequencies for Double Pump LOFs by Cause . . . . . . . . . 5 5-50

5.8 Annual Initiating Event Frequencies for Double Pump LOFs by Group . . . . . 5 5-51

5.9 EBR-II Scram and Shutdown History . . . . . . . . . . . . . . . . . 5 5-65

5.10 Initiating Event Frequencies and Error Factors for Quantification . . . . . . . . 5-67

5.11 Limiting Configuration of the Reactor Safety Shutdown System in the Operate Mode . . . . . . . . . . . . . . . . . . 5-71

5.12 Discrete Probability Density Function (pdf) and Cumulative Distribution

Function (cdf) for Scram Reactivity from a Single High Worth Control Rod . . . 5-73

5.13 Cumulative Distribution Functions for Reactivity from Scram of High-Worth Rods (Exact) . . . . . . . . . . . . . . . . . . . . . . 5-74

5.14 Number of Rods Required for Scram As a Function of Reactivity Insertion . . . 5-77

5.15 Decay Heat Removal Capabilities . . . . . . . . . . . . . . . . . . . . 5-80

5.16 Sources of Heat After Shutdown . . . . . . . . . . . . . . . . . . . 5-80

5.17 Net Heat Sinks for Reduced Heat Removal Capability . . . . . . . . . . . . . 5 5-82

5.18 Primary Tank Limits at Elevated Temperature . . . . . . . . . . . . . . . . . 5-84

5.19 Success and Failure Criteria for Decay Heat Removal (DHR) . . . . . . . . . 5-86

5.20 Success Criteria for Transients . . . . . . . . . . . . . . . . . . . . . . . 5-87

5.21 Definition of Plant Transient Categories . . . . . . . . . . . . . . 5-88

5.22 Plant Transient and Associated Fuel Damage Categories . . . . . . . . . . . 5-92

5.23 Core Damage Levels During Single Pump Faults . . . . . . . . . . . . . . 5-158

5.24 Core Damage Levels During Protected Double Pump Faults . . . . . . . . . . 5 5-163

5.25 Core Damage Levels During Unprotected Double Pump Faults . . . . . . . . . 5-166

5.26 Core Damage Levels During Slow Reactivity Insertion Accidents . . . . . . . 5-175

5.27 Reactor Conditions during Instantaneous Reactivity Insertion Accidents . . . . . 5-180

5.28 Steps in Passive Safety Analysis Procedure . . . . . . . . . . . . . . 5-187

5.29 Important Feedbacks . . . . . . . . . . . . . . . . . . . . . . . . . . 5-193

5.30 Feedback Uncertainties and Related Quantities . . . . . . . . . . . . . . 5-194

5.31 Discrete Probability Density Function for the Experimental

Full-Power PRD . . . . . . . . . . . . . . . . . . . . . . 5-196

5.32 Road Drop Feedback Quantities . . . . . . . . . . . . . . . . . . 5-196

5.33 Passive Safety Failure Probabilities for LONP-4 Accidents . . . . . . . . . . . 5-199 


\subsection{ACCIDENT SEQUENCE ANALYSIS}

This section contains the description of the accident sequence analysis. In Section 5.1, all the relevant initiating events are identified and categorized. Section 5.2 gives the description of the quantification of the initiating event frequency. Therefore, at the end of Section 5.2 there is the complete list of initiating events for the EBR-II PRA, the associated frequency and the basis for the estimation.

Section 5.3 describes the event trees development and includes the discussion of safety functions, their success criteria and the associated criteria for plant transient definition and damage category assignation. At the end of this section a full description of the event trees for each initiating event is available together with the damage assignation.

Sections 5.4 and 5.5 give further details on the behavior of EBR-II for certain key categories of initiating event. These calculations form the basis for the damage assignation of Section 5.3. Because the damage analysis of Section 5.4 is a best estimate analysis, the uncertainty in the calculations is analyzed in Section 5.5.

\subsection{Initiating Event Identification}

\subsubsection{Introduction}

This section discusses the identification and classification of Initiating Events (IE) for EBR-II. Initiating events are defined to include any event that leads to an accident sequence. Most initiating events, combined with safety system failures, have the potential to lead to some degree of core damage, (since even a routine shutdown involves challenges to plant safety systems). However, there are some events that can be shown to lead to a transient which will not damage fuel but are sufficiently important that they will be retained. An example of this type of event is a loss of normal power with the highly unlikely failure to scram, is 
predicted not to lead to fuel damage on a best estimate basis but the failure to scram would be occasion for serious concern. There are also some very rare initiating events which lead to damage, irrespective of safety system response. An example of this type of event is a very large, rapid reactivity insertion.

The process of core refueling is treated as an independent analysis and is documented in Appendix A. Events that occur while the system is shutdown are treated to the extent that for the shutdown decay heat removal process an extended mission time is considered, either 14 or 45 days (see 5.2). During this mission time failures of support systems and shutdown heat removal capability are considered. All other events are considered to be benign or not credible.

For example, once the system is shutdown, a flow transient has no effect (benign) and rod induced reactivity insertions are not credible since the control rods are unlatched. Accidents related to the transfer of subassemblies from EBR-II to HFEF (Hot Fuel Examination Facility) are not considered as they affect only one subassembly at a time, outside the reactor, and cannot lead to widespread core damage. Accidents of this type would be incorporated into an extended PRA or containment study because of the risk to on-site workers.

\subsubsection{Classes of Initiating Events}

EBR-II is one of two operating Liquid Metal Reactors (LMRs) in the USA, the other being FFTF. Other operating LMRs exist in the UK (PFR), France (Phenix and SuperPhenix), USSR (BOR-60, BN-350, BN-600), Japan (JOYO) and other countries. There are detailed design differences between EBR-II and other LMRs and significant differences compared to LWR reactors (PWR and BWR) as discussed in Section 3. EBR-II has been extensively characterized and studied over its 26 year operating history and a good basis for initiating event selection exists in the safety documentation for EBR-II [5.1]. In addition, despite the design differences, there are enough design similarities among all LMRs so as to make the initiating event lists used in the CRBRP PRAs, [5.2] and [5.3], JOYO, [5.4], and an EPRI study of comparative risk between LWR and LMFBR (LMR), [5.5], directly applicable. A review of the EBR-II UORs since 1976 was undertaken to try to identify any other plant-specific initiating events and to provide data for quantification. 
The generic classes of initiating events identified by the review of the EBR-II safety documents and the analysis of other LMRs are listed in Table 5.1. Each class was then developed into a comprehensive list of generic and EBR-II specific potential causes incorporating the events identified in [5.1], [5.2], [5.3], [5.4] and [5.5]. The resulting list is given in Table 5.2.

In the following sections each class of initiating event is discussed, the EBR-II-specific initiating events identified, and the basis for the quantification of the frequency is given.

Table 5.1. Generic Classes of LMR Initiating Events.

Loss of Flow

Reactivity Insertion

Loss of Primary Heat Sink

Overcooling

Loss of Decay Heat Removal Systems

Transients (including shutdown)

Loss of Sodium

Core Support Structural Failure

Support System Failures

Local Faults

External Events 


\section{Loss of Flow}

Loss of clutch power

Loss of primary pump power

Single pump seizure or coast down

Single pump seizure and coastdown of other pump

Total blockage of core inlet

Inlet pipe break (HPP)

Inlet pipe break (LPP)

Inlet pipe break (all inlet pipes)

HPP to LPP leakage excessive

Missing S/A's

Leakage past S/A's

$\underline{\text { Reactivity Insertion }}$

ACRDS insertion@12c/sec (Fast Speed)

ACRDS insertion @ 1c/sec (Slow Speed)

Control rod insertion (one or many)

Core support failure

Safety rod insertion

Voiding or gas bubble in core

Subassembly motion due to hydraulic force

Sudden core radial movement (step insertion)

\section{Loss of Primary Heat Sink}

Group 1

Condenser Cooling Water Low Flow

Loss of Condenser Vacuum

Group 2

Loss of Condenser System Flow

Loss of Feedwater Flow

Group 3

Major Steam Leak

Group 4

Secondary System Water-to-Sodium Leak

Secondary System Pump Leak

Sodium Leak in the Secondary Sodium System

Outlet Pipe Rupture

Inadvertent Drain of Secondary Sodium 
Table 5.2. EBR-II Potential Initiating Events Listed by Event Category (cont'd)

\section{Group 5}

Loss of Secondary Sodium Pump

Overcooling

Primary pump faults

BOP transients (steam leak)

Secondary pump faults

Loss of Decay Heat Removal Systems

Loss of shutdown cooler

Loss of shield cooling

$\underline{\text { Transients }}$

Scram, spurious

Anticipatory shutdown

Normal shutdown

Core characterization transients

\section{Loss of Sodium}

Siphon of $\mathrm{Na}$ (defeating siphon break system) through sodium purification system

Primary \& Guard Tank Failure and Leaks

Pumpout Na via FERD

Pumpout Na via BFTF

IHX Failure

Shutdown Cooler Failure

Core Support Failure

Core Distortion to more-reactive/less-coolable configuration

\section{Support System Failures}

Loss of Normal Power (13.8 kV)

Loss of Instrument Air

Loss of water systems

Loss of constant power

Loss of $125 \mathrm{~V} \mathrm{DC}$

Loss of $480 \mathrm{~V} \mathrm{AC}$ 
Table 5.2. EBR-II Potential Initiating Events Listed by Event Category (cont'd)

Local Faults

Driver fuel failure

Experiment failure

Local blockage (driver or experiment)

Others

External Events

Earthquake

Fire (sodium and non-sodium)

Aircraft Impact

Tornado/High Winds

Lightning

Flood

Volcanism 


\subsubsection{Loss-of-Flow}

Loss-of-flow transients must be divided into separate categories depending upon whether the faults are ones which affect one pump and its associated piping, termed single pump LOFs, faults which affect total flow or both pumps nearly simultaneously, referred to as double pump LOFs, loss of vital electrical power supplies which affect both pumps and possibly other systems and finally a loss of inlet piping integrity. This subdivision is required because of different degrees of damage possible and different responses of the mitigating systems in the various transients.

\subsubsection{Single Pump LOF}

The single pump LOF is discussed first since the double pump LOFs can be described as combinations of single pump LOFs. Factors leading to the loss of one or both primary pumps can be broadly classified as either electrical system faults or mechanical faults.

\subsection{Electrical Faults}

The major electrical support system to the primary pumps are the primary pump motor-generator sets, one set for each pump. Additional electrical faults can occur in the primary pump motors. The abbreviated schematic below shows the relationship of the motor-generator $(\mathrm{M} / \mathrm{G})$ set, the pump and the controller, Fig. 5.1. The electrical failures for the $M / G$ set and pump combination can be subdivided into three sets which are differentiated by the shape of their associated pump coastdown curves, Fig. 5.2.

Measurements of primary pump speeds from recent primary flow coastdown tests [5.6, 5.7] are used as being representative of coastdown conditions during electrical failure faults of the primary pumps. Figure 5.2 shows the pump speed coastdown curves for pump \#1 for the three types of electrical failure. Figure 5.2 also shows the assumed pump \#1 coastdown behavior during a pump shaft seizure LOF. The coastdown behavior of pump $\# 2$ is essentially 


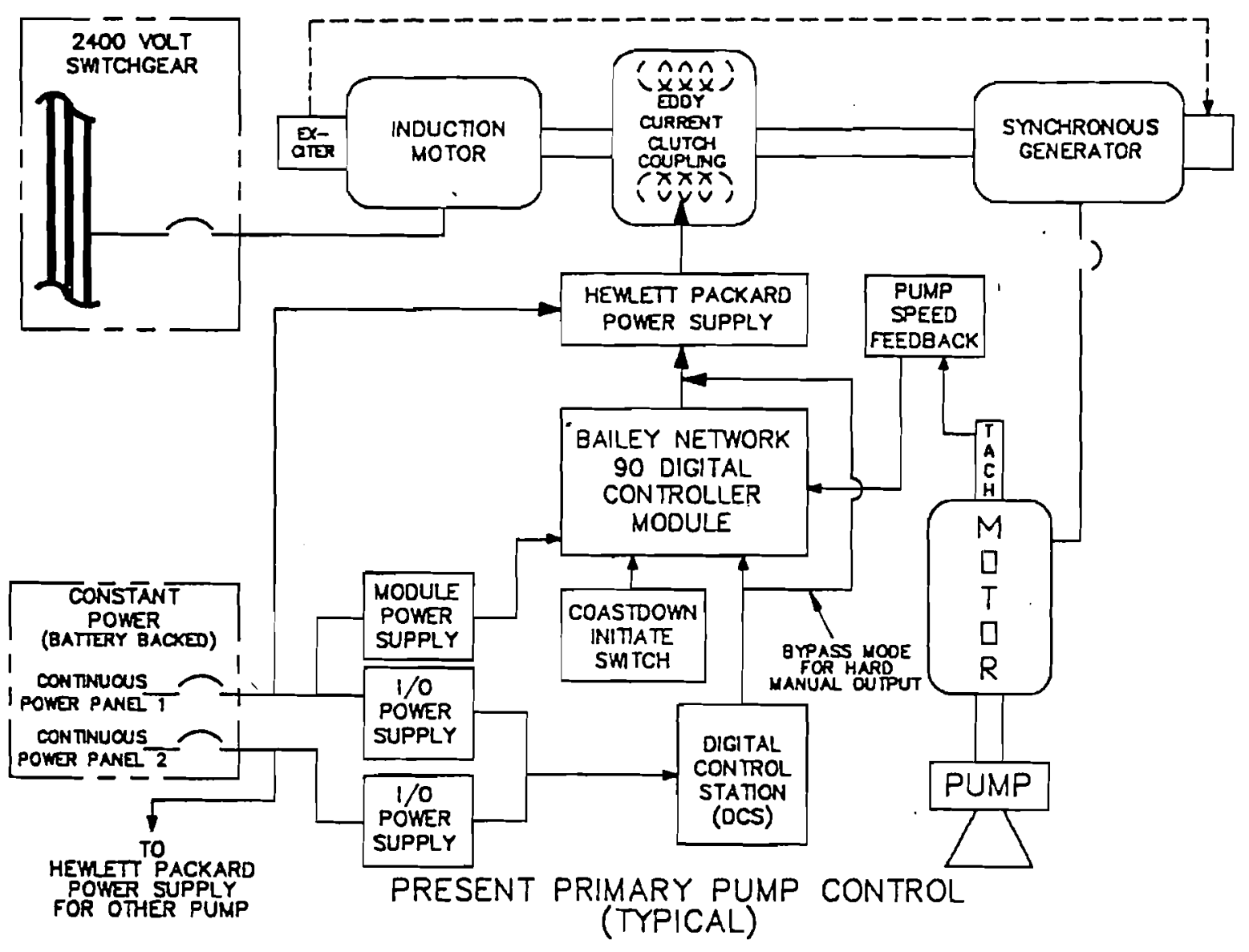

Fig. 5.1 Schematic of Pump and Motor Generator Set 


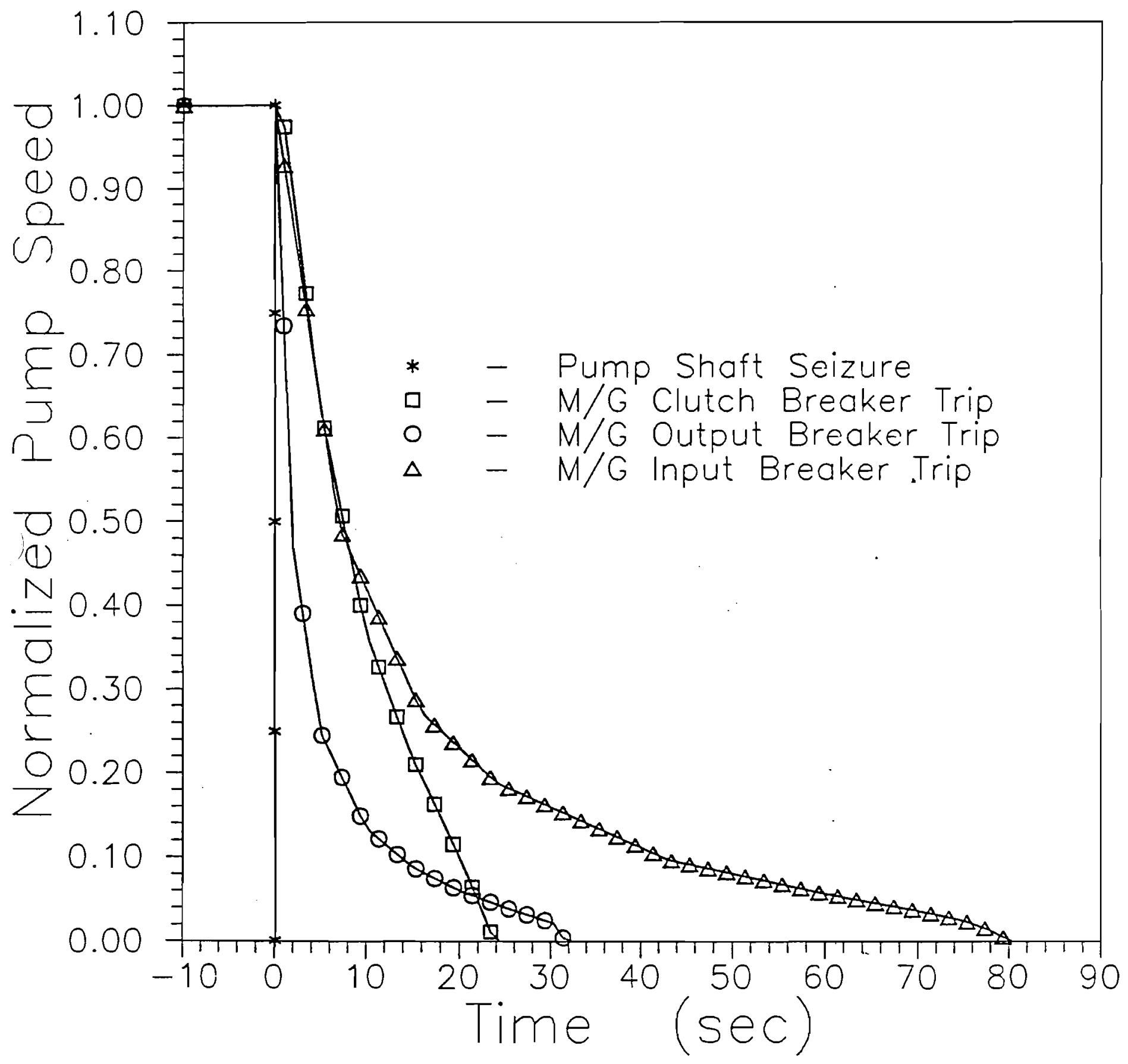

Fig. 5.2 Coastdowns for Four Types of Pump or Motor-Generator Set Failure 
the same as that of pump \#1, except that the stop time of the impeller of pump \#2 varies slightly from that of pump \#1, for a given coastdown type. For an $M / G$ input coastdown, the pump \#1 stop time is $82 \mathrm{~s}$, while the stop time of pump $\# 2$ is $87 \mathrm{~s}$.

\section{$\underline{\text { M/G Input Faults }}$}

A failure to the $\mathrm{M} / \mathrm{G}$ motor leads to the slowest coastdown considered. Failures of this type include the spurious trip of the input breaker to the $\mathrm{M} / \mathrm{G}$ set or a failure internal to the $\mathrm{M} / \mathrm{G}$ motor. These faults are developed in detail in the primary pump M/G set system notebook. The loss of normal power would also result in this slow coastdown but is considered separately. This coastdown has been referred to by a number of descriptive terms including input breaker trip, $M / G$ input breaker trip, $M / G$ input, $M / G$ motor. Here the term $M / G$ input will be used for the faults and the term slow will be used to describe the coastdown. The M/G input coastdown is the slowest of the electrical failure coastdowns, because the pump is driven by the combined rotational energy stored in the $\mathrm{M} / \mathrm{G}$ set, the pump motor, and the pump impeller.

\section{Clutch Faults}

Coastdowns associated with faults in the clutch control of the M/G sets have a faster coastdown than that of the $\mathrm{M} / \mathrm{G}$ input faults. The important faults in a clutch control initiated LOF are the loss of the continuous power supply associated with the respective pumps, faults in the clutch control circuits, spurious trip of the breaker between the continuous power supply and the clutch control circuits, failure of the clutch, and operator error. Operator error during reactor startup or power operation is excluded here because the normal means of tripping the primary pumps is through stop switches that are bypassed by relays powered from RSS control power in the clutch control circuits. Operator error would, therefore, enter the fault tree through an "AND gate" with the failure of either the stop switches, control power relays, or associated wiring. Since all of these hardware faults are modelled as direct clutch faults, operator error, even if explicitly modelled, would be subsumed into these hardware faults. 
There are other modes of clutch control failure that do not lead to a LOF but create undesirable operating conditions. These faults cause loss of the ability to change the clutch slip ratio and therefore the pump speed. The pumps, however, continue to operate. In such cases, operator action is necessary to change pump speed by going to either the manual or bypass mode of pump operation depending on the exact location of the fault. The frequency of these events is much less than that of clutch faults that cause a coastdown directly and the most severe outcome is bounded by a clutch failure. Therefore, these events are subsumed into this category.

The clutch failure coastdown is faster than the $M / G$ input coastdown, because the rotational energy of the $\mathrm{M} / \mathrm{G}$ motor is not involved in the coastdown. The clutch failure coastdown is driven by the combined rotational energy of the $M / G$ generator, the pump motor, and the pump impeller. As shown in Figure 5.2, the coastdown is initially the same as the M/G input coastdown. After about $10 \mathrm{~s}$, the coastdown curves diverge with the clutch failure coastdown decaying more rapidly. The stop time of each of the primary pumps during a clutch failure coastdown is about $23 \mathrm{~s}$. This coastdown decays rapidly because the generator field current, which is supplied by an exciter unit mounted on the output shaft of the M/G motor [5.8], is not affected by a clutch breaker trip. The constant electric field induced by the generator field current produces a braking force which slows the generator drive shaft, which in turn produces a rapidly decaying generator output current causing a rapid coastdown [5.9].

\section{M/G Output Faults}

The third subdivision of primary pump electrical faults includes $\mathrm{M} / \mathrm{G}$ set generator faults, pump motor faults, and trips of the circuit breaker connecting the two. These faults can be considered together because the rotational inertia involved is that of the pump only and they result in the same coastdown.

Other electrical faults are also associated with the primary pump motors since electrical power is used to run small air blowers on each pump which aid in cooling the primary pump motor by transferring heat from a self-contained argon cooling loop within the housing of each 
primary pump. These blowers are interlocked with the primary pump starting circuits but not with the run circuits and therefore do not cause an automatic primary pump shutdown if a blower fault occurs. Therefore the blowers are not essential to the primary pump motor operation. The long term effect of blower loss would be a higher operating temperature for the pump and shorten motor lifetime. For these reasons the argon blowers are not modeled.

As shown in Figure 5.2, the M/G output failure coastdown is initially faster than the clutch failure coastdown, because the rotational energy of the $M / G$ set is not involved. The M/G output failure coastdown is driven by the combined rotational energy of the pump motor and the pump impeller. The stop time of this coastdown is greater than the stop time of the clutch failure coastdown because the generator output current, which decays rapidly during a clutch failure coastdown, is not supplied to the pump motor. The stop time of pump \#1 during a M/G output failure coastdown is about $32 \mathrm{~s}$, while stop time of pump \#2 is about $37 \mathrm{~s}$.

\subsubsection{2 $\quad \underline{\text { Mechanical Faults }}$}

Mechanical faults are subdivided into binding events and bearing failure. Binding events result in either a limited reduction of pump speed, a postulated very slow coastdown of a pump, or a seizing of a pump that has already stopped so that it cannot be restarted. The much more abrupt seizure is the abrupt stoppage of a pump either due to shaft failure (fracture), shaft seizure, or a complete loss of the shaft coupling. Both of these types of failures have been the focus of an extensive evaluation with respect to degradation and wearout. During the study, a failure modes and effects analysis was performed and documented in the primary pump system notebook (Appendix C.2).

\section{Binding}

Primary pump binding could possibly occur due to failure of the upper or lower motor bearings, failure of the hydrostatic bearing, lubrication problems, mild shaft bowing, or buildup of material on the pump shaft. The problems with buildup of material is typical of the events that have been observed in EBR-II. In one case, a lubrication failure allowed lubricating 
material to leak onto the pump shaft where it formed a compound with sodium and caused a degradation of pump performance. The pump motor internals were modified to prevent recurrence of this problem. On two other occasions there was a buildup of sodium oxide on a primary pump shaft. The baffle section of the pump was modified to eliminate free interchange of aerosol-laden cover gas with the argon within the baffle by plugging existing upper drain holes and drilling new ones at the sodium level and the lower labyrinth was replaced with one incorporating a reverse spiral, which was designed to enhance clean argon flow down the shaft toward the new drain holes. The Primary-Tank Cover-Gas Sampling-Supply System (PTCGSSS) also supports the pumps by providing a small argon flow along the shafts of the pumps to prevent sodium vapor or sodium oxide from entering the pump motor. The use of the argon adds a weak dependence of the primary pumps on the PTCGSSS. However, the effects of the loss of support from the argon system would be long term and are modelled as binding of both pumps due to a common cause.

The term binding is used collectively to describe all of these slowly developing initiating events. It is also very difficult to assign a coastdown to any of these since the events do not result in a complete stoppage of even a single pump. Therefore, all of the binding events are assumed to be enveloped by a slow coastdown typified by the coastdown for $M / G$ input initiating events.

\section{$\underline{\text { Seizure }}$}

An abrupt seizure of a primary pump may occur due to catastrophic shaft failure in the form of a fracture. Shaft seizure due to extensive shaft bowing may occur but would probably be preceded by binding. Another failure mode would be a complete loss of the motor to shaft coupling caused by fracture of the drawbar or its keying mechanism. This failure would drop the pump impeller onto the pump casing and cause excessive vibration while the pump motor attempted to keep the pump rotating. The results of such an occurrence are difficult to predict and, for the PRA, it is assumed that an abrupt seizure would occur in order to envelop the possible outcomes. 
Although not all the abrupt failure modes may be a true seizure, the term seizure is used to group these initiating events. There have been no seizure initiating events in the 25 year history of EBR-II. Therefore, there is no known coastdown associated with a primary pump seizure. For calculational purposes, a primary pump seizure is assumed to be a nearly instantaneous stoppage which is modeled as 0.1 second ramp to zero pump speed in order to aid the numerical convergence of the accident analysis code.

\section{Hydrostatic Bearing Failure}

One failure mode considered was the hydrostatic bearing failure at low speed. The pump vendor does not specify a minimum speed at which damage may occur. During initial acceptance testing, the pumps were tested at $175 \mathrm{rpm}$ for 5200 hours. Testing during the 1982 \#2 pump repairs included operation at $50 \mathrm{rpm}$. EBR-II operational procedures prohibit operation below $260 \mathrm{rpm}$ and in the automatic mode of pump speed control, the controller has a lower setpoint. In addition, operation of a single primary pump below the minimum speed while at power would result in a flow below the scram setpoint. Therefore, the hydrostatic bearing failure at low speed is excluded.

\subsubsection{Double Pump LOF - General}

A double pump LOF is defined as the loss of one pump followed by the loss of a second pump within a time interval sufficiently short that the transient caused by the loss of the first pump is still in progress. This LOF includes both independent failures affecting the two pumps within a short time interval and common cause failures. In protected transients, the scram system would act to shut down the reactor following the loss of the first pump within a few seconds. In unprotected transients the reactivity feedbacks inherent in the EBR-II design have sufficient time to reduce power on a timescale commensurate with the full coastdown time thus reducing the effect of the loss of the second pump. Experimental data based on the SHRT tests using a controlled flow reduction has verified these feedback effects [5.10]. The unprotected LOFs studied in the SHRT tests included coastdowns from 95 seconds to as long as 600 seconds (10 minutes). 


\subsubsection{Double Pump LOF - Loss of Vital Electrical Power}

The loss of either normal power or loss of constant power are analyzed as a separate class due to certain unique aspects of these transients.

\subsection{Loss of Normal Power (LONP)}

The loss of normal power is defined to be loss of $13.8 \mathrm{kV}, 2400 \mathrm{~V}$, and $480 \mathrm{~V}$ normal AC supplies. Included in this event is the loss of $2400 \mathrm{~V}$ bus alone. For this case $480 \mathrm{~V}$ normal power is still available via the INEL loop, however the effects are similar so it is assumed that the fault also causes loss of $480 \mathrm{~V}$ normal power. There is an anticipatory trip in String A of the Reactor Shutdown System directly sensing undervoltage on the $2400 \mathrm{~V}$ supply which gives enhanced protection over the LOF trips.

The loss of normal power will result in the same double pump LOF as a double M/G input fault. It is considered separately because it also requires further plant-wide action which alter the means of decay heat removal. When the reactor is operating at power and parallel with the INEL loop (offsite power) there are two sources of power to the $2400 \mathrm{~V}$ bus. In circumstances where the INEL loop is lost or EBR-II separates from the INEL loop, the EBR-II generator will continue to supply electrical power to the $2400 \mathrm{~V}$ bus, this operating state is termed "on hotel load". Therefore loss of offsite power is not in itself an initiating event while the reactor is at power but it can lead to a loss of normal power. For example the loss of normal power can be caused by fluctuations on the INEL loop severe enough to cause the EBRII generator to trip as well as losing offsite power. These severe fluctuations have been caused principally by lightning. Another cause of LONP has been electrical transients when reconnecting to the INEL loop while operating on hotel load.

One additional route to a loss of normal power is a shutdown or scram when EBR-II is operating on hotel load (i.e. disconnected from the INEL loop). Since a turbine trip and a trip of the turbine generator output breaker accompany the scram, the generator, which is the sole source of $13.8 \mathrm{kV}$ power when on hotel load, is isolated. This will result in the loss of the 
$2400 \mathrm{~V}$ normal power bus and coastdown of the primary pumps. If the fault was a single pump LOF, a double pump LOF will result. This condition is included in the event tree analysis because operating on hotel load can lead to combinations of initiating events and different consequences.

\subsection{Double Pump LOF - Loss of Constant Power}

A loss of constant (continuous) power to a primary pump control circuit will result in a LOF involving that pump with the same coastdown as any other clutch failure. The loss of constant power is treated as a distinct LOF because of difference in the plant response. Also the nature of the interconnections between the two constant power static switches is such that there exists a possibility for a common cause power failure to both constant power busses.

The scram clutches on the control rods are held latched by constant power. Therefore, if both constant power supplies fail, the probability of a scram signal is 1.0. The failure to scram then becomes only the mechanical failure of the rods to drop. Thus, the branch point probabilities for failure to scram are lower than for a clutch or clutch control failure.

There are three related terms used to describe the constant power system. These are constant power, continuous power, and uninterruptible power. In a strict sense, the system is designated the constant power system but the other terms, especially continuous power, are used by EBR-II personnel and in some of the plant documentation. The two distribution busses are designated continuous power bus $\# 1$ and $\# 2$. The normal power supply to the continuous power busses are a pair of uninterruptible power supplies (UPS). Therefore, constant power is sometimes referred to as continuous power based on the bus designation and sometimes uninterruptible power based on the use of UPS to supply power to the bus.

The constant power system (CPS) provides $120 \mathrm{~V}, 60$ cycle grounded single phase uninterruptible power to critical instruments and components throughout EBR-II. The system is designed to reduce reactor scrams and forced shutdowns due to failure or fluctuations in the 
continuous power system. The continuous power system utilizes two Uninterruptible Power Supplies (UPS) to power two major distribution panels, Continuous Power Panels 1 and 2.

The UPS units were designed to meet the ANL policy on reactor safety which requires an uninterruptible power supply for the nuclear instruments. The intent of this policy is to ensure that nuclear instrumentation and detector capability is available to monitor the reactor during a station power outage until the reactor shutdown can be verified and the rest of the facility placed in a normal shutdown configuration.

Since UPS units 1 and 2 provide alternate power supplies for each other, reliability of the overall function of the system is increased by reducing the frequency of transients resulting from the failure of a single UPS such as a spurious scram or single pump LOF due to loss of clutch control power. However, this cross-connection of the two UPS units through the two static bypass switches allows the introduction of a potential common failure of both constant power panels.

Another way in which loss of both constant power panels can occur is if only one UPS is in operation. The minimum configuration allowed during normal power reactor operation and during unrestricted fuel handling is that one of the two continuous power supply batteries and its associated UPS system must be operable and normal feeder power must be available to at least the operable UPS unit. Therefore reactor operation with only one UPS is not precluded.

\subsubsection{Loss of Piping Integrity (LOPI)}

\subsection{Pipe Break}

The CRBR PRA gives a low frequency for this type of initiating event [5.3]. The combination of the low pressure differential and essentially no thermal gradient across the piping produces low pipe stresses from pressure. A break, other than a double guillotine rupture, of an inlet pipe can be bounded by a single pump seizure. A break in the outlet pipe would result in dumping hot sodium to the primary tank rather than the 
IHX. The effect on the plant would be that of a loss of primary heat sink rather than loss-offlow. The frequency of pipe breaks in the primary sodium circuit is, therefore, subsumed into the appropriate pump faults, or the loss of primary heat sink categories.

A double ended guillotine break of a section of inlet piping leads to a more severe transient than the pump seizure because of the ability for the sodium to bypass the core and flow directly from high pressure plenum to the tank through the ruptured loop and is thus identified as a separate initiating event (LOPI). Double ended guillotine breaks of both sets of high

pressure inlet piping can occur only due to total disruption of primary system geometry for which no internal initiating event has been identified. In addition it should be noted that thin walled pipes are not believed to exhibit guillotine breaks as a failure mode.

\subsection{Ball-Joint Bellows Failure}

Each primary pump outlet is connected to the fixed primary sodium piping by a ball-joint bellows which is attached to the outlet of each pump. Failure of these bellows due to either pressure or thermal cycling has been considered. Pressure cycles occur with the starting and stopping of the primary pumps. The normal differential pressure in the primary coolant is $56 \mathrm{psi}(386 \mathrm{kPa})$ and the design leakage paths where the bellows is connected to the fixed piping provides some pressure relief for starting surges. The thermal cycles are usually limited to two cycles from $700^{\circ} \mathrm{F}\left(371^{\circ} \mathrm{C}\right)$ to $350^{\circ} \mathrm{F}\left(177^{\circ} \mathrm{C}\right)$ and back to $700^{\circ} \mathrm{F}\left(371^{\circ} \mathrm{C}\right)$ each year. The role of temperature change is sufficiently slow to prevent stresses due to transient temperature changes. The bellows of \#1 pump have been inspected in 1970 and 1988. The bellows of $\# 2$ pump was inspected in 1982. No failures or cracks were detected in any of these inspections. If a crack did occur, its most likely consequence would be an increase in the leakage of the ball-joint bellows. Due to the small consequences and low frequency associated with this type of failure, it has been subsumed into the appropriate pump faults or loss of primary heat sink categories. 


\subsubsection{Other Primary System LOF Considerations}

\subsection{Auxiliary Pump}

For any appreciable amount of decay heat, EBR-II is designed to utilize natural circulation to transfer heat from the reactor core to the primary tank and/or the secondary sodium system. This natural circulation flow is initially $3.3 \%$ of full flow following reactor scram. The auxiliary pump is an electromagnetic pump placed in the outlet Z-pipe, whose function is simply to smooth the transition to natural circulation and can supply about $50 \%$ of the natural circulation primary system head increasing the available flow to $5 \%$. No credit is taken for operation of the auxiliary pump because analysis indicates that the auxiliary pump does not affect the accident progression and therefore the damage categorization.

\subsection{Primairy Flow Path Blockage}

Previous PRAs for CRBR and SNR300, as well as those for LWRs, exclude total blockage of large coolant pipes. The inlet piping from each pump to the high pressure plenum is 12 inches in diameter and the primary vessel outlet piping is 14 in. in diameter. No debris source has been identified nor is there an identified mechanism to cause debris collection in the primary piping. Furthermore the effects of an inlet blockage can be bounded by a single pump seizure LOF. It is possible to imagine outlet blockage being caused by pinching of the Z-pipe caused by relative movement of the components of the primary system but the only feasible cause for this would be a severe earthquake.

\subsubsection{Reactivity Insertion}

There are a variety of ways that reactivity might be inserted into the core of EBR-II. There are also ways which have been considered in other PRAs which are not possible in EBR-II. The CRBRP PRA [5.2] identified a class of events such as voiding or a gas bubble in the core. Because EBR-II has a negative void worth everywhere, these events can not lead to reactivity insertion. Overcooling of the primary system can also lead to reactivity insertion 
and is considered separately. The remaining possibilities require relative motion of core and control (or safety) rods. The EBR-II control rods are made of fuel with a poison follower above the fuel and are raised to make the reactor critical. Since during shutdown the rods are fully down, the restart involves raising the control rods. Motion of one or more control rods is a leading candidate for a reactivity insertion event (one which has occurred once at EBR-II through a human error). One specific control rod has the capability to be driven by the Automatic Control-Rod Drive System (ACRDS). The ACRDS was installed in order to produce limited transients for core characterization and fuel testing experiments [5.11]. The modes of operation of the ACRDS are slow-speed manual (i.e., just as a normal control rod), slow-speed automatic (up to $5 \mathrm{in.} / \mathrm{min}$.), and fast-speed automatic ( $72 \mathrm{in.} / \mathrm{min}$.). In the automatic modes the rod is computer controlled. Fast or slow speed automatic operation is not permitted during periods of normal reactor operations (in fact, the computer is disconnected). Nevertheless the ACRDS offers an additional, low probability, route to reactivity insertion.

All possible means of reactivity insertion by control rod motion can be classified as "slow" because of the drive speeds available. Slow indicates that the reactor will respond quasistatically to the reactivity input. The slow reactivity insertions are further subdivided into four separate events depending upon the total amount of reactivity to be inserted (and different success criteria in terms of the number of rods required to scram). The basic events identified which cause these four slow reactivity insertion events are:

Control Rod Insertion, (one or many),

Safety Rod Insertion,

ACRDS Insertion,

Combinations of these three events.

Fast reactivity insertion can be envisaged to arise from a sudden core radial movement to a more reactive configuration and internal rearrangement in an experimental subassembly. Technical specifications allow the PRD (Power Reactivity Decrement) to vary $15 \%$ from run to run. Assuming a PRD of $\leq 0.4 \$$, which has been true for all metal fuel dominated cores, this implies a $0.06 \$$ allowable change in reactivity from one run to the next. Therefore, even if all 
of this reactivity were available for insertion upon radial motion, the effects are limited. EBR-II safety analysis on the rearrangement of the fissile material in a subassembly indicates that reactivity insertion of $0.33 \$$ in 1.3 is a bounding estimate, [5.12]. The only identified mechanism for insertions of $0.35 \$$ to $\$ 1.00$ is limited motion of all control rods by slow motion of the control rod lifting platform. One event considered in other PRAs, loss of hydraulic holddown, is prevented by the design of the EBR-II vessel with its holddown fingers, one per assembly. Analysis also has shown that the flow is insufficient to levitate an assembly.

A very large fast reactivity insertion can only be caused by some core support failure. The core is supported directly upon the vessel bottom with a latticework of I-beams, and there is no redan or similar structure as found in other LMR designs. Therefore, the only mechanism identified to lead to fast reactivity insertion are primary vessel catastrophic failure, complete and circumferential, allowing a two inch drop of the vessel and core and $\sim \$ 1$ of reactivity input. While the most likely cause of vessel failure appears to be an earthquake the possibility of a random non-seismic failure is also considered.

\subsubsection{Loss of Primary Heat Sink}

Two forms of loss of primary heat sink are identified. These are the loss of secondary sodium heat transport and the loss of steam plant heat removal. The first type includes all faults in the secondary sodium flow paths, faults in the secondary pump and rupture of the primary sodium outlet Z-pipe in the primary tank leading to bypass of the IHX. All of these faults are modelled as an instantaneous loss of secondary sodium heat removal capability. These comprise groups 4 and 5 of Table 5.2. The second type includes all faults in the steam plant which ultimately lead to loss of heat removal capability. In practice the effects of this event are delayed because the heat capacity of the secondary sodium is available to remove heat but this is neglected in the damage analysis. In addition, in the event of loss of normal power, emergency electrical power is used to power the secondary pump and retard the secondary natural circulation flow and the heat load is placed upon the shutdown coolers. These events are those identified in groups 1 and 2 of Table 5.2. Group 3 in Table 5.2 is initially an overcooling event which transfers to a loss of primary heat sink upon operator action and is 
treated under overcooling. Water to sodium leaks in the steam generator are treated separately under Steam Generator Tube Rupture.

\subsubsection{Overcooling}

Overcooling of the primary system will result in a reactivity addition due to the negative temperature coefficient of the reactor if no scram takes place. The potential paths to overcooling were identified as inadvertent operation of the shutdown coolers, primary coolant pump run-up, secondary coolant pump run-up, and steam leak.

\subsubsection{Steam Leak}

Steam leaks which can occur differ in size over several orders of magnitude. Most of the leaks are minor in nature and present operational problems with no reactor safety consequences. These minor steam leaks are initiating events only in the sense that they result in a plant shutdown. Therefore, minor steam leaks are summed with transients as initiators. Major steam leaks will initially cause an overcooling event by removing excessive amounts of energy. If no operator or automatic actions take place, the steam generators will dry-out, or, if the secondary sodium is dumped by the operators to prevent steam generator dryout, the overcooling portion of the accident ends and a loss of primary heat sink sequence commences, due to the loss of the normal heat removal path. Nonetheless, it is theoretically possible to remove enough heat, given enough feedwater and no operator action or scram, to cause the reactor power to rise high enough to damage fuel.

\subsubsection{Primary Pump Run-up}

The effects of an increase of primary coolant pump speed were investigated during the Inherent Operability Tests (IOT) conducted in 1987 [5.13]. In this test, the primary pump speed and power were initially $30 \%$. Pump speed was then increased to $100 \%$ and the power was allowed to adjust through the inherent reactivity feedbacks in EBR-II. The final reactor power was $<100 \%$ and the power-to-flow ratio $<1$ for the whole transient. 
Since this test showed that the effects of primary pump run-up are minor, it could be removed from consideration as a separate initiating event and just included in the transient category. It will be retained as a distinct initiating event to allow consideration of the accident sequences, and the display of the above result.

\subsubsection{Secondary Pump Run-up}

The effects of an increase of secondary coolant pump speed were investigated during the PICT tests conducted in 1987. In these PICT tests, the secondary pump speed was varied to determine the effect on the primary system [5.14]. This test showed that the effects of secondary pump run-up are minor; it also could be removed from consideration as a separate initiating event, but is retained for the reasons stated in Section 5.1.6.2.

\subsubsection{Shutdown Coolers Inadvertent Operation}

Overcooling due to the inadvertent activation of the shutdown coolers has been removed from consideration due to the small percentage of heat removal relative to rated power, $\sim 1 / 2 \%$.

\subsubsection{Steam-Generator Failures}

The EBR-II steam generator transfers heat from secondary sodium on the shell side to water/steam on the tube side. The steam-generating system of EBR-II consists of seven parallel-connected natural-circulation evaporators, a steam drum, two parallel-connected superheaters, and interconnecting piping. The evaporators and superheaters are straight-tube heat exchangers. The only differences between the evaporators and superheaters are that core tubes are installed in the steam tubes of the superheaters to increase the steam velocity, and that the superheaters are inverted in relation to the evaporators. The evaporators and superheaters are designed to minimize the possibility of interaction between sodium and water/steam by using "duplex" tubes and double tubesheets. Therefore, there is no location where only a single weld, tube, or tubesheet separates the sodium from the water and/or steam [5.15]. 
A leak in the duplex tube would result in the injection of high pressure steam/water into liquid sodium. A failure in the welds which connect the duplex tubes to the duplex tube sheets results in leakage into the airspace which has leak detectors. An undetected failure in the outertube weld to its tube sheet and an undetected failure in the inner-tube weld to its tube sheet are required to obtain a sodium/water reaction in the airspace between the two tube sheets. The vigorous sodium/water reaction would produce heat, hydrogen, and sodium compounds. Left unchecked, the size of the original leak would increase due to self-wastage of the failed steam tube. Other steam-generator tubes in the vicinity of the leak could also corrode, producing extensive damage to the steam generator and possible damage to the IHX through a pressure pulse or corrosion due to reaction products.

The only detected leak in over 25 years of operation has been a small water leak due to a manufacturing defect in a water-tubesheet weld of an evaporator, which resulted in a small release of steam to the air gap between the tubesheets. The leaking steam was prevented from interacting with sodium by the intact sodium tubesheet and tubesheet-to-tube welds. At no time since have additional leaks been detected in any of the steam-generating equipment.

The only operational difficulty encountered with the steam generating system has been a degradation of heat transfer caused by separation of the duplex-tubing interface resulting from material creep in a superheater containing mechanically bonded tubes. One of the original eight evaporators, which contained metallurgically bonded tubes, was removed, converted into a superheater, and used to replace the original superheater. This operational problem had no safety consequences, [5.15].

\subsubsection{Loss of Primary Sodium Accidents (LOSA)}

The causes and effects of Loss of Primary Sodium Accidents (LOSA) in EBR-II are influenced greatly by the pool design of the EBR-II plant. The reactor, reactor vessel, and entire primary coolant system are submerged in approximately 90,000 gallons $\left(340 \mathrm{~m}^{3}\right)$ of liquid sodium with no penetrations in the side walls or bottom of the primary and guard vessels. All penetrations are through the top cover, above the bulk sodium surface level. Because the entire 
primary coolant system is submerged in the bulk sodium, primary system pipe breaks cannot cause a loss of the primary sodium inventory. A severe leak from a piping rupture would result in a loss of some forced coolant flow, but not a loss of coolant. Primary coolant system pipe breaks are, therefore, considered a Loss-of-Flow (LOF) accident, not a LOSA. Even with a major primary system piping rupture, some flow will be maintained through the core from natural circulation in the primary system and the built-in-leaks in the connections in inlet plena and system piping. Therefore, the definition of a LOSA for EBR-II is cast in terms of the sodium level in the primary vessel relative to that of the various components within the tank.

If the bulk sodium level were to fall 24 inches $(0.6 \mathrm{~m})$, the outlet Z-pipe would become uncovered. With the primary pumps operating there will be no effect, but once the pumps are tripped, natural circulation through the IHX can be compromised by the designed leakages at the junction of the Z-pipe and the IHX shell casing. This drop would also stop the operation of the Auxiliary Pump since the bulk sodium is used as part of its conduction circuit. If the pumps are tripped, once the pump coasts down, the sodium can drain out of the Z-pipe through the IHX casing holes, thus causing the sodium to find one level and effectively stopping natural circulation flow through the Z-pipe. The possibility of core damage arises only when the primary pumps are de-energized after a successful shutdown. This scenario is categorized as a loss of circulation, delayed (LCDL), and is produced by a limited loss of primary sodium.

For a LOSA to disrupt the forced flow in EBR-II, the level of the primary sodium must fall below the level of the primary pump inlets. The inlets are located approximately 5 feet $(1.5$ m.) below the nominal sodium level in the primary vessel. A LOSA of this magnitude would disrupt the primary flow circuit, preventing forced flow through both the inlet and outlet piping, and limit natural circulation to inside the reactor vessel and through the designed leakage paths. This is categorized as a loss of circulation, rapid (LCRA) caused by a large loss of primary sodium.

For the reactor core to actually become uncovered, the sodium level would have to drop approximately 14 feet $(4.3 \mathrm{~m}$.). In the analysis of the EBR-II plant, no accidents that could lead to an uncovering of the reactor core without the potential for a large reactivity addition were identified. 
Reference 5.16 categorizes the volume of sodium and the corresponding in primary sodium level in the primary associated with potential initiators. Based on the results of that study, eight potential initiating events were identified as sources of sodium of $\mathrm{NaK}$ for liquid metal fire considerations and potential LOSA initiating events. One of the events, a primary vessel catastrophic failure, has reactivity insertion consequences in addition to initiating a LOSA. Therefore this initiating event is considered as a large reactivity insertion event (RIFL, see 5.1.4.).

Two of the potential initiating events, the shutdown cooler system rupture and the Fuel Element Rupture Detection system piping leak, do not have the force necessary to remove the primary sodium from the primary vessel, and are therefore not considered as LOSA initiators. They have been included in considerations for liquid metal fire potential in Section 10. The shutdown cooler system failure is also included in the section on transients, since it represents a reduction of decay heat removal capability which could require a reactor shutdown.

Of the five remaining initiating events, all could cause a delayed loss of circulation. Only one, a rupture in both the primary and guard vessels, has the potential to cause a rapid loss of circulation, but only if the leak rate is large enough to lead to early uncovery of the primary pump skirts. The five initiating events which have been identified as potential paths to a LOSA are 1) primary vessel rupture, 2) primary and guard vessel rupture, 3) BFTF failure, 4) primary $\mathrm{Na}$ syphon break system failure, and 5) IHX/secondary Na system breach. Each event is considered below.

\subsubsection{Primary Vessel Rupture}

A rupture in the primary vessel allows primary sodium to enter the gas filled annulus between the primary and guard vessels. The drop in the primary bulk sodium level will be from 24 to 34.7 inches ( 0.6 to $0.9 \mathrm{~m}$.) depending on whether the lower deck on the guard vessel remains sealed, as designed and fabricated, or not. The annular gas filled space between the vessels includes detection systems designed to recognize changes in pressure and leak detectors. There is also a system to measure the level of the primary bulk sodium in the 
primary vessel. On receipt of signals corresponding to level changes in the sodium, the emergency procedures call for shutdown of the reactor. However, the leak will continue until the levels equilibrate in the tank and the primary-guard vessel gap.

\subsubsection{Primary and Guard Vessel Rupture}

In the event of a leak in the primary vessel, the failure of the guard vessel must also be considered. The same concerns as the primary vessel rupture apply here except that the predicted drop is 82.6 to 98.7 inches ( 2.1 to $2.5 \mathrm{~m}$.), depending on the guard vessel lower deck seal and the temperature of the sodium in the air baffle area. In this very improbable initiator the sodium will come into contact with the shield cooling air with the corresponding potential for a sodium fire.

\subsubsection{Breached Fuel Test Facility (BFTF) Failure}

The Breached Fuel Test Facility (BFTF) is a thimble assembly extending through the small rotating plug into the upper plenum of the reactor vessel and is closely coupled to the top of a subassembly in grid position 5A3. The flow from the subassembly is monitored by several diagnostic instruments, including flow meters and a delayed neutron detector. Two independent barriers must fail before sodium can flow out of the primary vessel. The maximum flow through the subassembly is about $20 \mathrm{gpm}\left(1.310^{-3} \mathrm{~m}^{3} \mathrm{~s}^{-1}\right)$, so leakage rate of any sodium loss would be lower. Any failure below the top cover would flow back into the bulk sodium, so only a limited portion of the system would allow sodium to leave the primary vessel. At this low flow rate, substantial time exists for corrective action to be taken, and the reactor shutdown. This failure does not contribute to the LOSA potential because of the low flow rate and multiple annunciator systems included to notify the control room of BFTF problems. 


\subsubsection{Primary Na Purification System Leak}

Because major sections of the primary sodium purification system and the radioactive sodium chemistry loop are located below the level of sodium in the primary tank, and the system draws primary sodium directly from the primary tank, the potential exists for siphoning primary sodium through a system piping break at a low elevation. To combat this possibility, there is an Engineered Safety Feature (ESF), the syphon break system, designed to prevent the syphoning of sodium through the purification system. A vacuum is necessary to raise the sodium to a surge tank in the system. If a leak should occur in the purification system, the syphon break system will admit argon gas into the surge tank, breaking the vacuum and removing the ability to draw sodium out of the primary vessel. If this system were to fail, primary sodium could be removed at the maximum rate of $100 \mathrm{gpm}\left(6.310^{-3} \mathrm{~m}^{3} \mathrm{~s}^{-1}\right)$, the rating of the EM pump in the system. All piping outside the purification and sodium chemistry cells is above bulk sodium levels, and the extensive detection systems in the cell mean this failure would not contribute to a LOSA, but is a liquid metal fire source.

\subsubsection{IHX Tube Failure}

During normal operation the secondary sodium system is operated at a pressure of about $10 \mathrm{psi}(69 \mathrm{kPa})$, which is higher than the primary sodium pressure of 3.4 psi $(23 \mathrm{kPa})$. A leak in the IHX would result in secondary sodium entering the primary vessel and a corresponding rise in the bulk sodium level. The action required by the emergency procedures to stop the in-leakage of sodium include shutting down the reactor, stopping the secondary pump, and reducing secondary system cover gas pressure to less than $0.1 \mathrm{psi}(1 \mathrm{kPa})$. If this action is not sufficient to stop the level increase, the secondary sodium is dumped. The secondary sodium dump to the storage tank in the presence of an IHX leak that provides a possible path for primary sodium to be syphoned out of the primary vessel. The secondary sodium system includes an argon vent system that maintains the secondary system pressure and allows for argon to fill the piping as the sodium is dumped. This system also acts to break any syphon, since it is connected to the inlet and outlet of the IHX at elevations above the bulk 
sodium level. Because of the number of independent system failures that must take place, this event does not contribute to the LOSA potential.

\subsubsection{Support System Failures}

Initiating events in this category arise from the malfunction of support systems which are not otherwise specifically assigned to another category. Normal plant shutdown and scrams are defined in section 5.1.11. Specifically excluded are plant experiments and tests such as the SHRT series [5.10]. The nature of these tests are such that a general envelope cannot be provided and the frequency of the tests is unpredictable from a statistical point of view since they are willful human actions. Therefore, experiments and testing are excluded from the PRA in the same sense that other willful acts such as sabotage are excluded. It should be noted that all plant tests are required to go through a review process and therefore are accompanied by a separate safety analysis. In addition the testing period is characterized as having heightened operator awareness so that all human actions, especially manual scram, will have a significantly different probability.

The principal support systems capable of causing plant transients are the electrical systems and the instrument air systems. Other systems such as the water systems may also contribute to transients but act on a timescale that is sufficiently long as to allow corrective action. Also included in this category is the unrecovered loss of a shutdown cooler.

\subsubsection{Instrument and Plant Air}

The plant and instrument air systems are normally cross-connected with the plant air compressor shutdown and isolated, with the instrument air compressors furnishing air to both systems. The plant air system serves as a partial backup because the plant air compressor cannot furnish enough air to maintain pressure in the entire instrument air system unless portions of the air systems are isolated. Therefore, the systems can be considered as one system from the viewpoint of initiating events. 
Numerous valves in the feedwater and steam systems are controlled with instrument air, including the steam drum level control. Loss of air supply to these valves can initiate a loss of primary heat sink accident.

Instrument air is also used to maintain the reactor building isolation valves open and the shutdown cooler louvers in the shut position. The set of isolation valves and the two shutdown coolers each have air accumulators and one directional isolation through check valves which prevent the spurious operation of these devices in the event of a temporary loss of the instrument air system. The reactor isolation valve accumulator is sized to allow for two complete cycles of the isolation valves after a loss of instrument air. Therefore this system should function normally. The accumulators for the shutdown coolers have no operational requirements because even if the shutdown coolers operated at full capacity due to the louvers opening at power, the heat removal would increase from $60 \mathrm{~kW}$ to $360 \mathrm{~kW}$ causing a $0.48 \%$ change in power. The only considerations in shutdown cooler inadvertent operation are operational ones at shutdown conditions which would require additional heat input from either the primary pumps or the primary tank heaters to compensate for heat loss.

The pneumatic-operated valves in the primary tank pressure control system will fail closed on a loss of instrument air; and argon cannot be made up to the floating head tank on a low level or pumped down on a high level. The primary tank pressure-vacuum relief system will maintain the cover gas pressure in a safe region between -4 and 13 in. of water. In addition, manual control of cover gas pressure is possible to maintain pressure near normal.

Loss of instrument and plant air is considered to be a separate initiating event, because a total air supply loss will require an anticipatory reactor shutdown and lead to a loss of feedwater, as well as affecting the state of the containment isolation. Thus, it is preserved as a separate event for consideration in a follow-on containment study. 


\title{
5.1.9.2 Reactor Auxiliaries and Plant Cooling Water Systems
}

The cooling water system supplies water for direct heat transfer to various components located in the power plant and the sodium boiler building. Cooling of components in the reactor building uses an intermediate heat transfer medium such as freon and silicone oil to transfer the heat to the cooling water system. The cooling water support system consists of the raw water system, the reactor auxiliaries cooling water system, the plant cooling water system and two cooling towers.

No accident initiating events have been identified that can result immediately from loss of the plant or reactor auxiliaries cooling water system. However, cooling water is required for normal operation of critical components such as the MG set clutches, secondary sodium main EM pump, air compressors and generator hydrogen coolers. Therefore, an extended total loss of cooling water would ultimately require an anticipatory reactor shutdown and is included in that category.

\subsubsection{Electrical Systems}

The electrical distribution system is divided into the following systems.

\author{
$138 \mathrm{kV}$ Distribution/Substation Equipment \\ $13.8 \mathrm{kV}$ Distribution System \\ 2400 V Distribution System \\ 480 V Main Distribution System \\ 480 V Emergency System \\ Continuous Power Supply System \\ 125 V DC Distribution System
}

Of these systems only the $2400 \mathrm{~V}$ distribution system and the continuous power supply system are directly involved in initiating events as described above. 


\section{V System}

The $2400 \mathrm{~V}$ system is directly connected with the control power relay string of the reactor. An undervoltage condition will cause the $2400 \mathrm{~V}$ undervoltage relay to operate contacts in string $\mathrm{A}$ of the reactor shutdown system, causing string $\mathrm{A}$ to de-energize, scramming the reactor in anticipation of a loss of primary cooling flow. This is discussed under Loss of Normal Power.

\section{Continuous Power Supply System}

The loss of either continuous power panel will directly cause a reactor scram and also loss of clutch control power to the primary pump connected to the continuous power panel that is lost. The scram occurs in anticipation of a reduction of primary cooling flow and is discussed in the LOF section. In the cáse where Continuous Power Panel No. 1 fails, the scram also occurs due to the loss of the control rod latch power bus. The simultaneous loss of both continuous power panels results in a total loss of primary flow and requires a reactor scram to limit fuel damage as described in the two pump LOF section. This can also happen if only one uninterruptible power supply (UPS) is in operation and both continuous power panels are supplied from this one source.

There are no other direct initiating events resulting from malfunctions of the continuous power system. If normal feeder power to both units is lost or if neither UPS unit is operable, an anticipatory shutdown of the reactor must be performed before the batteries are depleted. Normally, loss of a UPS does not cause a reactor scram, since the two Continuous Power Panels are then fed from the operable unit or normal power. 


\section{Balance of Electrical System}

Failures resulting from malfunctions of the $138 \mathrm{kV}$ Distribution/Substation Equipment, $13.8 \mathrm{kV}$ Distribution System (without loss of 2400 V), 480 V Normal Distribution System, 480 V Emergency System, or 125 V DC Distribution System are not direct initiating events.

The loss of all $480 \mathrm{~V}$ AC systems, including the emergency power from the diesel generators, does not cause an automatic reactor scram. Loss of $480 \mathrm{~V}$ power will cause a loss of secondary sodium surge tank level indication and a subsequent trip of the secondary pump. This event is included in the TSEC initiating event. If $480 \mathrm{~V} \mathrm{AC}$ feeder power is lost to the charging systems of the auxiliary pump battery, station battery or continuous power supply battery, an anticipatory reactor shutdown is required before required functions are jeopardized by excessive battery discharge.

A review of EBR-II Unusual Occurence Reports (UORs) indicated some LOFs originating from loss of $480 \mathrm{~V}$ power early in the plant history. This was because the original design of EBR-II powered the primary pump clutch control from $480 \mathrm{~V}$. A plant modification in 1986 changed the source for clutch control power from normal power to the constant power panels. Transients resulting from the loss of the constant power panels have been discussed earlier.

In the case of the loss of normal power, if the diesels fail to pick up the loads, emergency $480 \mathrm{~V}$ power is not available. As mentioned above, this station blackout is no different in its effect on core damage than loss of normal power because EBR-II is designed not to require $480 \mathrm{~V}$ AC for reactor operation. However, extended station blackout can have effects on certain key structures which would compromise the ability to restart the reactor. Specifically, the concerns are the temperatures of the concrete biological shield and reactor tank and plugs. Since the difficulties associated with these increased temperatures are operational (not safetyrelated) there is no unique initiating event associated with loss of $480 \mathrm{~V}$ power or complete station blackout. 
The only identified effect of the loss of $125 \mathrm{~V} \mathrm{DC}$ system are the inability to normally control the $13.8 \mathrm{kV}, 2400 \mathrm{~V}, 480 \mathrm{~V}$ normal, and $480 \mathrm{~V}$ emergency switch gear, selected motor control centers, as well as the emergency DC lighting system. While some protection functions for the switch gear is lost, the AC busses remain in operation. Manual operation of the switch gear would serve as a backup means of electrical distribution.

\subsubsection{Shutdown Coolers}

Under normal operation the shutdown cooler louvers are closed and each cooler removes $30 \mathrm{~kW}$ of heat from the primary tank due to natural circulation in the $\mathrm{NaK}$ loops and ambient losses through the piping and shutdown cooler box walls. The natural circulation flow of $\mathrm{NaK}$ with the louvers closed is monitored to assure that a minimum flow of $25 \mathrm{gpm}$ is maintained. The purpose of maintaining a minimum flow is three-fold: (1) to assure that the flow of $\mathrm{NaK}$ is not reduced by either leakage or blockage; (2) to maintain the proper temperature and flow fields so that the transition to natural circulation full flow with the cooler louvers open may be accomplished; and (3) to reduce the thermal shock to the system if the cooler louvers open. If the minimum $\mathrm{NaK}$ flow of $25 \mathrm{gpm}$ can not be obtained the reactor must be shutdown via a normal shutdown. Since the cause of below minimum flow is most likely due to an "unrecoverable" loss of a shutdown cooler, either a blockage or leak, the necessary procedure would be to plan to maintain decay heat removal with the balance of plant and keep the balance of plant available until repair of the affected cooler could be completed or until decay heat levels were sufficiently low to allow long term decay heat removal to continue with either a single cooler or the shield and thimble cooling systems. Thus since loss of a shutdown cooler assumes a plant response with reduced decay heat removal capacity, it is maintained as a separate initiating event.

The most likely malfunction of the shutdown coolers is the inadvertent opening of the louvers on one or both of the shutdown cooler boxes. The opening of both sets of louvers is possible due to faults in the primary tank bulk temperature sensing and alarm electronics or la shut valve in the portion of the instrument air system common to both coolers. Even if both cooler box louvers open for both of the shutdown coolers, the resulting increase in heat removal 
is a net $300 \mathrm{~kW}$ (180 kW each with louvers open, $30 \mathrm{~kW}$ each with louvers shut). This represents less than $1 / 2 \%$ of full power $(300 \mathrm{~kW} / 62.5 \mathrm{MW})$ and would not require a shutdown but only result in degraded plant efficiency.

If the opening was the result of the loss of instrument air, the initiating event would be based on the loss of BOP connected with the loss of instrument air and not the involvement of the shutdown coolers. Therefore, the inadvertent opening of the shutdown cooler louvers is excluded as a separate initiating event due to a lack of safety related consequences.

\subsubsection{Shield and Thimble Cooling}

The shield cooling system provides cooling to maintain the top and radial biological shields below $225^{\circ} \mathrm{F}$ and $150^{\circ} \mathrm{F}$ respectively. The EBR-II technical specifications require "shield temperatures satisfactory" with a minimum configuration of one temperature alarm channel and one exhaust fan operable. The emergency procedures place additional restrictions of one recirculating fan and one refrigeration system operable. The required action for loss of shield cooling temperature monitoring is a normal shutdown. Loss of the minimum required fans and refrigeration necessitates an anticipatory shutdown or termination of unrestricted fuel handling.

Only $3 \%$ of the heat transfer to the biological shields is due to gamma heating with the remainder being a combination of thermal radiation and convection. Therefore, even if the reactor is shutdown, a cooldown of the primary tank is required if the upper and radial biological shields are expected to exceed $225^{\circ} \mathrm{F}$ and $150^{\circ} \mathrm{F}$, respectively. With a total loss of shield cooling these limits are not expected to be exceeded for at least 24 hours, for the upper biological shield, and 100 hours for the radial biological shield as shown in Appendix C.5.

The thimble cooling system provides cooling of the nuclear detectors located in thimbles $\mathrm{J}-1, \mathrm{~J}-3$, and J-4. Since loss of the neutron detectors would degrade the RSS, it is required that an anticipatory reactor shutdown be performed within 15 minutes if thimble cooling flow is lost 
or if temperature $>135^{\circ} \mathrm{F}$ occurs in two of the three thimbles. Reduced flow or loss of temperature indication requires a normal shutdown.

The total heat removal from the shield cooling and thimble cooling systems is $150 \mathrm{~kW}$ which is approximately equivalent to the differential heat removal obtained by opening the louvers on one shutdown cooler at a primary tank temperature of $700^{\circ} \mathrm{F}\left(371^{\circ} \mathrm{C}\right)$.

In evaluating the plant response to loss of shield or thimble cooling, the decay heat removal capacity is reduced and, hence, is evaluated separately from other shutdowns. The initiating frequency for this event is calculated based on the loss of either part of the shield cooling system or the thimble cooling system. However, the plant response assumes that the entire heat removal capacity of both systems is lost. This conservatism is the result of the simplifying assumptions of categorizing the shield and thimble cooling as a single unit for decay heat removal comparable to a single shutdown cooler in capacity. This assumption allowed the use of a two of three logic for short term decay heat removal.

\subsubsection{Shutdown and Scram}

The number of shutdowns and scrams are based on plant operational data. Included in these categories are spurious trips and anticipatory shutdowns which occur because of faults in noncritical systems. A distinction is made between the normal shutdowns, unplanned or planned, and the annual long shutdown. In the long shutdown the secondary sodium is drained after the reactor is shutdown and the system temperature has been reduced to $350^{\circ} \mathrm{F}$ $\left(177^{\circ} \mathrm{C}\right)$. This process requires at least 18 hours for cooldown and several additional days for

reheat to $700^{\circ} \mathrm{F}$. Therefore the balance of plant is not immediately available for decay removal after this time. However, the decay heat generation is lower and the starting temperature for the sequence may be lower. 


\subsubsection{Local Faults}

The issue usually referred to as Local Faults has been a subject of research for LMRs for a considerable time. The specific concern is that given some form of local fault, random fuel pin failure or blockage in one subassembly, the fault might propagate until the whole core is involved in a damaging excursion. This issue has been considered in detail in [5.17]. EBR-II has a mission as a research reactor and routinely irradiates experiments to fuel pin failure and beyond without any impact upon driver fuel, i.e. local faults are deliberately initiated in EBR-II. Experimental irradiations which are judged to have the potential to cause problems are irradiated in special hardware (hexcans), designed to prevent damage propagation to the rest of the core. As a result a number of different local faults initiating events were considered, including inlet and outlet blockages, driver and experimental pin failure, and multiple pin failures.

\subsubsection{Initiating Event Categories}

Table 5.3 gives the EBR-II initiating event categories and the initiating events assigned to each category. This table lists all the considered initiating events. 
Table 5.3. Initiating Event Categories

\begin{tabular}{|c|c|c|}
\hline Initiating Event Category & Identifier & Initiating Events \\
\hline $\begin{array}{l}\text { Slow Flow Transient } \\
\text { (one loop) } \\
\text { Coastdown A }\end{array}$ & LF1A & $\begin{array}{l}\text { M/G set input breaker trip } \\
\text { Faults in the motor (of the motor generator } \\
\text { set) } \\
\text { Shaft binding due to excessive material build- } \\
\text { up } \\
\text { Slowly developing blockage in loop }\end{array}$ \\
\hline $\begin{array}{l}\text { Flow Transient due to clutch } \\
\text { problems at M-G set (one } \\
\text { loop) } \\
\text { Coastdown B }\end{array}$ & LF1B & $\begin{array}{l}\text { Loss of power to clutch } \\
\text { Clutch internal fault } \\
\text { Clutch control malfunction }\end{array}$ \\
\hline $\begin{array}{l}\text { Fast Flow Transient } \\
\text { (one loop) } \\
\text { Coastdown C }\end{array}$ & LF1C & $\begin{array}{l}\text { Fault in generator (of motor generator set) } \\
\text { Primary pump motor failure } \\
\text { M/G set output breaker trip }\end{array}$ \\
\hline $\begin{array}{l}\text { Immediate Flow Loss in } \\
\text { One Loop } \\
\text { Coastdown D }\end{array}$ & LF1D & $\begin{array}{l}\text { Shaft seizure } \\
\text { Instantaneous blockage of inlet piping } \\
\text { Inlet pipe rupture (partial) }\end{array}$ \\
\hline Loss of Primary Inlet Piping & LOPI & Inlet piping rupture - double ended guillotine \\
\hline Loss of Normal Power & LONP & $\begin{array}{l}\text { Loss of } 2400 \mathrm{~V} \text { bus } \\
\text { Loss of generator output while on hotel load } \\
\text { Loss of INEL loop while generator off-line }\end{array}$ \\
\hline Loss of Constant Power & LOCP & $\begin{array}{l}\text { Loss of both constant power sub-systems } \\
\text { Loss of the only operating constant power } \\
\text { subsystem while cross-connected }\end{array}$ \\
\hline $\begin{array}{l}\text { Slow Flow Transient in Both } \\
\text { Loops }\end{array}$ & LF2A & $\begin{array}{l}\text { As LF1A in both loops - allows common } \\
\text { mode evaluation }\end{array}$ \\
\hline $\begin{array}{l}\text { Clutch Disconnect on MG } \\
\text { Set in Both Loops }\end{array}$ & LF2B & $\begin{array}{l}\text { As LF1B in both loops - allows common } \\
\text { mode evaluation }\end{array}$ \\
\hline $\begin{array}{l}\text { Fast Flow Transient in Both } \\
\text { Loops }\end{array}$ & LF2C & $\begin{array}{l}\text { As LF1C in both loops - allows common } \\
\text { mode evaluation }\end{array}$ \\
\hline $\begin{array}{l}\text { Immediate Flow Loss in } \\
\text { Both Loops }\end{array}$ & LF2D & $\begin{array}{l}\text { As LF1D in both loops - allows common } \\
\text { mode evaluation }\end{array}$ \\
\hline $\begin{array}{l}\text { Immediate Flow Loss in } \\
\text { One Loop and Other Flow } \\
\text { Transient in Second Loop }\end{array}$ & LF2E & $\begin{array}{l}\text { Double pump faults of mixed type including } \\
\text { one seizure }\end{array}$ \\
\hline $\begin{array}{l}\text { Other Combination Flow } \\
\text { Transients }\end{array}$ & LF2F & $\begin{array}{l}\text { Double pump faults of mixed type - no } \\
\text { seizures }\end{array}$ \\
\hline
\end{tabular}


Table 5.3. Initiating Event Categories (cont'd)

\begin{tabular}{|c|c|c|}
\hline Initiating Event Category & Identifier & Initiating Events \\
\hline $\begin{array}{l}\text { Slow Reactivity Insertion of } \\
<0.20 \$\end{array}$ & RISA & $\begin{array}{l}\text { Control Rod Insertion } \\
\text { Safety Rods Insertion from shim position } \\
\text { Multiple Control Rod Insertion }\end{array}$ \\
\hline $0.20 \$$ to $0.65 \$$ & RISB & $\begin{array}{l}\text { ACRDS insertion fast speed mode or slow } \\
\text { speed mode } \\
\geq 2 \text { control rod insertions }\end{array}$ \\
\hline $0.65 \$$ to $\$ 1.00$ & RISC & $\begin{array}{l}\text { Multiple rod insertions } \\
\text { ACRDS + control rod insertion }\end{array}$ \\
\hline$>1.00 \$$ & RISD & Control rod bank insertion \\
\hline $\begin{array}{l}\text { Fast Reactivity Insertion of } \\
<0.35 \$\end{array}$ & RIFS & $\begin{array}{l}\text { Sudden core radial movement } \\
\text { Internal failure of experimental S/A }\end{array}$ \\
\hline $\begin{array}{l}\text { Fast Reactivity Insertion of } \\
\quad>1.00 \$\end{array}$ & RIFL & $\begin{array}{l}\text { Primary vessel catastrophic failure } \\
\text { Primary and guard vessel catastrophic failure }\end{array}$ \\
\hline $\begin{array}{l}\text { Loss of Secondary Sodium } \\
\text { Heat Removal }\end{array}$ & TSEC & $\begin{array}{l}\text { Inadvertent dump of secondary sodium } \\
\text { Loss of surge tank level indication (trip of } \\
\text { secondary pump) } \\
\text { Secondary system leak } \\
\text { Secondary pump controller error - flow to } \\
\quad \text { zero } \\
\text { IHX heat transfer degradation } \\
\text { Secondary sodium leak } \\
\text { Primary outlet piping rupture } \\
\text { Loss of secondary sodium pump }\end{array}$ \\
\hline $\begin{array}{l}\text { Loss of Steam Plant Heat } \\
\text { Removal }\end{array}$ & TBOP & $\begin{array}{l}\text { Loss of condenser system water flow } \\
\text { Loss of condenser vacuum } \\
\text { Loss of feedwater flow } \\
\text { Steam generator failure (no sodium } \\
\text { water interaction) } \\
\text { Turbine generator trip }\end{array}$ \\
\hline \multirow[t]{2}{*}{ Local Faults (Rapid) } & FLTA & $\begin{array}{l}\text { Complete subassembly inlet blockage } \\
\text { Complete subassembly outlet blockage }\end{array}$ \\
\hline & FLTB & Simultaneous failure of multiple pins \\
\hline Overcooling Steam Leak & OCSL & $\begin{array}{l}\text { Major steam leak } \\
\text { Relief valves stay open }\end{array}$ \\
\hline Overcooling Primary Pumps & OCPP & $\begin{array}{l}\text { Primary pump flow run up to maximum } \\
\text { speed of pump }\end{array}$ \\
\hline
\end{tabular}


Table 5.3. Initiating Event Categories (cont'd)

\begin{tabular}{|c|c|c|}
\hline Initiating Event Category & $\underline{\text { Identifier }}$ & Initiating Events \\
\hline $\begin{array}{l}\text { Overcooling Secondary } \\
\text { Pumps }\end{array}$ & OCSP & $\begin{array}{l}\text { Secondary pump flow run up to maximum } \\
\text { speed of pump }\end{array}$ \\
\hline $\begin{array}{l}\text { Steam Generator Tube } \\
\text { Rupture (sodium-water } \\
\text { interaction) }\end{array}$ & EVTR & $\begin{array}{l}\text { Steam generator tube rupture } \\
\text { Small leaks in duplex tube } \\
\text { Steam generator large leak, tube-to-tubesheet } \\
\quad \text { weld failure }\end{array}$ \\
\hline Rapid Loss of Circulation & LCRA & Primary and guard vessel rupture \\
\hline Delayed Loss of Circulation & LCDL & $\begin{array}{l}\text { Primary tank leak or rupture } \\
\text { Primary and guard vessel leak or rupture } \\
\text { FERD system leak } \\
\text { BFTF leak } \\
\text { Primary sodium purification system leak } \\
\text { IHX tube failure (combined with other } \\
\text { failures of secondary dump tank, etc.) }\end{array}$ \\
\hline $\begin{array}{l}\text { Transient - Unrecoverable } \\
\text { Loss of Shutdown Cooler }\end{array}$ & TSDC & $\begin{array}{l}\text { Break of cooler } \mathrm{NaK} \text { pipes } \\
\text { Leakage of } \mathrm{NaK} \\
\text { Plugging of } \mathrm{NaK} \text { loop }\end{array}$ \\
\hline $\begin{array}{l}\text { Transient - Loss of } \\
\text { Instrument Air }\end{array}$ & TAIR & Loss of Instrument Air \\
\hline $\begin{array}{l}\text { Transient - Unrecoverable } \\
\text { Loss of Shield Cooling }\end{array}$ & TSHC & $\begin{array}{l}\text { Loss of shield cooling if unrecoverable gives } \\
\text { a shutdown with impaired decay heat removal }\end{array}$ \\
\hline Long Shutdown & SHDL & Long shutdown - secondary sodium drained \\
\hline Short Shutdown & SHDS & $\begin{array}{l}\text { Shutdown- secondary sodium at normal } \\
\text { conditions }\end{array}$ \\
\hline Scram & SCRM & Scram, spurious \\
\hline Anticipatory & ANTC & Anticipatory shutdowns \\
\hline \multirow[t]{5}{*}{ Local Faults (Slow) } & MDLF & Driver subassembly pin failure \\
\hline & MELF & Experiment pin failure (metal) \\
\hline & OELF & Experiment pin failure (oxide) \\
\hline & SFPB & Partial subassembly blockage \\
\hline & SFLE & Loading error \\
\hline
\end{tabular}


Table 5.4 compares the risk-significant accident initiators in the reference LMFBR of [5.5] with the reference list for EBR-II. In the comparison some specific features are worth noting. EBR-II has a uniformly negative sodium void worth so that gas bubbles, etc., in the coolant cannot lead to reactivity insertion. This feature is important because recently PHENIX experienced (negative) reactivity transient attributed to a gas bubble. In addition the spurious, or otherwise, trip of the primary pumps does not apply to EBR-II because the primary pumps are not connected to the PPS.

Table 5.4. Comparison of EBR-II Initiating Events with Risk Significant Accident Initiators in the Reference LMFBR of [5.5]

EBR-II I.E.

1. Fuel failure caused by the formation of coolant blockages of SFPB fuel subassemblies due to crud, fuel particles, or external objects.

2. Fuel failure caused by fuel pin overenrichment errors.

MDLF

3: Voiding or gas bubbles in core.

N/A

4. Loss and re-establishment of hydraulic holddown (19c step

N/A for 30 assemblies moving) ${ }^{\mathrm{a}}$.

5. Failure of the core to be supported because of reactor vessel or core support failure.

RIFL

6. Single control assembly withdrawal at maximum design

RISA speed $(9 \mathrm{in} / \mathrm{min})(<10 \mathrm{c} / \mathrm{sec} \mathrm{ramp})^{\mathrm{a}}$.

7. Single control assembly withdrawal at maximum speed

RISA capability (i.e., $72 \mathrm{in} . / \mathrm{min}$ for primary rods) (10 to $50 \mathrm{c} / \mathrm{sec}$ ramp) ${ }^{\mathrm{a}}$.

8. Control assembly group withdrawal.

RISD

9. Earthquakes.

a. Sudden core radial movement initiated by an earthquake $(\leq 60 \mathrm{c} / \mathrm{step})^{\mathrm{a}}$-- stick-slip motion.

b. Core, radial blanket, and control rod movement due to a safe shutdown earthquake (60c step) ${ }^{\mathrm{a}}$.

EXTERNAL EVENT

c. Core, radial blanket, and control rod movement due to a safe shutdown earthquake (30c step) ${ }^{\mathrm{a}}$.

d. Other system failures. 
Table 5.4 Comparison of EBR-II Initiating Events with Risk Significant Accident Initiators in the Reference LMFBR of [5.5] (Cont'd)

10. Reactor vessel rupture.

EBR-II I.E. RIFL/LCRA/LCDL

11. Redan failure N/A

12. Loss of one intermediate heat transport loop. TSEC

13. Loss of flow in four primary pumps

LF2X

14. Pump trip in four primary loops due to plant protection N/Asignal.

15. Rupture of an intermediate loop in the steam generator bay.

TSEC

16. Internal failure of a steam generator module.

SGTR

17. Loss of the main condenser system.

TBOP

18. Loss of the main feedwater supply.

TBOP

19. Loss of a steam generator loop.

TBOP

20. Loss of offsite power.

LONP

21. Turbine-generator trip. TBOP

22. Normal reactor shutdown.

SHDS

23. Fires.

${ }^{a}$ CRBR - specific estimates of reactivity insertion potential. 


\subsection{Initiating Event Quantification}

Frequency estimates for each initiating event category involves use of a number of different techniques. The first source for initiating event frequencies is plant data. In common with other DOE facilities, EBR-II has been endeavoring to reduce the number of scrams experienced by the plant. A thorough review of the Reactor Shutdown System (RSS) led to many features being removed from the original scram string, the present alignment being in place since 1976, with some minor exceptions. Many other plant modifications were instituted in the period from first criticality, 1964, to 1976 and therefore the historical data used for initiating event quantification was selected to be 1975 to 1989 , the results then being collected into three five-year periods. In this way trends, either advantageous or disadvantageous, could be identified and the most appropriate time interval chosen. If the last five years showed a marked change in the number of occurrences, then this was the period adopted for quantification. The quantification uses a Bayesian update of a non-informative prior [5.18].

The review of the EBR-II Unusual Occurence Reports (UORs) for 1975-1989 gave estimates for the most frequent initiating events. The method used defines the frequency of failures, when $N$ have occurred in $T$ years, as $(2 N+1) / 2 T$. Use of this formula implies that data based frequencies will be greater than or equal to $3.310^{-2} \mathrm{yr}^{-1}$, because if there have been 0 failures in 15 years, $(T=15 \mathrm{yrs}, \mathrm{N}=0$ failures), the resulting frequency is given as $[2(0)$

$+1] /[2(15)]=3.310^{-2} \mathrm{yr}^{-1}$. Less frequent events must therefore be quantified using the fault tree models for EBR-II specific systems. This technique is used for slow reactivity insertion events, flow transients due to pump and motor generator set faults and support system failures such as loss of shutdown cooler when the reactor is at power. Frequencies for these events typically fall in the range of $10^{-2} \mathrm{yr}^{-1}$ or below. Finally, some very rare events are not amenable to quantified fault tree analysis because they rely on more subjective arguments. Examples of initiating events quantified in this way are fast large reactivity insertion, loss of coolant accidents due to catastrophic failure, and steam generator tube rupture.

It is important to bear in mind the distinction between initiating events quantified in different ways. Put simply, for those initiating events whose frequency is based upon historical 
data at EBR-II the numerical results can be viewed as "realistic". Those sequences that arise from initiating events whose frequency is derived from fault tree analysis can also be considered "realistic" but the quantification is more uncertain. Note that it is this category that includes most of the classic LMR accidents, particularly slow reactivity insertion and loss of flow events, that have been extensively studied. This feature does not arise by accident, the risk endemic in these events was recognized from the earliest days of the design of LMRs such as EBR-II. As a consequence the design, and later plant modifications, have been so carried out as to reduce the likelihood of events in this class. In fact when EBR-II experienced one control rod insertion event, caused by a human error, the response was to modify the control panel to preclude that particular error, and similarly, when one double pump loss-of-flow arose from a dependency between the clutches on the motor generator sets, the response was to eliminate the dependency. The final class of initiating events, whose frequency is evaluated by other techniques, usually represent a source of unmanageable and irreducible risk to the reactor. Typically the frequencies are derived by semi-quantitative arguments appealing to engineering judgment. Clearly there must be less confidence placed in the numerical results for these transients.

Figure 5.3 shows the operating history for the period 1984 to the long shutdown of 1989. This clearly demonstrates the improved performance achieved by EBR-II in 1987 and 1988. The period chosen ensures that the effects of earlier operation are included to some degree because the good performance of 1987 and 1988 cannot be guaranteed to continue.

\subsubsection{Flow Transients (Single Pump)}

The initiating event frequencies for each of the single pump LOFs are calculated in two ways. First the primary pump fault trees are combined with the $M / G$ set trees. Results are shown below for the contribution of each pump and for a combination of pumps, Tables 5.5 and 5.6. Secondly a direct data based estimate is evaluated where meaningful data can be found. 

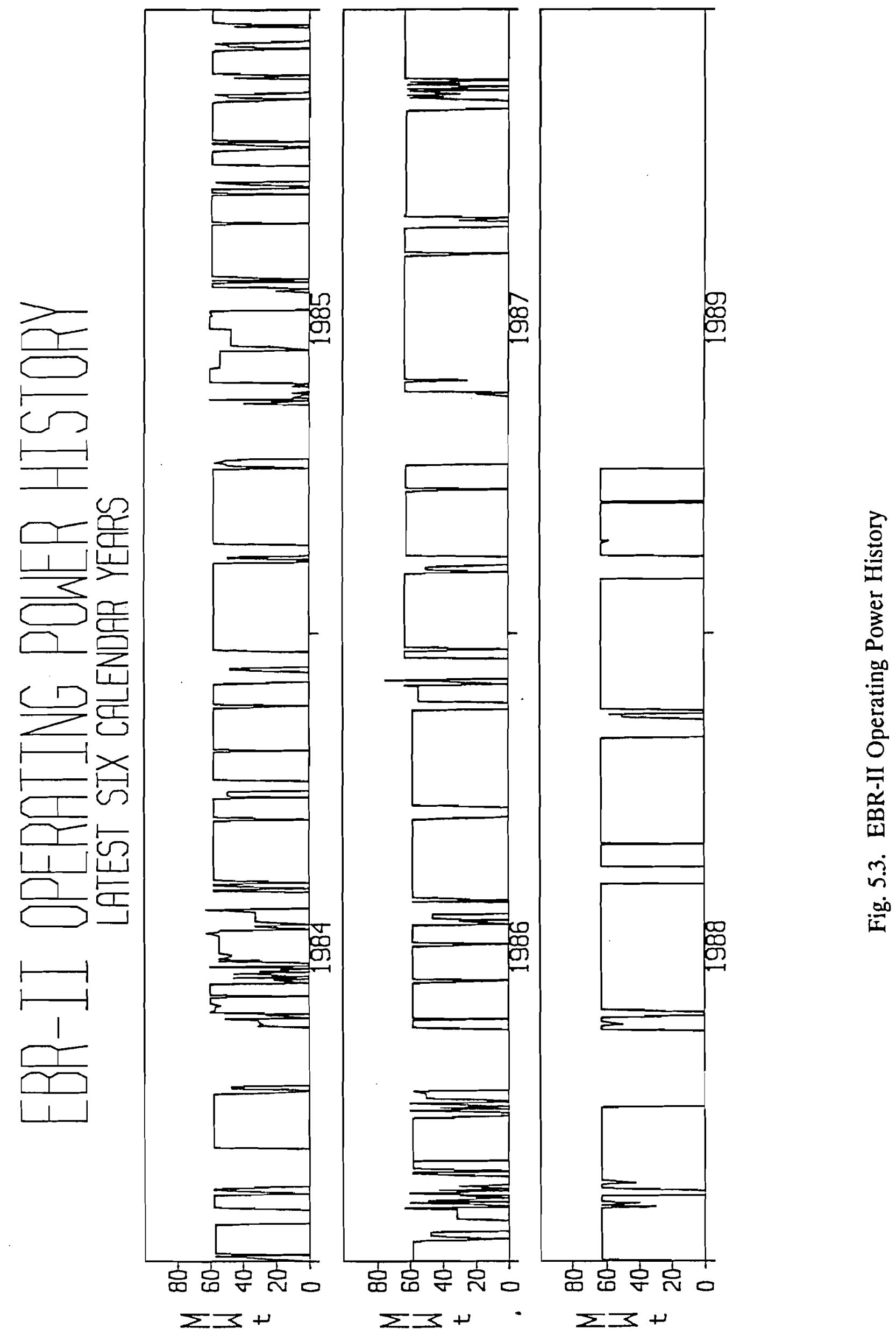
Table 5.5. Annual Initiating Frequencies for Single Pump LOFs by Cause

\begin{tabular}{|c|c|c|c|c|c|}
\hline \multirow{2}{*}{\multicolumn{2}{|c|}{ Initiating Event }} & Primary Pump 1 & Primary Pump 2 & Either Pump & Data Based Re \\
\hline & & $\begin{array}{l}\text { Annual Frequency } \\
\text { Error Factor }\end{array}$ & $\begin{array}{l}\text { Annual Frequency } \\
\text { Error Factor }\end{array}$ & $\begin{array}{l}\text { Annual Frequency } \\
\text { Error Factor }\end{array}$ & $\begin{array}{l}\text { Annual Freque } \\
\text { Error Factor }\end{array}$ \\
\hline \multirow{5}{*}{$\begin{array}{l}n \\
\dot{1} \\
a\end{array}$} & Binding & $\begin{array}{l}2.010^{-1} \\
2.6\end{array}$ & $\begin{array}{l}2.010^{-1} \\
2.6\end{array}$ & $\begin{array}{l}4.010^{-1} \quad[1] \\
2.0\end{array}$ & $\begin{array}{l}5.010^{-1}[1] \\
2.6\end{array}$ \\
\hline & M/G Input Faults & $\begin{array}{l}2.310^{-1} \\
2.8\end{array}$ & $\begin{array}{l}2.310^{-1} \\
2.8\end{array}$ & $\begin{array}{l}4.710^{-1} \\
2.2\end{array}$ & $\begin{array}{l}3.310^{-2} \\
8.4\end{array}$ \\
\hline & Clutch Faults & $\begin{array}{l}6.310^{-1} \\
4.6\end{array}$ & $\begin{array}{l}6.310^{-1} \\
4.5\end{array}$ & $\begin{array}{l}1.28 \\
4.1\end{array}$ & $\begin{array}{l}1.510^{-1} \\
3.3\end{array}$ \\
\hline & M/G Output Faults & $\begin{array}{l}4.210^{-1} \\
2.3\end{array}$ & $\begin{array}{l}4.210^{-1} \\
2.8\end{array}$ & $\begin{array}{l}8.310^{-1} \\
1.7\end{array}$ & $\begin{array}{l}2.510^{-1} \\
2.6\end{array}$ \\
\hline & Seizure & $\begin{array}{l}1.410^{-3} \\
8.4\end{array}$ & $\begin{array}{l}1.410^{-2} \\
8.4\end{array}$ & $\begin{array}{l}2.710^{-2} \quad[1] \\
5.1\end{array}$ & $\begin{array}{l}3.310^{-2} \quad[1] \\
8.4\end{array}$ \\
\hline
\end{tabular}

Note: [1] The data based results assume that the primary pump runs the entire year since the only time the pumps are usually off is during fuel handling. The fault tree data is based only on critical operation. Therefore, the value is smaller by a factor of $300 / 365 \approx 0.8$ 
Table 5.6. Annual Initiating Frequencies for Single Pump LOFs by Group

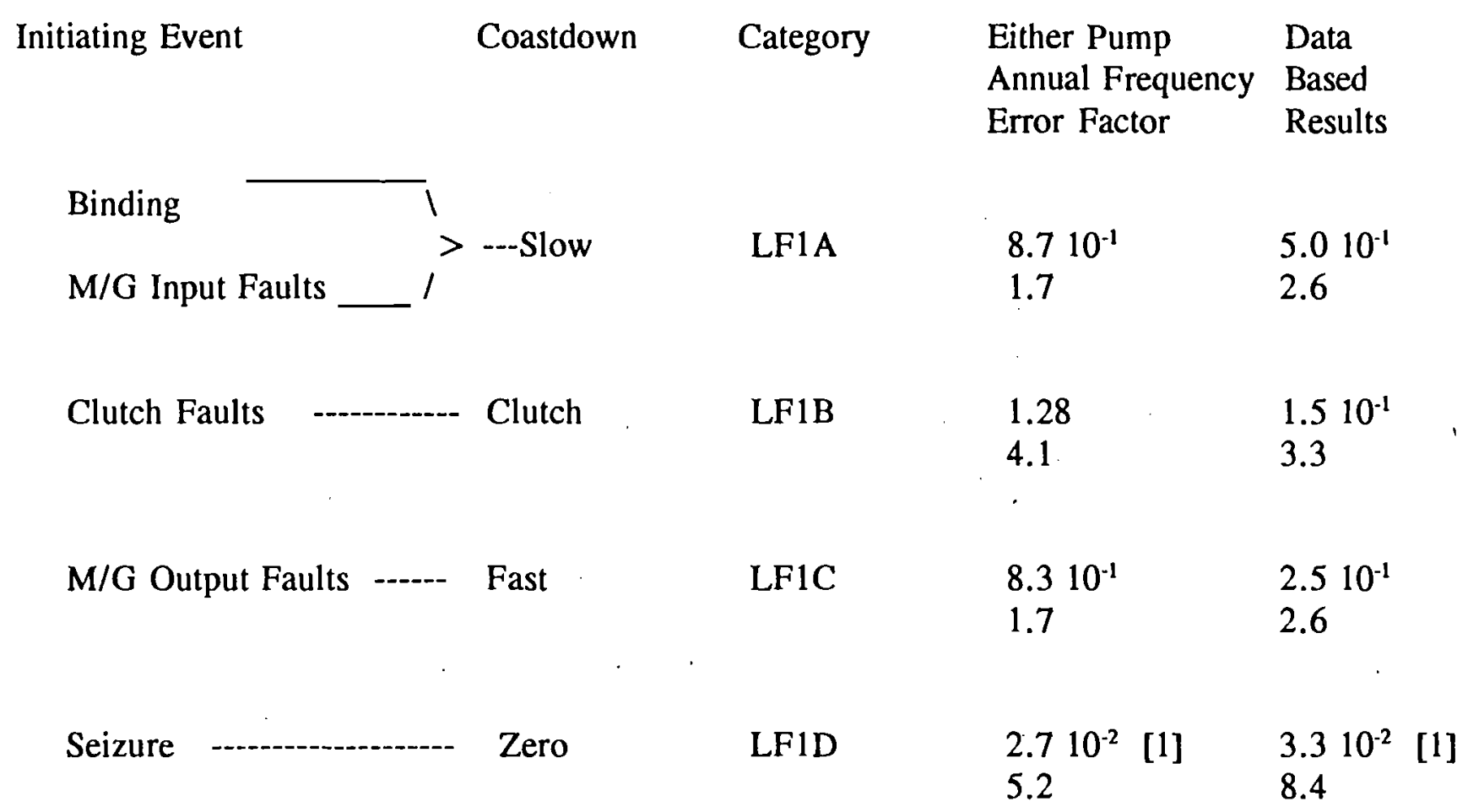

Note: [1] The data based results assume that the primary pump runs the entire year since the only time the pumps are usually off is during fuel handling. The fault tree data is based only on critical operation. Therefore, the value is smaller by a factor of $300 / 365 \approx 0.8$ 
Of the three primary pump binding incidents, only one required automatic protective action. The other binding at power was sufficiently slow that an anticipatory shutdown to 50 $\mathrm{kW}$ was achieved, followed by manual scram. The remaining binding occurred during startup. The data was grouped into three five year intervals to allow trending. There was one occurence in the 1975-1979 period, none between 1980 and 1984, and two in the 1985-1989 period. The data based results for the annual frequency using the last five, ten or fifteen years are 0.5, 0.25, and $0.23 \mathrm{yr}^{-1}$ respectively. Therefore the two incidents that occurred in the last five year period were used for estimating the frequency.

The minor differences between primary pump 1 and 2 as modeled in the fault trees are due to differences in the calculated reliability of the electrical busses supplying power to the respective $M / G$ sets. When the fault tree derived frequencies are compared to plant data the fault tree results are greater by factors of $14,8.5$, and 3.5 for faults of the $M / G$ input, clutch, and $\mathrm{M} / \mathrm{G}$ output respectively. This discrepancy is due to using generic data for the components involved. There is no specific plant data available at the component level. The five failure types of single pump faults discussed above are combined into four initiating events as shown in Table 5.6.

The annual frequency of initiating event LF1A, slow coastdown, is calculated to be 8.7 $10^{-1}$ which compares favorably with the data based result of $5.010^{-1}$. Initiating event LF1B, clutch coastdown, is calculated to be 1.28 which is an order of magnitude higher than the data based number of $1.510^{-1}$. For initiating event LF1C in which the fault tree analysis predicts an annual initiating event frequency of $8.310^{-1}$ compared to $2.510^{-1}$ for the data. Therefore, the initiating event frequencies for all single pump LOF's are directly based on the EBR-II plant data. Therefore, the initiating event frequencies, for all single pump LOF's are directly based on the EBR-II plant data. It should be recognized that the conservatisms introduced into the fault tree analysis by the use of generic data are carried into the calculations for double pump LOF's frequencies which are performed by combining fault trees. This is not a concern because the initiating event frequencies for various double pump LOFs are dominated by hypothesized common cause failures. 


\subsubsection{Flow Transients (Double Pump)}

The double pump LOF can be considered as a combination of single pump LOFs. The first pump failure is calculated based on a 100 day reactor run time to correspond with maintenance and testing of the system. The failure of the second pump is based on a mission time of 0.1 hour ( 6 minutes). This 6 minute interval is sufficient to separate the failure of the two pumps such that the second failure occurs as a separate event with respect to shutdown and flow transient. Although the second pump can fail any time within the 6 minute interval, it is assumed to fail simultaneously (i.e., before the scram system or feedbacks can act) in the damage calculations. This method reduces the total number of accident sequence calculations required. The annual frequency for loss of both primary pumps is calculated based on the results for a 100 day reactor run multiplied by 3.0 .

\subsubsection{Double Pump LOF Fault Tree}

Using the five categories of failure for a single pump LOF, a matrix of potential double pump LOFs can be constructed according to Table 5.7. Primary pump 1 is assumed to be the first pump to fail and primary pump 2 is assumed to fail within six minutes.

The lack of symmetry of the data in Table 5.7 for events involving the clutch is primarily due to the effect of the primary pump STOP switches for the second pump which are located at the control panel and the primary panel.

In order to reduce the number of accident sequence calculations required and eliminate manual cut set editing, the same approach as in the single pump LOF was taken to group the double pump LOFs according to their respective coastdown characteristics. The events on the diagonal of the matrix were retained for comparison with the corresponding single pump initiating events and to quantify the common cause aspects of the double pump LOFs. The remaining, mixed mode, double LOFs were grouped based on the commonality of the potential end states of the accident sequences (Table 5.8). All of the mixed mode LOFs which involve 

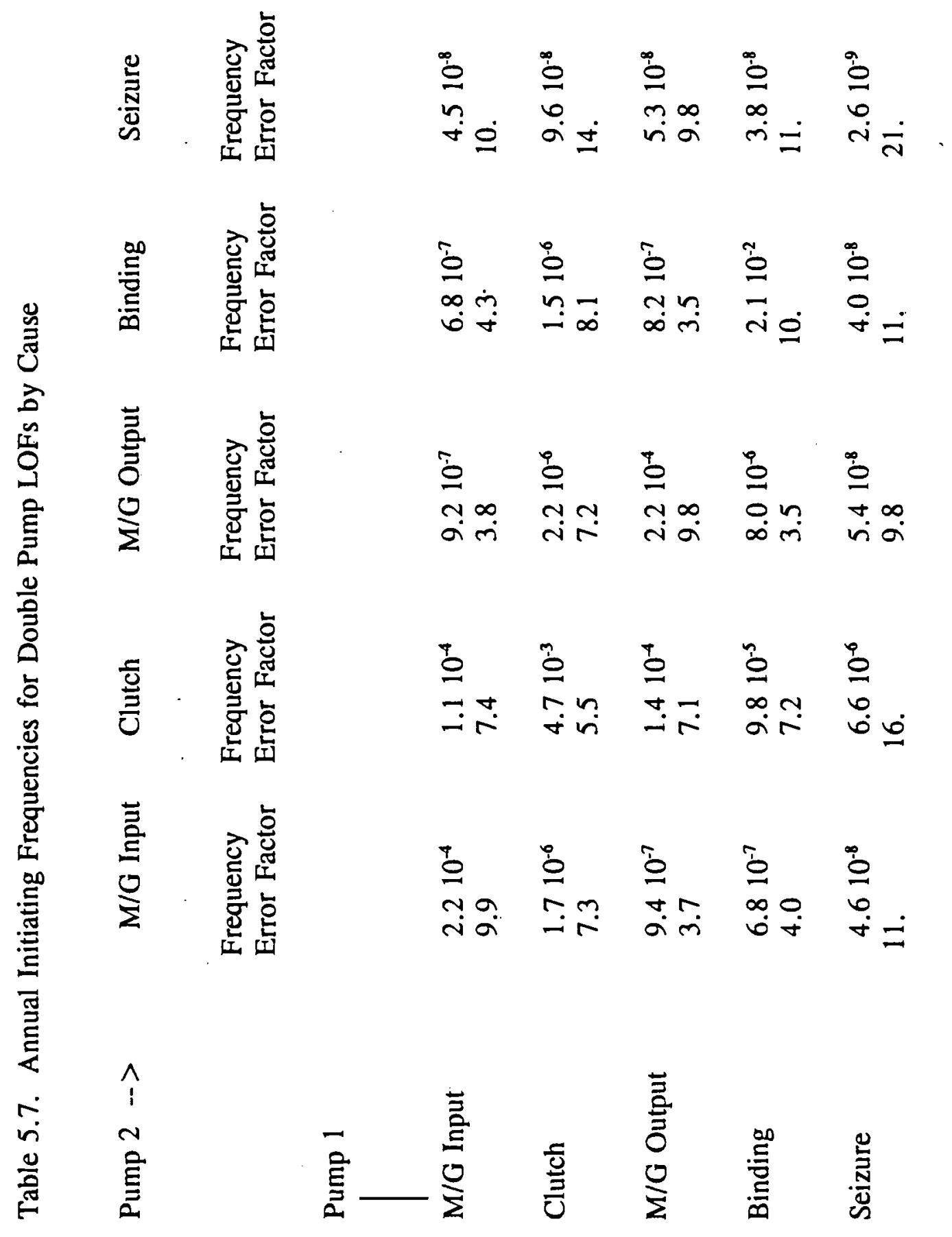

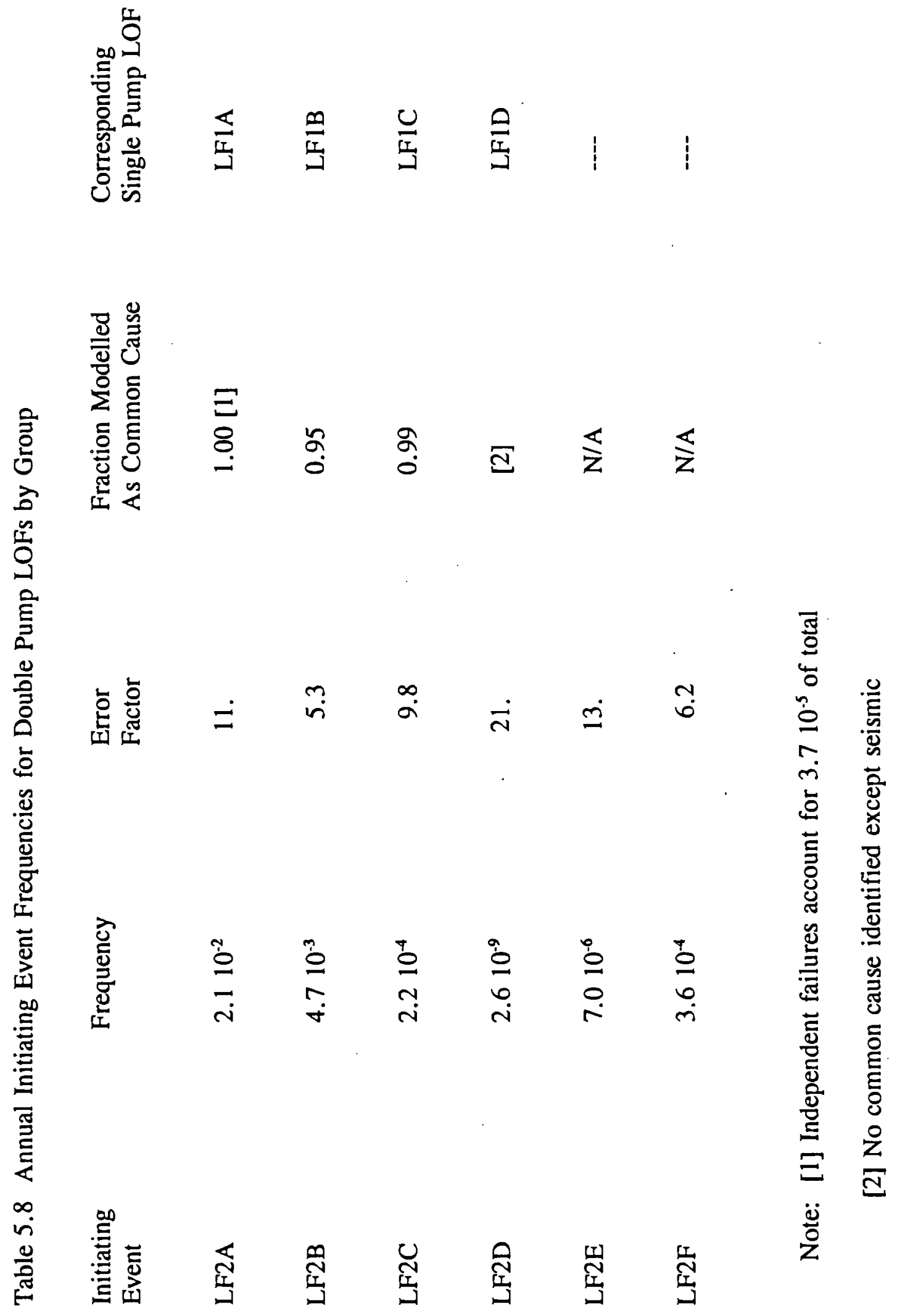
the seizure of one pump (zero time coastdown) and any non-seizure fault of the other pump lead to the same end states both in the protected and unprotected case. Therefore, these are all grouped together as LF2E meaning that a shaft seizure plus another pump fault is involved. The remaining mixed mode faults lead to the same states in the protected case and closely related cases in the unprotected case. Therefore, these are all grouped as LF2F, meaning any double pump faults not included in another group.

The common cause failures of both pumps identified, apart from dependence on electrical supply, were due to the STOP switches, binding, M/G input breakers, and M/G output breakers. Electrical supply common cause is considered separately as Loss of Normal Power (LONP) and Loss of Constant Power (LOCP).

The fault trees used for this analysis can be found in Appendix B.

\subsubsection{Pipe Breaks}

\section{Inlet Piping}

The inlet plena in EBR-II are fed by pipes from the two pumps. The accident of concern here is a leak or break in one or more of the inlet pipes. One obvious candidate common-cause mechanism for causing a pipe break is an earthquake. This is being treated separately by assigning a fragility to the piping and integrating into the overall seismic assessment. No other candidate common-cause mechanism can be identified.

The piping in the EBR-II primary tank does not have a large differential pressure across the walls. In a recent analysis of leaks in the primary piping for FFTF a leak frequency of $10^{-6} \mathrm{yr}^{-1}$ was proposed [5.19]. This estimate is based upon LWR estimates for long lengths of high pressure piping in the range of $10^{-4}$ to $10^{-5} \mathrm{yr}^{-1}$. The reasons for adopting a smaller frequency were 10 years of operation with no leaks, no source of stress corrosion cracking and the applicability of modern probabilistic fracture mechanics $\left(10^{-7}\right.$ to $10^{-8} \mathrm{yr}^{-1}$ calculated for LWRs). It was also noted that FFTF has $1500 \mathrm{ft}$ of primary piping compared to $3000 \mathrm{ft}$ in a 
typical PWR system. EBR-II has all the same advantages that FFTF possesses as well as a piping length an order of magnitude lower. Therefore for EBR-II a leak frequency of $10^{-7} \mathrm{yr}^{-1}$ has been adopted.

Studies on the aging effect of irradiation and thermal cycling have been carried out for the inlet piping [5.20]. The effects are predicted to be negligible.

A small leak in the inlet piping of EBR-II has no effect on the operation of the reactor. EBR-II has leaks designed into the primary circuit and a small extra flow path is irrelevant. The only concern is for a large rupture of the inlet piping. Recently, probabilistic fracture mechanics analyses have been performed for a typical LMR piping system [5.21]. The results presented indicate a cumulative leak probability (over the 40 year life of the system) of approximately $7.810^{-7}$ for small leaks (less than $3 \mathrm{gpm}$ ) and $1.610^{-8}$ for large leaks ( $\geq 3 \mathrm{gpm}$ ). The corresponding mean failure rates are $2.010^{-8}$ and $4.010^{-10}$ per year. These same analyses predicted a cumulative double ended guillotine break probability of approximately $10^{-12}$ to $10^{-14} \mathrm{yr}^{-1}$. The ratio of $10^{-2}$ between small and large leaks, and large leaks and guillotine ruptures is typical of those predicted in PWRs. This suggests using $10^{-9} \mathrm{yr}^{-1}$ for large leaks and $10^{-11} \mathrm{yr}^{-1}$ for double-ended guillotine break.

In summary; for EBR-II the frequencies of leaks has been estimated to be:

$\begin{array}{lll}\text { Small leak } & 10^{-7} \mathrm{yr}^{-1} & \\ \text { Large leak or rupture } & 10^{-9} \mathrm{yr}^{-1} & \\ \text { Guillotine Rupture } & 10^{-11} \mathrm{yr}^{-1} & \text { (LOPI) }\end{array}$

As a result small and large leaks are subsumed into the pump faults and the guillotine rupture is the frequency adopted for the LOPI initiating event. The LOPI initiating event frequency is below that used for screening events $\left(10^{-19}\right)$ and is thus not a credible initiator. 


\subsubsection{Loss of Vital Electrical Power}

\subsubsection{Loss of Normal Power (LONP)}

Loss of offsite power at EBR-II is a relatively frequent event, occurring typically once per year with a total unavailability of between $1 / 2$ and 1 day per year. As discussed earlier, if offsite power is lost the EBR-II reactor picks up the site load (hotel load) and no initiating event occurs. The Loss of Normal Power arises if the transfer is not made correctly, due to electrical transients when reconnecting to the INEL loop or transients on the loop which take down the generator as well. There has been a significant improvement in EBRII performance with respect to these events such that analysis of the UORs indicates seven events in 1975-1979, four in 1980-1984 but none in 1985-1989. Therefore the initiating event frequency chosen was based upon no events in five years, $0.1 \mathrm{yr}^{1}$.

\subsubsection{Loss of Constant Power (LOCP)}

The motor-generator set clutches have been powered by the constant power system since 1986 and no events attributable to problems in this system have occurred. On the other hand incidents connected with the constant power system have occurred, typically due to maintenance error. Analysis of the UORs identified a total of seven incidents in the last fifteen years, however it should be realized that this total included all UORs which are constant power related. Many occurred after a lightning strike in 1977. Fault tree analysis of the constant power panels gave a frequency of loss of each panel at $510^{-2} \mathrm{yr}^{-1}$ and operation with the two systems cross-tied is not limited in any way. In view of all of the above an estimate of the frequency of loss of all constant power was based upon no events in the last five years leading to $0.1 \mathrm{yr}^{-1}$.

\subsubsection{Reactivity Insertion}

Since the slow reactivity insertion initiating events have been classed by reactivity insertion potential rather than mechanical cause, two factors must be combined to give the 
frequency of each initiating event. The factors are the frequency of each mechanical fault and the probability that a given fault will lead to reactivity insertion of the four classes of insertion (RISA, 0-20c, RISB, 20-65c, RISC, 65c-1.00\$ and RISD, greater than $1 \$$ ).

Fault tree analysis, as documented in Appendix B, led to the following frequencies:

$$
\begin{aligned}
& \text { RISA }=3.010^{-2} \mathrm{yr}^{-1} \\
& \text { RISB }=7.710^{-3} \mathrm{yr}^{-1} \\
& \text { RISC }=1.710^{-6} \mathrm{yr}^{-1} \\
& \text { RISD }=4.410^{-7} \mathrm{yr}^{-1}
\end{aligned}
$$

The contribution to RISA is dominated by the frequency of the single rod insertion, RISC and RISD are dominated by two control rod and many control rod insertion events, respectively, while RISB has significant contributions from all events.

The only mechanism identified for a large, fast reactivity insertion (RIFL) arises if the core can drop away from the control rods. A rupture of the primary vessel would not cause such an accident because a failure would begin in one location on one side of the vessel, and as it progressed, if it did, the vessel would cock, jamming the control rods in their positions relative to the core. While some reactivity might be added, the amount would be limited.

However, if the vessel was to catastrophically fail, with an almost instantaneous circumferential failure, it would be possible for the primary vessel and core to fall away from the control rods, adding reactivity. The rupture frequency for the primary vessel was estimated to be $1.010^{-8} \mathrm{yr}^{-1}$. A instantaneous circumferential failure is not what is observed in higher pressure tanks with more corrosive materials, therefore it is not expected for the primary vessel. It is believed that a stainless steel vessel at low pressure cannot fail in this unique manner but it is difficult to prove that this is absolutely so and therefore an additional factor of $10^{-2}$ is applied because of the specialized nature of the failure. This yields a frequency of $1.010^{-10} \mathrm{yr}^{-1}$ for a rapid reactivity insertion from the catastrophic, circumferential failure of the primary vessel. 
One mechanism for rupture of the primary and secondary vessel is overpressurization of either the primary tank or annulus by a malfunction of the argon system. Although the addition of reactivity is uncertain, the accident sequence would lead to direct core damage due to uncovering the core. Therefore, the argon system overpressure frequency of $2.910^{-8}$ is added to RIFL for a total frequency of $2.910^{-8}$.

To estimate the frequency of small, fast reactivity insertion events of $-0.35 \$$ (RIFS) is more difficult because the causative mechanism is a gross material motion in an experimental subassembly. This has never happened and there is no basis for an estimate. EBR-II controls the design, fabrication and safety analyses for experiment in such a way that this event has to be considered "unlikely", [5.22], and unlikely is usually interpreted to be in the range $10^{-4}$ to $10^{-2}$. Consequently a screening number of $10^{-3} \mathrm{yr}^{-1}$ will be used. This is consistent with no events of this nature having occurred in approximately 500 experimental subassemblies.

\subsubsection{Loss of Primary Heat Sink}

The initiating event frequencies are derived from plant historical data. All events, however, minor, have been included in this evaluation. A more critical review of the events could reduce these numbers. The review of the UORs leads to initiating event frequencies of $6.310^{-1} \mathrm{yr}^{-1}$ for TSEC and $3.710^{-1} \mathrm{yr}^{-1}$ for TBOP.

\subsubsection{Overcooling}

The initiating event frequencies are derived for these events were derived directly from plant historical data. Review of the UORs for the last 15 years identified a number of events which correspond to these initiators or precursors to these events, 1, 3 (in the last 10 years) and 0 respectively for OCSL, OCPP and OCSP. The initiating event frequencies are derived from these using the standard formulation.

$$
\begin{aligned}
& \text { OCSL }=1.010^{-1} \mathrm{yr}^{-1} \\
& \text { OCPP }=3.010^{-1} \mathrm{yr}^{-1} \\
& \text { OCSP }=3.310^{-2} \mathrm{yr}^{-1}
\end{aligned}
$$




\subsubsection{Steam Generator Tube Rupture (SGTR)}

Estimation of an initiating frequency for the failure of a steam-tube in an EBR-II superheater is a difficult matter because these superheaters are a unique and conservative design with double-walled tubes, tube sheets, etc., so that it takes breach of two welds to bring sodium and water into contact. There have been no instances of sodium water reaction in the operating history of EBR-II although events have occurred at other reactors that have less conservative steam generator designs, particularly BN-600 in the USSR. In 1987 there was a steam generator failure at the Prototype Fast Reactor (PFR) in Dounreay, Scotland. The design and nature of the failure are not relevant to EBR II steam generators, however it does serve to demonstrate the possibility of rapid propagation given the right circumstances. Estimates available in the literature are given below for single wall tubed heat exchangers:

Eide et al [5.23] and CRBR [5.3]

\begin{tabular}{lllr} 
Small Leaks & $510^{-6} / \mathrm{hr}$-unit & $710^{-6}$ & $\sim 510^{-2} / \mathrm{yr}$-unit \\
Medium Leaks & $110^{-6}$ & $1.410^{-6}$ & $\sim 110^{-2} / \mathrm{yr}$-unit \\
Large Leaks & $310^{-7}$ & $2.810^{-7}$ & $\sim 210^{-3} / \mathrm{yr}$-unit \\
\multicolumn{4}{c}{+ Values for water/steam heat exchangers }
\end{tabular}

These values are much too conservative for EBR-II's duplex tube design

A detailed analysis of the thermal degradation of the superheaters suggests a frequency of $\leq 10^{-4} \mathrm{yr}^{-1}$ for all failures, Section 7. Since this latter argument is based upon physical arguments it is believed this estimate is more appropriate than those based upon water/steam heat exchangers which do not have the same double-tube design. Even then the dominant failure mode would not lead to immediate sodium-water reaction, nevertheless $10^{-4} \mathrm{yr}^{-1}$ with an error factor of 10 is adopted for the screening value for initiating events occuring in the evaporator, EVTR, or superheater, SHTR.

\subsubsection{Rapid Loss of Circulation}

For a rapid loss of primary sodium (LCRA) to occur, the sodium level must drop 
below the primary pump inlet, the pump skirt. Analysis [5.16] shows that this can only occur if both the primary and guard vessels rupture and sustain a rapid loss of primary sodium. In EBR-II, both these vessels were constructed as primary containment systems for the bulk sodium. The guard vessel was designed to hold the entire bulk sodium weight in case the primary vessel leaked.

While the FFTF plant and CRBR design contain significant difference from EBR-II in many aspects, the designs of the primary vessels contain significant similarities in size, design, and materials. All primary vessels are cylindrical containments. FFTF is about 20 feet in diameter and 43 feet high, CRBR was designed to be 20 feet in diameter and 59 feet high, and EBR-II is 26 feet in diameter and 26 feet high. FFTF and EBR-II are of all-welded construction, the same as was planned for CRBR. The main difference between EBR-II and other plants is that the guard vessel is designed as an additional primary vessel rather than just as a containment basin for leaked sodium coolant.

Because of these close similarities in primary vessel design and construction, it is appropriate to use the primary vessel rupture frequencies determined for FFTF and CRBR as the starting point for EBR-II. In the CRBR and FFTF analyses, primary tank rupture initiating event frequencies were determined to be $2.010^{-8} \mathrm{yr}^{-1}$ and $1.010^{-8} \mathrm{yr}^{-1,}$ respectively. These values include consideration of the experimental and operational observations of many systems and theoretical fracture mechanics calculations that all point to a leak-before-rupture behavior in current systems and materials, primarily based on water reactor analyses. Liquid metal cooled reactor systems contain other features which make this leak-before-rupture behavior even more likely, including high quality standards, low pressures, an inert atmosphere, isothermal conditions, sodium coolant, etc. The leak-before-rupture argument for CRBR was accepted by the USNRC.

Because EBR-II has been operating a significant period of time, about 25 years, the time frame for initial material and weld defects, which contribute to the majority of large tank failures, has passed. Therefore the tank rupture initiating event frequency from FFTF of 1.0 $10^{-8} \mathrm{yr}^{-1}$ will be used as the basis for EBR-II, since it also has passed the stage of initial material 
defect initiated failure. While FFTF has the capability to do in-service inspection and EBR-II does not, the annulus between the primary and guard vessels does include spark type sodium leak detectors and thermocouples that perform this same function, assuring no failure of the primary vessel has occurred. Therefore the value used for the EBR-II primary vessel rupture frequency is $1.010^{-8} \mathrm{yr}^{-1}$.

EBR-II has been operating for a significant period of time, so questions of aging effects and irradiation damage are of natural concern. Analyses have been performed and show that the thermal cycling fatigue limits of the primary vessel are orders of magnitude higher than has and would be experienced even under an aggressive transient operational program. Under the normal operation of EBR-II, there is very little thermal cycling because to the isothermal condition of the bulk primary sodium. Calculations of the fast fluence at the peak flux locations and examination of surveillance components also show that the flux is low enough to preclude concerns of metal embrittlement in the EBR-II primary vessel.

The FFTF analysis determined that the leak of a guard vessel in the presence of sodium is $1.010^{-4} \mathrm{yr}^{-1}$. However, for a rapid loss of primary sodium, the guard breach must be a rupture rather than a leak. The typical ratio of rupture to leak frequencies for higher pressure and more corrosive environments is $10^{-2}$ to $10^{-4}$. Taking the conservative ratio of $10^{-2}$ yields a rupture frequency for the guard vessel of $1.010^{-6} \mathrm{yr}^{-1}$. Since the guard vessel can not be visually inspected, it must be considered whether the guard vessel has already sustained cracking that would allow sodium to leak into the air baffle structure should the primary vessel rupture. While the guard vessel shows no indication of having sustained any leaking, there is no way to assure its integrity since the annulus gas system is not tested for influx of air or excessive argon makeup, so the failure frequency is increased another order of magnitude to $1.010^{-5} \mathrm{yr}^{-1}$.

Based on the necessity of the rupture of both the primary and guard vessels, the rapid Loss of Circulation frequency has been determined to be $1.010^{-13} \mathrm{yr}^{-1}$. For this initiator to contribute to core damage, its value would have to be increased by about six orders of magnitude, giving a significant margin of conservatism. 


\subsubsection{Delayed Loss of Circulation (LCDL)}

A delayed LOSA (LCDL) is caused when the sodium level in the primary tank drops below the Z-pipe entrance to the IHX, 24 inch $(0.6 \mathrm{~m})$ drop in bulk sodium level. This introduces the possibility of losing the natural circulation capability through the IHX if the pumps were to be turned off.

There are two potential initiators, the Fuel Element Rupture Detector (FERD) and the Shutdown Cooler System, that do not contribute to the initiator frequencies because it is not physically possible to move the quantity of bulk sodium needed out of the primary vessel. The primary sodium is drawn into the FERD loop by evacuating the loop and once the loop is filled, the vacuum is manually valved off. If a breach occurs in the FERD loop after the EM pump, the only way to continue to pump out primary sodium, the pump force is the only mechanism for removing the sodium. The 5 psi head produced by the pump is not enough to raise the amount of sodium necessary to keep the loop filled. The shutdown coolers work in natural circulation without any additional force. Even with the maximum temperature difference in the system, there is not enough force to raise sodium in the shutdown coolers to the level of the piping.

A rupture of the primary vessel alone could reduce the surface of the bulk sodium to a level where a LCDL could occur. Based on the discussion given above for the rapid loss of primary sodium, Section 5.2.8, the initiating event frequency is $1.010^{-8} \mathrm{yr}^{-1}$.

The Breached Fuel Test Facility (BFTF) could sustain a breach of the dry-well and then a breach of terminal box assembly, allowing sodium removal. There are numerous sensors in the system including flowmeters, Na leak detectors, and thermocouples which would give indications of primary sodium leaks. Since the flow through the subassembly under the BFTF is only ten to twenty gallons per minute, the source for leakage out of the primary system given these multiple breaches would be even less, requiring extensive time before enough sodium could be removed for a LCDL to occur. The frequency was determined to be $5.210^{-10} \mathrm{yr}^{-1}[5.16]$.

A leak in the piping of the primary sodium purification and sampling system could 
provide a path for the syphoning of primary sodium. There is a syphon break system on the surge tank that draws a vacuum on the primary sodium, drawing it into the surge tank. This break system must fail for any sodium syphoning to occur. If the leak in the piping occurs outside the purification or sodium sampling cell, it would be at an elevation above the nominal sodium level, and would therefore break the syphon even if the syphon break system failed. There is also a pump in the system that increases sodium flow in the piping. There are numerous detectors in the cells, where the elevation is below the critical bulk sodium elevation in the primary vessel. Detection and shutdown of the system, which does not operate continuously, could be performed with the pump and various system valves. The initiator frequency from a leak in the primary sodium purification system was calculated to be $4.410^{-10}$ $\mathrm{yr}^{-1}$.

The final initiator identified, a failure of IHX tubes and other component failures, was calculated to occur with a frequency of $1.210^{-12} \mathrm{yr}^{-1}$. For this to contribute to the LCDL, the IHX tubes must fail, the secondary sodium system must be dumped and the dump valves must remain open, the argon vent system, with mechanically blocked open values, must plug during the dump process, and the secondary sodium storage tank must rupture (or a vent valve must open and remain open) to spill sodium out on the basement floor.

The total of all LCDL initiators is therefore $1.110^{-8} \mathrm{yr}^{-1}$.

\subsubsection{Support System Failures}

The loss of shutdown cooler is based upon a fault tree analysis given in the system notebook. The failure mode here is break, leak or plugging of the NaK tube. PFR has had failures in its Air Dump Heat Exchangers which are of similar design but which are operated much differently. The frequency estimate is based upon estimates for liquid metal carrying pipes given in [5.23].

$$
\mathrm{TSDC}=5.810^{-3} \mathrm{yr}^{-1}
$$


The extended loss of shield cooling is based upon plant data.

$$
\mathrm{TSHC}=3.310^{-2} \mathrm{yr}^{-1}
$$

The loss of instrument air is based upon plant data.

TAIR $=1.810^{-1} \mathrm{yr}^{-1}$

\subsubsection{Shutdowns and Scrams}

There is one long shutdown each year where the sodium is drained after reactor shutdown, therefore the frequency of long shutdowns is one per year.

$$
\text { SHDL }=1.0 \mathrm{yr}^{-1}
$$

The number of other shutdowns is dominated by the planned end-of-run shutdowns and shutdowns after plant transient testing and operator training periods. Therefore an overall estimate is provided:

$$
\text { SHDS }=6.0 \mathrm{yr}^{-1}
$$

The frequency of scrams and anticipatory shutdowns has much improved in recent years and therefore the last five years data is used.

$$
\begin{aligned}
& \text { SCRM }=7.010^{-1} \mathrm{yr}^{-1} \\
& \text { ANTC }=5.010^{-1} \mathrm{yr}^{-1}
\end{aligned}
$$

\subsubsection{Local Faults}

Local faults can be divided into two categories, rapid and slow. Rapidly developing local faults require a mechanism which involves total subasssembly participation, such as a total blockage (FLTA) or simultaneous break of multiple pins (FLTB). Because of 
the subassembly design for EBR-II, with its multiple coolant entrance locations, a complete subassembly inlet blockage is not a credible event. Every subassembly loaded into EBR-II is flow tested. There is also no source of debris within the reactor system to plug either the inlet or outlet. These are discussed in detail in [5.17]. The simultaneous break of multiple pins in EBR-II is also not considered a credible event since no initiating mechanism has been identified aside from loading or orificing errors, which are considered separately. In [5.17] a discussion is given of the thermal effects of gas release from multiple pins breaking, and they are minimal.

Slow local fault generation may occur from several sources: (1) metal driver element breaches, (2) metal experiment element breaches, (3) non-metal experiment element breaches, (4) partially blocked subassemblies, and (5) subassembly loading errors. The metal driver pins are designed to operate up to a designated burnup goal, defined from experimentally determined data. The operating maximum burnup is then defined based on a statistical basis to give a $95 \%$ probability of less than one driver element breach in a core loading, which last about two years. This produces an initiating event frequency for MDLF of $1.3310^{-1} \mathrm{yr}^{-1}$, with and error factor of 10 .

In considering experimental pins, there have been a number of breaches on which to base an annual frequency. With about 90 metal experiment element breaches since about 1970, a yearly average of 4.5 (MELF) was determined with and error factor of 3 . The number of nonmetal experimental element breaches has varied significantly over the EBR-II operating history, depending on programmatic goals. A value of 4.5 per year (OELF) appears reasonable with an error factor of 3 , the same as the metal experiments. In the case of experimental pins and subassemblies, a separate safety package must be produced and reviewed by appropriate groups within EBR-II to assure the safety of the reactor and personnel. They may also be operated under special procedures.

Every subassembly which is loaded into EBR-II is argon flow tested which will detect a complete blockage; however, the discrimination may not detect a partial blockage such as might be caused by an orificing error. This has happened on three different occasions, providing an initiating frequency of $1.410^{-1} \mathrm{yr}^{-1}$ for the SFPB initiating event. During the 25 year 
operation of EBR-II, one loading error took place during routine handling, was detected before reactor operation, and not removed because the conditions were close to what was requested. This is the basis for the initiating event frequency of $6.010^{-2}$ for SFLE.

The initiating event frequencies are given below for the local fault considerations.

$\begin{array}{ll}\text { FLTA } & - \\ \text { FLTB } & - \\ \text { MDLF } & 1.33 \mathrm{10}^{-1} \mathrm{yr}^{-1} \\ \text { MELF } & 4.5 \mathrm{yr}^{-1} \\ \text { OELF } & 4.5 \mathrm{yr}^{-1} \\ \text { SFPB } & 1.410^{-1} \mathrm{yr}^{-1} \\ \text { SFLE } & 6.010^{-2} \mathrm{yr}^{-1}\end{array}$

\subsubsection{Final Initiating Event Frequencies}

The results of the analyses presented in the preceding sections are summarized in Table 5.9. Thirty-three initiating events have been identified with a frequency of $10^{-10}$ or greater. Those below $10^{-10}$ will not be considered further because their risk contribution is below the cutoff frequency applied in this study for any accident sequences. The total frequency of scrams and shutdowns is $11.11 \mathrm{yr}^{-1}$, Table $5.9,7.5 \mathrm{yr}^{-1}$ of which are anticipatory or normal shutdowns. The remaining $3.61 \mathrm{yr}^{-1}$ are dominated by balance of plant faults, in excess of one per year, primary pump faults, about one per year, spurious scrams, $0.7 \mathrm{yr}^{-1}$, and electrical supply faults, $0.2 \mathrm{yr}^{-1}$.

Although the initiating event frequencies for local faults have been derived in the above description they are not included in Table 5.9 which represents a shutdown history. Local faults represent spatially-dependent initiating events and are therefore treated separately. However, they will be grouped with other internal events as a special class of plant transients. Table 5.10 lists each initiating event, the frequency and error factors together with the basis for the determination, including local faults. 


\begin{tabular}{|c|c|c|c|c|c|c|c|c|c|}
\hline 1.010 .1 & -- & -- & I.0 & $68-S L 6 I$ & I & 0 & 0 & I & 7SDO \\
\hline 1.0I $L^{\prime} \varepsilon$ & -- & -- & $L \varepsilon^{\circ} 0$ & 68-SL6I & $S$ & I & $\varepsilon$ & I & dOQL \\
\hline ..0I $\varepsilon^{\prime} 9$ & -- & -- & E9.0 & $.68-S \angle 6 \mathrm{I}$ & 6 & $\mathcal{S}$ & $\mathcal{E}$. & I & วヨSI \\
\hline 8.016 .2 & 8.016 .2 & -- & -- & -- & 0 & 0 & 0 & 0 & 7Ald \\
\hline$\varepsilon .010 .1$ & $\varepsilon-0 I 0.1$ & -- & - & -- & 0 & 0 & 0 & 0 & SAly \\
\hline$t .01 t . t$ & -- & $\iota .01 t^{\circ} t$ & -- & -- & 0 & 0 & 0 & 0 & asid \\
\hline $.01 L^{\prime} I$ & $-\cdot$ &, $01 L^{\prime} \mathrm{I}$ & -- & -- & 0 & 0 & 0 & 0 & DSIX \\
\hline$\varepsilon .01 L^{\circ} L$ & -- & $\varepsilon .01 L^{\prime} L$ & -- & -- & 0 & 0 & 0 & 0 & gSIY \\
\hline$\tau^{2} .010^{\circ} \mathcal{E}$ & -- & $2.010 . \mathcal{E}$ & -- & -- & 0 & 0 & 0 & 0 & VSIY \\
\hline 1.010 .1 & - & -- & I.0 & q68-S86I & $L$ & 0 & $\tau$ & $s$ & dDOT \\
\hline I.0I & -- & $\cdots$ & $I^{\circ} 0$ & ${ }_{9} 68-586 \mathrm{I}$ & II & 0 & $t$ & $\iota$ & dNOT \\
\hline I I.0I 0.1 & ${ }^{11}-0 \mathrm{I}$ & -- & -- & - & 0 & 0 & 0 & 0 & IdOT \\
\hline $.019^{\circ} \varepsilon$ & -- & r.0I $9 . \varepsilon$ & -- & -- & 0 & 0 & 0 & 0 & $A 2 d 7$ \\
\hline $.010 . \mathrm{L}$ & -- & $.010 . \mathrm{L}$ & -- & -- & 0 & 0 & 0 & 0 & $32 \mathrm{~d} 7$ \\
\hline 6.019 .2 & - & 6.019 .2 & -- & - & 0 & 0 & 0 & 0 & aZsT \\
\hline $.012 z$ & -- & $.012 z$ & -- & - & 0 & 0 & 0 & 0 & วZA7 \\
\hline$\varepsilon .01 L^{\circ} b$ & -- & $\varepsilon .01 L^{\circ} b$ & -- & -- &.$\tau$ & 0 & I & I & gzd7 \\
\hline$z-011 \cdot z$ & -- & $z .011 \%$ & -- & - & 0 & 0 & 0 & 0 & $\forall Z \mathrm{AT}$ \\
\hline$z-0 I L \cdot Z$ & -- & $\tau .01 \iota^{\prime} z$ & - & - & 0 & 0 & 0 & 0 & aIs] \\
\hline I.0I $s^{\prime} Z$ & -- & †.0I $\varepsilon^{\cdot} 8$ & $\$ 2 \cdot 0$ & $68-086 \mathrm{I}$ & $\tau$ & I & I & 0 & DId] \\
\hline I-OI S'I & -- & $82 \cdot 1$ & SI'0 & $68-086 \mathrm{I}$ & $s$ & I & 0 & $t$ & 8IAT \\
\hline $1.010 . \mathrm{S}$ & -- & 1.010 .6 & $S^{\circ} 0$ & $68-S 861$ & $\varepsilon$ & $\tau$ & 0 & I & VIAา \\
\hline \multirow[t]{2}{*}{ ןEu! } & \multirow[t]{2}{*}{$\begin{array}{l}\text { spочю्W } \\
\text { Јәч10 }\end{array}$} & \multirow{2}{*}{ 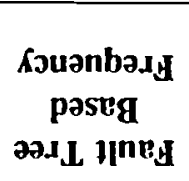 } & \multirow{2}{*}{ 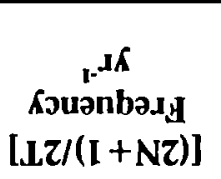 } & \multirow[t]{2}{*}{$\begin{array}{l}\text { uəsoYว } \\
\text { po!dəd }\end{array}$} & \multirow[t]{2}{*}{ [B]OJ } & $\begin{array}{c}686 \mathrm{I} \\
-586 \mathrm{I}\end{array}$ & $\begin{array}{l}\$ 86 I \\
-086 I\end{array}$ & $\begin{array}{c}6 L 6 I \\
-S L 6 I\end{array}$ & \multirow{2}{*}{ 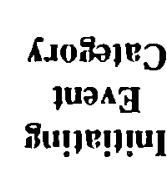 } \\
\hline & & & & & & \multicolumn{3}{|c|}{ po!.गəd } & \\
\hline
\end{tabular}

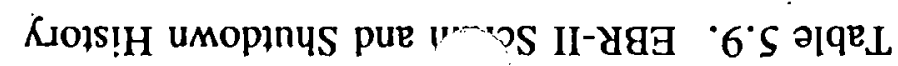


Table 5.9. EBR-II Scram and Shutdown History (Cont'd)

\begin{tabular}{|c|c|c|c|c|c|c|c|c|c|}
\hline \multirow{2}{*}{$\begin{array}{l}\text { Initiating } \\
\text { Event } \\
\text { Category }\end{array}$} & \multicolumn{3}{|c|}{ Period } & \multirow[b]{2}{*}{ Total } & \multirow{2}{*}{$\begin{array}{l}\text { Period } \\
\text { Chosen }\end{array}$} & \multirow{2}{*}{$\begin{array}{c}{[(2 \mathrm{~N}+1) / 2 \mathrm{~T}]} \\
\underset{\mathrm{yr}^{-1}}{\text { Frequency }}\end{array}$} & \multirow{2}{*}{$\begin{array}{l}\text { Fault Tree } \\
\text { Based } \\
\text { Frequency }\end{array}$} & \multirow{2}{*}{$\begin{array}{c}\text { Other } \\
\text { Methods }\end{array}$} & \multirow[b]{2}{*}{ Final } \\
\hline & $\begin{array}{l}1975- \\
1979\end{array}$ & $\begin{array}{l}1980- \\
1984\end{array}$ & $\begin{array}{l}1985- \\
1989\end{array}$ & & & & & & \\
\hline OCPP & 0 & 2 & 1 & 3 & $1985-89^{8}$ & 0.30 & -- & -- & $3.010^{-1}$ \\
\hline OCSP & 0 & 0 & 0 & 0 & $1975-89$ & 0.033 & -- &.- & $3.310^{-2}$ \\
\hline EVTR & 0 & 0 & 0 & 0 & -- & -- & -- & $1.010^{-4}$ & $1.010^{-4}$ \\
\hline SGTR & 0 & 0 & 0 & 0 & - & -- & -- & $1.010^{-4}$ & $1.010^{-4}$ \\
\hline LCRA & 0 & 0 & 0 & 0 & - & -- & -- & $1.110^{.13}$ & $1.010^{-13 i}$ \\
\hline $\mathrm{LCDL}$ & 0 & 0 & 0 & 0 & -- & -- & -- & $1.010^{-8}$ & $1.110^{-8}$ \\
\hline TSDC & 0 & 0 & 0 & 0 & - & -- & $5.810^{-3}$ & -- & $5.810^{-3}$ \\
\hline TSHC & 0 & 0 & 0 & 0 & $1975-89$ & 0.033 & -- & -- & $3.310^{-2}$ \\
\hline TAIR & 0 & 1 & 1 & 2 & $1980-89$ & 0.25 & -- & -- & $2.510^{-1}$ \\
\hline SHDL & 5 & 5 & 5 & 15 & - & -- & -- & $1.0^{d}$ & 1.0 \\
\hline $\begin{array}{c}\text { SHDS } \\
\text { (unplanned) }\end{array}$ & 4 & 3 & 0 & 7 & -- & -- & -- & $6.0^{\circ}$ & 6.0 \\
\hline SCRM & 29 & 9 & 3 & 41 & $1985-89$ & .7 & -- & -- & $7.010^{-1}$ \\
\hline ANTC & 3 & 13 & 2 & 18 & $1985-89$ & .5 & - & - & $5.010^{-1}$ \\
\hline TOTAL & & & & & & & & & 11.11 \\
\hline
\end{tabular}

a Cause removed by plant modification.

b Problems identified and cured by plant modification.

c Apparent increase in 1985-1989 caused by software error in secondary pump controller.

d One per year, each April.

- Includes planned and unplanned shutdowns - based upon 2 planned shutdowns at end of each 100 day run and 4 other shutdowns after plant transient tests at start of a run.

f Below screening frequency and not considered further.

s Control System modified in 1985. 
Table 5.10. Initiating Event Frequencies and Error Factors for Quantification

\begin{tabular}{|c|c|c|c|c|c|}
\hline Initiating & Frequency & Error & \multicolumn{3}{|c|}{ Basis for Quantification } \\
\hline LF1A & $5.010^{-1}$ & 2.6 & EBR-II Data & & \\
\hline LF1B & $1.510^{-1}$ & 3.3 & EBR-II Data & & \\
\hline LF1C & $2.510^{-1}$ & 2.6 & EBR-II Data & & \\
\hline LF1D & $2.710^{-2}$ & 5.3 & & Fault Tree & \\
\hline LF2A & $2.110^{-2}$ & 11. & & Fault Tree & \\
\hline LF2B & $4.710^{-3}$ & 5.3 & & Fault Tree & \\
\hline LF2C & $2.210^{-4}$ & 9.8 & & Fault Tree & \\
\hline LF2D & $2.610^{-9}$ & 21. & & Fault Tree & \\
\hline LF2E & $7.010^{-6}$ & 14. & & Fault Tree & \\
\hline LF2F & $3.610^{-4}$ & 6.2 & & Fault Tree & \\
\hline LONP & $1.010^{-1}$ & 8.4 & EBR-II Data & & \\
\hline LOCP & $1.010^{-1}$ & 8.4 & EBR-II Data & & \\
\hline RISA & $3.010^{-2}$ & 5.0 & & Fault Tree & \\
\hline RISB & $7.710^{-3}$ & 5.0 & & Fault Tree & \\
\hline RISC & $1.710^{-6}$ & 10. & & Fault Tree & \\
\hline RISD & $4.410^{-7}$ & 10. & & Fault Tree & \\
\hline RIFS & $1.010^{-3}$ & 10. & & & Other \\
\hline RIFL & $2.910^{-8}$ & 10. & & & Other \\
\hline TSEC & $6.310^{-1}$ & 2. & EBR-II Data & & \\
\hline TBOP & $3.710^{-1}$ & 2. & EBR-II Data & & \\
\hline OCSL & $1.010^{-1}$ & 3.3 & EBR-II Data & & \\
\hline OCPP & $3.010^{-1}$ & 8.4 & EBR-II Data & & \\
\hline OCSP & $3.310^{-2}$ & 8.4 & EBR-II Data & & \\
\hline EVTR & $1.010^{-4}$ & 10. & & & Other \\
\hline SGTR & $1.010^{-4}$ & 10. & & & Other \\
\hline LCDL & $1.110^{-8}$ & 10. & & & Other \\
\hline
\end{tabular}


Table 5.10. Initiating Event Frequencies and Error Factors for Quantification (cont'd)

\begin{tabular}{||l|c|c|l|l|l||}
\hline \hline $\begin{array}{l}\text { Initiating } \\
\text { Event } \\
\text { Category }\end{array}$ & $\begin{array}{c}\text { Frequency } \\
\mathrm{yr}^{-1}\end{array}$ & $\begin{array}{l}\text { Error } \\
\text { Factor }\end{array}$ & \multicolumn{3}{|c||}{ Basis for Quantification } \\
\hline TAIR & $2.510^{-1}$ & 2.6 & EBR-II Data & & \\
\hline TSDC & $5.810^{-3}$ & 4.6 & & Fault Tree & \\
\hline TSHC & $3.310^{-2}$ & 8.4 & EBR-II Data & & \\
\hline SHDL & 1.0 & 2.0 & EBR-II Data & & \\
\hline SHDS & 6.0 & 2.0 & EBR-II Data & & \\
\hline SCRM & $7.010^{-1}$ & 2.2 & EBR-II Data & & \\
\hline ANTC & $5.010^{-1}$ & 2.6 & EBR-II Data & & \\
\hline Subtotals & $11.11 \mathrm{yr}^{-1}$ & & 11.016 & $9.6810^{-2}$ & $1.2010^{-3}$ \\
\hline MDLF & $1.3310^{-1}$ & 10. & EBR-II Data & & \\
\hline MELF & 4.5 & 3.0 & EBR-II Data & & \\
\hline OELF & 4.5 & 3.0 & EBR-II Data & & \\
\hline SFPB & $1.410^{-1}$ & 2.2 & EBR-II Data & & \\
\hline SFLE & $6.010^{-2}$ & 3.3 & EBR-II Data & & \\
\hline Subtotal & $9.33 \mathrm{yr}^{-1}$ & & 9.33 & & \\
\hline \hline
\end{tabular}

\section{NOTES}

Minimum Error Factor set equal to 2.0.

Initiating Events $<10^{-10}$ omitted.

Fault trees appear in Appendix B. 


\subsection{Event Tree Development}

\subsubsection{Introduction}

This section presents the event tree development for the EBR-II PRA. Event trees provide a logical representation of the response of the plant to any abnormal event. The abnormal events are the initiating events discussed in the previous section. Event trees display each of the functions required to ensure safe shutdown, and the consequences of failure to perform the required functions. In order to establish the binary logic necessary to fit the progression of an accident sequence into this structure, success criteria for each system must be generated. The success criteria required of a single system may vary from sequence to sequence.

As described in Section 2 the methodology adopted is that usually characterized as small event tree - large fault tree. This is particularly suitable for EBR-II because of the relative lack of important active functions, when compared to LWRs, which in turn leads to a concise description of the accident sequences. The frequency of each of the accident sequences is quantified by transforming the sequence into a fault tree by linking the fault trees with an ANDgate and editing illogical cutsets.

\subsubsection{Safety Functions}

The safety functions that are required for safe and stable shutdown are as follows:

Reactivity control (shutdown)

Primary coolant system integrity and inventory maintained

Primary pumps operation (not required for safe shutdown but required by EBR-II Technical Specifications [5.24])

Decay heat removal 


\section{Success Criteria for Safety Functions}

\section{$\underline{\text { Reactivity Control }}$}

Reactivity control by the reactor shutdown system (RSS) requires sensing of an abnormal situation, generating a scram signal and releasing the control rods. The safety rods can also be released by operator action. The following table from the Technical Specifications, Table 5.11 gives the normal and limiting configuration of the RSS. The RSS provides overpower, underflow, and over temperature procedures. The subassembly outlet temperature high (SOT) trip is, in effect, automatic protection against core damage in all events, except those events local to a particular part of the core, i.e. local faults.

The issue of scram worth was resolved by establishing probability density functions of scram worth for control rods based upon analysis of 4 years of operating data. The analysis is discussed in more detail in Section 7.3. Fig. 5.4 is a plot of the discrete probability density function for single-rod scram worth. Table 5.12 shows, in tabular form, this probability density function and its integral, the cumulative distribution function (cdf). The cdf value for a reactivity interval is the probability that the scram reactivity is less than the upper limit of the interval. Thus, given a reactivity value that must be overcome, this cdf can be used to find the probability that scramming one rod will fail to shut down the fission chain reaction. In the absense of a reactivity insertion accident initiator, the reactivity to be overcome is the PRD (Power Reactivity Decrement - defined as the amount of reactivity needed to go from hot critical to full power). The PRD is typically about $30 \mathrm{c}$ and has never been more than $46 \mathrm{c}$. Using the cdf in Table 5.12, the probability of one rod failing to overcome the PRD is typically about $6.10^{-6}$ but can be as high as $10^{-2}$. However, scram worth cdfs in Table 5.13 (and probability density functions in Fig. 5.5) show that two rods will more than match even the largest PRD. To make the scram success criterion as simple as possible but not optimistic, scram of two rods will be taken as the success criterion when there is no reactivity insertion accident. In the case of an event which involved reactivity insertion, successful scram requires enough reactivity to be removed to overcome the insertion and the PRD. In most cases two rods are sufficient. In fact, the reactivity insertion initiating events can be classed according to the number of rods 
Table 5.11. Limiting Configuration of the Reactor Safety Shutdown System in the Operate Mode

\begin{tabular}{|c|c|c|c|}
\hline \multirow[b]{2}{*}{ Channel } & \multicolumn{2}{|c|}{ Trip Logic } & \multirow[b]{2}{*}{ Bypass Limits } \\
\hline & Limiting & Normal & \\
\hline \multicolumn{4}{|l|}{ Earthquake detectors ${ }^{1}$} \\
\hline Horizontal & 1 of 2 & 2 of 3 & \\
\hline Vertical & 1 of 2 & 2 of 3 & $\begin{array}{l}\text { No bypass permitted. A single } \\
\text { channel may be placed in tripped } \\
\text { condition. }\end{array}$ \\
\hline High flux level ${ }^{1}$ & 1 of 2 & 2 of 3 & \\
\hline Reactor period $^{2}$ & 1 of 2 & 2 of 3 & \\
\hline $\begin{array}{l}\text { Subassembly outlet } \\
\text { temperature high }\end{array}$ & 1 of 2 & 2 of 4 & $\begin{array}{l}\text { One channel may be bypassed. A } \\
\text { subsequent channel may be place in } \\
\text { tripped condition. }\end{array}$ \\
\hline Reactor flow low & 1 of 3 & $\begin{array}{l}2 \text { of } 5 \\
\text { or } \\
2 \text { of } 4\end{array}$ & $\begin{array}{l}\text { One channel may be bypassed or } \\
\text { tripped. Operation with two } \\
\text { channels failed is subject to review } \\
\text { and approval by the EBR-II } \\
\text { Division Director. }\end{array}$ \\
\hline $\begin{array}{l}\text { Loss of } 2400-\mathrm{V} \text { bus to } \\
\text { primary pumps }\end{array}$ & 1 of 1 & 1 of 1 & \\
\hline Control-rod manual trip & 1 of 1 & 1 of 1 & No bypass permitted \\
\hline Safety-rod manual trip ${ }^{3}$ & 1 of 1 & 1 of 1 & \\
\hline $\begin{array}{l}\text { Reactor-building exhaust } \\
\text { radiation level high }\end{array}$ & 1 of 2 & 1 of 2 & $\begin{array}{l}\text { Bypass of both channels permitted } \\
\text { when reactor building is isolated. }\end{array}$ \\
\hline
\end{tabular}

${ }^{1}$ These trips result in building isolation.

${ }^{2}$ Required only to $25 \mathrm{MWt}$.

${ }^{3}$ Also activates control-rod-scram. 


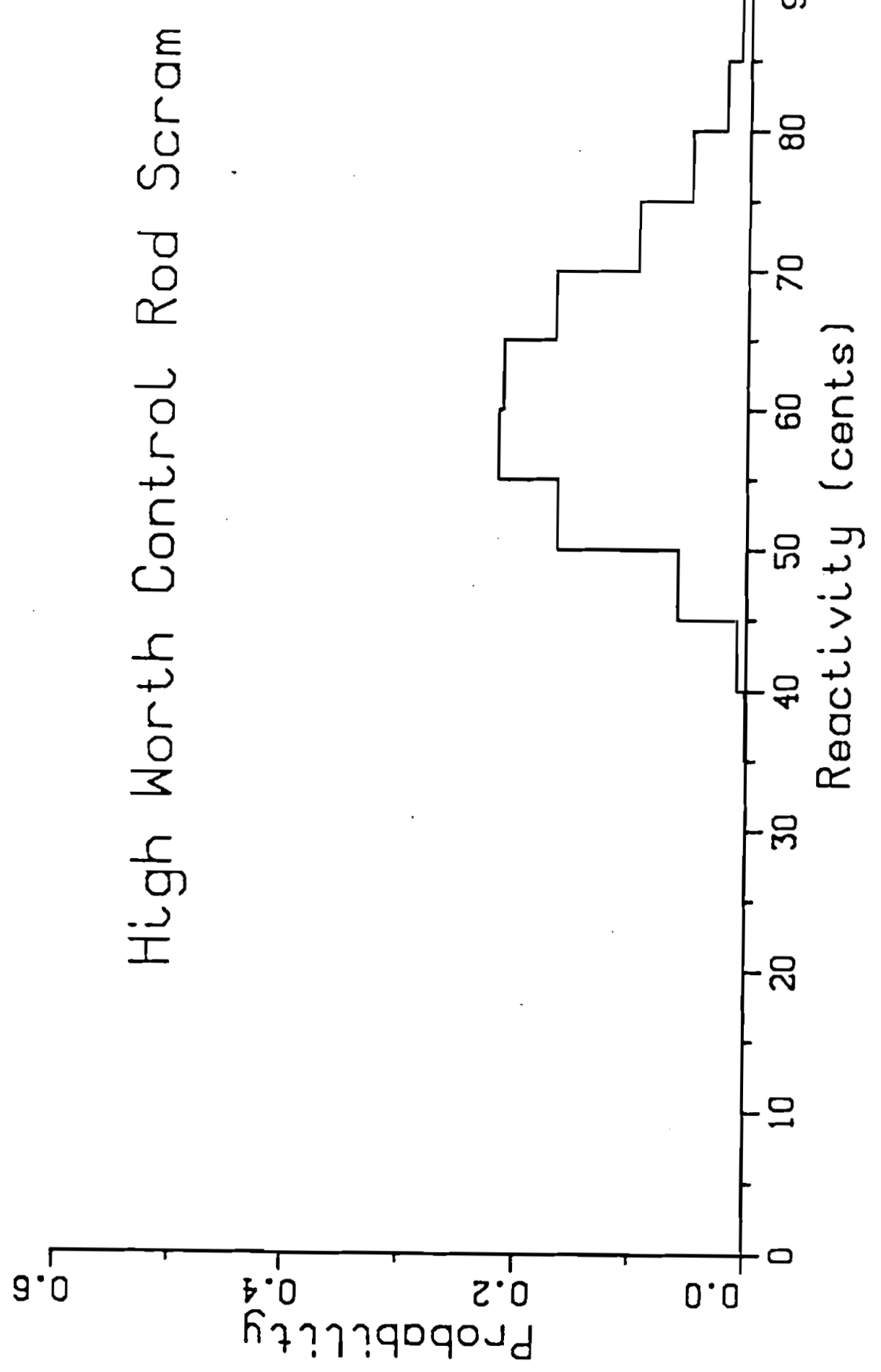


Table 5.12. Discrete Probability Density Function (pdf) and Cumulative Distribution Function (cdf) for Scram Reactivity from a Single High Worth Control Rod.

\begin{tabular}{|c|c|c|c|}
\hline \multicolumn{2}{|c|}{ Rho (cents) } & \multirow[t]{2}{*}{$p d f$} & \multirow[t]{2}{*}{$c d f$} \\
\hline MIN & MAX & & \\
\hline 0 & 5 & 0.000000000 & 0.000000000 \\
\hline 5 & 10 & 0.000000000 & 0.000000000 \\
\hline 10 & 15 & 0.000000000 & 0.000000000 \\
\hline 15 & 20 & 0.000000041 & 0.000000041 \\
\hline 20 & 25 & 0.000000691 & 0.000000732 \\
\hline 25 & 30 & 0.000004778 & 0.000005510 \\
\hline 30 & 35 & 0.000020446 & 0.000025957 \\
\hline 35 & 40 & 0.001042961 & 0.001068917 \\
\hline 40 & 45 & 0.007465519 & 0.008534436 \\
\hline 45 & 50 & 0.059038983 & 0.067573420 \\
\hline 50 & 55 & 0.164624479 & 0.232197899 \\
\hline 55 & 60 & 0.215690198 & 0.447888097 \\
\hline 60 & 65 & 0.212298742 & 0.660186840 \\
\hline 65 & 70 & 0.167526359 & 0.827713199 \\
\hline 70 & 75 & 0.095187643 & 0.922900842 \\
\hline 75 & 80 & 0.049038117 & 0.971938959 \\
\hline 80 & 85 & 0.020095220 & 0.992034179 \\
\hline 85 & 90 & 0.007965821 & 1.000000000 \\
\hline 90 & 95 & 0.000000000 & 1.000000000 \\
\hline 95 & 100 & 0.000000000 & 1.000000000 \\
\hline
\end{tabular}


Table 5.13. Cumulative Distribution Functions for Reactivity from Scram of High-Worth Rods (Exact).

Reactivitv (\&)

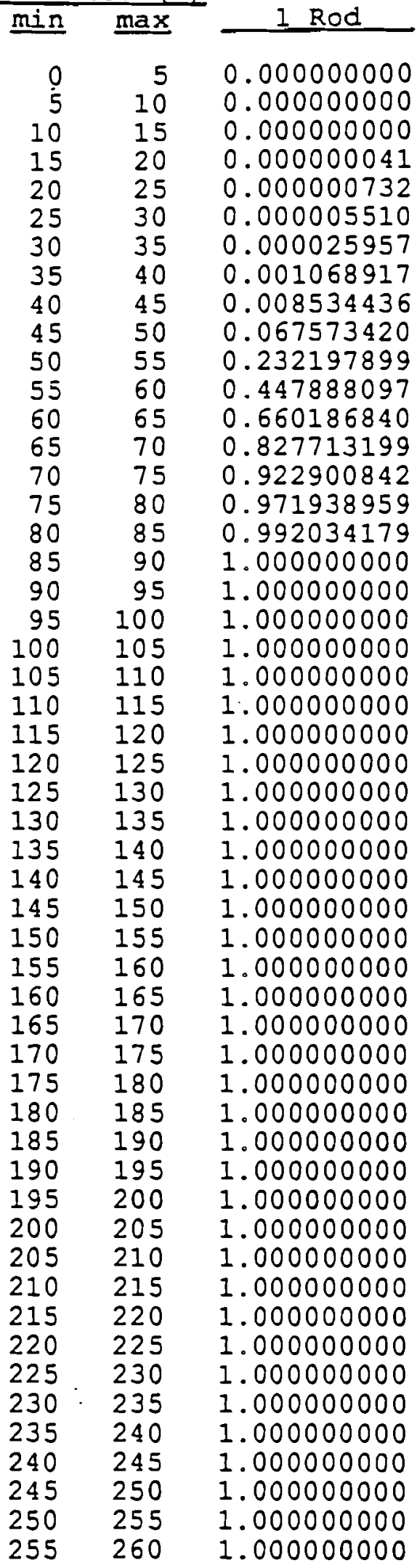

\begin{tabular}{c}
2 R0ds \\
\hline 0.000000000 \\
0.000000000 \\
0.000000000 \\
0.000000000 \\
0.000000000 \\
0.000000000 \\
0.000000000 \\
0.000000000 \\
0.000000000 \\
0.000000000 \\
0.000000000 \\
0.000000029 \\
0.000000299 \\
0.000000950 \\
0.000002103 \\
0.000582413 \\
0.002903764 \\
0.006612765 \\
0.013770516 \\
0.036788131 \\
0.083375613 \\
0.167729922 \\
0.289569650 \\
0.433270252 \\
0.573137178 \\
0.696419091 \\
0.791164600 \\
0.865813906 \\
0.919849040 \\
0.956679988 \\
0.977135270 \\
0.988947579 \\
0.995509071 \\
0.998757319 \\
0.999652079 \\
1.000000000 \\
1.000000000 \\
1.000000000 \\
1.000000000 \\
1.000000000 \\
1.000000000 \\
1.000000000 \\
1.000000000 \\
1.000000000 \\
1.000000000 \\
1.000000000 \\
1.000000000 \\
1.000000000 \\
1.000000000 \\
1.000000000 \\
1.000000000 \\
1.000000000
\end{tabular}

\begin{tabular}{c}
3 Rods \\
\hline 0.000000000 \\
0.000000000 \\
0.000000000 \\
0.000000000 \\
0.000000000 \\
0.000000000 \\
0.000000000 \\
0.000000000 \\
0.000000000 \\
0.000000000 \\
0.000000000 \\
0.000000000 \\
0.000000000 \\
0.000000000 \\
0.000000000 \\
0.000000000 \\
0.000000000 \\
0.000000000 \\
0.000000000 \\
0.000000008 \\
0.000000130 \\
0.000000522 \\
0.000001078 \\
0.000147700 \\
0.001619264 \\
0.003983677 \\
0.005343461 \\
0.006726602 \\
0.011165828 \\
0.024530602 \\
0.048488050 \\
0.086154355 \\
0.146082098 \\
0.224036213 \\
0.325329254 \\
0.433088989 \\
0.536989653 \\
0.626306504 \\
0.706362082 \\
0.774202001 \\
0.832638647 \\
0.881118054 \\
0.920147392 \\
0.948674534 \\
0.969223022 \\
0.981361677 \\
0.989695871 \\
0.994242018 \\
0.997375417 \\
0.998656610 \\
0.9999909911 \\
0.990
\end{tabular}

\begin{tabular}{c} 
4 R0ds \\
\hline 0.000000000 \\
0.000000000 \\
0.000000000 \\
0.000000000 \\
0.000000000 \\
0.000000000 \\
0.000000000 \\
0.000000000 \\
0.000000000 \\
0.000000000 \\
0.000000000 \\
0.000000000 \\
0.000000000 \\
0.000000000 \\
0.000000000 \\
0.000000000 \\
0.000000000 \\
0.000000000 \\
0.000000000 \\
0.000000000 \\
0.000000000 \\
0.000000000 \\
0.000000000 \\
0.000000000 \\
0.000000000 \\
0.000000000 \\
0.000000000 \\
0.000000000 \\
0.000000040 \\
0.000000251 \\
0.000000642 \\
0.000001101 \\
0.000594161 \\
0.002501629 \\
0.003955078 \\
0.004410833 \\
0.005268977 \\
0.007392493 \\
0.011162688 \\
0.018658693 \\
0.032955912 \\
0.055944611 \\
0.087996406 \\
0.132500100 \\
0.187082255 \\
0.259964613 \\
0.343038703 \\
0.432391521 \\
0.513186458 \\
0.587577849 \\
0.654726075 \\
0.715690890
\end{tabular}


Table 5.13. Cumulative Distribution Functions for Reactivity from Scram of High-Worth Rods (Exact). (Cont'd)

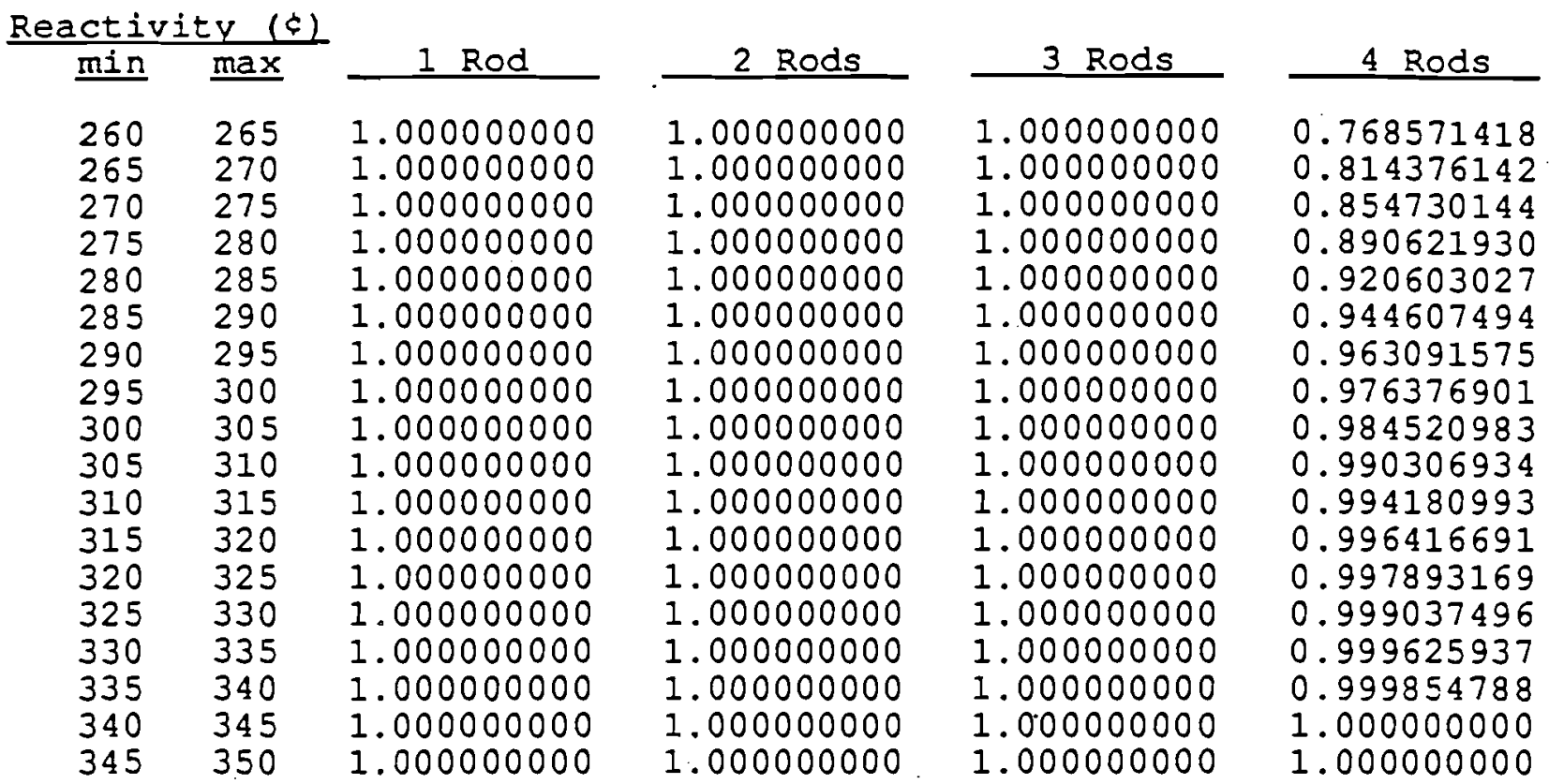



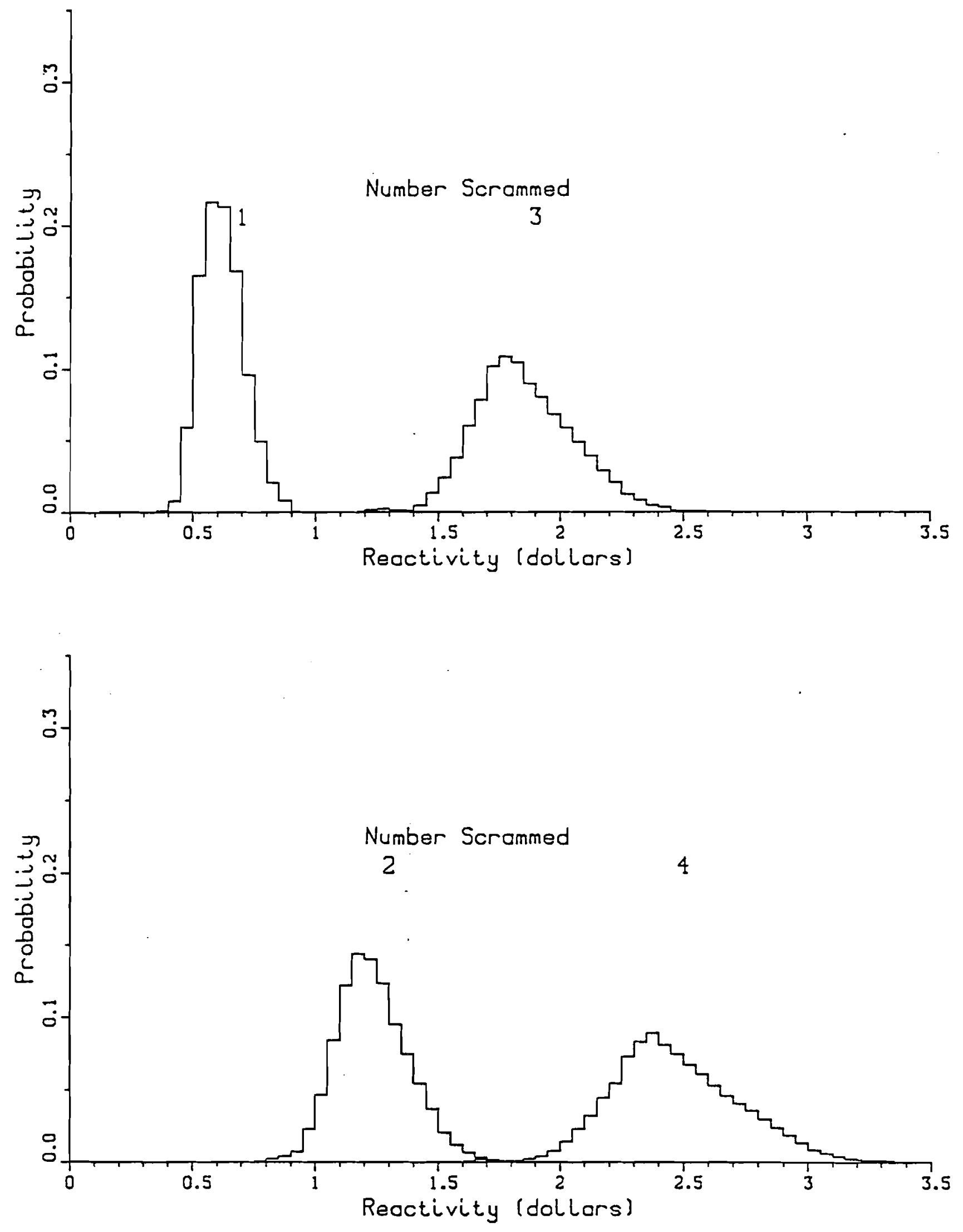

Fig. 5.5. Exact Probability Density Functions for Scram Worth 
required to scram to overcome the net reactivity as given in Table 5.14 below. Note that the RSS may sense that reactivity insertion is taking place through rising power and scram the reactor before the full insertion potential of the initiating event has been reached but the requirement for complete shutdown is that the full reactivity be overcome or, in the case of control rod insertion, that the drive mechanisms be detached from the power source and enough rods inserted to overcome the reactivity insertion that far into the transient.

Table 5.14. Number of Rods Required for Scram As a Function of Reactivity Insertion

$\begin{array}{lccc}\underline{\text { PRD }} & \frac{\text { Reactivity Insertion }}{\leq .35 \$} & \frac{\text { Neglecting Feedback }}{\text { Notal Required }} & \text { No. of Rods } \\ \leq .35 \$ & 0.20-0.65 \$ & \leq 0.55 \$ & 2 \\ \leq .35 \$ & 0.65-1.10 \$ & \leq 1.00 \$ & 3 \\ \leq .35 \$ & >1.10 \$ \$ & \leq 1.45 \$ & 4 \\ & & >1.45 \$ & 5 \text { or more }\end{array}$

A second form of reactivity control occurs in the case of failure to scram. In these unprotected events the reactivity feedbacks reduce the fission power and in many cases protect the fuel from damage. This issue is treated separately in the core damage evaluation.

\section{Primary Coolant Inventory}

Maintenance of the primary coolant inventory is required to sustain core cooling, however no internal initiating event has been identified, other than vessel failure, which can compromise this system. This event is of extremely low frequency and therefore this system is not included in the event trees except vessel failure is retained as an initiating event.

\section{Primary Pump Operation}

In contrast to other LMRs the EBR-II primary pumps are not tripped upon receipt of a scram signal. Primary pumping power is not necessary to achieve safe stable shutdown as long 
as the scram has taken place. Nonetheless simultaneous loss of pumping power and some other initiating event must be identified as a separate accident sequence because it has the potential for a greater challenge to fuel integrity if scram fails to occur. The occurrence of coincident but random initiating events is of very low probability. However, there is a mode of operation at EBR-II where the ANL-W site is separated from the INEL loop and EBR-II is supplying the electricity required to carry what is termed the "hotel load". In this eventuality any initiating event which leads to turbine trip leads to a combination of that initiating event and a double pump LOF due to loss of feeder power to the motor generator sets.

The Auxiliary Pump is an Engineered Safety Feature designed to smooth the transition to natural circulation. It is able to provide $3.3 \%$ of nominal flow when operating upon battery power and up to $5 \%$ on rectifier power. No credit is taken for the Auxiliary Pump and therefore it does not appear as an top event. This course of action was followed because analysis has shown that it does not affect the damage categories.

\section{Decay Heat Removal}

Decay Heat Removal is accomplished in a variety of ways. The main method of removing decay heat is via the Intermediate Heat Exchanger to the secondary sodium and balance of plant (BOP). The heat removal capability of the secondary side is sufficiently large that the operating procedures instruct the operator to reduce the secondary natural circulation flow to avoid overcooling the primary tank and to balance the heat removed with the decay heat being generated. In the case of loss of feedwater or loss of normal power the operators do not have a reliable method of determining and maintaining the water level in the steam drum. The decay heat is then rejected through the shutdown coolers and using alternate (emergency $480 \mathrm{~V}$ ) power to reduce the secondary flow to minimize water evaporation. Shutdown coolers also provide a diverse method of decay heat removal in all other cases. The shutdown coolers are passive NaK loops which remove either $60 \mathrm{~kW}$ or $360 \mathrm{~kW}$ depending on whether the louvers on the natural draft NaK/air heat exchangers are closed or open. The louvers open automatically at a bulk sodium temperature of $710^{\circ} \mathrm{F}\left(377^{\circ} \mathrm{C}\right)$ and close at $700^{\circ} \mathrm{F}\left(371^{\circ} \mathrm{C}\right)$. Finally, the shield cooling system and thimble cooling system combined can remove up to $130 \mathrm{~kW}$ but rely on 
electrical power for their operation. The decay heat removal capabilities are shown in Table 5.15 and the sources of heat in Table 5.16. For long term decay heat removal the decay heat of fuel in the storage basket may provide a significant heat source. The estimate in Table 5.16 is based upon half of a core, (basket inventory is 50 subassemblies) having been cooled for 7 days. Shutdown (or decay) heat removal has been demonstrated with a series of plant experiments [5.25, 5.26]. These experiments, the Shutdown Heat Removal Test, SHRT, series have demonstrated that the transition to natural circulation and long term decay heat removal will occur without fuel damage.

Comparison of Tables 5.15 and 5.16 allow simple bases for successful decay heat removal to be established. If successful shutdown is being accomplished with all decay heat removal systems available and electrical power available there is a maximum net source of heat of $80 \mathrm{~kW}$ apart from decay heat, due to the difference between primary pump heat and the counter-balancing sodium purification system. Taking no credit for the active systems i.e., louvers opening and the shield cooling operating, still gives a residual heat removal capability of $60 \mathrm{~kW}$ from the shutdown coolers, $25 \mathrm{~kW}$ from convection/radiation to the concrete blast shield and a minimum of $45 \mathrm{~kW}$ by the BOP, even if the secondary flow is retarded to minimum allowable. If electrical power is not available there are no extraneous heat sources. In both cases, therefore, the decay heat has to be counterbalanced by operation of the BOP, up to $-3800 \mathrm{~kW}$, opening of the shutdown cooler louvers (a net increase of $150 \mathrm{~kW}$ each) or operation of the shield cooling (a net increase of $105 \mathrm{~kW}$ ). Note that the figures above also show that for shutdown with any one of the shutdown coolers or the BOP completely unavailable through, for example, plugging of shutdown cooler NaK flow or dump of sodium, it is still true that sufficient residual heat losses exist to balance any pump heat if the pumps are not deenergized. This is summarized in Table 5.17. It should be noted that the heat removal capacities quoted are based upon measurements at the normal tank temperature of $700^{\circ} \mathrm{F}$ $\left(371^{\circ} \mathrm{C}\right)$. As the accident progresses and the tank temperature rises the heat removal capability will rise accordingly. No credit is taken for this in the calculations of heat loss from the shield cooling or thimble cooling. The shutdown cooler heat removal has been modelled [5.27]. 
Table 5.15. Decay Heat Removal Capabilities

\begin{tabular}{|c|c|c|c|}
\hline System & Mode & Support System Requirements & Heat Removal \\
\hline BOP & $\begin{array}{l}\text { Natural Circulation } \\
\text { Retarded } \\
\text { Minimum Retarded } \\
\quad 0.1-0.3 \%\end{array}$ & $\begin{array}{l}\text { Water makeup to steam drum } \\
\text { Electrical Power }+ \text { Water Makeup } \\
\text { Electrical Power ( } 480 \mathrm{~V})\end{array}$ & $\begin{array}{c}2350 \mathrm{~kW}-3800 \mathrm{~kW} \\
\text { As Required } \\
45 \mathrm{~kW}-135 \mathrm{~kW}\end{array}$ \\
\hline $\begin{array}{l}\text { Shutdown } \\
\text { Coolers }\end{array}$ & $\begin{array}{l}\text { Louvers Closed } \\
\text { Louvers Open }\end{array}$ & Instrument Air, Electrical Power & $\begin{array}{l}60 \mathrm{~kW} \\
360 \mathrm{~kW}\end{array}$ \\
\hline $\begin{array}{l}\text { Shield and } \\
\text { Thimble } \\
\text { Cooling }\end{array}$ & $\begin{array}{l}\text { Forced Air Cooling } \\
\text { Convection and } \\
\text { Radiation }\end{array}$ & $\begin{array}{l}\text { Electrical Power (480V) } \\
\text { (No shield cooling operational) }\end{array}$ & $25 \mathrm{~kW}$ \\
\hline $\begin{array}{l}\text { Sodium } \\
\text { Purifica- } \\
\text { tion }\end{array}$ & Forced Flow & Electrical Power (480V) & $50 \mathrm{~kW}$ \\
\hline
\end{tabular}

Table 5.16. Sources of Heat After Shutdown

\begin{tabular}{lccl}
\hline \multicolumn{1}{c}{ System } & $\underline{\text { Mode }}$ & $\begin{array}{c}\text { Support System Requirements } \\
\text { Electrical Power }(2400 \mathrm{~V})\end{array}$ & $\begin{array}{c}\text { Heat Addition } \\
200 \mathrm{~kW}^{*}\end{array}$ \\
Primary Pumps & Forced Flow & --- & See Fig. 5.6 \\
Core Decay Heat & --- & -- & $<100 \mathrm{~kW}$ \\
Basket Decay & --- & & \\
Heat & & & \\
\hline
\end{tabular}

*If pumps still energized 


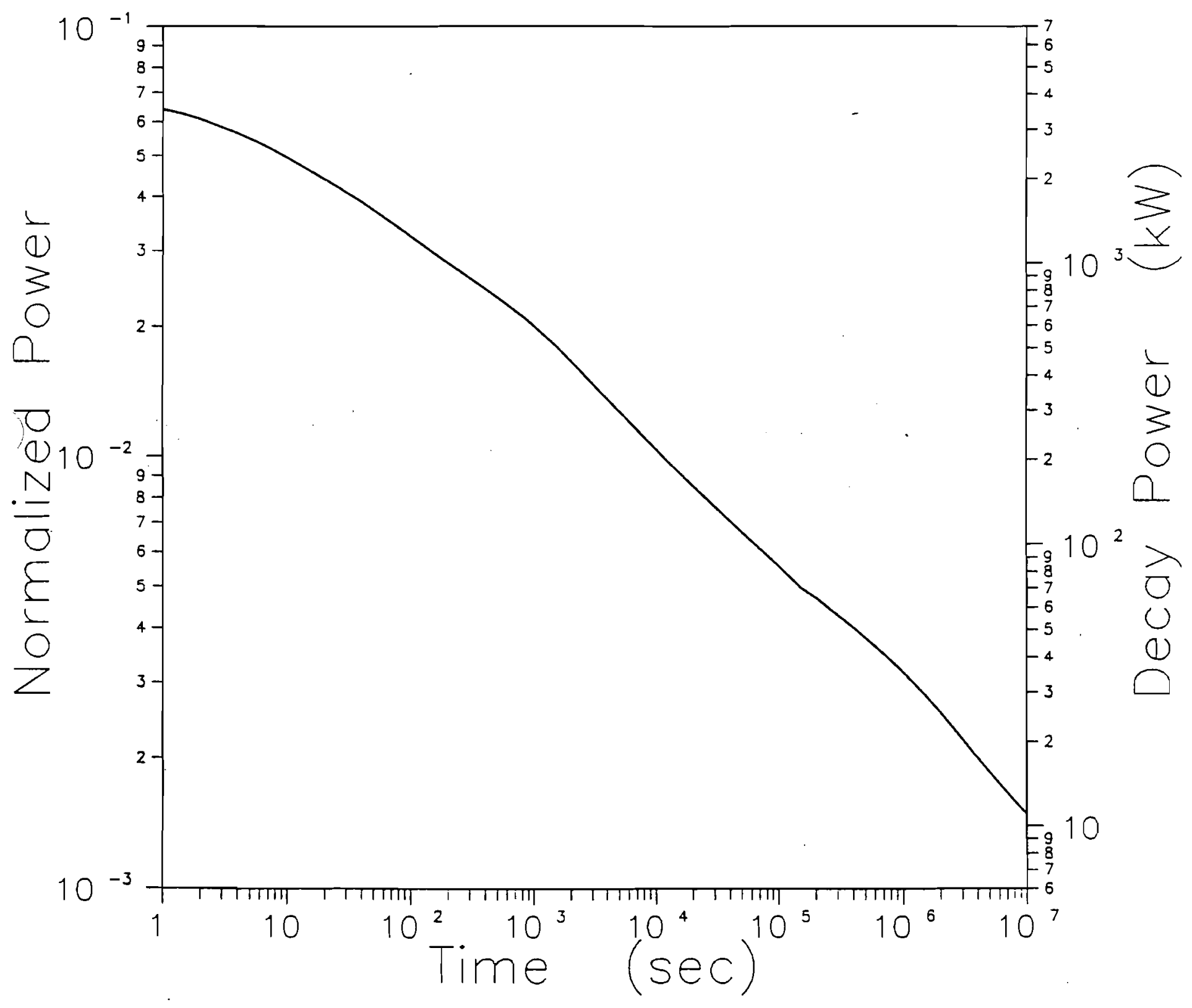

Fig. 5.6. EBR-II Decay Heat 


\section{With Electrical Power}

Heat Sources [1]

Heat Removal Path

Pumps $200 \mathrm{~kW}$

Shutdown Cooler

$30 \mathrm{~kW}$

(louvers closed)

$180 \mathrm{~kW} \quad$ (louvers open)

BOP Minimum [2]

$45 \mathrm{~kW}$

$(0.1 \%)$

Shield Cooling

$-130 \mathrm{~kW}$

Radiation/Convection

$25 \mathrm{~kW}$

Net Removal $\quad 5 \mathrm{~kW}$ (shield cooling and louvers closed)

$50 \mathrm{~kW}$ (no shield cooling and louvers open)

Without Electrical Power

Heat Sources [1] Heat Removal Path

None

Shutdown Cooler
BOP

$30 \mathrm{~kW}$

$180 \mathrm{~kW} \quad$ (louvers open)

Radiation/Convection

$3800 \mathrm{~kW} \quad$ (nat. circulation)

$25 \mathrm{~kW}$

Net Removal $3855 \mathrm{~kW}$ (BOP natural circulation and louvers closed) [3] $205 \mathrm{~kW}$ (No BOP heat sink and louvers open)

[1] Not including decay heat

[2] BOP heat removal increased as necessary

[3] With BOP heat removal, tank temperature remains below $710^{\circ} \mathrm{F}\left(377^{\circ} \mathrm{C}\right)$; therefore shutdown cooler louvers remain closed. 
Over the years a great deal of work has been performed establishing the performance of the decay heat removal systems, all of this work is summarized in [5.28] and as part of this work an effective heat capacity for the primary tank and associated structures has been established to be $80 \mathrm{~kW} \mathrm{hr} /{ }^{\circ} \mathrm{F}\left(5.1810^{8} \mathrm{~J} /{ }^{\circ} \mathrm{C}\right)$, for a full power of $62.5 \mathrm{MW}$. This equates to 4.6 full power seconds (fps) per ${ }^{\circ} \mathrm{F}$ of tank temperature, $\left(8.3 \mathrm{fps}\right.$ per $\left.{ }^{\circ} \mathrm{C}\right)$, which is a very large capacity.

The Technical Specifications include a structural temperature limit of $800^{\circ} \mathrm{F}\left(427^{\circ} \mathrm{C}\right)$ which is based upon a conservative evaluation of yield in the reactor tank [5.23] when the freeze seals in the reactor plug are not molten. If the bulk temperature increases by $100^{\circ} \mathrm{F}\left(56^{\circ} \mathrm{C}\right)$, melting of the freeze seals is required and is primarily an operational consideration to prevent deformation which would interfere with the operation of the large and small rotating plugs for refueling. From a safety viewpoint, the concern with elevated primary tank temperature is the structural integrity of the primary tank including its cover.

Additional analysis of the primary tank cover $[5.29,5.30]$ shows that bowing of the cover due to the temperature gradient across the cover causes a bending and a shear stress at the top of the inner vessel. The failure mechanism of the inner vessel is primarily due to creep stress. Fatigue damage is negligible. These results are summarized in Table 5.18. As can be seen from the table, the primary tank can remain at a temperature at or below $1000^{\circ} \mathrm{F}\left(538^{\circ} \mathrm{C}\right)$ for tens of years without creep failure. Therefore $1000^{\circ} \mathrm{F}$ was selected as the limit for primary tank temperature in this analysis.

A second reason for the $1000^{\circ} \mathrm{F}$ limit is that, assuming a $50^{\circ} \mathrm{F}\left(28^{\circ} \mathrm{C}\right)$ temperature difference between the primary tank temperature and the fuel cladding, the fuel remains within its operating temperature limit. Therefore, it is not necessary to perform additional analysis of the driver fuel at decay heat levels and below $1000^{\circ} \mathrm{F}$ primary tank temperature. 
Table 5.18. Primary Tank Limits at Elevated Temperature

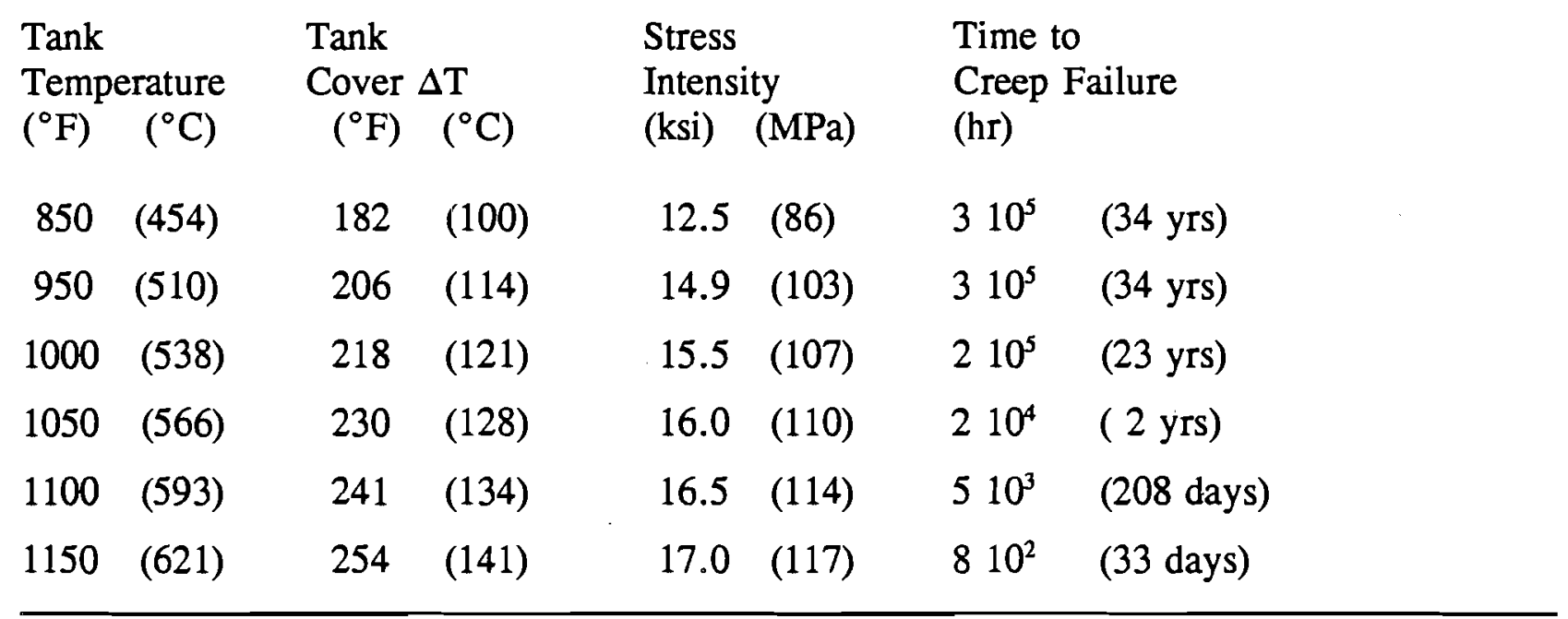

The decay heat removal scenario developed shows that normal mode decay heat removal is not required for the first 8 hours after shutdown. The shutdown cooler louvers are closed which limits the heat removal rate to $30 \mathrm{~kW}$ each. It is also stet that there is no air flow in either the shield or thimble cooling system. Thermal radiation to the concrete of the biological shields accounts for $25 \mathrm{~kW}$ of heat removal. Therefore, the maximum decay heat removal for the first 8 hours is $85 \mathrm{~kW}$ in this configuration. If the scenario includes an unrecovered loss of a shutdown cooler, the decay heat removal is limited to $55 \mathrm{~kW}$.

The overall decay heat removal scenario is divided into two time periods. Short term decay heat removal is defined as the first 14 days after shutdown. One of the two shutdown coolers is required to operate in the normal mode with its louvers open if the primary tank temperature is greater than $710^{\circ} \mathrm{F}\left(377^{\circ} \mathrm{C}\right)$ for the time from 8 hours after shutdown until 14 days. Decay heat is sufficiently reduced after 14 days that the shield and thimble cooling systems are able to remove the excess decay heat even if both shutdown cooler louvers are closed.

For the BOP to remove decay heat requires that sodium be present and system for providing the ultimate heat sink still exists, i.e., water can be provided to the steam drum. Only two general cases apply, (a) shutdown with the BOP available, in which case only some 
subsequent failure or operator action can render it unavailable, or (b) shutdown with the BOP unavailable because of some problem in the secondary or steam systems. The very short term behavior is not of any particular significance from a core damage perspective because of the large heat capacity of the sodium in the tank.

The distinction is made between periods of Short Term Decay Heat Removal, defined as up to 14 days and Long Term Decay Heat Removal defined to extend from 14 days to 45 days. The success and failure criteria associated with decay heat removal are given in Table 5.19. Failure of tong term decay heat removal requires the simultaneous failure of four systems and even then the temperature of the tank is readily controlled using parasitic losses (recovery action) and is only included for completeness. 
Table 5.19. Success and Failure Criteria for Decay Heat Removal (DHR)

Top Event BOP decay heat removal
maintained

Short term decay heat removal initiated and maintained

Long term decay heat removal maintained

\section{Success Criteria}

Heat sink available within 8 hours and maintained for 14 days

One of two shutdown coolers available within 8 hours and maintain cooling for 14 days

One of three methods, BOP, shutdown coolers, or shield cooling available from 14 to 45 days

\section{Failure Criteria}

Unrecoverable loss of heat sink within 14 days of shutdown

Less than one cooler available within 8 hours or DHR cannot be maintained for 14 days

All DHR systems fail in 14 to 45 days

Success criteria for the various systems are summarized in Table 5.20.

\subsubsection{Damage Definitions}

The EBR-II core may contain a variety of fuel types at any one time. There are various forms of driver fuel; Mk II, consisting of U-5 fissium fuel and SS316 clad, Mk III, U10Zr fuel and D-9 clad which have approved burnup limits and operating temperature limits specified in the Technical Specifications, [5.24]. In addition EBR-II will host a variety of irradiation experiments which might contain, for example, oxide fuel pins, deliberately faulted metal or oxide fuel pins, and structural materials. Clearly, the PRA cannot anticipate every type of experimental subassembly, therefore, the damage definitions applied must be general enough to encompass all these possibilities.

The approach adopted is to identify a plant transient category for each accident sequence and to treat the damage state of the core either by associating a specific fuel damage state or by consideration of the peak temperatures. The resulting set of accident sequences and plant transient states is then invariant under change of core, a very important attribute for EBR-II and its experimental mission. It also has the advantage of being most flexible in allowing different 
Table 5.20. Success Criteria for Transients

\begin{tabular}{|c|c|c|c|c|}
\hline \multirow[b]{2}{*}{ Reactivity Control } & \multicolumn{4}{|c|}{ Decay Heat Removal } \\
\hline & Primary Pumps & Via BOP & Shutdown Coolers & Shield Cooling \\
\hline $\begin{array}{l}\text { Sufficient control rods and } \\
\text { safety rods removed from } \\
\text { core in the case of } \\
\text { reactivity insertion. } \\
\text { (Number of rods depends } \\
\text { on magnitude of initiating } \\
\text { event) }\end{array}$ & $\begin{array}{l}\text { Remaining primary pump } \\
\text { continues to operate in the } \\
\text { case of a single pump fault }\end{array}$ & $\begin{array}{l}\text { One of decay heat re } \\
\text { days for long term de }\end{array}$ & $\begin{array}{l}\text { loval mechanisms is at } \\
\text { ay heat removal }\end{array}$ & iilable for up to 45 \\
\hline
\end{tabular}


definitions of core damage, which might result if the core were to change significantly. Passive safety is not included as a top event in the main tree. This approach prevents having to combine active systems such as removal of decay heat or scram, together with reactivity feedbacks, in to one tree. This view of the event trees for EBR-II is a systemic approach and corresponds to treating the top events as boundary conditions which completely specify the system thermalhydraulic state. The results of the accident sequence for any particular core can then be evaluated using an LMR system analysis code such as SASSYS [5.31].

All the accident sequences have been classified under six basic plant transient categories. These are described in Table 5.21.

Table 5.21. Definition of Plant Transient Categories

\section{Transient Definition}

Direct damage events resulting from vessel and primary piping leaks or rupture, structural failures.

Protected loss of heat sink events; reactor shutdown neutronically but decay heat removal capability fails leading to uniform overheating of system and a long term core vulnerability.

ATWS events leading to a short term core vulnerability (not necessarily leading to core damage, subject to passive safety evaluation).

Protected events of the class that still leads to some degree of damage, distinct from P1 in that protection saves core from core damage category. Leads to minor core damage or potential experimental damage.

Other faults which lead to a degraded containment function and where core damage cannot be ruled out, e.g., steam generator tube rupture.

Local Faults, i.e. events that occur within one subassembly and lead to damage of that subassembly and possibly its neighbors.

\section{Category}

P1

P2

P3

P4

P5

P6 
In this methodology the frequency of given sequences will be defined in the sequence event trees which will then only need to be updated with plant modifications. The role of passive safety and core damage definitions can then be depicted in a core response tree for each sequence. The basic core response tree forms a partition between degrees of damage. The partition is a direct result of the detailed accident evaluations. Another advantage of this method arises because a separate damage assessment is necessary for each analyzed sequence thereby allowing clear exposition of passive safety consistent with that described in ref. [5.32], where the emphasis is on "passively safe with respect to a particular accident" rather than just "passively safe". It should be noted that for many sequences the conditional probability of damage is 0 or 1 and a detailed analysis is not warranted.

The SASSYS systems analysis code has been used to analyze EBR-II transients using best estimate feedbacks, [5.33]. The fuel damage criteria against which results are judged are as follows:

(1) If an average driver subassembly reaches boiling, fuel melting or fuel pin failure then widespread core damage is presumed, (CD). (Average is defined by a subassembly whose outlet temperature is that of the core average).

(2) If the interior pins in the hottest allowed driver subassembly reach fuel pin failure, fuel melting or the sodium associated with these pins boils, but no damage occurs in average driver assemblies, then this is termed Minor Core Damage, (MCD).

In these criteria fuel pin failure is assessed using fuel pins at the peak allowed burnup for the driver fuel. Below this threshold (item 2) above no damage to driver fuel would occur but radionuclide release is not precluded because experiments may be irradiated more aggressively than driver fuel. Therefore the no damage region is subdivided into three regions based upon Technical Specification distinctions. 
(3) If the clad temperature in the hottest driver pins exceeds $1500^{\circ} \mathrm{F}\left(816^{\circ} \mathrm{C}\right)$ or lies between the eutectic temperature and $1500^{\circ} \mathrm{F}$ for greater than $60 \mathrm{~s}$ then there is the potential for damage to experiments, (PED).

(4) If the clad temperature in the hottest driver pins lies between the eutectic temperature and $1500^{\circ} \mathrm{F}$ for less than 60 s then no damage will occur, (ND). This is the limited capability operating regime.

5) If the temperature never exceeds the eutectic temperature no damage occurs, (ND), and this is the unlimited capability operating regime.

In the CDFR PRA reported in [5.34] the damage criterion adopted was a sodium temperature of $900^{\circ} \mathrm{C}$, including uncertainties. This roughly corresponds to classing PED, MCD and $\mathrm{CD}$ together as core damage. These criteria are summarized in the basic core response tree shown in Fig. 5.7. Note that the time ordering implied in Fig. 5.7 is only strictly valid for slow transients. Fast transients may go straight to fuel damage, $\mathrm{CD}$ or $\mathrm{MCD}$.

The core response is of most importance in the unprotected sequences where the reactivity feedbacks are known to limit the temperature rise and hence the resulting damage. In this case, the final state is not a completely subcritical state and there is the possibility that a subsequent separate event could occur which would alter this state. At the end of the transient where a stable state has been attained, the fission power will be defined by the heat sink available. The probability of attaining this stable but still critical state is low because the scram reliability is high. If the cause of failure to scram lies in the signal, mechanical (relays) or electrical components then the control rod drives will still be connected to the motors and positive and negative reactivity insertion by rod motion is still feasible. Currently no procedure exists which directs operator action under these circumstances, however, since the recovery action is trivially obvious and easy to carry out, it is not unreasonable to assume that the necessary signal, or release of stuck relay etc., can be carried out in a timescale of a few hours. Generally, if the control and safety rods failed to drop because they are all stuck then rodinduced reactivity insertion due to rod motion is equally not possible and the reactor will 
EVENT TREE FOR: Fuel Damage Sub-Tree

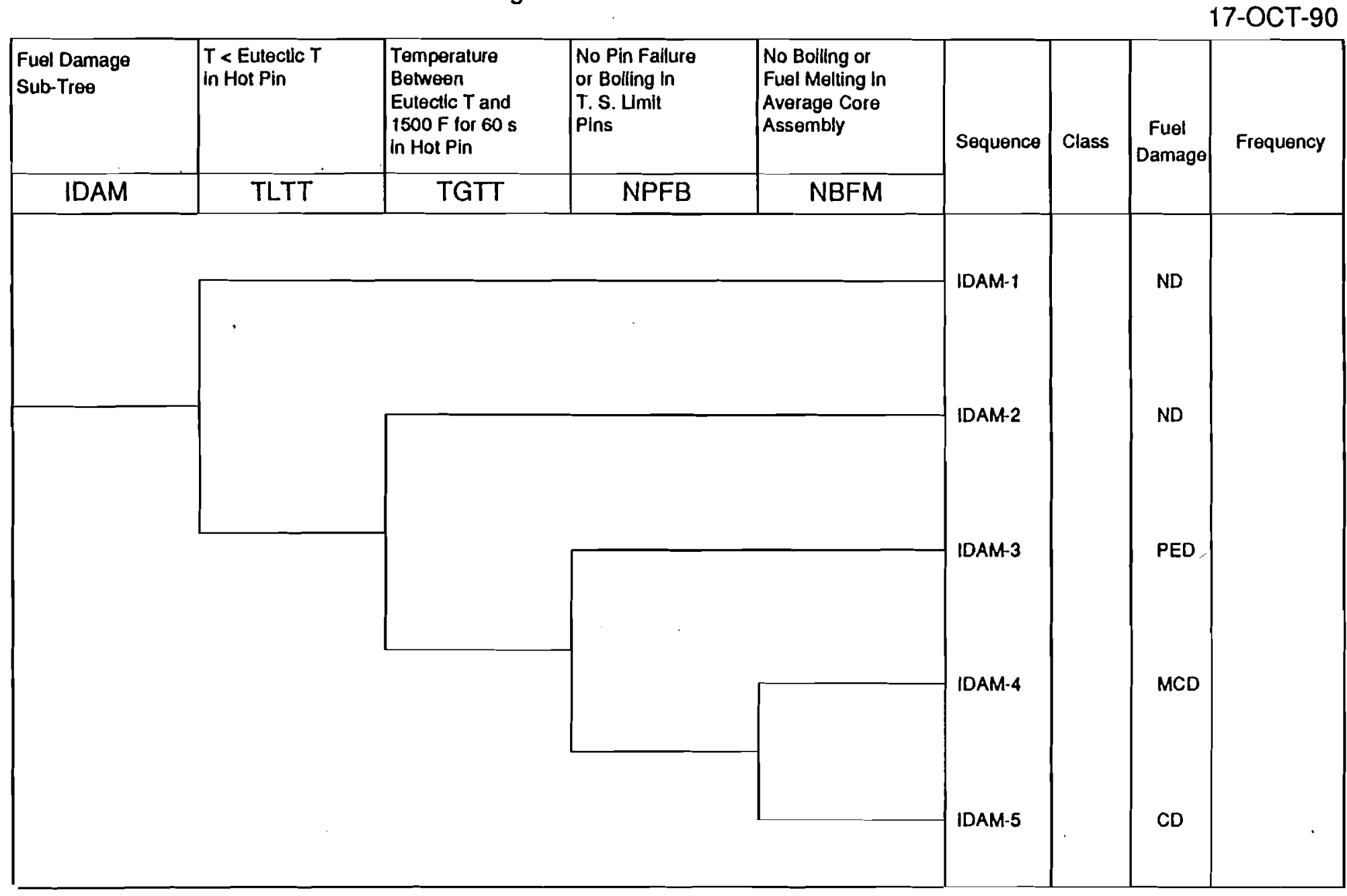

Fig. 5.7. Core Response Tree for Fuel Damage Definition. 
maintain its equilibrium power and temperature level until corrective measures are taken, meanwhile the power level will decrease as fuel is burned. All the potential events not involving positive reactivity insertion, e.g., LOF, LOHS, are benign. A basic assumption is that the recovery action to achieve reactor shutdown is assumed to be guaranteed and the accident is effectively terminated if the initial temperature transient does not lead to damage.

In the event trees, and the accident sequence descriptions that follow, the end states are assigned to a core damage bin on the basis of best estimate analysis. The uncertainties, and hence reliability associated with that assignment, are treated separately. In the case of long term heating of the core, tank, and structure through loss of decay heat removal a separate damage category is defined, which is associated with plant state P2. This category is specified by a single tank temperature limit above which some core and structural damage is assumed to take place. The temperature limit is chosen below the maximum allowable clad temperature because of uncertainties in the long term high temperature behavior of the various fuel-clad combinations in the EBR-II core. This category is denoted CSD (Core/Structural Damage). Table 5.22 combines the plant transient categories with the associated fuel damage possibilities.

Table 5.22 Plant Transient and Associated Fuel Damage Categories

\begin{tabular}{||l|l|}
\hline Plant Transient & Fuel Damage \\
\hline P1 & CD \\
\hline P2 & CSD \\
\hline P3 & All possible including no damage \\
\hline P4 & PED or MCD \\
\hline P5 & ND or CSD \\
\hline P6 & PED, MCD or CD \\
\hline
\end{tabular}




\subsubsection{EBR-II Event Trees}

A great deal is known about the details of accident sequence progression in EBR-II and this understanding is embodied in the various analysis tools that have been applied to EBR-II such as NATDEMO, DSNP and SASSYS, [5.31, 5.35, 5.36]. Event trees were developed using this understanding and the direct involvement of EBR-II Safety, Engineering and Operations personnel.

\subsubsection{Review of Event Trees in Other Relevant PRAs}

EBR-II, or any reactor, is designed with a very simple philosophy; in the event of a transient (initiating event) the first line of defense is to scram the reactor and, if scram is successful, to provide various means of removing the decay heat. The scram is achieved by removal of control rods (with safety rods as a backup). As described above, the decay (or shutdown) heat removal is carried out through the secondary sodium system (except for loss of normal power or loss of feedwater) and the shutdown coolers. The shield and thimble systems will also remove some heat if they are available. This design can be expressed as a simple event tree as follows, Fig. 5.8. The protected transients without decay heat removal can lead to some potential core vulnerability but this occurs in the long term only, because the lack of a heat sink can only affect the core on timescales which are long compared to scram actions (Plant Transient Type P2). Finally failure to scram will lead to a core vulnerability in the short term, irrespective of the heat sink available (Plant Transient Type P3).

It is instructive to review the event trees from other relevant PRAs as a guide to construction of those for EBR-II. Figure 5.9 shows the basic event tree for (non-LOCA) initiators in the N-Reactor PRA [5.37]. A very close analogy can be drawn with EBR-II, as is also indicated in Fig. 5.9. In the case of N-Reactor, the normal heat removal system is backed up by an Emergency Core Cooling System (ECCS). The Graphite and Shield Cooling System (GSCS) can also limit damage and is analogous to the EBR-II shield cooling. In Fig. 5.9 one top event was removed. This removed event allows for local effects in the ECCS which has no direct analogue at EBR-II. The only remaining endstates that admit damage are Z1(DEL) and 
EVENT TREE FOR: INITIATING EVENT

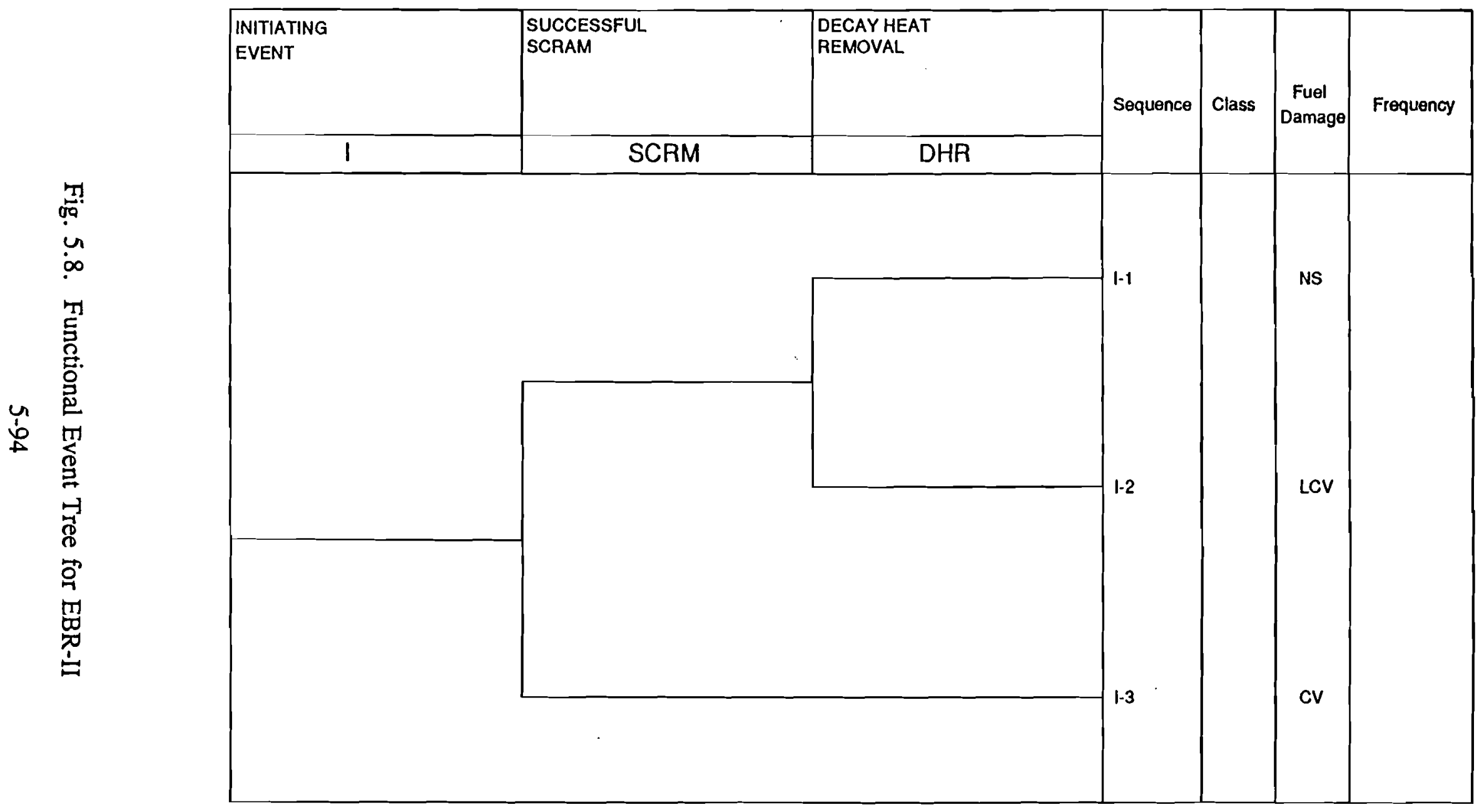




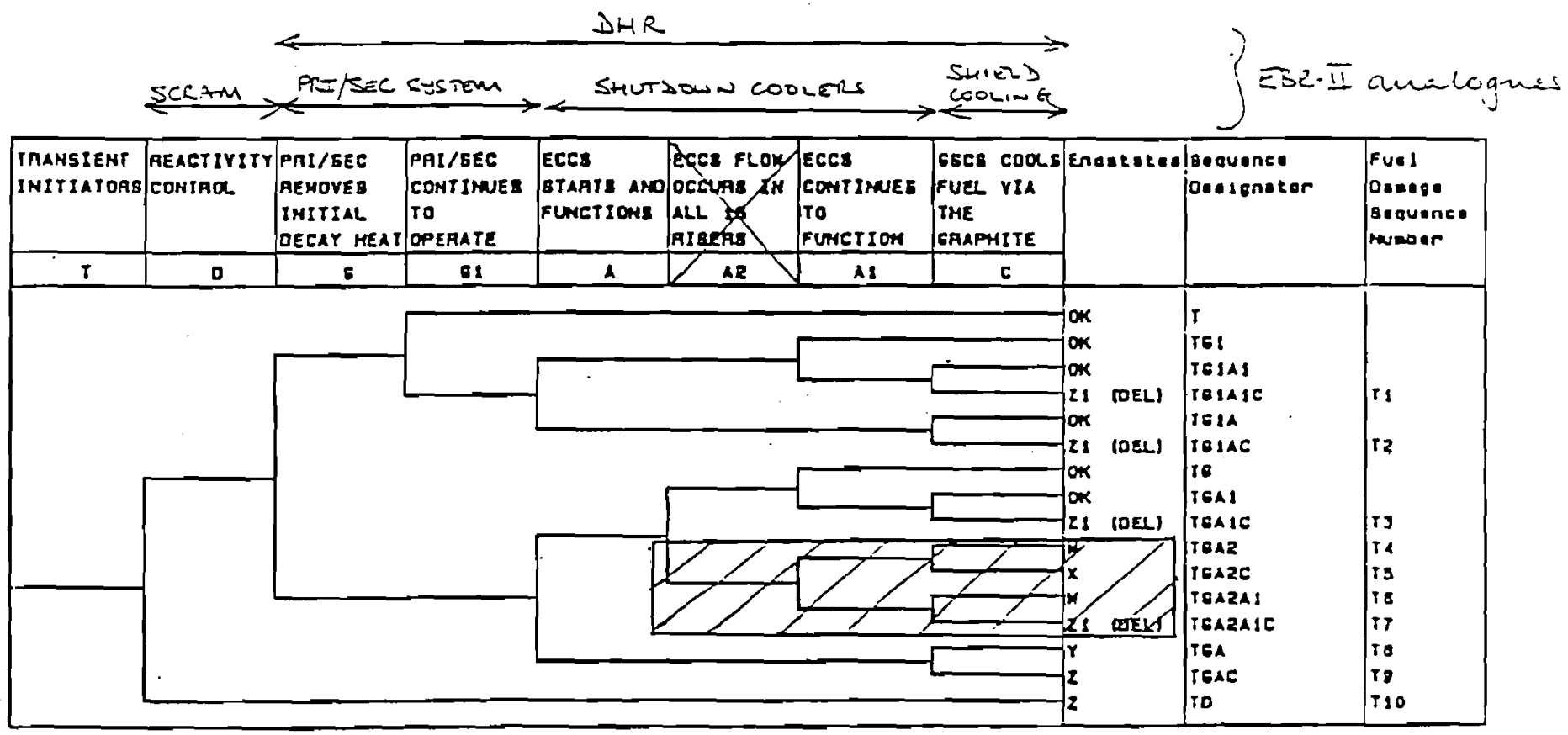

Fig. 5.9. Transient Event Tree for N-Reactor with EBR-II Analogues (Local Effects Removed) 
$Z$, which represent whole core damage delayed or immediate, and $Y$ which is damage limited by the GSCS heat removal when no primary decay heat removal or ECCS was available. No attempt is made to distinguish between failure to scram sequences and failure to remove decay heat sequences in the extent of core damage or to evaluate, by calculation, the details of damage, although N-Reactor shares many of the advantageous features that EBR-II possesses, (i.e., metallic fuel and large excess thermal capacity).

In the CRBR PRA, the bins were defined as accident sequences and not damage states. Figure 5.10 is the core damage event tree for internal initiators in the CRBR PRA [5.3]. This event tree considers short term and long term cooling via the steam generators and core cooling via the DHRS (analogous to Fig. 5.9). The additional elements in the CRBR event tree, $P, Q$ and I, are included as discriminators to allow specific sequences to be identified and evaluated within one overall tree. These discriminators are Pump Tripped (P), Initial Power Normal (Q) and Sodium Inventory (I). Again, it should be noted that all sequences which involve failure to scram have been assumed to lead to core damage, as does the Protected LOCA as well as the Protected Loss of Heat Sink and its delayed counterpart, (sequences TI, TSD, and TCD, respectively). For internal initiating events, only core damage sequences TKP, Unprotected Loss of Heat Sink (frequency $2.510^{-7}$ ), and TSD, Loss of Heat Sink, (frequency $3.410^{-6}$ ), exceeded a cut off frequency of $10^{-8}$, (indicated by $*$ in Fig. 5.10). For the EBR-II PRA an important issue is whether unprotected events do in fact lead to core damage.

\subsubsection{Event Tree Construction}

In the previous section definitions for fuel damage criteria, plant transient state and the success and failure criteria for the basic event tree top events were derived. In this section this information is used to present the event trees for each initiating event discussed in section 5.1 .

In unprotected events the details of the heat sink are generally unimportant because the damage, or lack of it, occurs on a very short timescale. In fact calculations can be made which demonstrate that for unprotected transients where the reactivity feedbacks lead to a benign 


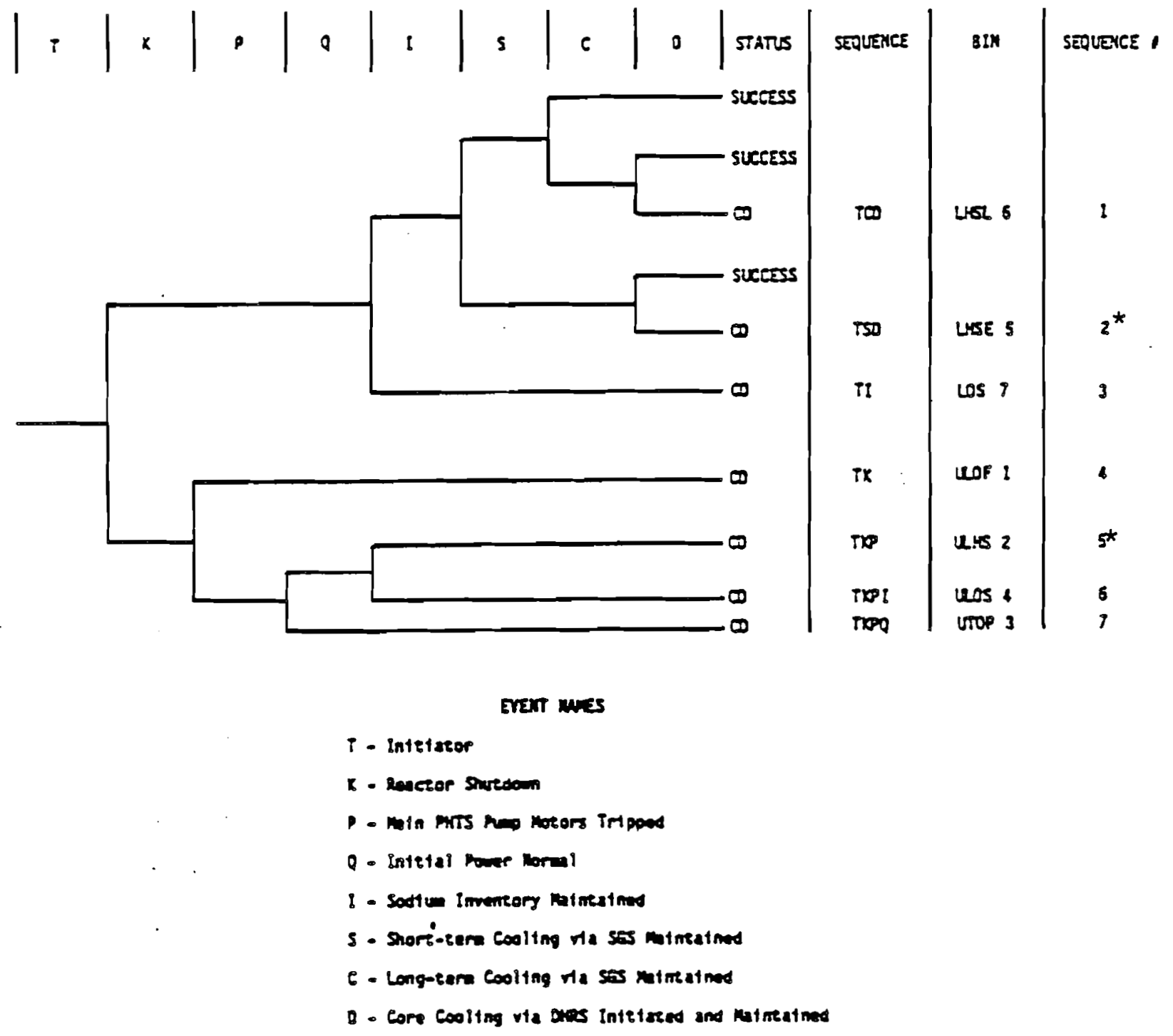

31183812

Fig. 5.10. Core-Damage Event Tree for Internal Initiators in the CRBR PRA. 
response to the accident the loss of the heat sink is beneficial. This surprising fact reflects the negative inlet temperature coefficient which leads to a negative reactivity input for any inlet temperature rise. All unprotected transients will therefore be assumed to have full heat sink available for the first few minutes except if the initiator itself affects the heat sink. Once the initial transient period is over, and if no damage has occurred the system approaches a state where the fission power matches the heat rejection capability and the transient is over. Any subsequent failure of the decay heat removal capability is covered by the protected case equivalent sequence and the use of the rare event approximation for successful scram, 1.0 instead of $(1.0-\epsilon)$.

In the simple functional event tree of Fig. 5.8 a top event of successful scram is used. Because the scram signal can have other effects other than causing the rods to drop this top event has been separated into scram signal generation and control or safety rods drop. The particular effect that the signal has is to trip the turbine which, in the case when the system is on hotel load, causes the pumps to coastdown.

A detailed discussion of the appropriate event tree for each initiating event now follows using the guidelines established here. 


\subsubsection{Event Trees for Flow Transients}

\subsubsection{Initiating Event Description}

Flow transients include all single or double pump faults, flow blockages and failures in the coolant circuit. Pump faults can occur in several ways which lead to characteristic coastdown times. The modes of failure and resulting coastdowns are analyzed in detail in the system notebooks for the pumps and motor generator sets, Appendices C.2 and C.3, and discussed earlier in Section 5.

\subsubsection{Normal Plant Response and Success Criteria}

Whatever the rate of coastdown and number of pumps involved, the normal plant response is the same. The RSS hàs a LOF protection subsystem which generates a scram signal, and in certain cases, the SOT subsystem may precede it in generating the scram signal. The scram signal causes the control rods to drop and after a $6 \mathrm{~s}$ delay, the secondary pump to trip. The turbine will drop off-line which normally has no effect on electric power except for the case when the EBR-II site is separated from the INEL loop and the turbine is providing hotel load. The success criteria for the two relevant systems are given in Table 5.20. Insertion of two control rods or both safety rods will shut the reactor down. The normal heat sink is available for removing decay heat. The operators must retard the secondary flow according to procedure to reduce excessive heat removal due to natural convection in the secondary system.

\subsubsection{Event Trees and Accident Sequences}

Figures 5.11 to 5.20 show the event trees for single and double pump faults. Core damage potential due to LOF accidents is dependent upon the duration of the pump coastdowns and the response of the reactor shutdown system and is discussed in detail in 5.4.2.2. 
EVENT TREE FOR: Single Pump Loss of Flow - Group A

31-MAY-91

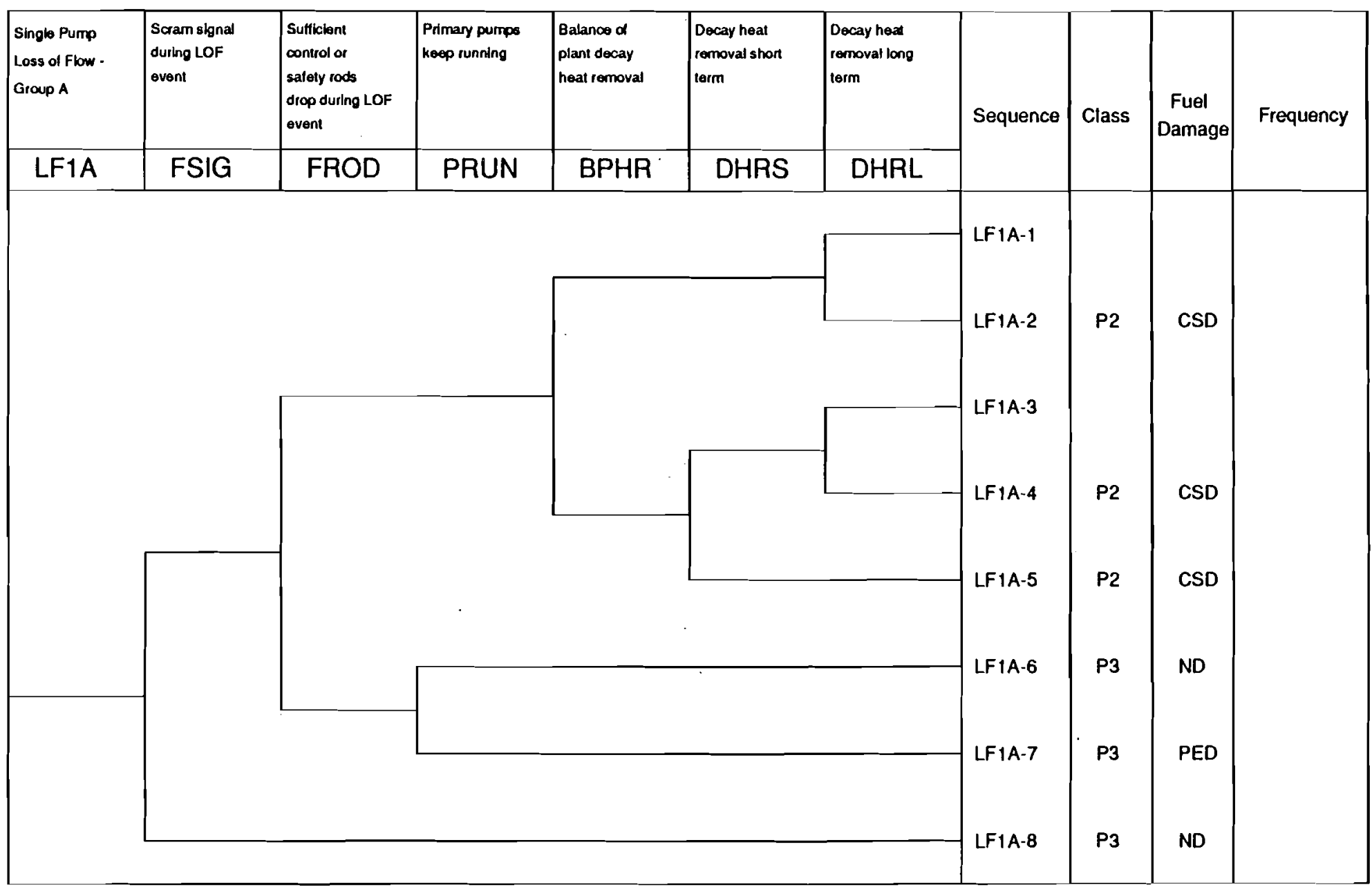

Fig. 5.11. Event Tree for Single Pump Fault - Group A 


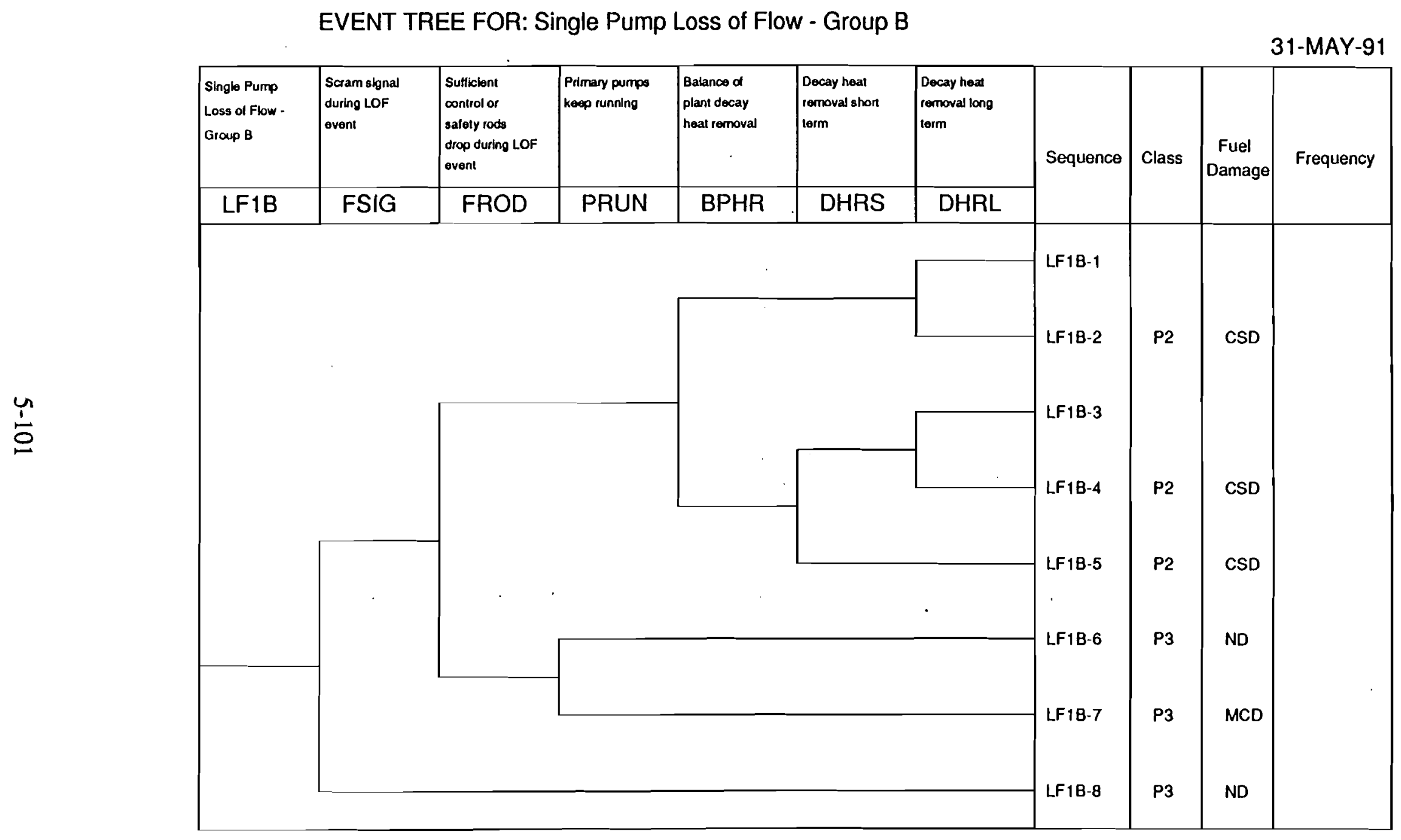

Fig. 5.12. Event Tree for Single Pump Fault - Group B 


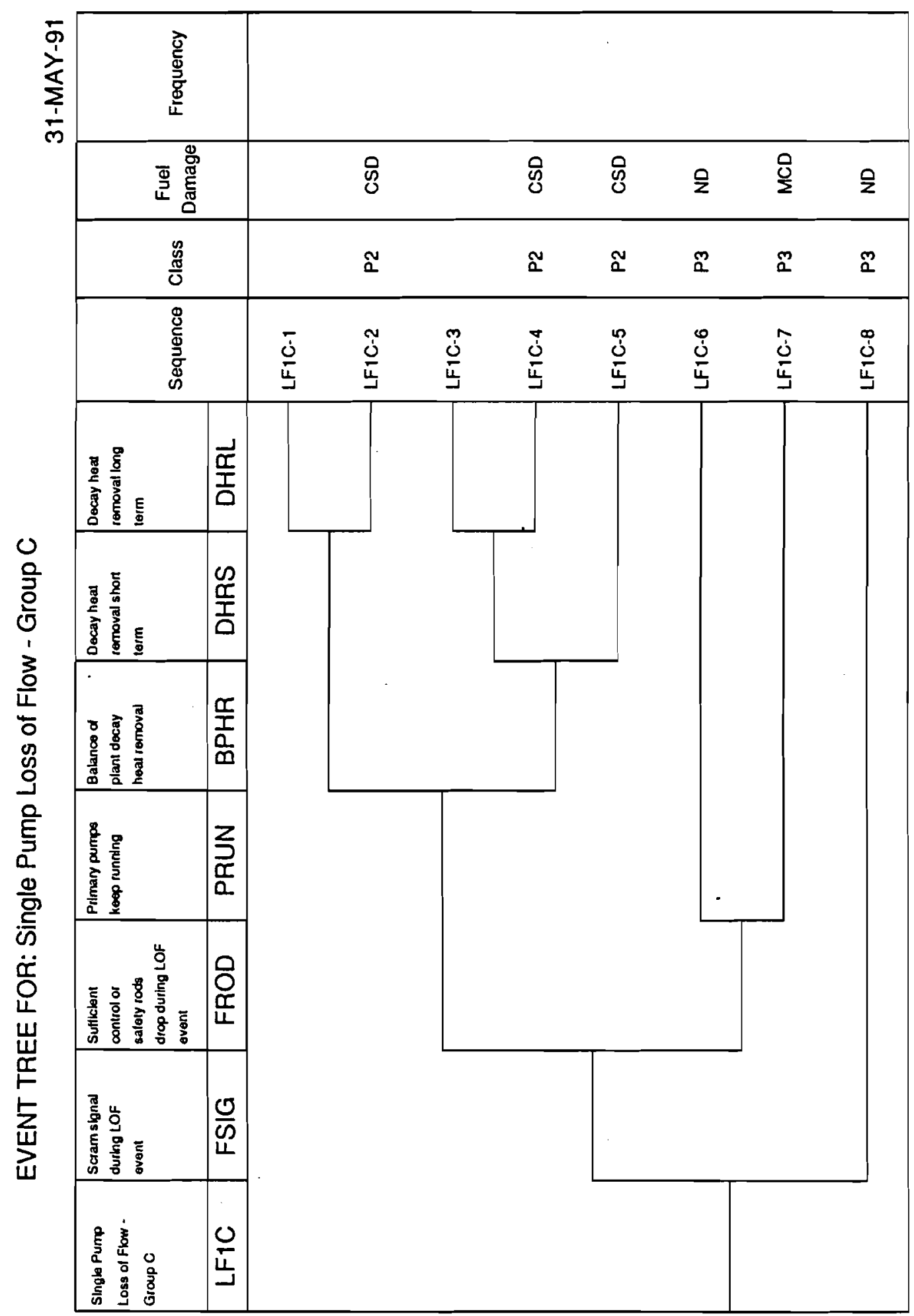

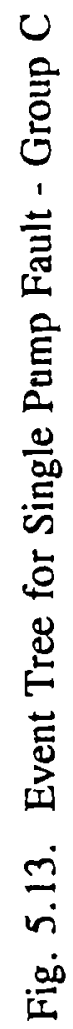




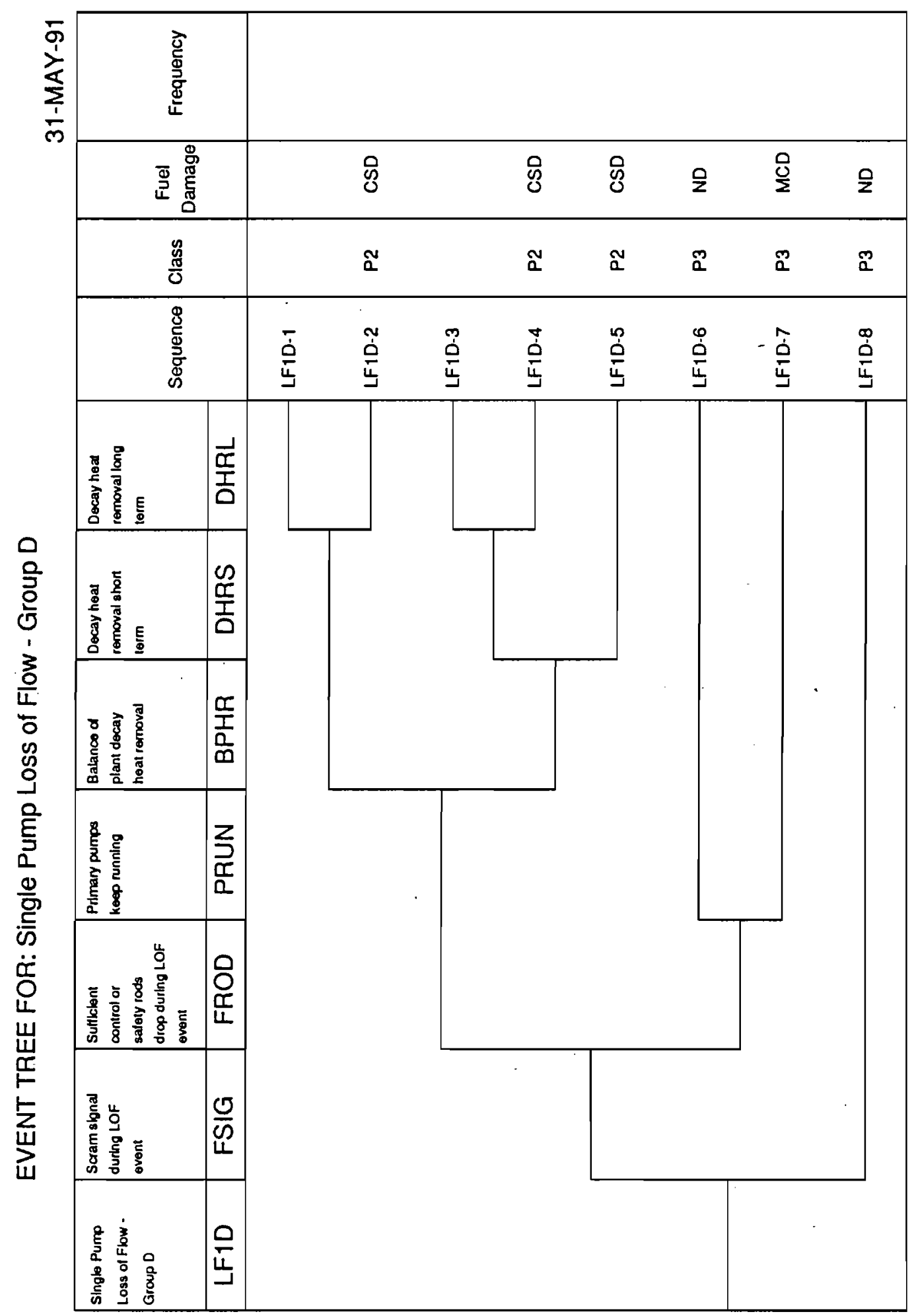

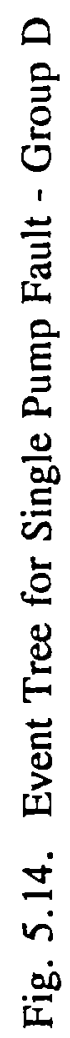




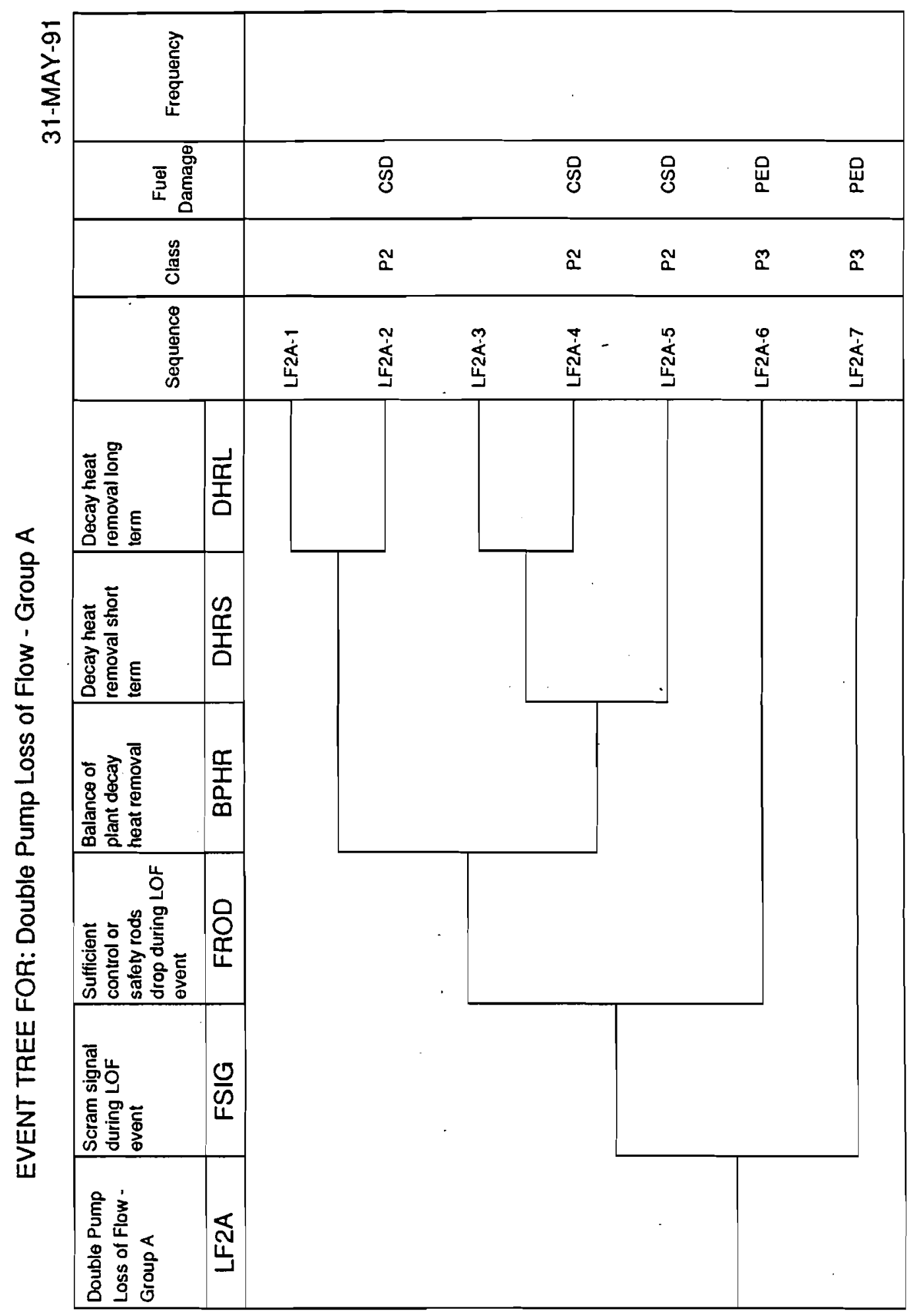

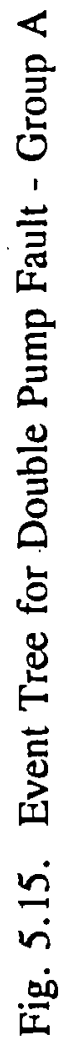


EVENT TREE FOR: Double Pump Loss of Flow - Group B .

31-MAY-91

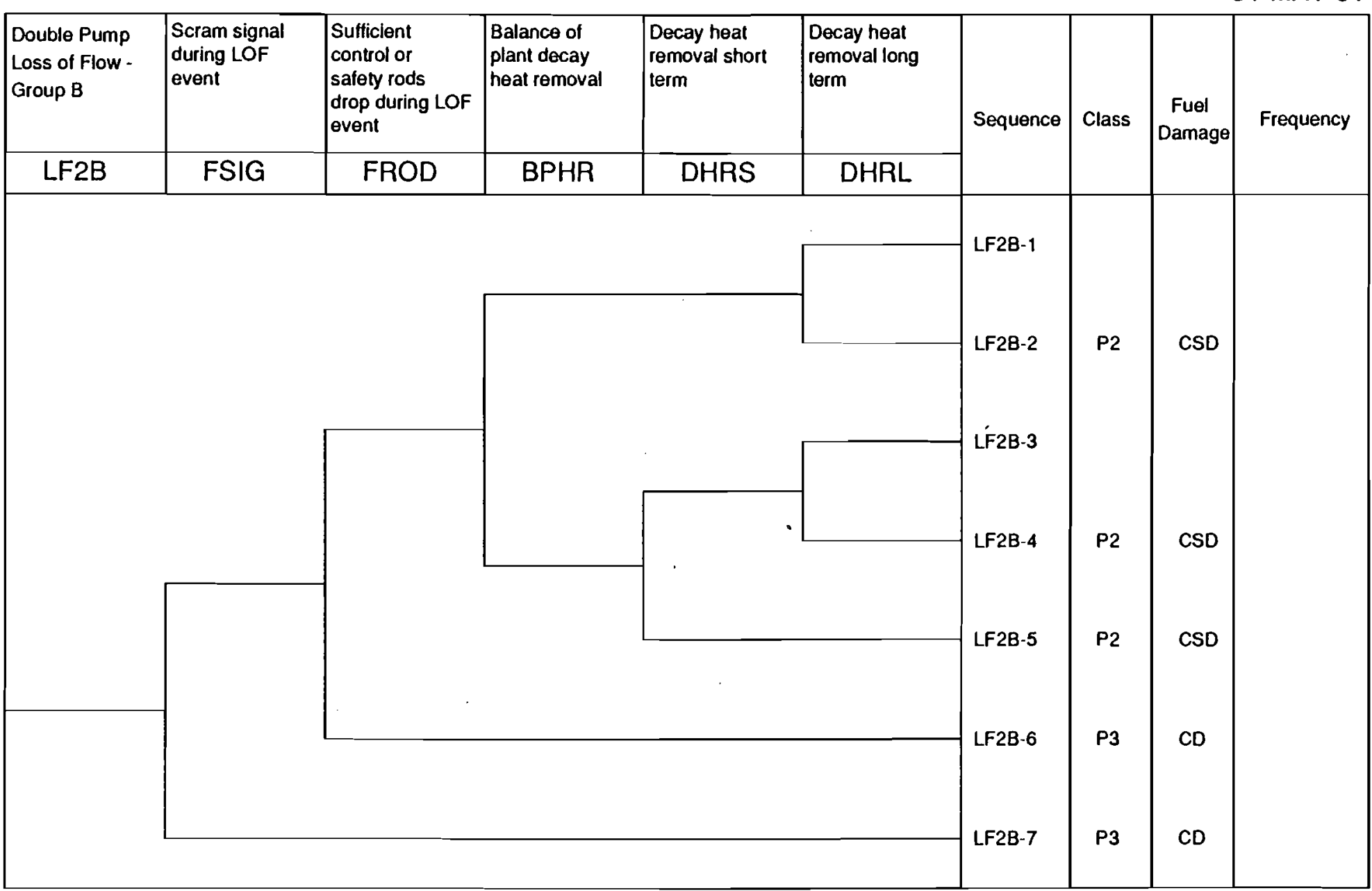

Fig. 5.16. Event Tree for Double Pump Fault - Group B 


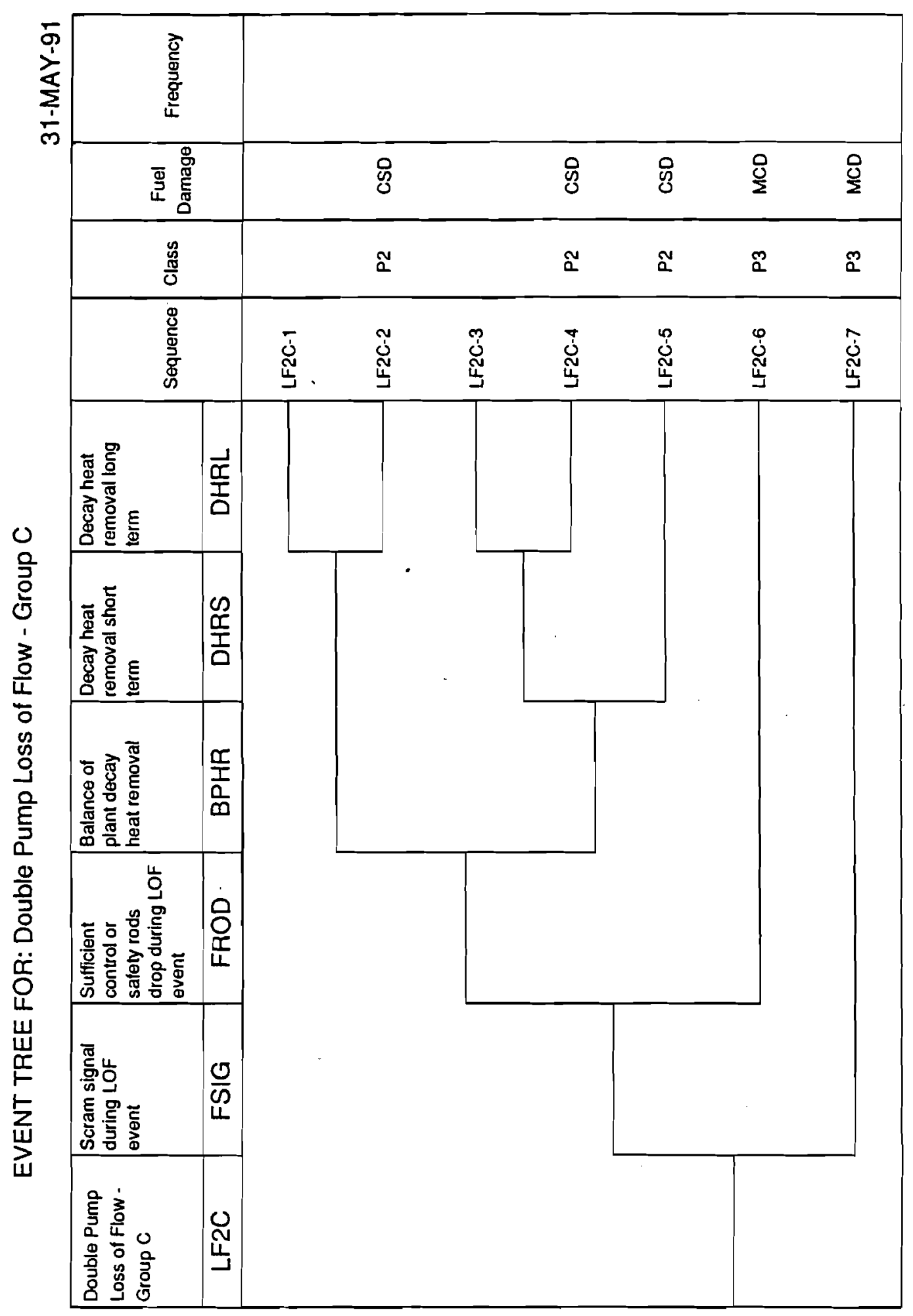

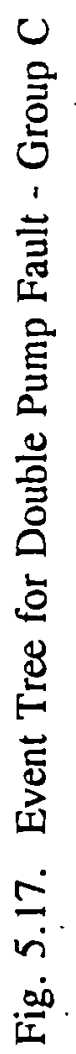




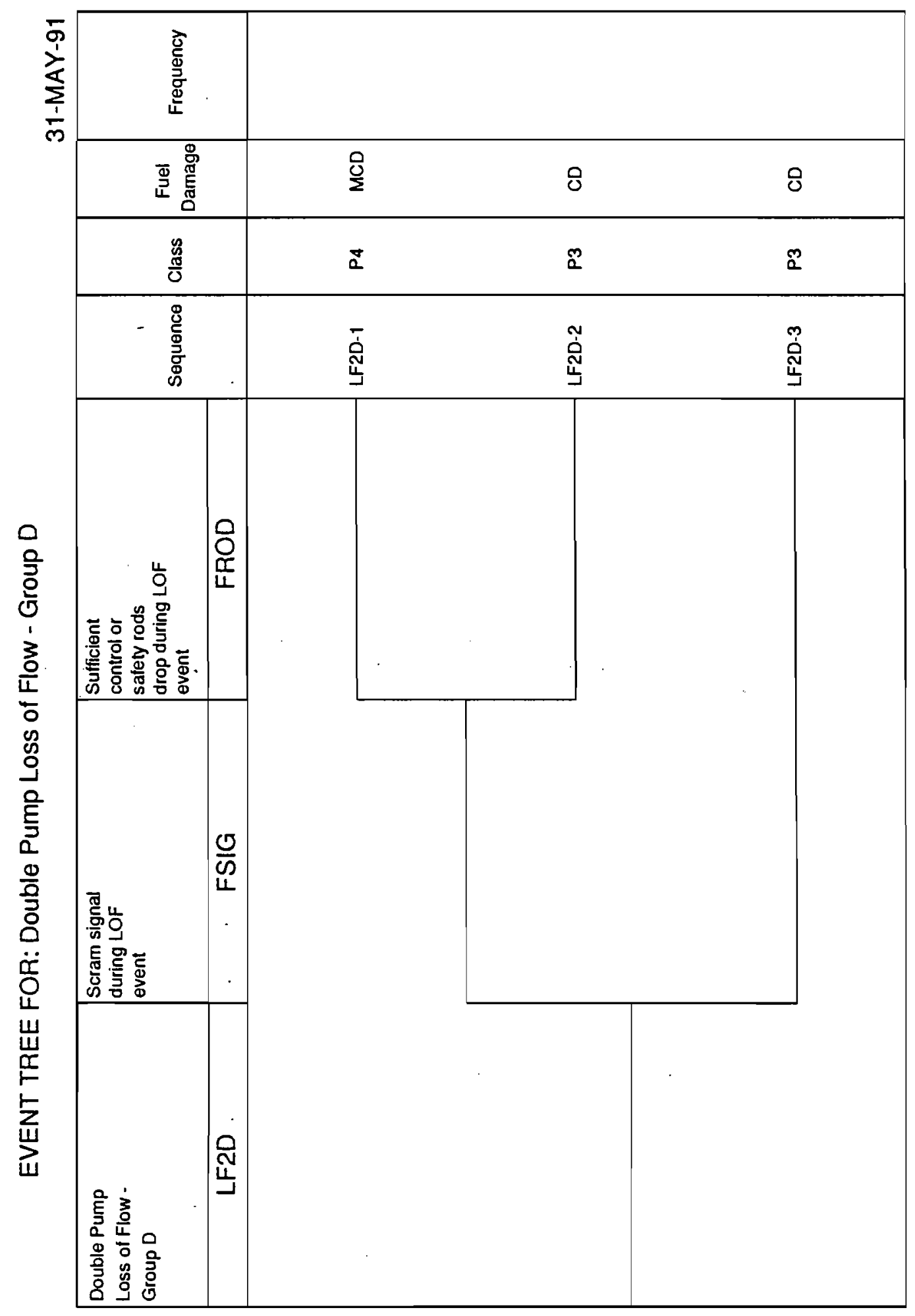

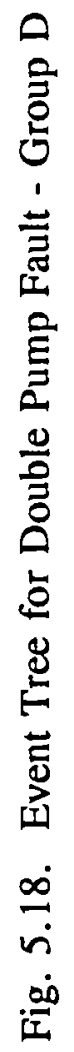




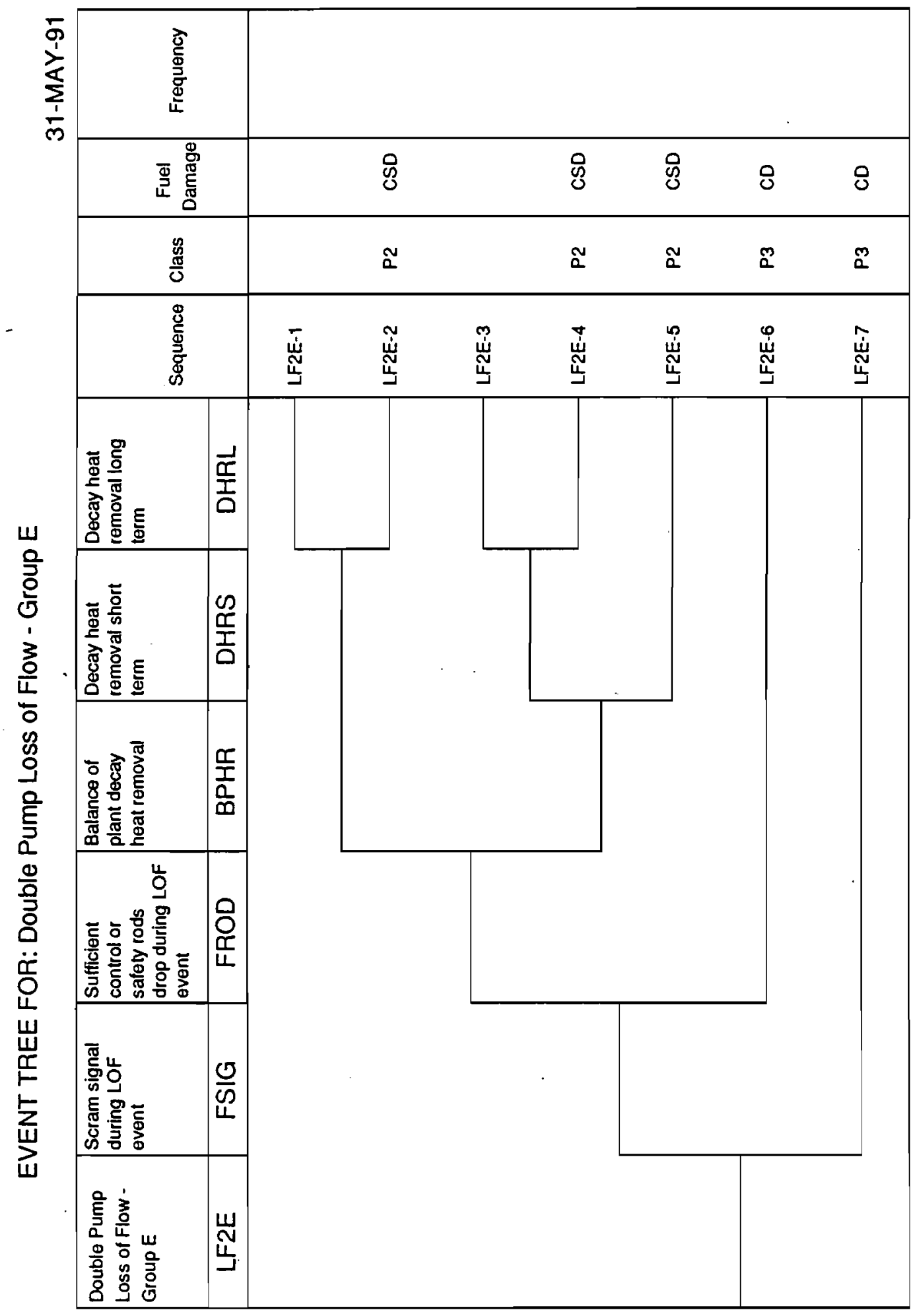

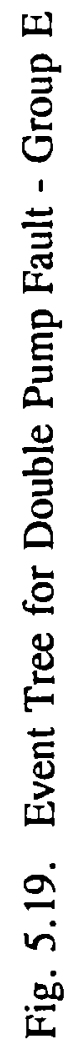




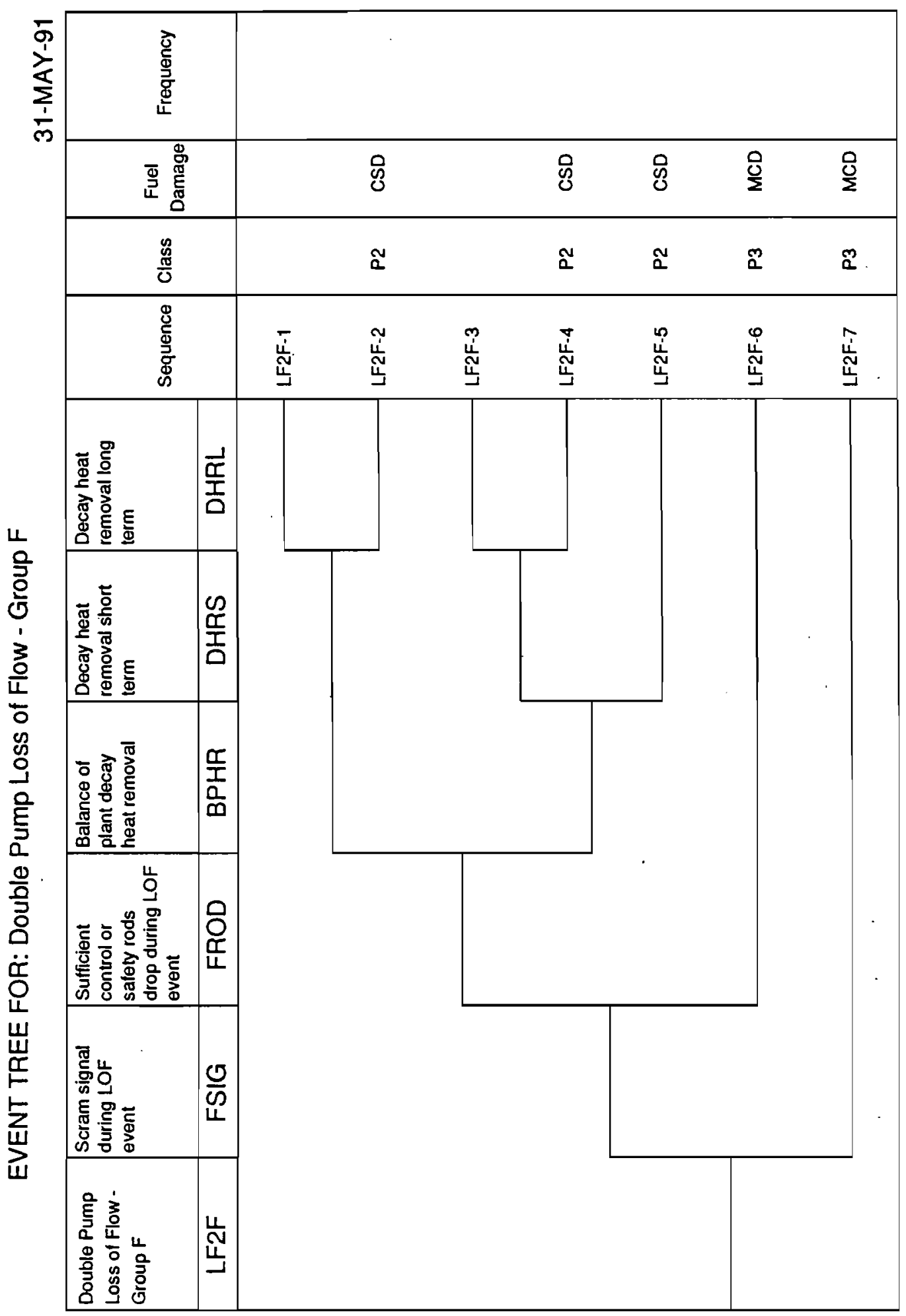

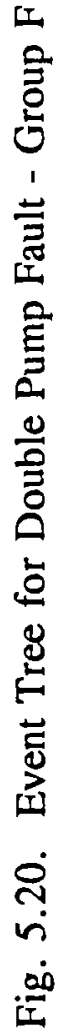




\subsubsection{Event Trees for Loss of Vital Electrical Power}

\subsubsection{Initiating Event Description}

There are two types of electrical power which when lost lead to scram of the reactor, normal power $(2400 \mathrm{~V})$ and constant power $(125 \mathrm{~V} \mathrm{AC})$. The effects of the loss of normal power are that both pumps will coast down with the full inertia of the motor generator set. The loss of constant power panels 1 and 2 leads to deenergizing of the clutches to motor generator sets 1 and 2 respectively. This is discussed in detail in the system notebooks for the pumps and motor generator sets.

\subsubsection{Normal Plant Response and Success Criteria}

In the case of loss of normal power there is a scram breaker in string A which directly initiates scram. In the case of loss of constant power panels the resulting loss of control power will cause any number of fail safe scram systems to give a scram signal. In both cases the LOF and SOT subsystems will also generate a scram signal because the direct effect is to cause a flow transient. In the case of Loss of Normal Power the emergency power is used to reduce the secondary flow to minimum conditions thus disabling the BOP as a decay heat removal mechanism.

\subsubsection{Event Trees and Accident Sequences}

Figures 5.21 and Fig. 5.22 show the event trees that apply. They are identical to those for flow transients. As shown in 5.4.2.3, the LONP transient will not cause core damage, for either protected or unprotected transients. In the protected case, the peak cladding temperature is well below the onset of eutectic attack threshold temperature, allowing the reactor to retain unlimited operating capabilities. In the unprotected case, the peak cladding temperature is great enough to lead to the reactor being in the unacceptable operating region with a potential for damage to experiments, PED; however, sodium boiling does not occur. 
III-S

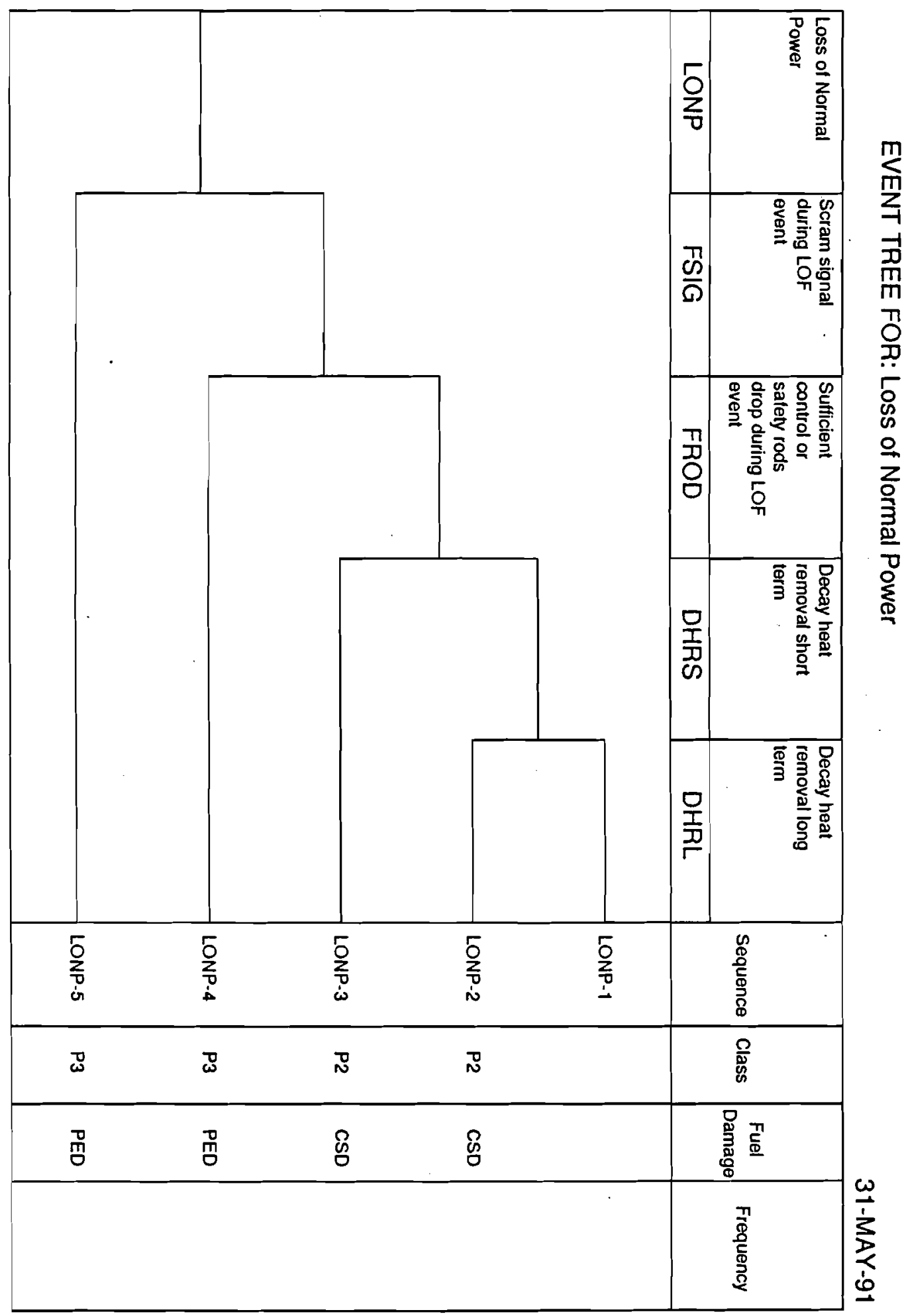




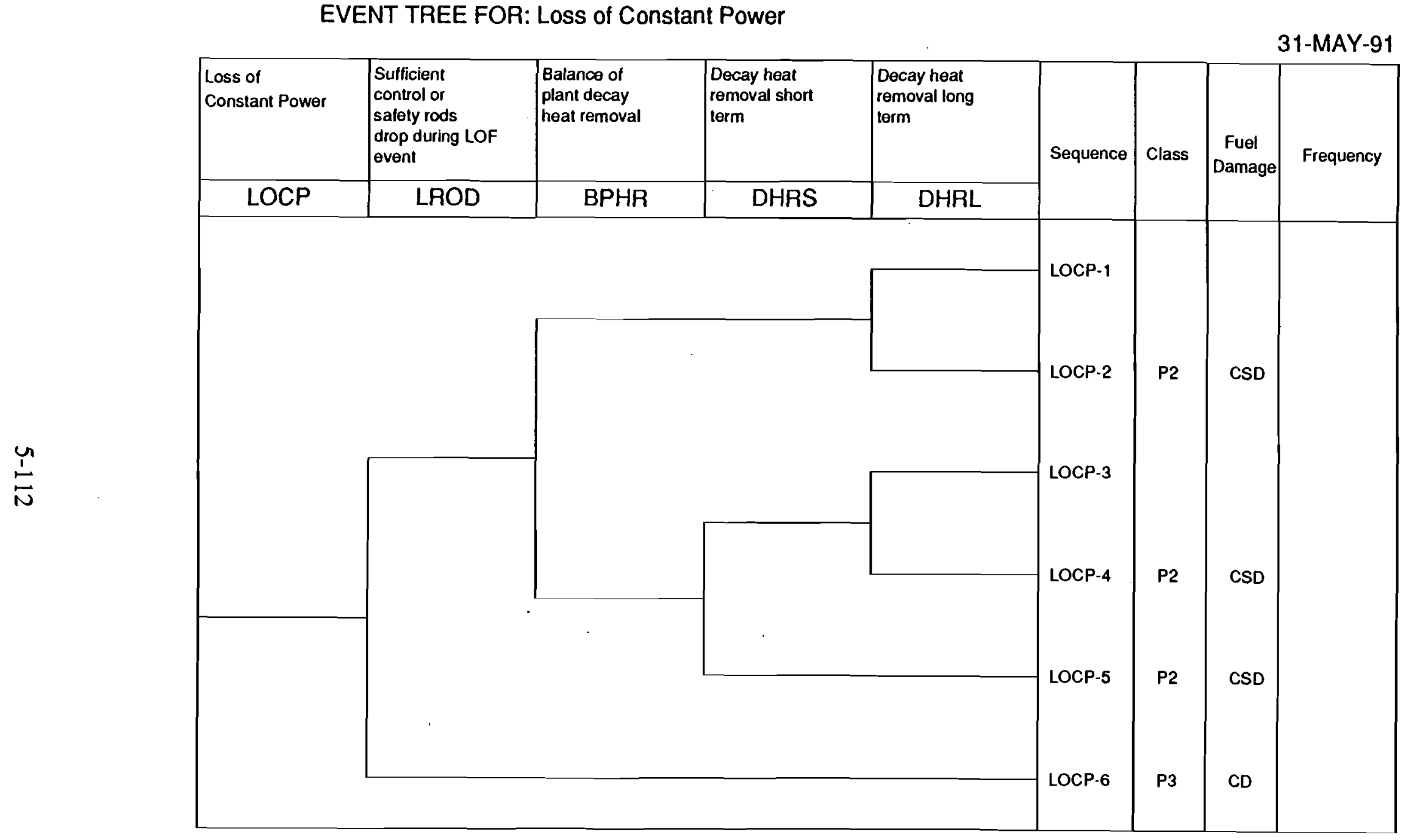

Fig. 5.22. Event Tree for Loss of Constant Power 


\subsubsection{Event Trees for Slow Reactivity Insertion}

\subsubsection{Initiating Event Description}

As discussed earlier the initiating events that lead to reactivity insertion are those due to motion of control or safety rods either singly or in combination. Other system faults which might result in reactivity insertion through reactivity feedback are treated separately (steam leak or pump overspeed leading to an overcooling and reactivity insertion via the temperature coefficient of reactivity). Other means of reactivity insertion which can be envisaged are some rearrangement of fuel on either the subassembly scale or a whole core motion (stick/slip).

\subsubsection{Normal Plant Response and Success Criteria}

Just as with other transients the normal plant response is to detect the transient, in this case with a 2 of 3 logic high flux monitoring system. The success criterion is to scram sufficient rods to shut the reactor down, sufficient being defined by the magnitude of the total insertion. If the scram signal is generated and the rods unlatch from the drive mechanism, a rod insertion initiator is terminated but it then requires rods to drop to achieve safe and stable shutdown. Decay heat removal is carried out in the usual way.

\subsubsection{Event Trees and Accident Sequences}

The event trees for reactivity insertion are shown in Figs. 5.23 to 5.26 . 
The definitions of the different slow reactivity insertion cases are given below with the associated damage category if unprotected.

$\begin{array}{llr}\text { RISA } & <20 c & \text { ND } \\ \text { RISB } & 20 c-65 c & \text { MCD } \\ \text { RISC } & 65 c-100 \$ & \mathrm{CD} \\ \text { RISD } & >1.00 \$ & \mathrm{CD}\end{array}$

The basis for these assignations is given in 5.4.2.5. 
EVENT TREE FOR: Slow Reactivity Insertion - Group A $\quad(<0.20 \$)$

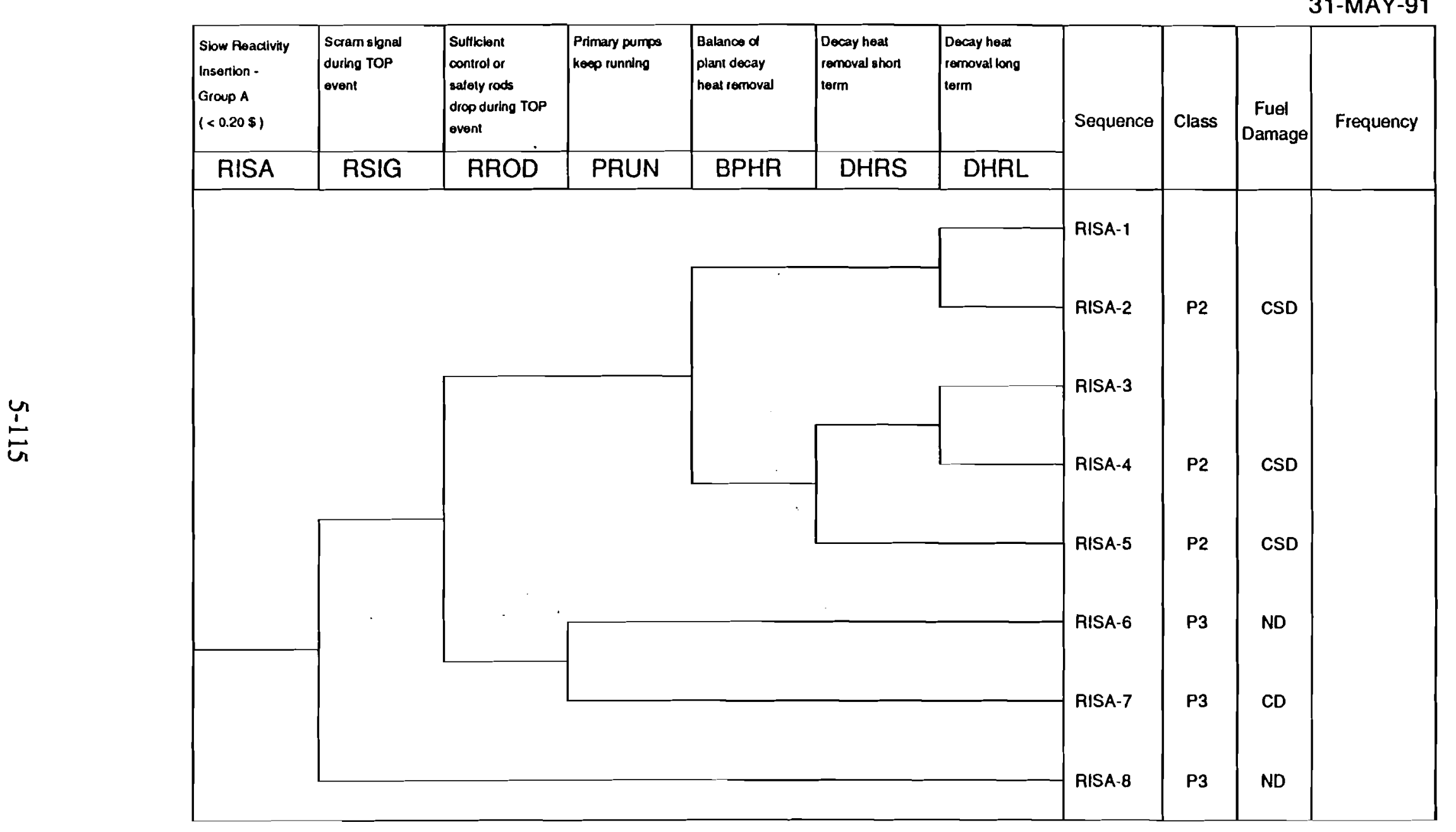

Fig. 5.23. Event Tree for Reactivity Insertion - RISA 


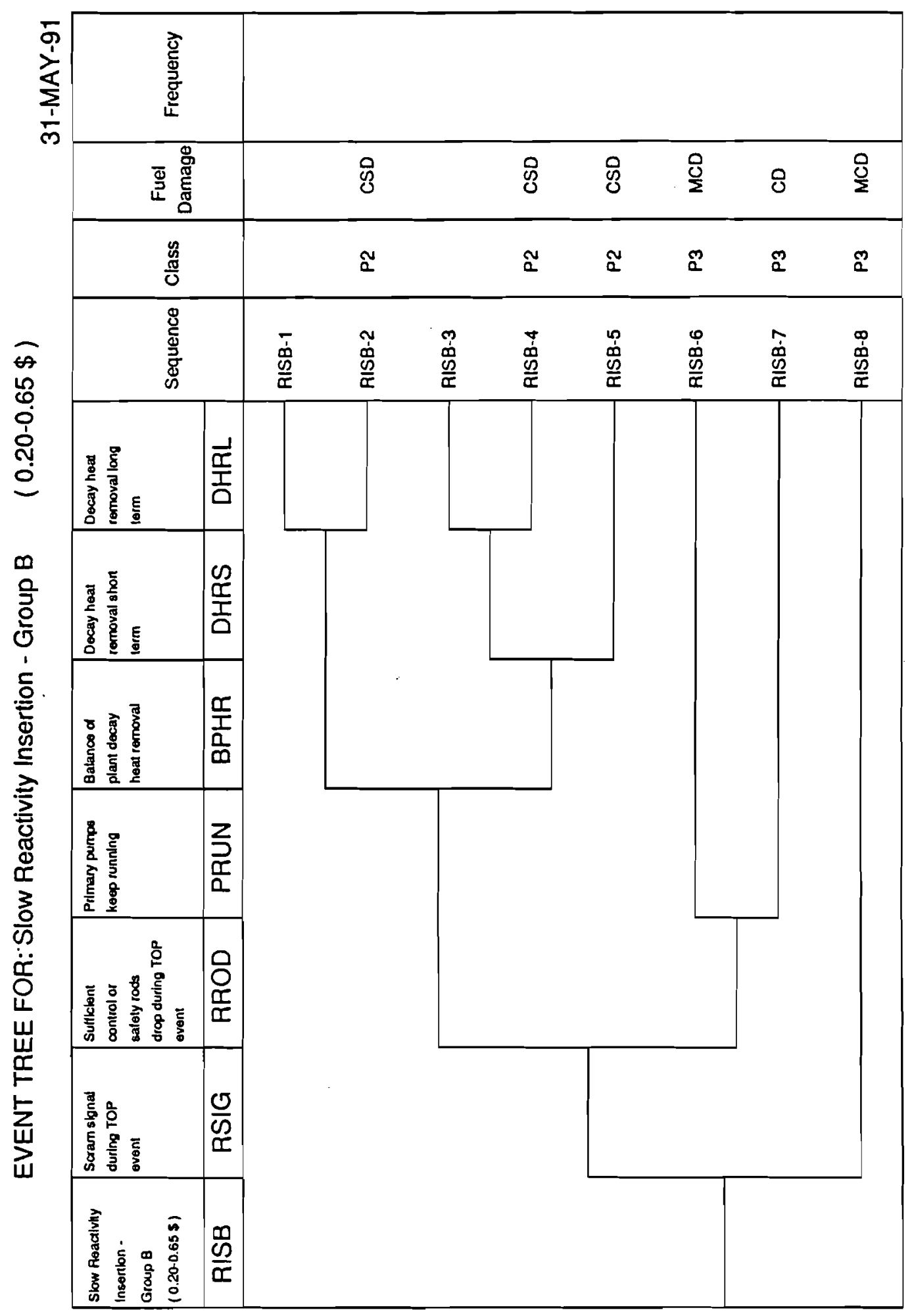

 


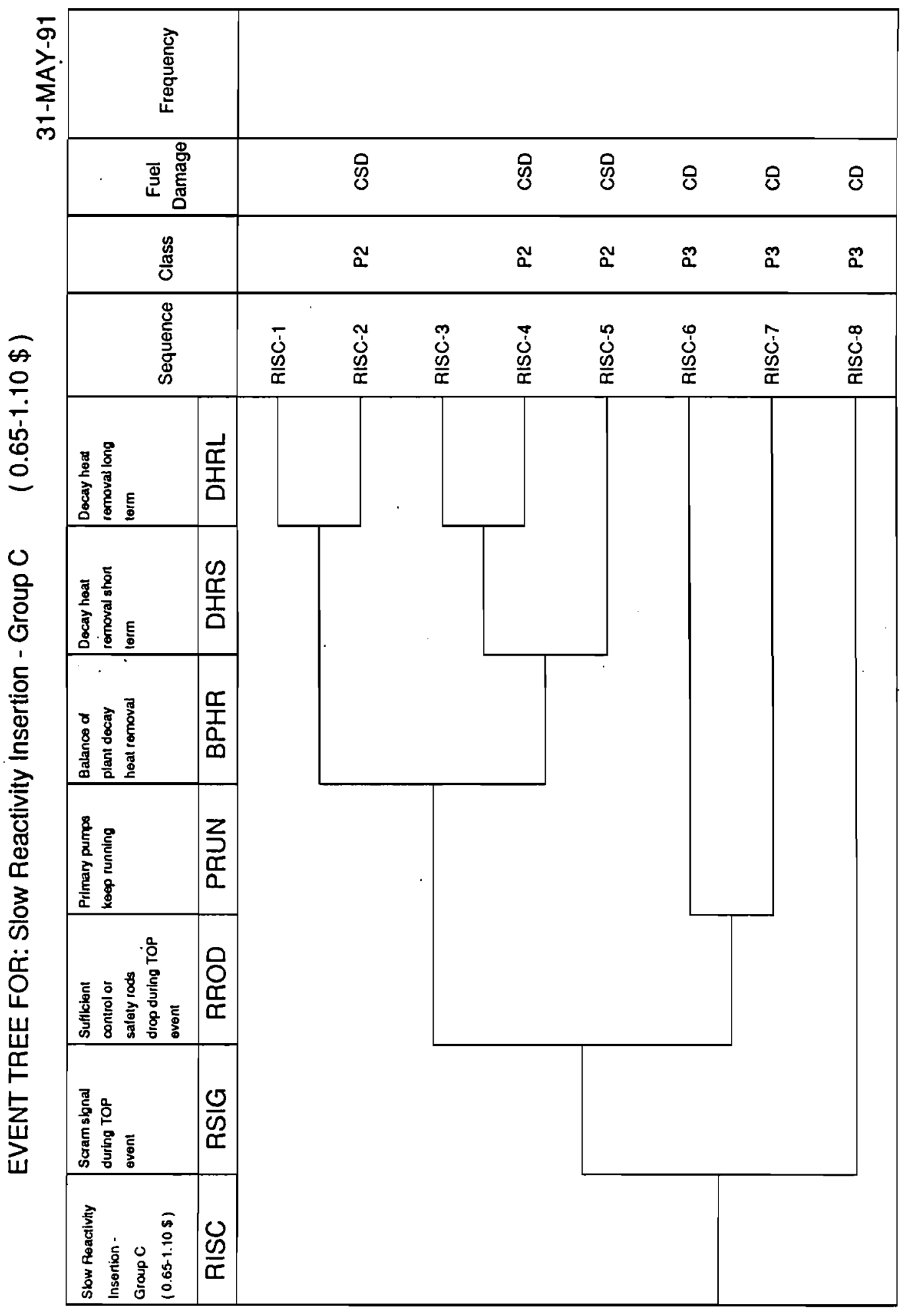

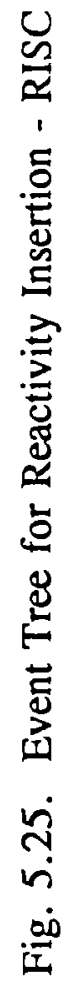




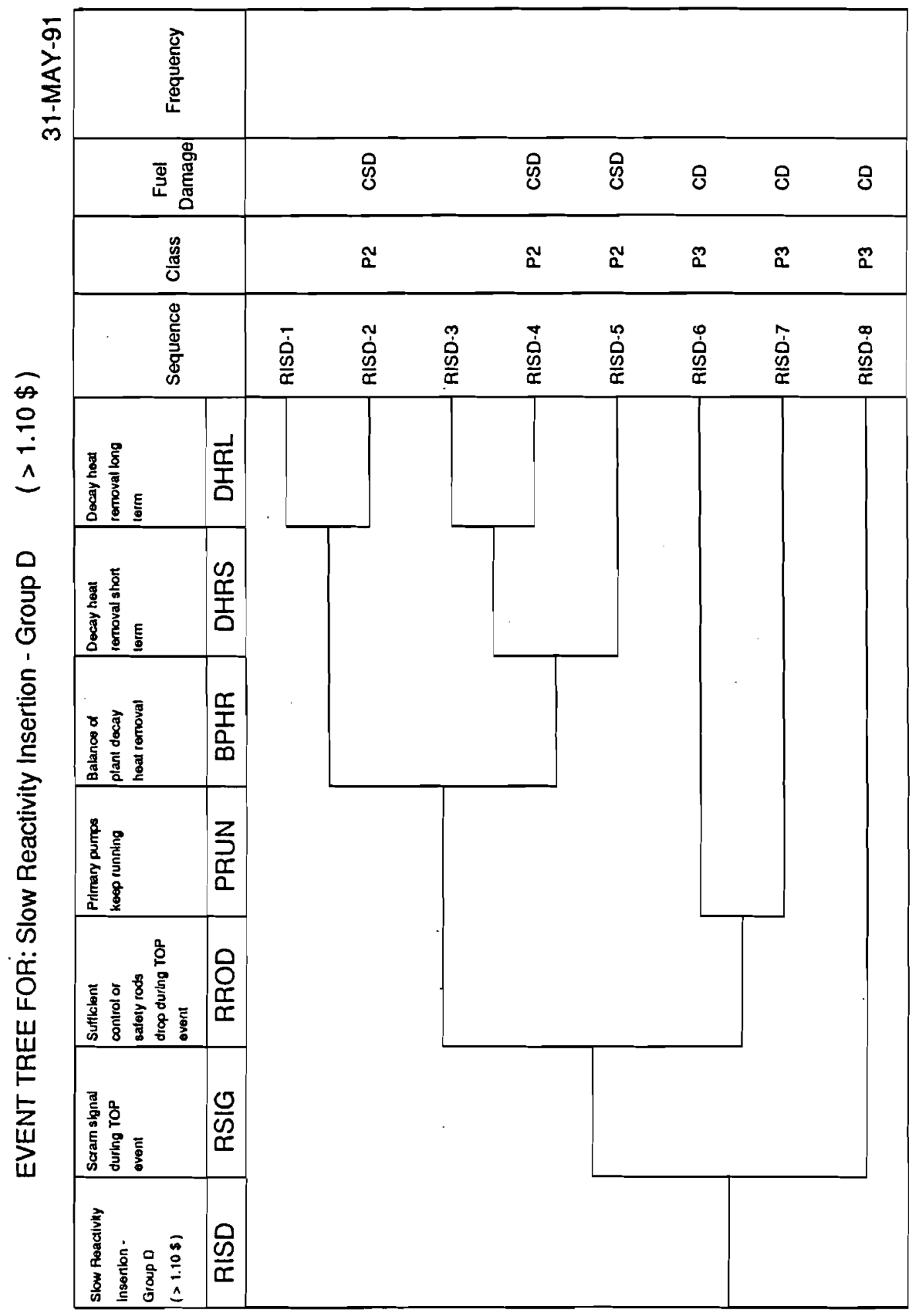

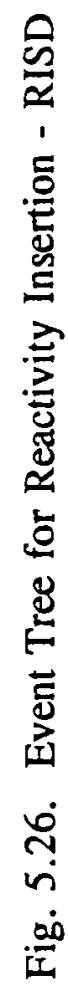




\subsubsection{Event Trees for Fast Reactivity Insertions}

\subsubsection{Initiating Event Definition}

The initiating event being considered here is any form of reactivity insertion that can insert sufficient reactivity quickly. In the case of a large reactivity input the amount is large enough to cause damage even if scram occurs. The only causative mechanisms that have been identified are vessel failures which lead to a 2-in $(50.8 \mathrm{~mm})$ drop in the core with respect to the control rods. The small reactivity insertion, of less than $0.35 \$$, can be identified with a substantial rearrangement of material in an experimental assembly.

\subsubsection{Normal Plant Response and Success Criteria}

The normal plant response is to detect power change, scram and remove the decay heat. The success criteria are shown in Table 5.20.

\subsubsection{Event Trees and Accident Sequences}

Fig. 5.27 gives the event tree for a small fast reactivity insertion. In the case of large fast reactivity insertion (RIFL) the event tree of Fig. 5.28 describes the accident sequence. By definition of the initiating event, damage occurs in both the protected and unprotected cases, and this belongs to the $\mathrm{P} 1$ category. 


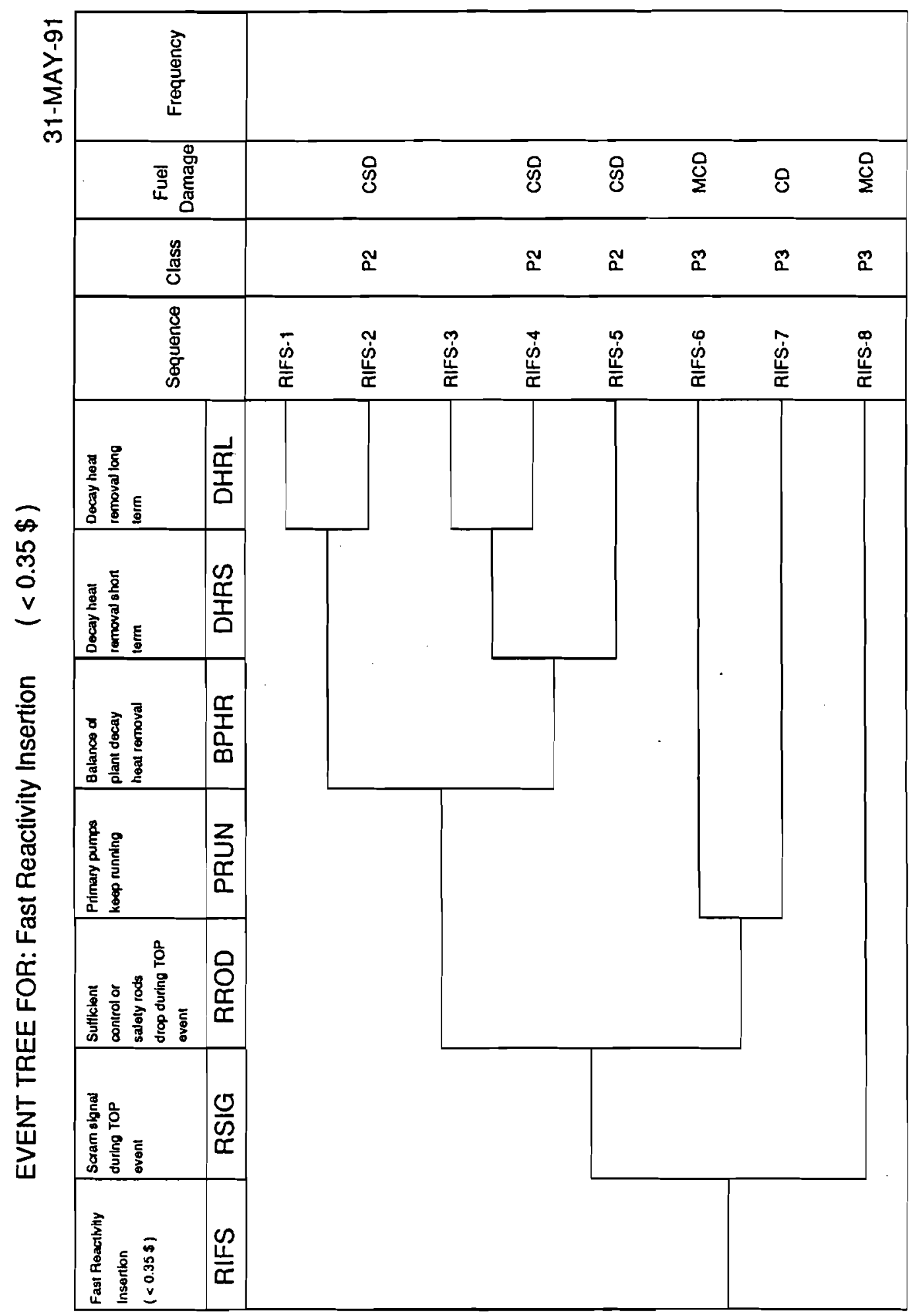

 
EVENT TREE FOR: Fast Reactivity Insertion $(>1.00 \$)$

31-MAY-91

$\stackrel{n}{n}$

\begin{tabular}{|r|r|r|r|l|}
\hline Fast Reactivity Insertion $(>1.00 \$)$ & Sequence & Class & $\begin{array}{c}\text { Fuet } \\
\text { Damage }\end{array}$ & Frequency \\
\hline RIFL & & & & \\
\hline & RIFL-1 & P1 & CD & \\
\hline & & & & \\
\end{tabular}

Fig. 5.28. Event Tree for Large Reactivity Insertion 


\subsubsection{Event Trees for Loss of Primary Heat Sink}

\subsubsection{Initiating Event Description}

This class of initiating events includes all faults on the secondary sodium and balance of plant that can be construed as leading to a significant reduction a total loss of the primary heat sink. Specifically excluded is the steam generator tube rupture, which is treated separately.

\subsubsection{Normal Plant Response and Success Criteria}

In the event of a fault on the balance of plant the operators perform a manual scram. Since the system response to an unprotected loss of heat sink is reduction of power no automatic scram should take place. The decay heat is removed by the shutdown coolers. For those faults which leave partial heat removal capability it is assumed that the operators dump the secondary sodium, others such as sodium leak and loss of feedwater result in mandatory dump of sodium.

\subsubsection{Event Trees and Accident Sequences}

The event trees associated with the loss of primary heat sink are shown in Figs. 5.29 and 5.30. In the event of scram the shutdown coolers must function to remove decay heat; failure to do so leads to protected loss of total heat sink. Failure to scram leads to the unprotected loss of primary or total heat sink and this transient has been shown by direct experimentation to lead to a benign outcome. In fact this then bears upon which automatic protection was removed from the EBR-II scram system. 


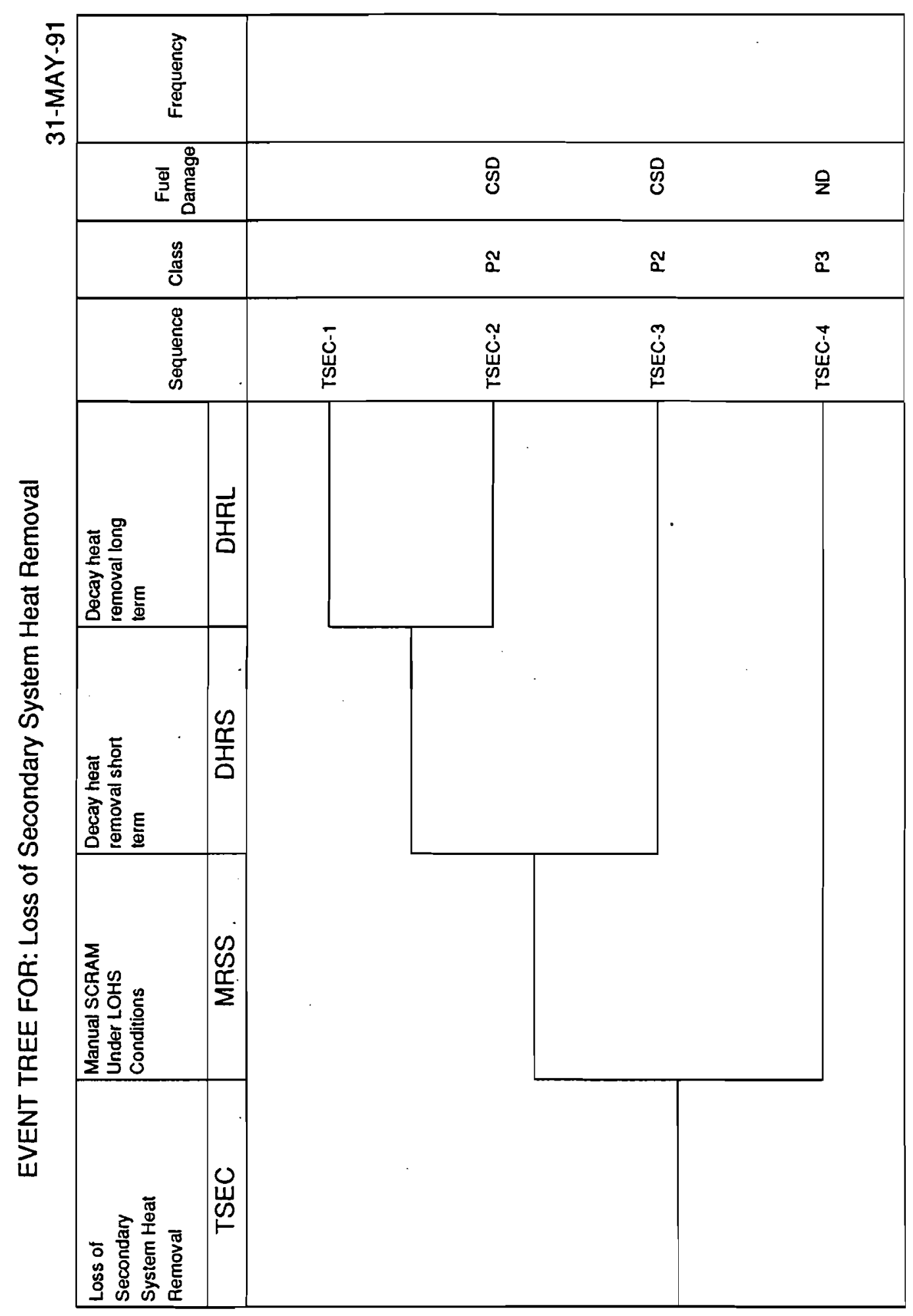

年 


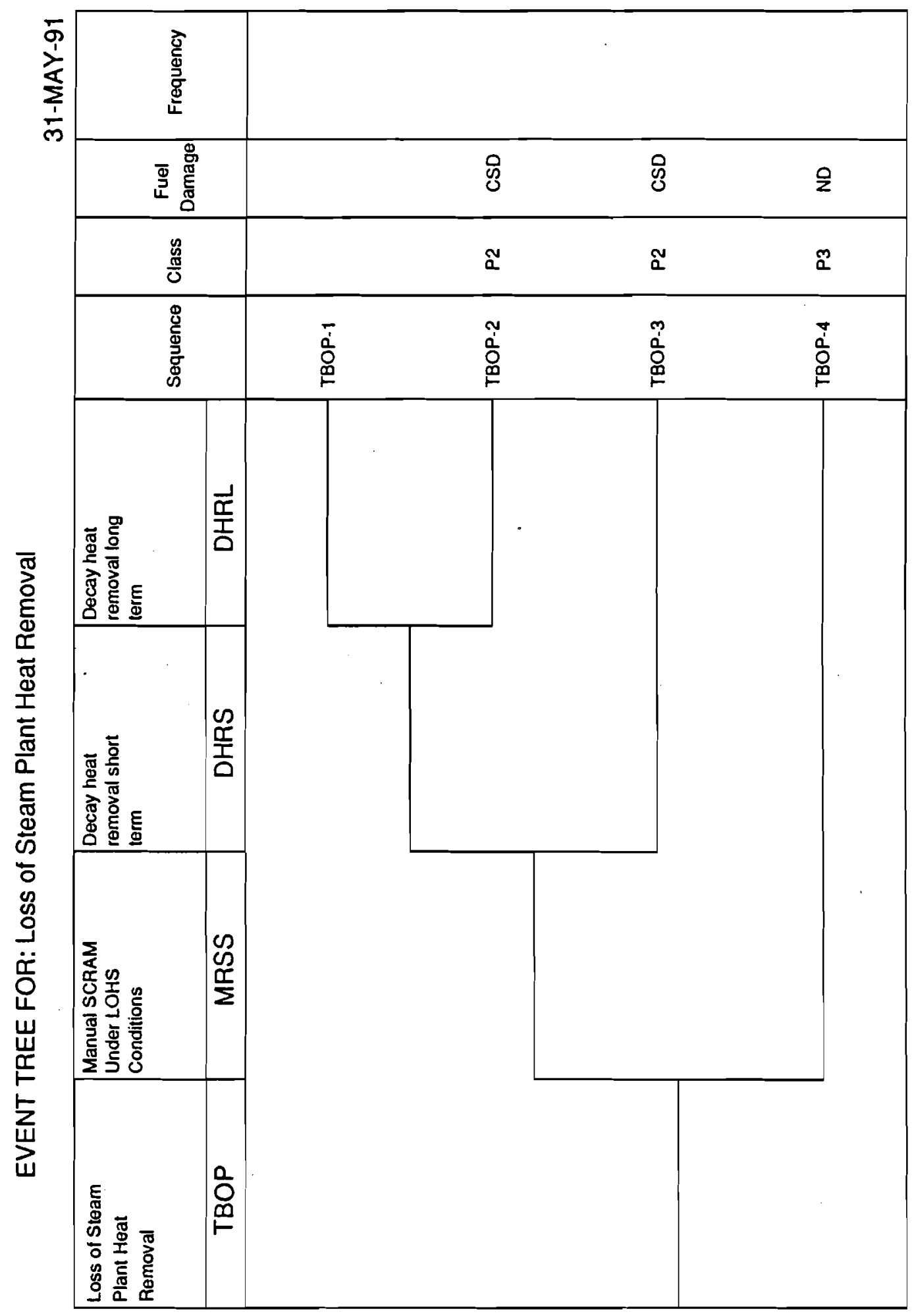

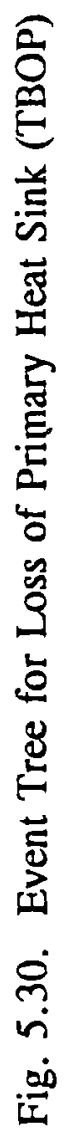




\subsubsection{Event Trees for Overcooling}

\subsubsection{Initiating Event Description}

This initiating event class includes those means by which the primary system could be cooled down. This represents a threat in the unscrammed case because the cooling of the inlet leads to reactivity insertion. There are three types of events that contribute to this category, but only the steam leak has the potential to lead to core damage. The other two events caused by either primary or secondary pump overspeed only lead to damage through the Loss of Heat Sink.

\subsubsection{Normal Plant Response and Success Criteria}

The response of the system to an overcooling event is that of any other overpower, that is to say the high flux protection should generate a scram signal. The scram signal can direct the accident to a loss of heat sink by one of two paths; either successfully scramming or by tripping the secondary pump.

\subsubsection{Event Trees and Accident Sequences}

The event tree for the overcooling sequence is exactly the same as that for reactivity insertion except that the additional possibility that the secondary pump fails to trip must be included. The three cases are given in Figs. 5.31, 5.32 and 5.33. 
EVENT TREE FOR: Overcooling by Major Steam Leak

31-MAY-91

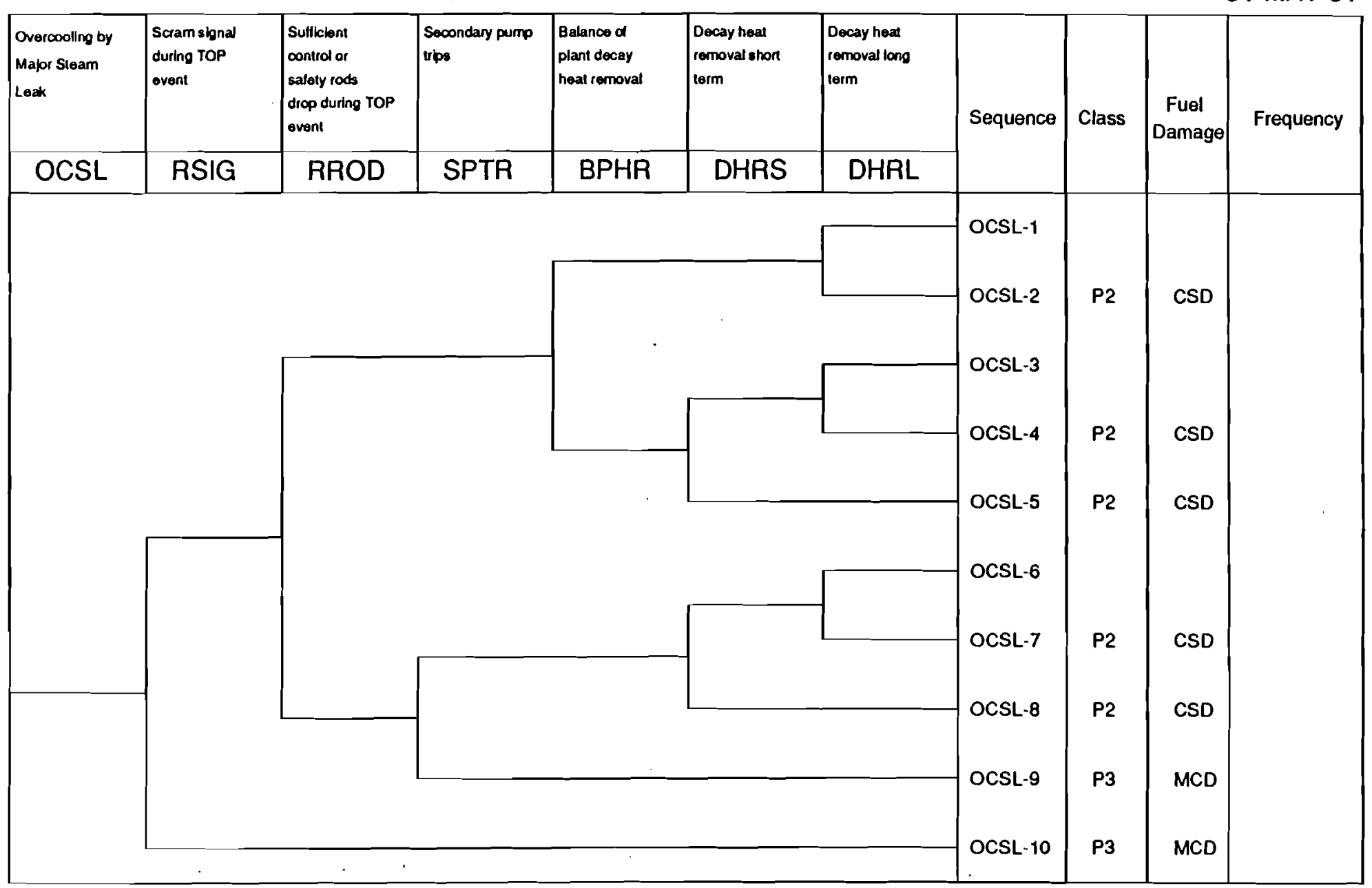

Fig. 5.31. Event Tree for Overcooling (Steam Lrak) 


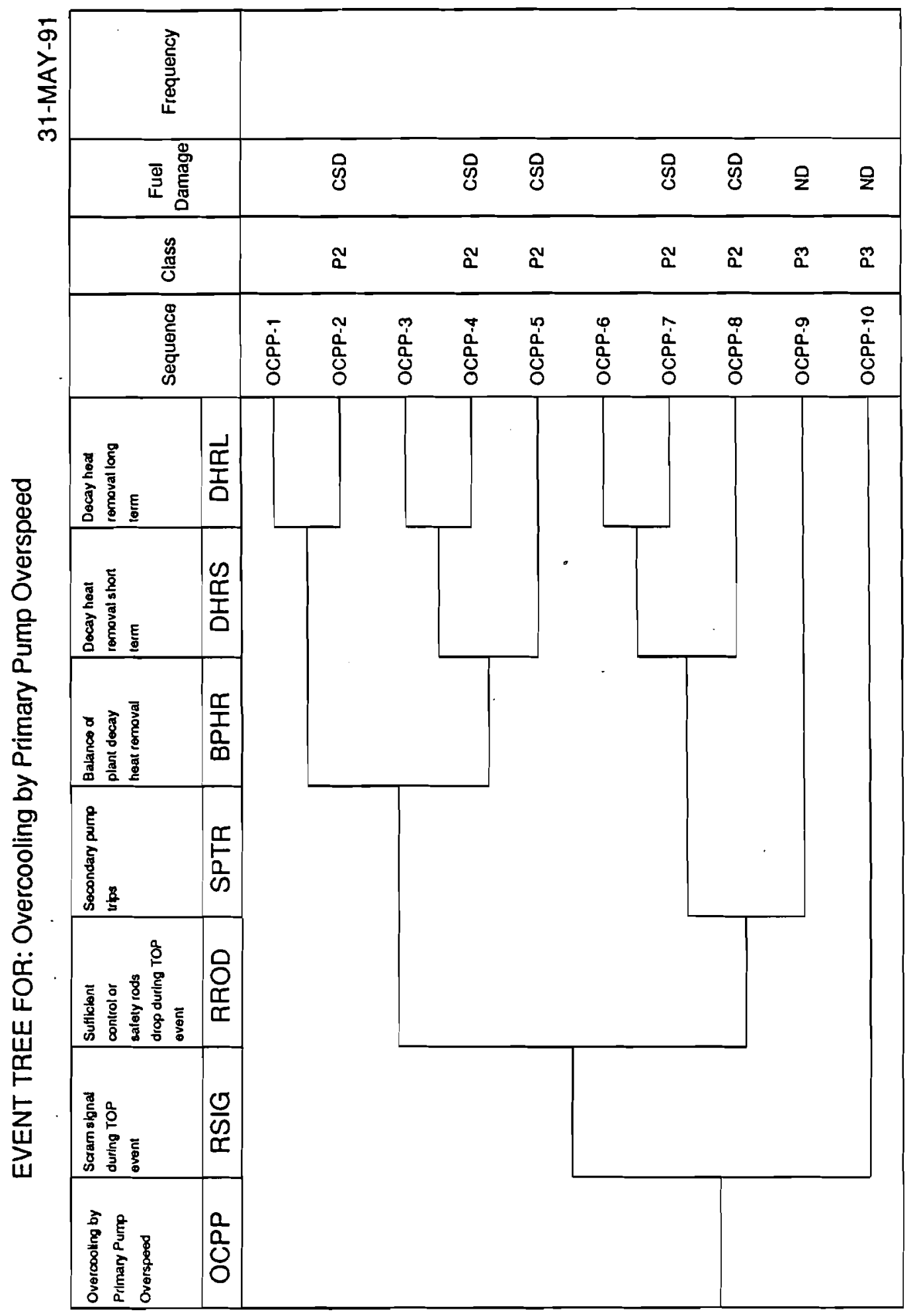

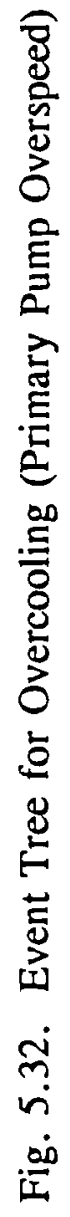




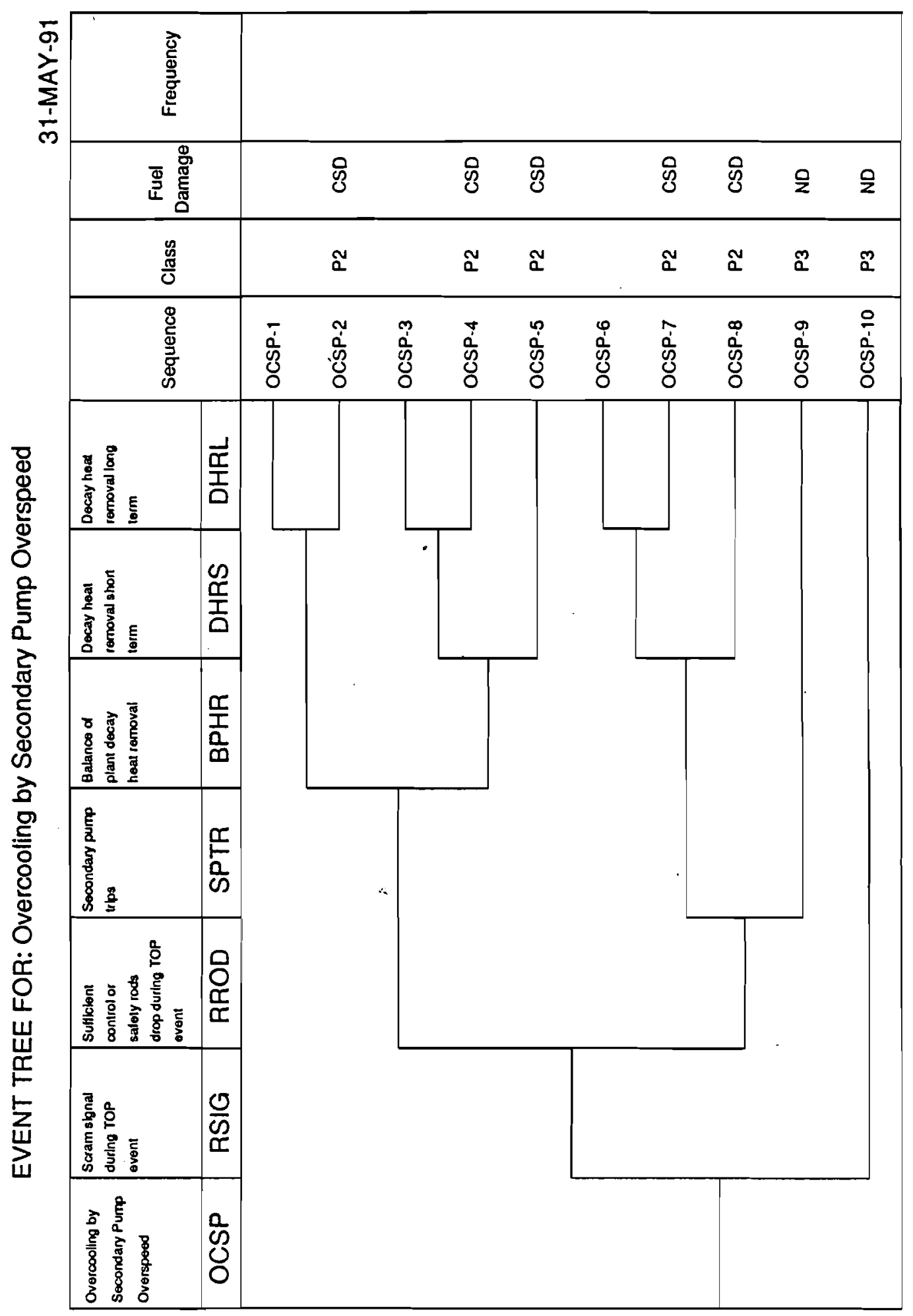

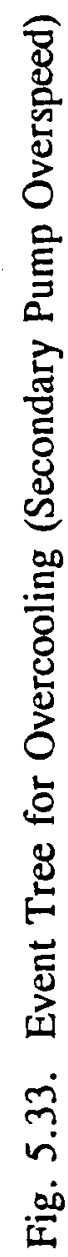




\subsubsection{Event Trees for Steam Generator Tube Rupture}

\subsubsection{Initiating Event Description}

The EBR-II steam generator transfers heat from secondary sodium on the shell side to water/steam on the tube side. The duplex heat transfer tube provides the barrier between the sodium and water. A leak in the duplex tube would result in the injection of high pressure steam/water into liquid sodium. A failure in the welds which connect the duplex tubes to the duplex tube sheets results in leakage into the airspace which has leak detectors. An undetected failure in the outer-tube weld to its tube sheet and an undetected failure in the inner-tube weld to its tube sheet are required to obtain a sodium/water reaction in the airspace between the two tube sheets. The vigorous sodium/water reaction would produce heat, hydrogen, and sodium compounds. Left unchecked, the size of the original leak would increase due to self-wastage of the failed steam tube, and other steam generator tubes in the vicinity of the leak would also corrode, producing extensive damage to the steam generator [5.38].

Besides possibly producing extensive damage to steam generator components, a steam generator failure accident in EBR-II could result in damage to other secondary and steam system components. Restoration of the secondary and steam systems could entail considerable repair costs and long-term plant unavailability. As regards to the PRA, more important considerations are the consequences of a steam generator failure accident to the integrity of the primary system. A possible consequence of an accident would be the loss of heat transfer capabilities of the IHX. A more remote outcome of an accident would be physical damage to the IHX due to pressure loading by acoustic pressures generated by the reaction or due to corrosive attack by reaction products.

\subsubsection{Normal Plant Response and Success Criteria}

The normal plant response consists of two parts - detection and mitigation. Detection of the steam generator tube rupture accident is accomplished by 
activation of the Hydrogen Meter Leak Detector (HMLD) system or by the activation of flow indicators in the sodium pressure relief system. Mitigation of the accident is achieved passively by the sodium pressure relief system or actively by dumping of sodium and water from the secondary system and the steam generator. The accident will be successfully terminated if detection and mitigation is accomplished before the IHX is damage. If the steam generator tube rupture endangers the integrity of the IHX by pressure loading or corrosive attack, the accident is classified as a P5 plant transient. Fuel damage classification is dependent upon the operating status of the reactor. If the IHX fails, contaminants will pass from the secondary into the primary system. The contaminants could conceivably cause plugging of subassembly coolant channels or control rod drivelines. If the reactor is scrammed before the IHX fails, the possible plugging of reactor coolant channels will not damage the fuel (fuel damage class ND). If the reactor is at power when the IHX fails, reactor coolant channels may become plugged creating local hot spots in the core. In this case, the accident is given a core/structural damage (CSD) classification.

\subsubsection{Event Trees and Accident Sequences}

The event trees for a steam tube rupture failure in a superheater module and an evaporator module are shown in Figs. 5.34 and 5.35, respectively. A tube rupture in a superheater module is different from a tube rupture in an evaporator module because there is a direct pressure relief path from the superheater modules that passes through the IHX, which facilitates the transfer of contaminants to the IHX. A detailed discussion of the top events and the accident sequences is found in Appendix D.3. 
EVENT TREE FOR: Superheater Tube Failure

31-MAY-91

\begin{tabular}{|c|c|c|c|c|c|c|c|c|c|c|c|}
\hline $\begin{array}{l}\text { Superheater } \\
\text { Tube Failure }\end{array}$ & $\begin{array}{l}\text { Less than } 2 \text { DEG } \\
\text { Fallures whinh } \\
\text { First } 0.2 \\
\text { Seconds }\end{array}$ & $\begin{array}{l}\text { Leak Escalales } \\
\text { whin First } \\
\text { Minure }\end{array}$ & \begin{tabular}{|l} 
LKDT \\
\end{tabular} & $\begin{array}{l}\text { Prossure Rellef } \\
\text { System } \\
\text { Activates }\end{array}$ & $\begin{array}{l}\text { Water and } \\
\text { Sodium Dump } \\
\text { Bofore Leak } \\
\text { Escalation }\end{array}$ & $\begin{array}{l}\text { Pressure Rellied } \\
\text { by Secondary } \\
\text { Boundary } \\
\text { Fallure }\end{array}$ & $\begin{array}{l}\text { Reactor } \\
\text { Shutdown Before } \\
\text { IHX Fallure }\end{array}$ & Sequence & Class & $\begin{array}{c}\text { Fuel } \\
\text { Damage }\end{array}$ & Frequency \\
\hline . & & & & & & & & $\begin{array}{l}\text { SPTR-1 } \\
\text { SPTR-2 } \\
\text { SPTR-3 } \\
\text { SPTR-4 } \\
\text { SPTR-5 } \\
\text { SPTR-6 } \\
\text { SPTR-7 } \\
\text { SPTR-8 } \\
\text { SPTR-9 } \\
\text { SPTR-10 } \\
\text { SPTR-11 } \\
\text { SPTR-12 } \\
\text { SPTR-13 } \\
\text { SPTR-14 } \\
\text { SPTR-15 } \\
\text { SPTR-16 } \\
\text { SPTR-17 } \\
\text { SPTR-18 } \\
\text { SPTR-19 } \\
\text { SPTR-20 } \\
\text { SPTR-21 } \\
\text { SPTR-22 } \\
\text { SPTR-23 }\end{array}$ & $\begin{array}{l}\text { P5 } \\
\text { P5 } \\
\text { P5 } \\
\text { P5 } \\
\text { P5 } \\
\text { P5 } \\
\text { P5 } \\
\text { P5 } \\
\text { P5 } \\
\text { P5 } \\
\text { P5 } \\
\text { P5 } \\
\text { P5 } \\
\text { P5 } \\
\text { P5 }\end{array}$ & $\begin{array}{l}\text { ND } \\
\text { CSD } \\
\text { ND } \\
\text { CSD } \\
\text { ND } \\
\text { CSD } \\
\text { CSD } \\
\text { ND } \\
\text { CSD } \\
\text { ND } \\
\text { CSD } \\
\text { ND } \\
\text { CSD } \\
\text { CSD } \\
\text { CSD }\end{array}$ & \\
\hline
\end{tabular}

Fig. 5.34. Event Tree for A Steam Tube Rupture in A Superheater Module 
EVENT TREE FOR: Evaporator Tube Rupture

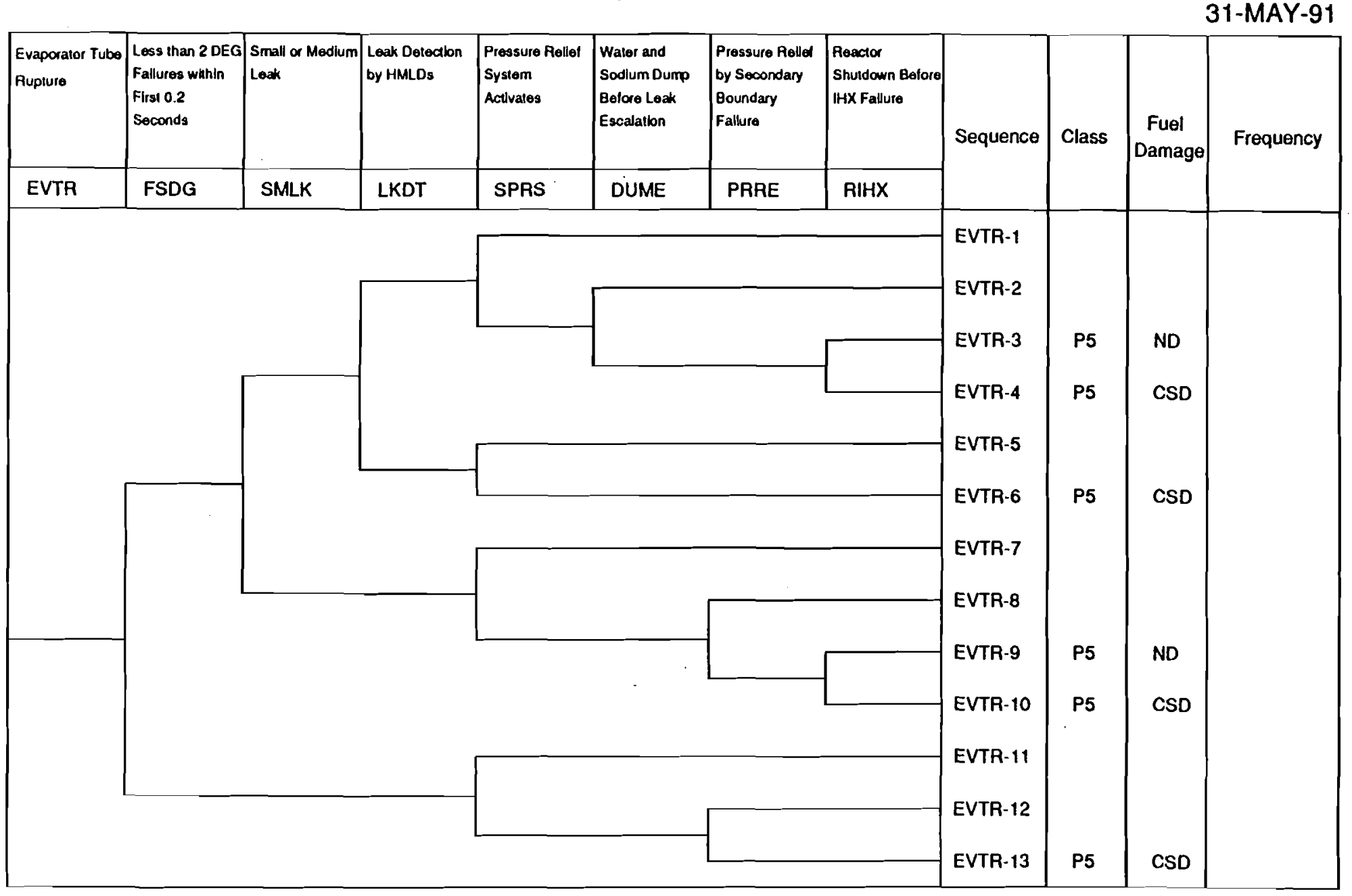

Fig. 5.35. Event Tree for A Steam Tube Rupture in An Evaporator Module 


\subsubsection{Event Tree for Delaved Loss of Circulation}

\subsubsection{Initiating Event Description}

This event includes all causes of detected ruptures and leaks of primary sodium sufficient to uncover the Z-pipe to IHX connection. The concern is that, having detected a sodium leak and having shutdown successfully (there can be many hours to shutdown before events of consequence occur), if the pumps stop operating an argon cover gas bubble forms in the outlet sodium Z-pipe thus stopping natural circulation heat removal.

\subsubsection{Normal Plant Response and Success Criteria}

The definition of the initiating event assumes that the reactor is successfully scrammed. The important question is whether the primary pumps are still operating for one hour after shutdown. Decay-heat removal is carried out by shutdown and shield cooling systems.

\subsubsection{Event Tree and Accident Sequences}

The event tree in Fig. 5.36 shows the sequences for this initiating event. Core damage can be avoided if deenergizing the pumps is delayed long enough after the reactor shutdown. If the loss of pumping power is delayed 1 hour after shutdown, the core will not be damaged however the peak coolant temperature predicted is only slightly less than the sodium boiling temperature. The Emergency Procedures allow the operators to remove the pumping power after a minimum of one hour. This will be assumed to take place at one hour. Therefore, even if decay heat removal is successful, there is potential for damage to experiments. 
EVENT TREE FOR: Loss of Circulation - Delayed

31-MAY-91

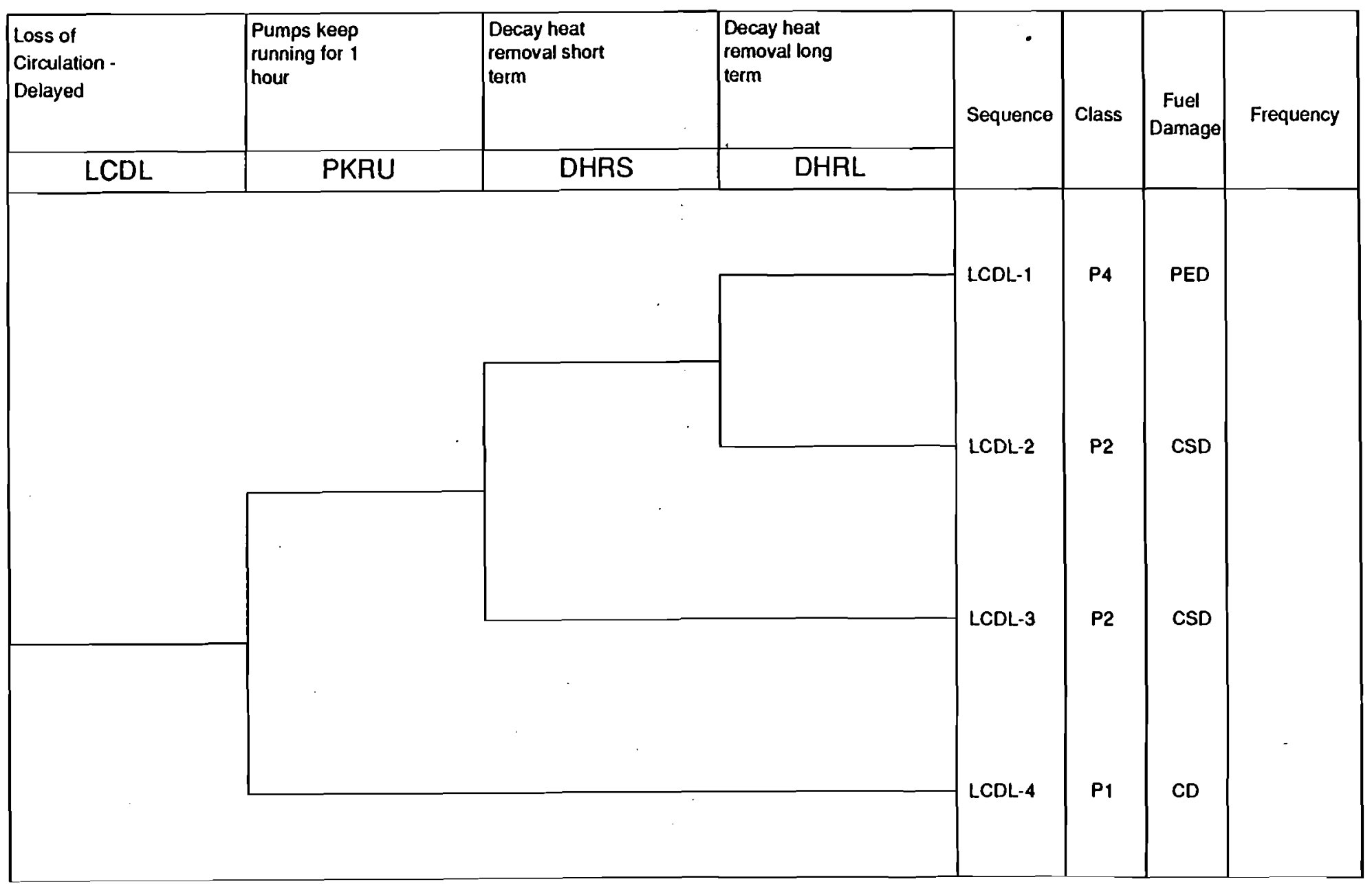

Fig. 5.36. Event Tree for Delayed Loss of Circulation 


\subsubsection{Event Trees for Support System Failures}

\subsubsection{Initiating Event Description}

This category includes all events which lead to shutdown with the decay heat removal function impaired. Included are loss of shutdown cooler (TSDC), loss of shield cooling (TSHC). Loss of instrument air is a more wide ranging transient whose primary effect is loss of feedwater control.

\subsubsection{Normal Plant Response and Accident Sequences}

The normal plant response is to remove the decay heat via the BOP unless the initiating event itself precludes this. In this eventuality the shutdown coolers are expected to carry the load. The success criteria are identical to those for the loss of primary heat sink.

\subsubsection{Event Trees and Accident Sequences}

The event tree for these events is given in Figs. 5.37, 5.38 and 5.39. The accident sequences revolve around either loss of primary heat sink or loss of heat sink. 


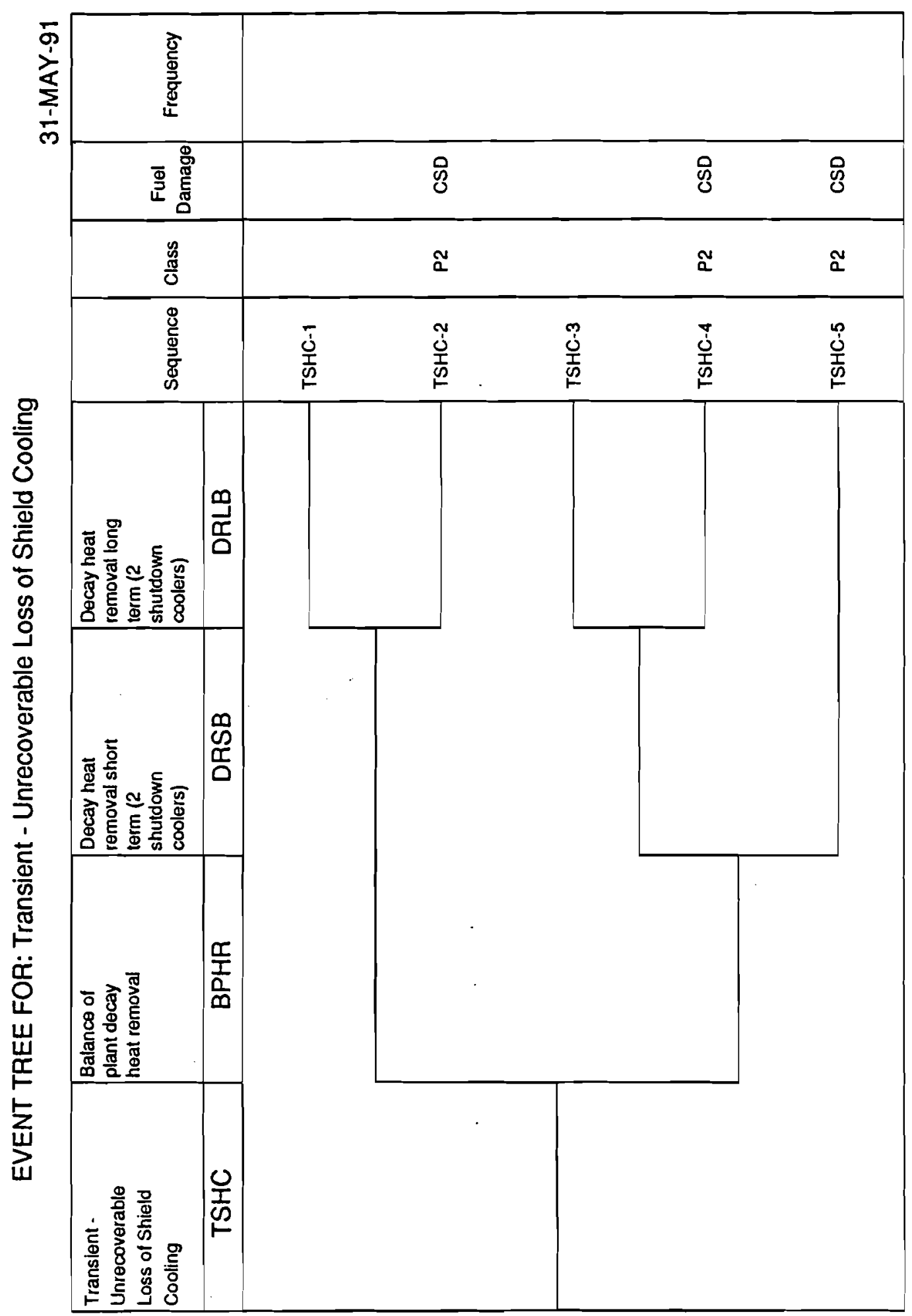

递 
EVENT TREE FOR: Transient - Unrecoverable Loss of a Shutdown Cooler

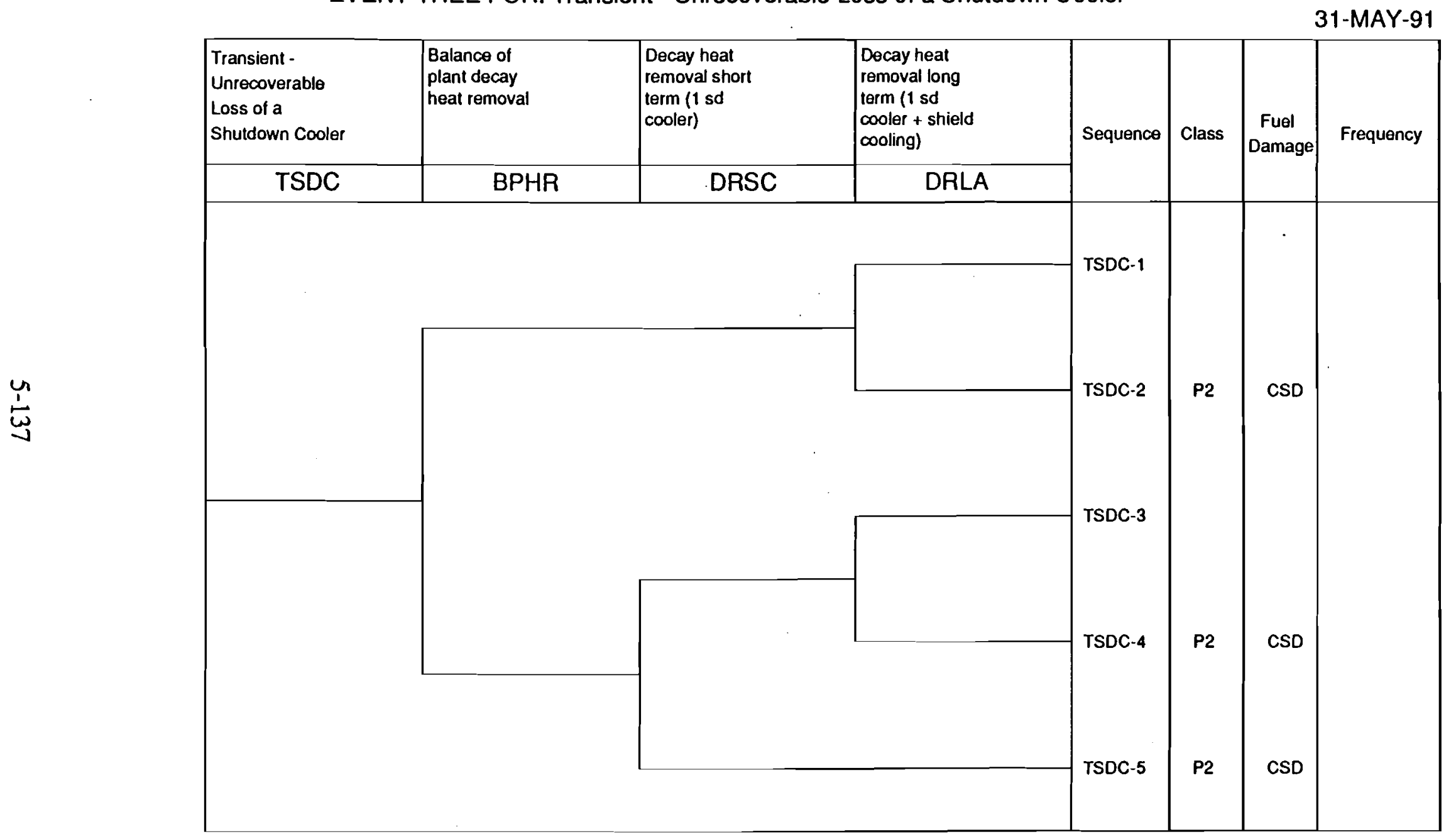

Fig. 5.38. Event Tree for Transient Loss of Shutdown Coolers 


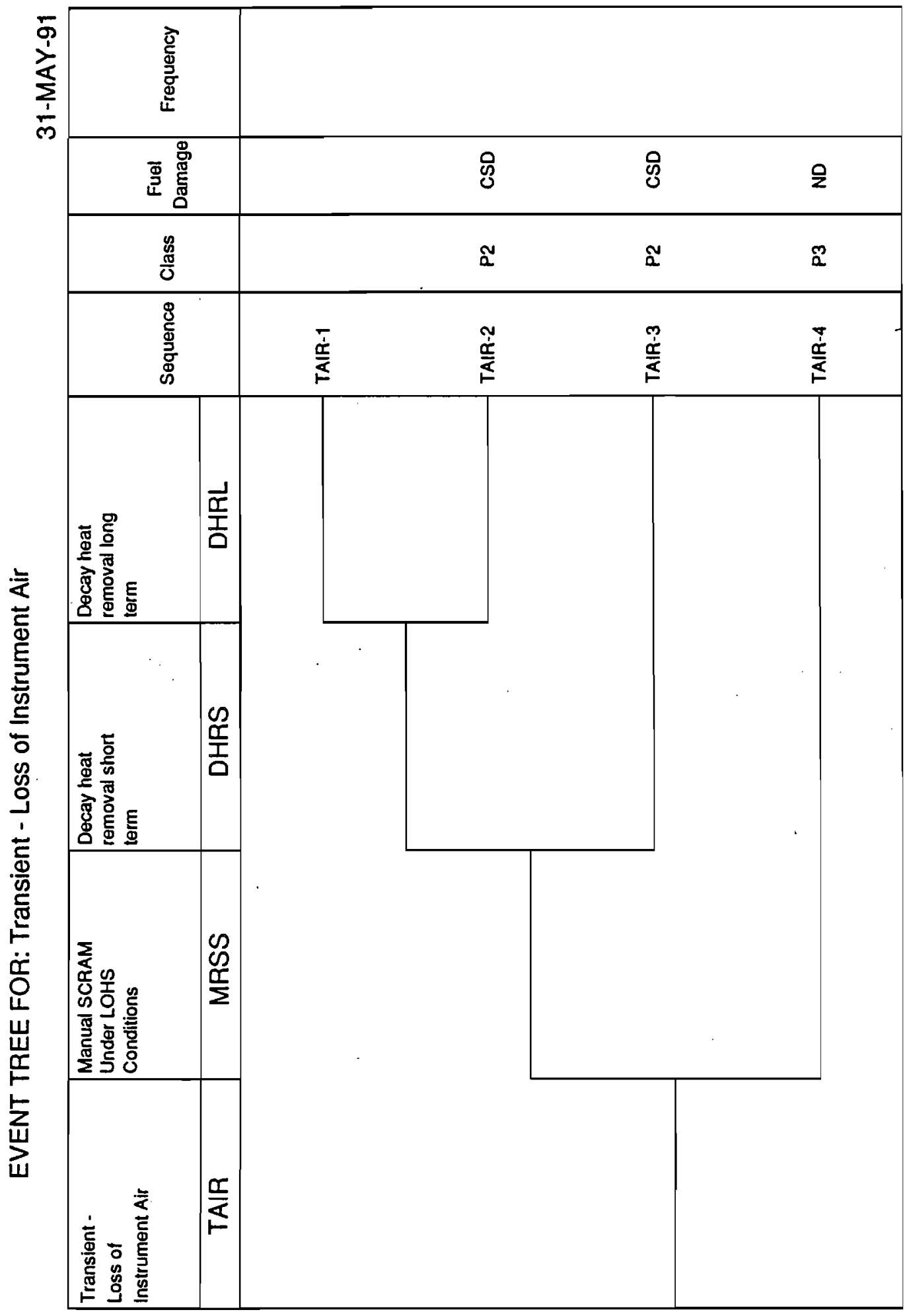

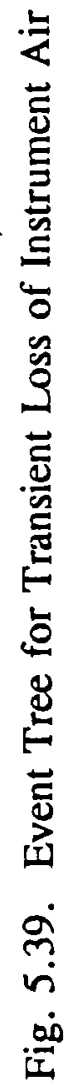




\subsubsection{Event Trees for Shutdowns}

\subsubsection{Initiating Event Description}

This category includes all events which call for normal shutdown, anticipatory shutdown and spurious scram where no safety threat exists. In all cases the threat is that having shutdown, the decay heat removal systems will fail to perform as required and core damage will occur. As before in the loss of primary heat sink description the damage is caused by elevated temperatures throughout the primary system and it is categorized as CSD (Core Structural Damage).

\subsubsection{Normal Plant Response and Success Criteria}

The normal plant response is to remove the decay heat via the BOP unless the initiating event itself precludes this. In this eventuality the shutdown coolers are expected to carry the load. The success criteria are identical to those for the loss of primary heat sink. In the case of the long shutdown an extended mission time is considered.

\subsubsection{Event Trees and Accident Sequences}

The event trees for these events are given in Figs. 5.40 to 5.43. The accident sequences revolve around either loss of primary heat sink or loss of heat sink. 


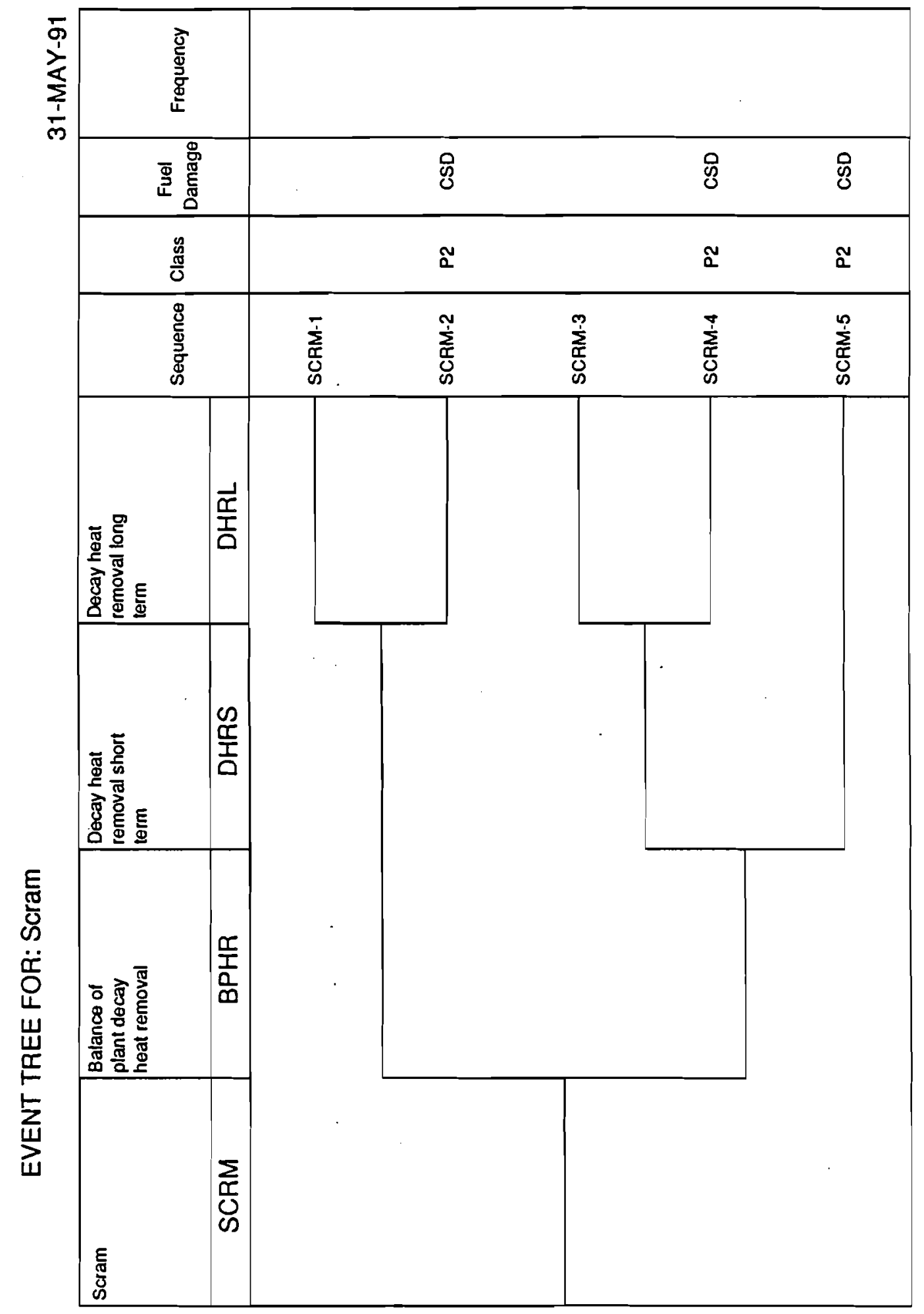

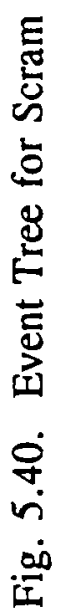


EVENT TREE FOR: Anticipatory Shutdown

31-MAY-91

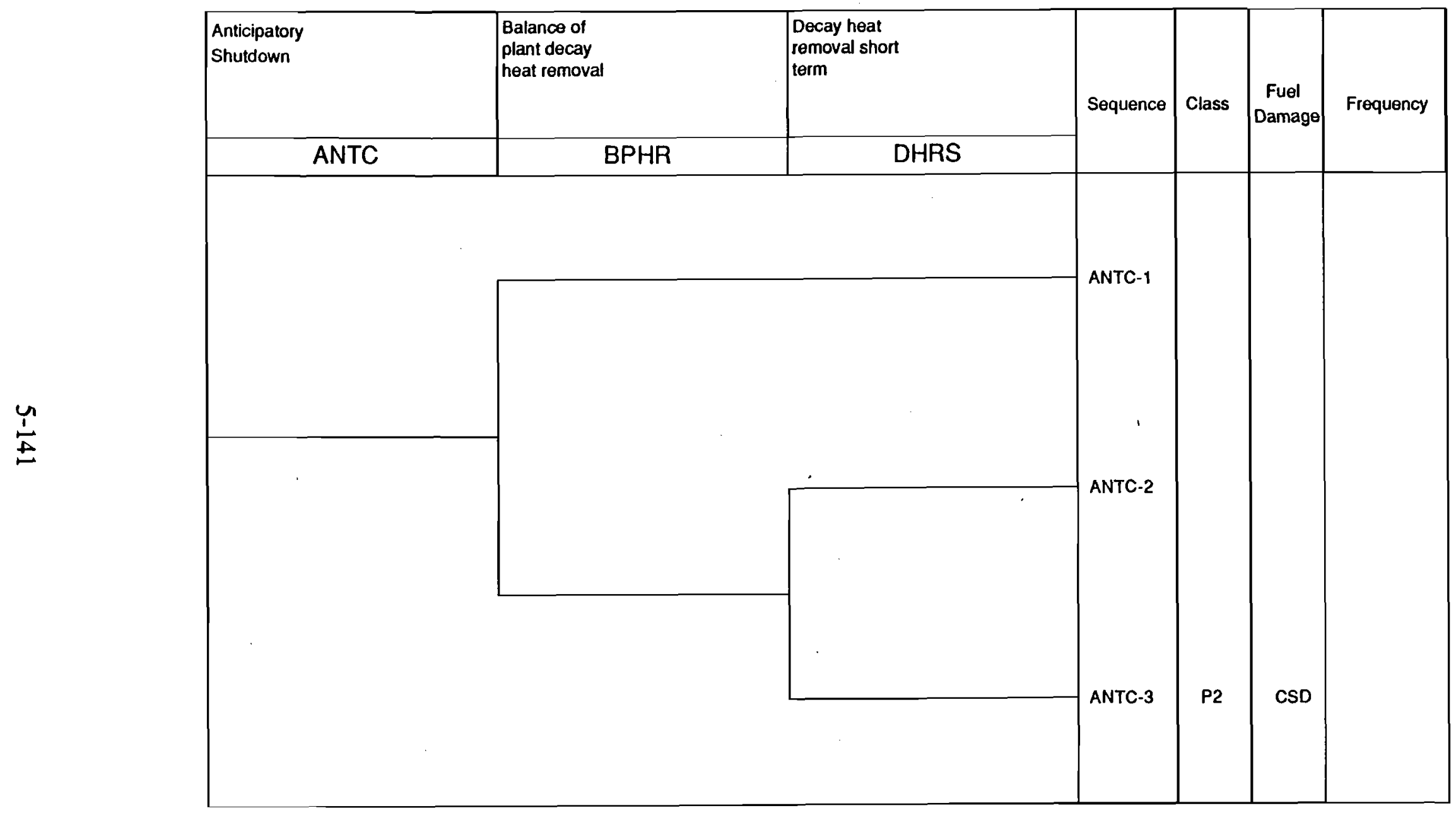

Fig. 5.41. Event Tree for Anticipatory Shutdown 


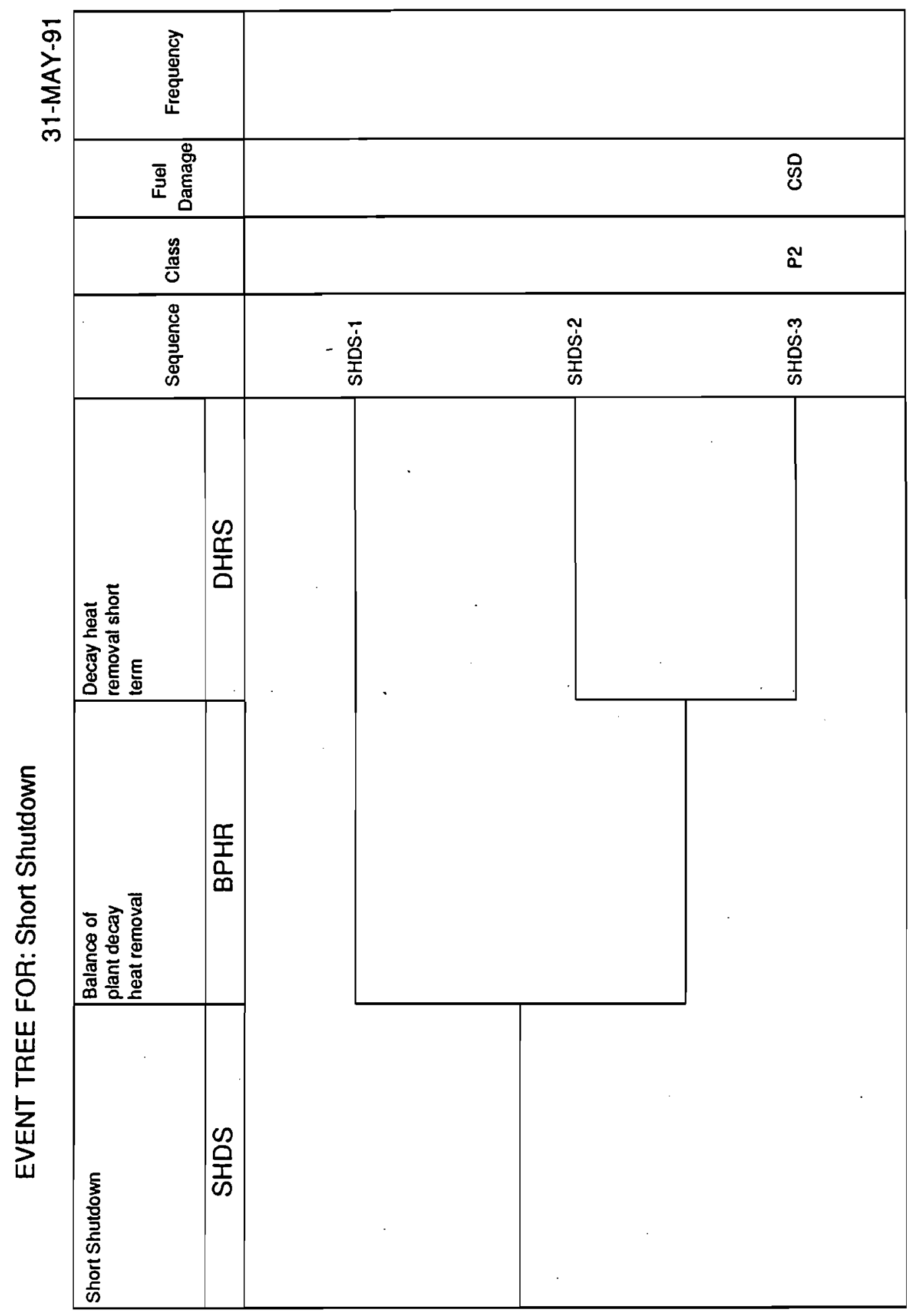

 


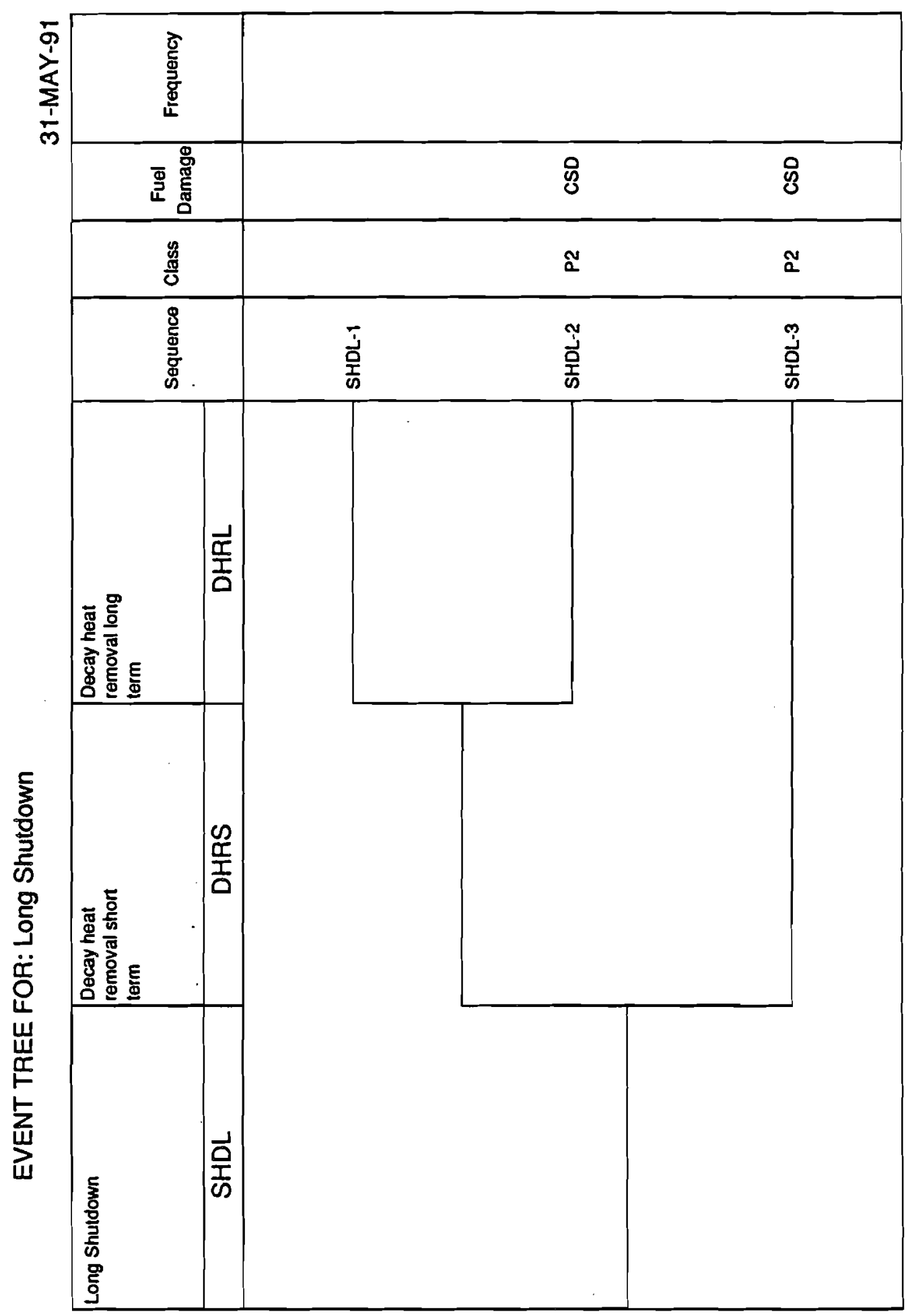

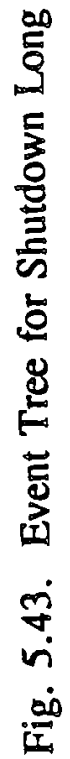




\subsubsection{Event Trees for Local Faults}

\subsubsection{Initiating Event Description}

This initiating event class includes all local faults, specifically, FLTA, complete subassembly inlet or outlet blockage, FLTB, simultaneous failure of large number of pins within a subassembly, MDLF, metal driver subassembly pin failure, MELF, metal experiment subassembly pin failure, OELF, non-metal experiment subassembly pin failure, SFLE, subassembly loading error, and SFPB, subassembly partial blockage. Based on the EBRII design and analyses, FLTA is not considered to be physically possible and FLTB does not lead to damage consequences, and is therefore not of concern.

\subsubsection{Normal Plant Response and Success Criteria}

The normal plant response is to detect failures using one of the FERD or GLASS signals or by reactivity change, whereupon shutdown is initiated if the operators deem it necessary. Certain conditions require scram, e.g. DN signal $>3000$ counts per second.

\subsubsection{Event Trees and Accident Sequences}

The event sequence from breach initiation in a fuel element to largescale damage is a complex series that involves phenomenological considerations and automatic and operator responses to conditions at specific times. Given the initiating event of an element breach, the next concern is the effect of the breach on neighboring elements, the classical pin-topin propagation concern. This is the first branch point considered in the event tree and is a phenomenological consideration. The general top event is No Pin-to-Pin Breach Propagation (NPPP). A positive response to this top event means cladding breach does not cause neighboring elements to breach, thereby leading to a state of no concern in the PRA context. 
If the breach of one element leads to a breach in neighboring elements, the nature of the effect leads to the next branch point, is the breach propagation self-limiting or not, another phenomenological consideration. This top event is designated as Self-Limiting Pin-to-Pin Breach Propagation (SLPP). A positive response to this top event means the initial breach would lead to the additional breach of at most three or four neighboring elements and is a state of no concern in the PRA context since it is limited in nature, a situation EBR-II is designed to accommodate.

A negative response to the SLPP top event is the path to continued propagation of cladding breaches within the subassembly. With each new breach, a breach of neighboring elements occurs. The next top event is a response to the proceeding phenomenological events. This top event addresses the detectability of this propagating breach state and is designated Failure Detection (DETK). A positive response means the breaches are detected by the systems included in EBR-II including the Fuel Element Rupture Detector (FERD), the GermaniumLithium Argon Scanning System (GLASS), and possible reactivity changes.

The consideration of whether a Reactor Shutdown Signal (SHTD) is initiated is the next top event. A reactor shutdown signal may be initiated by the reactor operator or an automatic system. In the case of an element breach initiated local fault, there are few systems that would automatically generate a scram signal. An increase in reactor power to the overpower trip setting ( $110 \%$ nominal) would provide an automatic scram. Other conditions will be governed by operator response and the associated operating and emergency procedures.

The final top event is again one of phenomenological considerations. If unlimited fuel element breach propagation occurs, the determination of subassembly-to-subassembly propagation must be made. The final top event is therefore No Subassembly-to-Subassembly Propagation (NSSP). If there is only a single breach or limited breach propagation, the integrity of the subassembly boundary is maintained.

The event trees for subassembly scale local faults, such as a partial blockage or orificing error and a loading error, are based on those for the single pin breach initiated local faults. The 
subassembly fault changes the operating conditions, lower flows or higher temperatures, and thereby changes the element operational lifetime. This change in conditions could then initiate element breaches within the subassembly. The first top event in this tree is therefore Subassembly Fault: No Pin Breach (SNPB). After this element breach consideration, the other top events are identical to the single element breach initiated local fault tree described above. Because the conditions are different than a normal driver or experimental subassemblies, the various branch ratios may be different, but the structure is the same.

The event trees are given in Figs. 5.44 to 5.48. Damage categories are assigned based on the state of the subassembly and its neighbors. Core Damage (CD) results if the subassembly boundary is breached and neighboring subassemblies are involved. If the subassembly boundary remains intact, but there are extensive pin breaches within the subassembly, the state is defined to be Minor Core Damage (MCD). If there are few pin breaches, the state is defined as No Damage (ND) since this is a normal operating state for EBR-II. 
EVENT TREE FOR: Local Faults: Metal Driver Breach Initiator

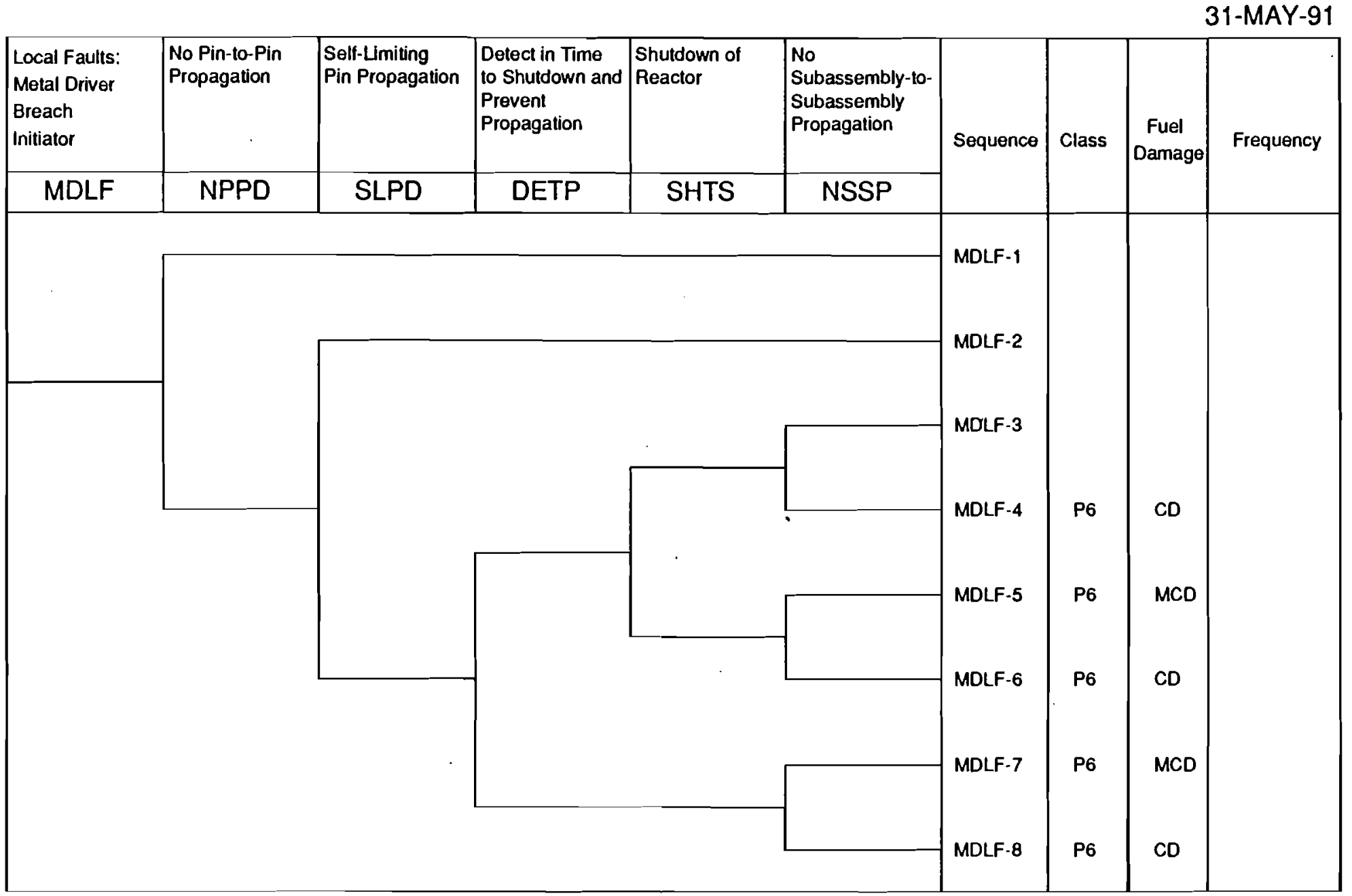

Fig. 5.44. Event Tree for Local Fault - Driver Pin Failure 


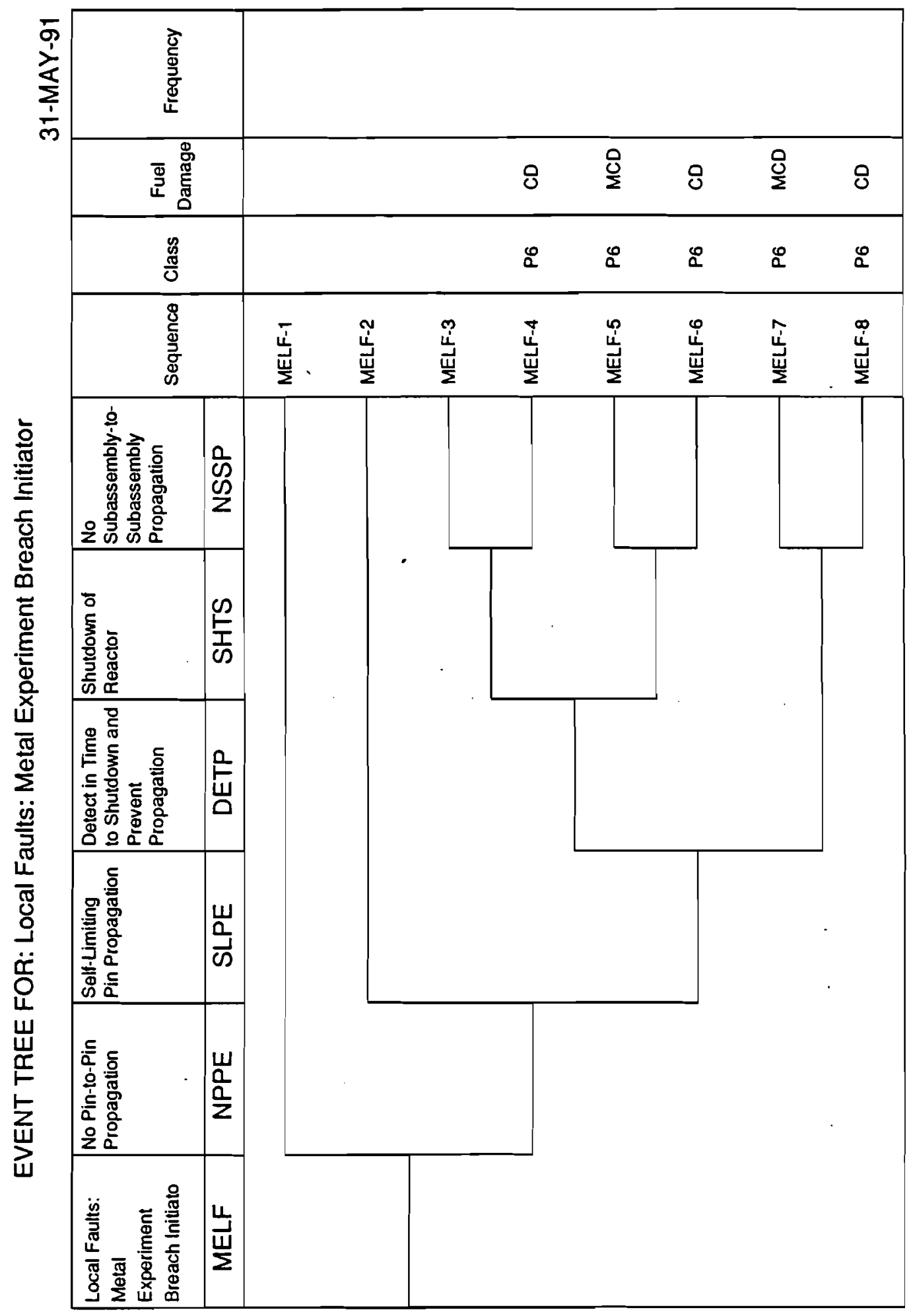

0. 


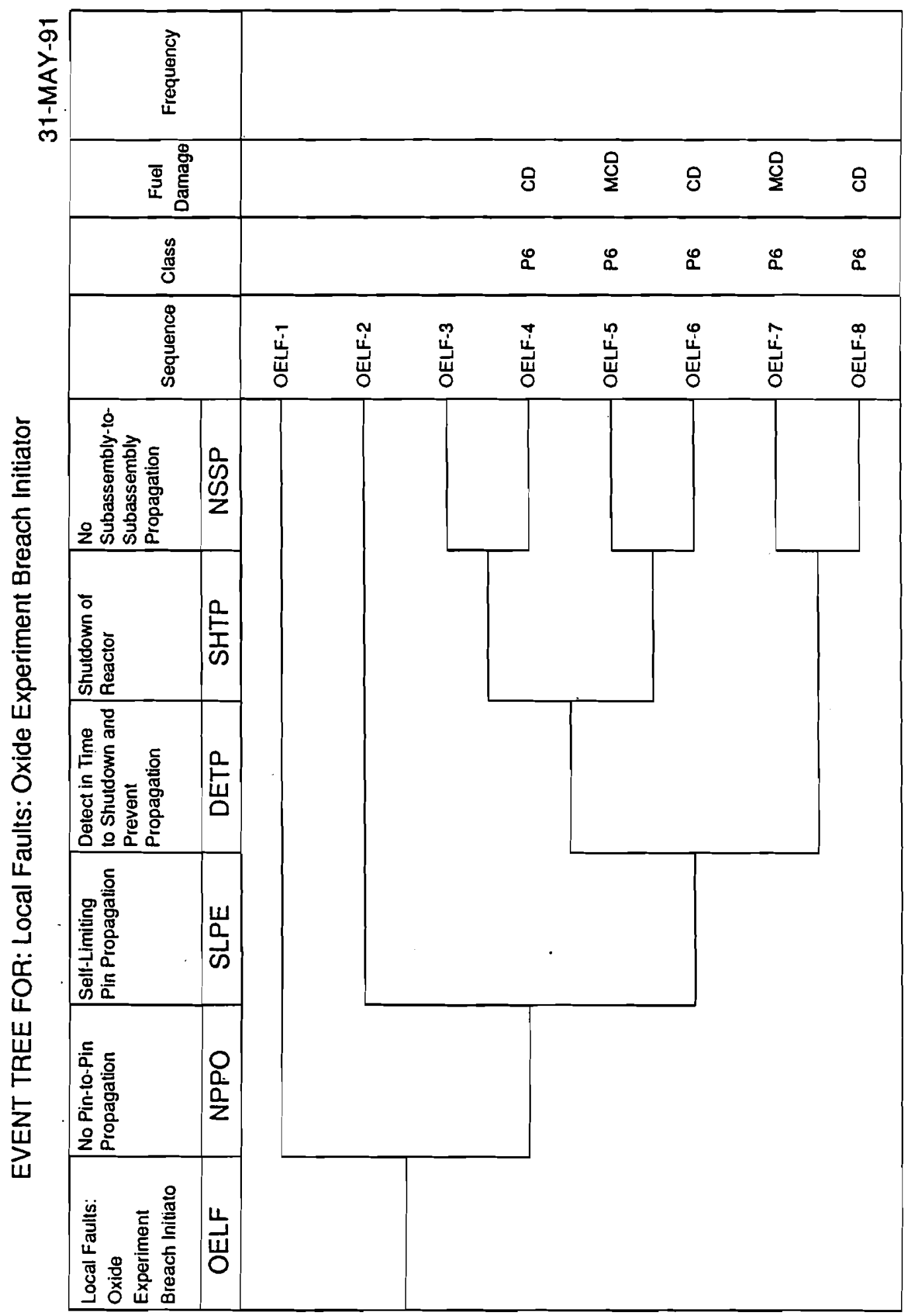

吾 


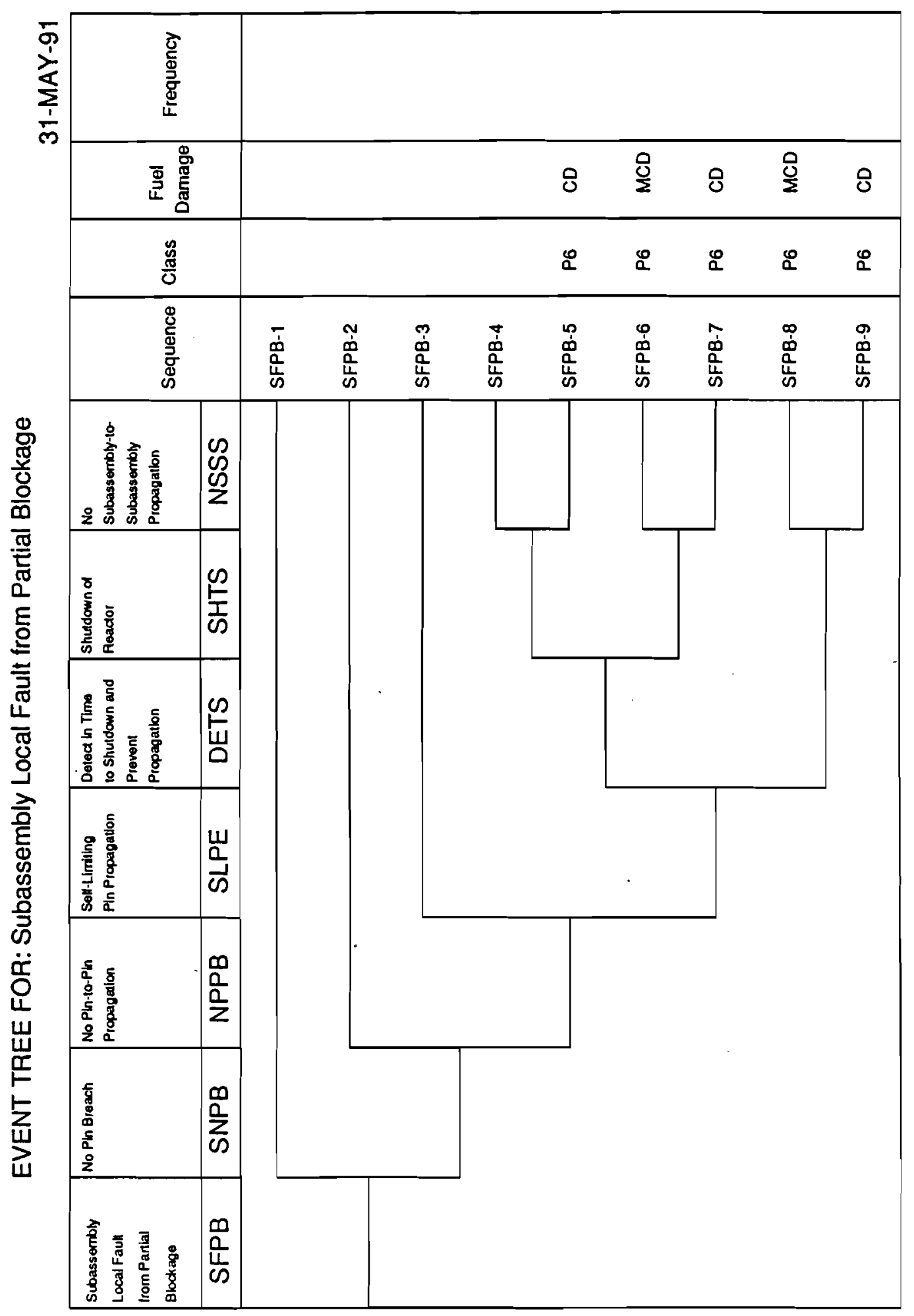

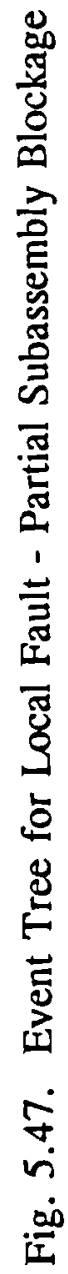




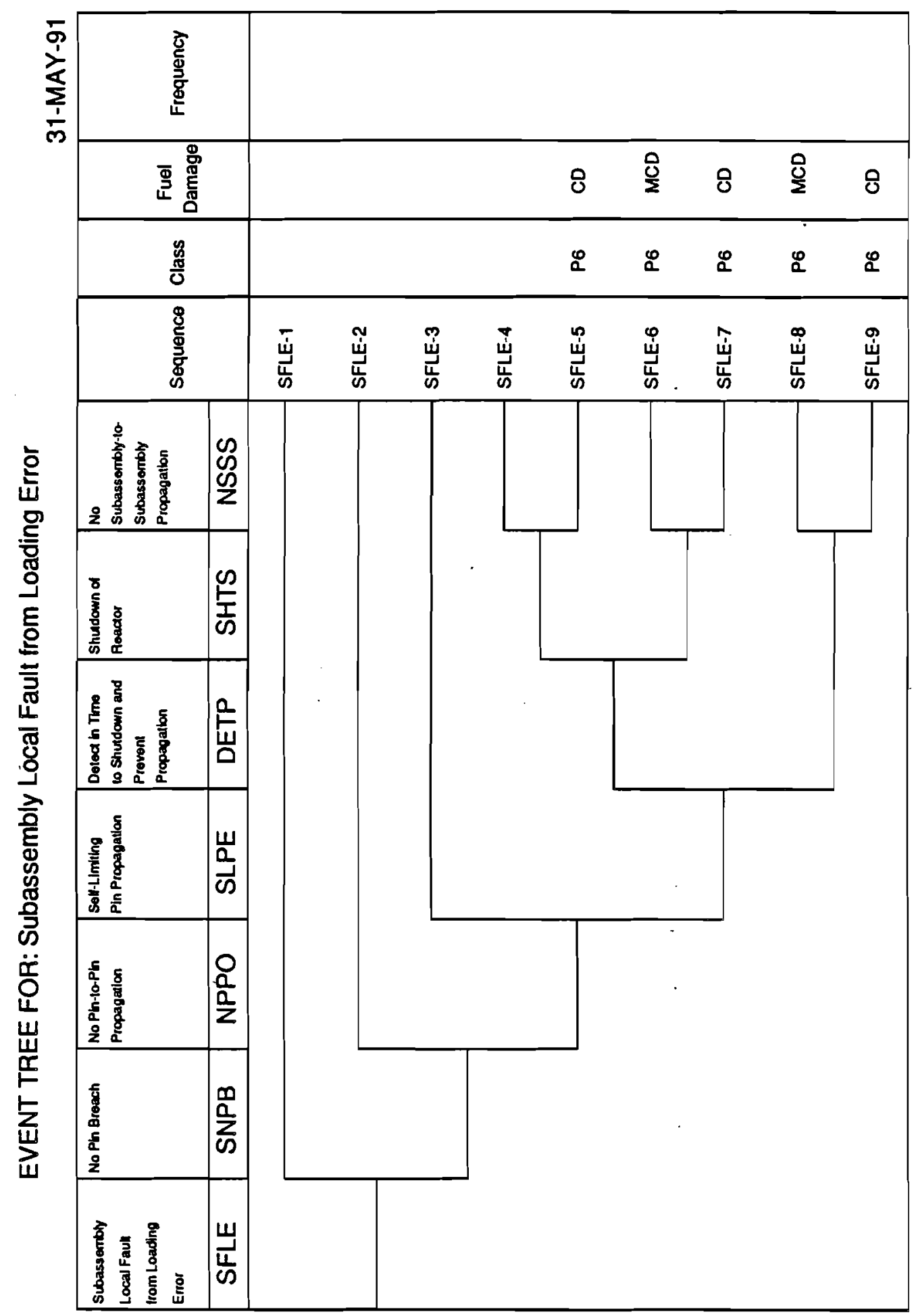

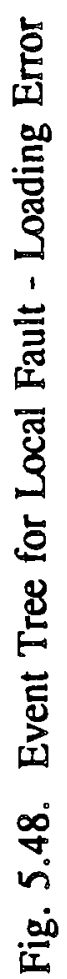




\subsection{Core Damage Analysis}

The assignation of damage categories in the previous section has been based upon calculations using a model of EBR-II with the SASSYS code. This enabled a consistent set of damage calculations to be undertaken for core loadings typical of the present loadings.

In the following, details of the SASSYS model of EBR-II are given together with results for various of the important transients.

\subsubsection{SASSYS Model of EBR-II}

The SASSYS model of EBR-II [5.41] has been extensively validated with the Shutdown Heat Removal Tests (SHRT) [5.41, 5.42, 5.43, 5.44]. Among the transients analyzed with SASSYS are protected LOF experiments (SHRT 8A [5.44] and SHRT 17 [5.42, 5.43]), mild reactor flow and inlet temperature perturbation experiments (SHRT 25 and SHRT 26 [5.43]), an unprotected LOF experiment (SHRT 45 [5.41, 5.43]), and an unprotected loss-ofheat-sink experiment (SHRT B302 [5.41]). The SASSYS model of EBR-II is based on a mixed Mark-II and Mark-III core loading and uses best estimate values for reactivity feedback characteristics [5.33].

The SASSYS model of EBR-II was modified to aid determination of core damage levels during upset conditions. Specifically, channels were modified to represent hot and core-average fuel pins. Hot channels represent the hottest fuel pin and coolant subchannel allowed in any driver-fuel subassembly by EBR-II Technical Specifications [5.24] (peak coolant temperature = $465^{\circ} \mathrm{C}=1050^{\circ} \mathrm{F}$ ). Only a few fuel pins operate near Technical Specifications limits in any core loading. If during an accident simulation, thermal conditions in hot channels are severe enough to produce localized boiling, fuel melting, or fuel pin failure, the accident is classified as causing minor core damage, (MCD). Only a few isolated pins would be damaged during the accident. If the accident is severe enough to produce boiling, fuel melting, or pin failure in average channels, the accident is classified as causing core damage, (CD). A core damage accident would result in large numbers of damaged fuel pins, and might result in core relocation. 
If hot channels are not damaged, the accident is classified as causing no core damage, (ND). This class of accidents is further divided into three subgroups based on operational considerations as stipulated by the Technical Specifications. If the peak clad temperature reached during the transient is below $710^{\circ} \mathrm{C}\left(1310^{\circ} \mathrm{F}\right)$, the reactor will retain unlimited capability to operate. This temperature is a conservative estimate of the onset temperature of eutectic formation between clad and metal fuel. If the hottest sections of cladding are predicted to reach temperatures within the range between $710^{\circ} \mathrm{C}$ and $816^{\circ} \mathrm{C}\left(1500^{\circ} \mathrm{F}\right)$ for less than $60 \mathrm{~s}$, the reactor is classified as operating in the limited capability region. If the clad temperature in the hottest pins exceeds $816^{\circ} \mathrm{C}$ or lies between the eutectic temperature and $816^{\circ} \mathrm{C}$ for greater than $60 \mathrm{~s}$, the reactor will pass into the unacceptable region of operation. In this case, no damage to driver fuel will occur, however there is the potential for damage to experiments, (PED). In the calculations shown in this section the effects of coolant boiling have been suppressed and the coolant temperature histories shown therefore continue into the boiling regime as if the sodium were still single phase. This is adopted as a convenience to display the severity of the temperature transient.

Core damage states are dependent upon the occurrence of coolant boiling, fuel melting, or fuel pin failure in hot or average channels. Fuel pin failure refers to breach of the cladding caused by eutectic attack, fission-gas pressure induced strain, or a combination of both. Fuel melting is considered to be a damage indicator although it is possible for fuel melting to occur without the subsequent release of fission products via fuel pin failure. In the case of fuel melting without cladding failure, the cladding will still be damaged enough by elevated cladding temperatures and fission-gas pressures to severely shorten the useful lifetime of the fuel pin. Thus although fuel pins may not actually "fail" during an accident that results in fuel melting, accident resolution would require the removal and examination of any subassemblies which are suspected to have experienced fuel melting. 


\subsubsection{Accident Calculations}

\subsubsection{Loss-of-Flow Events}

For calculational purposes, the pump coastdowns have been divided into four groups based on coastdown duration. The first group consists of $M / G$ input faults, which have a pump speed stop time of about $80 \mathrm{~s}$. Pump binding faults are included in this group and are assumed, for simplicity, to have the same coastdown behavior as the $M / G$ input faults. The second group consists of $M / G$ output faults, which have a stop time of about 30-35 s. The third coastdown group consists of clutch faults, which have a stop time of about $23 \mathrm{~s}$. The fourth group consists of pump seizures, which are assumed to have an essentially $0 \mathrm{~s}$ stop time. Figure 5.49 shows representative pump speed coastdown curves for the four groups. The response of the reactor shutdown system determines whether the LOF transient is protected (i.e., reactor scram is successful) or unprotected (reactor fails to scram).

In previous studies, the thermal response of cores loaded with Mark-II [5.45, 5.46] and Mark-III [5.47, 5.48] fuel elements to specific loss-of-flow transients has been investigated. Reactor experiments at full power and flow conditions have demonstrated the viability of natural circulation to adequately remove decay heat and reactor feedbacks to shutdown the reactor during protected [5.25] and unprotected [5.10], slow coastdown, LOF transients. In the current investigation, a systematic study of core damage potential for a core predominantly loaded with Mark-III fuel elements was performed for the various pump faults, under protected and unprotected conditions. The model utilizes measurements of primary pump speed made on actual pump coastdown experiments at EBR-II $[5.56,5.57]$ to simulate the flow coastdowns of all postulated pump faults except for pump seizures. The instantaneous stopping of the pump during a pump seizure is a conservative assumption that is physically impossible because it would require an instantaneous decrease in the momentum of the pump impeller. The auxiliary pump is assumed to be inoperative during the LOF transients. The secondary pump is assumed to be tripped $6 \mathrm{~s}$ into the transient. During protected transients, the reactor is assumed to be tripped $1.74 \mathrm{~s}$ after initiation of the transient. The control rods are assumed to 


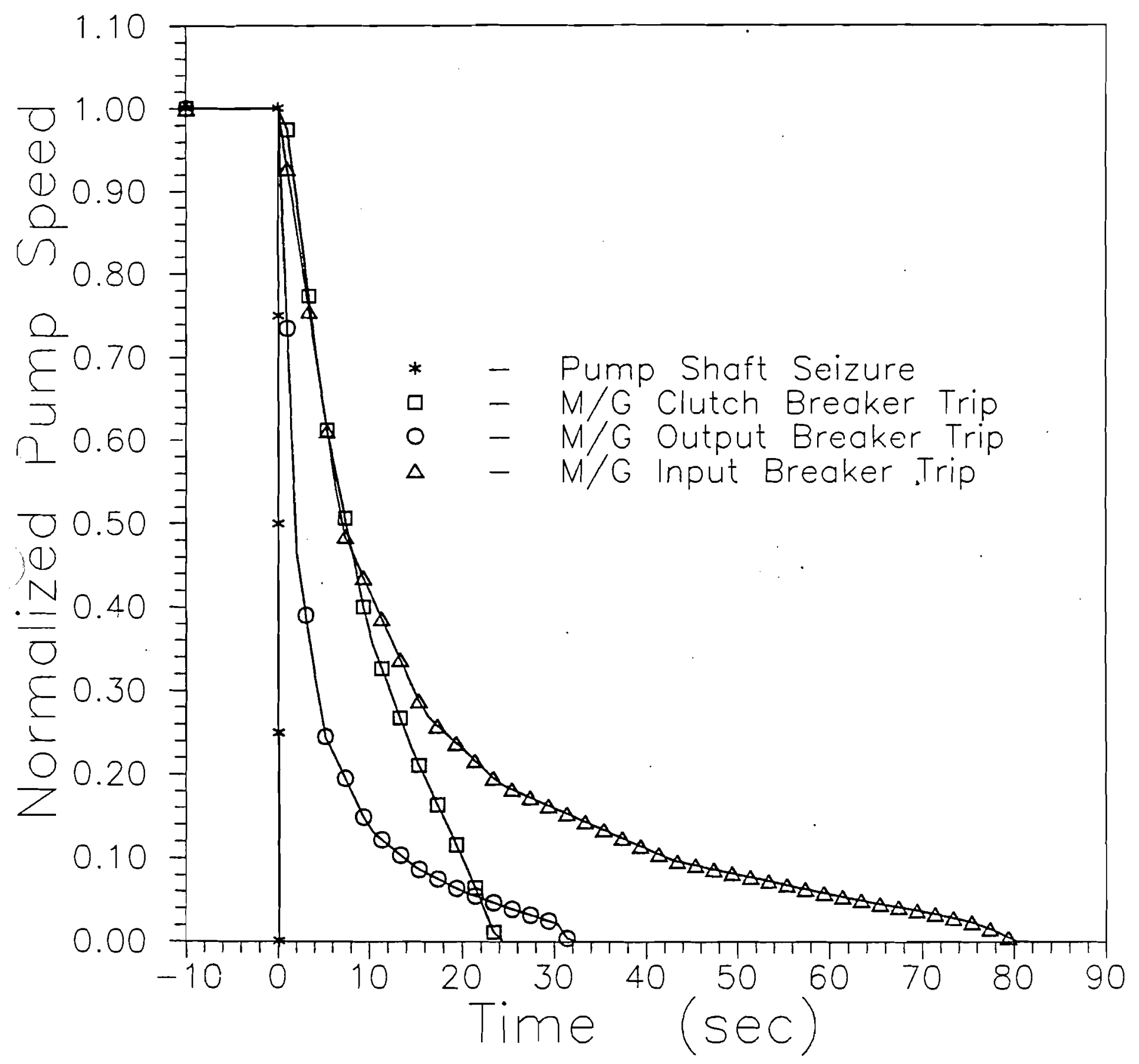

Fig. 5.49. Pump Coastdowns in EBR-II 
have a $0.252 \mathrm{~s}$ drop time. The reactor trip characteristics are typical of those used in previous calculations [5.47].

For the LOF calculations discussed here, representative coolant temperatures are shown in accompanying figures. Plots and data listings of reactor power and primary system flowrate profiles for the LOF calculations may be found in reference [5.49]. It should be noted that a calculational anomaly of SASSYS limits the representation of centrifugal pump coastdown curves to only ten points. This results in uncharacteristically rough temperature response during long coastdown LOF events (e.g. Fig. 5.50 and 5.51).

EBR-II Technical Specifications [5.24] require the operation of the auxiliary pump to guarantee that driver fuel element temperatures remain in the region of unlimited operating capability during an anticipated LOF event, which is defined as a protected, double clutch fault transient. The reactor remains in the unlimited operating capability region as long as the peak driver cladding temperature does not surpass the fuel/clad eutectic temperature limit $\left(710^{\circ} \mathrm{C}\right)$. The reactor can operate beyond the unlimited operating capability region (i.e. in the limited and unacceptable operating regions) without incurring fuel damage. Although the auxiliary pump acts to lower peak temperatures during LOF transients, this effect is not large enough to change the damage state of the reactor during any LOF event. Because the PRA is only concerned with the damage state of the driver fuel and not the operational capabilities of the reactor, no credit is taken for operation of the auxiliary pump in the accident analysis calculations. Because the auxiliary pump is assumed to be inoperative, it was hypothesized that the scenario in which a double pump LOF event occurs after the reactor is shutdown might pose a concern. In particular, if a LOF occurs when the reactor is nominally isothermal, natural circulation may take time to develop, during which local core temperatures may reach the sodium boiling point. This scenario, termed a delayed LOF event, has been analyzed (Appendix D.4) and found to not produce significantly elevated temperatures. 


\section{Single Pump Fault Events}

Single pump fault events are due to mechanical or electrical failures of one of the primary pumps. A special case arises if the reactor is operating on electrical power supplied by the turbine-generator with the EBR-II site disconnected from the INEL loop, i.e. the turbinegenerators is supplying the onsite, or hotel, load. In this case, the scram signal generated by the low flow indicators of the reactor shutdown system will generate a turbine trip and a trip of the turbine-generator output breaker. This results in a loss of normal power $(2400 \mathrm{~V})$ to the other pump which induces a $M / G$ input fault. Although the $M / G$ input fault of the second pump will occur a few seconds following the failure of the first pump, the second pump trip is conservatively assumed to occur at the same time as the failure of the first pump. A single pump failure with the reactor on hotel load is modeled in the same manner as any double pump fault which includes a $M / G$ input failure of one of the primary pumps.

Calculated core damage levels for protected and unprotected single pump faults are collected in Table 5.23. The columns in the table list the four types of single pump faults. The rows of the table divide the results into four cases, depending on whether the transient is protected or unprotected and whether the reactor is on hotel load or outside power. If the single pump failure occurs while the reactor is on outside power, the other pump continues to operate normally. If the reactor is successfully scrammed, a single pump fault does not produce core damage, even if the reactor is on hotel load. Peak cladding temperatures do not reach the eutectic limit $\left(710^{\circ} \mathrm{C}\right)$ during protected single pump faults, unless a pump seizure occurs while the reactor is on hotel load. For a single pump seizure with the reactor on hotel load, the cladding temperature in the hot channel momentarily $(<1 \mathrm{~s})$ surpasses the eutectic temperature limit and reaches a peak temperature of $725^{\circ} \mathrm{C}$. If the conservatism inherent in this calculation (instantaneous stopping of the seized pump and simultaneous $\mathrm{M} / \mathrm{G}$ input failure of the other pump) are replaced by more realistic conditions, the peak cladding temperature would not exceed the eutectic temperature. Figure 5.50 contains plots of the hot pin coolant temperature during a protected single pump seizure with the reactor operating either on hotel load or outside power, clad temperatures are typically $5-10^{\circ} \mathrm{C}$ hotter than the coolant. 
Table 5.23. Core Damage Levels During Single Pump Faults

\begin{tabular}{|c|c|c|c|c|}
\hline & Pump Seizure Fault & M/G Clutch Fault & M/G Output Fault & M/G Input Fault \\
\hline $\begin{array}{l}\text { Protected Single } \\
\text { Pump Fault, Reactor } \\
\text { on Outside Power }\end{array}$ & $\begin{array}{l}\text { No Core } \\
\text { Damage/Unlimited } \\
\text { Operating Capability }\end{array}$ & $\begin{array}{l}\text { No Core } \\
\text { Damage/Unlimited } \\
\text { Operating Capability }\end{array}$ & $\begin{array}{l}\text { No Core } \\
\text { Damage/Unlimited } \\
\text { Operating Capability }\end{array}$ & $\begin{array}{l}\text { No Core } \\
\text { Damage/Unlimited } \\
\text { Operating Capability }\end{array}$ \\
\hline $\begin{array}{l}\text { Protected Single } \\
\text { Pump Fault, Reactor } \\
\text { on Hotel Load }\end{array}$ & $\begin{array}{l}\text { No Core } \\
\text { Damage/Limited } \\
\text { Operating Capability }\end{array}$ & $\begin{array}{l}\text { No Core } \\
\text { Damage/Unlimited } \\
\text { Operating Capability }\end{array}$ & $\begin{array}{l}\text { No Core } \\
\text { Damage/Unlimited } \\
\text { Operating Capability }\end{array}$ & $\begin{array}{l}\text { No Core } \\
\text { Damage/Unlimited } \\
\text { Operating Capability }\end{array}$ \\
\hline $\begin{array}{l}\text { Unprotected Single } \\
\text { Pump Fault, Reactor } \\
\text { on Outside Power }\end{array}$ & $\begin{array}{l}\text { No Core } \\
\text { Damage/Unlimited } \\
\text { Operating Capability }\end{array}$ & $\begin{array}{l}\text { No Core } \\
\text { Damage/Unlimited } \\
\text { Operating Capability }\end{array}$ & $\begin{array}{l}\text { No Core } \\
\text { Damage/Unlimited } \\
\text { Operating Capability }\end{array}$ & $\begin{array}{l}\text { No Core } \\
\text { Damage/Unlimited } \\
\text { Operating Capability }\end{array}$ \\
\hline $\begin{array}{l}\text { Unprotected Single } \\
\text { Pump Fault, Reactor } \\
\text { On Hotel Load }\end{array}$ & Minor Core Damage & Minor Core Damage & Minor Core Damage & $\begin{array}{l}\text { No Core } \\
\text { Damage/Unaccept- } \\
\text { able Operating Region }\end{array}$ \\
\hline
\end{tabular}


Hot Pin Coolant Temperature during Protected Single Pump Faults

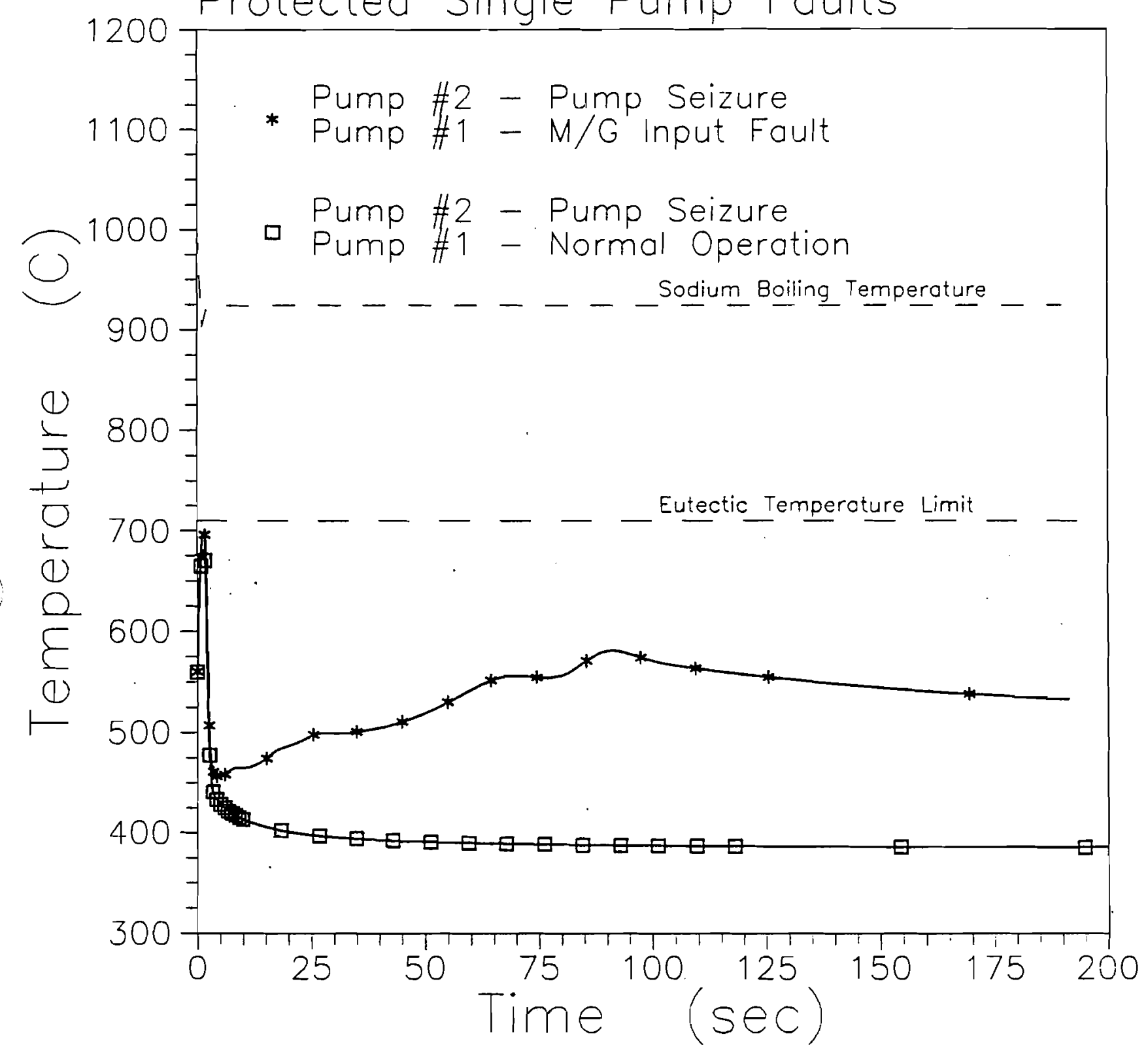

Fig. 5.50 Hot Pin Coolant Temperature during Protected Single Pump Faults 
For unprotected single pump faults, if the reactor is operating on hotel load, minor core damage occurs, unless the single pump failure is due to a $M / G$ input fault when only PED is predicted. Minor core damage occurs because the coolant temperature in the hot channels barely reaches the saturation temperature (see Fig. 5.51). If an unprotected single pump fault occurs while the reactor is operating on normal power, core damage levels will not be reached and the reactor will retain unlimited operating capabilities, for any coastdown type. Figure 5.51 contains plots of the hot pin coolant temperature during the most severe event, an unprotected single pump seizure with the reactor operating either on hotel load or outside power.

It should be noted that the calculated hot pin coolant temperature during an unprotected single pump seizure with the reactor on hotel load momentarily surpasses the sodium saturation temperature. Although SASSYS contains a model for two-phase sodium flow, it was not implemented during these calculations. Instead the coolant continued to be modeled as a singlephase fluid when the saturation temperature was reached. This simplification was taken because the main concern for this analysis was whether the system would reach a failure condition (i.e., boiling), not the effects of a failure on the system. Technically, the temperature plot in Fig. 5.51 should only be drawn up to when the boiling temperature is reached, since calculated temperatures beyond this time are not accurate. Yet calculated coolant temperatures that extend beyond the sodium boiling point are still presented (Figs. 5.51, 5.52, 5.53, 5.54, 5.55, and 5.57), because the degree to which the single phase coolant temperature surpasses the saturation temperature is an indication of the extent of boiling in the system. For instance, although the hot pin coolant at the top of the core reaches the boiling point during an unprotected single pump seizure with the reactor on hotel load, one would expect only localized vapor formation because the single phase coolant temperature rapidly falls below the boiling point.

In conclusion, all single pump faults, protected and unprotected do not in themselves lead to any form of core damage and all lie in the unlimited capability region unless coastdown of the second pump occurs. In the case of protected single pump faults with simultaneous slow coastdown of the other pump the only difference arises in the case of pump seizure where the conservative calculation causes the limited capability region to be entered for a time of $<1 \mathrm{~s}$. 
Hot Pin Coolant Temperature during

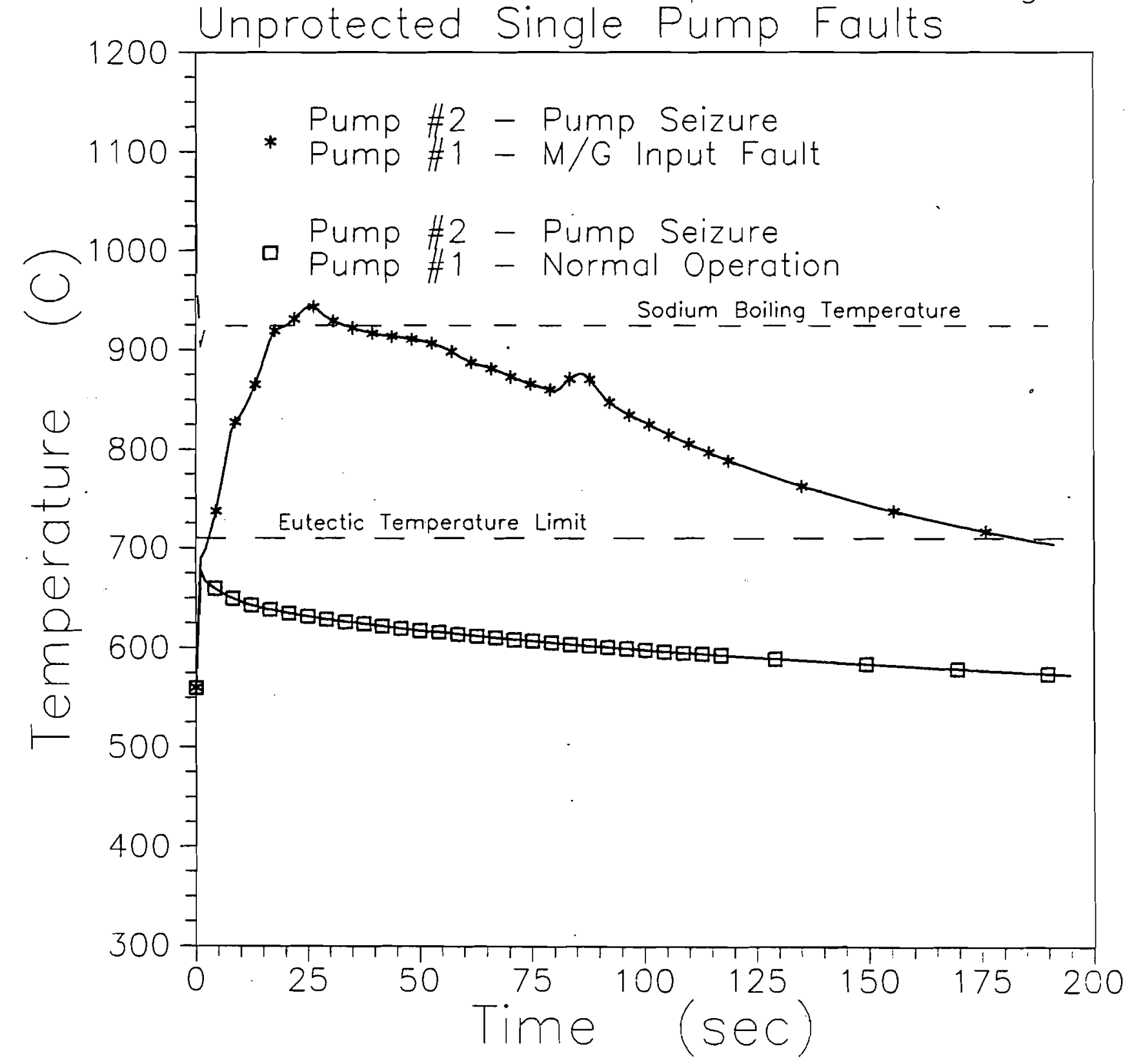

Fig. 5.51. Hot Pin Coolant Temperature during Unprotected Single Pump Faults 
The only cases where any form of damage can result are when the single pump fault is combined with an M/G input coastdown of the second pump due to operating on hotel load and failure to scram.

\section{Double Pump Fault Events}

In the analysis of initiating event frequencies, double pump faults have been defined as the loss of one primary pump followed by the loss of the second pump within a 6 minute interval. Although the failure of the second pump can occur anytime within the 6 minute interval, it is conservatively assumed to fail simultaneously with the first pump in the transient analysis.

Calculated core damage levels for protected double pump faults are collected in Table 5.24. The rows in the table list the coastdown behavior of one pump while the columns list the coastdown behavior of the other pump. Only a double pump seizure, which is an extremely low probability event (and a very conservative calculation), produces any level of core damage. The damage produced is minor because it is limited to hot channels. Coolant in average driver fuel pins approach saturation temperature (peak coolant temperature is about $880^{\circ} \mathrm{C}$ ). The other protected double pump faults do not produce core damage, but some produce peak cladding temperatures that place the reactor in the limited operating capability region as stipulated by EBR-II Technical Specifications. The peak cladding temperatures in the hot channels during these transients (double clutch faults and pump seizures with clutch, M/G output, or $\mathrm{M} / \mathrm{G}$ input faults), surpass the eutectic temperature limit $\left(710^{\circ} \mathrm{C}\right)$ by a few degrees $\left(<30^{\circ} \mathrm{C}\right)$ for a short period of time $(<7 \mathrm{~s})$. Calculations indicate that if the auxiliary pump is operating, peak cladding temperatures would not surpass the eutectic temperature limit for any protected double pump fault event, except for double pump seizures. Figure 5.52 contains plots of the peak coolant temperature in the hot pin channels for identical fault, protected double pump failure events (i.e., both pumps experience pump seizure, clutch, $M / G$ output, or $M / G$ input faults). 
Table 5.24. Core Damage Levels During Protected Double Pump Faults

\begin{tabular}{||l|l|l|l|l||}
\hline & Pump Seizure Fault & \multicolumn{1}{|c||}{ M/G Clutch Fault } & \multicolumn{1}{|c||}{ M/G Output Fault } & \multicolumn{1}{|c||}{ M/G Input Fault } \\
\hline Pump Seizure Fault & Minor Core Damage & $\begin{array}{l}\text { No Core } \\
\text { Damage/Limited } \\
\text { Operating Capability }\end{array}$ & $\begin{array}{l}\text { No Core } \\
\text { Damage/Limited } \\
\text { Operating Capability }\end{array}$ & $\begin{array}{l}\text { No Core } \\
\text { Damage/Limited } \\
\text { Operating Capability }\end{array}$ \\
\hline M/G Clutch Fault & $\begin{array}{l}\text { No Core } \\
\text { Damage/Limited } \\
\text { Operating Capability }\end{array}$ & $\begin{array}{l}\text { No Core } \\
\text { Damage/Limited } \\
\text { Operating Capability }\end{array}$ & $\begin{array}{l}\text { No Core } \\
\text { Damage/Unlimited } \\
\text { Operating Capability }\end{array}$ & $\begin{array}{l}\text { No Core } \\
\text { Damage/Unlimited } \\
\text { Operating Capability }\end{array}$ \\
\hline M/G Output Fault & $\begin{array}{l}\text { No Core } \\
\text { Damage/Limited } \\
\text { Operating Capability }\end{array}$ & $\begin{array}{l}\text { No Core } \\
\text { Damage/Unlimited } \\
\text { Operating Capability }\end{array}$ & $\begin{array}{l}\text { No Core } \\
\text { Damage/Unlimited } \\
\text { Operating Capability }\end{array}$ & $\begin{array}{l}\text { No Core } \\
\text { Damage/Unlimited } \\
\text { Operating Capability }\end{array}$ \\
\hline \multirow{5}{\text{M/GInputFault}}{} & $\begin{array}{l}\text { No Core } \\
\text { Damage/Limited } \\
\text { Operating Capability }\end{array}$ & $\begin{array}{l}\text { No Core } \\
\text { Damage/Unlimited } \\
\text { Operating Capability }\end{array}$ & $\begin{array}{l}\text { No Core } \\
\text { Damage/Unlimited } \\
\text { Operating Capability }\end{array}$ & $\begin{array}{l}\text { No Core } \\
\text { Damage/Unlimited } \\
\text { Operating Capability }\end{array}$ \\
\hline
\end{tabular}




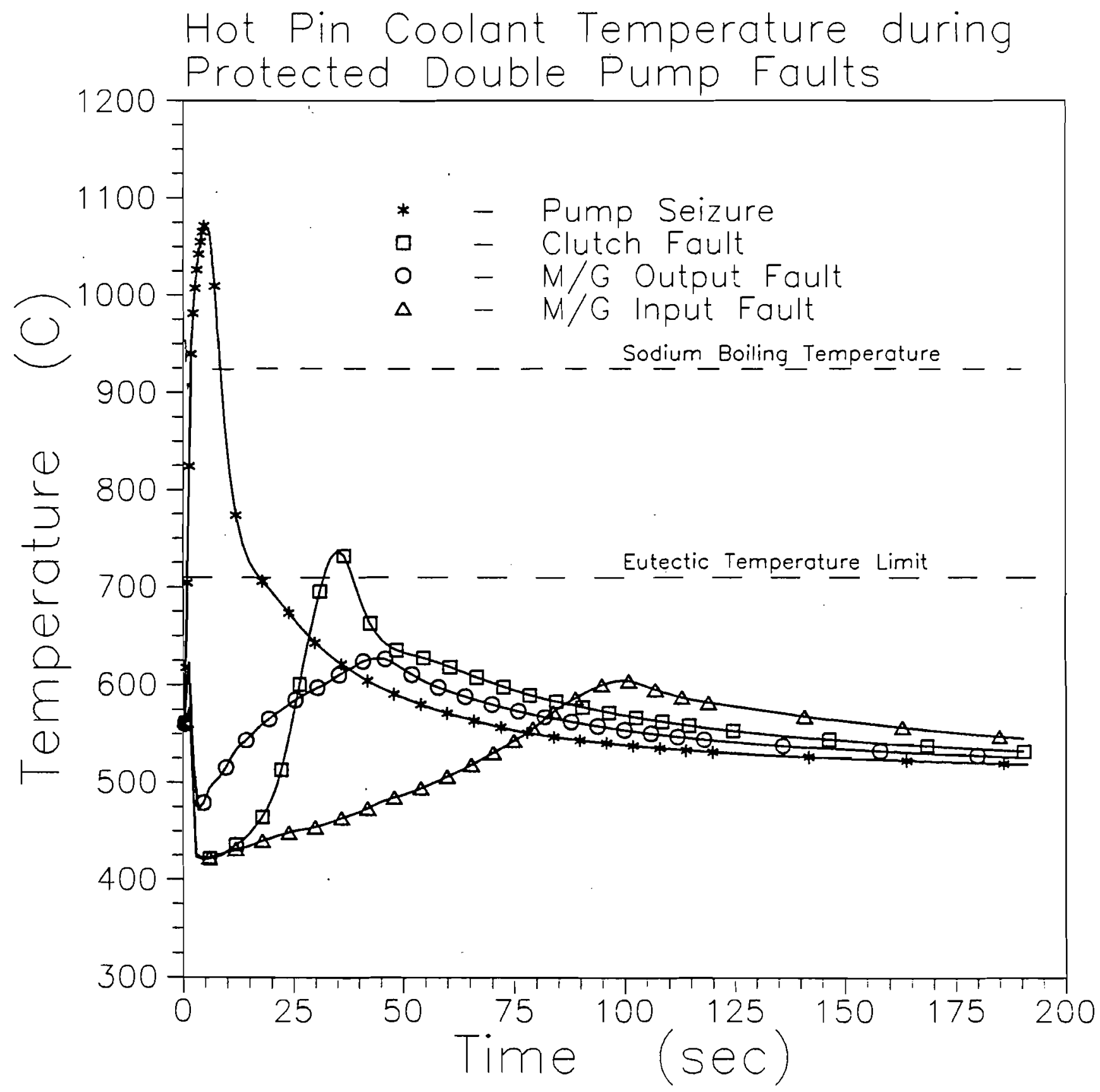

Fig. 5.52. Hot Pin Coolant Temperature during Protected Double Pump Faults 
Calculated core damage levels for unprotected double pump faults are collected in Table 5.25. All unprotected double pump faults result in some level of core damage except for a double MG input fault event. Although the double M/G input fault transient does not produce core damage, peak cladding temperatures are high enough to place the reactor in the unacceptable operating region as stipulated by EBR-II Technical Specifications. Figure 5.53 contains plots of the peak coolant temperature in the hot pin channels for identical fault, unprotected double pump failure events (i.e., both pumps experience clutch, $M / G$ output, or $\mathrm{M} / \mathrm{G}$ input faults). A calculation for an unprotected double pump seizure was not performed since it would undoubtedly lead to core damage given that the less severe unprotected double clutch failure leads to còre damage.

The LOF calculations are based on typical measurements of observable quantities such as reactivity feedback and pump speed coastdown characteristics. The core model is based on a mixed Mark-II and Mark-III core loading (Run 148B), except for the inclusion of a technical specifications limited hot pin channel to model hot spot conditions. Although by definition a double pump failure would occur anytime both pumps fail within a 6 minute period, the pumps are assumed to fail simultaneously since this produces the most damaging effects. Although the pump stopping time during a pump binding incident may be anywhere from a few minutes to a few hours, it is modelled as equivalent to the $M / G$ input fault stopping time. Likewise instantaneous pump stoppage is assumed to occur in a pump seizure, because it produces a worst-case. These assumptions reflect the uncertainty inherent in modeling a double pump failure, a pump binding, or a pump seizure incident.

\subsubsection{Loss of Normal Power}

One of the many reactor experiments performed as part of the Shutdown Heat Removal Test (SHRT) program simulated an unprotected LONP in EBR-II, which had the effect of a double $\mathrm{M} / \mathrm{G}$ input fault. Figure 5.54 provides a basis for comparing the results of the reactor experiment, SHRT 45 , with the unprotected double M/G input fault calculation. Three calculations were performed, and representative temperature plots from them are drawn in the figure. The first calculation consisted of the unprotected double $M / G$ input 
Table 5.25. Core Damage Levels During Unprotected Double Pump Faults

\begin{tabular}{||l|l|l|l|l||}
\hline & \multicolumn{1}{|c|}{ Pump Seizure Fault } & \multicolumn{1}{|c|}{ M/G Clutch Fault } & M/G Output Fault & \multicolumn{1}{|c|}{ M/G Input Fault } \\
\hline Pump Seizure Fault & Core Damage & Core Damage & Core Damage & Minor Core Damage \\
\hline M/G Clutch Fault & Core Damage & Core Damage & Minor Core Damage & Minor Core Damage \\
\hline M/G Output Fault & Core Damage & Minor Core Damage - & Minor Core Damage & Minor Core Damage \\
\hline M/G Input Fault & Minor Core Damage & Minor Core Damage & Minor Core Damage & $\begin{array}{l}\text { No Core } \\
\text { Damage/Unaccept- } \\
\text { able Operating Region }\end{array}$ \\
\hline
\end{tabular}




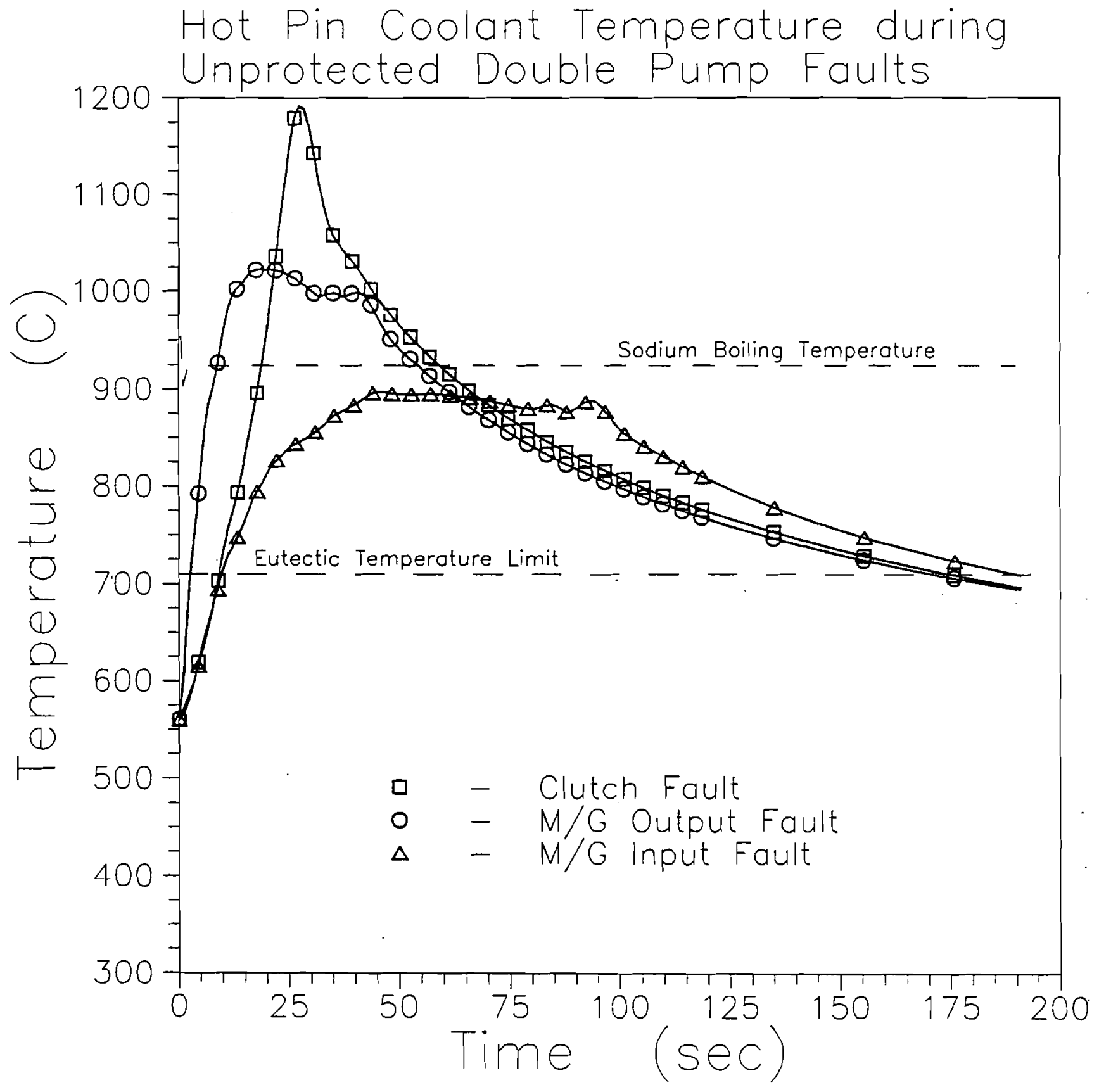

Fig. 5.53. Hot Pin Coolant Temperature during Unprotected Double Pump Faults 


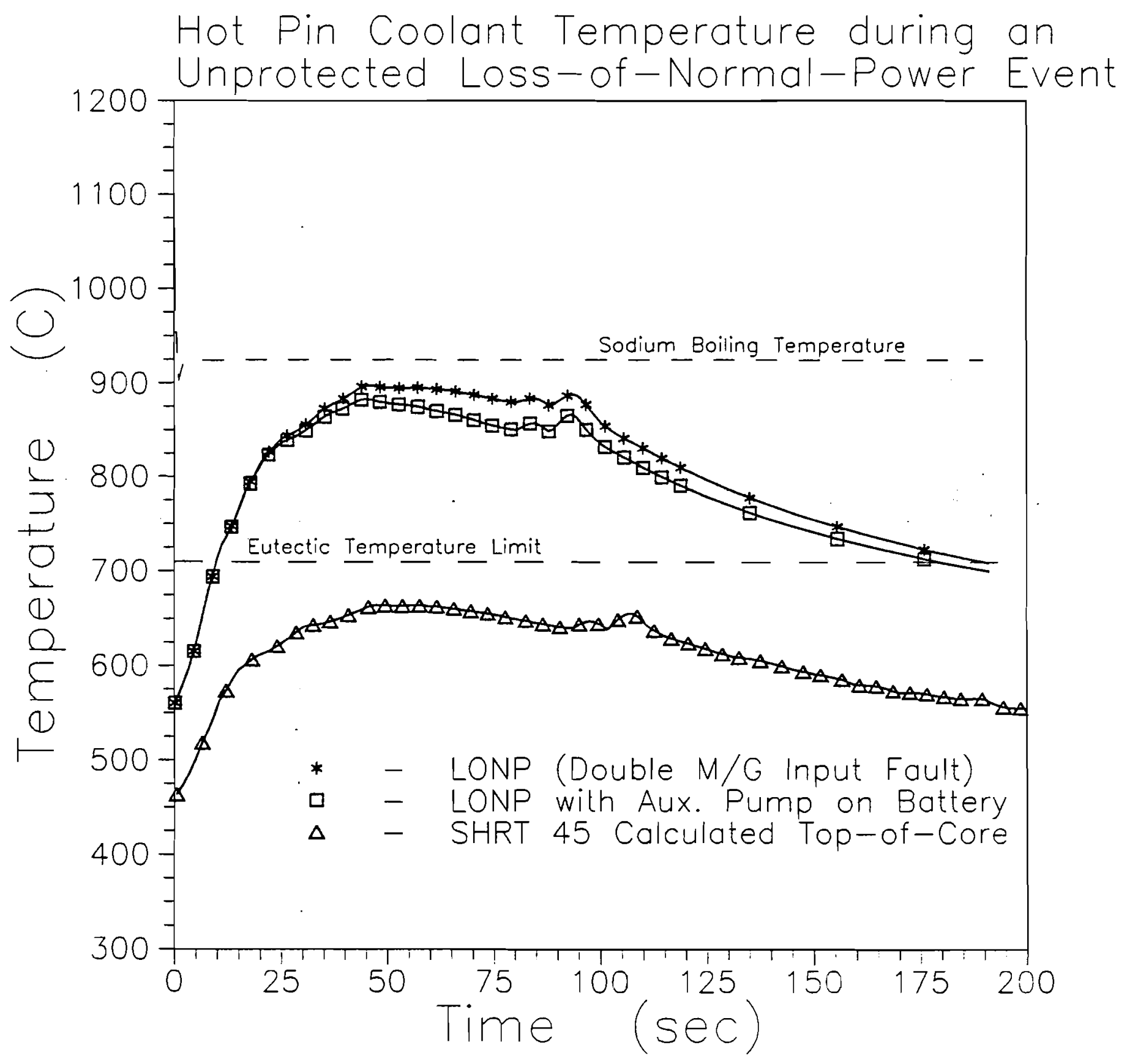

Fig. 5.54. Hot Pin Coolant Temperature during an Unprotected Loss-of-Normal-Power Event 5-168 
fault. This calculation was repeated for a second time, but this time the auxiliary pump was assumed to be operating with power supplied by battery. The second calculation matches more closely the conditions of SHRT 45 because the auxiliary pump was operating on battery during this test. The third calculation is a best estimate reproduction of SHRT 45 test conditions, and was used to validate the SASSYS model of EBR-II [5.41]. For the double M/G input fault calculations the hot pin coolant temperatures are shown in Figure 5.54, while for the SHRT 45 calculation the top-of-core coolant temperature in the instrumented subassembly XX09, which contained average power driver fuel elements, is shown. Also included in Figure 5.55 is the average top-of-core temperature for SHRT 45 as measured by the top-of-core thermocouples in XX09. The coolant temperature in XX09 during SHRT 45 is significantly lower than the coolant temperature in the hot channels of the double $\mathrm{M} / \mathrm{G}$ input fault calculations. The peak coolant temperature in the hottest subassembly during SHRT $45\left(730^{\circ} \mathrm{C}\right)[5.10]$ is still significantly lower than the coolant temperature in the hot channels of the double M/G input fault calculations $\left(900^{\circ} \mathrm{C}\right)$. The temperature difference occurs because the reactor core during SHRT 45 was specially configured to eliminate hot (near Technical Specifications limits) subassemblies and because the core inlet temperature of SHRT $45\left(343^{\circ} \mathrm{C}=650^{\circ} \mathrm{F}\right)$ is lower than that of the LOF calculations $\left(371^{\circ} \mathrm{C}=700^{\circ} \mathrm{F}\right)$. Also, the SHRT 45 core contained only Mark-II fuel elements, while the current model contains a large fraction of higher-powered Mark-III fuel elements.

\subsubsection{Loss of Constant Power}

Although a double primary pump clutch fault accompanies a loss of constant power (LOCP), the loss of $M / G$ generator field excitation uncouples the $M / G$ sets from the pump motors to produce a coastdown similar to a $M / G$ output coastdown but possibly with some minor breaking effect as the excitation field goes to zero. For this reason the consequences of a LOCP are considered to be the same as a M/G clutch fault as shown in Figs. 5.53 and 5.54, Tables 5.24 and 5.25. However, the branch point probabilities differ due to the response of the scram system. A LOCP causes a loss of power to the scram circuitry which due to its fail-safe design assures the generation of a scram signal (FSIG) with a probability of 1.0. The mechanical operation of the scram (FROD) also has a higher probability of success than other 
RSS elements since it is modelled to include the relays which supply power to the control rod clutches. Constant power is supplied to the clutches, hence an LOCP will remove power from the clutches even if the relays fail to open.

\subsubsection{Reactivity Insertion Events}

For purposes of accident sequence quantification the reactivity insertion initiating events (also called the transient overpower or TOP accidents) are divided into two classes. The first class, slow reactivity insertion events, is composed of single or multiple control rod and safety rod insertions. The second class, instantaneous reactivity insertion events, is composed of reactivity insertions which may be caused by structural failures or other rapid events. The division of the reactivity insertion events into two classes is based on the comparison of the rate of reactivity insertion to the response rate of reactor feedbacks. For the slow reactivity insertion events, the rate of reactivity insertion is much slower than the response rate of the dominant reactor feedback characteristics (reactor fuel, sodium, and steel expansion feedbacks) with the result that the system responds quasi-statistically. For the instantaneous reactivity insertion events, the reactivity is inserted into the reactor in a very short time span compared to the typical response time of reactor feedbacks.

The reactor is assumed to be operating at full power and flow conditions at the beginning of the transient. The transient is initiated by adding a time dependant, external source of reactivity to the core neutronics model. To a first approximation, the amount of reactivity inserted during a rod run-in varies linearly with time because the rod worths vary roughly linearly with rod position and the speed of the rod drive is constant [5.50]. Therefore for the slow reactivity insertion calculations, the rate of reactivity insertion is assumed to be constant. For the instantaneous reactivity insertion calculations, the reactivity is inserted as a $0.1 \mathrm{~s}$ ramp in order to aid the numerical convergence of the code. The transients described below are unprotected, because for protected transients a reactor scram will quickly halt the reactivity excursion and the undisturbed reactor coolant flow will quickly cool the core to safe conditions. 


\section{Slow Reactivity Insertion Events}

A safety rod run-in accident was simulated with SASSYS by using the median insertion rate for the accident $\left(2.910^{-3} \$ / \mathrm{s}\right)$. Figure 5.55 , which shows calculated hot pin fuel, cladding and coolant temperatures as functions of time and the amount of inserted reactivity, reveals that reactor temperatures are, to a first approximation, directly proportional to amount of inserted reactivity. As reactor temperatures increase, negative reactivity feedback effects due to thermal expansion of reactor components begin to compensate for the reactivity insertion. As shown in Figure 5.56, reactor feedbacks respond quickly to the reactivity insertion. The feedback response is rapid enough to keep the reactor nearly critical throughout the course of the transient. An analysis of a single control rod insertion accident was simulated by using the estimated median reactivity insertion rate $\left(4.210^{-3} \$ / \mathrm{s}\right)$ for such an accident. Calculated reactor temperatures for a single control rod insertion accident are plotted in Figure 5.57. The nearly identical behavior of reactor temperatures from the single control rod insertion calculation (Figure 5.57) and the safety rod insertion calculation (Figure 5.55) shows that the effects of slow reactivity insertion accidents are dependent upon the amount of reactivity inserted but independent upon the rate of reactivity insertion. For instance, referring to Figure 5.57, the reactor would remain in a state of no core damage/unlimited operating capability for an insertion of a control rod whose worth is less than $0.23 \$$. But for reactivity insertions worth more than $0.23 \$$, the reactor would enter the no core damage/limited operating capability state because the peak cladding temperature would exceed the eutectic temperature limit. A table was constructed (Table 5.26) which lists the minimum amounts of inserted reactivity that will produce the various core damage states for a given rate of reactivity insertion. The table indicates that for a single control rod insertion at the median rate of reactivity insertion (4.2 $\left.10^{-3} \$ / \mathrm{s}\right)$, a run-in of a rod worth $0.31 \$$ or more would cause the fuel in the high burn-up hot fuel pins to begin to melt, which is defined as minor core damage. If the control rod is worth more than $0.59 \$$, the accident would cause the fuel in average fuel pins to begin to melt. During the slow TOP simulations, minor core damage and core damage states are the result of peak fuel temperatures surpassing the fuel solidus temperature $\left(1214^{\circ} \mathrm{C}\right.$ for $\mathrm{U}-10 \mathrm{Zr}, 1133^{\circ} \mathrm{C}$ for $\mathrm{U}$ ) in hot and average fuel pins, respectively. This is in contrast to the LOF simulations, during which core damage states are produced by coolant boiling. 
Hot Pin Temperatures during a

Safety Rod Insertion at $0.0029 \$ / \mathrm{s}$

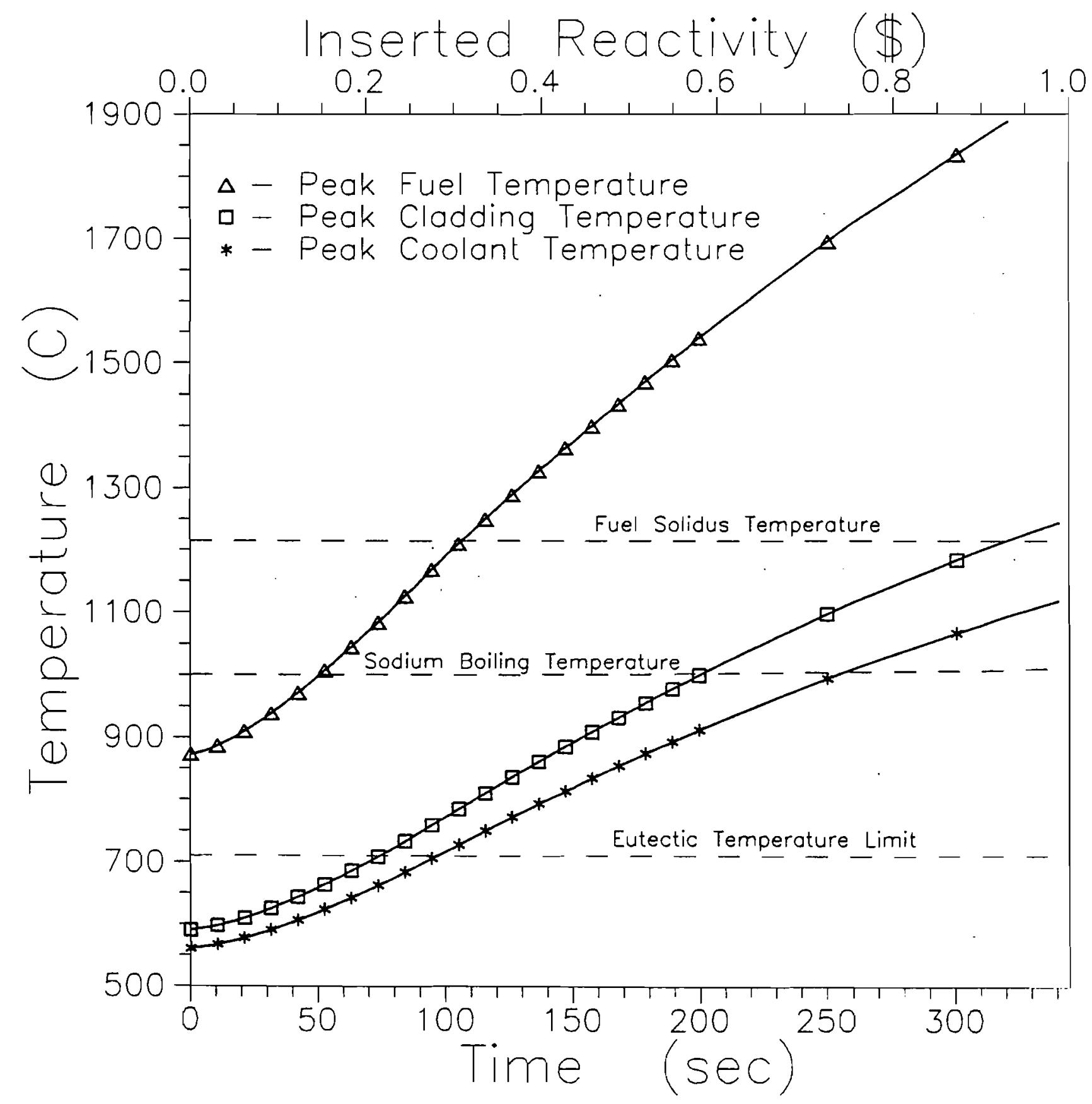

Fig. 5.55. Hot Pin Temperatures during a Safety Rod Insertion at $0.0029 \$ \mathrm{~s}$ 


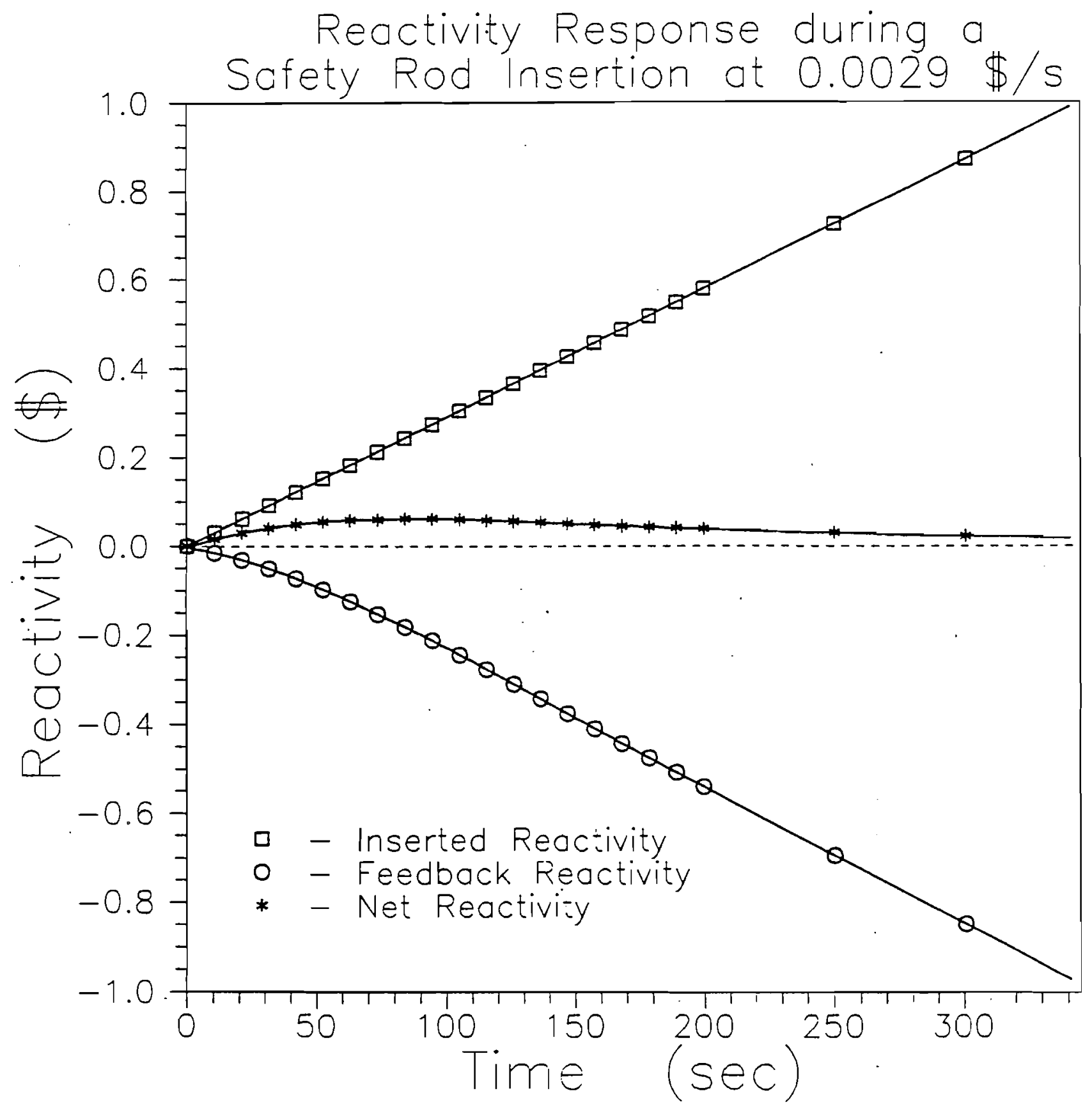

Fig. 5.56. Reactivity Response during a Safety Rod Insertion at $0.0029 \$ / \mathrm{s}$ 
Hot Pin Temperatures during a

Control Rod Insertion at 0.0042 \$/s

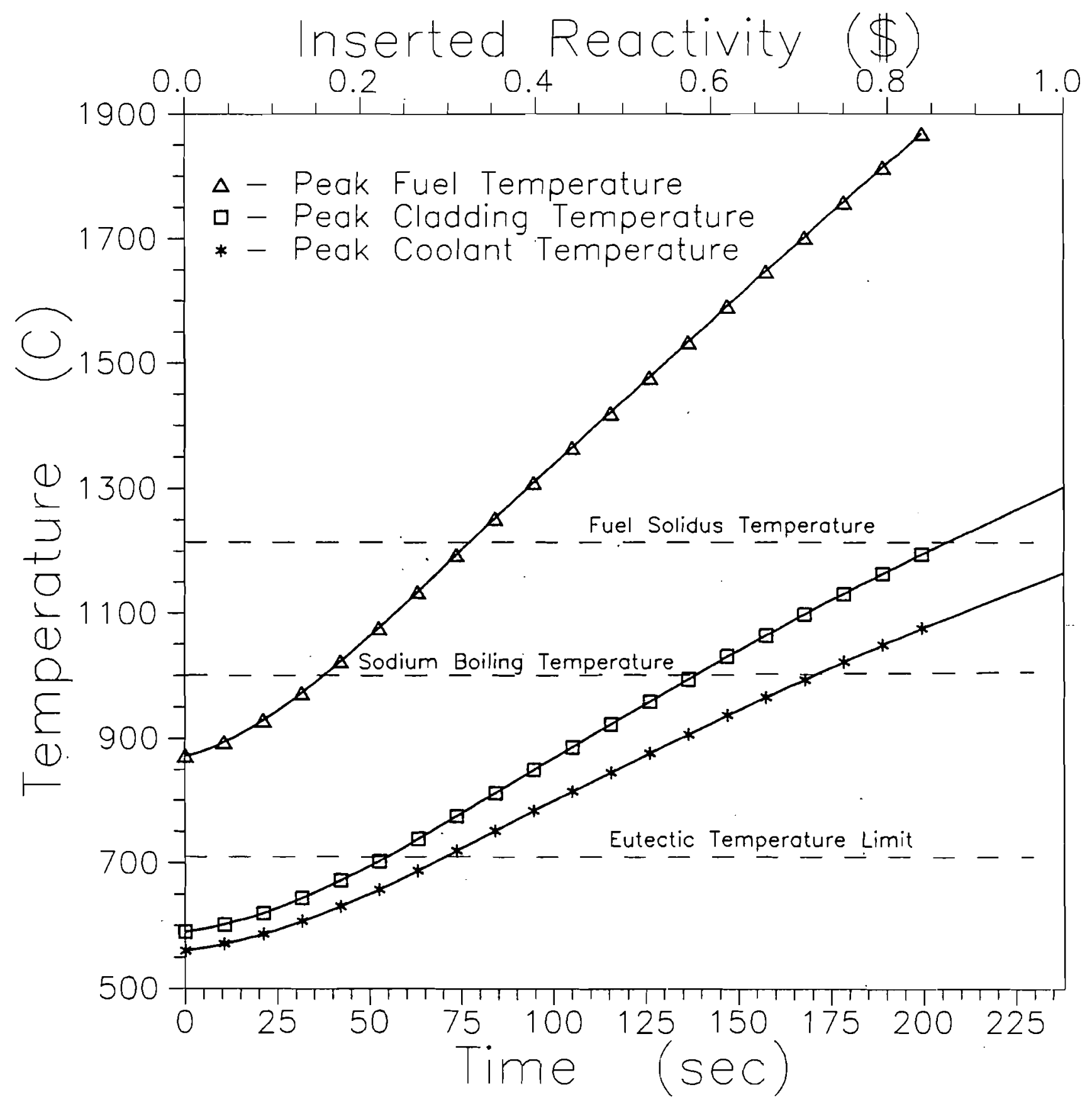

Fig. 5.57. Hot Pin Temperatures during a Control Rod Insertion at $0.0042 \$ / \mathrm{s}$ 
Table 5.26. Core Damage Levels during Slow Reactivity Insertion Accidents

\begin{tabular}{|c|c|c|c|}
\hline Reactivity Insertion Rate & $\begin{array}{l}\text { Reactivity Worth } \\
\text { Needed to Reach } \\
\text { No Core } \\
\text { Damage/Limited } \\
\text { Operation Level } \\
\text { (\$) } \\
\end{array}$ & $\begin{array}{l}\text { Reactivity Worth } \\
\text { Needed to Reach } \\
\text { Minor Core } \\
\text { Damage Level } \\
\qquad 1 \text { (\$) } \\
\end{array}$ & $\begin{array}{l}\text { Reactivity Worth } \\
\text { Needed to Reach } \\
\text { Core Damage Level } \\
\text { (\$) }\end{array}$ \\
\hline $\begin{array}{c}0.29 \\
\text { (Median Rate Safety Rod Insertion TOP) }\end{array}$ & 0.22 & 0.31 & 0.59 \\
\hline $\begin{array}{c}\quad 0.42 \\
\text { (Median Rate Single Control Rod Insertion TOP) }\end{array}$ & 0.23 & 0.32 & 0.59 \\
\hline $\begin{array}{c}0.64 \\
\text { (Maximum Rate Single Control Rod Insertion TOP) } \\
\end{array}$ & 0.25 & 0.34 & 0.60 \\
\hline $\begin{array}{c}\text { 0.84 } \\
\text { (Median Rate Double Control Rod Insertion TOP) }\end{array}$ & 0.26 & 0.36 & 0.62 \\
\hline $\begin{array}{c}1.22 \\
\text { (Maximum Rate Double Control Rod Insertion TOP) }\end{array}$ & 0.34 & 0.39 & 0.65 \\
\hline
\end{tabular}




\section{Instantaneous Reactivity Insertions}

A series of calculations were performed to investigate the effect of an instantaneous insertion of reactivity. An instantaneous TOP accident is a very low probability event which might be produced by a structural failure of the core support apparatus, for example, causing repositioning of the fueled subassemblies with respect to the control rods. Instantaneous TOP accidents were modeled with SASSYS by inserting a given amount of reactivity during the initial $0.1 \mathrm{~s}$ of a transient calculation. The external reactivity worth of the core was maintained at a constant, elevated level for the remainder of the calculation. At the beginning of the transient, reactor temperatures increase rapidly in response to the reactivity insertion. As shown in Figures 5.58 and 5.59, which contain plots of the hot pin peak fuel and cladding temperatures during three instantaneous TOP calculations (instantaneous insertions of $0.17 \$, 0,45 \$$ and $0.80 \$$ ), reactor temperatures soon begin to level off as prompt reactor feedback effects (reactor fuel, sodium and steel expansion feedbacks) [5.33] respond to the temperature rise. Figure 5.59 shows that for instantaneous reactivity insertions of more than $0.17 \$$, hot pin cladding temperatures would surpass the eutectic temperature limit. As with the slow reactivity insertion calculations, core damage levels are reached due to the onset of fuel melting instead of coolant boiling. Fuel melting occurs before cladding failure or the onset of boiling because the energy generated by the reactivity change is deposited directly into the fuel. Figure 5.60, which contains plots of the time to reach core damage conditions as a function of the amount of inserted reactivity, shows that for large reactivity insertions fuel melting would occur within fractions of a second. The figure also shows that fuel melting occurs before cladding failure. The reactor shutdown system will respond quickly, via the neutron power level trip, to a rapid reactivity insertion. But because the control rods require about $0.25 \mathrm{~s}$ to drop from the core upon receiving a trip signal [5.51], fuel melting and subsequent fuel pin failure would not be avoided during a large instantaneous reactivity insertion accident, regardless of the action of the reactor shutdown system. Reactor conditions for instantaneous reactivity insertion accidents as functions of the amount of inserted reactivity are listed in Table 5.27. This table shows that for instantaneous reactivity insertions worth less than about $0.3 \$$, core damage conditions would not be reached because the peak fuel temperature would not surpass the fuel solidus temperature. 
Hot Pin Cladding Temperature during

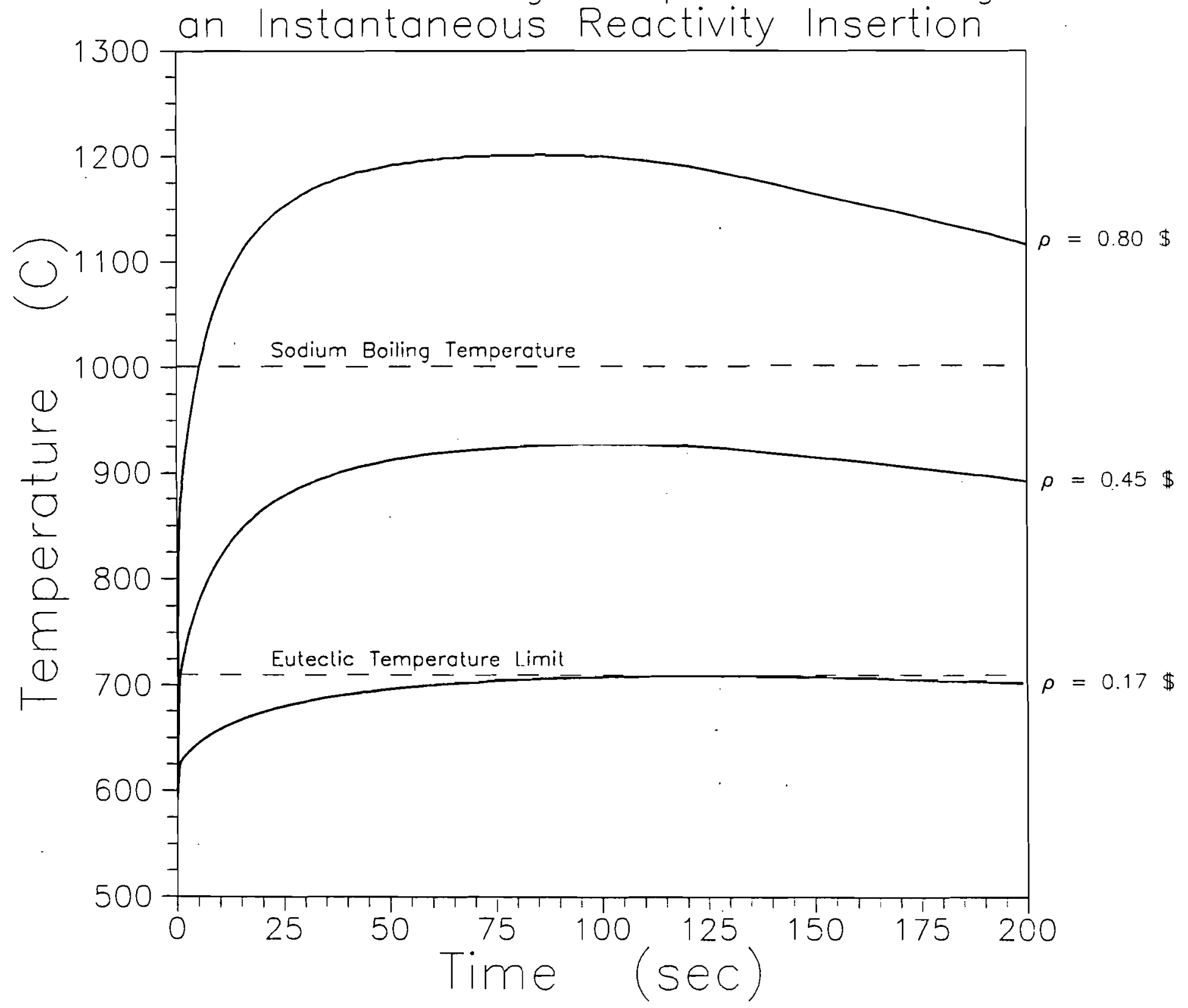

Fig. 5.58. Hot Pin Cladding Temperature during an Instantaneous Reactivity Insertion 


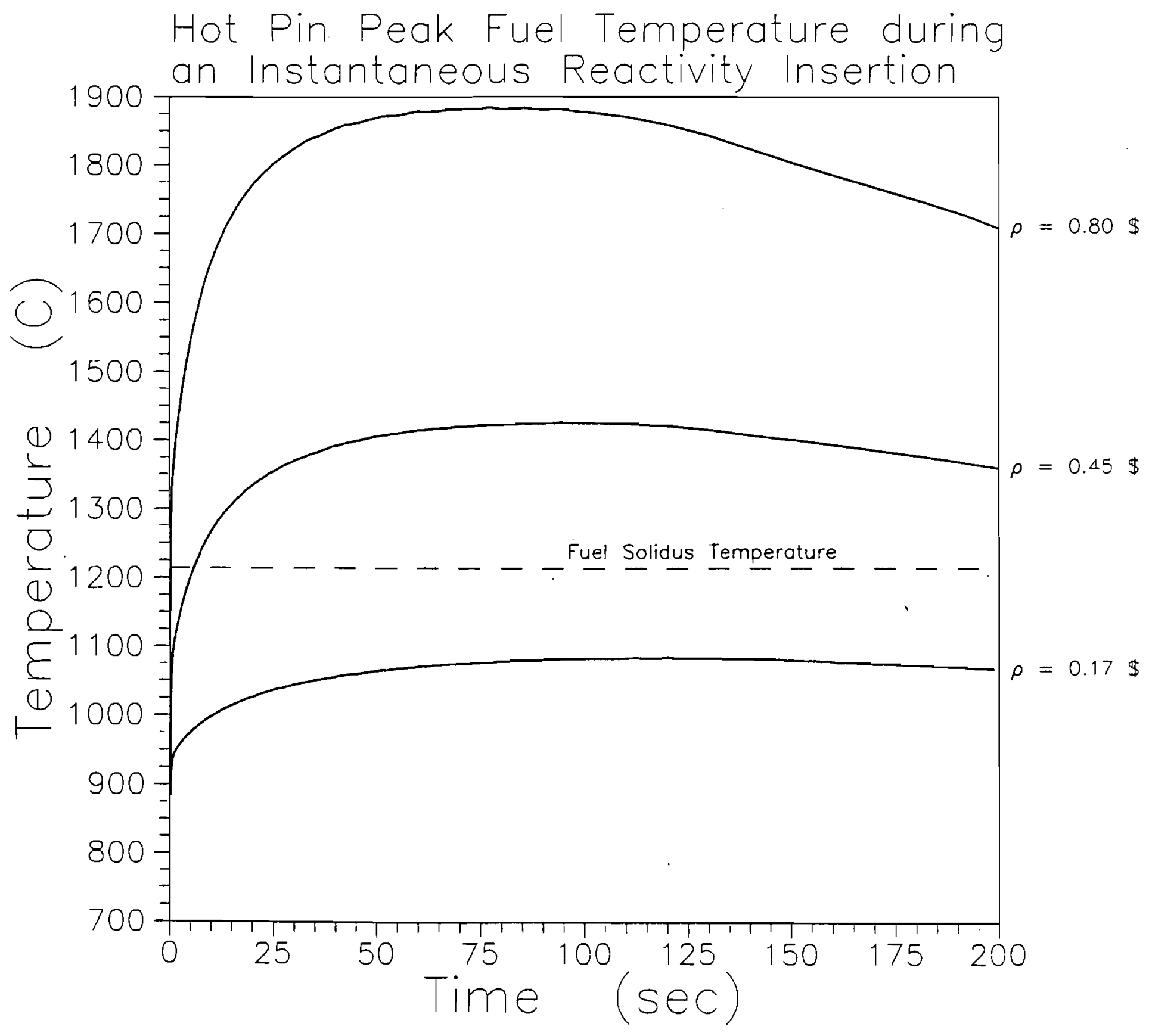

Fig. 5.59. Hot Pin Peak Fuel Temperature during an Instantaneous Reactivity Insertion 


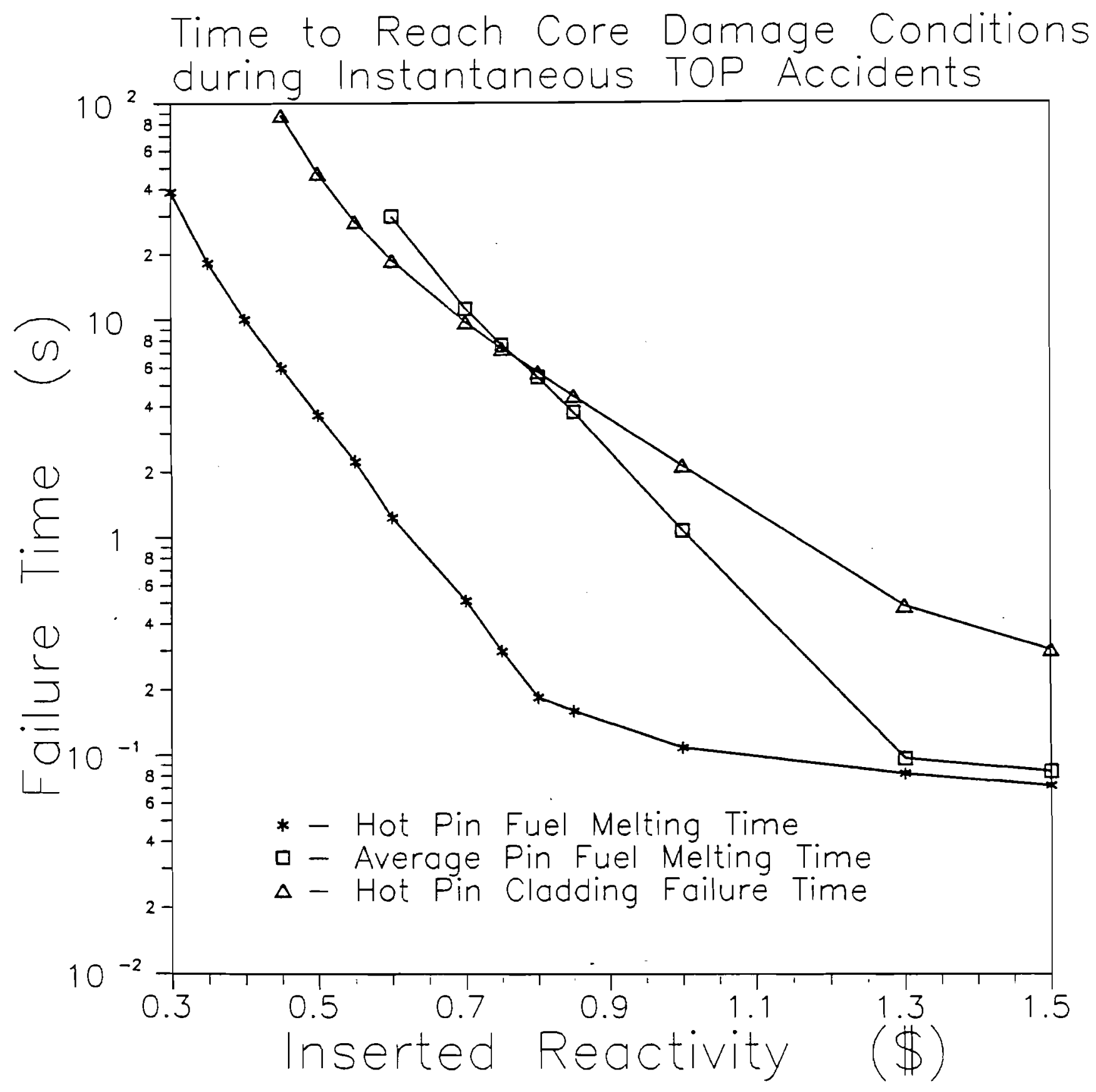

Fig. 5.60. Time to Reach Core Damage Conditions during Instantaneous TOP Accidents 
Table 5.27. Reactor Conditions during Instantaneous Reactivity Insertion Accidents

\begin{tabular}{|c|c|c|c|c|c|c|}
\hline $\begin{array}{l}\text { Inserted } \\
\text { Reactivity } \\
\text { (\$) }\end{array}$ & $\begin{array}{l}\text { Hot Pin Peak } \\
\text { Fuel Temperature } \\
\qquad\left({ }^{\circ} \mathrm{C}\right)\end{array}$ & $\begin{array}{l}\text { Hot Pin Peak } \\
\text { Cladding } \\
\text { Temperature } \\
\qquad\left({ }^{\circ} \mathrm{C}\right)\end{array}$ & $\begin{array}{l}\text { Hot Pin Peak } \\
\text { Coolant } \\
\text { Temperature } \\
\qquad\left({ }^{\circ} \mathrm{C}\right)\end{array}$ & $\begin{array}{l}\text { Hot Pin Fuel } \\
\text { Melting Time } \\
\text { (s) }\end{array}$ & $\begin{array}{l}\text { Average Pin Fuel } \\
\text { Melting Time } \\
\text { (s) }\end{array}$ & Core Damage State \\
\hline 0.17 & 1084 & 709 & 663 & -- & -- & No Core Damage/Unlimited Operation \\
\hline 0.20 & 1124 & 732 & 683 & -- & -- & No Core Damage/Limited Operation \\
\hline 0.25 & 1189 & 774 & 719 & -- & -- & No Core Damage/Limited Operation \\
\hline 0.30 & 1250 & 812 & 752 & 38.7 & -- & Minor Core Damage \\
\hline 0.40 & 1367 & 889 & 817 & 10.01 & -- & Minor Core Damage \\
\hline 0.50 & 1486 & 967 & 883 & 3.64 & - & Minor Core Damage \\
\hline 0.55 & 1549 & 1005 & 916 & 2.23 & - & Minor Core Damage \\
\hline 0.60 & 1613 & 1045 & 949 & 1.23 & 29.9 & Core Damage \\
\hline 0.70 & 1746 & 1123 & 1015 & 0.508 & 14.4 & Core Damage \\
\hline 0.80 & 1884 & 1202 & 1080 & 0.184 & 5.42 & Core Damage \\
\hline 0.85 & 1953 & 1241 & 1113 & 0.159 & 3.74 & Core Damage \\
\hline 1.00 & 2167 & 1359 & 1210 & 0.108 & 1.07 & Core Damage \\
\hline
\end{tabular}




\subsubsection{Loss of Primary Heat Sink}

For the unprotected loss of heat sink sequence the end state is well characterized. In fact this scenario was run as a plant experiment twice in early 1986 as part of the Inherent Safety Demonstration Tests, [5.10]. Fig. 5.61 shows the predicted and observed response for an instrumented subassembly in the second experiment, B302R. The response to the loss of heat sink is an inlet temperature rise which shuts the system down to a uniform elevated temperature. For the technical specifications limited hot-sub channel with an inlet temperature of $700^{\circ} \mathrm{F}$ the associated temperature rise is given by the PRD $(\leq 35 \mathrm{c})$ and the inlet temperature coefficient of reactivity $\left(\sim 0.7 \mathrm{c} /{ }^{\circ} \mathrm{C}\right)$ leading to $50^{\circ} \mathrm{C}$ rise $\left(90^{\circ} \mathrm{F}\right)$ and a final temperature of $790^{\circ} \mathrm{F}$.

\subsubsection{Loss-of-Sodium Events}

For purposes of accident sequence quantification the Loss-ofSodium Accidents (LOS) are divided into two classes, rapid and slow transients. During a rapid LOS, the primary system loses sodium at a rapid rate which results in pump inlet uncovery soon after initiation of the transient. During a slow LOS, the primary system loses sodium at a slower rate, which results in pump inlet uncovery that occurs a long time after the initiation of the transient. The slow LOS calculations consisted of determining the maximum rate of coolant loss that can be withstood without causing core damage levels to be reached anytime during the transient.

The loss-of-circulation transient calculations are based on the following sequence of events. The reactor, initially at full power and flow conditions, experiences a failure which causes the primary system to lose coolant. A scram signal is generated at the same time as or soon after initiation of the LOS and the reactor scram is assumed to be successful.

The first calculation performed was a simulation of a protected rapid LOS. The transient disrupts the normal flow circuit, limiting natural circulation flow paths to within the reactor vessel and through reactor vessel leakage paths. For simplicity, the speeds of both pumps were 


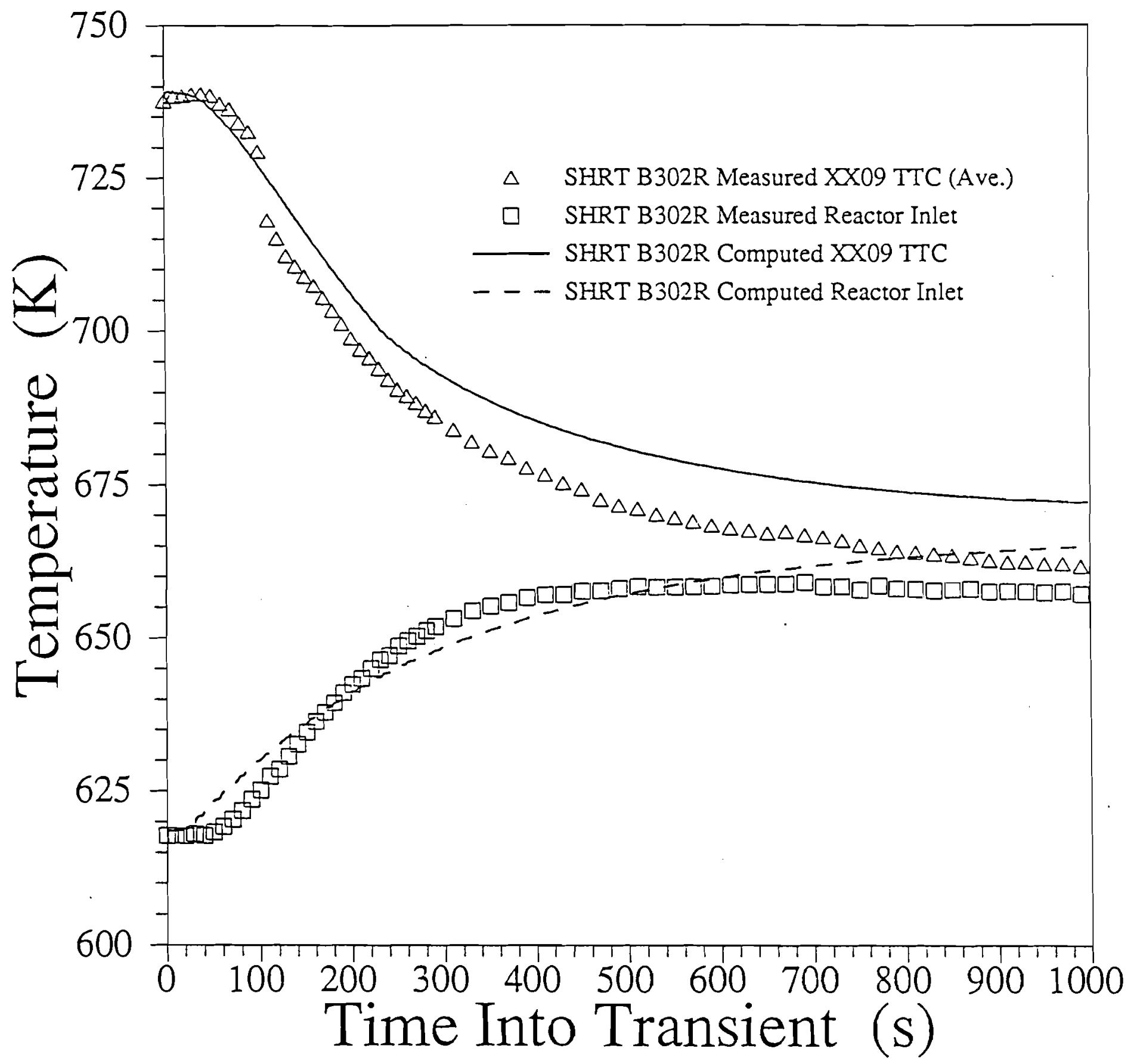

Fig. 5.61. SHRT B302R Top of Core Thermocouple and Reactor Inlet Temperatures 
assumed to decay with coastdown characteristics of a M/G clutch failure, which provides a 24 $s$ coastdown period. At the end of the coastdown phase, the pump inlets are assumed to become completely uncovered and sodium flow through the pump is disabled. In the model this is accomplished by giving the pumps an essentially infinite flow resistance. At the same time that flow through the primary pumps is disabled, coolant flow through the Z-pipe is stopped because, as the sodium level drops below the upper section of the Z-pipe, causing the upper section of the Z-pipe to drain in the absence of forced coolant flow. This also causes any heat removal through the IHX to cease.

Following the pump inlet uncovery, flow through the core region subassemblies, which are connected to the high pressure plenum, settles to a nearly constant rate that is slightly under $1 \%$ of the nominal core flowrate. The core region flowrate is balanced by a reverse direction flow through the blanket region subassemblies, which are connected to the low pressure plenum. A nearly steady flow circuit is established wherein natural circulation drives coolant flow through the core region subassemblies. The coolant flow then reverses. direction and travels downward past the cooler blanket region subassemblies. It then travels through the low pressure plenum and out through the low pressure piping, until it drains into the high pressure piping beneath the pump outlets, through which the flow returns to the high pressure plenum. This flow circuit is augmented by leakage flows which allow cooler sodium from the pool to enter the reactor vessel. Sodium enters the high pressure plenum through leakages in the subassembly adapters. This flow returns to the primary pool via leakages at the reactor cover, after traveling through the core region. The leakage flow accounts for roughly $20 \%$ of the total natural circulation flow through the core region following pump inlet uncovery. Although the bulk flow through the core region is relatively constant following the pump inlet uncovery, flow in individual channels oscillate, as shown in Figure 5.62. This figure shows the normalized coolant flowrate and coolant outlet temperature of the hot pin channel during a protected, rapid LOS. As the flowrate in the channel stagnates following pump inlet uncovery, the coolant temperature rises, which results in a larger natural circulation driving force and causes the flowrate to rebound. The rebounding flowrate cools the channel, lowering the temperature and natural circulation driving force. This process repeats resulting in oscillatory behavior within the 


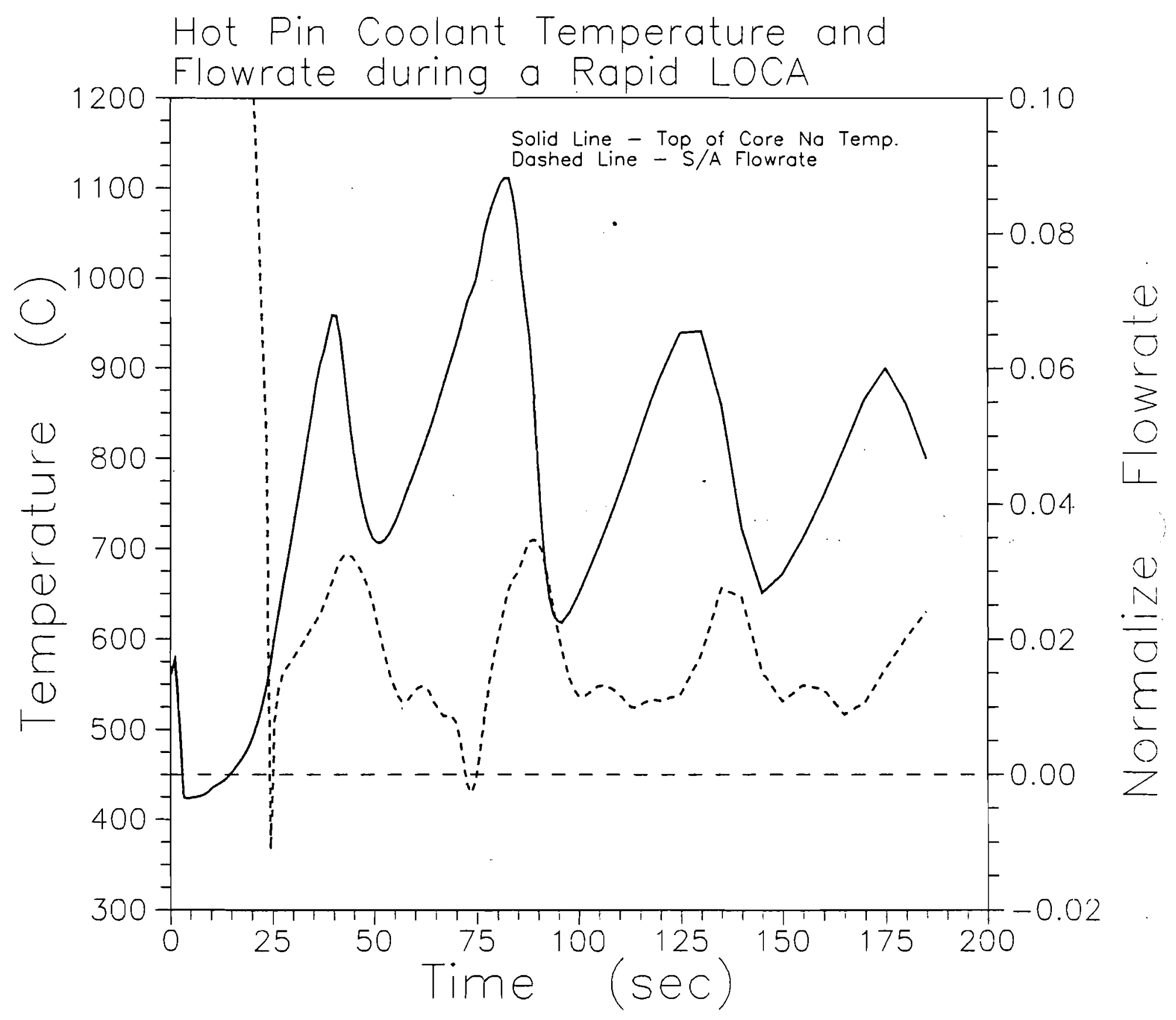

Fig. 5.62. Hot Pin Coolant Temperature and Flowrate for a Protected Rapid LOS 
channel. Driver fuel subassemblies which have a relatively low flowrate at the start of the transient exhibit a more pronounced oscillatory behavior, because they are more likely to experience flow stagnation. The temperature oscillations during a rapid LOS are great enough to produce boiling and pin failure in core channels, thus the rapid LOS is a direct core damage event, (plant transient category P1), even if protected.

For the delayed LOS calculations the beginning of the pump speed coastdown and the pump inlet uncovery time were delayed with respect to the initiation of the LOS and the scram signal. Core damage can be avoided if the pump inlet uncovery is delayed long enough after the reactor shutdown. If, during delayed LOS, the pump inlet uncovery is delayed 1 hour after initiation of the transient, the core will not be damaged because the peak coolant temperature $\left(918^{\circ} \mathrm{C}\right)$ is less than the sodium boiling temperature $\left(927^{\circ} \mathrm{C}\right)$. If the reactor has not been shutdown when the pump inlet uncovery occurs, reactor damage is unavoidable.

The definition of the delayed loss-of-sodium event is based on the assumption that the reactor will be successfully scrammed. Because the sodium leak may not be detected immediately, the reactor shutdown may be delayed from the initiation of the sodium leak. The period of time between accident initiation and reactor shutdown must be added to the 1 hour delay period between reactor shutdown and pump inlet uncovery to determine the time period that the sodium level must remain above the pump inlets. In other words, the sodium leak must be small enough to avoid pump inlet uncovery during the period needed to detect the leak, shutdown the reactor, and allow 1 hour of forced circulation cooling of reactor decay heat power. 


\subsection{Uncertainity Analysis For Reactivity Feedbacks}

\subsubsection{Introduction}

The reactivity feedbacks and their tendency to reestablish a stable state when the reactor is subjected to an accident initiator without scram were described earlier. The likelihood that this characteristic of EBR-II actually prevents core damage is the subject of this failure analysis. It therefore quantifies the conditional probability that reactivity feedbacks are not strong enough to keep temperature rises with safe bounds, given an accident initiator and failure to scram.

In essence the analysis consists of evaluating the ability to predict what the reactor behavior will be for each accident sequence. Calculational predictions are used and all the relevant experimental evidence is applied in the evaluation.

The main reason that calculated transient outcomes are uncertain is that the input data are uncertain. The uncertainty in each input parameter can be expressed by constructing a distribution of possible values called a probability density function (pdf). The pdf for the transient outcome quantity of interest, e.g. peak temperature, can be found by sampling from the input parameter pdfs and running transient calculations with these parameter values. The fraction of the peak temperature pdf that lies beyond a safe temperature limit is the passive safety failure probability. This is basically the method described by Mueller and Wade [5.52].

This uncertainty propagation process would be prohibitively expensive to carry out without taking measures to make it computationally efficient. There are several accident initiators to consider, many uncertain parameters on which a transient calculation depends, and many transient calculations (each with a different, randomly selected parameter value) required to propagate each parameter's uncertainty accurately.

Two techniques were used to make the process efficient. One is screening. The procedure is to sort through all possibilities, discarding those that are unimportant. The other 
efficiency measure is to use the response surface method [5.53]. The idea is to find a simple function, which is termed a response surface, that approximates the analytically unknown functional relationship between the outcome quantity and the input parameters. The parameter uncertainties can be propagated through this function inexpensively by Monte Carlo sampling, once the function is known.

The analysis method consists of the eight steps listed in Table 5.28. Several steps involve screening or the response surface approximation. EBR-II plant experiments play an important role in Steps 1 and 2, as well as Step 7. Steps are included that proved to be useful in other recent efforts to evaluate uncertainties in the outcome of reactor transients using probabilistic methods $[5.54,5.55]$.

Table 5.28. Steps in Passive Safety Analysis Procedure

1. Screen Accident Initiators

2. Calibrate EBR-II $\cdot$ Model in SASSYS Transient Code

3. Screen Parameters

4. Quantify Parameter Uncertainties

5. Develop Experimental Constraints

6. Compute Response Surfaces

7. Propagate Parameter Uncertainties Subject to Experimental Constraints

8. Assess Accuracy of Failure Probabilities

The presentation of the method and results in the next section is organized by step. Some of the descriptions are lengthy because the approach has not been described previously. The casual reader may be satisfied with the summary and discussion provided in Section 5.5.3. 


\subsubsection{Analysis Method and Results}

\section{Step 1 - Screen Initiators}

A screening analysis was done for each accident initiator. For initiators where this gives an unambiguous result, no further analysis is needed. The remaining accidents require a detailed analysis. The initiator screening was done principally using a quasi-static reactivity balance [5.56]. EBR-II plant experiments and past safety analyses supplemented the quasi-static evaluation.

The quasi-static reactivity balance can be written as

$$
0=\Delta \rho=A \cdot(P-1)+B \cdot(P / F-1)+C \cdot \delta T_{\text {in }}+\Delta \rho_{\text {exi }}
$$

Here $\mathrm{P}$ is the power (normalized to unity at full power), $\mathrm{P} / \mathrm{F}$ is the power-to-flow ratio (normalized to unity at normal power and flow), $\delta \mathrm{T}_{\text {in }}$ is the deviation from the normal inlet temperature and $\Delta \rho_{\text {ext }}$ is the change in externally-imposed reactivity. The coefficients $\mathrm{A}, \mathrm{B}$ and $\mathrm{C}$ reflect the reactivity feedbacks. An accident initiator influences the reactivity balance by changing $F, T_{\text {in }}$ and/or $\Delta \rho_{\text {ext }}$. Given the changes, Eq. 5.1 can be solved for the asymptotic power level that results when the feedbacks restore a reactivity balance. If the transient is one where worst conditions are reached asymptotically, then comparing the temperatures implied by the asymptotic power level against safe temperature limits tells whether there is damage in the

transient. The temperature limits used for screening purposes were the eutectic temperature to represent operational limitations and the sodium boiling temperature to represent radiological release potential.

Experiment-based data were used in the screening to the extent possible. The sum of A and $B$ is the power reactivity decrement from zero to full power (PRD), which is measured in every reactor run. The average from numerous runs, $-32.06 \pm 2.81 \mathrm{c}$ was used. The average of four calculated values of the ratio $\mathrm{A} / \mathrm{B}, 0.204 \pm 0.048$ [5.57], was used to separate $\mathrm{A}$ and $\mathrm{B}$ where needed. The average of experimental values for $\mathrm{C},-0.693 \pm 0.062 \mathrm{c} /{ }^{\circ} \mathrm{C}$, was used. 
Measured full power inlet and outlet temperatures provided initial conditions. The difference between inlet and outlet temperatures at any time, when multiplied by a peaking factor, gives the peak temperature rise at that time. Steady state peaking factors, 1.85 for coolant and 2.24 for cladding, were assumed to apply at all times.

For each ATWS event, using the average values of $\mathrm{A}, \mathrm{B}$ and $\mathrm{C}$ above yielded a best estimate of the peak temperature, which implied either a failure or a no-failure prediction. To evaluate the reliability of these predictions, A, B and C were treated as normally distributed probability density functions and the uncertainty quoted above was taken to be the standard deviation of the distribution. Propagating these distribution functions through Eq. 5-1 yielded a distribution of peak temperatures for a given ATWS event. The fraction of this distribution lying above the safe temperature limit gave the failure probability for that event.

The screening results for the ATWS events are as follows:

Loss Of Heat Sink - The failure probability is totally negligible, even by the eutectic formation criterion. This quasi-static result is consistent with the actual demonstration of the EBR-II plant response to a loss-of-heat-sink initiator [5.58], which had a completely benign outcome.

- Pump Overspeed - The failure probability again is totally negligible. The asymptotic $\mathrm{P} / \mathrm{F}$ does not exceed unity so the temperatures do not increase. The pump run-up tests in EBR-II confirm this prediction [5.59].

Loss Of Flow - A detailed analysis is needed. The quasi-static analysis shows that the asymptotic temperatures are benign with a very high probability. However, the peak temperatures in a loss-of-flow accident occurs during a transient peak, which is not addressed in the quasi static analysis. Past safety analyses suggest that the failure probability should be low for long pump coastdowns and high with short coastdowns. The SHRT plant experiments demonstrated that there is no damage if the coastdown time is sufficiently long [5.58]. 
- Loss Of Normal Power - A detailed analysis is needed. The accident is a long coastdown loss of flow combined with a loss of heat sink. The latter phase is benign and the former should have a low failure probability. The SHRT 45 experiment simulated this accident and there was no damage [5.58].

Transient Overpower - A detailed analysis is warranted. The probability of eutectic formation appears to be high and fuel melting could occur. This assumes the initiator is a rod run-in with a $\Delta \rho_{\text {ext }}$ that is based on probability tables derived from operating history.

All the remaining steps of the analysis have been carried out for LONP but Steps 6 and 7 need to be completed for loss-of-flow and transient overpower initiators.

\section{Step 2 - Calibrate Models}

The model constructed for the consequence analysis was adjusted to yield good agreement with key EBR-II passive safety demonstration experiments [5.58]. For this analysis the model was reevaluated based on data in the literature and additional experiments.

Three modest changes were made. One change was to the nominal values of several feedback reactivity coefficients. New values were chosen based on an evaluation of values reported in the literature $[5.60,5.61,5.62]$. The second change was to increase the metal fuel conductivities to best estimate values [5.63] from three-sigma-low (conservative) values; this had little effect on loss of flow and transient overpower predictions but significant impact on power reactivity decrement (PRD) and rod drop feedback reactivity predictions, which are used in the experimental constraint process.

The third change was to the bowing reactivity feedback model. This is an important feedback because it is the only one that can be positive and it can be more than $40 \%$ of net feedback (although it is typically $\sim 15 \%$ ). EBR-II's design is such that bowing is difficult to calculate from first principles and an empirical approach is usually taken. Typically the bowing 
feedback for a specific reactor run is inferred from the measurement of PRD vs. reactor power; it is assumed that the calculated nonbowing PRD is correct and any difference between it and the measured PRD is attributed to bowing. The traditional model treats bowing feedback vs. power as zero until some threshold power and then it grows at a constant, generally positive slope [5.63].

Physical reasoning [5.64] and evidence from fifty PRD measurements suggest a somewhat different behavior: the bowing feedback slope is initially negative, changes to a generally positive value around $20 \mathrm{MWt}$ and maintains that value until the vicinity of full power (62.5 MWt). In this vicinity the slope often starts to decrease and eventually it must become negative again. The new model incorporates the first two features but continues the second slope indefinitely beyond full power. This is conservatism prompted by a lack of unambiguous evidence for the location of the second slope change. Nominal values for the new bowing model input parameters were derived from the difference the nominal calculated nonbowing PRD vs. power and the average of the 50 PRD measurements.

The modified EBR-II model in SASSYS yields improved agreement with PRD, rod drop and passive safety demonstration experiments. Results for the SHRT 45 demonstration experiment [5.58] illustrate this. This experiment is directly relevant to unprotected LONP and other loss of flow accidents since it was a planned LONP event without scram. For this experiment, the prediction of peak feedback ractivity by the consequence analysis model is low by $10 \%$ and the model improvements reduced this error to less than $3 \%$. There have been no safety demonstration experiments explicitly simulating the other class of accidents requiring a detailed analysis, transient overpower events. However, PRD and rod drop experiments serve to validate the model indirectly since they involve the same feedbacks that control the behavior of the core in other transients. 


\section{Step 3 - Screen Parameters}

The number of transient calculations necessary for the analysis grows rapidly with the number of uncertain parameters. This provides a strong incentive to minimize the number of parameters that are treated as uncertain.

There are three classes of candidate parameters. One is modeling parameters, such as the number of channels and time step size. Selected values are such that parameters in this class are relatively unimportant. Modeling assumptions also should be considered here. None was identified as being likely to cause a substantially optimistic failure probability estimate. The second class is parameters that affect the predicted material expansions - such things as thermal conductivity and expansion coefficients. The third class is reactivity coefficients of expansion and bowing.

The first measure taken to reduce the number of uncertain parameters was to treat only the third class as uncertain and increase the uncertainty estimates to cover uncertainties that properly belong to the second class. The rationale for this approach is that 1) the feedback effect is the product of the calculated expansion and the reactivity coefficient of expansion, and 2) the reactivity coefficients often are the more uncertain factor.

The reactivity coefficients were screened using importance ranking. The importance of a parameter is the product of the parameter's uncertainty and the sensitivity of the transient outcome to a given change in the parameter. Screening uncertainty estimates for the feedback components were obtained from Ref. 5.52. Sensitivities were deducted from edits of component contributions to feedback reactivity histories in SASSYS calculations of each transient type. Of the ten feedbacks in the SASSYS EBR-II model, four of these were found to be unimportant to all transients relevant to the detailed failure analysis. The feedbacks that are important to a transient or constraining quantity are identified by an " $\mathrm{x}$ " in Table 5.29 . 
Table 5.29. Important Feedbacks

Core Na Fuel Up. Refl. $\underline{\text { Rad. Refl. }}$

\begin{tabular}{lllllll} 
LOF & $\mathrm{x}$ & & $\mathrm{x}$ & $\mathrm{x}$ & $\mathrm{x}$ & $\mathrm{x}$ \\
TOP & $\mathrm{x}$ & $\mathrm{x}$ & $\mathrm{x}$ & $\mathrm{x}$ & $\mathrm{x}$ & $\mathrm{x}$ \\
PRD & $\mathrm{x}$ & $\mathrm{x}$ & $\mathrm{x}$ & $\mathrm{x}$ & $\mathrm{x}$ & $\mathrm{x}$ \\
$\operatorname{RDF}(1.5)$ & $\mathrm{x}$ & $\mathrm{x}$ & $\mathrm{x}$ & & & \\
$\operatorname{RDF}(8.0)$ & $\mathrm{x}$ & $\mathrm{x}$ & $\mathrm{x}$ & & $\mathrm{x}$ & $\mathrm{x}$ \\
\hline
\end{tabular}

\section{Step 4 - Quantify Parameter Uncertainties}

A pdf must be specified for each important parameter. There are not enough data to trace out a distribution for any of the parameters. Rather, a physically reasonable statistical distribution form was assumed and the available evidence was used to determine the distribution parameters (e.g., mean and variance).

A normal distribution was selected for all of the parameters except fuel expansion. The parameters for all the feedbacks except bowing physically cannot change sign. This creates an asymmetry or skewness in the true distribution, which a normal distribution cannot reproduce. However, except for fuel expansion, neglect of this effect introduces only a minor conservatism because this physical limit is more than four standard deviations from the mean. The mean of the fuel expansion parameter is too close to zero ( 3.0 standard deviations) for a normal distribution to be reasonable choice. Consequently a log-normal distribution, which reflects this limit, was used for the fuel expansion parameter. Specification of the mean and standard deviation for each parameter is sufficient to complete the uncertainty quantification. Mean values are those developed in the evaluation of feedback parameters in Step 2.

The standard deviation estimates for the feedback components are based on an evaluation of the following sources: uncertainty estimates in the literature [5.61, 5.52, 5.65], the range of feedback values in the literature $[5.60,5.61,5.62]$ and evidence from critical experiments [5.66, 5.67]. Reference 5.61 values are smaller than the others, apparently because of an attempt to 
be consistent with the range of EBR-II PRD values. The spread in reported EBR-II feedback values primarily reflects the effect of core loading variations and some evolution in analysis methods, which make up only part of the total uncertainty. The C/Es (ratios of calculated to experimental values) are from a number of LMR mockups, none specifically of EBR-II. C/Es from [5.66] are based on more sophisticated neutronics analyses than those used to obtain EBRII feedbacks and they do not include thermo-mechanical uncertainties. Table 5.30 shows the standard deviation values used here as well as some related quantities from the literature.

Table 5.30. Feedback Uncertainties and Related Quantities

\begin{tabular}{|c|c|c|c|c|c|}
\hline \multirow[b]{2}{*}{ Feedback } & \multicolumn{3}{|c|}{ Standard Deviation } & & \multirow{2}{*}{$\begin{array}{c}\quad \underline{C / E} \\
\text { Ref. }[5.66\end{array}$} \\
\hline & this work & Ref. [5.61] & Ref. [5.52] & Ref. [5.65] & \\
\hline Core $\mathrm{Na}$ & $20 \%$ & $6 \%$ & $20 \%$ & $30 \%$ & $10 \%$ \\
\hline Fuel & $33 \%$ & $11 \%$ & $30 \%$ & $47 \% \rightarrow 58 \%$ & $1 \% \rightarrow 16 \%$ \\
\hline Up. Refl. & $30 \%$ & $7 \%$ & $20 \%$ & $30 \%$ & \\
\hline Rad. Refl. & $25 \%$ & & $20 \%$ & $30 \%$ & \\
\hline Control & $23 \%$ & $5 \%$ & $20 \%$ & & $10 \%$ \\
\hline Bowing & $70 \%$ & $25 \%$ & $15 \% \rightarrow 50 \%$ & & $1 \% \rightarrow 42 \%$ \\
\hline
\end{tabular}

Only a limited accounting for correlations among the uncertain parameters was made. The strongest correlation is among core $\mathrm{Na}$, upper reflector and radial reflector feedbacks because they all are sodium density effects. This correlation was treated by expressing each of these as a product of two factors, one of which is common to all three feedbacks. The common factor was estimated to be uncertain by $18 \%$, implying an uncertainty in the other factor of each feedback of $9 \%, 24 \%$ and $17 \%$, respectively. Other correlations are less clear and were neglected. 


\section{Step 5 - Develop Experimental Constraints}

Since the uncertain feedback parameters cannot be measured in EBR-II individually, the uncertainty distributions developed in Step 4 are not based on much experimental data specific to EBR-II. The net reactivity from all the feedbacks is measurable, however, and in fact has been monitored routinely over the life of the reactor. These data can be used to force the parameter uncertainty propagation to be consistent with the observed feedback characteristics. In this step the data are used to construct a pdf or a range for the net feedback under the various experiment conditions. These are used as constraints in Step 7.

The experimental constraints should allow the full distribution of feedback reactivities that EBR-II can exhibit but exclude feedback values that are unrealistic. Two types of experiments that reveal feedback properties have been performed often enough over the years to meet these criteria.

One is the PRD measurement, which yields the total feedback reactivity from hot zero power to full power. The PRD includes both prompt and slow feedbacks and has been measured at every subrun (a Technical Specification requirement). Fifty measurements made over the last ten years were selected to construct a probability density function (pdf) for the full-power PRD. The selection spans a wider range of core loadings than is anticipated for the future and includes the most extreme PRD values ever observed. The pdf is displayed in Table 5.31.

The other type of experiment used is the rod drop measurement of prompt feedback reactivity. This activity grows rapidly with time for a little more than a second, then the slope decreases and eventually an asymptotic value is approached. The feedback reactivities at 1.5 $s$ and $8.0 \mathrm{~s}$ divided by the magnitude of the rod drop reactivity $(\operatorname{RDF}(1.5)$ and $\operatorname{RDF}(8.0)$, respectively) were selected as constraining quantities. These two quantities and the PRD each emphasize feedbacks with different time constants. 
Table 5.31. Discrete Probability Density Function For The Experimental Full-Power PRDa

\begin{tabular}{|c|c|}
\hline 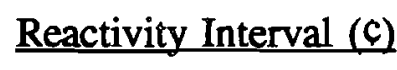 & Probability \\
\hline-46.8 to -42.3 & 0.06 \\
\hline-42.3 to -37.8 & 0.02 \\
\hline-37.8 to -33.3 & 0.30 \\
\hline-33.3 to -28.8 & 0.56 \\
\hline-28.8 to -24.2 & 0.06 \\
\hline
\end{tabular}

a The mean and standard deviation are $33.3 \pm 3.9 \mathrm{c}$

$\mathrm{RDF}(1.5)$ and $\mathrm{RDF}(8.0)$ values were available from twelve rod drop measurements. (Much more data are archived, but in a form that is very difficult to use.) These data are predominantly from the last three years but go back to 1978 . There are not enough data for constructing pdfs but they are sufficient to set limits on acceptable values of $\operatorname{RDF}(1.5)$ and $\operatorname{RDF}(8.0)$. From the twelve values, a mean and standard deviation were derived for each quantity. The acceptable limits were set at plus and minus three standard deviations from the mean. Some of these quantities are displayed in Table 5.32.

Table 5.32. Road Drop Feedback Quantities

\begin{tabular}{lccccc}
\hline & Lower Limit & & $\begin{array}{l}\text { Lowest } \\
\text { Observed }\end{array}$ & $\begin{array}{l}\text { Hean } \\
\text { Obsest }\end{array}$ & Upper Limit \\
RDF (1.5) & 0.146 & 0.165 & 0.193 & 0.217 & 0.240 \\
RDF (8.0) & 0.248 & 0.290 & 0.316 & 0.373 & 0.384 \\
& & & & & \\
\hline
\end{tabular}




\section{Step 6 - Compute Response Surfaces}

A response surface is generated by calculated transient responses for various combinations of input parameters and then fitting the results to a lower order, multivariate polynomial. This was done with the aid of the PROSA-2 code [5.68], which offers several parameter selection and fitting schemes. The single-quadrant scheme was found to be the most computationally efficient [5.69] and was used here. Parameter sets, called knot points, were selected by PROSA-2 for each output quantity. Next SASSYS transient calculations were run using each knot point. Finally the resulting output quantities corresponding to each knot point were supplied to PROSA-2, which used them to compute the response surfaces.

Response surfaces were computed for the LONP-4 sequence and for the PRD and rod drop transients used to constrain the sampling in Step 7. The computational requirements are illustrated by the PRD case. It has 6 uncertain feedbacks with 8 corresponding uncertain input parameters. The single-quadrant scheme identifies 45 knot points and requires 45 SASSYS calculations of the PRD to generate the 8-parameter PRD response surface.

The output quantities must behave smoothly enough to be described well by a quadratic for this approach to be reasonable. The PRD and RDF quantities easily satisfy this restriction. However, it did preclude using clad failure life fraction as an output quantity to monitor failure. Sodium, cladding or fuel temperatures, depending on the accident and damage category, did prove to be good choices of output quantities.

Three damage categories are defined in this PRA. The mildest category is Potential Experiment Damage (PED). This is taken to mean operation in the "Unacceptable Region" (Region 1) defined in Section 2.2 of the Technical Specifications. Cladding temperature exceeding $815^{\circ} \mathrm{C}$ anywhere in the core at any time was used to signal this "damage" state. This category implies a Technical Specification violation but actual damage is not necessarily implied. Next in severity is Minor Core Damage (MCD), which is indicated by fuel melting, cladding failure or sodium boiling in the hottest, highest-burnup driver subassemblies. Core Damage 
(CD), the most severe category, is indicated by fuel melting, cladding failure or boiling in an average driver assembly.

Response surface output quantities were selected for LONP-4 by examining the SASSYS model of EBR-II from the perspective of each damage category definition. The SASSYS code uses the concept of channels to represent different parts of the core. (A channel consists of a fuel pin, clad, coolant annulus and associated structure, and has characteristic physical properties.) The channels representing the hottest driver subassemblies are Channels 1 and 2, which are the same except for their burnups of $15 \%$ and $8 \%$, respectively. Accordingly, the peak clad temperature at the top of either of these channels served as the output quantity for PED. Examination of the results of the 28 SASSYS calculations for LONP-4 showed that boiling $\left(925^{\circ} \mathrm{C}\right)$ occurs long before cladding failure in Channel 2 and just after cladding failure in Channel 1. (Fuel melting does not occur in this accident.) A sodium temperature of $917^{\circ} \mathrm{C}$ at the top of Channel 1 (or 2) was determined to be a MCD threshold. Accordingly, the peak sodium temperature over the transient at the top of Channel 1 was used as the MCD output quantity. Average driver subassemblies are represented by Channels 6 and 8. Boiling occurs long before cladding failure at average burnups, and Channel 6 is always hotter than Channel 8. Accordingly, the output quantity used to monitor for $\mathrm{CD}$ was the peak sodium temperature at the top of Channel 6 , with the boiling point $\left(925^{\circ} \mathrm{C}\right)$ being the $\mathrm{CD}$ threshold.

\section{Step 7 - Propagate Uncertainties}

Once the response surfaces were known, computing transient outcomes corresponding to any set of input parameters (knot point) was simply a matter of evaluating the polynomial with that set of parameters. The input parameter pdfs were sampled about one million times and translated into transient outcomes. This knot point sampling was done randomly, i.e. by Monte Carlo, and yielded the distribution of possible transient outcomes. The fraction of an outcome distribution lying beyond the damage threshold is the failure probability.

The experimental constraints developed in Step 5 were imposed in this sampling process. A rejection technique was added to PROSA-2 such that the knot points retained to determine the 
transient outcome distributions reproduced the experimental PRD pdf and satisfied the limits on $\mathrm{RDF}(1.5)$ and $\mathrm{RDF}(8.0)$ values.

The parameter uncertainties were propagated both with and without the experimental constraints. The resulting conditional damage probabilities from LONP-4 are shown in Table 5.33 and the outcome distributions are shown in Figures 5.63 through 5.65. The abscissa label "Temperature" refers to a different output quantity in each of these figures, as explained under Step 6. The nominal SASSYS calculation of this accident indicated operation in the acceptable region and peak sodium temperature below, but close to boiling. Thus it is not surprising that the passive safety failure probability for PED is essentially unity and for MCD it is as large as 0.14 without experimental constraints. The imposition of the experimental constraints reduced the MCD failure probability by more than an order of magnitude. The RDF constraints added very little to the PRD effect for this accident, perhaps because of the long time scale of the accident.

Table 5.33. Passive Safety Failure Probabilities for LONP-4 Accidents ${ }^{\mathrm{a}}$

\begin{tabular}{llll}
\hline & PED $^{\mathrm{b}}$ & MCD & CD \\
\cline { 3 - 3 } No Experimental Constraints & 0.98 & $0.143 \pm 0.2 \%$ & $2.5 \times 10^{-6}$ \\
Only PRD Constraints & 0.99 & $1.3 \times 10-2$ & $<2.5 \times 10^{-6}$ \\
PRD and RDF Constraints & 1.00 & $1.2 \times 10-2$ & $<2.5 \times 10^{-6}$ \\
\hline
\end{tabular}

a The uncertainties shown reflect only Monte Carlo statistics.

b The PED statistical uncertainties are less than $0.1 \%$ 


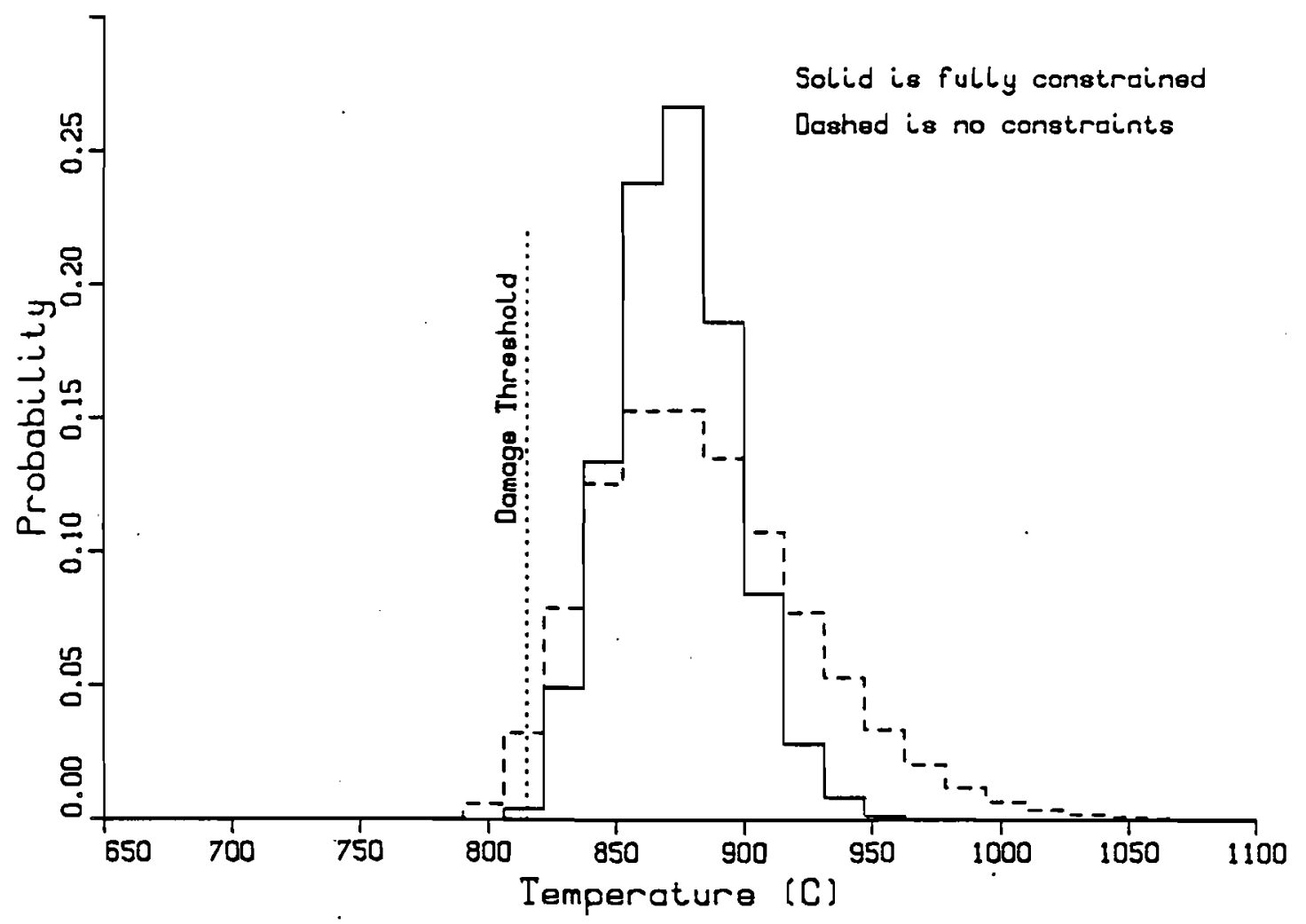

Fig. 5.63. LONP-4 Outcome Distribution For Potential Experiment Damage 


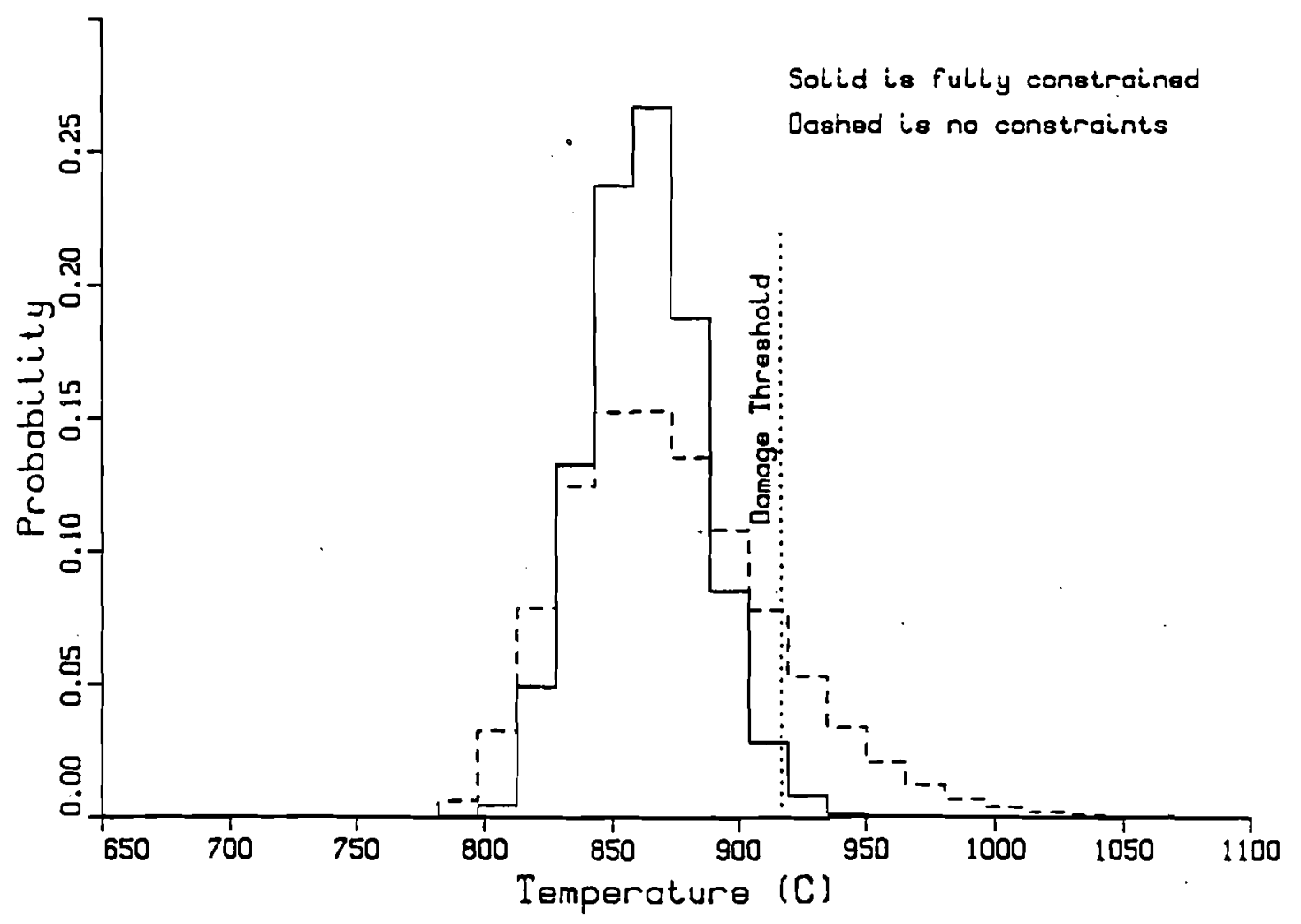

Fig. 5.64. LONP-4 Outcome Distribution for Minor Core Damage 


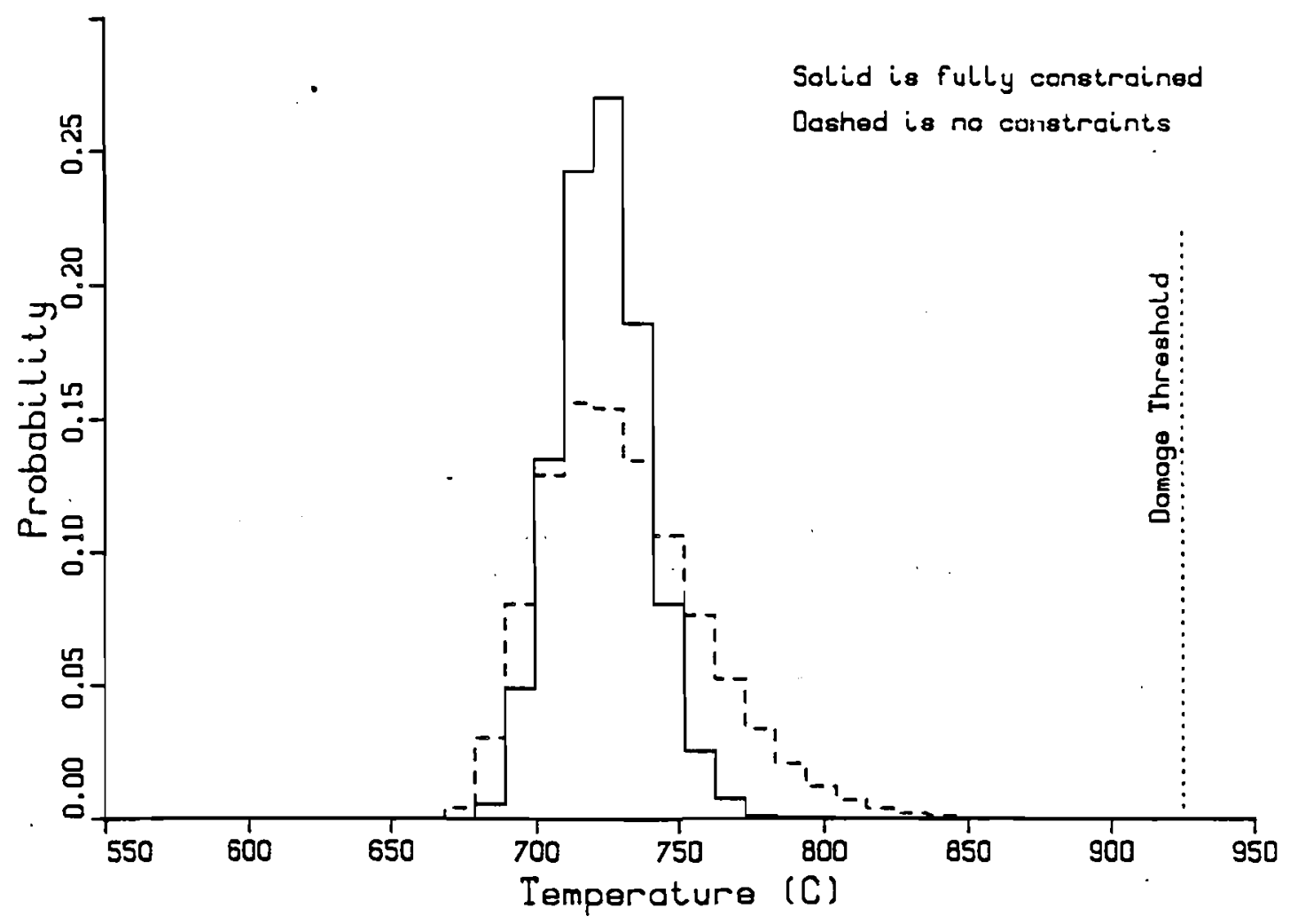

Fig. 5.65. LONP-4 Outcome Distribution for Core Damage 
Very few samples yielded $C D$ outcomes when there were no experimental constraints and none did with the constraints. Consequently, the non-constraint estimate of $2 \times 10^{-6}$ has a large statistical uncertainty. With none of the million samples exceeding the damage threshold, the explicit observation is simply that the constrained core damage probability is unlikely to be large compared to $10^{-6}$. Yet it is apparent from the way the temperature distributions are falling off in Fig. 5.65 that the fully constrained damage probability must be many orders of magnitude smaller than the unconstrained estimate of $2 \times 10^{-6}$. The much smaller bounds for the constrained cord damage probabilities shown in Table 5.33 were obtained from fits to the Monte Carlo data. The fitting scheme is discussed in [5.70]. Several statistical distributions functions were fit to the data and the damage probability was computed with these functions. This amounts to an extrapolation of the sampling data but, on physical grounds, that is reasonable approach in this case. The goal was simply to determine whether the probability is below the cutoff for consideration in the EBR-II PRA. All estimates are so many orders of magnitude below the $10^{-10}$ cutoff that core damage can be dismissed.

\section{Step 8 - Assess Accuracy}

Sensitivity calculations are done to assess the reliability of the failure probability predictions. These calculations evaluate the error due to parameter screening, the sensitivity to input parameter pdfs and the accuracy of the response surfaces. This analysis is discussed in more detail in [5.71].

A conservative upper bound was found for the error due to screening out parameters from the uncertainty propagation (Step 3). This was done by recomputing the failure probabilities with only the two most important parameters treated as uncertain and comparing these with the reference results ( 6 certain parameters). The bound for each damage category is $1 \%$ for PED, $19 \%$ for MCD and one order of magnitude for CD. (It is generally true that errors and uncertainties become a larger percentage of the failure probability as the failure probability gets smaller.) The actual error from the parameter screening is small compared to these bounds, since the four parameters dropped for this test are much more important than the ones screened out in Step 3. 
The sensitivity to assumptions about the parameter pdfs was computed with the aim of finding plausible upper bounds on failure probabilities. Assumptions as pessimistic as experimental evidence and common sense would allow were made. First, different statistical distribution functions (log-normal and beta) were tried, without changing the mean and standard deviation. The distributions were skewed to the pessimistic side by postulating a bound (for which there is no evidence) as close as three standard deviations from the mean.

Next, three cases were run where the mean or standard deviation was allowed to change. In one, the standard deviation of the most important parameter was increased by $30 \%$ of its original value. In another, all standard deviations were increased by $25 \%$ of their original values. Finally, all means were moved one standard deviation in the pessimistic direction. The last case was exposed by the experimental constraining process as being hopelessly in conflict with the PRD and rod drop data.

The following conclusions were drawn about the parameter pdf sensitivities. The PED probability is essentially unity no matter what plausible assumption is made. The uncertainty for the experimentally constrained MCD probability is less than a factor of 2. No CD failures were found in the experimentally constrained Monte Carlo sampling and fits to the data yield failure probabilities below the $10^{-11}$ cutoff [5.70].

The accuracy of the single-quadrant response surface approximation was investigated by comparing it to more accurate approximations on a simplified version of the LONP problem. The simplification was to treat only the two most important parameters as uncertain, as in the test of parameter screening. This made it feasible to trace out the true functional relationship with may more knot points and use higher order and more flexible polynomials.

The response surface test results are similar to those for parameter pdfs; the PED probability is essentially invariant, the MCD probability could increase by no more than a factor of two, and the experimentally constrained $C D$ probability remains below the $10^{-11}$ cutoff. 


\subsubsection{Summary and Discussion}

Reactivity feedbacks are a major element of passive safety, bringing heat production from fission into balance with heat removal. The EBR-II feedbacks are well understood and, with one exception, they are relatively easy to predict. There is no close competition between opposing feedbacks and even the sodium density effect, which is by far the strongest feedback, does not suffer from a close balance between opposing spectral and leakage effects. As a result, the best estimate feedback reactivity values and the uncertainty estimates should be reliable. A conservative model was used to deal with bowing, the one feedback that is quite difficult to predict accurately.

A screening analysis of ATWS events (Step 1) determined that reactivity feedbacks virtually guarantee a benign outcome in loss-of-heat-sink and pump-runup accidents. Loss-ofnormal-power (LONP), loss-of-flow and transient overpower transients were found to warrant a detailed analysis of the effectiveness of reactivity feedbacks.

In the detailed analysis the probability that the reactivity feedbacks are not strong enough to prevent core damage was computed by propagating parameter uncertainties through transient calculations. Plant transient data were used to calibrate calculational models (Step 2). Parameters were screened using importance ranking to reduce the number of uncertain parameters (Step 3). Transient calculations were run with different parameter values to construct response surfaces, approximate analytic functions describing the dependence of transient outcomes on the input parameters (Step 6). Then transient outcome distributions were found by evaluating the response surface functions for about a million parameter sets selected randomly (Step 7). In this Monte Carlo sampling from theoretical parameter distributions, the values selected were constrained to be consistent with PRD and rod drop experiments. A fitting procedure was used to obtain bounds on probabilities that are small compared to $10^{-6}$. Finally, an uncertainty analysis was done to evaluate the reliability of the failure probability estimates (Step 8). 
This method was used to analyze the LONP accident without scram. Operation in the Technical Specification unacceptable operating region, where damage to aggressive experimental subassemblies is possible, is a virtual certainty. The best estimate of the minor core damage probability is $1.210^{-2}$. The uncertainty in this estimate is a factor of 2 . The best estimate core damage probability is below the $10^{-11}$ cutoff for dismissing sequences, and the probability remains below this cutoff when uncertainties in the estimate are included. All of these are conditional probabilities; occurrence of a LONP initiator and failure to scram are given.

The analysis is firmly grounded in experimentally verified reality. Full advantage was taken of the EBR-II experimental database accumulated over its long operating history. The inherent safety demonstration experiments, in particular, provide compelling evidence of passive safety for the events they simulated. And they provided benchmarks for validating the computational tools used to predict responses that were not simulated explicitly. Information from experiments that measured net feedback reactivity made it possible to reduce them minor core damage probability estimate by one order of magnitude and the core damage probability by more than five orders of magnitude.

The reliability of the safety margins from the passive response compares favorably with those from engineered safety features. Since reactivity feedbacks are simply a manifestation of the laws of physics, primarily thermal expansion, there is virtually no chance that the passive response will fail to occur. The only exception is if there is a catastrophic accident initiator that disrupts the core geometry (severe earthquake), where it is reasonable to assume that the damage probability is unity. For the same reason, passive safety has advantages as a complement to primary safety systems. Compared to something like a secondary scram system, there is little chance of some dependent failure defeating both passive safety and the primary safety systems it complements.

The results demonstrate that reactivity feedbacks provide a large safety margin. The conditional damage probabilities would be unity for all the initiators retained in the screening if there were no feedbacks. A large margin is also anticipated for LOF and transient overpower. This margin is in addition to the large safety margin afforded by the scram system. 
The substantial safety benefit of EBR-II's reactivity feedback response is not typical of other reactors that have been the subject of PRAs. The vulnerability of LWR plants is such that core damage is generally assumed to occur if there is a failure to scram. Consequently, this is the first PRA to make a probabilistic assessment of reactivity feedbacks. Although this treatment is not a conventional system failure analysis, it is a well founded approach for producing a probabilistic failure quantification.

For application of the results of this analysis to future EBR-II cores, one anticipated reactor modification warrants some discussion. It is the substitution of plutonium for fissile uranium. The effect on reactivity feedbacks of such a substitution has been investigated for a few cores [5.56, 5.72, 5.73] including EBR-II [5.76]. The $\beta_{\text {eff }}$ would decrease by about $25 \%$, which would enhance the feedback reactivities in units of dollars by this amount if there were no other changes. Sodium density reactivity, which is by far the most important feedback in EBR-II, would get less negative in absolute units by a moderate amount - 10\%. (The sodium density feedback change is much less in EBR-II than in larger cores because of the large leakage component.) Fuel expansion feedback would be much larger with Mark-V (Pu) fuel and other significant feedbacks should change about $10 \%$ in absolute units. Asymptotic transient outcomes depend on reactivities in absolute units and there could be a slight degradation of the passive safety performance for most ATWS events. However, the changes are within the uncertainties used in the analysis. The peak temperature in LONP and LOF events depends on feedback in dollars, and therefore the passive safety performance in these transients should improve modestly with the fuel change. The overall conclusion is that the reactivity feedback reliability estimates presented in this section would be applicable to EBR-II loadings with plutonium driver fuel. 


\section{Section 5.0 References}

5.1 L. J. Koch et. al., "Hazard Summary Report, Experimental Breeder Reactor II (EBRII), "ANL-5719 (May 1957), and

L. J. Koch, W. B. Loewenstein, and H. O. Monson, "Addendum to Hazard Summary Report, Experimental Breeder Reactor-II," ANL-5719 (Addendum) (June 1962).

5.2 "CRBR Safety Study: An Assessment of Accident Risks in the CRBRP," CRBRP-1 Volumes 1 and 2 (1977).

5.3 Clinch River Breeder Reactor Plant Probabilistic Risk Assessment, CRBRP-4, Vols. 1-3 (September 1984).

5.4 Y. Kani and K. Aizawa, "Safety Evaluation for the LMFBR Plant Using Probabilistic Risk Assessment Techniques," in Implications, of PRAA, IAEA-SR-11/4, 1985.

5.5 C. Tzanos and N. Hanan, "A Comparison of Selected Risk Contributions of an LMFBR and an LWR, Phase A," EPRI-NP-2784 (January 1983).

5.6 E. E. Feldman, Argonne National Laboratory, unpublished information (1988).

5.7 J. Buzzell, private communication (April 1990).

5.8 ANL Publication, "EBR-II System Design Descriptions - Volume II Primary System," prepared by Argonne National Laboratory (May 1972).

5.9 D. Mohr, private communication (May 1990).

5.10 S. Fistedis (Ed.), "The Inherent Safety Demonstration," Nuclear Engineering and Design, 101, pp. 1-90 (1987). 
5.11 Final Safety Analysis Addendum to Hazards Summary Report, Experimental Breeder Reactor II: EBR-II Operation in the Limited Transient Mode, ANL-84-70, March 1985.

5.12 R. M. Fryer et al., "Efficacy of the EBR-II FERD System as an Automatic Trip Device" ANL-76-94, (August 1977).

5.13 W. K. Lehto et al., "Reactor Safety Implications of Pump Run-Up Tests in EBR-II," Nuclear Engineering and Design, 110, p. 47 (1988).

5.14 L. K. Chang et al., "Plant Inherent Control Testing in EBR-II," Topical Meeting on Anticipated and Abnormal Transients in Nuclear Plants, April 12-15, 1987, Atlanta, GA, USA.

5.15 J. P. Herzog, Argonne National Laboratory, unpublished information (1990).

5.16 K. J. Miles, Argonne National Laboratory, unpublished information (1990).

5.17 K. J. Miles, Argonne National Laboratory, unpublished information (1990).

5.18 U.S.N.R.C., "PRA Procedures Guide," NUREG/CR-2300, USNRC (1983).

5.19 T. Burke, private communication, 1990.

5.20 L. K. Chang, private communication, 1990.

5.21 J. Matsumoto et al., "Probabilistic Fracture Analysis for LMFBR Piping Integrity," 1987 SMIRT Proceedings. Vol. M.

5.22 Argonne National Laboratory, unpublished information.

5.23 S.A. Eide et al., "Generic Component Failure Data Base for Light Water and Liquid 
Sodium Reactor PRAs," EGG-SSRE-8875, Feb. 1990.

5.24 Argonne National Laboratory, unpublished information.

5.25 H. P. Planchon et al., "The Experimental Breeder Reactor II Inherent Shutdown and Heat Removal Tests - Results and Analysis," Nuclear Engineering and Design, 91 (1986).

5.26 R. M. Singer et al., "Studies of Thermal-Hydraulic Phenomena in EBR-II," Nuclear Engineering and Design, 62, pp. 219-232 (1980).

5.27 E. E. Feldman, Argonne National Laboratory, unpublished information (1991).

5.28 J.F. Koenig, Argonne National Laboratory, unpublished information (1984), and J.P. Herzog, Argonne National Laboratory, unpublished information (1989).

5.29 L. K. Chang, "Stress Analysis of the EBR-II Primary-Tank Inner Vessel," Intralaboratory Memo to R.M. Singer, Argonne National Laboratory, October 28, 1975.

5.30 L. K. Chang, Structural Evaluation of the EBR-II Primary Task Inner Vessel for Shutdown Cooler Failure Conditions," Intra-laboratory Memo to W. A. Ragland, Argonne National Laboratory, December 21, 1990.

5.31 F. E. Dunn et al., "The SASSYS-1 LMR Systems Analysis Code," ANL/RAS 84-14, Revision 1, Argonne National Laboratory, March (1987).

5.32 "Description of Passive Safety Related Terms," DRAFT IAEA Document, 622-I3-TC-633, October 3, 1988. 
5.33 L. K. Chang and D. Mohr, "The Effect of Primary Pump Coastdown Characteristics on Loss-Of-Flow Transients Without Scram in EBR-II," Nuclear Engineering and Deisgn, 97 (1986).

5.34 N. J. Holloway and P. N. Clough, "Primary Containment Source Term Analysis Accidents Involving Failure to Remove Decay Heat," Proc. of the Int. Conf. on the Science and Technology of Fast Reactor Safety, BNES, London (1986).

5.35 D. Mohr and E. E. Feldman, "A Dynamic Simulation of the EBR-II Plant During Natural Convection with the NATDEMO Code," Decay Heat Removal and Natural Convection in Fast Breeder Reactors, Hemisphere Publishing Corp., Washington, D.C., pp. 207-224 (1981).

5.36 H. A. Larson et al., "Dynamic Simulation of Nuclear Power Plants (DSNP): Development, Verification, and Expansion of Modules," Presented at International Conference on Power Plant Simulation, Cuernavaca, Morelos, Mexico, (Nov. 19-21, 1984).

5.37 M. Zentner et al., "N-Reactor PRA: Final Report," WHC-SP-0087, August 1988.

5.38 D. E. Vaden, "Chemical Leak Detection Experience at the Experimental Breeder Reactor II," ANL-88-34 (August 1988).

5.39 D. A. Greene, "The Leakage of Water into Sodium in Steam Generators and Test Rigs," Nuclear Technology, Vol. 18, p. 267 (June 1973).

5.40 E. E. Feldman et al., "EBR-II Unprotected Loss-of-Heat-Sink Predictions and Preliminary Test Results," Nuclear Engineering and Design, Vol. 101, No. 1, p. 57 (1987).

5.41 J. P. Herzog, "SASSYS Validation with the Shutdown Heat Removal Tests," Fifth Proc. of Nuclear Thermal-Hydraulics, San Francisco, California, Nov. 26-30, 1989. 
5.42 J. P. Herzog, Argonne National Laboratory, unpublished information (1988).

5.43 D. J. Hill, "SASSYS Validation Studies," ANL/RAS 88-4, June 1988.

5.44 D. K. Warinner, "A Preliminary Report on SASSYS-1 Computer Code Pretest Analyses of Selected EBR-II Shutdown-Heat-Removal Tests (SHRTs)," ANL/RAS 84-12, July 1984.

5.45 E. M. Dean and J. I. Sackett, "Response of EBR-II to Off-Normal Primary-Coolant Flow," ANL-75-40 (Aug. 1976).

5.46 J. F. Boland et al., "Design-Basis Document for Trips Related to Loss of Primary Flow in EBR-II Plant Protection System (PPS)," ANL-76-31 (Dec. 1980).

5.47 J. F. Koenig, Argonne National Laboratory, unpublished information (1988).

5.48 J. F. Koenig, Argonne National Laboratory, unpublished information (1989).

5.49 J. P. Herzog, Argonne National Laboratory, unpublished information (1991).

5.50 Argonne National Laboratory, unpublished information.

5.51 E. M. Dean and J. I. Sackett, "Response of EBR-II to Reactivity Insertion," ANL-75-41 (March 1978). 
5.52 C. J. Mueller and D. C. Wade, "Probabilities of Successful Inherent Shutdown of Unprotected Accidents in Innovative Liquid Meal Reactors, "Proc. Int. Topl. Mtg. Safety of Next Generation Power Reactors, Seattle, WA, May 1-5, 1988, p. 704, American Nuclear Society (1988).

5.53 R. H. Meyers, Response Surface Methodology, Allyn and Bacon, Inc., Boston (1971).

5.54 M. Khatib-Rahbar et al., "A Probabilistic Approach to Quantifying Uncertainties in the Progression of Severe Accidents," Nucl. Sci. Eng., 102, p. 219 (1989).

5.55 R.S. May et al., "Probabilistic Methods for Evaluating Operational Transient Margins and Uncertainties, Nucl. Sci Eng., 103, p. 81 (1989).

5.56 D.C. Wade and Y. I. Chang, "The Integral Fast Reactor (IFR) Concept: Physics of Operation and Safety," Proc. Int. Topl. Mtg. on Advances in Reactor Physics, Mathematics and Computation, Paris, France, April 27-30, 1987.

5.57 D. Meneghetti and D. A. Kucera, "Delineations of Power, Power-to-Flow and InletTemperature Feedbacks of EBR-II," Ann. Nucl. Energy, 17, No. 1, p. 19 (1990).

5.58 H. P. Planchon et al., "Implications of the EBR-II Inherent Safety Demonstration Test," Nucl. Engrg. Des., 101, p. 75 (1987).

5.59 W. K. Lehto et al., "Reactor Safety Implications of Pump Run-Up Test in EBR-II," Nucl. Engrg. Des., 110, p. 47 (1988).

5.60 D. Meneghetti and D. A. Kucera, "Comparisons of PRD Components For Various EBRII Configurations," Ann. Nucl. Energy, 14, No. 9, p. 473 (1987).

5.61 L. K. Chang and D. Mohr, "The Effect of Primary Pump Coastdown Characteristics on Loss-of-Flow Transients Without Scram in EBR-II," Nucl. Engrg. Des., 97, p. 49 (1986). 
5.62 M. L. Carboneau et al., " A Study of the Temperature Coefficients of Reactivity For EBR-II Run 93," ANL-80-85, Argonne National Laboratory, November 1980.

5.63 G. L. Hofman et al., "Metallic Fuels Handbook," ANL-IFR-29, Argonne National Laboratory, November 1985.

5.64 T. J. Moran, private communication, Argonne National Laboratory, June 1990.

5.65 Ph. Bergeonneau et al., "Uncertainty Analysis on the Measurements and Calculation of Feedback Reactivity Effects in LMFBR. Application of Super-Phenix-1 Startup Experiments," 30th NEACRP Meeting, Helsinki, Finland, September 1987, NEACRP-A833.

5.66 R. W. Schaefer, "Critical Experiment Tests of Bowing and Expansion Reactivity Calculations for Liquid-Metal-Cooled Fast Reactors," Nucl. Sci. Eng., 103, p. 196 (1989).

5.67 C. L. Beck et al., "Sodium Void Reactivity in LMFBRs: A Physics Assessment," Proc. Topl. Mtg. Advances in Reactor Physics and Core Thermal Hydraulics, Kiamesha Lake, NY, September 22-24, 1987, NUREG/CP-0034, Vol. 1, p. 78 (1982).

5.68 J. K. Vaurio, "PROSA-2: A Probabilistic Response-Surface Analysis and Simulation Code," ANL-81-33, Argonne National Laboratory, May 1981.

5.69 R. W. Schaefer, Argonne National Laboratory unpublished information (1990).

5.70 R. W. Schaefer, Argonne National Laboratory unpublished information (1991). 
5. 71 R. W. Schaefer, Argonne National Laboratory unpublished information (1990).

5.72 J. R. Liaw, Argonne National Laboratory unpublished information (1987).

5.73 D. Meneghetti and D. A. Kucera, "Feedback Components of a Pu-Fueled Compared to a U-Fueled 900 MWtLMR," Ann. Nucl. Energy, 17, No. 7, p. 353 (1990).

5.74 D. Meneghetti and D. A. Kucera, Argonne National Laboratory unpublished information (1991). 
6.0 SYSTEMS ANALYSIS 


\section{Table of Contents}

$\underline{\text { Page }}$

6.0 Systems Analysis $\ldots \ldots \ldots \ldots \ldots \ldots \ldots \ldots \ldots \ldots$

6.1 Systems Analysis Procedures . . . . . . . . . . . . . . . . . 6-1

6.1 .1 System Analysis Naming Conventions . . . . . . . . . . . . . 6-4

6.1.1.1 Gate Names . . . . . . . . . . . . . . . . . . 6-6

6.1 .1 .2 Basic Event Names $\ldots \ldots \ldots \ldots$. . . . . . . . . . . . . . . . . .

6.1 .1 .3 House Event Names . . . . . . . . . . . . . . . 6-6

6.1.1.4 Identifiers for Main and Support Systems . . . . . . . . . . . 6-6

6.1.1.5 Identifiers for Mechanical and Electrical Components . . . . . 6-6

6.1 .1 .6 Failure Mode Identifiers ... . . . . . . . . . . . . 6-11

6.1.1.7 Component Location Identifiers . . . . . . . . . . . . . . 6-11

6.2 Analysis of Frontline Systems . . . . . . . . . . . . . . . . . 6-13

6.2 .1 Reactor Shutdown System (RSS) $\ldots \ldots \ldots \ldots$. . . . . . . . . . . . . .

6.2.1.1 System Function and Description ............ 6-13

6.2.1.2 Support System Interfaces and Effects ........... . 6-16

6.2.1.3 Fault Tree Development ... . . . . . . . . . . . . . 6-16

6.2.1.3.1 LOF Fault Tree . . . . . . . . . . . . . 6 6-17

6.2 .1 .3 .2 TOP Fault Tree $\ldots \ldots \ldots \ldots$. . . . . . . . . . . . . . .

6.2.1.3.3 Contribution of SOT Channels ......... . 6-18

6.2.1.4 Component Failure Data, Common Cause Failures, Human

Reliability ... . . . . . . . . . . . . . . . . 6-19

6.2.1.5 Quantification - Results ... . . . . . . . . . . . . 6-21

6.2.1.6 Conclusions and Special Insights ... . . . . . . . 6 6-26

6.2 .2 Primary Pumps ... . . . . . . . . . . . . . . . . 6 6-27

6.2.2.1 System Function and Description . . . . . . . . . . . 6-27

6.2.2.2 Support System Interfaces and Effects . . . . . . . . . . . 6-30

6.2.2.3 Fault Tree Development ... . . . . . . . . . . . 6-30

6.2.2.4 Component Failure Data, Common Cause, Human Personality 6-30

6.2.2.5 Quantification - Results ................ 6-32

6.2.2.6 Initiating Events and Special Insights . . . . . . . . . . . 6-32

6.2.3 Primary Pumps - Motor Generator Sets . . . . . . . . . . . . . . . 6- . . . . . . . . 6-32

6.2.3.1 System Function and Description . . . . . . . . . . 6-32

6.2.3.2 Support System Interfaces and Effects (Table 6.9) . . . . . . 6-32

6.2.3.3 Fault Tree Development ............... . 6-35

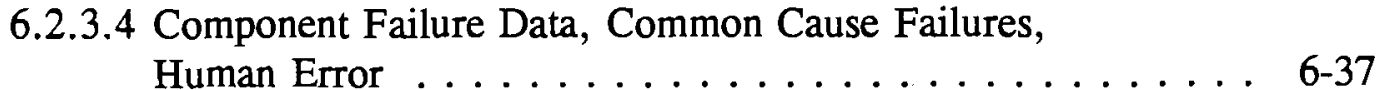

6.2.3.5 Quantification/Results . . . . . . . . . . . . . 6-37

6.2.3.6 Initiating Events and Special Insights . . . . . . . . 6- 6-38

6.2 .4 Shutdown Coolers . . . . . . . . . . . . . . . . . . . 6- 6 . . . . . . . .

6.2.4.1 System Function and Description . . . . . . . . . . . 6 6-40

6.2.4.2 Support System Interfaces ... . . . . . . . . . . 6- 6-40

6.2.4.3 Fault Tree Development ... . . . . . . . . . . . 6-42 
Page

6.2.4.4 Component Failure Data, Common Cause Failures, Human Reliability . . . . . . . . . . . . . . . 6-42

6.2.4.5 Quantification - Results . . . . . . . . . . . . . 6-42

6.2.4.6 Initiating Events and Special Insights . . . . . . . . . 6 6-44

6.2 .5 Shield Cooling System . . . . . . . . . . . . . . . . . . . 6-44

6.2.5.1 System Function and Description . . . . . . . . . . . 6-44

6.2.5.2 Support System Interfaces $\ldots \ldots \ldots \ldots \ldots \ldots$. . . . 6-48

6.2.5.3 Fault Tree Development . . . . . . . . . . . . . 6-48

6.2.5.4 Component Failure Data, Common Cause Failures, Human Reliability . . . . . . . . . . . . . . . . . . . . . 6-49

6.2.5.5 Quantification - Results . . . . . . . . . . . . . . 6-49

6.2.5.6 Initiating Events and Special Insights . . . . . . . . 6 6-49

6.3 Analysis of Support Systems . . . . . . . . . . . . . . . . . . . 6 6-49

6.3.1 Thimble Cooling/Stack Exhaust . . . . . . . . . . . . . 6-51

6.3.1.1 System Function and Description . . . . . . . . . 6-51

6.3.1.2 Support System Interfaces $\ldots \ldots \ldots \ldots \ldots \ldots$. . . 6-54

6.3.1.3 Fault Tree Development . . . . . . . . . . . . . 6-54

6.3.1.4 Component Failure Data, Common Cause Failures, Human

Reliability . . . . . . . . . . . . . 6- 6-55

6.3.1.5 Quantification . . . . . . . . . . . . . . 6-55

6.3.1.6 Initiating Events and Special Insights . . . . . . . . 6-55

6.3.2 Secondary Sodium System . . . . . . . . . . . . . . . 6-57

6.3.3 Balance-of-Plant (BOP) . . . . . . . . . . . . . . . . . 6-57

6.3 .4 Electrical System . . . . . . . . . . . . . . . . . 6 6-57

6.3.4.1 System Function and Description . . . . . . . . . . . 6-57

6.3.4.2 Support System Interfaces $\ldots \ldots \ldots$. . . . . . . 6-63

6.3.4.3 Fault Tree Development . . . . . . . . . . . . . . 6-63

6.3.4.4 Component Failure Data, Common Cause Failures, Human

Reliability . . . . . . . . . . . . . . . 6-64

6.3.4.5 Quantification . . . . . . . . . . . . . . . . . 6-64

6.3.4.6 Initiating Events and Special Insights . . . . . . . . 6-64

6.3.5 Instrument and Plant Air . . . . . . . . . . . . . . . 6-66

6.3.5.1 System Function and Description . . . . . . . . . 6-66

6.3.5.1.1 Instrument Air Compressors . . . . . . . . . . 6 6-68

6.3.5.1.2 Plant Air Compressor . . . . . . . . . . . . 6-68

6.3.5.2 Support System Interfaces $\ldots \ldots \ldots \ldots \ldots \ldots . \ldots .6 .68$

6.3.5.3 Fault Tree Development . . . . . . . . . . . . . . . . 6-69

6.3.5.4 Component Failure Data, Common Cause Failures, Human

Reliability . . . . . . . . . . . . . . 6-70 
6.3.5.5 Quantification . . . . . . . . . . . . . . . . . 6-70

6.3.5.5.1 Instrument Air System . . . . . . . . . . . 6 6-70

6.3.5.5.2 Plant Air System . . . . . . . . . . . . . 6-71

6.3.5.5.3 Emergency Air System . . . . . . . . . . . 6-71

6.3.5.6 Initiating Events and Special Insights . . . . . . . . . 6-71

6.3.6 Cooling Water System . . . . . . . . . . . . . . . . . 6-72

6.3.6.1 System Function and Description . . . . . . . . . 6 6-72

6.3.6.1.1 Reactor Auxiliaries Cooling Water System . . . . . 6 6-73

6.3.6.1.2 Plant Cooling Water System . . . . . . . . . . . 6-73

6.3.6.2 Support System Interfaces . . . . . . . . . . . . . 6-77

6.3.6.3 Fault Tree Development . . . . . . . . . . . . . 6 6-77

6.3.6.3.1 Reactor Auxiliaries Cooling Water System . . . . . 6-77

6.3.6.3.2 Plant Cooling Water System . . . . . . . . . . . 6-77

6.3.6.4 Component Failure Data, Common Cause Failures, Human Reliability . . . . . . . . . . . . . 6 6-78

6.3.6.5 Quantification - Results . . . . . . . . . . . . 6-78

6.3.6.6 Initiating Events and Special Insights . . . . . . . . 6-78

Section 6.0 References . . . . . . . . . . . . . . . . . . . . . . . . . . . . . 6-79 


\section{$\underline{\text { List of Figures }}$}

Page

6.1 Gate and Basic/House Event Formats for Fault Tree Inputs . . . . . . . . . 6-5

6.2 Reactor Shutdown System (RSS) Simplified Schematic . . . . . . . . . . . . . 6-14

6.3 Main Primary Coolant Pump . . . . . . . . . . . . . . . . . . . . . 6 6-29

6.4 Primary Pump Control . . . . . . . . . . . . . . . . . . . . . . 6-33

6.5 Shutdown Cooling System . . . . . . . . . . . . . . . . . . 6-41

6.6 Shield Cooling System Pictorial Flow Diagram . . . . . . . . . . . . . . . 6-46

6.7 Instrument Thimble Cooling System Flow and Equipment Diagram . . . . . . . 6-52

6.8 Single Line Electrical Diagram EBR-II Electrical Power Distribution . . . . . . . 6-59

6.9 Instrument and Plant Air Systems . . . . . . . . . . . . . . . . 6-67

6.10 Reactor Auxiliaries Cooling Water System . . . . . . . . . . . . . 6 6-74

6.11 Plant Cooling Water System . . . . . . . . . . . . . . . . 6-76 
List of Tables

$\underline{\text { Page }}$

6.1 Front Line and Support Systems Interface/Dependency Matrix . . . . . . . . 6-2

6.2 System Identifiers . . . . . . . . . . . . . . . . . . . . . 6-7

6.3 Mechanical and Electrical Component Identifiers . . . . . . . . . . . . 6-8

6.4 Component Location Identifiers . . . . . . . . . . . . . . . . . . 6-11

6.5 Failure Mode Identifiers . . . . . . . . . . . . . . . . . . . 6-12

6.6 Scram Failure Probabilities for LOF Events . . . . . . . . . . . . . . . 6-22

6.7 Scram Failure Probabilities for TOP Events . . . . . . . . . . . . . 6-23

6.8 FMEA Analysis - Loss of Pumps . . . . . . . . . . . . . . 6-31

6.9 Motor Generator Support System Dependencies . . . . . . . . . . . . . . . . . 6-34

6.10 Primary Pump Failure Modes/Coastdown Times . . . . . . . . . . . . . . . . 6-36

6.11 Annual Frequencies for MG Sets Failure Modes . . . . . . . . . . . . . 6-39

6.12 Shutdown Cooler System - Failure Probabilities in a One-Year Period . . . . . . . 6-43

6.13 Shield Cooling-Probability of Failure in a One-Year Period . . . . . . . . . . . 6-50

6.14 Thimble Cooling-System Failure Probabilities in a One-Year Period . . . . . . . 6-56

6.15 Failure Probabilities for Electrical Systems in a One-Year (300 day) Period . . . 6-65 


\subsection{SYSTEMS ANALYSIS}

This section presents a summary description of the systems analysis methodology used to estimate the probability of the failure of EBR-II frontline and support systems which may lead to core damage states as described by the accident sequences in Section 5.0 of this report. Included are brief descriptions of the systems presented in Appendix $\mathrm{C}$, the mean failure probabilities for each system, possible initiating event occurrences, and the results of sensitivity and uncertainty analyses.

\subsection{Systems Analysis Procedures}

In the early phases (1989) of this study, the set of systems requiring analysis were selected along the traditional division of reactor systems into (1) frontline systems (i.e., mitigating systems important to safety in terms of core damage) and (2) support systems (systems required to ensure the success of one or more of the frontline systems or support systems). A matrix showing the interfaces and dependencies among the frontline and support systems is shown in Table 6.1.

Following the selection of these systems, a methodology for analysis was created specific to the needs of the EBR-II PRA by utilizing the procedures recommended in a number of PRA reactor studies and references, particularly the USNRC PRA Procedures Guide [6.1] and the IDCOR Technical Report 86.3B1, Appendix B [6.2].

In addition, the collaboration of ANL staff and outside PRA consultants was required to ensure completeness of the technical effort.

The results of the systems analysis effort are documented in system notebooks (Appendix C) which contain the following information (as recommended in ref. 6.2):

- System function 
Table 6.1. Front Line and Support Systems Interface/Dependency Matrix

\begin{tabular}{|c|c|c|c|c|c|c|c|}
\hline Support Systems $\rightarrow$ & 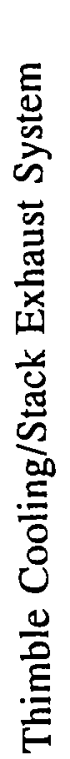 & 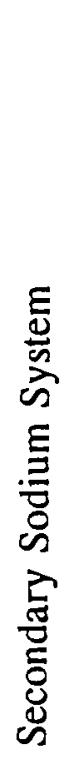 & 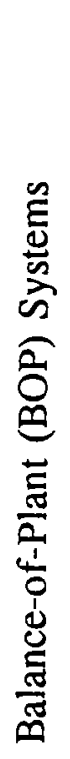 & 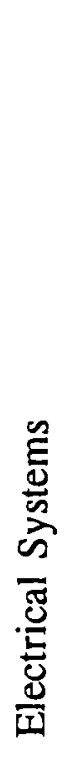 & 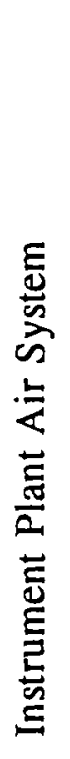 & 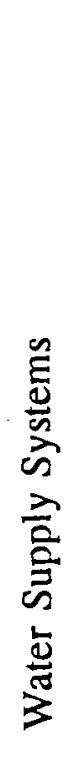 & 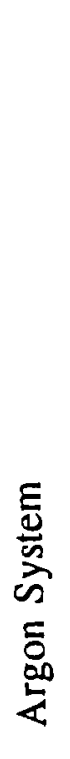 \\
\hline Reactor Shutdown System (RSS) & & & $\mathrm{X}$ & $\mathrm{X}$ & & & \\
\hline Primary Pumps & $\mathrm{X}$ & & & $\mathrm{X}$ & & & $\mathrm{X}$ \\
\hline Primary Pumps Motor-Generator Sets & & & & $\mathrm{X}$ & & $\mathrm{X}$ & \\
\hline Shutdown Cooling System & & & & $\mathrm{X}$ & & $\mathrm{X}$ & \\
\hline Shield Cooling System & $\mathrm{X}$ & & & $\mathrm{X}$ & $\mathrm{X}$ & $\mathrm{X}$ & \\
\hline $\begin{array}{c}\text { Support Systems } \\
\downarrow\end{array}$ & & & & & & & \\
\hline Thimble Cooling/Stack Exhaust System & & & & $\mathrm{X}$ & $\mathrm{X}$ & & \\
\hline Secondary Sodium System & & & $\mathrm{X}$ & $\mathrm{x}$ & & $\mathrm{X}$ & $\mathrm{X}$ \\
\hline Balance-of-Plant (BOP) Systems & & $\mathrm{X}$ & & $\mathrm{X}$ & $\mathrm{X}$ & $\mathrm{X}$ & \\
\hline Electrical Systems & $\mathrm{X}$ & $\mathrm{X}$ & $\mathrm{X}$ & & $\mathrm{X}$ & $\mathrm{X}$ & $\mathrm{X}$ \\
\hline Instrument and Plant Air System & $\mathrm{X}$ & & $\mathrm{x}$ & $\mathrm{X}$ & & $\mathrm{X}$ & \\
\hline Water Supply Systems & & $\mathrm{X}$ & $\mathrm{X}$ & $\mathrm{X}$ & $\mathrm{x}$ & & \\
\hline Argon System & & $\mathrm{X}$ & & $\mathrm{X}$ & & & \\
\hline
\end{tabular}




$\begin{array}{ll}\text { - } & \text { System description } \\ \text { - } & \text { System operation } \\ \text { - } & \text { Performance during accident conditions } \\ \text { - } & \text { Oocation within the plant } \\ \text { - } & \text { Operating experience } \\ \text { - } & \text { Initiating event review } \\ \text { - } & \text { Quantification } \\ \text { - } & \text { Summary of key findings } \\ \text { - } & \text { Summary of review comments } \\ \text { - } & \text { References }\end{array}$

This summary section presents information from the system notebooks for the frontline and support systems with emphasis on the quantification results as pertains to the core damage frequency.

The systems analysis procedures included several phases:
- Plant familiarization, including several walkdowns
- System modeling and fault trees
- Integration (and critique) of the data base
- Quantification - failure frequency

Concomitant with these phases was the evolution of a set of guidelines and system descriptors particularly oriented to computer storage and documentation requirements as outlined in the Quality Assurance Plan [6.3].

Following the plant familiarization task, which included the accumulation of information from safety documents, current drawings, maintenance and operating procedures and extensive interaction with EBR-II management and operating personnel, the system modeling and fault tree development proceeded under the following rules: 
The system models reflect the plant configuration as of October, 1989.

For reactor operational data, there are three (3) 100-day runs per year (capacity factor $\sim 82 \%$ ).

The truncation values for the fault tree models are a maximum of $10^{-11}$ failure probability and 7 terms of the minimal cutsets.

System models include random failures, mandated operator actions, test and maintenance unavailability, and human error.

System models do not include errors of commission or recovery actions, with some special exceptions.

Events in the final fault trees are of two types: basic events and house events.

The database (see Section 7.0) used for quantification is derived from generic data, the CREDO LMR database, and EBR-II plant-specific data.

Failures due to intersystem dependencies, effects of initiating events and common-cause failures are modeled explicitly in the fault trees.

\subsubsection{System Analysis Naming Conventions}

The construction of fault trees requires naming conventions for identification of main systems, support systems, components, failure modes, component locations, gates (ANDs and ORs), basic events, and house events. Figure 6.1 shows the formats for gates and basic/house events. The sections presented here (6.1.1) are intended to demonstrate the depth and completeness of the systems analysis task. (For specific examples of the usage of naming conventions, refer to the system notebooks in Appendix C). 


\section{Gate Names:}

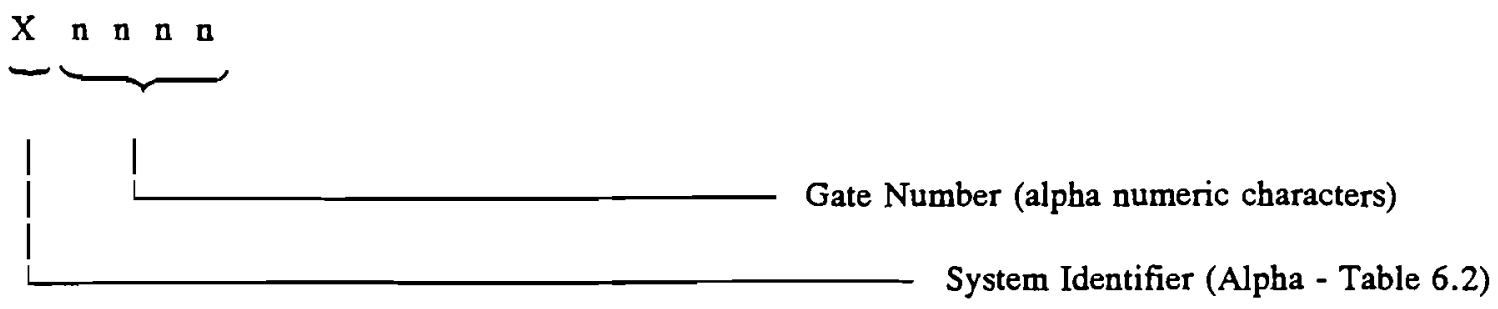

\section{Basic Events:}

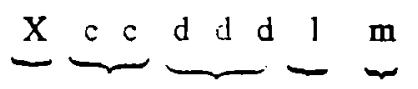

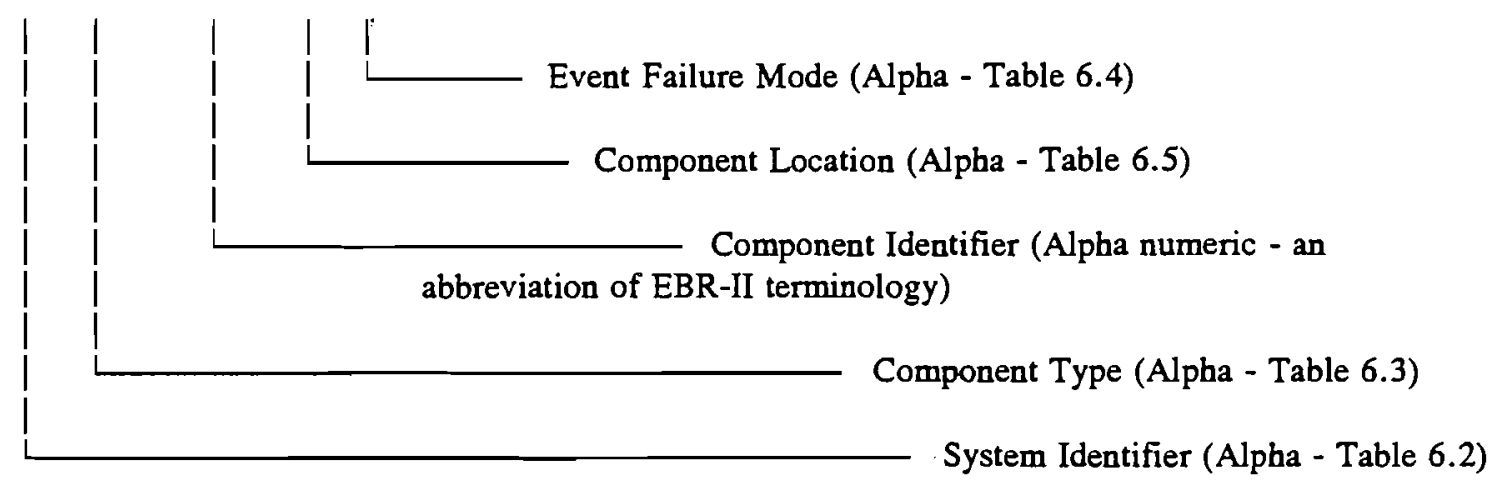

House Events:

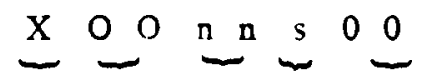

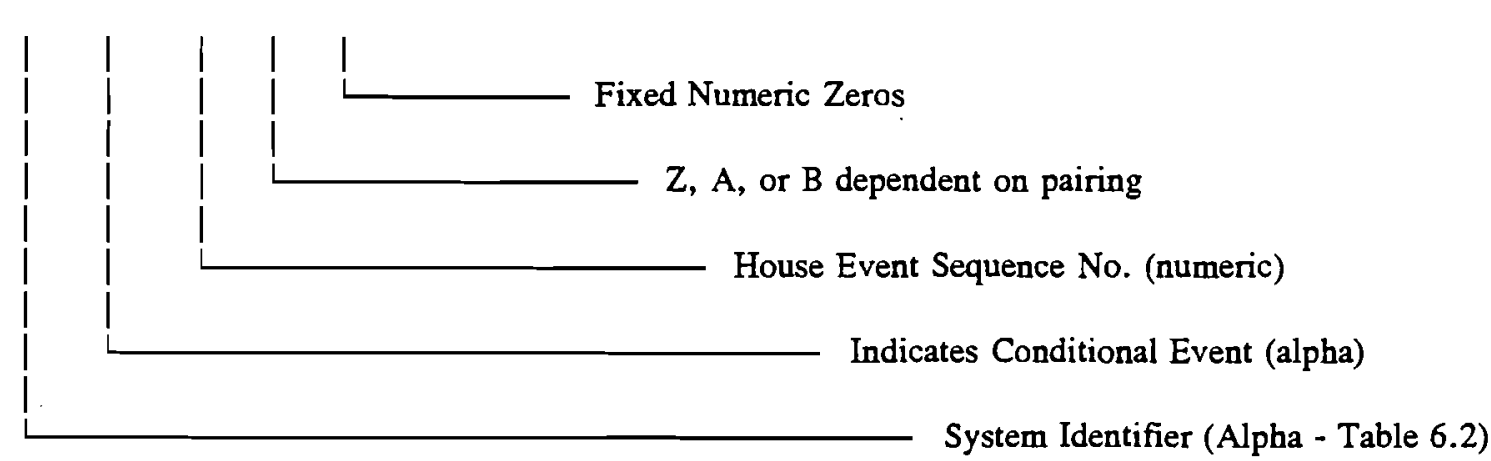

(Note: See Appendix C for specific examples.)

Fig. 6.1. Gate and Basic/House Event Formats for Fault Tree Inputs 


\subsubsection{Gate Names}

As shown in Figure 6.1, gate names are formatted as Xnnnn, where $\mathrm{X}$ is the system identifier and $\mathrm{nnnn}$ is a unique four-character (alphanumeric) set designated by the system analyst. Where feasible, nnnn is numeric and these numbers are in ascending order as the gates appear more deeply nested in the fault trees.

\subsubsection{Basic Event Names}

As shown in Figure 6.1, basic event names are formatted as $X$ ccdddlm, where $X$ is the system identifier, cc is the component type identifier, ddd is a component identifier specific to EBR-II plant terminology, 1 is the location code, and $\mathrm{m}$ is the failure mode for the basic event.

\subsubsection{House Event Names}

As shown in Figure 6.1, house events are formatted as XOOnns00, where $\mathrm{X}$ is the system identifier, $\mathrm{OO}$ is the component identifier for a conditional event (identical for all house events), $\mathrm{nn}$ is a two-digit (numeric) house event sequence identifier, $\mathrm{s}$ assumes a $\mathrm{Z}, \mathrm{A}$, or $\mathrm{B}$ value dependent upon pairing, and 00 are fixed numeric zeros.

\subsubsection{Identifiers for Main and Support Systems}

The main systems and the support systems are designated in the fault trees by a one-character alpha identifier as listed in Table 6.2. This identifier always appears as the first character of a gate name, a basic event name, or a house event name.

\subsubsection{Identifiers for Mechanical and Electrical Components}

The system component type is designated in the fault trees by a two-character alpha identifier as listed in Table 6.3. This identifier always appears as the second 
Table 6.2. System Identifiers

\section{MAIN SYSTEMS}

Auxiliary Pump

Balance of Plant

Condensate

Steam

Control Rods

Fuel and Experimental Pins and Subassemblies

$\mathrm{V}$

C

Primary Pumps

Reactor Shutdown System

Safety Rods

Secondary Pump

Shield Cooling

Shutdown Coolers

\section{SUPPORT SYSTEMS}

Argon

Containment Isolation

K

Cover Gas

G

Cover Gas Cleaner

M

DC Power

L

Emergency Power

Instrument Air

Normal Power

$\mathrm{E}$

Plant Cooling Water

Reactor Cooling Water

Primary Sodium and Siphon Break

Thimble Cooling

Uninterruptible Power Supply (UPS) 
Table 6.3. Mechanical and Electrical Component Identifiers

\begin{tabular}{llcl} 
IDENTIFIER & MECHANICAL COMPONENT & IDENTIFIER & ELECTRICAL COMPONENT \\
\cline { 2 - 3 } AU & Accumulator & AC & AC Power \\
CA & Cable & AM & Amplifier \\
CD & Safety/Control Rod Drive Unit & AN & Annunciator \\
DL & Diesel & BY & Battery Charger \\
FL & Filter or Strainer & BS & Bus \\
GB & Gas Bottle & CA & Cable \\
HE & Heat Exchanger & CB & Circuit Breaker \\
IG & Igniter & CL & Clutch \\
OR & Orifice & DC & DC Power Supply \\
PF & Flexible Hose & IV & Inverter (Solid State) \\
PP & Pipe & LT & Light \\
PE & Expansion Joint & LS & Limit Switch \\
PC & Pipe Cap & MO & Motor \\
PV & Pressure Vessel & ND & Neutron Detector \\
PT & Pump, Turbine-Driven & DE & Diode or Rectifier \\
PM & Pump, Motor-Driven & RC & Recorder \\
TK & Tank & LA & Light Arrester \\
CR & Cooler & GS & Ground Switch \\
TG & Tubing & RE & Relay \\
CV & Valve, Check & RT & Resistor, Temperature \\
XV & Valve, Manual & & Device \\
MV & Valve, Motor-Operated & PS & Switch, Process \\
RV & Valve, Relief & VR & Voltage Regulator \\
SV & Valve, Solenoid & PI & Process Indicator \\
DV & Valve, Diaphragm & GC & Governor Valve \\
AV & Valve, Pneumatic & & Controller \\
HV & Valve, Hydraulic & ST & Solid State Device \\
GV & Valve, Regulator & & \\
VV & Valve System & HR & Heater \\
FE & Flow Element & SW & Switch, Manual \\
SD & Steam Drum & KS & Switch, Lock-out \\
MF & Motor Fan (Blower) & AT & Automatic Transfer \\
FN & Fan & & Switch \\
RD & Rupture Disc & DP & Distribution Panel \\
CH & Chiller & IN & Instrumentation \\
CM & Compressor & CO & Connector \\
CN & Condenser & FU & Fuse \\
LC & Control Logic & GE & Generator \\
SL & Seals & HT & Heat Tracing \\
TV & Turbine & & \\
& & &
\end{tabular}


Table 6.3. (Continued)

\begin{tabular}{|c|c|c|c|}
\hline IDENTIFIER & MECHANICAL COMPONENT & IDENTIFIER & ELECTRICAL COMPONENT \\
\hline $\mathrm{BE}$ & Bearing & TR & Transformer, Power \\
\hline GG & GN Generator & $\mathrm{XT}$ & Transformer, Voltage \\
\hline FM & Flow Meter & SC & Speed Controllers \\
\hline $\mathrm{BO}$ & Boiler & WR & Wire \\
\hline GE & Generator & $\mathrm{CC}$ & Capacitor Gear \\
\hline $\mathrm{DM}$ & Damper & RS & Resistor \\
\hline $\mathrm{TP}$ & Power Turbine & $\mathrm{TZ}$ & Position Transmitter \\
\hline $\mathrm{HU}$ & Human Error & EX & Exciter (of Motor \\
\hline SZ & Position Sensor & & Pump Generator) \\
\hline TE & Temperature Element & SG & Switch Gear \\
\hline ME & Meter & OO & Conditional Event \\
\hline DC & DC Power & $\mathrm{TC}$ & Thermocouple \\
\hline LE & Level Sensor & $\mathrm{CZ}$ & Electrical Contacts \\
\hline PR & DC Power & BI & Bistable \\
\hline LE & Level Sensor & VI & MVI Converter \\
\hline PR & Pressure Sensor & BA & Battery \\
\hline TM & Tachometer & TS & Pressure Transmitter \\
\hline DR & Dryer & & \\
\hline RR & Receiver & & \\
\hline LK & Linkage & & \\
\hline $\mathrm{CP}$ & Coupling & & \\
\hline IP & Impeller & & \\
\hline LB & Lubrication Subsystem & & \\
\hline AR & Actuator & & \\
\hline SR & Safety/Control Rod & & \\
\hline TW & Tower/Chimney & & \\
\hline HA & Primary Tank Hangers & & \\
\hline BD & Containment Building & & \\
\hline SB & Fuel Storage Basket & & \\
\hline DK & Primary Tank Deck & & \\
\hline NA & Not Available, Other & & \\
\hline $\mathrm{OO}$ & Conditional Event & & \\
\hline
\end{tabular}


and third characters of a basic event name. The system component identifier is an alphanumeric abbreviation derived from EBR-II terminology as used on drawings, etc. This identifier always appears as the fourth, fifth, and sixth characters of a basic event name.

\subsubsection{Component Location Identifiers}

System component locations are designated in the fault trees by onecharacter alpha identifiers as listed in Table 6.4. This identifier always appears as the seventh character of a basic event name.

\subsubsection{Failure Mode Identifiers}

Failure modes for system components are designated in the fault trees by a one-character alpha identifier as listed in Table 6.5. This identifier always appears as the eighth character of a basic event name. 
Table 6.4. Component Location Identifiers

$\begin{array}{clll}\text { IDENTIFIER } & \text { BUILDING } & \text { LOCATION } \\ \text { R } & \text { Reactor } & \text { Reactor/Reactor Top, Tank } \\ \text { A } & \text { Reactor } & \text { Batteries; Control Rod Instrument Air Tank } \\ \text { F } & \text { Reactor } & \text { Fuel Handling Machine Area; Argon Cell; } \\ & & \text { CGCS Area } \\ \text { L } & \text { Reactor } & \text { RSLC; Electrical Equipment } \\ \text { S } & \text { Reactor } & \text { Subbasement Area } \\ \text { W } & \text { Boiler } & \text { Boiler Wing } \\ \text { N } & \text { Boiler } & \text { Sodium Storage Tank } \\ \text { E } & \text { Boiler } & \text { Electrical Equipment Room } \\ \text { I } & \text { Boiler } & \text { Electrical Equipment Room } \\ \text { J } & \text { Boiler } & \text { Operations Area } \\ \text { P } & \text { Power Plant } & \text { Power Plant Corridor } \\ \text { B } & \text { Power Plant } & \text { Battery Room } \\ \text { V } & \text { Power Plant } & \text { 2400 V / 480 V Switch Gear } \\ \text { G } & \text { Power Plant } & \text { 100 kW Diesel Generator } \\ \text { H } & \text { Power Plant } & \text { 400 kW Diesel Generator, Boilers } \\ \text { Y } & \text { Power Plant } & \text { Rest of First Floor Power Plant Building } \\ \text { T } & \text { Power Plant/ } & \\ & \text { Reactor } & \text { Cable Tunnel } \\ \text { D } & \text { Power Plant } & \text { DAS Room } \\ \text { Q } & \text { Power Plant } & \text { Cable Routing Room } \\ \text { X } & \text { Power Plant } & \text { DAS Expansion Room } \\ \text { K } & \text { Power Plant } & \text { Condenser, Pumps, Rest of Mezzanine } \\ & & \text { Floor Power Plant } \\ \text { C } & \text { Power Plant } & \text { Control Room } \\ \text { Z } & \text { Power Plant } & \text { Turbine, 13.8 kV Switch Gear, Rest of } \\ & & \text { Operating Floor Power Plant } \\ \text { M } & \text { Power Plant } & \text { Diesel Oil Day Tanks Area } \\ \text { I } & \text { Power Plant } & \text { Auxiliary Cooling Tower (Roof) } \\ 2 & - & \text { Main Cooling Tower } \\ \text { O } & - & \text { Outside Main Buildings (Shutdown Coolers, } \\ & & \text { Piping, CGCS Building...) } \\ \text { U } & & \text { Other } \\ & & \end{array}$


Table 6.5. Failure Mode Identifiers

\begin{tabular}{|c|c|c|c|}
\hline IDENTIFIER & FAILURE MODE (ACTIVE) & $\underline{\text { IDENTIFIER }}$ & FAILURE MODE (PASSIVE) \\
\hline $\mathrm{K}$ & Does Not Reclose & A & Short to Power \\
\hline $\mathrm{L}$ & Latent Human Error & B & Open Circuit \\
\hline M & Calibration & $\mathrm{C}$ & Short to Ground \\
\hline $\mathbf{N}$ & Does Not Close & D & Shorts \\
\hline 0 & Does Not Remain Closed & $\mathrm{E}$ & Plugged \\
\hline $\mathbf{P}$ & Does Not Open & $\mathrm{F}$ & Leakage/Rupture \\
\hline Q & Does Not Remain Open- & G & Distortion/Binding \\
\hline & Fails Closed & $\mathrm{H}$ & Leak \\
\hline $\mathbf{R}$ & Does Not Continue to Run & I & Dirt/Foreign Material \\
\hline$S$ & Does Not Start & $\mathrm{J}$ & Vibration \\
\hline $\mathrm{T}$ & $\begin{array}{l}\text { Multi-Position Switch/Valve } \\
\text { in Wrong Position }\end{array}$ & & \\
\hline $\mathrm{U}$ & Common Cause Failure & & \\
\hline V & Grip Failure & & \\
\hline $\mathrm{W}$ & Loss of Function & & \\
\hline $\mathrm{X}$ & Maintenance Unavailability & & \\
\hline $\mathrm{Y}$ & Test Unavailability & & \\
\hline $\mathrm{Z}$ & Dynamic Human Error & & \\
\hline
\end{tabular}


The frontline systems include the Reactor Shutdown System (RSS), the Primary Pumps, the Primary Pumps Motor-Generator Sets, the Shutdown Cooling System, and the Shield Cooling System.

\subsubsection{Reactor Shutdown System (RSS)}

This section presents the analysis used to determine the probability of scram failure of the RSS in the unlikely events of loss-of-flow (LOF) or transient-over-power (TOP). Either of the two events is assumed to occur at any reactor steady power level. The RSS and the fault trees for the LOF and TOP events are described in detail in Appendix C.1.

\subsubsection{System Function and Description}

The EBR-II Reactor Shutdown System (RSS) is designed to automatically shut down the reactor in the event of equipment or operator malfunctions or environmental problems that could damage the reactor, injure personnel, or release contamination to the environment. The shutdown system will cause the control rods (and in some cases, the safety rods) to automatically scram to their least reactive position to shutdown reactor operation, if any of the selected plant parameters exceed established limits.

In the event of an LOF or TOP, an automatic scram signal normally opens a sufficient number of contacts in shutdown strings A and B (Figure 6.2) to deenergize the corresponding Control Power relays (CP-relays) which in turn deenergize the clutches of the individual control rods and thus allow rapid insertion of all the control rods. Each control rod has its own release and scram mechanism and drops into the core by gravity assisted by a pneumatic force acting on a piston at the top of the rod. Scram can also be initiated manually by the reactor operators. The operators are instructed first to scram the control rods and if the shutdown can not be verified, the operators should then scram the safety rods. The two safety rods share the same 


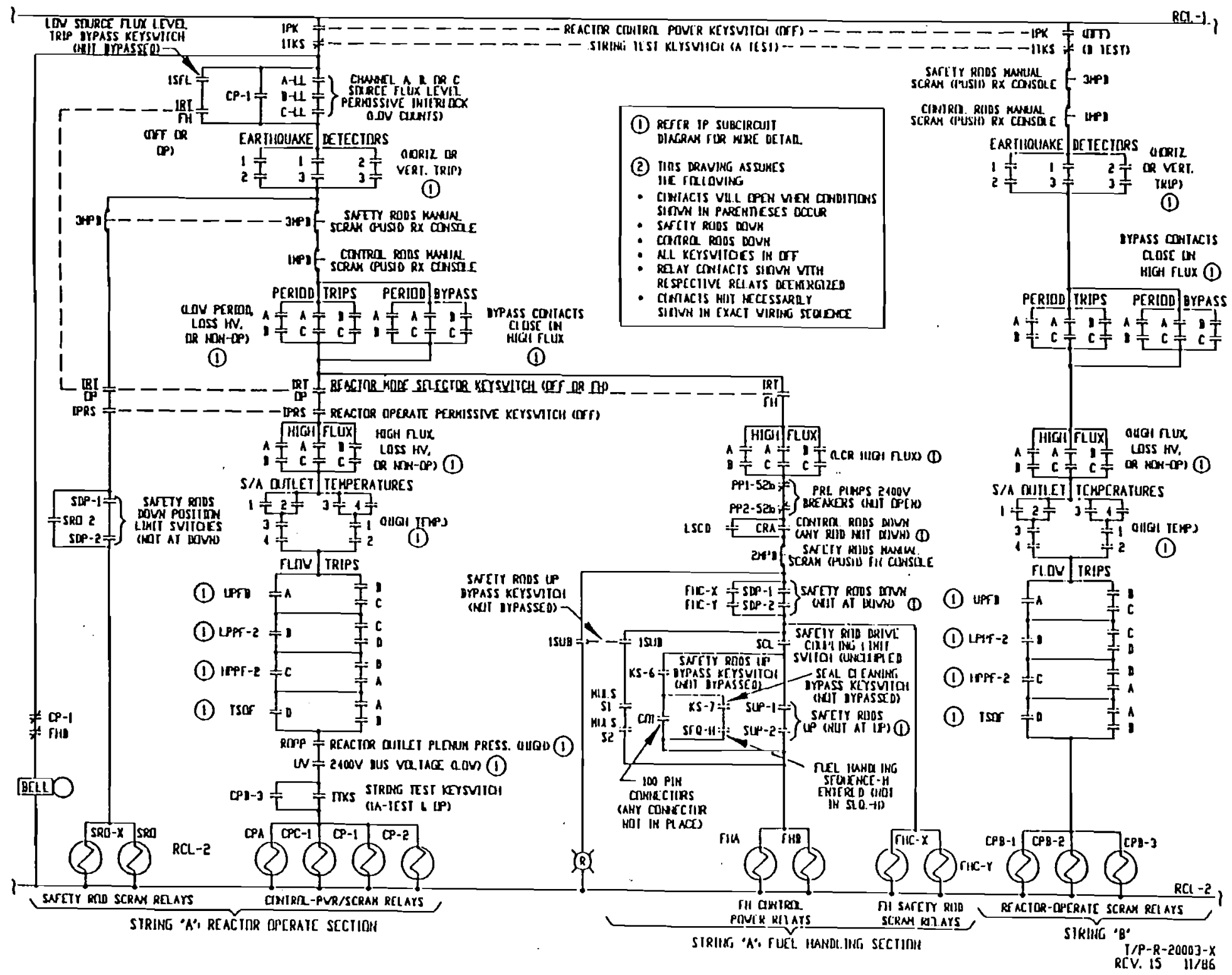

Fig. 6.2. Reactor Shutdown System (RSS) Simplified Schematic 
scram mechanism and are laterally connected so that they can only move together vertically. The safety rods drop into the core by gravity assisted by a torsional spring force.

The EBR-II core can be brought subcritical from any authorized power level by fully inserting a single high worth control rod or the two safety rods. Therefore, a failure to scram the reactor would arise when all the high worth control rods (there are currently nine high worth rods in the core) fail to drop in the core and also the two safety rods fail to drop. Specifically, a failure to scram can take place due to one of three reasons: (i) failure to send a scram signal to the shutdown strings, (ii) failure to release the control rods and safety rods, and (iii) failure to insert the control rods and safety rods. All potential causes and mechanisms that would lead to the above scram failures have been delineated in the fault trees. 


\subsubsection{Support System Interfaces and Effects}

The electrical systems (Appendix C.9) provide the only direct interface system for the RSS. In particular the Continuous Power Supply (CPS) provides power to the electrical buses RCL-1 and RCL-2 for both shutdown strings. There are a number of interfaces with the Balance-of-Plant (BOP) systems that provide reactor scram signals.

\subsubsection{Fault Tree Development}

The fault tree analyses have been developed based on the background material provided in the RSS notebook (Appendix C.1). Some of the highlights of these fault trees are presented in this subsection. Since a scram failure occurs only when all the high-worth control rods fail to drop, most of the independent mechanical failure modes of the control rods have been eliminated from the fault trees. Earlier analyses have shown that these independent failure modes have insignificant contribution to the overall scram failure probability. As system understanding developed, more emphasis was placed on the mechanical common cause failures of the control rods and common cause failures of similar electrical components in redundant channels and strings.

The mechanical failure of the control rods are represented in terms of five different failure modes which cover potential causes and locations of binding. Distinct failure modes, for instance, are assigned to foreign materials in sodium entering the assemblies and in sodium exiting the assemblies. Such a detailed description is useful for understanding the system and how its components interact with each other and also for developing future data base. As a result of this detailed representation, two failure modes are found to have potential common cause mechanisms between the control rods and safety rods. The two modes are: failure of the rods to move within their thimbles as a result of foreign material in sodium entering the assemblies and as a result of cross section distortion and bowing of the assemblies relative to their thimbles. 


\subsection{LOF Fault Tree}

The LOF event considered in the fault tree analysis reduces the primary sodium flow below the specified set points of the low-flow channels. There are presently four low-flow channels with scram capability. The four channels are designated according to the location of the flow sensor as follows: HPPF-2 with a flow sensor at the high pressure plenum of pump No. 2, LPPF-2 with a sensor at the low pressure plenum of pump No. 2, TSOF with a sensor at the reactor coolant outlet pipe, and UPFB with a sensor at the reactor upper plenum. Channels HPPF-2 and LPPF-2 use magnetic type of flow sensors whereas channels TSOF and UPFB use pressure difference type of sensors in conjunction with $\mathrm{NaK}$-pressure and strain-gauge signal transducers. In an LOF event that could be detected by all the four sensors, the flow channels in a 2-out-of-4 trip logic would initiate a scram signal by eliminating the voltage across shutdown strings A and B. Because of the locations of the flow sensors, not all types of LOF can be detected by all the four sensors. For example, shaft binding of primary pump No. 1 would be directly detected by TSOF and UPFB channels and indirectly by LPPF-2 channel. The HPPF-2 channel, because of its sensor location, can not detect such an LOF condition and thus the trip logic of the flow channels becomes 2-out-of-3. To accommodate this type of LOF events, two mutually-exclusive house events are used with the HPPF- 2 channel to indicate at the outset whether this channel can detect the LOF condition under consideration.

In addition to the automatic scram, the reactor operators are trained and instructed to quickly initiate a manual scram when they realize that an LOF condition exists. The human action in this case will rely on annunciators, alarms and indicators that are independent of the low-flow channels and SOT channels. The operators continuously monitor the reactor delta-T along with the primary system flow. The reactor delta-T has two annunciators that should alert the operators in the event of an LOF. With regard to the primary flow condition, there is an annunciator for Pump No. 1 or No. 2 low rpm and an annunciator for Pump No. 1 low discharge pressure. In addition there are DAS alarms for reactor power, speed of the two primary pumps and array of bulk sodium temperatures and upper plenum probe temperatures. 


\subsection{TOP Fault Tree}

For the purpose of assessing the reactor shutdown capability, the TOP can be described simply as an abnormal event that increases the neutron flux level above the specified set points of the wide-range nuclear channels. There are presently three independent wide-range nuclear channels, designated channels $\mathrm{A}, \mathrm{B}$, and $\mathrm{C}$, with scram capability. In the event of a TOP, the wide-range nuclear channels in a 2-out-of-3 trip logic would initiate a scram by eliminating the voltage across shutdown strings $A$ and $B$. Each of the wide-range channel also trips at loss of DC voltage to the nuclear detector and when one of the non-operate conditions exists in the channel.

In addition to the automatic scram capability, the reactor operators can initiate a manual scram when they realize that a TOP condition exists and that the automatic scram has failed. Reactor delta-T with its two annunciators should alert the operators of the abnormal core condition. Digital indicator of the compensated ion chambers (channel 7 or $7 \mathrm{a}$ ) should confirm that a TOP has actually occurred. As in the case of LOF, the DAS provides an array of temperature alarms as well as alarms from channels 7 and $7 \mathrm{a}$.

\subsection{Contribution of SOT Channels}

There are four independent SOT channels that provide a redundant and diversified automatic scram capability in the event of LOF, TOP, or any core overheating. The SOT channels scram has a 2-out-of-4 trip logic in both reactor shutdown strings. The thermocouples used in the SOT channels are located in the reactor vessel upper plenum above driver assemblies where they sense the temperature of sodium as it exits these assemblies. The modeling of the SOT channels covers the failure to initiate an automatic scram signal in terms of the failures of the electrical components and a common human error in setting the trip points of all the channels. This SOT branch is then combined with the failure to initiate a scram signal by the low-flow channels in the LOF fault tree and with the wide-range nuclear channels in the TOP fault tree. It is important to mention that there are a number of components that are similar in design and made by the same manufacturer that are used in the 
SOT channels and the low-flow channels. Similar MV/I converters, trip bistables, and trip relays are used in both types of channels. The SOT channels and TOP wide-range nuclear channels, however, only have similar trip relays. Common cause failure between these similar components are considered in the fault trees.

\subsubsection{Component Failure Data, Common Cause Failures, Human Reliability}

The components failure data used in the fault tree analyses are given in Section 7 and the system notebook (Appendix C.1). The common cause failures of the electrical and mechanical components and human errors have been treated in detail. Generally, conservatism is applied when the failure data is selected either from generic data sources or derived from plant operating records. For instance, two failure modes are considered for each relay and its contacts, one failure per demand of $3.010^{-4}$ and another failure per hour of operation equal to $2.010^{-7}$. The latter failure mode is particularly important as it simulates the effect of extended run times. Also, component failure rate corresponding to all modes are used when failure rates for individual modes can not be determined. Generic data [6.4] and EBR-II CREDO [6.5] data are used in conjunction with more recently available EBR-II specific data.

In almost all parts of the EBR-II RSS, redundancy has been provided in order to reduce scram failure probability. As a result, common cause failures (CCFs) of similar components, rather than independent failures, are expected to dominate the probability of scram failure. There are few mechanisms, however, that can lead to CCFs among the similar and independent components in the RSS. For instance, the detection channels rely in their normal operation on AC voltage supplied from the UPS system. But a failure of the UPS would directly lead to an automatic scram as a result of deenergizing the magnetic clutches of all the control rods as well as the magnetic clutch of the two safety rods. Also, the RSS of EBR-II has been successfully operating for more than 25 years and any design or maintenance procedure defects are excluded as CCF mechanisms. One of the mechanisms of CCF that is postulated is a hardware failure among similar components made by the same manufacturer. This CCF can arise as a result of extended operations of components or simultaneous replacement of old components with new ones that are either defective or are not compatible with other components. 
CCFs among similar components are considered in the fault tree analyses in terms of the beta-factor method [6.6] by two types of analyses. Analysis " $A$ " utilizes relatively large and fixed beta-factors, since the emphasis is on common causes among components that share the same location and operate within the same channel or string. For instance, all the CP-relays on string A that affect both the control and safety rods are assumed to fail in a common mode with a relatively large beta-factor since the relays are located in the same cabinet. Analysis "B" utilizes beta-factors that depend on the number of redundant components in the system (cf. Section 7.4). In both types of analyses, each redundant component is represented by two basic events: an independent component failure and common failure among all the redundant components. The probability of the CCF is set equal to the independent failure probability multiplied by the beta factor. To account for the number of redundant components in the system, a beta-factor of 0.10 is used for less than four redundant components, 0.02 for four or five redundant components, and 0.008 for six or more redundant components. As an example, in the wide-range nuclear channels the beta-factor for the three detectors is 0.10 , whereas the beta-factor for the six high-flux bistables is 0.008 . Comparing the results of the two types of analyses illustrates the sensitivity of scram failure probability to CCFs.

Human errors are addressed in the fault trees in two general areas: errors that would prevent the automatic scram channels from detecting the abnormal transient events and human errors that would prevent utilization of the manual scram capability. (Details of the human reliability analysis, which is based on the methodology of Ref. [6.7], are given in Section 8.) Human errors in the first area include errors in calibrating the electrical components, in setting the specified trip points, and in performing channel operability tests. The potential for common errors in performing each of the above tasks has been taken into account. Such a common human error is found to be the dominant failure mode in the case of the SOT channels. The same type of common error has some effect on the wide-range nuclear channels but insignificant effect on the low-flow channels.

In the second area, two different human errors are considered: (i) reactor operators failure to manually scram the reactor when the automatic scram fails, and (ii) reactor operators failure to scram the safety rods when the control rods fail to drop for mechanical reasons. With 
regard to the manual scram simulation in the fault trees, the approach taken is that the operators should be able to diagnose the abnormal transient condition and realize the need to initiate a scram based on annunciators and alarms independent of the automatic scram channels. It is important to mention that the DAS, which is usually in operation with the reactor at steady power more than $99 \%$ of the time, provides a useful and fast means for alerting the operators in the event of LOF or TOP. The DAS, however, has alarms for more than 900 parameters and these parameters appear on the terminal, up to 20 alarming channels at one time, according to the order that the limits are exceeded without any channel priority system. The display of scram related alarms in the middle of a list of less important parameters could delay the operator response.

Human reliability analysis of the second operator error, failure to scram the safety rods, shows that the operators in this situation will be aware of the need to scram and would normally follow the manual-scram emergency procedure. The probability of failure to perform this task is found to be small. However, as a conservative approach, the fault tree modeling accounts for possible dependencies between the two types of human errors.

\subsubsection{Quantification - Results}

The probabilities of scram failure in the event of LOF and TOP are obtained for a set of analyses that cover different transient scenarios and equipment testing intervals and illustrate the effect of potential common cause failures. The results of these analyses are summarized in Tables 6.6 and 6.7. Each table shows the mean probability of scram failure in the abnormal event for a total of eight cases. The probability values listed in the tables include correction for uncertainty coupling. To demonstrate the effect of the SOT channels, the results of the first four cases do not include the automatic scram capability of the SOT channels, whereas the second four cases include the capability of the SOT channels. The probability of scram failure is presented in terms of the three major contributors to failure: signal, mechanical, and electrical. The signal failure represents the failure to send a scram signal to the shutdown strings by either the automatic scram channels or the manual scram channels. 
Table 6.6. Scram Failure Probabilities for LOF Events

\begin{tabular}{|c|c|c|c|c|c|}
\hline \multirow{2}{*}{$\operatorname{Case}^{(1)}$} & \multirow{2}{*}{ Total } & \multicolumn{2}{|c|}{ Signal Failure ${ }^{(2)}$} & \multirow[b]{2}{*}{$\begin{array}{l}\text { Mechanical } \\
\text { Failure }\end{array}$} & \multirow[b]{2}{*}{$\begin{array}{l}\text { Electrical } \\
\text { Failure }\end{array}$} \\
\hline & & $\mathrm{A} / \mathrm{S} \& \mathrm{M} / \mathrm{S}$ & A/S Only & & \\
\hline \multicolumn{6}{|c|}{ Without SOT Channels } \\
\hline I-A & $2.5310^{-7}$ & $7.0010^{-10}$ & $1.4010^{-8}$ & $2.2610^{-7}$ & $2.6310^{-8}$ \\
\hline $\mathrm{I}-\mathrm{B}$ & $4.9710^{-6}$ & $4.2410^{-7}$ & $8.4810^{-6}$ & $2.2610^{-7}$ & $4.3210^{-6}$ \\
\hline II-A & $6.7010^{-7}$ & $4.1810^{-7}$ & $8.3510^{-6}$ & $2.2610^{-7}$ & $2.6310^{-8}$ \\
\hline II-B & $5.3510^{-6}$ & $8.0410^{-7}$ & $1.6110^{-5}$ & $2.2610^{-7}$ & $4.3210^{-6}$ \\
\hline \multicolumn{6}{|c|}{ With SOT Channels } \\
\hline I-A & $2.5210^{-7}$ & $1.7510^{-11}$ & $3.5010^{-10}$ & $2.2610^{-7}$ & $2.6310^{-8}$ \\
\hline I-B & $4.7210^{-6}$ & $1.7410^{-7}$ & $3.4810^{-6}$ & $2.2610^{-7}$ & $4.3210^{-6}$ \\
\hline II-A & $2.6310^{-7}$ & $1.0510^{-8}$ & $2.0910^{-7}$ & $2.2610^{-7}$ & $2.6310^{-8}$ \\
\hline II-B & $4.7310^{-6}$ & $1.8410^{-7}$ & $3.6810^{-6}$ & $2.2610^{-7}$ & $4.3210^{-6}$ \\
\hline
\end{tabular}

Notes:

(1)

Case I: $2 / 4$ channel trip configuration

Case II: $2 / 3$ channel trip configuration without channel HPPF-2

Case A: Limited CCFs are considered

Case B: More extensive CCFs are considered

(2) A/S: Automatic Scram

$\mathrm{M} / \mathrm{S}: \quad$ Manual Scram; HEP $=5.010^{-2}$ 
Table 6.7. Scram Failure Probabilities for TOP Events

\begin{tabular}{|c|c|c|c|c|c|}
\hline \multirow{2}{*}{ Case $^{(1)}$} & \multirow{2}{*}{ Total } & \multicolumn{2}{|c|}{ Signal Failure ${ }^{(2)}$} & \multirow{2}{*}{$\begin{array}{l}\text { Mechanical } \\
\text { Failure }\end{array}$} & \multirow{2}{*}{$\begin{array}{l}\text { Electrical } \\
\text { Failure }\end{array}$} \\
\hline & & $\mathrm{A} / \mathrm{S} \& \mathrm{M} / \mathrm{S}$ & A/S Only & & \\
\hline \multicolumn{6}{|c|}{ Without SOT Channels } \\
\hline I-A & $7.6410^{-5}$ & $7.6210^{-5}$ & $7.6210^{-4}$ & $2.2610^{-7}$ & $2.6310^{-8}$ \\
\hline I-B & $1.6910^{-4}$ & $1.6410^{-4}$ & $1.6410^{-3}$ & $2.2610^{-7}$ & $4.3210^{-6}$ \\
\hline II-A & $8.3510^{-7}$ & $5.8310^{-7}$ & $5.8310^{-6}$ & $2.2610^{-7}$ & $2.6310^{-8}$ \\
\hline II-B & $1.1810^{-5}$ & $7.2610^{-6}$ & $7.2610^{-5}$ & $2.2610^{-7}$ & $4.3210^{-6}$ \\
\hline \multicolumn{6}{|c|}{ With SOT Channels } \\
\hline I-A & $2.1610^{-6}$ & $1.9110^{-6}$ & $1.9110^{-5}$ & $2.2610^{-7}$ & $2.6310^{-8}$ \\
\hline $\mathrm{I}-\mathrm{B}$ & $9.1010^{-6}$ & $4.5510^{-6}$ & $4.5510^{-5}$ & $2.2610^{-7}$ & $4.3210^{-6}$ \\
\hline II-A & $2.6710^{-7}$ & $1.4610^{-8}$ & $1.4610^{-7}$ & $2.2610^{-7}$ & $2.6310^{-8}$ \\
\hline II-B & $4.9810^{-6}$ & $4.3410^{-7}$ & $4.3410^{-6}$ & $2.2610^{-7}$ & $4.3210^{-6}$ \\
\hline
\end{tabular}

Notes:

(1)

Case I: Test Interval of HF Channel Components $=100$ days

Case II: Test Interval of HF Channel Components $=7$ days

Case A: Limited CCFs are considered

Case B: More extensive CCFs are considered

$\begin{array}{ll}\text { A/S: } & \text { Automatic Scram } \\ \text { M/S: } & \text { Manual Scram; HEP }=1.010^{-1}\end{array}$ 
The mechanical failure represents the failure to release or insert all the control rods and the two safety rods. It has a fixed magnitude of $2.2610^{-7}$ in all the LOF and TOP cases with a major portion of $2.010^{-7}$ due to the CCF of the control and safety rods. The other portion represents the probability of the control rods mechanical failure (including the failure of magnetic clutches) multiplied by the human error of failing to scram the safety rods. The electrical failure represents the failure of the CP-relays to open and prevent deenergizing the clutches of the control and safety rods. This part of the scram failure depends heavily on the extent of the common cause failure that one postulates and models in the analysis.

As an independent scram system, the SOT channels would normally have a mean failure probability that ranges from $1.010^{-5}$ to $2.510^{-2}$ per demand based on the beta-factors assumed among similar components, test interval of the components, and human error in setting the trip points of the channels. The upper value of $2.510^{-2}$ is determined by a human error in setting the trip points of all the four channels higher than the specified temperatures in the reactor-run plan. The effect of including the automatic scram capability of the SOT channels can be simply viewed in Tables 6.6 and 6.7 as multiplying the failure probability of the signal part by $2.510^{-2}$. In addition, analysis B takes into consideration potential common cause failures between similar components in the SOT channels and either LOF or TOP channels. The mechanical and electrical parts remain the same since the SOT channels can only affect transient detection and scram signal initiation.

The results of the LOF and TOP analyses show that the modeling of the CCFs has a very large effect on the electrical contribution to the scram failure probability. The electrical failure probability increases by almost two orders of magnitude to $4.3210^{-6}$ in analysis B with more extensive CCFs. This effect dominates the total failure probability and thus minimizes the effect of any improvement in the detection capability introduced by the SOT channels for example.

The LOF results in Table 6.6 show the effect of the number of channels that can detect the transient event by considering two cases. Case I assumes that all the low-flow channels can detect the transient and case II assumes that the sensor of channel HPPF-2 cannot detect the transient. The effect of number of channels is more evident in the signal analysis without the 
SOT channels, particularly in analysis A. The results of analysis B, however, converge to almost the same value of about $5.010^{-6}$ irrespective of the number of channels. The essentially identical results of analysis B with the SOT channels indicates that the failure probability is largely determined by the mechanical and electrical failures and by the CCFs among similar components in the low-flow channels and SOT channels.

The TOP results in Table 6.7 show the effect of the test interval of the electrical components by considering two cases. Case I has all the components of the wide-range channels tested every reactor run (100 days) without any monitoring, and case II has all the components tested every week. Case I conservatively models the channels based on the current operation and maintenance procedures. Case II is an alternative that would require weekly operability checking of the channels. The failure probability of the signal part drops from $1.610^{-4}$ to 7.3 $10^{-6}$, in analysis B without the SOT channels, as a result of decreasing the test interval. This significant improvement in the failure probability of the signal part is an incentive for periodic testing of the wide-range nuclear channel components. It is important, however, to assess any system improvement in terms of the total failure probability which shows significant improvement in both analyses A and B without the SOT channels. Such an improvement becomes much less significant when the analyses take into consideration the SOT channels as the effect of the signal contribution diminishes. The results of Case I are used in the current analysis. 


\subsubsection{Conclusions and Special Insights}

- The mean probability of scram failure in the event of LOF ranges from $2.510^{-7}$ to 5.0 $10^{-6}$ per demand when all the four low-flow channels can detect the low flow condition. The probability of failure ranges from $2.510^{-7}$ to $5.410^{-6}$ per demand when channel HPPF-2 can not detect the LOF condition. The probability of the scram failure varies based on the extent of the CCFs and whether the capability of the SOT channels is taken into account. The higher failure probability in both cases is determined by the CCF among all the CP-relays in the shutdown strings.

- The mean probability of scram failure in the event of TOP ranges from $2.610^{-7}$ to 1.7 $10^{-4}$ per demand based on the assumptions used in the analysis with regard to CCFs, test interval of components and whether the redundant capability of SOT channels is included. The higher failure probability is determined by the signal-failure contribution with a test interval of 100 days. This limit is reduced to $9.110^{-6}$ by taking into account the scram capability of SOT channels.

Any one of five CP-relays in shutdown strings A and B can release the latches of four or more control rods, allowing the control rods to drop into the core, bringing the reactor core subcritical. Similarly, any one of the two CP-relays on the safety-rod branch of string A can release the clutch of the two safety rods. A common cause failure among all the seven CP-relays sets a lower limit on the scram failure probability of about 4.3 $10^{-6}$ per demand.

The failure rate due to mechanical common modes between all the control rods is estimated at $2.010^{-6}$ per demand and the mechanical failure rate of the two safety rods is estimated at $3.010^{-4}$ per demand. A common mode failure is postulated between the two types of rods and is estimated at $2.010^{-7}$ failure per demand. These failure rates are based on a generic single-rod failure rate of $3.010^{-5}$ per demand and supported by plant specific data for 25 years of reactor operation and a daily test of each control rod that is capable of detecting any potential rod binding. 
- The effect of human error in initiating a manual scram in the event of an automatic scram failure is significant. In the absence of scram annunciators, the DAS alarms (not always online) can be a useful means to alert the operators to the possibility of LOF or TOP, but such scram alarms are not currently distinguishable from other causes of DAS alarms (see Section 14).

- Human errors during calibration and trip point setting are found to have small effect on the LOF and TOP channels. Human error in setting the trip points of the SOT channels based on the present startup procedure is relatively large and as a result limits the effectiveness of the SOT channels (see Section 14).

\subsubsection{Primary Pumps}

\subsubsection{System Function and Description}

Two single-stage centrifugal pumps circulate primary sodium to cool the reactor core. The two pumps are identical and operate in parallel. The pumps are designed to operate in a liquid sodium environment and meet several functional criteria:

(1) Flow and differential pressure requirements are sufficient to provide cooling for the reactor.

(2) Leak tightness to prevent leakage of the radioactive primary blanket gas to the building atmosphere, and liquid sodium into the motor.

(3) Effective biological shielding to contain radiation within the primary tank. 
(4) Accessibility. The assembly is physically arranged to submerge the pump in the sodium. The driver motor is located at the operating floor level, completely out of the radiation field. Removal is accomplished with a simple vertical lift.

(5) Bearing reliability. The hydrostatic pump bearing is reliable and does not require an external pressure source. Motor bearings support imposed thrust and static loads.

(6) Flow variation. Pump flow rate is variable from 10 to $100 \%$, with specified acceleration/deceleration rates, during steady-state operation, speed is constant within $0.2 \%$ of rated speed.

During full-power operation, 62.5 megawatts of heat is continuously removed from the reactor. To remove this heat, each main pump supplies sodium coolant at about $4500 \mathrm{gpm}$ $\left(0.284 \mathrm{~m}^{3} / \mathrm{s}\right)$. Both pumps must operate against the total pressure drop of the primary cooling system, which is $56 \mathrm{psi}(0.37 \mathrm{MPa})$ (from pump outlet to heat exchanger outlet at maximum flow).

Each pump (Figure 6.3) is a vertically mounted structure consisting of a centrifugal sodium pump, a stilling baffle assembly, a shield plug, a hermetically-sealed 350hp ( $262 \mathrm{~kW}$ ) drive motor, and a pump shaft. The entire assembly is approximately $27 \mathrm{ft} 7$ inches $(8.4 \mathrm{~m})$ long and weighs 27 tons $(24,545 \mathrm{Kg})$. The pump shaft transfers power from the drive motor at the top of the structure to the pump. This shaft passes through the shielding and baffle sections, and connects to the pump impeller.

Each pump is a four-volute 27 -inch $(0.67 \mathrm{~m})$ centrifugal pump designed for liquid sodium service. The 26 -inch $(0.66 \mathrm{~m})$ pump impeller is of conventional design, similar to water pumps of equal capacity. Type 304 stainless steel is used for impeller material (as well as all other 


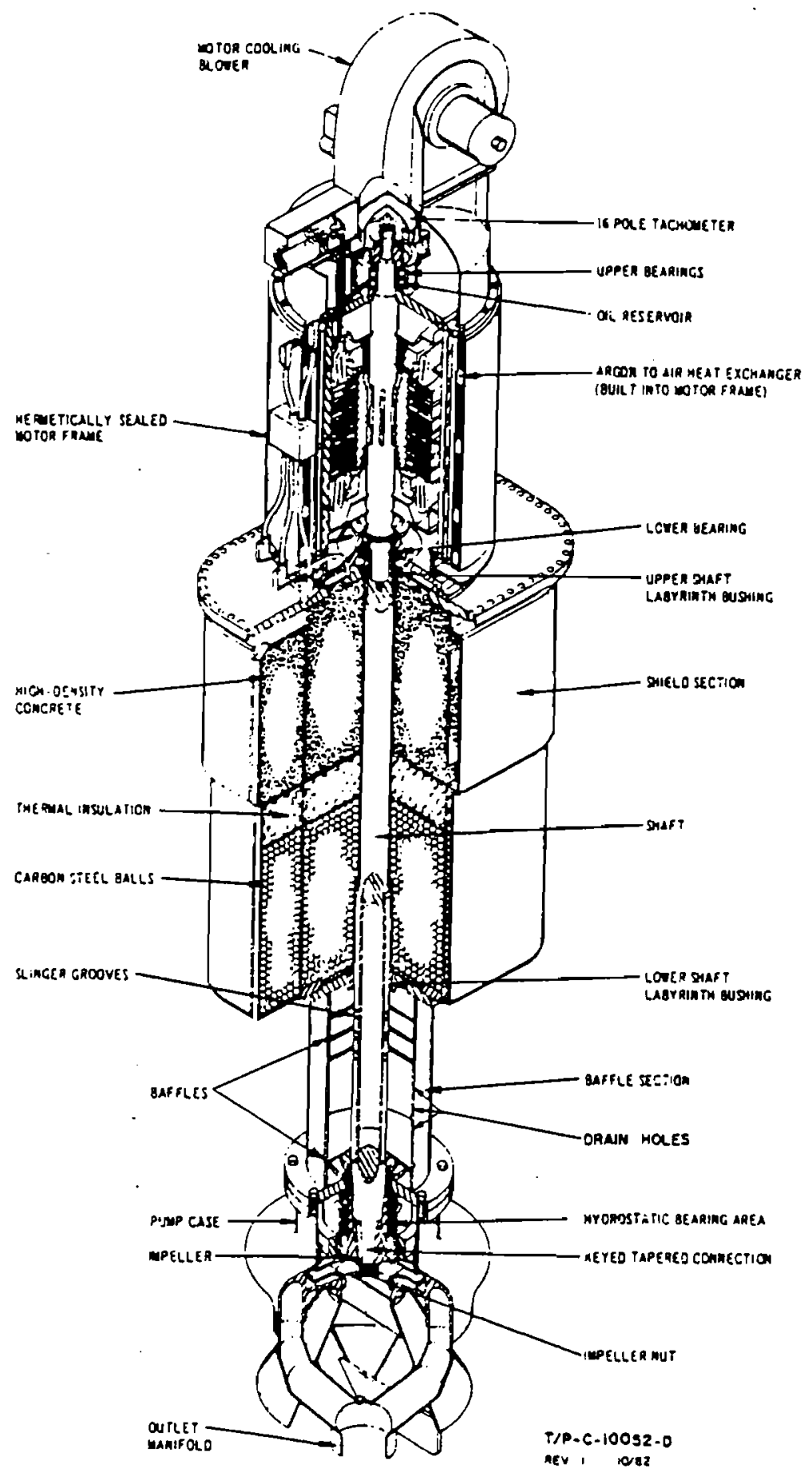

Fig. 6.3. Main Primary Coolant Pump 
parts submerged in sodium) due to its compatibility with the high-temperature coolant. The hydrostatic bearing surfaces are faced with Colmonoy to reduce wear and galling that could occur during startup and low-speed operation.

Further details of the pump assemblies are contained in Appendix C.2.

\subsubsection{Support System Interfaces and Effects}

Two support systems are required for proper pump operation:

(1) Electrical systems - the pump motors require a $480 \mathrm{~V}$ input from the MG sets which operate from the $2400 \mathrm{~V}$ switchgear.

Argon systems - the low pressure argon system provides argon flow along the pump shafts to prevent sodium vapor or sodium oxide from migrating up the pump shaft.

\subsubsection{Fault Tree Development}

The top event for the pump system is loss of both primary pumps. The tree is developed from a failure mode and effects analysis (FMEA). This detailed analysis is discussed in Appendix C.2. The results of the FMEA are presented in Table 6.8 shown below.

\subsubsection{Component Failure Data, Common Cause, Human Reliability}

The data for the pumps is extremely limited; there have been no severe failures of the EBR-II primary pumps and the unique pump design precludes the use of generic data (e.g., from LWRs). Some shaft binding problems have occurred. Hence, the FMEA described above was used as the basic input failure data. Common cause data is not available for similar reasons. 


\section{Table 6.8. FMEA Analysis - Loss of Pumps}

\section{$\underline{\text { Basic Event }}$}

Loss of electrical power

to pump 1

Loss of electrical power

to pump 2

Pump 1 motor fails to run

Pump 2 motor fails to run

Pump 1 shaft binding

Pump 2 shaft binding

Pump 1 shaft broken

or seized

Pump 2 shaft broken

or seized
Failure Rate

$9.410^{-1 *}$

$9.310^{-1 *}$

$2.010^{-5} / \mathrm{h}$

$2.010^{-5} / \mathrm{h}$

$2.910^{-5} / \mathrm{h}$

$2.910^{-5} / \mathrm{h}$

$1.910^{-6} / \mathrm{h}$

$1.910^{-6} / \mathrm{h}$

8.4

3.

3.

3.

3.

2.6

2.6

8.5
Error

Factor

*annual frequency 


\subsubsection{Quantification-Results}

The top level fault tree (Appendix C.2) has been quantified for loss of both pumps, including the MG sets. The probability of losing both primary pumps in a 7200 hr (300 day) period is estimated at $6.510^{-2}$ with an error factor of 4.0.

\subsubsection{Initiating Events and Special Insights}

The loss of one or both primary pumps can be considered an initiating event in the loss-of-flow (LOF) category discussed in Section 5.

\subsubsection{Primary Pumps - Motor Generator Sets}

\subsubsection{System Function and Description}

Each of the two primary cooling pumps is powered by an associated motor-generator (MG) set (see Figure 6.4). The MG sets provide power to the primary pumps and are used to control the pump speed through the clutch connecting the motor and generator. Failure of the MG sets to provide power to the primary pumps is an initiating event for a loss of cooling accident.

\subsubsection{Support System Interfaces and Effects (Table 6.9)}

In the event of loss of normal power, specifically the $2400 \mathrm{~V}$ supply to the motors of the MG sets, both pumps will coast down with a time determined by the inertia of the entire MG set. This result is identical to that for motor malfunction. A worse case initiating event is the failure of one or both clutches. If coupling is lost, only the inertia of the generator will determine the coastdown period. Clutch coupling has been lost in the past, but only for one pump at a time. The shortest coastdown initiating event caused by failure of the MG set is loss of electrical output from both of the generators. In this case only the inertia of the pump motor and impeller is available to promote flow. 


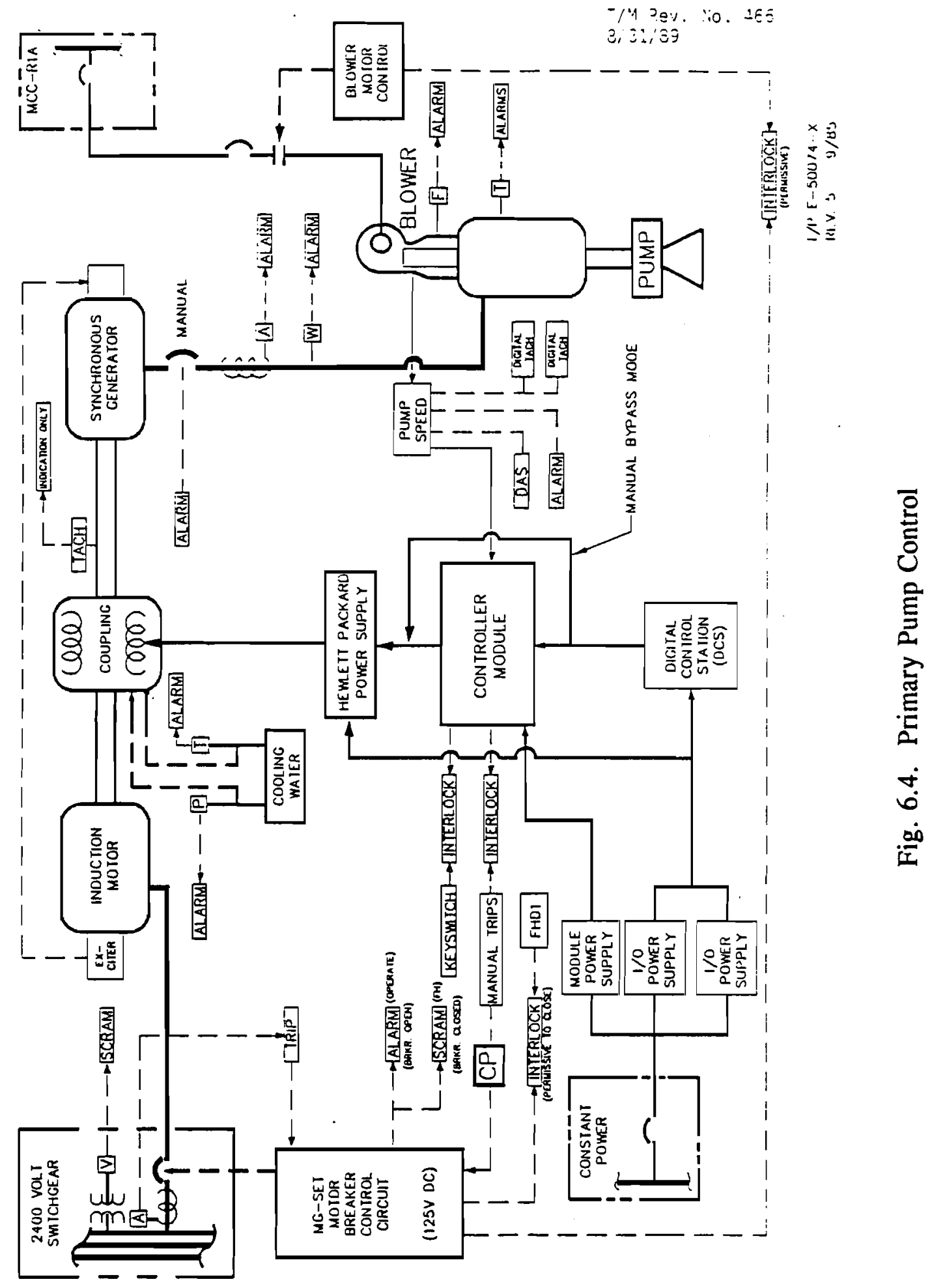


Table 6.9. Motor Generator Support System Dependencies

\begin{tabular}{|c|c|c|c|}
\hline & Support & Support System & Effect on \\
\hline Component & System & Failure Mode & MG System \\
\hline \multirow[t]{2}{*}{ Motor } & $2400 \mathrm{VAC}$ & Loss of voltage & $\begin{array}{l}\text { Motor coastdown } \\
\text { reactor shutdown }\end{array}$ \\
\hline & $125 \mathrm{VDC}$ & Loss of voltage & $\begin{array}{l}\text { No control of } \\
\text { motor input } \\
\text { breakers }\end{array}$ \\
\hline \multirow[t]{2}{*}{ Clutch } & 480 VAC & Loss of voltage & $\begin{array}{l}\text { Cooling water } \\
\text { sump pumps stop }\end{array}$ \\
\hline & $\begin{array}{l}\text { Cooling } \\
\text { water }\end{array}$ & No water flow & $\begin{array}{l}\text { Clutch overheats, } \\
\text { alarm condition } \\
\text { anticipatory } \\
\text { reactor shutdown }\end{array}$ \\
\hline $\begin{array}{l}\text { Clutch Power } \\
\text { Supply }\end{array}$ & CPS & Loss of voltage & $\begin{array}{l}\text { Loss of HP power } \\
\text { supply output, } \\
\text { loss of MG } \\
\text { coupling, pump } \\
\text { coastdown }\end{array}$ \\
\hline $\begin{array}{l}\text { Clutch } \\
\text { Controller }\end{array}$ & CPS & Loss of voltage & $\begin{array}{l}\text { No primary pump } \\
\text { speed control }\end{array}$ \\
\hline Instrumentation & CPS & Loss of Voltage & $\begin{array}{l}\text { No alarms for } \\
\text { off-normal } \\
\text { conditions }\end{array}$ \\
\hline
\end{tabular}


In addition to electrical systems needed for monitor and control instrumentation, the primary support systems associated with the MG sets are the $2400 \mathrm{~V}$ power connection, Continuous Power Supply (CPS) connections, the $125 \mathrm{~V}$ DC supply, and the reactor auxiliaries cooling water supply for the clutch. As described above, loss of $2400 \mathrm{~V}$ (or the motor exciter circuits) causes both pumps to stop and causes a reactor scram. Loss of cooling water to the clutches causes an alarm condition, which if not corrected results in an anticipatory shutdown of the reactor. Failure of either one of the two CPS panels results in loss of clutch coupling for the associated MG set, and a dual CPS panel failure causes a dual-pump loss.

Under other accident conditions, the pumps are expected to function normally, provided power is available for the pumps and the clutch control systems, and provided the sodium level in the reactor remains above the pump inlets.

\subsubsection{Fault Tree Development}

The fault tree analysis evolved from the information and data collected and organized in the system notebook in Appendix C.3.

Table 6.10 lists the three major failure modes for each MG set. The top event is defined as a loss of power to the primary pump. For each pump, loss of power results from either loss of motor output, loss of clutch coupling, or a failure associated with the generator. Motor output is lost only if the input power $(2400 \mathrm{~V})$ is lost or the motor subsystem has failed. The clutch output is lost either because the motor output is lost or a failure has occurred in the clutch system. The third failure mode is loss of generator output even though the motor and clutch are operating normally.

Assuming that the reactor is operating at full power, and that both primary pumps are operating normally, the longest coastdown time occurs if a motor fails, either because of a power loss or mechanical problem. In this case, the inertia of the motor, generator and pump generally leads to the longest pump stopping time, depending on operating conditions at the time 
Table 6.10. Primary Pump Failure Modes/Coastdown Times

$50 \%$
Coastdown

(s)

7

7

Loss of clutch

coupling

Loss of generator

output
$100 \%$
Coastdown

(s)

$81-84$

23

23 
of failure. If the coupling between the motor and generator is lost, the pump will coastdown in a shorter time since the motor inertia is no longer in the system. The shortest case occurs when only the inertia of the primary pump is available to extend the coastdown time, leading to the potentially shortest pump stop time, depending upon conditions at the time of failure. Table 6.10 lists the approximate times to reach $50 \%$ flow and full pump coastdown for the three cases described above. In the development of the fault trees, it is assumed that the reactor is operating at normal power, that the clutch excitation key switch is locked in the "on" position, and that all necessary support systems are functioning normally.

\subsubsection{Component Failure Data, Common Cause Failures, Human Error}

A review of the fault trees shows that each MG set is for the most part independent of the other. However, several common cause failures can occur which will lead to a coastdown of both pumps. Loss of the $2400 \mathrm{~V}$ bus, which powers the motors, causes both MG sets to stop, in addition to being an automatic scram condition. A loss of cooling water would cause both clutches to overheat. A high temperature alarm or low water pressure alarm calls for an anticipatory reactor shutdown but does not lead to an initiating event. Failure of the Module Power Supply which powers both clutch controller modules causes both controllers to automatically switch to bypass mode. Failure of both I/O power supplies causes a loss of pump control, but the HP power supplies remain at the set level, not causing a loss of pump output condition. Failure of the Constant Power Supply (both panels 1 and 2) causes power to be lost to the controller modules, the DCSs and the HP power supplies, resulting in loss of clutch coupling on both MG sets.

\subsubsection{Quantification/Results}

The failure rate data for various components of the EBR-II motorgenerator systems as identified in the fault trees of Appendix C. 3 were selected from standard PRA references and refined to reflect the operating experience at EBR-II. 
The annual frequency of failure of MG Set 1 through any of the three failure modes is 0.94 ; for MG set 2 , the value is 0.93 . The difference reflects the commonality of feeder circuits to the I/O and Module power supplies. Analysis of the fault trees for each of the three failure modes results in the data shown in Table 6.11.

If the two individual MG set fault trees are combined, the annual frequency of one of the two pumps failing according to the MODULE analysis is 1.842 with an error factor of 2.8 while the annual frequency of both MG sets failing at the same time is 0.04 with an error factor of 5 .

\subsubsection{Initiating Events and Special Insights}

Loss of output from one or both of the MG sets is an accident initiator. In the event of loss of normal power, specifically $2400 \mathrm{~V}$ supply to the motors of the MG sets, both pumps will coast down with a time determined by the inertia of the entire MG set. This is the same as if the motor malfunctions. A worse case initiating event is the failure of one or both clutches. If coupling is lost only the inertia of the generator will determine the coastdown period. Clutch coupling has been lost in the past, but only for one pump at a time. The worst initiating event is loss of electrical output from one or both of the generators. In this case only the inertia of the pump motor and impeller is available to slow the pump coastdown. 
Table 6.11. Annual Frequencies for MG Sets Failure Modes

$\begin{array}{lccc}\text { Event } & \text { Gate } & \text { Point Mean Value } & \text { Error Factor } \\ \begin{array}{c}\text { Loss of MG Set 1 } \\ \text { Motor Output }\end{array} & \text { P0010 } & 0.238 & 3 \\ \begin{array}{c}\text { Loss of MG Set 1 } \\ \text { Clutch Coupling }\end{array} & \text { P0020 } & 0.560 & 6 \\ \begin{array}{c}\text { Loss of MG Set 1 } \\ \text { Generator Output }\end{array} & \mathrm{P} 0040 & 0.194 & 3 \\ \begin{array}{c}\text { Loss of MG Set 2 } \\ \text { Motor Output }\end{array} & \mathrm{P} 0110 & 0.238 & 3 \\ \begin{array}{c}\text { Loss of MG Set 2 } \\ \text { Clutch Coupling }\end{array} & \mathrm{P} 0120 & 0.555 & 6 \\ \begin{array}{c}\text { Loss of MG Set 2 } \\ \text { Generator Output }\end{array} & \mathrm{P} 0140 & 0.194 & 3\end{array}$




\subsubsection{Shutdown Coolers}

\subsubsection{System Function and Description}

The shutdown cooling system removes heat from the primary sodium tank through two natural convection NaK loops to the atmosphere. The primary purpose of the system is the removal of decay heat from the primary sodium when the secondary sodium system is inoperable. The system is one of the EBR-II "sensitive" systems, i.e., it is critical to the integrity of the primary tank and internals and therefore is an engineered safety feature (ESF). In addition to its function as an ESF, the system is also used to accelerate the reactor cooldown rate in conjunction with the main sodium and BOP systems.

The shutdown cooling system consists of two identical sub-systems. Each of the cooling sub-systems consists of a shutdown cooler plug, a shutdown cooler nozzle, a NaK-air heat exchanger, and associated piping and equipment (Fig. 6.5). Flow occurs in a closed loop between the heat exchangers and individually mounted L1 and L2 nozzles extending into the primary tank. The heat exchanger cores are mounted in two box assemblies on the outside wall of the reactor building. The natural convection flow of $\mathrm{NaK}$ in the closed loop systems is completely independent of any electrical power source and functions whenever the bulk sodium in the primary tank is hotter than the outside air. A small expansion tank blanketed with argon gas and part of the box assembly permits thermal expansion of the system. The transfer of decay heat from the $\mathrm{NaK}$ coolant to the atmosphere is accomplished by the natural convection of air past a finned-tube heat exchanger. The rate of air flow through the fins is controlled by dampers positioned above and below the heat exchanger and by a sheet metal chimney located above the upper damper. A detailed description of the system is found in Appendix C.4.

\subsubsection{Support System Interfaces}

The shutdown cooling system under normal conditions requires only two support systems, instrument air system (Appendix C.10), and the continuous power system (Appendix C.9). It should be noted that the shutdown cooling system does not rely on any 


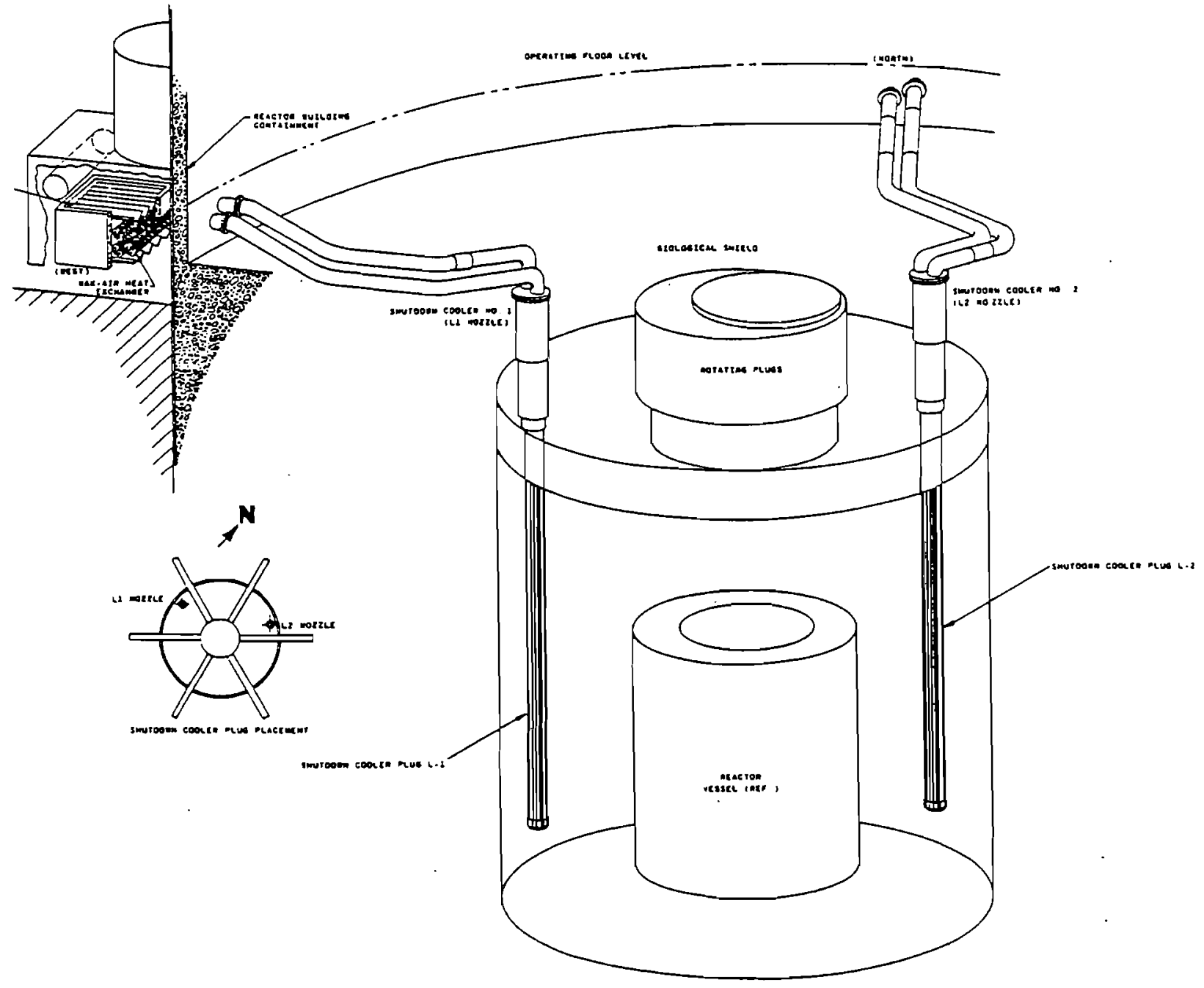

Fig. 6.5. Shutdown Cooling System 
sources of power for its operation during an emergency cooling system situation. However, during normal operation when the shutdown coolers are in standby, it is required that both instrument air and continuous power supplies be available to the system to prevent excessive heat loss from the bulk sodium.

\subsubsection{Fault Tree Development}

The top event (Appendix C.4) for the system is entitled "Any Shutdown Cooling System Malfunction" has two major branches: (1) undercooling by either shutdown cooler, or (2) overcooling (excess heat removal). Each cooler removes $165 \mathrm{~kW}$ of power and system failure is defined by either the inability to remove $330 \mathrm{~kW}$ or an excessive cooling rate for the current reactor operation.

\subsubsection{Component Failure Data, Common Cause Failures, Human Reliability}

With the exception of the damper actuator mechanism, no failures of the shutdown cooler system have occurred in the lifetime of the reactor. Generic data and some EBR-II experimental data (e.g., the actuators) are used for calculations. Common cause data is considered only for the damper actuators. Because of the passive nature of system operation, proper operation has a low vulnerability to human error. In fact, human error is possible only if the instrument air supply valve is manually closed or if the temperature and flow off-normal alarms are ignored.

\subsubsection{Quantification - Results}

The failure rates and method of calculating unavailabilities can be found in Appendix C.4. Five cases were quantified, as shown in Table 6.12. 
Table 6.12. Shutdown Cooler System - Failure Probabilities in a One-Year Period

\begin{tabular}{lccccc} 
Case: & \multicolumn{2}{c}{ System } & \multicolumn{2}{c}{ Undercooling } & \multicolumn{2}{c}{ Overcooling } \\
Point mean value: & $3.1010^{-1}$ & $6.9510^{-2}$ & $1.4510^{-1}$ & $1.6010^{-1}$ & $1.6510^{-1}$ \\
Error factor: & 2. & 5. & 2. & 3. & 3.
\end{tabular}


For the overall failure study the assumption that both overcooling and undercooling could occur is used. The most restrictive of the subtrees is used. One cooler is needed for success in the undercooling case and one cooler is considered to cause excess heat removal.

The calculations are based on a 2400 hour (100 day) reactor run length and 3 runs per year.

\subsubsection{Initiating Events and Special Insights}

Neither failure of the shutdown coolers nor occurrence of any of the basic events in the fault tree is considered to be an initiating event, provided corrective action occurs within $\sim 8$ hours. As shown by the analysis in Appendix C.4, the technical specification limits on primary tank temperature would not be exceeded within $\sim 8$ hours, if no other heat transfer mechanism is available. Beyond the 8 hour limit, an anticipatory reactor shutdown is required, which is an initiating event (see Section 5).

\subsubsection{Shield Cooling System}

\subsubsection{System Function and Description}

The system cools the reactor shielding and recirculates air through the air-baffle tank surrounding the primary tank inside of the blast shield and draws in air around openings near the nozzles in the rotating plugs and boot seals of the nozzles of the primary tank cover. The shield cooling system also maintains the reactor building pressure at slightly less than atmospheric by exhausting air from the reactor building through the plant suspect exhaust system.

The shield cooling system removes heat lost from the primary system bulk sodium before it reaches the shielding which surrounds the primary tank. This cooling is necessary to prevent the concrete shielding from losing its water content resulting in a loss of structural strength and effective shielding. Another purpose of the shield cooling system is to provide circulation of air from the reactor building main floor through the depressed area and the space above the 
primary tank. Shield cooling also forms an integral part of the reactor building ventilation system.

Secondary functions of the shield cooling system are to provide a cross connected, partial backup for the thimble cooling system in the event of a failure in the thimble cooling system, to provide a means of air removal from the exhaust hood over the shield plug at the storage pit workstation.

The shield cooling system consists of two recirculation fans, two exhaust fans, three refrigeration systems, manual and automatic dampers, and inter-connecting duct work. An overview of the system is shown in Figure 6.6. The exhaust fans draw approximately $5000 \mathrm{cfm}$ of air into the system from the reactor building atmosphere. This air enters twelve manual dampers located on the rotating plugs. Six of the dampers control flow through the small plug and the other six control flow through the large plug. The air being drawn through these plugs provides cooling around the rotating plug nozzles and boot seals in the primary tank cover. The combined flow from both plugs mixes in the annulus outside the large plug and cools the large plug freeze seal trough. Ducts adjacent to the primary auxiliary pumps busbars direct cooling air along the busbars and into the pump nozzle cooling annuli. There are also six inlet dampers above the biological shields to direct air to cool the area under the operating floor deck plates. Each of these inlets has an inlet filter and a four inch manual damper. A single large inlet filter and 10 inch manual damper is located in the depressed area near the ACS vapor trap which helps cool the depressed area by removing $750 \mathrm{cfm}$ of air. Another $300 \mathrm{cfm}$ of air is normally drawn from the exhaust hood over the shield plug at the storage pit workstation. The manual damper located just downstream of the hood controls the amount of exhaust flow to the system between zero to $530 \mathrm{cfm}$. In addition to the $5000 \mathrm{cfm}$ of air being drawn into the system there is an additional 15,000 cfm being recirculated through the air baffle tank which surrounds the primary tank. These two flows mix in a common header; the flow then divides and approximately 5000 $\mathrm{cfm}$ of air from the return duct passes through the absolute filters which are arranged in two banks. The filter bank consists of eight two inch thick cleanable filters mounted in interlocking frames in a straight bank arrangement. The resistance to air flow of a clean filter does not exceed 0.175 inches w.g. at an air velocity of 628 feet per minute. Only one bank of filters is 


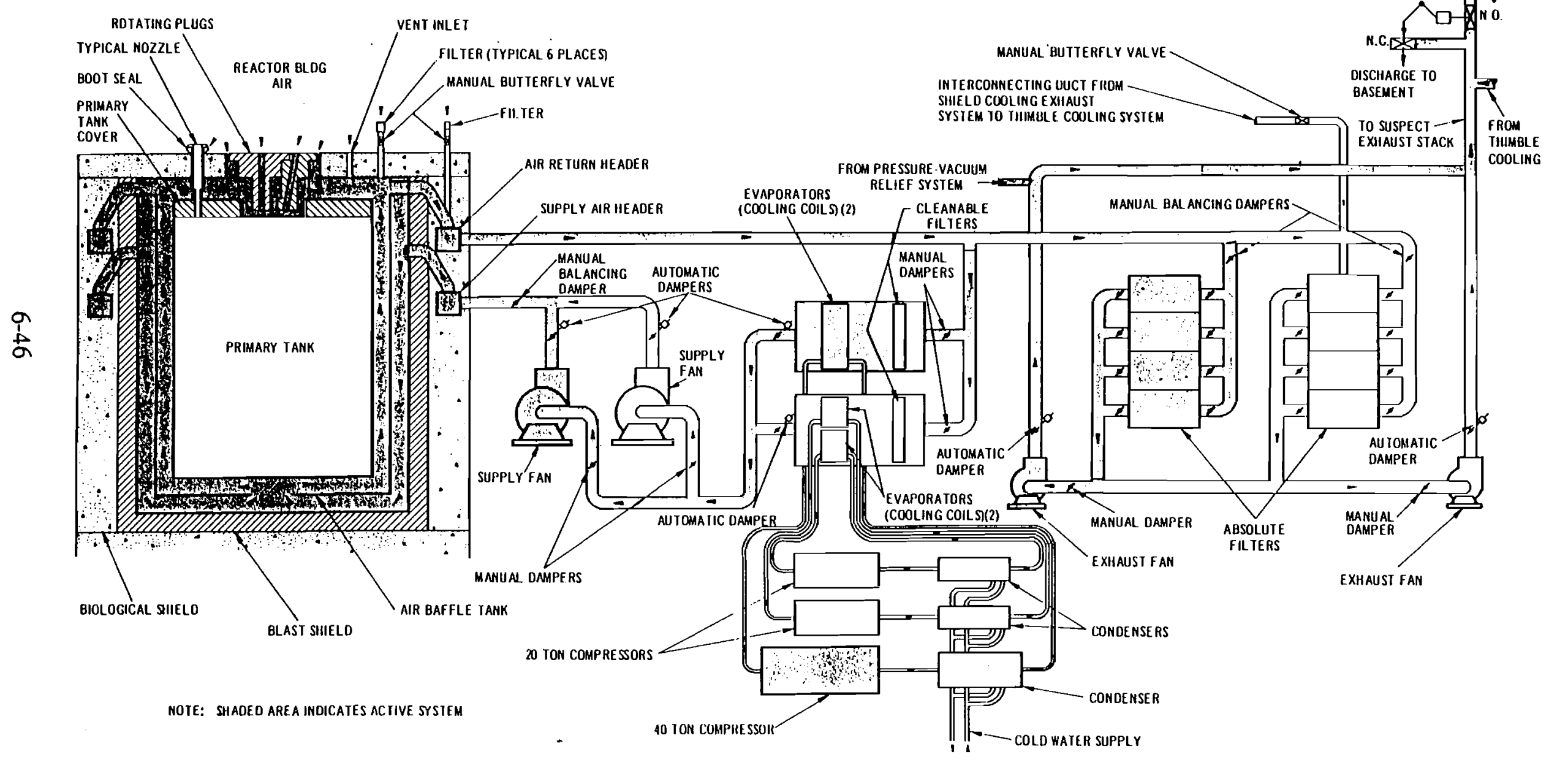

Fig. 6.6. Shield Cooling System Pictorial Flow Diagram 
in service and the second bank remains in standby to be used in the event of an excessive pressure differential across the operating bank filters caused by build up of dirt and other foreign particles. The operation of the filters is such that usually the operating filters need only be changed one time per year.

Cooling of the circulating air in the shield cooling system is provided by freon refrigeration systems. The normal refrigeration system consists of a 40-ton compressor, a water cooler condenser, alternate air cooling condensing coils, and two normal cooling units with two cooling coils in each unit. The standby refrigeration system consists of two 20-ton units similar in design to the 40 ton unit. The two smaller units serve a dual function and are normally used for a space cooling of the reactor building basement and sub-basement. Each one has two sets of cooling coils, normal and standby. The normal sets of cooling coils for the 20-ton unit are located in the basement and sub-basement space cooling units. The standby sets of cooling coils are located in a branch duct of the shield cooling recirculation line. The water-cooled condensers are located outside of the reactor building in order to minimize the possibility of sodium water reactions. In addition all of the freon lines penetrating the reactor building containment may be isolated by the containment isolation system.

Heat is removed from the refrigeration system by one of two methods: (1) by condensers in the reactor auxiliary cooling water system; or (2) by air cooled condensing coils located in the reactor building inlet air supply. During the cooler months of the year the refrigerant can be lined up to be condensed by air cooled condensing coils. This recycles the heat removal by the shield cooling system back to the supply air to the reactor building and power plant building, saving energy needed by plant systems to heat the supply air.

When the ambient humidity in the reactor building is high, moisture collects on the normal shield cooling coils, the standby shield cooling coils, the basement space cooling unit coils, and the sub-basement space cooling unit coils. This condensate is drained from below the coolant coils to a collection tank in the sub-basement. In order to minimize the volume of water present within the reactor building this collection tank is limited in volume to between one and two liters. A condensate pump activated by a float switch discharges the condensate from the 
collection tank to the suspect exhaust tank via the thimble cooling system exhaust duct. The piping from pump to the thimble cooling duct is maintained at $220^{\circ} \mathrm{F}$ by trace heaters to vaporize water prior to entering the exhaust duct. These heaters aid in the evaporation of condensate in the thimble cooling air flow.

\subsubsection{Support System Interfaces}

The shield cooling system requires electrical systems (to $480 \mathrm{~V}$ ) and the instrument air system.

The electrical system powers the exhaust and recirculating fans, the normal and standby refrigeration compressors, the motor-operated dampers, the shield cooling condensate pump and line heater, auxiliary and corridor panels for $\mathrm{I} / \mathrm{C}$ and the annunciators, and the building refrigeration and full isolation relays.

The reactor building instrument air operates the automatic outlet dampers on the exhaust fans.

\subsubsection{Fault Tree Development}

The top level event in the shield cooling system is "Failed or Reduced Heat Removal by Shield Cooling". For purposes of constructing the fault tree (Appendix C.5), the system contains two major subsystems:

(1) cooling of the rotating plug and nozzle area

(2) cooling of the primary tank walls and bottom and the biological shield using recirculating heat removal.

The only part of the system which the two subsystems share is a common section of ducting which contains no valves or other active components. The failure of this common ducting is 
excluded in this analysis. This failure of either part of the shield cooling system would not adversely affect the operation of the other part of the shield cooling system. In fact, if fans were not operating in one section there may be some beneficial, residual flow in the other section but this has not been completely verified and no credit is taken in this analysis.

\subsubsection{Component Failure Data, Common Cause Failures, Human Reliability}

The failure rates (as listed in Appendix C.5) are selected from standard PRA literature (IREP, CRBRP-4, generic) and refined to reflect EBR-II experience.

\subsubsection{Quantification - Results}

The system has been quantified for the system as a whole, and also for plug and nozzle area cooling and for recirculation cooling as shown in Table 6.13. These results assume a 100 day reactor run cycle, a 72 hour mission time to allow reactor cooldown, and 3 cycles per year.

\subsubsection{Initiating Events and Special Insights}

No initiating events can be caused by the independent failure of the shield cooling system, provided the failure is recoverable within $\sim 30$ hours. As shown by the analysis in Appendix C.5, the thermal inertia of the reactor shielding is large enough so that $\sim 30$ hours are available to initiate corrective actions prior to exceeding technical specification limits. Beyond the 30 hour limit, an anticipatory reactor shutdown is required, which is an initiating event (see Section 5).

\subsection{Analysis of Support Systems}

The support systems include the Thimble Cooling/Stack Exhaust System, the Secondary Sodium System, the Balance-of-Plant (BOP) Systems, the Electrical System, the Instrument and Plant Air System, Water Supply Systems, and the Argon System. 
Table 6.13. Shield Cooling-Probability of Failure in a One-Year Period

Point Mean Value $\quad$ Error Factor

\section{Shield Cooling}

System

0.519

5.

Recirculation

Cooling

0.236

8.

Plug \& Nozzle

Cooling

0.285

6. 


\subsubsection{Thimble Cooling/Stack Exhaust}

\subsubsection{System Function and Description}

The instrument thimble cooling system provides cooling for the reactor neutron detectors and the FERD system Benelex neutron moderator. The thimble cooling system also aids in maintaining reactor building pressure, controlled by balanced operation of the reactor building ventilation supply system and the reactor building exhaust. The thimble cooling system exhaust and the shield cooling system exhaust provide the total reactor building exhaust flow of about $7000 \mathrm{cfm}$. Therefore the thimble cooling system is maintained in continuous operation. If the reactor building ventilation supply or the shield cooling exhaust or thimble cooling system shuts down, the reactor building must be partially isolated to control reactor building pressure.

The instrument thimble cooling system is designed: (1) to cool nuclear detectors that monitor reactor power level, (2) to provide about 2000-cfm reactor building exhaust ventilation flow, (3) to provide cooling for the Fuel Element Rupture Detector (FERD) System Benelex neutron moderator, and (4) to provide low vacuum flow for the primary pumps shield-plug dehydration system.

The thimble cooling system consists of the following major components: Eight instrument thimbles, two turbocompressors, thimble intake dust filters, in-line absolute filters, sound absorbers, and air flow regulating valves. The thimble intake dust filters and the upper ends of the instrument thimbles are located just under the operating floor level of the reactor building; the remainder of the major system components are located in the reactor building subbasement.

There are eight instrument thimbles designated $\mathrm{J} 1, \mathrm{~J} 2, \mathrm{~J} 3, \mathrm{~J} 4, \mathrm{O} 1, \mathrm{O} 2, \mathrm{O} 3$, and $\mathrm{O} 4$. The thimbles extend from the reactor building operating floor downwards to the reactor core radial centerline and are submerged in $700^{\circ} \mathrm{F}\left(371^{\circ} \mathrm{C}\right)$ primary bulk sodium. Refer to Fig. 6.7, "Instrument Thimble Cooling System Flow and Equipment Diagram," and detailed figures in Appendix C.6. 


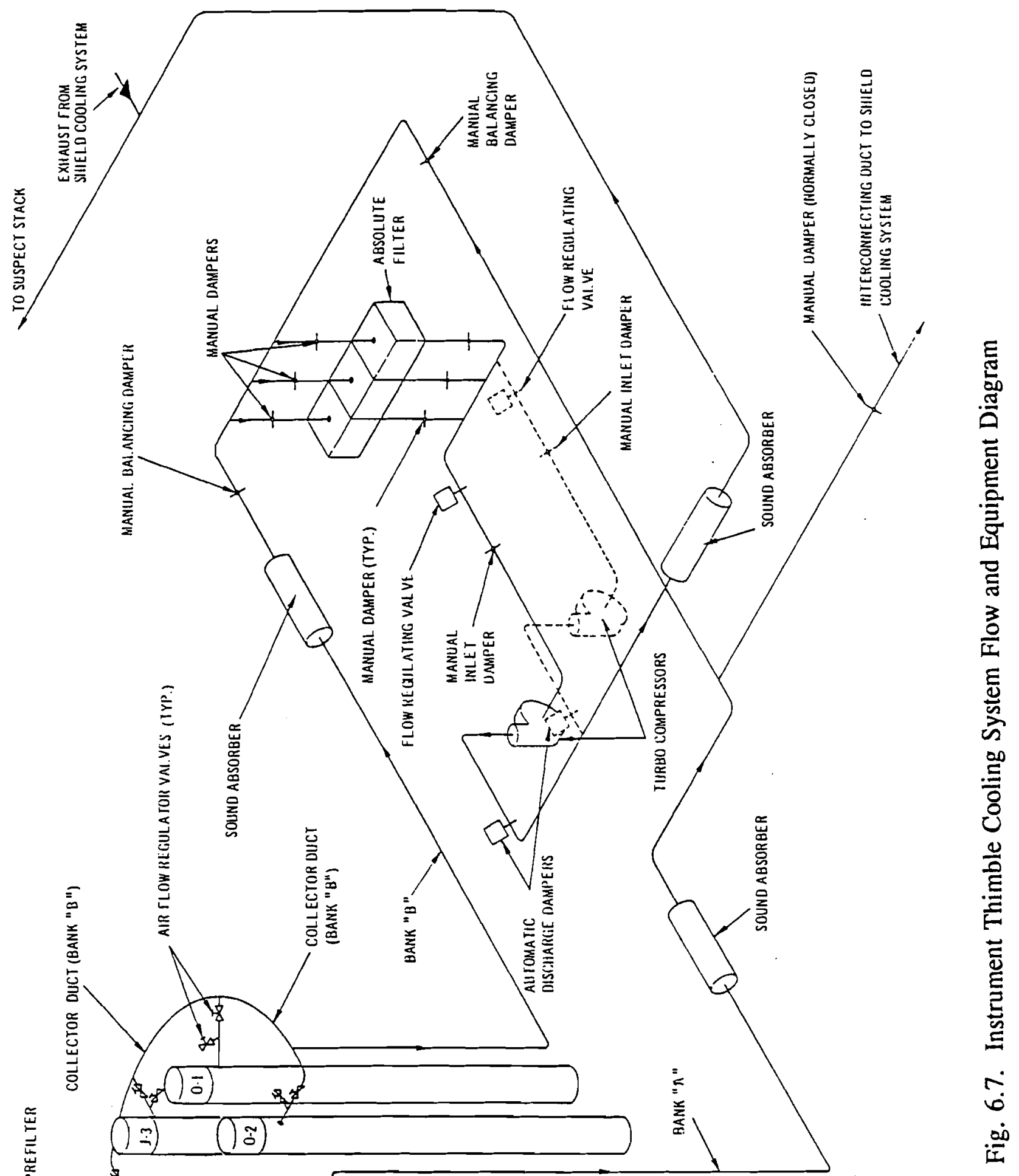


Thimble cooling system flow is provided by either of two identical turbocompressors. One compressor operates continuously with the other in automatic standby. Air enters the 0-1, O-2, O-3, and O-4 thimbles through filters located under the reactor building deck plates. The air inlet for thimbles $\mathrm{J}-1, \mathrm{~J}-2, \mathrm{~J}-3$, and $\mathrm{J}-4$ is located at grates in the deck plates, where the air passes through double filters. For all the thimbles the air flow is channeled downwards through the center annulus of the thimble to provide cooling for the instruments such as the nuclear detectors. The air then flows up the outer annulus and out through branch $\mathrm{A}$ and $\mathrm{B}$ piping which routes the air exhaust through individual sound absorbers before entering a common bank of absolute filters, located in the subbasement. The filter bank is monitored by a beta/gamma radiation monitor which indicates and alarms locally and in the main control room. Air flow continues into the operating compressor, discharges through another sound absorber and continues into the reactor building ventilation exhaust system. Thimble cooling system flow is controlled by motor operated manually-positioned valves located at the inlet of each compressor.

The following is a tabulation showing which nuclear detectors are located in each thimble.

$\begin{array}{ll}\text { J-1 } & \text { Channel "A" WR Fission Chamber/Channel } 7 \text { (CIC) } \\ \text { J-2 } & \text { Empty } \\ \text { J-3 } & \text { Channel "B" WR Fission Chamber/Channel 7A (CIC) } \\ \text { J-4 } & \text { Channel "C" WR Fission Chamber/DAS (CIC) } \\ \text { O-1 } & \text { Empty } \\ \text { O-2 } & \text { Empty } \\ \text { O-3 } & \text { Empty } \\ \text { O-4 } & \text { Empty }\end{array}$

If the thimble cooling compressors fail, the system can be cross-connected to the shield cooling exhaust system. The shield cooling exhaust system can draw about 500-cfm total flow through the thimbles. This prevents the nuclear detector cable temperatures (most sensitive component) from exceeding temperature limits for at least 8 hours. 
Since the thimble cooling system provides cooling for the reactor neutron detectors and the FERD system Benelex neutron moderator, the thimble cooling system is maintained in continuous operation. Reactor building pressure is controlled by balanced operation of the reactor building ventilation supply system and the reactor building ventilation air exhaust flow. Exhaust flow is provided by the shield cooling exhaust and thimble cooling systems; this exhaust flow is channeled to the suspect stack through the motor operated suspect stack dampers.

\subsubsection{Support System Interfaces}

The instrument thimble cooling system depends on the electrical systems $480 \mathrm{~V}$ power for operation of the turbocompressors and the (inlet) flow regulating valves; the system also requires CPS power for the panel instrumentation in the control room. The system also requires the instrument air system for operation of the automatic discharge dampers.

\subsubsection{Fault Tree Development}

The top level event for thimble cooling system failure is "Thimble Cooling Less Than Adequate". The three major branches to the top level event correspond to inadequate cooling in any one of the three thimbles currently in use, i.e., J1, J3, J4. The cooling for the FERD system Benelex neutron moderator is not included in the tree since the FERD is not part of the reactor safety circuits.

Each thimble has an isolation valve between the thimble and its exhaust line. There is also a bypass tap which allows some form of forced thimble cooling. The bypass is modeled as mode of failure to "short circuit" air flow around the thimble but recovery using the bypass is not modeled. Each thimble has a filter at its inlet. These filters are not the HEPA type filters used in the exhaust section but rather commercial air filters used to prevent dirt and debris from entering the thimbles. Clogging of individual filters as well as common cause clogging is considered. There are two branches ("legs") for exhaust flow. 
For this system, "recovery" and "no recovery" are considered since the shielding cooling system can be used as backup and therefore shield cooling failure modes are considered in the fault tree construction.

\subsubsection{Component Failure Data, Common Cause Failures, Human Reliability}

The component failure data is based on standard PRA literature (CRBRP-4, IREP, and generic), combined with EBR-II operating experience. A common cause failure is considered for failure of the commercial inlet filters to the thimbles.

\subsubsection{Quantification}

The thimble cooling system has been quantified for the individual thimbles and for the system as a whole. Due to the symmetry of the system there is no difference in numerical failure values for any of the individual thimbles when recovery by crossconnecting to the shield cooling system is not considered. If the cross-connect is considered, the J-3 thimble as a flow path through two additional valves which will make its failure rate slightly higher than that of the other two thimbles. The results summarized in Table 6.14 are for the no recovery case only. The recovery case is analyzed when the shield cooling and stack exhaust trees are linked with the thimble cooling system (see Section C.5, "Shield Cooling System").

The results assume a 100 day reactor run cycle, an 8 hour mission time to allow reactor cooldown, and 3 cycles per year.

\subsubsection{Initiating Events and Special Insights}

The potential for the thimble cooling system to be an initiating event is considered in that the loss of thimble cooling for an extended period of time would result in 
Table 6.14. Thimble Cooling-System Failure Probabilities in a One-Year Period

\section{Point Mean Value $\quad$ Error Factor}

Thimble cooling system failure

$3.0710^{-1}$

6.

(any of three thimbles)

Failure of J-1 thimble

$2.7110^{-1}$

6.

Failure of J-3 thimble

$2.7110^{-1}$

6.

Failure of J-4 thimble

$2.7110^{-1}$

6. 
the neutron detector cable and connector temperatures increasing beyond the maximum allowable limit of $230^{\circ} \mathrm{F}$. If this condition is undetected there is a possibility of erroneous signals from the neutron detectors. Since EBR-II uses a variety of scram functions, this could only lead to a problem if the reactor was being controlled, either manually or automatically, based on the high flux channel readings. To prevent this, the thimble cooling system is designed to alarm on several abnormal conditions including high temperature. Emergency procedure EP 3-7 of the operating procedures outlines the operator action required in the event of abnormal thimble cooling. Thus a failure of the thimble cooling system has to be combined with a failure of the thimble cooling alarms system or operator error to fail to respond correctly to the alarm condition, and a condition of controlling on the flux level instruments in order to lead to an initiating event.

\subsubsection{Secondary Sodium System}

The failure of the secondary sodium system is one of the loss-of-heat-sink (LOHS) accident sequences (see Section 5). Direct plant data was used to estimate failure frequencies for initiation event quantification.

\subsubsection{Balance-of-Plant (BOP)}

A BOP failure is one of the LOHS accidents (Section 5).

\subsubsection{Electrical System}

\subsubsection{System Function and Description}

The electrical distribution system provides power to all components and equipment of the EBR-II facility. The EBR-II substation steps down the $138 \mathrm{kV}$ power from the Idaho National Engineering Laboratory (INEL) loop to $13.8 \mathrm{kV}$ and matches the $13.8 \mathrm{kV}$ turbine generator output voltage to the $13.8 \mathrm{kV}$ from the main power transformers. Circuit breakers, motor starters and disconnect switches located in switchgear cubicles, motor control 
centers and distribution panels provide individual feeder controls. Numerous relays guard against over-voltage, under-voltage and over-current to provide protection for most circuits. Figure 6.8 shows a summary single-line diagram of the EBR-II electrical distribution system.

EBR-II is a power producing reactor, and the turbine generator has a power output capability of $20 \mathrm{MW}$ at $13.8 \mathrm{kV}$. The nominal generator output is $20 \mathrm{MW}$, but is also given on certain diagrams and some manuals as $25600 \mathrm{kVA}$. The electrical distribution system is designed to utilize the turbine-generator-produced power to satisfy power requirements of the EBR-II facility when the reactor is operating. When the turbine generator is producing more power than needed by the ANL-W facility, the excess $13.8 \mathrm{kV}$ power is stepped up by the substation power transformers and fed to the INEL power loop.

The electrical distribution system is divided essentially into the following systems.

$138 \mathrm{kV}$ Distribution/Substation Equipment

$13.8 \mathrm{kV}$ Distribution System

2400 V Distribution System

480 V Main Distribution System

480 V Emergency System

Continuous Power Supply System

125 V DC Distribution System

The detailed system analysis (Appendix C.9) considers each of the subsystems separately and calculates a subsystem failure rate for seven top fault tree levels. The general system description that follows indicates the major functions of each subsystem.

Under normal operating conditions, power utilized in the EBR-II facility is distributed from the $13.8 \mathrm{kV}$ switchgear. The $2400 \mathrm{~V}$ distribution system supplies $2400 \mathrm{~V}$ power for water pumps, induction heaters, sodium pump motor generator sets, and other facilities at the ANL-W site. 


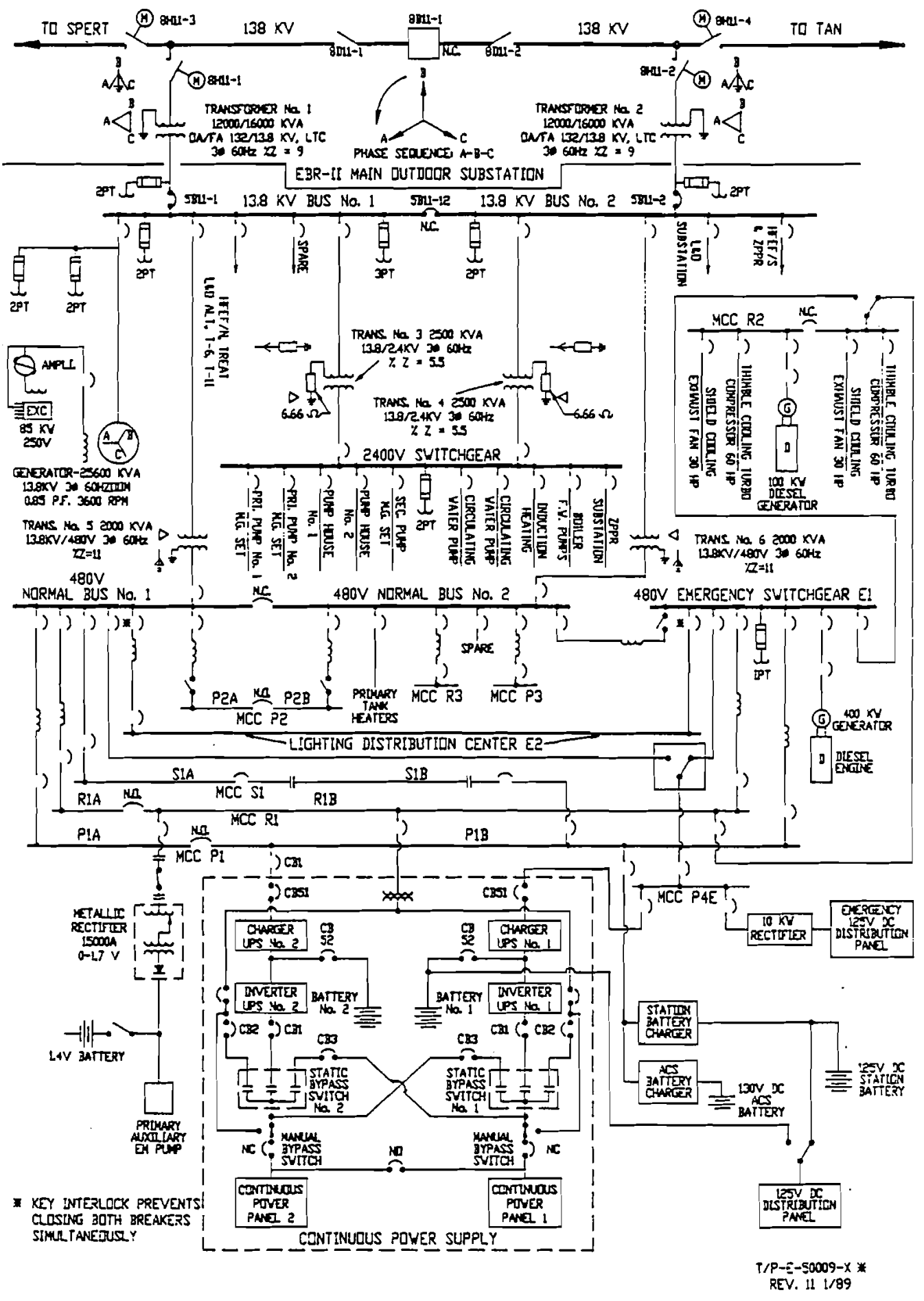

Fig. 6.8. Single Line Electrical Diagram EBR-II Electrical Power Distribution 
The $480 \mathrm{~V}$ distribution system is divided into a normal power distribution system and an emergency power distribution system. The system supplies power to all EBR-II equipment and components requiring 480,208 , or $120 \mathrm{~V}$ for operation. The $480 \mathrm{~V}$ emergency system furnishes power at $480 \mathrm{~V}$ from emergency diesel generators to components that must continue to operate if the normal source of power fails and a reactor shutdown is to be avoided.

The continuous power supply system supplies constant regulated $120 \mathrm{~V}$ AC power to the nuclear and process instrumentation and control equipment even after normal power and the emergency diesel generators fail. The continuous power system utilizes two uninterruptible power supplies (UPS) to power two major distribution panels, Continuous Power Panels 1 and 2.

The 125 V DC Distribution System provides control power for electrically operated air circuit breakers, isolation valves, emergency lighting, and essential turbine generator auxiliaries and controls.

The $2400 \mathrm{~V}$ system is directly connected with the control power chain of the reactor. An undervoltage condition will cause the $2400 \mathrm{~V}$ undervoltage relay to operate contacts in string A of the reactor shutdown system, causing string A to de-energize, scramming the reactor in anticipation of a loss of primary cooling flow. The reactor primary pumps enter into a coastdown mode.

The loss of all $480 \mathrm{~V}$ AC systems, including the emergency power from the diesel generators, does not cause a reactor scram. If $480 \mathrm{~V} \mathrm{AC}$ feeder power is lost to the charging systems of the auxiliary pump battery, station battery or continuous power supply battery, an anticipatory reactor shutdown is required before required functions are jeopardized by excessive battery discharge.

Personnel safety is ensured without availability of the emergency diesels. The emergency diesels are required only for protection of equipment, particularly the radiological and blast shields. If the radiological and blast shields are overheated, reactor operation would have to be 
delayed until necessary analysis and/or modifications could be completed. Normal and backup means of supplying power are considered satisfactory to avoid such damage.

The core and primary tank are adequately cooled without the availability of the diesels via convective cooling of the reactor core and the shutdown coolers providing cooling to remove decay heat, maintaining bulk sodium temperatures below limits associated with the primary tank.

The loss of either Continuous Power Panel will cause a reactor scram and also loss of clutch control power to the primary pump connected to the Continuous Power Panel that is lost. The scram occurs in anticipation of a reduction of primary cooling flow and is caused by a control power interruption because of various fail-safe scram instrumentation systems on each Continuous Power Panel and, in the case where Continuous Power Panel No. 1 fails, also with the loss of the control rod latch power bus. To ensure availability of continuous power in the event of an outage of normal power during reactor operation, one of the two continuous power supply batteries and its associated UPS system must be operable and normal feeder power must be available to at least the operable UPS unit. If normal feeder power to both units is lost or if neither UPS unit is operable, an anticipatory shutdown of the reactor must be performed. Normally, loss of a UPS does not cause a reactor scram, since the two Continuous Power Panels are then fed from the operable unit or normal power.

The simultaneous loss of both Continuous Power Panels is an unlikely but significant initiating event, since it results in a total loss of primary flow and requires a reactor scram to limit fuel damage.

Loss of any $125 \mathrm{~V} \mathrm{DC}$ bus does not cause a reactor scram. During the total loss of $125 \mathrm{~V}$ DC power, several critical loads will lose power. These include the control power for the $13.8 \mathrm{kV}, 2400 \mathrm{~V}$, and $480 \mathrm{~V}$ switchgears, support systems for the turbine generator, and selected motor control center functions. If the station battery $125 \mathrm{~V} \mathrm{DC}$ system fails or normal feeder power to the station battery charger is lost, an anticipatory reactor shutdown is required. 
The major concerns with a complete loss of $125-\mathrm{V}$ DC are:

1. The turbine generator loses automatic transfer control, field rheostat control, remote control of the governor and the load limit, the DC-powered seal oil pump, electrical trip protection for the turbine stop valve, and trip protection for the generator output breaker and the field breaker. The turbine generator cannot be tripped from the control room and must be shut down manually. To prevent possible damage to the generator armature due to overvoltage, the generator field breaker must be opened immediately following opening of the generator output breaker.

2. There is no trip protection for any of the breakers in the $13.8-\mathrm{kV}$ and the 2400-V switchgears. Most 480-V breakers have thermal overload and instantaneous short circuit protection, but all other fault protection is lost, including the ability to automatically switch to emergency diesel power.

3. Power to the DC-powered seal-oil pump is lost. If the seal-oil pressure to the $13.8-\mathrm{kV}$ generator is lost, hydrogen will leak out and create an explosion hazard and fire hazard in the power plant building. Normal AC power or the $400-\mathrm{kW}$ diesel generator provide power to the AC seal-oil pump and the tuming-gear oil pump. The AC-powered pump will provide sufficient oil to the hydrogen seals of the generator to prevent loss of hydrogen if the $125 \mathrm{~V}$ DC-powered seal-oil pump is lost.

4. The reactor-building-isolation relay deenergizes causing reactor building isolation.

The electrical distribution systems are expected to function normally under postulated accident conditions, unless physical damage interrupts service. External causes such as fire or seismic events can cause loss of various electrical systems. 


\subsubsection{Support System Interfaces}

The main support system interface is with the BOP, essentially at the turbine generator. The generator output is connected to the $13.8 \mathrm{kV}$ switchgear through an air circuit breaker. The generator operates in parallel with the INEL system supplying the power to operate the EBR-II site and also feeding additional power to the INEL loop.

\subsubsection{Fault Tree Development}

The top events of the electrical subsystems are as follows:

(1) Failure of the $138 \mathrm{kV}$ Distribution/Substation Equipment

(2) Loss of $13.8 \mathrm{kV}$ Bus Power

(3) Loss of 2400 V Bus Power

(4) Loss of 480 VAC - Main Distribution System (10 buses)

(5) Loss of 480 VAC - Emergency System ( 8 buses)

(6) Loss of Continuous Power Panels (No. 1 and No. 2)

(7) Loss of $125 \mathrm{~V}$ DC Distribution System (8 buses) 


\subsubsection{Component Failure Data, Common Cause Failures, Human Reliability}

Failure rate data for the system fault trees were selected from standard PRA references (CRBRP-4, IREP, generic) and refined to reflect EBR-II operating history. Throughout the electrical system fault trees, a number of common mode failures are considered.

\subsubsection{Quantification}

As a first step, each of the seven major subsystems (and the buses) was analyzed for mean failure probability, as shown in Table 6.15.

\subsubsection{Initiating Events and Special Insights}

Loss of the electrical power subsystems can produce several potential initiating events:

(1) Loss of $13.8 \mathrm{kV}$ power produces a reactor scram.

(2) Loss of the $2400 \mathrm{~V}$ distribution system causes a reactor scram and immediately puts the primary pumps into a coastdown mode.

(3) Loss of all $480 \mathrm{~V}$. AC systems does not cause a reactor scram. However, the EBR-II Technical Specifications require some of the $480 \mathrm{~V}$ electrical equipment to be available for continued reactor operation. The required actions and anticipatory shutdowns are detailed in Appendix C.9.

(4) Loss of either Continuous Power Panel (120 V AC) will cause a reactor scram and loss of control power to the primary pumps. Details of UPS requirements and anticipatory reactor shutdown requirements are found in Appendix C.9. 
Table 6.15. Failure Probabilities for Electrical Systems in a One-year (300 day) Period

\section{System}

$138 \mathrm{kV}$ Distr./Substation

Equipment

$13.8 \mathrm{kV}$ Bus Power

2400 V Bus Power

480 V Main Distr. System

(10 buses)

$480 \mathrm{~V}$ Emergency System

(7 buses)

Continuous Power System

(Panels 1 and 2)

125 V DC Distr. System

(8 buses)
Failure Rate Per Year

$5.110^{-1}$

$2.210^{-3}$

$4.210^{-3}$

$4.210^{-3}-1.110^{-2}$

$6.010^{-3}-1.610^{-2}$

No. $1-5.410^{-2}$

No. 2 - $5.410^{-2}$

$5.110^{-2}-9.610^{-2}$

3-4
3-4

Error Factor

2

4

3

3

5

5 
(5) The loss of $125 \mathrm{~V} \mathrm{DC}$ power does not cause a reactor scram. However, the $125 \mathrm{~V}$ DC system buses provide power for several critical loads of EBR-II. The detailed requirements prior to anticipatory reactor shutdown are found in Appendix C.9.

\subsubsection{Instrument and Plant Air}

\subsubsection{System Function and Description}

The instrument air system is the primary air system for EBR-II components and systems and provides a source of cool, dry, filtered and oil-free service air to all control equipment via the instrument air distribution system and the plant air distribution system under normal operations.

The plant and instrument air systems are cross-connected. Figure 6.9 shows a diagram of the combined systems. Under normal conditions, the plant air compressor is shut down and isolated and one of the two instrument air compressors furnishes air to both systems. The two instrument air compressors are operated as one on-line compressor and one on standby (alternated weekly).

The plant compressor is used as a high quality backup system when both instrument air compressors are inoperative, although the plant compressor cannot provide enough air to maintain the entire system, i.e., some portion must be isolated.

In addition, there is a reactor building emergency air compressor (air-cooled) which automatically activates when the reactor building is isolated or when there is a loss of air pressure to the reactor building. This system also supplies air to the ACS during fuel handling operations. (See Appendix C.10 for detailed descriptions.) 


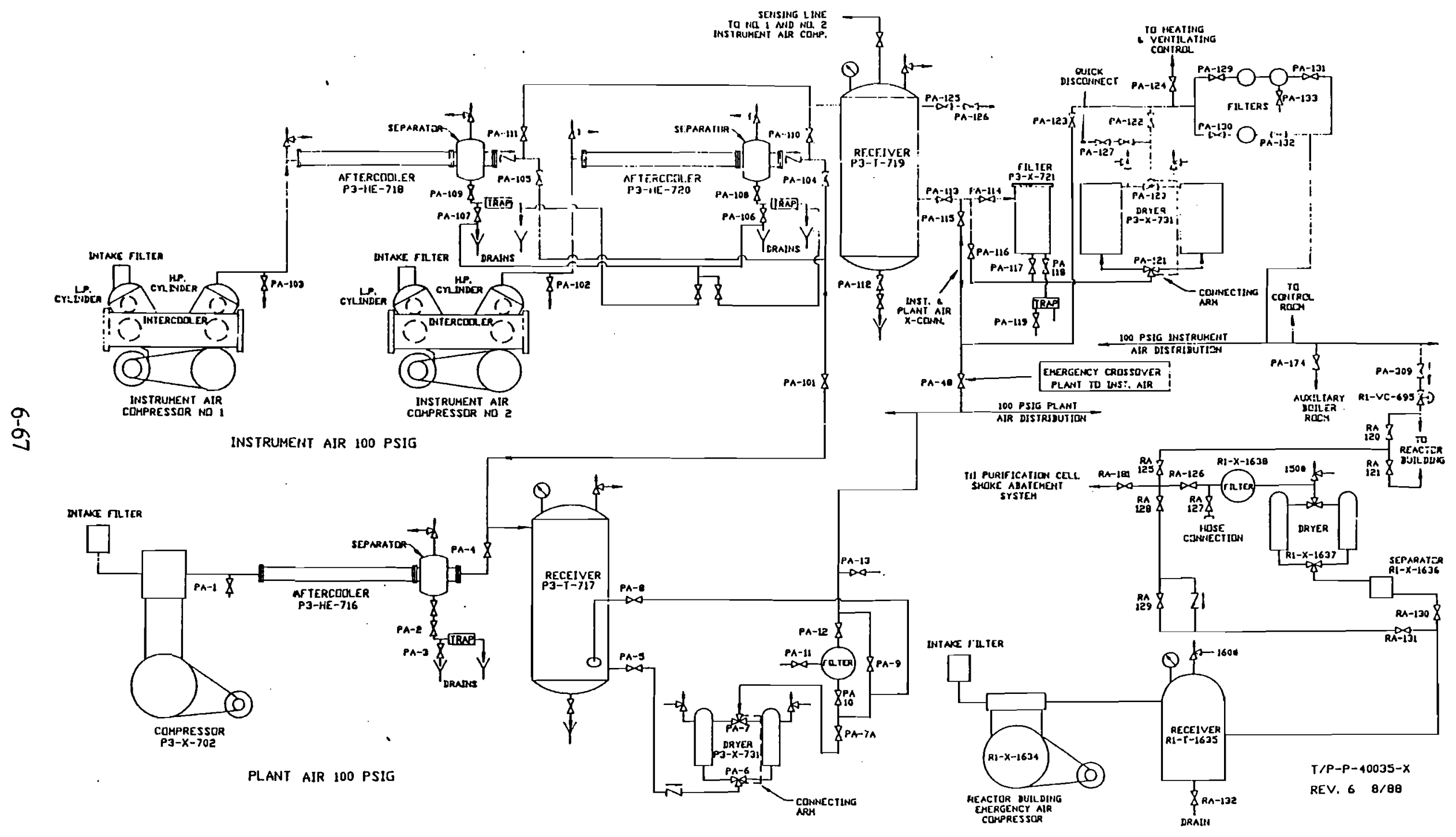

Fig. 6.9. Instrument and Plant Air Systems 


\subsection{Instrument Air Compressors}

The instrument air compressors are Joy Class WNOL-112B type compressors. They are two-cylinder, two-stage units constructed for continuous heavy duty service. The cylinders are double acting and operate from a common single-throw crankshaft. Each compressor is rated $398 \mathrm{cfm}$ at $5000 \mathrm{ft}$ elevation, $110 \mathrm{psig}$ discharge pressure, while operating at normal speed $(570 \mathrm{rpm})$.

The compressor cylinders are water cooled and require no lubrication. Teflon piston rings are used to ensure clean, oil-free air is furnished to the instrument air system.

\subsection{Plant Air Compressor}

The plant air compressor is a vertical Ingersoll Rand unit belt-driven by a $50 \mathrm{HP}$ motor. The compressor is rated $200 \mathrm{cfm}$ at $100 \mathrm{psig}$. Teflon piston rings are used to eliminate the requirement for cylinder oil lubrication. This serves to minimize possible air system oil contamination. Chassis oil lubrication is provided by a gear pump off the compressor crankshaft. The compressor and aftercooler are normally cooled by the raw water system.

\subsubsection{Support System Interfaces}

In addition to the cross-connection between the two air systems, a limited amount of air can be supplied to both systems from the FCF and the HFEF facilities via a normally closed valve in the FCF facility basement.

The Raw Water System is used to provide the normal cooling water to the plant and instrument compressors. The Plant and Reactor Auxiliary Cooling Water Systems can be lined up as backup cooling sources to both compressor systems. The electrical system supplies power to both systems and the reactor building emergency air compressor. 


\subsubsection{Fault Tree Development}

Three fault trees are developed in Appendix C.10. The top level events are:

$$
\text { Loss of Plant Air Distribution }
$$

\section{Loss of Instrument Air System Output}

For the plant air system, the analysis assumes that the system is operating in normal mode, with the plant air compressor shut down and the plant air distribution system supplied by the instrument air system. It is assumed that valves used for maintenance, such as drains, have been returned to their operating positions, and that manual valves do not fail from the operational positions.

For the instrument air system, the analysis assumes that the system is operating normally, with one instrument air compressor running with the controller in the MAN position, and the other in the AUTO position. Since the plant air compressor cannot provide a complete backup of the instrument air system, it is not included as a backup in the fault trees. It is assumed that valves used for maintenance have all been returned to their operating position, and that normal valves will not fail from their operational position. The distinction between the primary and standby compressor systems is artificial, since the roles are reversed on a regular basis. The analysis is identical for both combinations of primary/standby assignments except for the $480 \mathrm{~V}$ emergency power circuit. Since the two circuits have nearly the same failure probability, little difference in the final result will be seen between the two configurations.

The reactor building emergency air distribution system is considerably simpler than the normal instrument air system, but its failure modes are comparable. The fault tree assumes that 
the emergency compressor controller is in the STANDBY mode and is started by a low pressure signal.

\subsubsection{Component Failure Data, Common Cause Failures, Human Reliability}

For all three air systems, the failure rate data was selected from standard PRA references (CRBRP-4, IREP, generic) and refined to reflect EBR-II operating experience.

\subsubsection{Quantification}

\subsection{Instrument Air System}

Because of the dual-compressor nature of the system, the mission time for the primary (i.e., operating) system was set to one week. Since the roles of operating and standby compressors are routinely reversed each week, this is a more accurate assumption than requiring one compressor to run for the entire reactor run time. Similarly, a conservative estimate of one week was used for the standby system. On the average, it is expected that the standby system would be called on to function one-half of the mission time of the primary system, and using the full mission time produces a conservative failure probability.

The point mean probability for the loss of instrument air distribution in a 300-day period is 0.216 with an error factor of 8 . 


\subsection{Plant Air System}

Since the plant air system is not normally in operation, it is difficult to assign a mission time for failure calculations. The system is tested each week, and is used only as a backup to the instrument air system, which cycles compressors on a one-week basis. It is assumed that the plant air system would have to function for no longer than one week before the instrument air system is restored to operational status.

The point mean probability for the loss of plant air distribution in a 300-day period is 0.321 with an error factor of 5 .

\subsection{Emergency Air System}

The emergency air system is used only on a temporary basis in the event of reactor building isolation. The duration of its average mission time has been set to one day.

The point mean probability for loss of the emergency air system in a 300-day period is 0.026 with an error factor of 3 .

\subsubsection{Initiating Events and Special Insights}

For the three air systems, no initiating events can be caused by system failures.

It should be noted that the conditions following certain initiating events can be mitigated or degraded by the presence or absence of the instrument air system (see System Interconnections in Appendix C.10). Total failure of the instrument air system would necessitate manual cross-connections to the plant and could require an anticipatory reactor shutdown. 


\subsubsection{Cooling Water System}

\subsubsection{System Function and Description}

The cooling water system supplies water for heat transfer to various components located in the power plant, the sodium boiler building and the reactor building. After heat transfer occurs, the cooling water is returned to a cooling tower where the water is cooled through evaporation and conduction and again recirculated through the system.

The cooling water support system consists of the reactor auxiliaries cooling water system and the plant cooling water system. These systems are supported by the raw water system and two cooling towers.

The reactor auxiliaries cooling water system provides the required cooling for various components associated with the primary coolant systems. The system may be cross-connected to supply the plant cooling water system, but only if sufficient cooling is available to the reactor auxiliaries components. Interconnection of the two systems is made by opening the appropriate manually operated cross-connection valves.

The plant cooling water system furnishes cooling water to the power plant building, sodium boiler building, FCF, laboratory and office building and the fuel assembly and storage building. This system can be cross-connected as backup to the reactor auxiliaries cooling water system.

The raw water system provides make-up water for all systems and can provide a backup cooling water source for the reactor auxiliaries and plant cooling water systems via a backflow preventer assembly (emergency cross-connect). The backflow preventer blocks inadvertent contamination of the raw water system by the chemically treated plant or reactor auxiliary cooling water systems. System details are provided in Appendix C.11. 


\subsection{Reactor Auxiliaries Cooling Water System}

The reactor auxiliaries cooling water system consists of the auxiliary cooling tower, two cooling water pumps, a Cuno filter, piping and valves. Figure 6.10 shows a schematic of the system. This system provides heat removal for the following components:

Primary pumps MG eddy current clutches (2)

Primary purification silicone/water heat exchangers (2)

Reactor shield cooling freon condenser (40-ton unit)

Reactor building basement air freon condenser (20-ton unit)

Reactor building sub-basement air freon condenser (20-ton unit)

Reactor building ventilation freon condenser

Plant and instrument air compressors (backup supply)

In addition, the system may be cross-connected to supply the plant cooling water system, but only if sufficient cooling is available to the reactor auxiliaries components. The system also provides a backup, via a check valve, to the auxiliary boiler feedwater pump clutches.

Cooling water is pumped from the auxiliary cooling tower basin by either of two 275 gpm centrifugal reactor auxiliary cooling water pumps. The water is then discharged at approximately $55 \mathrm{psig}$ and $65^{\circ} \mathrm{F}$ through a Cuno filter in the power plant pump pit to a distribution pipe. Several parallel flow paths are provided at this point through the primary systems components and coolers. Cooling water is then collected in a return pipe which goes directly back to the auxiliary cooling tower.

\subsection{Plant Cooling Water System}

The major elements of the plant cooling water system are the two cooling water pumps, miscellaneous valves, the Cuno filter and the auxiliary 


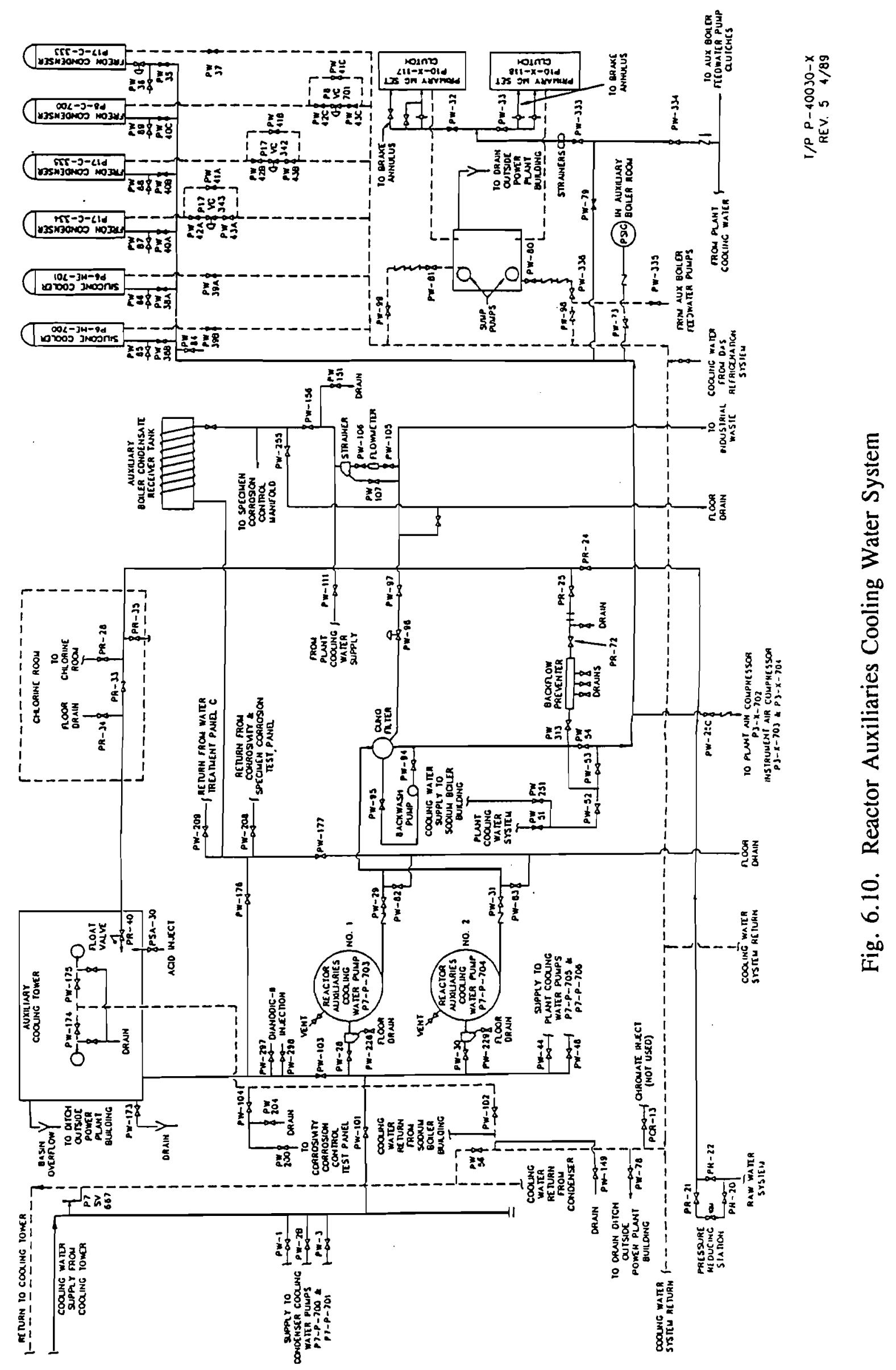


cooling tower. Figure 6.11 shows the plant cooling water system configuration. Cooling water is supplied from the auxiliary cooling tower basin by either of two $1080 \mathrm{gpm}$ centrifugal plant cooling water pumps. The water is then discharged through the Cuno filter at approximately $70 \mathrm{psig}$ and $65^{\circ} \mathrm{F}$ into a distribution header located in the power plant building. Several parallel flow paths are provided at this point to various components and coolers. Cooling water is then collected in a return pipe which goes directly back to the auxiliary cooling tower.

The plant cooling water system provides heat removal for the following EBR-II components:

(1) Sodium boiler building:

Secondary sodium main EM pump

Dowtherm/water heat exchanger

Sodium recirculating pumps (2)

Dowtherm pumps (2)

(2) Power plant building:

Sample station sample coolers (5)

Feedwater pump no. 1

Feedwater pump no. 2

Blowdown after-cooler

Turbine-generator lube oil Coolers (2)

Generator hydrogen coolers (4)

Instrument air compressor no. 1 - alternate supply

Instrument air compressor no. 2 - alternate supply

Plant air compressor - alternate supply

Freon condenser - control room air conditioning

Freon condenser - DAS room

Freon condenser - room 14 (Computer Development Lab.)

Feedwater heater level alarm panel cooler 


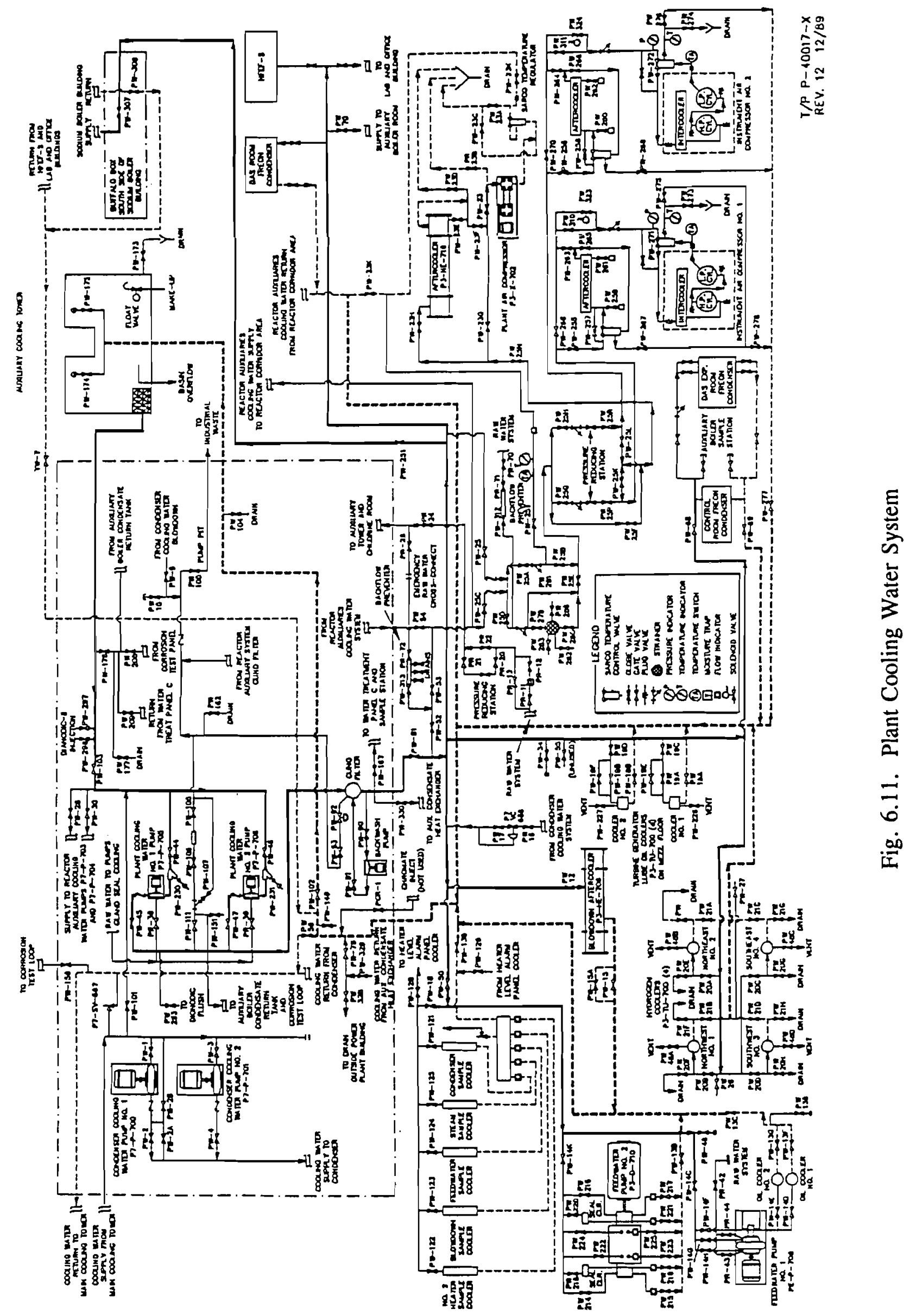




\subsubsection{Support System Interfaces}

Both of the above systems are supported by the raw water system, the instrument air system and the electrical system.

\subsubsection{Fault Tree Development}

\subsection{Reactor Auxiliaries Cooling Water System}

The top level event for this system is "Loss of Reactor Auxiliaries Cooling Water". The two main branches of the fault tree reflect the loss of the centrifugal pumps output or blockage of the Cuno filters.

It is assumed that the reactor auxiliaries cooling water system is operating normally using one operating auxiliary cooling water pump, and the other pump is in standby mode. Since cross connections to the plant water or raw water systems are manually activated and are not the normal operating procedure, these systems are not included as backups to the reactor auxiliaries system. Manual valves that are in their correct operating position are assumed to remain so, and no human errors of commission in changing valve positions are taken into account.

\subsection{Plant Cooling Water System}

The top level event for this system is "Loss of Plant Cooling Water". The two main branches of the fault tree reflect the loss of centrifugal pumps output or blockage of the Cuno filters.

It is assumed that the plant cooling water system is operating normally using one operating plant cooling water pump, and the other pump is in standby mode. Since cross connections to the reactor auxiliaries water or raw water systems are manually activated and are not the normal operating procedure, these systems are not included as backups to the plant cooling water system. Manual valves that are in their correct operating position are assumed 
to remain so, and no human errors of commission in changing valve positions are taken into account.

\subsubsection{Component Failure Data, Common Cause Failures, Human $\underline{\text { Reliability }}$}

The component failure data were selected from standard PRA references (CRBRP-4, IREP, generic) and refined to reflect EBR-II operating experience.

\subsubsection{Quantification - Results}

Because of the dual-pump nature of both systems, the mission time for each of the primary (i.e., operating) pumps was set to one week. Since the roles of operating and standby pumps are routinely reversed, this is a more accurate assumption than requiring one pump to run for the entire reactor run time. Similarly, a conservative estimate of one week was used for the standby pump system. On the average, it is expected that the standby system would be called on to function one-half of the mission time of the primary pump, and using the full mission time produces a conservative failure probability.

The point mean probability for the loss of the reactor auxiliaries cooling water system in a 300-day period is 0.203 with an error factor of 9.

The point mean probability for the loss of the plant cooling water system in a 300-day period is 0.202 with an error factor of 9 .

\subsubsection{Initiating Events and Special Insights}

No initiating events can be caused by the failure of either the reactor auxiliaries cooling water system or the plant cooling water system. In the event of a total failure of either system without rapid cross-connection to backup supplies, the event would require an anticipatory reactor shutdown. 


\section{Section 6.0 References}

6.1 U.S.N.R.C., "PRA Procedures Guide," NUREG/CR-2300, USNRC (1983).

6.2 Industry Degraded Core Rulemaking Program (IDCOR) Technical Report 86.3B1, "Individual Plant Evaluation Methodology for Boiling Water Reactors," Vol. I, Appendix B, April 1987.

6.3 Argonne National Laboratory unpublished information (1990).

6.4 S. A. Eide, S. V. Chmielewski, and T. D. Swantz, "Generic Component Failure Data Base for Light Water and Liquid Metal Reactor PRAs," Informal Report, EGG-SSRE8875, February 1990.

6.5 ORNL/TM-11091, "The Centralized Reliability Data Organization (CREDO) Handbook of Component Reliability," January 1989.

6.6 A. Mosleh et al., "Procedures for Treating Common Failures in Safety and Reliability Studies," NUREG/CR-4780, Vols. 1 and 2, January 1989.

6.7 A. D. Swain and H. E. Guttmann, "Handbook of Human Reliability Analysis with Emphasis on Nuclear Power Plant Applications," NUREG/CR-1278, August 1983. 
7.0 DATA BASE DEVELOPMENT 

Table of Contents

Page

7.0 Data Base Development $\ldots \ldots \ldots \ldots \ldots \ldots \ldots$. . . . . . . . . . . . .

7.1 Discussion . . . . . . . . . . . . . . . . 7-1

7.1 .1 Sources of Data . . . . . . . . . . . 7-1

7.1.2 Failure Probability Calculation Methodology . . . . . . . . 7-2

7.2 Data . . . . . . . . . . . . . . . . . . 7-5

7.2.1 Data Used in Quantification ... . . . . . . . 7-5

7.2 .2 Plant Specific Data . . . . . . . . . . . . . 7-5

7.2.3 Special Data Considerations . . . . . . . . . . 7-6

7.2 .4 Reactivity Insertion Data . . . . . . . . . . . . 7-78

7.2.4.1 Introduction . . . . . . . . . . . . . . 7-78

7.2.4.2 Administrative and Physical Reactivity Limits . . . . . . 7-78

7.2.4.2.1 Reactivity Insertion Magnitude . . . . . . . 7-78

7.2.4.2.2 Rate of Insertion . . . . . . . . . . . . . 7-83

7.2.4.2.3 Relative Initiator Frequencies . . . . . . . . 7-85

7.2.4.3 Analysis of DAS Data . . . . . . . . . . . 7-86

7.2.4.3.1 Processing the Data . . . . . . . . . . 7-86

7.2.4.3.2 Results . . . . . . . . . . . . . . . 7-88

7.2.4.3.3 Scram Reactivity . . . . . . . . . 7-96

7.2.4.4 Other Operating Modes . . . . . . . . . . . . 7-108

7.2.4.4.1 Startup and Shutdown . . . . . . . . . 7-108

7.2.4.4.2 Transient Experiments . . . . . . . . . . 7-110

7.2.4.5 Summary . . . . . . . . . . . . . . 7-113

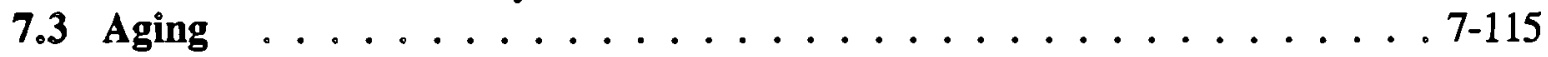

7.3.1 Aging Effects in Initiating Events .......... 7-115

7.3.1.1 Initiating Events Quantification ......... . 7-115

7.3.1.2 Structural Failures . . . . . . . . . . . . . 7-116

7.3.1.2.1 Materials Aging Assessment . . . . . . . . 7-116

7.3.1.2.2 Structural Assessment . . . . . . . . . . 7-120

7.3.1.2.3 Damage Criteria . . . . . . . . . . . 7-122

7.3.2 Aging Effects at the Component Level . . . . . . . . . . 7-127

7.3.2.1 Reactor Shutdown System (RSS) . . . . . . . . . 7-128

7.3.2.2 Primary Pumps . . . . . . . . . . . . . . . 7-128

7.4 Dependency Analysis . . . . . . . . . . . . . . . . . . 7-128

Section 7.0 References . . . . . . . . . . . . . . . . . . . . 7-148 


\section{List of Figures}

Page

7.1 Rod Worth Profiles . . . . . . . . . . . . . . . . . . 7-84

7.2 Probability Density Functions for Single-Rod Run-In Reactivity . . . . . . 7 7-91

7.3 Probability Density Function for Double-Rod Run-In Reactivity . . . . . . . 7-92

7.4 Exact Probability Density Functions for Scram Worth . . . . . . . . . . 7-99

7.5 Exact and Approximate Probability Density Functions for Scram Worth . . . 7-103

7.6 Schematic View of Superheater, Showing Regions for Which Thermal-Analysis Models Were Developed . . . . . . . . . . . . . 7-126

7.7 Comparison of Redundant Component Failure Probability According to Beta (n) and MGL Methods . . . . . . . . . . . . . 7-136 
List of Tables

Page

7.1 Failure Data Calculation Models . . . . . . . . . . . . . . 7-5

7.2 Reactor Shutdown System Data . . . . . . . . . . . . . 7-7

7.3 Reactor Control System Data . . . . . . . . . . . . . . 7-17

7.4 Primary Pump and Primary Pump M/G Set Data . . . . . . . . . . 7-19

7.5 Shutdown Cooler Data . . . . . . . . . . . . . . . . . . 7-23

7.6 Shield Cooling System Data . . . . . . . . . . . . . . 7-26

7.7 Thimble Cooling System Data . . . . . . . . . . . . . 7-35

7.8 Stack Exhaust System Data . . . . . . . . . . . . . . 7-38

7.9 Secondary Sodium System Data . . . . . . . . . . . . . . . 7-39

7.10 Balance of Plant Data - Steam System Data . . . . . . . . . . . . 7-40

7.11 Balance of Plant Data - General Data . . . . . . . . . . 7-41

7.12 Electrical Normal Power System Data . . . . . . . . . . . . . . . 7-42

7.13 Electrical Emergency Power System Data . . . . . . . . . . . . . . 7-47

7.14 Electrical Continuous Power System Data . . . . . . . . . . . . 7-53

7.15 Electrical 115V DC System Data . . . . . . . . . . . . 7-56

7.16 Air Systems Data . . . . . . . . . . . . . . . . 7-60

7.17 Reactor Water Systems Data . . . . . . . . . . . . . 7-65

7.18 Plant Water Systems Data . . . . . . . . . . . . . 7-67

7.19 Argon Systems Data . . . . . . . . . . . . . . . . 7-68

7.20 Reactor Building Isolation Data . . . . . . . . . . . . . 7-69

7.21 Safety Rod Data . . . . . . . . . . . . . . . . . . 7-70

7.22 Test and Maintenance Unavailabilities ... . . . . . . . . . . 7-71

7.23 Plant-Specific Component Failure Data for Reactor Shutdown System . . . 7-72

7.24 Special Data . . . . . . . . . . . . . . . . . . . 7-74

7.25 Rod Worth by Type from Run 139A to Run 153A (c) . . . . . . . . . . 7-80

7.26 Discrete Probability Density Functions for Rod Run-in Reactivity . . . . . . 7-90

7.27 Discrete Cumulative Distribution Functions for Rod Run-in Reactivity . . . . 7-93

7.28 Average Rod Position for Each Reactivity Bin and Rod Category . . . . . 7-94

7.29 Polynomial Coefficients for the Worth Profiles, Eq.2 . . . . . . . . 7-95

7.30 Probability Density Functions for Reactivity from Scram of High-worth Rods (Exact) . . . . . . . . . . . . . . . . 7-97

7.31 Cumulative Distribution Functions for Reactivity from Scram of High-worth Rods (Exact) . . . . . . . . . . . . . . 7-100

7.32 Probability Density Functions for Reactivity from Scram of High-worth Rods (Approximate) . . . . . . . . . . . . . . 7-104

7.33 Cumulative Distribution Functions for Reactivity from Scram of High-worth Rods (Approximate) . . . . . . . . . . . . . . 7-106

7.34 Summary of Rod Run-in Characteristics . . . . . . . . . . . . . . . 7-114

7.35 Structural Analysis of Major EBR-II Components . . . . . . . . . . 7-125

7.36 Parameters Used in Modeling CCR . . . . . . . . . . . . . . . 7-134

7.37 Comparison of Redundant Component Failure Methods . . . . . . . . 7-135 


\section{List of Tables (Cont'd)}

Page

7.38 Common Cause Failures Among RSS Electrical Components . . . . . . . . 7-138

7.39 Common Cause Failures Among RSS Mechanical Components . . . . . . 7-146 


\subsection{DATA BASE DEVELOPMENT}

\subsection{Discussion}

\subsubsection{Sources of Data}

The development of a data base for the EBR-II PRA presents some difficulties because, in common with other DOE Class A facilities, EBR-II has a large number of unique components. The data which can be derived from EBR-II records, even assuming impeccable record keeping, is limited to 25 reactor years of operation. This position can be improved somewhat because EBR-II FFTF and the Japanese LMR program have been contributing data to a LMR data base, CREDO, based at Oak Ridge National Lab [7.1], which can be used to augment this database. The component data used in this PRA have been developed as follows:

(1) Establish an initial data estimate for a given event using generic data [7.2], the IREP data base [7.3] and the data given in the Clinch River Breeder Reactor PRA [7.4]. This estimate will be the only data gathered in most cases where the fault is not specific to EBR-II or there are no identified concerns about the relative performance of the EBR-II equipment.

(2) CREDO data was used to improve the estimates for LMR-specific equipment. This data was confirmed against EBR-II records for the failures deemed to be particularly important.

(3) For the RSS system, a detailed survey of plant specific data was undertaken because of the importance of the system and the sensitivity of the results to common mode failures in this system.

Many of the systems analyses performed were used to evaluate initiating event frequencies and in some of these cases the results could be directly compared to the historical frequency of faults derived from a survey of EBR-II UORs. In general the frequencies derived 
from plant data have been used, the insight gained from the comparison being incorporated into the fault tree quantification as plant specific data.

The basic data in the following tables can therefore be characterized as plant specific or generic. In addition, there are human errors identified and quantified, as discussed in Section 8 , and dependent failures which are discussed in the following sections.

\subsubsection{Failure Probability Calculation Methodology}

Both generic and plant specific data normally provide information on failure rates or demand failure probabilities for selected components. In addition, plant records provide information on test, repair, and maintenance periods and frequencies. Fault tree analysis, however, requires input data in the form of failure probability for operating components, and unavailability for standby components. Failure probabilities and unavailabilities must be calculated using the raw data on failure rates and test and maintenance information.

Different models can be used in calculating the failure probability or unavailability of a component, depending upon the component type and its operating condition. Table 7.1 lists the different models available in the EBR-II PRA. The calculation models apply as follows:

1. Type 0 - this model applies to components that can fail on a failure per demand basis. The raw data for such components is already in the form of a probability of failure per demand, and no further correction is needed when several demands are required of the component during an accident sequence. This input mode can also be used to input probability and error factor results from external calculations which fall outside these models.

2. Types 1 and 2 - this is the standard failure probability model for an operating component without repair. The failure rate is known from generic sources or it has been estimated from plant data. Two types based on the same model are

provided to differentiate between the operating time of the component: type 2 
Table 7.1. Failure Data Calculation Models

\begin{tabular}{|c|c|c|c|c|c|}
\hline Type & Description & F1 & $\mathrm{F} 2$ & F3 & Probability Model \\
\hline \multicolumn{6}{|l|}{ DT } \\
\hline $\mathrm{O}$ & $\begin{array}{l}\text { Probability } \\
\text { Standby component, failure per demand }\end{array}$ & $\begin{array}{l}\mathrm{P} \\
\delta_{\mathrm{d}}\end{array}$ & $\begin{array}{l}\text { EF } \\
\text { EF }\end{array}$ & (d) & $\mathrm{P}=\delta_{\mathrm{d}}^{*}(\mathrm{~d})$ \\
\hline 1 & Operating component without repair & $\delta_{\mathrm{o}}$ & EF & $\cdot$ & $\mathrm{P}=1-\exp \left(-\delta_{\mathrm{o}} \mathrm{TMIS}\right)$ or $\mathrm{P}=\delta_{\mathrm{o}} \mathrm{TMIS}$ \\
\hline 2 & Operating component without repair & $\delta_{0}$ & EF & - & $\mathrm{P}=1-\exp \left(-\delta_{\mathrm{o}} \mathrm{TRUN}\right)$ or $\mathrm{P}=\delta_{\mathrm{o}} \mathrm{TRUN}$ \\
\hline 3 & Operating component with repair & $\delta_{\mathrm{o}}$ & $\mathrm{EF}$ & $\tau$ & $\begin{array}{l}\mathrm{P}=\left(1-\exp \left(-\left(\delta_{\mathrm{o}}+1 / \tau\right) \mathrm{TMIS}\right)\right) * \delta_{\mathrm{o}} \tau /\left(1+\delta_{\mathrm{o}} \tau\right) \\
\text { or } \mathrm{P}=\delta_{\mathrm{o}} \min (\mathrm{TMIS}, \tau)\end{array}$ \\
\hline 4 & Operating component with repair & $\delta_{\mathrm{o}}$ & EF & $\tau$ & $\begin{array}{l}\mathrm{P}=\left(1-\exp \left(-\left(\delta_{\mathrm{o}}+1 / \tau\right) \mathrm{TRUN}\right)\right)^{*} \delta_{\mathrm{o}} \tau /\left(1+\delta_{\mathrm{o}} \tau\right) \\
\text { or } \mathrm{P}=\delta_{0} \min (\mathrm{TRUN}, \tau)\end{array}$ \\
\hline 5 & Standby component, failure in time & $\delta_{0}$ & EF & $\mathrm{T}_{\mathrm{t}}$ & $\mathrm{P}=1+\left(\exp \left(-\delta_{\mathrm{s}} \mathrm{T} \mathrm{\nu}\right)-1\right) / \delta_{\mathrm{s}} \mathrm{T}^{\mathrm{t}}$ or $\mathrm{P}=\delta_{\mathrm{s}} \mathrm{T} / 2$ \\
\hline 6 & Standby component, test/repair outage & $\tau_{1}$ & $\mathrm{EF}$ & $\mathrm{T}_{\mathrm{t}}$ & $\mathrm{P}=\tau_{\mathrm{t}} / \mathrm{T}_{\mathrm{t}}$ \\
\hline 7 & Standby component, maintenance outage & $\tau_{\mathrm{m}}$ & EF & $\mathrm{T}_{\mathrm{m}}$ & $\mathrm{P}=\left(\tau_{\mathrm{m}} / \mathrm{T}_{\mathrm{m}}\right) /\left(1+\tau_{\mathrm{m}} / \mathrm{T}_{\mathrm{m}}\right)$ \\
\hline $\mathrm{T}$ & True house event & - & - & - & $P=1.0$ \\
\hline $\mathrm{F}$ & False house event & - & - & - & $P=0.0$ \\
\hline
\end{tabular}

$\begin{array}{lll}\text { Definitions: } & \mathrm{P} & \text { Basic Event Probability } \\ & \mathrm{EF} & \text { Error factor } \\ & \delta_{\mathrm{d}} & \text { Failure rate per demand } \\ \mathrm{d} & \text { Number of demands } \\ & \delta_{\mathrm{o}} & \text { Operating failure rate, hr-1 } \\ & \text { TMIS } & \text { Mission time, hr } \\ & \text { TRUN } & \text { Reactor Run time, hr }\end{array}$

$\tau \quad$ Average repair time, $\mathrm{hr}$

$\delta$ Standby failure rate, $\mathrm{hr}^{-1}$

$\mathrm{T}$, Test interval, hr

$\tau_{\mathrm{t}}$ Average test/repair time, hr

$\tau_{\mathrm{m}}$ Maintenance time, $\mathrm{hr}$

$\mathrm{T}_{\mathrm{m}}$ Average maintenance interval, $\mathrm{hr}$

TRUN Reaçtor Run time, hr $\quad \mathrm{m}_{\mathrm{m}}$ (inverse of maintenance frequency)

Notes: $\quad$ An optional third variable can be entered for types 1 and 2, to be used instead of TMIS or TRUN. If F3 is 0 or is not provided, then TMIS and TRUN are used in the formulae.

The rare event approximations for types $1,2,3,4$ and 5 are used when $\delta t$ is smaller than 0.01 . 
applying to components in continuous operation during a reactor run, and type 1 for those components that are only in operation during off-normal conditions.

3. Types 3 and 4 - these models are the unavailability models of types 1 and 2 corrected for the possibility of repair of the component. Plant data supplies the information on repair times. Again the distinction is made between components operating in normal or offnormal conditions.

4. Type 5 - this model provides the unavailability of a standby component that can fail in time. The standby component failure is undetected until the component is demanded or tested. Generic or plant-specific data are used in calculating the standby failure rate, while plant specifications supply information on test intervals.

5. Type 6 - this is the model to estimate the unavailability of a component due to test or repair outage. In the case of test outage, $T_{\mathfrak{t}}$ is the average test outage time, while for repair outage unavailability, $T_{t}$ is interpreted as:

$$
\begin{array}{ll}
T_{t}=T_{R} F_{R}, \quad & \text { where } T_{R} \text { is the average component } \\
& \text { repair time, and } F_{R} \text { is the expected } \\
& \text { repair frequency per test period. }
\end{array}
$$

Plant data and records must be used in estimating all the variables involved in this model.

6. Type 7 - model for the unavailability of a component due to maintenance outage. Again, plant data provides the necessary information. 
7. Types $\mathrm{T}$ and $\mathrm{F}$ - these two models simply set the failure probability of an event to $O$ or 1 , for its use as a house event in a fault tree to trigger a branch of a fault tree on or off.

Data types $1,2,3,4$, and 5 can be simplified to their rare event approximation when the product of the failure rate and the corresponding operating or standby time is smaller than $10^{-2}$. The applicable rare event approximations are:

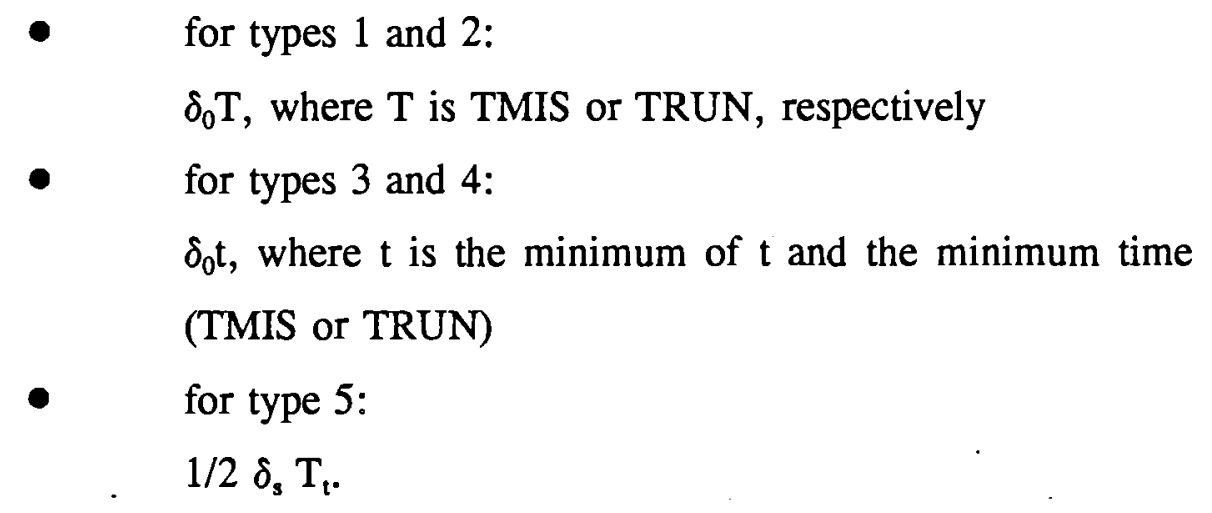

\subsection{Data}

\subsubsection{Data Used in Quantification}

The data used in the quantification of the fault trees is given in the various system notebooks and the tables of data repeated here system by system, Tables $7.2-7.21$ and 7.24 .

\subsection{2 $\quad$ Plant Specific Data}

Plant-specific data relates to such data as test and maintenance, and specific pieces of equipment. There is almost no test and maintenance related unavailability of safety significant equipment to be considered. This arises because of the EBR-II operating philosophy. Maintenance is performed with the reactor shutdown and all safety significant systems are tested 
Maintenance is performed with the reactor shutdown and all safety significant systems are tested before returning to power. Test and maintenance unavailabilities are summarized in Table 7.22.

Plant specific component failure data was generated in areas where the system possesses attributes which did not have direct analogies in the generic database or was deemed to be significantly important. The plant specific data used in the EBR-II PRA are summarized in Table 7.23.

\subsubsection{Special Data Considerations}

Several top events in the event trees were not developed into fault trees. In certain cases they represent subjectively evaluated probabilities related to the occurrence of different physical phenomena. This includes most of the evaluation of the local fault and steam generator tube rupture event trees. In addition, there is one important undeveloped top event whose quantification can be related to plant data - this is the probability that the system is operating on hotel load. These special data are listed in Table 7.24. A number of initiating event frequencies, particularly those relating to very rare events, are evaluated as special cases using engineering judgement. These events are discussed on a case-by-case basis in the treatment of initiating events. 
Table 7.2. Reactor Shutdown System Data

PAGE 1

\begin{tabular}{|c|c|c|c|c|c|c|c|c|c|}
\hline \multirow{2}{*}{$\begin{array}{l}\text { EVENT } \\
\text { NAME }\end{array}$} & \multirow[b]{2}{*}{ DESCRIPTION } & \multicolumn{2}{|c|}{ VALUE USED 391} & \multicolumn{2}{|c|}{ CRBAP.4 } & \multicolumn{2}{|c|}{ IREP } & \multicolumn{2}{|c|}{ GENERIC } \\
\hline & & $\begin{array}{c}\text { Failure } \\
\text { Pate }\end{array}$ & $\begin{array}{l}\text { Error } \\
\text { Factor }\end{array}$ & $\begin{array}{c}\text { Failure } \\
\text { Rate }\end{array}$ & $\begin{array}{l}\text { Error } \\
\text { Factor }\end{array}$ & $\begin{array}{c}\text { Failure } \\
\text { Rate }\end{array}$ & $\begin{array}{l}\text { Error } \\
\text { Factor }\end{array}$ & $\begin{array}{c}\text { Failure } \\
\text { Rate }\end{array}$ & $\begin{array}{l}\text { Error } \\
\text { Factor }\end{array}$ \\
\hline RAM2A1OW & $\begin{array}{l}\text { Current Ampltr } \\
\text { Reduces Signal to } \\
\text { Trip unit }\end{array}$ & $\begin{array}{c}1.0 E-6 / h \\
{[1]}\end{array}$ & 10 & $\begin{array}{c}1.0 E-6 / \mathrm{h} \\
{[2]}\end{array}$ & 10 & & & $\begin{array}{c}5.0 \mathrm{E}-6 / \mathrm{h} \\
{[3]}\end{array}$ & 20 \\
\hline RAM2B1OW & $\begin{array}{l}\text { Current Ampitr } \\
\text { Reduces Signal to } \\
\text { Trip unit }\end{array}$ & $\begin{array}{c}1.0 \mathrm{E}-6 / \mathrm{h} \\
{[1]}\end{array}$ & 10 & $\begin{array}{c}1.0 \mathrm{E}-6 / \mathrm{h} \\
{[2]}\end{array}$ & 10 & & & $\begin{array}{c}5.0 \mathrm{E}-6 / \mathrm{h} \\
{[3]}\end{array}$ & 20 \\
\hline RAM2C1QW & $\begin{array}{l}\text { Current Amplfr } \\
\text { Reduces Signal to } \\
\text { Trip unit }\end{array}$ & $\begin{array}{c}1.0 E-6 / \mathrm{h} \\
{[1]}\end{array}$ & 10 & $\begin{array}{c}1.0 E-6 / \mathrm{h} \\
{[2]}\end{array}$ & 10 & & & $\begin{array}{c}5.0 \mathrm{E}-6 \mathrm{~h} \\
{[3]}\end{array}$ & 20 \\
\hline RAM2Z1QU & $\begin{array}{l}\text { CCF - All Current } \\
\text { Ampli Reduce } \\
\text { Signals to Trip unit }\end{array}$ & $\begin{array}{c}1.0 \mathrm{E}-7 / \mathrm{h} \\
{[4]}\end{array}$ & 10 & & & & & & \\
\hline RBI1AOOW & $\begin{array}{l}\text { Trip Unit of Strings } \\
\text { A \& B Fails }\end{array}$ & $\begin{array}{c}5.0 \mathrm{E}-7 / \mathrm{h} \\
{[1]}\end{array}$ & 10 & $\begin{array}{c}1.0 \mathrm{E}-6 / \mathrm{h} \\
{[2]}\end{array}$ & 10 & $3.0 E-7 / d$ & 10 & $\begin{array}{c}3.0 E-7 / h \\
{[5]}\end{array}$ & 10 \\
\hline RBI1BOQW & $\begin{array}{l}\text { Trip Unit of Strings } \\
\text { A \& B Fails }\end{array}$ & $\begin{array}{c}5.0 \mathrm{E}-7 \mathrm{~h} \\
{[1]}\end{array}$ & 10 & $\begin{array}{c}1.0 \mathrm{E}-6 / \mathrm{h} \\
{[2]}\end{array}$ & 10 & $3.0 \mathrm{E}-7 / \mathrm{d}$ & 10 & $\underset{[5]}{3.0 \mathrm{E} \cdot 7 \mathrm{~h}}$ & 10 \\
\hline RBIICOOW & $\begin{array}{l}\text { Trip Unit of Strings } \\
\text { A \& } 8 \text { Fails }\end{array}$ & $\begin{array}{c}5.0 \mathrm{E} \cdot 7 \mathrm{~m} \\
{[1]}\end{array}$ & 10 & $\begin{array}{c}1.0 E-6 / \mathrm{h} \\
{[2]}\end{array}$ & 10 & $3.0 E-7 / d$ & 10 & $\begin{array}{c}3.0 E-7 / \mathrm{h} \\
{[5]}\end{array}$ & 10 \\
\hline RBI1DOQW & $\begin{array}{l}\text { Trip Unit of Strings } \\
\text { A \& B Fails }\end{array}$ & $\begin{array}{c}5.0 \mathrm{E}-7 \mathrm{~m} \\
{[1]}\end{array}$ & 10 & $\begin{array}{c}1.0 E-6 / \mathrm{h} \\
{[2]}\end{array}$ & 10 & $3.0 \mathrm{E}-7 / \mathrm{d}$ & 10 & $\begin{array}{c}3.0 \mathrm{E}-7 / \mathrm{h} \\
{[5]}\end{array}$ & 10 \\
\hline RBI1ZOQU & $\begin{array}{l}\text { CCF - All Trip Units } \\
\text { in All Channels Fail }\end{array}$ & $\begin{array}{c}4.0 \bar{E}-9 / \mathrm{h} \\
{[6]}\end{array}$ & 10 & & & & & & \\
\hline $\mathrm{RBI} 2 \mathrm{AOQU}$ & $\begin{array}{l}\text { CCF - All Bistables } \\
\text { of Channel A Fail }\end{array}$ & $\begin{array}{c}1.0 \mathrm{E}-7 / \mathrm{h} \\
{[7]}\end{array}$ & 10 & & & & & & \\
\hline RBI2A1OW & $\begin{array}{l}\text { Bistable of String A } \\
\text { Fails to dtet High } \\
\text { Flux }\end{array}$ & $\begin{array}{c}5.0 \mathrm{E}-7 \mathrm{~h} \\
{[8]}\end{array}$ & 10 & $\begin{array}{c}1.0 E-6 / \mathrm{h} \\
{[2]}\end{array}$ & 10 & $3.0 \mathrm{E}-7 / \mathrm{d}$ & 10 & $\begin{array}{c}3.0 E-7 / \mathrm{h} \\
{[5]}\end{array}$ & 10 \\
\hline RBI2A2QW & $\begin{array}{l}\text { Bistable of String B } \\
\text { Fails to dtct High } \\
\text { Flux }\end{array}$ & $\underset{[8]}{5.0 \mathrm{E}-7 \mathrm{~h}}$ & 10 & $\begin{array}{c}1.0 \mathrm{E}-6 \mathrm{~h} \\
{[2]}\end{array}$ & 10 & $3.0 \mathrm{E}-7 / \mathrm{d}$ & 10 & $\begin{array}{c}3.0 \mathrm{E}-7 \mathrm{~h} \\
{[5]}\end{array}$ & 10 \\
\hline RBI2A3OW & $\begin{array}{l}\text { Bistable of String A } \\
\text { Fails to dtct Low } \\
\text { Voltage }\end{array}$ & $\begin{array}{c}5.0 \mathrm{E}-7 \mathrm{~h} \\
{[8]}\end{array}$ & 10 & $\begin{array}{c}1.0 \mathrm{E}-6 / \mathrm{h} \\
{[2]}\end{array}$ & 10 & $3.0 \mathrm{E}-7 / \mathrm{d}$ & 10 & $\begin{array}{c}3.0 \mathrm{E} \cdot 7 \mathrm{~h} \\
{[5]}\end{array}$ & 10 \\
\hline RBI2A4QW & $\begin{array}{l}\text { Bistable of String B } \\
\text { Fails to dtet Low } \\
\text { Voltage }\end{array}$ & $\begin{array}{c}5.0 \mathrm{E}-7 \mathrm{~m} \\
{[8]}\end{array}$ & 10 & $\begin{array}{c}1.0 E-6 / \mathrm{h} \\
{[2]}\end{array}$ & 10 & $3.0 E-7 / d$ & 10 & $\begin{array}{c}3.0 \mathrm{E}-7 \mathrm{~m} \\
{[5]}\end{array}$ & 10 \\
\hline RBI2BOQU & $\begin{array}{l}\text { CCF - All Bistables } \\
\text { of Channel B Fail }\end{array}$ & $\begin{array}{c}1.0 \mathrm{E}-7 / \mathrm{h} \\
{[7]}\end{array}$ & 10 & & & & & & \\
\hline $\mathrm{ABI} 2 \mathrm{~B} 1 \mathrm{OW}$ & $\begin{array}{l}\text { Bistable of String A } \\
\text { Fails to dtct High } \\
\text { Flux }\end{array}$ & $\begin{array}{c}5.0 \mathrm{E}-7 \mathrm{~h} \\
{[8]}\end{array}$ & 10 & $\begin{array}{c}1.0 \mathrm{E}-6 / \mathrm{h} \\
{[2]}\end{array}$ & 10 & $3.0 E-7 / d$ & 10 & $\begin{array}{c}3.0 \mathrm{E}-7 \mathrm{~h} \\
{[5]}\end{array}$ & 10 \\
\hline $\mathrm{RB} 12 \mathrm{~B} 2 \mathrm{OW}$ & $\begin{array}{l}\text { Bistable of String B } \\
\text { Fails to dtct High } \\
\text { Flux }\end{array}$ & $\begin{array}{c}5.0 \mathrm{E}-7 \mathrm{~h} \\
{[8]}\end{array}$ & 10 & $\begin{array}{c}1.0 E-6 / \mathrm{h} \\
{[2]}\end{array}$ & 10 & $3.0 \mathrm{E}-7 / \mathrm{d}$ & 10 & $\begin{array}{c}3.0 \mathrm{E}-7 \mathrm{~h} \\
{[5]}\end{array}$ & 10 \\
\hline RBI2B3OW & $\begin{array}{l}\text { Bistable of String A } \\
\text { Fails to dict Low } \\
\text { Voltage }\end{array}$ & $\begin{array}{c}5.0 \mathrm{E} \cdot 7 \mathrm{~h} \\
{[8]}\end{array}$ & 10 & $\begin{array}{c}1.0 \mathrm{E}-6 / \mathrm{h} \\
{[2]}\end{array}$ & 10 & $3.0 \mathrm{E}-7 / \mathrm{d}$ & 10 & $\begin{array}{c}3.0 \mathrm{E}-7 \mathrm{~h} \\
{[5]}\end{array}$ & 10 \\
\hline$R B 12 B 4 Q W$ & $\begin{array}{l}\text { Bistable of String B } \\
\text { Fails to dtct Low } \\
\text { Voltage }\end{array}$ & $\begin{array}{c}5.0 \mathrm{E} \cdot 7 / \mathrm{h} \\
{[8]}\end{array}$ & 10 & $\begin{array}{c}1.0 \mathrm{E}-6 \mathrm{M} \\
{[2]}\end{array}$ & 10 & $3.0 \mathrm{E}-7 / \mathrm{d}$ & 10 & $\underset{[5]}{3.0 E-7 / h}$ & 10 \\
\hline RBI2COOU & $\begin{array}{l}\text { CCF - All Bistables } \\
\text { of Channel C Fail }\end{array}$ & $\begin{array}{c}1.0 \mathrm{E}-7 \mathrm{~h} \\
{[7]}\end{array}$ & 10 & & & & & & \\
\hline RBI2C1OW & $\begin{array}{l}\text { Bistable of String A } \\
\text { Fails to dtet High } \\
\text { Flux }\end{array}$ & $\begin{array}{c}5.0 \mathrm{E}-7 \mathrm{~h} \\
{[8]}\end{array}$ & 10 & $\begin{array}{c}1.0 \mathrm{E}-6 / \mathrm{h} \\
{[2]}\end{array}$ & 10 & $3.0 \mathrm{E}-7 / \mathrm{d}$ & 10 & $\begin{array}{c}3.0 \mathrm{E} \cdot 7 \mathrm{~h} \\
{[5]}\end{array}$ & 10 \\
\hline $\mathrm{RB12C2 \textrm {QW }}$ & $\begin{array}{l}\text { Bistable of String B } \\
\text { Fails to dict High } \\
\text { Flux }\end{array}$ & $\begin{array}{c}5.0 E-7 / \mathrm{M} \\
{[8]}\end{array}$ & 10 & $\begin{array}{c}1.0 \mathrm{E}-6 / \mathrm{h} \\
{[2]}\end{array}$ & 10 & $3.0 \mathrm{E}-7 / \mathrm{d}$ & 10 & $\underset{[5]}{3.0 \mathrm{E}-7 / \mathrm{h}}$ & 10 \\
\hline
\end{tabular}


Table 7.2. Reactor Shutdown System Data (Cont'd)

PAGE 2

\begin{tabular}{|c|c|c|c|c|c|c|c|c|c|}
\hline \multirow{2}{*}{$\begin{array}{l}\text { EVENT } \\
\text { NAME }\end{array}$} & \multirow[b]{2}{*}{ DESCRIPTION } & \multicolumn{2}{|c|}{ VALUE USED 391} & \multicolumn{2}{|c|}{ CABRP-4 } & \multicolumn{2}{|c|}{ IREP } & \multicolumn{2}{|c|}{ GENERIC } \\
\hline & & $\begin{array}{c}\text { Failure } \\
\text { Rate }\end{array}$ & $\begin{array}{l}\text { Error } \\
\text { Factor }\end{array}$ & $\begin{array}{l}\text { Failure } \\
\text { Rate }\end{array}$ & $\begin{array}{l}\text { Error } \\
\text { Factor }\end{array}$ & $\begin{array}{c}\text { Failure } \\
\text { Rate }\end{array}$ & $\begin{array}{l}\text { Error } \\
\text { Factor }\end{array}$ & $\begin{array}{l}\text { Failure } \\
\text { Rate }\end{array}$ & $\begin{array}{l}\text { Error } \\
\text { Factor }\end{array}$ \\
\hline $\mathrm{RBI} 2 \mathrm{C} 3 \mathrm{OW}$ & $\begin{array}{l}\text { Bistable of String A } \\
\text { Fails to dtct Low } \\
\text { Voltage }\end{array}$ & $\begin{array}{c}5.0 \mathrm{E}-7 / \mathrm{h} \\
{[8]}\end{array}$ & 10 & $\begin{array}{c}1.0 E-6 / \mathrm{h} \\
{[2]}\end{array}$ & 10 & $3.0 \mathrm{E}-7 / \mathrm{d}$ & 10 & $\underset{[5]}{3.0 \mathrm{E}-7 \mathrm{~h}}$ & 10 \\
\hline RBI2C4OW & $\begin{array}{l}\text { Bistable of String B } \\
\text { Fails to dtct Low } \\
\text { Voltage }\end{array}$ & $\begin{array}{c}5.0 \mathrm{E}-7 / \mathrm{h} \\
{[8]}\end{array}$ & 10 & $\begin{array}{c}1.0 \mathrm{E}-6 / \mathrm{h} \\
{[2]}\end{array}$ & 10 & $3.0 \mathrm{E}-7 / \mathrm{d}$ & 10 & $\begin{array}{c}3.0 \mathrm{E}-7 \mathrm{~h} \\
{[5]}\end{array}$ & 10 \\
\hline RBI2Z1QU & $\begin{array}{l}\text { CCF - All HF } \\
\text { Bistables of All } \\
\text { Channels Fail }\end{array}$ & $\begin{array}{c}4.0 \mathrm{E}-9 / \mathrm{h} \\
{[6]}\end{array}$ & 10 & & & & & & \\
\hline RBI2Z2QU & $\begin{array}{l}\text { CCF - All LV } \\
\text { Bistables of All } \\
\text { Channels Fail }\end{array}$ & $\begin{array}{c}4.0 \mathrm{E}-9 / \mathrm{h} \\
{[6]}\end{array}$ & 10 & & & & & & \\
\hline RBI3C1QW & $\begin{array}{l}\text { SOT Trip Unit of } \\
\text { Strings A \& B Fails }\end{array}$ & $\begin{array}{c}5.0 \mathrm{E}-7 \mathrm{~h} \\
{[1]}\end{array}$ & 10 & $\begin{array}{c}1.0 \mathrm{E}-6 / \mathrm{h} \\
{[2]}\end{array}$ & 10 & $3.0 E-7 / d$ & 10 & $\begin{array}{c}3.0 \mathrm{E}-7 / \mathrm{h} \\
{[5]}\end{array}$ & 10 \\
\hline RBI3D1QW & $\begin{array}{l}\text { SOT Trip Unit of } \\
\text { Strings A \& B Fails }\end{array}$ & $\begin{array}{c}5.0 E-7 / \mathrm{h} \\
{[1]}\end{array}$ & 10 & $\begin{array}{c}1.0 E-6 / \mathrm{h} \\
{[2]}\end{array}$ & 10 & $3.0 \mathrm{E}-7 / \mathrm{d}$ & 10 & $\begin{array}{c}3.0 E-7 / \mathrm{h} \\
{[5]}\end{array}$ & 10 \\
\hline RBI3F1QW & $\begin{array}{l}\text { SOT Trip Unit of } \\
\text { Strings A \& B Fails }\end{array}$ & $\begin{array}{c}\text { 5.0E-7/h } \\
{[1]}\end{array}$ & 10 & $\begin{array}{c}1.0 \mathrm{E}-6 / \mathrm{h} \\
{[2]}\end{array}$ & 10 & $3.0 E-7 / d$ & 10 & $\begin{array}{c}3.0 \mathrm{E}-7 / \mathrm{h} \\
{[5]}\end{array}$ & 10 \\
\hline RBI3H1OW & $\begin{array}{l}\text { SOT Trip Unit of } \\
\text { Strings A \& B Fails }\end{array}$ & $\begin{array}{c}5.0 \mathrm{E}-7 / \mathrm{h} \\
{[1]}\end{array}$ & 10 & $\begin{array}{c}1.0 \mathrm{E}-6 / \mathrm{h} \\
{[2]}\end{array}$ & 10 & $3.0 \mathrm{E}-7 / \mathrm{d}$ & 10 & $\begin{array}{c}3.0 \mathrm{E}-7 / \mathrm{h} \\
{[5]}\end{array}$ & 10 \\
\hline RBI3ZOQU & $\begin{array}{l}\text { CCF - All Trip Units } \\
\text { in All SOT Channels } \\
\text { Fail }\end{array}$ & $\begin{array}{c}1.0 \mathrm{E}-8 / \mathrm{h} \\
{[9]}\end{array}$ & 10 & & & & & & \\
\hline RBSOOAOP & $\begin{array}{l}\text { REACTOR SHUTDOWN } \\
\text { SYSTEM FAILURE }\end{array}$ & $1.0 \mathrm{E}-3 / \mathrm{d}$ & 10 & & & & & & \\
\hline RBS007QP & $\begin{array}{l}\text { REACTOR SHUTDOWN } \\
\text { SYSTEM FAILURE }\end{array}$ & $1.0 \mathrm{E}-4 / \mathrm{d}$ & 10 & & & & & & \\
\hline RCA2AORW & $\begin{array}{l}\text { Cable and Connector } \\
\text { of Channel A Fail }\end{array}$ & $\begin{array}{c}4.0 E-7 / \mathrm{h} \\
{[8]}\end{array}$ & 10 & $1.0 \mathrm{E}-7 \mathrm{~h}$ & 10 & $3.0 \mathrm{E}-7 \mathrm{~m}$ & 10 & $\begin{array}{c}1.0 E-7 / \mathrm{h} \\
{[10]}\end{array}$ & 10 \\
\hline RCA2BORW & $\begin{array}{l}\text { Cable and Connector } \\
\text { of Channel B Fail }\end{array}$ & $\begin{array}{c}4.0 \mathrm{E}-7 \mathrm{~m} \\
{[8]}\end{array}$ & 10 & $1.0 \mathrm{E}-7 \mathrm{~h}$ & 10 & $3.0 \mathrm{E}-7 / \mathrm{h}$ & 10 & $\begin{array}{c}1.0 \mathrm{E}-7 / \mathrm{h} \\
{[10]}\end{array}$ & 10 \\
\hline RCA2CORW & $\begin{array}{l}\text { Cable and Connector } \\
\text { of Channel C Fail }\end{array}$ & $\begin{array}{c}4.0 \mathrm{E}-7 \mathrm{~h} \\
{[8]}\end{array}$ & 10 & $1.0 \mathrm{E}-7 \mathrm{~h}$ & 10 & $3.0 \mathrm{E}-7 / \mathrm{h}$ & 10 & $\begin{array}{c}1.0 E-7 / h \\
{[10]}\end{array}$ & 10 \\
\hline RCA2ZORU & $\begin{array}{l}\text { CCF - All Cabies and } \\
\text { Connectors Fail }\end{array}$ & $\begin{array}{c}4.0 \mathrm{E} \cdot 8 / \mathrm{h} \\
{[4]}\end{array}$ & 10 & & & & & & \\
\hline RCZ1AOOU & $\begin{array}{l}\text { CCF - Contacts of } \\
\text { both Strings Fail } \\
\text { Closed }\end{array}$ & $\begin{array}{c}6.0 E-5 / d \\
{[7]}\end{array}$ & 10 & & & & & & \\
\hline RCZ1A1QP & $\begin{array}{l}\text { Contact } 3,4 \text { or } 5 \text { in } \\
\text { String A Fails } \\
\text { Closed }\end{array}$ & $\begin{array}{c}3.0 \text { 드.4/d } \\
{[10]}\end{array}$ & 10 & $1.0 E \cdot 4 / d$ & 10 & $3.0 E-4 / d$ & 10 & $\begin{array}{c}3.0 E-4 / \mathrm{d} \\
{[10]}\end{array}$ & 10 \\
\hline RCZ1AZQP & $\begin{array}{l}\text { Contact } 3,4 \text { or } 5 \text { in } \\
\text { String B Fails } \\
\text { Closed }\end{array}$ & $\begin{array}{c}3.0 \mathrm{E}-4 / \mathrm{d} \\
{[10]}\end{array}$ & 10 & $1.0 E-4 / d$ & 10 & $3.0 \mathrm{E}-4 / \mathrm{d}$ & 10 & $\begin{array}{c}3.0 \mathrm{E}-4 / \mathrm{d} \\
{[10]}\end{array}$ & 10 \\
\hline RCZ1BOQU & $\begin{array}{l}\text { CCF - Contacts of } \\
\text { both Strings Fail } \\
\text { Closed }\end{array}$ & $\begin{array}{c}6.0 E-5 / d \\
{[7]}\end{array}$ & 10 & & & & & & \\
\hline$\overline{R C Z 1 B 1 Q P}$ & $\begin{array}{l}\text { Contact } 3,4 \text { or } 5 \text { in } \\
\text { String A Fails } \\
\text { Closed }\end{array}$ & $\begin{array}{c}3.0 \mathrm{E}-4 / \mathrm{d} \\
{[10]}\end{array}$ & 10 & $1.0 \mathrm{E}-4 / \mathrm{d}$ & 10 & $3.0 E-4 / d$ & 10 & $\begin{array}{c}3.0 E-4 / d \\
{[10]}\end{array}$ & 10 \\
\hline RCZ182QP & $\begin{array}{l}\text { Contact } 3,4 \text { or } 5 \text { in } \\
\text { String } B \text { Fails } \\
\text { Closed }\end{array}$ & $\begin{array}{c}3.0 E-4 / d \\
{[10]}\end{array}$ & 10 & $1.0 E-4 / d$ & 10 & $3.0 E-4 / d$ & 10 & $\begin{array}{c}3.0 E-4 / d \\
{[10]}\end{array}$ & 10 \\
\hline RCZ1COOU & $\begin{array}{l}\text { CCF - Contacts of } \\
\text { both Strings Fail } \\
\text { Closed }\end{array}$ & $\begin{array}{c}6.0 E-5 / d \\
{[7]}\end{array}$ & 10 & & & & & & \\
\hline RCZ1C1OP & $\begin{array}{l}\text { Contact } 3,4 \text { or } 5 \text { in } \\
\text { String A Fails } \\
\text { Closed }\end{array}$ & $\begin{array}{c}3.0 \mathrm{E}-4 / \mathrm{d} \\
{[10]}\end{array}$ & 10 & $1.0 \mathrm{E}-4 / \mathrm{d}$ & 10 & $3.0 E-4 / d$ & 10 & $\begin{array}{c}3.0 E-4 / d \\
{[10]}\end{array}$ & 10 \\
\hline
\end{tabular}


Table 7.2. Reactor Shutdown System Data (Cont'd)

PAGE 3

\begin{tabular}{|c|c|c|c|c|c|c|c|c|c|}
\hline \multirow{2}{*}{$\begin{array}{l}\text { EVENT } \\
\text { NAME }\end{array}$} & \multirow[b]{2}{*}{ DESCRIPTION } & \multicolumn{2}{|c|}{ VALUE USED $3 / 91$} & \multicolumn{2}{|c|}{ CRBRP-4 } & \multicolumn{2}{|c|}{ IREP } & \multicolumn{2}{|c|}{ GENERIC } \\
\hline & & $\begin{array}{l}\text { Failure } \\
\text { Rate }\end{array}$ & $\begin{array}{l}\text { Error } \\
\text { Factor }\end{array}$ & $\begin{array}{c}\text { Failure } \\
\text { Rate }\end{array}$ & $\begin{array}{l}\text { Error } \\
\text { Factor }\end{array}$ & $\begin{array}{c}\text { Failure } \\
\text { Rate }\end{array}$ & $\begin{array}{l}\text { Error } \\
\text { Factor }\end{array}$ & $\begin{array}{c}\text { Failure } \\
\text { Rate }\end{array}$ & $\begin{array}{l}\text { Error } \\
\text { Factor }\end{array}$ \\
\hline RCZ1C2QP & $\begin{array}{l}\text { Contact } 3,4 \text { or } 5 \text { in } \\
\text { String B Fails } \\
\text { Closed }\end{array}$ & $\begin{array}{c}3.0 \mathrm{E} \cdot 4 / \mathrm{d} \\
{[10]}\end{array}$ & 10 & $1.0 E-4 / d$ & 10 & $3.0 E-4 / d$ & 10 & $\begin{array}{c}3.0 \mathrm{E}-4 / \mathrm{d} \\
{[10]}\end{array}$ & 10 \\
\hline RCZ1DOQU & $\begin{array}{l}\text { CCF - Contacts of } \\
\text { both Strings Fail } \\
\text { Closed }\end{array}$ & $\begin{array}{c}6.0 \mathrm{E}-5 / \mathrm{d} \\
{[7]}\end{array}$ & 10 & & & & & & \\
\hline RCZ1D1QP & $\begin{array}{l}\text { Contact } 3,4 \text { or } 5 \text { in } \\
\text { String A Fails } \\
\text { Closed }\end{array}$ & $\begin{array}{c}3.0 E-4 / d \\
{[10]}\end{array}$ & 10 & $1.0 \mathrm{E}-4 / \mathrm{d}$ & 10 & $3.0 \mathrm{E}-4 / \mathrm{d}$ & 10 & $\begin{array}{c}3.0 E-4 / d \\
{[10]}\end{array}$ & 10 \\
\hline RCZ1D2QP & $\begin{array}{l}\text { Contact } 3,4 \text { or } 5 \text { in } \\
\text { String B Fails } \\
\text { Closed }\end{array}$ & $\begin{array}{c}3.0 E-4 / \mathrm{d} \\
{[10]}\end{array}$ & 10 & $1.0 \mathrm{E}-4 / \mathrm{d}$ & 10 & $3.0 E-4 / d$ & 10 & $\begin{array}{c}3.0 E-4 / d \\
{[10]}\end{array}$ & 10 \\
\hline RCZ1Z0QU & $\begin{array}{l}\text { CCF - All Trip } \\
\text { Contacts of both } \\
\text { Stings Fail Closed }\end{array}$ & $\begin{array}{c}2.4 E-6 / d \\
{[6]}\end{array}$ & 10 & & & & & & \\
\hline RCZ2AOOU & $\begin{array}{l}\text { CCF - Contacts of } \\
\text { both Stings Fail } \\
\text { Closed }\end{array}$ & $\begin{array}{c}6.0 E-5 / d \\
{[7]}\end{array}$ & 10 & & & & & & \\
\hline RCZ2A1OP & $\begin{array}{l}\text { Contact } 7 \text { or } 8 \text { in } \\
\text { String A Fails } \\
\text { Closed }\end{array}$ & $\begin{array}{c}3.0 E-4 / d \\
{[10]}\end{array}$ & 10 & $1.0 E-4 / d$ & 10 & $3.0 E-4 / d$ & 10 & $\begin{array}{c}3.0 \mathrm{E}-4 / \mathrm{d} \\
{[10]}\end{array}$ & 10 \\
\hline RCZ2A2QP & $\begin{array}{l}\text { Contact } 7 \text { or } 8 \text { in } \\
\text { String A Fails } \\
\text { Closed }\end{array}$ & $\begin{array}{c}3.0 E-4 / d \\
{[10]}\end{array}$ & 10 & $1.0 E-4 / d$ & 10 & $3.0 \mathrm{E}-4 / \mathrm{d}$ & 10 & $\begin{array}{c}3.0 E-4 / d \\
{[10]}\end{array}$ & 10 \\
\hline RCZ2A3QP & $\begin{array}{l}\text { Contacts of K3 Relay } \\
\text { Fail Closed }\end{array}$ & $\begin{array}{c}3.0 E-4 / d \\
{[10]}\end{array}$ & 10 & $1.0 E-4 / d$ & 10 & $3.0 \mathrm{E}-4 / \mathrm{d}$ & 10 & $\begin{array}{c}3.0 E-4 / d \\
{[10]}\end{array}$ & 10 \\
\hline RCZ2A4QP & $\begin{array}{l}\text { Contacts of K4 Relay } \\
\text { Fail Closed }\end{array}$ & $\begin{array}{c}3.0 E-4 / d \\
{[10]}\end{array}$ & 10 & $1.0 E-4 / d$ & 10 & $3.0 E-4 / d$ & 10 & $\begin{array}{c}.0 E-4 / d \\
{[10]}\end{array}$ & 10 \\
\hline RCZ2A5OP & $\begin{array}{l}\text { Contacts of K5 Relay } \\
\text { Fail Closed }\end{array}$ & $\begin{array}{c}3.0 E-4 / d \\
{[10]}\end{array}$ & 10 & $1.0 E-4 / d$ & 10 & $3.0 E-4 / \mathrm{d}$ & 10 & $\begin{array}{c}3.0 E-4 / d \\
{[10]}\end{array}$ & 10 \\
\hline RCZ2AGOP & $\begin{array}{l}\text { Contacts of K6 Relay } \\
\text { Fail Closed }\end{array}$ & $\begin{array}{c}3.0 E-4 / \mathrm{d} \\
{[10]}\end{array}$ & 10 & $1.0 \mathrm{E}-4 / \mathrm{d}$ & 10 & $3.0 \mathrm{E}-4 / \mathrm{d}$ & 10 & $\begin{array}{c}3.0 E-4 / d \\
{[10]}\end{array}$ & 10 \\
\hline RCZ2A9QU & $\begin{array}{l}\text { CCF - Contacts of } \\
\text { All K-Rolays Fail } \\
\text { Closed }\end{array}$ & $\begin{array}{c}6.0 E-5 / d \\
{[7]}\end{array}$ & 10 & & & & & & \\
\hline RCZ2BOQU & $\begin{array}{l}\text { CCF - Contacts of } \\
\text { both Strings Fail } \\
\text { Closed }\end{array}$ & $\begin{array}{c}6.0 E-5 / d \\
{[7]}\end{array}$ & 10 & & & & & & \\
\hline ACZ2B1QP & $\begin{array}{l}\text { Contact } 7 \text { or } 8 \text { in } \\
\text { String A Fails } \\
\text { Closed }\end{array}$ & $\begin{array}{c}3.0 E-4 / d \\
{[10]}\end{array}$ & 10 & $1.0 \mathrm{E} \cdot 4 / \mathrm{d}$ & 10 & $3.0 \mathrm{E}-4 / \mathrm{d}$ & 10 & $\begin{array}{c}3.0 E-4 / d \\
{[10]}\end{array}$ & 10 \\
\hline RCZ2B2QP & $\begin{array}{l}\text { Contact } 7 \text { or } 8 \text { in } \\
\text { String A Fails } \\
\text { Closed }\end{array}$ & $\begin{array}{c}3.0 E-4 / \mathrm{d} \\
{[10]}\end{array}$ & 10 & $1.0 \mathrm{E}-4 / \mathrm{d}$ & 10 & $3.0 E-4 / d$ & 10 & $\begin{array}{c}3.0 E-4 / d \\
{[10]}\end{array}$ & 10 \\
\hline ACZ2B3QP & $\begin{array}{l}\text { Contacts of K3 Relay } \\
\text { Fail Closed }\end{array}$ & $\begin{array}{c}\text { 3.0E-4/d } \\
{[10]}\end{array}$ & 10 & $1.0 E-4 / d$ & 10 & $3.0 \mathrm{E}-4 / \mathrm{d}$ & 10 & $\begin{array}{c}3.0 E-4 / d \\
{[10]}\end{array}$ & 10 \\
\hline $\mathrm{RCZ2B4QP}$ & $\begin{array}{l}\text { Contacts of K4 Relay } \\
\text { Fail Closed }\end{array}$ & $\begin{array}{c}3.0 \mathrm{E} \cdot 4 / \mathrm{d} \\
{[10]}\end{array}$ & 10 & $1.0 E-4 / d$ & 10 & $3.0 \mathrm{E}-4 / \mathrm{d}$ & 10 & $\begin{array}{c}3.0 E-4 / d \\
{[10]}\end{array}$ & 10 \\
\hline RCZ2B5OP & $\begin{array}{l}\text { Contacts of K5 Relay } \\
\text { Fail Closed }\end{array}$ & $\begin{array}{c}\text { 3.0E-4/d } \\
{[10]}\end{array}$ & 10 & $1.0 \mathrm{E}-4 / \mathrm{d}$ & 10 & $3.0 \mathrm{E}-4 / \mathrm{d}$ & 10 & $\begin{array}{c}3.0 E-4 / d \\
{[10]}\end{array}$ & 10 \\
\hline ACZ2B6QP & $\begin{array}{l}\text { Contacts of K6 Relay } \\
\text { Fail Closed }\end{array}$ & $\begin{array}{c}3.0 E-4 / d \\
{[10]}\end{array}$ & 10 & $1.0 E-4 / d$ & 10 & $3.0 E-4 / d$ & 10 & $\begin{array}{c}3.0 \mathrm{E}-4 / \mathrm{d} \\
{[10]}\end{array}$ & 10 \\
\hline RCZ2B9QU & $\begin{array}{l}\text { CCF - Contacts of } \\
\text { All K-Relays Fail } \\
\text { Closed }\end{array}$ & $\begin{array}{c}6.0 \mathrm{E}-5 / \mathrm{d} \\
{[7]}\end{array}$ & 10 & & & & & & \\
\hline RCZ2COQU & $\begin{array}{l}\text { CCF - Contacts of } \\
\text { both Strings Fail } \\
\text { Closed }\end{array}$ & $\begin{array}{c}6.0 E-5 / d \\
{[7]}\end{array}$ & 10 & & & & & & \\
\hline
\end{tabular}


Table 7.2. Reactor Shutdown System Data (Cont'd)

PAGE 4

\begin{tabular}{|c|c|c|c|c|c|c|c|c|c|}
\hline \multirow{2}{*}{$\begin{array}{l}\text { EVENT } \\
\text { NAME }\end{array}$} & \multirow[b]{2}{*}{ DESCAIPTION } & \multicolumn{2}{|c|}{ VALUE USED 391} & \multicolumn{2}{|c|}{ CRBRP-4 } & \multicolumn{2}{|c|}{ IREP } & \multicolumn{2}{|c|}{ GENERIC } \\
\hline & & $\begin{array}{l}\text { Failure } \\
\text { Rate }\end{array}$ & $\begin{array}{l}\text { Error } \\
\text { Factor }\end{array}$ & $\begin{array}{l}\text { Failure } \\
\text { Rate }\end{array}$ & $\begin{array}{l}\text { Error } \\
\text { Factor }\end{array}$ & $\begin{array}{c}\text { Failure } \\
\text { Rate }\end{array}$ & $\begin{array}{l}\text { Error } \\
\text { Factor }\end{array}$ & $\begin{array}{l}\text { Failure } \\
\text { Rate }\end{array}$ & $\begin{array}{l}\text { Error } \\
\text { Factor }\end{array}$ \\
\hline $\mathrm{RCZ2C} 1 \mathrm{QP}$ & $\begin{array}{l}\text { Contact } 7 \text { or } 8 \text { in } \\
\text { String A Fails } \\
\text { Closed }\end{array}$ & $\begin{array}{c}3.0 \mathrm{E}-4 / \mathrm{d} \\
{[10]}\end{array}$ & 10 & $1.0 E-4 / d$ & 10 & $3.0 \mathrm{E}-4 / \mathrm{d}$ & 10 & $\begin{array}{c}3.0 E-4 / d \\
{[10]}\end{array}$ & 10 \\
\hline $\mathrm{ACZ2C2OP}$ & $\begin{array}{l}\text { Contact } 7 \text { or } 8 \text { in } \\
\text { String A Fails } \\
\text { Closed }\end{array}$ & $\begin{array}{c}3.0 E-4 / d \\
{[10]}\end{array}$ & 10 & $1.0 E-4 / d$ & 10 & $3.0 \mathrm{E}-4 / \mathrm{d}$ & 10 & $\begin{array}{c}3.0 \mathrm{E}-4 / \mathrm{d} \\
{[10]}\end{array}$ & 10 \\
\hline $\mathrm{RCZ2C3QP}$ & $\begin{array}{l}\text { Contacts of K3 Relay } \\
\text { Fail Closed }\end{array}$ & $\begin{array}{c}3.0 \mathrm{E}-4 / \mathrm{d} \\
{[10]}\end{array}$ & 10 & $1.0 E-4 / \mathrm{d}$ & 10 & $3.0 \mathrm{E}-4 / \mathrm{d}$ & 10 & $\begin{array}{c}3.0 \mathrm{E} \cdot 4 / \mathrm{d} \\
{[10]}\end{array}$ & 10 \\
\hline $\mathrm{RCZ2C4OP}$ & $\begin{array}{l}\text { Contacts of K4 Relay } \\
\text { Fail Closed }\end{array}$ & $\begin{array}{c}3.0 \mathrm{E}-4 / \mathrm{d} \\
{[10]}\end{array}$ & 10 & $1.0 \mathrm{E}-4 / \mathrm{d}$ & 10 & $3.0 \mathrm{E}-4 / \mathrm{d}$ & 10 & $\begin{array}{c}3.0 E-4 / d \\
{[10]}\end{array}$ & 10 \\
\hline ACZ2C5OP & $\begin{array}{l}\text { Contacts of K5 Relay } \\
\text { Fail Closed }\end{array}$ & $\begin{array}{c}3.0 \mathrm{E}-4 / \mathrm{d} \\
{[10]}\end{array}$ & 10 & $1.0 E-4 / d$ & 10 & $3.0 \mathrm{E} \cdot 4 / \mathrm{d}$ & 10 & $\begin{array}{c}3.0 E-4 / d \\
{[10]}\end{array}$ & 10 \\
\hline $\mathrm{RCZ2C6QP}$ & $\begin{array}{l}\text { Contacts of K6 Relay } \\
\text { Fail Closed }\end{array}$ & $\begin{array}{c}3.0 \mathrm{E}-4 / \mathrm{d} \\
{[10]}\end{array}$ & 10 & $1.0 \mathrm{E}-4 / \mathrm{d}$ & 10 & $3.0 \mathrm{E}-4 / \mathrm{d}$ & 10 & $\begin{array}{c}3.0 E-4 / d \\
{[10]}\end{array}$ & 10 \\
\hline RCZ2CSOU & $\begin{array}{l}\text { CCF - Contacts of } \\
\text { All K-Relays Fail } \\
\text { Closed }\end{array}$ & $\begin{array}{c}6.0 \mathrm{E}-5 / \mathrm{d} \\
{[7]}\end{array}$ & 10 & & & & & & \\
\hline RCZ2ZOQU & $\begin{array}{l}\text { CCF - All Trip } \\
\text { Contacts of both } \\
\text { Strings Fail Closed } \\
\end{array}$ & $\begin{array}{c}2.4 E-6 / d \\
{[6]}\end{array}$ & 10 & & & & & & \\
\hline RCZ2Z8OU & $\begin{array}{l}\text { CCF - All Contacts } \\
\text { of LV K-Relays Fail } \\
\text { Closed }\end{array}$ & $\begin{array}{c}2.4 \mathrm{E}-6 / \mathrm{d} \\
{[6]}\end{array}$ & 10 & & & & & & \\
\hline ACZ2Z9QU & $\begin{array}{l}\text { CCF - All Contacts } \\
\text { of HF K-Relays Fail } \\
\text { Closed }\end{array}$ & $\begin{array}{c}2.4 \mathrm{E}-6 / \mathrm{d} \\
{[6]}\end{array}$ & 10 & & & & . & & \\
\hline RCZ3C1QP & $\begin{array}{l}\text { Contact } 7 \text { or } 8 \text { in } \\
\text { String A Fails } \\
\text { Closed }\end{array}$ & $\begin{array}{c}3.0 E-4 / d \\
{[10]}\end{array}$ & 10 & $1.0 \mathrm{E}-4 / \mathrm{d}$ & 10 & $3.0 \mathrm{E}-4 / \mathrm{d}$ & .10 & $\begin{array}{c}3.0 \mathrm{E}-4 / \mathrm{d} \\
{[10]}\end{array}$ & 10 \\
\hline $\mathrm{ACZ3C2OP}$ & $\begin{array}{l}\text { Contact } 7 \text { or } 8 \text { in } \\
\text { String B Fails } \\
\text { Closed }\end{array}$ & $\begin{array}{c}3.0 \mathrm{E}-4 / \mathrm{d} \\
{[10]}\end{array}$ & 10 & $1.0 \mathrm{E}-4 / \mathrm{d}$ & 10 & $3.0 \mathrm{E}-4 / \mathrm{d}$ & 10 & $\begin{array}{c}3.0 \mathrm{E}-4 / \mathrm{d} \\
{[10]}\end{array}$ & 10 \\
\hline ACZ3D1QP & $\begin{array}{l}\text { Contact } 7 \text { or } 8 \text { in } \\
\text { String A Fails } \\
\text { Closed }\end{array}$ & $\begin{array}{c}3.0 E-4 / d \\
{[10]}\end{array}$ & 10 & $1.0 E-4 / d$ & 10 & $3.0 E-4 / d$ & 10 & $\begin{array}{c}.0 E-4 / d \\
{[10]}\end{array}$ & 10 \\
\hline ACZ3D2QP & $\begin{array}{l}\text { Contact } 7 \text { or } 8 \text { in } \\
\text { String } B \text { Fails } \\
\text { Closed }\end{array}$ & $\begin{array}{c}3.0 \mathrm{E}-4 / \mathrm{d} \\
{[10]}\end{array}$ & 10 & $1.0 \mathrm{E}-4 / \mathrm{d}$ & 10 & $3.0 \mathrm{E}-4 / \mathrm{d}$ & 10 & $\begin{array}{c}3.0 \mathrm{E}-4 / \mathrm{d} \\
{[10]}\end{array}$ & 10 \\
\hline ACZ3FIOP & $\begin{array}{l}\text { Contact } 7 \text { or } 8 \text { in } \\
\text { String A Fails } \\
\text { Closed }\end{array}$ & $\begin{array}{c}3.0 E-4 / d \\
{[10]}\end{array}$ & 10 & $1.0 \mathrm{E}-4 / \mathrm{d}$ & 10 & $3.0 \mathrm{E}-4 / \mathrm{d}$ & 10 & $\begin{array}{c}3.0 \mathrm{E}-4 / \mathrm{d} \\
{[10]}\end{array}$ & 10 \\
\hline ACZ3F2OP & $\begin{array}{l}\text { Contact } 7 \text { or } 8 \text { in } \\
\text { String B Fails } \\
\text { Closed }\end{array}$ & $\begin{array}{c}3.0 E-4 / d \\
{[10]}\end{array}$ & 10 & $1.0 \mathrm{E}-4 / \mathrm{d}$ & 10 & $3.0 E-4 / d$ & 10 & $\begin{array}{c}3.0 \mathrm{E}-4 / \mathrm{d} \\
{[10]}\end{array}$ & 10 \\
\hline RCZ3H1QP & $\begin{array}{l}\text { Contact } 7 \text { or } 8 \text { in } \\
\text { String A Fails } \\
\text { Closed }\end{array}$ & $\begin{array}{c}3.0 E-4 / d \\
{[10]}\end{array}$ & 10 & $1.0 \mathrm{E}-4 / \mathrm{d}$ & 10 & $3.0 E-4 / d$ & 10 & $\begin{array}{c}3.0 \mathrm{E}-4 / \mathrm{d} \\
{[10]}\end{array}$ & 10 \\
\hline $\mathrm{ACZ3H} 2 \mathrm{OP}$ & $\begin{array}{l}\text { Contact } 7 \text { or } 8 \text { in } \\
\text { String B Fails } \\
\text { Closed } \\
\end{array}$ & $\begin{array}{c}3.0 E-4 / d \\
{[10]}\end{array}$ & 10 & $1.0 \mathrm{E}-4 / \mathrm{d}$ & 10 & $3.0 E-4 / d$ & 10 & $\begin{array}{c}3.0 \mathrm{E}-4 / \mathrm{d} \\
{[10]}\end{array}$ & 10 \\
\hline$R C Z 401 Q P$ & $\begin{array}{l}\text { Relay UV Contact } 1 \\
\text { in String A Fails } \\
\text { Closed }\end{array}$ & $\begin{array}{c}3.0 \mathrm{E}-4 / \mathrm{d} \\
{[10]}\end{array}$ & 10 & $1.0 \mathrm{E}-4 / \mathrm{d}$ & 10 & $3.0 E-4 / d$ & 10 & $\begin{array}{c}3.0 E-4 / d \\
{[10]}\end{array}$ & 10 \\
\hline $\mathrm{RCZ402OU}$ & $\begin{array}{l}\text { CCF - UV Trip Relay } \\
\text { Contacts Fail Closed }\end{array}$ & $\begin{array}{c}3.0 \mathrm{E}-5 / \mathrm{d} \\
{[4]}\end{array}$ & 10 & & & & & & \\
\hline RCZCOOQU & $\begin{array}{l}\text { CCF - Contacts of } \\
\text { All CP-Relays Fail } \\
\text { Closed }\end{array}$ & $\begin{array}{c}2.4 \mathrm{E}-6 / \mathrm{d} \\
{[6]}\end{array}$ & 10 & & & & & & \\
\hline
\end{tabular}


Table 7.2. Reactor Shutdown System Data (Cont'd)

PAGE 5

\begin{tabular}{|c|c|c|c|c|c|c|c|c|c|}
\hline \multirow{2}{*}{$\begin{array}{l}\text { EVENT } \\
\text { NAME }\end{array}$} & \multirow[b]{2}{*}{ DESCRIPTION } & \multicolumn{2}{|c|}{ VALUE USED $3 / 91$} & \multicolumn{2}{|c|}{ CRBRP-4 } & \multicolumn{2}{|c|}{ IREP } & \multicolumn{2}{|c|}{ GENERIC } \\
\hline & & $\begin{array}{l}\text { Failure } \\
\text { Rate }\end{array}$ & $\begin{array}{l}\text { Error } \\
\text { Factor }\end{array}$ & $\begin{array}{c}\text { Failure } \\
\text { Rate }\end{array}$ & $\begin{array}{l}\text { Error } \\
\text { Factor }\end{array}$ & $\begin{array}{c}\text { Failure } \\
\text { Rate }\end{array}$ & $\begin{array}{l}\text { Error } \\
\text { Factor }\end{array}$ & $\begin{array}{c}\text { Failure } \\
\text { Rate }\end{array}$ & $\begin{array}{l}\text { Error } \\
\text { Factor }\end{array}$ \\
\hline RCZCO1QU & $\begin{array}{l}\text { CCF - Contacts of } \\
\text { All String A Relays } \\
\text { Fail Closed }\end{array}$ & $\begin{array}{c}9.0 E-5 / d \\
{[11]}\end{array}$ & 10 & & & & & & \\
\hline RCZCO2OU & $\begin{array}{l}\text { CCF - Contacts of } \\
\text { All String B Relays } \\
\text { Fail Closed }\end{array}$ & $\begin{array}{c}9.0 \mathrm{E}-5 / \mathrm{d} \\
{[11]}\end{array}$ & 10 & & & & & & \\
\hline RCZC81QP & Contacts Fail Closed & $\begin{array}{c}3.0 \mathrm{E}-4 / \mathrm{d} \\
{[10]}\end{array}$ & 10 & $1.0 E-4 / d$ & 10 & $3.0 E-4 / d$ & 10 & $\begin{array}{c}3.0 E-4 / d \\
{[10]}\end{array}$ & 10 \\
\hline RCZCB2QP & Contacts Fail Closed & $\begin{array}{c}3.0 E-4 / d \\
{[10]}\end{array}$ & 10 & $1.0 \mathrm{E}-4 / \mathrm{d}$ & 10 & $3.0 E-4 / d$ & 10 & $\begin{array}{c}\text { 3.0E-4/d } \\
{[10]}\end{array}$ & 10 \\
\hline RCZCC1QP & Contacts Fail Closed & $\begin{array}{l}3.0 \mathrm{E}-4 / \mathrm{d} \\
\cdot[10]\end{array}$ & 10 & $1.0 E-4 / d$ & 10 & $3.0 \mathrm{E}-4 / \mathrm{d}$ & 10 & $\begin{array}{c}3.0 E-4 / d \\
{[10]}\end{array}$ & 10 \\
\hline RCZCP2QP & Contacts Fail Closed & $\begin{array}{c}3.0 \mathrm{E}-4 / \mathrm{d} \\
{[10]}\end{array}$ & 10 & $1.0 E-4 / d$ & 10 & $3.0 E-4 / d$ & 10 & $\begin{array}{c}3.0 E-4 / d \\
{[10]}\end{array}$ & 10 \\
\hline RCZCPAQP & Contacts Fail Closed & $\begin{array}{c}3.0 \mathrm{E}-4 / \mathrm{d} \\
{[10]}\end{array}$ & 10 & $1.0 \mathrm{E}-4 / \mathrm{d}$ & 10 & $3.0 \mathrm{E}-4 / \mathrm{d}$ & 10 & $\begin{array}{c}\text { 3.0E-4/d } \\
{[10]}\end{array}$ & 10 \\
\hline RCZCPYQP & Contacts Fail Closed & $\begin{array}{c}3.0 \mathrm{E}-4 / \mathrm{d} \\
{[10]}\end{array}$ & 10 & $1.0 E-4 / d$ & 10 & $3.0 \mathrm{E}-4 / \mathrm{d}$ & 10 & $\begin{array}{c}3.0 \mathrm{E}-4 / \mathrm{d} \\
{[10]}\end{array}$ & 10 \\
\hline RCZSROQP & Contacts Fail Closed & $\begin{array}{c}3.0 \mathrm{E}-4 / \mathrm{d} \\
{[10]}\end{array}$ & 10 & $1.0 \mathrm{E}-4 / \mathrm{d}$ & 10 & $3.0 \mathrm{E}-4 / \mathrm{d}$ & 10 & $\begin{array}{c}3.0 \mathrm{E}-4 / \mathrm{d} \\
{[10]}\end{array}$ & 10 \\
\hline RCZSRXQP & Contacts Fail Closed & $\begin{array}{c}3.0 E-4 / \mathrm{d} \\
{[10]}\end{array}$ & 10 & $1.0 \mathrm{E}-4 / \mathrm{d}$ & 10 & $3.0 \mathrm{E}-4 / \mathrm{d}$ & 10 & $\begin{array}{c}3.0 \mathrm{E}-4 / \mathrm{d} \\
{[10]}\end{array}$ & 10 \\
\hline RDC1C1AW & $\begin{array}{l}\text { Transmitter DC Power } \\
\text { Supply Fails. }\end{array}$ & $\begin{array}{c}3.0 \mathrm{E} \cdot 6 \mathrm{~m} \\
{[3]}\end{array}$ & .10 & & & & & $\begin{array}{c}3.0 \mathrm{E} \cdot 6 / \mathrm{h} \\
{[3]}\end{array}$ & 10 \\
\hline RDC1D1RW & $\begin{array}{l}\text { Transmitter DC Power } \\
\text { Supply Fails }\end{array}$ & $\begin{array}{c}3.0 \mathrm{E}-6 / \mathrm{h} \\
{[3]}\end{array}$ & 10 & & & & & $\begin{array}{c}3.0 \mathrm{E}-6 / \mathrm{h} \\
{[3]}\end{array}$ & 10 \\
\hline RDC1D2QW & $\begin{array}{l}\text { Bistable DC Power } \\
\text { Supply Fails }\end{array}$ & $\begin{array}{c}3.0 \mathrm{E} \cdot 6 / \mathrm{h} \\
{[3]}\end{array}$ & 10 & & & & & $\begin{array}{c}3.0 \mathrm{E}-6 / \mathrm{h} \\
{[3]}\end{array}$ & 10 \\
\hline RDC2A1OW & $\begin{array}{l}+15 \text { V DC Power } \\
\text { Supply Fails Low }\end{array}$ & $\begin{array}{c}3.0 \mathrm{E}-6 \mathrm{~h} \\
{[8]}\end{array}$ & 10 & & & & & $\begin{array}{c}3.0 \mathrm{E}-6 / \mathrm{h} \\
{[3]}\end{array}$ & 10 \\
\hline RDC2A2OW & $\begin{array}{l}\text { High Volt DC Power } \\
\text { Supply Fails Low }\end{array}$ & $\begin{array}{c}3.0 E-6 / \mathrm{h} \\
{[8]}\end{array}$ & 10 & & & & & $\begin{array}{c}.0 E-6 / \mathrm{h} \\
{[3]}\end{array}$ & 10 \\
\hline RDC2B1OW & $\begin{array}{l}+15 \text { V DC Power } \\
\text { Supply Fails Low }\end{array}$ & $\begin{array}{c}.0 E-6 / \mathrm{h} \\
{[8]}\end{array}$ & 10 & & & & & $\begin{array}{c}3.0 E-6 / \mathrm{h} \\
{[3]}\end{array}$ & 10 \\
\hline RDC2B2aW & $\begin{array}{l}\text { High Volt DC Power } \\
\text { Supply Fails Low }\end{array}$ & $\begin{array}{c}3.0 \mathrm{E}-6 / \mathrm{h} \\
{[8]}\end{array}$ & 10 & & & & & $\begin{array}{c}3.0 \mathrm{E}-6 \mathrm{~h} \\
{[3]}\end{array}$ & 10 \\
\hline $\mathrm{RDC} 2 \mathrm{C} 1 \mathrm{OW}$ & $\begin{array}{l}+15 \text { V DC Power } \\
\text { Supply Fails Low }\end{array}$ & $\begin{array}{c}.0 E-6 / \mathrm{h} \\
{[8]}\end{array}$ & 10 & & & & & $\begin{array}{c}3.0 \mathrm{E}-6 / \mathrm{h} \\
{[3]}\end{array}$ & 10 \\
\hline RDC2C2OW & $\begin{array}{l}\text { High Volt DC Power } \\
\text { Supply Fails Low }\end{array}$ & $\begin{array}{c}3.0 \mathrm{E}-6 / \mathrm{h} \\
{[8]}\end{array}$ & 10 & & & & & $\begin{array}{c}3.0 E-6 / \mathrm{h} \\
{[3]}\end{array}$ & 10 \\
\hline RDC2Z1QU & $\begin{array}{l}\text { ALL +15 V DC Power } \\
\text { Supplies Fail Low }\end{array}$ & $\begin{array}{c}3.0 E-7 / \mathrm{h} \\
{[4]}\end{array}$ & 10 & & & & & & \\
\hline RDC2Z2QU & $\begin{array}{l}\text { CCF - All High Volt } \\
\text { DC Power Supplies } \\
\text { Fail Low }\end{array}$ & $\underset{[4]}{3.0 E-7 / h}$ & 10 & & & & & & \\
\hline RFM1AORW & $\begin{array}{l}\text { Flow Sensor Fails } \\
\text { High or Fails to } \\
\text { Respond }\end{array}$ & $\begin{array}{c}3.0 \mathrm{E}-6 / \mathrm{h} \\
{[12]}\end{array}$ & 10 & $\begin{array}{c}3.0 E-5 / h \\
{[13]}\end{array}$ & 10 & $\begin{array}{c}3.0 E-6 / h \\
{[13]}\end{array}$ & 10 & $\begin{array}{c}1.0 \mathrm{E}-6 / \mathrm{h} \\
{[10]}\end{array}$ & 10 \\
\hline RFM1BORW & $\begin{array}{l}\text { Flow Sensor Fails } \\
\text { High or Fails to } \\
\text { Respond }\end{array}$ & $\begin{array}{c}3.0 \mathrm{E}-6 / \mathrm{h} \\
{[12]}\end{array}$ & 10 & $\begin{array}{c}3.0 \mathrm{E}-5 / \mathrm{h} \\
{[13]}\end{array}$ & 10 & $\begin{array}{c}3.0 \mathrm{E}-6 / \mathrm{h} \\
{[13]}\end{array}$ & 10 & $\begin{array}{c}1.0 \mathrm{E}-6 / \mathrm{h} \\
{[10]}\end{array}$ & 10 \\
\hline RFM1CORW & $\begin{array}{l}\text { Flow Sensor Fails } \\
\text { High or Fails to } \\
\text { Respond }\end{array}$ & $\begin{array}{c}3.0 \mathrm{E}-6 \mathrm{~h} \\
{[12]}\end{array}$ & 10 & $\begin{array}{c}3.0 \mathrm{E}-5 / \mathrm{h} \\
{[13]}\end{array}$ & 10 & $\begin{array}{c}3.0 \mathrm{E}-6 / \mathrm{h} \\
{[13]}\end{array}$ & 10 & $\begin{array}{c}1.0 \mathrm{E}-6 / \mathrm{h} \\
{[10]}\end{array}$ & 10 \\
\hline RFM1DORW & $\begin{array}{l}\text { Flow Sensor Fails } \\
\text { High or Fails to } \\
\text { Respond }\end{array}$ & $\begin{array}{c}3.0 \mathrm{E}-6 / \mathrm{h} \\
{[12]}\end{array}$ & 10 & $\begin{array}{c}3.0 \mathrm{E}-5 / \mathrm{h} \\
{[13]}\end{array}$ & 10 & $\begin{array}{c}3.0 \mathrm{E}-6 / \mathrm{h} \\
{[13]}\end{array}$ & 10 & $\begin{array}{c}1.0 \mathrm{E}-6 / \mathrm{h} \\
{[10]}\end{array}$ & 10 \\
\hline
\end{tabular}


Table 7.2. Reactor Shutdown System Data (Cont'd)

PAGE 6

\begin{tabular}{|c|c|c|c|c|c|c|c|c|c|}
\hline \multirow{2}{*}{$\begin{array}{l}\text { EVENT } \\
\text { NAME }\end{array}$} & \multirow[b]{2}{*}{ DESCRIPTION } & \multicolumn{2}{|c|}{ VALUE USED 391 } & \multicolumn{2}{|c|}{ CRBRP-4 } & \multicolumn{2}{|c|}{ IREP } & \multicolumn{2}{|c|}{ GENERIC } \\
\hline & & $\begin{array}{c}\text { Failure } \\
\text { Rate }\end{array}$ & $\begin{array}{l}\text { Error } \\
\text { Factor }\end{array}$ & $\begin{array}{l}\text { Failure } \\
\text { Rate }\end{array}$ & $\begin{array}{l}\text { Error } \\
\text { Factor }\end{array}$ & $\begin{array}{c}\text { Failure } \\
\text { Rate }\end{array}$ & $\begin{array}{l}\text { Error } \\
\text { Factor }\end{array}$ & $\begin{array}{c}\text { Failure } \\
\text { Rate }\end{array}$ & $\begin{array}{l}\text { Error } \\
\text { Factor }\end{array}$ \\
\hline RFM1Z1RU & $\begin{array}{l}\text { CCF - } \\
\text { Electro-Magnetic } \\
\text { Flow Sensors Fail }\end{array}$ & $\begin{array}{c}3.0 \mathrm{E}-7 \mathrm{M} \\
{[4]}\end{array}$ & 10 & & & - & & & \\
\hline RHU001CL & $\begin{array}{l}\text { Opert Error - } \\
\text { Failure to Push } \\
\text { SCRAM Buttons }\end{array}$ & $\begin{array}{c}5.0 \mathrm{E}-2 / \mathrm{d} \\
{[14]}\end{array}$ & 10 & & & & & & \\
\hline RHU002CL & $\begin{array}{l}\text { Opert Error - } \\
\text { Failure to Push } \\
\text { SCRAM Buttons }\end{array}$ & $\begin{array}{c}1.0 \mathrm{E}-1 / \mathrm{d} \\
{[14]}\end{array}$ & 9 & & & & & & \\
\hline RHU006CL & $\begin{array}{l}\text { Operator Failure to } \\
\text { Push SCRAM Buttons } \\
\text { WLOHS Conditions }\end{array}$ & $\begin{array}{c}1.0 \mathrm{E}-6 / \mathrm{d} \\
{[15]}\end{array}$ & 10 & & & & & & \\
\hline RHUOOZCL & $\begin{array}{l}\text { Opert Error - } \\
\text { Failure to Push 3MPB } \\
\text { SCRAM Button }\end{array}$ & $\begin{array}{c}7.5 \mathrm{E}-3 / \mathrm{d} \\
{[16]}\end{array}$ & 10 & & & & & & \\
\hline RHU201QL & $\begin{array}{l}\text { CCF Human Error - } \\
\text { Failure to Set HF } \\
\text { Trip Points Correct }\end{array}$ & $\begin{array}{c}1.5 \mathrm{E}-6 / \mathrm{d} \\
{[16]}\end{array}$ & 10 & & & & & & \\
\hline RHU202QL & $\begin{array}{l}\text { CCF Human Error - } \\
\text { Failure to Set LV } \\
\text { Trip Points Correct }\end{array}$ & $\begin{array}{c}1.5 \mathrm{E}-6 / \mathrm{d} \\
{[16]}\end{array}$ & 10 & & & & & & \\
\hline RHU302QL & $\begin{array}{l}\text { CCF - Human Error - } \\
\text { SOT Trip Points Set } \\
\text { Too High }\end{array}$ & $\begin{array}{c}2.5 \mathrm{E}-2 / \mathrm{d} \\
{[16]}\end{array}$ & 10 & & & & & & \\
\hline RMOOO1RR & $\begin{array}{l}\text { Primary Pump } 1 \text { Motor } \\
\text { Fails to Run }\end{array}$ & $2.0 \mathrm{E}-5 / \mathrm{h}$ & 3 & $1.0 \mathrm{E}-5 \mathrm{~h}$ & 10 & & & $3.0 \mathrm{E}-5 / \mathrm{h}$ & 3 \\
\hline RMOOO2RR & $\begin{array}{l}\text { Primary Pump } 2 \text { Motor } \\
\text { Fails to Run }\end{array}$ & $2.0 \mathrm{E}-5 / \mathrm{h}$ & 3 & $1.0 \mathrm{E}-5 / \mathrm{h}$ & 10 & & & $3.0 \mathrm{E}-5 / \mathrm{h}$ & 3 \\
\hline RND2AORW & $\begin{array}{l}\text { Grded Fission } \\
\text { Chamber in } \mathrm{J} 1 \\
\text { Thimble Fails }\end{array}$ & $\begin{array}{c}3.0 \mathrm{E} \cdot 6 / \mathrm{h} \\
{[1]}\end{array}$ & 10 & & & & & $\begin{array}{c}6.0 \mathrm{E}-6 / \mathrm{h} \\
{[3]}\end{array}$ & 3 \\
\hline RND2BORW & $\begin{array}{l}\text { Grded Fission } \\
\text { Chamber in J3 } \\
\text { Thimble Fails }\end{array}$ & $\begin{array}{c}3.0 \mathrm{E}-6 / \mathrm{h} \\
{[1]}\end{array}$ & 10 & & & & & $\begin{array}{c}6.0 \mathrm{E}-6 / \mathrm{h} \\
{[3]}\end{array}$ & 3 \\
\hline RND2CORW & $\begin{array}{l}\text { Grded Fission } \\
\text { Chamber in J44 } \\
\text { Thimble Fails }\end{array}$ & $\begin{array}{c}3.0 \mathrm{E}-6 / \mathrm{h} \\
{[1]}\end{array}$ & 10 & & & & & $\begin{array}{c}6.0 \mathrm{E}-6 / \mathrm{h} \\
{[3]}\end{array}$ & 3 \\
\hline RND2ZORU & $\begin{array}{l}\text { All Grded Fission } \\
\text { Chambers Fail }\end{array}$ & $\begin{array}{c}3.0 \mathrm{E}-7 \mathrm{~h} \\
{[4]}\end{array}$ & 10 & & & & & & \\
\hline ROO1AA00 & $\begin{array}{l}\text { Flow Sensor Can Not } \\
\text { Detect LOF Condition } \\
\text { (Location) }\end{array}$ & 0.0 & 1 & & & & & & \\
\hline$R O 01 A B 00$ & $\begin{array}{l}\text { Flow Sensor Can } \\
\text { Detect LOF Condition } \\
\text { (Location) }\end{array}$ & 1.0 & 1 & & & & & & \\
\hline ROOS9A00 & $\begin{array}{l}\text { Reactor Operation } \\
\text { Permitted }\end{array}$ & 1.0 & 1 & & & & & & \\
\hline$R 0099800$ & $\begin{array}{l}\text { Reactor Operation } \\
\text { Not Permitted }\end{array}$ & 0.0 & 1 & & & & & & \\
\hline RREIAOQU & $\begin{array}{l}\text { CCF - Trip Relays of } \\
\text { both Strings Fail to } \\
\text { Move }\end{array}$ & $\begin{array}{c}4.0 \mathrm{E}-8 / \mathrm{h} \\
{[7]}\end{array}$ & 10 & & & & & & \\
\hline RRE 1A1QP & $\begin{array}{l}\text { String A Relay Fails } \\
\text { to Change Position }\end{array}$ & $\begin{array}{c}2.0 \mathrm{E}-7 \mathrm{M} \\
{[8]}\end{array}$ & 10 & $1.0 \mathrm{E}-6 / \mathrm{h}$ & 10 & $3.0 \mathrm{E}-6 / \mathrm{h}$ & 10 & $\begin{array}{c}3.0 \mathrm{E}-6 / \mathrm{h} \\
{[5]}\end{array}$ & \\
\hline RRE1A2QP & $\begin{array}{l}\text { String B Relay Fails } \\
\text { to Change Position }\end{array}$ & $\begin{array}{c}2.0 \mathrm{E}-7 \mathrm{~m} \\
{[8]}\end{array}$ & 10 & $1.0 \mathrm{E}-6 / \mathrm{h}$ & 10 & $3.0 \mathrm{E}-6 / \mathrm{h}$ & 10 & $\begin{array}{c}3.0 \mathrm{E}-6 / \mathrm{h} \\
{[5]}\end{array}$ & \\
\hline RRE1BOOU & $\begin{array}{l}\text { CCF - Trip Relays of } \\
\text { both Strings Fail to } \\
\text { Move }\end{array}$ & $\begin{array}{c}4.0 \mathrm{E}-8 / \mathrm{h} \\
{[7]}\end{array}$ & 10 & & & & & & \\
\hline
\end{tabular}


Table 7.2. Reactor Shutdown System Data (Cont'd)

PAGE 7

\begin{tabular}{|c|c|c|c|c|c|c|c|c|c|}
\hline \multirow{2}{*}{$\begin{array}{l}\text { EVENT } \\
\text { NAME }\end{array}$} & \multirow[b]{2}{*}{ DESCRIPTION } & \multicolumn{2}{|c|}{ VALUE USED 391} & \multicolumn{2}{|c|}{ CRBRP-4 } & \multicolumn{2}{|c|}{ IREP } & \multicolumn{2}{|c|}{ GENERIC } \\
\hline & & $\begin{array}{c}\text { Failure } \\
\text { Rate }\end{array}$ & $\begin{array}{l}\text { Error } \\
\text { Factor }\end{array}$ & $\begin{array}{l}\text { Failure } \\
\text { Rate }\end{array}$ & $\begin{array}{l}\text { Error } \\
\text { Factor }\end{array}$ & $\begin{array}{l}\text { Failure } \\
\text { Rate }\end{array}$ & $\begin{array}{l}\text { Error } \\
\text { Factor }\end{array}$ & $\begin{array}{c}\text { Failure } \\
\text { Rate }\end{array}$ & $\begin{array}{l}\text { Error } \\
\text { Factor }\end{array}$ \\
\hline RRE1B1QP & $\begin{array}{l}\text { String A Relay Fails } \\
\text { to Change Position }\end{array}$ & $\underset{[8]}{2.0 E-7 / h}$ & 10 & $1.0 \mathrm{E}-6 / \mathrm{h}$ & 10 & $3.0 \mathrm{E}-6 / \mathrm{h}$ & 10 & $\begin{array}{c}3.0 \mathrm{E}-6 / \mathrm{h} \\
{[5]}\end{array}$ & \\
\hline RRE182QP & $\begin{array}{l}\text { String B Relay Fails } \\
\text { to Change Position }\end{array}$ & $\begin{array}{c}2.0 \mathrm{E}-7 / \mathrm{h} \\
{[8]}\end{array}$ & 10 & $1.0 \mathrm{E}-6 / \mathrm{h}$ & 10 & $3.0 \mathrm{E}-6 / \mathrm{h}$ & 10 & $\begin{array}{c}3.0 \mathrm{E}-6 / \mathrm{h} \\
{[5]}\end{array}$ & \\
\hline RRE1COOU & $\begin{array}{l}\text { CCF - Trip Relays of } \\
\text { both Strings Fail to } \\
\text { Move }\end{array}$ & $\begin{array}{c}4.0 \mathrm{E} \cdot 8 / \mathrm{h} \\
{[7]}\end{array}$ & 10 & & & & & & \\
\hline RRE1C1QP & $\begin{array}{l}\text { String A Relay Fails } \\
\text { to Change Position }\end{array}$ & $\begin{array}{c}2.0 \mathrm{E}-7 \mathrm{~h} \\
{[8]}\end{array}$ & 10 & $1.0 \mathrm{E}-6 / \mathrm{h}$ & 10 & $3.0 \mathrm{E}-6 / \mathrm{h}$ & 10 & $\begin{array}{c}3.0 \mathrm{E}-6 / \mathrm{h} \\
{[5]}\end{array}$ & \\
\hline RRE1C2OP & $\begin{array}{l}\text { String B Relay Fails } \\
\text { to Change Position }\end{array}$ & $\underset{[8]}{2.0 \mathrm{E}-7 / \mathrm{h}}$ & 10 & $1.0 \mathrm{E}-6 / \mathrm{h}$ & 10 & $3.0 \mathrm{E}-6 / \mathrm{h}$ & 10 & $\begin{array}{c}3.0 \mathrm{E}-6 / \mathrm{h} \\
{[5]}\end{array}$ & \\
\hline RRE 1DOQU & $\begin{array}{l}\text { CCF - Trip Relays of } \\
\text { both Strings Fail to } \\
\text { Move }\end{array}$ & $\begin{array}{c}4.0 \mathrm{E}-8 \mathrm{~h} \\
{[7]}\end{array}$ & 10 & & & & & & \\
\hline RRE1D1QP & $\begin{array}{l}\text { String A Relay Fails } \\
\text { to Change Position }\end{array}$ & $\begin{array}{c}2.0 E-7 / h \\
{[8]}\end{array}$ & 10 & $1.0 \mathrm{E}-6 / \mathrm{h}$ & 10 & $3.0 \mathrm{E}-6 / \mathrm{h}$ & 10 & $\begin{array}{c}3.0 \mathrm{E}-6 / \mathrm{h} \\
{[5]}\end{array}$ & \\
\hline RRE1D2QP & $\begin{array}{l}\text { String B Relay Fails } \\
\text { to Change Position }\end{array}$ & $\begin{array}{c}2.0 \mathrm{E}-7 \mathrm{~h} \\
{[8]}\end{array}$ & 10 & $1.0 \mathrm{E}-6 / \mathrm{h}$ & 10 & $3.0 \mathrm{E}-6 / \mathrm{h}$ & 10 & $\begin{array}{c}3.0 \mathrm{E}-6 / \mathrm{h} \\
{[5]}\end{array}$ & \\
\hline RRE1ZOQU & $\begin{array}{l}\text { CCF - All Trip } \\
\text { Relays Fail to Move } \\
\end{array}$ & $\begin{array}{c}1.6 \mathrm{E}-9 / \mathrm{h} \\
{[6]}\end{array}$ & 10 & & & & & & \\
\hline RRE2AOQU & $\begin{array}{l}\text { CCF - Trip Relays of } \\
\text { both Strings Fail to } \\
\text { Move }\end{array}$ & $\begin{array}{c}4.0 \mathrm{E}-8 / \mathrm{h} \\
{[7]}\end{array}$ & 10 & & & & & & \\
\hline RRE2A1OP & $\begin{array}{l}\text { String A Relay Fails } \\
\text { to Move to Open } \\
\text { Position }\end{array}$ & $\begin{array}{c}2.0 \mathrm{E}-7 / \mathrm{h} \\
{[8] .}\end{array}$ & 10 & $1.0 \mathrm{E}-6 / \mathrm{h}$ & 10 & $3.0 \mathrm{E}-6 / \mathrm{h}$ & 10 & $\begin{array}{c}3.0 \mathrm{E}-6 / \mathrm{h} \\
{[5]}\end{array}$ & \\
\hline RRE2A2QP & $\begin{array}{l}\text { String B Relay Fails } \\
\text { to Move to Open } \\
\text { Position }\end{array}$ & $\begin{array}{c}2.0 \mathrm{E}-7 \mathrm{~h} \\
{[8]}\end{array}$ & 10 & $1.0 \mathrm{E}-6 / \mathrm{h}$ & 10 & $3.0 \mathrm{E} \cdot 6 / \mathrm{h}$ & 10 & $\begin{array}{c}3.0 \mathrm{E}-6 / \mathrm{h} \\
{[5]}\end{array}$ & \\
\hline RRE2A3QP & $\begin{array}{l}\text { K3 Relay Fails to } \\
\text { Move to Open } \\
\text { Position }\end{array}$ & $\begin{array}{c}2.0 \mathrm{E}-7 / \mathrm{h} \\
{[8]}\end{array}$ & 10 & $1.0 \mathrm{E}-6 / \mathrm{h}$ & 10 & $3.0 \mathrm{E}-6 / \mathrm{h}$ & 10 & $\begin{array}{c}3.0 \mathrm{E}-6 / \mathrm{h} \\
{[5]}\end{array}$ & \\
\hline RRE2A4QP & $\begin{array}{l}\text { K4 Relay Fails to } \\
\text { Move to Open } \\
\text { Position }\end{array}$ & $\begin{array}{c}2.0 \mathrm{E}-7 \mathrm{~h} \\
{[8]}\end{array}$ & 10 & $1.0 \mathrm{E}-6 / \mathrm{h}$ & 10 & $3.0 \mathrm{E}-6 / \mathrm{h}$ & 10 & $\begin{array}{c}3.0 \mathrm{E}-6 / \mathrm{h} \\
{[5]}\end{array}$ & \\
\hline RRE2A5QP & $\begin{array}{l}\text { K5 Relay Fails to } \\
\text { Move to Open } \\
\text { Position }\end{array}$ & $\begin{array}{c}2.0 \mathrm{E}-7 \mathrm{~h} \\
{[8]}\end{array}$ & 10 & $1.0 \mathrm{E} \cdot 6 / \mathrm{h}$ & 10 & $3.0 E-6 / \mathrm{h}$ & 10 & $\begin{array}{c}3.0 \mathrm{E}-6 / \mathrm{h} \\
{[5]}\end{array}$ & \\
\hline RRE2A6QP & $\begin{array}{l}\text { K6 Relay Fails to } \\
\text { Move to Open } \\
\text { Position }\end{array}$ & $\begin{array}{c}2.0 E-7 / \mathrm{h} \\
{[8]}\end{array}$ & 10 & $1.0 \mathrm{E}-6 \mathrm{~h}$ & 10 & $3.0 \mathrm{E}-6 / \mathrm{h}$ & 10 & $\begin{array}{c}3.0 E-6 / h \\
{[5]}\end{array}$ & \\
\hline RRE2A9QU & $\begin{array}{l}\text { CCF - All K-Relays } \\
\text { of Channel A Fail to } \\
\text { Move }\end{array}$ & $\begin{array}{c}4.0 \mathrm{E}-8 / \mathrm{h} \\
{[7]}\end{array}$ & 10 & & & & - & & \\
\hline RRE2BOQU & $\begin{array}{l}\text { CCF - Trip Relays of } \\
\text { both Strings Fail to } \\
\text { Move }\end{array}$ & $\begin{array}{c}4.0 E-8 / h \\
{[7]}\end{array}$ & 10 & & & & & & \\
\hline RRE2B1QP & $\begin{array}{l}\text { String A Relay Fails } \\
\text { to Move to Open } \\
\text { Position }\end{array}$ & $\begin{array}{c}2.0 \mathrm{E}-7 \mathrm{~h} \\
{[8]}\end{array}$ & 10 & $1.0 \mathrm{E}-6 / \mathrm{h}$ & 10 & $3.0 \mathrm{E}-6 / \mathrm{h}$ & 10 & $\begin{array}{c}\text { 3.0E-6/h } \\
{[5]}\end{array}$ & \\
\hline RRE2B2QP & $\begin{array}{l}\text { String B Relay Fails } \\
\text { to Move to Open } \\
\text { Position }\end{array}$ & $\begin{array}{c}2.0 \mathrm{E}-7 \mathrm{M} \\
{[\mathrm{g}]}\end{array}$ & 10 & $1.0 \mathrm{E}-6 / \mathrm{h}$ & 10 & $3.0 \mathrm{E}-6 / \mathrm{h}$ & 10 & $\begin{array}{c}3.0 E \cdot 6 / \mathrm{h} \\
{[5]}\end{array}$ & \\
\hline RRE2B3QP & $\begin{array}{l}\text { K3 Relay Fails to } \\
\text { Move to Open } \\
\text { Position }\end{array}$ & $\begin{array}{c}2.0 \mathrm{E}-7 \mathrm{~h} \\
{[8]}\end{array}$ & 10 & $1.0 \mathrm{E}-6 / \mathrm{h}$ & 10 & $3.0 \mathrm{E}-6 / \mathrm{h}$ & 10 & $\begin{array}{c}3.0 \mathrm{E}-6 / \mathrm{h} \\
{[5]}\end{array}$ & \\
\hline RRE2B4QP & $\begin{array}{l}\text { K4 Relay Fails to } \\
\text { Move to Open } \\
\text { Position }\end{array}$ & $\begin{array}{c}2.0 \mathrm{E}-7 / \mathrm{h} \\
{[8]}\end{array}$ & 10 & $1.0 \mathrm{E}-6 / \mathrm{h}$ & 10 & $3.0 \mathrm{E} \cdot 6 / \mathrm{h}$ & 10 & $\begin{array}{c}3.0 \mathrm{E}-6 / \mathrm{h} \\
{[5]}\end{array}$ & \\
\hline
\end{tabular}


Table 7.2. Reactor Shutdown System Data (Cont'd)

PAGE 8

\begin{tabular}{|c|c|c|c|c|c|c|c|c|c|}
\hline \multirow{2}{*}{$\begin{array}{l}\text { EVENT } \\
\text { NAME }\end{array}$} & \multirow[b]{2}{*}{ DESCRIPTION } & \multicolumn{2}{|c|}{ VALUE USED $3 / 91$} & \multicolumn{2}{|c|}{ CRBRP-4 } & \multicolumn{2}{|c|}{ IREP } & \multicolumn{2}{|c|}{ GENERIC } \\
\hline & & $\begin{array}{c}\text { Failure } \\
\text { Rate }\end{array}$ & $\begin{array}{l}\text { Error } \\
\text { Factor }\end{array}$ & $\begin{array}{c}\text { Failure } \\
\text { Rate }\end{array}$ & $\begin{array}{l}\text { Error } \\
\text { Factor }\end{array}$ & $\begin{array}{c}\text { Failure } \\
\text { Rate }\end{array}$ & $\begin{array}{l}\text { Error } \\
\text { Factor }\end{array}$ & $\begin{array}{c}\text { Failure } \\
\text { Pate }\end{array}$ & $\begin{array}{l}\text { Error } \\
\text { Factor }\end{array}$ \\
\hline RRE2B5OP & $\begin{array}{l}\text { K5 Relay Fails to } \\
\text { Move to Open } \\
\text { Position }\end{array}$ & $\begin{array}{c}2.0 \mathrm{E}-7 \mathrm{~h} \\
{[8]}\end{array}$ & 10 & $1.0 \mathrm{E}-6 \mathrm{~h}$ & 10 & $3.0 E-6 / \mathrm{h}$ & 10 & $\begin{array}{c}3.0 \mathrm{E}-6 \mathrm{~h} \\
{[5]}\end{array}$ & \\
\hline RRE2B6QP & $\begin{array}{l}\text { K6 Relay Fails to } \\
\text { Move to Open } \\
\text { Position }\end{array}$ & $\begin{array}{c}2.0 \mathrm{E}-7 \mathrm{~h} \\
{[8]}\end{array}$ & 10 & $1.0 \mathrm{E}-6 / \mathrm{h}$ & 10 & $3.0 \mathrm{E}-6 / \mathrm{h}$ & 10 & $\begin{array}{c}3.0 \mathrm{E}-6 / \mathrm{h} \\
{[5]}\end{array}$ & \\
\hline RRE2B9QU & $\begin{array}{l}\text { CCF - All K-Relays } \\
\text { of Channel B Fail to } \\
\text { Move }\end{array}$ & $\begin{array}{c}4.0 E-8 / \mathrm{h} \\
{[7]}\end{array}$ & 10 & & & & & & \\
\hline RRE2COOU & $\begin{array}{l}\text { CCF - Trip Relays of } \\
\text { both Strings Fail to } \\
\text { Move }\end{array}$ & $\begin{array}{c}4.0 E-8 / \mathrm{h} \\
{[7]}\end{array}$ & 10 & & & & & & \\
\hline RRE2C1QP & $\begin{array}{l}\text { String A Relay Fails } \\
\text { to Move to Open } \\
\text { Position }\end{array}$ & $\begin{array}{c}2.0 E-7 / h \\
{[8]}\end{array}$ & 10 & $1.0 \mathrm{E}-6 / \mathrm{h}$ & 10 & $3.0 \mathrm{E}-6 / \mathrm{h}$ & 10 & $\begin{array}{c}3.0 \mathrm{E}-6 / \mathrm{h} \\
\cdot[5]\end{array}$ & \\
\hline RRE2C2OP & $\begin{array}{l}\text { String B Relay Fails } \\
\text { to Move to Open } \\
\text { Position }\end{array}$ & $\begin{array}{c}2.0 E-7 \mathrm{M} \\
{[8]}\end{array}$ & 10 & $1.0 \mathrm{E}-6 / \mathrm{h}$ & 10 & $3.0 \mathrm{E}-6 / \mathrm{h}$ & 10 & $\begin{array}{c}3.0 \mathrm{E}-6 / \mathrm{h} \\
{[5]}\end{array}$ & \\
\hline RRE2C3OP & $\begin{array}{l}\text { K3 Relay Fails to } \\
\text { Move to Open } \\
\text { Position }\end{array}$ & $\begin{array}{c}2.0 E-7 / \mathrm{h} \\
{[8]}\end{array}$ & 10 & $1.0 \mathrm{E} \cdot 6 \mathrm{~m}$ & 10 & $3.0 \mathrm{E}-6 / \mathrm{h}$ & 10 & $\begin{array}{c}3.0 E-6 / \mathrm{h} \\
{[5]}\end{array}$ & \\
\hline RRE2C4QP & $\begin{array}{l}\text { K4 Relay Fails to } \\
\text { Move to Open } \\
\text { Position }\end{array}$ & $\begin{array}{c}2.0 E-7 / \mathrm{h} \\
{[8]}\end{array}$ & 10 & $1.0 \mathrm{E}-6 / \mathrm{h}$ & 10 & $3.0 E-6 / \mathrm{h}$ & 10 & $\begin{array}{c}3.0 \mathrm{E}-6 / \mathrm{h} \\
{[5]}\end{array}$ & \\
\hline RRE2C5OP & $\begin{array}{l}\text { K5 Relay Fails to } \\
\text { Move to Open } \\
\text { Position }\end{array}$ & $\begin{array}{c}2.0 \mathrm{E}-7 \mathrm{~h} \\
{[8]}\end{array}$ & 10 & $1.0 \mathrm{E}-6 / \mathrm{h}$ & 10 & $3.0 \mathrm{E}-6 / \mathrm{h}$ & 10 & $\begin{array}{c}3.0 \mathrm{E}-6 \mathrm{~h} \\
{[5]}\end{array}$ & \\
\hline RRE2C6QP & $\begin{array}{l}\text { K6 Relay Fails to } \\
\text { Move to Open } \\
\text { Position }\end{array}$ & $\begin{array}{c}2.0 \mathrm{E}-7 \mathrm{~h} \\
{[8]}\end{array}$ & 10 & $1.0 \mathrm{E}-6 / \mathrm{h}$ & 10 & $3.0 E-6 / \mathrm{h}$ & 10 & $\begin{array}{c}3.0 \mathrm{E}-6 / \mathrm{h} \\
{[5]}\end{array}$ & \\
\hline RRE2C9OU & $\begin{array}{l}\text { CCF-All K-Relays } \\
\text { of Channel C Fail to } \\
\text { Move }\end{array}$ & $\begin{array}{c}4.0 \mathrm{E}-8 \mathrm{~h} \\
{[7]}\end{array}$ & 10 & & & & & & \\
\hline RRE2ZOQU & $\begin{array}{l}\text { CCF - All Trip } \\
\text { Relays of both } \\
\text { Strings Fail to Move }\end{array}$ & $\begin{array}{c}1.6 E-9 / h \\
{[6]}\end{array}$ & 10 & & & & & & \\
\hline RRE2Z8QU & $\begin{array}{l}\text { CCF - LV K-Relays of } \\
\text { All Channels Fail to } \\
\text { Move }\end{array}$ & $\begin{array}{c}1.6 E-9 / h \\
{[6]}\end{array}$ & 10 & & & & & - & \\
\hline RRE2ZSOU & $\begin{array}{l}\text { CCF - HF K-Relays of } \\
\text { All Channels Fail to } \\
\text { Move }\end{array}$ & $\begin{array}{c}1.6 E-9 / h \\
{[6]}\end{array}$ & 10 & & & & & . & \\
\hline RRE3C1OP & $\begin{array}{l}\text { String A Relay Fails } \\
\text { to Change Position }\end{array}$ & $\begin{array}{c}2.0 \mathrm{E}-7 \mathrm{~m} \\
{[8]}\end{array}$ & 10 & $1.0 \mathrm{E}-6 / \mathrm{h}$ & 10 & $3.0 E-6 / h$ & 10 & $\begin{array}{c}3.0 \mathrm{E}-6 / \mathrm{h} \\
{[5]}\end{array}$ & \\
\hline RRE3C2OP & $\begin{array}{l}\text { String } 8 \text { Relay Fails } \\
\text { to Change Position }\end{array}$ & $\begin{array}{c}2.0 E-7 / \mathrm{h} \\
{[8]}\end{array}$ & 10 & $1.0 \mathrm{E}-6 / \mathrm{h}$ & 10 & $3.0 \mathrm{E}-6 / \mathrm{h}$ & 10 & $\begin{array}{c}.0 \mathrm{E}-6 / \mathrm{h} \\
{[5]}\end{array}$ & \\
\hline RRE3D1OP & $\begin{array}{l}\text { String A Relay Fails } \\
\text { to Change Position }\end{array}$ & $\begin{array}{c}2.0 \mathrm{E}-7 \mathrm{~h} \\
{[8]}\end{array}$ & 10 & $1.0 E-6 / h$ & 10 & $3.0 \mathrm{E}-6 / \mathrm{h}$ & 10 & $\begin{array}{c}3.0 \mathrm{E}-6 / \mathrm{h} \\
{[5]}\end{array}$ & \\
\hline RRE3D2OP & $\begin{array}{l}\text { String } 8 \text { Relay Fails } \\
\text { to Change Position }\end{array}$ & $\begin{array}{c}2.0 E-7 / \mathrm{h} \\
{[8]}\end{array}$ & 10 & $1.0 \mathrm{E}-6 / \mathrm{h}$ & 10 & $3.0 \mathrm{E}-6 / \mathrm{h}$ & 10 & $\begin{array}{c}3.0 \mathrm{E}-6 / \mathrm{h} \\
{[5]}\end{array}$ & \\
\hline RRE3F1QP & $\begin{array}{l}\text { String A Relay Fails } \\
\text { to Change Position }\end{array}$ & $\begin{array}{c}2.0 \mathrm{E}-7 / \mathrm{h} \\
{[8]}\end{array}$ & 10 & $1.0 \mathrm{E}-6 / \mathrm{h}$ & 10 & $3.0 E-6 / \mathrm{m}$ & 10 & $\begin{array}{c}.0 E-6 / \mathrm{h} \\
{[5]}\end{array}$ & \\
\hline RRE3FZOP & $\begin{array}{l}\text { String B Relay Fails } \\
\text { to Change Position }\end{array}$ & $\begin{array}{c}2.0 \mathrm{E}-7 / \mathrm{h} \\
{[8]}\end{array}$ & 10 & $1.0 \mathrm{E}-6 \mathrm{~h}$ & 10 & $3.0 \mathrm{E}-6 \mathrm{~m}$ & 10 & $\begin{array}{c}3.0 E-6 / \mathrm{h} \\
{[5]}\end{array}$ & \\
\hline RRE3H1QP & $\begin{array}{l}\text { String A Relay Fails } \\
\text { to Change Position }\end{array}$ & $\begin{array}{c}2.0 \mathrm{E}-7 \mathrm{~h} \\
{[8]}\end{array}$ & 10 & $1.0 \mathrm{E}-6 \mathrm{~h}$ & 10 & $3.0 \mathrm{E}-6 \mathrm{~h}$ & 10 & $\begin{array}{c}3.0 E-6 / \mathrm{h} \\
{[5]}\end{array}$ & \\
\hline RRE3H2QP & $\begin{array}{l}\text { String } 8 \text { Relay Fails } \\
\text { to Change Position }\end{array}$ & $\begin{array}{c}2.0 E-7 / \mathrm{h} \\
{[8]}\end{array}$ & 10 & $1.0 \mathrm{E}-6 / \mathrm{h}$ & 10 & $3.0 \mathrm{E}-6 \mathrm{~h}$ & 10 & $\begin{array}{c}3.0 E-6 / \mathrm{h} \\
{[5]}\end{array}$ & \\
\hline
\end{tabular}


Table 7.2. Reactor Shutdown System Data (Cont'd)

PAGE 9

\begin{tabular}{|c|c|c|c|c|c|c|c|c|c|}
\hline \multirow{2}{*}{$\begin{array}{l}\text { EVENT } \\
\text { NAME }\end{array}$} & \multirow[b]{2}{*}{ DESCRIPTION } & \multicolumn{2}{|c|}{ VALUE USED $3 / 91$} & \multicolumn{2}{|c|}{ CRBRP-4 } & \multicolumn{2}{|c|}{ IREP } & \multicolumn{2}{|c|}{ GENERIC } \\
\hline & & $\begin{array}{l}\text { Failure } \\
\text { Rate }\end{array}$ & $\begin{array}{l}\text { Error } \\
\text { Factor }\end{array}$ & $\begin{array}{c}\text { Failure } \\
\text { Rate }\end{array}$ & $\begin{array}{l}\text { Error } \\
\text { Factor }\end{array}$ & $\begin{array}{c}\text { Failure } \\
\text { Rate }\end{array}$ & $\begin{array}{l}\text { Error } \\
\text { Factor }\end{array}$ & $\begin{array}{c}\text { Failure } \\
\text { Rate }\end{array}$ & $\begin{array}{l}\text { Error } \\
\text { Factor }\end{array}$ \\
\hline RRE $\$ 01 Q P$ & $\begin{array}{l}\text { Relay UV (Device } \\
21-\text { RAI) Fails to } \\
\text { Change Position }\end{array}$ & $\begin{array}{c}2.0 \mathrm{E}-7 \mathrm{~h} \\
{[8]}\end{array}$ & 10 & $1.0 \mathrm{E}-6 / \mathrm{h}$ & 10 & $3.0 \mathrm{E}-6 / \mathrm{h}$ & 10 & $\begin{array}{c}3.0 \mathrm{E}-6 \mathrm{~h} \\
{[5]}\end{array}$ & \\
\hline RRE402QU & $\begin{array}{l}\text { CCF - UV Trip Relays } \\
\text { (Devices } 27-A \text { \& -C) }\end{array}$ & $\begin{array}{c}2.0 \mathrm{E}-8 \mathrm{~h} \\
{[4]}\end{array}$ & 10 & & & & & & \\
\hline RRECOOOU & $\begin{array}{l}\text { CCF - All CP-Relays } \\
\text { on Strings A \& B } \\
\text { Fail }\end{array}$ & $\begin{array}{c}1.6 \mathrm{E}-9 / \mathrm{h} \\
{[6]}\end{array}$ & 10 & & & & & & \\
\hline RRECO1QU & $\begin{array}{l}\text { CCF - All Relays on } \\
\text { String A Fail }\end{array}$ & $\begin{array}{c}6.0 \mathrm{E}-8 \mathrm{~m} \\
{[11]}\end{array}$ & 10 & & & & & & \\
\hline RAECO2QU & $\begin{array}{l}\text { CCF - All Relays on } \\
\text { String B Fail }\end{array}$ & $\begin{array}{c}6.0 \mathrm{E}-8 / \mathrm{h} \\
{[11]}\end{array}$ & 10 & & & & & & \\
\hline RRECB1QP & $\begin{array}{l}\text { CPB-1 Relay Fails to } \\
\text { Change Position }\end{array}$ & $\begin{array}{c}2.0 \mathrm{E}-7 / \mathrm{h} \\
{[8]}\end{array}$ & 10 & $1.0 \mathrm{E}-6 / \mathrm{h}$ & 10 & $3.0 \mathrm{E}-6 \mathrm{~m}$ & 10 & $\begin{array}{c}3.0 \mathrm{E} \cdot 6 / \mathrm{h} \\
{[5]}\end{array}$ & \\
\hline RREC82QP & $\begin{array}{l}\text { CPB-2 Relay Fails to } \\
\text { Change Position }\end{array}$ & $\begin{array}{c}2.0 \mathrm{E}-7 / \mathrm{h} \\
{[8]}\end{array}$ & 10 & $1.0 \mathrm{E}-6 / \mathrm{h}$ & 10 & $3.0 \mathrm{E}-6 / \mathrm{h}$ & 10 & $\begin{array}{c}3.0 \mathrm{E}-6 \mathrm{~h} \\
{[5]}\end{array}$ & \\
\hline RRECC1QP & $\begin{array}{l}\text { CPC-1 Relay Fails to } \\
\text { Change Position }\end{array}$ & $\begin{array}{c}2.0 \mathrm{E}-7 / \mathrm{h} \\
{[8]}\end{array}$ & 10 & $1.0 \mathrm{E}-6 / \mathrm{h}$ & 10 & $3.0 \mathrm{E}-6 / \mathrm{h}$ & 10 & $\begin{array}{c}3.0 \mathrm{E}-6 / \mathrm{h} \\
{[5]}\end{array}$ & \\
\hline RRECP2OP & $\begin{array}{l}\text { CP-2 Relay Fails to } \\
\text { Change Position }\end{array}$ & $\begin{array}{c}2.0 \mathrm{E}-7 \mathrm{~h} \\
{[8]}\end{array}$ & 10 & $1.0 \mathrm{E}-6 / \mathrm{h}$ & 10 & $3.0 \mathrm{E}-6 / \mathrm{h}$ & 10 & $\begin{array}{c}3.0 \mathrm{E}-6 / \mathrm{h} \\
{[5]}\end{array}$ & \\
\hline RRECPAQP & $\begin{array}{l}\text { CPA Relay Fails to } \\
\text { Change Position }\end{array}$ & $\begin{array}{c}2.0 \mathrm{E}-7 / \mathrm{h} \\
{[8]}\end{array}$ & 10 & $1.0 \mathrm{E}-6 / \mathrm{h}$ & 10 & $3.0 \mathrm{E}-6 / \mathrm{h}$ & 10 & $\begin{array}{c}3.0 \mathrm{E}-6 / \mathrm{h} \\
{[5]}\end{array}$ & \\
\hline RRECPYQP & $\begin{array}{l}\text { CPY Relay Fails to } \\
\text { Change Position }\end{array}$ & $\begin{array}{c}2.0 E-7 / \mathrm{h} \\
{[8]}\end{array}$ & 10 & $1.0 \mathrm{E}-6 / \mathrm{h}$ & 10 & $3.0 \mathrm{E}-6 / \mathrm{h}$ & 10 & $\begin{array}{c}3.0 E-6 / \mathrm{h} \\
{[5]}\end{array}$ & \\
\hline RRESROQP & $\begin{array}{l}\text { SRO Relay Fails to } \\
\text { Change Position }\end{array}$ & $\begin{array}{c}2.0 E-7 / \mathrm{h} \\
{[8]}\end{array}$ & 10 & $1.0 \mathrm{E}-6 / \mathrm{h}$ & 10 & $3.0 \mathrm{E}-6 / \mathrm{h}$ & 10 & $\begin{array}{c}3.0 E-6 / \mathrm{M} \\
{[5]}\end{array}$ & \\
\hline RRESRXOP & $\begin{array}{l}\text { SRO-X Relay Fails to } \\
\text { Change Position }\end{array}$ & $\begin{array}{c}2.0 E-7 / \mathrm{h} \\
{[8]}\end{array}$ & 10 & $1.0 \mathrm{E}-6 / \mathrm{h}$ & 10 & $3.0 \mathrm{E}-6 / \mathrm{h}$ & 10 & $\begin{array}{c}3.0 E-6 / \mathrm{h} \\
{[5]}\end{array}$ & \\
\hline RRT300AU & $\begin{array}{l}\text { CCF - All Ref } \\
\text { Junctions Fail High }\end{array}$ & $\begin{array}{c}4.0 E-8 / h \\
{[9]}\end{array}$ & 10 & & & & & & \\
\hline RRT3COAW & $\begin{array}{l}\text { T/C 503C Ref } \\
\text { Junction Fails High }\end{array}$ & $\begin{array}{c}2.0 E-6 / \mathrm{h} \\
{[14]}\end{array}$ & 10 & & & & & $\begin{array}{c}3.0 E-6 / \mathrm{h} \\
{[10]}\end{array}$ & 10 \\
\hline RAT3DOAW & $\begin{array}{l}\text { T/C 503D Ref } \\
\text { Junction Fails High }\end{array}$ & $\begin{array}{c}2.0 E-6 / \mathrm{h} \\
{[14]}\end{array}$ & 10 & & & & & $\begin{array}{c}3.0 \mathrm{E}-6 / \mathrm{M} \\
{[10]}\end{array}$ & 10 \\
\hline RRT3FOAW & $\begin{array}{l}\text { T/C 503F Ref } \\
\text { Junction Fails High }\end{array}$ & $\begin{array}{c}2.0 E-6 / \mathrm{M} \\
{[14]}\end{array}$ & 10 & & & & & $\begin{array}{c}3.0 E-6 / \mathrm{M} \\
{[10]}\end{array}$ & 10 \\
\hline RRT3HOAW & $\begin{array}{l}\text { T/C 503H Ref } \\
\text { Junction Fails High }\end{array}$ & $\begin{array}{c}2.0 E-6 / h \\
{[14]}\end{array}$ & 10 & & & & & $\begin{array}{c}3.0 \mathrm{E}-6 / \mathrm{h} \\
{[10]}\end{array}$ & 10 \\
\hline RTC300RU & $\begin{array}{l}\text { CCF - All T/C's Fail } \\
\text { Low }\end{array}$ & $\begin{array}{c}2.0 \mathrm{E}-8 \mathrm{M} \\
{[9]}\end{array}$ & 10 & & & & & & \\
\hline RTC3COAW & $\begin{array}{l}\text { T/C 503C Fails Low - } \\
\text { Short/Open }\end{array}$ & $\begin{array}{c}1.0 \mathrm{E}-6 \mathrm{~h} \\
{[10]}\end{array}$ & 10 & $1.0 \mathrm{E}-6 \mathrm{~m}$ & 10 & & & $\begin{array}{c}1.0 \mathrm{E}-6 \mathrm{~h} \\
{[10]}\end{array}$ & 10 \\
\hline RTC3DOAW & $\begin{array}{l}\text { T/C 503D Fails Low - } \\
\text { Short/Open }\end{array}$ & $\begin{array}{c}1.0 \mathrm{E}-6 \mathrm{M} \\
\text { [10] }\end{array}$ & 10 & $1.0 \mathrm{E}-6 \mathrm{~m}$ & 10 & & & $\begin{array}{c}1.0 \mathrm{E}-6 / \mathrm{h} \\
{[10]}\end{array}$ & 10 \\
\hline RTC3FORW & $\begin{array}{l}\text { T/C 503F Fails Low - } \\
\text { Short/Open }\end{array}$ & $\begin{array}{c}1.0 E-6 / \mathrm{h} \\
{[10]}\end{array}$ & 10 & $1.0 \mathrm{E}-6 / \mathrm{h}$ & 10 & & & $\begin{array}{c}1.0 \mathrm{E}-6 / \mathrm{h} \\
{[10]}\end{array}$ & 10 \\
\hline RTC3HORW & $\begin{array}{l}\text { T/C 503H Fails Low - } \\
\text { Short/Open }\end{array}$ & $\begin{array}{c}1.0 E-6 / \mathrm{h} \\
{[10]}\end{array}$ & 10 & $1.0 E-6 / \mathrm{h}$ & 10 & & & $\begin{array}{c}1.0 E-6 / \mathrm{h} \\
{[10]}\end{array}$ & 10 \\
\hline RTS1C1AW & \begin{tabular}{|l|} 
Pressure \\
Transmitters Fail
\end{tabular} & $\begin{array}{c}2.0 \mathrm{E}-5 \mathrm{~h} \\
{[14]}\end{array}$ & 10 & & & & & $\begin{array}{c}3.0 \mathrm{E}-5 \mathrm{~m} \\
{[3]}\end{array}$ & 12 \\
\hline RTS1D1RW & $\begin{array}{l}\text { Pressure } \\
\text { Transmitters Fail }\end{array}$ & $\begin{array}{c}2.0 \mathrm{E}-5 / \mathrm{h} \\
{[14]}\end{array}$ & 10 & & & & & $\begin{array}{c}3.0 \mathrm{E}-5 / \mathrm{h} \\
{[3]}\end{array}$ & 12 \\
\hline RVI1A1QW & MV/I Converter Fails & $\begin{array}{c}1.0 E-6 / \mathrm{h} \\
{[8]}\end{array}$ & 10 & $\begin{array}{c}1.0 \mathrm{E}-6 \mathrm{~h} \\
{[2]}\end{array}$ & 10 & $\begin{array}{c}3.0 \mathrm{E}-6 / \mathrm{h} \\
{[2]}\end{array}$ & 10 & $\begin{array}{c}1.0 E-6 \mathrm{M} \\
{[3]}\end{array}$ & 10 \\
\hline
\end{tabular}


Table 7.2. Reactor Shutdown System Data (Cont'd)

PAGE 10

\begin{tabular}{|c|c|c|c|c|c|c|c|c|c|}
\hline \multirow{2}{*}{$\begin{array}{l}\text { EVENT } \\
\text { NAME }\end{array}$} & \multirow[b]{2}{*}{ DESCRIPTION } & \multicolumn{2}{|c|}{ VALUE USED 3/91 } & \multicolumn{2}{|c|}{ CRBRP-4 } & \multicolumn{2}{|c|}{ IREP } & \multicolumn{2}{|c|}{ GENERIC } \\
\hline & & $\begin{array}{l}\text { Failure } \\
\text { Rate }\end{array}$ & $\begin{array}{l}\text { Error } \\
\text { Factor }\end{array}$ & $\begin{array}{l}\text { Failure } \\
\text { Rate }\end{array}$ & $\begin{array}{l}\text { Error } \\
\text { Factor }\end{array}$ & $\begin{array}{l}\text { Failure } \\
\text { Rate }\end{array}$ & $\begin{array}{l}\text { Error } \\
\text { Factor }\end{array}$ & $\begin{array}{l}\text { Failure } \\
\text { Pate }\end{array}$ & $\begin{array}{l}\text { Error } \\
\text { Factor }\end{array}$ \\
\hline RVI1A2OW & $\begin{array}{l}\text { DMVA Converter } \\
\text { Fails }\end{array}$ & $\begin{array}{c}1.0 \mathrm{E}-6 \mathrm{M} \\
{[8]}\end{array}$ & 10 & $\begin{array}{c}1.0 E-6 / h \\
{[2]}\end{array}$ & 10 & $\begin{array}{c}3.0 E-6 / \mathrm{M} \\
{[2]}\end{array}$ & 10 & $\begin{array}{c}1.0 \mathrm{E}-6 \mathrm{~h} \\
{[3]}\end{array}$ & 10 \\
\hline RVI1B1QW & MV/I Converter Fails & $\begin{array}{c}1.0 E-6 / h \\
{[8]}\end{array}$ & 10 & $\begin{array}{c}1.0 \mathrm{E}-6 / \mathrm{h} \\
{[2]}\end{array}$ & 10 & $\begin{array}{c}3.0 E-6 / h \\
{[2]}\end{array}$ & 10 & $\begin{array}{c}1.0 \mathrm{E}-6 / \mathrm{h} \\
{[3]}\end{array}$ & 10 \\
\hline RVI1C1QW & MV/I Converter Fails & $\begin{array}{c}.0 E-6 / \mathrm{h} \\
{[8]}\end{array}$ & 10 & $\begin{array}{c}1.0 \mathrm{E}-6 / \mathrm{h} \\
{[2]}\end{array}$ & 10 & $\begin{array}{c}3.0 E-6 / \mathrm{h} \\
{[2]}\end{array}$ & 10 & $\begin{array}{c}1.0 \mathrm{E}-6 / \mathrm{h} \\
{[3]}\end{array}$ & 10 \\
\hline RVI1ZOQU & $\begin{array}{l}\text { CCF - All MVI } \\
\text { Comverters Fail }\end{array}$ & $\begin{array}{c}.0 E-9 / \mathrm{h} \\
{[6]}\end{array}$ & 10 & & & & & & \\
\hline RVI3COOW & MV/I Converter Fails & $\begin{array}{c}1.0 E-6 / \mathrm{h} \\
{[8]}\end{array}$ & 10 & $\begin{array}{c}1.0 E-6 / h \\
{[2]}\end{array}$ & 10 & $\begin{array}{c}\text { 3.0E-6/h } \\
{[2]}\end{array}$ & 10 & $\begin{array}{c}1.0 \mathrm{E}-6 / \mathrm{h} \\
{[3]}\end{array}$ & 10 \\
\hline RVI3DOOW & MV/I Converter Fails & $\begin{array}{c}1.0 E-6 / \mathrm{h} \\
{[8]}\end{array}$ & 10 & $\begin{array}{c}.0 E-6 / \mathrm{h} \\
{[2]}\end{array}$ & 10 & $\begin{array}{c}3.0 E-6 / \mathrm{M} \\
{[2]}\end{array}$ & 10 & $\begin{array}{c}1.0 \mathrm{E}-6 / \mathrm{M} \\
{[3]}\end{array}$ & 10 \\
\hline RVI3FOOW & MV/I Converter Fails & $\begin{array}{c}1.0 E-6 / \mathrm{h} \\
{[8]}\end{array}$ & 10 & $\begin{array}{c}1.0 \mathrm{E}-6 / \mathrm{h} \\
{[2]}\end{array}$ & 10 & $\begin{array}{c}.0 \mathrm{E}-6 / \mathrm{h} \\
{[2]}\end{array}$ & 10 & $\begin{array}{c}1.0 E-6 / \mathrm{h} \\
{[3]}\end{array}$ & 10 \\
\hline RVI3HOOW & MV/I Converter Fails & $\begin{array}{c}1.0 E-6 / h \\
{[8]}\end{array}$ & 10 & $\begin{array}{c}1.0 \mathrm{E}-6 \mathrm{M} \\
{[2]}\end{array}$ & 10 & $\begin{array}{c}3.0 \mathrm{E}-6 / \mathrm{h} \\
{[2]}\end{array}$ & 10 & $\begin{array}{c}1.0 \mathrm{E}-6 / \mathrm{h} \\
{[3]}\end{array}$ & 10 \\
\hline RVI3ZOQU & $\begin{array}{l}\text { CCF - All MVII } \\
\text { Convertors in SOT } \\
\text { Channels Fail }\end{array}$ & $\begin{array}{c}\text { 2.0E-8/h } \\
{[9]}\end{array}$ & 10 & & & & & & \\
\hline
\end{tabular}

Notes: 1. Based on Plant-Specific Data

2. Low power solid state device value

3. IEEE Standard 500, 1984

4. $0.1 \times$ independent Failure Value

5. Taken from Table 7-3, SAND-87-7138

6. $0.008 \times$ independent Failure Vaiue

7. $0.2 \times$ Independent Failure Value

8. Based on Plant-Specific Data and Engineering Judgement

9. $0.02 \times$ Independent Failure Value

10. Recommended value tables, EGG-SSRE-8875, Feb. 1990

11. $0.3 \times$ Independent Failure Value

12. CREDO Data for EBR-ll Flow Sensors, 7-1-64 to 12-21-89

13. Instrumentation in general

14. Based on Engineering Judgement

15. Directed by Procedures; Long Time Available

16. Estimate provided by D. Sherman, SAIC, Idaho Falls 
Table 7.3. Reactor Control System Data

PAGE 1

\begin{tabular}{|c|c|c|c|c|c|c|c|c|c|}
\hline \multirow{2}{*}{$\begin{array}{l}\text { EVENT } \\
\text { NAME }\end{array}$} & \multirow[b]{2}{*}{ DESCRIPTION } & \multicolumn{2}{|c|}{ VALUE USED 391} & \multicolumn{2}{|c|}{ CRBRP.4 } & \multicolumn{2}{|c|}{ IREP } & \multicolumn{2}{|c|}{ GENERIC } \\
\hline & & $\begin{array}{l}\text { Failure } \\
\text { Rate }\end{array}$ & $\begin{array}{l}\text { Error } \\
\text { Factor }\end{array}$ & $\begin{array}{l}\text { Failure } \\
\text { Rate }\end{array}$ & $\begin{array}{l}\text { Error } \\
\text { Factor }\end{array}$ & $\begin{array}{c}\text { Failure } \\
\text { Rate }\end{array}$ & $\begin{array}{l}\text { Error } \\
\text { Factor }\end{array}$ & $\begin{array}{c}\text { Failure } \\
\text { Rate }\end{array}$ & $\begin{array}{l}\text { Error } \\
\text { Factor }\end{array}$ \\
\hline CARCRPRD & $\begin{array}{l}\text { LIFTING PLATFORM } \\
\text { RAISED } 1 / 4 \text { IN }\end{array}$ & $\underset{[1]}{6.0 E-4 / d}$ & 5 & & & & & & \\
\hline CCDIOGRU & $\begin{array}{l}\text { CCF - Bowing and } \\
\text { Binding / Inside } \\
\text { Tank }\end{array}$ & $\begin{array}{c}1.3 E-6 / d \\
{[2]}\end{array}$ & 20 & & & & & & \\
\hline CCO20WRU & $\begin{array}{l}\text { CCF - Bowing and } \\
\text { Binding/Above } \\
\text { Rotating Plug }\end{array}$ & $\begin{array}{c}1.0 \mathrm{E}-7 / \mathrm{d} \\
{[3]}\end{array}$ & 20 & & & & & & \\
\hline CCO3OIRU & $\begin{array}{l}\text { CCF - Forces due to } \\
\text { Foreign Materials in } \\
\text { Sodium }\end{array}$ & $\begin{array}{l}\text { 3.0E-7/d } \\
{[3]}\end{array}$ & 20 & & & & & & \\
\hline CCLOOPRU & $\begin{array}{l}\text { CCF - Magnetic } \\
\text { Clutcties Fail to } \\
\text { Release CRs }\end{array}$ & $\begin{array}{c}1.6 \mathrm{E}-6 / \mathrm{d} \\
{[4]}\end{array}$ & 20 & & & & & & - \\
\hline CHUOPICL & $\begin{array}{l}\text { OPERATOR DECIDES TO } \\
\text { COMPENSATE WITH 2nd } \\
\text { ROD }\end{array}$ & $\begin{array}{c}1.0 \mathrm{E}-1 / \mathrm{d} \\
{[5]}\end{array}$ & 5 & & & & & & \\
\hline CRECSUQP & $\begin{array}{l}\text { CU OR SU CONTACT } \\
\text { FAILS TO REOPEN }\end{array}$ & $\begin{array}{c}6.6 \mathrm{E}-7 / \mathrm{d} \\
{[6]}\end{array}$ & 8 & & & & & & \\
\hline CRECSUQR & $\begin{array}{l}\text { CU OR SU RELAY } \\
\text { CLOSES SPONTANEOUSLY }\end{array}$ & $\begin{array}{c}1.4 \mathrm{E}-6 / \mathrm{h} \\
{[6]}\end{array}$ & 8 & & & & & & \\
\hline CREDUMQI & $\begin{array}{l}\text { RELAY EVENT MUST } \\
\text { OCCUR WITHIN } 0.1 \mathrm{~h}\end{array}$ & $\begin{array}{c}1.4 E-5 / d \\
{[7]}\end{array}$ & 1 & & & & & & \\
\hline CREKD7QP & $\begin{array}{l}\text { K7 RELAY FAILS TO } \\
\text { REOPEN }\end{array}$ & $\begin{array}{c}3.0 \mathrm{E}-6 / \mathrm{d} \\
{[8]}\end{array}$ & 2 & & & & & & \\
\hline CREKD7OQ & $\begin{array}{l}\text { K7 RELAY CLOSES } \\
\text { SPONTANEOUSLY }\end{array}$ & $\begin{array}{c}5.0 \mathrm{E}-7 / \mathrm{h} \\
{[9]}\end{array}$ & 10 & & . & & & & \\
\hline CSR10GRU & $\begin{array}{l}\text { CCF - Forces due to } \\
\text { Bowing and Binding }\end{array}$ & $\begin{array}{c}1.0 \mathrm{E}-7 / \mathrm{d} \\
{[3]}\end{array}$ & 20 & & & & & & \\
\hline CSR30IRU & $\begin{array}{l}\text { CCF - Forces due to } \\
\text { Foreign Materials in } \\
\text { Sodium }\end{array}$ & $\underset{[3]}{1.0 \mathrm{E}-7 / \mathrm{d}}$ & 20 & & & & & & \\
\hline CSTMC1QD & $\begin{array}{l}\text { CONTROLLER SENDS } \\
\text { UP-VOLTAGE } \\
\text { SPONTANEOUSLY }\end{array}$ & $\begin{array}{c}4.0 \mathrm{E}-7 / \mathrm{h} \\
{[10]}\end{array}$ & 4 & & & & & & \\
\hline CSTMC2OD & $\begin{array}{l}\text { CONTROLLER FAILS } \\
\text { AFTER SWITCH OR } \\
\text { RELAY }\end{array}$ & $\begin{array}{c}4.0 \mathrm{E}-7 \mathrm{~h} \\
{[10]}\end{array}$ & 4 & & & & $\cdot$ & & \\
\hline CSW100CP & 1MPB Mech Parts Fail & $\underset{[5]}{2.0 E-6 / d}$ & 10 & . & & & & & \\
\hline CSW1OACP & $\begin{array}{l}\text { 1MPB Switch of } \\
\text { String A Fails to } \\
\text { Open }\end{array}$ & $\begin{array}{c}1.0 \mathrm{E}-5 / \mathrm{d} \\
{[9]}\end{array}$ & 5 & $1.0 \mathrm{E}-4 / \mathrm{d}$ & 10 & $3.0 \mathrm{E}-5 / \mathrm{d}$ & 10 & $\begin{array}{c}1.0 \mathrm{E}-5 / \mathrm{d} \\
{[9]}\end{array}$ & 5 \\
\hline CSW10BCP & $\begin{array}{l}\text { 1MPB Switch of } \\
\text { String B Fails to } \\
\text { Open }\end{array}$ & $\begin{array}{c}1.0 \mathrm{E}-5 / \mathrm{d} \\
{[9]}\end{array}$ & 5 & $1.0 E-4 / d$ & 10 & $3.0 \mathrm{E}-5 / \mathrm{d}$ & 10 & $\begin{array}{c}1.0 \mathrm{E}-5 / \mathrm{d} \\
{[9]}\end{array}$ & 5 \\
\hline CSWCONCZ & $\begin{array}{l}\text { CONSOLE ACCESS } \\
\text { RESTRICTION VIOLATED }\end{array}$ & $\begin{array}{c}5.0 \mathrm{E}-2 / \mathrm{d} \\
{[11]}\end{array}$ & 5 & & & & & & \\
\hline CSWOUMCl & $\begin{array}{l}\text { SWITCH EVENT MUST } \\
\text { OCCUR WITHIN } 0.1 \mathrm{~h}\end{array}$ & $\begin{array}{c}1.4 \mathrm{E}-5 / \mathrm{d} \\
{[7]}\end{array}$ & 1 & & & & & & \\
\hline CSWMSICL & $\begin{array}{l}\text { OPERATORS FAIL TO } \\
\text { FREE MSW FRM } \\
\text { UNAUTHORIZED PERSON }\end{array}$ & $\begin{array}{c}5.0 \mathrm{E}-2 / \mathrm{d} \\
{[11]}\end{array}$ & 2 & & & & & & \\
\hline CSWMS1CO & $\begin{array}{l}\text { MSW-1 CLOSES } \\
\text { SPONTANEOUSLY }\end{array}$ & $\begin{array}{c}7.0 \mathrm{E}-8 / \mathrm{h} \\
{[8]}\end{array}$ & 2 & & & & & & \\
\hline CSWMS1CZ & $\begin{array}{l}\text { MSW SWITCH HELD UP } \\
\text { BY UNAUTHORIZED } \\
\text { PERSON }\end{array}$ & $\begin{array}{c}2.1 E-1 / d \\
{[6]}\end{array}$ & 2 & & & & & & \\
\hline
\end{tabular}


Table 7.3. Reactor Control System Data (Cont'd)

PAGE 2

\begin{tabular}{|c|c|c|c|c|c|c|c|c|c|}
\hline \multirow{2}{*}{$\begin{array}{l}\text { EVENT } \\
\text { NAME }\end{array}$} & \multirow[b]{2}{*}{ DESCRIPTION } & \multicolumn{2}{|c|}{ VALUE USED 391} & \multicolumn{2}{|c|}{ CRBRP-4 } & \multicolumn{2}{|c|}{ IREP } & \multicolumn{2}{|c|}{ GENERIC } \\
\hline & & $\begin{array}{l}\text { Failure } \\
\text { Rate }\end{array}$ & $\begin{array}{l}\text { Error } \\
\text { Factor }\end{array}$ & $\begin{array}{l}\text { Failure } \\
\text { Rate }\end{array}$ & $\begin{array}{l}\text { Error } \\
\text { Factor }\end{array}$ & $\begin{array}{l}\text { Failure } \\
\text { Rate }\end{array}$ & $\begin{array}{l}\text { Error } \\
\text { Factor }\end{array}$ & $\begin{array}{l}\text { Failure } \\
\text { Rate }\end{array}$ & $\begin{array}{l}\text { Error } \\
\text { Factor }\end{array}$ \\
\hline CSWMS2CL & $\begin{array}{l}\text { OPERATORS FAIL TO } \\
\text { RELEASE MSW WITHIN } 1 \\
\text { MIN AFTER ALARM }\end{array}$ & $\begin{array}{c}1.0 E-3 / d \\
{[11]}\end{array}$ & 5 & & & & & & \\
\hline CSWMS2CP & $\begin{array}{l}\text { INTERLOCK BETWEEN } \\
\text { MSWS FAILS }\end{array}$ & $\begin{array}{c}1.0 \mathrm{E} \cdot 4 / \mathrm{d} \\
{[5]}\end{array}$ & 10 & & & & & & \\
\hline CSWMS2CO & $\begin{array}{l}\text { MSW-2 CLOSES } \\
\text { SPONTANEOUSLY }\end{array}$ & $\begin{array}{c}7.0 \mathrm{E}-8 \mathrm{~m} \\
{[8]}\end{array}$ & 2 & & & & & & \\
\hline CSWMS2CZ & $\begin{array}{l}\text { OPERATOR HOLDS UP } \\
\text { MSW UNTIL ALARMS }\end{array}$ & $\begin{array}{c}2.0 E-2 / d \\
{[6]}\end{array}$ & 8 & & & & & & \\
\hline CSWMS3CZ & $\begin{array}{l}\text { OP. CLOSES RELAY } \\
\text { FAILS TO COMPENSATE } \\
\text { WICONTROLLING ROD }\end{array}$ & $\begin{array}{c}1.1 \mathrm{E}-1 / \mathrm{d} \\
{[11]}\end{array}$ & 3 & & & & & & \\
\hline CSWMS4CZ & $\begin{array}{l}\text { OPERATOR HOLDS MSW } \\
\text { UP INSTEAD OF DOWN }\end{array}$ & $\begin{array}{c}2.5 E-2 / d \\
{[11]}\end{array}$ & 2 & & & & & & \\
\hline CSWMSSCL & $\begin{array}{l}\text { MSSW NOT SET TO } \\
\text { NEUTRAL POSITION }\end{array}$ & $\begin{array}{c}1.7 E-4 / h \\
{[11]}\end{array}$ & 3 & & & & & & \\
\hline CSWMSWCP & $\begin{array}{l}\text { MSW STAYS UP WHEN } \\
\text { RELEASED }\end{array}$ & $\begin{array}{c}\text { 1.4E-6/d } \\
{[6]}\end{array}$ & 3 & & & & & & \\
\hline CSWMSWCZ & $\begin{array}{l}\text { OPERATOR FAILS TO } \\
\text { FREE STUCK SWITCH }\end{array}$ & $\begin{array}{c}3.0 \mathrm{E}-1 / \mathrm{d} \\
{[5]}\end{array}$ & 2 & & & & & & \\
\hline CSWPSBCL & $\begin{array}{l}\text { OPERATOR FAILS TO } \\
\text { PUSH PSB2 }\end{array}$ & $\begin{array}{c}1.7 E-4 / h \\
{[11]}\end{array}$ & 3 & & & & & & \\
\hline CSWPSBCP & $\begin{array}{l}\text { PSB2 SWITCH FAILS TO } \\
\text { OPEN }\end{array}$ & $\begin{array}{c}1.0 \mathrm{E}-5 / \mathrm{d} \\
{[9]}\end{array}$ & 5 & & & & & & \\
\hline CSWPSBCQ & $\begin{array}{l}\text { PSB2 SWITCH CLOSES } \\
\text { SPONTANEOUSLY }\end{array}$ & $\begin{array}{c}8.5 \mathrm{E}-8 / \mathrm{h} \\
{[8]}\end{array}$ & 10 & & & & & & \\
\hline CSWSS2CD & $\begin{array}{l}\text { 2-ROD SHORT OF } \\
\text { SELECTOR SWITCH }\end{array}$ & $\begin{array}{c}1.5 E-6 / \mathrm{h} \\
{[8]}\end{array}$ & 1 & & & & & & \\
\hline CSWSS3CD & $\begin{array}{l}\text { MANY-ROD SHORT OF } \\
\text { SELECTOR SWITCH }\end{array}$ & $\begin{array}{c}1.8 \mathrm{E}-8 \mathrm{~m} \\
{[5]}\end{array}$ & 10 & & & & & & \\
\hline
\end{tabular}

Notes: 1. From Fault Tree Analysis for the CRD Platform

2. Ind. Prob. of 1.2E-6/d due to RV Cover Tilting

3. Based on combined CCF Prob of $7.0 E-7 / d$ for all CRs

4. $0.008 \times$ Independent Failure Value

5. Based on Engineering Judgement

6. Based on Plant Data

7. Based on a 6-minute Window

8. IEEE Standard 500, 1984

9. Recommended value tables, EGG-SSRE-8875, Feb. 1990

10. IEEE Standard 500 plus Engineering Judgement

11. Based on NUREG/CR-1278 and Engineering Judgement 
Table 7.4. Primary Pump and Primary Pump M/G Set Data

PAGE 1

\begin{tabular}{|c|c|c|c|c|c|c|c|c|c|}
\hline \multirow{2}{*}{$\begin{array}{l}\text { EVENT } \\
\text { NAME }\end{array}$} & \multirow[b]{2}{*}{ DESCRIPTION } & \multicolumn{2}{|c|}{ VALUE USED $3 / 91$} & \multicolumn{2}{|c|}{ CRBRP-4 } & \multicolumn{2}{|c|}{ IREP } & \multicolumn{2}{|c|}{ GENERIC } \\
\hline & & $\begin{array}{c}\text { Failure } \\
\text { Rate }\end{array}$ & $\begin{array}{l}\text { Error } \\
\text { Factor }\end{array}$ & $\begin{array}{l}\text { Failure } \\
\text { Rate }\end{array}$ & $\begin{array}{l}\text { Error } \\
\text { Factor }\end{array}$ & $\begin{array}{l}\text { Failure } \\
\text { Rate }\end{array}$ & $\begin{array}{l}\text { Error } \\
\text { Factor }\end{array}$ & $\begin{array}{c}\text { Failure } \\
\text { Rate }\end{array}$ & $\begin{array}{l}\text { Eeror } \\
\text { Factor }\end{array}$ \\
\hline PCB001PO & $\begin{array}{l}\text { MG Set } 1 \text { Output } \\
\text { Breaker Fails Open }\end{array}$ & $3.0 \mathrm{E}-7 / \mathrm{h}$ & 10 & $1.0 \mathrm{E}-5 / \mathrm{h}$ & 10 & $3.0 \mathrm{E}-5 / \mathrm{d}$ & 10 & $3.0 E-5 / d$ & 3 \\
\hline PC8002PO & $\begin{array}{l}\text { MG Set } 2 \text { Output } \\
\text { Breaker Fails Open }\end{array}$ & $3.0 \mathrm{E}-7 / \mathrm{h}$ & 10 & $1.0 \mathrm{E}-5 / \mathrm{h}$ & 10 & $3.0 \mathrm{E}-5 / \mathrm{d}$ & 10 & $3.0 \mathrm{E}-5 / \mathrm{d}$ & 3 \\
\hline PCB012PU & $\begin{array}{l}\text { MG Set } 1 \text { and } 2 \\
\text { Output Breaker Fails } \\
\text { Open, Common Cause }\end{array}$ & $3.0 \mathrm{E}-8 / \mathrm{h}$ & 10 & & & & & - & \\
\hline PCL001PW & $\begin{array}{l}\text { MG Set } 1 \text { Clutch } \\
\text { Failure }\end{array}$ & $5.0 \mathrm{E}-5 / \mathrm{h}$ & 30 & $3.0 E-4 / d$ & 3 & & & $3.0 \mathrm{E}-4 / \mathrm{d}$ & 3 \\
\hline PCLO02PW & $\begin{array}{l}\text { MG Set } 2 \text { Clutch } \\
\text { Failure }\end{array}$ & $5.0 E-5 / h$ & 30 & $3.0 \mathrm{E}-4 / \mathrm{d}$ & 3 & & & $3.0 \mathrm{E}-4 / \mathrm{d}$ & 3 \\
\hline PCZCP6OB & $\begin{array}{l}\text { Contacts } 6 \text { of Relay } \\
\text { CPC-2 Fail Open }\end{array}$ & $1.0 \mathrm{E}-7 / \mathrm{h}$ & 10 & $1.0 \mathrm{E}-7 \mathrm{~h}$ & 10 & & & & \\
\hline PCZCP7OB & $\begin{array}{l}\text { Contacts } 7 \text { of Relay } \\
\text { CPC-2 Fail Open }\end{array}$ & $1.0 \mathrm{E}-7 / \mathrm{h}$ & 10 & $1.0 \mathrm{E}-7 \mathrm{~h}$ & 10 & & & & \\
\hline PCZPB1PB & $\begin{array}{l}\text { Contacts } 1 \text { of Relay } \\
\text { PBS Fail Open }\end{array}$ & $1.0 \mathrm{E}-7 / \mathrm{h}$ & 10 & $1.0 \mathrm{E} \cdot 7 / \mathrm{h}$ & 10 & & & & \\
\hline PCZPBOPB & $\begin{array}{l}\text { Contacts } 8 \text { of Relay } \\
\text { PBS Fail Open }\end{array}$ & $1.0 \mathrm{E}-7 / \mathrm{h}$ & 10 & $1.0 \mathrm{E}-7 / \mathrm{h}$ & 10 & & & & \\
\hline PCZX15PB & $\begin{array}{l}\text { Contacts } 5 \text { of Relay } \\
\text { EX1 Fail OPen }\end{array}$ & $1.0 \mathrm{E}-7 / \mathrm{h}$ & 10 & $\begin{array}{c}1.0 \mathrm{E}-7 / \mathrm{h} \\
{[1 \cdot]}\end{array}$ & 10 & & & & \\
\hline PCZX24PB & $\begin{array}{l}\text { Contacts } 4 \text { of Relay } \\
\text { EX2 Fail Open }\end{array}$ & $1.0 \mathrm{E}-7 \mathrm{~h}$ & 10 & $\begin{array}{c}1.0 \mathrm{E}-7 / \mathrm{h} \\
{[1]}\end{array}$ & 10 & & & & \\
\hline PCZX25PB & $\begin{array}{l}\text { Contacts } 5 \text { of Relay } \\
\text { EX2 Fail Open }\end{array}$ & $1.0 \mathrm{E}-7 / \mathrm{h}$ & 10 & $\begin{array}{c}1.0 \mathrm{E}-7 \mathrm{~h} \\
{[1]}\end{array}$ &.$^{10}$ & & & & \\
\hline PDC001PB & $\begin{array}{l}\text { HP Power Supply No. } \\
1 \text { Failure }\end{array}$ & $3.0 \mathrm{E}-6 \mathrm{~h}$ & 10 & $\begin{array}{c}1.0 \mathrm{E}-6 / \mathrm{h} \\
{[2]}\end{array}$ & 10 & $\begin{array}{c}3.0 \mathrm{E}-6 / \mathrm{h} \\
{[2]}\end{array}$ & 10 & $\begin{array}{c}3.0 \mathrm{E}-6 / \mathrm{h} \\
{[2]}\end{array}$ & 10 \\
\hline PDC002PB & $\begin{array}{l}\text { HP Power Supply No. } \\
2 \text { Failure }\end{array}$ & $3.0 \mathrm{E}-6 / \mathrm{h}$ & 10 & $\begin{array}{c}1.0 E-6 / h \\
{[2]}\end{array}$ & 10 & $\begin{array}{c}3.0 \mathrm{E}-6 \mathrm{~h} \\
{[2]}\end{array}$ & 10 & $\begin{array}{c}3.0 \mathrm{E}-6 / \mathrm{h} \\
{[2]}\end{array}$ & 10 \\
\hline PDCDO6PB & $\begin{array}{l}\text { Exciter No. } 1 \text { Power } \\
\text { Supply Failure }\end{array}$ & $3.0 \mathrm{E}-6 / \mathrm{h}$ & 10 & $\begin{array}{c}1.0 E-6 / h \\
{[2]}\end{array}$ & 10 & $\begin{array}{c}3.0 \mathrm{E}-6 / \mathrm{h} \\
{[2]}\end{array}$ & 10 & $\begin{array}{c}3.0 \mathrm{E} \cdot 6 / \mathrm{h} \\
{[2]}\end{array}$ & 10 \\
\hline PDCOOTPB & $\begin{array}{l}\text { Exciter No. } 2 \text { Power } \\
\text { Supply Failure }\end{array}$ & $3.0 \mathrm{E}-6 \mathrm{~h}$ & 10 & $\begin{array}{c}1.0 \mathrm{E}-6 / \mathrm{h} \\
{[2]}\end{array}$ & 10 & $\begin{array}{c}3.0 \mathrm{E}-6 / \mathrm{h} \\
{[2]}\end{array}$ & 10 & $\begin{array}{c}3.0 \mathrm{E} \cdot 6 / \mathrm{h} \\
{[2]}\end{array}$ & 10 \\
\hline PDC012PU & $\begin{array}{l}\text { HP Power Supply No. } \\
1 \text { and } 2 \text { Fails, } \\
\text { Common Cause }\end{array}$ & $3.0 \mathrm{E}-7 \mathrm{~h}$ & 10 & & & & & & \\
\hline PEX001PW & $\begin{array}{l}\text { MG Set } 1 \text { Exciter } \\
\text { Failure }\end{array}$ & $3.0 \mathrm{E}-6 / \mathrm{h}$ & 10 & $\begin{array}{c}1.0 \mathrm{E}-6 / \mathrm{h} \\
{[2]}\end{array}$ & 10 & $\begin{array}{c}3.0 \mathrm{E}-6 / \mathrm{h} \\
{[2]}\end{array}$ & 10 & $\begin{array}{c}3.0 \mathrm{E}-6 / \mathrm{h} \\
{[2]}\end{array}$ & 10 \\
\hline PEX002PW & $\begin{array}{l}\text { MG Set } 2 \text { Exciter } \\
\text { Failure }\end{array}$ & $3.0 \mathrm{E}-6 / \mathrm{h}$ & 10 & $\begin{array}{c}1.0 \mathrm{E}-6 / \mathrm{h} \\
{[2]}\end{array}$ & 10 & $\begin{array}{c}3.0 E-6 / \mathrm{h} \\
{[2]}\end{array}$ & 10 & $\begin{array}{c}3.0 \mathrm{E} \cdot 6 / \mathrm{h} \\
{[2]}\end{array}$ & 10 \\
\hline PGE001PW & $\begin{array}{l}\text { MG Set } 1 \text { Generator } \\
\text { Failure }\end{array}$ & $3.0 E-6 / \mathrm{h}$ & 10 & $\begin{array}{c}1.0 E-6 / \mathrm{h} \\
{[3]}\end{array}$ & 10 & $\begin{array}{c}3.0 \mathrm{E}-6 \mathrm{~h} \\
{[3]}\end{array}$ & 10 & . & \\
\hline PGE002PW & $\begin{array}{l}\text { MG Set } 2 \text { Generator } \\
\text { Fajilure }\end{array}$ & $3.0 \mathrm{E}-6 / \mathrm{h}$ & 10 & $\begin{array}{c}1.0 E-6 / \mathrm{h} \\
\text { [3] }\end{array}$ & 10 & $\begin{array}{c}3.0 \mathrm{E}-6 / \mathrm{h} \\
{[3]}\end{array}$ & 10 & & \\
\hline PKS001PB & $\begin{array}{l}\text { Primary Pump Key } \\
\text { Switch Fails Open }\end{array}$ & $1.0 \mathrm{E} \cdot 7 \mathrm{~h}$ & 10 & $1.0 E-7 / h$ & 10 & & & & \\
\hline PMO001PW & $\begin{array}{l}\text { MG Set } 1 \text { Motor } \\
\text { Failure }\end{array}$ & $3.0 \mathrm{E}-5 / \mathrm{h}$ & 3 & $1.0 \mathrm{E}-5 / \mathrm{h}$ & 10 & & & $3.0 \mathrm{E}-5 / \mathrm{h}$ & 3 \\
\hline PMO002PW & $\begin{array}{l}\text { MG Set } 2 \text { Motor } \\
\text { Failure }\end{array}$ & $3.0 \mathrm{E}-5 / \mathrm{h}$ & 3 & $1.0 \mathrm{E}-5 / \mathrm{h}$ & 10 & & & $3.0 \mathrm{E}-5 / \mathrm{h}$ & 3 \\
\hline PPMO01RG & $\begin{array}{l}\text { Primary Pump } 1 \text { Shaft } \\
\text { Binding }\end{array}$ & $2.9 E-5 / \mathrm{h}$ & 2 & & & & & & \\
\hline PPMO01RR & $\begin{array}{l}\text { Primary Pump } 1 \text { Shaft } \\
\text { Broken or Seized }\end{array}$ & $1.9 \mathrm{E}-6 / \mathrm{h}$ & 8 & & & & & & \\
\hline
\end{tabular}


Table 7.4. Primary Pump and Primary Pump M/G Set Data (Cont'd)

PAGE 2

\begin{tabular}{|c|c|c|c|c|c|c|c|c|c|}
\hline \multirow{2}{*}{$\begin{array}{l}\text { EVENT } \\
\text { NAME }\end{array}$} & \multirow[b]{2}{*}{ DESCAIPTION } & \multicolumn{2}{|c|}{ VALUE USED $3 / 91$} & \multicolumn{2}{|c|}{ CRBRP-4 } & \multicolumn{2}{|c|}{ IREP } & \multicolumn{2}{|c|}{ GENERIC } \\
\hline & & $\begin{array}{c}\text { Failure } \\
\text { Rate }\end{array}$ & $\begin{array}{l}\text { Error } \\
\text { Factor }\end{array}$ & $\begin{array}{c}\text { Failure } \\
\text { Rate }\end{array}$ & $\begin{array}{l}\text { Error } \\
\text { Factor }\end{array}$ & $\begin{array}{c}\text { Failure } \\
\text { Rate }\end{array}$ & $\begin{array}{l}\text { Error } \\
\text { Factor }\end{array}$ & $\begin{array}{c}\text { Failure } \\
\text { Rate }\end{array}$ & $\begin{array}{l}\text { Error } \\
\text { Factor }\end{array}$ \\
\hline PPM002RG & $\begin{array}{l}\text { Primary Pump } 2 \text { Shaft } \\
\text { Binding }\end{array}$ & $2.9 \mathrm{E}-5 \mathrm{~h}$ & 2 & & & & & & \\
\hline PPM002RR & $\begin{array}{l}\text { Primary Pump } 2 \text { Shaft } \\
\text { Broken or Selzed }\end{array}$ & $1.9 \mathrm{E}-6 / \mathrm{h}$ & 8 & & & & & & \\
\hline PPMO12RU & $\begin{array}{l}\text { Primary Pump } 1 \text { and } 2 \\
\text { Shaft Binding, } \\
\text { Common Cause }\end{array}$ & $2.9 \mathrm{E} \cdot 6 / \mathrm{h}$ & 10 & & & & & & \\
\hline PPMO21RU & $\begin{array}{l}\text { Primary Pump } 2 \text { Shaft } \\
\text { Binding, Common } \\
\text { Cause }\end{array}$ & $8.0 \mathrm{E}-7 / \mathrm{h}$ & 30 & & & & & & \\
\hline PREEX1PB & $\begin{array}{l}\text { Relay EX1 Coil Fails } \\
\text { Open }\end{array}$ & $5.0 \mathrm{E}-7 \mathrm{~h}$ & 10 & $1.0 \mathrm{E}-6 \mathrm{~h}$ & 10 & $3.0 E-6 / h$ & 10 & $3.0 E-6 / \mathrm{h}$ & 10 \\
\hline PREEX2PB & $\begin{array}{l}\text { Relay EX2 Coil Fails } \\
\text { Open }\end{array}$ & $5.0 \mathrm{E}-7 / \mathrm{h}$ & 10 & $1.0 \mathrm{E}-6 / \mathrm{h}$ & 10 & $3.0 \mathrm{E}-6 \mathrm{~h}$ & 10 & $3.0 \mathrm{E}-6 / \mathrm{h}$ & 10 \\
\hline PREP2ACB & $\begin{array}{l}\text { Relay CPC-2 Coil } \\
\text { Fails Open }\end{array}$ & $5.0 \mathrm{E}-7 \mathrm{~h}$ & 10 & $1.0 \mathrm{E}-\mathrm{\sigma} / \mathrm{h}$ & 10 & $3.0 \mathrm{E} \cdot 6 / \mathrm{h}$ & 10 & $3.0 \mathrm{E}-6 / \mathrm{h}$ & 10 \\
\hline PREPBSPB & $\begin{array}{l}\text { Relay PBS Coil Fails } \\
\text { Open }\end{array}$ & $5.0 \mathrm{E}-7 \mathrm{~m}$ & 10 & $1.0 \mathrm{E}-6 / \mathrm{h}$ & 10 & $3.0 \mathrm{E}-6 / \mathrm{h}$ & 10 & $3.0 \mathrm{E}-6 / \mathrm{h}$ & 10 \\
\hline PSC001PB & $\begin{array}{l}\text { MG Set Controller } 1 \\
\text { Overspeed Cutoff }\end{array}$ & $3.0 \mathrm{E}-6 / \mathrm{h}$ & 10 & $\begin{array}{c}1.0 \mathrm{E}-6 / \mathrm{h} \\
{[4]}\end{array}$ & 10 & $\begin{array}{c}3.0 \mathrm{E}-6 \mathrm{~h} \\
{[4]}\end{array}$ & 10 & $\begin{array}{c}2.0 E-6 / h \\
{[4]}\end{array}$ & 10 \\
\hline PSC002PB & $\begin{array}{l}\text { MG Set Controller } 2 \\
\text { Overspeed Cutoff }\end{array}$ & $3.0 \mathrm{E}-6 / \mathrm{h}$ & 10 & $\begin{array}{c}1.0 E-6 / \mathrm{h} \\
{[4]}\end{array}$ & 10 & $\begin{array}{c}3.0 E-6 / h \\
{[4]}\end{array}$ & 10 & $\begin{array}{c}2.0 E-6 / h \\
{[4]}\end{array}$ & 10 \\
\hline PSC01BPW & $\begin{array}{l}\text { MG Set } 1 \text { Controller } \\
\text { Faulty Demand Signal }\end{array}$ & $3.0 \mathrm{E}-6 / \mathrm{h}$ & 10 & $\begin{array}{c}1.0 E-6 / \mathrm{h} \\
{[4]}\end{array}$ & 10 & $\begin{array}{c}3.0 E-6 / h \\
{[4]}\end{array}$ & 10 & $\begin{array}{c}2.0 \mathrm{E} \cdot 6 / \mathrm{h} \\
{[4]}\end{array}$ & 10 \\
\hline PSC01CPW & $\begin{array}{l}\text { DCS No. } 1 \text { Fails with } \\
\text { Low Demand Signal } \\
\end{array}$ & $3.0 \mathrm{E}-6 / \mathrm{h}$ & 10 & $\begin{array}{c}1.0 \mathrm{E}-6 / \mathrm{h} \\
{[4]}\end{array}$ & 10 & $\begin{array}{c}3.0 E-6 / \mathrm{h} \\
{[4]}\end{array}$ & 10 & $\begin{array}{c}2.0 E-6 / h \\
{[4]}\end{array}$ & 10 \\
\hline PSC02BPW & $\begin{array}{l}\text { MG Set } 2 \text { Controller } \\
\text { Faulty Demand Signal }\end{array}$ & $3.0 \mathrm{E} \cdot 6 / \mathrm{h}$ & 10 & $\begin{array}{c}1.0 \mathrm{E}-6 / \mathrm{h} \\
{[4]}\end{array}$ & 10 & $\begin{array}{c}3.0 \mathrm{E}-6 / \mathrm{h} \\
{[4]}\end{array}$ & 10 & $\begin{array}{c}2.0 \mathrm{E}-6 / \mathrm{h} \\
{[4]}\end{array}$ & 10 \\
\hline PSC02CPW & $\begin{array}{l}\text { DCS No. } 2 \text { Fails with } \\
\text { Low Demand Signal }\end{array}$ & $3.0 \mathrm{E}-6 / \mathrm{h}$ & 10 & $\begin{array}{c}1.0 \mathrm{E}-6 / \mathrm{h} \\
{[4]}\end{array}$ & 10 & $\begin{array}{c}3.0 \mathrm{E}-6 \mathrm{Mh} \\
{[4]}\end{array}$ & 10 & $\begin{array}{c}2.0 E-6 / h \\
{[4]}\end{array}$ & 10 \\
\hline PST001PW & $\begin{array}{l}\text { No. } 1 \text { Feedback } \\
\text { Signal Circuit Fails } \\
\text { With High Signal }\end{array}$ & $3.0 \mathrm{E}-6 / \mathrm{h}$ & 10 & $\begin{array}{c}1.0 \mathrm{E}-6 / \mathrm{h} \\
{[4]}\end{array}$ & 10 & $\begin{array}{c}3.0 E-6 / \mathrm{h} \\
{[4]}\end{array}$ & 10 & $\begin{array}{c}2.0 E-6 / \mathrm{h} \\
{[4]}\end{array}$ & 10 \\
\hline PST002PW & $\begin{array}{l}\text { No. } 2 \text { Feedback } \\
\text { Signal Circuit Fails } \\
\text { With High Signal }\end{array}$ & $3.0 \mathrm{E}-6 / \mathrm{h}$ & 10 & $\begin{array}{c}1.0 E-6 / h \\
{[4]}\end{array}$ & 10 & $\begin{array}{c}3.0 E-6 / \mathrm{h} \\
{[4]}\end{array}$ & 10 & $\begin{array}{c}2.0 E-6 / h \\
{[4]}\end{array}$ & 10 \\
\hline PSW012CU & $\begin{array}{l}\text { Control Room STOP } \\
\text { Switch Short, Common } \\
\text { Cause Loss of CPS }\end{array}$ & $1.0 \mathrm{E}-8 \mathrm{~h}$ & 10 & & & & & & \\
\hline PSW012PU & $\begin{array}{l}\text { Primary Panel STOP } \\
\text { Switch Short, Common } \\
\text { Cause Loss of CPS }\end{array}$ & $1.0 \mathrm{E}-8 \mathrm{~h}$ & 10 & & & & & & \\
\hline PSW01APB & $\begin{array}{l}\text { Primary Panel STOP } \\
\text { Switch Fails Open, } \\
\text { Pump } 1\end{array}$ & $1.0 \mathrm{E}-7 \mathrm{~m}$ & 10 & $1.0 \mathrm{E}-7 / \mathrm{h}$ & 10 & & & & \\
\hline PSW01APD & $\begin{array}{l}\text { Primary Panel STOP } \\
\text { Switch Short, Loss } \\
\text { of CPS, Pump } 1\end{array}$ & $1.0 \mathrm{E}-7 / \mathrm{h}$ & 10 & $1.0 E-7 / h$ & 10 & & & & \\
\hline PSW01BPB & $\begin{array}{l}\text { Primary Panel STOP } \\
\text { Switch Fails Open, } \\
\text { Pump } 2\end{array}$ & $1.0 \mathrm{E}-7 / \mathrm{h}$ & 10 & $1.0 \mathrm{E}-7 / \mathrm{h}$ & 10 & & & & \\
\hline PSW01BPD & $\begin{array}{l}\text { Primary Panel STOP } \\
\text { Switch Short, Loss } \\
\text { of CPS, Pump } 2\end{array}$ & $1.0 \mathrm{E}-7 / \mathrm{h}$ & 10 & $1.0 \mathrm{E}-7 / \mathrm{h}$ & 10 & & & & \\
\hline PSW02ACB & $\begin{array}{l}\text { Control Room STOP } \\
\text { Switch Fails Open, } \\
\text { Pump } 1\end{array}$ & $1.0 \mathrm{E}-7 / \mathrm{h}$ & 10 & $1.0 E-7 / h$ & 10 & & & & \\
\hline
\end{tabular}


Table 7.4 Primary Pump and Primary Pump M/G Set Data (Cont'd)

PAGE 3

\begin{tabular}{|c|c|c|c|c|c|c|c|c|c|}
\hline \multirow{2}{*}{$\begin{array}{l}\text { EVENT } \\
\text { NAME }\end{array}$} & \multirow[b]{2}{*}{ DESCRIPTION } & \multicolumn{2}{|c|}{ VALUE USED 391} & \multicolumn{2}{|c|}{ CRBRP-4 } & \multicolumn{2}{|c|}{ IREP } & \multicolumn{2}{|c|}{ GENERIC } \\
\hline & & $\begin{array}{c}\text { Failure } \\
\text { Rate }\end{array}$ & $\begin{array}{l}\text { Error } \\
\text { Factor }\end{array}$ & $\begin{array}{c}\text { Failure } \\
\text { Rate }\end{array}$ & $\begin{array}{l}\text { Error } \\
\text { Factor }\end{array}$ & $\begin{array}{c}\text { Failure } \\
\text { Rate }\end{array}$ & $\begin{array}{l}\text { Error } \\
\text { Factor }\end{array}$ & $\begin{array}{c}\text { Failure } \\
\text { Rate } \\
\end{array}$ & $\begin{array}{l}\text { Error } \\
\text { Factor }\end{array}$ \\
\hline PSW02ACD & $\begin{array}{l}\text { Control Room STOP } \\
\text { Switch Short, Loss } \\
\text { of CPS, Pump } 1\end{array}$ & $1.0 \mathrm{E}-7 / \mathrm{h}$ & 10 & $1.0 \mathrm{E}-7 \mathrm{~h}$ & 10 & & & & \\
\hline PSW02BCB & $\begin{array}{l}\text { Control Room STOP } \\
\text { Switch Fails Open, } \\
\text { Pump } 2\end{array}$ & $1.0 \mathrm{E}-7 / \mathrm{h}$ & 10 & $1.0 \mathrm{E}-7 / \mathrm{h}$ & 10 & & & & \\
\hline PSWO2BCD & $\begin{array}{l}\text { Control Room STOP } \\
\text { Switch Short, Loss } \\
\text { of CPS, Pump 2 } \\
\end{array}$ & $1.0 \mathrm{E}-7 / \mathrm{h}$ & 10 & $1.0 \mathrm{E}-7 / \mathrm{h}$ & 10 & & & & \\
\hline PWR012PU & $\begin{array}{l}\text { Pumps } 1 \text { \& } 2 \text { START/ST } \\
\text { OP Circuit Wiring Fa } \\
\text { it Open Common Cause }\end{array}$ & $\begin{array}{c}3.0 \mathrm{E}-7 / \mathrm{h} \\
{[5]}\end{array}$ & 10 & & & & & & \\
\hline PWRO1APB & $\begin{array}{l}\text { Primary Pump } 1 \\
\text { START/STOP Circuit } \\
\text { Wiring Fails Open } \\
\end{array}$ & $3.0 \mathrm{E}-6 / \mathrm{h}$ & 10 & $1.0 \mathrm{E}-6 / \mathrm{h}$ & 10 & $3.0 \mathrm{E} \cdot 6 / \mathrm{h}$ & 10 & $3.0 \mathrm{E}-6 \mathrm{~h}$ & 10 \\
\hline PWRO1BPB & $\begin{array}{l}\text { Primary Pump } 2 \\
\text { START/STOP Circuit } \\
\text { Wiring Fails Open }\end{array}$ & $3.0 \mathrm{E}-6 / \mathrm{h}$ & 10 & $1.0 \mathrm{E} \cdot 6 / \mathrm{h}$ & 10 & $3.0 E-6 / h$ & 10 & $3.0 \mathrm{E}-6 / \mathrm{h}$ & 10 \\
\hline PWR022PU & $\begin{array}{l}\text { Wiring between STOP } \\
\text { Switches Fails Open, } \\
\text { Pumps } 1 \text { \& } 2 \text { Common }\end{array}$ & $\begin{array}{c}3.0 \mathrm{E}-7 / \mathrm{h} \\
{[5]}\end{array}$ & 10 & & & & & & \\
\hline PWR022YB & $\begin{array}{l}\text { CPS Feeder to HP } \\
\text { Power Supply No. } 1 \\
\text { Fails Open } \\
\end{array}$ & $3.0 \mathrm{E}-6 / \mathrm{h}$ & 10 & $1.0 \mathrm{E}-6 / \mathrm{h}$ & 10 & $3.0 E-6 / h$ & 10 & $3.0 \mathrm{E}-6 / \mathrm{h}$ & 3 \\
\hline PWRO22YC & $\begin{array}{l}\text { CPS Feeder to HP } \\
\text { Power Supply No. } 1 \\
\text { Short Circuit }\end{array}$ & $3.0 \mathrm{E}-7 / \mathrm{h}$ & 10 & $\begin{array}{l}1.0 \mathrm{E}-7 \mathrm{~h} \\
. \quad[6]\end{array}$ & 10 & $\begin{array}{c}3.0 \mathrm{E}-7 / \mathrm{h} \\
{[6]}\end{array}$ & 10 & $\begin{array}{c}3.0 \mathrm{E}-7 / \mathrm{h} \\
{[6]}\end{array}$ & 10 \\
\hline PWR02APB & $\begin{array}{l}\text { Wiring between STOP } \\
\text { Switches Fails Open, } \\
\text { Pump } 1\end{array}$ & $3.0 \mathrm{E}-6 / \mathrm{h}$ & 10 & $1.0 \mathrm{E}-6 / \mathrm{h}$ & 10 & $3.0 \mathrm{E}-6 / \mathrm{h}$ & 10 & $3.0 \mathrm{E}-6 / \mathrm{h}$ & 10 \\
\hline PWRO2BPB & $\begin{array}{l}\text { Wiring between STOP } \\
\text { Switches Fails Open, } \\
\text { Pump } 2\end{array}$ & $3.0 \mathrm{E}-6 / \mathrm{h}$ & 10 & $1.0 \mathrm{E}-6 / \mathrm{h}$ & 10 & $3.0 \mathrm{E} \cdot 6 / \mathrm{h}$ & 10 & $3.0 \mathrm{E}-6 / \mathrm{h}$ & 10 \\
\hline PWR032QU & $\begin{array}{l}\text { Wiring to/from CPC-2 } \\
\text { Relay Fails Open, } \\
\text { Pumps } 1 \text { \& } 2 \text { Common }\end{array}$ & $\begin{array}{c}6.0 \mathrm{E-7} / \mathrm{h} \\
{[7]}\end{array}$ & 10 & & & & & & \\
\hline PWRO3AQB & $\begin{array}{l}\text { Wiring to/from CPC-2 } \\
\text { Relay Fails Open, } \\
\text { Pump } 1\end{array}$ & $6.0 \mathrm{E}-6 / \mathrm{h}$ & 10 & $1.0 E-6 / h$ & 10 & $3.0 \mathrm{E}-6 / \mathrm{h}$ & 10 & $3.0 \mathrm{E}-6 / \mathrm{h}$ & 10 \\
\hline PWR03BQB & $\begin{array}{l}\text { Wiring to/trom CPC-2 } \\
\text { Relay Fails Open, } \\
\text { Pump } 2\end{array}$ & $6.0 E-6 / h$ & 10 & $1.0 \mathrm{E}-6 / \mathrm{h}$ & 10 & $3.0 \mathrm{E}-6 / \mathrm{h}$ & 10 & $3.0 \mathrm{E}-6 / \mathrm{h}$ & 10 \\
\hline PWR042PB & $\begin{array}{l}\text { Exciter No. } 1 \text { Wiring } \\
\text { to Generator Fails } \\
\text { Open }\end{array}$ & $3.0 \mathrm{E}-6 / \mathrm{h}$ & 10 & $1.0 \mathrm{E}-6 / \mathrm{h}$ & 10 & $3.0 \mathrm{E}-6 / \mathrm{h}$ & 10 & $3.0 \mathrm{E}-6 / \mathrm{h}$ & 3 \\
\hline PWR042PC & $\begin{array}{l}\text { Exciter No. } 1 \text { Wiring } \\
\text { to Generator Short } \\
\text { Circuit }\end{array}$ & $3.0 \mathrm{E}-7 / \mathrm{h}$ & 10 & $\begin{array}{c}1.0 \mathrm{E}-7 / \mathrm{h} \\
{[6]}\end{array}$ & 10 & $\begin{array}{c}3.0 \mathrm{E}-7 / \mathrm{h} \\
{[6]}\end{array}$ & 10 & $\begin{array}{c}3.0 \mathrm{E}-7 \mathrm{~h} \\
{[6]}\end{array}$ & 10 \\
\hline PWR044PB & $\begin{array}{l}\text { CPS Feeder to } \\
\text { Exciter No. } 1 \text { PS } \\
\text { Fails Open }\end{array}$ & $3.0 \mathrm{E}-6 / \mathrm{h}$ & 10 & $1.0 \mathrm{E}-6 / \mathrm{h}$ & 10 & $3.0 \mathrm{E}-6 / \mathrm{h}$ & 10 & $3.0 \mathrm{E}-6 / \mathrm{h}$ & 3 \\
\hline PWR044PC & $\begin{array}{l}\text { CPS Feeder to } \\
\text { Exciter No. } 1 \text { PS } \\
\text { Short Circuit } \\
\end{array}$ & $3.0 \mathrm{E}-7 / \mathrm{h}$ & 10 & $\begin{array}{c}1.0 E-7 / \mathrm{h} \\
{[6]}\end{array}$ & 10 & $\begin{array}{c}3.0 \mathrm{E}-7 \mathrm{~h} \\
{[6]}\end{array}$ & 10 & $\begin{array}{c}3.0 \mathrm{E}-7 / \mathrm{h} \\
{[6]}\end{array}$ & 10 \\
\hline PWR051TB & $\begin{array}{l}\text { MG Set } 1 \text { Wiring to } \\
\text { Pump } 1 \text { Fails Open }\end{array}$ & $3.0 \mathrm{E}-6 / \mathrm{h}$ & 10 & $1.0 \mathrm{E}-6 / \mathrm{h}$ & 10 & $3.0 \mathrm{E}-6 / \mathrm{h}$ & 10 & $3.0 \mathrm{E}-6 / \mathrm{h}$ & 3 \\
\hline PWR051TC & $\begin{array}{l}\text { MG Set } 1 \text { Wiring to } \\
\text { Pump } 1 \text { Short Circuit }\end{array}$ & $3.0 \mathrm{E}-7 / \mathrm{h}$ & 10 & $\begin{array}{c}1.0 \mathrm{E}-7 \mathrm{~h} \\
{[6]}\end{array}$ & 10 & $\begin{array}{c}3.0 \mathrm{E}-7 / \mathrm{h} \\
{[6]}\end{array}$ & 10 & $\begin{array}{c}3.0 \mathrm{E} \cdot 7 / \mathrm{h} \\
{[6]}\end{array}$ & 10 \\
\hline PWR122YB & $\begin{array}{l}\text { CPS Feeder to HP } \\
\text { Power Supply No. } 2 \\
\text { Fails Open }\end{array}$ & $3.0 \mathrm{E}-6 / \mathrm{h}$ & 10 & $1.0 \mathrm{E}-6 / \mathrm{h}$ & 10 & $3.0 \mathrm{E}-6 / \mathrm{h}$ & 10 & $3.0 \mathrm{E}-6 / \mathrm{h}$ & 3 \\
\hline
\end{tabular}


Table 7.4 Primary Pump and Primary Pump M/G Set Data (Cont'd)

PAGE 4

\begin{tabular}{|c|c|c|c|c|c|c|c|c|c|}
\hline \multirow{2}{*}{$\begin{array}{l}\text { EVENT } \\
\text { NAME }\end{array}$} & \multirow[b]{2}{*}{ DESCRIPTION } & \multicolumn{2}{|c|}{ VALUE USED $3 / 91$} & \multicolumn{2}{|c|}{ CRBRP-4 } & \multicolumn{2}{|c|}{ IREP } & \multicolumn{2}{|c|}{ GENERIC } \\
\hline & & $\begin{array}{l}\text { Failure } \\
\text { Rate }\end{array}$ & $\begin{array}{l}\text { Error } \\
\text { Factor }\end{array}$ & $\begin{array}{l}\text { Failure } \\
\text { Rate }\end{array}$ & $\begin{array}{l}\text { Error } \\
\text { Factor }\end{array}$ & $\begin{array}{l}\text { Failure } \\
\text { Rate }\end{array}$ & $\begin{array}{l}\text { Error } \\
\text { Factor }\end{array}$ & $\begin{array}{l}\text { Failure } \\
\text { Rate }\end{array}$ & $\begin{array}{l}\text { Error } \\
\text { Factor }\end{array}$ \\
\hline PWR122YC & $\begin{array}{l}\text { CPS Feeder to HP } \\
\text { Power Supply No. } 2 \\
\text { Short Circuit }\end{array}$ & $3.0 \mathrm{E}-7 / \mathrm{h}$ & 10 & $\underset{[6]}{1.0 \mathrm{E}-7 \mathrm{~h}}$ & 10 & $\begin{array}{c}3.0 \mathrm{E} \cdot 7 \mathrm{~h} \\
{[6]}\end{array}$ & 10 & $\begin{array}{c}3.0 \mathrm{E}-7 / \mathrm{h} \\
{[6]}\end{array}$ & 10 \\
\hline PWR142PB & $\begin{array}{l}\text { Exciter No. } 2 \text { Wiring } \\
\text { to Generator Fails } \\
\text { Open }\end{array}$ & $3.0 \mathrm{E}-6 / \mathrm{h}$ & 10 & $1.0 \mathrm{E} \cdot 6 / \mathrm{h}$ & 10 & $3.0 \mathrm{E}-6 / \mathrm{h}$ & 10 & $3.0 \mathrm{E}-6 / \mathrm{h}$ & 3 \\
\hline PWR142PC & $\begin{array}{l}\text { Exciter No. } 2 \text { Wiring } \\
\text { to Generator Short } \\
\text { Circuit }\end{array}$ & $3.0 \mathrm{E}-7 / \mathrm{h}$ & 10 & $\underset{[6]}{1.0 E-7 / h}$ & 10 & $\begin{array}{c}3.0 \mathrm{E} \cdot \mathrm{7} / \mathrm{h} \\
{[6]}\end{array}$ & 10 & $\begin{array}{c}3.0 \mathrm{E}-7 / \mathrm{h} \\
{[6]}\end{array}$ & 10 \\
\hline PWR144PB & $\begin{array}{l}\text { CPS Feeder to } \\
\text { Exciter No. } 2 \text { PS } \\
\text { Fails Open }\end{array}$ & $3.0 \mathrm{E}-6 / \mathrm{h}$ & 10 & $1.0 \mathrm{E} \cdot 6 / \mathrm{h}$ & 10 & $3.0 \mathrm{E}-6 / \mathrm{h}$ & 10 & $3.0 \mathrm{E}-6 / \mathrm{h}$ & 3 \\
\hline PWR144PC & $\begin{array}{l}\text { CPS Feeder to } \\
\text { Exciter No. } 2 \text { PS } \\
\text { Short Circuit }\end{array}$ & $3.0 \mathrm{E}-7 / \mathrm{h}$ & 10 & $\underset{[6]}{1.0 \mathrm{E}-7 / \mathrm{h}}$ & 10 & $\begin{array}{c}3.0 \mathrm{E} \cdot 7 \mathrm{~h} \\
{[6]}\end{array}$ & 10 & $\begin{array}{c}3.0 \mathrm{E}-7 / \mathrm{h} \\
{[6]}\end{array}$ & 10 \\
\hline PWR151TB & $\begin{array}{l}\text { MG Set } 2 \text { Wiring to } \\
\text { Pump } 2 \text { Fails Open }\end{array}$ & $3.0 \mathrm{E}-6 / \mathrm{h}$ & 10 & $1.0 \mathrm{E}-6 / \mathrm{h}$ & 10 & $3.0 \mathrm{E}-6 / \mathrm{h}$ & 10 & $3.0 \mathrm{E}-6 / \mathrm{h}$ & 3 \\
\hline PWR151TC & $\begin{array}{l}\text { MG Set } 2 \text { Wiring to } \\
\text { Pump } 2 \text { Short Circuit }\end{array}$ & $3.0 \mathrm{E}-7 / \mathrm{h}$ & 10 & $\begin{array}{c}1.0 \mathrm{E}-7 / \mathrm{h} \\
{[6]}\end{array}$ & 10 & $\begin{array}{c}3.0 \mathrm{E}-7 \mathrm{~h} \\
{[6]}\end{array}$ & 10 & $\begin{array}{c}3.0 \mathrm{E} \cdot 7 \mathrm{~h} \\
{[6]}\end{array}$ & 10 \\
\hline PWR2O1YB & $\begin{array}{l}2400 \text { V Feeder to MG } \\
\text { Set } 1 \text { Fails Open }\end{array}$ & $3.0 \mathrm{E} \cdot 6 / \mathrm{h}$ & 10 & $1.0 \mathrm{E}-6 / \mathrm{h}$ & 10 & $3.0 \mathrm{E} \cdot 6 / \mathrm{h}$ & 10 & $3.0 \mathrm{E}-6 / \mathrm{h}$ & 3 \\
\hline PWR201YC & $\begin{array}{l}2400 \text { V Feeder to MG } \\
\text { Set } 1 \text { Short Circuit }\end{array}$ & $3.0 \mathrm{E}-7 / \mathrm{h}$ & 10 & $\begin{array}{c}1.0 \mathrm{E} \cdot 7 \mathrm{~h} \\
{[6]}\end{array}$ & 10 & $\begin{array}{c}3.0 \mathrm{E}-7 / \mathrm{h} \\
{[6]}\end{array}$ & 10 & $\begin{array}{c}3.0 \mathrm{E} \cdot 7 / \mathrm{h} \\
{[6]}\end{array}$ & 10 \\
\hline PWR202YB & $\begin{array}{l}2400 \text { V Feeder to MG } \\
\text { Set } 2 \text { Fails Open }\end{array}$ & $3.0 \mathrm{E}-6 / \mathrm{h}$ & 10 & $1.0 \mathrm{E}-6 / \mathrm{h}$ & 10 & $3.0 \mathrm{E}-6 / \mathrm{h}$ & 10 & $3.0 \mathrm{E}-6 / \mathrm{h}$ & 3 \\
\hline PWRZO2YC & $\begin{array}{l}2400 \text { V Feeder to MG } \\
\text { Set } 2 \text { Short Circuit }\end{array}$ & $3.0 \mathrm{E}-7 / \mathrm{h}$ & 10 & $\begin{array}{c}1.0 \mathrm{E}-7 / \mathrm{h} \\
{[6]}\end{array}$ & .10 & $\begin{array}{c}3.0 \mathrm{E}-7 / \mathrm{h} \\
{[6]}\end{array}$ & 10 & $\begin{array}{c}3.0 \mathrm{E}-7 / \mathrm{h} \\
{[6]}\end{array}$ & 10 \\
\hline
\end{tabular}

Notes: 1. Taken from CRBRP-4 column

2. High power solid state device value

3. DC motor-generator failure value

4. Low power solid state device value

5. Value for $100 \mathrm{ft}$ open circuit with beta factor of 0.1

6. Short to ground value

7. Value for $200 \mathrm{ft}$ open circuit with beta factor of 0.1 
Table 7.5. Shutdown Cooler Data

PAGE 1

\begin{tabular}{|c|c|c|c|c|c|c|c|c|c|}
\hline \multirow{2}{*}{$\begin{array}{l}\text { EVENT } \\
\text { NAME }\end{array}$} & \multirow[b]{2}{*}{ DESCRIPTION } & \multicolumn{2}{|c|}{ VALUE USED $3 / 91$} & \multicolumn{2}{|c|}{ CRBRP-4 } & \multicolumn{2}{|c|}{ IREP } & \multicolumn{2}{|c|}{ GENERIC } \\
\hline & & $\begin{array}{c}\text { Failure } \\
\text { Rate } \\
\end{array}$ & $\begin{array}{l}\text { Error } \\
\text { Factor }\end{array}$ & $\begin{array}{c}\text { Failure } \\
\text { Rate }\end{array}$ & $\begin{array}{l}\text { Error } \\
\text { Factor }\end{array}$ & $\begin{array}{c}\text { Failure } \\
\text { Rate } \\
\end{array}$ & $\begin{array}{l}\text { Error } \\
\text { Factor }\end{array}$ & $\begin{array}{c}\text { Failure } \\
\text { Rate } \\
\end{array}$ & $\begin{array}{l}\text { Error } \\
\text { Factor } \\
\end{array}$ \\
\hline DAMAAHAW & $\begin{array}{l}\text { AV BULK NA TEMP RONA } \\
\text { N X81MV/MAILS HIGH } \\
\text { ELEC P1-TMV/I-504A }\end{array}$ & $3.0 \mathrm{E} \cdot 6 \mathrm{~h}$ & 10 & $1.0 E-6 / h$ & 10 & $3.0 \mathrm{E} \cdot 6 / \mathrm{h}$ & 10 & $2.0 \mathrm{E} \cdot 6 / \mathrm{h}$ & 10 \\
\hline DAMAALAW & $\begin{array}{l}\text { AV BULKNA TEMP RONA } \\
\text { N X81MV/ FAILS LOW } \\
\text { ELEC P1-TMVII-504A }\end{array}$ & $3.0 \mathrm{E}-6 / \mathrm{h}$ & 10 & $1.0 \mathrm{E}-6 / \mathrm{h}$ & 10 & $3.0 \mathrm{E}-6 / \mathrm{h}$ & 10 & $2.0 E-6 / \mathrm{h}$ & 10 \\
\hline DAMABHQW & $\begin{array}{l}\text { EXPN RANGE TEMP RONA } \\
\text { NX81MV/I FAILS HIGH } \\
\text { ELEC P I-TMV/1-504B }\end{array}$ & $3.0 \mathrm{E}-6 / \mathrm{h}$ & 10 & $1.0 \mathrm{E}-6 / \mathrm{h}$ & 10 & $3.0 \mathrm{E}-6 / \mathrm{h}$ & 10 & $2.0 \mathrm{E}-6 / \mathrm{h}$ & 10 \\
\hline DAMABLOW & $\begin{array}{l}\text { EXPN RANGE TEMP RONA } \\
N \times 81 \text { MVII FAILS LOW } \\
\text { ELEC P1-TMVII-504B }\end{array}$ & $3.0 \mathrm{E}-6 / \mathrm{h}$ & 10 & $1.0 \mathrm{E}-6 / \mathrm{h}$ & 10 & $3.0 \mathrm{E}-6 / \mathrm{h}$ & 10 & $2.0 \mathrm{E}-6 / \mathrm{h}$ & 10 \\
\hline DAN501AW & $\begin{array}{l}\text { T/C REFERENCE JUNCT } \\
\text { ION HIGH ANNUN FAIL } \\
\text { S FOR R1-CRJ-50-1 }\end{array}$ & $3.0 \mathrm{E}-6 / \mathrm{h}$ & 10 & $1.0 \mathrm{E}-6 / \mathrm{h}$ & 10 & $3.0 E-6 / \mathrm{h}$ & 10 & $2.0 \mathrm{E}-6 / \mathrm{h}$ & 10 \\
\hline DAN502AW & $\begin{array}{l}\text { T/C REFERENCE JUNCT } \\
\text { ION HIGH ANNUN FAIL } \\
\mathrm{S} \text { FOR R1-CRJ-50-2 }\end{array}$ & $3.0 \mathrm{E}-6 / \mathrm{h}$ & 10 & $1.0 \mathrm{E}-6 \mathrm{~m}$ & 10 & $3.0 \mathrm{E}-6 / \mathrm{h}$ & 10 & $2.0 \mathrm{E}-6 / \mathrm{h}$ & 10 \\
\hline DAN504AW & $\begin{array}{l}\text { T/C REFERENCE JUNCT } \\
\text { ION HIGH ANNUN FAIL } \\
\text { S FOR R1-CRJ-50-4 }\end{array}$ & $3.0 \mathrm{E}-6 \mathrm{~h}$ & 10 & $1.0 E \cdot 6 / \mathrm{h}$ & 10 & $3.0 \mathrm{E}-6 \mathrm{~h}$ & 10 & $2.0 \mathrm{E}-6 / \mathrm{h}$ & 10 \\
\hline DAN505AW & $\begin{array}{l}\text { T/C REFERENCE JUNCT } \\
\text { ION HIGH ANNUN FAIL } \\
\mathrm{S} \text { FOR R1-CRJ-50-5 }\end{array}$ & $3.0 \mathrm{E}-6 / \mathrm{h}$ & 10 & $1.0 \mathrm{E}-6 / \mathrm{h}$ & 10 & $3.0 \mathrm{E}-6 / \mathrm{h}$ & 10 & $2.0 \mathrm{E}-6 / \mathrm{h}$ & 10 \\
\hline $\begin{array}{c}\text { DAN751AW } \\
.\end{array}$ & $\begin{array}{l}\text { T/C REFERENCE JUNCT } \\
\text { ION HIGH ANNUN FAIL } \\
\text { S FOR R1-CRJ-75-1 }\end{array}$ & $3.0 \mathrm{E}-6 / \mathrm{h}$ & 10 & $1.0 \mathrm{E}-6 / \mathrm{h}$ & 10 & $3.0 \mathrm{E}-6 / \mathrm{h}$ & 10 & $2.0 E-6 / h$ & 10 \\
\hline DAN82LAW & $\begin{array}{l}\text { BULK SODIUM LOW } \\
\text { TEMPERATURE ANNUN } \\
\text { FAILS }\end{array}$ & $3.0 \mathrm{E}-6 / \mathrm{h}$ & 10 & $1.0 \mathrm{E} \cdot 6 / \mathrm{h}$ & 10 & $3.0 \mathrm{E}-6 \mathrm{~h}$ & 10 & $2.0 \mathrm{E}-6 / \mathrm{h}$ & 10 \\
\hline DARX82QB & $\begin{array}{l}\text { RONAN X82 ALARM } \\
\text { CIRCUIT } \\
\text { HIGH FAILS } \\
\text { P1-TA-504A }\end{array}$ & $3.0 \mathrm{E} \cdot 6 \mathrm{~m}$ & 10 & $1.0 E-6 / \mathrm{h}$ & 10 & $3.0 \mathrm{E}-6 / \mathrm{h}$ & 10 & $2.0 \mathrm{E} \cdot 6 / \mathrm{h}$ & 10 \\
\hline DAR $\times 8200$ & $\begin{array}{l}\text { RONAN X82 ALARM } \\
\text { RELAY (COIL) FAILS } \\
\text { OPEN P1-TA-504A }\end{array}$ & $3.0 E-7 / \mathrm{h}$ & 10 & $1.0 \mathrm{E}-6 / \mathrm{h}$ & 10 & $3.0 \mathrm{E}-6 / \mathrm{h}$ & 10 & $3.0 \mathrm{E}-6 / \mathrm{h}$ & 10 \\
\hline DARX82QP & $\begin{array}{l}\text { RONAN X82 ALARM } \\
\text { RELAY FAILS } \\
\text { TO OPEN P1-TA-504A }\end{array}$ & $3.0 \mathrm{E}-4 / \mathrm{d}$ & 10 & $1.0 \mathrm{E}-4 / \mathrm{d}$ & 10 & $3.0 \mathrm{E}-4 / \mathrm{d}$ & 10 & $3.0 E-4 / d$ & 10 \\
\hline DARX82OW & $\begin{array}{l}\text { RONAN X82 ALARM } \\
\text { CIRCUIT FAILS } \\
\text { LOW P1-TA-504A } \\
\end{array}$ & $3.0 E-6 / h$ & 10 & $1.0 E-6 / \mathrm{h}$ & 10 & $3.0 E-6 / \mathrm{h}$ & 10 & $2.0 \mathrm{E} \cdot 6 \mathrm{~h}$ & 10 \\
\hline DATO1LOO & $\begin{array}{l}\text { COOLER } 1 \text { LOWER ACTUA } \\
\text { TOR OR LINKAGE FAILS } \\
\text { TO REMAIN CLOSED }\end{array}$ & $3.0 E-7 / \mathrm{h}$ & 10 & $1.0 \mathrm{E}-7 / \mathrm{h}$ & 3 & & & & \\
\hline DATO1LOP & $\begin{array}{l}\text { COOLER I LOWER ACTUA } \\
\text { TOR OR UNKAGE FA } \\
\text { ILS TO OPEN DAMPER }\end{array}$ & $3.0 E-3 / d$ & 5 & $1.0 \mathrm{E}-3 / \mathrm{d}$ & 10 & $3.0 E-3 / d$ & 10 & $3.0 E-3 / d$ & 3 \\
\hline DATO1UOO & $\begin{array}{l}\text { COOLER I UPPER ACTUA } \\
\text { TOR OR LINKAGE FAILS } \\
\text { TO REMAIN CLOSED }\end{array}$ & $3.0 E-7 / \mathrm{h}$ & 10 & $1.0 \mathrm{E}-7 \mathrm{~h}$ & 3 & & & & \\
\hline DAT01UOP & $\begin{array}{l}\text { COOLER I UPPER ACTUA } \\
\text { TOR OR LINKAGE FA } \\
\text { ILS TO OPEN DAMPER }\end{array}$ & $3.0 E-3 / d$ & 5 & $1.0 \mathrm{E}-3 / \mathrm{d}$ & 10 & $3.0 \mathrm{E}-3 / \mathrm{d}$ & 10 & $3.0 \mathrm{E}-3 / \mathrm{d}$ & 3 \\
\hline DAT02LOO & $\begin{array}{l}\text { COOLER } 2 \text { LOWER ACTUA } \\
\text { TOR OR LINKAGE FAILS } \\
\text { TO REMAIN CLOSED }\end{array}$ & $3.0 \mathrm{E}-7 \mathrm{~h}$ & 10 & $1.0 \mathrm{E}-7 / \mathrm{h}$ & 3 & & & & \\
\hline DATO2LOP & $\begin{array}{l}\text { COOLER } 2 \text { LOWER ACTUA } \\
\text { TOR OR LINKAGE FA } \\
\text { ILS TO OPEN DAMPER }\end{array}$ & $3.0 E-3 / d$ & 5 & $1.0 \mathrm{E}-3 / \mathrm{d}$ & 10 & $3.0 \mathrm{E}-3 / \mathrm{d}$ & 10 & $3.0 \mathrm{E}-3 / \mathrm{d}$ & 3 \\
\hline
\end{tabular}


Table 7.5. Shutdown Cooler Data (Cont'd)

PAGE 2

\begin{tabular}{|c|c|c|c|c|c|c|c|c|c|}
\hline \multirow{2}{*}{$\begin{array}{l}\text { EVENT } \\
\text { NAME }\end{array}$} & \multirow[b]{2}{*}{ DESCRIPTION } & \multicolumn{2}{|c|}{ VALUE USED $3 / 91$} & \multicolumn{2}{|c|}{ CRBRP-4 } & \multicolumn{2}{|c|}{ IREP } & \multicolumn{2}{|c|}{ GENERIC } \\
\hline & & $\begin{array}{c}\text { Failure } \\
\text { Rate }\end{array}$ & $\begin{array}{l}\text { Error } \\
\text { Factor }\end{array}$ & $\begin{array}{l}\text { Failure } \\
\text { Rate }\end{array}$ & $\begin{array}{l}\text { Error } \\
\text { Factor }\end{array}$ & $\begin{array}{c}\text { Failure } \\
\text { Rate }\end{array}$ & $\begin{array}{l}\text { Error } \\
\text { Factor }\end{array}$ & $\begin{array}{c}\text { Failure } \\
\text { Rate }\end{array}$ & $\begin{array}{l}\text { Error } \\
\text { Factor }\end{array}$ \\
\hline DAT02U0O & $\begin{array}{l}\text { COOLER } 2 \text { UPPER ACTUA } \\
\text { TOR OR LINKAGE FAILS } \\
\text { TO REMAIN CLOSED }\end{array}$ & $3.0 \mathrm{E} \cdot 7 \mathrm{~m}$ & 10 & $1.0 \mathrm{E} \cdot 7 / \mathrm{h}$ & 3 & & & & \\
\hline DAT02UOP & $\begin{array}{l}\text { COOLER } 2 \text { UPPER ACTUA } \\
\text { TOR OR LINKAGE FA } \\
\text { ILS TO OPEN DAMPER }\end{array}$ & $3.0 E-3 / d$ & 5 & $1.0 \mathrm{E}-3 / \mathrm{d}$ & 10 & $3.0 \mathrm{E}-3 / \mathrm{d}$ & 10 & $3.0 \mathrm{E} \cdot 3 / \mathrm{d}$ & 3 \\
\hline DCR001OE & $\begin{array}{l}\text { COOLER \#1 NAK } \\
\text { BLOCKAGE }\end{array}$ & $1.0 \mathrm{E}-7 / \mathrm{h}$ & 10 & $1.0 \mathrm{E}-9 \mathrm{~m}$ & & & & $8.0 \mathrm{E}-9 / \mathrm{h}$ & 30 \\
\hline DCROO1OF & $\begin{array}{l}\text { COOLER \#1 NAK } \\
\text { LEAKAGE }\end{array}$ & $3.0 \mathrm{E}-7 / \mathrm{h}$ & 10 & $1.0 \mathrm{E}-9 / \mathrm{h}$ & & & & $8.0 \mathrm{E} \cdot 9 / \mathrm{h}$ & 30 \\
\hline DCR002OE & $\begin{array}{l}\text { COOLER \#2 NAK } \\
\text { BLOCKAGE } \\
\end{array}$ & $1.0 \mathrm{E} \cdot 7 \mathrm{~m}$ & 10 & & & & & & \\
\hline DCR002OF & $\begin{array}{l}\text { COOLER \#2 NAK } \\
\text { LEAKAGE }\end{array}$ & $3.0 \mathrm{E}-7 / \mathrm{h}$ & 10 & $1.0 \mathrm{E}-9 \mathrm{~h}$ & & & & 8.0E-9/h & 30 \\
\hline DHR501AW & $\begin{array}{l}\text { T/C REFERENCE } \\
\text { JUNCTION OVEN FAILS } \\
\text { HIGH R1-CRJ-50-1 } \\
\end{array}$ & $3.0 \mathrm{E}-6 / \mathrm{h}$ & 10 & $1.0 \mathrm{E}-6 \mathrm{~m}$ & 10 & $3.0 \mathrm{E}-6 / \mathrm{h}$ & 10 & $2.0 \mathrm{E}-6 / \mathrm{h}$ & 10 \\
\hline DHR502AW & $\begin{array}{l}\text { T/C REFERENCE } \\
\text { JUNCTION OVEN FAILS } \\
\text { HIGH R1-CRJ-50-2 } \\
\end{array}$ & $3.0 \mathrm{E}-6 / \mathrm{h}$ & 10 & $1.0 \mathrm{E}-6 / \mathrm{h}$ & 10 & $3.0 \mathrm{E} \cdot 6 / \mathrm{h}$ & 10 & $2.0 \mathrm{E}-6 / \mathrm{h}$ & 10 \\
\hline DHR504AW & $\begin{array}{l}\text { T/C REFERENCE } \\
\text { JUNCTION OVEN FAILS } \\
\text { HIGH R1-CRJ-50-4 } \\
\end{array}$ & $3.0 \mathrm{E} \cdot 6 / \mathrm{h}$ & 10 & $1.0 \mathrm{E}-6 / \mathrm{h}$ & 10 & $3.0 \mathrm{E} \cdot 6 / \mathrm{h}$ & 10 & $2.0 E-6 / h$ & 10 \\
\hline DHF505AW & $\begin{array}{l}\text { T/C REFERENCE } \\
\text { JUNCTION OVEN FAILS } \\
\text { HIGH R1-CRJ-50-5 } \\
\end{array}$ & $3.0 \mathrm{E}-6 / \mathrm{h}$ & 10 & $1.0 \mathrm{E}-6 / \mathrm{h}$ & 10 & $3.0 \mathrm{E}-6 / \mathrm{h}$ & 10 & $2.0 \mathrm{E}-6 / \mathrm{h}$ & 10 \\
\hline DHR751AW & $\begin{array}{l}\text { T/C REFERENCE } \\
\text { JUNCTION OVEN FAILS } \\
\text { HIGH R1-CRJ-75-1 } \\
\end{array}$ & $3.0 \mathrm{E} \cdot 6 / \mathrm{h}$ & 10 & $1.0 \mathrm{E}-6 / \mathrm{h}$ & 10 & $3.0 \mathrm{E}-6 / \mathrm{h}$ & 10 & $2.0 \mathrm{E}-6 / \mathrm{h}$ & 10 \\
\hline DHU000OL & $\begin{array}{l}\text { HUMAN ERROR - } \\
\text { FAILURE TO RECOVER } \\
\text { COOLERS }\end{array}$ & $4.0 \mathrm{E}-6 / \mathrm{d}$ & 10 & & & & & & \\
\hline DHU154PZ & $\begin{array}{l}\text { HUMAN ERROR } \\
\text { IMPROPER VALVE } \\
\text { OPERATION } \quad \text { PA-15 } \\
\end{array}$ & $1.0 \mathrm{E}-4 / \mathrm{d}$ & 10 & & & & & & \\
\hline DHU500CZ & $\begin{array}{l}\text { HUMAN ERROR ANY T } \\
\text { IC REF JUNCT >152 } \\
\text { F ALARM IGNORED } \\
\end{array}$ & $1.0 \mathrm{E}-2 / \mathrm{d}$ & 10 & & & & & & \\
\hline DHU82LCZ & $\begin{array}{l}\text { HUMAN ERROR BULK } \\
\text { SODIUM LOW TEMP } \\
\text { ALARM IGNORED }\end{array}$ & $1.0 \mathrm{E} \cdot 2 / \mathrm{d}$ & 10 & & & & & & \\
\hline 00011200 & $\begin{array}{l}\text { FORCE IS ENOUGH TO } \\
\text { UFT SODIUM }\end{array}$ & 1.0 & 1 & & & & & & \\
\hline 00022200 & $\begin{array}{l}\text { HOT LEG BREACH BELOW } \\
\text { PUMP INLET }\end{array}$ & 1.0 & 1 & & & & & & \\
\hline 00033200 & $\begin{array}{l}\text { BREACH OUTSIDE } \\
\text { REACTOR AFTER HEAT } \\
\text { EXCHANGER }\end{array}$ & 1.0 & 1 & & & & & - & \\
\hline DPPCOLRF & COLD LEG BREACH & $\begin{array}{c}3.0 \mathrm{E}-7 / \mathrm{h} \\
{[1]}\end{array}$ & 30 & & & & & & \\
\hline DPPINNRF & INNER PIPE BREACH & $\begin{array}{c}1.0 E-1 / d \\
{[2]}\end{array}$ & 3 & & & & & & \\
\hline DPPOUTRF & OUTER PIPE BREACH & $\begin{array}{c}1.0 E-6 / \mathrm{h} \\
{[3]}\end{array}$ & 10 & & & & & & \\
\hline DPVO2AOQ & $\begin{array}{l}\text { AIR REGULATOR COOLE } \\
\text { R } 1 \text { FAILS TO REMAIN } \\
\text { OPEN R4-PVC-682A }\end{array}$ & $3.3 E-7 / \mathrm{h}$ & 10 & $1.0 \mathrm{E}-7 \mathrm{~m}$ & 3 & & & & \\
\hline
\end{tabular}


Table 7.5. Shutdown Cooler Data (Cont'd)

PAGE 3

\begin{tabular}{|c|c|c|c|c|c|c|c|c|c|}
\hline \multirow{2}{*}{$\begin{array}{l}\text { EVENT } \\
\text { NAME }\end{array}$} & \multirow[b]{2}{*}{ DESCRIPTION } & \multicolumn{2}{|c|}{ VALUE USED $3 / 91$} & \multicolumn{2}{|c|}{ CRBRP -4} & \multicolumn{2}{|c|}{ IREP } & \multicolumn{2}{|c|}{ GENERIC } \\
\hline & & $\begin{array}{c}\text { Failure } \\
\text { Rate }\end{array}$ & $\begin{array}{l}\text { Error } \\
\text { Factor }\end{array}$ & $\begin{array}{c}\text { Failure } \\
\text { Rate }\end{array}$ & $\begin{array}{l}\text { Error } \\
\text { Factor }\end{array}$ & $\begin{array}{l}\text { Failure } \\
\text { Rate }\end{array}$ & $\begin{array}{l}\text { Error } \\
\text { Factor }\end{array}$ & $\begin{array}{c}\text { Failure } \\
\text { Rate }\end{array}$ & $\begin{array}{l}\text { Error } \\
\text { Factor }\end{array}$ \\
\hline DPVO2BOQ & $\begin{array}{l}\text { AIR REGULATOR COOLE } \\
\text { R } 2 \text { FAILS TO REMAIN } \\
\text { OPEN R4-PVC-682B }\end{array}$ & $3.3 \mathrm{E}-7 \mathrm{~h}$ & 10 & $1.0 \mathrm{E}-7 \mathrm{~h}$ & 3 & & & & \\
\hline DRE53ACO & $\begin{array}{l}\text { AUX ANN RELAY PT } 2 \\
\text { (COIL) FAILS OPEN } \\
\text { ITEM 53A-PR2A }\end{array}$ & $3.0 \mathrm{E}-7 / \mathrm{h}$ & 10 & $1.0 \mathrm{E}-6 / \mathrm{h}$ & 10 & $3.0 \mathrm{E}-6 / \mathrm{h}$ & 10 & $3.0 \mathrm{E}-6 / \mathrm{h}$ & 10 \\
\hline DRE53ACP & $\begin{array}{l}\text { AUXANN RELAY PT } 2 \\
\text { (CONTACD,FAILS TO } \\
\text { OPEN ITEM 53A-PR2A }\end{array}$ & $3.0 \mathrm{E}-4 / \mathrm{d}$ & 10 & $1.0 \mathrm{E}-4 / \mathrm{d}$ & 10 & $3.0 \mathrm{E}-4 / \mathrm{d}$ & 10 & $3.0 \mathrm{E}-4 / \mathrm{d}$ & 10 \\
\hline DSV01AON & $\begin{array}{l}\text { SOLENOID } \\
\text { COOLER \#1 FAILS } \\
\text { TO CLOSE R4-VS-681A }\end{array}$ & $3.0 \mathrm{E}-3 / \mathrm{d}$ & 5 & $1.0 \mathrm{E}-3 / \mathrm{d}$ & 3 & $1.0 \mathrm{E}-3 / \mathrm{d}$ & 3 & $1.0 \mathrm{E}-3 / \mathrm{d}$ & 3 \\
\hline DSV01ACQ & $\begin{array}{l}\text { SOLENOID FAILS DOES } \\
\text { NOT REMAIN } \\
\text { OPEN R4-VS-681A }\end{array}$ & $5.0 \mathrm{E}-8 / \mathrm{h}$ & 10 & $1.0 \mathrm{E}-7 / \mathrm{m}$ & 3 & & & & \\
\hline DSV01BON & $\begin{array}{l}\text { SOLENOID } \\
\text { COLLER \#2 FAILS } \\
\text { TO CLOSE R4-VS-691B }\end{array}$ & $3.0 \mathrm{E}-3 / \mathrm{d}$ & 5 & $1.0 \mathrm{E}-3 / \mathrm{d}$ & 3 & $1.0 \mathrm{E}-3 / \mathrm{d}$ & 3 & $1.0 \mathrm{E}-3 / \mathrm{d}$ & 3 \\
\hline DSV01BOO & $\begin{array}{l}\text { SOLENOID FAILS DOES } \\
\text { NOT REMAIN } \\
\text { OPEN R4-VS-681B }\end{array}$ & $5.0 \mathrm{E}-8 / \mathrm{h}$ & 10 & $1.0 \mathrm{E}-7 / \mathrm{h}$ & 3 & & & & \\
\hline DTC4OHAB & $\begin{array}{l}\text { BULK SODIUM } \\
\text { THERMOCOUPLE FAILS } \\
\text { OPEN RI-TC-540H }\end{array}$ & $1.0 \mathrm{E}-6 \mathrm{~h}$ & 10 & $1.0 \mathrm{E}-6 / \mathrm{h}$ & 10 & & & $3.0 \mathrm{E}-6 \mathrm{~h}$ & 10 \\
\hline DTC4OHRD & $\begin{array}{l}\text { BULK SODIUM THERM } \\
\text { OCOUPLE SHORTED } \\
\text { AI-TC-540H }\end{array}$ & $1.0 \mathrm{E}-6 \mathrm{~h}$ & 10 & $1.0 \mathrm{E}-6 / \mathrm{h}$ & 10 & & & $3.0 \mathrm{E}-6 / \mathrm{h}$ & 10 \\
\hline DTC4OKRB & $\begin{array}{l}\text { BULK SODIUM } \\
\text { THERMOCOUPLE FAILS } \\
\text { OPEN RI-TC-540K }\end{array}$ & $1.0 \mathrm{E}-6 \mathrm{~h}$ & 10 & $1.0 \mathrm{E}-6 / \mathrm{h}$ & 10 & & & $3.0 E-6 / \mathrm{h}$ & 10 \\
\hline DTC4OKRD & $\begin{array}{l}\text { BULK SODIUM THERM } \\
\text { OCOUPLE SHORTED } \\
\text { RH-TC-540K }\end{array}$ & $1.0 \mathrm{E}-6 \mathrm{~h}$ & 10 & $1.0 \mathrm{E}-6 / \mathrm{h}$ & 10 & & & $3.0 \mathrm{E}-6 / \mathrm{h}$ & 10 \\
\hline DTC4OMRB & $\begin{array}{l}\text { BULK SODIUM } \\
\text { THERMOCOUPLE FAILS } \\
\text { OPEN RI-TC-540M } \\
\end{array}$ & $1.0 \mathrm{E}-6 / \mathrm{h}$ & 10 & $1.0 \mathrm{E}-6 / \mathrm{h}$ & 10 & & & $3.0 \mathrm{E}-6 / \mathrm{h}$ & 10 \\
\hline DTC4OMRD & $\begin{array}{l}\text { BULK SODIUM THERM } \\
\text { OCOUPLE SHORTED } \\
\text { RI-TC-540M }\end{array}$ & $1.0 \mathrm{E}-6 \mathrm{~h}$ & 10 & $1.0 E-6 / \mathrm{h}$ & 10 & & & $3.0 \mathrm{E}-6 / \mathrm{h}$ & 10 \\
\hline DTC4ONRB & $\begin{array}{l}\text { BULK SODIUM } \\
\text { THERMOCOUPLE FAILS } \\
\text { OPEN RI-TC-540N }\end{array}$ & $1.0 \mathrm{E}-6 \mathrm{~h}$ & 10 & $1.0 \mathrm{E} \cdot 6 \mathrm{~h}$ & 10 & & & $3.0 \mathrm{E}-6 / \mathrm{h}$ & 10 \\
\hline DTC4ONRD & $\begin{array}{l}\text { BULK SODIUM THERM } \\
\text { OCOUPLE SHORTED } \\
\text { RI-TC-54ON }\end{array}$ & $1.0 \mathrm{E}-6 \mathrm{~h}$ & 10 & $1.0 \mathrm{E}-6 / \mathrm{h}$ & 10 & & & $3.0 \mathrm{E}-6 / \mathrm{h}$ & 10 \\
\hline DTC4OPRB & $\begin{array}{l}\text { BULK SODIUM } \\
\text { THERMOCOUPLE FAILS } \\
\text { OPEN RIIC-TC-54OP }\end{array}$ & $.1 .0 \mathrm{E}-6 / \mathrm{h}$ & 10 & $1.0 \mathrm{E}-6 / \mathrm{h}$ & 10 & & & $3.0 E-6 / h$ & 10 \\
\hline DTC4OPRD & $\begin{array}{l}\text { BULK SODIUM THERM } \\
\text { OCOUPLE SHORTED } \\
\text { RI-TC-54OP }\end{array}$ & $1.0 \mathrm{E}-6 \mathrm{~h}$ & 10 & $1.0 E-6 / \mathrm{h}$ & 10 & & & $3.0 \mathrm{E}-6 / \mathrm{h}$ & 10 \\
\hline DTWOO1OE & $\begin{array}{l}\text { COOLER \#1 } \\
\text { CHIMNEY } \\
\text { BLOCKED }\end{array}$ & $1.1 \mathrm{E}-6 / \mathrm{h}$ & 10 & & & & & & \\
\hline DTWOO2OE & $\begin{array}{l}\text { COOLER \#2 } \\
\text { CHIMNEY } \\
\text { BLOCKED }\end{array}$ & $1.1 \mathrm{E}-6 / \mathrm{h}$ & 10 & & & & & & \\
\hline
\end{tabular}

Notes: 1. Recommended Value Tables, EGG-SSRE-8875, Feb. $1990-100 \mathrm{ft}$

2. Based on Engineering Judgement

3. Recommended value tables, EGG-SSRE-8875, Feb. 1990 
Table 7.6. Shield Cooling System Data

PAGE 1

\begin{tabular}{|c|c|c|c|c|c|c|c|c|c|}
\hline \multirow{2}{*}{$\begin{array}{l}\text { EVENT } \\
\text { NAME }\end{array}$} & \multirow[b]{2}{*}{ DESCRIPTION } & \multicolumn{2}{|c|}{ VALUE USED 391} & \multicolumn{2}{|c|}{ CRBRP-4 } & \multicolumn{2}{|c|}{ IREP } & \multicolumn{2}{|c|}{ GENERIC } \\
\hline & & $\begin{array}{c}\text { Failure } \\
\text { Rate }\end{array}$ & $\begin{array}{l}\text { Error } \\
\text { Factor }\end{array}$ & $\begin{array}{c}\text { Failure } \\
\text { Rate }\end{array}$ & $\begin{array}{l}\text { Error } \\
\text { Factor }\end{array}$ & $\begin{array}{c}\text { Failure } \\
\text { Rate }\end{array}$ & $\begin{array}{l}\text { Error } \\
\text { Factor }\end{array}$ & $\begin{array}{l}\text { Failure } \\
\text { Rate }\end{array}$ & $\begin{array}{l}\text { Error } \\
\text { Factor }\end{array}$ \\
\hline HAV346PQ & $\begin{array}{l}\text { RX BLD FREON ISOLA } \\
\text { TION FAILS TO REMAIN } \\
\text { OPEN P17-VC-346B }\end{array}$ & $1.0 \mathrm{E}-7 / \mathrm{h}$ & 10 & $1.0 \mathrm{E}-7 / \mathrm{h}$ & 3 & & & & \\
\hline HAV347PO & $\begin{array}{l}\text { RX BLD FREON ISOLA } \\
\text { TION FAILS TO REMAIN } \\
\text { OPEN P17-VC-3478 }\end{array}$ & $1.0 \mathrm{E} \cdot 7 / \mathrm{h}$ & 10 & $1.0 \mathrm{E}-7 / \mathrm{h}$ & 3 & & & & \\
\hline HAV348PO & $\begin{array}{l}\text { RX BLD FREON ISOLA } \\
\text { TION FAILS TO REMAIN } \\
\text { OPEN P17-VC-348B }\end{array}$ & $1.0 \mathrm{E}-7 / \mathrm{h}$ & 10 & $1.0 \mathrm{E} \cdot 7 \mathrm{~h}$ & 3 & & & & \\
\hline HAV349PO & $\begin{array}{l}\text { RX BLD FREON ISOLA } \\
\text { TION FAILS TO REMAIN } \\
\text { OPEN P17-VC-3498 }\end{array}$ & $1.0 \mathrm{E}-7 / \mathrm{h}$ & 10 & $1.0 \mathrm{E} \cdot 7 / \mathrm{h}$ & 3 & & & & \\
\hline HAV702PO & $\begin{array}{l}\text { RX BLD FREON ISOLA } \\
\text { TION FAILS TO REMAIN } \\
\text { OPEN P17-VC-702B }\end{array}$ & $1.0 \mathrm{E}-7 / \mathrm{h}$ & 10 & $1.0 \mathrm{E}-7 / \mathrm{h}$ & 3 & & & & \\
\hline HAV703PO & $\begin{array}{l}\text { RX BLD FREON ISOLA } \\
\text { TION FAILS TO REMAIN } \\
\text { OPEN P17-VC-7038 }\end{array}$ & $1.0 \mathrm{E}-7 / \mathrm{h}$ & 10 & $1.0 \mathrm{E}-7 / \mathrm{h}$ & 3 & & & & \\
\hline HAV727SO & $\begin{array}{l}\text { AUTO DAMPER } 727 \\
\text { FAILS TO REMAIN } \\
\text { CLOSED R8-DM-727 }\end{array}$ & $1.0 \mathrm{E}-7 / \mathrm{h}$ & 10 & $1.0 \mathrm{E}-7 / \mathrm{h}$ & 3 & & & & \\
\hline HCMOAOSR & $\begin{array}{l}40 \text { TON } \\
\text { COMPRESSOR } \\
\text { FAILURE TO RUN }\end{array}$ & $6.0 \mathrm{E}-6 / \mathrm{h}$ & 10 & & & & & & \\
\hline HCM201SR & $\begin{array}{l}20 \text { TON } \\
\text { COMPRESSOR \#1 } \\
\text { FAILURE TO RUN }\end{array}$ & $6.0 \mathrm{E} \cdot 6 / \mathrm{h}$ & 10 & & & & & & \\
\hline HCMZO2SR & $\begin{array}{l}20 \text { TON } \\
\text { COMPRESSOR \#2 } \\
\text { FAILURE TO RUN }\end{array}$ & $6.0 \mathrm{E}-6 / \mathrm{h}$ & 10 & . & & & & & ? \\
\hline HDM705SW & $\begin{array}{l}\text { VORTEX DAMPER FAN } \\
705 \text { INTERNAL FAILURE }\end{array}$ & $3.6 E-8 / \mathrm{h}$ & 8 & & & & & & \\
\hline HDM706SP & $\begin{array}{l}\text { VORTEX DAMPER ON' } \\
\text { FAN 706 DOES NOT } \\
\text { OPEN FN-706 }\end{array}$ & $3.0 \mathrm{E}-3 / \mathrm{d}$ & 10 & $1.0 \mathrm{E}-3 / \mathrm{d}$ & 10 & $3.0 \mathrm{E}-3 / \mathrm{d}$ & 10 & $3.0 \mathrm{E}-3 / \mathrm{d}$ & 10 \\
\hline HDM706SO & $\begin{array}{l}\text { VORTEX DAMPER FAN } \\
706 \text { DOES NOT } \\
\text { REMAIN OPEN FN-706 }\end{array}$ & $1.0 \mathrm{E}-7 / \mathrm{h}$ & 10 & $1.0 \mathrm{E}-7 \mathrm{~m}$ & 3 & $1.0 \mathrm{E}-7 / \mathrm{h}$ & 3 & $1.0 \mathrm{E}-7 / \mathrm{h}$ & 3 \\
\hline HDM706SW & $\begin{array}{l}\text { DAMPER 706 INTERNAL } \\
\text { FAILURE R\&-DM-706 }\end{array}$ & $3.6 \mathrm{E}-8 / \mathrm{h}$ & 8 & & & & & & \\
\hline HDM722SO & $\begin{array}{l}\text { DAMPER } 722 \text { FAILS TO } \\
\text { REMAIN CLOSED } \\
\text { R8-DM-722 }\end{array}$ & $1.0 \mathrm{E}-7 \mathrm{~m}$ & 10 & $1.0 \mathrm{E} \cdot 7 \mathrm{~h}$ & 3 & $1.0 \mathrm{E}-7 \mathrm{~m}$ & 3 & $1.0 \mathrm{E}-7 / \mathrm{h}$ & 3 \\
\hline HDM722SP & $\begin{array}{l}\text { DAMPER } 722 \\
\text { MECHANICAL FAILS } \\
\text { TO OPEN R8-DM-722 }\end{array}$ & $3.0 E-3 / d$ & 10 & $1.0 \mathrm{E}-3 / \mathrm{d}$ & 10 & $3.0 E-3 / d$ & 10 & $3.0 E-3 / d$ & 10 \\
\hline HDM722SO & $\begin{array}{l}\text { DAMPER } 722 \text { FAILS } \\
\text { TO REMAIN OPEN } \\
\text { R8-DM-722 }\end{array}$ & $1.0 \mathrm{E}-7 \mathrm{~m}$ & 10 & $1.0 \mathrm{E}-7 \mathrm{~m}$ & 3 & $1.0 E-7 / h$ & 3 & $1.0 \mathrm{E}-7 \mathrm{~m}$ & 3 \\
\hline HDM722SW & $\begin{array}{l}\text { DAMPER } 722 \text { INTERNAL } \\
\text { FAILURE R8-DM-722 }\end{array}$ & $3.6 \mathrm{E}-8 \mathrm{~h}$ & 8 & & & & & & \\
\hline HDM723SN & $\begin{array}{l}\text { DAMPER 723 } \\
\text { MECHANICAL FAILS } \\
\text { TO OPEN R8-DM-723 }\end{array}$ & $3.0 E-3 / d$ & 10 & $1.0 \mathrm{E}-3 / \mathrm{d}$ & 10 & $3.0 \mathrm{E}-3 / \mathrm{d}$ & 10 & $3.0 \mathrm{E}-3 / \mathrm{d}$ & 10 \\
\hline HDM723SO & $\begin{array}{l}\text { DAMPER } 723 \text { FAILS TO } \\
\text { REMAIN CLOSED } \\
\text { R8-DM-723 }\end{array}$ & $1.0 \mathrm{E}-7 / \mathrm{h}$ & 10 & $1.0 E-7 / \mathrm{h}$ & 3 & $1.0 \mathrm{E} \cdot 7 / \mathrm{h}$ & 3 & $1.0 E-7 / \mathrm{h}$ & 3 \\
\hline HDM723SO & $\begin{array}{l}\text { DAMPER 723 FAILS } \\
\text { TO REMAIN OPEN } \\
\text { R8-DM-723 }\end{array}$ & $1.0 \mathrm{E}-7 \mathrm{~h}$ & 10 & $1.0 \mathrm{E}-7 / \mathrm{h}$ & 3 & $1.0 \mathrm{E} \cdot 7 / \mathrm{h}$ & 3 & $1.0 E-7 / h$ & 3 \\
\hline
\end{tabular}


Table 7.6. Shield Cooling System Data (Cont'd)

PAGE 2

\begin{tabular}{|c|c|c|c|c|c|c|c|c|c|}
\hline \multirow{2}{*}{$\begin{array}{l}\text { EVENT } \\
\text { NAME }\end{array}$} & \multirow[b]{2}{*}{ DESCRIPTION } & \multicolumn{2}{|c|}{ VALUE USED $3 / 91$} & \multicolumn{2}{|c|}{ CRBRP-4 } & \multicolumn{2}{|c|}{ IREP } & \multicolumn{2}{|c|}{ GENERIC } \\
\hline & & $\begin{array}{c}\text { Failure } \\
\text { Rate }\end{array}$ & $\begin{array}{l}\text { Error } \\
\text { Factor }\end{array}$ & $\begin{array}{l}\text { Failure } \\
\text { Rate }\end{array}$ & $\begin{array}{l}\text { Error } \\
\text { Factor }\end{array}$ & $\begin{array}{c}\text { Failure } \\
\text { Rate }\end{array}$ & $\begin{array}{l}\text { Error } \\
\text { Factor }\end{array}$ & $\begin{array}{l}\text { Failure } \\
\text { Rate }\end{array}$ & $\begin{array}{l}\text { Error } \\
\text { Factor }\end{array}$ \\
\hline HDM723SW & $\begin{array}{l}\text { DAMPER } 723 \text { INTERNAL } \\
\text { FAILURE R8-DM-723 }\end{array}$ & $3.6 \mathrm{E}-8 / \mathrm{h}$ & 8 & & & & & & \\
\hline HDM724SQ & $\begin{array}{l}\text { DAMPER } 724 \text { FAILS } \\
\text { TO REMAIN OPEN } \\
\text { R8-DM-724 }\end{array}$ & $1.0 \mathrm{E}-7 \mathrm{~h}$ & 10 & $1.0 \mathrm{E}-7 / \mathrm{h}$ & 3 & $1.0 \mathrm{E}-7 / \mathrm{h}$ & 3 & $1.0 \mathrm{E}-7 / \mathrm{h}$ & 3 \\
\hline HDM725SP & $\begin{array}{l}\text { DAMPER } 725 \\
\text { MECHANICAL FAILS } \\
\text { TO OPEN R8-DM-725 }\end{array}$ & $3.0 \mathrm{E}-3 / \mathrm{d}$ & 10 & $1.0 \mathrm{E}-3 / \mathrm{d}$ & 10 & $3.0 \mathrm{E}-3 / \mathrm{d}$ & 10 & $3.0 \mathrm{E}-3 / \mathrm{d}$ & 10 \\
\hline HDM725SQ & $\begin{array}{l}\text { DAMPER } 725 \text { FAILS } \\
\text { TO REMAIN OPEN } \\
\text { R8-DM-725 }\end{array}$ & $1.0 \mathrm{E} \cdot 7 / \mathrm{h}$ & 10 & $1.0 \mathrm{E}-7 / \mathrm{h}$ & 3 & $1.0 \mathrm{E}-7 / \mathrm{h}$ & 3 & $1.0 \mathrm{E}-7 / \mathrm{h}$ & 3 \\
\hline HDM725SW & $\begin{array}{l}\text { DAMPER } 725 \text { INTERNAL } \\
\text { FAILURE R8-DM-725 }\end{array}$ & $3.6 \mathrm{E}-8 / \mathrm{h}$ & 8 & & & & & & \\
\hline HDM726SN & $\begin{array}{l}\text { DAMPER } 726 \\
\text { MECHANICAL FAILS TO } \\
\text { CLOSE R8-DM-726 }\end{array}$ & $3.0 \mathrm{E}-3 / \mathrm{d}$ & 10 & $1.0 \mathrm{E}-3 / \mathrm{d}$ & 10 & $3.0 E-3 / d$ & 10 & $3.0 \mathrm{E}-3 / \mathrm{d}$ & 10 \\
\hline HDM726SO & $\begin{array}{l}\text { DAMPER } 726 \text { FAILS TO } \\
\text { REMAIN CLOSED } \\
\text { R8-DM-726 }\end{array}$ & $1.0 \mathrm{E} \cdot 7 / \mathrm{h}$ & 10 & $1.0 \mathrm{E}-7 \mathrm{~m}$ & 3 & $1.0 \mathrm{E}-7 / \mathrm{h}$ & 3 & $1.0 \mathrm{E}-7 / \mathrm{h}$ & 3 \\
\hline HDM726SO & $\begin{array}{l}\text { DAMPER } 726 \text { FAILS } \\
\text { TO REMAIN OPEN } \\
\text { R8-DM-726 }\end{array}$ & $1.0 \mathrm{E}-7 \mathrm{~h}$ & 10 & $1.0 \mathrm{E}-7 / \mathrm{h}$ & 3 & $1.0 \mathrm{E}-7 / \mathrm{h}$ & 3 & $1.0 \mathrm{E}-7 / \mathrm{h}$ & 3 \\
\hline HDM726SW & $\begin{array}{l}\text { DAMPER } 726 \text { INTERNAL } \\
\text { FAILURE R8-DM-726 }\end{array}$ & $3.6 \mathrm{E}-8 / \mathrm{h}$ & 8 & & & & & & \\
\hline HDM727SP & $\begin{array}{l}\text { DAMPER } 727 \\
\text { MECHANICAL FAILS } \\
\text { TO OPEN R8-DM-727 }\end{array}$ & $3.0 \mathrm{E}-3 / \mathrm{d}$ & 10 & $1.0 \mathrm{E}-3 / \mathrm{d}$ & 10 & $3.0 \mathrm{E}-3 / \mathrm{d}$ & 10 & $3.0 \mathrm{E}-3 / \mathrm{d}$ & 10 \\
\hline HDM727SQ & $\begin{array}{l}\text { DAMPER } 727 \text { FAILS } \\
\text { TO REMAIN OPEN } \\
\text { R8-DM-727 }\end{array}$ & $1.0 \mathrm{E}-7 / \mathrm{h}$ & 10 & $1.0 \mathrm{E} \cdot 7 / \mathrm{h}$ & 3 & $1.0 \mathrm{E}-7 \mathrm{~h}$ & 3 & $1.0 \mathrm{E} \cdot 7 / \mathrm{h}$ & 3 \\
\hline HDM727SW & $\begin{array}{l}\text { DAMPER } 727 \text { INTERNAL } \\
\text { FAILURE RQ-DM-727 }\end{array}$ & $3.6 \mathrm{E}-8 \mathrm{~h}$ & 8 & & & & & & \\
\hline HOM741SQ & $\begin{array}{l}\text { ISOL DAMPER } 741 \text { DOES } \\
\text { NOT REMAIN OPEN } \\
\text { R8-DM-741 }\end{array}$ & $3.6 \mathrm{E}+8 / \mathrm{h}$ & 8 & $1.0 \mathrm{E}-7 \mathrm{~h}$ & 3 & $1.0 \mathrm{E}-7 / \mathrm{h}$ & 3 & $1.0 \mathrm{E}-7 / \mathrm{h}$ & 3 \\
\hline HDM742SL & $\begin{array}{l}\text { ISOLATION DAMPER } 742 \\
\text { LEFT CLOSED } \\
\text { R8-DM-742 }\end{array}$ & $1.0 \mathrm{E}-4 / \mathrm{d}$ & 10 & $1.0 E-4 / d$ & 10 & $1.0 \mathrm{E}-4 / \mathrm{d}$ & 3 & $1.0 \mathrm{E}-4 / \mathrm{d}$ & 3 \\
\hline HDM742SQ & $\begin{array}{l}\text { ISOL DAMPER } 742 \text { DOES } \\
\text { NOT REMAIN OPEN } \\
\text { R8-DM-742 }\end{array}$ & $3.6 \mathrm{E}-8 / \mathrm{h}$ & 8 & $1.0 \mathrm{E}-7 \mathrm{~h}$ & 3 & $1.0 \mathrm{E}-7 \mathrm{~h}$ & 3 & $1.0 \mathrm{E}-7 / \mathrm{h}$ & 3 \\
\hline HDM745SQ & $\begin{array}{l}\text { ISOL DAMPER } 745 \text { DOES } \\
\text { NOT REMAIN OPEN } \\
\text { R8-DM-745 }\end{array}$ & $3.6 E-8 / \mathrm{h}$ & 8 & $1.0 \mathrm{E}-7 / \mathrm{h}$ & 3 & $1.0 \mathrm{E}-7 \mathrm{~h}$ & 3 & $1.0 \mathrm{E}-7 \mathrm{~h}$ & 3 \\
\hline HDM746SL & $\begin{array}{l}\text { ISOLATION DAMPER } 746 \\
\text { LEFT CLOSED } \\
\text { R8-DM-746 }\end{array}$ & $1.0 \mathrm{E}-4 / \mathrm{d}$ & 10 & $1.0 \mathrm{E}-4 / \mathrm{d}$ & 10 & $1.0 \mathrm{E}-4 / \mathrm{d}$ & 3 & $1.0 \mathrm{E}-4 / \mathrm{d}$ & 3 \\
\hline HDM746SO & $\begin{array}{l}\text { ISOL DAMPER 746 DOES } \\
\text { NOT REMAIN OPEN } \\
\text { R8-DM-746 }\end{array}$ & $3.6 \mathrm{E} \cdot 8 / \mathrm{h}$ & 8 & $1.0 \mathrm{E}-7 / \mathrm{h}$ & 3 & $1.0 \mathrm{E}-7 \mathrm{~h}$ & 3 & $1.0 \mathrm{E}-7 / \mathrm{h}$ & 3 \\
\hline HOM747SO & $\begin{array}{l}\text { FLTR } 717 \text { INLET OMPR } \\
747 \text { FAILS TO REMAIN } \\
\text { OPEN FL717/R8-DM-747 }\end{array}$ & $3.6 \mathrm{E}-8 / \mathrm{h}$ & 8 & $1.0 \mathrm{E}-7 / \mathrm{h}$ & 3 & $1.0 \mathrm{E}-7 \mathrm{~h}$ & 3 & $1.0 \mathrm{E}-7 / \mathrm{h}$ & 3 \\
\hline HDM74\&SP & $\begin{array}{l}\text { FLTR } 718 \text { INLET DAMPE } \\
\text { R 748 FAILS TO O } \\
\text { PEN FL718/R8-DM-748 }\end{array}$ & $2.0 E-4 / d$ & 10 & $1.0 \mathrm{E}-3 / \mathrm{d}$ & 10 & $3.0 \mathrm{E}-3 / \mathrm{d}$ & 10 & $3.0 \mathrm{E}-3 / \mathrm{d}$ & 10 \\
\hline HDM748SQ & $\begin{array}{l}\text { FLTR } 718 \text { INLET DMPR } \\
748 \text { FAILS TO REMAIN } \\
\text { OPEN FL718/R8-DM-748 }\end{array}$ & $3.6 \mathrm{E}-8 / \mathrm{h}$ & 8 & $1.0 \mathrm{E}-7 / \mathrm{h}$ & 3 & $1.0 \mathrm{E}-7 / \mathrm{h}$ & 3 & $1.0 \mathrm{E}-7 \mathrm{~h}$ & 3 \\
\hline
\end{tabular}


Table 7.6. Shield Cooling System Data (Cont'd)

PAGE 3

\begin{tabular}{|c|c|c|c|c|c|c|c|c|c|}
\hline \multirow{2}{*}{$\begin{array}{l}\text { EVENT } \\
\text { NAME }\end{array}$} & \multirow[b]{2}{*}{ DESCRIPTION } & \multicolumn{2}{|c|}{ VALUE USED $3 / 91$} & \multicolumn{2}{|c|}{ CRBRP.4 } & \multicolumn{2}{|c|}{ IREP } & \multicolumn{2}{|c|}{ GENERIC } \\
\hline & & $\begin{array}{c}\text { Failure } \\
\text { Rate } \\
\end{array}$ & $\begin{array}{l}\text { Error } \\
\text { Factor }\end{array}$ & $\begin{array}{l}\text { Failure } \\
\text { Rate }\end{array}$ & $\begin{array}{l}\text { Error } \\
\text { Factor }\end{array}$ & $\begin{array}{l}\text { Failure } \\
\text { Rate }\end{array}$ & $\begin{array}{l}\text { Error } \\
\text { Factor }\end{array}$ & $\begin{array}{c}\text { Failure } \\
\text { Rate }\end{array}$ & $\begin{array}{l}\text { Error } \\
\text { Factor }\end{array}$ \\
\hline HOM764SO & $\begin{array}{l}\text { ISOL DAMPER } 764 \text { DOES } \\
\text { NOT REMAIN OPEN } \\
\text { R8-DM-764 }\end{array}$ & $3.6 \mathrm{E}-8 \mathrm{~h}$ & 8 & $1.0 \mathrm{E}-7 / \mathrm{h}$ & 3 & $1.0 \mathrm{E}-7 \mathrm{~m}$ & 3 & $1.0 \mathrm{E}-7 \mathrm{~m}$ & 3 \\
\hline HDM765SL & $\begin{array}{l}\text { ISOLATION DAMPER } 765 \\
\text { LEFT CLOSED } \\
\text { R8-DM-765 }\end{array}$ & $1.0 E-4 / d$ & 10 & $1.0 \mathrm{E}-4 / \mathrm{d}$ & 10 & $1.0 \mathrm{E}-4 / \mathrm{d}$ & 3 & $1.0 \mathrm{E}-4 / \mathrm{d}$ & 3 \\
\hline HDM7655O & $\begin{array}{l}\text { ISOL DAMPER } 765 \text { DOES } \\
\text { NOT REMAIN OPEN } \\
\text { R8-DM-765 }\end{array}$ & $3.6 \mathrm{E}-8 / \mathrm{h}$ & 8 & $1.0 \mathrm{E}-7 \mathrm{~h}$ & 3 & $1.0 \mathrm{E}-7 \mathrm{~h}$ & 3 & $1.0 \mathrm{E}-7 / \mathrm{h}$ & 3 \\
\hline HENOO1RO & $\begin{array}{l}\text { RX BUILDING } \\
\text { AMBIENT TEMP TOO } \\
\text { HIGH }\end{array}$ & & & & & & & & \\
\hline HFL717SE & $\begin{array}{l}\text { ABSOLUTE FILTE } \\
\text { RS } 717 \text { CLOGGED } \\
\text { R8-FL-717 }\end{array}$ & $3.0 \mathrm{E}-5 / \mathrm{h}$ & 10 & $1.0 \mathrm{E}-5 / \mathrm{h}$ & & $3.0 \mathrm{E}-5 \mathrm{~m}$ & 10 & & \\
\hline HFL718SE & $\begin{array}{l}\text { ABSOLUTE FILTE } \\
\text { RS 718 CLOGGED } \\
\text { R8-FL-718 }\end{array}$ & $3.0 \mathrm{E}-5 / \mathrm{h}$ & 10 & $1.0 \mathrm{E}-5 / \mathrm{h}$ & & $3.0 \mathrm{E} \cdot 5 \mathrm{~h}$ & 10 & & \\
\hline HFN706SS & $\begin{array}{l}\text { EXHAUST } \text { FAN } \\
706 \text { FAlLS TO } \\
\text { START RE-F.706 }\end{array}$ & $1.0 \mathrm{E}-3 / \mathrm{d}$ & 10 & $3.0 \mathrm{E}-4 / \mathrm{d}$ & 3 & & & $3.0 E-4 / d$ & 3 \\
\hline HFN708SR & $\begin{array}{l}\text { RECIRCULATION FAN } \\
708 \text { FAILS TO } \\
\text { RUN R8-F-708 }\end{array}$ & $3.0 E-5 / h$ & 10 & $1.0 \mathrm{E}-5 \mathrm{~m}$ & & & & $1.0 \mathrm{E}-5 / \mathrm{h}$ & 10 \\
\hline HFN708SS & $\begin{array}{l}\text { RECIRCULATION FAN } \\
708 \text { FAILS TO } \\
\text { START R\&-F-708 }\end{array}$ & $1.0 \mathrm{E} \cdot 3 / \mathrm{d}$ & 10 & $3.0 \mathrm{E}-4 / \mathrm{d}$ & 3 & & & $3.0 \mathrm{E}-4 / \mathrm{d}$ & 3 \\
\hline HGV346PQ & $\begin{array}{l}\text { REGULATOR FOR VS-34 } \\
\text { 6A FAILS TO REMAIN } \\
\text { OPEN P17-VS-346A }\end{array}$ & $1.0 \mathrm{E}-7 / \mathrm{h}$ & 10 & $1.0 \mathrm{E}-7 / \mathrm{h}$ & 3 & & & & \\
\hline HGV347PQ & $\begin{array}{l}\text { REGULATOR FOR VS-34 } \\
\text { 7A FAILS TO REMAIN } \\
\text { OPEN P17-VS-347A }\end{array}$ & $1.0 \mathrm{E}-7 \mathrm{~m}$ & 10 & $1.0 \mathrm{E}-7 \mathrm{~m}$ & 3 & & & & \\
\hline HGV348PQ & $\begin{array}{l}\text { REGULATOR FOR VS-34 } \\
\text { 8A FAILS TO REMAIN } \\
\text { OPEN P17-VS-348A }\end{array}$ & $1.0 E-7 / \mathrm{h}$ & 10 & $1.0 \mathrm{E}-7 \mathrm{~m}$ & 3 & & & & \\
\hline HGV349PQ & $\begin{array}{l}\text { REGULATOR FOR VS-34 } \\
\text { 9A FAILS TO REMAIN } \\
\text { OPEN P17-VS-349A }\end{array}$ & $1.0 \mathrm{E}-7 / \mathrm{h}$ & 10 & $1.0 \mathrm{E}-7 / \mathrm{h}$ & 3 & & & & \\
\hline HGV702PQ & $\begin{array}{l}\text { REGULATOR FOR VS-70 } \\
\text { 2A FAILS TO REMAIN } \\
\text { OPEN P17-VS-702A }\end{array}$ & $1.0 \mathrm{E}-7 \mathrm{~h}$ & .10 & $1.0 \mathrm{E}-7 / \mathrm{h}$ & 3 & & & & \\
\hline HGV703PQ & $\begin{array}{l}\text { REGULATOR FOR VS-70 } \\
\text { 3A FAILS TO REMAIN } \\
\text { OPEN P17-VS-703A }\end{array}$ & $1.0 \mathrm{E}-7 / \mathrm{h}$ & 10 & $1.0 \mathrm{E}-7 / \mathrm{h}$ & 3 & & & & \\
\hline HGV735SL & $\begin{array}{l}\text { COIL } 715 \# 2 \\
\text { EXPANSION VALVE LEFT } \\
\text { CLOSED RA-VC-735 }\end{array}$ & $1.0 E-4 / d$ & 10 & $1.0 \mathrm{E}-4 / \mathrm{d}$ & 10 & $1.0 \mathrm{E}-4 / \mathrm{d}$ & 3 & $1.0 E-4 / d$ & \\
\hline HGV735SQ & $\begin{array}{l}\text { COIL } 715 \text { \#2 EXP VALV } \\
\text { E DOES NOT REMAIN OP } \\
\text { EN R8-VC-735 }\end{array}$ & $1.0 \mathrm{E} \cdot 7 \mathrm{~h}$ & 10 & $1.0 \mathrm{E}-7 / \mathrm{h}$ & 3 & $1.0 \mathrm{E}-7 \mathrm{~h}$ & 3 & $1.0 \mathrm{E}-7 \mathrm{~h}$ & 3 \\
\hline HGV736SL & $\begin{array}{l}\text { COIL } 715 \# 1 \\
\text { EXPANSION VALVE LEFT } \\
\text { CLOSED R8-VC-736 }\end{array}$ & $1.0 \mathrm{E}-4 / \mathrm{d}$ & 10 & $1.0 E-4 / d$ & 10 & $1.0 \mathrm{E}-4 / \mathrm{d}$ & 3 & $1.0 \mathrm{E}-4 / \mathrm{d}$ & \\
\hline HGV736SQ & $\begin{array}{l}\text { COIL } 715 \text { \#1 EXP VALV } \\
\text { E DOES NOT REMAIN OP } \\
\text { EN R8-VC-736 }\end{array}$ & $1.0 \mathrm{E}-7 / \mathrm{h}$ & 10 & $1.0 \mathrm{E}-7 / \mathrm{h}$ & 3 & $1.0 \mathrm{E}-7 / \mathrm{h}$ & 3 & $1.0 \mathrm{E}-7 \mathrm{~h}$ & 3 \\
\hline HGV737SL & $\begin{array}{l}\text { COIL } 718 \# 1 \\
\text { EXPANSION VALVE LEFT } \\
\text { CLOSED R8-VC-737 }\end{array}$ & $1.0 \mathrm{E}-4 / \mathrm{d}$ & 10 & $1.0 \mathrm{E}-4 / \mathrm{d}$ & 10 & $1.0 E-4 / d$ & 3 & $1.0 \mathrm{E}-4 / \mathrm{d}$ & \\
\hline
\end{tabular}


Table 7.6. Shield Cooling System Data (Cont'd)

PAGE 4

\begin{tabular}{|c|c|c|c|c|c|c|c|c|c|}
\hline \multirow{2}{*}{$\begin{array}{l}\text { EVENT } \\
\text { NAME }\end{array}$} & \multirow[b]{2}{*}{ DESCRIPTION } & \multicolumn{2}{|c|}{ VALUE USED $3 / 91$} & \multicolumn{2}{|c|}{ CRBRP-4 } & \multicolumn{2}{|c|}{ IREP } & \multicolumn{2}{|c|}{ GENERIC } \\
\hline & & $\begin{array}{l}\text { Failure } \\
\text { Rate }\end{array}$ & $\begin{array}{l}\text { Error } \\
\text { Factor }\end{array}$ & $\begin{array}{l}\text { Failure } \\
\text { Rate }\end{array}$ & $\begin{array}{l}\text { Error } \\
\text { Factor }\end{array}$ & $\begin{array}{c}\text { Failure } \\
\text { Rate }\end{array}$ & $\begin{array}{l}\text { Error } \\
\text { Factor }\end{array}$ & $\begin{array}{l}\text { Failure } \\
\text { Rate }\end{array}$ & $\begin{array}{l}\text { Error } \\
\text { Factor }\end{array}$ \\
\hline HGV737SO & $\begin{array}{l}\text { COIL } 718 \text { \#1 EXP VALV } \\
\text { EDOES NOT REMAIN OP } \\
\text { EN R8-VC-737 }\end{array}$ & $1.0 \mathrm{E}-7 / \mathrm{h}$ & 10 & $1.0 \mathrm{E}-7 / \mathrm{h}$ & 3 & $1.0 \mathrm{E}-7 / \mathrm{h}$ & 3 & $1.0 \mathrm{E}-7 / \mathrm{h}$ & 3 \\
\hline HGV738SL & $\begin{array}{l}\text { COIL } 718 \text { \#2 } \\
\text { EXPANSION VALVE LEFT } \\
\text { CLOSED R8-VC-738 }\end{array}$ & $1.0 \mathrm{E}-4 / \mathrm{d}$ & 10 & $1.0 \mathrm{E}-4 / \mathrm{d}$ & 3 & $1.0 E-4 / d$ & 10 & $1.0 \mathrm{E}-4 / \mathrm{d}$ & 3 \\
\hline HGV738SQ & $\begin{array}{l}\text { COIL } 718 \text { \#2 EXP VALV } \\
\text { EDOES NOT REMAIN OP } \\
\text { EN RQVC- } 738\end{array}$ & $1.0 \mathrm{E}-7 / \mathrm{h}$ & 10 & $1.0 \mathrm{E} \cdot 7 / \mathrm{h}$ & 3 & $1.0 \mathrm{E}-7 / \mathrm{h}$ & 3 & $1.0 \mathrm{E}-7 / \mathrm{h}$ & 3 \\
\hline HGV754SQ & $\begin{array}{l}\text { COIL } 713 \text { \#1 EXP VALV } \\
\text { EDOES NOT REMAIN OP } \\
\text { EN RQVC-754 }\end{array}$ & $1.0 \mathrm{E}-7 / \mathrm{h}$ & 10 & $1.0 \mathrm{E}-7 / \mathrm{h}$ & 3 & $1.0 \mathrm{E}-7 \mathrm{~m}$ & 3 & $1.0 \mathrm{E}-7 / \mathrm{h}$ & 3 \\
\hline HHU002YL & $\begin{array}{l}\text { NO RECOVERY ACTION } \\
\text { TO RESET CIRCUIT } \\
\text { BREAKER }\end{array}$ & $2.0 \mathrm{E}-2 / \mathrm{d}$ & 10 & & & & & & \\
\hline HHU003SL & $\begin{array}{l}\text { NO RECOVERY ACTION } \\
\text { TO USE DAMPER MANUAL } \\
\text { HANDWHEEL }\end{array}$ & $2.0 \mathrm{E}-2 / \mathrm{d}$ & 10 & & & & & & \\
\hline HHU004SL & $\begin{array}{l}\text { NO RECOVERY ACTION } \\
\text { TO USE MOVE DAMPER } \\
\text { MANUALLY }\end{array}$ & $2.0 \mathrm{E}-2 / \mathrm{d}$ & 10 & & & & & & \\
\hline HHU01BSZ & $\begin{array}{l}\text { NO RECOVERY ACTION } \\
\text { FOR CLOGGED FILTER } \\
\text { R8-FL-717 }\end{array}$ & $2.0 \mathrm{E}-2 / \mathrm{d}$ & 10 & & . & & & & \\
\hline HLC641SW & $\begin{array}{l}\text { PRESSURE } \\
\text { CONTROLLER FAILS } \\
\text { R13-1564-1 }\end{array}$ & $3.0 E-6 / \mathrm{h}$ & 10 & $1.0 \mathrm{E}-6 / \mathrm{h}$ & 10 & $3.0 \mathrm{E}-6 / \mathrm{h}$ & 10 & $2.0 \mathrm{E} \cdot 6 / \mathrm{h}$ & 10 \\
\hline HLC642SW & $\begin{array}{l}\text { PRESSURE } \\
\text { CONTROLLER FAILS } \\
\text { R13-1564-2 }\end{array}$ & $1.0 \mathrm{E}-7 \mathrm{~h}$ & 10 & $1.0 \mathrm{E}-7 / \mathrm{h}$ & 3 & $1.0 \mathrm{E}-7 / \mathrm{h}$ & 3 & $1.0 \mathrm{E}-7 \mathrm{~m}$ & 3 \\
\hline HPR641SW & $\begin{array}{l}\text { PRESSURE } \\
\text { TRANSDUCER FAILS } \\
\text { R13-1564-1 } \\
\end{array}$ & $3.0 \mathrm{E}-6 / \mathrm{h}$ & 10 & $1.0 \mathrm{E}-6 / \mathrm{h}$ & & & & $3.0 \mathrm{E}-6 / \mathrm{h}$ & 10 \\
\hline HPR642SW & $\begin{array}{l}\text { PRESSURE } \\
\text { TRANSDUCER FAILS } \\
\text { R13-1564-2 } \\
\end{array}$ & $3.0 \mathrm{E}-6 / \mathrm{h}$ & 10 & $1.0 \mathrm{E}-6 / \mathrm{h}$ & & & & $3.0 \mathrm{E}-6 / \mathrm{h}$ & 10 \\
\hline HREOOCSO & $\begin{array}{l}\text { CONTROL RELAY CR-10 } \\
\text { O COIL FAILURE } \\
\text { DM-722/CR-100 }\end{array}$ & $3.0 \mathrm{E}-6 / \mathrm{h}$ & 10 & $1.0 \mathrm{E}-6 / \mathrm{h}$ & 10 & $3.0 \mathrm{E}-6 / \mathrm{h}$ & 10 & $3.0 \mathrm{E}-6 / \mathrm{h}$ & 10 \\
\hline HREOOCSW & $\begin{array}{l}\text { CONTROL RELAY CR-10 } \\
\text { O CONTACT FAILURE } \\
\text { DN-722/CR-100 }\end{array}$ & $3.0 E-4 / d$ & 10 & $1.0 \mathrm{E}-4 / \mathrm{d}$ & 10 & $3.0 \mathrm{E}-4 / \mathrm{d}$ & 10 & $3.0 E-4 / d$ & 10 \\
\hline HREOOMSW & $\begin{array}{l}\text { MOTOR OPERATOR RM-10 } \\
\text { O FAILS TO OPERATE } \\
\text { DM-722/RM-100 }\end{array}$ & $3.0 \mathrm{E}-3 / \mathrm{d}$ & 10 & $1.0 \mathrm{E}-3 / \mathrm{d}$ & 10 & $3.0 \mathrm{E}-3 / \mathrm{d}$ & 10 & $3.0 \mathrm{E}-3 / \mathrm{d}$ & 3 \\
\hline HRE01CSW & $\begin{array}{l}\text { CONTROL RELAY CR-10 } \\
1 \text { CONTACT FAILURE } \\
\text { DM-724/CR-101 }\end{array}$ & $3.0 E-4 / d$ & 10 & $1.0 E-4 / d$ & 10 & $3.0 \mathrm{E}-4 / \mathrm{d}$ & 10 & $3.0 \mathrm{E}-4 / \mathrm{d}$ & 10 \\
\hline HRE02CSO & $\begin{array}{l}\text { CONTROL RELAY CR-10 } \\
2 \text { COIL FAILURE } \\
\text { DM-725/CR-102 }\end{array}$ & $3.0 \mathrm{E}-6 / \mathrm{h}$ & 10 & $1.0 \mathrm{E}-6 / \mathrm{h}$ & 10 & $3.0 \mathrm{E}-6 / \mathrm{h}$ & 10 & $3.0 \mathrm{E}-6 / \mathrm{h}$ & 10 \\
\hline HRE02CSW & $\begin{array}{l}\text { CONTROL RELAY CR-10 } \\
2 \text { CONTACT FAILURE } \\
\text { DM-725/CR-102 } \\
\end{array}$ & $3.0 \mathrm{E}-4 / \mathrm{d}$ & 10 & $1.0 E-4 / d$ & 10 & $3.0 E-4 / d$ & 10 & $3.0 E-4 / d$ & 10 \\
\hline HRE02MSW & $\begin{array}{l}\text { MOTOR OPERATOR RM-10 } \\
2 \text { FAILS TO OPERATE } \\
\text { DM-725/RM-102 }\end{array}$ & $3.0 \mathrm{E}-3 / \mathrm{d}$ & 10 & $1.0 \mathrm{E}-3 / \mathrm{d}$ & 10 & $3.0 \mathrm{E}-3 / \mathrm{d}$ & 10 & $3.0 \mathrm{E}-3 / \mathrm{d}$ & 3 \\
\hline HRE051SO & $\begin{array}{l}\text { MOTOR RELAY RM-51 } \\
\text { COIL FAILU } \\
\text { RE FN-705/RM-51 }\end{array}$ & $3.0 \mathrm{E}-6 / \mathrm{h}$ & 10 & $1.0 \mathrm{E}-6 / \mathrm{h}$ & 10 & $3.0 \mathrm{E}-6 / \mathrm{h}$ & 10 & $3.0 \mathrm{E}-6 / \mathrm{h}$ & 10 \\
\hline
\end{tabular}


Table 7.6. Shield Cooling System Data (Cont'd)

PAGE 5

\begin{tabular}{|c|c|c|c|c|c|c|c|c|c|}
\hline \multirow{2}{*}{$\begin{array}{l}\text { EVENT } \\
\text { NAME }\end{array}$} & \multirow[b]{2}{*}{ DESCRIPTION } & \multicolumn{2}{|c|}{ VALUE USED $3 / 91$} & \multicolumn{2}{|c|}{ CRBRP-4 } & \multicolumn{2}{|c|}{ IREP } & \multicolumn{2}{|c|}{ GENERIC } \\
\hline & & $\begin{array}{l}\text { Failure } \\
\text { Rate }\end{array}$ & $\begin{array}{l}\text { Error } \\
\text { Factor }\end{array}$ & $\begin{array}{l}\text { Failure } \\
\text { Rate } \\
\end{array}$ & $\begin{array}{l}\text { Error } \\
\text { Factor }\end{array}$ & $\begin{array}{c}\text { Failure } \\
\text { Rate }\end{array}$ & $\begin{array}{l}\text { Error } \\
\text { Factor }\end{array}$ & $\begin{array}{c}\text { Failure } \\
\text { Rate }\end{array}$ & $\begin{array}{l}\text { Error } \\
\text { Factor }\end{array}$ \\
\hline HRE097SW & $\begin{array}{l}\text { MOTOR OPERATOR } \\
\text { VORTEX DAMPEA FAILS } \\
\text { FN-706/RM-9 }\end{array}$ & $3.0 E-3 / d$ & 10 & $1.0 \mathrm{E}-3 / \mathrm{d}$ & 10 & $3.0 \mathrm{E}-3 / \mathrm{d}$ & 10 & $3.0 \mathrm{E}-3 / \mathrm{d}$ & 3 \\
\hline HRE99CSN & $\begin{array}{l}\text { CONTROL RELAY CR-99 } \\
\text { FAILS TO CLOSE } \\
\text { DM-723/CR-99 }\end{array}$ & $3.0 E-4 / d$ & 10 & $1.0 \mathrm{E}-4 / \mathrm{d}$ & 10 & $3.0 \mathrm{E}-4 / \mathrm{d}$ & 10 & $3.0 \mathrm{E}-4 / \mathrm{d}$ & 10 \\
\hline HRE99CSO & $\begin{array}{l}\text { CONTROL RELAY CR-99 } \\
\text { COIL FAILURE } \\
\text { DM-723/CR-99 }\end{array}$ & $3.0 \mathrm{E}-6 / \mathrm{h}$ & 10 & $1.0 \mathrm{E} \cdot 6 / \mathrm{h}$ & 10 & $3.0 \mathrm{E}-6 / \mathrm{h}$ & 10 & $3.0 \mathrm{E}-6 / \mathrm{h}$ & 10 \\
\hline HRE99MSW & $\begin{array}{l}\text { MOTOR OPERATOR RM-99 } \\
\text { FAILS TO OPERATE } \\
\text { DM-723/RM-99 }\end{array}$ & $3.0 \mathrm{E}-3 / \mathrm{d}$ & 10 & $1.0 \mathrm{E}-3 / \mathrm{d}$ & 10 & $3.0 \mathrm{E}-3 / \mathrm{d}$ & 10 & $3.0 \mathrm{E}-3 / \mathrm{d}$ & 3 \\
\hline HSVO95SO & $\begin{array}{l}\text { SOLENOID VALVE RSV-9 } \\
5 \text { FAILS TO REMAIN CL } \\
\text { OSED DM-727/RSV-95 }\end{array}$ & $1.0 \mathrm{E}-7 \mathrm{~m}$ & 3 & $1.0 \mathrm{E}-7 / \mathrm{h}$ & 3 & & & & \\
\hline HSV095SP & $\begin{array}{l}\text { SOLENOID VALVE RSV-9 } \\
5 \\
\text { PAILS TOO DM-727/RSV-95 }\end{array}$ & $1.0 \mathrm{E}-3 / \mathrm{d}$ & 3 & $1.0 \mathrm{E}-3 / \mathrm{d}$ & 3 & $1.0 \mathrm{E}-3 / \mathrm{d}$ & 30 & $1.0 \mathrm{E}-3 / \mathrm{d}$ & 3 \\
\hline HSV095SQ & $\begin{array}{l}\text { SOLENOID VALVE RSV-9 } \\
5 \text { FAILS TO REMAIN OP } \\
\text { EN DM-727/RSV-95 }\end{array}$ & $1.0 \mathrm{E}-7 \mathrm{~h}$ & 3 & $1.0 \mathrm{E}-7 / \mathrm{h}$ & 3 & & & & \\
\hline HSV096SN & $\begin{array}{l}\text { SOLENOID VALVE } \\
\text { RSV-96 FAILS TO } \\
\text { CLOSE DM-726/RSV-96 }\end{array}$ & $1.0 \mathrm{E}-3 / \mathrm{d}$ & 3 & $1.0 \mathrm{E}-3 / \mathrm{d}$ & 3 & $1.0 \mathrm{E}-3 / \mathrm{d}$ & 30 & $1.0 \mathrm{E}-3 / \mathrm{d}$ & 3 \\
\hline HSV096SO & $\begin{array}{l}\text { SOLENOID VALVE RSV-9 } \\
6 \text { FAILS TO REMAIN CL } \\
\text { OSED DM-726/RSV-96 }\end{array}$ & $1.0 \mathrm{E}-7 / \mathrm{h}$ & 3 & $1.0 \mathrm{E}-7 \mathrm{~h}$ & 3 & & & & \\
\hline HSV096SO & $\begin{array}{l}\text { SOLENOID VALVE RSV-9 } \\
6 \text { FAILS TO REMAIN } \\
\text { OPEN DM-726/RSV-96 }\end{array}$ & $1.0 \mathrm{E}-7 / \mathrm{h}$ & 3 & $1.0 \mathrm{E}-7 / \mathrm{h}$ & 3 & & & & \\
\hline HSV346PQ & $\begin{array}{l}\text { SOLENOID VALVE VS-34 } \\
\text { 6A FAILS TO REMAIN } \\
\text { OPEN P17-VS-346A }\end{array}$ & $1.0 \mathrm{E} \cdot 7 \mathrm{~h}$ & 3 & $1.0 \mathrm{E}-7 / \mathrm{h}$ & 3 & & & & \\
\hline HSV347PQ & $\begin{array}{l}\text { SOLENOID VALVE VS-34 } \\
\text { 7A FAILS TO REMAIN } \\
\text { OPEN P17-VS-347A }\end{array}$ & $1.0 \mathrm{E}-7 / \mathrm{h}$ & 3 & $1.0 \mathrm{E}-7 \mathrm{~h}$ & 3 & & & & \\
\hline HSV348PQ & $\begin{array}{l}\text { SOLENOID VALVE VS-34 } \\
\text { BA FAILS TO REMAIN } \\
\text { OPEN P17-VS-348A } \\
\end{array}$ & $1.0 \mathrm{E}-7 \mathrm{~h}$ & 3 & $1.0 \mathrm{E} \cdot 7 \mathrm{~h}$ & 3 & & & . & \\
\hline HSV349PQ & $\begin{array}{l}\text { SOLENOID VALVE VS-34 } \\
\text { 9A FAILS TO REMAIN } \\
\text { OPEN P17-VS-349A }\end{array}$ & $1.0 \mathrm{E}-7 \mathrm{~m}$ & 3 & $1.0 \mathrm{E}-7 \mathrm{~m}$ & 3 & & & . & \\
\hline HSV356SN & $\begin{array}{l}\text { SPACE COOL } 309 \\
\text { SOLENOID FAILS TO } \\
\text { CLOSE R\&-VS-356 }\end{array}$ & $1.0 E-3 / d$ & 3 & $1.0 \mathrm{E}-3 / \mathrm{d}$ & 3 & $1.0 \mathrm{E}-3 / \mathrm{d}$ & 3 & $1.0 \mathrm{E}-3 / \mathrm{d}$ & 3 \\
\hline HSV356SO & $\begin{array}{l}\text { SPACE COOL } 309 \text { SOLEM } \\
\text { OID FAILS TO REMAIN } \\
\text { CLOSED R8-VS-356 }\end{array}$ & $1.0 \mathrm{E}-7 \mathrm{~h}$ & 3 & $1.0 \mathrm{E}-7 / \mathrm{h}$ & 3 & & & . & \\
\hline HSV357SP & $\begin{array}{l}\text { STBY COILS } 715 \\
\text { SOLENOID XFER FAILS } \\
\text { TO OPEN R8.VS-357 }\end{array}$ & $1.0 \mathrm{E}-3 / \mathrm{d}$ & 3 & $1.0 \mathrm{E}-3 / \mathrm{d}$ & 3 & $1.0 \mathrm{E}-3 / \mathrm{d}$ & 3 & $1.0 \mathrm{E}-3 / \mathrm{d}$ & 3 \\
\hline HSV357SQ & $\begin{array}{l}\text { STBY COILS } 715 \\
\text { SOLENOID FAILS TO } \\
\text { REMAIN OPEN R8-VS-35 }\end{array}$ & $1.0 \mathrm{E}-7 / \mathrm{h}$ & 3 & $1.0 \mathrm{E}-7 / \mathrm{h}$ & 3 & & & & \\
\hline HSV358LN & $\begin{array}{l}\text { SPACE COOL } 308 \\
\text { SOLENOID FAILS TO } \\
\text { CLOSE R\&-VS-358 }\end{array}$ & $1.0 \mathrm{E}-3 / \mathrm{d}$ & 3 & $1.0 E-3 / d$ & 3 & $1.0 \mathrm{E}-3 / \mathrm{d}$ & 3 & $1.0 E-3 / d$ & 3 \\
\hline HSV358LO & $\begin{array}{l}\text { SPACE COOL } 308 \text { SOLEN } \\
\text { OID FAILS TO REMAIN } \\
\text { CLOSED R8-VS-358 }\end{array}$ & $1.0 \mathrm{E}-7 \mathrm{~h}$ & 3 & $1.0 \mathrm{E}-7 / \mathrm{h}$ & 3 & & & & \\
\hline
\end{tabular}


Table 7.6. Shield Cooling System Data (Cont'd)

PAGE 6

\begin{tabular}{|c|c|c|c|c|c|c|c|c|c|}
\hline \multirow{2}{*}{$\begin{array}{l}\text { EVENT } \\
\text { NAME }\end{array}$} & \multirow[b]{2}{*}{ DESCRIPTION } & \multicolumn{2}{|c|}{ VALUE USED 391} & \multicolumn{2}{|c|}{ CRBRP-4 } & \multicolumn{2}{|c|}{ IREP } & \multicolumn{2}{|c|}{ GENERIC } \\
\hline & & $\begin{array}{l}\text { Failure } \\
\text { Rate }\end{array}$ & $\begin{array}{l}\text { Error } \\
\text { Factor }\end{array}$ & $\begin{array}{c}\text { Failure } \\
\text { Rate }\end{array}$ & $\begin{array}{l}\text { Error } \\
\text { Factor }\end{array}$ & $\begin{array}{l}\text { Failure } \\
\text { Rate }\end{array}$ & $\begin{array}{l}\text { Error } \\
\text { Factor }\end{array}$ & $\begin{array}{l}\text { Failure } \\
\text { Rate }\end{array}$ & $\begin{array}{l}\text { Error } \\
\text { Factor }\end{array}$ \\
\hline HSV359SP & $\begin{array}{l}\text { STBY COILS } 718 \\
\text { SOLENOID XFER FAILS } \\
\text { TO OPEN R8-VS-359 }\end{array}$ & $1.0 \mathrm{E}-3 / \mathrm{d}$ & 3 & $1.0 \mathrm{E}-3 / \mathrm{d}$ & 3 & $1.0 E-3 / d$ & 3 & $1.0 E-3 / d$ & 3 \\
\hline HSV359SO & $\begin{array}{l}\text { STBY COILS } 718 \\
\text { SOLENOID FAILS TO } \\
\text { REMAIN OPEN R8-VS-35 }\end{array}$ & $1.0 \mathrm{E}-7 / \mathrm{h}$ & 3 & $1.0 \mathrm{E}-7 \mathrm{~h}$ & 3 & & & & \\
\hline HSV $400 L N$ & $\begin{array}{l}\text { CLR } 308 \text { COIL } 2 \\
\text { SOLENOID FAILS TO } \\
\text { CLOSE RB-VS } 400\end{array}$ & $1.0 E-3 / d$ & 3 & $1.0 E-3 / d$ & 3 & $1.0 \mathrm{E}-3 / \mathrm{d}$ & 3 & $1.0 E-3 / d$ & 3 \\
\hline HSV 400 LO & $\begin{array}{l}\text { CLR } 308 \text { COIL } 2 \text { SOLEN } \\
\text { OID FAILS TO REMAIN } \\
\text { CLOSED R8-VS-400 }\end{array}$ & $1.0 \mathrm{E}-7 \mathrm{~m}$ & 3 & $1.0 \mathrm{E} \cdot 7 / \mathrm{h}$ & 3 & & & & \\
\hline HSV702PQ & $\begin{array}{l}\text { SOLENOID VALVE VS-70 } \\
\text { 2A FAILS TO REMAIN } \\
\text { OPEN P17-VS-702A }\end{array}$ & $1.0 \mathrm{E}-7 / \mathrm{h}$ & 3 & $1.0 \mathrm{E}-7 / \mathrm{h}$ & 3 & & & & \\
\hline HSV703PQ & $\begin{array}{l}\text { SOLENOID VALVE VS-70 } \\
\text { 3A FAILS TO REMAIN } \\
\text { OPEN P17-VS-703A }\end{array}$ & $1.0 \mathrm{E}-7 \mathrm{~h}$ & 3 & $1.0 \mathrm{E}-7 / \mathrm{h}$ & 3 & & & & \\
\hline HSV755SQ & $\begin{array}{l}\text { COIL } 714 \text { \#1 } \\
\text { SOLENOID FAILS TO } \\
\text { REMAIN OPEN R8-VS-75 }\end{array}$ & $1.0 \mathrm{E}-7 / \mathrm{h}$ & 3 & $1.0 \mathrm{E}-7 / \mathrm{h}$ & 3 & & & & \\
\hline HSV756SQ & $\begin{array}{l}\text { COIL } 713 \text { \#2 } \\
\text { SOLENOID FAILS TO } \\
\text { REMAIN OPEN R8-VS.75 }\end{array}$ & $1.0 \mathrm{E}-7 / \mathrm{h}$ & 3 & $1.0 \mathrm{E}-7 / \mathrm{h}$ & 3 & & & & \\
\hline HSV757SP & $\begin{array}{l}\text { COIL } 718 \# 1 \\
\text { SOLENOID FAILS } \\
\text { TOOPEN R8-VS-757 }\end{array}$ & $1.0 \mathrm{E}-3 / \mathrm{d}$ & 3 & $1.0 \mathrm{E}-3 / \mathrm{d}$ & 3 & $1.0 \mathrm{E} \cdot 3 / \mathrm{d}$ & 3 & $1.0 \mathrm{E}-3 / \mathrm{d}$ & 3 \\
\hline HSV757SQ & $\begin{array}{l}\text { COIL } 718 \# 1 \\
\text { SOLENOID FAILS TO } \\
\text { REMAIN OPEN R8-VS-75 }\end{array}$ & $1.0 . E-7 / \mathrm{h}$ & 3 & $1.0 \mathrm{E}-7 / \mathrm{h}$ & 3 & & & & \\
\hline HSV758SP & $\begin{array}{l}\text { COIL } 715 \# 2 \\
\text { SOLENOID FAILS } \\
\text { TOOPEN R8-VS-758 }\end{array}$ & $1.0 \mathrm{E}-3 / \mathrm{d}$ & 3 & $1.0 \mathrm{E}-3 / \mathrm{d}$ & 3 & $1.0 \mathrm{E}-3 / \mathrm{d}$ & 3 & $1.0 \mathrm{E}-3 / \mathrm{d}$ & 3 \\
\hline HSV758SQ & $\begin{array}{l}\text { COIL } 715 \text { \%2 } \\
\text { SOLENOID FAILS TO } \\
\text { REMAIN OPEN R8-VS-75 }\end{array}$ & $1.0 \mathrm{E}-7 \mathrm{M}$ & 3 & $1.0 \mathrm{E}-7 / \mathrm{h}$ & 3 & & & & \\
\hline HSV759SN & $\begin{array}{l}\text { CLR } 309 \text { COIL } 1 \\
\text { SOLENOID FAILS TO } \\
\text { CLOSE R8-VS-759 }\end{array}$ & $1.0 \mathrm{E}-3 / \mathrm{d}$ & 3 & $1.0 \mathrm{E}-3 / \mathrm{d}$ & 3 & $1.0 E-3 / d$ & 3 & $1.0 \mathrm{E}-3 / \mathrm{d}$ & 3 \\
\hline HSV759SO & $\begin{array}{l}\text { CLR } 309 \text { COIL } 1 \text { SOLEN } \\
\text { OID FAILS TO REMAIN } \\
\text { CLOSED R8-VS-759 }\end{array}$ & $1.0 E-7 / \mathrm{h}$ & 3 & $1.0 \mathrm{E}-7 \mathrm{~h}$ & 3 & & & & \\
\hline HSV766SN & $\begin{array}{l}\text { CLR } 309 \text { COIL } 2 \\
\text { SOLENOID FAILS TO } \\
\text { CLOSE R\&-VS-766 }\end{array}$ & $1.0 E-3 / d$ & 3 & $1.0 \mathrm{E}-3 / \mathrm{d}$ & 3 & $1.0 \mathrm{E}-3 / \mathrm{d}$ & 3 & $1.0 \mathrm{E}-3 / \mathrm{d}$ & 3 \\
\hline HSV766SO & $\begin{array}{l}\text { CLR } 309 \text { COIL } 2 \text { SOLEN } \\
\text { OID FAILS TO REMAIN } \\
\text { CLOSED R8-VS-766 }\end{array}$ & $1.0 \mathrm{E}-7 / \mathrm{h}$ & 3 & $1.0 \mathrm{E}-7 / \mathrm{h}$ & 3 & & & & \\
\hline HSV767SP & $\begin{array}{l}\text { COIL } 715 \# 1 \\
\text { SOLENOID FAILS } \\
\text { TOOPEN R8-VS-767 }\end{array}$ & $1.0 \mathrm{E}-3 / \mathrm{d}$ & 3 & $1.0 \mathrm{E}-3 / \mathrm{d}$ & 3 & $1.0 \mathrm{E}-3 / \mathrm{d}$ & 3 & $1.0 \mathrm{E}-3 / \mathrm{d}$ & 3 \\
\hline HSV767SO & $\begin{array}{l}\text { COIL } 715 \# 1 \\
\text { SOLENOID FAILS TO } \\
\text { REMAIN OPEN R8-VS-76 }\end{array}$ & $1.0 \mathrm{E}-7 / \mathrm{h}$ & 3 & $1.0 \mathrm{E}-7 / \mathrm{h}$ & 3 & & & & \\
\hline HSV768SO & $\begin{array}{l}\text { COIL } 714 \text { \#2 } \\
\text { SOLENOID FAILS TO } \\
\text { REMAIN OPEN R8-VS-76 }\end{array}$ & $1.0 \mathrm{E}-7 \mathrm{M}$ & 3 & $1.0 \mathrm{E}-7 \mathrm{~h}$ & 3 & & & & \\
\hline HSV769SO & $\begin{array}{l}\text { COIL } 713 \text { \#1 } \\
\text { SOLENOID FAILS TO } \\
\text { REMAIN OPEN R8-VS-76 }\end{array}$ & $1.0 \mathrm{E} \cdot 7 \mathrm{~h}$ & 3 & $1.0 \mathrm{E}-7 / \mathrm{h}$ & 3 & & & & \\
\hline
\end{tabular}


Table 7.6. Shield Cooling System Data (Cont'd)

PAGE 7

\begin{tabular}{|c|c|c|c|c|c|c|c|c|c|}
\hline \multirow{2}{*}{$\begin{array}{l}\text { EVENT } \\
\text { NAME }\end{array}$} & \multirow[b]{2}{*}{ DESCRIPTION } & \multicolumn{2}{|c|}{ VALUE USED $3 / 91$} & \multicolumn{2}{|c|}{ CRBRP-4 } & \multicolumn{2}{|c|}{ IREP } & \multicolumn{2}{|c|}{ GENERIC } \\
\hline & & $\begin{array}{l}\text { Failure } \\
\text { Rate }\end{array}$ & $\begin{array}{l}\text { Error } \\
\text { Factor }\end{array}$ & $\begin{array}{l}\text { Failure } \\
\text { Rate }\end{array}$ & $\begin{array}{l}\text { Error } \\
\text { Factor }\end{array}$ & $\begin{array}{c}\text { Failure } \\
\text { Rate }\end{array}$ & $\begin{array}{l}\text { Error } \\
\text { Factor }\end{array}$ & $\begin{array}{l}\text { Failure } \\
\text { Rate }\end{array}$ & $\begin{array}{l}\text { Error } \\
\text { Factor }\end{array}$ \\
\hline HSVT7OSP & $\begin{array}{l}\text { COIL } 718 * 2 \\
\text { SOLENOID FAILS } \\
\text { TO OPEN R8-VS-770 }\end{array}$ & $1.0 \mathrm{E}-3 / \mathrm{d}$ & 3 & $1.0 \mathrm{E}-3 / \mathrm{d}$ & 3 & $1.0 E-3 / d$ & 3 & $1.0 \mathrm{E}-3 / \mathrm{d}$ & 3 \\
\hline HSVT7OSO & $\begin{array}{l}\text { COIL } 718 \text { \#2 } \\
\text { SOLENOID FAILS TO } \\
\text { REMAIN OPEN R8-VS-77 }\end{array}$ & $1.0 \mathrm{E}-7 / \mathrm{h}$ & 3 & $1.0 \mathrm{E}-7 / \mathrm{h}$ & 3 & & & & \\
\hline HSV771LN & $\begin{array}{l}\text { CLR } 308 \text { COIL } 1 \\
\text { SOLENOID FAILS TO } \\
\text { CLOSE R\&-VS- } 771\end{array}$ & $1.0 \mathrm{E}-3 / \mathrm{d}$ & 3 & $1.0 \mathrm{E}-3 / \mathrm{d}$ & 3 & $1.0 \mathrm{E}-3 / \mathrm{d}$ & 3 & $1.0 \mathrm{E}-3 / \mathrm{d}$ & 3 \\
\hline HSVT71LO & $\begin{array}{l}\text { CLR } 308 \text { COIL } 1 \text { SOLEN } \\
\text { OID FAILS TO REMAIN } \\
\text { CLOSED R8-VS-771 }\end{array}$ & $1.0 \mathrm{E}-7 / \mathrm{h}$ & 3 & $1.0 \mathrm{E}-7 / \mathrm{h}$ & 3 & & & & \\
\hline HSW201ST & $\begin{array}{l}20 \text { TON COMP \#1 } \\
\text { SWITCH IN WRONG } \\
\text { POSITION }\end{array}$ & $1.0 \mathrm{E}-4 / \mathrm{d}$ & 10 & & & & & & \\
\hline HSW202ST & $\begin{array}{l}20 \text { TON COMP \#2 } \\
\text { SWITCHIN WRONG } \\
\text { POSITION }\end{array}$ & $1.0 \mathrm{E}-4 / \mathrm{d}$ & 10 & & & & & & \\
\hline HTZ096SW & $\begin{array}{l}\text { DM726 POSITION INDIC } \\
\text { ATOR ERROR } \\
\text { DM-726/CR-96-7 }\end{array}$ & $3.0 \mathrm{E}-6 / \mathrm{h}$ & 10 & $1.0 \mathrm{E}-6 / \mathrm{h}$ & 10 & & & $3.0 \mathrm{E}-6 / \mathrm{h}$ & 10 \\
\hline HTZ099SW & $\begin{array}{l}\text { DM723 POSITION INDIC } \\
\text { ATOR ERROR } \\
\text { DM-723/ES-99-1 }\end{array}$ & $3.0 \mathrm{E}-6 / \mathrm{h}$ & 10 & $1.0 \mathrm{E}-6 / \mathrm{h}$ & 10 & & & $3.0 \mathrm{E}-6 / \mathrm{h}$ & 10. \\
\hline HTZ741SW & $\begin{array}{l}\text { DM741 POSITION } \\
\text { INDICATOR } \text { ERROR }\end{array}$ & $3.0 \mathrm{E}-6 / \mathrm{h}$ & 10 & $1.0 \mathrm{E}-6 / \mathrm{h}$ & 10 & & & $3.0 \mathrm{E}-6 / \mathrm{h}$ & 10 \\
\hline HTZ742SW & $\begin{array}{l}\text { DM742 POSITION } \\
\text { INDICATOR }\end{array}$ & $3.0 \mathrm{E}-6 / \mathrm{h}$ & 10 & $1.0 \mathrm{E}-6 / \mathrm{h}$ & 10 & & & $3.0 \mathrm{E}-6 / \mathrm{h}$ & 10 \\
\hline HTZ745SW & $\begin{array}{l}\text { DM745 POSITION INDIC } \\
\text { ATOR ERROR } \\
\text { DM } 745 / M D-51-1\end{array}$ & $3.0 \mathrm{E}-6 / \mathrm{h}$ & 10 & $1.0 \mathrm{E}-6 / \mathrm{h}$ & 10 & & & $3.0 \mathrm{E}-6 / \mathrm{h}$ & 10 \\
\hline HTZ746SW & $\begin{array}{l}\text { DM746 POSITION INDIC } \\
\text { ATOR ERROR } \\
\text { DM-746/MD-50-1 }\end{array}$ & $3.0 \mathrm{E} \cdot 6 \mathrm{~h}$ & 10 & $1.0 \mathrm{E} \cdot 6 / \mathrm{h}$ & 10 & & & $3.0 \mathrm{E}-6 / \mathrm{h}$ & 10 \\
\hline HWR705TB & $\begin{array}{l}\text { FAN } 705 \text { POWER CABLE } \\
\text { FAILS: OPEN CIRCUIT }\end{array}$ & $3.0 \mathrm{E}-6 / \mathrm{h}$ & 10 & & & & & & \\
\hline HWR705TC & $\begin{array}{l}\text { FAN } 705 \text { POWER CABLE } \\
\text { FAILS: SHORT TO } \\
\text { GROUND }\end{array}$ & $3.0 \mathrm{E}-7 \mathrm{~h}$ & 10 & & & & & & \\
\hline HWR706TB & $\begin{array}{l}\text { FAN } 706 \text { POWER CABLE } \\
\text { FAILS: OPEN CIRCUIT }\end{array}$ & $3.0 \mathrm{E}-6 / \mathrm{h}$ & 10 & & & & & & \\
\hline HWR706TC & $\begin{array}{l}\text { FAN } 706 \text { POWER CABLE } \\
\text { FAILS: SHORT TO } \\
\text { GROUND }\end{array}$ & $3.0 \mathrm{E}-7 \mathrm{~h}$ & 10 & & & & & & \\
\hline HWR70TTB & $\begin{array}{l}\text { FAN } 707 \text { POWER CABLE } \\
\text { FAILS: OPEN CIRCUIT }\end{array}$ & $3.0 \mathrm{E}-6 / \mathrm{h}$ & 10 & & & & & & \\
\hline HWR707TC & $\begin{array}{l}\text { FAN } 707 \text { POWER CABLE } \\
\text { FAILS: SHORT TO } \\
\text { GROUND }\end{array}$ & $3.0 \mathrm{E}-7 \mathrm{~h}$ & 10 & & & & & & \\
\hline HWR708TB & $\begin{array}{l}\text { FAN } 708 \text { POWER CABLE } \\
\text { FAILS: OPEN CIRCUIT }\end{array}$ & $3.0 \mathrm{E}-6 \mathrm{~h}$ & 10 & & & & & & \\
\hline HWR708TC & $\begin{array}{l}\text { FAN } 708 \text { POWER CABLE } \\
\text { FAILS: SHORT TO } \\
\text { GROUND }\end{array}$ & $3.0 \mathrm{E}-7 \mathrm{~h}$ & 10 & & & & & & \\
\hline HXT048SW & $\begin{array}{l}\text { RM48 } 480 \mathrm{~V} / 120 \mathrm{~V} 250 \mathrm{~V} \\
\text { STARTER TRANSFORMER } \\
\text { FAILS }\end{array}$ & $1.0 \mathrm{E}-6 / \mathrm{h}$ & 3 & $1.0 \mathrm{E} \cdot 6 \mathrm{~h}$ & 3 & $1.0 \mathrm{E}-6 \mathrm{~h}$ & 3 & $1.0 \mathrm{E}-6 \mathrm{~h}$ & 3 \\
\hline HXT050SW & $\begin{array}{l}\text { RM50 } 480 \mathrm{~V} / 120 \mathrm{~V} 250 \mathrm{~V} \\
\text { STARTER TRANSFORMER } \\
\text { FAILS }\end{array}$ & $1.0 \mathrm{E}-6 / \mathrm{h}$ & 3 & $1.0 \mathrm{E}-6 / \mathrm{h}$ & 3 & $1.0 \mathrm{E}-6 \mathrm{~m}$ & 3 & $1.0 \mathrm{E}-6 / \mathrm{h}$ & 3 \\
\hline
\end{tabular}


Table 7.6. Shield Cooling System Data (Cont'd)

PAGE 8

\begin{tabular}{|c|c|c|c|c|c|c|c|c|c|}
\hline \multirow{2}{*}{$\begin{array}{l}\text { EVENT } \\
\text { NAME }\end{array}$} & \multirow[b]{2}{*}{ DESCRIPTION } & \multicolumn{2}{|c|}{ VALUE USED $3 / 91$} & \multicolumn{2}{|c|}{ CRBRP-4 } & \multicolumn{2}{|c|}{ IREP } & \multicolumn{2}{|c|}{ GENERIC } \\
\hline & & $\begin{array}{c}\text { Failure } \\
\text { Rate }\end{array}$ & $\begin{array}{l}\text { Error } \\
\text { Factor }\end{array}$ & $\begin{array}{l}\text { Failure } \\
\text { Rate }\end{array}$ & $\begin{array}{l}\text { Error } \\
\text { Factor }\end{array}$ & $\begin{array}{l}\text { Failure } \\
\text { Rate }\end{array}$ & $\begin{array}{l}\text { Error } \\
\text { Factor }\end{array}$ & $\begin{array}{l}\text { Failure } \\
\text { Rate }\end{array}$ & $\begin{array}{l}\text { Error } \\
\text { Factor }\end{array}$ \\
\hline HXTOS1SW & $\begin{array}{l}\text { RM51 } 480 \mathrm{~V} / 120 \mathrm{~V} 250 \mathrm{~V} \\
\text { STARTER TRANSFORMER } \\
\text { FAILS }\end{array}$ & $1.0 \mathrm{E}-6 / \mathrm{h}$ & 3 & $1.0 \mathrm{E}-6 / \mathrm{h}$ & 3 & $1.0 \mathrm{E}-6 / \mathrm{h}$ & 3 & $1.0 \mathrm{E}-6 / \mathrm{h}$ & 3 \\
\hline HXT054SW & $\begin{array}{l}\text { RM54 480V/120V 500VA } \\
\text { STARTER TRANSFORMER } \\
\text { FAILS }\end{array}$ & $1.0 \mathrm{E}-6 / \mathrm{h}$ & 3 & $1.0 \mathrm{E}-6 / \mathrm{h}$ & 3 & $1.0 \mathrm{E}-6 / \mathrm{h}$ & 3 & $1.0 \mathrm{E} \cdot 6 / \mathrm{h}$ & 3 \\
\hline HXT098SW & $\begin{array}{l}\text { AM98 120V/24V } \\
\text { TRANSFORMER FAILS }\end{array}$ & $1.0 \mathrm{E}-6 / \mathrm{h}$ & 3 & $1.0 \mathrm{E}-6 / \mathrm{h}$ & 3 & $1.0 \mathrm{E}-6 / \mathrm{h}$ & 3 & $1.0 \mathrm{E}-6 / \mathrm{h}$ & 3 \\
\hline HXTOG9SW & $\begin{array}{l}\text { CR99 120V/24V } \\
\text { TRANSFORMER FAILS }\end{array}$ & $1.0 \mathrm{E}-6 / \mathrm{h}$ & 3 & $1.0 \mathrm{E}-6 / \mathrm{h}$ & 3 & $1.0 E-6 / \mathrm{h}$ & 3 & $1.0 \mathrm{E}-6 \mathrm{~h}$ & 3 \\
\hline HXT100SW- & $\begin{array}{l}\text { CR100 120V/24V } \\
\text { TRANSFORMER FAILS }\end{array}$ & $1.0 \mathrm{E}-6 / \mathrm{h}$ & 3 & $1.0 \mathrm{E}-6 / \mathrm{h}$ & 3 & $1.0 \mathrm{E}-6 / \mathrm{h}$ & 3 & $1.0 \mathrm{E}-6 / \mathrm{h}$ & 3 \\
\hline HXV953SQ & $\begin{array}{l}\text { COILS } 713 / 714 \\
\text { ISOLATION FAILS TO } \\
\text { REMAIN OPEN R8-V-953 }\end{array}$ & $1.0 \mathrm{E}-7 / \mathrm{h}$ & 10 & $1.0 \mathrm{E}-7 / \mathrm{h}$ & 3 & $1.0 \mathrm{E}-7 / \mathrm{h}$ & 3 & $1.0 \mathrm{E}-7 / \mathrm{h}$ & 3 \\
\hline HXV954SQ & $\begin{array}{l}\text { COIL } 713 \text { \#2 } \\
\text { ISOLATION FAILS TO } \\
\text { REMAIN OPEN R8-V-954 }\end{array}$ & $1.0 \mathrm{E}-7 / \mathrm{h}$ & 10 & $1.0 \mathrm{E}-7 / \mathrm{h}$ & 3 & $1.0 \mathrm{E}-7 \mathrm{~h}$ & 3 & $1.0 \mathrm{E} \cdot 7 \mathrm{~h}$ & 3 \\
\hline HXV955SO & $\begin{array}{l}\text { COIL } 713 \text { \#1 } \\
\text { ISOLATION FAILS TO } \\
\text { REMAIN OPEN R8-V-955 }\end{array}$ & $1.0 \mathrm{E}-7 / \mathrm{h}$ & 10 & $1.0 \mathrm{E}-7 / \mathrm{h}$ & 3 & $1.0 \mathrm{E}-7 / \mathrm{h}$ & 3 & $1.0 \mathrm{E}-7 / \mathrm{h}$ & 3 \\
\hline HXV956SO & $\begin{array}{l}\text { COIL } 714 \text { \#2 } \\
\text { ISOLATION FAILS TO } \\
\text { REMAIN OPEN R8-V-956 }\end{array}$ & $1.0 \mathrm{E}-7 / \mathrm{h}$ & 10 & $1.0 \mathrm{E}-7 / \mathrm{h}$ & 3 & $1.0 \mathrm{E}-7 \mathrm{~h}$ & 3 & $1.0 \mathrm{E}-7 / \mathrm{h}$ & 3 \\
\hline HXV957SQ & $\begin{array}{l}\text { COIL } 714 \text { \#1 } \\
\text { ISOLATION FAILS TO } \\
\text { REMAIN OPEN R8-V-957 }\end{array}$ & $1.0 \mathrm{E}-7 / \mathrm{h}$ & 10 & $1.0 \mathrm{E}-7 / \mathrm{h}$ & 3 & $1.0 \mathrm{E}-7 \mathrm{~h}$ & 3 & $1.0 \mathrm{E} \cdot 7 \mathrm{~h}$ & 3 \\
\hline HXV958SL & $\begin{array}{l}\text { COIL } 715 \text { \#2 } \\
\text { ISOLATION LEFT } \\
\text { CLOSED R8-V-958 }\end{array}$ & $1.0 \mathrm{E}-4 / \mathrm{d}$ & 10 & $1.0 E-4 / d$ & 3 & $1.0 \mathrm{E}-4 / \mathrm{d}$ & 10 & $1.0 \mathrm{E}-4 / \mathrm{d}$ & 3 \\
\hline HXV958SQ & $\begin{array}{l}\text { COIL } 715 \text { \#2 } \\
\text { ISOLATION FAILS TO } \\
\text { REMAIN OPEN R8-V-958 }\end{array}$ & $1.0 \mathrm{E}-7 / \mathrm{h}$ & 10 & $1.0 \mathrm{E}-7 / \mathrm{h}$ & 3 & $1.0 \mathrm{E} \cdot 7 / \mathrm{h}$ & 3 & $1.0 \mathrm{E}-7 / \mathrm{h}$ & 3 \\
\hline HXV959SL & $\begin{array}{l}\text { STBY COILS 718 } \\
\text { ISOLATION LEFT } \\
\text { CLOSED R8-V-959 }\end{array}$ & $1.0 \mathrm{E}-4 / \mathrm{d}$ & 10 & $1.0 \mathrm{E}-4 / \mathrm{d}$ & 3 & $1.0 \mathrm{E}-4 / \mathrm{d}$ & 10 & $1.0 E-4 / d$ & 3 \\
\hline HXV959SQ & $\begin{array}{l}\text { STBY COILS } 718 \\
\text { ISOLATION FAILS TO } \\
\text { REMAIN OPEN R8-V-959 }\end{array}$ & $1.0 \mathrm{E}-7 / \mathrm{h}$ & 10 & $1.0 \mathrm{E}-7 / \mathrm{h}$ & 3 & $1.0 \mathrm{E}-7 \mathrm{~m}$ & 3 & $1.0 \mathrm{E}-7 \mathrm{~h}$ & 3 \\
\hline HXV960SL & $\begin{array}{l}\text { COIL 715 \#1 } \\
\text { ISOLATION LEFT } \\
\text { CLOSED R8-V-960 }\end{array}$ & $1.0 \mathrm{E}-4 / \mathrm{d}$ & 10 & $1.0 \mathrm{E}-4 / \mathrm{d}$ & 3 & $1.0 E-4 / d$ & 10 & $1.0 E-4 / d$ & 3 \\
\hline HXV960SQ & $\begin{array}{l}\text { COIL } 715 \text { \#1 } \\
\text { ISOLATION FAILS TO } \\
\text { REMAIN OPEN R8-V-960 }\end{array}$ & $1.0 \mathrm{E}-7 / \mathrm{h}$ & 10 & $1.0 \mathrm{E}-7 / \mathrm{h}$ & 3 & $1.0 \mathrm{E}-7 \mathrm{~h}$ & 3 & $1.0 E-7 / \mathrm{h}$ & 3 \\
\hline HXV961SL & $\begin{array}{l}\text { COIL } 718 \# 1 \\
\text { ISOLATION LEFT } \\
\text { CLOSED R8-V-961 }\end{array}$ & $1.0 E-4 / d$ & 10 & $1.0 \mathrm{E}-4 / \mathrm{d}$ & 3 & $1.0 \mathrm{E}-4 / \mathrm{d}$ & 10 & $1.0 \mathrm{E}-4 / \mathrm{d}$ & 3 \\
\hline HXV961SQ & $\begin{array}{l}\text { COIL 718 \#1 } \\
\text { ISOLATION FAILS TO } \\
\text { REMAIN OPEN R8-V-961 }\end{array}$ & $1.0 \mathrm{E}-7 \mathrm{~h}$ & 10 & $1.0 \mathrm{E}-7 \mathrm{~h}$ & 3 & $1.0 \mathrm{E}-7 / \mathrm{h}$ & 3 & $1.0 \mathrm{E} \cdot 7 / \mathrm{h}$ & 3 \\
\hline HXV962SL & $\begin{array}{l}\text { COIL } 718 \text { \#2 } \\
\text { ISOLATION LEFT } \\
\text { CLOSED R8-V.962 }\end{array}$ & $1.0 \mathrm{E}-4 / \mathrm{d}$ & 10 & $1.0 \mathrm{E}-4 / \mathrm{d}$ & 3 & $1.0 \mathrm{E}-4 / \mathrm{d}$ & 10 & $1.0 \mathrm{E}-4 / \mathrm{d}$ & 3 \\
\hline HXV962SQ & $\begin{array}{l}\text { COIL } 718 \# 2 \\
\text { ISOLATION FAILS TO } \\
\text { REMAIN OPEN R8-V-962 }\end{array}$ & $1.0 \mathrm{E} \cdot 7 / \mathrm{h}$ & 10 & $1.0 \mathrm{E}-7 \mathrm{~h}$ & 3 & $1.0 \mathrm{E}-7 / \mathrm{h}$ & 3 & $1.0 \mathrm{E}-7 / \mathrm{h}$ & 3 \\
\hline HXV963SL & $\begin{array}{l}\text { STBY COILS 715 } \\
\text { ISOLATION LEFT } \\
\text { CLOSED A8-V-963 }\end{array}$ & $1.0 \mathrm{E}-4 / \mathrm{d}$ & 10 & $1.0 E-4 / d$ & 3 & $1.0 \mathrm{E}-4 / \mathrm{d}$ & 10 & $1.0 \mathrm{E}-4 / \mathrm{d}$ & 3 \\
\hline
\end{tabular}


Table 7.6. Shield Cooling System Data (Cont'd)

PAGE 9

\begin{tabular}{|c|c|c|c|c|c|c|c|c|c|}
\hline \multirow{2}{*}{$\begin{array}{l}\text { EVENT } \\
\text { NAME }\end{array}$} & \multirow[b]{2}{*}{ DESCAIPTION } & \multicolumn{2}{|c|}{ VALUE USED $3 / 91$} & \multicolumn{2}{|c|}{ CRBAP-4 } & \multicolumn{2}{|c|}{ IREP } & \multicolumn{2}{|c|}{ GENERIC } \\
\hline & & $\begin{array}{c}\text { Failure } \\
\text { Rate }\end{array}$ & $\begin{array}{l}\text { Error } \\
\text { Factor }\end{array}$ & $\begin{array}{c}\text { Failure } \\
\text { Pate }\end{array}$ & $\begin{array}{l}\text { Error } \\
\text { Factor }\end{array}$ & $\begin{array}{c}\text { Failure } \\
\text { Rate }\end{array}$ & $\begin{array}{l}\text { Error } \\
\text { Factor }\end{array}$ & $\begin{array}{c}\text { Failure } \\
\text { Pate }\end{array}$ & $\begin{array}{l}\text { Error } \\
\text { Factor }\end{array}$ \\
\hline HXV963SQ & $\begin{array}{l}\text { STBY COILS } 715 \\
\text { ISOLATION FAILS TO } \\
\text { REMAIN OPEN R8-V-963 }\end{array}$ & $1.0 \mathrm{E}-7 \mathrm{~h}$ & 10 & $1.0 \mathrm{E}-7 \mathrm{~h}$ & 3 & $1.0 E-7 / \mathrm{h}$ & 3 & $1.0 \mathrm{E}-7 / \mathrm{h}$ & 3 \\
\hline
\end{tabular}


Table 7.7. Thimble Cooling System Data

PAGE 1

\begin{tabular}{|c|c|c|c|c|c|c|c|c|c|}
\hline \multirow{2}{*}{$\begin{array}{l}\text { EVENT } \\
\text { NAME }\end{array}$} & \multirow[b]{2}{*}{ DESCRIPTION } & \multicolumn{2}{|c|}{ VALUE USED 391} & \multicolumn{2}{|c|}{ CRBRP-4 } & \multicolumn{2}{|c|}{ IREP } & \multicolumn{2}{|c|}{ GENERIC } \\
\hline & & $\begin{array}{l}\text { Failure } \\
\text { Rate }\end{array}$ & $\begin{array}{l}\text { Error } \\
\text { Factor }\end{array}$ & $\begin{array}{l}\text { Failure } \\
\text { Rate }\end{array}$ & $\begin{array}{l}\text { Error } \\
\text { Factor }\end{array}$ & $\begin{array}{l}\text { Failure } \\
\text { Rate }\end{array}$ & $\begin{array}{l}\text { Error } \\
\text { Factor }\end{array}$ & $\begin{array}{l}\text { Failure } \\
\text { Rate }\end{array}$ & $\begin{array}{l}\text { Error } \\
\text { Factor }\end{array}$ \\
\hline TDM728SN & $\begin{array}{l}\text { DISCHARGE DAMPER } 728 \\
\text { FAILS TO CLOSE } \\
\text { R\&-DM-728 }\end{array}$ & $3.0 \mathrm{E}-3 / \mathrm{d}$ & 10 & $1.0 \mathrm{E}-3 / \mathrm{d}$ & 10 & $3.0 \mathrm{E}-3 / \mathrm{d}$ & 10 & $3.0 \mathrm{E}-3 / \mathrm{d}$ & 3 \\
\hline TDM728SO & $\begin{array}{l}\text { DISC DAMPER } 728 \\
\text { FAILS TO REMAIN } \\
\text { CLOSED R8-DM-728 } \\
\end{array}$ & $1.0 \mathrm{E}-7 \mathrm{~h}$ & 10 & $1.0 \mathrm{E}-7 / \mathrm{h}$ & 3 & & & & \\
\hline TDM728SQ & $\begin{array}{l}\text { DISC DAMPER } 728 \\
\text { FAILS TO REMAIN } \\
\text { OPEN RB-DM-728 } \\
\end{array}$ & $1.0 \mathrm{E}-7 / \mathrm{h}$ & 10 & $1.0 \mathrm{E}-7 / \mathrm{h}$ & 3 & & & & \\
\hline TOM728SW & $\begin{array}{l}\text { DAMPER } 728 \text { INTERNAL } \\
\text { FAILURE R8-DM-728 }\end{array}$ & $3.6 \mathrm{E}-8 / \mathrm{h}$ & 8 & & & & & & \\
\hline TDM729SO & $\begin{array}{l}\text { DISC DAMPER } 729 \\
\text { FAILS TO REMAIN } \\
\text { CLOSED R8-DM-729 } \\
\end{array}$ & $1.0 \mathrm{E}-7 / \mathrm{h}$ & 10 & $1.0 \mathrm{E}-7 / \mathrm{h}$ & 3 & & & & \\
\hline TDM729SP & $\begin{array}{l}\text { DISCHARGE } \\
\text { DAMPER 729 FAILS } \\
\text { TO OPEN R8-DM-729 } \\
\end{array}$ & $3.0 \mathrm{E}-3 / \mathrm{d}$ & 10 & $1.0 \mathrm{E}-3 / \mathrm{d}$ & 10 & $3.0 E-3 / d$ & 10 & $3.0 \mathrm{E}-3 / \mathrm{d}$ & 3 \\
\hline TDM729SQ & $\begin{array}{l}\text { DISC DAMPER } 729 \\
\text { FAILS TO REMAIN } \\
\text { OPEN R8-DM-729 } \\
\end{array}$ & $1.0 \mathrm{E}-7 / \mathrm{h}$ & 10 & $1.0 \mathrm{E}-7 / \mathrm{h}$ & 3 & & & & \\
\hline TDM729SW & $\begin{array}{l}\text { DAMPER } 729 \text { INTERNAL } \\
\text { FAILURE R8-DM729 }\end{array}$ & $3.6 \mathrm{E}-8 / \mathrm{h}$ & 8 & & & & & & \\
\hline TDM749SQ & $\begin{array}{l}\text { ISOL DAMPER } 749 \\
\text { FAILS TO REMAIN } \\
\text { OPEN R\&-DM-749 } \\
\end{array}$ & $1.0 \mathrm{E}-7 / \mathrm{h}$ & 10 & $1.0 \mathrm{E}-7 / \mathrm{h}$ & 10 & $1.0 \mathrm{E}-7 \mathrm{~h}$ & 3 & $1.0 \mathrm{E}-7 / \mathrm{h}$ & 3 \\
\hline TDM751SL & $\begin{array}{l}\text { ISOLATION DAMPER } 751 \\
\text { LEFT CLOSED } \\
\text { R8-DM-751 }\end{array}$ & $1.0 \mathrm{E}-4 / \mathrm{d}$ & 10 & $1.0 E-4 / d$ & 3 & $1.0 \mathrm{E}-4 / \mathrm{d}$ & 10 & $1.0 \mathrm{E}-4 / \mathrm{d}$ & 3 \\
\hline TDM751SQ & $\begin{array}{l}\text { ISOL DAMPER } 751 \\
\text { FAILS TO REMAIN } \\
\text { OPEN R8-DM-751 } \\
\end{array}$ & $1.0 \mathrm{E}-7 / \mathrm{h}$ & 10 & $1.0 \mathrm{E}-7 / \mathrm{h}$ & 10 & $1.0 \mathrm{E} \cdot 7 \mathrm{~h}$ & 3 & $1.0 \mathrm{E}-7 / \mathrm{h}$ & 3 \\
\hline TDM761SQ & $\begin{array}{l}\text { THROTTL DMP } 761 \\
\text { FAILS TO REMAIN } \\
\text { OPEN } \\
\text { R8-DM-761 }\end{array}$ & $1.0 \mathrm{E}-7 \mathrm{~h}$ & 10 & $1.0 \mathrm{E}-7 \mathrm{~h}$ & 3 & $1.0 \mathrm{E}-7 / \mathrm{h}$ & 3 & $1.0 \mathrm{E} \cdot 7 \mathrm{~h}$ & \\
\hline TDM762SQ & $\begin{array}{l}\text { THROTTL DMP } 762 \\
\text { FAILS TO REMAIN } \\
\text { OPEN } \\
\text { R\&-DM-762 }\end{array}$ & $1.0 \mathrm{E}-7 / \mathrm{h}$ & 10 & $1.0 \mathrm{E}-7 / \mathrm{h}$ & 3 & $1.0 \mathrm{E}-7 / \mathrm{h}$ & 3 & $1.0 \mathrm{E}-7 / \mathrm{h}$ & \\
\hline TFLOO1SE & $\begin{array}{l}\text { HEPA FILTER \#1 } \\
\text { PLUGGED }\end{array}$ & $3.0 \mathrm{E}-5 / \mathrm{h}$ & 10 & $1.0 \mathrm{E}-5 / \mathrm{h}$ & & $1.0 \mathrm{E} \cdot 5 / \mathrm{h}$ & 10 & $3.0 \mathrm{E}-5 / \mathrm{h}$ & \\
\hline TFLOO2SE & $\begin{array}{c}\text { HEPA FILTER \#2 } \\
\text { PLUGGED }\end{array}$ & $3.0 \mathrm{E}-5 / \mathrm{h}$ & 10 & $1.0 \mathrm{E}-5 / \mathrm{h}$ & & $3.0 \mathrm{E}-5 / \mathrm{h}$ & 10 & & \\
\hline TFLOO3SE & $\begin{array}{l}\text { HEPA FILTER \#3 } \\
\text { PLUGGED }\end{array}$ & $3.0 \mathrm{E}-5 \mathrm{~h}$ & 10 & $1.0 \mathrm{E}-5 / \mathrm{h}$ & & $3.0 \mathrm{E}-5 / \mathrm{h}$ & 10 & & \\
\hline TFLOU1FE & $\begin{array}{l}\text { J-1 INLET } \\
\text { FILTER PLUGGED }\end{array}$ & $3.0 \mathrm{E}-5 / \mathrm{h}$ & 10 & $1.0 \mathrm{E} \cdot 5 \mathrm{~h}$ & & $3.0 \mathrm{E}-5 / \mathrm{h}$ & 10 & & \\
\hline TFLOJ3FE & $\begin{array}{l}\text { J-3 INLET } \\
\text { FILTER PLUGGED }\end{array}$ & $3.0 \mathrm{E}-5 / \mathrm{h}$ & 10 & $1.0 \mathrm{E}-5 / \mathrm{h}$ & & $3.0 \mathrm{E}-5 / \mathrm{h}$ & 10 & & \\
\hline TFLOJ4FE & $\begin{array}{l}\text { J-4 INLET } \\
\text { FILTER PLUGGED }\end{array}$ & $3.0 \mathrm{E}-5 / \mathrm{h}$ & 10 & $1.0 \mathrm{E}-5 / \mathrm{h}$ & & $3.0 \mathrm{E}-5 / \mathrm{h}$ & 10 & & \\
\hline TFLHEPSU & $\begin{array}{l}\text { HEPA FILTERS } \\
\text { PLUGGED DUE TO } \\
\text { COMMON CAUSE } \\
\end{array}$ & $3.0 \mathrm{E}-6 / \mathrm{h}$ & 10 & & & & & & \\
\hline TFLلJINFU & $\begin{array}{l}\text { THIMBLE INLET FILTE } \\
\text { RS PLUGGED DUE T } \\
\text { O COMMON CAUSE } \\
\end{array}$ & $3.0 \mathrm{E}-6 / \mathrm{h}$ & 10 & & & & & & \\
\hline TFN709SR & $\begin{array}{l}\text { TURBO FAN } 709 \\
\text { FAILS TO RUN } \\
\text { R8-FN-709 }\end{array}$ & $3.0 \mathrm{E}-5 / \mathrm{h}$ & 10 & $1.0 \mathrm{E}-5 / \mathrm{h}$ & & & & $1.0 \mathrm{E}-5 / \mathrm{h}$ & 10 \\
\hline
\end{tabular}


Table 7.7. Thimble Cooling System Data (Cont'd)

PAGE 2

\begin{tabular}{|c|c|c|c|c|c|c|c|c|c|}
\hline \multirow{2}{*}{$\begin{array}{l}\text { EVENT } \\
\text { NAME }\end{array}$} & \multirow[b]{2}{*}{ DESCRIPTION } & \multicolumn{2}{|c|}{ VALUE USED $3 / 91$} & \multicolumn{2}{|c|}{ CRBRP-4 } & \multicolumn{2}{|c|}{ IREP } & \multicolumn{2}{|c|}{ GENERIC } \\
\hline & & $\begin{array}{c}\text { Failure } \\
\text { Rate }\end{array}$ & $\begin{array}{l}\text { Error } \\
\text { Factor }\end{array}$ & $\begin{array}{c}\text { Failure } \\
\text { Rate }\end{array}$ & $\begin{array}{l}\text { Error } \\
\text { Factor }\end{array}$ & $\begin{array}{l}\text { Failure } \\
\text { Rate }\end{array}$ & $\begin{array}{l}\text { Error } \\
\text { Factor }\end{array}$ & $\begin{array}{c}\text { Failure } \\
\text { Rate }\end{array}$ & $\begin{array}{l}\text { Error } \\
\text { Factor } \\
\end{array}$ \\
\hline TFN71OSR & $\begin{array}{l}\text { TURBO FAN } 710 \\
\text { FAILS TO RUN } \\
\text { R8-FN-710 }\end{array}$ & $3.0 \mathrm{E}-5 \mathrm{~h}$ & 10 & $1.0 \mathrm{E}-5 / \mathrm{h}$ & & & & $1.0 \mathrm{E}-5 / \mathrm{h}$ & 10 \\
\hline TFN710SS & $\begin{array}{l}\text { TURBO FAN } 710 \\
\text { FAILS TO START } \\
\text { R8-FN-710 }\end{array}$ & $1.0 \mathrm{E}-3 / \mathrm{d}$ & 10 & $3.0 E-4 / d$ & 3 & & & $3.0 E-4 / d$ & 3 \\
\hline THU002YL & $\begin{array}{l}\text { NO RECOVERY ACTION } \\
\text { TO RESET BREAKER }\end{array}$ & $2.0 \mathrm{E}-2 / \mathrm{d}$ & 10 & & & & & & \\
\hline THU01BSZ & $\begin{array}{l}\text { NO RECOVERY ACTION } \\
\text { TO CROSS.CONNECT } \\
\text { SHIELD COOLING }\end{array}$ & $2.0 \mathrm{E}-2 / \mathrm{d}$ & 10 & & & & & & \\
\hline TPR01ASW & $\begin{array}{ll}\text { PRESSURE } & \\
\text { TRANSDUCER } & \text { FAILS } \\
\text { DP-PS-1A } & \end{array}$ & $3.0 \mathrm{E}-6 / \mathrm{h}$ & 10 & $1.0 \mathrm{E}-6 \mathrm{~m}$ & 10 & & & $3.0 \mathrm{E}-6 / \mathrm{h}$ & 10 \\
\hline TREO91SO & $\begin{array}{l}\text { CONTROL RELAY RM-91 } \\
\text { COIL FAILURE } \\
\text { FN-709/CR-91 }\end{array}$ & $3.0 \mathrm{E} \cdot 6 / \mathrm{h}$ & 10 & $1.0 \mathrm{E}-6 \mathrm{~m}$ & 10 & $3.0 \mathrm{E}-6 / \mathrm{h}$ & 10 & $3.0 \mathrm{E}-6 / \mathrm{h}$ & 10 \\
\hline TREO92SO & $\begin{array}{l}\text { CONTROL RELAY RM-92 } \\
\text { COIL FAILURE } \\
\text { FN-710/CR-92 }\end{array}$ & $3.0 \mathrm{E}-6 / \mathrm{h}$ & 10 & $1.0 \mathrm{E}-6 / \mathrm{h}$ & 10 & $3.0 \mathrm{E} \cdot 6 / \mathrm{h}$ & 10 & $3.0 \mathrm{E}-6 / \mathrm{h}$ & 10 \\
\hline TRES2XSO & $\begin{array}{l}\text { CONTROL RELAY RM-52 } \\
\text { X COIL FAILURE } \\
\text { FN-709/CR-52X }\end{array}$ & $3.0 \mathrm{E}-6 / \mathrm{h}$ & 10 & $1.0 \mathrm{E}-6 / \mathrm{h}$ & 10 & $3.0 E-6 / h$ & 10 & $3.0 \mathrm{E}-6 / \mathrm{h}$ & 10 \\
\hline TRE53TSN & $\begin{array}{l}\text { TIME DELAY RELAY } \\
\text { TO-53A CONTACT FAILS } \\
\text { TO CLOSE }\end{array}$ & $3.0 E-4 / d$ & 10 & $1.0 \mathrm{E} \cdot 4 / \mathrm{d}$ & 10 & $3.0 E-4 / d$ & 10 & $3.0 \mathrm{E}-4 / \mathrm{d}$ & 10 \\
\hline TRE53XSO & $\begin{array}{l}\text { CONTROL RELAY RM-53 } \\
\text { X COIL FAILURE } \\
\text { FN-710/CR-53X }\end{array}$ & $3.0 \mathrm{E}-6 / \mathrm{h}$ & 10 & $1.0 \mathrm{E}-6 \mathrm{~h}$ & 10 & $3.0 \mathrm{E}-6 \mathrm{~h}$ & 10 & $3.0 \mathrm{E}-6 / \mathrm{h}$ & 10 \\
\hline TSVO91SQ & $\begin{array}{l}\text { SOLENOID VALVE } \\
\text { RSV-91 FAILS TO } \\
\text { REMAIN OPEN RSV-91 }\end{array}$ & $1.0 \mathrm{E}-7 / \mathrm{h}$ & $\cdot 3$ & $1.0 \mathrm{E}-7 \mathrm{~h}$ & 3 & & & & \\
\hline TSVO92SP & \begin{tabular}{|ll} 
SOLENOID VALVE \\
RSV-92 & FAILS \\
TO OPEN & RSV-92
\end{tabular} & $1.0 \mathrm{E}-7 \mathrm{~m}$ & 3 & $1.0 \mathrm{E}-7 \mathrm{~m}$ & 3 & & & & \\
\hline TSVO92SQ & $\begin{array}{l}\text { SOLENOID VALVE } \\
\text { RSV-92 FAILS TO } \\
\text { REMAIN OPEN RSV-92 }\end{array}$ & $1.0 \mathrm{E}-3 / \mathrm{d}$ & 3 & $1.0 E-3 / d$ & 3 & $1.0 \mathrm{E}-3 / \mathrm{d}$ & 3 & $1.0 E-3 / d$ & 3 \\
\hline TSW761ST & $\begin{array}{l}\text { THROTTL DMR 761 SWIT } \\
\text { CH IN WRONG POSITIO } \\
\text { N DM-761/RM93 }\end{array}$ & $1.0 E-4 / d$ & 10 & $1.0 \mathrm{E}-4 / \mathrm{d}$ & 3 & $1.0 \mathrm{E}-4 / \mathrm{d}$ & 3 & $1.0 \mathrm{E}-4 / \mathrm{d}$ & 3 \\
\hline TTZ728SW & $\begin{array}{l}\text { DM728 POSITION } \\
\text { INDICATOR ERROR } \\
\text { DM-728 }\end{array}$ & $3.0 \mathrm{E}-6 / \mathrm{h}$ & 10 & $1.0 \mathrm{E}-6 / \mathrm{h}$ & 10 & & & $3.0 \mathrm{E}-6 / \mathrm{h}$ & 10 \\
\hline TT2729sw & $\begin{array}{l}\text { DM729 POSITION } \\
\text { INDICATOR ERROR } \\
\text { DM-729 }\end{array}$ & $3.0 E-6 / h$ & 10 & $1.0 \mathrm{E} \cdot 6 / \mathrm{h}$ & 10 & & & $3.0 \mathrm{E}-6 \mathrm{~h}$ & 10 \\
\hline TTZ749SW & $\begin{array}{l}\text { DM749 POSITION INDIC } \\
\text { ATOR ERROR } \\
\text { DM-749/RSV-91 }\end{array}$ & $3.0 \mathrm{E}-6 / \mathrm{h}$ & 10 & $1.0 \mathrm{E}-6 / \mathrm{h}$ & 10 & & & $3.0 \mathrm{E}-6 / \mathrm{h}$ & 10 \\
\hline TTZ751SW & $\begin{array}{l}\text { DM751 POSITION INDIC } \\
\text { ATOR ERROR } \\
\text { DM-751/RSV-92 }\end{array}$ & $3.0 \mathrm{E}-6 / \mathrm{h}$ & 10 & $1.0 \mathrm{E}-6 / \mathrm{h}$ & 10 & & & $3.0 \mathrm{E}-6 / \mathrm{h}$ & 10 \\
\hline TWR709TB & $\begin{array}{l}\text { FAN } 709 \text { POWER CABLE } \\
\text { FAILS: OPEN CIRCUIT }\end{array}$ & $3.0 \mathrm{E}-6 / \mathrm{h}$ & 10 & & & & & & . \\
\hline TWR709TC & $\begin{array}{l}\text { FAN } 709 \text { POWER CABLE } \\
\text { FAILS: SHORT TO } \\
\text { GROUND }\end{array}$ & $3.0 \mathrm{E}-7 / \mathrm{h}$ & 10 & & & & & & \\
\hline TWA710TB & $\begin{array}{l}\text { FAN 710 POWER CABLE } \\
\text { FAILS: OPEN CIRCUIT }\end{array}$ & $3.0 \mathrm{E}-6 / \mathrm{h}$ & 10 & & & & & & \\
\hline
\end{tabular}


Table 7.7. Thimble Cooling System Data (Cont'd)

PAGE 3

\begin{tabular}{|c|c|c|c|c|c|c|c|c|c|}
\hline \multirow{2}{*}{$\begin{array}{l}\text { EVENT } \\
\text { NAME }\end{array}$} & \multirow[b]{2}{*}{ DESCRIPTION } & \multicolumn{2}{|c|}{ VALUE USED $3 / 91$} & \multicolumn{2}{|c|}{ CABRP-4 } & \multicolumn{2}{|c|}{ IREP } & \multicolumn{2}{|c|}{ GENERIC } \\
\hline & & $\begin{array}{l}\text { Failure } \\
\text { Rate }\end{array}$ & $\begin{array}{l}\text { Error } \\
\text { Factor }\end{array}$ & $\begin{array}{l}\text { Failure } \\
\text { Rate }\end{array}$ & $\begin{array}{l}\text { Error } \\
\text { Factor }\end{array}$ & $\begin{array}{l}\text { Failure } \\
\text { Rate }\end{array}$ & $\begin{array}{l}\text { Error } \\
\text { Factor }\end{array}$ & $\begin{array}{l}\text { Failure } \\
\text { Rate }\end{array}$ & $\begin{array}{l}\text { Error } \\
\text { Factor }\end{array}$ \\
\hline TWR710TC & $\begin{array}{l}\text { FAN } 710 \text { POWER CABLE } \\
\text { FAILS: SHORT TO } \\
\text { GROUND }\end{array}$ & $3.0 \mathrm{E}-7 \mathrm{~h}$ & 10 & & & & & & \\
\hline TXT052SW & $\begin{array}{l}\text { RM52 48OV/120V } 250 V A \\
\text { STARTER TRANSFORMER } \\
\text { FAILS }\end{array}$ & $1.0 \mathrm{E}-6 / \mathrm{h}$ & 3 & $1.0 \mathrm{E}-6 / \mathrm{h}$ & 3 & $1.0 \mathrm{E}-6 / \mathrm{h}$ & 3 & $1.0 \mathrm{E}-6 / \mathrm{h}$ & 3 \\
\hline TXT0535W & $\begin{array}{l}\text { RM53 48OV/12OV } 250 V A \\
\text { STARTER TRANSFORMER } \\
\text { FAILS }\end{array}$ & $1.0 \mathrm{E}-6 / \mathrm{h}$ & 3 & $1.0 \mathrm{E}-6 / \mathrm{h}$ & 3 & $1.0 \mathrm{E}-6 / \mathrm{h}$ & 3 & $1.0 \mathrm{E}-6 / \mathrm{h}$ & 3 \\
\hline TXVOO5FQ & $\begin{array}{l}\text { J-1 ISOLATION VALVE } \\
\text { FAILS TO REMAIN OPEN } \\
\text { RSA-5 }\end{array}$ & $1.0 \mathrm{E} \cdot 7 / \mathrm{h}$ & 10 & $1.0 \mathrm{E}-7 / \mathrm{h}$ & 3 & $1.0 \mathrm{E}-7 / \mathrm{h}$ & 3 & $1.0 \mathrm{E}-7 / \mathrm{h}$ & 3 \\
\hline TXVOO7FQ & $\begin{array}{l}\text { J-3 ISOLATION VALVE } \\
\text { FAILS TO REMAIN OPEN } \\
\text { RSA-7 }\end{array}$ & $1.0 \mathrm{E}-7 / \mathrm{h}$ & 10 & $1.0 \mathrm{E}-7 / \mathrm{h}$ & 3 & $1.0 \mathrm{E}-7 \mathrm{~h}$ & 3 & $1.0 \mathrm{E} \cdot 7 / \mathrm{h}$ & 3 \\
\hline TXVOO8FQ & $\begin{array}{l}\text { J-4 ISOLATION VALVE } \\
\text { FAILS TO REMAIN OPEN } \\
\text { RSA-8 }\end{array}$ & $1.0 \mathrm{E}-7 / \mathrm{h}$ & 10 & $1.0 \mathrm{E} \cdot 7 \mathrm{~h}$ & 3 & $1.08-7 / \mathrm{h}$ & 3 & $1.0 \mathrm{E}-7 / \mathrm{h}$ & 3 \\
\hline TXV01ISQ & $\begin{array}{l}\text { HEPA FILTER \#1 INLET } \\
\text { FAILS TO REMAIN OPEN }\end{array}$ & $1.0 E-7 / \mathrm{h}$ & 10 & $1.0 \mathrm{E} \cdot 7 \mathrm{~h}$ & 3 & $1.0 \mathrm{E}-7 / \mathrm{h}$ & 3 & $1.0 \mathrm{E}-7 / \mathrm{h}$ & 3 \\
\hline TXV01OSQ & $\begin{array}{l}\text { HEPA FILTER \#1 } \\
\text { OUTLET FAILS TO } \\
\text { REMAIN OPEN }\end{array}$ & $1.0 E-7 / \mathrm{h}$ & 10 & $1.0 \mathrm{E}-7 / \mathrm{h}$ & 3 & $1.0 E-7 / \mathrm{h}$ & 3 & $1.0 E-7 / \mathrm{h}$ & 3 \\
\hline TXV02ISQ & $\begin{array}{l}\text { HEPA FILTER \#2 INLET } \\
\text { FAILS TO REMAIN OPEN }\end{array}$ & $1.0 \mathrm{E} \cdot 7 / \mathrm{h}$ & 10 & $1.0 \mathrm{E}-7 / \mathrm{h}$ & 3 & $1.0 \mathrm{E}-7 / \mathrm{h}$ & 3 & $1.0 \mathrm{E} \cdot 7 / \mathrm{h}$ & 3 \\
\hline TXV02OSQ & $\begin{array}{l}\text { HEPA FILTER \#2 } \\
\text { OUTLET FAILS TO } \\
\text { REMAIN OPEN }\end{array}$ & $1.0 \mathrm{E}-7 / \mathrm{h}$ & 10 & $1.0 E-7 / \mathrm{h}$ & 3 & $1.0 \mathrm{E}-7 / \mathrm{h}$ & 3 & $1.0 \mathrm{E}-7 \mathrm{~m}$ & 3 \\
\hline TXVO3ISQ & $\begin{array}{l}\text { HEPA FILTER \#3 INLET } \\
\text { FAILS TO REMAIN OPEN }\end{array}$ & $1.0 \mathrm{E}-7 / \mathrm{h}$ & 10 & $1.0 \mathrm{E}-7 / \mathrm{h}$ & 3 & 1.OE-7/h & 3 & $1.0 \mathrm{E}-7 / \mathrm{M}$ & 3 \\
\hline TXV03OSO & $\begin{array}{l}\text { HEPA FILTER \#3 } \\
\text { OUTLET FAILS TO } \\
\text { REMAIN OPEN }\end{array}$ & $1.0 \mathrm{E}-7 / \mathrm{h}$ & 10 & $1.0 E-7 / h$ & 3 & $1.0 \mathrm{E} \cdot 7 \mathrm{~h}$ & 3 & $1.0 \mathrm{E}-7 / \mathrm{h}$ & 3 \\
\hline TXV05AFO & $\begin{array}{l}\text { J-1 BYPASS VALVE } \\
\text { FAILS TO REMAIN } \\
\text { CLOSED RSA-5A }\end{array}$ & $1.0 \mathrm{E}-7 / \mathrm{m}$ & 10 & $1.0 E-7 / \mathrm{h}$ & 3 & $1.0 \mathrm{E}-7 \mathrm{~h}$ & 3 & $1.0 \mathrm{E}-7 / \mathrm{h}$ & 3 \\
\hline TXVOTAFO & $\begin{array}{l}\text { J-3 BYPASS VALVE } \\
\text { FAILS TO REMAIN } \\
\text { CLOSED RSA-7A }\end{array}$ & $1.0 \mathrm{E}-7 / \mathrm{h}$ & 10 & $1.0 \mathrm{E}-7 / \mathrm{h}$ & 3 & $1.0 \mathrm{E}-7 / \mathrm{h}$ & 3 & $1.0 \mathrm{E}-7 / \mathrm{h}$ & 3 \\
\hline TXVOBAFO & $\begin{array}{l}\text { J-4 BYPASS VALVE } \\
\text { FAILS TO REMAIN } \\
\text { CLOSED RSA-8A }\end{array}$ & $1.0 \mathrm{E-7/h}$ & 10 & $1.0 \mathrm{E}-7 / \mathrm{h}$ & 3 & $1.0 \mathrm{E}-7 \mathrm{~h}$ & 3 & $1.0 \mathrm{E}-7 / \mathrm{h}$ & 3 \\
\hline TXV752SQ & $\begin{array}{l}\text { BALANCE DAMPER LEG B } \\
\text { FAILS TO REMAIN OPEN } \\
\text { R8-DM-752 }\end{array}$ & $1.0 \mathrm{E} \cdot 7 \mathrm{~m}$ & 10 & $1.0 \mathrm{E}-7 / \mathrm{h}$ & 3 & & & & \\
\hline TXV753SQ & $\begin{array}{l}\text { BALANCE DAMPER LEG A } \\
\text { FAILS TO REMAIN OPEN } \\
\text { R8-DM-753 }\end{array}$ & $1.0 \mathrm{E}-7 / \mathrm{h}$ & 10 & $1.0 \mathrm{E}-7 \mathrm{~h}$ & 3 & & & & \\
\hline TXV77OSP & $\begin{array}{l}\text { SHIELD COOLING } \\
\text { X-CONN FAILS TO } \\
\text { OPEN } \quad \text { R8-DM-77 }\end{array}$ & $2.0 \mathrm{E}-4 / \mathrm{d}$ & 10 & $1.0 \mathrm{E}-3 / \mathrm{d}$ & 10 & $3.0 \mathrm{E}-3 / \mathrm{d}$ & 10 & $3.0 E-3 / d$ & 10 \\
\hline TXV77OSQ & $\begin{array}{l}\text { SHIELD COOLING X-CON } \\
\text { N FAILS TO REMAIN OP } \\
\text { EN R8-OM-770 }\end{array}$ & $1.0 \mathrm{E}-7 \mathrm{~h}$ & 10 & $1.0 \mathrm{E} \cdot 7 / \mathrm{h}$ & 3 & $1.0 \mathrm{E}-7 / \mathrm{h}$ & 3 & $1.0 E-7 / \mathrm{h}$ & 3 \\
\hline TXVBYPFU & $\begin{array}{l}\text { J AND O BYPASS VALVE } \\
\text { S FAIL TO REMAIN CLO } \\
\text { SED - COMMON CAUSE }\end{array}$ & $1.0 \mathrm{E}-8 / \mathrm{h}$ & 10 & & & & & & \\
\hline TXVISOFU & $\begin{array}{l}\text { INLET ISOLATION VALV } \\
\text { ES FAIL TO REMAIN OP } \\
\text { EN - COMMON CAUSE }\end{array}$ & $1.0 \mathrm{E}-8 / \mathrm{h}$ & 10 & & & & & & \\
\hline
\end{tabular}


Table 7.8. Stack Exhaust System Data

PAGE 1

\begin{tabular}{|c|c|c|c|c|c|c|c|c|c|}
\hline \multirow{2}{*}{$\begin{array}{l}\text { EVENT } \\
\text { NAME }\end{array}$} & \multirow[b]{2}{*}{ DESCRIPTION } & \multicolumn{2}{|c|}{ VALUE USED $3 / 91$} & \multicolumn{2}{|c|}{ CRBRP-4 } & \multicolumn{2}{|c|}{ IREP } & \multicolumn{2}{|c|}{ GENERIC } \\
\hline & & $\begin{array}{c}\text { Failure } \\
\text { Rate }\end{array}$ & $\begin{array}{l}\text { Error } \\
\text { Factor }\end{array}$ & $\begin{array}{l}\text { Fallure } \\
\text { Rate }\end{array}$ & $\begin{array}{l}\text { Error } \\
\text { Factor }\end{array}$ & $\begin{array}{c}\text { Failure } \\
\text { Rate }\end{array}$ & $\begin{array}{l}\text { Error } \\
\text { Factor }\end{array}$ & $\begin{array}{l}\text { Failure } \\
\text { Rate }\end{array}$ & $\begin{array}{l}\text { Error } \\
\text { Factor }\end{array}$ \\
\hline YDMDIAOO & $\begin{array}{l}\text { DAMPER DA- } 1 \text { A FAILS } \\
\text { TO REMAIN OPEN } \\
\text { EXTERNALIY }\end{array}$ & $\begin{array}{c}6.4 E-8 / h \\
{[1]}\end{array}$ & 10 & $1.0 \mathrm{E}-7 / \mathrm{h}$ & 3 & $1.0 \mathrm{E}-7 / \mathrm{h}$ & 3 & $1.0 \mathrm{E}-7 / \mathrm{h}$ & \\
\hline YDMDIAOW & $\begin{array}{l}\text { DAMPER DA-1A FAILS } \\
\text { TO REMAIN OPEN } \\
\text { INTERNALY }\end{array}$ & $3.6 \mathrm{E}-8 \mathrm{~h}$ & 8 & & & & & & \\
\hline YDMD1BOQ & $\begin{array}{l}\text { FAN VANES D-1B FAIL } \\
\text { REMAIN OPEN } \\
\text { EXTERNALYY }\end{array}$ & $\begin{array}{c}6.4 \mathrm{E}-8 / \mathrm{h} \\
{[1]}\end{array}$ & 10 & $1.0 \mathrm{E}-7 / \mathrm{h}$ & 3 & $1.0 \mathrm{E}-7 / \mathrm{h}$ & 3 & $1.0 \mathrm{E}-7 / \mathrm{h}$ & \\
\hline YDMD1BOW & $\begin{array}{l}\text { FAN VANES D-1B FAIL } \\
\text { TO ROMAIN } \\
\text { OPEN INTERNALLY }\end{array}$ & $3.6 \mathrm{E}-8 \mathrm{~h}$ & 8 & & & & & & \\
\hline YDMD1COQ & $\begin{array}{l}\text { DAMPER DA-1C FAILS } \\
\text { TO REMAIN OPEN } \\
\text { EXTERNALY }\end{array}$ & $\begin{array}{c}6.4 E-8 \mathrm{~h} \\
{[1]}\end{array}$ & 10 & $1.0 \mathrm{E}-7 / \mathrm{h}$ & 3 & $1.0 \mathrm{E}-7 / \mathrm{h}$ & 3 & $1.0 \mathrm{E}-7 / \mathrm{h}$ & \\
\hline YDMD1COW & $\begin{array}{l}\text { DAMPER DA-1C FAILS } \\
\text { TO REMAIN OPEN } \\
\text { INTERNALIY }\end{array}$ & $3.6 E \cdot 8 / \mathrm{h}$ & 8 & & & & & & \\
\hline YDMD2AOP & $\begin{array}{l}\text { DAMPER DA-2A FAILS } \\
\text { TO OPEN EXTERNALIY }\end{array}$ & $3.0 \mathrm{E}-3 / \mathrm{d}$ & 10 & $1.0 \mathrm{E}-3 / \mathrm{d}$ & 10 & $3.0 \mathrm{E}-3 / \mathrm{d}$ & 10 & $3.0 \mathrm{E}-3 / \mathrm{d}$ & 3 \\
\hline YOMO2AOQ & $\begin{array}{l}\text { DAMPER DA-2A FAILS } \\
\text { TO REMAIN OPEN } \\
\text { EXTERNALY }\end{array}$ & $\begin{array}{c}6.4 \mathrm{E}-8 / \mathrm{h} \\
{[1]}\end{array}$ & 10 & & & & & & \\
\hline YDMD2AOW & $\begin{array}{l}\text { DAMPER DA-2A FAILS } \\
\text { TO REMAIN OPEN } \\
\text { INTERNALYY }\end{array}$ & $\begin{array}{c}3.6 \mathrm{E}-8 / \mathrm{h} \\
{[2]}\end{array}$ & 10 & & & & & & \\
\hline YDMD2BOP & $\begin{array}{l}\text { FAN VANES D-2B FAIL } \\
\text { TO OPEN EXTERNALLY }\end{array}$ & $3.0 \mathrm{E}-3 / \mathrm{d}$ & 10 & $1.0 \mathrm{E}-3 / \mathrm{d}$ & 10 & $3.0 \mathrm{E}-3 / \mathrm{d}$ & 10 & $3.0 \mathrm{E}-3 / \mathrm{d}$ & 3 \\
\hline YDMD2BOO & $\begin{array}{l}\text { FAN VANES D-2B FAIL } \\
\text { TO REMAIN OPEN } \\
\text { EXTERNALY }\end{array}$ & $\begin{array}{c}6.4 E-8 / h \\
{[1]}\end{array}$ & 10 & & & & & & \\
\hline YDMD2BOW & $\begin{array}{l}\text { FAN VANES D-2B FAIL } \\
\text { TO REMAIN } \\
\text { OPEN INTERALLY }\end{array}$ & $\begin{array}{c}3.6 \mathrm{E}-8 / \mathrm{h} \\
{[2]}\end{array}$ & 10 & & & & & & \\
\hline YDMD2COP & $\begin{array}{l}\text { DAMPER DA-2C FAILS } \\
\text { TO OPEN EXTERNALIY }\end{array}$ & $3.0 \mathrm{E}-3 / \mathrm{d}$ & 10 & $1.0 \mathrm{E}-3 / \mathrm{d}$ & 10 & $3.0 \mathrm{E}-3 / \mathrm{d}$ & 10 & $3.0 E-3 / d$ & 3 \\
\hline YOMD2COQ & $\begin{array}{l}\text { DAMPER DA-2C FAILS } \\
\text { TO REMAIN OPEN } \\
\text { EXTERNALY }\end{array}$ & $\begin{array}{c}\text { 6.4E-8/h } \\
{[1]}\end{array}$ & 10 & & & & & & \\
\hline YFN056OR & $\begin{array}{l}\text { FAN FM-56 FAILS } \\
\text { TO RUN } \\
\text { FM-56 }\end{array}$ & $2.0 \mathrm{E}-5 / \mathrm{h}$ & 3 & $1.0 \mathrm{E}-5 / \mathrm{h}$ & & & & $1.0 \mathrm{E}-5 \mathrm{~h}$ & 10 \\
\hline YFNOS8OR & $\begin{array}{l}\text { FAN FM-58 } \\
\text { FAILS TO } \\
\text { RUN FM-58 }\end{array}$ & $2.0 \mathrm{E}-5 / \mathrm{h}$ & 3 & $1.0 \mathrm{E}-5 / \mathrm{h}$ & & & & $1.0 \mathrm{E}-5 \mathrm{~h}$ & 10 \\
\hline YFNO58OS & $\begin{array}{l}\text { FAN FM-58 } \\
\text { FAILS TO } \\
\text { START FM-58 }\end{array}$ & $3.0 \mathrm{E}-4 / \mathrm{d}$ & 3 & $3.0 \mathrm{E}-4 / \mathrm{d}$ & 3 & & & $3.0 \mathrm{E}-4 / \mathrm{d}$ & 3 \\
\hline YHU001OL & $\begin{array}{l}\text { NO RECOVERY ACTION } \\
\text { TO MANUALY OPEN } \\
\text { DAMPER }\end{array}$ & $\begin{array}{c}1.0 E-1 / d \\
{[3]}\end{array}$ & 10 & & & & & & \\
\hline
\end{tabular}

Notes: 1. Motor Operated Valve Minus Internal HVAC Valve

2. Manual HVAC Damper (IAEA)

3. Lower Recovery; non-EBR-ll personnel 
Table 7.9. Secondary Sodium System Data

PAGE 1

\begin{tabular}{|c|c|c|c|c|c|c|c|c|c|}
\hline \multirow{2}{*}{$\begin{array}{l}\text { EVENT } \\
\text { NAME }\end{array}$} & \multirow[b]{2}{*}{ DESCRIPTION } & \multicolumn{2}{|c|}{ VALUE USED $3 / 91$} & \multicolumn{2}{|c|}{ CRBRP-4 } & \multicolumn{2}{|c|}{ IREP } & \multicolumn{2}{|c|}{ GENERIC } \\
\hline & & $\begin{array}{l}\text { Failure } \\
\text { Rate }\end{array}$ & $\begin{array}{l}\text { Error } \\
\text { Factor }\end{array}$ & $\begin{array}{l}\text { Failure } \\
\text { Rate }\end{array}$ & $\begin{array}{l}\text { Error } \\
\text { Factor }\end{array}$ & $\begin{array}{c}\text { Failure } \\
\text { Rate }\end{array}$ & $\begin{array}{l}\text { Error } \\
\text { Factor }\end{array}$ & $\begin{array}{l}\text { Failure } \\
\text { Rate }\end{array}$ & $\begin{array}{l}\text { Error } \\
\text { Factor }\end{array}$ \\
\hline NOOOOOUE & $\begin{array}{l}\text { SECONDARY } \\
\text { SODIUM BLOCKAGE }\end{array}$ & $2.0 E-7 / h$ & 10 & & & & & & \\
\hline NOOOOOUF & $\begin{array}{l}\text { SECONDARY } \\
\text { SODIUM LEAK } \\
\text { ANDJOR FIRE }\end{array}$ & $6.0 E-7 / \mathrm{h}$ & 10 & & & & & & \\
\hline NPMOVCWN & \begin{tabular}{|l|} 
Probability of \\
Secondary Pump \\
Failing to Trip
\end{tabular} & $1.0 \mathrm{E}-3 / \mathrm{d}$ & 10 & & & & & & \\
\hline NSWOOOCZ & $\begin{array}{l}\text { INADVERTANT OPERA } \\
\text { TOR ACTION TO DUMP } \\
\text { SECONDARY Na }\end{array}$ & $1.0 E-4 / d$ & 10 & & & & & & \\
\hline
\end{tabular}


Table 7.10. Balance of Plant Data - Steam System Data

PAGE 1

\begin{tabular}{|c|c|c|c|c|c|c|c|c|c|}
\hline $\begin{array}{l}\text { EVENT } \\
\text { NAME }\end{array}$ & DESCRIPTION & $\begin{array}{l}\text { Failure } \\
\text { Rate }\end{array}$ & $\begin{array}{l}\text { Error } \\
\text { Factor }\end{array}$ & $\begin{array}{l}\text { Failure } \\
\text { Rate }\end{array}$ & $\begin{array}{l}\text { Error } \\
\text { Factor }\end{array}$ & $\begin{array}{c}\text { Failure } \\
\text { Rate }\end{array}$ & $\begin{array}{l}\text { Error } \\
\text { Factor }\end{array}$ & $\begin{array}{l}\text { Failure } \\
\text { Rate }\end{array}$ & $\begin{array}{l}\text { Error } \\
\text { Factor }\end{array}$ \\
\hline VDLOOOUW & $\begin{array}{l}\text { DIESEL DRIVEN FIRE } \\
\text { PUMPS NOT } \\
\text { AVAILABLE }\end{array}$ & $1.0 E-2 / d$ & -5 & & & & & & \\
\hline vo0000uW & $\begin{array}{l}\text { OTHEF BOP } \\
\text { FAILURES }\end{array}$ & $5.1 E-5 / \mathrm{h}$ & 10 & & & & & & \\
\hline VRV161YP & $\begin{array}{l}\text { STEAM HEADER } \\
\text { RELIEF DOES NOT OPEN } \\
\text { B3-VC- } 1610\end{array}$ & $3.0 E-3 / d$ & 5 & . & & & & & \\
\hline VRV $161 Y O$ & $\begin{array}{l}\text { STEAM HEADER RELIE } \\
\text { F DOES NOT REMAIN OP } \\
\text { EN B3-VC-1610 }\end{array}$ & $5.0 E-6 / h$ & 10 & & & & & & \\
\hline
\end{tabular}


Table 7.11. Balance of Plant Data - General Data

PAGE 1

\begin{tabular}{|c|c|c|c|c|c|c|c|c|c|}
\hline \multirow{2}{*}{$\begin{array}{l}\text { EVENT } \\
\text { NAME }\end{array}$} & \multirow[b]{2}{*}{ DESCRIPTION } & \multicolumn{2}{|c|}{ VALUE USED $3 / 91$} & \multicolumn{2}{|c|}{ CRBRP-4 } & \multicolumn{2}{|c|}{ IREP } & \multicolumn{2}{|c|}{ GENERIC } \\
\hline & & $\begin{array}{c}\text { Failure } \\
\text { Rate }\end{array}$ & $\begin{array}{l}\text { Error } \\
\text { Factor }\end{array}$ & $\begin{array}{l}\text { Failure } \\
\text { Rate }\end{array}$ & $\begin{array}{l}\text { Error } \\
\text { Factor }\end{array}$ & $\begin{array}{l}\text { Failure } \\
\text { Rate }\end{array}$ & $\begin{array}{l}\text { Error } \\
\text { Factor }\end{array}$ & $\begin{array}{l}\text { Failure } \\
\text { Rate }\end{array}$ & $\begin{array}{l}\text { Error } \\
\text { Factor }\end{array}$ \\
\hline$B 0001200$ & $\begin{array}{l}\text { BOP Not Available: } \\
\text { Sodium Drained }\end{array}$ & 1.0 & 1 & & & & & & \\
\hline 80002700 & $\begin{array}{l}\text { BALANCE OF PLANT NOT } \\
\text { AVAILABLE FOR LONG } \\
\text { TERM HEAT REMOVAL }\end{array}$ & 1.0 & 1 & & & & & & \\
\hline BO099ZUW & $\begin{array}{l}\text { Operating on Hotel } \\
\text { Mode }\end{array}$ & $2.0 E-3 / d$ & 10 & & & & & & \\
\hline
\end{tabular}


Table 7.12. Electrical Normal Power System Data

PAGE

\begin{tabular}{|c|c|c|c|c|c|c|c|c|c|}
\hline \multirow{2}{*}{$\begin{array}{l}\text { EVENT } \\
\text { NAME }\end{array}$} & \multirow[b]{2}{*}{ DESCAIPTION } & \multicolumn{2}{|c|}{ VALUE USED $3 / 91$} & \multicolumn{2}{|c|}{ CRBAP.4 } & \multicolumn{2}{|c|}{ IREP } & \multicolumn{2}{|c|}{ GENERIC } \\
\hline & & $\begin{array}{c}\text { Failure } \\
\text { Rate }\end{array}$ & $\begin{array}{l}\text { Error } \\
\text { Factor }\end{array}$ & $\begin{array}{l}\text { Failure } \\
\text { Rate }\end{array}$ & $\begin{array}{l}\text { Error } \\
\text { Factor }\end{array}$ & $\begin{array}{c}\text { Failure } \\
\text { Rate }\end{array}$ & $\begin{array}{l}\text { Error } \\
\text { Factor }\end{array}$ & $\begin{array}{l}\text { Failure } \\
\text { Rate }\end{array}$ & $\begin{array}{l}\text { Error } \\
\text { Factor }\end{array}$ \\
\hline JBSOO1OB & Loss of $138 \mathrm{kV}$ Bus & $\begin{array}{c}1.0 \mathrm{E}-4 / \mathrm{h} \\
{[1]}\end{array}$ & 10 & $1.0 \mathrm{E}-8 / \mathrm{h}$ & 3 & $1.0 \mathrm{E}-8 \mathrm{~h}$ & 3 & $1.0 E \cdot 8 / h$ & 3 \\
\hline JBS001ZD & $\begin{array}{l}\text { Overload Fault on } \\
13.8 \mathrm{kV} \text { Bus } 1\end{array}$ & $\begin{array}{c}1.0 \mathrm{E} \cdot 7 / \mathrm{h} \\
{[2]}\end{array}$ & 10 & $\underset{[3]}{1.0 E-7 / h}$ & 10 & $\begin{array}{c}3.0 \mathrm{E} \cdot 7 / \mathrm{h} \\
{[3]}\end{array}$ & 10 & $\begin{array}{c}3.0 \mathrm{E} \cdot 7 \mathrm{~h} \\
{[3]}\end{array}$ & 10 \\
\hline JBS002ZD & $\begin{array}{l}\text { Overload Fault on } \\
13.8 \mathrm{kV} \text { Bus } 2\end{array}$ & $\begin{array}{c}1.0 \mathrm{E}-7 / \mathrm{h} \\
{[2]}\end{array}$ & 10 & $\begin{array}{c}1.0 \mathrm{E}-7 \mathrm{~m} \\
{[3]}\end{array}$ & 10 & $\begin{array}{c}3.0 \mathrm{E}-7 / \mathrm{h} \\
{[3]}\end{array}$ & 10 & $\begin{array}{c}3.0 \mathrm{E}-7 / \mathrm{h} \\
{[3]}\end{array}$ & 10 \\
\hline JBS200YD & $\begin{array}{l}2400 \text { V Bus Overload } \\
\text { Fault }\end{array}$ & $\underset{[2]}{1.0 \mathrm{E} \cdot 7 / \mathrm{h}}$ & 10 & $\begin{array}{c}1.0 \mathrm{E}-7 / \mathrm{h} \\
{[3]}\end{array}$ & 10 & $\begin{array}{c}.0 \mathrm{E}-7 / \mathrm{h} \\
{[3]}\end{array}$ & 10 & $\begin{array}{c}.0 E-7 / \mathrm{h} \\
{[3]}\end{array}$ & 10 \\
\hline JBS480ZU & $\begin{array}{l}480 \text { Vac Normal Bus } \\
\text { No. } 1 \text { and Bus No. } 2 \\
\text { Common Mode Failure }\end{array}$ & $\begin{array}{c}1.0 \mathrm{E}-8 / \mathrm{h} \\
{[4]}\end{array}$ & 10 & & & & & & \\
\hline JBSN1LVC & $\begin{array}{l}\text { Uncleared Overload } \\
\text { on Normal Bus No. } 1 \\
\text { Load Side }\end{array}$ & $\begin{array}{c}1.0 \mathrm{E}-7 / \mathrm{h} \\
{[2]}\end{array}$ & 10 & $\begin{array}{c}1.0 \mathrm{E}-7 / \mathrm{h} \\
{[3]}\end{array}$ & 10 & $\begin{array}{c}3.0 \mathrm{E}-7 / \mathrm{h} \\
{[3]}\end{array}$ & 10 & $\begin{array}{c}3.0 \mathrm{E}-7 \mathrm{~m} \\
{[3]}\end{array}$ & 10 \\
\hline JBSN2LVC & $\begin{array}{l}\text { Uncleared Overload } \\
\text { on Normal Bus No. } 2 \\
\text { Load Side }\end{array}$ & $\begin{array}{c}1.0 E-7 / h \\
{[2]}\end{array}$ & 10 & $\begin{array}{c}1.0 \mathrm{E}-7 / \mathrm{h} \\
{[3]}\end{array}$ & 10 & $\begin{array}{c}3.0 \mathrm{E}-7 / \mathrm{h} \\
{[3]}\end{array}$ & 10 & $\begin{array}{c}3.0 E-7 / \mathrm{h} \\
{[3]}\end{array}$ & 10 \\
\hline JBSP1AYC & $\begin{array}{l}\text { Uncleared Overload } \\
\text { on Normal Bus P-1A }\end{array}$ & $\begin{array}{c}1.0 \mathrm{E}-7 / \mathrm{h} \\
{[2]}\end{array}$ & 10 & $\begin{array}{c}1.0 \mathrm{E}-7 / \mathrm{h} \\
{[3]}\end{array}$ & 10 & $3.0 \underset{[3]}{E-7 / h}$ & 10 & $\begin{array}{c}3.0 E-7 / \mathrm{h} \\
{[3]}\end{array}$ & 10 \\
\hline JBSP2AYC & $\begin{array}{l}\text { Uncleared Overioad } \\
\text { on Normal Bus P.2A }\end{array}$ & $\begin{array}{c}1.0 \varepsilon-7 / \mathrm{h} \\
{[2]}\end{array}$ & 10 & $\begin{array}{c}1.0 \mathrm{E}-7 / \mathrm{h} \\
{[3]}\end{array}$ & 10 & $\begin{array}{c}3.0 \mathrm{E}-7 / \mathrm{h} \\
{[3]}\end{array}$ & 10 & $\begin{array}{c}3.0 \mathrm{E} \cdot 7 / \mathrm{h} \\
{[3]}\end{array}$ & 10 \\
\hline JBSP2BYC & $\begin{array}{l}\text { Uncleared Overload } \\
\text { on Normal Bus P-2B }\end{array}$ & $\begin{array}{c}1.0 E-7 / h \\
{[2]}\end{array}$ & 10 & $\begin{array}{c}1.0 \mathrm{E}-7 / \mathrm{h} \\
{[3]}\end{array}$ & 10 & $\begin{array}{c}3.0 E-7 / \mathrm{h} \\
{[3]}\end{array}$ & 10 & $\begin{array}{c}3.0 E-7 / \mathrm{M} \\
{[3]}\end{array}$ & 10 \\
\hline JBSP3XYC & $\begin{array}{l}\text { Uncleared Overload } \\
\text { on Normal Bus P-3 }\end{array}$ & $\begin{array}{c}1.0 E \cdot 7 / \mathrm{h} \\
{[2]}\end{array}$ & 10 & $\begin{array}{c}1.0 \mathrm{E}-7 / \mathrm{h} \\
{[3]}\end{array}$ & 10 & $\begin{array}{c}3.0 \mathrm{E}-7 / \mathrm{h} \\
{[3]}\end{array}$ & 10 & $\begin{array}{c}3.0 E-7 / \mathrm{h} \\
{[3]}\end{array}$ & 10 \\
\hline JBSR1AYC & $\begin{array}{l}\text { Uncleared Overload } \\
\text { on Normal Bus R-1A }\end{array}$ & $\begin{array}{c}1.0 \mathrm{E}-7 \mathrm{~m} \\
{[2]}\end{array}$ & 10 & $\begin{array}{c}1.0 \mathrm{E}-7 / \mathrm{h} \\
{[3]}\end{array}$ & 10 & $\begin{array}{c}3.0 \mathrm{E}-7 / \mathrm{h} \\
{[3]}\end{array}$ & 10 & $\begin{array}{c}3.0 \mathrm{E}-7 / \mathrm{h} \\
{[3]}\end{array}$ & 10 \\
\hline JBSA3XYC & $\begin{array}{l}\text { Uncleared Overload } \\
\text { on Normal Bus } \mathrm{A}-3\end{array}$ & $\begin{array}{c}1.0 \mathrm{E}-7 / \mathrm{h} \\
{[2]}\end{array}$ & 10 & $\begin{array}{c}1.0 \mathrm{E}-7 / \mathrm{h} \\
{[3]}\end{array}$ & 10 & $\begin{array}{c}3.0 \mathrm{E}-7 / \mathrm{h} \\
{[3]}\end{array}$ & 10 & $\begin{array}{c}3.0 \mathrm{E}-7 / \mathrm{h} \\
{[3]}\end{array}$ & 10 \\
\hline JESSIAYC & $\begin{array}{l}\text { Uncleared Overload } \\
\text { on Normal Bus. S-1A }\end{array}$ & $\begin{array}{c}1.0 \mathrm{E} \cdot 7 / \mathrm{h} \\
{[2]}\end{array}$ & 10 & $\begin{array}{c}1.0 \mathrm{E}-7 / \mathrm{h} \\
{[3]}\end{array}$ & 10 & $\begin{array}{c}3.0 \mathrm{E}-7 / \mathrm{h} \\
{[3]}\end{array}$ & 10 & $\begin{array}{c}3.0 \mathrm{E}-7 \mathrm{~h} \\
{[3]}\end{array}$ & 10 \\
\hline JCA131ZB & $\begin{array}{l}\text { Supply Wiring Fails } \\
\text { Open, Transt. No. } 5\end{array}$ & $\begin{array}{c}1.0 \mathrm{E}-7 / \mathrm{h} \\
{[2]}\end{array}$ & 10 & $1.0 \mathrm{E}-6 / \mathrm{h}$ & 10 & $3.0 \mathrm{E}-6 / \mathrm{h}$ & 10 & $3.0 \mathrm{E}-6 / \mathrm{h}$ & 3 \\
\hline JCA132ZB & $\begin{array}{l}\text { Supply Wiring Fails } \\
\text { Open, Transf. No. } 6\end{array}$ & $\begin{array}{c}1.0 \mathrm{E} \cdot 7 / \mathrm{h} \\
{[2]}\end{array}$ & 10 & $1.0 \mathrm{E}-6 / \mathrm{h}$ & 10 & $3.0 \mathrm{E}-6 / \mathrm{h}$ & 10 & $3.0 \mathrm{E}-6 / \mathrm{h}$ & 3 \\
\hline JCAN1SVC & $\begin{array}{l}\text { Overload Fault } \\
\text { Between Breaker 1CD } \\
\text { and Normal Bus No. } 1\end{array}$ & $\begin{array}{c}1.0 \mathrm{E}-7 / \mathrm{h} \\
{[2]}\end{array}$ & 10 & $\begin{array}{c}1.0 \mathrm{E}-7 / \mathrm{h} \\
{[3]}\end{array}$ & 10 & $\begin{array}{c}3.0 \mathrm{E}-7 / \mathrm{h} \\
{[3]}\end{array}$ & 10 & $\begin{array}{c}3.0 \mathrm{E}-7 \mathrm{~m} \\
{[3]}\end{array}$ & 10 \\
\hline JCAN2SVC & $\begin{array}{l}\text { Overload Fault } \\
\text { Between Breaker 7CD } \\
\text { and Normal Bus No. } 2\end{array}$ & $\begin{array}{c}1.0 \mathrm{E}-7 / \mathrm{h} \\
{[2]}\end{array}$ & 10 & $\begin{array}{c}1.0 E-7 / h \\
{[3]}\end{array}$ & 10 & $\begin{array}{c}3.0 \mathrm{E}-7 / \mathrm{h} \\
{[3]}\end{array}$ & 10 & $\begin{array}{c}3.0 \mathrm{E}-7 \mathrm{~h} \\
{[3]}\end{array}$ & 10 \\
\hline JCAT5OVC & $\begin{array}{l}\text { Overload Fault } \\
\text { Occurs Before } \\
\text { Breaker 1CD }\end{array}$ & $\begin{array}{c}1.0 \mathrm{E}-7 / \mathrm{h} \\
{[2]}\end{array}$ & 10 & $\begin{array}{c}1.0 \mathrm{E} \cdot 7 / \mathrm{h} \\
{[3]}\end{array}$ & 10 & $\begin{array}{c}3.0 \mathrm{E}-7 \mathrm{~h} \\
{[3]}\end{array}$ & 10 & $\begin{array}{c}3.0 \mathrm{E}-7 \mathrm{~m} \\
{[3]}\end{array}$ & 10 \\
\hline JCAT6OVC & $\begin{array}{l}\text { Overload Fault } \\
\text { Occurs Before } \\
\text { Breaker 7CD }\end{array}$ & $\begin{array}{c}1.0 \mathrm{E}-7 / \mathrm{h} \\
{[2]}\end{array}$ & 10 & $\begin{array}{c}1.0 E-7 / h \\
{[3]}\end{array}$ & 10 & $\begin{array}{c}3.0 E-7 / h \\
{[3]}\end{array}$ & 10 & $\begin{array}{c}3.0 \mathrm{E} \cdot 7 / \mathrm{h} \\
{[3]}\end{array}$ & 10 \\
\hline JCB002ZO & $\begin{array}{l}13.8 \text { KV Breaker No. } \\
2 \text { Fails Open }\end{array}$ & $\begin{array}{c}3.0 \mathrm{E}-7 / \mathrm{h} \\
{[2]}\end{array}$ & 10 & $1.0 \mathrm{E} \cdot 5 / \mathrm{h}$ & 10 & $3.0 \mathrm{E} \cdot 5 / \mathrm{d}$ & 10 & $3.0 E-5 / d$ & 3 \\
\hline JCB0132O & $\begin{array}{l}13.8 \text { KV Breaker No. } \\
13 \text { Fails Open }\end{array}$ & $\begin{array}{c}3.0 E-7 / \mathrm{h} \\
{[2]}\end{array}$ & 10 & $1.0 \mathrm{E}-5 / \mathrm{h}$ & 10 & $3.0 E-5 / d$ & 10 & $3.0 E-5 / d$ & 3 \\
\hline $\mathrm{JCBO2CYO}$ & $\begin{array}{l}\text { MCC-P2A Circuit } \\
\text { Breaker 2C Fails } \\
\text { Open }\end{array}$ & $\begin{array}{c}.0 E-7 / \mathrm{h} \\
{[2]}\end{array}$ & 10 & $1.0 \mathrm{E}-5 / \mathrm{h}$ & 10 & $3.0 E-5 / d$ & 10 & $3.0 E-5 / d$ & 3 \\
\hline JCBO3BVO & $\begin{array}{l}\text { Normal Bus No. } 1 \\
\text { Breaker } 38 \text { Fails } \\
\text { Open }\end{array}$ & $\begin{array}{c}3.0 \mathrm{E}-7 / \mathrm{h} \\
{[2]}\end{array}$ & 10 & $1.0 \mathrm{E}-5 / \mathrm{h}$ & 10 & $3.0 \mathrm{E}-5 / \mathrm{d}$ & 10 & $3.0 E-5 / d$ & 3 \\
\hline
\end{tabular}


Table 7.12. Electrical Normal Power System Data (Cont'd)

PAGE 2

\begin{tabular}{|c|c|c|c|c|c|c|c|c|c|}
\hline \multirow{2}{*}{$\begin{array}{l}\text { EVENT } \\
\text { NAME }\end{array}$} & \multirow[b]{2}{*}{ DESCRIPTION } & \multicolumn{2}{|c|}{ VALUE USED 3/91 } & \multicolumn{2}{|c|}{ CABRP-4 } & \multicolumn{2}{|c|}{ IREP } & \multicolumn{2}{|c|}{ GENERIC } \\
\hline & & $\begin{array}{c}\text { Failure } \\
\text { Rate } \\
\end{array}$ & $\begin{array}{l}\text { Error } \\
\text { Factor }\end{array}$ & $\begin{array}{c}\text { Failure } \\
\text { Rate }\end{array}$ & $\begin{array}{l}\text { Error } \\
\text { Factor }\end{array}$ & $\begin{array}{c}\text { Failure } \\
\text { Rate } \\
\end{array}$ & $\begin{array}{l}\text { Error } \\
\text { Factor }\end{array}$ & $\begin{array}{c}\text { Failure } \\
\text { Rate }\end{array}$ & $\begin{array}{l}\text { Error } \\
\text { Factor }\end{array}$ \\
\hline JcBoscyo & $\begin{array}{l}\text { Normal Bus No. } 2 \\
\text { Breaker 5C Fails } \\
\text { Open }\end{array}$ & $\begin{array}{c}3.0 \mathrm{E}-7 \mathrm{~h} \\
{[2]}\end{array}$ & 10 & $1.0 \mathrm{E}-5 / \mathrm{h}$ & 10 & $3.0 \mathrm{E}-5 / \mathrm{d}$ & 10 & $3.0 \mathrm{E}-5 / \mathrm{d}$ & 3 \\
\hline JCB11AYO & $\begin{array}{l}\text { Breaker } 11 \mathrm{~A} \text { of } \mathrm{MCC} \\
\text { P2B Fails Open }\end{array}$ & $\begin{array}{c}\text { 3.0E-7/h } \\
{[2]}\end{array}$ & 10 & $1.0 \mathrm{E}-5 / \mathrm{h}$ & 10 & $3.0 \mathrm{E}-5 / \mathrm{d}$ & 10 & $3.0 \mathrm{E}-5 / \mathrm{d}$ & 3 \\
\hline JCB11CYO & $\begin{array}{l}\text { MCC-P2B Circuit } \\
\text { Breaker } 11 C \text { Fails } \\
\text { Open }\end{array}$ & $\begin{array}{c}3.0 \mathrm{E} \cdot 7 / \mathrm{h} \\
{[2]}\end{array}$ & 10 & $1.0 \mathrm{E}-5 / \mathrm{h}$ & 10 & $3.0 \mathrm{E}-5 / \mathrm{d}$ & 10 & $3.0 \mathrm{E}-5 / \mathrm{d}$ & 3 \\
\hline JCB12AYO & $\begin{array}{l}\text { Normal Bus P-2B } \\
\text { Breaker } 12 \mathrm{~A} \text { Fails } \\
\text { Open }\end{array}$ & $\underset{[2]}{3.0 E \cdot 7 / \mathrm{h}}$ & 10 & $1.0 \mathrm{E} \cdot 5 / \mathrm{h}$ & 10 & $3.0 \mathrm{E}-5 / \mathrm{d}$ & 10 & $3.0 \mathrm{E}-5 / \mathrm{d}$ & 10 \\
\hline JCB1A1YO & $\begin{array}{l}\text { Normal Bus R-1A } \\
\text { Breaker } 1 \text { A Fails } \\
\text { Open }\end{array}$ & $\begin{array}{c}3.0 \mathrm{E} \cdot 7 / \mathrm{h} \\
{[2]}\end{array}$ & 10 & $1.0 \mathrm{E}-5 / \mathrm{h}$ & 10 & $3.0 \mathrm{E}-5 / \mathrm{d}$ & 10 & $3.0 \mathrm{E}-5 / \mathrm{d}$ & 10 \\
\hline JCB1A2YO & $\begin{array}{l}\text { Normal Bus P-2A } \\
\text { Breaker 1A Fails } \\
\text { Open }\end{array}$ & $\underset{[2]}{3.0 \mathrm{E}-7 \mathrm{~m}}$ & 10 & $1.0 \mathrm{E}-5 / \mathrm{h}$ & 10 & $3.0 \mathrm{E}-5 / \mathrm{d}$ & 10 & $3.0 \mathrm{E}-5 / \mathrm{d}$ & 10 \\
\hline JCB1A3YO & $\begin{array}{l}\text { Normal Bus P-1A } \\
\text { Breaker 1A Fails } \\
\text { Open }\end{array}$ & $\begin{array}{c}3.0 \mathrm{E}-7 \mathrm{~m} \\
{[2]}\end{array}$ & 10 & $1.0 \mathrm{E}-5 / \mathrm{h}$ & 10 & $3.0 \mathrm{E}-5 / \mathrm{d}$ & 10 & $3.0 \mathrm{E}-5 / \mathrm{d}$ & 10 \\
\hline JCB1A4YO & $\begin{array}{l}\text { Normal Bus R-3 } \\
\text { Breaker 1A Fails } \\
\text { Open }\end{array}$ & $\begin{array}{c}3.0 \mathrm{E}-7 \mathrm{~m} \\
{[2] \cdot}\end{array}$ & 10 & $1.0 \mathrm{E}-5 \mathrm{~h}$ & 10 & $3.0 \mathrm{E}-5 / \mathrm{d}$ & 10 & $3.0 \mathrm{E}-5 / \mathrm{d}$ & 10 \\
\hline JCB1CDVO & $\begin{array}{l}480 \text { Vac Breaker } 1 \mathrm{CD} \\
\text { Fails Open }\end{array}$ & $\begin{array}{c}3.0 \mathrm{E}-7 / \mathrm{h} \\
{[2]}\end{array}$ & 10 & $1.0 \mathrm{E}-5 / \mathrm{h}$ & 10 & $3.0 \mathrm{E}-5 / \mathrm{d}$ & 10 & $3.0 \mathrm{E}-5 / \mathrm{d}$ & 3 \\
\hline JCB1CDVP & $\begin{array}{l}\text { Breaker } 1 C D \text { Fails to } \\
\text { Open on Demand }\end{array}$ & $\begin{array}{c}5.0 \mathrm{E}-4 / \mathrm{d} \\
{[2]}\end{array}$ & 10 & $1.0 \mathrm{E}-3 / \mathrm{d}$ & 10 & $3.0 \mathrm{E}-3 / \mathrm{d}$ & 10 & $3.0 \mathrm{E}-3 / \mathrm{d}$ & 10 \\
\hline JC81D1YO & $\begin{array}{l}\text { Normal Bus S-1A } \\
\text { Breaker } 10 \text { Fails } \\
\text { Open }\end{array}$ & $\begin{array}{c}3.0 \mathrm{E}-7 \mathrm{~h} \\
{[2]}\end{array}$ & 10 & $1.0 \mathrm{E}-5 / \mathrm{h}$ & 10 & $3.0 \mathrm{E}-5 / \mathrm{d}$ & 10 & $3.0 \mathrm{E}-5 / \mathrm{d}$ & 10 \\
\hline JC82B1VO & $\begin{array}{l}\text { Normal Bus No. } 1 \\
\text { Breaker } 28 \text { Fails } \\
\text { Open }\end{array}$ & $\begin{array}{c}3.0 \mathrm{E}-7 / \mathrm{h} \\
{[2]}\end{array}$ & 10 & $1.0 \mathrm{E}-5 / \mathrm{h}$ & 10 & $3.0 \mathrm{E}-5 / \mathrm{d}$ & 10 & $3.0 \mathrm{E}-5 / \mathrm{d}$ & 3 \\
\hline JCB2C1VO & $\begin{array}{l}\text { Normal Bus No. } 1 \\
\text { Breaker 2C Fails } \\
\text { Open }\end{array}$ & $\begin{array}{c}3.0 \mathrm{E}-7 \mathrm{~m} \\
{[2]}\end{array}$ & 10 & $1.0 \mathrm{E}-5 / \mathrm{h}$ & 10 & $3.0 \mathrm{E}-5 / \mathrm{d}$ & 10 & $3.0 \mathrm{E}-5 / \mathrm{d}$ & 3 \\
\hline JCB2C1YO & $\begin{array}{l}\text { Normal Bus P-3 } \\
\text { Breaker 2C Fails } \\
\text { Open }\end{array}$ & $\begin{array}{c}3.0 \mathrm{E}-7 \mathrm{~h} \\
{[2]}\end{array}$ & 10 & $1.0 \mathrm{E}-5 / \mathrm{h}$ & 10 & $3.0 \mathrm{E} \cdot 5 / \mathrm{d}$ & 10 & $3.0 \mathrm{E}-5 / \mathrm{d}$ & 10 \\
\hline JCB2D1VO & $\begin{array}{l}\text { Normal Bus No. } 1 \\
\text { Breaker } 20 \text { Fails } \\
\text { Open }\end{array}$ & $\begin{array}{c}3.0 \mathrm{E}-7 / \mathrm{h} \\
{[2]}\end{array}$ & 10 & $1.0 \mathrm{E}-5 / \mathrm{h}$ & 10 & $3.0 \mathrm{E}-5 / \mathrm{d}$ & 10 & $3.0 \mathrm{E}-5 / \mathrm{d}$ & 3 \\
\hline JC83C1Vo & $\begin{array}{l}\text { Normal Bus No. } 1 \\
\text { Breaker 3C Fails } \\
\text { Open }\end{array}$ & $\begin{array}{c}3.0 \mathrm{E}-7 / \mathrm{h} \\
{[2]}\end{array}$ & 10 & $1.0 \mathrm{E}-5 / \mathrm{h}$ & 10 & $3.0 \mathrm{E}-5 / \mathrm{d}$ & 10 & $3.0 \mathrm{E}-5 / \mathrm{d}$ & 3 \\
\hline JCB4BCVO & $\begin{array}{l}\text { Tie Breaker } 4 B C \\
\text { Fails Open }\end{array}$ & $\begin{array}{c}3.0 \mathrm{E}-7 \mathrm{~h} \\
{[2]}\end{array}$ & 10 & $1.0 \mathrm{E}-5 / \mathrm{h}$ & 10 & $3.0 \mathrm{E}-5 / \mathrm{d}$ & 10 & $3.0 \mathrm{E}-5 / \mathrm{d}$ & 3 \\
\hline JCBABCVP & $\begin{array}{l}\text { Breaker } 4 B C \text { Fails to } \\
\text { Open on Demand }\end{array}$ & $\begin{array}{c}5.0 \mathrm{E}-4 / \mathrm{d} \\
{[2]}\end{array}$ & 10 & $1.0 \mathrm{E}-3 / \mathrm{d}$ & 10 & $3.0 \mathrm{E}-3 / \mathrm{d}$ & 10 & $3.0 \mathrm{E}-3 / \mathrm{d}$ & 10 \\
\hline JCB5B1VO & $\begin{array}{l}\text { Normal Bus No. } 2 \\
\text { Breaker 5B Fails } \\
\text { Open }\end{array}$ & $\begin{array}{c}3.0 \mathrm{E}-7 / \mathrm{h} \\
{[2]}\end{array}$ & 10 & $1.0 \mathrm{E}-5 / \mathrm{h}$ & 10 & $3.0 \mathrm{E}-5 / \mathrm{d}$ & 10 & $3.0 \mathrm{E}-5 / \mathrm{d}$ & 3 \\
\hline JC86C1VO & $\begin{array}{l}\text { Normal Bus No. } 2 \\
\text { Breaker } 6 \mathrm{C} \text { Fails } \\
\text { Open }\end{array}$ & $\underset{[2]}{3.0 \mathrm{E}-7 \mathrm{~h}}$ & 10 & $1.0 \mathrm{E}-5 / \mathrm{h}$ & 10 & $3.0 E-5 / d$ & 10 & $3.0 \mathrm{E}-5 / \mathrm{d}$ & 3 \\
\hline JCB7CDVO & $\begin{array}{l}480 \text { Vac } 8 \text { reaker } 7 C D \\
\text { Fails Open }\end{array}$ & $\begin{array}{c}3.0 E-7 / \mathrm{h} \\
{[2]}\end{array}$ & 10 & $1.0 \mathrm{E}-5 / \mathrm{h}$ & 10 & $3.0 \mathrm{E}-5 / \mathrm{d}$ & 10 & $3.0 E-5 / \mathrm{d}$ & 3 \\
\hline JCB7CDVP & $\begin{array}{l}\text { Breaker 7CD Fails to } \\
\text { Open on Demand }\end{array}$ & $\begin{array}{c}5.0 E \cdot 4 / \mathrm{d} \\
{[2]}\end{array}$ & 10 & $1.0 \mathrm{E}-3 / \mathrm{d}$ & 10 & $3.0 \mathrm{E}-3 / \mathrm{d}$ & 10 & $3.0 E-3 / d$ & 10 \\
\hline
\end{tabular}


Table 7.12. Electrical Normal Power System Data (Cont'd)

PAGE 3

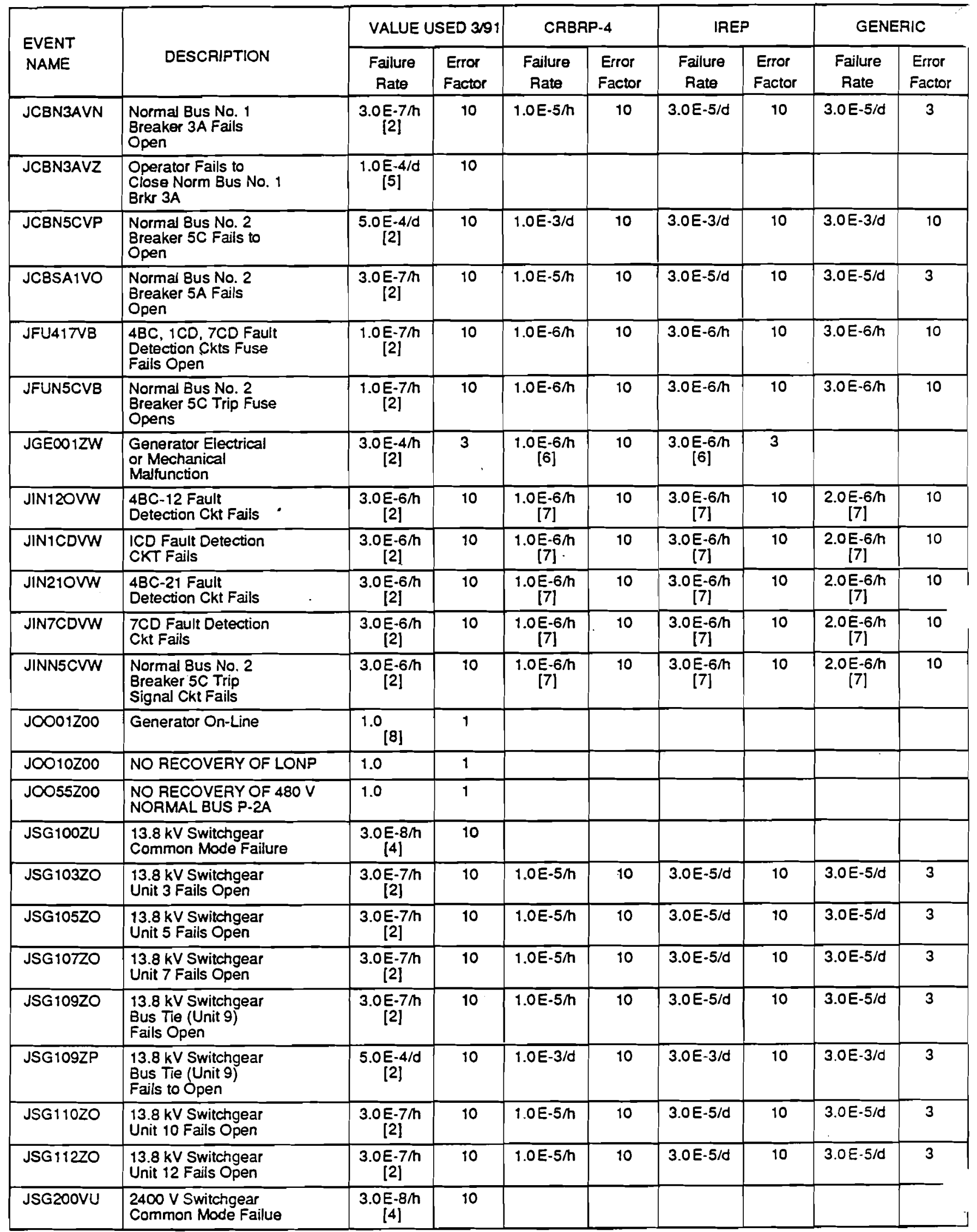


Table 7.12. Electrical Normal Power System Data (Cont'd)

PAGE 4

\begin{tabular}{|c|c|c|c|c|c|c|c|c|c|}
\hline \multirow{2}{*}{$\begin{array}{l}\text { EVENT } \\
\text { NAME }\end{array}$} & \multirow[b]{2}{*}{ DESCRIPTION } & \multicolumn{2}{|c|}{ VALUE USED $3 / 91$} & \multicolumn{2}{|c|}{ CRBRP-4 } & \multicolumn{2}{|c|}{ IREP } & \multicolumn{2}{|c|}{ GENERIC } \\
\hline & & $\begin{array}{l}\text { Failure } \\
\text { Rate }\end{array}$ & $\begin{array}{l}\text { Error } \\
\text { Factor }\end{array}$ & $\begin{array}{l}\text { Failure } \\
\text { Rate }\end{array}$ & $\begin{array}{l}\text { Error } \\
\text { Factor }\end{array}$ & $\begin{array}{l}\text { Failure } \\
\text { Rate }\end{array}$ & $\begin{array}{l}\text { Error } \\
\text { Factor }\end{array}$ & $\begin{array}{c}\text { Failure } \\
\text { Rate }\end{array}$ & $\begin{array}{l}\text { Error } \\
\text { Factor }\end{array}$ \\
\hline JSG201VO & $\begin{array}{l}\text { MG Set } 1 \text { Input } \\
\text { Breaker Fails Open }\end{array}$ & $\begin{array}{c}3.0 \mathrm{E}-7 / \mathrm{h} \\
{[9]}\end{array}$ & 10 & $1.0 \mathrm{E}-5 / \mathrm{h}$ & 10 & $3.0 E-5 / d$ & 10 & $3.0 E-5 / d$ & 3 \\
\hline JSG2O2VO & $\begin{array}{l}\text { MG Set } 2 \text { Input } \\
\text { Breaker Fails Open }\end{array}$ & $\begin{array}{c}3.0 \mathrm{E}-7 \mathrm{~h} \\
{[9]}\end{array}$ & 10 & $1.0 \mathrm{E}-5 / \mathrm{h}$ & 10 & $3.0 \mathrm{E}-5 / \mathrm{d}$ & 10 & $3.0 \mathrm{E}-5 / \mathrm{d}$ & 3 \\
\hline JSG203VO & $\begin{array}{l}2400 \text { V Switchgear } \\
\text { Unit } 3 \text { Fails Open }\end{array}$ & $\begin{array}{c}3.0 \mathrm{E}-7 \mathrm{~h} \\
{[2]}\end{array}$ & 10 & $1.0 \mathrm{E} \cdot 5 / \mathrm{h}$ & 10 & $3.0 \mathrm{E}-5 / \mathrm{d}$ & 10 & $3.0 \mathrm{E}-5 / \mathrm{d}$ & 3 \\
\hline JSG209VO & $\begin{array}{l}2400 \text { V Switchgear } \\
\text { Unit } 9 \text { Fails Open }\end{array}$ & $\begin{array}{c}3.0 \mathrm{E}-7 \mathrm{~h} \\
{[2]}\end{array}$ & 10 & $1.0 \mathrm{E}-5 / \mathrm{h}$ & 10 & $3.0 \mathrm{E}-5 / \mathrm{d}$ & 10 & $3.0 \mathrm{E}-5 / \mathrm{d}$ & 3 \\
\hline JSG212VU & $\begin{array}{l}\text { MG Set } 1 \text { and } 2 \text { Input } \\
\text { Breaker Fails Open, } \\
\text { Common Cause }\end{array}$ & $\begin{array}{c}3.0 \mathrm{E}-8 / \mathrm{h} \\
{[4]}\end{array}$ & 10 & & & & & & \\
\hline JSG221VU & $\begin{array}{l}\text { MG Set } 2 \text { Input } \\
\text { Breaker Fails Open, } \\
\text { Common Cause }\end{array}$ & $\begin{array}{c}3.0 \mathrm{E}-8 / \mathrm{h} \\
{[4]}\end{array}$ & 10 & & & & & & \\
\hline JTRO010W & $\begin{array}{l}138 / 13.8 \mathrm{kV} \\
\text { Transformer } 1 \text { Fails }\end{array}$ & $\begin{array}{c}1.0 \mathrm{E}-6 / \mathrm{h} \\
{[2]}\end{array}$ & 10 & $1.0 \mathrm{E}-6 / \mathrm{h}$ & 3 & $1.0 E-6 / \mathrm{h}$ & 3 & $1.0 \mathrm{E}-6 / \mathrm{h}$ & 3 \\
\hline JTR002OW & $\begin{array}{l}138 / 13.8 \mathrm{kV} \\
\text { Transformer } 2 \text { Fails }\end{array}$ & $\begin{array}{c}1.0 \mathrm{E}-6 / \mathrm{h} \\
{[2]}\end{array}$ & 10 & $1.0 \mathrm{E}-6 / \mathrm{h}$ & 3 & $1.0 E-6 / h$ & 3 & $1.0 \mathrm{E}-6 / \mathrm{h}$ & 3 \\
\hline JTRO03OW & $\begin{array}{l}13.8 / 2.4 \mathrm{kV} \\
\text { Transformer } 3 \text { Fails }\end{array}$ & $\begin{array}{c}1.0 \mathrm{E}-6 / \mathrm{h} \\
{[2]}\end{array}$ & 10 & $1.0 \mathrm{E}-6 / \mathrm{h}$ & 3 & $1.0 \mathrm{E}-6 / \mathrm{h}$ & 3 & $1.0 \mathrm{E}-6 / \mathrm{h}$ & 3 \\
\hline JTR004OW & $\begin{array}{l}13.8 / 2.4 \mathrm{kV} \\
\text { Transformer } 4 \text { Fails }\end{array}$ & $\begin{array}{c}1.0 \mathrm{E}-6 / \mathrm{h} \\
{ }_{[2]}\end{array}$ & 10 & $1.0 \mathrm{E}-6 / \mathrm{h}$ & 3 & $1.0 \mathrm{E}-6 / \mathrm{h}$ & 3 & $1.0 E-6 / \mathrm{h}$ & 3 \\
\hline JTROO5OB & $\begin{array}{l}\text { Transf. No. } 5 \text { Fails } \\
\text { Open }\end{array}$ & $\begin{array}{c}1.0 \mathrm{E}-6 / \mathrm{h} \\
{[2]}\end{array}$ & 10 & $1.0 E-6 / \mathrm{h}$ & 3 & $1.0 \mathrm{E}-6 / \mathrm{h}$ & 3 & $1.0 \mathrm{E}-6 / \mathrm{h}$ & 3 \\
\hline JTROOSOB & $\begin{array}{l}\text { Transt. No. } 6 \text { Fails } \\
\text { Open }\end{array}$ & $\begin{array}{c}1.0 E-6 / h \\
{[2]}\end{array}$ & 10 & $1.0 \mathrm{E}-6 / \mathrm{h}$ & 3 & $1.0 \mathrm{E}-6 / \mathrm{h}$ & 3 & $1.0 \mathrm{E}-6 / \mathrm{h}$ & 3 \\
\hline JTR012OU & $\begin{array}{l}138 / 13.8 \mathrm{kV} \\
\text { Transformers Common } \\
\text { Mode Failure }\end{array}$ & $\begin{array}{c}1.0 \mathrm{E}-7 \mathrm{~h} \\
{[4]}\end{array}$ & 10 & & & & & & \\
\hline JTRO34OU & $\begin{array}{l}13.8 / 2.4 \mathrm{kV} \\
\text { Transformers Common } \\
\text { Mode Failure }\end{array}$ & $\begin{array}{c}1.0 \mathrm{E}-7 / \mathrm{h} \\
{[4]}\end{array}$ & 10 & & & & & & \\
\hline JTRT560U & $\begin{array}{l}13.8 \mathrm{KV} / 480 \text { Vac } \\
\text { Transf } 5 \text { and } 6 \\
\text { Common Mode Failure }\end{array}$ & $\begin{array}{c}1.0 \mathrm{E}-7 \mathrm{~m} \\
{[4]}\end{array}$ & 10 & & & & & & \\
\hline JWROOOOU & $\begin{array}{l}138 \mathrm{kV} \text { Feed Wires } \\
\text { Common Mode Failure }\end{array}$ & $\begin{array}{c}1.0 \mathrm{E}-8 / \mathrm{h} \\
{[4]}\end{array}$ & 10 & & & & & & \\
\hline JWR001OB & $\begin{array}{l}138 \mathrm{kV} \text { Feed Wire to } \\
\text { Transformer } 1 \text { Fails }\end{array}$ & $\begin{array}{c}1.0 \mathrm{E}-7 \mathrm{~h} \\
{[2]}\end{array}$ & 10 & $\begin{array}{c}1.0 \mathrm{E}-6 \mathrm{~h} \\
{[10]}\end{array}$ & 10 & $\begin{array}{c}3.0 \mathrm{E}-6 / \mathrm{h} \\
{[10]}\end{array}$ & 10 & $\begin{array}{c}\begin{array}{c}3.0 E-6 / \mathrm{h} \\
{[10]}\end{array} \\
\end{array}$ & 3 \\
\hline JWR0O2OB & $\begin{array}{l}138 \text { kV Feed Wire to } \\
\text { Transformer } 2 \text { Fails }\end{array}$ & $\begin{array}{c}1.0 \mathrm{E}-7 \mathrm{~h} \\
{[2]}\end{array}$ & 10 & $\begin{array}{c}1.0 E-6 / \mathrm{h} \\
{[10]}\end{array}$ & 10 & $\begin{array}{c}3.0 \mathrm{E}-6 / \mathrm{h} \\
{[10]}\end{array}$ & 10 & $\begin{array}{c}.0 E-6 / \mathrm{h} \\
{[10]}\end{array}$ & 3 \\
\hline JWR100OU & $\begin{array}{l}\text { 13.8 kV Feed Wires } \\
\text { to Switchgear Common } \\
\text { Mode Failure }\end{array}$ & $\begin{array}{c}1.0 \mathrm{E}-8 / \mathrm{h} \\
{[4]}\end{array}$ & 10 & & & & & & \\
\hline JWR103ZB & $\begin{array}{l}13.8 \mathrm{kV} \text { Feed Wire to } \\
\text { Transformer } 3 \text { Fails }\end{array}$ & $\begin{array}{c}1.0 \mathrm{E}-7 / \mathrm{h} \\
{[2]}\end{array}$ & 10 & $\begin{array}{c}1.0 \mathrm{E}-6 / \mathrm{h} \\
{[10]}\end{array}$ & 10 & $\begin{array}{c}3.0 \mathrm{E}-6 / \mathrm{h} \\
{[10]}\end{array}$ & 10 & $\begin{array}{c}3.0 \mathrm{E}-6 / \mathrm{h} \\
{[10]}\end{array}$ & 3 \\
\hline JWR1050B & $\begin{array}{l}\text { 13.8 kV Feed Wire to } \\
\text { Switchgear Unit } 5 \\
\text { Fails }\end{array}$ & $\begin{array}{c}1.0 \mathrm{E}-7 \mathrm{~h} \\
{[2]}\end{array}$ & 10 & $\begin{array}{c}1.0 \mathrm{E}-6 \mathrm{~h} \\
{[10]}\end{array}$ & 10 & $\begin{array}{c}3.0 \mathrm{E}-6 \mathrm{~h} \\
{[10]}\end{array}$ & 10 & $\begin{array}{c}3.0 \mathrm{E}-6 / \mathrm{h} \\
{[10]}\end{array}$ & 3 \\
\hline JWR107OB & $\begin{array}{l}\text { Feed Wire to } 13.8 \mathrm{kV} \\
\text { Switchgear Unit } 7 \\
\text { Fails }\end{array}$ & $\begin{array}{c}1.0 \mathrm{E} \cdot 7 \mathrm{~h} \\
{[2]}\end{array}$ & 10 & $\begin{array}{c}1.0 E-6 / h \\
{[10]}\end{array}$ & 10 & $\begin{array}{c}3.0 \mathrm{E}-6 / \mathrm{h} \\
{[10]}\end{array}$ & 10 & $\begin{array}{c}3.0 \mathrm{E}-6 / \mathrm{h} \\
{[10]}\end{array}$ & 3 \\
\hline JWR1 $100 B$ & $\begin{array}{l}\text { 13.8 kV Feed Wire to } \\
\text { Switchgear Unit } 10 \\
\text { Fails }\end{array}$ & $\begin{array}{c}1.0 \mathrm{E}-7 / \mathrm{h} \\
{[2]}\end{array}$ & 10 & $\begin{array}{c}1.0 \mathrm{E}-6 / \mathrm{h} \\
{[10]}\end{array}$ & 10 & $\begin{array}{c}3.0 \mathrm{E}-6 / \mathrm{h} \\
{[10]}\end{array}$ & 10 & $\begin{array}{c}3.0 \mathrm{E}-6 / \mathrm{h} \\
{[10]}\end{array}$ & 3 \\
\hline JWR112ZB & $\begin{array}{l}13.8 \mathrm{kV} \text { Feed Wire to } \\
\text { Transformer } 4 \text { Fails }\end{array}$ & $\begin{array}{l}1.0 E-7 / \mathrm{h} \\
{[2]}\end{array}$ & 10 & $\begin{array}{c}1.0 \mathrm{E}-6 / \mathrm{h} \\
{[10]}\end{array}$ & 10 & $\begin{array}{c}\text { 3.0E-6/h } \\
{[10]}\end{array}$ & 10 & $\begin{array}{c}3.0 \mathrm{E}-6 / \mathrm{h} \\
{[10]}\end{array}$ & 3 \\
\hline
\end{tabular}


Table 7.12. Electrical Normal Power System Data (Cont'd)

PAGE 5

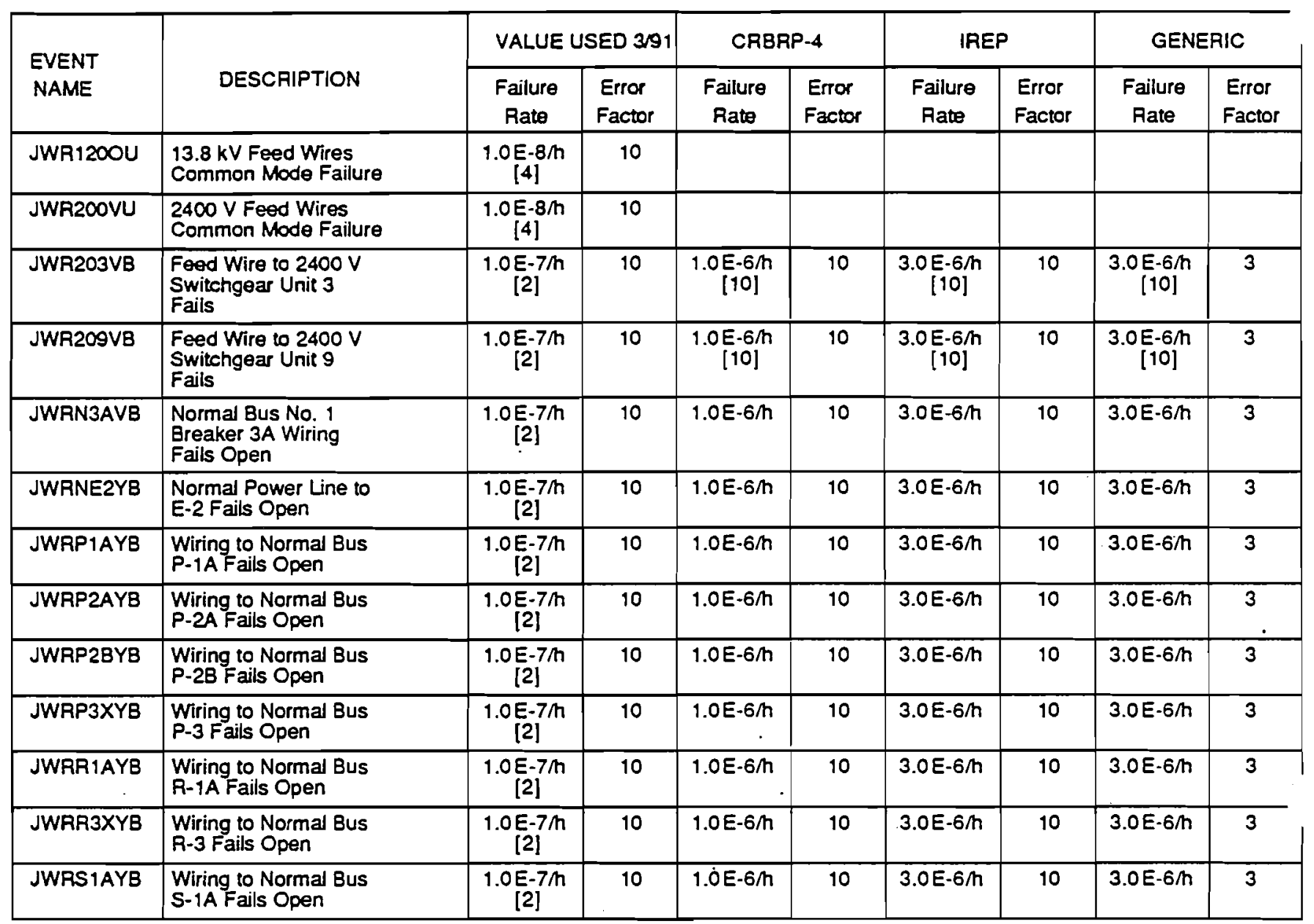

Notes: 1. Estimate based on loss of off-site power, $\sim$ once per year

2. Recommended value tables, EGG-SSRE-8875, Feb. 1990

3. Short to ground value

4. $0.1 \times$ individual component value

5. Est. value; time to perform action, lack of action obvious

6. DC motor-generator failure value

7. Low power solid state device value

8. Assumed on-line when malfunction occurs

9. Recommended value tables, EEG-SSRE-8875, Feb. 1990

10. Open circuit value 
Table 7.13. Electrical Emergency Power System Data

PAGE 1

\begin{tabular}{|c|c|c|c|c|c|c|c|c|c|}
\hline \multirow{2}{*}{$\begin{array}{l}\text { EVENT } \\
\text { NAME }\end{array}$} & \multirow[b]{2}{*}{ DESCAIPTION } & \multicolumn{2}{|c|}{ VALUE USED $3 / 91$} & \multicolumn{2}{|c|}{ CRBRP.4 } & \multicolumn{2}{|c|}{ IREP } & \multicolumn{2}{|c|}{ GENERIC } \\
\hline & & $\begin{array}{l}\text { Failure } \\
\text { Rate }\end{array}$ & $\begin{array}{l}\text { Error } \\
\text { Factor }\end{array}$ & $\begin{array}{l}\text { Failure } \\
\text { Rate }\end{array}$ & $\begin{array}{l}\text { Error } \\
\text { Factor }\end{array}$ & $\begin{array}{l}\text { Failure } \\
\text { Rate }\end{array}$ & $\begin{array}{l}\text { Error } \\
\text { Factor }\end{array}$ & $\begin{array}{c}\text { Failure } \\
\text { Rate }\end{array}$ & $\begin{array}{l}\text { Error } \\
\text { Factor }\end{array}$ \\
\hline EBSO01UW & $\begin{array}{l}\text { NO POWER AT OUTPUT } \\
\text { OF BREAKER MCC EI } 1 \mathrm{~B}\end{array}$ & $\begin{array}{c}1.6 \mathrm{E}-6 / \mathrm{h} \\
{[1]}\end{array}$ & 3 & & & & & & \\
\hline EBSOO2UW & $\begin{array}{l}\text { NO POWER AT OUTPUT } \\
\text { OF BREAKER MCC E2 } 18\end{array}$ & $\begin{array}{c}1.6 E-6 / h \\
{[1]}\end{array}$ & 3 & . & & & & & \\
\hline EBSE11YC & $\begin{array}{l}\text { Uncleared Overload } \\
\text { on Emergency Bus E-1 }\end{array}$ & $\begin{array}{c}1.0 \mathrm{E}-7 / \mathrm{h} \\
{[2]}\end{array}$ & 10 & $\begin{array}{c}1.0 \mathrm{E}-7 / \mathrm{h} \\
{[3]}\end{array}$ & 10 & $\begin{array}{c}3.0 \mathrm{E}-7 / \mathrm{h} \\
{[3]}\end{array}$ & 10 & $\begin{array}{c}\text { 3.0 E-7/h } \\
{[3]}\end{array}$ & 10 \\
\hline EBSEE2YC & $\begin{array}{l}\text { Uncleared Overload } \\
\text { on Emergency Bus E-2 }\end{array}$ & $\begin{array}{c}1.0 \mathrm{E} \cdot 7 / \mathrm{h} \\
{[2]}\end{array}$ & 10 & $\begin{array}{c}1.0 \mathrm{E}-7 \mathrm{~m} \\
{[3]}\end{array}$ & 10 & $\begin{array}{c}3.0 \mathrm{E}-7 / \mathrm{h} \\
{[3]}\end{array}$ & 10 & $\begin{array}{c}3.0 \mathrm{E} \cdot 7 / \mathrm{h} \\
{[3]}\end{array}$ & 10 \\
\hline EBSP1BYC & $\begin{array}{l}\text { Uncleared Overload } \\
\text { on Emergency Bus } \\
P \cdot 1 B\end{array}$ & $\begin{array}{c}1.0 \mathrm{E}-7 / \mathrm{h} \\
{[2]}\end{array}$ & 10 & $\begin{array}{c}1.0 \mathrm{E} \cdot 7 / \mathrm{h} \\
{[3]}\end{array}$ & 10 & $\begin{array}{c}3.0 \mathrm{E} \cdot 7 / \mathrm{h} \\
{[3]}\end{array}$ & 10 & $\begin{array}{c}3.0 \mathrm{E}-7 / \mathrm{h} \\
{[3]}\end{array}$ & 10 \\
\hline EBSP4EYC & $\begin{array}{l}\text { Uncleared Oveload } \\
\text { Fault on Bus P-4E }\end{array}$ & $\begin{array}{c}1.0 E-7 / \mathrm{h} \\
{[2]}\end{array}$ & 10 & $\begin{array}{c}1.0 \mathrm{E} \cdot 7 / \mathrm{h} \\
{[3]}\end{array}$ & 10 & $\begin{array}{c}3.0 \mathrm{E}-7 / \mathrm{h} \\
{[3]}\end{array}$ & 10 & $\begin{array}{c}3.0 \mathrm{E}-7 / \mathrm{h} \\
{[3]}\end{array}$ & 10 \\
\hline EBSR1BYC & $\begin{array}{l}\text { Uncleared Overload } \\
\text { on Emergency Bus } \\
\text { R-1B }\end{array}$ & $\begin{array}{c}1.0 \mathrm{E}-7 \mathrm{~m} \\
{[2]}\end{array}$ & 10 & $\begin{array}{c}1.0 \mathrm{E}-7 / \mathrm{h} \\
{[3]}\end{array}$ & 10 & $\begin{array}{c}3.0 E-7 / \mathrm{m} \\
{[3]}\end{array}$ & 10 & $\begin{array}{c}3.0 \mathrm{E}-7 / \mathrm{h} \\
{[3]}\end{array}$ & 10 \\
\hline EBSA2AYC & $\begin{array}{l}\text { Uncleared Overload } \\
\text { on Emergency Bus } \\
\text { R-2A }\end{array}$ & $\begin{array}{c}1.0 \mathrm{E}-7 / \mathrm{h} \\
{[2]}\end{array}$ & 10 & $\begin{array}{c}1.0 \mathrm{E} \cdot 7 / \mathrm{h} \\
{[3]}\end{array}$ & 10 & $\underset{[3]}{3.0 E-7 / \mathrm{h}}$ & 10 & $\begin{array}{c}3.0 \mathrm{E}-7 / \mathrm{h} \\
{[3]}\end{array}$ & 10 \\
\hline EBSR2BYC & $\begin{array}{l}\text { Uncleared Overload } \\
\text { on Emergency Bus } \\
\mathrm{R}-2 \mathrm{~B}\end{array}$ & $\begin{array}{c}1.0 \mathrm{E} \cdot 7 \mathrm{~h} \\
{[2]}\end{array}$ & 10 & $\begin{array}{c}1.0 \mathrm{E}-7 \mathrm{~m} \\
{[3]}\end{array}$ & 10 & $\begin{array}{c}3.0 \mathrm{E}-7 / \mathrm{h} \\
{[3]}\end{array}$ & 10 & $\begin{array}{c}3.0 \mathrm{E}-7 / \mathrm{h} \\
{[3]}\end{array}$ & 10 \\
\hline EBSS1BYC & $\begin{array}{l}\text { Uncleared Overload } \\
\text { on Emergency Bus } \\
S-1 B\end{array}$ & $\begin{array}{c}1.0 \mathrm{E} \cdot 7 / \mathrm{h} \\
{[2]}\end{array}$ & 10 & $\begin{array}{c}1.0 \mathrm{E}-7 \mathrm{~m} \\
{[3]}\end{array}$ & 10 & $\begin{array}{c}3.0 \mathrm{E}-7 / \mathrm{h} \\
{[3]}\end{array}$ & 10 & $\begin{array}{c}3.0 \mathrm{E}-7 \mathrm{~h} \\
{[3]}\end{array}$ & 10 \\
\hline ECB02AYB & $\begin{array}{l}\text { CIRCUIT BREAKER } \\
\text { SPURIOUS TRIP MCC } \\
\text { R2-2A }\end{array}$ & $1.0 \mathrm{E}-5 / \mathrm{h}$ & 10 & & & & & & \\
\hline ECB02CYB & $\begin{array}{l}\text { CIRCUIT BREAKER } \\
\text { SPURIOUS TRIP } \\
\text { MCC R2-4C }\end{array}$ & $1.0 E-5 / \mathrm{h}$ & 10 & & & & & & \\
\hline ECBO2EYO & $\begin{array}{l}\text { Breaker } 2 E \text { of } M C C \\
\text { P4E Fails Open }\end{array}$ & $\begin{array}{c}3.0 \mathrm{E}-7 / \mathrm{h} \\
{[2]}\end{array}$ & 10 & $1.0 \mathrm{E}-5 / \mathrm{h}$ & 10 & $3.0 \mathrm{E}-5 / \mathrm{d}$ & 10 & $3.0 \mathrm{E}-5 / \mathrm{d}$ & 3 \\
\hline ECBO3AYB & $\begin{array}{l}\text { CIRCUIT BREAKER } \\
\text { SPURIOUS TRIP } \\
\text { MCC R2-3A }\end{array}$ & $1.0 \mathrm{E}-5 / \mathrm{h}$ & 10 & $1.0 \mathrm{E}-5 / \mathrm{h}$ & 10 & $3.0 \mathrm{E}-5 / \mathrm{d}$ & 10 & $3.0 \mathrm{E}-5 / \mathrm{d}$ & 3 \\
\hline ECB03CYO & $\begin{array}{l}\text { Breaker } 3 C \text { of } M C C \\
\text { P4E Fails Open }\end{array}$ & $\begin{array}{c}3.0 \mathrm{E}-7 / \mathrm{h} \\
{[2]}\end{array}$ & 10 & $1.0 \mathrm{E}-5 / \mathrm{h}$ & 10 & $3.0 \mathrm{E}-5 / \mathrm{d}$ & 10 & $3.0 \mathrm{E}-5 / \mathrm{d}$ & 3 \\
\hline ECBO4AYB & $\begin{array}{l}\text { CIRCUIT BREAKER } \\
\text { SPURIOUS TRIP }\end{array}$ & $1.0 \mathrm{E}-5 / \mathrm{h}$ & 10 & & & & & & \\
\hline ECB04CYB & $\begin{array}{l}\text { CIRCUIT BAEAKER } \\
\text { SPUAIOUS TRIP MCC } \\
\text { R2B-4C }\end{array}$ & $1.0 \mathrm{E}-5 / \mathrm{h}$ & 10 & & & & & & \\
\hline ECB10CYO & $\begin{array}{l}\text { MCC-R1B Circuit } \\
\text { Breaker 10C Fajls } \\
\text { Open }\end{array}$ & $\begin{array}{c}3.0 \mathrm{E}-7 / \mathrm{h} \\
{[2]}\end{array}$ & 10 & $1.0 \mathrm{E}-5 / \mathrm{h}$ & 10 & $3.0 \mathrm{E}-5 / \mathrm{d}$ & 10 & $3.0 \mathrm{E}-5 / \mathrm{d}$ & 3 \\
\hline ECB10DYO & $\begin{array}{l}\text { MCC-A1B Circuit } \\
\text { Breaker 10D Fails } \\
\text { Open }\end{array}$ & $\begin{array}{c}3.0 \mathrm{E}-7 / \mathrm{h} \\
{[2]}\end{array}$ & 10 & $1.0 E-5 / \mathrm{h}$ & 10 & $3.0 \mathrm{E}-5 / \mathrm{d}$ & 10 & $3.0 \mathrm{E}-5 / \mathrm{d}$ & 3 \\
\hline ECB11BYZ & $\begin{array}{l}\text { Operator Error in } \\
\text { Connecting } E-1 \text { to } \\
R-1 B\end{array}$ & $\begin{array}{c}1.0 E-4 / d \\
{[4]}\end{array}$ & 10 & & & & & & \\
\hline ECB130YB & $\begin{array}{l}\text { CIRCUIT BREAKER } \\
\text { SPURIOUS TRIP MCC } \\
\text { R1B-130 }\end{array}$ & $1.0 \mathrm{E} \cdot 5 / \mathrm{h}$ & 10 & & & & & & \\
\hline EC813EY8 & $\begin{array}{l}\text { CIRCUIT BREAKER } \\
\text { SPURIOUS TRIP MCC } \\
\text { R1B-13E }\end{array}$ & $1.0 E-5 / \mathrm{h}$ & 10 & & & & & & \\
\hline
\end{tabular}


Table 7.13. Electrical Emergency Power System Data (Cont'd)

PAGE 2

\begin{tabular}{|c|c|c|c|c|c|c|c|c|c|}
\hline \multirow{2}{*}{$\begin{array}{l}\text { EVENT } \\
\text { NAME }\end{array}$} & \multirow[b]{2}{*}{ DESCRIPTION } & \multicolumn{2}{|c|}{ VALUE USED $3 / 91$} & \multicolumn{2}{|c|}{ CRBRP-4 } & \multicolumn{2}{|c|}{ IREP } & \multicolumn{2}{|c|}{ GENERIC } \\
\hline & & $\begin{array}{c}\text { Failure } \\
\text { Rate }\end{array}$ & $\begin{array}{l}\text { Error } \\
\text { Factor }\end{array}$ & $\begin{array}{l}\text { Failure } \\
\text { Rate }\end{array}$ & $\begin{array}{l}\text { Error } \\
\text { Factor }\end{array}$ & $\begin{array}{c}\text { Failure } \\
\text { Rate }\end{array}$ & $\begin{array}{l}\text { Error } \\
\text { Factor }\end{array}$ & $\begin{array}{c}\text { Failure } \\
\text { Rate }\end{array}$ & $\begin{array}{l}\text { Error } \\
\text { Factor }\end{array}$ \\
\hline EC814AYO & $\begin{array}{l}\text { Emergency 8us P-18 } \\
\text { Breaker 14AA Fails } \\
\text { Open }\end{array}$ & $\begin{array}{c}1.0 \mathrm{E}-7 \mathrm{~m} \\
{[2]}\end{array}$ & 10 & $1.0 E-5 / \mathrm{h}$ & 10 & $3.0 E-5 / d$ & 10 & $3.0 \mathrm{E}-5 / \mathrm{d}$ & 3 \\
\hline EC815CYO & $\begin{array}{l}\text { Bus R-1B Breaker } 15 \mathrm{C} \\
\text { Fails Open }\end{array}$ & $\begin{array}{c}3.0 \mathrm{E}-7 / \mathrm{h} \\
{[2]}\end{array}$ & 10 & $1.0 \mathrm{E}-5 / \mathrm{h}$ & 10 & $3.0 E-5 / d$ & 10 & $3.0 \mathrm{E}-5 / \mathrm{d}$ & 3 \\
\hline EC815CYP & $\begin{array}{l}\text { Emergency Bus R-1B } \\
\text { Brkr 15C Fails to } \\
\text { Open on Demand }\end{array}$ & $\begin{array}{c}5.0 \mathrm{E} \cdot 4 / \mathrm{d} \\
{[2]}\end{array}$ & 10 & $1.0 \mathrm{E}-3 / \mathrm{d}$ & 10 & $3.0 \mathrm{E}-3 / \mathrm{d}$ & 10 & $3.0 E-3 / d$ & 10 \\
\hline ECB16AYO & $\begin{array}{l}\text { Emergency Bus R-18 } \\
\text { Breaker 16A Fails } \\
\text { Open }\end{array}$ & $\begin{array}{c}3.0 \mathrm{E}-7 \mathrm{~m} \\
{[2]}\end{array}$ & 10 & $1.0 \mathrm{E}-5 / \mathrm{h}$ & 10 & $3.0 E-5 / d$ & 10 & $3.0 E-5 / d$ & 3 \\
\hline ECB16AYP & $\begin{array}{l}\text { Bus R-1 Breaker 16A } \\
\text { Fais to Open on } \\
\text { Demand }\end{array}$ & $\begin{array}{c}5.0 E-4 / d \\
{[2]}\end{array}$ & 10 & $1.0 \mathrm{E}-3 / \mathrm{d}$ & 10 & $3.0 E-3 / d$ & 10 & 3.0E-3/d & 10 \\
\hline ECB18AYN & $\begin{array}{l}\text { Bus R-1 Breaker 8A } \\
\text { Fails to Close on } \\
\text { Demand }\end{array}$ & $\begin{array}{c}5.0 E-4 / d \\
{[2]}\end{array}$ & 10 & $1.0 E-3 / d$ & 10 & $3.0 \mathrm{E}-3 / \mathrm{d}$ & 10 & $3.0 E-3 / d$ & \\
\hline ECB19AYN & $\begin{array}{l}\text { Bus P-1 Breaker 9A } \\
\text { Fails to Close on } \\
\text { Demand }\end{array}$ & $\begin{array}{c}5.0 \mathrm{E}-4 / \mathrm{d} \\
{[2]}\end{array}$ & 10 & $1.0 \mathrm{E}-3 / \mathrm{d}$ & 10 & $3.0 \mathrm{E}-3 / \mathrm{d}$ & 10 & $3.0 \mathrm{E}-3 / \mathrm{d}$ & \\
\hline ECB1O8GO & $\begin{array}{l}100 \mathrm{KW} \text { Generator } \\
\text { Output Breaker Fails } \\
\text { Open }\end{array}$ & $\begin{array}{c}3.0 \mathrm{E}-7 \mathrm{~h} \\
{[2]}\end{array}$ & 10 & $1.0 \mathrm{E}-5 \mathrm{~h}$ & 10 & $3.0 \mathrm{E}-5 / \mathrm{d}$ & 10 & $3.0 \mathrm{E}-5 / \mathrm{d}$ & 3 \\
\hline ECBIPBYZ & $\begin{array}{l}\text { Operator Error in } \\
\text { Connecting } E-1 \text { to } \\
P .18\end{array}$ & $\begin{array}{c}1.0 \mathrm{E}-4 / \mathrm{d} \\
{[4]}\end{array}$ & 10 & . & & & & & 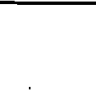 \\
\hline ECB21AYN & $\begin{array}{l}\text { R-2A Breaker 1A } \\
\text { Fails To Close }\end{array}$ & $\begin{array}{c}5.0 E-4 / d \\
{[2]}\end{array}$ & 10 & $1.0 E-3 / d$ & 10 & $3.0 \mathrm{E}-3 / \mathrm{d}$ & 10 & $3.0 E-3 / d$ & 10 \\
\hline ECB23BYO & $\begin{array}{l}\text { Emergency Bus R-2 } \\
\text { Tie Breaker } 38 \text { Fails } \\
\text { Open }\end{array}$ & $\begin{array}{c}3.0 \mathrm{E}-7 / \mathrm{h} \\
{[2]}\end{array}$ & 10 & $1.0 \mathrm{E}-5 / \mathrm{h}$ & 10 & $3.0 E \cdot 5 / d$ & 10 & $3.0 \mathrm{E}-5 / \mathrm{d}$ & 3 \\
\hline ECB23PYP & $\begin{array}{l}\text { Bus R-2 Tie Breaker } \\
\text { 3B Fails to Open on } \\
\text { Overload }\end{array}$ & $\begin{array}{c}5.0 \mathrm{E}-4 / \mathrm{d} \\
{[2]}\end{array}$ & 10 & $1.0 \mathrm{E}-3 / \mathrm{d}$ & 10 & $3.0 E-3 / d$ & 10 & $3.0 \mathrm{E}-3 / \mathrm{d}$ & 10 \\
\hline ECB2BAYP & $\begin{array}{l}\text { Bus E-1 Breaker 2B } \\
\text { Fails to Open on } \\
\text { Demand }\end{array}$ & $\begin{array}{c}5.0 E-4 / d \\
{[2]}\end{array}$ & 10 & $1.0 \mathrm{E}-3 / \mathrm{d}$ & 10 & $3.0 \mathrm{E}-3 / \mathrm{d}$ & 10 & $3.0 E-3 / d$ & 10 \\
\hline ECB2BBYO & $\begin{array}{l}\text { Emergency Bus E-1 } \\
\text { Breaker } 2 B \text { Fails } \\
\text { Open }\end{array}$ & $\begin{array}{c}3.0 \mathrm{E} \cdot 7 \mathrm{~h} \\
{[2]}\end{array}$ & 10 & $1.0 E-5 / h$ & 10 & $3.0 \mathrm{E}-5 / \mathrm{d}$ & 10 & $3.0 \mathrm{E}-5 / \mathrm{d}$ & 3 \\
\hline ECB2CAYP & $\begin{array}{l}\text { Bus E-1 Breaker 2C } \\
\text { Fails to Open on } \\
\text { Demand }\end{array}$ & $\begin{array}{c}5.0 E-4 / d \\
{[2]}\end{array}$ & 10 & $1.0 \mathrm{E}-3 / \mathrm{d}$ & 10 & $3.0 E-3 / d$ & 10 & $3.0 \mathrm{E}-3 / \mathrm{d}$ & 10 \\
\hline ECB2CBYO & $\begin{array}{l}\text { Emergency Bus E-1 } \\
\text { Breaker 2C Fails } \\
\text { Open }\end{array}$ & $\begin{array}{c}3.0 \mathrm{E}-7 / \mathrm{h} \\
{[2]}\end{array}$ & 10 & $1.0 E-5 / h$ & 10 & $3.0 \mathrm{E}-5 / \mathrm{d}$ & 10 & $3.0 \mathrm{E}-5 / \mathrm{d}$ & 3 \\
\hline ECB2DHYZ & $\begin{array}{l}\text { Operator Error Fails } \\
\text { to Close E-2 Breaker } \\
2 D\end{array}$ & $\begin{array}{c}1.0 \mathrm{E}-4 / \mathrm{d} \\
{[4]}\end{array}$ & 10 & & & & & & \\
\hline ECB2DMYN & $\begin{array}{l}\text { E-2 Breaker 2D Fails } \\
\text { to Remain Closed }\end{array}$ & $\begin{array}{c}.0 \mathrm{E}-7 \mathrm{~m} \\
{[2]}\end{array}$ & 10 & $1.0 \mathrm{E}-5 / \mathrm{h}$ & 10 & $3.0 \mathrm{E}-5 / \mathrm{d}$ & 10 & $3.0 \mathrm{E}-5 / \mathrm{d}$ & 3 \\
\hline ECB2FFYO & $\begin{array}{l}\text { Bus P-4E Breaker } 2 F \\
\text { Fails Open }\end{array}$ & $\begin{array}{c}3.0 \mathrm{E}-7 \mathrm{M} \\
{[2]}\end{array}$ & 10 & $1.0 \mathrm{E}-5 / \mathrm{h}$ & 10 & $3.0 \mathrm{E}-5 / \mathrm{d}$ & 10 & $3.0 \mathrm{E}-5 / \mathrm{d}$ & 3 \\
\hline ECBЗAAYO & $\begin{array}{l}\text { Breaker 3AA of } \\
\text { MCC-R2A Fails Open }\end{array}$ & $\begin{array}{c}3.0 \mathrm{E} \cdot 7 / \mathrm{h} \\
{[2]}\end{array}$ & 10 & $1.0 \mathrm{E}-5 / \mathrm{h}$ & 10 & $3.0 \mathrm{E}-5 / \mathrm{d}$ & 10 & $3.0 \mathrm{E}-5 / \mathrm{d}$ & 3 \\
\hline EC83D2YN & $\begin{array}{l}\text { E-1 Breaker 30 } \\
\text { Failure to Close }\end{array}$ & $\begin{array}{c}5.0 \mathrm{E}-4 / \mathrm{d} \\
{[2]}\end{array}$ & 10 & $1.0 \mathrm{E}-3 / \mathrm{d}$ & 10 & $3.0 \mathrm{E}-3 / \mathrm{d}$ & 10 & $3.0 \mathrm{E}-3 / \mathrm{d}$ & 10 \\
\hline ECBЗEEYO & $\begin{array}{l}\text { Bus P.4E Breaker 3EE } \\
\text { Fails Open }\end{array}$ & $\begin{array}{c}3.0 \mathrm{E}-7 \mathrm{~h} \\
{[2]}\end{array}$ & 10 & $1.0 \mathrm{E}-5 / \mathrm{h}$ & 10 & $3.0 \mathrm{E}-5 / \mathrm{d}$ & 10 & $3.0 E-5 / d$ & 3 \\
\hline
\end{tabular}


Table 7.13. Electrical Emergency Power System Data (Cont'd)

PAGE 3

\begin{tabular}{|c|c|c|c|c|c|c|c|c|c|}
\hline \multirow{2}{*}{$\begin{array}{l}\text { EVENT } \\
\text { NAME }\end{array}$} & \multirow[b]{2}{*}{ DESCRIPTION } & \multicolumn{2}{|c|}{ VALUE USED $3 / 91$} & \multicolumn{2}{|c|}{ CRBRP-4 } & \multicolumn{2}{|c|}{ IREP } & \multicolumn{2}{|c|}{ GENERIC } \\
\hline & & $\begin{array}{l}\text { Failure } \\
\text { Rate }\end{array}$ & $\begin{array}{l}\text { Error } \\
\text { Factor }\end{array}$ & $\begin{array}{l}\text { Failure } \\
\text { Rate }\end{array}$ & $\begin{array}{l}\text { Error } \\
\text { Factor }\end{array}$ & $\begin{array}{l}\text { Failure } \\
\text { Rate }\end{array}$ & $\begin{array}{l}\text { Error } \\
\text { Factor }\end{array}$ & $\begin{array}{l}\text { Failure } \\
\text { Rate }\end{array}$ & $\begin{array}{l}\text { Error } \\
\text { Factor }\end{array}$ \\
\hline ECB42EYO & $\begin{array}{l}\text { Emergency Bus S-1B } \\
\text { Contractor } 42 \mathrm{E} \text { Fails } \\
\text { Open }\end{array}$ & $\begin{array}{c}3.0 \mathrm{E}-7 \mathrm{~h} \\
{[2]}\end{array}$ & 10 & $1.0 \mathrm{E}-5 \mathrm{~h}$ & 10 & $3.0 E-5 / d$ & 10 & $3.0 \mathrm{E}-5 / \mathrm{d}$ & 3 \\
\hline ECB4OBHO & $\begin{array}{l}400 \mathrm{Kw} \text { Generator } \\
\text { Output Breaker Fails } \\
\text { Open }\end{array}$ & $\begin{array}{c}3.0 \mathrm{E}-7 / \mathrm{h} \\
{[2]}\end{array}$ & 10 & $1.0 \mathrm{E}-5 \mathrm{~h}$ & 10 & $3.0 \mathrm{E}-5 / \mathrm{d}$ & 10 & $3.0 \mathrm{E}-5 / \mathrm{d}$ & 3 \\
\hline ECB6AAYO & $\begin{array}{l}\text { Emergency Bus S-1 } \\
\text { Breaker 6A Fails } \\
\text { Open }\end{array}$ & $\begin{array}{c}1.0 \mathrm{E}-7 / \mathrm{h} \\
{[2]}\end{array}$ & 10 & $1.0 \mathrm{E}-5 / \mathrm{h}$ & 10 & $3.0 E-5 / d$ & 10 & $3.0 \mathrm{E}-5 / \mathrm{d}$ & 3 \\
\hline ECB8EAYO & $\begin{array}{l}\text { Emergency Bus S-1B } \\
\text { Breaker 8E Fails } \\
\text { Open }\end{array}$ & $\begin{array}{c}1.0 \mathrm{E}-7 / \mathrm{h} \\
{[2]}\end{array}$ & 10 & $1.0 E-5 / \mathrm{h}$ & 10 & $3.0 \mathrm{E}-5 / \mathrm{d}$ & 10 & $3.0 \mathrm{E}-5 / \mathrm{d}$ & 3 \\
\hline ECBA16YO & $\begin{array}{l}\text { Emergency Bus P-1B } \\
\text { Breaker } 16 \dot{A} \text { Fails } \\
\text { Open }\end{array}$ & $\begin{array}{c}3.0 E-7 / \mathrm{h} \\
{[2]}\end{array}$ & 10 & $1.0 \mathrm{E}-5 / \mathrm{h}$ & 10 & $3.0 \mathrm{E}-5 / \mathrm{d}$ & 10 & $3.0 \mathrm{E}-5 i \mathrm{~d}$ & 3 \\
\hline ECBA16YP & $\begin{array}{l}\text { Bus P-1 Breaker 16A } \\
\text { Fails to Open on } \\
\text { Demand }\end{array}$ & $\begin{array}{c}5.0 E \cdot 4 / d \\
{[2]}\end{array}$ & 10 & $1.0 E-3 / d$ & 10 & $3.0 \mathrm{E}-3 / \mathrm{d}$ & 10 & $3.0 \mathrm{E}-3 / \mathrm{d}$ & 10 \\
\hline ECBB9BYB & $\begin{array}{l}\text { CIRCUIT BREAKER } \\
\text { SPURIOUS TRIP MCC } \\
\text { R1B-9B }\end{array}$ & $1.0 \mathrm{E}-5 / \mathrm{h}$ & 10 & $\cdot$ & & & & & \\
\hline ECBB9CYB & $\begin{array}{l}\text { CIRCUIT BREAKER } \\
\text { SPURIOUS TRIP R1B-9C }\end{array}$ & $1.0 \mathrm{E}-5 / \mathrm{h}$ & 10 & & & & & & \\
\hline ECBB9DYB & $\begin{array}{l}\text { CIRCUIT BREAKER } \\
\text { SPURIOUS TRIP MCC } \\
\text { R1B-9D }\end{array}$ & $1.0 \mathrm{E}-5 / \mathrm{h}$ & 10 & & & & & & \\
\hline ECBD15YO & $\begin{array}{l}\text { Bus P-1B Breaker 15D } \\
\text { Fails Open }\end{array}$ & $\begin{array}{c}3.0 \mathrm{E}-7 / \mathrm{h} \\
{[2]}\end{array}$ & 10 & $1.0 \mathrm{E}-5 / \mathrm{h}$ & 10 & $3.0 \mathrm{E}-5 / \mathrm{d}$ & 10 & $3.0 \mathrm{E}-5 / \mathrm{d}$ & 3 \\
\hline ECBE1AYO & $\begin{array}{l}\text { Emergency Bus E-1 } \\
\text { Bieaker } 1 \text { A Fails } \\
\text { Open }\end{array}$ & $\begin{array}{c}3.0 \mathrm{E}-7 \mathrm{~h} \\
{[2]}\end{array}$ & 10 & $1.0 \mathrm{E}-5 \mathrm{~m}$ & 10 & $3.0 \mathrm{E}-5 / \mathrm{d}$ & 10 & $3.0 \mathrm{E}-5 / \mathrm{d}$ & 3 \\
\hline ECBE1BYO & $\begin{array}{l}\text { Bus E-1 Breaker 1-B } \\
\text { Fails Open }\end{array}$ & $\begin{array}{c}3.0 E-7 / h \\
{[2]}\end{array}$ & 10 & $1.0 \mathrm{E}-5 \mathrm{~h}$ & 10 & $3.0 \mathrm{E}-5 / \mathrm{d}$ & 10 & $3.0 \mathrm{E}-5 / \mathrm{d}$ & 3 \\
\hline ECBE1XYP & $\begin{array}{l}\text { Selected Emergency } \\
\text { Power Loads are not } \\
\text { Shed }\end{array}$ & $\begin{array}{c}3.0 \mathrm{E}-6 / \mathrm{h} \\
{[2]}\end{array}$ & 10 & $\begin{array}{c}1.0 \mathrm{E}-6 / \mathrm{h} \\
{[5]}\end{array}$ & 10 & $\begin{array}{c}3.0 \mathrm{E}-6 / \mathrm{h} \\
{[5]}\end{array}$ & 10 & $\begin{array}{c}2.0 \mathrm{E}-6 / \mathrm{h} \\
{[5]}\end{array}$ & 10 \\
\hline ECBER2YP & $\begin{array}{l}\text { Selected } 100 \mathrm{KW} \\
\text { Emergency Power } \\
\text { Loads Are Not Shed }\end{array}$ & $\begin{array}{c}3.0 E-6 / \mathrm{h} \\
{[2]}\end{array}$ & 10 & $\begin{array}{c}1.0 \mathrm{E}-6 / \mathrm{h} \\
{[5]}\end{array}$ & 10 & $\begin{array}{c}3.0 \mathrm{E}-6 / \mathrm{h} \\
{[5]}\end{array}$ & 10 & $\begin{array}{c}2.0 \mathrm{E} \cdot 6 / \mathrm{h} \\
{[5]}\end{array}$ & 10 \\
\hline ECBH1AYO & $\begin{array}{l}\text { E-2 Bus Breaker } 1 \mathrm{~A} \\
\text { Fails Open }\end{array}$ & $\begin{array}{c}3.0 \mathrm{E}-7 / \mathrm{h} \\
{[2]}\end{array}$ & 10 & $1.0 \mathrm{E}-5 / \mathrm{h}$ & 10 & $3.0 \mathrm{E}-5 / \mathrm{d}$ & 10 & $3.0 \mathrm{E}-5 / \mathrm{d}$ & 10 \\
\hline ECBP 42YO & $\begin{array}{l}\text { P-4E Supply Breaker } \\
2 C \text { Fails Open }\end{array}$ & $\begin{array}{c}3.0 \mathrm{E}-7 / \mathrm{h} \\
{[2]}\end{array}$ & 10 & $1.0 \mathrm{E}-5 / \mathrm{h}$ & 10 & $3.0 \mathrm{E} \cdot 5 / \mathrm{d}$ & 10 & $3.0 E-5 / d$ & 3 \\
\hline ECBT8AYO & $\begin{array}{l}\text { Bus R-1 Breaker } 8 \mathrm{~A} \\
\text { Trips Open }\end{array}$ & $\begin{array}{c}3.0 \mathrm{E}-7 / \mathrm{h} \\
{[2]}\end{array}$ & 10 & $1.0 \mathrm{E}-5 / \mathrm{h}$ & 10 & $3.0 \mathrm{E}-5 / \mathrm{d}$ & 10 & $3.0 \mathrm{E}-5 / \mathrm{d}$ & 3 \\
\hline ECBT9AYO & $\begin{array}{l}\text { Bus P.1 Breaker 9A } \\
\text { Trips Open }\end{array}$ & $\begin{array}{c}3.0 \mathrm{E}-7 / \mathrm{h} \\
{[2]}\end{array}$ & 10 & $1.0 \mathrm{E}-5 / \mathrm{h}$ & 10 & $3.0 \mathrm{E}-5 / \mathrm{d}$ & 10 & $3.0 \mathrm{E}-5 / \mathrm{d}$ & 3 \\
\hline ECBU15YO & $\begin{array}{l}\text { Bus R-1B Breaker } 15 \\
\text { A Fails Open }\end{array}$ & $\begin{array}{c}3.0 \mathrm{E} \cdot 7 / \mathrm{h} \\
{[2]}\end{array}$ & 10 & $1.0 \mathrm{E}-5 / \mathrm{h}$ & 10 & $3.0 \mathrm{E}-5 / \mathrm{d}$ & 10 & $3.0 \mathrm{E}-5 / \mathrm{d}$ & 3 \\
\hline EDL1SFGS & $\begin{array}{l}100 \mathrm{KW} \text { Diesel Fails } \\
\text { to Start on Command }\end{array}$ & $\begin{array}{c}1.0 \mathrm{E}-2 / \mathrm{d} \\
{[6]}\end{array}$ & 5 & & & & & & \\
\hline EDL41OHU & $\begin{array}{l}400 / 100 \mathrm{KW} \text { Diesel } \\
\text { Fuel Supply Common } \\
\text { Mode Failure }\end{array}$ & $\begin{array}{c}5.0 \mathrm{E}-4 / \mathrm{h} \\
{[7]}\end{array}$ & 10 & & & & & & \\
\hline EDL4SFHS & $\begin{array}{l}400 \mathrm{KW} \text { Diesel Fails } \\
\text { to Start on Command }\end{array}$ & $\begin{array}{c}1.0 E-2 / d \\
{[2]}\end{array}$ & 5 & $3.0 \mathrm{E}-2 / \mathrm{d}$ & 3 & $3.0 \mathrm{E} \cdot 2 / \mathrm{d}$ & 3 & $3.0 \mathrm{E}-2 / \mathrm{d}$ & 3 \\
\hline EFU12BBB & $\begin{array}{l}\text { Bus E-1 Breaker 2B } \\
\text { Trip Fuse Opens }\end{array}$ & $\begin{array}{c}1.0 E-7 / h \\
{[2]}\end{array}$ & 10 & $1.0 \mathrm{E} \cdot 6 / \mathrm{h}$ & 10 & $3.0 \mathrm{E}-6 / \mathrm{h}$ & 10 & $3.0 \mathrm{E}-6 / \mathrm{h}$ & 10 \\
\hline
\end{tabular}


Table 7.13. Electrical Emergency Power System Data (Cont'd)

PAGE 4

\begin{tabular}{|c|c|c|c|c|c|c|c|c|c|}
\hline \multirow{2}{*}{$\begin{array}{l}\text { EVENT } \\
\text { NAME }\end{array}$} & \multirow[b]{2}{*}{ DESCRIPTION } & \multicolumn{2}{|c|}{ VALUE USED 391} & \multicolumn{2}{|c|}{ CRBRP-4 } & \multicolumn{2}{|c|}{ IREP } & \multicolumn{2}{|c|}{ GENERIC } \\
\hline & & $\begin{array}{c}\text { Failure } \\
\text { Rate }\end{array}$ & $\begin{array}{l}\text { Error } \\
\text { Factor }\end{array}$ & $\begin{array}{l}\text { Failure } \\
\text { Rate }\end{array}$ & $\begin{array}{l}\text { Error } \\
\text { Factor }\end{array}$ & $\begin{array}{l}\text { Failure } \\
\text { Rate }\end{array}$ & $\begin{array}{l}\text { Error } \\
\text { Factor }\end{array}$ & $\begin{array}{c}\text { Failure } \\
\text { Rate } \\
\end{array}$ & $\begin{array}{l}\text { Error } \\
\text { Factor }\end{array}$ \\
\hline EFU12C8B & $\begin{array}{l}\text { Bus E-1 Breaker 2C } \\
\text { Trip Fuse Opens }\end{array}$ & $\begin{array}{c}1.0 \mathrm{E}-7 / \mathrm{h} \\
{[2]}\end{array}$ & 10 & $1.0 \mathrm{E}-6 / \mathrm{h}$ & 10 & $3.0 \mathrm{E}-6 / \mathrm{h}$ & 10 & $3.0 \mathrm{E} \cdot 6 / \mathrm{h}$ & 10 \\
\hline EFU15CYB & $\begin{array}{l}\text { Emergency Bus R-1B } \\
\text { Brks 15C Trip Fuse } \\
\text { Opens }\end{array}$ & $\begin{array}{c}1.0 \mathrm{E}-7 / \mathrm{h} \\
{[2]}\end{array}$ & 10 & $1.0 \mathrm{E}-6 / \mathrm{h}$ & 10 & $3.0 \mathrm{E} \cdot 6 / \mathrm{h}$ & 10 & $3.0 \mathrm{E}-6 / \mathrm{h}$ & 10 \\
\hline EFU16ABB & $\begin{array}{l}\text { Bus R-1 Breaker 16A } \\
\text { Trip Fuse Opens }\end{array}$ & $\begin{array}{c}1.0 \mathrm{E}-7 \mathrm{~h} \\
{[2]}\end{array}$ & 10 & $1.0 \mathrm{E}-6 / \mathrm{h}$ & 10 & $3.0 \mathrm{E}-6 / \mathrm{h}$ & 10 & $3.0 \mathrm{E}-6 / \mathrm{h}$ & 10 \\
\hline EFU $16 P 88$ & $\begin{array}{l}\text { Bus P-1 Breaker } 16 \mathrm{~A} \\
\text { Trip Fuse Opens }\end{array}$ & $\begin{array}{c}1.0 \mathrm{E}-7 / \mathrm{h} \\
{[2]}\end{array}$ & 10 & $1.0 \mathrm{E}-6 / \mathrm{h}$ & 10 & $3.0 \mathrm{E}-6 / \mathrm{h}$ & 10 & $3.0 \mathrm{E} \cdot 6 / \mathrm{h}$ & 10 \\
\hline EFUDBABB & $\begin{array}{l}\text { Bus R-1 Breaker } 8 A \\
\text { Close Fuse Fails } \\
\text { Open }\end{array}$ & $\begin{array}{c}1.0 \mathrm{E}-7 / \mathrm{h} \\
{[2]}\end{array}$ & 10 & $1.0 \mathrm{E}-6 / \mathrm{h}$ & 10 & $3.0 \mathrm{E}-6 / \mathrm{h}$ & 10 & $3.0 \mathrm{E} \cdot 6 / \mathrm{h}$ & 10 \\
\hline EFUD9ABB & $\begin{array}{l}\text { Bus P-1 Breaker 9A } \\
\text { Close Fuse Fails } \\
\text { Open }\end{array}$ & $\underset{[2]}{1.0 E-7 / h}$ & 10 & $1.0 E-6 / h$ & 10 & $3.0 \mathrm{E} \cdot 6 \mathrm{~m}$ & $10^{\circ}$ & $3.0 \mathrm{E}-6 / \mathrm{h}$ & 10 \\
\hline EFUES280 & $\begin{array}{l}480 \text { Vac Emerg Switch } \\
\text { gear } 125 \text { Vdc Bus } 1 \mathrm{t} \\
\text { O Bus } 2 \text { Fuse Opens }\end{array}$ & $\begin{array}{c}1.0 \mathrm{E}-7 / \mathrm{h} \\
{[6]}\end{array}$ & 10 & $1.0 \mathrm{E} \cdot 6 / \mathrm{h}$ & 10 & $3.0 \mathrm{E}-6 / \mathrm{h}$ & 10 & $3.0 \mathrm{E}-6 / \mathrm{h}$ & 10 \\
\hline EGE1EFGW & $\begin{array}{l}100 \mathrm{KW} \text { Diesel Fails } \\
\text { to Run }\end{array}$ & $\begin{array}{c}5.0 \mathrm{E}-3 / \mathrm{h}^{\circ} \\
{[2]}\end{array}$ & 10 & $1.0 \mathrm{E}-3 / \mathrm{h}$ & 10 & $3.0 \mathrm{E}-3 \mathrm{~m}$ & 10 & $3.0 \mathrm{E}-3 / \mathrm{h}$ & 10 \\
\hline EGE4EFHW & $\begin{array}{l}400 \mathrm{Kw} \text { Diesel Fails } \\
\text { to Run }\end{array}$ & $\begin{array}{c}5.0 E-3 / h \\
{[2]}\end{array}$ & 10 & $1.0 \mathrm{E}-3 / \mathrm{h}$ & 10 & $3.0 \mathrm{E}-3 / \mathrm{h}$ & 10 & $3.0 \mathrm{E}-3 / \mathrm{h}$ & 10 \\
\hline EIN12BWW & $\begin{array}{l}\text { Bus E-1 Breaker Trip } \\
\text { Signal Ckt Fails }\end{array}$ & $\begin{array}{c}3.0 E-6 / \mathrm{h} \\
{[2]}\end{array}$ & 10 & $\begin{array}{c}1.0 \mathrm{E}-6 \mathrm{~h} \\
{[5]}\end{array}$ & 10 & $\begin{array}{c}3.0 \mathrm{E}-6 / \mathrm{h} \\
{[5]}\end{array}$ & 10 & $\begin{array}{c}2.0 \mathrm{E}-6 / \mathrm{h} \\
{[5]}\end{array}$ & 10 \\
\hline EIN12CYW & $\begin{array}{l}\text { Bus E-1 Breaker 2C } \\
\text { Trip Signal Ckt } \\
\text { Fails }\end{array}$ & $\begin{array}{c}3.0 \mathrm{E}-6 / \mathrm{h} \\
{[2]}\end{array}$ & 10 & $\begin{array}{c}1.0 \mathrm{E}-6 / \mathrm{h} \\
{[5]}\end{array}$ & 10 & $\begin{array}{c}3.0 \mathrm{E}-6 / \mathrm{h} \\
{[5]}\end{array}$ & 10 & $\begin{array}{c}2.0 \mathrm{E}-6 / \mathrm{h} \\
{[5]}\end{array}$ & 10 \\
\hline EIN151YW & $\begin{array}{l}\text { Brkr 15C - Brkr } 1 \mathrm{~A} \\
\text { Interlock Fails and } \\
1 \mathrm{~A} \text { Closes }\end{array}$ & $\begin{array}{c}3.0 \mathrm{E}-6 / \mathrm{h} \\
{[2]}\end{array}$ & 10 & $\begin{array}{c}1.0 \mathrm{E}-6 / \mathrm{h} \\
{[5]}\end{array}$ & 10 & $\begin{array}{c}3.0 \mathrm{E}-6 / \mathrm{h} \\
{[5]}\end{array}$ & 10 & $\begin{array}{c}2.0 \mathrm{E}-6 / \mathrm{h} \\
{[5]}\end{array}$ & 10 \\
\hline EIN15CWW & $\begin{array}{l}\text { Emergency Bus R-1B } \\
\text { Brkt 15C Trip Signal } \\
\text { Ckt Fails }\end{array}$ & $\begin{array}{c}3.0 \mathrm{E}-6 / \mathrm{h} \\
{[2]}\end{array}$ & 10 & $\begin{array}{c}3.0 \mathrm{E}-6 / \mathrm{h} \\
{[5]}\end{array}$ & 10 & $\begin{array}{c}3.0 E-6 / \mathrm{h} \\
{[5]}\end{array}$ & 10 & $\begin{array}{c}2.0 \mathrm{E}-6 / \mathrm{h} \\
{[5]}\end{array}$ & 10 \\
\hline EIN16AYW & $\begin{array}{l}\text { Bus R-1 Breaker 16A } \\
\text { Trip Signal Ckt } \\
\text { Fails }\end{array}$ & $\begin{array}{c}3.0 \mathrm{E}-6 / \mathrm{h} \\
{[2]}\end{array}$ & 10 & $\begin{array}{c}1.0 \mathrm{E}-6 / \mathrm{h} \\
{[5]}\end{array}$ & 10 & $\begin{array}{c}3.0 \mathrm{E}-6 / \mathrm{h} \\
{[5]}\end{array}$ & 10 & $\begin{array}{c}2.0 \mathrm{E}-6 / \mathrm{h} \\
{[5]}\end{array}$ & 10 \\
\hline EIN16PYW & $\begin{array}{l}\text { Bus P-1 Breaker 16A } \\
\text { Trip Signal Ckt } \\
\text { Fails }\end{array}$ & $\begin{array}{c}3.0 \mathrm{E}-6 / \mathrm{h} \\
{[2]}\end{array}$ & 10 & $\begin{array}{c}1.0 \mathrm{E}-6 / \mathrm{h} \\
{[5]}\end{array}$ & 10 & $\begin{array}{c}3.0 \mathrm{E}-6 / \mathrm{h} \\
{[5]}\end{array}$ & 10 & $\begin{array}{c}2.0 \mathrm{E}-6 / \mathrm{h} \\
{[5]}\end{array}$ & 10 \\
\hline EIN1SSGW & $\begin{array}{l}100 \mathrm{KW} \text { Diesel Start } \\
\text { Signal Ckt Fails }\end{array}$ & $\begin{array}{c}3.0 \mathrm{E}-6 / \mathrm{h} \\
{[2]}\end{array}$ & 10 & $\begin{array}{c}1.0 \mathrm{E}-6 / \mathrm{h} \\
{[5]}\end{array}$ & 10 & $\begin{array}{c}3.0 \mathrm{E}-6 / \mathrm{h} \\
{[5]}\end{array}$ & 10 & $\begin{array}{c}2.0 \mathrm{E}-6 / \mathrm{h} \\
{[5]}\end{array}$ & 10 \\
\hline EIN21AYW & $\begin{array}{l}\text { R-2A Breaker 1A } \\
\text { Close Signal Ckt } \\
\text { Fails }\end{array}$ & $\begin{array}{c}3.0 \mathrm{E}-6 / \mathrm{h} \\
{[2]}\end{array}$ & 10 & $\begin{array}{c}1.0 \mathrm{E}-6 / \mathrm{h} \\
{[5]}\end{array}$ & 10 & $\begin{array}{c}3.0 \mathrm{E} \cdot 6 / \mathrm{h} \\
{[5]}\end{array}$ & 10 & $\begin{array}{c}2.0 \mathrm{E}-6 / \mathrm{h} \\
{[5]}\end{array}$ & 10 \\
\hline EIN3D3YW & $\begin{array}{l}\text { E-1 Breaker 30 Close } \\
\text { Signal Ckt Fails }\end{array}$ & $\begin{array}{c}3.0 E-6 / \mathrm{h} \\
{[2]}\end{array}$ & 10 & $\begin{array}{c}1.0 \mathrm{E}-6 / \mathrm{h} \\
{[5]}\end{array}$ & 10 & $\begin{array}{c}3.0 \mathrm{E}-6 / \mathrm{h} \\
{[5]}\end{array}$ & 10 & $\begin{array}{c}2.0 \mathrm{E}-6 / \mathrm{h} \\
{[5]}\end{array}$ & 10 \\
\hline EIN42NYW & $\begin{array}{l}\text { Emergency Bus S-1B } \\
\text { Cntcr } 42 N \text { Close } \\
\text { Signal Ckt Fails }\end{array}$ & $\begin{array}{c}3.0 \mathrm{E}-6 / \mathrm{h} \\
{[2]}\end{array}$ & 10 & $\begin{array}{c}1.0 E-6 / h \\
{[5]}\end{array}$ & 10 & $\begin{array}{c}3.0 \mathrm{E}-6 / \mathrm{h} \\
{[5]}\end{array}$ & 10 & $\begin{array}{c}2.0 \mathrm{E}-6 / \mathrm{h} \\
{[5]}\end{array}$ & 10 \\
\hline EIN4SSHW & $\begin{array}{l}400 \text { Diesel Start } \\
\text { Signal Ckt Fails }\end{array}$ & $\begin{array}{c}3.0 \mathrm{E}-6 \mathrm{~h} \\
{[2]}\end{array}$ & 10 & $\begin{array}{c}1.0 E-6 / h \\
{[5]}\end{array}$ & 10 & $\begin{array}{c}3.0 \mathrm{E}-6 / \mathrm{h} \\
{[5]}\end{array}$ & 10 & $\begin{array}{c}2.0 E \cdot 6 / \mathrm{h} \\
{[5]}\end{array}$ & 10 \\
\hline EINDBAYW & $\begin{array}{l}\text { Bus R-1 Breaker } 8 A \\
\text { Close Signal Ckt } \\
\text { Fails }\end{array}$ & $\begin{array}{c}3.0 E-6 / \mathrm{h} \\
{[2]}\end{array}$ & 10 & $\begin{array}{c}1.0 E-6 / \mathrm{h} \\
{[5]}\end{array}$ & 10 & $\begin{array}{c}3.0 \mathrm{E}-6 / \mathrm{h} \\
{[5]}\end{array}$ & 10 & $\begin{array}{c}2.0 E-6 / h \\
{[5]}\end{array}$ & 10 \\
\hline EINDSAYW & $\begin{array}{l}\text { Bus P-1 Breaker 9A } \\
\text { Close Signal Ckt } \\
\text { Fails }\end{array}$ & $\begin{array}{c}3.0 E-6 / \mathrm{h} \\
{[2]}\end{array}$ & 10 & $\begin{array}{c}1.0 \mathrm{E}-6 / \mathrm{h} \\
{[5]}\end{array}$ & 10 & $\begin{array}{c}3.0 \mathrm{E}-6 / \mathrm{h} \\
{[5]}\end{array}$ & 10 & $\begin{array}{c}2.0 E-6 / \mathrm{h} \\
{[5]}\end{array}$ & 10 \\
\hline
\end{tabular}


Table 7.13. Electrical Emergency Power System Data (Cont'd)

PAGE 5

\begin{tabular}{|c|c|c|c|c|c|c|c|c|c|}
\hline \multirow{2}{*}{$\begin{array}{l}\text { EVENT } \\
\text { NAME }\end{array}$} & \multirow[b]{2}{*}{ DESCRIPTION } & \multicolumn{2}{|c|}{ VALUE USED $3 / 91$} & \multicolumn{2}{|c|}{ CRBRP.4 } & \multicolumn{2}{|c|}{ IREP } & \multicolumn{2}{|c|}{ GENERIC } \\
\hline & & $\begin{array}{l}\text { Failure } \\
\text { Rate }\end{array}$ & $\begin{array}{l}\text { Error } \\
\text { Factor }\end{array}$ & $\begin{array}{l}\text { Failure } \\
\text { Rate }\end{array}$ & $\begin{array}{l}\text { Error } \\
\text { Factor }\end{array}$ & $\begin{array}{l}\text { Failure } \\
\text { Rate }\end{array}$ & $\begin{array}{l}\text { Error } \\
\text { Factor }\end{array}$ & $\begin{array}{l}\text { Failure } \\
\text { Rate }\end{array}$ & $\begin{array}{l}\text { Error } \\
\text { Factor }\end{array}$ \\
\hline EINE IXYW & $\begin{array}{l}\text { Brkr 5C - Brkr 3D } \\
\text { Interlock Fails and } \\
30 \text { Closes }\end{array}$ & $\begin{array}{c}3.0 \mathrm{E} \cdot 6 \mathrm{~h} \\
{[2]}\end{array}$ & 10 & $\begin{array}{c}1.0 \mathrm{E}-6 \mathrm{M} \\
{[5]}\end{array}$ & 10 & $\begin{array}{c}3.0 \mathrm{E}-6 \mathrm{~h} \\
{[5]}\end{array}$ & 10 & $\begin{array}{c}2.0 \mathrm{E}-6 / \mathrm{h} \\
{[5]}\end{array}$ & 10 \\
\hline 80012700 & $\begin{array}{l}\text { NO RECOVERY OF } 480 \mathrm{~V} \\
\text { EMERGENCY BUS P.4E }\end{array}$ & 1.0 & 1 & & & & & & \\
\hline$E 0020200$ & $\begin{array}{l}\text { NO RECOVERY OF } 480 \mathrm{~V} \\
\text { EMERGENCY BUS P-1B }\end{array}$ & 1.0 & 1 & & & & & & \\
\hline ERE42NYO & $\begin{array}{l}\text { Emergency Bus S-1B } \\
\text { Cntcr } 42 \mathrm{~N} \text { Fails to } \\
\text { Close on Demand }\end{array}$ & $\begin{array}{c}5.0 E-4 / d \\
{[2]}\end{array}$ & 10 & $1.0 E-3 / d$ & 10 & $3.0 E-3 / d$ & 10 & $3.0 \mathrm{E}-3 / \mathrm{d}$ & 10 \\
\hline ESGE2EYW & $\begin{array}{l}\text { Emergency Bus E-2 } \\
\text { Interlock Fails Gen. } \\
\text { On, 3B, } 2 \mathrm{D} \text { closed }\end{array}$ & $\begin{array}{c}3.0 \mathrm{E}-6 / \mathrm{h} \\
{[2]}\end{array}$ & 10 & $\begin{array}{c}1.0 \mathrm{E} \cdot 6 / \mathrm{h} \\
{[5]}\end{array}$ & 10 & $\begin{array}{c}3.0 \mathrm{E}-6 / \mathrm{h} \\
{[5]}\end{array}$ & 10 & $\begin{array}{c}2.0 \mathrm{E}-6 / \mathrm{h} \\
{[5]}\end{array}$ & 10 \\
\hline ESW0O4YO & $\begin{array}{l}\text { E-1 Bus Disconn. } \\
\text { Switch } 4 \text { Fails Open }\end{array}$ & $\begin{array}{c}1.0 \mathrm{E}-6 / \mathrm{h} \\
{[2]}\end{array}$ & 10 & $1.0 E-7 \mathrm{~m}$ & 10 & & & & \\
\hline ESWE1RYO & $\begin{array}{l}\text { Bus R-1B Manual } \\
\text { Trans. Switch E-1 } \\
\text { Side Fails Open }\end{array}$ & $\begin{array}{c}1.0 E-5 / d \\
{[2]}\end{array}$ & 10 & $1.0 \mathrm{E}-7 \mathrm{~h}$ & 10 & & & & \\
\hline ESWE1RYZ & $\begin{array}{l}\text { Operator Fails to } \\
\text { Effect } E-1 \text { to R-2B } \\
\text { Line on Demand }\end{array}$ & $\underset{[4]}{1.0 E-4 / d}$ & 10 & & & & & & \\
\hline ESWP 4EYB & $\begin{array}{l}\text { E-1 Side ofP.4E Man. } \\
\text { Trans. Switch Fails } \\
\text { Open }\end{array}$ & $\underset{[2]}{1.0 E-6 / \mathrm{h}}$ & 10 & $1.0 \mathrm{E}-7 / \mathrm{h}$ & 10 & & & & \\
\hline ESWP4EYW & $\begin{array}{l}\text { Total Failure of } \\
\text { P.4E Man Transf } \\
\text { Switch }\end{array}$ & $\begin{array}{c}1.0 \mathrm{E}-7 / \mathrm{h} \\
{[8]}\end{array}$ & 10 & . & & & & . & \\
\hline ESWP 4NYB & $\begin{array}{l}\text { Normal Pwr Side of } \\
\text { P-4E Man. Transf. } \\
\text { Switch Fails Open }\end{array}$ & $\begin{array}{c}1.0 \mathrm{E}-6 \mathrm{~h} \\
{[2]}\end{array}$ & 10 & $1.0 \mathrm{E}-7 / \mathrm{h}$ & 10 & & & & \\
\hline ESWP ANYZ & $\begin{array}{l}\text { Operator Fails to Sw } \\
\text { itch P-4E Man. Trans } \\
\text { f. Sw to Norm PwT }\end{array}$ & $\begin{array}{c}T .0 E-4 / d \\
{[4]}\end{array}$ & 10 & & & & & & \\
\hline ESWR1BYO & $\begin{array}{l}\text { Bus R-1B Manual } \\
\text { Trans. Switch R-1B } \\
\text { Side Fails Open }\end{array}$ & $\begin{array}{c}1.0 E-6 / \mathrm{h} \\
{[2]}\end{array}$ & 10 & $1.0 E-7 / \mathrm{h}$ & 10 & & & & \\
\hline EWR118YB & $\begin{array}{l}\text { E-1 to R-1B Line } \\
\text { Fails Open }\end{array}$ & $\begin{array}{c}1.0 \mathrm{E}-7 \mathrm{~m} \\
{[2]}\end{array}$ & 10 & $1.0 \mathrm{E}-6 \mathrm{~h}$ & 10 & $3.0 E-6 / \mathrm{h}$ & 10 & $3.0 \mathrm{E} \cdot 6 \mathrm{~h}$ & 3 \\
\hline EWR118YC & $\begin{array}{l}\text { Overload Fault on } \\
E-1 \text { to R-1B Line }\end{array}$ & $\begin{array}{c}1.0 E-7 / \mathrm{h} \\
{[2]}\end{array}$ & 10 & $\begin{array}{c}1.0 \mathrm{E}-7 / \mathrm{h} \\
{[3]}\end{array}$ & 10 & $\begin{array}{c}3.0 \mathrm{E}-7 \mathrm{~m} \\
{[3]}\end{array}$ & 10 & $\begin{array}{c}3.0 \mathrm{E}-7 / \mathrm{h} \\
{[3]}\end{array}$ & 10 \\
\hline EWR12BYB & $\begin{array}{l}\text { Bus E-1 Breaker 2B } \\
\text { Trip Ckt Sup. Wiring } \\
\text { Fals Open }\end{array}$ & $\begin{array}{c}1.0 \mathrm{E}-7 / \mathrm{h} \\
{[2]}\end{array}$ & 10 & $1.0 \mathrm{E}-6 / \mathrm{h}$ & 10 & $3.0 \mathrm{E}-6 / \mathrm{h}$ & 10 & $3.0 \mathrm{E}-6 \mathrm{~h}$ & 3 \\
\hline EWR12CYB & $\begin{array}{l}\text { Bus E-1 Breaker 2C } \\
\text { Trip Ckt Sup. Wiring } \\
\text { Fails Open }\end{array}$ & $\begin{array}{c}1.0 \mathrm{E}-7 / \mathrm{h} \\
{[2]}\end{array}$ & 10 & $1.0 E-6 / \mathrm{h}$ & 10 & $3.0 \mathrm{E}-6 \mathrm{M}$ & 10 & $3.0 \mathrm{E}-6 \mathrm{~m}$ & 3 \\
\hline EWR15CYB & $\begin{array}{l}\text { Emerg Bus R-1B Brkr } \\
15 \mathrm{C} \text { Trip Ckt Sup. } \\
\text { Wiring Fails Open }\end{array}$ & $\begin{array}{c}1.0 \mathrm{E} \cdot 7 / \mathrm{h} \\
{[2]}\end{array}$ & 10 & $1.0 \mathrm{E}-6 / \mathrm{h}$ & 10 & $3.0 \mathrm{E}-6 / \mathrm{h}$ & 10 & $3.0 \mathrm{E}-6 / \mathrm{h}$ & 3 \\
\hline EWR16AYB & $\begin{array}{l}\text { Bus R-1 Breaker 16A } \\
\text { Trip Ckt Sup. Wiring } \\
\text { Fals Open }\end{array}$ & $\begin{array}{c}1.0 \mathrm{E}-7 \mathrm{~h} \\
{[2]}\end{array}$ & 10 & $1.0 \mathrm{E}-6 \mathrm{~h}$ & 10 & $3.0 \mathrm{E}-6 / \mathrm{h}$ & 10 & $3.0 \mathrm{E}-5 / \mathrm{h}$ & 3 \\
\hline EWR16PYB & $\begin{array}{l}\text { Bus P-1 Breaker 16A } \\
\text { Trip Ckt Sup. Wiring } \\
\text { Fails OPen }\end{array}$ & $\begin{array}{c}1.0 \mathrm{E}-7 / \mathrm{h} \\
{[2]}\end{array}$ & 10 & $1.0 \mathrm{E}-6 / \mathrm{h}$ & 10 & $3.0 E \cdot 6 / \mathrm{h}$ & 10 & $3.0 \mathrm{E}-6 / \mathrm{h}$ & 3 \\
\hline EWR1SWGB & $\begin{array}{l}100 \mathrm{KW} \text { Generator } \\
\text { Supply Wiring Fails } \\
\text { Open }\end{array}$ & $\begin{array}{c}1.0 \mathrm{E}-7 / \mathrm{h} \\
{[2]}\end{array}$ & 10 & $1.0 \mathrm{E} \cdot 6 \mathrm{~h}$ & 10 & $3.0 E-6 / \mathrm{h}$ & 10 & $3.0 \mathrm{E}-6 / \mathrm{h}$ & 3 \\
\hline
\end{tabular}


Table 7.13. Electrical Emergency Power System Data (Cont'd)

PAGE 6

\begin{tabular}{|c|c|c|c|c|c|c|c|c|c|}
\hline \multirow{2}{*}{$\begin{array}{l}\text { EVENT } \\
\text { NAME }\end{array}$} & \multirow[b]{2}{*}{ DESCRIPTION } & \multicolumn{2}{|c|}{ VALUE USED 391} & \multicolumn{2}{|c|}{ CRBRP-4 } & \multicolumn{2}{|c|}{ IREP } & \multicolumn{2}{|c|}{ GENERIC } \\
\hline & & $\begin{array}{c}\text { Failure } \\
\text { Pate }\end{array}$ & $\begin{array}{l}\text { Error } \\
\text { Factor }\end{array}$ & $\begin{array}{c}\text { Failure } \\
\text { Pate }\end{array}$ & $\begin{array}{l}\text { Error } \\
\text { Factor }\end{array}$ & $\begin{array}{l}\text { Failure } \\
\text { Rate }\end{array}$ & $\begin{array}{l}\text { Error } \\
\text { Factor }\end{array}$ & $\begin{array}{l}\text { Failure } \\
\text { Pate }\end{array}$ & $\begin{array}{l}\text { Error } \\
\text { Factor }\end{array}$ \\
\hline EWR2E1YB & $\begin{array}{l}480 \text { Vac Normal Bus } \\
\text { No. } 2 \text { to E-1 Wiring } \\
\text { Fails Open }\end{array}$ & $\underset{[2]}{1.0 E-7 / h}$ & 10 & $1.0 \mathrm{E}-6 \mathrm{~h}$ & 10 & $3.0 \mathrm{E}-6 / \mathrm{h}$ & 10 & $3.0 \mathrm{E}-6 / \mathrm{h}$ & 3 \\
\hline EWR2E1YC & $\begin{array}{l}\text { Overioad on Normal } \\
\text { Bus No. } 2 \text { to E-1 } \\
\text { Line }\end{array}$ & $\begin{array}{c}1.0 \mathrm{E}-7 \mathrm{~h} \\
{[2]}\end{array}$ & 10 & $\begin{array}{c}1.0 E-7 / \mathrm{h} \\
{[3]}\end{array}$ & 10 & $\begin{array}{c}3.0 \mathrm{E}-7 / \mathrm{h} \\
{[3]}\end{array}$ & 10 & $\begin{array}{c}3.0 \mathrm{E} \cdot 7 \mathrm{~h} \\
{[3]}\end{array}$ & 10 \\
\hline EWR4SWHB & $\begin{array}{l}400 \mathrm{Kw} \text { Generator } \\
\text { Supply Wiring Fails } \\
\text { Open }\end{array}$ & $\begin{array}{c}1.0 \mathrm{E}-7 \mathrm{~h} \\
{[2]}\end{array}$ & 10 & $1.0 \mathrm{E}-6 / \mathrm{h}$ & 10 & $3.0 \mathrm{E} \cdot 6 / \mathrm{h}$ & 10 & $3.0 \mathrm{E}-6 / \mathrm{h}$ & 3 \\
\hline EWRD8AYB & $\begin{array}{l}\text { Bus R-1 Breaker } 8 A \\
\text { Close Ckt Sup. } \\
\text { Wiring Fails Open }\end{array}$ & $\begin{array}{c}1.0 E-7 / \mathrm{h} \\
{[2]}\end{array}$ & 10 & $1.0 \mathrm{E}-6 / \mathrm{h}$ & 10 & $3.0 \mathrm{E}-6 / \mathrm{h}$ & 10 & $3.0 \mathrm{E} \cdot 6 / \mathrm{h}$ & 3 \\
\hline EWRDSAYB & $\begin{array}{l}\text { Bus P-1 Breaker } 9 A \\
\text { Close Ckt Sup. } \\
\text { Wiring Fails Open }\end{array}$ & $\begin{array}{c}1.0 E-7 / \mathrm{h} \\
{[2]}\end{array}$ & 10 & $1.0 \mathrm{E}-6 / \mathrm{h}$ & 10 & $3.0 \mathrm{E}-6 / \mathrm{h}$ & 10 & $3.0 \mathrm{E} \cdot 6 / \mathrm{h}$ & 3 \\
\hline EWRE12YB & $\begin{array}{l}\text { E-1 to R-2B Line } \\
\text { Fails Open }\end{array}$ & $\begin{array}{c}1.0 \mathrm{E}-7 / \mathrm{h} \\
{[2]}\end{array}$ & 10 & $1.0 \mathrm{E}-6 / \mathrm{h}$ & 10 & $3.0 \mathrm{E}-6 / \mathrm{h}$ & 10 & $3.0 \mathrm{E}-6 / \mathrm{h}$ & 3 \\
\hline EWRE1AYB & $\begin{array}{l}\text { Emergency Bus E-1 } \\
\text { Breaker } 1 \mathrm{~A} \text { Wiring } \\
\text { Fails Open }\end{array}$ & $\begin{array}{c}1.0 \mathrm{E}-7 \mathrm{~h} \\
{[2]}\end{array}$ & 10 & $1.0 \mathrm{E}-6 \mathrm{~h}$ & 10 & $3.0 \mathrm{E}-6 / \mathrm{h}$ & 10 & $3.0 \mathrm{E}-6 / \mathrm{h}$ & 3 \\
\hline EWREE2YB & $\begin{array}{l}\text { Emergency Pwr Line } \\
\text { to E-2 Fails Open }\end{array}$ & $\begin{array}{c}1.0 \mathrm{E} \cdot 7 / \mathrm{h} \\
{[2]}\end{array}$ & 10 & $1.0 \mathrm{E}-6 \mathrm{~h}$ & 10 & $3.0 \mathrm{E}-6 / \mathrm{h}$ & 10 & $3.0 \mathrm{E}-6 / \mathrm{h}$ & 3 \\
\hline EWREE2YC & $\begin{array}{l}\text { Uncleared Overload } \\
\text { on Emergency Pwr } \\
\text { Line to E-2 }\end{array}$ & $\begin{array}{c}1.0 \mathrm{E}-7 / \mathrm{h} \\
{[2]}\end{array}$ & 10 & $\begin{array}{c}1.0 \mathrm{E}-7 / \mathrm{h} \\
{[3] .}\end{array}$ & 10 & $\begin{array}{c}3.0 \mathrm{E}-7 / \mathrm{h} \\
{[3]}\end{array}$ & 10 & $\begin{array}{c}3.0 \mathrm{E}-7 / \mathrm{h} \\
{[3]}\end{array}$ & 10 \\
\hline EWRER1YC & $\begin{array}{l}\text { Overload Fault on } \\
\text { E-1 to R-2B Line }\end{array}$ & $\begin{array}{c}1.0 E_{[2]} 7 / \mathrm{h} \\
{[2]}\end{array}$ & 10 & $\begin{array}{c}1.0 \mathrm{E}-7 \mathrm{~h} \\
{[3]}\end{array}$ & 10 & $\underset{[3]}{3.0 \mathrm{E} \cdot 7 / \mathrm{h}}$ & 10 & $\begin{array}{c}3.0 \mathrm{E}-7 \mathrm{~h} \\
{[3]}\end{array}$ & 10 \\
\hline EWRP12YB & $\begin{array}{l}\text { Line From Bus P-1B } \\
\text { to Ups } 2 \text { Fails }\end{array}$ & $\begin{array}{c}1.0 \mathrm{E}-7 / \mathrm{h} \\
{[2]}\end{array}$ & 10 & $1.0 \mathrm{E}-6 / \mathrm{h}$ & 10 & $3.0 \mathrm{E} \cdot 6 / \mathrm{h}$ & 10 & $3.0 \mathrm{E}-6 / \mathrm{h}$ & 3 \\
\hline EWRP1BYC & $\begin{array}{l}\text { Overioad Fault on } \\
\text { E-1 to P-1B Line }\end{array}$ & $\begin{array}{c}1.0 \mathrm{E}-7 \mathrm{~h} \\
{[2]}\end{array}$ & 10 & & & & & & \\
\hline EWRP4EYB & $\begin{array}{l}\text { Line From P-4E to } 10 \\
\mathrm{KW} \text { Rectifier Fails }\end{array}$ & $\begin{array}{c}1.0 \mathrm{E} \cdot 7 / \mathrm{h} \\
{[2]}\end{array}$ & 10 & $1.0 \mathrm{E}-6 / \mathrm{h}$ & 10 & $3.0 \mathrm{E}-6 / \mathrm{h}$ & 10 & $3.0 \mathrm{E}-6 / \mathrm{h}$ & 3 \\
\hline EWRPAUYB & $\begin{array}{l}\text { Line From Bus P-4E } \\
\text { to UPS } 1 \text { Fails }\end{array}$ & $\begin{array}{c}1.0 E-7 / \mathrm{h} \\
{[2]}\end{array}$ & 10 & $1.0 \mathrm{E} \cdot 6 / \mathrm{h}$ & 10 & $3.0 \mathrm{E} \cdot 6 / \mathrm{h}$ & 10 & $3.0 \mathrm{E}-6 / \mathrm{h}$ & 3 \\
\hline EWRPS1YB & $\begin{array}{l}\text { P-1B to S-1B Line } \\
\text { Fails Open }\end{array}$ & $\underset{[2]}{1.0 E-7 / h}$ & 10 & $1.0 \mathrm{E}-6 / \mathrm{h}$ & 10 & $3.0 \mathrm{E}-6 / \mathrm{h}$ & 10 & $3.0 \mathrm{E}-6 / \mathrm{h}$ & 3 \\
\hline EWRPS1YC & $\begin{array}{l}\text { Overload Fault on } \\
P-1 B \text { to } S-1 B \text { Line }\end{array}$ & $\begin{array}{c}1.0 E-7 / h \\
{[2]}\end{array}$ & 10 & $\begin{array}{c}1.0 E-7 / \mathrm{h} \\
{[3]}\end{array}$ & 10 & $\begin{array}{c}3.0 \mathrm{E}-7 \mathrm{~h} \\
{[3]}\end{array}$ & 10 & $\begin{array}{c}3.0 \mathrm{E} \cdot 7 / \mathrm{h} \\
{[3]}\end{array}$ & 10 \\
\hline EWRR12YB & $\begin{array}{l}\text { R-1B to R-2B Line } \\
\text { Fails Open }\end{array}$ & $\begin{array}{c}1.0 E-7 / \mathrm{h} \\
{[2]}\end{array}$ & 10 & $1.0 \mathrm{E}-6 / \mathrm{h}$ & 10 & $3.0 \mathrm{E}-6 / \mathrm{h}$ & 10 & $3.0 \mathrm{E}-6 / \mathrm{h}$ & 3 \\
\hline EWRR12YC & $\begin{array}{l}\text { Overload Fault on } \\
R-1 B \text { to R-2B Line }\end{array}$ & $\begin{array}{c}1.0 \mathrm{E}-7 \mathrm{~h} \\
{[2]}\end{array}$ & 10 & 1.0E-7/h & 10 & $\begin{array}{c}3.0 \mathrm{E}-7 / \mathrm{h} \\
{[3]}\end{array}$ & 10 & $\begin{array}{c}3.0 \mathrm{E}-7 / \mathrm{h} \\
{[3]}\end{array}$ & 10 \\
\hline EWASS1YB & $\begin{array}{l}\text { S-1A to } S-1 B \text { Line } \\
\text { Fails Open }\end{array}$ & $\begin{array}{c}1.0 E-7 / \mathrm{h} \\
{[2]}\end{array}$ & 10 & $1.0 \mathrm{E}-6 / \mathrm{h}$ & 10 & $3.0 \mathrm{E}-6 / \mathrm{h}$ & 10 & $3.0 \mathrm{E}-6 / \mathrm{h}$ & 3 \\
\hline EWRSS1YC & $\begin{array}{l}\text { Overload Fault on } \\
S-1 A \text { to } S-1 B \text { Lin }\end{array}$ & $\begin{array}{c}1.0 \mathrm{E}-7 / \mathrm{h} \\
{[2]}\end{array}$ & 10 & $\begin{array}{c}1.0 \mathrm{E} \cdot 7 / \mathrm{h} \\
{[3]}\end{array}$ & 10 & $\begin{array}{c}3.0 \mathrm{E}-7 \mathrm{~h} \\
{[3]}\end{array}$ & 10 & $\begin{array}{c}3.0 E-7 \mathrm{~h} \\
{[3]}\end{array}$ & 10 \\
\hline
\end{tabular}

Notes: 1. Based on highest bus value for 100 days divided by $2400 \mathrm{~h}$

2. Recommended value tables, EGG-SSRE-8875, Feb. 1990

3. Short to ground value

4. Est. value; time to perform action, lack of action obvious

5. Low power solid state device value

6. Recommended value tables, EEG-SSRE-8875, Feb. 1990

7. $0.1 \times$ failure to run value

8. $0.1 \times$ switch failure value 
Table 7.14. Electrical Continuous Power System Data

PAGE 1

\begin{tabular}{|c|c|c|c|c|c|c|c|c|c|}
\hline \multirow{2}{*}{$\begin{array}{l}\text { EVENT } \\
\text { NAME }\end{array}$} & \multirow[b]{2}{*}{ DESCRIPTION } & \multicolumn{2}{|c|}{ VALUE USED $3 / 91$} & \multicolumn{2}{|c|}{ CRBRP-4 } & \multicolumn{2}{|c|}{ IAEP } & \multicolumn{2}{|c|}{ GENERIC } \\
\hline & & $\begin{array}{c}\text { Failure } \\
\text { Rate }\end{array}$ & $\begin{array}{l}\text { Error } \\
\text { Factor }\end{array}$ & $\begin{array}{l}\text { Failure } \\
\text { Rate }\end{array}$ & $\begin{array}{l}\text { Error } \\
\text { Factor }\end{array}$ & $\begin{array}{l}\text { Failure } \\
\text { Rate }\end{array}$ & $\begin{array}{l}\text { Error } \\
\text { Factor }\end{array}$ & $\begin{array}{c}\text { Failure } \\
\text { Rate }\end{array}$ & $\begin{array}{l}\text { Error } \\
\text { Factor }\end{array}$ \\
\hline UBAU2IBW & $\begin{array}{l}135 \text { VDC Ups } 2 \\
\text { Battery Loses } \\
\text { Capacity }\end{array}$ & $\begin{array}{c}1.0 \mathrm{E}-5 / \mathrm{h} \\
{[1]}\end{array}$ & 5 & $1.0 \mathrm{E} \cdot 6 \mathrm{~h}$ & 3 & $1.0 \mathrm{E}-6 / \mathrm{h}$ & 3 & $1.0 \mathrm{E}-6 / \mathrm{h}$ & 3 \\
\hline UBAUP1BW & $\begin{array}{l}\text { Charged } 135 \text { VDC UPS } \\
1 \text { Battery Fails } \\
\text { Suddenly }\end{array}$ & $\begin{array}{c}1.0 \mathrm{E}-5 / \mathrm{h} \\
{[1]}\end{array}$ & 5 & $1.0 \mathrm{E}-6 / \mathrm{h}$ & 3 & $1.0 \mathrm{E}-6 / \mathrm{h}$ & 3 & $1.0 \mathrm{E}-6 / \mathrm{h}$ & 3 \\
\hline UBAUP2BW & $\begin{array}{l}\text { Charged } 135 \text { VDC Ups } \\
\text { No. } 2 \text { Battery Fails } \\
\text { Suddenly }\end{array}$ & $\begin{array}{c}1.0 E-5 / h \\
{[1]}\end{array}$ & 5 & $1.0 \mathrm{E}-6 / \mathrm{h}$ & 3 & $1.0 \mathrm{E}-6 / \mathrm{h}$ & 3 & $1.0 \mathrm{E}-6 / \mathrm{h}$ & 3 \\
\hline UBAUPIBW & $\begin{array}{l}135 \text { VDC UPS } 1 \\
\text { Battery Loses } \\
\text { Capacity }\end{array}$ & $\begin{array}{c}1.0 \mathrm{E}-5 / \mathrm{h} \\
{[1]}\end{array}$ & 5 & $1.0 \mathrm{E}-6 / \mathrm{h}$ & 3 & $1.0 \mathrm{E}-6 \mathrm{~h}$ & 3 & $1.0 \mathrm{E}-6 / \mathrm{h}$ & 3 \\
\hline UBSCP1QC & $\begin{array}{l}\text { Uncleared Overload } \\
\text { on Cont Power Panel } \\
\text { No. } 1\end{array}$ & $\begin{array}{c}1.0 E-7 / h \\
{[1]}\end{array}$ & 10 & $\begin{array}{c}1.0 \mathrm{E}-7 \mathrm{~h} \\
{[2]}\end{array}$ & 10 & $\begin{array}{c}3.0 \mathrm{E}-7 / \mathrm{h} \\
{[2]}\end{array}$ & 10 & $\begin{array}{c}3.0 \mathrm{E}-7 \mathrm{~h} \\
{[2]}\end{array}$ & 10 \\
\hline UBSCP2QC & $\begin{array}{l}\text { Uncleared Overload } \\
\text { on Cont. Power Panel } \\
\text { No. } 2\end{array}$ & $\begin{array}{c}1.0 E-7 / \mathrm{h} \\
{[1]}\end{array}$ & 10 & $\begin{array}{c}1.0 E-7 / h \\
{[2]}\end{array}$ & 10 & $\begin{array}{c}3.0 \mathrm{E}-7 \mathrm{~h} \\
{[2]}\end{array}$ & 10 & $\begin{array}{c}3.0 \mathrm{E}-7 \mathrm{~h} \\
{[2]}\end{array}$ & 10 \\
\hline UBYUP1BW & $\begin{array}{l}135 \text { VDC UPS } 1 \\
\text { Battery Has Low } \\
\text { Charge Rate }\end{array}$ & $\begin{array}{c}1.0 \mathrm{E}-5 / \mathrm{h} \\
{[1]}\end{array}$ & 5 & $1.0 \mathrm{E}-6 / \mathrm{h}$ & & $1.0 \mathrm{E}-6 / \mathrm{h}$ & 3 & & \\
\hline UBYUP1YW & $\begin{array}{l}\text { UPS } 1 \text { Charger } \\
\text { Circuit Faits }\end{array}$ & $\begin{array}{c}1.0 E-5 / h \\
{[1]}\end{array}$ & 5 & $1.0 \mathrm{E}-6 / \mathrm{h}$ & & $1.0 E-6 / \mathrm{h}$ & 3 & & \\
\hline UBYUP2BW & $\begin{array}{l}135 \text { VDC Ups } 2 \\
\text { Battery Has Low } \\
\text { Charge Rate }\end{array}$ & $\begin{array}{c}1.0 E-5 / \mathrm{h} \\
{[1]}\end{array}$ & 5 & $1.0 \mathrm{E}-6 / \mathrm{h}$ & & $1.0 \mathrm{E}-6 / \mathrm{h}$ & 3 & & \\
\hline UBYUP2YW & $\begin{array}{l}\text { Ups } 2 \text { Charger } \\
\text { Circuit Fails }\end{array}$ & $\begin{array}{c}1.0 \mathrm{E}-5 / \mathrm{h} \\
{[1]}\end{array}$ & 5 & $1.0 E-6 / \mathrm{h}$ & & $1.0 \mathrm{E}-6 / \mathrm{h}$ & 3 & $\cdot$ & \\
\hline UC80130W & $\begin{array}{l}\text { CIRCUIT BREAK } \\
\text { ER FAULT SPURIOUS T } \\
\text { RIP CPP } 1 \text { BKR } 3\end{array}$ & $3.0 \mathrm{E}-7 / \mathrm{h}$ & 10 & $1.0 \mathrm{E}-5 / \mathrm{h}$ & 10 & $3.0 E-5 / d$ & 10 & $3.0 E-5 / d$ & 3 \\
\hline UCB022QW & $\begin{array}{l}\text { CIRCUIT BREAK } \\
\text { ER FAULT SPURIOUS T } \\
\text { RIP CPP } 2 \text { BKR } 2 \\
\end{array}$ & $3.0 \mathrm{E}-7 / \mathrm{h}$ & 10 & $1.0 E-5 / h$ & 10 & $3.0 \mathrm{E}-5 / \mathrm{d}$ & 10 & $3.0 \mathrm{E}-5 / \mathrm{d}$ & 3 \\
\hline UCBO23OW & $\begin{array}{l}\text { CIRCUIT BREAK } \\
\text { ER FAULT SPURIOUS T } \\
\text { RIP CPP } 2 \text { BKR } 3\end{array}$ & $3.0 \mathrm{E}-7 / \mathrm{h}$ & 10 & $1.0 \mathrm{E}-5 / \mathrm{h}$ & 10 & $3.0 \mathrm{E}-5 / \mathrm{d}$ & 10 & $3.0 \mathrm{E}-5 / \mathrm{d}$ & 3 \\
\hline UC8103FW & $\begin{array}{l}\text { CIRCUIT BREAK } \\
\text { ER FAULT SPURIOUS T } \\
\text { RIP CPP R } 10 \text { BKR } 3\end{array}$ & $3.0 \mathrm{E}-7 / \mathrm{h}$ & 10 & $1.0 \mathrm{E}-5 \mathrm{~h}$ & 10 & 3.0E-5/d & 10 & $3.0 E-5 / d$ & 3 \\
\hline UC81250O & $\begin{array}{l}\text { CPS Panel } 1 \text { Breaker } \\
25 \text { Fails Open }\end{array}$ & $\begin{array}{c}3.0 E-7 / \mathrm{h} \\
{[1]}\end{array}$ & 10 & $1.0 \mathrm{E} \cdot 5 / \mathrm{h}$ & 10 & $3.08-5 / d$ & 10 & $3.0 E-5 / d$ & 3 \\
\hline UCB217QO & $\begin{array}{l}\text { CPS Panel } 2 \text { Breaker } \\
17 \text { Fails Open }\end{array}$ & $\begin{array}{c}3.0 E-7 / \mathrm{h} \\
{[1]}\end{array}$ & 10 & $1.0 \mathrm{E}-5 / \mathrm{h}$ & 10 & $3.0 \mathrm{E}-5 / \mathrm{d}$ & 10 & $3.0 \mathrm{E}-5 / \mathrm{d}$ & 3 \\
\hline UC8811YO & $\begin{array}{l}\text { Ups No. } 1 \text { Bypass Ckt } \\
\text { Brkr No. } 1 \text { Fails }\end{array}$ & $\begin{array}{c}3.0 E-7 / \mathrm{h} \\
{[1]}\end{array}$ & 10 & $1.0 \mathrm{E} \cdot 5 / \mathrm{h}$ & 10 & 3.0E-5/d & 10 & $3.0 \mathrm{E}-5 / \mathrm{d}$ & 3 \\
\hline UCBB12YO & $\begin{array}{l}\text { Bypass Power Input } \\
\text { Ckt Breaker to Sbs } \\
\text { No.1 Fails }\end{array}$ & $\begin{array}{c}3.0 E-7 / h \\
{[1]}\end{array}$ & 10 & $1.0 \mathrm{E}-6 / \mathrm{h}$ & 10 & $3.0 E-6 / \mathrm{h}$ & 10 & $3.0 \mathrm{E}-6 / \mathrm{h}$ & 3 \\
\hline UCBB21YO & $\begin{array}{l}\text { Bypass Power Input } \\
\text { Ckt Breaker to SBS } \\
\text { No. } 2 \text { Fails }\end{array}$ & $\begin{array}{c}3.0 \mathrm{E}-7 / \mathrm{h} \\
{[1]}\end{array}$ & 10 & $1.0 \mathrm{E}-6 / \mathrm{h}$ & 10 & $3.0 \mathrm{E}-6 / \mathrm{h}$ & 10 & $3.0 \mathrm{E}-6 / \mathrm{h}$ & 3 \\
\hline UCBB22YO & $\begin{array}{l}\text { Ups No. } 2 \text { Bypass Ckt } \\
\text { Brks No. } 2 \text { Fails }\end{array}$ & $\begin{array}{c}3.0 E-7 / h \\
{[1]}\end{array}$ & 10 & $1.0 \mathrm{E}-5 / \mathrm{h}$ & 10 & $3.0 E-5 / d$ & 10 & $3.0 E-5 / d$ & 3 \\
\hline UCBIB1YO & $\begin{array}{l}\text { Ups No. } 1 \text { Isolation } \\
\text { Breaker Fails Open }\end{array}$ & $\begin{array}{c}3.0 \mathrm{E} \cdot 7 / \mathrm{h} \\
{[1]}\end{array}$ & 10 & $1.0 \mathrm{E}-5 / \mathrm{h}$ & 10 & $3.0 \mathrm{E}-5 / \mathrm{d}$ & 10 & $3.0 E-5 / d$ & 3 \\
\hline UCBIB2YO & $\begin{array}{l}\text { Ups No. } 2 \text { Isolation } \\
\text { Breaker Fails Open }\end{array}$ & $\begin{array}{c}3.0 E-7 / \mathrm{h} \\
{[1]}\end{array}$ & 10 & $1.0 E-5 / h$ & 10 & $3.0 E-5 / d$ & 10 & $3.0 \mathrm{E} \cdot 5 / \mathrm{d}$ & 3 \\
\hline
\end{tabular}


Table 7.14. Electrical Continuous Power System Data (Cont'd)

PAGE 2

\begin{tabular}{|c|c|c|c|c|c|c|c|c|c|}
\hline \multirow{2}{*}{$\begin{array}{l}\text { EVENT } \\
\text { NAME }\end{array}$} & \multirow[b]{2}{*}{ DESCRIPTION } & \multicolumn{2}{|c|}{ VALUE USED $3 / 91$} & \multicolumn{2}{|c|}{ CRBRP-4 } & \multicolumn{2}{|c|}{ IREP } & \multicolumn{2}{|c|}{ GENERIC } \\
\hline & & $\begin{array}{l}\text { Failure } \\
\text { Rate }\end{array}$ & $\begin{array}{l}\text { Error } \\
\text { Factor }\end{array}$ & $\begin{array}{l}\text { Failure } \\
\text { Rate }\end{array}$ & $\begin{array}{l}\text { Error } \\
\text { Factor }\end{array}$ & $\begin{array}{l}\text { Failure } \\
\text { Rate }\end{array}$ & $\begin{array}{l}\text { Error } \\
\text { Factor }\end{array}$ & $\begin{array}{l}\text { Failure } \\
\text { Rate }\end{array}$ & $\begin{array}{l}\text { Error } \\
\text { Factor }\end{array}$ \\
\hline UCBS12YO & $\begin{array}{l}\text { SBS No. } 1 \text { to SBS No. } \\
2 \text { Circuit Breaker } \\
\text { Fails Open }\end{array}$ & $\begin{array}{c}3.0 \mathrm{E}-7 \mathrm{M} \\
{[1]}\end{array}$ & 10 & $1.0 \mathrm{E}-5 / \mathrm{h}$ & 10 & $3.0 \mathrm{E}-5 / \mathrm{d}$ & 10 & $3.0 E-5 / d$ & 3 \\
\hline UCBS21YO & $\begin{array}{l}\text { SBS No. } 2 \text { to SBS No. } \\
1 \text { Circuit Breaker } \\
\text { Fails Open }\end{array}$ & $\begin{array}{c}3.0 \mathrm{E}-7 \mathrm{~m} \\
{[1]}\end{array}$ & 10 & $1.0 \mathrm{E}-5 / \mathrm{h}$ & 10 & $3.0 \mathrm{E}-5 / \mathrm{d}$ & 10 & $3.0 \mathrm{E}-5 / \mathrm{d}$ & 3 \\
\hline UCBU11YO & $\begin{array}{l}135 \text { VDC UPS } 1 \\
\text { 8atery Output Ckt } \\
\text { Brks Fails Open }\end{array}$ & $\begin{array}{c}1.0 \mathrm{E}-7 \mathrm{~h} \\
{[3]}\end{array}$ & 10 & $1.0 \mathrm{E}-5 / \mathrm{h}$ & 10 & $3.0 \mathrm{E}-5 / \mathrm{d}$ & 10 & $3.0 \mathrm{E}-5 / \mathrm{d}$ & 3 \\
\hline UCBU22YO & $\begin{array}{l}135 \text { VDC Ups } 2 \\
\text { Battery Output Ckt } \\
\text { Brks Fails Open }\end{array}$ & $\begin{array}{c}3.0 \mathrm{E} \cdot \mathrm{7m} \\
{[1]}\end{array}$ & 10 & $1.0 \mathrm{E}-5 / \mathrm{h}$ & 10 & $3.0 \mathrm{E}-5 / \mathrm{d}$ & 10 & $3.0 \mathrm{E}-5 / \mathrm{d}$ & 3 \\
\hline UCBUP1YO & $\begin{array}{l}\text { Ups No. } 1 \text { Inverter } \\
\text { Output Breaker Fails } \\
\text { Open }\end{array}$ & $\begin{array}{c}3.0 \mathrm{E}-7 \mathrm{~h} \\
{[1]}\end{array}$ & 10 & $1.0 \mathrm{E}-5 / \mathrm{h}$ & 10 & $3.0 \mathrm{E}-5 / \mathrm{d}$ & 10 & $3.0 \mathrm{E}-5 / \mathrm{d}$ & 3 \\
\hline UCBUP2YO & $\begin{array}{l}\text { Ups No. } 2 \text { Inverter } \\
\text { Output Breaker Fails } \\
\text { Open }\end{array}$ & $\begin{array}{c}3.0 \mathrm{E}-7 \mathrm{~h} \\
{[1]}\end{array}$ & 10 & $1.0 \mathrm{E}-5 / \mathrm{h}$ & 10 & $3.0 \mathrm{E}-5 / \mathrm{d}$ & 10 & $3.0 \mathrm{E}-5 / \mathrm{d}$ & 3 \\
\hline UINU12YU & $\begin{array}{l}\text { Ups No. } 1 \text { and Ups } \\
\text { No. } 2 \text { Common Mode } \\
\text { Failure }\end{array}$ & $\begin{array}{c}3.0 \mathrm{E} \cdot 6 \mathrm{~h} \\
{[4]}\end{array}$ & 10 & $\begin{array}{c}1.0 E-6 / h \\
{[5]}\end{array}$ & 10 & $\begin{array}{c}3.0 \mathrm{E} \cdot 6 / \mathrm{h} \\
{[5]}\end{array}$ & 10 & $\begin{array}{c}2.0 \mathrm{E} \cdot 6 \mathrm{~h} \\
{[5]}\end{array}$ & 10 \\
\hline UINU2BYW & $\begin{array}{l}135 \text { VDC Ups } 2 \\
\text { Battery Low Charge } \\
\text { Rate Undetected }\end{array}$ & $\begin{array}{c}3.0 \mathrm{E}-6 / \mathrm{h} \\
{[1]}\end{array}$ & 10 & $\begin{array}{c}1.0 \mathrm{E}-6 \mathrm{~h} \\
{[5]}\end{array}$ & 10 & $\begin{array}{c}3.0 \mathrm{E}-6 / \mathrm{h} \\
{[5]}\end{array}$ & 10 & $\begin{array}{c}2.0 \mathrm{E}-6 \mathrm{~h} \\
{[5]}\end{array}$ & 10 \\
\hline UINV18YL & $\begin{array}{l}135 \text { VDC UPS } 1 \\
\text { Battery Capacity } \\
\text { Loss Not Detected }\end{array}$ & $\begin{array}{c}1.0 \mathrm{E}-3 / \mathrm{d} \\
{[6]}\end{array}$ & 30 & & & & & & \\
\hline UINV18YW & $\begin{array}{l}135 \text { VDC UPS } 1 \\
\text { Battery Low Charge } \\
\text { Rate Not Detected }\end{array}$ & $\begin{array}{c}3.0 \mathrm{E}-6 / \mathrm{h} \\
{[1]}\end{array}$ & 10 & $\begin{array}{c}1.0 \mathrm{E}-6 \mathrm{~h} \\
{[5]}\end{array}$ & 10 & $\begin{array}{c}3.0 \mathrm{E}-6 / \mathrm{h} \\
{[5]}\end{array}$ & 10 & $\begin{array}{c}2.0 \mathrm{E}-6 \mathrm{~h} \\
{[5]}\end{array}$ & 10 \\
\hline UINV2BYL & $\begin{array}{l}135 \text { VDC Ups } 2 \\
\text { 8attery Capacity } \\
\text { Loss Not Detected }\end{array}$ & $\begin{array}{c}1.0 \mathrm{E}-3 / \mathrm{d} \\
{[6]}\end{array}$ & 30 & & & & & & \\
\hline UIVUP 1YW & $\begin{array}{l}\text { Ups No. } 1 \text { Inverter } \\
\text { Fails }\end{array}$ & $\begin{array}{c}5.0 \mathrm{E}-6 / \mathrm{h} \\
{[1]}\end{array}$ & 5 & & & $1.0 \mathrm{E}-4 / \mathrm{h}$ & 3 & & \\
\hline UIVUP2YW & $\begin{array}{l}\text { Ups No. } 2 \text { Inverter } \\
\text { Fails }\end{array}$ & $\begin{array}{c}5.0 \mathrm{E}-6 / \mathrm{h} \\
{[1]}\end{array}$ & 5 & & & $1.0 \mathrm{E}-4 / \mathrm{h}$ & 3 & & \\
\hline USWSS1YW & $\begin{array}{l}\text { Ups No. } 1 \text { Static } \\
\text { Bypass Switch or SBS } \\
\text { Control Fails }\end{array}$ & $\begin{array}{c}3.0 \mathrm{E}-6 / \mathrm{h} \\
{[1]}\end{array}$ & 10 & $\begin{array}{c}1.0 E-6 / \mathrm{h} \\
{[7]}\end{array}$ & 10 & $\begin{array}{c}3.0 E-6 \mathrm{~m} \\
{[7]}\end{array}$ & 10 & $\begin{array}{c}3.0 E-6 / \mathrm{h} \\
{[7]}\end{array}$ & 10 \\
\hline USWSS2YW & $\begin{array}{l}\text { Ups No. } 2 \text { Static } \\
\text { Bypass Switch or SBS } \\
\text { Control Fails }\end{array}$ & $\begin{array}{c}3.0 \mathrm{E}-6 / \mathrm{h} \\
{[1]}\end{array}$ & 10 & $\begin{array}{c}1.0 \mathrm{E}-6 \mathrm{~h} \\
{[7]}\end{array}$ & 10 & $\begin{array}{c}3.0 E-6 / \mathrm{h} \\
{[7]}\end{array}$ & 10 & $\begin{array}{l}3.0 E-6 \mathrm{~m} \\
{[7]}\end{array}$ & 10 \\
\hline USWUP1YO & $\begin{array}{l}\text { Ups No. } 1 \text { Manual } \\
\text { Bypass Switch Fails } \\
\text { Open }\end{array}$ & $\begin{array}{c}1.0 \mathrm{E} \cdot 6 \mathrm{~h} \\
{[1]}\end{array}$ & 10 & $1.0 \mathrm{E}-7 \mathrm{~h}$ & 10 & & & & \\
\hline USWUP2YO & $\begin{array}{l}\text { Ups No. } 2 \text { Manual } \\
\text { Bypass Switch Fails } \\
\text { Open } \\
\end{array}$ & $\begin{array}{c}1.0 \mathrm{E}-6 \mathrm{~h} \\
{[1]}\end{array}$ & 10 & $1.0 \mathrm{E}-7 \mathrm{~h}$ & 10 & & & & \\
\hline UTRUBTYW & $\begin{array}{l}\text { Ups Bypass } 480 \mathrm{~V} / \\
120 \mathrm{~V} \text { Transformer } \\
\text { Fails } \\
\end{array}$ & $\begin{array}{c}1.0 \mathrm{E}-6 \mathrm{~h} \\
{[1]}\end{array}$ & 10 & $1.0 \mathrm{E}-6 / \mathrm{h}$ & 3 & $1.0 \mathrm{E}-6 / \mathrm{h}$ & 3 & $1.0 \mathrm{E}-6 / \mathrm{h}$ & 3 \\
\hline UVRBSSYW & $\begin{array}{l}\text { Ups Bypass Surge } \\
\text { Suppresor Fails }\end{array}$ & $\begin{array}{c}3.0 \mathrm{E}-6 \mathrm{~h} \\
{[1]}\end{array}$ & 10 & $\begin{array}{c}1.0 \mathrm{E}-6 / \mathrm{h} \\
{[7]}\end{array}$ & 10 & $\begin{array}{c}3.0 E-6 / h \\
{[7]}\end{array}$ & 10 & $\begin{array}{c}3.0 \mathrm{E}-6 / \mathrm{h} \\
{[7]}\end{array}$ & 10 \\
\hline UWRS12YB & $\begin{array}{l}\text { SBS No. } 1 \text { to SBS No. } \\
2 \text { Wiring Fails }\end{array}$ & $\begin{array}{c}1.0 \mathrm{E}-7 \mathrm{~h} \\
{[1]}\end{array}$ & 10 & $1.0 \mathrm{E}-6 / \mathrm{h}$ & 10 & $3.0 \mathrm{E}-6 / \mathrm{h}$ & 10 & $3.0 \mathrm{E}-6 / \mathrm{h}$ & 3 \\
\hline UWRS21YB & $\begin{array}{l}\text { S8S No. } 2 \text { to SBS } \\
\text { No.1 Wiring Fails }\end{array}$ & $\begin{array}{c}1.0 \mathrm{E}-7 / \mathrm{h} \\
{[1]}\end{array}$ & 10 & $1.0 \mathrm{E}-6 / \mathrm{h}$ & 10 & $3.0 \mathrm{E}-6 / \mathrm{h}$ & 10 & $3.0 \mathrm{E}-6 / \mathrm{h}$ & 3 \\
\hline
\end{tabular}


- Table 7.14. Electrical Continuous Power System Data (Cont'd)

PAGE 3

\begin{tabular}{|c|c|c|c|c|c|c|c|c|c|}
\hline \multirow{2}{*}{$\begin{array}{l}\text { EVENT } \\
\text { NAME }\end{array}$} & \multirow[b]{2}{*}{ DESCRIPTION } & \multicolumn{2}{|c|}{ VALUE USED $3 / 91$} & \multicolumn{2}{|c|}{ CRBRP-4 } & \multicolumn{2}{|c|}{ IREP } & \multicolumn{2}{|c|}{ GENERIC } \\
\hline & & $\begin{array}{c}\text { Failure } \\
\text { Rate }\end{array}$ & $\begin{array}{l}\text { Error } \\
\text { Factor }\end{array}$ & $\begin{array}{c}\text { Failure } \\
\text { Rate }\end{array}$ & $\begin{array}{l}\text { Error } \\
\text { Factor }\end{array}$ & $\begin{array}{c}\text { Failure } \\
\text { Rate }\end{array}$ & $\begin{array}{l}\text { Error } \\
\text { Factor }\end{array}$ & $\begin{array}{c}\text { Failure } \\
\text { Rate }\end{array}$ & $\begin{array}{l}\text { Error } \\
\text { Factor }\end{array}$ \\
\hline UWRUBWYB & $\begin{array}{l}\text { Ups Bypass Supply } \\
\text { Wiring Fails }\end{array}$ & $\underset{[1]}{1.0 \mathrm{E}-7 \mathrm{~h}}$ & 10 & $1.0 \mathrm{E}-6 / \mathrm{h}$ & 10 & $3.0 \mathrm{E}-6 / \mathrm{h}$ & 10 & $3.0 \mathrm{E}-6 / \mathrm{h}$ & 3 \\
\hline UWRUCIYB & $\begin{array}{l}\text { Ups No. } 1 \text { to CPP No. } \\
1 \text { Wiring Fails }\end{array}$ & $\begin{array}{c}1.0 E-7 / \mathrm{m} \\
{[1]}\end{array}$ & 10 & $1.0 \mathrm{E}-6 / \mathrm{h}$ & 10 & $3.0 \mathrm{E}-6 / \mathrm{h}$ & 10 & $3.0 \mathrm{E}-6 \mathrm{M}$ & 3 \\
\hline UWRUC2YB & $\begin{array}{l}\text { Ups No. } 2 \text { to CPP No. } \\
2 \text { Wiring Fails }\end{array}$ & $\begin{array}{c}1.0 E-7 / \mathrm{h} \\
{[1]}\end{array}$ & 10 & $1.0 \mathrm{E}-6 / \mathrm{h}$ & 10 & $3.0 \mathrm{E}-6 / \mathrm{h}$ & 10 & $3.0 \mathrm{E}-6 / \mathrm{h}$ & 3 \\
\hline
\end{tabular}

Notes: 1. Recommended value tables, EGGSSRE-8875, Feb. 1990

2. Short to ground value

3. Recommended value tables, EEG-SSRE-8875, Feb. 1990

4. EEG-SSRE-8875, sync logic module, low power solid state

5. Low power solid state device value

6. Est. value; operator does not detect deficiency upon testing

7. High power solld state device value 
Table 7.15. Electrical 115V DC System Data

PAGE 1

\begin{tabular}{|c|c|c|c|c|c|c|c|c|c|}
\hline \multirow{2}{*}{$\begin{array}{l}\text { EVENT } \\
\text { NAME }\end{array}$} & \multirow[b]{2}{*}{ DESCRIPTION } & \multicolumn{2}{|c|}{ VALUE USED $3 / 91$} & \multicolumn{2}{|c|}{ CRBRP.4 } & \multicolumn{2}{|c|}{ IREP } & \multicolumn{2}{|c|}{ GENEAIC } \\
\hline & & $\begin{array}{l}\text { Failure } \\
\text { Rate }\end{array}$ & $\begin{array}{l}\text { Error } \\
\text { Factor }\end{array}$ & $\begin{array}{l}\text { Failure } \\
\text { Rate }\end{array}$ & $\begin{array}{l}\text { Error } \\
\text { Factor }\end{array}$ & $\begin{array}{l}\text { Failure } \\
\text { Rate }\end{array}$ & $\begin{array}{l}\text { Error } \\
\text { Factor }\end{array}$ & $\begin{array}{l}\text { Failure } \\
\text { Rate }\end{array}$ & $\begin{array}{l}\text { Error } \\
\text { Factor }\end{array}$ \\
\hline LBA12CBW & $\begin{array}{l}125 \text { VDC Station } \\
\text { Battery Loses } \\
\text { Capacity }\end{array}$ & $\begin{array}{c}1.0 E-5 / h \\
{[1]}\end{array}$ & 5 & $1.0 E-6 / \mathrm{h}$ & 3 & $1.0 \mathrm{E}-6 \mathrm{~h}$ & 3 & $1.0 \mathrm{E}-6 / \mathrm{h}$ & 3 \\
\hline LBA12MBW & $\begin{array}{l}\text { Charged } 125 \text { VDC } \\
\text { Station Battery } \\
\text { Fails Suddenly }\end{array}$ & $\begin{array}{c}1.0 \mathrm{E}-5 / \mathrm{h} \\
{[1]}\end{array}$ & 5 & $1.0 \mathrm{E}-6 \mathrm{~h}$ & 3 & $1.0 \mathrm{E}-6 / \mathrm{h}$ & 3 & $1.0 \mathrm{E}-6 / \mathrm{h}$ & 3 \\
\hline LBSO01BU & $\begin{array}{l}125 \text { VDC Distr. Cente } \\
\text { r Bus Pwr Sources Co } \\
\text { mmon Mode Failure }\end{array}$ & $\begin{array}{c}1.0 \mathrm{E}-7 \mathrm{~m} \\
{[2]}\end{array}$ & 10 & & & & & & \\
\hline LSS002BU & $\begin{array}{l}125 \text { VDC/480 Vac Norm } \\
\text { Bus Common Mode Loss } \\
\text { of Power Sources }\end{array}$ & $\underset{[2]}{1.0 E \cdot 7 / \mathrm{h}}$ & 10 & & & & & & \\
\hline LSS003BU & $\begin{array}{l}125 \text { VDC/480 Vac Emer } \\
\text { Bus Common Mode Loss } \\
\text { of Power Sources }\end{array}$ & $\begin{array}{c}1.0 \mathrm{E}-7 / \mathrm{h} \\
{[2]}\end{array}$ & 10 & & & & . & & \\
\hline LBSO04BU & $\begin{array}{l}125 \text { VDC/2.4 KVAC Bus } \\
\text { Common Mode Loss of } \\
\text { Power Sources }\end{array}$ & $\begin{array}{c}1.0 \mathrm{E}-7 / \mathrm{h} \\
{[2]}\end{array}$ & 10 & & & & & & \\
\hline LBSO05BU & $\begin{array}{l}125 \text { VDC/13.8 KVAC } \\
\text { Bus Common Mode Loss } \\
\text { of Power Sources }\end{array}$ & $\begin{array}{c}1.0 \mathrm{E}-7 / \mathrm{h} \\
{[2]}\end{array}$ & 10 & & & & & & \\
\hline LBS121BC & $\begin{array}{l}\text { First } 125 \text { VDC Ground } \\
\text { Fault Occurs }\end{array}$ & $\begin{array}{c}1.0 \mathrm{E} \cdot 7 \mathrm{~m} \\
{[1]}\end{array}$ & 10 & $\begin{array}{c}1.0 \mathrm{E}-7 / \mathrm{h} \\
{[3]}\end{array}$ & 10 & $\begin{array}{c}3.0 \mathrm{E}-7 \mathrm{M} \\
{[3]}\end{array}$ & 10 & $\begin{array}{c}3.0 E-7 / \mathrm{h} \\
{[3]}\end{array}$ & 10 \\
\hline LBS122BC & $\begin{array}{l}\text { Second } 125 \text { VDC } \\
\text { Ground Fault Oळurs }\end{array}$ & $\begin{array}{c}1.0 E-7 \mathrm{~m} \\
{[1]}\end{array}$ & 10 & $\begin{array}{c}1.0 \mathrm{E} \cdot 7 \mathrm{~h} \\
{[3]}\end{array}$ & 10 & $\begin{array}{c}3.0 E-7 / \mathrm{m} \\
{[3]}\end{array}$ & 10 & $\begin{array}{c}3.0 \mathrm{E}-7 \mathrm{~h} \\
{[3]}\end{array}$ & 10 \\
\hline LBS124BC & $\begin{array}{l}\text { Uncleared Overload } \\
\text { on } 125 \text { VDC } / 480 \text { Vac } \\
\text { Bus }\end{array}$ & $\begin{array}{c}1.0 \mathrm{E}-7 \mathrm{~m} \\
{[1]}\end{array}$ & 10 & $1.0 \mathrm{E}-7 \mathrm{~m}$ & 10 & $\begin{array}{c}3.0 \mathrm{E}-7 / \mathrm{M} \\
{[3]}\end{array}$ & 10 & $\underset{[3]}{3.0 \mathrm{E}-7 \mathrm{~h}}$ & 10 \\
\hline LBS12EBC & $\begin{array}{l}\text { Uncleared Overload } \\
\text { on } 125 \text { VDC Emergency } \\
\text { Bus }\end{array}$ & $\begin{array}{c}1.0 \mathrm{E}-7 / \mathrm{h} \\
{[1]}\end{array}$ & 10 & $\begin{array}{c}1.0 \mathrm{E}-7 \mathrm{~h} \\
{[3]}\end{array}$ & 10 & $\begin{array}{c}3.0 \mathrm{E}-7 / \mathrm{h} \\
{[3]}\end{array}$ & 10 & $\begin{array}{c}3.0 \mathrm{E}-7 / \mathrm{h} \\
{[3]}\end{array}$ & 10 \\
\hline LBS12M8A & $\begin{array}{l}\text { Line-to-Line Short } \\
\text { on } 125 \text { VDC Dist } \\
\text { Center Bus }\end{array}$ & $\begin{array}{c}1.0 \mathrm{E}-7 \mathrm{~m} \\
{[1]}\end{array}$ & 10 & $1.0 \mathrm{E}-8 / \mathrm{h}$ & 10 & $3.0 \mathrm{E}-8 \mathrm{~m}$ & 10 & $3.0 \mathrm{E}-8 \mathrm{~h}$ & 10 \\
\hline LBS133BC & $\begin{array}{l}\text { Uncleared Overload } \\
\text { on } 125 \mathrm{VDC} / 13.8 \mathrm{KV} \\
\text { Bus }\end{array}$ & $\begin{array}{c}1.0 E \cdot 7 / \mathrm{h} \\
{[1]}\end{array}$ & 10 & $\begin{array}{c}1.0 \mathrm{E}-7 / \mathrm{h} \\
{[3]}\end{array}$ & 10 & $\begin{array}{c}3.0 \mathrm{E}-7 / \mathrm{h} \\
{[3]}\end{array}$ & 10 & $\begin{array}{c}3.0 \mathrm{E}-7 / \mathrm{h} \\
{[3]}\end{array}$ & 10 \\
\hline LBS244BC & $\begin{array}{l}\text { Uncleared Overioad } \\
\text { on } 125 \mathrm{VDC} / 2.4 \mathrm{KV} \\
\text { Bus }\end{array}$ & $\begin{array}{c}1.0 E-7 / \mathrm{m} \\
{[1]}\end{array}$ & 10 & $\underset{[3]}{1.0 E-7 / \mathrm{h}}$ & 10 & $\begin{array}{c}3.0 \mathrm{E}-7 \mathrm{~h} \\
{[3]}\end{array}$ & 10 & $\begin{array}{c}3.0 \mathrm{E}-7 / \mathrm{h} \\
{[3]}\end{array}$ & 10 \\
\hline LBSEB1BC & $\begin{array}{l}\text { Uncleared Overload } \\
\text { on } 125 \text { VDC/480 Vac } \\
\text { Emerg Bus No. } 1\end{array}$ & $\begin{array}{c}1.0 E-7 / \mathrm{h} \\
{[1]}\end{array}$ & 10 & $\begin{array}{c}1.0 E-7 \mathrm{~m} \\
{[3]}\end{array}$ & 10 & $\begin{array}{c}3.0 E \cdot 7 / \mathrm{h} \\
{[3]}\end{array}$ & 10 & $\begin{array}{c}3.0 \mathrm{E} \cdot 7 \mathrm{~h} \\
{[3]}\end{array}$ & $\cdot 10$ \\
\hline LBSEB2BC & $\begin{array}{l}\text { Uncleared Overload } \\
\text { on } 125 \text { VDC } / 480 \text { Vac } \\
\text { Emerg Bus No. } 2\end{array}$ & $\begin{array}{c}1.0 \mathrm{E} \cdot 7 / \mathrm{h} \\
{[1]}\end{array}$ & 10 & $\begin{array}{c}1.0 \mathrm{E}-7 \mathrm{~m} \\
{[3]}\end{array}$ & 10. & $\begin{array}{c}3.0 \mathrm{E}-7 / \mathrm{h} \\
{[3]}\end{array}$ & 10 & $\begin{array}{c}3.0 \mathrm{E}-7 / \mathrm{h} \\
{[3]}\end{array}$ & 10 \\
\hline LBY12CBW & $\begin{array}{l}125 \text { VDC Station } \\
\text { Battery Has Failed } \\
\text { Charger }\end{array}$ & $\begin{array}{c}1.0 E-5 / h \\
{[1]}\end{array}$ & 5 & $1.0 \mathrm{E}-6 / \mathrm{h}$ & & $1.0 \mathrm{E}-6 / \mathrm{h}$ & 3 & & \\
\hline LC8D068O & $\begin{array}{l}125 \text { VDC Distr Center } \\
\text { Bus Breaker } 6 \text { Fails } \\
\text { Open }\end{array}$ & $\begin{array}{c}3.0 \mathrm{E}-7 \mathrm{~m} \\
{[1]}\end{array}$ & 10 & $1.0 E-5 / \mathrm{h}$ & 10 & $3.0 \mathrm{E}-5 / \mathrm{d}$ & 10 & $3.0 E-5 / d$ & 3 \\
\hline LC8D07BO & $\begin{array}{l}125 \text { VDC Distr Center } \\
\text { Bus Breaker } 7 \text { Fails } \\
\text { Open }\end{array}$ & $\begin{array}{c}3.0 \mathrm{E} \cdot 7 / \mathrm{h} \\
{[1]}\end{array}$ & 10 & $1.0 \mathrm{E}-5 / \mathrm{h}$ & 10 & $3.0 \mathrm{E}-5 / \mathrm{d}$ & 10 & $3.0 \mathrm{E}-5 / \mathrm{d}$ & 3 \\
\hline LCBD10BO & $\begin{array}{l}125 \text { VDC Distr Center } \\
\text { Bus Breaker } 10 \text { Fails } \\
\text { Open }\end{array}$ & $\begin{array}{c}3.0 \mathrm{E} \cdot 7 / \mathrm{h} \\
{[1]}\end{array}$ & 10 & $1.0 \mathrm{E}-5 / \mathrm{h}$ & 10 & $3.0 \mathrm{E}-5 / \mathrm{d}$ & 10 & $3.0 \mathrm{E}-5 / \mathrm{d}$ & 3 \\
\hline LCBD11BO & $\begin{array}{l}125 \text { VDC Dist Center } \\
\text { Bus Breaker } 11 \text { Fails } \\
\text { Open }\end{array}$ & $\begin{array}{c}3.0 \mathrm{E}-7 / \mathrm{h} \\
{[1]}\end{array}$ & 10 & $1.0 \mathrm{E}-5 / \mathrm{h}$ & 10 & $3.0 \mathrm{E}-5 / \mathrm{d}$ & 10 & $3.0 \mathrm{E}-5 / \mathrm{d}$ & 3 \\
\hline
\end{tabular}


Table 7.15. Electrical 115V DC System Data (Cont'd)

PAGE 2

\begin{tabular}{|c|c|c|c|c|c|c|c|c|c|}
\hline \multirow{2}{*}{$\begin{array}{l}\text { EVENT } \\
\text { NAME }\end{array}$} & \multirow[b]{2}{*}{ DESCRIPTION } & \multicolumn{2}{|c|}{ VALUE USED $3 / 91$} & \multicolumn{2}{|c|}{ CRBRP-4 } & \multicolumn{2}{|c|}{ IREP } & \multicolumn{2}{|c|}{ GENERIC } \\
\hline & & $\begin{array}{l}\text { Failure } \\
\text { Rate }\end{array}$ & $\begin{array}{l}\text { Error } \\
\text { Factor }\end{array}$ & $\begin{array}{c}\text { Failure } \\
\text { Rate }\end{array}$ & $\begin{array}{l}\text { Error } \\
\text { Factor }\end{array}$ & $\begin{array}{l}\text { Failure } \\
\text { Rate }\end{array}$ & $\begin{array}{l}\text { Error } \\
\text { Factor }\end{array}$ & $\begin{array}{l}\text { Failure } \\
\text { Rate }\end{array}$ & $\begin{array}{l}\text { Error } \\
\text { Factor }\end{array}$ \\
\hline LCBE11BO & $\begin{array}{l}125 \text { VDC Emergency } \\
\text { Bus Breaker } 1 \text { Fails } \\
\text { Open }\end{array}$ & $\begin{array}{c}3.0 \mathrm{E}-7 / \mathrm{h} \\
{[1]}\end{array}$ & 10 & $1.0 \mathrm{E}-5 / \mathrm{h}$ & 10 & $3.0 \mathrm{E}-5 / \mathrm{d}$ & 10 & $3.0 \mathrm{E}-5 / \mathrm{d}$ & 3 \\
\hline LCBE 12BO & $\begin{array}{l}125 \text { VDC Emergency } \\
\text { Bus Breaker } 2 \text { Fails } \\
\text { Open }\end{array}$ & $\begin{array}{c}3.0 \mathrm{E}-7 / \mathrm{h} \\
{[1]}\end{array}$ & 10 & $1.0 \mathrm{E}-5 / \mathrm{h}$ & 10 & $3.0 E-5 / d$ & 10 & $3.0 E-5 / d$ & 3 \\
\hline LCBE13BO & $\begin{array}{l}125 \text { VDC Emergency } \\
\text { Bus Breaker } 3 \text { Fails } \\
\text { Open }\end{array}$ & $\begin{array}{c}3.0 \mathrm{E}-7 / \mathrm{h} \\
{[1]}\end{array}$ & 10 & $1.0 \mathrm{E}-5 / \mathrm{h}$ & 10 & $3.0 \mathrm{E}-5 / \mathrm{d}$ & 10 & $3.0 \mathrm{E}-5 / \mathrm{d}$ & 3 \\
\hline LCBE 14BO & $\begin{array}{l}125 \text { VDC Emergency } \\
\text { Bus Breaker } 4 \text { Fails } \\
\text { Open }\end{array}$ & $\begin{array}{c}3.0 \mathrm{E}-7 / \mathrm{h} \\
{[1]}\end{array}$ & 10 & $1.0 \mathrm{E}-5 / \mathrm{h}$ & 10 & $3.0 E-5 / d$ & 10 & $3.0 E-5 / d$ & 3 \\
\hline LCBMCCBC & $\begin{array}{l}\text { Uncleared Overload } \\
\text { on } 125 \text { VDC MCC Bus }\end{array}$ & $\begin{array}{c}1.0 \mathrm{E}-7 / \mathrm{h} \\
{[1]}\end{array}$ & 10 & $\begin{array}{c}1.0 \mathrm{E}-7 / \mathrm{h} \\
{[3]}\end{array}$ & 10 & $\begin{array}{c}3.0 E-7 / h \\
{[3]}\end{array}$ & 10 & $\begin{array}{c}3.0 E-7 / h \\
{[3]}\end{array}$ & 10 \\
\hline LCBMCCBO & $\begin{array}{l}125 \text { VDC Dist Center } \\
\text { Bus Breaker } 5 \text { Fails } \\
\text { Open }\end{array}$ & $\begin{array}{c}3.0 \mathrm{E}-7 / \mathrm{h} \\
{[1]}\end{array}$ & 10 & $1.0 \mathrm{E}-5 / \mathrm{h}$ & 10 & $3.0 \mathrm{E}-5 / \mathrm{d}$ & 10 & $3.0 \mathrm{E}-5 / \mathrm{d}$ & 3 \\
\hline LDC12EBW & $\begin{array}{l}10 \mathrm{KW} \text { Rectifier } \\
\text { Fails }\end{array}$ & $\begin{array}{c}1.0 \mathrm{E}-5 / \mathrm{h} \\
{[1]}\end{array}$ & 5 & $\begin{array}{c}1.0 \mathrm{E}-6 / \mathrm{h} \\
{[4]}\end{array}$ & 10 & $\begin{array}{c}3.0 E-6 / \mathrm{h} \\
{[4]}\end{array}$ & 10 & $\begin{array}{c}3.0 E-6 / h \\
{[4]}\end{array}$ & 10 \\
\hline LFU100BB & $\begin{array}{l}100 \mathrm{KW} \text { Diesel Start } \\
\text { Sup. Fuse Open }\end{array}$ & $\begin{array}{c}1.0 \mathrm{E}-7 / \mathrm{h} \\
{[1]}\end{array}$ & 10 & $1.0 \mathrm{E}-6 / \mathrm{h}$ & 10 & $3.0 \mathrm{E}-6 / \mathrm{h}$ & 10 & $3.0 \mathrm{E}-6 / \mathrm{h}$ & 10 \\
\hline LFU1CDVB & $\begin{array}{l}\text { Breaker 1CD Trip Ckt } \\
\text { Fuse Fails Open }\end{array}$ & $\begin{array}{c}1.0 \mathrm{E} \cdot \mathrm{7} / \mathrm{h} \\
{[1]}\end{array}$ & 10 & $1.0 \mathrm{E}-6 / \mathrm{h}$ & 10 & $3.0 \mathrm{E}-6 / \mathrm{h}$ & 10 & $3.0 \mathrm{E}-6 / \mathrm{h}$ & 10 \\
\hline LFU21ABB & $\begin{array}{l}\text { R-2A Breaker } 1 A 125 \\
\text { VDC Sup. Wire/Fuse } \\
\text { Opens }\end{array}$ & $\begin{array}{c}1.0 \mathrm{E}-7 / \mathrm{h} \\
{[1]}\end{array}$ & 10 & $1.0 \mathrm{E}-6 / \mathrm{h}$ & 10 & $3.0 \mathrm{E}-6 / \mathrm{h}$ & 10 & $3.0 \mathrm{E}-6 / \mathrm{h}$ & 10 \\
\hline LFU3D4BB & $\begin{array}{l}\text { E-1 Breaker 3D } 125 \\
\text { Vdc Sup. Wire/Fuse } \\
\text { Opens }\end{array}$ & $\begin{array}{c}1.0 \mathrm{E}-7 / \mathrm{h} \\
{[1]}\end{array}$ & 10 & $1.0 \mathrm{E}-6 / \mathrm{h}$ & 10 & $3.0 \mathrm{E}-6 / \mathrm{h}$ & 10 & $3.0 \mathrm{E}-6 / \mathrm{h}$ & 10 \\
\hline LFU400BB & $\begin{array}{l}400 \text { Kw Diesel Start } \\
\text { Sup. Fuse Open }\end{array}$ & $\underset{[1]}{1.0 E-7 / h}$ & 10 & $1.0 \mathrm{E}-6 / \mathrm{h}$ & 10 & $3.0 \mathrm{E}-6 / \mathrm{h}$ & 10 & $3.0 \mathrm{E}-6 / \mathrm{h}$ & 10 \\
\hline LFU4BCVB & $\begin{array}{l}\text { Breaker 4BC Trip Ckt } \\
\text { Fuse Fails Open }\end{array}$ & $\begin{array}{c}1.0 \mathrm{E} \cdot 7 / \mathrm{h} \\
{[1]}\end{array}$ & 10 & $1.0 \mathrm{E}-6 / \mathrm{h}$ & 10 & $3.0 \mathrm{E}-6 / \mathrm{h}$ & 10 & $3.0 \mathrm{E}-6 / \mathrm{h}$ & 10 \\
\hline LFU7CDVB & $\begin{array}{l}\text { Breaker 7CD Trip Ckt } \\
\text { Fuse Fails Open }\end{array}$ & $\begin{array}{c}1.0 \mathrm{E}-7 / \mathrm{h} \\
{[1]}\end{array}$ & 10 & $1.0 \mathrm{E}-6 / \mathrm{h}$ & 10 & $3.0 \mathrm{E}-6 / \mathrm{h}$ & 10 & $3.0 \mathrm{E}-6 / \mathrm{h}$ & 10 \\
\hline UN12CBL & $\begin{array}{l}125 \text { VDC Station } \\
\text { Battery Capacity } \\
\text { Loss Not Detected }\end{array}$ & $\begin{array}{c}1.0 \mathrm{E}-3 / \mathrm{d} \\
{[5]}\end{array}$ & 30 & & & & & & \\
\hline UN12CBW & $\begin{array}{l}125 \text { VDC Station } \\
\text { Battery Low Charge } \\
\text { Rate Not Detected }\end{array}$ & $\begin{array}{c}3.0 \mathrm{E} \cdot 6 / \mathrm{h} \\
{[1]}\end{array}$ & 10 & $\begin{array}{c}1.0 \mathrm{E}-6 / \mathrm{h} \\
{[6]}\end{array}$ & 10 & $\begin{array}{c}3.0 E-6 / h \\
.6]\end{array}$ & 10 & $\begin{array}{c}2.0 \mathrm{E}-6 / \mathrm{h} \\
{[6]}\end{array}$ & 10 \\
\hline UN12MBW & $\begin{array}{l}\text { First } 125 \text { VDC Ground } \\
\text { Fault Alarm CKT } \\
\text { Fails }\end{array}$ & $\begin{array}{c}3.0 \mathrm{E}-6 / \mathrm{h} \\
{[1]}\end{array}$ & 10 & $\begin{array}{c}1.0 \mathrm{E}-6 / \mathrm{h} \\
{[6]}\end{array}$ & 10 & $\begin{array}{c}3.0 \mathrm{E}-6 / \mathrm{h} \\
{[6]}\end{array}$ & 10 & $\begin{array}{c}2.0 \mathrm{E}-6 / \mathrm{h} \\
{[6]}\end{array}$ & 10 \\
\hline UN12MBZ & $\begin{array}{l}\text { Operator Error } \\
\text { Ignores First } 125 \\
\text { VDC Gnd Fault Alarm }\end{array}$ & $\begin{array}{c}1.0 E-4 / d \\
{[7]}\end{array}$ & 10 & & & & & & \\
\hline LSW12TBB & $\begin{array}{l}125 \text { VDC Throwover } \\
\text { Sw. Sta. Battery } \\
\text { Side Fails }\end{array}$ & $\begin{array}{c}1.0 \mathrm{E}-6 / \mathrm{h} \\
{[1]}\end{array}$ & 10 & $1.0 \mathrm{E} \cdot 7 / \mathrm{h}$ & 10 & & & & \\
\hline LSWMD1BO & $\begin{array}{l}125 \text { VDC MTS } 1 \text { Distr } \\
\text { Cent Bus Side Fails } \\
\text { Open }\end{array}$ & $\frac{1.0 \mathrm{E}-6 / \mathrm{h}}{[1]}$ & 10 & $1.0 \mathrm{E}-7 / \mathrm{h}$ & 10 & & & & \\
\hline LSWMD2BO & $\begin{array}{l}125 \text { VDC MTS } 2 \text { Distr } \\
\text { Cent Bus Side Fails } \\
\text { Open }\end{array}$ & $\begin{array}{c}1.0 \mathrm{E}-6 / \mathrm{h} \\
{[1]}\end{array}$ & 10 & $1.0 \mathrm{E}-7 / \mathrm{h}$ & 10 & & & & \\
\hline LSWMD3BO & $\begin{array}{l}125 \text { VDC MTS } 3 \text { Distr } \\
\text { Cent Bus Side Fails } \\
\text { Open }\end{array}$ & $\begin{array}{c}1.0 \mathrm{E}-6 / \mathrm{h} \\
{[1]}\end{array}$ & 10 & $1.0 \mathrm{E}-7 / \mathrm{h}$ & 10 & & & & \\
\hline
\end{tabular}


Table 7.15. Electrical 115V DC System Data (Cont'd)

PAGE 3

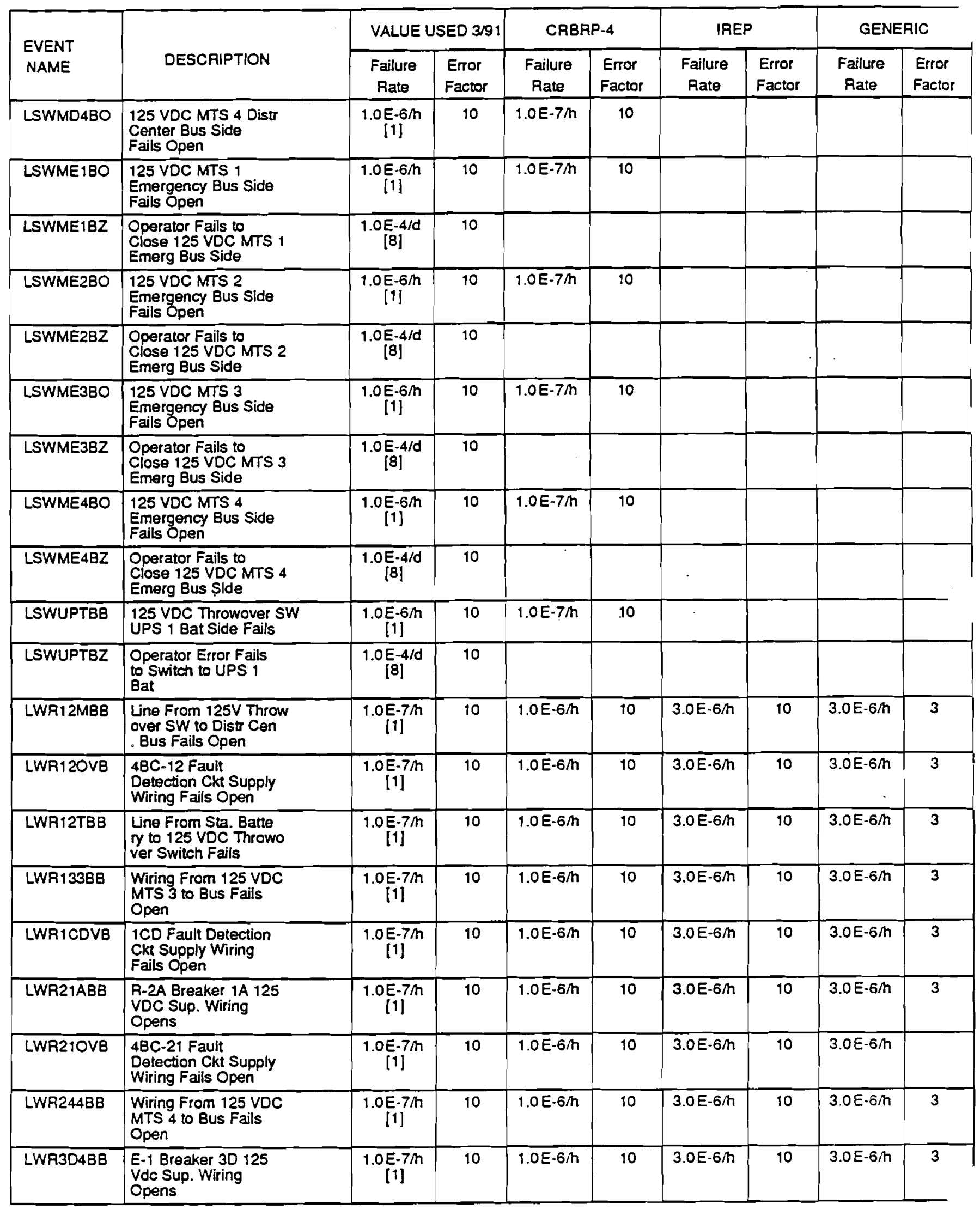


Table 7.16. Air System Data

PAGE 1

\begin{tabular}{|c|c|c|c|c|c|c|c|c|c|}
\hline \multirow{2}{*}{$\begin{array}{l}\text { EVENT } \\
\text { NAME }\end{array}$} & \multirow[b]{2}{*}{ DESCRIPTION } & \multicolumn{2}{|c|}{ VALUE USED $3 / 91$} & \multicolumn{2}{|c|}{ CRBRP-4 } & \multicolumn{2}{|c|}{ IREP } & \multicolumn{2}{|c|}{ GENERIC } \\
\hline & & $\begin{array}{l}\text { Failure } \\
\text { Rate }\end{array}$ & $\begin{array}{l}\text { Error } \\
\text { Factor }\end{array}$ & $\begin{array}{l}\text { Failure } \\
\text { Rate }\end{array}$ & $\begin{array}{l}\text { Error } \\
\text { Factor }\end{array}$ & $\begin{array}{c}\text { Failure } \\
\text { Rate }\end{array}$ & $\begin{array}{l}\text { Error } \\
\text { Factor }\end{array}$ & $\begin{array}{l}\text { Failure } \\
\text { Rate }\end{array}$ & $\begin{array}{l}\text { Error } \\
\text { Factor }\end{array}$ \\
\hline IAV695LQ & $\begin{array}{l}\text { Isolation Valve } \\
\text { R1-VC-695 Fails } \\
\text { Closed }\end{array}$ & $2.0 \mathrm{E}-6 / \mathrm{h}$ & 3 & & & & & & \\
\hline ICMOO1YW & $\begin{array}{l}\text { Primary Inst. Air Co } \\
\text { mpressor, Aftercoole } \\
r \text { or Motor Failure }\end{array}$ & $\begin{array}{c}3.0 \mathrm{E}-5 / \mathrm{h} \\
{[1]}\end{array}$ & 10 & & & & & $\begin{array}{c}6.0 \mathrm{E} \cdot 6 / \mathrm{h} \\
{[2]}\end{array}$ & \\
\hline ICMO02YW & $\begin{array}{l}\text { Standby Inst. Air Co } \\
\text { mpressor, Aftercoole } \\
\text { r of Motor Failure }\end{array}$ & $\begin{array}{c}3.0 \mathrm{E}-5 / \mathrm{h} \\
{[1]}\end{array}$ & 10 & & & & & $\begin{array}{c}6.0 E-6 / h \\
{[2]}\end{array}$ & \\
\hline ICMO02YX & $\begin{array}{l}\text { Standby Inst. Air } \\
\text { Compressor Down for } \\
\text { Maintenance }\end{array}$ & $\begin{array}{c}\text { 6.0 E-3/d } \\
{[3]}\end{array}$ & 10 & & & & & & \\
\hline ICMOO3YW & $\begin{array}{l}\text { Plant Air Compressor } \\
\text { or Motor Failure }\end{array}$ & $\begin{array}{c}6.0 E-6 / \mathrm{h} \\
{[1]}\end{array}$ & 10 & & & & & $\begin{array}{c}6.0 E-6 / \mathrm{h} \\
{[2]}\end{array}$ & ${ }^{\circ}$ \\
\hline ICMO03YX & $\begin{array}{l}\text { Plant Air Compressor } \\
\text { Down for Maintenance }\end{array}$ & $\begin{array}{c}6.0 \mathrm{E}-3 / \mathrm{d} \\
{[3]}\end{array}$ & 10 & & & & & & \\
\hline ICM004LW & $\begin{array}{l}\text { Emergency Air } \\
\text { Compressor or Motor } \\
\text { Failure }\end{array}$ & $\begin{array}{c}3.0 \mathrm{E}-5 / \mathrm{h} \\
{[1]}\end{array}$ & 10 & & & & & $\begin{array}{c}6.0 \mathrm{E}-6 / \mathrm{h} \\
{[2]}\end{array}$ & \\
\hline ICMOOALX & $\begin{array}{l}\text { Emergency Air } \\
\text { Compressor Down for } \\
\text { Maintenance }\end{array}$ & $\begin{array}{c}6.0 \mathrm{E}-3 / \mathrm{d} \\
{[3]}\end{array}$ & 10 & & & & & & \\
\hline ICZ011YO & $\begin{array}{l}\text { Primary Cont. Main } \\
\text { Switch MAN Contacts } \\
\text { Fail Open }\end{array}$ & $\begin{array}{c}1.0 \mathrm{E}-7 / \mathrm{h} \\
{[4]}\end{array}$ & 10 & $1.0 \mathrm{E}-7 / \mathrm{h}$ & 10 & $3.0 E-4 / d$ & 10 & $3.0 \mathrm{E}-4 / \mathrm{d}$ & 3 \\
\hline ICZO12YO & $\begin{array}{l}\text { Primary Cont. } \\
\text { Overload Contacts } \\
\text { (1/3) Fail Open }\end{array}$ & $\begin{array}{c}3.0 \mathrm{E}-7 / \mathrm{h} \\
{[5]}\end{array}$ & 10 & $1.0 \mathrm{E}-7 / \mathrm{h}$ & 10 & $3.0 E-4 / d$ & 10 & $3.0 \mathrm{E}-4 / \mathrm{d}$ & 3 \\
\hline ICZ021YO & $\begin{array}{l}\text { Slandby Cont. Main } \\
\text { Switch AUTO Contacts } \\
\text { Fail Open }\end{array}$ & $\begin{array}{c}1.0 \mathrm{E}-7 \mathrm{~h} \\
{[4]}\end{array}$ & 10 & $1.0 \mathrm{E}-7 / \mathrm{m}$ & 10 & $3.0 E-4 / d$ & 10 & $3.0 \mathrm{E}-4 / \mathrm{d}$ & 3 \\
\hline ICZO22YO & $\begin{array}{l}\text { Standby Cont. } \\
\text { Overload Contacts } \\
\text { (1/3) Fail Open }\end{array}$ & $\begin{array}{c}3.0 \mathrm{E}-7 / \mathrm{h} \\
{[5]}\end{array}$ & 10 & $1.0 \mathrm{E}-7 / \mathrm{h}$ & 10 & $3.0 E-4 / d$ & 10 & $3.0 E \cdot 4 / d$ & 3 \\
\hline ICZ031YO & $\begin{array}{l}\text { Controller Main } \\
\text { Switch Contacts Fail } \\
\text { Open }\end{array}$ & $\begin{array}{c}1.0 \mathrm{E}-7 \mathrm{~m} \\
{[4]}\end{array}$ & 10 & $1.0 \mathrm{E}-7 / \mathrm{h}$ & 10 & $3.0 \mathrm{E}-4 / \mathrm{d}$ & 10 & $3.0 \mathrm{E} \cdot 4 / \mathrm{d}$ & 3 \\
\hline ICZO32YO & $\begin{array}{l}\text { Controiler Overload } \\
\text { Contacts(1/2) Fail } \\
\text { Open }\end{array}$ & $\begin{array}{c}2.0 \mathrm{E}-7 \mathrm{~h} \\
{[6]}\end{array}$ & 10 & $1.0 \mathrm{E}-7 / \mathrm{h}$ & 10 & $3.0 E-4 / d$ & 10 & $3.0 E-4 / d$ & 3 \\
\hline ICZO41LO & $\begin{array}{l}\text { Controller Main } \\
\text { Switch STANDBY } \\
\text { Contacts Fail Open }\end{array}$ & $\begin{array}{c}1.0 \mathrm{E}-7 / \mathrm{h} \\
{[4]}\end{array}$ & 10 & $1.0 \mathrm{E} \cdot 7 \mathrm{~h}$ & 10 & $3.0 E-4 / d$ & 10 & $3.0 \mathrm{E} \cdot 4 / \mathrm{d}$ & 3 \\
\hline ICZO42LO & $\begin{array}{l}\text { Controller Overloaid } \\
\text { Contacts (1/2) Fail } \\
\text { Open }\end{array}$ & $\begin{array}{c}2.0 \mathrm{E}-7 / \mathrm{h} \\
{[6]}\end{array}$ & 10 & $1.0 \mathrm{E}-7 / \mathrm{h}$ & 10 & $3.0 E-4 / d$ & 10 & $3.0 \mathrm{E}-4 / \mathrm{d}$ & 3 \\
\hline ICZO92YO & $\begin{array}{l}\text { Controller } \\
\text { Compressor Motor } \\
\text { Contacts Fail Open }\end{array}$ & $\begin{array}{c}1.0 \mathrm{E}-7 / \mathrm{h} \\
{[4]}\end{array}$ & 10 & $1.0 \mathrm{E} \cdot 7 / \mathrm{h}$ & 10 & $3.0 E-4 / d$ & 10 & $3.0 E-4 / d$ & 3 \\
\hline ICZO94LO & $\begin{array}{l}\text { Controller } \\
\text { Compressor Motor } \\
\text { Contacts Fail Open }\end{array}$ & $\begin{array}{c}1.0 \mathrm{E} \cdot 7 / \mathrm{h} \\
{[4]}\end{array}$ & 10 & $1.0 \mathrm{E}-7 / \mathrm{h}$ & 10 & $3.0 \mathrm{E}-4 / \mathrm{d}$ & 10 & $3.0 \mathrm{E}-4 / \mathrm{d}$ & 3 \\
\hline ICZ19OYO & $\begin{array}{l}\text { Primary Cont. Comp. } \\
\text { Motor Contacts Fail } \\
\text { Open }\end{array}$ & $\begin{array}{c}1.0 \mathrm{E}-7 / \mathrm{h} \\
{[4]}\end{array}$ & 10 & $1.0 \mathrm{E}-7 / \mathrm{h}$ & 10 & $3.0 \mathrm{E}-4 / \mathrm{d}$ & 10 & $3.0 \mathrm{E}-4 / \mathrm{d}$ & \\
\hline ICZ29OYO & $\begin{array}{l}\text { Standby Cont. Comp. } \\
\text { Motor Contacts Fail } \\
\text { Open }\end{array}$ & $\begin{array}{c}1.0 \mathrm{E}-7 / \mathrm{h} \\
{[4]}\end{array}$ & 10 & $1.0 \mathrm{E}-7 / \mathrm{h}$ & 10 & $3.0 \mathrm{E} \cdot 4 / \mathrm{d}$ & 10 & $3.0 \mathrm{E}-4 / \mathrm{d}$ & 3 \\
\hline IMSO02YS & $\begin{array}{l}\text { Standby Compressor } \\
\text { Motor Fails to Start }\end{array}$ & $\begin{array}{c}3.0 \mathrm{E}-5 / \mathrm{d} \\
{[1]}\end{array}$ & 10 & $1.0 \mathrm{E}-3 / \mathrm{d}$ & 10 & $3.0 \mathrm{E}-3 / \mathrm{d}$ & 10 & $3.0 \mathrm{E}-3 / \mathrm{d}$ & 3 \\
\hline
\end{tabular}


Table 7.16. Air System Data (Cont'd)

PAGE 2

\begin{tabular}{|c|c|c|c|c|c|c|c|c|c|}
\hline \multirow{2}{*}{$\begin{array}{l}\text { EVENT } \\
\text { NAME }\end{array}$} & \multirow[b]{2}{*}{ DESCRIPTION } & \multicolumn{2}{|c|}{ VALUE USED $3 / 91$} & \multicolumn{2}{|c|}{ CRBRP-4 } & \multicolumn{2}{|c|}{ IREP } & \multicolumn{2}{|c|}{ GENERIC } \\
\hline & & $\begin{array}{l}\text { Failure } \\
\text { Rate }\end{array}$ & $\begin{array}{l}\text { Error } \\
\text { Factor }\end{array}$ & $\begin{array}{l}\text { Failure } \\
\text { Rate }\end{array}$ & $\begin{array}{l}\text { Error } \\
\text { Factor }\end{array}$ & $\begin{array}{l}\text { Failure } \\
\text { Pate }\end{array}$ & $\begin{array}{l}\text { Error } \\
\text { Factor }\end{array}$ & $\begin{array}{c}\text { Failure } \\
\text { Rate }\end{array}$ & $\begin{array}{l}\text { Error } \\
\text { Factor }\end{array}$ \\
\hline IMSO03YS & $\begin{array}{l}\text { Plant Air Compressor } \\
\text { Motor Fails to Start }\end{array}$ & $\begin{array}{c}3.0 E-5 / d \\
{[1]}\end{array}$ & 10 & $1.0 \mathrm{E}-3 / \mathrm{d}$ & 10 & $3.0 E-3 / d$ & 10 & $3.0 \mathrm{E}-3 / \mathrm{d}$ & 3 \\
\hline IMSOOALS & $\begin{array}{l}\text { Emergency Air } \\
\text { Compressor Motor } \\
\text { Fails to Start }\end{array}$ & $\begin{array}{c}3.0 E-5 / d \\
{[1]}\end{array}$ & 10 & $1.0 E-3 / d$ & 10 & $3.0 \mathrm{E}-3 / \mathrm{d}$ & 10 & $3.0 E-3 / d$ & 3 \\
\hline $10001 A 00$ & $\begin{array}{l}\text { Plant/lnstrument Air } \\
\text { Emergency Crossover } \\
\text { Open }\end{array}$ & 1.0 & 1 & & & & & & \\
\hline 10001800 & $\begin{array}{l}\text { Plant/nstrument Air } \\
\text { Emergency Crossover } \\
\text { Closed }\end{array}$ & 0.0 & 1 & & & & & & \\
\hline 10002700 & $\begin{array}{l}\text { Instrument Air } \\
\text { System Partially } \\
\text { Isolated }\end{array}$ & 1.0 & 1 & & & & - & & \\
\hline IPPOO1YF & $\begin{array}{l}\text { Main Instrument Air } \\
\text { System Distribution } \\
\text { Line Failure }\end{array}$ & $\begin{array}{c}8.0 E-9 / \mathrm{h} \\
{[7]}\end{array}$ & 30 & $\begin{array}{c}1.0 E-9 / \mathrm{h} \\
{[8]}\end{array}$ & & & & $\begin{array}{c}8.0 E-9 / \mathrm{M} \\
{[8]}\end{array}$ & 30 \\
\hline IPPO04YF & $\begin{array}{l}\text { Plant Air } \\
\text { Aftercooler to } \\
\text { Receive Line Failure }\end{array}$ & $\begin{array}{c}8.0 E-9 / \mathrm{h} \\
{[7]}\end{array}$ & 30 & $\underset{[8]}{1.0 E-9 / \mathrm{h}}$ & & & & $\begin{array}{c}8.0 E-9 / \mathrm{h} \\
{[8]}\end{array}$ & 30 \\
\hline IPPOO5YF & $\begin{array}{l}\text { Plant Air Receiver } \\
\text { to Dryer Line } \\
\text { Failure }\end{array}$ & $\begin{array}{c}8.0 E-9 / \mathrm{h} \\
{[7]}\end{array}$ & 30 & $\begin{array}{c}1.0 \mathrm{E}-9 / \mathrm{h} \\
{[8]}\end{array}$ & & & & $\begin{array}{c}8.0 \mathrm{E}-9 / \mathrm{h} \\
{[8]}\end{array}$ & 30 \\
\hline IPP012YF & $\begin{array}{l}\text { Plant Air Filter to } \\
\text { Distribution Line } \\
\text { Failure }\end{array}$ & $\begin{array}{c}8.0 E-9 / h \\
{[7]}\end{array}$ & 30 & $\begin{array}{c}1.0 E \cdot 9 / \mathrm{h} \\
{[8] .}\end{array}$ & . & & & $\begin{array}{c}8.0 \mathrm{E}-9 / \mathrm{h} \\
{[8] .}\end{array}$ & 30 \\
\hline IPP110YF & $\begin{array}{l}\text { Standby Aftercooler } \\
\text { to Receiver Line } \\
\text { Failure }\end{array}$ & $\begin{array}{c}8.0 E-9 / \mathrm{h} \\
{[7]}\end{array}$ & 30 & $\begin{array}{c}1.0 E-9 / \mathrm{h} \\
{[8]}\end{array}$ & & & & $\begin{array}{c}8.0 E-9 / \mathrm{h} \\
{[8]}\end{array}$ & 30 \\
\hline IPP111YF & $\begin{array}{l}\text { Primary Aftercooler } \\
\text { to Reciever Line } \\
\text { Failure }\end{array}$ & $\begin{array}{c}8.0 E-9 / \mathrm{h} \\
{[7]}\end{array}$ & 30 & $\begin{array}{c}1.0 \mathrm{E}-9 / \mathrm{h} \\
{[8]}\end{array}$ & & & & $\begin{array}{c}8.0 E-9 / \mathrm{h} \\
{[8]}\end{array}$ & 30 \\
\hline IPP113YF & $\begin{array}{l}\text { Instrument Air } \\
\text { Receiver to Main } \\
\text { Filter Line Failure }\end{array}$ & $\begin{array}{c}8.0 \mathrm{E}-9 \mathrm{~h} \\
{[7]}\end{array}$ & 30 & $\begin{array}{c}1.0 \mathrm{E} \cdot 9 \mathrm{~h} \\
{[8]}\end{array}$ & & & & $\begin{array}{c}8.0 E-9 \mathrm{~h} \\
{[8]}\end{array}$ & 30 \\
\hline IPP11TYF & $\begin{array}{l}\text { Instrument Air Main } \\
\text { Filter to Dryer Line } \\
\text { Failure }\end{array}$ & $\begin{array}{c}8.0 E-9 / h \\
{[7]}\end{array}$ & 30 & $\begin{array}{c}1.0 E-9 / h \\
{[8]}\end{array}$ & & & & $\begin{array}{c}8.0 E-9 / \mathrm{h} \\
{[8]}\end{array}$ & 30 \\
\hline IPP 122YF & $\begin{array}{l}\text { Instrument Air Dryer } \\
\text { to Filter Line } \\
\text { Failure }\end{array}$ & $\begin{array}{c}8.0 E-9 / h \\
{[7]}\end{array}$ & 30 & $\begin{array}{c}1.0 E-9 / h \\
{[8]}\end{array}$ & & & & $\begin{array}{c}8.0 E-9 / \mathrm{h} \\
{[8]}\end{array}$ & 30 \\
\hline IPP125LF & $\begin{array}{l}\text { Emergency Air } \\
\text { Filler/Dryer Bypass } \\
\text { Line Failure }\end{array}$ & $\begin{array}{c}8.0 E-9 / \mathrm{h} \\
{[7]}\end{array}$ & 30 & $\begin{array}{c}1.0 \mathrm{E}-9 / \mathrm{h} \\
{[8]}\end{array}$ & & & & $\begin{array}{c}8.0 E-9 / \mathrm{h} \\
{[8]}\end{array}$ & 30 \\
\hline IPP132YF & $\begin{array}{l}\text { Sec. Filter to Instr } \\
\text { ument Air Distributi } \\
\text { on Line Failure }\end{array}$ & $\begin{array}{c}8.0 E-9 / \mathrm{h} \\
{[7]}\end{array}$ & 30 & $\begin{array}{c}1.0 \varepsilon-9 / \mathrm{h} \\
{[8]}\end{array}$ & & & & $\begin{array}{c}8.0 E-9 / \mathrm{h} \\
{[8]}\end{array}$ & 30 \\
\hline IPP2OIYF & $\begin{array}{l}\text { Secondary Instrument } \\
\text { Air Distribution } \\
\text { Line Leakage }\end{array}$ & $\begin{array}{c}8.0 \mathrm{E}-9 / \mathrm{h} \\
{[7]}\end{array}$ & 30 & $\begin{array}{c}1.0 \mathrm{E}-9 / \mathrm{h} \\
{[8]}\end{array}$ & & & & $\begin{array}{c}8.0 \mathrm{E}-9 \mathrm{~h} \\
{[8]}\end{array}$ & 30 \\
\hline IPP301YF & $\begin{array}{l}\text { Main Plant Air } \\
\text { Distribution Line } \\
\text { Failure }\end{array}$ & $\begin{array}{c}8.0 E-9 / \mathrm{h} \\
{[7]}\end{array}$ & 30 & $\begin{array}{c}1.0 E-9 / \mathrm{h} \\
{[8]}\end{array}$ & & & & $\begin{array}{c}8.0 E-9 / \mathrm{M} \\
{[8]}\end{array}$ & 30 \\
\hline IPP501LF & $\begin{array}{l}\text { Emergency Air System } \\
\text { Distribution Line } \\
\text { Failure }\end{array}$ & $\begin{array}{c}8.0 E \cdot 9 / \mathrm{h} \\
{[7]}\end{array}$ & 30 & $\begin{array}{c}1.0 \mathrm{E}-9 / \mathrm{h} \\
{[8]}\end{array}$ & & & & $\begin{array}{c}8.0 E-9 / h \\
{[8]}\end{array}$ & 30 \\
\hline IPP634LF & $\begin{array}{l}\text { Emergency Air } \\
\text { Compressor to } \\
\text { Receiver Line Failur }\end{array}$ & $\begin{array}{c}8.0 E-9 / h \\
{[7]}\end{array}$ & 30 & $\begin{array}{c}1.0 E-9 / \mathrm{h} \\
{[8]}\end{array}$ & & & & $\begin{array}{c}8.0 \mathrm{E}-9 / \mathrm{h} \\
{[8]}\end{array}$ & 30 \\
\hline
\end{tabular}


Table 7.16. Air System Data (Cont'd)

PAGE 3

\begin{tabular}{|c|c|c|c|c|c|c|c|c|c|}
\hline \multirow{2}{*}{$\begin{array}{l}\text { EVENT } \\
\text { NAME }\end{array}$} & \multirow[b]{2}{*}{ DESCRIPTION } & \multicolumn{2}{|c|}{ VALUE USED $3 / 91$} & \multicolumn{2}{|c|}{ CRBRP.4 } & \multicolumn{2}{|c|}{ IREP } & \multicolumn{2}{|c|}{ GENERIC } \\
\hline & & $\begin{array}{c}\text { Failure } \\
\text { Rate }\end{array}$ & $\begin{array}{l}\text { Error } \\
\text { Factor }\end{array}$ & $\begin{array}{l}\text { Failure } \\
\text { Rate }\end{array}$ & $\begin{array}{l}\text { Error } \\
\text { Factor }\end{array}$ & $\begin{array}{c}\text { Failure } \\
\text { Rate }\end{array}$ & $\begin{array}{l}\text { Error } \\
\text { Factor }\end{array}$ & $\begin{array}{c}\text { Failure } \\
\text { Rate }\end{array}$ & $\begin{array}{l}\text { Error } \\
\text { Factor }\end{array}$ \\
\hline IPP716YF & $\begin{array}{l}\text { Plant Air Compressor } \\
\text { to Aftercooler Line } \\
\text { Failure }\end{array}$ & $\begin{array}{c}8.0 \mathrm{E}-9 \mathrm{~h} \\
{[7]}\end{array}$ & 30 & $\underset{[8]}{1.0 \mathrm{E}-9 / \mathrm{h}}$ & & & & $\begin{array}{c}8.0 \mathrm{E}-9 \mathrm{~m} \\
{[8]}\end{array}$ & 30 \\
\hline IPP718YF & $\begin{array}{l}\text { Primary Compressor } \\
\text { to Aftercooler Line } \\
\text { Failure }\end{array}$ & $\begin{array}{c}8.0 E-9 / \mathrm{h} \\
{[7]}\end{array}$ & 30 & $\begin{array}{c}1.0 \mathrm{E}-9 / \mathrm{h} \\
{[8]}\end{array}$ & & & & $\begin{array}{c}8.0 \mathrm{E} \cdot 9 / \mathrm{h} \\
{[8]}\end{array}$ & 30 \\
\hline IPP720YF & $\begin{array}{l}\text { Standby Compressor } \\
\text { to Aftercooler Line } \\
\text { Failure }\end{array}$ & $\begin{array}{c}8.0 \mathrm{E}-9 / \mathrm{h} \\
{[7]}\end{array}$ & 30 & $\begin{array}{c}1.0 \mathrm{E}-9 / \mathrm{h} \\
{[8]}\end{array}$ & & & & $\begin{array}{c}8.0 \mathrm{E}-9 / \mathrm{h} \\
{[8]}\end{array}$ & 30 \\
\hline IPP731YF & $\begin{array}{l}\text { Plant Air Dryer to } \\
\text { Filter Line Failure }\end{array}$ & $\begin{array}{c}8.0 \mathrm{E}-9 \mathrm{~h} \\
{[7]}\end{array}$ & 30 & $\begin{array}{c}1.0 \mathrm{E}-9 / \mathrm{h} \\
{[8]}\end{array}$ & & & & $\begin{array}{c}8.0 \mathrm{E}-9 / \mathrm{h} \\
{[8]}\end{array}$ & 30 \\
\hline IPROO4LB & $\begin{array}{l}\text { Emergency Air } \\
\text { Controller Pressure } \\
\text { Regulator Fails }\end{array}$ & $\begin{array}{c}1.0 \mathrm{E}-6 / \mathrm{h} \\
{[1]}\end{array}$ & 10 & $\begin{array}{c}\text { 1.0E-3/d } \\
{[9]}\end{array}$ & 3 & $\begin{array}{c}1.0 \mathrm{E}-3 / \mathrm{d} \\
{[9]}\end{array}$ & 3 & $\begin{array}{c}1.0 \mathrm{E}-3 / \mathrm{d} \\
{[9]}\end{array}$ & 3 \\
\hline IPRO11YB & $\begin{array}{l}\text { Primary Compressor } \\
\text { Oil Pressure Sensor } \\
\text { Fails Low }\end{array}$ & $\begin{array}{c}1.0 \mathrm{E}-6 / \mathrm{h} \\
{[1]}\end{array}$ & 10 & $1.0 \mathrm{E}-7 / \mathrm{h}$ & 10 & & & & \\
\hline IPRo12YB & $\begin{array}{l}\text { Primary Comp. } \\
\text { Controller Pressure } \\
\text { Regulator Fails }\end{array}$ & $\begin{array}{c}1.0 E-6 / \mathrm{h} \\
{[1]}\end{array}$ & 10 & $\begin{array}{c}1.0 \mathrm{E}-3 / \mathrm{d} \\
{[9]}\end{array}$ & 3 & $\begin{array}{c}1.0 \mathrm{E} \cdot 3 / \mathrm{d} \\
{[9]}\end{array}$ & 3 & $\begin{array}{c}1.0 \mathrm{E}-3 / \mathrm{d} \\
{[9]}\end{array}$ & 3 \\
\hline IPR021YB & $\begin{array}{l}\text { Standby Compressor } \\
\text { Oil Pressure Sensor } \\
\text { Fails Low }\end{array}$ & $\begin{array}{c}1.0 \mathrm{E}-6 / \mathrm{h} \\
{[1]}\end{array}$ & 10 & $1.0 \mathrm{E}-7 / \mathrm{h}$ & 10 & & & & \\
\hline IPR022YB & $\begin{array}{l}\text { Standby Comp. } \\
\text { Controller Pressure } \\
\text { Regulator Fails }\end{array}$ & $\begin{array}{c}1.0 \mathrm{E}-6 \mathrm{~m} \\
{[1]}\end{array}$ & 10 & $\begin{array}{c}1.0 \mathrm{E}-3 / \mathrm{d} \\
{[9]}\end{array}$ & 3 & $\begin{array}{c}1.0 E-3 / d \\
{[9]}\end{array}$ & 3 & $\begin{array}{c}1.0 E-3 / d \\
{[S]}\end{array}$ & 3 \\
\hline IPR031YB & $\begin{array}{l}\text { Compressor Oil } \\
\text { Pressure Sensor } \\
\text { Fails Low }\end{array}$ & $\begin{array}{c}1.0 \mathrm{E}-6 / \mathrm{h} \\
{[1]}\end{array}$ & 10 & $1.0 \mathrm{E}-7 / \mathrm{h}$ & 10 & & & & . \\
\hline IPR032YB & $\begin{array}{l}\text { Plant Air Controller } \\
\text { Pressure Regulator } \\
\text { Fails }\end{array}$ & $\begin{array}{c}1.0 \mathrm{E}-6 / \mathrm{h} \\
{[1]}\end{array}$ & 10 & $\begin{array}{c}1.0 \mathrm{E}-3 / \mathrm{d} \\
{[9]}\end{array}$ & 3 & $\begin{array}{c}1.0 E-3 / d \\
{[9]}\end{array}$ & 3 & $\begin{array}{c}1.0 \mathrm{E} \cdot 3 / \mathrm{d} \\
{[9]}\end{array}$ & 3 \\
\hline IPRO42LB & $\begin{array}{l}\text { Emergency Air Oil } \\
\text { Pressure Sensor } \\
\text { Fails Low }\end{array}$ & $\begin{array}{c}1.0 E-6 / \mathrm{h} \\
{[1]}\end{array}$ & 10 & $1.0 \mathrm{E}-7 / \mathrm{h}$ & 10 & & & & \\
\hline IRR635LF & $\begin{array}{l}\text { Emergency Air } \\
\text { Receiver R1-T-1635 } \\
\text { Tank Failure }\end{array}$ & $\begin{array}{c}1.0 \mathrm{E}-10 / \mathrm{h} \\
{[10]}\end{array}$ & 30 & & & & & & \\
\hline IRR717YF & $\begin{array}{l}\text { Plant Air Receiver } \\
\text { P3-T-717 Tank } \\
\text { Failure }\end{array}$ & $\begin{array}{c}1.0 \mathrm{E}-10 / \mathrm{h} \\
{[10]}\end{array}$ & 30 & & & & & & \\
\hline IRR719YF & $\begin{array}{l}\text { Instrument Air } \\
\text { Receiver P3-T-719 } \\
\text { Tank Failure }\end{array}$ & $\begin{array}{c}1.0 \mathrm{E}-10 / \mathrm{h} \\
{[10]}\end{array}$ & 30 & & & & & & \\
\hline IRVDOIYN & $\begin{array}{l}\text { Instrument Air Dryer } \\
\text { Relief Valve Fails } \\
\text { to Close }\end{array}$ & $\begin{array}{c}3.0 \mathrm{E}-3 / \mathrm{d} \\
{[1]}\end{array}$ & 5 & $2.0 \mathrm{E}-2 / \mathrm{d}$ & 3 & $2.0 \mathrm{E}-2 / \mathrm{d}$ & 3 & $1.0 \mathrm{E}-2 / \mathrm{d}$ & 3 \\
\hline IRVOO3YN & $\begin{array}{l}\text { Instrument Air } \\
\text { Receiver Relief } \\
\text { Valve Fails to Close }\end{array}$ & $\begin{array}{c}3.0 \mathrm{E}-3 / \mathrm{d} \\
{[1]}\end{array}$ & 5 & $2.0 \mathrm{E}-2 / \mathrm{d}$ & 3 & $2.0 \mathrm{E} \cdot 2 / \mathrm{d}$ & 3 & $1.0 \mathrm{E}-2 / \mathrm{d}$ & 3 \\
\hline IRVOOLYN & $\begin{array}{l}\text { Primary Separator } \\
\text { Relief Valve Fails } \\
\text { to Close }\end{array}$ & $\begin{array}{c}3.0 E-3 / d \\
{[1]}\end{array}$ & 5 & $2.0 \mathrm{E}-2 / \mathrm{d}$ & 3 & $2.0 \mathrm{E}-2 / \mathrm{d}$ & 3 & $1.0 \mathrm{E}-2 / \mathrm{d}$ & 3 \\
\hline IRVOOSYN & $\begin{array}{l}\text { Primary Compressor } \\
\text { Relief Vahve Fails } \\
\text { to Close }\end{array}$ & $\begin{array}{c}3.0 E-3 / d \\
{[1]}\end{array}$ & 5 & $2.0 E-2 / d$ & 3 & $2.0 \mathrm{E}-2 / \mathrm{d}$ & 3 & $1.0 \mathrm{E}-2 / \mathrm{d}$ & 3 \\
\hline IRVDO6YN & $\begin{array}{l}\text { Plant Air Receiver } \\
\text { Relief Valve Fails } \\
\text { to Close }\end{array}$ & $\begin{array}{c}3.0 \mathrm{E}-3 / \mathrm{d} \\
{[1]}\end{array}$ & 5 & $2.0 E-2 / d$ & 3 & $2.0 E-2 / d$ & 3 & $1.0 \mathrm{E}-2 / \mathrm{d}$ & 3 \\
\hline
\end{tabular}


Table 7.16. Air System Data (Cont'd)

PAGE 4

\begin{tabular}{|c|c|c|c|c|c|c|c|c|c|}
\hline \multirow{2}{*}{$\begin{array}{l}\text { EVENT } \\
\text { NAME }\end{array}$} & \multirow[b]{2}{*}{ DESCRIPTION } & \multicolumn{2}{|c|}{ VALUE USED $3 / 91$} & \multicolumn{2}{|c|}{ CRBRP-4 } & \multicolumn{2}{|c|}{ IREP } & \multicolumn{2}{|c|}{ GENERIC } \\
\hline & & $\begin{array}{c}\text { Failure } \\
\text { Rate }\end{array}$ & $\begin{array}{l}\text { Error } \\
\text { Factor }\end{array}$ & $\begin{array}{l}\text { Failure } \\
\text { Rate }\end{array}$ & $\begin{array}{l}\text { Error } \\
\text { Factor }\end{array}$ & $\begin{array}{c}\text { Failure } \\
\text { Rate } \\
\end{array}$ & $\begin{array}{l}\text { Error } \\
\text { Factor }\end{array}$ & $\begin{array}{c}\text { Failure } \\
\text { Rate } \\
\end{array}$ & $\begin{array}{l}\text { Error } \\
\text { Factor }\end{array}$ \\
\hline IRVOOTYN & $\begin{array}{l}\text { Standby Compressor } \\
\text { Relief Vahv Fails } \\
\text { to Close }\end{array}$ & $\begin{array}{c}3.0 \mathrm{E}-3 / \mathrm{d} \\
{[1]}\end{array}$ & 5 & $2.0 \mathrm{E}-2 / \mathrm{d}$ & 3 & $2.0 E-2 / d$ & 3 & $1.0 \mathrm{E}-2 / \mathrm{d}$ & 3 \\
\hline IRVOO9YN & $\begin{array}{l}\text { Plant Air Moisture } \\
\text { Separator Relief } \\
\text { Valve Fails to Close }\end{array}$ & $\begin{array}{c}3.0 \mathrm{E}-3 / \mathrm{d} \\
{[1]}\end{array}$ & 5 & $2.0 \mathrm{E}-2 / \mathrm{d}$ & 3 & $2.0 \mathrm{E}-2 / \mathrm{d}$ & 3 & $1.0 \mathrm{E}-2 / \mathrm{d}$ & 3 \\
\hline IRV013LN & $\begin{array}{l}\text { Emergency Air } \\
\text { Recenver Relief } \\
\text { Valve Fails to Close }\end{array}$ & $\begin{array}{c}3.0 \mathrm{E}-3 / \mathrm{d} \\
{[1\}}\end{array}$ & 5 & $2.0 \mathrm{E}-2 / \mathrm{d}$ & 3 & $2.0 E-2 / d$ & 3 & $1.0 \mathrm{E}-2 / \mathrm{d}$ & 3 \\
\hline ITE011YB & $\begin{array}{l}\text { Primary Comp. Water } \\
\text { Temp. Sensor Fails } \\
\text { High }\end{array}$ & $\begin{array}{c}3.0 E-6 / \mathrm{h} \\
{[1]}\end{array}$ & 10 & & & & & $\begin{array}{c}6.0 \mathrm{E}-5 / \mathrm{h} \\
{[2]}\end{array}$ & \\
\hline ITE012YB & $\begin{array}{l}\text { Primary Compressor } \\
\text { Air Temp. Sensor } \\
\text { Fails High }\end{array}$ & $\begin{array}{c}3.0 \mathrm{E}-6 / \mathrm{h} \\
{[1]}\end{array}$ & 10 & & & . & & $\begin{array}{c}6.0 \mathrm{E}-5 \mathrm{~h} \\
{[2]}\end{array}$ & \\
\hline ITE021YB & $\begin{array}{l}\text { Standby Comp. Water } \\
\text { Temp. Sensor Fails } \\
\text { High }\end{array}$ & $\begin{array}{c}3.0 E-6 / \mathrm{h} \\
{[1]}\end{array}$ & 10 & & & & & $\begin{array}{c}6.0 \mathrm{E}-5 / \mathrm{h} \\
{[2]}\end{array}$ & \\
\hline ITE022YB & $\begin{array}{l}\text { Standby Compressor } \\
\text { Air Temp. Sensor } \\
\text { Fails High }\end{array}$ & $\begin{array}{c}3.0 E-6 / h \\
{[1]}\end{array}$ & 10 & & & & & $\begin{array}{c}6.0 \mathrm{E}-5 / \mathrm{h} \\
{[2]}\end{array}$ & \\
\hline ITE031YB & $\begin{array}{l}\text { Compressor Water } \\
\text { Temp. Sensor Fails } \\
\text { High }\end{array}$ & $\begin{array}{c}3.0 \mathrm{E}-6 / \mathrm{h} \\
{[11]}\end{array}$ & 10 & & & & & $\begin{array}{c}6.0 \mathrm{E}-5 / \mathrm{h} \\
{[2]}\end{array}$ & \\
\hline IXT001YW & $\begin{array}{l}\text { Primary Comp. } \\
480 / 120 \mathrm{~V} \\
\text { Transformer Fails }\end{array}$ & $\begin{array}{c}1.0 E-6 / h \\
{[1]}\end{array}$ & 10 & $1.0 \mathrm{E}-6 \mathrm{~h}$ & 3 & $1.0 \mathrm{E}-6 / \mathrm{h}$ & 3 & $1.0 \mathrm{E}-6 / \mathrm{h}$ & 3 \\
\hline IXT002YW & $\begin{array}{l}\text { Standby Comp. } \\
480 / 120 \mathrm{~V} \\
\text { Transformer Fails }\end{array}$ & $\begin{array}{c}1.0 \mathrm{E}-6 / \mathrm{h} \\
{[1]}\end{array}$ & 10 & $1.0 \mathrm{E}-6 / \mathrm{h}$ & 3 & $1.0 \mathrm{E}-6 / \mathrm{h}$ & 3 & $1.0 \mathrm{E}-6 / \mathrm{h}$ & 3 \\
\hline IXT003YW & $\begin{array}{l}\text { Controller } 480 / 120 \mathrm{~V} \\
\text { Transformer Fails }\end{array}$ & $\begin{array}{c}1.0 \mathrm{E}-6 / \mathrm{h} \\
{[1]}\end{array}$ & 3 & $1.0 \mathrm{E}-6 / \mathrm{h}$ & 3 & $1.0 \mathrm{E}-6 / \mathrm{h}$ & 3 & $1.0 \mathrm{E}-6 / \mathrm{h}$ & 3 \\
\hline IXTOOALW & $\begin{array}{l}\text { Controller } 480 / 120 \mathrm{~V} \\
\text { Transformer Fails }\end{array}$ & $\begin{array}{c}1.0 \mathrm{E}-6 / \mathrm{h} \\
{[1]}\end{array}$ & 10 & $1.0 \mathrm{E}-6 / \mathrm{h}$ & 3 & $1.0 \mathrm{E}-6 / \mathrm{h}$ & 3 & $1.0 \mathrm{E}-6 / \mathrm{h}$ & 3 \\
\hline IXV12OLQ & $\begin{array}{l}\text { Instrument Air Valve } \\
\text { RA- } 120 \text { Fails to } \\
\text { Remain Open }\end{array}$ & $1.0 \mathrm{E} \cdot 7 \mathrm{~m}$ & 3 & & & & & & \\
\hline IXV120LX & $\begin{array}{l}\text { Instrument Air Valve } \\
\text { RA-120 Closed }\end{array}$ & 0.0 & 1 & & & & & & \\
\hline IXV121LQ & $\begin{array}{l}\text { Instrument Air Valve } \\
\text { RA-121 Fails to } \\
\text { Remain Open }\end{array}$ & $.1 .0 \mathrm{E} \cdot 7 \mathrm{~h}$ & 3 & & & & & & \\
\hline IXV121LX & $\begin{array}{l}\text { Instrument Air Valve } \\
\text { RA-121 Closed }\end{array}$ & 0.0 & 1 & & & & & & \\
\hline IXV134YQ & $\begin{array}{l}\text { Instrument Air Vaive } \\
\text { PA-134 Fails to } \\
\text { Remain Open }\end{array}$ & $1.0 \mathrm{E} \cdot 7 / \mathrm{h}$ & 3 & & & . & & & \\
\hline IXV134YX & $\begin{array}{l}\text { Instrument Air Vaive } \\
\text { PA-134 Closed }\end{array}$ & 0.0 & 1 & & & & & & \\
\hline IXV149YQ & $\begin{array}{l}\text { Instrument Air Valve } \\
\text { PA-149 Fails to } \\
\text { Remain Open }\end{array}$ & $1.0 \mathrm{E}-7 \mathrm{~h}$ & 3 & & & & & & \\
\hline IXV149YX & $\begin{array}{l}\text { Instrument Air Valve } \\
\text { PA-149 Closed }\end{array}$ & 0.0 & 1 & & & & & & \\
\hline IXV309YQ & $\begin{array}{l}\text { Instrument Air Valve } \\
\text { PA-309 Fails to } \\
\text { Remain Open }\end{array}$ & $1.0 \mathrm{E}-7 / \mathrm{h}$ & 3 & & & & & & \\
\hline IXV309YX & $\begin{array}{l}\text { Instrument Air Valve } \\
\text { PA-309 Closed }\end{array}$ & 0.0 & 1 & & & & & & \\
\hline
\end{tabular}


Table 7.16. Air System Data (Cont'd)

PAGE 5

Notes: 1. Recommended value tables, EGG-SSRE-8875, Feb. 1990

2. Taken from Table 7-3, SAND-87-7138

3. Probability based on 1 hr/week maintenance

4. From CRBRP

5. From CRBRP; $3 x$ individual component value

6. From CRBRP; $2 x$ individual component value

7. Generic

8. Value for pipe $<3$ in. diameter

9. Solenoid valve failure to operate

10. Estimated value

11. Recommended value tables, EGG-SSRE-8875. Feb. 1990 
Table 7.17. Reactor Water Systems Data

PAGE 1

\begin{tabular}{|c|c|c|c|c|c|c|c|c|c|}
\hline \multirow{2}{*}{$\begin{array}{l}\text { EVENT } \\
\text { NAME }\end{array}$} & \multirow[b]{2}{*}{ DESCRIPTION } & \multicolumn{2}{|c|}{ VALUE USED $3 / 91$} & \multicolumn{2}{|c|}{ CRBRP-4 } & \multicolumn{2}{|c|}{ IREP } & \multicolumn{2}{|c|}{ GENERIC } \\
\hline & & $\begin{array}{l}\text { Failure } \\
\text { Rate }\end{array}$ & $\begin{array}{l}\text { Error } \\
\text { Factor }\end{array}$ & $\begin{array}{l}\text { Failure } \\
\text { Rate }\end{array}$ & $\begin{array}{l}\text { Error } \\
\text { Factor }\end{array}$ & $\begin{array}{l}\text { Failure } \\
\text { Rate }\end{array}$ & $\begin{array}{l}\text { Ertor } \\
\text { Factor }\end{array}$ & $\begin{array}{l}\text { Failure } \\
\text { Rate }\end{array}$ & $\begin{array}{l}\text { Error } \\
\text { Factor }\end{array}$ \\
\hline WCV001YO & $\begin{array}{l}\text { Reactor Aux. Primary } \\
\text { Pump Check Valve } \\
\text { Fails Closed }\end{array}$ & $\begin{array}{c}5.0 E-9 / h \\
{[1]}\end{array}$ & 10 & $1.0 E-7 / h$ & 10 & $3.0 \mathrm{E}-7 / \mathrm{h}$ & 10 & $1.0 E-4 / d$ & 3 \\
\hline WCV0O2YQ & $\begin{array}{l}\text { Reactor Aux. Standby } \\
\text { Pump Check Valve } \\
\text { Faiis Closed }\end{array}$ & $\begin{array}{c}5.0 \mathrm{E}-9 \mathrm{~h} \\
{[1]}\end{array}$ & 10 & $1.0 \mathrm{E}-7 / \mathrm{h}$ & 10 & $3.0 \mathrm{E}-7 / \mathrm{h}$ & 10 & $1.0 \mathrm{E}-4 / \mathrm{d}$ & 3 \\
\hline WCZ101YO & $\begin{array}{l}\text { Primary Controller } \\
\text { RUN Contacts Fail } \\
\text { Open }\end{array}$ & $\begin{array}{c}1.0 \mathrm{E} \cdot 7 \mathrm{~h} \\
{[2]}\end{array}$ & 10 & $1.0 \mathrm{E} \cdot 7 \mathrm{~h}$ & 10 & $3.0 E-4 / d$ & 10 & $3.0 \mathrm{E}-4 / \mathrm{d}$ & 3 \\
\hline WCZ102YO & $\begin{array}{l}\text { Primary Controller } \\
\text { OL Contacts }(1 / 2) \\
\text { Fail Open }\end{array}$ & $\begin{array}{c}2.0 \mathrm{E}-7 \mathrm{~h} \\
{[3]}\end{array}$ & 10 & $1.0 \mathrm{E}-7 \mathrm{~h}$ & 10 & $3.0 E-4 / d$ & 10 & $3.0 E-4 / d$ & 3 \\
\hline WCZ115YO & $\begin{array}{l}\text { Primary Controller } \\
\text { Pump Motor Contacts } \\
\text { Fail Open }\end{array}$ & $\begin{array}{c}1.0 \mathrm{E}-7 \mathrm{~h} \\
{[2]}\end{array}$ & 10 & $1.0 \mathrm{E}-7 \mathrm{~h}$ & 10 & $3.0 \mathrm{E}-4 / \mathrm{d}$ & 10 & $3.0 E-4 / d$ & 3 \\
\hline WCZ201YO & $\begin{array}{l}\text { Standby Cont. } \\
\text { STANDBY Contacts } \\
\text { Fail Open }\end{array}$ & $\begin{array}{c}1.0 \mathrm{E}-7 \mathrm{~h} \\
{[2]}\end{array}$ & 10 & $1.0 \mathrm{E}-7 / \mathrm{h}$ & 10 & $3.0 E-4 / d$ & 10 & $3.0 \mathrm{E}-4 / \mathrm{d}$ & 3 \\
\hline WCZ2O2YO & $\begin{array}{l}\text { Standby Controller } \\
\text { OL Contacts }(1 / 2) \\
\text { Fail Open }\end{array}$ & $\begin{array}{c}2.0 E-7 \mathrm{~h} \\
{[3]}\end{array}$ & 10 & $1.0 \mathrm{E}-7 / \mathrm{h}$ & 10 & $3.0 \mathrm{E}-4 / \mathrm{d}$ & 10 & $3.0 E-4 / d$ & 3 \\
\hline WCZ215YO & $\begin{array}{l}\text { Standby Controller } \\
\text { Pump Motor Contacts } \\
\text { Fail Open }\end{array}$ & $\underset{[2]}{1.0 \mathrm{E}-7 \mathrm{~h}}$ & 10 & $1.0 E-7 / \mathrm{h}$ & 10 & $3.0 E-4 / d$ & 10 & $3.0 \mathrm{E}-4 / \mathrm{d}$ & 3 \\
\hline WFLOSSYE & $\begin{array}{l}\text { Reactor Aux. Water } \\
\text { CUNO Filter Blocked }\end{array}$ & $\begin{array}{c}3.0 E-5 / h \\
{[4]}\end{array}$ & 10 & $1.0 \mathrm{E}-5 \mathrm{~h}$ & 10 & $3.0 \mathrm{E}-5 / \mathrm{h}$ & 10 & & \\
\hline WGV342PQ & $\begin{array}{l}\text { RX AUX WATER FLOW } \\
\text { CONTAOL FAILURE } \\
\text { P17-VC-342 }\end{array}$ & $3.0 \mathrm{E}-6 / \mathrm{h}$ & 10 & $1.0 \mathrm{E}-6 / \mathrm{h}$ & 10 & $3.0 \mathrm{E}-6 / \mathrm{h}$ & 10 & $2.0 \mathrm{E}-6 / \mathrm{h}$ & 10 \\
\hline WGV343PQ & $\begin{array}{l}\text { RX AUX WATER FLOW } \\
\text { CONTROL FAILURE } \\
\text { P17-VC-343 }\end{array}$ & $3.0 \mathrm{E} \cdot 6 / \mathrm{h}$ & 10 & $1.0 \mathrm{E}-6 / \mathrm{h}$ & 10 & $3.0 \mathrm{E}-6 / \mathrm{h}$ & 10 & $2.0 E-6 / \mathrm{h}$ & 10 \\
\hline WGV701PQ & $\begin{array}{l}\text { RX AUX WATER FLOW } \\
\text { CONTROL FAILURE } \\
\text { P\&-VC-701 }\end{array}$ & $3.0 \mathrm{E}-6 / \mathrm{h}$ & 10 & $1.0 \mathrm{E}-6 / \mathrm{h}$ & 10 & $3.0 \mathrm{E}-6 / \mathrm{h}$ & 10 & $2.0 \mathrm{E}-6 / \mathrm{h}$ & 10 \\
\hline WMS002YW & $\begin{array}{l}\text { Standby Pump Motor } \\
\text { Starter Failure }\end{array}$ & $\begin{array}{c}3.0 E-5 / d \\
{[1]}\end{array}$ & 10 & $1.0 E-3 / d$ & 10 & $3.0 \mathrm{E}-3 / \mathrm{d}$ & 10 & $3.0 \mathrm{E}-3 / \mathrm{d}$ & 3 \\
\hline WPM001YW & $\begin{array}{l}\text { Reactor Aux. Primary } \\
\text { Pump Fails }\end{array}$ & $\begin{array}{c}3.0 \mathrm{E}-5 / \mathrm{h} \\
{[4]}\end{array}$ & 10 & $1.0 \mathrm{E}-5 / \mathrm{h}$ & 10 & $3.0 \mathrm{E}-5 / \mathrm{h}$ & 10 & $3.0 \mathrm{E}-5 / \mathrm{h}$ & 10 \\
\hline WPMOO2YW & $\begin{array}{l}\text { Reactor Aux. Standby } \\
\text { Pump Fails }\end{array}$ & $\begin{array}{c}3.0 E-5 / h \\
{[4]}\end{array}$ & 10 & $1.0 \mathrm{E}-5 / \mathrm{h}$ & 10 & $3.0 \mathrm{E}-5 / \mathrm{h}$ & 10 & $3.0 \mathrm{E}-5 / \mathrm{h}$ & 10 \\
\hline WPM0O2YX & $\begin{array}{l}\text { Reactor Aux. Standby } \\
\text { Pump Down for } \\
\text { Maintenance }\end{array}$ & $\begin{array}{c}6.0 \mathrm{E}-3 \\
{[5]}\end{array}$ & 10 & & - & & & & \\
\hline WPPOO6OF & $\begin{array}{l}\text { Break in Supply Line } \\
\text { from Cooling Tower }\end{array}$ & $\begin{array}{c}8.0 \mathrm{E}-9 / \mathrm{h} \\
{[6]}\end{array}$ & 30 & $\begin{array}{c}1.0 \mathrm{E}-9 / \mathrm{h} \\
{[7]}\end{array}$ & & & & $\begin{array}{c}8.0 \mathrm{E}-9 / \mathrm{h} \\
{[7]}\end{array}$ & 30 \\
\hline WPPO7AOF & $\begin{array}{l}\text { Raw Water Supply } \\
\text { Line Failure }\end{array}$ & $\begin{array}{c}8.0 \mathrm{E}-9 / \mathrm{h} \\
{[6]}\end{array}$ & 30 & $\begin{array}{c}1.0 \mathrm{E}-9 / \mathrm{h} \\
{[7]}\end{array}$ & & & & $\begin{array}{c}8.0 E-9 / h \\
{[7]}\end{array}$ & 30 \\
\hline WPP07BOF & $\begin{array}{l}\text { Insufficient Water } \\
\text { Return to Cooling } \\
\text { Tower }\end{array}$ & $\begin{array}{c}8.0 \mathrm{E}-9 / \mathrm{h} \\
{[8]}\end{array}$ & 30 & $\begin{array}{c}\text { 1.0E-9/h } \\
{[7]}\end{array}$ & & & & $\begin{array}{c}8.0 \mathrm{E}-9 \mathrm{~m} \\
{[7]}\end{array}$ & 30 \\
\hline WPP211YF & $\begin{array}{l}\text { Primary Air } \\
\text { Compressor Cooling } \\
\text { Water Line Failure }\end{array}$ & $\begin{array}{c}8.0 E-9 / \mathrm{h} \\
{[6]}\end{array}$ & 30 & $\begin{array}{c}1.0 \mathrm{E}-9 / \mathrm{h} \\
{[7]}\end{array}$ & & & & $\begin{array}{c}8.0 \mathrm{E} \cdot 9 \mathrm{~h} \\
{[7]}\end{array}$ & 30 \\
\hline WPP238YF & $\begin{array}{l}\text { Standby Air } \\
\text { Compressor Cooling } \\
\text { Water Line Failure }\end{array}$ & $\begin{array}{c}8.0 E-9 / h \\
{[6]}\end{array}$ & 30 & $\begin{array}{c}1.0 E-9 / h \\
{[7]}\end{array}$ & & & & $\begin{array}{c}8.0 E-9 / \mathrm{h} \\
{[7]}\end{array}$ & 30 \\
\hline WPP410YF & $\begin{array}{l}\text { Plant Air Compressor } \\
\text { Cooling Water Line } \\
\text { Failure }\end{array}$ & $\begin{array}{c}8.0 \mathrm{E}-9 / \mathrm{h} \\
{[6]}\end{array}$ & 30 & $\begin{array}{c}1.0 \mathrm{E}-9 / \mathrm{h} \\
{[7]}\end{array}$ & & & & $\begin{array}{c}8.0 \mathrm{E}-9 / \mathrm{h} \\
{[7]}\end{array}$ & 30 \\
\hline
\end{tabular}


Table 7.17. Reactor Water Systems Data (Cont'd)

PAGE 2

\begin{tabular}{|c|c|c|c|c|c|c|c|c|c|}
\hline \multirow{2}{*}{$\begin{array}{l}\text { EVENT } \\
\text { NAME }\end{array}$} & \multirow[b]{2}{*}{ DESCRIPTION } & \multicolumn{2}{|c|}{ VALUE USED $3 / 91$} & \multicolumn{2}{|c|}{ CRBRP-4 } & \multicolumn{2}{|c|}{ IREP } & \multicolumn{2}{|c|}{ GENERIC } \\
\hline & & $\begin{array}{c}\text { Failure } \\
\text { Rate }\end{array}$ & $\begin{array}{l}\text { Error } \\
\text { Factor }\end{array}$ & $\begin{array}{l}\text { Failure } \\
\text { Rate }\end{array}$ & $\begin{array}{l}\text { Efror } \\
\text { Factor }\end{array}$ & $\begin{array}{c}\text { Failure } \\
\text { Rate }\end{array}$ & $\begin{array}{l}\text { Error } \\
\text { Factor }\end{array}$ & $\begin{array}{c}\text { Failure } \\
\text { Rate }\end{array}$ & $\begin{array}{l}\text { Error } \\
\text { Factor }\end{array}$ \\
\hline WPROO1YO & $\begin{array}{l}\text { Water Pressure Low } \\
\text { Switch Fails to } \\
\text { Remain Closed }\end{array}$ & $\begin{array}{c}1.0 E-6 / \mathrm{h} \\
{[1]}\end{array}$ & 10 & $1.0 \mathrm{E}-7 \mathrm{~h}$ & 10 & & & & \\
\hline WPROO2YN & $\begin{array}{l}\text { Water Pressure Low } \\
\text { Switch Fails to } \\
\text { Close }\end{array}$ & $\begin{array}{c}3.0 \mathrm{E}-7 \mathrm{~h} \\
{[1]}\end{array}$ & 10 & $1.0 E-4 / d$ & 3 & $1.0 \mathrm{E}-4 / \mathrm{d}$ & 3 & $1.0 E-4 / d$ & 3 \\
\hline WPR0O2YO & $\begin{array}{l}\text { Water Pressure Low } \\
\text { Switch Fails to } \\
\text { Remain Closed }\end{array}$ & $\begin{array}{c}1.0 \varepsilon \cdot 6 / \mathrm{h} \\
{[1]}\end{array}$ & 10 & $1.0 \mathrm{E}-7 \mathrm{~h}$ & 10 & & & & \\
\hline WXT101YW & $\begin{array}{l}\text { Primary Cont. } \\
480 / 120 \mathrm{~V} \\
\text { Transformer Fails }\end{array}$ & $\begin{array}{c}1.0 \mathrm{E} \cdot 6 / \mathrm{h} \\
{[1]}\end{array}$ & 3 & $1.0 \mathrm{E}-6 \mathrm{~h}$ & 3 & $1.0 \mathrm{E}-6 \mathrm{~h}$ & 3 & $1.0 E-6 / \mathrm{h}$ & 3 \\
\hline WXT201YW & $\begin{array}{l}\text { Standby Cont. } \\
480 / 120 \mathrm{~V} \\
\text { Transformer Fails }\end{array}$ & $\begin{array}{c}1.0 \mathrm{E}-6 / \mathrm{h} \\
{[1]}\end{array}$ & 10 & $1.0 \mathrm{E}-6 \mathrm{~h}$ & 3 & $1.0 \mathrm{E}-6 \mathrm{~h}$ & 3 & $1.0 E-6 / \mathrm{h}$ & 3 \\
\hline
\end{tabular}

Notes: 1. Recommended value tables, EGG-SSRE-8875, Feb. 1990

2. From CRBRP

3. From CRBRP; $2 x$ individual component value

4. From IREP

5. Probability based on 1 hrweek maintenance

6. Generic

7. Value for pipe < 3 in. diameter

8. Estimated value, generic, for pipes $<3$ in. diameter 
Table 7.18. Plant Water Systems Data

PAGE 1

\begin{tabular}{|c|c|c|c|c|c|c|c|c|c|}
\hline \multirow{2}{*}{$\begin{array}{l}\text { EVENT } \\
\text { NAME }\end{array}$} & \multirow[b]{2}{*}{ DESCRIPTION } & \multicolumn{2}{|c|}{ VALUE USED $3 / 91$} & \multicolumn{2}{|c|}{ CRBRP-4 } & \multicolumn{2}{|c|}{ IREP } & \multicolumn{2}{|c|}{ GENERIC } \\
\hline & & $\begin{array}{c}\text { Failure } \\
\text { Rate }\end{array}$ & $\begin{array}{l}\text { Error } \\
\text { Factor }\end{array}$ & $\begin{array}{c}\text { Failure } \\
\text { Rate }\end{array}$ & $\begin{array}{l}\text { Error } \\
\text { Factor }\end{array}$ & $\begin{array}{c}\text { Failure } \\
\text { Rate }\end{array}$ & $\begin{array}{l}\text { Error } \\
\text { Factor } \\
\end{array}$ & $\begin{array}{c}\text { Failure } \\
\text { Rate }\end{array}$ & $\begin{array}{l}\text { Error } \\
\text { Factor }\end{array}$ \\
\hline QCV001YQ & $\begin{array}{l}\text { Plant Water Primary } \\
\text { Pump Check Vave } \\
\text { Plugs }\end{array}$ & $\begin{array}{c}5.0 \mathrm{E}-9 / \mathrm{h} \\
{[1]}\end{array}$ & 10 & $1.0 \mathrm{E}-7 / \mathrm{h}$ & 10 & $3.0 \mathrm{E}-7 \mathrm{~h}$ & 10 & $1.0 \mathrm{E}-4 / \mathrm{d}$ & 3 \\
\hline QCV002YP & $\begin{array}{l}\text { Plant Water Standby } \\
\text { Pump Checx Valve } \\
\text { Fails to Open }\end{array}$ & $\begin{array}{c}5.0 E-5 / d \\
{[1]}\end{array}$ & 10 & $1.0 \mathrm{E}-4 / \mathrm{d}$ & 3 & $1.0 \mathrm{E}-4 / \mathrm{d}$ & 3 & $1.0 \mathrm{E}-4 / \mathrm{d}$ & 3 \\
\hline QCV0O2YO & $\begin{array}{l}\text { Plant Water Standby } \\
\text { Pump Check Valve } \\
\text { Piugs }\end{array}$ & $\begin{array}{c}5.0 \mathrm{E}-9 \mathrm{~h} \\
{[1]}\end{array}$ & 10 & $1.0 \mathrm{E} \cdot 7 / \mathrm{h}$ & 10 & $3.0 \mathrm{E}-7 \mathrm{~h}$ & 10 & $1.0 \mathrm{E}-4 / \mathrm{d}$ & 3 \\
\hline OCZ101YO & $\begin{array}{l}\text { Primary Controller } \\
\text { RUN Contacts Fail } \\
\text { Open }\end{array}$ & $\begin{array}{c}1.0 \mathrm{E}-7 \mathrm{~h} \\
{[2]}\end{array}$ & 10 & $1.0 \mathrm{E}-7 \mathrm{~h}$ & 10 & $3.0 E-4 / d$ & 10 & $3.0 E-4 / d$ & 3 \\
\hline QCZ102YO & $\begin{array}{l}\text { Primary Controller } \\
\text { OL Contacts (1/2) } \\
\text { Fail Open }\end{array}$ & $\begin{array}{c}2.0 E-7 / \mathrm{h} \\
{[3]}\end{array}$ & 10 & $1.0 \mathrm{E}-7 / \mathrm{h}$ & 10 & $3.0 E-4 / d$ & 10 & $3.0 E-4 / d$ & 3 \\
\hline OCZ117YO & $\begin{array}{l}\text { Primary Controller } \\
\text { Pump Motor Contacts } \\
\text { Fail Open }\end{array}$ & $\begin{array}{c}1.0 \mathrm{E}-7 / \mathrm{h} \\
{[2]}\end{array}$ & 10 & $1.0 \mathrm{E}-7 / \mathrm{h}$ & 10 & $3.0 E-4 / d$ & 10 & $3.0 E-4 / d$ & 3 \\
\hline OCZ201YO & $\begin{array}{l}\text { Standby Cont. } \\
\text { STANDBY Contacts } \\
\text { Fail Open }\end{array}$ & $\begin{array}{c}1.0 \mathrm{E}-7 / \mathrm{h} \\
{[2]}\end{array}$ & 10 & $1.0 \mathrm{E} \cdot 7 \mathrm{~h}$ & 10 & $3.0 \mathrm{E}-4 / \mathrm{d}$ & 10 & $3.0 \mathrm{E}-4 / \mathrm{d}$ & 3 \\
\hline OCZZO2YO & $\begin{array}{l}\text { Standby Controller } \\
\text { OL Contacts (1/2) } \\
\text { Fail Open }\end{array}$ & $\begin{array}{c}2.0 E-7 / \mathrm{h} \\
{[3]}\end{array}$ & 10 & $1.0 \mathrm{E}-7 / \mathrm{h}$ & 10 & $3.0 \mathrm{E}-4 / \mathrm{d}$ & 10 & $3.0 E-4 / d$ & 3 \\
\hline OCZ217YO & $\begin{array}{l}\text { Standby Controller } \\
\text { Pump Motor Contacts } \\
\text { Fail Open }\end{array}$ & $\begin{array}{c}1.0 \mathrm{E}-7 / \mathrm{h} \\
{[2]}\end{array}$ & 10 & $1.0 \mathrm{E}-7 / \mathrm{h}$ & 10 & $3.0 \mathrm{E}-4 / \mathrm{d}$ & 10 & $3.0 \mathrm{E}-4 / \mathrm{d}$ & 3 \\
\hline QFLO92YE & $\begin{array}{l}\text { Plant Water CUNO } \\
\text { Filter Blocked }\end{array}$ & $\begin{array}{c}3.0 \mathrm{E}-5 / \mathrm{h} \\
{[4]}\end{array}$ & 10 & $1.0 \mathrm{E}-5 / \mathrm{h}$ & 10 & $3.0 \mathrm{E}-5 / \mathrm{h}$ & 10 & & \\
\hline QMS002YW & $\begin{array}{l}\text { Standby Pump Motor } \\
\text { Starter Fajlure }\end{array}$ & $\begin{array}{c}3.0 E-5 / d \\
{[1]}\end{array}$ & 10 & $1.0 \mathrm{E}-3 / \mathrm{d}$ & 10 & $3.0 \mathrm{E}-3 / \mathrm{d}$ & 10 & $3.0 \mathrm{E}-3 / \mathrm{d}$ & 3 \\
\hline OPM001YW & $\begin{array}{l}\text { Plant Water Primary } \\
\text { Pump Fails }\end{array}$ & $\begin{array}{c}3.0 E-5 / h \\
{[4]}\end{array}$ & 10 & $1.0 \mathrm{E}-5 / \mathrm{h}$ & 10 & $3.0 \mathrm{E}-5 / \mathrm{h}$ & 10 & $3.0 \mathrm{E}-5 / \mathrm{h}$ & 10 \\
\hline QPM002YW & $\begin{array}{l}\text { Plant Water Standby } \\
\text { Pump Fails }\end{array}$ & $\begin{array}{c}3.0 E-5 / h \\
{[4]}\end{array}$ & 10 & $1.0 \mathrm{E}-5 \mathrm{~h}$ & 10 & $3.0 \mathrm{E}-5 / \mathrm{h}$ & 10 & $3.0 \mathrm{E}-5 / \mathrm{h}$ & 10 \\
\hline QPMO02YX & $\begin{array}{l}\text { Plant Water Standby } \\
\text { Pump Down for } \\
\text { Maintenance }\end{array}$ & $\begin{array}{c}6.0 E-3 \\
{[5]}\end{array}$ & 10 & & & & & & \\
\hline QPPOO6OF & $\begin{array}{l}\text { Break in Supply Line } \\
\text { fom Cooling Tower }\end{array}$ & $\begin{array}{c}8.0 \mathrm{E}-9 / \mathrm{h} \\
{[6]}\end{array}$ & 30 & $\begin{array}{c}1.0 \mathrm{E}-9 / \mathrm{h} \\
{[7]}\end{array}$ & & & & $\begin{array}{c}8.0 \mathrm{E}-9 / \mathrm{h} \\
{[7]}\end{array}$ & 30 \\
\hline QPR0O1YO & $\begin{array}{l}\text { Water Pressure Low } \\
\text { Switch Fails to } \\
\text { Remain Closed }\end{array}$ & $\begin{array}{c}1.0 \bar{E}-6 / \mathrm{h} \\
{[1]}\end{array}$ & 10 & $1.0 \mathrm{E}-7 / \mathrm{h}$ & 10 & & & & \\
\hline QPR002YN & $\begin{array}{l}\text { Water Pressure Low } \\
\text { Switch Fails to } \\
\text { Close }\end{array}$ & $\begin{array}{c}3.0 \mathrm{E}-7 / \mathrm{d} \\
{[1]}\end{array}$ & 10 & $1.0 E-4 / d$ & 3 & $1.0 \mathrm{E}-4 / \mathrm{d}$ & 3 & $1.0 \mathrm{E}-4 / \mathrm{d}$ & 3 \\
\hline OPR002YO & $\begin{array}{l}\text { Water Pressure Low } \\
\text { Switch Fails to } \\
\text { Remain Closed }\end{array}$ & $\begin{array}{c}1.0 \mathrm{E}-6 / \mathrm{h} \\
{[1]}\end{array}$ & 10 & $1.0 \mathrm{E}-7 / \mathrm{h}$ & 10 & & & & \\
\hline QXT101YW & $\begin{array}{l}\text { Primary Cont. } \\
480 / 120 \mathrm{~V} \\
\text { Transformer Fails }\end{array}$ & $\begin{array}{c}1.0 E-6 / \mathrm{h} \\
{[1]}\end{array}$ & 10 & $1.0 \mathrm{E} \cdot 6 / \mathrm{h}$ & 3 & $1.0 \mathrm{E}-6 / \mathrm{h}$ & 3 & $1.0 E-6 / \mathrm{h}$ & 3 \\
\hline QXT201YW & $\begin{array}{l}\text { Standby Cot. } 480 / 120 \\
V \text { Transformer Fails }\end{array}$ & $\begin{array}{c}1.0 E-6 / \mathrm{h} \\
{[1]}\end{array}$ & 10 & $1.0 \mathrm{E}-6 / \mathrm{h}$ & 3 & $1.0 \mathrm{E} \cdot 6 / \mathrm{h}$ & 3 & $1.0 \mathrm{E}-6 / \mathrm{h}$ & 3 \\
\hline
\end{tabular}

Notes: 1. Recommended value tables, EGG-SSRE-8875, Feb. 1990

2. From CRBRP

3. From CRBRP; $2 x$ individual component value

4. From IREP

5. Probability based on 1 hr/week maintenance

6. Generic

7. Value lor pipe $<3$ in. diameter 
Table 7.19. Argon Systems Data

PAGE 1

\begin{tabular}{|c|c|c|c|c|c|c|c|c|c|}
\hline \multirow{2}{*}{$\begin{array}{l}\text { EVENT } \\
\text { NAME }\end{array}$} & \multirow{2}{*}{ DESCRIPTION } & \multicolumn{2}{|c|}{ VALUE USED $3 / 91$} & \multicolumn{2}{|c|}{ CRBRP-4 } & \multicolumn{2}{|c|}{ IREP } & \multicolumn{2}{|c|}{ GENERIC } \\
\hline & & $\begin{array}{l}\text { Failure } \\
\text { Rate }\end{array}$ & $\begin{array}{l}\text { Error } \\
\text { Factor }\end{array}$ & $\begin{array}{c}\text { Failure } \\
\text { Rate }\end{array}$ & $\begin{array}{l}\text { Error } \\
\text { Factor }\end{array}$ & $\begin{array}{c}\text { Failure } \\
\text { Rate }\end{array}$ & $\begin{array}{l}\text { Error } \\
\text { Factor }\end{array}$ & $\begin{array}{l}\text { Failure } \\
\text { Rate }\end{array}$ & $\begin{array}{l}\text { Error } \\
\text { Factor }\end{array}$ \\
\hline OTGOO1OE & $\begin{array}{l}\text { VENT LINE TO IHX } \\
\text { OUTLET CLOSED }\end{array}$ & $2.5 \mathrm{E}-7 / \mathrm{h}$ & 10 & & & & & & \\
\hline OTG002OE & $\begin{array}{l}\text { VENT LINE TO IHX } \\
\text { INLET CLOSED }\end{array}$ & $1.0 \mathrm{EO} / \mathrm{d}$ & 1 & & & & & & \\
\hline
\end{tabular}


Table 7.20. Reactor Building Isolation Data

PAGE 1

\begin{tabular}{|c|c|c|c|c|c|c|c|c|c|}
\hline \multirow{2}{*}{$\begin{array}{l}\text { EVENT } \\
\text { NAME }\end{array}$} & \multirow[b]{2}{*}{ DESCRIPTION } & \multicolumn{2}{|c|}{ VALUE USED $3 / 91$} & \multicolumn{2}{|c|}{ CRBRP-4 } & \multicolumn{2}{|c|}{ IREP } & \multicolumn{2}{|c|}{ GENERIC } \\
\hline & & $\begin{array}{c}\text { Failure } \\
\text { Rate }\end{array}$ & $\begin{array}{l}\text { Error } \\
\text { Factor }\end{array}$ & $\begin{array}{l}\text { Failure } \\
\text { Rate }\end{array}$ & $\begin{array}{l}\text { Error } \\
\text { Factor }\end{array}$ & $\begin{array}{l}\text { Failure } \\
\text { Rate }\end{array}$ & $\begin{array}{l}\text { Error } \\
\text { Factor }\end{array}$ & $\begin{array}{l}\text { Failure } \\
\text { Rate }\end{array}$ & $\begin{array}{l}\text { Error } \\
\text { Factor }\end{array}$ \\
\hline KAUOOOPH & $\begin{array}{l}\text { REACTOR BLD } \\
\text { ISOLATION VALVE } \\
\text { ACCUMULATORS FAIL }\end{array}$ & $5.0 \mathrm{E}-6 \mathrm{~m}$ & 10 & & & & & & \\
\hline KAV23ASQ & $\begin{array}{l}\text { ISOLATION VALVE 323A } \\
\text { FAILS TO REMAIN OPEN } \\
\text { R13-VR-323A }\end{array}$ & $\begin{array}{c}6.4 E-8 / h \\
{[1]}\end{array}$ & 10 & $1.0 E-7 / \mathrm{h}$ & 3 & & & & \\
\hline KAV23BSP & $\begin{array}{l}\text { ISOLATION VALVE } \\
\text { 323B FAILS TO } \\
\text { OPEN R13-VR-3238 }\end{array}$ & $2.0 \mathrm{E}-3 / \mathrm{d}$ & 10 & $1.0 E-3 / d$ & 10 & $3.0 \mathrm{E} \cdot 3 / \mathrm{d}$ & 10 & $3.0 \mathrm{E}-3 / \mathrm{d}$ & 3 \\
\hline KAV23BSO & $\begin{array}{l}\text { ISOLATION VALVE } 3238 \\
\text { FAILS TO REMAIN OPEN } \\
\text { R13-VR-323B }\end{array}$ & $1.0 \mathrm{E}-7 / \mathrm{h}$ & 10 & $1.0 \mathrm{E}-7 / \mathrm{h}$ & 3 & & & & \\
\hline KIN003UW & $\begin{array}{l}\text { REACTOR BUILDING } \\
\text { TOTAL ISOLATION }\end{array}$ & $\begin{array}{c}2.3 E-6 / \mathrm{h} \\
{[3]}\end{array}$ & 10 & & & & & & \\
\hline
\end{tabular}

Notes: 1. Air Operated Valve Minus Internal HVAC Valve

2. Assumes One Partial Isolation in Four 100 Day Runs

3. Based on no inadvertent actuations in $25 \mathrm{yr}$ 
Table 7.21. Safety Rod Data

PAGE 1

\begin{tabular}{|c|c|c|c|c|c|c|c|c|c|}
\hline \multirow{2}{*}{$\begin{array}{l}\text { EVENT } \\
\text { NAME }\end{array}$} & \multirow[b]{2}{*}{ DESCRIPTION } & \multicolumn{2}{|c|}{ VALUE USED 391} & \multicolumn{2}{|c|}{ CRBRP-4 } & \multicolumn{2}{|c|}{ IREP } & \multicolumn{2}{|c|}{ GENERIC } \\
\hline & & $\begin{array}{l}\text { Failure } \\
\text { Rate }\end{array}$ & $\begin{array}{l}\text { Error } \\
\text { Factor }\end{array}$ & $\begin{array}{l}\text { Failure } \\
\text { Rate }\end{array}$ & $\begin{array}{l}\text { Error } \\
\text { Factor }\end{array}$ & $\begin{array}{l}\text { Failure } \\
\text { Rate }\end{array}$ & $\begin{array}{l}\text { Error } \\
\text { Factor }\end{array}$ & $\begin{array}{l}\text { Failure } \\
\text { Rate }\end{array}$ & $\begin{array}{l}\text { Error } \\
\text { Factor }\end{array}$ \\
\hline SCD100RG & $\begin{array}{l}\text { Forces due to Bowing } \\
\text { and Binding }\end{array}$ & $\begin{array}{c}6.0 E-5 / d \\
{[1]}\end{array}$ & 20 & & & & & & \\
\hline SCD200RW & $\begin{array}{l}\text { Failure of Rise } \\
\text { Mechanism, Connctng } \\
\text { Shafts, Bearngs }\end{array}$ & $\begin{array}{c}6.0 E-5 / d \\
{[1]}\end{array}$ & 20 & & & & & & \\
\hline SCD300RI & $\begin{array}{l}\text { Forces due to } \\
\text { Foreign Materials in } \\
\text { Sodium }\end{array}$ & $\begin{array}{c}6.0 E-5 / d \\
{[1]}\end{array}$ & 20 & & & & & & \\
\hline SCLOOORP & $\begin{array}{l}\text { Magnetic Clutch } \\
\text { Fails to Release SRs }\end{array}$ & $\begin{array}{c}2.0 E-4 / d \\
{[2]}\end{array}$ & 20 & & & & & $\begin{array}{c}3.0 E-4 / d \\
{[3]}\end{array}$ & 5 \\
\hline SSR100RG & $\begin{array}{l}\text { Forces due to Bowing } \\
\text { and Binding }\end{array}$ & $\begin{array}{c}6.0 E-5 / d \\
{[1]}\end{array}$ & 20 & & & & & & \\
\hline SSW300CP & 3MPB Mech Parts Fail & $\begin{array}{c}2.0 E-6 / d \\
{[4]}\end{array}$ & 10 & & & & & & \\
\hline SSW3OACP & $\begin{array}{l}\text { 3MPB Switch of } \\
\text { String A Fails to } \\
\text { Open }\end{array}$ & $\begin{array}{c}1.0 \mathrm{E}-5 / \mathrm{d} \\
{[3]}\end{array}$ & 5 & $1.0 E-4 / d$ & 10 & $3.0 E-5 / d$ & 10 & $\begin{array}{c}1.0 E-5 / d \\
{[3]}\end{array}$ & 5 \\
\hline SSW $30 B C P$ & $\begin{array}{l}\text { 3MPB Switch of } \\
\text { String B Fails to } \\
\text { Open }\end{array}$ & $\underset{[3]}{1.0 E-5 / d}$ & 5 & $1.0 E-4 / d$ & 10 & $3.0 \mathrm{E}-5 / \mathrm{d}$ & 10 & $\begin{array}{c}1.0 E-5 / d \\
{[3]}\end{array}$ & 5 \\
\hline
\end{tabular}

Notes: 1. Based on combined CCF Prob of $3.0 E-4 / d$ for the 2 SRs

2. Clutch failure to release SRs less probable than to engage

3. Recommended value tables, EGG-SSRE-8875, Feb. 1990

4. Based on Engineering Judgement 
Table 7.22. Test and Maintenance Unavailabilities

\begin{tabular}{||c|l|c|c||}
\hline \multicolumn{2}{|c|}{} & \multicolumn{2}{|c|}{ Value Used 3/90 } \\
\hline $\begin{array}{c}\text { Event } \\
\text { Name }\end{array}$ & \multicolumn{1}{|c|}{ Description } & $\begin{array}{c}\text { Failure } \\
\text { Rate }\end{array}$ & $\begin{array}{c}\text { Error } \\
\text { Rate }\end{array}$ \\
\hline ICM002YX & $\begin{array}{l}\text { Standby Inst. Air Compressor Down for } \\
\text { Maintenance }\end{array}$ & $6.010^{-3}$ & 10 \\
\hline ICM004LX & $\begin{array}{l}\text { Emergency Air Compressor Down for } \\
\text { Maintenance }\end{array}$ & $6.010^{-3}$ & 10 \\
\hline QPM002YX & Plant Water Standby & $6.010^{-3}$ & 10 \\
\hline WPM002YX & $\begin{array}{l}\text { Reactor Aux. Standby Pump } \\
\text { Maintenance }\end{array}$ & $6.010^{-3}$ & 10 \\
\hline
\end{tabular}

Notes: Probability based on $1 \mathrm{hr} /$ week maintenance. 
Table 7.23. Plant-Specific Component Failure Data For Reactor Shutdown System

\begin{tabular}{||l|c|l|l||}
\hline Event Name ${ }^{(2)}$ & Failure Rate ${ }^{(1)}$ & \multicolumn{1}{|c|}{ Event Description } & \multicolumn{1}{c|}{ Operation Experience } \\
\hline $\begin{array}{l}\text { RRE1A1QP, etc. } \\
\text { RRE2A1QP, etc. } \\
\text { RRE3C1QP, etc. } \\
\text { RRECC1QP, etc. } \\
\text { RRECB1QP, etc. } \\
\text { RRESROQP, etc. }\end{array}$ & $1.010^{-7} \mathrm{hr}^{-1}$ & $\begin{array}{l}\text { Relay Armature jams and fails to move } \\
\text { to open position }\end{array}$ & $\begin{array}{l}\text { There are about 250 clark relays, model } \\
\text { 5UU, in the plant. Five unsafe relay } \\
\text { failures have been noticed. }\end{array}$ \\
\hline $\begin{array}{l}\text { RND2AORW, } \\
\text { RND2BORW, } \\
\text { RND2CORW }\end{array}$ & $3.010^{-6} \mathrm{hr}^{-1}$ & $\begin{array}{l}\text { Guarded fission chamber of a wide-range } \\
\text { channel fails low }\end{array}$ & $\begin{array}{l}\text { There are 3 similar chambers in the } \\
\text { plant. One unsafe failure was recorded, } \\
\text { CREDO \#EBR850006. }\end{array}$ \\
\hline $\begin{array}{l}\text { RCA2AORW, } \\
\text { RCA2BORW, } \\
\text { RCA2CORW }\end{array}$ & $9.010^{-7} \mathrm{hr}^{-1}$ & $\begin{array}{l}\text { Cables and connectors of a wide-range } \\
\text { channel reduce or eliminate signal from } \\
\text { detector }\end{array}$ & $\begin{array}{l}\text { There are 3 similar cables in the plant. } \\
\text { No failure. }\end{array}$ \\
\hline $\begin{array}{l}\text { RBI2A1QW, etc. } \\
\text { RB2A2QW, etc. }\end{array}$ & $2.010^{-7} \mathrm{hr}^{-1}$ & $\begin{array}{l}\text { High-flux and low-volt bistables of a } \\
\text { wide-range channel fail }\end{array}$ & $\begin{array}{l}\text { There are 12 similar components in the } \\
\text { plant circuits. No failure }\end{array}$ \\
\hline $\begin{array}{l}\text { RAM2AOQW, } \\
\text { RAM2BOQW, } \\
\text { RAM2COQW }\end{array}$ & $1.010^{-6} \mathrm{hr}^{-1}$ & $\begin{array}{l}\text { Current amplifier of a wide-range } \\
\text { channel fails }\end{array}$ & $\begin{array}{l}\text { There are 3 similar components in the } \\
\text { plant. One failure was detected and } \\
\text { overcome during operation. }\end{array}$ \\
\hline $\begin{array}{l}\text { RDC2A1QW, } \\
\text { RDC2B1QW, } \\
\text { RDC2C1QW }\end{array}$ & $3.010^{-6} \mathrm{hr}^{-3}$ & $\begin{array}{l} \pm 15 \text { Vdc power supply in a wide-range . } \\
\text { channel fails }\end{array}$ & $\begin{array}{l}\text { There is a large number of these } \\
\text { components in the wide-range channels. } \\
\text { Failures have occurred but not in the } \\
\text { scram portion of the channels. Estimate. }\end{array}$ \\
\hline $\begin{array}{l}\text { RDC2A2QW, } \\
\text { RDC2B2QW, } \\
\text { RDC2C2QW, }\end{array}$ & $9.010^{-7} \mathrm{hr}^{-1}$ & $\begin{array}{l}\text { High Volt DC power supply in a wide- } \\
\text { range channel fails }\end{array}$ & $\begin{array}{l}\text { There are 3 similar units in the plant. No } \\
\text { failure. }\end{array}$ \\
\hline
\end{tabular}


Table 7.23. Plant-Specific Component Failure Data For Reactor Shutdown System (Cont'd)

\begin{tabular}{||l|l|l|l||}
\hline $\begin{array}{l}\text { RVIIA1QW, etc. } \\
\text { RVI3COQW, etc. }\end{array}$ & $5.010^{-7} \mathrm{hr}^{-1}$ & $\begin{array}{l}\text { MV/I converter in a low-flow channel or } \\
\text { SOT channel fails }\end{array}$ & $\begin{array}{l}\text { There is a large number of this } \\
\text { component, Ronan X-81, in the plant } \\
\text { circuits. Estimated accum. hrs of } \\
\text { operation up to } 1987=9 ., 75 \mathrm{E}+5 \mathrm{H} . \\
\text { No failure. }\end{array}$ \\
\hline \hline RBIIAOQW, etc. & $5.010^{-7} \mathrm{hr}^{-1}$ & $\begin{array}{l}\text { Trip bistable in a low-flow channel or } \\
\text { SOT channel fails }\end{array}$ & $\begin{array}{l}\text { There is a large number of this } \\
\text { component, Ronan X-82, in the plant } \\
\text { circuits. Estimated accum. hrs. of } \\
\text { operation up to } 1987=9.32 \mathrm{E}+5 \mathrm{H} . \text { No } \\
\text { failure. }\end{array}$ \\
\hline
\end{tabular}

\section{NOTES:}

(1) Mean failure rate $=(2 \mathrm{~N}+1) /(2 \mathrm{~T})$

$\mathrm{N}$ is number of failures,

$\mathrm{T}$ is estimated hours of power operation, with $\mathrm{T}=1.80 \mathrm{E}+5 \mathrm{H}$ for one component

(2) An example for a basic event from each channel or string is provided. 
Table 7.24. Special Data

PAGE 1

\begin{tabular}{|c|c|c|c|c|c|c|c|c|c|}
\hline \multirow{2}{*}{$\begin{array}{l}\text { EVENT } \\
\text { NAME }\end{array}$} & \multirow[b]{2}{*}{ DESCRIPTION } & \multicolumn{2}{|c|}{ VALUE USED $3 / 91$} & \multicolumn{2}{|c|}{ CRBRP-4 } & \multicolumn{2}{|c|}{ IREP } & \multicolumn{2}{|c|}{ GENERIC } \\
\hline & & $\begin{array}{c}\text { Failure } \\
\text { Rate }\end{array}$ & $\begin{array}{l}\text { Error } \\
\text { Factor }\end{array}$ & $\begin{array}{c}\text { Failure } \\
\text { Rate }\end{array}$ & $\begin{array}{l}\text { Error } \\
\text { Factor }\end{array}$ & $\begin{array}{l}\text { Failure } \\
\text { Rate }\end{array}$ & $\begin{array}{l}\text { Error } \\
\text { Factor }\end{array}$ & $\begin{array}{c}\text { Failure } \\
\text { Rate }\end{array}$ & $\begin{array}{l}\text { Error } \\
\text { Factor }\end{array}$ \\
\hline XHEOO1RF & IXH TUBE FAILURE & $\begin{array}{c}1.0 \mathrm{E}-6 / \mathrm{h} \\
{[1]}\end{array}$ & 10 & & & & & & \\
\hline$x 0000200$ & DRAIN VALVES OPENNED & 1.0 & 1 & & & & & & \\
\hline $\mathrm{x} 0011200$ & $\begin{array}{l}\text { IHX OUTLET DRAIN } \\
\text { VALVE OPEN }\end{array}$ & 1.0 & 1 & & & & & & \\
\hline $\mathrm{x} 0022200$ & $\begin{array}{l}\text { SURGE TANK DRAIN } \\
\text { VALVE OPEN }\end{array}$ & 1.0 & 1 & & & & & & \\
\hline$x 0033200$ & $\begin{array}{l}\text { EM PUMP DRAIN VALVE } \\
\text { OPEN }\end{array}$ & 1.0 & 1 & & & & & & \\
\hline XOOPMZOO & PUMP REMAINS ON & 1.0 & 1 & & & & & & \\
\hline XPP09OLF & $\begin{array}{l}\text { PIPE BREACH IN } \\
\text { PURIFICATION SYSTEM }\end{array}$ & $\begin{array}{c}3.0 \mathrm{E}-7 / \mathrm{h} \\
{[1]}\end{array}$ & 10 & & & & & & \\
\hline XPP100RF & $\begin{array}{l}\text { OUTER BOUNDARY } \\
\text { BREACH }\end{array}$ & $\begin{array}{c}1.0 \mathrm{E}-6 / \mathrm{h} \\
{[2]}\end{array}$ & 10 & & & & & & \\
\hline XPP 101RF & $\begin{array}{l}\text { INNER BOUNDARY } \\
\text { BREACH }\end{array}$ & $\begin{array}{c}1.0 \mathrm{E}-1 / \mathrm{d} \\
{[1]}\end{array}$ & 3 & & & & & & \\
\hline XRVOO1NK & $\begin{array}{l}\text { PRESSURE RELIEF ALLO } \\
\text { WS SODIUMOVERFLOW: } \\
\text { OPENS AND REMAINS SO }\end{array}$ & $\begin{array}{c}1.0 E-3 / d \\
{[1]}\end{array}$ & 10 & & & & & & \\
\hline XTKOO1NF & $\begin{array}{l}\text { SODIUM STORAGE TANK } \\
\text { RUPTURES }\end{array}$ & $\begin{array}{c}1.0 \mathrm{E}-6 / \mathrm{h} \\
{[1]}\end{array}$ & 10 & . & & & & & \\
\hline XVVOO1LP & $\begin{array}{l}\text { FAILURE OF VALVE } 1 \\
\text { TO OPEN }\end{array}$ & $\begin{array}{c}1.0 \mathrm{E}-2 / \mathrm{d} \\
{[1]}\end{array}$ & 10 & & & & & & \\
\hline XVV002LP & $\begin{array}{l}\text { FAILURE OF VALVE } 2 \\
\text { TO OPEN }\end{array}$ & $\begin{array}{c}1.0 \mathrm{E}-2 / \mathrm{d} \\
{[1]}\end{array}$ & 10 & & & & & & \\
\hline XVVOORLP & $\begin{array}{l}\text { FAILURE OF REDUNDANT } \\
\text { SYSTEM; VALVE FAILS } \\
\text { TO OPEN }\end{array}$ & $\begin{array}{c}1.0 \mathrm{E}-2 / \mathrm{d} \\
{[1]}\end{array}$ & 10 & & & & & & \\
\hline
\end{tabular}

Notes: 1. Recommended value tables, EGG-SSRE-8875, Feb. 1990

2. Engineering Judgement for CCF 
Table 7.24. Special Data (Cont'd)

PAGE 1

\begin{tabular}{|c|c|c|c|c|c|c|c|c|c|}
\hline \multirow{2}{*}{$\begin{array}{l}\text { EVENT } \\
\text { NAME }\end{array}$} & \multirow[b]{2}{*}{ DESCRIPTION } & \multicolumn{2}{|c|}{ VALUE USED $3 / 91$} & \multicolumn{2}{|c|}{ CABRP.4 } & \multicolumn{2}{|c|}{ IREP } & \multicolumn{2}{|c|}{ GENERIC } \\
\hline & & $\begin{array}{l}\text { Failure } \\
\text { Rate }\end{array}$ & $\begin{array}{l}\text { Error } \\
\text { Factor }\end{array}$ & $\begin{array}{l}\text { Failure } \\
\text { Rate }\end{array}$ & $\begin{array}{l}\text { Error } \\
\text { Factor }\end{array}$ & $\begin{array}{l}\text { Failure } \\
\text { Rate }\end{array}$ & $\begin{array}{l}\text { Error } \\
\text { Factor }\end{array}$ & $\begin{array}{l}\text { Failure } \\
\text { Rate }\end{array}$ & $\begin{array}{l}\text { Error } \\
\text { Factor }\end{array}$ \\
\hline VHEDMIWF & $\begin{array}{l}\text { WATER AND SODIUM } \\
\text { DUMP AFTER LEAK } \\
\text { ESCALATES }\end{array}$ & $1.0 \mathrm{E}-1 / \mathrm{d}$ & 5 & & & & & & \\
\hline VHEDM2WF & $\begin{array}{l}\text { WATER AND SODIUM } \\
\text { DUMP AFTER LEAK } \\
\text { ESCALATES }\end{array}$ & $9.0 \mathrm{E}-1 / \mathrm{d}$ & 1 & & & & & & \\
\hline VHEFSDWF & $\begin{array}{l}\text { PROBABILITY OF } 2 \\
\text { SIMULTANEOUS DEG } \\
\text { FAILURES }\end{array}$ & $3.2 \mathrm{E} \cdot 3 / \mathrm{d}$ & 10 & & & & & & \\
\hline VHELKDWF & $\begin{array}{l}\text { PROBABILITY OF LEAK } \\
\text { DETECTION SYSTEM } \\
\text { FAILURE }\end{array}$ & $3.5 E-4 / d$ & 10 & & & & & & \\
\hline VHEMOIWF & $\begin{array}{l}\text { LEAK ESCALATES } \\
\text { WITHIN FIRST MINUTE }\end{array}$ & $1.6 E-1 / d$ & 3 & & & & & & \\
\hline VHENO2WF & $\begin{array}{l}\text { LEAKESCALATES } \\
\text { WITHIN FIRST MINUTE }\end{array}$ & $8.4 E-1 / d$ & 1 & & & & & & \\
\hline VHEPF2WF & $\begin{array}{l}\text { PRESSURE RELIEF DOES } \\
\text { NOT OCCUR }\end{array}$ & $5.0 E-1 / d$ & 10 & & & & & & \\
\hline VHEPF3WF & $\begin{array}{l}\text { PRESSURE RELIEF DOES } \\
\text { NOT OCCUR }\end{array}$ & $1.0 \mathrm{E}-1 / \mathrm{d}$ & 10 & & & & & & \\
\hline VHEPFAWF & $\begin{array}{l}\text { PRESSURE RELIEF DOES } \\
\text { NOT OCCUR }\end{array}$ & $9.0 \mathrm{E}-1 / \mathrm{d}$ & 1 & & & & & & \\
\hline VHERIXWF & $\begin{array}{l}\text { REACTOR SHUTDOWN } \\
\text { AFTER IHX FAILURE }\end{array}$ & $1.0 \mathrm{E}-2 / \mathrm{d}$ & 10 & & & & & & \\
\hline VHESLKWF & SMALL OA MEDIUM LEAK & $3.2 \mathrm{E}-2 / \mathrm{d}$ & 10 & & & & & & \\
\hline VHESPRWW & $\begin{array}{l}\text { SODIUM PRESSURE } \\
\text { RELIEF SYSTEM FAILS }\end{array}$ & $4.4 E-4 / d$ & 10 & & & . & & & \\
\hline
\end{tabular}


Table 7.24. Special Data (Cont'd)

PAGE 1

\begin{tabular}{|c|c|c|c|c|c|c|c|c|c|}
\hline \multirow{2}{*}{$\begin{array}{l}\text { EVENT } \\
\text { NAME }\end{array}$} & \multirow[b]{2}{*}{ DESCAIPTION } & \multicolumn{2}{|c|}{ VALUE USED $3 / 91$} & \multicolumn{2}{|c|}{ CRBRP-4 } & \multicolumn{2}{|c|}{ IREP } & \multicolumn{2}{|c|}{ GENERIC } \\
\hline & & $\begin{array}{c}\text { Failure } \\
\text { Rate }\end{array}$ & $\begin{array}{l}\text { Error } \\
\text { Factor }\end{array}$ & $\begin{array}{c}\text { Failure } \\
\text { Rate }\end{array}$ & $\begin{array}{l}\text { Error } \\
\text { Factor }\end{array}$ & $\begin{array}{c}\text { Failure } \\
\text { Rate }\end{array}$ & $\begin{array}{l}\text { Error } \\
\text { Factor }\end{array}$ & $\begin{array}{c}\text { Failure } \\
\text { Rate }\end{array}$ & $\begin{array}{l}\text { Error } \\
\text { Factor }\end{array}$ \\
\hline FPPDO1RF & $\begin{array}{l}\text { PIN-TO-PIN BREACH } \\
\text { PROPAGATION }\end{array}$ & $1.0 \mathrm{E}-4 / \mathrm{d}$ & 10 & & & & & & \\
\hline FPPOO2RF & $\begin{array}{l}\text { PIN-TO-PIN BREACH } \\
\text { PROPAGATION }\end{array}$ & $1.0 \mathrm{E}-2 / \mathrm{d}$ & 10 & & & & & & \\
\hline FPPOO3RF & $\begin{array}{l}\text { PIN-TO-PIN BREACH } \\
\text { PROPAGATION }\end{array}$ & $1.0 \mathrm{E}-1 / \mathrm{d}$ & 3 & & & & & & \\
\hline FPPOO4RF & $\begin{array}{l}\text { PIN-TO-PIN BREACH } \\
\text { PROPAGATION }\end{array}$ & $1.0 \mathrm{E}-3 / \mathrm{d}$ & 10 & & & & & & \\
\hline FPP111RF & $\begin{array}{l}\text { PIN-TO-PIN BREACH } \\
\text { PROPAGATION NOT } \\
\text { SELF-LIMITING }\end{array}$ & $1.0 \mathrm{E}-4 / \mathrm{d}$ & 10 & & & & & & \\
\hline FPP112RF & $\begin{array}{l}\text { PIN-TO-PIN BREACH } \\
\text { PROPAGATION NOT } \\
\text { SELF-LIMITING }\end{array}$ & $1.0 \mathrm{E} \cdot 3 / \mathrm{d}$ & 10 & & & & & & \\
\hline FPPQ01RF & $\begin{array}{l}\text { SUB-TO-SUB DAMAGE } \\
\text { PROPAGATION }\end{array}$ & $1.0 \mathrm{E}-4 / \mathrm{d}$ & 10 & & & & & & \\
\hline FPPSO2RF & $\begin{array}{l}\text { SUB-TO-SUB DAMAGE } \\
\text { PROPAGATION }\end{array}$ & $1.0 \mathrm{E} \cdot 3 / \mathrm{d}$ & 10 & & & & & & \\
\hline FPP903RF & $\begin{array}{l}\text { SUB-TO-SUB DAMAGE } \\
\text { PROPAGATION }\end{array}$ & $1.0 \mathrm{E}-2 / \mathrm{d}$ & 10 & & & & & & \\
\hline FPP991RF & $\begin{array}{l}\text { PIN BREACH IN } \\
\text { FAULTED SUBASSEMBLY }\end{array}$ & 9.0E-1/d & 3 & & & & & & \\
\hline
\end{tabular}


Table 7.24. Special Data (Cont'd)

\begin{tabular}{|c|c|c|c|c|c|c|c|c|c|}
\hline \multirow{2}{*}{$\begin{array}{l}\text { EVENT } \\
\text { NAME }\end{array}$} & \multirow[b]{2}{*}{ DESCRIPTION } & \multicolumn{2}{|c|}{ VALUE USED $3 / 91$} & \multicolumn{2}{|c|}{ CRBRP-4 } & \multicolumn{2}{|c|}{ IREP } & \multicolumn{2}{|c|}{ GENERIC } \\
\hline & & $\begin{array}{c}\text { Failure } \\
\text { Rate }\end{array}$ & $\begin{array}{l}\text { Error } \\
\text { Factor }\end{array}$ & $\begin{array}{c}\text { Failure } \\
\text { Rate }\end{array}$ & $\begin{array}{l}\text { Error } \\
\text { Factor }\end{array}$ & $\begin{array}{c}\text { Failure } \\
\text { Rate }\end{array}$ & $\begin{array}{l}\text { Error } \\
\text { Factor }\end{array}$ & $\begin{array}{c}\text { Failure } \\
\text { Rate }\end{array}$ & $\begin{array}{l}\text { Error } \\
\text { Factor }\end{array}$ \\
\hline GNDOO2RW & $\begin{array}{l}\text { BREACH DETECTION } \\
\text { FAILURE }\end{array}$ & $1.0 \mathrm{E}-5 / \mathrm{d}$ & 10 & & & & & & \\
\hline GND003RW & $\begin{array}{l}\text { BREACH DETECTION } \\
\text { FAILURE }\end{array}$ & $1.0 \mathrm{E} \cdot 4 / \mathrm{d}$ & 10 & & & & & & \\
\hline
\end{tabular}




\subsubsection{Reactivity Insertion Data}

\subsubsection{Introduction}

One important class of events in the EBR-II PRA is the reactivity insertion events which arise from inadvertent motion of control or safety rods. The relative importance depends on two factors, 1) the frequency of a given event and 2) the amount of reactivity inserted. The purpose of this discussion is to present the assessment of reactivity available for insertion from accidental rod run-in in EBR-II. Estimates are generated for the magnitude of reactivity inserted, the insertion rate and the relative frequency for different classifications of rods.

These estimates were developed from administrative and physical limits, operating procedures and actual operating history. Technical Specifications [7.5] impose upper bounds on rod worths. Rod and core designs constitute additional limitations on rods worths and reactivity insertion rates. Operating Procedures [7.6] imply patterns for axial rod positioning and movement. Finally, data files from the Digital Acquisition System (DAS) provide an extensive history of rod positions and, implicitly, reactivity insertion potential. Probability density functions (pdfs) for reactivity insertion magnitudes, and estimates of insertion rates and relative frequencies were constructed from these sources.

\subsubsection{Administrative and Physical Reactivity Limits}

\subsection{Reactivity Insertion Magnitude}

The reactivity that a rod can insert in a TOP accident depends on the total worth of the rod and how far the rod was inserted before the event. Since rod worths vary roughly linearly with insertion over their 14 inch travel, the TOP reactivity is approximately

$$
\mathrm{R}_{\mathrm{i}}=\mathrm{R}_{\mathrm{t}}^{*}(1-\mathrm{Z} / 14) \text {, }
$$


where $R_{\mathbf{l}}$ is the total worth and $Z$ is the pre-accident rod position, in inches, measured from full out. (Recall that the EBR-II rods are fueled, so reactivity increases as they are inserted.)

The administrative and physical limits on rod worths $\left(R_{t}\right)$ and positions $(Z)$ are discussed in the following paragraphs. These limits are then used with Eq. 7.1 to estimate reactivity insertion magnitudes.

\section{Range of TOP Reactivity, $R_{\imath}$}

There are three basic rod types, each with different characteristic values of $R_{t}$. The control rods are referred to as "normal" or "high-worth" types, depending on their design. In recent years, each loading has had only one normal worth rod and seven to nine high-worth rods. The third type is safety rods, and every loading has two of them. They are driven as a pair, so their worths are combined for this assessment.

The total worth of a rod is limited by the design of the rod and the surrounding core. It is unlikely that these parameters will change significantly and this analysis assumes that they will not. The grid locations of the rods, the fuel pin and boron follower components of the rods and even the basic core layout are all invariant. The rod worths vary due to such factors as the burnup of the fuel in the rod and the types of subassemblies in the vicinity of the rod.

The range of $R_{t}$ values that occurred over the 29 subruns from Run 139A (May 1986) through Run 153A (March 1990) are shown in Table 7.23. The ratio of maximum to minimum total worth of a rod is about 1.5 for all three types. To confirm that the upper limits in Table 7.25 are accurate, the maximum rod worth for each rod type was checked for every fifth numbered run starting with Run 100A (1979). A slightly higher worth was found for the normal type, 44.95 cents, but not for the others. Thus, the upper bounds for rod worths are $\$ 1.43$ for safety rods, $\$ 0.45$ for normal-type control rods and $\$ 0.99$ for high worth-type control rods.

Control rod worths are also limited by Technical Specifications. No control rod is allowed to be worth more than $\$ 1.30$, but this limit is of no practical concern since that value 
Table 7.25. Rod Worth ${ }^{\mathrm{a}}$ By Type From Run 139A Through Run 153A (c)

\begin{tabular}{|c|c|c|c|}
\hline Type & $\underline{\text { Safety Rods }}$ & Normal CR & High Worth $\mathrm{CR}$ \\
\hline Minimum Worth (cents) & 96.2 & 31.2 & 64.0 \\
\hline Average Worth (cents) & 116.2 & 38.2 & 79.0 \\
\hline Maximum Worth (cents) & 143.1 & 44.0 & 99.0 \\
\hline
\end{tabular}

${ }^{\text {a }}$ For a single control rod or the pair of safety rods 
cannot be approached with the current design. There is a limit of practical interest, $\$ 0.90$, when a control rod is operated under computer control (automatic control rod drive system, or ACRDS) at the fast drive speed. However, this mode of operation is only allowed as part of an approved plant transient experiment. Thus, these two Technical Specification limits are not really relevant to steady-power operation.

\section{Range of Rod Position, $\mathrm{Z}$}

There is a Technical Specification that implies a minimum control rod bank position. It is that the control rods must be able to withdraw at least $\$ 3.70$ when scrammed during full power operation. Assuming that 1) the scram worth is proportional to the distance moved, 2) control rod worths are the averages in Table 7.22, and 7.3) the number of control rods is the maximum used in the last 10 years (one normal and nine high worth), this Specification implies a minimum control rod bank position $(\mathrm{Z})$ of 6.9 inches.

The practical minimum control rod bank position is about an inch higher that this, 8.0 inches. It is the policy of the Chief Physicist to reject any proposed core loading that would require a control bank position less than 8 inches [7.7]. The reason for this policy is to keep

the boron portion of high worth control rods out of the active core region. A survey of beginning-of-run bank positions (every fifth numbered run from Run 100 to Run 135 and every subrun starting from Run 139A) showed that the minimum bank position that has actually been used is 8.0 inches.

There are two specifications that restrict the safety rod position. One is an Operating Instruction prescribing an absolute minimum insertion of 7.5 inches. The other is a Technical Specification that the safety rods must be able to withdraw reactivity equal to at least $120 \%$ of the power reactivity decrement (PRD). This second limit is almost always less constraining than the 7.5 inch minimum; with an average safety rod worth (\$1.16) and the average PRD (32 cents) it implies a bank position of only 4.6 inches. In the most extreme case since 1978 (94 cent rod worth and 46 cent PRD in Run 103B), the PRD constraint was the limiting one, with a limit of 8.3 inches. In recent years, the safety rods have been positioned near the 7.5 inch minimum 
on a number of occasions to allow more control rod insertion when there was a high fissile loading.

The Operating Instructions prescribe additional limits on the rod positions during steady power operation. Control rods are divided into two categories in these instructions. The Operations Physicist designates a particular control rod to be the "controlling" rod for the duration of a subrun. This rod is used to make the short-term reactivity adjustments necessary to compensate for burnup. The allowable range of positions for the controlling rod is 9 to 12 inches if it is a high worth rod and 5 to 9.5 inches if it is the normal worth rod. The remaining control rods are all banked at a common position.

Once every few days the rods must be rebanked. When the controlling rod approaches its upper position limit, either the control rod bank or the safety rod pair is moved up a short distance ( $\sim 1 / 4$ inch) as the controlling rod is lowered to approximately its lower limit. Which set of rods is moved up depends on how much excess reactivity is present in the core at the time. Assuming a large initial excess, the current procedure calls for rebanking the control rods until they reach 10 inches, then rebanking the safety rods until they are fully inserted, and then rebanking the control rods again.

Putting these facts together yields the following position histories for each rod category. For the controlling rod, the position varies uniformly between 9 and 12 inches if it is a high worth rod or 5 and 9.5 inches if it is a normal worth rod. For the control rod bank, the position varies uniformly between a lower limit, determined by the initial excess, and an upper limit, determined by the final burnup. The lower limit is at least 8 inches and the upper limit can be as large as full insertion (14 inches). For the safety rods, the history in general is a bimodal curve with a low plateau between the peaks. The position of the first peak is determined by the initial excess and can be as little as 7.5 inches. Because the safety rods are worth less than $1 / 5$ as much as the control rod bank, the safety rods spend relatively little time between this lower position and the second peak, which is at the, fully inserted, 14 inch position. The safety rods stay at 14 inches for the remainder of the run. 
Combining these position histories with the average rod worths from Table 7.23 implies an $\mathbf{R}_{\mathbf{i}}$ distribution for each rod category. For the controlling rod, the $\mathbf{R}_{\mathbf{i}}$ varies uniformly between 28 and 11 cents if it is a high worth rod or between 24 and 12 cents if it is a normal worth rod. For a rod in the control rod bank, $R_{i}$ varies uniformly from as much as 34 cents to as little as zero. For the safety rods, there can be a peak where $R_{i}$ is as much as 54 cents, and then a short time that is spread uniformly from there to $R_{i}=0$, which is the value maintained for the remainder of the run.

The Operating Instructions prescribe one other kind of rod motion during steady power operation, a daily control rod motion test. In this test, each control rod must be moved at least three inches, either up or down, to verify that it is working properly. This implies that, for a small fraction of one hour per day, some control rods are down at least three inches from the bank position. This adds a low frequency, higher-than-normal worth contribution to the control rod insertion potential. This phenomenon is treated in Section 7.2.4.3.

\subsection{Rate of Insertion}

For a given value of $R_{i}$, the rate of reactivity insertion depends on the initial rod position $\mathrm{Z}$ and the drive speed. The drive speed is 2.11 inches/minute for safety rods and 4.76 inches/minute for control rods. (The ACRDS drives control rod \#4 at 72 inches/minute in high speed mode but this is only done as part of a plant experiment, not during steady power operation.) Again assuming linear worth variation with rod position, it is easy to estimate reactivity insertion rates. For example, an average safety rod run in from the 7.5 inch position would introduce a total of $\sim 54$ cents at $\sim 0.29$ cents/s, an average high-worth control rod starting at 8 inches would introduce a total of $\sim 34$ cents at $\sim 0.45$ cents/s, and an average normal worth rod run in from 8 inches would introduce a total of $\sim 16$ cents at $\sim 0.22$ cents/s.

The linear worth variation approximation underestimates the insertion rate over part of the rod travel. The actual worth profiles are shown in Fig. 7.1. For high-worth control rods, the maximum insertion rate is $14 \%$ larger than the average and it is essentially constant at this 


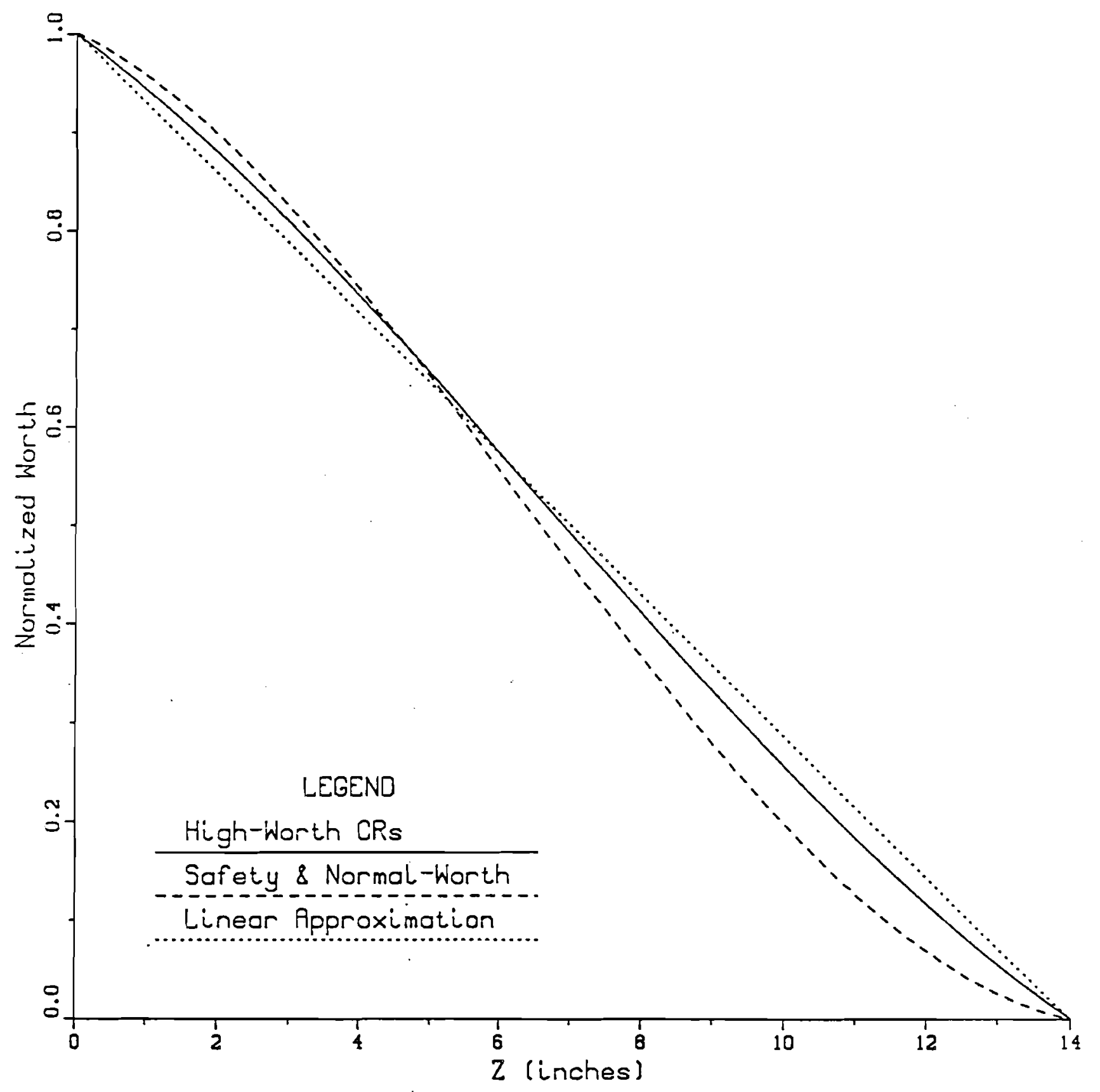

Fig. 7.1. Rod Worth Profiles 
maximum value between 5 and 10 inches. For safety rods and normal-worth control rods, the maximum insertion rate is $32 \%$ larger than the average and it is essentially constant at this maximum value between 4 and 9 inches.

It should be noted that insertion rate bounds in the Technical Specifications are quite conservative. Actual bounds can be found using maximum rod worths from Table 7.23, physical drive speeds, and nonlinearity factors from the previous paragraph. The maximum insertion rate for the highest worth control rod is 0.64 cents/s and for the highest worth safety rods it is 0.47 cents/s. These are much smaller than the Technical Specification limit of 1.0 cents/s. Similarly, the maximum insertion rate for the highest-allowed-worth control rod (90 cents), driven by the ACRDS at high speed, is 8.8 cents/s, compared to the Technical Specification limit of 12 cents/s.

\subsection{Relative Initiator Frequencies}

The occurrence frequencies for rod run-in events depends, of course, on the postulated failure modes. No attempt is made here to make a complete evaluation of failure modes, but to point out the relative frequency for the various rod categories for what appears to be the most likely failure mode. Run-in of a single rod is considered first, followed by simultaneous run-in of multiple rods.

The most likely scenario for a single rod run-in is failure of a rod to stop moving in when the reactor operator releases the up motion control switch. The spring-loaded control rod motion switch could fail closed in the up position, for example.

For this mode, the failure frequency is proportional to the frequency of up-motion demands. The demand frequency depends on the rod category. The controlling control rod is the one moved most frequently. Both discussions with Operations [7.8] and observation of controlling rod motions recorded by the DAS indicate that this rod is moved up three times per hour on average. The other control rods are moved up at most once every few days to follow burnup, but are moved up once per day during the daily motion check ( 0.04 times per hour). 
The safety rods are not involved in daily motion tests and are moved only at one stage of burnup, as described in Section II.A.2. From May 1986 through February 1990, the safety rods were moved up 86 times in the 22,486 hours of full-power operation. This implies an average up motion frequency of 0.004 times per hour.

Simultaneous run-in of more than one rod is much less likely than a single-rod run-in. Interlocks prevent more than one rod from being driven in at a time. A failure mode such as a short must occur before more than one rod could move in at a time. The number of rods that could move up at one time and the frequency at which this could occur has been determined by a failure analysis. Only the upward motion of one rod and two rods is considered in Section 7.2.4.3.

\subsubsection{Analysis of DAS Data}

\subsection{Processing the Data}

The DAS records of rod positions were used to construct more precise descriptions of rod run-in reactivity. The results in Section 7.2.2.2 provided guidance for development of a procedure to process the DAS data.

Data going back to May 1986 were analyzed, since that is the time at which the current format of DAS records was implemented. The most recent data included are from February 28, 1990. These data cover 15 numbered runs, 29 subruns and 22486 hours of full-power operation. This history is extensive enough to represent the range of conditions expected to occur in EBR-II and to provide good statistical precision for the probability density functions derived from it.

A DAS record contains time averages of the power level and position of each control and safety rod. One minute, 10 minute, and one hour time averages are available. One hour records were used here to make it feasible to process many years worth of data.

The disadvantage of using one-hour averages is that short-duration departures from an 
average position are not apparent. However, movements at steady power occur much less frequently than once per hour with two exceptions. One is adjustment of the controlling rod. This occurs about three times per hour but the total movement over an hour is only about 0.03 inches, which is much less than the resolution of interest.

The other exception is the daily control rod motion check. This is an important departure from the average that must be accounted for. It was treated by modelling a typical daily motion test in a separate subroutine of the processing code written for this analysis. A typical rod motion pattern was established by interviewing Operations management and recording the actual motion test on March 20, 1990. Typically, each rod is moved 3.5 inches from its normal position and is immediately returned to the normal position. To keep the power level steady, pairs of rods are moved simultaneously in opposite directions. Thus only half the rods go lower than their normal position in a test. Only the motion of these rods was modeled, a slight conservatism that saved considerable effort. The motion-test subroutine was called to process the records corresponding to the first hour of each day, since that is then motion tests actually are done.

Only records corresponding to steady power operation were accepted when processing the data. Records with a power level outside the range 52 to $65 \mathrm{MWt}$ were rejected, which eliminated all but 11 records of unacceptable data. This residual was identified by having the program flag records with extraordinary rod position values, and then hand-checking documentation to determine whether a plant transient experiment was run at that time.

A rod position, $\mathrm{Z}$, was converted to a reactivity insertion potential, $\mathrm{R}_{\mathrm{i}}$, using the measured total worth of that rod and the appropriate axial worth distribution (profile). Accordingly, the measured total worth of each rod at each subrun was supplied to the processing code. Actual worth profiles depart modestly from the linear profile assumed in most of Section II. Four different rod worth profiles, in the form of quartic polynomials, were available. By testing these profiles against actual rod calibration data from Runs 144 and 149, one profile was found to be adequate for the safety and normal rods, and another profile was shown to be adequate for high worth rods. These are the two profiles shown in Fig. 7.1. 
Discrete probability density functions were constructed from the $R_{i}$ values. The range of possible $R_{i}$ values was divided into five cent intervals or bins. For each $R_{i}$ value encountered, the bin encompassing that value was identified and then a weight was added to that bin. The weight was one (hour) except when the hour represented by a record was subdivided to simulate the daily motion test. This process produced a frequency distribution. To convert this to a probability density function, the weight in each bin was divided by the total of all bin weights.

A separate pdf for single-rod run-in was constructed for each rod category. Three categories were defined, each with a characteristic rod drive speed and relative frequency for initiating a TOP accident, as identified in Section 7.2.4.2. The categories are safety rods, the controlling rod and the banked control rods.

Simultaneous run-in of two control rods also was analyzed. The $R_{i}$ from each pair of control rods was formed, producing a two-control-rod pdf.

\subsubsection{2 $\underline{\text { Results }}$}

The pdfs are shown in Table 7.26 and Figures 7.2 and 7.3. The pdfs show the conditional probability that the magnitude of the TOP initiator will be within a 5 cent interval given that there is an accidental run-in of a rod of a particular type. The results are also shown as cumulative distribution functions (cdfs) in Table 7.25.

The distributions are consistent with the estimates made in Section 7.2.4.2. For example, the safety rod pdf shows there is about a $20 \%$ probability that $R_{i}$ will be around 50 cents, a $60 \%$ probability that $\mathbf{R}_{\mathbf{i}}$ will be nearly zero cents, and a small, nearly uniform probability that $\mathbf{R}_{\mathbf{i}}$ will have some intermediate value. The control rod pdfs have a low probability tail at the high worth end, which is the result of the daily motion tests. The controlling rod pdf is narrower than the pdf for the banked control rods, reflecting the more restricted range of $\mathrm{Z}$ positions prescribed by the Operating Instructions. The two-rod run-in pdf is the broadest and it bears some resemblance to a normal distribution function. The cdfs show that the 99.99th percentile is below 55 cents for safety rods, 40 cents for any control rod and 75 cents for two control rods. 
Safety rods are treated as having two or three states, because of their uneven pattern of activity. About $58 \%$ of the time they are fully inserted and inactive. Therefore, the time when safety rods could run-in is $42 \%$ of the 6900 hours of steady power operation per year ( 2900 $\mathrm{hr} / \mathrm{yr})$. When the number of hours exposed to an insertion potential is the dominant consideration for the failure frequency, then the safety rod columns in Tables 7.26 and 7.27 are used after adjustment. The adjustment consists of subtracting 0.58 from the first bin and renormalizing. When, however, the number of up-motion demands are the key, then it must be recognized that the safety rods are inactive for almost all the time they are at their lowest position. A flat pdf spanning the range from 0 to 55 cents is then the most appropriate one to use, since the safety rods are moved up uniformly from their startup position to 14 inches. (The variations in the middle portion of the safety rod pdf in Table 7.26 are largely just statistical fluctuations.)

The rate of reactivity insertion, as well as the total magnitude, is needed to do transient calculations. The rate can be determined by combining the reactivity magnitude, a corresponding $\mathrm{Z}$ value, a rod worth profile and a drive speed. The average $\mathrm{Z}$ value corresponding to each reactivity bin is given in Table 7.28. The drive speed, $\mathrm{S}$, is 0.0352 inches/s for the safety rods and 0.0793 inches/s for control rods. The worth profile is expressed as a quartic polynomial,

$$
\mathrm{P}(\mathrm{z})=\mathrm{A} 0+\mathrm{A} 1 * \mathrm{Z}+\mathrm{A} 2 * \mathrm{Z} * * 2+\mathrm{A} 3 * \mathrm{Z}^{* * 3}+\mathrm{A} 4 * \mathrm{Z} * * 4,
$$

and the coefficients for safety rods and control rods are given in Table 7.29. 
Table 7.26. Discrete Probability Density Functions For Rod Run-in Reactivity

\begin{tabular}{|c|c|c|c|c|c|}
\hline \multirow{2}{*}{\multicolumn{2}{|c|}{ Rho (cents) }} & \multicolumn{3}{|c|}{ Single Rod Run-in } & \multirow[b]{2}{*}{ Two CRs } \\
\hline & & \multirow[t]{2}{*}{ Safety } & \multirow[t]{2}{*}{$C n \pm 1 C R$} & \multirow[t]{2}{*}{ Banked CRs } & \\
\hline MIN & MAX & & & & \\
\hline 0 & 5 & 0.606822023 & 0.000132345 & 0.051044466 & 0.010040040 \\
\hline 5 & 10 & 0.040736458 & 0.075747506 & 0.150779352 & 0.020520435 \\
\hline 10 & 15 & 0.007782620 & 0.448401672 & 0.183909602 & 0.038487127 \\
\hline$\$ 5$ & 20 & 0.032804894 & 0.381694672 & 0.231532430 & 0.077445187 \\
\hline 20 & 25 & 0.010984613 & 0.080360714 & 0.231616327 & $0.1070487 \delta \varepsilon$ \\
\hline 25 & 30 & 0.014364493 & 0.011718298 & 0.101984695 & 0.145214671 \\
\hline 30 & 35 & 0.040780930 & 0.001677569 & 0.033815610 & $0 .: 52012425$ \\
\hline 35 & 40 & 0.027439296 & 0.000255383 & 0.005253354 & 0.154911625 \\
\hline 40 & 45 & 0.016098906 & 0.000008715 & 0.000038014 & 0.143157532 \\
\hline 45 & 50 & 0.180201014 & 0.000002369 & 0.000017457 & 0.073531546 \\
\hline 50 & 55 & 0.021924753 & 0.000000747 & $0: 000006374$ & 0.051334064 \\
\hline 55 & 60 & 0.000000000 & 0.000000010 & 0.000002124 & 0.031193260 \\
\hline 60 & 65 & 0.000000000 & 0.000000000 & 0.000000133 & 0.008349918 \\
\hline 65 & 70 & 0.000000000 & 0.000000000 & 0.000000000 & C. $00395893 i$ \\
\hline 70 & 75 & 0.000000000 & 0.000000000 & 0.000000000 & 0.002769025 \\
\hline 75 & 80 & 0.000000000 & 0.000000000 & 0.000000000 & 0.000005152 \\
\hline 80 & $\varepsilon 5$ & 0.000000000 & 0.000000000 & 0.000000000 & C. 000002127 \\
\hline 85 & 90 & 0.000000000 & 0.000000000 & 0.000000000 & 0.000000880 \\
\hline 90 & $\Phi=$ & 0.000000000 & 0.000000000 & 0.000000000 & C. 000000320 \\
\hline 95 & 100 & 0.000000000 & 0.000000000 & c. 000000000 & 0.000000042 \\
\hline
\end{tabular}



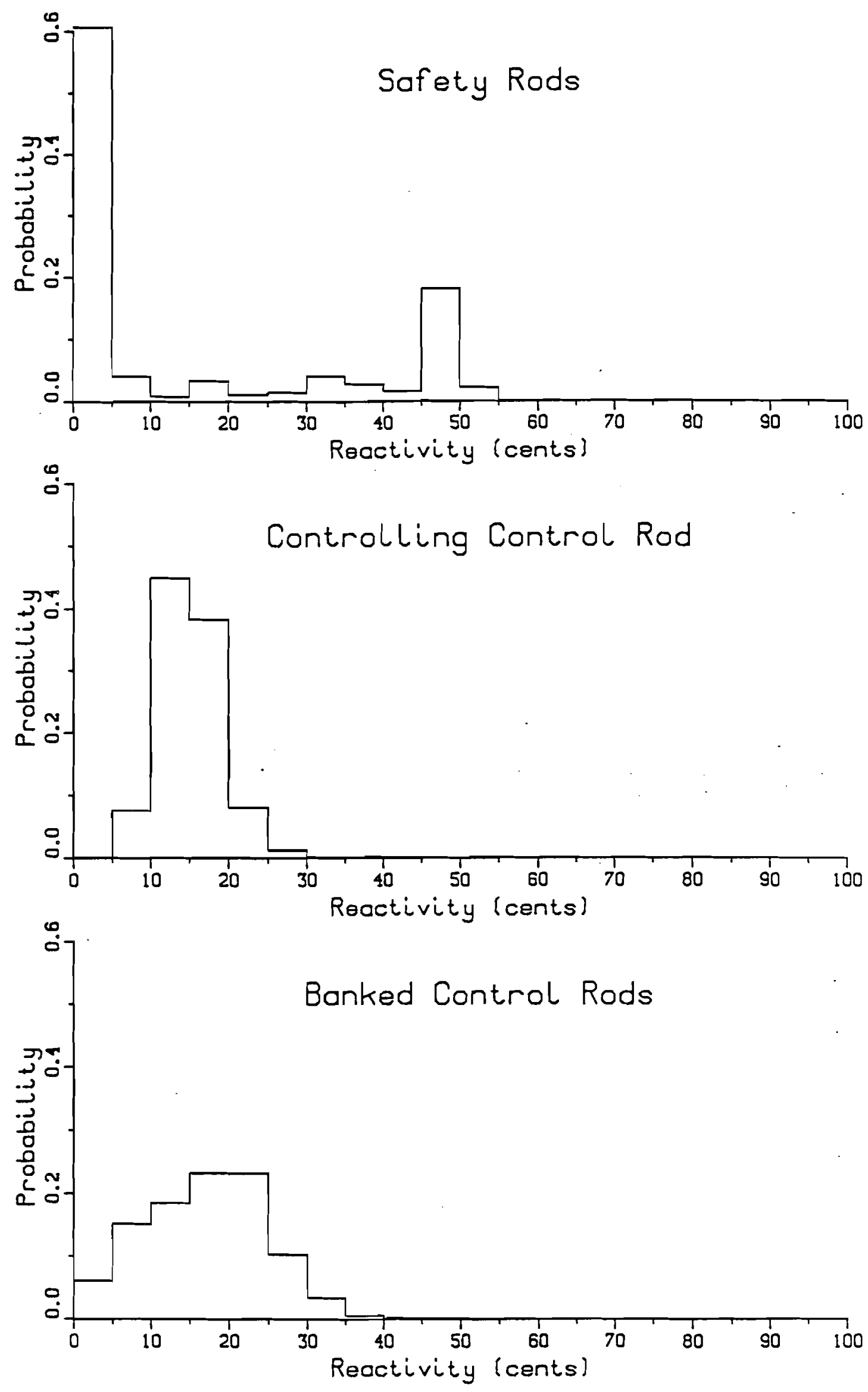

Fig. 7.2. Probability Density Functions for Single-Rod Run-In Reactivity 


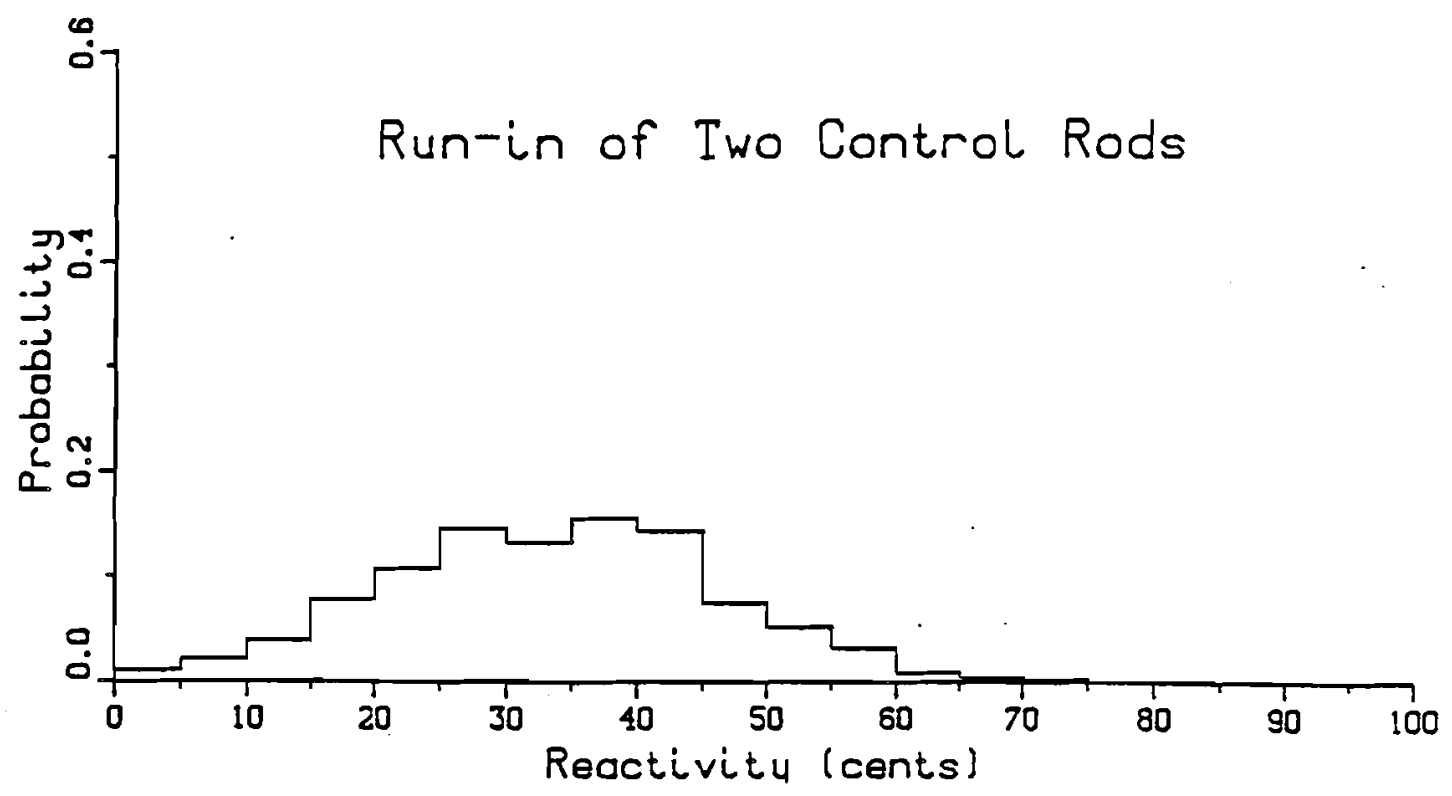

Fig. 7.3. Probability Density Function for Double-Rod Run-In Reactivity 
Table 7.27. Discrete Cumulative Distribution Functions For Rod Run-in Reactivity

\begin{tabular}{|c|c|c|c|c|c|}
\hline \multirow{2}{*}{\multicolumn{2}{|c|}{ Rho (cents) }} & \multicolumn{3}{|c|}{ Single Rod Run-in } & \multirow{3}{*}{ Two CRs } \\
\hline & & \multirow[t]{2}{*}{$\underline{\text { Safety }}$} & \multirow[t]{2}{*}{ Cnt] CR } & \multirow[t]{2}{*}{ Banked CRs } & \\
\hline MIN & $\underline{\text { MAX }}$ & & & & \\
\hline 0 & 5 & 0.606822023 & 0.000132345 & 0.061044466 & 0.010040046 \\
\hline 5 & 10 & 0.647558481 & 0.075879851 & 0.211823818 & 0.030566481 \\
\hline 10 & 15 & 0.655341101 & 0.524281523 & 0.395733480 & 0.069053608 \\
\hline 15 & 20 & 0.688205995 & 0.905976195 & 0.627265910 & 0.146498795 \\
\hline 20 & 25 & 0.699190607 & 0.986336909 & 0.858882237 & 0.253547563 \\
\hline 25 & 30 & 0.713555101 & 0.998055208 & 0.960866932 & 0.398762234 \\
\hline 30 & 35 & 0.754336031 & 0.999732777 & 0.994682542 & 0.530774659 \\
\hline 35 & 40 & 0.781775327 & 0.999988160 & 0.999935897 & 0.685686285 \\
\hline 40 & 45 & 0.797874233 & 0.999996875 & 0.999973911 & 0.828843816 \\
\hline 45 & 50 & 0.978075247 & 0.999999244 & 0.999991369 & 0.902375362 \\
\hline 50 & 55 & 1.000000000 & 0.999999990 & 0.999997743 & 0.953709426 \\
\hline 55 & 60 & 1.000000000 & 1.000000000 & 0.999999867 & 0.984902695 \\
\hline 60 & 65 & 1.000000000 & 1.000000000 & 1.000000000 & 0.993252613 \\
\hline 65 & 70 & 1.000000000 & 1.000000000 & 1.000000000 & 0.997221544 \\
\hline 70 & 75 & 1.000000000 & 1.000000000 & 1.000000000 & 0.999991479 \\
\hline 75 & 80 & 1.000000000 & 1.000000000 & 1.000000000 & 0.999996631 \\
\hline 80 & 85 & 1.000000000 & 1.000000000 & 1.000000000 & 0.999998758 \\
\hline 85 & 90 & 1.000000000 & 1.000000000 & 1.000000000 & 0.999999638 \\
\hline 90 & 95 & 1.000000000 & 1.000000000 & 1.000000000 & 0.999999958 \\
\hline 0 a & n & 1. חกחกno0000 & 1.000000000 & 1.000000000 & 1.000000000 \\
\hline
\end{tabular}


Table 7.28. Average Rod Position For Each Reactivity Bin and Rod Category

Single Rod Run-in

\begin{tabular}{|c|c|c|c|c|c|}
\hline \multicolumn{2}{|c|}{ Rho (cents) } & \multirow[t]{2}{*}{ Safety } & \multirow[t]{2}{*}{ Cntl CR } & \multirow[t]{2}{*}{ Banked CRs } & \multirow[t]{2}{*}{ Two CRs } \\
\hline MIN & MAX & & & & \\
\hline 0 & 5 & 14.006 & 13.023 & 12.450 & 13.555 \\
\hline 5 & 10 & 11.996 & 11.658 & 11.402 & 12.860 \\
\hline 10 & 15 & 11.227 & 10.914 & 11.058 & 12.241 \\
\hline 15 & 20 & 10.690 & 9.896 & 10.338 & 11.678 \\
\hline 20 & 25 & 9.857 & 9.349 & 9.721 & 11.200 \\
\hline 25 & 30 & 9.597 & 8.954 & 9.091 & 10.688 \\
\hline 30 & 35 & 9.044 & 8.857 & 8.743 & 10.382 \\
\hline 35 & 40 & 7.868 & 7.837 & 7.706 & 10.007 \\
\hline 40 & 45 & 7.832 & 6.950 & 6.794 & 9.817 \\
\hline 45 & 50 & 7.541 & 6.575 & 6.333 & 9.465 \\
\hline 50 & 55 & 7.517 & 5.991 & 5.916 & 9.077 \\
\hline 55 & 60 & & 5.561 & 5.507 & 8.879 \\
\hline 60 & 65 & & & 5.180 & 8.607 \\
\hline 65 & 70 & & & & 7.997 \\
\hline 70 & 75 & & & & 7.566 \\
\hline 75 & 80 & & & & 7.393 \\
\hline 80 & 85 & & & & 7.075 \\
\hline 85 & 90 & & & & 6.664 \\
\hline 90 & 95 & & & & 6.291 \\
\hline 95 & 100 & & & & 6.136 \\
\hline
\end{tabular}


Table 7.29. Polynomial Coefficients for the Worth Profiles, Eq. 2.

\begin{tabular}{ccc}
\hline & \multicolumn{2}{c}{ Value } \\
\cline { 2 - 3 } Coefficient & Safety or & Hormal \\
A0 & 1.001092 & 0.9997367 \\
A1 & $-3.472015 \mathrm{E}-2$ & $-5.249227 \mathrm{E}-2$ \\
A2 & $-9.896010 \mathrm{E}-3$ & $-4.656591 \mathrm{E}-3$ \\
A3 & $5.692658 \mathrm{E}-4$ & $2.734573 \mathrm{E}-4$ \\
A4 & $-3.577061 \mathrm{E}-6$ & $-2.653448 \mathrm{E}-6$ \\
\hline
\end{tabular}




\subsection{Scram Reactivity}

While processing the DAS data for rod run-in, it was also possible to resolve the question of how many control rods must successfully scram to achieve neuronic shutdown. A pdf for reactivity removal by one or more high worth control rods was constructed to answer this question.

The pdfs are shown in Table 7.30 and Fig. 7.4. Corresponding cumulative distribution functions are displayed in Table 7.29. From Fig. 7.4 one notes that even one rod scramming will usually be sufficient, but examination of the tables shows that each distribution has a long, low probability tail at the low worth end. This tail arises from the few minutes each day that some control rods are well below the rod bank position during the daily rod motion test. (There should be a corresponding high worth tail from rods above the bank position, but this was not modeled because it is of no consequence here.) Although this produces a significant probability, $310^{-5}$, that one rod will not be enough, two rods are clearly sufficient to overcome the PRD.

The most likely source of external reactivity, which would also have to be overcome by scramming rods, is accidental control rod run-in. Since the rod moving in is obviously not jammed, it is very likely to be one of the rods that scrams. Then its run-in reactivity will be withdrawn, making scram of two rods still sufficient. If for some reason it does not scram, as many as three rods might be necessary for shutdown, considering the probable run-in reactivity magnitudes.

In the case of scramming more than four rods, a variation on the analysis was done. Instead of tallying all possible combinations of $\mathrm{N}$ rods, $\mathrm{N}$ times the average scram worth was used. This ignores the fluctuations at any given hour, but it has the property that the pdf for $\mathrm{N}$ rods scramming can be found by scaling the abscissa of the single-rod pdf. (There is a modest discretization error from dividing the worths into five cent intervals.) If the pdfs based 
Table 7.30. Probability Density Functions for Reactivity From Scram of High-Worth Rods (Exact)

Reactivity (ह) min$$
110
$$

$\begin{array}{rrr}0 & 5 & 0.000000000 \\ 5 & 10 & 0.000000000 \\ 10 & 15 & 0.000000000 \\ 15 & 20 & 0.000000041 \\ 20 & 25 & 0.000000691 \\ 25 & 30 & 0.000004778 \\ 30 & 35 & 0.000020446 \\ 35 & 40 & 0.001042961 \\ 40 & 45 & 0.007465519\end{array}$

$45 \quad 50$

$.50 \quad 55$

$55 \quad 60$

$60 \quad 65$

$65 \quad 70$

$70 \quad 75$

$75 \quad 80$

$80 \quad 85$

8590

$90 \quad 95$

95
$100 \quad 100$

$105 \quad 110$

$110 \quad 115$

$115 \quad 120$

$120 \quad 125$

$125 \quad 130$

$130 \quad 135$

$\$ 35 \quad i 40$

$140 \quad i 45$

$145 \quad 150$

$150 \quad 155$

$1 \equiv 5 \quad 160$

$160 \quad 165$

$165 \quad 170$

$170 \quad 175$

$175 \quad 180$

$180 \quad 185$

$185 \quad 190$

$190 \quad 195$

195200

$200 \quad 205$

$205 \quad 210$

$210 \quad 215$

$215 \quad 220$

$220 \quad 225$

$225 \quad 230$

$230 \quad 235$

$235 \cdot 240$

$240 \quad 245$

$245 \quad 250$

$250 \quad 255$

$255 \quad 260$
0.059038983

0.164624479

0.215690198

0.212298742

0.167526359

0.095187643

0.049038117

0.020095220

0.007965821

0.000000000

0.000000000

0.000000000

0.000000000

0.000000000

0.000000000

0.000000000

0.000000000

0.000000000

0.000000000

0.000000000

0.000000000

0.000000000

0.000000000

0.000000000

0.000000000

0.000000000

0.000000000

0.000000000

0.000000000

0.000000000

0.000000000

0.000000000

0.000000000

0.000000000

0.000000000

0.000000000

0.000000000

0.000000000

0.000000000

0.000000000

0.000000000

0.000000000

0.000000000

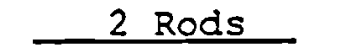

0.000000000

0.000000000

0.000000000

0.000000000

0.000000000

0.000000000

0.000000000

0.000000000

0.000000000

- 0.000000000

0.000000000

0.000000029

0.000000270

0.000000651

0.000001153

0.000580310

0.002321351

0.003709001

0.007157751

0.023017615

0.046587482

0.084354309

0.121839728

0.143700601

0.139866926

0.123281913

0.094745509

0.074649307

0.054035134

0.036830947

0.020455282

0.011812309

0.006561492

0.003248248

0.000894760

0.000347921

0.000000000

0.000000000

0.000000000

0.000000000

0.000000000

0.000000000

0.000000000

0.000000000

0.000000000

0.000000000

0.000000000

0.000000000

0.000000000

0.000000000

0.000000000

0.000000000

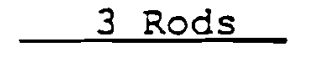

0.000000000

0.000000000

0.000000000

0.000000000

0.000000000

0.000000000

0.000000000

0.000000000

0.000000000

0.000000000

0.000000000

0.000000000

0.000000000

0.000000000

0.000000000

0.000000000

0.000000000

0.000000000

0.000000000

0.000000008

0.000000122

0.000000392

0.000000556

0.000146622

0.001471564

0.002364413

0.001359784

0.001383141

0.004439226

0.013364774

0.023957448

0.037666305

0.059927742

0.077954115

0.101293041

0.107759736

0.103900663

0.089316851

0.080055579

0.067839918

0.058436646

0.048479407

0.039029338

0.028527142

0.020548488

0.012138655

0.008334194

0.004546147

0.003133398

0.001281194

0.001064301

0.000188618 \begin{tabular}{c}
4 Rods \\
\hline 0.000000000 \\
0.000000000 \\
0.000000000 \\
0.000000000 \\
0.000000000 \\
0.000000000 \\
0.000000000 \\
0.000000000 \\
0.000000000 \\
0.000000000 \\
0.000000000 \\
0.000000000 \\
0.000000000 \\
0.000000000
\end{tabular}

0.000000000

0.000000000

0.000000000

0.000000000

0.000000000

0.000000000

0.000000000

0.000000000

0.000000000

0.000000000

0.000000000

0.000000000

0.000000000

0.000000000

0.000000040

0.000000211

0.000000391

0.000000459

0.000593059

0.001907468

0.001453449

0.000455755

0.000858144

0.002123516

0.003770195

0.007496005

0.014297219

0.022988700

0.032051795

0.044503694

0.054582154

0.072882359

0.083074090

0.089352818

0.080794937

0.074391391

0.067148227

0.060964815 
Table 7.30. Probability Density Functions for Reactivity From Scram of High-Worth Rods (Exact) (Cont'd)

\begin{tabular}{ccc} 
Reactivitv ( $)$ & 1 Rod \\
\cline { 1 - 1 } min & max & \\
260 & 265 & 0.000000000 \\
265 & 270 & 0.000000000 \\
270 & 275 & 0.000000000 \\
275 & 280 & 0.000000000 \\
280 & 285 & 0.000000000 \\
285 & 290 & 0.000000000 \\
290 & 295 & 0.000000000 \\
295 & 300 & 0.000000000 \\
300 & 305 & 0.000000000 \\
305 & 310 & 0.000000000 \\
310 & 315 & 0.000000000 \\
315 & 320 & 0.000000000 \\
320 & 325 & 0.000000000 \\
325 & 330 & 0.000000000 \\
330 & 335 & 0.000000000 \\
335 & 340 & 0.000000000 \\
340 & 345 & 0.000000000 \\
345 & 350 & 0.000000000
\end{tabular}

\begin{tabular}{c}
2 Rods \\
\hline 0.000000000 \\
0.000000000 \\
0.000000000 \\
0.000000000 \\
0.000000000 \\
0.000000000 \\
0.000000000 \\
0.000000000 \\
0.000000000 \\
0.000000000 \\
0.000000000 \\
0.000000000 \\
0.000000000 \\
0.000000000 \\
0.000000000 \\
0.000000000 \\
0.000000000 \\
0.000000000
\end{tabular}

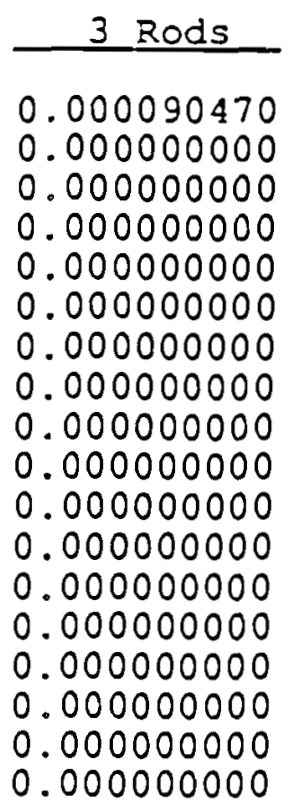

\begin{tabular}{c}
4 Rods \\
\hline 0.052880528 \\
0.045804723 \\
0.040354003 \\
0.035891786 \\
0.029981097 \\
0.024004467 \\
0.018484081 \\
0.013285326 \\
0.008144082 \\
0.005785951 \\
0.003874059 \\
0.002235698 \\
0.001476479 \\
0.001144326 \\
0.000588441 \\
0.000228851 \\
0.000145212 \\
0.000000000
\end{tabular}



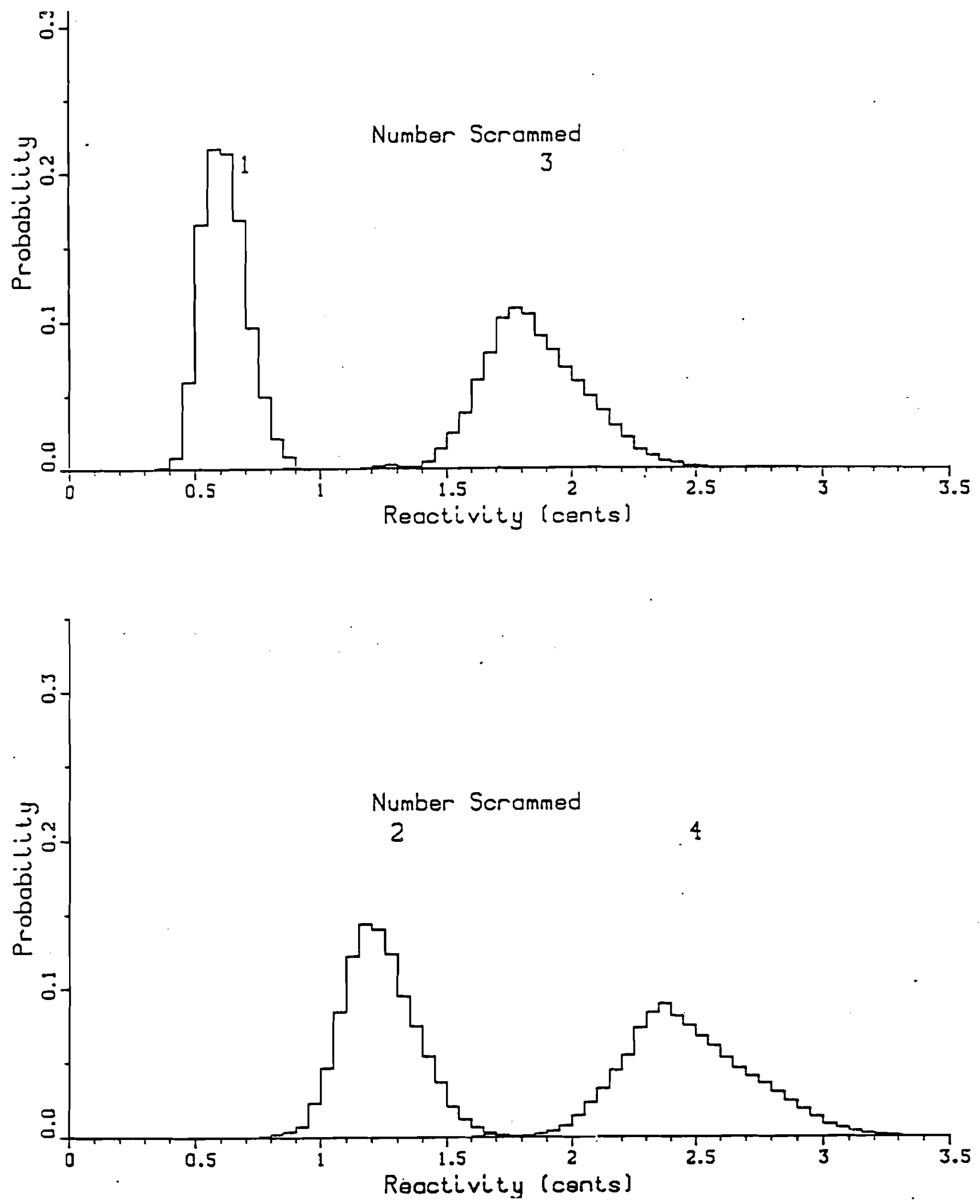

Fig. 7.4. Exact Probability Density Functions for Scram Worth 
Table 7.31. Cumulative Distribution Functions for Reactivity From Scram

of High-Worth Rods (Exact)

Reactivitv (द)

$\min \max$

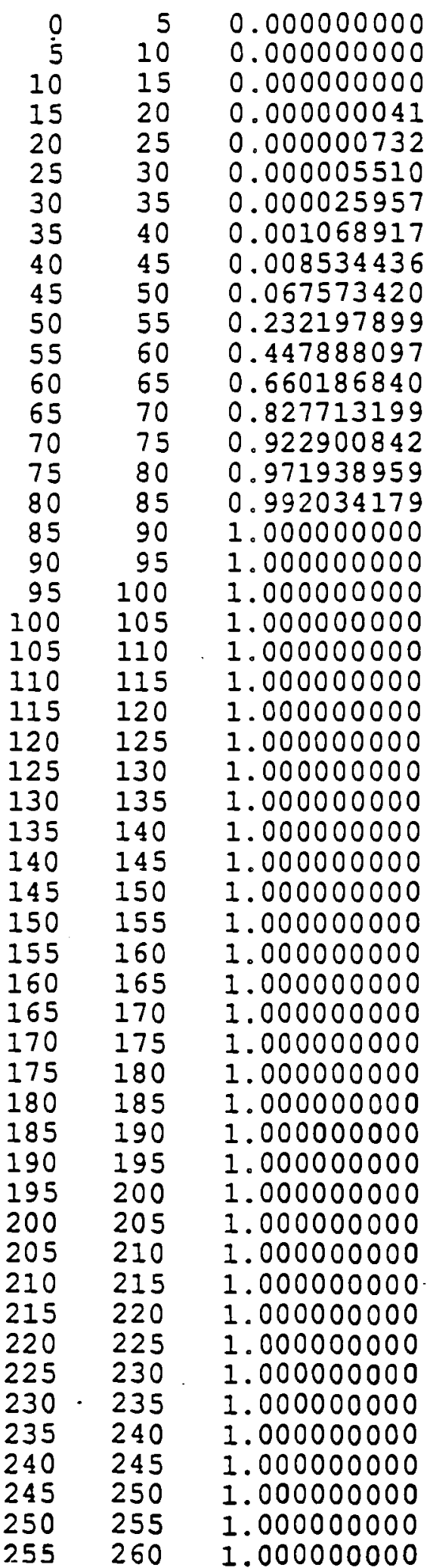

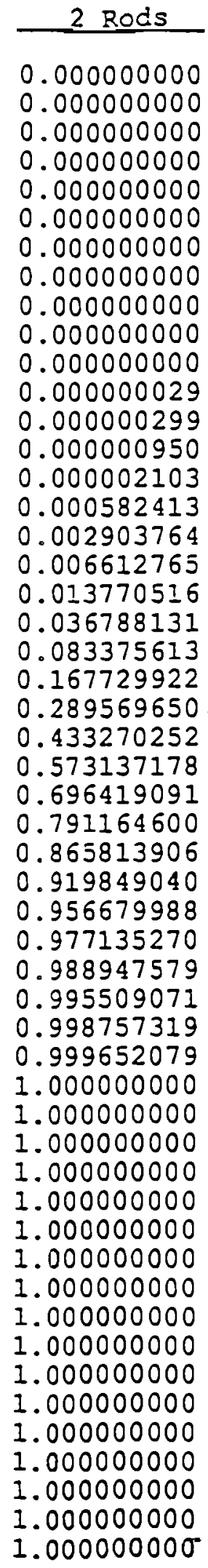

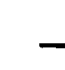

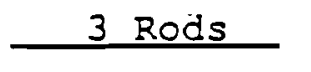

0.000000000

0.000000000

0.000000000

0.000000000

0.000000000

0.000000000

0.000000000

0.000000000

0.000000000

0.000000000

0.000000000

0.000000000

0.000000000

0.000000000

0.000000000

0.000000000

0.000000000

0.000000000

0.000000000

0.000000008

0.000000130

0.000000522

0.000001078

0.000147700

0.001619264

0.003983677

0.005343461

0.006726602

0.011165828

0.024530602

0.048488050

0.086154355

0.146082098

0.224036213

0.325329254

0.433088989

0.536989653

0.626306504

0.706362082

0.774202001

0.832638647

0.881118054

0.920147392

0.948674534

0.969223022

0.981361677

0.989695871

0.994242018

0.997375417

0.998656610

0.999720911

0.999909530 \begin{tabular}{c} 
4 Rods \\
\hline 0.000000000 \\
0.000000000 \\
0.000000000 \\
0.000000000 \\
0.000000000 \\
0.000000000 \\
0.000000000 \\
0.000000000 \\
0.000000000 \\
0.000000000 \\
0.000000000 \\
0.000000000 \\
0.000000000 \\
0.000000000 \\
0.000000000 \\
0.000000000 \\
0.000000000 \\
0.000000000 \\
0.000000000 \\
0.000000000 \\
0.000000000 \\
0.000000000 \\
0.000000000 \\
0.000000000 \\
0.000000000 \\
0.000000000 \\
0.000000000 \\
0.000000000 \\
0.000000040 \\
0.000000251 \\
0.000000642 \\
0.000001101 \\
0.000594161 \\
0.002501629 \\
0.003955078 \\
0.004410833 \\
0.005258977 \\
0.007392493 \\
0.011162688 \\
0.018658693 \\
0.032955912 \\
0.055944611 \\
0.087996406 \\
0.132500100 \\
0.187082255 \\
0.259964613 \\
0.343038703 \\
0.432391521 \\
0.513186458 \\
0.587577849 \\
0.654726075 \\
0.715690890
\end{tabular} 
Table 7.31. Cumulative Distribution Functions for Reactivity From Scram of High-Worth Rods (Exact) (Cont'd)

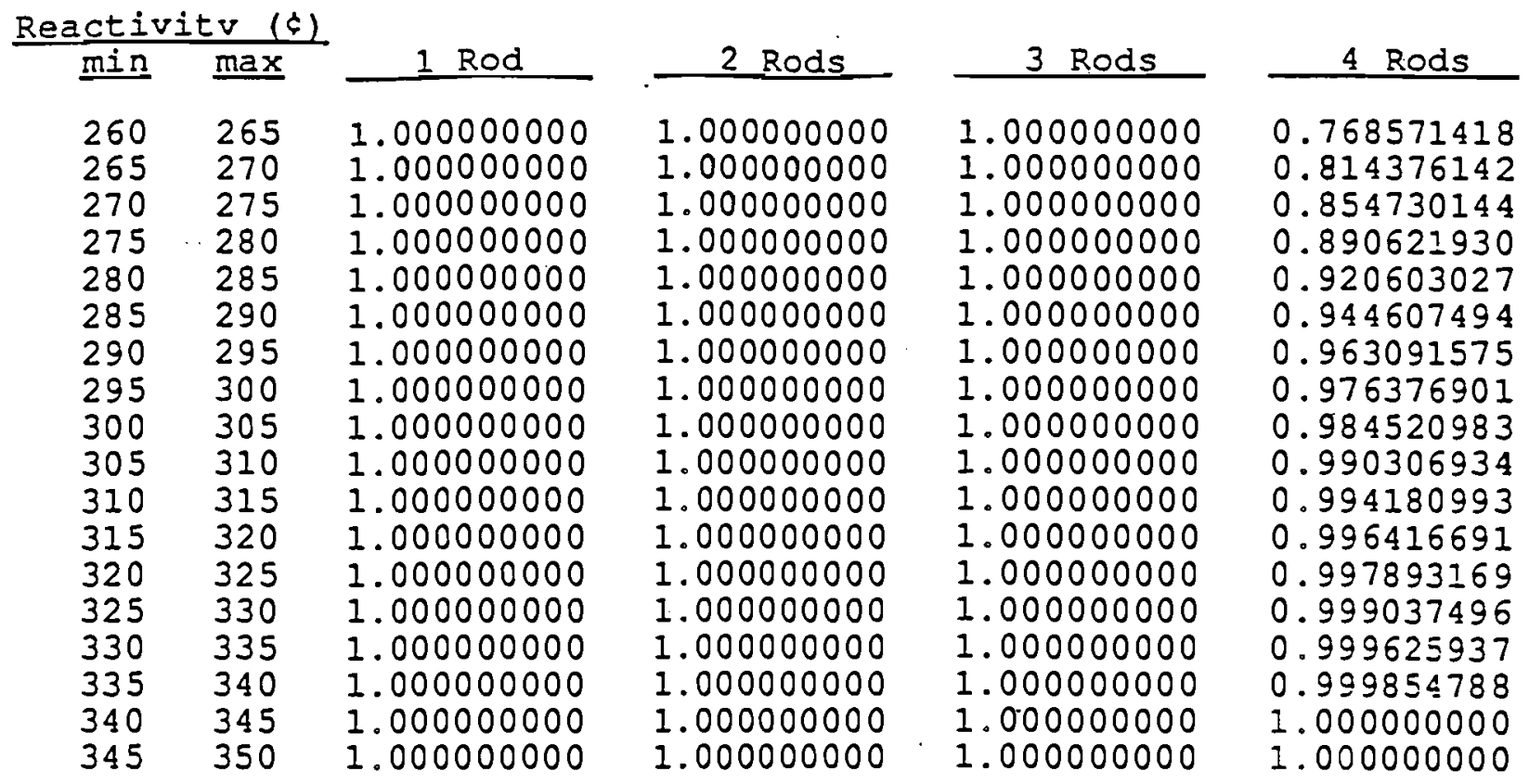


on the average worth closely match the exact ones, then it should be reasonable to get the pdfs for more than four rods by scaling.

The exact and approximate pdfs are displayed graphically in Fig. 7.5 and the approximate pdf and cdf values are given in Tables 7.32 and 7.33. The agreement improves as $\mathrm{N}$ increases and is quite good by $N=4$. The only significant deficiency there is the missing low worth tail, which was removed by the averaging. At most one rod is below the bank at any moment, so the missing tail is about the same for any $\mathrm{N}$. Therefore, a reasonably accurate procedure is to strip off the tail from the exact pdf, scale the remainder and then add back the tail.

The worth distributions for scramming between one and four high-worth control rods have been determined by analyzing control rod data from 22486 hours of operation at steady power (four years). With a probability precision of $10^{-9}$, the minimum scram worth from one, two, three and four rods is $\$ 0.15, \$ 0.55, \$ 0.95$ and $\$ 1.40$, respectively. With a precision of $10^{-7}$, the minimum scram worths are five cents higher.

Shutdown is achieved when the reactivity removed is greater than the sum of the PRD and any external reactivity. A survey of PRD measurements over many years showed that the average PRD is about 32 cents and the most extreme values are 26 and 46 cents [7.9]. Table 7.30 shows that there is only about a $1 \%$ probability that a single control rod would not overcome even the largest PRD ever observed. Combining the scram pdf and the PRD magnitude pdf would give a more general probability that a single rod scram would overcome the PRD. Successful scram of two rods will certainly overcome the PRD. 

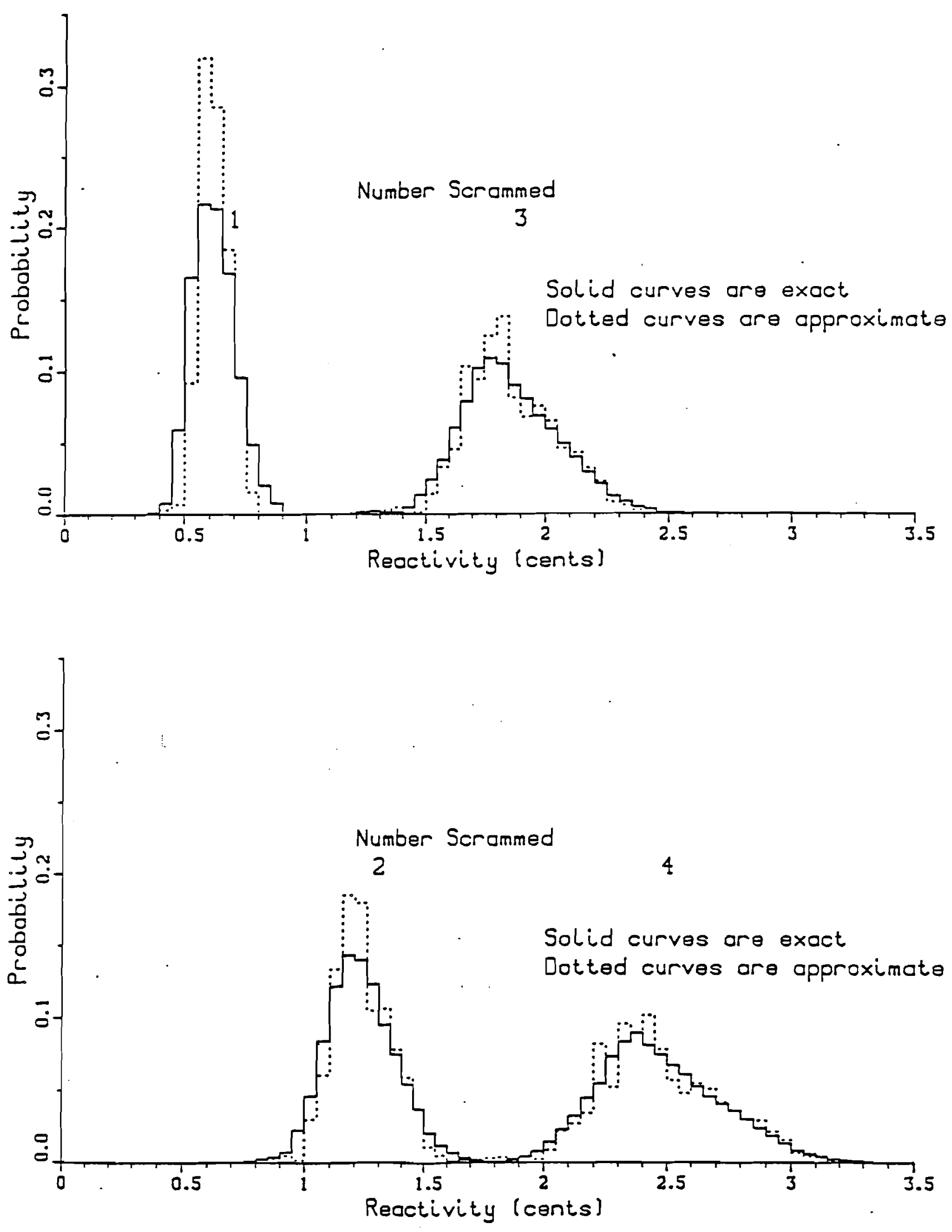

Fig. 7.5. Exact and Approximate Probability Density Functions for Scram Worth 
Table 7.32. Probability Density Functions for Reactivity From Scram of High-Worth Rods (Approximate)

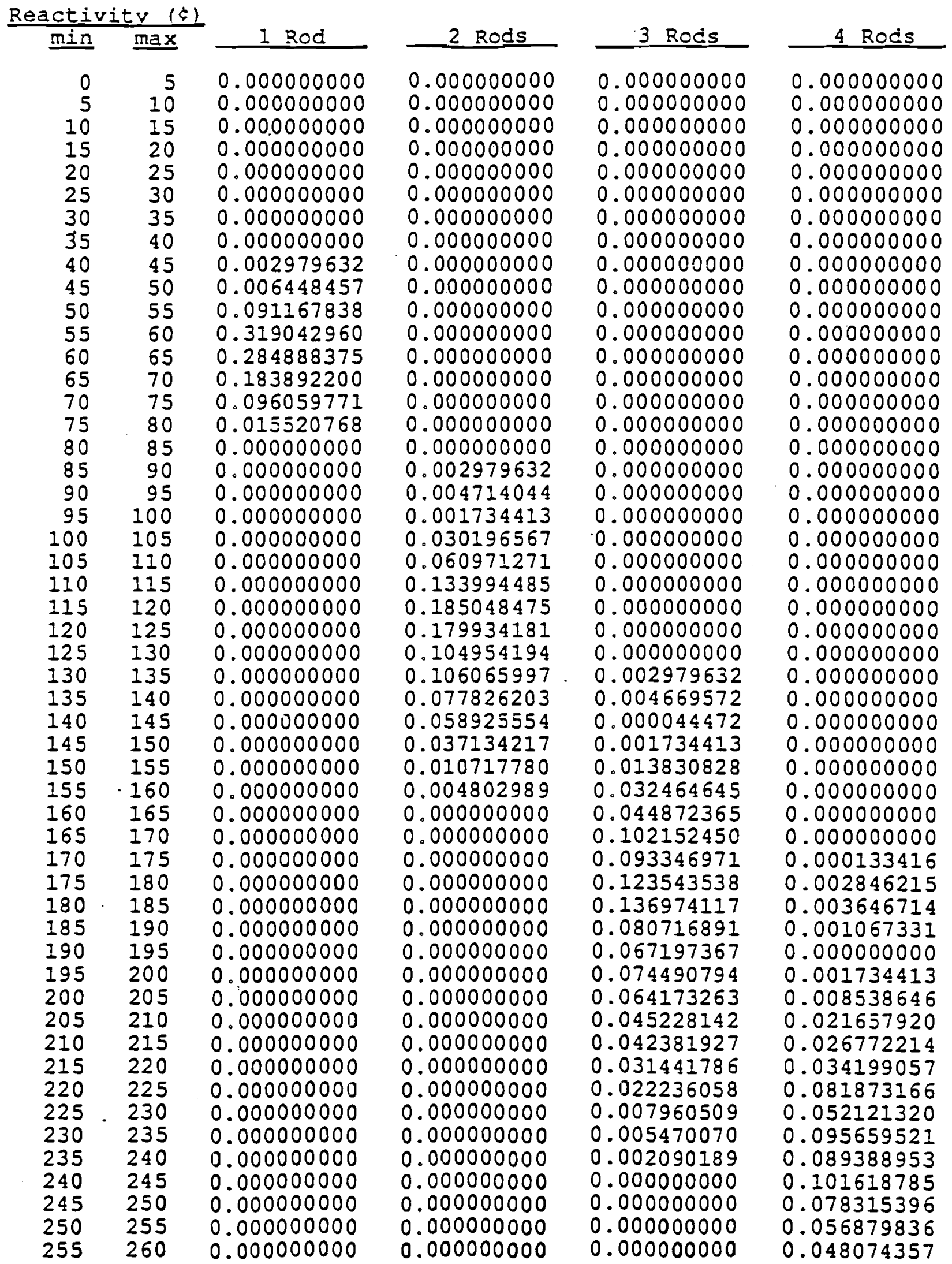


Table 7.32. Probability Density Functions for Reactivity From Scram of High-Worth Rods (Approximate) (Cont'd)

\begin{tabular}{ccc} 
Reactivity ( $)$ & 1 Rod \\
\cline { 1 - 2 } min & max & \\
260 & 265 & 0.000000000 \\
265 & 270 & 0.000000000 \\
270 & 275 & 0.000000000 \\
275 & 280 & 0.000000000 \\
280 & 285 & 0.000000000 \\
285 & 290 & 0.000000000 \\
290 & 295 & 0.000000000 \\
295 & 300 & 0.000000000 \\
300 & 305 & 0.000000000 \\
305 & 310 & 0.000000000 \\
310 & 315 & 0.000000000 \\
315 & 320 & 0.000000000 \\
320 & 325 & 0.000000000 \\
325 & 330 & 0.000000000 \\
330 & 335 & 0.000000000 \\
335 & 340 & 0.000000000 \\
340 & 345 & 0.000000000 \\
345 & 350 & 0.000000000
\end{tabular}

\begin{tabular}{c}
2 Rods \\
\hline 0.000000000 \\
0.000000000 \\
0.000000000 \\
0.000000000 \\
0.000000000 \\
0.000000000 \\
0.000000000 \\
0.000000000 \\
0.000000000 \\
0.000000000 \\
0.000000000 \\
0.000000000 \\
0.000000000 \\
0.000000000 \\
0.000000000 \\
0.000000000 \\
0.000000000 \\
0.000000000
\end{tabular}

\begin{tabular}{c}
3 Rods \\
\hline 0.000000000 \\
0.000000000 \\
0.000000000 \\
0.000000000 \\
0.000000000 \\
0.000000000 \\
0.000000000 \\
0.000000000 \\
0.000000000 \\
0.000000000 \\
0.000000000 \\
0.000000000 \\
0.000000000 \\
0.000000000 \\
0.000000000 \\
0.000000000 \\
0.000000000 \\
0.00 .0000000
\end{tabular}

\begin{tabular}{c}
4 Rods \\
\hline 0.054745175 \\
0.051320822 \\
0.041848261 \\
0.035977942 \\
0.029796318 \\
0.029129236 \\
0.021480032 \\
0.015654185 \\
0.006537401 \\
0.004180379 \\
0.004313795 \\
0.000489193 \\
0.000000000 \\
0.000000000 \\
0.000000000 \\
0.000000000 \\
0.000000000 \\
0.000000000
\end{tabular}


Table 7.33. Cumulative Distribution Functions for Reactivity From Scram of High-Worth Rods (Approximate)

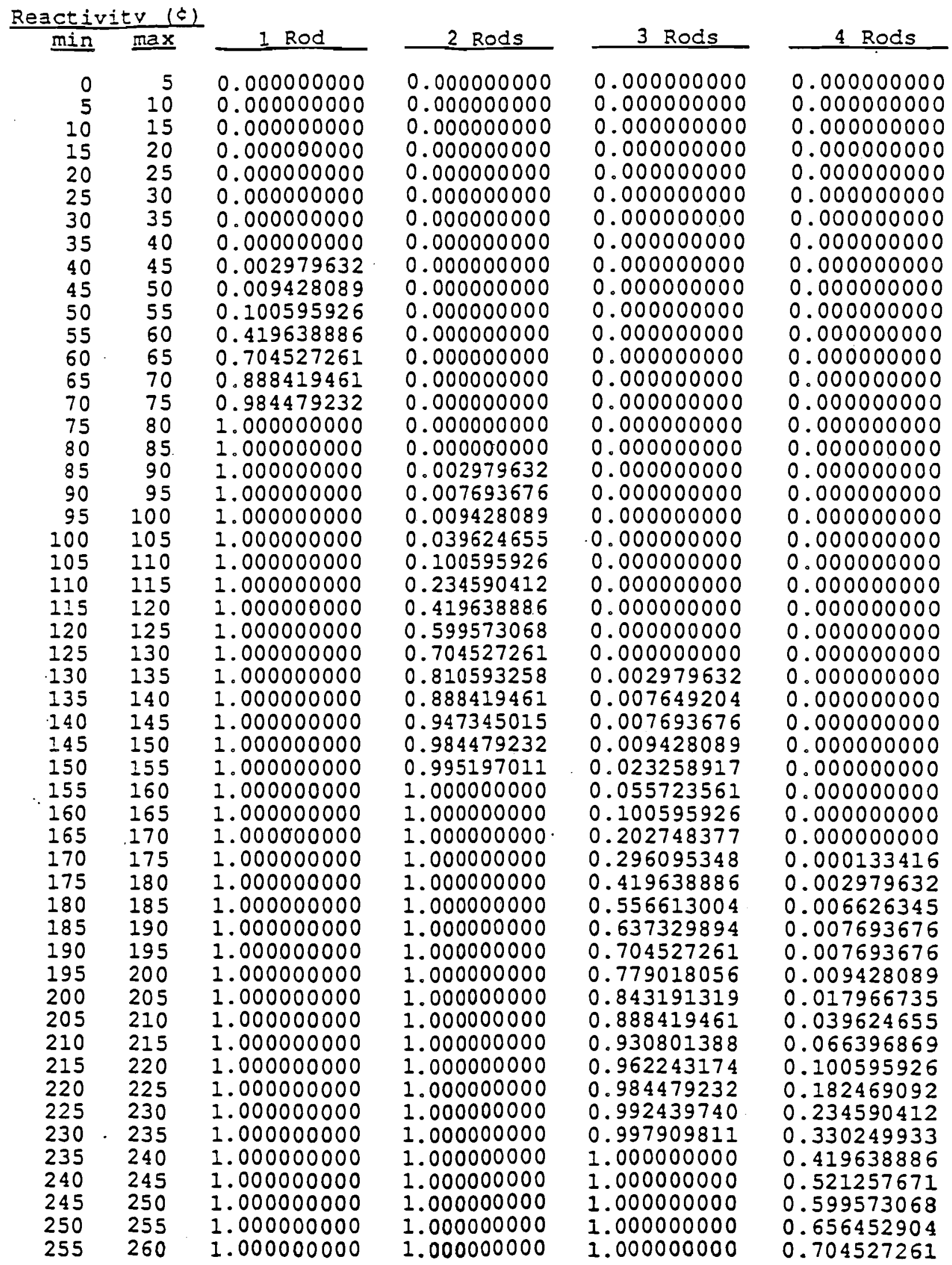


Table 7.33. Cumulative Distribution Functions for Reactivity From Scram of High-Worth Rods (Approximate) (Cont'd)

\begin{tabular}{ccc}
$\frac{\text { Reactivity ( })}{\text { min }}$ & max & 1 Rod \\
\cline { 1 - 2 } 260 & 265 & 1.000000000 \\
265 & 270 & 1.000000000 \\
270 & 275 & 1.000000000 \\
275 & 280 & 1.000000000 \\
280 & 285 & 1.000000000 \\
285 & 290 & 1.000000000 \\
290 & 295 & 1.000000000 \\
295 & 300 & 1.000000000 \\
300 & 305 & 1.000000000 \\
305 & 310 & 1.000000000 \\
310 & 315 & 1.000000000 \\
315 & 320 & 1.000000000 \\
320 & 325 & 1.000000000 \\
325 & 330 & 1.000000000 \\
330 & 335 & 1.000000000 \\
335 & 340 & 1.000000000 \\
340 & 345 & 1.000000000 \\
345 & 350 & 1.000000000
\end{tabular}

\begin{tabular}{c}
2 Rods \\
\hline 1.000000000 \\
1.000000000 \\
1.000000000 \\
1.000000000 \\
1.000000000 \\
1.000000000 \\
1.000000000 \\
1.000000000 \\
1.000000000 \\
1.000000000 \\
1.000000000 \\
1.000000000 \\
1.000000000 \\
1.000000000 \\
1.000000000 \\
1.000000000 \\
1.000000000 \\
1.000000000
\end{tabular}

\begin{tabular}{c}
3 Rods \\
\hline 1.000000000 \\
1.000000000 \\
1.000000000 \\
1.000000000 \\
1.000000000 \\
1.000000000 \\
1.000000000 \\
1.000000000 \\
1.000000000 \\
1.000000000 \\
1.000000000 \\
1.000000000 \\
1.000000000 \\
1.000000000 \\
1.000000000 \\
1.000000000 \\
1.000000000 \\
1.000000000
\end{tabular}

\begin{tabular}{c}
4 Rods \\
\hline 0.759272436 \\
0.810593258 \\
0.852441519 \\
0.888419461 \\
0.918215779 \\
0.947345015 \\
0.968825047 \\
0.984479232 \\
0.991016633 \\
0.995197011 \\
0.999510807 \\
1.000000000 \\
1.000000000 \\
1.000000000 \\
1.000000000 \\
1.000000000 \\
1.000000000 \\
1.000000000
\end{tabular}




\subsubsection{Other Operating Modes}

\subsection{Startup and Shutdown}

Rod positions and/or frequency of rod motion during startup and shutdown can be different than when at steady power. This makes the reactivity insertion potential different. An understanding of the rod characteristics during these modes of operation was obtained from the Operating Instructions and interviews with Operations personnel [7.10, 7.11, 7.12]. Operation reports for Runs 139 through 152 were reviewed to estimate the frequency of startups, restarts and shutdowns. The five month wait for DOE restart approval in 1989 was excluded for this purpose.

The goal was to construct a very simple, but not overly conservative, representation of reactivity insertion potential. Accordingly, the focus was strictly on lumping events into categories that have similar rod position and motion frequency characteristics. Two categories were identified. For each category, the parameters of interest are 1) magnitude of the potential reactivity insertion, 2) frequency of rod activation, 3) duration of the operation, and 4) frequency of the operation.

The most important category is termed the 14-in. bank configuration. In this configuration, the safety rods and several control rods are at 14 inches (fully inserted), several control rods are at 0 inches and one control rod is partially inserted. Rods at 14 inches obviously cannot insert any reactivity and rods at 0 inches can insert their full worth. (Table 7.25 shows the average and range of full rod worths.)

The number of control rods at 14 and 0 inches depends on the reactivity excess of the core loading. For the startup of Run 152A, which had a large initial excess, there were five control rods at 14 inches and four control rods at zero inches. This configuration may be approximated by four high worth control rods with their full worths available for a TOP accident, and the rest of the rods with no insertion potential. 
The main operation where the rods are in this configuration is the startup period from first attainment of criticality to the time when rods are banked in a steady power configuration for the ascent to full power. This period typically lasts about four hours, but it can be as short as two hours and as long as 24 hours. This period includes rod calibration, which is not done in every startup but sufficiently often that it will be assumed always to occur. During this period the number of up-motion demands made for rods at 0 inches is approximately 15 .

There are two other operations that can be represented by the 14-in. bank configuration. One is a period near the end of a normal shutdown when rods are rebanked from the steady power configuration to the 14-in. configuration. About 7 normal shutdowns are performed per year. Because of the burnup, few if any rods are at 0 inches and the reactor is kept critical in this configuration for only minutes. Thus this condition can be accounted for by an appropriate increase in the time estimate for the startup. The other operation to include here is the measurement of the prompt power coefficient. In recent runs, this has been done at every startup. The relevant event in this measurement is that Rod \#1 is at 0 inches and latched for several minutes. The other rods are generally at their steady power positions. Rod \#1 is a normal worth rod, so it is worth much less than the typical rod. Thus again an increase in the startup time estimate would account for this operation.

In summary, the 14-in. bank configuration has a relatively large reactivity magnitude potential but constitutes a very small fraction of the reactor's time at critical. On the order of 80 cents could be introduced and about four rods have this potential. About 15 up-motion demands are placed on these rods over a period of about 4 hours. This condition occurs about 10 times per year, the frequency of reactor startups. The roughly 40 hours per year in this state are to be compared with approximately 6900 hours per year in the steady-power mode.

The other category is characterized by a low controlling rod position, a steady-power configuration for the other rods and a high frequency of up-motion demands on the controlling rod. Included in this category are the ascent to authorized power during startups and restarts. The power ascent takes about 6 hours during a startup and 2 to 3 hours during a restart. During each of these operations there may be as many as 600 up-motion demands made on the 
controlling rod. It takes about 200 hours of steady-power operation to equal this demand. The controlling rod is below its steady power position range much of this time. A typical 80 cent rod is capable of inserting as much as 60 cents at the beginning of this period if the final position is 9 inches. Its average insertion potential during these operations is about 36 cents, however. Startups occur about 10 times per year and restarts occur about 3 times per year.

The importance of this category depends on the principal failure mode leading to rod runin. When the key parameter is hours of exposure, this category is unimportant since it accounts for just $1 \%$ of the time at critical. This feature is simply added to the steady-power pdfs in a manner similar to that used for the daily motion tests. When the number of up motion demands is the key, then it is quite important; the number of demands per year is about $40 \%$ as large as at steady power and the magnitude of the reactivity insertion is almost twice as large as at steady power.

Other operating modes present no significant rod run-in threat. Only down motion demands are made during normal power descent, anticipatory shutdown and scram. The reactor is made subcritical in about 2 hours during normal shutdown and much more quickly in the other shutdown operations. In restarts, the previous steady power bank position is established before the reactor is critical, so that this phase of restarts is not relevant.

\subsection{Transient Experiments}

This mode of operation is less constrained by fixed specifications than any other mode. Still, a reasonably clear picture of the rod run-in potential can be developed. The description presented here was developed from the Operations Reports for Runs 139 through 152, five Approved Procedures for experiments run in that period, and interviews with Operations personnel $[7.10,7.13,7.14,7.15]$.

In Runs 139 through 152 there was an average of five transient experiments per year. The ACRDS was used in more than half of these, with high speed mode used in one case and slow speed mode in the others. High-worth rods were always used in controlling the transients. 
The transients always lasted less than two hours, usually much less.

The ACRDS was used in slow speed mode to produce either reactivity oscillations or a nearly step increase in power. There is only one ACRDS-controlled up-motion demand for the step power increase but there could be hundreds for reactivity oscillations. In either case Rod \#4, which is the one the ACRDS controls, was positioned between 6 and 8 inches because the worth slope is constant in that vicinity. It is assumed that the rod would always be at least at 5.5 inches because of the desirability of having a constant worth slope. From 5.5 inches it could insert up to 55 cents at a maximum rate of 0.58 cents/s.

The ACRDS can be programmed to respond to reactor power or to rod position. When programmed to maintain a steady power, the ACRDS could, in principle, defeat passive feedbacks if a LOF or LOHS accident were to occur during a transient experiment [7.16]. Two things protect against this, a bulk sodium high temperature interlock and an Emergency * Procedures requirement for manual scram if either of these accidents occurs. Thus, for there to be an accidental rod run-in where the ACRDS defeats passive safety, the probabilities must be combined for 1) LOF or LOHS occurrence during a transient experiment, 2) accidental runin, 3) interlock failure, and 4) scram failure. Experiments are excluded from the EBR-II PRA, but it is expected the combined probabilities would be below the threshold of interest.

The one use of the high speed mode is described because there is a possibility that the procedure will become a routine way of measuring prompt reactivity feedback, replacing rod drop measurements. In this experiment, EX-234, a control rod worth 87 cents was moved in $\mathrm{a}+-0.24$ inch range about the 7 inch position. In a run-in accident from this position, it would have had the potential to insert about 44 cents at a maximum rate of 8.6 cents/s. The power oscillated between 57 and 59 MWt. The ACRDS was programmed to respond to rod position. The experiment lasted about one hour, during which time the rod was moved up and down roughly 500 times. If this becomes a routine measurement, it would occur up to 10 times per year. 
As in the slow speed case, it is reasonable to assume that the high-speed rod would be positioned above 5.5 inches in order to keep it in the range of the constant worth slope. From there, a 90 cent rod could introduce 55 cents. Hard-wired high and low position limit switches exist that trip the rod to manual control; a requirement to keep the rod above 5.5 inches would therefore be easy to ensure.

In the experiments where all rods were under manual control, there were no extraordinary rod positions or up motion demand rates. The lowest position seems to have been 8 inches.

With the exception of high-speed ACRDS use, it is reasonable to argue that plant transient experiments are covered by the treatments of steady-power and startup operations. The reactivity insertion rates are the same as in those operations. Compared to the 14-in. bank configuration in startups, the rod positions are higher and the frequency is less for transients. The failure probability for the ACRDS control system is not compared to that for manual activation of control rods, since plant experiments are excluded.

High speed operation of the ACRDS has to be treated separately because of the uniquely high reactivity insertion rate. Currently this mode is used very infrequently but it might be desirable to anticipate that it could be used $\sim 10$ times/year in the future. A maximum insertion of 55 cents should be assumed, as discussed earlier. It would have to be stated clearly in the PRA that the analysis is valid only for Rod \#4 positioned higher than 5.5 inches.

In estimating a rod run-in frequency for plant experiments, it should be recognized that each such experiment is reviewed and approved before it is run. It must be shown that no damage would occur in protected accidents to gain approval. Fast-speed ACRDS experiments require a safety analysis demonstrating a damage-free outcome if a $12 \mathrm{cent} / \mathrm{s}$ rod run-in (with scram) were to occur (Sections 3.18 and 7 of the Technical Specifications [7.5]). 


\subsubsection{Summary}

Most of the important results are summarized in Table 7.32. The parameters include the initial rod position $(\mathrm{Z})$, the insertion magnitude $\left(\mathrm{R}_{\mathrm{i}}\right)$ and the insertion rate. Both median and 99.99 percentile values of the insertion magnitude and insertion rate are given. (When these were taken from pdfs, they are the upper limit of the appropriate bin.) Updemands and hours per year should be useful in a failure analysis to determine run-in frequencies. The situations are listed across the top.

In steady-power operation, there are three categories of rods, which are distinguished by the rod design and/or the way they are used. Table 7.34 shows just two characteristic insertion magnitudes from the pdfs developed in Section 7.2.4.3. Safety rods have two entries for some parameters; the first applies if exposure time determines the failure frequency and the second applies if up-demands do. In either case, the safety rods have a lower run-in frequency than the other two types but they have a larger insertion magnitude. The controlling rod is by far the most likely one to run-in if up-demand frequency is key.

Run-in of multiple rods is anticipated to be a very rare event. Consequently, the only situation considered in this analysis is run-in of two control rods during steady power operation. (The pair of safety rods is treated as a single rod because the two rods are driven as a unit.) This situation has a higher insertion magnitude and rate than any other at steady power.

Although not analyzed in the EBR-II PRA, two situations were identified for startup and shutdown operations. The 14 in. bank configuration occurs most often in the initial critical phase of startups. It has a low occurrence frequency but the largest insertion magnitude of any operation. It is reasonably conservative to treat this configuration as having four rods at 0 inches and the rest at 14 inches. The normal bank configuration occurs in the next phase of startups and during restarts. It is distinguished from the true steady-power configuration by the controlling rod characteristics, which are what is shown in the table; the controlling rod has a lower position, so a larger $\mathrm{R}_{\mathrm{i}}$, and a large number of up-demands per hour. 
Table 7.34. Summary of Rod Run-in Characteristics

\begin{tabular}{|c|c|c|c|c|c|c|c|}
\hline & \multirow{2}{*}{\multicolumn{3}{|c|}{$\begin{array}{l}\text { Steady-Power Operation } \\
\text { Single Rod Run-in }\end{array}$}} & & \multicolumn{2}{|c|}{ Startup \& Shutdown } & \multirow{3}{*}{$\begin{array}{l}\text { Transients } \\
\text { Fast ACRDS }\end{array}$} \\
\hline & & & & & \multirow{2}{*}{$\begin{array}{l}\text { 14-in. Bank } \\
\text { O-in. Rod }\end{array}$} & \multirow{2}{*}{$\begin{array}{l}\text { Normal Bank } \\
\text { Cntl CR }\end{array}$} & \\
\hline & $\underline{\text { Safety }}$ & Cntl CR & Banked CRs & Two CRs & & & \\
\hline Median $\mathrm{Z}$ (in.) & $7.5,10.8$ & 10.9 & 10.3 & 10.4 & 0.0 & 7.5 & - \\
\hline Median $R_{i}(\mathcal{C})$ & 50,27 & 15 & 20 & 30 & 79 & 35 & - \\
\hline $99.99 \% \mathrm{R}_{\mathrm{i}}(\mathrm{c})$ & 55 & 35 & 40 & 70 & 99 & 81 & 55 \\
\hline Median Rate (c/s) & 0.29 & 0.42 & 0.42 & 0.84 & 0.44 & 0.42 & 7.7 \\
\hline Max. Rate (c/s) & 0.47 & 0.64 & 0.64 & 1.22 & 0.64 & 0.64 & 8.8 \\
\hline Up-Demands/yr & 26 & 20700 & 288 & - & 150 & 7800 & 5000 \\
\hline Oper. Hours/yr & 2900 & 6900 & 6900 & 6900 & 40 & 68 & 10 \\
\hline
\end{tabular}




\section{3 $\quad$ Aging}

Aging of vital pieces of equipment, as a concern at EBR-II, has been and continues to be addressed by a comprehensive program of analysis, tracking, and plant modification. This is exemplified by the report, "Extending the Operating Lifetime of EBR-II to 30 Years and Beyond" [7.17], which addressed all the concerns associated with aging of major pieces of equipment as well as other issues such as ensuring continuity of staff knowledge as knowledgeable engineers retire. Currently, (1990), in progress is an extension of this study to a 40 year life [7.18]. Results presented in this section will draw upon work completed for this project. In general we one asks whether there are aging effects to be accounted for in the initiating event quantification, and in the safety system response (systems analyses). These will be discussed separately below.

\subsubsection{Aging Effects in Initiating Events}

In Section 5, the initiating event frequencies were derived using a number of methods, historical data based occurrences, fault tree analysis, and expert judgement. The aging factors for the initiating events have been considered differently depending on the method of deriving the initiating event frequency. Section 7.3.1.1 describes the method used for historical data. Section 7.3.2 evaluates aging at a system level for those initiating events derived by system fault trees and for the response of mitigations system. Section 7.3.1.2 is focused on the aging component of structural failures which have never happened and are not tractable using fault tree analysis and therefore rely on expert judgement supported by other means of analysis. Some initiating events, especially local facts, since these involve fuel and or experimental subassemblies which are in essence consumables and replaced at regular intervals, have no aging effects.

\subsubsection{Initiating Events Quantification}

The initiating event frequencies based upon direct observation of EBR-II experience were grouped into 5 year bins in order to detect any trends. In all cases, except one, 
the trend was downwards (less events in recent history), or no trend was discernable. The apparent exception being in the secondary sodium systems (TSEC). On closer inspection the cause of the apparent increase was two software errors in loading a controller for a test program which drove the secondary flow to zero. Therefore no specific aging concerns were identified by data-based initiating event frequencies.

\subsubsection{Structural Failures}

As part of the EBR-II PRA, the likelihood and consequences of structural failures must be considered. As part of the analysis required to support the extension of the operating life of EBR-II to 40 years, structural analysis has been performed on most of the major components [7.19]. The methodology applied is described in an earlier work [7.20], upon which many of the conclusions are based. In this section, the methodology of the above analyses is presented briefly in conjunction with a simple interpretation in a probabilistic sense. The results are then given as an annual frequency of failure for all major components. Finally the results and their implications are discussed.

\subsection{Materials Aging Assessment}

A forty-year lifetime for the EBR-II plant requires that structural and irreplaceable components perform in service for more time than has been experienced elsewhere. This requires a careful study of the materials aging factors of these components. The structures within the primary system are largely constructed of austenitic stainless steel, primarily Type-304. The austenitic stainless steels are particularly resistant to mechanical property degradation and environmental factors. The particular conditions to which the materials are subjected are a long-term thermal environment, irradiation-induced modification of materials properties, and long-term compatibility with sodium. Therefore, the property of primary interest is mechanical integrity. Of additional interest is the geometrical stability of the grid plate in that it fixes the position of all fueled assemblies within the primary system. 
Embrittlement and Impact Properties

A potential safety concern for nuclear reactors is the inservice embrittlement of reactor vessel materials. While there are problems of this kind of both water-cooled and liquid-metalcooled systems, the origin and degree of these concerns are quit different for each system type. Liquid-metal-cooled reactors (such as EBR-II) are constructed from stainless austenitic steels while water-cooled reactor vessels are constructed using ferritic steels. The chemical composition of the two types of steel, and resultant crystal structure are very different, which results in differences in the embrittlement phenomenon observed for each system.

Reactor vessel embrittlement, manifest as a reduction in ductility when subjected to longterm loading, sudden loading (impact), or cyclic loading (fatigue), is caused by several factors. Neutron irradiation creates atomic displacements in vessel materials that result in increases in dislocation densities similar to work hardening. These dislocation densities tend to saturate with neutron dose and, therefore, the associated embrittlement phenomenon also tends to saturate at some given loss of ductility. The degree of ductility loss is often dependent upon temperature because dislocations are removed by application of high temperatures (annealing), the higher the temperature, the faster is the removal, and thus the saturation in ductility loss is less severe.

Austenitic steels are more susceptible to ductility loss caused by work-hardening, so liquid-metal-cooled reactors are more subject to this type of embrittlement. Austenitic steels, however, often have inherently high initial ductilities and therefore observed ductility losses result in a material that retains adequate ductility as not to present a safety concern. Moreover, neutron doses required to cause the embrittlement saturation will not occur at reactor vessel locations over the usual lifetime of a nuclear reactor. Reactor vessel embrittlement is therefore not likely to be severe or to pose safety implications for liquid-metal-cooled systems.

A similar embrittlement phenomenon occurs in the ferritic materials used in water-cooled reactors, where certain tramp or chemical-alloying elements (copper, nickel, phosphorus) tend to cluster and create crystal defects which impede plastic flow. This effect tends to occur at relatively low neutron doses when these chemical elements are present. Of paramount 
importance to safety concerns is that the embrittlement phenomenon strongly affects the ability of the material to withstand impact loading. Ferritic steels are subject to a phenomenon where the material suddenly loses all ductility and its ability to absorb impact energy without breaking. The temperature where this occurs is normally quite low, and is called the nil ductility temperature (NDT). Irradiation causes the NDT to rise to values within the nuclear reactor operational ranges. Austenitic steels used in liquid-metal-cooled reactors do not experience NDT.

Other embrittling phenomena are related to absorbed gases (helium and hydrogen) in the metal. Austenitic steels have a high nickel content, which can produce helium in a neutron irradiation environment. Because the irradiation causes both helium embrittlement and dislocation embrittlement concurrently, the effects are not easily separated and are always measured together: Therefore, the previous discussion concerning the lack of safety issues regarding the embrittlement of austenitic stainless steel vessel components in liquid-metal-cooled reactors remains valid for irradiation hardening and He-embrittlement effects combined. Moreover, recent evidence has shown that helium embrittlement is of no concern for irradiation temperatures of less than $932-1022^{\circ} \mathrm{F}$; EBR-II inlet and outlet temperatures are $700^{\circ} \mathrm{F}$ and $883^{\circ} \mathrm{F}$, respectively. Therefore, no major core structural components will be affected. This finding is supported by the data from the surveillance capsules and control-rod-thimble replacement.

Another embrittling phenomenon that may become important to a 40 -year lifetime is thermal embrittlement of Type-304 stainless steel, and particularly of welds mating Type-304 to Type-304 using Type-308 stainless steel as a filler material. The primary tank is constructed of 304 plate sections welded together using 308 welding electrodes.

The embrittlement phenomenon is characterized by degradation of the free ferrite in cast 304 or in the $304 / 308$ welds after long periods of time due to phase transformations and formation of precipitates. EBR-II did not use a pure case form of 304 (CR-8 or CF-8A) in the construction of the primary tank so this should not be a problem. In addition, the potential embrittlement problem was anticipated by the designers of the reactor and weld criteria were 
specified to limit the ferrite content in both weld rods used and in the welds themselves by adhering to ASTM Specification A240-57T with an additions requirement the maximum carbon content be 0.08 percent, with 0.04 to 0.06 percent preferred. By working in close cooperation with the primary tank fabricator, all steel used met the desired requirements.

\section{Void Swelling}

Neutron-irradiation damage of stainless steel components eventually creates a densitychange in the stainless steel. Voids that form in the material lowers the density, and the result is that the component swells. The behavior has been studied extensively, and the amount of swelling can be fairly accurately predicted by knowing the fast $(E>0.1 \mathrm{MeV})$ neutron fluence exposure and the irradiation temperature.

The core contains many different types of stainless steel and the swelling behavior of each, and allowable dimensional changes of each component are used to provide exposure limitations. In the core, where the neutron flux is high, these exposure limits are used to assess when a component assembly needs to be replaced. Fuel handling force surveillance is eventually utilized to monitor whether an exposure limit has been properly assigned and prevent unexpected problems from occurring.

The areas surrounding the main core contain structures which are irreplaceable such as the grid plate assembly, the reactor vessel, and the neutron shield. The only steel used within this region is Type-304: Because the components are irreplaceable, the swelling behavior is assessed to determine the useful lifetime of these components as a measure of the reactor lifetime. The neutron flux to which these components are exposed is much less than that of incore components.

The most critical of these components, in terms of swelling-related problems is the grid plates. There is a higher neutron exposure to the upper than lower plate. It was proposed that differential swelling may cause crowning of the upper plate and/or misalignment of the holes in the plates. A program to assess the problem and monitor the hole alignment was instituted in 
1974, and no problems have been found during periodic gauging of the grid. As a precaution, the diameter of the lower part of the pole piece of some subassemblies has been reduced to insure that no binding occurs.

The expected peak fast neutron exposure on the grid plate will be less than $2 \times 10^{22} \mathrm{n} / \mathrm{cm}^{2}$ in the year 2004. The predicted void swelling $\left(\Delta V / V_{o}\right)$ at that point is predicted to less than $0.1 \%$. This indicates that grid plate crowning is not likely the problem, but the monitoring program will insure detection if it should occur.

Other irreplaceable components are not exposed to as high a neutron flux, and their dimensional changes are not as critical. Therefore, the void swelling assessment will be limited to the grid plate.

Sodium Compatibility

The exposure of steel to sodium has also been widely studied. The high-temperature corrosion effects have been observed characterized by changes in the near-surface chemistry of the steel (nickel loss, etc.) and also carbon transfer out of the steel with perhaps a carburization of other components in the reactor. The operating temperature within the EBR-II reactor systems and the materials used do not create a situation where major sodium compatibility effects would be seen.

\subsubsection{2 $\quad \underline{\text { Structural Assessment }}$}

The basic structural analysis is described in [7.20]. Briefly, standard codes are used for thermal hydraulic and structural analysis, THTB is used for thermal analysis and ANSYS and SAP6 used for structural and piping analyses. The components considered include the reactor vessel, the reactor vessel cover, the intermediate heat exchanger (IHX), the superheater, the primary piping system, and some of the secondary piping between the IHX and the superheater. 
The total fluence per MWd at the reactor cover bottom plate above the core rows 1 and 16 locations are given in Ref. 7.31 and they are $1.4 \times 10^{16}$ and $5.3 \times 10^{15} \mathrm{n} / \mathrm{cm}^{2}$ for year reactor operation assuming a plant factor of $80 \%$. The expected fatigue damage factor of the thermal baffle is $1.5 \times 10^{-3}$ which is considerably less than 1 , and the fatigue damage is within the limit specified by the ASME code [7.21].

\section{Reactor Vessel}

The creep and fatigue damage of the reactor vessel was found to have negligible effect for reactor normal and transient operations [7.17]. The irradiation damage of the reactor vessel is small, and the stress and strain ranges of the reactor vessel are within the design limit specified by the ASME code for EBR-II 40 year operation [7.19].

$$
\text { Intermediate Heat Exchanger (IHX) }
$$

The irradiation damage of components in the IHX is small and can be neglected. Analysis in [7.20] indicated that the creep damage of the IHX during reactor normal operation is negligible. A reactor scram from $123 \%$ overpower was identified as the most severe transient for the IHX components due to fatigue damage, and 1000 duty cycles are allowed for the transient through the life of the reactor. The structural damage for steady-state and other transient operations are small and can be neglected. If we conservatively use this transient to represent all protected overpower transient occurring during EBR-II operation, then the IHX can survive at least 1000 protected overpower transients. In the past, the EBR-II experienced 249 reactor scram transients [7.22]. If we conservatively assume that 250 additional overpower transients are expected in the future reactor operation, then the total number of the protected overpower transients throughout the life of the reactor is about 500, which is less than 1000 (corresponds to damage factor of 0.5 ), and the design criterion is satisfied. 
The analysis in [7.20] indicated that the fatigue and creep damage to each of the primary-inlet, the primary-outlet (Z-pipe), and the secondary piping systems are very small for transient and steady-state reactor operations. To extend the EBR-II life to 40 years will not affect the structural performance of piping systems.

$$
\text { Primary Tank }
$$

The primary tank includes an inner vessel and an outer vessel. The primary tank inner vessel is suspended form the primary-tank cover, and the load of the primary tank is transmitted from the six radial beams in the primary-tank cover to the six radial beams in the top support structure through six stainless steel hangers. The average primary-tank sodium temperature, is $700^{\circ} \mathrm{F}$ during normal operation, and a temperature gradient across the cover occurs. As a result, a downward bowing in the primary-tank cover is induced, which causes a moment and a shear force at the top of the inner-vessel tank.

During reactor normal and transient operations, a temperature difference between top and bottom of the shell occurs due to stratification. A study of the measured data recorded by the primary-tank probes for the SHRT tests and the plant inherent control test (PICT) series [7.23] indicated that the maximum axial temperature difference in the inner vessel will not exceed $200^{\circ} \mathrm{F}$, and the maximum temperature across the primary cover is below $750^{\circ} \mathrm{F}$. For an axial vessel temperature difference and the temperature across the cover are $200^{\circ} \mathrm{F}$ and $750^{\circ} \mathrm{F}$, respectively, the stress intensities at the top and the bottom of the inner vessel are 15,000 and $14,800 \mathrm{psi}$, respectively. These stress intensities are considerably less than the $3 \mathrm{~S}_{\mathrm{m}}(44,000 \mathrm{psi})$ allowed by the ASME code and the design criterion is satisfied.

\subsection{Damage Criteria}

Creep and fatigue damage were evaluated in the preceding analysis using the ASME code [7.19], a total creep fatigue damage, D, being 
evaluated. A design life is defined as $D=1$, but this does not give a failure probability.

Consider first the case when the damage is being caused by transients, either protected overpower or loss-of-flow transients and assume that $\mathrm{N}$ of the limiting transients are required to give $\mathrm{D}=1$.

Hence: $\quad \mathrm{Nd}_{\mathrm{T}}=1$

where $d_{T}$ is the damage in each transient. Further, let us conservatively assume that the effects of every scram at EBR-II gives damage $d_{T}$ and that there are $n_{s}$ scrams per year. Therefore, using (7.3) the design life of a component, $\mathrm{A}$, is given by

$$
\mathrm{An}_{3} \mathrm{~d}_{\mathrm{T}}=1 \quad \text { or } \mathrm{A}=1 /\left(\mathrm{n}_{\mathrm{s}} \mathrm{d}_{\mathrm{T}}\right)
$$

To transform this into an estimation of probability requires an interpretation of design life.

Here the assumption is made that design life can be interpreted as a "2- $\sigma$ " probability of failure, i.e., if $\mathrm{D}=1$ then, using a normal distribution

$$
\operatorname{Pr}(\text { component failure })=2.310^{-2}
$$

Consultation with structural engineering consultants indicated that this assumption is conservative [7.24]. 
Now from Table 5.6, the number of scrams is $\sim 4.4 / \mathrm{yr}$ and if a uniform failure rate model is assumed the annual frequency of failure can be estimated as

$$
\begin{aligned}
& \left(2.310^{-1} \mathrm{~d}_{\mathrm{T}}\right) \times\left(4.4 \mathrm{yr}^{-1}\right) \\
& \simeq 0.1 \mathrm{~d}_{\mathrm{T}} \mathrm{yr}^{-1}
\end{aligned}
$$


Table 7.35. Structural Analyses of Major EBR-II Components

\begin{tabular}{|c|c|c|c|}
\hline & & $\mathrm{d}_{\mathrm{T}}$ & $\begin{array}{c}\text { Mean Failure Freq. } \\
\mathrm{yr}^{-1}\end{array}$ \\
\hline Reactor Vessel Core & & $6.6710^{-6}$ & $6.710^{-7}$ \\
\hline Reactor Vessel & & $<10^{-6}$ & $<10^{-7}$ \\
\hline IHX & & $110^{-3}$ & $110^{-4}$ \\
\hline $\begin{array}{r}\text { Super Heater (Fig. } 7 . \\
\text { Region } 1 \\
2 \\
3 \\
4\end{array}$ & $\begin{array}{l}\text { Shield Transition } \\
\text { Duplex Tube in air } \\
\text { Duplex Tube in sodium } \\
\text { Steam Reducer }\end{array}$ & $\begin{aligned} & 10^{-6} \\
1.4 & 10^{-5} \\
< & 10^{-6} \\
< & 10^{-6}\end{aligned}$ & 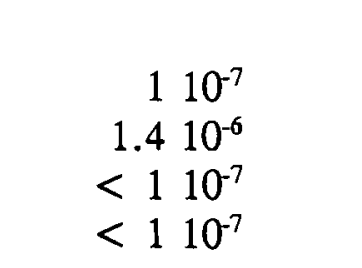 \\
\hline Piping & & $<10^{-6}$ & $<10^{-7}$ \\
\hline Tank & & $<10^{-6}$ & $<10^{-7}$ \\
\hline Evaporator & & $<10^{-6}$ & $<10^{-7}$ \\
\hline
\end{tabular}




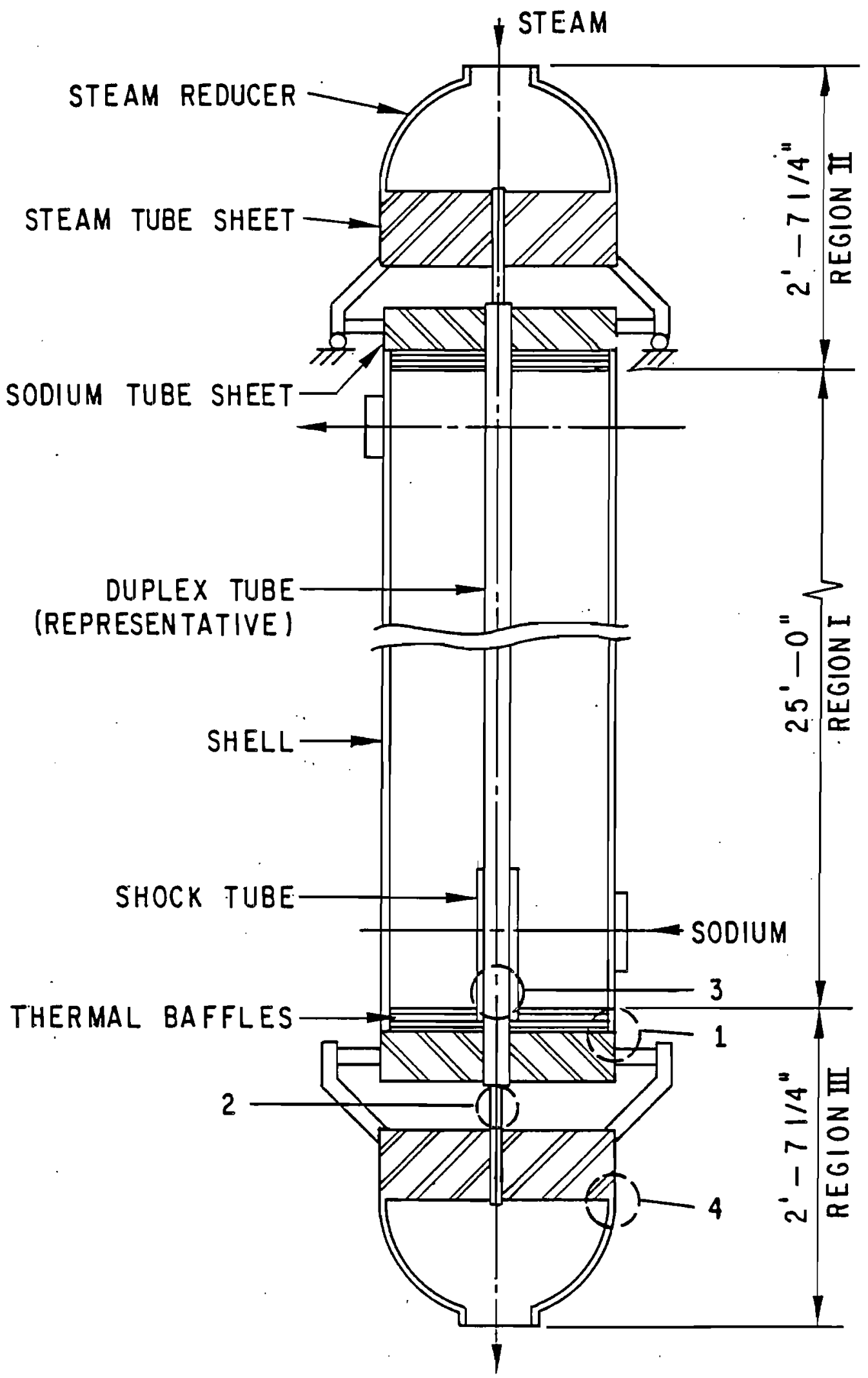

Fig. 7.6. Schematic View of Superheater, Showing Regions for Which Thermal-analysis Models Were Developed 
The table shows that the IHX is most vulnerable to failure due to repeated thermal cycling. IHX failure is treated in the LOCA analysis, where a higher failure rate than that derived above is used.

Additional studies have been carried out upon the grid plate and no embrittlement or swelling concerns have been identified as shown in the preceeding section. This conclusion is based upon the performance of surveillance materials irradiated to fluxes greater than experienced by any component in the reactor tank.

The concern for superheater problems lies in sodium-water reactions. This is considered to be an independent initiating event (SGTR). A generic estimate for a tube failure of $10^{-6}$ failure $\mathrm{hr}^{-1}, 7.210^{-3} \mathrm{yr}^{-1}$, is available [7.25]. This generic estimate is based upon data from sodium steam generators of the 1960 s and steam/water steam generators.

The same structural evaluation identified creep in steady operation as the life limiting damage mechanism affecting the superheaters. The analysis of [7.20] identifies a creep damage factor of 0.43 over 40 years with an $80 \%$ capacity factor, or a 93 year design life, for the limiting case of the transition region where the creep damage is greatest. If a design life is interpreted, as above, as a " $2 \sigma$ " probability of failure then the failure rate can be estimated as $2.510^{-4} \mathrm{yr}^{-1}$. The failure rates evaluated in this way for the other regions of the superheater give the following values $7.510^{-6} \mathrm{yr}^{-1}, 2.510^{-6} \mathrm{yr}^{-1}$ and $7.510^{-6} \mathrm{yr}^{-1}$.

Failure in the transition would not lead to immediate sodium-water contact, nevertheless in view of the simplicity of the arguments presented here, an estimated value of $10^{-4} \mathrm{yr}^{-1}$ will be adopted for steam generator tube rupture with sodium water interaction.

\subsubsection{Aging Effects at the Component Level}

Aging effects at the component level can affect the reliability of the safety systems. In most cases the EBR-II systems are undergoing continual upgrading. Therefore, 
where aging concerns might have existed, these have been addressed. The issue of aging is addressed in a system by system basis below.

\subsubsection{Reactor Shutdown System (RSS)}

One common cause failure in the RSS was identified which can be considered as due to an aging mechanism. This event is the failure of the relays to act upon demand. The specific mechanism is wear due to $60 \mathrm{~Hz}$ vibration for relays power by AC. The relays in the RSS are to be replaced with new relays with hardened shafts that guard against this failure.

Another symptom of aging in EBR-II is reflected in the steady loss of flow instrumentation. This is reflected in the PRA by the various possible trip configurations and specifically in that a flow transient on loop \#1 will not be sensed directly because there is no remaining flow instrumentation there.

\subsubsection{Primary Pumps}

Primary pump \#1 was removed for repair in December 1970 and again in may 1988. Primary pump $\# 2$ was removed for repair in May 1982. Primary pump $\# 2$ was removed for repair in May 1982. The impellers of both pumps were replaced during their last removal from the primary tank. Inspection of the pump shafts and associated reveled no degradation of the pumps. The pump motors were inspected and found to be in "like new" conditions. This is primarily the result of the argon atmosphere within the motor housings. Thus, there appears to be no aging effects other than impeller wear which has been accommodated by replacement of worn parts.

\subsection{Dependency Analysis}

Dependent failures are extremely important in PRA and must be carefully treated in order to avoid underestimation of risk $[7.26,7.27]$. Dependent failures are those failures that defeat 
the redundancy or diversity which is usually utilized to improve the availability of critical plant functions such as reactor abnormal-transient detection. In the absence of dependent failures, separate trains of a redundant system, or diverse methods of providing the same function are regarded as independent so that unavailability of the function is basically the product of the unavailabilities of the separate trains or diverse systems. However, a dependent failure arises from some cause that fails more than one system, or more than one train of a system, simultaneously. Accordingly, the effect of dependent failures is to largely increase (by several orders of magnitudes in some cases) the unavailability of the system with respect to the situation of true independence.

Generally, dependent failures could result from initiating events (external events), interassembly dependencies, and intercomponents dependencies [7.26]. Intersystem and intercomponent dependencies cover functional dependence (for example, when a system is designed to function only in conjunction with the successful operation of other systems), shared equipment dependence, physical interaction dependence (for example, extreme environmental stresses created by the failure of one system), and human interaction dependence. Most of these dependencies are usually accounted for explicitly in the PRA logic models (event trees and fault trees). For example, intersystem dependence arising from shared equipments, such as the undervoltage CP-relays in the shutdown strings shared by the low-flow channels and subassembly outlet temperature channels, are included in the logic model by introducing basic events, which represent component failure modes associated with the shared equipments. Failures resulting from the failure of another component (cascading or propagating failures) are also modeled explicitly. Similarly, errors made during maintenance and testing of component operability in redundant channels are modeled explicitly in fault trees.

However, many dependencies among component failures can not be accounted for explicitly in the logic models, and this means that some basic events are not truly independent. This is addressed by introducing the concept of common cause basic events, which represent the class of residual dependent failures whose root caused are not explicitly modeled. Thus, a common cause event is defined as the failure or unavailable state of more than one component at the same time or within a short interval and due to some shared cause. 
There are three important concepts in understanding and modeling common cause events [7.27]. The first is the root cause of failure or unavailability of the component. A root cause is a mechanism of a transition of state from operating or functionally available to that of failed or unavailable. Given the existence of the root cause, the second concept of importance is that of linking or coupling mechanism, which is what leads to multiple equipment failure. Whereas, each component fails because of its susceptibility to the conditions created by the root cause, the coupling mechanism makes those conditions common to several components. The third factor that enters into determining the potential for dependent failures is the existence of engineered or operational defenses against unanticipated equipment failures. Some of the tactics adopted in defense against CCFs include design control, physical separation of equipments, well-designed test and inspection procedures, maintenance procedures, and training of personnel.

Common cause failures among components can result from various root causes and by different mechanisms. The following list broadly categorizes possible root causes of component failure. This list has been used in identifying and assessing potential CCFs in EBR-II systems.

1. Design Inadequacy - This category covers actions and decisions during design, manufacturing, or installation of components both before and after the reactor is operational.

2. Procedure Error - This category refers to the ambiguity, incompleteness, or errors in procedures for operation and maintenance of equipment.

3. Human Actions - This represents causes related to errors of omission and commission on the part of the plant staff, such as failure to follow a correct procedure.

4. Abnormal Environmental Stress - This includes all causes related to a harsh environment that is not within the specified design criteria of the components.

5. Internal Failure - This encompasses malfunctioning of something internal within the component boundary as a result of normal wear-out or other intrinsic failures.

6. Unknown - The cause of the failure can not be identified. 
The key output based on the previous guidelines is the identification of the groups of components for which CCFs may be important. The objective of the next stage is to modify the logic model to incorporate common cause events, convert this logic model to a probability estimation model, and to analyze the data for quantifying the parameters of the selected probability model [7.27]. The number of parameters in the model, single or multiple, determines the number of the basic events required in the logic model and obviously the number of the parameters to be derived from the data analysis. The most widely used single parameter model is known as the beta-factor model. According to this model a function (beta) of the component failure rate can be associated with common cause events shared by the other components in that group. Whenever a common cause event occurs, all components within the common cause component group are assumed to fail. Although data collected from the operation of nuclear power plants (mainly LWR plants) indicate that common cause events do not always fail all redundant components, experience from using this model shows that, in many cases it gives reasonably accurate but slightly conservative results for redundancy levels up to about three or four items. Beyond such a redundancy level, this model generally yields results that are conservative. A more accurate analysis model for systems with higher level of redundancy is the Multiple Greek Letter (MGL) model. This multiple parameter model is an extension of the beta method. For $\mathrm{n}$ redundant components the model generally requires $\mathrm{n}$ different parameters which are used to quantify all possible ways of CCFs.

Ideally, the numerical values of the model parameters (only a single parameter is required with the beta-factor method) should be estimated in a manner that makes the maximum possible use of plant specific event data. This would include data screening and classification of event data and developing uncertainty distributions and point estimate of the parameters. However, due to virtually non-existent common cause events in the EBR-II operating history, such a detailed data analysis becomes futile and one might need to consider data from the LWR operating experience and a variety of sources to make statistical inferences about the frequencies of common cause events [7.28, 7.29, 7.30]. It is worth noting in this regard that the procedures for treating common cause failures described in Ref. 7.27 recognized the need in some analyses to bypass the event screening step and use instead published numerical values of common cause event parameters. Accordingly, the common cause failures in the RSS fault trees have been 
addressed in terms of generic beta factors as follows:

The total failure probability of each redundant component is expanded into dependent and independent failure contributions. The probability of dependent failure is assumed equal to the total failure probability of each component multiplied by the beta factor. The independent failure probability of each component would then normally be the difference between the total failure probability and dependent failure probability. But since independent component failures in the RSS do not contribute any significant amounts to the system unavailability, it was found convenient (data checking) in the analyses to have the independent failure probability the same as the total failure probability.

The generic beta factors are applied to similar components in any subsystem or in redundant subsystems or strings. These similar components have to meet the following CCF requirements: (i) same initial conditions, (ii) same failure mode, (iii) same use or function, and (iv) same design or same manufacturer.

To account for the effect of number of redundancy, a generic beta factor of 0.100 is used for redundant components numbered less than four, 0.020 for redundant components numbered four or five, and 0.008 for redundant components numbered six or more. Combined with this method of modeling CCFs is a substantial effort to explicitly model most of the unsafe dependent events. Any remaining dependent failures can be viewed as CCF with weak linkage between redundant components. An example of this weak linkage is wear-out of parts of components by extended usage. Weak linkage implies that although a linkage mechanism can be postulated, its probability of occurrence in all components at the same time gets smaller with increase in redundancy level.

The main advantage of the above method of addressing CCFs, to be referred to herein as beta(n) method, is that it is much simpler to use with fault trees than the MGL method. The 
beta(n) method requires only one basic event to model the CCFs irrespective of the number of redundant components. Whereas the MGL method requires a much larger number of basic events to model the CCFs. For instance, with four redundant components eleven different CCF events have to be modeled in addition to the independent failures of the components. One should notice, however, that the beta(n) method is an approximate method for taking account of redundancy level. It is a convenient means for assessing the CCFs among redundant components when there are no CCF data available and the alternatives would be either to ignore the CCFs all together or use a fixed beta factor that does not vary with the redundancy level. It is needless to say that the beta(n) method is not a substitute for the more precise MGL method when reliable CCF data are available.

A better perspective of the present beta(n) method can be gained when one compares the failure probabilities of redundant components determined by this method and by the alternative MGL method. Three different generic models (labeled Model 1, 2, and 3) are selected for the MGL method calculations, see Table 7.36. The CCF parameters used in the three models are based on the beta and gamma values given in Table 3-5 of Ref 7.31 and Table 11 of Ref 7.32. The parameter delta is assigned a value of 0.8 in Models 1 and 2 to provide a smooth transition from gamma $=0.5$ to epsilon=1. A more gradual transition is assumed in Model 3 with gamma $=0.2$, delta $=0.4$, and epsilon $=1$. Although any of the three models could be justified as a generic CCF model for a group of redundant components, Model 1 appears to be an over-pessimistic model for components that have not shown in the past clear CCFs. Either Model 2 or Model 3 would more appropriately represent postulated CCFs for this type of redundant components or for redundant components with weak linkage. Model 3 is particularly important in view of a recent evaluation of CCFs among relays (PLG proprietary data). The MGL parameters of the relays indicate that Model 3 is not only realistic but conservative as well. Table 7.37 and Fig. 7.7 show the probability of failure of $m$ components among $n$ redundant components $(\mathrm{Q}(\mathrm{m} / \mathrm{n}))$ according to the beta( $\mathrm{n})$ method and according to the three models based on the MGL method. The results of the beta(n) method compares very favorably with the results of Models 2 and 3 in almost all cases. It is interesting to notice that the two alternative methods yield identical results for $n=6$ and $m=n-1$. Common cause failure at this high level of redundancy is particularly important for the EBR-II Reactor Shutdown System, see 
Table 7.36. Parameters Used in Modeling CCF by $\beta(\mathrm{n})$ Method and MGL Method

\begin{tabular}{||cc|cccc||}
\hline \multicolumn{2}{|c|}{$\gamma(\mathrm{n})$ Method $^{(2)}$} & \multicolumn{5}{|c||}{ MGL Method $^{(2)}$} \\
\hline $\mathrm{n}$ & $\underline{\beta(\mathrm{n})}$ & $\underline{\text { Parameter }}$ & $\underline{1}$ & $\underline{2}$ & $\underline{3}$ \\
\hline 2 & 0.100 & $\beta$ & 0.10 & 0.02 & 0.10 \\
\hline 3 & 0.100 & $\gamma$ & 0.50 & 0.50 & 0.20 \\
\hline 4 & 0.020 & $\gamma$ & 0.80 & 0.80 & 0.40 \\
\hline 5 & 0.020 & $\epsilon$ & 1.00 & 1.00 & 1.00 \\
\hline 6 & 0.008 & & & & \\
\hline
\end{tabular}

Notes

(1) $\mathrm{Q}_{\mathrm{t}}=\beta(\mathrm{n}) \mathrm{Q}_{\mathrm{s}}+\mathrm{Q}_{\mathrm{s}}$

$\mathrm{Q}_{\mathrm{t}}=$ Total Failure Probability of a Redundant Component

$\mathrm{Q}_{\mathbf{s}}=$ Single Component Failure Probability

$\mathrm{n}=$ Number of Redundant Components

(2) Provides $\mathrm{Q}(\mathrm{m} / \mathrm{n})$, Probability of $m$ Components failing among $n$

Redundant Components. As an example:

$$
Q(2 / 3)=\beta \gamma Q_{s}+(3 / 2) \beta(1-\gamma) Q_{s}+3(1-\beta)^{2} Q_{s}^{2}
$$


Table 7.37. Comparison of Redundant Component Failures According to $\gamma(\mathrm{n})$ Method and MGL Methods

\begin{tabular}{|c|c|c|c|c|c|c|c|}
\hline \multirow{3}{*}{$(\mathrm{m} / \mathrm{n})$} & \multicolumn{7}{|c|}{$\frac{\mathrm{Q}(\mathrm{m} / \mathrm{n})(1)}{\mathrm{Qs}}$} \\
\hline & \multirow{2}{*}{$\beta(\mathrm{n})$} & \multicolumn{3}{|c|}{ MGL(2) } & \multicolumn{3}{|c|}{$\beta(\mathrm{n}) / \mathrm{MGL}$} \\
\hline & & $\underline{1}$ & 2 & $\underline{3}$ & $\underline{1}$ & $\underline{2}$ & $\underline{3}$ \\
\hline$(2 / 2)$ & 0.101 & 0.101 & 0.021 & 0.101 & 1.00 & 4.81 & 1.00 \\
\hline$(2 / 3)$ & 0.103 & 0.127 & 0.028 & 0.142 & 0.81 & 3.68 & 0.73 \\
\hline$(3 / 3)$ & 0.100 & 0.050 & 0.010 & 0.020 & 2.00 & 10.00 & 5.00 \\
\hline$(4 / 4)$ & 0.020 & 0.054 & 0.011 & 0.024 & 0.37 & 1.82 & 0.83 \\
\hline$(4 / 5)$ & 0.020 & 0.040 & 0.008 & 0.008 & 0.50 & 2.50 & 2.50 \\
\hline$(5 / 5)$ & 0.020 & 0.040 & 0.008 & 0.008 & 0.50 & 2.50 & 2.50 \\
\hline$(5 / 6)$ & 0.008 & 0.040 & 0.008 & 0.008 & 0.20 & 1.00 & 1.00 \\
\hline$(6 / 6)$ & 0.008 & 0.040 & 0.008 & 0.008 & 0.20 & 1.00 & 1.00 \\
\hline
\end{tabular}

Notes:

(1) Qs: Single Component Failure Probability $(\mathrm{Q} s=10-3$, used only with nonlinear terms)

$\mathrm{Q}(\mathrm{m} / \mathrm{n})$ : Probability of $\mathrm{m}$ components failing among $\mathrm{n}$ redundant components

(2) See Table 7.36 for the MGL parameters of each case. 


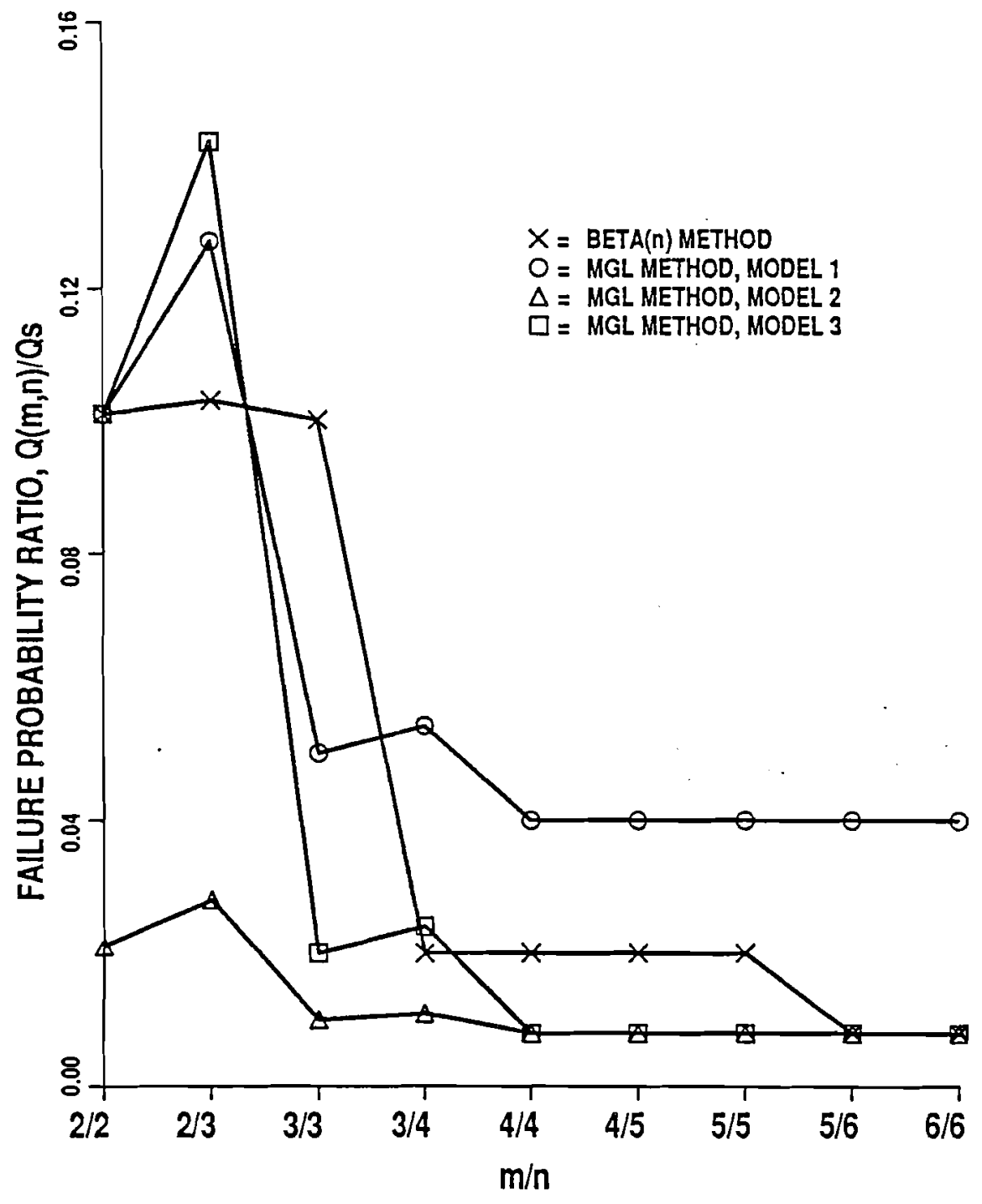

Fig. 7.7. Comparison of Redundant Component Failure Probability According to Beta(n) and MGL Methods 
Section 6.2.1 and Appendix C.1. The beta(n) method, however, appears to under-predict failure probability when one compares its results with Model 1 for $n$ 4. As mentioned earlier Model 1 is an over-pessimistic model for redundant components that have not shown CCF during their service in the past. The inability of the beta(n) method to differentiate between the various $\mathrm{m}$ values for a given $n$ value appears to be significant only with $n=3$. The three MGL models show, as one would expect, larger failure probabilities with $Q(2 / 3)$ than with $Q(3 / 3)$; whereas the beta(n) method has virtually the same value for both types of failure logic. For redundant components that have 2-out-of-3 failure logic, this situation can be simply rectified by raising the beta-factor from 0.10 to 0.15 . Because of the relatively small difference in the failure probability and in order to keep the beta(n) method as simple as possible it was found unnecessary to modify the method for this special case.

Table 7.38 shows the beta factors among the various electrical components that are used in the LOF and TOP fault trees of the RSS. The beta factors used in the accident sequence evaluations are the values listed under Analysis Model B. These beta factors are based on the previously described beta(n) method and cover all similar components in various channels or strings. Table 7.38 also shows beta factors for a different analysis model, designated Analysis Model A, with less extensive CCF modeling. In Analysis Model A, one assumes relatively large beta factors for similar components within the same channel or same shutdown string (larger than would have been used with beta(n) method) but ignores CCF between similar components in different channels or shutdown strings. The comparison between the results of Analyses A and B given in Section 6.2.1.5 provides insights into the effect of CCFs among the RSS critical components.

In addition to CCFs among electrical components, CCFs among the nine high-worth control and among the two safety rods have been given careful assessment. Table 7.39 shows the common cause events that are postulated and used in both fault trees to model the mechanical failure of the control and safety rods. The failure probability calculations begin with a generic failure per demand of $3.10^{-5}$ for a single rod [7.25]. This is the failure probability of a control rod or safety exclusive of the failure of the magnetic clutch to release the rod. In the case of control rods, this generic failure probability is increased by a factor of three, to $9 .{ }^{10-5}$ per 
Table 7.38

LIST OF COMMON CAUSE FAILURES AMONG ELECTRICAL COMPONENTS FAULT TREE ANALYSIS OF SCRAM FAILURE UNDER LOF OR TOP CONDITION

\begin{tabular}{|l|c|c|c|l||}
\hline \multicolumn{1}{|c|}{ Description of CCF } & $\begin{array}{c}\text { Analysis } \\
\text { Model }\end{array}$ & $\begin{array}{c}\beta \\
\text { Factor }\end{array}$ & $\begin{array}{c}\text { CCF } \\
\text { Event }\end{array}$ & $\begin{array}{l}\text { Corresponding } \\
\text { Independent } \\
\text { Events }\end{array}$ \\
\hline $\begin{array}{l}\text { ALL FAULT TREES } \\
\text { 1. Armatures/coils of all CP-relays } \\
\text { on shutdown string A(1) }\end{array}$ & A & 0.30 & RREC01QU & $\begin{array}{l}\text { RRECPAQP, } \\
\text { RRECC1QP, } \\
\text { RRECP2QP, } \\
\text { RRECPYQP, } \\
\text { RRESR0QP, } \\
\text { RRESRXQP }\end{array}$ \\
\hline $\begin{array}{l}\text { Contact of all CP-relays on } \\
\text { shutdown string A(1) }\end{array}$ & A & 0.30 & RCZC01QU & $\begin{array}{l}\text { RCZCPAQP, } \\
\text { RCZCC1QP, } \\
\text { RCZCP2QP, } \\
\text { RCZCPYQP, } \\
\text { RCZSR0QP, } \\
\text { RCZSRXQP }\end{array}$ \\
\hline 3. $\begin{array}{l}\text { Armatures/coils of all CP-relays } \\
\text { on shutdown string B }\end{array}$ & A & 0.30 & RREC01QU & $\begin{array}{l}\text { RRECB1QP, } \\
\text { RRECB2QP, } \\
\text { RRECB3QP }\end{array}$ \\
\hline $\begin{array}{l}\text { 4. Contacts of all CP-relays on } \\
\text { shutdown string B }\end{array}$ & A & 0.30 & RCZC02QU & $\begin{array}{l}\text { RCZCB1QP, } \\
\text { RCZCB2QP, } \\
\text { RCZCB3QP }\end{array}$ \\
\hline $\begin{array}{l}\text { 5. Armatures/coils of all CP-relays } \\
\text { on both shutdown strings }\end{array}$ & B & 0.008 & RREC00QU & $\begin{array}{l}\text { RRECPAQP, etc } \\
\text { RRECB1QP, etc }\end{array}$ \\
\hline $\begin{array}{l}\text { Contacts of all CP-relays on } \\
\text { both shutdown strings }\end{array}$ & B & 0.008 & RCZC00QU & $\begin{array}{l}\text { RCZCPAQP, etc } \\
\text { RCZCB1QP, etc }\end{array}$ \\
\hline
\end{tabular}


Table 7.38

LIST OF COMMON CAUSE FAILURES AMONG ELECTRICAL COMPONENTS -

FAULT TREE ANALYSIS OF SCRAM FAILURE UNDER LOF OR TOP CONDITION (Cont'd)

\begin{tabular}{|c|c|c|c|c|}
\hline Description of $\mathrm{CCF}$ & $\begin{array}{l}\text { Analysis } \\
\text { Model }\end{array}$ & $\begin{array}{c}\beta \\
\text { Factor }\end{array}$ & $\begin{array}{l}\text { CCF } \\
\text { Event }\end{array}$ & $\begin{array}{l}\text { Corresponding } \\
\text { Independent } \\
\text { Events }\end{array}$ \\
\hline $\begin{array}{l}\text { LOF FAULT TREE } \\
\text { (i) Low-Flow Channels } \\
\text { 1. Armatures/coils of two trip } \\
\text { relays for both shutdown strings } \\
\text { - each channel separate }\end{array}$ & A & 0.20 & $\begin{array}{l}\text { RRE1A0QU } \\
\text { RRE1B0QU } \\
\text { RRE1C0QU } \\
\text { RRE1D0QU }\end{array}$ & $\begin{array}{l}\text { RRE1A1QP, } \\
\text { RRE1A2QP } \\
\text { RRE1B1QP, } \\
\text { RRE1B2QP } \\
\text { RRE1C1QP, } \\
\text { RRE1C2QP } \\
\text { RRE1D1QP, } \\
\text { RRE1D2QP }\end{array}$ \\
\hline $\begin{array}{l}\text { 2. Contacts of two trip relays for } \\
\text { both shutdown strings - each } \\
\text { channel separate }\end{array}$ & A & 0.20 & $\begin{array}{l}\text { RCZ1A0QU } \\
\text { RCZ1B0QU } \\
\text { RCZ1C0QU } \\
\text { RCZ1D0QU }\end{array}$ & $\begin{array}{l}\text { RCZ1A1QP, } \\
\text { RCZ1A2QP } \\
\text { RCZ1B1QP, } \\
\text { RCZ1B2QP } \\
\text { RCZ1C1QP, } \\
\text { RCZ1C2QP } \\
\text { RCZ1D1QP, } \\
\text { RCZ1D2QP }\end{array}$ \\
\hline $\begin{array}{l}\text { 3. Armatures/coils of all trip } \\
\text { relays in all channels }\end{array}$ & B & 0.008 & RRE1Z0QU & $\begin{array}{l}\text { RRE1A1QP, etc } \\
\text { RRE1B1QP, etc } \\
\text { RRE1C1QP, etc } \\
\text { RRE1D1QP, etc }\end{array}$ \\
\hline $\begin{array}{l}\text { 4. Contacts of all trip relays in all } \\
\text { channels }\end{array}$ & B & 0.008 & RCZ1Z0QU & $\begin{array}{l}\text { RCZ1A1QP, etc } \\
\text { RCZ1B1QP, etc } \\
\text { RCZ1C1QP, etc } \\
\text { RCZ1D1QP, etc }\end{array}$ \\
\hline
\end{tabular}


Table 7.38

LIST OF COMMON CAUSE FAILURES AMONG ELECTRICAL COMPONENTS -

FAULT TREE ANALYSIS OF SCRAM FAILURE UNDER LOF OR TOP CONDITION (Cont'd)

\begin{tabular}{|c|c|c|c|c|}
\hline Description of CCF & $\begin{array}{l}\text { Analysis } \\
\text { Model }\end{array}$ & $\begin{array}{c}\beta \\
\text { Factor }\end{array}$ & $\begin{array}{l}\mathrm{CCF} \\
\text { Event }\end{array}$ & $\begin{array}{l}\text { Corresponding } \\
\text { Independent } \\
\text { Events }\end{array}$ \\
\hline $\begin{array}{l}\text { 5. Bistables for both shutdown } \\
\text { strings - each channel separate }\end{array}$ & A & 1.0 & $\begin{array}{l}\text { RBI1A0QW } \\
\text { RBI1B0QW } \\
\text { RBI1C0QW } \\
\text { RBI1D0QW }\end{array}$ & $\begin{array}{l}\text { N/A } \\
\text { N/A } \\
\text { N/A } \\
\text { N/A }\end{array}$ \\
\hline $\begin{array}{l}\text { 6. All bistables (Ronan X-82) in } \\
\text { all channels }(2,),(4)\end{array}$ & B & 0.10 & RBI1Z0QU & $\begin{array}{l}\text { RBI1A0QW, } \\
\text { RBI1B0QW, } \\
\text { RBI1C0QW }\end{array}$ \\
\hline $\begin{array}{l}\text { 7. All MV/I converters (Ronan X- } \\
\text { 81) in all channels(4) }\end{array}$ & B & 0.02 & RVI1Z0QU & $\begin{array}{l}\text { RVI1A1QW, } \\
\text { RVI1A2QW, } \\
\text { RVI1B1QW, } \\
\text { RVI1C1QW }\end{array}$ \\
\hline $\begin{array}{l}\text { 8. Electro-magnetic flow sensor of } \\
\text { channels HPPF-2 and LPPF-2 }\end{array}$ & B & 0.10 & RFM1Z1RU & $\begin{array}{l}\text { RFM1A0RW, } \\
\text { RFM1B0RW }\end{array}$ \\
\hline (ii) SOT Channels & & & & \\
\hline $\begin{array}{l}\text { 1. Armatures/coils of two trip } \\
\text { relays for both shutdown strings } \\
\text { - each channel separate(5) }\end{array}$ & A & 0.20 & $\begin{array}{l}\text { RRE3C0QU } \\
\text { RRE3D0QU } \\
\text { RRE3F0QU } \\
\text { RRE3H0QU }\end{array}$ & $\begin{array}{l}\text { RRE3C1QP, } \\
\text { RRE3C2QP } \\
\text { RRE3D1QP, } \\
\text { RRE3D2QP } \\
\text { RRE3F1QP, } \\
\text { RRE3F2QP } \\
\text { RRE3H1QP, } \\
\text { RRE3H2QP }\end{array}$ \\
\hline
\end{tabular}


Table 7.38

LIST OF COMMON CAUSE FAILURES AMONG ELECTRICAL COMPONENTS -

FAULT TREE ANALYSIS OF SCRAM FAILURE UNDER LOF OR TOP CONDITION (Cont'd)

\begin{tabular}{|c|c|c|c|c|}
\hline Description of CCF & $\begin{array}{c}\text { Analysis } \\
\text { Model }\end{array}$ & $\begin{array}{c}\beta \\
\text { Factor }\end{array}$ & $\begin{array}{l}\text { CCF } \\
\text { Event }\end{array}$ & $\begin{array}{l}\text { Corresponding } \\
\text { Independent } \\
\text { Events }\end{array}$ \\
\hline $\begin{array}{l}\text { 2. Contacts of two trip relays for } \\
\text { both shutdown strings - each } \\
\text { channel separate(5) }\end{array}$ & A & 0.20 & $\begin{array}{l}\text { RCZ3C0QU } \\
\text { RCZ3D0QU } \\
\text { RCZ3F0QU } \\
\text { RCZ3H0QU }\end{array}$ & $\begin{array}{l}\text { RCZ3C1QP, } \\
\text { RCZ3C2QP } \\
\text { RCZ3D1QP, } \\
\text { RCZ3D2QP } \\
\text { RCZ3F1QP, } \\
\text { RCZ3F2QP } \\
\text { RCZ3H1QP, } \\
\text { RCZ3H2QP }\end{array}$ \\
\hline $\begin{array}{l}\text { 3. Armatures/coils of all trip } \\
\text { relays in all channels }\end{array}$ & B & 0.008 & RRE1Z0QU & $\begin{array}{l}\text { RRE3C1QP, etc } \\
\text { RRE3D1QP, etc } \\
\text { RRE3F1QP, etc } \\
\text { RRE3H1QP, etc }\end{array}$ \\
\hline $\begin{array}{l}\text { 4. Contacts of all trip relays in all } \\
\text { channels }\end{array}$ & B & 0.008 & RCZ1Z0QU & $\begin{array}{l}\text { RCZ3C1QP, etc } \\
\text { RCZ3D1QP, etc } \\
\text { RCZ3F1QP, etc } \\
\text { RCZ3H1QP, etc }\end{array}$ \\
\hline $\begin{array}{l}\text { 5. All bistables (Ronan } \mathrm{X}-82 \text { ) in } \\
\text { all channels }\end{array}$ & B & 0.008 & RBI1Z0QU & $\begin{array}{l}\text { RBI3C0QW, } \\
\text { RBI3D0QW, } \\
\text { RBI3F0QW, } \\
\text { RBI3H0QW }\end{array}$ \\
\hline $\begin{array}{l}\text { 6. All MV/I converters (Ronan X- } \\
\text { 81) in all channels }\end{array}$ & B & 0.008 & RVI1Z0QU & $\begin{array}{l}\text { RVI3C0QW, } \\
\text { RVI3D0QW, } \\
\text { RVI3F0QW, } \\
\text { RVI3H0QW }\end{array}$ \\
\hline $\begin{array}{l}\text { 7. Thermocouples in all } \\
\text { channels }(5)\end{array}$ & B & 0.02 & RTC300RU & $\begin{array}{l}\text { RTC3C0RW, } \\
\text { RTC3D0RW, } \\
\text { RTC3F0RW, } \\
\text { RTC3H0RW }\end{array}$ \\
\hline
\end{tabular}


Table 7.38

\section{LIST OF COMMON CAUSE FAILURES AMONG ELECTRICAL COMPONENTS - FAULT TREE ANALYSIS OF SCRAM FAILURE UNDER LOF OR TOP CONDITION (Cont'd)}

\begin{tabular}{|c|c|c|c|c|}
\hline Description of CCF & $\begin{array}{c}\text { Analysis } \\
\text { Model }\end{array}$ & $\begin{array}{c}\beta \\
\text { Factor }\end{array}$ & $\begin{array}{l}\text { CCF } \\
\text { Event }\end{array}$ & $\begin{array}{l}\text { Corresponding } \\
\text { Independent } \\
\text { Events }\end{array}$ \\
\hline $\begin{array}{l}\text { 8. Reference junctions in all } \\
\text { channels }(5)\end{array}$ & B & 0.02 & $\cdot$ & $\begin{array}{l}\text { RRT3C0AW, } \\
\text { RRT3D0AW, } \\
\text { RRT3F0AW, } \\
\text { RRT3H0AW }\end{array}$ \\
\hline $\begin{array}{l}\text { Armatures/coils of two trip } \\
\text { relays for both shutdown strings } \\
\text { - each channel separate }\end{array}$ & A & 0.20 & $\begin{array}{l}\text { RRE2A0QU } \\
\text { RRE2B0QU } \\
\text { RRE2C0QU }\end{array}$ & $\begin{array}{l}\text { RRE2A1QP, } \\
\text { RRE2A2QP } \\
\text { RRE2B1QP, } \\
\text { RRE2B2QP } \\
\text { RRE2C1QP, } \\
\text { RRE2C2QP }\end{array}$ \\
\hline $\begin{array}{l}\text { 2. Contacts of two trip relays for } \\
\text { both shutdown strings - each } \\
\text { channel separate }\end{array}$ & A & 0.20 & $\begin{array}{l}\text { RCZ2A0QU } \\
\text { RCZ2B0QU } \\
\text { RCZ2C0QU }\end{array}$ & $\begin{array}{l}\text { RCZ2A1QP, } \\
\text { RCZ2A2QP } \\
\text { RCZ2B1QP, } \\
\text { RCZ2B2QP } \\
\text { RCZ2C1QP, } \\
\text { RCZ2C2QP }\end{array}$ \\
\hline $\begin{array}{l}\text { 3. Armatures/coils of all trip } \\
\text { relays in all channels }\end{array}$ & B & 0.008 & RRE2Z0QU & $\begin{array}{l}\text { RRE2A1QP, etc } \\
\text { RRE2B1QP, etc } \\
\text { RRE2C1QP, etc }\end{array}$ \\
\hline $\begin{array}{l}\text { 4. Contacts of all trip relays in all } \\
\text { channels }\end{array}$ & B & 0.008 & RCZ2Z0QU & $\begin{array}{l}\text { RCZ2A1QP, etc } \\
\text { RCZ2B1QP, etc } \\
\text { RCZ2C1QP, etc }\end{array}$ \\
\hline
\end{tabular}


Table 7.38

LIST OF COMMON CAUSE FAILURES AMONG ELECTRICAL COMPONENTS FAULT TREE ANALYSIS OF SCRAM FAILURE UNDER LOF OR TOP CONDITION (Cont'd)

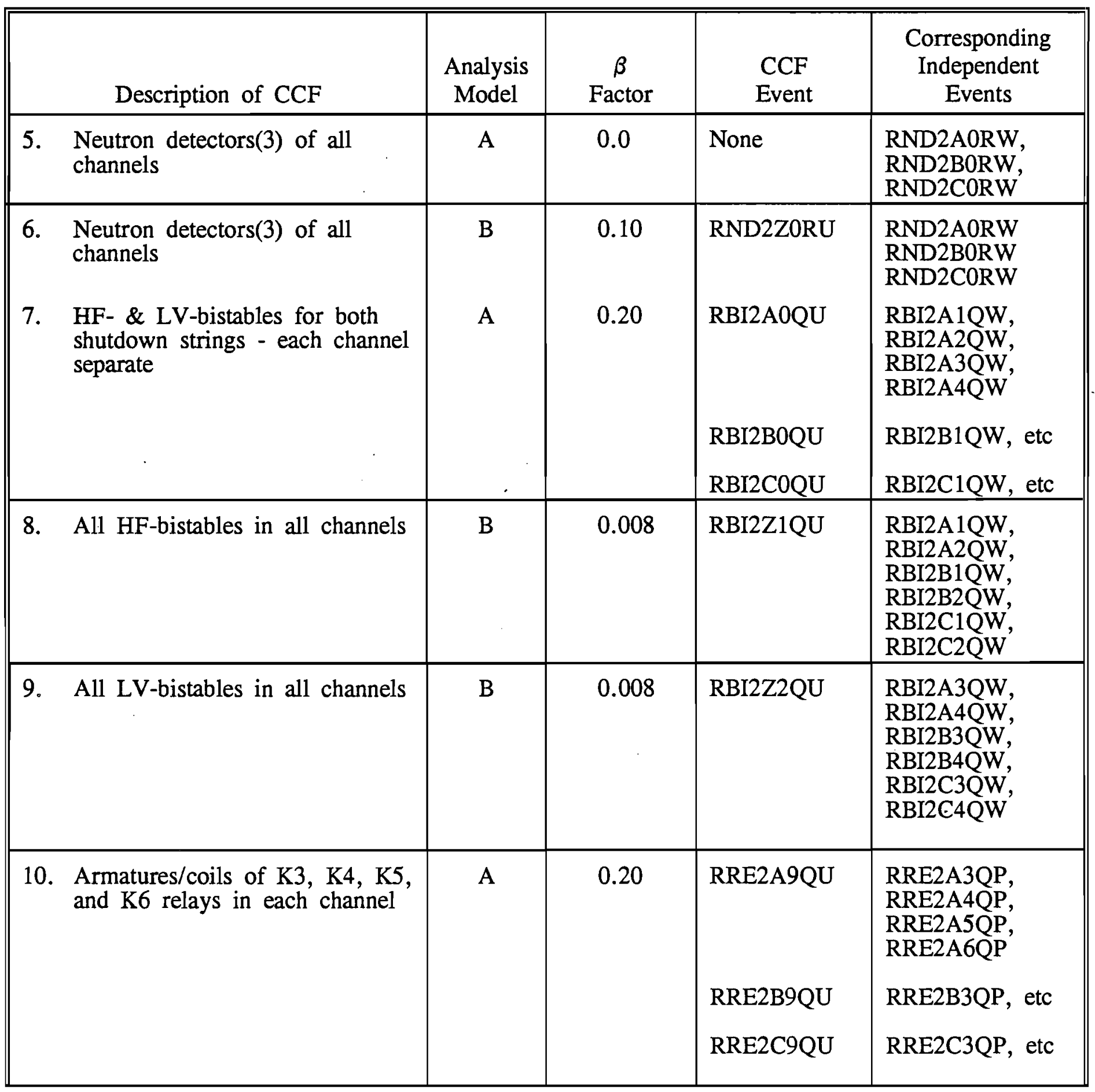


Table 7.38

LIST OF COMMON CAUSE FAILURES AMONG ELECTRICAL COMPONENTS FAULT TREE ANALYSIS OF SCRAM FAILURE UNDER LOF OR TOP CONDITION (Cont'd)

\begin{tabular}{|c|c|c|c|c|}
\hline Description of $\mathrm{CCF}$ & $\begin{array}{l}\text { Analysis } \\
\text { Model }\end{array}$ & $\begin{array}{c}\beta \\
\text { Factor }\end{array}$ & $\begin{array}{l}\text { CCF } \\
\text { Event }\end{array}$ & $\begin{array}{l}\text { Corresponding } \\
\text { Independent } \\
\text { Events }\end{array}$ \\
\hline $\begin{array}{l}\text { 11. Contacts of } \mathrm{K} 3, \mathrm{~K} 4, \mathrm{~K} 5 \text {, and } \\
\mathrm{K} 6 \text { relays in each channel }\end{array}$ & A & 0.20 & $\begin{array}{l}\text { RCZ2A9QU } \\
\text { RCZ2B9QU } \\
\text { RCZ2C9QU }\end{array}$ & $\begin{array}{l}\text { RCZ2A3QP, } \\
\text { RCZ2A4QP, } \\
\text { RCZ2A5QP, } \\
\text { RCZ2A6QP } \\
\text { RCZ2B3QP, etc } \\
\text { RCZ2C3QP, etc }\end{array}$ \\
\hline $\begin{array}{l}\text { 12. Armatures/coils of } \mathrm{K} 3 \text { and } \mathrm{K} 4 \\
\text { relays }(\mathrm{HF}) \text { in all channels }\end{array}$ & B & 0.008 & RRE2Z9QU & $\begin{array}{l}\text { RRE2A3QP, } \\
\text { RRE2A4QP, } \\
\text { RRE2B3QP, } \\
\text { RRE2B4QP, } \\
\text { RRE2C3QP, } \\
\text { RRE2C4QP }\end{array}$ \\
\hline $\begin{array}{l}\text { 13. Armatures/coils of } \mathrm{K} 5 \text { and } \mathrm{K} 6 \\
\text { relays }(\mathrm{LV}) \text { in all channels }\end{array}$ & B & 0.008 & RRE2Z8QU & $\begin{array}{l}\text { RRE2A5QP, } \\
\text { RRE2A6QP, } \\
\text { RRE2B5QP, } \\
\text { RRE2B6QP, } \\
\text { RRE2C5QP, } \\
\text { RRE2C6QP }\end{array}$ \\
\hline $\begin{array}{l}\text { 14. Contacts of } K 3 \text { and } K 4 \text { relays } \\
(\mathrm{HF}) \text { in all channels }\end{array}$ & B & 0.008 & RCZ2Z9QU & $\begin{array}{l}\text { RCZ2A3QP, } \\
\text { RCZ2A4QP, } \\
\text { RCZ2B3QP, } \\
\text { RCZ2B4QP, } \\
\text { RCZ2C3QP, } \\
\text { RCZ2C4QP }\end{array}$ \\
\hline $\begin{array}{l}\text { 15. Contacts of } \mathrm{K} 5 \text { and } \mathrm{K} 6 \text { relays } \\
\text { (LV) in all channels }\end{array}$ & B & 0.008 & RCZ2Z8QU & $\begin{array}{l}\text { RCZ2A5QP, } \\
\text { RCZ2A6QP, } \\
\text { RCZ2B5QP, } \\
\text { RCZ2B6QP, } \\
\text { RCZ2C5QP, } \\
\text { RCZ2C6QP }\end{array}$ \\
\hline
\end{tabular}


Table 7.38

LIST OF COMMON CAUSE FAILURES AMONG ELECTRICAL COMPONENTS -

FAULT TREE ANALYSIS OF SCRAM FAILURE UNDER LOF OR

TOP CONDITION (Cont'd)

\begin{tabular}{|c|c|c|c|c|}
\hline Description of CCF & $\begin{array}{l}\text { Analysis } \\
\text { Model }\end{array}$ & $\begin{array}{c}\beta \\
\text { Factor }\end{array}$ & $\begin{array}{l}\text { CCF } \\
\text { Event }\end{array}$ & $\begin{array}{l}\text { Corresponding } \\
\text { Independent } \\
\text { Events }\end{array}$ \\
\hline (ii) SOT Channels & & & & \\
\hline $\begin{array}{l}\text { 1. Armatures/coils of all trip } \\
\text { relays in all channels }\end{array}$ & B & 0.008 & RRE2Z0QU & $\begin{array}{l}\text { RRE3C1QP, etc } \\
\text { RRE3D1QP, etc } \\
\text { RRE3F1QP, etc } \\
\text { RRE3H1QP, etc }\end{array}$ \\
\hline $\begin{array}{l}\text { 2. Contacts of all trip relays in all } \\
\text { channels }\end{array}$ & B & 0.008 & RCZ2Z0QU & $\begin{array}{l}\text { RCZ3C1QP, etc } \\
\text { RCZ3D1QP, etc } \\
\text { RCZ3F1QP, etc } \\
\text { RCZ3H1QP, etc }\end{array}$ \\
\hline $\begin{array}{l}\text { 3. All bistables (Ronan X-82) in } \\
\text { all channels }\end{array}$ & B & 0.02 & RBI3Z0QU & $\begin{array}{l}\text { RBI3C1QW, } \\
\text { RBI3D1QW, } \\
\text { RBI3F1QW, } \\
\text { RBI3H1QW }\end{array}$ \\
\hline $\begin{array}{l}\text { 4. All MV/I Converters (Ronan } \\
\mathrm{X}-81 \text { ) in all channels }\end{array}$ & B & 0.02 & RVI3Z0QU & $\begin{array}{l}\text { RVI3COQW, } \\
\text { RVI3D0QW, } \\
\text { RVI3F0QW, } \\
\text { RVI3H0QW }\end{array}$ \\
\hline
\end{tabular}

Notes:

(1) In Analysis Model A, relatively large $\beta$-Factors are used to emphasize CCFs between similar components on the same Shutdown STring or same Channel. In Analysis Model B, $\beta$-Factors are based only on the number of redundant components.

(2) The bistables of channel UPFB are made by a different manufacturer.

(3) Similar $\beta$-Factors are used with:

- Cables and Connectors

- Current amplifiers

- $\quad+15 \mathrm{~V}$ dc power supplies

- $\quad-680 \mathrm{~V}$ dc power supplies

(4) The $\beta$-Factors are reduced to 0.008 when the SOT channels are part of LOF detection.

(5) Similar $\beta$-Factors are used with the SOT channels in the TOP Fault Tree. 
Table 7.39

BASIC EVENTS USED IN REPRESENTING MECHANICAL FAILURE OF CONTROL AND SAFETY RODS

\begin{tabular}{|c|c|c|}
\hline Event Name & $\begin{array}{c}\text { Failure } \\
\text { per } \\
\text { Demand }\end{array}$ & Description and Causes of Binding \\
\hline \multicolumn{3}{|l|}{ CRs \& CRDs } \\
\hline 1 CCD3OIRU & $3.10^{-7}$ & CR Drives; Foreign material or particulates in $\mathrm{Na}$ exiting assemblies \\
\hline 2 CCD20WRV & $1.10^{-7}$ & CR Drives; Failure of components located above rotating plug level \\
\hline 3 CCDI0GRU & $1.310^{-6}$ & $\begin{array}{l}\text { CR Drives; Failure of components located inside tank, mainly reactor vessel } \\
\text { cover locking locking mechanism }\end{array}$ \\
\hline 4 CSR30IRU & $1.10^{-7}$ & $\begin{array}{l}\text { CCF among CRs \& SRs; Foreign materials or particulates in Na entering } \\
\text { assemblies }\end{array}$ \\
\hline 5 CSR10GRU & $1.10^{-7}$ & $\begin{array}{l}\text { CCF among CRs \& SRs; Rods and Thimbles: Cross section distortion and } \\
\text { bowing }\end{array}$ \\
\hline 6 CCLOOPRU & $1.610^{-7}$ & Màgnetic Clutches Fail to Release CRs \\
\hline \multicolumn{3}{|l|}{ SRs \& SRDs } \\
\hline 7 SCD300RI & $6.10^{-5}$ & SR Drive; Foreign materials or particulates in $\mathrm{Na}$ \\
\hline 8 SCD200RW & $6.10^{-5}$ & SR Drive; Failure of components located above rotating plug level \\
\hline 9 SCD100RG & $6.10^{-5}$ & SR Drive; Failure of components located inside vessel \\
\hline 10 SSR300RI & $6.10^{-5}$ & SRs and Thimbles; Foreign materials or particulate in Na existing assemblies \\
\hline 11 SSR100RG & $6.10^{-5}$ & SRs and Thimbles; Cross section distortion, bowing \\
\hline 12 SSR100RG & $2.10^{-4}$ & Magnetic Clutch Fails to Release SRs \\
\hline
\end{tabular}


demand, reflecting the experimental role of EBR-II operation. [This failure probability of a single control rod agrees with the probability that one would obtain based on the number of previous EBR-II scrams.] With the generic beta factor for a nine-rod redundancy, beta $(9)=0.008$, the total failure probability becomes about $7 . .^{10-7}$ per demand. As for the two safety rods, since they are laterally connected and are inserted in the core with the same drive mechanism, the failure rate of the two rods combined is conservatively estimated at $3.10^{-4}$, which is 10 times larger than the generic value. Table 7.37 breaks down the common cause events of both the control rods and safety rods to their respective components. The purpose of this expansion, which is based on engineering judgement and familiarity with the system, is to arrive at a potential CCF between the two different types of rods. Among the five failure modes of each rod type, two have been identified as potential CCFs between the two types of rods with a combined failure probability of $2.10^{7}$ per demand. Obviously this CCF among the two types of rods sets a lower limit on the RSS availability. types of rods. Among the five failure modes of each rod type, two have been identified as potential CCFs between the two types of rods with a combined failure probability of $210^{-7}$ per demand. Obviously this CCF among the two types of rods sets a lower limit on the RSS availability. 


\section{Section 7,0 References}

7.1 J. J. Manning et al., "A Guide for Completing Input Data Forms for CREDO, A Centralized Reliability, Availability and Maintainability Analysis Center for LiquidMetal-Cooled Reactor and Test Facility Components," ORNL/TM-9892, November 1986.

7.2 "IEEE Guide to the Collection and Presentation of Electrical, Electronic, Sensing Component, and Mechanical Equipment Reliability Data for Nuclear-Power Generating Stations," IEEE Std. 500-1984, 1983.

7.3 W. K. Kahl and R. J. Borkowski, "The In-Plant Reliability Data Base for Nuclear Plant Components," NUREG/CR-3831, January 1985.

7.4 "Clinch River Breeder Reactor Plant Probabilistic Risk Assessment," CRBRP-4. (September 1984).

7.5 Argonne National Laboratory unpublished information.

7.6 Argonne National Laboratory unpublished information.

7.7 D. C. Cutforth, private communication, March 1990 .

7.8 Roger Evans, private communication, March 1990.

7.9 R. W. Schaefer, Argonne National Laboratory unpublished information (1989).

7.10 G. L. Lentz, private communication, March 1990.

7.11 R. A. Evans, private communication, March 1990. 
7.12 C. E. Matthews, private communication, March 1990.

7.13 J. R. Armstrong, private communication, March 1990.

7.14 W. F. Booty, private communication, March 1990.

7.15 E. M. Dean, private communication, March 1990.

7.16 R. W. Schaefer, Argonne National Laboratory unpublished information (1990).

7.17 R. W. King, E. C. Filewicz, J. Poloncsik, and W. H. Radtke, Argonne National Laboratory (1985).

7.18 Ron King, private communication, 1990.

7.19 L. K. Chang, Argonne National Laboratory unpublished information (1989).

7.20 L. K. Chang, M. J. Lee, and J. G. Gale, Argonne National Laboratory unpublished information (1982).

7.21 K. N. Grimm, Argonne National Laboratory unpublished information (1989).

7.22 W. H. Perry, Argonne National Laboratory unpublished information (1989).

7.23 L. K. Chang et al., "Demonstration of EBR-II Power Maneuver without Control Rod Movement," Proceeding, International Topical Meeting on Safety of Next Generation Power Reactors," Seattle, Washington, May 1-5, 1988. 
7.24 Bob Kennedy, private communication (1990).

7.25 S. A. Eide et al., "Generic Component Failure Data Base for Light Water Reactor and Liquid Sodium Reactor PRAs," EGG-SSRE-8875, prepared for the U.S. DOE by EG\&G Idaho, Inc., February 1990.

7.26 'PRA Procedure Guide', NUREG/CR-2300, Vol. 1, January 1983.

7.27 'Procedures for Treating Common Failures in Safety and Reliability Studies,' NUREG/CR-4780, Vols. 1 and 2, A. Mosleh et al., January 1989.

7.28 'Classification and Analysis of Reactor Operating Experience Involving Dependent Events,' EPRI NP-3967, K. N. Flemming and A. Mosleh, June 1985.

7.29 'Common Cause Fault Rates for Instrumentation and Control Assemblies, NUREG/CR-3289, T. R. Meachum and C. L. Atwood, May 1983.

7.30 'Data Summaries of Licensee Event Reports of Control and Drive Mechanisms at U.S. Commercial Nuclear Power Plants, January 1, 1972 to April 30, 1978,' NUREG/CR-1331, W. H. Hubble and C. F. Miller, February 1980.

7.31 B. J. Garrick et al., "Three Mile Island Unit 1 Probabilistic Risk Assessment - Data Analysis Report," Vol 5, PLG-0525, November 1987.

7.32 H. J. Reilly et al., "A Review of the Three Mile Island 1 Probabilistic Risk Assessment," NUREG/CR-5457, EG\&G Idaho, November 1989. 
8.0 HUMAN RELIABILITY ANALYSIS 


\section{-}


Table of Contents

Page

8.0 Human Reliability Analysis $\ldots \ldots \ldots \ldots \ldots \ldots \ldots \ldots$ 8 $\ldots \ldots \ldots$

8.1 General Evaluation of EBR-II Human Factors $\ldots \ldots \ldots \ldots$. . . . . .

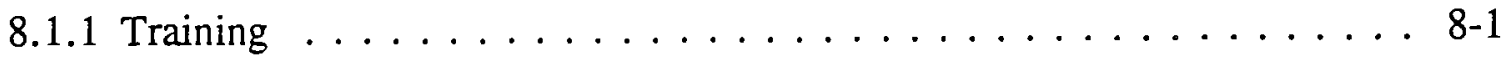

8.1 .2 Procedures . . . . . . . . . . . . . . . 8-1

8.1 .3 Maintenance . . . . . . . . . . . . . . . . . . . 8-2

8.1 .4 Maintenance History . . . . . . . . . . . . . . . . 8-2

8.2 Human Reliability Assessment $\ldots \ldots \ldots \ldots \ldots \ldots \ldots$. . . . . . . . . .

8.2.1 Initiating Events . . . . . . . . . . . . . 8-3

8.2.2 Human Error Contribution to Scram Failures . . . . . . . . . . . . . 8 8-4

8.2.2.1 Miscalibration and Misadjustment of Safety-Significant

Instrumentation . . . . . . . . . . . . 8-5

8.2.2.2 Flow Sensors . . . . . . . . . . . . . . . . . 8-5

8.2.2.3 Sub-assembly Outlet Temperature Sensor Calibrations . . . . 8-23

8.2.2.4 Wide Range Nuclear Channel Trip Points . . . . . . . . . . 8-31

8.2.2.5 Subassembly Outlet Temperature Instruments . . . . . . . . 8 8-39

8.2.2.6 Failure to Remove Mechanical Latches from Control Rod

Release Rollers . . . . . . . . . . . . . . . . . . 8-39

8.2.2.7 Manual Scram . . . . . . . . . . . . . . . . 8 8-40

8.3 Insufficient Subcriticality Margin during Refueling . . . . . . . . . 8 8-45

8.4 Other Human Errors During Operation . . . . . . . . . . . . . 8-58

8.4 .1 Valves and Dampers . . . . . . . . . . . . . . 8-58

8.4 .2 Alarms . . . . . . . . . . . . . . . 8 8-63

8.4.3 Maintenance and Testing . . . . . . . . . . . 8-63

8.4 .4 Acts of Commission . . . . . . . . . . . . . . 8-64

8.5 Human Errors During Recovery . . . . . . . . . . . . . . 8-66

8.5.1 Recovery of Electrical Systems . . . . . . . . . . . 8-66

8.5.2 Recovery of Decay Heat Removal Systems . . . . . . . . . . . 8 8-67

8.5.3 Recovery of Control, Rod Malfunctions . . . . . . . . . . . . 8 8-74

8.6 Summary . . . . . . . . . . . . . . . . . . 8-76

Section 8.0 References $\ldots \ldots \ldots \ldots \ldots \ldots$. . . . . . . . . . . . . . . . . . 
$\underline{\text { List of Figures }}$

$\underline{\text { Page }}$

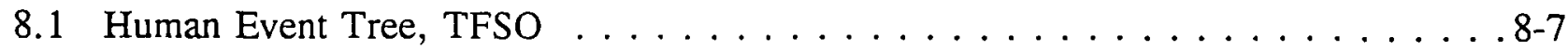

8.2 Human Event Tree, HPPF . . . . . . . . . . . . . . . . . 8-11

8.3 Human Event Tree, LPPF . . . . . . . . . . . . . . . . . . 8-14

8.4 Human Event Tree, $\mathrm{TSOF} \ldots \ldots \ldots \ldots \ldots \ldots \ldots \ldots \ldots \ldots$

8.5 Human Event Tree, UPFB . . . . . . . . . . . . . . . . . . . . . . . . . . . . . . . . . . . . . . . . . .

8.6 Human Event Tree, SOTC . . . . . . . . . . . . . . . . . . . . . . . . . . . . . . . . . . . . . . . . . . . . . . .

8.7 Human Event Tree, SOTT . . . . . . . . . . . . . . . . . . 8-28

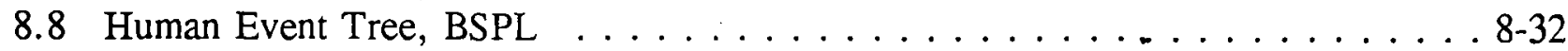

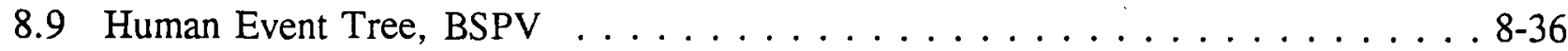

8.10 Human Event Tree, SCRM . . . . . . . . . . . . . . . . . 8-41

8.11 Human Event Tree, CLPE . . . . . . . . . . . . . . . . . . . . 8-47

8.12 Human Event Tree, FHHU . . . . . . . . . . . . . . . . . . . . . . . . . . . . . . . . . . . . . . . .

8.13 Human Event Tree, FHCR . . . . . . . . . . . . . . . . . . . . . . . . . . . . . . . . . . . . . . . . . . . .

8.14 Human Event Tree, FHSP . . . . . . . . . . . . . . . . . . 8-59

8.15 Human Event Tree, ARGI . . . . . . . . . . . . . . . . . 8-67 
List of Tables

$\underline{\text { Page }}$

8.1 HEPs for Human Event Tree TFSO . . . . . . . . . . . . . . . . . . 8-8

8.2 Failure Paths and Total Failure/Success Probabilities

for Human Event Tree TFSO . . . . . . . . . . . . . . . . . . . . . . . . . 8 8-9

8.3 HEPs for Human Event Tree HPPF . . . . . . . . . . . . . . . . . . . 8 8-12

8.4 Failure Paths and Total Failure/Success Probabilities

for Human Event Tree HPPF . . . . . . . . . . . . . . . . . . . . . 8-13

8.5 HEPs for Human Event Tree LPPF . . . . . . . . . . . . . . . . . . . . 8-15

8.6 Failure Paths and Total Failure/Success Probabilities

for Human Event Tree LPPF . . . . . . . . . . . . . . . . . . . . . . 8 8-16

8.7 HEPs for Human Event Tree TSOF . . . . . . . . . . . . . . . . . . 8-18

8.8 Failure Paths and Total Failure/Success Probabilities

for Human Event Tree TSOF . . . . . . . . . . . . . . . . . . . . . . . . 8-19

8.9 HEPs for Human Event Tree UPFB . . . . . . . . . . . . . . . . . . . . 8-21

8.10 Failure Paths and Total Failure/Success Probabilities

for Human Event Tree UPFB . . . . . . . . . . . . . . . . . . . . . . . 8 8-22

8.11 HEPs for Human Event Tree SOTC . . . . . . . . . . . . . . . . . . . 8-25

8.12 Failure Paths and Total Failure/Success Probabilities

8.13 HEPs for Human Event Tree SOTT . . . . . . . . . . . . . . . . . . . . . 8-29

8.14 Failure Paths and Total Failure/Success Probabilities

8.15 HEPs for Human Event Tree BSPL . . . . . . . . . . . . . . . . . . . . 8 8-33

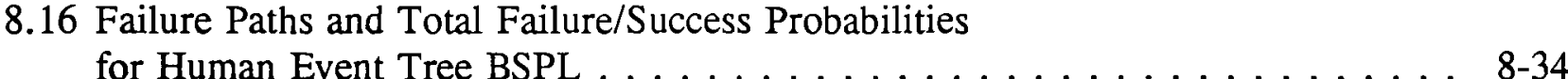

8.17 HEPs for Human Event Tree BSPV . . . . . . . . . . . . . . . . 8-37

8.18 Failure Paths and Total Failure/Success Probabilities
for Human Event Tree BSPV

8.19 HEPs for Human Event Tree SCRM . . . . . . . . . . . . . . . . . . 8-42

8.20 Failure Paths and Total Failure/Success Probabilities

for Human Event Tree SCRM . . . . . . . . . . . . . . . . . . . . . . 8 8-43

8.21 HEPs for Human Event Tree CLPE . . . . . . . . . . . . . . . . . . . . 8-48

8.22 Failure Paths and Total Failure/Success Probabilities

for Human Event Tree CLPE . . . . . . . . . . . . . . . . . . . . . . . . 8 8-49

8.23 HEPs for Human Event Tree FHHU . . . . . . . . . . . . . . . . . . 8 8-51

8.24 Failure Paths and Total Failure/Success Probabilities

for Human Event Tree FHHU . . . . . . . . . . . . . . . . . . . . . . 8-53

8.25 HEPs for Human Event Tree FHCR . . . . . . . . . . . . . . . . . . . 8 8-56

8.26 Failure Paths and Total Failure/Success Probabilities
for Human Event Tree FHCR . . . . . . . . . . . . . . . . . 8-57

8.27 HEPs for Human Event Tree FHSP . . . . . . . . . . . . . . . . . . . . 8 8-60 
8.28 Failure Paths and Total Failure/Success Probabilities for Human Event Tree FHSP $\ldots \ldots \ldots \ldots \ldots$. . . . . . . . . . . . . . .

8.29 HEPs for Human Event Tree ARGI . . . . . . . . . . . . . . . . . . . . . 8-69

8.30 Failure Paths and Total Failure/Success Probabilities for Human Event Tree ARGI . . . . . . . . . . . . . . . 8-70 


\subsection{HUMAN RELIABILITY ANALYSIS}

Because of the passive safety features of the EBR-II design, the contributions of human error to core damage sequences are those associated with failure to scram and failure to recover decay heat removal. The events which can effect scram reliability can be promulgated by: 1) errors in calibration and setting of trip points and 2) failure to effect manual scram. The human failure sequences lending to these errors have been examined in detail; the resulting relatively high human reliability is primarily due to reactor design, with strong contributions from EBR-II operator training, operating and maintenance procedures, and a high degree of professionalism demonstrated by EBR-II personnel. There are a few events which influence the decay heat removal capability. These events are predominantly failure to recover events because of the passive nature of the decay heat removal function.

\subsection{General Evaluation of EBR-II Human Factors}

\subsubsection{Training}

Operator training at EBR-II is extensive in terms of system and reactor knowledge presented. For example, current training emphasizes a thorough knowledge of functional design and characteristics of the Reactor Shutdown System (RSS). This knowledge is tested in both new and requalification training by random selection of test items that require operators to state consequences of failure of components and cascade effects of those failures,

as well as expected indications associated with those failures. The operators of EBR-II have an extensive knowledge of the physics, operation, and design of the plant.

\subsubsection{Procedures}

Currently, the format and layout of operating and maintenance procedures are in line with updated MIL Standards [8.1] and NUREG-0899 [8.2]. The procedures are 
excellent in terms of their requirements for check-off and sign-off actions. Observation indicates that these sign-off provisions are usable and are used. Extensive cross-checking of system operability required during start-up and approach-to-power sequences add to the safety of both these sequences and full-power operation. The net result of these procedural requirements is to make reactor startup of the EBR-II a cautious and deliberate enhancement to safety.

\subsubsection{Maintenance}

- The EBR-II facilities are well lighted and spacious in general, especially when compared with most LWR facilities. Most areas in which maintenance personnel work have ample space and generally good environmental conditions, including low radiation levels. It is worth noting that most calibration and setting of trip-points for safety significant instrumentation occur in the cable-routing room. This room is limited access, with notification of the control room required. It is spacious and well lighted. The instruments, relays, etc. are located within locked cabinets in this room. The instruments themselves are clearly labeled and color coded. Instruments themselves are easily accessible with ample room for maintenance or replacement activities.

\subsubsection{Maintenance History}

A review of Unusual Occurrence Reports (UORs) for the last ten years was performed. The overall rate of human error-related UORs is low, on the order of 4 per year. There has been only an average of about one scram per year that was attributable to maintenance/equipment failures related to maintenance personnel error. The philosophy of performing most maintenance in shut-down conditions reduces the amount of lost power and challenges to the safety systems due to maintenance errors. The low number of maintenance errors that contribute to either UORs or scrams supports the observation that maintenance procedures are adequate and contribute minimal risk to the operation of this reactor. 


\subsection{Human Reliability Assessment}

The investigation of human errors that could initiate core damage sequences at EBR-II was performed by means of personal interviews, reviews of relevant operating and maintenance procedures, reviews of the control console, review of internal documentation related to the PRA effort, and reviews of EBR-II Technical Specifications. Interviews were held with personnel from Training and Procedures, Critical Systems Maintenance, Instrumentation and Control Computer Applications and Program Development, and Operations Analysis. The analyses were performed by following the procedures outlined in NUREG/CR-1278, the "THERP Handbook". [8.3]. In particular, a human error probability of $10^{-6}$ was selected as a lower limit, as suggested in THERP.

\subsubsection{Initiating Events}

Three major classes of transient events related to human error were isolated as being most likely to lead to core vulnerability or plant unavailability. These events are:

1. Loss of Flow (LOF)

2. Loss of Heat Sink (LOHS)

3. Transient Over Power (TOP).

The first human reliability analysis that was performed was the evaluation of the contribution of human error to the initiation of these events. Operator actions are one of the primary contributors to risk in most reactor PRAs. The analysis was performed by review of operating procedures and discussions with operators and shift supervisors. The conclusion is that human error does not contribute to initiating sequences that are included in the cutsets. 
There are several reasons for the lack of human contribution to initiation of these sequences. Most of the reasons are a result of the design of the reactor, while the remaining reasons derive from the operating philosophy and practices as embodied in the procedures. For example human error contribution to initiation of LOF or TOP events is largely precluded by the reactor design. Operators and maintainers cannot inadvertently trip the main pumps because switches and controls that shutoff these pumps are electrically locked out of operation when RSS is activated at reactor startup. Although operators can (and have) inadvertently added reactivity at full power, only one control rod can be inserted at one time, due to electrical interlocks.

The EBR-II reactor startup is a slow and deliberate procedure that is very safe. Startup is preceded by a full interlock check of all safety and operating systems. The startup procedure begins slowly at minimal $(50 \mathrm{~W})$ power and has hold points at every $10 \mathrm{MW}$ stage for further checklist and calibration checks until reaching authorized power (typically $62.5 \mathrm{MW}$ ). These repeated hold-and-check points makes startup a lengthy process, but reduces risk to a very low value. These repeated checks also reduce the probability of undetected and uncorrected human errors to a very low value.

\subsubsection{Human Error Contribution to Scram Failures}

Human error during maintenance activities can contribute to failure of actuation of the automatic reactor shutdown system (scram system). Once the automatic system has failed, human operator actions may effect recovery actions (primarily manual scram).

There are two categories in which maintenance activities can contribute to failure of the scram system: 1) Instrumentation, and 2) Mechanical. Maintenance activities on instrumentation can cause or contribute to instrumentation failure through miscalibration and/or misadjustment of trip setpoints. Maintenance activities can cause or contribute to mechanical failure through faulty mechanical latching of control rod latch (release) rollers. These two categories will be treated separately below. 


\subsubsection{Miscalibration and Misadjustment of Safety-Significant Instrumentation}

The primary instrumentation to initiate a scram following an LOF event are the Flow Sensors, which are backed up by the Sub-Assembly Outlet Temperature (SOT) instruments. That is, if flow is reduced or lost, sub-assembly outlet temperatures would immediately begin to rise. Thus, if flow indication was inaccurate or disabled, sub-assembly outlet temperatures would give indications of low or lost flow and initiate a scram. The primary instrumentation to initiate scram in case of a TOP event is the Wide Range Nuclear Channels (A, B, and C) - again, backed up by the SOT instruments. Increases in power will also increase temperatures within the core, leading to increased SOT indications. All calibration and adjustment procedures are performed in the cable routing room, a limited access (keycard) room, where telephone notification to the control room of entry and purpose is required.

\subsubsection{Flow Sensors}

\section{Failure to Properly Calibrate Sensors, $p=\sim 0$}

The flow sensors in the scram strings are the Low Pressure Plenum Flow for pump \#2, High Pressure Plenum Flow for pump \#2, the Total Reactor Outlet Flow at the outlet of the reactor coolant pipe, and the Upper Plenum Flow at the reactor upper plenum. The calibration procedures for these instruments were examined, but detailed analysis was not performed. The probability of undetected miscalibration was judged to be nearly zero for the following reasons:

1. The flow sensor instruments are set to relative readings $(100 \%)$. Miscalibration would result in a power mismatch for the flow ratings at $100 \%$, which would cause a halt in the approach to power and re-examination of several instrument calibrations. 
2. The flow is checked during start up and again during approach to power and, finally, after reaching full power operation.

3. Flow readings are taken every four hours during normal operation.

Event RHU1010L, Failure to Properly Perform Flow Sensor Operability Check: $p=$ $1.510^{-3}$ (Ref. Human Event Tree TFSO, Figure 8.1, Tables 8.1 and 8.2)

Sensor operability and trip point verification procedures were reviewed for these flow sensor instruments and a detailed Human Reliability Analysis (HRA) was judged to be warranted.

The operability check is performed at startup, and weekly while operating at authorized power. To perform this procedure, the technician requests that the operators increase flow by $2 \%$. The technician observes and records the change in flow readings on all four channels. The probability of error in this check on one channel is $1.510^{-3}$. This value is deemed appropriate because the operability test is repeated weekly during normal operations, reducing the probability of unnoticed loss of operability. This human error essentially increases the test interval of the checked components from one week to two weeks. As a result, the net effect on the failure probability of flow channels is insignificant. 


\section{HRA TREE DIAGRAM}

10: TFSO I\& C Tedh icres flow sensor operability. (STM-T-35(7) 10189)

Sequence RHUIOIQL

Date modilied: 26-MLAR-90

Analyst: Sc/urmion

NOTES: This is e tech. spec. start up item Perfomied in sable rouring room which has aceess controlled by operators. It is a two-man job with one man acting as a checker on each step and signing off on the complesion

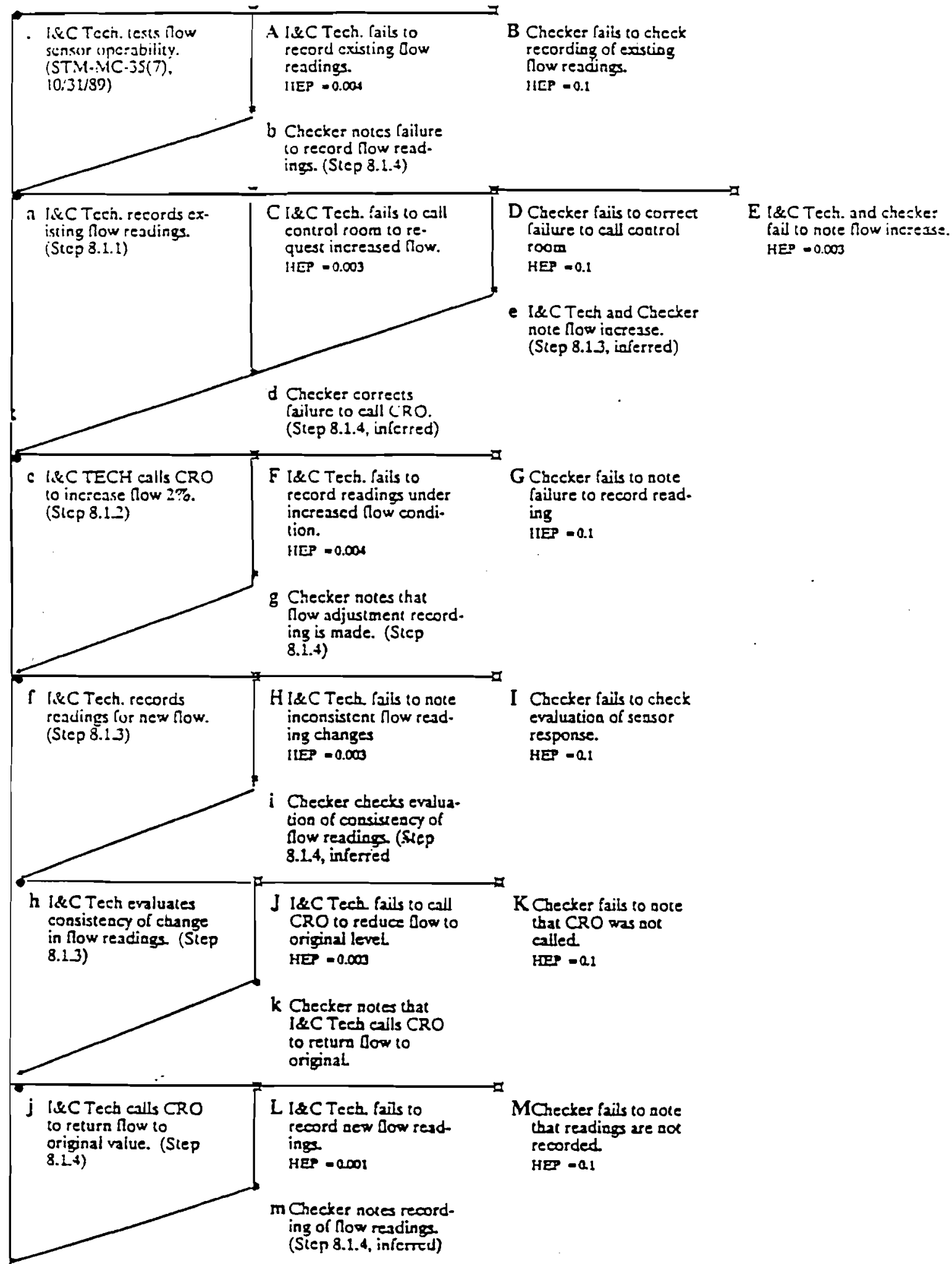

I likC Tech reeords new now readings

Fig. 8.1. Human Event Tree, TFSO 
Table 8.1. HEPs for Human Event Tree TFSO

\begin{tabular}{|c|c|c|c|c|c|c|c|c|}
\hline & Iluman Acllon/Error & $\begin{array}{l}\text { Music llEP } \\
\text { (Error Fuclor) }\end{array}$ & $\begin{array}{c}\text { TIIEKP } \\
\text { Source } \\
\text { Tuble (IIem) }\end{array}$ & $\begin{array}{l}\text { Siep-loy- } \\
\text { Siep (Sffs) } \\
\text { Dynumic } \\
\text { (I)YN) }\end{array}$ & $\begin{array}{l}\text { Modilier } \\
\text { for Sirtess, } \\
\text { Expperienter. } \\
\text { SIIIS or IIYN } \\
\end{array}$ & 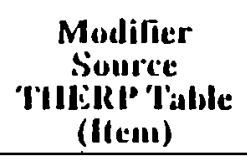 & Dependency & $\begin{array}{l}\text { Nominal llEP - } \\
\text { (Error Faclor) }\end{array}$ \\
\hline $\mathbf{A}$ & $\begin{array}{l}\text { I\&CTecly. fails to record existing } \\
\text { nlow readings. }\end{array}$ & $0.0 \times 1) 4$ & l'able 20-10, (9) & S1IS & 1 & & $21)$ & $0.004(3)$ \\
\hline B & $\begin{array}{l}\text { Checker fails lo check recording of } \\
\text { existing now readings. }\end{array}$ & 0.1 & Table 20-22, (1) & SIISS & 1 & & $2(1)$ & $0.1(5)$ \\
\hline $\mathbf{C}$ & $\begin{array}{l}\text { 1\&C Tech. fails to call conlrol room } \\
\text { lo request increased flow. }\end{array}$ & 0.003 & Table 20-7, (3) & SIIS & 1 & & $\angle O$ & $0.0 \times 13(.3)$ \\
\hline D & $\begin{array}{l}\text { Checker fails to correct failure to call } \\
\text { control room }\end{array}$ & 0.1 & Table 20-22 (1) & DYN & 1 & & $\mathrm{ZI})$ & $0.1(5)$ \\
\hline IC & $\begin{array}{l}\text { I\& C Tech. and checker fail to note } \\
\text { flow increase. }\end{array}$ & 0.003 & 'l'able 20-7 (3) & DYN & 1 & & $\mathrm{Z1)}$ & $(0.0 \times 13(3)$ \\
\hline $\mathbf{F}$ & $\begin{array}{l}\text { I\&C Tech. fails lo record readings } \\
\text { under increased flow condition. }\end{array}$ & 0.004 & Table 20-10 (9) & SBS & 1 & & $\mathrm{ZI}$ & $0.1 \times 144(3)$ \\
\hline G & $\begin{array}{l}\text { Checker fails to note failure to } \\
\text { record reading }\end{array}$ & 0.1 & Table 20-22(1) & SIBS & 1 & & $\mathrm{ZI})$ & $0.1(5)$ \\
\hline 11 & $\begin{array}{l}\text { I\&C Tech. fails } 10 \text { note inconsistent } \\
\text { flow reading claanges }\end{array}$ & $0 .(x) .3$ & I'able 20-10 (1) & SisS & 1 & & $\mathrm{ZII}$ & $0.0 \times 13(3)$ \\
\hline I & $\begin{array}{l}\text { Checker fails to check evaluation of } \\
\text { sensor response. }\end{array}$ & 0.1 & I'alslc 20-22(1) & SBS & 1 & & $2(1)$ & $0.1(5)$ \\
\hline J & $\begin{array}{l}\text { 1\&CTech. fails to call C.R() 10 } \\
\text { reduce flow to original level: }\end{array}$ & $0.0 \times 13$ & 'Iable 20-7 (3) & SIBS & $\mathbf{I}$ & & $\mathrm{ZI}$ & $0 .(x) .3(5)$ \\
\hline K & $\begin{array}{l}\text { Checker fails to note that CR() was } \\
\text { not called. }\end{array}$ & 0.1 & 'lable 20-22 (1) & I)YN & 1 & & $\mathrm{ZID}$ & $0.1(5)$ \\
\hline 1. & $\begin{array}{l}\text { I\& CTech. fails to record new flow } \\
\text { readings. }\end{array}$ & $0 .(\mathbf{0}) 1$ & Table 20-7 (1) & SIBS & 1 & & $\mathrm{ZI)}$ & $0 .(x) 1(3)$ \\
\hline & $\begin{array}{l}\text { (heck ar.. fails lo note that reatlings } \\
\text { ance corded. }\end{array}$ & 0.1 & l'able 20-22 (1) & 1)YN & 1 & & $(1)$ & 1) 1 (5) \\
\hline
\end{tabular}




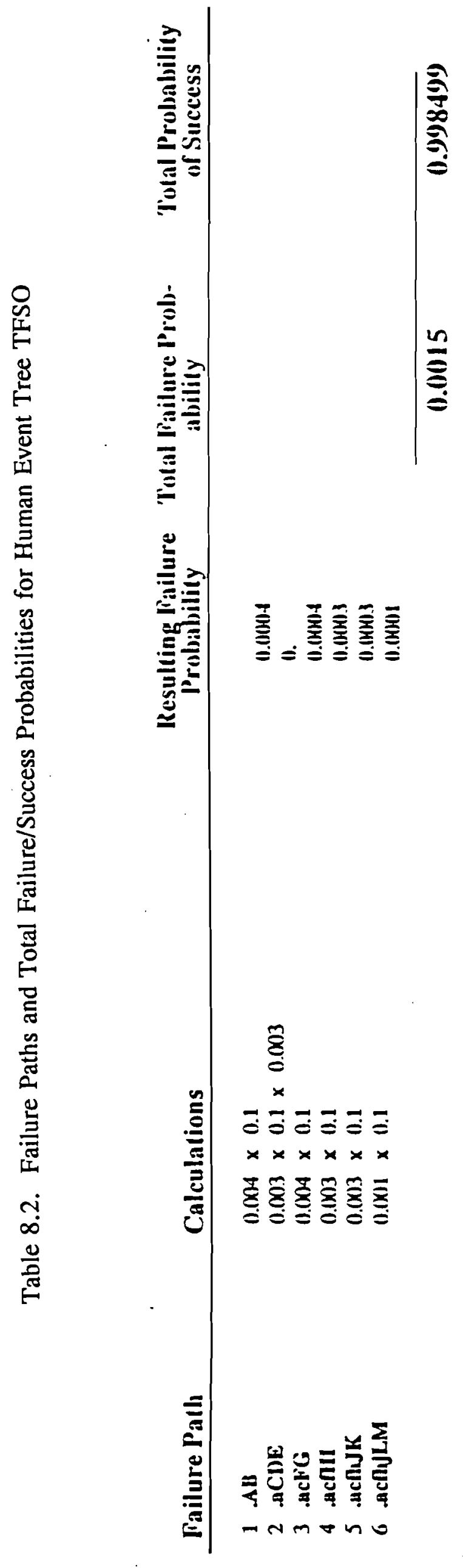


Events RHU1A1OL, RHU1B1OL, RHU1C1OL and RHU1D1OL, Failure to Properly Verify Flow Sensor Trip Point Operability: $p=310^{-6}$ (Ref. Human Event Trees LPPF, HPPF, TSOF, UPFB, Figures 8.2 to 8.5 . Tables 8.3 to 8.10 )

The flow sensor trip points are calibrated annually and are also verified during startup and weekly during authorized power. These verifications are performed by switching each instrument to CALIBRATE position and back to NORMAL, sequentially. Verification is performed by means of built-in test equipment. When any channel is in CALIBRATE position, it is tripped, and a TRIP signal is displayed in the control room - thus, the system is working on a 1 of 3 trip logic during this period. If the technician places a channel in CALIBRATE without returning the previous channel to NORMAL, the plant will trip on a low flow condition. Prior to startup, the trip points are operationally checked by lowering flow to $85 \%$ and verifying that the sensors all trip. Verification is made by means of a built-in test circuit on each channel when the reactor is critical. The probability of failure to verify trip points for each channel is $310^{-6}$. These failures are judged to be independent, since each instrument is in a separate locked cabinet and one channel at a time is adjusted, then the cabinet relocked and the next cabinet unlocked. Credit was not taken for the fact that there is a checker assigned to observe the technician during these adjustments. Similar to the above, these human errors would essentially increase the test interval of the checked components from one week to two weeks. As a result, their net effect on the failure probability of their respective channels is insignificant. In the present LOF fault tree, these human errors are conservatively modeled as events that lead to channel failure but at a reduced probability of $110^{-6}$. 


\title{
HRA TREE DIAGRAM
}

\author{
ID: $\quad$ HPPF Tech. fails to accuratehy set pump \#2 HP plenum trip-point \\ Sequence: RHULA1QL \\ Date modiried: $\quad 18-\pi N-90$ \\ Analyst: Scrumar \\ NOTES: I\& C Tech. fails to set the pump \#2 HP plenum trip point at proper value. $\quad$ (STM-T-35, 10/31:S9)
}

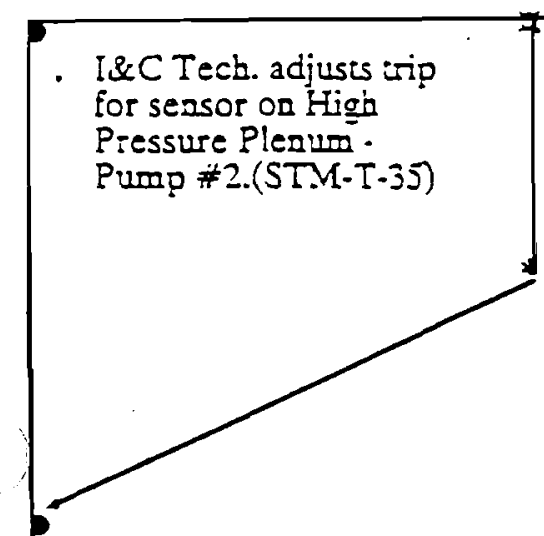

A Tech. fails to accurateIy set trip point during Annual Calibration.

HEP $=0.003$

b Pre-Startup Operational Interlock check dis. covers misadjustment.(STMMC-92(39))

a I느 Tech. accurately sets trip point. (St=0 8.2.3)

B Pie-Starrp Operation-

al Interlock check fails to discover misadjustmeat.

HEP $=0.001$

Fig. 8.2. Human Event Tree, HPPF 


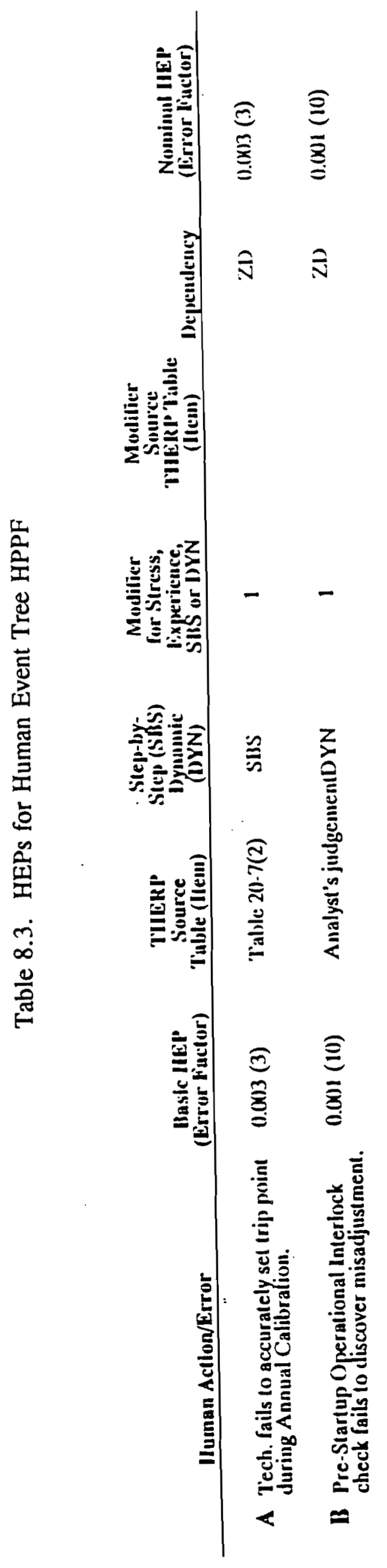




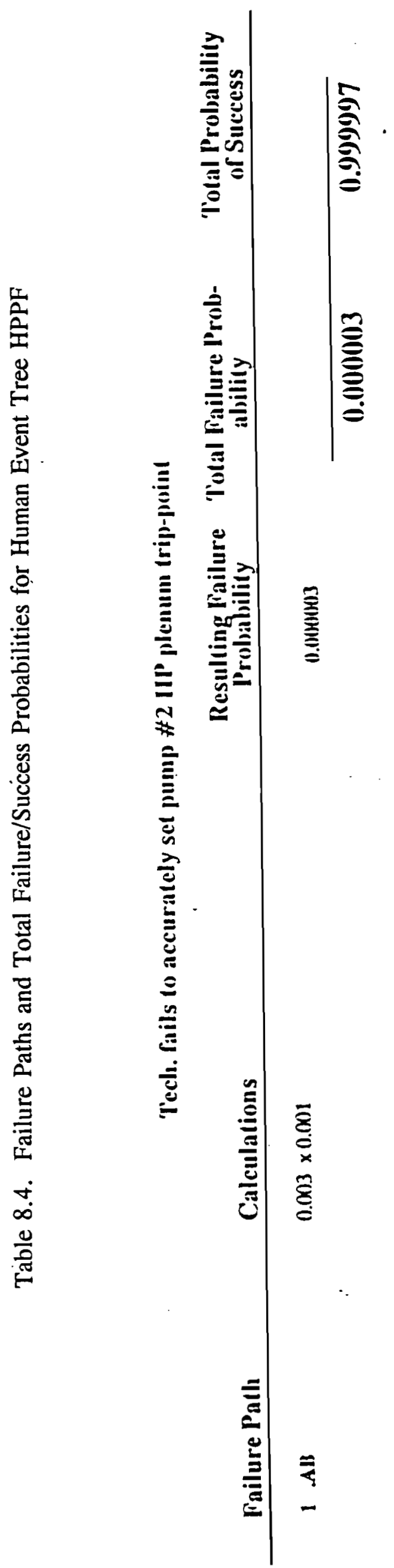




\title{
HRA TREE DIAGRAM
}

\author{
ID: LPPF Tech. fails to accurately set pump \#2 LP plenum trip-point \\ Sequence: $\quad R H U 1 B I Q L$ \\ Date modified: $\quad I O A A P R-90$ \\ Analyst: Schurmen
}

NOTES: I\& C Tech. fails to set the pump $\stackrel{\|}{\pi} 2 \mathrm{LP}$ plenum tip point at proper value.

(ST.M-T-35(7) 10i31/69)

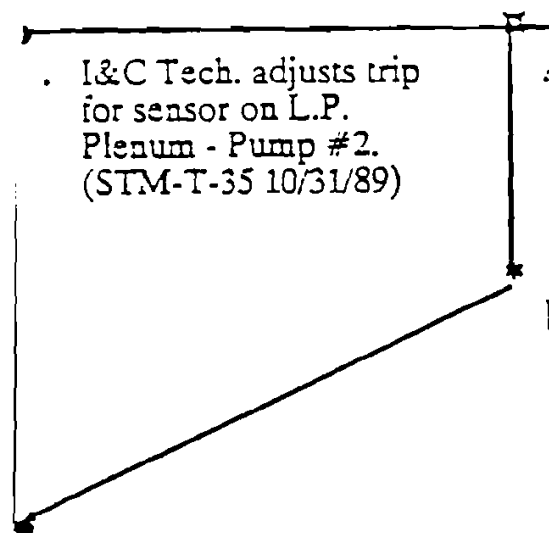

A I\&C Tech. fails to accurately set trip for Low Pressure Plenum on Purnp \#2.

HEP $=0.003$

b Pre-Startup Operabiliry Interlock check discovers misad. justment.(STM-MC. 92(39)

a I\&C Tech. accurately sets trip for $L$. P. Plequm - Pump \#2. (Step 8.2.2)

B Pre-Startup

Operabiiiry Interlock check fails to discover misadjustment.

HEP $=0.001$

Fig. 8.3 Human Event Tree, LPPF 


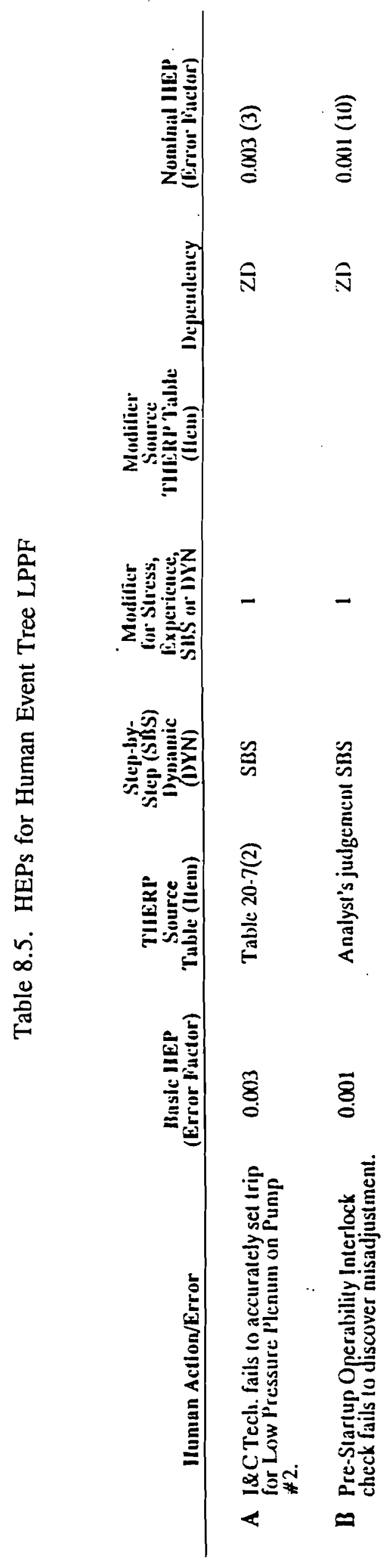




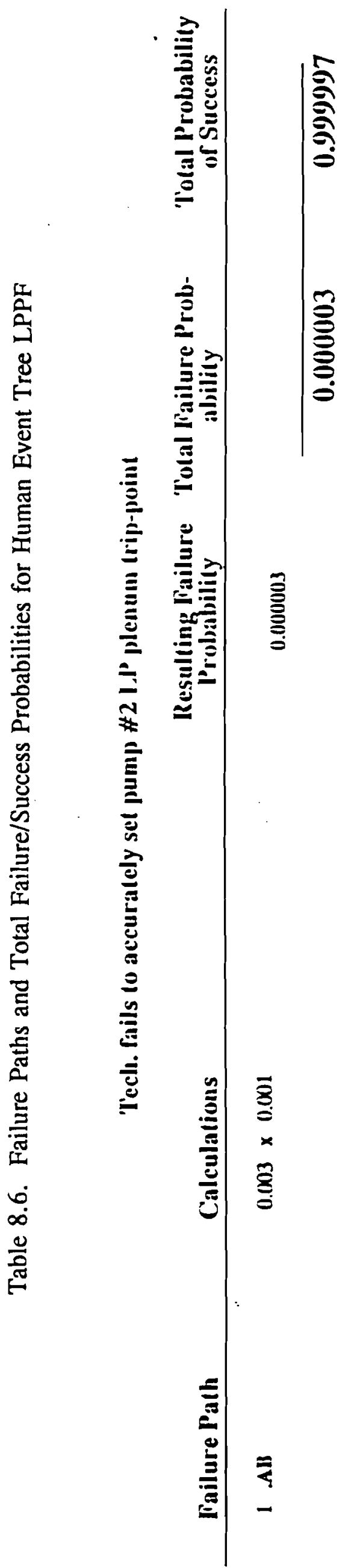




\section{HRA TREE DIAGRAM}

ID: TSOF Tech. fails to accurately set total flow trip-point

Sequence: $R H U I C I Q L$

Date modified: $\quad$ 10-APR-90

Analyst: Schumnan

NOTES: I\& C Tech. fails to set the trip point for the total-flow sensor.

$(S T M-T-35(7) 10 ; 31 / 89)$

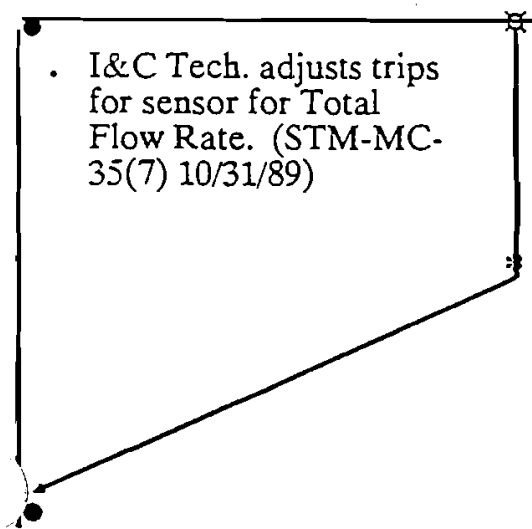

A I\&C Tech. fails to accurately adjust trips for Total Flow Rate.

HEP $=0.003$

b Pre-Startup Operability Interlock check discovers misadjustment.(STM-MC92(39))
B Pre-Startup Operability Interlock check fails to discover misadjustment. HEP $=0.001$

a I\&C Tech. accurately adjusts trips for Total

Flow Rate. (Step 8.2.4)

Fig. 8.4. Human Event Tree, TSOF 


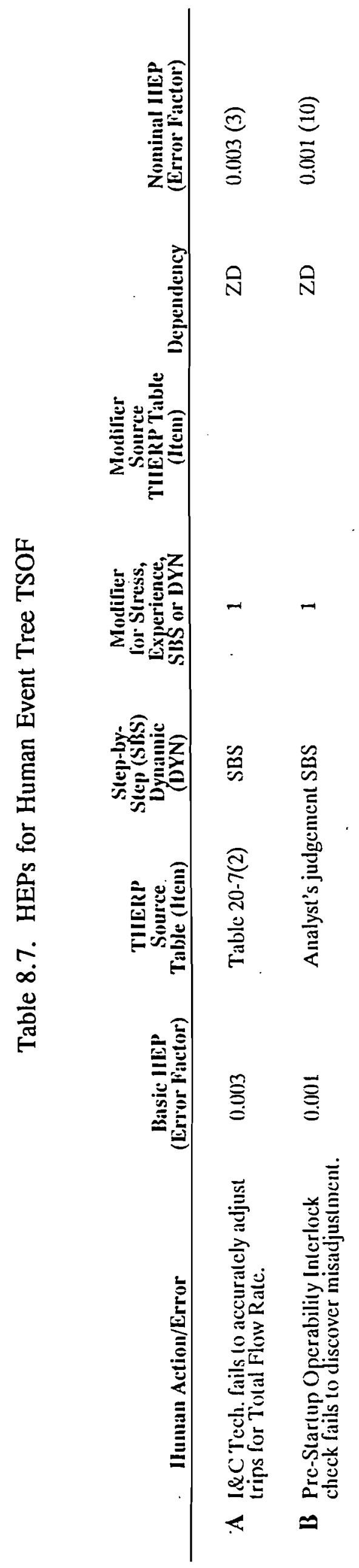




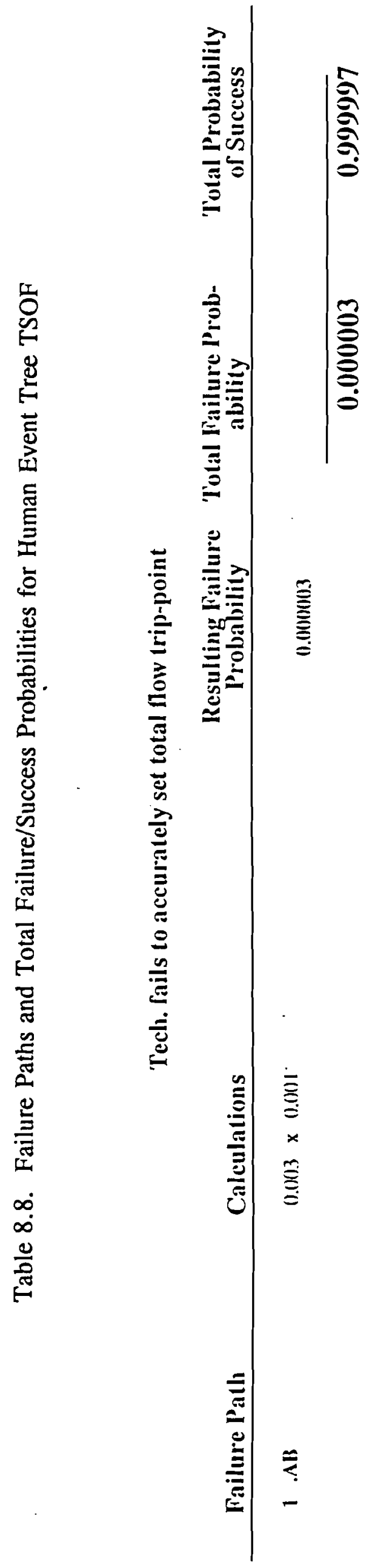




\title{
HRA TREE DIAGRAM
}

\author{
ID: UPFB Tech. fails to accurately set upper plenum trip-point \\ Sequence: $\quad R H U 1 D I Q L$ \\ Date modified: $\quad 10-A P R-90$ \\ Analyst: Schumnan \\ NOTES: I\& C Tech. fails to set the trip point for the upper plenum sensor.
}

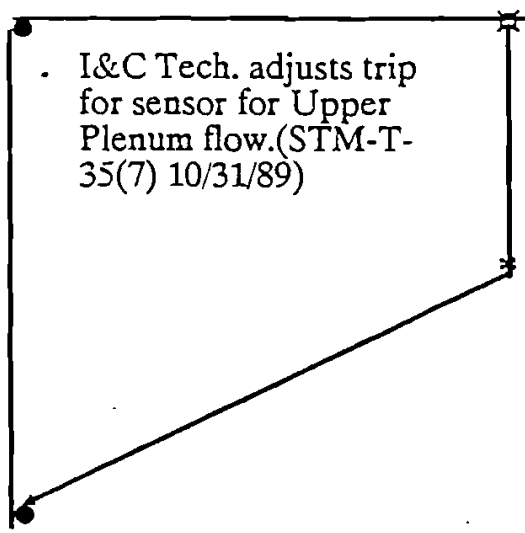
A I\&C Tech. fails to ac- curately adjust trip for Upper Plenum flow. HEP $=0.003$
b Pre-Startisp
Operability Interlock
check discovers misad- justment.(STM-MC- 92(39))

B Pre-Startup Operability Interlock check fails to discover misadjustment.

HEP $=0.001$

a I\&C Tech. accurately adjusts trip for Upper Plenum flow. (Step 8.2.1)

Fig. 8.5. Human Event Tree, UPFB 
Table 8.9. HEPs for Human Event Tree UPFB

\begin{tabular}{|c|c|c|c|c|c|c|c|c|}
\hline & Iluman Action/Error & $\begin{array}{c}\text { Bassic IIEP } \\
\text { (Error l'actor) }\end{array}$ & $\begin{array}{c}\text { TIIERP } \\
\text { Source } \\
\text { Table (Item) } \\
\end{array}$ & $\begin{array}{l}\text { Siep-lyy- } \\
\text { Step (SBSS) } \\
\text { Dymanic } \\
\text { (I)YN) }\end{array}$ & $\begin{array}{l}\text { Modilier } \\
\text { for Stress, } \\
\text { fixperience, } \\
\text { SIBS or DYYN } \\
\end{array}$ & $\begin{array}{l}\text { Moditier } \\
\text { Source } \\
\text { TIIERR'Table } \\
\text { (Item) }\end{array}$ & Dependency & $\begin{array}{l}\text { Nominal IIEP } \\
\text { (Error liattor) }\end{array}$ \\
\hline A & $\begin{array}{l}\text { I\&C Tech. fails to accurately adjust } \\
\text { trip for Upper Plenum flow. }\end{array}$ & $0.003(3)$ & Table $20-7(2)$ & SBS & 1 & & $\mathrm{ZD}$ & $0.003(3)$ \\
\hline B & $\begin{array}{l}\text { Pre-Startup Operability Interlock } \\
\text { check fails to discover misadjustment. }\end{array}$ & $0.001(10)$ & \multicolumn{2}{|c|}{ Analyst's judgement SBS } & 1 & & $\mathrm{ZD}$ & $0.001(10)$ \\
\hline
\end{tabular}




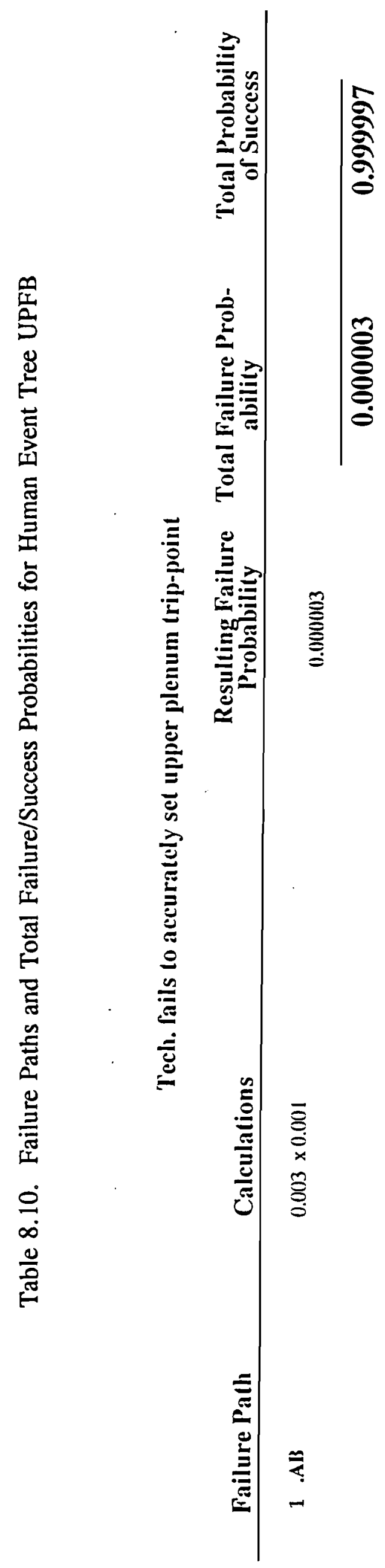




\subsubsection{Sub-assembly Outlet Temperature Sensor Calibrations}

The calibration and adjustment procedures were reviewed for the Sub-assembly Outlet Temperature (SOT) instruments. These procedures were found to be complex and required the calibration of several subunits for successful completion; furthermore no operability check is possible. Therefore detailed HRA of the calibration procedures was judged to be necessary.

Event RHU3AQL Calibration, Time Response Test of Core Subassembly Thermocouples: $p=610^{-4}$ (Ref. Human Event Tree SOTC in Figure 8.6. Tables 8.11 and 8.12)

The calibration procedure is performed annually. The procedure requires the adjustment of a number of amplifiers and detectors in series. Subsequent to calibration, timed tests of trip function are performed. The estimated probability of failing to perform the calibration accurately, and that subsequent check steps would not catch the error, is $610^{-4}$. Since these calibration steps are performed separately on the four channels, three of four must be miscalibrated for consequences to occur, and since each channel is located in a separate, locked cabinet - the four channels are assumed to be independently calibrated.

Event RHU302QL, Set Sub-assembly Outlet Temperature Sensor Trip-points: $\mathrm{p}=2.510^{-2}$ (Ref. Human Event Tree SOTT in Figure 8.7. Tables 8.13 and 8.14)

The setting of the trip points for the SOT channels consists of making a setting of each of the Ronan High Temperature Alarms to a value calculated by the control room operators and checked against estimates in the operating plan. It is performed at start up, at $50 \%$ power, and again when authorized power is reached. This activity is covered by a one-sentence procedure in the Startup Procedure (II-B). It is unlikely that the technician actually reads that procedure for each setting. There is often a second technician present who acts as a checker, but the checker is not procedurally required. The settings are made quickly and in order, so they cannot 


\section{HRA TREE DLAGRAM}

II): SOTC Calibrate SOT Channeis (one at a time)

Se'luence: RHL3AIQL, RHU3BIQL, RHU 3CIQL, RHCIDIQL

Date inodilicd: SS-JUN-90

Anilyst: Schumnan

NOTES: Calibration of Sub-4ssembly Outed Tenuperanure Clanuds (4 ea.) one at a time. (STM-MC-61 Rew. 7. $2 / 21 /(\$))$

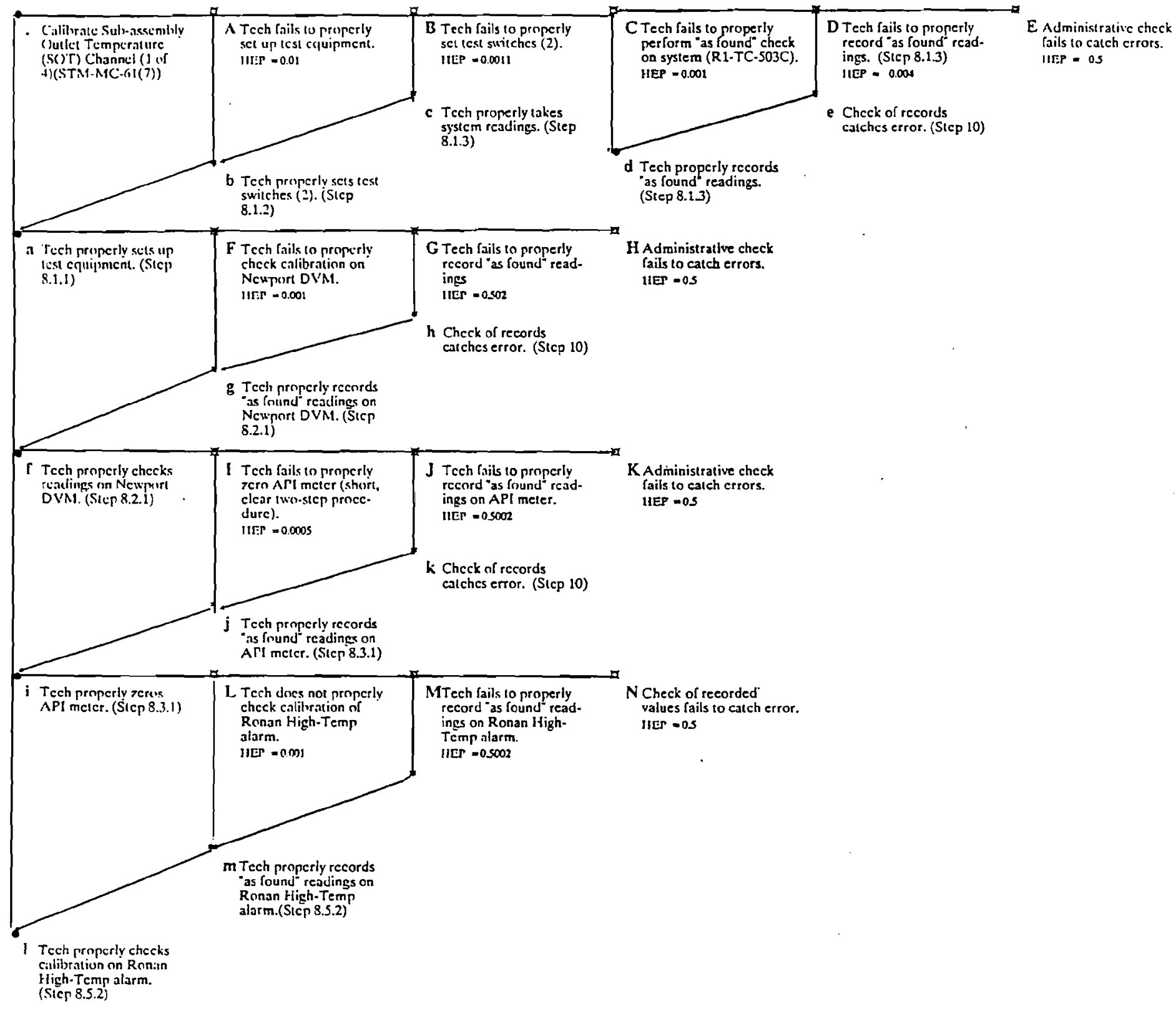

Fig. 8.6. Human Event Tree, SOTC 


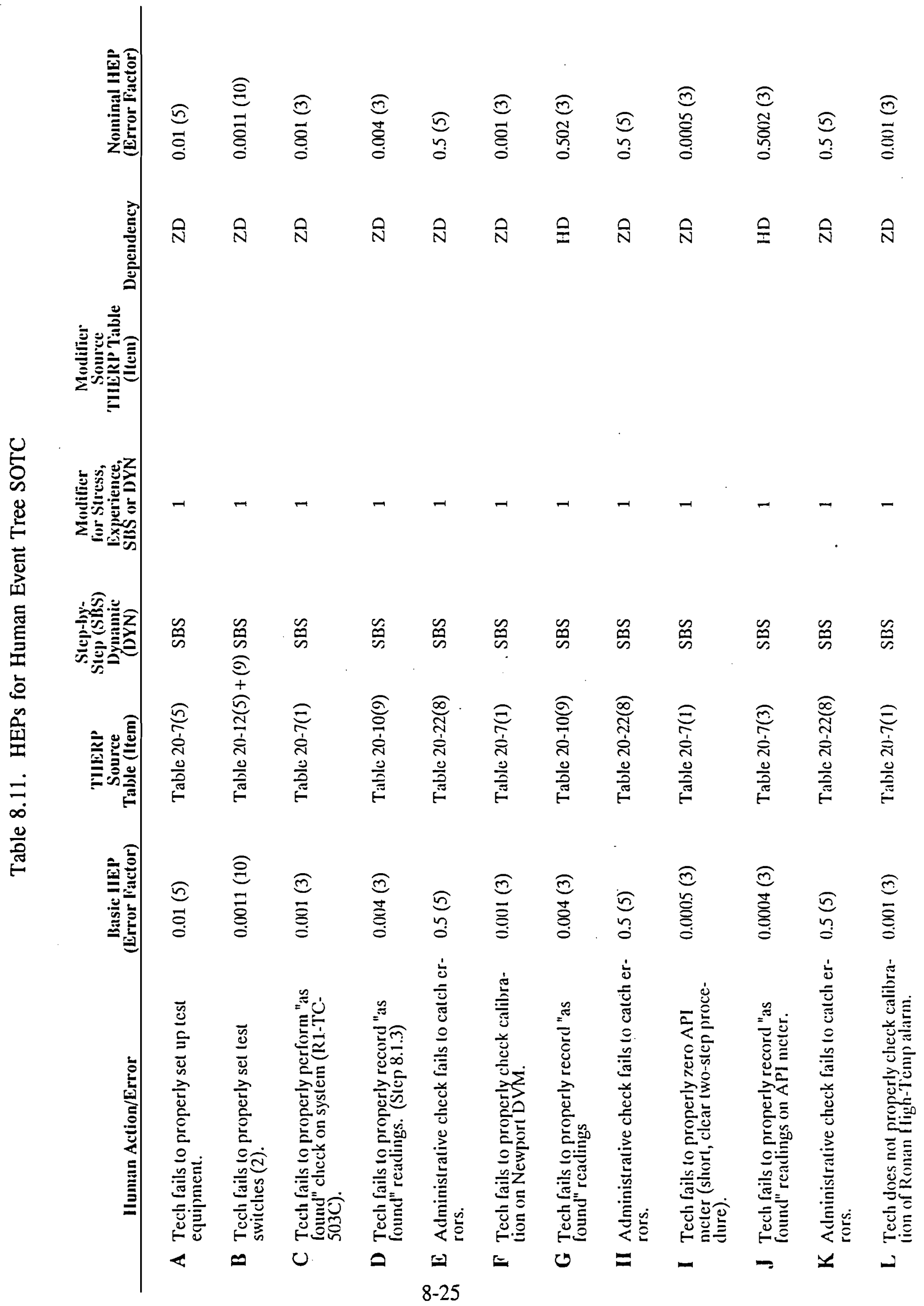



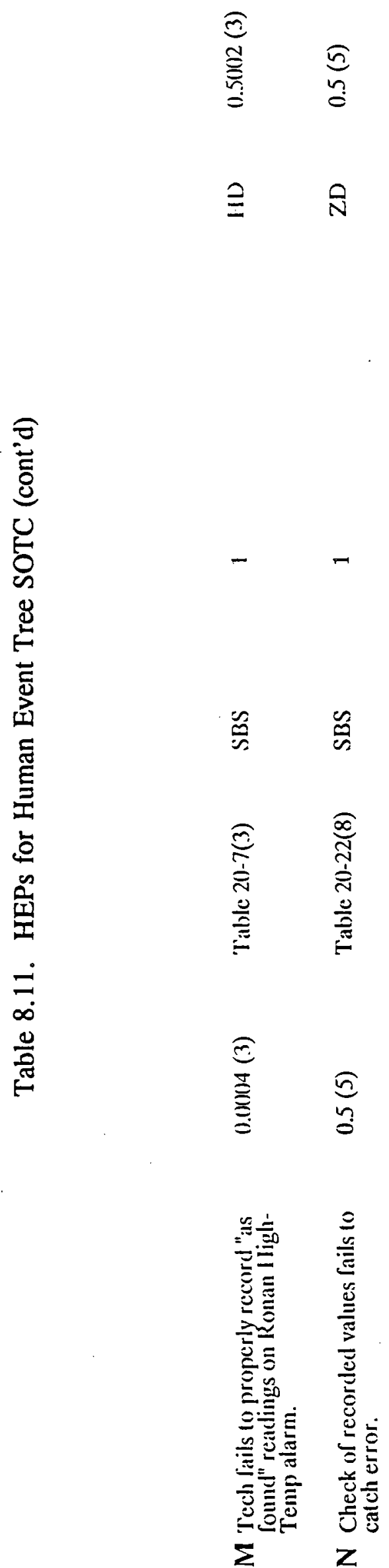
Table 8.12. Failure Paths and Total Failure/Success Probabilities for Human Event Tree SOTC

\section{Calibrate SOT Channels (one at a time)}

\begin{tabular}{|c|c|c|c|c|}
\hline Failure Patlı & Calculations & $\begin{array}{l}\text { Resulting Failure } \\
\text { Probability }\end{array}$ & $\begin{array}{c}\text { Total Failure Prob- } \\
\text { ability }\end{array}$ & $\begin{array}{c}\text { Total Probability } \\
\text { of Success }\end{array}$ \\
\hline \multirow{3}{*}{$\begin{array}{ll}1 & \text {. } \\
2 & \text { afGil } \\
3 & \text {.nfiLMN }\end{array}$} & $0.001 \times 0.502 \times 0.5$ & 0.000251 & & \\
\hline & $0.0005 \times 0.50(1) 2 \times 0.5$ & $\begin{array}{l}0.01001251 \\
0.000125\end{array}$ & & \\
\hline & & & 0.0006261 & 0.9993738 \\
\hline
\end{tabular}

N 


\title{
HRA TREE DIAGRAM
}

\author{
ID: $\quad$ SOTT Adjust trips for Subassembly Outlet Tcmperature (SOT) \\ Sequence: $\quad R H U 302 Q L$ \\ Date modified: $\quad 18-J U N-90$ \\ Analyst: Schurman \\ NOTES: Tech. sets trip points for Subassembly Outlet Temperature Instruments RI-TC-503 D, F, H, and C at \\ temperatures calculated by operators.
}

- I\&C Tech. adjusts trips for Subassembly Outlet Temperatures (SOT)

a I\&C Tech. adjusts trips for Subassembly Outlet Temperatures (SOT) R1-TC-503D
A Tech. adjusts trips

'High' on SOT Instruments R1-TC-503 C, $D, F$, and $\mathrm{H}$.

HEP $=0.025$

Fig. 8.7. Human Event Tree, SOTT 


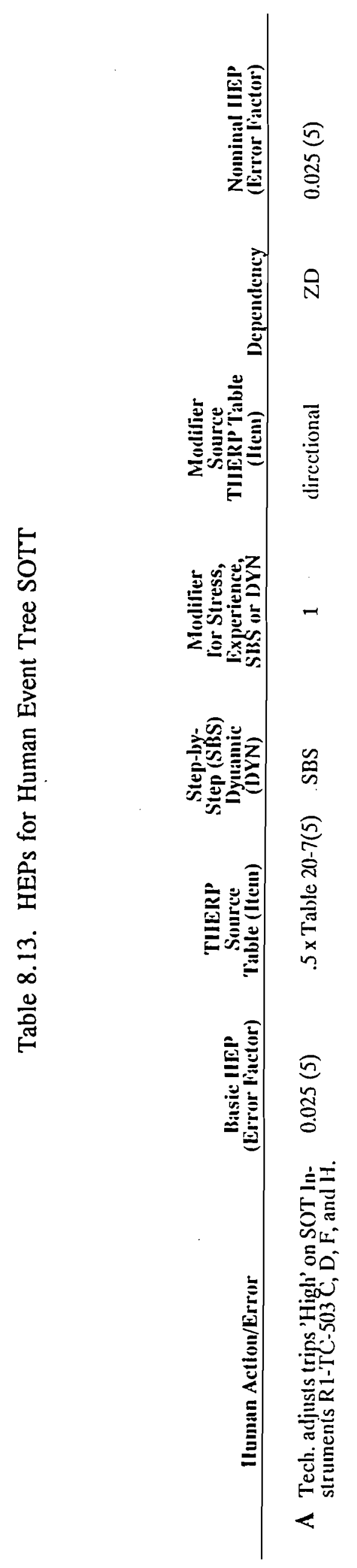




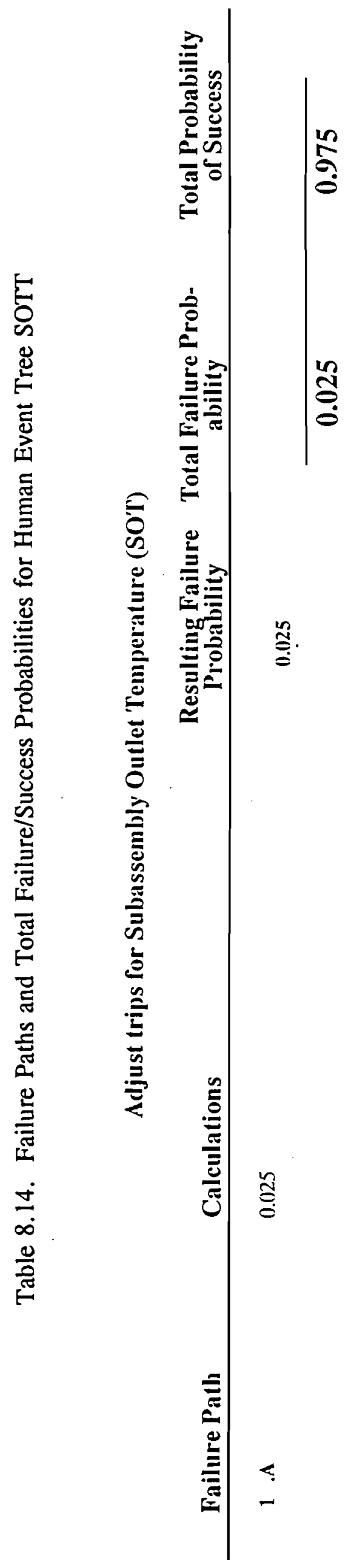


be presumed to be independent. The probability of directional error in adjusting each channel is assumed to be $2.510^{-2}$, which is one-half of the value given in NUREG/CR-1278, Table 20-7, item (5), [8.3]. One-half of the table value is used because misadjustment can occur in one direction only for an unsafe trip setting to result.

Since the SOT systems are designed to be a backup signal to primary trip systems, the procedures reflect this philosophy. However, if it is desired that this probability be reduced, procedurally requiring the checker to be present and making this adjustment a sign-off step would lower the estimated error probability by approximately two orders of magnitude.

\subsubsection{Wide Range Nuclear Channel Trip Points}

The linear drawer sections of each of the three wide-range nuclear channels (A, B, and C) provide flux measurement for the system. Since these channels are set to relative values (100\%), are checked and reset several times during startup, and readings are checked and recorded every four hours during normal operation, it was determined that it would not be necessary to perform a detailed analysis on the calibration procedures.

The setting of trip points on these channels require that the scram trip points be set for "overpower" (high flux greater than 110\%) and for loss of high voltage. An alarm trip point is also set for flux exceeding $104 \%$ of full power. This alarm trip point is important in providing additional time for the operators to respond to power increases (TOP events) but does not otherwise contribute to control of the event, so it was not included in the HRA event tree.

Event RHU201QL, Failure to Accurately Set High Flux Trip Point at $110 \%$ of Authorized Power: $p=1.510^{-6}$ (Ref. Human Event Tree BSPL, Figure 8.8, Tables 8.15 and 8.16$)$ 


\title{
HRA TREE DIAGRAM
}

\author{
ID: $\quad B S P L$ Tech. sets level trip points for linear drawers $A, B, \& C$. \\ Sequence: RHU201QL \\ Date modified: $\quad 18$-JUN-90 \\ Analyst: Schumnan \\ NOTES: $\quad$ Technician sets high level bistable trip points for linear amplifiers of channel $A, B$, and $C$. These set- \\ tings are checked by the Interlock checks before start up of the reactor. (Proc. STM-MC-77 Rev. S)
}

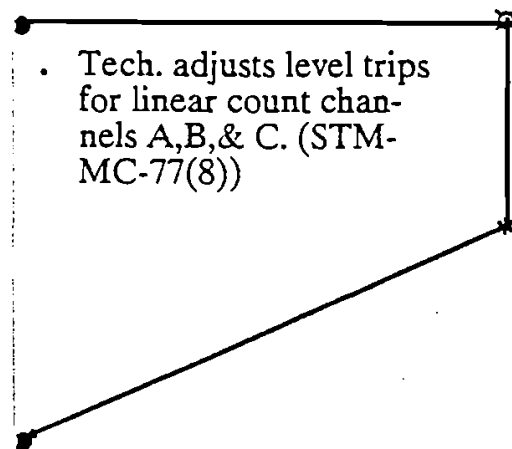
A Tech. fails to set high level trip level to $110 \%$ $+0 \%$ or $-2 \%$
HEP $=0.0015$ find misadjustment of high level setpoint.
HEP $=0.001$
B Interlock check fails to
b Interlock check dis- covers high level trip point misadjustment. (STM-MC-92(39))

a Tech sets low High Level trip to $110 \%$. (Step 10.7) 
Table 8.15. HEPs for Human Event Tree BSPL

\begin{tabular}{|c|c|c|c|c|c|c|c|c|}
\hline & Iluman Action/Error & $\begin{array}{c}\text { Basic IIEP } \\
\text { (Error Factor) }\end{array}$ & $\begin{array}{c}\text { THERP } \\
\text { Source } \\
\text { Table (IleII) }\end{array}$ & $\begin{array}{l}\text { Step-by- } \\
\text { Step (SBS) } \\
\text { Dynamic } \\
\text { (DYN) }\end{array}$ & $\begin{array}{l}\text { Modifier } \\
\text { for Stress, } \\
\text { Experience, } \\
\text { SIBS or DYN }\end{array}$ & $\begin{array}{c}\text { Modifier } \\
\text { Source } \\
\text { TIIERP Table } \\
\text { (ltem) } \\
\end{array}$ & Dependency & $\begin{array}{l}\text { Nominal HEP } \\
\text { (Error Factor) }\end{array}$ \\
\hline A & $\begin{array}{l}\text { Tech. fails to set high level trip level } \\
\text { to } 110 \%+0 \% \text { or }-2 \%\end{array}$ & $0.0015(3)$ & $.5 \times$ Table $20-7(3)$ & SBS & 1 & directional & $\mathrm{ZD}$ & $0.0015(3)$ \\
\hline B & $\begin{array}{l}\text { Interlock check fails to find misad- } \\
\text { justment of highl level setpoint. }\end{array}$ & $0.001(10)$ & Analyst's judgeme & ent SBS & 1 & & $\mathrm{ZD}$ & $0.001(10)$ \\
\hline
\end{tabular}




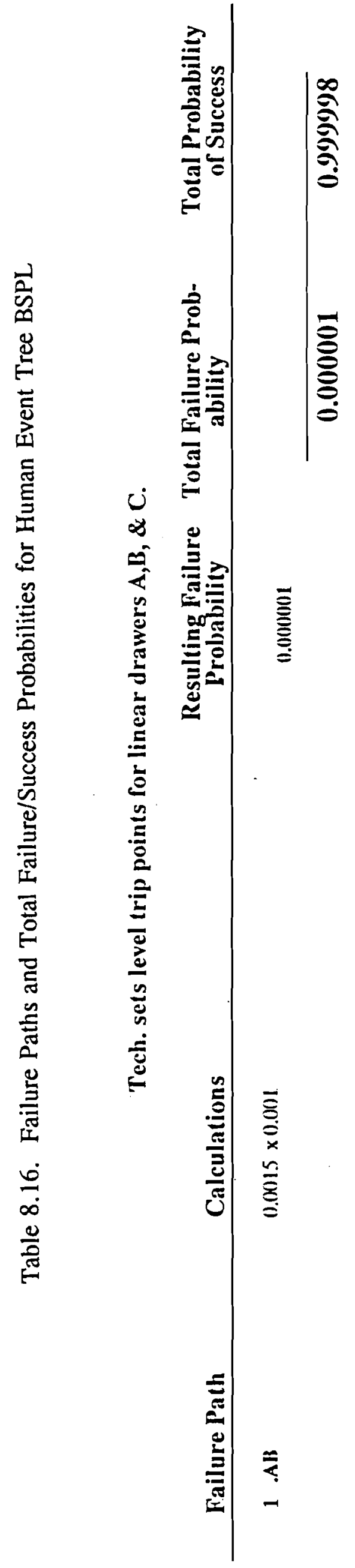


This procedure requires that the bistable trip on each channel (A,B \& $\mathrm{C}$ ) be set, by means of gain controls, to trip at $90 \%$ of authorized power during startup until power reaches $30 \mathrm{MW}$. At $30 \mathrm{MW}$ the gain controls are reset to trip at $110 \%$ of authorized power (calculated). The gain control accuracy is verified one hour after full power has been attained. The accuracy of the trip point settings at $110 \%,+0 \% /-2 \%$, is verified by built in tests prior to each numbered run. The probability that the bistable trips will be set inaccurately in either direction is $310^{-6}$. This is a conservative estimate, since there is a 50:50 chance of setting the trip point too low (producing a scram or alarm before it is needed) rather than too high (failing to produce a scram or alarm when it is needed).

The probability of misadjusting scram trip points for the channels is conservatively assumed to be non-independent. Although the drawers are in separate locked cabinets, the cabinets are transparent and the trip points are set one after the other.

Event RHU2020L, Failure to Accurately Set High Voltage-Low Trip Point: $p=1.510^{-6}$ (Ref. Human Event Tree BSPV, Figure 8.9. Tables 8.17 and 8.18)

This procedure requires that the bistable trip on each channel $(\mathrm{A}, \mathrm{B} \& \mathrm{C}$ ) be set to $-680 \pm 20 \mathrm{VdC}$. The probability that the bistable trips will be set inaccurately in either direction is $310^{-6}$. This is a conservative estimate, since there is a 50:50 chance of setting the trip point too high (producing a scram or alarm before it is needed) rather than too low (failing to produce a scram or alarm when it is needed).

The probability of misadjusting scram trip points for the channels is conservatively assumed to be non-independent. Although the drawers are in separate locked cabinets, the cabinets are transparent and the trip points are set one after the other. 


\section{HRA TREE DIAGRAM}

ID: $\quad B S P V$ Tech. sets voltage trip points for linear drawers $A, B, \& C$.

Sequence: $\quad R H U 202 Q L$

Date modified: $\quad 18-J U N-90$

Analyst: Schuman

NOTES: $\quad$ Technician sets high voltage bistable trip points for linear amplifiers of channels $A, B$, and $C$. These settings are checked by the Interlock checks before startup of the reactor. (Proc. STM-MC-77 Rev. 8).

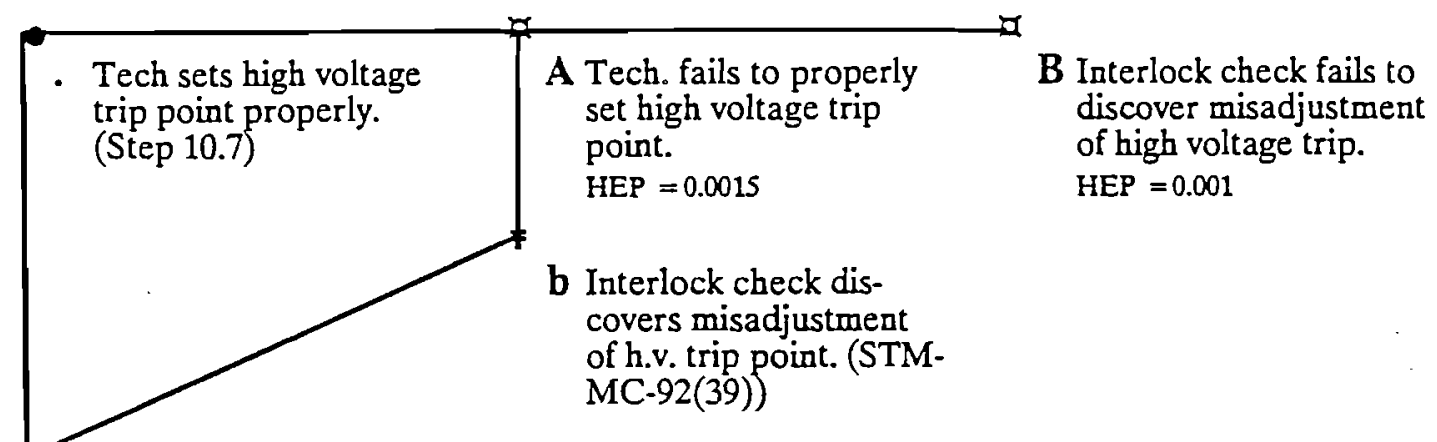

a Tech sets high voltage trip point properly. (Step 10.7)

Fig. 8.9. Human Event Tree, BSPV 


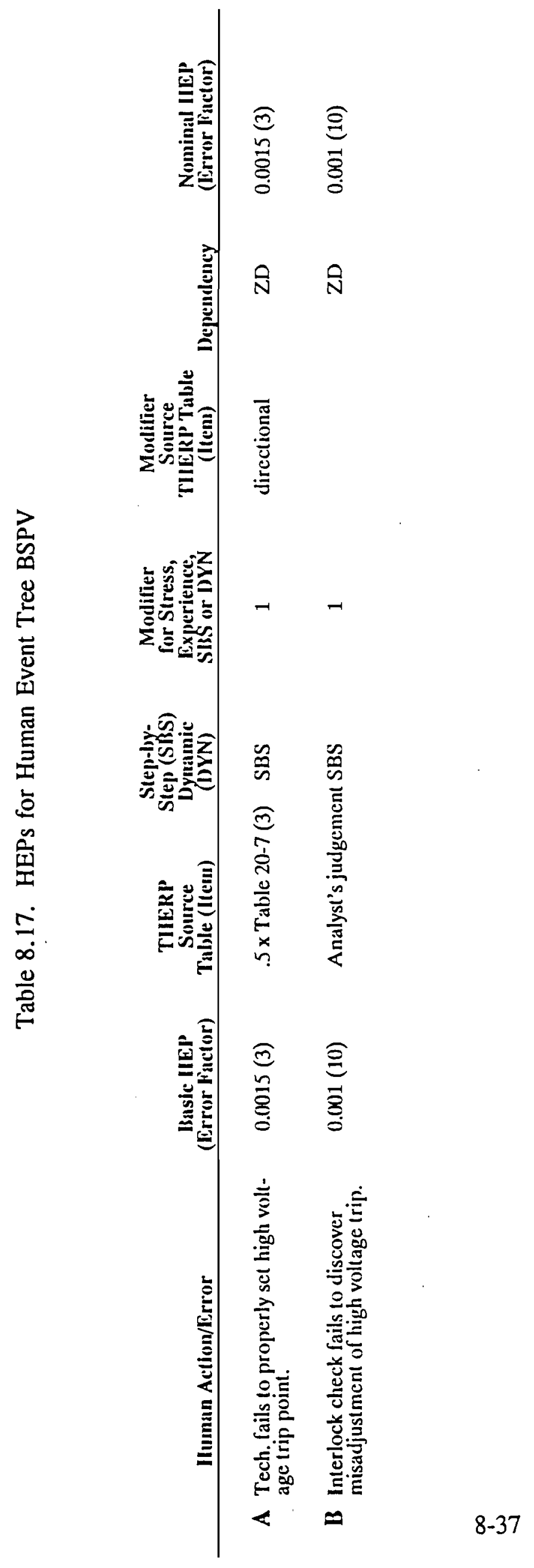




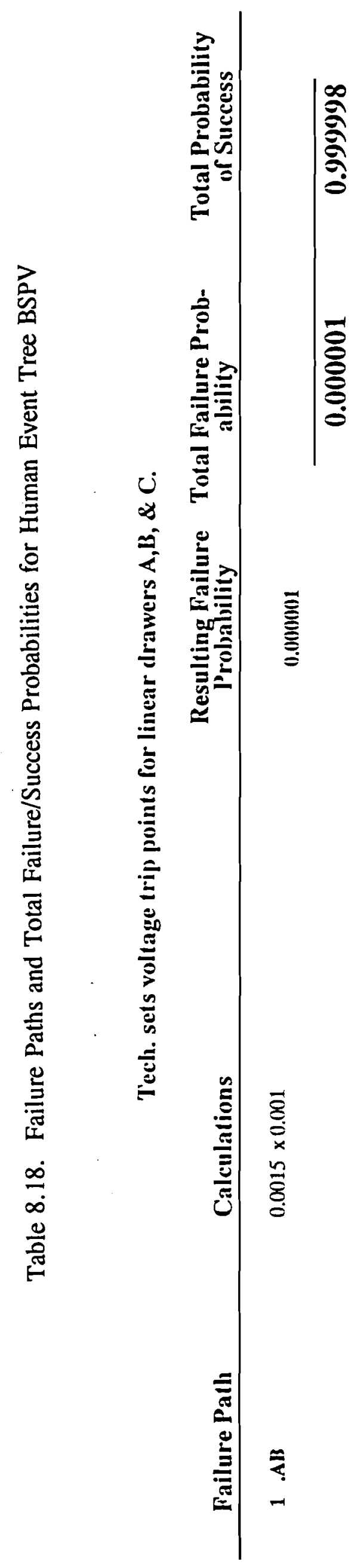




\subsubsection{Subassembly Outlet Temperature Instruments}

The SOT system is designed as a backup signal to the wide range nuclear channels as well as for the flow sensors. The calibration and trip point errors are the same as discussed, above, under the paragraphs on Flow Sensor instruments.

\subsubsection{Failure to Remove Mechanical Latehes from Control Rod Release Rollers}

There are several procedures, having to do with maintenance on the control rod drive systems, that call for placing mechanical latches on the roller latches (usually held closed by magnetic clutches). The roller latches are spring-loaded against the shaft of the control rod drive mechanism and are held closed by the action of magnetic clutches. Loss or removal of power allows the weight of the control rod drive mechanisms (assisted by pneumatic pressure) to open the roller latches. The control rods then drop from the force of gravity - assisted by an air driven piston. In order to perform maintenance on the control rod drive assemblies, these roller latches are mechanically held closed by U-shaped mechanical latches. In each of these procedures, the placement and removal of the mechanical latches are called out in separate steps. Procedures calling for mechanical latching of one or more control rod roller latches occur an average of four times per year (approximately 96 times in the 25 years of operation). In most procedures, the control rod drive being worked on and two adjacent drives are latched at the same time. Supervision procedures (and good mechanical practice) call for work on only one control rod drive assembly at a time.

Nonetheless, it is conceivable, if unlikely, that two control rod drive assemblies could be worked on at the same time. In such case, as many as six assemblies could be mechanically held closed and disabled from dropping. In order for failure to scram to occur, all but two of the control rods would have to be mechanically latched, since any two control rods will reduce reactivity to subcritical (see Section 7 for a more detailed explanation). The post-maintenance procedures call for a performance check of the control rod drop system. The performance check is performed by timing control rod drop without the air assist. Failure of one or more rods to 
fall to the bottom is indicated by a red light and annunciator in the control room. The drop times are recorded on a pre-startup interlock checksheet and must be less than $0.45 \mathrm{sec}$.

The probability of any control rod remaining mechanically latched after maintenance and that latching being undetected is judged to be negligible.

\subsubsection{Manual Scram}

Event RHUO0ZCL, Failure to Manually Scram Safety Rods after Scram Signal: $p=7.5$ $10^{-3}$ (Ref. Human Event Tree SCRM. Figure 8.10. Tables 8.19 and 8.20.)

The operators are trained to press the control rod scram button immediately upon receiving a scram alarm and the probability of one of the two console operators failing to take this action approaches zero. The event of concern here, however, is the operator failure to scram the safety rods in the case the control rods fail to shutdown the reactor. An emergency procedure for manual or automatic scram of EBR-II requires the operators to verify that a reactor scram has actually occurred after the operators press the control rod scram pushbutton. If the operators can not verify the shutdown, they are instructed to immediately press the safety rod scram pushbutton. This is failure path .abD in Figure 8.20 and has probability $7.510^{-3}$.

Event RHU001CL, Failure to Manually Scram in the Absence of a Scram Signal: $\underline{\text { Screening } p=510^{-2}}$

Event RHU002CL, Failure to Manually Scram in the Absence of a Scram Signal: Screening $p=110^{-1}$ 


\title{
HRA TREE DIAGRAM
}

\author{
ID: SCRM Operators use full manual SCRAM procedure on failure of auto \\ Sequence: $\quad R H U O O Z C L$ \\ Date modified: $\quad I 6-A P R-90$ \\ Analyst: Schumnan \\ NOTES: Operators go through full mantal SCRAM procedure given that autonatic SCRAM mechanisms \\ fail, but SCRAM is annunciated. (Proc. EP 2-1 $\delta / \delta, \delta 9)$
}

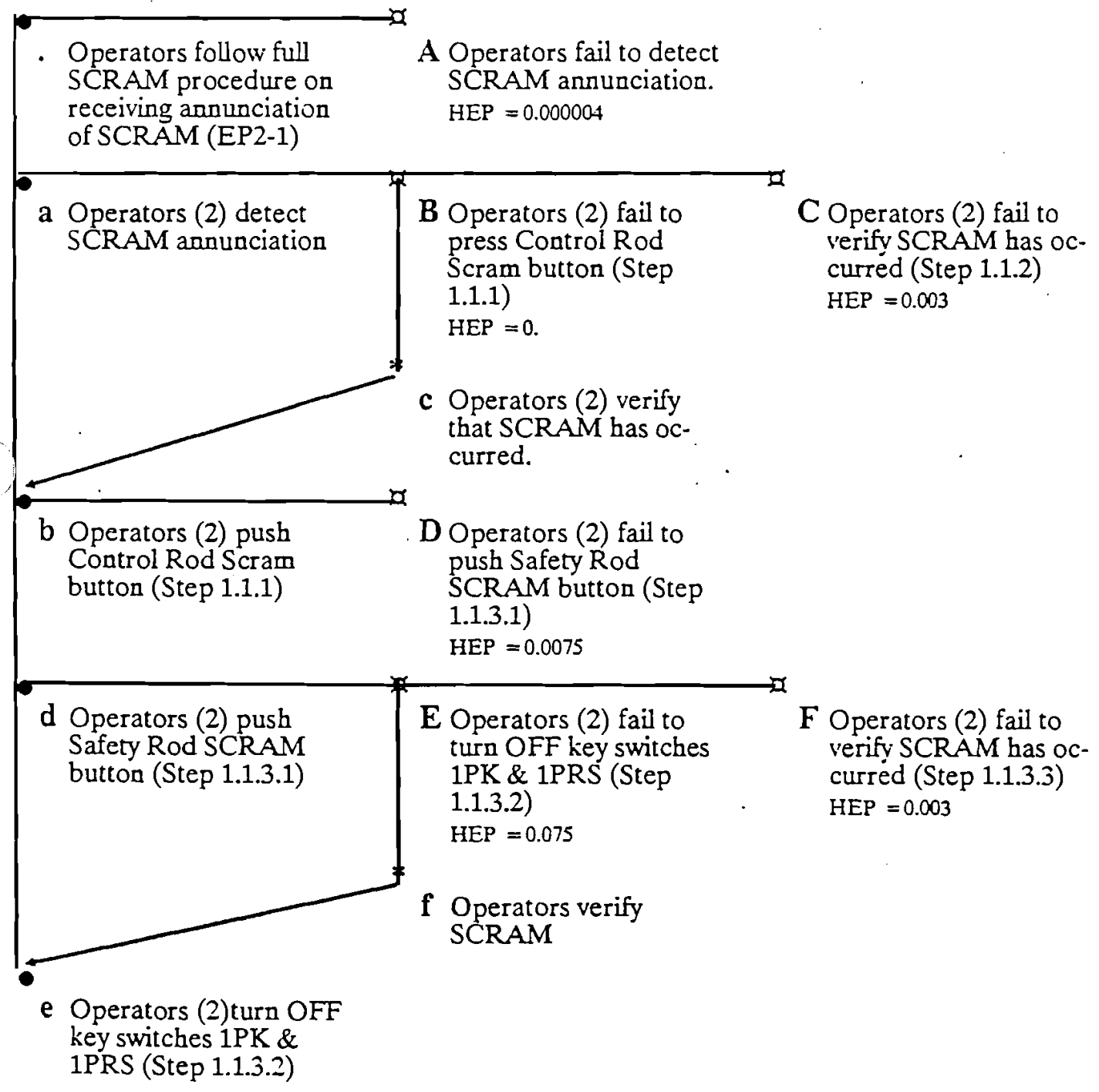

Fig. 8.10. Human Event Tree, SCRM 


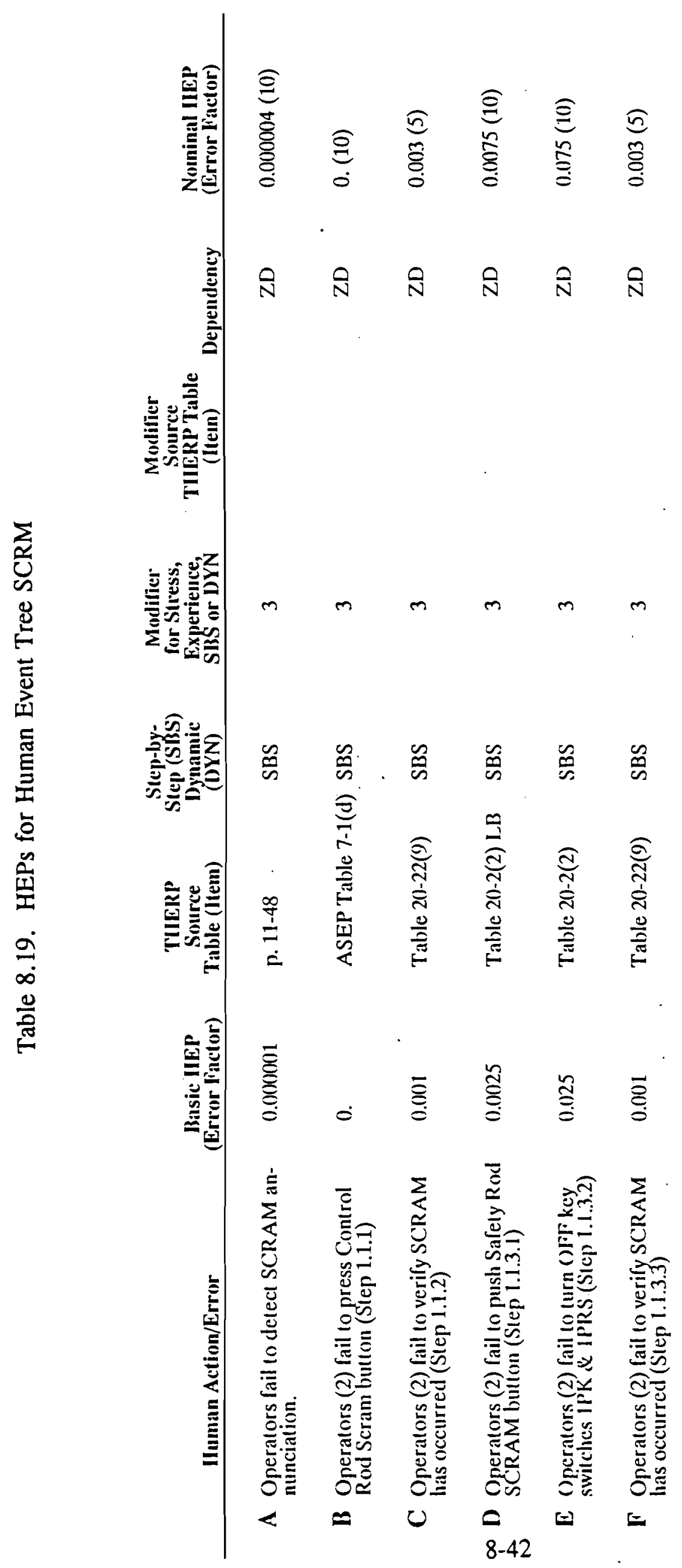




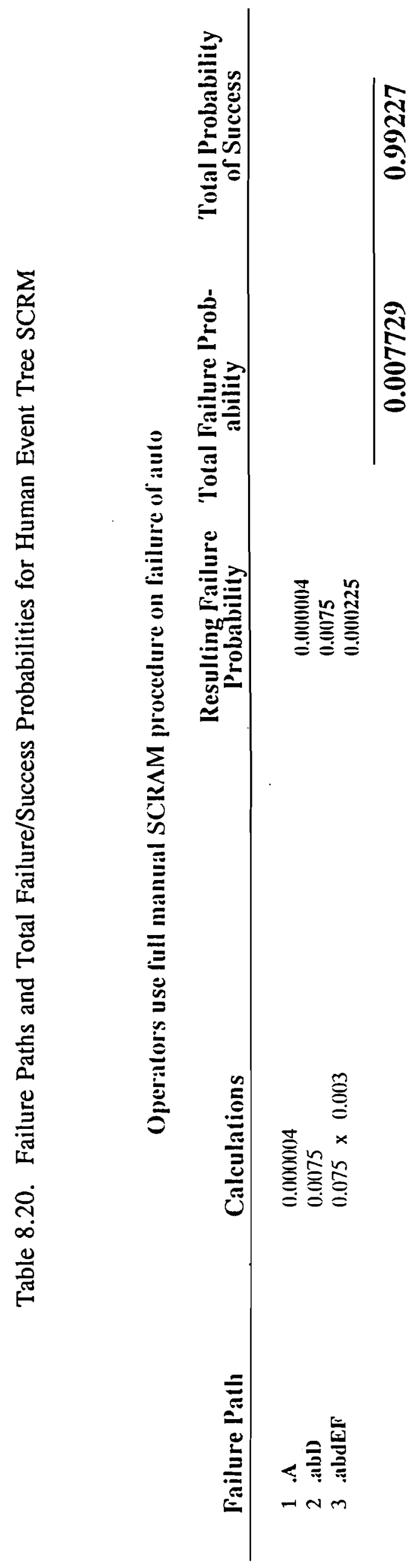


In the case of failure of all scram logic, no scram signal occurs at the control console. Determination of the precise human error probability for this scenario is situation-dependent, since different sets of alarms will be present in the control room for each of the top events in the fault trees. A screening value of $110^{-4}$, using the ASEP values from NUREG/CR-4772 [8.4], Table 8.2, was initially assigned using two assumptions:

1) the operators at EBR-II, unlike commercial operators, are thoroughly trained in the philosophy, "When in doubt, scram the reactor!"

2) all of the annunciators, therefore, signal the same action - scram the reactor and can be assumed to be equivalent to a single unequivocal annunciation.

However, more conservative values can be more appropriate, particularly for the stressful situation in which the operators have to determine the need to scram. For instance the DAS, which could be useful in alerting the operators, keeps track of more than 900 parameters and shows on a terminal the parameters that exceed their specified range. However, if a large number of less important parameters appears on the terminal, the operators could be distracted from the parameters that indicate the need to scram. Accordingly, a failure to manually scram in the event of LOF, RHU001CL, is conservatively estimated to be $510^{-2}$, whereas, in the event of TOP, RHU002CL, the failure is estimated to be $110^{-1}$. The availability of an emergency procedure for abnormal operation of the primary pumps supports the use of a smaller value in the event of LOF.

In the contrast, the situtation for the Loss of Heat Sink is quite different. In this case there is no scram signal that is to be expected and the procedures dictate a manual scram upon diagnosis of the event. The symptoms are clear, slowly developing and the operators are trained to recognize the characteristics of such an event. Since there are two operators the value used is the minimum of $110^{-6}$ for the event; failure to manually scram in a LOHS, RHU006CL. 


\subsection{Insufficient Subcriticality Margin during Refueling}

A possible vulnerability is evaluated for the EBR-II when it is not at power. The event that can occur during reactor shutdown is criticality occurring during the "Unrestricted Fuel Handling" process. During this activity, fuel and experimental subassemblies are moved around or replaced in the reactor core between almost every reactor run due largely to the research nature of this reactor. Although the reactor is shut down, care must be taken to maintain the core at a subcritical level of reactivity, plus a safety margin of subcriticality.

Subcriticality margins are maintained, according to the Technical Specifications, in order to preclude reactivity of the core attaining criticality while refueling is in progress. Technical Specifications require that the core remain subcritical with both safety rods fully inserted by at least 3.0 \$. There are two major Human Errors that could be involved in a criticality event during refueling: 1) Errors in developing the core loading plan; and, 2) Errors in observing neutron count rates that could lead to failure to terminate fuel handling prior to actuation of the engineered safety systems.

In addition, the engineered safety systems and the operator observation (and tracking) of changes depend on the wide-range nuclear channels $\mathrm{A}, \mathrm{B}$, and $\mathrm{C}$. The lower level measuring systems of these channels, Log Count Rate (LCR) drawers, provide information regarding reactivity increases. These instruments also provide safety rod trips in case of high count rates or low count rates from a calibrated neutron source (indicating loss of detector function). Thus, calibration of the LCR drawers of the wide-range systems and setting of the low and high count rate trips are important parts of the safety system for unrestricted fuel handling. For this reason, evaluation of the human error probabilities in performing these functions was required for this analysis. 
Event CFA005RL, Errors in Developing Core Loading Plan: Screening $p=410^{-5}$, (Ref. Human Event Tree CLPE, Figure 8.11, Tables 8.21 and 8.22)

The development of the core loading plans are tightly controlled, technically and administratively. The purpose of the plan is to accomplish refueling in such a manner that the reactivity and flux envelope of the core is known and appropriate after the removal and insertion of each subassembly. This refueling activity is categorized as "Unrestricted Fuel Handling" and may involve movement of fuel subassemblies from one location to another within the core, removal of spent fuel subassemblies, and/or insertion of fresh fuel subassemblies. The reactivity worth, burn-up level, and other characteristics of the constituent subassemblies are calculated and balanced in the core "Loading Plan" by the EBR-II Chief Physicist and checked by the Operations Physicist. This loading plan is indirectly checked by the calculation of fissiles and fission rates using a proprietary program in the Argonne, IL, location.

The Operations Physicist performs an independent calculation of expected reactivity at each step of the loading plan. The loading plan is loaded into the Fuel Handling computer at EBR-II and copies are distributed to the main personnel involved in the fuel handling sequence. The operations managers involved in the fuel handling sequence, including the Fuel. Management Supervisor and the Reactor Neutronics and Chemistry Manager, independently review and check the loading plan before the fuel handling begins. An ASEP screening value analysis was performed on this sequence of activities and the screening value for human error in the development and implementation of the loading plan was found to be on the order of 4 $10^{-5}$, as an upper confidence bound. This value was judged to be too low to warrant further analysis.

Event FHU001RZ, Errors in Observing Neutron Countrates: $p=7.310^{-4}$ (Ref. Human Event Tree FHHU, Figure 8.12, Tables 8.23 and 8.24)

During the Unrestricted Fuel Handling process, a Control Room Operator (CRO) is stationed in the control room with the sole job of tracking neutron countrates to ensure that the core does not trend toward criticality. The CRO has the authority to halt the fuel handling 


\title{
HRA TREE DIAGRAM
}

\author{
ID: CLPE Error is made in developing Core Loading Plan \\ Sequence: unk \\ Date modified: $\quad 08-J U N-90$ \\ Analyst: Schurman \\ NOTES: ASEP screening values used.
}

\begin{tabular}{|c|c|}
\hline - Core Loading Plan & $\begin{array}{l}\text { A Core Loading Plan mis- } \\
\text { calculated by physicists. } \\
\text { HEP }=0.00003\end{array}$ \\
\hline $\begin{array}{l}\text { a Core Loading Plan cal- } \\
\text { culated correctly by } \\
\text { physicists. }\end{array}$ & $\begin{array}{l}\text { B Core Loading Plan in- } \\
\text { correctly translated } \\
\text { into loading instruc- } \\
\text { tions. } \\
\text { HEP }=0.00001\end{array}$ \\
\hline $\begin{array}{l}\text { b Core Loading Plan } \\
\text { properly translated } \\
\text { into loading instruc- } \\
\text { tions. }\end{array}$ & \\
\hline
\end{tabular}

Fig. 8.11. Human Event Tree, CLPE 


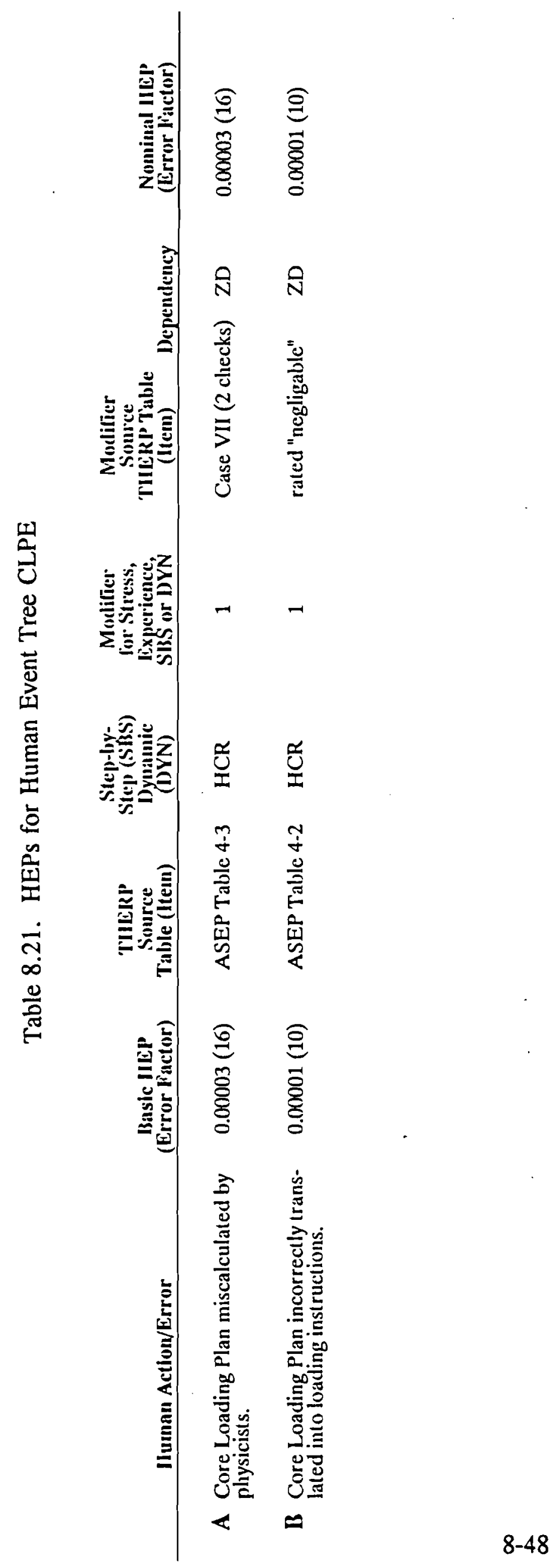




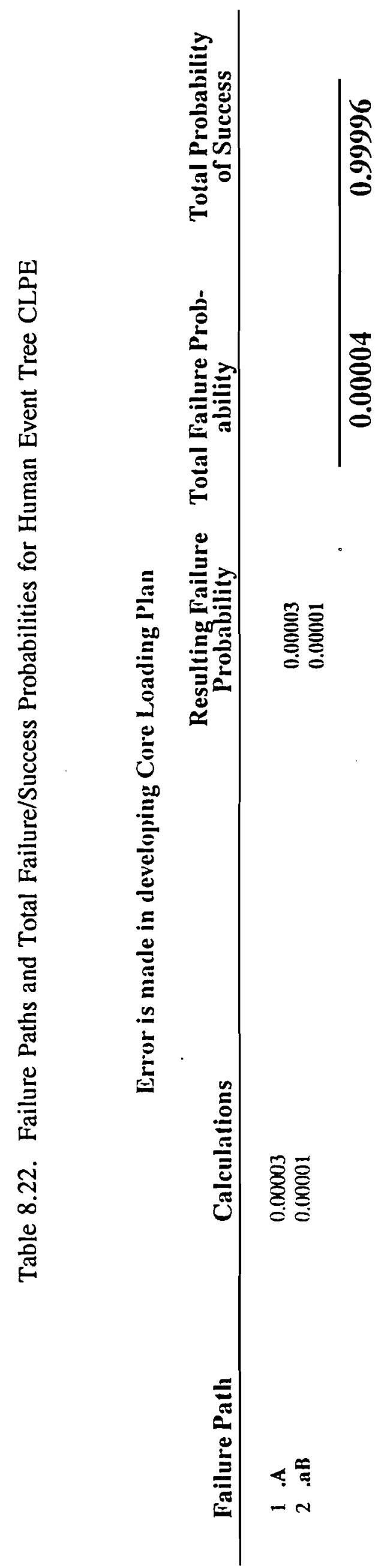


FHIYU Opcrator fails in follow fuel handling Counsrase Procedure.

Se(juence:

Unkinuwn

Diste modificd:

$0+-16+4\}=90$

Anilyst:

Schurnan

NOTES:

IIhen notificd by Fucl-Hundling Console Operutor - Control Room Operalor fauils lo use and follow Coumtrare Suncillance Procedure.

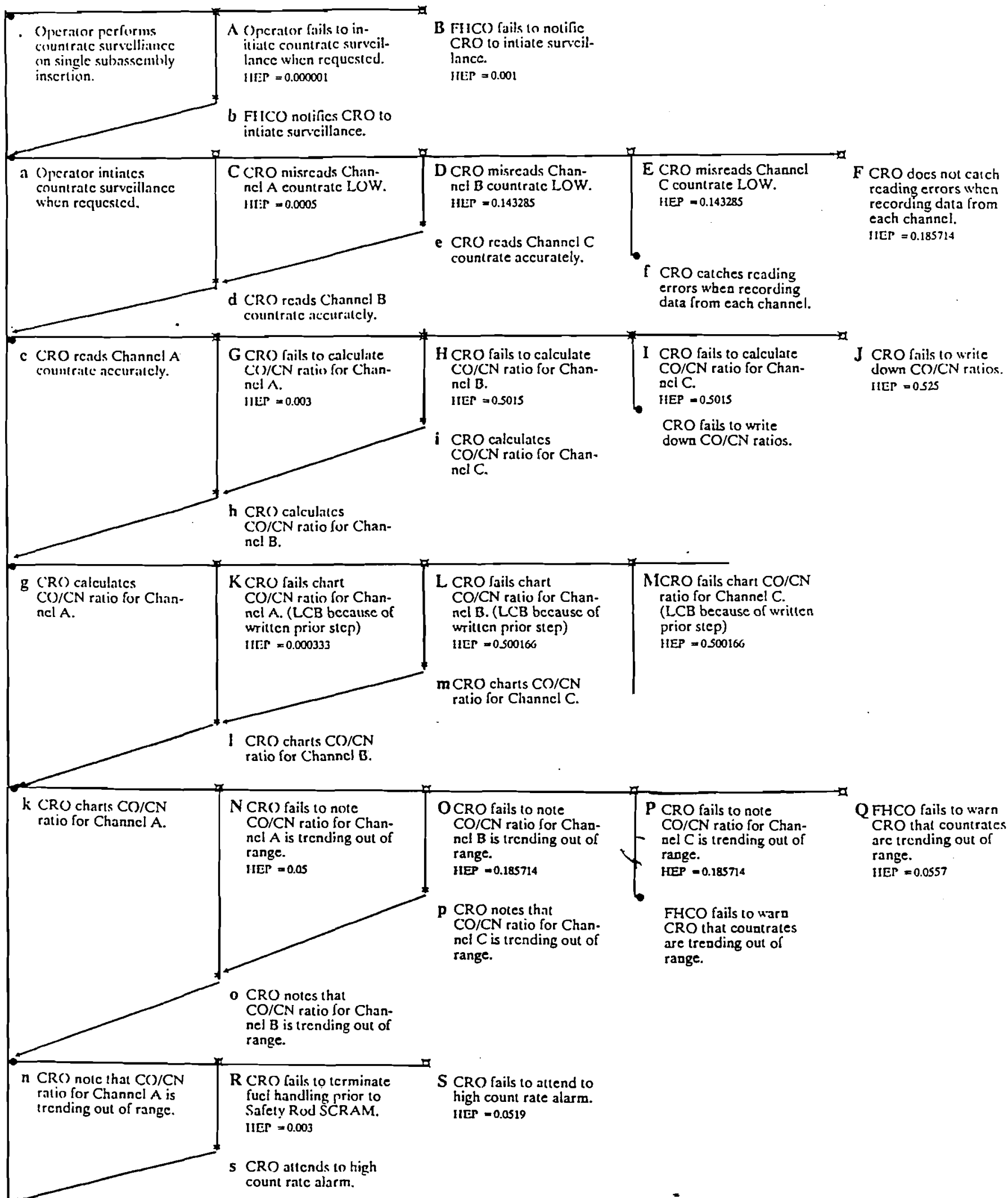

Fig. 8.12. Human Event Tree, FHHU

C.RO tcrminatcs fucl handling prior to Safcty Rud SCRAM 
Table 8.23. HEPs for Human Event Tree FHHU

\begin{tabular}{|c|c|c|c|c|c|c|c|c|}
\hline & Iluman Action/Error & $\begin{array}{c}\text { Basic IIEP } \\
\text { (Error Factor) } \\
\end{array}$ & $\begin{array}{c}\text { TIEIRP } \\
\text { Source } \\
\text { Table (Item) } \\
\end{array}$ & $\begin{array}{l}\text { Step-by- } \\
\text { Step (SisS) } \\
\text { Dynamic } \\
\text { (DYN) }\end{array}$ & $\begin{array}{c}\text { Modilier } \\
\text { for Stress, } \\
\text { Experience, } \\
\text { SBSS or DYN } \\
\end{array}$ & $\begin{array}{l}\text { Modilier } \\
\text { Soln'ce } \\
\text { TIISRP Table } \\
\text { (ltem) }\end{array}$ & Dependency & $\begin{array}{c}\text { Nominal IIEP } \\
\text { (Error Factor) }\end{array}$ \\
\hline $\mathbf{A}$ & $\begin{array}{l}\text { Operator fails to initiate countrate } \\
\text { surveillance when requested. }\end{array}$ & $0.000001(3)$ & Table 20-5(2) & SBS & 1 & & $\mathrm{ZD}$ & $0.0000001(3)$ \\
\hline $\mathbf{B}$ & $\begin{array}{l}\text { FHCO fails to notifie CRO to intiate } \\
\text { surveillance. }\end{array}$ & $0.001(5)$ & Table 20-6(2) & SBS & 1 & & $\mathrm{ZD}$ & $0.001(5)$ \\
\hline $\mathbf{C}$ & $\begin{array}{l}\text { CRO misreads Channel A countrate } \\
\text { LOW. }\end{array}$ & $0.0005(5)$ & $.5 \times$ Table $20-10(2)$ & SBS & 1 & & $\mathrm{ZD}$ & $0.0005(5)$ \\
\hline D & $\begin{array}{l}\text { CRO misreads Channel B countrate } \\
\text { LOW. }\end{array}$ & $0.0005(5)$ & $.5 \times$ Table $20-10(2)$ & SBS & 1 & & MD & $0.143285(5)$ \\
\hline $\begin{array}{l}\mathbf{E} \\
\mathfrak{u}^{\prime}\end{array}$ & $\begin{array}{l}\text { CRO misreads Channel C countrate } \\
\text { LOW. }\end{array}$ & $0.0005(5)$ & $.5 \times$ Table $20-10(2)$ & SBS & 1 & & MD & $0.143285(5)$ \\
\hline $\mathbf{F}$ & $\begin{array}{l}\text { CRO does not catch reading errors } \\
\text { when recording data from each chan- } \\
\text { nel. }\end{array}$ & $0.05(10)$ & Page 11-13/An. Jdg & $\operatorname{lgnt} B S$ & 1 & & $\mathrm{MD}$ & $0.185714(10)$ \\
\hline $\mathbf{G}$ & $\begin{array}{l}\text { CRO fails to calculate } \mathrm{CO} / \mathrm{CN} \text { ratio } \\
\text { for Channel } \mathrm{A} \text {. }\end{array}$ & $0.003(3)$ & Table 20-5(1) & SBS & 1 & & $\mathrm{ZD}$ & $0.003(3)$ \\
\hline II & $\begin{array}{l}\text { CRO fails to calculate } \mathrm{CO} / \mathrm{CN} \text { ratio } \\
\text { for Channel } \mathrm{B} \text {. }\end{array}$ & $0.003(3)$ & Table $20-5(1)$ & SBS & 1 & & $\mathrm{HD}$ & $0.5015(3)$ \\
\hline 1 & $\begin{array}{l}\text { CRO fails to calculate } \mathrm{CO} / \mathrm{CN} \text { ratio } \\
\text { for Channel } \mathrm{C} \text {. }\end{array}$ & $0.003(3)$ & Table 20-5(1) & SBS & 1 & & HD & $0.5015(3)$ \\
\hline $\boldsymbol{J}$ & $\begin{array}{l}\text { CRO fails to write down } \mathrm{CO} / \mathrm{CN} \\
\text { ratios. }\end{array}$ & $0.05(3)$ & Table 20-10(10) & SBS & 1 & & HD & $0.525(3)$ \\
\hline $\mathbf{K}$ & $\begin{array}{l}\text { CRO fails chart } \mathrm{CO} / \mathrm{CN} \text { ratio for } \\
\text { Channel A. (LCB because of written } \\
\text { prior step) }\end{array}$ & $0.000333(10)$ & Table 20-7(LCB) & SBS & 1 & & $\mathrm{ZD}$ & $0.000333(10)$ \\
\hline $\mathbf{L}$ & $\begin{array}{l}\text { CRO fails chart CO/CN ratio for } \\
\text { Channel B. (LCB because of written } \\
\text { prior step) }\end{array}$ & $0.000333(10)$ & Table 20-7(LCB) & SBS & 1 & & HD & $0.500166(10)$ \\
\hline
\end{tabular}




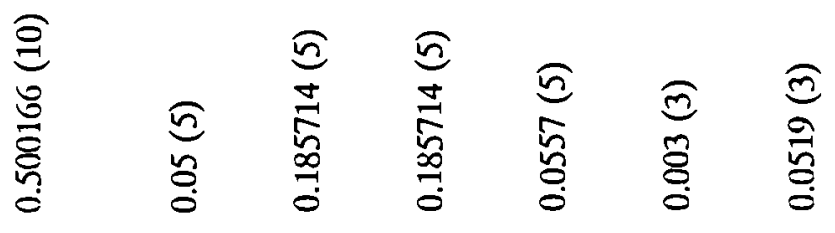

ฏ

옹

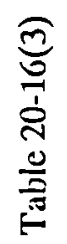

胥

密

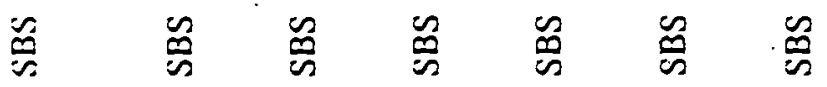

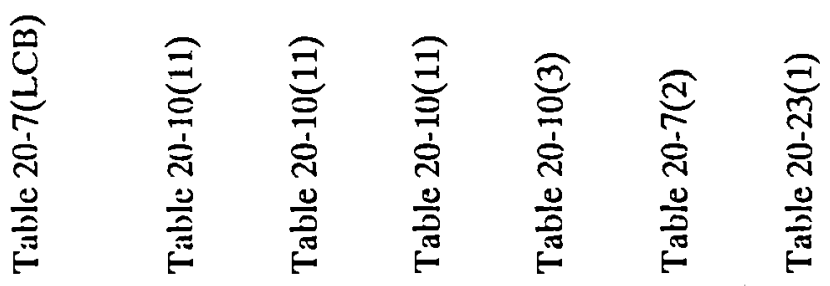

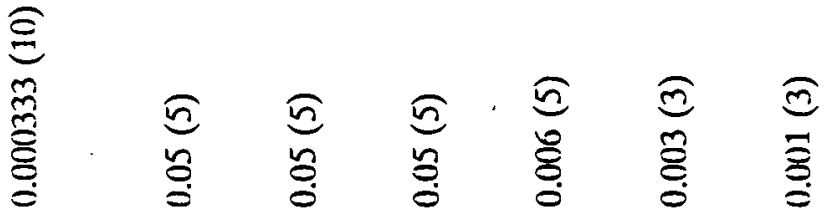

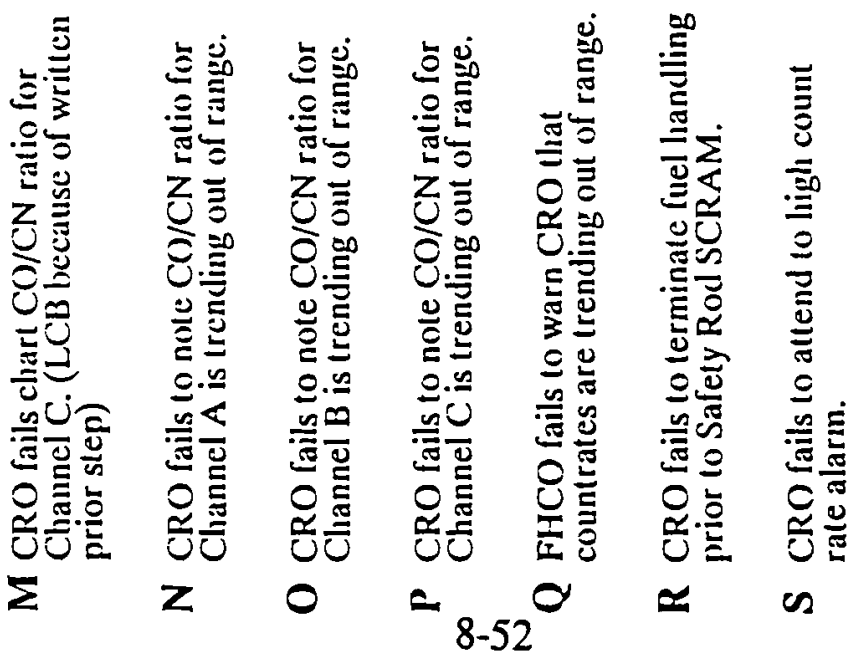




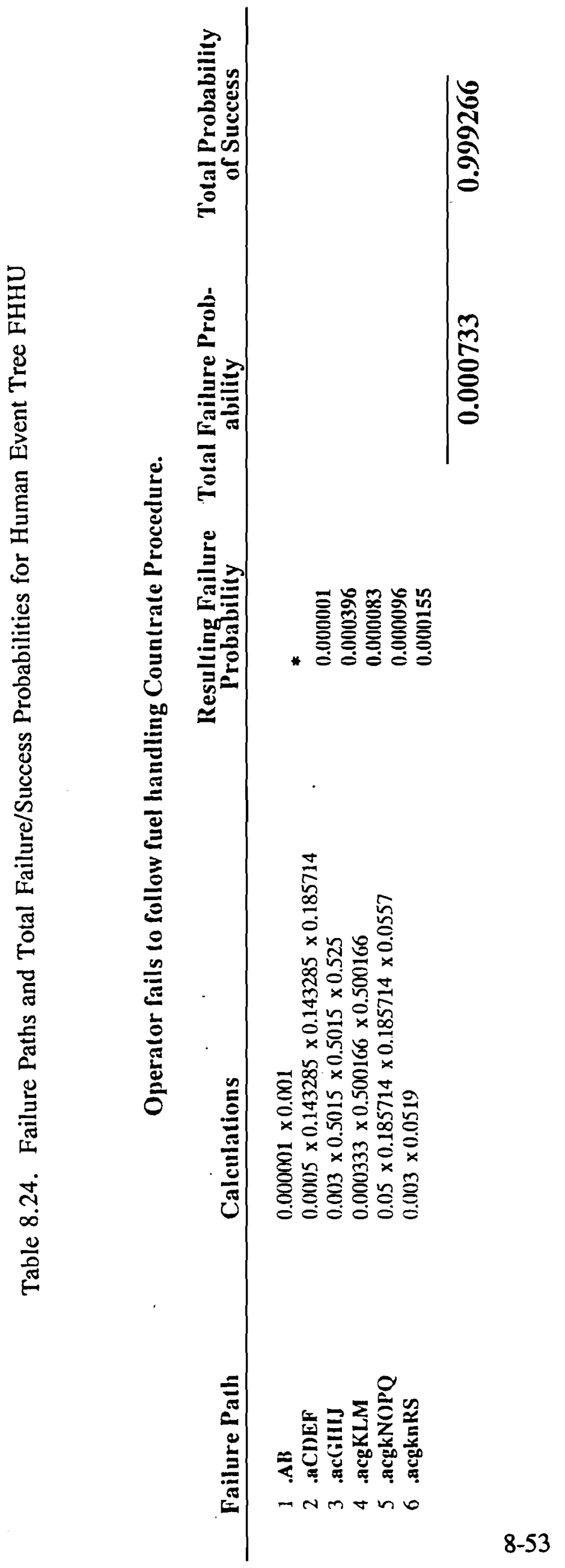


process if reactivity changes are not occurring according to the loading plan. He/She communicates with the Fuel Handling Console Operator (FHCO) who is in charge of the actual fuel-handling procedure.

The CRO takes a baseline count (referred to as $\mathrm{C}_{0}$, in the procedures) that identifies the reactivity of the core before fuel loading begins. He then takes a three-minute countrate reading from each of the wide-range nuclear channels after each insertion of a subassembly (referred to as $C_{n}$, in the procedures). The $C R O$ records these readings, then calculates the $C_{0} / C_{n}$ ratio. The CRO then plots these readings, for each channel, on a graph and visually verifies that the ratio is not tending toward less than 0.7 on the graph. Thus, this CRO serves as a safety barrier to terminate fuel handling before core reactivity increases to the point that would actuate the automatic safety systems (at 1500 - 2000 counts per second). The human reliability probability for this task is approximately $7.310^{-4}$.

Although this task appears simple and straightforward, there are a number of error-likely steps involved, such as accurate transcription of meter readings, accurate calculations, and accurate transfer of the calculations to graphs. Therefore, estimated human error probability is higher than was found for the rest of the analyzed tasks.

\section{Event CND001RM, Errors in Calibration for Log Count Rate Instrumentation:} $\mathrm{p}=310^{-5}$ (Ref. Human Event Tree FHCR, Figure 8.13. Tables 8.25 and 8.26)

Calibration of the wide-range nuclear channels Log Count Rate (LCR) drawers is very important, since both the accuracy of information upon which to perform countrate surveillance and the actuation of automatic safety systems are both dependent upon these instruments.

The calibration of the LCR instruments is part of the calibration of all ranges of the widerange nuclear channels (STM-MC-77, Rev. 8). However, the calibration for this portion of the channels is separately checked by use of the "Unrestricted Fuel Handling Interlock Checks" 


\section{HRA TREE DIAGRAM}

1D: FHCR Calibrate Log Count Ratc on channels $A, B, \& C$ fuel handlin

Sequence: Unknown

Date modilied: $\quad 02-A P R-90$

Anilyst: Schumant

NOTE:S: Tech. calibrates instrumcnts for log count ratc on Wide Range Nuclear Channels $A$, $B$, and $C$. Checkcd by the Intcrlock check prior to Fucl Handling.

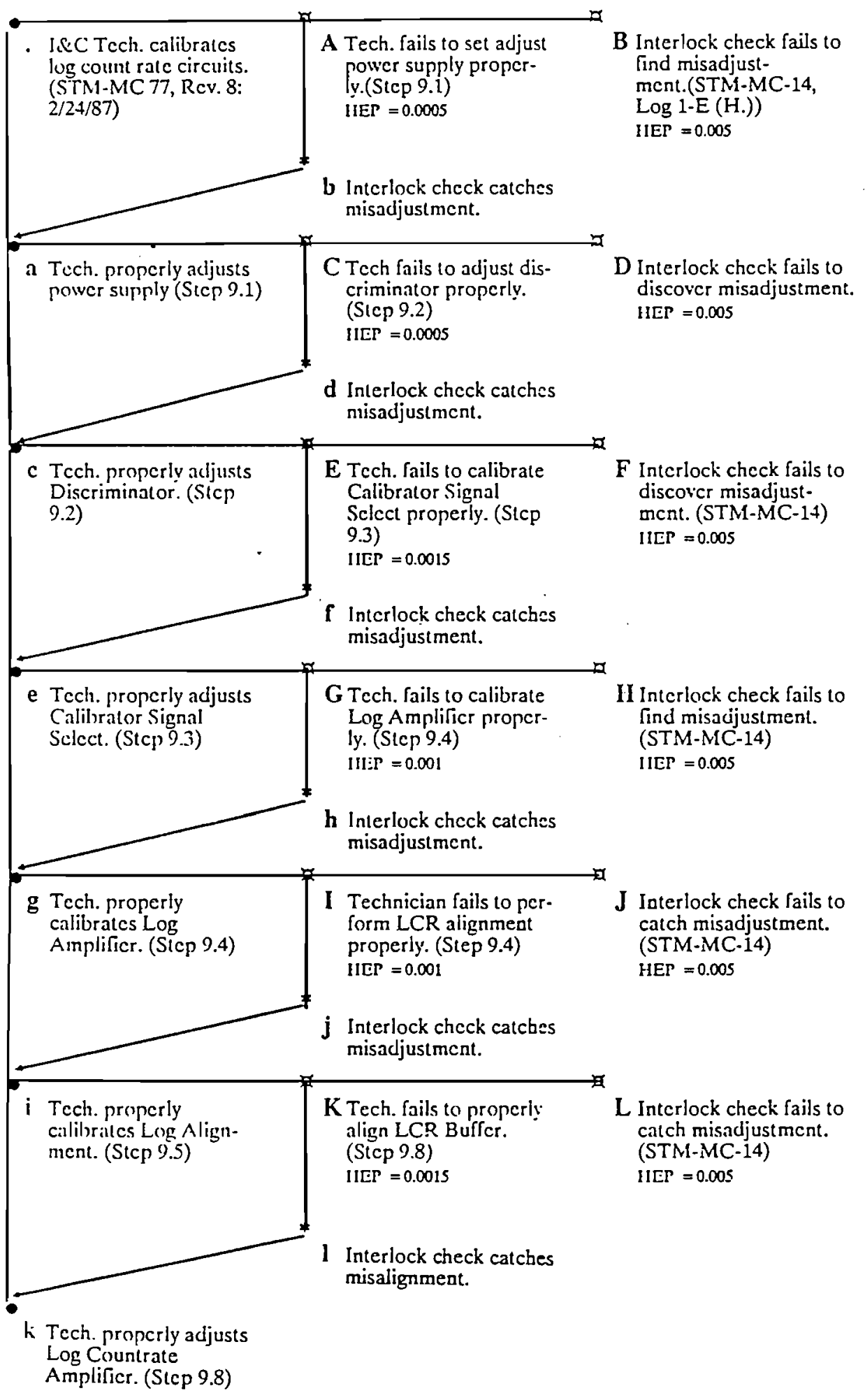

Fig. 8.13. Human Event Tree, FHCR 


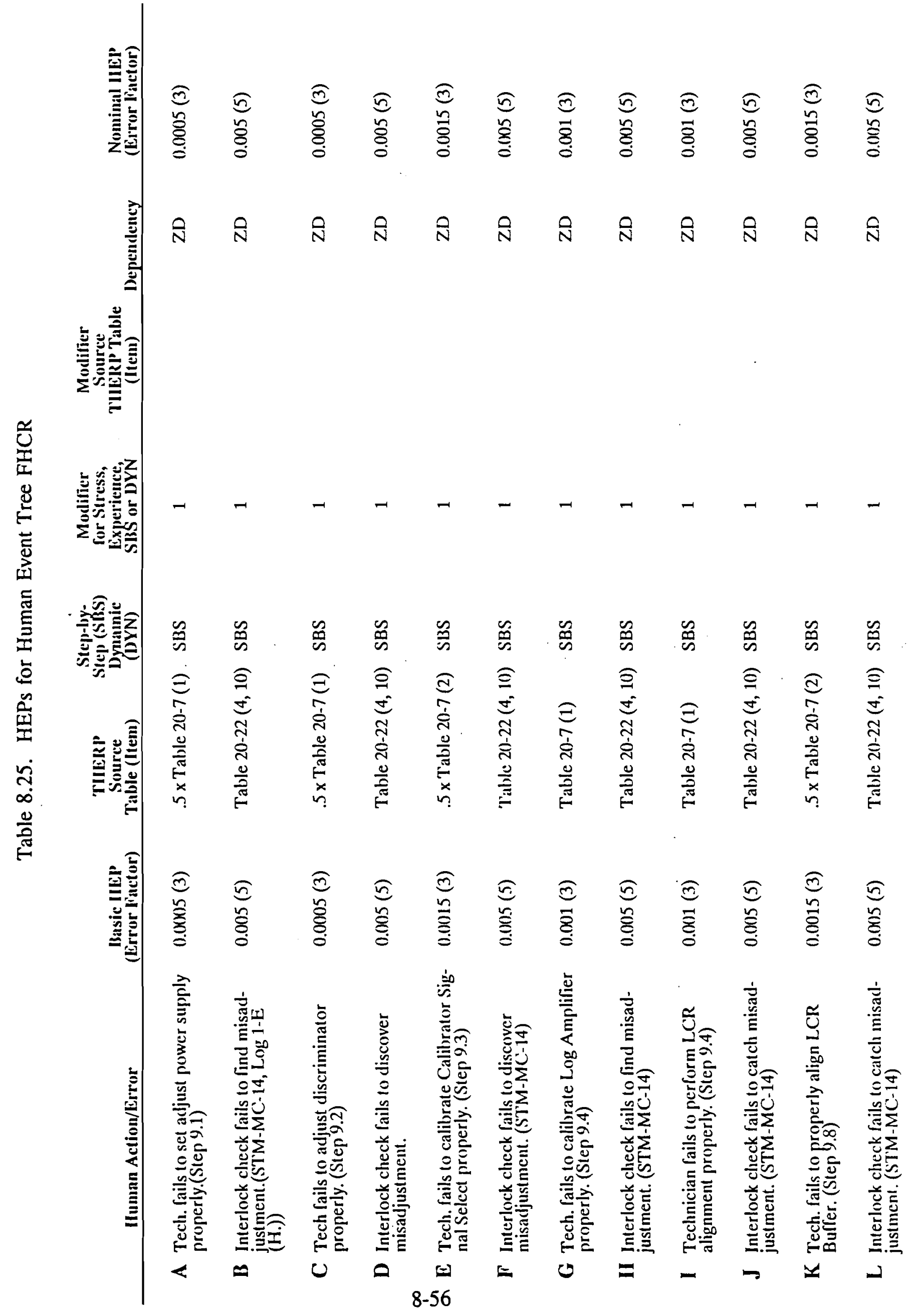




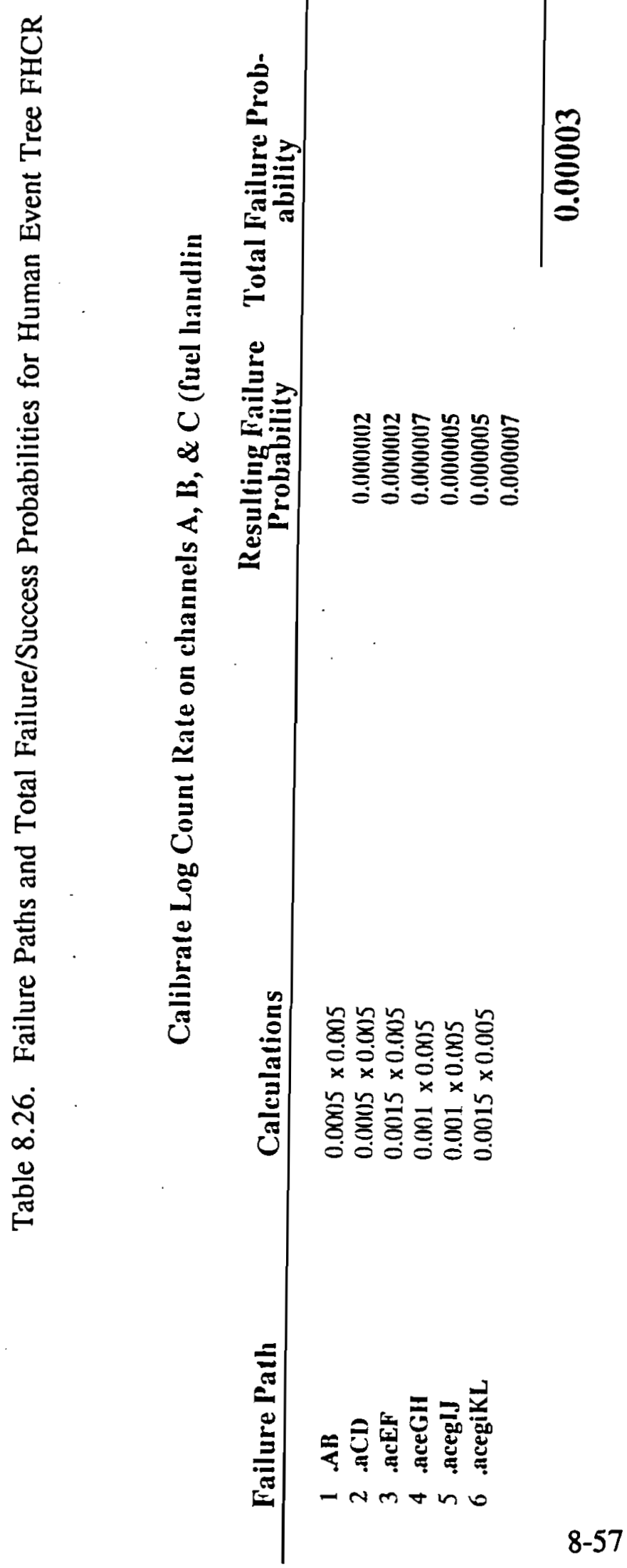


(STM-MC 14, Rev. 22) and checked again by the Technical Specification Compliance Log No. 1-E (Rev. 28). The probability of an undetected error in performing this procedure is low $\left(310^{-5}\right)$.

Event FHU002RZ, Error in Setting Trip Points for High and Low Log Count Rates: $\mathrm{p}=510^{-6}$ (Ref. Human Event Tree FHSP, Figure 8.14, Tables 8.27 and 8.28)

Similarly, the actuation of the automatic safety systems is dependent upon the accurate setting of the trip points for high count rate. Knowledge of instrument failure is dependent upon the accuracy of the low count rate settings. The trip point settings of the LCR instruments is part of the procedure for calibration of all ranges of the wide-range nuclear channels (STM-MC77, Rev. 8). However, the setting of trip points for this portion of the channels is separately checked by use of the "Unrestricted Fuel Handling Interlock Checks" (STM-MC 14, Rev. 22) and checked again by the Technical Specification Compliance Log No. 1-E (Rev. 28). The probability of an undetected error in setting the trip points is low $\left(510^{-6}\right)$.

\subsection{Other Human Errors During Operation}

In the systems analyses a number of other human errors were identified. Since these errors do not constitute safety significant errors, screening values are used.

\subsubsection{Valves and Dampers}

In the Shield Cooling System, the basic events listed below concern manual isolation valves which are located in the freon flowpath for backup cooling and are subject to a latent human error of improper valve line-up by being left closed. Since there is no flow in these freon lines during normal operation, the error is assumed to be undetectable until the backup cooling is in operation. 


\title{
HRA TREE DIAGRAM
}

\author{
ID: $\quad$ FHSP Tech. sets bistable trip points for $L C R A, B, \&$ C. (ficel hand) \\ Sequence: Unknown \\ Date modified: $\quad 03-A P R-90$ \\ Analyst: Schuman \\ NOTES: $\quad$ Technician sets $L C R$ bistable trip points for log count rate of channels $A, B$, and $C$. These settings are \\ checked by the Interlock checks before start up of the reactor. (Proc. STM-MC-77 Rev: 8:2/24/87)
}

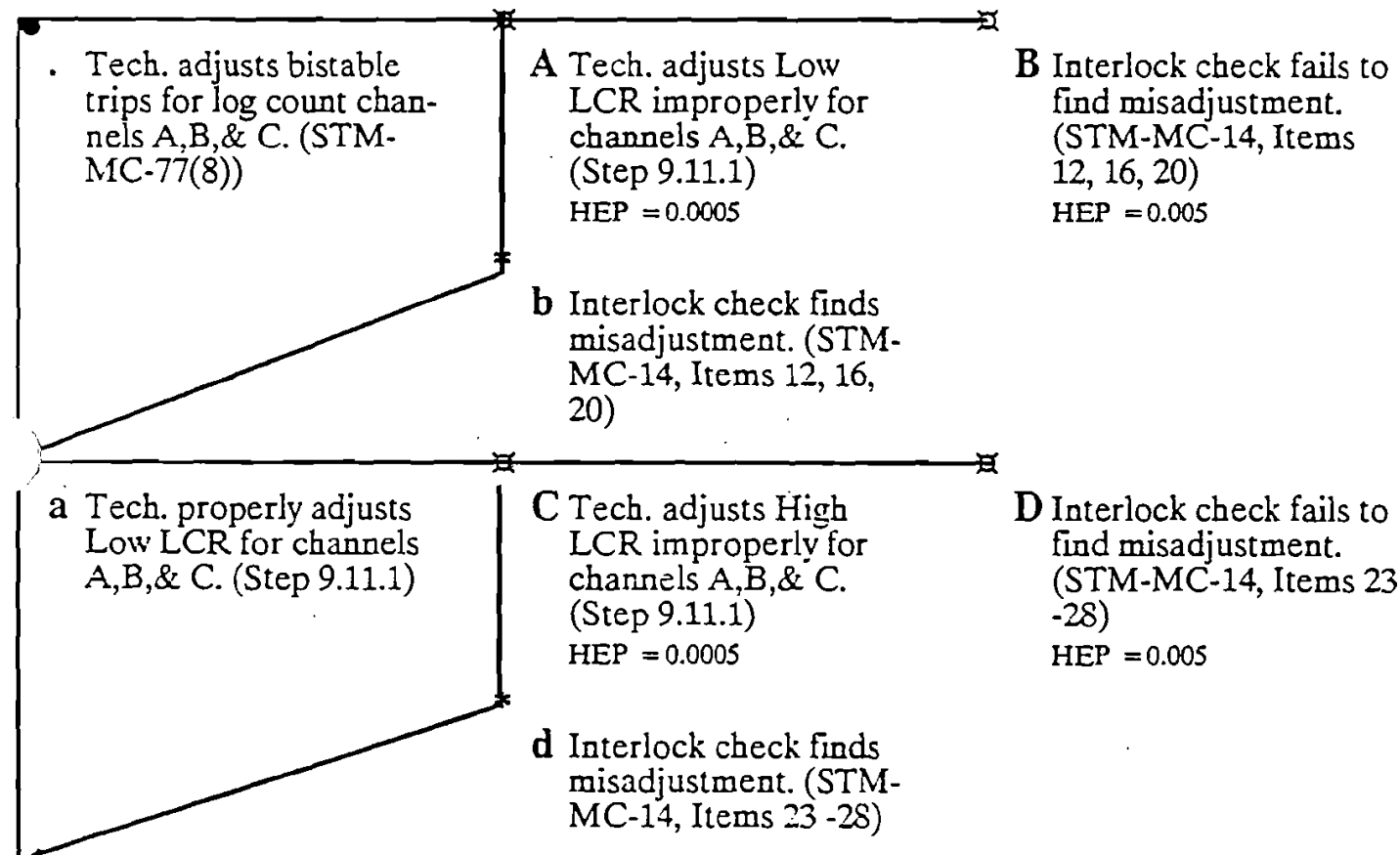

c Tech. adjusts High

LCR properly for chan-

nels A,B,\& C. (Step

9.11.1)

Fig. 8.14. Human Event Tree, FHSP 


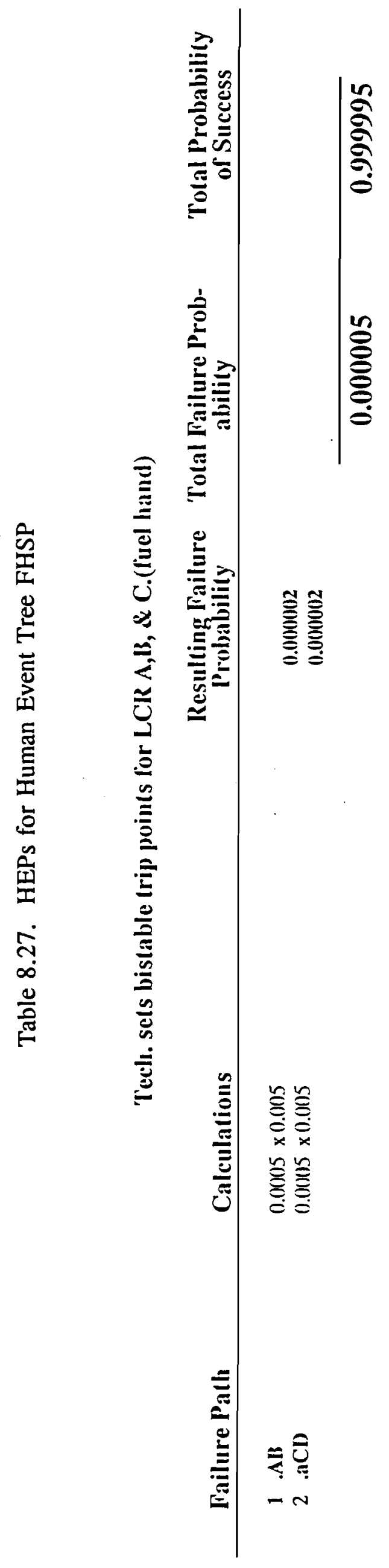




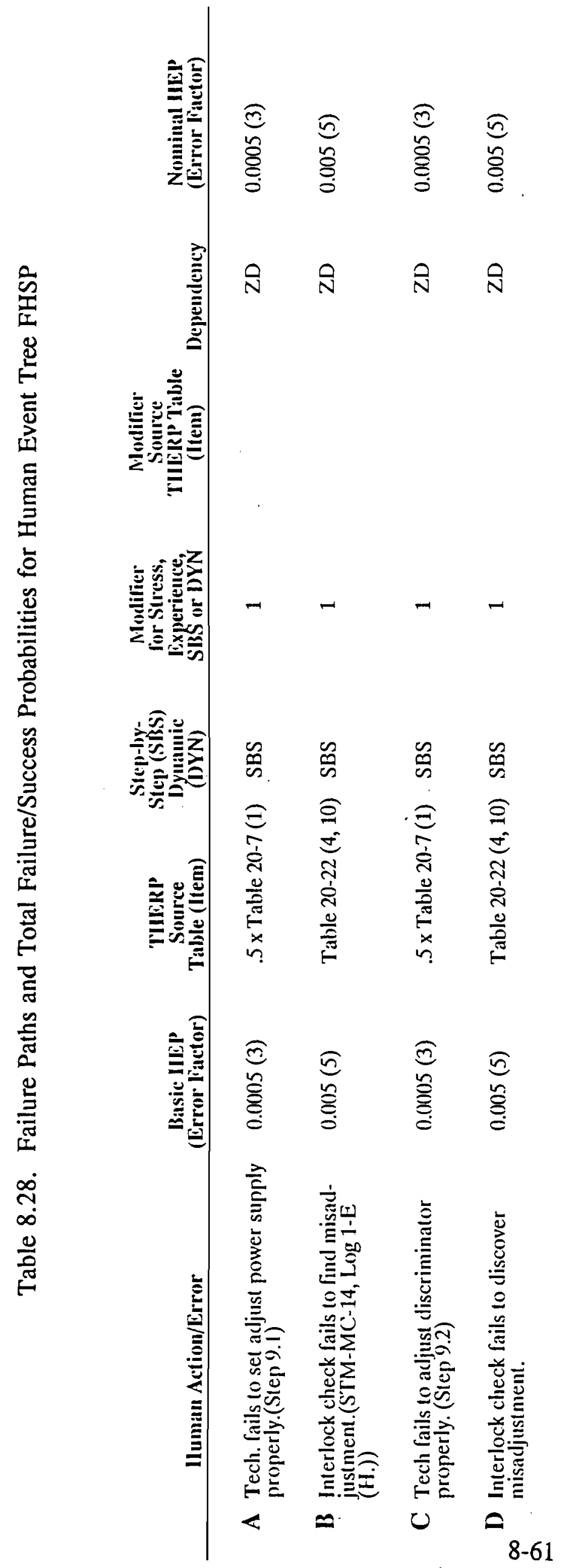




$\begin{array}{ll}\text { HXV958SL } & \text { COIL } 715 \text { \#2 ISOLATION LEFT CLOSED R8-V-958 } \\ \text { HXV959SL } & \text { STBY COILS 718 ISOLATION LEFT CLOSED R8-V-959 } \\ \text { HXV960SL } & \text { COIL } 715 \text { \#1 ISOLATION LEFT CLOSED R8-V-960 } \\ \text { HXV961SL } & \text { COIL } 718 \text { \# ISOLATION LEFT CLOSED R8-V-961 } \\ \text { HXV962SL } & \text { COIL 718 \#2 ISOLATION LEFT CLOSED R8-V-962 } \\ \text { HXV963SL } & \text { STBY COILS 715 ISOLATION LEFT CLOSED R8-V-963 } \\ \text { HGV735SL } & \text { COIL 715 \#2 EXPANSION VALVE LEFT CLOSED R8-VC-735 } \\ \text { HGV736SL } & \text { COIL 715 \#1 EXPANSION VALVE LEFT CLOSED R8-VC-736 } \\ \text { HGV737SL } & \text { COIL 718 \#1 EXPANSION VALVE LEFT CLOSED R8-VC-737 } \\ \text { HGV738SL } & \text { COIL } 718 \# 2 \text { EXPANSION VALVE LEFT CLOSED R8-VC-738 }\end{array}$

The mean value used is $1.010^{-4}$ based on the IREP number for manual valves failure [8.5] to operate which was identified as being dominated by human error (error factor 3). Since the data did not divide the human error and mechanical failures, an error factor of 10 was used. This same number was also applied to the basic events for manual isolation dampers in the shield cooling system listed below.

$\begin{array}{ll}\text { HDM741SL } & \text { ISOLATION DAMPER 741 LEFT CLOSED R8-DM-741 } \\ \text { HDM742SL } & \text { ISOLATION DAMPER 742 LEFT CLOSED R8-DM-742 } \\ \text { HDM746SL } & \text { ISOLATION DAMPER 746 LEFT CLOSED R8-DM-746 } \\ \text { HDM765SL } & \text { ISOLATION DAMPER 765 LEFT CLOSED R8-DM-765 } \\ \text { XV962SL } & \text { COIL 718 \#2 ISOLATION LEFT CLOSED R8-V-962 }\end{array}$

There is also a basic event for one isolation damper in the Thimble Cooling System which falls in this category.

TDM751SL ISOLATION DAMPER 751 LEFT CLOSED R8-DM-751 


\title{
8.4.2 Alarms
}

The human error of failing to respond to a control room alarm is $310^{-6}$ based on NUREG/CR-1278 (THERP handbook) chapter 11, page 48, [8.3] with two operators present. However, recent PRA's have used a value of $110^{-4}$ for this type of error. Therefore, the value used for event LIN12MBZ is $110^{-4}$.

\section{CSWMS2CL OPERATORS FAIL TO RELEASE MSW WITHIN 1 MINUTE AFTER ALARM}

The value in NUREG/CR-1278 page $10-15$ is $10^{-5}$ for two operators failing to repsond to an annunciator. This value is increased by a factor of 10 to account for the alarm being caused by a previous error by one of the operators involved and by a second factor of 10 for the possibility that the secondary operator may not be a qualified primary operator. The value used for CSWMS2CL is $110^{-3}$ per demand.

If the alarm is a low priority alarm which persists, it may be considered by the operators to be a nuisance alarm. In that case, the probability to fail to respond other than to silence the alarm may be as high as $110^{-2}$ for the two basic events:

\author{
DAN82LCZ \\ HUMAN ERROR BULK SODIUM LOW TEMP ALARM IGNORED \\ DAN500CZ HUMAN ERROR ANY T/C REF JUNCT > 152F ALARM IGNORED
}

\subsubsection{Maintenance and Testing}

The Continuous Power System is subject to a human error in the testing of the UPS batteries and the DC electrical system is subject to the same type of error in the testing of the station battery. The IREP value for this type of maintenance activity of $110^{-3}$ is applied to the basic events: 
$\begin{array}{ll}\text { UINV1BYL } & 135 \text { VDC UPS } 1 \text { BATTERY CAPACITY LOSS NOT DETECTED } \\ \text { UINV2BYL } & 135 \text { VDC UPS } 2 \text { BATTERY CAPACITY LOSS NOT DETECTED } \\ \text { LIN12CBL } & 125 \text { VDC STATION BATTERY CAPACITY LOSS NOT DETECTED }\end{array}$

\subsubsection{Acts of Commission}

In general actions of commission are not included in a PRA but selected inadvertent actions are included here due to potential consequences, particularly in delaying recovery actions.

In the Balance of Plant ( $\mathrm{Sec} \mathrm{Na} \&$ Steam), a possible human error action is an inadvertent operator action to dump secondary sodium. This basic event is:

\section{NSWOOCZ · INADVERTENT OPERATOR ACTION TO DUMP SECONDARY NA}

A mean of $110^{-4}$ is used. This is the result of starting with the screening number of $110^{-3}$ and reducing it one order of magnitude to account for the effect of the procedural requirements.

Another area where acts of commission are considered is the reactor control console. Operation of some of the switches has occurred and based on plant data [8.6], the following events are considered:

\section{CSWMSICZ MSW SWITCH HELD UP BY UNAUTHORIZED PERSON}

The frequency of CSWMS1CZ is 0.21 per year or $2.410^{-5} \mathrm{hr}^{-1}$. For this to happen the console access restriction must be violated.

CSWCONCZ CONSOLE ACCESS RESTRICTION VOLATED 
The probability for CSWCONCZ used in the EBR-II PRA is $510^{-2}$ demand. This value is based on the NUREG/CR-1278 Table 20-20 [8.3] value of $110^{-2}$ and an estimate of 5 demands per year for EBR-II.

\section{CSWMSZCZ OPERATOR HOLDS UP MSW UNTIL ALARM OCCURS}

A value of $210^{-2} \mathrm{yr}^{-1}$ or $2.310^{-6} \mathrm{hr}^{-1}$ is based on plant data [8.6].

\section{CSWMSCCL MSSW NOT SET TO NEUTRAL POSITION}

NUREG/CR-1278 page 13-9 has a value of $310^{-3}$ per demand for a similar error [8.3]. This value is converted to an hourly value of $1.6710^{-4} \mathrm{hr}^{-1}$ to account for the demand to set the switch to neutral every 18 hours.

In the Shutdown Cooler System there is an air operated valve, PA-154, which could cause inadvertent excess heat removal through the shield cooling system if inadvertently operated. This valve appears in the system notebook only and not in the accident sequence evaluation since excess shield cooling heat removal is an operational problem affecting plant efficiency and not an initiating event. The basic event is:

\section{DHU154PZ HUMAN ERROR IMPROPER VALVE OPERATION PA-154}

The value used is $110^{-4}$ which is a screening number. 


\subsection{Human Errors During Recovery}

The design of LMRs allows significant time for recovery action, particularly of decay heat removal systems. The minimal dependance on electrical systems allows recovery of many of these systems also.

\subsubsection{Recovery of Electrical Systems}

In the electrical systems, there are a number of alternate power supplies to various busses which involve either the closing of manual breakers or the switching of manual transfer switches. The human error in these cases is dominated mostly by the ability of the operator to diagnose the problem rather than to take the specific action. Loss of the normal power to an electrical bus is annunciated and the cause is usually obvious. For this reason the screening value of $110^{-4}$ was used. The breaker related human error basic events are as follows:

ECB11BYZ OPERATOR ERROR IN CONNECTING E-1 TO R-1B

ECB1PBYZ OPERATOR ERROR IN CONNECTING E-1 TO P-1B

ECB2DHYZ OPERATOR ERROR FAILS TO CLOSE E-1 BREAKER 2D

JCBN3AVZ OPERATOR FAILS TO CLOSE NORM BUS NO.1 BRKR 3A

The manual transfer switch basic events are:

ESWE1RYZ OPERATOR FAILS TO EFFECT E-1 TO R-2B LINE ON DEMAND

ESWP4NYZ OPERATOR FAILS TO SWITCH P-4E MAN. TRANSF. SW TO NORM. PWR

LSWME1BZ OPERATOR FAILS TO CLOSE 125 VDC MTS 1 EMERG BUS SIDE

LSWME2BZ OPERATOR FAILS TO CLOSE 125 VDC MTS 2 EMERG BUS SIDE

LSWME3BZ OPERATOR FAILS TO CLOSE 125 VDC MTS 3 EMERG BUS SIDE 


\section{LSWME4BZ OPERATOR FAILS TO CLOSE 125 VDC MTS 4 EMERG BUS SIDE \\ LSWUPTBZ OPERATOR ERROR FAILS TO SWITCH TO UPS 1 BAT.}

\subsubsection{Recovery of Decay Heat Removal Systems}

The primary recovery action considered is in the event of the irrecoverable loss of both shutdown coolers while the secondary system is drained.

Event BOO01ZOO. Failure to Recover from loss of Decay Heat Removal $\left(p=410^{-6}\right)$ (Ref. Human Event Tree, Figure 8.15. Tables 8.29 and 8.30)

This event is presumed to result from the loss of both shutdown coolers during shutdown, with the secondary sodium loop drained. There are two operator actions that will recover from this situation: refill secondary loop to institute cooling from Balance of Plant (requires 48 hours to complete from cold pipes - or about $31 / 2$ hours if the system is heated) or start shield/thimble cooling. Any one of these two actions will serve to delay heat-up to unacceptable temperatures while other measures are instituted or additional problem-solving help (engineering staff) is obtained.

These two actions provide opportunity for two human errors of omission in addition to failure to respond to the annunciators, failure to perform these actions could result in overheating. The Human Error Probability (HEP) for each of two three actions is listed below. The total failure probability, given above, is the product of these two individual probabilities. These probabilities are estimated conservatively from Table 20-1 of NUREG-1278 [8.3], using the assumption that only 60 minutes is available for diagnosis and action. 


\section{HRA TREE DIAGRAM}

ID: ARGI OPERATORS FAII TO RECOVER FROM LOSS OF CORE COOLING

Sequence: [TBD]

Date modined: $25-S E P-90$

Aaslyst: SCHURMAN

NOTES: THIS EVENT OCCURS WTH THE LOSS OF BOTH SHUTDOWN COOLERS WITH THE SECONDARY SODIUM LOOP DRAINED

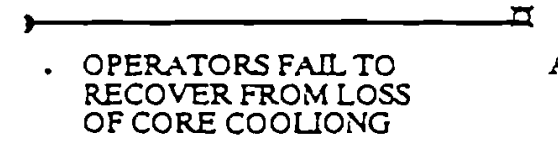

A OPERATORS FAII TO DLAGNOSE PROBLEM WITHIN $60 \mathrm{MIN}$. AFTER LOSS IS ANNUNCLATED HEP $=Q 000000 \mathrm{~s}$

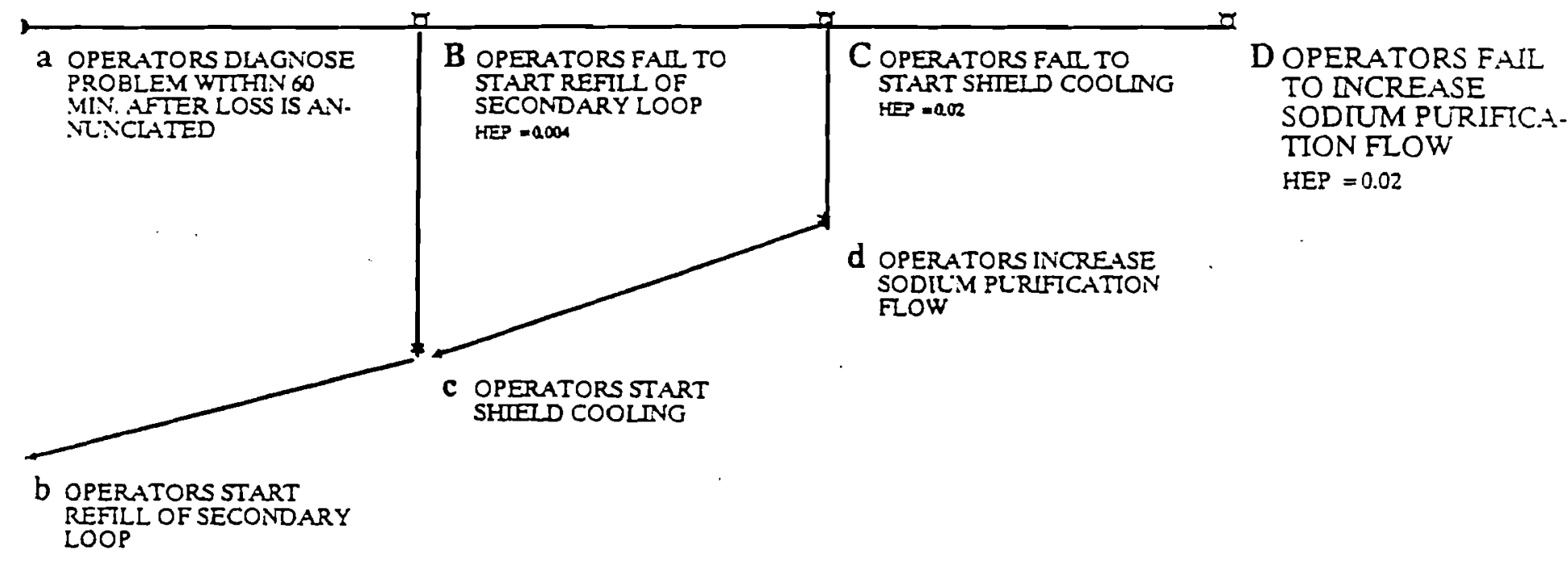

Fig. 8.15. Human Event Tree, ARGI 
Table 8.29. HEPs for Human Event Tree ARGI

\begin{tabular}{|c|c|c|c|c|c|c|c|c|}
\hline & Iluman Aclion/Error & $\begin{array}{c}\text { Basic IIEP } \\
\text { (Error Factor) }\end{array}$ & $\begin{array}{c}\text { TIIERP } \\
\text { Source } \\
\text { Table (Item) } \\
\end{array}$ & $\begin{array}{c}\text { Slep-by: } \\
\text { Slep (SHS) } \\
\text { Dynanic } \\
\text { (DYN) }\end{array}$ & $\begin{array}{l}\text { Modifier } \\
\text { for Stress, } \\
\text { Lxperience, } \\
\text { SIIS or DYN } \\
\end{array}$ & $\begin{array}{c}\text { Modifier } \\
\text { Source } \\
\text { TIIERP Table } \\
\text { (Item) } \\
\end{array}$ & Dependency & $\begin{array}{l}\text { Nominal IIEP } \\
\text { (Error Factor) }\end{array}$ \\
\hline A & $\begin{array}{l}\text { OPERATORS PAIL TO DIAGNOSE } \\
\text { PROMINM WTIIIN } 60 \text { MIN. AI'IIR LOSS } \\
\text { IS ANNUNCIA'TIII) }\end{array}$ & $0.000003(30)$ & \multicolumn{2}{|c|}{ TABLE 20-3 (5) LBDYN } & 1 & CLEAR SIG. & $\mathrm{ZD}$ & $0.000003(30)$ \\
\hline B & $\begin{array}{l}\text { OPRRATORS PAII TO START REPLL } \\
\text { OPSECONISARY LOOP }\end{array}$ & $0.004(5)$ & \multicolumn{2}{|c|}{ TABLE 8-5 (3) LB DYN } & 1 & PRACTICED & $\mathrm{ZD}$ & $0.004(5)$ \\
\hline & $\begin{array}{l}\text { OPRRATORS PAIL TO START SIIIELD } \\
\text { COOLING }\end{array}$ & $0.02(5)$ & TABLE 8-5 (3) & DYN & 1 & & $\mathrm{ZD}$ & $0.02(5)$ \\
\hline & $\begin{array}{l}\text { OPRRATORS PAIL TO INCREASE } \\
\text { SODIUM PURIPICAITONIR.OW }\end{array}$ & $0.02(5)$ & TABLE 8-5 (3) & DYN & 1 & . & $\mathrm{ZD}$ & $0.02(5)$ \\
\hline
\end{tabular}

$\infty$
1
0 


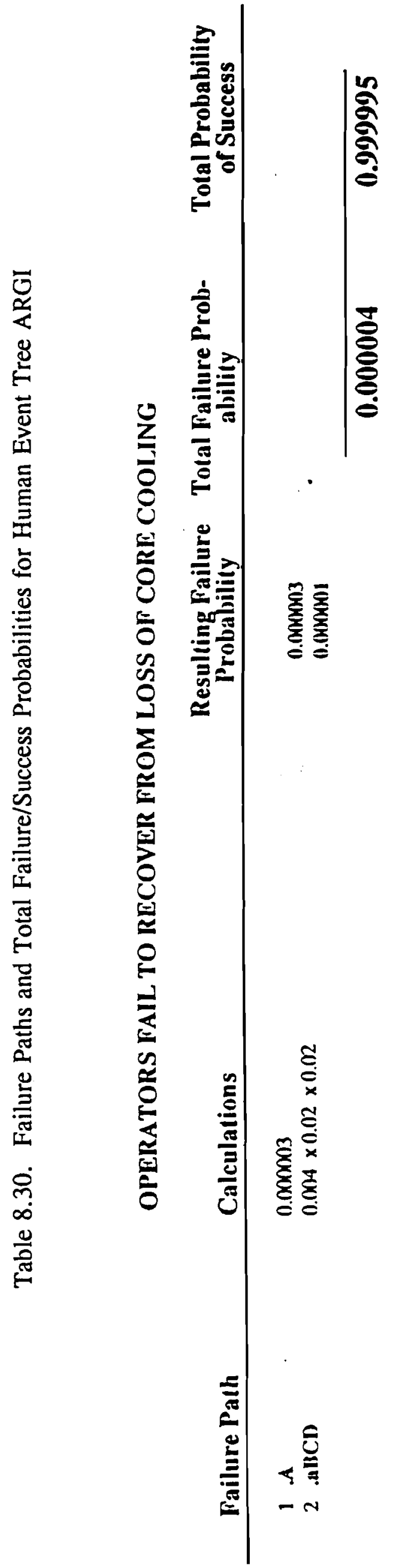


The operators are trained on this situation and trained to use shield cooling and the cooling effects from increased sodium purification flow to maintain shutdown cooling margins. They are trained and practiced on refilling the secondary loop and viewing the secondary loop as a cooling factor in controlling core temperature (although the usual case is throttling secondary loop circulation to prevent overcooling during normal shutdowns). Nonetheless, the operators are trained, in case of loss of cooling, to use or return to use the secondary sodium system.

Operators fail to diagnose problem within 60 minutes after loss of shutdown cooling is annunciated, $\left(p=310^{-6}\right.$. Table 20-3(5), Lower Bound)

The lower bound is used because operators, in fact, have much more than 60 minutes to respond and make a diagnosis and because the diagnosis is quite clear.

Operators fail to refill Secondary Loop. $\left(p=410^{-3}\right.$, ASEP (NUREG/CR-4772) Table 8-5 Item (3), Lower Bound)

This action is practiced and the time required is limited by the temperature of the secondary loop pipes and their heat-up time. The lower bound was used because the action is well-learned.

Operators fail to start Shield Cooling, $\left(\mathrm{p}=210^{-2}\right.$, ASEP (NUREG/CR-4772) Table 8-5 Item (3))

The median value is used in this case because the task is trained during initial training and discussed periodically, but not retrained or practiced.

The recoverable loss of shutdown coolers is modeled in the decay heat removal trees and dominated by the failure to diagnose the problem $10^{-6}$ as shown in the analysis above.

DHU000OL HUMAN ERROR - FAILURE TO RECOVER COOLERS 
Action to restore the recoverable loss of shutdown coolers is simple and may be accomplished by a number of diverse actions (e.g. disconnecting electric power to air solenoids, closing off instrument air to the cooler actuators, inputting high temperature test signal, venting air from shutdown cooler actuators, or removing linkage pins). Many of these actions are practiced. The temperature test signal is input before every reactor run. The closing of instrument air and venting actuators is done at least twice per year during the cooldowns associated with the long shutdown.

In other systems such as shield cooling, a spurious breaker trip of a component resulting in a signal based on current, voltage, or power has a higher probability of noncorrective action than an alarm based on flow which indicates a more urgent need for action. In both cases, the values used depend on the existence of specific procedures as opposed to operating in the troubleshooting mode. The following values are used: (1) Flow alarm with recovery procedure, $110^{-2}$; (2) current, voltage, power alarm with recovery procedure, $110^{-1}$; (3) Flow alarm in troubleshooting mode, $510^{-1}$; (4) current, voltage, power alarm in troubleshooting mode, $510^{-1}$.

In the Shield Cooling System and Thimble Cooling System the value of $210^{2}$ is used for the basic events,

HHU002YL NO RECOVERY ACTION TO RESET CIRCUIT BREAKER

THU002YL NO RECOVERY ACTION TO RESET CIRCUIT BREAKER

Failure of recovery actions for shifting the filters in the event of a clogged filter is 2 $10^{-2}$ since this action is performed on an annual basis for filter replacement.

\section{HHU01BSZ NO RECOVERY ACTION FOR CLOGGED FILTER R8-FL-717}

The motor dampers in both the Shield Cooling System, the Thimble Cooling System, and the Stack Exhaust System have integral handwheels in the motor operators for use in the event that the motor fails. The actions to use these handwheels are taught during operator qualification on the systems. There is no repeat training on these values but there is training on the steam 
isolation valve located in the sodium boiler building which is of similar construction and operation but different in size. The value used therefore is $110^{-2}$ for the basic events for Shield and Thimble Cooling dampers.

\section{HHU003SL NO RECOVERY ACTION TO USE DAMPER MANUAL HANDWHEEL \\ HHU003YL NO RECOVERY ACTION TO USE DAMPER MANUAL HANDWHEEL \\ THU003SL NO REOVERY ACTION TO USE DAMPER MANUAL HANDWHEEL}

Dampers in the stack exhaust system are operated by non-EBR-II personnel and a higher failure rate of $110^{-1}$ was used.

\section{HHU004SL NO RECOVERY ACTION TO USE MOVE DAMPER MANUALLY}

The pneumatic dampers in both the Shield Cooling System and the Thimble Cooling System require either a connecting pin or bolt to be removed for manual operation if the pneumatic operators fails. These actions to remove the pin or bolt are taught during operator qualification on the systems and there is annual training on the actions. The value used for failure to recover is $110^{-1}$ for the basic event,

\section{HHU004SL NO RECOVERY ACTION TO USE MOVE DAMPER MANUALLY}

In the event that flow in the Thimble Cooling System is lost due to failure of either the exhaust fans or exhaust flowpath, the system can be cross-connected to the Shield Cooling System by opening a manual damper. For failure to perform this recovery action a number of $210^{-2}$ is used for the basic event, 


\subsubsection{Recovery of ControL Rod Malfunctions}

There are several recovery actions associated with control rod malfunctions.

\section{CSWMSWCZ OPERATOR FAILS TO FREE STUCK SWITCH}

A value of $310^{-1}$ per demand is used although a failure such that the operator would be physically unable to open the up contacts appears to be extremely unlikely, based on examination of the switch. The operator did free the switch in the one event when it stuck [8.6]. It is a natural reaction for him to try.

\section{CSWPSBCL OPERATOR FAILS TO PUSH PSB2}

A value of $1.6710^{-4} \mathrm{hr}^{-1}$ is used based on NUREG/CR-1278 page 13-9 [8.3] value of 3 $10^{-3}$ per demand for a similar error and converted to $1 \mathrm{hr}^{-1}$ to use the code input option that can account for the demand to set the switch to neutral every 18 hours.

\section{CSWMS4CZ OPERATOR HOLDS MSW UP INSTEAD OF DOWN}

CMWMS4CZ is based on the NUREG/CR-1278 Table 12-1 value of $5 \times 10^{-4}$ with a Performance Shaping Factor (PSF) of 50 to account for a stress level between moderately high and extremely high page 17-17 [8.3]. This gives a mean for CSWMS4CZ of $2.510^{-2}$ per demand.

CSWMSICL OPERATORS FAIL TO FREE MSW FROM UNAUTHORIZED PERSON

A value of $5 \times 10^{-2}$ is used.

NUREG/CR-1278 Table 20-16 states that fialure to detect an error of commission is the basic HEP $\mathrm{x}$ 10. Since CSWCONCZ is based on $0.01 / \mathrm{d}$, this implies 0.1 . However, the high 
flux alarms should reduce the failure probability by at least a factor of 2 . No credit is taken for the fact that two operators are in a position to intervene.

\section{CSWMS3CZ OPERATOR CLOSES RELAY, THEN FAILS TO COMPENSATE WITH CONTROLLING ROD}

The probability of compensation depends on whether the controlling rod is \#4. Assuming the operator does not take the unusual action of bringing another rod into play if \#4 is the controlling rod. For \#4 to be the controlling rod the probability is $110^{-1}$. If \#4 is not the controlling rod (probability $910^{-1}$ ) the operator will be expecting to balance rod \#4 with another rod. Under normal conditions this action is similar to item 7 on page 13-6 of NUREG/CP-1278, which has an HEP of 0.003 . But rod \#4 will move before it is expected to, which can be accounted for by a PSF. The stress level is judged to be moderately high, which introduces a PSF of 5. The failure role is therefore:

$$
(0.1)(1)+(0.9)\left(310^{-3}\right)(5)=1.110^{-1}
$$

\section{CHUOP1CL OPERATOR DECIDES TO COMPENSATE WITH 2ND ROD}

Value used is 0.1 per demand. There is no procedure for such action. It is inconsistent to make this probability large when corrective action has been assumed not to occur with the causes that dominate run-in frequency for the first rod.

\subsection{Summary}

The human error probabilities derived in this section have been used in quantifying the accident sequence trees. The results of the quantification are given in Sections 11, 12 and 13 while a complete listing of the fault tree used in the quantification can be found in Appendix B.

The results of the human reliability analysis described here show a very limited dependence of EBR-II on human error. Those events which do have significance are related to failure to scram, one maintenance error possibility and one dynamic error possibility, and failure 
to recover from a failure of decay heat removal. The last event has a low proability but is important nonetheless because the decay heat removal system is otherwise reliable. 


\section{Section 8.0 References}

8.1 Military Specifications Civil/Construction: C004, C005, C006, C007, C015, C017, C019, C021, C022.

8.2 "Guidelines for the Preparation of Emergency Procedures," NUREG-0899, USNRC, August 1982.

8.3 A.D. Swain and H. E. Guttman, "Handbook of Human Reliability with Emphasis on Nuclear Power Plant Applications, " NUREG/CR-1278, Sandia Laboratories draft report, October 1980.

8.4 A. D. Swain, "Accident Sequence Evaluation Program: Human Reliability Analysis Procedure," NURGEG/CR-4772, Sandia Laboratories, February 1987.

8.5 R. J. Borkowski, et.al., "The In-Plant Reliability Dața Base for Nuclear Plant Components: Interim Data Report - The Valve Component," NUREG/CR-3154, December 1983.

8.6 R. W. Schaefer, Argonne National Laboratory, unpublished information (1991). 
9.0 SEISMIC ANALYSIS 


\subsection{SEISMIC ANALYSIS}

(Preparation of this section in progress; publication date is TBD) 
10.0 EXTERNAL EVENTS 


\section{Table of Contents}

Page

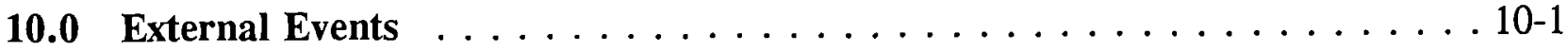

10.1 Introduction . . . . . . . . . . . . . . . . . . 10-1

10.1.1 Identification of External Events . . . . . . . . . 10-2

10.1.2 Screening Methodology . . . . . . . . . . . . 10-2

10.2 Sodium Fires $\ldots \ldots \ldots \ldots \ldots \ldots \ldots \ldots \ldots \ldots$

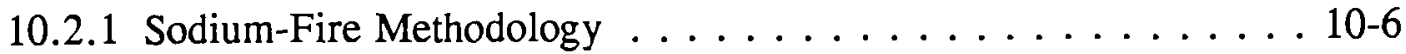

10.2.2 Screening by Location . . . . . . . . . . . . . 10-8

10.2.2.1 Negative Reactivity Functional Failure . . . . . . . 10-8

10.2.2.2 Primary Coolant Inventory (LOSA) Functional Failure . 10-9

10.2.2.3 Decay Heat Removal Functional Failure . . . . . . 10-10

10.2.2.4 Screening Location Results . . . . . . . . . . . . 10 10-10

10.2.3 Quantification . . . . . . . . . . . . . . . 10-11

10.2.3.1 Initiating Event Identification . . . . . . . . . . 10-11

10.2.3.2 Initiating Event Frequency . . . . . . . . . . . . 10-15

10.2.3.3 Sodium Fire Detection . . . . . . . . . . . 10-17

10.2.3.4 Reactor Shutdown . . . . . . . . . . . . 10-18

10.2.3.5 Limited Leak vs Rupture . . . . . . . . . . . . . 10 10-20

10.2.3.6 Prevention of Cable Damage . . . . . . . . . 10-21

10.2.3.7 Operation of Unaffected Systems . . . . . . . . . 10-21

10.2.4 Analysis Results . . . . . . . . . . . . . . . . . . 10-22

10.2.5 Planned Modifications to Address Vulnerability . . . . . . . 10-23

10.3 Fires . . . . . . . . . . . . . . . . . . . . . 10-27

10.3.1 Plant Impacts From Fire Initiators . . . . . . . . . . . 10-28

10.3.2 Ongoing Fire Safety Improvements . . . . . . . . . . 10-29

10.4 Tornadoes and High Winds . . . . . . . . . . . . . 10-30

10.4.1 Tornadoes . . . . . . . . . . . . . . . . 10-30

10.4 .2 High Winds . . . . . . . . . . . . . 10-33

10.5 Volcanism . . . . . . . . . . . . . . . . 10-34

10.6 External Floods . . . . . . . . . . . . . . . . . . 10-36

10.7 Lightning . . . . . . . . . . . . . . . . . . . . 10-37

10.8 Aircraft Impact . . . . . . . . . . . . . . . . . . . 10-38

10.9 Accidents at Other INEL Facilities (Industrial and Military) . . . 10-40

10.10 Turbine Missiles . . . . . . . . . . . . . . . . . . . 10-40

10.11 Internal Floods $\ldots \ldots \ldots \ldots \ldots \ldots \ldots \ldots \ldots$. . . . . . . . . . . . . .

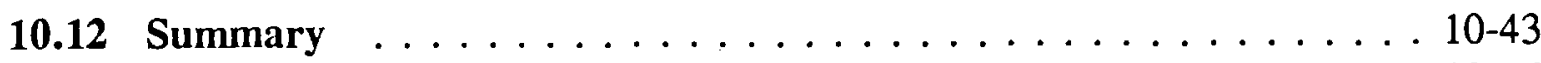

Section 10.0 References . . . . . . . . . . . . . . . . . . . . . . . 10-46 


\section{$\underline{\text { List of Figures }}$}

Page

10.1 Event Tree for Sodium Fire Sequences . . . . . . . . . . . . . . . . . 10-12

10.2 Reactor Building (767) Operating Floor Fire Detectors . . . . . . . . . . . . 10-16

10.3 Event Tree Results for Secondary Sodium Fire in Containment . . . . . . . . 10 10-24

10.4 Shutdown Cooler NaK Fire in Containment . . . . . . . . . . . . . . . 10-25

10.5 Event Tree for Tornado . . . . . . . . . . . . . . . . . . . . . 10-32

10.6 Volcanic Structures and Rift Zones near the INEL Site . . . . . . . . . . 10-35 


\section{List of Tables}

Page

10.1 Natural and Man-Induced External Events to be Considered in PRA Studies . . . 10-3

10.2 Summary of External Event Initiating Events and Sequences . . . . . . . . . . 10-44 


\subsection{EXTERNAL EVENTS}

\subsection{Introduction}

External events, as assessed in PRAs, typically include seismic activity, weather-related phenomena such as tornadoes, high winds, and lightning along with floods and aircraft impact, to evaluate their potential contribution to plant risk. In addition to these natural and maninduced hazards, the scope for some PRAs is extended to include internal flooding, fires, and turbine missiles. Although these latter events are not external events in the strict sense, they have the potential to defeat multiple plant safety systems, and are therefore, generally included under the external events category.

\subsubsection{Identification of External Events}

Selection of the external events to be considered for the EBR-II PRA involved a two-tier screening process. The first level of screening was based upon guidance in the PRA Procedures Guide (NUREG/CR-2300) [10.1], which provides a suggested list of natural and man-made external events that should be considered in a PRA study. It also suggests applying a set of screening criteria to identify only those significant external events to be included for further analyses. Table 10.1 is the list from the PRA procedures guide. The screening criteria suggested by the PRA procedures guide were:

1. The event is of equal or lesser damage potential than the events for which the plant has been designed.

2. The event has a significantly lower mean frequency of occurrence than other events with similar uncertainties and could not result in worse consequences than those events.

3. The event cannot occur close enough to the plant to affect it.

4. The event is included in the definition of another event. 
After applying these criteria as shown in Table 10.1, the following external events were subjected to the second tier of the screening process:

\begin{tabular}{ll} 
- & Fires (Liquid Metal and Conventional) \\
- & Tornadoes and High Winds \\
- & Volcanism \\
- & External Floods \\
- & Lightning \\
- & Aircraft Impact \\
- & Accident at Other INEL Facilities \\
- & Turbine Missiles \\
- & Internal Floods \\
\hline
\end{tabular}

\subsubsection{Screening Methodology}

Although external initiating events may impact facility operation and result in plant unavailability, they are only considered important initiating events if they can require plant shutdown, either automatically or manually, in response to the external event, and lead to a core vulnerable condition at a frequency commensurate with those sequences initiated by internal events. While seismic events are generally expected to dominate the risk contribution from external events, some PRA studies have found events such as fires and internal floods to significantly contribute to overall plant risk usually through the total loss of electrical zones.

The following sections describe the specific analysis approach used to address each external event except seismic. Seismic events, due to their relative importance, are covered in Section 9. 
Table 10.1. Natural and Man-induced External Events to be Considered in PRA Studies

\begin{tabular}{|c|c|c|}
\hline Event & $\begin{array}{l}\text { Applicable } \\
\text { screening } \\
\text { criterion } \\
\text { [10.1] }\end{array}$ & Remarks \\
\hline Aircraft impact & -- & Requires further analysis \\
\hline Avalanche & 3 & Can be excluded for EBR-II site \\
\hline Coastal erosion & 3 & Can be excluded for EBR-II site \\
\hline Drought & 1 & $\begin{array}{l}\text { Excluded since the ultimate heat sink } \\
\text { (shutdown coolers) is not affected by } \\
\text { drought }\end{array}$ \\
\hline External flooding & -- & Requires further analysis \\
\hline Extreme winds and tornadoes & -- & Requires further analysis \\
\hline Fire & -- & Requires further analysis \\
\hline Fog & 4 & $\begin{array}{l}\text { Considered under man-made hazards } \\
\text { involving aircraft }\end{array}$ \\
\hline Forest fire & 1,3 & $\begin{array}{l}\text { Fire cannot propagate to the site } \\
\text { because the site is cleared }\end{array}$ \\
\hline Frost & 1 & Snow and ice govern \\
\hline Hail & 1 & Other missiles govern \\
\hline $\begin{array}{l}\text { High tide, high lake level, or } \\
\text { high river stage }\end{array}$ & 4 & Included under external flooding \\
\hline High summer temperature & 1 & $\begin{array}{l}\text { Ultimate heat sink is designed for at } \\
\text { least } 45 \text { days of operation, even } \\
\text { under high summer temperatures }\end{array}$ \\
\hline Hurricane & 3 & Can be excluded for EBR-II site \\
\hline Ice cover & 1,4 & $\begin{array}{l}\text { Ice blockage of river included in } \\
\text { flood }\end{array}$ \\
\hline $\begin{array}{l}\text { Industrial or military facility } \\
\text { accident }\end{array}$ & -- & Requires further analysis \\
\hline Internal flooding & -- & Requires further analysis \\
\hline Landslide & 3 & Can be excluded for EBR-II site \\
\hline Lightning & 1 & $\begin{array}{l}\text { Considered in plant design and in } \\
\text { analysis }\end{array}$ \\
\hline
\end{tabular}


Table 10.1. Natural and Man-induced External Events

to be Considered in PRA Studies (Cont'd)

\begin{tabular}{|c|c|c|}
\hline Event & $\begin{array}{l}\text { Applicable } \\
\text { screening } \\
\text { criterion } \\
\text { [10.1] }\end{array}$ & Remarks \\
\hline Low lake or river water level & 1 & $\begin{array}{l}\text { Ultimate heat sink is designed for at } \\
\text { least } 45 \text { days of operation; does not } \\
\text { require cooling water }\end{array}$ \\
\hline Low winter temperature & 1 & $\begin{array}{l}\text { Ultimate heat sink standby operation } \\
\text { precludes effects of icing and thermal } \\
\text { stresses }\end{array}$ \\
\hline Meteorite & 2 & Insignificant frequency of occurrence \\
\hline Pipeline accident (gas, etc.) & 3 & Can be excluded for EBR-II site \\
\hline Intense precipitation & 4 & $\begin{array}{l}\text { Included under external and internal } \\
\text { flooding }\end{array}$ \\
\hline $\begin{array}{l}\text { Release of chemicals in onsite } \\
\text { storage }\end{array}$ & 1,3 & $\begin{array}{l}\text { Limited quantities of hazardous } \\
\text { chemicals quantities which could } \\
\text { effect plant safety stored onsite. } \\
\text { Chemical processing plant several } \\
\text { miles distant }\end{array}$ \\
\hline River diversion & 1,4 & $\begin{array}{l}\text { Considered in the evaluation of } \\
\text { external flooding }\end{array}$ \\
\hline Sandstorm & 1 & $\begin{array}{l}\text { Potential blockage of air intakes with } \\
\text { particulate matter is generally } \\
\text { considered in plant design }\end{array}$ \\
\hline Seiche & 3 & Can be excluded for EBR-II site \\
\hline Seismic activity & -- & Requires detailed study \\
\hline Snow & 1,4 & $\begin{array}{l}\text { Ultimate heat sink standby operation } \\
\text { precludes effects of icing and thermal } \\
\text { stresses; snow melt causing river } \\
\text { flooding is included under external } \\
\text { flooding }\end{array}$ \\
\hline Soil shrink-swell consolidation & 1 & $\begin{array}{l}\text { Site-suitability evaluation and site } \\
\text { development for the plant are } \\
\text { designed to preclude the effects of } \\
\text { this hazard }\end{array}$ \\
\hline
\end{tabular}


Table 10.1. Natural and Man-induced External Events to be Considered in PRA Studies (Cont'd)

\begin{tabular}{lcl}
\hline & $\begin{array}{c}\text { Applicable } \\
\text { screening } \\
\text { criterion } \\
{[\mathbf{1 0 . 1 ]}}\end{array}$ & \multicolumn{1}{c}{ Rvent } \\
\hline Storm surge & 4 & Included under external flooding \\
Transportation accidents & 2,3 & $\begin{array}{l}\text { Vehicle traffic on-site controlled, } \\
\text { closest highway over } 3 \text { miles away }\end{array}$ \\
Tsunami & 3 & Can be excluded for EBR-II site \\
Toxic gas & 3 & Can be excluded for EBR-II site \\
Turbine-generated missile & -- & Requires further analysis \\
Volcanic activity & -- & Requires further analysis \\
Waves & 3 & Can be excluded for EBR-II site \\
\hline
\end{tabular}




\subsection{Sodium Fires}

Sodium is the primary coolant in EBR-II, and is also used for secondary system heat transport to the plant steam evaporators and superheaters. $\mathrm{NaK}$ (sodium-potassium) coolant is used for the shutdown cooler natural circulation loops and in the cold trap cooling system.

Although of different chemical compositions, both sodium and $\mathrm{NaK}$ may burn in air. At room temperature, $\mathrm{NaK}$ is a liquid which ignites on contact with air. Sodium is a solid which does not undergo spontaneous combustion at room temperature. The elevated temperatures in EBR-II during normal operation are such that the sodium remains in a molten state, and may ignite on contact with oxygen, if a leak should occur.

Therefore, leaks or spills of sodium or $\mathrm{NaK}$ coolant in areas of the plant where the atmosphere is not inerted, may result in a fire and the release of sodium aerosols and reaction product aerosols, principly sodium oxide. Sodium oxide reacts when in contact with air and water vapor in air to form sodium carbonate and caustic sodium hydroxide which may cause corrosion of structural materials. Sodium oxide will also react when in contact with the water present in concrete to create sodium hydroxide, which is highly corrosive in nature, and hydrogen, [10.2]. Incidences of sodium fires have occurred at EBR-II, as discussed further in Section 10.2.3.5.

\subsubsection{Sodium-Fire Methodology}

The purpose of the analytical method discussed in this section is to aid in the identification of a list of the dominant accident sequences that are initiated by fire and then to assess the frequency of occurrence for each. This process requires information about several important aspects of a fire (e.g., ignition, progression, detection and suppression, characteristics of materials under fire conditions) as well as the plant safety functions and their behavior under accident conditions. 
The objective of a PRA-based fire-risk analysis is to estimate the frequency of fireinduced radionuclide releases of varying magnitudes. These releases would occur as the result of core damage and containment failure, both of which, potentially, could be initiated by the same fire.

Following the lead of NUREG/CR-2300, "PRA Procedures Guide" [10.1], the method for quantifying the risk due to fires can be divided into four somewhat independent steps: the fire-hazard analysis, where critical plant areas are identified and the frequency of fires assessed; the fire-propagation analysis, where the behavior of fires in the critical areas is modeled; the plant-systems analysis, where the likelihood of the fires leading to plant-damage states is estimated; and the release-frequency analysis, where the frequencies of accident sequences leading to radionuclide releases are derived from the results of the preceding analyses.

The first step in any risk analysis is to identify the specific sequences of concern. In the case of fires, sequences of concern are those in which a fire has caused an initiating event and in addition resulted in a sufficient amount of failures to cause some degree of core damage. Release of radionuclides outside of the plant containment can then be estimated based upon an analysis of fire-induced damage to the containment boundary.

Given that a fire-related initiating event requiring plant shutdown has occurred, failure which could result in core damage can be divided into three general classifications of critical safety function failure, as follows:

1) Failure to reach and maintain a condition of negative reactivity.

2) Failure to monitor and control primary system coolant inventory.

3) Failure to remove decay heat.

A working knowledge of the plant and its system interactions is necessary to ensure that a thorough investigation of failures which fall into one of these three categories is performed. 
Thus, the fire-risk analysis is generally performed in parallel with or near the end of the event tree and fault tree analysis performed for the internal events portions of the PRA. That is, once the event tree and fault tree models have been completed to represent the expected plant response to internal initiating events and resulting dominant accident sequences, these models can be utilized to determine the impact (e.g., fragility analyses) of the external initiating events.

\subsubsection{Screening by Location}

In many of the previous PRA studies that analyzed external events (e.g., Zion [10.21], and Indian Point [10.22]), the fire-induced loss of safety system instrumentation or control was judged to dominate fire-induced hardware losses. Therefore, the critical locations investigated should be those which contain electric cables and/or switchgear for many safety and non-safety systems, the failures of which initiate a requirement for reactor shutdown and fall into one of the three critical safety function failure categories previously described.

In order to greatly reduce the effort required for detailed external initiating event analysis, e.g., for fire or internal floods, a screening approach can be used to limit the events that have to be examined in detail. This approach relies upon PRA-based core vulnerability arguments. That is, although external initiating events may impact facility operation and result in plant downtime, they are only considered important initiating events as assessed by the PRA if they can require plant shutdown either automatically and/or manually in response to the external event, and lead to a core vulnerable condition at a frequency commensurate with those sequences initiated by internal events.

\subsubsection{Negative Reactivity Functional Failure}

The inability to trip the reactor, i.e., in the case of the EBR-II reactor, drop the control rods out of the core through automatic action of the reactor protection system during a rapid loss of flow (LOF) or transient overpower condition (TOP) could result in a short-term fuel vulnerable condition. Therefore, fire-related initiating events which could affect the ability to scram the reactor, and/or result in a positive reactivity insertion or loss of 
flow are potentially significant initiators for EBR-II. To achieve this type of impact, a sodiumfire initiator would have to occur within containment in the vicinity of the control rod drive mechanisms, reactor shutdown system circuitry, or primary pump control cables.

\subsubsection{Primary Coolant Inventory (LOSA) Functional Failure}

There are no credible mechanisms by which a sodium fire could induce any of the postulated LOSA initiators discussed previously in Section 5 of the internal initiating events analyses. However, the converse case of fire resulting from postulated_LOSA initiators has been considered. These fall into the categories of air ingress, small leak, and large rupture.

In the case of air ingress, the oxidation of sodium at the sodium surface in the primary tank is inhibited by the argon cover gas so that the reaction is slowed. The problem then becomes one of high sodium plugging temperature which is an operational problem if the amount of oxygen exceeds the capacity of the cold trap.

Small leaks in the sampling system or test equipment are limited by the systems pumping capacity and would occur in confined spaces, and therefore would not lead to core damage. A small leak from the primary vessel would be limited to the annulus space between the primary vessel and guard vessel which is filled with argon gas. In order for a small leak to react in air, both primary and guard vessels would have to breach. The probability of primary tank leak event is $110^{-8}$. Breach of both primary and guard vessel is below the $10^{-10}$ cutoff.

The only remaining LOSA initiator is the large rupture (e.g. one caused by overpressuration of the primary tank and/or annulus. This initiator leads to direct core damage and therefore is not included in external events to avoid double counting. 


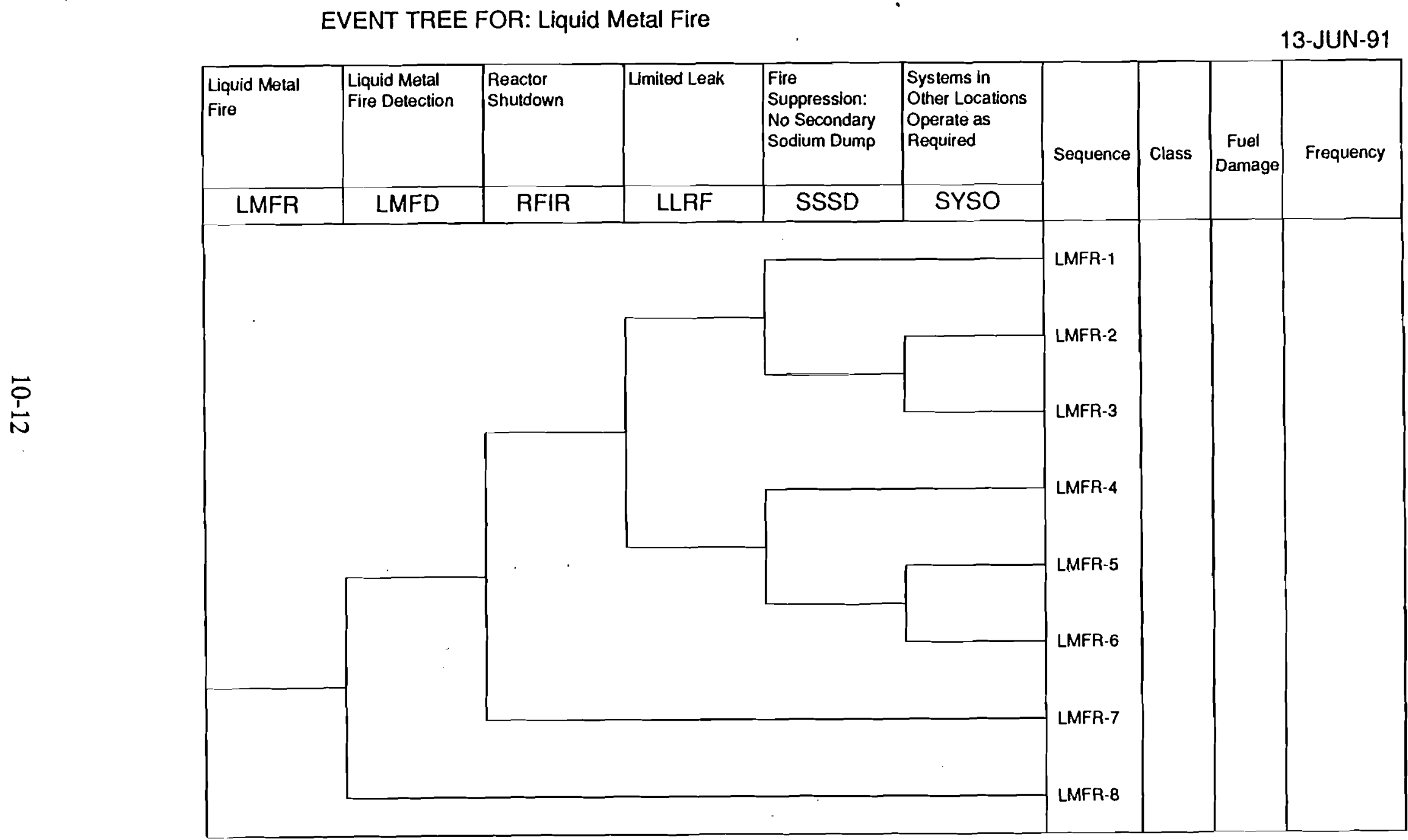

Fig. 10.1. Event Tree For Sodium Fire Sequences 
In particular, this would include the piping beneath the reactor building operating floor deck plates for the sodium primary purification system and Radioactive Sodium Chemistry Loop (RSCL), the secondary system sodium piping to/from the IHX within containment, the shutdown cooler NaK piping, the Fuel Element Rupture Detection (FERD) system piping and the Breached Fuel Test Facility (BFTF).

The Breached Fuel Test Facility (BFTF) uses primary system sodium for cooling and for transport of fission products from breached fuel experiments to the neutron detector that is at an elevation above the bottom of the primary tank. The head is provided by the primary system coolant pumps. In order to pump sodium out of the primary vessel, two barriers would have to be breached. The first is the detector dry well and the second is the seal in the instrument box. Both the dry well and the instrument box have leak detectors. Corrective action in case of a leak would be to shut down the reactor and trip the primary coolant pumps. The instrument box is also pressurized and monitored so that a leak would be detected early. The pressure at the seal in the instrument box for a high flow rate subassembly, e.g., XY24, would be about 8.8 psig after correcting for elevation loss. . The leak rate would be from 1.4 to $14 \mathrm{gpm}$ depending on the area of the breach.

The Fuel Element Rupture Detection (FERD) system removes primary sodium from the outlet region of the IHX for the purpose of monitoring the level of delayed neutron emitting fission products in the primary coolant as a measure of fuel element failure. Piping carries the sodium out of the primary system through the E-3 nozzle plug to the detector system located in the depressed area of the reactor building operating floor. The flow is through a magnetic flowmeter, the detector assembly, a DC electromagnetic pump, and back to the primary tank, discharging below the sodium surface. The head for the system flow is provided solely by the EM pump which is designed to provide a 5 psig head at the rated $100 \mathrm{gpm}$ flow. A leak in the piping carrying the primary sodium to the detectors would not provide a means of sodium pumpout if the leak was located before the EM pump. Sodium could possibly spill out, but there would be no pumping force to remove additional sodium from the tank. A leak after the pump would continue the sodium flow as long as the pump was powered. The reactor building smoke 
detectors would respond to a large sodium spill in the depressed area, and the system could be shutdown by turning off the pump.

The primary sodium purification system provides for the continuous purification of the primary sodium to control impurities to lessen the corrosion rate of reactor containment and reactor materials. The primary sodium is drawn to a surge tank in the purification system by applying a vacuum at the top of the surge tank. The sodium flows from the primary tank, out the piping through the "V" nozzle, and enters the side of the surge tank. The sodium leaves the bottom of the surge tank and flows to the primary purification cell, via a DC powered EM pump which provides the flow through the purification system before returning the sodium to the primary tank through the " $\mathrm{V}$ " nozzle. A syphon-break system prevents syphoning of primary tank sodium should a leak occur since other than the surge tank, most of the primary purification system and RSCL piping is located below the sodium level in the primary tank.

The secondary sodium system provides for heat transport from the primary sodium via the IHX to the superheaters and evaporators, and then into a sodium surge tank. The secondary flow is provided by an electromagnetic pump in the secondary system which returns cooled sodium from the surge tank back to the IHX. A leak in the secondary system would require the operators to scram the reactor, trip the secondary system EM pump and dump the secondary sodium to the storage tank.

There are two shutdown coolers in the EBR-II system, each completely independent and providing for heat removal from the primary tank when the secondary sodium system is not in operation. NaK coolant flows by natural circulation from a bayonet-type heat exchanger that extends through a nozzle in the primary tank cover down into the bulk sodium, to an outside air heat exchanger where it is cooled and returned to the bayonet heat exchanger. Since this system is completely sealed and driven by natural circulation, any breach removes the driving force that provides the circulation. 


\subsubsection{Initiating Event Frequency}

The probability of piping failures in these systems leading to a lossof-coolant-accident from the primary vessel was examined previously in Section 5 of the internal initiating event discussion. The LOSA analysis was primarily concerned with events that could lower sodium in the primary tank below the suction intakes of the primary coolant circulation pumps. However, initiating event frequencies corresponding to leaks in the piping systems exiting the primary tank cover region as discussed previously were determined based upon a piping failure rate of $310^{-9} \mathrm{hr}^{-1} \mathrm{ft}^{-1}$ per foot of piping and a typical reactor run time of 2400 hours, assuming that there are approximately three reactor runs per year. This yielded initiating event frequencies for a piping leak for each of the previous systems described above as follows: BFTF at $7.210^{-4}$ per year; FERD at $9.110^{-4}$ per year; primary sodium purification system (excluding in-cell piping) at $2.210^{-3}$ per year; secondary sodium system piping within containment at $1.510^{-3}$ per year; and the shutdown coolers system piping had a frequency of 4.3 $10^{-3}$ per year for a leak in either system.

However, as noted earlier, for a primary sodium leak to occur in the BFTF requires failure of two barriers: first the detector dry well and then the seal in the instrument box. Should this occur, the dry-well leak detector provided in the system should alert the operators to the problem. Because the only driving force for the flow in the BFTF are the primary coolant pumps, any action that would stop or reduce the primary coolant flow will also stop or reduce the flow in the BFTF and therefore the LOSA potential. For these reasons, the BFTF leak is not considered a significant initiator for a sodium fire that would threaten the integrity of the RSS and primary pump cabling.

Most fire studies assume that if a fire is of sufficient magnitude, that all components or cabling will eventually be damaged due to either the heat generation of the fire or propagation of the fire within that fire zone. The radial beams supporting the reactor vessel as shown in Fig. 10.2 effectively divide the area below the reactor building operating floor deck plates into various sectors, and serve as a barrier to limit the effect of a sodium fire to that sector. As shown by Fig. 10.2, the relative location of the FERD and primary sodium purification system 


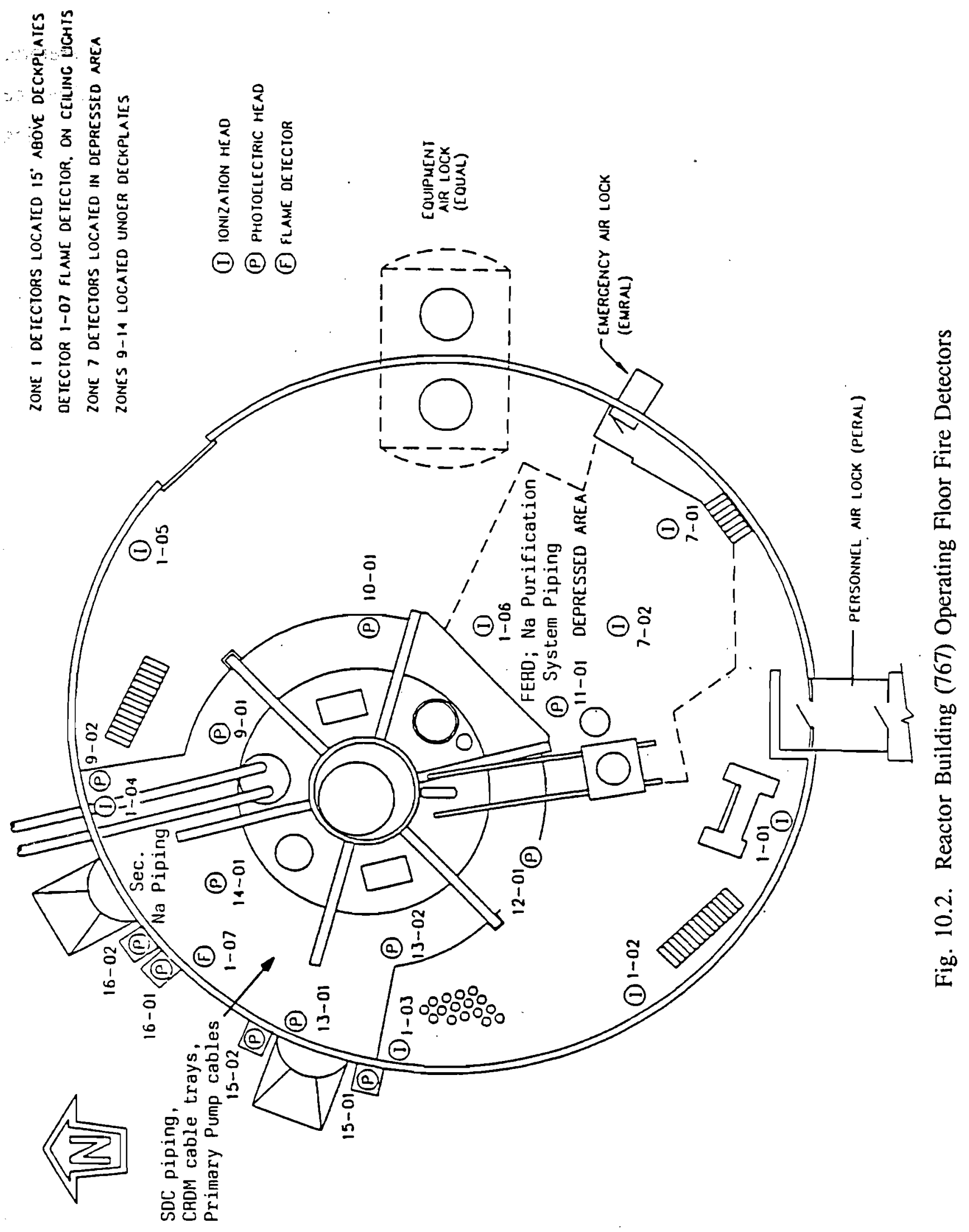


piping is opposite the sector containing cables for the CRDM's and primary pumps. Therefore, a fire due to a leak in the FERD or primary sodium purification system piping would not be expected to propagate or effect the cables for the CRDM's and primary pumps due to shielding from the thermal effects of the fire provided by the radial beam structures.

However, since the piping for the secondary sodium system and the shutdown coolers exits the primary tank cover region in the same relative location as the CRDM's and primary pump cables, the effect of a sodium or $\mathrm{NaK}$ fire due to a leak in a piping system in this area could fail these cables due to the burning of cable insulation and shorting of circuit conductors. While there is separation of cables in this sector of the compartment below the reactor building operating floor deck plates, the intensity of the fire due to a sodium or NaK leak is assumed to affect all cabling within this compartment area. This simplifying assumption is conservative since it implies that any fire which starts in this area will propagate and affect all safety-related cables in this area unless detected and extinguished. It is also conservative in that it bases the shutdown coolers $\mathrm{NaK}$ leak on the total length of piping rather than the part of piping within containment.

The initiating event frequency is therefore, based upon the occurrence of a leak in either of the shutdown coolers system piping $\left(4.310^{-3}\right.$ per year) or the secondary sodium system piping (1.5 $10^{-3}$ per year) that could impact components of the reactor shutdown system, primary pumps, or decay heat removal systems.

\subsubsection{Sodium Fire Detection}

Referring to the event tree presented as Figure 10.1 for analyzing the effect of a sodium fire, it can be seen that separate events are included to account for detection and suppression methods for limiting the impact of fire.

The two obvious methods of detection in any area are manual (i.e., visual) or automatic (with smoke/flame detection). To estimate the probability of visual detection requires the determination of the frequency of routine walkthroughs, test or maintenance, in this case, in the 
reactor building. Since this frequency is not readily determined, the availability of automatic smoke' detectors is relied upon to determine if adequate detection capability exists. Eight redundant photoelectric smoke detectors are located in six separate zones underneath the reactor building operating floor (see figure 10.2). This fire detection system was installed in 1980 [10.3] and provides alarms in the main control room and at the fire station to alert the operators if a fire in any of these areas should occur. The sensors are smoke tested and calibrated every six months [10.4]. None have failed since the original installation. There are also two ionization detectors in the depressed area and six ionization detectors and one flame detector above the operation floor to provide backup means of sodium fire detection in the reactor building (See Fig. 10.2). In addition to the fire detectors, increasing levels of airborne radioactive contaminants in the reactor building would provide a diverse means of indication. Based upon the various number of redundant smoke detectors to alarm the operators to a fire in the reactor building, the probability of detection for a significant sodium or $\mathrm{NaK}$ fire is estimated to be approximately 1.0. A screening value of $1.010^{-6}$ for failure to detect a liquid metal fire prior to core and structural damage is used in the sequence quantification.

\subsubsection{Reactor Shutdown}

If detection of the fire under the operating deck plates is successful, the operators are procedurally instructed by Emergency Procedure 1-4 [10.5] to scram the reactor and monitor the reactor building radiation monitors. Failure of the operators to scram the reactor could potentially result in a core vulnerable condition due to a Loss-of-Flow condition when the power cables to the primary coolant pumps fail, if the reactor shutdown system does not automatically respond to the LOF condition in time to prevent exceeding clad temperature limits. In 1981, MCN-387 [10.6] provided for the replacement of the primary pump cables with $500 \mathrm{MCM}, 600$ volt cables with an asbestos braid rated at $125^{\circ} \mathrm{C}$. The cable runs under the deck plates in the reactor building were placed in flexible conduit, where each conduit holds the three electrical cables for the pump. However, both conduits are routed together for a portion of the routing under the operating floor deck plates next to the piping for one of the shutdown coolers. Since the fire rating of the cables is low compared to temperatures expected from a sodium or $\mathrm{NaK}$ fire $\left(\sim 900^{\circ} \mathrm{F}\right)$, the cable insulation would be expected to fail 
eventually, shorting out electrical power to the primary pumps. If this were to occur, pumping power would be lost resulting in a rapid pump coastdown similar to that of opening the $\mathbf{M G}$, set output circuit breakers.

The postulated initiating event could also cause similar effects to the cables for the CRDM's and reactor shutdown circuitry, since these cables are routed in cable trays that are not protected, and may in fact, result in a reactor trip due to loss of power to the CRDM's. A study conducted by Factory Mutual Research Corporation in December 1987 [10.7] concluded that the only situation that would result in an unsafe condition would be a burn through of the cable to the control rods in such a manner that each of the two conductors of the circuit came in contact with conductors of an approximately equal DC power source. There are only three DC power cables in the reactor building cable trays. The possibility of this occurring to one control rod is extremely low. It would not be credible for this to occur to enough control rods at the same time to prevent safe shutdown, since normally only one or two control rods are required to shutdown the reactor and four (see Section 7) control rods are the limiting condition.

Since the deck plates are normally in place except during outages for maintenance, the effect of the sodium fire would not affect the ability to scram the reactor using the control rods, and/or safety rods. Although sodium aerosols would be released during the fire, there is no credible mechanism that could be identified to prevent the control rods from being inserted during the initial time period when the fire has been detected. Failure to achieve manual reactor shutdown following detection of the sodium fire is dominated by the human error for the operators failing to scram the reactor as required by procedure EP 1-4. However, in the event of failure of the cables to primary pumps, the reactor shutdown system would automatically respond to the loss-of-flow condition. Failure of both manual and automatic response to scram in the event of a LOF has been calculated in Section 6 to be $4.010^{6}$. 


\subsubsection{Limited Leak vs Rupture}

The initiating frequency for liquid metal fires is based on the leak rates for the associated system piping. Due to the low pressure in the systems and in the case of shutdown coolers lack of even a pump head other than natural convection, a differentiation is made between limited leaks and rupture. A leak in a low pressure system would be a weeping type of leak. The liquid metal could oxidize as smoke if it reacted with air in the insulation or seeped out of the insulation. A rupture would release liquid metal at a sufficient rate to allow rapid oxidation and/or fire. Analysis of pipe breaks using probabilistic fracture mechanics and LWR experience [10.8] shows a leak to rupture ratio of 10:1 for 1" pipes and 1000:1 for pipes >10". NUREG/CR-4407 [10.9] gives a leak to guillotine ratio break of 1000:1. A slightly older reference [10.10] gives a leak to rupture ratio of $30: 1$ for all size piping. Combining these references, a leak to rupture ratio of $99: 1$ was selected to give the branch point conditional probability of $1.010^{-2}$ that the initiator is a rupture rather than a pipe leak. The experience at EBR-II has been that all breaches have been leaks. A sodium fire in 1960 in the boiler building involved an inventory of approximately 80 gallons of sodium. The primary effect of this fire was sodium aerosols coating the metal surfaces of the secondary pump controls and other instrumentation and controls in the control wing of the SBB. An incident involving a small leak from a sodium-filled instrumentation line under the operating floor deck plates in the reactor building resulted in a small, self-limiting fire and smoke. No damage to other components occurred from this fire. UOR $81-4$, February 22,1981 , was a leak in the radioactive chemistry loop which resulted in only instrument failure with no fire. UOR 81-8, June 19, 1981 was a leak from the secondary sodium sampling loop which was sufficient to cause a limited sodium

fire which was contained. The most recent leak (UOR 89-8) was of NaK from a pressure instrument which produced only a wisp of smoke but no fire. A secondary sodium leak (UOR 90-5) from a vent valve caused an alarm indication but was so small that it was found only after hourly surveillance had been established. Thus the EBR-II operation experience supports the assumption that sodium and $\mathrm{NaK}$ piping failures lead to leaks and not ruptures. 


\subsubsection{Prevention of Cable Damage}

A sodium leak inside of the reactor building requires the operators to perform the applicable steps of Procedure EP 3-1 [10.11] in conjunction with the previously mentioned EP 1-4 procedure. Procedure EP 3-1 instructs the operators for actions to be taken to terminate leaks in the primary purification system, RSCL or FERD systems. In addition, it cautions the plant operators that entry into the reactor building will require Scott Air-Paks and anti-C's. Radiation Safety Technician coverage is required and visibility may be impaired due to sodium aerosol and smoke. Since remote capability for suppression of a fire in this location is not provided at this time, the requirements for reactor building entry make it unlikely that manual fire suppression actions could be taken in time to prevent cable damage. Even if the fire is successfully extinguished, extensive damage to cables or other equipment and secondary sodium dump may have already occurred. Therefore, limited credit is given for the ability for early fire suppression to limit cable and instrumentation damage, and failure of suppression is estimated to be $5.010^{-1}$ for pipe rupture. For pipe leak, a value of $1.010^{-2}$ is used.

In the event of significant damage to cabling that is required to allow the operators to monitor the status of the reactor and locate the system which initiated the fire, it is assumed that the secondary system sodium may be dumped, precluding the use of the normal balance-oif-plant system for decay heat removal. Also, if the leak is from the secondary sodium system; the secondary sodium is assumed to be dumped regardless of the status of the cabling.

\subsubsection{Operation of Unaffected Systems}

The operation of the decay heat removal systems in the event of a secondary sodium dump is dependent on the effect of aerosols generated by the liquid metal fire. The shield/thimble cooling system is considered inoperable due to the clogging of the system intake filters due to aerosols released by an unsuppressed rupture. However, short-term decay heat removal relies only on the successful operation of the remaining shutdown cooler(s). For long-term decay heat removal, fourteen days are available for recovery of the shield and thimble cooling systems by removal and/or replacement of the clogged thimble inlet filters and the 
HEPA filters once the fire has been extinguished. Shutdown cooler system failure is dominated by the lowvers failing to open, which is an easily recoverable event outside containment. Plant Operations indicates that the BOP could be restored for long-term decay heat removal (14-45 days) but could not be recovered within 8 hours for short-term decay heat removal if the secondary sodium and steam plant water are dumped.

For any leak or suppressed rupture of secondary sodium, both shutdown coolers, are initially available. One of the two shutdown coolers is required for fourteen days to assure success with a probability of $3.310^{-8}$. For a rupture of the secondary sodium with an unsuppressed sodium fire, the shutdown cooler piping next to the secondary sodium piping is assumed to be affected to an extent to render that cooler inoperative. Also the effectiveness of the second cooler may be reduced due to thermal effects from the fire that may reduce natural circulation. Therefore, the probability of failure of the secondary coolers is increased from 3.3 $10^{-8}$ to a single cooler value of $1.3510^{-4}$ and then further increased to $1.010^{-1}$ to account for possible thermal effects.

In the case of a suppressed shutdown cooler $\mathrm{NaK}$ leak, the remaining shutdown cooler is required for short-term decay heat removal. The one of one logic gives a failure probability of $1.35 \cdot 10^{-4}$. For the case of an unsuppressed rupture of shutdown cooler piping, the probability of failure of the remaining cooler is increased from $1.3510^{-4}$ to $1.010^{-1}$ to account for possible thermal effects.

\subsubsection{Analysis Results}

The simplying assumptions employed in examination of the postulated sodium

fire accident scenario were conservative. Therefore, several potentially significant $\left(\geq 10^{-10}\right)$ core vulnerable sequences result. 
The event tree shown in Fig. 10.3 assumes the initiating event is a secondary sodium fire in containment (SSFR). The significant sequences are SSFR-6, 7, and 8. SSFR-6 is an uncontained rupture with a frequency of $1.510^{-6} \mathrm{yr}^{-1}$. Failure of suppression is considered to be 1.0 since, for this initiating event, the operators are assumed to dump secondary sodium even if suppression is successful. Thus sequences SSFR- 1 and SSFR-4 are not allowed and no credit is taken for recovery of the BOP. SSFR-7 and SSFR-8 are failure to scram or detect, respectively, with frequencies of $6.010^{-9} \mathrm{yr}^{-1}$ and $1.510^{-9} \mathrm{yr}^{-1}$.

The event tree as shown by Fig. 10.4 assumes the initiating event is a leak in a shutdown cooler NaK piping loop which causes a fire underneath the operating floor deck plates. For sequences SDFR-3 and SDFR-6, the fire is immediately detected and the operators scram the reactor as required by procedure but fail to manually suppress the fire before extensive cable damage occurs. The balance-of-plant heat removal is either purposely or inadvertently rendered unavailable by dumping the secondary system sodium. In the case of a leak, SDFR-3, the remaining shutdown cooler is not affected due to the small size of the fire. The sequence frequency is $5.810^{-9} \mathrm{yr}^{-1}$. In the case of a rupture, the remaining cooler failure probability is increased from $1.3510^{-4}$ to $1.010^{-1}$. This sequence (SDFR-6) has a frequency of $2.110^{-6} \mathrm{yr}^{-1}$. For sequence SDFR-7, the fire is detected but the operators fail to scram the reactor. The frequency for this sequence is $1.710^{-8} \mathrm{yr}^{-1}$. For SDFR-8, the failure to detect, the frequency is $4.310^{-9} \mathrm{yr}^{-1}$.

The above sequences, although not likely to result in core damage, illustrate the relative importance for not being able to immediately suppress the fire upon detection. Even if decay heat removal is successful, the damage to plant cables and other non-safety equipment could preclude future operation of the plant.

\subsubsection{Planned Modifications to Address Vulnerability}

Modifications to reduce plant vulnerability to cable fires and sodium fires in the reactor building have been previously identified and are described in the February 1988 report, "Addendum \#1 to Conceptual Design Report for Fire Safety Improvements" [10.12]. 


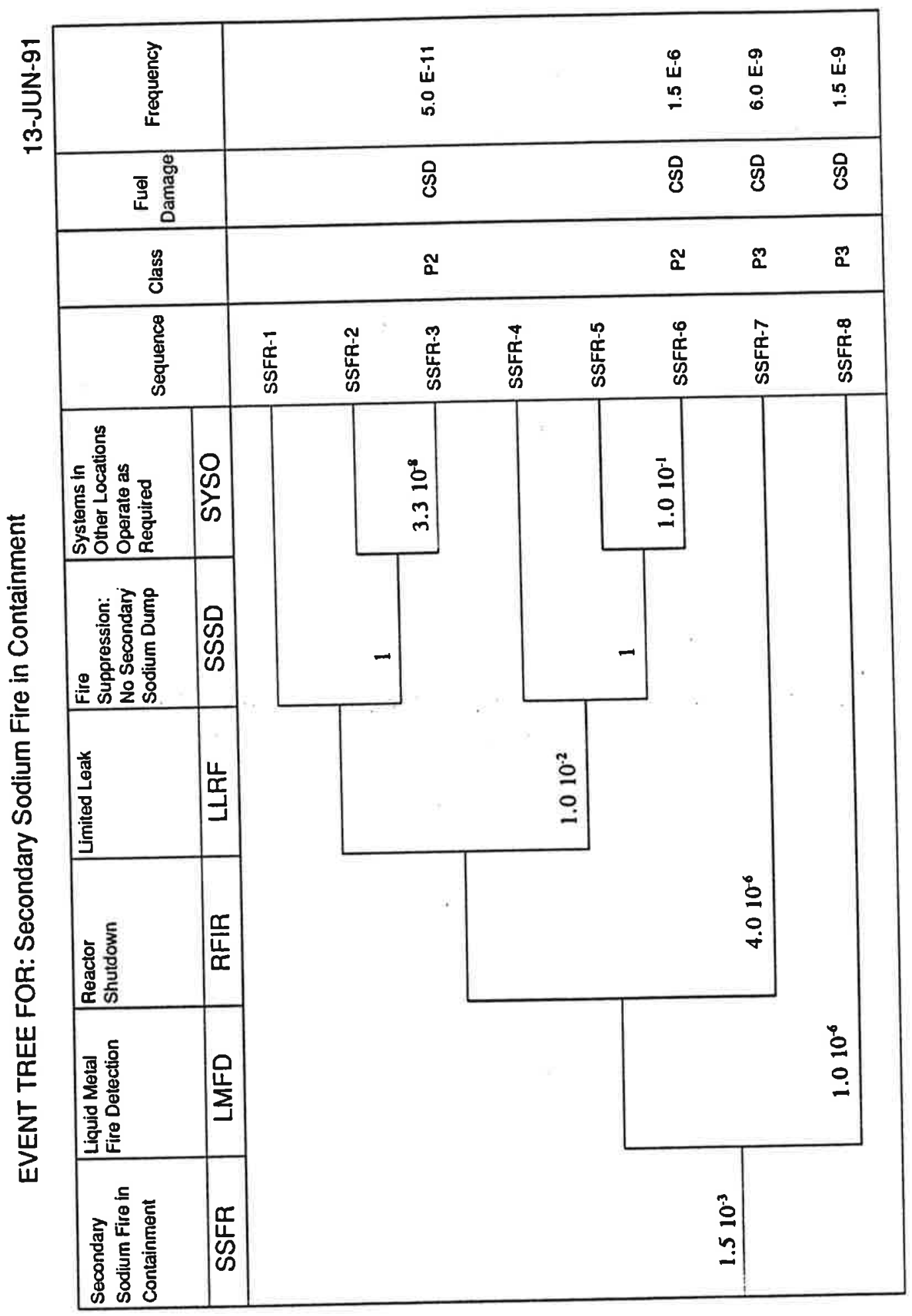

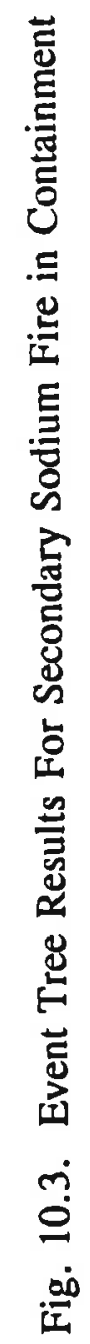




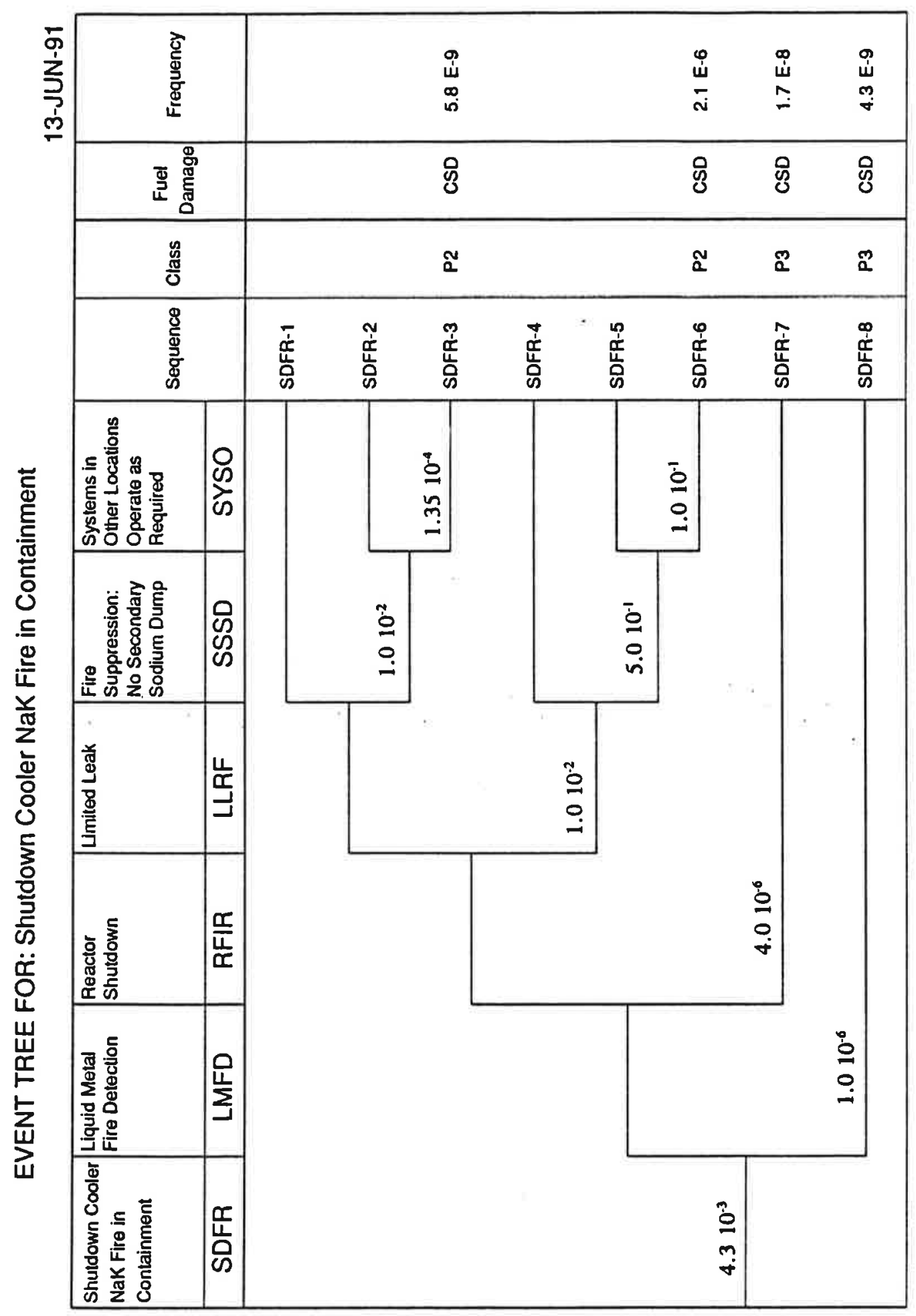

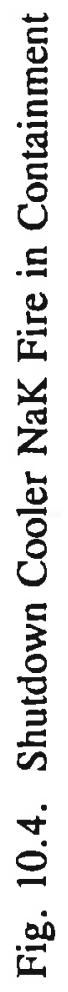


The Fire Safety Improvements, Addendum \#1 to the document W7500-0343-ES, entitled "Conceptual Design Report for Fire Safety Improvements", dated April, 1987 consists of alterations and additions to existing Argonne-West facilities that will upgrade them to meet the Department of Energy's Improved Risk Criteria, incorporate Technical Safety Appraisal items, Factory Mutual Research Corporation recommendations, and satisfy Life Safety Code recommendations.

Two of these modifications that pertain to this analysis are described below:

\section{EBR-II Cable Protection}

This project provides for protection of cables in grouped cable trays in the cable tunnel, the power plant, and reactor building. As recommended by Factory Mutual Research Corporation the protection will be accomplished by covering cable trays with solid covers top and bottom and installing fire stops at $15 \mathrm{ft}$ intervals. Flammastic cable coating may also be used where covering the trays is not practical. Protecting the cables in this manner will limit the potential damage in these areas to an acceptable level.

\section{Reactor Building - ARGON System for Under the Operation Floor Deck Plates, Bldg. 767}

This project provides for the installation of a remote-manually actuated fixed pipe inert gas (argon) system which will service each of the six sectors formed by the primary tank support structure beneath the operating floor deck plates. The volume to be inerted is estimated to be 2300-cu ft. However, because of the impracticality of isolating the support structure sectors from an adjacent area in the northwest quadrant of the reactor building and the depressed area, an additional volume of 4700-cu $\mathrm{ft}$ will be inerted (depressed area: 3900-cu $\mathrm{ft}$, northwest quadrant: $800-\mathrm{cu} \mathrm{ft}$ ). Each sector will be provided with fire detectors that will alarm in the reactor building and the EBR-II control room. In the event of a fire, all of the sectors will be inerted. Because of the asphyxiating hazard of argon to personnel, actuation of the inert gas system will only be permitted after all personnel have exited the reactor building. Actuation of the inert gas system will be from the EBR-II control room. The area underneath the deck plates 
contain instrumentation and control cabling and mechanical components that are essential to the operation of the EBR-II reactor. This project will minimize the potential fire damage and the impact on reactor operation.

These modifications are scheduled to be completed over the next two years. Completion of the modification for the ARGON system would significantly improve the probability for suppression of the fire since detection is highly reliable. Failure to remotely actuate the ARGON system from the EBR-II control room given detection should be no worse than RHU001CL in the RSS fault tree for failure to manually scram in the absence of a SCRAM signal, i.e., $510^{-2}$. Reducing the probability of fire suppression failure to $510^{-2}$ from $510^{-1}$ reduces SDFR-6 sequence frequency to $2.610^{-7}$, sequence SSFR-6 to $1.510^{-7}$ and sequence SDFR-3 to $\leq 10^{-10}$. Additional analysis, once the system has been installed, should allow a further reduction in these sequence probabilities. As stated previously, this illustrates the relative importance of the proposed modification.

\section{3 $\underline{\text { Fires }}$}

In the previous section, the risk to EBR-II from sodium-related fires was examined and quantified. Non-sodium related fires also have the potential to affect multiple plant systems and must be considered as a separate initiating event. The methodology to examine plant vulnerabilities due to fires is the same as that considered previously in Section 10.2.1 for sodium fires. That is, identifying those locations which contain electrical cables for instrumentation, power, and control for multiple systems whose failure would initiate a requirement for reactor shutdown and impact plant safety by impacting the ability to: reach and maintain a condition of negative reactivity; monitor and control primary coolant system inventory; or remove decay heat. As stated previously, although an external initiating event such as a fire can impact facility operation and result in plant downtime, it is only considered as an important initiator as assessed by the PRA if it requires plant shutdown and leads to a core vulnerable condition at a frequency commensurate with those sequences initiated by internal events. It can be shown that the potential for core damage due a non-sodium fire initiator is bounded by the previous analysis for sodium fires, or by internal initiating events that have the same effect and are more frequent 
initiators than fires involving safety-related equipment. Due to the low dependence on electrical power and other support systems, the worst case non-sodium fire is assumed to be a fire that disables the constant power supply to the primary pump clutches. Loss of constant power has a frequency of 0.1 per year which bounds the case for fire.

\subsubsection{Plant Impacts From Fire Initiators}

The most important reason for the conclusion that non-sodium fires are insignificant contributors to plant risk is that there are no credible fire initiating events that can impact the ability to remove decay heat with the shutdown cooling system following reactor shutdown. The shutdown cooling system removes heat from the primary sodium tank through two natural convection NaK loops to the atmosphere. The shutdown cooling system does not require support systems for its operation. The system does not contain any active components for system operation other than louvers which open to increase heat removal capacity. Instrumentation and control for automatic opening of the louvers on high primary bulk sodium tank temperature is not required since the louvers can be opened manually outside the reactor building, and at least 8 hours are available to take this action. While there are a number of plant locations where a fire could impact loss of the primary heat sink, i.e., the balance-of-plant system, e.g., fires associated with electrical switchgear or components in the sodium boiler building, the power plant, cooling towers, etc., a fire in these locations can not affect the ability of the plant to remove decay heat with the shutdown cooler system, and would be expected to occur much less frequently than other internal initiators that cause loss of the primary heat sink.

Similarly, there are no credible fire initiators that could affect the ability to shutdown the reactor with the control rods either automatically or manually in response to a fire. Other than the cables themselves, there are no combustibles in the reactor containment building that could ignite in the vicinity of the control rod drive mechanisms, reactor shutdown system circuitry or primary pump control cables. Since there are no ignition sources, the frequency of a cable fire starting and propagating to affect several cables is less likely than a sodium fire initiator in this area. A sodium fire due to a leak as analyzed previously also has potentially more severe consequence due the effect on multiple plant safety systems and is therefore, more limiting than 
a cable fire in the reactor building. As discussed in Section 10.2.3.4 of the sodium fire analysis, a fire in a cable tray containing cables for the CRDM's and reactor shutdown circuitry would most likely result in a reactor trip due to loss of power to the CRDM's. As referenced in that section, a study conducted by Factory Mutual Research Corporation in December 1987 [10.10] concluded that the only unsafe condition would be a burn through of a cable to the control rods in such a manner that each of the two conductors of the circuit came in contact with conductors of an approximately equal DC power source, since power is required to keep the rods latched. The possibility of this occurring to one control rod is extremely low. It would not be credible for this to occur to enough control rods at the same time to prevent safe shutdown, since for normal core loadings only two control rods are required to shutdown the reactor. In the limiting case, four control rods are sufficient to shutdown the reactor.

Areas that were considered vulnerable to the effects of a fire due to the impact on operations were identified in the Factory Mutual Research Corporation study as the cable routing room, the cable tunnel, and the vertical cable tray runs between them, since a fire in one cable tray could affect all trays in that area. Nuclear system and primary sodium instrumentation important to the plant operators for control and surveillance during operation and after shutdown would be affected. If undetected, a fire in these areas would most likely lead to a loss of normal power and reactor scram, and inability to control heat removal with the BOP. However, the result would be a LOF/LOHS sequence similar to that described for LONP but much less frequent as an initiator. The shutdown cooler system would be required for decay heat removal and would not be impacted by a cable fire in these areas. Although status of the primary system and reactor may be unknown in this situation, decay heat would continue to be removed via natural circulation within the primary system and the shutdown coolers.

\subsubsection{Ongoing Fire Safety Improvements}

While risk to EBR-II from a fire is relatively low compared to other initiators, previous studies and appraisals conducted at the facility have resulted in a number of improvements to address fire protection. The report entitled "Addendum No. 1 To Conceptual Design Report for Fire Safety Improvements" [10.12] identified a number of modifications that 
will upgrade ANL-W facilities to meet DOE's Improved Risk Criteria, incorporate items from the Technical Safety Appraisal and fire protection survey by Factory Mutual Research Corporation that were conducted in 1987, and satisfy Life Safety Code recommendations.

There are eleven subprojects which will require the utilization of wet sprinkler systems; protection of cable trays in the cable tunnel, power plant and reactor building with solid covers, fire stops and/or use of Flammastic cable coating; Halon gas, argon gas and dry chemical fire suppression systems; fire detection additions; redundant water supply systems, and diesel fire pump or a combination of these methods to establish an "improved risk" level of fire protection. The basic philosophy has been to provide automatic systems where possible for fire protection for those components required for reactor operation and increased emphasis on automatic fire suppression systems. Some of these items have already been incorporated on a priority basis, e.g., the addition of automatic wet-pipe sprinkler system in the cable tunnel and cable routing room, while others are scheduled for completion during plant outages over the next few years. The work that is already underway and scheduled will ensure that risk from fires at EBR-II continues to be maintained at a low level.

\subsection{Tornadoes and High Winds}

This section describes the evaluation of the hazard to EBR-II from tornadoes and high wind. Tornadoes and high winds can affect structures at the ANL-W site in two ways. If the wind forces exceed the load capacity of the structure, the walls might collapse or the structure may fall on other buildings. Also, the winds may be capable of lifting objects against the walls of structures. If a wind-propelled missile broke through a structure's wall, critical equipment inside the structure or the structure itself might be damaged [10.2].

\subsubsection{Tornadoes}

The INEL is in the region of the country where tornado frequency and damage potential are the lowest in the 48 contiguous states. In more than 23 years of observation at the INEL, no tornadoes have been reported within its boundaries. Two low-intensity tornadoes and 
10 vortex clouds (where the funnel does not touch the ground) have been sighted within 10 miles of the INEL. A tornado was sighted near the Pocatello airport (approximately 50 miles distant from ANL-W) in August, 1990.

Studies conducted for other INEL facilities indicate that the annual probability of a tornado striking the INEL is low, $7.810^{-5}$ per year [10.14]. The Environmental Assessment for the Fuel Processing Restoration Project at the Idaho Chemical Processing Plant [10.17] referenced studies that indicated the probability of a Design Basis Tornado with a maximum windspeed of $175 \mathrm{mph}$ striking that facility was estimated at $510^{-7}$ per year. A tornado with the associated windspeeds and missiles could potentially result in loss of the primary heat sink by causing failure of the secondary sodium yard piping or condenser cooling tower. Depending upon the path of the tornado, the outside containment portion of one of the shutdown coolers is also assumed to be damaged. Only one shutdown cooler is assumed to fail because they are oriented approximately $90^{\circ}$ apart around the containment and are not susceptible to the same missile. Only a missile generated by a Design Basis Tornado could damage a shutdown cooler since the shutdown cooler chimney and boxes are constructed of 1/4-inch steel and are structurally sound, as noted in the seismic analysis in Section 9. The reactor containment building shell, consisting of a carbon steel cylinder with a hemispherical top, which is up to one inch thick and lined with reinforced concrete inside the cylindrical portion of the shell to a depth of 12 inches, would not be impacted by high winds or missiles. Components within the reactor containment building would not be impacted. Short-term decay heat removal would be dependent upon operation of the remaining shutdown cooler system. Figure 10.5 depicts the accident sequences.

The initiator probability for the tornado is $510^{-7}$ per year. Reactor shutdown is initiated manually or is assumed to occur upon loss of normal power. Short-term decay heat removal requires that the remaining shutdown cooler be operable within the 8 hour to 14 day time phase.

Quantification of the event tree for the postulated tornado scenario does not yield any significant core damage sequences as shown in Fig. 10.5. Failure either to shutdown or failure of the remaining shutdown cooler result in sequences below the $110^{-10}$ cutoff frequency. 


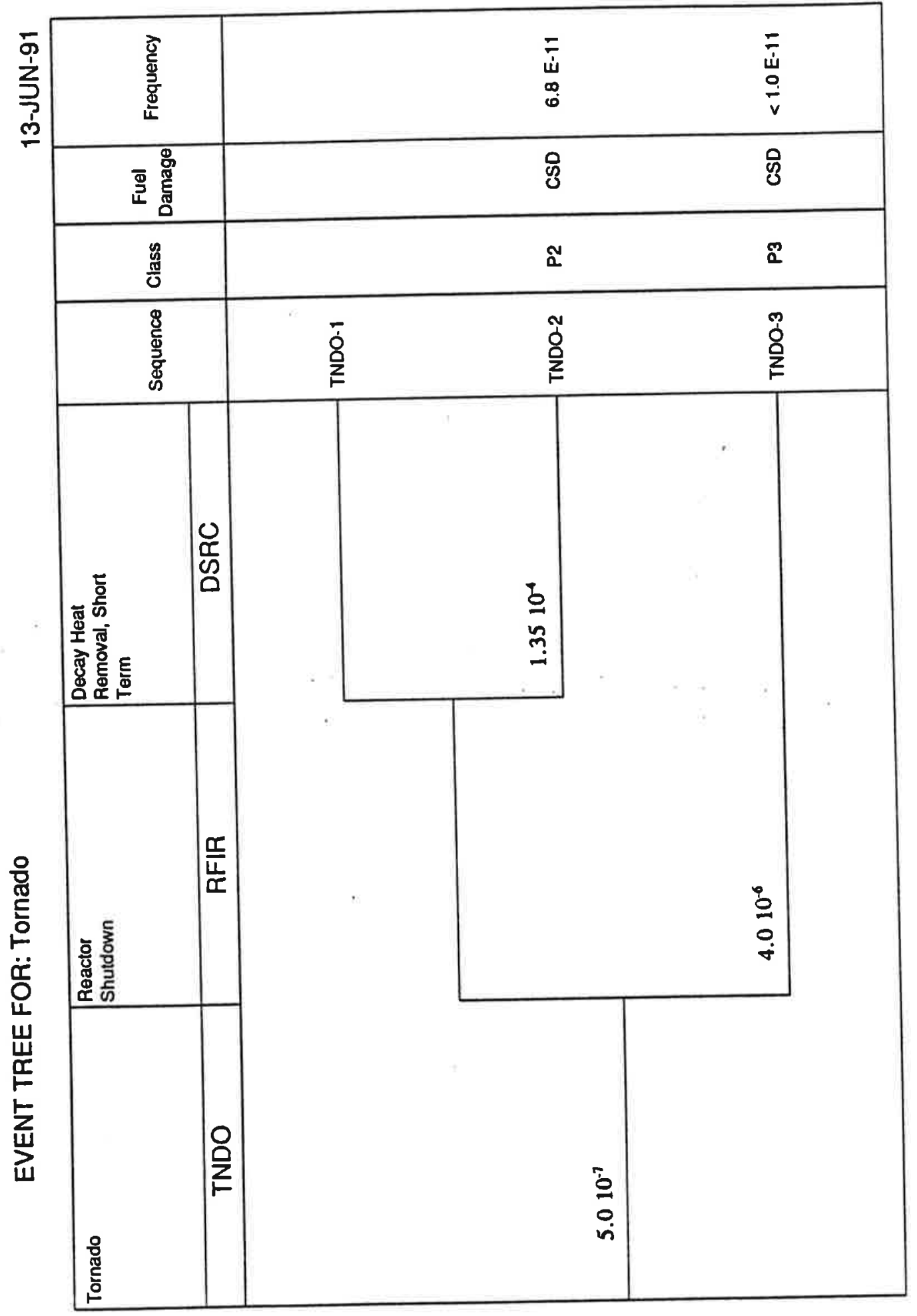

总 
Therefore, the risk to EBR-II from an external event initiator due to a tornado is considered to be an insignificant contributor.

\subsubsection{High Winds}

The report "Design and Evaluation Guidelines for Department of Energy Facilities Subjected to Natural Phenomena Hazards", [10.18], provides guidance and criteria for design of new facilities and for evaluation, modification, or upgrade of existing facilities to ensure that DOE facilities can safely withstand the effects of natural phenomena. This report indicates that rather than tornadoes, the viable wind hazard to ANL-W specifically, comes from straight winds and associated gusts or missiles carried by these winds. The Design Basis Wind (DBW) for a high hazard facility (defined as those which pose a potential hazard to the safety of general public and/or the environment due to the presence of radioactive or toxic materials within the facility) such as EBR-II was determined to be $95 \mathrm{mph}$ for ANL-W based upon a 10,000 year return period [10.18]. This is in general agreement with the wind speed records that have been accumulated for 32 years at the Central Facilities Area (CFA). These records indicate that peak wind gusts of $78 \mathrm{mph}$ at a height of $20 \mathrm{ft}$, and $89 \mathrm{mph}$ at $250 \mathrm{ft}$ have occurred. The extreme values for wind speeds for a 100-year return period for INEL correlate to a $82 \mathrm{mph}$ gust at 20 feet and a $91 \mathrm{mph}$ gust at 250 feet [10.13].

Site Engineering for EBR-II indicates that the reinforced concrete of the sodium boiler building and steel siding/masonry block walls of the power plant were designed to resist these wind forces.

The missile criteria specified for objects or debris that could be picked up by high winds is a $2 \times 4$ inch timber plank weighing $15 \mathrm{lbs}$. Its impact speed is $50 \mathrm{mph}$ at a maximum height of 50 feet above the ground. This missile is expected to perforate sheet metal siding, or wood siding up to $3 / 4$ inch thick, but will not perforate unreinforced concrete masonry or brick veneer walls [10.18]. The safety assessment at ANL-W concluded that missiles which can cause significant damage to the facility will not be generated at windspeeds below $100 \mathrm{mph}$ [10.13]. Small missiles light in weight could be generated at velocities which would penetrate a light 
building shell, but these missiles would not have the energy required to do structural damage or breach containment. Therefore, damage from such a missile would be limited to the power plant or cooling tower resulting in the loss of the balance-of-plant system as the primary heat sink. Since the loss of primary heat sink as an initiator (TSEC and TBOP) is more frequent $(-1 / \mathrm{yr})$ than the probability of exceeding the DBW in an 10,000-year return period $\left(10^{-4} \mathrm{yr}\right)$, the hazard to EBR-II from high winds is bounded by the analysis for the normal Loss of Primary Heat Sink event tree.

\subsection{Volcanism}

The INEL is located in an area built principally by volcanic eruptions; therefore, there is a potential for resumption of volcanic activity. Volcanic processes that might affect facilities at the INEL are lava flows, earthquakes associated with volcanism, and explosive eruptions.

Volcanics on the Eastern Portion of the Snake River Plain (ESRP) consists of rhyolites and basalts. As shown by Fig. 10.6, rhyolite domes on the ESRP include Big Southern Butte, East Butte, Middle Butte and Cedar Butte. Vents that produced surface basalt flows occurred along a series of rift zones. Fig. 10.6 shows three well-defined rift zones (Arco - Big Southern Butte, Lava Ridge - Hell's Half Acre, and Circular Butte - Kettle Butte), as well as the (offINEL) Great Rift [10.13].

Explosive rhyolitic volcanism associated with the large calderas of the ESRP has not occurred in the vicinity of the INEL for about 4-6 million years. The possibility of flows from the potentially more active Yellowstone region reaching the INEL is essentially nonexistent because of the distances involved and intervening topographic barriers. The possibility of airfall ash from such an eruption impacting the ANL-W site is also remote because the recurrence interval of the major eruptions is on the order of 0.5-1 million years and the prevailing winds have generally distributed the ash to the east or southeast of Yellowstone [10.14].

The age of several basalt flows located on, and adjacent to, the INEL have been evaluated and range from 12,000 to 400,000 years old. Just off the east boundary of the INEL, 


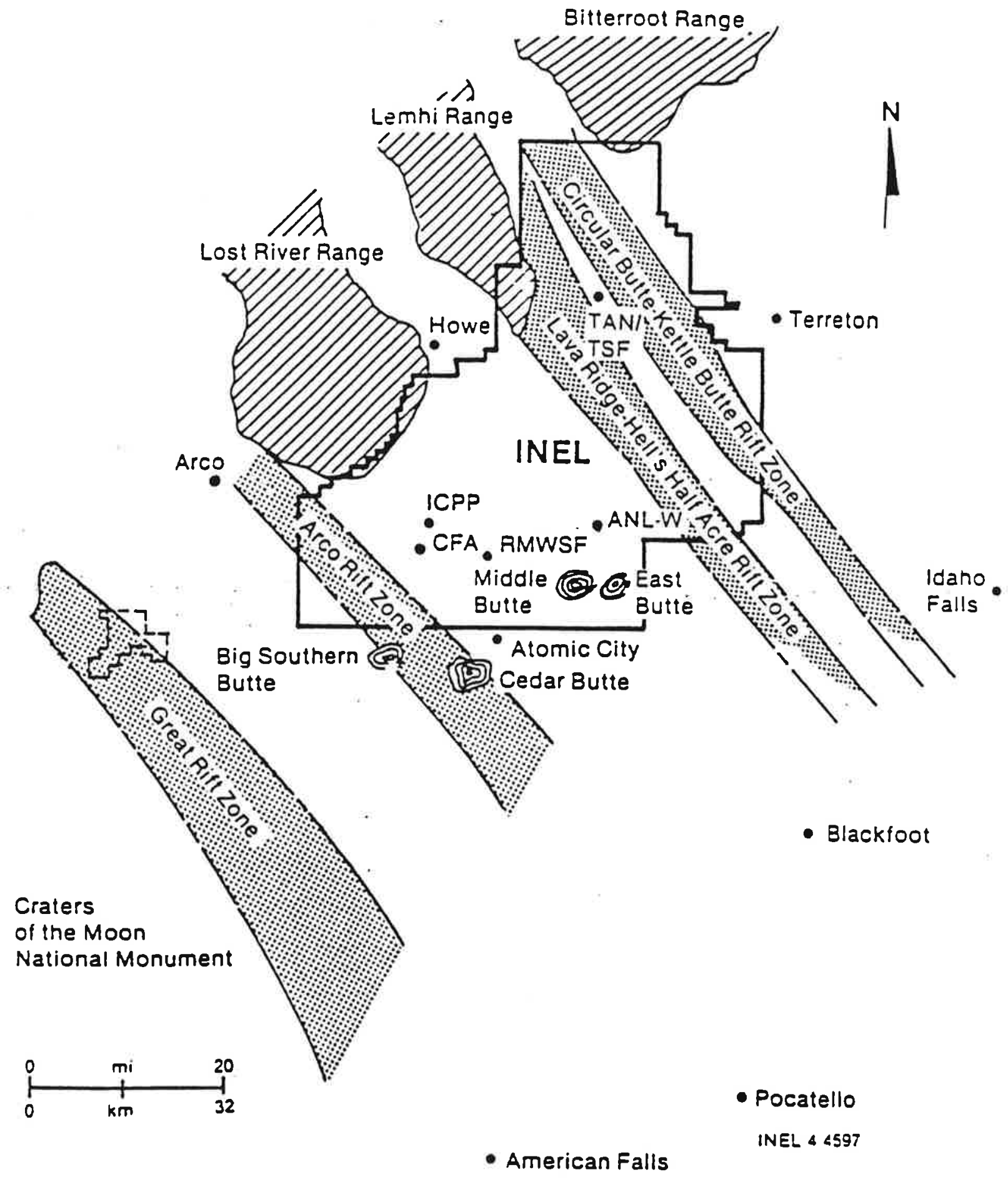

Fig. 10.6. Volcanic Structures and Rift Zones near the INEL Site 
the most recent Hell's Half Acre flow has been dated at 4100 years [10.13]. Away from the rift zones, individual areas are inundated at less frequent intervals. Studies of cores from wells at ANL-W have determined that the ANL-W site has experienced 5 flows during the past 500,000 years. The likelihood of a given eruption reaching the site is once every $80,000-100,000$ years $[10.14]$.

Earthquakes that might be expected in association with volcanic eruptions at the INEL are of two types: (1) earthquakes due to magma movement and (2) sympathetic tectonic earthquakes. Earthquakes due to magma movement are typically of low magnitude ( 2 to 5 Richter magnitude), and restricted to areas near the magma source. Sympathetic tectonic earthquakes are caused by forceful injection of magma into existing rift zones and as such are generally confined to the rift areas. Because of the low magnitude and localized effects from these types of earthquakes, safety-related problems due to sympathetic tectonic earthquakes and earthquakes due to magma movement are not anticipated [10.14].

The recurrence interval for lava flows would suggest that the ANL-W site is susceptible to hazards from lava flows since recurrence frequencies are in the range of $10^{-5}$. Precursory events such as ground deformation, seismic activity, and fuming would likely occur near vents prior to any possible future volcanic activity on the ESRP. At that time, geologic and geophysical monitoring should help to predict the likelihood and probable location of an eruption as well as the possible threat to individual facilities at the INEL. If a particular facility like EBR-II were in danger, orderly shutdown procedures could be initiated, including removing the fuel from the reactor. Lava flow control or diversion methods could be instituted to allow six to twelve months for defueling the reactor. Therefore the likelihood of any release due to volcanic activity would be insignificant.

\subsection{External Floods}

The INEL is situated on a high arid plain with flat or gently rolling terrain. These characteristics, combined with the low precipitation and the permeable soils that are prevalent, result in little or no concern for external hazards from flooding. The waters of the Big and 
Little Lost Rivers and Birch Creek drain through the Eastern Snake River Plain to sinks in the vicinity of the Test Area North (TAN), significantly contributing to the Snake River Aquifer. The Big Lost River, as the most significant water source, has two major flood control features, the Mackay Dam and the INEL flood-diversion system. The INEL flood-control system reduces the threat of floods at INEL from any abnormally large stream flows in the Big Lost River, by diverting stream flow out of the main channel to INEL spreading areas.

There is no history of flooding at the ANL-W site. The afore-mentioned rivers do not constitute a flood threat to the EBR-II facility since the elevation above mean sea level (MSL) of ANL-W is approximately $5120 \mathrm{ft}$. At its closest approach to the site, the Big Lost River is 11 miles away at a lower elevation of $4850 \mathrm{ft}$ MSL. This elevation differential ( $+270 \mathrm{ft}$ higher than the river channel) and distance from the river protect the site from even a worst case scenario involving complete failure of the Mackay Dam due to a seismic event [10.13].

\subsection{Lightning}

Air-to-ground lightning strikes occur infrequently at the INEL. However, the lack of natural targets and the poor conductivity of the lava rock and desert soil cause manmade structures at the INEL to be highly susceptible to lightning strikes. Therefore, lightning protection systems are required for structures that could be susceptible to damage from lightning.

On two separate occasions, the EBR-II plant has tripped due to lightning strikes. While at power in June of 1977 , a lightning strike caused both $2400 \mathrm{~V}$ and $480 \mathrm{~V}$ transformer secondary circuit breakers to open and the $13.8 \mathrm{kV}$ incoming and feeder breakers to open. Voltage and current transients associated with the lightning strike also caused the constant uninterruptible power system (UPS) units No. 1 and No. 2 to transfer to the 480V AC bypass power which was failed since the $480 \mathrm{~V}$ power line had already failed. This resulted in an automatic reactor scram

and loss of power to the reactor control room. However, the emergency diesel generators started and assumed the loads. It was later determined that significant ground currents were flowing in the power plant's grounding network and substantial overcurrent flowed through the UPS inverter and control logic as a result of the lightning surge. A later incident occurred in 
1984 as power was lost to the main feedwater pumps when a EBR-II $13.8 \mathrm{kV}$ incoming line breaker failed to open upon an electric power system transient due to lightning. The reactor was manually scrammed after the operators received a steam drum low-level alarm.

Lightning rods above the power plant stack and weather tower, as well as for the metal surfaces of the power plant and sodium boiler buildings, are connected by large grounding cables to the site ground system. It was determined by plant engineering that lightning strikes which hit these rods or structures could eventually reach the grounding system and create disturbances similar to those observed during the June 1977 incident. Therefore, Engineering Change Notice 161 and 161B [10.15] provided for the installation of surge suppression devices on the input and bypass power lines for continuous power UPS No. 1 and 2, and the DAS UPS. The continuous power system provides $120 \mathrm{~V}$ AC power to critical instruments and components throughout EBRII. The surge suppressors are installed on the $480 \mathrm{~V}$ AC input power feeders for the UPS units. These surge suppressors prevent damage to the UPS components due to spikes on the 480V AC distribution system, caused primarily by lightning strikes on the INEL loop transmission lines.

Even with the design change, lightning strikes can still result in the requirement for reactor shutdown, either automatically or manually, due to the effect of loss of normal power to operating components, and therefore, contribute to the frequency for the Loss of Normal Power initiating event. However, since the modification ensures reliable power for the nuclear and process instrumentation and control equipment in the event of a lightning strike, and the shutdown coolers do not require power to remove decay heat, any accident sequences initiated by lightning are bounded by the evaluation for LONP, in Section 11.

\subsection{Aircraft Impact}

One possible threat to EBR-II is from accidents associated with aircraft. There are five commercial airports located within 100 miles of INEL: Pocatello (50 miles), Idaho Falls (29 miles), Twin Falls (80 miles), Hailey (70 miles), and Jackson, Wyoming ( 85 miles). 
Several smaller, gravel-surface landing strips near INEL are used for emergency landings, charter flights, crop dusting, and other such intermittent activities. The closest such landing strip to ANL-W is at Atomic City, just outside the south boundary of INEL. Only one large commercial airline flight (Salt Lake to Calgary) is known to over-fly the INEL routinely, although several commuter flights (Idaho Falls to Boise) may be routed to traverse the southern portion of the INEL. Aircraft are warned away by high-intensity strobe lights on ANL-W facilities (such as the 240-ft meteorological tower). The location of these lights and other restrictions are designated on current aeronautical charts. Increased security surveillance at all INEL sites, and the need to upgrade security forces, resulted in helicopter pads being installed at most INEL sites. The ANL-W helipad is just south of the main parking lot. Routine helicopter overflights over ANL-W are not allowed [10.13].

Because overflying aircraft activity is very limited and airports are relatively distant from the site, hazards to EBR-II due to aircraft are not a significant threat. An upper bound estimate on the total frequency of aircraft impact hazards for EBR-II can be estimated based upon criteria established in the U.S. NRC Standard Review Plan (SRP), Section 3.5.1.6, which addresses the frequency of aircraft crashing into a nuclear plant [10.16].

According to the guidelines in the SRP, the crash frequency at a nuclear plant for aircraft using a federal airway can be estimated from the expression:

$$
\mathrm{P}_{\mathrm{FA}}=\mathrm{C} * \mathrm{~N} * \mathrm{~A} / \mathrm{W}
$$

where

$\mathrm{P}_{\mathrm{FA}}=$ the frequency of aircraft crashing into a nuclear power plant structure (crashes/year),

$\mathrm{C}=$ the inflight crash rate for aircraft using the airway (crashes/aircraft-mile),

$\mathbf{N}=$ the annual number of flights along that segment of the airway (aircraft/year),

$A=$ the effective area of the plant, i.e., the area that includes all plant structures which, if struck, could have an adverse affect on safety (square miles), and

$\mathrm{W}=$ the width of the federal airway (miles). 
The SRP recommends using an inflight crash rate of $4.010^{-10}$ crashes per aircraft-mile. The Federal Aviation Administration, Idaho Falls, projects that 2920 aircraft per year operating under instrument flight rules (IFR) use a fixed route between Salt Lake City and Calgary, Canada which passes over the INEL. There are also approximately 100 Vortex Test Flights conducted by the NOAA-FAA at the site per year. Additionally, visual flight rules (VFR) smaller aircraft, while restricted to maintaining 8000 ' MSL elevation, are allowed to pass over the INEL. The FAA estimates there are approximately 100 such operations per month, giving a total number of flights of 4220 . The effective area of the EBR-II structures is estimated to be 0.01 square miles. The width of the fixed airway route was estimated by the FAA to be approximately 2 miles. All of these operations are assumed to take place within this fixed route. Therefore, the estimated frequency of an aircraft flying this route and crashing into EBR-II is approximately $8.410^{-9}$ crashes per year. Since this evaluation only considers the frequency of aircraft impacts without investigating the capabilities of the EBR-II plant to withstand crashes, it can be assumed that this hazard poses a negligible risk to EBR-II.

\subsection{Accidents at Other INEL Facilities (Industrial and Military)}

Activities or accidents at other INEL facilities do not pose a physical hazard to ANL-W since the nearest facility is nine miles distant. However, airborne release of radioactive materials during emergency conditions at these other facilities could conceivably pose a hazard to ANL-W site personnel. The DOE Warning Communications Center (WCC) in Idaho Falls maintains continuous site-wide surveillance of all INEL facilities, and transmits site-wide wamings of any unsafe conditions. Upon receipt of such warnings, the ANL-W Emergency Plan would be activated. Operations at EBR-II can be safely terminated, and the personnel evacuated almost immediately [10.13].

\subsection{Turbine Missiles}

An analysis of external events often includes an examination of the impact of a missile from the turbine-generator striking a critical plant component. The postulated missile is a result

of mechanical components being hurled at high velocity from self-destruction and breakup of the 
turbine-generator during plant operation. The failure mechanism for such an initiator is based upon either metallurgical and/or design errors, environmental effects such as stress corrosion, overspeed, or a combination of these factors. The probability of significant damage to critical nuclear reactor components due to turbine failure has been assessed in previous studies of commercial reactors by determining the combined probabilities of turbine failure and ejection of an energetic missile that escapes the turbine casing; the probability the missile strikes a critical component, and the probability that significant damage occurs to the component [10.19]. An example of such a scenario would be a missile that strikes the reactor containment, and penetrates and damages key components in the primary coolant system. Studies conducted for Westinghouse and General Electric LWRs indicate the overall probability of serious consequences from such an initiator is very low, on the range of $10^{-10}$ to $10^{-12}$ per year, and is even further reduced if the turbine axis is oriented on a radius of the containment, since missile direction would be radial about the axis of the turbine [10.19].

The turbine generator for EBR-II is located on the turbine deck floor of the power plant, as shown previously by Figure 3.8 in Section 3 of this report. The positioning of the turbinegenerator relative to the reactor building precludes missile damage to the containment or the shutdown coolers. It would be expected that balance-of-plant heat removal would be impacted requiring reactor shutdown but the ability to remove decay heat with the shutdown coolers would not be affected. However, since the EBR-II main control room is adjacent to the turbine, a more immediate concern would be missiles that could strike the main control room since there are no barriers to such a missile other than the concrete wall providing separation. Control room personnel and equipment would be vulnerable to the effects of such an event. The probability of a turbine generator missile initiator was determined to be in the order of $3.110^{4}$ to $3.510^{-5}$ per turbine year for failures observed in turbine population (nuclear as well as nonnuclear) corrected to be relevant to nuclear reactors [10.20]. The previously referenced study indicated that the probability of striking a critical target is approximately $1.010^{-3}$. This would result in a worst-case probability on the order of $3.110^{7} / \mathrm{yr}$ for a turbine missile striking the control room. The effect of such a missile upon the ability to achieve reactor scram would require further analysis, but the engineering judgement of plant operations indicate that most likely, either due to the initiator effect on the electrical grid or the loss of control room 
instrumentation or control power by the missile would result in an automatic reactor scram, and that manual scram would not necessarily be precluded, resulting in safe shutdown and decay heat removal via the shutdown coolers. Therefore, turbine missiles are considered to be an insignificant contributor to the overall frequency of core damage at EBR-II, but do pose a risk to plant personnel should they occur.

\subsection{Internal Floods}

The reactor containment building does not contain any water sources such as tanks or piping. Water sources are purposely excluded in the reactor building to prevent any possibility of a sodium-water reaction from occurring due to a spill or accident. An internal flood initiator could occur in the power plant since the balance-of-plant power conversion cycle involves the condensate and feedwater systems and the condenser and associated cooling water systems. An internal flood initiator involving these systems would only serve to disrupt normal operations by disabling balance-of-plant heat removal, requiring plant shutdown. There are no credible internal flood events that can impact the ability to remove decay heat with the shutdown cooling system following reactor shutdown. Plant shutdown with the primary heat sink unavailable would be expected to occur more frequently due to other internal initiators, e.g., loss of feedwater flow, than the frequency for a flood-induced event, and therefore, would not significantly contribute to plant risk.

There is a potential for water accumulation in the cable tunnel between the main control room and the reactor building due to groundwater seepage or inadvertent actuation of the wet pipe fire protection sprinkler system that has been recently installed. The most likely consequence of water accumulation in the cable tunnel would be an inadvertent reactor scram due to electrical shorts of seismic detectors located on the floor of the cable tunnel. The cables would not be affected by water immersion or spray since they are sealed and routed in cable trays a minimum of two to three feet above the floor of the tunnel. Cable connections may be susceptible to immersion but are located a minimum of 4-5 feet above the floor. The cable tunnel itself has sumps in the event of water accumulation with sump pumps that automatically start to transfer water out of the sumps. Operation of the sump pumps is annunciated in the 
main control room. Inadvertent operation of the wet pipe fire protection system would result in a trouble alarm code at the site fire department indicating water delivery flow in the cable tunnel. Operations personnel would be dispatched to determine the cause for the alarm and water flow would be terminated.

Inadvertent actuation of the wet pipe fire protection sprinkler system in the remainder of the plant other that the BOP could impact the 2400 VAC switchgear and the primary pump clutch control panel. The spray shields above the 2400 VAC switchgear are designed to prevent water intrusion. The failure rate of sprinkler heads for inadvertent actuation is calculated based on three environmental failures (high temperature due to steam) and five due to maintenance in a population of 60,000 for 30 years [10.23]. The calculated value is $4.710^{-6} \mathrm{yr}^{-1}$. Even if several sprinklers could effect the switchgear, the LONP resulting would be much smaller than the value of $10^{-1}$ from other causes. Therefore, this event is subsumed into LONP.

Water in primary pump clutch control could cause a loss of both primary pumps due to loss of power to the clutches, resulting in a LOF condition. Since there is no spray shield on the clutch control cabinet, the initiating frequency is assumed to be the same as the failure of the single sprinkler in the area, $4.710^{-6} \mathrm{yr}^{-1}$. This is three orders of magnitude below the LF2B internal initiating event frequency of $4.710^{-3} \mathrm{yr}^{-1}$. The LF2B conditional probabilities of core and structural damage (CSD) and core damage (CD) are $8.310^{-9}$ and $4.610^{-6}$, respectively. Therefore the core damage frequency for inadvertent sprinkler actuation is below the $10^{-10}$ threshold.

\subsection{Summary}

Of the external events considered in this section, only liquid metal fires have sequence frequencies which are above the $10^{-10}$ screening cutoff. The total frequency for secondary sodium fire damage of any type is $1.510^{-6} \mathrm{yr}^{-1}$. The total frequency for shutdown cooler $\mathrm{NaK}$ fire is $2.110^{-6} \mathrm{yr}^{-1}$. Detailed results for all sequences are given in Table 10.2. All other sodium fires have sequences which do no lead to core damage or which fall significantly below the screening cutoff. 


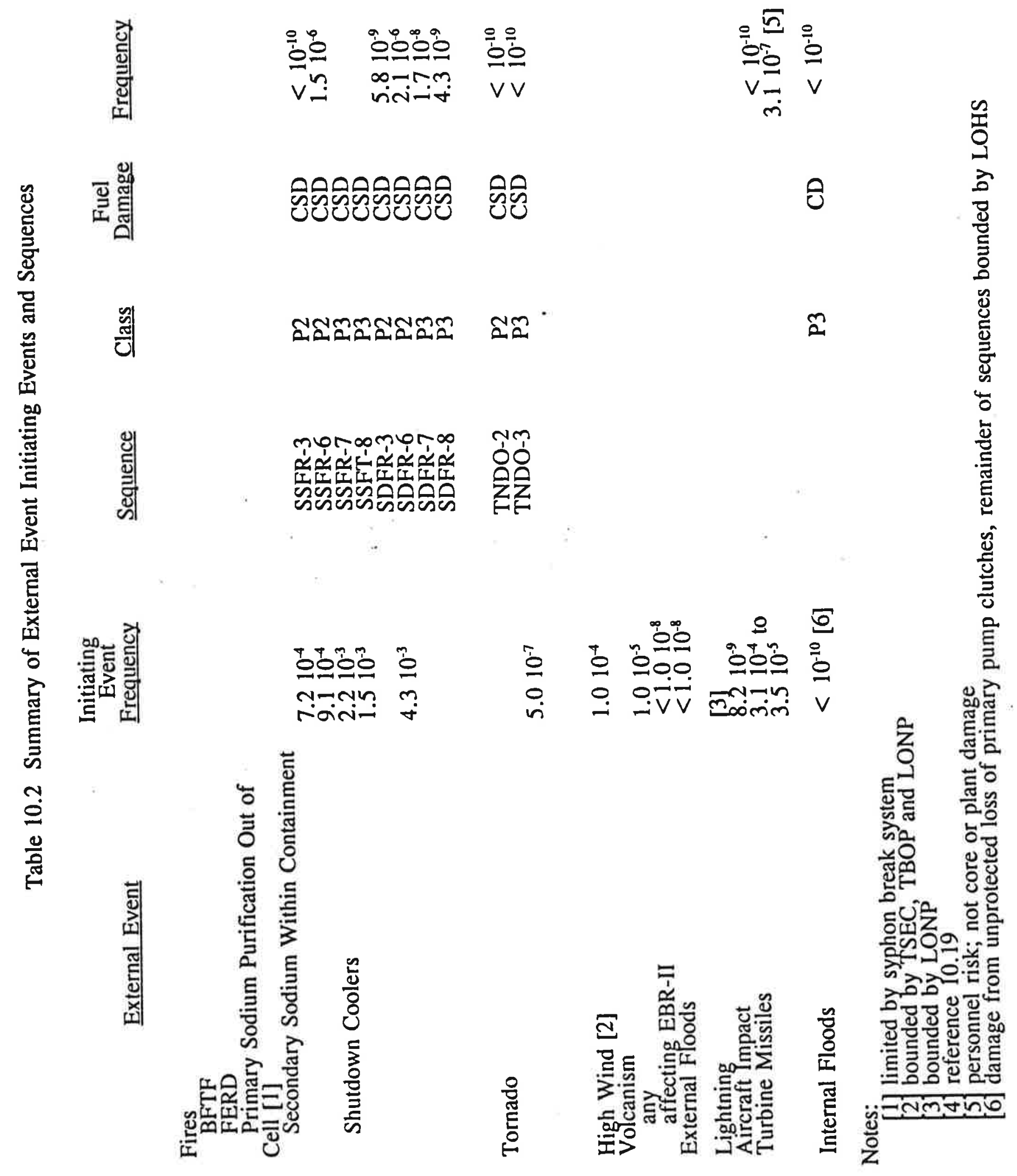


The only other sequence which is above the $10^{-10}$ cutoff is turbine missiles. Depending on the reference, a value of $3.110^{-7} \mathrm{yr}^{-1}$ is found but this value is limited to personnel injury only. A lower value is assumed for equipment damage. Another reference for equipment damage [10.19] gives a value below the cutoff of $10^{-10}$. 


\section{Section 10.0 References}

sverif

10.1 USNRC, "PRA Procedures Guide: A Guide to the Performance of Probabilistic Risk Assessments for Nuclear Power Plants", NUREG/CR-2300, 1981.

10.2 Clinch River Breeder Reactor Plant Probabilistic Risk Assessment, September, 1984.

10.3 Argonne National Laboratory, unpublished information (1980).

10.4 Argonne National Laboratory, unpublished information (1990).

10.5 Argonne National Laboratory, unpublished information (1990).

10.6 ANL, "Replacement of \#1 and \#2 Primary Pump Motor Cables", MCN No. 387(I), July 1981:

10.7 Factory Mutual Research Corporation, "Fire Protection Survey for EBR-II", FMRCJ.I.1P1N9.CY, December 1987.

$$
\text { ¿i: }
$$

10.8 "The Probability of Leaking in Piping Systems of Pressurized Water Reactors on the Basis of Fracture Mechanics and Operating Experience", S. Beliczey and H. Shultz, Nuclear Engineering and Design, Volume 102, 1987, pp. 431-438.

10.9 USNRC, "Pipe Break Frequency Estimates for Nuclear Power Plants", R. E. Wright et al., NUREG/CR-4407, May 1987.

10.10 "Pipe and Vessel Failure Probability", H. M. Thomas, Reliability Engineering, Volume 2, 1981,pp. 83-124. 
10.11 Argonnè National Laboratory, unpublishèd information (1989).

10.12 Argonnē National Laboratory, unpublishèd information (1988).

10.13 Argonnē National Laboratory, unpublishèd information (1988).

10.14. DOE, "Final Environmental Impact Statement - Special Isotope Separation Project", INEL, DOE/EIS-0136, Volume I, November 1988.

10.15 Argonnè National Laboratory, unpublishèd information (1978).

10.16 "Aircraft Hazards", U.S. NRC Standard Review Plan, Section 3.5.1.6, NUREG-0800, Rev. 2, Washington, D.C., July 1981.

10.17 "Environmental Assessment: Fuel Processing Restoration at the Idaho National Engineering Laboratory", DOE/EA-0306, August 1987.

10.18 "Design and Evaluation Guidelines For Department of Energy Facilities Subjected to Natural Phenomena Hazards", UCRL-15910, May 1989.

10.19 S. H. Bush, "Probability of Damage to Nuclear Components Due to Turbine Failure", Nuclear Safety, Volume 14, No. 3, May-June 1973.

10.20 "A Reassessment of Turbine-Generator Failure Probability", S. H. Bush, Nuclear Safety, Volume 19, No. 6, November-December 1978.

10.21 Zion Probabilistic Safety Study, Section II.7.2 
10.22 Indian Point Probabilistic Safety Study, Power Authority of the State of New York, Consolidated Edison Company of New York, Section 0.19.2, 1982.

10.23 D. J. Keigher, "Water and Electronics Can Mix", Fire Journal, Volume 62, No. 6, November 1968, pp. 68-72. 
11.0 SEQUENCE QUANTIFICATION 


\section{Table of Contents}

Page

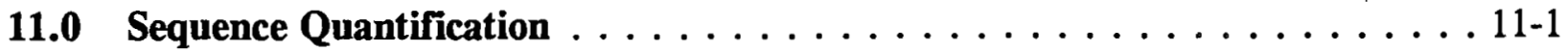

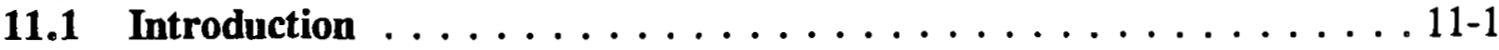

11.2 Sequence Quantification Methodology . . . . . . . . . . . 11-2

11.3 Sequence Quantification Results . . . . . . . . . . . 11-10

11.3.1 Results by Accident Sequence . . . . . . . . . 11-12

11.3.2 Results by Plant Damage Class . . . . . . . . . . . . . 11-21

11.3.3 Results by Damage Category . . . . . . . . . . . . 11-27

11.3.4 Results by Initiating Event $\ldots \ldots \ldots \ldots \ldots \ldots \ldots \ldots . \ldots \ldots$

11.3.5 Results by Event Tree . . . . . . . . . . . 11-47 


\section{List of Figures}

11.1 Decay Heat Removal Sub-Tree . . . . . . . . . . . . . . . . 11-11

11.2 Contribution of Dominant Sequences to Damage Frequency in EBR-II . . . . 11-16

11.3 Dominant Sequences in Plant Transient Class P1 . . . . . . . . . . . . . 11-28

11.4 Dominant Sequences in Plant Transient Class P2 . . . . . . . . . . . . . . . 11-29

11.5 Dominant Sequences in Plant Transient Class P3 . . . . . . . . . . . . . . 11-30

11.6 Dominant Sequences in Plant Transient Class P4 . . . . . . . . . . . . . . 11-31

11.7 Dominant Sequences in Plant Transient Class P5 . . . . . . . . . . . . 11-32

11.8 Dominant Sequences in Plant Transient Class P6 . . . . . . . . . . . . . . 11-33

11.9 Comparison of Plant Transient Classes by their Contribution to

Total Frequency (damage + ND) . . . . . . . . . . . . . . . . . . . 11-34

11.10 Dominant Sequences in the Core Damage Category . . . . . . . . . . . . . 11-40

11.11 Dominant Sequences in the Minor Core Damage Category . . . . . . . . . . . 11-41

11.12 Dominant Sequences in the Core/Structural Damage Category . . . . . . . . 11-42

11.13 Dominant Sequences in the Potential Experiment Damage Category . . . . . . 11-43

11.14 Dominant Sequences in the No Damage Category . . . . . . . . . . . . . . 11-44

11.15 Comparison of Damage Categories by their Contribution to the Total

Damage Frequency . . . . . . . . . . . . . . . . . . . . 11-45

11.16 Comparison of Damage Categories by their Contribution to the Total (damage + ND) Frequency . . . . . . . . . . . . . . . 11-48

11.17 Comparison of Initiating Events by their Contribution to the

Damage Frequency . . . . . . . . . . . . . . . . . . 11-51

11.18 Comparison of Initiating Events by their Contribution to the Total (damage + ND) Frequency . . . . . . . . . . . . . . . . 11-52

11.19 Event Tree for a Delayed Loss of Circulation with Sequence Frequency Results . . . . . . . . . . . . . . . . . . . . . 11-53

11.20 Event Tree for a Single Pump Loss of Flow (Group A) with Sequence Frequency Results . . . . . . . . . . . . . . . . . . 11-54

11.21 Event Tree for a Single Pump Loss of Flow (Group B) with Sequence Frequency Results . . . . . . . . . . . . . . . . . . 11-55

11.22 Event Tree for a Single Pump Loss of Flow (Group C) with Sequence Frequency Results . . . . . . . . . . . . . . . . 11-56

11.23 Event Tree for a Single Pump Loss of Flow (Group D) with Sequence Frequency Results . . . . . . . . . . . . . . . . . . 11-57

11.24 Event Tree for a Double Pump Loss of Flow (Group A) with Sequence Frequency Results . . . . . . . . . . . . . . . . 11-58

11.25 Event Tree for a Double Pump Loss of Flow (Group B) with Sequence Frequency Results . . . . . . . . . . . . . . . . . . . . . 11-59

11.26 Event Tree for a Double Pump Loss of Flow (Group C) with Sequence Frequency Results . . . . . . . . . . . . . . . . . . 11-60

11.27 Event Tree for a Double Pump Loss of Flow (Group D) with Sequence Frequency Results . . . . . . . . . . . . . . . 11-61 
11.28 Event Tree for a Double Pump Loss of Flow (Group E) with Sequence Frequency Results . . . . . . . . . . . . . . . . . . 11-62

11.29 Event Tree for a Double Pump Loss of Flow (Group F) with Sequence Frequency Results . . . . . . . . . . . . . . . . . . 11-63

11.30 Event Tree for the Loss of Normal Power with Sequence Frequency Results . . . . . . . . . . . . . . . . . . . . . . . . . 11-64

11.31 Event Tree for the Loss of Constant Power with Sequence Frequency Results . . . . . . . . . . . . . . . . . . . 11-65

11.32 Event Tree for an Overcooling by Secondary Pump Overspeed with Sequence Frequency Results . . . . . . . . . . . . . . . . . 11-66

11.33 Event Tree for an Overcooling by Primary Pump Overspeed with Sequence Frequency Results . . . . . . . . . . . . . . . . . . 11-67

11.34 Event Tree for an Overcooling by Major Steam Leak with Sequence Frequency Results . . . . . . . . . . . . . . . . . . . . . 11-68

11.35 Event Tree for a Slow Reactivity Insertion (Group A) with Sequence Frequency Results . . . . . . . . . . . . . . . . . . . . . . 11-69

11.36 Event Tree for a Slow Reactivity Insertion (Group B) with Sequence Frequency Results . . . . . . . . . . . . . . . . . . . . 11-70

11.37 Event Tree for a Slow Reactivity Insertion (Group C) with Sequence Frequency Results . . . . . . . . . . . . . . . . . 11-71

11.38 Event Tree for a Slow Reactivity Insertion (Group D) with Sequence Frequency Results . . . . . . . . . . . . . . . . . . 11-72

11.39 Event Tree for a Fast Reactivity Insertion $(>1.0 \$)$ with Sequence Frequency Results . . . . . . . . . . . . . . . . . . . . 11-73

11.40 Event Tree for a Fast Reactivity Insertion $(<0.35 \$)$ with Sequence Frequency Results . . . . . . . . . . . . . . . . . . . 11-74

11.41 Event Tree for an Anticipatory Shutdown with Sequence Frequency Results . . . . . . . . . . . . . . . . . . . . 11-75

11.42 Event Tree for an Scram with Sequence Frequency Results . . . . . . . . . 11-76

11.43 Event Tree for a Long Shutdown with Sequence Frequency Results . . . . . 11-77

11.44 Event Tree for a Short Shutdown with Sequence Frequency Results . . . . . . 11-78

11.45 Event Tree for the Loss of Instrument Air with Sequence Frequency Results . . . . . . . . . . . . . . . . . . . . . . . . . 11-79

11.46 Event Tree for the Loss of Steam Plant Heat Removal with Sequence Frequency Results . . . . . . . . . . . . . . . . . . . . . 11-80

11.47 Event Tree for the Unrecoverable Loss of a Shutdown Cooler with Sequence Frequency Results . . . . . . . . . . . . . . . . . . . 11-81

11.48 Event Tree for the Unrecoverable Loss of Shield Cooling with Sequence Frequency Results . . . . . . . . . . . . . . . . . 11-82

11.49 Event Tree for the Loss of Secondary System Heat Removal with Frequency Results . . . . . . . . . . . . . . . . . . . 11-83 
11.50 Event Tree for the an Evaporator Tube Rupture with

Sequence Frequency Results . . . . . . . . . . . . . . . . . . . . 11-84

11.51 Event Tree for a Superheater Tube Failure with Sequence

Frequency Results

11.52 Event Tree for Metal Driver Fuel Breach Local Fault with

Sequence Frequency Results . . . . . . . . . . . . . . . . . . . . 11-86

11.53 Event Tree for Metal Experiment Fuel Breach Local Fault with

Sequence Frequency Results . . . . . . . . . . . . . . . . . . . . . 11-87

11.54 Event Tree for a Oxide Experiment Fuel Breach Local Fault with Sequence Frequency Results . . . . . . . . . . . . . . . . . . . 11-88

11.55 Event Tree for a Subassembly Local Fault from Partial Blockage with Sequence Frequency Results . . . . . . . . . . . . . . . . . . . . 11-89

11.56 Event Tree for a Subassembly Local Fault from Loading Error with Sequence Frequency Results . . . . . . . . . . . . . . . . . . 11-90 


\section{List of Tables}

\section{Page}

11.1 Accident Sequences Analyzed, Listed by Damage Category . . . . . . . . . 11-3

11.2 Description of the Event Tree Top Events . . . . . . . . . . . . . . . . . . 11-9

11.3 Summary of Dominant Sequences Contributing to Fuel and Experiment Damage 11-13

11.4 Dominant Sequences Grouped by Transient Class . . . . . . . . . . . . . . 11-22

11.5 Dominant Sequences Grouped by Damage Category . . . . . . . . . . . . . . 11-35

11.6 Damage Accident Sequences Quantification Results Grouped by Initiating Event 11-49 


\section{SEQUENCE QUANTIFICATION}

\subsection{Introduction}

The accident sequence quantifieation task consists in integrating the models developed for plant systems, the models developed for accident progression, and the data compiled for the basic events and the initiating events. The frequency of an accident sequence is calculated by determining the dominant minimal cutsets of the sequence and evaluating their frequency using the initiator frequency and the probabilities of the basic events. The sum of the frequencies of the minimal cutsets is the point estimate of the sequence frequency, and the sum of the sequence frequencies is the point estimate of the overall frequency of core damage.

The products of the accident sequence analysis are the estimated frequencies of each sequence, a list of the sequences and their relative contribution to the overall damage frequency, and a list of the most significant minimal cutsets and basic events contributing to the dominant sequences. The results reported in this section refer only to the sequences from internal initiating events. Although uncertainty coupling was studied in some systems, the calculation for accident sequences have been performed without correction for uncertainty coupling among basic event probability distributions.

The process of quantifying accident sequences, using the event tree and fault tree models developed, in practice becomes a complex task when all the system dependencies and interconnections are taken into account. The most important aspects to be considered during sequence quantifications are:

- $\quad$ Effects of the initiating event on the different plant systems and their response. 
- $\quad$ Logical loops that appear when a system depends upon a support system that in turn depends upon the original system.

- $\quad$ Effect of linking faulted and successful systems in a sequence, with the possible appearance of incompatibilities, such as minimal cutsets in the faulted systems that are supersets of those of the systems that have not failed.

- Dependencies and commonalities between systems, such as failure of a common support system or the failure of a shared component.

- Inclusion of recovery actions.

\subsection{Sequence Quantification Methodology}

With the exception of two direct damage initiators, the accident sequences in EBR-II are formed by a combination of an.initiating event and one or more system failures and/or successes. Following the criteria developed in Section 5, the accident sequences leading to some sort of fuel damage in EBR-II have been selected and analyzed. Table 11.1 provides a listing of all the sequences that have been quantified, grouped by fuel damage category. It lists the potential sequences leading to fuel damage (CD, CSD $M C D, P E D)$ plus those sequences that, although not resulting in fuel damage, have the potential for major plant disruption (ND). For each sequence listed in the tables, the failed and success top events are identified by the 4-letter acronyms that have been used in the event trees. Table 11.2 provides a description of these event tree top events. The initiating event has not been explicitly listed under the failed top events because it is part of the sequence identification, which also provides direct correspondence between the sequences and the event trees of Section 5 .

When all the relevant sequences had been identified and properly described, the quantification process began by building the composite fault trees. This task consisted primarily in including the support system trees in the main system trees, in particular the decay heat 
Table 11.1 Accident Sequences Analyzed, Listed by Damage Category

DAMAGE CLASS: CD

SEQUENCE FAILED TOP EVENTS: SUCCESSFUL TOP EVENTS:

\begin{tabular}{|c|c|c|c|c|c|c|c|}
\hline LCDL-4 & PKRU & & & & & & \\
\hline LF2B-6 & FROD & & & & & FSIG & \\
\hline LF2B-7 & FSIG & & & & & & \\
\hline LF $2 \mathrm{D}-2$ & FROD & & & & & FSIG & \\
\hline$L F 2 D-3$ & FSIG & & & & & & \\
\hline LF $2 E-6$ & FROD & & & & & FSIG & \\
\hline LF $2 E-7$ & FSIG & & & & & & \\
\hline LOCP -6 & LROD & & & & & & \\
\hline MDLF-4 & NPPD & SLPD & NSSP & & & DETP & SHTS \\
\hline MDLF-6 & NPPD & SLPD & SHTS & NSSP & & DETP & \\
\hline$M D L F-8$ & NPPD & SLPD & DETP & NSSP & & & \\
\hline MELF-4 & NPPE & SLPE & NSSP & & & DETP & SHTS \\
\hline MELF-6 & NPPE & SLPE & SHTS & NSSP & & DETP & \\
\hline MELF-8 & NPPE & SLPE & DETP & NSSP & & & \\
\hline OELF-4 & NPPO & SLPE & NSSP & & & DETP & SHTP \\
\hline OELF-6 & NPPO & SLPE & SHTP & NSSP & & DETP & \\
\hline OELF-8 & NPPO & SLPE & DETP & NSSP & & & \\
\hline \multicolumn{8}{|l|}{ RIFL-1 } \\
\hline RIFS -7 & RROD & PRUN & & & & RS IG & \\
\hline RISA-7 & RROD & PRUN & & & & RS IG & \\
\hline RISB -7 & RROD & PRUN & & & & RSIG & \\
\hline RISC- 6 & RROD & & & & & RSIG & PRUN \\
\hline RISC-7 & RROD & PRUN & & & & RS IG & \\
\hline RISC-8 & RSIG & & & & & & \\
\hline RISD- 6 & RROD & & & & & RSIG & PRUN \\
\hline RISD -7 & RROD & PRUN & & & & RSIG & \\
\hline RISD-8 & RSIG & & & & & & \\
\hline SFLE-5 & SNPB & NPPO & SLPE & NSSS & & DETP & SHTS \\
\hline SFLE-7 & SNPB & NPPO & SLPE & SHTS & NSSS & DETP & \\
\hline SFLE-9 & SNPB & NPPO & SLPE & DETP & NSSS & & \\
\hline SFPB -5 & SNPB & NPPB & SLPE & NSSS & & DETS & SHTS \\
\hline SFPB -7 & SNPB & NPPB & SLPE & SHTS & NSSS & DETS & \\
\hline SFPB-9 & SNPB & NPPB & SLPE & DETS & NSSS & & \\
\hline
\end{tabular}


Table 11.1 Accident Sequences Analyzed, Listed by Damage Category (Cont'd)

DAMAGE CLASS: MCD

SEQUENCE FAILED TOP EVENTS:

\begin{tabular}{|c|c|c|c|}
\hline$L F 1 B-7$ & FROD & PRUN & \\
\hline LF $1 \mathrm{C}-7$ & FROD & PRUN & \\
\hline$L F 1 D-7$ & FROD & PRUN & \\
\hline$L F 2 C-6$ & FROD & & \\
\hline $\mathrm{LF} 2 \mathrm{C}-7$ & FSIG & & \\
\hline LF2D-1 & & & \\
\hline$L F 2 F-6$ & FROD & & \\
\hline$L F 2 F-7$ & FSIG & & \\
\hline MDLF-5 & NPPD & SLPD & SHTS \\
\hline MDLF -7 & NPPD & SLPD & DETP \\
\hline MELF-5 & NPPE & SLPE & SHTS \\
\hline MELF-7 & NPPE & SLPE & DETP \\
\hline OCsL-9 & RROD & SPTR & \\
\hline OCSL-10 & RSIG & & \\
\hline OELF-5 & NPPO & SLPE & SHTP \\
\hline OELF-7 & NPPO & SLPE & DETP \\
\hline RIFS- 6 & RROD & & \\
\hline RIFS- 8 & RSIG & & \\
\hline RISB- 6 & RROD & & \\
\hline RISB-8 & RSIG & & \\
\hline SFLE-6 & SNPB & NPPO & SLPE \\
\hline SFLE-8 & SNPB & NPPO & SLPE \\
\hline SFPB -6 & SNPB & NPPB & SLPE \\
\hline SFPB-8 & SNPB & NPPB & SLPE \\
\hline
\end{tabular}

SUCCESSFUL TOP EVENTS:

FSIG
FSIG
FSIG
FSIG
FSIG FROD
FSIG
DETP NSSP
NSSP
DETP NSSP
NSSP
RSIG
DETP NSSP
NSSP
RSIG PRUN
RSIG PRUN
DETP NSSS
NSSS
DETS NSSS
NSSS


Table 11.1 Accident Sequences Analyzed, Listed by Damage Category (Cont'd)

DAMAGE CLASS: CSD

SEQUENCE FAILED TOP EVENTS:

ANTC-3 BPHR DHRS

EVTR-4 SPRS DUME RIHX

EVTR-6 LKDT SPRS

EVTR-10 SMLK SPRS PRRE RIHX

EVTR-13 FSDG SPRS PRRE

LCDL -2 DHRL

LCDL -3 DHRS

LFIA-2 DHRL

LF1A-4 BPHR DHRL

LF1A-5 BPHR DHRS

LF1B-2 DHRL

LF1B-4 BPHR DHRL

LF1B-5 BPHR DHRS

LF1C-2 DHRL

LF1C-4 BPHR DHRL

LF1C-5 BPHR DHRS

LF1D-2 DHRI

LF1D-4 BPHR DHRL

LF1D-5 BPHR DHRS

LF 2A-2 DHRL

LF2A-4 BPHR DHRL

LF2A-5 BPHR DHRS

LF2B-2 DHRL

LF2B-4 BPHR DHRL

LF2B-5 BPHR DHRS

LF2C-2 DHRL

LF2C-4 BPHR DHRL

LF2C-5 BPHR DHRS

LF2E-2 DHRL

LF2E-4 BPHR DHRL

LF 2E-5 BPHR DHRS

LF 2F-2 DHRL

LF2F-4 BPHR DHRL

LF 2F-5 BPHR DHRS

LOCP-2 DHRL

LOCP-4 BPHR DHRL
SUCCESSFUL TOP EVENTS:
FSDG SMLK LKDT

FSDG SMLK

FSDG

PKRU DHRS

PKRU

FSIG FROD BPHR

FSIG FROD DHRS

FSIG FROD

FSIG FROD BPHR

FSIG FROD DHRS

FSIG FROD

FSIG FROD BPHR

FSIG FROD DHRS

FSIG FROD

FSIG FROD BPHR

FSIG FROD DHRS

FSIG FROD

FSIG FROD BPHR

FSIG FROD DHRS

FSIG FROD

FSIG FROD BPHR

FSIG FROD DHRS

FSIG . FROD

FSIG FROD BPHR

FSIG FROD DHRS

FSIG FROD

FSIG FROD BPHR

FSIG FROD DHRS

FSIG FROD

FSIG FROD BPHR

FSIG FROD DHRS

FSIG FROD

LROD BPHR

LROD DHRS 
Table 11.1 Accident Sequences Analyzed, Listed by Damage Category (Cont'd)

DAMAGE CLASS: CSD (cont'd)

SEQUENCE FAILED TOP EVENTS:

SUCCESSFUL TOP EVENTS:

\begin{tabular}{|c|c|c|c|c|c|}
\hline LOCP -5 & BPHR & DHRS & LROD & & \\
\hline LONP-2 & DHRL & & FSIG & FROD & DHRS \\
\hline LONP-3 & DHRS & & FSIG & FROD & \\
\hline OCPP-2 & DHRL & & RSIG & RROD & BPHR \\
\hline OCPP-4 & BPHR & DHRL & RSIG & RROD & DHRS \\
\hline OCPP-5 & BPHR & DHRS & RSIG & RROD & \\
\hline OCPP-7 & RROD & DHRL & RS IG & SPTR & DHRS \\
\hline OCPP-8 & RROD & DHRS & RS IG & SPTR & \\
\hline OCSL-2 & DHRL & & RSIG & RROD & BPHR \\
\hline OCSL-4 & BPHR & DHRL & RSIG & RROD & DHRS \\
\hline OCSL -5 & BPHR & DHRS & RSIG & RROD & \\
\hline OCSL -7 & RROD & DHRL & RSIG & SPTR & DHRS \\
\hline OCSL-8 & RROD & DHRS & RSIG & SPTR & \\
\hline OCSP-2 & DHRL & & RSIG & RROD & BPHR \\
\hline OCSP- 4 & BPHR & DHRL & RS IG & RROD & DHRS \\
\hline OCSP-5 & BPHR & DHRS & RS IG & RROD & \\
\hline OCSP 7 & RROD & DHRL & RSIG & SPTR & DHRS \\
\hline OCSP-8 & RROD & DHRS. & RS IG & SPTR & \\
\hline RIFS-2 & DHRL & & RS IG & RROD & BPHR \\
\hline RIFS -4 & BPHR & DHRL & RSIG & RROD & DHRS \\
\hline RIFS-5 & BPHR & DHRS & RSIG & RROD & \\
\hline RISA-2 & DHRL & & RS IG & RROD & BPHR \\
\hline RISA-4 & BPHR & DHRL & RSIG & RROD & DHRS \\
\hline RISA-5 & BPHR & DHRS & RSIG & RROD & \\
\hline RISB-2 & DHRL & & RSIG & RROD & BPHR \\
\hline RISB-4 & BPHR & DHRL & RS IG & RROD & DHRS \\
\hline RISB-5 & BPHR & DHRS & RSIG & RROD & \\
\hline RISC-2 & DHRL & & RSIG & RROD & BPHR \\
\hline RISC-4 & BPHR & DHRL & RS IG & RROD & DHRS \\
\hline RISC- 5 & BPHR & DHRS & RSIG & RROD & \\
\hline RISD-2 & DHRL & & RS IG & RROD & BPHR \\
\hline RISD-4 & BPHR & DHRL & RSIG & RROD & DHRS \\
\hline RISD-5 & BPHR & DHRS & RSIG & RROD & \\
\hline SCRM-2 & DHRL & & BPHR & & \\
\hline SCRM-4 & BPHR & DHRL & DHRS & & \\
\hline SCRM -5 & BPHR & DHRS & & & \\
\hline
\end{tabular}


Table 11.1 Accident Sequences Analyzed, Listed by Damage Category (Cont'd)

DAMAGE CLASS: CSD (cont'd)

SEQUENCE FAILED TOP EVENTS:

SUCCESSFUL TOP EVENTS:

$\begin{array}{llll}\text { SHDL-2 } & \text { DHRL } & \\ \text { SHDL-3 } & \text { DHRS } & & \\ \text { SHDS-3 } & \text { BPHR DHRS } & \\ \text { SPTR-3 } & \text { DUMS RIHX } & \\ \text { SPTR-6 } & \text { SPRS DUMS RIHX } \\ \text { SPTR-9 } & \text { LKDT DUMS RIHX } \\ \text { SPTR-10 } & \text { LKDT SPRS } & \\ \text { SPTR-13 } & \text { MODS PRRS RIHX } & \\ \text { SPTR-16 } & \text { MODS SPRS PRRS RIHX } \\ \text { SPTR-19 } & \text { MODS LKDT PRRS RIHX } \\ \text { SPTR-21 } & \text { MODS LKDT SPRS PRRS } \\ \text { SPTR-23 } & \text { FSDG PRRS } & \\ \text { TAIR-2 } & \text { DHRL } & & \\ \text { TAIR-3 } & \text { DHRS } & & \\ \text { TBOP-2 } & \text { DHRL } & & \\ \text { TBOP-3 } & \text { DHRS } & & \\ \text { TSDC-2 } & \text { DRLA } & & \\ \text { TSDC-4 } & \text { BPHR DRLA } & \\ \text { TSDC-5 } & \text { BPHR DRSC } & \\ \text { TSEC-2 } & \text { DHRL } & \\ \text { TSEC-3 } & \text { DHRS } & \\ \text { TSHC-2 } & \text { DRLB } & \\ \text { TSHC-4 } & \text { BPHR DRLB } & \\ \text { TSHC-5 } & \text { BPHR DRSB } & \end{array}$

DHRS

FSDG MODS LKDT SPRS

FSDG MODS LKDT

FSDG MODS SPRS

FSDG MODS

FSDG LKDT SPRS

FSDG LKDT

FSDG SPRS

FSDG

MRSS DHRS

MRSS

MRSS DHRS

MRSS

BPHR

DRSC

MRSS DHRS

MRSS

BPHR

DRSB 
Table 11.1 Accident Sequences Analyzed, Listed by Damage Category (Cont'd)

DAMAGE CLASS: PED

SEQUENCE FAILED TOP EVENTS:

SUCCESSFUL TOP EVENTS:

\begin{tabular}{lll}
\hline & & \\
LCDL-1 & & \\
LF1A-7 & FROD PRUN & PKRU DHRS DHRL \\
LF2A-6 & FROD & FSIG \\
LF2A-7 & FSIG & FSIG \\
LONP-4 & FROD & \\
LONP-5 & FSIG & FSIG \\
\hline
\end{tabular}

DAMAGE CLASS : ND

\begin{tabular}{|c|c|c|c|c|c|c|c|c|}
\hline SEQUENCE & FAILEI & D TOP & EVENTS: & SUCCESS & SEUL $T$ & COP EV & JENTS : & \\
\hline EVTR-3 & SPRS I & DUME & & FSDG & SMLK & LKDT & RIHX. & \\
\hline EVTR-9 & SMLK & SPRS & PRRE & FSDG & RIHX & & & \\
\hline LF $1 A-6$ & FROD & & & FSIG & PRUN & & & \\
\hline LF $1 A-8$ & FSIG & & & & & & & \\
\hline LF $1 B-6$ & FROD & & & FSIG & PRUN & & & $\cdot$ \\
\hline LF $1 B-8$ & FSIG & & & & & & & \\
\hline$L F 1 C-6$ & FROD & & & FSIG & PRUN & & & \\
\hline $\mathrm{LF} 1 \mathrm{C}-8$ & FSIG & & & & & & & \\
\hline LF $1 D-6$ & FROD & & & FSIG & PRUN & & & \\
\hline LF $1 D-8$ & FSIG & & & & & & & \\
\hline OCPP-9 & RROD & SPTR & & RSIG & & & & \\
\hline OCPP-10 & RSIG & & & & & & & \\
\hline OCSP-9 & RROD & SPTR & & RSIG & & & & \\
\hline OCSP-10 & RS IG & & & & & & & \\
\hline RISA-6 & RROD & & & RSIG & PRUN & & & \\
\hline RISA-8 & RSIG & & & & & & & \\
\hline SPTR-2 & DUMS & & & FSDG & MODS & LKDT & SPRS & RIHX \\
\hline SPTR- 5 & SPRS I & DUMS & & FSDG & MODS & LKDT & RIHX & \\
\hline SPTR-8 & LKDT & DUMS & & FSDG & MODS & SPRS & RIHX & \\
\hline SPTR-12 & MODS & PRRS & & FSDG & LKDT & SPRS & RIHX & \\
\hline SPTR-15 & MODS & SPRS & PRRS & FSDG & LKDT & RIHX & & \\
\hline SPTR-18 & MODS 1 & LKDT & PRRS & FSDG & SPRS & RIHX & & \\
\hline TAIR-4 & MRSS & & & & & & & \\
\hline TBOP-4 & MRSS & & & & & & & \\
\hline TSEC-4 & MRSS & & & & & & & \\
\hline
\end{tabular}


Table 11.2 Description of the Event Tree Top Events

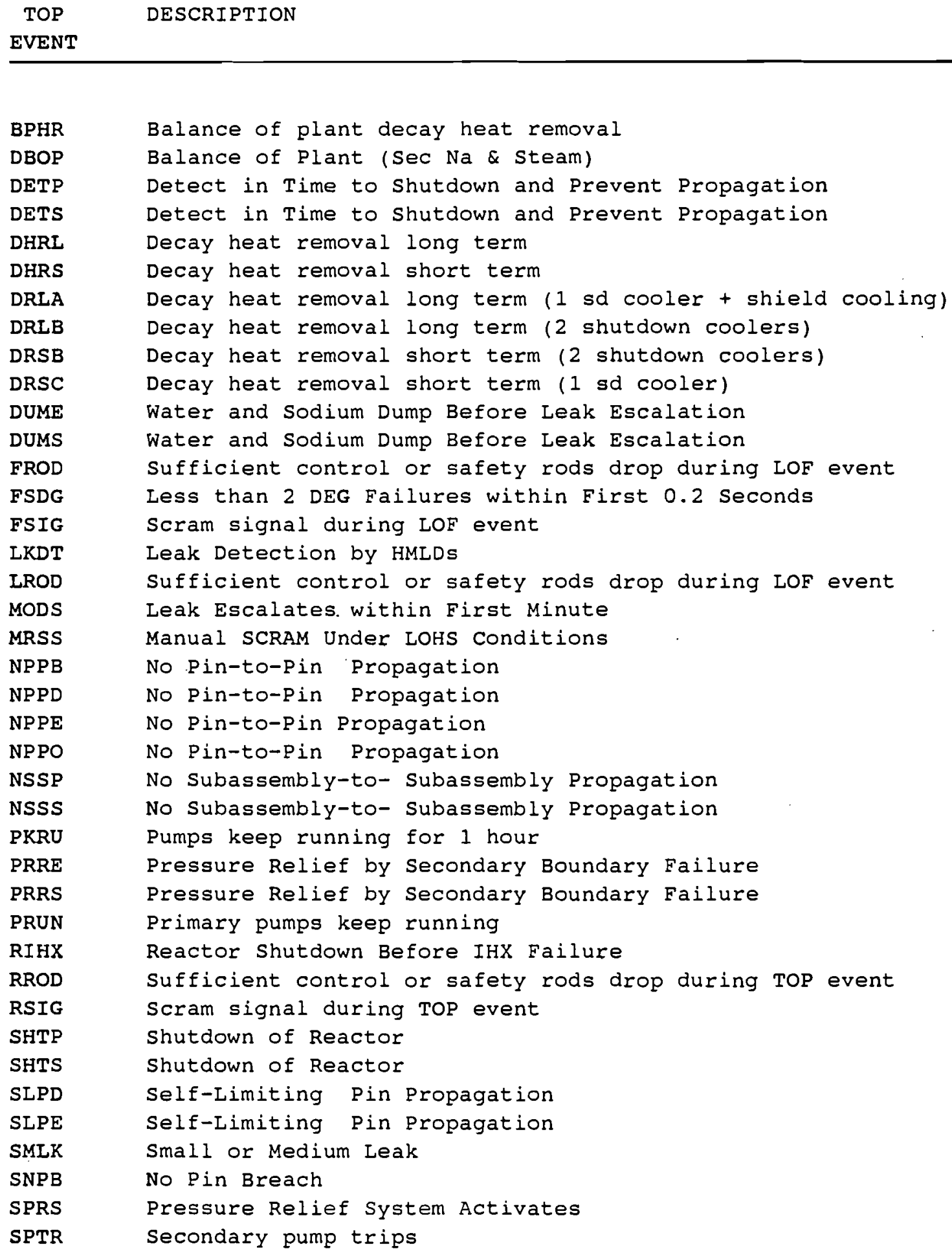


removal trees. A logic loop had to be broken in the electrical support system trees. The next step was the generation of the minimal cutsets for each sequence. Because the balance of plant (BPHR) and the decay heat removal trees (DHRS and DHRL) are the largest in terms of minimal cutsets and appear in many different sequences, an intermediate sequence evaluation (qualitative determination of minimal cutsets only) was performed for the heat removal sequences. The heat removal sequences evaluated are depicted in the event tree of Fig. 11.1. Only three of the intermediate sequences were analyzed (DKHR-2, DKHR-4, DKHR-5), since the other two lead to successful decay heat removal. The minimal cutsets for the three relevant partial sequences were found and then used in the calculations for the complete accident sequences.

A fault tree linking method was used in generating the minimal cutsets of the accident sequences. Logically inconsistent minimal cutsets, those sequence cutsets of failure trees that were also supersets of a minimal cutset of the success trees, were automatically eliminated. The cutset generation was truncated by the use of a probability cutoff. The probability cutoff was selected at $10^{-15}$ for all the sequence calculations except for those of the local faults and evaporator and superheater tube rupture event trees, where no probability cutoff was used at all. The cutsets of the fault trees involved in the accident sequences had previously been generated with a probability cutoff of $10^{-11}$ or $10^{-12}$, depending on the top event probability and the computation times involved. After the minimal cutsets were determined the frequency was estimated. Importance and uncertainty calculations were also performed for each sequence, as reported in the next section. For two initiators (loss of piping integrity, LOPI, and rapid loss of circulation, LCRA) the frequency of the initiating events was less than $1.010^{-11} \mathrm{yr}^{-1}$, and their sequences were not quantified.

\subsection{Sequence Quantification Results}

The accident sequence frequency values reported in this section are point mean values, estimated using only mean values for the initiator frequencies and the basic event 


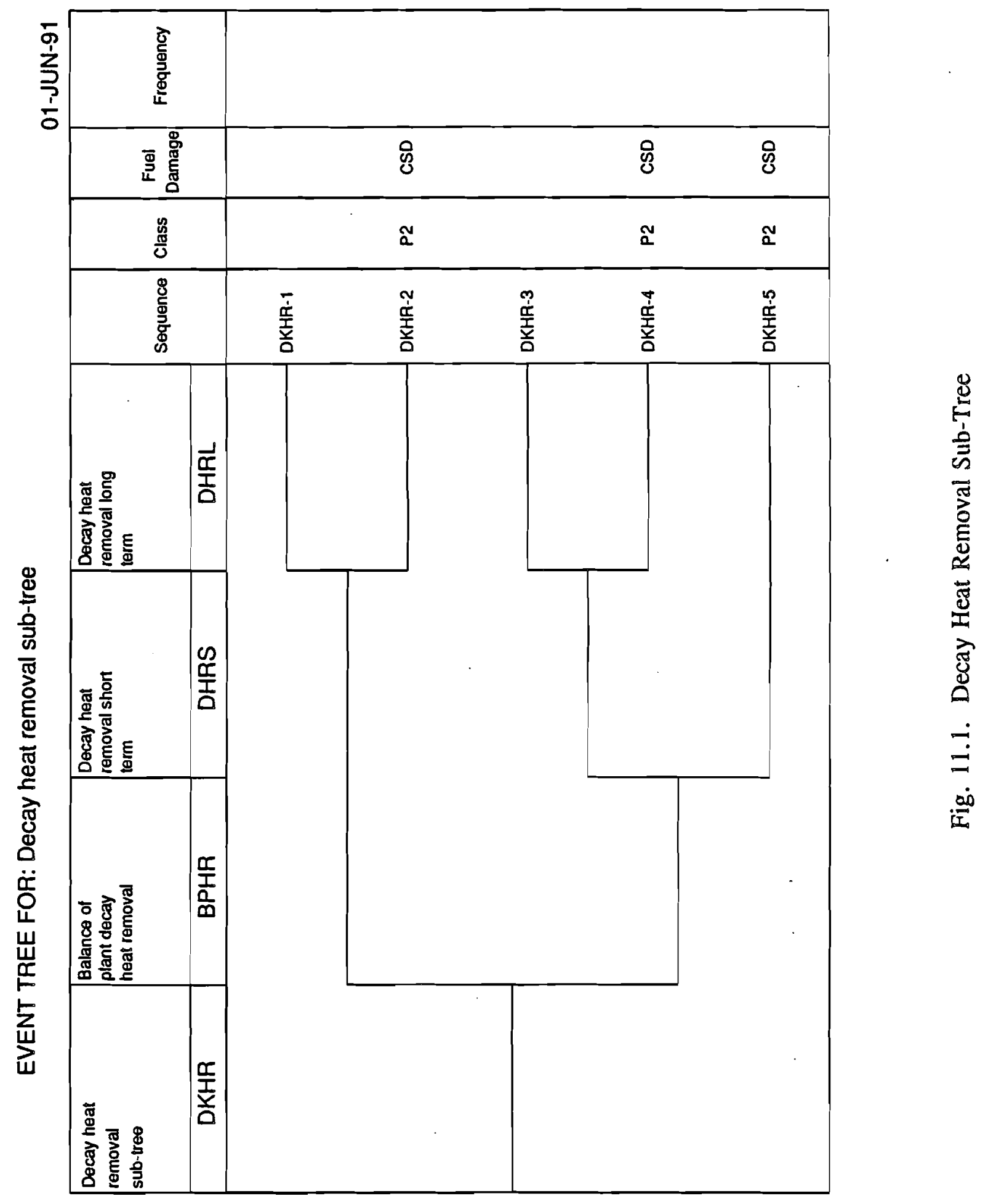


probabilities. Section 12 , on the other hand, reports the frequency distributions. Depending on the type of transient and damage produced, the accident sequences have been classified in different bins, as defined in Section 5 .

There are sequences that lead to some form of fuel damage or core and structural damage, grouped under classes $C D, M C D$, and $C S D$, sequences that lead only to potential experiment damage, assembled under PED, and finally sequences that, without causing damage to the core, are severe or disruptive enough as to be included in the frequency evaluation (collected under class ND). Within the ND class, a clear distinction can be made among sequences belonging to two different transient types. Sequences in the ATWS (P3) and ND class represent the unprotected LOF, TOP, and LOHS transients that result in passive shutdown and heat removal in EBR-II. The ND sequences in class P5 (evaporator or superheater tube rupture) are those that, although a safe shutdown is achieved, lead to potential severe damage or contamination of the IHX.

The following subsections summarize the annual frequency estimates for the accident sequences. The sequence annual frequencies are compared on the bases of their relative contribution to the different types of transient and to the different types of damage. When the frequency results are presented, the differentiation between damage and total annual frequency is made; damage sequences refer to those sequences that lead to either fuel or structural damage or potential experiment damage (classes $C D, M C D$, CSD, and PED). Similarly, the damage frequency is the annual frequency of classes $\mathrm{CD}, \mathrm{MCD}, \mathrm{CSD}$, and PED combined, while total frequency refers to the annual damage frequency plus the frequency of the ND class.

\subsubsection{Results by Accident Sequence}

The dominant sequences have been defined as those with a point mean frequency above $1.010^{-11} \mathrm{yr}^{-1}$. The dominant sequences leading to fuel or experiment damage are listed in Table 11.3 and the contribution of the most important sequences is graphically shown in Fig. 11.2. The first 10 sequences account for over $82 \%$ of 
Table 11.3 Summary of Dominant Sequences Contributing to Fuel and Experiment Damage

\begin{tabular}{|c|c|c|}
\hline \multirow{2}{*}{$\begin{array}{l}\text { TOTAL D } \\
\text { ACCIDENT } \\
\text { SEQUENCE }\end{array}$} & & $1.5710^{-6} \mathrm{yr}^{-1}$ \\
\hline & $\begin{array}{c}\text { FREQUENCY } \\
\mathrm{yr}^{-1}\end{array}$ & $\begin{array}{c}\text { PERCENTAGE } \\
z\end{array}$ \\
\hline LONP-4 & $4.5510^{-7}$ & 28.9 \\
\hline OCsL-10 & $3.28 \quad 10^{-7}$ & 20.9 \\
\hline TSDC-2 & $1.25 \quad 10^{-7}$ & 8.0 \\
\hline LF $2 A-6$ & $9.5510^{-8}$ & 6.1 \\
\hline SPTR-3 & $8.4010^{-8}$ & 5.3 \\
\hline SPTR-13 & $8.0010^{-8}$ & 5.1 \\
\hline RISB-6 & $3.5010^{-8}$ & 2.2 \\
\hline SHDL -3 & $3.3310^{-8}$ & 2.1 \\
\hline SPTR-23 & $3.2010^{-8}$ & 2.0 \\
\hline RIFL-1 & $2.9110^{-8}$ & 1.9 \\
\hline RISB-8 & $2.5310^{-8}$ & 1.6 \\
\hline LF2B-6 & $2.1410^{-8}$ & 1.4 \\
\hline TSEC-3 & $2.1010^{-8}$ & 1.3 \\
\hline LOCP- 6 & $2.0210^{-8}$ & 1.3 \\
\hline LONP-5 & $1.7710^{-8}$ & 1.1 \\
\hline TSDC-5 & $1.3710^{-8}$ & 0.9 \\
\hline SFPB -5 & $1.2610^{-8}$ & 0.8 \\
\hline SFPB- 6 & $1.2610^{-8}$ & 0.8 \\
\hline TBOP-3 & $1.2310^{-8}$ & 0.8 \\
\hline LCDL-1 & $1.1010^{-8}$ & 0.7 \\
\hline SHDL-2 & $8.4010^{-9}$ & 0.5 \\
\hline TAIR-3 & $8.3210^{-9}$ & 0.5 \\
\hline SCRM-2 & $5.8810^{-9}$ & 0.4 \\
\hline TSEC-2 & $5.2910^{-9}$ & 0.3 \\
\hline$L F 1 A-7$ & $4.5510^{-9}$ & 0.3 \\
\hline RIFS- 6 & $4.5510^{-9}$ & 0.3 \\
\hline MELF-5 & $4.5010^{-9}$ & 0.3 \\
\hline$O E L F-4$ & $4.5010^{-9}$ & 0.3 \\
\hline OELF-5 & $4.5010^{-9}$ & 0.3 \\
\hline$L F \perp A-2$ & $4.2010^{-9}$ & 0.3 \\
\hline TSHC-2 & $3.92 \quad 10^{-9}$ & 0.2 \\
\hline LF $2 A-7$ & $3.7210^{-9}$ & 0.2 \\
\hline SHDS-3 & $3.5110^{-9}$ & 0.2 \\
\hline LONP-3 & $3.33 \quad 10^{-9}$ & 0.2 \\
\hline RIFS-8 & $3.2810^{-9}$ & 0.2 \\
\hline TBOP-2 & $3.11 \quad 10^{-9}$ & 0.2 \\
\hline
\end{tabular}


Table 11.3 Summary of Dominant Sequences Contributing to Fuel and Experiment Damage (Cont'd)

\begin{tabular}{|c|c|c|}
\hline $\begin{array}{l}\text { ACCIDENT } \\
\text { SEQUENCE }\end{array}$ & $\begin{array}{c}\text { FREQUENCY } \\
\mathrm{Y}^{-1}\end{array}$ & $\underset{8}{\text { PERCENTAGE }}$ \\
\hline LF 2D-1 & $2.6010^{-9}$ & 0.2 \\
\hline OCPP-2 & $2.5210^{-9}$ & 0.2 \\
\hline LF1C-7 & $2.27 \quad 10^{-9}$ & 0.1 \\
\hline TSDC-4 & $2.2010^{-9}$ & 0.1 \\
\hline $\operatorname{LF} 1 \mathrm{C}-2$ & $2.1010^{-9}$ & 0.1 \\
\hline TAIR-2 & $2.1010^{-9}$ & 0.1 \\
\hline$L F 2 F-6$ & $1.64 \quad 10^{-9}$ & 0.1 \\
\hline LF 1B-7 & $1.3610^{-9}$ & 0.1 \\
\hline SFPB 8 & $1.2610^{-9}$ & 0.1 \\
\hline$L F 1 B-2$ & $1.2610^{-9}$ & 0.1 \\
\hline$L F 2 C-6$ & $1.0010^{-9}$ & 0.1 \\
\hline LONP-2 & $8.4010^{-10}$ & 0.1 \\
\hline OCSL -2 & $8.40 \quad 10^{-10}$ & 0.1 \\
\hline LOCP-2 & $8.4010^{-10}$ & 0.1 \\
\hline LF $2 B-7$ & $8.3310^{-10}$ & 0.1 \\
\hline SFLE-5 & $5.4010^{-10}$ & 0.0 \\
\hline SFLE-6 & $5.4010^{-10}$ & 0.0 \\
\hline OCSL-9 & $4.5510^{-10}$ & 0.0 \\
\hline MELF - 4 & $4.5010^{-10}$ & 0.0 \\
\hline OELE-7 & $4.50 \quad 10^{-10}$ & 0.0 \\
\hline SCRM-5 & $4.09 \quad 10^{-10}$ & 0.0 \\
\hline EVTR-4 & $3.9610^{-10}$ & 0.0 \\
\hline SPTR-6 & $3.3310^{-10}$ & 0.0 \\
\hline LF $1 A-5$ & $2.9210^{-10}$ & 0.0 \\
\hline ANTC -3 & $2.9210^{-10}$ & 0.0 \\
\hline OCSP-2 & $2.77 \quad 10^{-10}$ & 0.0 \\
\hline RISA-7 & $2.7310^{-10}$ & 0.0 \\
\hline RISA-2 & $2.5210^{-10}$ & 0.0 \\
\hline LF 1D-7 & $2.4510^{-10}$ & 0.0 \\
\hline LF1D-2 & $2.27 \quad 10^{-10}$ & 0.0 \\
\hline$L F 2 A-2$ & $1.7610^{-10}$ & 0.0 \\
\hline OCPP-5 & $1.7510^{-10}$ & 0.0 \\
\hline$L F 1 C-5$ & $1.4610^{-10}$ & 0.0 \\
\hline SFPB-7 & $1.2610^{-10}$ & 0.0 \\
\hline SCRM-4 & $1.0310^{-10}$ & 0.0 \\
\hline LF 1B-5 & $8.7610^{-11}$ & 0.0 \\
\hline$L F 1 A-4$ & $7.3510^{-11}$ & 0.0 \\
\hline RISB-7 & $7.0010^{-11}$ & 0.0 \\
\hline
\end{tabular}


Table 11.3 Summary of Dominant Sequences Contributiong to Fuel and Experiment Damage (Cont'd)

\begin{tabular}{|c|c|c|}
\hline $\begin{array}{l}\text { ACCIDENT } \\
\text { SEQUENCE }\end{array}$ & $\begin{array}{c}\text { FREQUENCY } \\
y^{-1} x^{-1}\end{array}$ & $\begin{array}{c}\text { PERCENTAGE } \\
8\end{array}$ \\
\hline TSHC -4 & $6.8710^{-11}$ & 0.0 \\
\hline RISB-2 & $6.47 \quad 10^{-11}$ & 0.0 \\
\hline LF $2 F-7$ & $6.3810^{-11}$ & 0.0 \\
\hline LOCP-5 & $5.8310^{-11}$ & 0.0 \\
\hline OCSL-5 & $5.8310^{-11}$ & 0.0 \\
\hline MELF - 7 & $4.5010^{-11}$ & 0.0 \\
\hline$O C P P-4$ & $4.4110^{-11}$ & 0.0 \\
\hline$L F 2 B-2$ & $3.9510^{-11}$ & 0.0 \\
\hline$L F 2 C-7$ & $3.90 \quad 10^{-11}$ & 0.0 \\
\hline$L F 1 C-4$ & $3.6710^{-11}$ & 0.0 \\
\hline SPTR-16 & $3.5210^{-11}$ & 0.0 \\
\hline$L F 2 E-6$ & $3.18 \quad 10^{-11}$ & 0.0 \\
\hline SPTR-9 & $2.9410^{-11}$ & 0.0 \\
\hline SPTR-19 & $2.8010^{-11}$ & 0.0 \\
\hline$L F 1 B-4$ & $2.2010^{-11}$ & 0.0 \\
\hline $\operatorname{OCSP}-5$ & $1.9210^{-11}$ & 0.0 \\
\hline TSHC -5 & $1.9210^{-11}$ & 0.0 \\
\hline RISA-5 & $1.7510^{-11}$ & 0.0 \\
\hline$L F 1 D-5$ & $1.57 \quad 10^{-11}$ & 0.0 \\
\hline EVTR-6 & $1.5410^{-11}$ & 0.0 \\
\hline LOCP -4 & $1.4710^{-11}$ & 0.0 \\
\hline OCSL-4 & $1.47 \quad 10^{-11}$ & 0.0 \\
\hline EVTR-13 & $1.4110^{-11}$ & 0.0 \\
\hline SPTR-10 & $1.29 \quad 10^{-11}$ & 0.0 \\
\hline SFPB-9 & $1.2610^{-11}$ & 0.0 \\
\hline$L F 2 A-5$ & $1.2210^{-11}$ & 0.0 \\
\hline
\end{tabular}




\section{DAMAGE SEQUENCES}

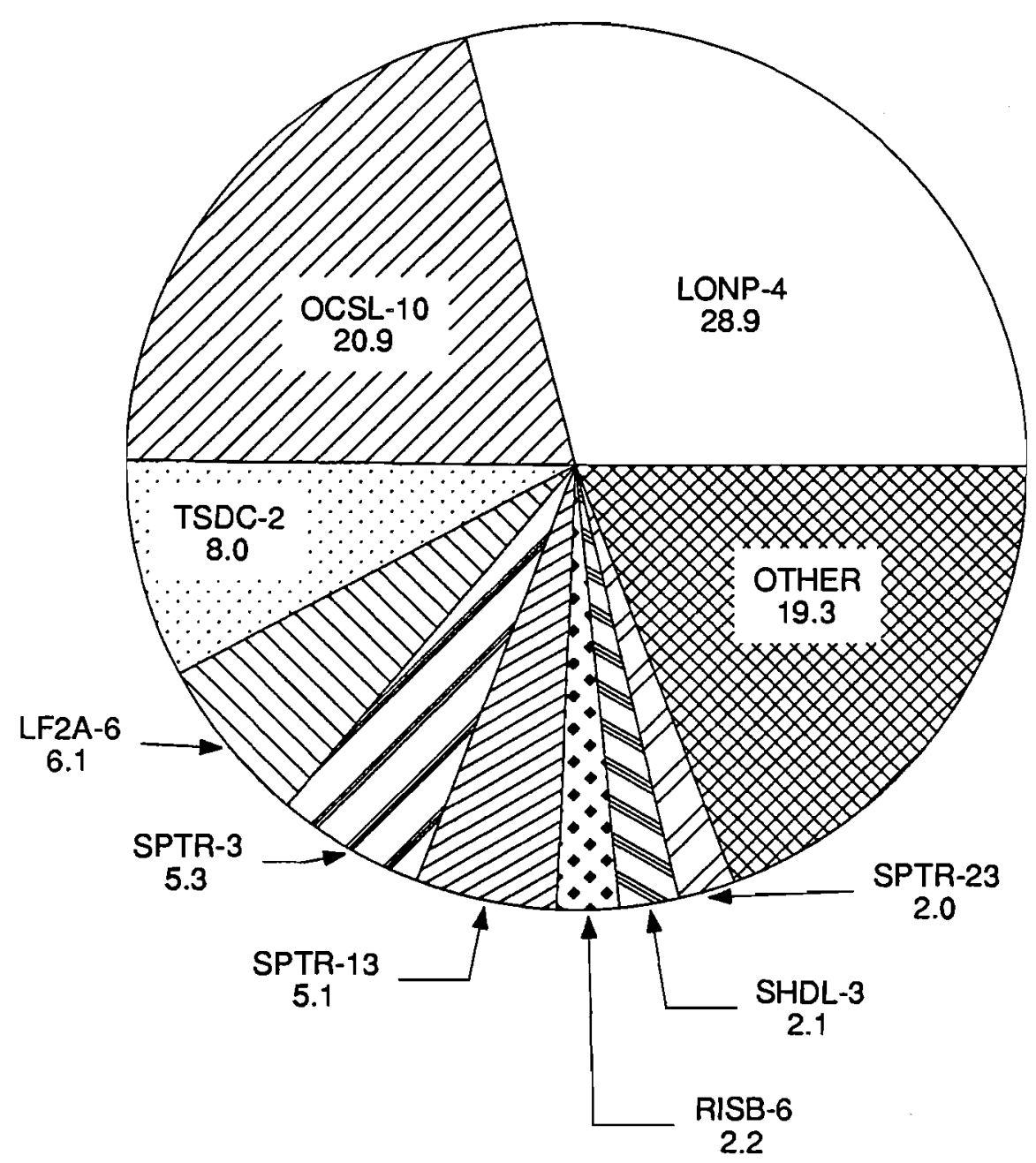

Fig. 11.2. Contribution of Dominant Sequences to Damage Frequency in EBR-II 
frequency of damage, while the contribution of the first 20 sequences is about $93 \%$ of the total. The frequencies of the two most dominant sequences, LONP-4 and OCSL-10, are $4.5510^{-7} \mathrm{yr}^{-1}$ and $3.2810^{-7} \mathrm{yr}^{-1}$ respectively, and together they account for almost 50 $\%$ of the damage frequency. The sequences with the highest frequency are briefly described below.

\section{Sequence LONP-4}

This sequence is initiated by the loss of normal power and continues with the failure of the scram system under LOF conditions. The scram signal is successfully generated, but the control or safety rods are not inserted. The contributions to the scram failure are dominated by the common cause failures of the CP relays to open their contacts, the common cause failures of safety and control rods to drop, and the operator failure to push the 3MPB button. This sequence leads to potential experiment damage, because of the availability of constant power, which maintains excitation to the motorgenerator clutches and does not result in the most severe coastdown of the primary

pumps. This sequence accounts for about $28.9 \%$ of the total damage frequency, and over $77 \%$ of its damage class. Common cause failures are almost entirely responsible for the high frequency of this sequence.

\section{$\underline{\text { Sequence OCSL-10 }}$}

The initiator for this sequence is an overcooling caused by a major steam leak, and continues with a failure of the reactor safety system to produce a scram signal under TOP conditions. The dominant events in this sequence are human errors (failure to push scram button and a common cause miscalibration of the SOT sensors), common cause failures of the trip relays, and common mode failure of the power supply components for the detection system. The sequence, which leads to minor core damage and accounts for $20.9 \%$ of the total damage frequency, is particularly sensitive to common cause and human error. This sequence frequency represents over $76 \%$ of the minor core damage class frequency. 


\section{Sequence TSDC-2}

The unrecoverable loss of a shutdown cooler initiates this sequence. The balance of plant is available for decay heat removal in the short term, but heat removal is insufficient in the long term, when either the remaining shutdown cooler or the shield and thimble cooling must be available. The failure of the long term heat removal is dominated by the combination of a single failure in the remaining shutdown cooler and a single failure in the shield or thimble cooling system that makes it inoperable. The single failures in the shutdown cooler are either a NaK leakage or a NaK blockage, while the most important failures in the shield/thimble cooling are the blockage of a shield cooling system filter, the human failure to recover it, the common cause blockage of the thimble cooling filters, and the unavailability of reactor water because of a water filter blockage. As a loss of decay heat removal this sequence contributes to the core/structural damage class, CSD, and is responsible for $27 \%$ of the frequency in its class and about $8 \%$ of the damage frequency.

\section{Sequence LF2A-6}

A double pump loss of flow sequence that proceeds in an identical manner to that of the LONP-4 sequence described above. The only difference is in the initiating event, which in this case is a loss of both primary pumps due to binding or to MG input faults instead of the loss of normal power. Since the LOF signal generation is successful, the minimal cutsets of the sequence are again dominated by the common cause failures of the CP relays, the common cause failures of the rods to drop and the failure of the operator to push the 3MPB scram button. Similarly, this sequence results in potential experiment damage, being responsible for about $16 \%$ of the annual frequency of this damage class. The contribution of LF2A-6 to the damage frequency is of $6.1 \%$. 


\section{Sequence SPTR-3}

The sequence is characterized by an initially small tube leak in a superheater module that gradually grows by self wastage and wastage of adjacent tubes. The leak becomes large enough to force backflow of water and reaction products to the IHX. The operator fails to mitigate the accident by dumping sodium and water in the secondary system and the steam generator before the leak grows to the critical size, four times the cross sectional area of a steam tube. The operator also fails to shutdown the reactor before the IHX fails, thus resulting in potential core/structural damage. This sequence accounts for approximately $18 \%$ of the frequency in its damage class, and $5.3 \%$ of the total damage frequency.

\section{Sequence SPTR-13}

The sequence is characterized by a rapid progression of a moderately sized tube leak in a superheater module to a leak size greater than four times the cross sectional area of a steam tube. This accident is not vigorous enough to cause failure of the superheater shell or secondary piping, which would lead to rapid pressure relief. The accident results in the transportation of water and reaction products to the IHX, causing its eventual failure. As in the previous sequence, the operator fails to shutdown the reactor before the IHX failure, so that the sequence is also a contributor to the CSD class, in which it is responsible for $17 \%$ of the annual frequency. The frequency of SGTR-13 is about $5 \%$ of the damage frequency in EBR-II.

\section{Sequence RISB-6}

A reactivity insertion between 20 and $65 c$ initiates a TOP transient. The reactor safety system generates a scram signal under TOP conditions, but the scram fails. Although the scram signal has been generated, the rods fail to drop. Since the reasons for the rods not dropping are independent of the conditions under which the scram signal is generated, the minimal cutsets for this TOP sequence are dominated by the same 
events as for the LOF sequences LONP-4 and LF2A-6, namely the common cause failure of the CP relays, the common cause failure of the rods to move, and the operator error to push the scram button. Contrary to the two LOF sequences, RISB-6 results in minor core damage (it accounts for about $8 \%$ of the frequency for this damage class) and is responsible for $2.2 \%$ of the annual damage frequency.

\section{Sequence SHDL-3}

This sequence is characterized by the short term failure of the decay heat removal when the annual long shutdown is initiated. The loss of the decay heat removal in the short term involves the failure of both shutdown coolers. The dominant minimal cutsets for the sequence consist of the simultaneous failure of the two coolers by $\mathrm{NaK}$ leakage or blockage and the failure of the electronic controller circuits combined with the human failure to recover the shutdown coolers. Like other loss of decay heat removal sequences, SHDL-3 leads to core/structural damage. It accounts for over $7 \%$ of the annual frequency of the CSD class and for over $2 \%$ of the damage frequency.

\section{Sequence SPTR-23}

The sequence is initiated by the simultaneous (within $0.2 \mathrm{~s}$ ) double-ended guillotine failure of two or more steam tubes in a superheater module. The pressure wave generated by the accident causes direct failure of the IHX, resulting in a potential core/structural damage. The superheater shell or secondary piping do not fail immediately, allowing the pressure wave to travel to the IHX. This sequence is responsible for almost $7 \%$ of the core/structural damage annual frequency and for $2 \%$ of the total damage frequency.

\section{Sequence RIFL-1}

RIFL-1 is one of the direct damage sequences. Its frequency is therefore that of the initiator. The initiating event is a fast reactivity insertion caused by the rupture of 
the primary tank. The rupture of the tank results in the reactor core dropping away from the control rods, with the consequent large and fast reactivity insertion. The major contributor to this mechanism is an overpressurization of the argon above the sodium level in the primary tank. The annual frequency of this accident sequence represents a contribution of about $32 \%$ to the frequency of the core damage (CD) class, and $1.9 \%$ to the total damage frequency.

All other sequences contribute less than $2 \%$ to the sum of the frequencies of all the dominant damage sequences. Among those, only five have a contribution in excess of $1 \%$, for a combined frequency of $6.7 \%$.

\subsubsection{Results by Plant Damage Class}

The accident sequence results are shown in Table 11.4 grouped by damage class. This table also includes the sequences leading to major plant disruption with no fuel damage (ND sequences). Class P1 is made up of only one dominant sequence, the direct damage sequence RIFL-1.

Almost $60 \%$ of the contribution to the total frequency of class P2 comes from two sequences described above, namely TSDC-2 and SHDL-3. One of this sequences involves the failure of the short term decay heat removal system, while the other involves the failure in the long term. Among the remaining sequences, 12 of them contribute more than $1 \%$ each to the total for class $\mathrm{P} 2$, and they represent a mixture of failure of the decay heat removal in the short and in the long term.

The dominant sequences in class P3 are essentially no damage (ND) sequences, mainly from loss of heat sink, single pump loss of flow, and overcooling transients. Three of the sequences, TSEC-4, TBOP-4, and TAIR- 4 , are caused by the failure of manually scramming the reactor under a LOHS transient. They account for more than $50 \%$ of the total frequency for this class. The other top ND sequences contribute over 
Table 11.4 Dominant Sequences Grouped by Transient Class

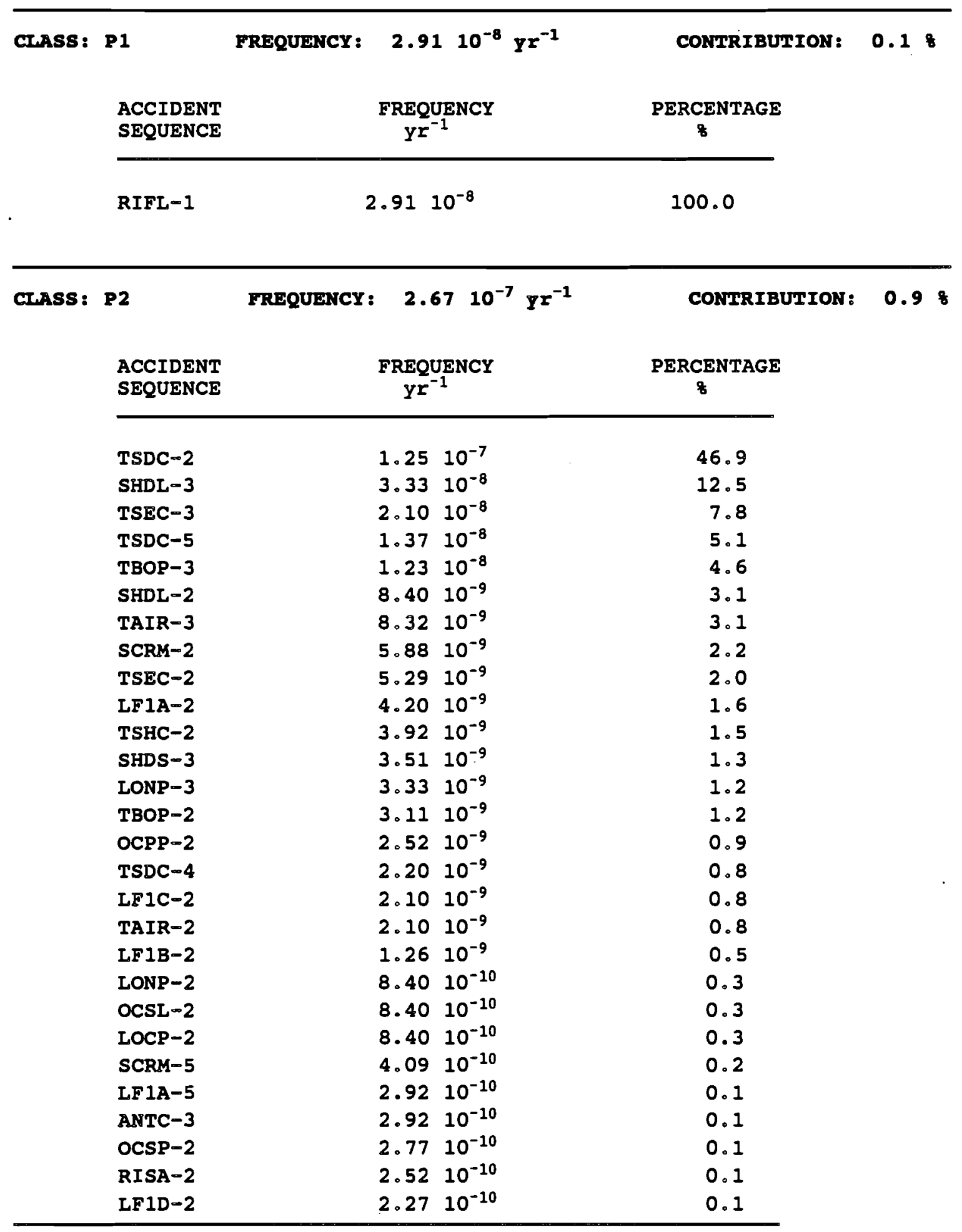


Table 11.4 Dominant Sequences Grouped by Transient Class (Cont'd)

Chass: P2 (cont'd)

\begin{tabular}{|c|c|c|}
\hline $\begin{array}{l}\text { ACCIDENT } \\
\text { SEQUENCE }\end{array}$ & $\begin{array}{c}\text { FREQUENCY } \\
\mathbf{y}^{-1}\end{array}$ & $\begin{array}{c}\text { PERCENTAGE } \\
8\end{array}$ \\
\hline LF $2 A-2$ & $1.7610^{-10}$ & 0.1 \\
\hline OCPP -5 & $1.7510^{-10}$ & 0.1 \\
\hline$L F 1 C-5$ & $1.4610^{-10}$ & 0.1 \\
\hline SCRM-4 & $1.0310^{-10}$ & 0.0 \\
\hline$L F 1 B-5$ & $8.7610^{-11}$ & 0.0 \\
\hline LF $1 A-4$ & $7.3510^{-11}$ & 0.0 \\
\hline TSHC- 4 & $6.8710^{-11}$ & 0.0 \\
\hline RISB-2 & $6.4710^{-11}$ & 0.0 \\
\hline LOCP-5 & $5.8310^{-11}$ & 0.0 \\
\hline OCSL -5 & $5.8310^{-11}$ & 0.0 \\
\hline OCPP-4 & $4.4110^{-11}$ & 0.0 \\
\hline$L F 2 B-2$ & $3.9510^{-11}$ & 0.0 \\
\hline LF $1 C-4$ & $3.6710^{-11}$ & 0.0 \\
\hline$L F 1 B-4$ & $2.2010^{-11}$ & 0.0 \\
\hline OCSP-5 & $1.9210^{-11}$ & 0.0 \\
\hline TSHC- 5 & $1.9210^{-11}$ & 0.0 \\
\hline RISA-5 & $1.7510^{-11}$ & 0.0 \\
\hline LF 1D-5 & $1.5710^{-11}$ & 0.0 \\
\hline LOCP-4 & $1.4710^{-11}$ & 0.0 \\
\hline OCSL-4 & $1.4710^{-11}$ & 0.0 \\
\hline LF $2 A-5$ & $1.2210^{-11}$ & 0.0 \\
\hline
\end{tabular}

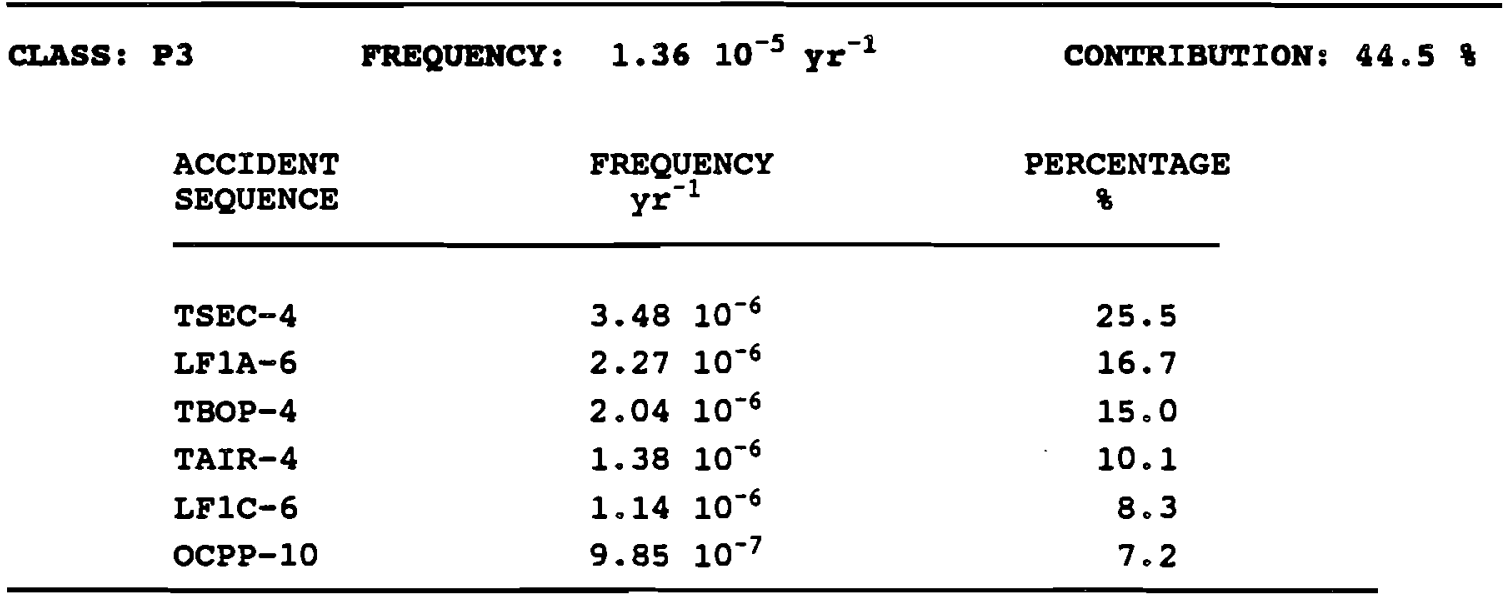


Table 11.4 Dominant Sequences Grouped by Transient Class (Cont'd)

\section{CLASS: P3 (cont'd)}

\begin{tabular}{|c|c|c|}
\hline $\begin{array}{l}\text { ACCIDENT } \\
\text { SEQUENCE }\end{array}$ & $\begin{array}{c}\text { FREQUENCY } \\
y x^{-1}\end{array}$ & $\begin{array}{c}\text { PERCENTAGE } \\
8\end{array}$ \\
\hline LF $1 B-6$ & $6.8210^{-7}$ & 5.0 \\
\hline LONP-4 & $4.5510^{-7}$ & 3.3 \\
\hline OCsL-10 & $3.2810^{-7}$ & 2.4 \\
\hline RISA-6 & $1.3610^{-7}$ & 1.0 \\
\hline LF1D-6 & $1.2310^{-7}$ & 0.9 \\
\hline OCSP-10 & $1.08 \quad 10^{-7}$ & 0.8 \\
\hline RISA-8 & $9.85 \quad 10^{-8}$ & 0.7 \\
\hline LF $2 A-6$ & $9.5510^{-8}$ & 0.7 \\
\hline LF $1 A-8$ & $8.8610^{-8}$ & 0.7 \\
\hline LF $1 C-8$ & $4.43 \quad 10^{-8}$ & 0.3 \\
\hline RISB-6 & $3.5010^{-8}$ & 0.3 \\
\hline LF 1B-8 & $2.6610^{-8}$ & 0.2 \\
\hline RISB-8 & $2.5310^{-8}$ & 0.2 \\
\hline LF2B-6 & $2.14 \quad 10^{-8}$ & 0.2 \\
\hline LOCP-6 & $2.0210^{-8}$ & 0.1 \\
\hline LONP-5 & $1.7710^{-8}$ & 0.1 \\
\hline LF $1 D-8$ & $4.7910^{-9}$ & 0.0 \\
\hline LF $1 A-7$ & $4.55 \quad 10^{-9}$ & 0.0 \\
\hline RIFS- 6 & $4.5510^{-9}$ & 0.0 \\
\hline LF 2A-7 & $3.72 \quad 10^{-9}$ & 0.0 \\
\hline RIFS-8 & $3.28 \quad 10^{-9}$ & 0.0 \\
\hline LF $1 C-7$ & $2.27 \quad 10^{-9}$ & 0.0 \\
\hline LF2F-6 & $1.6410^{-9}$ & 0.0 \\
\hline LF $1 B-7$ & $1.3610^{-9}$ & 0.0 \\
\hline OCPP-9 & $1.3610^{-9}$ & 0.0 \\
\hline LF $2 C-6$ & $1.0010^{-9}$ & 0.0 \\
\hline LF $2 B-7$ & $8.3310^{-10}$ & 0.0 \\
\hline OCSL-9 & $4.5510^{-10}$ & 0.0 \\
\hline RISA-7 & $2.7310^{-10}$ & 0.0 \\
\hline LF $1 D-7$ & $2.4510^{-10}$ & 0.0 \\
\hline OCSP-9 & $1.5010^{-10}$ & 0.0 \\
\hline RISB-7 & $7.0010^{-11}$ & 0.0 \\
\hline LF $2 F-7$ & $6.3810^{-11}$ & 0.0 \\
\hline LF $2 C-7$ & $3.9010^{-11}$ & 0.0 \\
\hline LF $2 E-6$ & $3.1810^{-11}$ & 0.0 \\
\hline
\end{tabular}


Table 11.4 Dominant Sequences Grouped by Transient Class (Cont'd)

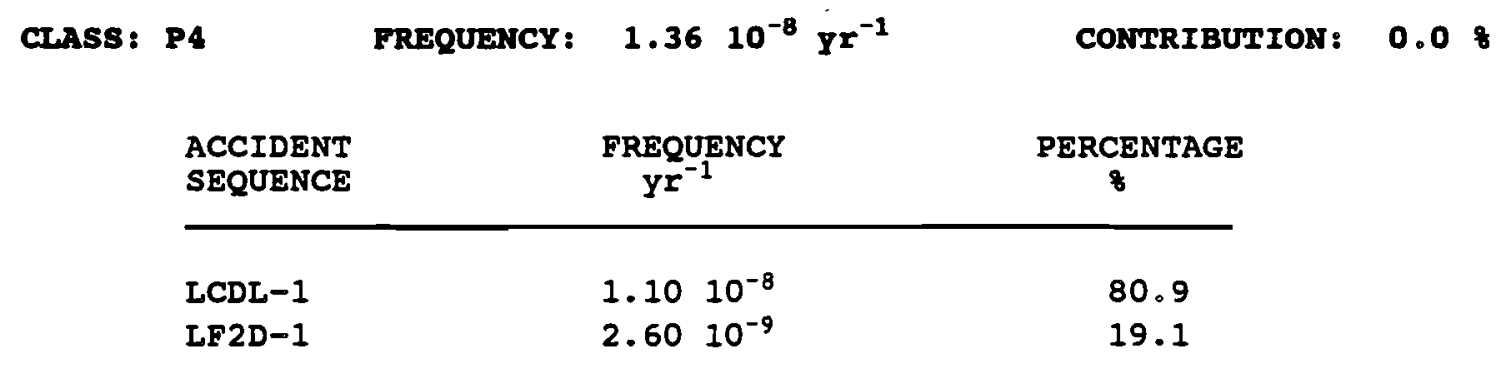

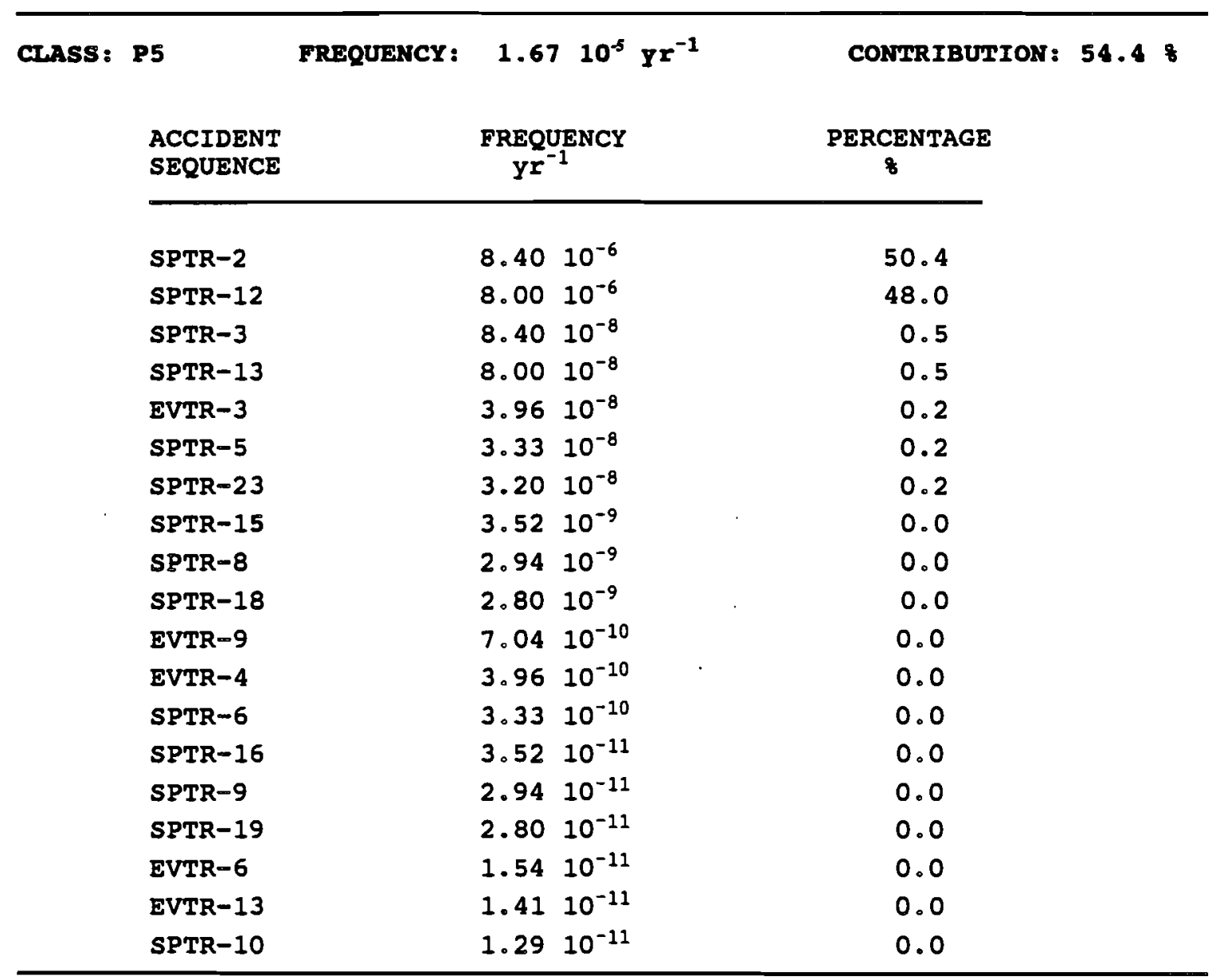


Table 11.4 Dominant Sequences Grouped by Transient Class (Cont'd)

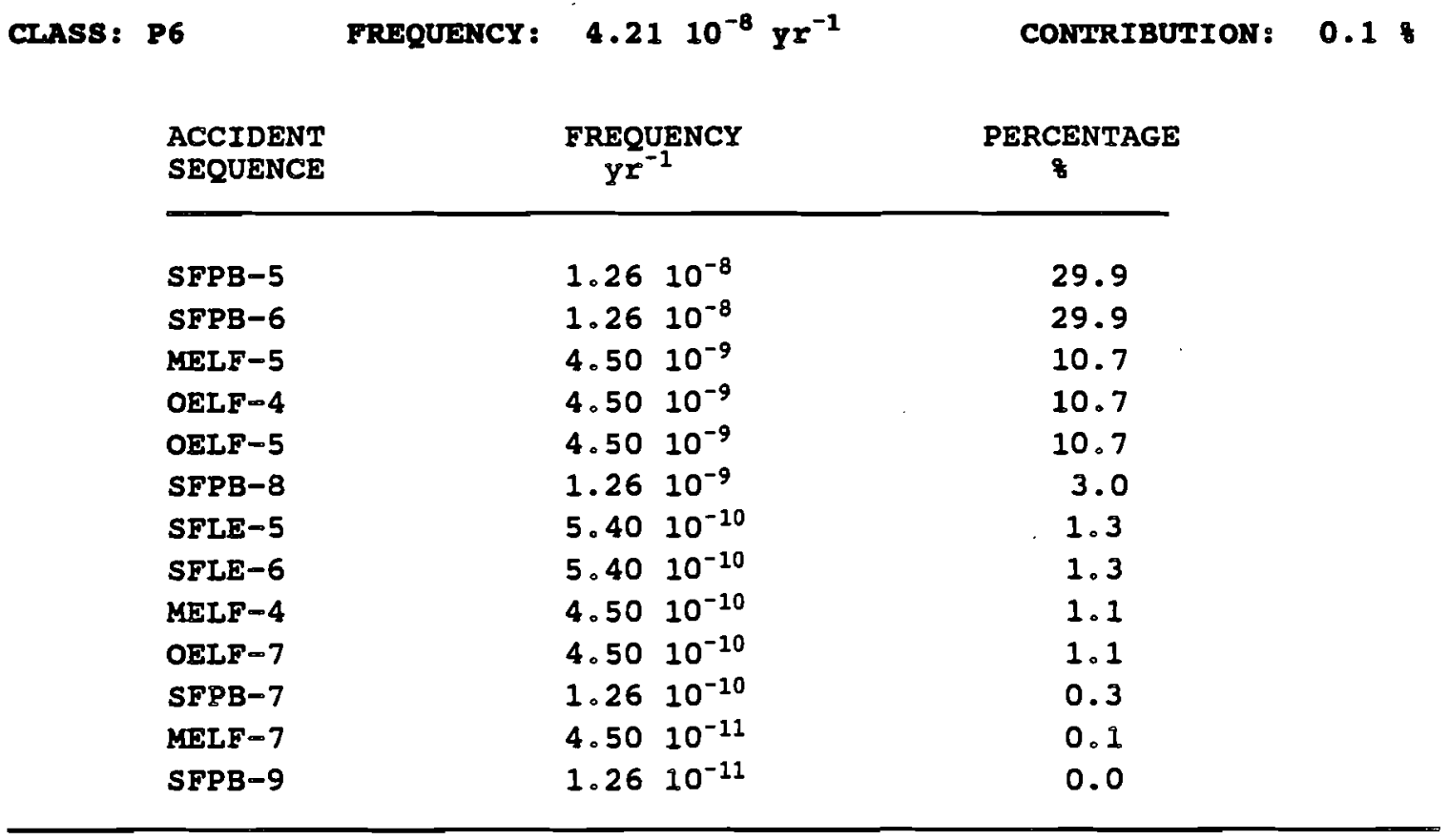


$40 \%$ of the frequency of P3. The first damage sequence is LONP-4, with a contribution of $3.3 \%$.

There are only two sequences in class P4, and they are both direct damage sequences due to a delayed loss of circulation (LCDL) and a double pump loss of flow (group D, LF2D) initiating events. The delayed loss of circulation frequency is close to one order of magnitude above that of the LF2D, and it accounts for almost $81 \%$ of the annual frequency for this class.

The steam generator tube rupture class, P5, is dominated by two ND sequences, SPTR-2 and SPTR-12, which together represent over $98 \%$ of the annual frequency for the class. In class P6, reserved for local faults, the main contributors are sequences of the subassembly partial blockage initiating event. The top two sequences have the same annual frequency and one contributes to core damage while the other is in the minor core damage class. Together, their frequency is about $60 \%$ of the frequency of P6. Experiment local faults (both metal and oxide fuel) have a significant contribution to the P6 annual frequency (more than $30 \%$ ), while no sequences of the metal driver fuel local fault initiator appear among the dominant sequences.

A graphical representation of the results by transient class, showing the fractions of the total class frequency carried by the most dominant sequences, is shown in Figures 11.3 to 11.8. Figure 11.9 displays the contribution of each transient class to the total (damage plus ND) annual frequency in EBR-II.

\subsubsection{Results by Damage Category}

The results of the accident sequence quantification assembled by damage type are shown in Table 11.5, and graphically in Figures 11.10 to 11.14 . Figure 11.15 shows the contribution by damage class to the annual damage frequency in EBR-II, excluding the no damage category, ND. 
CLASS: P1

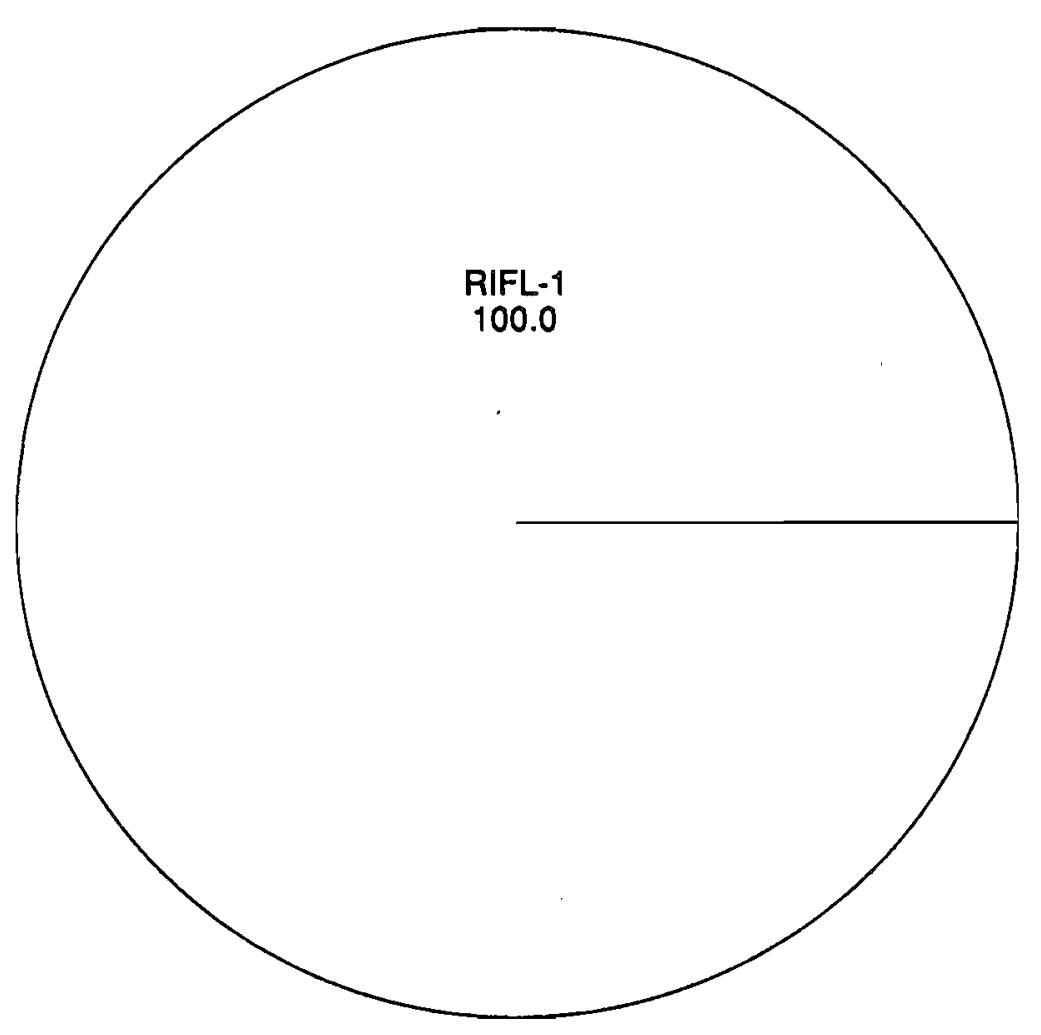

Fig. 11.3. Dominant Sequences in Plant Transient Class P1 


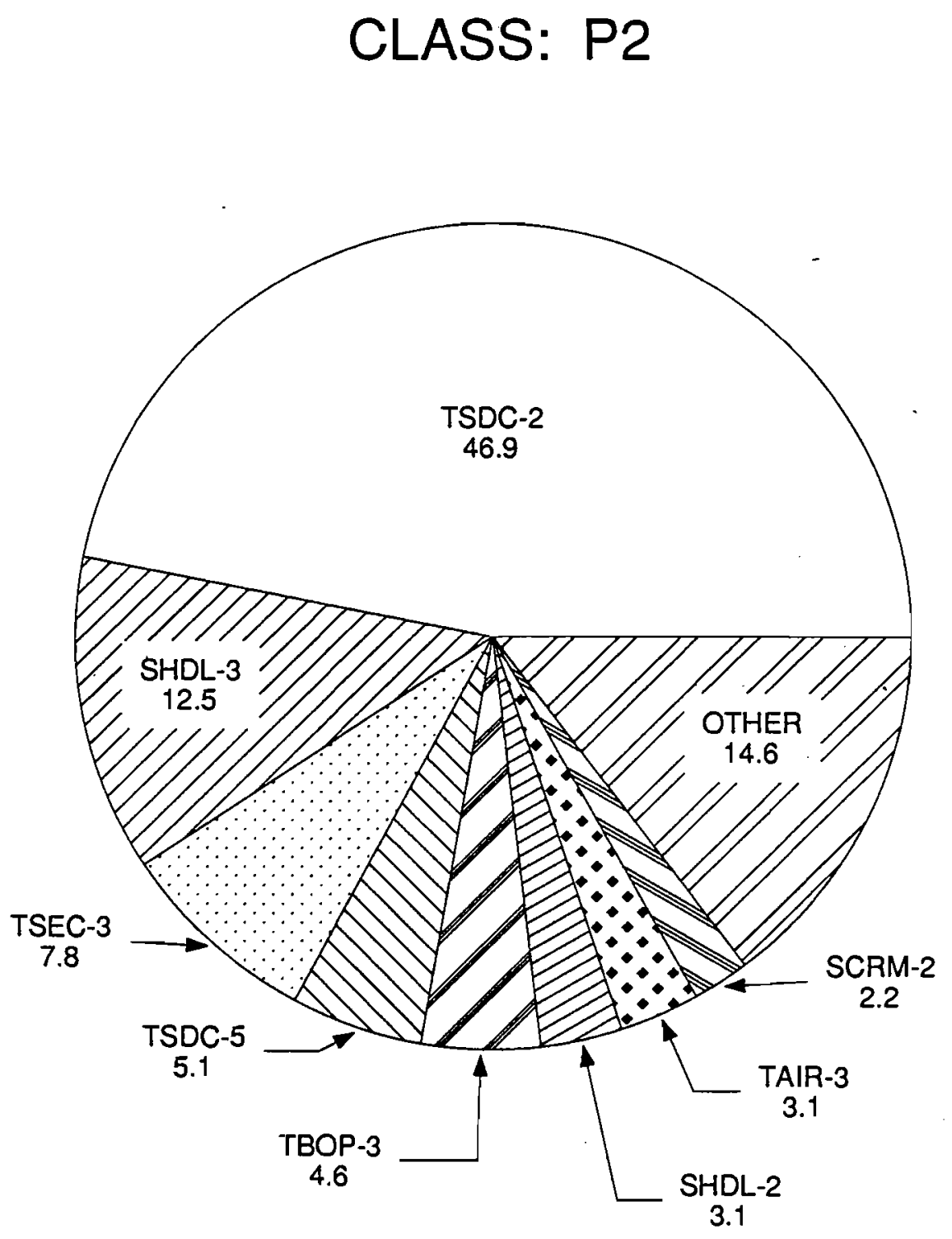

Fig. 11.4. Dominant Sequences in Plant Transient Class P2 


\section{CLASS: P3}

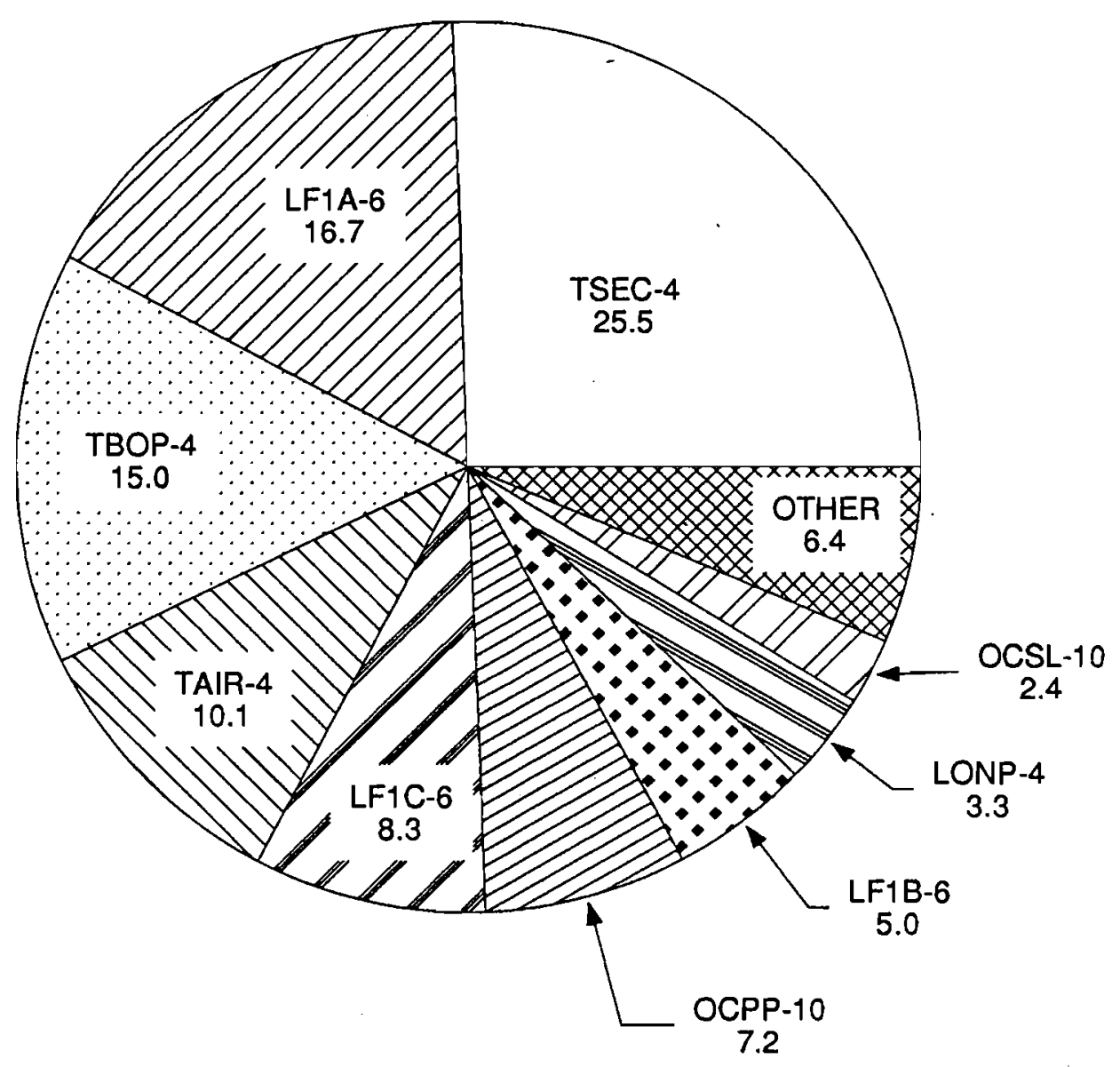

Fig. 11.5. Dominant Sequences in Plant Transient Class P3 
CLASS: P4

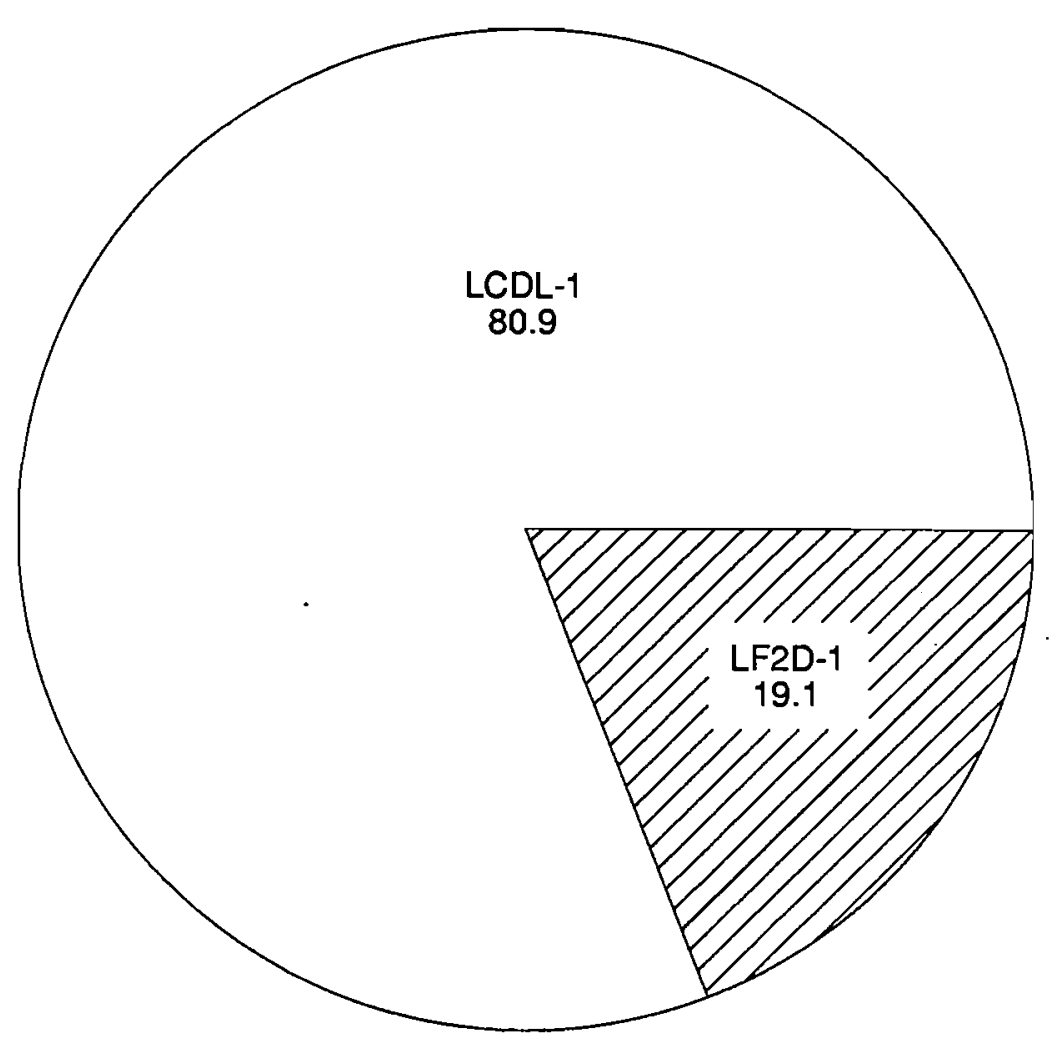

Fig. 11.6. Dominant Sequences in Plant Transient Class P4 


\section{CLASS: P5}

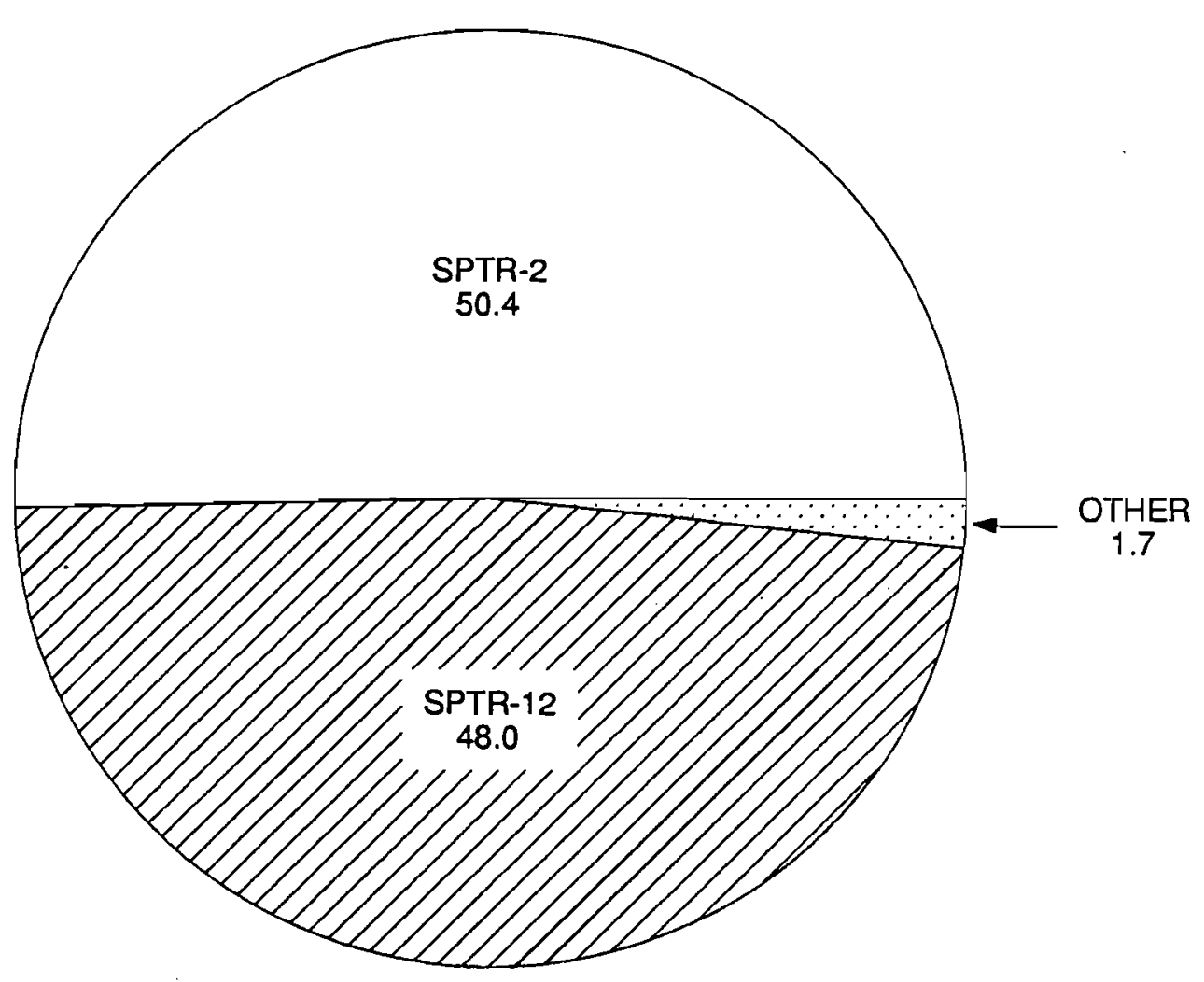

Fig. 11.7. Dominant Sequences in Plant Transient Class P5 


\section{CLASS: P6}

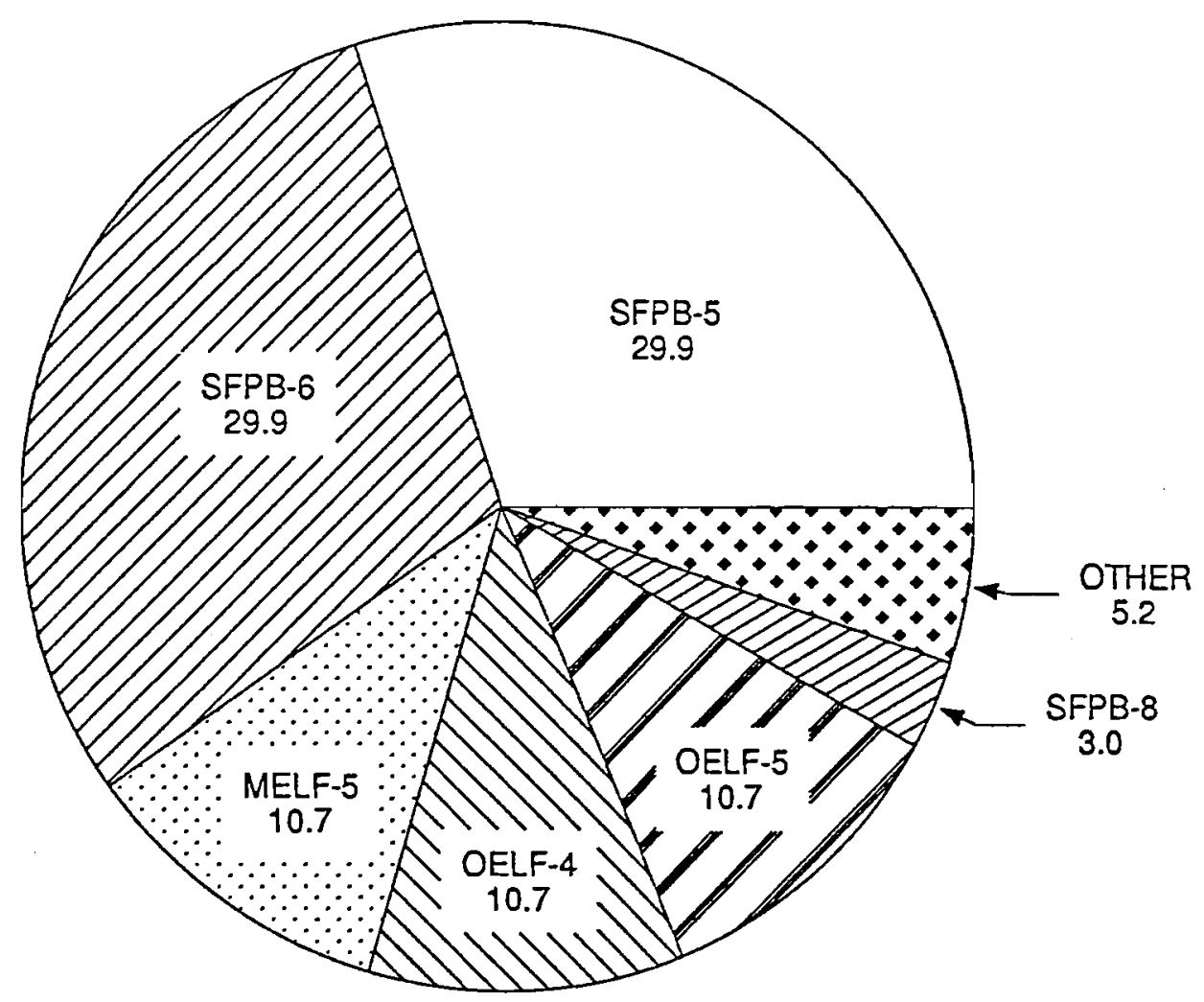

Fig. 11.8. Dominant Sequences in Plant Transient Class P6 


\section{CONTRIBUTION BY CLASS}

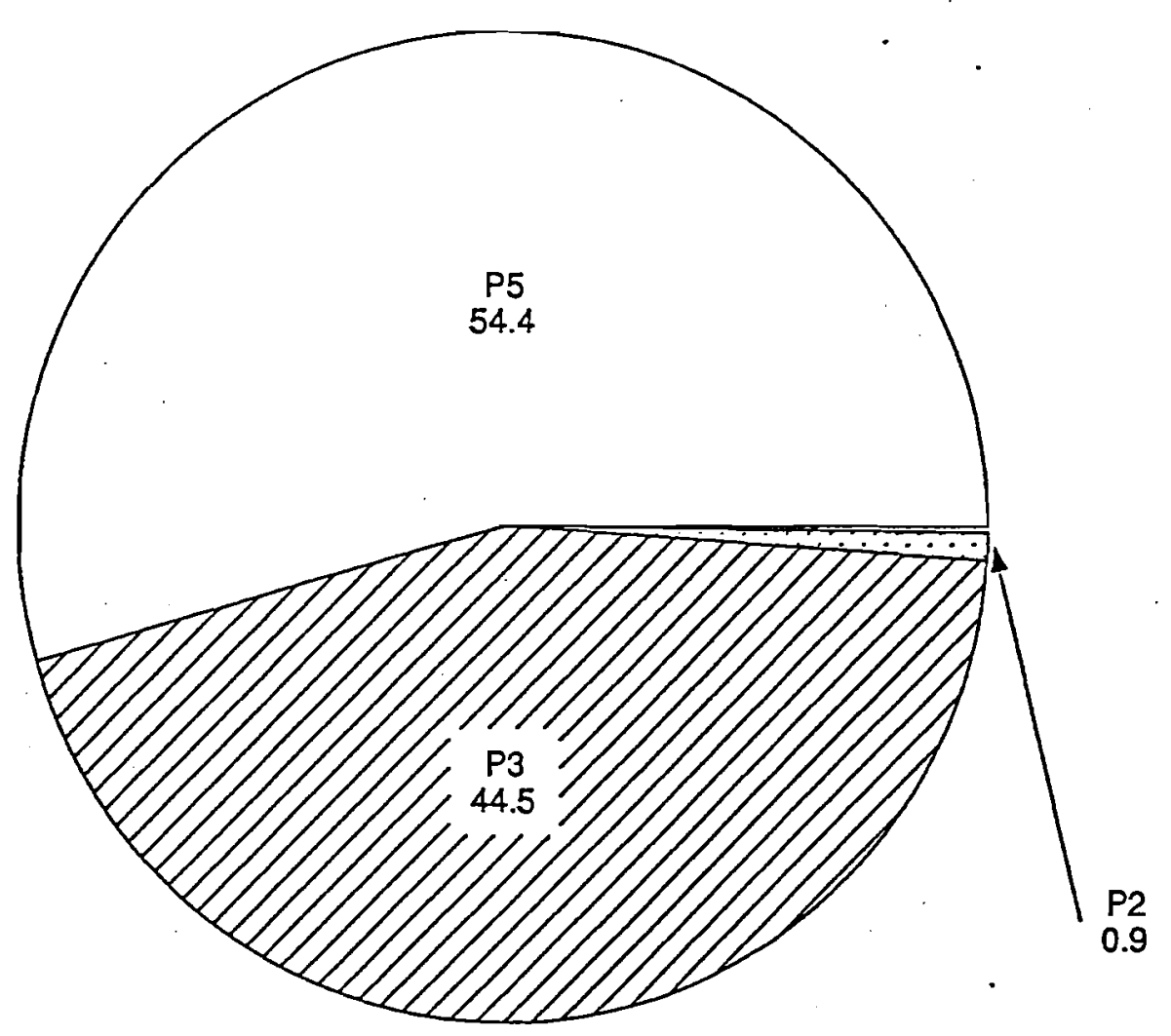

Fig. 11.9. Comparison of Plant Transient Classes by their Contribution to Total Frequency (damage $+\mathrm{ND}$ ) 
Table 11.5 Dominant Sequences Grouped by Damage Category

DAYAGE CLASS: CD

PREQUENCY :

$9.0110^{-8} Y^{-1}$

CONIRIBUTION TO DAMAGE: 5.7 \&

CONIRIBUTION TO TOTAL: 0.38

ACCIDENT

SEQUENCE

FREQUENCY

$$
\mathrm{yx}^{-1}
$$

PERCENTAGE

8

RIFL-1

$2.9110^{-8}$

32.3

LF2B-6

$2.1410^{-8}$

23.7

LOCP-6

$2.0210^{-8}$

22.4

SFPB-5

$1.2610^{-8}$

14.0

OELF-4

$4.5010^{-9}$

5.0

LF 2B-7

$8.33 \quad 10^{-10}$

0.9

SFLE-5

$5.4010^{-10}$

0.6

MELF-4

$4.5010^{-10}$

0.5

RISA-7

$2.7310^{-10}$

0.3

SFPB-7

RISB-7

$1.2610^{-10}$

0.1

LF 2E-6

$7.00 \quad 10^{-11}$

0.1

3. $18 \quad 10^{-11}$

0.0

SFPB-9

$1.2610^{-11}$

0.0

DAYAGE CLASS: MCD

ACCIDENT SEQUENCE

\begin{tabular}{l}
\hline OCSL-10 \\
RISB-6 \\
RISB-8 \\
SFPB-6 \\
RIFS-6 \\
MELF-5 \\
OELF-5 \\
RIFS-8 \\
LF 2D-1 \\
LF 1C-7 \\
LF 2F-6
\end{tabular}

FREQUENCY

$$
\mathrm{Yx}^{-1}
$$

$3.2810^{-7}$

$3.5010^{-8}$

$2.5310^{-8}$

$1.2610^{-8}$

$4.5510^{-9}$

$4.5010^{-9}$

$4.5010^{-9}$

$3.2810^{-9}$

$2.6010^{-9}$

$2.2710^{-9}$

$1.6410^{-9}$
FREQUENCY: $\quad 4.3010^{-7} \mathrm{Yr}^{-1}$ CONIRIBUTION TO DAMAGE: 27.48 CONTRIBUTION TO TOTAL: 1.4 ह

PERCENTAGE

8

76.3

8.1

5.9

2.9

1.1

1.0

1.0

0.8

0.6

0.5

0.4 
Table 11.5 Dominant Sequences Grouped by Damage Category (Cont'd)

DAMAGE CLASS: MCD (cont'd)

\begin{tabular}{lcc}
$\begin{array}{c}\text { ACCIDENT } \\
\text { SEQUENCE }\end{array}$ & $\begin{array}{c}\text { FREQUENCY } \\
\mathrm{YY}^{-1}\end{array}$ & $\begin{array}{c}\text { PERCENTAGE } \\
8\end{array}$ \\
\hline & & \\
LF1B-7 & $1.3610^{-9}$ & 0.3 \\
SFPB-8 & $1.2610^{-9}$ & 0.3 \\
LF2C-6 & $1.0010^{-9}$ & 0.2 \\
SFLE-6 & $5.4010^{-10}$ & 0.1 \\
OCSL-9 & $4.5510^{-10}$ & 0.1 \\
OELF-7 & $4.5010^{-10}$ & 0.1 \\
LF1D-7 & $2.4510^{-10}$ & 0.1 \\
LF2F-7 & $6.3810^{-11}$ & 0.0 \\
MELF-7 & $4.5010^{-11}$ & 0.0 \\
LF2C-7 & $3.9010^{-11}$ & 0.0
\end{tabular}

DAMAGE CLASS: CSD

FREQUENCY: $\quad 4.6410^{-7} \mathrm{Yx}^{-1}$ CONTRIBUTION TO DAMAGE: 29.5 \& CONTRIBUTION TO TOTAL: $1.5 \%$

\begin{tabular}{|c|c|c|}
\hline $\begin{array}{l}\text { ACCIDENT } \\
\text { SEQUENCE }\end{array}$ & $\begin{array}{c}\text { FREQUENCY } \\
\mathrm{yr}^{-1}\end{array}$ & $\begin{array}{c}\text { PERCENTAGE } \\
8\end{array}$ \\
\hline TSDC-2 & $1.2510^{-7}$ & 27.0 \\
\hline SPTR-3 & $8.4010^{-8}$ & 18.1 \\
\hline$S P T R-13$ & $8.0010^{-8}$ & 17.2 \\
\hline SKDL -3 & $3.3310^{-8}$ & 7.2 \\
\hline SPTR-23 & $3.2010^{-8}$ & 6.9 \\
\hline TSEC-3 & $2.1010^{-8}$ & 4.5 \\
\hline TSDC-5 & $1.3710^{-8}$ & 3.0 \\
\hline TBOP-3 & $1.2310^{-8}$ & 2.7 \\
\hline SHDL -2 & $8.4010^{-9}$ & 1.8 \\
\hline TAIR-3 & $8.32 \quad 10^{-9}$ & 1.8 \\
\hline$S C R M-2$ & $5.88 \quad 10^{-9}$ & 1.3 \\
\hline TSEC -2 & $5.29 \quad 10^{-9}$ & 1.1 \\
\hline$L F 1 A-2$ & $4.20 \quad 10^{-9}$ & 0.9 \\
\hline TSHC -2 & $3.92 \quad 10^{-9}$ & 0.8 \\
\hline SHDS -3 & $3.5110^{-9}$ & 0.8 \\
\hline LONP-3 & $3.33 \quad 10^{-9}$ & 0.7 \\
\hline
\end{tabular}


Table 11.5 Dominant Sequences Grouped by Damage Category (Cont'd)

DAMAGE CLASS: CSD (cont'd)

\begin{tabular}{|c|c|c|}
\hline $\begin{array}{l}\text { ACCIDENT } \\
\text { SEQUENCE }\end{array}$ & $\begin{array}{c}\text { FREQUENCY } \\
\mathrm{Yr}^{-1}\end{array}$ & $\begin{array}{c}\text { PERCENTAGE } \\
z\end{array}$ \\
\hline TBOP-2 & $3.1110^{-09}$ & 0.7 \\
\hline OCPP-2 & $2.5210^{-09}$ & 0.5 \\
\hline TSDC-4 & $2.2010^{-09}$ & 0.5 \\
\hline$L F 1 C-2$ & $2.1010^{-09}$ & 0.5 \\
\hline TAIR-2 & $2.1010^{-09}$ & 0.5 \\
\hline$L F 1 B-2$ & $1.2610^{-09}$ & 0.3 \\
\hline LONP-2 & $8.4010^{-10}$ & 0.2 \\
\hline OCSL-2 & $8.4010^{-10}$ & 0.2 \\
\hline LOCP -2 & $8.4010^{-10}$ & 0.2 \\
\hline SCRM-5 & $4.0910^{-10}$ & 0.1 \\
\hline EVTR-4 & $3.9610^{-10}$ & 0.1 \\
\hline SPTR- 6 & $3.3310^{-10}$ & 0.1 \\
\hline$L F 1 A-5$ & $2.9210^{-10}$ & 0.1 \\
\hline ANTC-3 & $2.9210^{-10}$ & 0.1 \\
\hline OCSP-2 & $2.7710^{-10}$ & 0.1 \\
\hline RISA-2 & $2.5210^{-10}$ & 0.1 \\
\hline$L F 1 D-2$ & $2.2710^{-10}$ & 0.0 \\
\hline LF $2 A-2$ & $1.7610^{-10}$ & 0.0 \\
\hline OCPP-5 & $1.7510^{-10}$ & 0.0 \\
\hline$L F 1 C-5$ & $1.4610^{-10}$ & 0.0 \\
\hline SCRM-4 & $1.0310^{-10}$ & 0.0 \\
\hline$L F 1 B-5$ & $8.7610^{-11}$ & 0.0 \\
\hline$L F 1 A-4$ & $7.3510^{-11}$ & 0.0 \\
\hline TSHC-4 & $6.8710^{-11}$ & 0.0 \\
\hline RISB-2 & $6.4710^{-11}$ & 0.0 \\
\hline LOCP-5 & $5.8310^{-11}$ & 0.0 \\
\hline OCSL-5 & $5.8310^{-11}$ & 0.0 \\
\hline OCPP-4 & $4.4110^{-11}$ & 0.0 \\
\hline$L F 2 B-2$ & $3.9510^{-11}$ & 0.0 \\
\hline LF IC-4 & $3.6710^{-11}$ & 0.0 \\
\hline SPTR-16 & $3.5210^{-11}$ & 0.0 \\
\hline SPTR-9 & $2.9410^{-11}$ & 0.0 \\
\hline SPTR-19 & $2.8010^{-11}$ & 0.0 \\
\hline LF 1B-4 & $2.2010^{-11}$ & 0.0 \\
\hline OCsP-5 & $1.9210^{-11}$ & 0.0 \\
\hline
\end{tabular}


Table 11.5 Dominant Sequences Grouped by Damage Category (Cont'd)

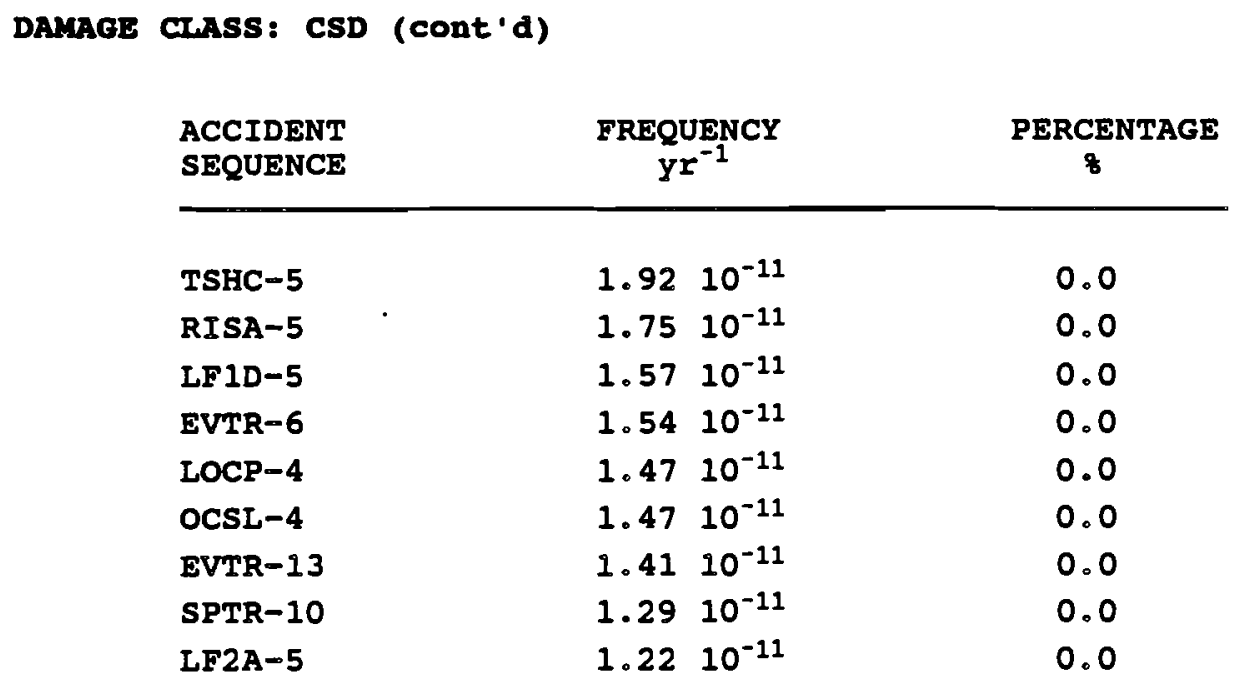

DAMAGE Class: PED

FREQUENCY: $\quad 5.8710^{-07} \mathrm{YF}^{-1}$ CONIRIBUTION TO DAMAGE: 37.48 CONTRIBUTION TO TOTAL: 1.9 \%

\begin{tabular}{|c|c|c|}
\hline $\begin{array}{l}\text { ACCIDENT } \\
\text { SEQUENCE }\end{array}$ & $\begin{array}{c}\text { FREQUENCY } \\
y r^{-1}\end{array}$ & $\begin{array}{c}\text { PERCEN } \\
8\end{array}$ \\
\hline LONP-4 & $4.5510^{-07}$ & 77.4 \\
\hline LF $2 A-6$ & $9.5510^{-08}$ & 16.3 \\
\hline LONP-5 & $1.7710^{-08}$ & 3.0 \\
\hline$L C D L-1$ & $1.1010^{-08}$ & 1.9 \\
\hline LF $1 A-7$ & $4.5510^{-09}$ & 0.8 \\
\hline LF $2 A-7$ & $3.72 \quad 10^{-09}$ & 0.6 \\
\hline
\end{tabular}

DAMAGE CHASS: ND

ACCIDENT SEQUENCE
FREOUENCY :

CORTR CONHRIBUTION TO TOTAL:

94.98
FREQUENCY $y \mathbf{r}^{-1}$
PERCENTAGE

8

28.9

27.5

12.0 
Table 11.5 Dominant Sequences Grouped by Damage Category (Cont'd)

DAMAgE chass: ND (cont'd)

\begin{tabular}{lccc} 
ACCIDENT & \multicolumn{2}{c}{ FREQUENCY } \\
SEQUENCE & Y & $\begin{array}{c}\text { PERCENTAGE } \\
\text { ( }\end{array}$ \\
\hline LF1A-6 & 2.27 & $10^{-06}$ & 7.8 \\
TBOP-4 & 2.04 & $10^{-06}$ & 7.0 \\
TAIR-4 & 1.38 & $10^{-06}$ & 4.7 \\
LF1C-6 & 1.14 & $10^{-06}$ & 3.9 \\
OCPP-10 & 9.85 & $10^{-07}$ & 3.4 \\
LF1B-6 & 6.82 & $10^{-07}$ & 2.3 \\
RISA-6 & 1.36 & $10^{-07}$ & 0.5 \\
LF1D-6 & 1.23 & $10^{-07}$ & 0.4 \\
OCSP-10 & 1.08 & $10^{-07}$ & 0.4 \\
RISA-8 & 9.85 & $10^{-08}$ & 0.3 \\
LF1A-8 & 8.86 & $10^{-08}$ & 0.3 \\
LF1C-8 & 4.43 & $10^{-08}$ & 0.2 \\
EVTR-3 & 3.96 & $10^{-08}$ & 0.1 \\
SPTR-5 & 3.33 & $10^{-08}$ & 0.1 \\
LF1B-8 & 2.66 & $10^{-08}$ & 0.1 \\
LF1D-8 & 4.79 & $10^{-09}$ & 0.0 \\
SPTR-15 & 3.52 & $10^{-09}$ & 0.0 \\
SPTR-8 & 2.94 & $10^{-09}$ & 0.0 \\
SPTR-18 & $2.8010^{-09}$ & 0.0 \\
OCPP-9 & 1.36 & $10^{-09}$ & 0.0 \\
EVTR-9 & 7.04 & $10^{-10}$ & 0.0 \\
OCSP-9 & $1.5010^{-10}$ & 0.0 \\
\hline
\end{tabular}




\section{FUEL DAMAGE: CD}

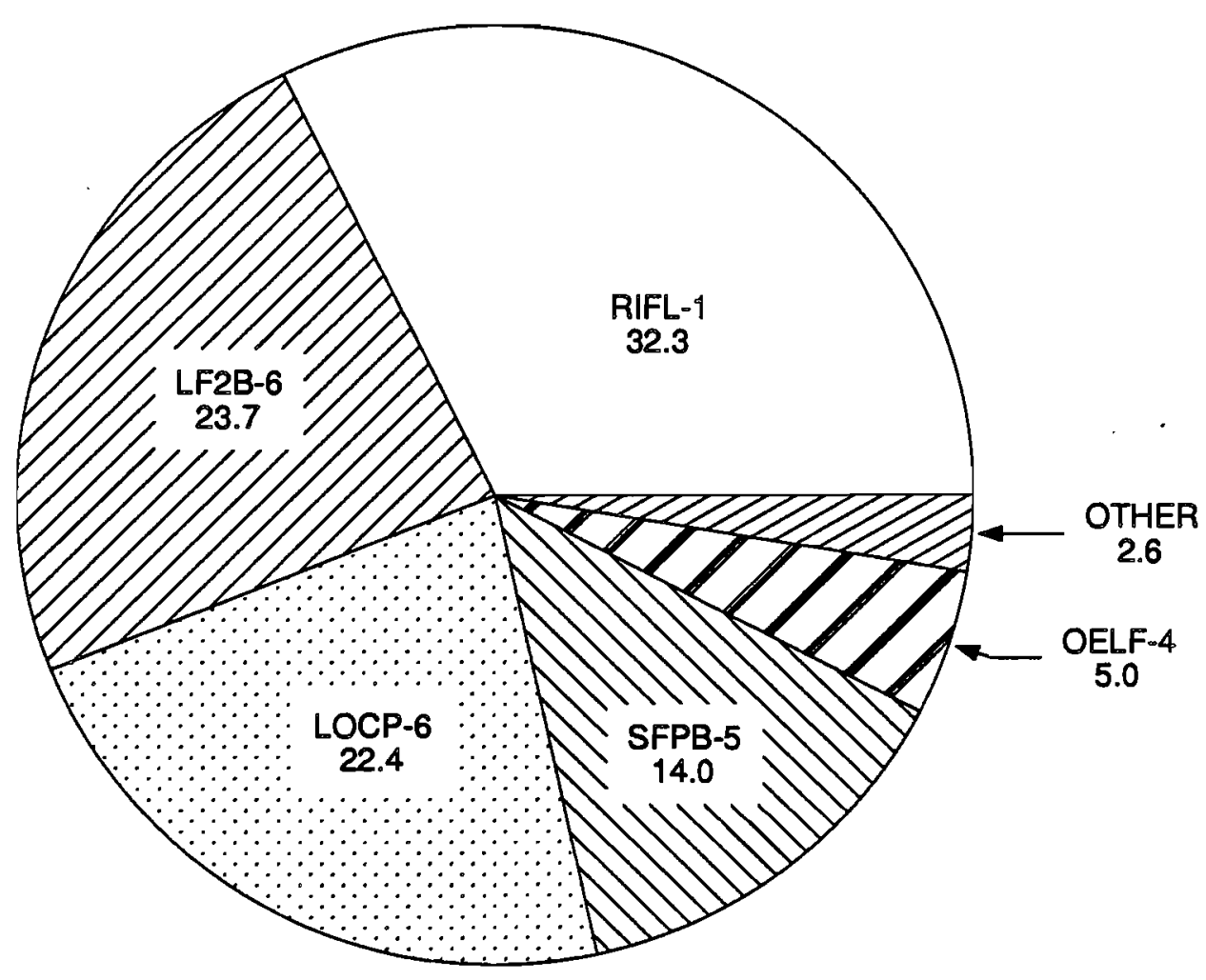

Fig. 11.10. Dominant Sequences in the Core Damage Category 
FUEL DAMAGE: MCD

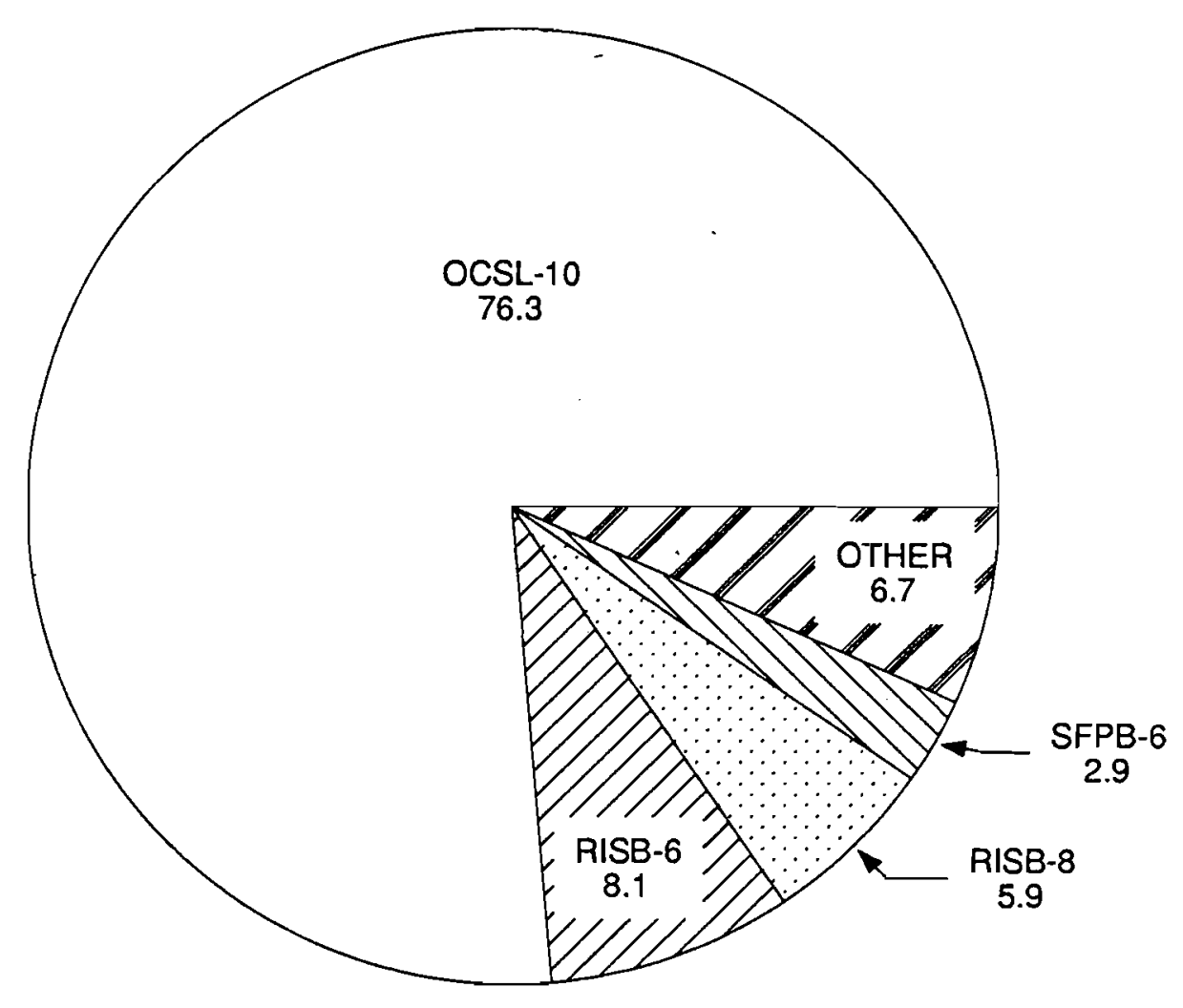

Fig. 11.11. Dominant Sequences in the Minor Core Damage Category 


\section{FUEL DAMAGE: CSD}

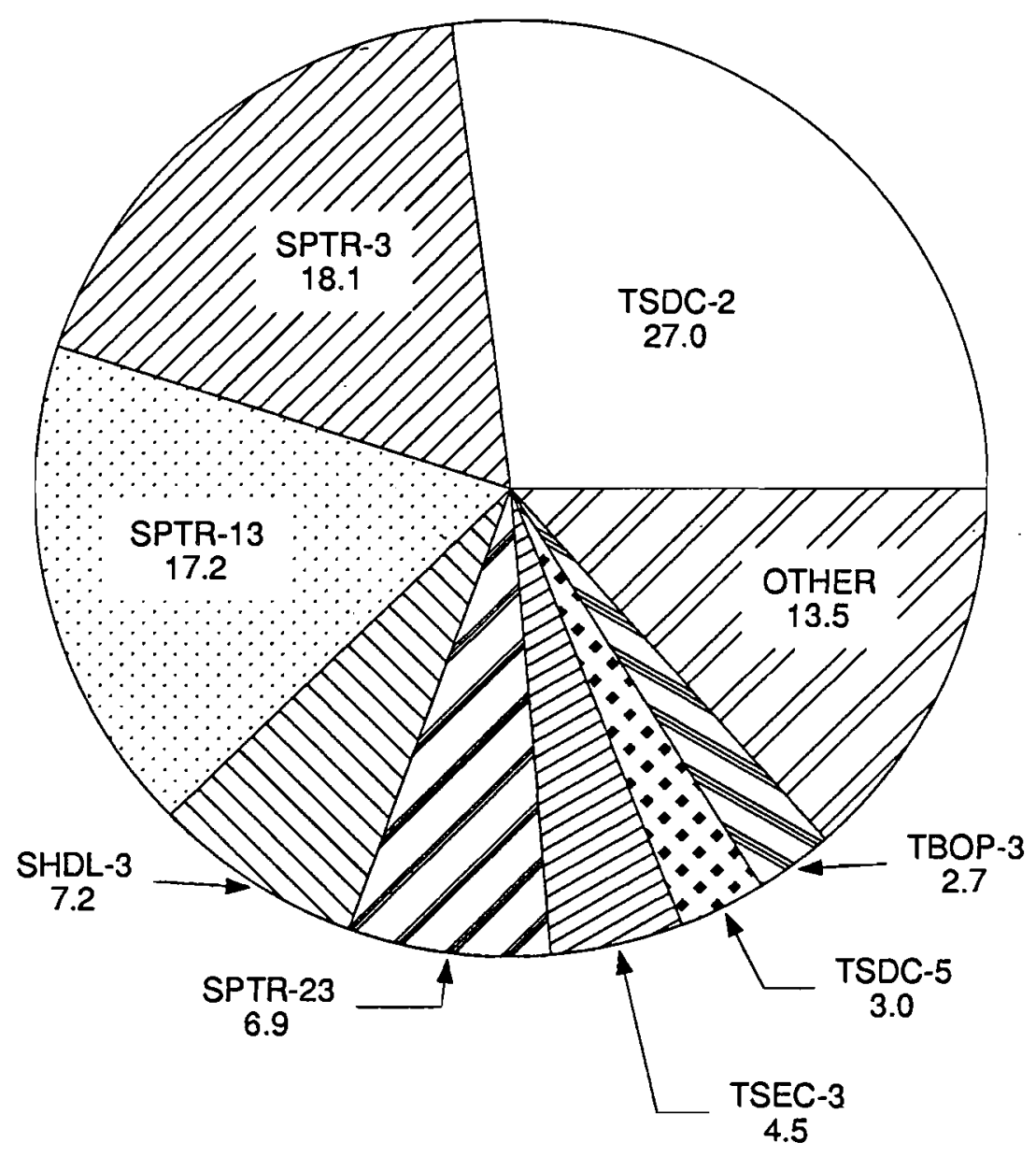

Fig. 11.12. Dominant Sequences in the Core/Structural Damage Category 
FUEL DAMAGE: PED

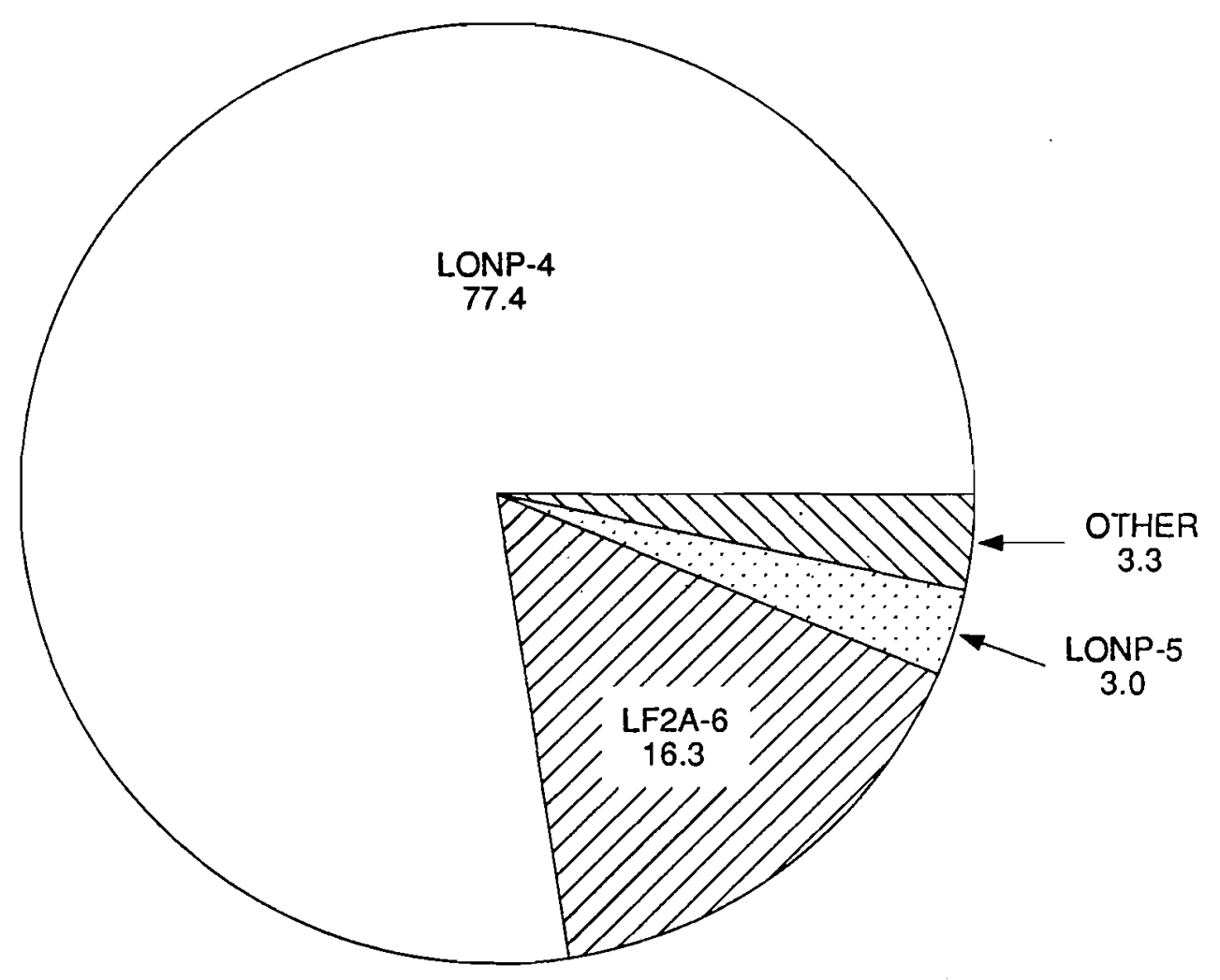

Fig. 11.13. Dominant Sequences in the Potential Experiment Damage Catetory 


\section{FUEL DAMAGE: ND}

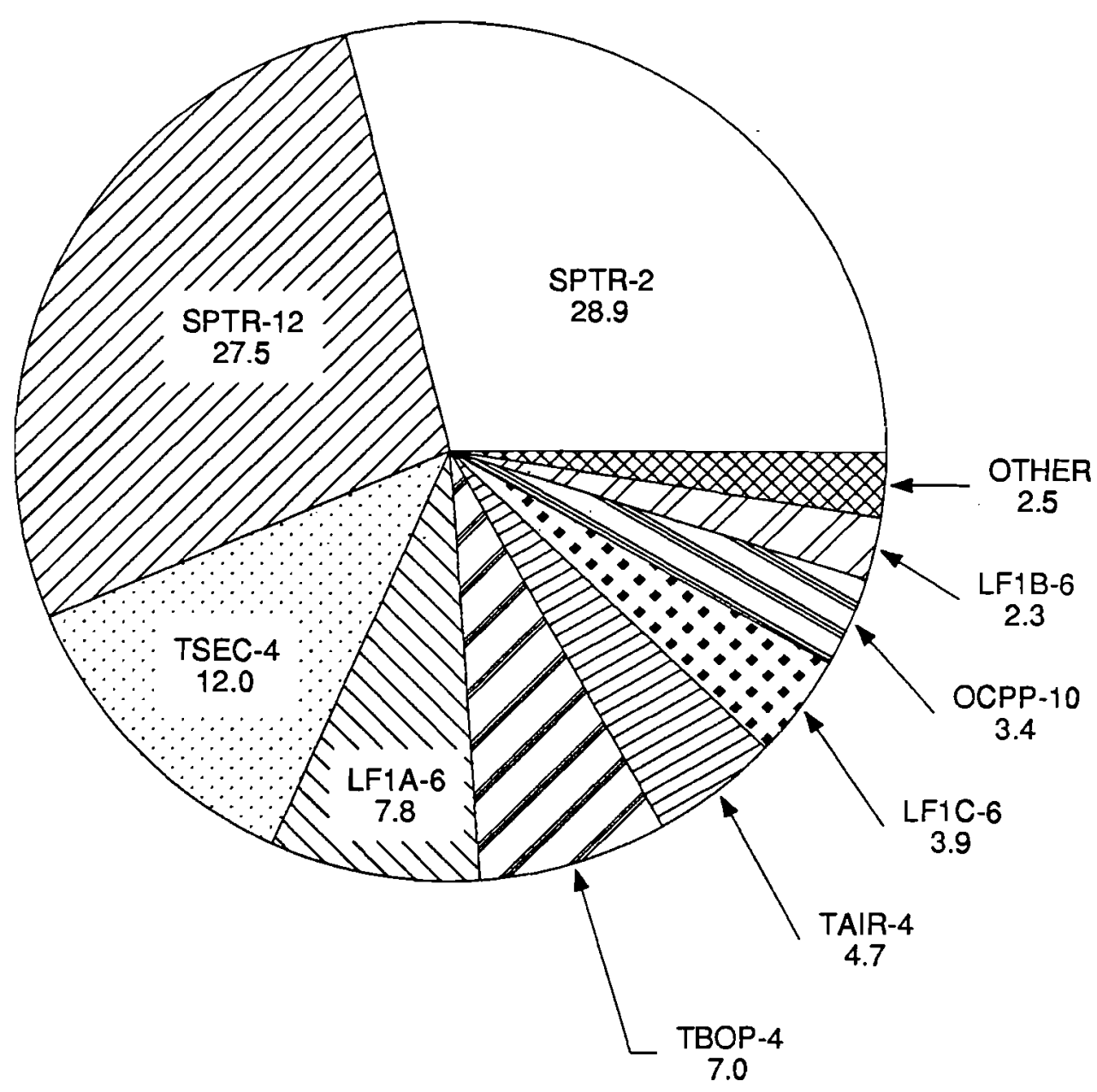

Fig. 11.14. Dominant Sequences in the No Damage Category 


\section{CONTRIBUTION BY DAMAGE TYPE}

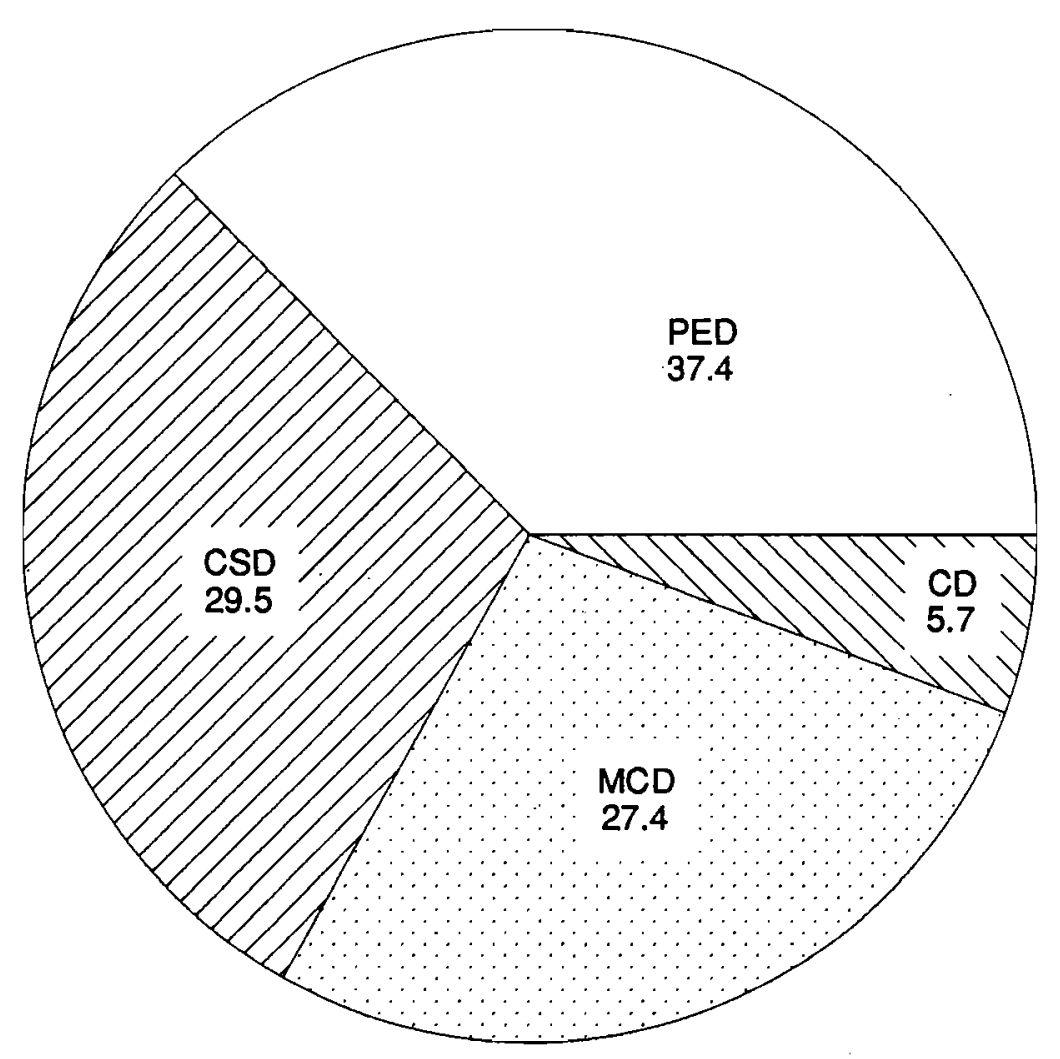

Fig. 11.15. Comparison of Damage Categories by their Contribution to the Total Damage Frequency 
The most severe damage class, core damage (CD), accounts for less than $6 \%$ of the damage frequency. The most important sequence in this category is RIFL-1 (over $32 \%$ of the frequency) followed by two sequences that involve a double clutch LOF with failure to scram (LF2B-6 and LOCP-6). Local fault sequences also carry a significant fraction of the annual frequency of $\mathrm{CD}$.

More than $76 \%$ of the total frequency in the minor core damage (MCD) category is due to the sequence OCSL-10, followed by other smaller contributions mostly from reactivity insertion and local faults initiators. Because the frequency of OCSL-10 is not only large within its class, but also relative to the entire damage frequency, the minor core damage class accounts for over $27 \%$ of the annual damage frequency in EBR-II.

In the core/structural damage category (CSD) the dominant sequences involve the short term decay heat removal (those in the P2 class), and some of the superheater tube rupture sequences. The loss of a shutdown cooler has three dominant sequences in this class, in particular TSDC-2 (loss of a shutdown cooler and later failure of the long term decay heat removal), which accounts for $27 \%$ of the CSD annual frequency. Three superheater tube rupture sequences carry more than $42 \%$ of the frequency in this class. Other significant sequences in CSD are among the shutdown and loss of heat sink initiating events. The core/structural damage class is responsible for almost $30 \%$ of the annual damage frequency.

The two most dominant sequences in the potential experiment damage category (PED) are initiated with a double pump loss of flow with similar coastdown characteristics (LONP-4 and LF2A-6). Over $77 \%$ of the frequency of the PED class can be attributed to the loss of normal power sequence, with the LF2A sequence being a distant second with $16 \%$ of the PED annual frequency. Another, minor, sequence in this class is delayed loss of circulation, LCDL1 , which is a direct damage sequence. Because of the comparatively high frequency of the LONP-4 sequence, the PED class represents more than $37 \%$ of the annual damage frequency in EBR-II.

The no-damage (ND) class has the highest annual frequency, close to $310^{-5}$. More than $56 \%$ of this frequency is attributed to the superheater tube rupture initiator. The result is a potential severe damage and contamination of the IHX. A distant second group of sequences 
in this class are formed by the three unprotected loss of heat sink sequences (TSEC-4, TBOP-4, TAIR-4), together accountable for almost $25 \%$ of the ND annual frequency. Although the ND class does not contribute to fuel or experiment damage, it eclipses the damage classes when their frequencies are compared. As shown in Figure 11.16, the ND class is responsible for almost $95 \%$ of the total (damage plus ND) annual frequency.

\subsubsection{Results by Initiating Event}

Table 11.6 presents the results of the accident sequence quantification associated by initiating event. The sequences leading to no fuel damage (ND) have been excluded from the table and from the calculation of the percentage contributions. The most important single initiator is the loss of normal power (LONP) with $30 \%$ of the damage frequency. The next dominant initiators are the overcooling by major steam leak and the superheater tube rupture, which account for $21 \%$ and $12 \%$ of the damage frequency respectively. The unrecoverable loss of a shutdown cooler is another significant initiating event, responsible for $9 \%$ of the damage frequency, and double pump loss of flow due to binding/MG input faults carries an additional $6.3 \%$. In summary, $32 \%$ of the damage is due to loss of power initiators, $21 \%$ due to overcooling transients, $13 \%$ due to steam generator tube failures, $9 \%$ due to loss of a decay heat removal system, $9 \%$ due to the loss of flow initiators, $6 \%$ is contributed by reactivity insertions, $7 \%$ is due to shutdown and loss of heat sink initiators,

$2 \%$ is attributed to local faults, and less than $1 \%$ can be imputed to the loss of circulation initiators. The relative contributions of the different initiators to the annual damage frequency in EBR-II is also shown in Fig. 11.17, while Fig. 11.18 depicts the contribution of the main initiators to the total frequency of all the dominant sequences (including the no damage, ND, sequences).

\subsubsection{Results by Event Tree}

The accident sequence quantification results are also listed by event tree in Figures 11.19 to 11.56. The value in the frequency column is listed for all sequences that were quantified. A blank is left for those sequences that were not analyzed because they did not lead to any damage state. The sequences that, after quantification, were found to have an annual frequency below the cutoff value of $1.010^{-11} \mathrm{yr}^{-1}$ are noted by specifying that the frequency was below the cutoff. 


\section{CONTRIBUTION BY DAMAGE TYPE}

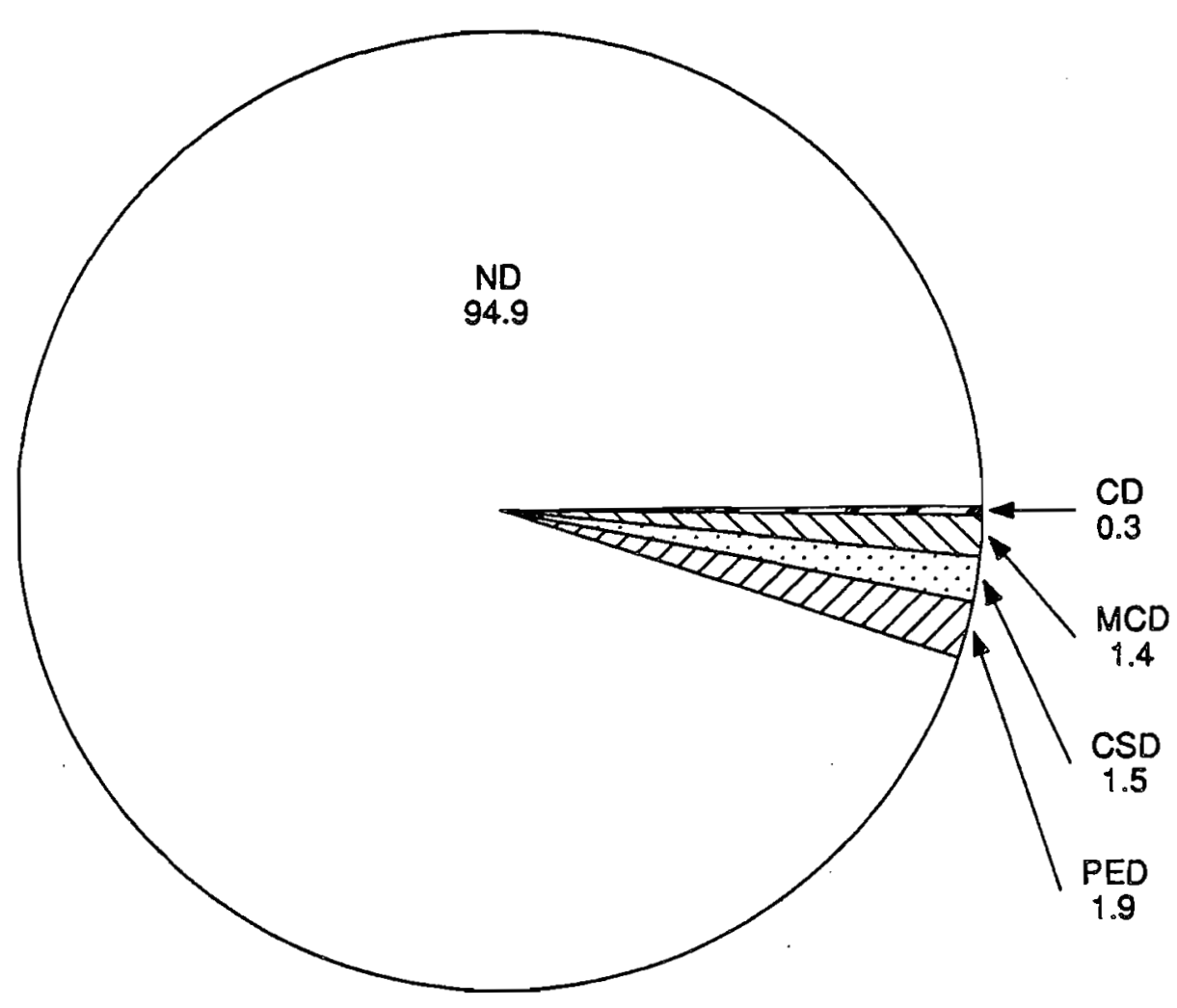

Fig. 11.16. Comparison of Damage Categories by their Contribution to the Total (damage + ND) Frequency 
Table 11.6 Damage Accident Sequence Quantification Results Grouped by Initiating Event

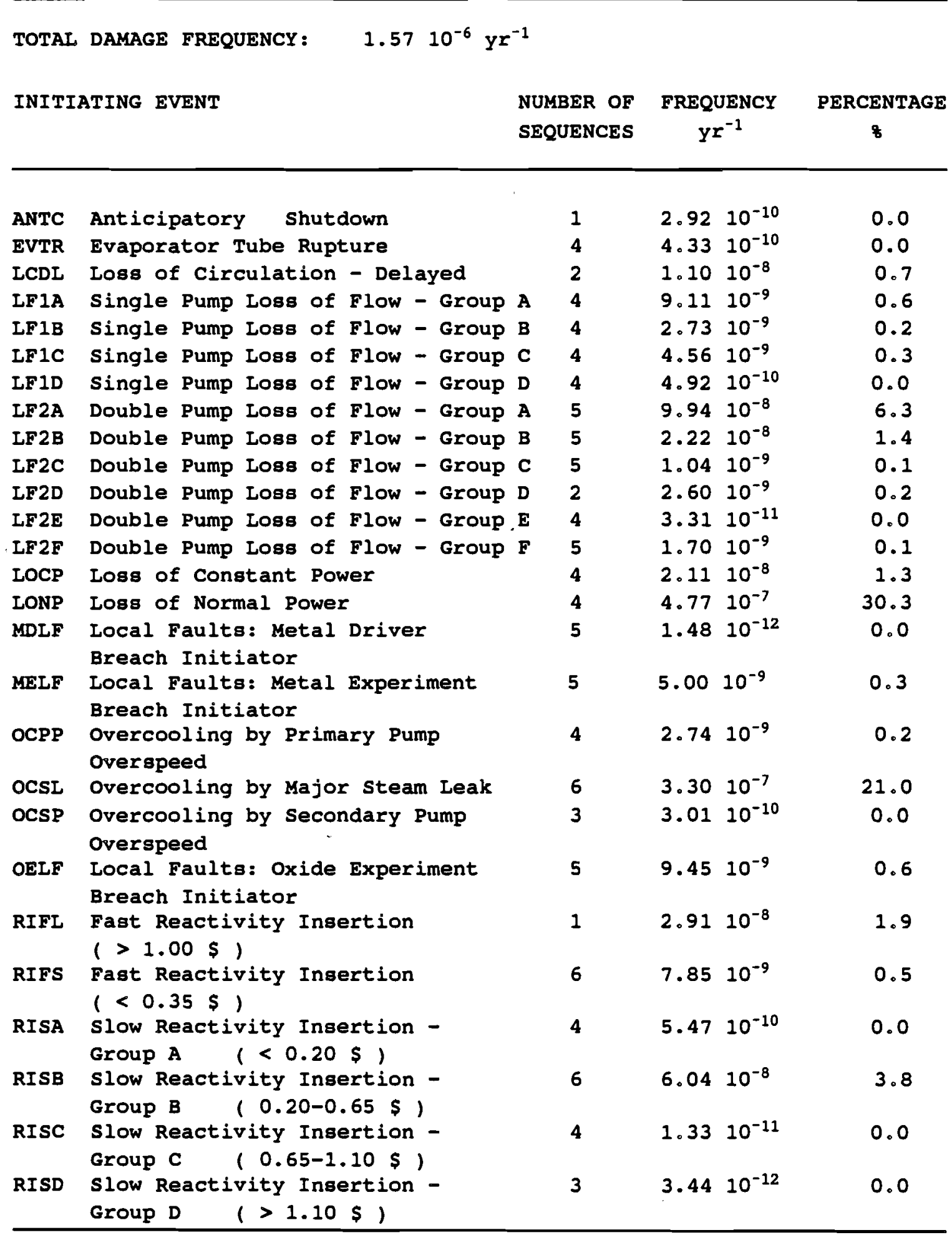


Table 11.6 Damage Accident Sequence Quantification Results Grouped by Initiating Event (Cont'd)

\begin{tabular}{|c|c|c|c|c|}
\hline \multicolumn{2}{|c|}{ INITIATING EVENT } & \multirow{2}{*}{$\begin{array}{c}\text { NUMBER OF } \\
\text { SEQUENCES } \\
3\end{array}$} & $\begin{array}{c}\text { FREQUENCY } \\
\mathrm{yr}^{-1}\end{array}$ & \multirow{2}{*}{$\begin{array}{c}\text { PERCENTAGE } \\
8 \\
0.4\end{array}$} \\
\hline SCRM & Scram & & $6.3910^{-9}$ & \\
\hline SFLE & $\begin{array}{l}\text { Subassembly Local Fault from } \\
\text { Loading Error }\end{array}$ & 5 & $1.0910^{-9}$ & 0.1 \\
\hline SFPB & $\begin{array}{l}\text { Subassembly Local Fault from } \\
\text { Partial Blockage }\end{array}$ & 5 & $2.6610^{-8}$ & 1.7 \\
\hline SHDL & Long shutdown & 2 & $4.1710^{-8}$ & 2.7 \\
\hline SHDs & short shutdown & 1 & $3.5110^{-9}$ & 0.2 \\
\hline SPTR & Superheater Tube Failure & 9 & $1.9610^{-7}$ & 12.5 \\
\hline TAIR & Transient - Loss of Instrument Air & 2 & $1.04 \quad 10^{-8}$ & 0.7 \\
\hline TBOP & Loss of Steam Plant Heat Removal & 2 & $1.5410^{-8}$ & 1.0 \\
\hline TSDC & $\begin{array}{l}\text { Transient - Unrecoverable Loss } \\
\text { of a Shutdown Cooler }\end{array}$ & 3 & $1.4110^{-7}$ & 9.0 \\
\hline TSEC & $\begin{array}{l}\text { Loss of Secondary system Heat } \\
\text { Removal }\end{array}$ & 2 & $2.6310^{-8}$ & 1.7 \\
\hline TSHC & $\begin{array}{l}\text { Transient - Unrecoverable Loss } \\
\text { of Shield Cooling }\end{array}$ & 3 & $4.01 \quad 10^{-9}$ & 0.3 \\
\hline
\end{tabular}




\section{DAMAGE SEQUENCES}

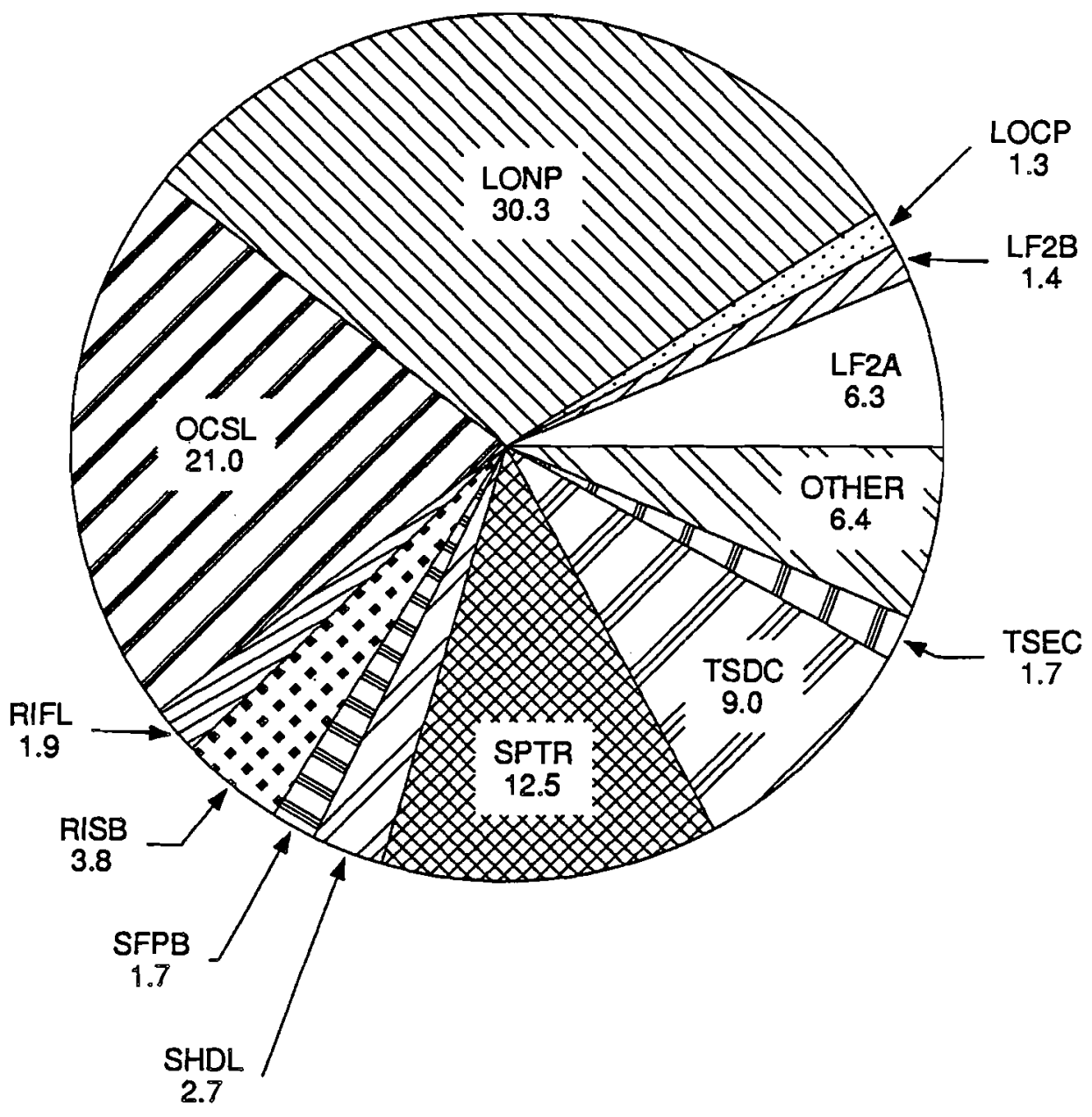

Fig. 11.17. Comparison of Initiating Events by their Contribution to the Damage Frequency 


\section{ALL DOMINANT SEQUENCES}

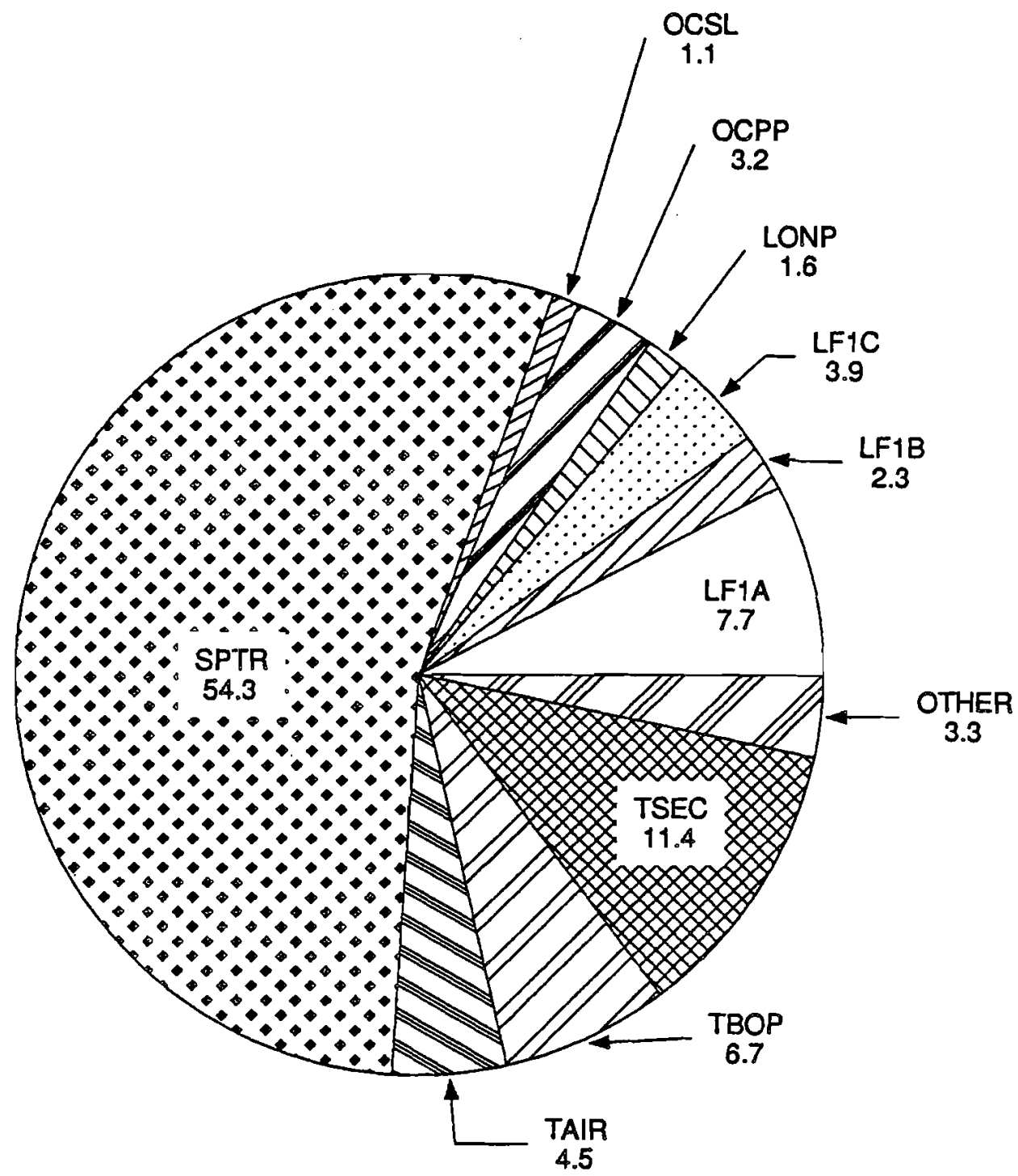

Fig. 11.18. Comparison of Initiating Events by their Contribution to the Total (damage + ND) Frequency 


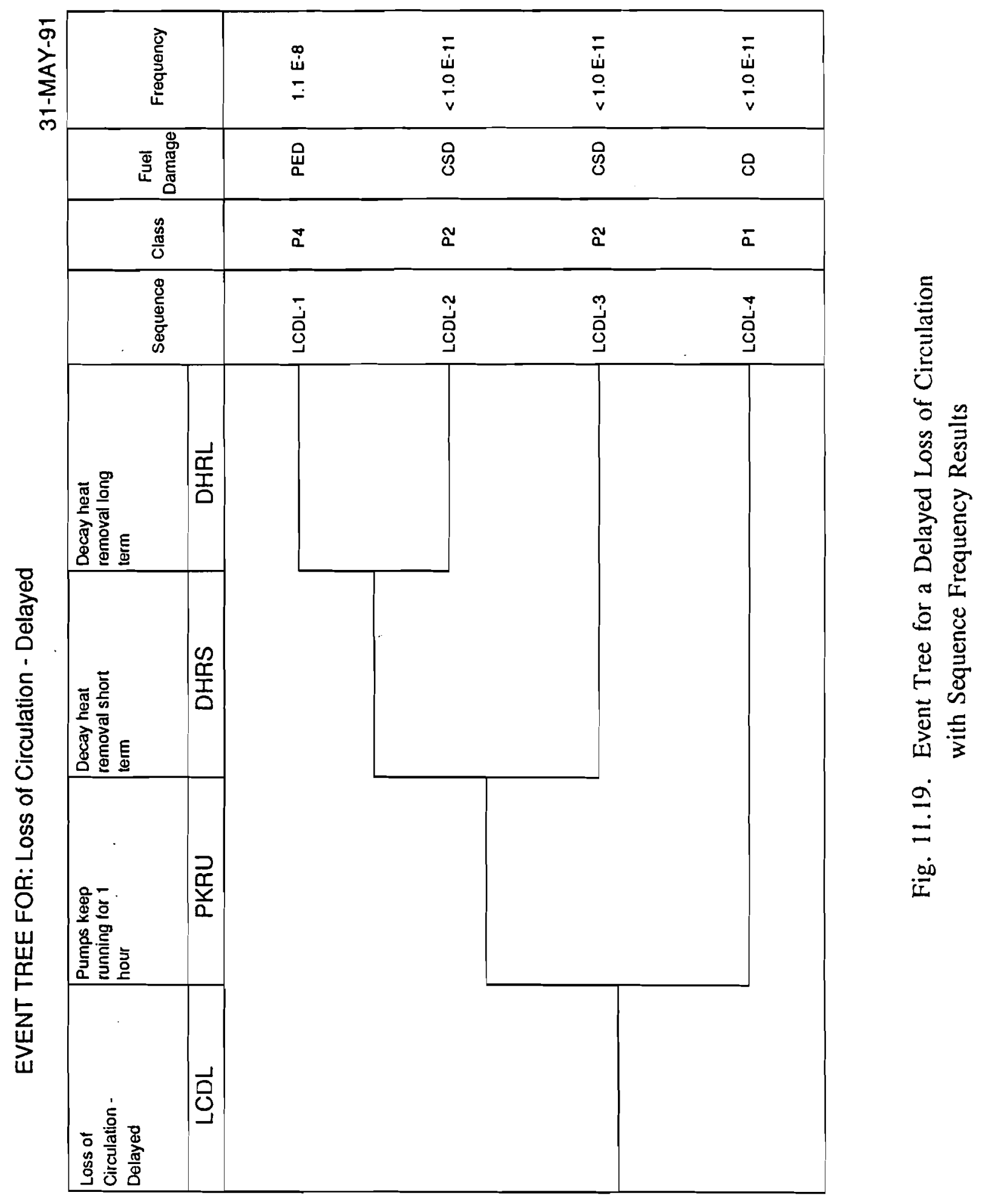




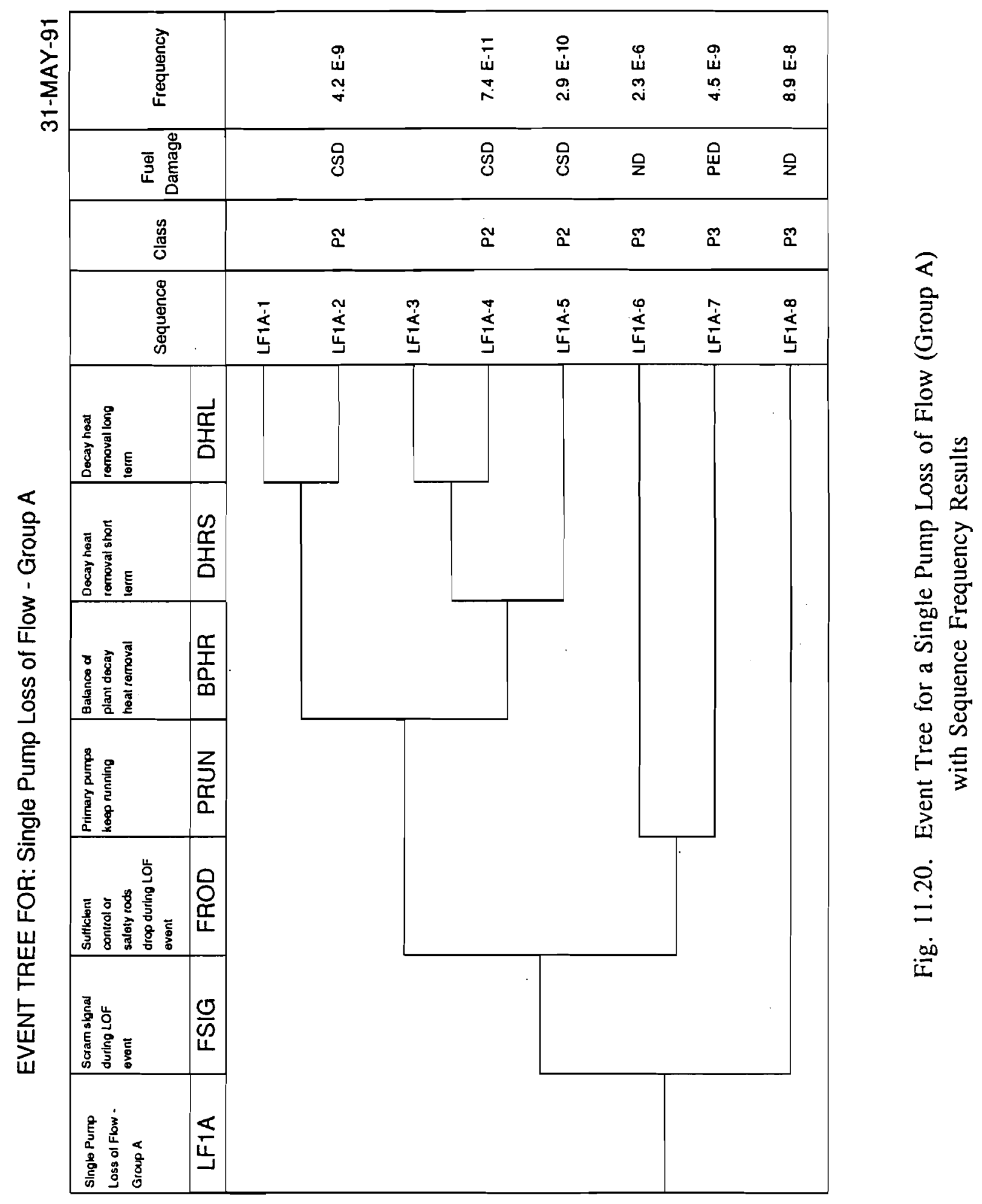


EVENT TREE FOR: Single Pump Loss of Flow - Group B

31-MAY-91

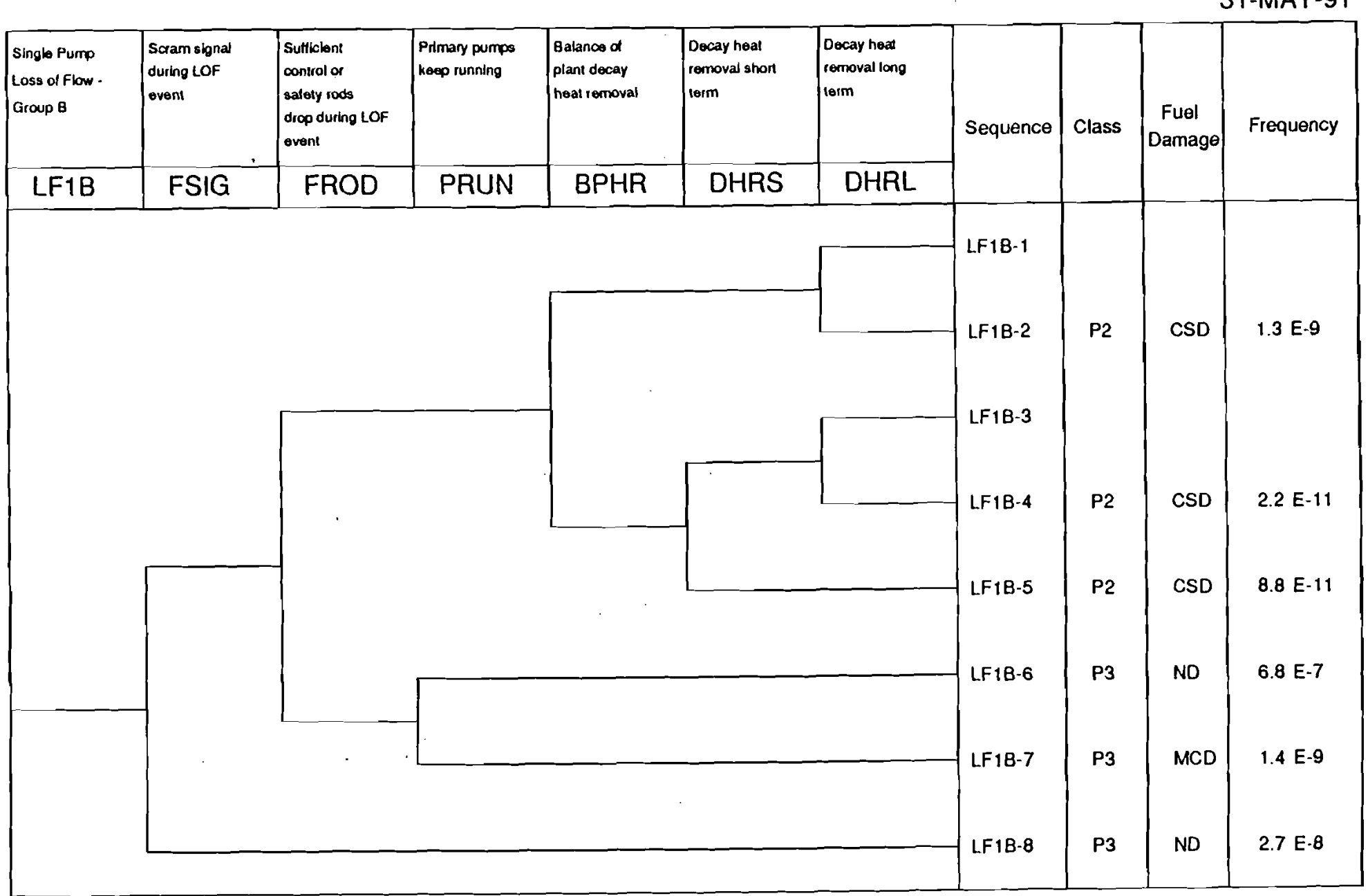

Fig. 11.21. Event Tree for a Single Pump Loss of Flow (Group B) with Sequence Frequency Results 


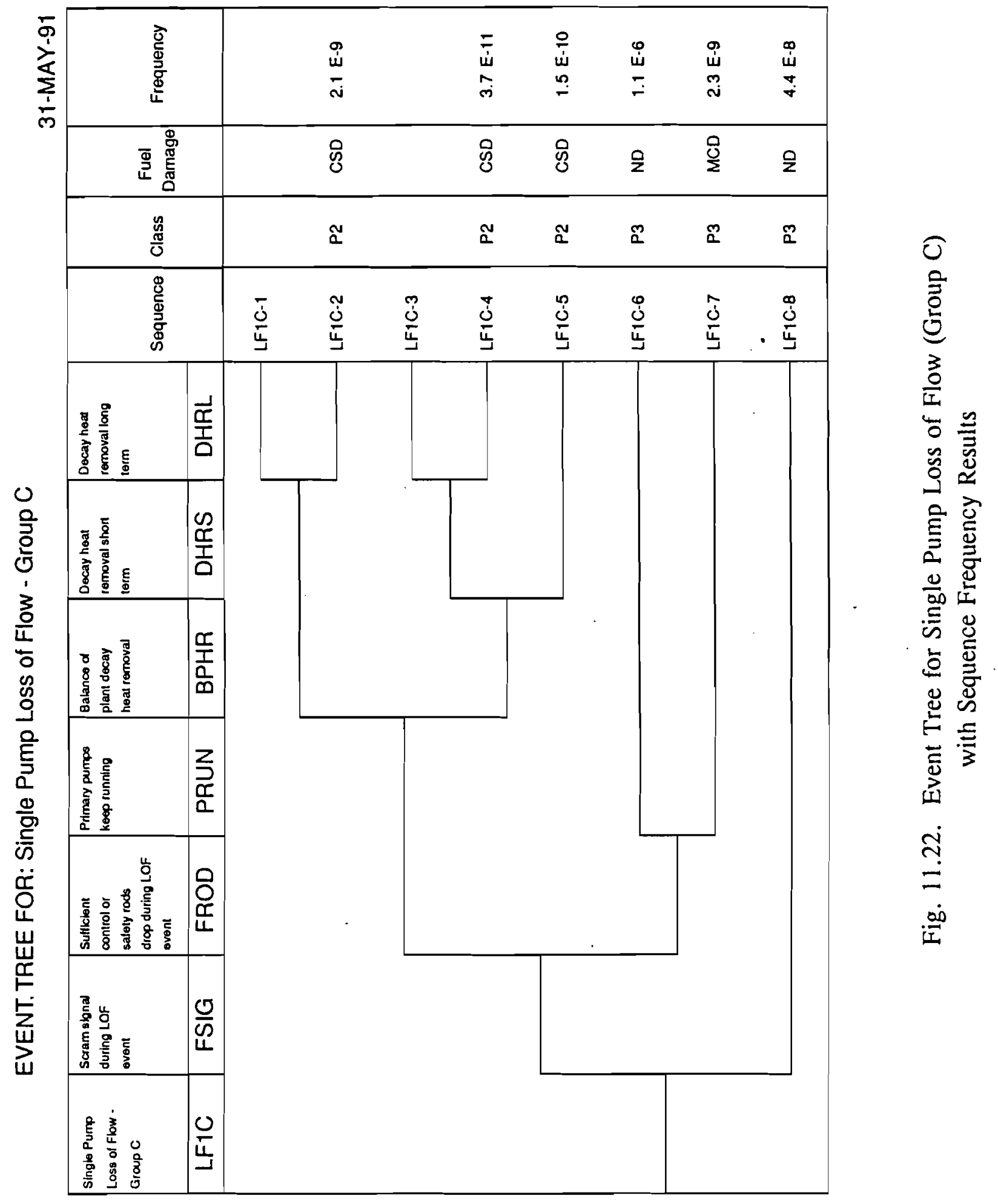




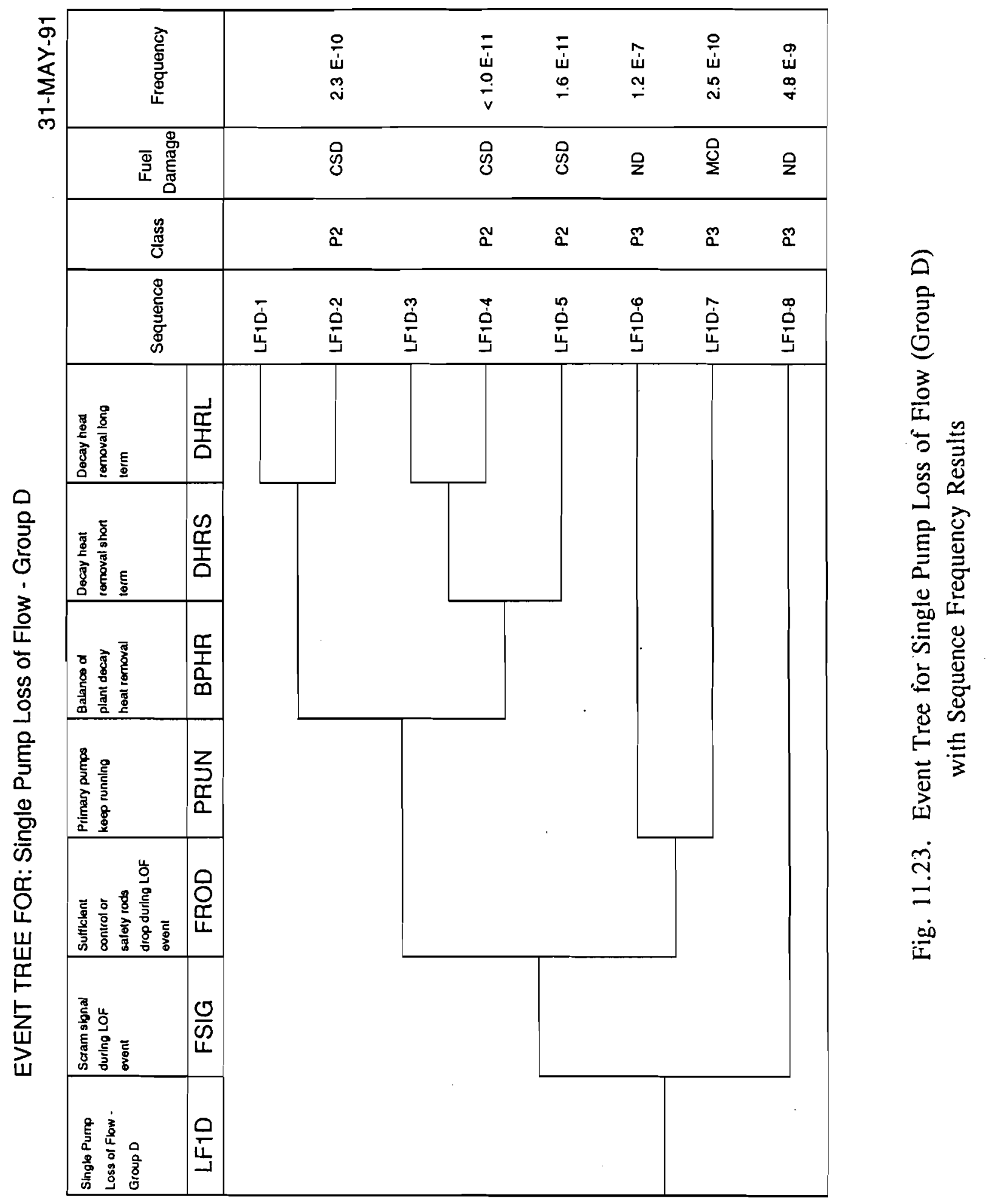




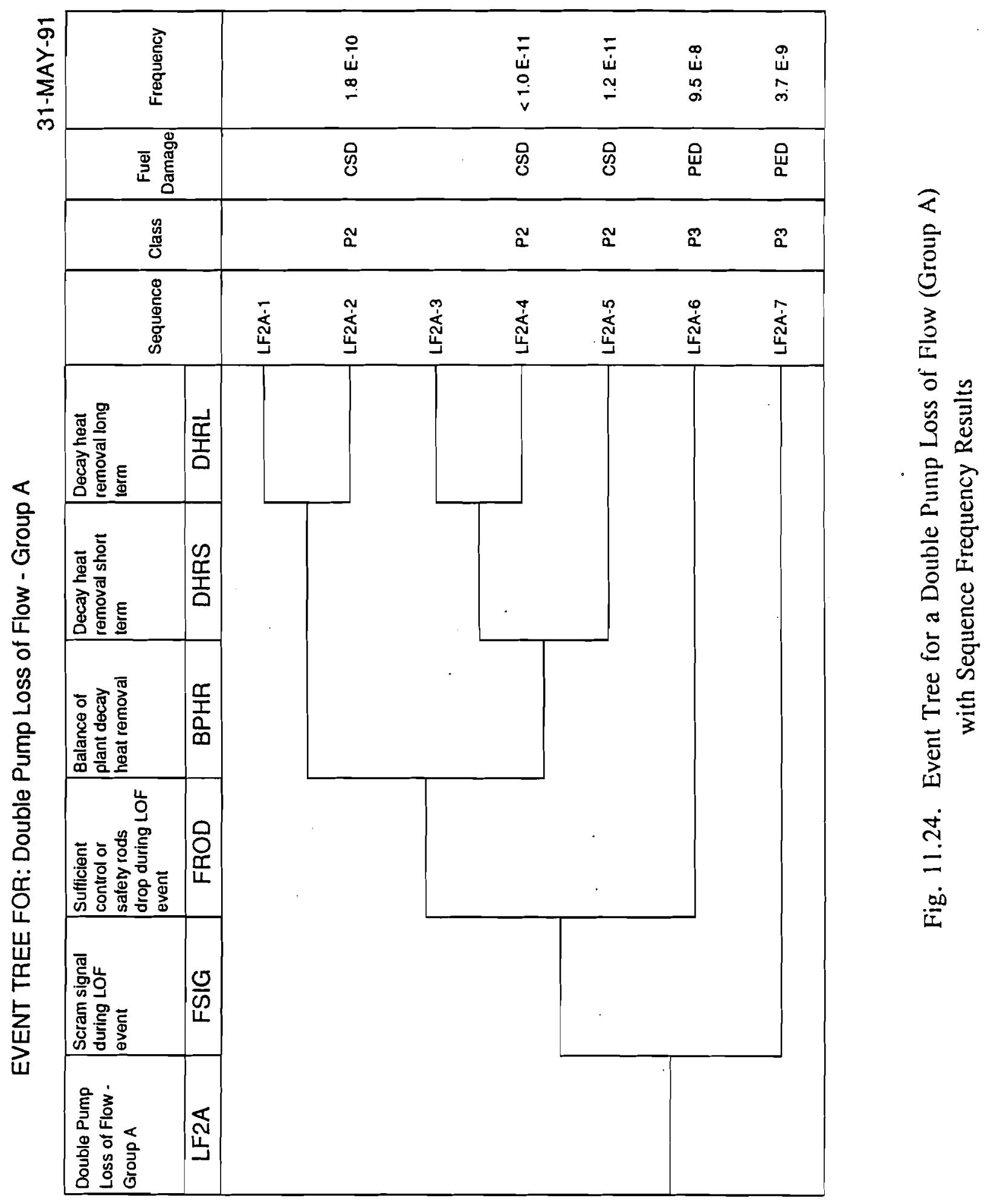


EVENT TREE FOR: Double Pump Loss of Flow - Group B

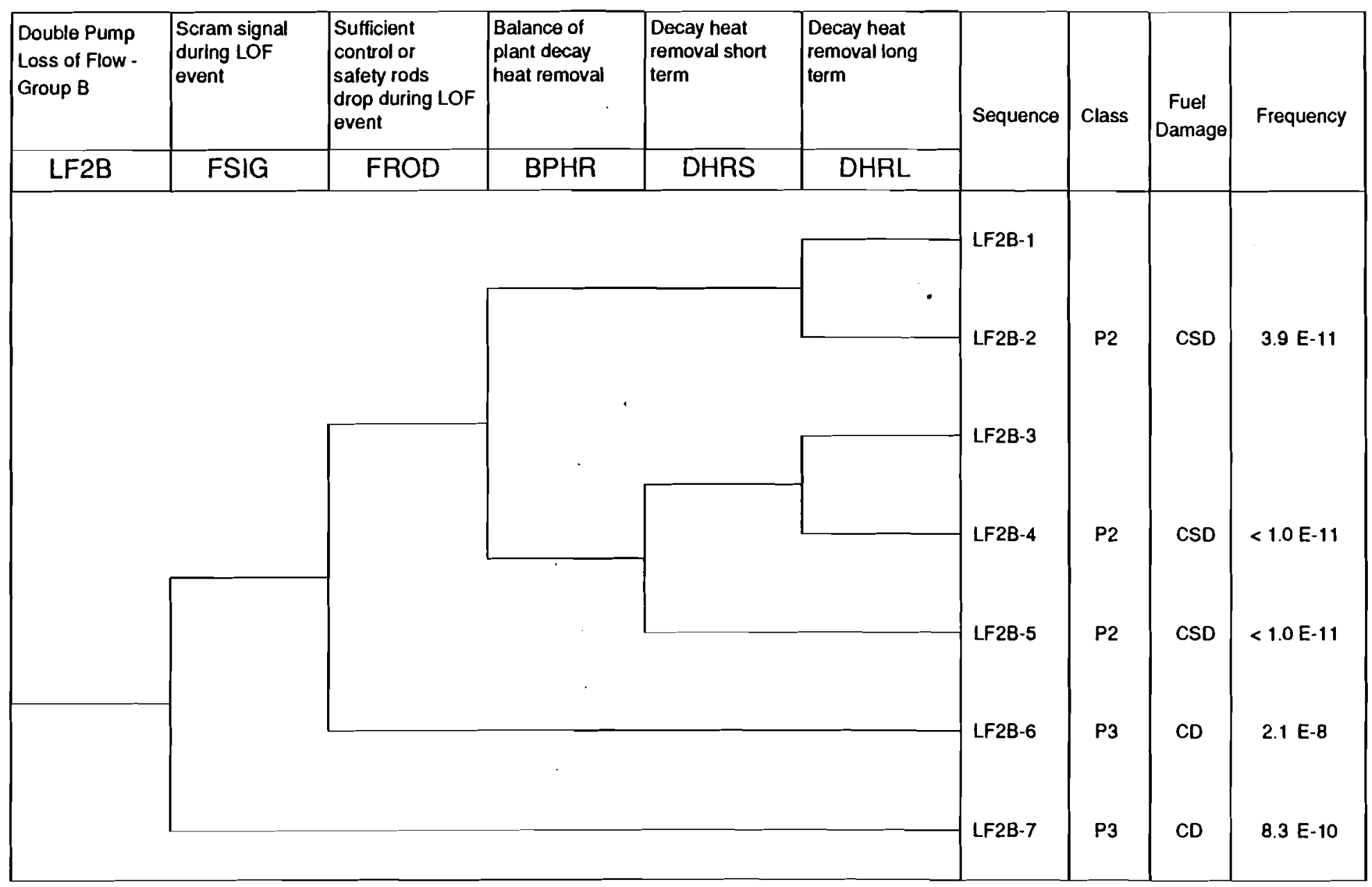

Fig. 11.25. Event Tree for a Double Pump Loss of Flow (Group B) with Sequence Frequency Results 
EVENT TREE FOR: Double Pump Loss of Flow - Group C

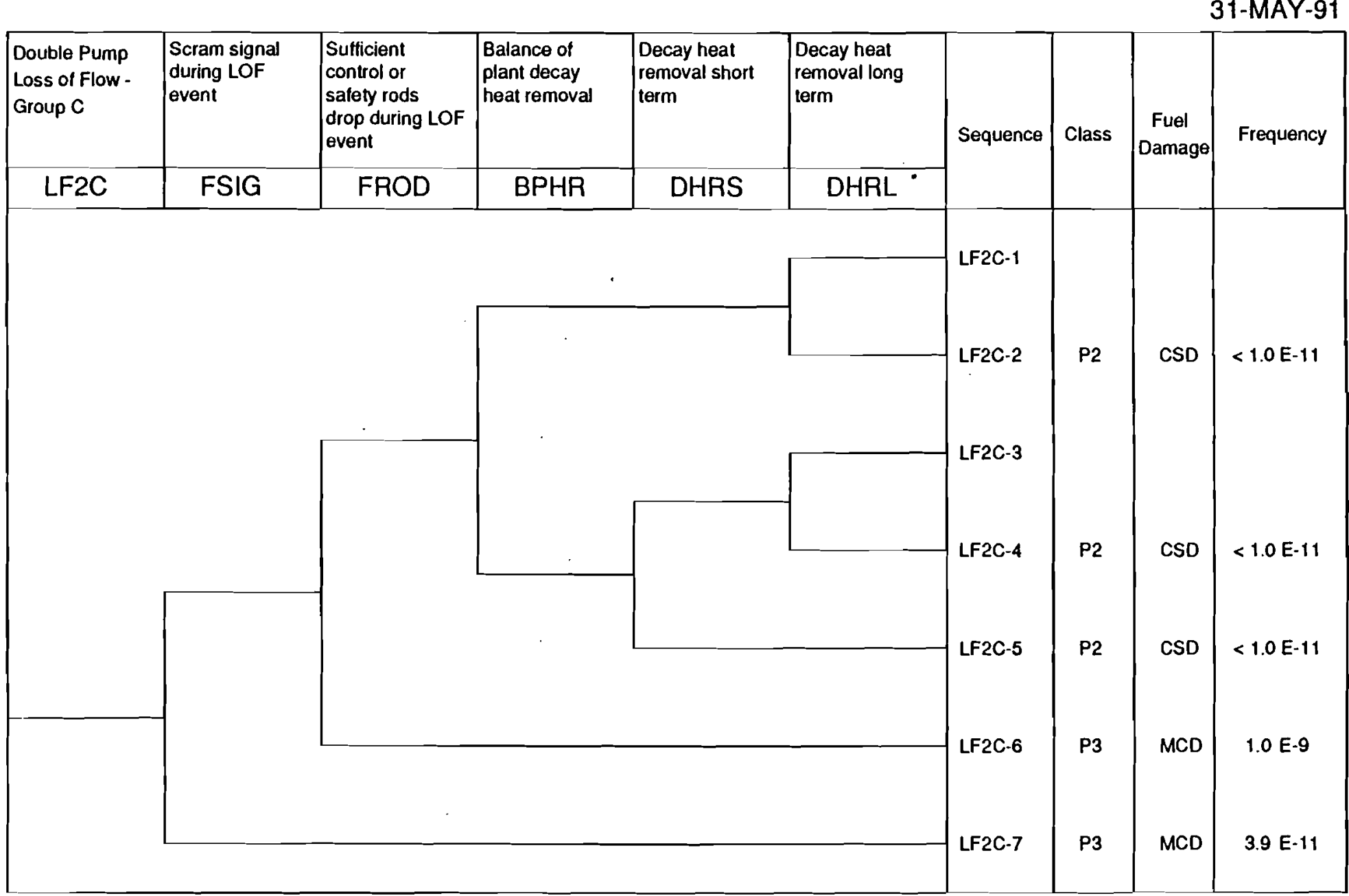

Fig. 11.26. Event Tree for a Double Pump Loss of Flow (Group C)

with Sequence Frequency Results 


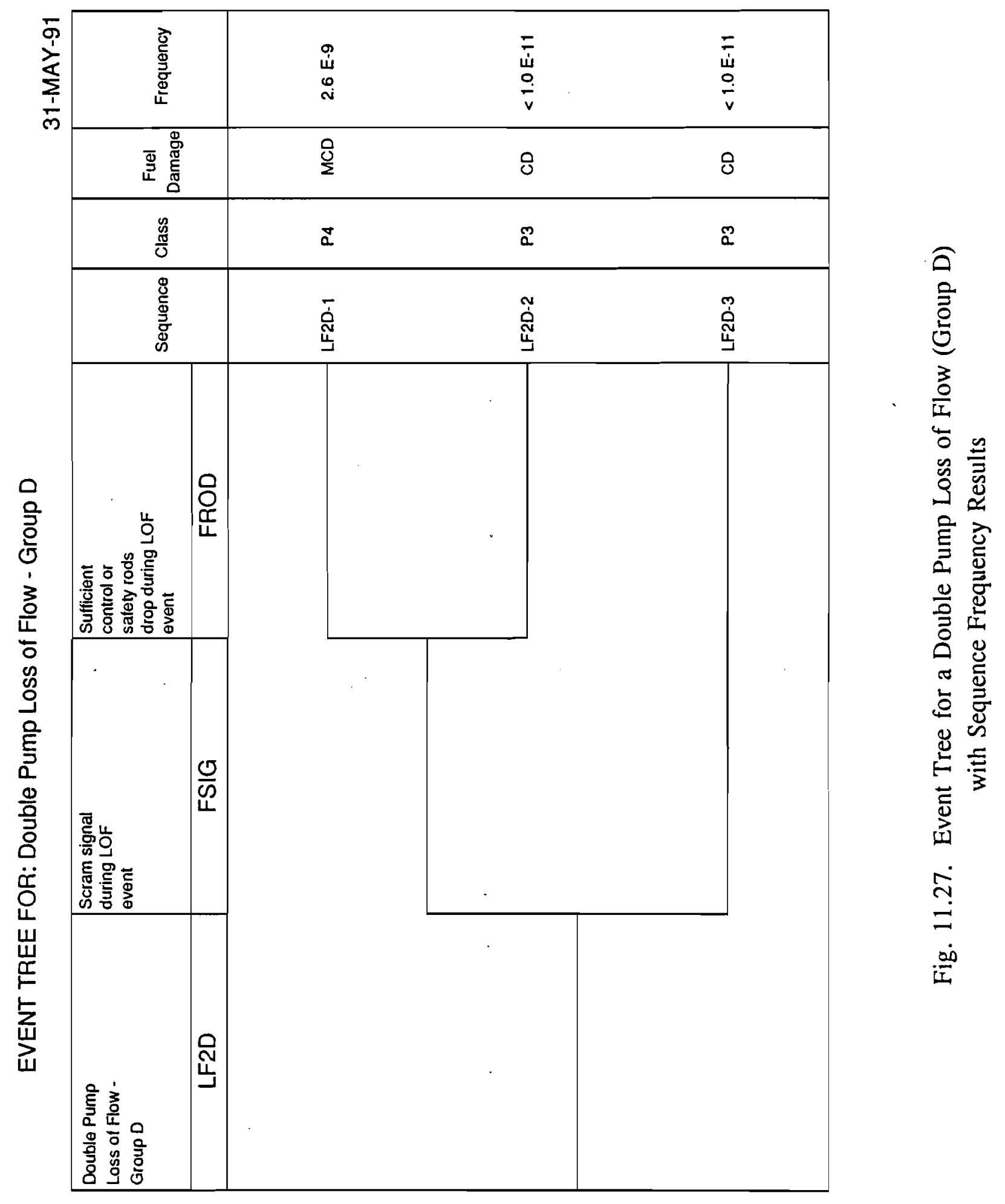


EVENT TREE FOR: Double Pump Loss of Flow - Group E

31-MAY-91

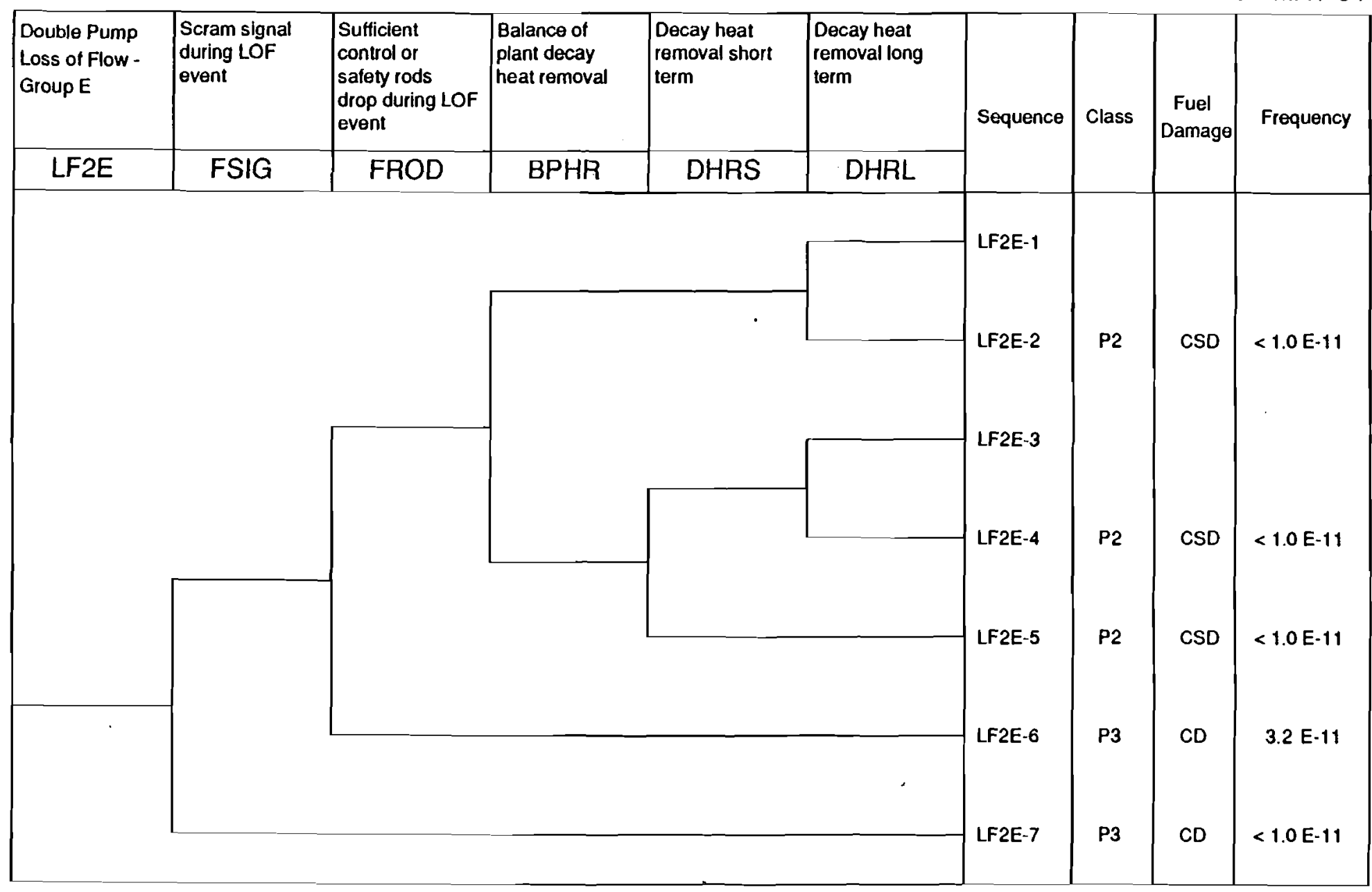

Fig. 11.28. Event Tree for a Double Pump Loss of Flow (Group E) with Sequence Frequency Results 


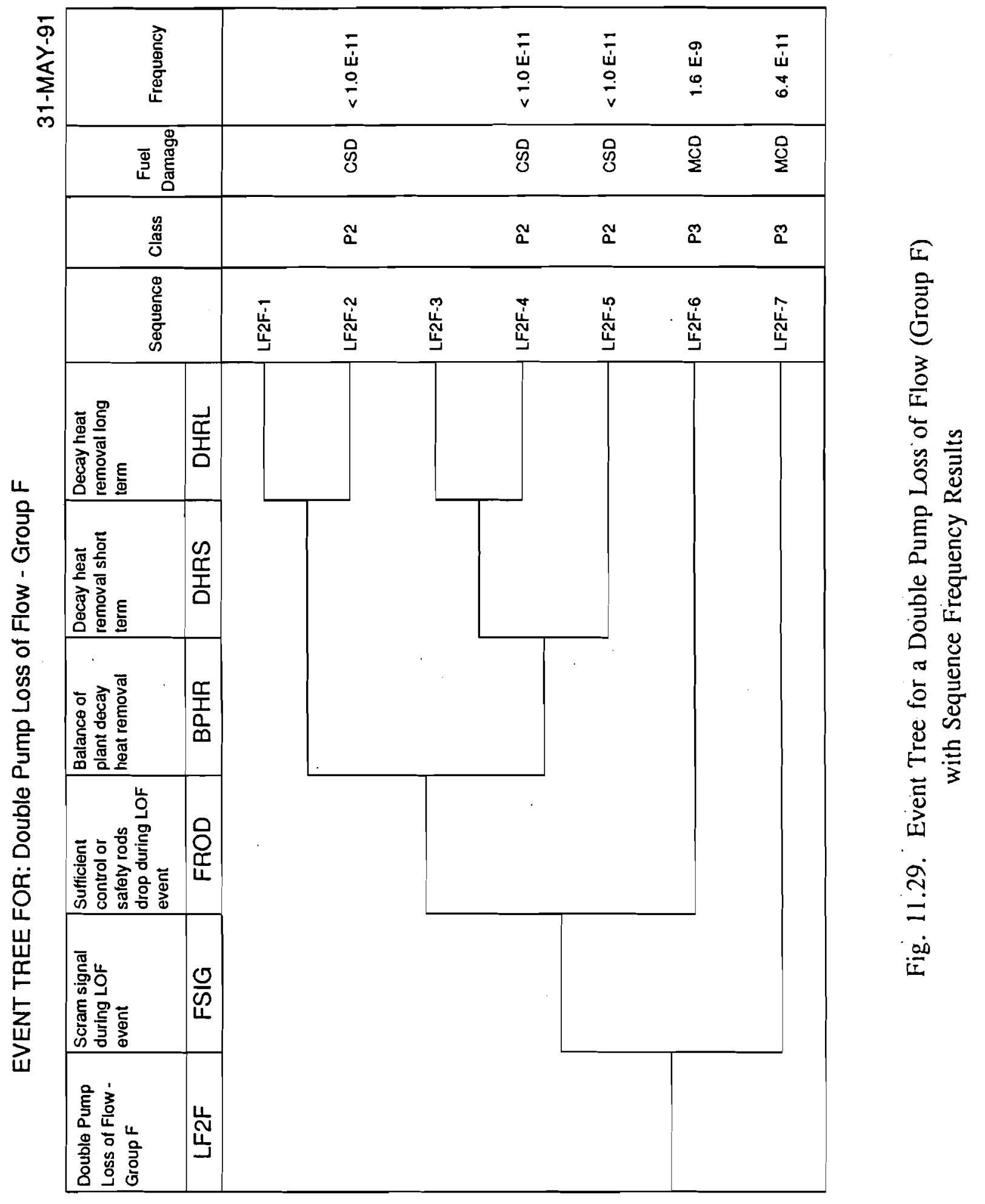


EVENT TREE FOR: Loss of Normal Power

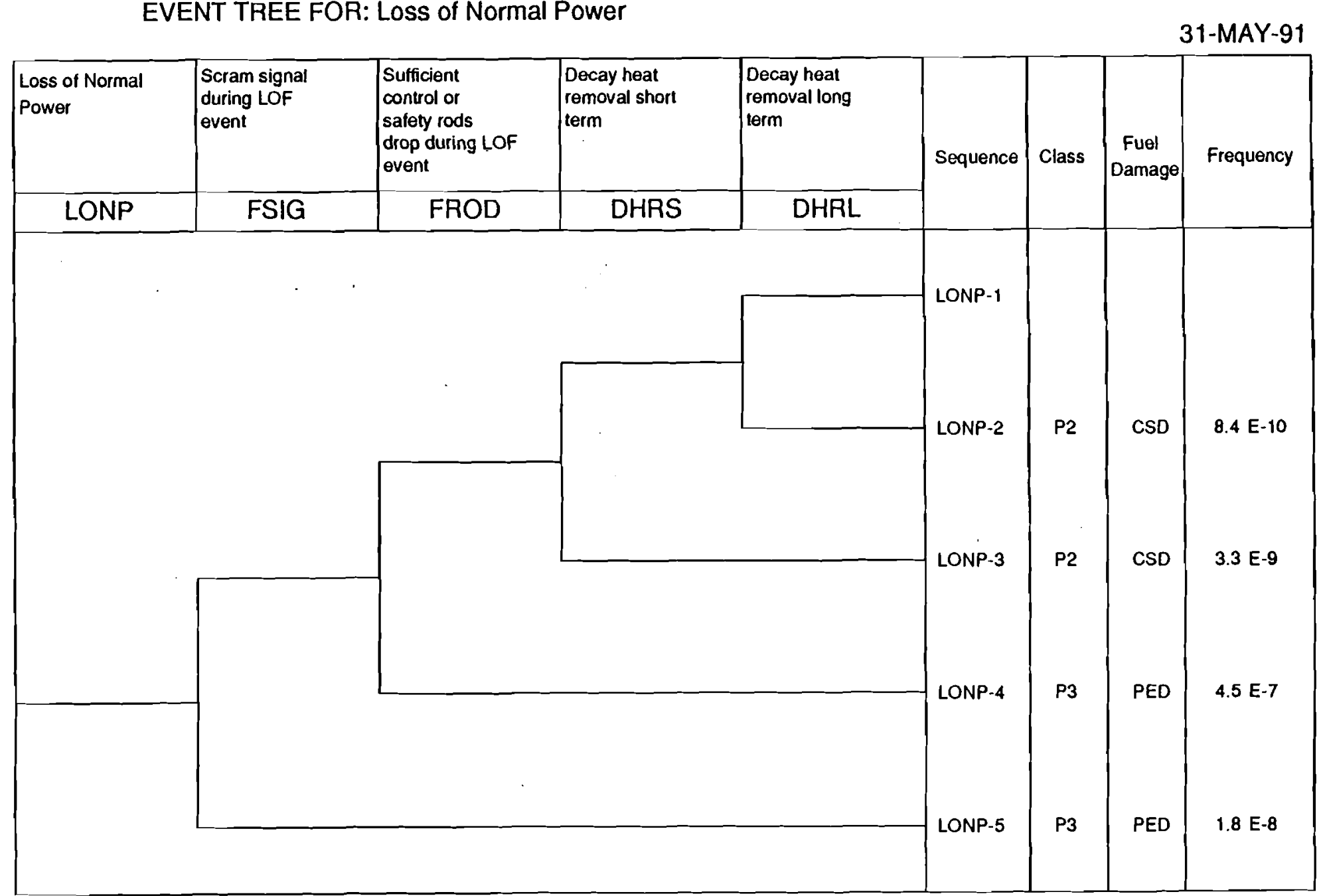

Fig. 11.30. Event Tree for the Loss of Normal Power with Sequence Freqeucny Results 


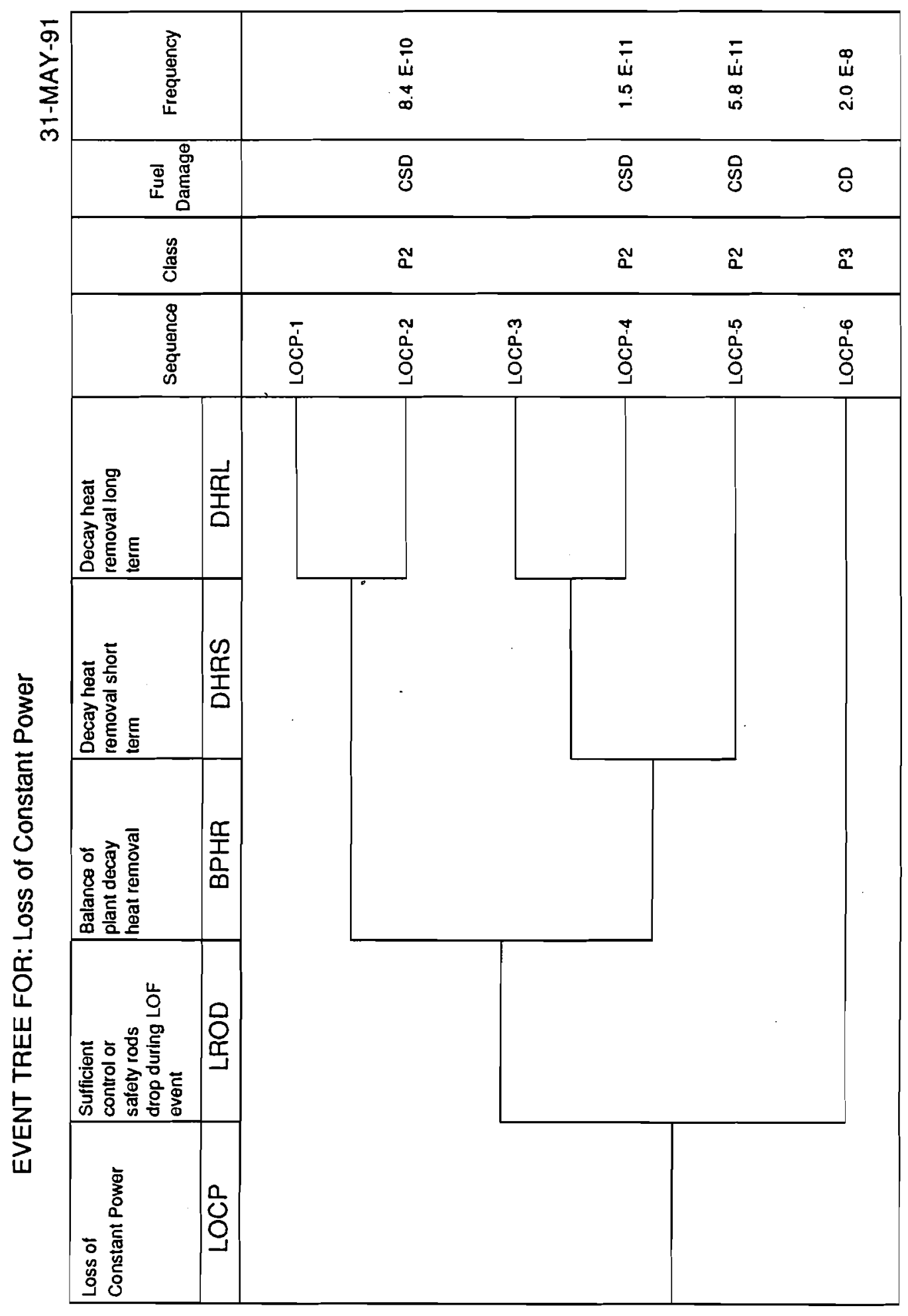

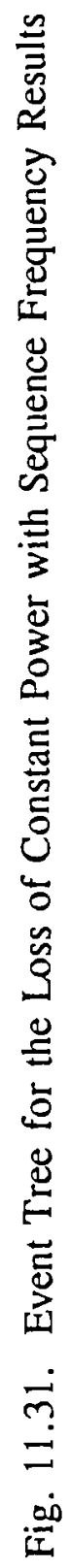


EVENT TREE FOR: Overcooling by Secondary Pump Overspeed

31-MAY-91

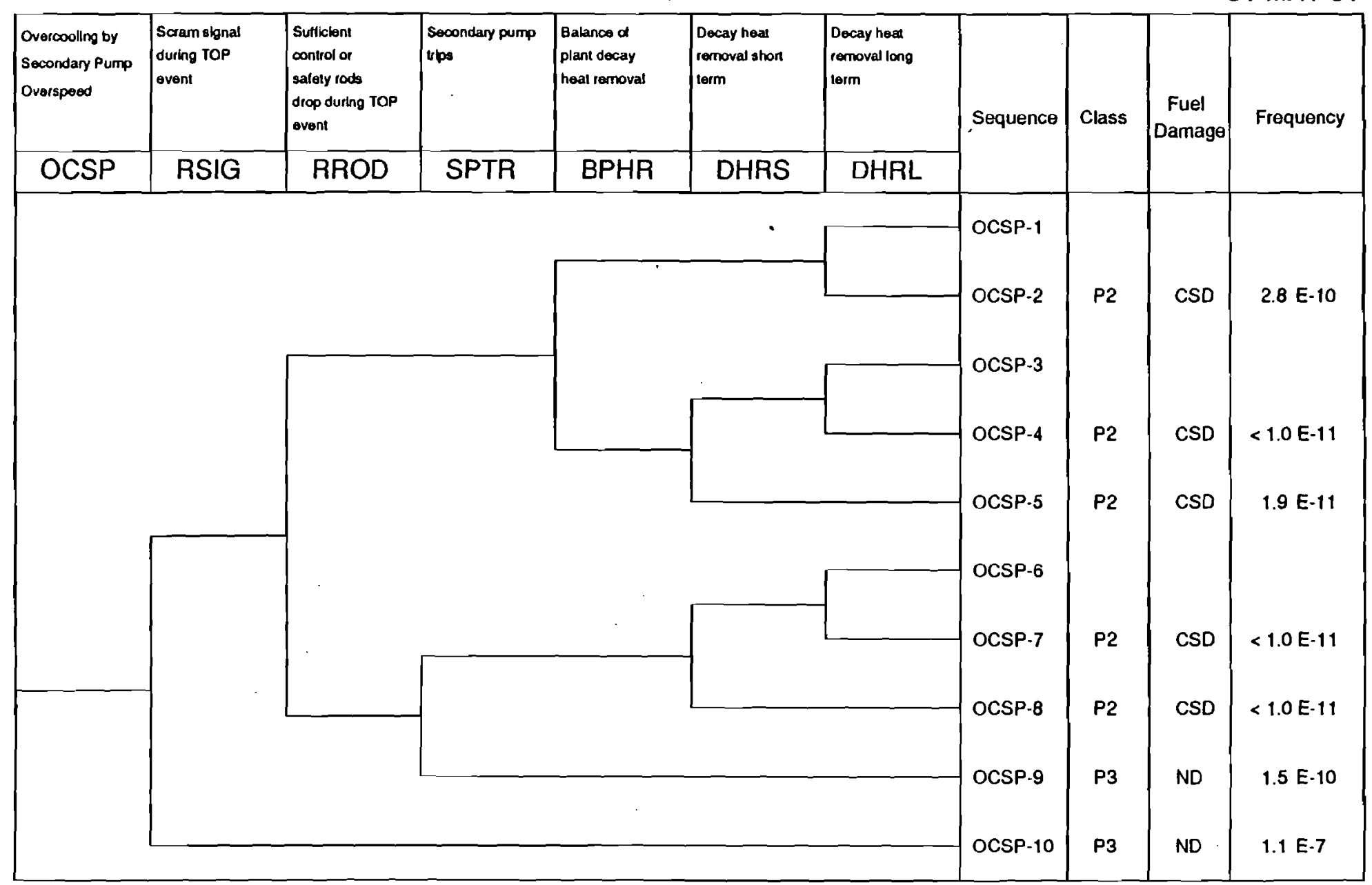

Fig. 11.32. Event Tree for an Overcooling by Secondary Pump Overspeed with Sequence Frequency Results 


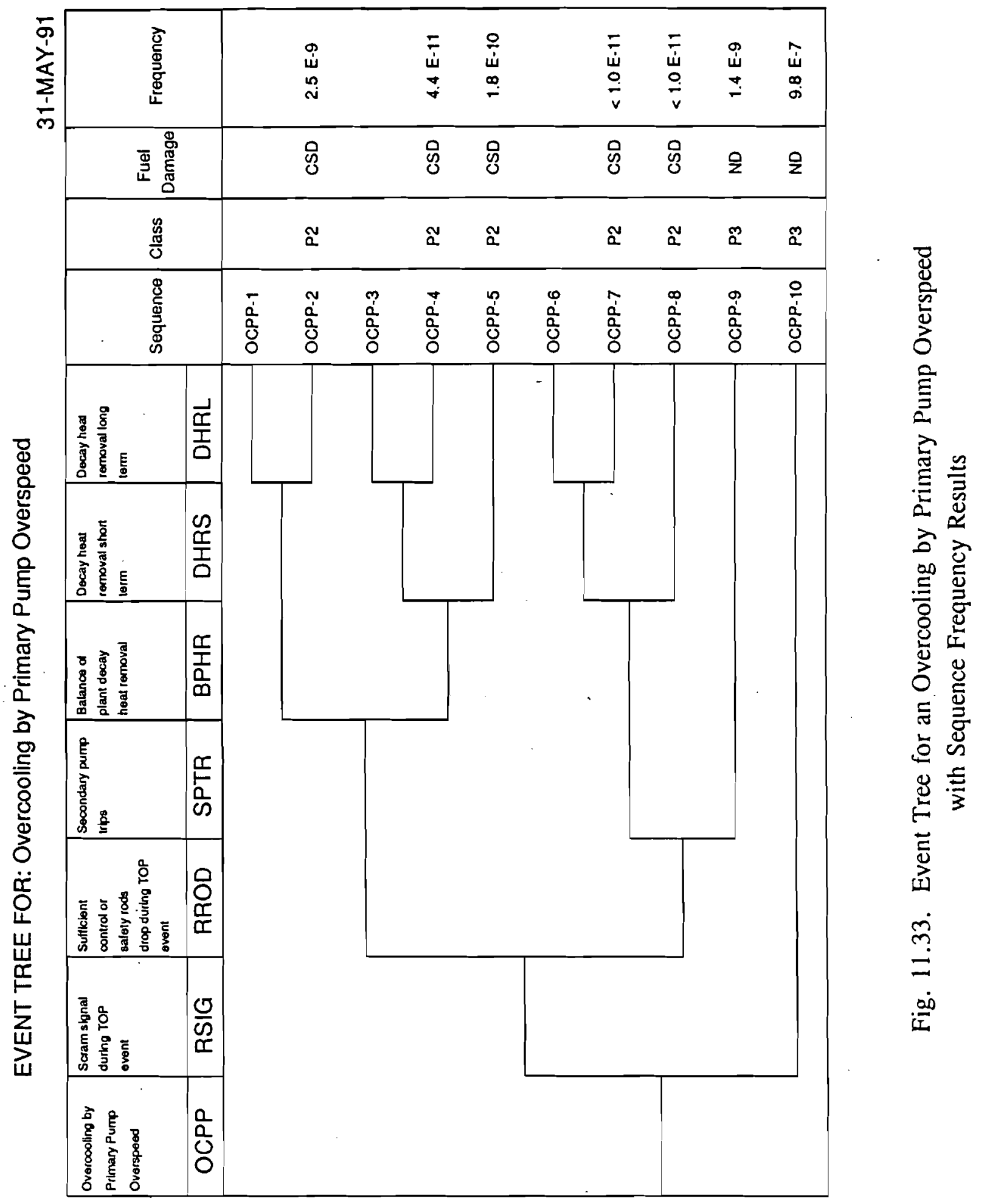




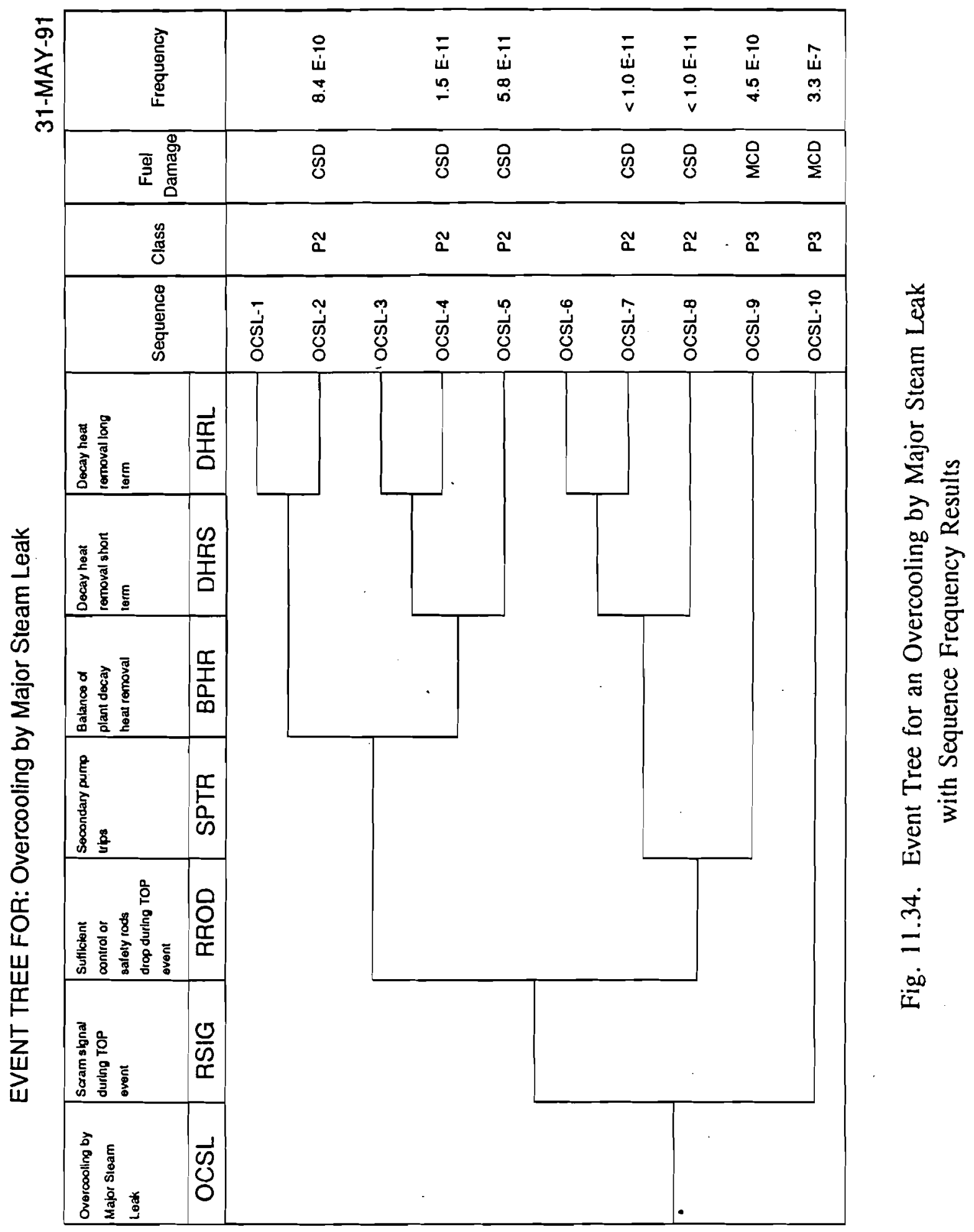




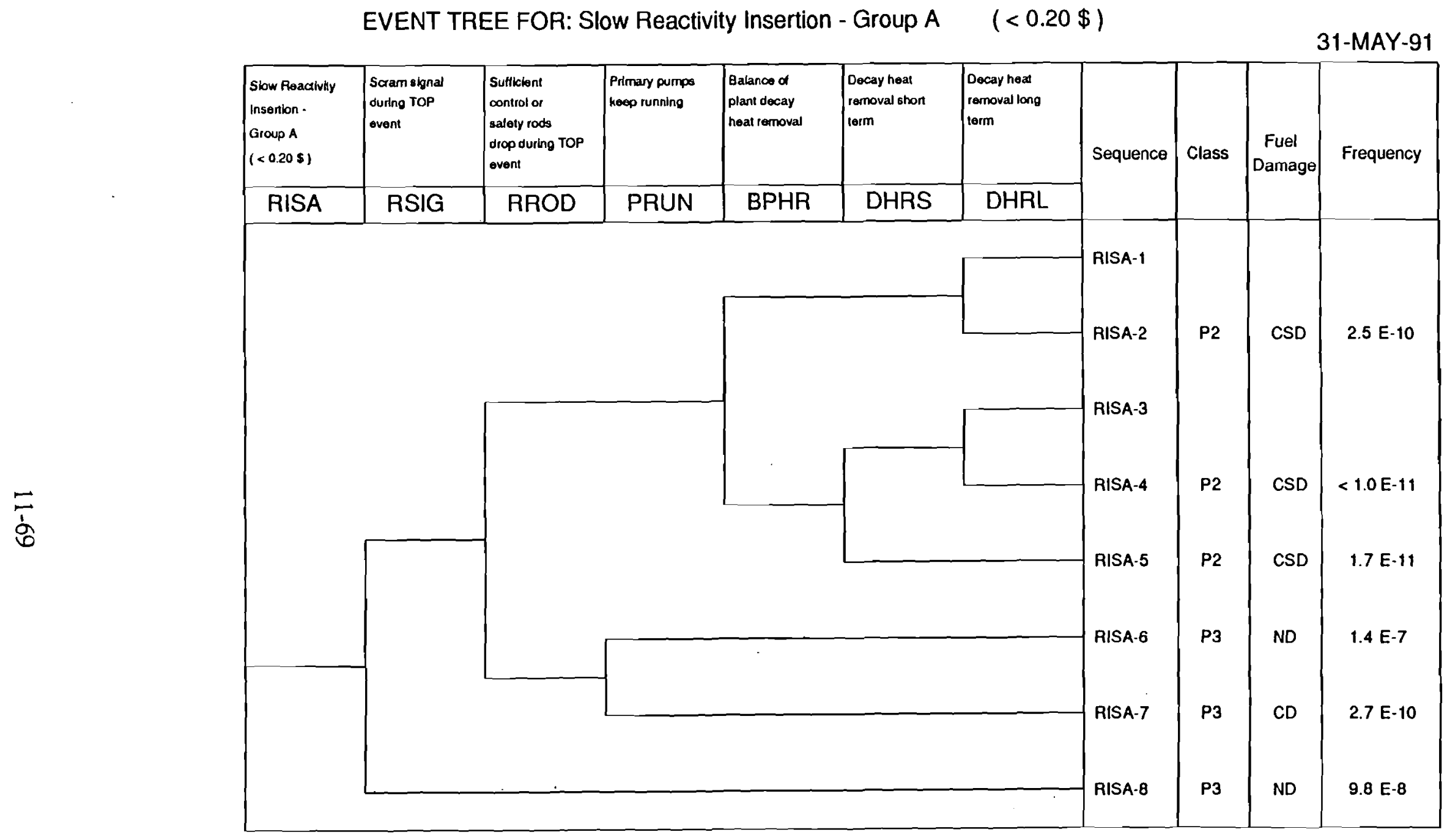

Fig. 11.35. Event Tree for a Slow Reactivity Insertion (Group A) with Sequence Frequency Results 


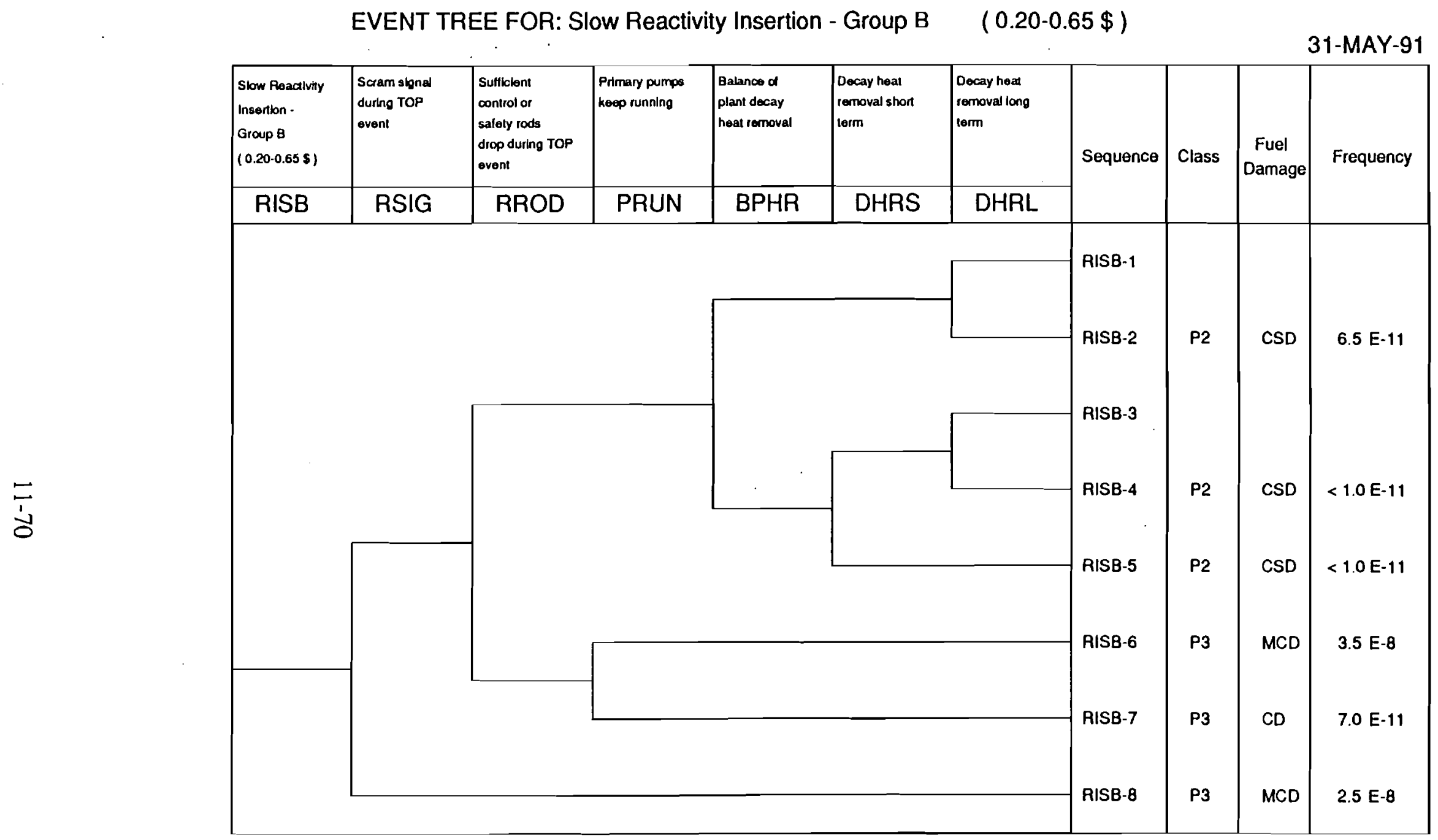

Fig. 11.36. Event Tree for a Slow Reactivity Insertion (Group B) with Sequence Frequency Results 


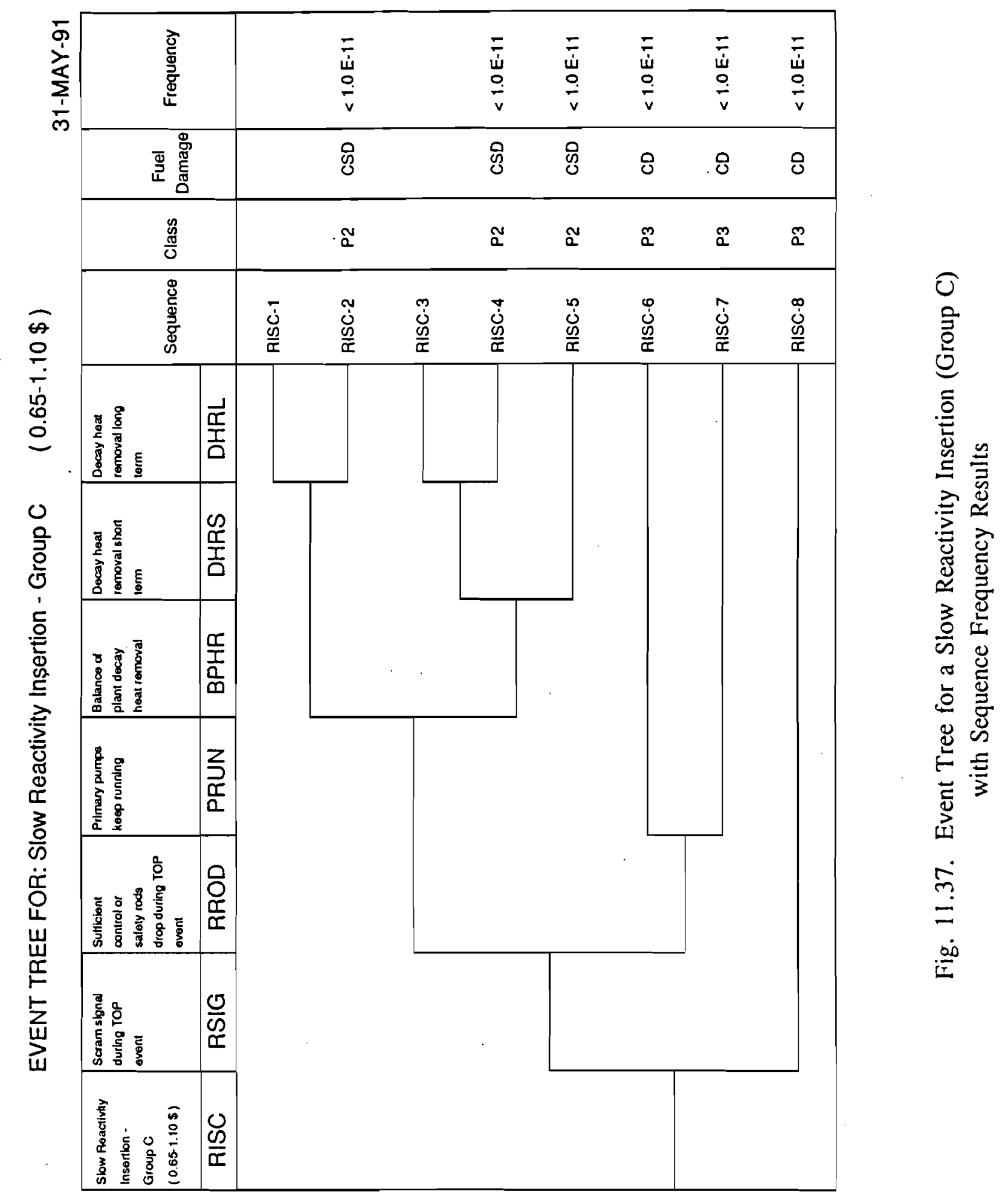




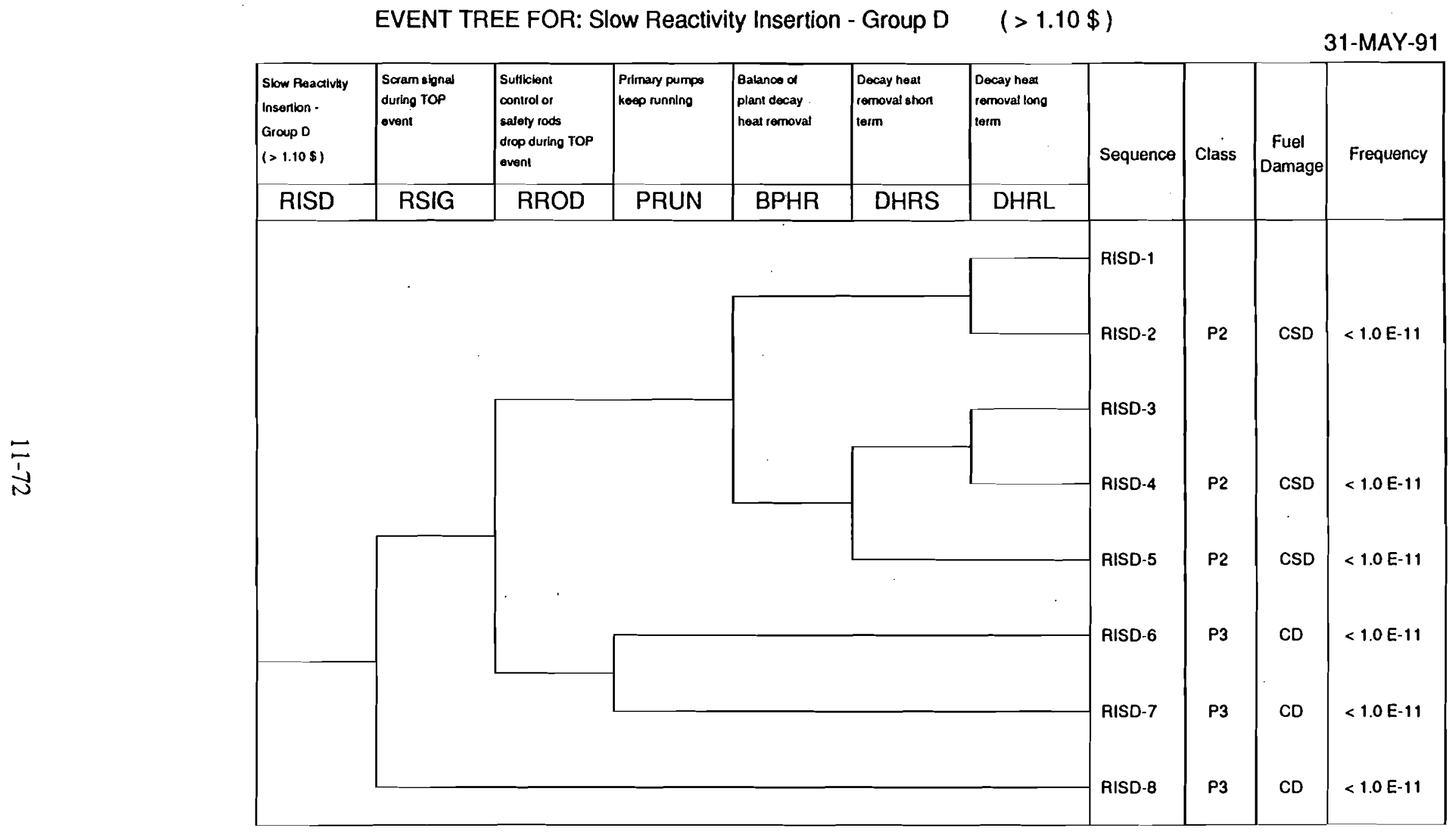

Fig. 11.38. Event Tree for a Slow Reactivity Insertion (Group D) with Sequence Frequency Results 


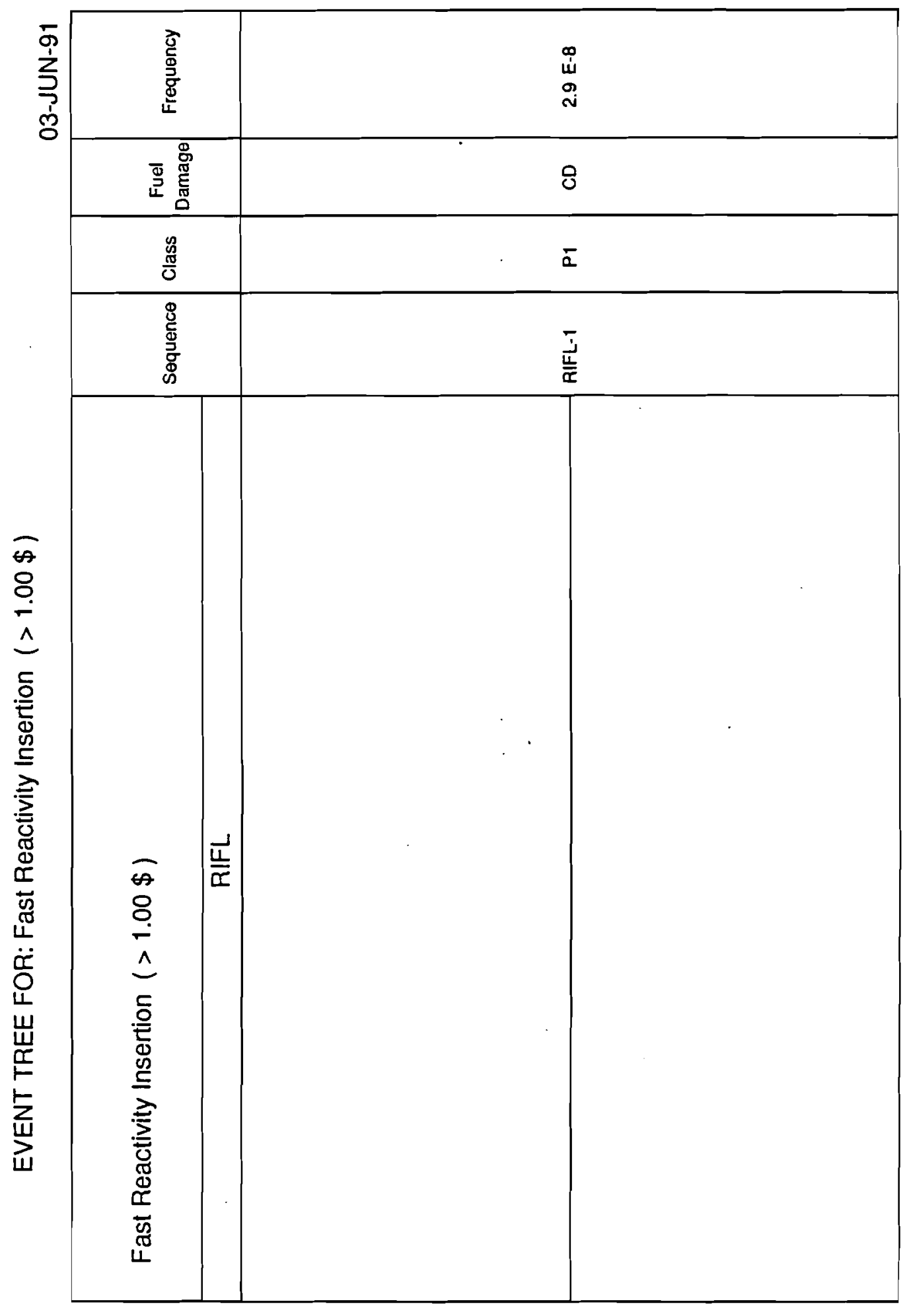

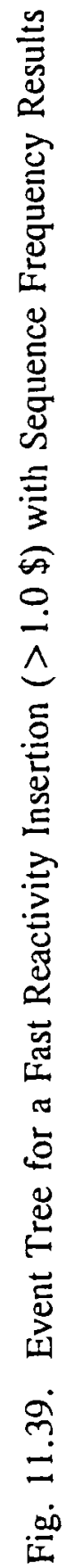




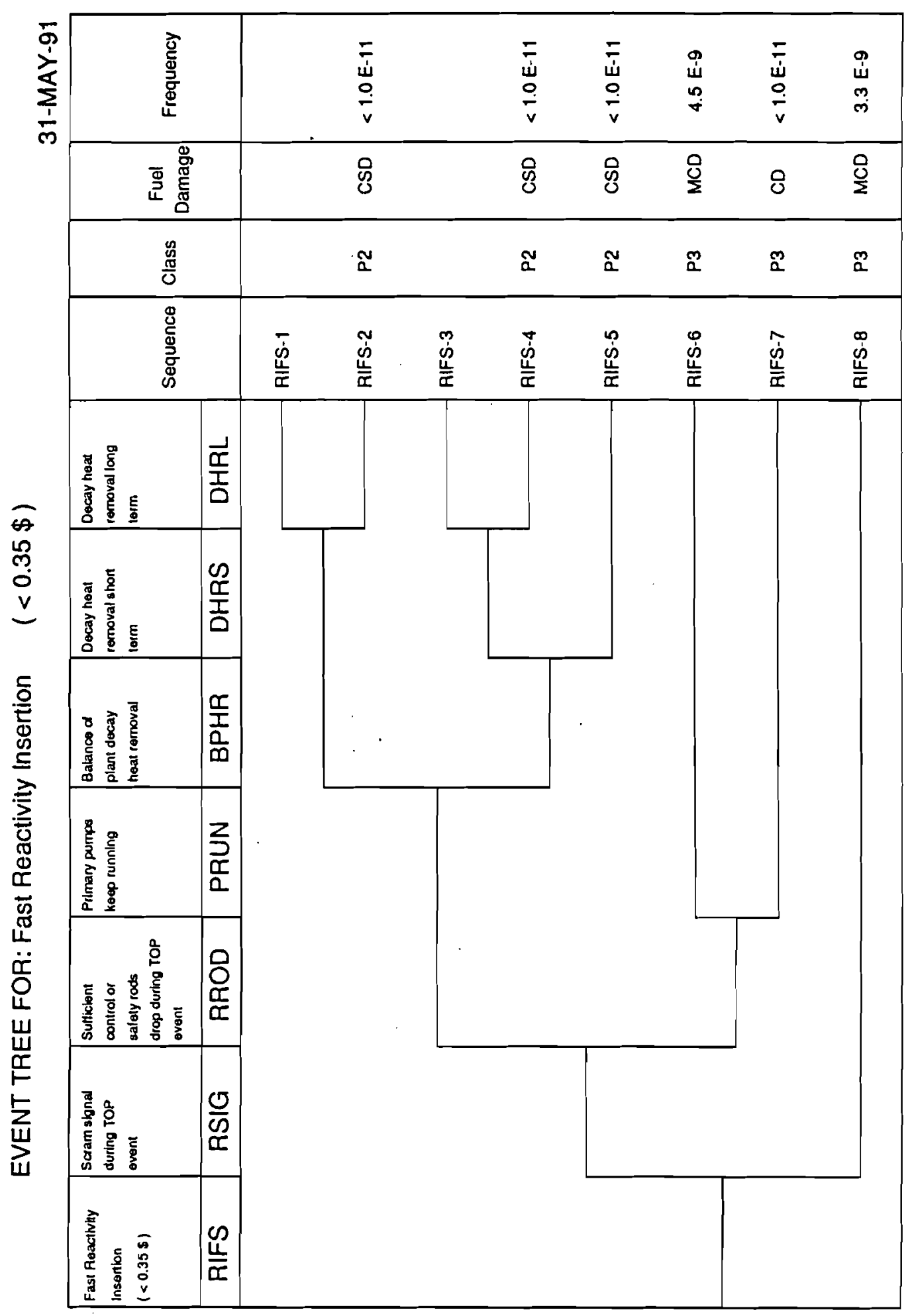

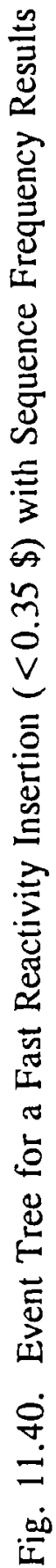


EVENT TREE FOR: Anticipatory Shutdown

31-MAY-91

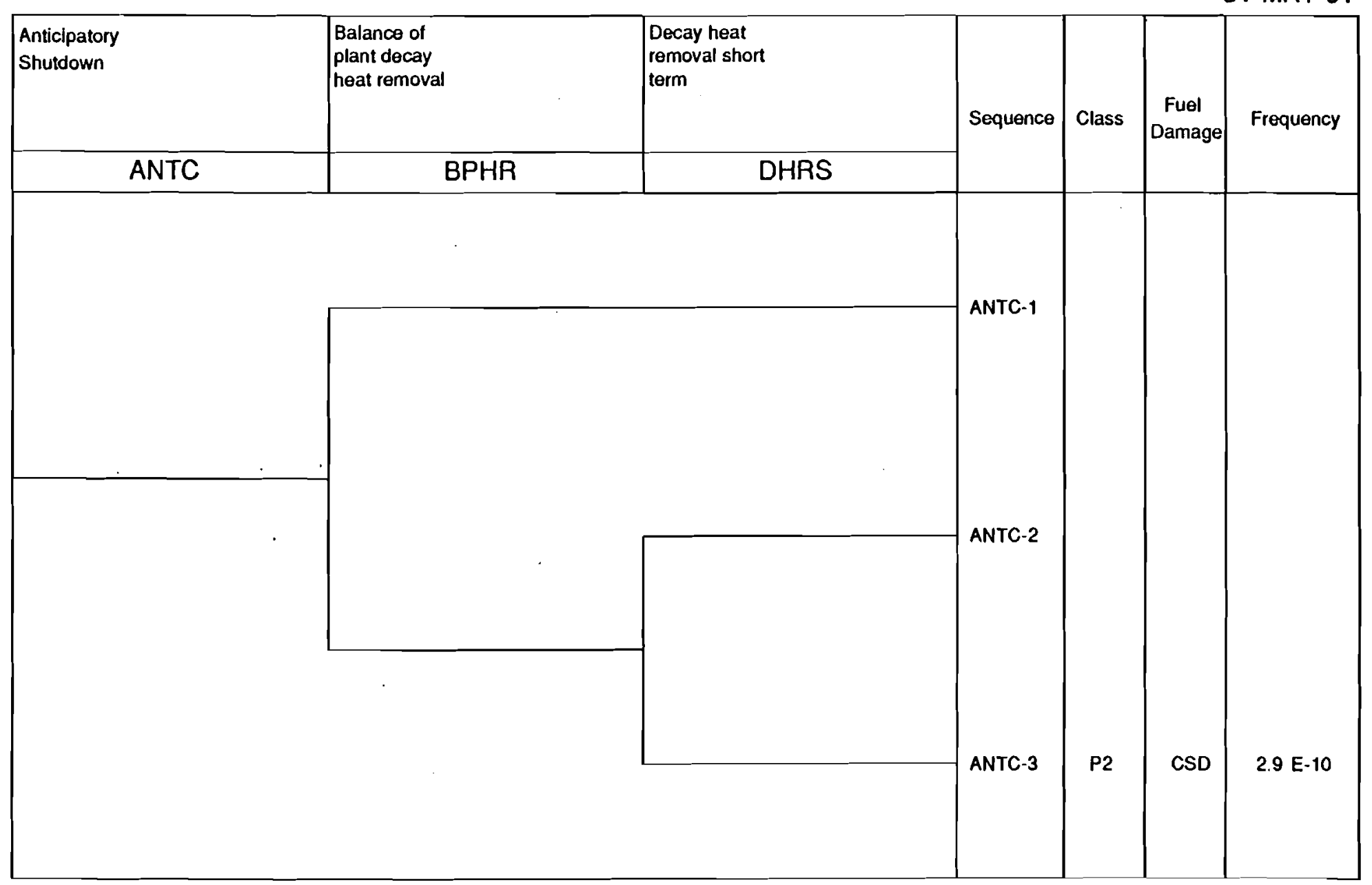

Fig. 11.41. Event Tree for an Anticipatory Shutdown with Seqeunce Frequency Results 


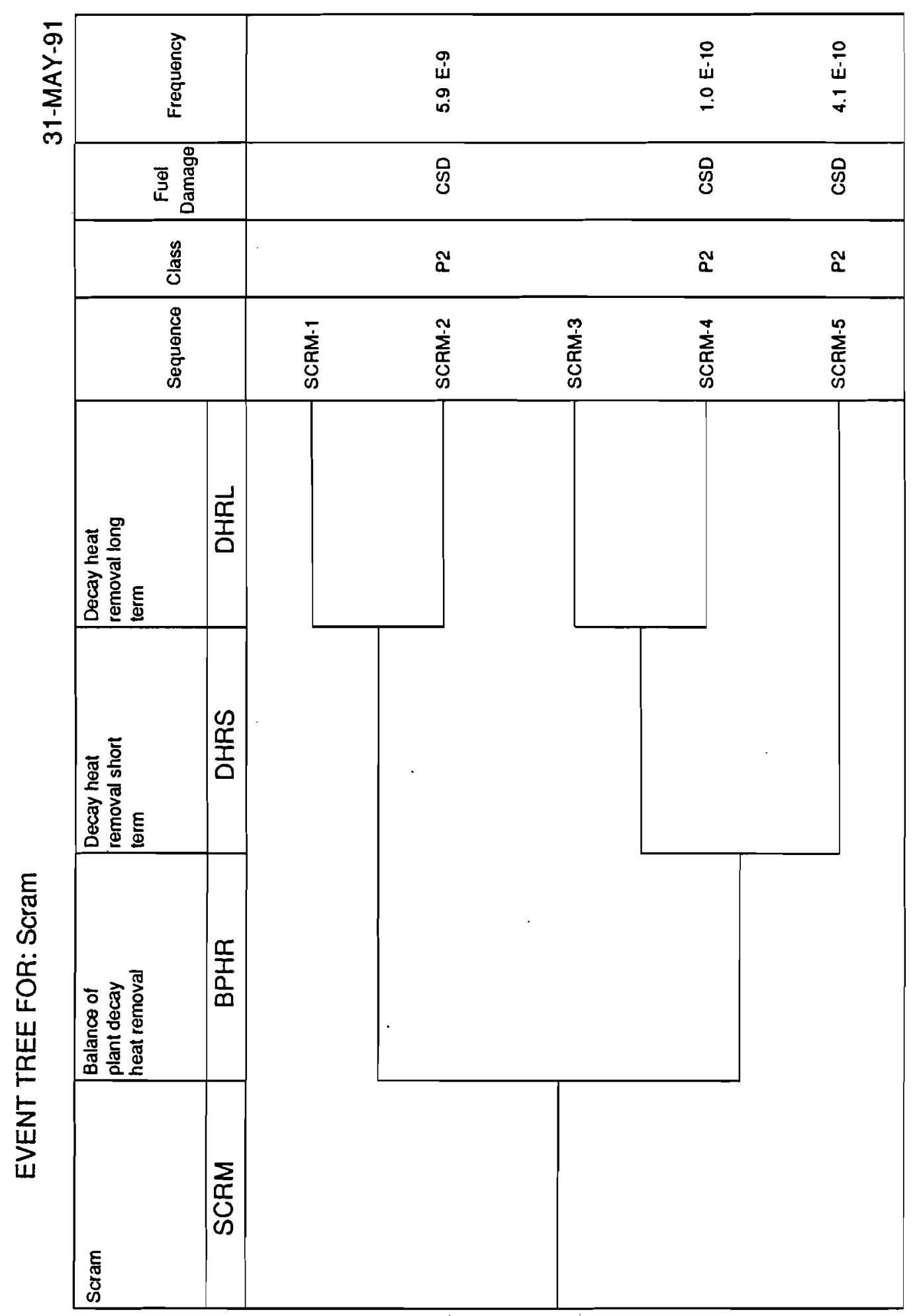

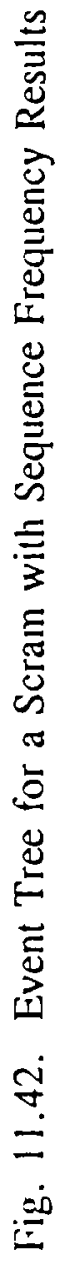




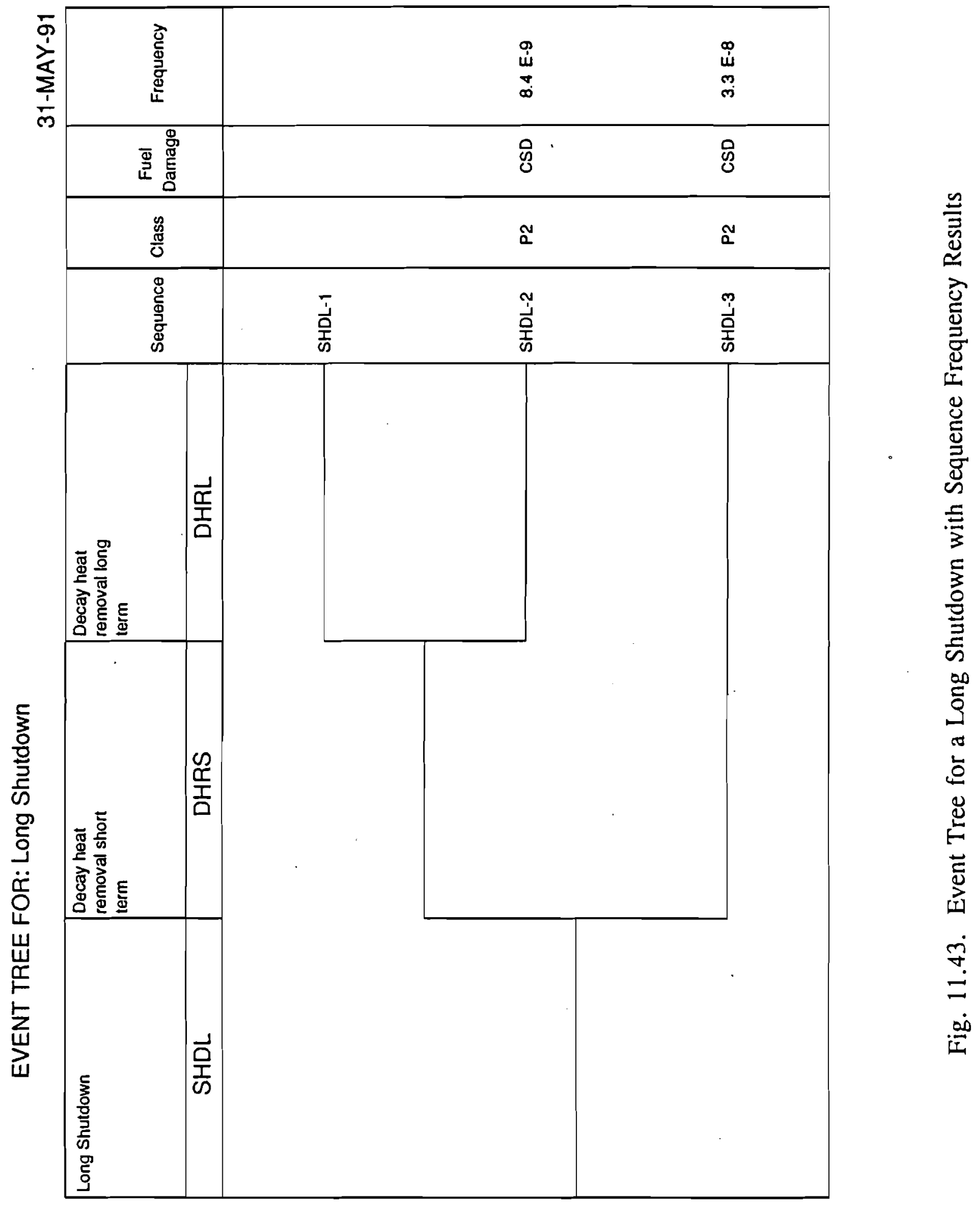


EVENT TREE FOR: Short Shutdown

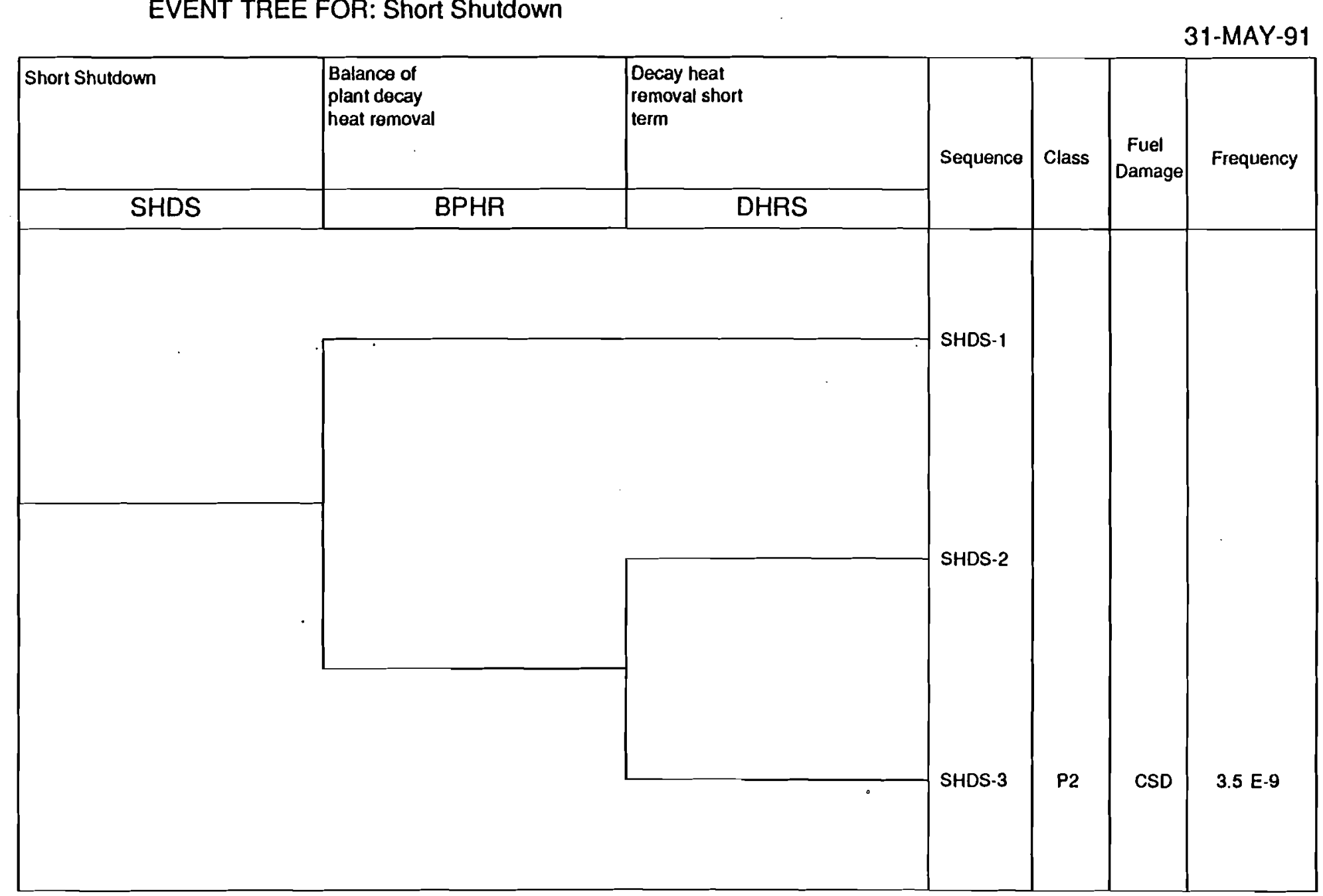

Fig. 11.44. Event Tree for a Short Shutdown with Sequence Frequency Results 


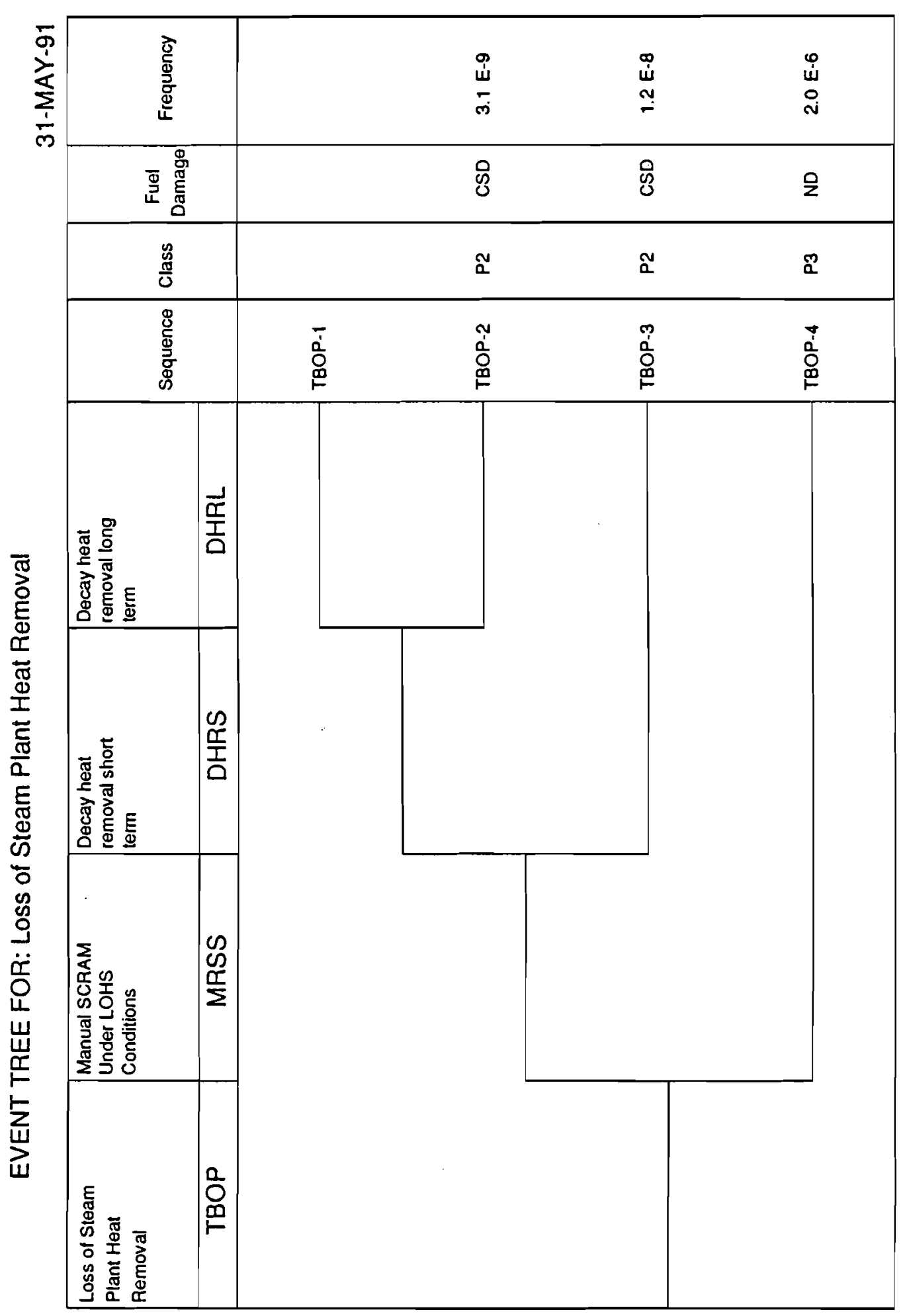

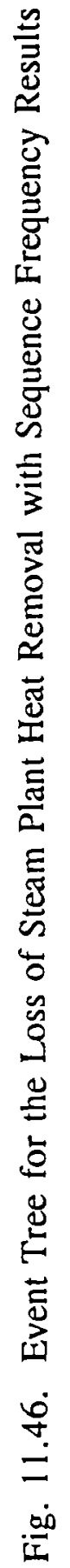




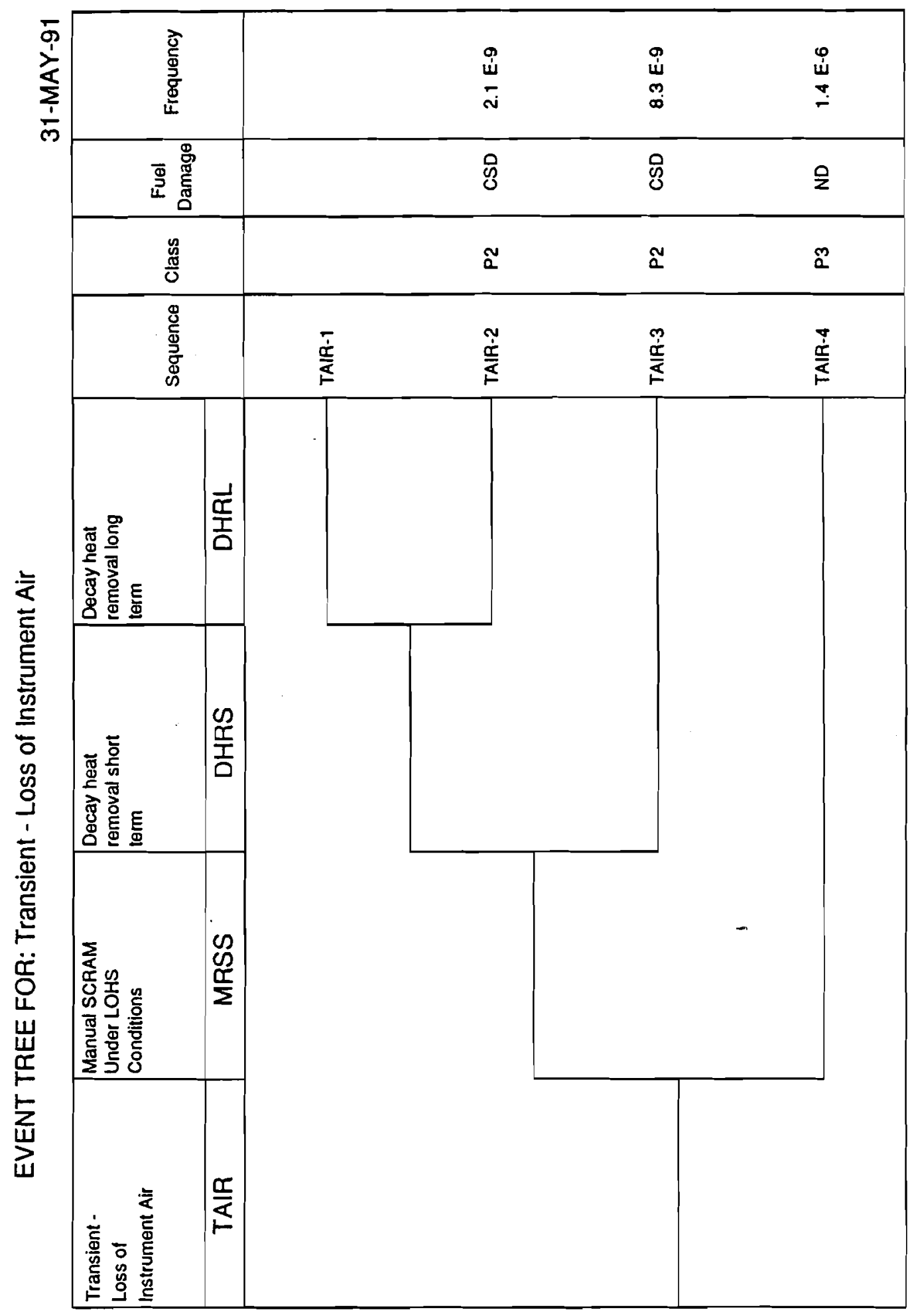

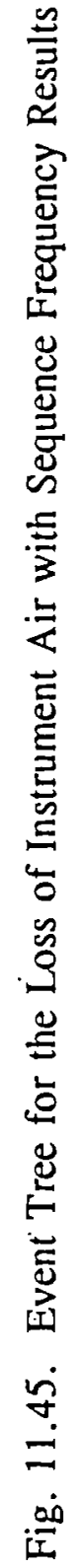




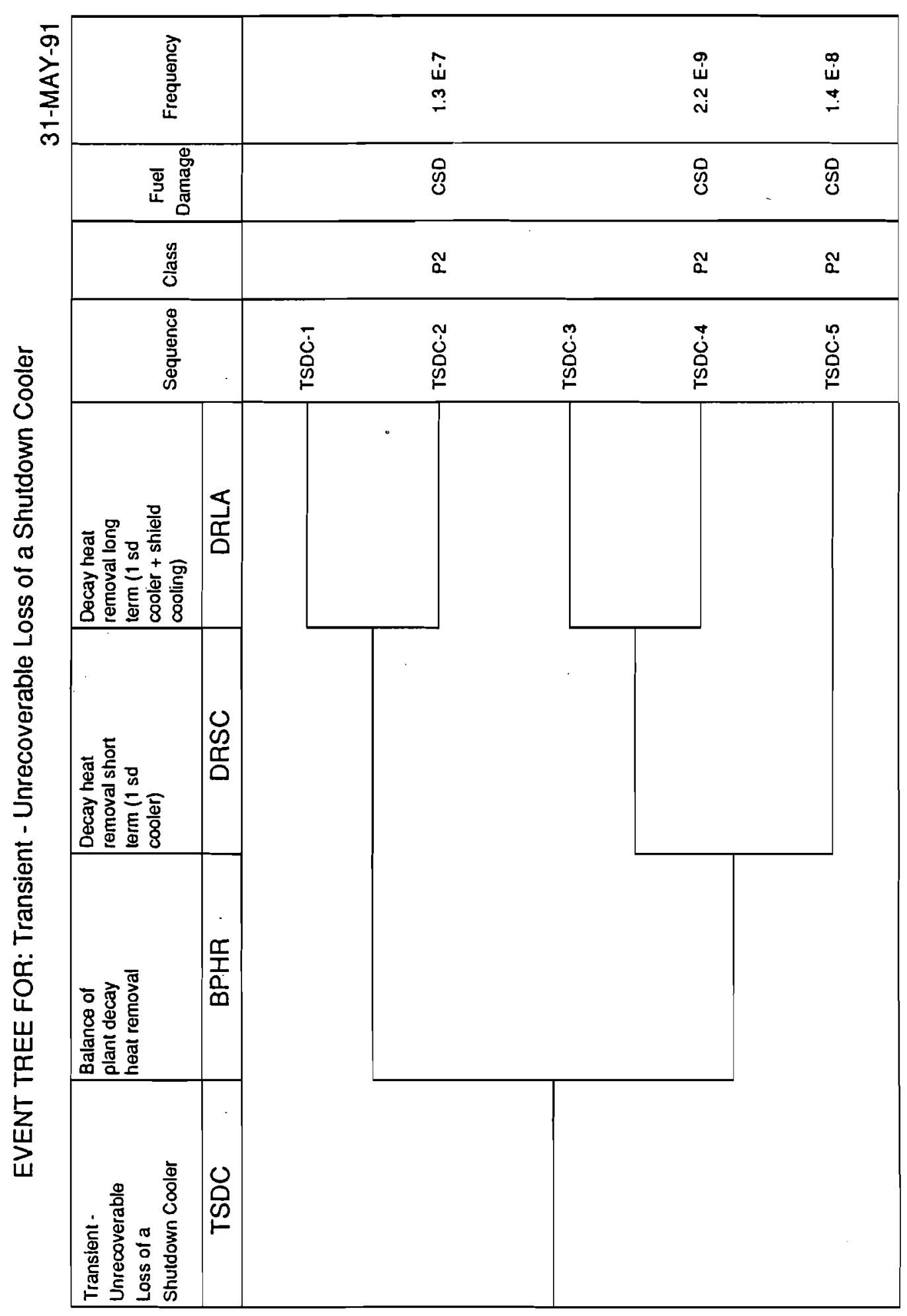

$\frac{5}{0}$

方

4

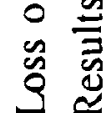

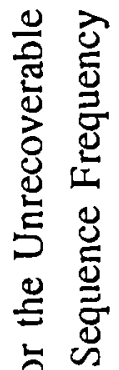

运

E

혼

$\dot{\sigma}$
$\dot{0}$
$\dot{0}$ 
EVENT TREE FOR: Transient - Unrecoverable Loss of Shield Cooling

31-MAY-91

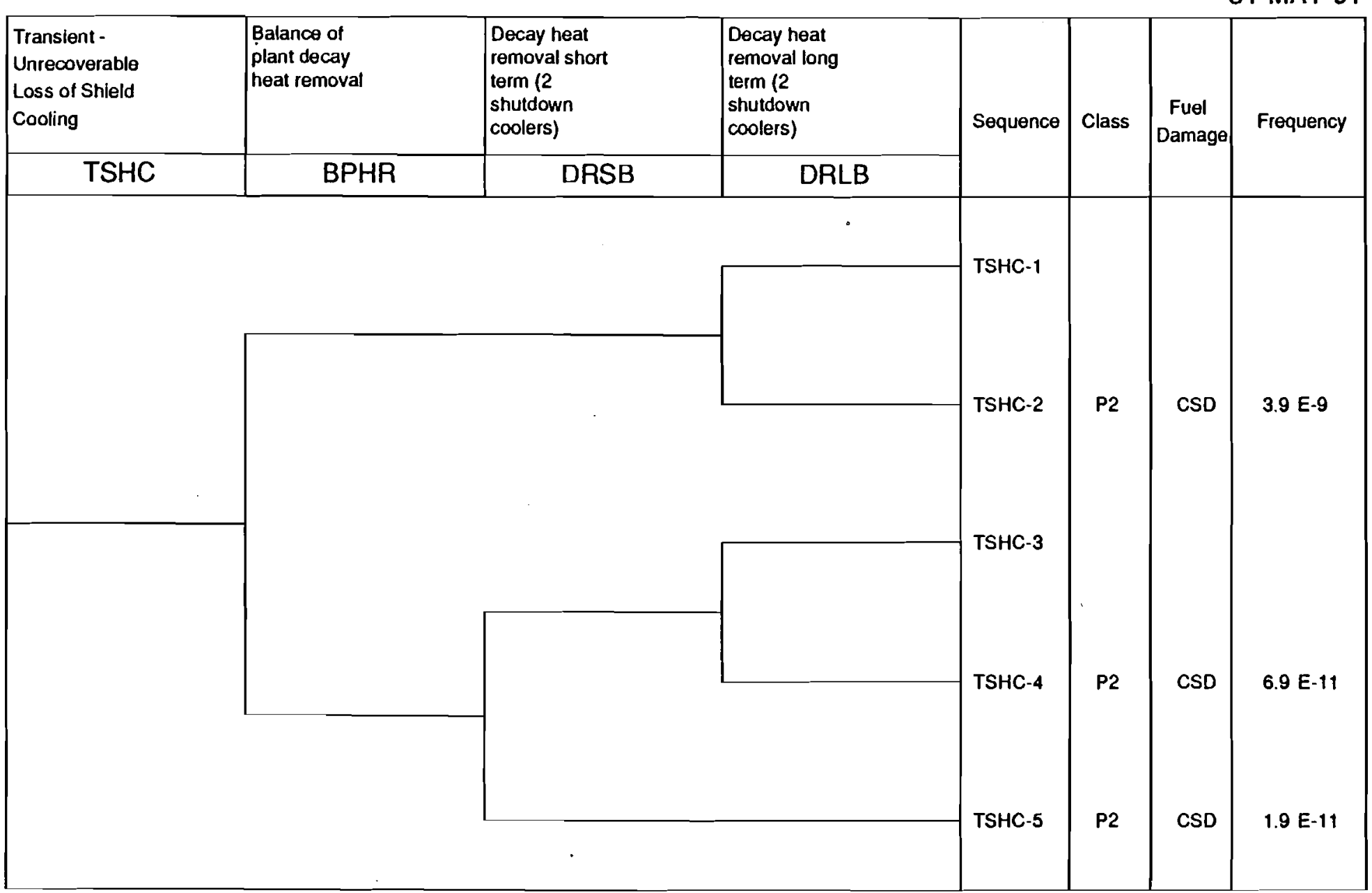

Fig. 11.48. Event Tree for the Unrecoverable Loss of Shield Cooling with Sequence Frequency Results 


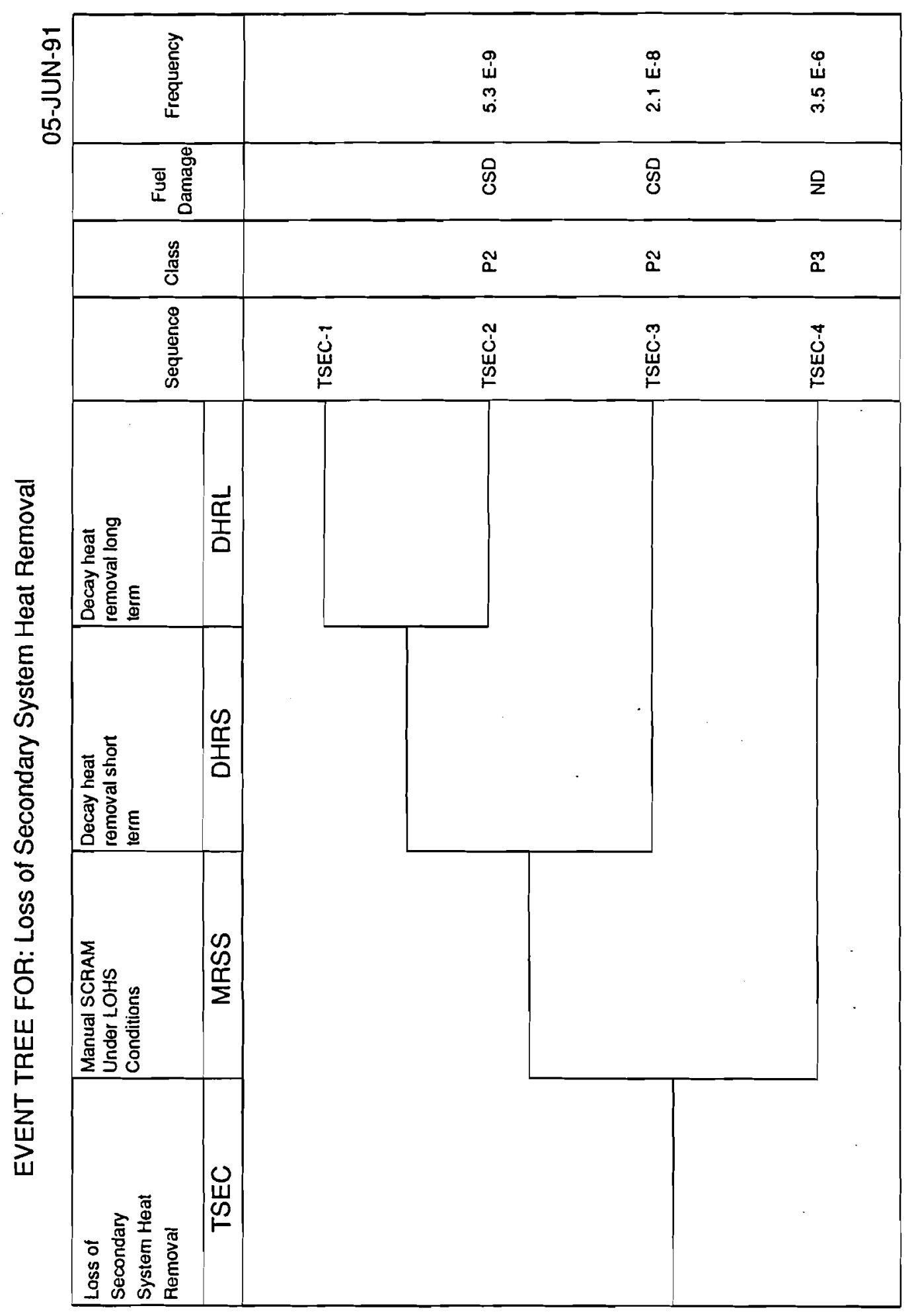

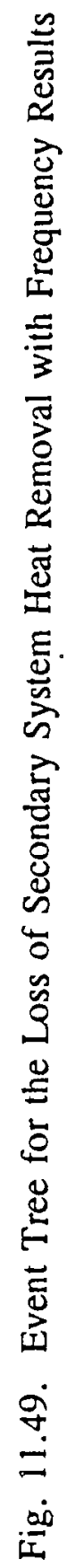




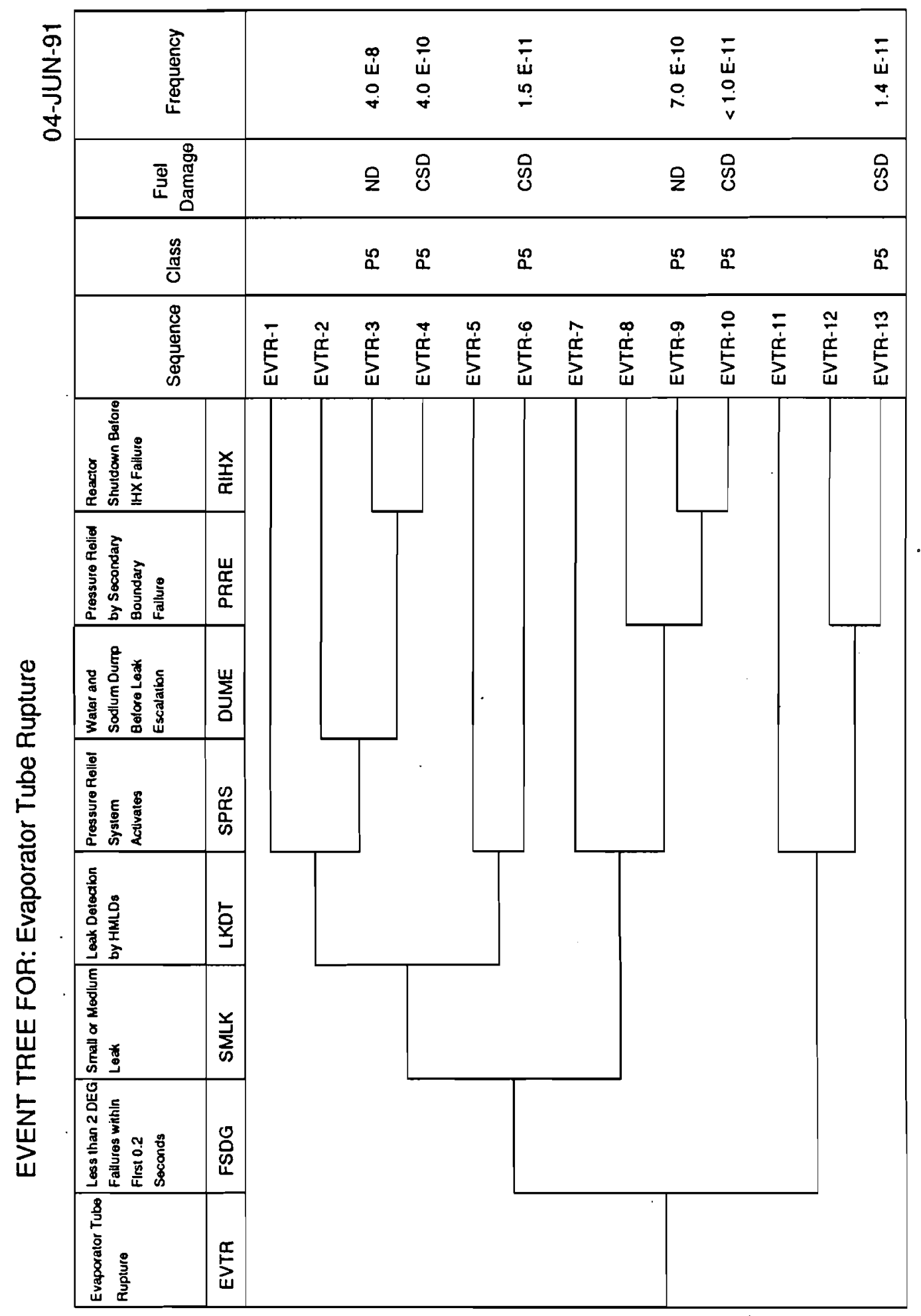

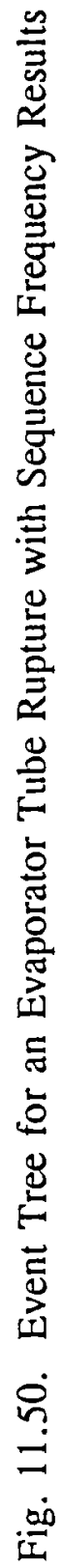




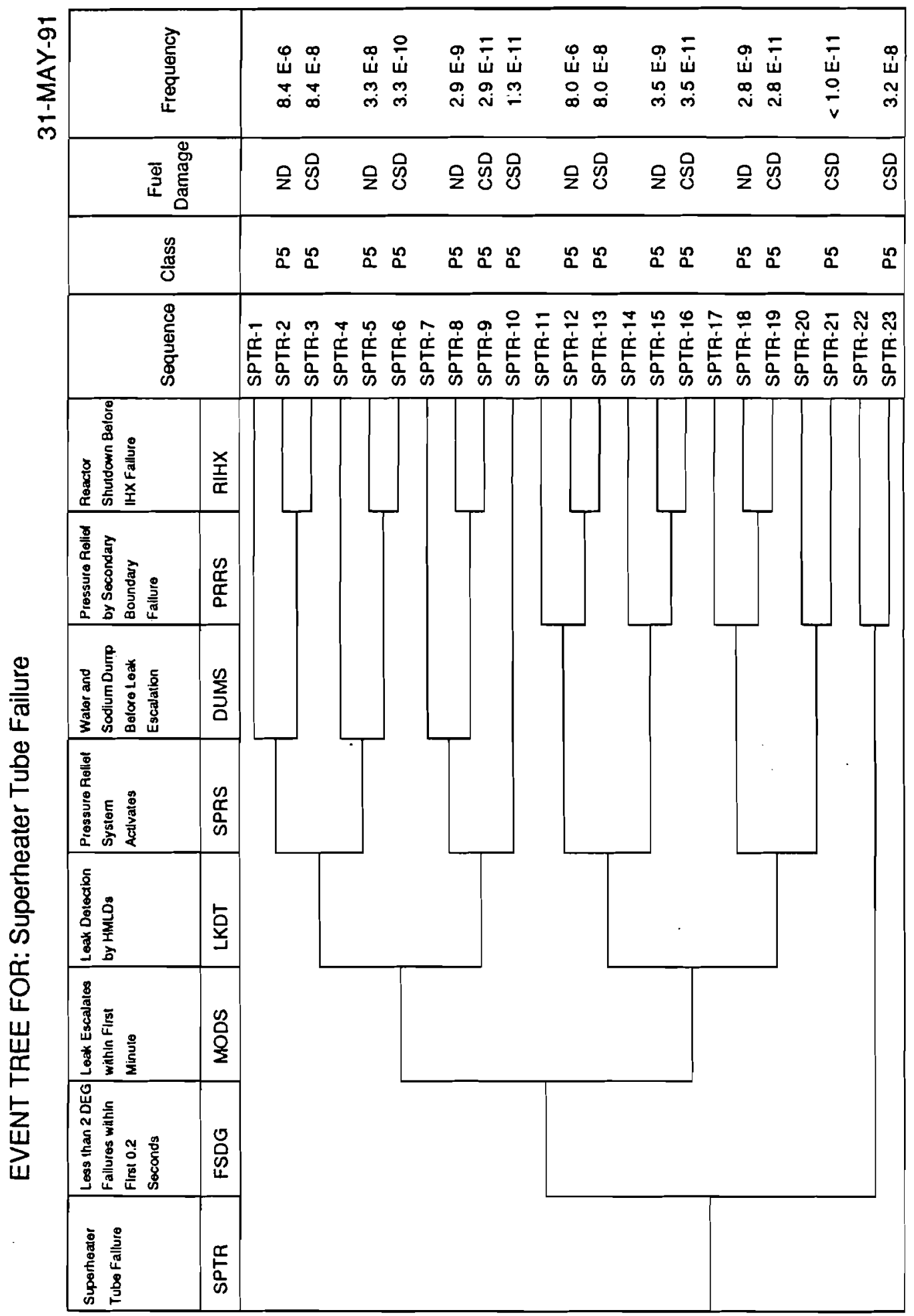

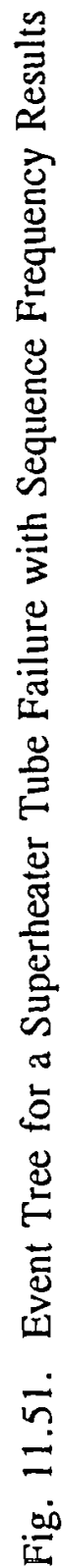


EVENT TREE FOR: Local Faults: Metal Driver Breach Initiator

31-MAY-91

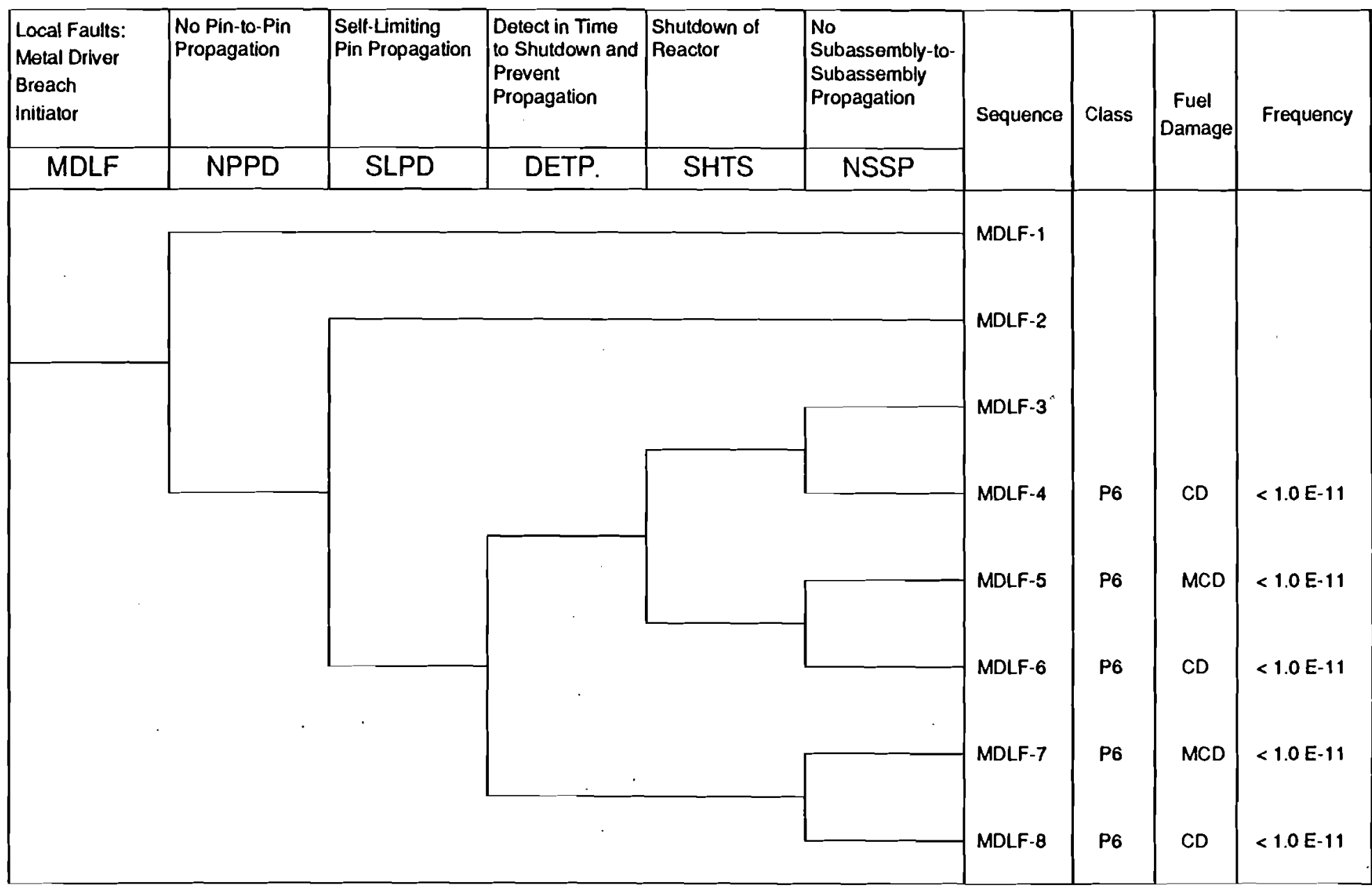

Fig. 11.52. Event Tree for Metal Driver Fuel Breach Local Fault with Sequence Frequency Results 
EVENT TREE FOR: Local Faults: Metal Experiment Breach Initiator

31-MAY-91

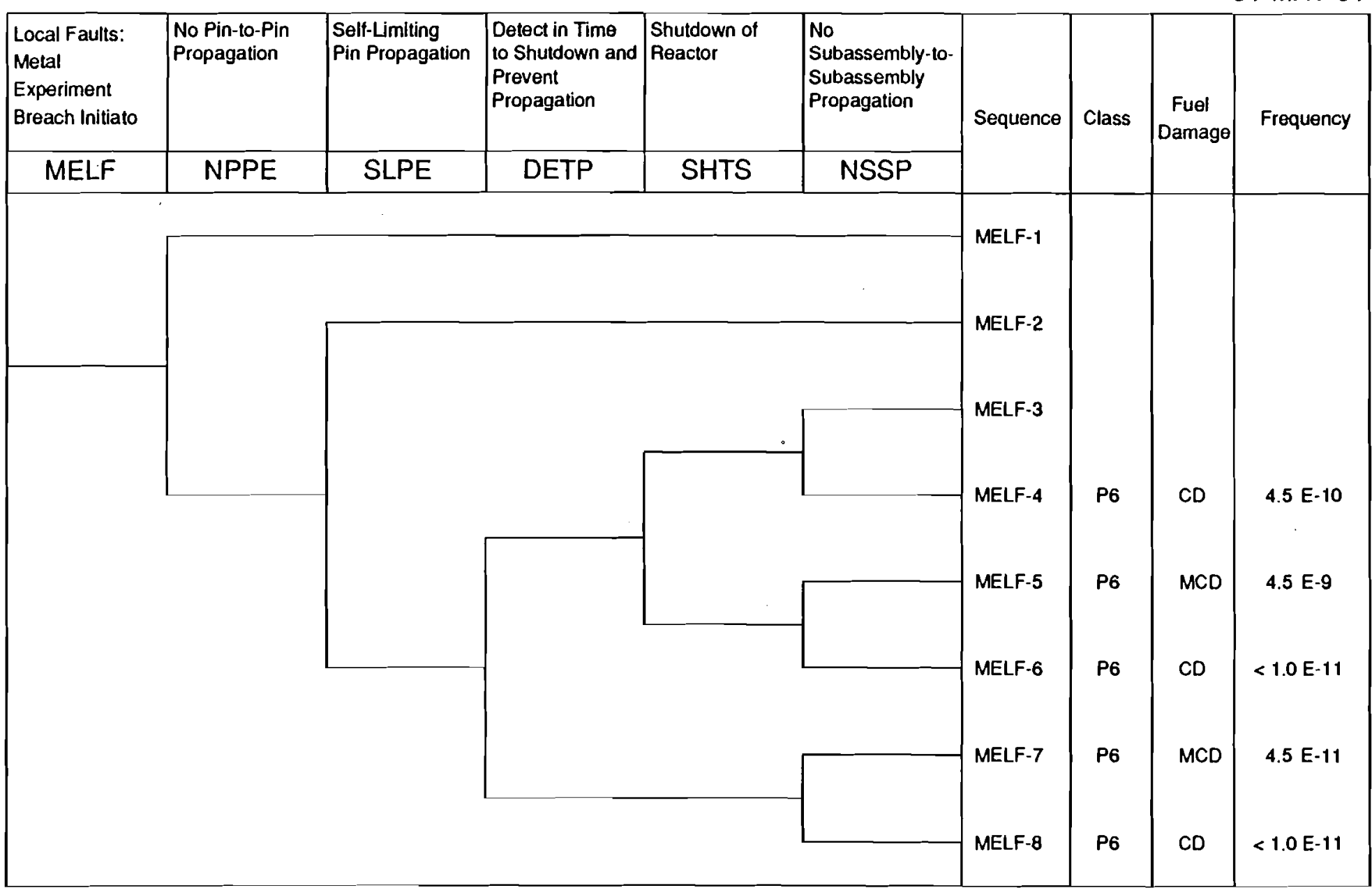

Fig. 11.53. Event Tree for a Metal Experiment Fuel Breach Local Fault with Sequence Frequency Results 


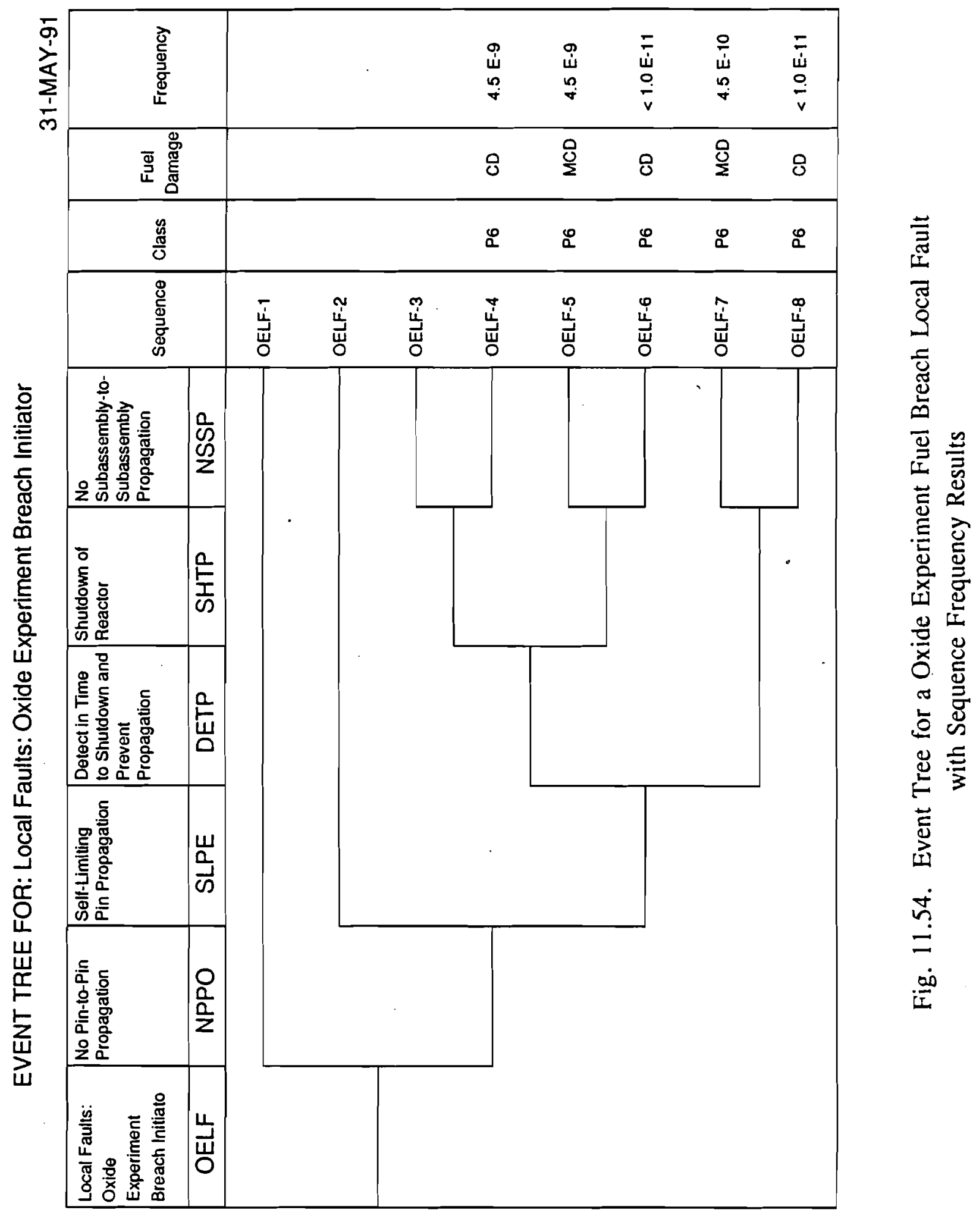


EVENT TREE FOR: Subassembly Local Fault from Partial Blockage

31-MAY-91

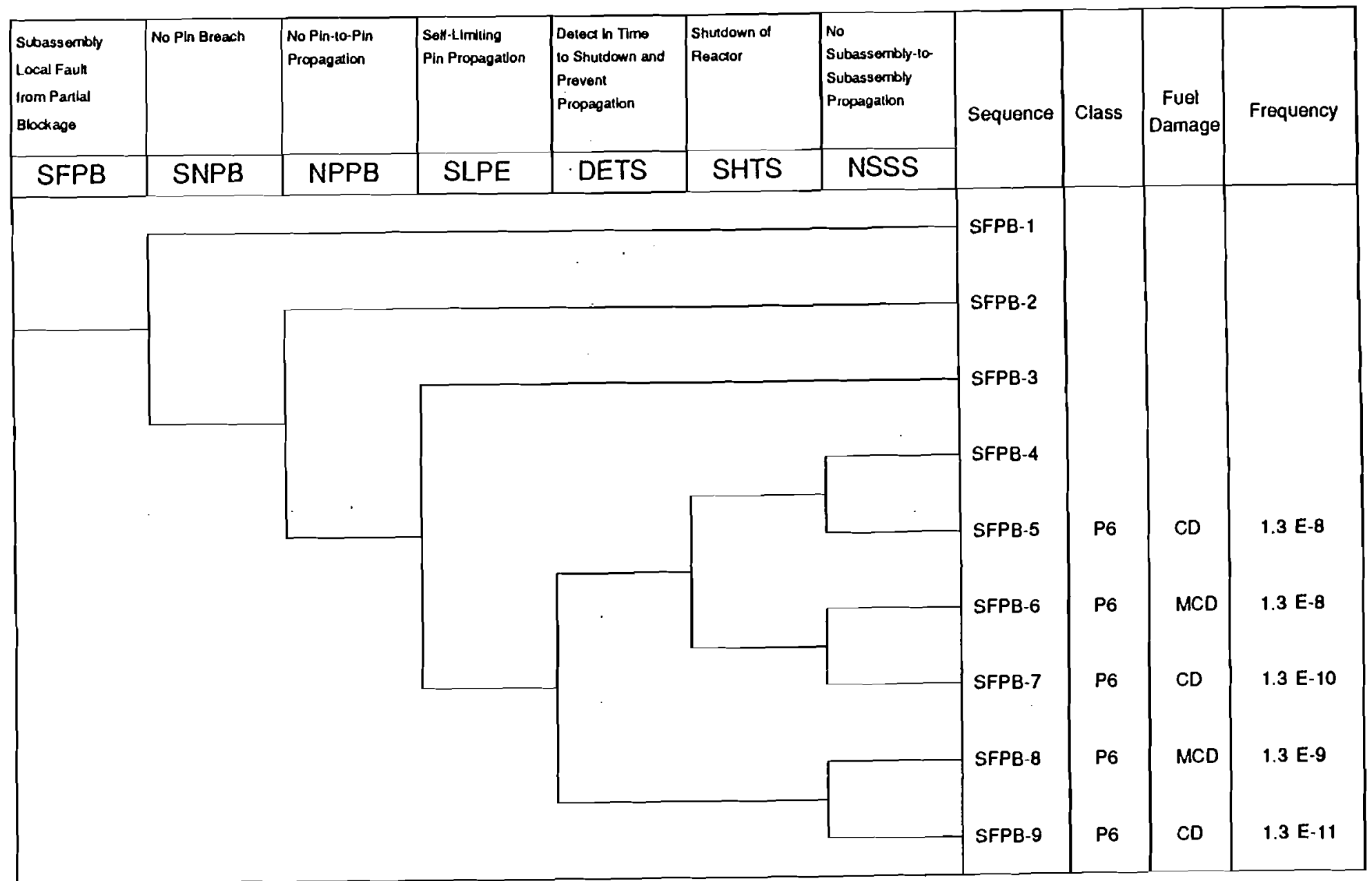

Fig. 11.55. Event Tree for a Subassembly Local Fault from Partial Blockage with Sequence Frequency Results 
EVENT TREE FOR: Subassembly Local Fault from Loading Error

31-MAY-91

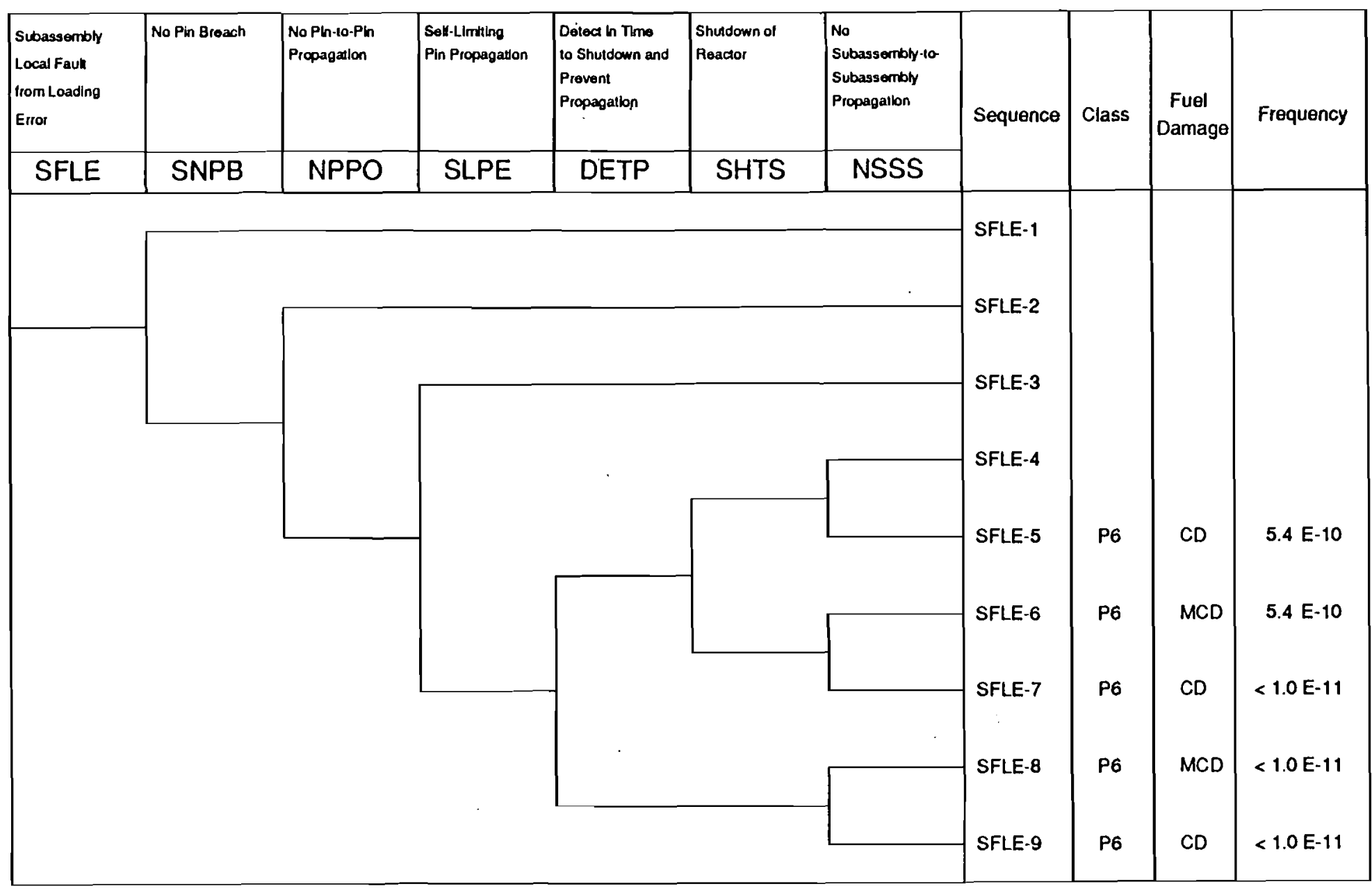

Fig. 11.56. Event Tree for a Subassembly Local Fault from Loading Error with Sequence Frequency Results 
12.0 UNCERTAINTY, SENSITIVITY, AND IMPORTANCE ANALYSES 

12.0 Uncertainty, Sensitivity, and Importance Analyses . . . . . . . . . . . . 12-1

12.1 Introduction . . . . . . . . . . . . . . . . . 12-1

12.2 Uncertainty Analysis . . . . . . . . . . . . . . . . 12-1

12.2.1 Data Uncertainties . . . . . . . . . . . . . . . 12-1

12.2.2 Model Uncertainties . . . . . . . . . . . . . . . . . . 12-8

12.2.3 Completeness Uncertainty . . . . . . . . . . . . 12-8

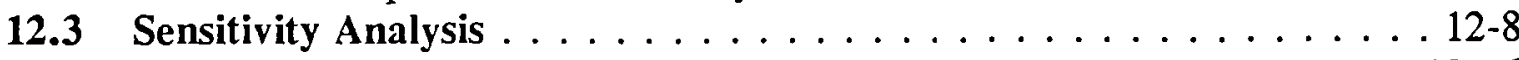

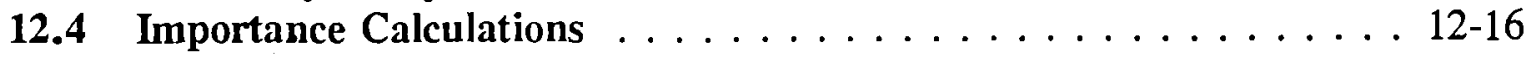




\section{List of Tables}

Page

12.1 Uncertainty Analysis Results by Plant Damage State and Transient Type . . . . . . . . . . . . . . . . . . . . 12-3

12.2 Uncertainty Analysis Results for the Dominant Sequences . . . . . . . . . . . 12-4

12.3 Results of the Sensitivity Analysis; Comparison of Point Mean Values of the Annual Damage Frequency . . . . . . . . . . . . 12-11

12.4 Distributions of the Sensitivity Analysis Cases . . . . . . . . . . . . . . . 12-12

12.5 Results of the Importance Calculations . . . . . . . . . . . . . . . . 12-17 


\section{UNCERTAINTY, SENSITIVITY, AND IMPORTANCE ANALYSES}

\subsection{Introduction}

The previous section presented the results of the accident sequence frequencies in terms of a point mean value estimate. The certainty about these results, however, is an important issue. This section provides a summary of the analyses performed to assess the certainty about the point estimates. The analyses performed cover an analysis of the uncertainty associated with the reliability data, an analysis of the sensitivity of the results to variations in the data for some relevant basic events, and finally an importance evaluation of the basic events involved in the dominant sequences. With the exception of Table 12.3, the tables in this section provide a value labelled "MEAN" for sequence and damage bin frequencies. This mean value is that of the annual frequency distribution obtained with a Monte Carlo sampling method, and does not necessarily coincide, although it is close to, with the point mean value estimate presented in the previous section.

\subsection{Uncertainty Analysis}

In a PRA study, uncertainty can be divided into three principal types according to its source: data uncertainty, model uncertainty, and completeness uncertainty. Some of the uncertainties associated with a PRA can be quantified, but most of the contributions to uncertainty are not amenable to accurate quantitative estimation or calculation.

\subsubsection{Data Uncertainties}

The values of the input parameters are not exactly known, and probability distributions are required to describe the failure rates and unavailabilities of the systems components, the probabilities of human error and the probability of common cause failures. 
Although plant-specific data has been used whenever possible in estimating the probability distributions for the failure rates and unavailabilities of the components, generic sources have been widely used.

Uncertainty is also associated with the estimated frequency of the initiating events. When these estimates have been obtained from plant records, statistical methods have been used to quantify the uncertainty, as explained in Section 5. For those initiating events whose frequency was estimated with the use of systems fault trees, the uncertainty of the basic events in the fault tree was propagated through the fault tree model using a Monte Carlo simulation.

Each basic event in the plant models has an uncertainty associated with the unreliability or unavailability that it represents. Throughout the analysis, a lognormal distribution has always been assumed for the probability of the basic events. The two parameters of choice to describe a lognormal distribution are usually a mean or a median value and the error factor, defined as the ratio of the 95th percentile of the distribution to the median. The error factors used in describing the lognormal distributions of the basic events have been listed in Section 7 along with the remaining basic event data, and are not repeated here.

The results of the uncertainty analysis presented below represent essentially the propagation of the data uncertainties through the fault tree and event tree models to quantify the uncertainty of the sequences, damage bins, and total plant damage estimates. The distributions have been estimated by using a Monte Carlo simulation of 5000 samples. In quantifying the sequence distributions, random values were drawn for all events appearing in the minimal cutsets of the sequence. Similarly, in estimating the damage bin distributions, the samples were drawn from the events distributions rather than from the sequence distributions previously estimated. Table 12.1 provides the uncertainty results by plant damage state. Table 12.2 list the uncertainty analysis results for the dominant accident sequences. 
Table 12.1 Uncertainty Analysis Results by Plant Damage State and Transient Type

PLANT TRANSIENT:

\begin{tabular}{|c|c|c|c|c|c|c|c|c|c|}
\hline \multirow{2}{*}{$\begin{array}{l}\text { P1 } \\
\text { P2 }\end{array}$} & & 2.86 & $10^{-8}$ & 1.09 & $10^{-9}$ & 1.10 & $10^{-8}$ & 1.10 & $10^{-7}$ \\
\hline & & 2.70 & $10^{-7}$ & 2.31 & $10^{-8}$ & 1.19 & $10^{-7}$ & 9.28 & $10^{-7}$ \\
\hline & P3-CD & 4.49 & $10^{-8}$ & 2.25 & $10^{-9}$ & 1.59 & $10^{-8}$ & 1.48 & $10^{-7}$ \\
\hline & P3-MCD & 3.63 & $10^{-7}$ & 1.29 & $10^{-8}$ & 9.06 & $10^{-8}$ & 1.24 & $10^{-6}$ \\
\hline & P3-PED & 5.68 & $10^{-7}$ & 1.80 & $10^{-8}$ & 1.77 & $10^{-7}$ & 2.10 & $10^{-6}$ \\
\hline & P3-ND & 1.27 & $10^{-5}$ & 1.91 & $10^{-6}$ & 7.59 & $10^{-6}$ & 3.77 & $10^{-5}$ \\
\hline P3 & & 1.41 & $10^{-5}$ & 2.11 & $10^{-6}$ & 8.06 & $10^{-6}$ & 4.18 & $10^{-5}$ \\
\hline & P4-MCD & 2.77 & $10^{-9}$ & 2.23 & $10^{-11}$ & 4.51 & $10^{-10}$ & 1.02 & $10^{-8}$ \\
\hline & P4-PED & 1.12 & $10^{-8}$ & 4.13 & $10^{-10}$ & 4.01 & $10^{-9}$ & 4.23 & $10^{-8}$ \\
\hline P4 & & 1.40 & $10^{-8}$ & 8.36 & $10^{-10}$ & 5.96 & $10^{-9}$ & 5.05 & $10^{-8}$ \\
\hline & P5-CSD & 2.04 & $10^{-7}$ & 1.15 & $10^{-9}$ & 2.77 & $10^{-8}$ & 7.02 & $10^{-7}$ \\
\hline & P5-ND & 1.55 & $10^{-5}$ & 3.75 & $10^{-7}$ & 4.91 & $10^{-6}$ & 6.19 & $10^{-5}$ \\
\hline P5 & & 1.83 & $10^{-5}$ & 3.96 & $10^{-7}$ & 5.24 & $10^{-6}$ & 6.44 & $10^{-5}$ \\
\hline & P6-CD & 1.57 & $10^{-8}$ & 1.00 & $10^{-10}$ & 2.25 & $10^{-9}$ & 5.45 & $10^{-8}$ \\
\hline & P6-MCD & 2.83 & $10^{-8}$ & 1.52 & $10^{-10}$ & 3.36 & $10^{-9}$ & 7.82 & $10^{-8}$ \\
\hline P6 & & 4.80 & $10^{-8}$ & 3.77 & $10^{-10}$ & 7.30 & $10^{-9}$ & 1.52 & $10^{-7}$ \\
\hline
\end{tabular}

\section{DAMAGE CLASS :}

\begin{tabular}{llllllll} 
CD & $9.3610^{-8}$ & $1.0710^{-8}$ & $4.8410^{-8}$ & $2.9210^{-7}$ \\
$M C D$ & $4.4510^{-7}$ & $1.8610^{-8}$ & $1.1410^{-7}$ & $1.2410^{-6}$ \\
CSD & $4.4410^{-7}$ & $3.9810^{-8}$ & $2.0010^{-7}$ & $1.4810^{-6}$ \\
PED & $5.8010^{-7}$ & $2.6010^{-8}$ & $1.8610^{-7}$ & $2.1810^{-6}$ \\
DAMAGE & $1.5310^{-6}$ & $2.4610^{-7}$ & $8.4810^{-7}$ & $4.7410^{-6}$ \\
ND & $2.8410^{-5}$ & $4.0810^{-6}$ & $1.6010^{-5}$ & $8.8110^{-5}$ \\
TOTAL & $2.9610^{-5}$ & $4.8810^{-6}$ & $1.7410^{-5}$ & $9.2210^{-5}$ \\
\hline
\end{tabular}


Table 12.2 Uncertainty Analysis Results for the Dominant Sequences

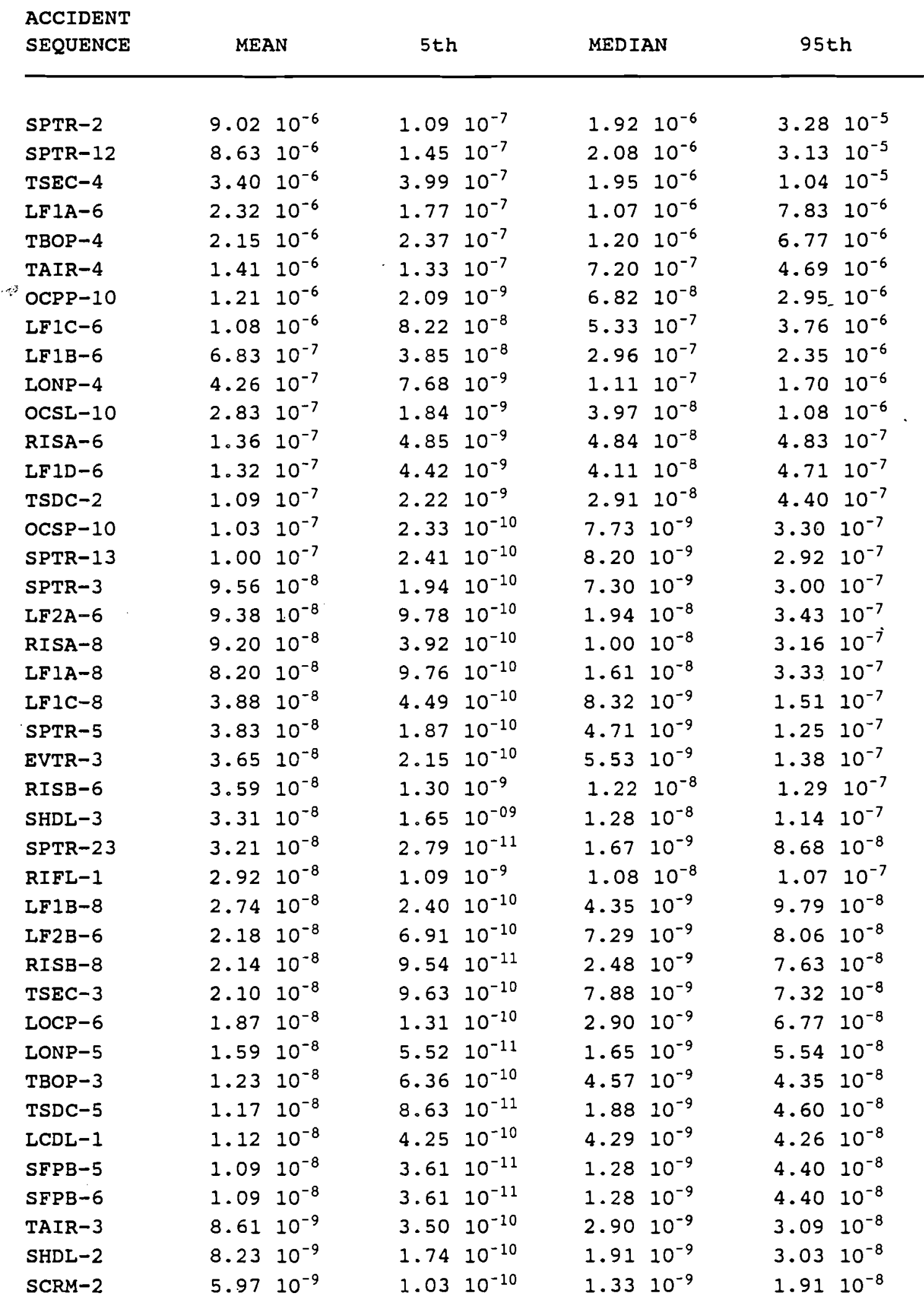


Table 12.2 Uncertainty Analysis Results for the Dominant Sequences (Cont'd)

\begin{tabular}{|c|c|c|c|c|c|c|c|c|}
\hline $\begin{array}{l}\text { ACCIDENT } \\
\text { SEQUENCE }\end{array}$ & \multicolumn{2}{|c|}{ MEAN } & \multicolumn{2}{|c|}{5 th } & \multicolumn{2}{|c|}{ MEDIAN } & \multicolumn{2}{|l|}{$95 t h$} \\
\hline RIFS- 6 & 5.29 & $10^{-9}$ & 5.84 & $10^{-11}$ & 9.87 & $10^{-10}$ & 1.81 & $10^{-8}$ \\
\hline TSEC-2 & 5.05 & $10^{-9}$ & 1.00 & $10^{-10}$ & 1.25 & $10^{-9}$ & 1.86 & $10^{-8}$ \\
\hline LF $1 D-8$ & 4.15 & $10^{-9}$ & 2.70 & $10^{-11}$ & 6.32 & $10^{-10}$ & 1.60 & $10^{-8}$ \\
\hline SPTR-15 & 4.07 & $10^{-9}$ & 1.06 & $10^{-11}$ & 3.64 & $10^{-10}$ & 1.27 & $10^{-8}$ \\
\hline$L F 1 A-7$ & 4.07 & $10^{-9}$ & 3.92 & $10^{-11}$ & 7.84 & $10^{-10}$ & 1.58 & $10^{-8}$ \\
\hline OELF-4 & 3.82 & $10^{-9}$ & 3.19 & $10^{-12}$ & 1.77 & $10^{-10}$ & 1.11 & $10^{-8}$ \\
\hline OELF-5 & 3.82 & $10^{-9}$ & 3.19 & $10^{-12}$ & 1.77 & $10^{-10}$ & 1.11 & $10^{-8}$ \\
\hline TSHC-2 & 3.63 & $10^{-9}$ & 3.57 & $10^{-11}$ & 6.22 & $10^{-10}$ & 1.37 & $10^{-8}$ \\
\hline$L F 1 A-2$ & 3.62 & $10^{-9}$ & 6.53 & $10^{-11}$ & 9.12 & $10^{-10}$ & 1.39 & $10^{-8}$ \\
\hline RIFS- 8 & 3.55 & $10^{-9}$ & 5.55 & $10^{-12}$ & 2.02 & $10^{-10}$ & 8.81 & $10^{-9}$ \\
\hline LONP-3 & 3.47 & $10^{-9}$ & 3.18 & $10^{-11}$ & 5.77 & $10^{-10}$ & 1.18 & $10^{-8}$ \\
\hline MELF-5 & 3.45 & $10^{-9}$ & 3.19 & $10^{-12}$ & 1.87 & $10^{-10}$ & 1.23 & $10^{-8}$ \\
\hline$L F 2 A-7$ & 3.36 & $10^{-9}$ & 7.92 & $10^{-12}$ & 2.68 & $10^{-10}$ & 1.12 & $10^{-8}$ \\
\hline SPTR-8 & 3.28 & $10^{-9}$ & 6.91 & $10^{-12}$ & 2.59 & $10^{-10}$ & .9 .76 & $10^{-9}$ \\
\hline SPTR- 18 & 3.24 & $10^{-9}$ & 8.43 & $10^{-12}$ & 2.90 & $10^{-10}$ & 1.01 & $10^{-8}$ \\
\hline SHDS-3 & 3.14 & $10^{-9}$ & 2.96 & $10^{-11}$ & 5.10 & $10^{-10}$ & 1.26 & $10^{-8}$ \\
\hline TBOP-2 & 2.75 & $10^{-9}$ & 6.43 & $10^{-11}$ & 7.45 & $10^{-10}$ & 1.08 & $10^{-8}$ \\
\hline OCPP-2 & 2.48 & $10^{-9}$ & 1.17 & $10^{-11}$ & 2.82 & $10^{-10}$ & 8.35 & $10^{-9}$ \\
\hline$L F 2 D-1$ & 2.42 & $10^{-9}$ & 2.10 & $10^{-11}$ & 4.50 & $10^{-10}$ & 9.34 & $10^{-9}$ \\
\hline $\mathrm{LF} 1 \mathrm{C}-7$ & 2.30 & $10^{-9}$ & 2.12 & $10^{-11}$ & 3.94 & $10^{-10}$ & 8.74 & $10^{-9}$ \\
\hline TSDC-4 & 2.13 & $10^{-9}$ & 8.32 & $10^{-12}$ & 2.14 & $10^{-10}$ & 7.76 & $10^{-9}$ \\
\hline $\mathrm{LF} 1 \mathrm{C}-2$ & 1.84 & $10^{-9}$ & 3.51 & $10^{-11}$ & 4.60 & $10^{-10}$ & 6.95 & $10^{-9}$ \\
\hline TAIR-2 & 1.79 & $10^{-9}$ & 3.57 & $10^{-11}$ & 4.59 & $10^{-10}$ & 6.85 & $10^{-9}$ \\
\hline$L F 2 F-6$ & 1.66 & $10^{-9}$ & 4.24 & $10^{-11}$ & 5.13 & $10^{-10}$ & 6.07 & $10^{-9}$ \\
\hline LF1B-7 & 1.29 & $10^{-9}$ & 1.00 & $10^{-11}$ & 2.21 & $10^{-10}$ & 5.22 & $10^{-9}$ \\
\hline OCPP-9 & 1.24 & $10^{-9}$ & 3.64 & $10^{-12}$ & 1.26 & $10^{-10}$ & 4.79 & $10^{-9}$ \\
\hline LF 1B-2 & 1.19 & $10^{-9}$ & 1.67 & $10^{-11}$ & 2.54 & $10^{-10}$ & 4.25 & $10^{-9}$ \\
\hline SFPB-8 & 1.09 & $10^{-9}$ & 3.61 & $10^{-12}$ & 1.28 & $10^{-10}$ & 4.40 & $10^{-9}$ \\
\hline LF $2 C-6$ & 1.03 & $10^{-9}$ & 1.29 & $10^{-11}$ & 2.22 & $10^{-10}$ & 3.54 & $10^{-9}$ \\
\hline LOCP-2 & 8.79 & $10^{-10}$ & 3.77 & $10^{-12}$ & 9.36 & $10^{-11}$ & 2.88 & $10^{-9}$ \\
\hline $\mathrm{L} F 2 \mathrm{~B}-7$ & 8.41 & $10^{-10}$ & 4.44 & $10^{-12}$ & 1.13 & $10^{-10}$ & 2.67 & $10^{-9}$ \\
\hline ocsL-2 & 8.26 & $10^{-10}$ & 1.11 & $10^{-11}$ & 1.65 & $10^{-10}$ & 2.77 & $10^{-9}$ \\
\hline LONP-2 & 7.44 & $10^{-10}$ & 3.71 & $10^{-12}$ & 9.14 & $10^{-11}$ & 2.75 & $10^{-9}$ \\
\hline EVTR-9 & 7.30 & $10^{-10}$ & 5.45 & $10^{-13}$ & 3.32 & $10^{-11}$ & 2.06 & $10^{-9}$ \\
\hline SFLE-5 & 6.74 & $10^{-10}$ & 3.08 & $10^{-13}$ & 2.19 & $10^{-11}$ & 1.38 & $10^{-9}$ \\
\hline SFLE-6 & 6.74 & $10^{-10}$ & 3.08 & $10^{-13}$ & 2.19 & $10^{-11}$ & 1.38 & $10^{-9}$ \\
\hline EVTR-4 & 4.94 & $10^{-10}$ & 4.08 & $10^{-13}$ & 2.08 & $10^{-11}$ & 1.08 & $10^{-9}$ \\
\hline
\end{tabular}


Table 12.2 Uncertainty Analysis Results for the Dominant Sequences (Cont'd)

\begin{tabular}{|c|c|c|c|c|c|c|c|c|}
\hline \multirow{2}{*}{$\begin{array}{l}\text { ACCIDENT } \\
\text { SEQUENCE }\end{array}$} & \multirow{2}{*}{\multicolumn{2}{|c|}{ MEAN }} & \multirow{2}{*}{\multicolumn{2}{|c|}{5 th }} & \multirow{2}{*}{\multicolumn{2}{|c|}{ MEDIAN }} & \multirow{2}{*}{\multicolumn{2}{|c|}{95 th }} \\
\hline & & & & & & & & \\
\hline OCSL-9 & 4.67 & $10^{-10}$ & 3.26 & $10^{-12}$ & 7.13 & $10^{-11}$ & 1.69 & $10^{-9}$ \\
\hline OELF- 7 & 3.82 & $10^{-10}$ & 3.19 & $10^{-13}$ & 1.77 & $10^{-11}$ & 1.11 & $10^{-9}$ \\
\hline SCRM-5 & 3.71 & $10^{-10}$ & 3.24 & $10^{-12}$ & 6.35 & $10^{-11}$ & 1.44 & $10^{-9}$ \\
\hline SPTR- 6 & 3.57 & $10^{-10}$ & 3.03 & $10^{-13}$ & 1.91 & $10^{-11}$ & 9.40 & $10^{-10}$ \\
\hline MELF-4 & 3.45 & $10^{-10}$ & 3.19 & $10^{-13}$ & 1.87 & $10^{-11}$ & 1.23 & $10^{-9}$ \\
\hline RISA-2 & 3.19 & $10^{-10}$ & 2.09 & $10^{-12}$ & 3.96 & $10^{-11}$ & 8.06 & $10^{-10}$ \\
\hline ANTC-3 & 3.07 & $10^{-10}$ & 1.95 & $10^{-12}$ & 3.97 & $10^{-11}$ & 1.15 & $10^{-9}$ \\
\hline OCSP-2 & 2.70 & $10^{-10}$ & 1.34 & $10^{-12}$ & 3.09 & $10^{-11}$ & 9.18 & $10^{-10}$ \\
\hline LF $1 A-5$ & 2.62 & $10^{-10}$ & 2.10 & $10^{-12}$ & 4.12 & $10^{-11}$ & 1.02 & $10^{-9}$ \\
\hline RISA-7 & 2.58 & $10^{-10}$ & 1.39 & $10^{-12}$ & 3.76 & $10^{-11}$ & 9.85 & $10^{-10}$ \\
\hline LF $1 D-7$ & 2.41 & $10^{-10}$ & 1.27 & $10^{-12}$ & 3.16 & $10^{-11}$ & 8.72 & $10^{-10}$ \\
\hline$L F 1 D-2$ & 1.97 & $10^{-10}$ & 2.00 & $10^{-12}$ & 3.65 & $10^{-11}$ & 7.22 & $10^{-10}$ \\
\hline OCPP -5 & 1.57 & $10^{-10}$ & 3.86 & $10^{-13}$ & 1.27 & $10^{-11}$ & 4.65 & $10^{-10}$ \\
\hline$L F 1 C-5$ & 1.53 & $10^{-10}$ & 1.09 & $10^{-12}$ & 1.96 & $10^{-11}$ & 4.93 & $10^{-10}$ \\
\hline$L F 2 A-2$ & 1.50 & $10^{-10}$ & 4.95 & $10^{-13}$ & 1.57 & $10^{-11}$ & 5.72 & $10^{-10}$ \\
\hline OCSP-9 & 1.48 & $10^{-10}$ & 4.02 & $10^{-13}$ & 1.32 & $10^{-11}$ & 4.87 & $10^{-10}$ \\
\hline SCRM-4 & 1.34 & $10^{-10}$ & 3.90 & $10^{-13}$ & 9.51 & $10^{-12}$ & 3.25 & $10^{-10}$ \\
\hline SFPB-7 & 1.27 & $10^{-10}$ & 6.48 & $10^{-14}$ & 4.63 & $10^{-12}$ & 3.11 & $10^{-10}$ \\
\hline $\mathrm{L} F 1 \mathrm{~B}-5$ & 8.65 & $10^{-11}$ & 5.70 & $10^{-13}$ & 1.07 & $10^{-11}$ & 3.10 & $10^{-10}$ \\
\hline$L F \perp A-4$ & 8.36 & $10^{-11}$ & 2.61 & $10^{-13}$ & 6.20 & $10^{-12}$ & 2.23 & $10^{-10}$ \\
\hline TSHC-4 & 7.99 & $10^{-11}$ & 1.26 & $10^{-13}$ & 4.52 & $10^{-12}$ & 2.05 & $10^{-10}$ \\
\hline RISB-7 & 6.66 & $10^{-11}$ & 3.49 & $10^{-13}$ & 9.20 & $10^{-12}$ & 2.31 & $10^{-10}$ \\
\hline RISB-2 & 6.26 & $10^{-11}$ & 5.58 & $10^{-13}$ & 1.02 & $10^{-11}$ & 2.12 & $10^{-10}$ \\
\hline OCsL -5 & 5.44 & $10^{-11}$ & 3.53 & $10^{-13}$ & 7.37 & $10^{-12}$ & 1.95 & $10^{-10}$ \\
\hline$L F 2 F-7$ & 5.21 & $10^{-11}$ & 2.63 & $10^{-13}$ & 7.27 & $10^{-12}$ & 1.97 & $10^{-10}$ \\
\hline LOCP -5 & 5.17 & $10^{-11}$ & 1.34 & $10^{-13}$ & 4.57 & $10^{-12}$ & 1.66 & $10^{-10}$ \\
\hline $\mathrm{LF} 2 \mathrm{C}-7$ & 4.50 & $10^{-11}$ & 9.73 & $10^{-14}$ & 3.40 & $10^{-12}$ & 1.40 & $10^{-10}$ \\
\hline OCPP -4 & 4.37 & $10^{-11}$ & 4.37 & $10^{-14}$ & 2.00 & $10^{-12}$ & 1.11 & $10^{-10}$ \\
\hline $\mathrm{L} F \perp \mathrm{C}-4$ & 4.25 & $10^{-11}$ & 1.18 & $10^{-13}$ & 3.19 & $10^{-12}$ & 1.01 & $10^{-10}$ \\
\hline SPTR-16 & 3.90 & $10^{-11}$ & 1.80 & $10^{-14}$ & 1.36 & $10^{-12}$ & 8.77 & $10^{-11}$ \\
\hline$L F 2 B-2$ & 3.55 & $10^{-11}$ & 3.32 & $10^{-13}$ & 6.07 & $10^{-12}$ & 1.28 & $10^{-10}$ \\
\hline MELF - 7 & 3.45 & $10^{-11}$ & 3.19 & $10^{-14}$ & 1.87 & $10^{-12}$ & 1.23 & $10^{-10}$ \\
\hline$L F 2 E-6$ & 3.18 & $10^{-11}$ & 2.15 & $10^{-13}$ & 4.80 & $10^{-12}$ & 1.16 & $10^{-10}$ \\
\hline SPTR-19 & 3.11 & $10^{-11}$ & 1.43 & $10^{-14}$ & 1.08 & $10^{-12}$ & 6.98 & $10^{-11}$ \\
\hline SPTR-9 & 2.98 & $10^{-11}$ & 1.23 & $10^{-14}$ & 9.80 & $10^{-13}$ & 7.16 & $10^{-11}$ \\
\hline OCSP-5 & 2.10 & $10^{-11}$ & 4.13 & $10^{-14}$ & 1.32 & $10^{-12}$ & 5.49 & $10^{-11}$ \\
\hline TSHC- 5 & 1.90 & $10^{-11}$ & 3.98 & $10^{-14}$ & 1.44 & $10^{-12}$ & 5.76 & $10^{-11}$ \\
\hline EVTR-13 & 1.66 & $10^{-11}$ & 2.70 & $10^{-15}$ & 2.82 & $10^{-13}$ & 2.85 & $10^{-11}$ \\
\hline
\end{tabular}


Table 12.2 Uncertainty Analysis Results for the Dominant Sequences (Cont'd)

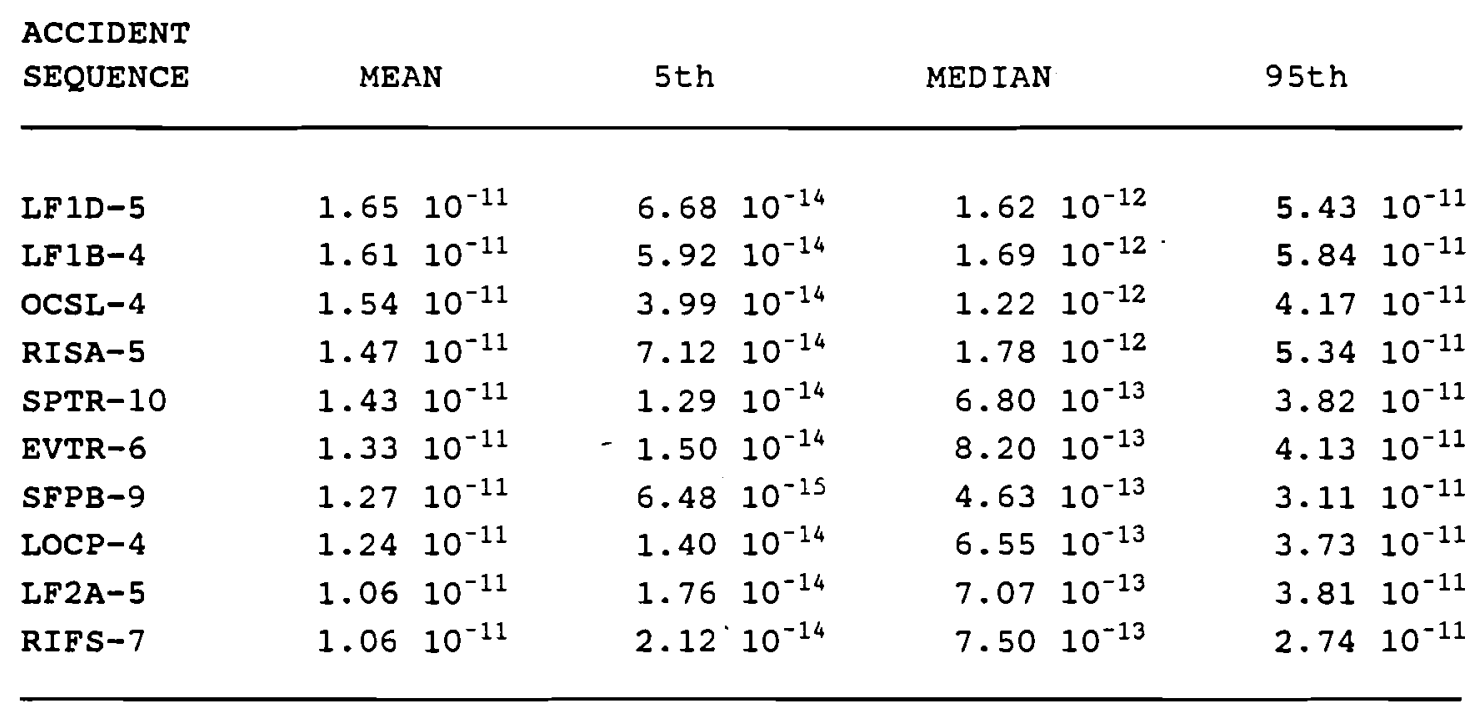




\subsubsection{Model Uncertainties}

Model uncertainties appear with regard to how well the models developed represent the actual plant and its behavior under accident conditions. Uncertainty has been introduced during model development in the form of simplifying, conservative assumptions and approximations, properly explained in the event tree and system description sections. The models have been thoroughly reviewed by plant personnel. The use of the rare event approximation to sum large number of cutsets results in a conservative approach. Its use is also appropriate given the absence of events with large probability of occurrence. Other model uncertainties such as human errors and common mode failure are specifically addressed in the sensitivity analysis that has been performed. The results are listed in Section 12.3 below.

\subsubsection{Completeness Uncertainty}

This source of uncertainty deals with the possibility of using incomplete models. Commercial PRA experience could only be used to a very limited extent because of the uniqueness of the EBR-II plant. An extensive search of plant records was conducted to identify all possible initiating events, and comparisons were made with commercial nuclear plants and existing PRAs for other fast reactors. The system models were developed to the level of detail needed to identify the appropriate relationships between the modelled system and the support systems.

Exclusion of significant numbers of low-probability accident sequences or minimal cutsets has been avoided by defining very low truncation setpoints in both the accident sequence analysis and the damage bin calculations. Because of the steps taken to ensure completeness in modelling task, the PRA is deemed to be complete in addressing the scope of the project.

\subsection{Sensitivity Analysis}

The sensitivity of the results to several important issues has been analyzed. In particular, the cases that have been studied address the modeling uncertainties related to human error 
quantification and common cause failures, the effect of the direct damage initiators, the effect of the special transient classes P5 (steam generator tube rupture) and P6 (local faults), and combinations of the different issues. The following cases have been analyzed;

1 Human error probability increased: the probability of the basic events representing human failures has been increased by a factor on 10 .

2 Human error probability decreased: the probability of the basic events representing human failures has been decreased by a factor of 10 .

3 Common cause probability increased: the probability of the basic events representing common cause failures has been increased by a factor of 3 .

4 Common cause probability decreased: the probability of the basic events representing common cause failures has been decreased by a factor of 3 .

5 Direct damage initiator frequency increased: the frequency of the initiating events leading to direct damage hạs been increased by a factor of 10 .

6 Direct damage initiator frequency decreased: the frequency of the initiating events leading to direct damage has been decreased by a factor of 10 .

7 Local Faults initiator frequency increased: the frequency of the local faults initiating events has been increased by a factor of 10 .

8 Local Faults initiator frequency decreased: the frequency of the local faults initiating events has been decreased by a factor of 10 .

9 Steam generator tube rupture initiator frequency increased: the frequency of the evaporator and superheater tube rupture initiating events has been increased by a factor of 10 .

10 Steam generator tube rupture initiator frequency decreased: the frequency of the evaporator and superheater tube rupture initiating events has been decreased by a factor of 10 .

11 Human error and common cause failure probabilities increased: combination of cases 1 and 3; human error probabilities increased by a factor of 10 and common cause failures by a factor of 3 .

12 Human error and common cause failure probabilities decreased: combination of cases 2 and 4; human error probabilities decreased by a factor of 10 and common cause failure by a factor of 3 . 
13 Direct damage, local faults, and steam generator tube rupture initiator frequencies increased: combination of cases 5,7 , and 9 ; all of these frequencies increased by a factor of 10 .

14 Direct damage, local faults, and steam generator tube rupture initiator frequencies decreased: combination of cases 6,8 , and 10 ; all of these frequencies decreased by a factor of 10 .

15 Human error and common cause failure probabilities and direct damage, local faults, and steam generator tube rupture initiator frequency increased: combination of cases 11 and 13; common cause failure probabilities increased by a factor of 3 , and the other items - by a factor of 10 .

16 Human error and common cause failure probabilities and direct damage, local faults, and steam generator tube rupture initiator frequency decreased: combination of cases 12 and 14; common cause failure probabilities decreased by a factor of 3 , and the other items by a factor of 10 .

A summary of the results, compared with the baseline case, is presented in Table 12.3. Only the point mean values of the damage frequency are compared in this table, while the distributions are compared in Table 12.4. Note that the mean value of the distribution does not necessarily coincide with the point mean value estimate. It must be pointed out that only the damage sequences have been included in the sensitivity calculations; the ND category has been left out.

\section{Human Error}

There are 44 different human error events in the dominant minimal cutsets of the dominant sequences, mostly from the reactor safety system and recovery actions in the decay heat removal systems. An increase by a factor of 10 in the human error probabilities results in an increase by a factor of almost 22 in the annual damage frequency. The effect is so important because several of the human failures already have a large probability in the baseline case, placing them in the most dominant cutsets. Furthermore, some of the top cutsets include two human errors, namely the failure to push the scram button and the miscalibration of the SOT 
Table 12.3 Results of the Sensitivity Analysis; Comparison of Point Mean Values of the Annual Damage Frequency

\begin{tabular}{|c|c|c|c|c|}
\hline \multicolumn{2}{|c|}{ CASE } & FREQUENCY & $\begin{array}{l}\text { BASELINE } \\
\text { FREQUENCY }\end{array}$ & \multirow{2}{*}{ RATIO } \\
\hline 1 & Human error increased by 10 & $3.4310^{-5}$ & $1.5710^{-6}$ & \\
\hline 2 & Human error decreased by 10 & $1.1210^{-6}$ & $1.5710^{-6}$ & 0.71 \\
\hline 3 & Common cause increased by 3 & $3.5010^{-6}$ & $1.5710^{-6}$ & 2.23 \\
\hline 4 & Common cause decreased by 3 & $9.2910^{-7}$ & $1.5710^{-6}$ & 0.59 \\
\hline 5 & Direct damage increased by 10 & $1.9610^{-6}$ & $1.5710^{-6}$ & 1.25 \\
\hline 6 & Direct damage decreased by 10 & $1.5310^{-6}$ & $1.5710^{-6}$ & 0.97 \\
\hline 7 & Local faults increased by 10 & $1.9510^{-6}$ & $1.5710^{-6}$ & 1.24 \\
\hline 8 & Local faults decreased by 10 & $1.5310^{-6}$ & $1.5710^{-6}$ & 0.97 \\
\hline 9 & $\begin{array}{l}\text { Steam generator tube rupture } \\
\text { increased by } 10\end{array}$ & $3.3410^{-6}$ & $1.5710^{-6}$ & 2.13 \\
\hline 10 & $\begin{array}{l}\text { Steam generator tube rupture } \\
\text { decreased by } 10\end{array}$ & $1.3910^{-6}$ & $1.5710^{-6}$ & 0.89 \\
\hline 11 & $\begin{array}{l}\text { Human error and common cause } \\
\text { increased (cases } 1 \text { and } 3 \text { ) }\end{array}$ & $8.5910^{-5}$ & $1.5710^{-6}$ & 54.71 \\
\hline 12 & $\begin{array}{l}\text { Human error and common cause } \\
\text { decreased (cases } 2 \text { and } 4 \text { ) }\end{array}$ & $6.7910^{-7}$ & $1.5710^{-6}$ & 0.43 \\
\hline 13 & $\begin{array}{l}\text { Direct damage, local faults and } \\
\text { steam generator tube rupture } \\
\text { increased by } 10 \text { (cases } 5,7 \text {, and 9) }\end{array}$ & $4.1110^{-6}$ & $1.5710^{-6}$ & 2.62 \\
\hline 14 & $\begin{array}{l}\text { Direct damage, local faults and } \\
\text { steam generator tube rupture } \\
\text { decreased by } 10 \text { (cases } 6,8 \text {, and } 10 \text { ) }\end{array}$ & $1.3210^{-6}$ & $1.5710^{-6}$ & 0.84 \\
\hline 15 & $\begin{array}{l}\text { Direct damage, local faults, } \\
\text { steam generator tube rupture, } \\
\text { human error and common cause } \\
\text { increased (cases } 11 \text { and } 13 \text { ) }\end{array}$ & $8.8510^{-5}$ & $1.5710^{-6}$ & 56.37 \\
\hline 16 & $\begin{array}{l}\text { Direct damage, local faults, } \\
\text { steam generator tube rupture, } \\
\text { human error and common cause } \\
\text { decreased (cases } 12 \text { and } 14 \text { ) }\end{array}$ & $4.2610^{-7}$ & $1.5710^{-6}$ & 0.27 \\
\hline
\end{tabular}


Table 12.4 Distributions of the Sensitivity Analysis Cases

\begin{tabular}{|c|c|c|c|c|}
\hline SEQUENCE & MEAN & $5 t h$ & MEDIAN & $95 \mathrm{th}$ \\
\hline BASELINE & $1.5310^{-6}$ & $2.4610^{-7}$ & $8.48 \quad 10^{-7}$ & $4.7410^{-6}$ \\
\hline 1 & $2.8210^{-5}$ & $1.8110^{-6}$ & $1.0010^{-5}$ & $1.0710^{-4}$ \\
\hline 2 & $1.1210^{-6}$ & $1.67 \quad 10^{-7}$ & $5.77 \quad 10^{-7}$ & $3.2410^{-6}$ \\
\hline 3 & $3.5510^{-6}$ & $4.6010^{-7}$ & $1.7010^{-6}$ & $1.0310^{-5}$ \\
\hline 4 & $9.13 \quad 10^{-7}$ & $1.68 \quad 10^{-7}$ & $5.3010^{-7}$ & $2.6410^{-6}$ \\
\hline 5 & $1.9410^{-6}$ & $3.89 \quad 10^{-7}$ & $1.2110^{-6}$ & $5.4710^{-6}$ \\
\hline 6 & $1.5110^{-6}$ & $2.23 \quad 10^{-7}$ & $8.10 \quad 10^{-7}$ & $4.7210^{-6}$ \\
\hline 7 & $1.9210^{-6}$ & $3.10 \quad 10^{-7}$ & $1.0810^{-6}$ & $6.0210^{-6}$ \\
\hline 8 & $1.5110^{-6}$ & $2.3710^{-7}$ & $8.13 \quad 10^{-7}$ & $4.7110^{-6}$ \\
\hline 9 & $3.1210^{-6}$ & $3.48 \quad 10^{-7}$ & $1.4110^{-6}$ & $9.6710^{-6}$ \\
\hline 10 & $1.3910^{-6}$ & $2.1910^{-7}$ & $7.45 \quad 10^{-7}$ & $4.3010^{-6}$ \\
\hline 11 & $6.9010^{-5}$ & $3.4610^{-6}$ & $2.20 \quad 10^{-5}$ & $2.6210^{-4}$ \\
\hline 12 & $6.68 \quad 10^{-7}$ & $1.13 \quad 10^{-7}$ & $3.77 \quad 10^{-7}$ & $2.0210^{-6}$ \\
\hline 13 & $4.28 \quad 10^{-6}$ & $5.9410^{-7}$ & $2.0310^{-6}$ & $1.2610^{-5}$ \\
\hline 14 & $1.28 \quad 10^{-6}$ & $1.77 \quad 10^{-7}$ & $6.56 \quad 10^{-7}$ & $3.8610^{-6}$ \\
\hline 15 & $7.21 \quad 10^{-5}$ & $4.58 \quad 10^{-6}$ & $2.4210^{-5}$ & $2.5810^{-4}$ \\
\hline 16 & $4.14 \quad 10^{-7}$ & $6.0310^{-8}$ & $2.30 \quad 10^{-7}$ & $1.2410^{-6}$ \\
\hline
\end{tabular}


sensors. Decreasing the human failure probabilities by a factor of 10 , in contrast, only reduces the damage frequency to $71 \%$ of the baseline.

\section{Common Cause}

There are 58 common cause failures that appear in the dominant cutsets of the damage sequences. For the common cause failures, the analysis is equivalent to a sensitivity study to the beta factors used in defining the probabilities of the common cause failures. The probability has been increased (case 3 ) and decreased (case 4 ) by a factor of 3 instead of 10 as in the other sensitivity cases. The factor of three has been chosen to keep the common cause failure probability of a component below the probability of independent failure (typical beta factors used were between 0.1 and 0.3 ). As in the case of the human failures, the results are more sensitive to increases than decreases in the probabilities of the common cause failures. An increase by a factor of 2.23 occurs when the common causes were increased by a factor of 3 , and a $40 \%$ reduction in the damage frequency is observed when the common cause is decreased by the same factor. An increase in the common cause events probabilities is fairly important because several of the common cause failures of the reactor safety system appear in the top minimal cutsets. Several top minimal cutsets are single term, common cause events cutsets. Several other dominant cutsets, the ones containing two human errors, contain also a common cause event. A disproportionately large effect in the damage frequency is observed when raising the human error probabilities because of the minimal cutsets with the two human error terms. On the other hand, lowering the common cause probability (by a factor of 3) has a larger impact on the frequency than lowering the human error probability (by a factor of 10 ) because the common cause affect both the single term, common cause dominant cutsets and the human error-common cause dominant cutsets.

\section{Direct Damage Initiators}

Increasing the frequency of the three direct damage initiators (RIFL, LCDL, and 
LF2D) by a factor of 10 results in an increase by $25 \%$ of the damage frequency, mostly due to the RIFL initiator, which has the largest frequency of the three. The results of case 6 , however, indicate that reducing their frequency by a factor of 10 only reduces. the damage frequency by about $3 \%$. The small effect observed by decreasing their frequency is due to the fact that, in the baseline case, the first direct damage minimal cutset (RIFL) appears with a frequency almost an order of magnitude below that of the most dominant cutsets.

\section{Local Faults Initiators}

A similar effect to that observed in the direct damage initiators is seen in the case of the local faults. Local faults sequences are about an order of magnitude in frequency lower than the dominant cutsets in the baseline case. Therefore, an increase by a factor of 10 is significant ( $25 \%$ increase in damage frequency) and a decrease is essentially insignificant ( $3 \%$ difference).

\section{Steam Generator Tube Rupture Initiators}

Three of the minimal cutsets for superheater tube rupture sequences (those of SPTR-3, SPTR-13, and SPTR-23) are among the dominant cutsets in the baseline case. This results in a high sensitivity of the damage frequency to changes in the frequency of the steam generator tube rupture initiators. Increasing their frequency by a factor of 10 raises the damage frequency by a factor of 2.13 , while decreasing their frequency by the same amount lowers the damage frequency by a factor of 0.89 . The superheater tube rupture initiator is almost entirely responsible for the changes.

\section{Human Error and Common Cause}

When combining these two types of failure the results change more considerably, especially when increasing the probability of both types of failure. The annual frequency of damage increases by a factor of almost 55 in response to increasing the human error probabilities by 10 and the common cause failures by 3 , while a $60 \%$ reduction in 
damage frequency is observed when the probabilities are decreased by the same amounts. As it was noted above, both human error and common cause failures appear in the dominant cutsets of the damage sequences. Some of the top minimal cutsets combine both types of failures, and there is one particular event, miscalibration of the SOT sensors, which is a common cause human error. Consequently, the damage frequency is very sensitive to changes in the probabilities of the two types of failures.

\section{Direct Damage, Local Faults, and Steam Generator Tube Rupture Initiators}

The combination of changes in this three types of initiating event frequencies produces considerable changes in the annual frequency of damage. When increasing the initiator frequency by a factor of 10 , an increase by a factor of 2.62 appears in the damage frequency. Decreasing the initiator frequency by 10 , lowers the damage frequency $16 \%$. The superheater tube rupture initiating event dominates the changes in the damage frequency, and the sensitivity results for this combined case are close to those obtained in cases 9 and 10 , where the steam generator tube rupture initiators were considered alone.

\section{Human Error, Common Cause, and Direct Damage, Local Faults and Steam Generator Tube Rupture Initiators}

This combination does not differ much from the combination cases of human error and common cause failure, only in this case the changes in damage frequency are somewhat more drastic. The human error and common cause events (but specially the human errors) are the prevalent factor in increasing the damage frequency in case 15 (by a factor of over 56). The initiator frequency changes, most notably that of the superheater tube rupture initiating event, play a more important role in case 16, when probabilities and frequencies are lowered, resulting in a damage frequency that is only $27 \%$ of the original. This effect occurs because most of the dominant cutsets include at least one of the initiators or basic events whose frequency or probability is being decreased. The direct damage and specially the local faults initiators have a moderate effect overall. 


\subsection{Importance Calculations}

An importance analysis has been carried out for the basic events that appear in the minimal cutsets of the dominant sequences. The importance calculations provide a ranking of the basic events ordered by their contribution to the damage frequency. The summary of the importance results appears in Table 12.5. Three measures of importance have been calculated for each basic event: the Fussell-Vesely importance (labeled F-V IMPORTANCE in the table), the risk achievement worth (RAW), and the risk reduction worth (RRW).

The Fussell-Vesely importance represents the fraction of the total damage frequency that involves the basic event. It is calculated as the difference between the damage frequency with the basic event probability at its nominal value and the frequency with the event probability set to 0.0 , divided by the nominal damage frequency. The risk achievement worth importance measure represents the increase in damage frequency expected if the basic event were a true event. It is calculated as the ratio of the damage frequency with the basic event probability set to 1.0 to the frequency with the nominal event probability. The risk reduction worth importance measure indicates the reduction in damage frequency if the basic event in question were a false event. It is computed as the ratio of the nominal frequency to the damage frequency calculated with the basic event probability set to 0.0 . Only the most important (Fussell-Vesely, F-V, criterion) basic events are listed in Table 12.5. Among the basic events with the highest F-V importance are the common cause and human error events, as could be expected from the sensitivity analyses. Although some human errors are more important than the common cause failures under the F-V measure, it is noticeable that the 6 events with the largest RAW measure importance are all common cause failures (all in the reactor safety system). The increase that would result in the damage frequency in EBR-II if any of this

common cause events were a true event would be of the order between $10^{4}$ and $10^{5}$. This very large RAW measure for these events occurs because they appear in single term minimal cutsets in several accident sequences. 
Table 12.5 Results of the Importance Calculations

\begin{tabular}{|c|c|c|c|c|}
\hline $\begin{array}{l}\text { EVENT } \\
\text { NAME }\end{array}$ & $\begin{array}{c}\text { MEAN } \\
\text { PROBABILITY }\end{array}$ & $\begin{array}{c}F-V \\
\text { IMPORTANCE }\end{array}$ & RAW & RRW \\
\hline RHU002CL & $1.00010^{-1}$ & 0.2270 & 3.0433 & 1.2937 \\
\hline RCzCOOQU & $2.40010^{-6}$ & 0.2093 & $8.7210^{4}$ & 1.2647 \\
\hline RHU302QL & $2.50010^{-2}$ & 0.1963 & 8.6572 & 1.2443 \\
\hline RRECOOQU & $1: 92010^{-6}$ & 0.1674 & $8.7210^{4}$ & 1.2011 \\
\hline VHERIXWF & $1.00010^{-2}$ & 0.1049 & 11.3849 & 1.1172 \\
\hline DCR001OF & $2.23010^{-4}$ & 0.0762 & 342.6879 & 1.0825 \\
\hline RND2 ZORU & $3.60010^{-4}$ & 0.0625 & 174.6201 & 1.0667 \\
\hline RDC2 Z1QU & $3.60010^{-4}$ & 0.0625 & 174.6201 & 1.0667 \\
\hline VHEMO2WF & $8.40010^{-1}$ & 0.0537 & 1.0102 & 1.0567 \\
\hline VHEDM1WF & $1.00010^{-1}$ & 0.0535 & 1.4813 & 1.0565 \\
\hline VHEPF2WF & $5.00010^{-1}$ & 0.0510 & 1.0510 & 1.0537 \\
\hline VHEMO1WF & $1.60010^{-1}$ & 0.0509 & 1.2675 & 1.0537 \\
\hline WFL095YE & $2.21010^{-2}$ & 0.0323 & 2.4310 & 1.0334 \\
\hline HFL7 17SE & $2.21010^{-2}$ & 0.0323 & 2.4310 & 1.0334 \\
\hline HHUO 1BSZ & $2.00010^{-2}$ & 0.0293 & 2.4341 & 1.0301 \\
\hline 9CR001OF & $1.01010^{-4}$ & 0.0282 & 280.2044 & 1.0290 \\
\hline FPP112RF & $1.00010^{-3}$ & 0.0268 & 27.7885 & 1.0276 \\
\hline DCR001OE & $7.44010^{-5}$ & 0.0254 & 341.7316 & 1.0260 \\
\hline 9HUOOOOL & $4.00010^{-6}$ & 0.0242 & 6044.3315 & 1.0248 \\
\hline 9CR0O2OF & $1.010 \cdot 10^{-4}$ & 0.0217 & 215.4129 & 1.0221 \\
\hline RAM2 Z1QU & $1.20010^{-4}$ & 0.0208 & 174.6252 & 1.0213 \\
\hline VHEFSDWF & $3.20010^{-3}$ & 0.0204 & 7.3446 & 1.0208 \\
\hline VHEPF 3WF & $1.00010^{-1}$ & 0.0204 & 1.1833 & 1.0208 \\
\hline FPP991RF & $9.00010^{-1}$ & 0.0176 & 1.0020 & 1.0179 \\
\hline FPPO03RF & $1.00010^{-1}$ & 0.0169 & 1.1523 & 1.0172 \\
\hline RCz2 zOQU & $2.40010^{-6}$ & 0.0166 & 6918.5205 & 1.0169 \\
\hline DCR002OF & $2.23010^{-4}$ & 0.0154 & 69.8630 & 1.0156 \\
\hline CSR3OIRU & $1.000 \quad 10^{-7}$ & 0.0151 & $1.5110^{5}$ & 1.0153 \\
\hline CSR10GRU & $1.00010^{-7}$ & 0.0151 & $1.5110^{5}$ & 1.0153 \\
\hline RHUO01CL & $5.00010^{-2}$ & 0.0142 & 1.2707 & 1.0145 \\
\hline RRE2 ZOQU & $1.92010^{-6}$ & 0.0133 & 6919.4282 & 1.0135 \\
\hline vo0000Uw & $1.71010^{-2}$ & 0.0133 & 1.7618 & 1.0134 \\
\hline RBS004QP & $1.00010^{-3}$ & 0.0113 & 12.3030 & 1.0114 \\
\hline RDC2A1QW & $3.60010^{-3}$ & 0.0111 & 4.0730 & 1.0112 \\
\hline RDC2B1QW & $3.60010^{-3}$ & 0.0111 & 4.0730 & 1.0112 \\
\hline
\end{tabular}


Table 12.5 Results of the Importance Calculations (Cont'd)

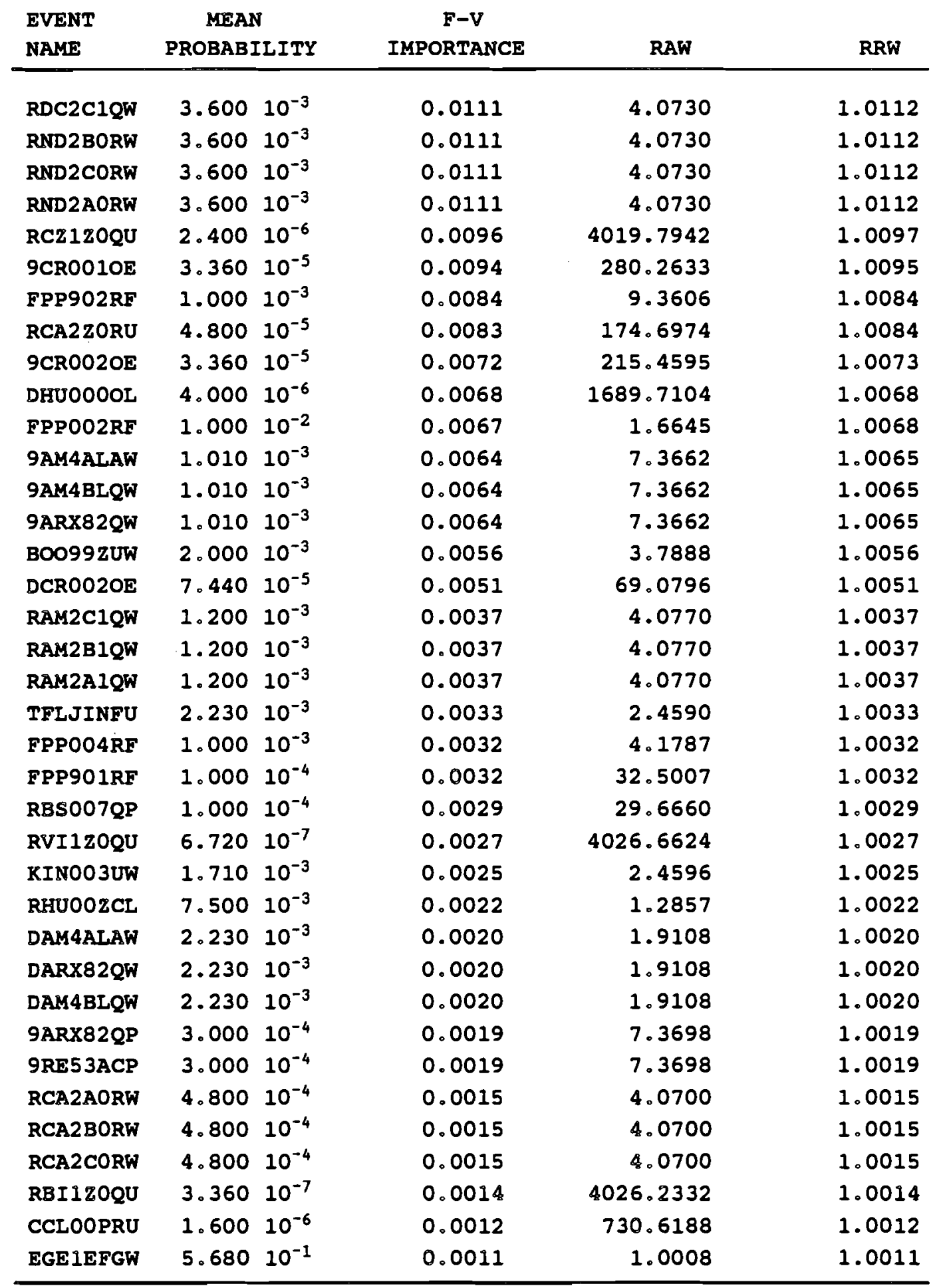




\subsection{INTERPRETATION OF RESULTS}




\section{Table of Contents}

Page

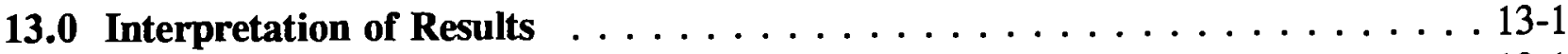

13.1 Internal Events . . . . . . . . . . . . . . . . . 13-1

13.1.1 Plant Transients . . . . . . . . . . . . . . . 13-1

13.1.2 Damage Sequences . . . . . . . . . . . . . . . 13-3

13.1.3 General Insights . . . . . . . . . . . . . . . 13-7

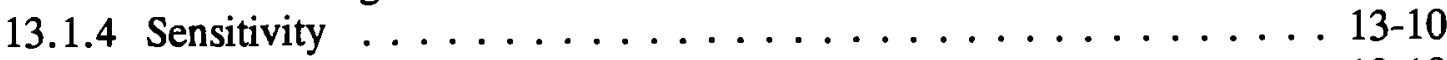

13.1.5 Comparison to Other PRAs . . . . . . . . . . . . . 13-10

13.1.5.1 Clinch River Breeder Reactor (CRBR) . . . . . . . 13-10

13.1.5.2 NUREG-1150 . . . . . . . . . . . . . . 13-11

13.1.5.3 Other USDOE Reactors . . . . . . . . . . . 13-11

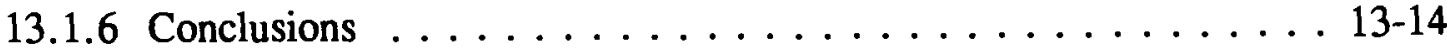

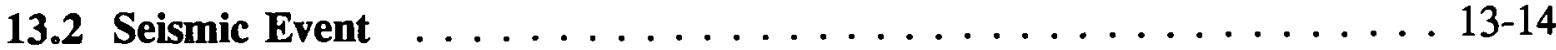

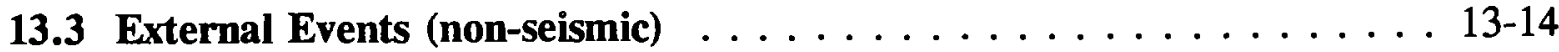

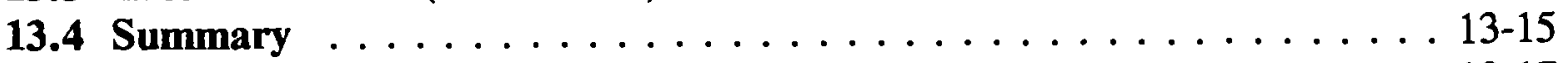

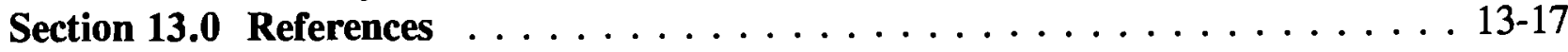




\section{$\underline{\text { List of Figures }}$}

$\underline{\text { Page }}$

13.1 Relative Contribution of Initiating Events to the No Damage Category . . . . . . 13-2

13.2 Relative Contribution of Initiating Events to All Damage Sequences . . . . . . . . 13-4

13.3 Relative Contribution of Initiating Events to the CSD Category . . . . . . . . 13-5

13.4 Relative Contribution of Initiating Events to the PED Category . . . . . . . . 13-6

13.5 Relative Contribution of Initiating Events to the MCD Category . . . . . . . . 13-8

13.6 Relative Contribution of Initiating Events to the CD Category . . . . . . . . . 13-9

13.7 Comparison of Internal Core Damage Frequency Distributions . . . . . . . . . 13-12

13.8 Comparison of Internal Core Damage Frequency Ranges

(5th and 95th percentiles) from NUREG-1150 and EBR-II $\ldots \ldots \ldots$ 13-13 


\section{List of Tables}

Page

13.1 Mean Core Damage Frequencies for USDOE Facilities $\ldots \ldots \ldots \ldots$. . . . . . . 


\subsection{INTERPRETATION OF RESULTS}

\subsection{Internal Events}

The most obvious result of the EBR-II PRA is that the frequency of plant transients is low, $-3.110^{-5}$ per year, and less than $7 \%$ of these sequences leads to any form of damage. If EBR-II were operated only as a power reactor (i.e., without radiation experiments), then the PED damage category could be excluded and the estimated damage frequency would be further reduced.

\subsubsection{Plant Transients}

The total frequency of sequences arising from internal initiating events which are classified as plant transient states, i.e., P1 through P6, has been calculated to be 3.07 $10^{-5} \mathrm{yr}^{-1}$. However the frequency of sequences leading to all forms of damage is only $1.5710^{-6}$. The difference is a result of the fact that there are many sequences that lead to plant transients which can be shown not to lead to any fuel or experiment damage but are important in that they could lead to administrative shutdown of EBR-II. For example, the P3 plant transient category, which includes all of the sequences with failure to scram, has a frequency of $1.3610^{-5} \mathrm{yr}^{-1}$ and $93 \%$ of these have been identified as leading to no damage because of the inherent response of the system. Figure 13.1 shows the relative contribution of initiating events to those plant transients which lead to no damage. Almost $24 \%$ of the frequency in the no damage class comes from loss of heat sink events (TSEC, TBOP, TAIR). A total of $18 \%$ of the initiating events are faults on a single pump, (LF1A, LF1B, LF1C and OCPP), while a further $56 \%$ arise from a steam generator tube rupture. Two sequences which result from superheater tube rupture, SPTR-2 and SPTR-12, which model a propagating sodium-water reaction which does not fail the IHX due to overpressure, are included as plant transients, P5, with frequencies of $8.410^{6}$ and $8.010^{-6}$ respectively, but assigned to the no damage category. 


\section{FUEL DAMAGE: ND}

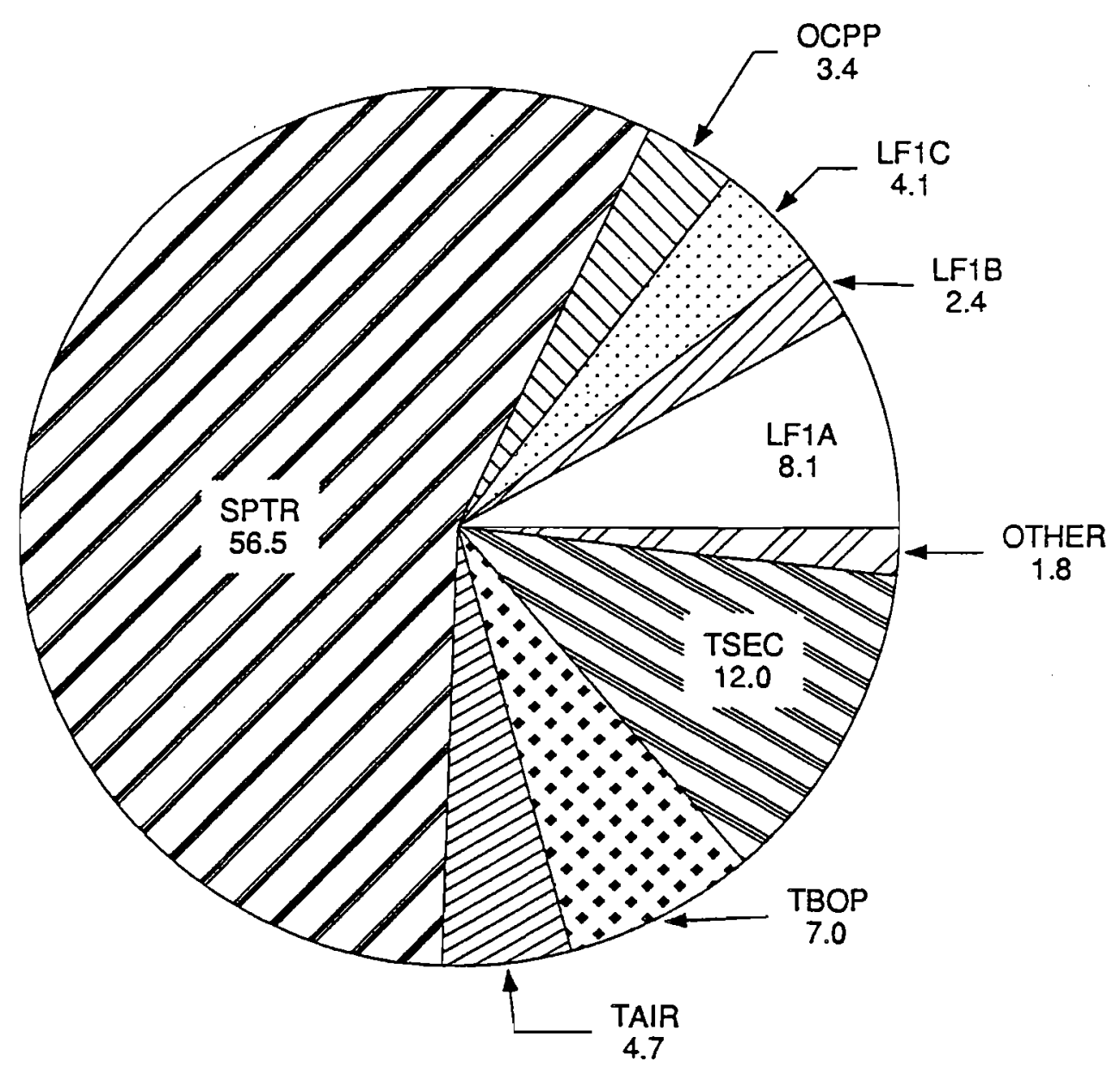

Fig. 13.1. Relative Contribution of Initiating Events to the No Damage Category 
There is a threat to the IHX in these sequences but the threat is stress corrosion cracking due to unreacted water, sodium hydroxide etc. remaining in the IHX for a few hours. The system is shutdown and no route to core damage can be identified.

\subsubsection{Damage Sequences}

Figure 13.2 gives the relative contribution of initiating events to all forms of damage. The largest contributor is the loss of normal power, LONP, followed by overcooling from a major steam leak (OCSL) and the rupture of a superheater module tube. The direct damage events do not appear to be significant. The only event which does appear, representing $1.9 \%$ of the risk, is RIFL, the fast reactivity insertion which occurs only because of the contribution of the argon overpressurization event. The loss of normal power appears by virtue of inclusion of the Potential Experiment Damage Category and would not lead to damage in a power reactor.

Figures 13.3 to 13.6 give the relative contribution of initiating events to each damage category. Figure 13.3 shows that the loss of a shutdown cooler and the superheater tube rupture are the dominant contributors to the CSD category. The long shutdown is also important, because the secondary sodium is drained and unavailable. Figure 13.4 gives the contribution of initiating events to the Potential Experiment Damage Category. Damage to driver fuel is precluded and there is at most damage to one assembly occurring in these sequences. The major contributors to this category are the loss of normal power, $\sim 80 \%$, and a double pump failure (LF2A) $\sim 17 \%$. Events in this class would not lead to radionuclide release of any consequence but would be undesirable from a plant viewpoint and would certainly lead to a lengthy administrative shutdown. 


\section{DAMAGE SEQUENCES}

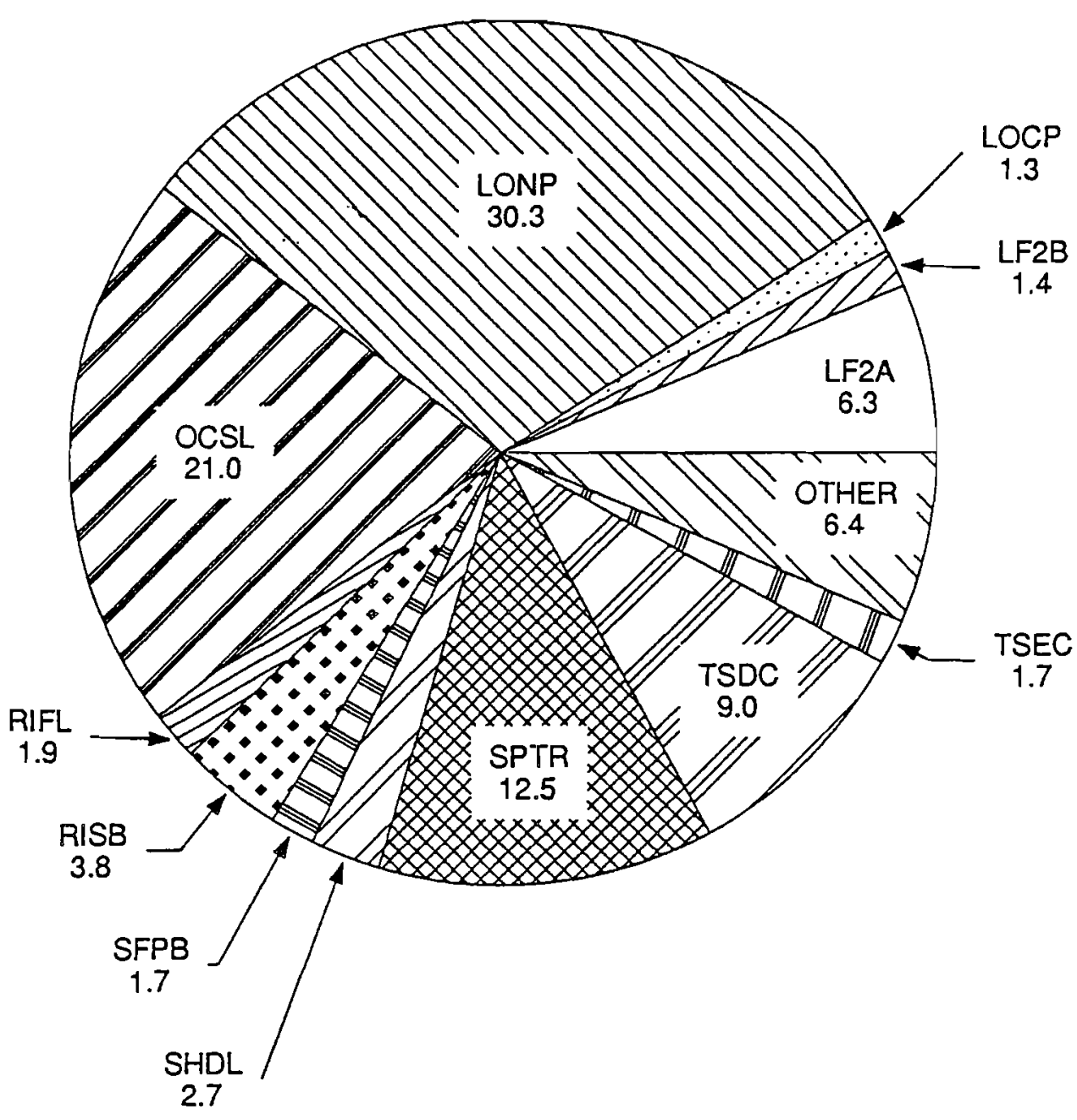

Fig. 13.2. Relative Contribution of Initiating Events to All Damage Sequences 


\section{FUEL DAMAGE: CSD}

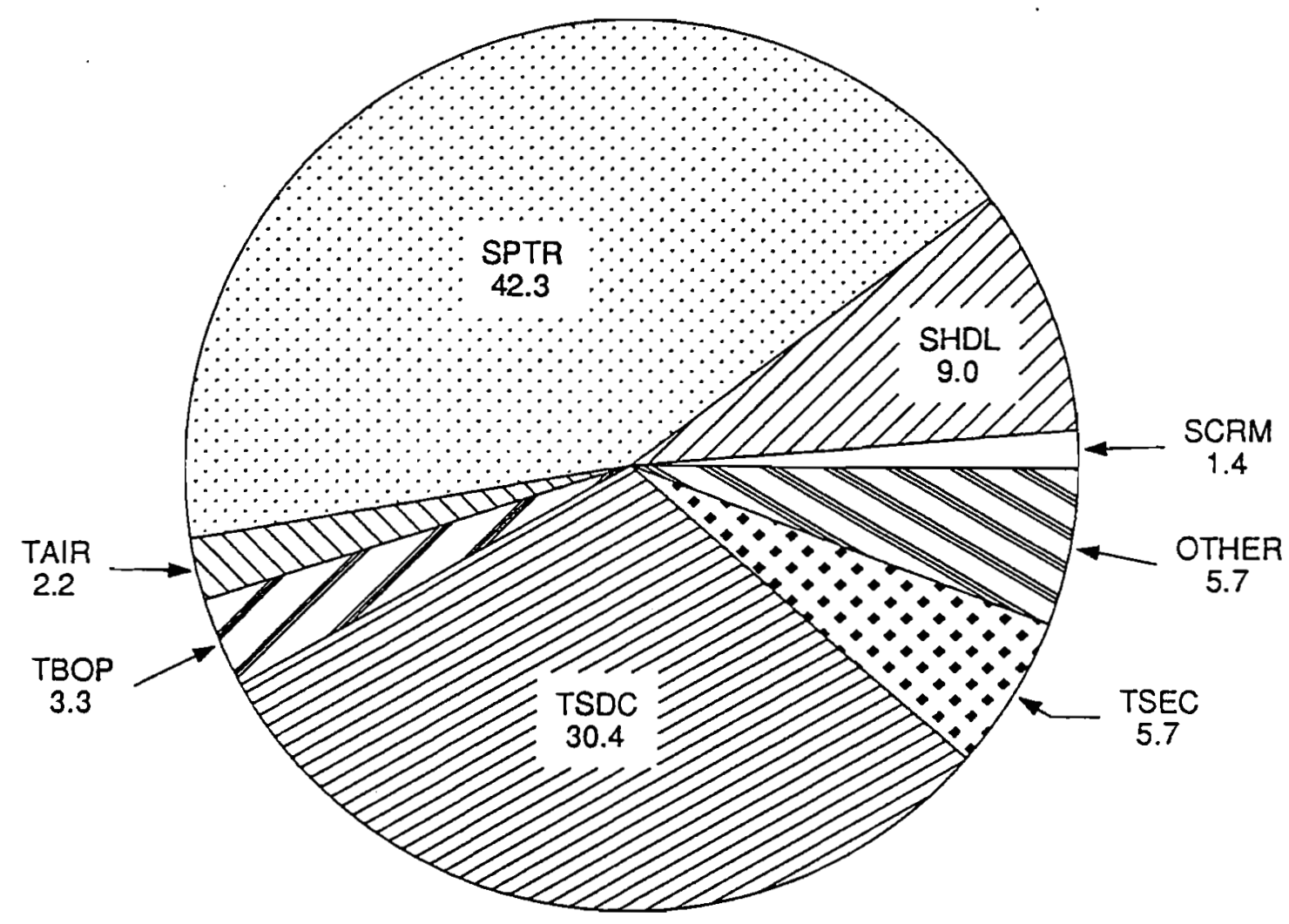

Fig. 13.3. Relative Contribution of Initiating Events to the CSD Category 


\section{FUEL DAMAGE: PED}

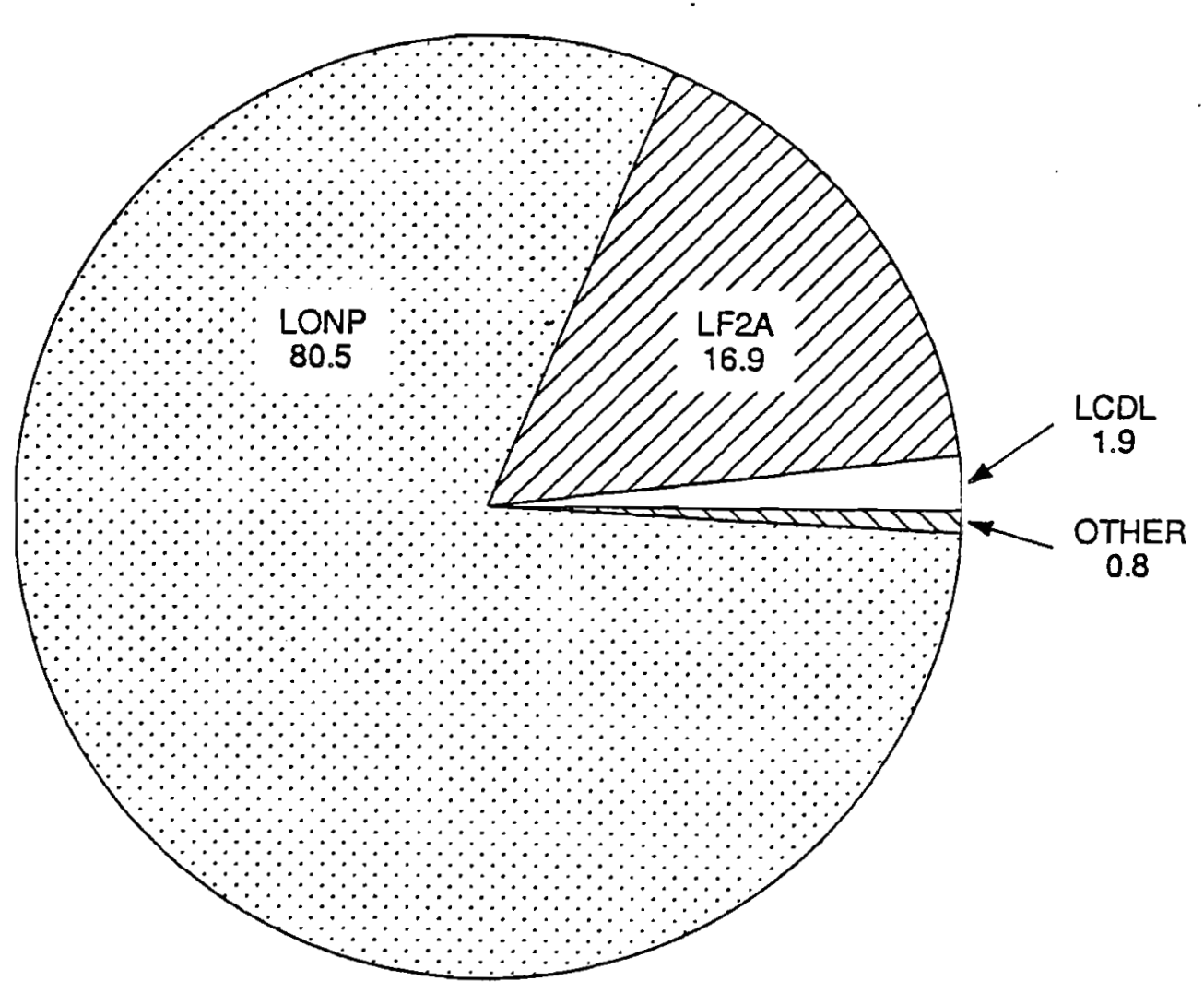

Fig. 13.4. Relative Contribution of Initiating Events to the PED Category 
Minor Core Damage, Fig. 13.5, is dominated by an overcooling event, OCSL, $\sim 77 \%$, and a reactivity insertion sequence. The overcooling sequence has been modeled without recovery being applied. This accident is slowly developing, where uncontrolled release of steam extracts sufficient energy to cool the primary tank which causes the reactor power to rise until some damage cannot be ruled out. Simply deenergizing the secondary pump is sufficient to prevent damage.

Core Damage, Fig. 13.6, is not dominated by any single initiating event: two double clutch failures (LOCP and LF2B), the RIFL direct damage sequence, and local faults (SFPB and OELF) contribute similar fractions. However the frequency of the class is $910^{-8} \mathrm{yr}^{-1}$, which is very low.

\subsubsection{General Insights}

Generally the reactivity insertion events have been found to be fairly unimportant, with the plant transient categories being dominated by pump faults and decay heat removal faults. Direct damage events and local faults are not significant, however the other special plant transient category, evaporator and superheater tube rupture, has significant implications. Furthermore all three of these families of sequences are quantified in essentially a subjective manner relying on expert judgement and simple calculations for end state assignation and are therefore more uncertain.

The important systems from a mitigative point of view are the scram system and shutdown coolers and therefore the postulated common cause and human error events are most important in these systems. 


\section{FUEL DAMAGE: MCD}

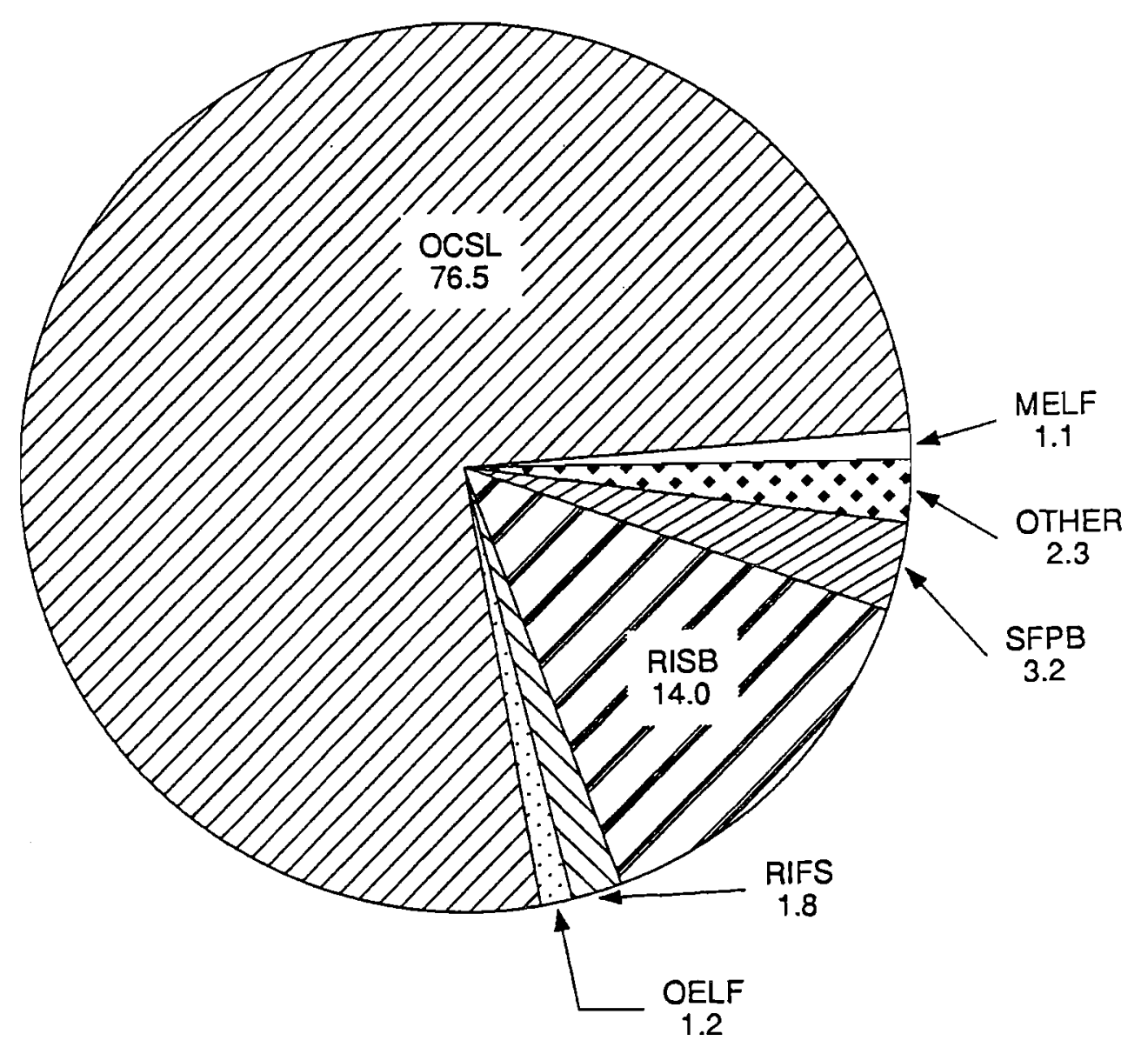

Fig. 13.5. Relative Contribution of Initiating Events to the MCD Category 


\section{FUEL DAMAGE: CD}

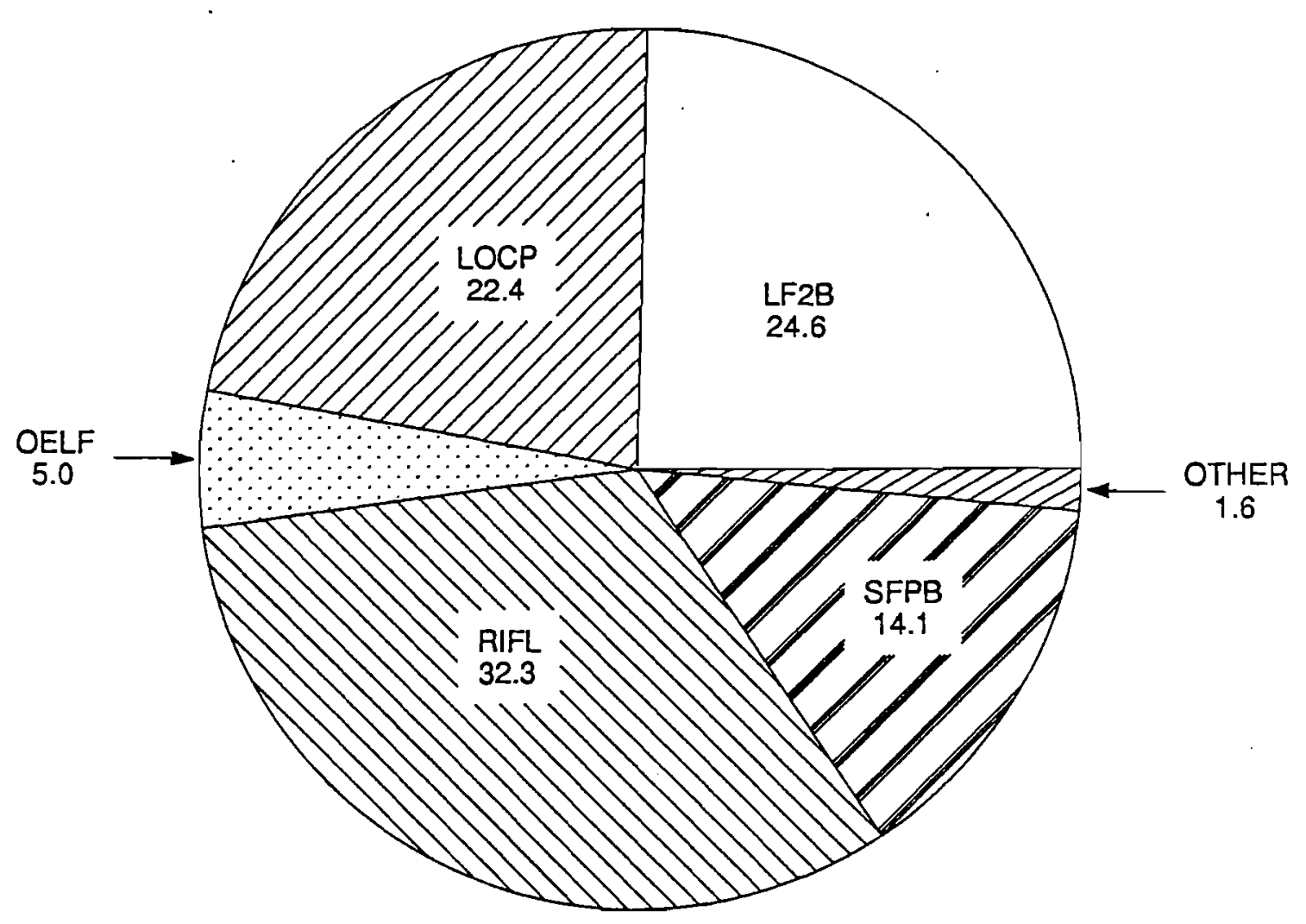

Fig. 13.6. Relative Contribution of Initiating Events to the CD Category 


\subsubsection{Sensitivity}

Since the overall frequency of damage is sufficiently small and there is a lack of dominant event, the sensitivity analysis assumes a complexity that is important in interpreting the results.

Fortunately, sensitivity studies show a very weak dependence on initiating event frequencies for those direct damage events which are difficult to quantify. There is a large sensitivity to human error events which arises mainly through manual scram actions. Common cause is extensively modeled in the scram system which affects the ATWS sequences and thus tends to be very important. The numerical sensitivity studies undertaken and the importance calculations show that results are not very sensitive to local faults, while the superheater tube rupture event has a more significant impact. In general, the sensitivity to those events whose initiating event frequencies were subjectively evaluated is not significant.

Significant human errors (of omission) have been identified, most notably the failure to manually scram, miscalibration of the SOT set points, and recovery failures in the heat removal systems. The SOT setpoint miscalibration can be dealt with by an improved procedure. Common cause effects are important in the scram system especially through the relays. These are examined in the sensitivity studies of Section 12 . The results of that study indicate a high sensitivity to the combination of human error and common cause because of the existence of cutsets containing two human errors and a common cause.

\subsubsection{Comparison to Other PRAs}

\subsubsection{Clinch River Breeder Reactor (CRBR)}

The CRBR PRA [13.1] found only two classes of sequence above a $10^{-8}$ cutoff for the annual frequency, the protected and unprotected Loss of Heat Sink transients at $1.410^{-6}$ and $2.510^{-7} \mathrm{yr}^{-1}$ respectively. The EBR-II PRA has a detailed operating history to draw upon for fault characterization whereas CRBR was never built. For EBR-II the 
scram system has estimated probabilities of failure to scram of $-510^{-6}$ per demand. In contrast, for the CRBR, diverse and redundant primary and secondary scram systems were used with the result that the total failure to scram was estimated to be $<10^{7}$ per demand and therefore only frequent initiating events, i.e. heat sink faults, gave any contribution above the cutoff of $10^{-8} \mathrm{yr}^{-1}$ when unprotected. The contribution from protected heat sink faults is comparable in CRBR and EBR-II. CRBR did not consider local faults except as a means of reactivity insertion, and the whole category of Potential Experimental Damage does not apply to CRBR because it was to be a power reactor. With these facts in mind the results of the two PRAs are broadly consistent.

\subsubsection{NUREG-1150}

Core damage frequencies for the EBR-II are compared with relevant results from NUREG-1150 [13.2] in Figs. 13.7 and 13.8. It can readily be seen that the core damage frequency is in the same range as the two BWR plants, Peach Bottom and Grand Gulf, and significantly below the other plants. This comparison may be misleading because of the different definitions of damage applied and the existence of conservatisms in the EBR-II results. Further detailed analysis can be expected to reduce the EBR-II value whereas the NUREG-1150 results have been extensively reviewed and are considered to be final.

\subsubsection{Other USDOE Reactors}

PRAs have been issued for HFIR, ATR, N-Reactor, SRP K-Reactor, and HFBR, results have been presented in [13.3, 13.4, 13.5, 13.6, 13.7, and 13.8]. The results for these plants give the frequency of core damage from internal events in the range of $410^{-4}$ to $710^{-5} \mathrm{yr}^{-1}$. In many of the Category $\mathrm{A}$ reactors direct damage events, through failure of pressure vessels, has been found to be important. These types of events have been found to be unimportant in EBR-II and therefore the lower frequencies described here should not be surprising. 
Comparison of EBR-II Damage Frequency with Core Damage at Commercial LWRS (LWR data from NUREG-1150)

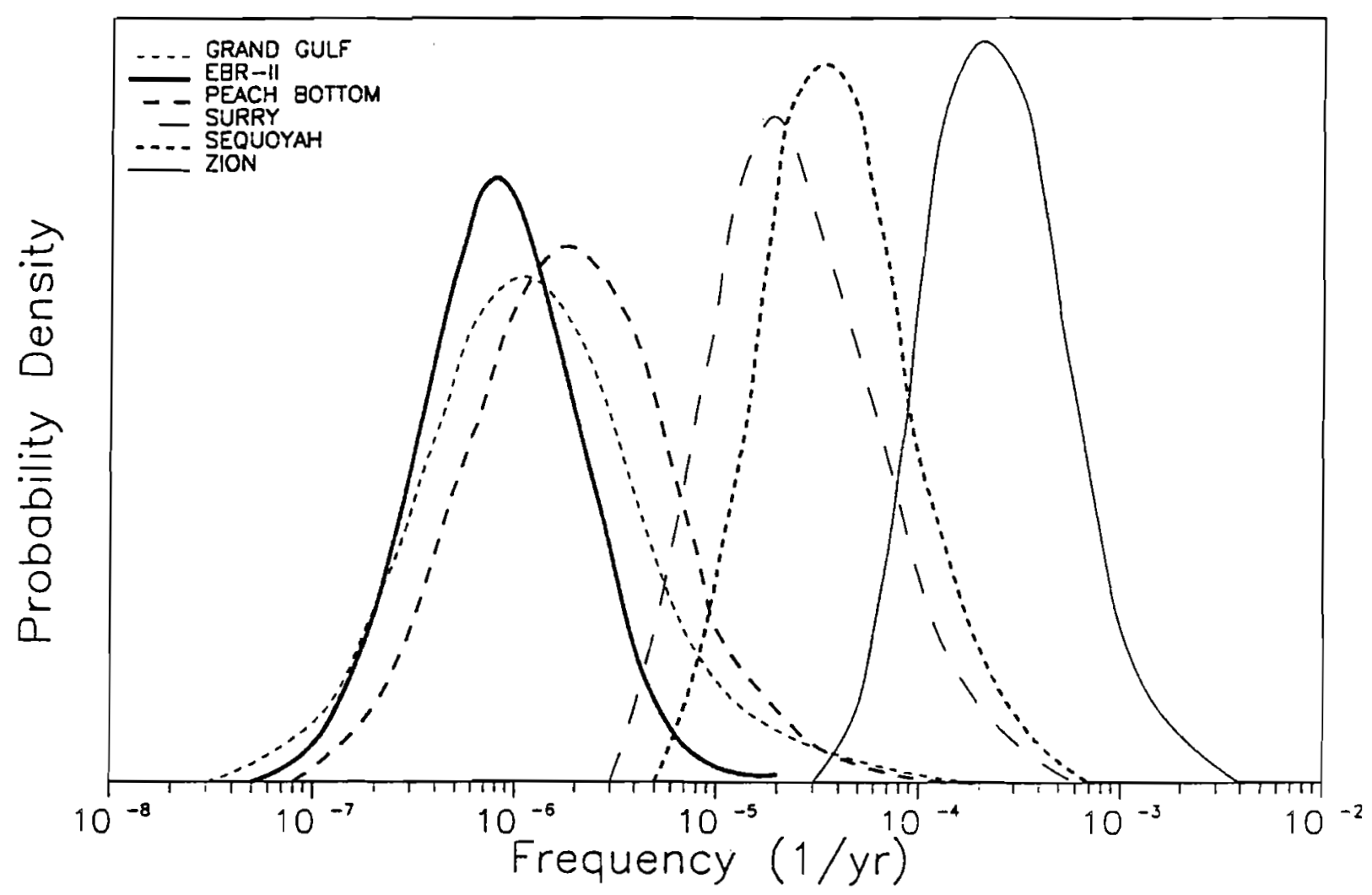

Fig. 13.7 Comparison of Internal Core Damage Frequency Distributions 


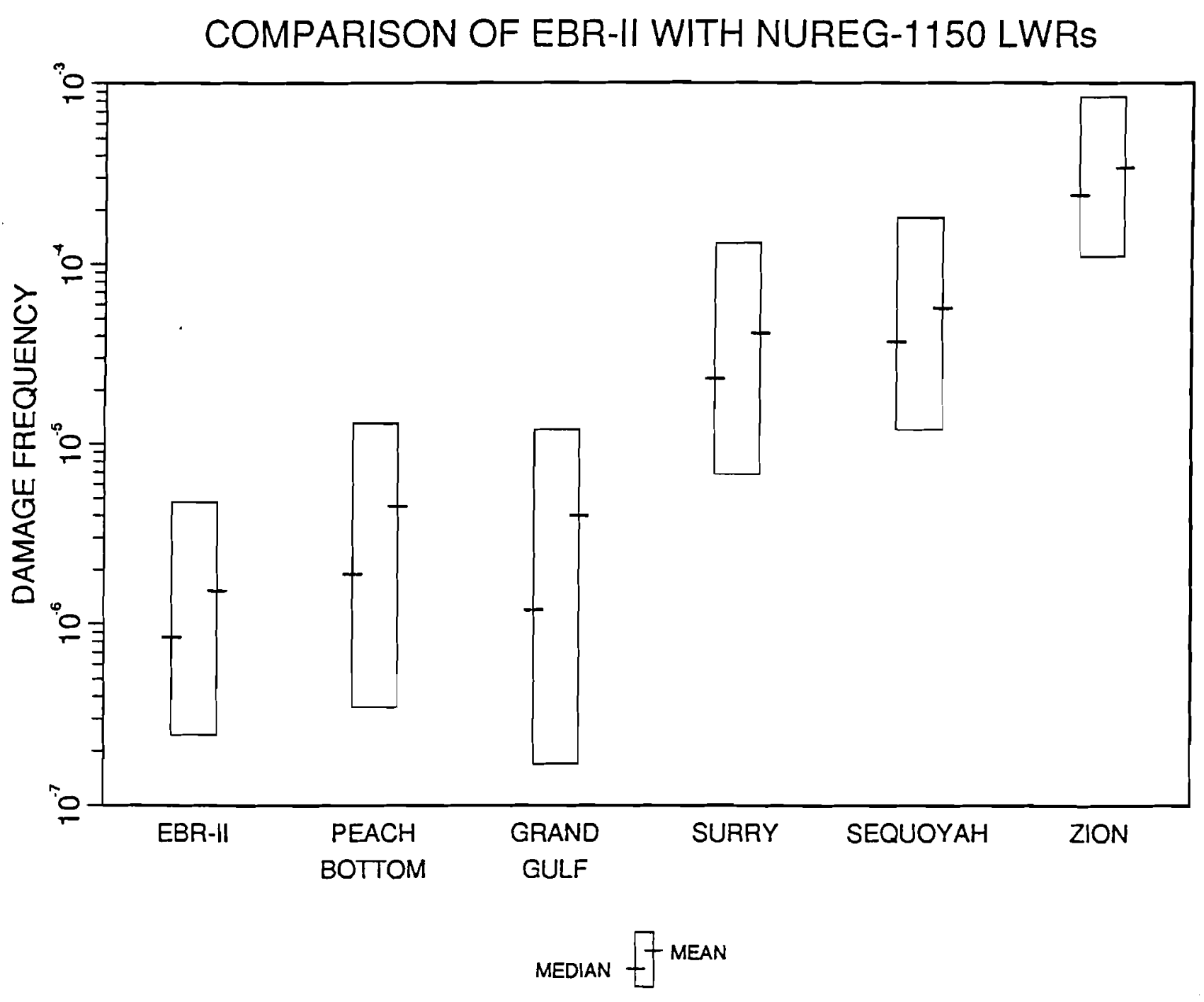

Fig. 13.8. Comparison of Internal Core Damage Frequency Ranges (5th and 95th percentiles) from NUREG-1150 and EBR-II 


\subsubsection{Conclusions}

The results presented above describe a very low risk for operation of EBRII when internal events alone are considered. This is not particularly surprising since EBR-II has a mission which is directly safety related and much effort has been expended to ensure that EBR-II is demonstrably safe. In fact EBR-II is ANL's prototype for the Integral Fast Reactor which bases part of its appeal on its passive safety characteristics.

\subsection{Seismic Event}

Not included at this time.

\subsection{External Events (non-seismic)}

A screening analysis of external (non-seismic) events has identified three sequences of note. Two sequences arise from a rupture in a NaK-filled shutdown cooler under the deck plates, SDFR-3 and SDFR-6. In SDFR-6 a rupture, rather than a small leak, is presumed to lead to a large liquid metal fire and the operators dump the secondary sodium after shutting down the reactor. It is assumed that the fire renders the remaining shutdown cooler less reliable. The result is a sequence where the $1000^{\circ} \mathrm{F}$ structural limit is exceeded. Clearly a large fire is required. The sequence has an estimated frequency of $2.110^{-6} \mathrm{yr}^{-1}$. SDFR-3 is an unsuppressed leak. The operators dump secondary sodium in this case also and cooling failure is due to failure of the remaining cooler. This sequence frequency is $5.810^{-9} \mathrm{yr}^{-1}$. A similar sodium fire sequence arises from a rupture of secondary sodium piping within containment. The fire is assumed to disable one shutdown cooler and render the other cooler less reliable. The frequency of this sequence, SSFR-6, is calculated to be $1.510^{-6} \mathrm{yr}^{-1}$. The external events analysis was done as a screening analysis to identify the important sequences using generic numbers. Detailed analysis is required to refine the quantification and to better establish the relative importance of these sequences. It should be noted that the external events sequences identified here dominate the internal events contributions to damage frequency. 


\subsection{Summary}

Table 13.1 compares published results from PRAs on USDOE Category A reactors. Where comparisons can be made it can be seen that EBR-II compares favorably with the other facilities. Comparisons of this sort should be viewed with caution as the widely varying reactor types and definitions of damage could lead to a bias in the results. Nevertheless, the favorable comparison is not surprising as the EBR-II mission has been centered upon demonstrating the safety of LMRs for commercial application and EBR-II is viewed as a prototype of a particular design concept for LMRs, the Integral Fast Reactor or IFR. 
Table 13.1 Mean Core Damage Frequencies for USDOE Facilities

\begin{tabular}{||l|l|c|c|c|c|c|c||}
\hline & & \multicolumn{2}{|c|}{ Mean Core Damage Frequencies $\left(\right.$ Yr. $\left.^{-1}\right)$} & & \\
\hline & & $\begin{array}{c}\text { Total } \\
\text { Internal }\end{array}$ & Seismic & Fire & $\begin{array}{c}\text { Wind/ } \\
\text { Tornado }\end{array}$ & $\begin{array}{c}\text { Total } \\
\text { External }\end{array}$ & Total \\
\hline \hline & EBR-II & $1.610^{-6}$ & & $3.610^{-6}$ & - & & \\
\hline$[13.4]$ & ATR & $1.810^{-4}$ & - & - & - & - & - \\
\hline$[13.5]$ & HFBR & $3.510^{-4}$ & - & - & - & - & \\
\hline$[13.3]$ & HFIR & $3.110^{-4}$ & $1.210^{-4}$ & $1.910^{-5}$ & $2.910^{-4}$ & $4.310^{-4}$ & $7.410^{-4}$ \\
\hline$[13.6]$ & $\begin{array}{l}\text { N- } \\
\text { Reactor }\end{array}$ & $6.710^{-5}$ & $1.710^{-4}$ & $1.710^{-5}$ & - & $1.910^{-4}$ & $2.510^{-4}$ \\
\hline$[13.7]$ & $\begin{array}{l}\text { SRP K- } \\
{[13.8]}\end{array}$ & $2.110^{-4}$ & $1.210^{-4}$ & $1.410^{-7}$ & - & $2.210^{-4}$ & $4.310^{-4}$ \\
\hline
\end{tabular}




\subsection{References}

13.1 "Clinch River Breeder Plant Probabilistic Risk Assessment", CRBRP-4, 1984.

13.2 "Severe Accident Risks: An Assessment for Five U.S. Nuclear Power Plant", NUREG1150.

13.3 G. F. Flanagan, "The Use of PRA in Management of Safety Issues at the High Flux Isotope Reactor", Proc. of the ANS Conference on The Safety, Status and Future of Noncommercial Reactors and Irradiation Facilities, Boise, Idaho, September 31 - October 4, 1990.

13.4 S. A. Eide, D. M Henry, and T. A. Thatcher, "Advanced Test Reactor Level 1 Probabilistic Risk Assessment", ibid.

13.5 T. L. Chu, M. A. Azarm, R. Bari. J. Lehner, W. Luckas, "Quantification of the Probabilistic Risk Assessment (PRA) of the High Flux Beam Reactor (HFBR) at Brookhaven National Laboratory", ibid.

13.6 G. D. Wyss, D. M. Kunsman, A. L. Camp, "Results and Insights from the N Reactor Level II/III PRA for Application to Advanced Reactor Design", ibid.

13.7 S. P. Tinnes, D. S. Cramer, V. E. Logan, S. V. Topp, J. A. Smith, M. D. Brandyberry, "Results of the Level 1 Probabilistic Risk Assessment (PRA) of Internal Events for Heavy Water Production Reactors (U)", ibid.

13.8 M. D. Brandyberry, H. E. Wingo, "External Events Analysis for the Savannah River Site K Reactor (U)", ibid. 
14.0 SUMMARY AND CONCLUSIONS 
Table of Contents

Page

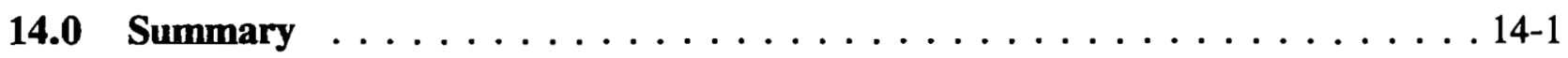

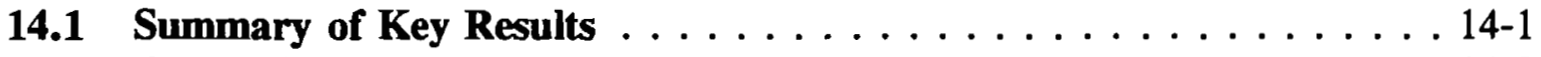

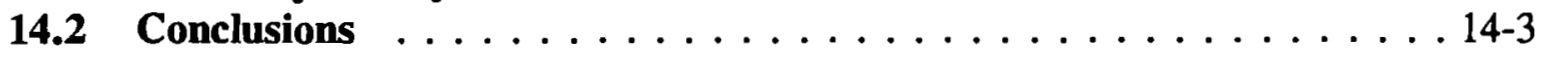




\subsection{SUMMARY AND CONCLUSIONS}

\subsection{Summary of Key Results}

The most obvious result of the EBR-II PRA is that the risk arising from the operation of EBR-II is very low. This result holds when both internal and external events are considered. While this reflects a great deal of credit upon those responsible for the design and operation of EBR-II, the results of the PRA can be used to determine where improvements might be made that could improve the risk profile for the plant. Potential improvements can then be evaluated for cost, amount of risk reduction and for any reduction in plant availability that might arise. Furthermore the insight provided will assist in applying the lessons learned from this study to future IFR design studies.

This discussion identifies those characteristics of EBR-II that result in the low estimate of risk found in this study.

(1) Initiating Event Frequency - The initiating event frequency is low. Roughly four initiating events per year require a scram, manual or automatic, and the scram reliability has been estimated to be between $510^{-6}$ and $10^{-5}$ per demand dependent on the initiating event.

(2) Reactivity feedbacks for those accident sequences in which failure to scram occurs - The results clearly demonstrate the effects of the reactivity feedbacks in limiting the temperature excursion in most of the more likely ATWS events $93 \%$ do not lead to any form of damage.

(3) Decay Heat Removal - EBR-II possesses multiple passive decay heat removal paths and a large heat capacity. Therefore, in contrast to more conventional reactor systems, the loss of decay heat removal systems is only a small factor in the EBR-II risk profile. 
(4) Support Systems - The loss of support systems is relatively unimportant. Loss of Normal or Constant Power systems do challenge the reactor shutdown system to some degree. There is no dependence on electrical power for short term decay heat removal. For long term decay heat removal ( $>14$ days) mitigating systems do require electrical power.

(5) Human Factors - The sensitivity to human error is low because there are relatively few actions required of the operator. Latent errors identified involve maintenance and the improper setting of trip points, however the risk can be reduced with straightforward procedural modifications.

(6) External Events - The sensitivity to a wide range of external events is low because of the wide physical separation between the means of decay heat removal and their relative robustness in the face of external hazard. No external events have been identified which can lead to failure to scram.

In general terms, the risk of operation of EBR-II has important contributions from the relatively rare events of large consequence whose estimated frequency is commensurate with failure-to-scram probabilities. These events, such as primary tank failure or steam generator tube ruptures, have frequencies whose estimation is at best subjective and open to debate. Therefore the engineering approach to these events should not be solely probabilistically based but more in the traditional defense-in-depth manner, including inservice inspection, detectability (ideally on precursor events) and automatic protection (scram, sodium and water dumps, etc.).

One class of events which show up as contributors to risk are the so-called Local Faults due to performing experimental irradiations in EBR-II. However, it is not that these experiments are particularly risky in themselves, it is more that the overall level of risk for EBR-II is low and the uncertainty on the physical phenomena involved in these experiments leads to some level of risk. Two points need to be made here. Firstly, EBR-II is a research reactor and these experiments are being performed to investigate many of the uncertain phenomena. Secondly, the results are uncertainty driven, and should be compared to the other class of risk whose 
numerical estimation is uncertainty driven, the seismic risk. When viewed in that context, the risk from these experiments is very low.

\subsection{Conclusions}

The conclusions reached by the Level 1 study are:

- The frequency of damage at EBR-II is generally lower than that reported for commercial LWRs and the USDOE Category A reactors.

- There is no single dominant source or cause of the various accident sequences.

- The passive decay heat removal function and the ability of reactivity feedbacks to limit the temperature excursion in a transient lead to low risk.

- The passive decay heat removal function and ability of reactivity feedbacks to limit temperature excursion in a transient also lead to a sparse set of requirements for active safety systems or operator intervention.

- EBR-II is relatively insensitive to human error and only a few important human actions were identified.

- EBR-II has little or no requirement for electrical power to prevent fuel damage. 
Appendix A AN ASSESSMENT OF RISK

DURING CORE REFUELING

AT EBR-II 


\section{Table of Contents}

Page

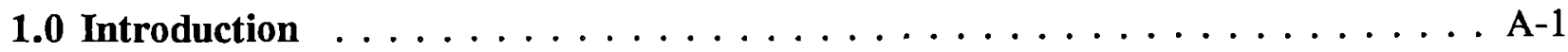

2.0 The Core Refueling Process $\ldots \ldots \ldots \ldots \ldots \ldots \ldots \ldots \ldots$. . . . . . . . . . . .

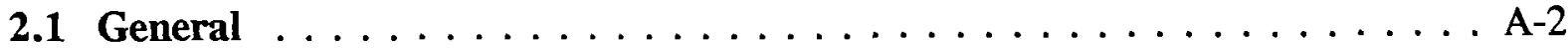

2.1 .1 Gripper Mechanism . . . . . . . . . . . . . . . . A

2.1 .2 Holddown Mechanism $\ldots \ldots \ldots \ldots \ldots \ldots \ldots \ldots$ A $4 \ldots \ldots$

2.1.3 Large and Small Rotating Shield Plugs . . . . . . . . . . . . A A-4

2.1 .4 Transfer Arm . . . . . . . . . . . . . . . . . . A

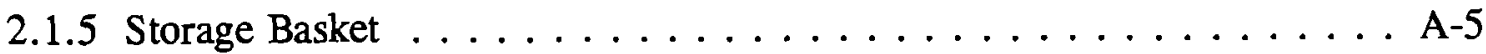

2.1 .6 Description of a Transfer . . . . . . . . . . . . . . A A 6

2.2 Fuel Handling Control System . . . . . . . . . . . . . . . . A A-7

2.2.1 Numerically Controlled Positioning . . . . . . . . . . . . . A-9

2.2.2 Control and Verification . . . . . . . . . . . . . . A-9

2.3 Preparation for Fuel Handling . . . . . . . . . . . . . . A-10

2.3.1 System Preparation $\ldots \ldots \ldots \ldots \ldots \ldots \ldots \ldots \ldots$

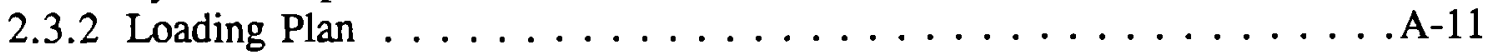

2.4 Summary Observations on Refueling . . . . . . . . . . . . . A-14

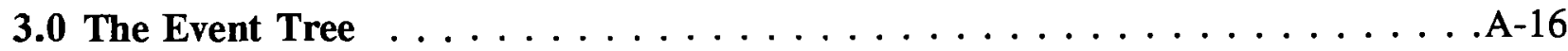

4.0 The Initiating Event Sequence $\ldots \ldots \ldots \ldots \ldots \ldots \ldots \ldots \ldots$

5.0 Assessment of Event Probabilities . . . . . . . . . . . . . . . . . . A-20

5.1 The Fault Trees . . . . . . . . . . . . . . . . . . . . A $\ldots \ldots$

5.2 Analysis of the Fault Trees . . . . . . . . . . . . . . A $\ldots \ldots$

5.2 .1 The Initiating Event Tree . . . . . . . . . . . . . A A 25

5.2 .2 The Protective Surveillance Tree . . . . . . . . . . . . . . A-26

5.2 .3 The Trip Signal Fault Tree . . . . . . . . . . . . . . A A-26

5.2 .4 Safety Rod Trip . . . . . . . . . . . . . . . . A . . . . . . . . . . . . . . . . . . . .

5.3 Analysis of the Event Tree . . . . . . . . . . . . . . . A-27

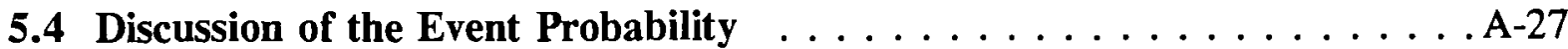

5.5 Secondary Events . . . . . . . . . . . . . . . . . . . . . A .29

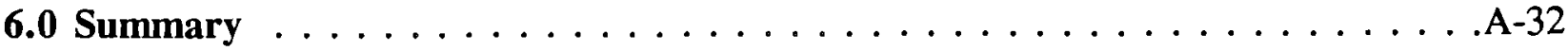

Appendix A References . . . . . . . . . . . . . . . . . . . A-32

Appendix A.1 Human Factors Trees . . . . . . . . . . . . . . A.1-i

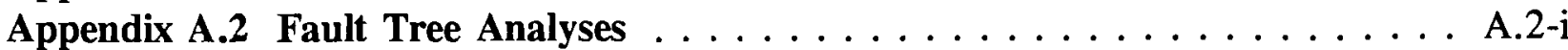

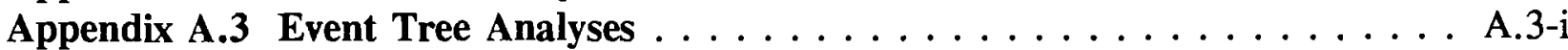




\section{List of Figures}

Page

A.1 - Fuel-Handling Mechanisms in Primary Tank ................ A-3

A.2 - Creation of Loading Instructions . . . . . . . . . . . . . . . A-12

A.3 - Execution of Loading Instructions . . . . . . . . . . . . . . . A-13

A.4 - Core Refueling Accident Event Tree . . . . . . . . . . . . . . . A-17

A.5 - State of Subcriticality During Core Refueling . . . . . . . . . . . . . . A-19

A.6 - Initiating Event Fault Tree . . . . . . . . . . . . . . . . . A-21

A.7 - Protective Surveillance Fault Tree . . . . . . . . . . . . . . . . A-22

A.8 - Trip Signal Fault Tree . . . . . . . . . . . . . . . . . . . . A A-23

A.9 - Safety Rod Trip Fault Tree . . . . . . . . . . . . . . . . . . . A-24 


\section{An Assessment of Risk during Core Refueling at EBR-II}

\subsection{Introduction}

The Experimental Breeder Reactor II (EBR-II) is a liquid-metal cooled, fast neutron nuclear reactor located on the Idaho National Engineering Laboratory [A.1, A.2]. The driver fuel for EBR-II is a uranium alloy encapsulated in stainless steel. However, because the reactor is an experimental facility, it routinely contains a complement of both fueled and structural experiments of widely varying compositions. The heterogenous nature of the core results in individual subassembly reactivity worths that vary from slightly negative for some structural experiments to approximately $1.8 \$$ positive for core drivers being loaded in central positions. A typical core is about $6-1 / 2$ rows across and contains 100 subassemblies.

During the core refueling process, the reactor is taken subcritical by fully withdrawing and unlatching the fueled control rods. Typically, the reactor is five to eight dollars subcritical during refueling. Two safety rods, having a combined worth of $\sim 1 \$$, are fully inserted in the core during the refueling phase and are automatically tripped out by a two-out-of-three neutron detector system should the refueling process cause the system state to approach too close to critical. Forced convective cooling of the reactor by the primary pumps is not allowable during refueling so achieving criticality under these conditions is very undesirable.

The purpose of this document is to present an assessment of the risks associated with the core refueling process in EBR-II. A simplified event tree and its corresponding fault trees using the methods of reference [A.3] are developed and analyzed to yield an estimate of the overall probability of sustaining an inadvertent criticality, and therefore possible core damage, during a reactor operating year. The systems that support core refueling will be described to the extent necessary to impart a basic understanding of the process. 


\subsection{The Core Refueling Process}

\subsection{General}

The fuel handling system includes the various mechanisms required for the remote manipulation of reactor subassemblies in the reactor vessel, together with the control equipment necessary for the safe operation of the mechanisms. The fuel-handling operation involving the movement of the subassemblies between the reactor and the storage basket is termed unrestricted fuel-handling. Unrestricted fuel-handling requires that the reactor be shut down. The operations are performed within the primary tank with the subassemblies completely submerged in sodium.

The principal components used for core refueling are the storage basket, the transfer arm, and the core subassembly gripper and holddown mechanisms, which are positioned by two rotating plugs. Figure A.1 displays the global arrangement of these components on a cross section of the primary system. Brief descriptions of the major components follow.

\subsubsection{Gripper Mechanism}

The gripper mechanism is the electro-mechanical component used to insert or to extract subassemblies from the reactor vessel grid. It is mounted on the small rotating shield plug and extends through a nozzle in the small rotating plug into the sodium in the primary tank, and through a sleeve in the reactor vessel top cover into the reactor vessel. The gripper mechanism grasps the upper adaptor of the subassembly and moves the subassembly vertically out of or into the reactor vessel. The gripper cannot physically engage more than one subassembly at a time. 


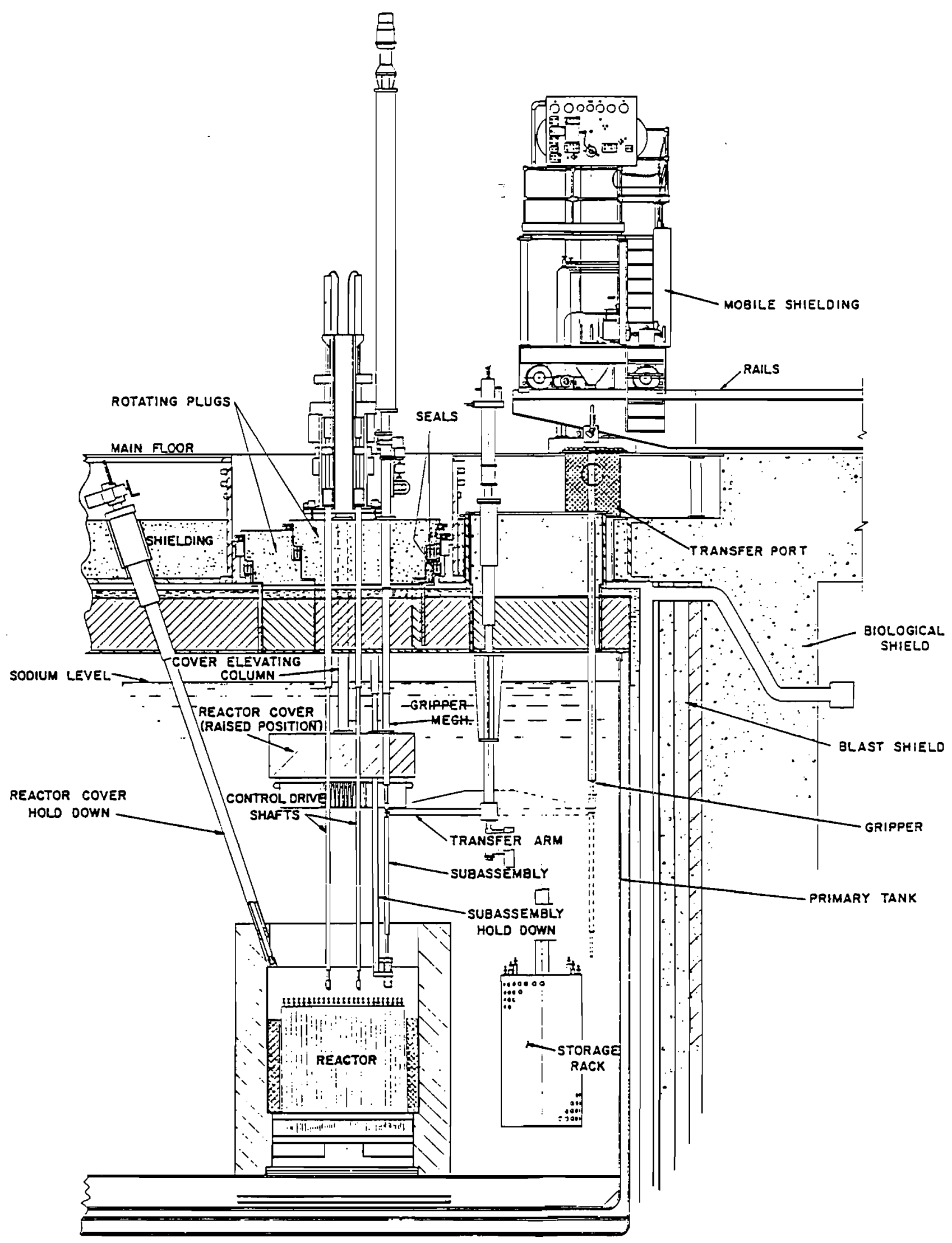

Figure A.1 - Fuel-Handling Mechanisms In Primary Tank 


\subsubsection{Holddown Mechanism}

The holddown mechanism is an electro-mechanical device which operates in conjuction with the gripper mechanism. It is also mounted on the small rotating shield plug, and extends through a nozzle into the reactor vessel. During the insertion or removal of a subassembly from the reactor vessel, the holddown mechanism functions as a guide funnel and prevents inadvertent extraction or movement of adjacent subassemblies. The holddown mechanism also functions as a guide sleeve to steady the subassembly while it is being moved through the primary tank bulk sodium to the transfer position by the rotating plugs.

\subsubsection{Large and Small Rotating Shield Plugs}

Two rotating shield plugs, the large plug and the small plug, are located in the top part of the primary tank cover. The large plug is mounted in the primary tank cover and supported by the T-1 central ring with its vertical axis coinciding with the vertical axis of the reactor core. The small plug is positioned eccentrically within the large plug with the small plug vertical axis offset 15.2 in. from the large plug vertical axis. Both plugs rotate about their respective vertical axes and serve two basic purposes:

(1)

Provide a means of positioning the gripper mechanism over any desired point on the reactor when extracting or inserting any of the 637 subassemblies in the reactor vessel, ${ }^{1}$ and to position the gripper to the operating position or the transfer position.

(2) To position the control rod drives over their respective subassemblies for reactor operation.

An important function of the two plugs is maintaining a positioning accuracy of 1/64 in. by either rotating plug with respect to any of the 637 grid locations.

1 Included are stainless reflector assemblies and breeder blanket assemblies. 


\subsubsection{Transfer Arm}

The transfer arm is an " $\mathrm{L}$ " shaped structure that is manually rotated to transfer subassemblies between the core gripper and the storage basket. Transfer arm motion is both vertical and horizontal. The arm rotates through an arc of approximately $180^{\circ}$ and a vertical motion of 12 in. to allow the fuel handling operator to make manual contacts between the transfer arm and associated equipment. The fuel handling area traversed by the transfer arm carrier block (outside the reactor vessel wall) is provided with a subassembly catch basin.

\subsubsection{Storage Basket}

The storage basket is a cylindrical structure with two basic purposes: (1) provide storage for irradiated subassemblies allowing radioactive decay and decay heat reduction prior to transfer from the primary tank, and (2) provide storage of nonirradiated subassemblies prior to installation in the reactor grid [A.2].

The basket contains 75 storage tubes arranged in three concentric rows. The outer two rows each contain 30 tubes and the inner row contains 15 .

The basket has both rotary and vertical drive systems. The rotary drive has 30 positions, one for each storage location (only even location numbers are used for the inner row). The vertical drive is provided with numerous stop elevations to facilitate receiving or transferring subassemblies.

The basket is wholly contained within the primary tank, and the subassemblies are cooled by conduction and natural convection to the pool sodium. 


\subsubsection{Description of a Transfer}

To transfer a subassembly from the core to the storage basket, the gripper and holddown are positioned over the proper core location. The holddown is lowered around the adapter of the subassembly being removed. The gripper is then lowered onto the upper adapter of the subassembly in the holddown funnel and the gripper jaws are closed. The holddown, as its name implies, prevents any adjacent subassemblies from being lifted with the one being raised by the gripper. When the gripper engagement is properly effected, the sense rod within the gripper shaft is lifted approximately $3 / 4 \mathrm{in}$. This motion is indicated on the fuel handling console. The gripper jaws are then closed by vertical shaft motion. This information is displayed to the fuel handling operator by an indicator on the fuel handling console.

The gripper is raised to its full-up position, approximately $10 \mathrm{ft}$. above the core, with the subassembly removed from the reactor vessel, but still completely immersed in sodium. The holddown mechanism is then raised to its up position and serves as a guide for the lower portion of the subassembly during plug rotation. The shield plugs are rotated to the "transfer" position where the subassembly is picked up by the transfer arm. The transfer arm is manually rotated to engage the lower (rectangular) portion of the subassembly upper adapter and then locked to the subassembly. A "wiggle test" is performed at this point to assure that a proper transfer has been made. When the transfer verification is complete, the gripper jaws are opened and the transfer arm is lowered to remove the subassembly adapter from the gripper jaws.

To complete the transfer of the subassembly to its programmed storage basket position requires rotation of the transfer arm to one of three storage basket rows. The basket is rotated to the coordinates programmed in the Numerically Controlled Positioning System, and then raised until the subassembly's lower adapter almost enters the storage hole. The subassembly is started into the storage hole manually by lowering the transfer arm, and then the basket is raised until the subassembly is seated. Another wiggle test verifies that the transfer is complete, and the transfer arm is unlocked and moved clear of the subassembly. This process is reversed to remove a fresh subassembly from the storage basket and transfer it to the core. Under optimum conditions, one complete interchange can be completed in about 45 minutes. 
Certain special operations are also performed with the fuel handling equipment. These operations include removal and replacement of safety rods, safety and control-rod thimbles, and neutron source rods. These operations involve manual rotation of the core gipper to lock and unlock bayonet fittings in the reactor grid.

Two important facts should be emphasized at this point. The gripper mechanism is the only device capable of engaging and removing a subassembly from, or inserting one into, the reactor grid. Its design prevents it from engaging more than one subassembly. In fact, the capacity of all of the EBR-II equipment that transfers subassemblies is limited to a single subassembly.

\subsection{Fuel Handling Control System}

The fuel handling control system consists of the fuel handling control console, the interlock and repetitive motion controls, and the numerically controlled positioning system. Also part of the fuel handling control system are the limit switches and relays that are integrated with the various fuel handling mechanisms, the motors to operate the mechanisms, and the motor controllers and contactors that control operation of the mechanism's motor.

The purpose of the fuel handling control system is to coordinate the operation of all associated fuel handling mechanisms in a sequentially prescribed manner when removing or replacing reactor subassemblies. The system functions to automatically direct and limit the mechanical motions initiated by an operator at the fuel handling control center to prevent a potentially dangerous or damaging condition from developing.

The EBR-II fuel handling operation is essentially an automatically sequenced series of repetitive operations. The basic control philosophy consists of a permissive system. The operator must initiate each step in the process from the fuel handling control console, or refueling machine control panel, but the control system will permit the step to proceed only if all prerequisite conditions have been satisified. 
In the event an abnormal condition develops, the causative action is stopped automatically and in many cases an identifying alarm light is illuminated. Overall supervision of the fuel handling operations is effected by a qualified fuel handling operator at the fuel handling console.

Nuclear instrumentation is provided for automatically scramming the safety rods during fuel handling operations if the neutron flux level exceeds a preset maximum, or if the reactor period is less than a preset minimum. A trip from the neutron monitors also arrests the vertical motion of the core gripper, thereby stopping any fuel movement. The neutron flux alarms if the signal on any one of the three neutron channels exceeds the trip setpoint. The alarm provides an additional alerting device that signals a low subcriticality margin. The neutron flux level is also visually monitored during the fuel handling operation by means of the log count rate recorder located on the fuel handling control console. Safety rod scram can be manually initiated if necessary by a pushbutton on the fuel handling console or on the main control room console. Additionally, after each subassembly insertion in the reactor grid, an operator in the reactor control room takes three-minute counts on all three nuclear channels and records, plots, and interprets the results. He will notify the Shift Supervisor if any unusual condition is observed. The fuel handling operator is provided with a specific count rate threshold at which he is instructed to terminate fuel handling.

A large part of the fuel handling control system is concerned with control of the angular position of the large rotating plug, small rotating plug, and gripper; the angular positioning and elevation of the storage basket; and the manual positioning of the transfer arm over the storage basket or under the core gripper. This part of the system is known as the Numerically Controlled Positioning System (NCPS). It positions and supervises the positioning of the plugs, gripper, and basket in accordance with coordinate information available in a reference library. 


\subsubsection{Numerically Controlled Positioning}

The major components of the NCPS are: a microprocessor computer, plug and gripper position synchro-transmitters, synchro-to-digital converters, digitalto-analog converters, drive motor controllers, and drive motor units.

Rotating plug and gripper (plus storage basket, as appropriate) command coordinates are transmitted to the microprocesser computer by the FHANDLE program on the Data Acquisition System Computer (DAS). Analog output (digital-to-analog) units provide signal voltages to the motor controllers for the plug and gripper drives to position the core gripper over the prescribed grid position.

The FHANDLE program on the DAS provides input (type of transfer, sequence number, coordinates, basket position etc.) to the NCPS computer. This program also provides a check of each fuel handling sequence to verify it can be performed, maintains a record of the current reactor and basket loadings, and provides a record of each transfer (including time and operator name). The computer operations consist primarily of three basic functions: (1) reading input data from the DAS FHANDLE program, (2) measuring and controlling the rotation (and elevation) of the storage basket, and (3) controlling the angular rotation of the rotating plugs and the core gripper.

\subsubsection{Control \& Verification}

In fuel handling Sequence B, the operator tells the DAS to transmit the proper coordinates to the NCPS computer. These data are then displayed on the fuel handling console. The plugs and gripper are then rotated to the destination angles. When the

plugs and gripper reach the destination angles, a green completion light is received. The operator then visually verifies that the location and destination displays are correct. The actual angles of the plugs and gripper are then manually checked and compared to the displayed angles on the fuel handling console. If these agree, the fuel handling console operator then enters the angles into the terminal and these data are sent to DAS. A coordcheck subroutine in the 
FHANDLE program verifies that the coordinates are correct. An interlock prevents further fuel handling until this verification sequence is complete.

The same routine is used to place a new subassembly in an open core position during Sequence $\mathrm{G}$ of fuel handling.

\subsection{Preparation for Fuel Handling}

Preparation for unrestricted fuel handling involves two major efforts. The first is to shut the reactor down and place the reactor and the primary system in the proper configuration for fuel handling. The second is the preparation, verification, and approval of the fuel handling loading plan. The following two subsections describe these processes.

\subsubsection{System Preparation}

The reactor is shut down by lowering all of the fueled control and safety rods to their least reactive (down) positions. During this process, the Operations Physicist establishes the degree of subcriticality of the reactor.

Immediately after shut down, the reactor is prepared for core refueling. A fuel handling checksheet is completed and fuel handling interlocks are checked. To prepare the reactor vessel for fuel handling, the control rods are released from their drive mechanisms. All 12 drives are then raised 3 in. to preclude their interference with the subassembly upper adapters when the shield plugs are rotated.

The reactor vessel cover is unlocked and raised until three torque pins mounted to the top of the cover engage sockets on the underside of the small rotating plug. Each rotating plug employs a eutectic alloy freeze seal that must be fully molten before plug rotation can be attempted. To fulfill the required interlocks, the safety rods are driven into the core to their maximum reactivity position $(\sim 1 \$)$. When the above conditions have been met and the gripper and holddown operation have been checked, fuel handling may be started. 


\subsubsection{Loading Plan}

Conducting refueling requires the preparation of a carefully considered loading plan, which results in the creation of loading instructions. A basic tenet of core refueling is that subassemblies are always replaced on a one-for-one exchange basis except during replacement of control and safety rod thimbles, when up to six fueled subassemblies may be removed.

A flow chart for creation of loading instructions [A.4] is shown in Figure A.2. Reactivity predictions used in developing a loading plan are made by computing changes from the known conditions of the previous loading. This minimizes the reliance placed on predictive capabilities and thereby reduces the chances of a serious reactivity prediction error. The Chief Physicist has the responsibility for developing the loading plan. In the course of this work he predicts the reactivity at each step of the loading and the Operations Physicist assures that Technical Specification restrictions on reactivity are not violated. The Fuel Management Supervisor prepares the unrestricted-loading instructions which are reviewed by the Chief Physicist, the Operations Physicist, and the Reactor Shift Supervisor, and approved by the Reactor Neutronics Manager and the Plant Operatons Manager. Restricted-loading instructions are reviewed by the Reactor Shift Superivsor and approved by the Plant Operations Manager.

A flow chart for implementation of the loading instructions [A.4] is shown in Figure A.3. The steps shown there apply to both restricted and unrestricted refueling. Several operations personnel are involved in the process to provide built-in cross checks. The procedure requires a number of sequential steps and intermediate checks, e.g., the preprogramming of instructions before the actual loading is done. These features preclude a single operation from causing a series of errors. The process of transferring the actual coordinate locations via a hard-wired computer eliminates operator input errors. 


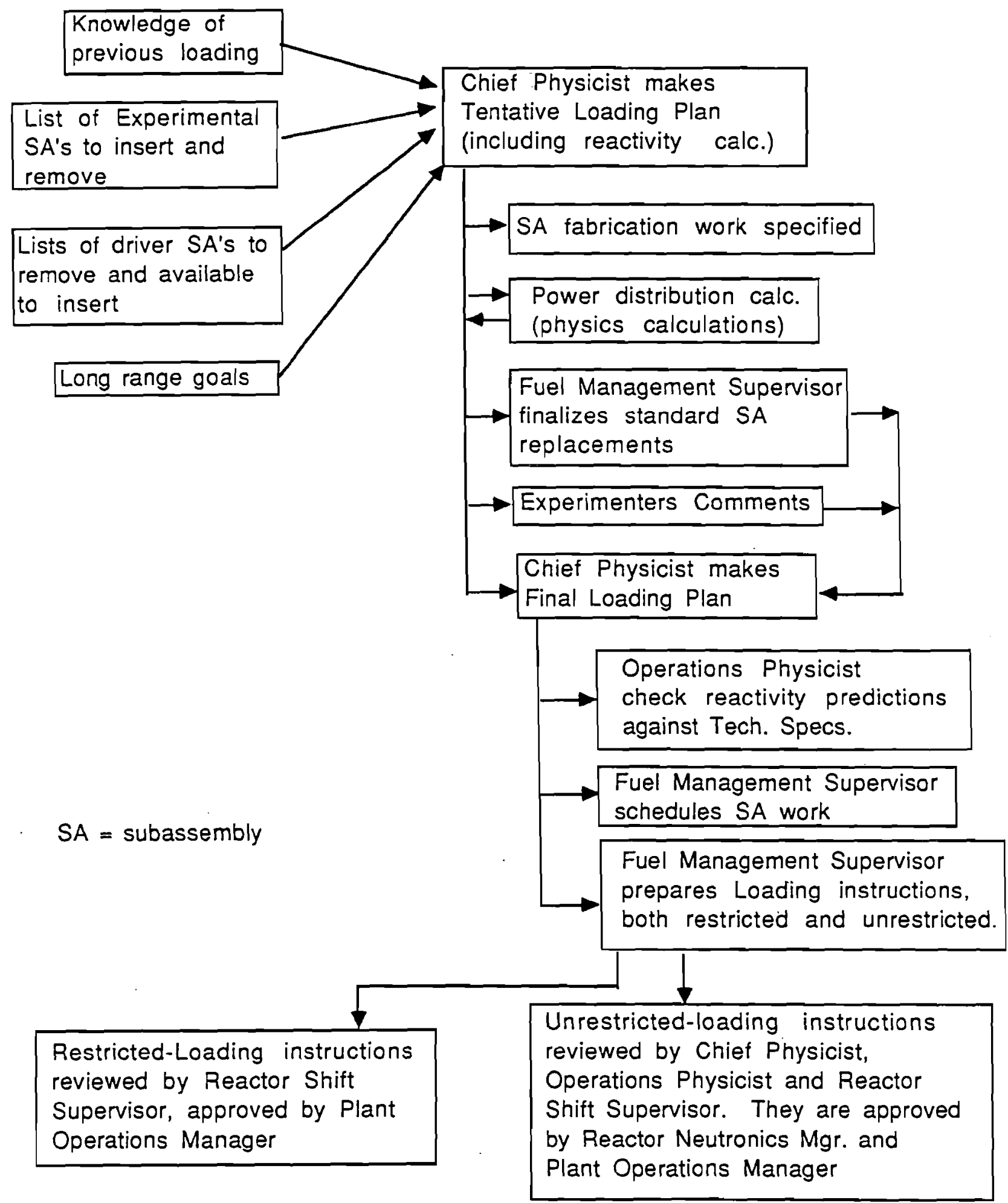

Figure A.2 - Creation of Loading Instructions 
Check that loading instructions are properly approved.

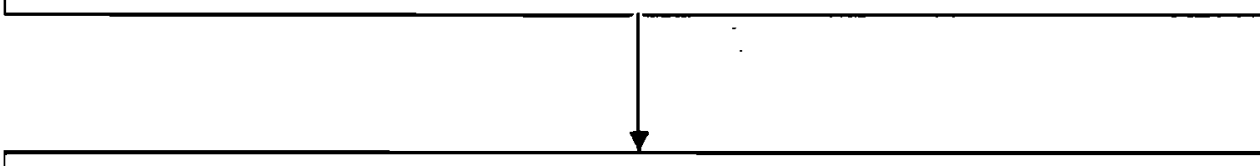

Load electronic copy of loading instructions into FHAN code. Code checks validity.

Follow fuel handling control checksheet.

Fuel handling console (FHC) operator initiates instructions for FHAN to transmit coordinates to numerically-controlled positioning system (NCPS).

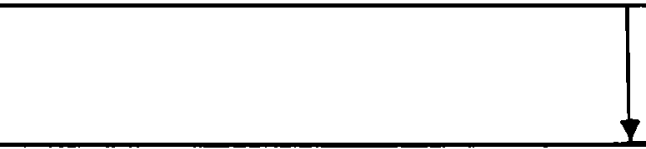

Execute each transfer:

a) FHC operator intiates steps with NCPS

b) FHAN program and operator independently verify grid coordinates

c) Third operator (in control room) checks count rate change (unrestricted refueling only).

Figure A.3 - Execution of Loading Instructions 


\subsection{Summary Observations on Refueling}

This section summarizes the basic principles, facts, and crosschecks attendant to the EBR-II core refueling process.

(1) Only one subassembly can be handled at a time.

(2) Subassemblies are replaced on a one-for-one exchange basis, i.e., multiple consecutive removals are not allowed. Even if an insertion positioning error is made, it is not be possible to insert the subassembly, (with the exception of control and safety rods) because the position already contains an assembly.

(3) Neutron countrate surveillance is formally required of the reactor console operator after each subassembly insertion. The operator is required to notify the fuel handling console operator and Shift Supervisor to terminate fuel handling if the count rate ratios fall to a prescribed standard. The fuel handling console operator also checks neutron count rates.

(4) The loading plan is prepared by the Chief Physicist and checked by the Operations Physicist.

(5) After the actual loading instructions are prepared by the Fuel Management Supervisor, they are checked by the Chief Physicist, the Operations Physicist, and a Reactor Shift Supervisor.

(6) The loading instruction is approved by the Reactor Neutronics Manager and the Plant Operations Manager.

(7) The loading instruction is computer-checked by a standing program - FHAN to ensure that physically improper placements are not undetected.

(8) After these checks, the approved loading instruction is, by the process, already stored in the DAS computer.

(9) Actual core destination coordinates are withdrawn from a standard reference library and transmitted down to the NCPS by a hard-wired computer connection from the DAS. 
(10) After each subassembly transfer, multiple cross checks of the core position, both by the fuel handling computer and by the operator, must be satisfied before further fuel handling steps are allowed.

(11) An automatic system is in place to prevent an inadvertent criticality. Based on a coincidence signal from two-out-of-three neutron detector channels, the safety rods are tripped if the neutron count rate or the reactor period approaches trip set points. 


\subsection{The Event Tree}

The sequence of events during which errors may occur leading to an unplanned criticality during refueling has been described in the previous section. These events can be simplified and summarized.

- Initiating events leading towards a critical configuration which may originate either from loading plan errors or system-induced mispositioning errors.

- Failure to complete the count rate surveillance activity.

- $\quad$ Either of:

- $\quad$ Failure of two-out-of-three neutron channels to respond either to high neutron count rate levels or short reactor period.

Failure of safety rods to remove fuel from the reactor given a trip signal from the neutron detector channels.

Figure A.4 is the event diagram for this accident sequence. It has been simplified in the following ways. No credit is taken for the period trips for two reasons. First, they derive their signals from the same neutron detectors as the level trips, so there is commonality. Second, for normal insertion or removal speeds, the reactor period is not suffiently short to reach the period trip points. Another item that is not included in the tree is interruption of the gripper vertical motion by the neutron channels when they trip (conservative assumption). Additionally, the secondary accident of dropping a fueled subassembly into a just-subcritical core is omitted from the tree. No subassembly has been dropped into the core in 20,700 subassembly insertions and removals, so its probability of occurrence is of the order of $5 \times 10^{-5}$ less than any sequence leading to a critical core. ${ }^{2}$ The probability of this secondary event sequence is discussed in Section 5.5 below.

2

The EBR-II fuel transfer history includes two subassemblies that were dropped in the primary tank. These were dropped by the transfer arm; it is impossible for subassemblies released from the transfer arm to interact with the reactor core. The two cases that occurred were completely benign. 


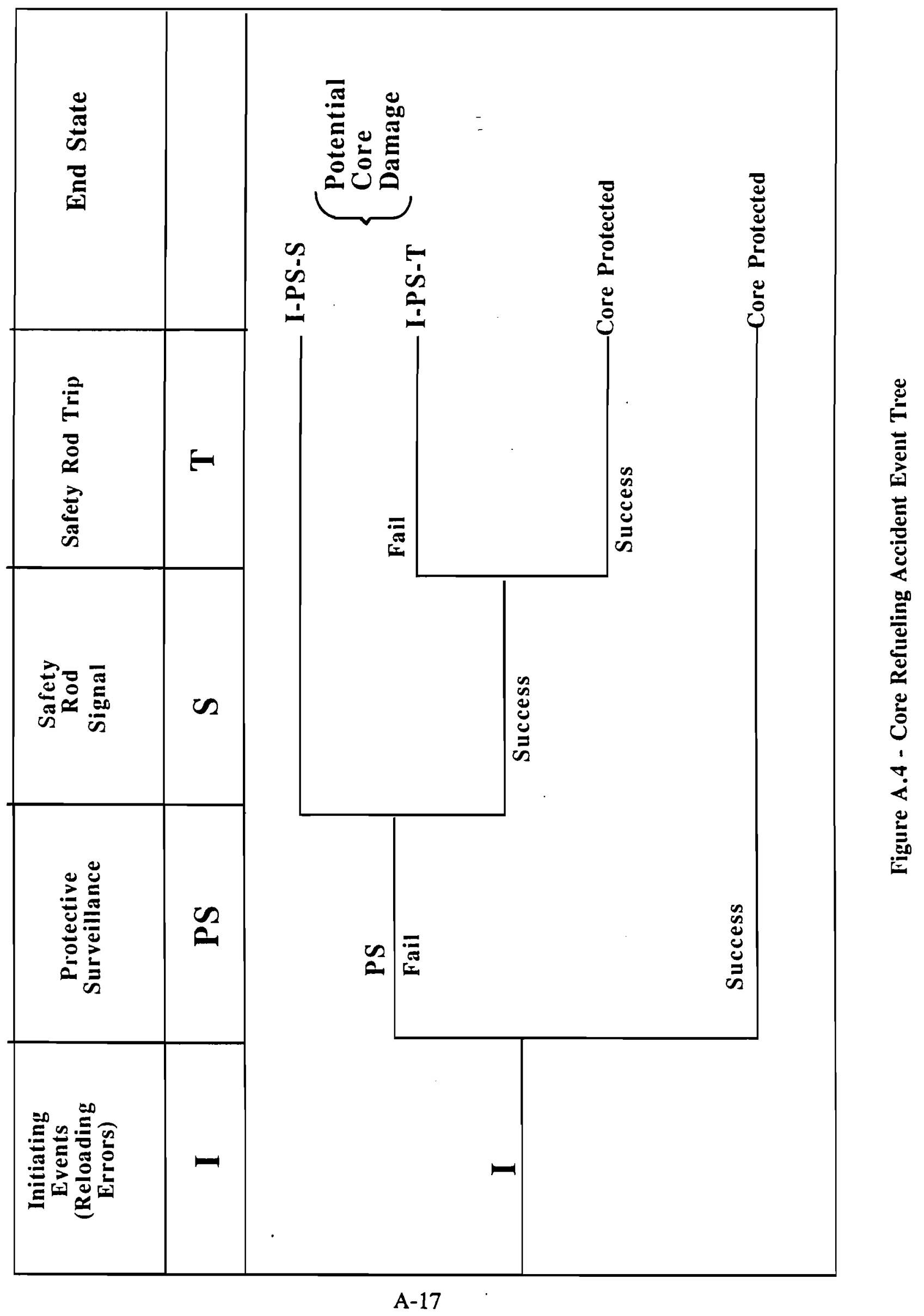




\subsection{The Initiating Event Sequence}

Prior to initiating the refueling sequence, the reactor is taken to a subcritical condition by fully withdrawing the control and safety rods. During this process, the degree of subcriticality is established; subsequently the safety rods are cocked (inserted) to their full worth of 1.0\$. The EBR-II Technical Specifications require that at least $4.0 \$$ of reactivity be vested in the safety and control rods during the refueling process. This means that the absolute minimum subcritical position allowed during refueling is $-3.0 \$$. This relationship is shown in Fig. A.5.

In practice, refueling must be conducted at a level more subcritical than $-3.0 \$$. Analysis of 304 sub-runs gave an average shut-down reactivity margin of approximately $6.1 \$$ at the conclusion of fuel handling. Normally, the worth of subassemblies range from $-0.1 \$$ to $+1.8 \$$. From the $3.0 \$$ position, ignoring the trippable reactivity vested in the safety rods, it would take two errors averaging $1.5 \$$ each to just reach critical, or three of $1.0 \$$ each or five of $0.6 \$$, etc. However, considering that reloading occurs at a subcriticality margin substantially greater than $3.0 \$$ and that the safety rods provide a response of $-1.0 \$$, in practice it would take four or more reloading errors of $1.5 \$$ each, five to seven errors of $1.0 \$$ each, and a large number of positive insertion errors for subassemblies having worths less than $1.0 \$$ to approach critical.

Considering that, in general, the refueling process involves replacing one subassembly with another that has only marginally greater reactivity worth, the relative probability of producing a sequence of errors (within any loading plan) having sufficient additional reactivity to reach critical is very low when compared to the bounding cases described above. In the case of control rod or safety rod thimble replacement, the fueled subassemblies (up to six) temporarily removed and replaced represent a small increase in reactivity. 


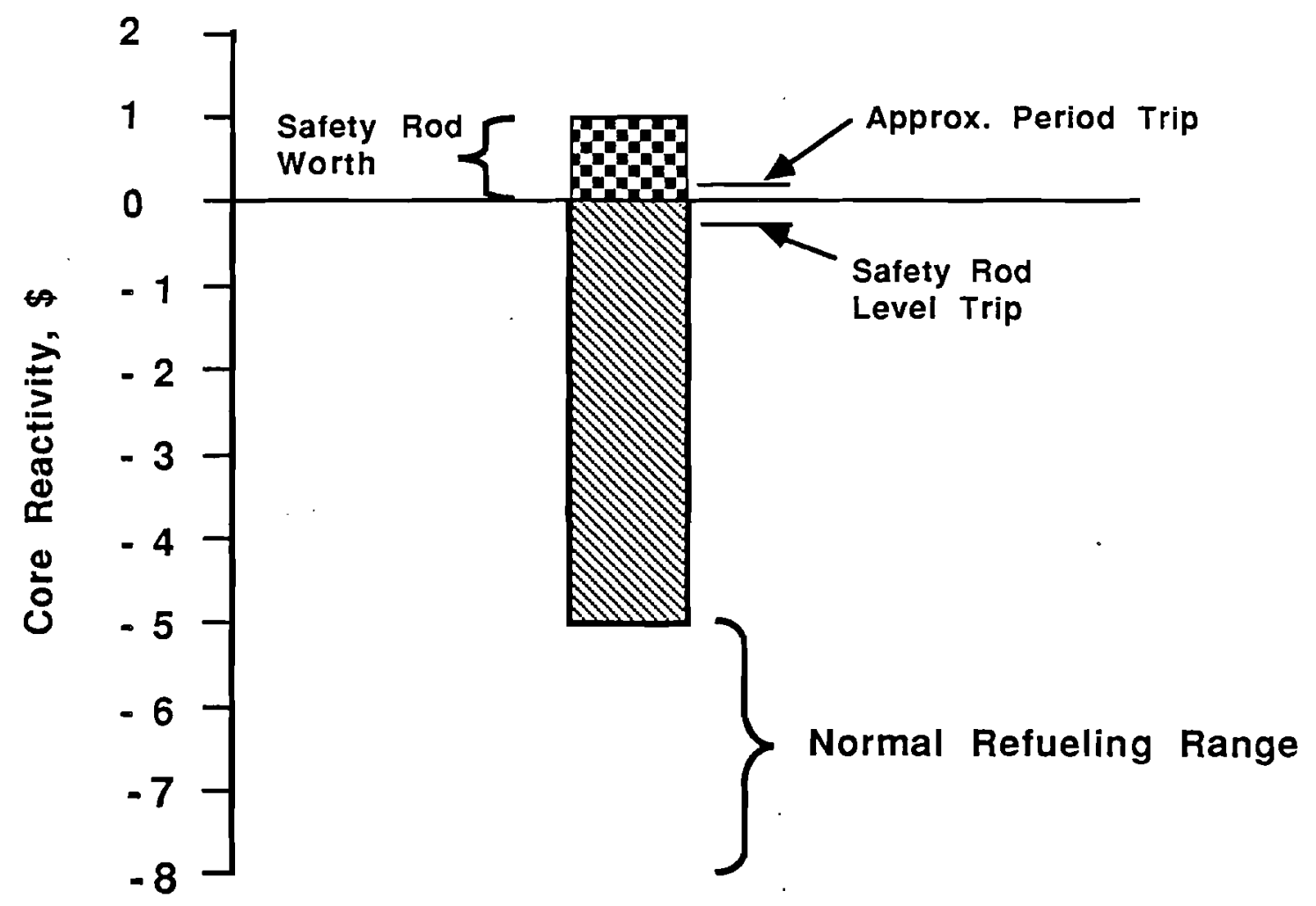

Figure A.5 - State of Subcriticality During Core Refueling 


\subsection{Assessment of Event Probabilities}

\subsection{The Fault Trees}

The event tree shown in Fig. A.4 indicates four fault trees, one for initiating events, one to evaluate the protective surveillance activity, one for neutron detector trip signal response, and one for safety rod trip.

Figures A.6, A.7, A.8, and A.9 show the four fault trees. The human error probabilities (HEPs) were prepared by a consultant to ANL for the PRA effort [A.5]. A few remarks are appropriate. The effect of common mode, that is, planning errors on the initiating event sequence is directly accounted for in the initiating event fault tree by a human factors analysis. The process of count rate surveillance is required after each subassembly insertion in the core. However, credit is taken in the protective surveillance tree only once -- when the count rate response threshold is reached -- for this activity. Calibration errors which could impact surveillance effectiveness are specifically accounted for in the protective surveillance tree.

However, a review of the protective system and the potential effects of calibration errors make it clear that calibration errors will not invalidate the generation of trip signals. The trip level may be altered by errors in the calibration process, but as the reactor approaches or reaches critical, the large increase in neutron population ensures that trip levels will be reached. Note also that interlock checks require both low-level and high-level trip tests on the neutron channels prior to conducting core refueling. 
员品
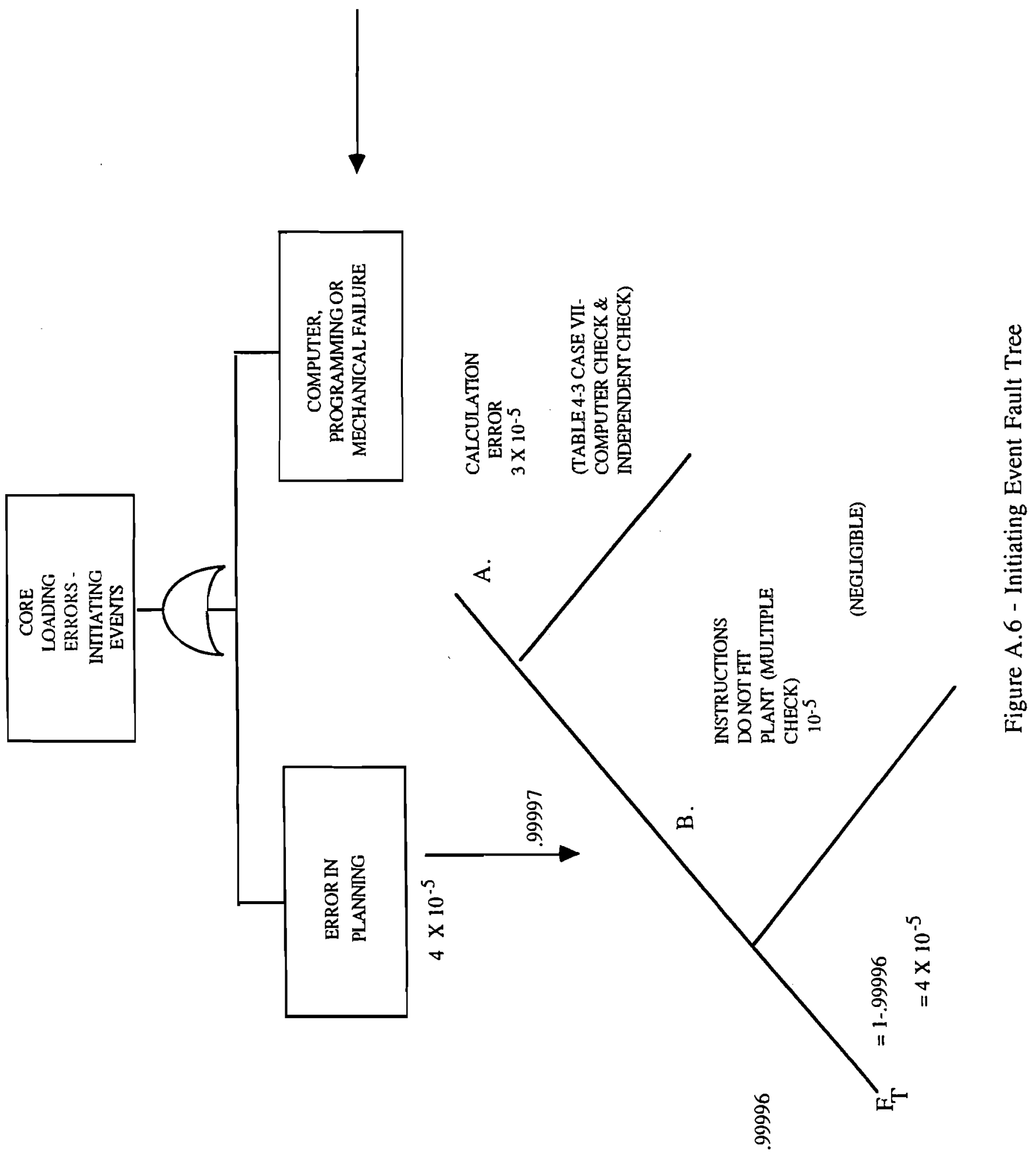


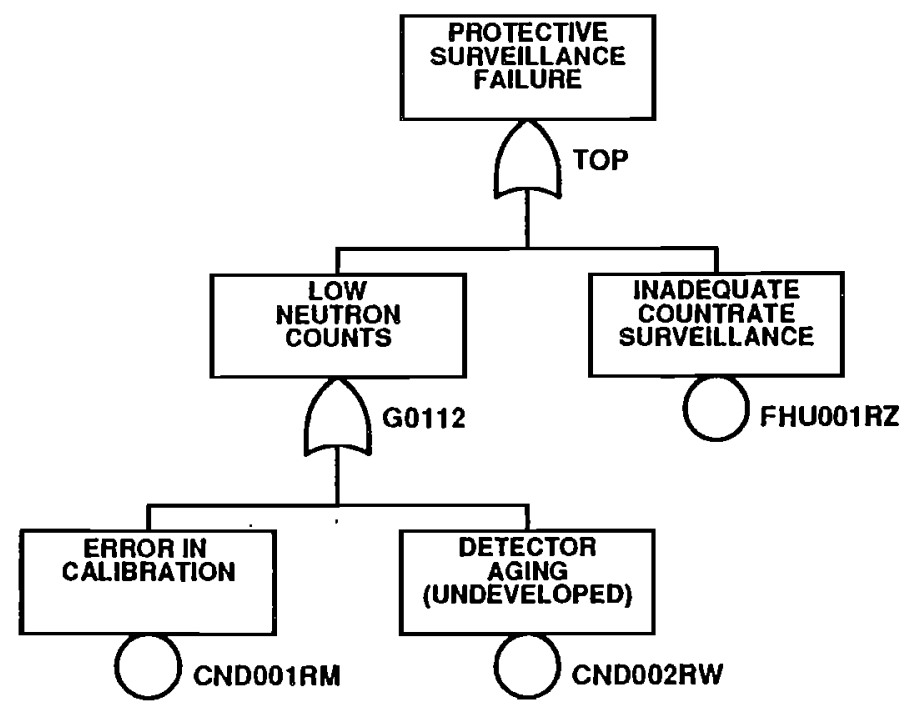

\begin{tabular}{|l|l|}
\hline \multicolumn{2}{|l|}{ PROTECTIVE SURVEILLANCE FAULT TREE } \\
\hline CORE REFUELING & PAGE 1 OF 1 \\
\hline 09-MAY-90
\end{tabular}

Figure A.7 - Protective Surveillance Fault Tree 


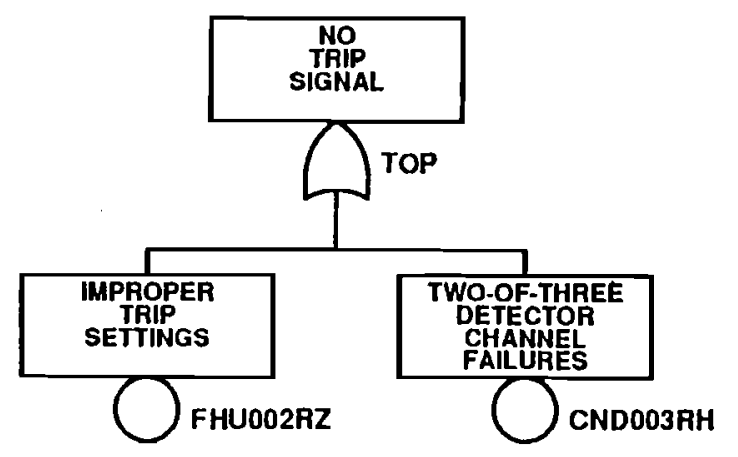

离

\begin{tabular}{|l|l|}
\hline \multicolumn{1}{|l|}{ TRIP SIGNAL FAULT TREE } \\
\hline CORE REFUELING \\
\hline 09-MAY-90 & PAGE 1 OF 1 \\
\hline
\end{tabular}

Figure A.8 - Trip Signal Fault Tree 


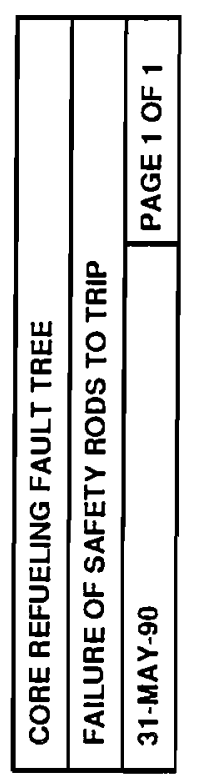

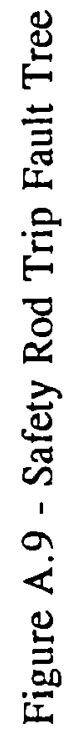




\subsection{Analysis of The Fault Trees}

\subsubsection{The Initiating Event Tree}

The details of human error and fault tree probability analyses are presented in appendices A.1 and A.2. For the fuel reloading planning process, process errors were evaluated using ASEP techniques as shown on Fig. A.6. The value estimated by this technique $\left(41^{-5}\right)$ was sufficiently low that it was not refined by further analysis. No direct estimates were found for computer or data transmission errors, so these and hardware misposition errors were conservatively estimated from plant data.

Regarding the computer and hardware systems; the current software has been operating for the past ten (10) loading sequences (subruns) and the hardware system has been in place since the reactor was constructed. No failures have been observed. Assume that a loading error occurs on the next use. Then, the probability of an error is $1 / 11$ or 0.0909 . This number only represents the probability of a random error, without regard to reactivity magnitude. With the reactor $3.0 \$$ or more subcritical, two loading errors having a net positive reactivity change of $1.5 \$$ each must occur for criticality to be approached. This means that two $1.5 \$$ assemblies must replace, by mistake, two null-worth assemblies. The net probability for one mistake of this magnitude is $0.0909 \mathrm{x}$ frequency of $1.5 \$$ insertions $\mathrm{x}$ freq. of null-worth removals or $0.0909 \mathrm{x}$ $0.161 \times 0.091=1.3410^{-3}$. A minimum of two errors is required to overcome the $3.0 \$$ margin. If one loading error has been made, it is assumed that a companion error is highly probable, so the net probability of a second $1.5 \$$ error is taken as $1 \times 0.161 \times 0.091$ or $1.4710^{-2}$. The maximum probability of system-induced positioning errors just leading to critical is thus $\left(1.3410^{-3}\right) \times\left(1.4710^{-2}\right)$ or $2.010^{-5}$ per subrun.

These data were input to the MODULE code [A.6], which analyzes the fault tree. The mean probability (per reactor subrun) of incurring the initiating event sequence is $5.610^{-5}$. (These values are from the modularized analytical method in MODULE). 


\subsubsection{The Protective Surveillance Tree}

The effect of neutron detector aging is nominally indicated on the fault tree for this activity (Fig. A.7). However, the detector failure rate is not developed because of the manner in which the detectors are managed. Each detector is inserted years apart, providing a gradient in age, so that multiple simultaneous failures are extremely unlikely. Further, there are a number of self-check devices, including low count rate trips which stop the fuel handling process if any one channel trips on low count rate.

The calibration error and operator surveillance failure events were assigned probabilities of $3.010^{-5}$ and $7.3310^{-4}$ per opportunity, e.g., per subrun (see HEP analysis in Appendix A.1). The MODULE code combines these effects to yield a mean probability of failure of the protective surveillance activity of $7.410^{-4}$ per subrun.

\subsubsection{The Trip Signal Fault Tree}

This fault tree is composed of two basic events: failure of two-outof-three of the neutron detector channels and improper setting of the trip points. For the trip settings, the HEP analysis took credit for interlock checks prior to refueling (as recovery actions). For this event, the probability was computed as $510^{6}$ per subrun. Published data exist for the neutron detector channels [A.7]. A conservative fuel loading time span of 3 days was assumed.

For the three detector channels, the failure probability in a three day refueling sequence is given from

$$
\begin{aligned}
& \begin{aligned}
\mathrm{P}_{\mathrm{f}} & =3(\lambda \tau)^{2}-2(\lambda \tau)^{3} \\
& =3\left(5.310^{-6} / \mathrm{hr} \times 72 \mathrm{hr}\right)^{2}-2\left(5.310^{-6} \times 72\right)^{3} \\
& =4.4 \times 10^{-7} \text { per loading sequence. }
\end{aligned} \\
& \text { The failure rate data are given in reference [A.7]. }
\end{aligned}
$$


With these inputs, the predicted rate of failure to generate a trip signal is $5.3 \times 10^{-6} \mathrm{per}$ sub run. Detector channel failure represents, again, only a small fraction of this rate.

\subsubsection{Safety Rod Trip}

The probability of the safety rods failing to scram is calculated as $1 / 2 \times(1 / 475)$ or $1.110^{-3}$ per demand (zero failure in 475 demands). Noting that the demand rate is about one use per subrun, the failure rate is $1.110^{-3}$ per subrun.

\subsection{Analysis of the Event Tree}

Referring back to the event tree (Fig. A.4), there are two paths leading to an unprotected criticality during core refueling, I-PS-S and I-PS-T. These were computer-analyzed with Monte-Carlo techniques [A.8]. The probabilities per subrun for these two branches are:

I-PS-S: $2.610^{-13}$ with $5 \% / 95 \%$ Confidence Limits of $2.910^{-15} / 8.110^{-13}$

I-PS-T: $1.010^{-10}$ with $5 \% / 95 \%$ Confidence Limits of $1.610^{-12} / 3.5510^{-10}$

Because of the high reliability of the three-channel detector system, the probability of the I-PS-S event is sufficiently low compared to the I-PS-T event that it can be dismissed. Considering that there are 7.5 subruns per year, the mean probability of incurring an unprotected criticality is $1.010^{-10} \times 7.5=7.510^{-10}$ per reactor year.

\subsection{Discussion of the Event Probability}

Some of the factors that could modify, or affect, the above conclusion are reviewer herein. When a trip signal is generated, it interrupts core gripper motion, thereby stopping any further reactivity insertion. Clearly, this action reduces those branches in the event tree for which trip signal success is indicated; e.g., the I-PS-T event would create lessened consequences because fuel insertion is stopped. 
Another factor of conservatism is that count rate surveillance is credited only once in the structure of the fault and event trees. In practice, count rate surveillance is required after every insertion. The operators at both the fuel handling console and the reactor console are changed every two hours; since an average round trip (core to basket to core) takes 45 minutes, only two loading changes typically occur for each pair of operators. Even if surveillance were omitted by one set of operators, it is highly likely that the next set would record, and respond to, system neutron count rates.

An experience rated value of $\sim .110^{-3}$ per demand for safety rod trip failure is used in the I-PS-T event branch. Another member of the EBR-II PRA team has judged that this value is less than $110^{-4}$ [A.9]. Note also that the loading plan error rate input to the initiating event tree was derived by the ASEP method. It appears that a more detailed analysis of the loading plan preparation process would produce a lower failure rate estimate for loading plan errors. In the same tree, since core refueling is conducted from a subcritical position considerably greater than the required minimum of $-3 \$$, it would take more mechanical or computer loading errors than is assumed to actually challenge the protective system.

Finally, no attempt was made to take credit for manual safety rod trip by the operators. Note that if a single detector channel reaches its trip point, an alarm is triggered in the control room. This would provide the basis for a manual trip even before the automatic trip which is based on two-out-of-three coincidence.

Arrayed against the above factors of conservatism are uncertainties in some of the estimates; for example, more recent, detector channel failure rates and more definitive estimates of computer and mechanical failures. Note, however, that a search of the EBR-II incident and UOR records did not produce any evidence of a mechanical (NCPS) error in over 20,000 core fuel movements.

This discussion is concluded by saying that there is an array of conservative factors that will offset any uncertainties or significant underestimates in the numerical 
probabilities. It is concluded that the event estimates are reasonable, but conservative, representations of the process and that the probability of incurring plant damage during core refueling is very low.

\subsection{Secondary Events}

Secondary events of two other types related to core refueling can be postulated. One is the rapid insertion of a fueled assembly into the core (failure of speed interlocks or a dropped subassembly). The second is an unplanned startup of the primary pumps with core refueling in progress.

Regarding the first of these events, the event has safety significance only if it occurs with the core near critical.

At lower subcritical levels, a dropped assembly will simply serve to replace the reactivity of the previously removed assembly without further effect (note, however, that it will set off the period trips which will initiate protective action). Since this event has significance only if the initiating event sequence and protective surveillance activities have failed, it is a very low probability scenario. The probability of dropping an assembly is less than $510^{-5}$ per demand ( $<1$ in 20,690 transfers). Inputting this value to the event tree analysis code while taking no credit for automatic protective response, ${ }^{3}$ the mean probability per subrun is: $2.710^{-11}$ with $5 \% / 95 \%$ confidence limits of 3.810 $13 / 1.0 \times 10^{-10}$. The dropped subassembly is considered to be by far the worst of the two rapid insertion scenarios, as the other requires multiple interlock failures, and the safety system can respond to it because the insertion occurs at lower speeds. The mean probability for this case over a reactor year is thus $7.5 \times 2.710^{-11}$ or $2.010^{-10}$.

3 For a dropped assembly, system automatic trip is not sufficiently fast to prevent core criticality, assuming that the core is just subcritical [A.10]. 
Startup of the primary pumps would be a significant event if there were any possibility that hydraulic forces could lift the fueled control rods into the core. An enumeration of factors related to this event serves to demonstrate that this event is not possible.

First, the pumps are interlocked out during core refueling and the pump motor power is off. But more directly, the control rods, within their thimbles, are specifically designed to achieve positive hydraulic holddown. Technical analysis demonstrates that they have positive holddown over the entire range of flow provided by the primary pumps. Therefore, control rod lift due to pump startup is precluded.

The contribution of these two secondary scenarios is thus either zero (pump induced lift of the control rods) or very low ( $210^{-10}$ for a subassembly dropped into a just-subcritical core). The event tree analyses are attached as Appendix A.3. 


\subsection{Summary}

The events leading to an unprotected criticality during core refueling have been separated into four fault trees.

Evaluation of the components contributing to each tree leads to the final conlusion that the probability of incurring such an event is $810^{-10}$ per reactor year or less. Although there are always uncertainties associated with the genesis of such a number, these uncertainties tend to be offset by conservative factors inherent in the analysis.

Two secondary events potentially leading to an unplanned criticality were also reviewed. One of these is considered to be impossible - that is, prevented by physical design - and the other has a predicted probability of $210^{-10}$ per reactor year.

It is concluded that the risks presented by the core refueling process at EBR-II are substantially below any threshold that requires further analysis. 


\section{Appendix A References}

A.1. L. J. Koch, et al., "Hazard Summary Report, Experimental Breeder Reactor II," ANL5719 (May 1957).

A.2. L. J. Koch, W. B. Loewenstein, and H. O. Monson, "Addendum to Hazard Summary Report, Experimental Breeder Reactor II (EBR-II)," ANL-5719 (Addendum) (June 1962).

A.3. W. E. Vesely, et. al., Fault Tree Handbook, NUREG-0492, USNRC, January 1981.

A.4. R. W. Schaefer, private communication (1990).

A.5. D. Schurman, private communication (1990).

A.6. S. H. Han, T. W. Kim, and K. J. Yoo, "Description and Input Manual of an Integrated Fault Tree Analysis Computer Code, MODULE," Reactor Safety Department, Korea Advanced Energy Research Institute, Daeduk-Danji, Choongnam, Korea.

A.7. J. I. Sackett and N. L. Gale, "FSAA to Hazards Summary Report, EBR-II: Upgrading of Plant Protective System," Vol. I, Section 3, ANL-76-34, August 1977.

A.8. J. Roglans - Ribas, private communication (1990).

A.9. S. A. Kamal, private communication (1990).

A.10. H. A. Larson and J. I. Sackett, "Safety Considerations for Trips Related to Reactivity Change in the EBR-II PPS (In The Fuel Handling Mode), ANL-77-32, Feb. 1983. 


\section{APPENDIX A.1}

\section{HUMAN RELIABILITY TREES \\ (Human Factors)}

- Detector Reliability

- Countrate Surveillance

- Trip Setting

A.1-i 


\section{HRA TREE DIAGRAM}

ID:

FHCR Calibrate Log Count Rate on channels $A, B, \& C$ fucl handlin

Sequence: Unknown

Date modified: $\quad 02-A P R-90$

Atialyst: Sc/umnan

NOTES: Tech. calibrates instrumcnts for log count ratc on thide Range Nuclear Chamels $A, B$, and $C$. Checkcd by the liticrlock check prior to Fucl Handing.

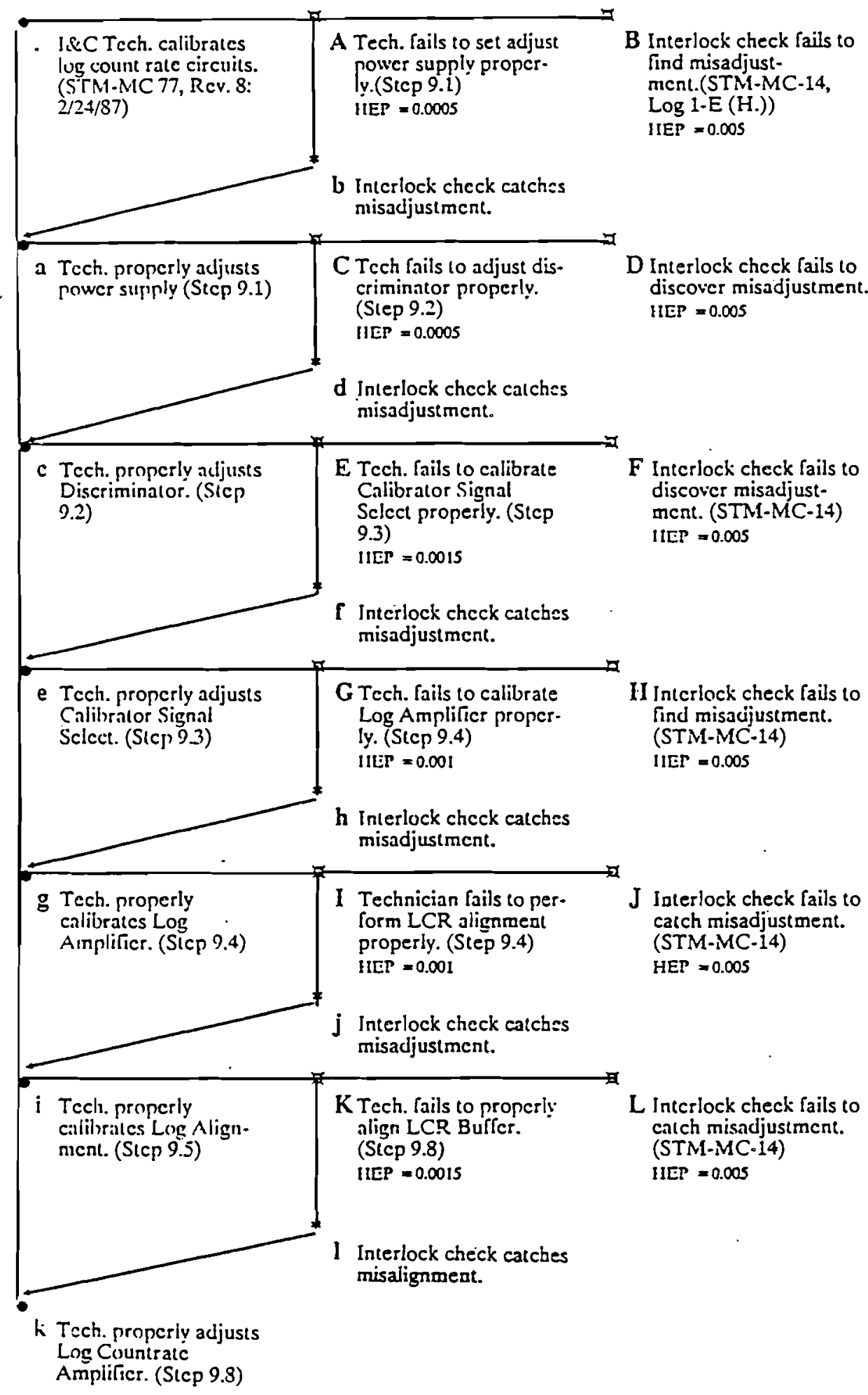




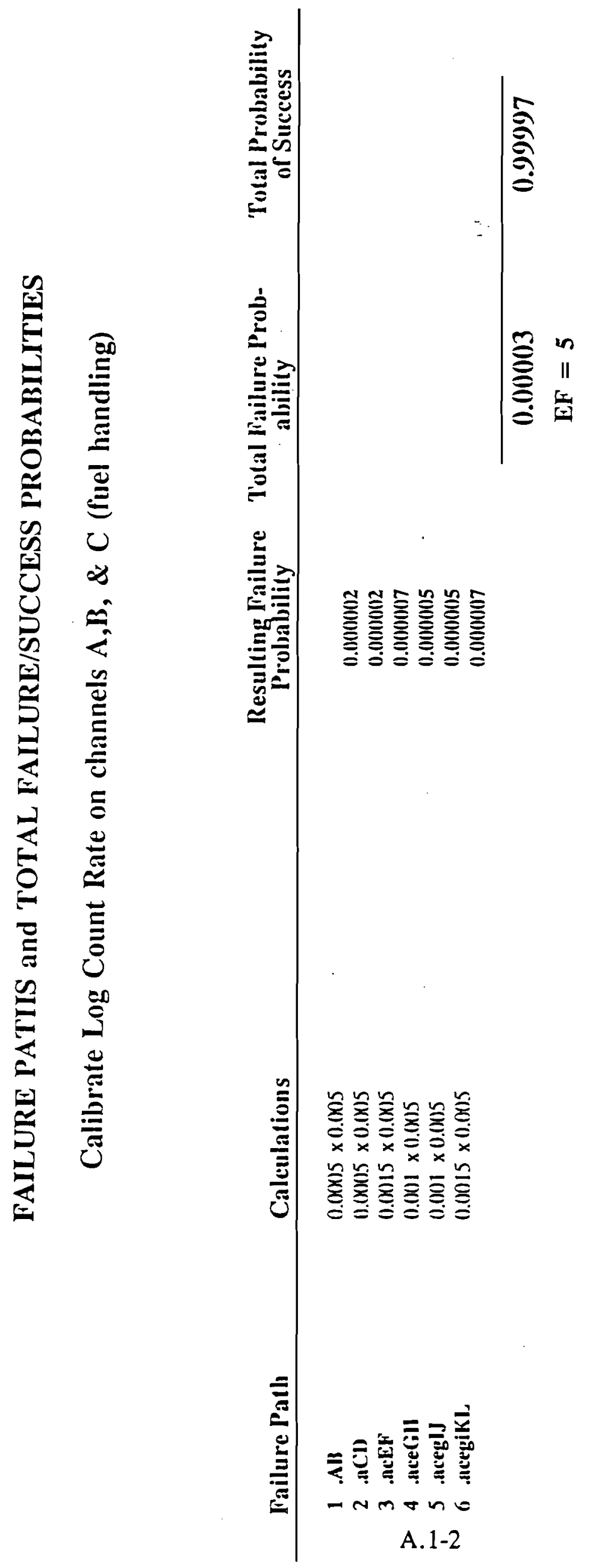




\section{HRA TREE DLAGRAM}

ID:

FHIHU Opcrator fails in follow fuel handling Countrate Procedure.

Sequence: Uinknown

Date modilicd: 0 - Blf-4Y-90

Analyst: Sciumman

NOTES: IIten norificd by Fuct-Handling Cunsolc Of'crotor - Control Room Operator fatils to use and follow Countrate Suncillones Procedure.

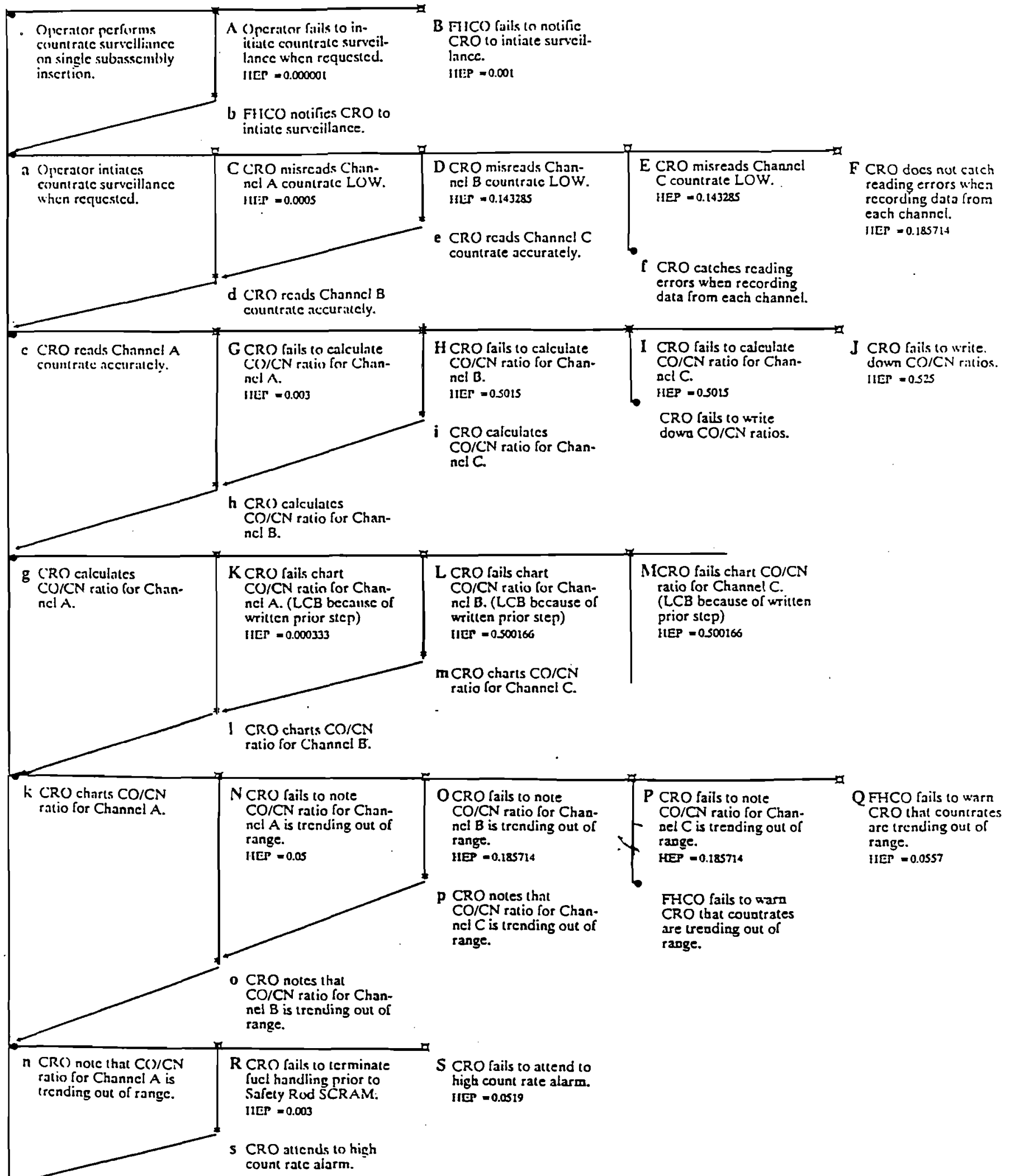

$r$ CRO icrminates fucl handling prior to Safety 


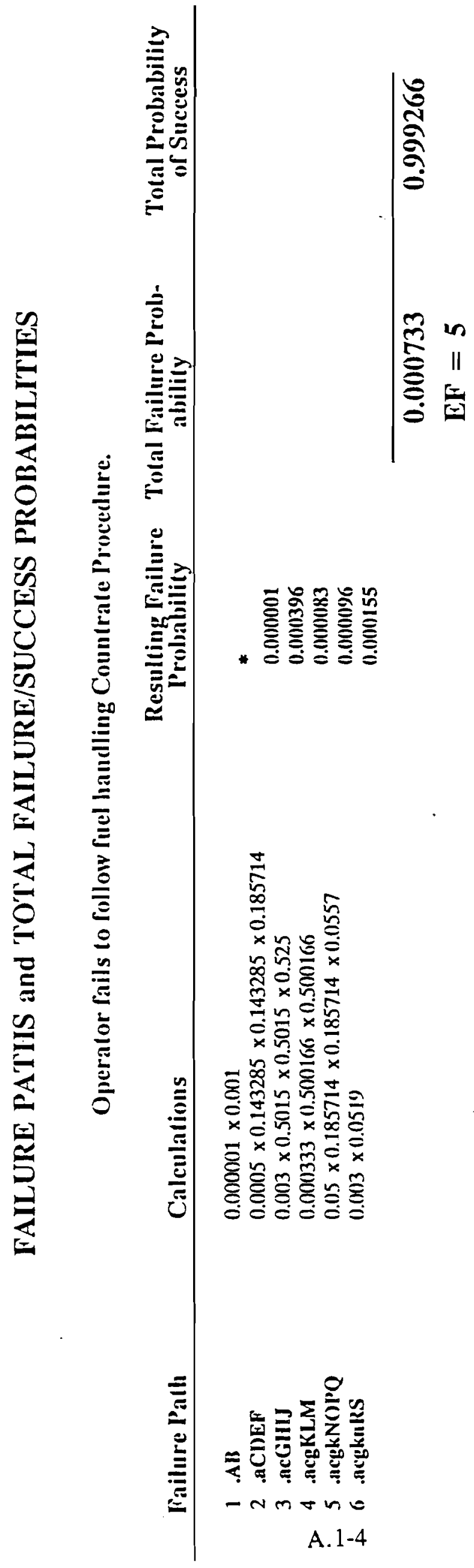




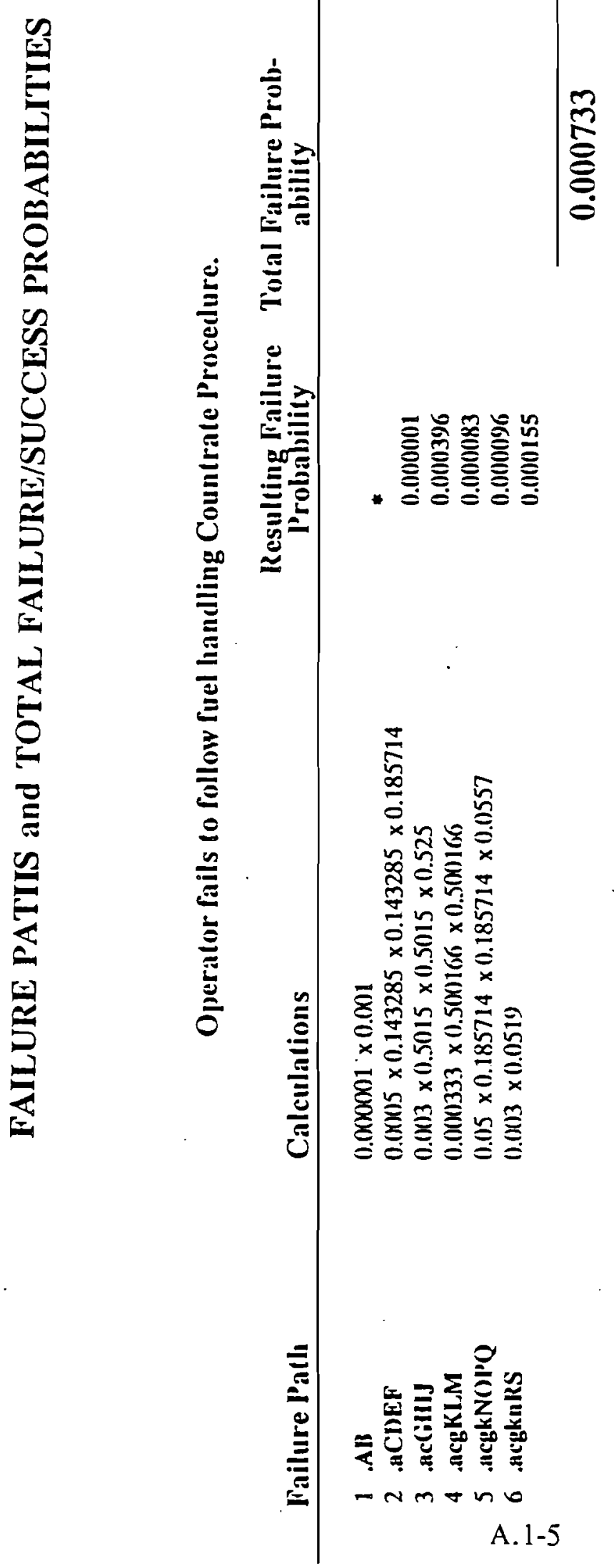




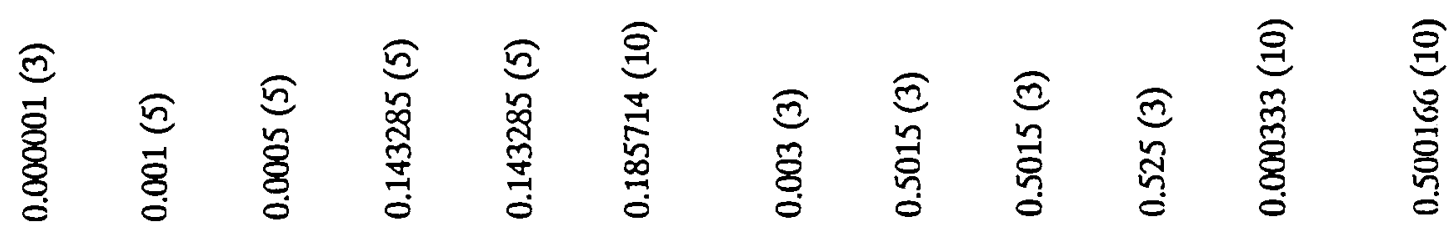

ล จ ล

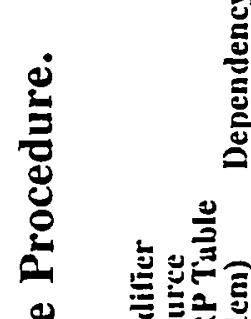

离 A

选

然

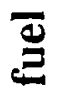

实瓷

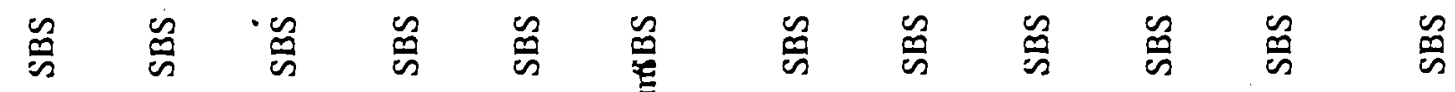

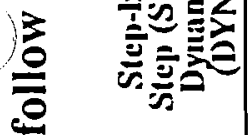

$-1$

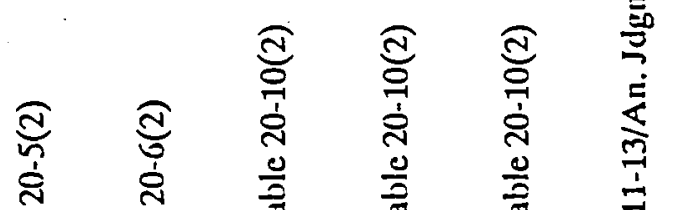

s

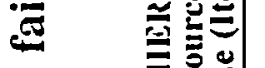

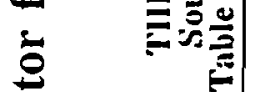

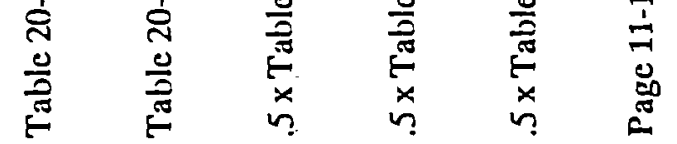

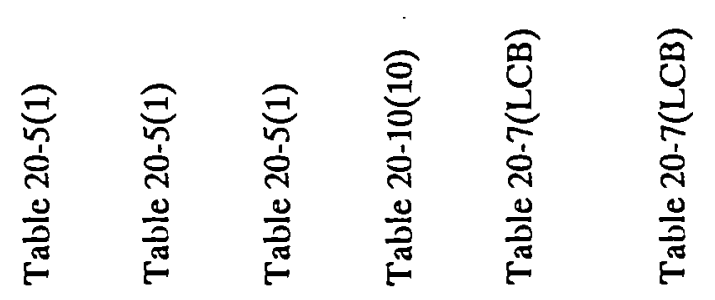

官

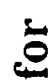

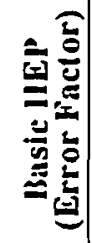

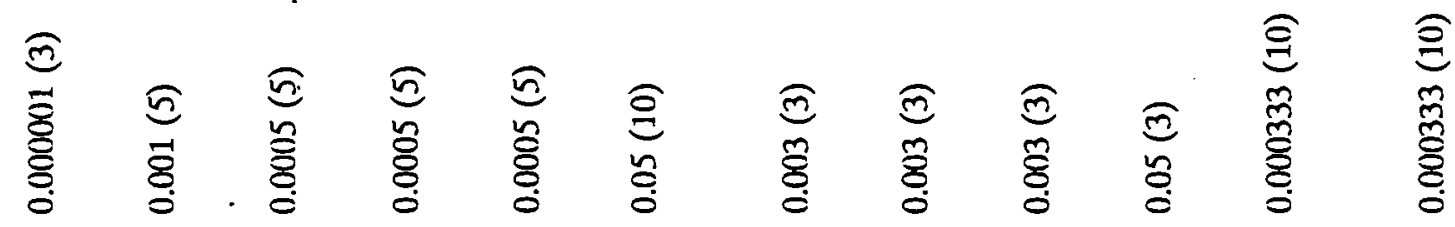

国

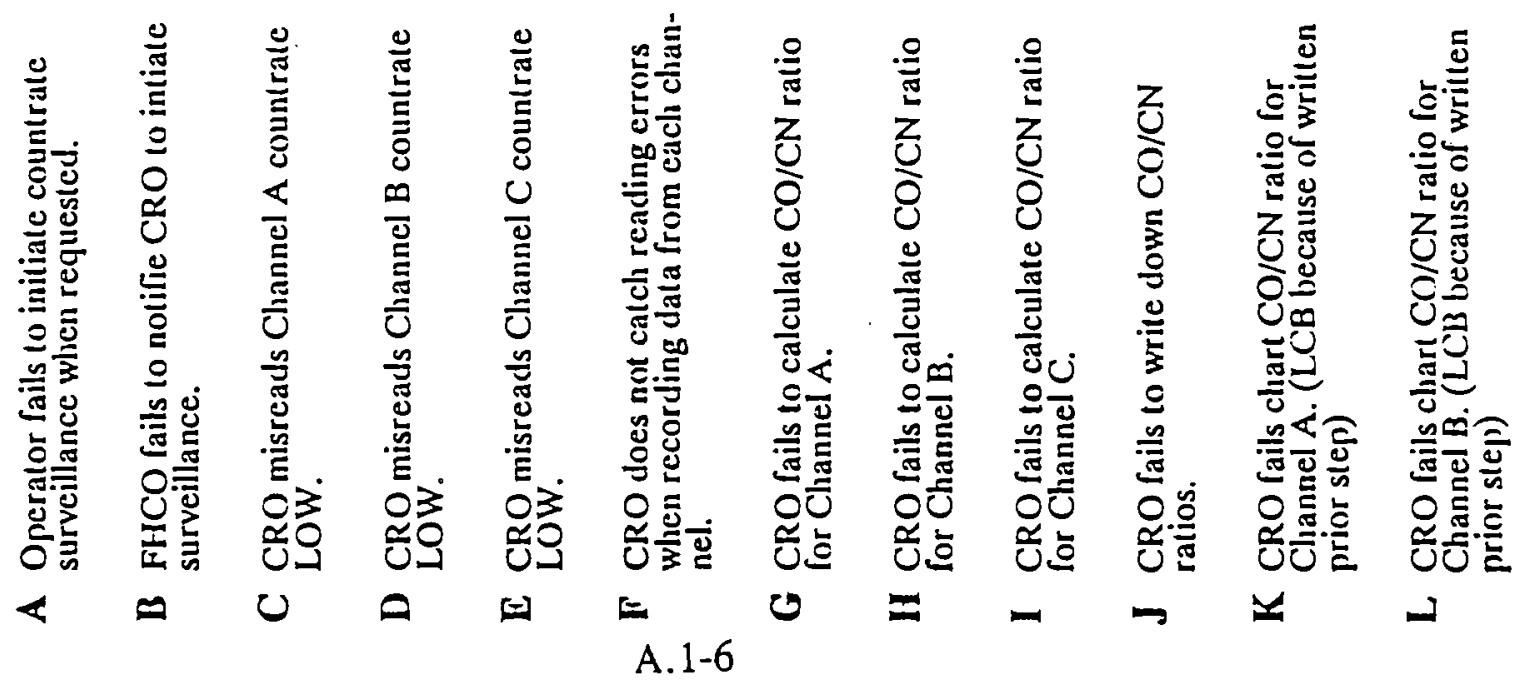




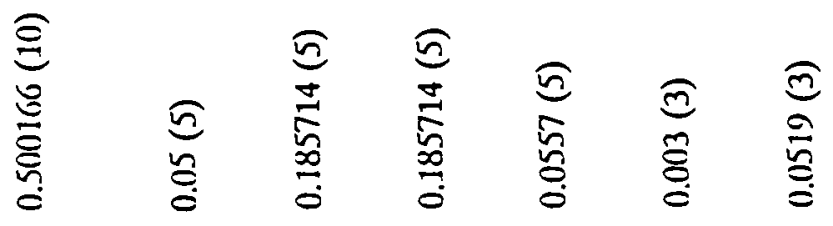

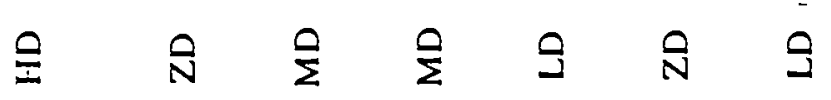

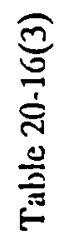

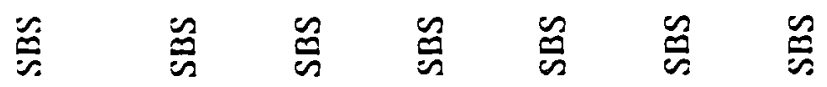

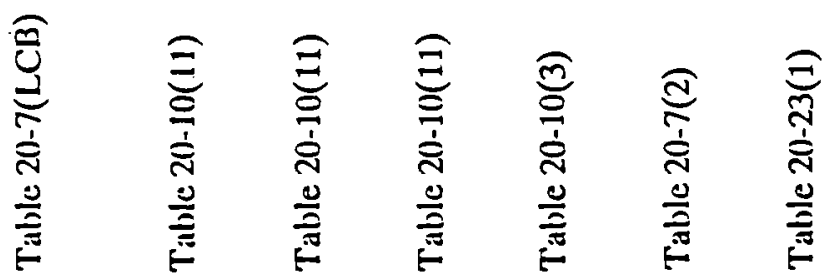

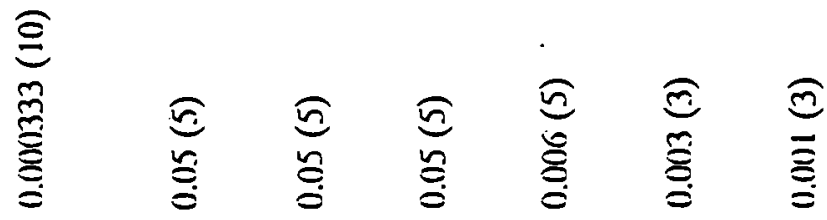

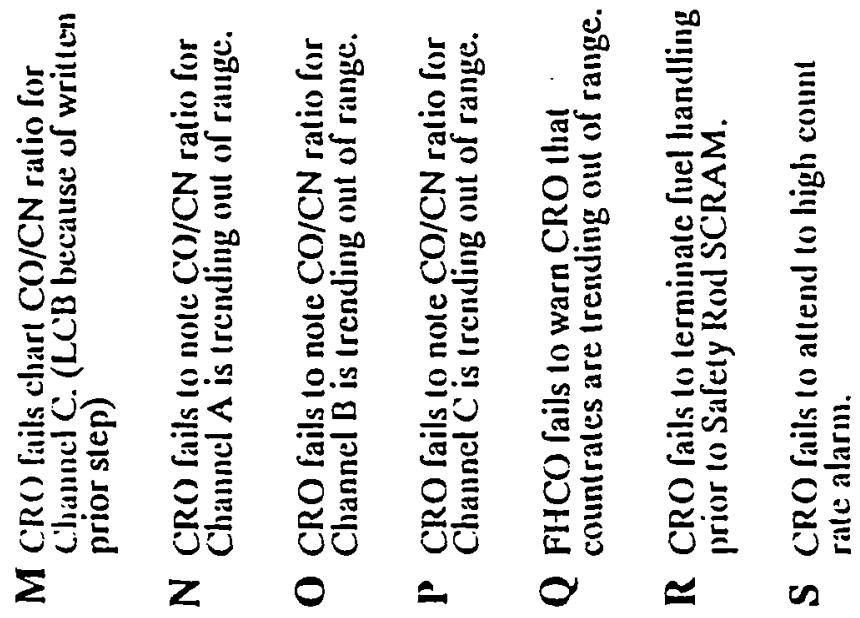

A. 1-7 


\title{
HRA TREE DIAGRAM
}

\author{
ID: $\quad F H S P$ Tech. sets bistable trip points for $L C R A, B, \&$ C. (fuel hand) \\ Sequence: Unknown \\ Date modified: $\quad 03-A P R-90$ \\ Analyst: Schuman \\ NOTES: $\quad$ Technician sets $L C R$ bistable trip points for log count rate of channels $A, B$, and $C$. These settings are \\ checked by the Interlock checks before start up of the reactor. (Proc. STM-MC-77Rev. 8: 2/24/ST)
}

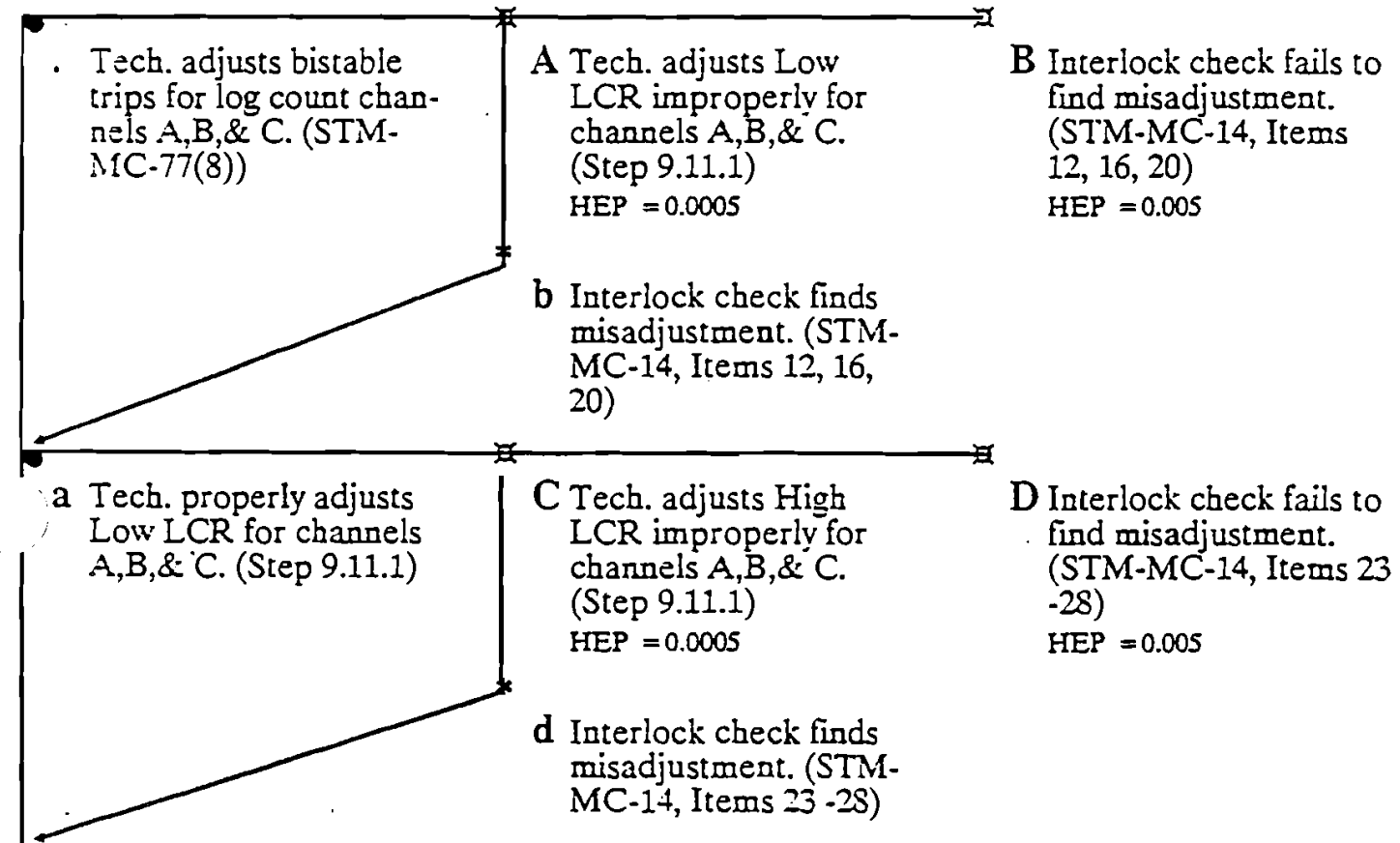

c. Tech. adjusts High LCR properly for channels A,B,\& C. (Step

9.11.1) 


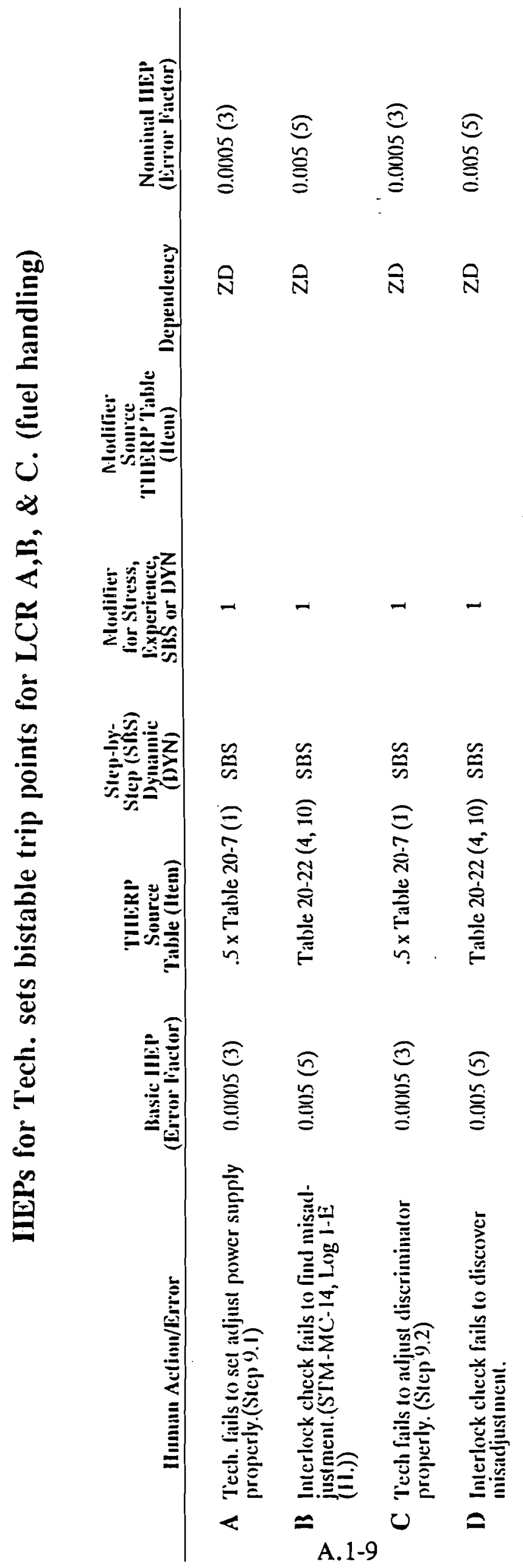




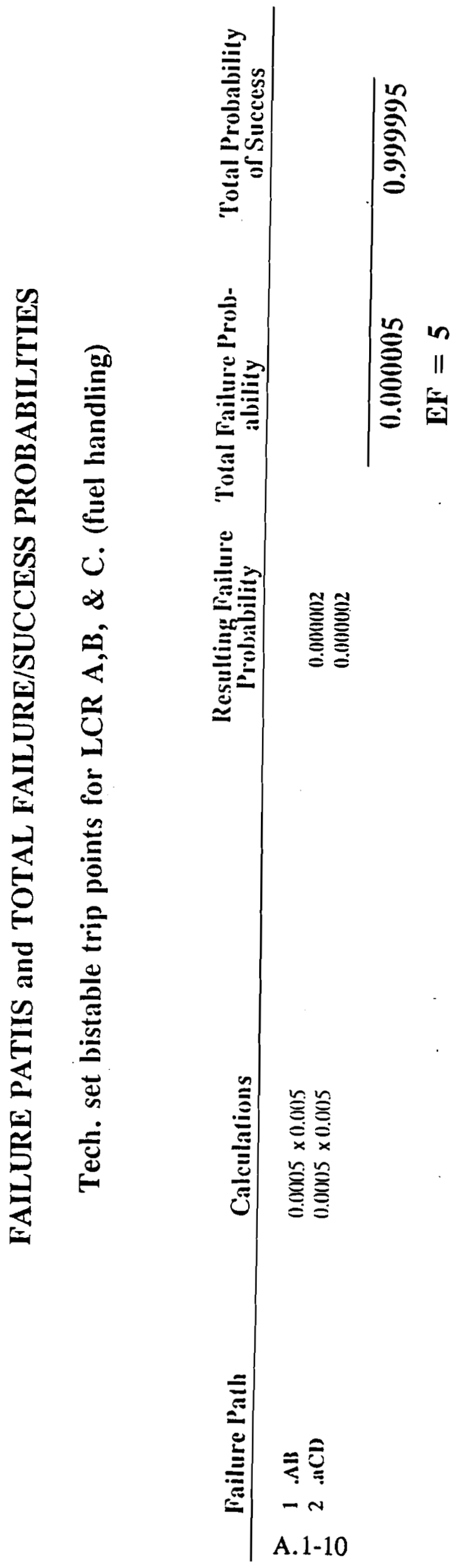


APPENDIX A.2

\section{FAULT TREE ANALYSES}

(Module)

- Initiating Events

- Protective (Countrate) Surveillance

- Trip Setting

- Safety Rod Trip

- Dropped Subassembly 
$5 / 9 / 902: 56$ PM

\begin{tabular}{|c|c|c|c|c|}
\hline \multicolumn{5}{|l|}{ \$TREE } \\
\hline \multirow{2}{*}{$\begin{array}{l}\text { STREE } \\
\text { TOP }\end{array}$} & + & CFA005RL & $05 R G$ & \multirow[b]{2}{*}{ ERRORS } \\
\hline & $"$ & CORE & RELOADING & \\
\hline CEA005RL & c & ERRORS IN & PLANNING & \\
\hline CCG005RG & $\mathrm{C}$ & COMPUTER OR & MECHANICAL & FAILURE \\
\hline \multicolumn{5}{|l|}{ \$END } \\
\hline \multicolumn{5}{|l|}{ SDATA } \\
\hline \multirow{2}{*}{ 4. $000 E-05$} & 16.0 & CFA005RL & & \\
\hline & 510.0 & CCG005RG & SEND D D & \\
\hline
\end{tabular}




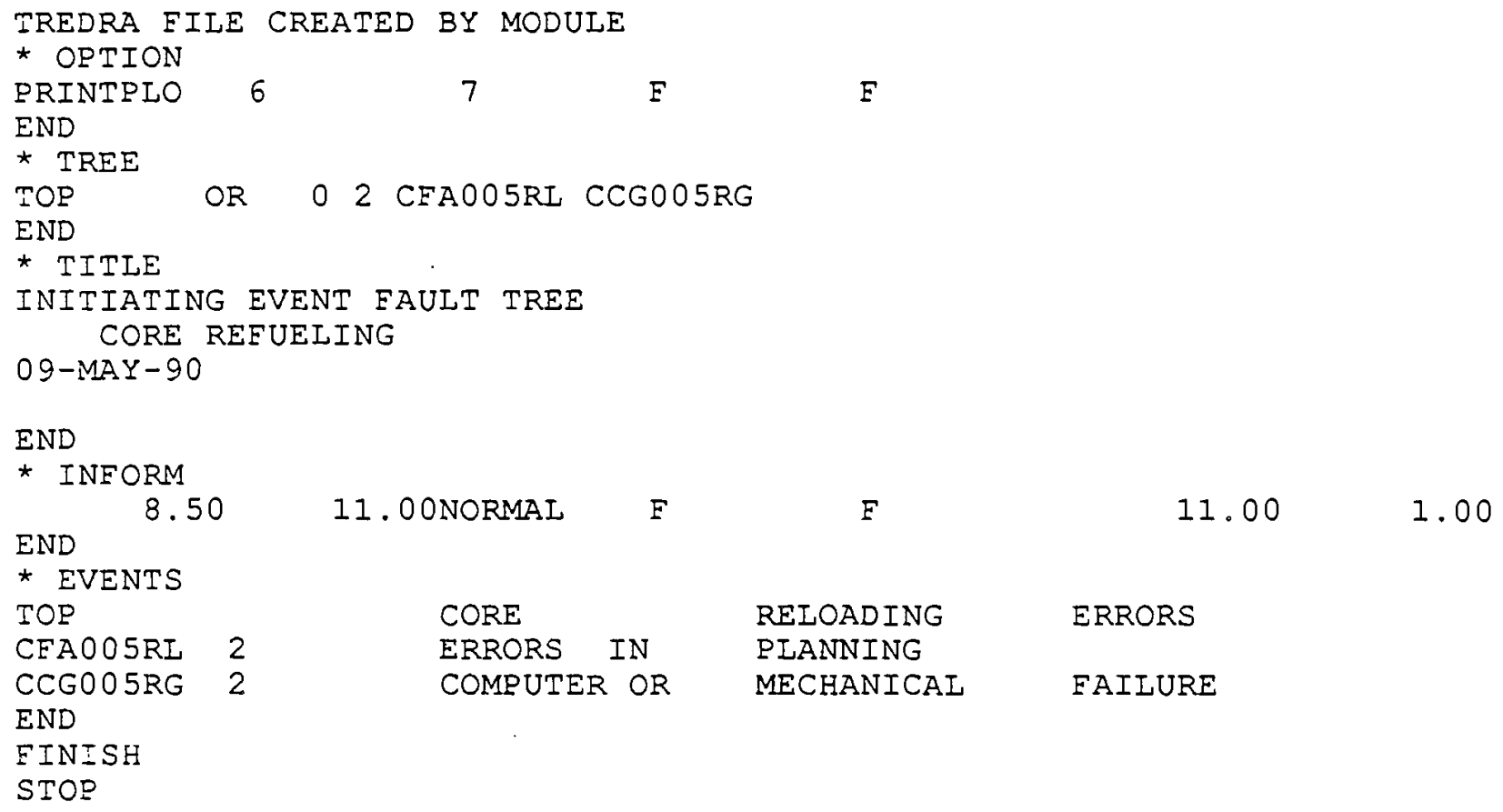

$\begin{array}{lll}\text { CORE } & \text { RELOADING } & \text { ERRORS } \\ \text { ERRORS IN } & \text { PLANNING } & \\ \text { COMPUTER OR } & \text { MECHANICAL } & \text { FAILURE }\end{array}$


ENTER THE INPUT FILE NAME :

INI EV

ENTER THE OUTPUT FILE NAME :

IE OUT

ENTER TOP EVENT NAME :

READ DATA : $\quad 43.0$ SEC REQUIRED

TOP

WRITE TREDRA INPUT : $\quad 8.0$ SEC REQUIRED

ENTER CUT-OFF PROBA. :

MODULARIZATION : 0.0 SEC REQUIRED

1. OE-07

ENTER MAX. TERM NUM. :

4

MCS : 1 TERMS GENERATED

Y

IMPORTANCE : $\quad 3.0$ SEC REQUIRED

DO YOU WANT TO ANALYZE UNCERTAINTY ?

$Y$

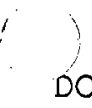

BOTTOM-UP :

SORTING : 1.0 SEC REQUIRED

8.0 SEC REQUIRED

Y

MCS : 2 TERMS GENERATED

DO YOU WANT TO ANALYZE IMPORTANCE ?:

Y

IMPORTANCE : $\quad 3.0$ SEC REQUIRED

Y

DO YOU WANT TO ANALYZE UNCERTAINTY ?:

\author{
SAMPLING : $\quad 13.0$ SEC REQUIRED \\ SORTING : 0.0 SEC REQUIRED \\ UNCERTAINTY : 1.0 SEC REQUIRED \\ MODULE : $\quad 105.0$ SEC REQUIRED
}

END OF MODULE RUN

STOP 


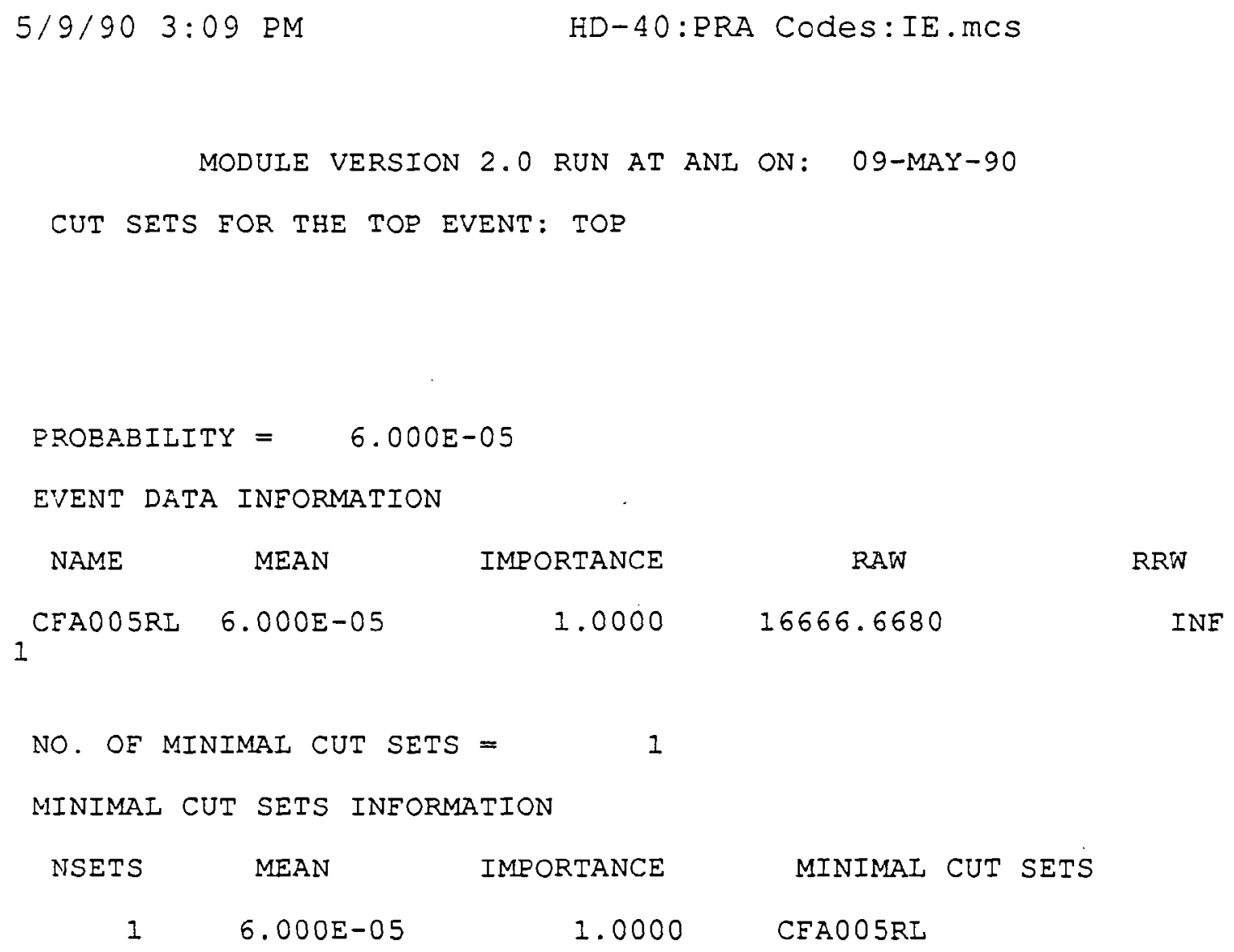


MODULE VERSION 2.0 RUN AT ANL ON: 09-MAY-90

FAULT TREE INPUT

$\begin{array}{llllll}\text { TOP } & + & \text { CFAO05RL } & \text { CCG005RG } & \\ & " & \text { CORE } & \text { RELOADING } & \text { ERRORS } \\ \text { CFA005RL } & C & \text { ERRORS IN } & \text { PLANNING } & \\ \text { CCG005RG } & C & \text { COMPUTER OR } & \text { MECHANICAL } & \text { FAILURE }\end{array}$

1

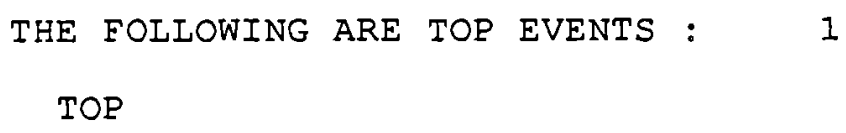




$$
\text { PROBABILITY }=6.000 \mathrm{E}-05
$$

EVENT DATA INFORMATION

$\begin{array}{ccrrr}\text { NAME } & \text { MEAN } & \text { IMPORTANCE } & \text { RAW } & \text { RRW } \\ \text { CFA005RL } & 6.000 E-05 & 1.0000 & 16666.6680 & \text { INF }\end{array}$

NO. OF MINIMAL CUT SETS =

MINIMAL CUT SETS INFORMATION

$\begin{array}{rrrr}\text { NSETS } & \text { MEAN } & \text { IMPORTANCE } & \text { MINIMAL CUT SETS } \\ 1 & 6.000 E-05 & 1.0000 & \text { CFA005RL }\end{array}$

R E S U L T S S U M MA R I Z E D

$\begin{aligned} \text { DISTRIBUTION VALUES } & \\ \text { NUMBER OF SAMPLE } & = \\ \text { ACCURACY ON 95\% CONFIDENCE INTERVAL } & =1.03496 \mathrm{E}+00 \\ \text { POINT MEAN VALUE } & =6.00000 \mathrm{E}-05 \\ \text { MEDIAN VALUE } & =1.97560 \mathrm{E}-05 \\ \text { ERROR FACTOR } & =1.21363 \mathrm{E}+01 \\ \text { MEAN VALUE } & =5.60293 \mathrm{E}-05 \\ \text { STANDARD DEVIATION } & =1.41182 \mathrm{E}-04 \\ 5 \% \text { BOUND } & =1.62785 \mathrm{E}-06 \\ 95 \% \text { BOUND } & =2.15119 \mathrm{E}-04\end{aligned}$

0

DISTRIBUTION CONEIDENCE LIMITS

$\begin{array}{rr}\begin{array}{r}\text { CONFIDENCE } \\ \text { (PERRCENT) }\end{array} & \text { FUNCTION } \\ \text { VALUE } \\ 0.5 & 3.89317 \mathrm{E}-07 \\ 1.0 & 5.50815 \mathrm{E}-07 \\ 2.5 & 1.01425 \mathrm{E}-06 \\ 5.0 & 1.62785 \mathrm{E}-06 \\ 10.0 & 2.96571 \mathrm{E}-06 \\ 20.0 & 5.86514 \mathrm{E}-06 \\ 25.0 & 7.59584 \mathrm{E}-06 \\ 30.0 & 9.41912 \mathrm{E}-06 \\ 40.0 & 1.40493 \mathrm{E}-05 \\ 50.0 & 1.97560 \mathrm{E}-05 \\ 60.0 & 2.86400 \mathrm{E}-05 \\ 70.0 & 4.41414 \mathrm{E}-05 \\ 75.0 & 5.39243 \mathrm{E}-05 \\ 80.0 & 6.52844 \mathrm{E}-05 \\ 90.0 & 1.24124 \mathrm{E}-04 \\ 95.0 & 2.15119 \mathrm{E}-04 \\ 97.5 & 3.28589 \mathrm{E}-04 \\ 99.0 & 5.68533 \mathrm{E}-04 \\ 99.5 & 7.87373 \mathrm{E}-04\end{array}$


PROBABILITY $=6.000 E-05$

EVENT DATA INEORMATION

NAME

MEAN

IMPORTANCE

RAW

RRW

$\begin{array}{rr}\text { CFAO05RL } & 4.000 E-05 \\ \text { CCGOO5RG } & 2.000 E-05\end{array}$

1

NO. OF MINIMAL CUT SETS =

$0.6667 \quad 16667.0000$

$0.3333 \quad 16667.3340$

3.0000

1.5000

MINIMAI CUT SETS INEORMATION

$\begin{array}{rlrr}\text { NSETS } & \text { MEAN } & \text { IMPORTANCE } & \text { MINIMAL CUT SETS } \\ 1 & 4.000 E-05 & 0.6667 & \text { CFAOO5RL } \\ 2 & 2.000 E-05 & 0.3333 & \text { CCGOO5RG }\end{array}$

$$
\begin{aligned}
& \text { RES ULTS S U M M A I Z E D } \\
& \text { DISTRIBUTION VALUES } \\
& \text { NUMBER OF SAMPIE }= \\
& \text { ACCURACY ON 95\% CONEIDENCE INTERVAL }=1.03496 \mathrm{E}+00 \\
& \text { POINT MEAN VALUE }=6.00000-05 \\
& \text { MEDIAN VALUE }=2.42780 \mathrm{E}-05 \\
& \text { ERROR FACTOR }=9.37351 \mathrm{E}+00 \\
& \text { MEAN VALUE }=6.38393 \mathrm{E}-05 \\
& \text { STANDARD DEVIATION }=1.75463 \mathrm{E}-04 \\
& 5 \% \text { BOUND }=3.62710 \mathrm{E}-06 \\
& 95 \% \text { BOUND }=2.27570 \mathrm{E}-04
\end{aligned}
$$

\section{DISTRIBUTION CONFIDENCE LIMITS}

$\begin{array}{rr}\begin{array}{r}\text { CONEIDENCE } \\ \text { (PERRCENT) }\end{array} & \text { FUNCTION } \\ & \text { VALUE } \\ 0.5 & 1.24301 E-06 \\ 1.0 & 1.73254 E-06 \\ 2.5 & 2.63171 E-06 \\ 5.0 & 3.62710 E-06 \\ 10.0 & 5.44619 E-06 \\ 20.0 & 9.16734 E-06 \\ 25.0 & 1.10777 E-05 \\ 30.0 & 1.32602 E-05 \\ 40.0 & 1.82556 E-05 \\ 50.0 & 2.42780 E-05 \\ 60.0 & 3.31054 E-05 \\ 70.0 & 4.89109 E-05 \\ 75.0 & 5.78617 E-05 \\ 80.0 & 7.35172 E-05 \\ 90.0 & 1.34821 E-04 \\ 95.0 & 2.27570 E-04 \\ 97.5 & 3.17953 E-04 \\ 99.0 & 5.16274 E-04\end{array}$

$99.0 \quad 5.16274 \mathrm{E}-04$

A. $2-7$ 


\section{$99.5 \quad 1.10218 E-03$ \\ MODULE : $\quad 105.0$ SEC EXECUTION TIME}

END OF MODULE RUN 


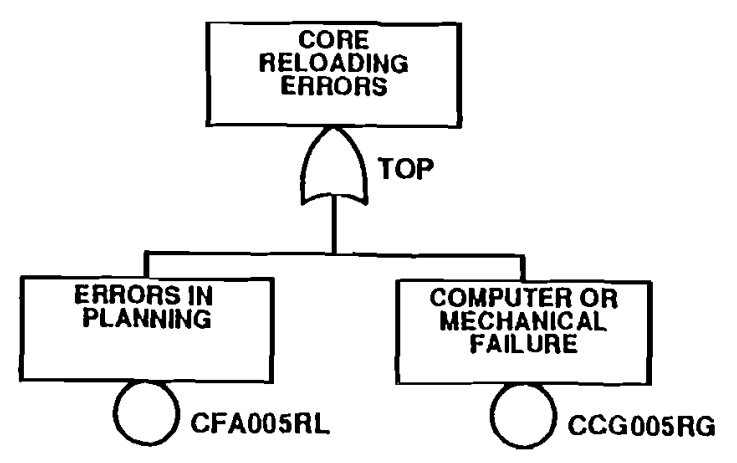

\begin{tabular}{|l|l|}
\hline \multicolumn{2}{|l|}{ INITIATING EVENT FAULT TREE } \\
\hline CORE REFUELING \\
\hline 09-MAY-90 & PAGE 1 OF 1 \\
\hline
\end{tabular}




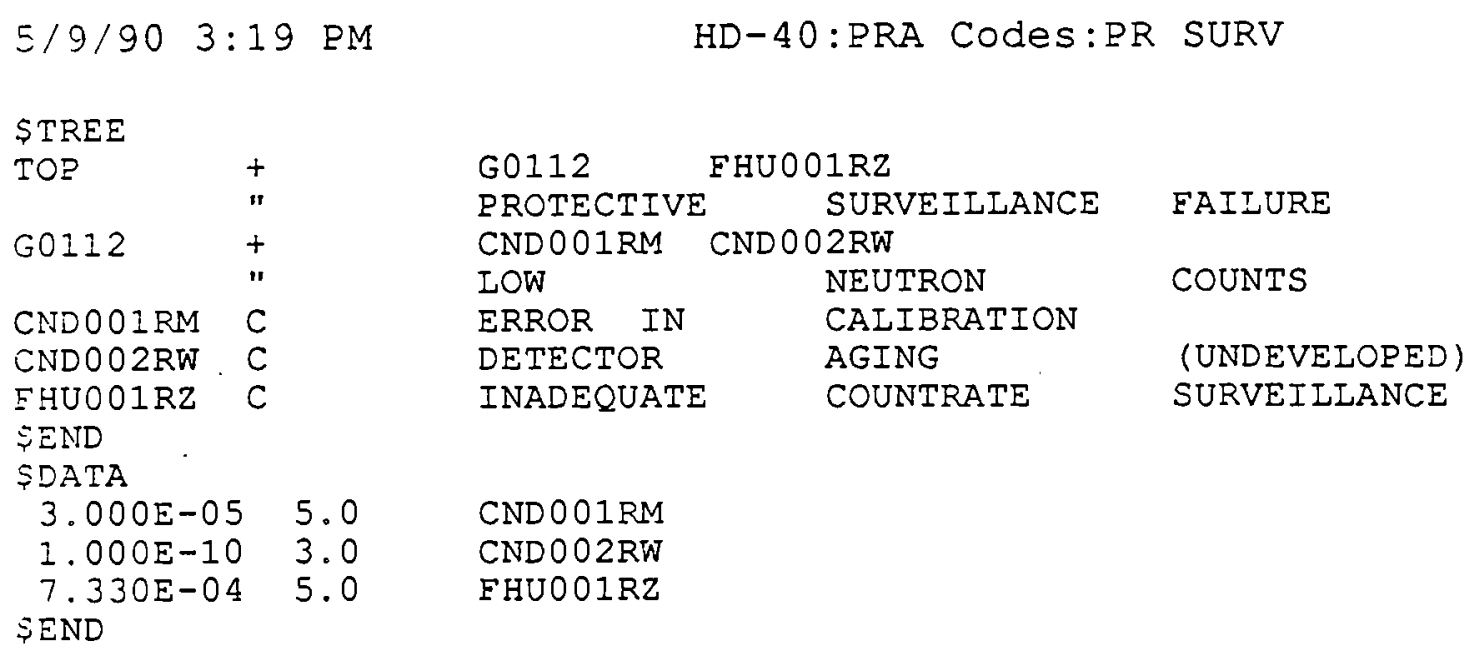

CND 001RM

CNDO02RW

$7.330 E-04 \quad 5.0 \quad$ FHU001R2 
TREDRA FILE CREATED BY MODULE

* OPTION

$\begin{array}{lllll}\text { PRINTPLO } & 6 & 7 & \text { F } & \text { F }\end{array}$

END

* TREE

TOP OR I I GOII2 FHUOOIRZ

G0112 OR 02 CNDOOIRM CNDO02RW

END

* TITLE

EROTECTIVE SURVEILIANCE FAULT TREE CORE REFUELING

$O S-M A Y-9 O$

END

* INFORM

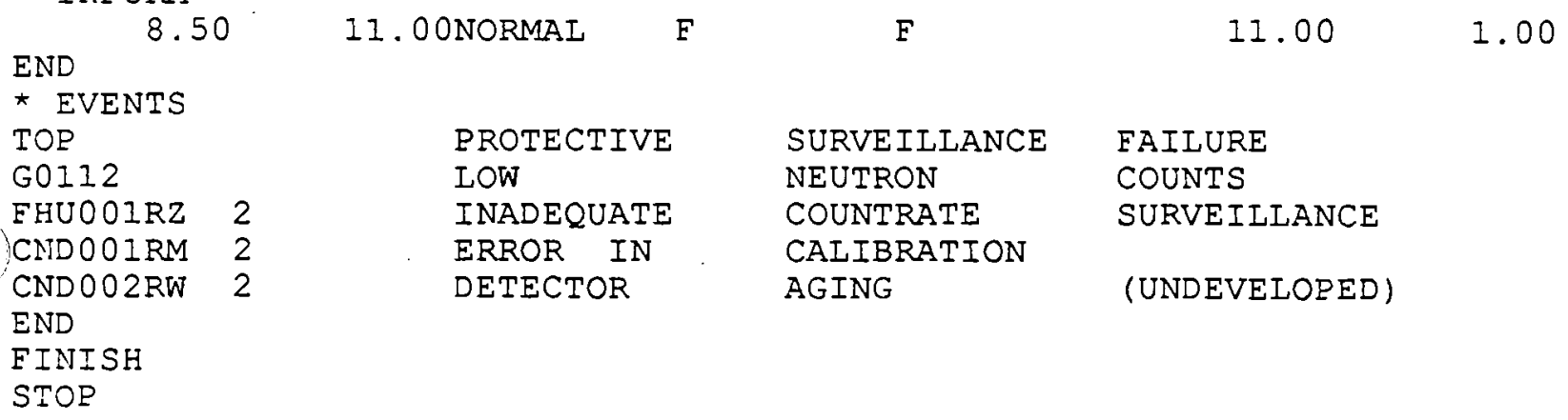


PROGRAM MODULE ENTERED: O9-MAY-90

ENTER THE INPUT FILE NAME :

PR SURV

ENTER THE OUTPUT FILE NAME :

PS.OUT

ENTER TOP EVENT NAME :

READ DATA : $\quad 34.0$ SEC REQUIRED

TOP

WRITE TREDRA INPUT :
MODULARIZATION :

ENTER CUT-OFF PROBA. :

0.0 SEC REQUIRED

1. $0 E-08$

ENTER MAX. TERM NUM. :

5

MCS : 1 TERMS GENERATED

DO YOU WANT TO ANALYZE IMPORTANCE ?

Y

IMPORTANCE : $\quad 2.0$ SEC REQUIRED

DO YOU WANT TO ANAIYZE UNCERTAINTY ? $Y$

53.0 SEC REQUIRED

DO YOU WANT TO EXPAND MODULARIZED MCS ?:

SAMPLING : $\quad 7.0$ SEC REQUIRED

SORTING : $\quad 1.0$ SEC REQUIRED

UNCERTAINTY : 0.0 SEC REQUIRED

Y

MCS : $\quad 2$ TERMS GENERATED

$Y$

DO YOU WANT TO ANALYZE IMPORTANCE ?:

CUTSET : 4.0 SEC REQUIRED

IMPORTANCE : 4.0 SEC REQUIRED

$Y$

DO YOU WANT TO ANALYZE UNCERTAINTY ?:

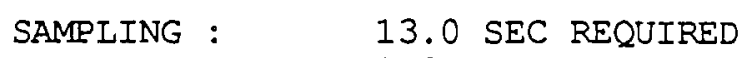

SAMPIING : $\quad 13.0$ SEC REQUIRED

0.0 SEC REQUIRED

EXPAND : $\quad 22.0$ SEC REQUIRED

MODULE : $\quad 113.0$ SEC REQUIRED

END OF MODULE RUN

STOP 
MODULE VERSION 2.0 RUN AT ANL ON: 09-MAY-90

CUT SETS FOR THE TOP EVENT: TOP

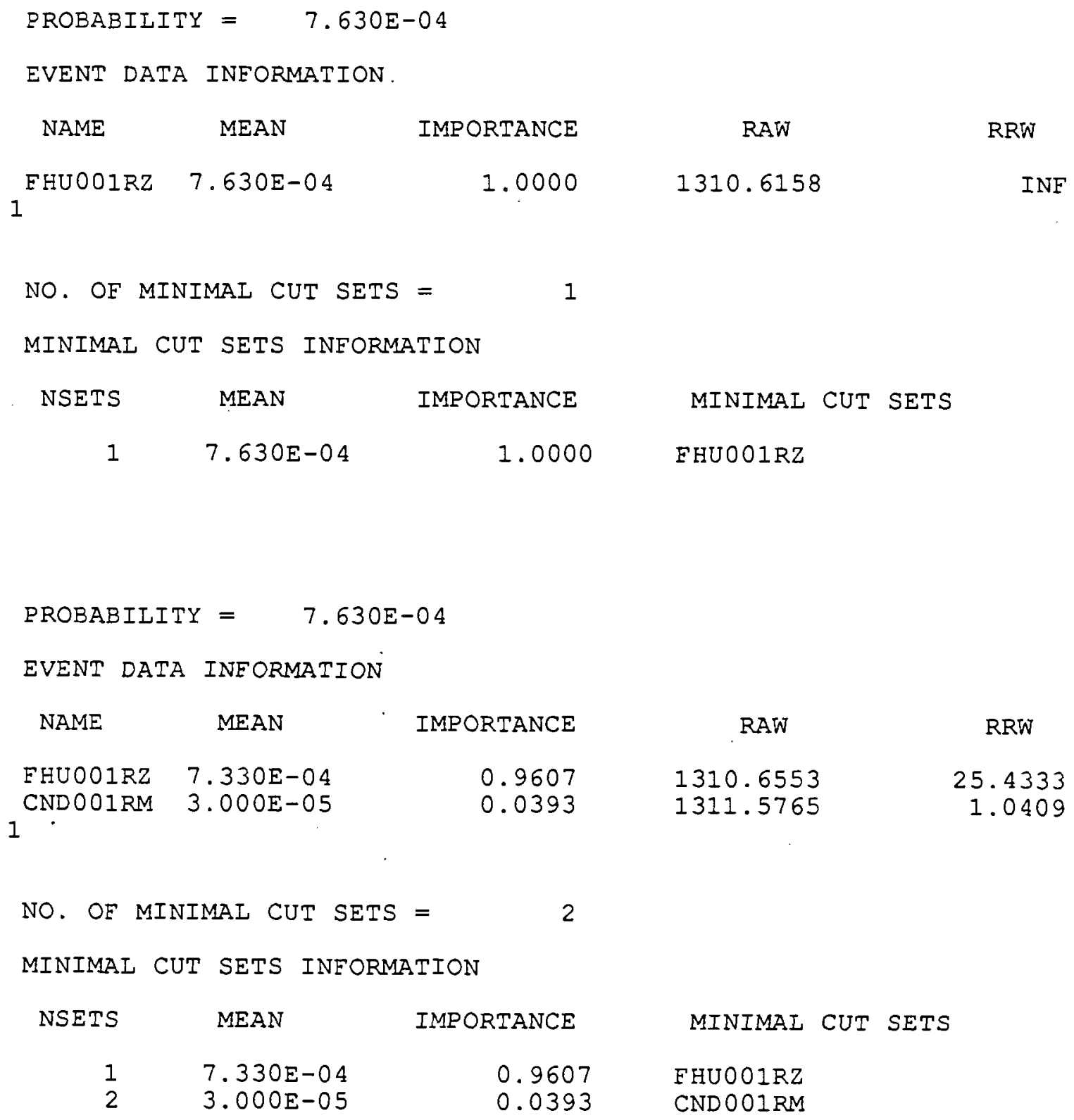


MODULE VERSION 2.0 RUN AT ANL ON: 09-MAY-90

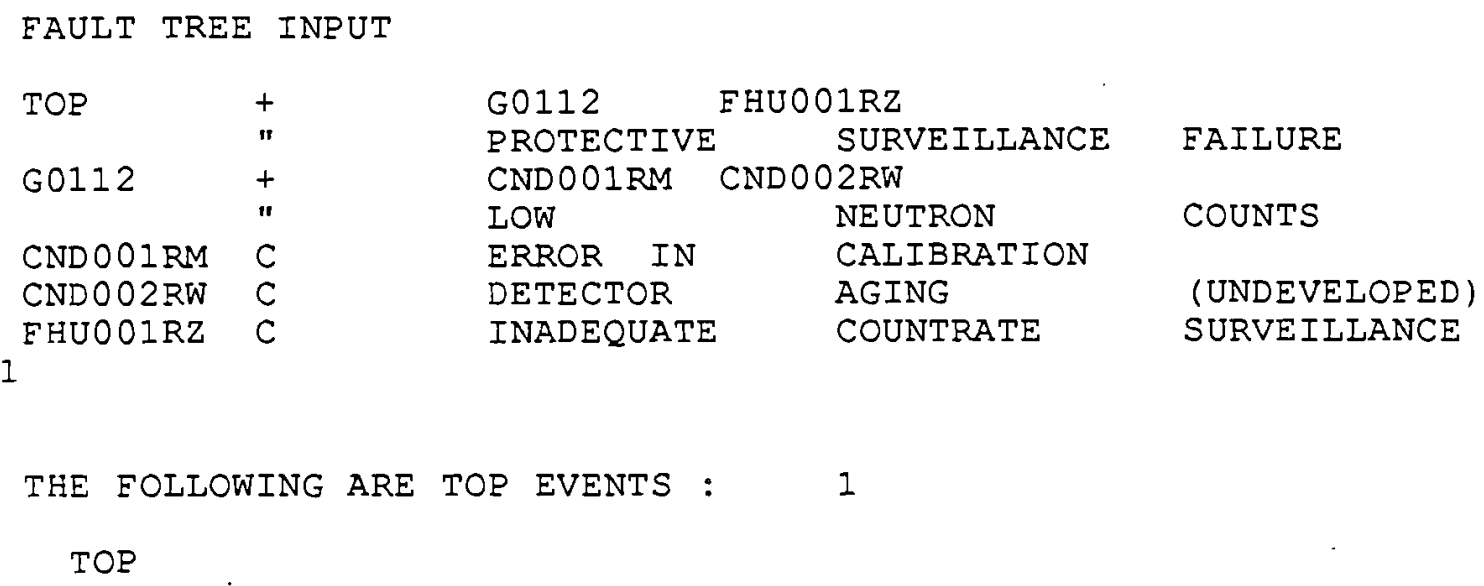

THE FOLLOWING ARE GATES : 2

TOP G0112

THE FOLLOWING ARE EVENTS : 3

FHU001RZ CND001RM CND002RW

RELIABILITY INPUT DATA
$3.000 E-05 \quad 5.0$
CND001RM
$1.000 E-10 \quad 3.0$
CND 002RW
7. $330 E-04$
5.0
FHUO01RZ

1

MODULARIZED FAULT TREE

TOR $\quad+$ FHUOO1RZ

MODULES INFORMATION

G0112+ CND001RM CND002RW

FHU001RZ + G0112 FHU001RZ

CUT SETS FOR THE TOP EVENT: TOP 

EHUO01RZ
$7.630 E-04$
4.801
CUTSETS
43.0 SEC REQUIRED
1
PROBABILITY $=7.630 \mathrm{E}-04$

EVENT DATA INFORMATION

NAME MEAN IMPORTANCE RAW RRW
FHU001RZ
$7.630 E-04$
1.0000
1310.6158
INE

1

NO. OF MINIMAI CUT SETS =

1

MINIMAI CUT SETS INFORMATION

NSETS MEAN IMPORTANCE MINIMAL CUT SETS

1

$1 \quad 7.630 \mathrm{E}-04$

1.0000

FHUO01RZ

RES U I T S S U M M A R I Z E D

DISTRIBUTION VALUES

NUMBER OF SAMPLE $=1200$

ACCURACY ON 95\% CONFIDENCE INTERVAL $=1.03496 \mathrm{E}+00$

POINT MEAN VALUE $=7.63000 \mathrm{E}-04$

MEDIAN VALUE $=4.77689 \mathrm{E}-04$

ERROR FACTOR $=5.01491 \mathrm{E}+00$

MEAN VALUE $=7.40154 \mathrm{E}-04$

STANDARD DEVIATION $=8.61753 \mathrm{E}-04$

5 움 BOUND $=9.52537 \mathrm{E}-05$

95 BOUND $=2.23346 \mathrm{E}-03$

0

\section{DISTRIBUTION CONFIDENCE LIMITS}

$\begin{array}{rr}\begin{array}{r}\text { CONFIDENCE } \\ \text { (PERRCENT) }\end{array} & \begin{array}{r}\text { FUNCTION } \\ \text { VALUE }\end{array} \\ 0.5 & 3.78049 \mathrm{E}-05 \\ 1.0 & 4.73035 \mathrm{E}-05 \\ 2.5 & 7.01712 \mathrm{E}-05 \\ 5.0 & 9.52537 \mathrm{E}-05 \\ 10.0 & 1.40333 \mathrm{E}-04 \\ 20.0 & 2.18001 \mathrm{E}-04 \\ 25.0 & 2.57630 \mathrm{E}-04 \\ 30.0 & 2.96040 \mathrm{E}-04 \\ 40.0 & 3.83280 \mathrm{E}-04 \\ 50.0 & 4.77689 \mathrm{E}-04 \\ 60.0 & 6.07181 \mathrm{E}-04 \\ 70.0 & 8.02925 \mathrm{E}-04 \\ 75.0 & 9.13761 \mathrm{E}-04 \\ 80.0 & 1.03386 \mathrm{E}-03 \\ 90.0 & 1.56571 \mathrm{E}-03\end{array}$

A. $2-15$ 


$$
\begin{array}{ll}
95.0 & 2.23346 E-03 \\
97.5 & 2.93641 E-03 \\
99.0 & 4.18426 E-03 \\
99.5 & 5.16382 E-03
\end{array}
$$

1

$$
\text { PROBABILITY }=7.630 \mathrm{E}-04
$$

EVENT DATA INFORMATION

$\begin{array}{lcrrr}\text { NAME } & \text { MEAN } & \text { IMPORTANCE } & \text { RAW } & \text { RRW } \\ \text { EHUO01RZ } & 7.330 E-04 & 0.9607 & 1310.6553 & 25.4333 \\ \text { CNDO01RM } & 3.000 E-05 & 0.0393 & 1311.5765 & 1.0409\end{array}$

1

NO. OF MINIMAI CUT SETS $=2$

MINIMAL CUT SETS INEORMATION

$\begin{array}{rlrr}\text { NSETS } & \text { MEAN } & \text { IMPORTANCE } & \text { MINIMAL CUT SETS } \\ 1 & 7.330 E-04 & 0.9607 & \text { FHU001RZ } \\ 2 & 3.000 E-05 & 0.0393 & \text { CND001RM }\end{array}$

R E S U L T S S U M M A R I Z E D

$\begin{aligned} \text { DISTRIBUTION VALUES } & \\ \text { NUMBER OF SAMPLE } & = \\ \text { ACCURACY ON 95\% CONFIDENCE INTERVAL } & =1.03496 \mathrm{E}+00 \\ \text { POINT MEAN VALUE } & =7.63000 \mathrm{E}-04 \\ \text { MEDIAN VALUE } & =4.79150 \mathrm{E}-04 \\ \text { ERROR FACTOR } & =5.32981 \mathrm{E}+00 \\ \text { MEAN VALUE } & =7.77385 \mathrm{E}-04 \\ \text { STANDARD DEVIATION } & =1.02385 \mathrm{E}-03 \\ 5 \% \text { BOUND } & =1.11516 \mathrm{E}-04 \\ 95 \% \text { BOUND } & =2.55378 \mathrm{E}-03\end{aligned}$

0

\section{DISTRIBUTION CONFIDENCE LIMITS}

$\begin{array}{rr}\begin{array}{r}\text { CONEIDENCE } \\ \text { (PERRCENT) }\end{array} & \begin{array}{r}\text { EUNCTION } \\ \text { VALUE }\end{array} \\ 0.5 & 4.89684 \mathrm{E}-05 \\ 1.0 & 6.13423 \mathrm{E}-05 \\ 2.5 & 7.99520 \mathrm{E}-05 \\ 5.0 & 1.11516 \mathrm{E}-04 \\ 10.0 & 1.52970 \mathrm{E}-04 \\ 20.0 & 2.18788 \mathrm{E}-04 \\ 25.0 & 2.52932 \mathrm{E}-04 \\ 30.0 & 2.83635 \mathrm{E}-04 \\ 40.0 & 3.65183 \mathrm{E}-04 \\ 50.0 & 4.79150 \mathrm{E}-04 \\ 60.0 & 6.10710 \mathrm{E}-04 \\ 70.0 & 7.74163 \mathrm{E}-04 \\ 75.0 & 8.79451 \mathrm{E}-04\end{array}$

A. $2-16$ 
$80.0 \quad 1.06862 \mathrm{E}-03$

$90.0 \quad 1.67896 \mathrm{E}-03$

$95.0 \quad 2.55378 E-03$

$97.53 .18339 E-03$

$99.0 \quad 4.53526 \mathrm{E}-03$

$99.57 .09643 \mathrm{E}-03$

MODULE :

113.0 SEC EXECUTION TIME

END OF MODULE RUN 


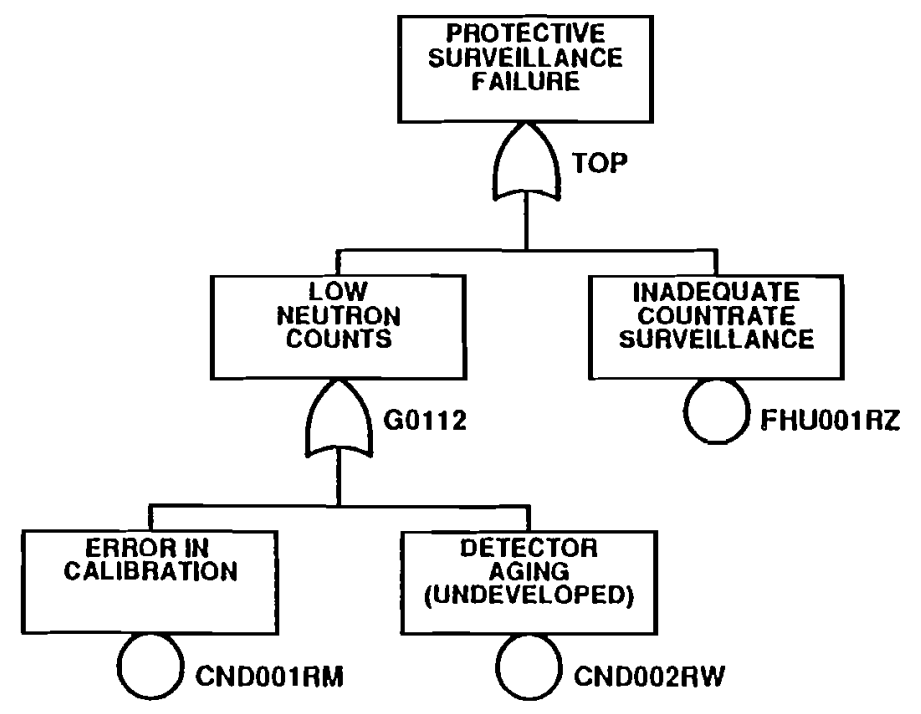

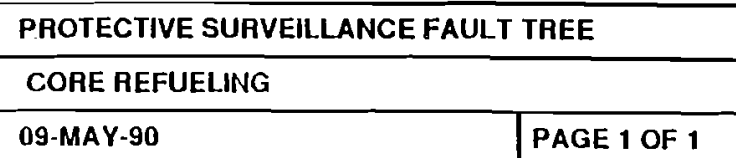




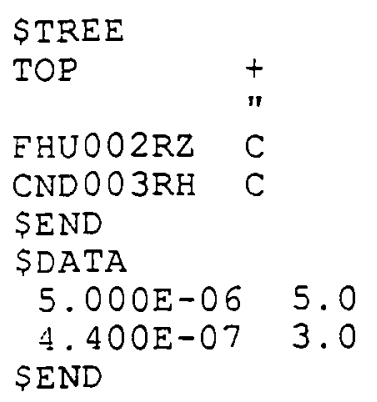

$\begin{array}{lc}\text { FHU002RZ } & \text { CNDO03RH } \\ \text { NO } & \text { TRIP } \\ \text { IMPROPER } & \text { TRIP } \\ \text { TWO-OF-THREE } & \text { DETECTOR }\end{array}$

SIGNAL SETTINGS CHANNEL
FAIIURES

\section{FHUO02RZ}

CNDO0 3RH

\section{A. 2-19}


TREDRA FILE CREATED BY MODULE

* OPTION

PRINTPLO

6

7

F

F

END

* TREE

TOP

OR 02 FHU002RZ CND003RH

END

* TITLE

TRIP SIGNAI FAULT TREE CORE REFUELING

O $9-M A Y-90$

END

* INFORM

8.50

11.0ONORMAI F

F

11.00

1.00

* EVENTS

TOP

FHU0 02RZ 2

CNDO03RH 2

NO

IMPROPER

TWO-OF-THREE
TRIP

TRIP

DETECTOR
SIGNAI SETTINGS CHANNEL

FAILURES

END

FINISH

STOP 
PROGRAM MODULE ENTERED: O9-MAY-90

ENTER THE INPUT FILE NAME :

TR SIG

ENTER THE OUTRUT FILE NAME :

TS.OUT

ENTER TOP EVENT NAME :

READ DATA : $\quad 19.0$ SEC REQUIRED

TOP

$$
\begin{aligned}
& \text { WRITE TREDRA INPUT : } \\
& \text { MODULARIZATION : }
\end{aligned}
$$

ENTER CUT-OEE PROBA. :

1. $0 E-08$

ENTER MAX. TERM NUM. :

4

MCS : 1 TERMS GENERATED

DO YOU WANT TO ANALYZE IMPORTANCE ?

$Y$

DO YOU WANT TO ANALYZE UNCERTAINTY ?

IMPORTANCE : $\quad 3.0$ SEC REQUIRED $Y$

SAMPIING : $\quad 7.0$ SEC REQUIRED SORTING : 1.0 SEC REQUIRED

BOTTOM-UP : 29.0 SEC REQUIRED

DO YOU WANT TO EXPAND MODULARIZED MCS ?:

$Y$

$\begin{array}{rrrr}\text { MCS : } & 2 \text { TERMS GENERATED } \\ & \text { CUTSET } & : & 6.0 \text { SEC REQUIRED }\end{array}$

$Y$

$\begin{array}{rrr}\text { MCS : } & 2 \text { TERMS GENERATED } \\ \text { DO YOU WANT TO ANALYZE IMPORTANCE } & : & 6.0 \text { SEC REQUIRED }\end{array}$

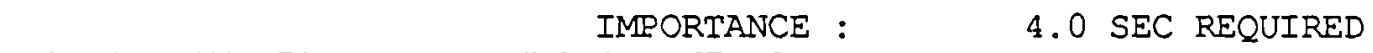

DO YOU WANT TO ANALYZE UNCERTAINTY ?:

\author{
SAMPIING : $\quad 13.0$ SEC REQUIRED \\ SORTING : $\quad 0.0$ SEC REQUIRED \\ EXPAND : $\quad 24.0$ SEC REQUIRED \\ MODULE : $\quad 76.0$ SEC REQUIRED
}

END OF MODULE RUN

STOP 
MODULE VERSION 2.0 RUN AT ANL ON: 09-MAY-90

CUT SETS FOR THE TOP EVENT: TOP

$$
\text { PROBABILITY }=5.440 \mathrm{E}-06
$$

EVENT DATA INFORMATION

$\begin{array}{crrrr}\text { NAME } & \text { MEAN } & \text { IMPORTANCE } & \text { RAW } & \text { RRW } \\ \text { FHU002R2 } & 5.440 E-06 & 1.0000 & * \star \star * \star \star \star \star \star * * & \text { INF }\end{array}$

NO. OF MINIMAL CUT SETS $=1$

MINIMAL CUT SETS INFORMATION

$\begin{array}{rrrr}\text { NSETS } & \text { MEAN } & \text { IMPORTANCE } & \text { MINIMAL CUT SETS } \\ 1 & 5.440 \mathrm{E}-06 & 1.0000 & \text { FHUO02RZ }\end{array}$

PROBABILITY $=5.440 \mathrm{E}-06$

EVENT DATA INFORMATION

\begin{tabular}{|c|c|c|c|c|}
\hline NAME & MEAN & IMPORTANCE & RAW & RRW \\
\hline $\begin{array}{l}\text { FHUO02RZ } \\
\text { CNDO03RH }\end{array}$ & $\begin{array}{l}5.000 E-06 \\
4.400 E-07\end{array}$ & 0.9191 & 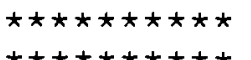 & 12.3636 \\
\hline
\end{tabular}

1

NO. OF MINIMAL CUT SETS $=2$

MINIMAL CUT SETS INFORMATION

$\begin{array}{rrrr}\text { NSETS } & \text { MEAN } & \text { IMPORTANCE } & \text { MINIMAI CUT SETS } \\ 1 & 5.000 E-06 & 0.9191 & \text { FHUO02RZ } \\ 2 & 4.400 E-07 & 0.0809 & \text { CND003RH }\end{array}$


MODULE VERSION 2.0 RUN AT ANL ON: 09-MAY-90

FAULT TREE INPUT

$\begin{array}{lllcl}\text { TOP } & + & \text { FHUOO2RZ } & \text { CNDOO3RH } & \\ & " & \text { NO } & \text { TRIP } & \text { SIGNAL } \\ \text { FHU002RZ } & \text { C } & \text { IMPROPER } & \text { TRIP } & \text { SETTINGS } \\ \text { CNDO03RH } & \text { C } & \text { TWO-OF-THREE } & \text { DETECTOR } & \text { CHANNEL }\end{array}$

TOP

THE FOLLOWING ARE GATES : I

TOP

THE FOLLOWING ARE EVENTS : 2

EHU002RZ CND003RH

\section{RELIABILITY INPUT DATA}

$$
\begin{array}{lll}
5.000 E-06 & 5.0 & \text { FHUO02RZ } \\
4.400 E-07 & 3.0 & \text { CNDO03RH }
\end{array}
$$

MODULARIZED FAULT TREE

TOP + EHUOO2RZ

MODULES INFORMATION

FHU002RZ + FHU002RZ CND003RH

CUT SETS FOR THE TOP EVENT: TOP

NO. OF EVENTS IN FAULT TREE = 1

FHUOO2RZ $\quad 5.440 E-06 \quad 4.590$

CUTSETS : $\quad 18.0$ SEC REQUIRED 


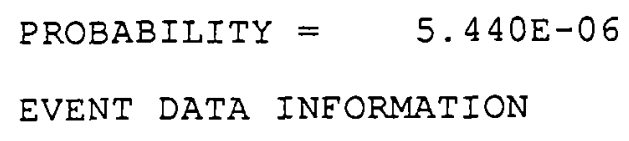

1.0000

INF 1

NO. OF MINIMAL CUT SETS =

MINIMAL CUT SETS INFORMATION

NSETS MEAN IMPORTANCE MINIMAL CUT SETS

CONF IDENCE (PERRCENT)
FUNCTION

VALUE

$99.5 \quad 3.53003 E-05$




$$
\text { PROBABILITY }=5.440 \mathrm{E}-06
$$

EVENT DATA INEORMATION

\begin{tabular}{|c|c|c|c|c|}
\hline NAME & MEAN & IMPORTANCE & RAW & RRW \\
\hline$F \mathrm{HUOO} 2 \mathrm{RZ}$ & $5.000 E-06$ & 0.9191 & 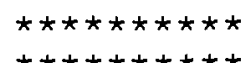 & 12.3636 \\
\hline CND 003RH & $4.400 E-07$ & 0.0809 & 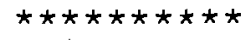 & 1.0880 \\
\hline
\end{tabular}
1

NO. OF MINIMAL CUT SETS =

MINIMAI CUT SETS INFORMATION

$\begin{array}{rlrl}\text { NSETS } & \text { MEAN } & \text { IMPORTANCE } & \text { MINIMAL CUT SETS } \\ 1 & 5.000 E-06 & 0.9191 & \text { FHUO02RZ } \\ 2 & 4.400 E-07 & 0.0809 & \text { CNDO03RH }\end{array}$

$R$ E S U I I S S S U M $M A A R$

$\begin{aligned} \text { DISTRIBUTION VALUES } & \\ \text { NUMBER OF SAMPLE } & = \\ \text { ACCURACY ON 95\% CONFIDENCE INTERVAL } & =1.03496 \mathrm{E}+00 \\ \text { POINT MEAN VALUE } & =5.44000 \mathrm{E}-06 \\ \text { MEDIAN VALUE } & =3.51559 \mathrm{E}-06 \\ \text { ERROR FACTOR } & =5.04508 \mathrm{E}+00 \\ \text { MEAN VALUE } & =5.53629 \mathrm{E}-06 \\ \text { STANDARD DEVIATION } & =6.98674 \mathrm{E}-06 \\ 5 \% \text { BOUND } & =9.50156 \mathrm{E}-07 \\ 95 \% \text { BOUND } & =1.77364 \mathrm{E}-05\end{aligned}$

0

\section{DISTRIBUTION CONFIDENCE LIMITS}

$\begin{array}{rr}\text { CONEIDENCE } & \text { FUNCTION } \\ \text { (PERRCENT) } & \text { VALUE } \\ 0.5 & 4.83732 E-07 \\ 1.0 & 5.94763 E-07 \\ 2.5 & 7.57991 E-07 \\ 5.0 & 9.50156 E-07 \\ 10.0 & 1.26117 E-06 \\ 20.0 & 1.70644 E-06 \\ 25.0 & 1.97069 E-06 \\ 30.0 & 2.19928 E-06 \\ 40.0 & 2.75084 E-06 \\ 50.0 & 3.51559 E-06 \\ 60.0 & 4.39291 E-06 \\ 70.0 & 5.49891 E-06 \\ 75.0 & 6.24136 E-06 \\ 80.0 & 7.57784 E-06 \\ 90.0 & 1.16374 E-05 \\ 95.0 & 1.77364 E-05 \\ 97.5 & 2.20581 E-05 \\ 99.0 & 3.12171 E-05\end{array}$




\section{$99.5 \quad 4.86015 E-05$}

MODULE : $\quad 76.0$ SEC EXECUTION TIME

END OF MODULE RUN 

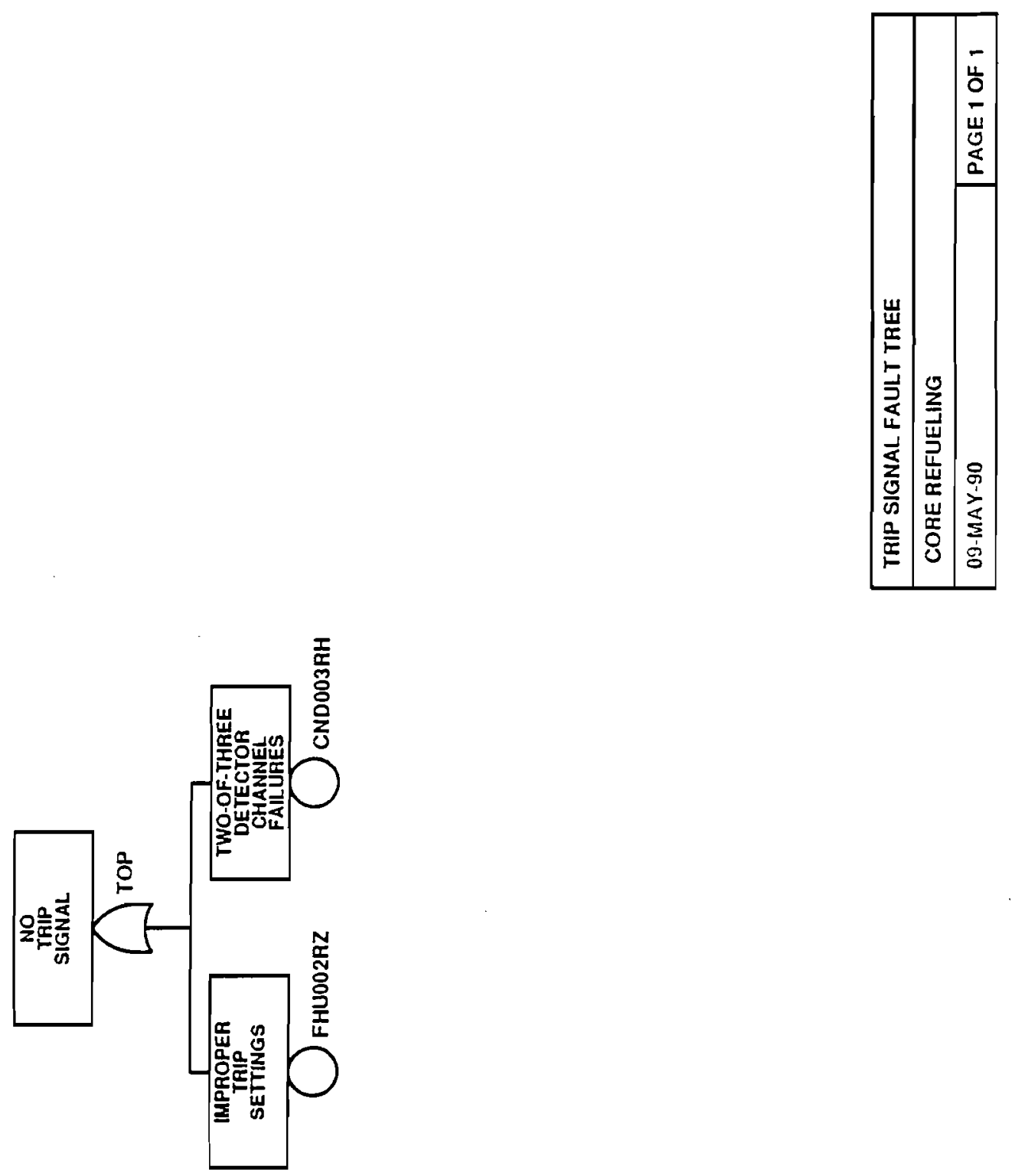


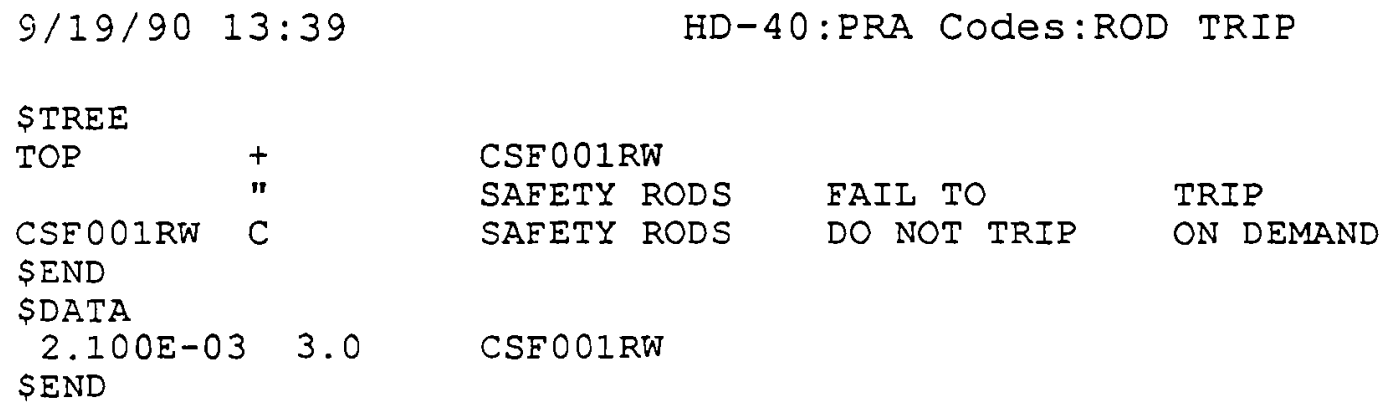

CSF001RW

SAFETY RODS FAIL TO

SAFETY RODS DO NOT TRIP ON DEMAND 
TREDRA FILE CREATED BY MODULE

* OPTION

PRINTPIO

END

* TREE

TOP OR O I CSFOOIRW

END

* TITIE

CORE REFUELING FAULT TREE

FAILURE OF SAFETY RODS TO TRIP

$31-M A Y-90$

END

* INEORM

8.
END
EVENTS

TOP

CSFOO1RW 2

END

TNISH

$\therefore O P$
11. OONORMAL F

F

SAFETY RODS

SAFETY RODS
F

F 
PROGRAM MODULE ENTERED: 31-MAY-90

ENTER THE INPUT FILE NAME :

ROD TRIP

ENTER THE OUTPUT FILE NAME :

RT. OUT

ENTER TOP EVENT NAME :

READ DATA : $\quad 45.0$ SEC REQUIRED

TOP

WRITE TREDRA INPUT :
MODULARIZATION :

ENTER CUT-OFF PROBA. :

0.0 SEC REQUIRED

1. 0 E-06

ENTER MAX. TERM NUM. : 5

MCS : 1 TERMS GENERATED

DO YOU WANT TO ANALYZE IMPORTANCE ? $Y$

IMPORTANCE : $\quad 2.0$ SEC REQUIRED

DO YOU WANT TO ANALYZE UNCERTAINTY ? $Y$

$\begin{array}{ccc} & \text { SAMPLING : } & 7.0 \text { SEC REQUIRED } \\ \text { SORTING : } & 1.0 \text { SEC REQUIRED } \\ \text { UNCERTAINTY : } & 0.0 \text { SEC REQUIRED } \\ \text { BOTTOM-UP : } & 29.0 \text { SEC REOUIRED } & \end{array}$

DO YOU WANT TO EXPAND MODULARIZED MCS ?:

$Y$

MCS : $\quad 1$ TERMS GENERATED

Y

DO YOU WANT TO ANALYZE IMPORTANCE ?:

CUTSET : 7.0 SEC REQUIRED

IMPORTANCE : $\quad 3.0$ SEC REQUIRED

Y

DO YOU WANT TO ANALYZE UNCERTAINTY ?:

\author{
SAMPLING : $\quad 8.0$ SEC REQUIRED \\ SORTING : $\quad 1.0$ SEC REQUIRED \\ UNCERTAINTY : $\quad 1.0$ SEC REQUIRED \\ EXPAND : $\quad 20.0$ SEC REQUIRED \\ MODULE : $\quad 98.0$ SEC REQUIRED
}

END OF MODULE RUN

STOP 
MODULE VERSION 2.0 RUN AT ANL ON: 31-MAY-90

CUT SETS FOR THE TOP EVENT: TOP

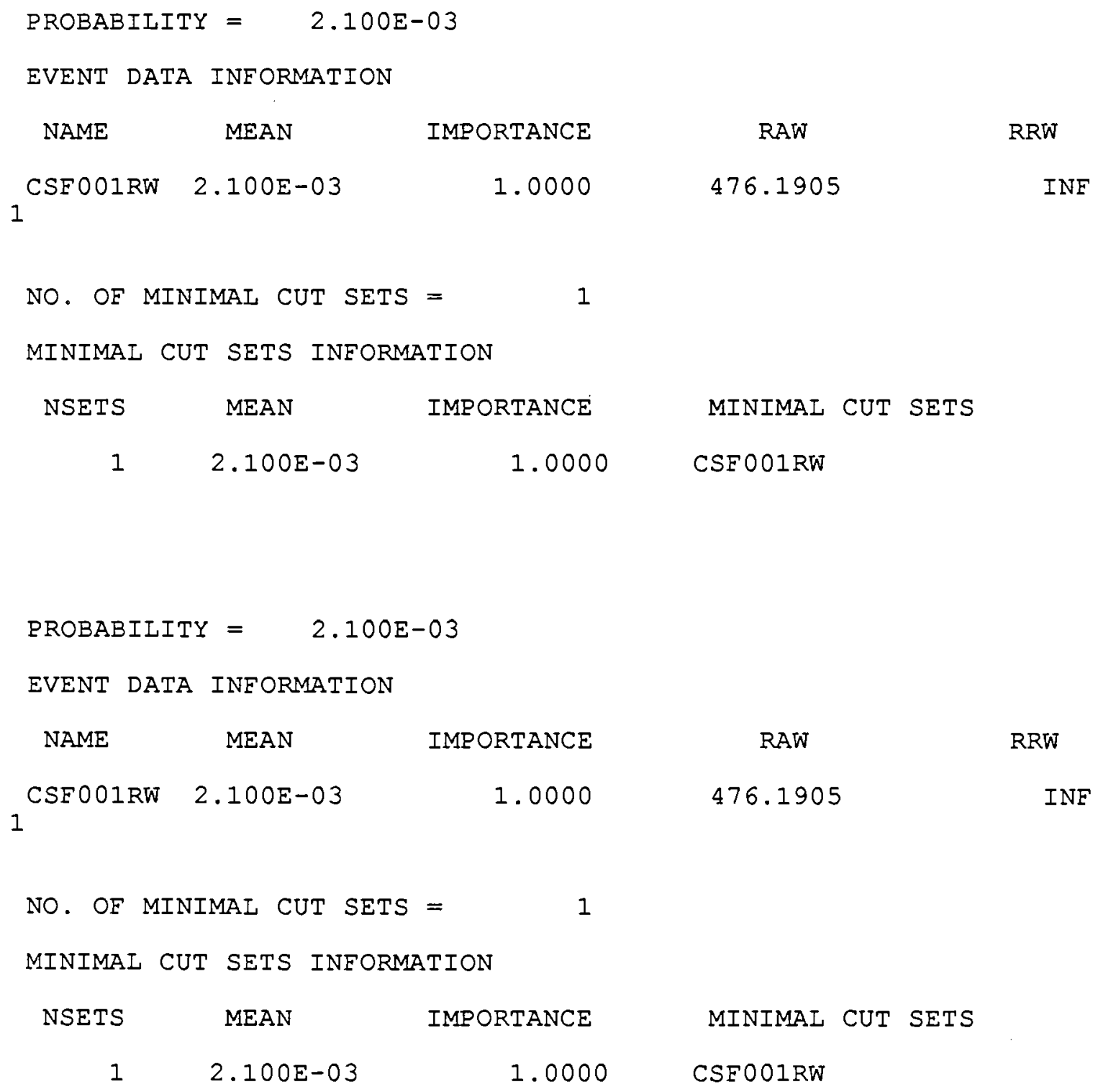


MODULE VERSION 2.0 RUN AT ANL ON: 31-MAY-90

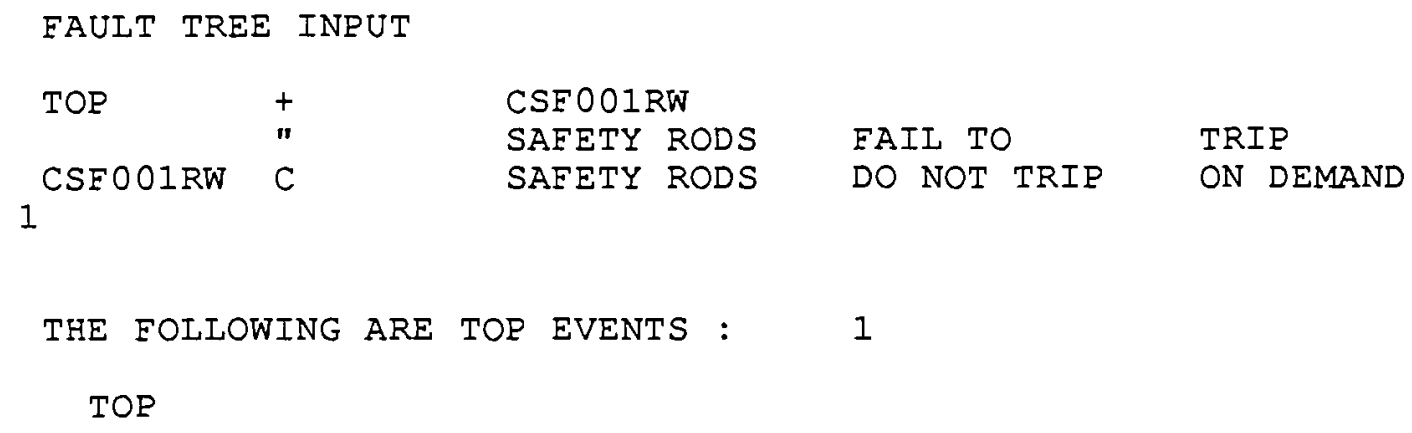


NO. OF MINIMAL CUT SETS =

1

MINIMAL CUT SETS INFORMATION

NSETS MEAN IMPORTANCE MINIMAL CUT SETS

1
1
$2.100 \mathrm{E}-03$
1.0000
CSE001RW

$\begin{aligned} & \text { RE S U I T S U M M A I Z E D } \\ & \text { DISTRIBUTION VALUES } \\ & \text { NUMBER OF SAMPIE }= \\ & \text { ACCURACY ON 95\% CONFIDENCE INTERVAL }=1.03496 \mathrm{E}+00 \\ & \text { POINT MEAN VALUE }=2.10000 \mathrm{E}-03 \\ & \text { MEDIAN VALUE }=1.66440 \mathrm{E}-03 \\ & \text { ERROR FACTOR }=3.09291 \mathrm{E}+00 \\ & \text { MEAN VALUE }=2.06506 \mathrm{E}-03 \\ & \text { STANDARD DEVIATION }=1.49934 \mathrm{E}-03 \\ & \text { 5\% BOUND }=5.38134 \mathrm{E}-04 \\ & \text { 95\% BOUND }=4.90135 \mathrm{E}-03\end{aligned}$

0

DISTRIBUTION CONFIDENCE LIMITS

$\begin{array}{rr}\begin{array}{r}\text { CONFIDENCE } \\ \text { (PERRCENT) }\end{array} & \text { FUNCTION } \\ \text { VALUE } \\ 0.5 & 2.81743 E-04 \\ 1.0 & 3.29625 E-04 \\ 2.5 & 4.34460 E-04 \\ 5.0 & 5.38134 \mathrm{E}-04 \\ 10.0 & 7.05878 \mathrm{E}-04 \\ 20.0 & 9.60922 \mathrm{E}-04 \\ 25.0 & 1.08015 \mathrm{E}-03 \\ 30.0 & 1.19055 \mathrm{E}-03 \\ 40.0 & 1.42657 \mathrm{E}-03 \\ 50.0 & 1.66440 \mathrm{E}-03 \\ 60.0 & 1.96882 \mathrm{E}-03 \\ 70.0 & 2.39435 \mathrm{E}-03 \\ 75.0 & 2.62127 \mathrm{E}-03 \\ 80.0 & 2.85803 \mathrm{E}-03 \\ 90.0 & 3.82198 \mathrm{E}-03 \\ 95.0 & 4.90135 \mathrm{E}-03 \\ 97.5 & 5.93653 \mathrm{E}-03 \\ 99.0 & 7.60737 \mathrm{E}-03 \\ 99.5 & 8.81465 \mathrm{E}-03\end{array}$

1

PROBABILITY $=2.100 \mathrm{E}-03$

EVENT DATA INFORMATION

NAME MEAN IMPORTANCE RAW RRW

$\begin{array}{lllll}\text { CSF 001RW } 2.100 E-03 & 1.0000 & 476.1905 & \text { INF }\end{array}$ 
NO. OF MINIMAL CUT SETS =

MINIMAL CUT SETS INFORMATION

NSETS

MEAN

IMPORTANCE

MINIMAL CUT SETS

1

$12.100 \mathrm{E}-03$

1.0000

CSF 001RW

$\begin{array}{lllllllllllllllll}R & E & S & U & L & T & S & S & U & M & M & A & R & I & Z & E & D\end{array}$

DISTRIBUTION VALUES

$\begin{aligned} \text { NUMBER OF SAMPLE } & = \\ \text { ACCURACY ON 95\% CONEIDENCE INTERVAL } & =1.03496 \mathrm{E}+00 \\ \text { POINT MEAN VALUE } & =2.10000 \mathrm{E}-03 \\ \text { MEDIAN VALUE } & =1.68540 \mathrm{E}-03 \\ \text { ERROR FACTOR } & =3.02220 \mathrm{E}+00 \\ \text { MEAN VALUE } & =2.08947 \mathrm{E}-03 \\ \text { STANDARD DEVIATION } & =1.60316 \mathrm{E}-03 \\ 5 \% \text { BOUND } & =5.57672 \mathrm{E}-04 \\ 95 \% \text { BOUND } & =5.05613 \mathrm{E}-03\end{aligned}$

DISTRIBUTION CONEIDENCE LIMITS

CONFIDENCE (PERRCENT)

0.5

1.0

2.5

5.0

10.0

20.0

25.0

30.0

40.0

50.0

60.0

70.0

75.0

80.0

90.0

95.0

97.5

99.0

99.5

FUNCTION

VALUE
$3.08592 \mathrm{E}-04$
3. $52549 \mathrm{E}-04$
4. $48240 \mathrm{E}-04$
$5.57672 \mathrm{E}-04$
$6.90646 \mathrm{E}-04$
$9.28801 \mathrm{E}-04$
$1.03674 \mathrm{E}-03$
1. $14591 \mathrm{E}-03$
$1.38 .548 E-03$
1. $68540 \mathrm{E}-03$
1. $97055 \mathrm{E}-03$
$2.31367 \mathrm{E}-03$
$2.55980 E-03$
3. $00483 E-03$
3. $96435 E-03$
$5.05613 \mathrm{E}-03$
$6.36085 E-03$
8. 57124E-03
$9.97013 E-03$

MODULE : $\quad 98.0$ SEC EXECUTION TIME

END OF MODULE RUN 


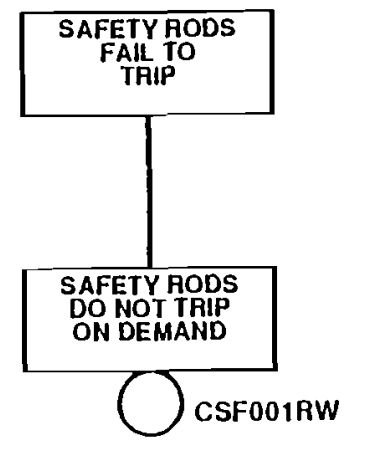

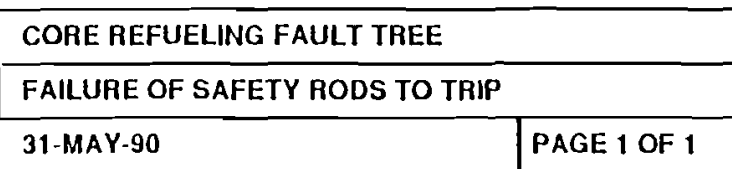




$\begin{array}{ll}\text { \$TREE } & \\ \text { TOP } & + \\ & \quad \\ \text { CCGOO1RV } \quad C & \\ \text { \$END } & \\ \text { \$DATA } & \\ \text { 5.50OE-04 } & 3.0 \\ \text { \$END } & \end{array}$

CCGO01RV SUBASSEMBLY SUBASSEMBLY

$\begin{array}{lll}\text { DROPPED } & & \text { INTO CORE } \\ \text { DROPPED } & \text { BY } & \text { CORE GRIPPER }\end{array}$

CCG001RV 
$6 / 21 / 90 \quad 14: 05$

HD-40:PRA Codes : DROP . trd

Page 1

TREDRA FILE CREATED BY MODULE

* OPTION

$\begin{array}{llllll}\text { PRINTPLO } & 6 & 7 & \text { F } & \text { F }\end{array}$

END

* TREE

TOP OR 01 CCGOOIRV

END

* TITLE

CORE REFUELING ANALYSIS

SUBASSEMBLY DROPPED INTO CORE

21-JUN-90

END

* INFORM

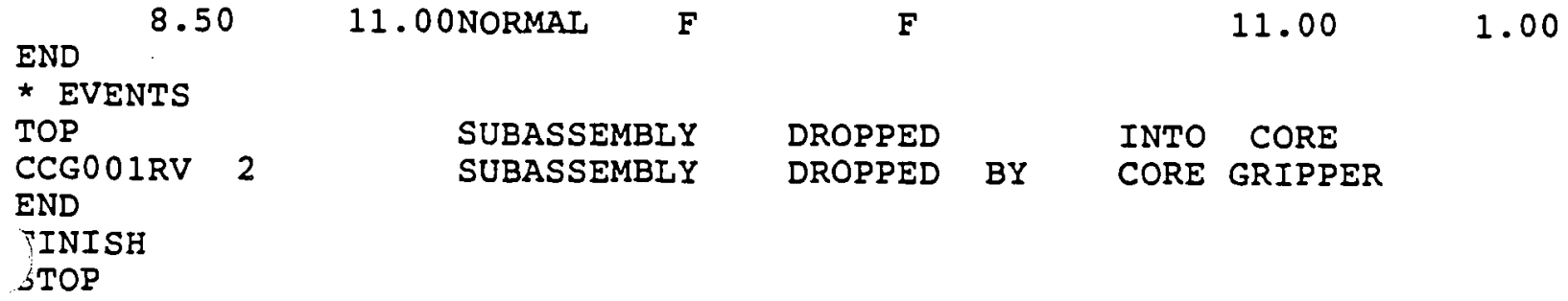


MODULE VERSION 2.0 RUN AT ANL ON: 21-JUN-90

CUT SETS FOR THE TOP EVENT: TOP

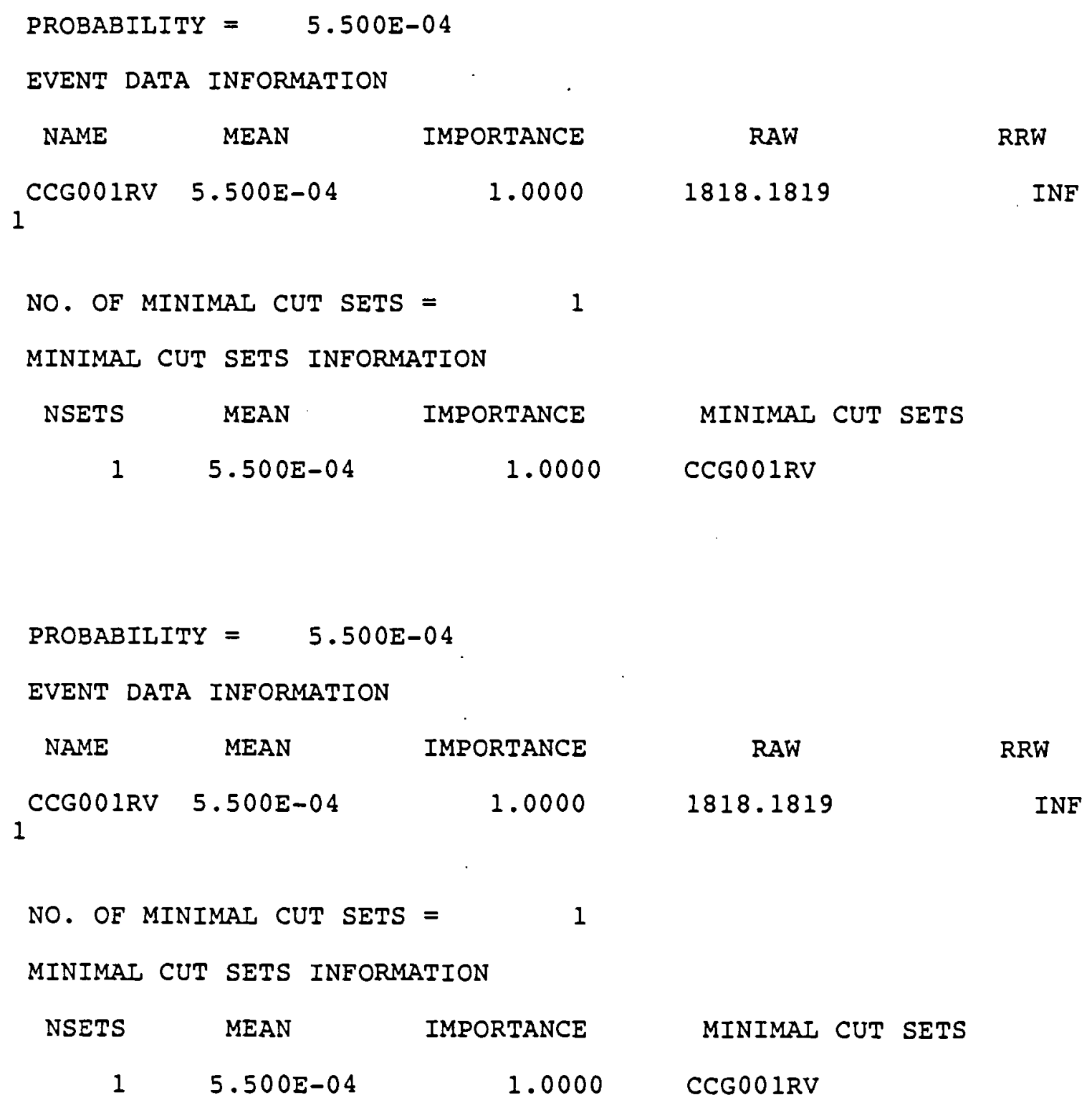


MODULE VERSION 2.0 RUN AT ANL ON: 21-JUN-90

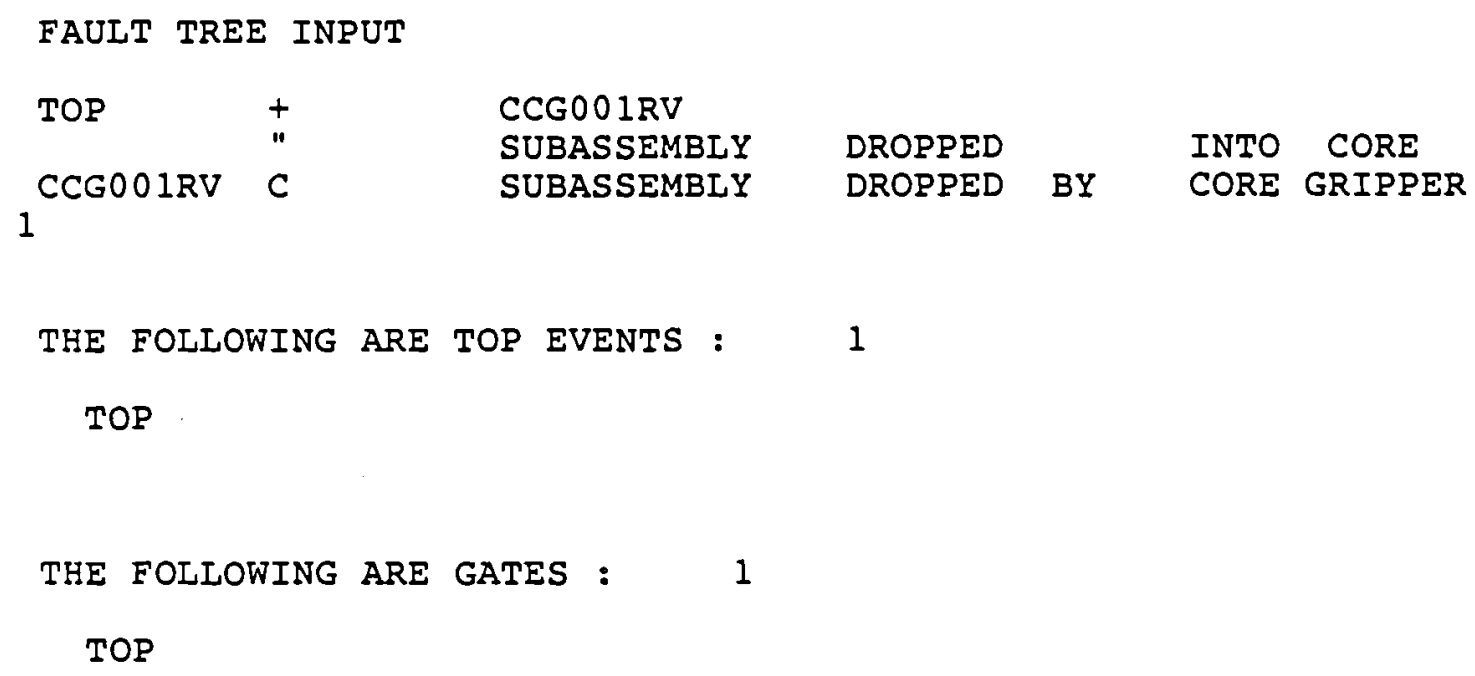


NO. OF MINIMAL CUT SETS $=1$

MINIMAI CUT SETS INFORMATION

$\begin{array}{rrrr}\text { NSETS } & \text { MEAN } & \text { IMPORTANCE } & \text { MINIMAL CUT SETS } \\ 1 & 5.500 E-04 & 1.0000 & \text { CCG001RV }\end{array}$

$\begin{aligned} & \text { RE S U L T S S U M M A R I E D } \\ & \text { DISTRIBUTION VALUES } \\ & \text { NUMBER OF SAMPLE }= \\ & \text { ACCURACY ON 958 CONEIDENCE INTERVAI }=1.03496 \mathrm{E}+00 \\ & \text { POINT MEAN VALUE }=5.50000 \mathrm{E}-04 \\ & \text { MEDIAN VALUE }=4.35914 \mathrm{E}-04 \\ & \text { ERROR FACTOR }=3.09291 \mathrm{E}+00 \\ & \text { MEAN VALUE }=5.40850 \mathrm{E}-04 \\ & \text { STANDARD DEVIATION }=3.92685 \mathrm{E}-04 \\ & 58 \text { BOUND }=1.40940 \mathrm{E}-04 \\ & 958 \text { BOUND }=1.28369 \mathrm{E}-03\end{aligned}$

0

DISTRIBUTION CONFIDENCE LIMITS

$\begin{array}{rr}\text { CONEIDENCE } & \text { FUNCTION } \\ \text { (PERRCENT) } & \text { VALUE } \\ 0.5 & 7.37897 \mathrm{E}-05 \\ 1.0 & 8.63303 \mathrm{E}-05 \\ 2.5 & 1.13787 \mathrm{E}-04 \\ 5.0 & 1.40940 \mathrm{E}-04 \\ 10.0 & 1.84873 \mathrm{E}-04 \\ 20.0 & 2.51670 \mathrm{E}-04 \\ 25.0 & 2.82896 \mathrm{E}-04 \\ 30.0 & 3.11810 \mathrm{E}-04 \\ 40.0 & 3.73625 \mathrm{E}-04 \\ 50.0 & 4.35914 \mathrm{E}-04 \\ 60.0 & 5.15643 \mathrm{E}-04 \\ 70.0 & 6.27091 \mathrm{E}-04 \\ 75.0 & 6.86523 \mathrm{E}-04 \\ 80.0 & 7.48532 \mathrm{E}-04 \\ 90.0 & 1.00099 \mathrm{E}-03 \\ 95.0 & 1.28369 \mathrm{E}-03 \\ 97.5 & 1.55480 \mathrm{E}-03 \\ 99.0 & 1.99240 \mathrm{E}-03 \\ 99.5 & 2.30860 \mathrm{E}-03\end{array}$

1

PROBABILITY $=\quad 5.500 \mathrm{E}-04$

EVENT DATA INFORMATION

$\begin{array}{llll}\text { NAME MEAN IMPORTANCE RAW } & \text { RRW }\end{array}$

CCG001RV 5.500E-04 1.0000 A.2-41 1818.1819 INF 
NO. OF MINIMAL CUT SETS = 1

MINIMAI CUT SETS INFORMATION

$\begin{array}{rlrr}\text { NSETS } & \text { MEAN } & \text { IMPORTANCE } & \text { MINIMAI CUT SETS } \\ 1 & 5.500 E-04 & 1.0000 & \text { CCG001RV }\end{array}$

1

R E S U L T S S U M M A R I Z E D

\section{DISTRIBUTION VALUES}

NUMBER OF SAMPLE $=1200$

ACCURACY ON $95 \%$ CONFIDENCE INTERVAL $=1.03496 \mathrm{E}+00$

POINT MEAN VALUE $=5.50000 \mathrm{E}-04$

MEDIAN VALUE $=4.41413 \mathrm{E}-04$

ERROR FACTOR $=3.02220 \mathrm{E}+00$

MEAN VALUE $=5.47243 \mathrm{E}-04$

STANDARD DEVIATION $=4.19874 \mathrm{E}-04$

$58 \mathrm{BOUND}=1.46057 \mathrm{E}-04$

958 BOUND $=1.32422 \mathrm{E}-03$

0

\section{DISTRIBUTION CONFIDENCE LIMITS}

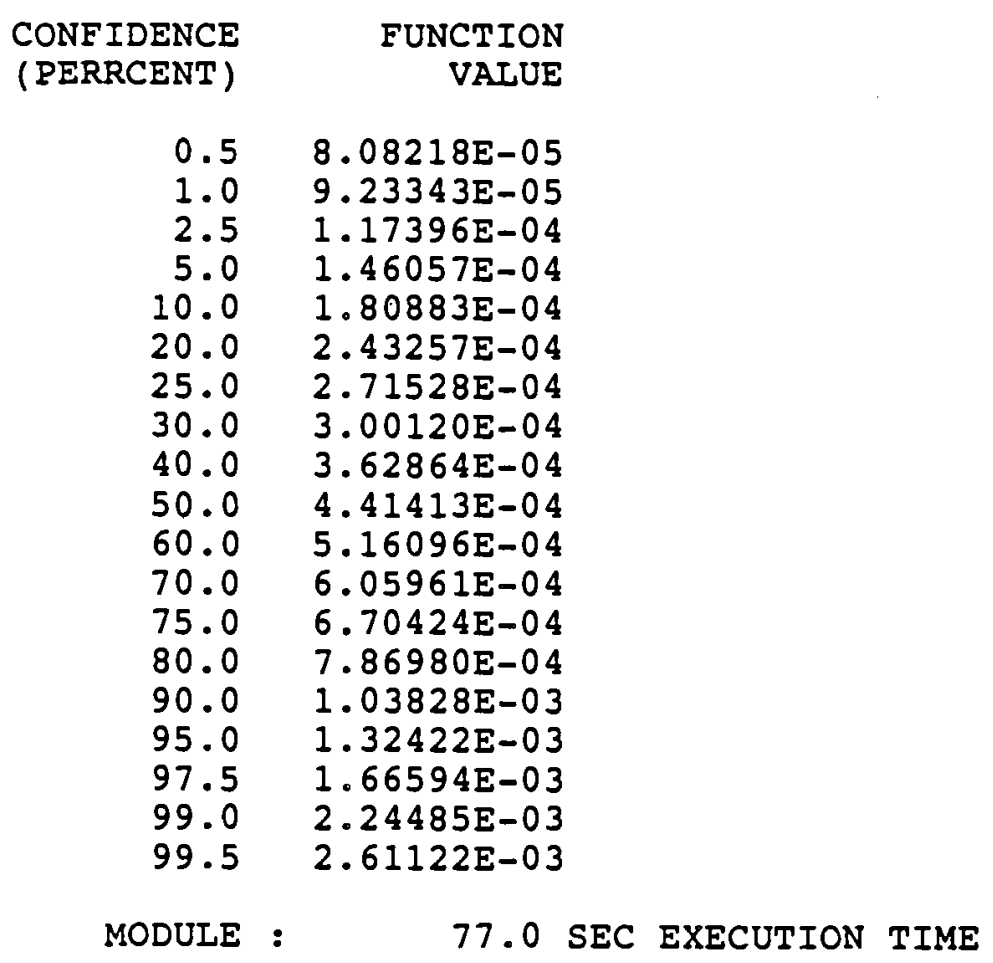

END OF MODULE RUN 


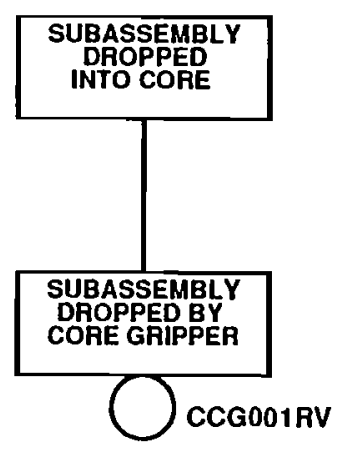

CORE REFUELING ANALYSIS

SUBASSEMBLY DROPPED INTO CORE

\begin{tabular}{|l|l}
\hline 21-JUN-90 & PAGE 1 OF 1 \\
\hline
\end{tabular} 


\section{APPENDIX A.3}

\section{EVENT TREE ANALYSES}

- Dropped Subassembly

- Trip Signal Failure

- Safety Rod Trip Failure

A.3-i 


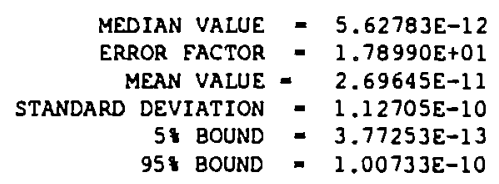

0

DISTRIBUTION CONFIDENCE LIMITS

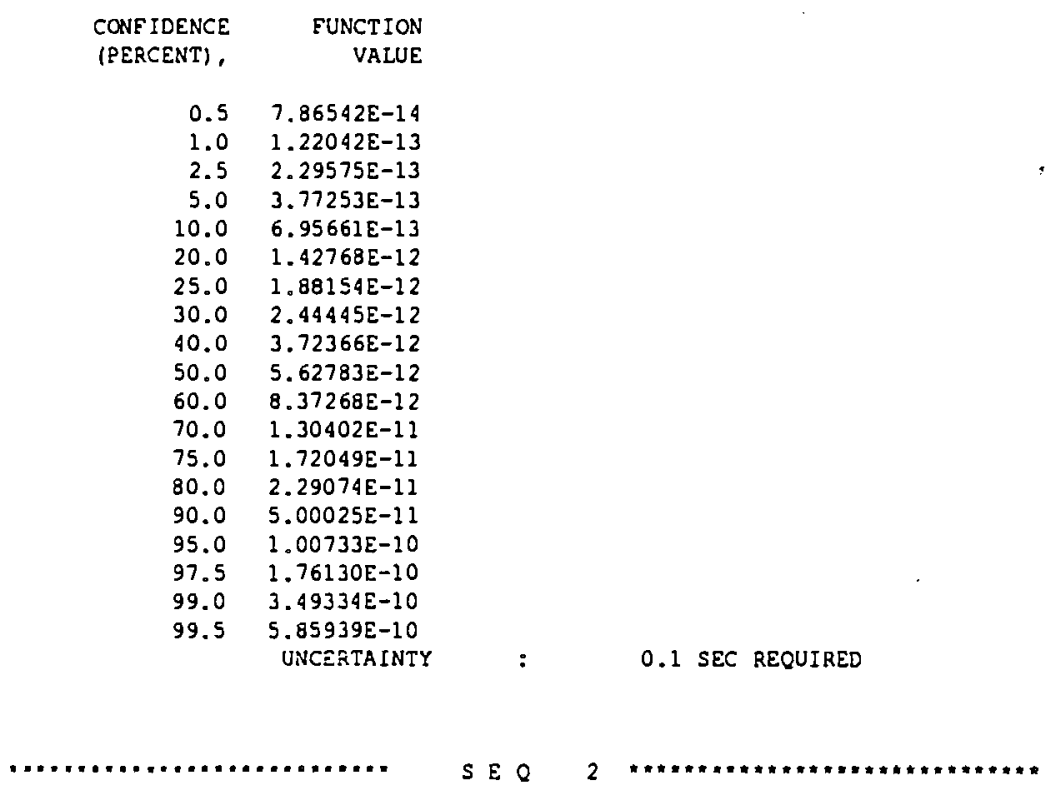

FILE: SEQS ROCLANS AI ANLMM VM/SP 508 CMS

SEQUE:ACE ECR T:IE INITIATING EVENT lel

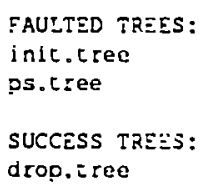

\begin{tabular}{|c|c|c|c|c|}
\hline NANE & MA:: & IM:PCRTANCE & RAW & RRW \\
\hline ie: & 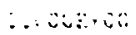 & . . OCDC & i. 0000 & 0.0000 \\
\hline Fitce:.̈. & $1.3,3=3-0$ & 0.9607 & 1310.6552 & 25.4332 \\
\hline Cticos: & $\therefore$, & 0.6067 & 16 6́) 7.0020 & 3.0000 \\
\hline cre:os:..: & $\therefore \therefore:-\because$ & 6.3333 & $: 506 \% .3359$ & 1.5000 \\
\hline
\end{tabular}




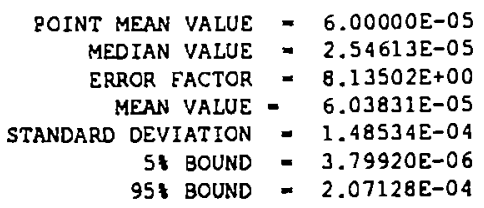

0

DISTRIBUTION CCNFIDENCE LIMITS

$\begin{array}{lr}\text { CONEIDENCE } & \text { FUNCTION } \\ \text { (PERCENT), } & \text { VALUE }\end{array}$

FILE: SEQS ROGLANS AI ANLVM VM/SP 508 CMS

PAGE 00006

$\begin{array}{rr}0.5 & 1.22796 E-06 \\ 1.0 & 1.74866 E-06 \\ 2.5 & 2.69552 E-06 \\ 5.0 & 3.79920 E-06 \\ 10.0 & 5.82636 E-06 \\ 20.0 & 9.53138 E-06 \\ 25.0 & 1.14114 E-05 \\ 30.0 & 1.36944 E-05 \\ 40.0 & 1.86214 E-05 \\ 50.0 & 2.54613 E-05 \\ 60.0 & 3.46341 E-05 \\ 70.0 & 4.88213 E-05 \\ 75.0 & 5.76464 E-05 \\ 80.0 & 7.16117 E-05 \\ 90.0 & 1.23354 E-04 \\ 95.0 & 2.07128 E-04 \\ 97.5 & 3.40122 E-04 \\ 99.0 & 5.71166 E-04 \\ 99.5 & 8.46995 E-04 \\ & \text { UNCERTAINTY }\end{array}$




\begin{tabular}{|c|c|c|c|c|}
\hline CFA005RL & 4.000E-05 & 0.6667 & 16666.9980 & 3.0000 \\
\hline CCG005RG & $2.000 \varepsilon-05$ & 0.3333 & 16667.3301 & 1.5000 \\
\hline CND003RH & $4.400 \varepsilon-07$ & 0.0809 & 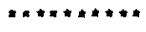 & 1.0880 \\
\hline CND001RM & $3.000 E-05$ & 0.0393 & 1311.5730 & 1.0409 \\
\hline
\end{tabular}

FILE: SEQS ROGLANS AI ANLMM VM/SP SOQ CMS

PAGE 00007

No. CF MINIMAL CUT SETS - 12

MINIMAL CUT SETS INFORMATION

\begin{tabular}{|c|c|c|c|c|c|c|c|}
\hline NSETS & MEAN & \multicolumn{2}{|c|}{ IMPORTANCE } & \multicolumn{3}{|c|}{ MINIMAL CUT SETS } & \multirow[b]{2}{*}{ EHU0O2R2 } \\
\hline 1 & $1.466 E-13$ & 0.5887 & 1e2 & & CFA00SRL & FHU001R2 & \\
\hline 2 & $7.330 E-14$ & 0.2943 & 1e2 & & CCGOOSRG & FHUO01R2 & FHU002R2 \\
\hline 3 & $1.290 E-14$ & 0.0518 & $1 \mathrm{e} 2$ & & CFA005RL & FHUO01RZ & CND003RH \\
\hline 4 & $6.450 E-15$ & $0.0259^{\circ}$ & ie2 & & CCGOOSRG & FHUOO1R2 & CND003RH \\
\hline 5 & $6.000 E-15$ & 0.0241 & le2 & & CFA005RL & CND001RM & FHU002R2 \\
\hline 6 & $3.000 E-15$ & 0.0120 & $1 \mathrm{e} 2$ & & CCG005RG & CND001RM & FHUOO2R2 \\
\hline 7 & $5.280 E-16$ & 0.0021 & le2 & & CEA005RL & CND001RM & CND003RH \\
\hline 8 & $2.640 E-16$ & 0.0011 & le2 & & CCG005RG & CND001RM & CND003RH \\
\hline 9 & $2.000 E-20$ & 0.0000 & 1e2 & & CFA005RL & CNDO02RH & FHUOO2R2 \\
\hline 10 & $1.000 \varepsilon-20$ & 0.0000 & 1e2 & & CCG005RG & CND002RW & FHU0O2R2 \\
\hline 11 & $1.760 \varepsilon-21$ & 0.0000 & 1e2 & & CFA005RL & CNDO02RW & CND003RH \\
\hline \multirow[t]{4}{*}{12} & $8.800 E-22$ & 0.0000 & le2 & & CCGO05RG & CND002RW & CND003RH \\
\hline & & \multicolumn{2}{|c|}{ IMPORTANCE } & : & \multicolumn{3}{|c|}{ 1.2 SEC REQUIRED } \\
\hline & & \multicolumn{2}{|l|}{ SAMP LING } & : & 7.3 & \multicolumn{2}{|c|}{ SEC REQUIRED } \\
\hline & & \multicolumn{2}{|l|}{ SORTING } & : & 0.5 & SEC REQUI & RED \\
\hline
\end{tabular}

- RESULTS SUMAARIZE D

DISTRIBUTION VALUES

MEAN (Calculaced with 99 of the points) $0.159 \mathrm{E}-12$

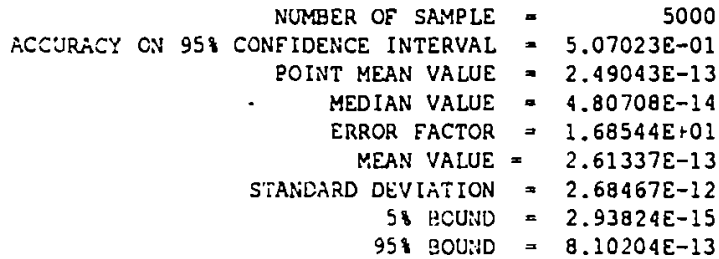

0

DISTRIBUTICN CONEIOENCE LIMITS

\begin{tabular}{|c|c|}
\hline 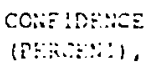 & $\begin{array}{r}\text { YהIOION } \\
\text { VIUE }\end{array}$ \\
\hline 0.5 & b. $5700=0-16$ \\
\hline 1.0 & 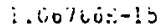 \\
\hline 2.5 & a.73:il:z-15 \\
\hline
\end{tabular}

A. 3-4 


\begin{tabular}{|c|c|c|c|c|c|c|c|}
\hline$i$ & 1. 20uc-12 & U. U.jil & i 42 & & CCLOOSRG & CNDOOIRM & CSEO01RW \\
\hline 5 & 8. $400 E-18$ & 0.0000 & Le2 & & CEA0O5RL & CND002RW & CSF001RW \\
\hline 6 & 4. $200 E-18$ & 0.0000 & le2 & & CCGOOSRG & CNDO02RW & CSFOOIRW \\
\hline & & \multicolumn{2}{|c|}{ IMPORTANCE } & : & \multicolumn{3}{|c|}{0.1 SEC REQUIRED } \\
\hline & & \multicolumn{2}{|l|}{ SAMPLING } & : & 5.8 & \multicolumn{2}{|c|}{ SEC REQUIRED } \\
\hline & & \multicolumn{2}{|l|}{ SORT ING } & : & 0.5 & SEC REQUI & RED \\
\hline
\end{tabular}

\author{
RESULTS SUMMARIZED
}

DISTRIBUTION VALUES

$\begin{aligned} & \text { MEAN (Calculated with } 99 \text { of the polnts) } 0.676 \mathrm{E}-10 \\ & \text { NUMBER OF SAMPLE }= \\ & \text { CONFIDENCE INTERVAL }=5.07023 \mathrm{E}-01 \\ & \text { POINT MEAN VALUE }=9.61380 \mathrm{E}-11 \\ & \text { MEDIAN VALUE }=2.15358 \mathrm{E}-11 \\ & \text { ERROR FACTOR }=1.64629 \mathrm{E}+01 \\ & \text { MEAN VALUE }=1.01555 \mathrm{E}-10 \\ & \text { STANDARD DEVIATION }=4.82961 \mathrm{E}-10 \\ & \text { 5E BOUND }=1.56976 \mathrm{E}-12 \\ & \text { 95 BOUND }=3.54531 \mathrm{E}-10\end{aligned}$

0

DISTRIBUTION CONFIDENCE LIMITS

$\begin{array}{rr}\text { CCNFIDENCE } & \text { FUNCTION } \\ \text { (PERCENT), } & \text { VALUE } \\ & \\ 0.5 & 3.63275 E-13 \\ 1.0 & 5.17699 \mathrm{E}-13 \\ 2.5 & 9.43252 \mathrm{E}-13 \\ 5.0 & 1.56976 \mathrm{E}-12 \\ 10.0 & 2.67717 \mathrm{E}-12 \\ 20.0 & 5.34060 \mathrm{E}-12 \\ 25.0 & 6.98678 \mathrm{E}-12 \\ 30.0 & 8.86005 \mathrm{E}-12 \\ 40.0 & 1.40628 \mathrm{E}-11 \\ 50.0 & 2.15358 \mathrm{E}-11 \\ 60.0 & 3.17958 \mathrm{E}-11 \\ 70.0 & 4.88775 \mathrm{E}-11 \\ 75.0 & 6.17467 \mathrm{E}-11\end{array}$

FILE: SEQS ROGLANS AI ANLVM VM/SP 508 CMS

PAGE 00010
$80.0 \quad 8.04972 \mathrm{E}-11$
90.3 i. $7,3 \pm 0 \equiv-10$
95.0 3.5:531E-10
97.5 E.7i $33 \pm \equiv-1 C$
$99.0 \quad 1.91 / 9 j \mathrm{E}-0 \mathrm{z}$
$99.5 \quad 2.522: 8=-69$
VIEERTAIITY : 0.1 SEC REQUIRED 
FILE: SECS ROGLANS AI ANLMM VM/SP 500 CMS

PAGE $0000 B$

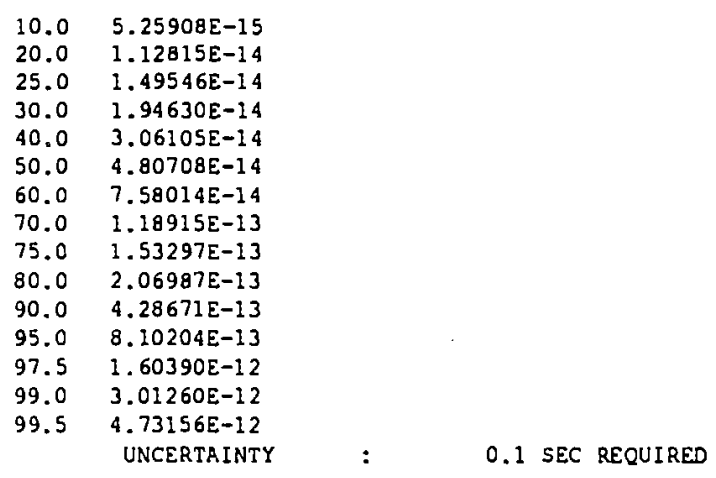

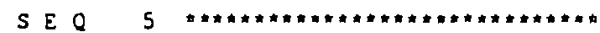

sequence gor the inttiating event ter I-PS-T Sequence

FAULTED TREES:

Init.cree

ps.tree

rt.tree

SUCCESS TREES:

tr.tree

1

PROBABILITY $=9.614 E-11$

EVENT DATA INEORMATION

\begin{tabular}{lcrrr} 
NAME & MEAN & IMPORTANCE & \multicolumn{1}{c}{ RAH } & \multicolumn{1}{c}{ RRW } \\
CSFO01RH & $2.100 E-03$ & 1.0000 & 476.1905 & 0.0000 \\
Le2 & $1.000 E+00$ & 1.0000 & 1.0000 & 0.0000 \\
FHUO0192 & $7.330 E-04$ & 0.9607 & 1310.6552 & 25.4332 \\
CFAO05RL & $4.000 E-05$ & 0.6667 & 16667.0000 & 3.0000 \\
CCGO05RG & $2.000 E-05$ & 0.3333 & 16667.3340 & 1.5000 \\
CNDO01RY & $3.000 E-05$ & 0.0393 & 1311.5757 & 1.0409 \\
CNDCO2RH & $1.000 E-10$ & 0.0000 & 1444.5277 & 1.0000
\end{tabular}

NO. OF M!IVUAL CU'T SËIS =

6

MINIVAL CUT SETS INECHYATION

FILE: SERE ROCIA.NS AI ANLVM VM/SP 500 CMS

\begin{tabular}{|c|c|c|c|c|c|c|}
\hline NSt:.:S & MEAd & $1 \because A P C$ & RTALICE & MINIMAI & CUT SETS & \\
\hline$i$ & $6.1575-11$ & C. 6405 & $i \in 2$ & CFA005RL & EHUO01R2 & CSF001RW \\
\hline 2 & $3.6,10-11$ & 2. .320? & \{e 2 & CCGC05RG & FHUOOIRZ & CSFOOIRW \\
\hline$\vdots$ & $2 . \therefore \therefore$ t. -2 & $\therefore, \quad \therefore 2 i, 2$ & $i \in: 2$ & Cf $\cap 005 R L$ & CND001RM & CSFOOJRW \\
\hline
\end{tabular}


Appendix B

FAULT TREES 
Appendix B contains all of the fault trees and input data used for quantification of the EBR-II PRA. The figures on pages B.1-nnn are associated with the initiating events. The figures on pages B.2-nnn are configurations associated with top events. The table on pages B.3-n contain the minimum cutsets of the dominant sequences.

\section{B. 1 - INITIATING EVENTS}

ASSOCIATED TOP EVENT(S) $\quad$ PAGE

\section{LCRA, LCDL}

BFTF

B. $1-1$

Primary sodium purification system siphon

B. 1-3

IHX tube and secondary storage tank failure

B. $1-5$

LOFs

LF1D

P3055

B. 1-7

LF2A

P2001

B. 1-9

LF2B

P1022

B. $1-14$

LF2C

P1033

B. $1-26$

LF2D

P1055

B. 1-33

LF2E

P2002

B. 1-35

LF2F

P2003

B. $1-56$

\section{Reactivity Insertions}

RISA, RISB, RISC, RISD

$\begin{array}{ll}\text { C9010 } & \text { B. } 1-87 \\ \text { C9020 } & \text { B. } 1-92 \\ \text { C9030 } & \text { B. } 1-105\end{array}$




\section{B.2 - TOP EVENTS}

Fault Tree TOP Events

Event Tree Top Events

Page

Reactor Safety System (RSS)

R00A2

$\mathrm{R} 00 \mathrm{C} 2$

R01A1

$\mathrm{R} 00 \mathrm{C} 1$

$\mathrm{R} 00 \mathrm{C} 3$

$\mathrm{R} 0003$

Primary Pumps

$\mathrm{P} 1000$

P7777

Secondary $\mathrm{Na}$ and Balance of Plant

B1000L

N7000

Decay Heat Removal Trees

D1104L

D1104

D5000

D4000

D6000L
RSIG

RROD

FSIG

FROD

LROD

MRSS
B. 2-1

B. $2-52$

B. 2-114

B. 2-155

B. 2-204

B.2-206

PKRU

B. 2-222

PRUN

B. 2-245

BPHR

B. $2-247$

SPTR

B. $2-254$

DHRS,DRSB B.2-256

DRLB B.2-268

DRLA B. $2-280$

DHRL B.2-289

DRSC B.2-291

Electrical Systems

E0100

B. 2-299

E0102

B. 2-301

E0105

B. 2-303

E0120

B. 2-306

E0132

B. 2-308

E0150

B. 2-311

E0200

B. 2-319

E0250

B. 2-327

E0300

B. $2-330$

E0306

B. 2-332

E0308

B. 2-335

E0360

B. 2-338

B. -ii 


\section{B.2 - TOP EVENTS (Cont'd)}

Fault Tree TOP Events

Page

J0100

B. 2-340

J0101

B. $2-342$

J0201

B. $2-348$

$\mathrm{J} 0301$

B. $2-354$

$\mathrm{J} 0500$

B. 2-359

J0530

B. $2-364$

J0550

B. 2-369

J0555

B. 2-371

J0560

B. 2-373

J0565

B. $2-375$

J0570

B. $2-377$

J0575

B. $2-379$

J0580

B. 2-381

L0100

B. 2-383

L0200

B. 2-388

L0300

B. $2-390$

L0400

B. 2-392

L0500

B. 2-394

L0510

B. 2-396

L0520

B. $2-398$

L0530

B. $2-400$

U0050

B. $2-402$

U0084

B. 2-404

U0101

B. $2-406$

U0150

B. $2-408$

U0184

B. $2-410$

U0201

B. 2-412

ED100

B. 2-414

ED102

B. $2-416$

ED105

B. $2-418$

JD500

B. $2-421$

JD530

B. $2-426$

LD100

B. 2-431

LD200

B. $2-436$

LD500

B. $2-438$

LD510

B. $2-440$

B.-iii 


\section{B.2 - TOP EVENTS (Cont'd)}

Fault Tree TOP Events $\quad \underline{\text { Page }}$

Example of Short Term Electrical System Tree

E0200L

B. $2-442$

$\underline{\text { Instrument and Plant Air Systems }}$

I0001

B. $2-450$

I0200

B. $2-453$

I0301

B. $2-461$

I0310

B. $2-463$

I0501

B. $2-468$

I0900

B. 2-472

Water Systems

w0001

B. 2-474

Shield Cooling System

H1000

B. $2-479$

H8100

B. 2-537

Stack Exhaust System

Y1000

B. 2-553

Y1001

B.2-561

Thimble Cooling System

$\mathrm{T} 1000$

B. 2-569

B.3 - MINIMAL CUT SETS OF THE DOMINANT

B.3-1 DAMAGE SEQUENCES

B.-iv 


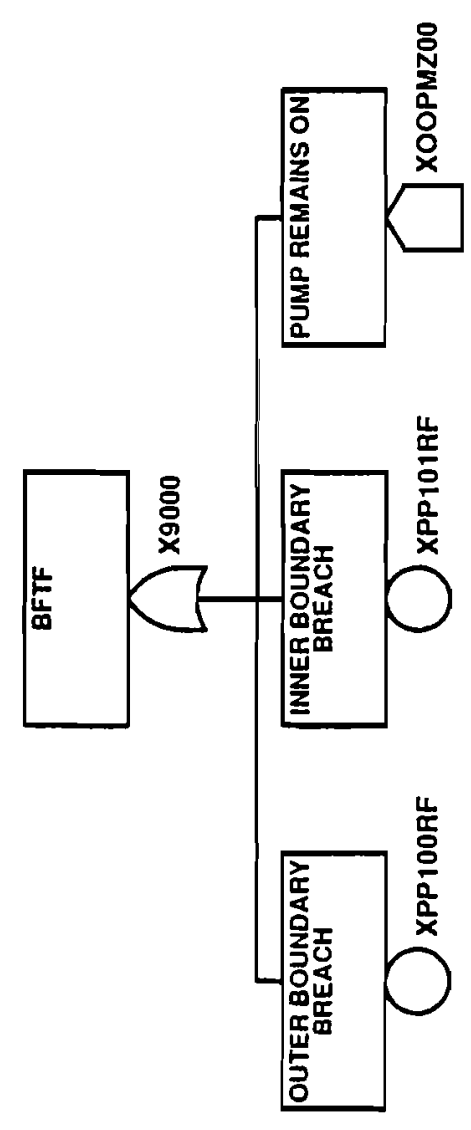

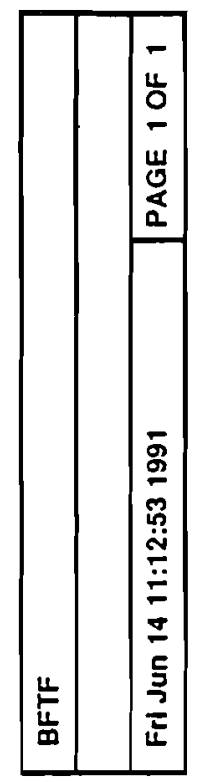

B. 1-1 


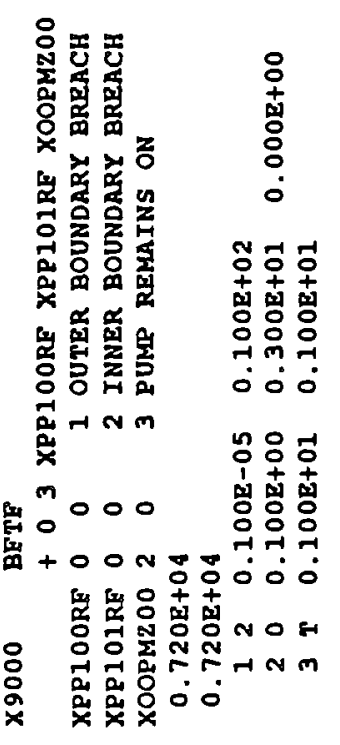

B. 1-2 


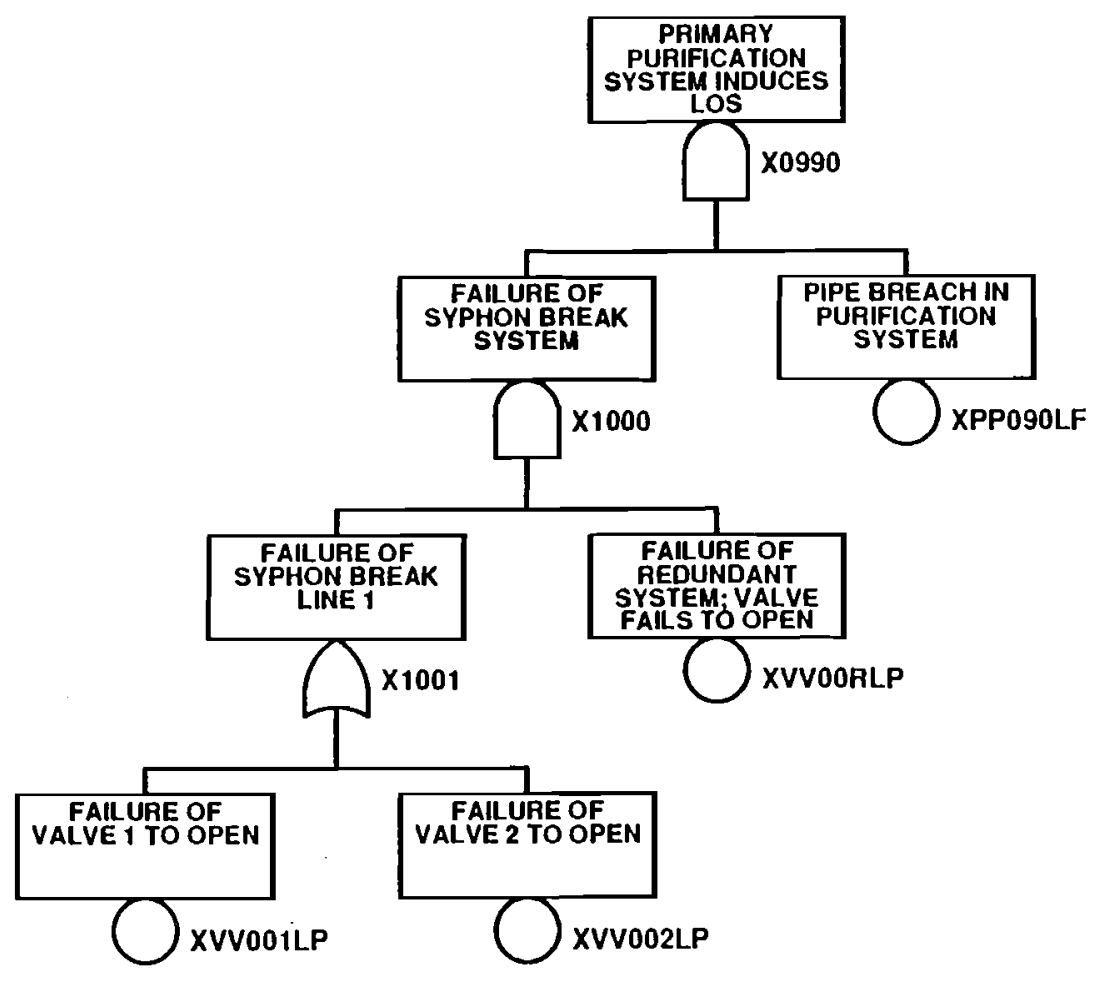

\begin{tabular}{|c|c|}
\hline \multicolumn{2}{|c|}{ PRIMARY SODIUM PURIFICATION SYSTEM SYPHON } \\
\hline BREAK & \\
\hline Fri Jun 14 11:12:27 1991 & PAGE 1 OF 1 \\
\hline
\end{tabular}


344

PRIMARY SODIUM PURIEICATION SYSTEM SYPHON BRRAK

X0990 PRIMARY PURIFICATION SYSTEM INDUCES LOS

$\star 11 \times 1000$ XPP090LF

X1000 EAILURE OF SYPHON BREAK SYSTEM

$\star 11$ X1001 XVVOORIP
$X 1001$ EAILURE OF SYPHON BRRAK LINE 1

+02 XVV001LP XVV002L

XPPO90LE 0 - 0 1 PIPE BREACH IN PURIFICATION SYSTEM

XVVOORLP 0 O 02 FAILURE OF REDUNDANT SYSTEM; VALVE FAILS TO OPEN

XVVOOILP 0 O 3 FAILURE OF VALVE 1 TO OPEN

XVV002LP 0 O 4 FAILURE OF VALVE 2 TO OPEN

$0.720 \mathrm{E}+04$
$0.720 \mathrm{E}+0$
$\begin{array}{llll}1 & 2 & 0.300 \mathrm{z}-06 & 0.100 \mathrm{E}+02\end{array}$
$200.100 \mathrm{E}-01 \quad 0.100 \mathrm{E}+02 \quad 0.000 \mathrm{E}+00$
$\begin{array}{lllll}30 & 0.100 \mathrm{E}-01 & 0.100 \mathrm{E}+02 & 0.000 \mathrm{E}+00\end{array}$
40 o $0.100 z-01 \quad 0.100 \mathrm{E}+02 \quad 0.000 \mathrm{E}+00$ 


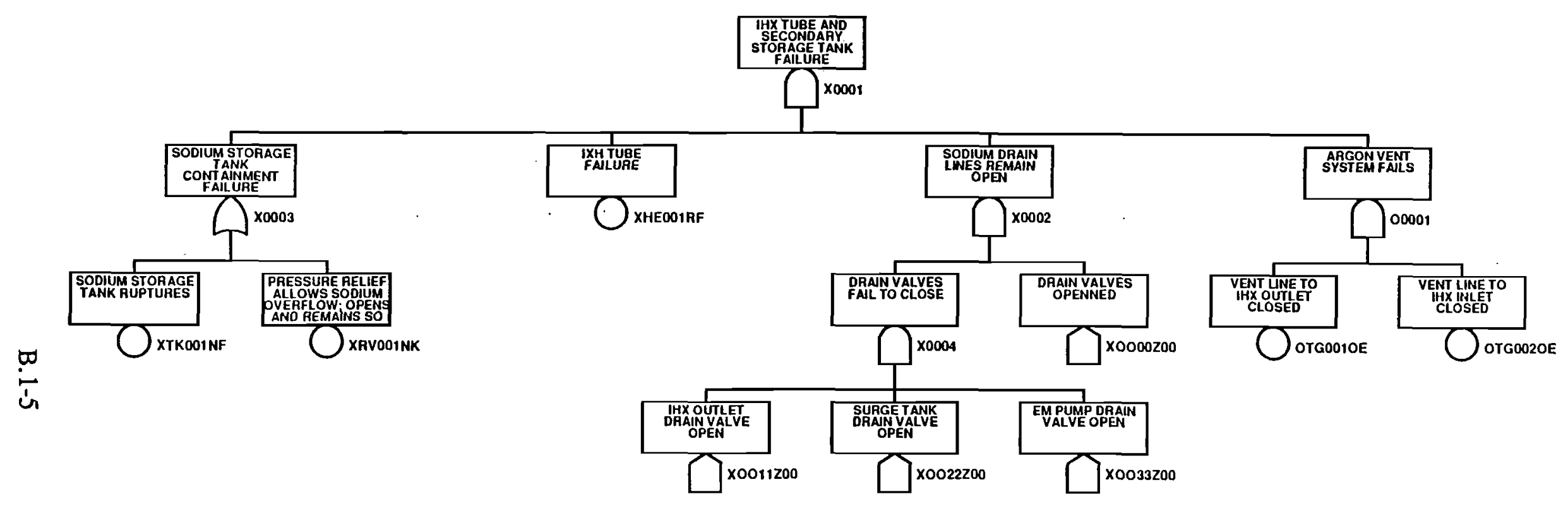

\begin{tabular}{|l|l|}
\hline \multicolumn{2}{|l|}{ IHX Tube and Secondary Storage Tank Failure } \\
\hline Fri Jun 14 11:12:06 1991 & PAGE 1 OF 1 \\
\hline
\end{tabular}




$$
5 \quad 9 \quad 9
$$

IHX Tube and Secondary Storage Tank Fallure

X0001 IHX TUBE AND SECONDARY STORAGE TANK FAILURE

X0002 $31 \times 0002$ X0003 00001 XHE001R

SODIUM DRAIN LINES REMAIN
\$ 11 X0004 XOO00200

X0003 SODIUM STORAGE TANK CONTAINMENT FAILURE

+ 02 XTK001NE XRV001NK

O0001 ARGON VENT SYSTEM FAILS

ARGON VENT SYSTEM FAILS

X0004 DRAIN VALVES FAIL TO CLOSE

* 03 X0011200 X0022200. X0033200

XHE001RF 0 O 0 1 IXH TUBE FAILURR

X0000200 2002 DRAIN VALVES OPENNED

XTKOO1NE 0 O 3 SODIUM STORAGE TANK RUPTURES

XRVO01NK 0 O 4 PRESSURE RELIEF ALLOWS SODIUM OVERFLOW: OPENS AND REMAINS SO

OTGO01OE 0 O 5 VENT LINE TO IHX OUTLET CLOSED

OTG002OE 0 O 6 VENT LINE TO IHX INLET CLOSED

X0011200 2007 IHX OUTLET DRAIN VALVE OPEN

XO022200 2 0 8 SURGE TANK DRAIN VALVE OPEN

X00332002 09 EM PUMP DRAIN VALVE OPEN

$0.720 \mathrm{E}+04$

$0.720 \mathrm{E}+04$
$\begin{array}{llll}12 & 0.100 \mathrm{E}-05 & 0.100 \mathrm{E}+02\end{array}$
2 T $0.100 \mathrm{E}+01 \quad 0.100 \mathrm{E}+01$
$32 \quad 0.100 \mathrm{E}-05 \quad 0.100 \mathrm{E}+02$
$\begin{array}{lllll}3 & 2 & 0.100 \mathrm{E}-05 & 0.100 \mathrm{E}+02 & 0.000 \mathrm{E}+00\end{array}$
52 0.250E-06 $0.100 \mathrm{E}+02 \quad 0.830 \mathrm{E}-01$
$\begin{array}{lllll}6 & 0 & 0.100 \mathrm{E}+01 & 0.100 \mathrm{E}+01 & 0.000 \mathrm{E}+00\end{array}$
7 T $0.100 \mathrm{E}+01 \quad 0.100 \mathrm{E}+01$
$8 \mathrm{~T} 0.100 \mathrm{~B}+010.100 \mathrm{~B}+01$
9 T $0.100 \mathrm{E}+01 \quad 0.100 \mathrm{E}+01$ 

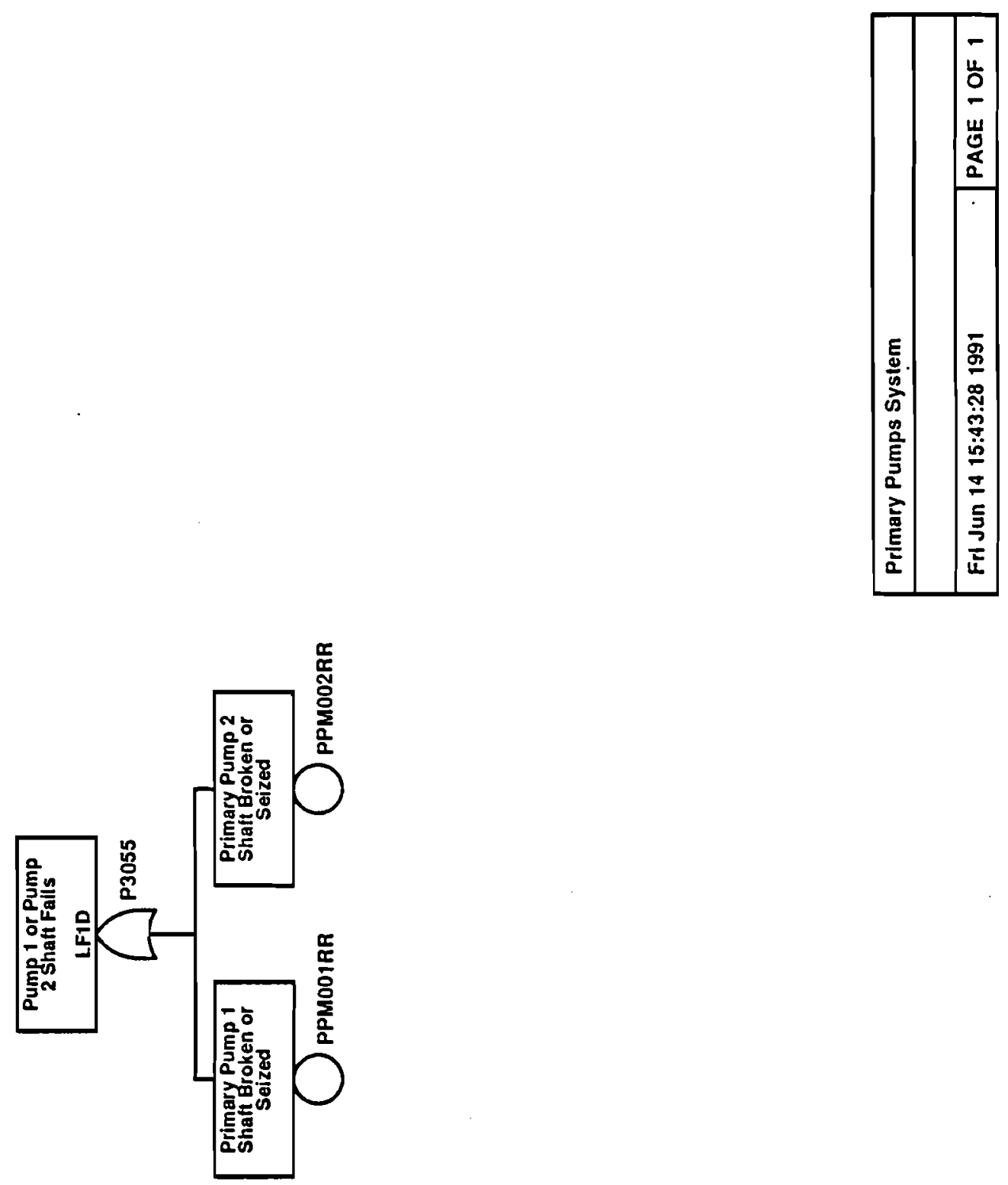

B. 1-7 
옥

क्:

मै

总咅

出出

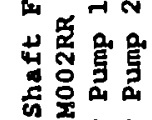

$N$ 㟧

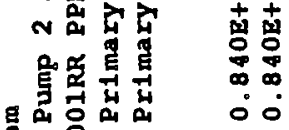

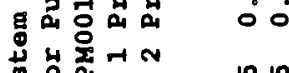

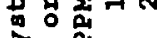
होन म

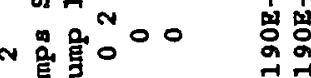

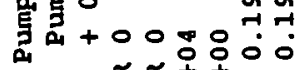

N

约

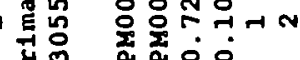

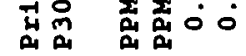

B. 1-8 


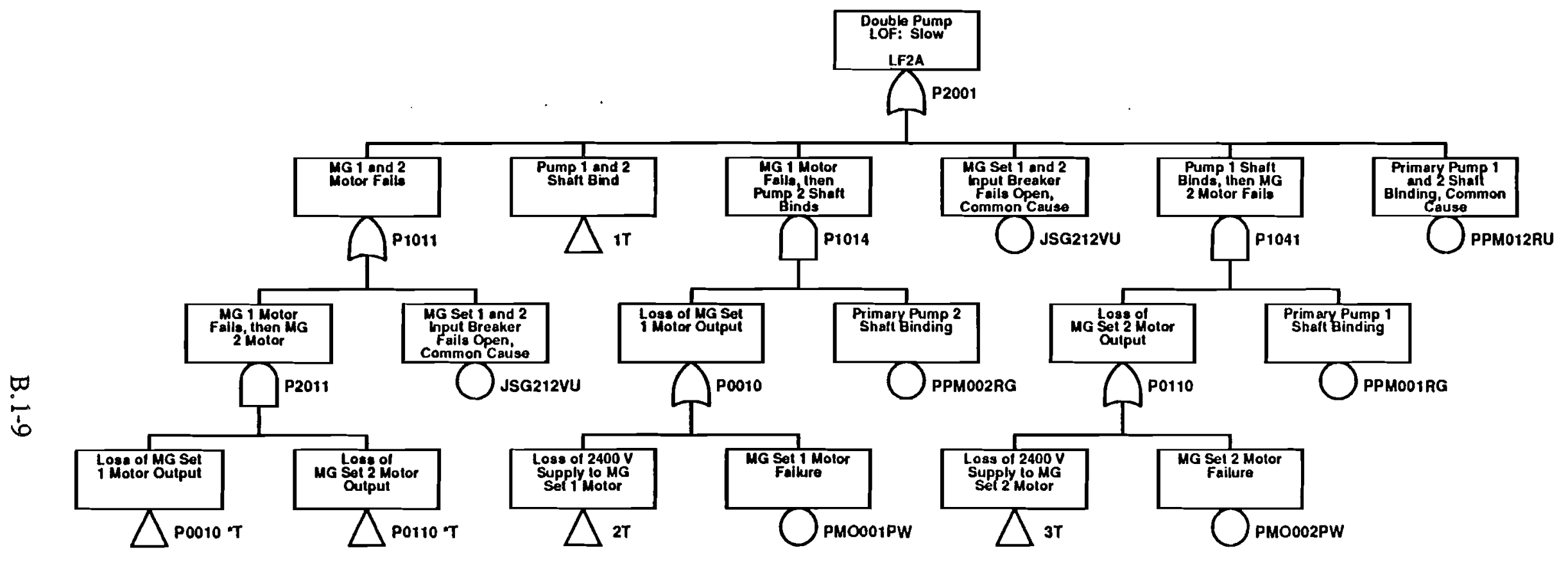

Primary Pumps System

Frl Jun 14 15:43:51 1991

PAGE 1 OF 4 


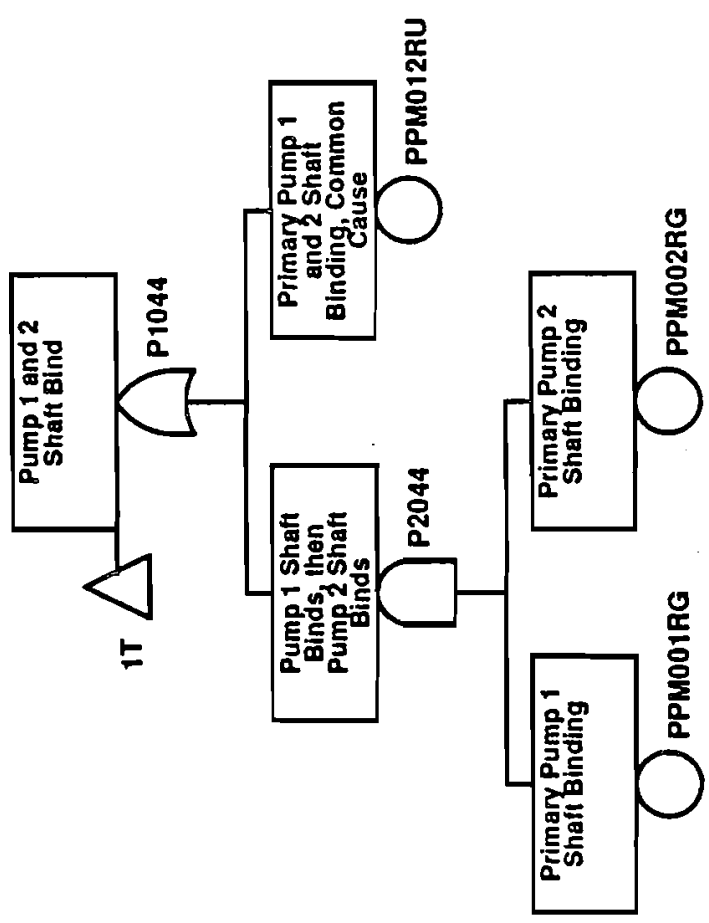

B. $1-10$ 


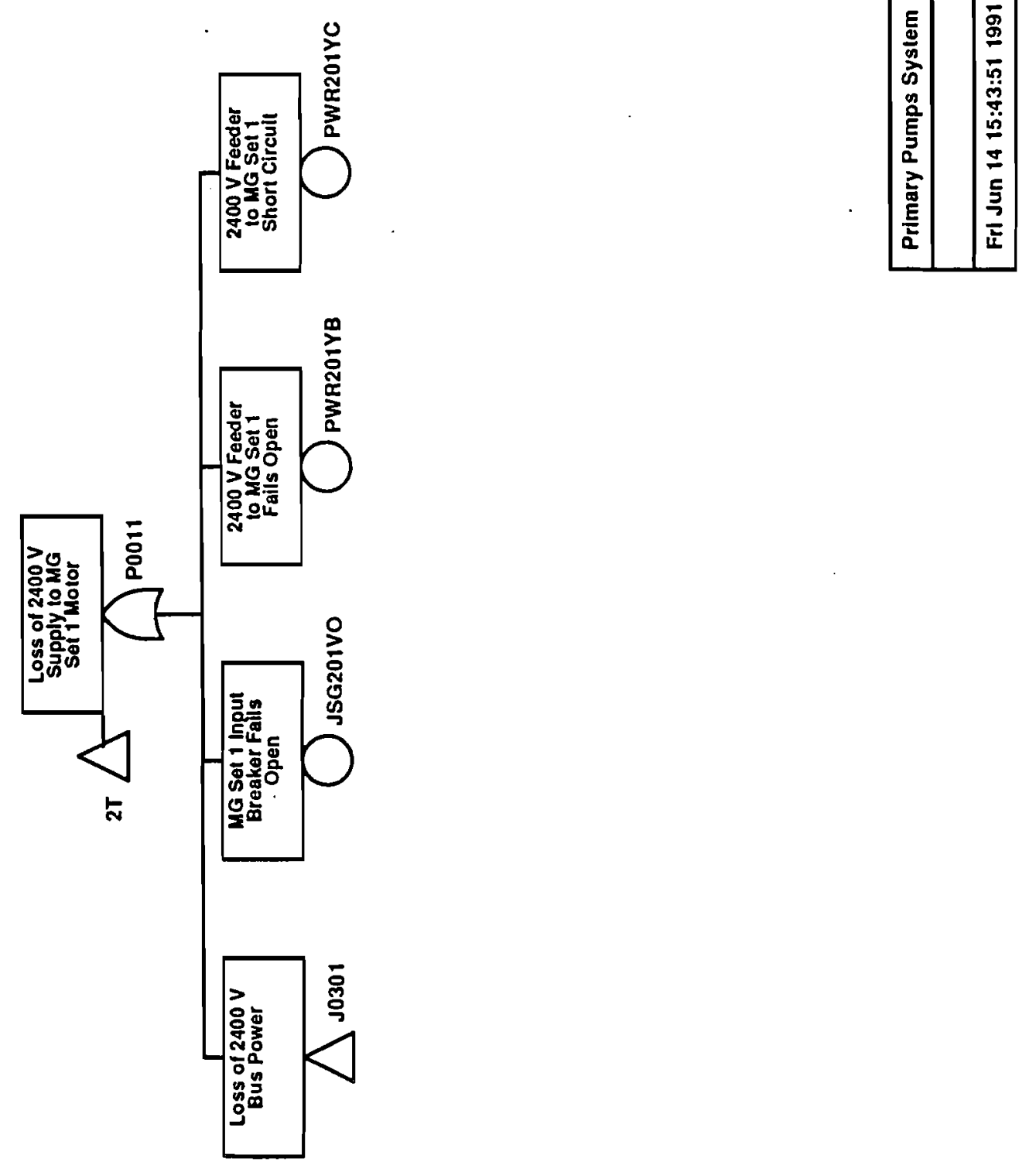

B. 1-11 


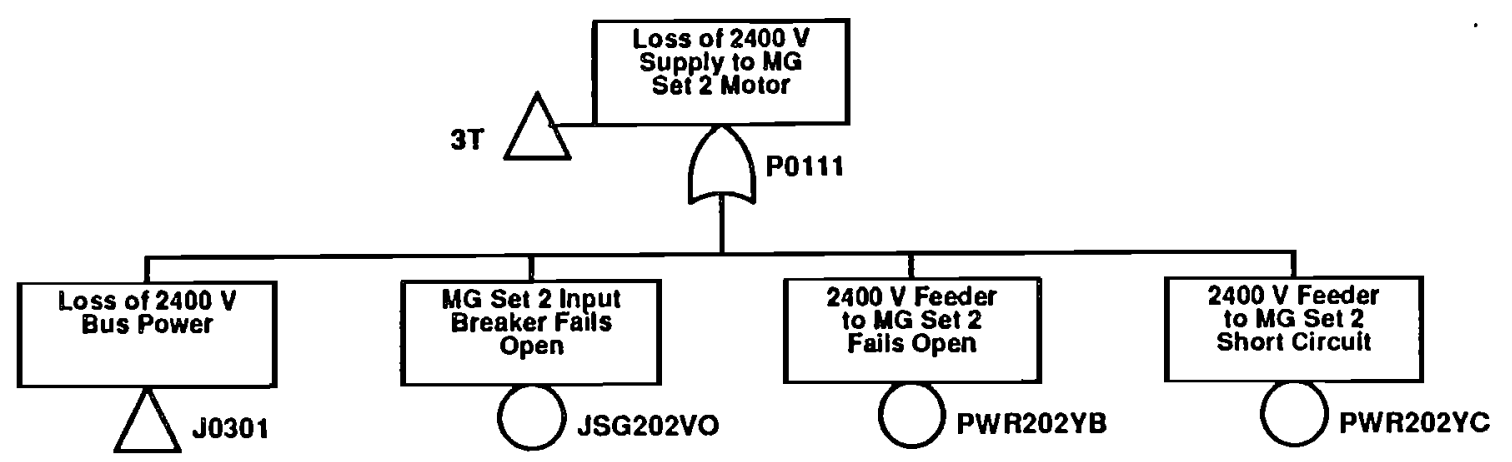


$11 \quad 13 \quad 13$

Primary Pumps system

P0010 LOBs of MG Set 1 Motor Output

Loss of MG Set 1 Motor Out
+11 P0011 PMO001PW

P0011 Loss of $2400 \mathrm{~V}$ Supply to MG Set 1 Motor

+04 J0301 JSG201VO PHR201YB PHR201YC

P0110 Loss of MG Set 2 Motor output

+11 P0111 PMO002PW

P0111 Loss of $2400 \mathrm{~V}$ Supply to MG Set 2 Motor

P1011 +04 J0301 JSG202VO PWR202Y日 PWR202YC

+11 P2011 JSG212VO

P1014 MG 1 Motor Falls, then Pump 2 Shaft Blnde

$\star 11$ P0010 PPM002RG

P1041 Pump 1 Shaft B1nds, then MG 2 Motor Fall.

P1044 Pump 1 and 2 shaft Bind

+11 P2044 PPMO12RO

P2001 Double Pump LOF: S10W LF2A

JSG212VU PPMO12RU

P2011 MG 1 Motor Falla, then MG 2 Motor

P2044 Pump 1 Shaft Binds, then Pump 2 shaft Binds

J0301 302 PPMOO1RG PPMO02RG

PMO001PW 0 o 2 MG Set 1 Motor Fallure

JSG201VO 0 O 3 MG Set 1 Input Breaker Falls Open

PWR201YB 0 o 42400 V Feeder to MG Set 1 Fall open

PWR201YC 0 o 52400 V Feeder to MG set 1 short circult

PMO002PW 0 0 6 MG Set 2 Motor Eallure

JSG202VO 0 0 7 MG Set 2 Input Breaker Falls Open

PWR202Y日 0 0 82400 V Feeder to MG Set 2 Fall Open

PWR202YC 0 o $92400 \mathrm{~V}$ Feeder to MG Set 2 Short Circult

PPMODIRG 0 o 10 Primary Pump 1 Shaft Binding

PPMO12RU 0 O 11 Primary Pump 1 and 2 Shaft Binding, Common Cause

PPMO02RG 0 o 12 Primary Pump 2 Shaft Binding

JSG212VO 0013 MG Set 1 and 2 Input Breaker Falls Open, Comtnon Cause

$0.720 \mathrm{E}+04$

$0.100 \mathrm{E}+00$

$\begin{array}{rrrll}1 & 0 & 0.100 \mathrm{E}-09 & 0.100 \mathrm{E}+02 & 0.000 \mathrm{E}+00 \\ 2 & 2 & 0.300 \mathrm{E}-04 & 0.300 \mathrm{E}+01 & \\ 3 & 2 & 0.300 \mathrm{E}-06 & 0.100 \mathrm{E}+02 & \\ 4 & 2 & 0.300 \mathrm{E}-05 & 0.100 \mathrm{E}+02 & \\ 5 & 2 & 0.300 \mathrm{E}-06 & 0.100 \mathrm{E}+02 & \\ 6 & 1 & 0.300 \mathrm{E}-04 & 0.300 \mathrm{E}+01 & \\ 7 & 1 & 0.300 \mathrm{E}-06 & 0.100 \mathrm{E}+02 & \\ 8 & 1 & 0.300 \mathrm{E}-05 & 0.100 \mathrm{E}+02 & \\ 9 & 1 & 0.300 \mathrm{E}-06 & 0.100 \mathrm{E}+02 & \\ 10 & 2 & 0.290 \mathrm{E}-04 & 0.260 \mathrm{E}+01 & \\ 11 & 2 & 0.290 \mathrm{E}-05 & 0.100 \mathrm{E}+02 & \\ 12 & 1 & 0.290 \mathrm{E}-04 & 0.260 \mathrm{E}+01 & \\ 13 & 2 & 0.300 \mathrm{E}-07 & 0.100 \mathrm{E}+02\end{array}$




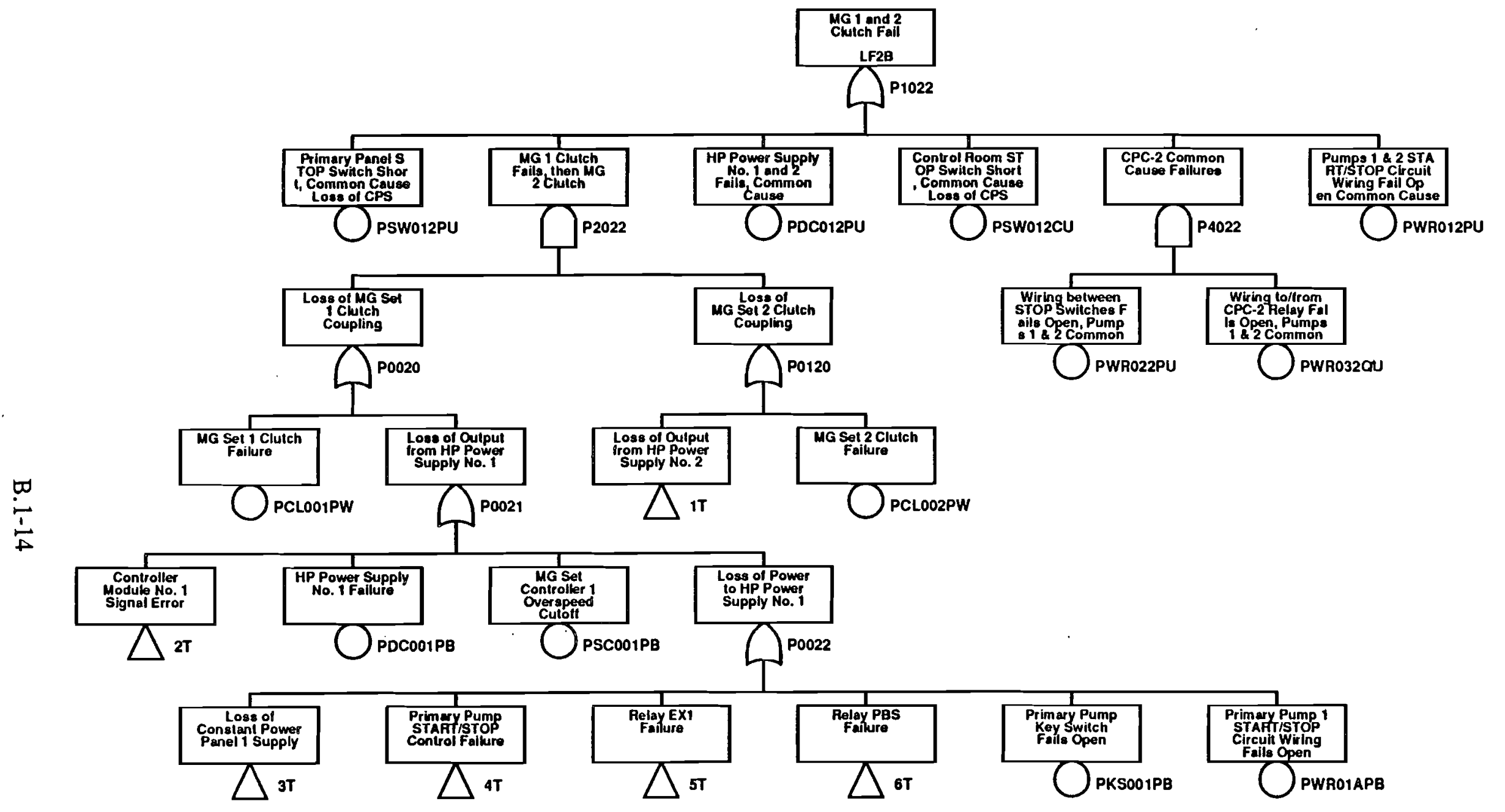

\begin{tabular}{|c|c|}
\hline Primary Pumps System & \\
\hline Fri Jun 14 15:56:04 1991 & PAGE 1 OF 9 \\
\hline
\end{tabular}




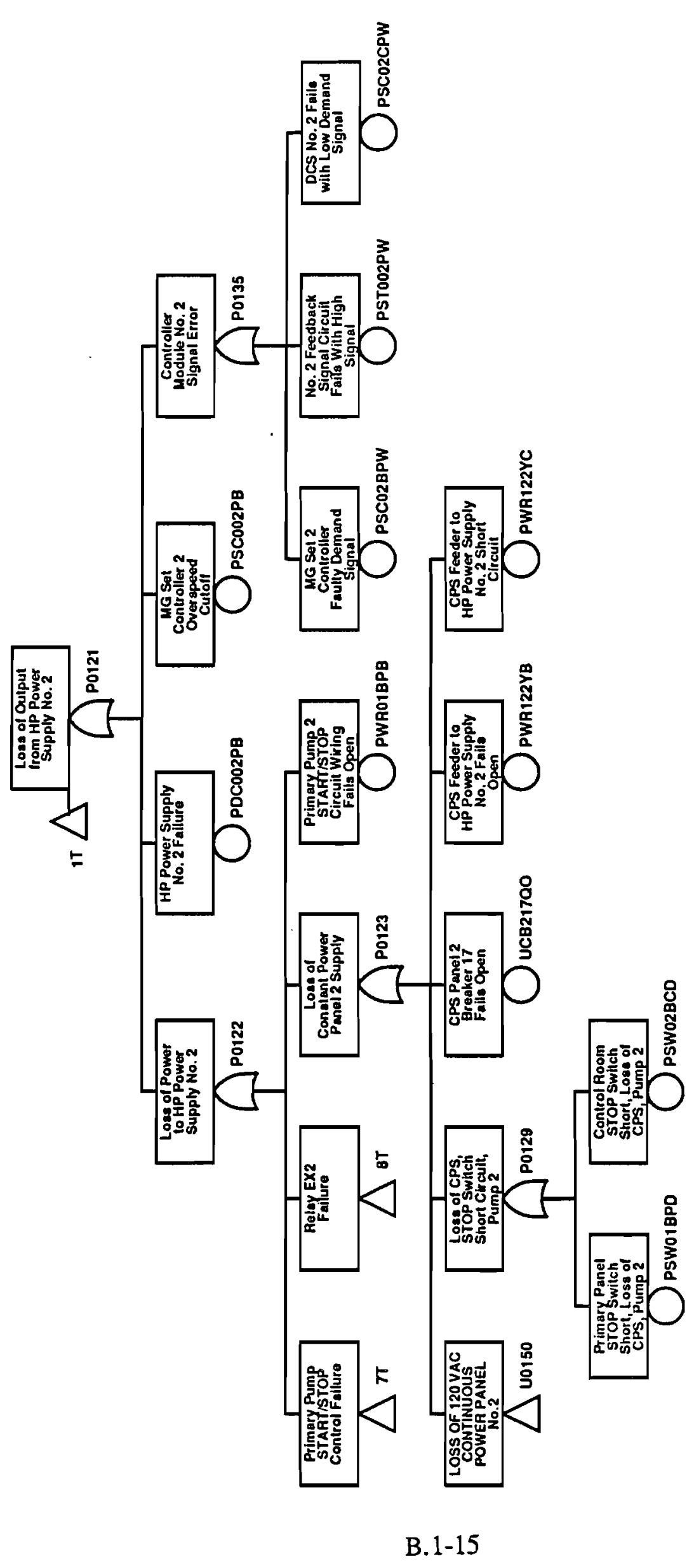

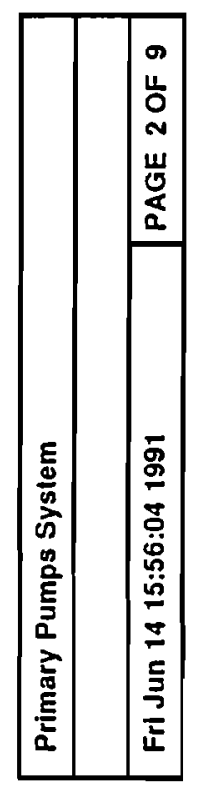




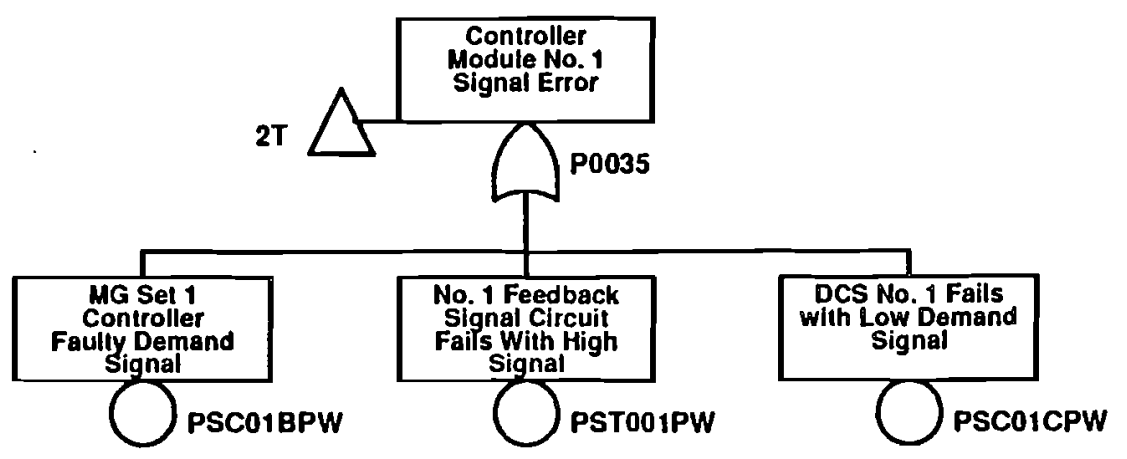

Primary Pumps System

Fri Jun 14 15:56:04 1991 


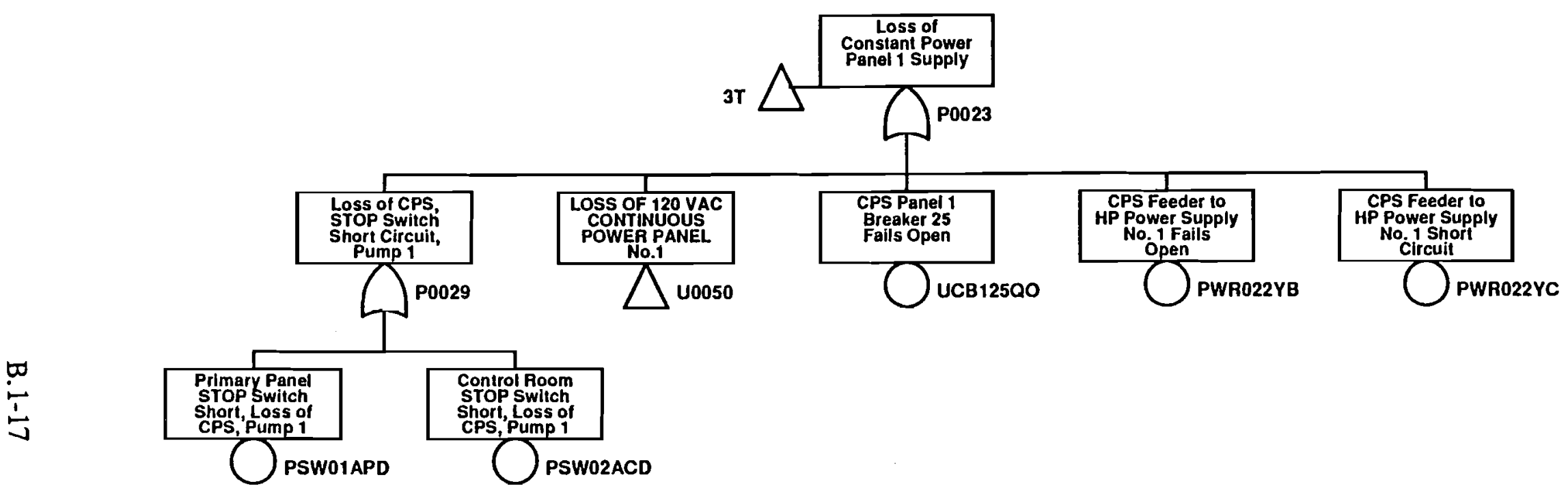

PrImarý Pumps System

Fri Jun 14 15:56:04 1991

PAGE 4 OF 9 


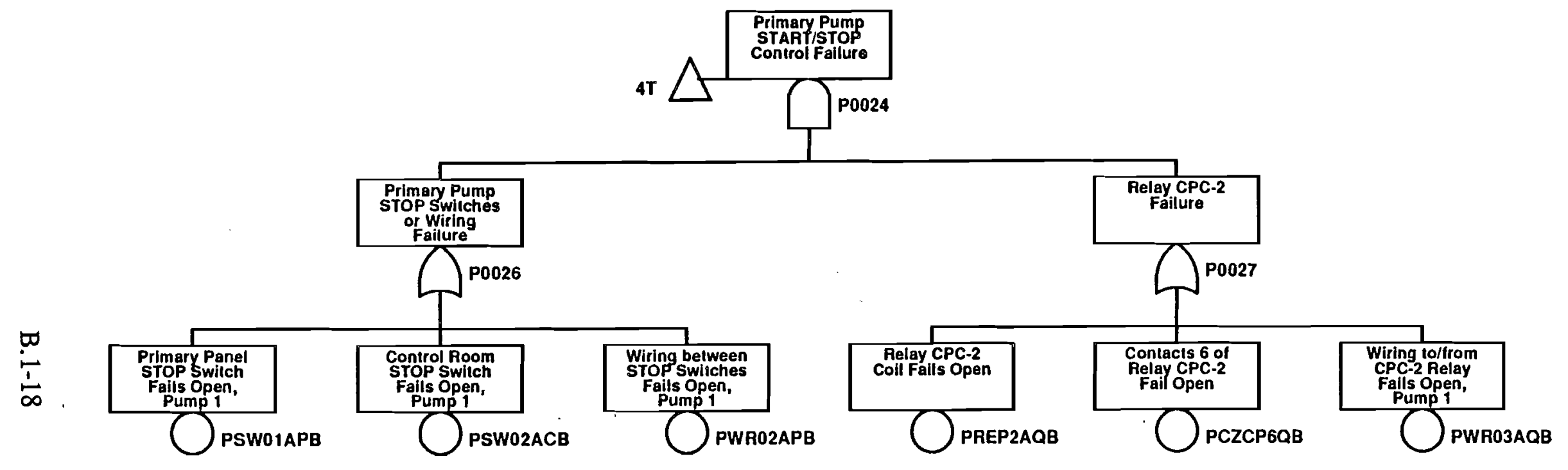

Primary Pumps System

\begin{tabular}{|l|l|}
\hline Frl Jun 14 15:56:04 1991 & PAGE 5 OF 9 \\
\hline
\end{tabular} 

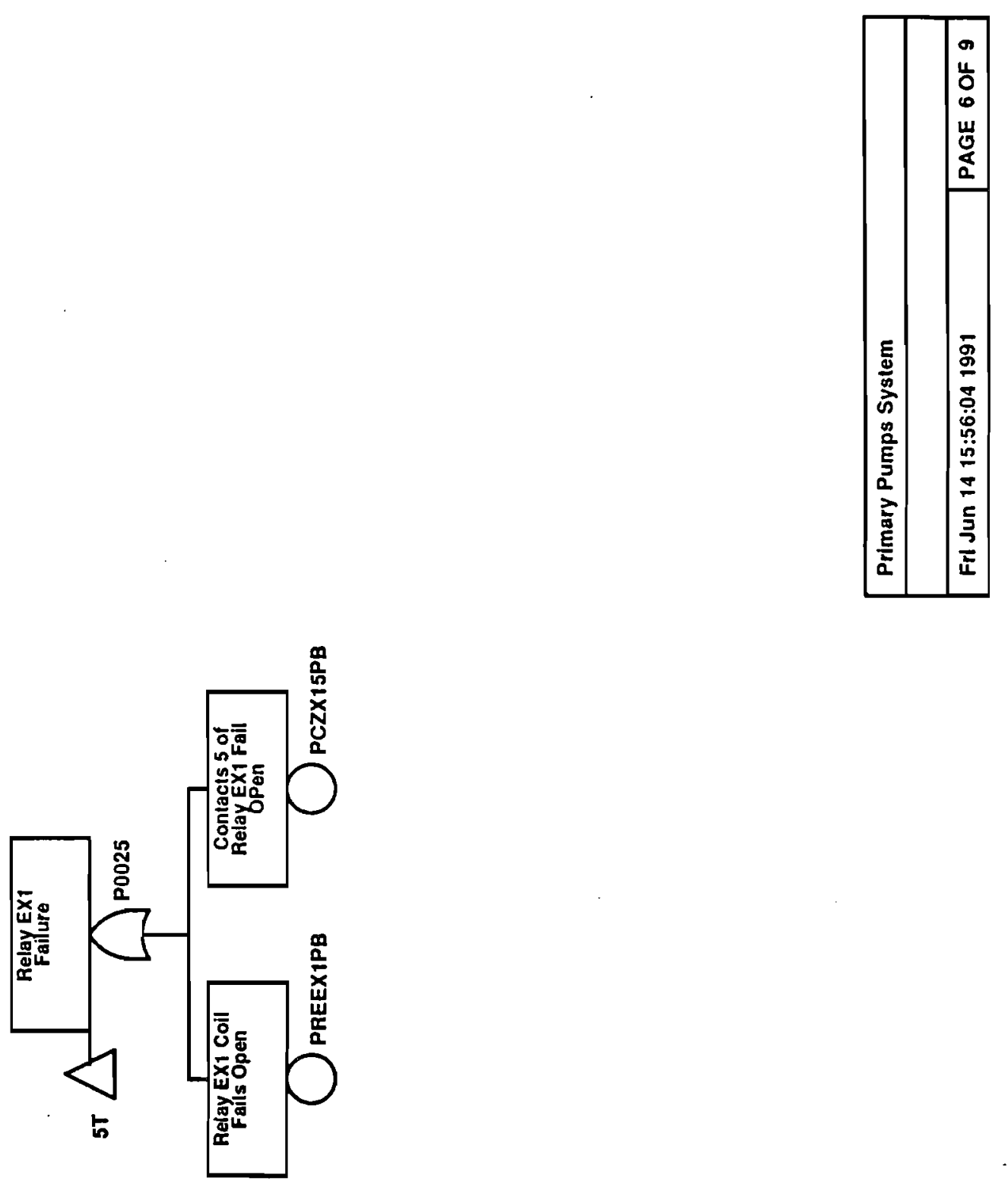

B. 1-19 


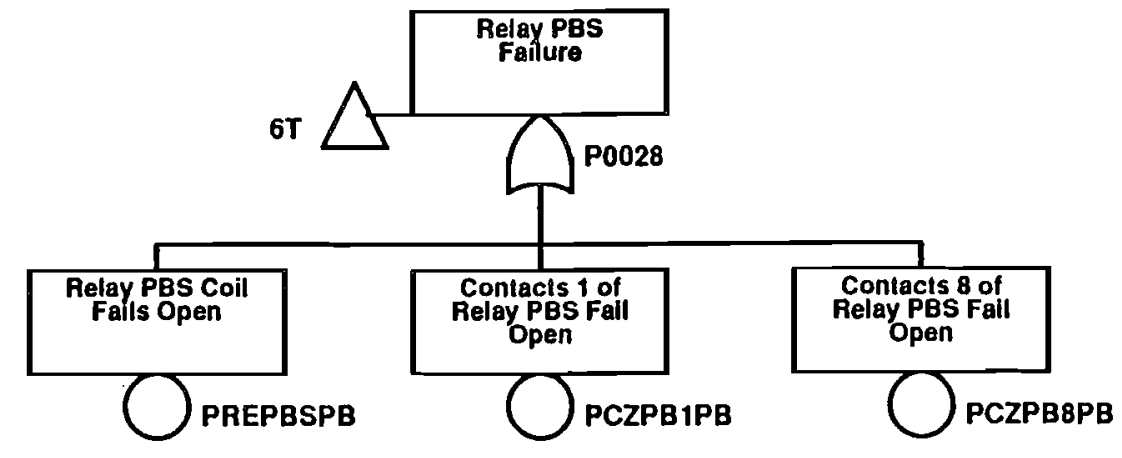

Primary Pumps System

Frl Jun 14 15:56:04 1991

PAGE 7 OF 9 


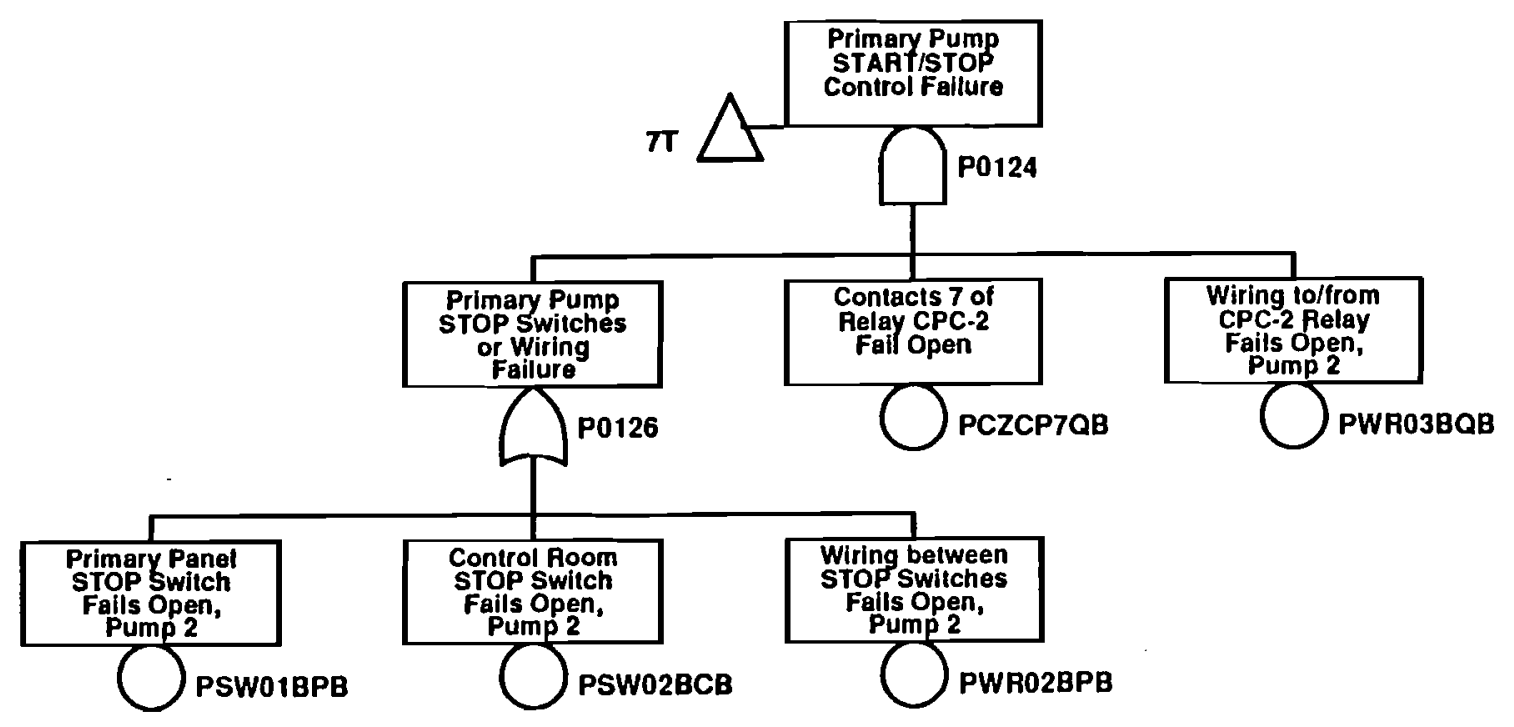

Primary Pumps System 

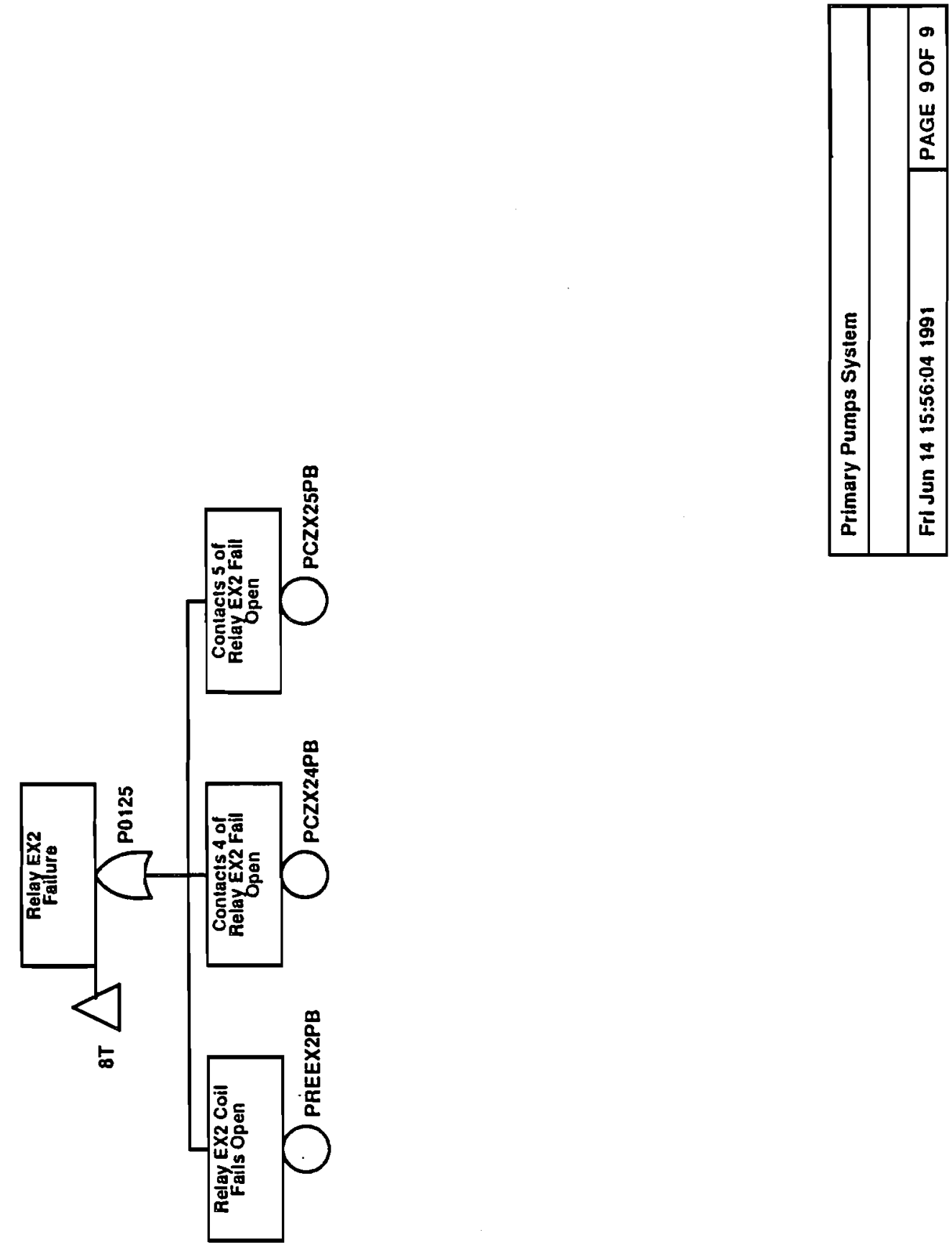

B. 1-22 


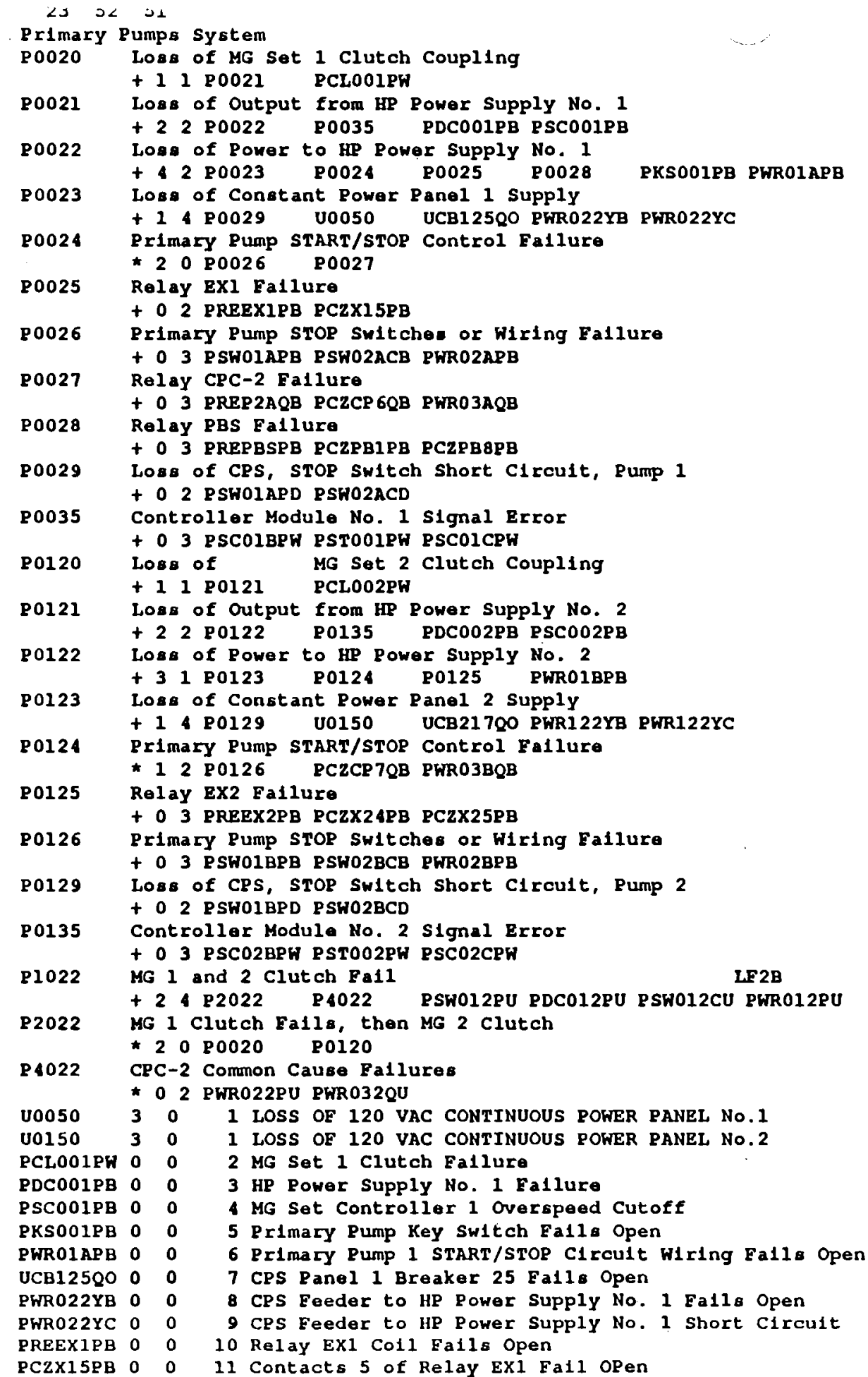


PSWOIAPB 0 o 12 Primary Panel STOP Swltch Falls Open, Pump 1

PSW02ACB 0 o 13 Control Room STOP Switch Falls Open, Pump 1

PWR02APB 0 o 14 W1ring between STOP Switches Falls Open, Pump 1

PREP2AQB 0 o 15 Relay CPC-2 Coll Falle Open

PC2CP 6QB 00016 Contacts 6 of Relay CPC-2 Fall Open

PWR03AQB 0 o 17 Wiring to/from CPC-2 Relay Fall Open, Pump 1

PREPBSPB 0 o 18 Relay PBS Coll Falls Open

PC2PB1PB $0 \quad 0 \quad 19$ Contact 1 of Relay PBS Fall Open

PC2PB8PB 0020 Contact 8 of Relay PBS Fall Open

PSWOLAPD $0 \quad 0 \quad 21$ Primary Panel STOP Switch Short, Loss of CPS, Pump 1

PSWO2ACD 0 o 22 Control Room STOP Sw1tch Short, Loss of CPS, Pump 1

PDC012PU 0 o 23 HP Power Supply No. 1 and 2 Falls, Common Cause

PSC01BPW 0 o 24 MG Set 1 Controller Faulty Demand Signal

PST001PW $0 \quad 0 \quad 25$ No. 1 Peodback Signal Clrcult Falls With High Signal

PSC01CPW 00026 DCS No. 1 Palls with Low Demand Signal

PCLO02PW 0 o 27 MG Set 2 Clutch Pallure

PDC002PB 0 0 28 HP Power Supply No. 2 Fallure

PSC002PB 0 o 29 MG Sat Controller 2 Overspeed Cutoff

PWRO1BPB 00030 Primary Pump 2 START/STOP Circult wiring Falls Open

UCB217g0 00031 CPS Panel 2 Breaker 17 Eatls Open

PWR122YB 0 o 32 CPS Feeder to HP Power Supply No. 2 Falls open

PWR122YC 00033 CPS Feeder to HP Power Supply No. 2 Short C1rcult

PCZCP7QB 0 o 34 Contacts 7 of Relay CPC-2 Eall Open

PWR03BQB 0 o 35 Wiring to/from CPC-2 Relay Falls Open, Purm 2

PREEX2PB 0 o 36 Relay EX2 Coll Fall. Open

PC2X24PB 0 o 37 Contact of Relay Ex2 Fall Open

PC2X25PB 00038 Contact 5 of Relay EX2 Fall Open

PSWO1BPB 00039 Primary Panel STOP Switch Falls Open, Purm 2

PSW02BCB 0 o 40 Control Room STOP Switch Falls Open, Pump 2

PWR02BPB 0 o 41 Wlring botween STOP Switches Falls Open, Purmp

PSWO1BPD 0 o 42 Primary Panel STOP Switch Short, Loss of CPS, Pump 2

PSW02BCD $0 \quad 0 \quad 43$ Control Room STOP Switch Short, Lose of CPS, PurtP 2

PSC02BPW 0 o 44 MG Set 2 Controller Faulty Demand Signal

PST002PW 0045 No. 2 Feodback Signal Circult Fails with High signal

PSC02CPW 0 o 46 DCS No. 2 Palla with Low Demand Signal

PSW012CU 0 o 47 Control Room STOP Sw1tch Short, Common Cause Lose of CPS

PSW012PU 0 o 48 Primary Panel STOP Switch Short, Common Cause Loss of CPS

PHR012PU O 0 49 Pump I 2 START/STOR Circult Wiring Fall Open Common Cause

PWR022PU 00 50 wiring between STOP Swltches Falls Open, Pumpe 1

PWR0320U 0 o 51 wiring to/from CPC-2 Relay Falls Open, Pumps 1 a 2 Comonon

$0.720 \mathrm{E}+04$

$0.100 \mathrm{~B}+00$

$\begin{array}{rlll}1 & 0 & 0.100 \mathrm{E}-09 & 0.100 \mathrm{E}+02 \\ 2 & 2 & 0.500 \mathrm{E}-04 & 0.300 \mathrm{E}+02 \\ 3 & 2 & 0.300 \mathrm{E}-05 & 0.100 \mathrm{E}+02 \\ 1 & 2 & 0.300 \mathrm{E}-05 & 0.100 \mathrm{E}+02 \\ 5 & 2 & 0.100 \mathrm{E}-06 & 0.100 \mathrm{E}+02 \\ 6 & 2 & 0.300 \mathrm{E}-05 & 0.100 \mathrm{E}+02 \\ 7 & 2 & 0.300 \mathrm{E}-06 & 0.100 \mathrm{E}+02 \\ 8 & 2 & 0.300 \mathrm{E}-05 & 0.100 \mathrm{E}+02 \\ 9 & 2 & 0.300 \mathrm{E}-06 & 0.100 \mathrm{E}+02 \\ 10 & 2 & 0.500 \mathrm{E}-06 & 0.100 \mathrm{E}+02 \\ 11 & 2 & 0.100 \mathrm{E}-06 & 0.100 \mathrm{E}+02 \\ 12 & 2 & 0.100 \mathrm{E}-06 & 0.100 \mathrm{E}+02 \\ 13 & 2 & 0.100 \mathrm{E}-06 & 0.100 \mathrm{E}+02 \\ 14 & 2 & 0.300 \mathrm{E}-05 & 0.100 \mathrm{E}+02 \\ 15 & 2 & 0.500 \mathrm{E}-06 & 0.100 \mathrm{E}+02 \\ 16 & 2 & 0.100 \mathrm{E}-06 & 0.100 \mathrm{E}+02 \\ 17 & 2 & 0.600 \mathrm{E}-05 & 0.100 \mathrm{E}+02 \\ 18 & 2 & 0.500 \mathrm{E}-06 & 0.100 \mathrm{E}+02\end{array}$

$0.000 \mathrm{E}+00$ 
$\begin{array}{llll}192 & 0.100 \mathrm{E}-06 & 0.100 \mathrm{E}+02\end{array}$

$\begin{array}{llll}202 & 0.100 \mathrm{E}-06 & 0.100 \mathrm{E}+02\end{array}$

$\begin{array}{llll}21 & 2 & 0.100 \mathrm{E}-06 & 0.100 \mathrm{E}+02\end{array}$

$\begin{array}{llll}22 & 0.100 \mathrm{E}-06 & 0.100 \mathrm{E}+02\end{array}$

$\begin{array}{llll}232 & 0.300 \mathrm{E}-06 & 0.100 \mathrm{E}+02\end{array}$

$2420.300 \mathrm{E}-05 \quad 0.100 \mathrm{E}+02$

$\begin{array}{llll}25 & 2 & 0.300 \mathrm{E}-05 & 0.100 \mathrm{E}+02\end{array}$

$\begin{array}{llll}26 & 2 & 0.300 \mathrm{E}-05 & 0.100 \mathrm{E}+02\end{array}$

$\begin{array}{llll}27 & 1 & 0.500 \mathrm{E}-04 & 0.300 \mathrm{E}+02\end{array}$

$\begin{array}{llll}28 & 1 & 0.300 \mathrm{E}-05 & 0.100 \mathrm{E}+02\end{array}$

$2910.300 \mathrm{E}-05 \quad 0.100 \mathrm{E}+02$

$\begin{array}{llll}30 & 1 & 0.300 \mathrm{E}-05 & 0.100 \mathrm{E}+02\end{array}$

$\begin{array}{llll}31 & 1 & 0.300 \mathrm{E}-06 & 0.100 \mathrm{E}+02\end{array}$

$\begin{array}{llll}32 & 1 & 0.300 \mathrm{E}-05 & 0.100 \mathrm{E}+02\end{array}$

$\begin{array}{llll}33 & 1 & 0.300 \mathrm{E}-06 & 0.100 \mathrm{E}+02\end{array}$

342 0.100E-06 $0.100 \mathrm{E}+02$

$\begin{array}{llll}352 & 0.600 \mathrm{E}-05 & 0.100 \mathrm{E}+02\end{array}$

$\begin{array}{llll}361 & 0.500 \mathrm{E}-06 & 0.100 \mathrm{E}+02\end{array}$

$\begin{array}{llll}371 & 0.100 \mathrm{E}-06 & 0.100 \mathrm{E}+02\end{array}$

$\begin{array}{llll}38 & 1 & 0.100 \mathrm{E}-06 & 0.100 \mathrm{E}+02\end{array}$

$\begin{array}{llll}39 & 2 & 0.100 \mathrm{E}-06 & 0.100 \mathrm{E}+02\end{array}$

$4020.100 \mathrm{E}-06 \quad 0.100 \mathrm{E}+02$

$4120.300 \mathrm{E}-05 \quad 0.100 \mathrm{E}+02$

$4220.100 \mathrm{E}-06 \quad 0.100 \mathrm{E}+02$

$4320.100 \mathrm{E}-06 \quad 0.100 \mathrm{E}+02$

$4410.300 \mathrm{E}-05 \quad 0.100 \mathrm{E}+02$

$4510.300 \mathrm{E}-05 \quad 0.100 \mathrm{E}+02$

$4610.300 \mathrm{E}-05 \quad 0.100 \mathrm{E}+02$

472 0.100E-07 0.100E+02

$4820.100 \mathrm{E}-07 \quad 0.100 \mathrm{E}+02$

$4920.300 \mathrm{E}-06 \quad 0.100 \mathrm{E}+02$

$\begin{array}{llll}502 & 0.300 \mathrm{E}-06 & 0.100 \mathrm{E}+02\end{array}$

$\begin{array}{llll}512 & 0.600 \mathrm{E}-06 & 0.100 \mathrm{E}+02\end{array}$ 


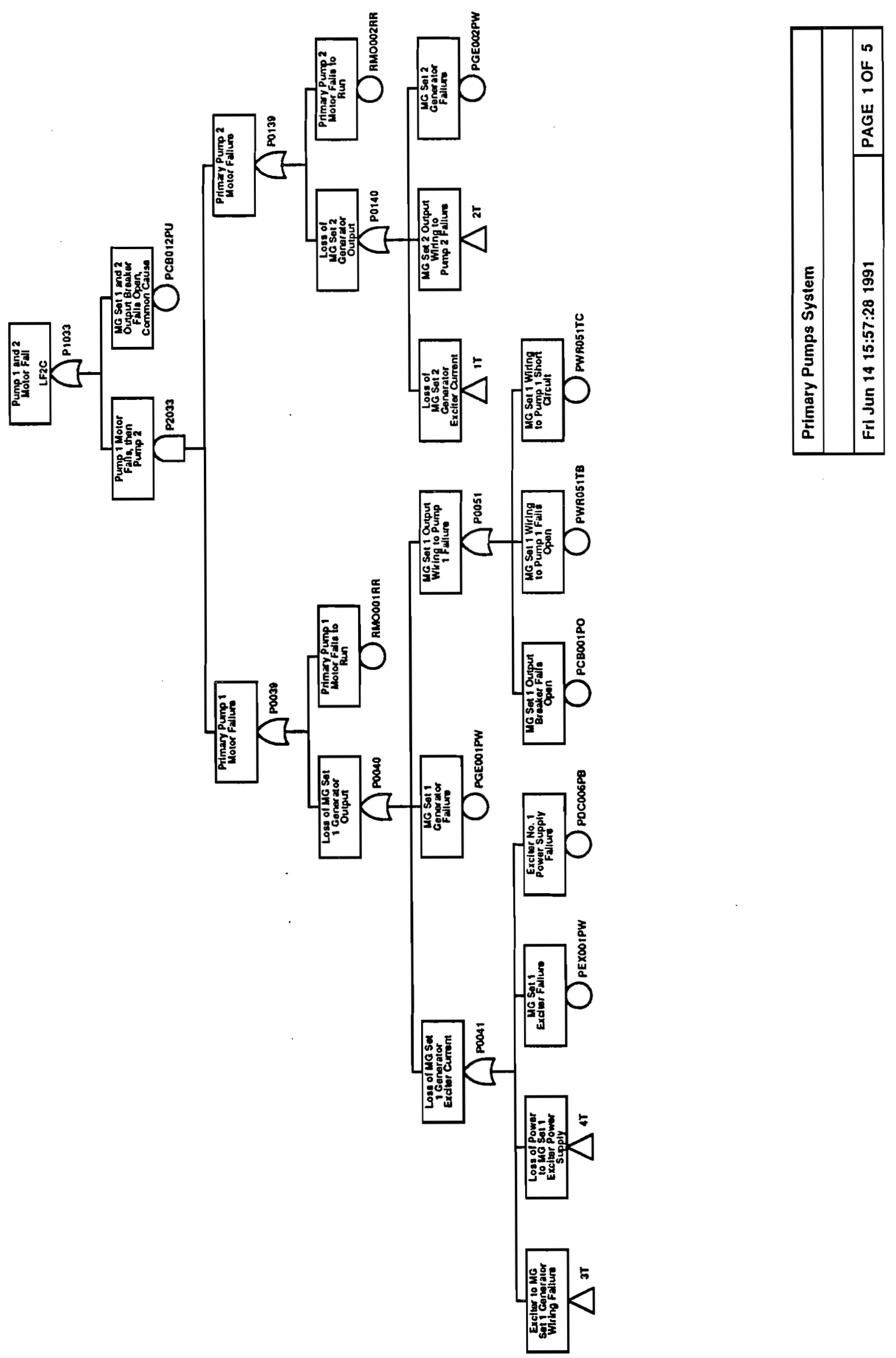

B. $1-26$ 


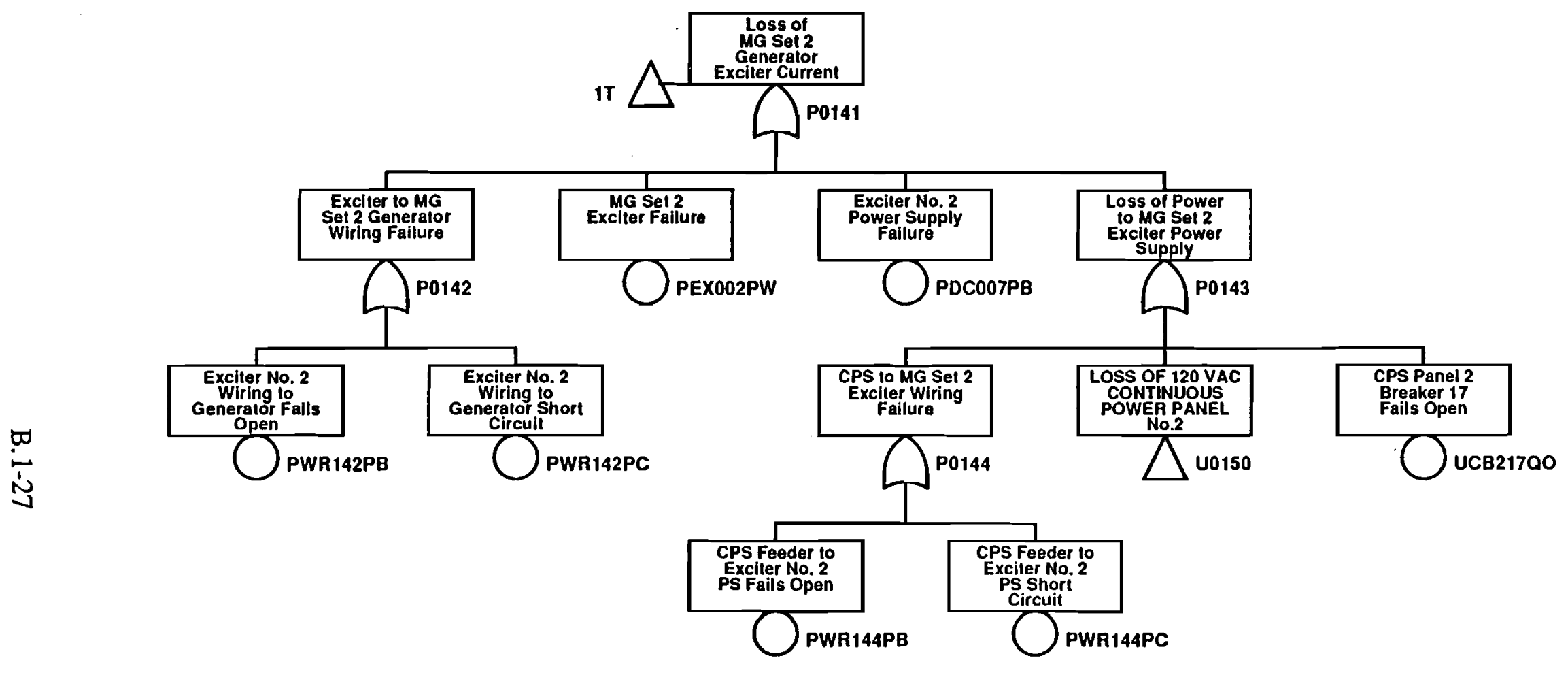

Primary Pumps System

Frl Jun 14 15:57:28 1991

PAGE 2 OF 5 

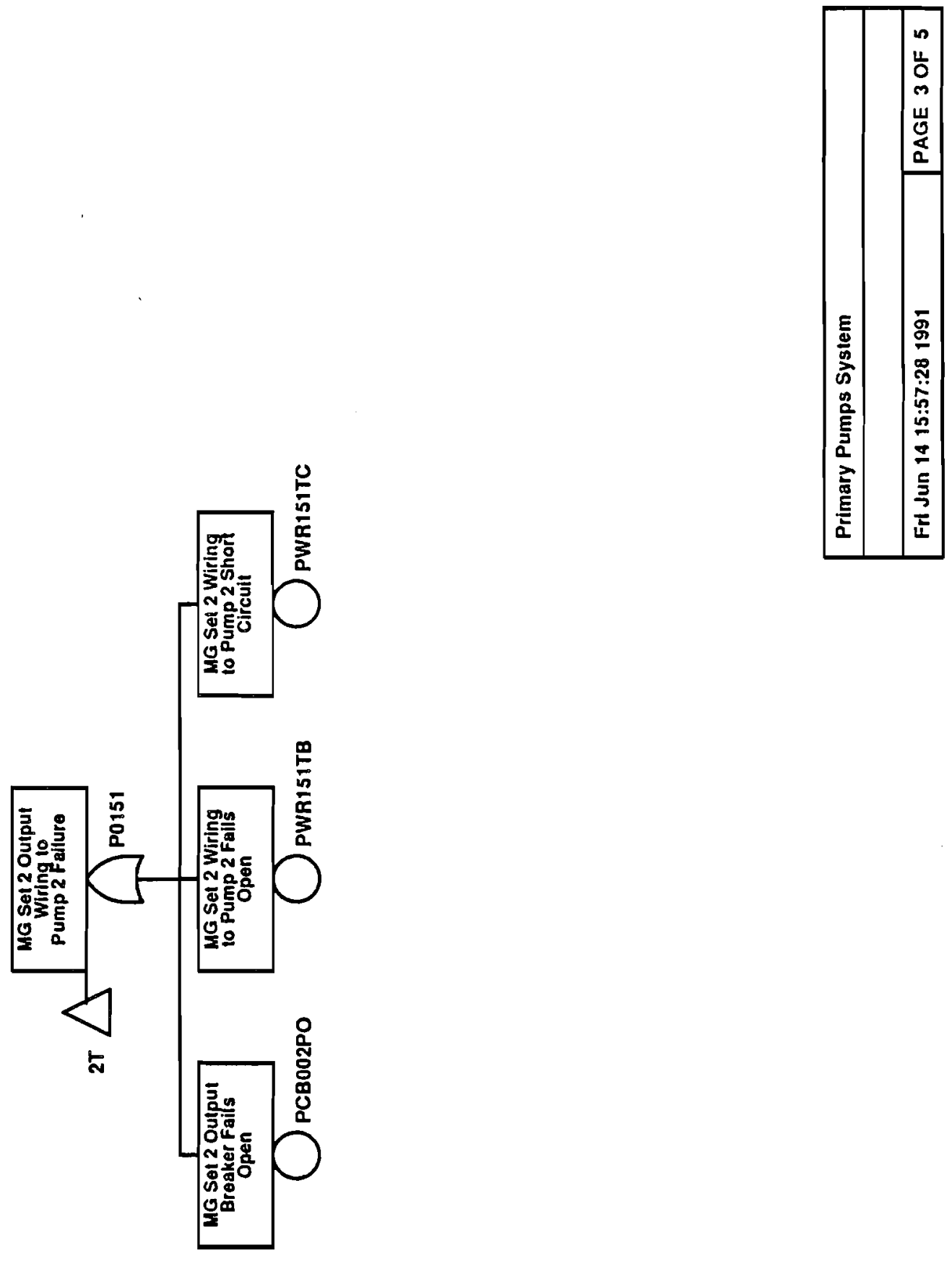

B. 1-28 


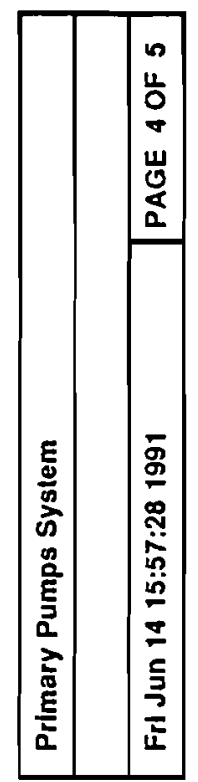

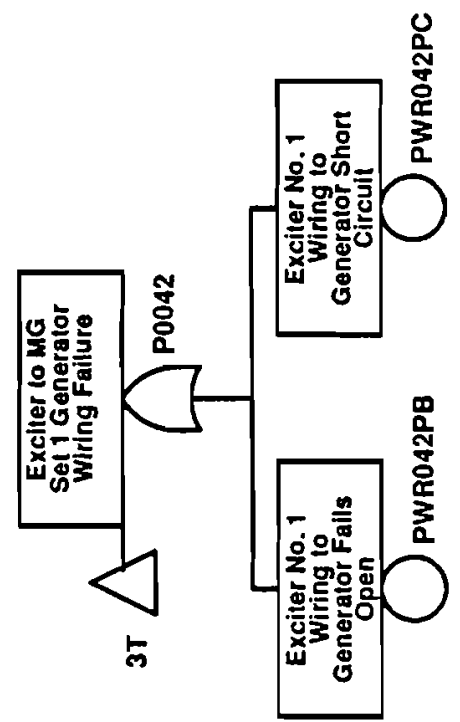




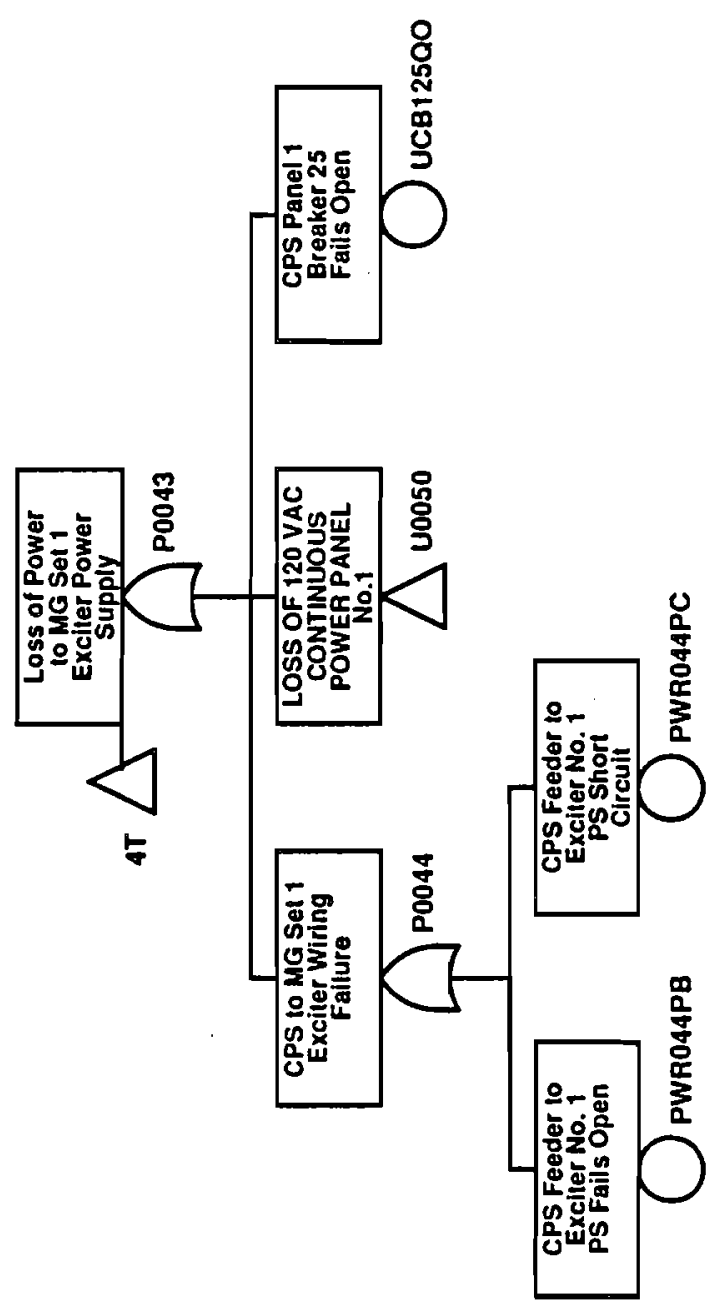

B. $1-30$ 
$16 \quad 27 \quad 26$

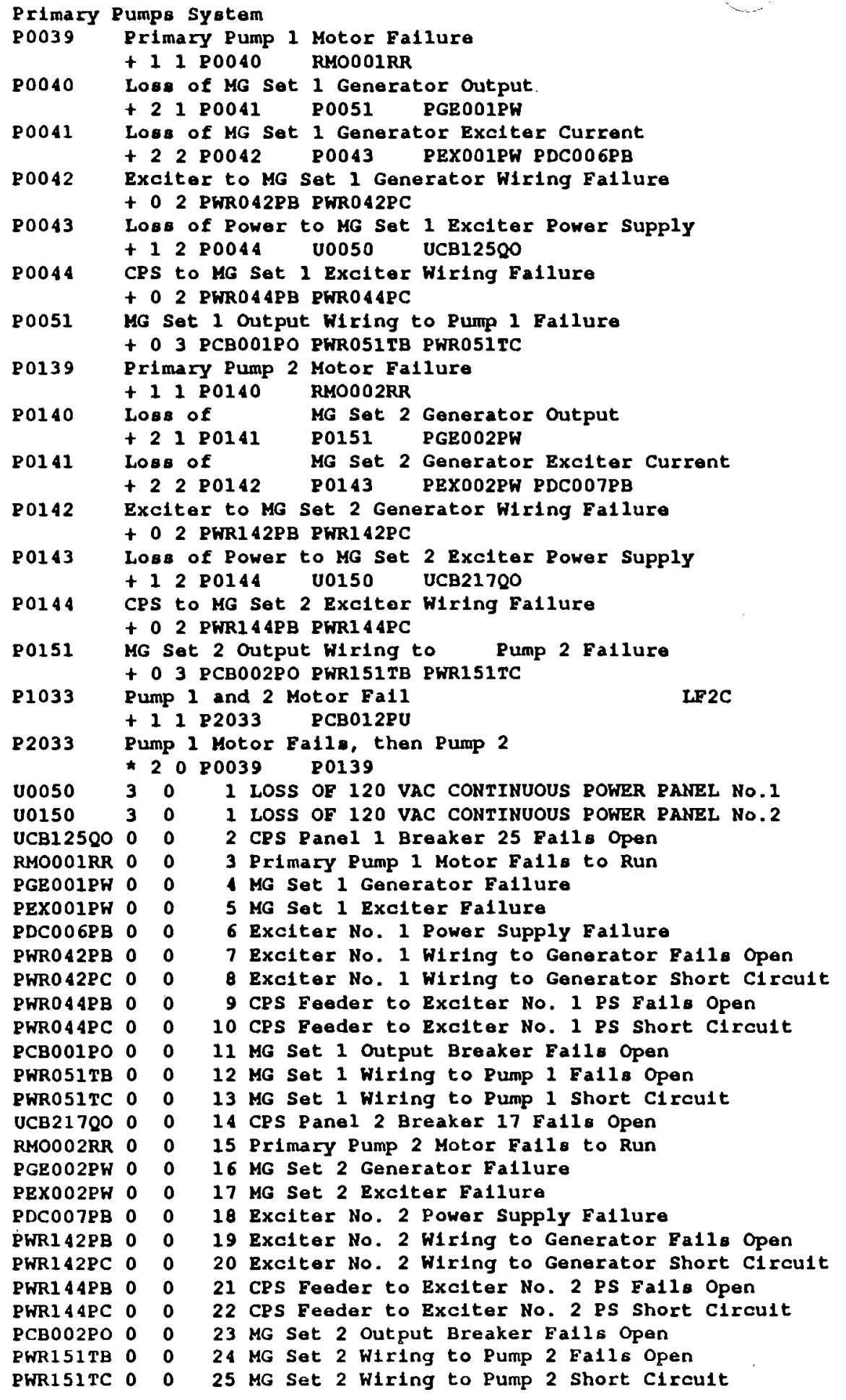


PCB012PU 0 o 26 MG Set 1 and 2 Output Breaker Falls Open, Common Cause $0.720 \mathrm{E}+04$

$0.100 \mathrm{E}+00$
$\begin{array}{llll}1 & 0 & 0.100 \mathrm{E}-09 & 0.100 \mathrm{E}+02\end{array}$
$220.300 \mathrm{E}-06 \quad 0.100 \mathrm{E}+02$
$\begin{array}{llll}32 & 0.200 \mathrm{E}-04 & 0.300 \mathrm{E}+01\end{array}$
$12 \quad 0.300 \mathrm{E}-05 \quad 0.100 \mathrm{E}+02$
$520.300 \mathrm{E}-05 \quad 0.100 \mathrm{E}+02$
$\begin{array}{llll}62 & 0.300 \mathrm{E}-05 & 0.100 \mathrm{E}+02\end{array}$
$\begin{array}{llll}72 & 0.300 \mathrm{E}-05 & 0.100 \mathrm{~B}+02\end{array}$
$820.300 \mathrm{E}-06 \quad 0.100 \mathrm{E}+02$
$\begin{array}{llll}9 & 2 & 0.300 \mathrm{E}-05 & 0.100 \mathrm{E}+02\end{array}$
$\begin{array}{llll}10 & 2 & 0.300 \mathrm{E}-06 & 0.100 \mathrm{E}+02\end{array}$
$\begin{array}{llll}11 & 2 & 0.300 \mathrm{E}-06 & 0.100 \mathrm{E}+02\end{array}$
$1220.300 \mathrm{E}-05 \quad 0.100 \mathrm{E}+02$
$\begin{array}{llll}132 & 0.300 \mathrm{E}-06 & 0.100 \mathrm{E}+02\end{array}$

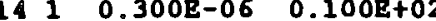
$1510.200 \mathrm{E}-04 \quad 0.300 \mathrm{E}+01$
$\begin{array}{llll}16 & 1 & 0.300 \mathrm{E}-05 & 0.100 \mathrm{E}+02\end{array}$
$1710.300 \mathrm{E}-05 \quad 0.100 \mathrm{E}+02$
$\begin{array}{llll}18 & 1 & 0.300 \mathrm{E}-05 & 0.100 \mathrm{E}+02\end{array}$
$\begin{array}{llll}191 & 0.300 \mathrm{E}-05 & 0.100 \mathrm{E}+02\end{array}$
$\begin{array}{llll}20 & 1 & 0.300 \mathrm{E}-06 & 0.100 \mathrm{E}+02\end{array}$
$2110.300 \mathrm{E}-050.100 \mathrm{E}+02$
$2210.300 \mathrm{E}-06 \quad 0.100 \mathrm{E}+02$
$\begin{array}{llll}22 & 1 & 0.300 \mathrm{E}-06 & 0.100 \mathrm{E}+02 \\ 23 & 1 & 0.300 \mathrm{E}-06 & 0.100 \mathrm{E}+02\end{array}$
$\begin{array}{llll}23 & 1 & 0.300 \mathrm{E}-06 & 0.100 \mathrm{E}+02 \\ 24 & 1 & 0.300 \mathrm{E}-05 & 0.100 \mathrm{E}+02\end{array}$
251 0.300E-06 $0.100 \mathrm{E}+02$
$0.000 \mathrm{E}+00$
$\begin{array}{llll}26 & 2 & 0.300 \mathrm{E}-07 & 0.100 \mathrm{E}+02\end{array}$ 

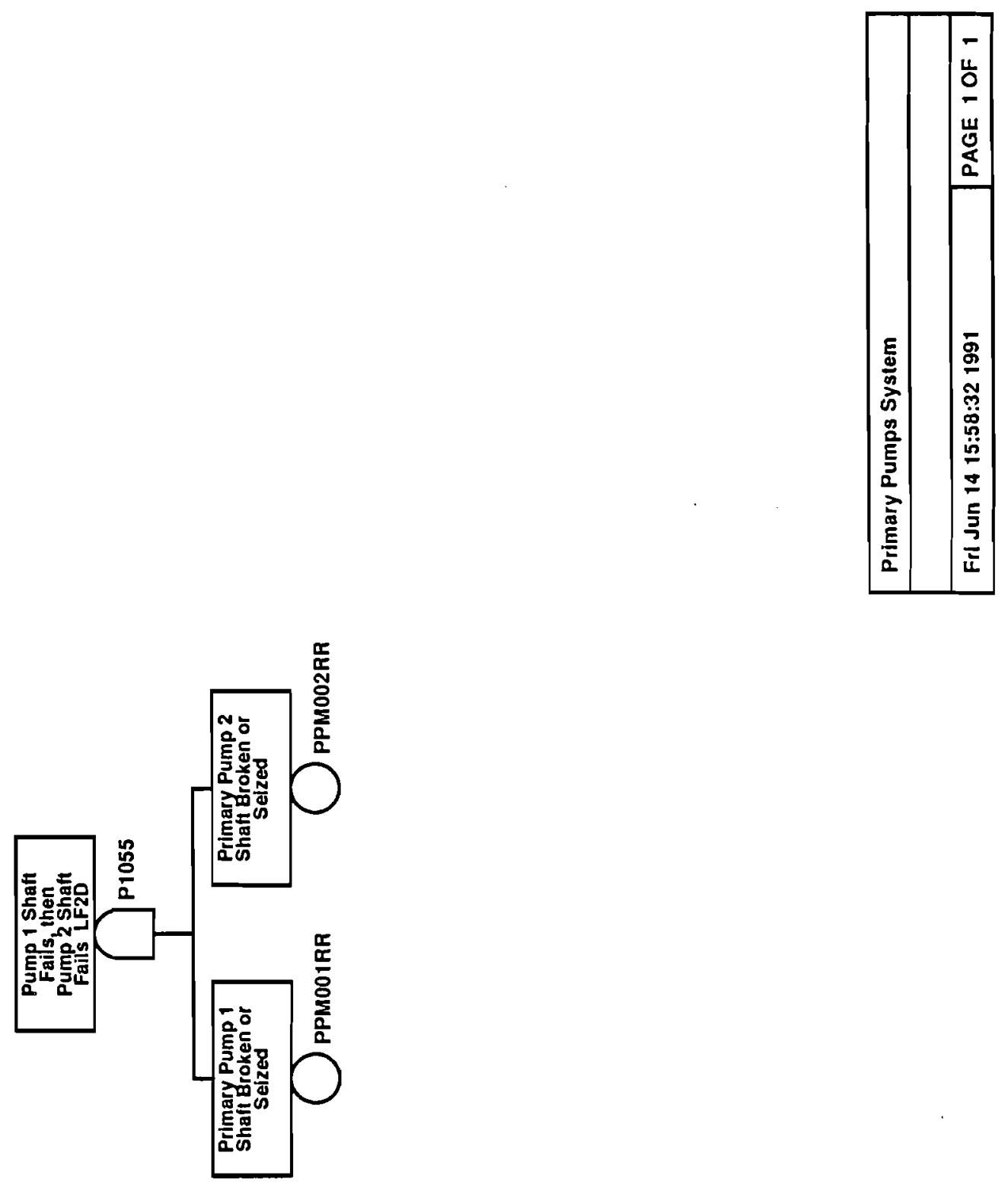

B. 1-33 


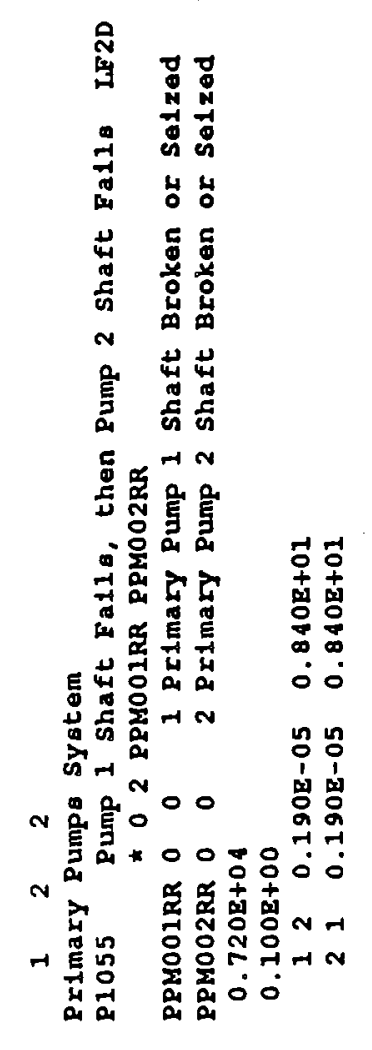

B. 1-34 

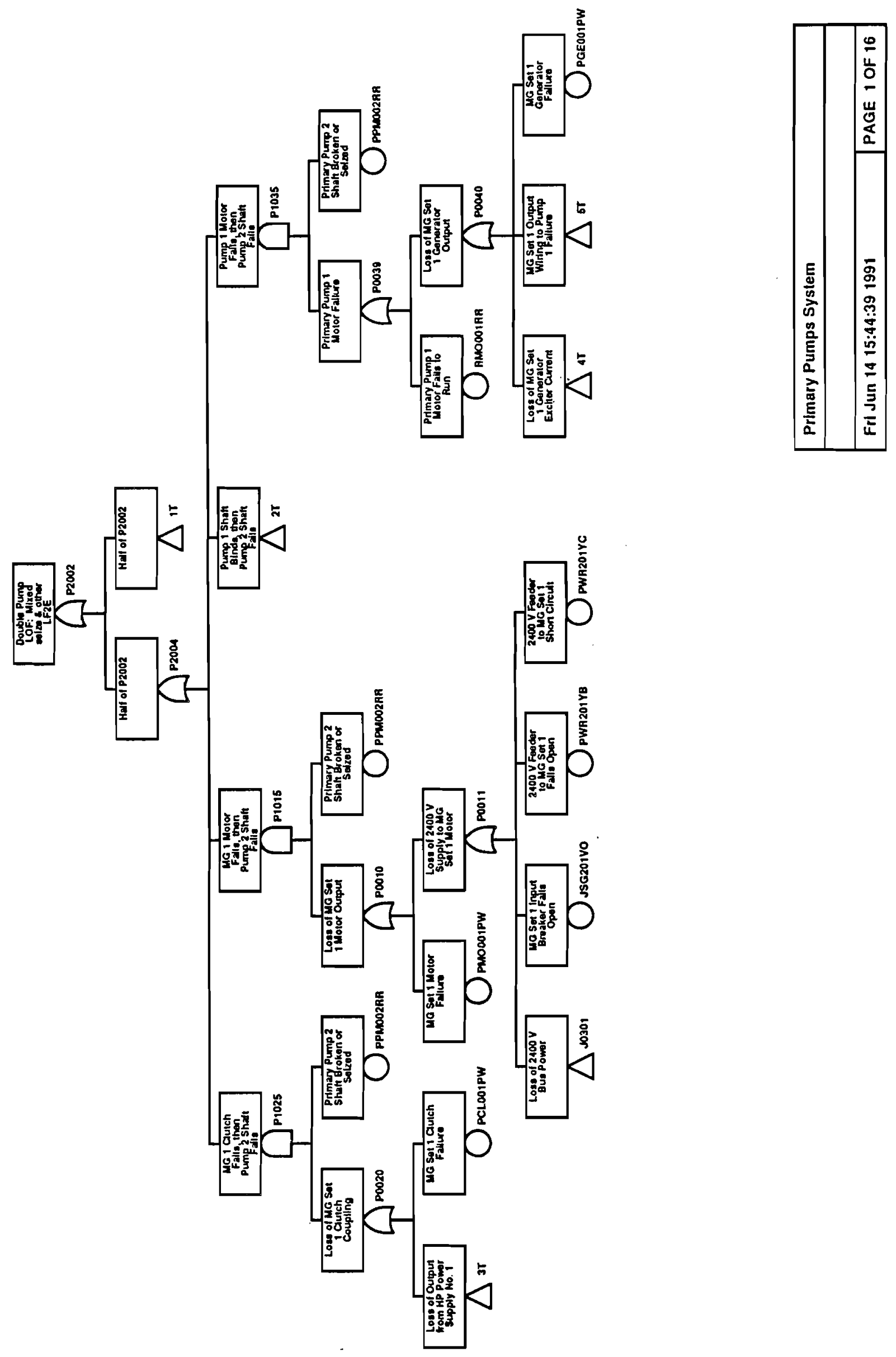

B. 1-35 

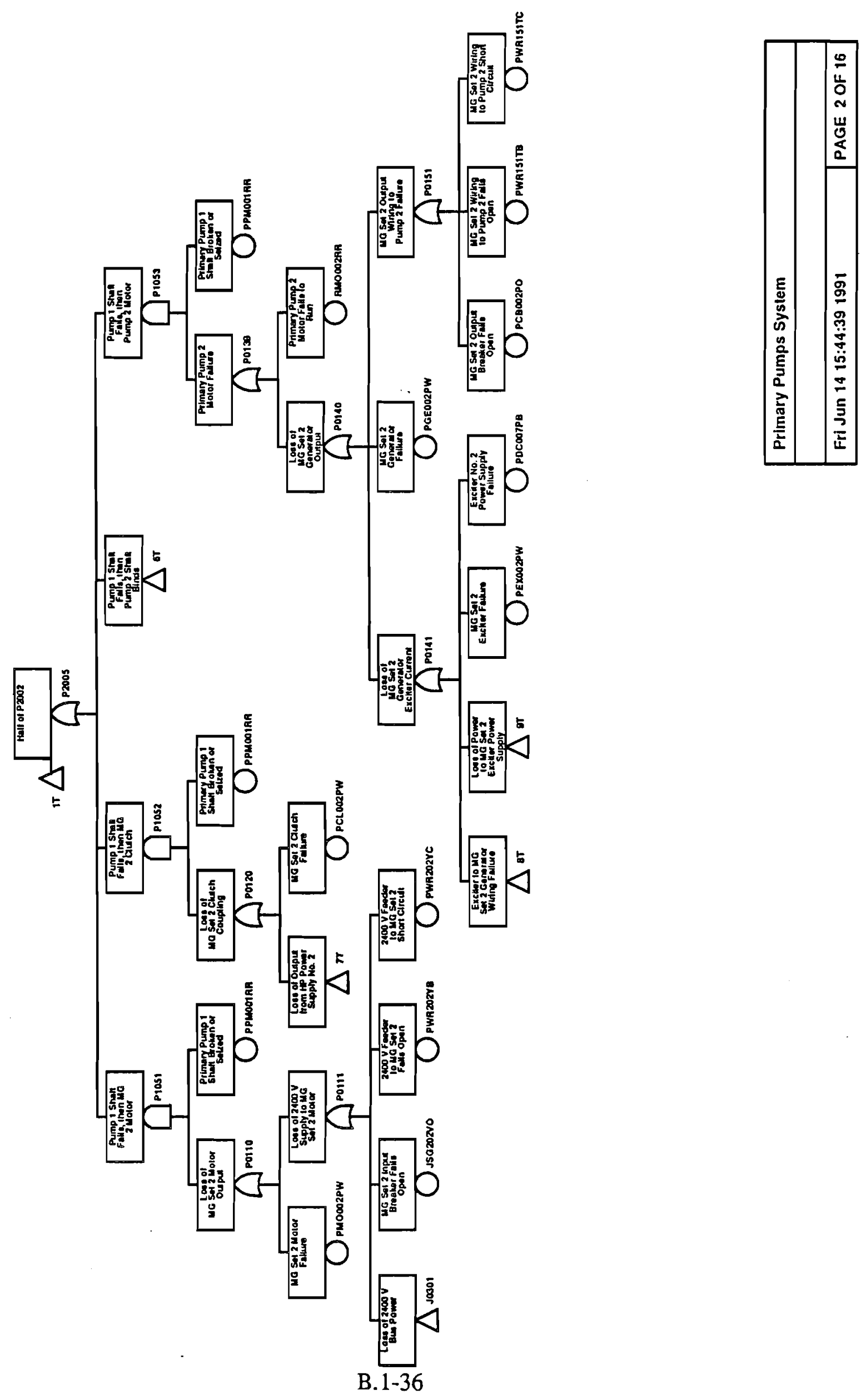

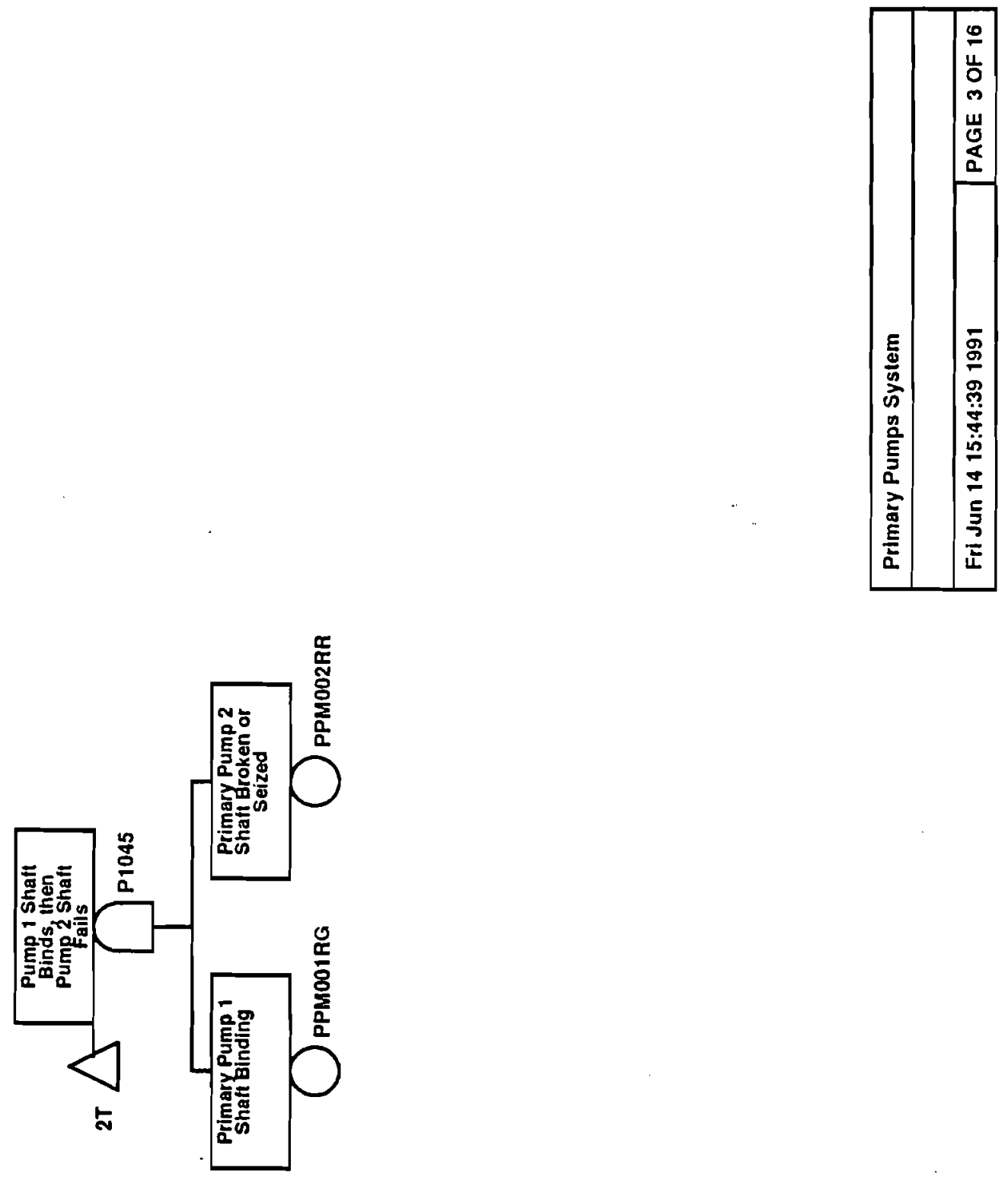

B. 1-37 


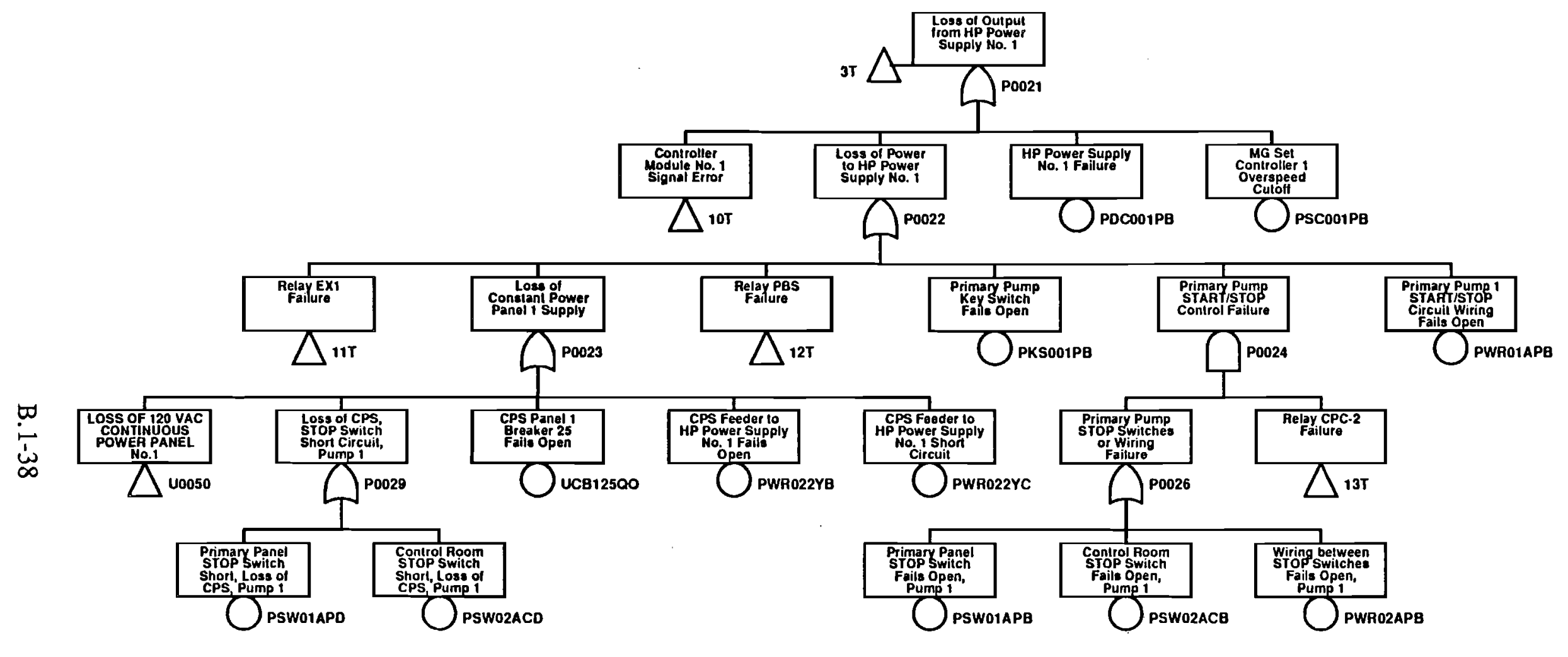

Primary Pumps System

\begin{tabular}{|l|l|}
\hline Fri Jun 14 15:44:39 1991 & PAGE 4 OF 16 \\
\hline
\end{tabular}




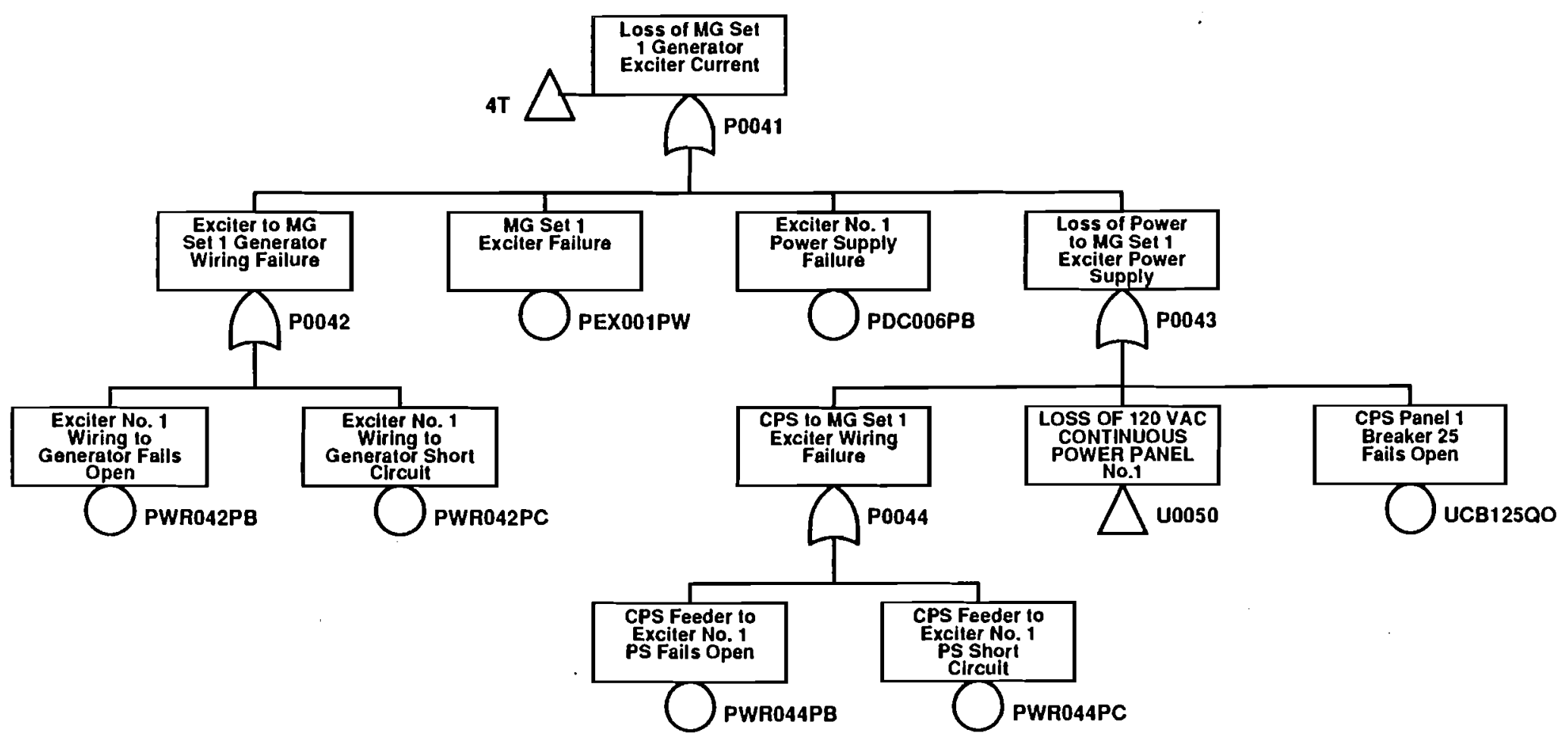

Primary Pumps System 

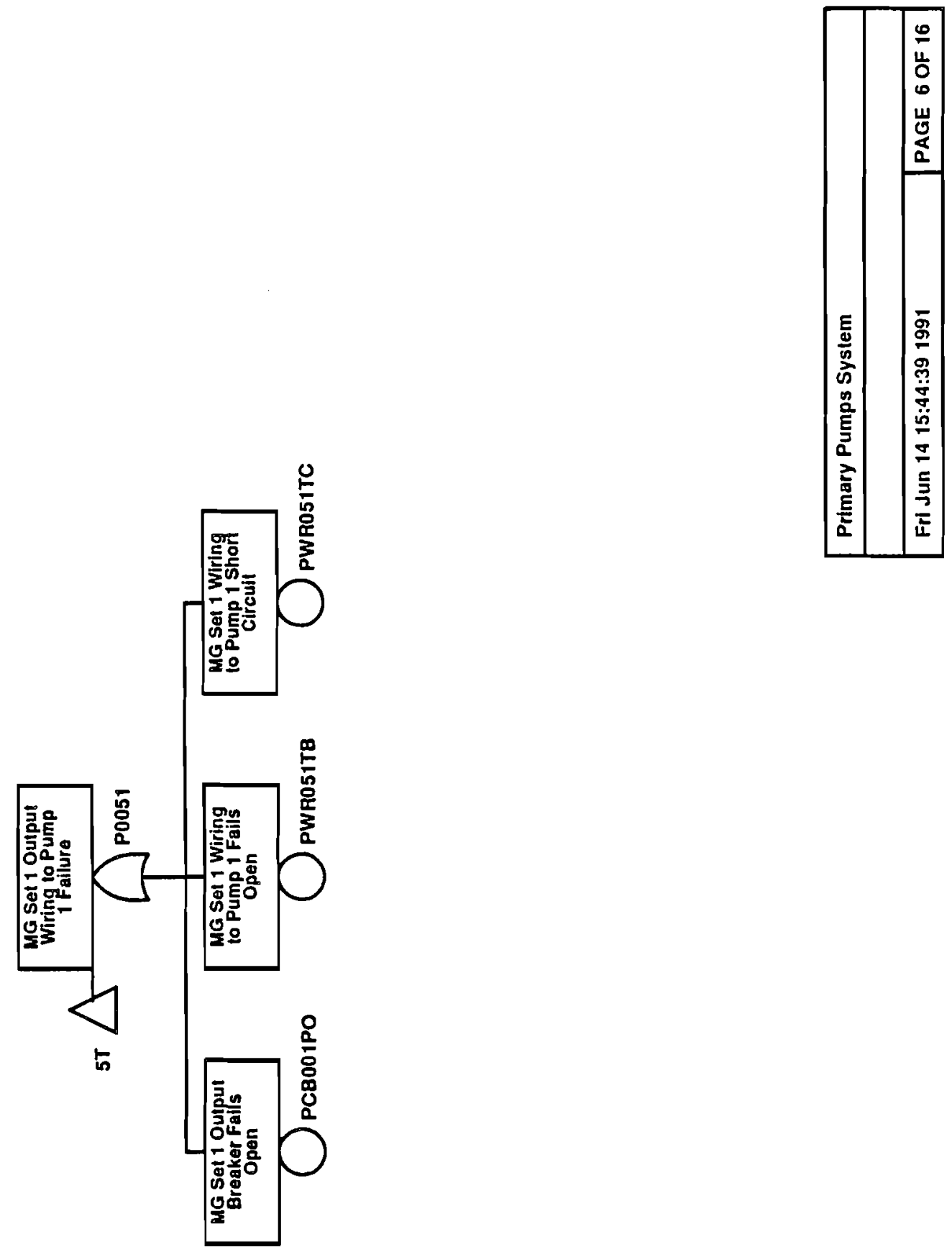

B. $1-40$ 

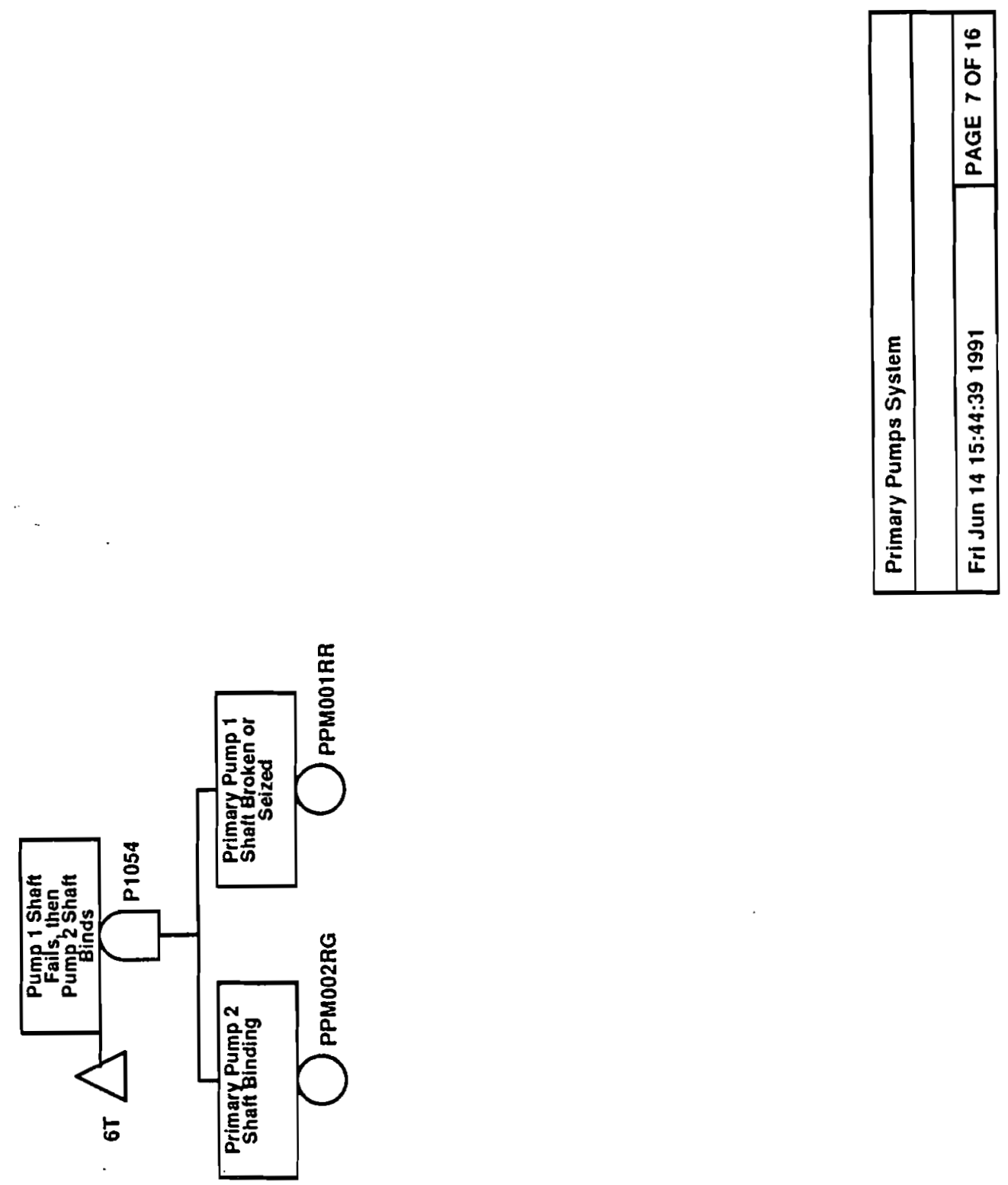

B. $1-41$ 


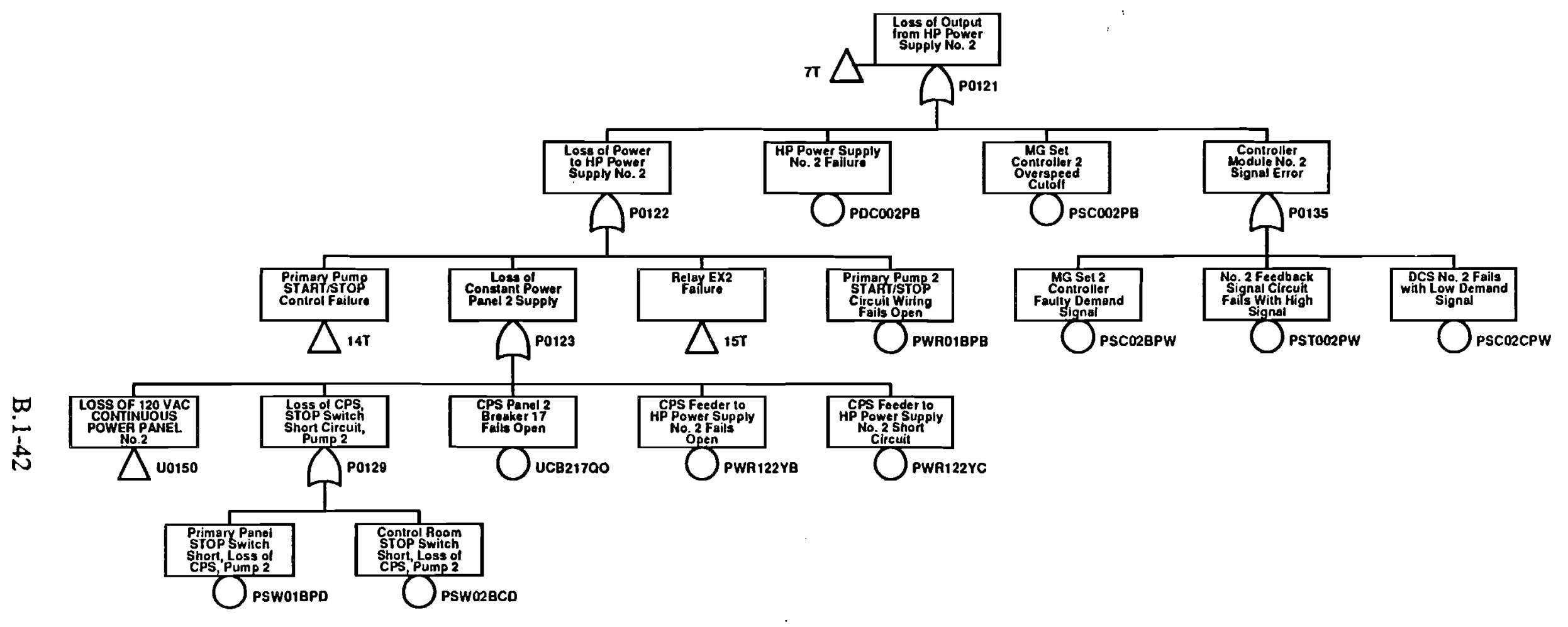

Primary Pumps System

Frl Jun 14 15:44:39 1991

PAGE 8 OF 16 


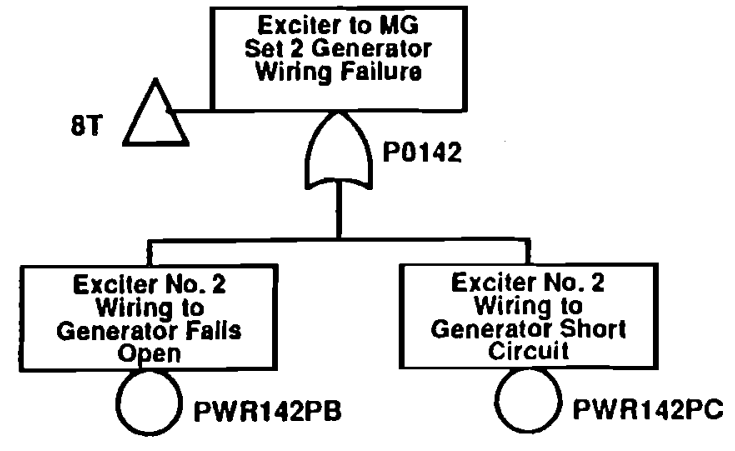

Primary Pumps System 


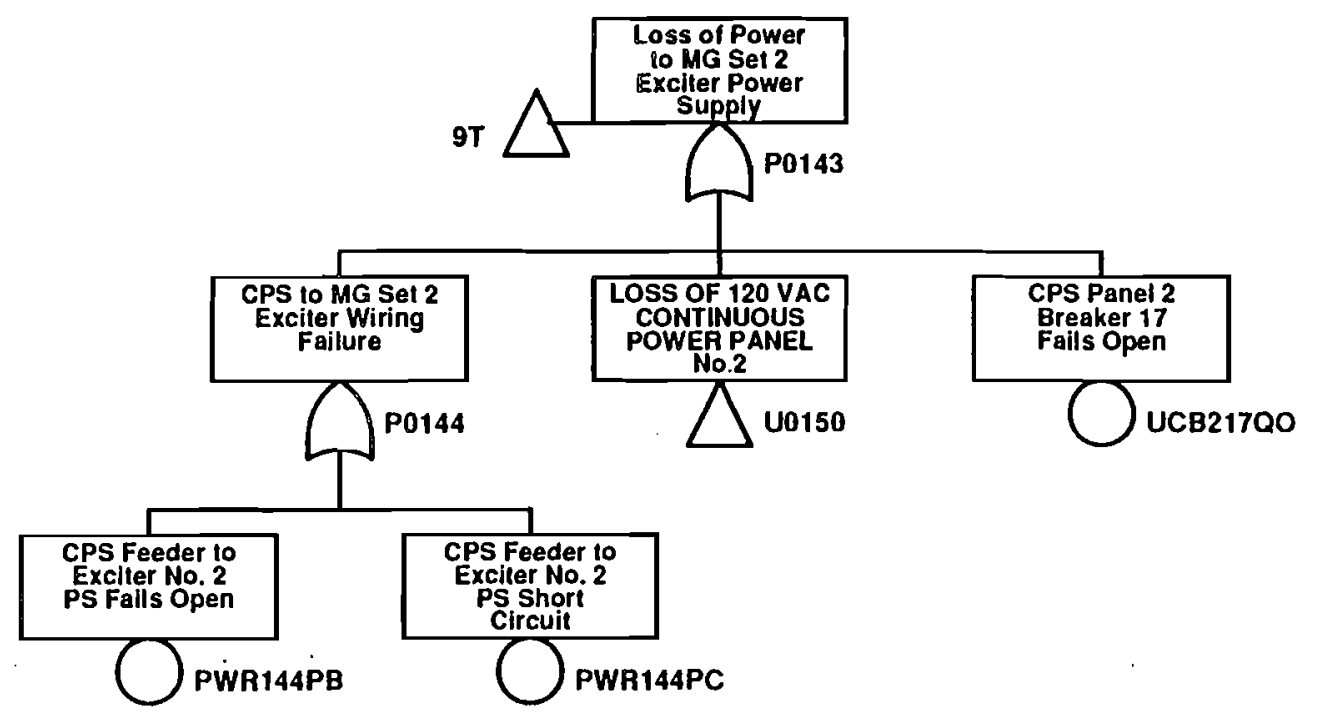

Primary Pumps System

Fri Jun 14 15:44:39 1991

PAGE 10 OF 16 


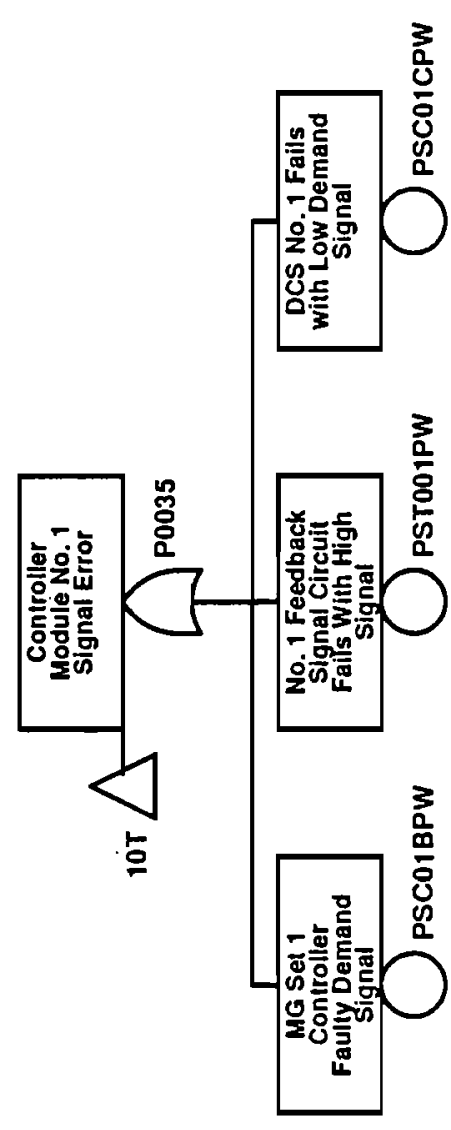

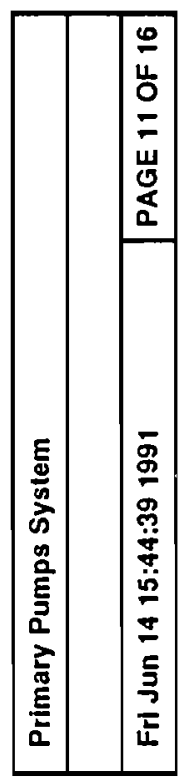

B. 1-45 

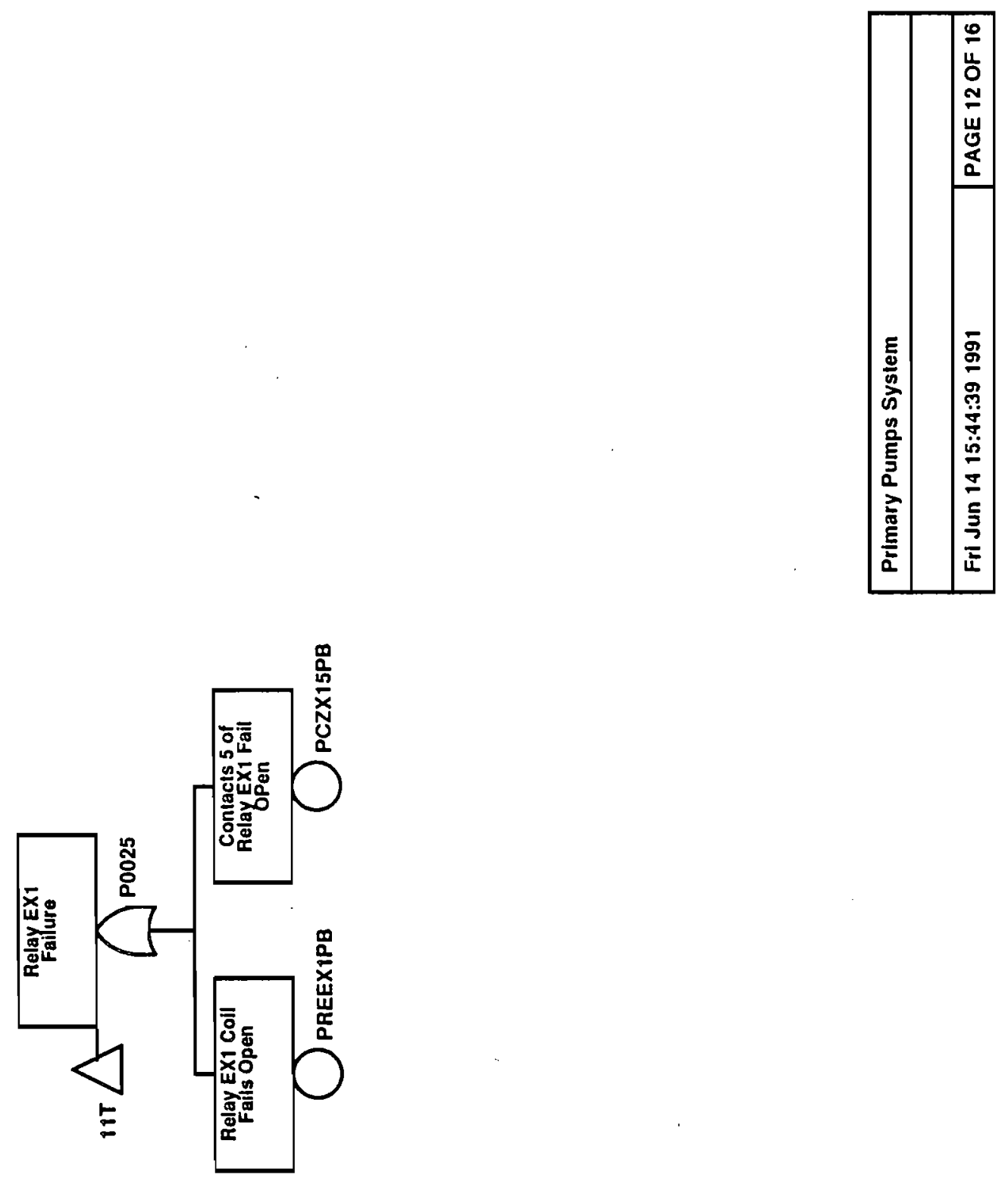

B. 1-46 

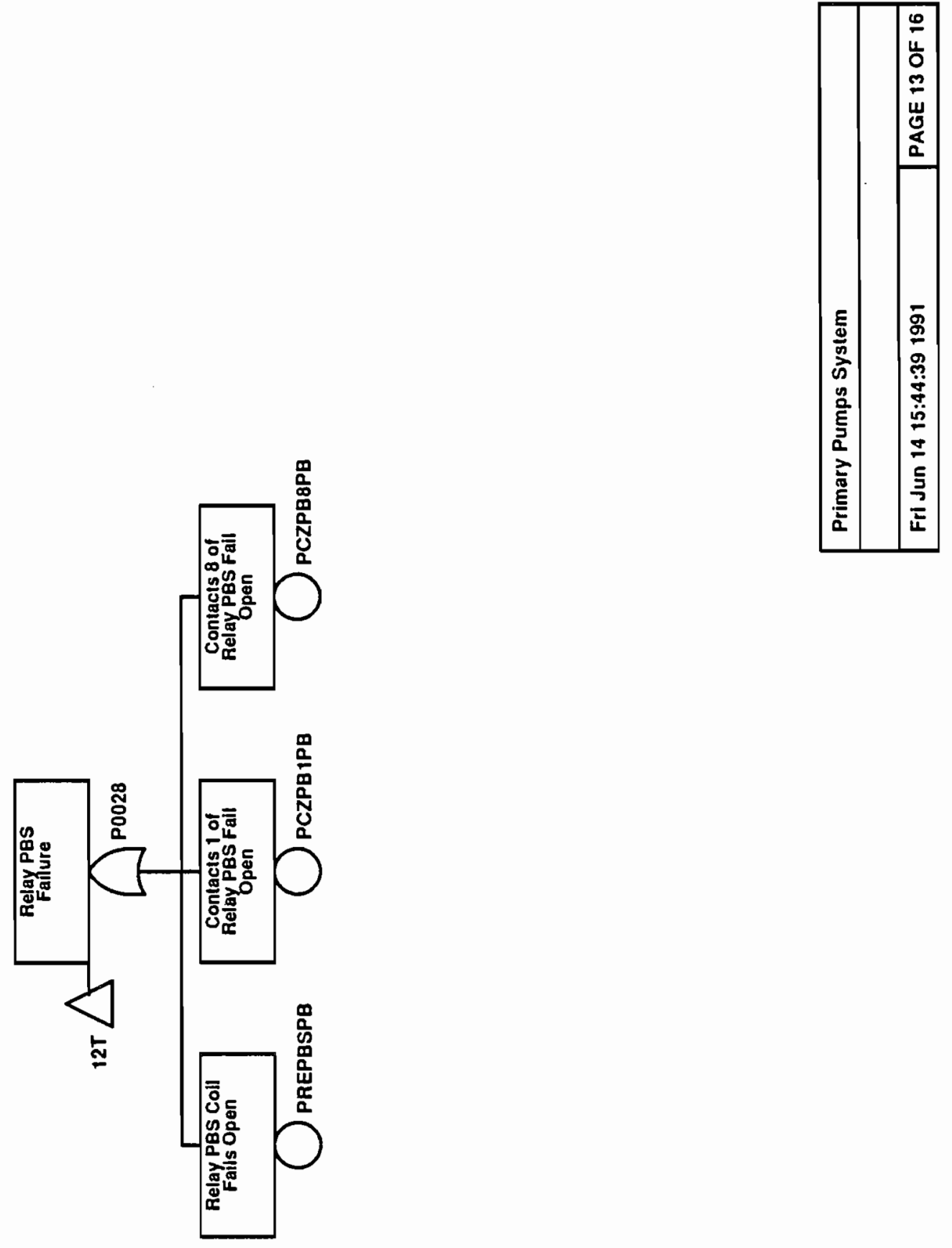

B. $1-47$ 


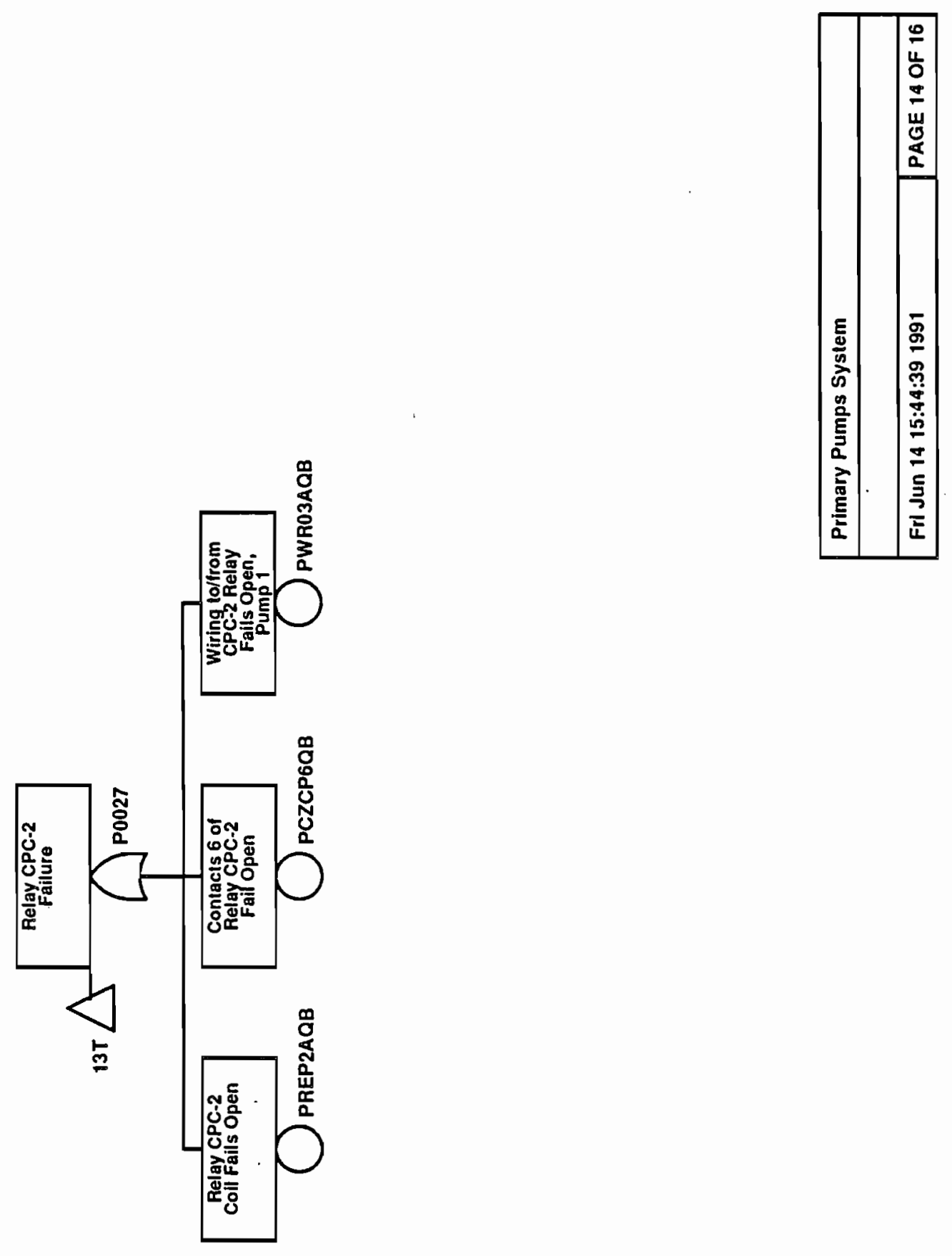

B. 1-48 


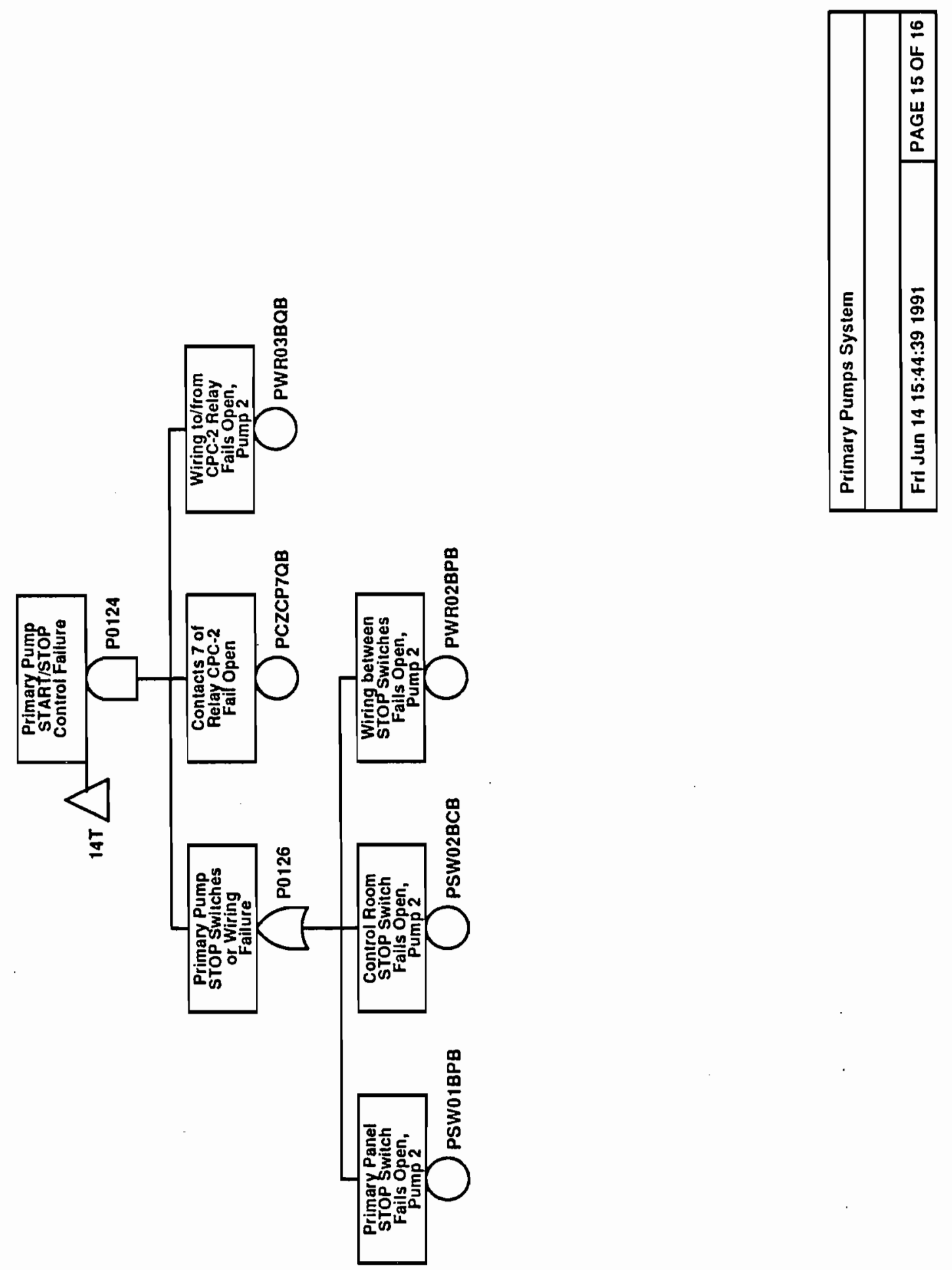

B. 1-49 


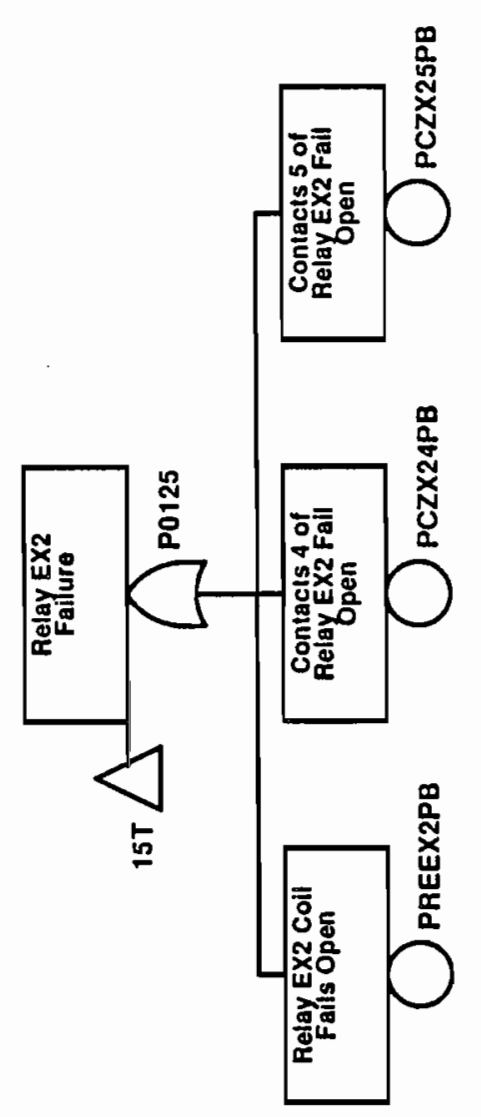

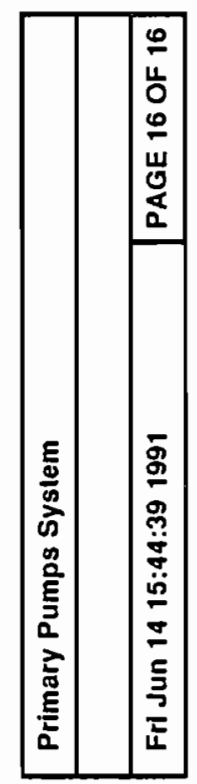




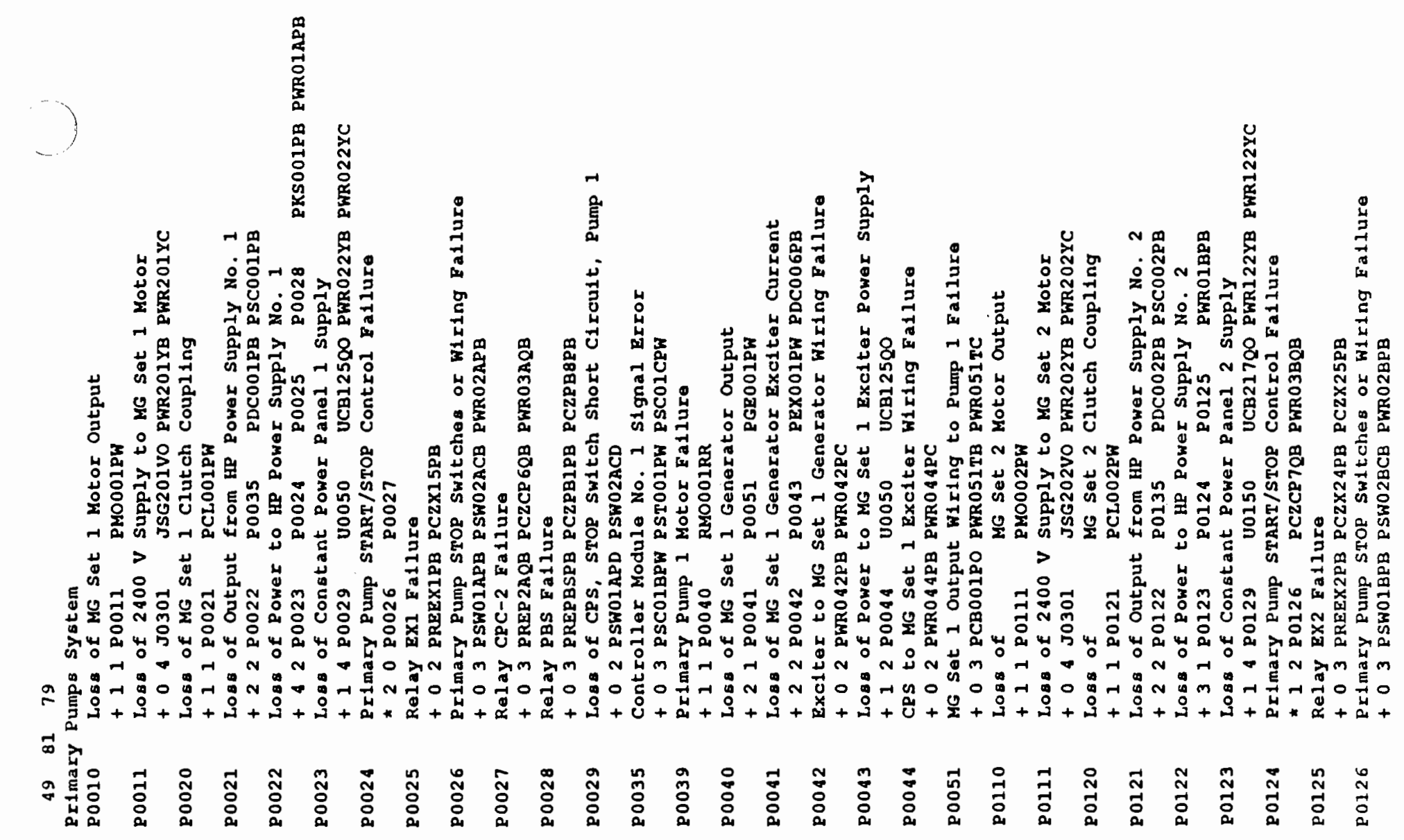

B. 1-51 


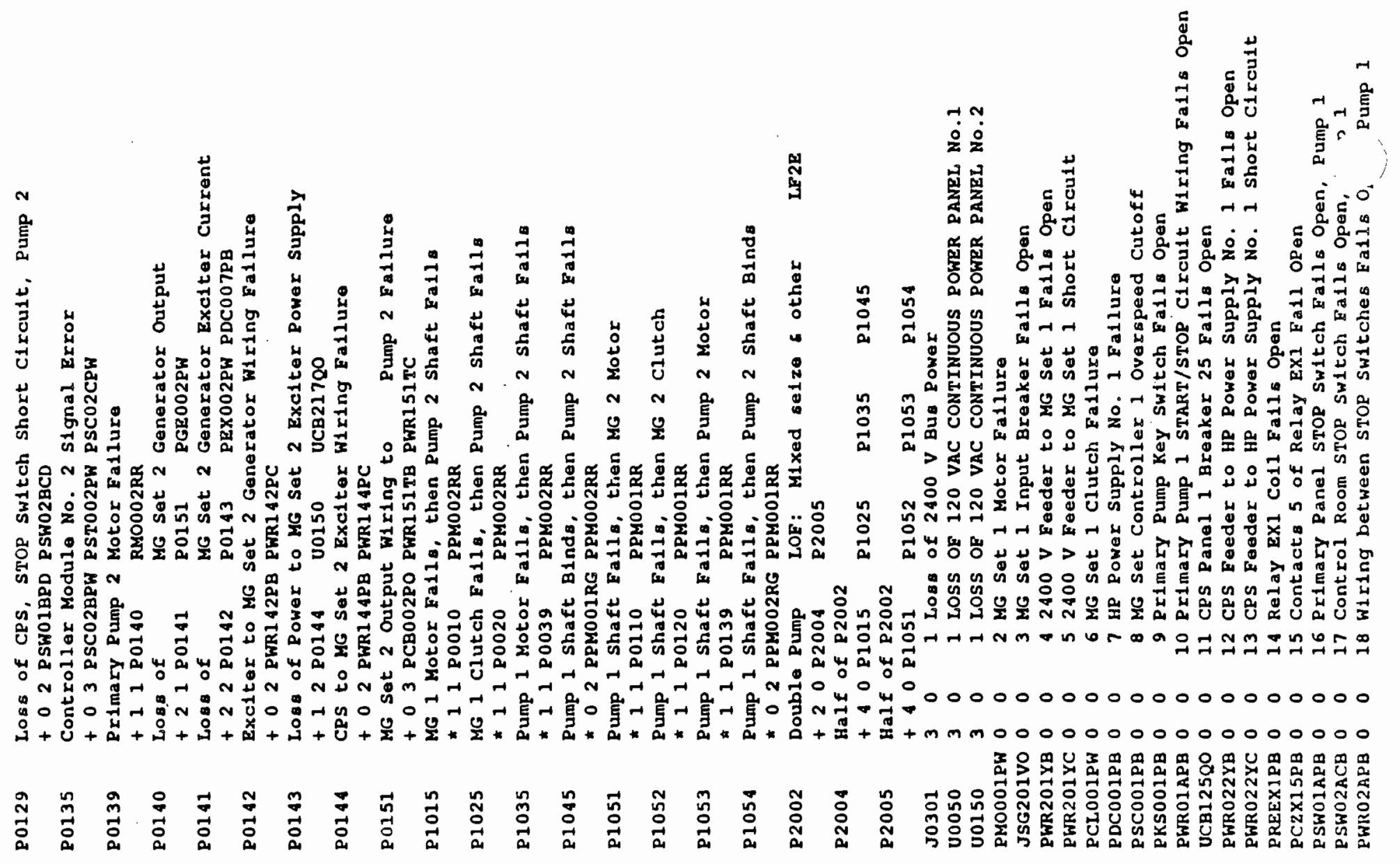




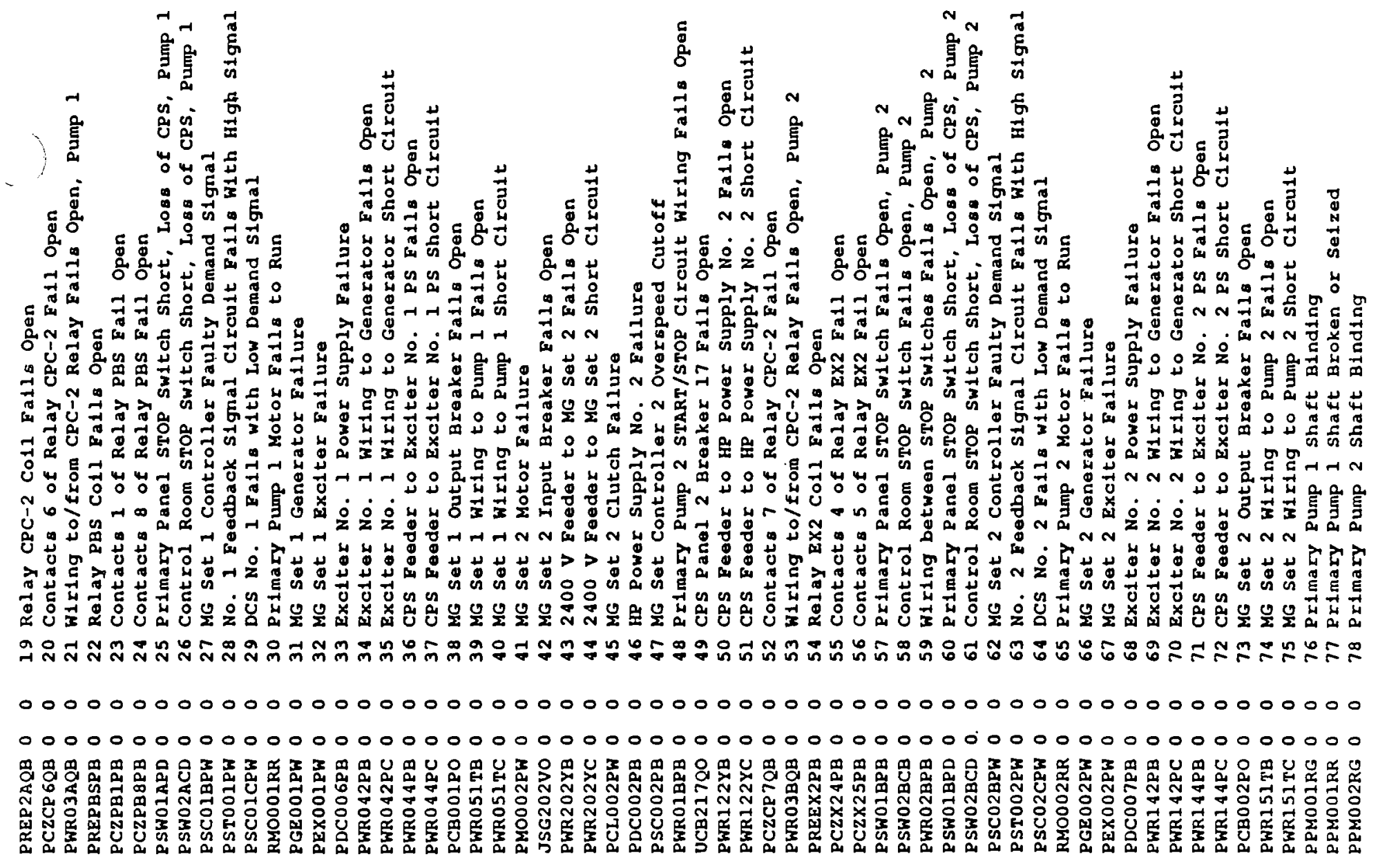




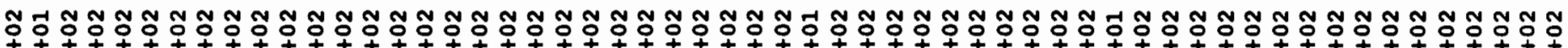

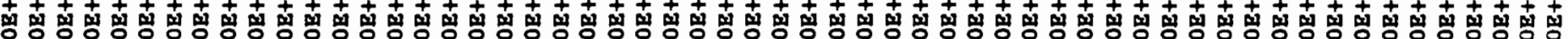

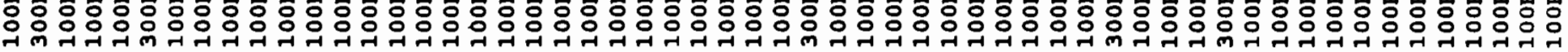

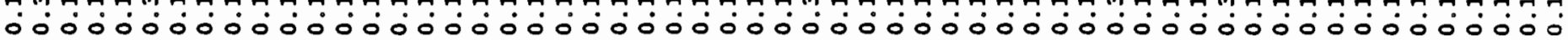

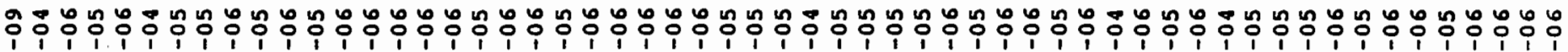

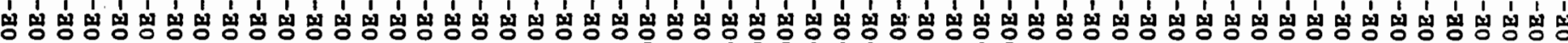

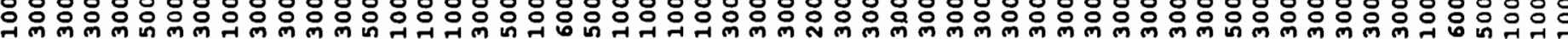

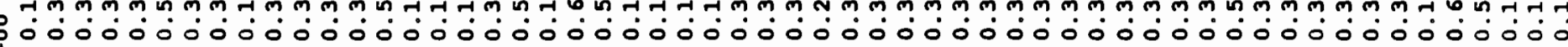
우요

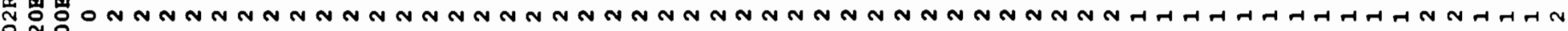

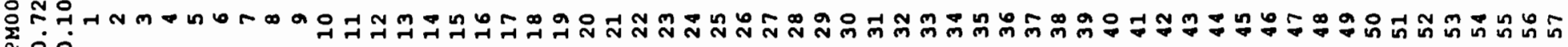
00

B. $1-54$ 


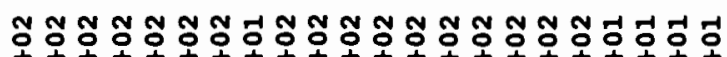

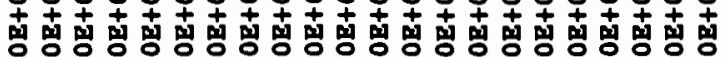

응 ㅇㅇㅇㅇㅇㅇㅇㅇㅇㅇㅇㅇㅇㅇㅇㅇㅇㅇㅇㅇㅇㅇㅇㅇㅇㅇㅇㅇㅇㅇㅇㅇㅇㅇㅇㅇㅇㅇㅇㅇㅇㅇㅇㅇㅇㅇ

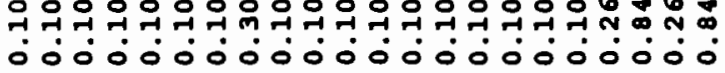

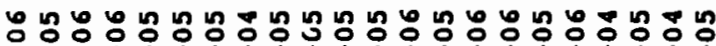

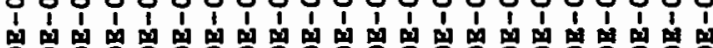

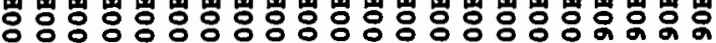

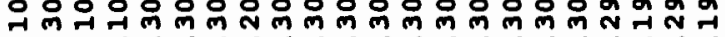

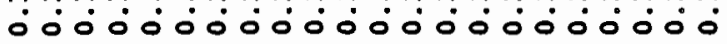

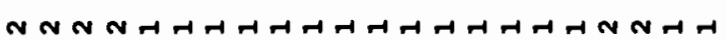

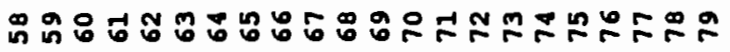



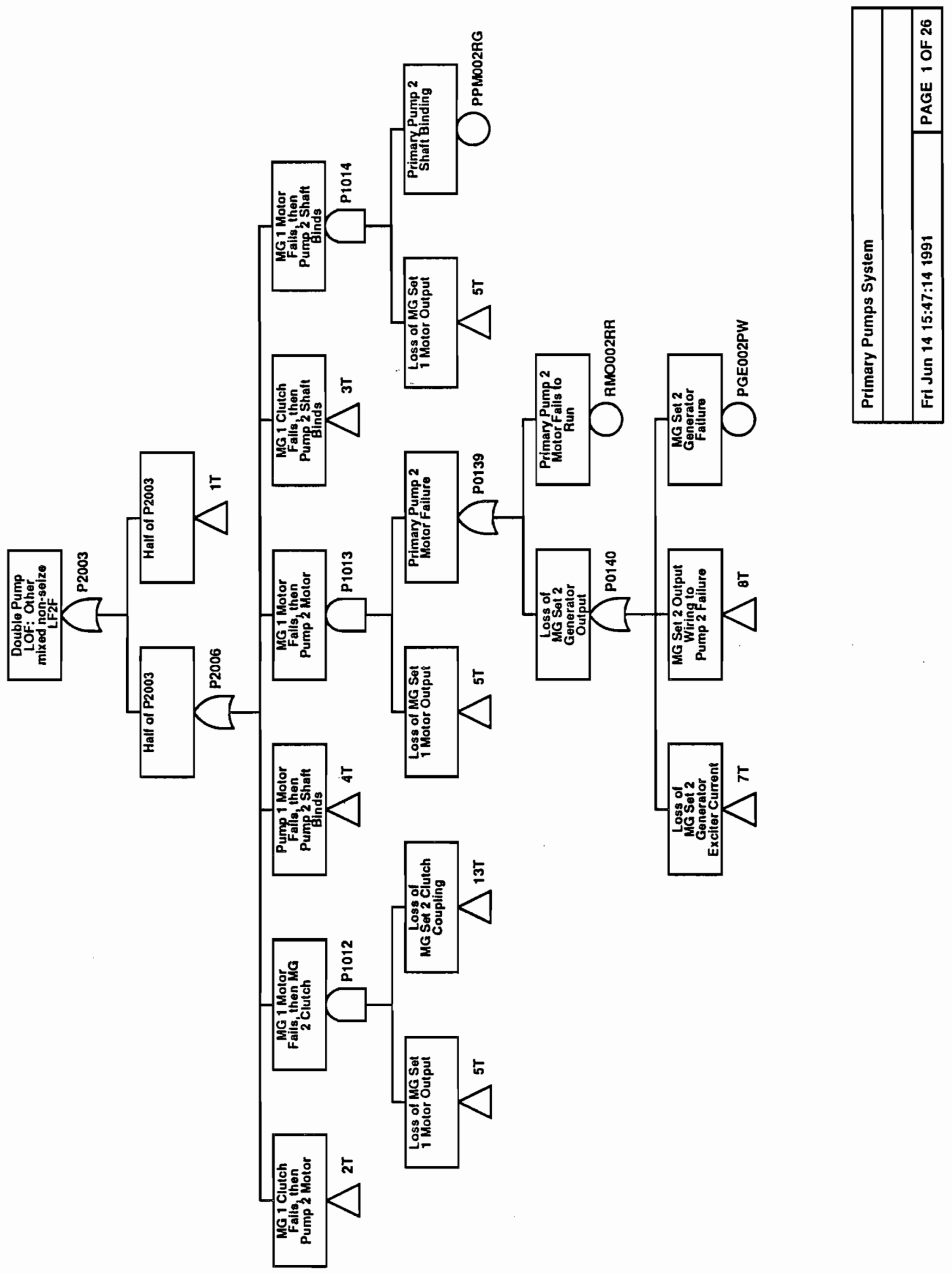

B. 1-56 


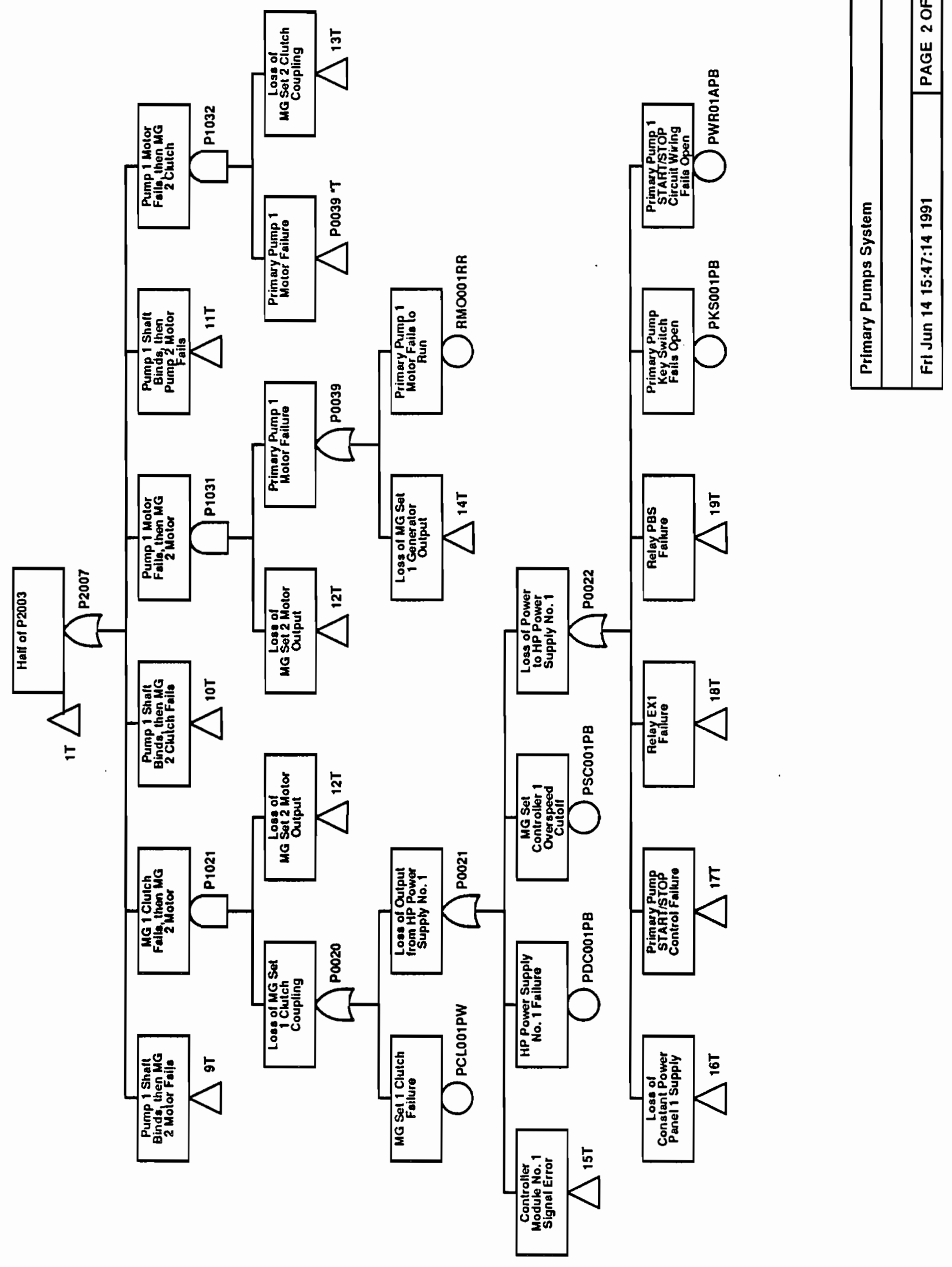

B. 1-57 

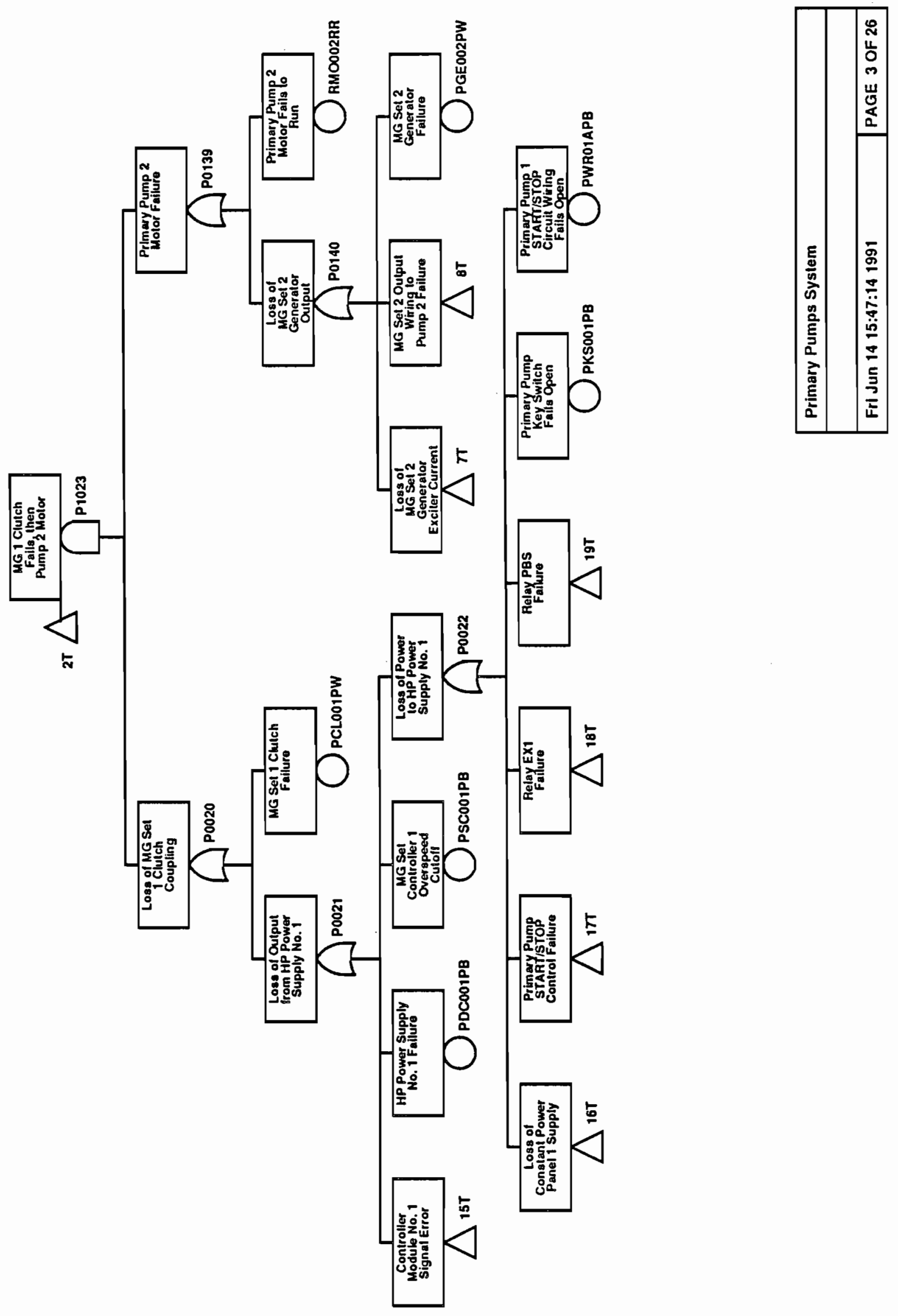

B. $1-58$ 


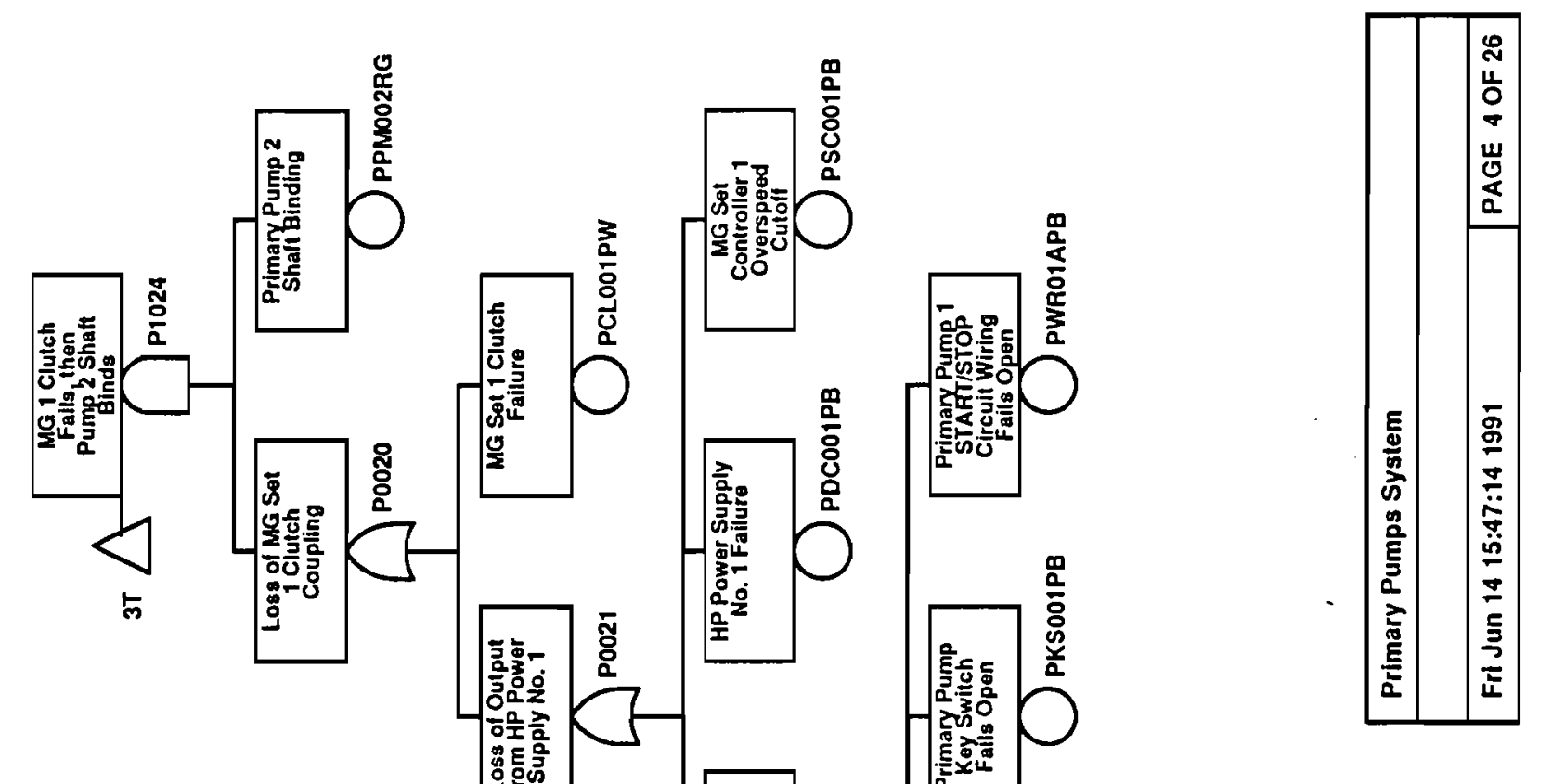




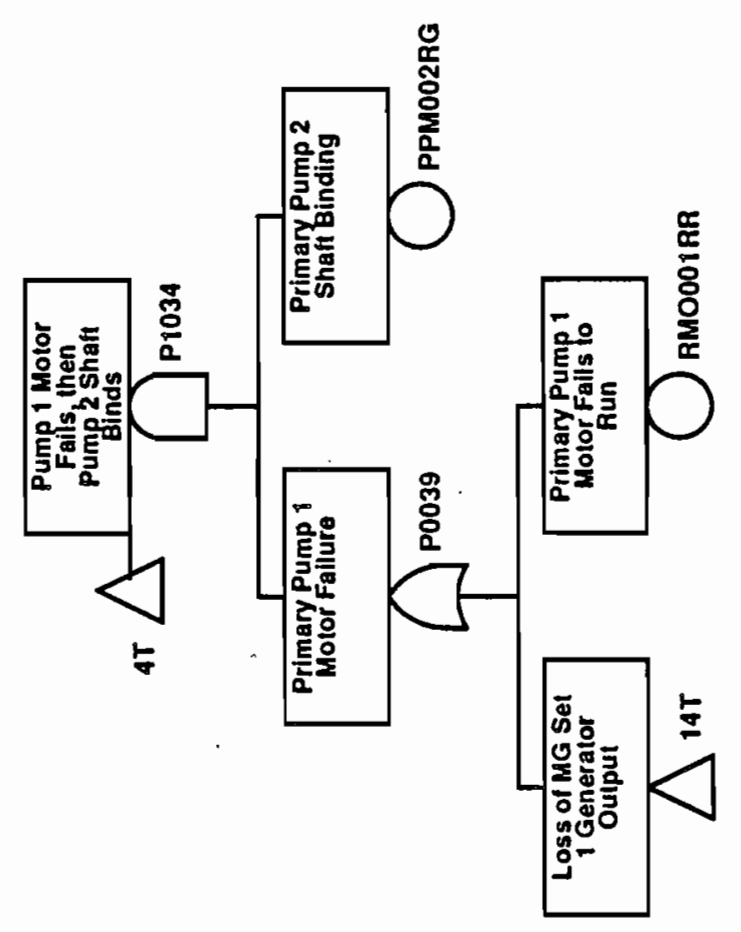

B. $1-60$ 


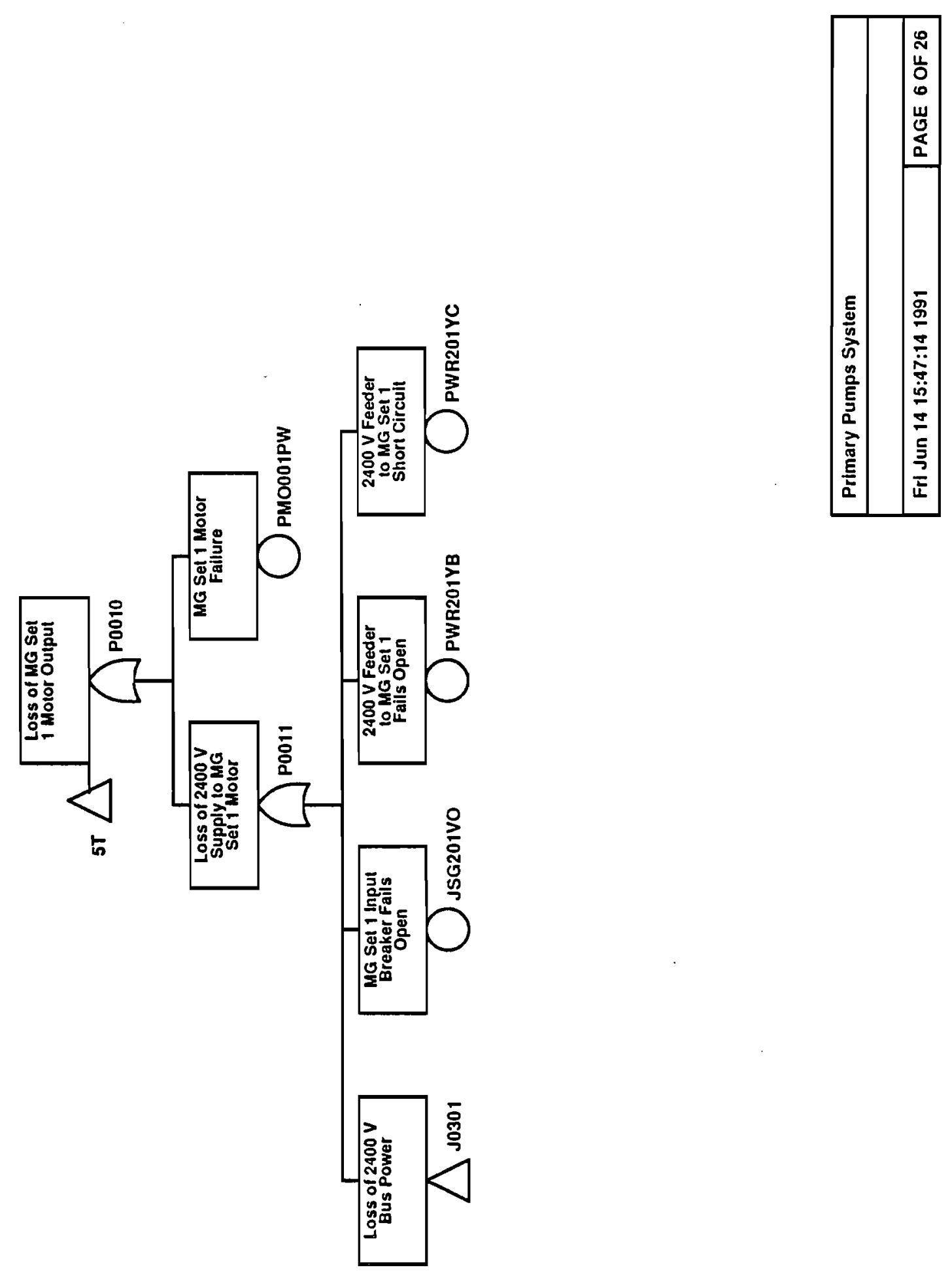

B. 1-61 

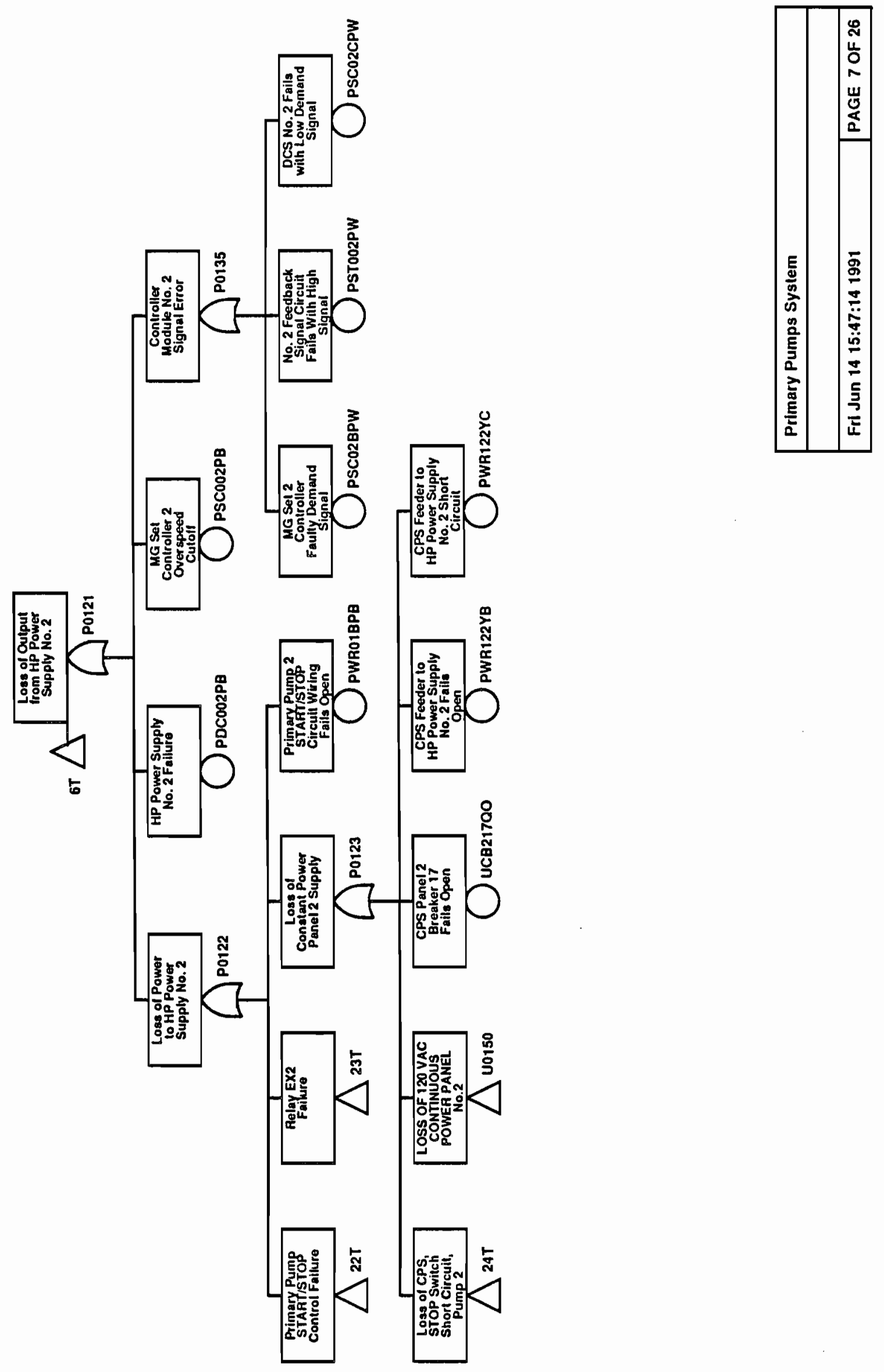

B. 1-62 

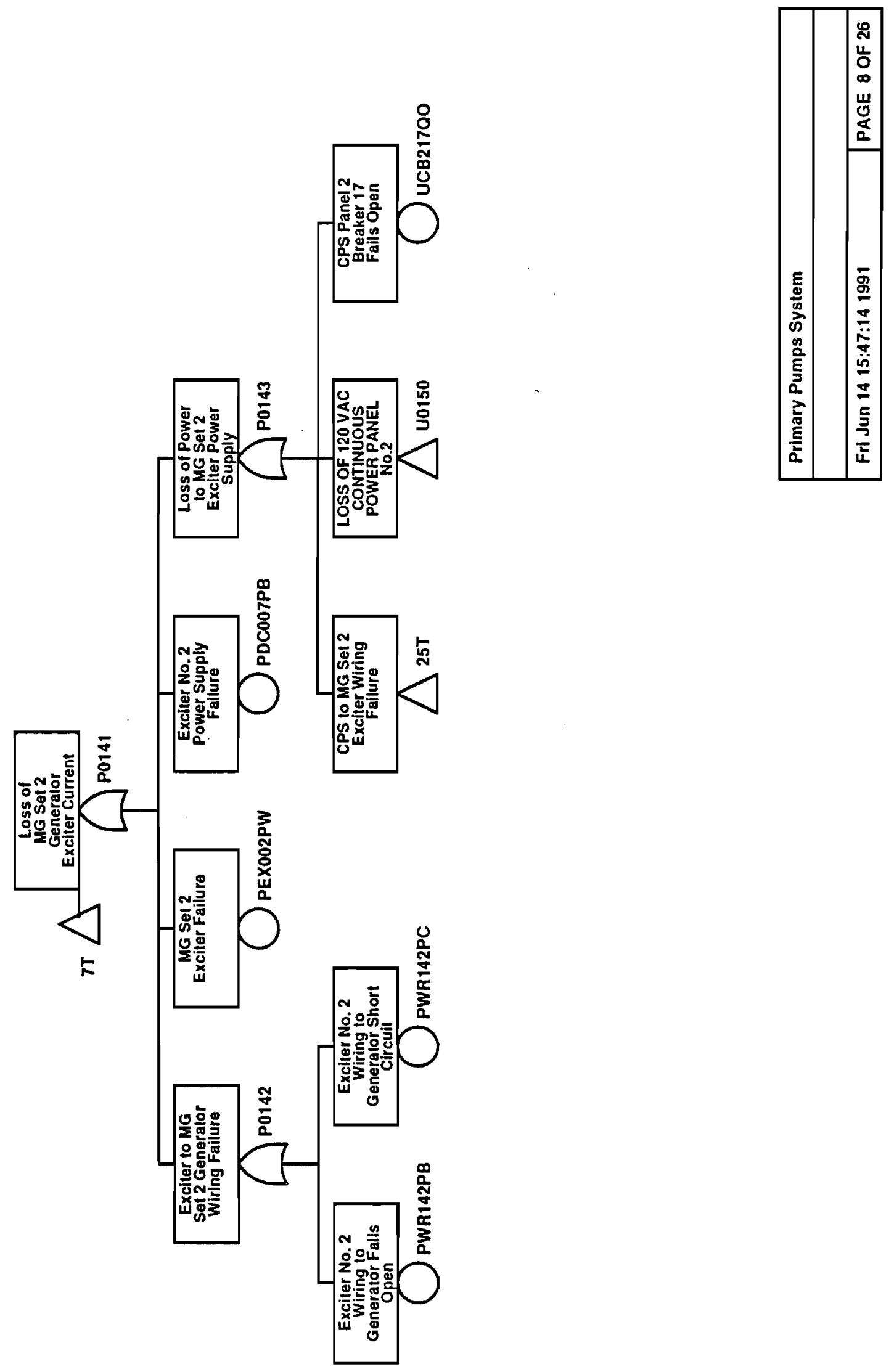

B. 1-63 


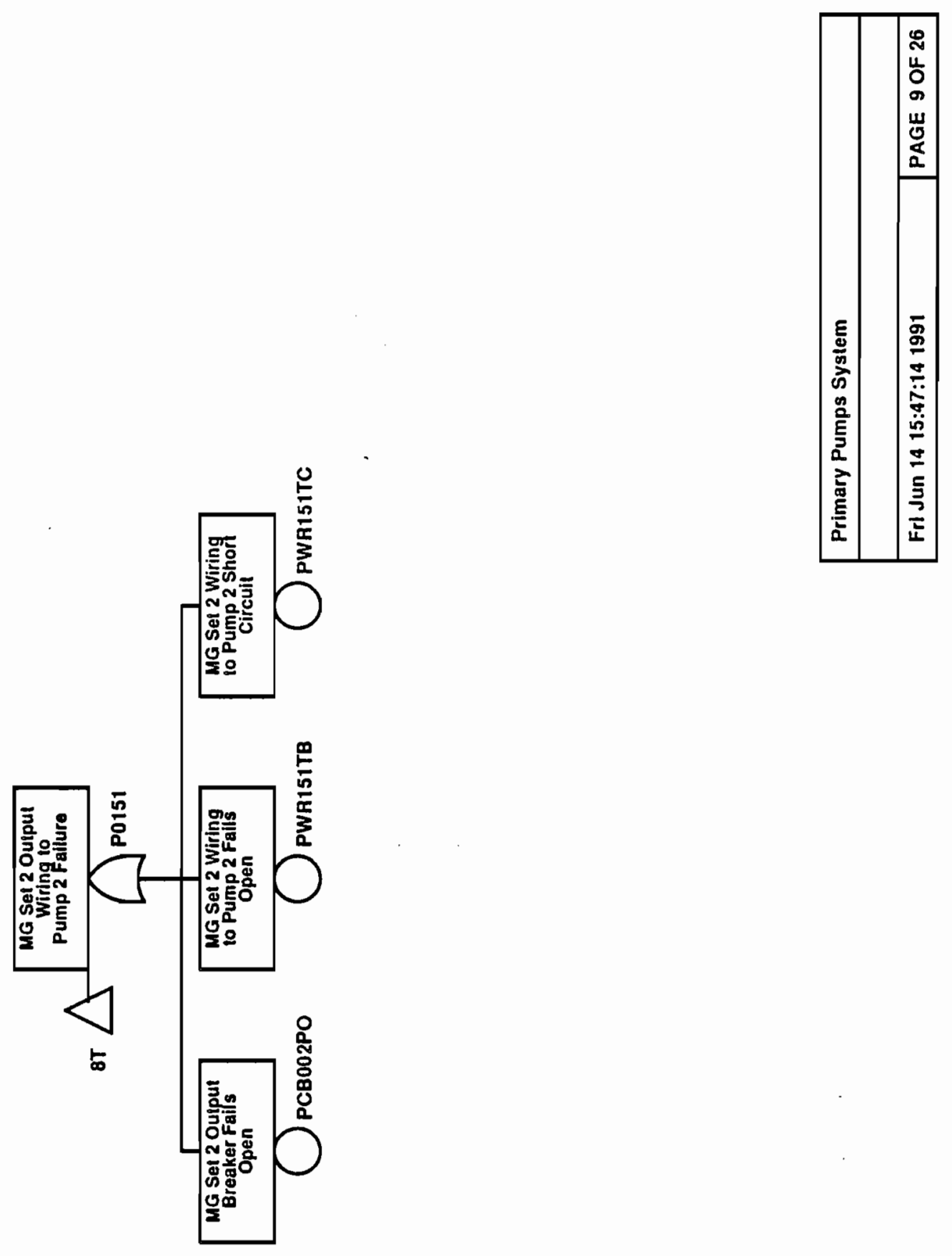

B. 1-64 

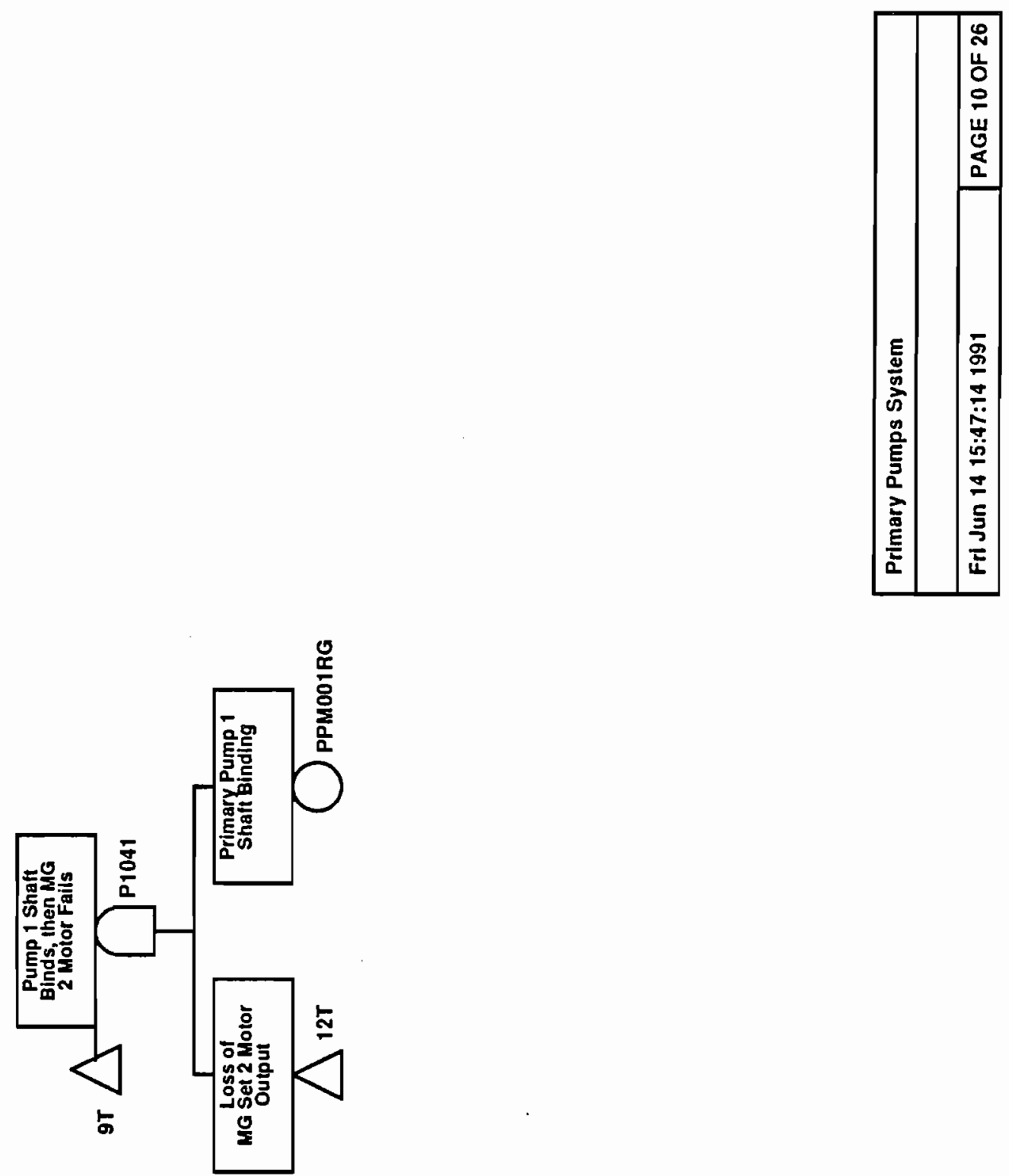

B. 1-65 


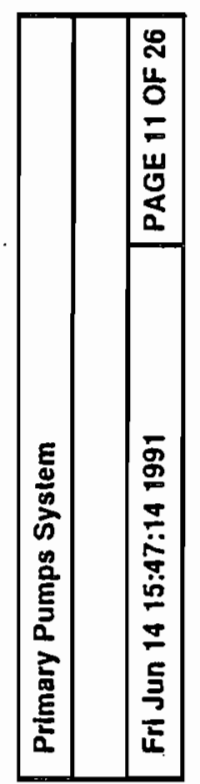

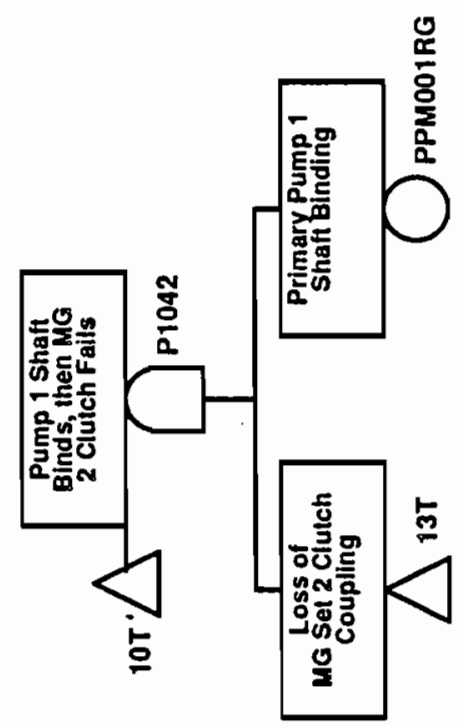

B. 1-66 


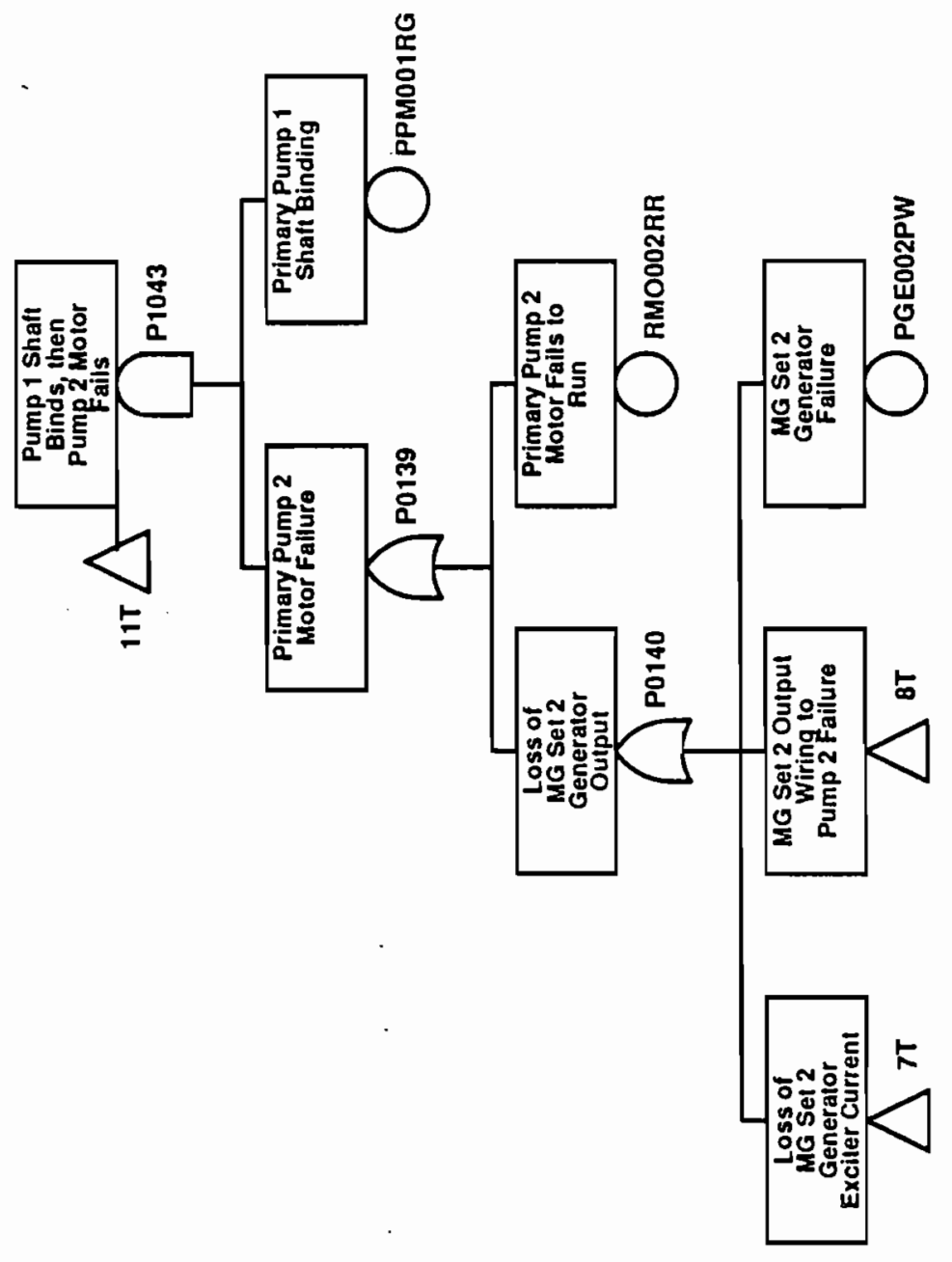

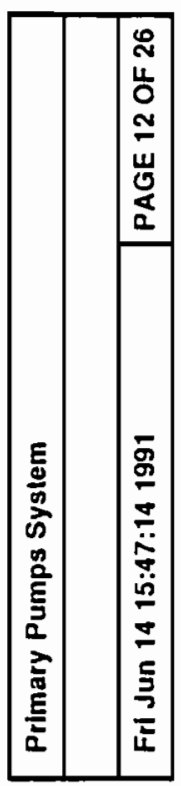

B. 1-67 


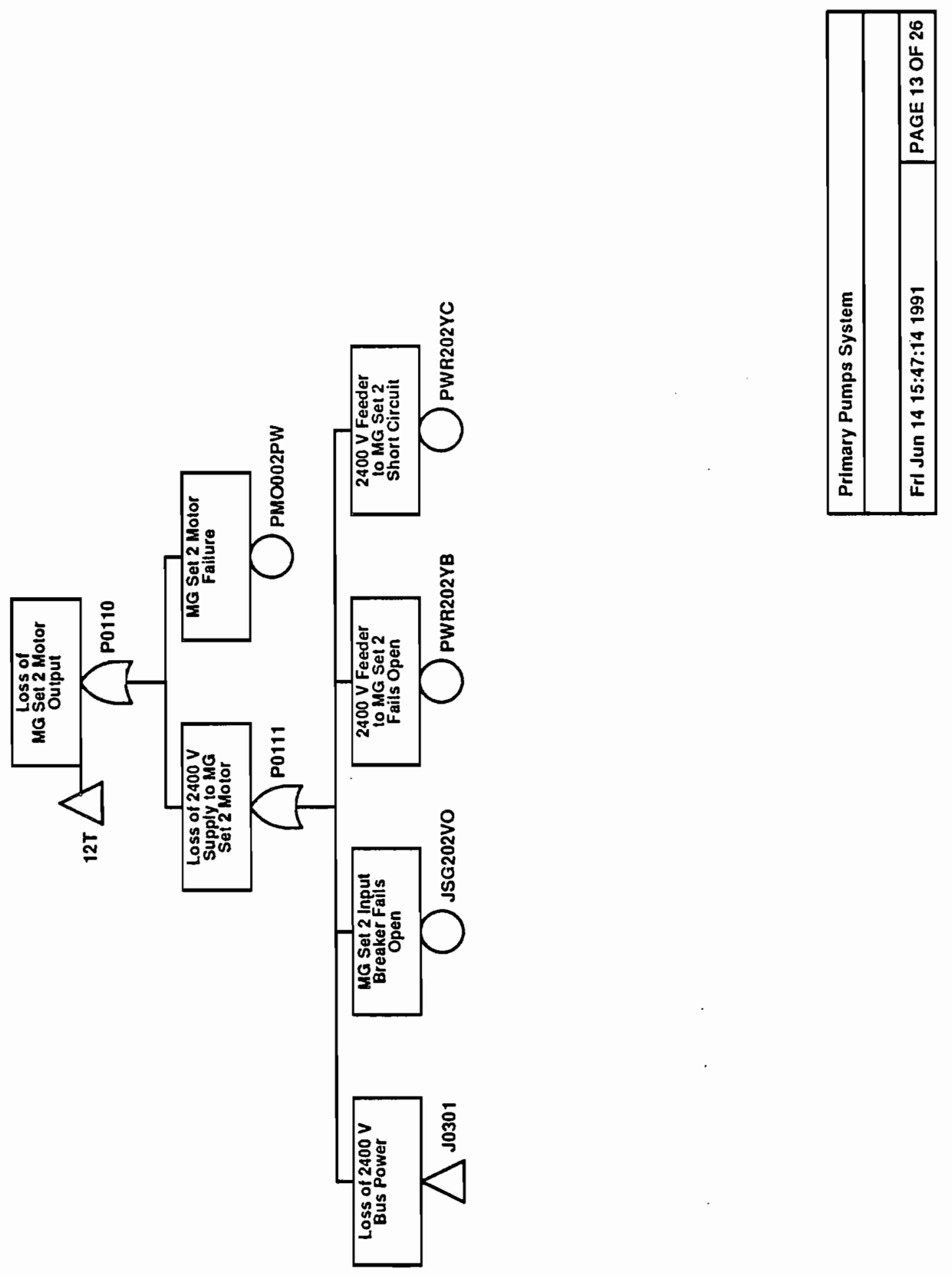

B-1-68 

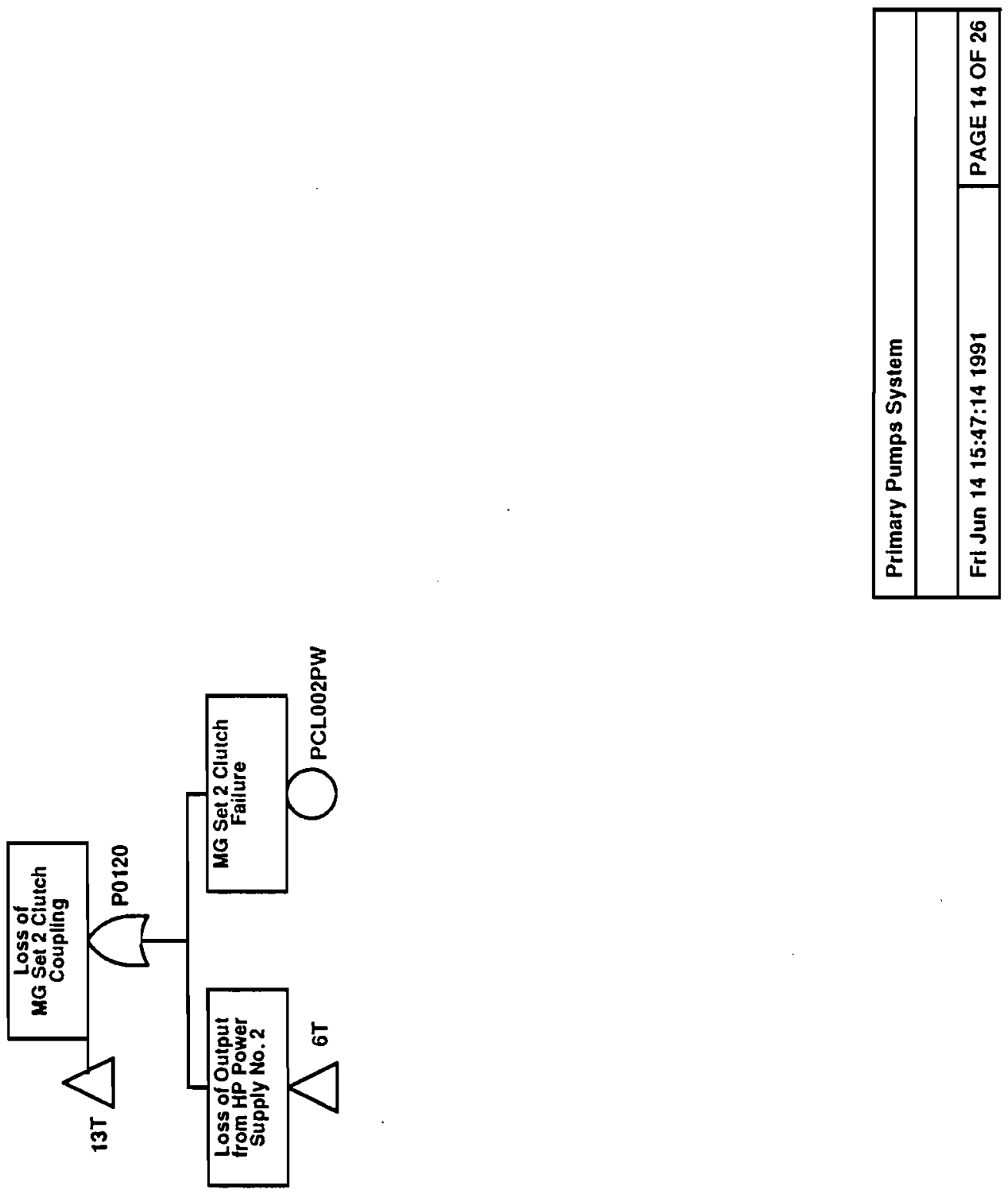

B. 1-69 

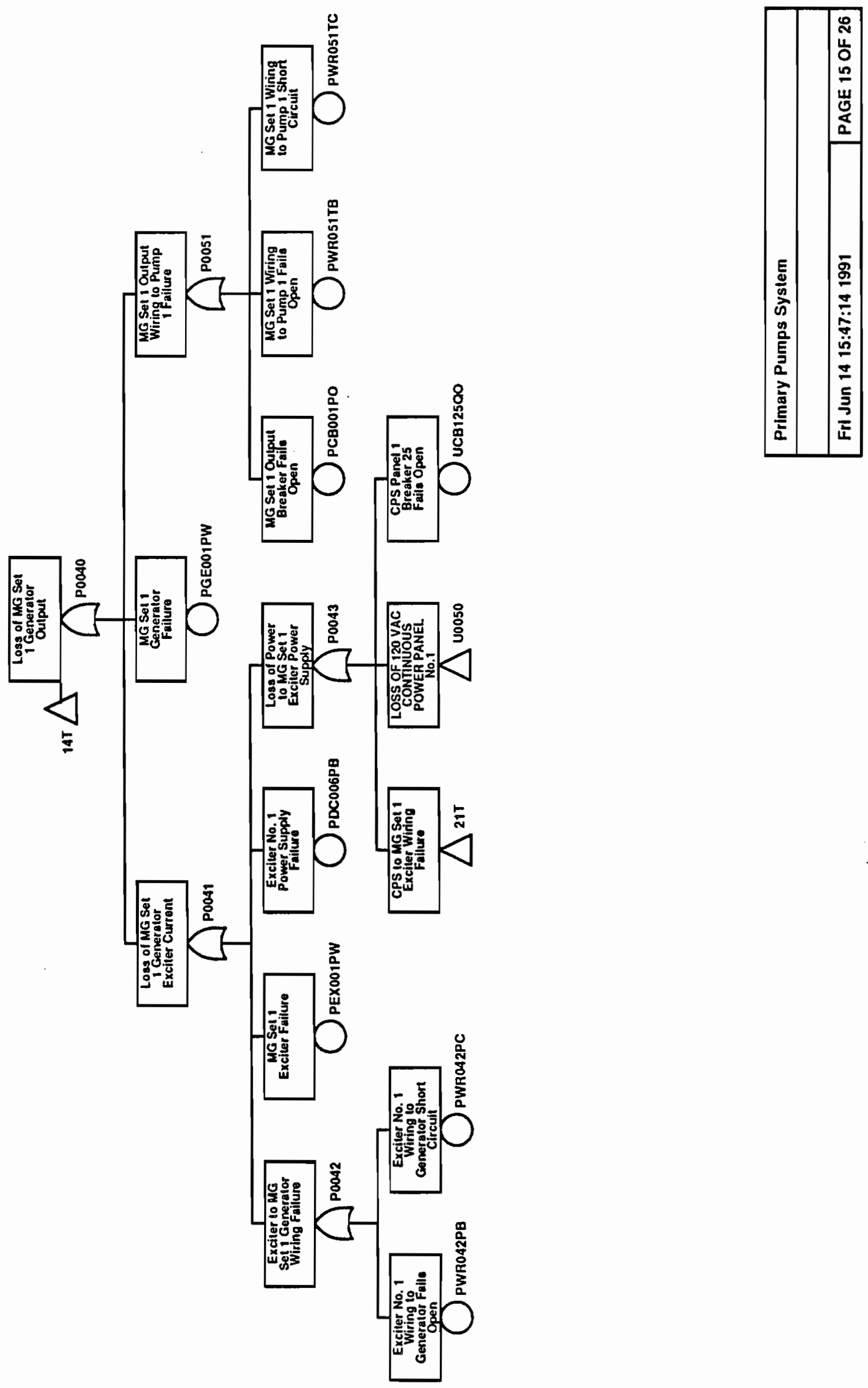

B. $1-70$ 


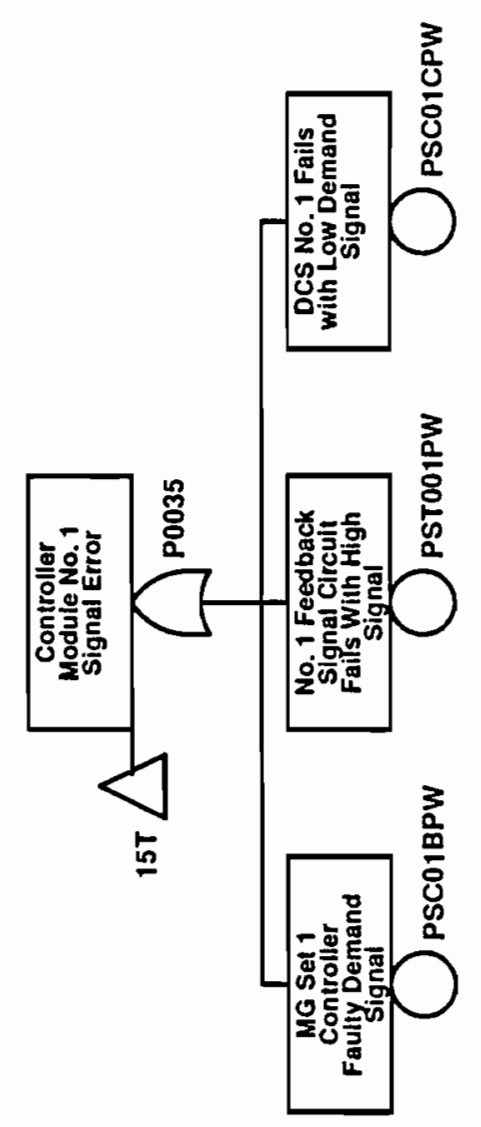

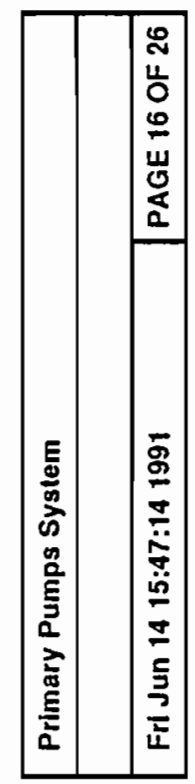

B. 1-71 


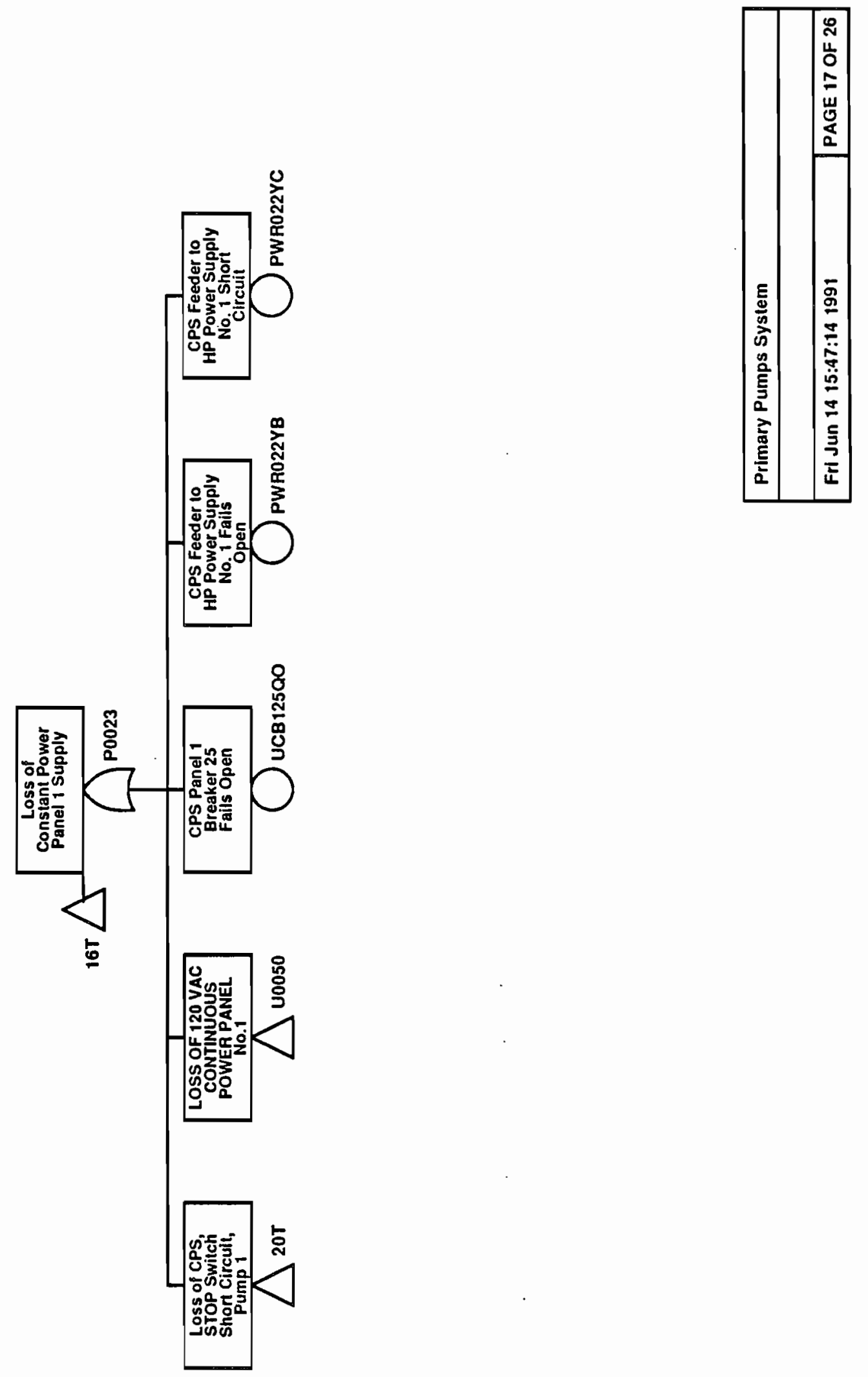

B. 1-72 


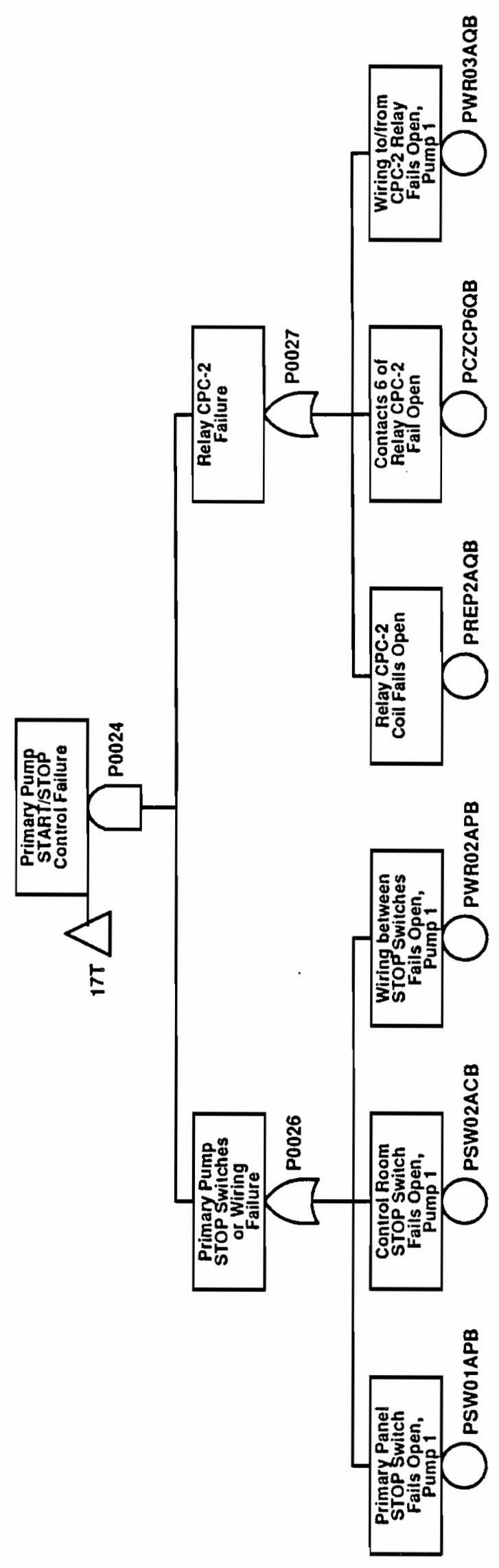

B. 1-73 


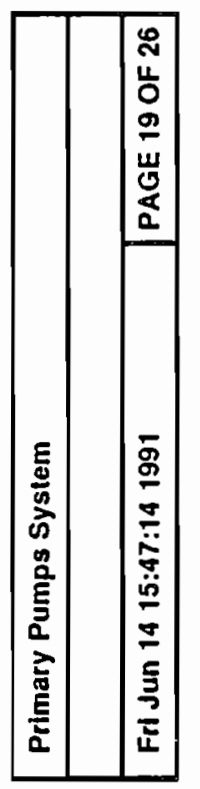

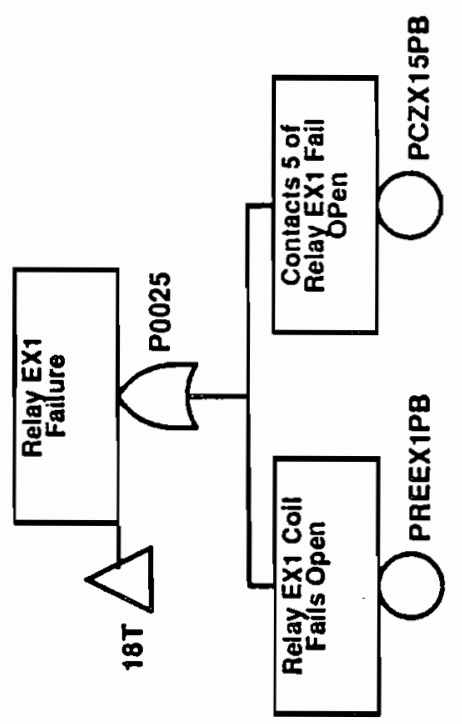

B. 1-74 


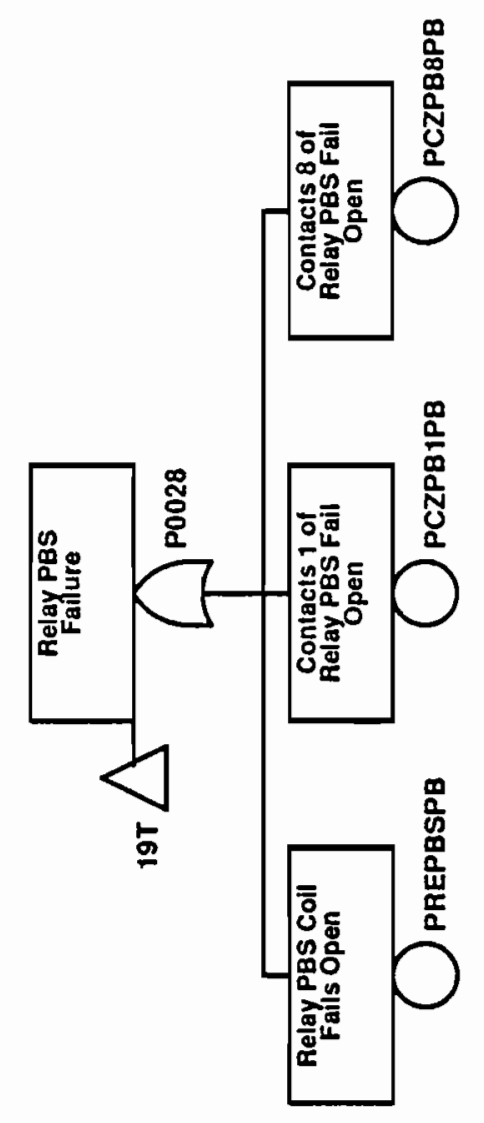

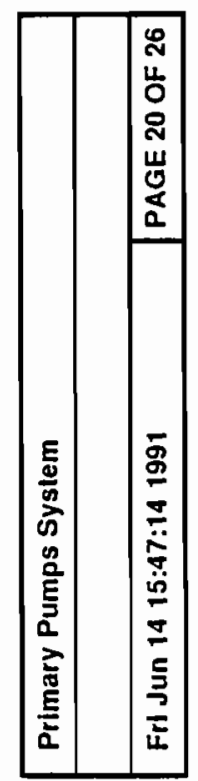

B. 1-75 


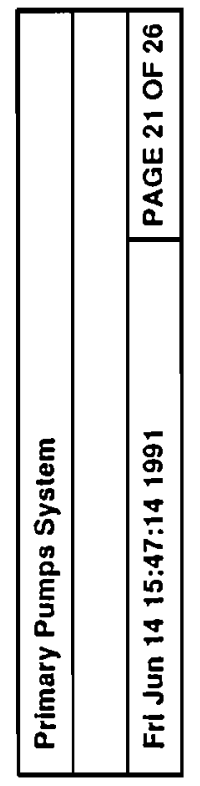

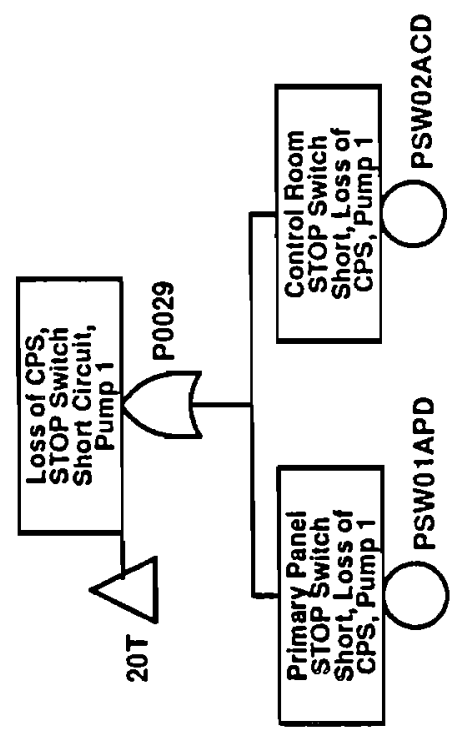

B. 1-76 

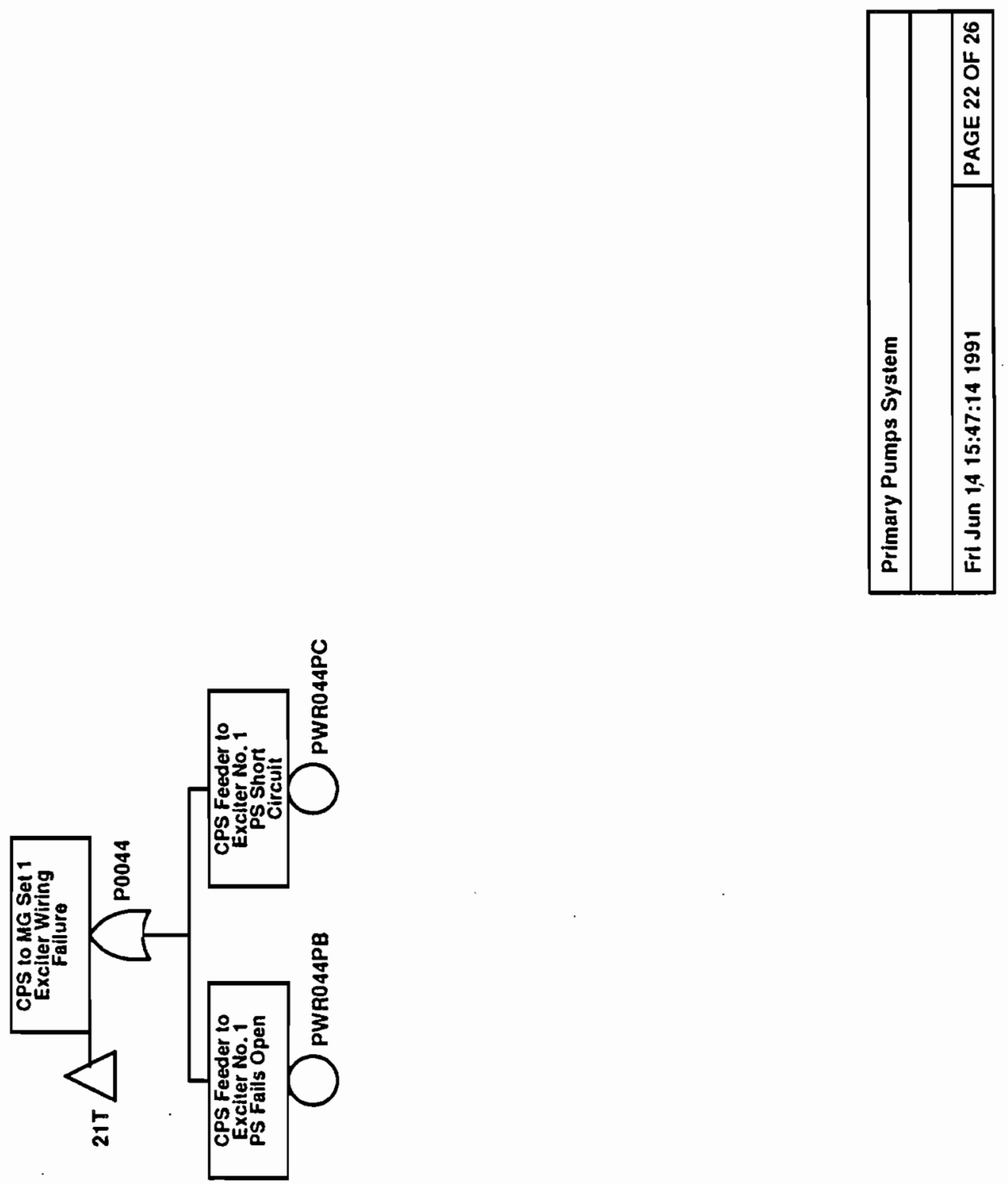

B. 1-77 


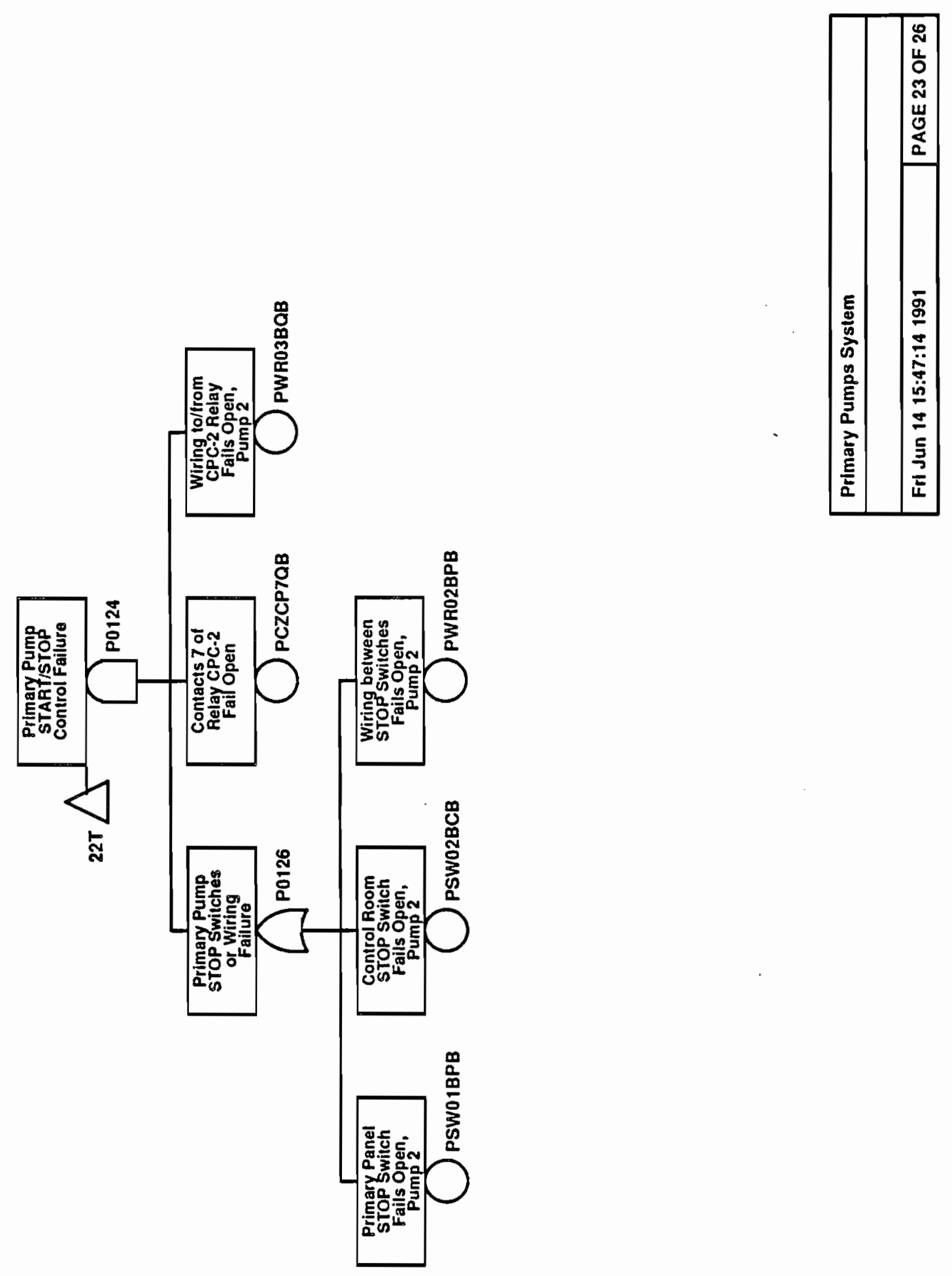

B. 1-78 


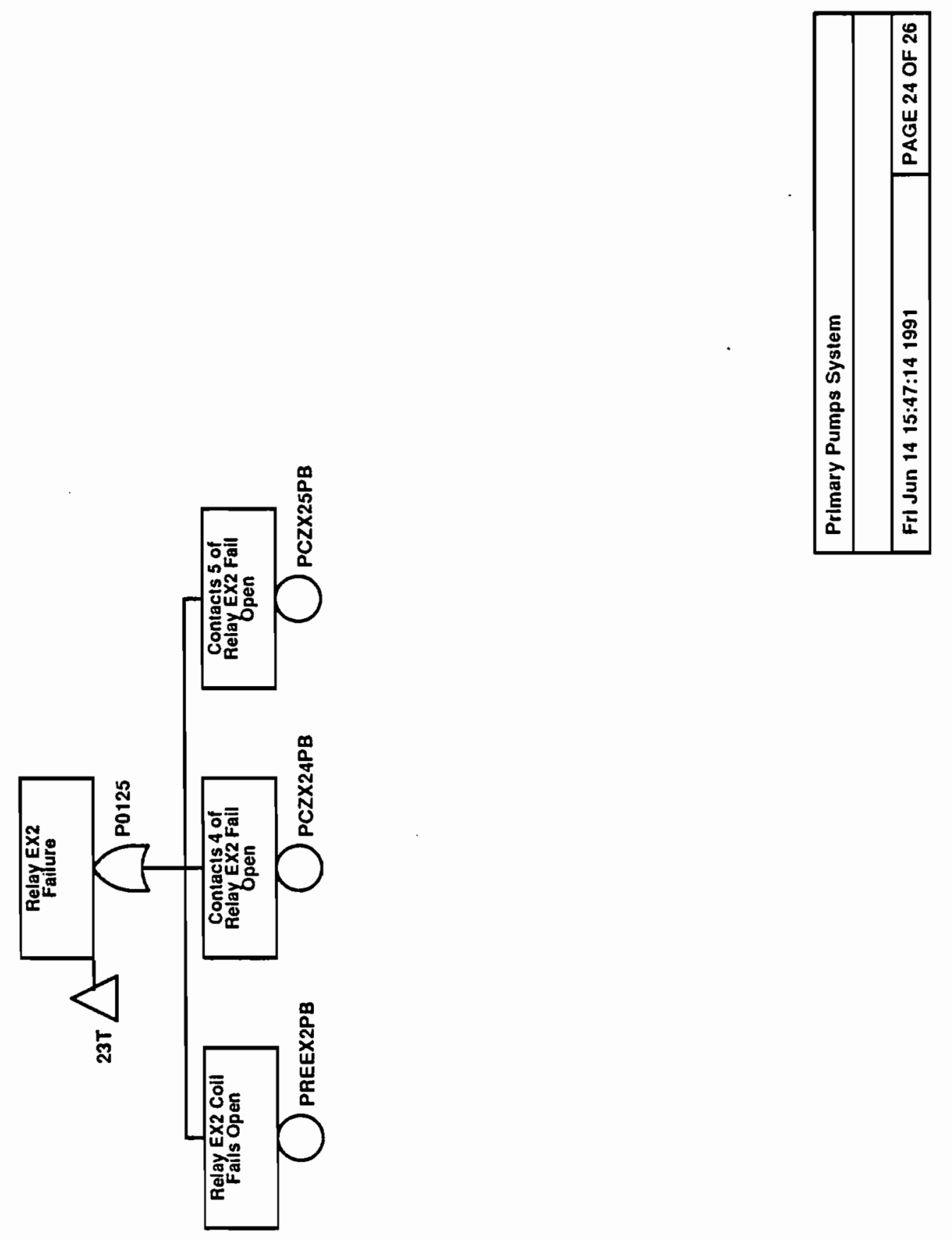

B. 1-79 


\begin{tabular}{|c|c|}
\hline & 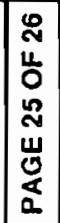 \\
\hline 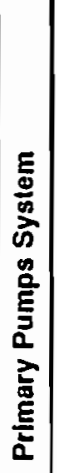 & 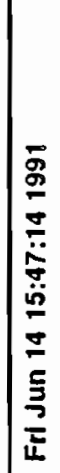 \\
\hline
\end{tabular}

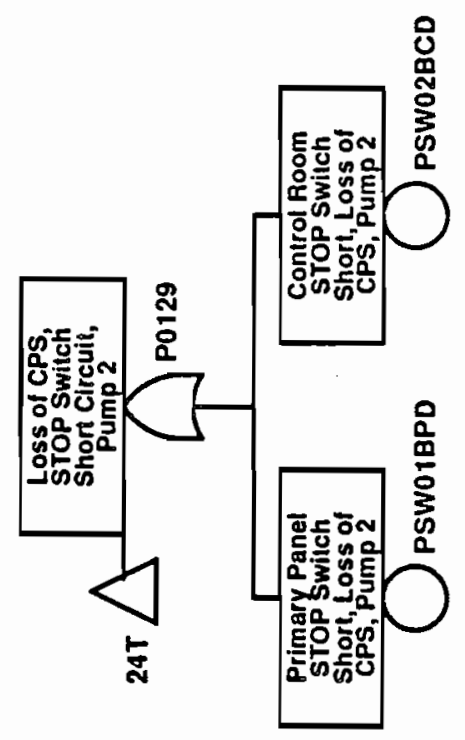

B. $1-80$ 


\begin{tabular}{|c|c|}
\hline & 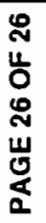 \\
\hline 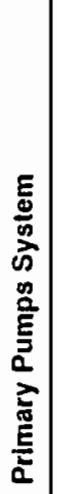 & 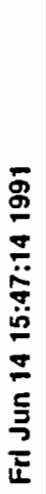 \\
\hline
\end{tabular}

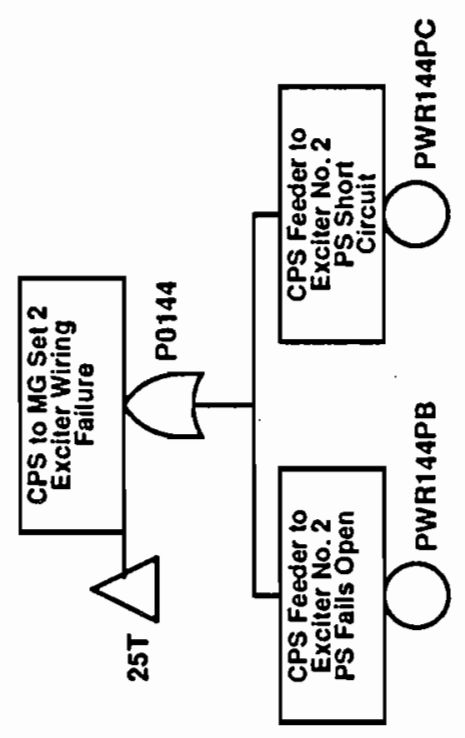

B. 1-81 


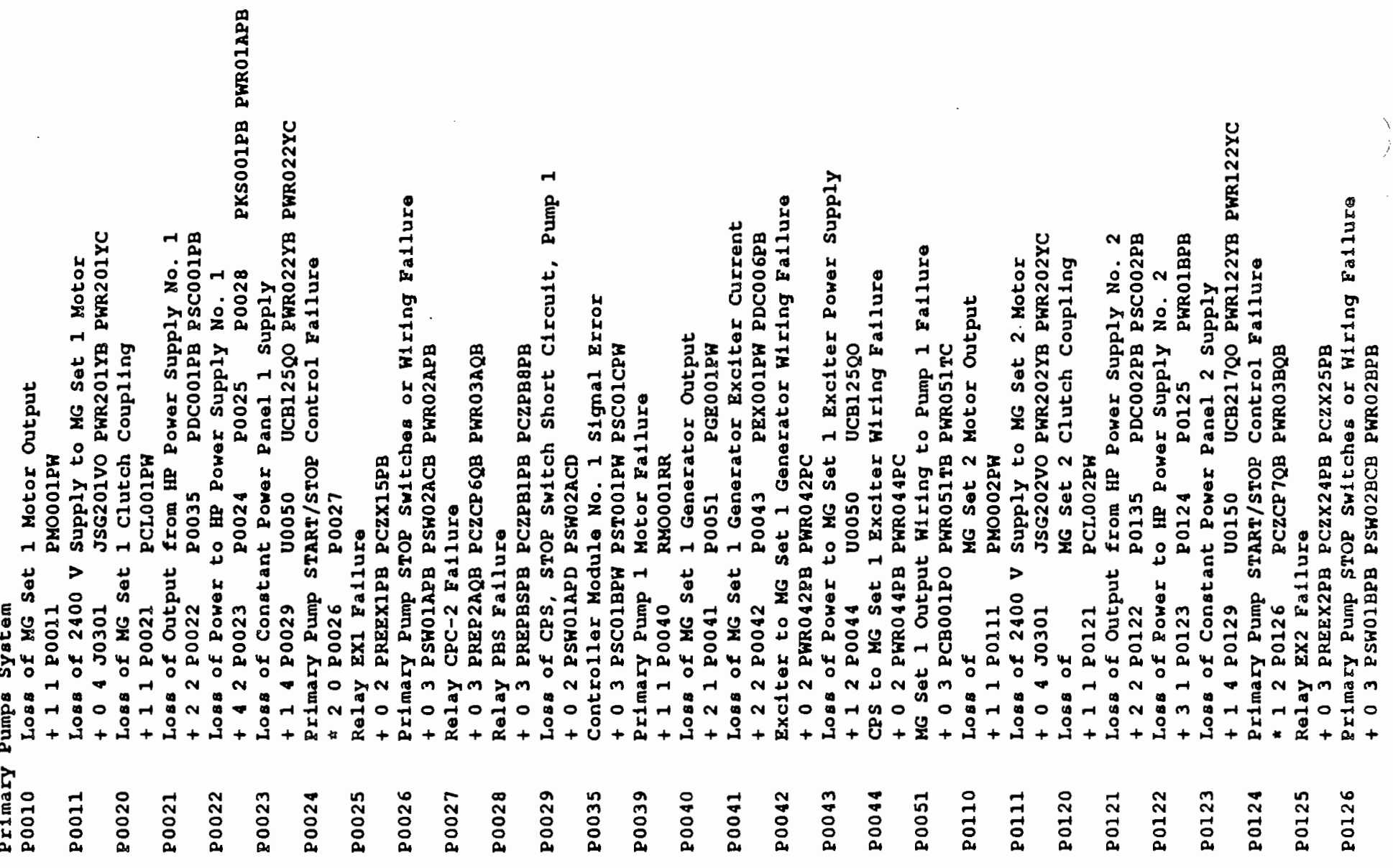

B. 1-82 


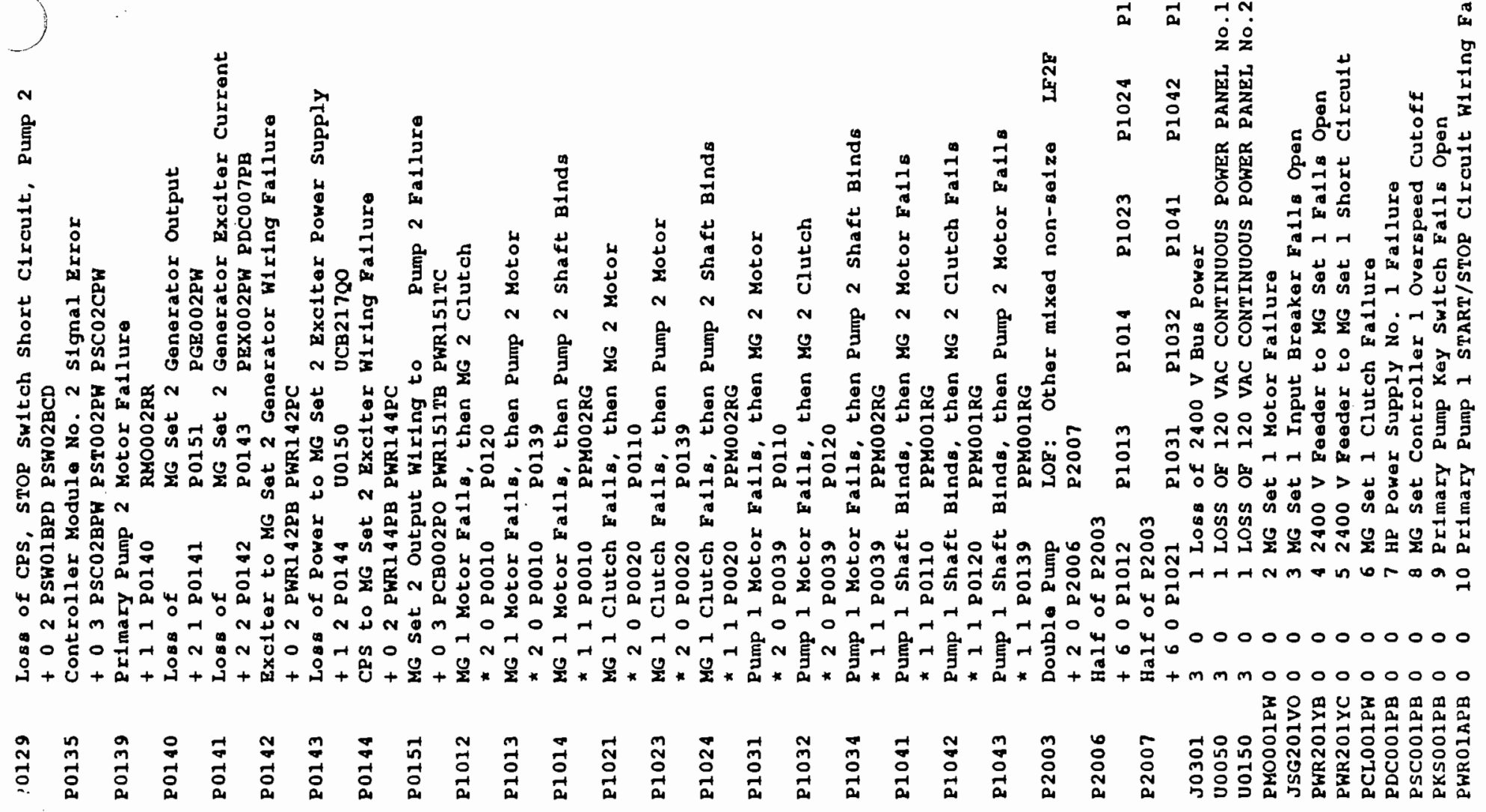




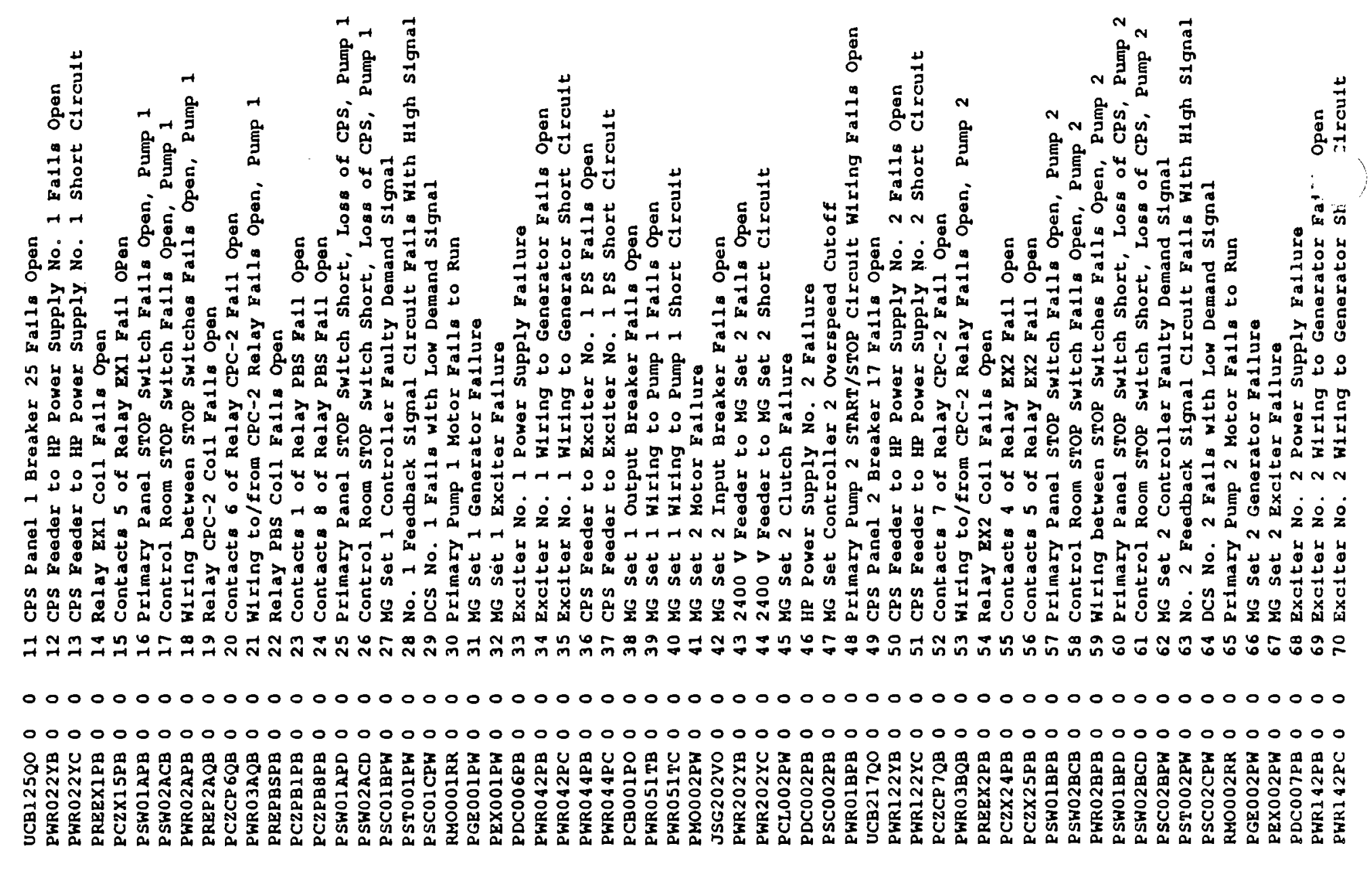

B. 1- 84 


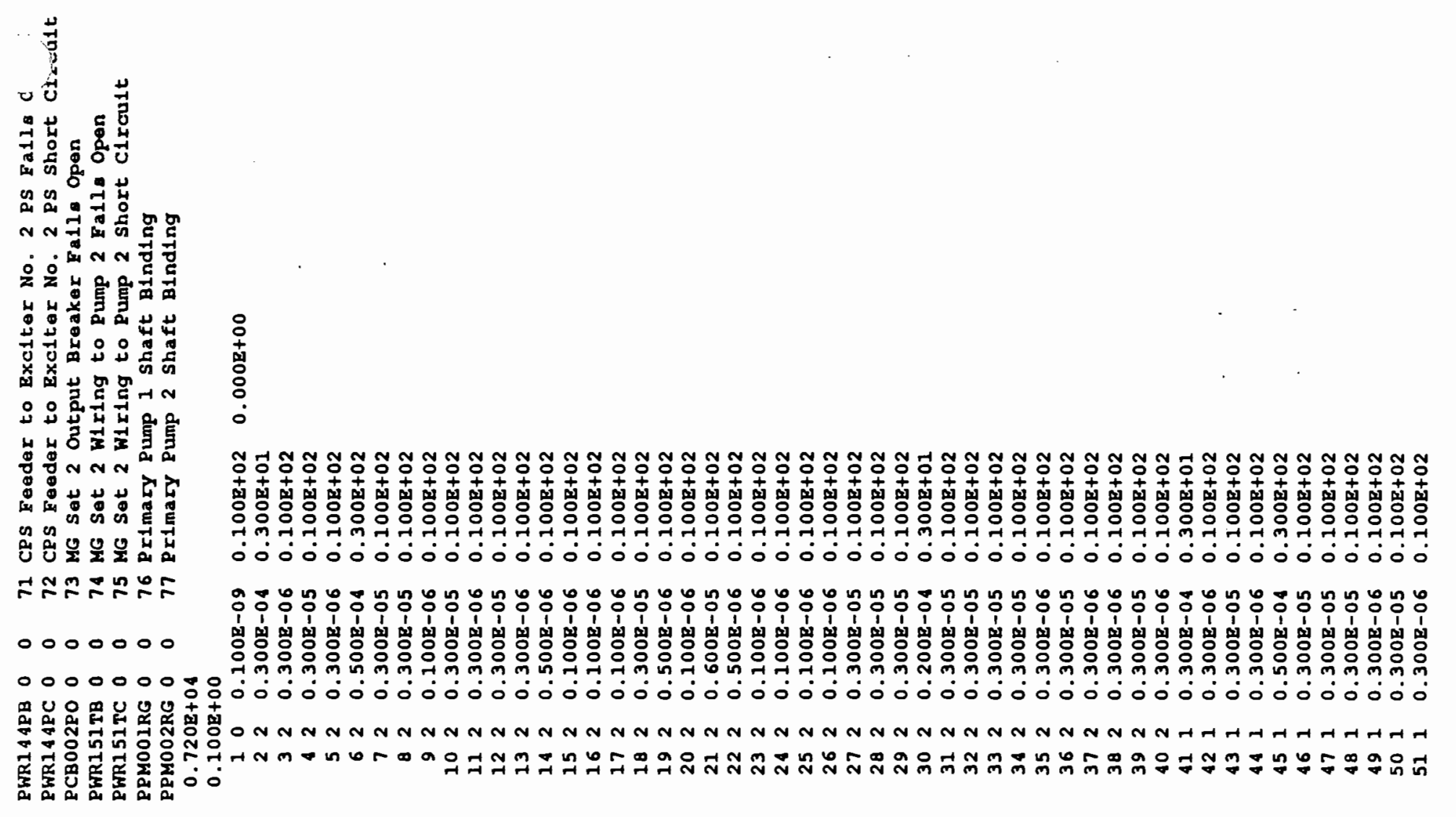

B. 1-85 


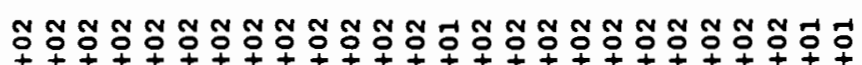

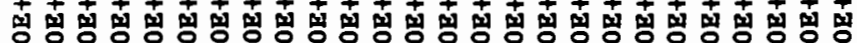

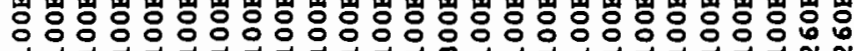

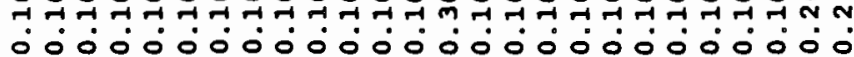

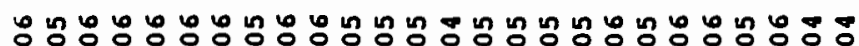
i i i i i i i i i i i i i i i i i i i i

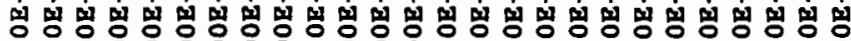

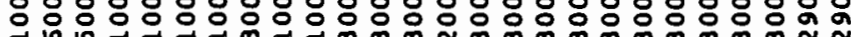

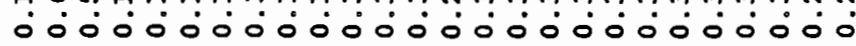

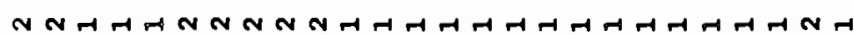

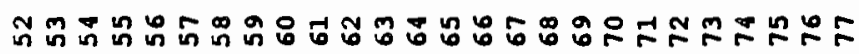




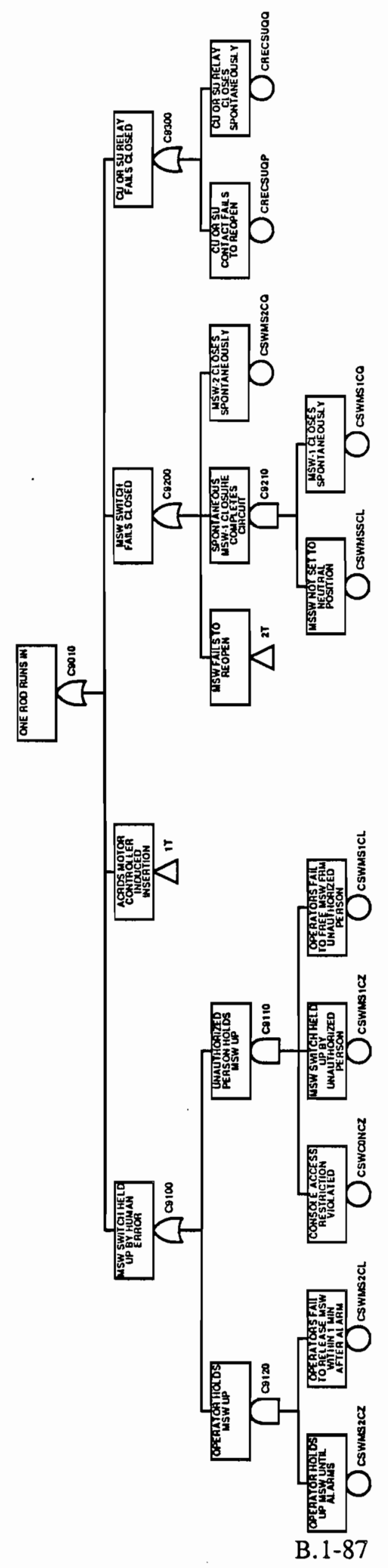



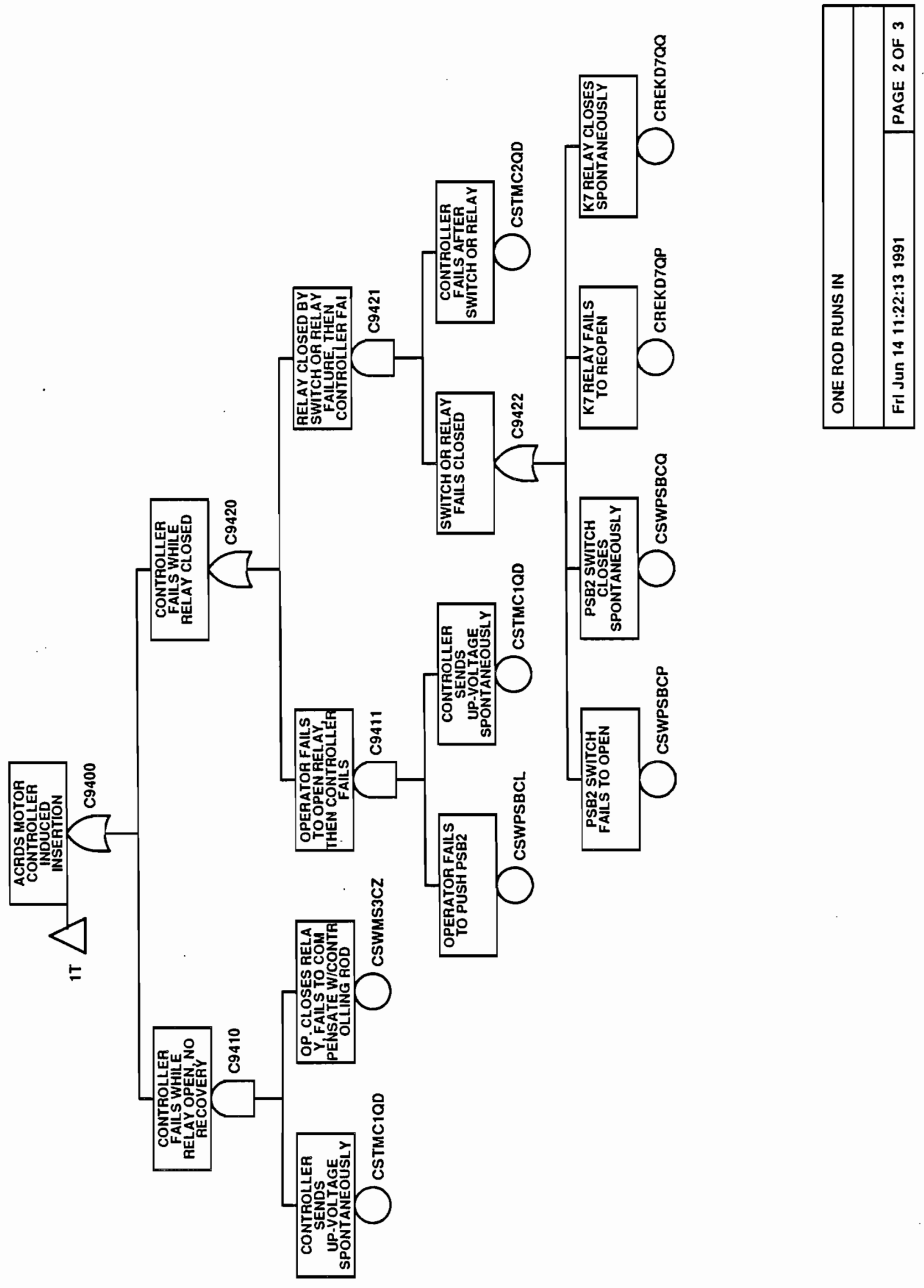

\section{B. $1-88$}




\begin{tabular}{|c|c|}
\hline & 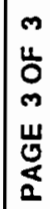 \\
\hline 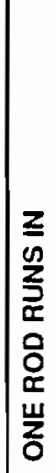 & | \\
\hline
\end{tabular}

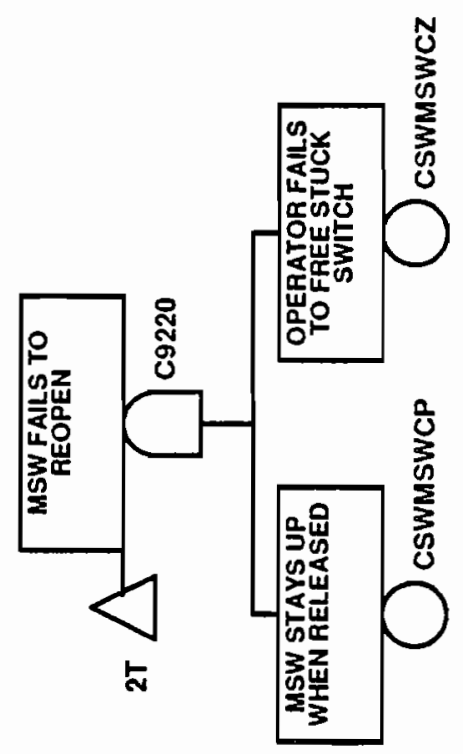

B. 1-89 


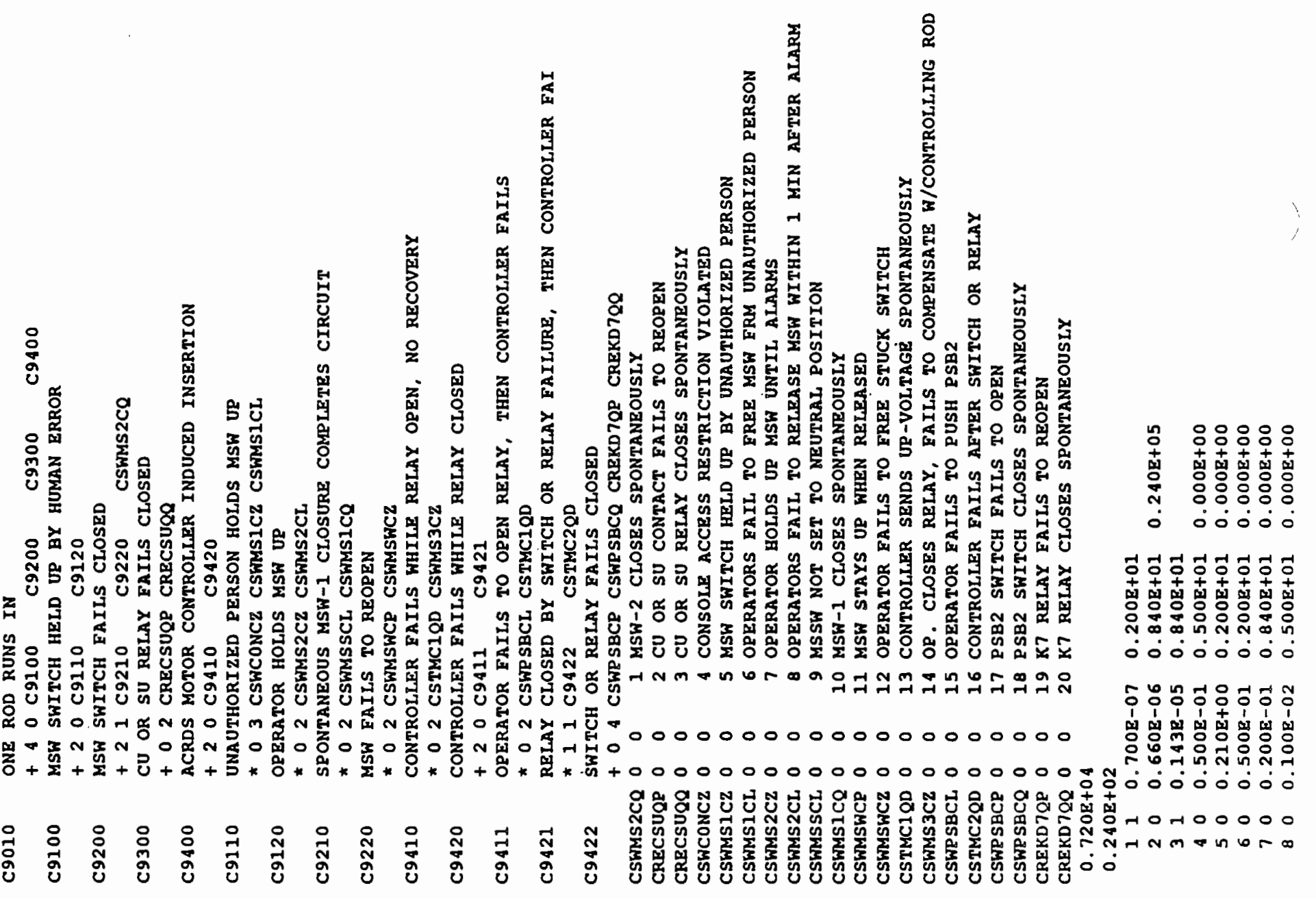

B. $1-90$ 


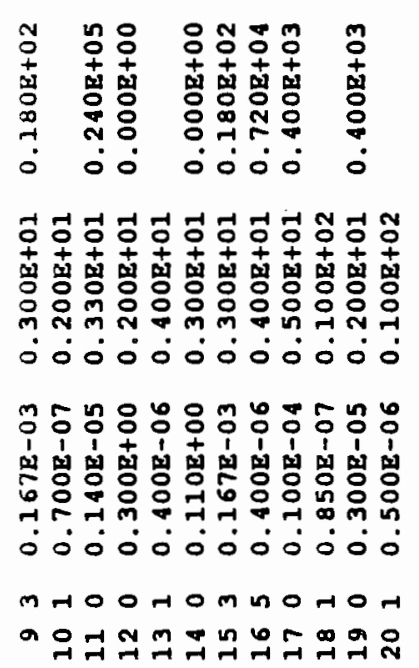

B. 1-91 

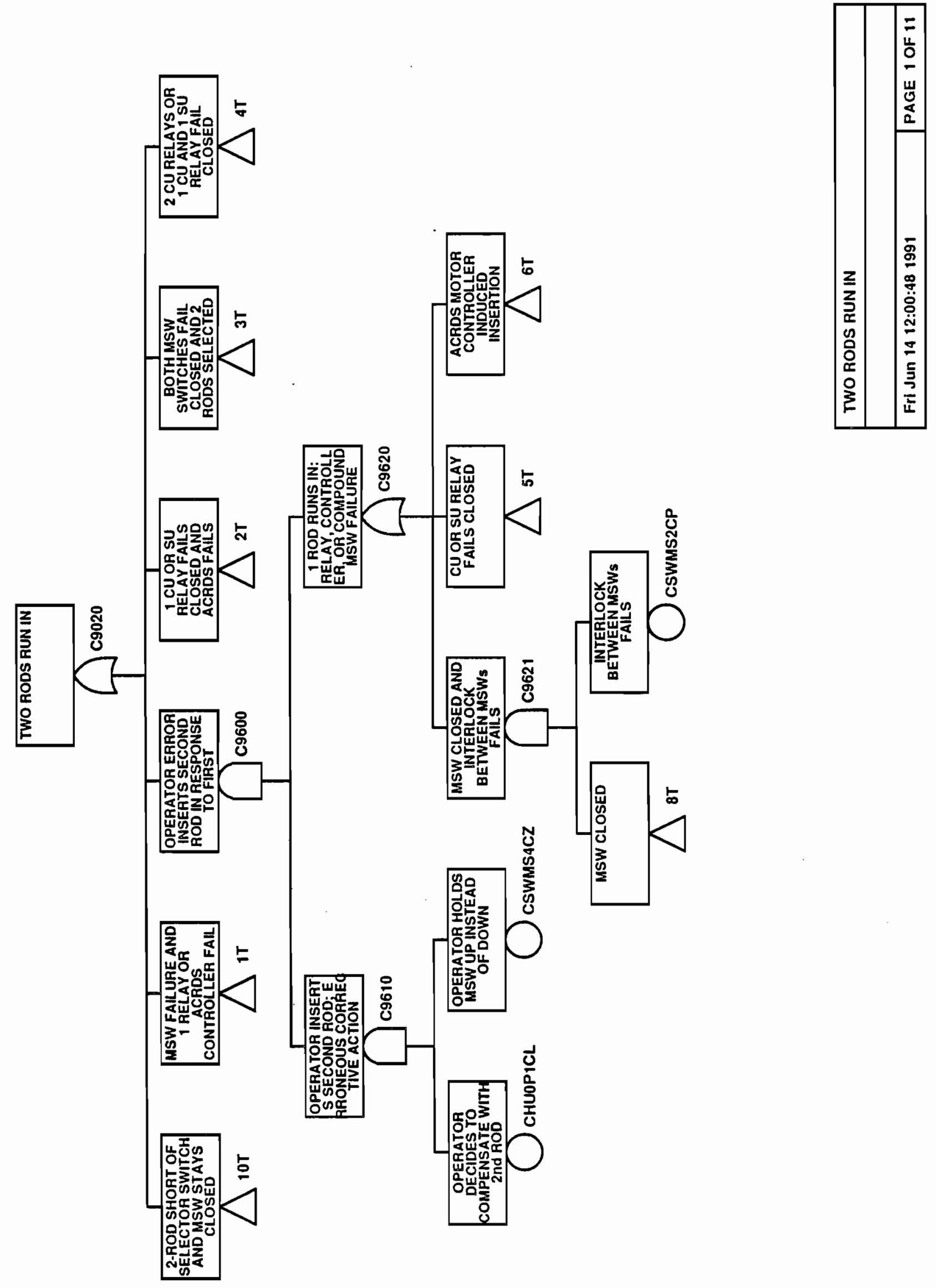

B. 1-92 


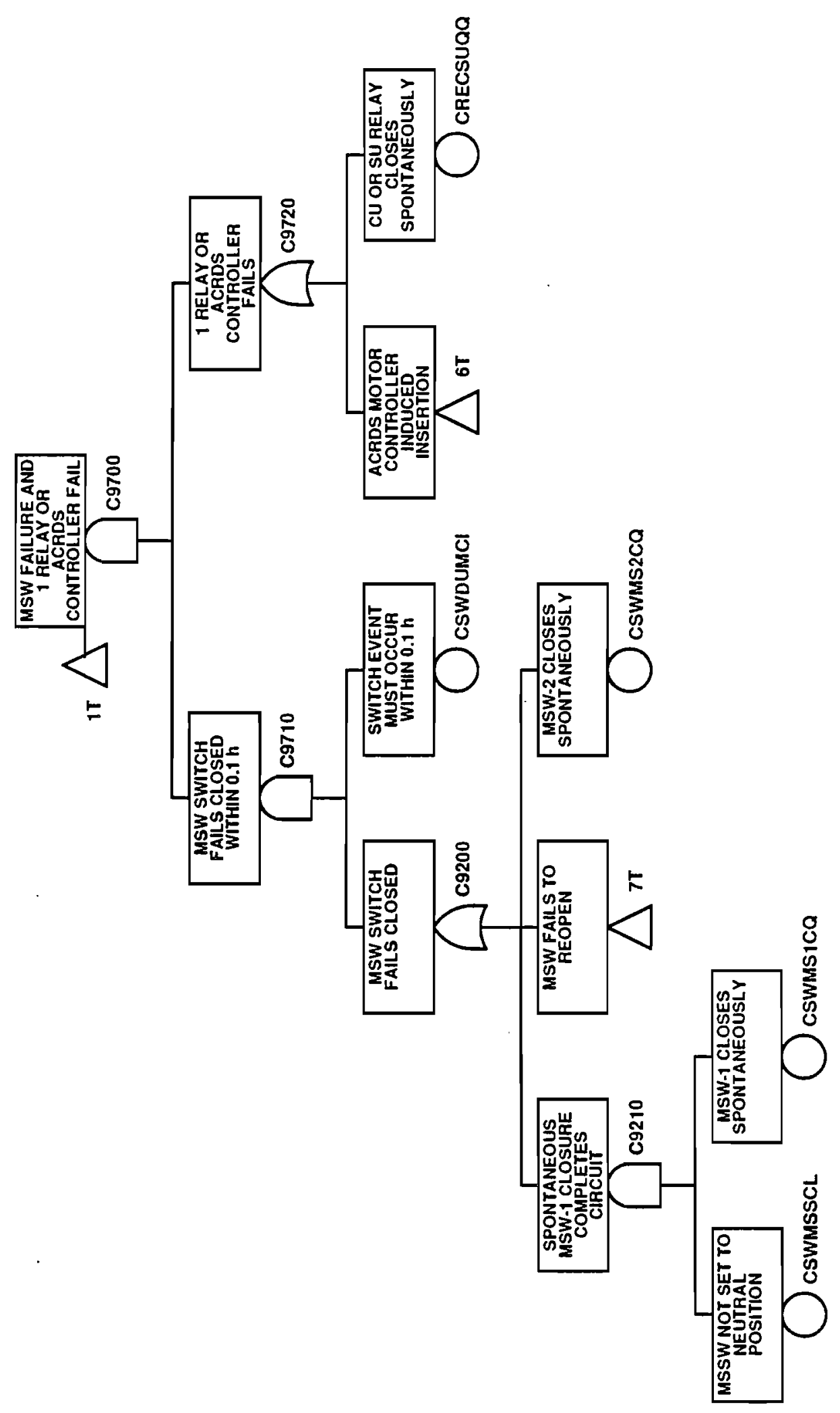

B. 1-93 


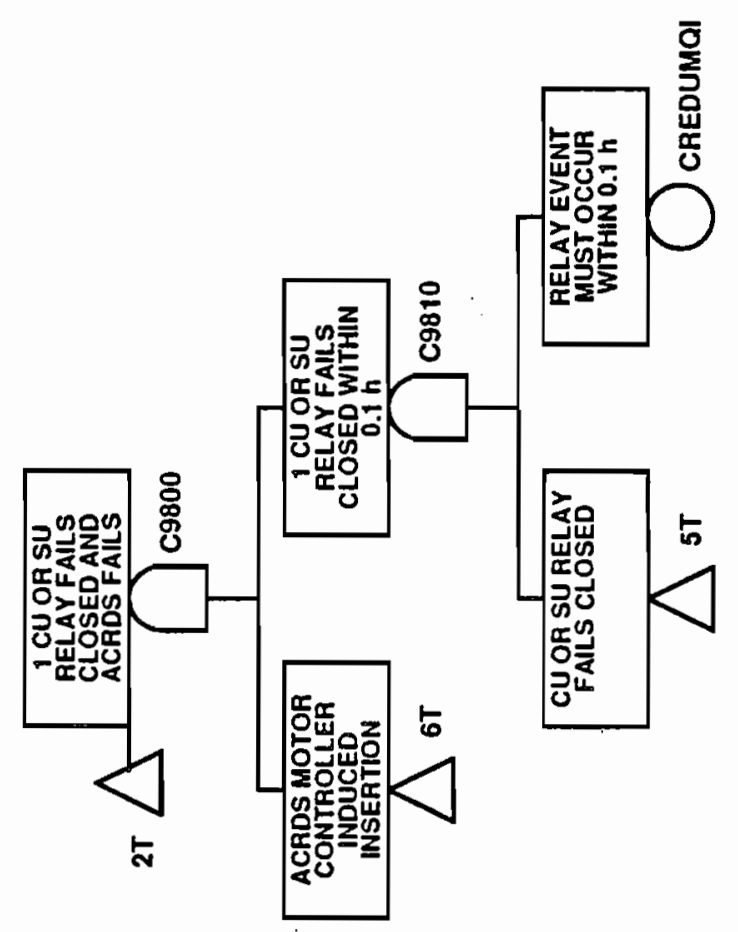

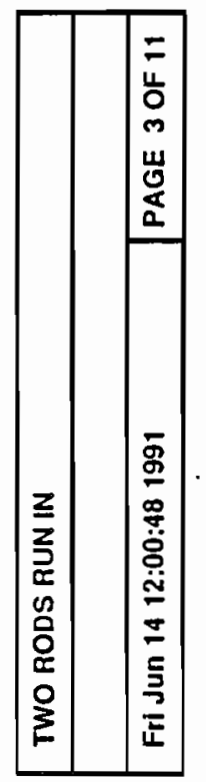

B. 1-94 

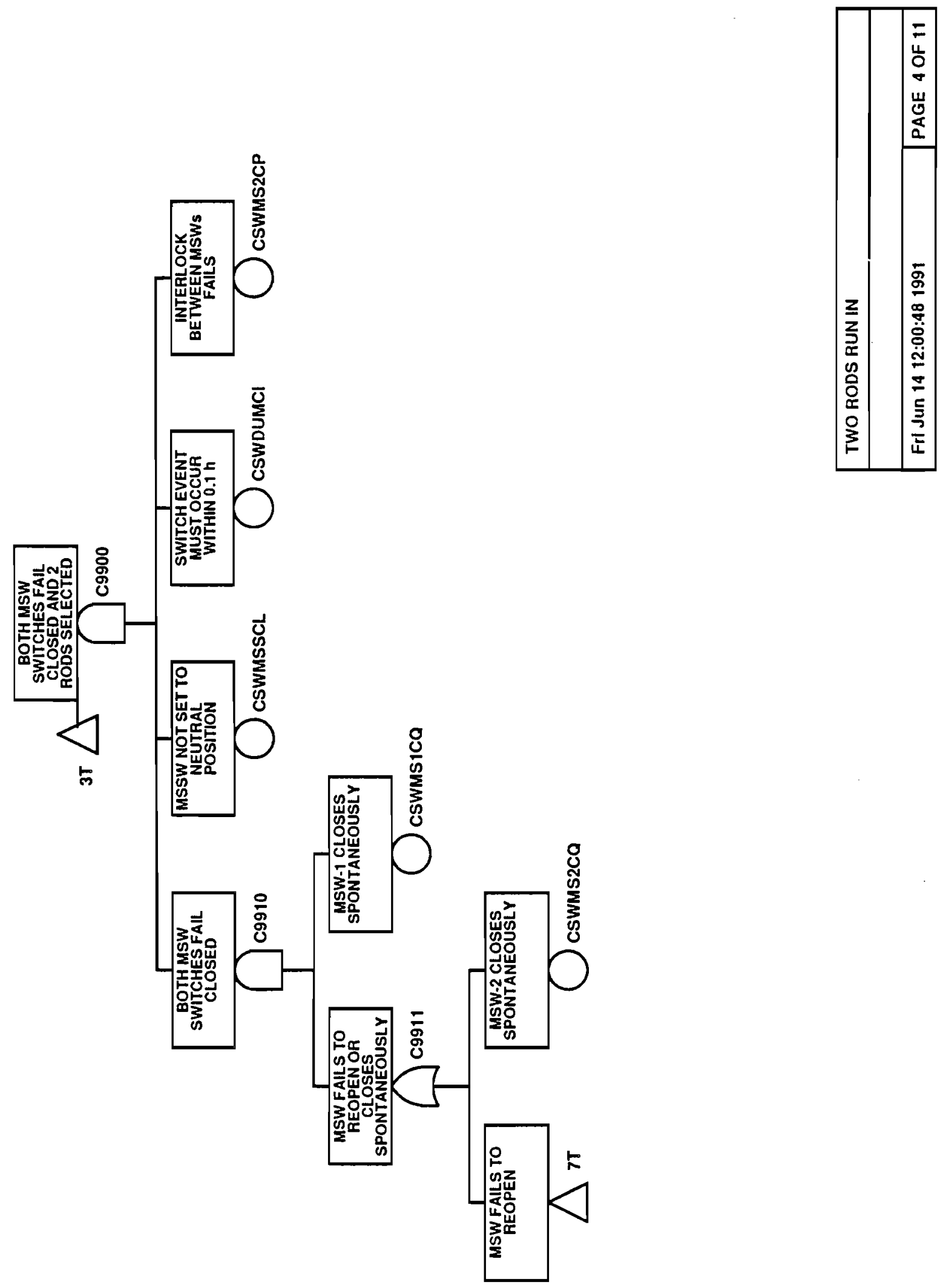

B. 1-95 


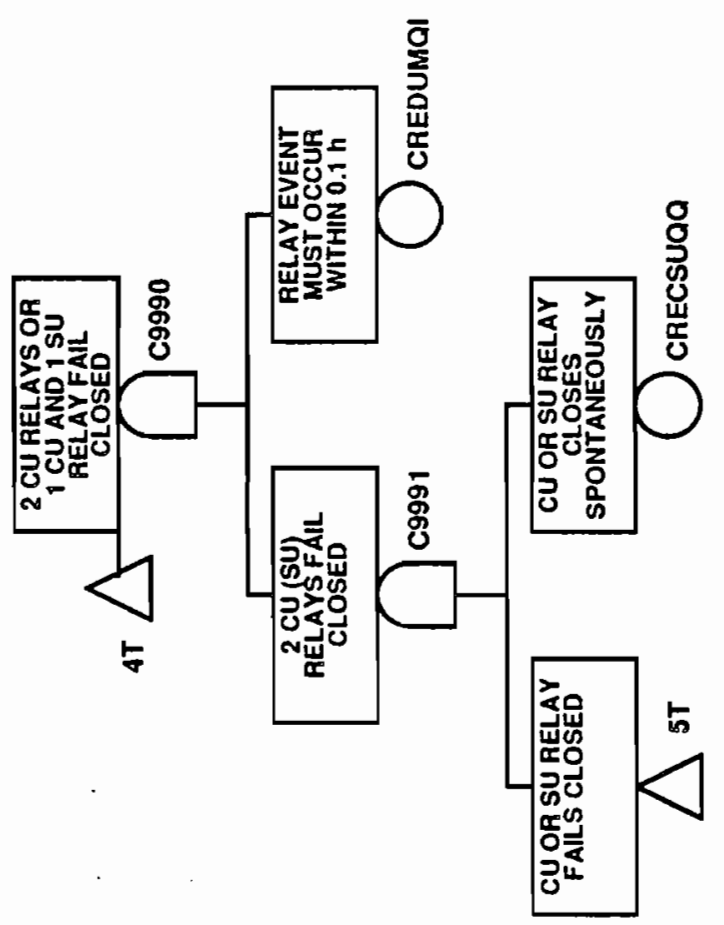

B. 1-96 

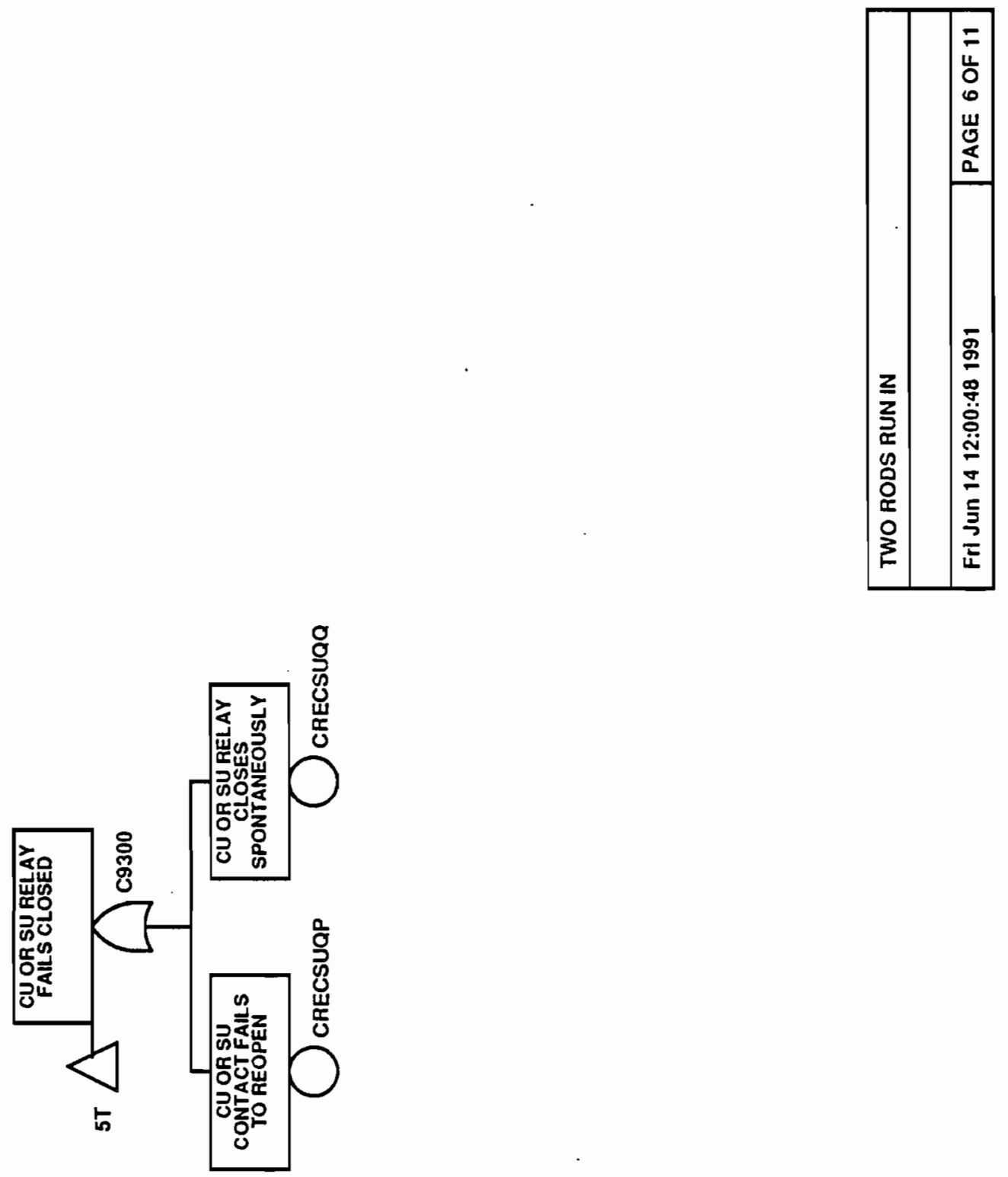

B. 1-97 


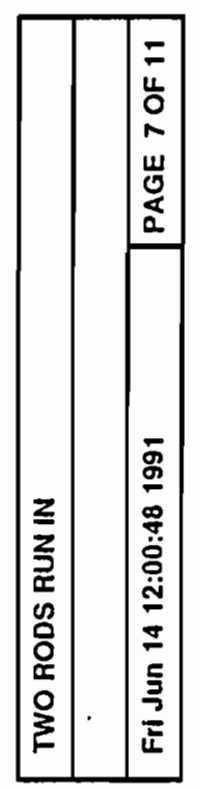

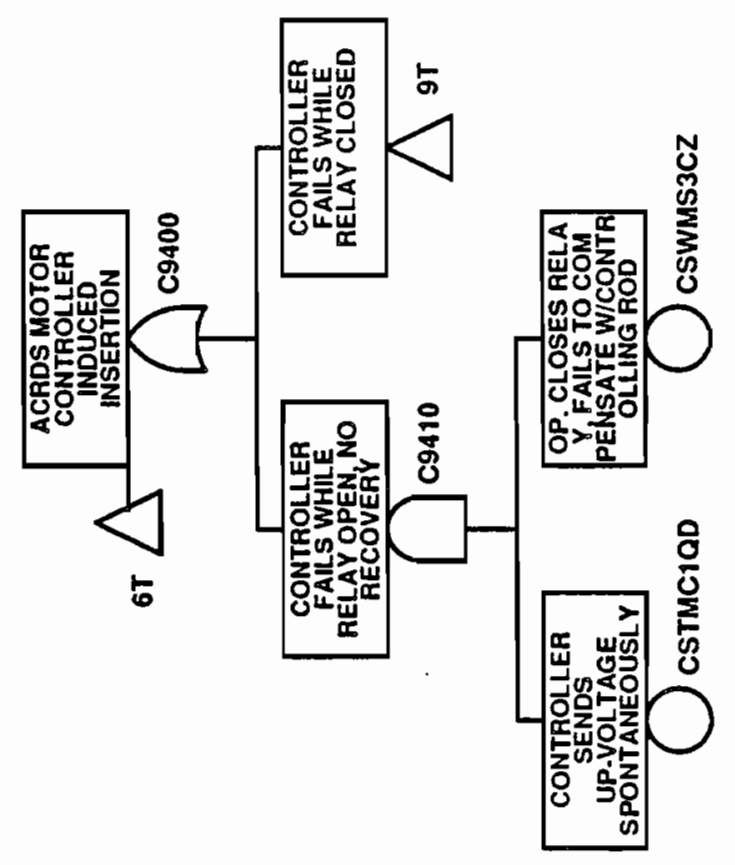

B. 1-98 


|

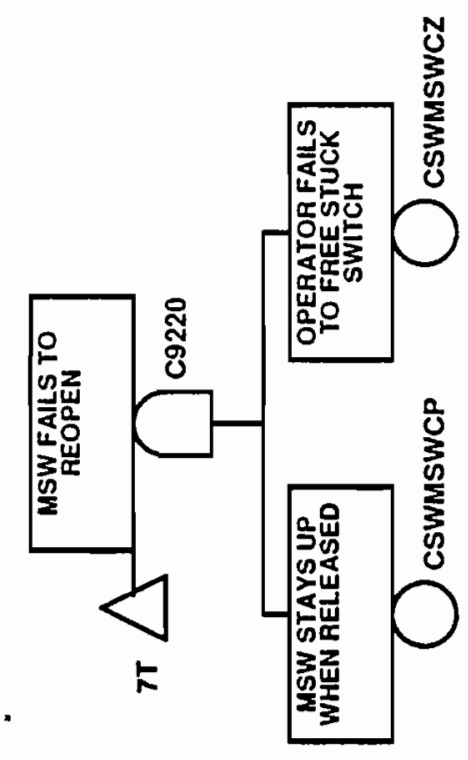

B. 1-99 

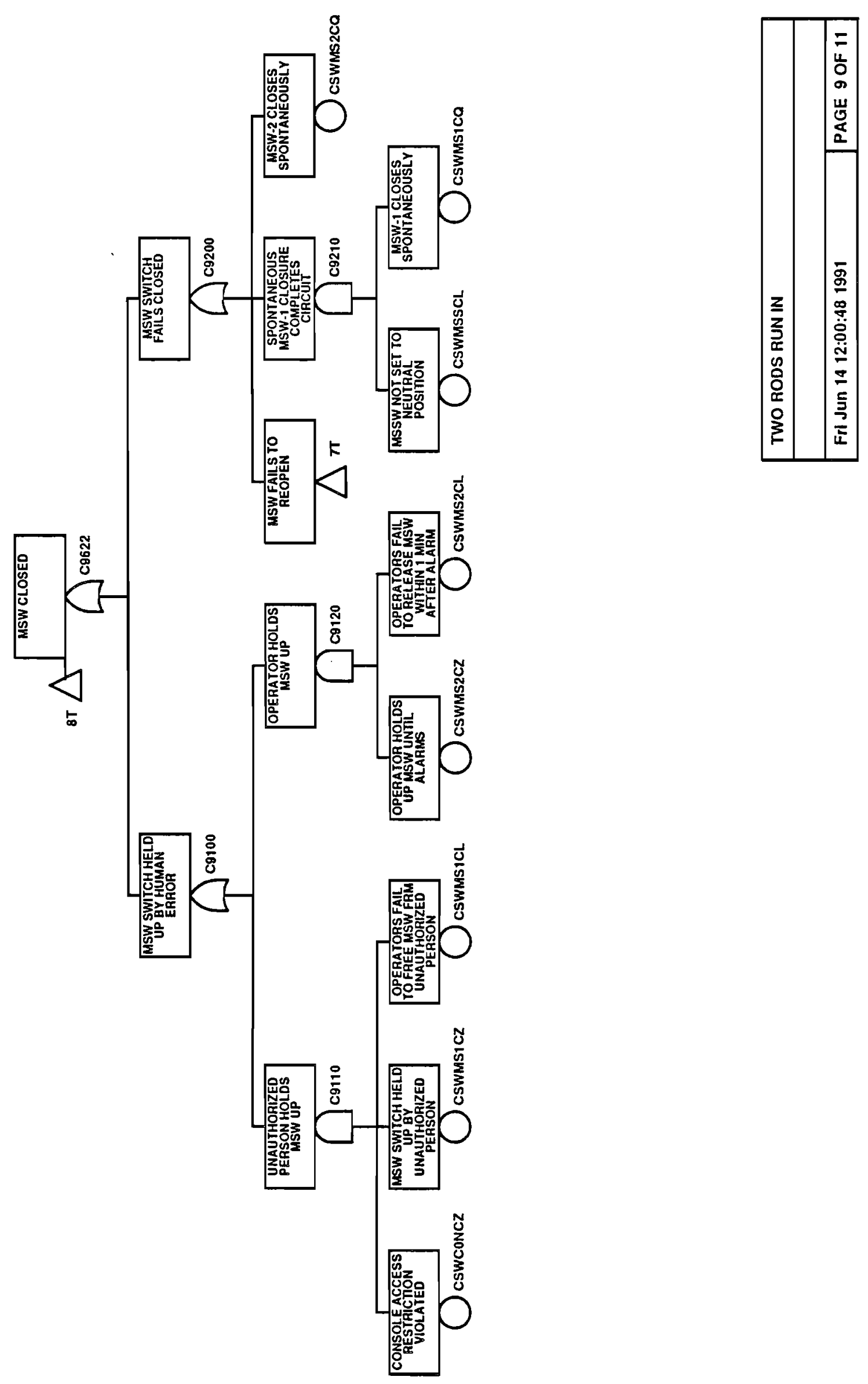

B. $1-100$ 


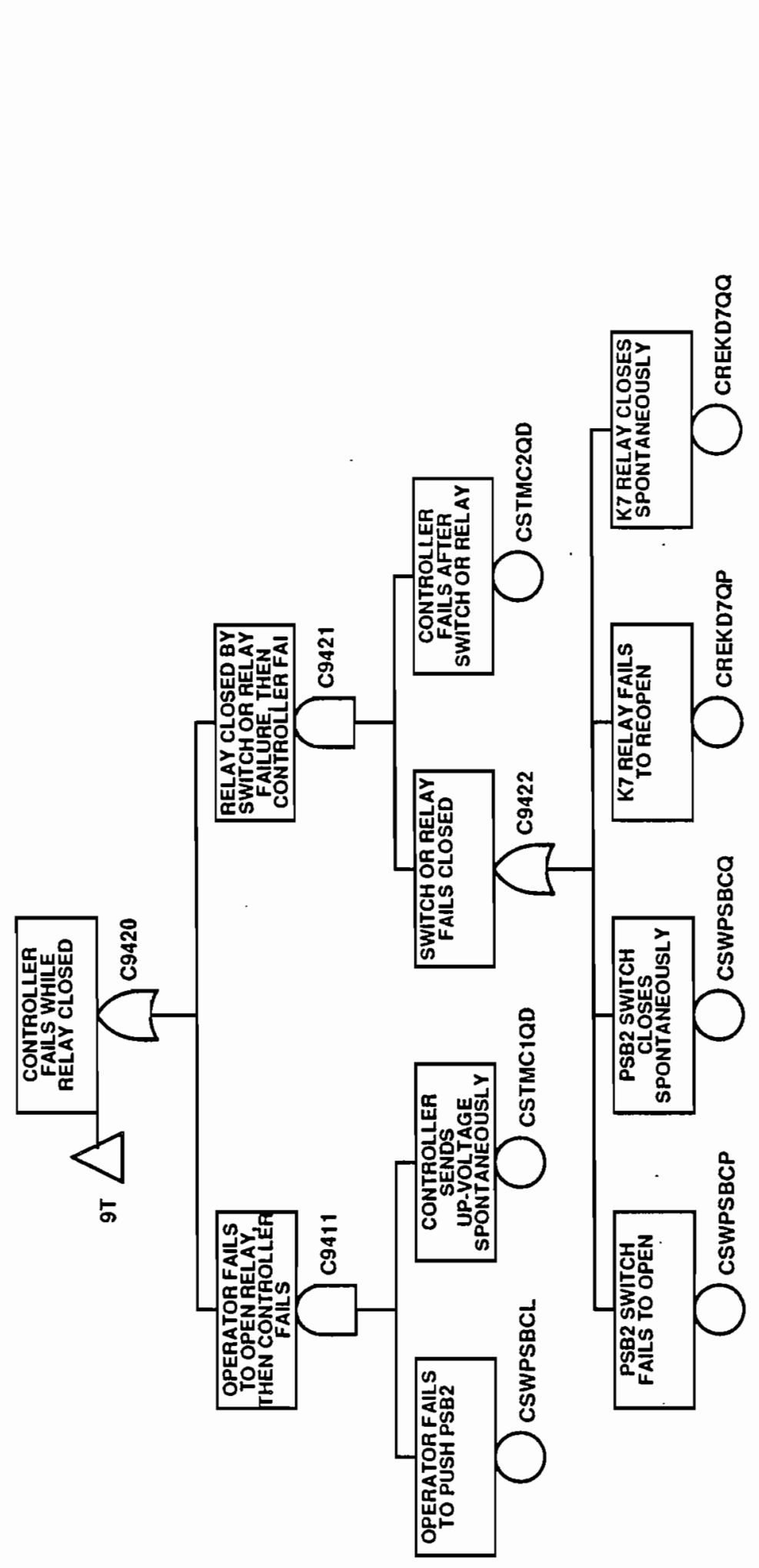

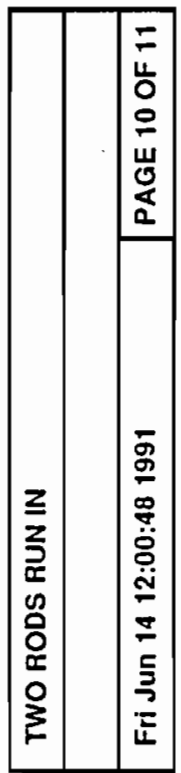

B. 1-101 


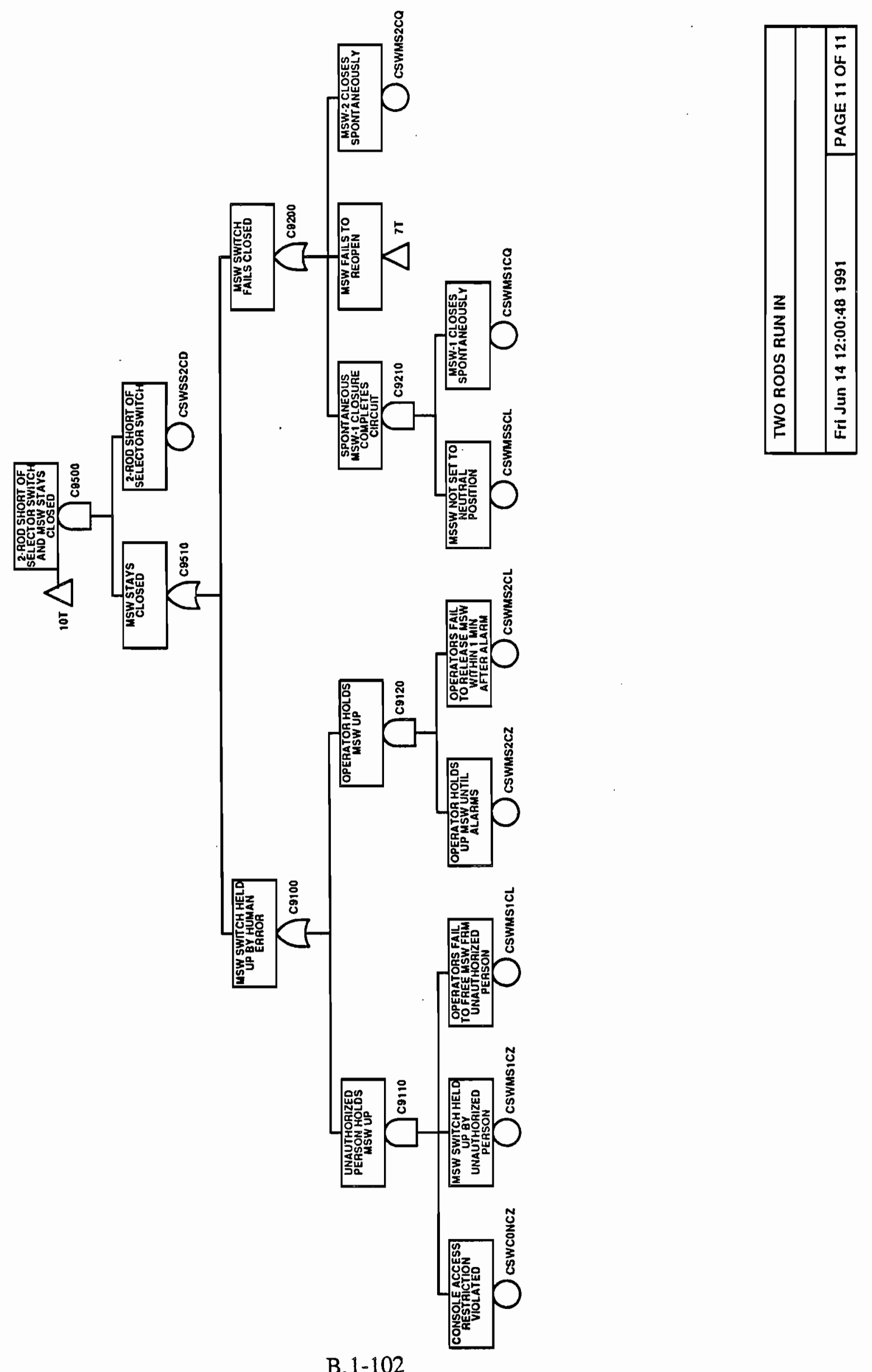




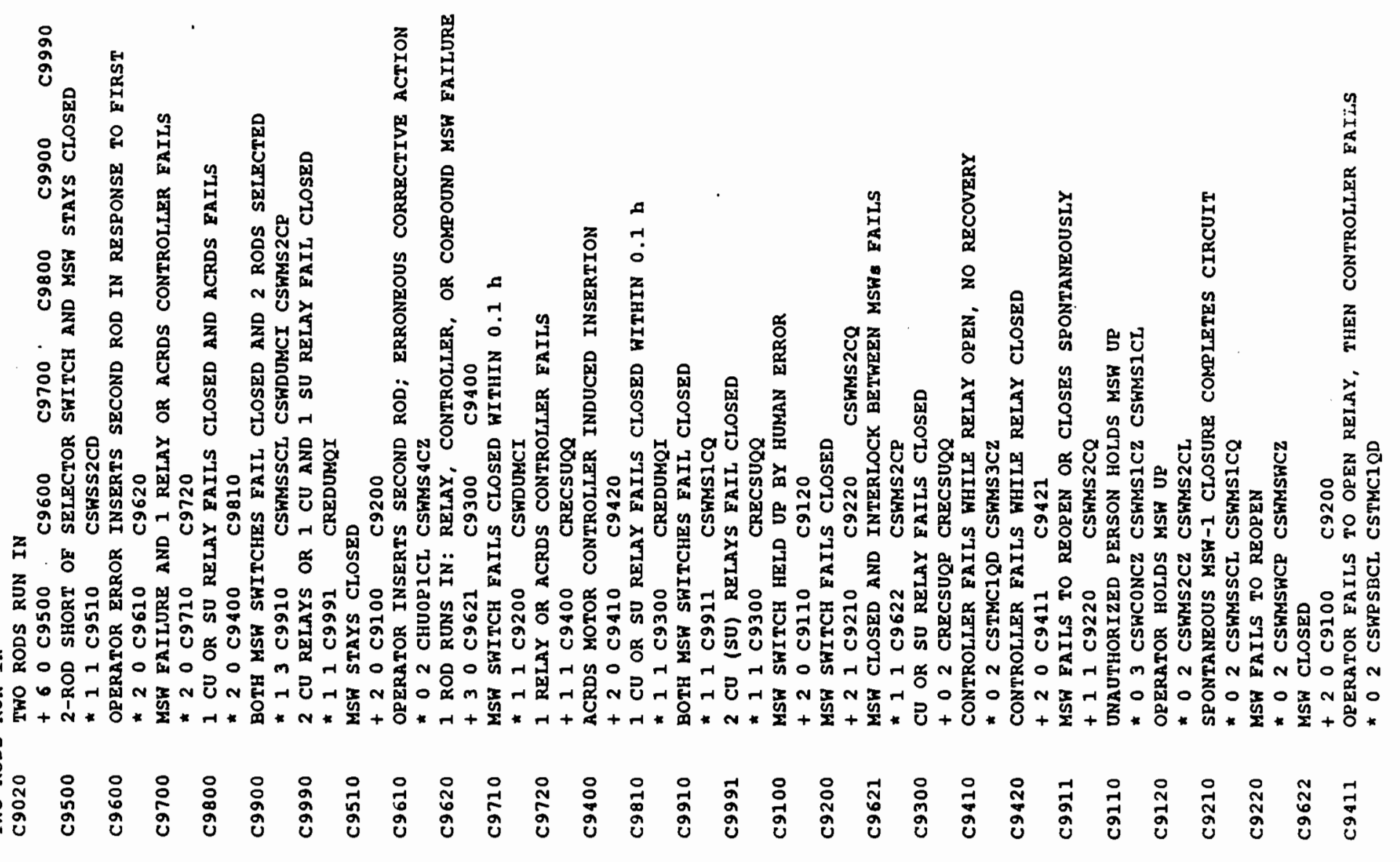

B. 1-103 


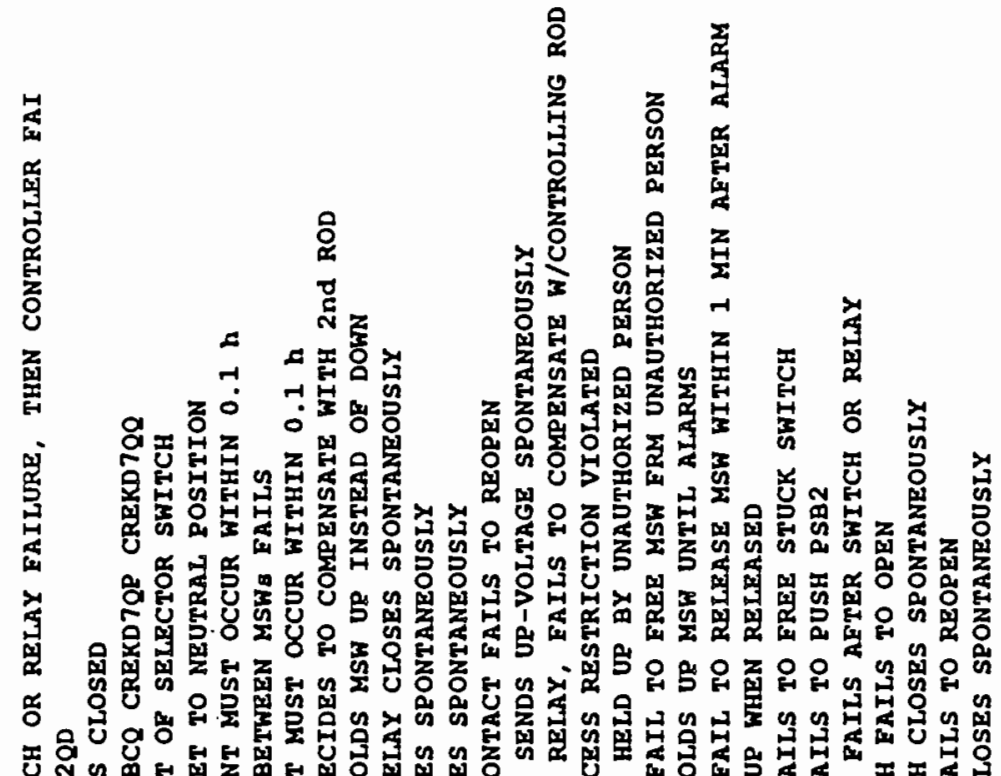

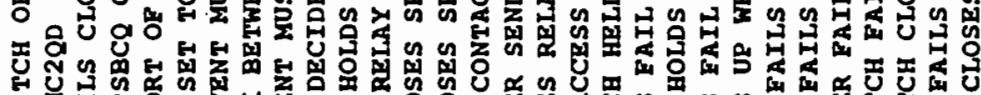

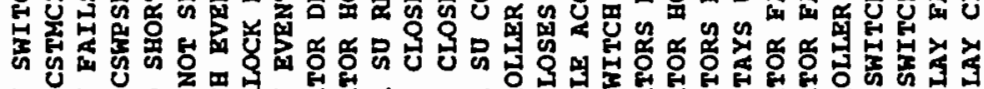

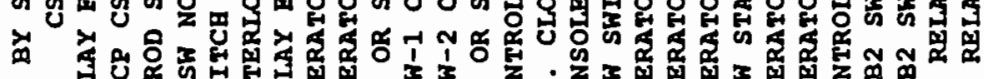

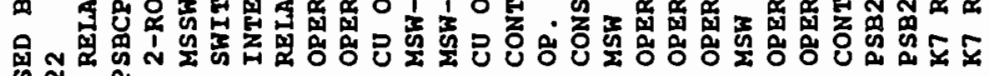

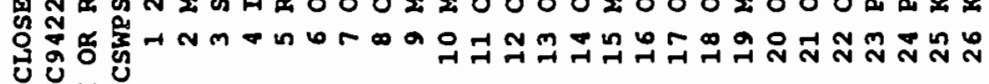

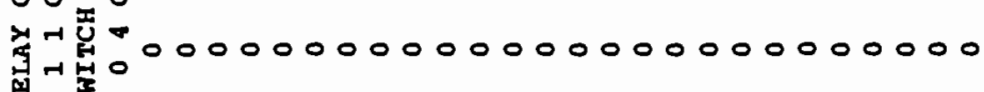
* *

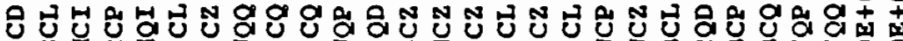

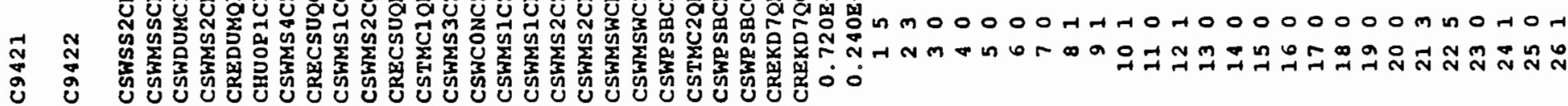

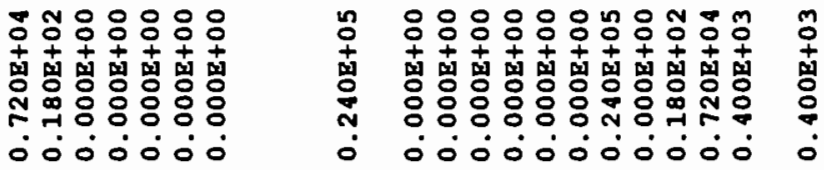

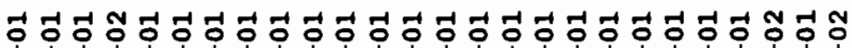

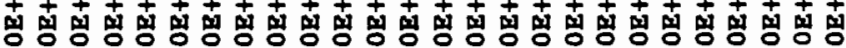

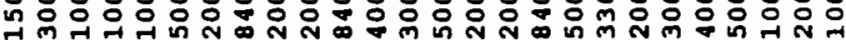

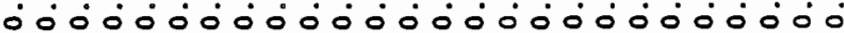

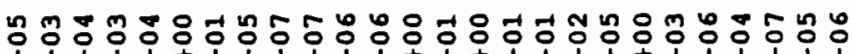

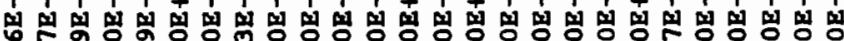

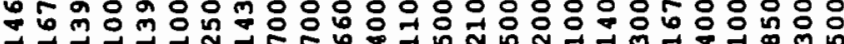

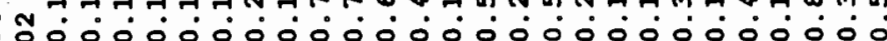




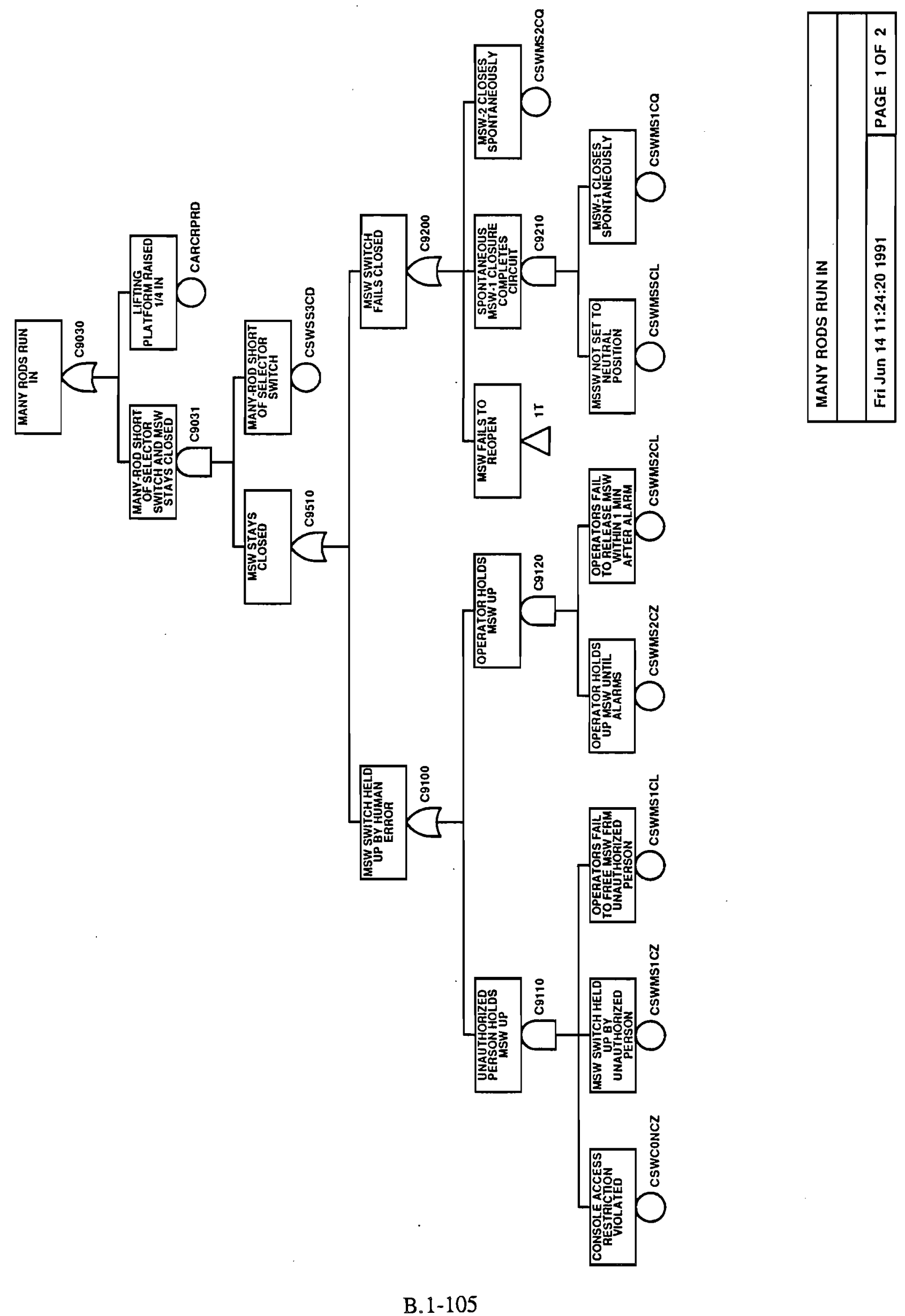




\begin{tabular}{|c|c|}
\hline & 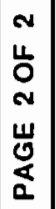 \\
\hline 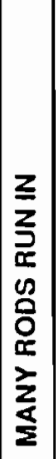 & 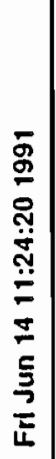 \\
\hline
\end{tabular}

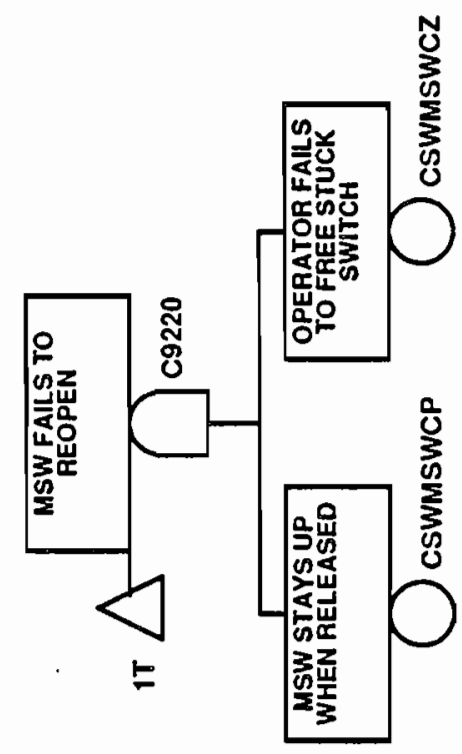

B. 1-106 


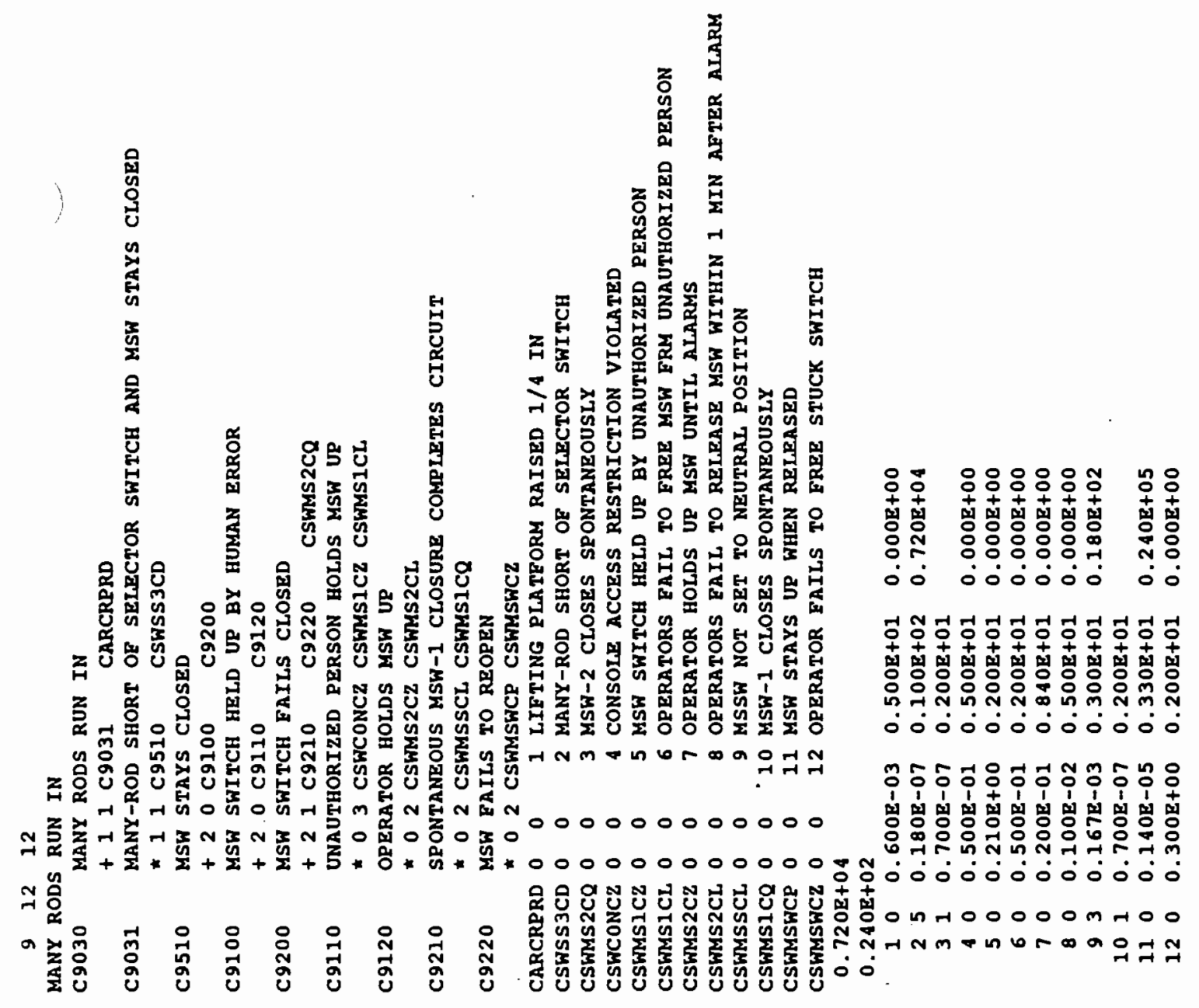

B. 1-107 


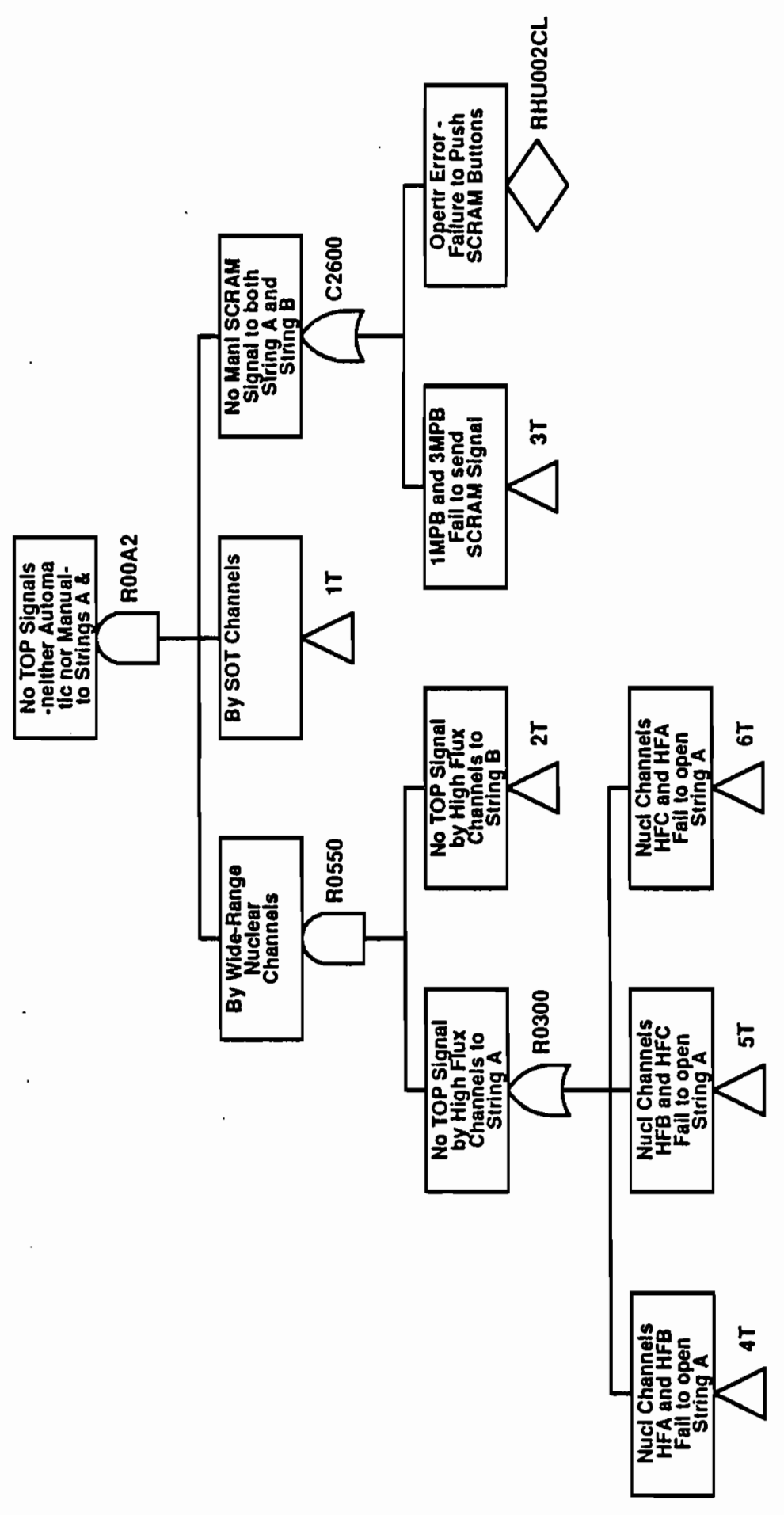




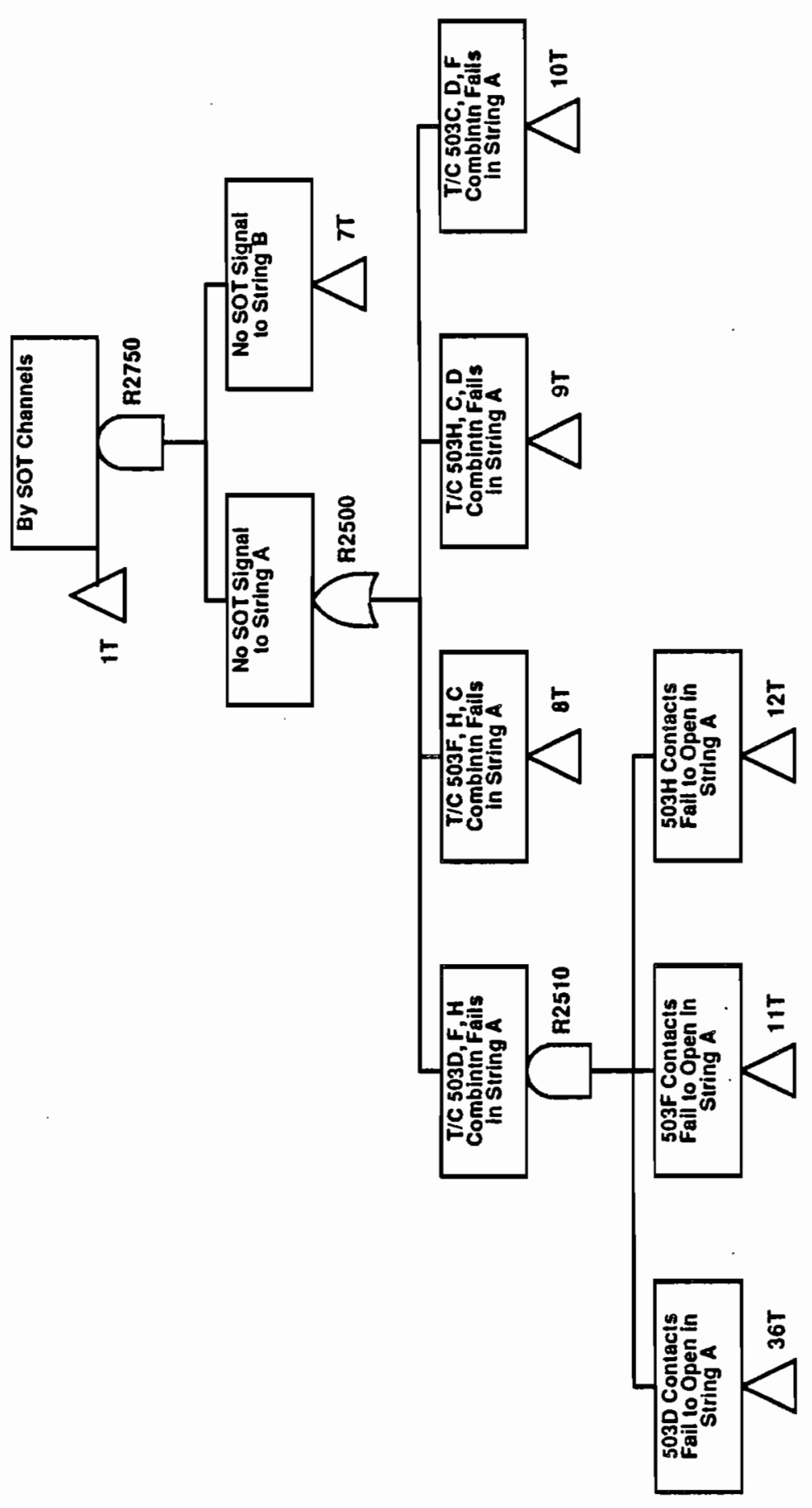

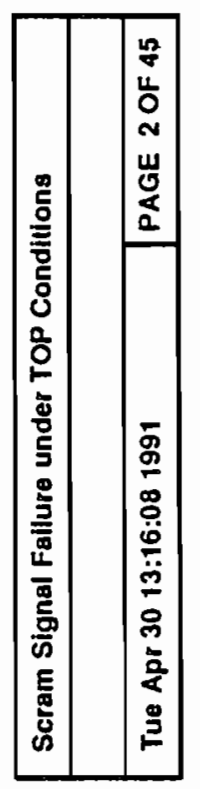




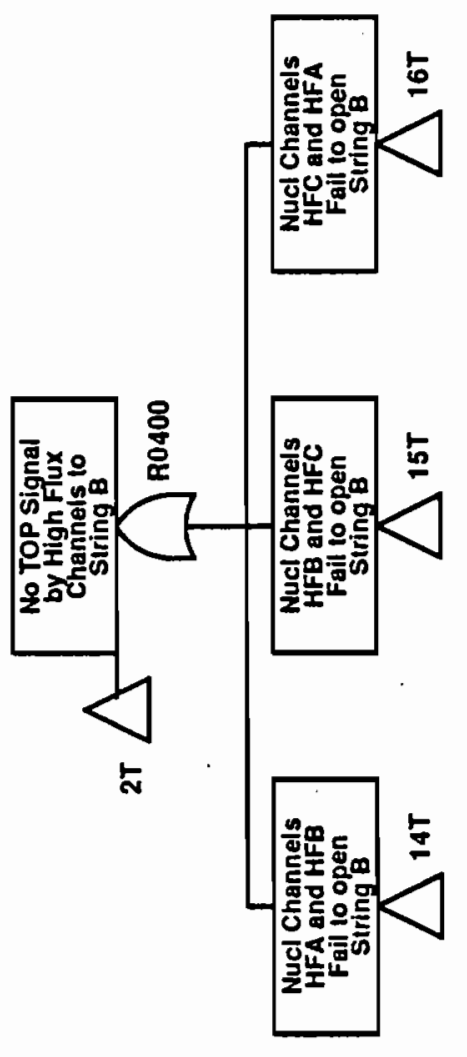

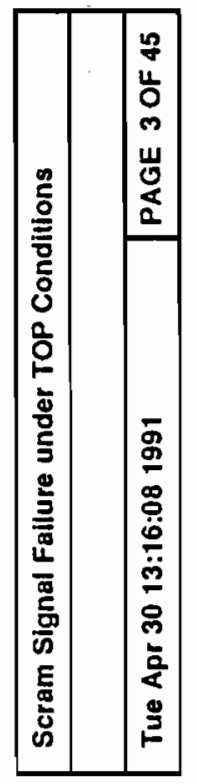




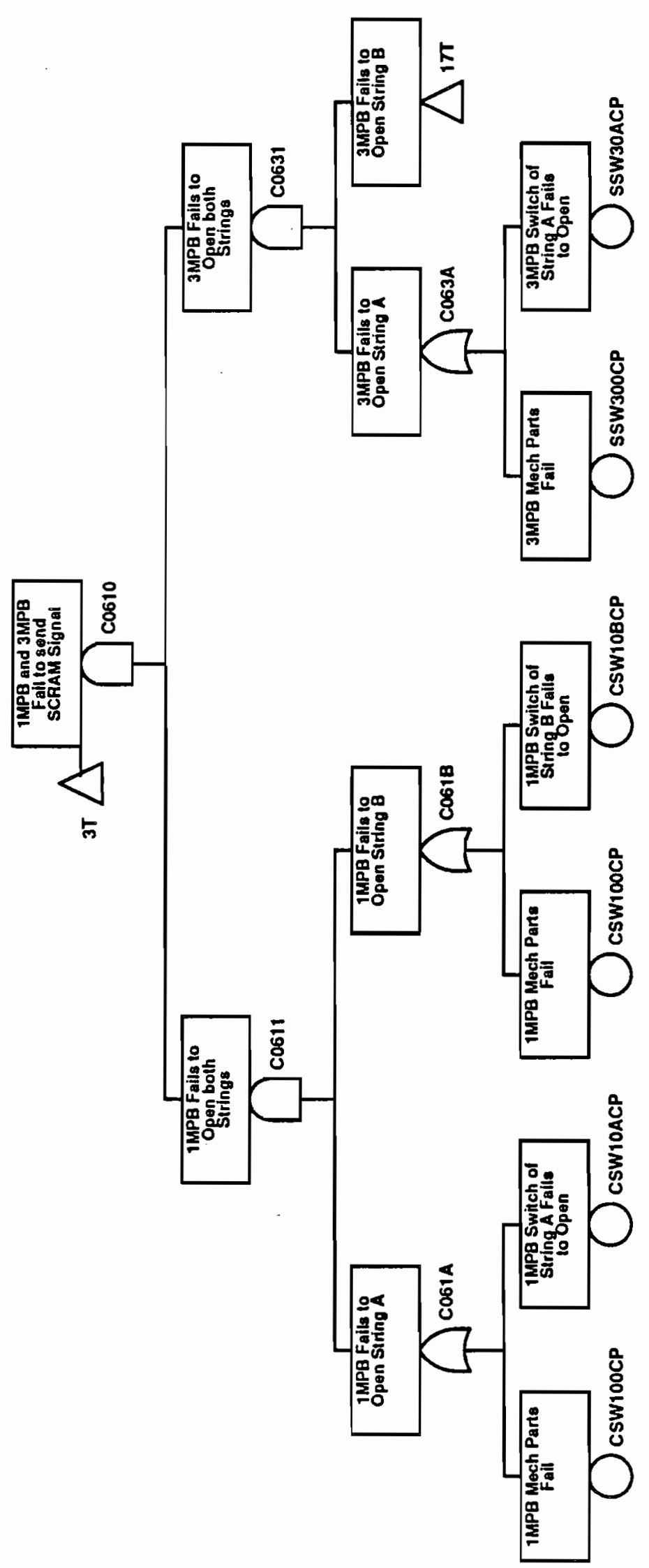




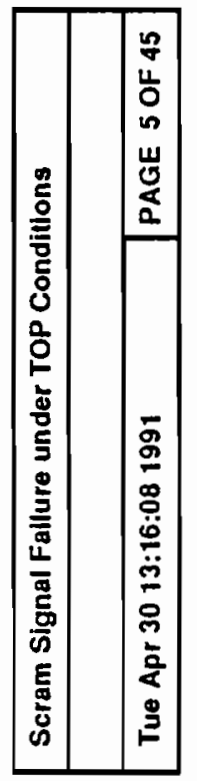

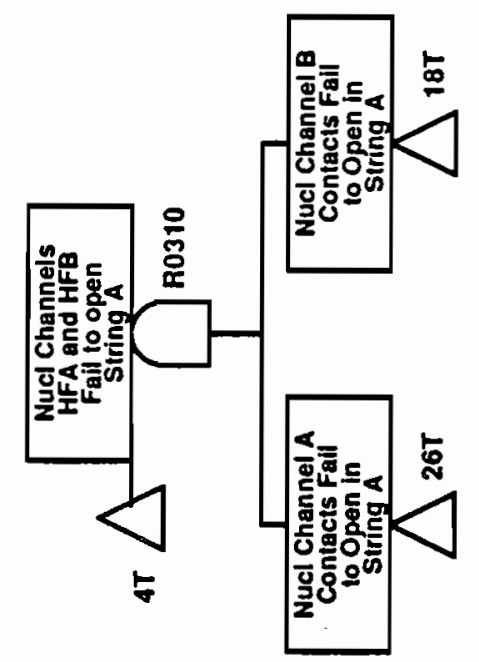

B. $2-5$ 


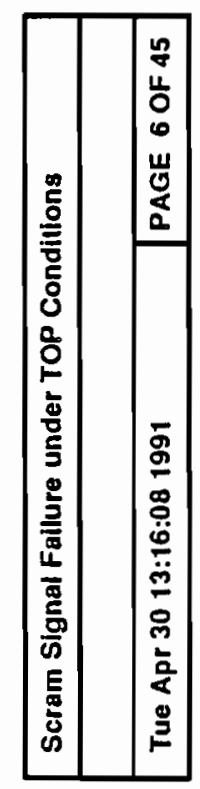

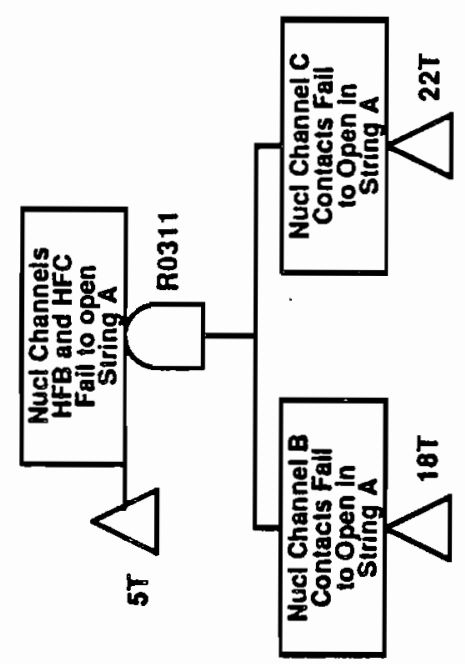

B. $2-6$ 


\begin{tabular}{|c|c|}
\hline$\stackrel{\infty}{\stackrel{\infty}{0}} \stackrel{0}{\equiv}$ & 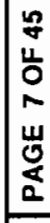 \\
\hline 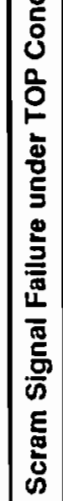 & 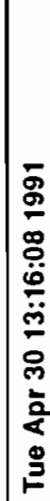 \\
\hline
\end{tabular}

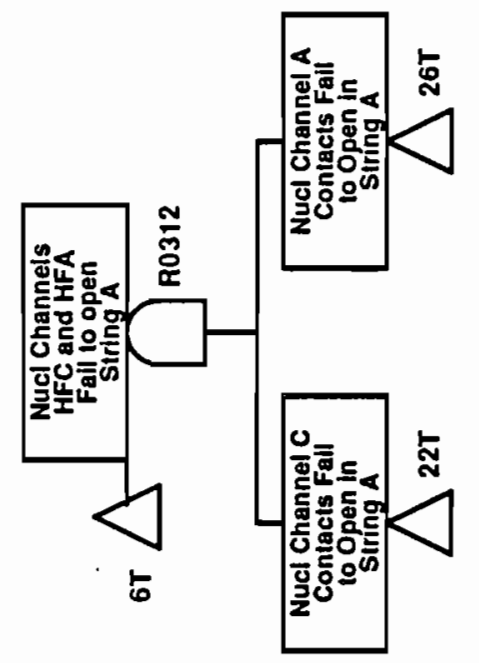

B. 2-7 


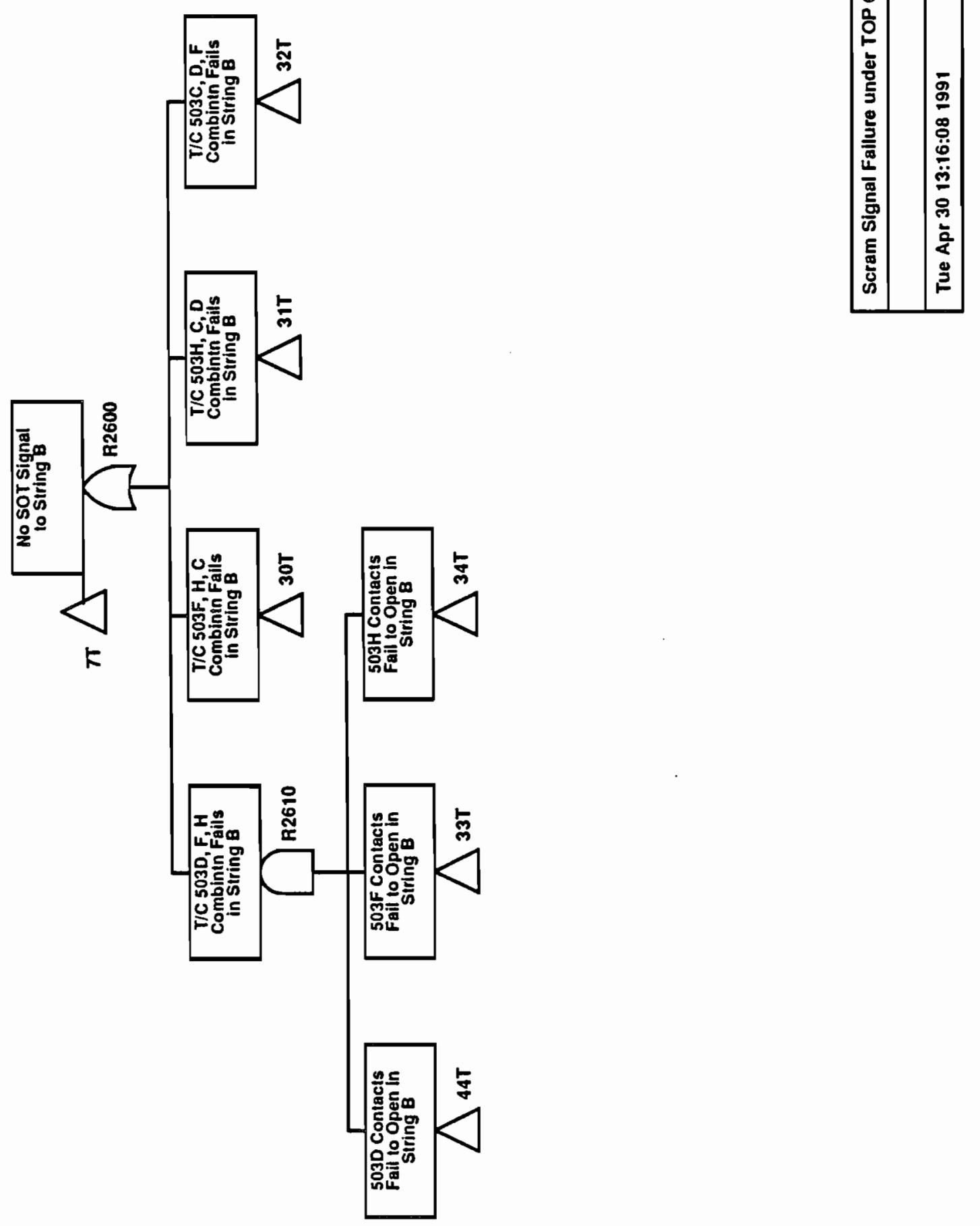




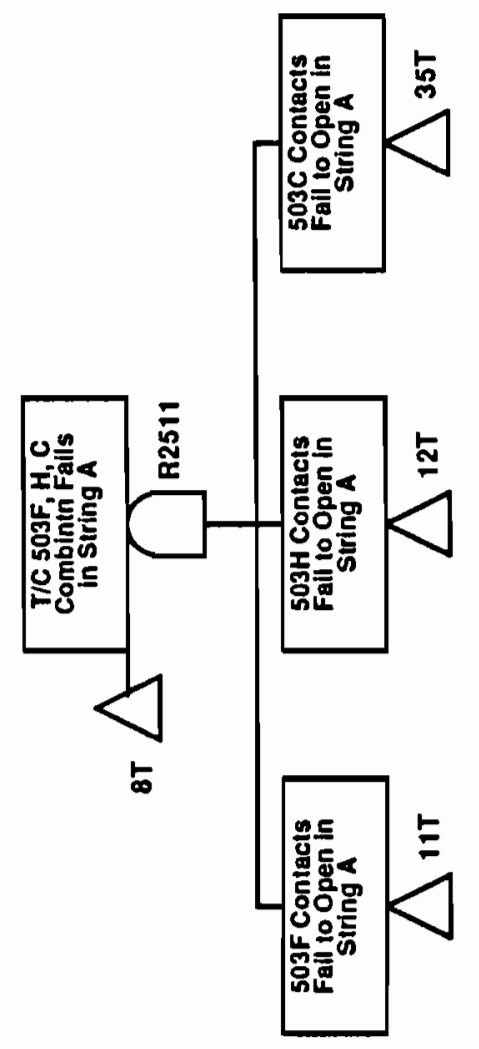

\begin{tabular}{|c|c|}
\hline$\stackrel{\infty}{\stackrel{0}{0}}$ & 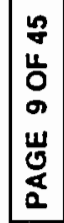 \\
\hline 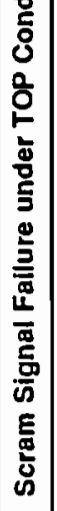 & 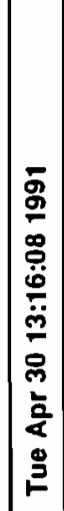 \\
\hline
\end{tabular}



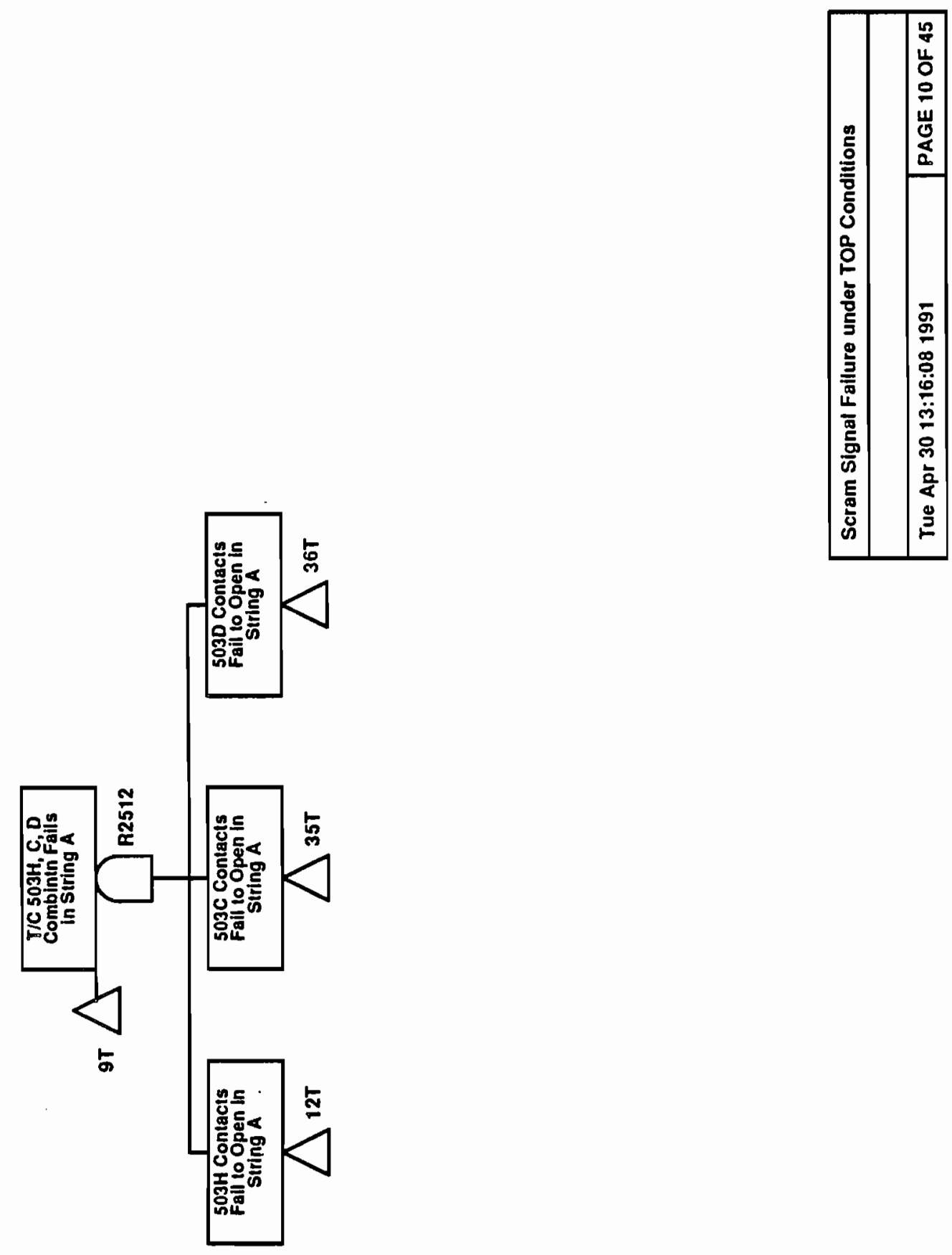

B. $2-10$ 


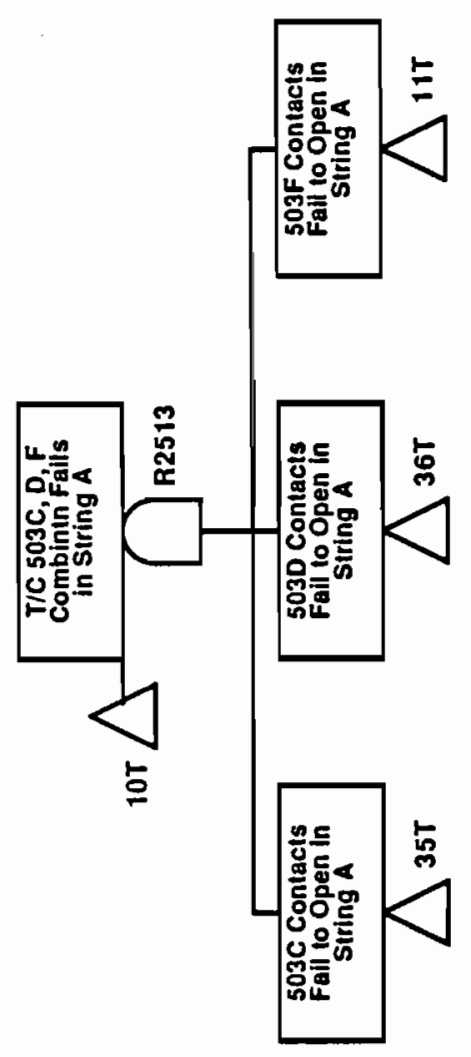

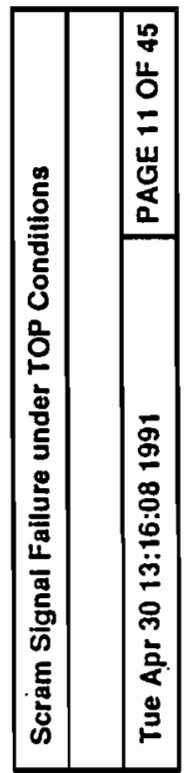

B. 2-11 


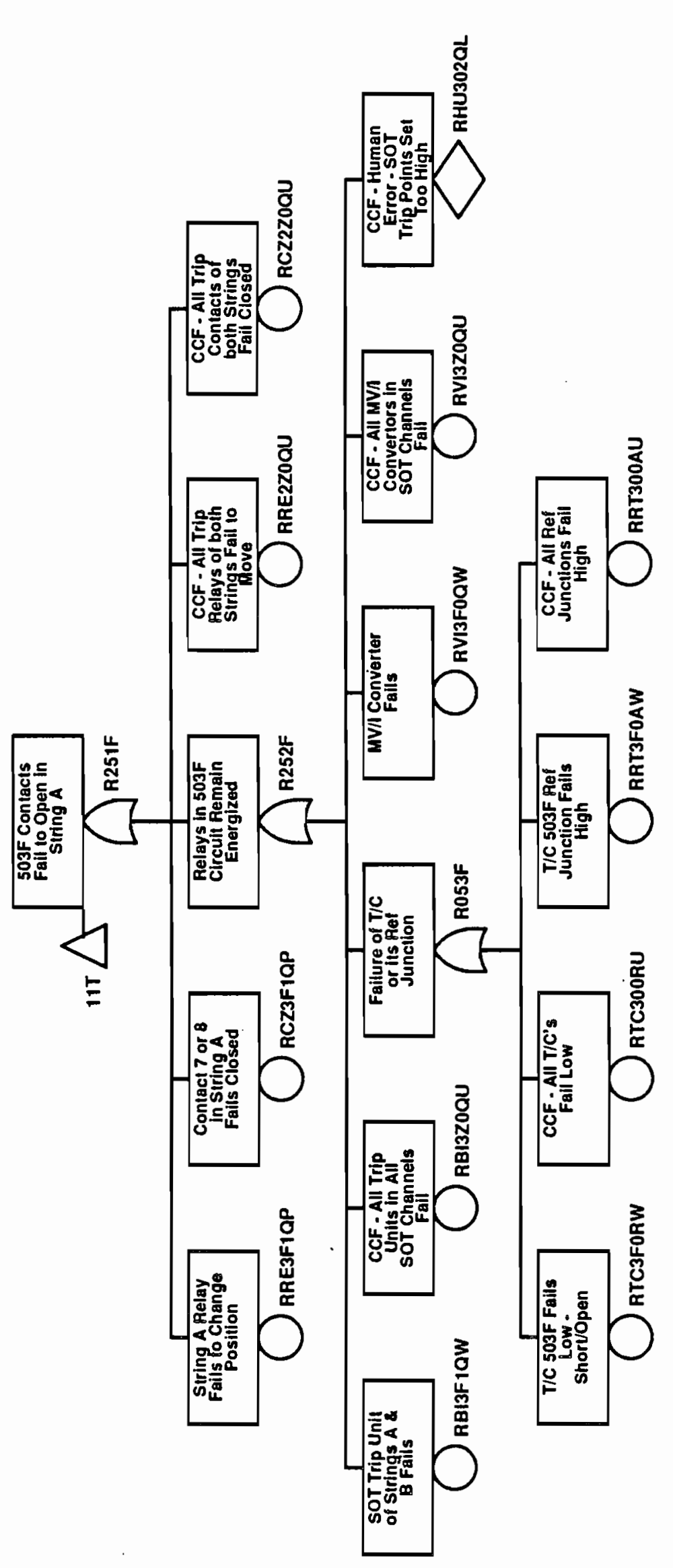

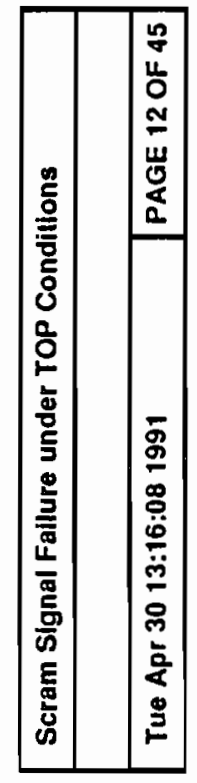



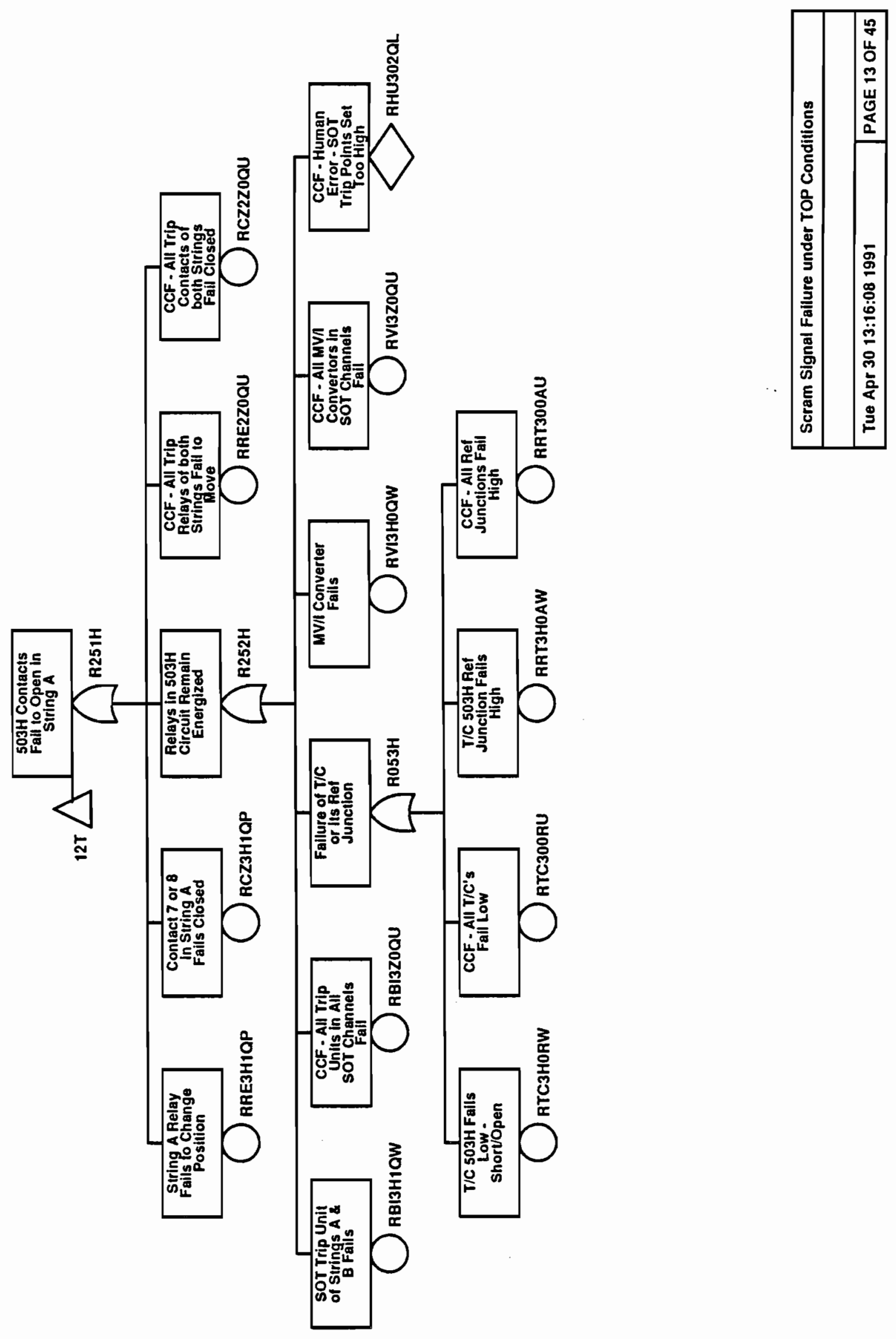

B. 2-13 


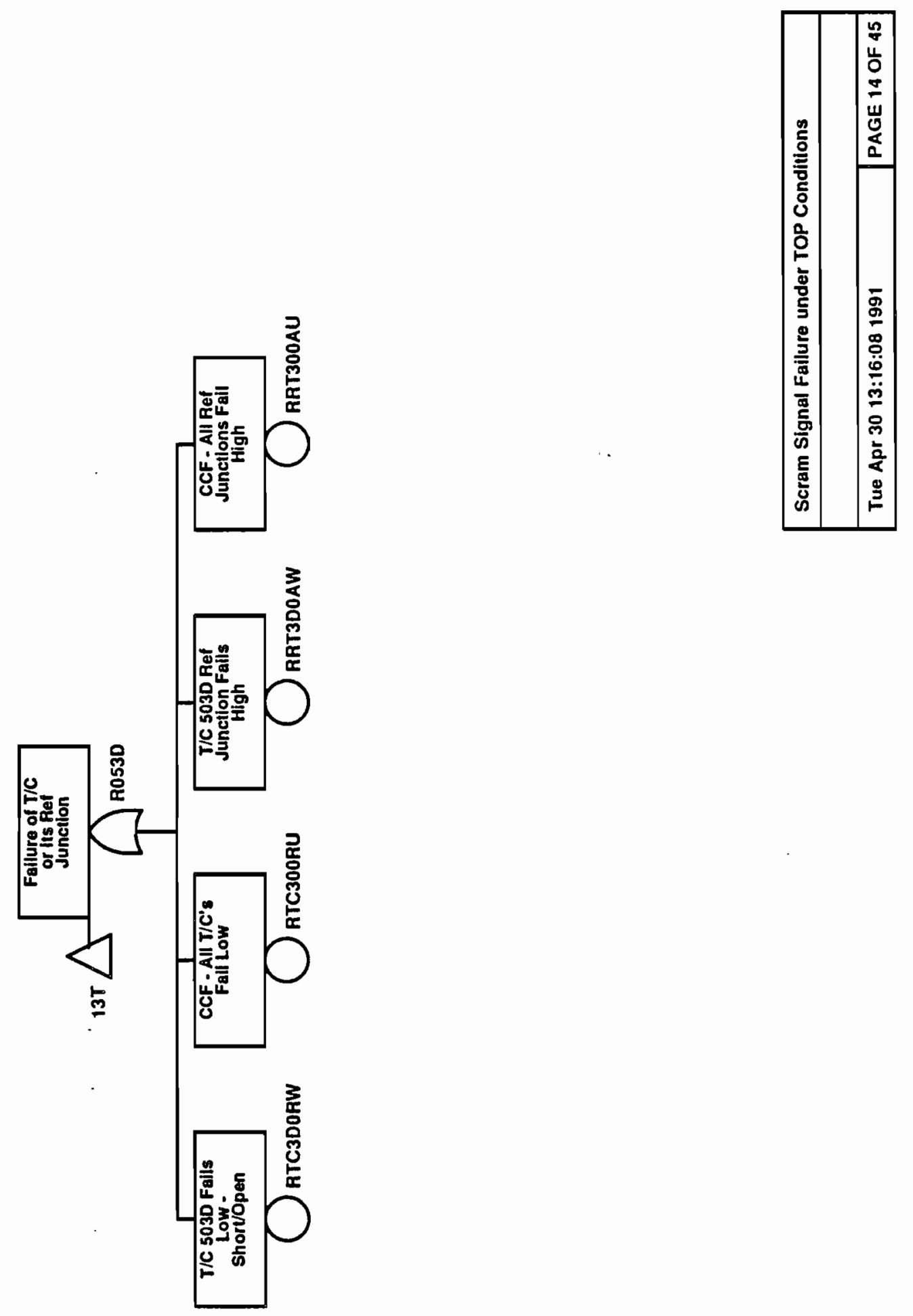




\begin{tabular}{|c|c|}
\hline 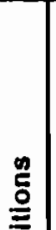 & 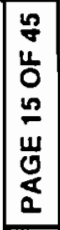 \\
\hline 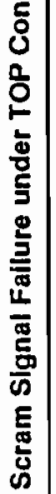 & 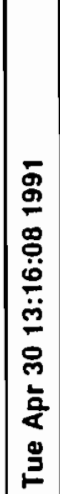 \\
\hline
\end{tabular}

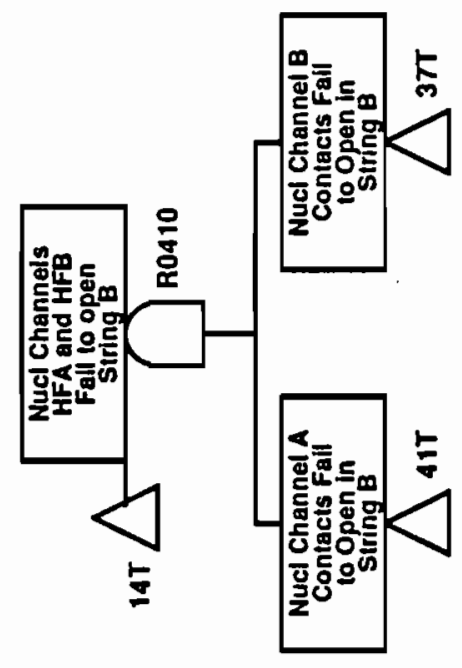

B. 2-15 


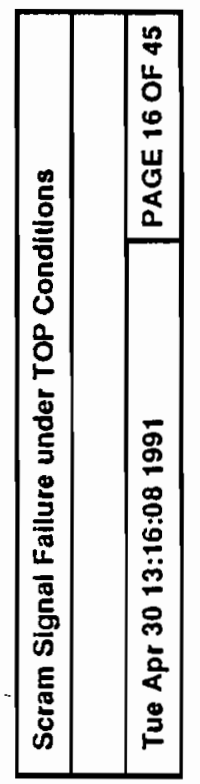

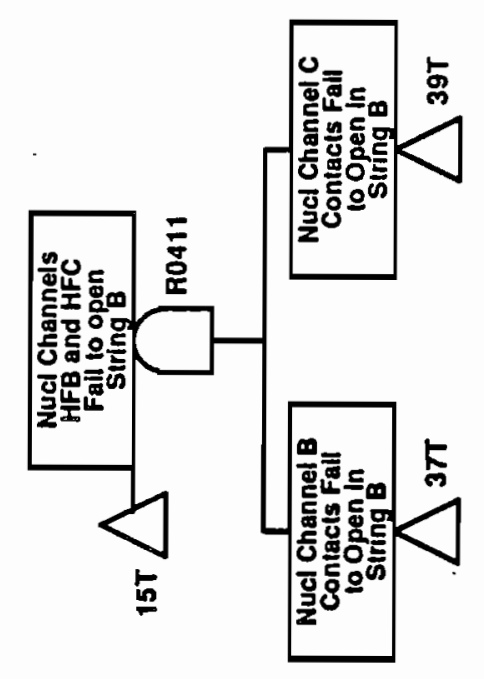

B. 2-16 


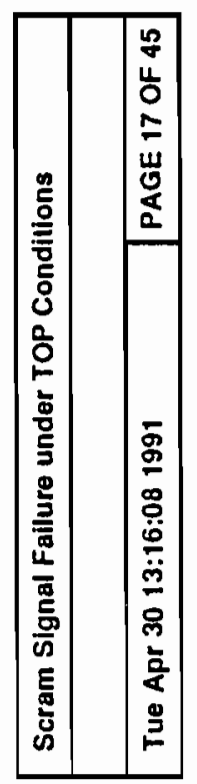

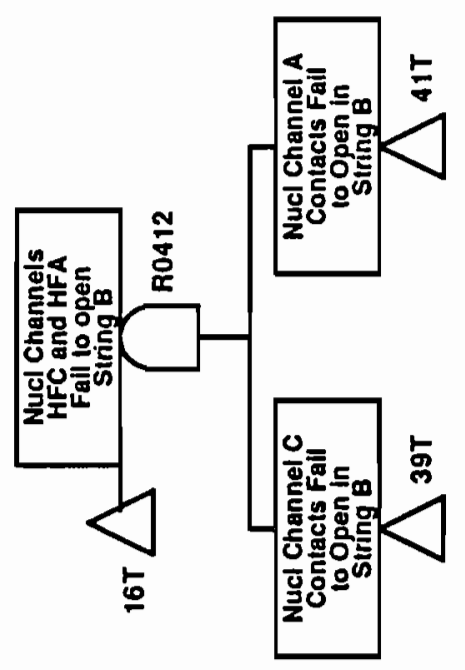




\begin{tabular}{|c|c|}
\hline 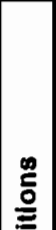 & 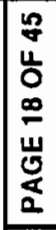 \\
\hline 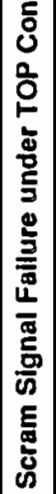 & 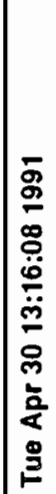 \\
\hline
\end{tabular}

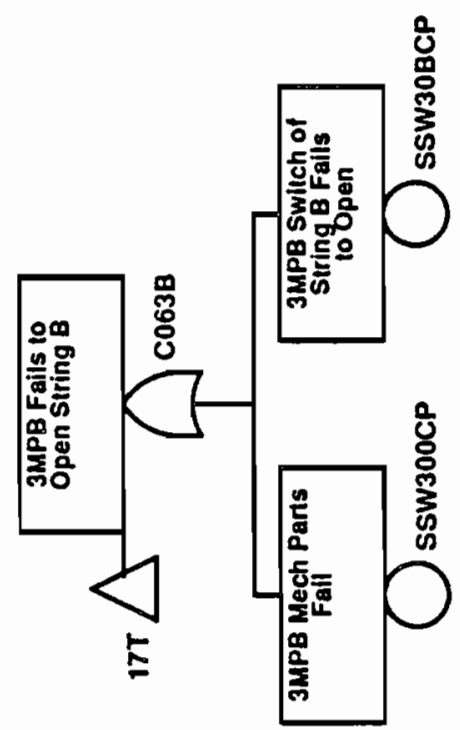

B. $2-18$ 


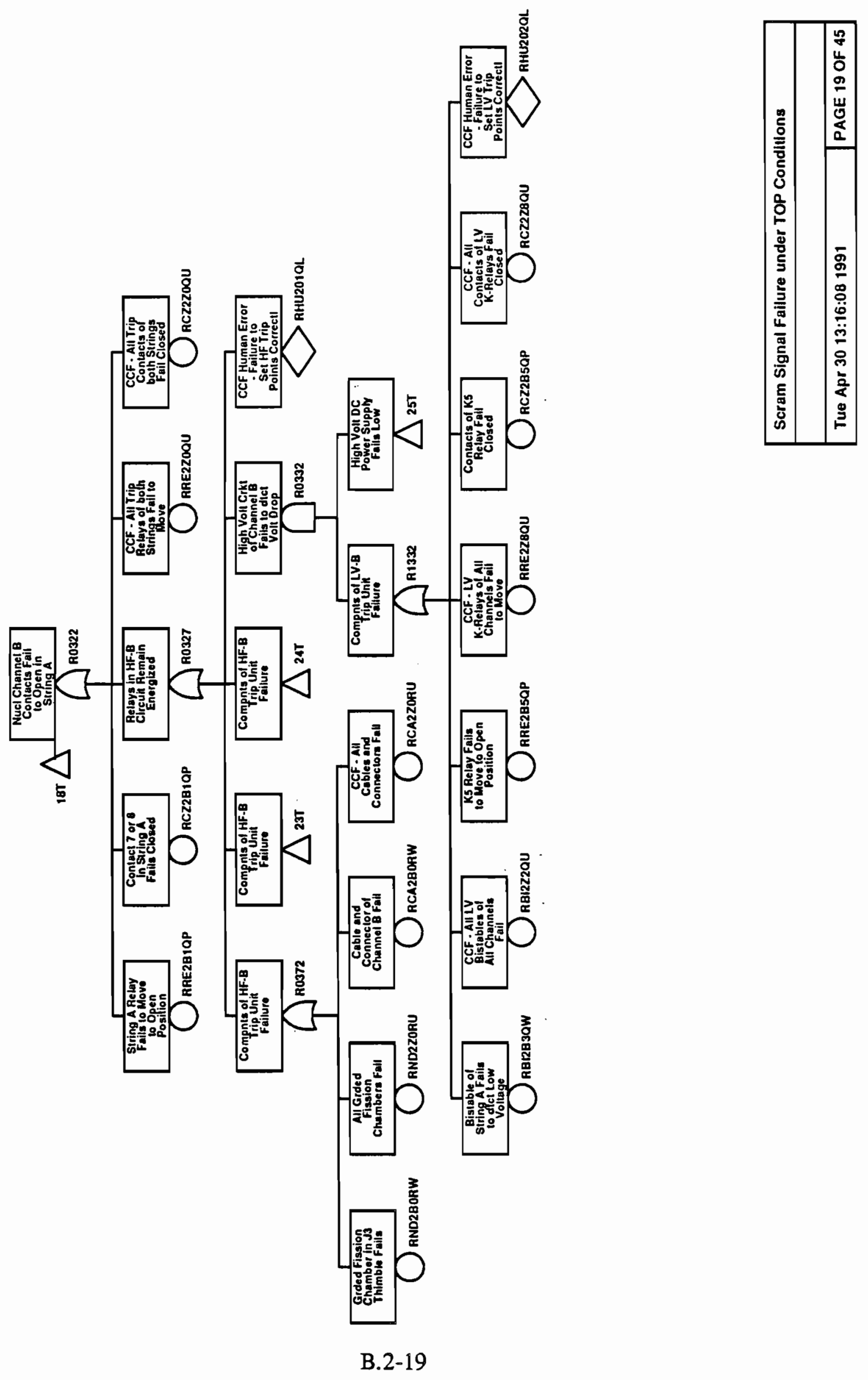




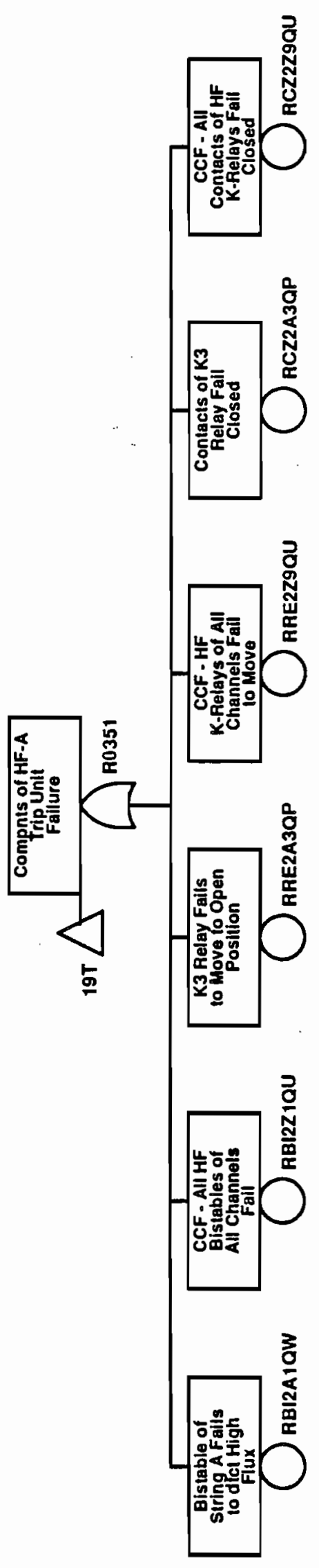

B. $2-20$ 

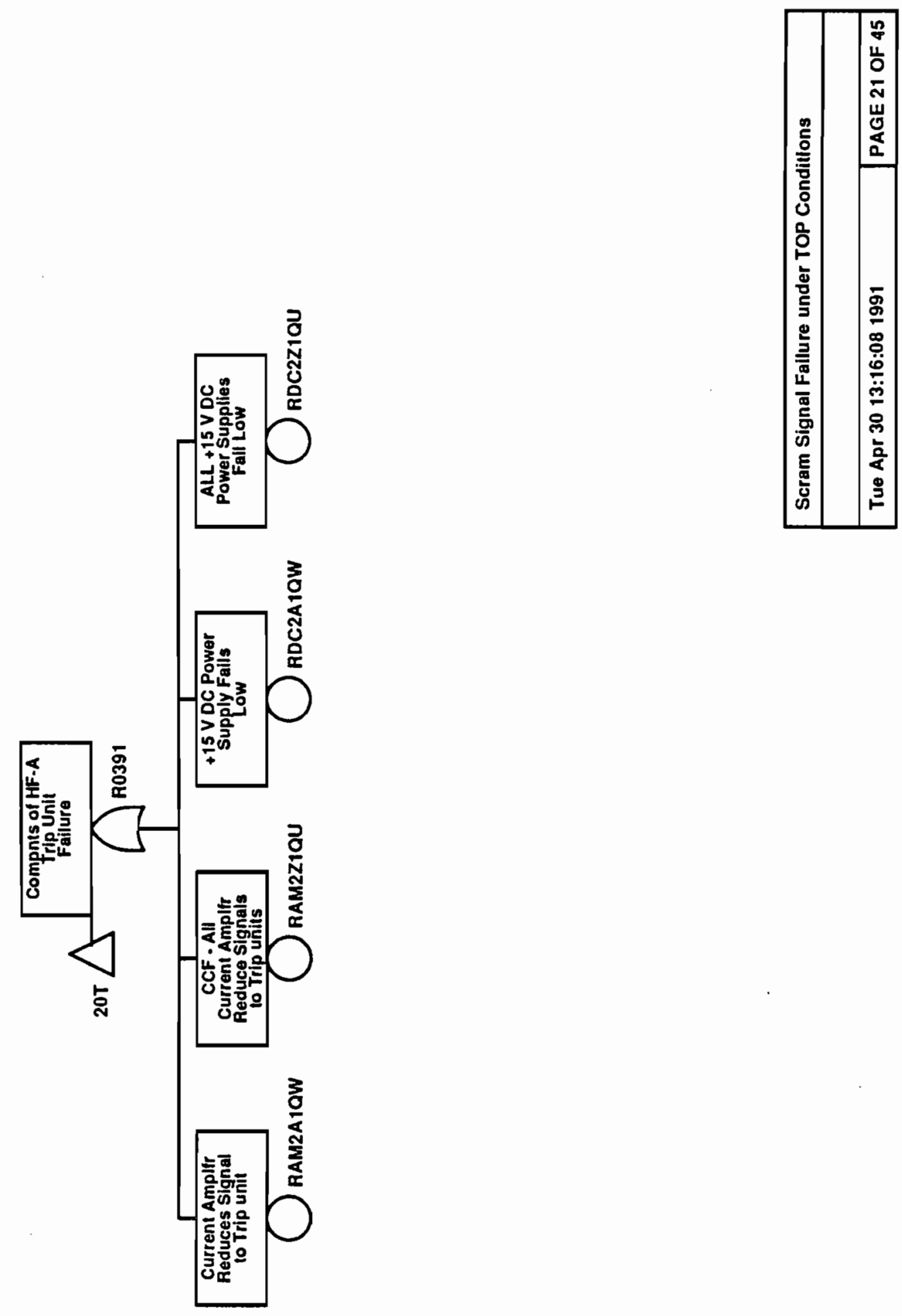


\begin{tabular}{|c|c|}
\hline 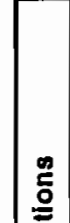 & 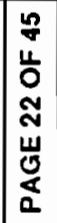 \\
\hline 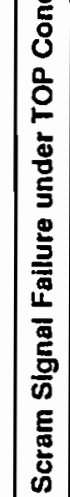 & 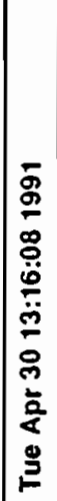 \\
\hline
\end{tabular}

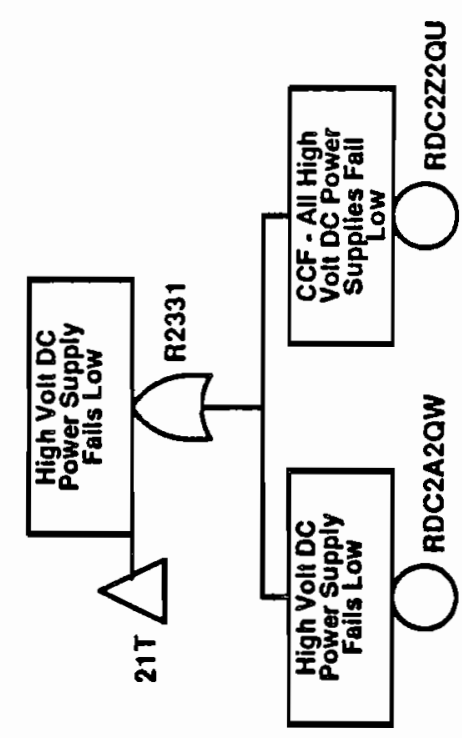




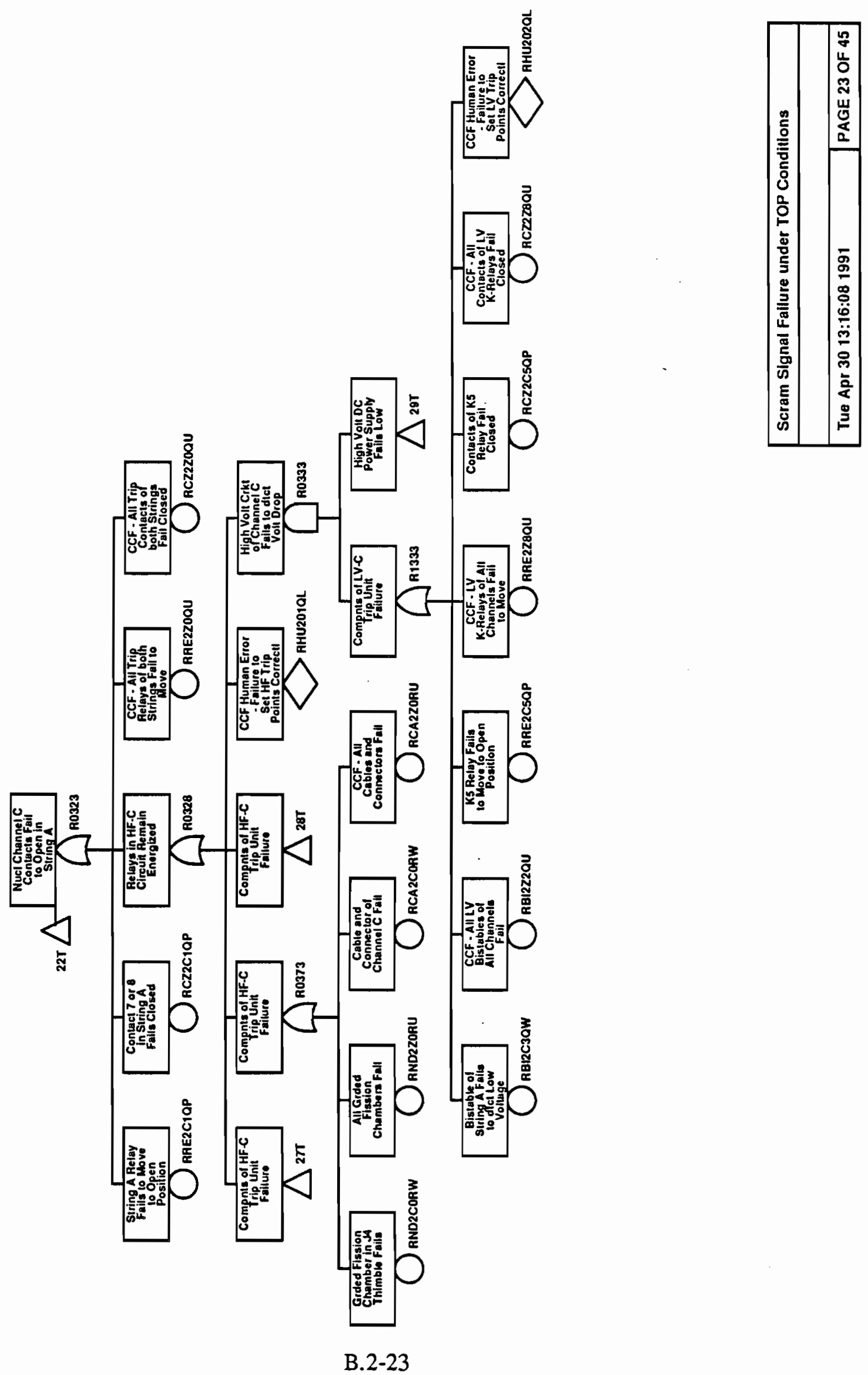



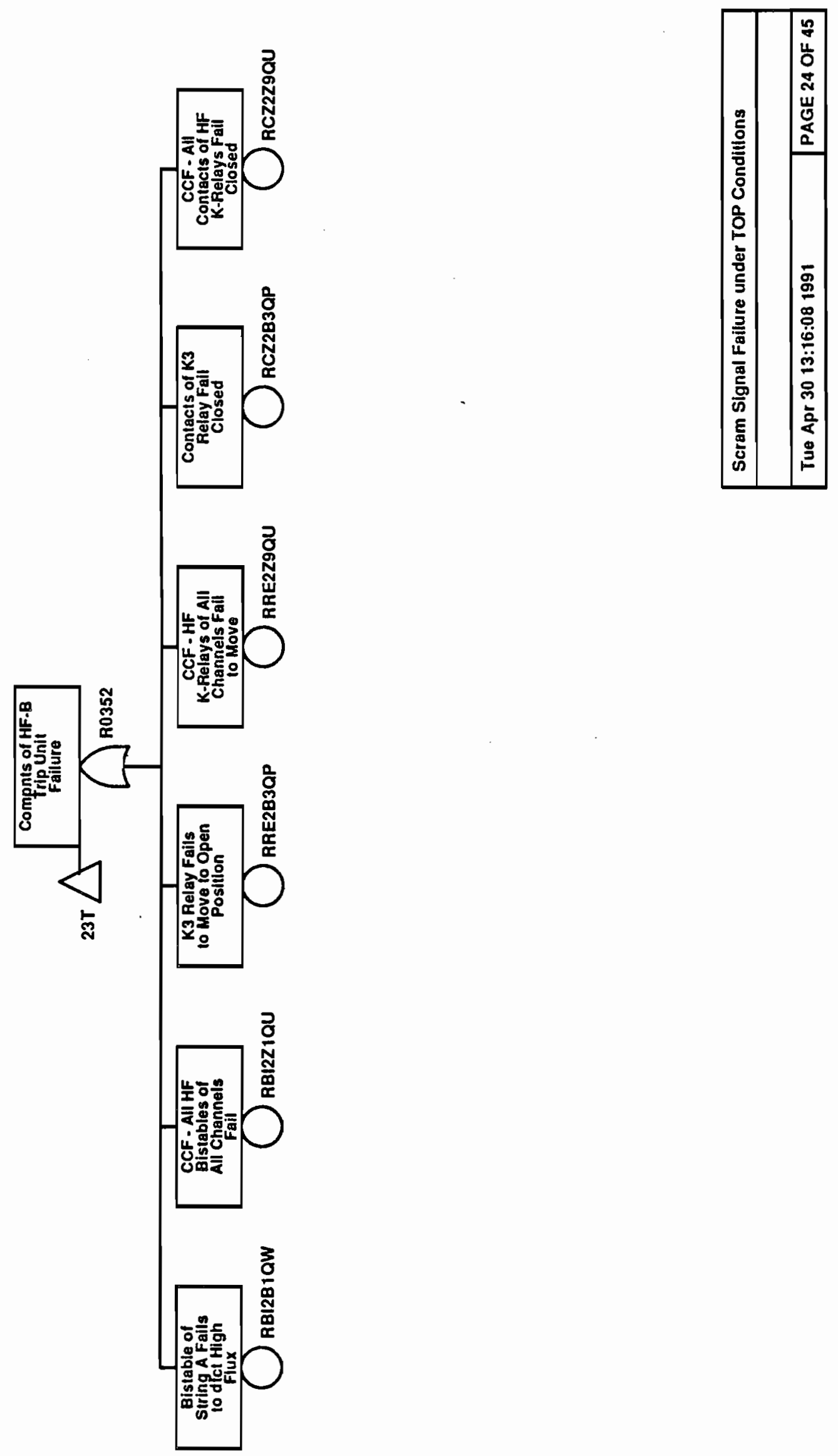

B. 2-24 


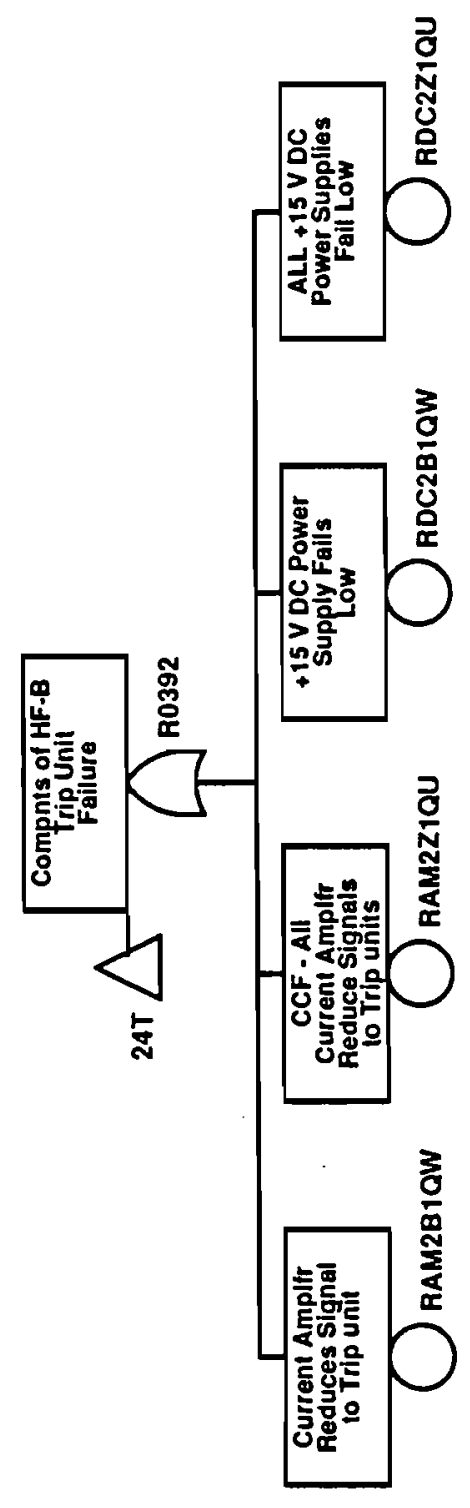

\begin{tabular}{|c|c|}
\hline 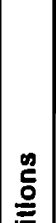 & 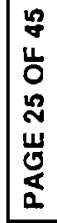 \\
\hline 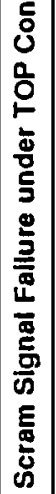 & 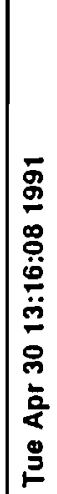 \\
\hline
\end{tabular}




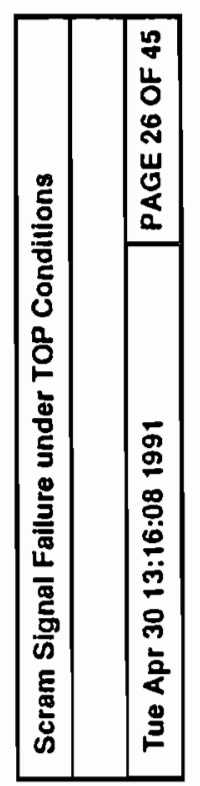

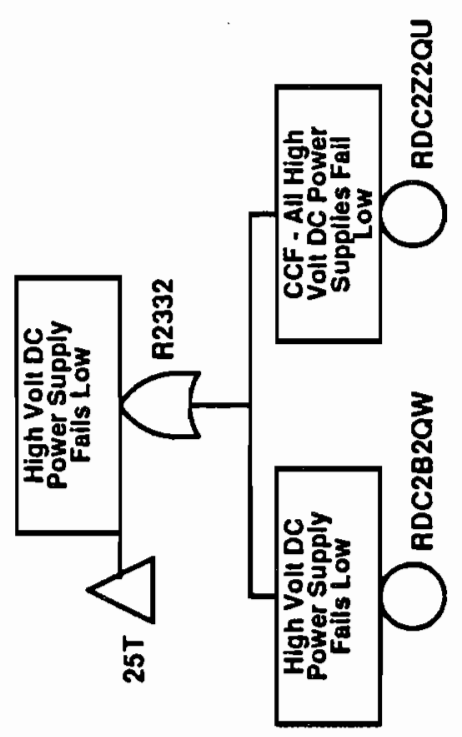




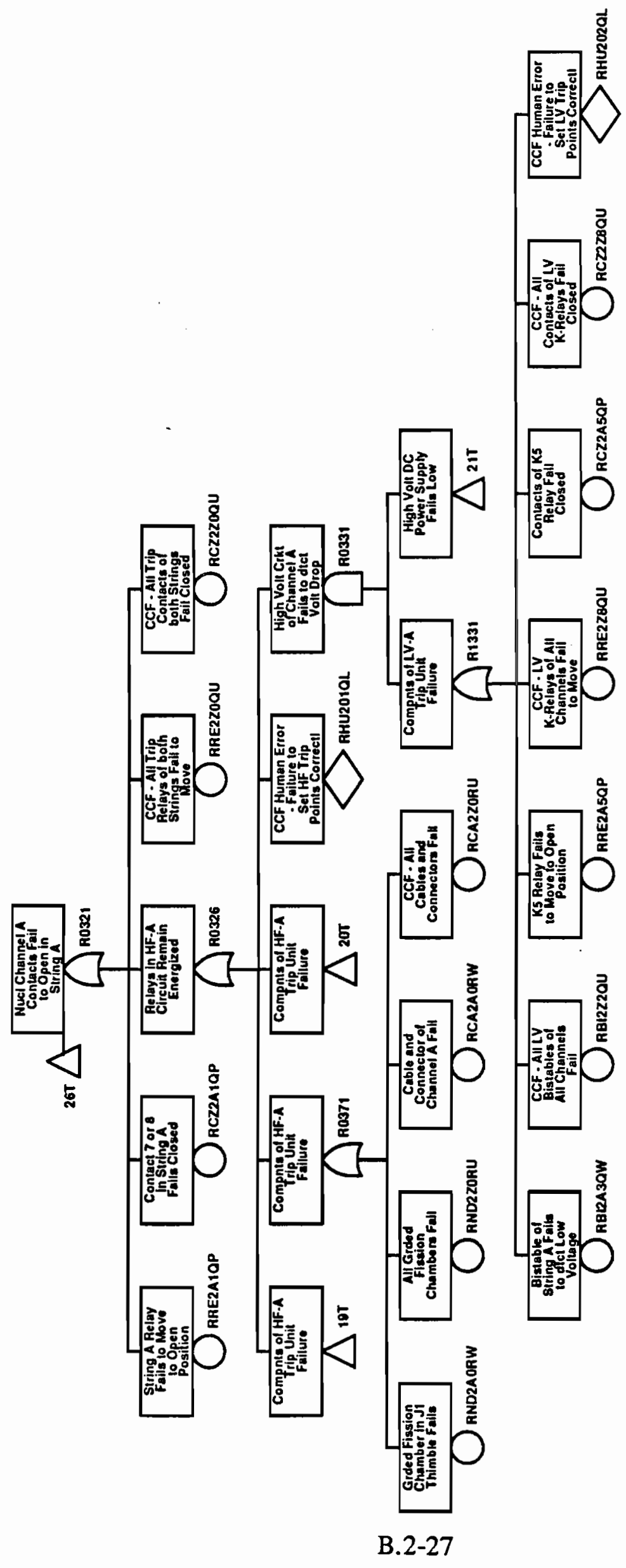

\begin{tabular}{|c|c|}
\hline 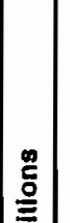 & 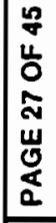 \\
\hline 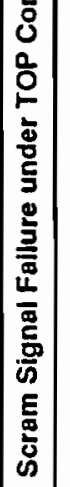 & 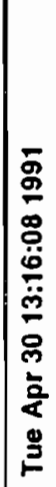 \\
\hline
\end{tabular}




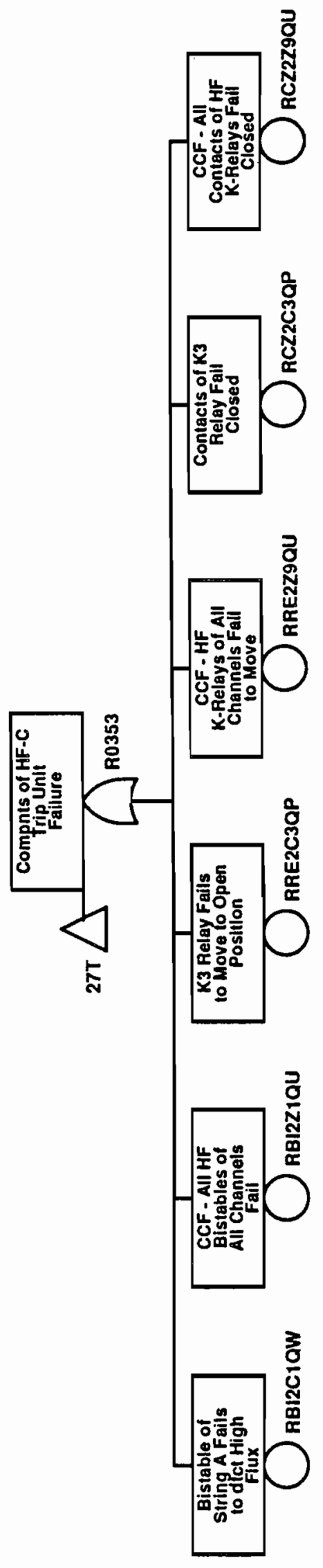

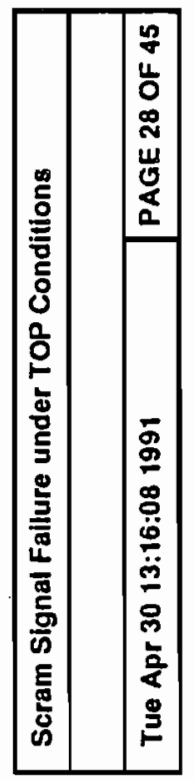

\section{B. $2-28$}




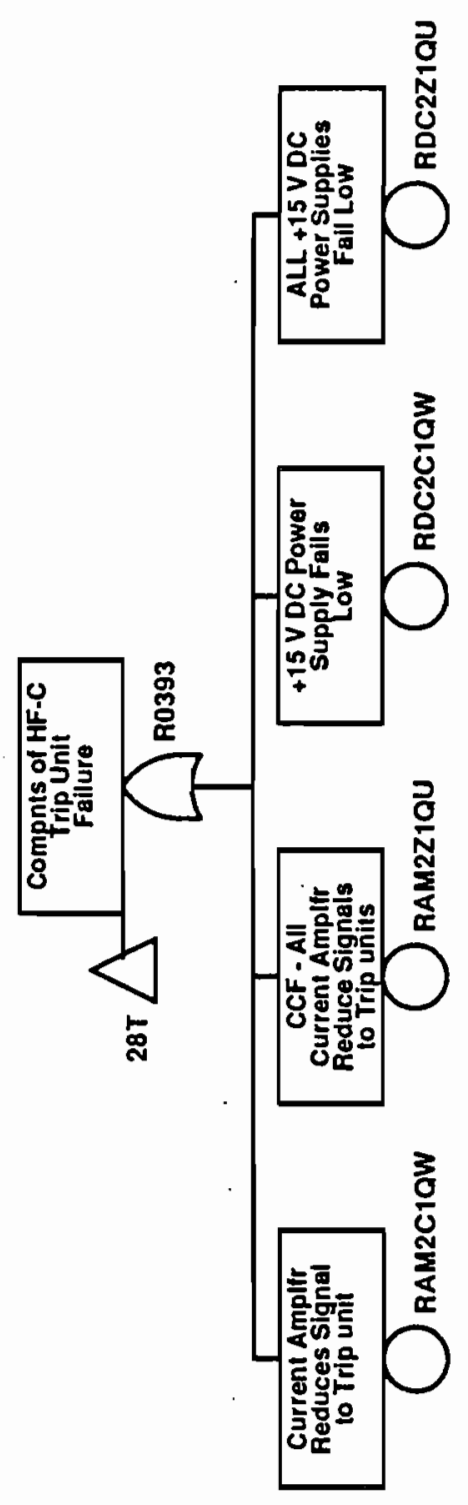

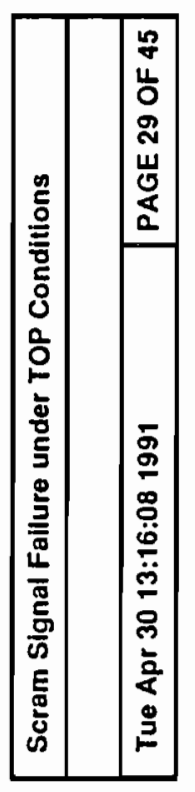




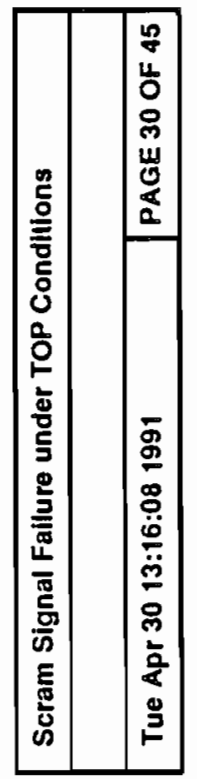

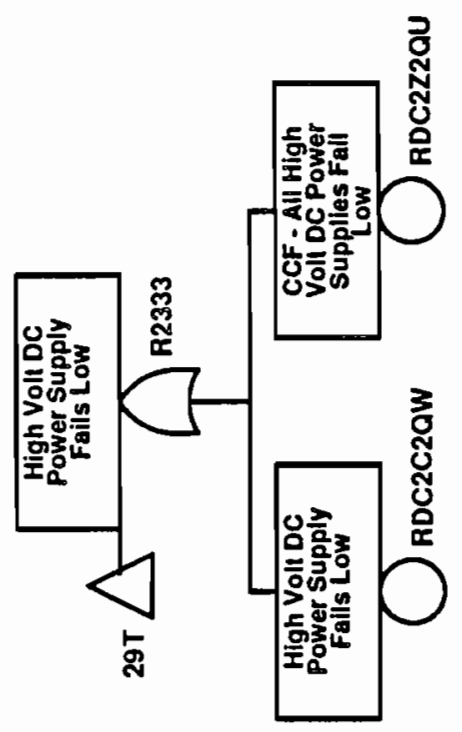




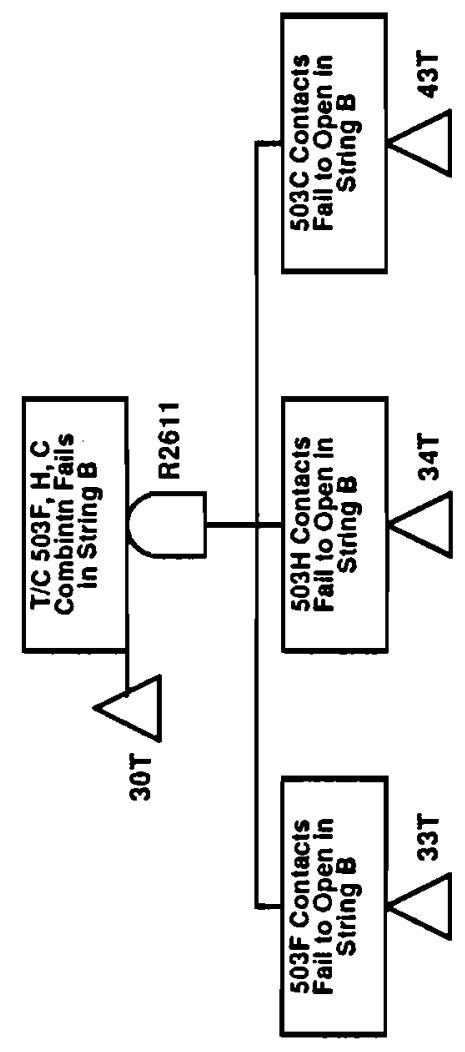

\begin{tabular}{|c|c|}
\hline$\stackrel{n}{o}$ & 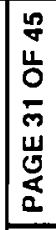 \\
\hline 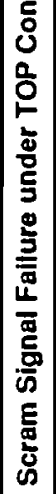 & 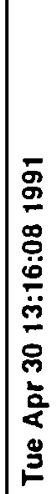 \\
\hline
\end{tabular}




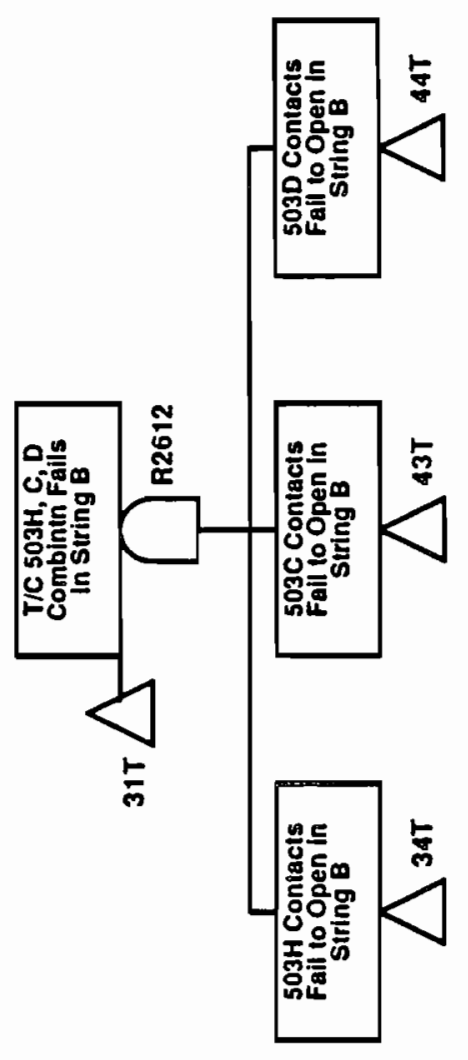

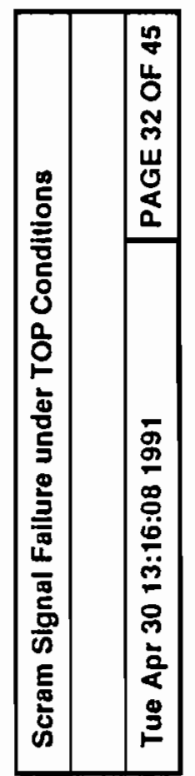




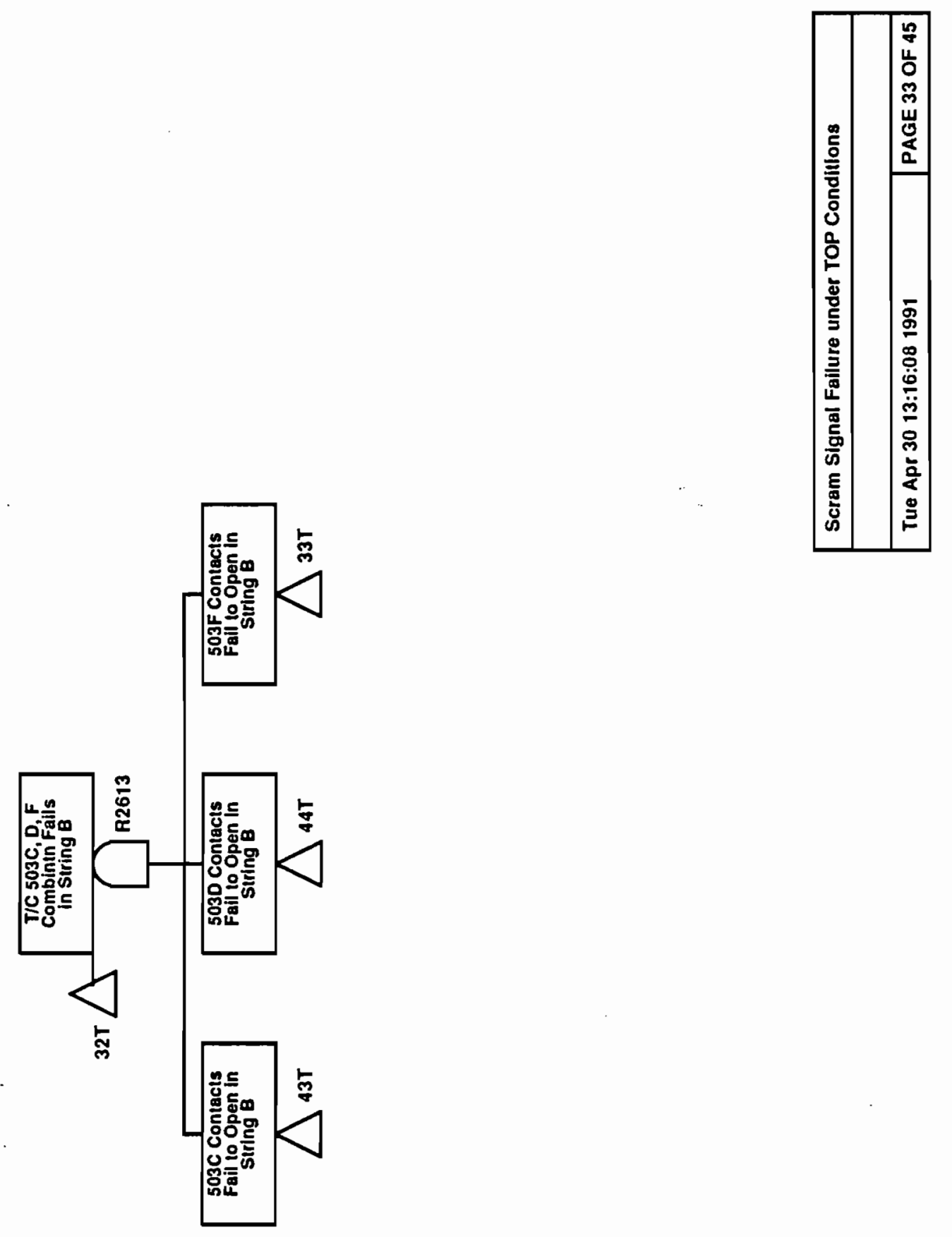




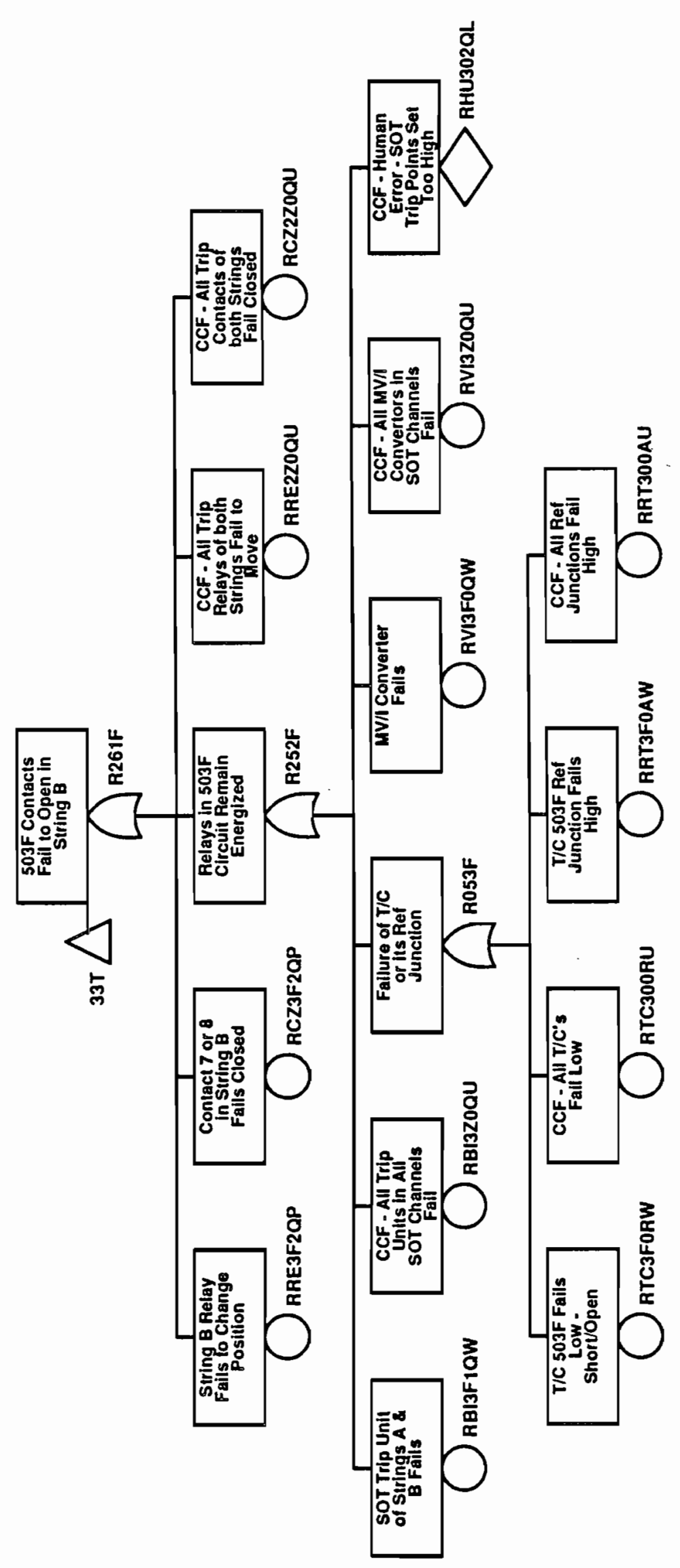



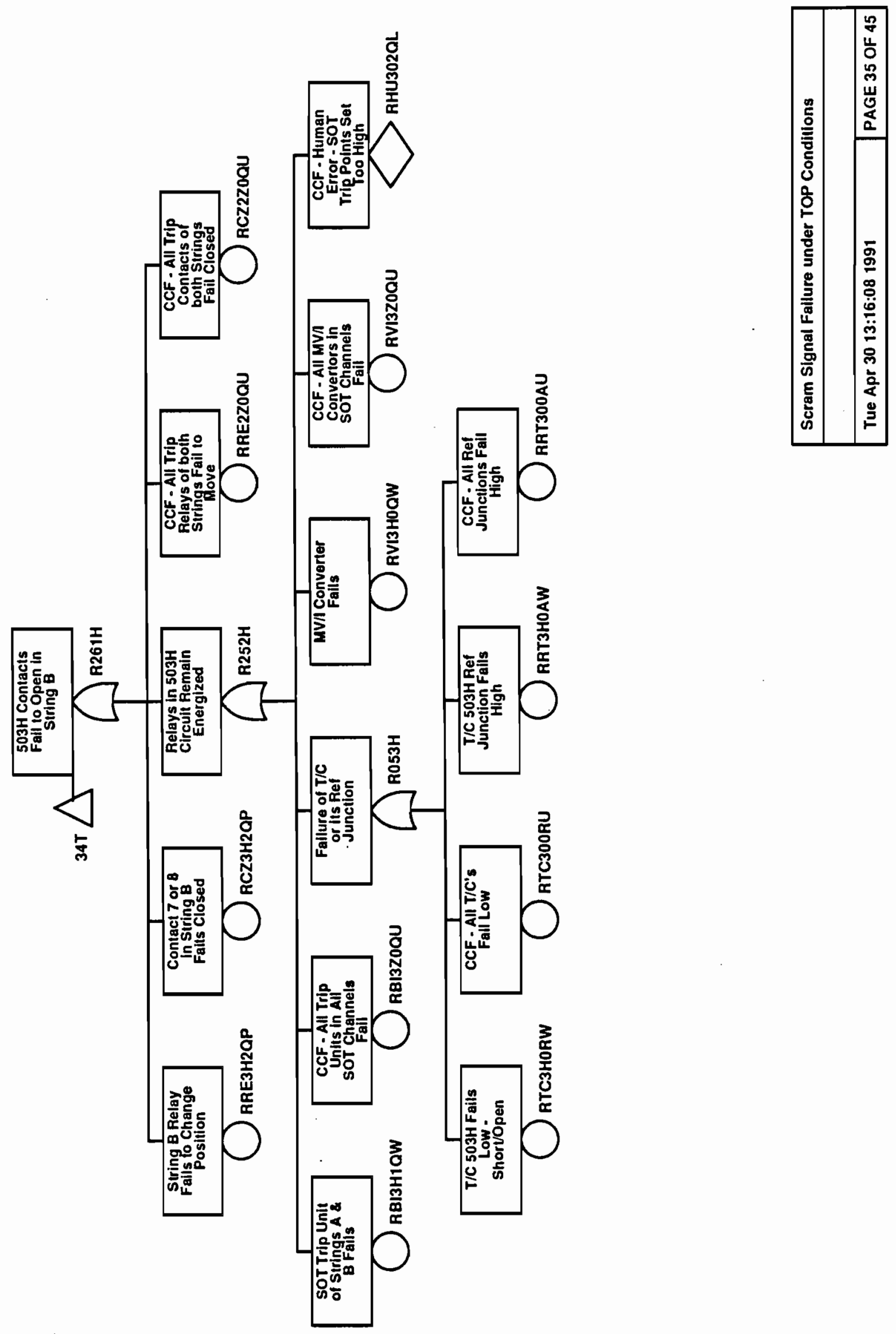

B. 2-35 

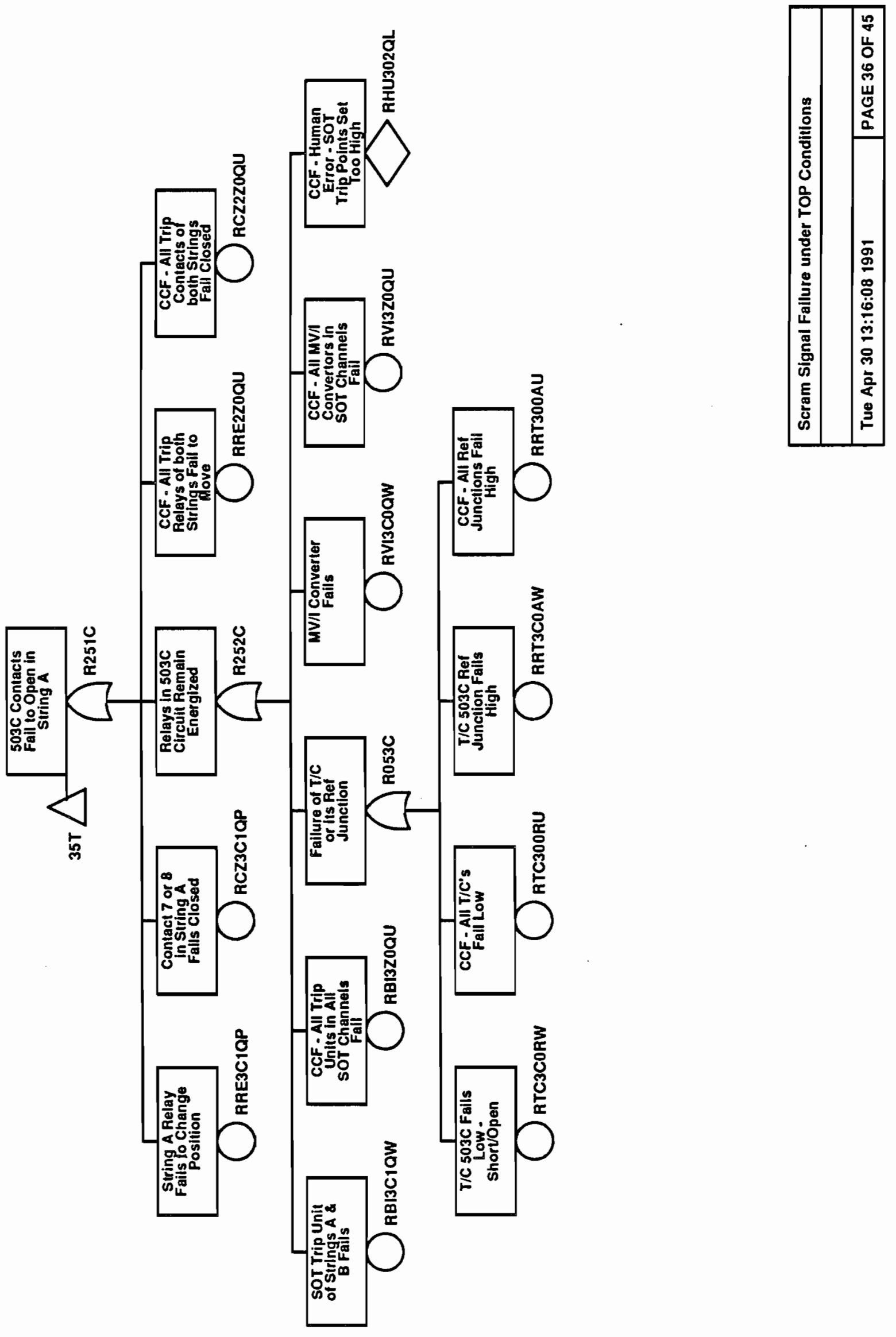

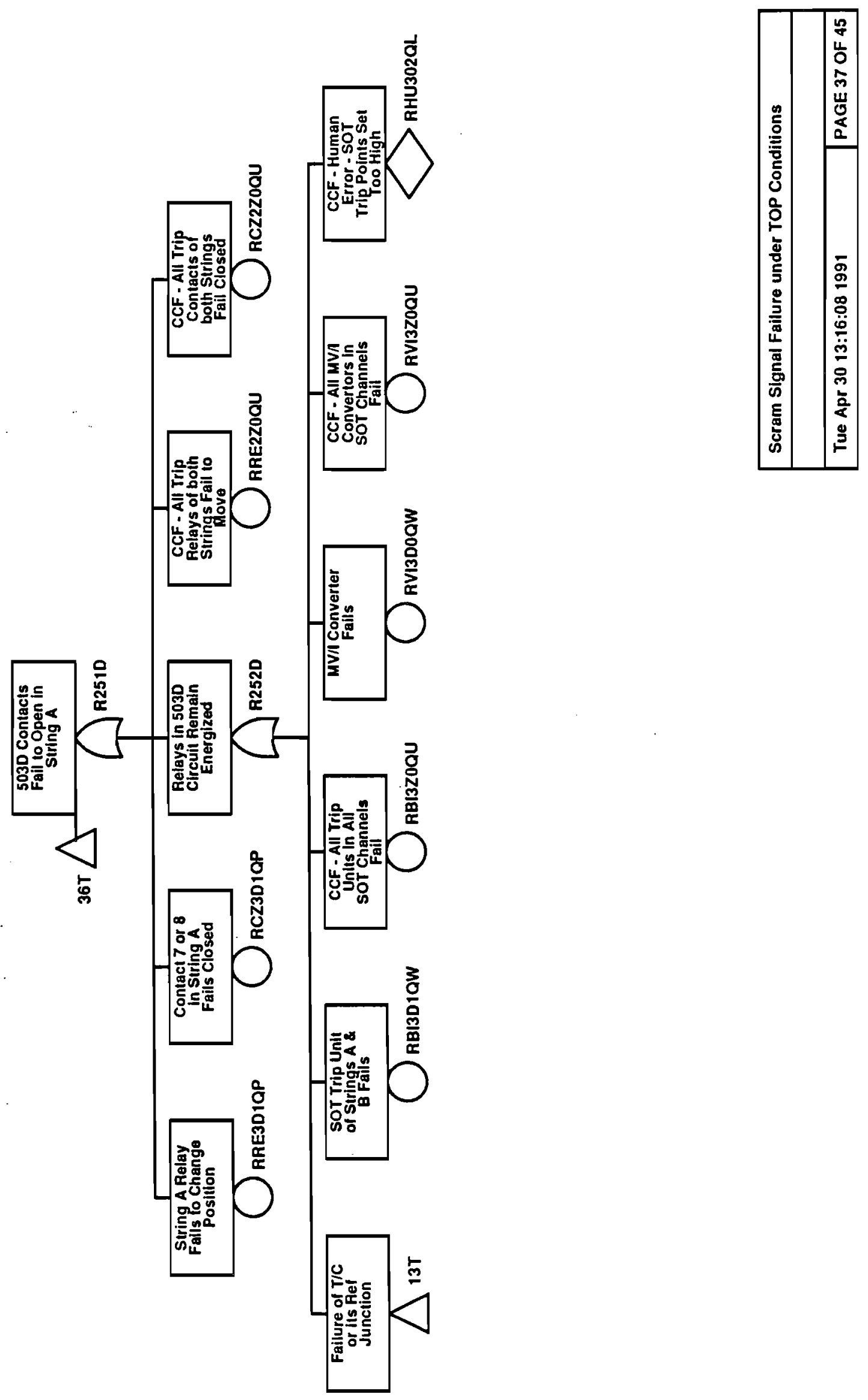

B. 2-37 


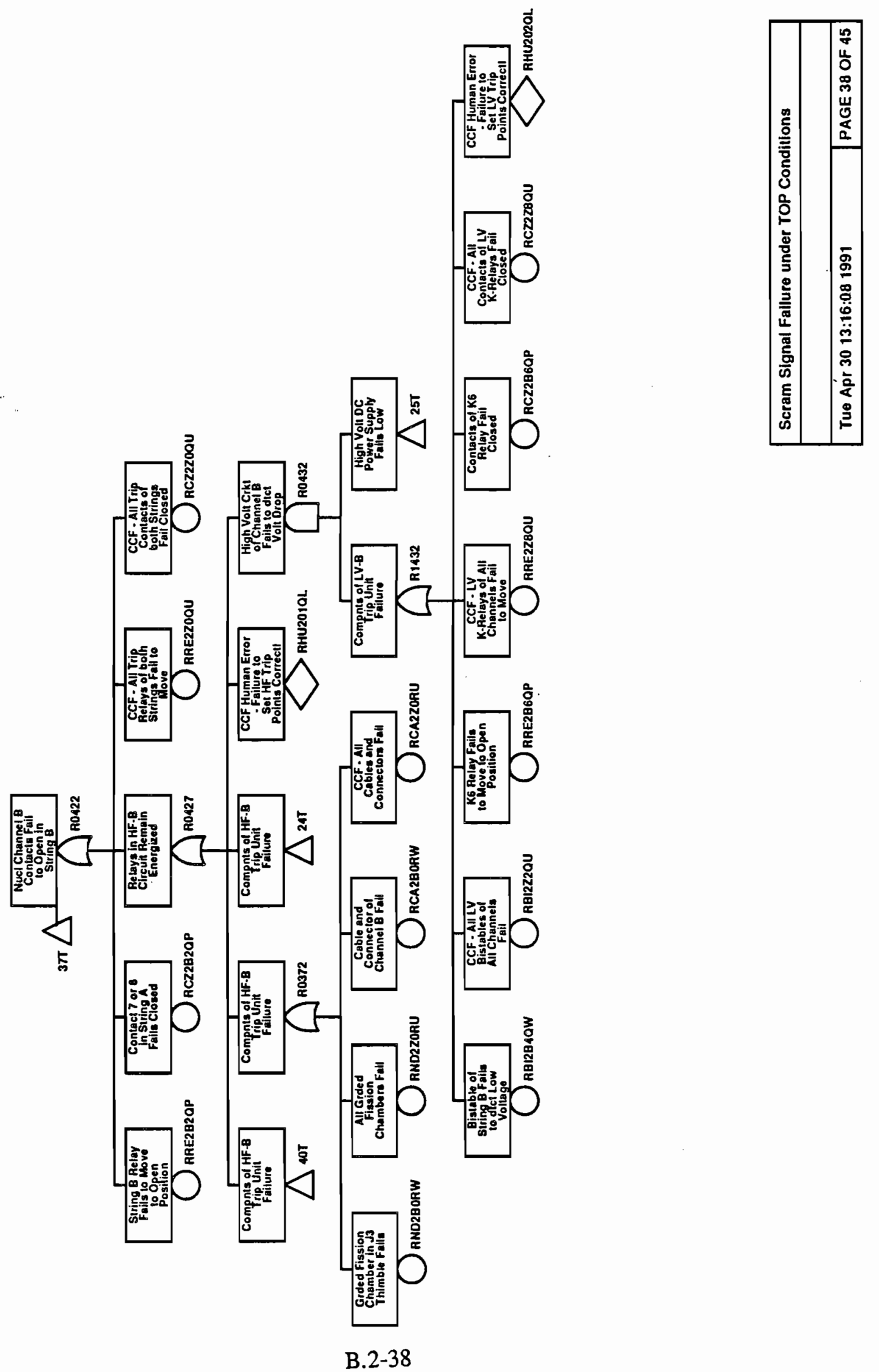




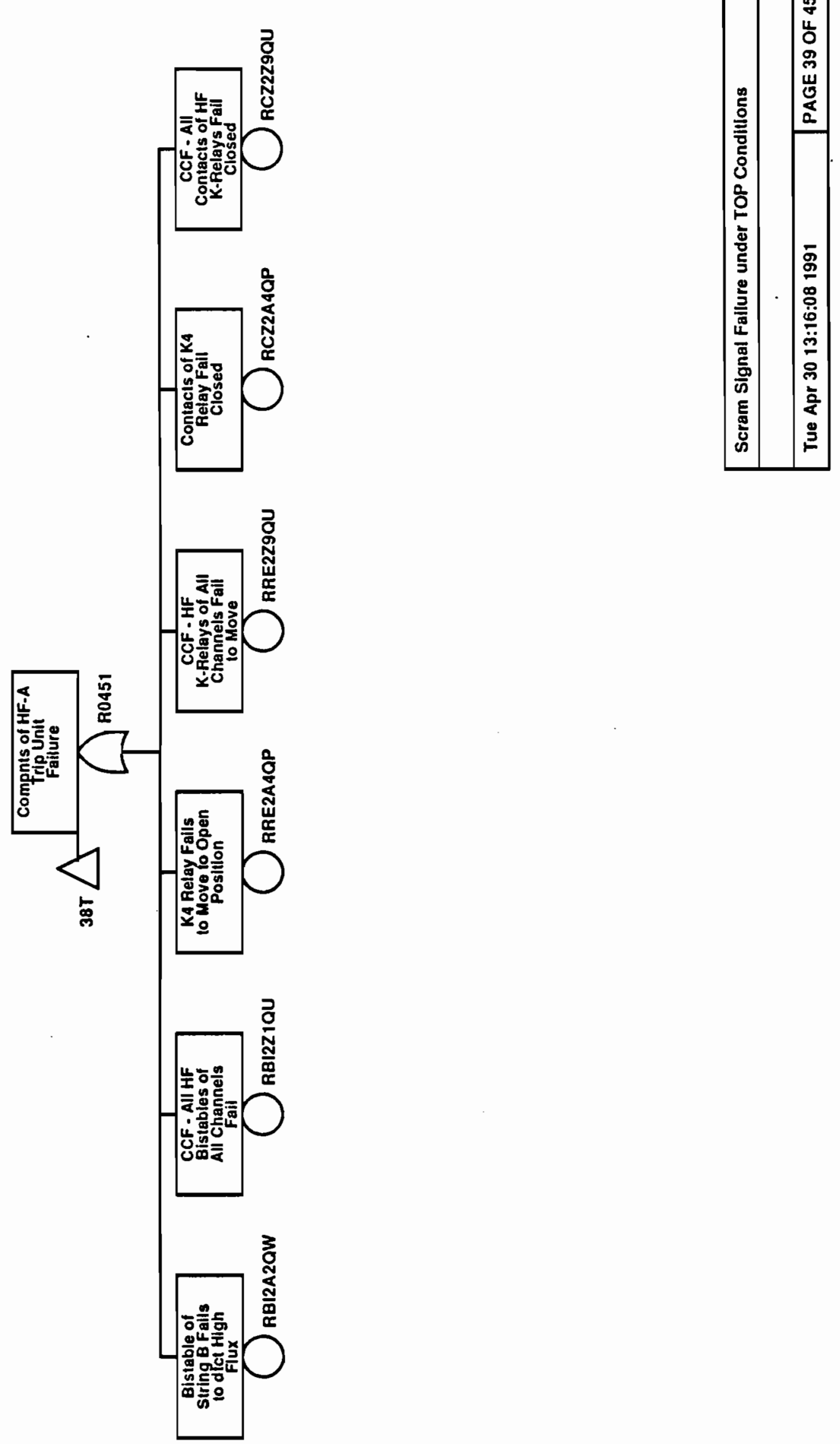

B. 2-39 


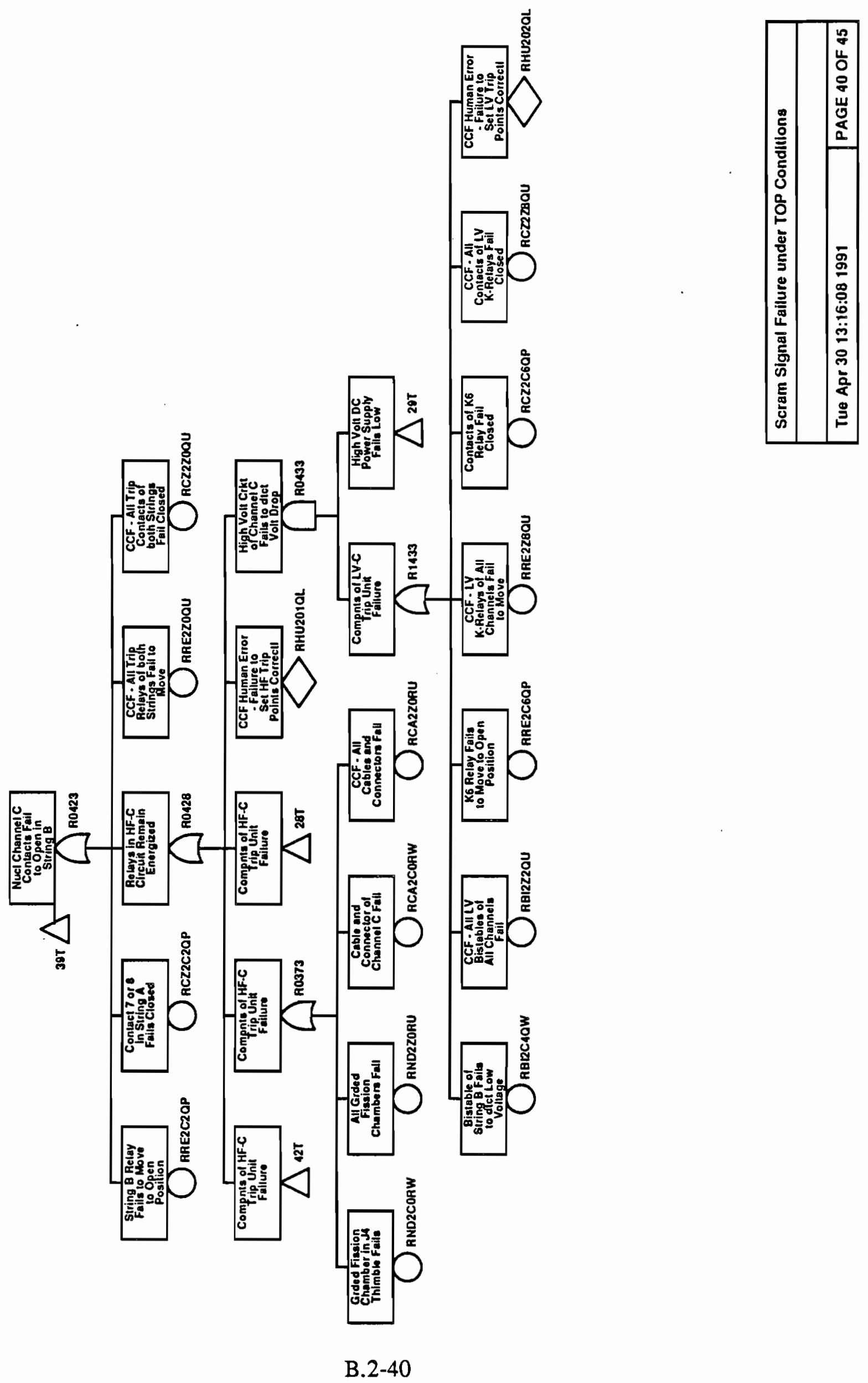




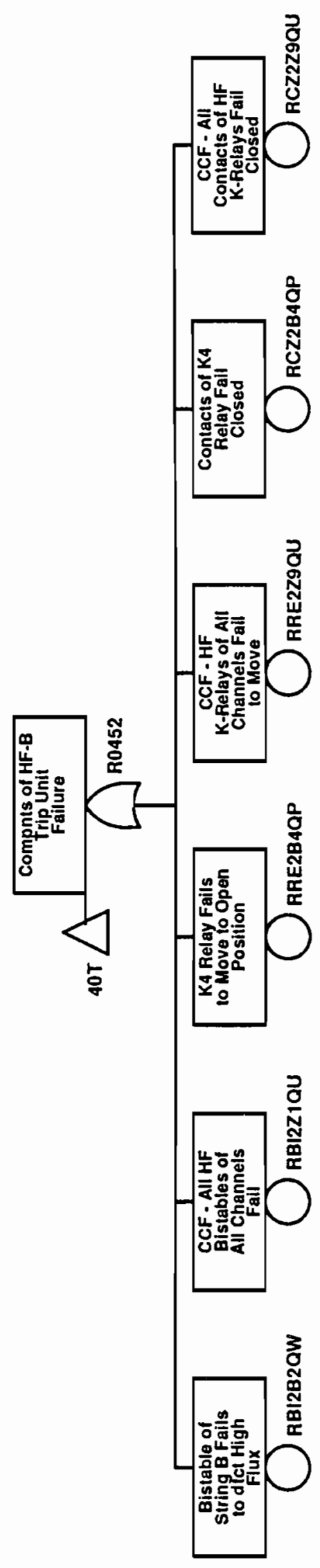

B. 2-41 


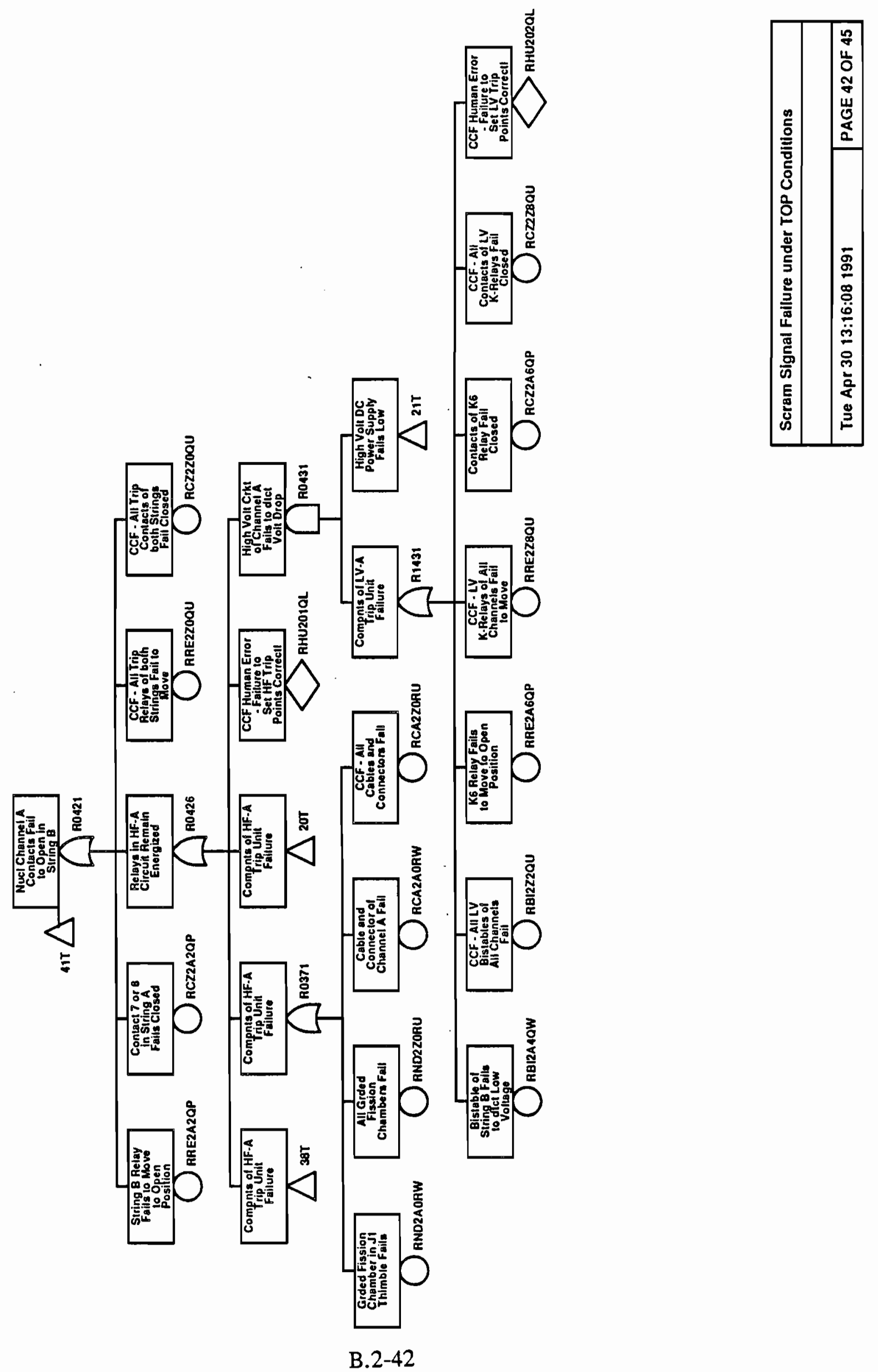




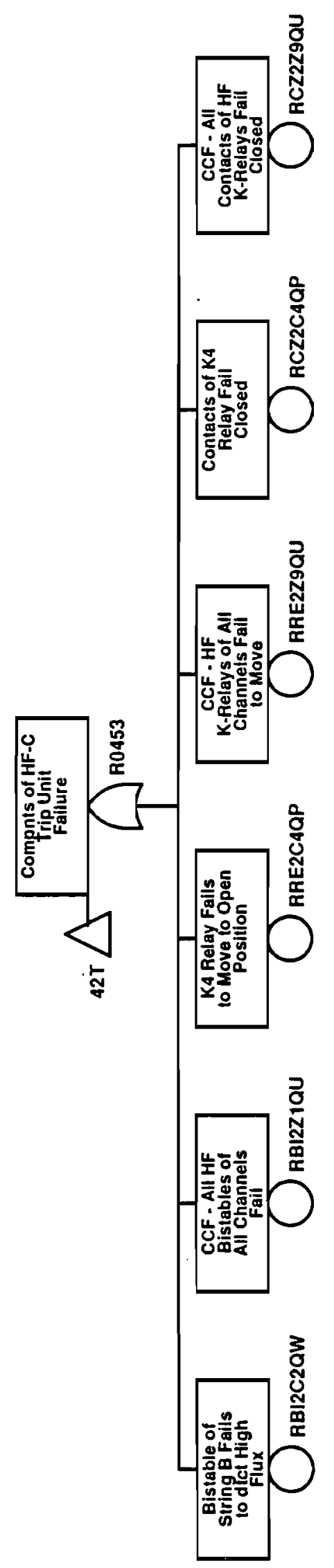




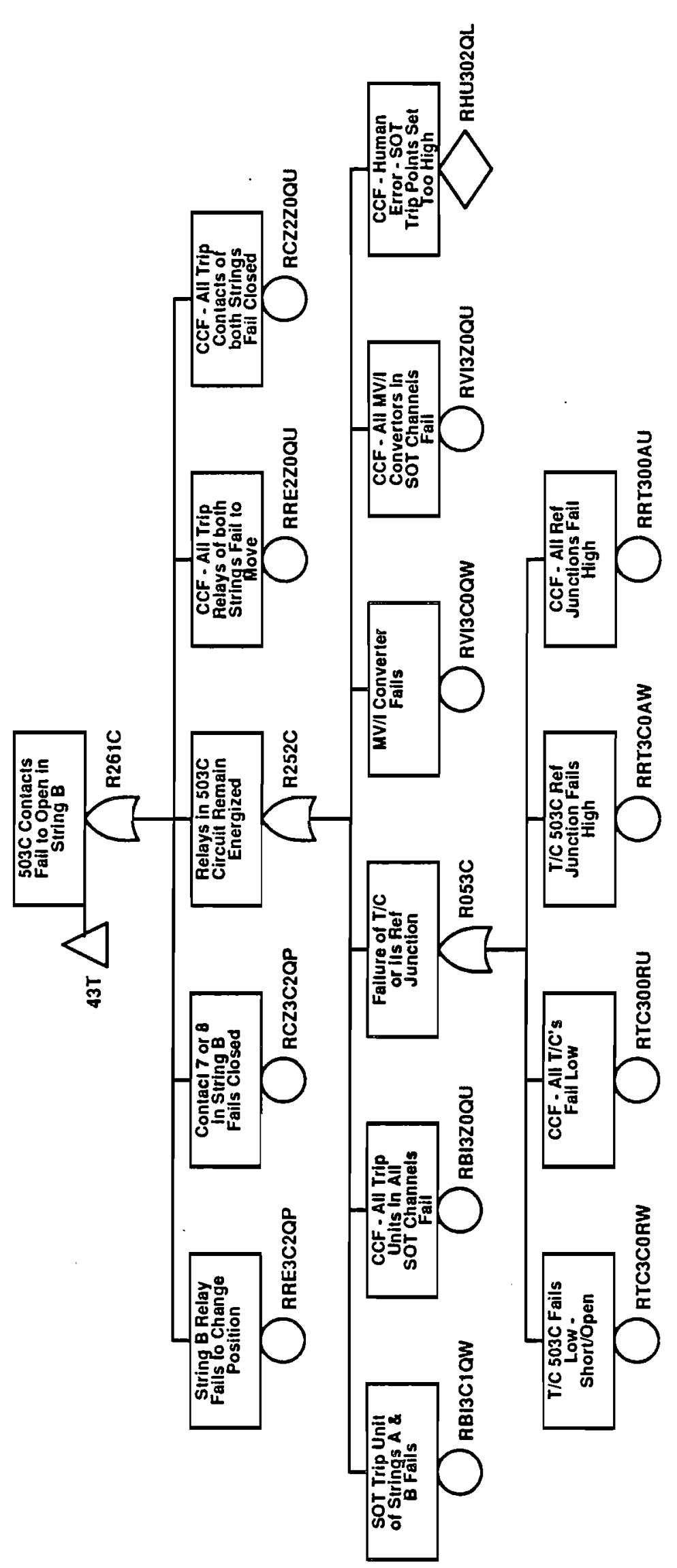




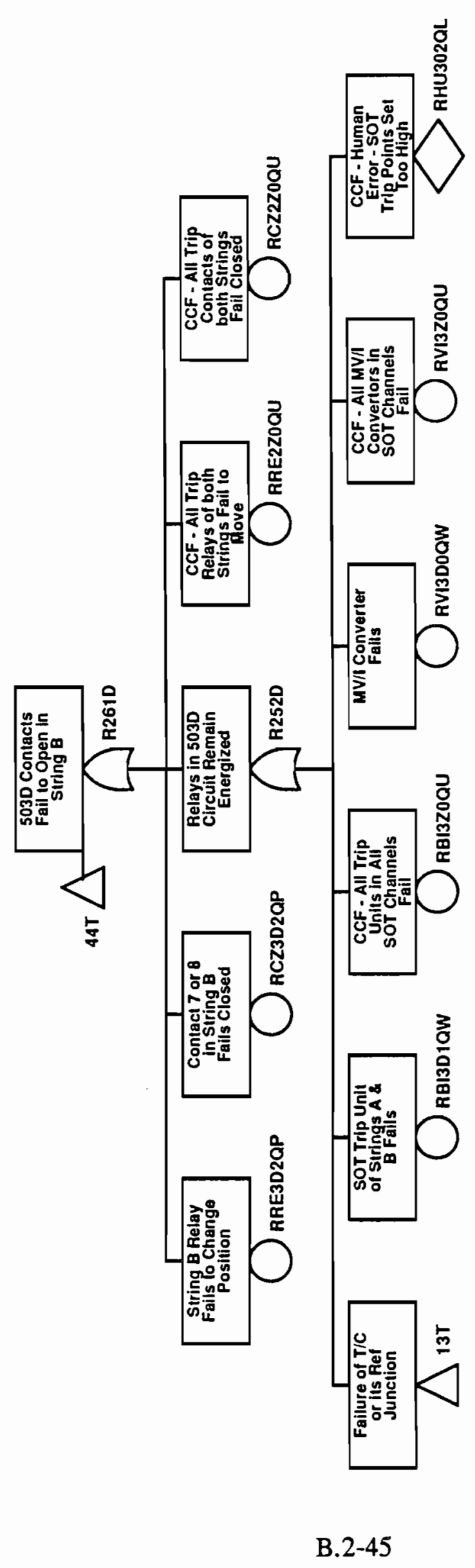




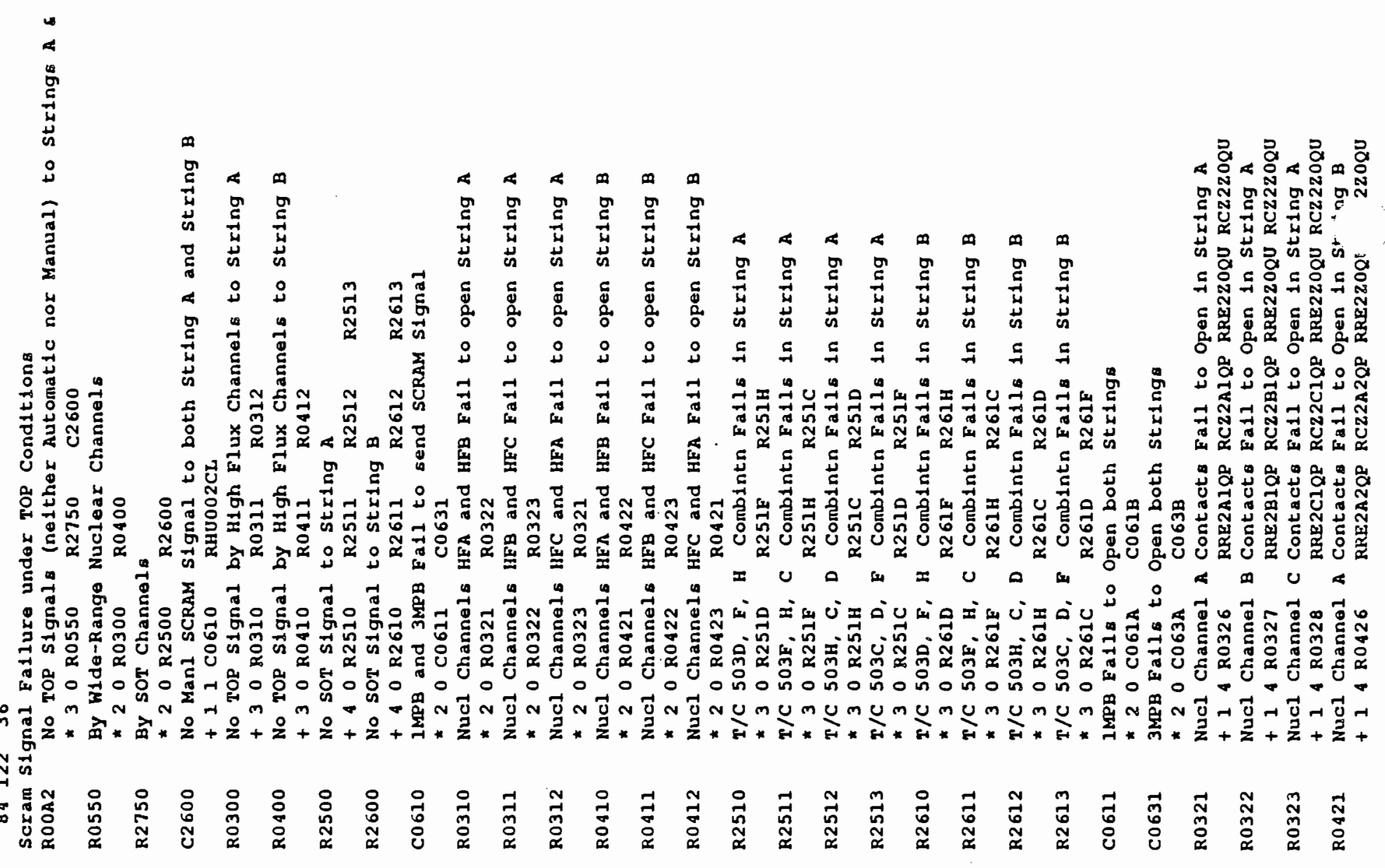




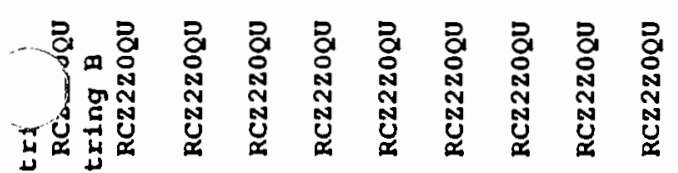
a

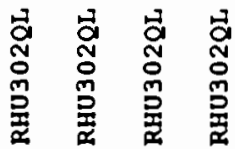

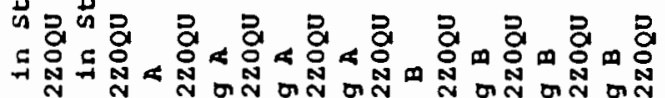

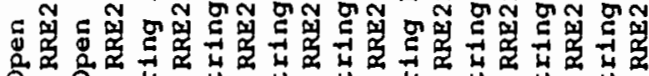

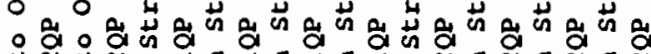

+

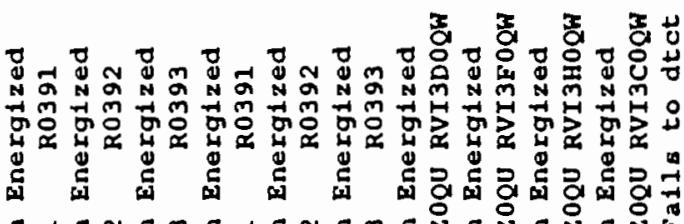

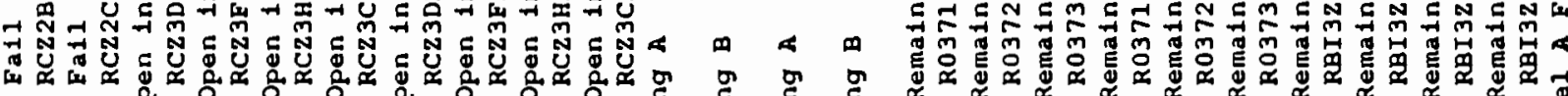

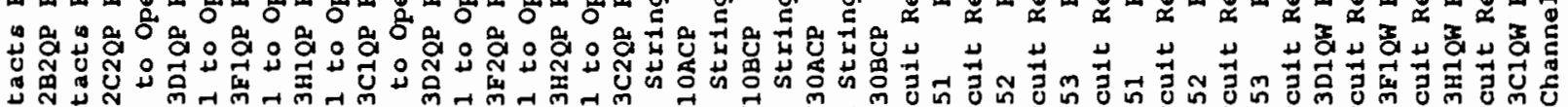

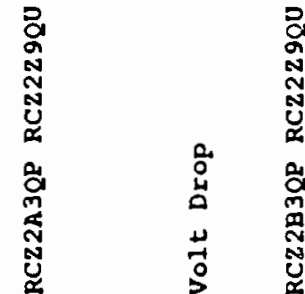

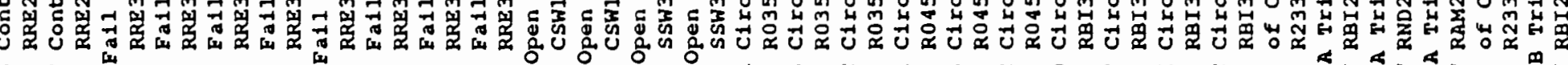

-

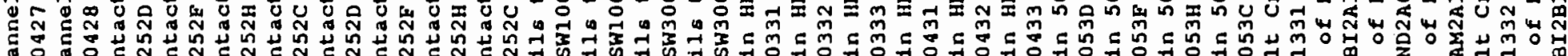

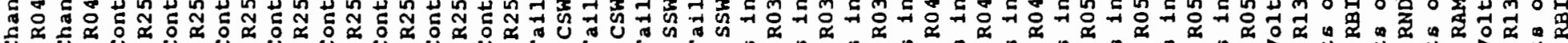

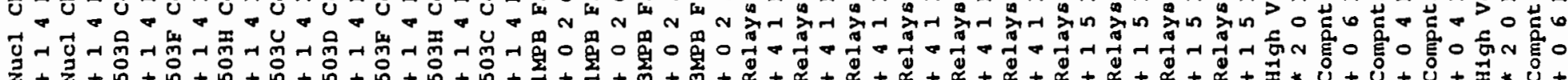

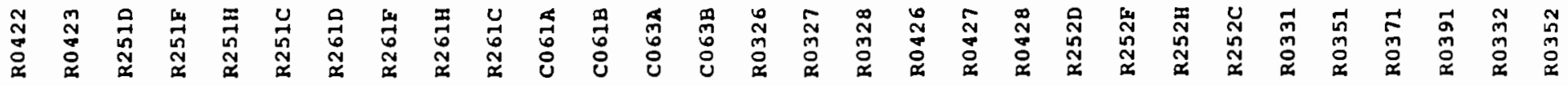




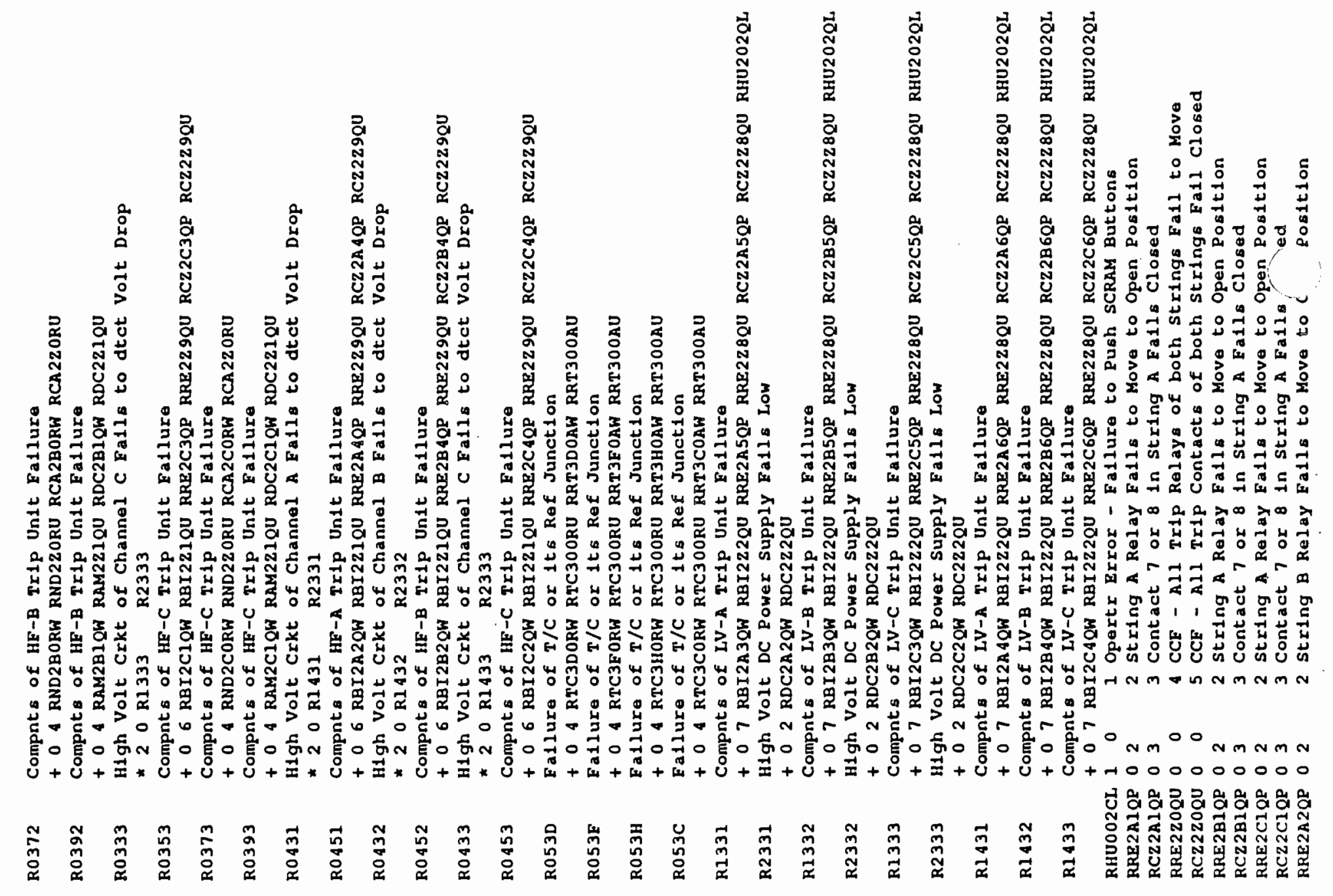




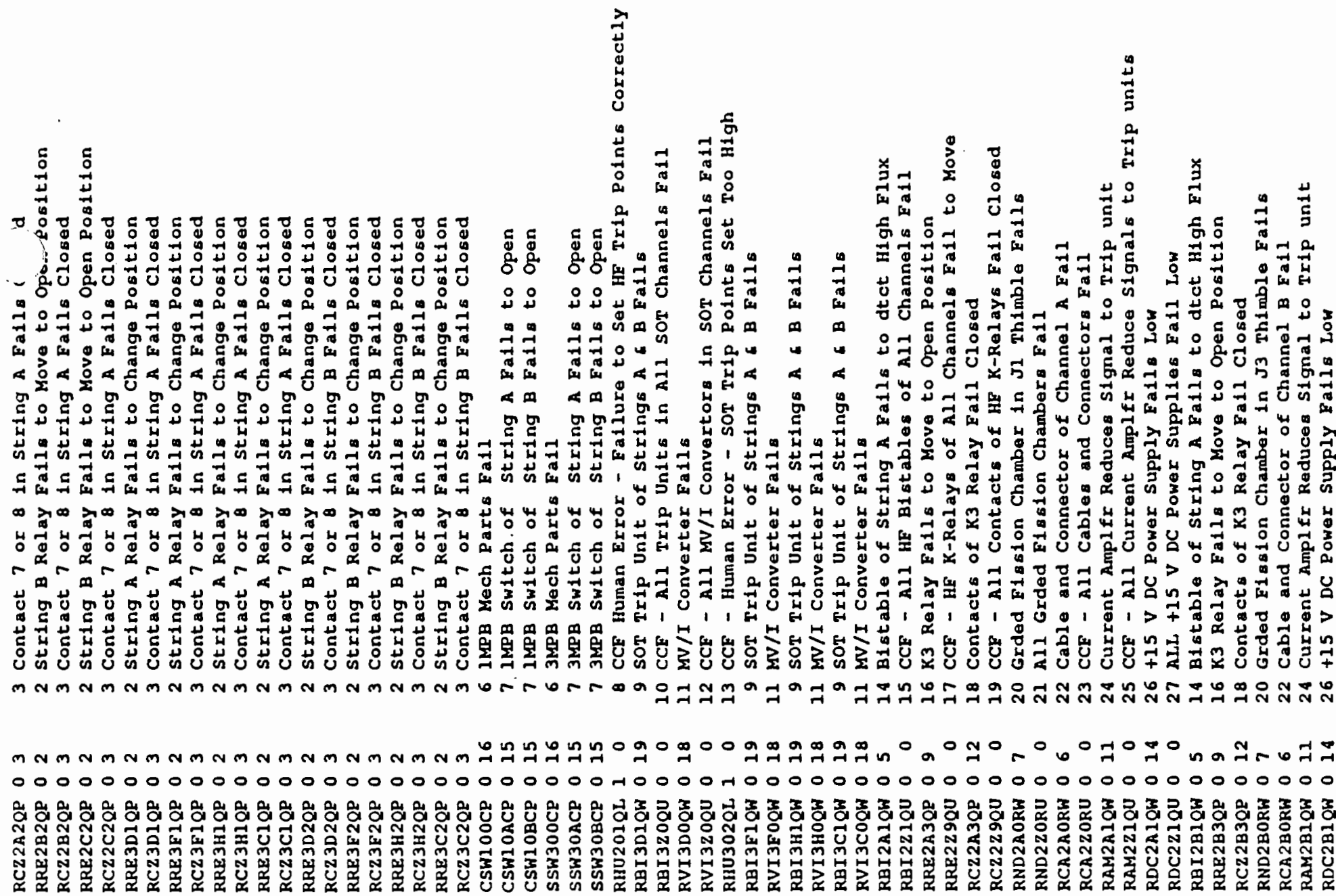




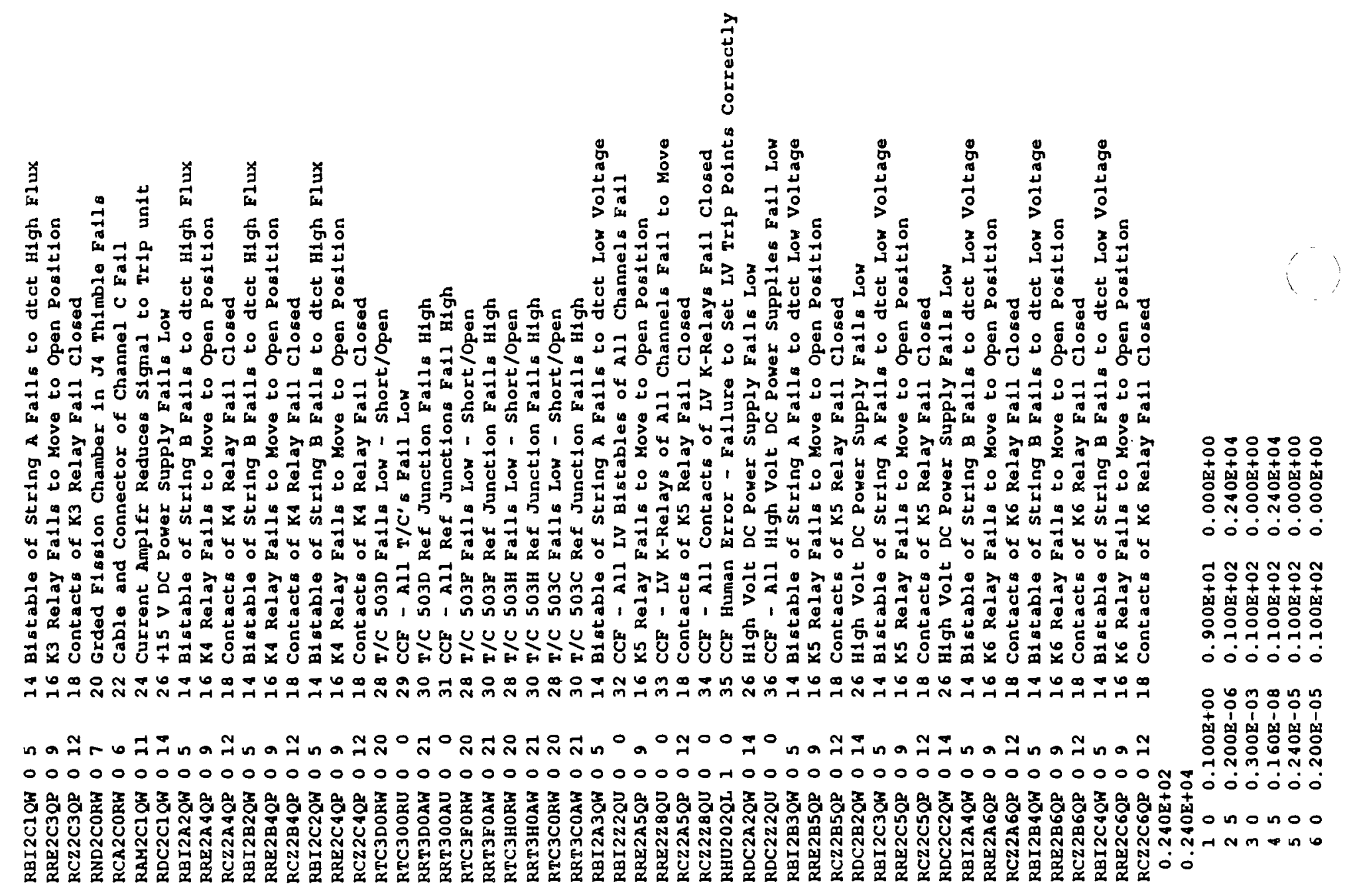

B. $2-50$ 


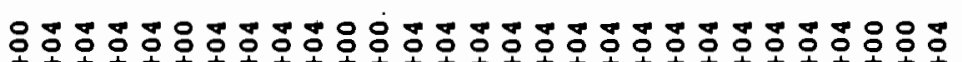

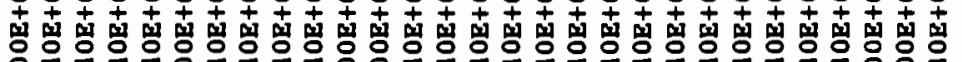

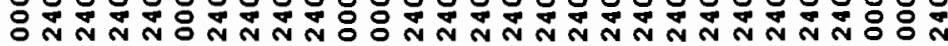

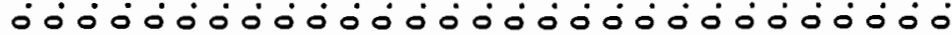

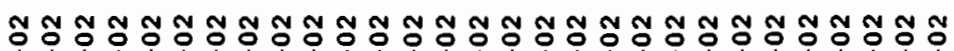

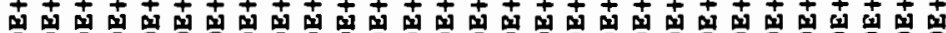

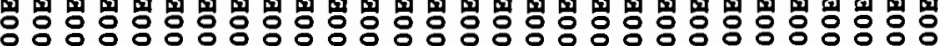

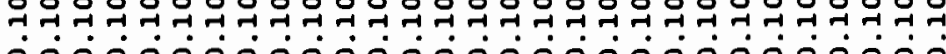

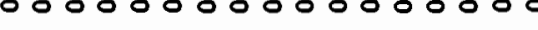

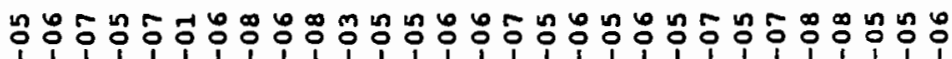

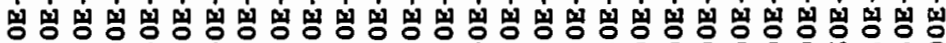

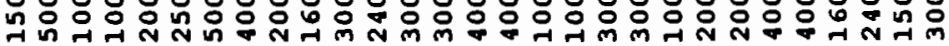

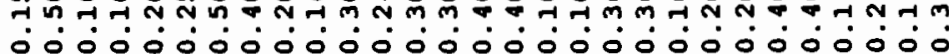
O ก

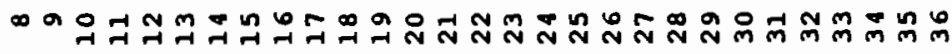




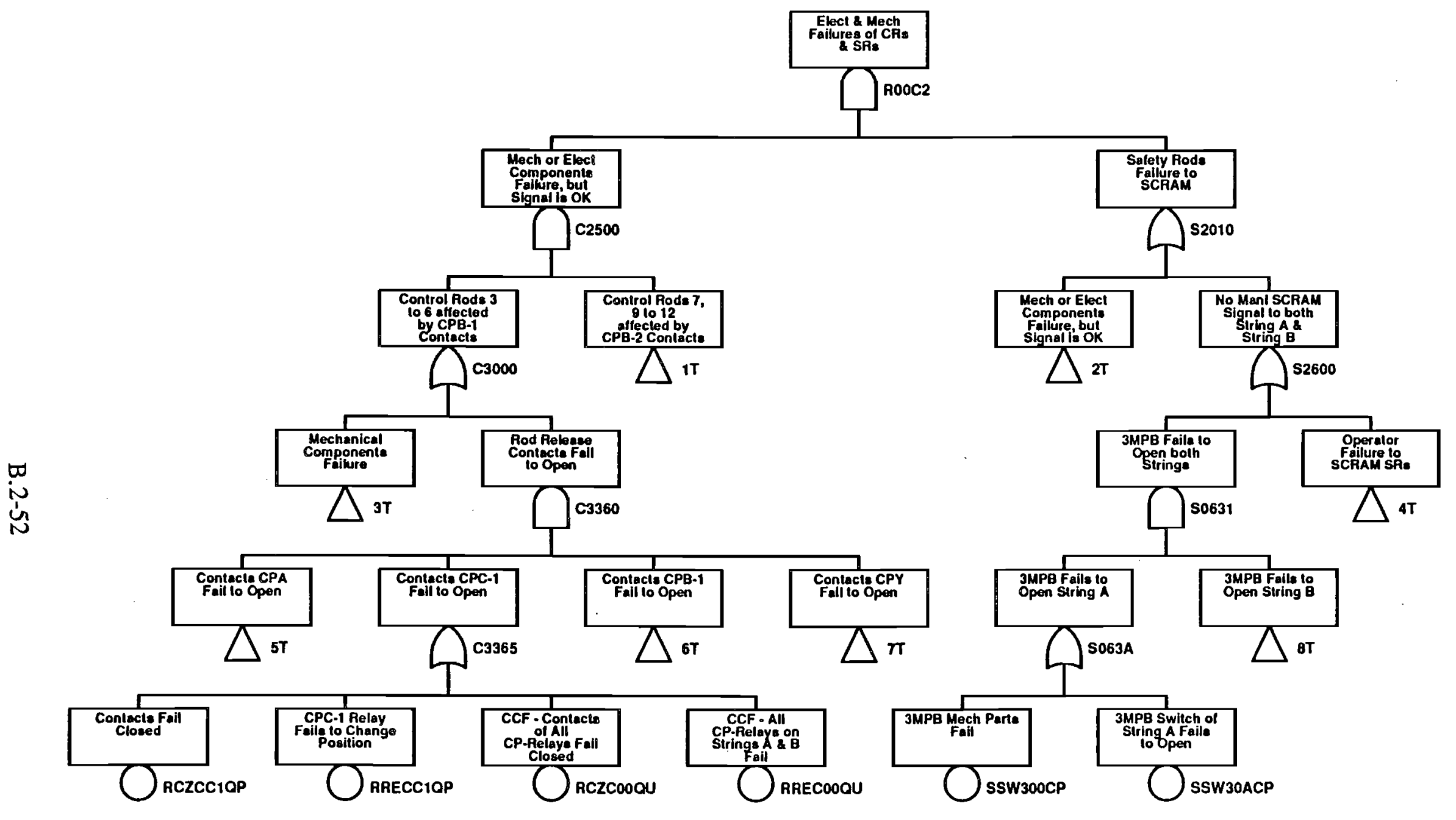

\begin{tabular}{|c|c|}
\hline Rod Drop Fallure under TC & \\
\hline Tue Apr 30 12:36:12 1991 & PAGE 1 OF 54 \\
\hline
\end{tabular}




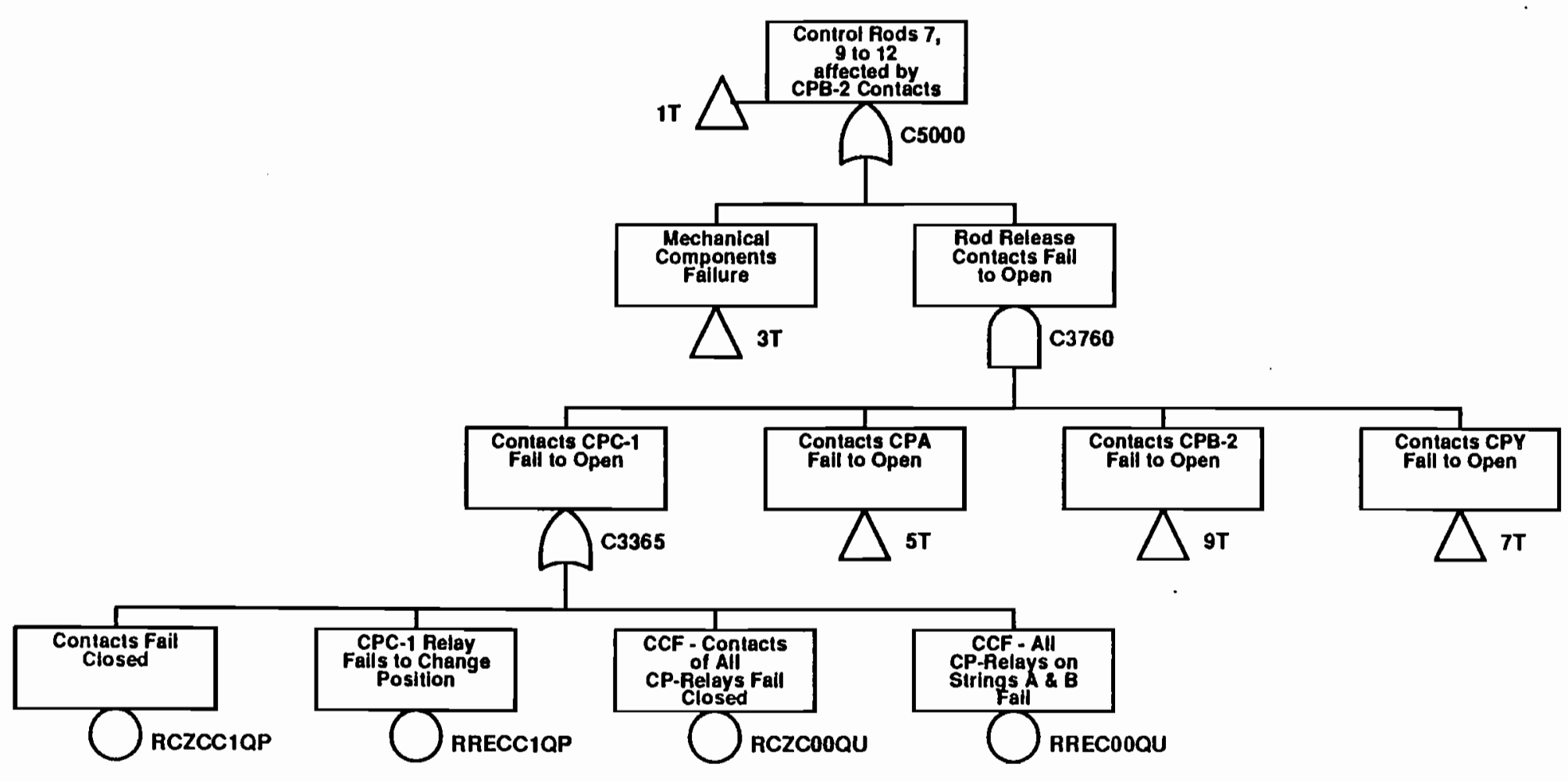

Rod Drop Failure under TOP Conditions 


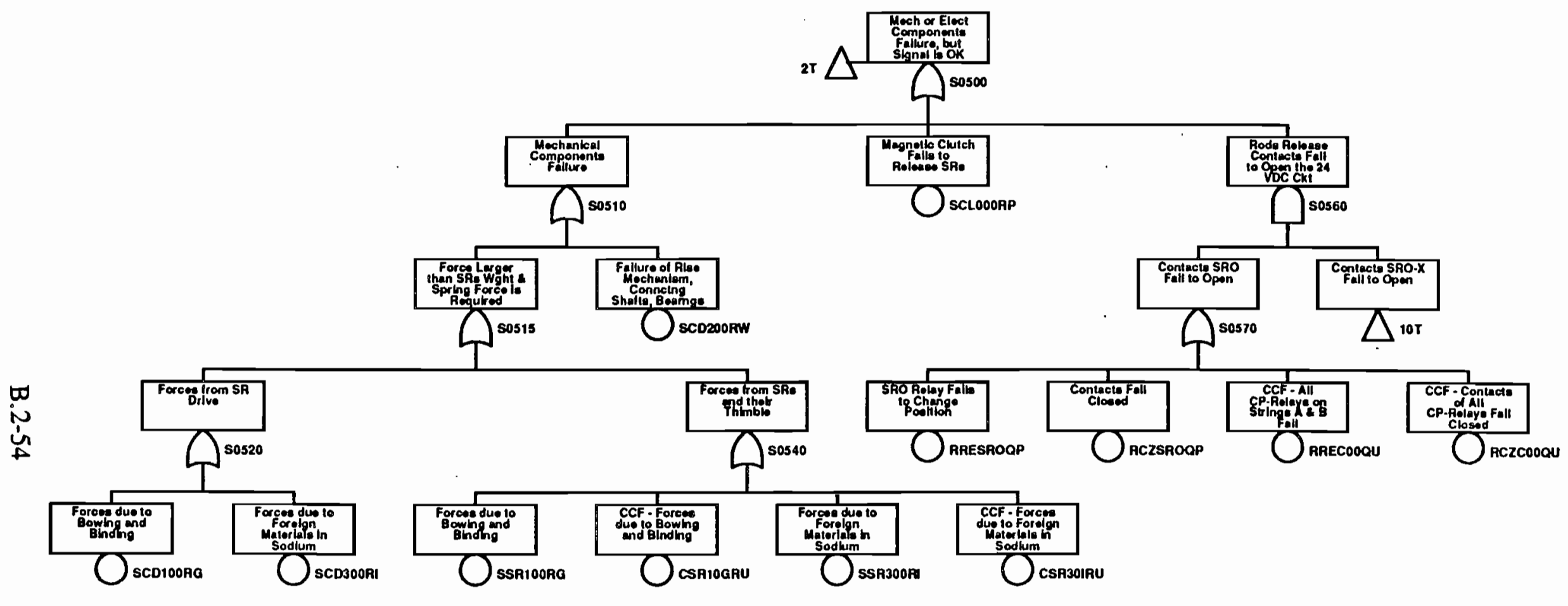

Rod Drop Fallure under TOP Condilions 


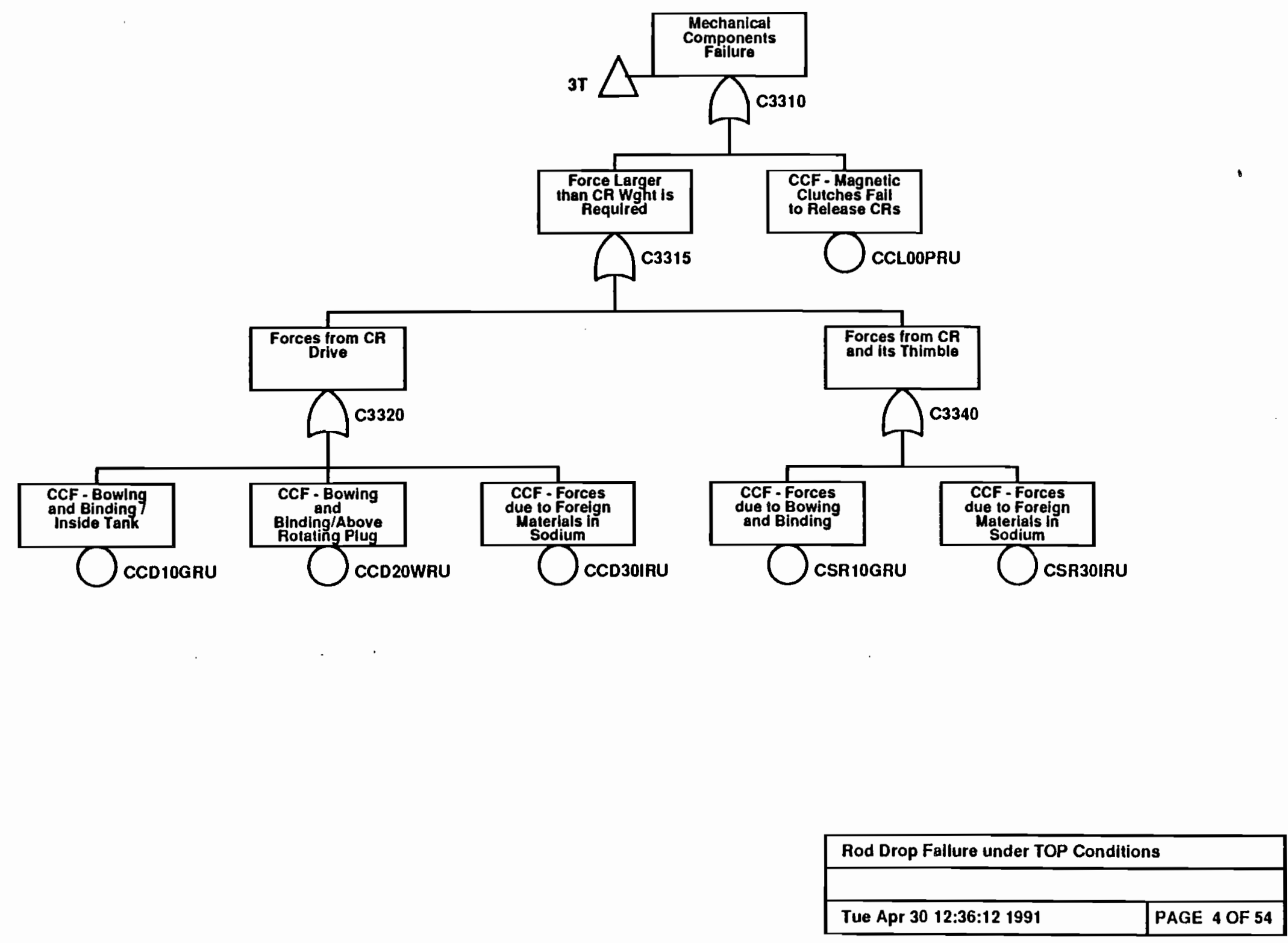


$\infty$

un

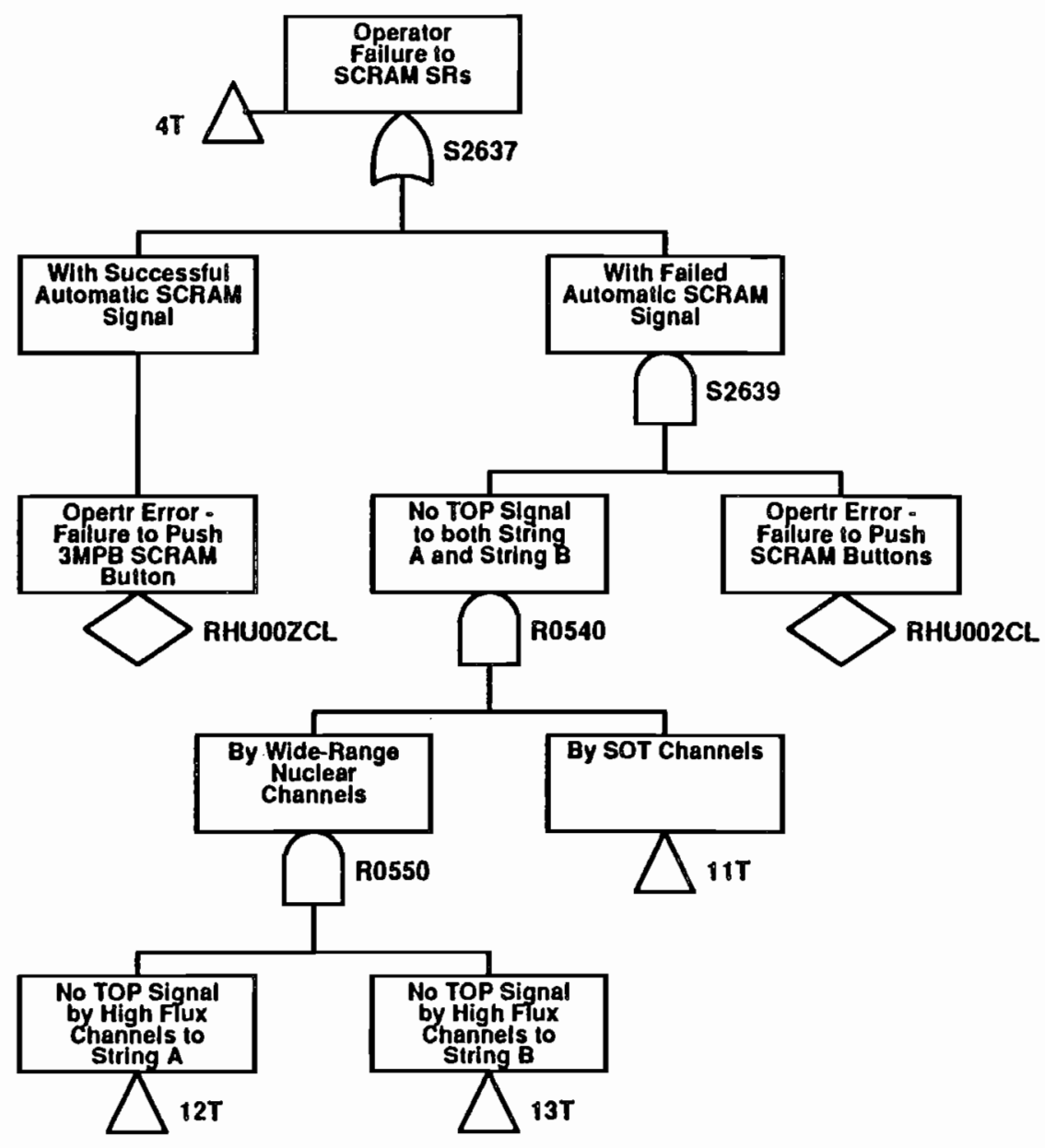

Rod Drop Fallure under TOP Condillons

Tue Apr 30 12:36:12 1991

PAGE 5 OF 54 


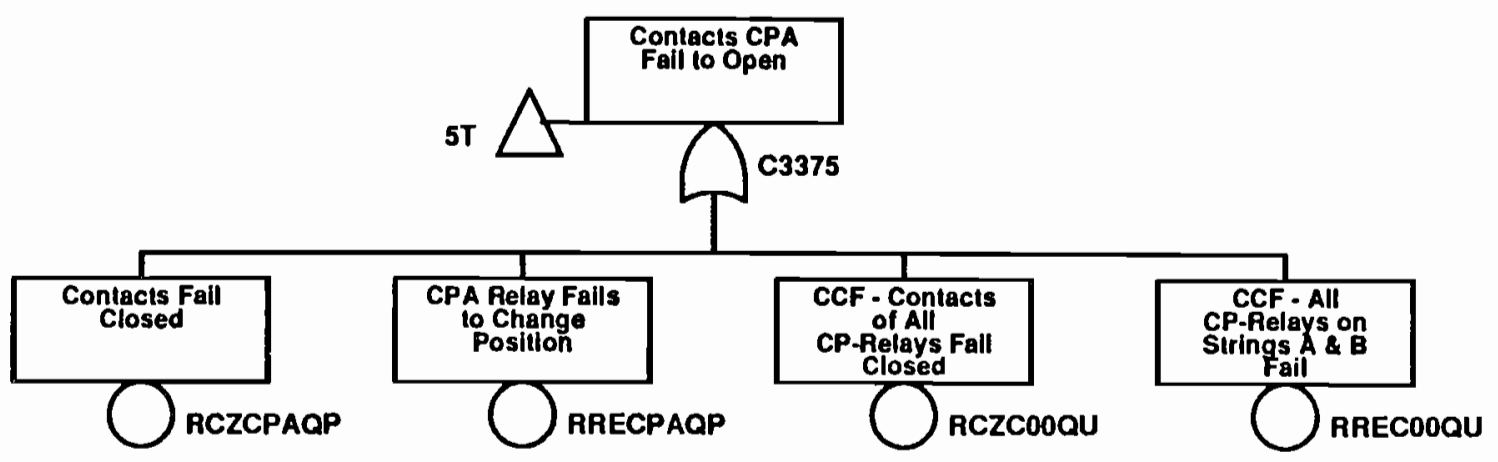

Rod Drop Failure under TOP Conditions 


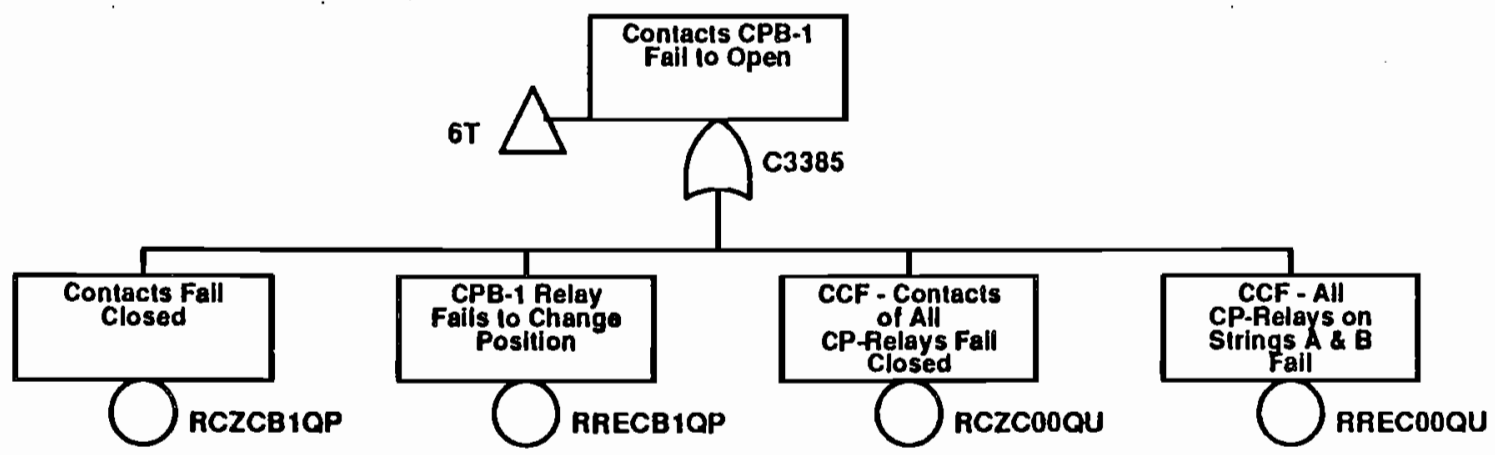

Rod Drop Failure under TOP Condilions 


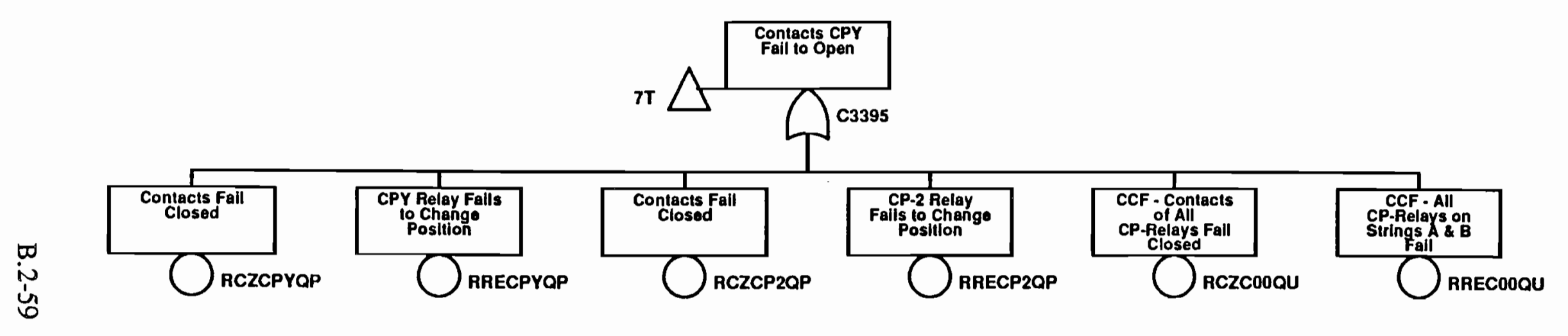

Rod Drop Fallure under TOP Conditions 


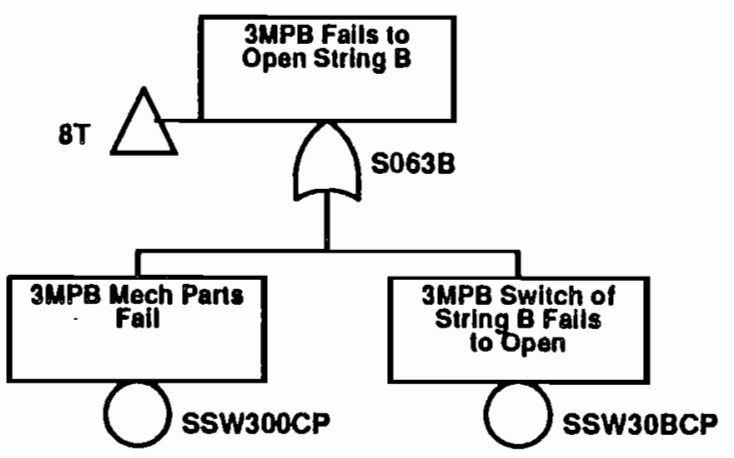

Rod Drop Failure under TOP Conditlons 


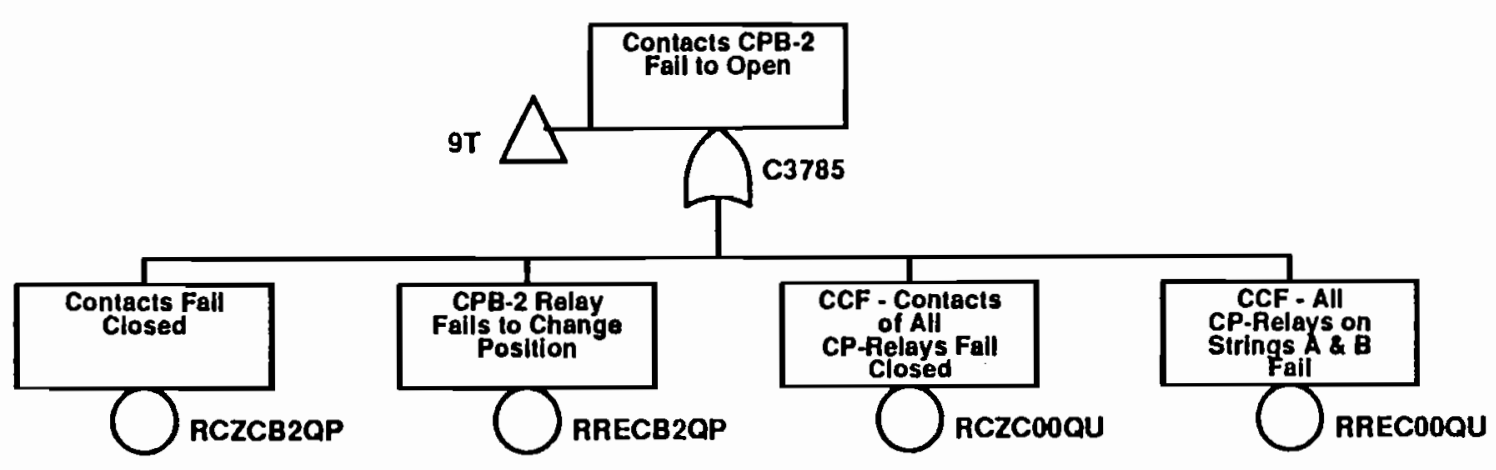

Rod Drop Failure under TOP Conditions

Tue Apr 30 12:36:12 1991 


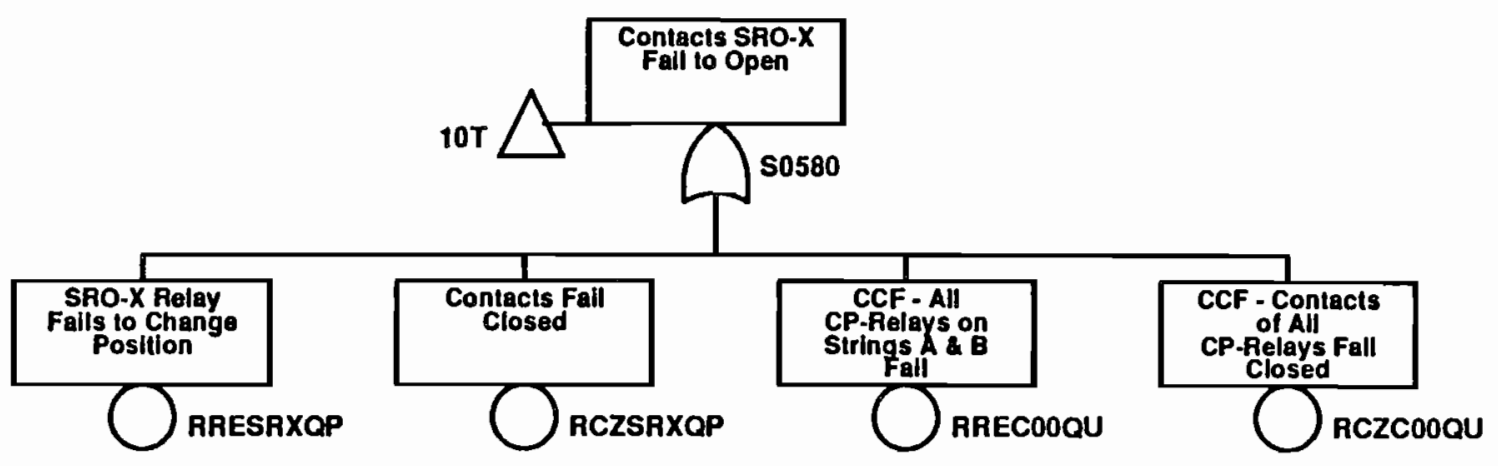

Rod Drop Fallure under TOP Conditions 
্ֻৰ

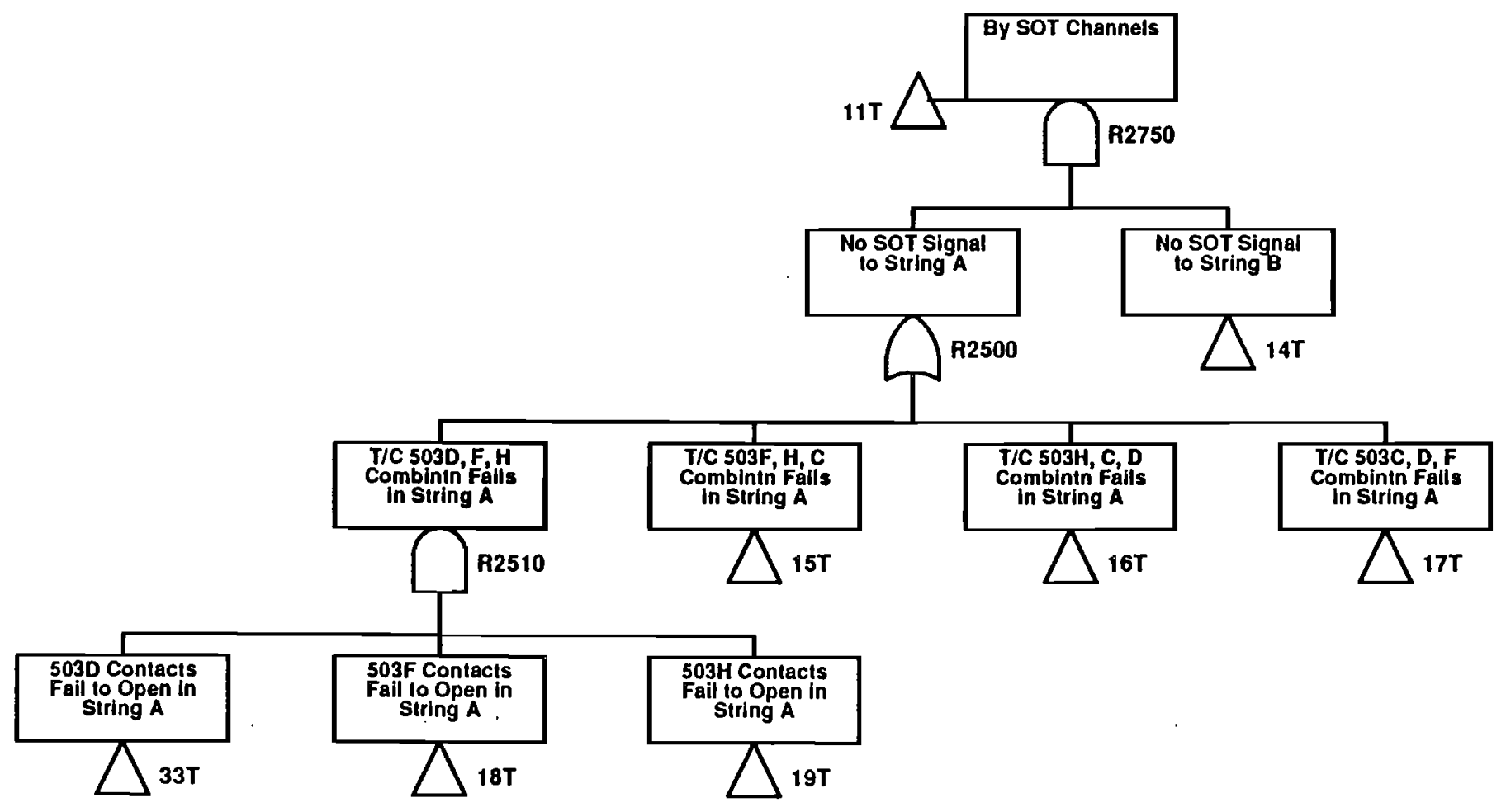

Rod Drop Failure under TOP Conditions

Tue Apr 30 12:36:12 1991 


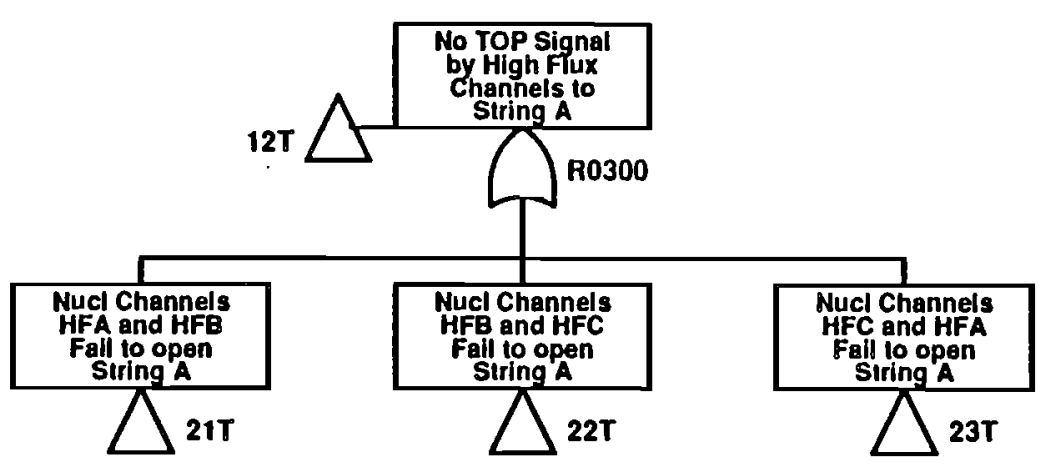

Rod Drop Failure under TOP Conditions

Tue Apr 30 12:36:12 1991 


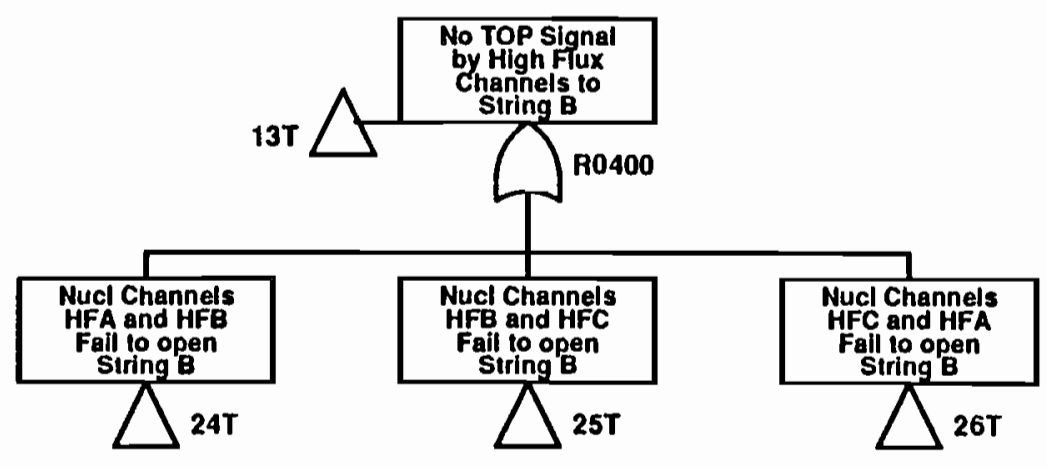

Rod Drop Fallure under TOP Condlitions

Tue Apr 30 12:36:12 1991 
必

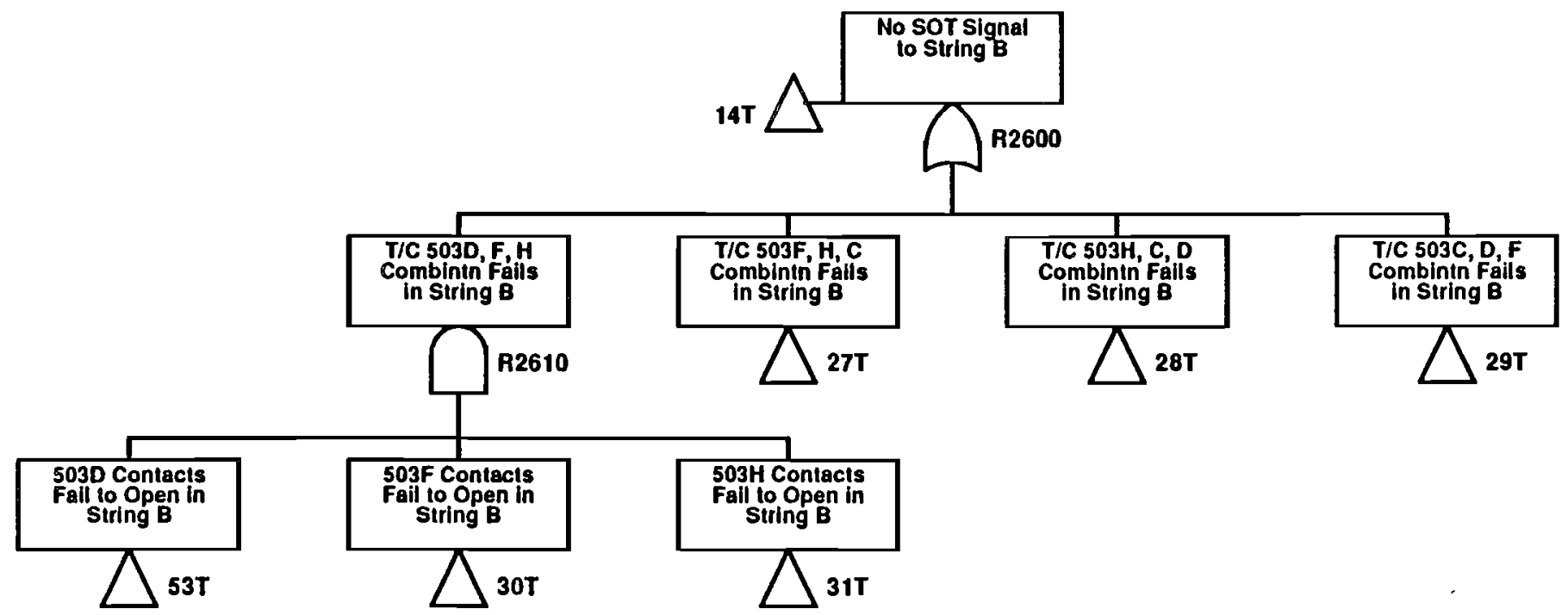


0
$\vdots$
$\vdots$
$\vdots$

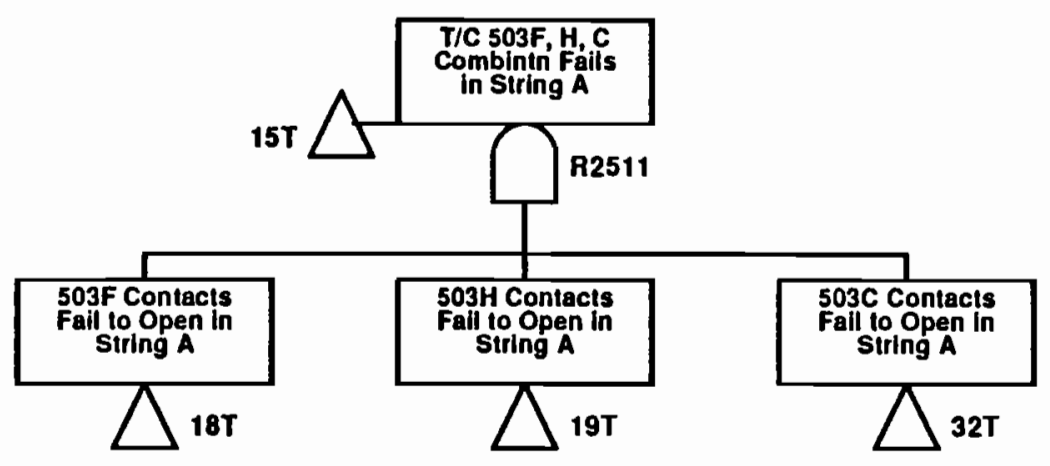

Rod Drop Failure under TOP Conditions

Tue Apr 30 12:36:12 1991 


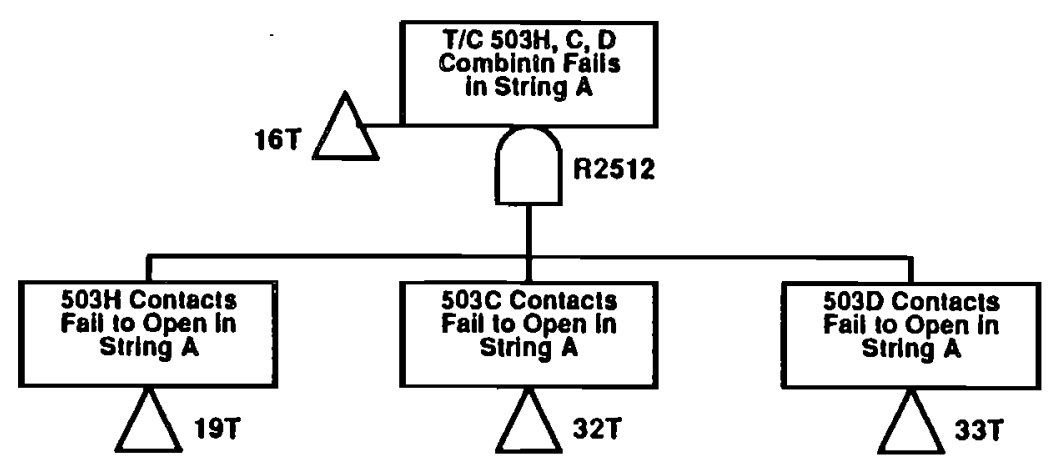

Rod Drop Failure under TOP Condilions 


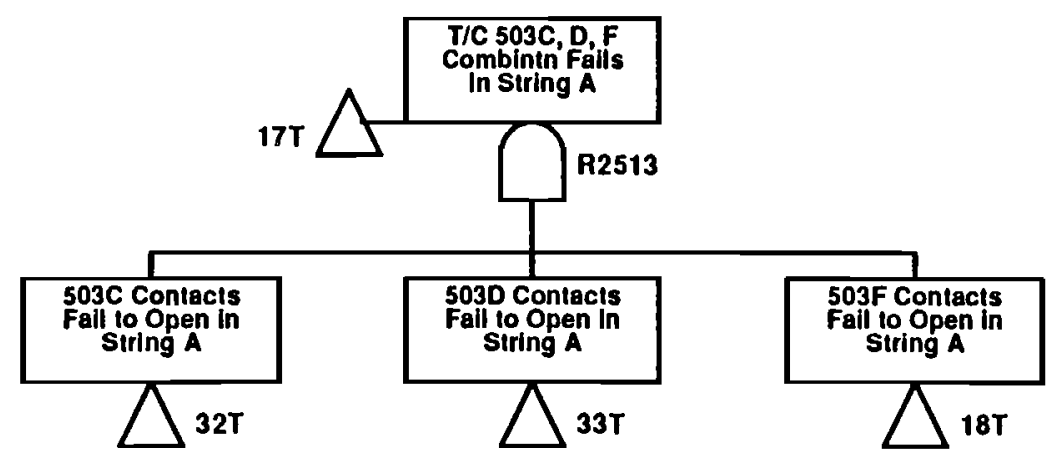

Rod Drop Failure under TOP Conditions 


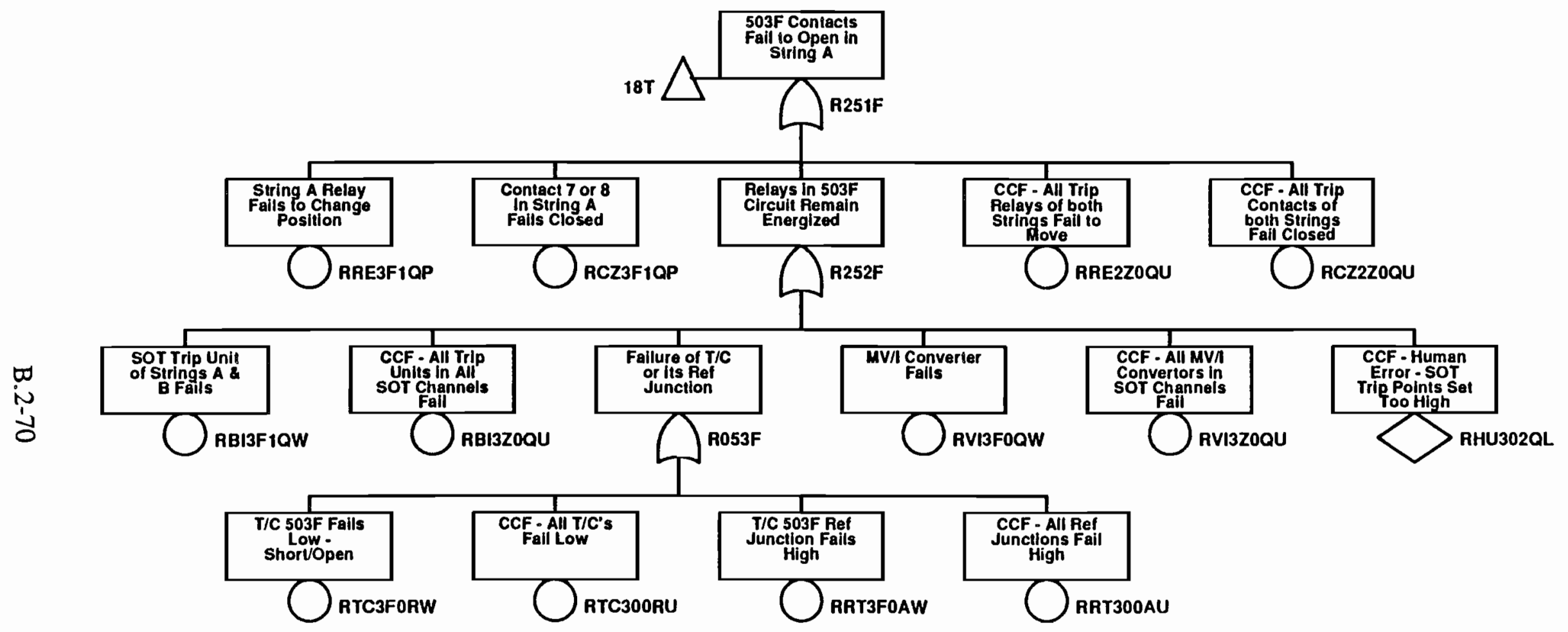

Rod Drop Failure under TOP Conditions 


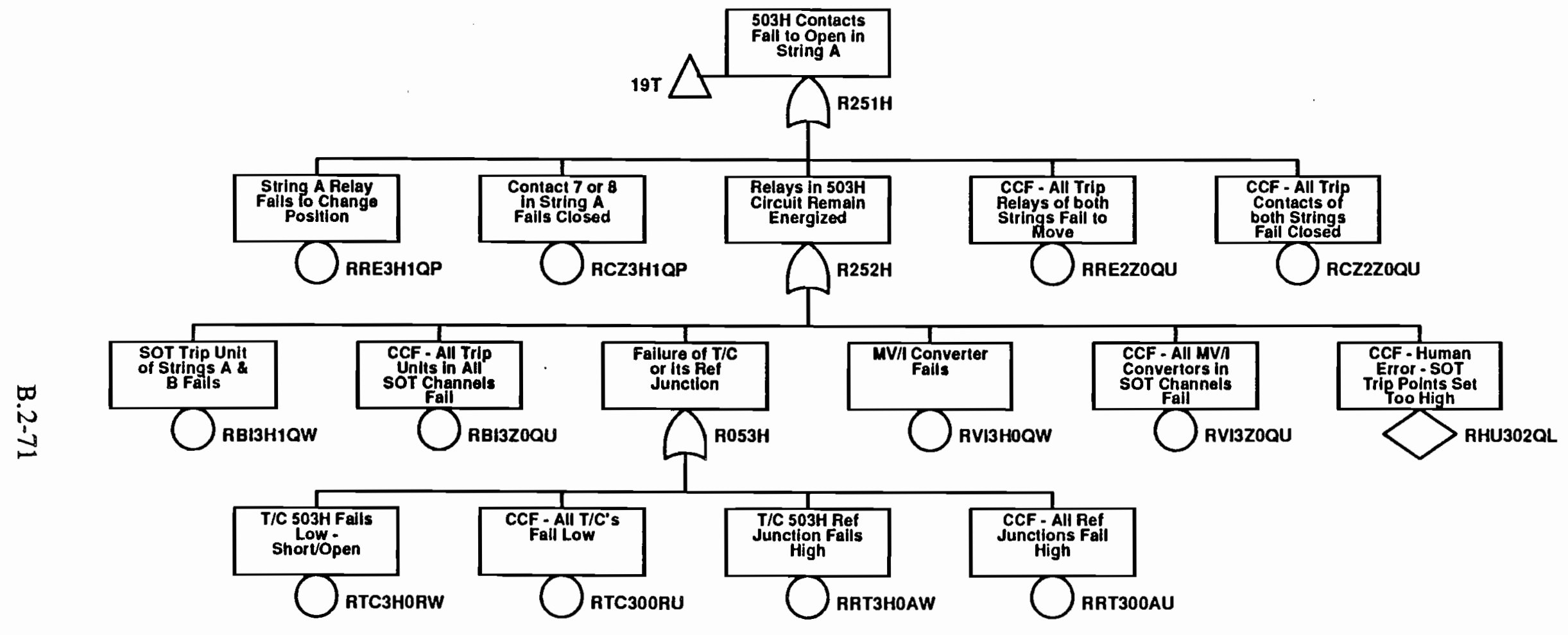

Rod Drop Failure under TOP Conditions

Tue Apr 30 12:36:12 1991

PAGE 20 OF 54 


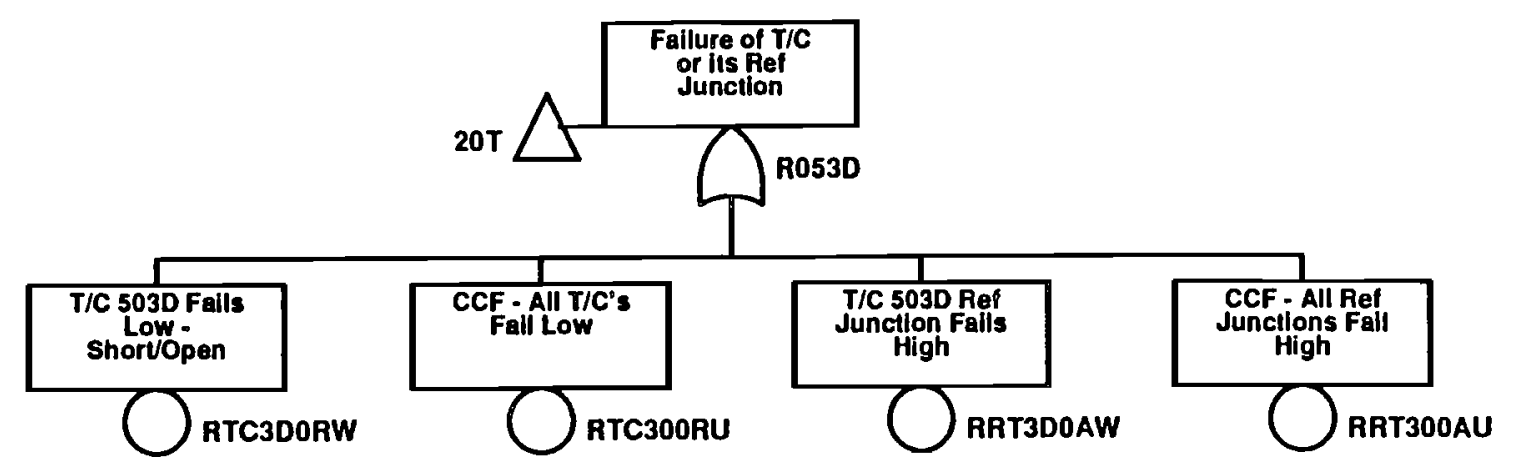

Rod Drop Fallure under TOP Conditions 


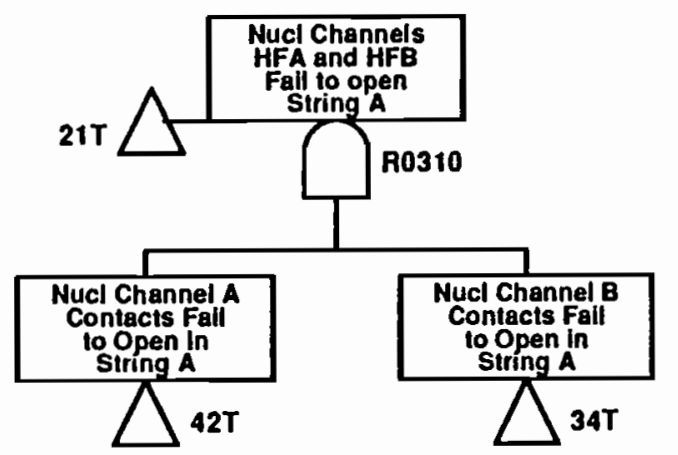




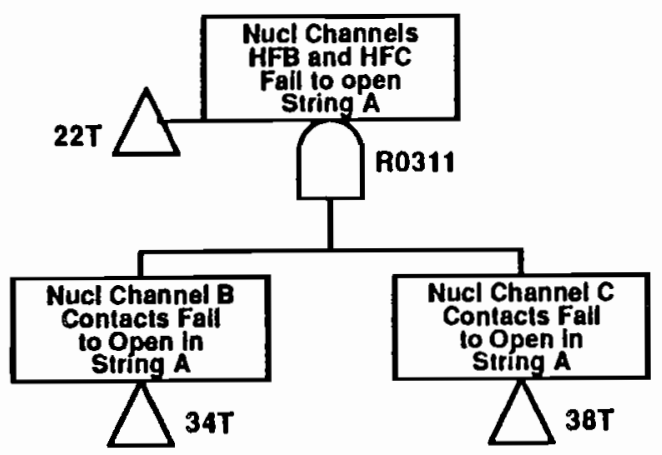

Rod Drop Fallure under TOP Condilions

Tue Apr 30 12:36:12 1991 


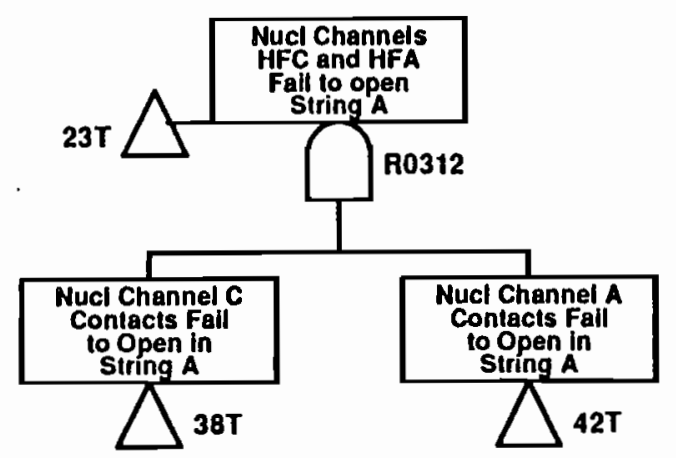

Rod Drop Fallure under TOP Conditions 


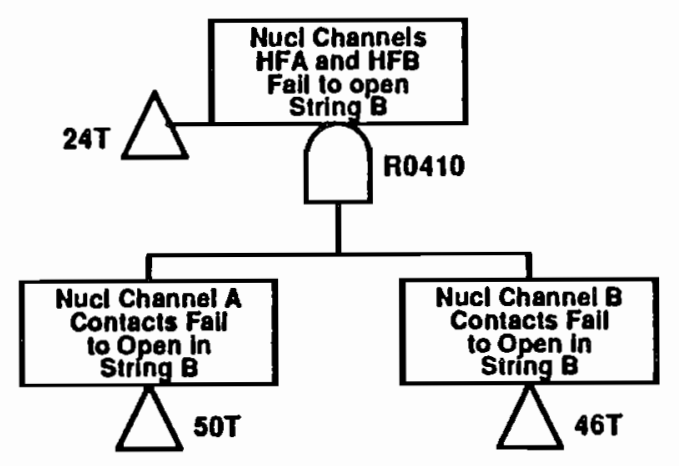

Rod Drop Fallure under TOP Conditions

Tue Apr 30 12:36:12 1991 


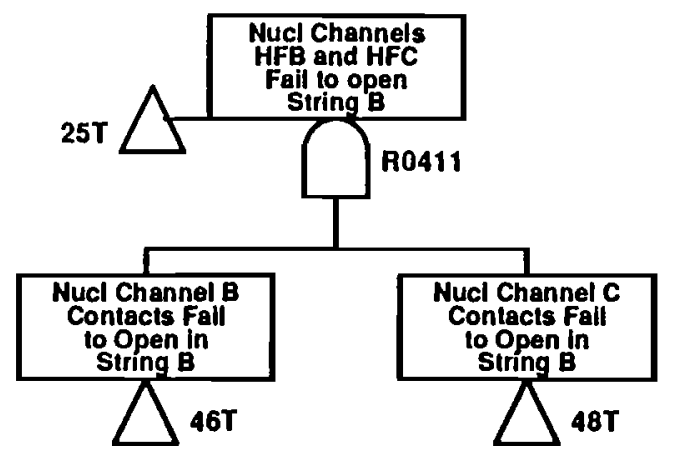

Rod Drop Fallure under TOP Conditions

Tue Apr 30 12:36:12 1991 


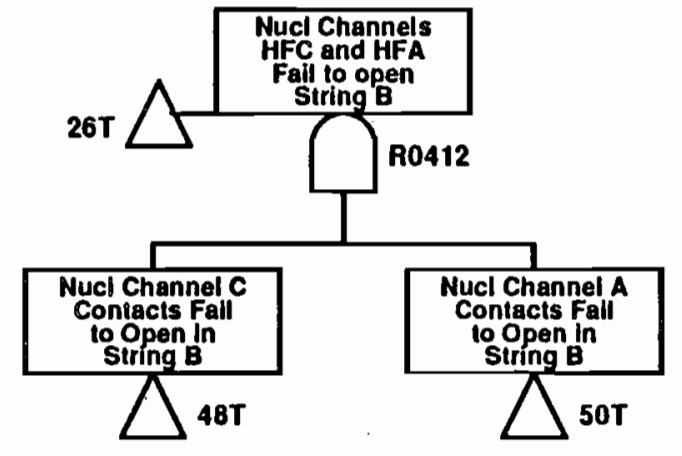

Rod Drop Fallure under TOP Conditions

Tue Apr 30 12:36:12 1991 


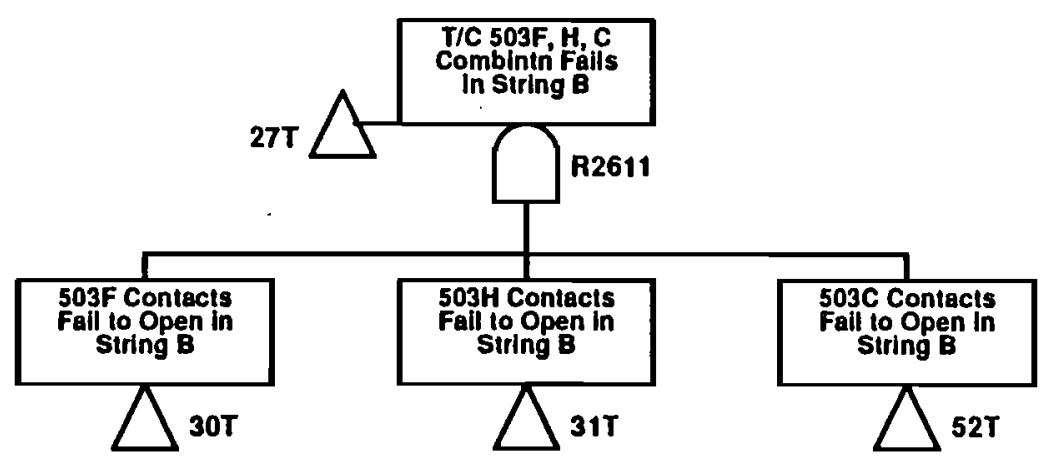

Rod Drop Failure under TOP Conditions 


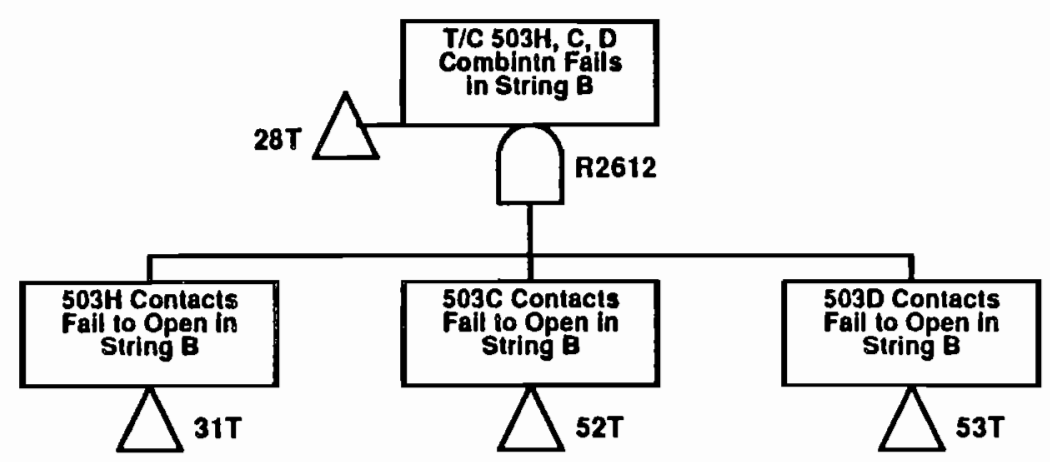

Rod Drop Failure under TOP Conditions

Tue Apr 30 12:36:12 1991 


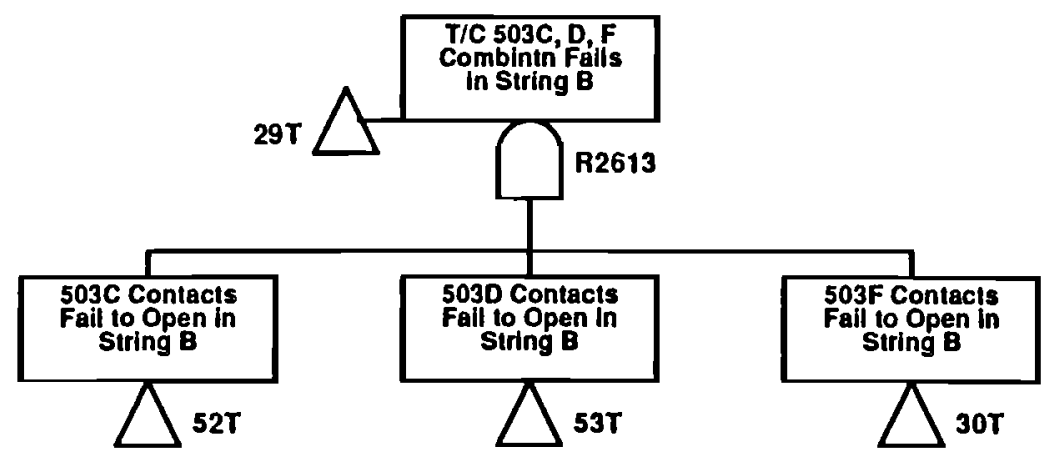

Rod Drop Fallure under TOP Conditions 


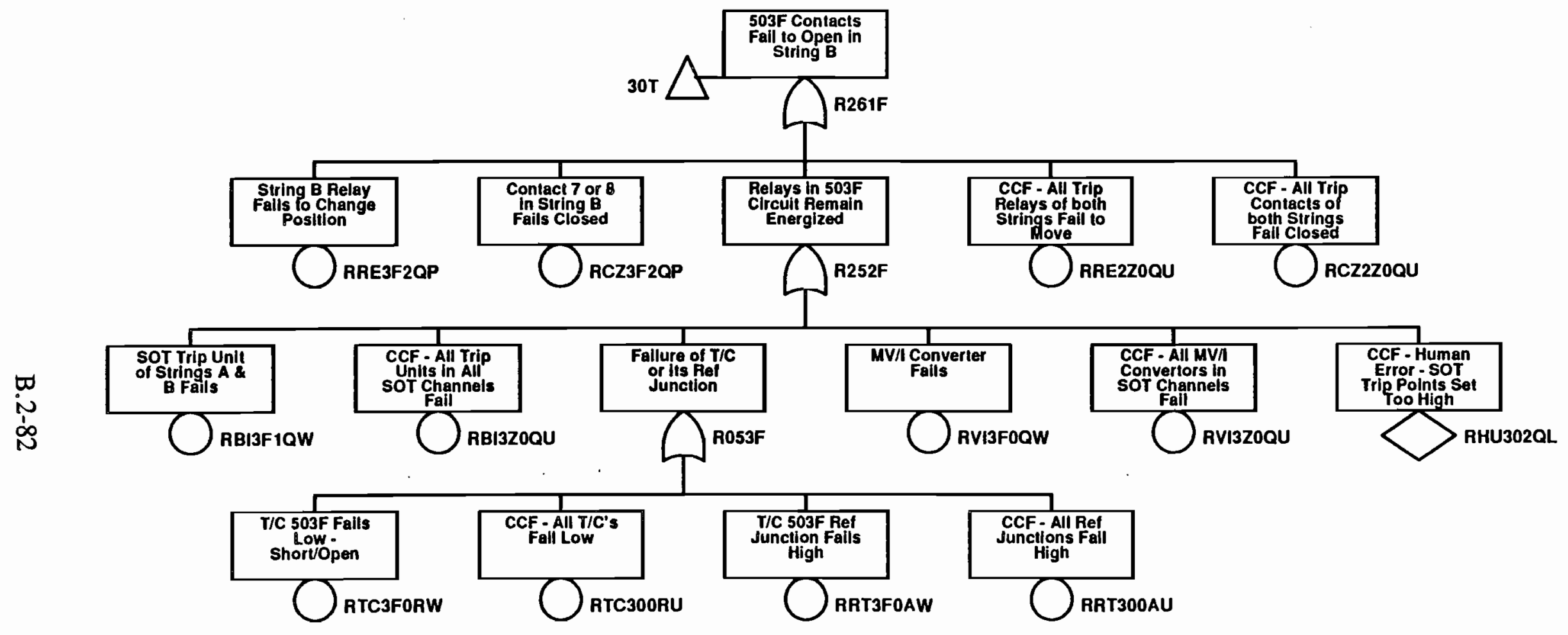

\begin{tabular}{|l|l|}
\hline \multicolumn{2}{|l|}{ Rod Drop Faiłure under TOP Conditions } \\
\hline Tue Apr 30 12:36:12 1991 & PAGE 31 OF 54 \\
\hline
\end{tabular}




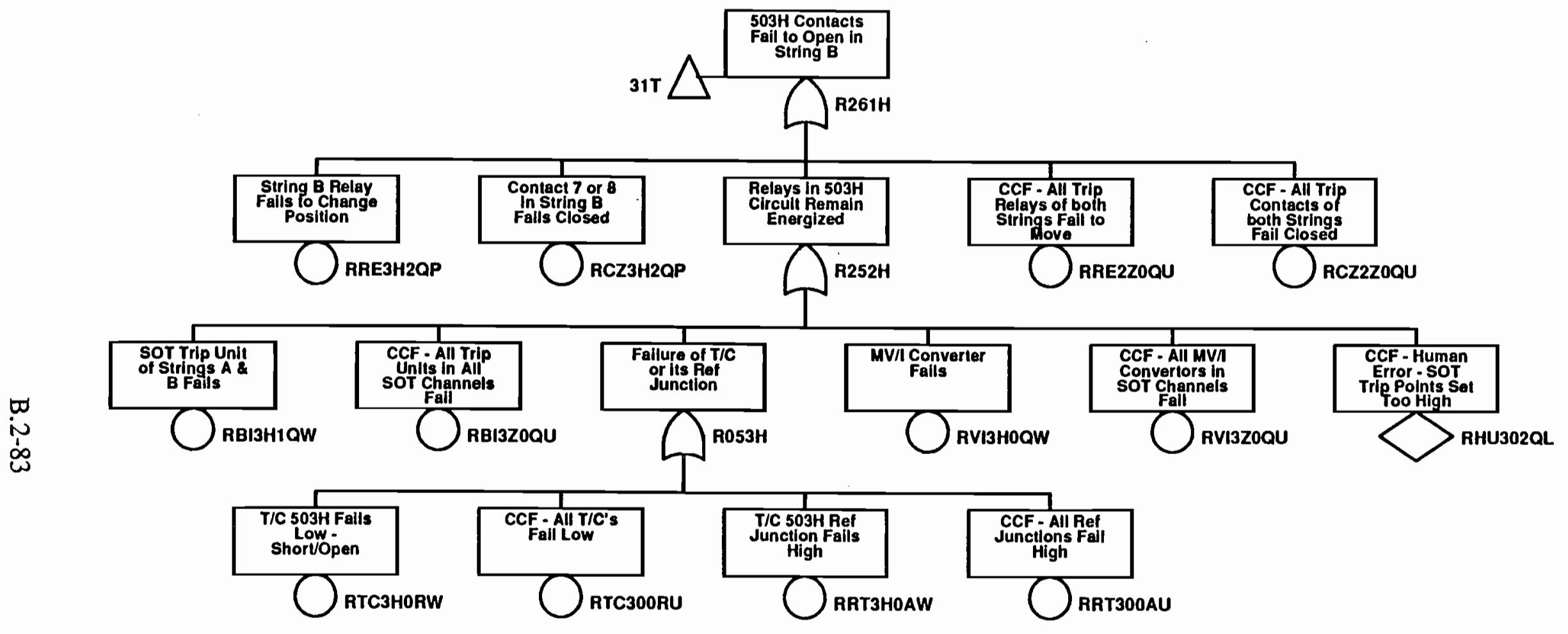

Rod Drop Fallure under TOP Conditions

Tue Apr 30 12:36:12 1991

PAGE 32 OF 54 


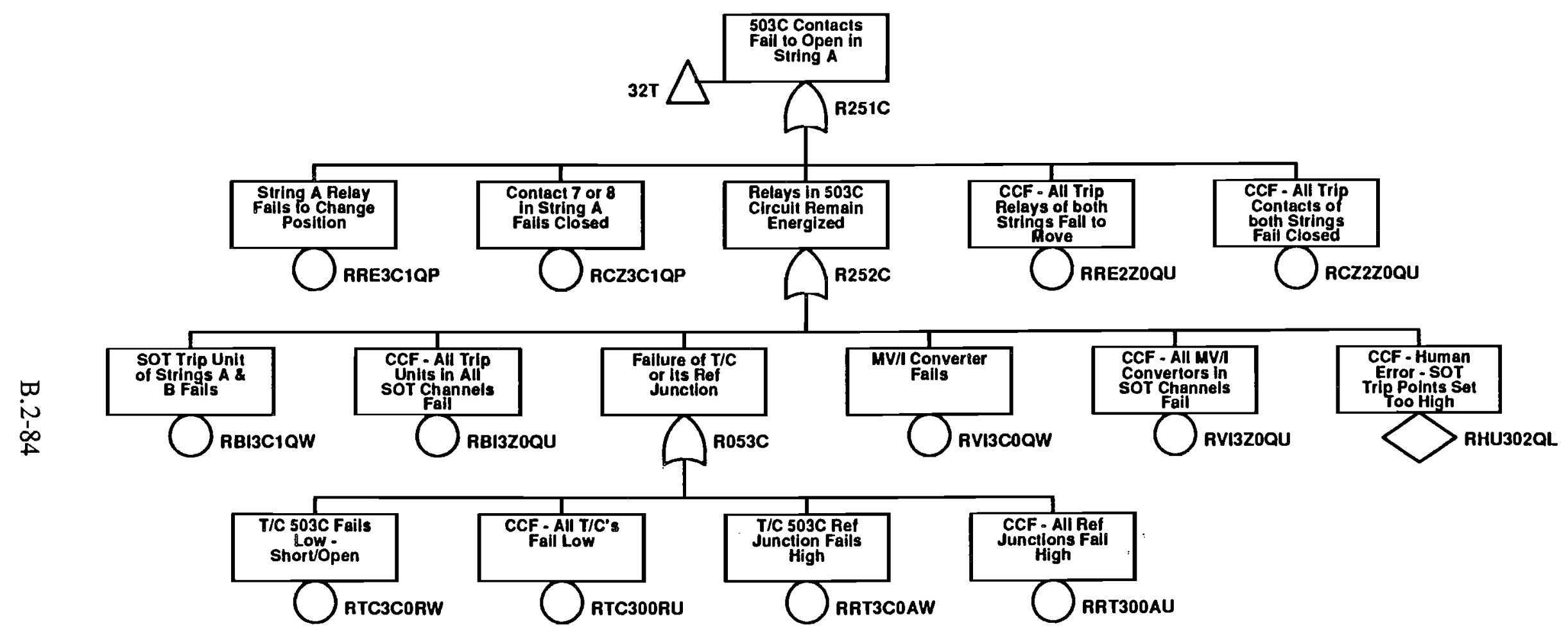

\begin{tabular}{|l|l|}
\hline \multicolumn{2}{|l|}{ Rod Drop Failure under TOP Conditions } \\
\hline \multicolumn{2}{|l|}{} \\
\hline Tue Apr 30 12:36:12 1991 & PAGE 33 OF 54 \\
\hline
\end{tabular}




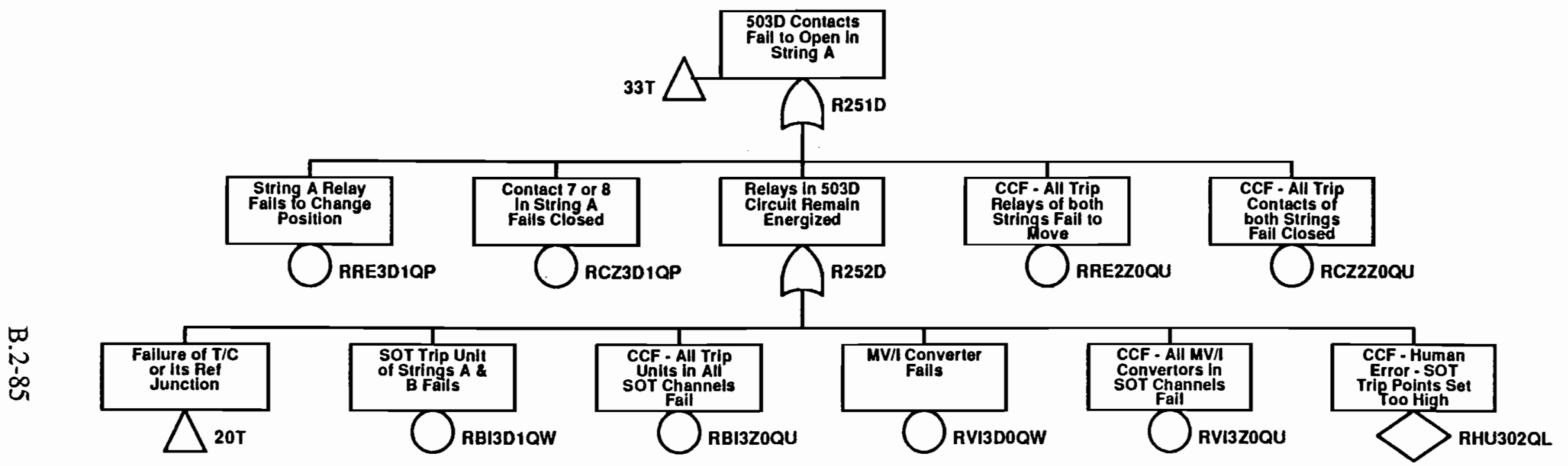

Rod Drop Failure under TOP Conditions

Tue Apr 30 12:36:12 1991 


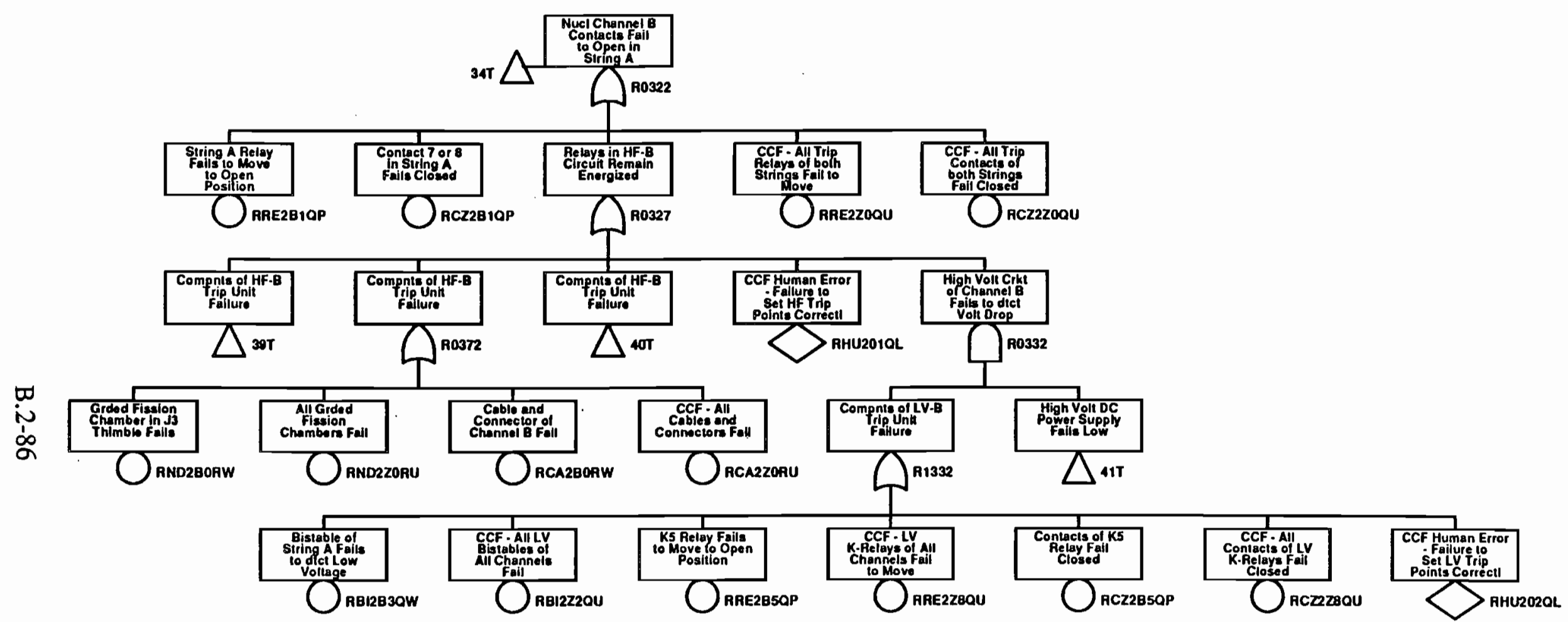

Rod Drop Fallure under TOP Condilions

Tue Apr 30 12:36:12 1991

PAGE 35 OF 54 


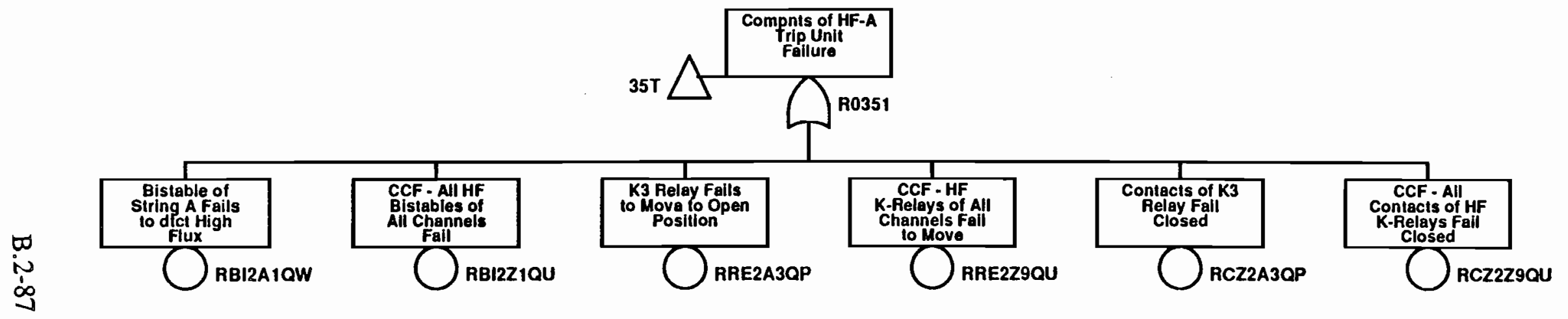

Rod Drop Failure under TOP Condilions

Tue Apr 30 12:36:12 1991

PAGE 36 OF 54 


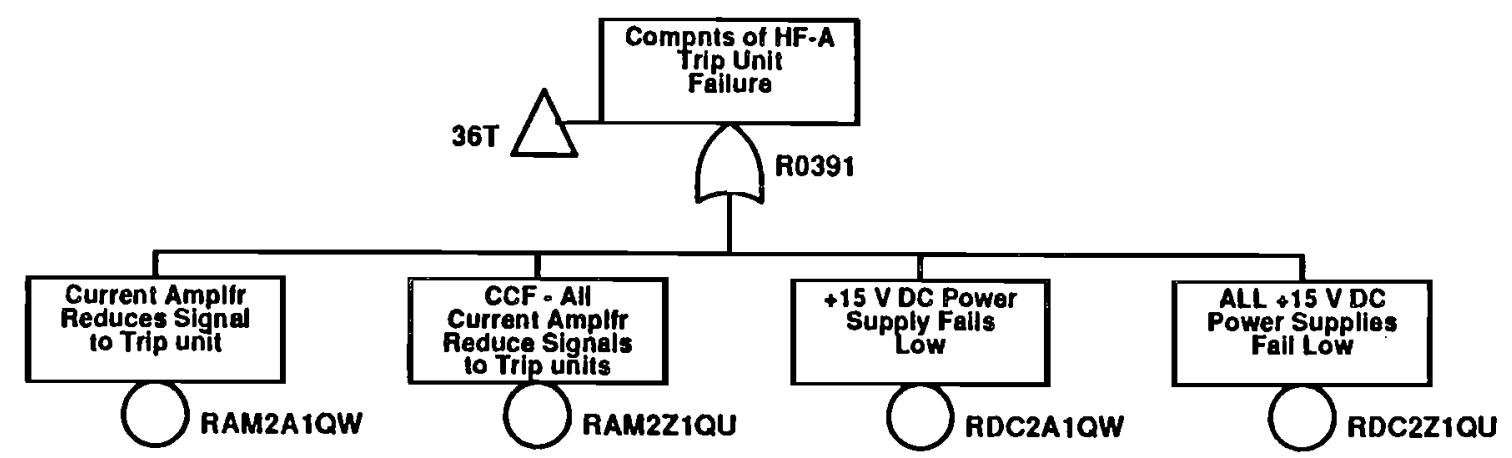

Rod Drop Fallure under TOP Conditions

Tue Apr 30 12:36:12 1991 


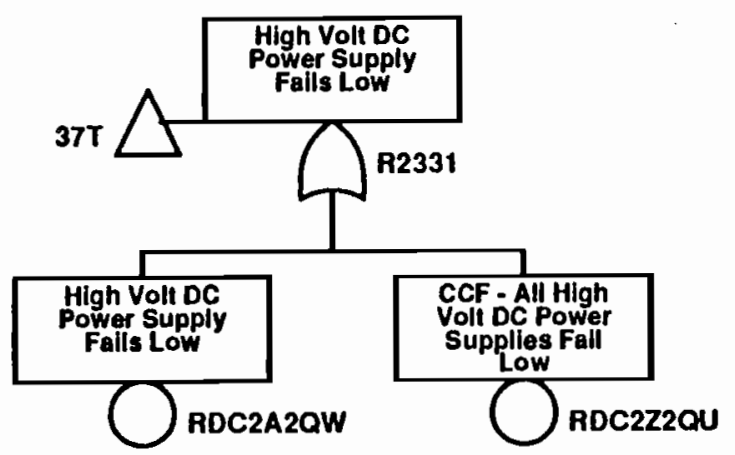

Rod Drop Failure under TOP Conditions

Tue Apr 30 12:36:12 1991 


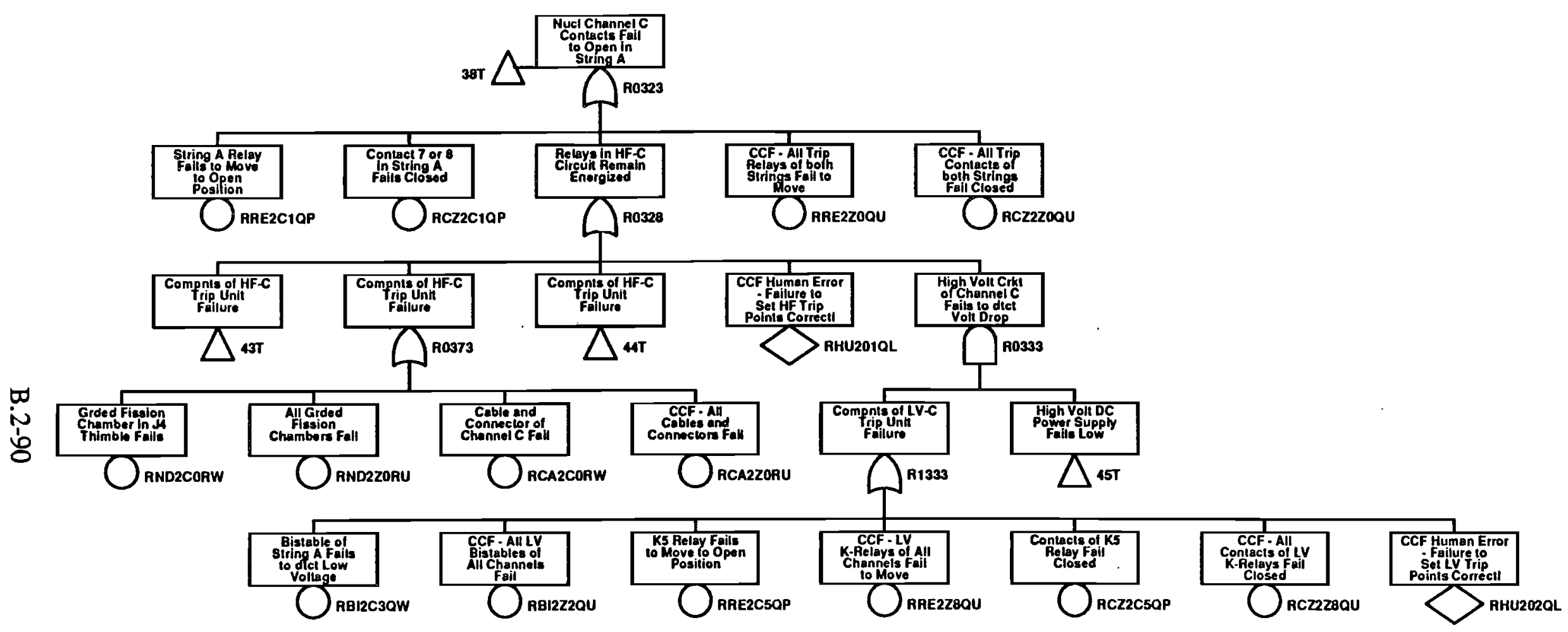

Rod Drop Fallure under TOP Conditions

Tue Apr 30 12:36:12 1991 


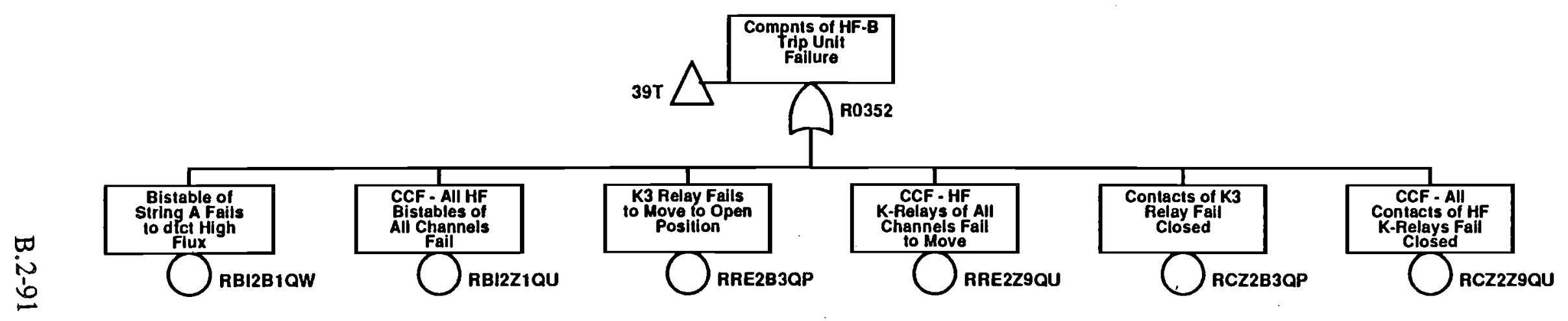

Rod Drop Fallure under TOP Conditions 


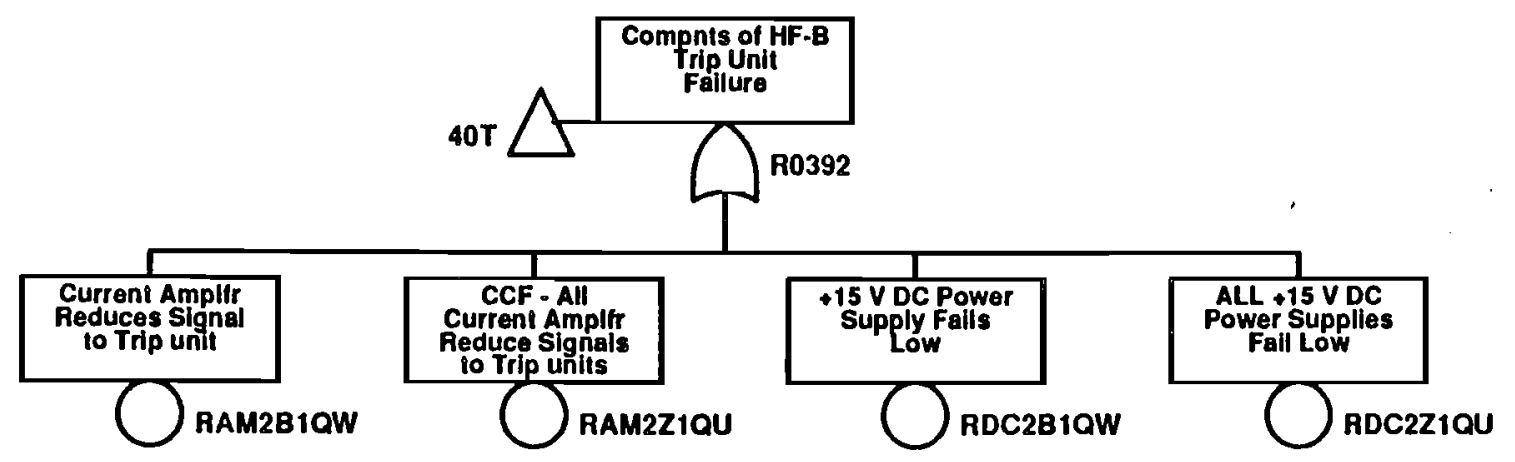

Rod Drop Fallure under TOP Conditiong 


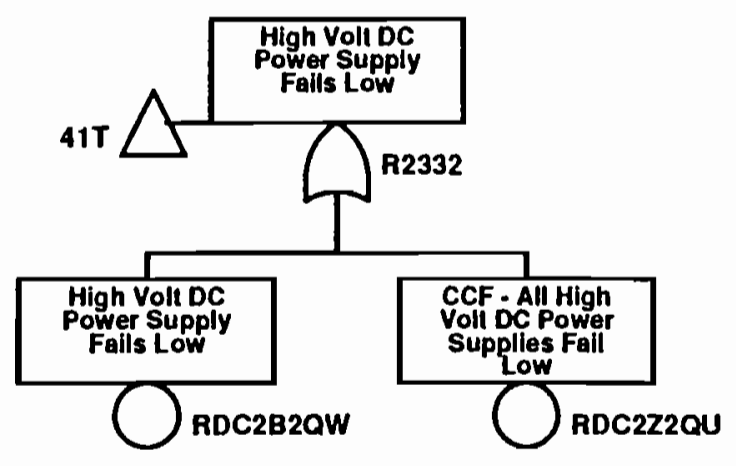

Rod Drop Failure under TOP Condilions

Tue Apr 30 12:36:12 1991 


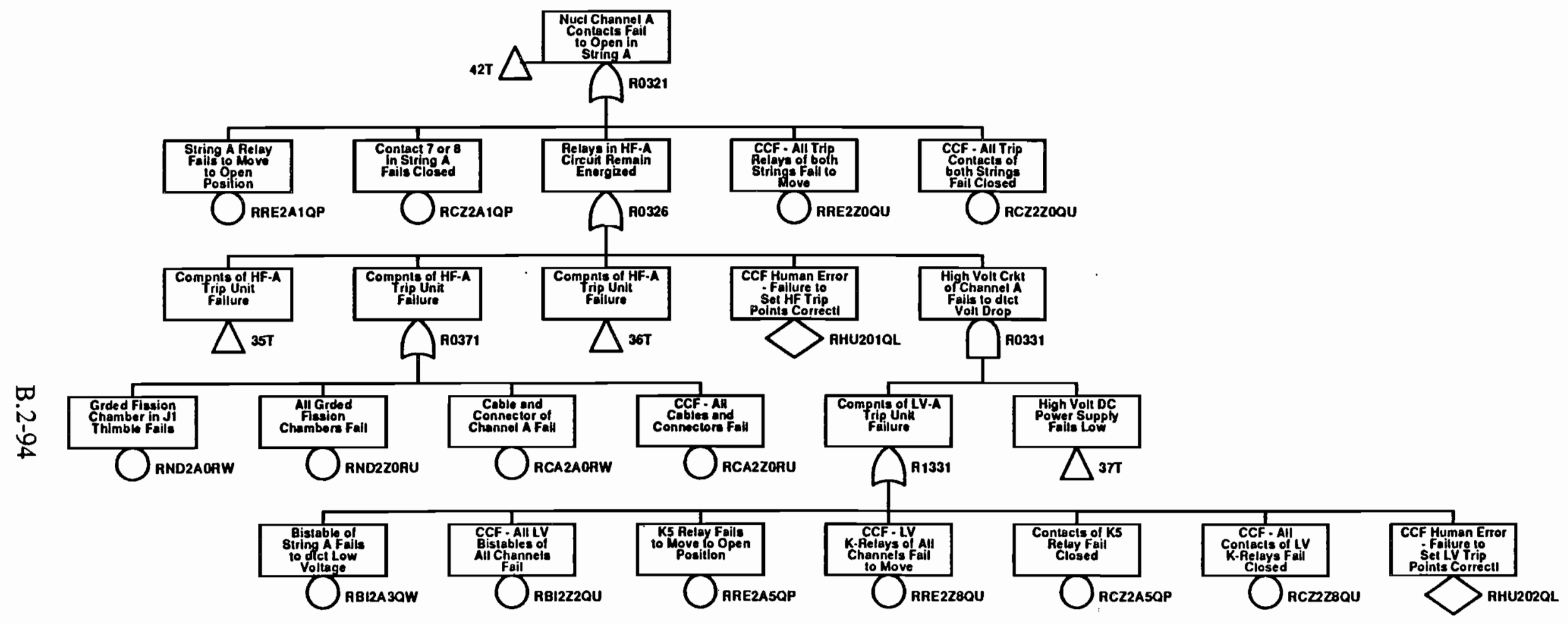

Rod Drop Failure under TOP Condillons

Tue Apr 30 12:36:12 1991

PAGE 43 OF 54 


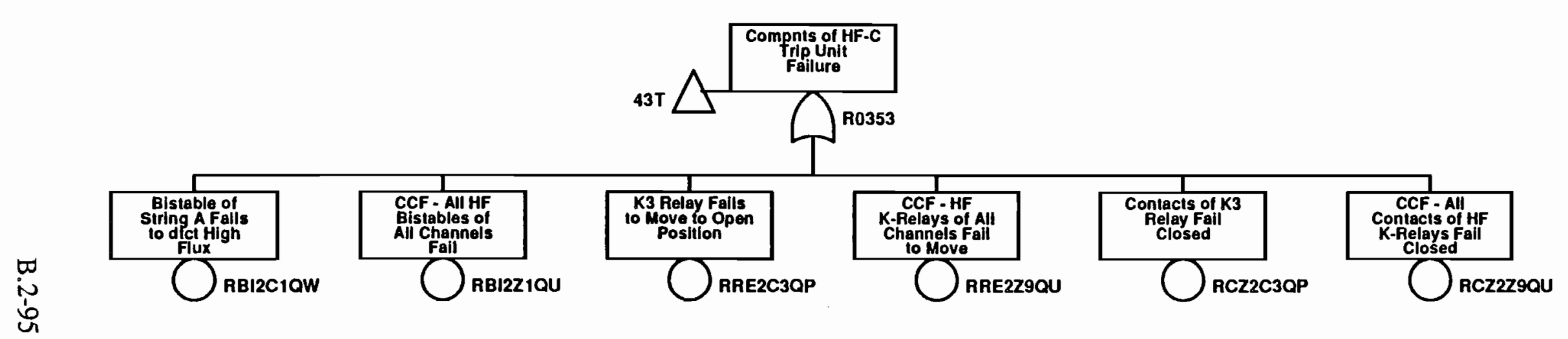

Rod Drop Failure under TOP Conditions

Tue Apr 30 12:36:12 1991 


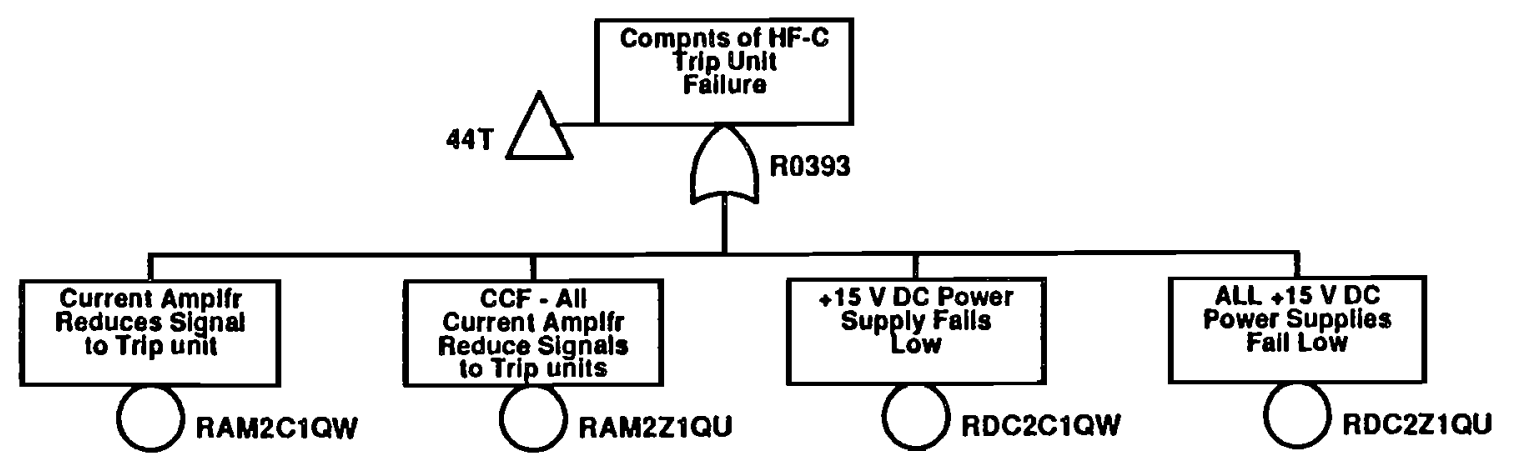

Rod Drop Fallure under TOP Conditions

Tue Apr 30 12:36:12 1991 


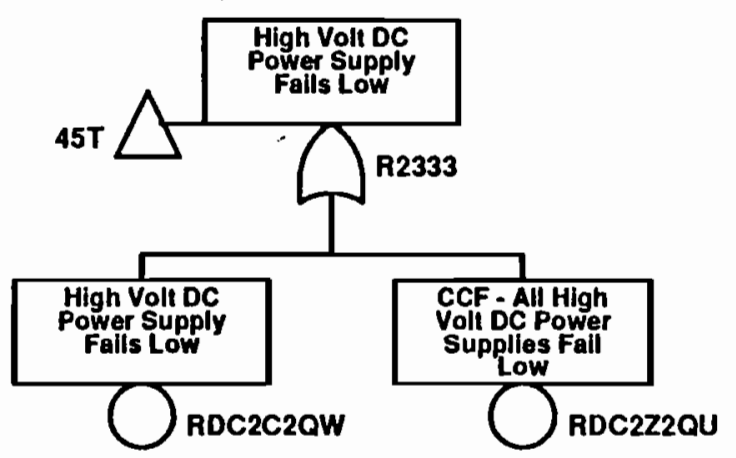

Rod Drop Fallure under TOP Conditions

\begin{tabular}{|l|l|}
\hline Tue Apr 30 12:36:12 1991 & PAGE 46 OF 54 \\
\hline
\end{tabular} 


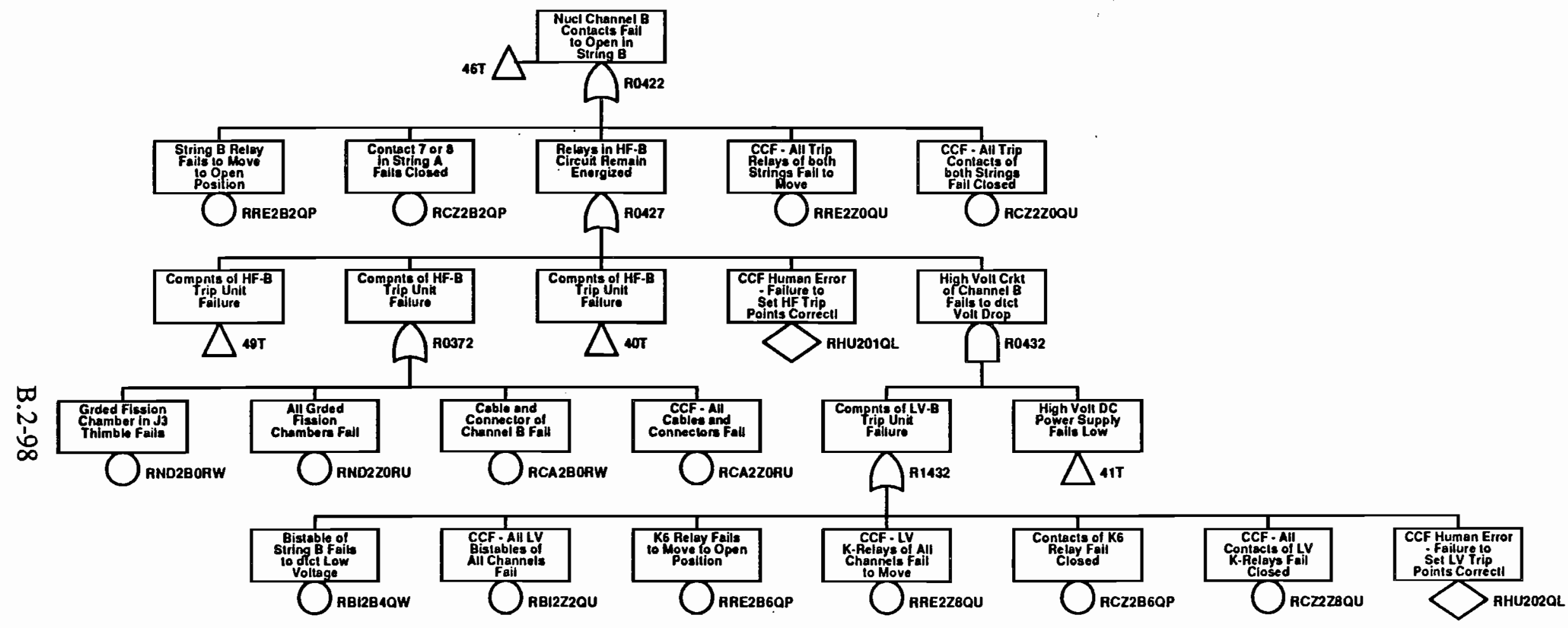

Rod Drop Failure under TOP Conditions 


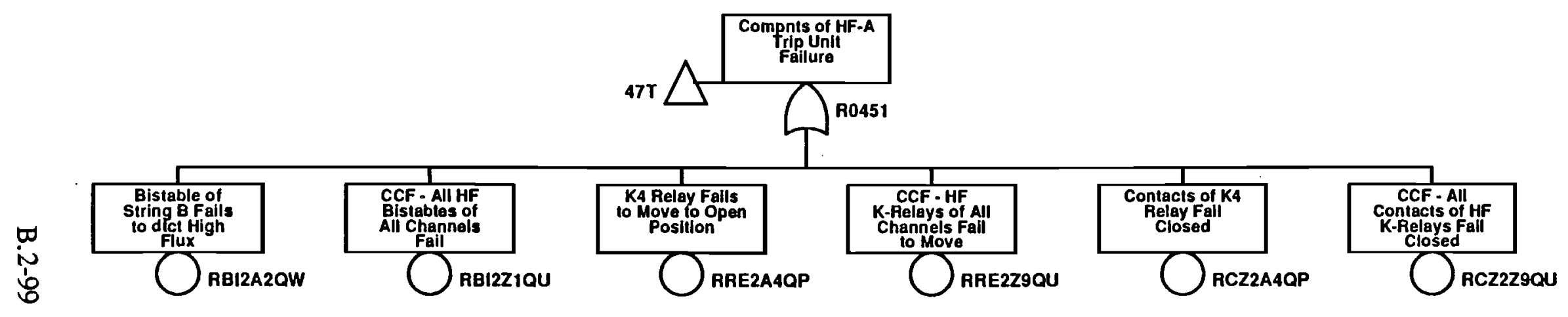

Rod Drop Fallure under TOP Conditions

Tue Apr 30 12:36:12 1991

PAGE 48 OF 54 


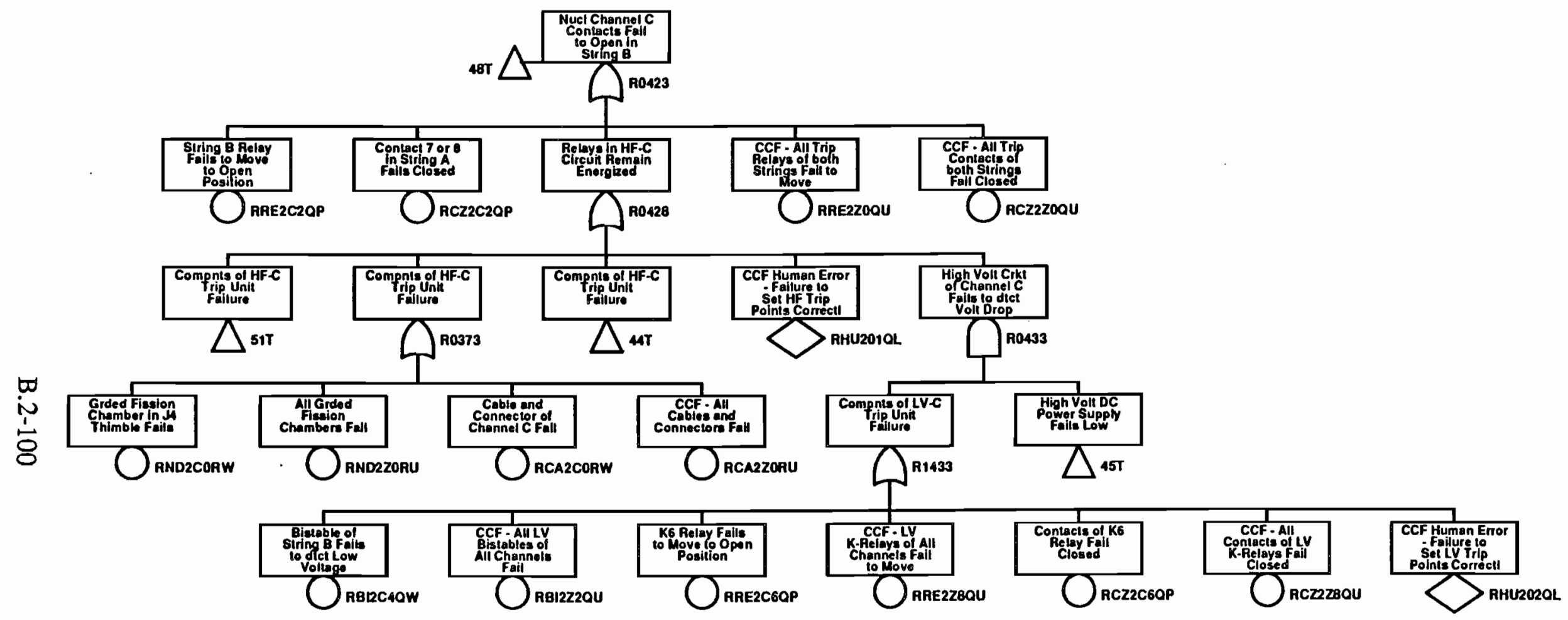

Rod Drop Falture under TOP Conditions 


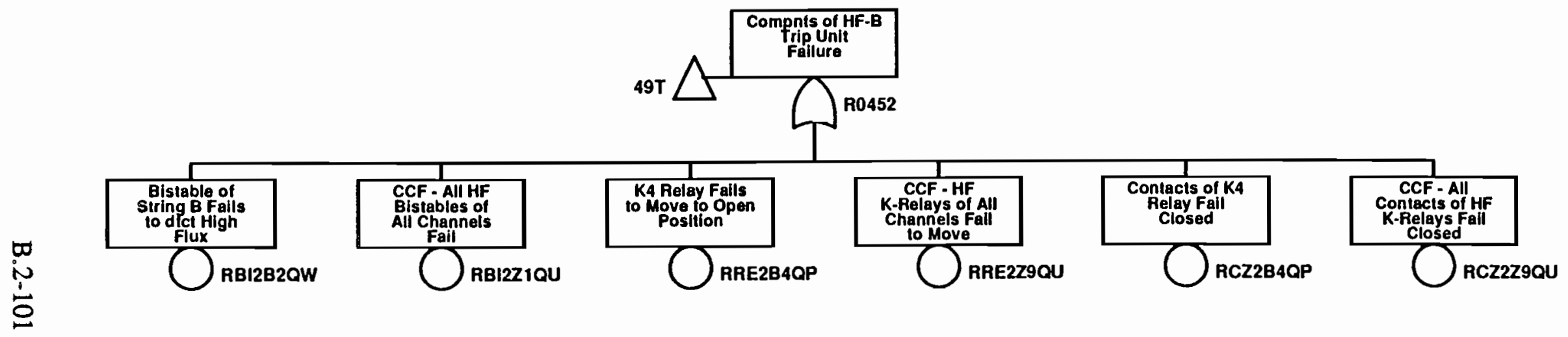

Rod Drop Fallure under TOP Conditions

Tue Apr 30 12:36:12 1991

PAGE 50 OF 54 


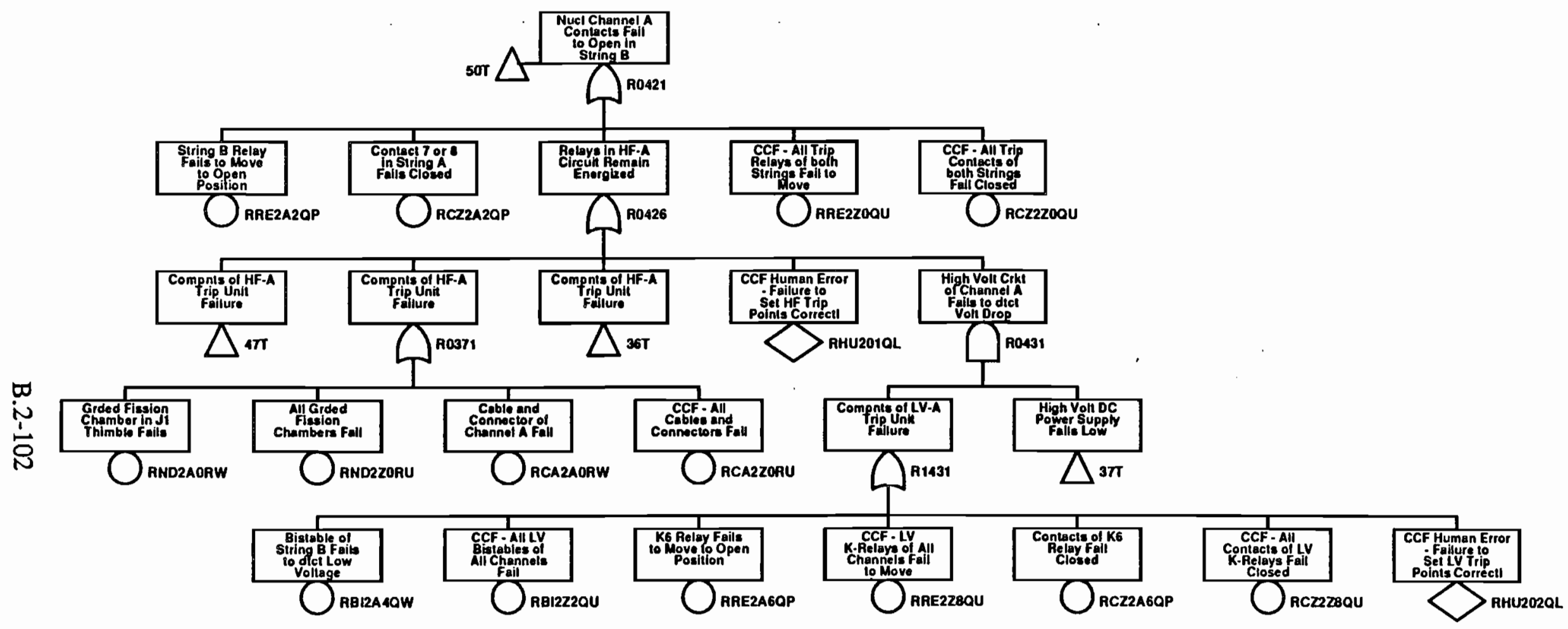

Rod Drop Failure under TOP Conditions 


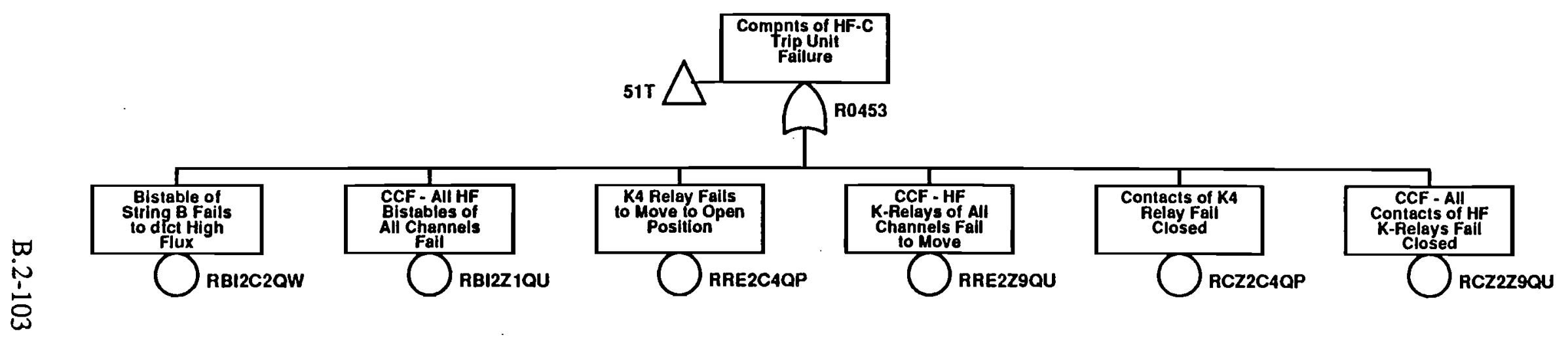

Rod Drop Fallure under TOP Conditions

Tue Apr 30 12:36:12 1991

PAGE 52 OF 54 


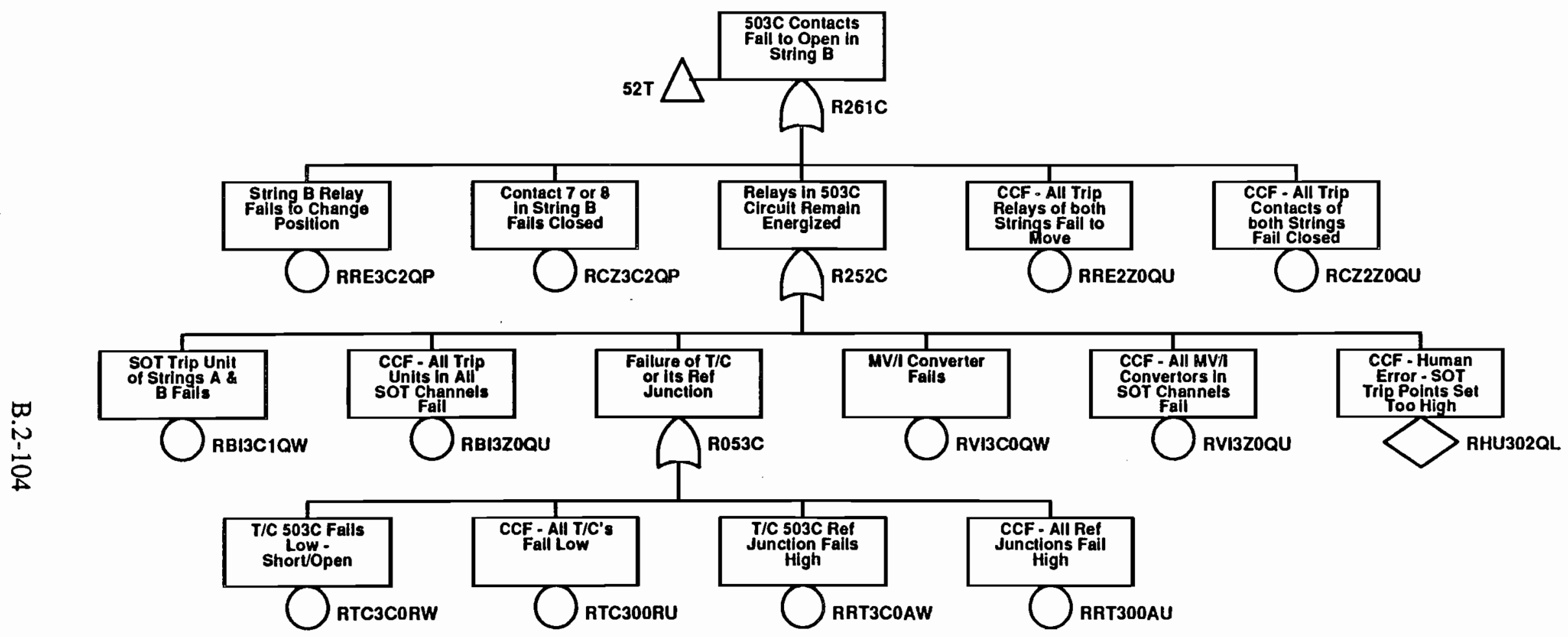

Rod Drop Fallure under TOP Conditions

Tue Apr 30 12:36:12 1991

PAGE 53 OF 54 


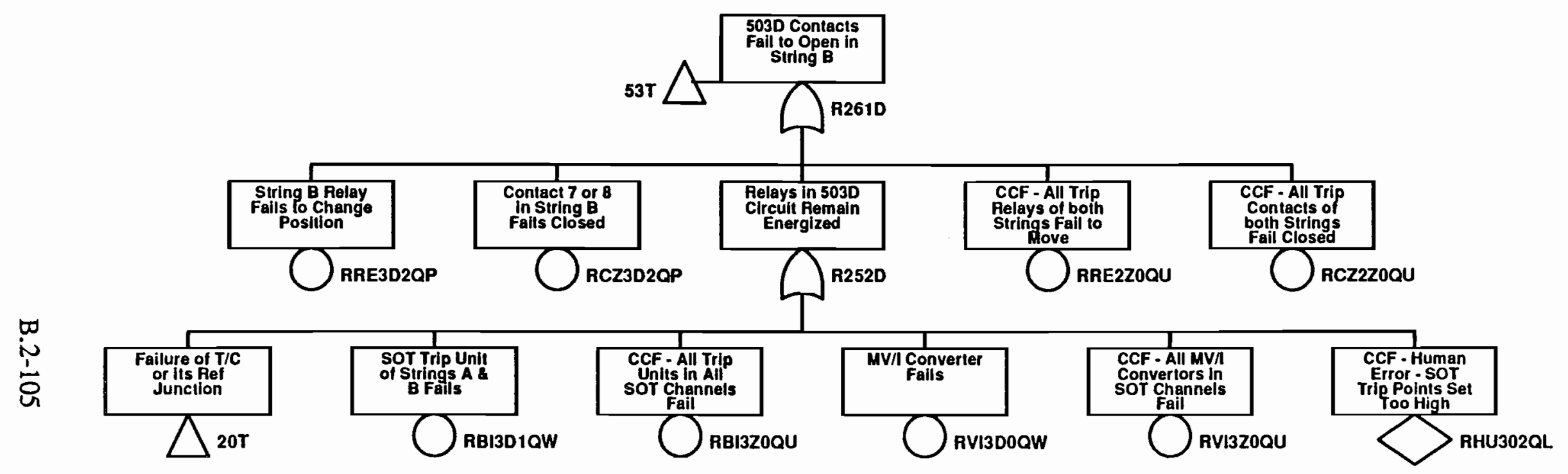

Rod Drop Fallure under TOP Conditions

Tue Apr 30 12:36:12 1991

PAGE 54 OF 54 


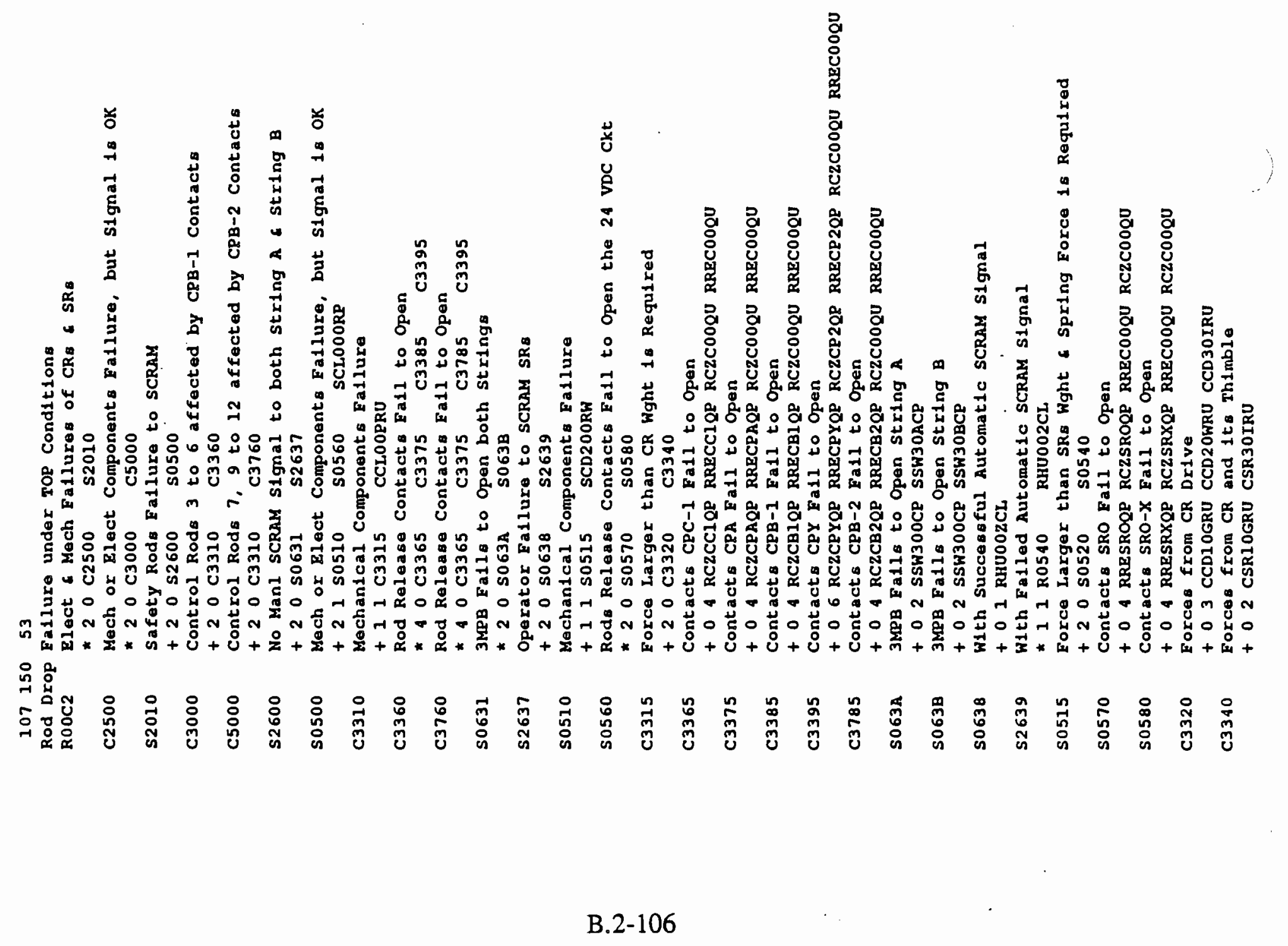




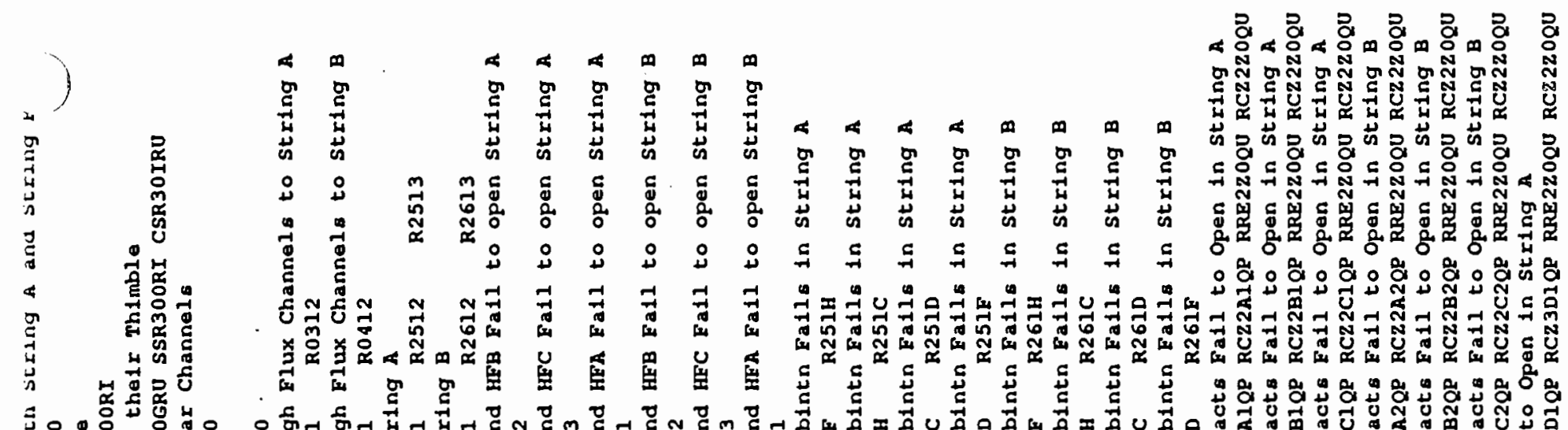

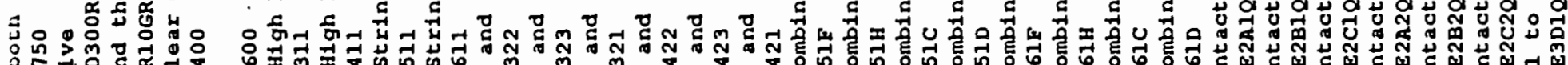

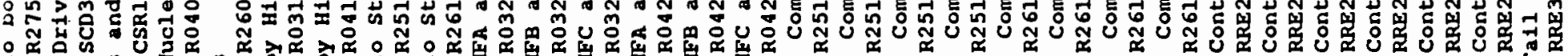

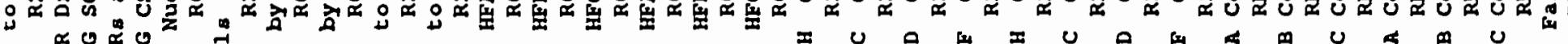

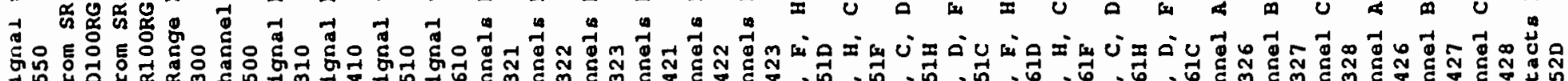

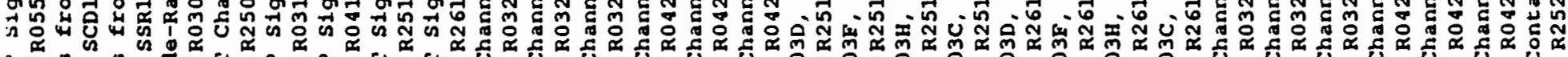

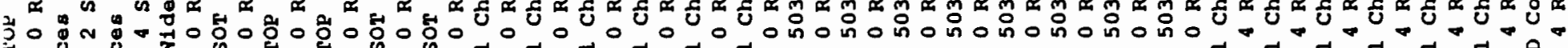
N

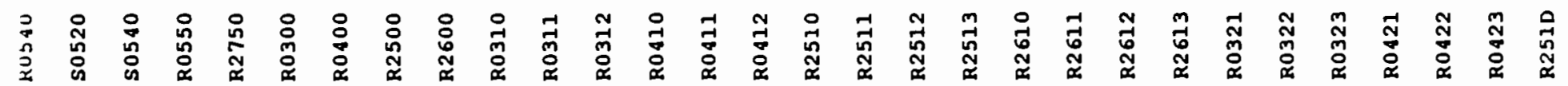




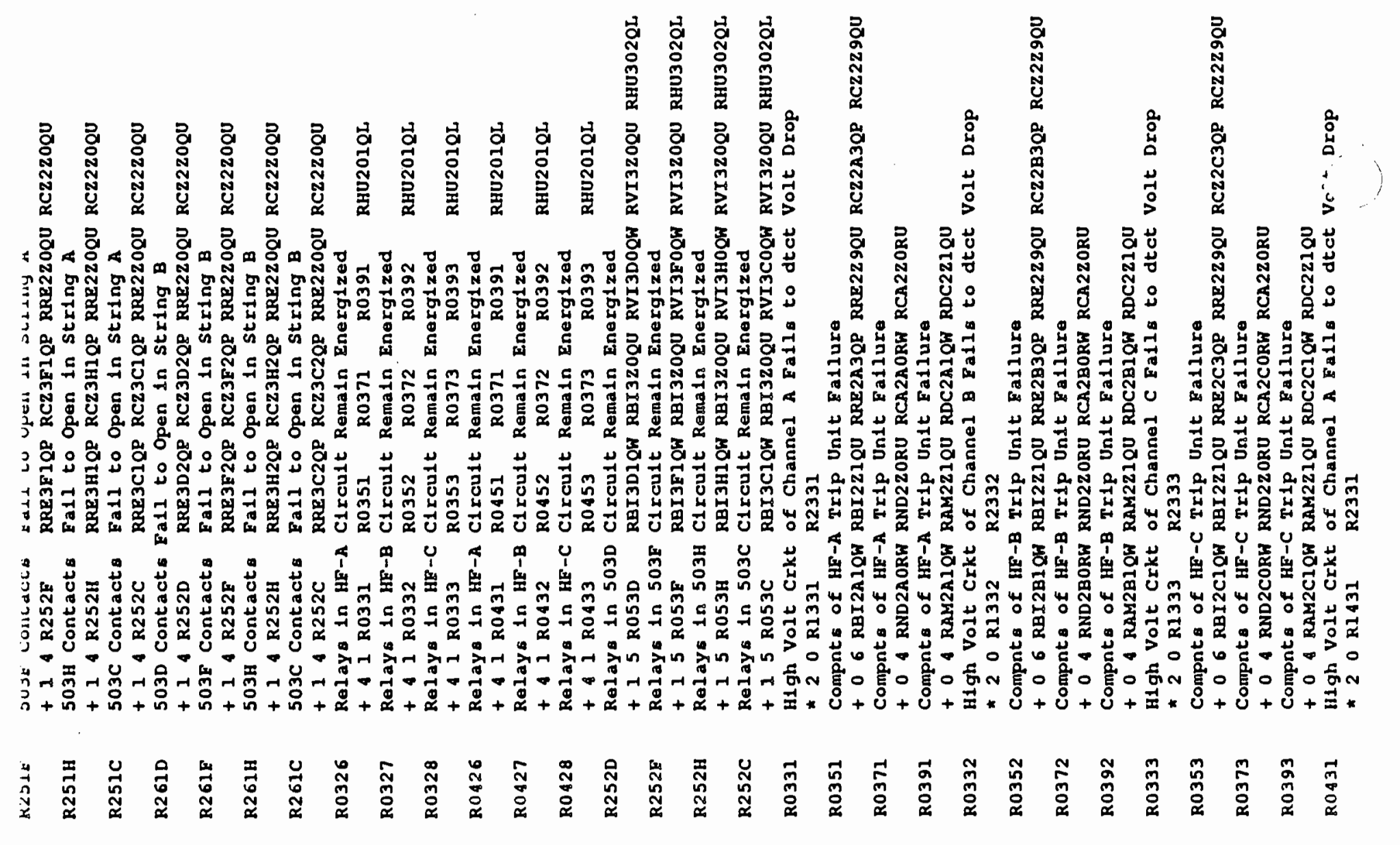




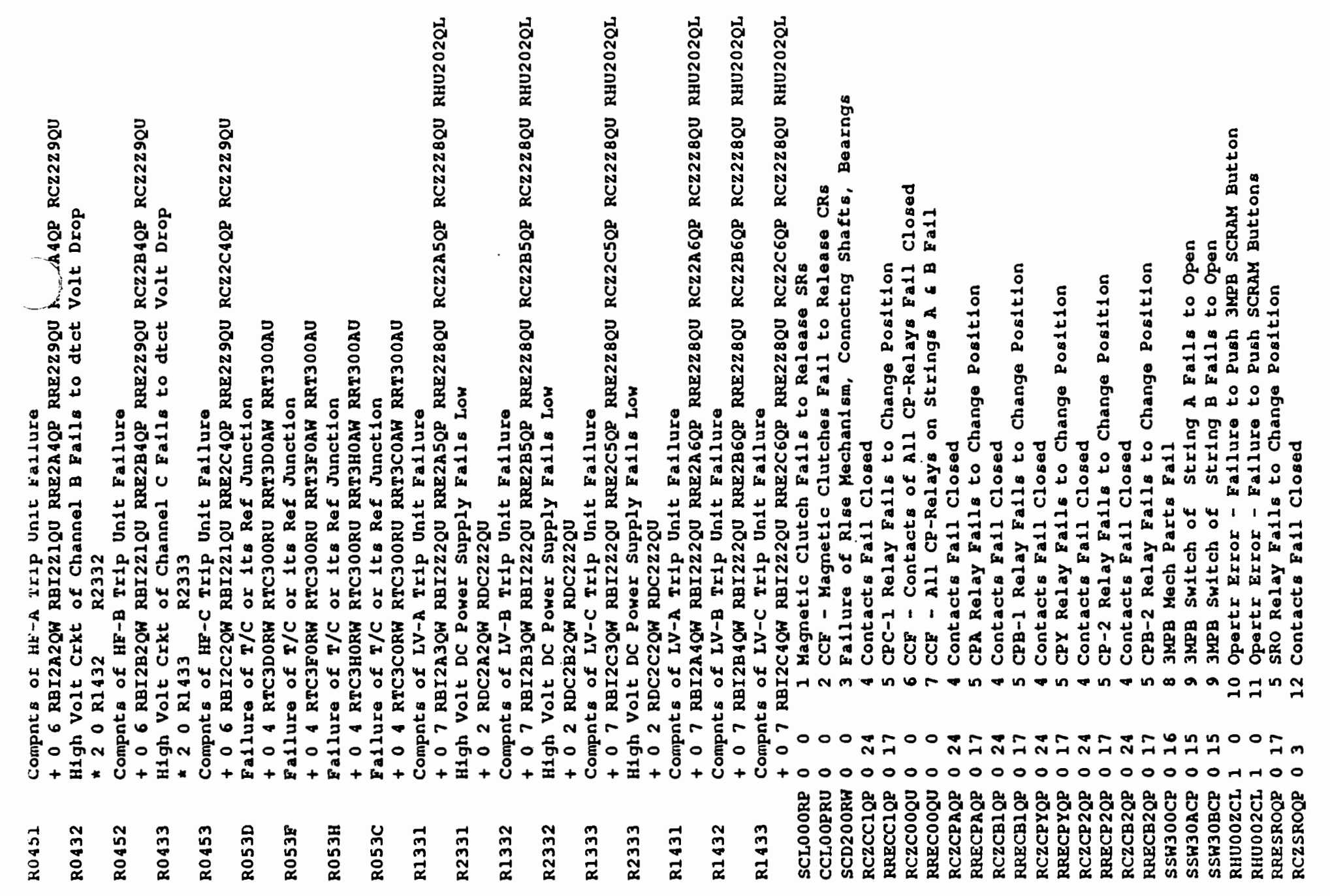




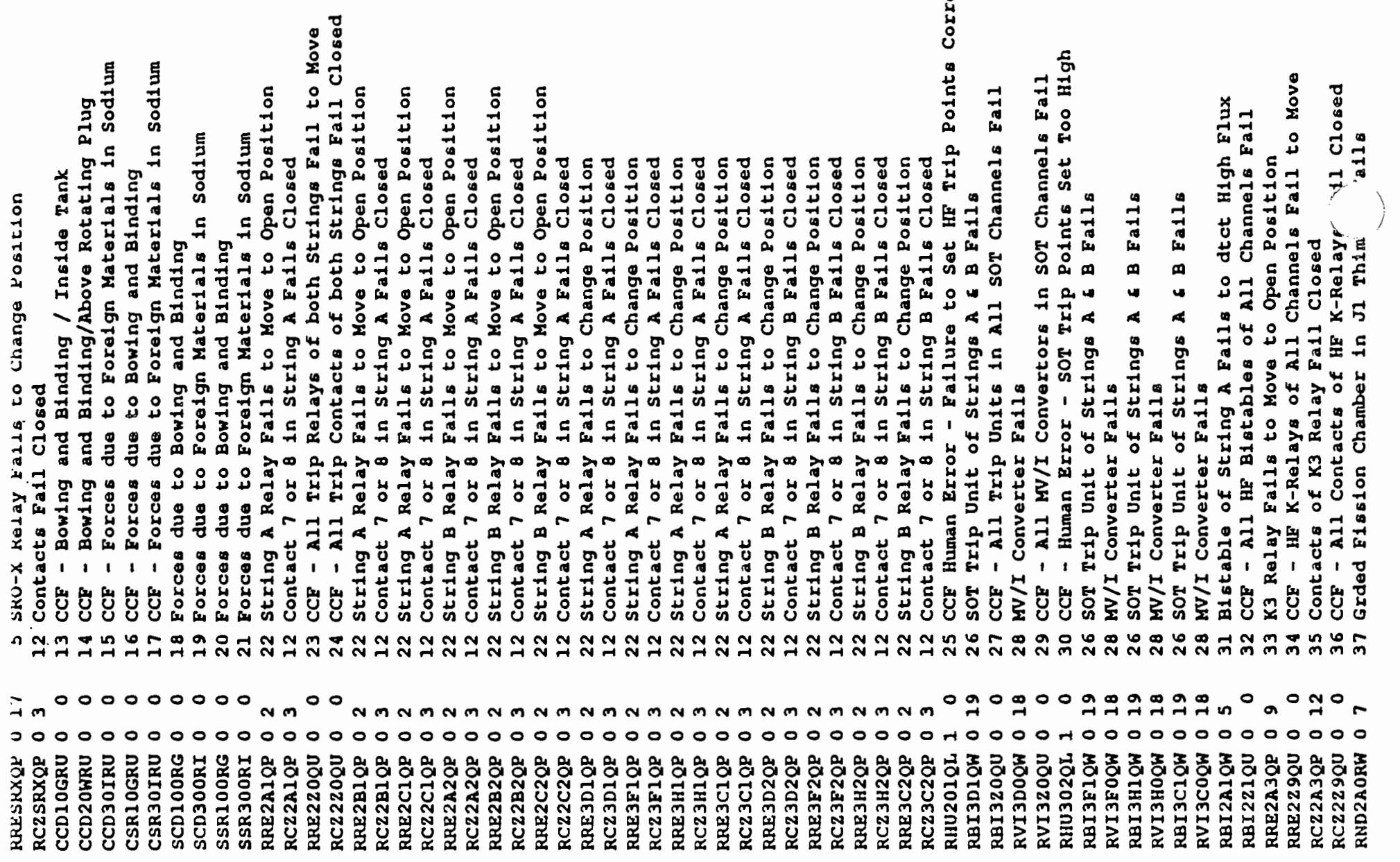




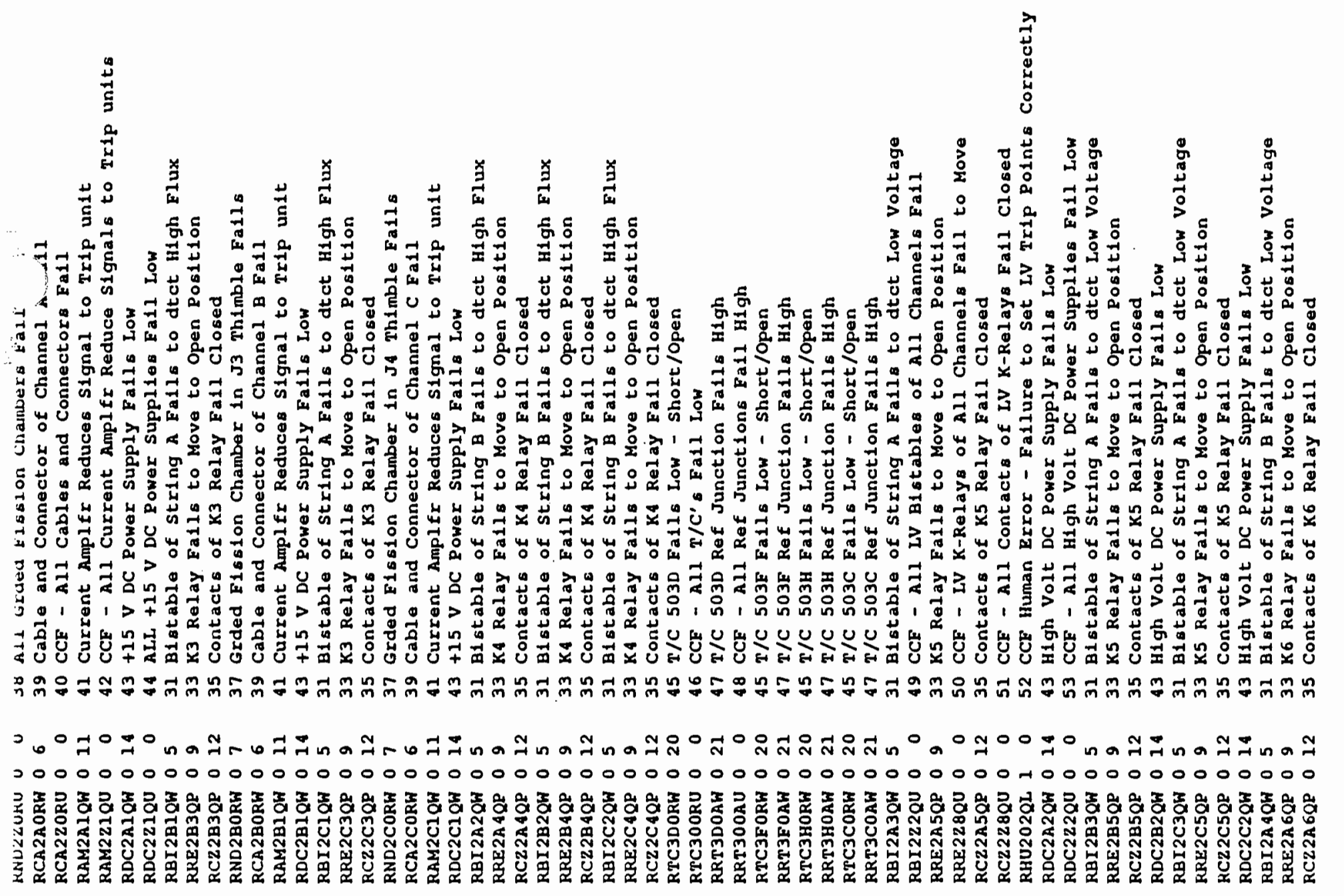




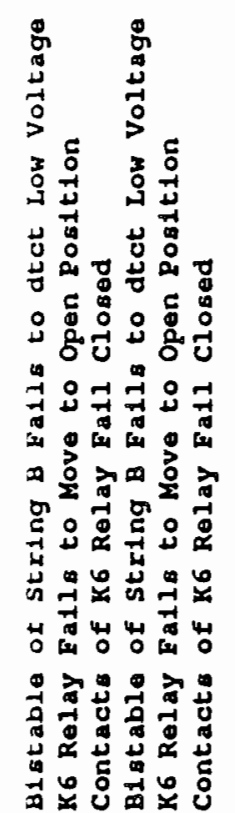

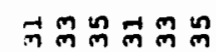

ภดสนกับ 000000

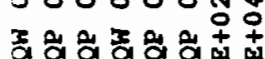

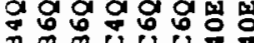

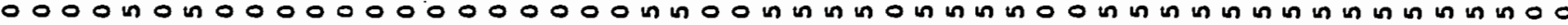

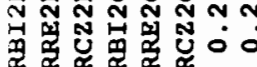

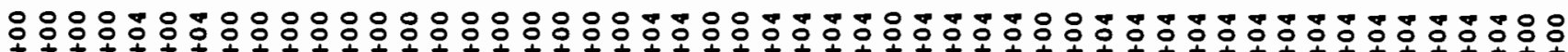

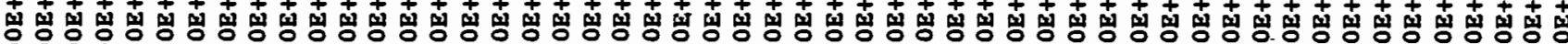

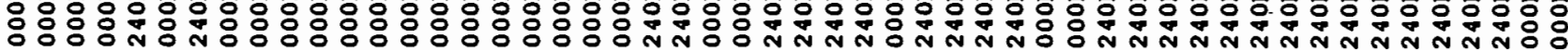

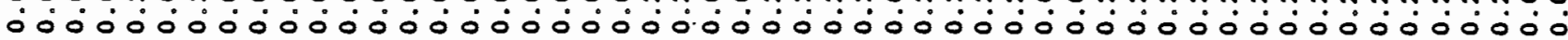

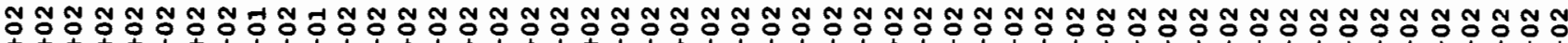

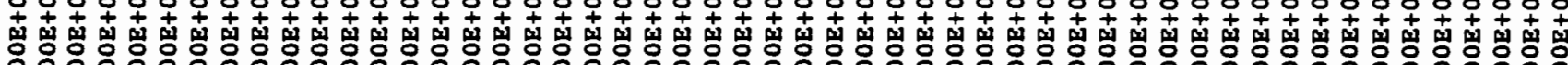

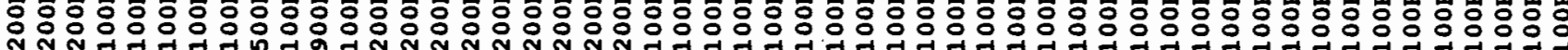

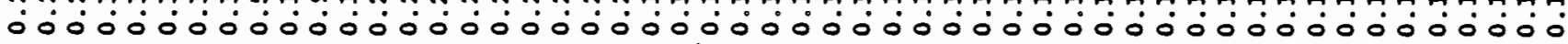

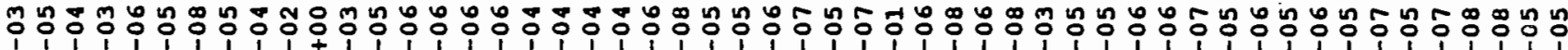

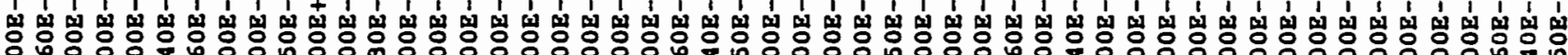

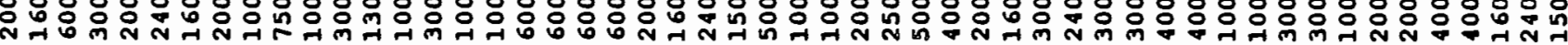




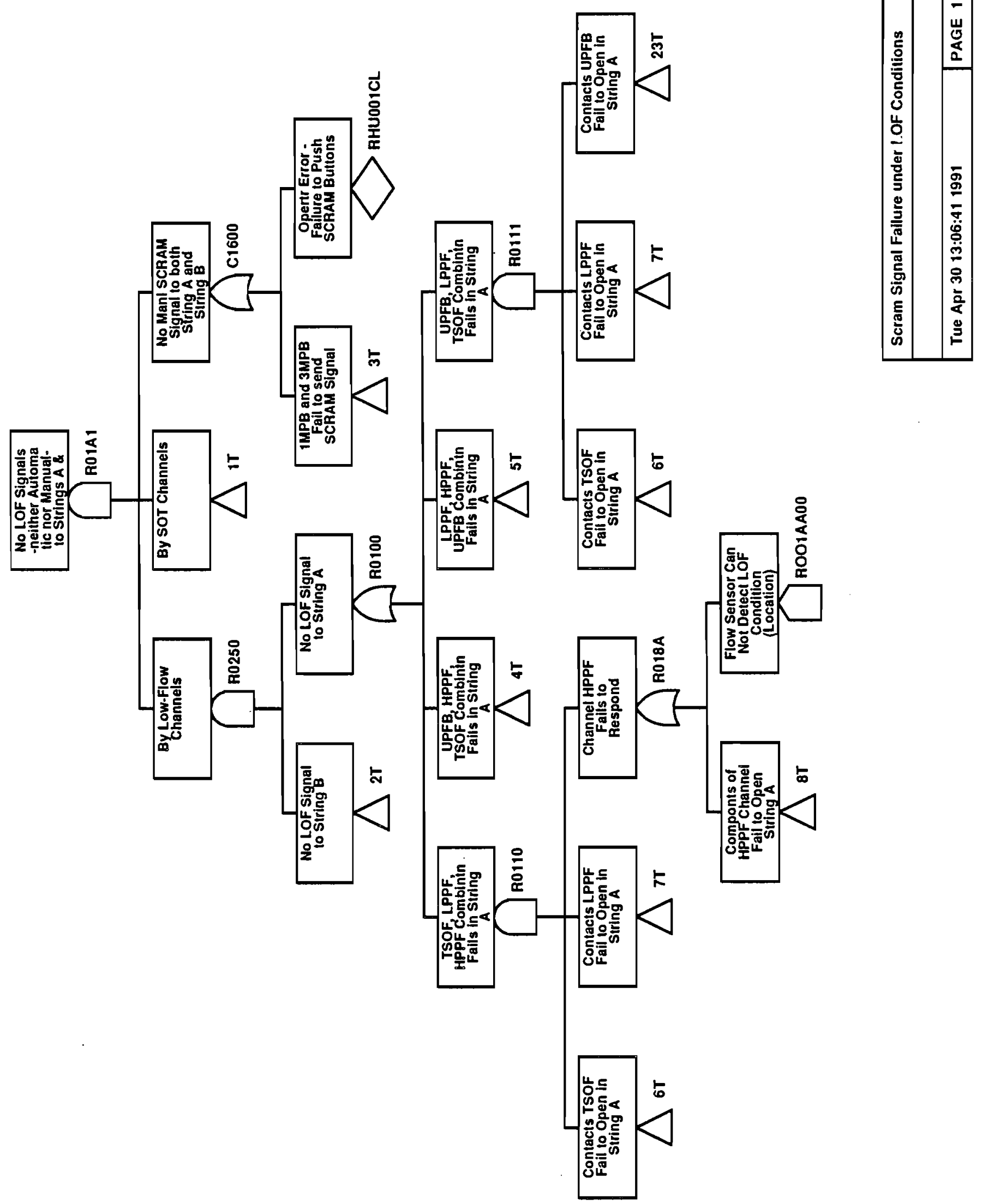




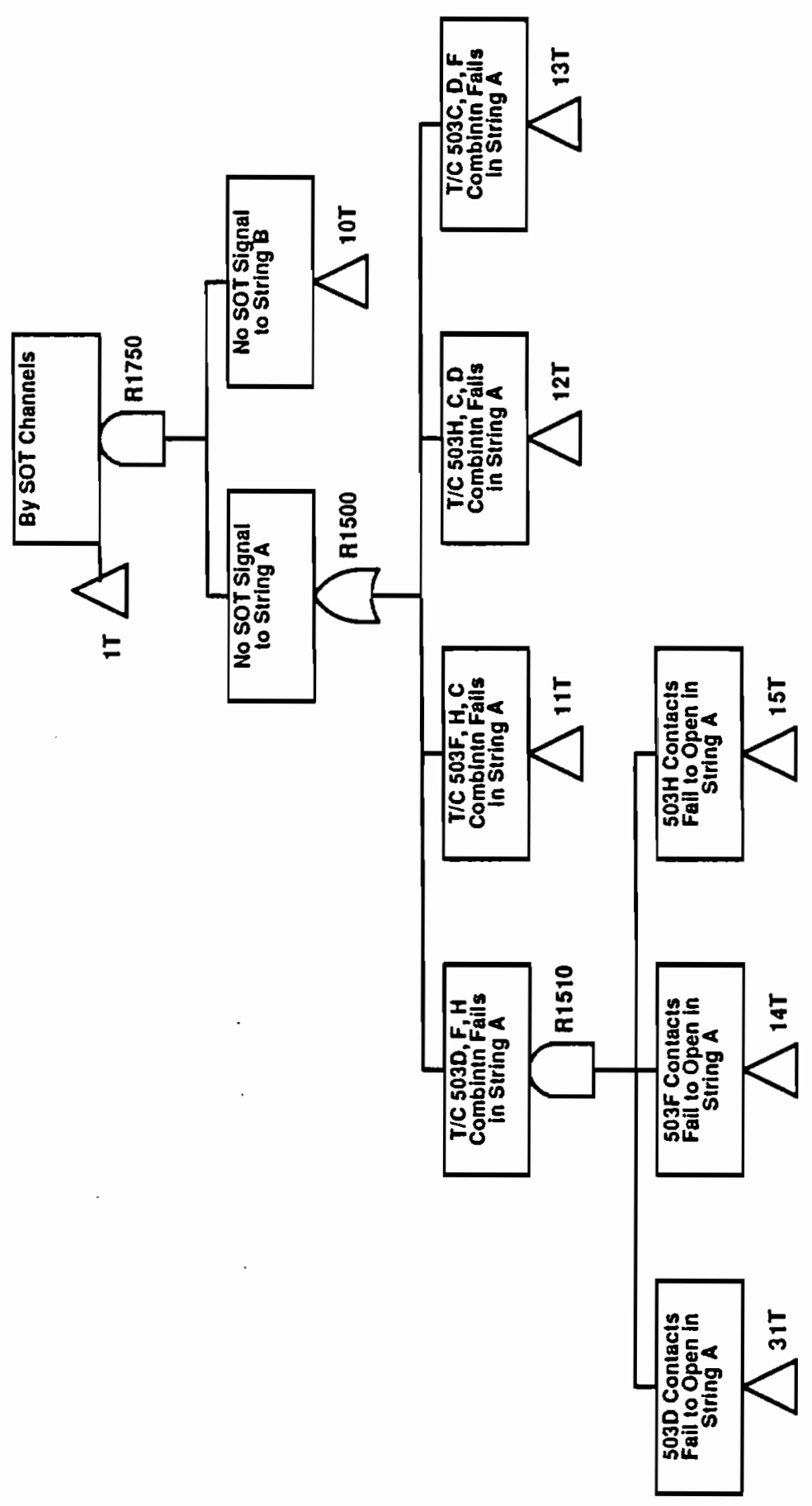




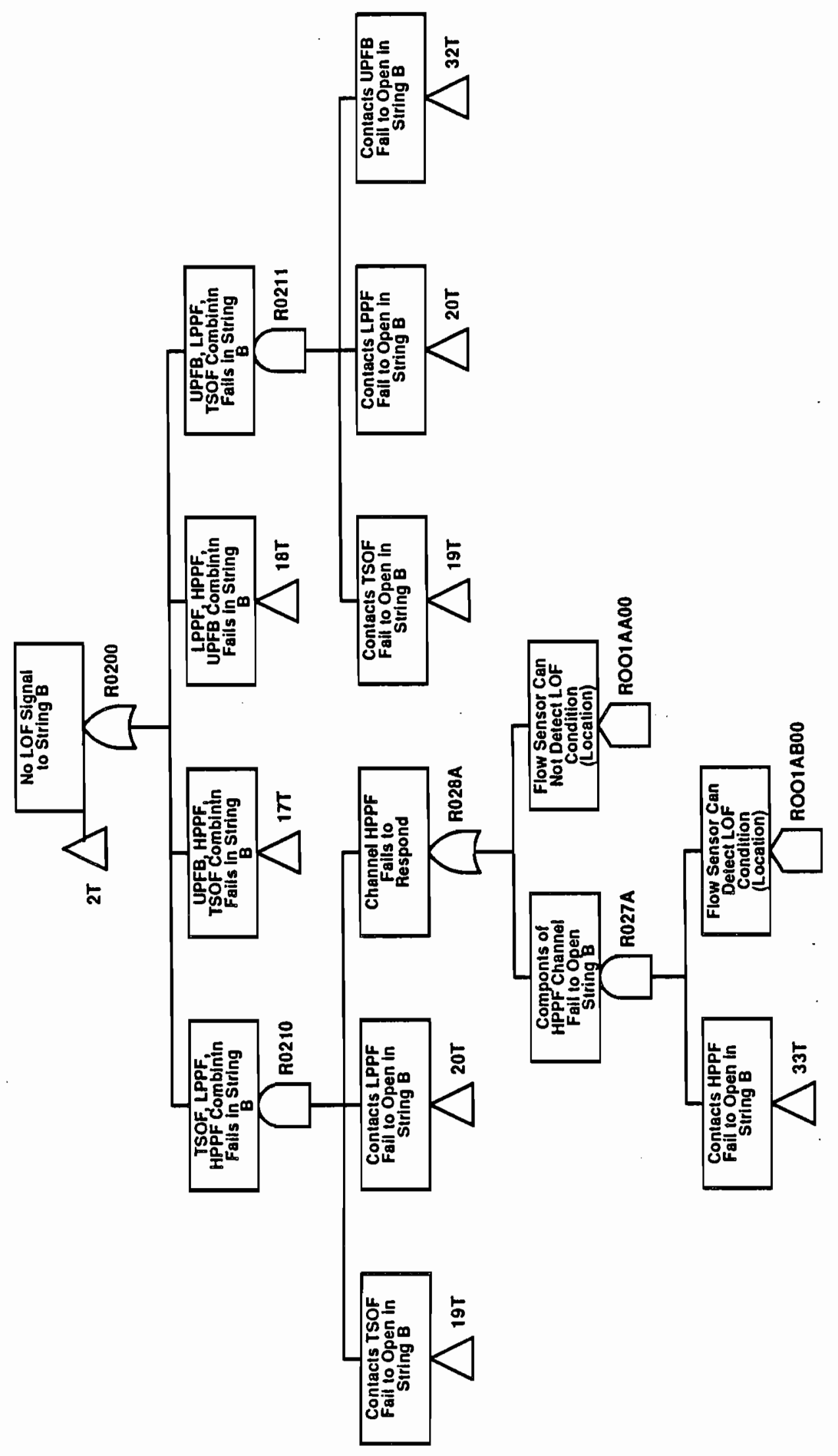

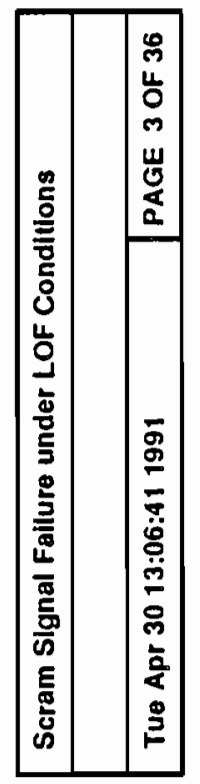

B. $2-116$ 


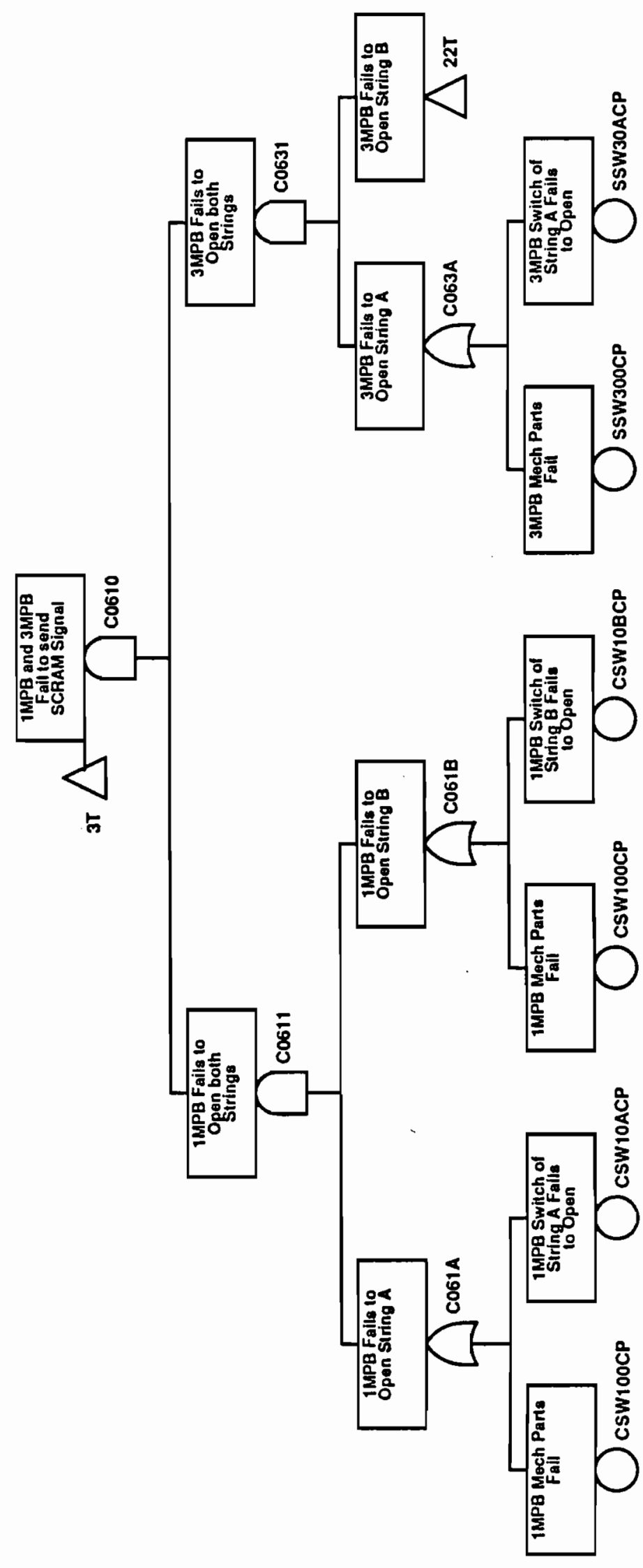




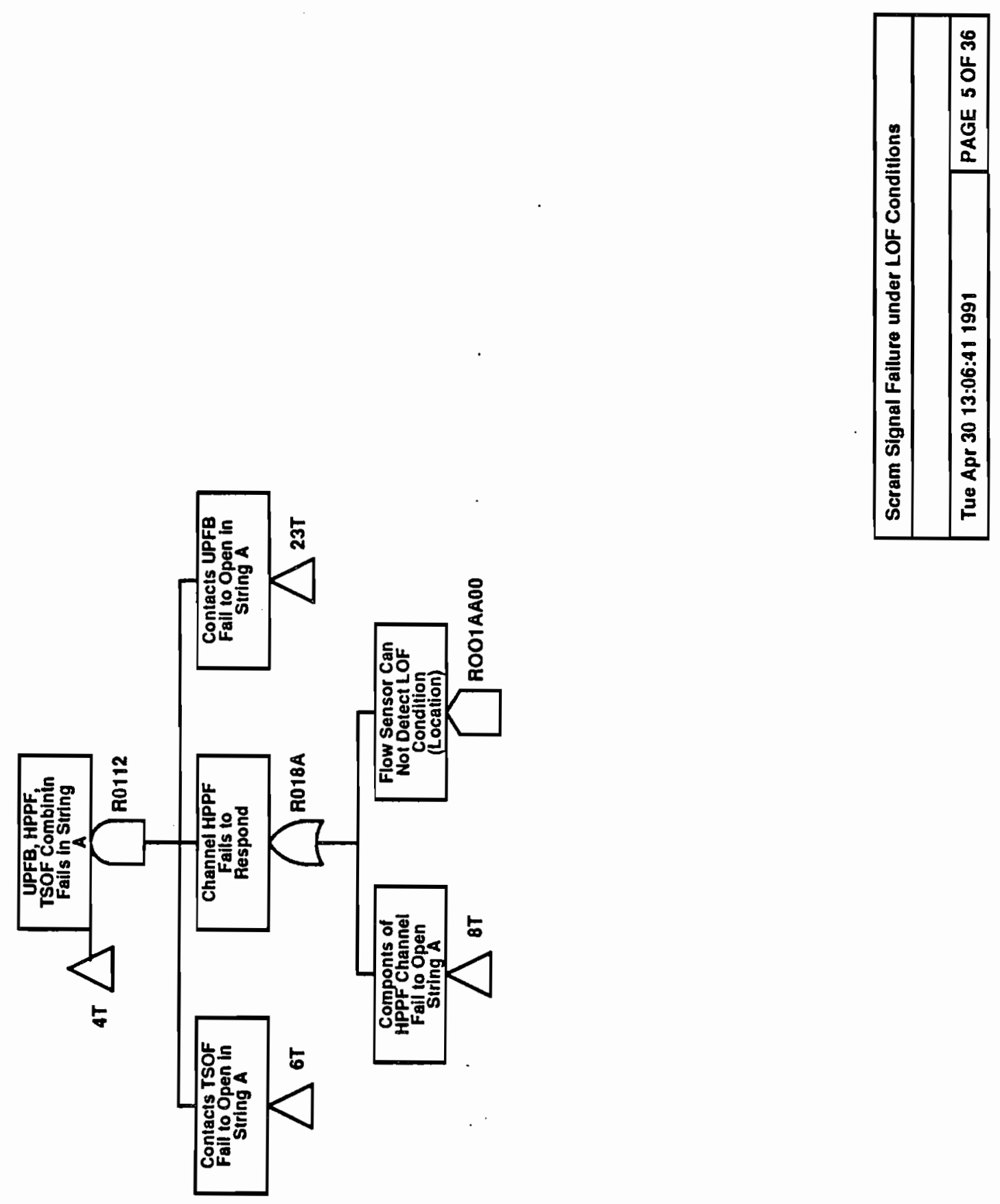




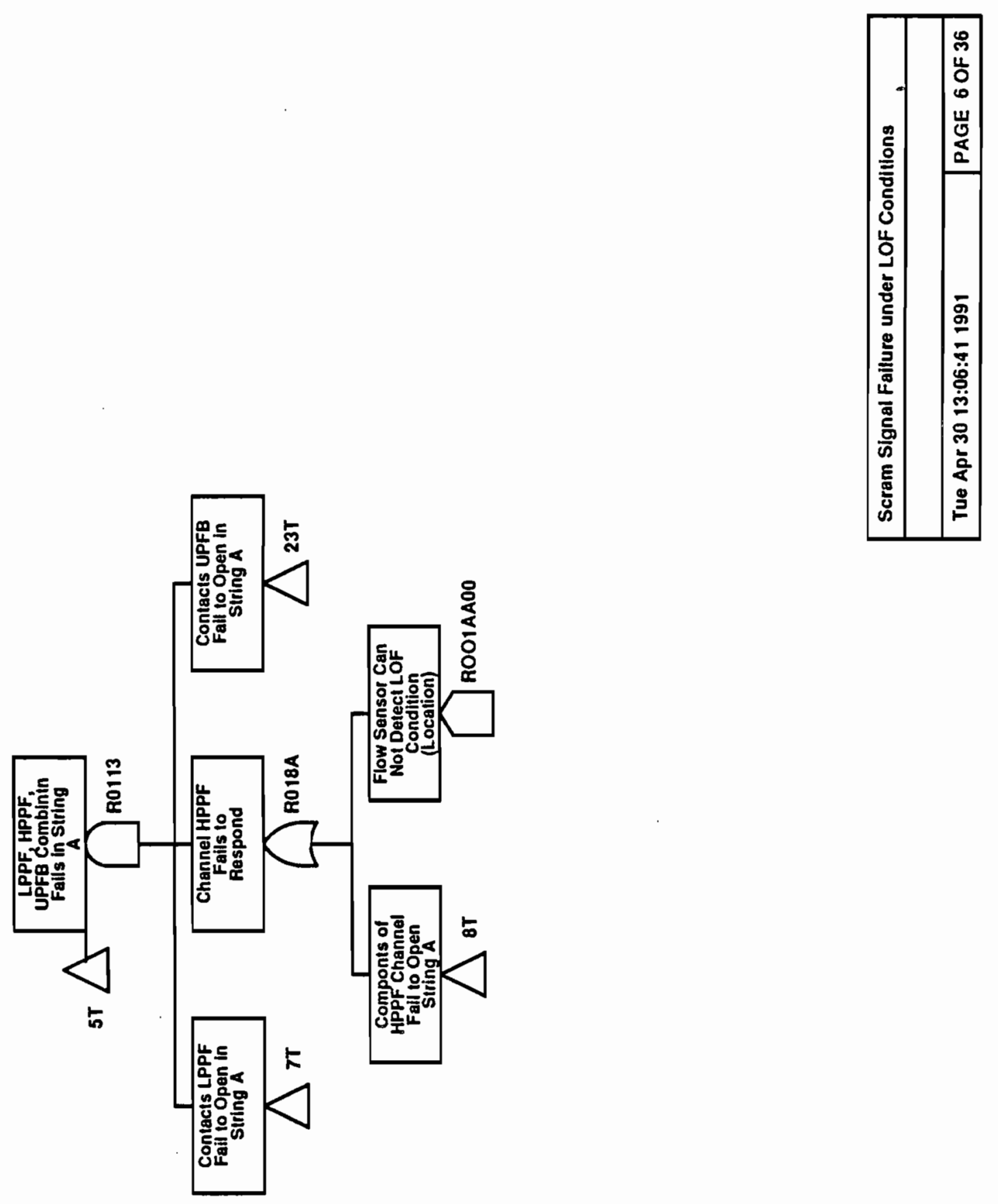



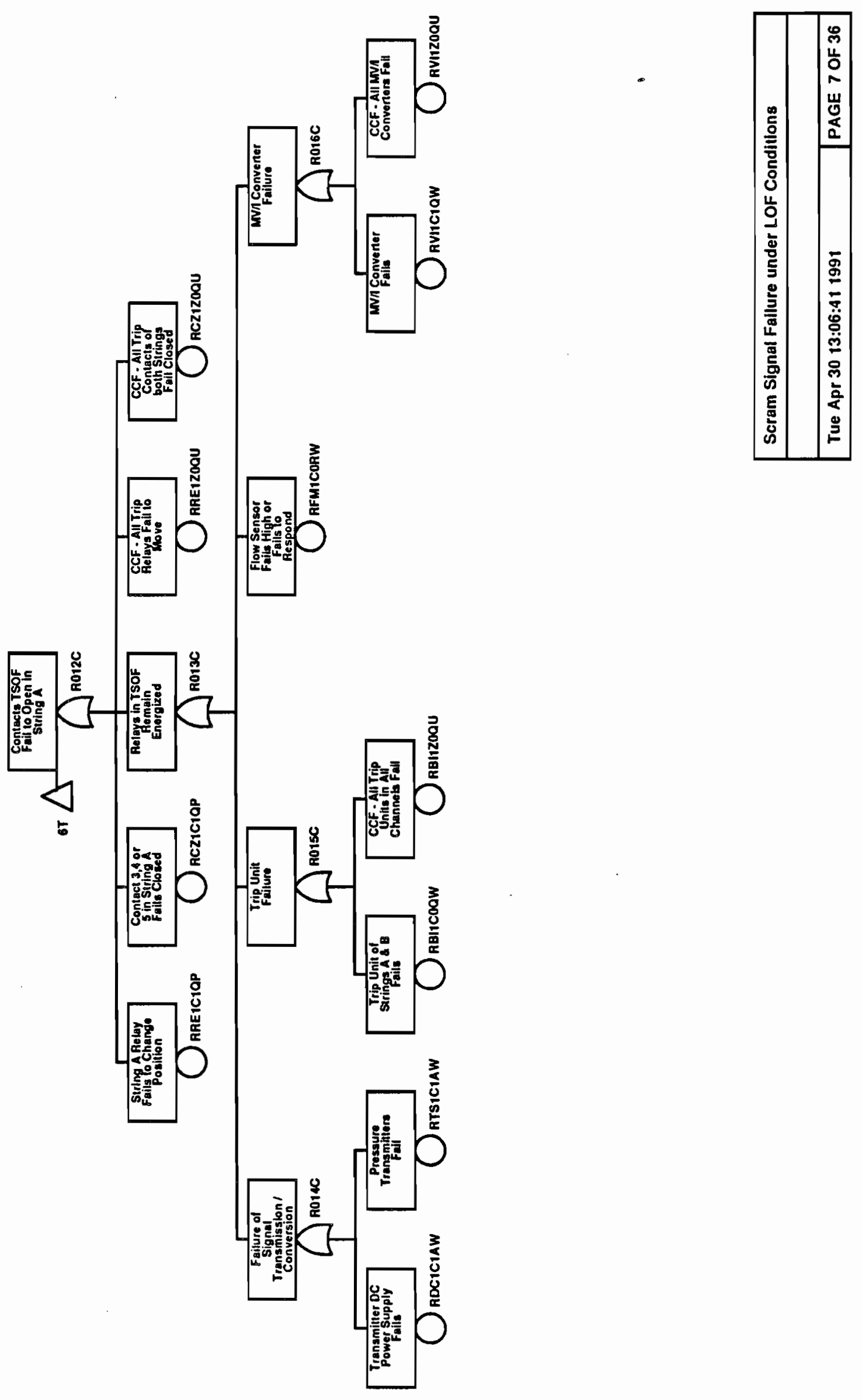

B. $2-120$ 


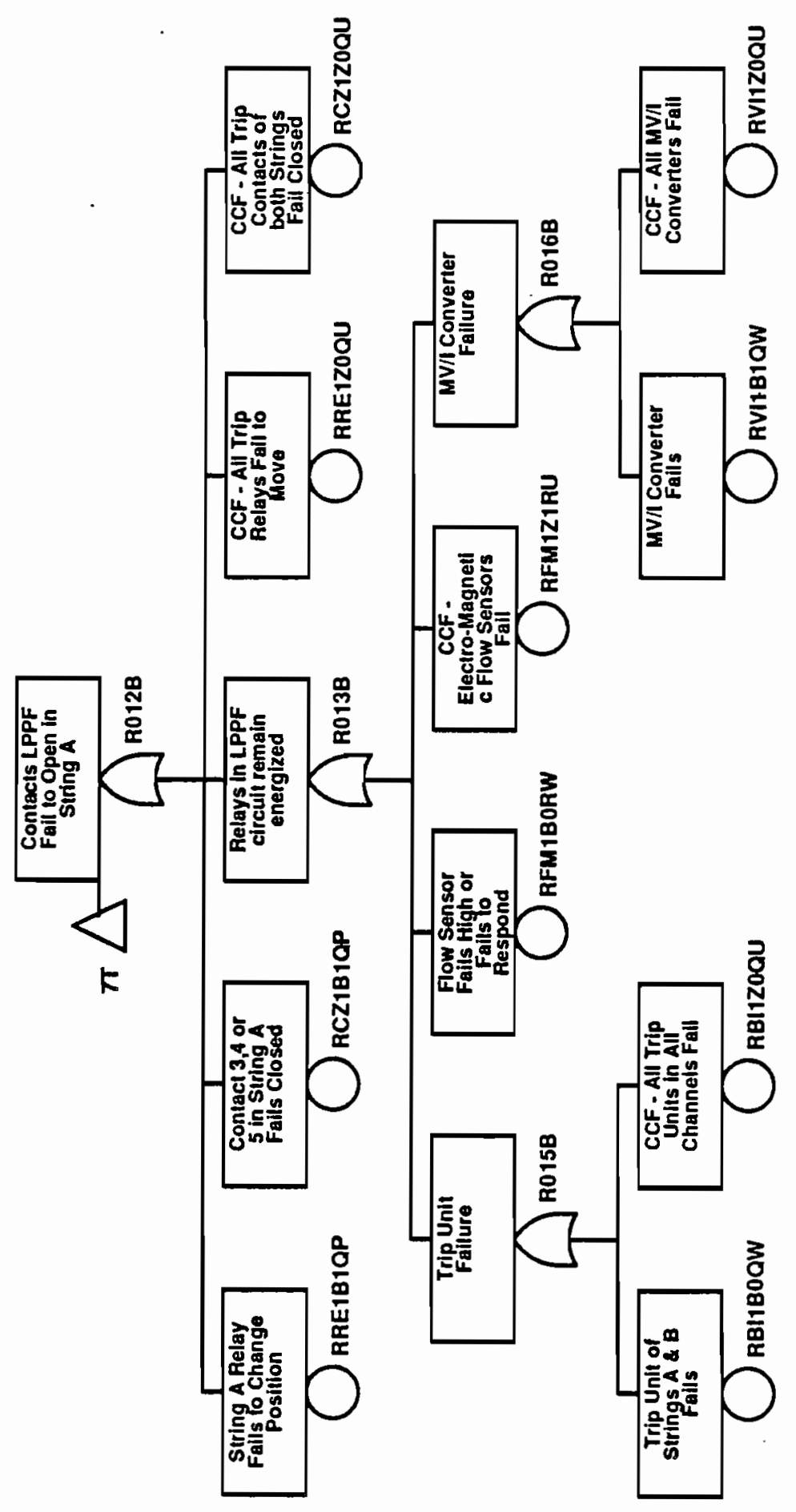

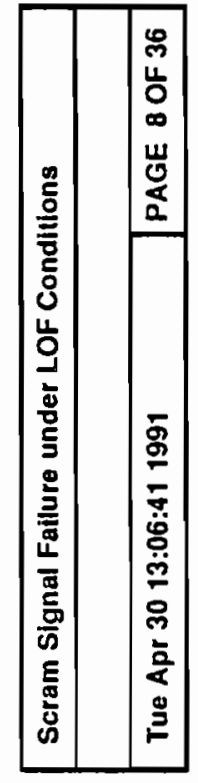




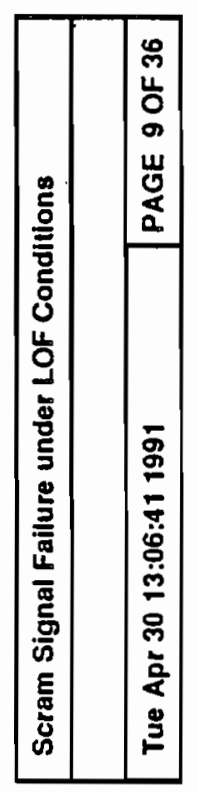

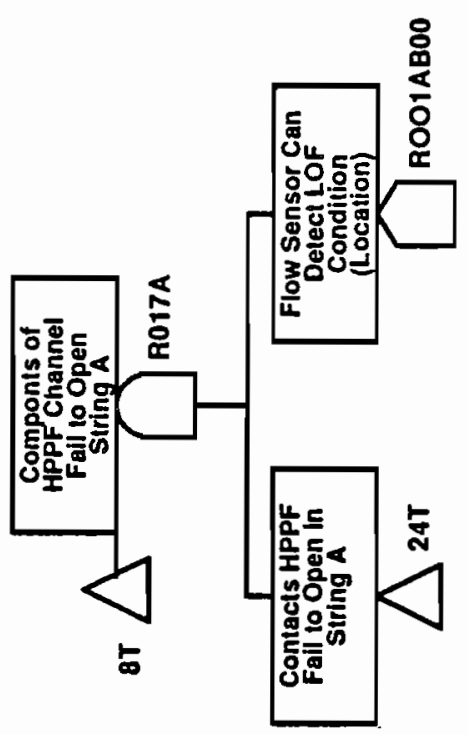




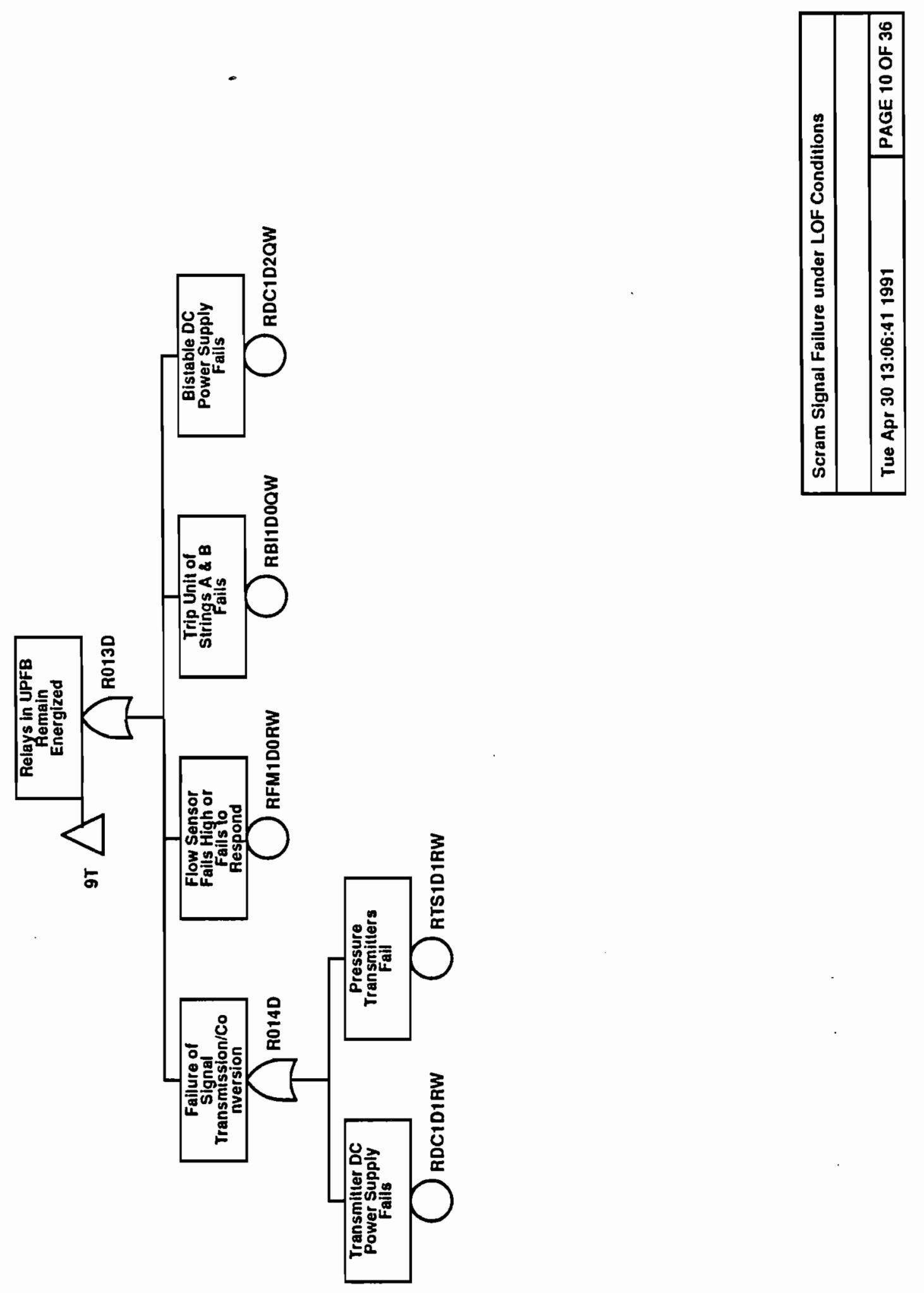

\section{B. 2-123}




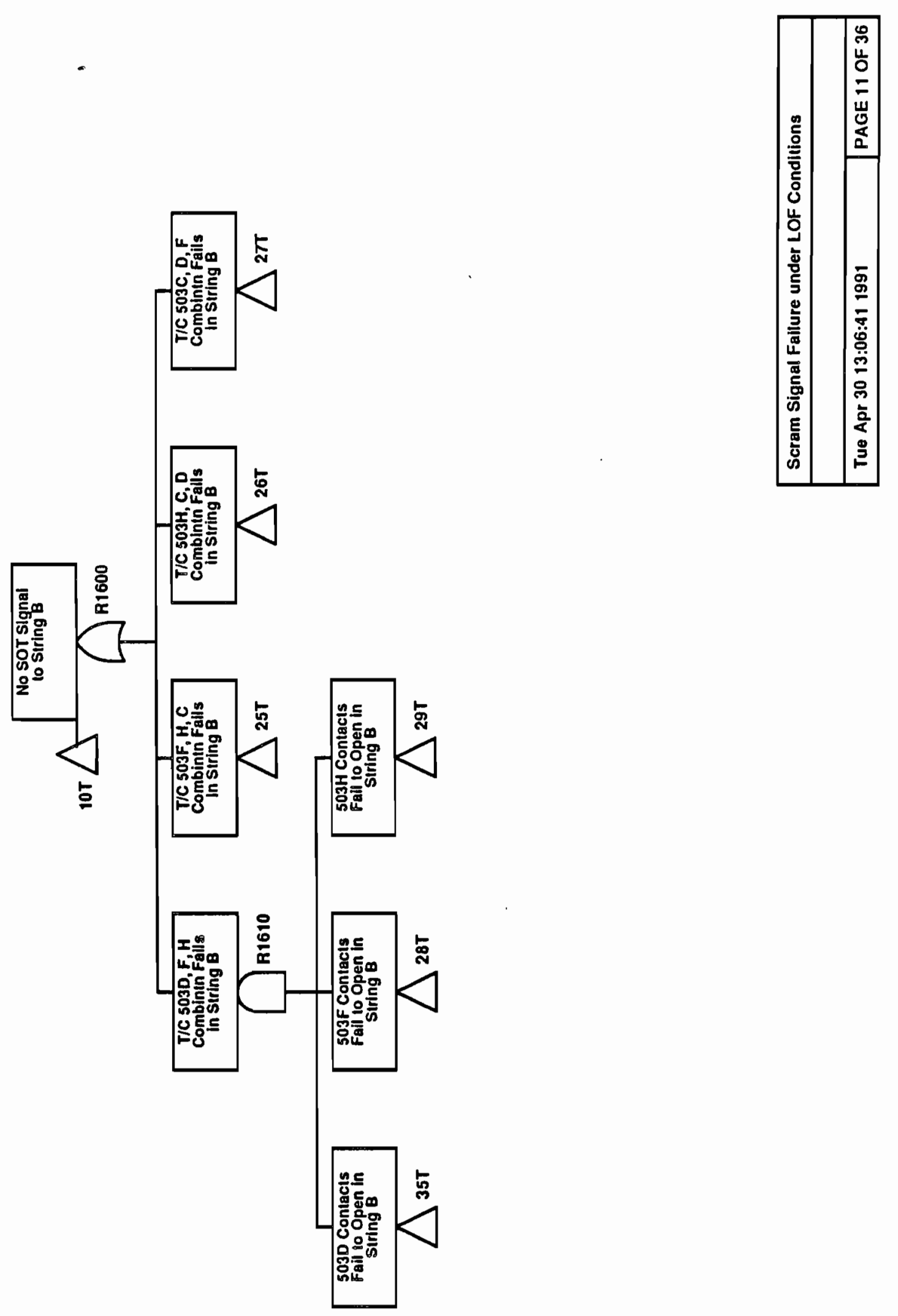

\section{B. $2-124$}




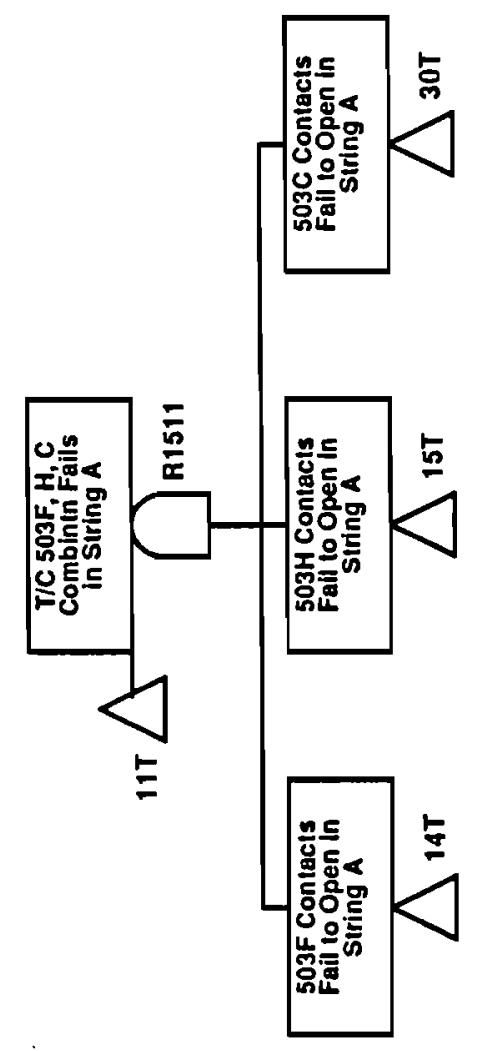

\begin{tabular}{|c|c|}
\hline$\stackrel{n}{0}$ & 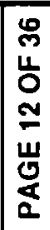 \\
\hline 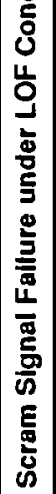 & 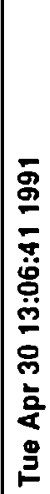 \\
\hline
\end{tabular}




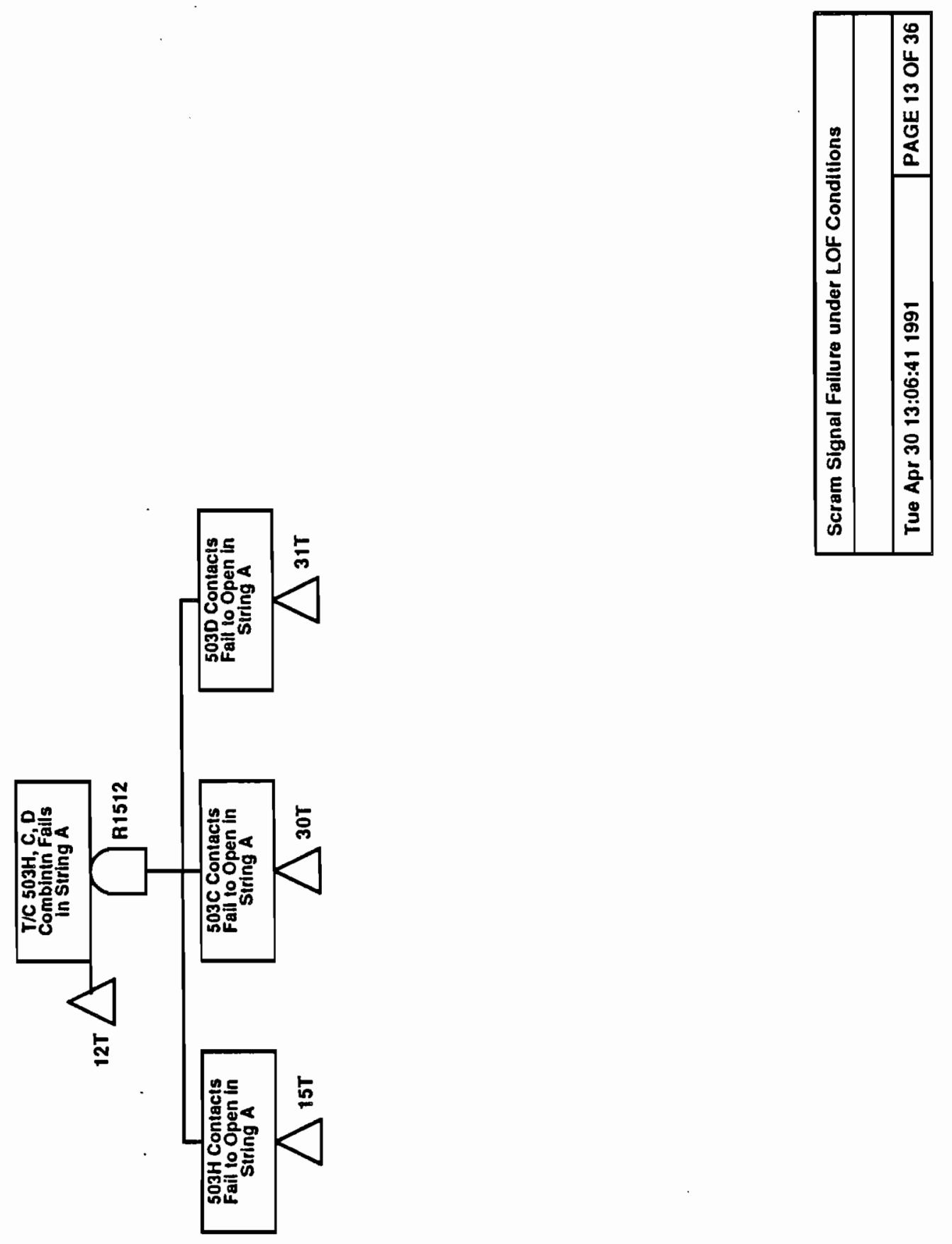




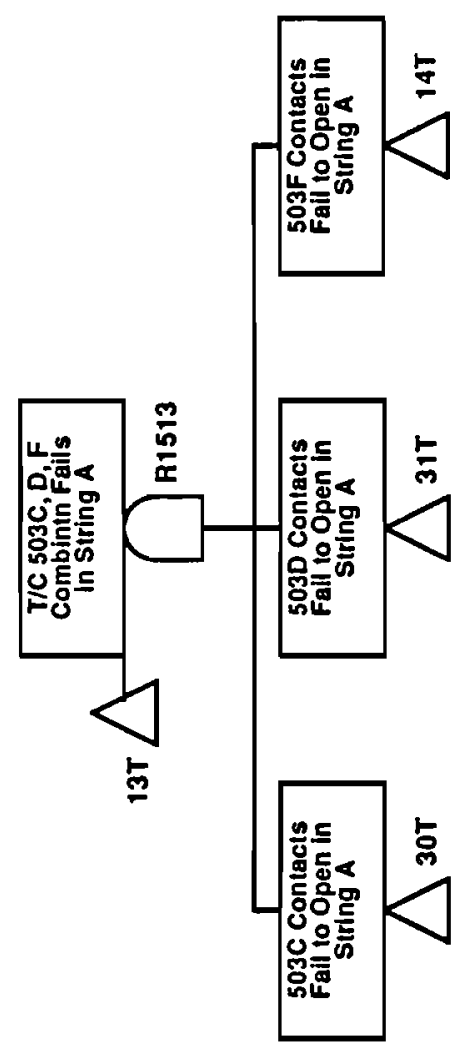

\begin{tabular}{|c|c|}
\hline 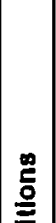 & 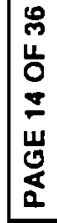 \\
\hline 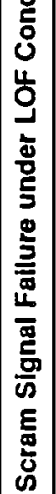 & 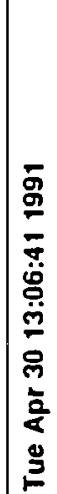 \\
\hline
\end{tabular}




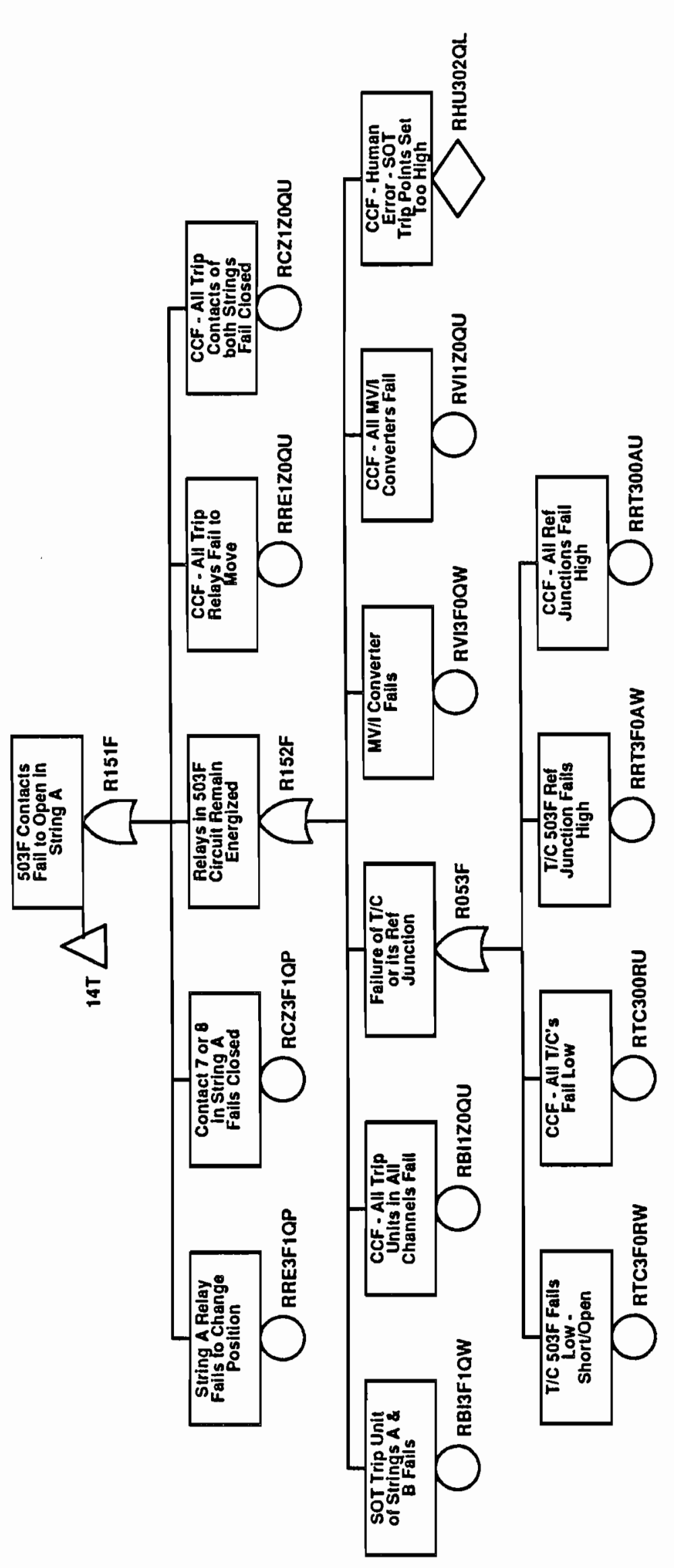

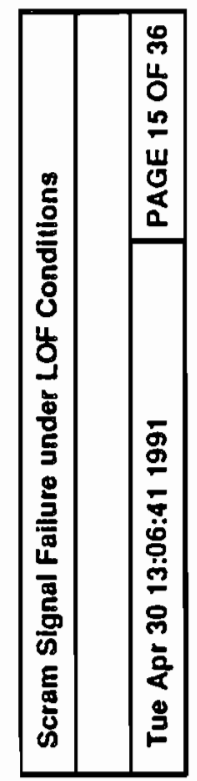

B. 2-128 


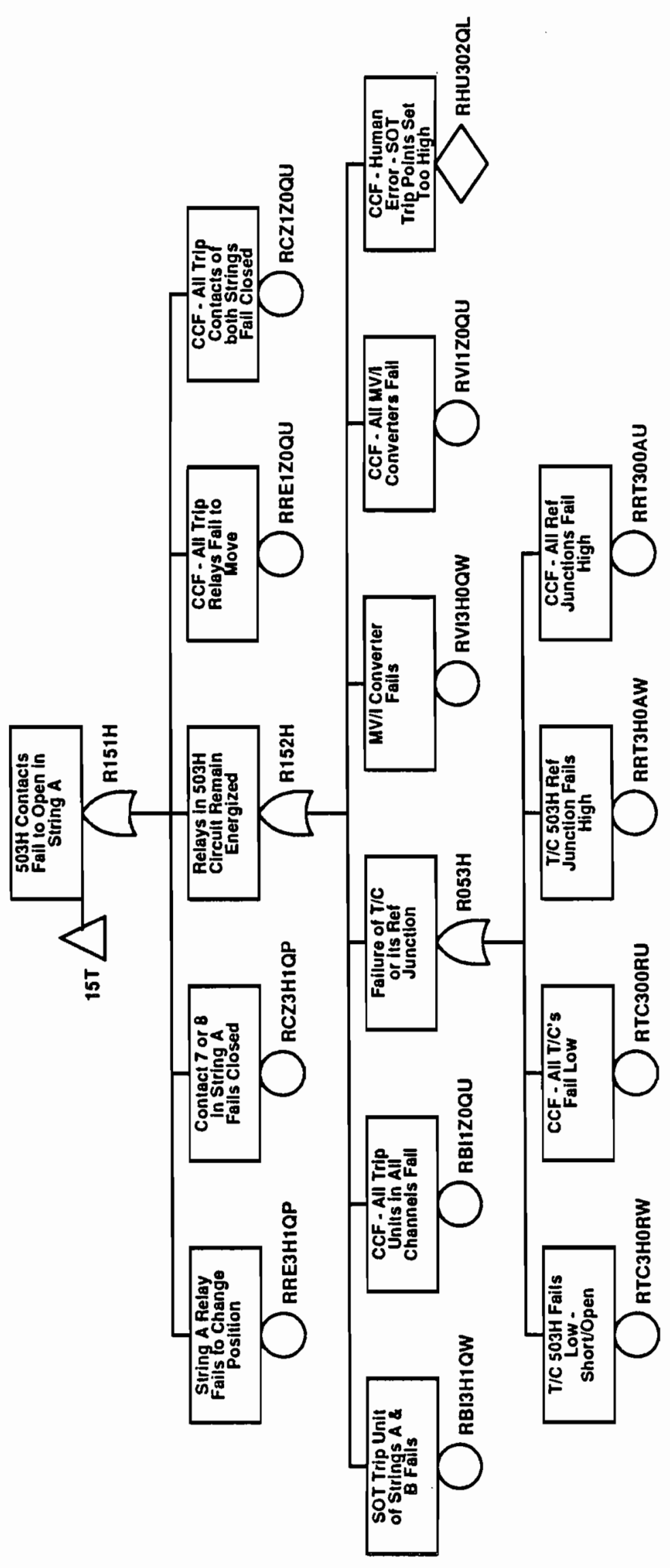

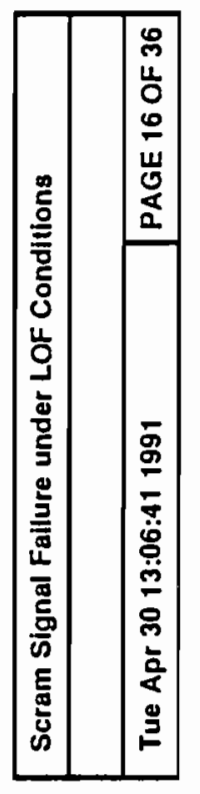

B. 2-129 


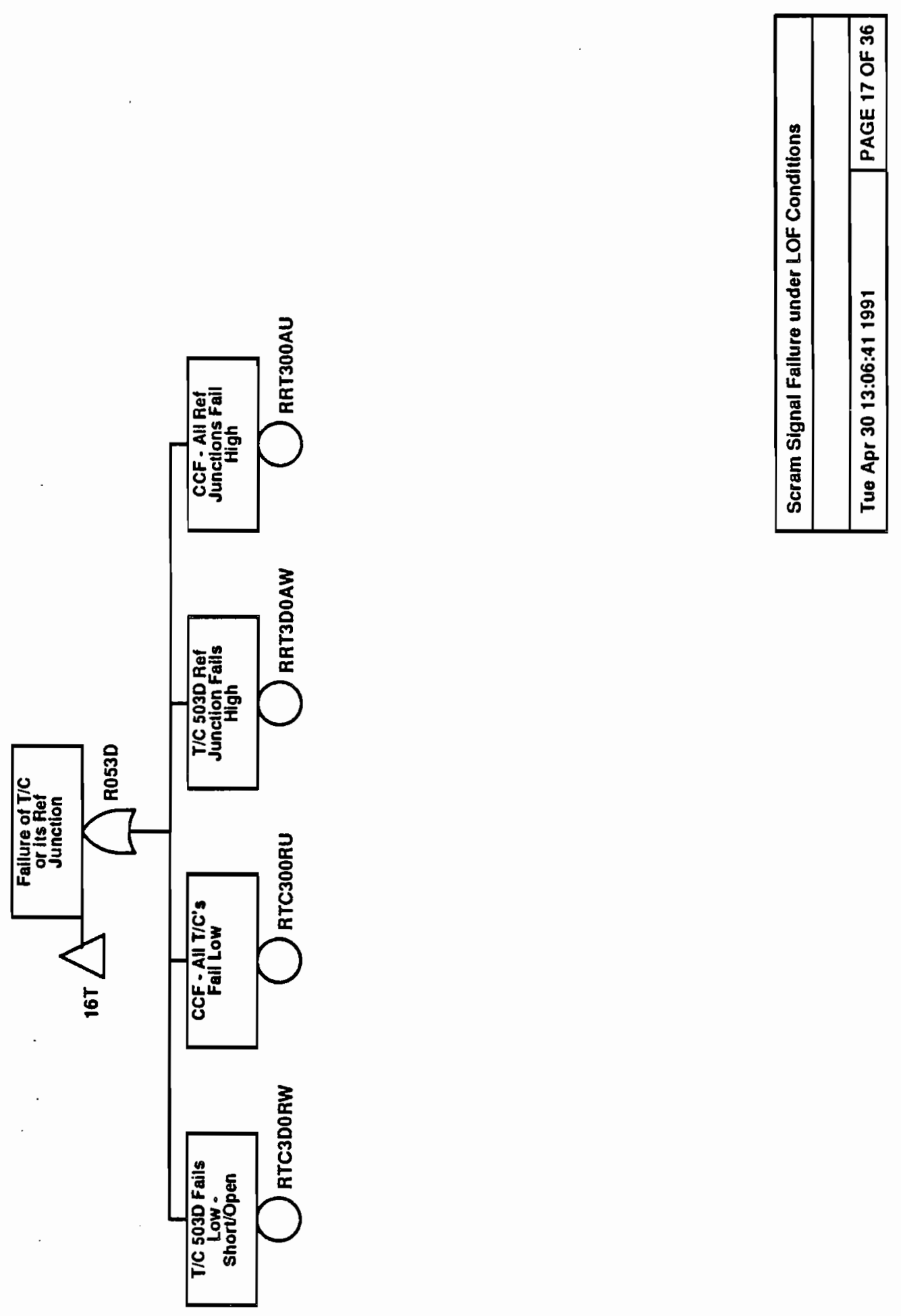




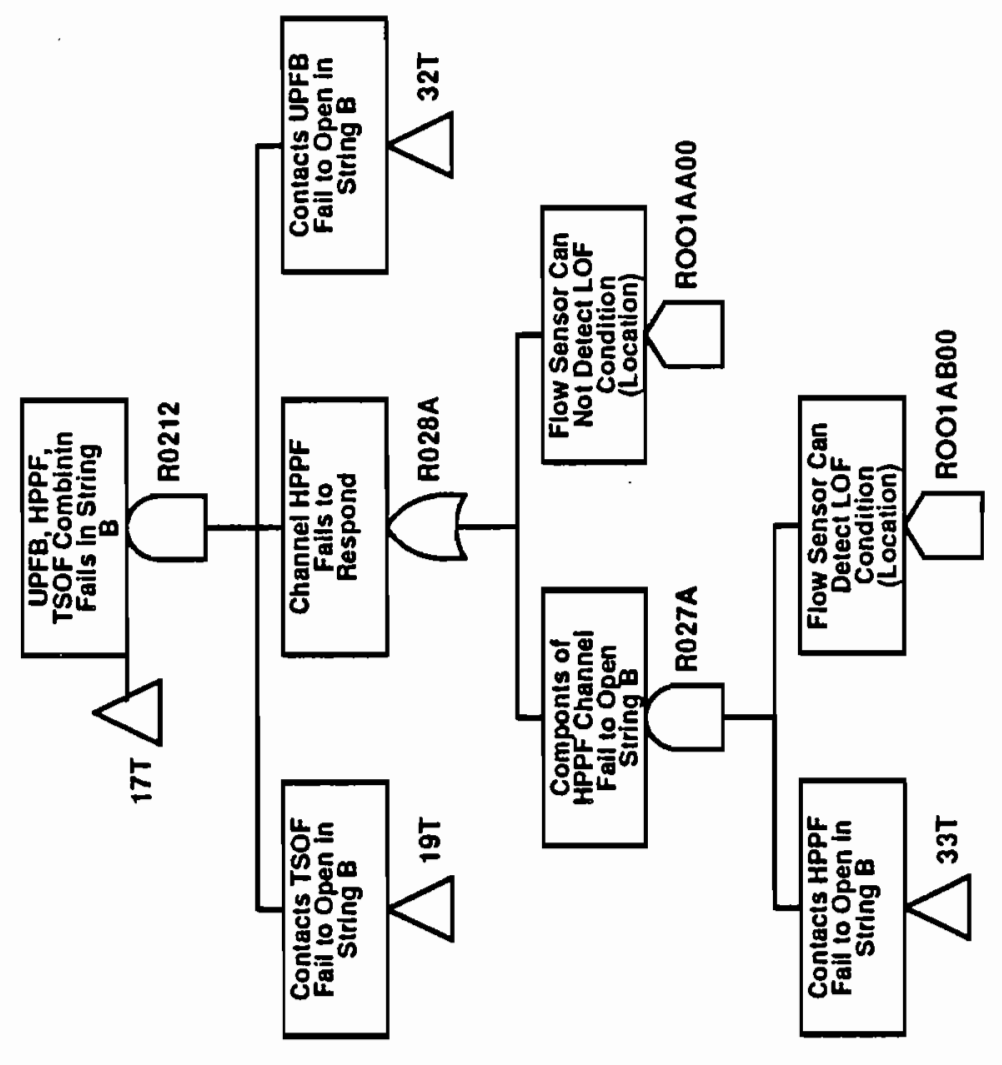

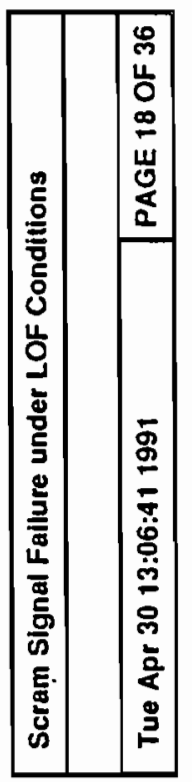




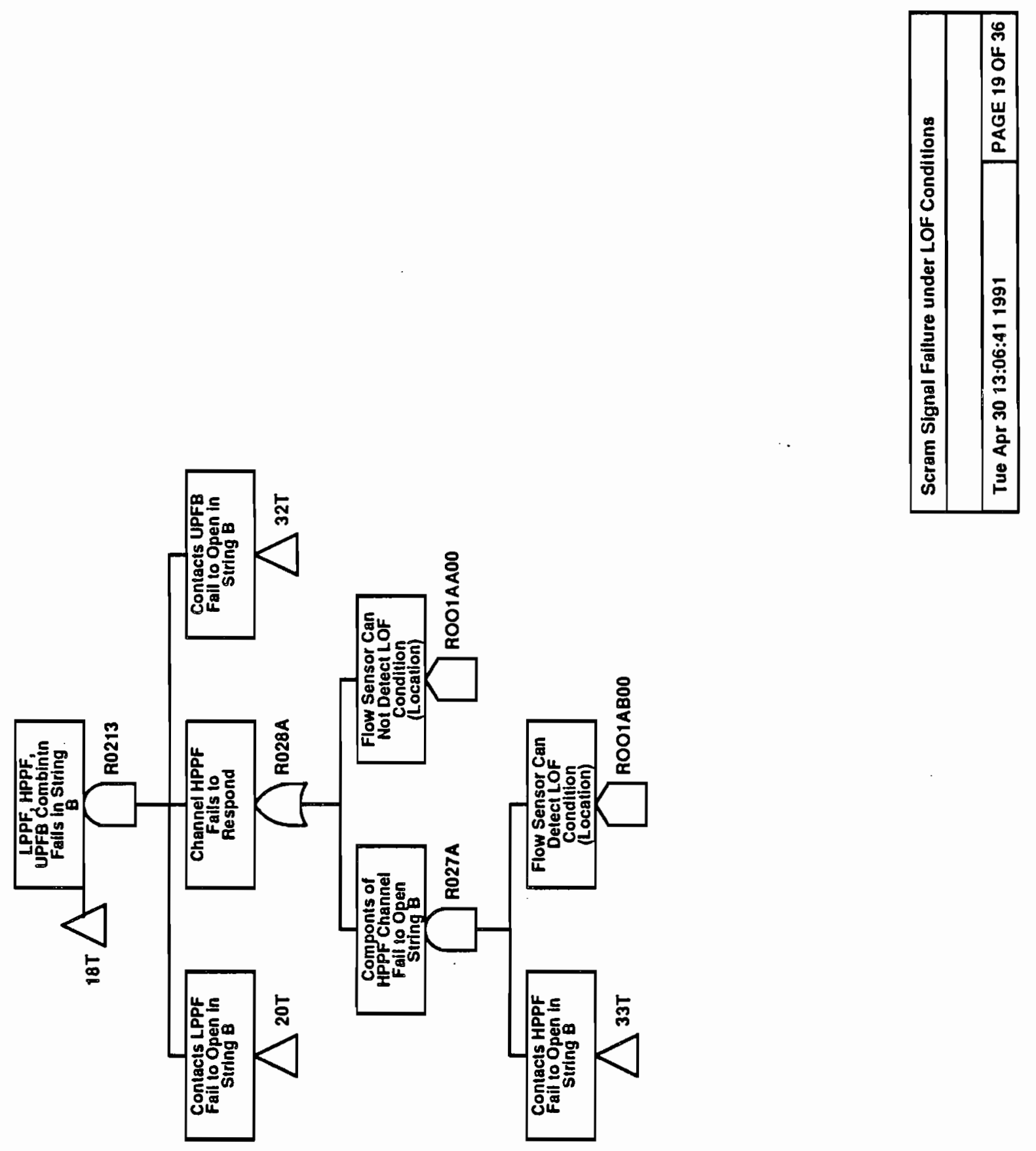



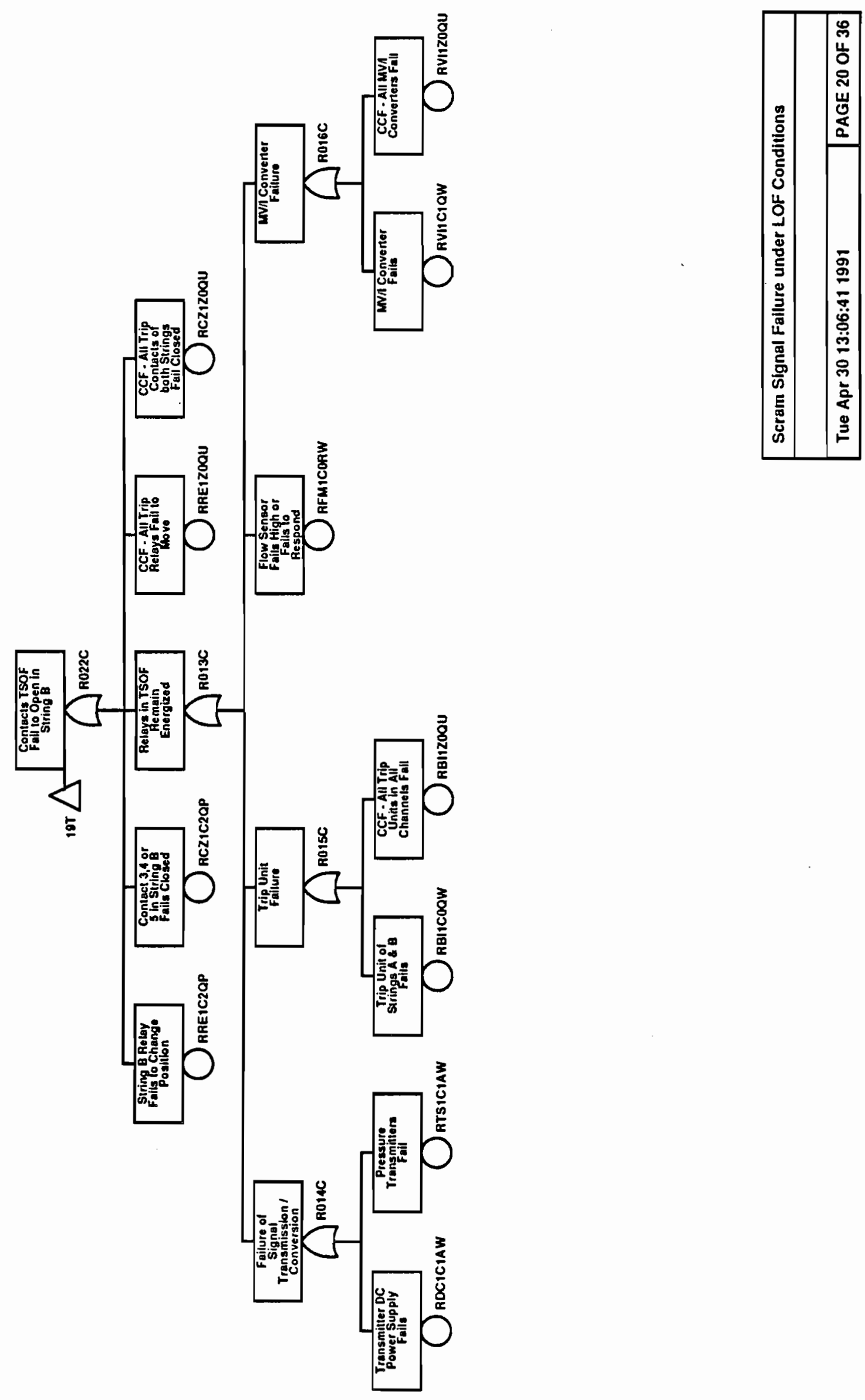

B. 2-133 

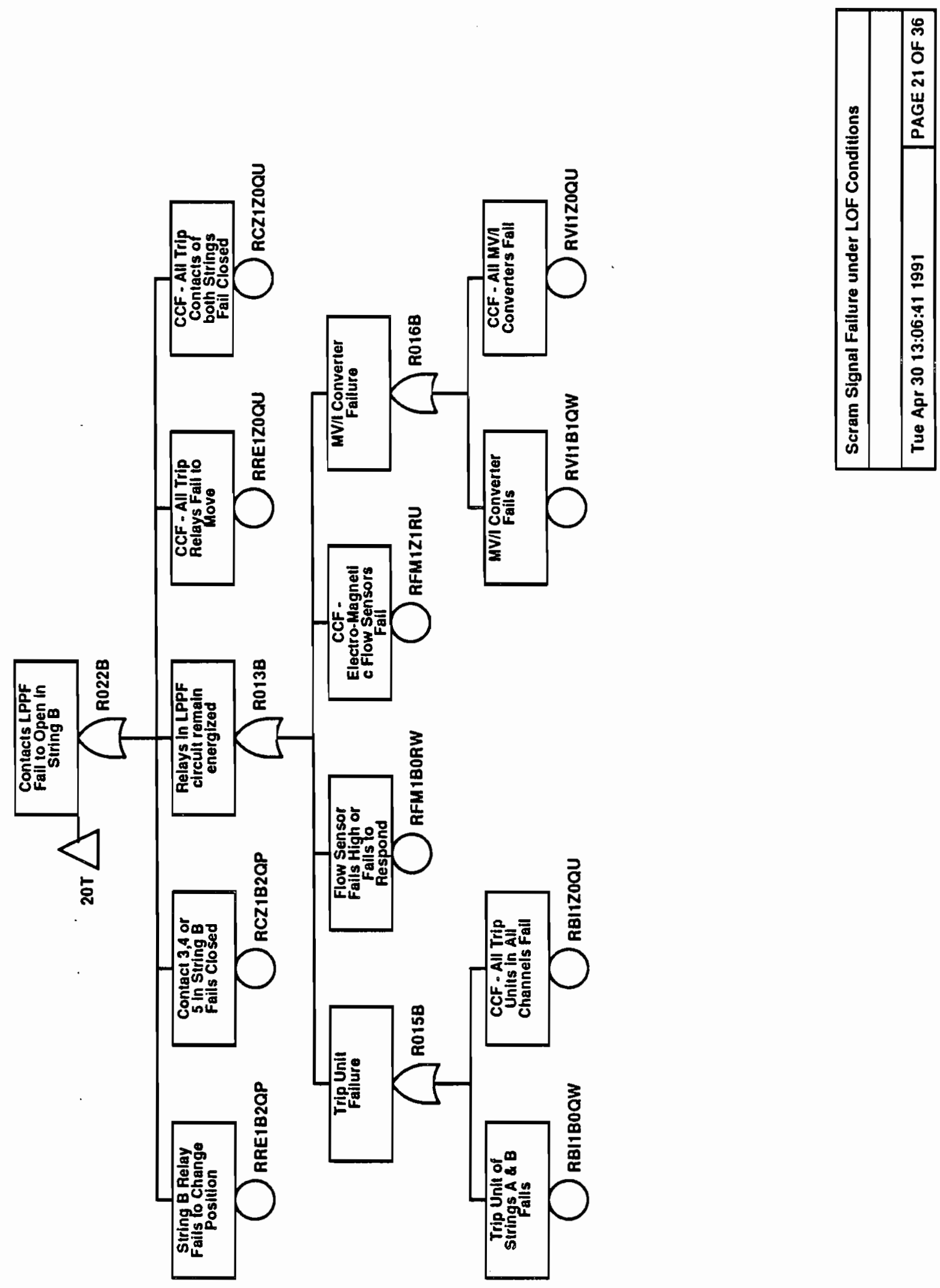


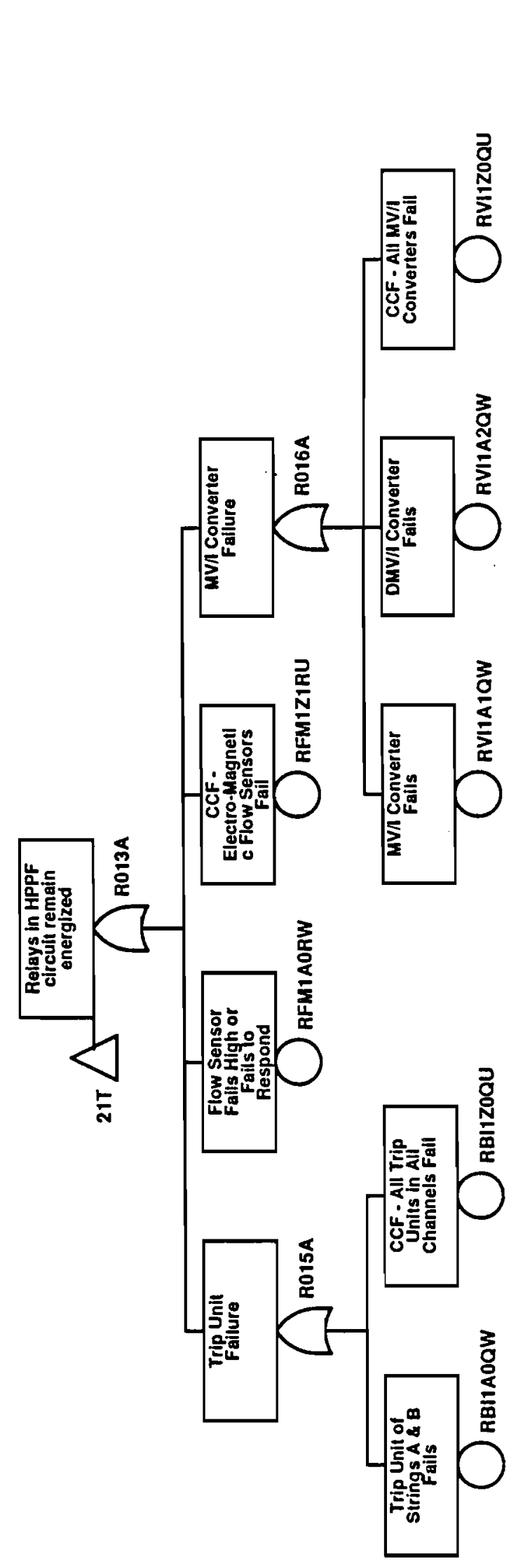

B. 2-135 

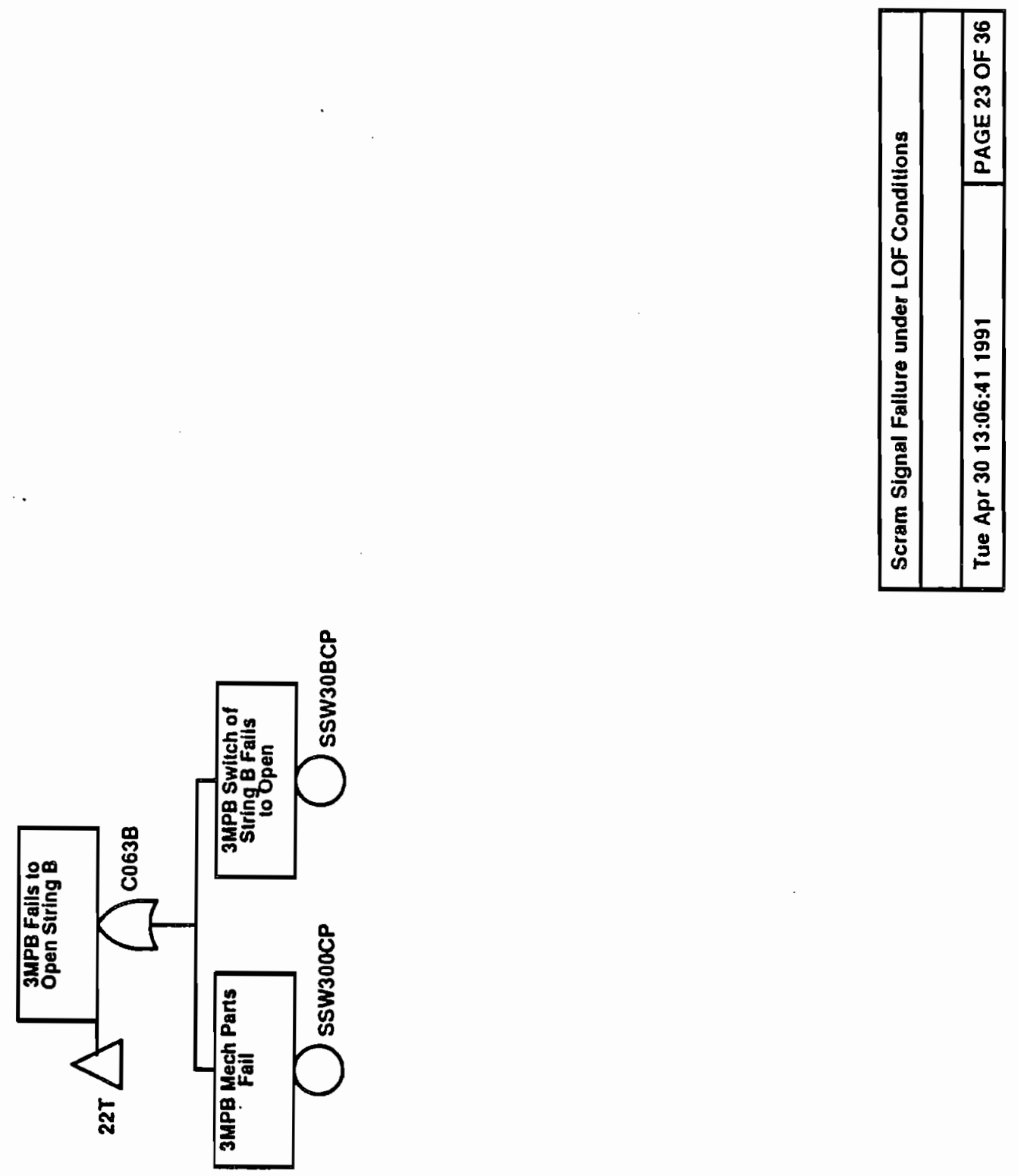

B. 2-136 


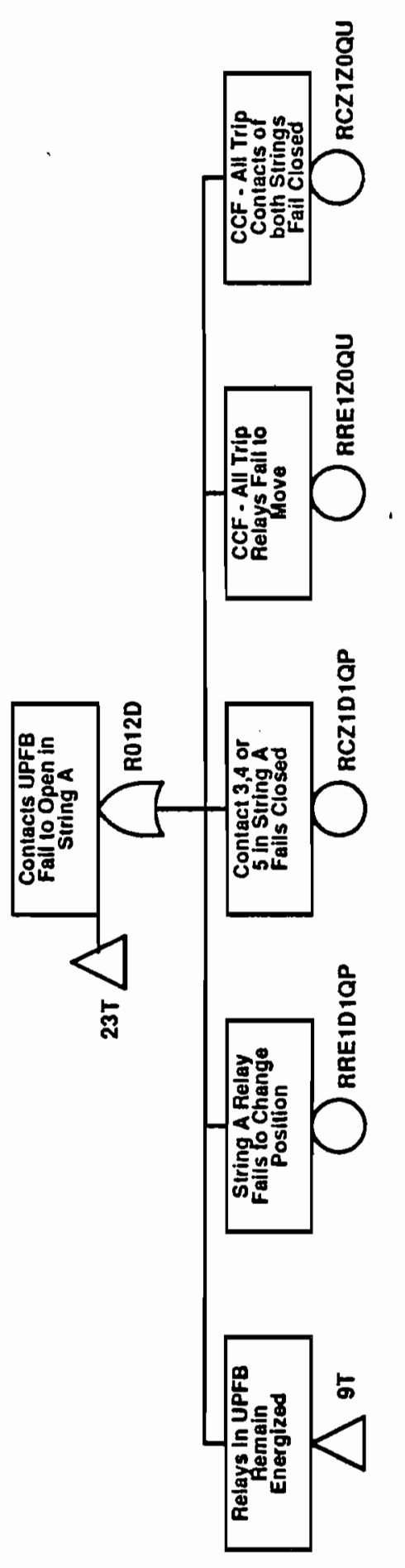

\begin{tabular}{|c|c|}
\hline$\stackrel{\infty}{0}$ & 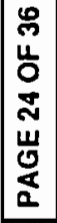 \\
\hline 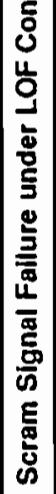 & 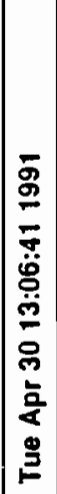 \\
\hline
\end{tabular}




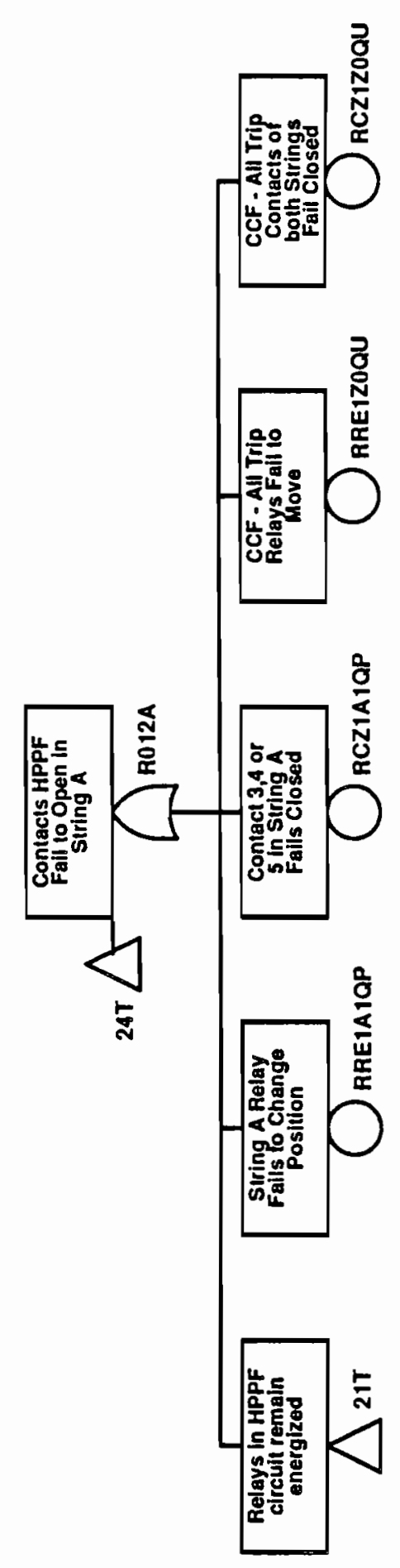




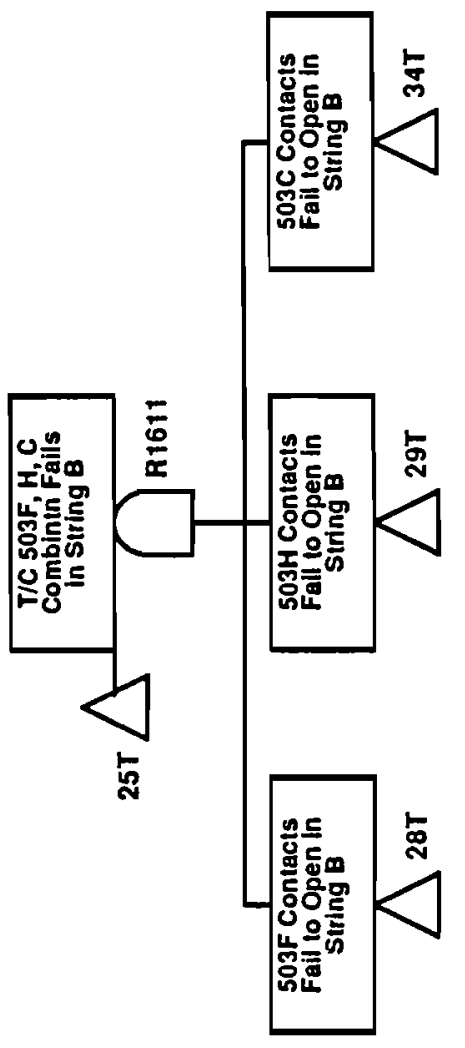

\begin{tabular}{|c|c|}
\hline 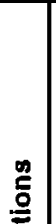 & 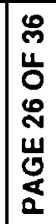 \\
\hline 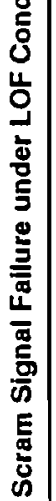 & 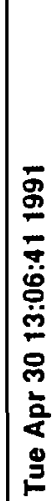 \\
\hline
\end{tabular}

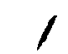




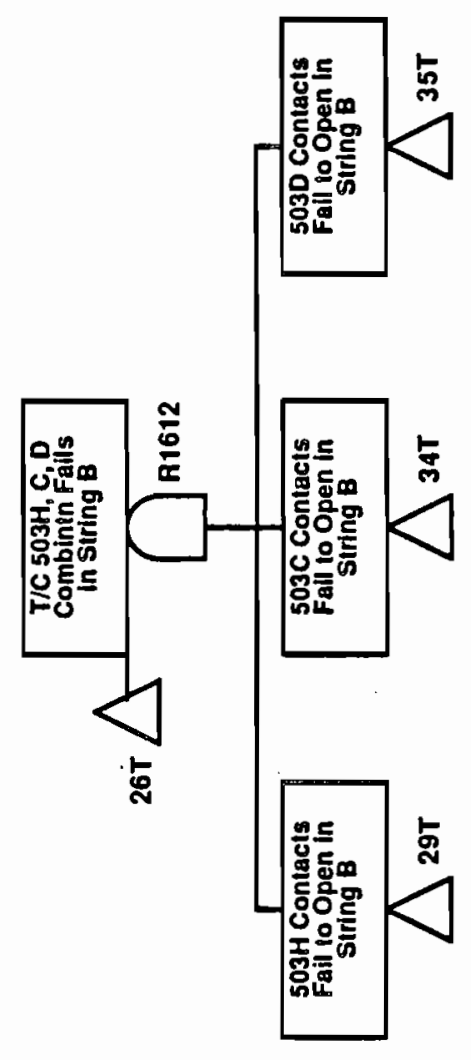

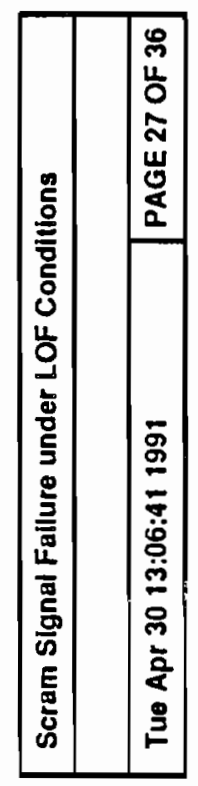




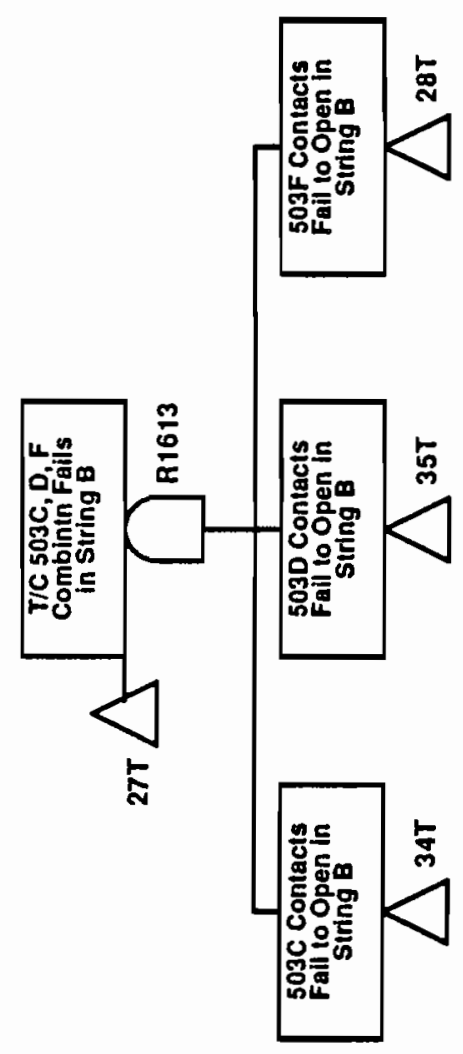

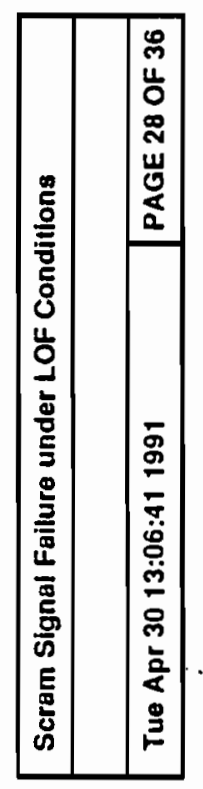



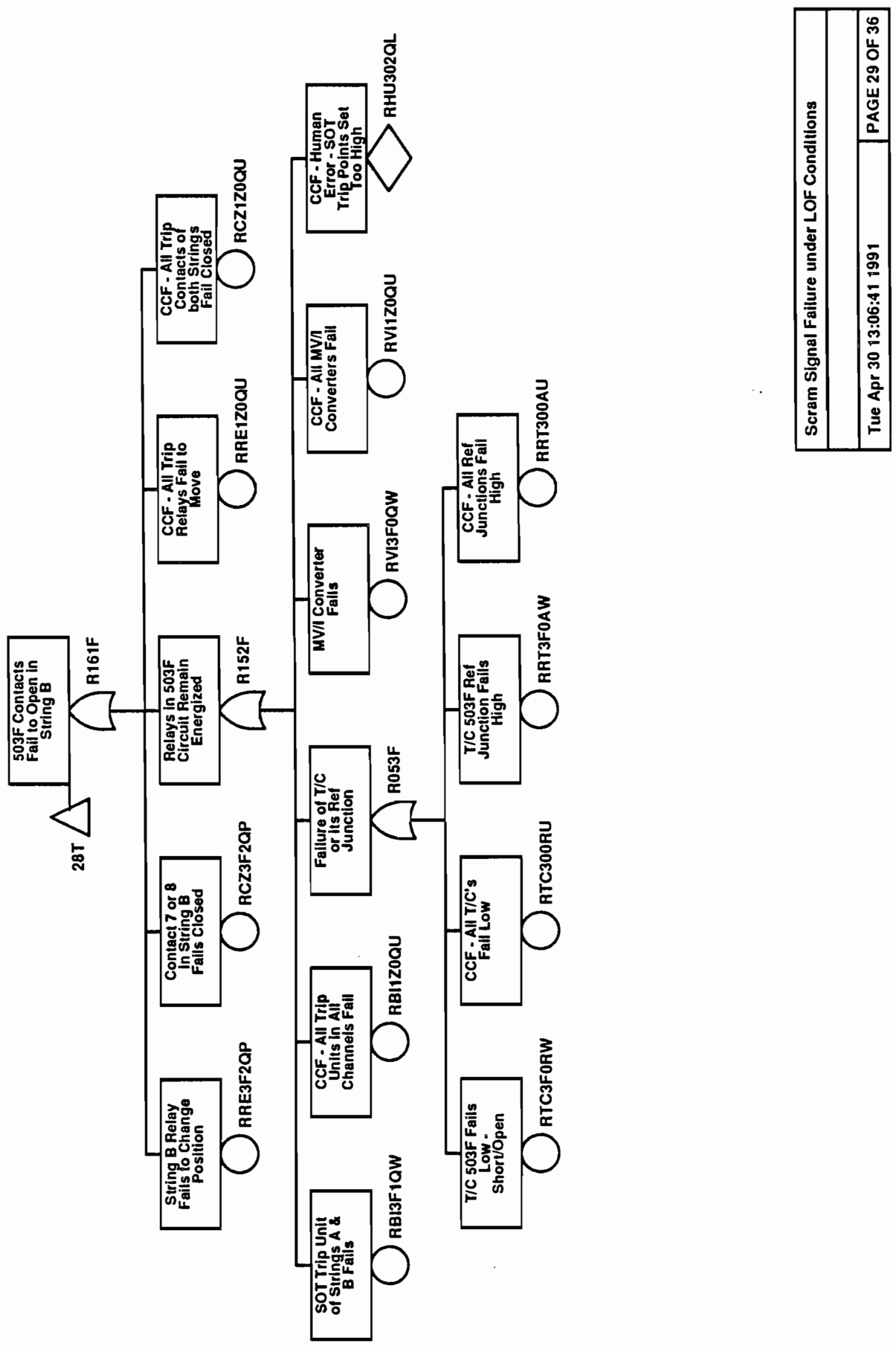

B. 2-142 

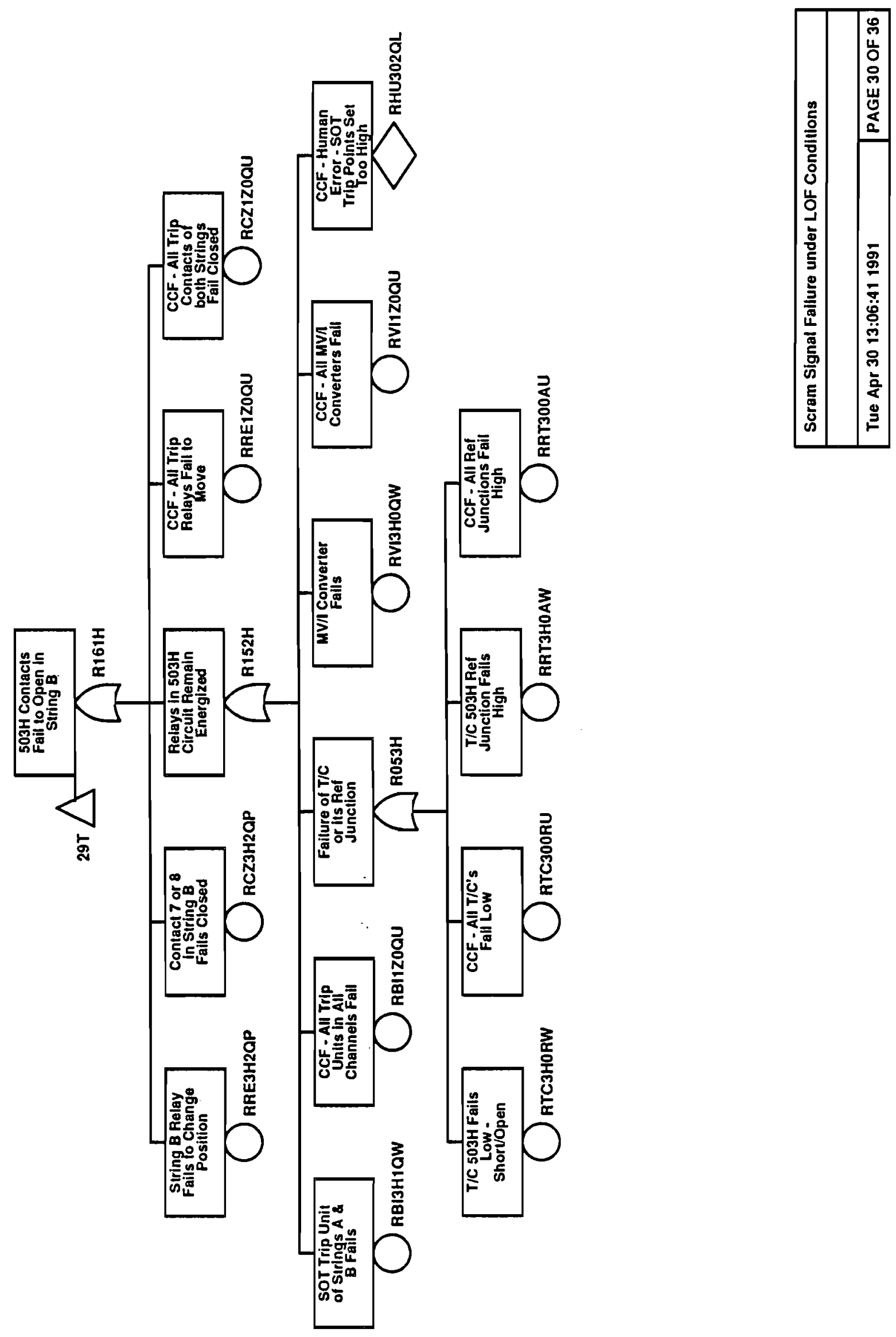

B. 2-143 

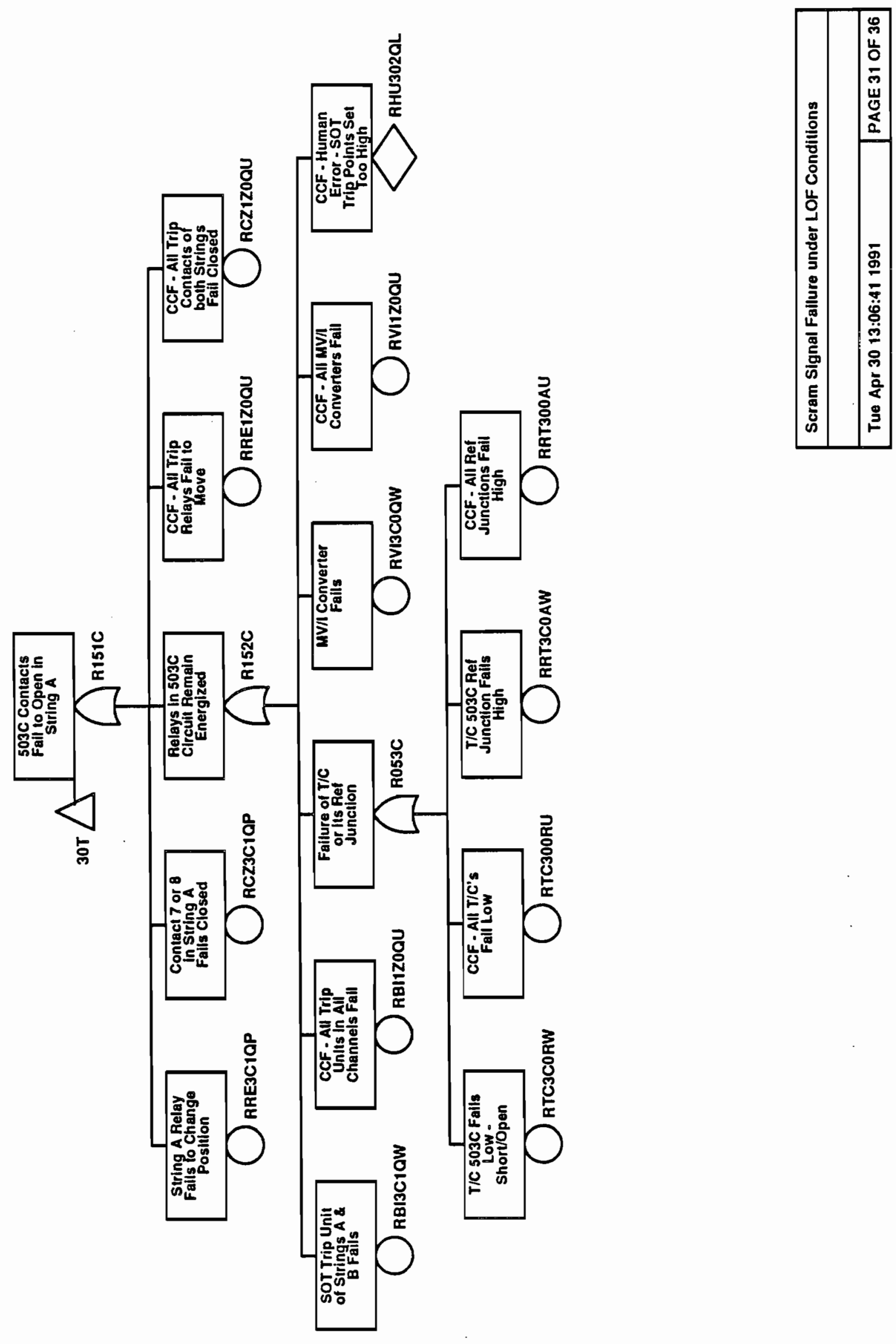

B. 2-144 

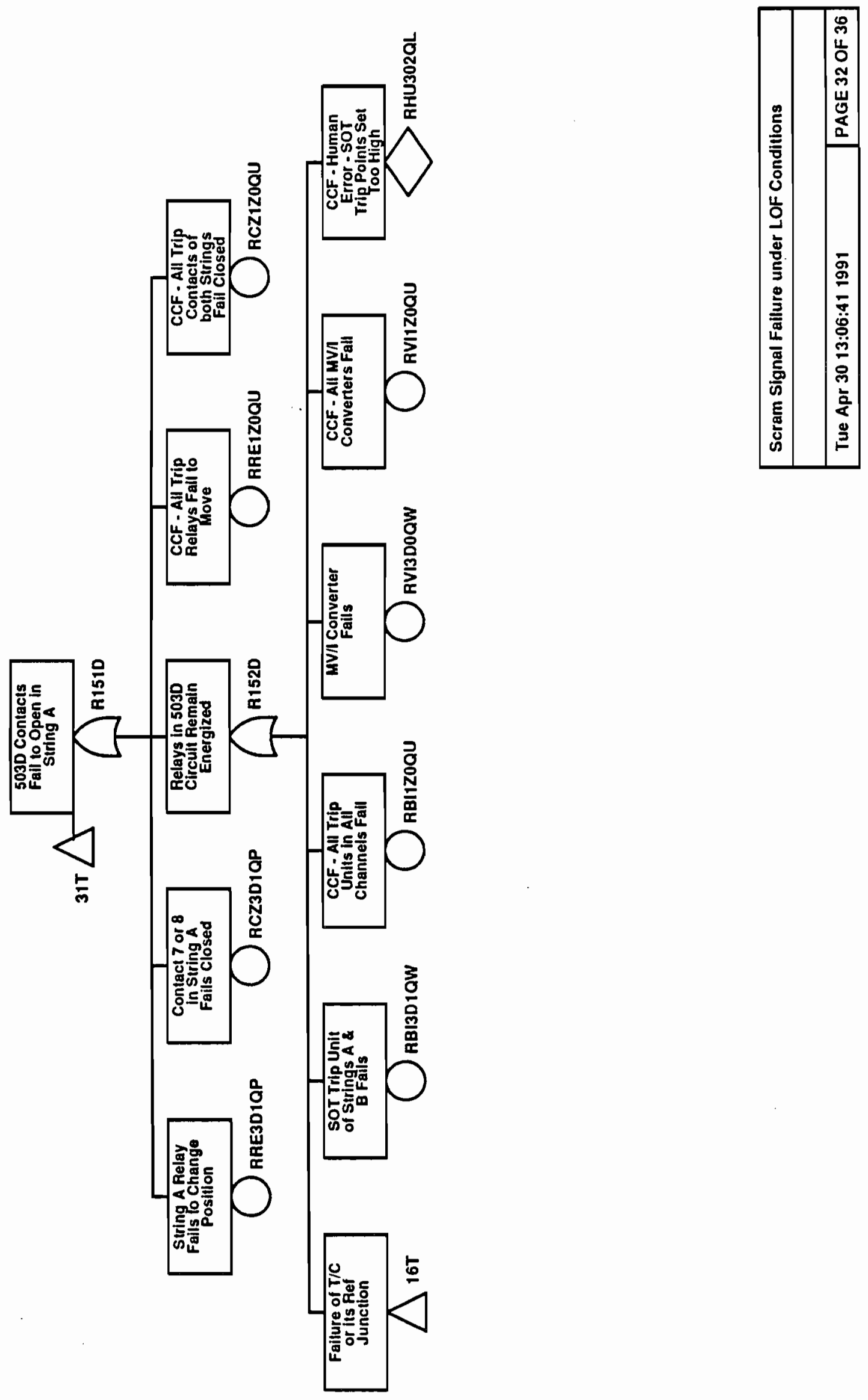

B. 2-145 


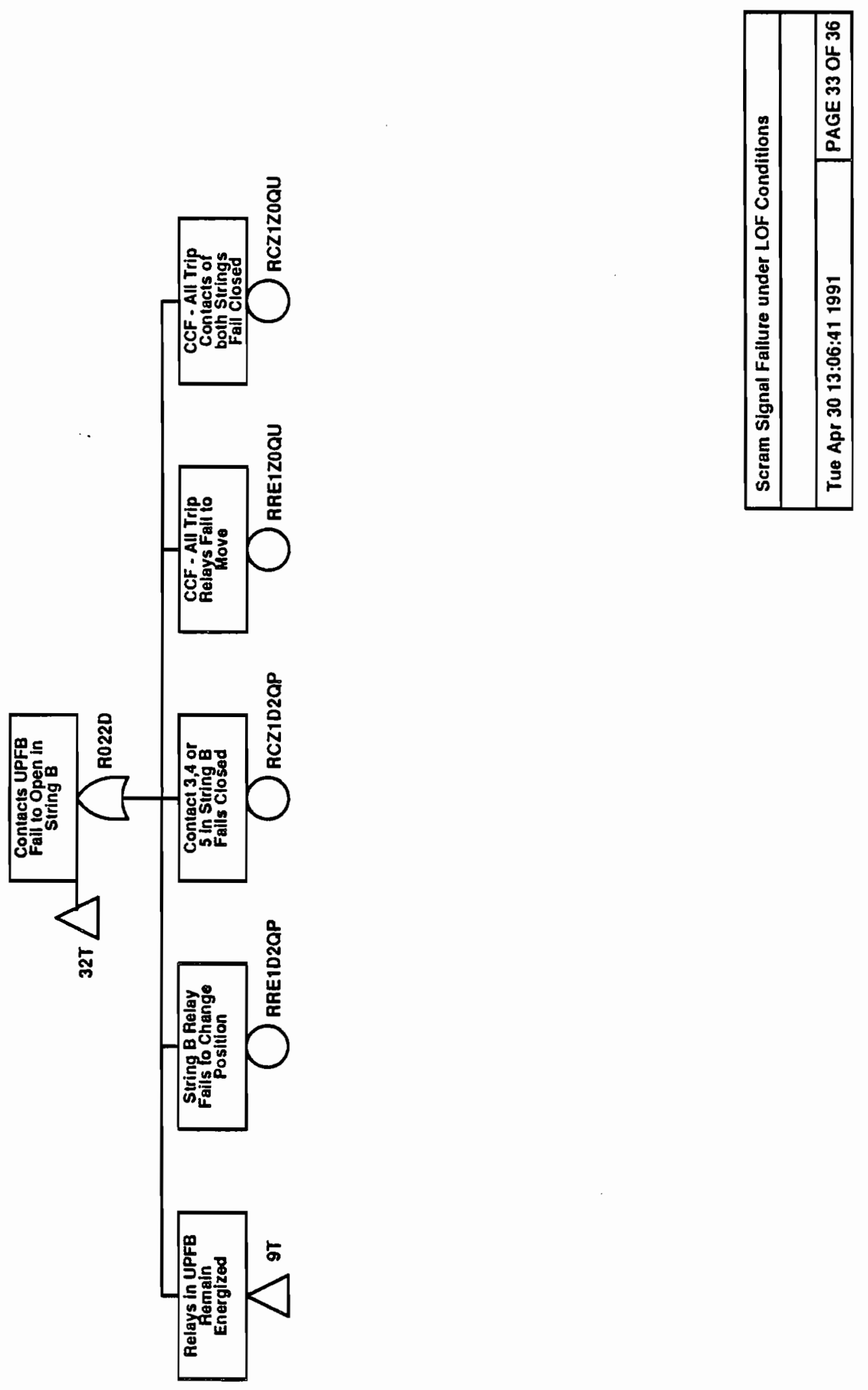



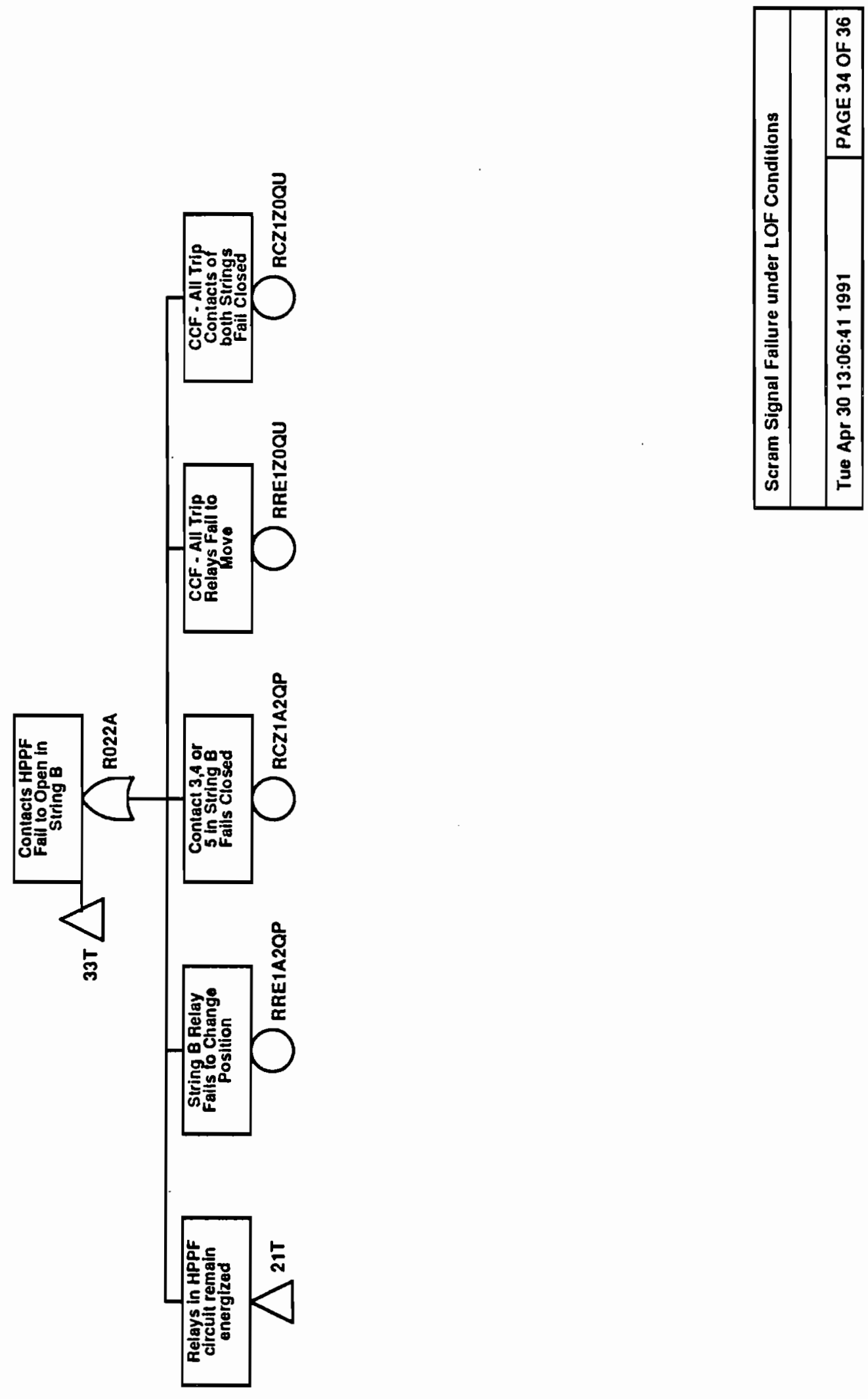

\section{B. 2-147}



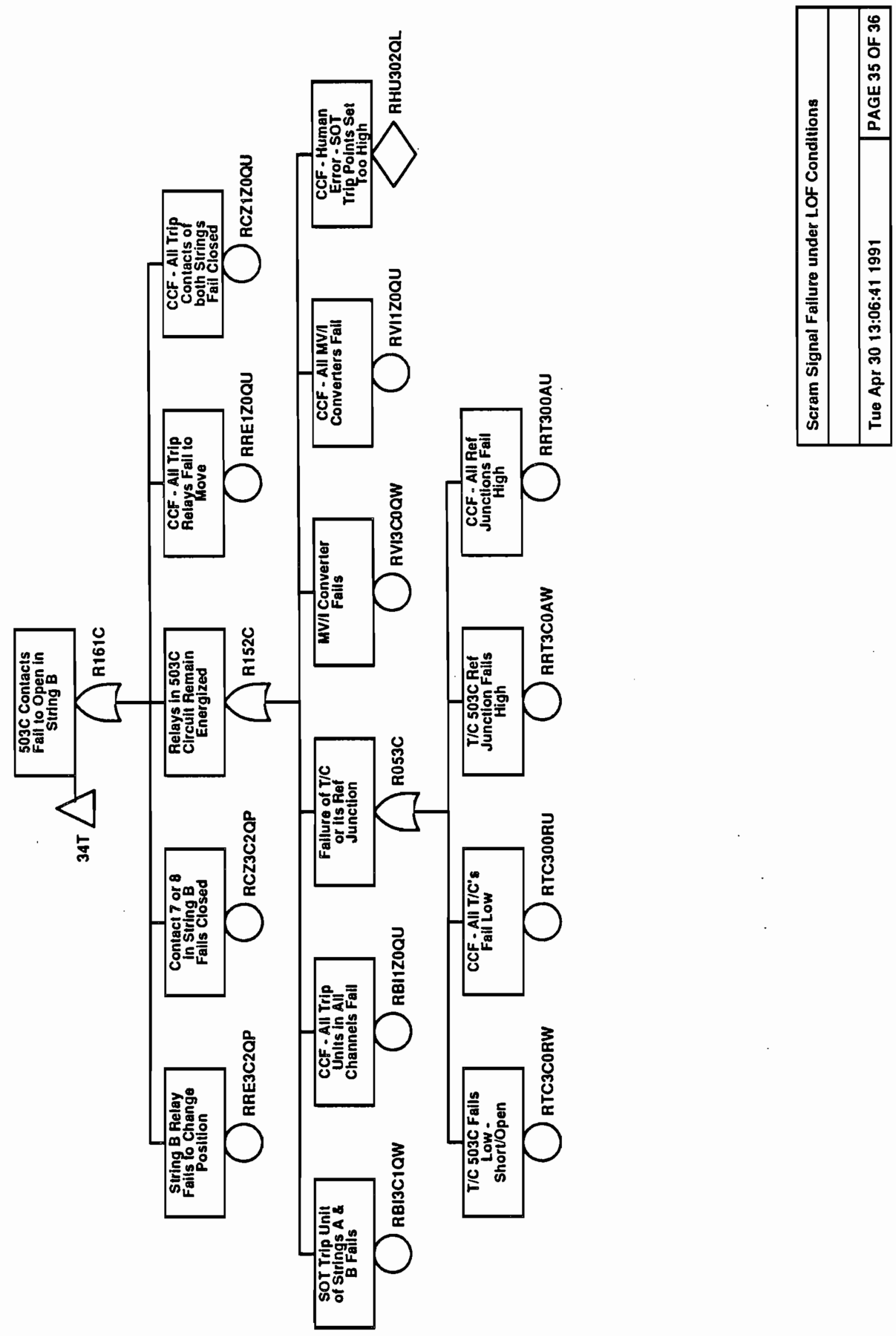

B. $2-148$ 


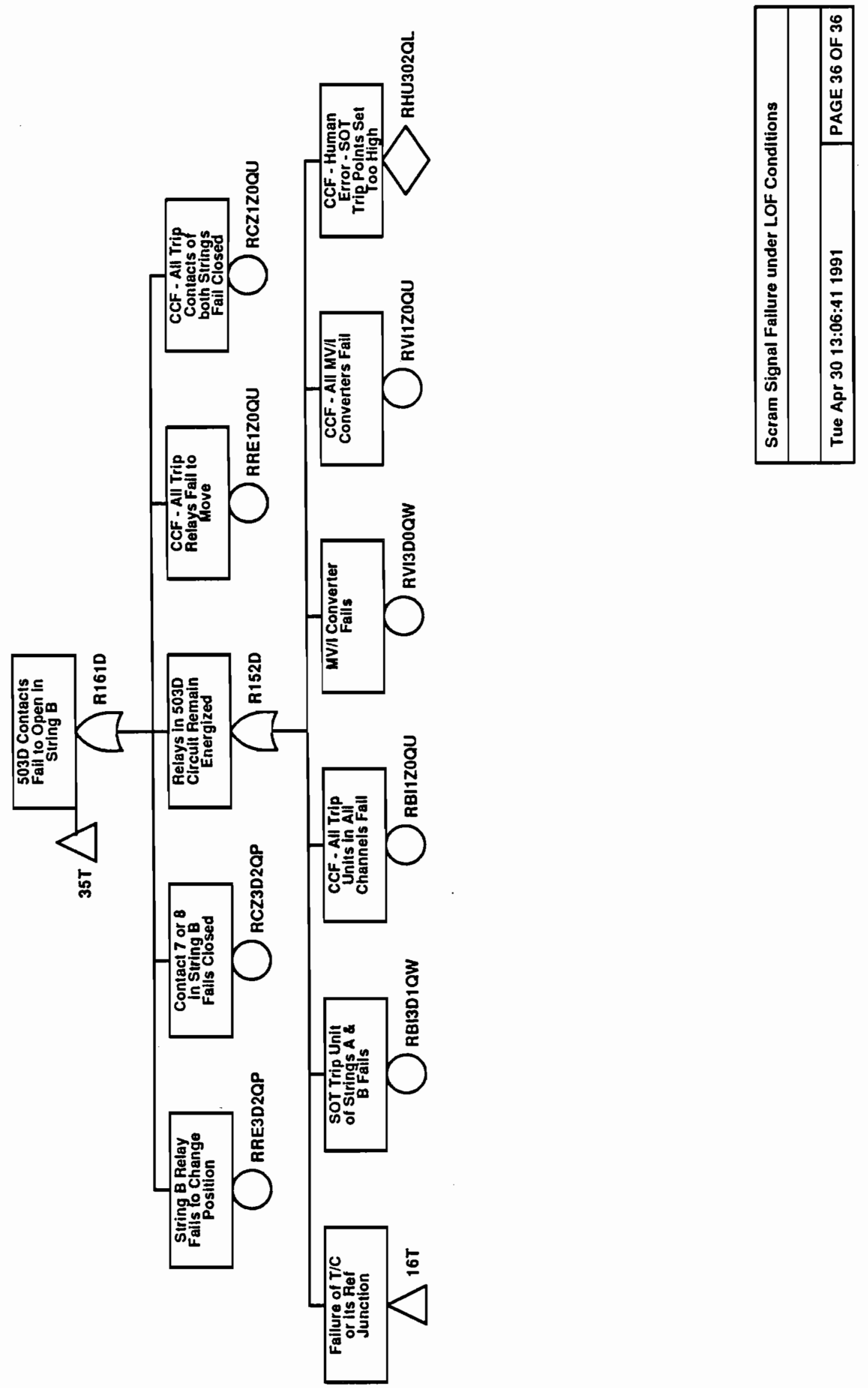

B. $2-149$ 


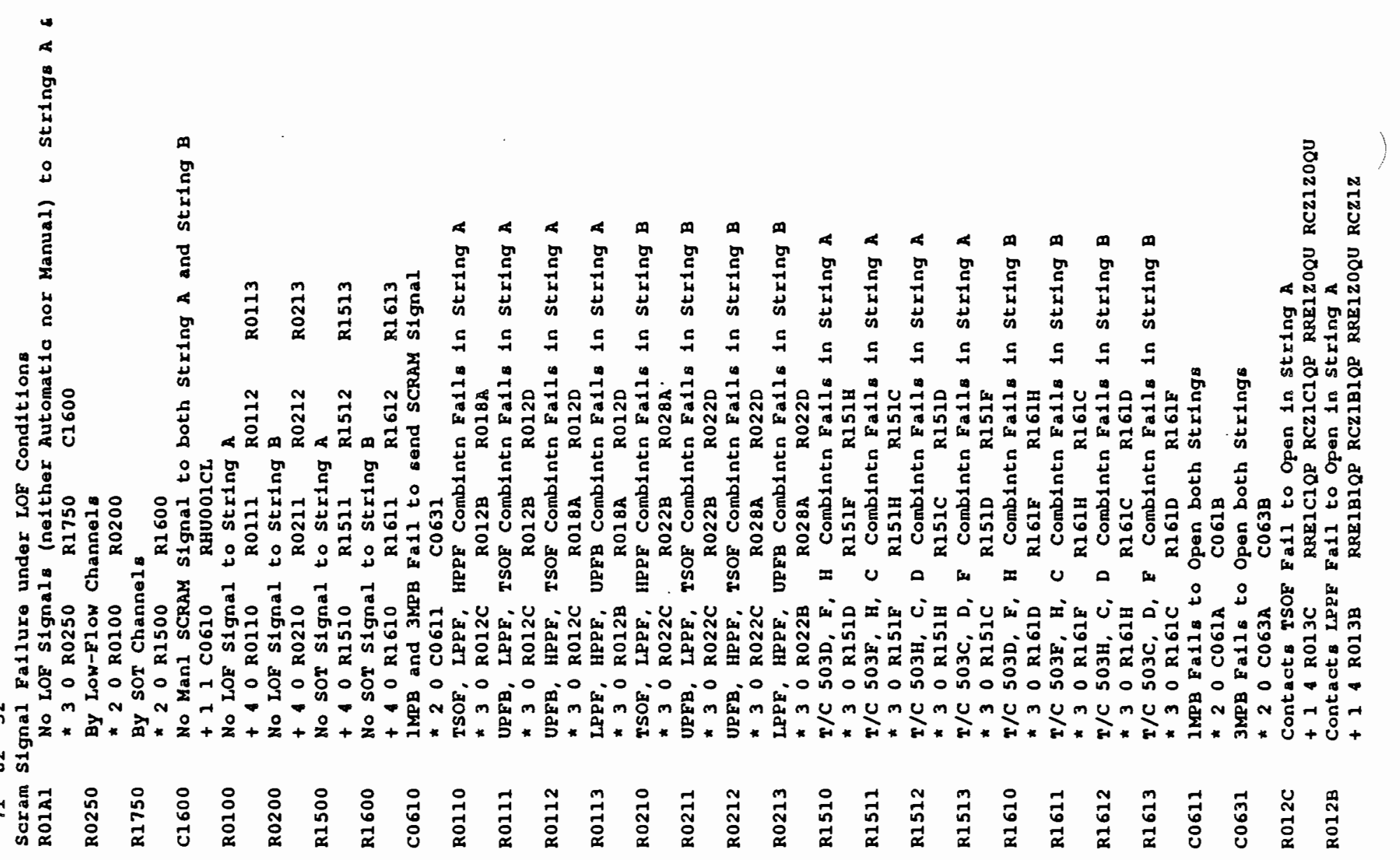




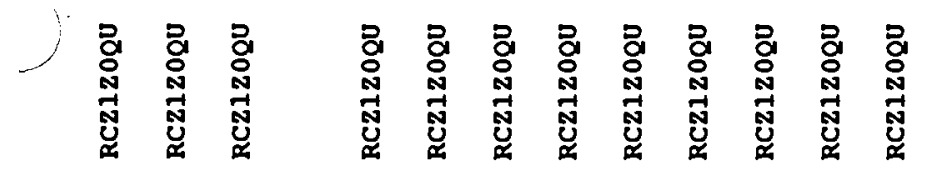

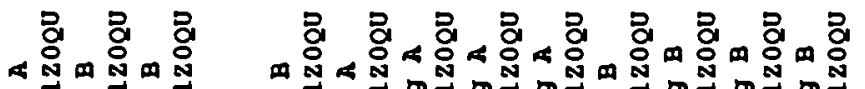

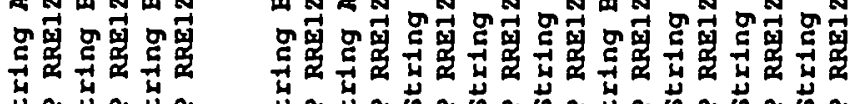

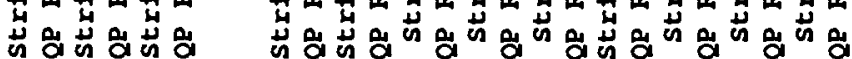

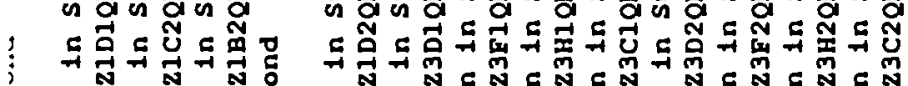

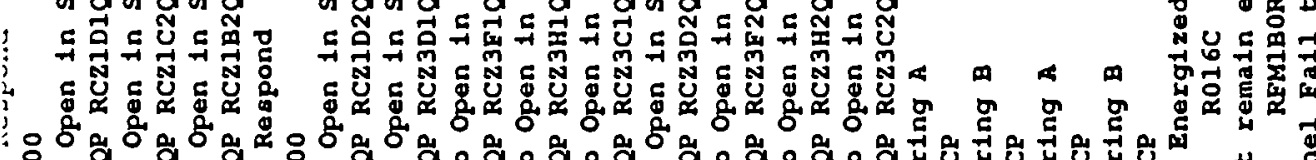

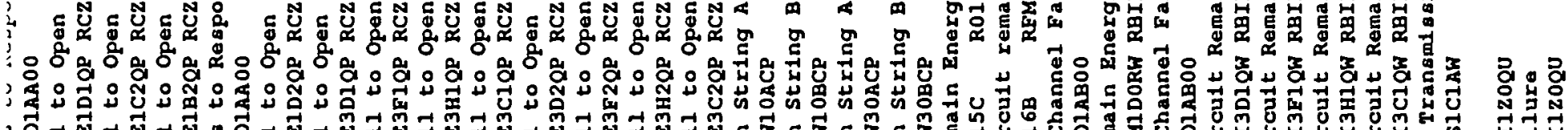

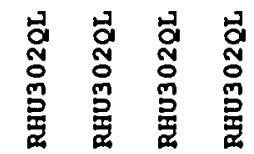

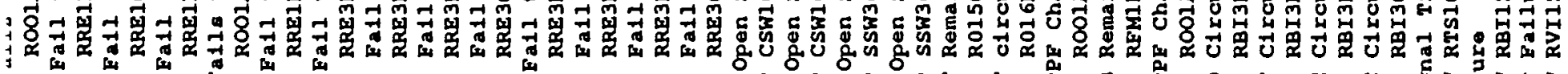

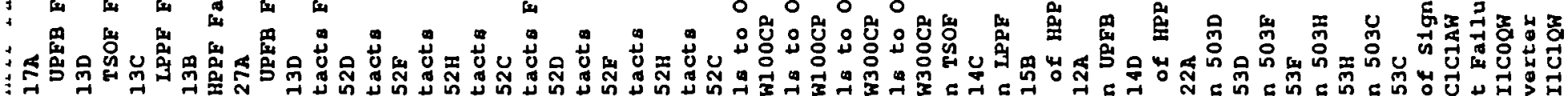

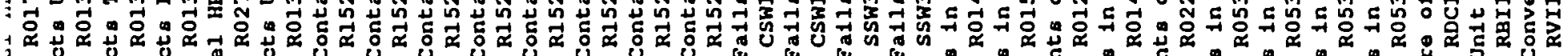
H

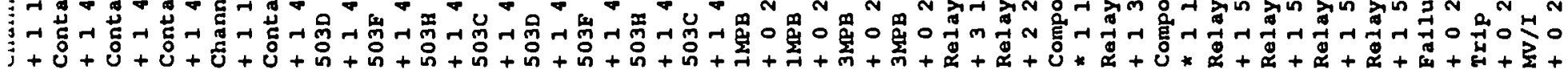

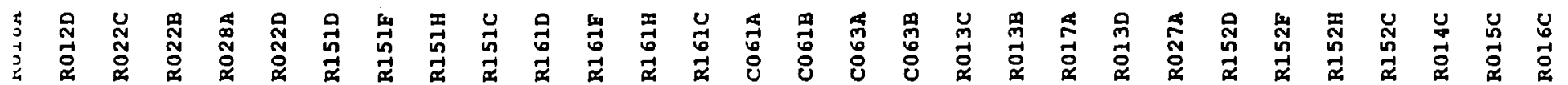



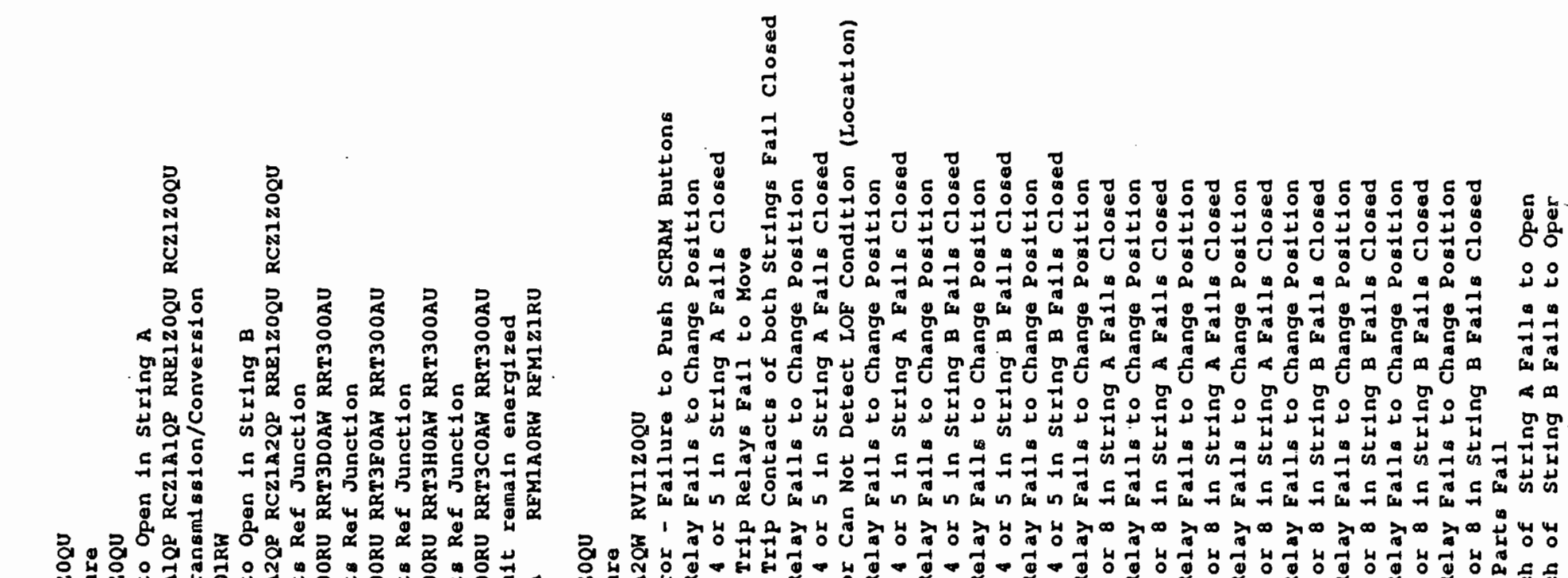

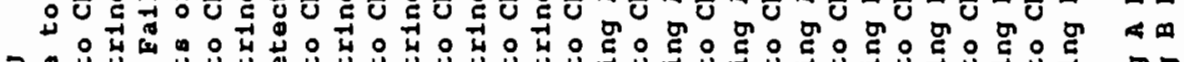

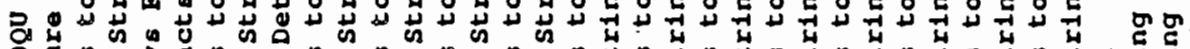

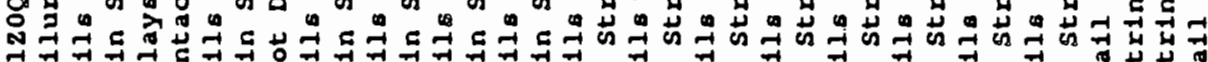

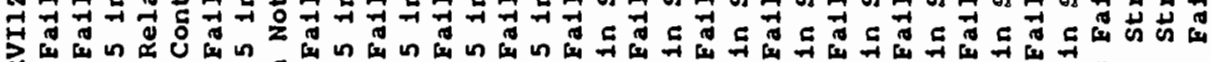

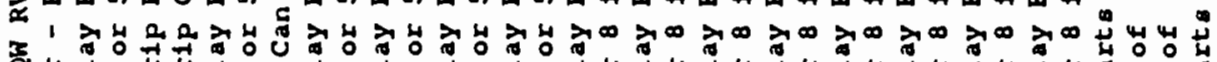

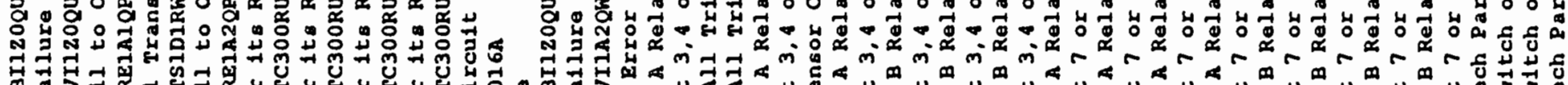

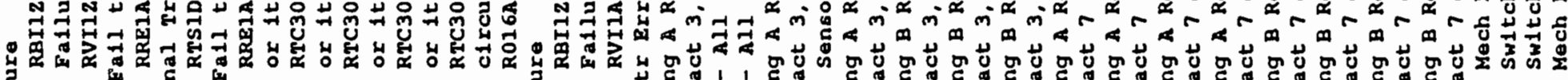

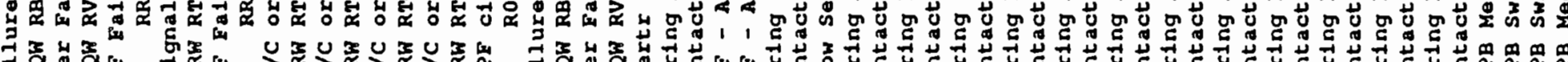

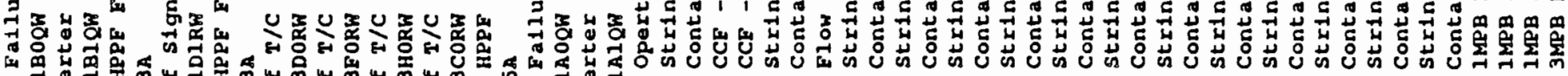

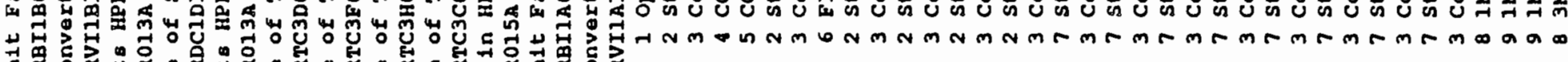

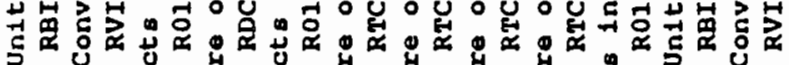
NoNo

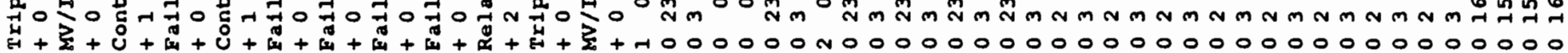

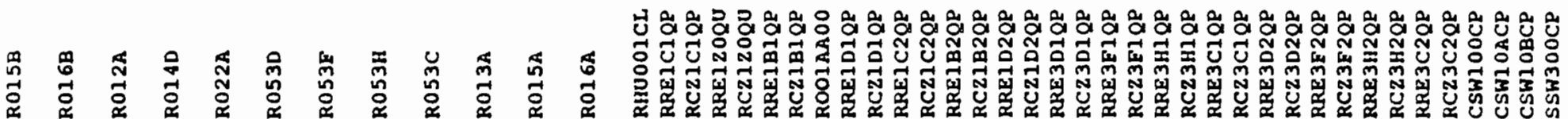




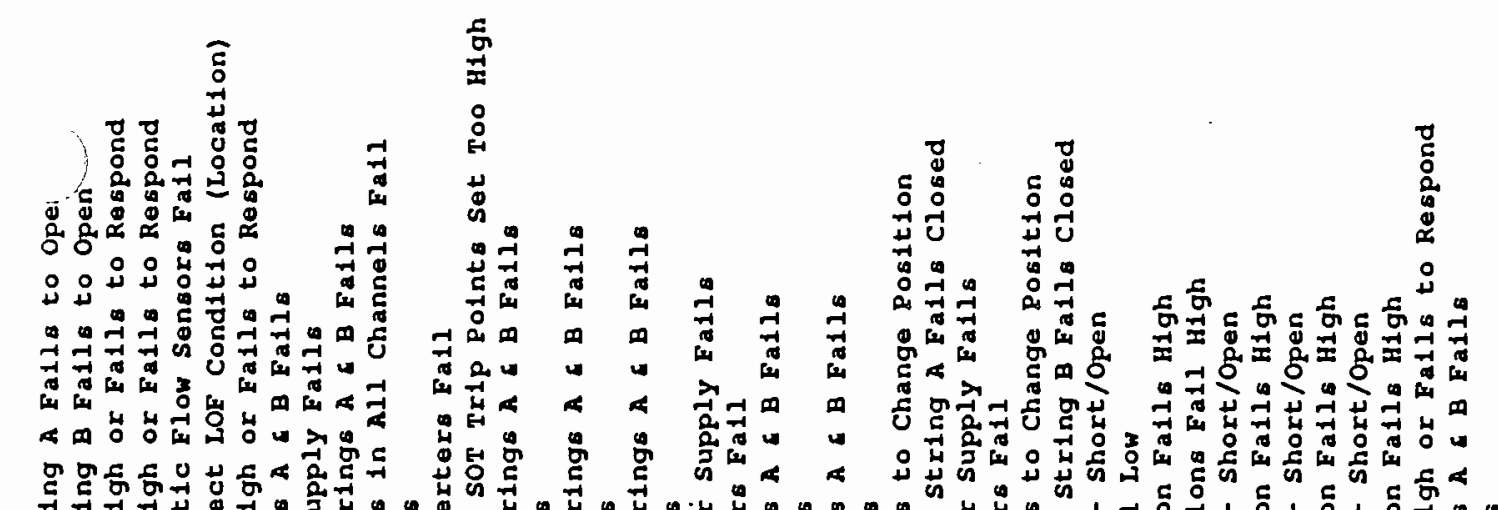

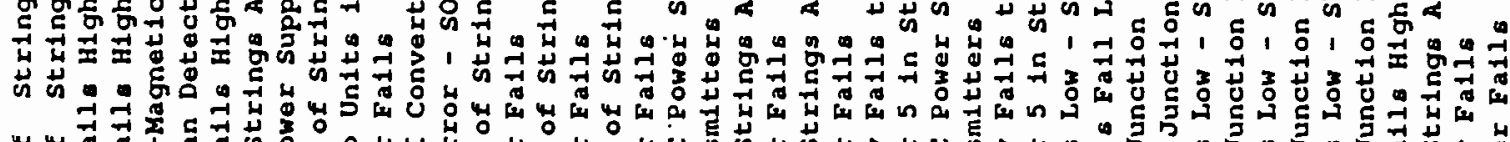

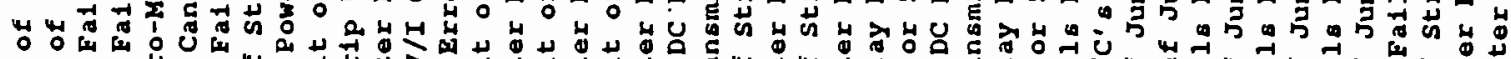

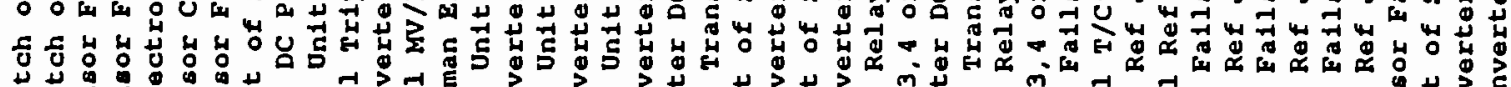

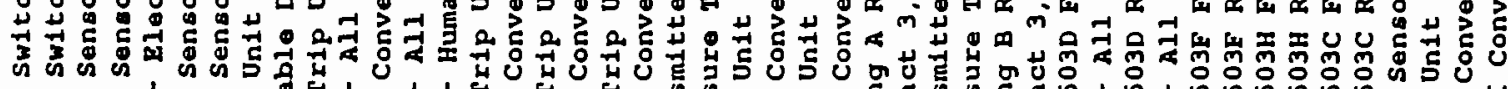

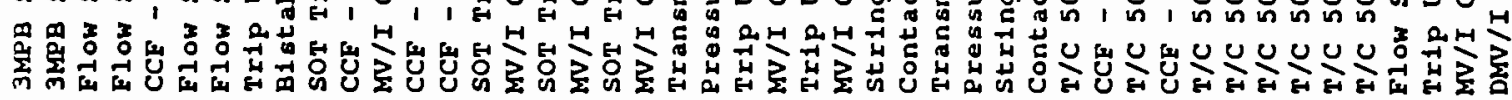

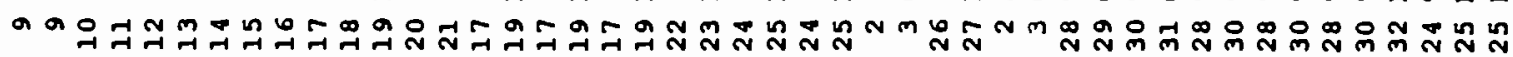
ก n

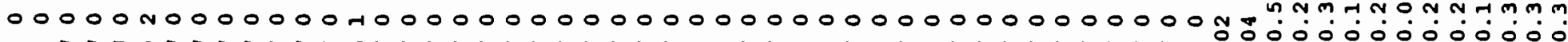

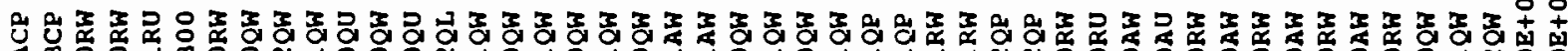

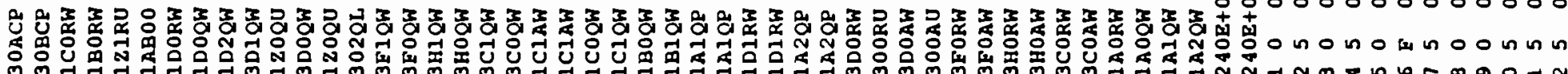

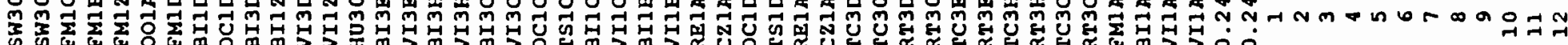

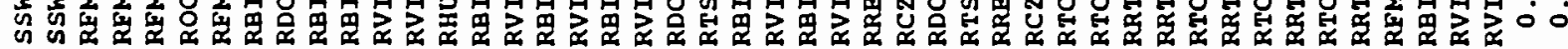

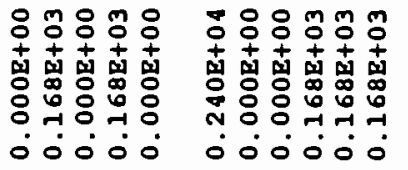

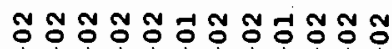

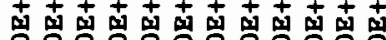

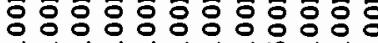
1.-1

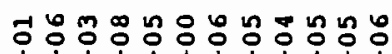

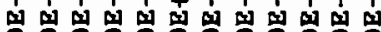

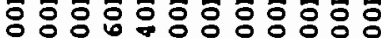

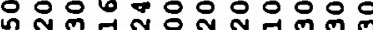




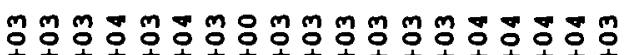

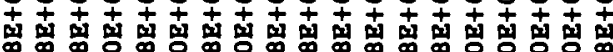

:

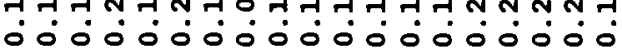

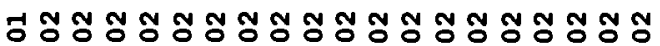

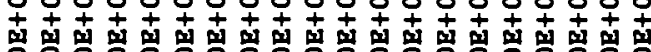

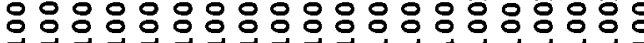
구

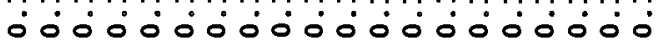

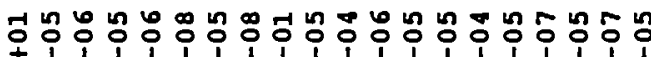

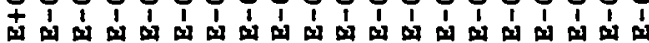

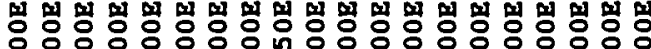

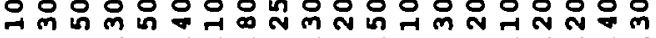
00000000000000000000 H in แ

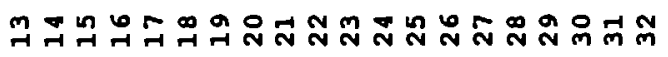



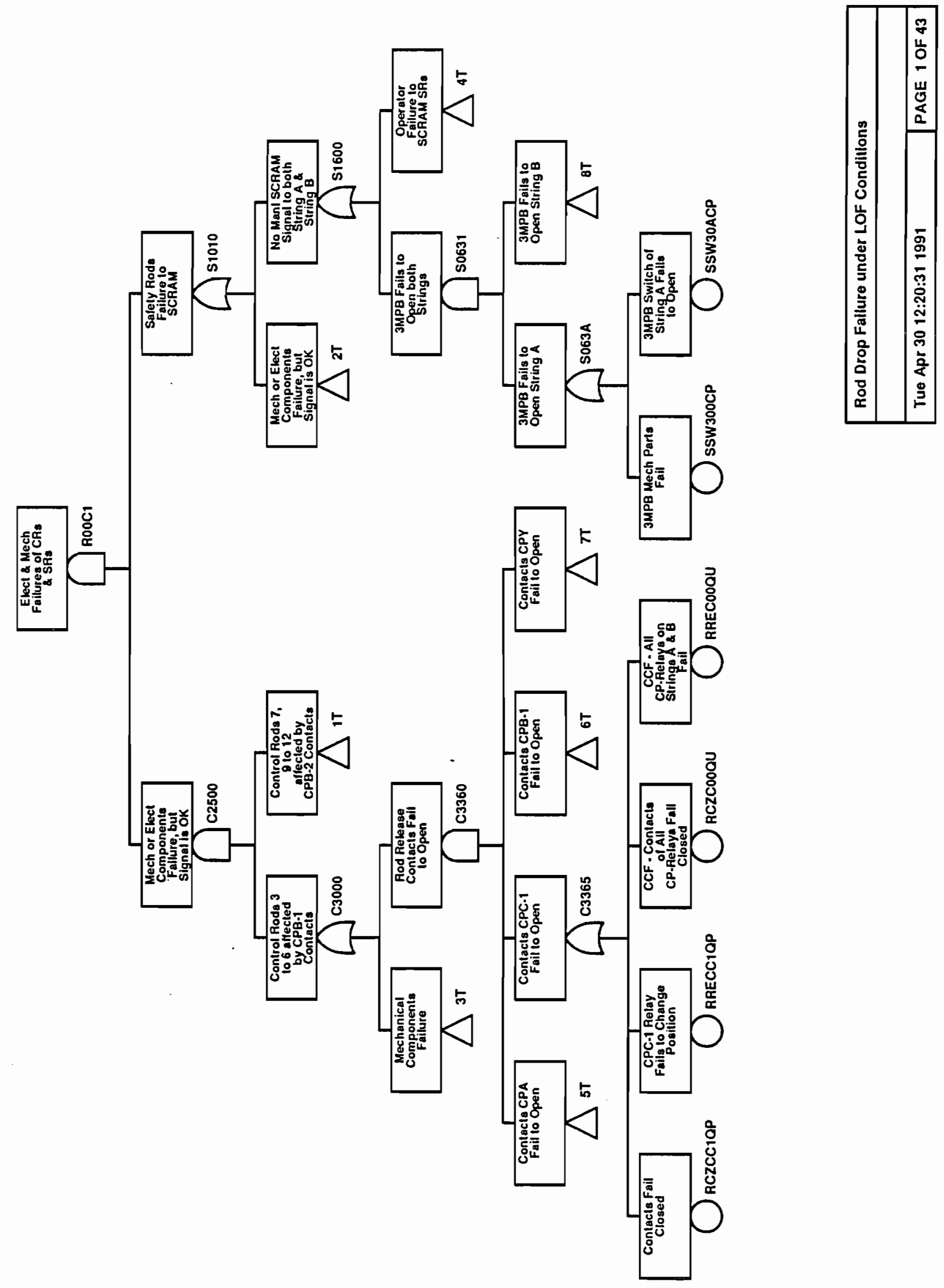


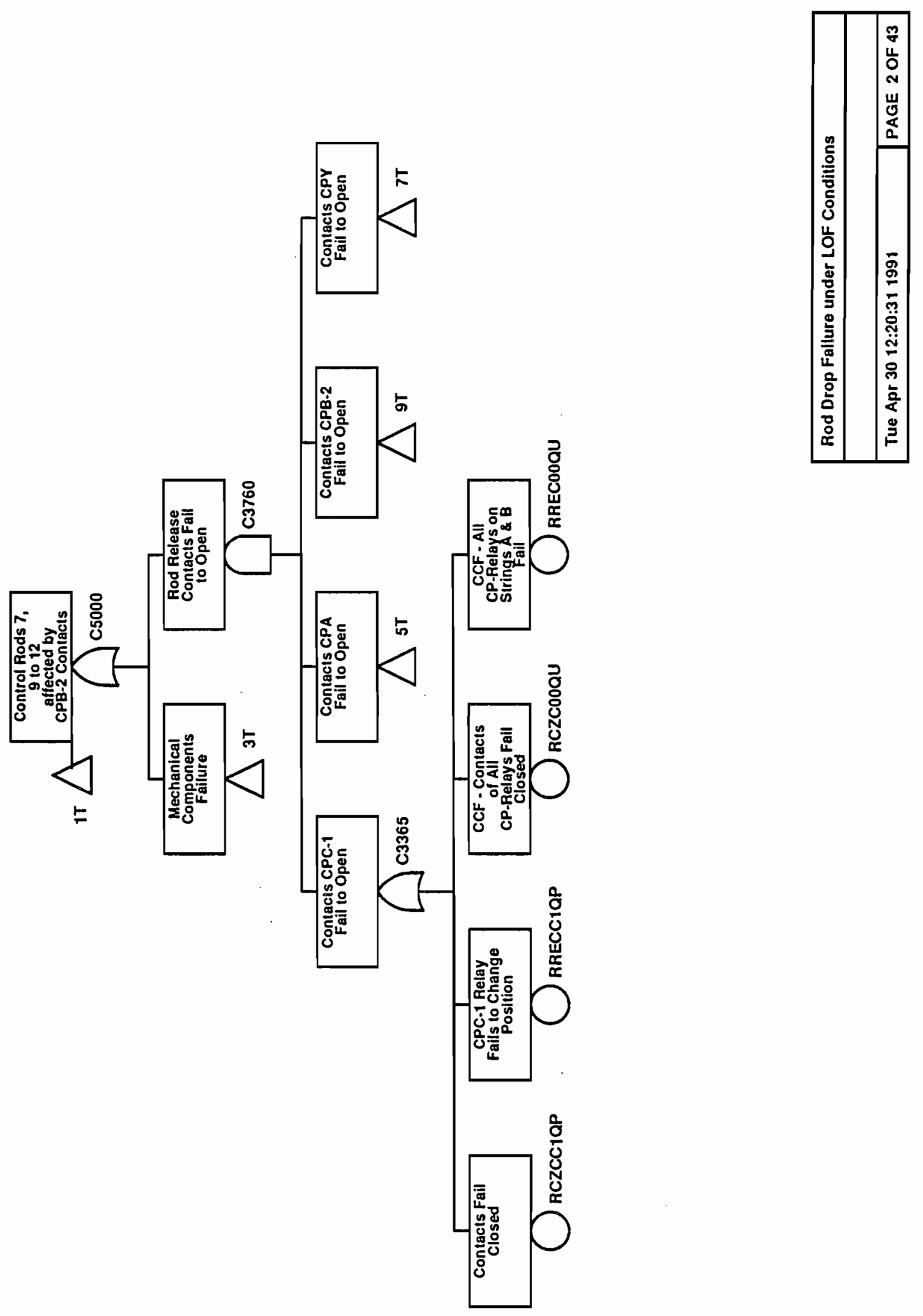




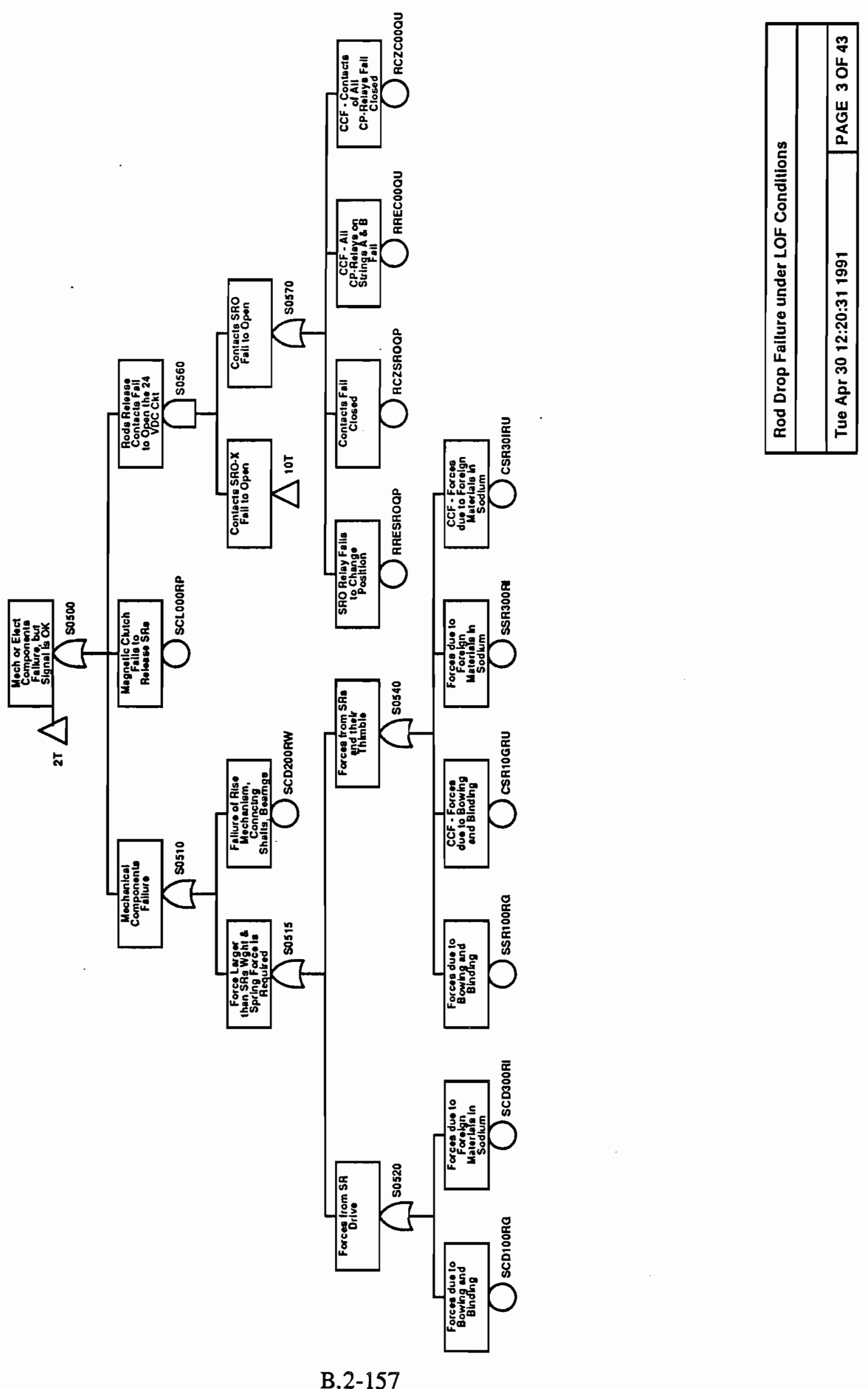




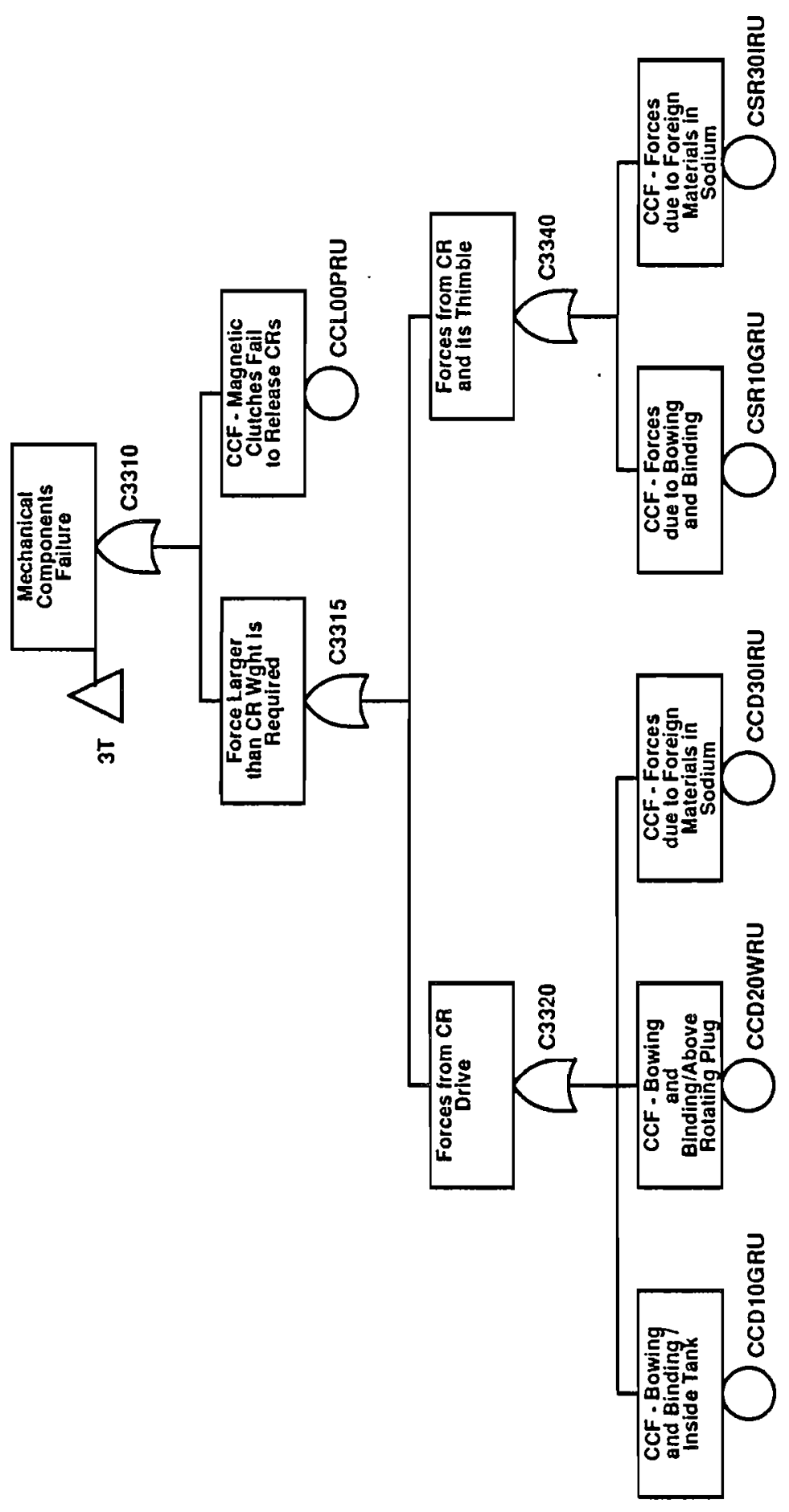

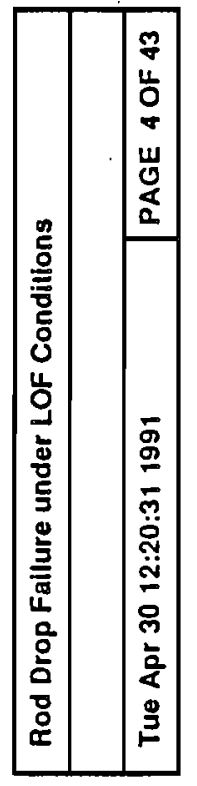




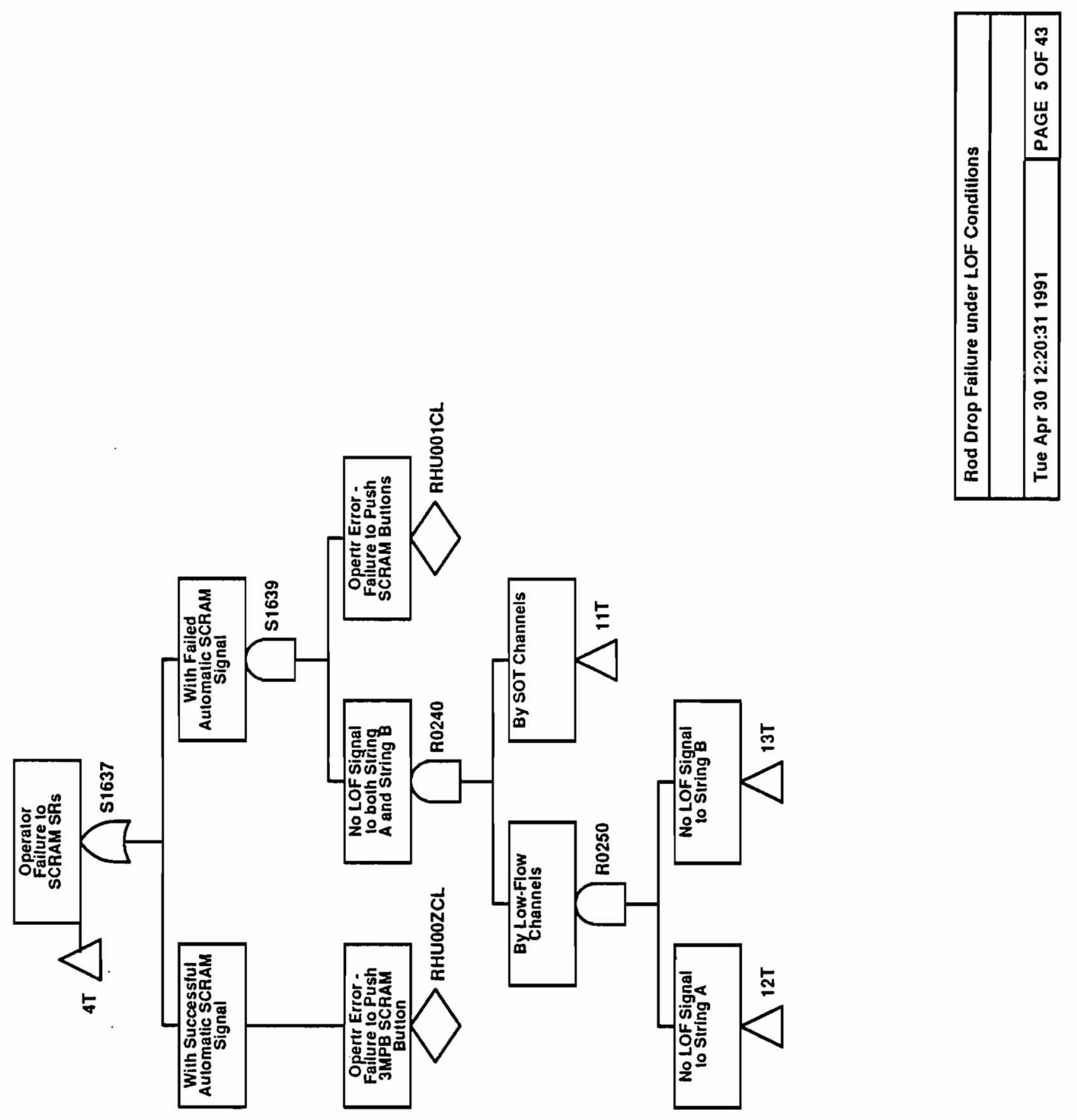




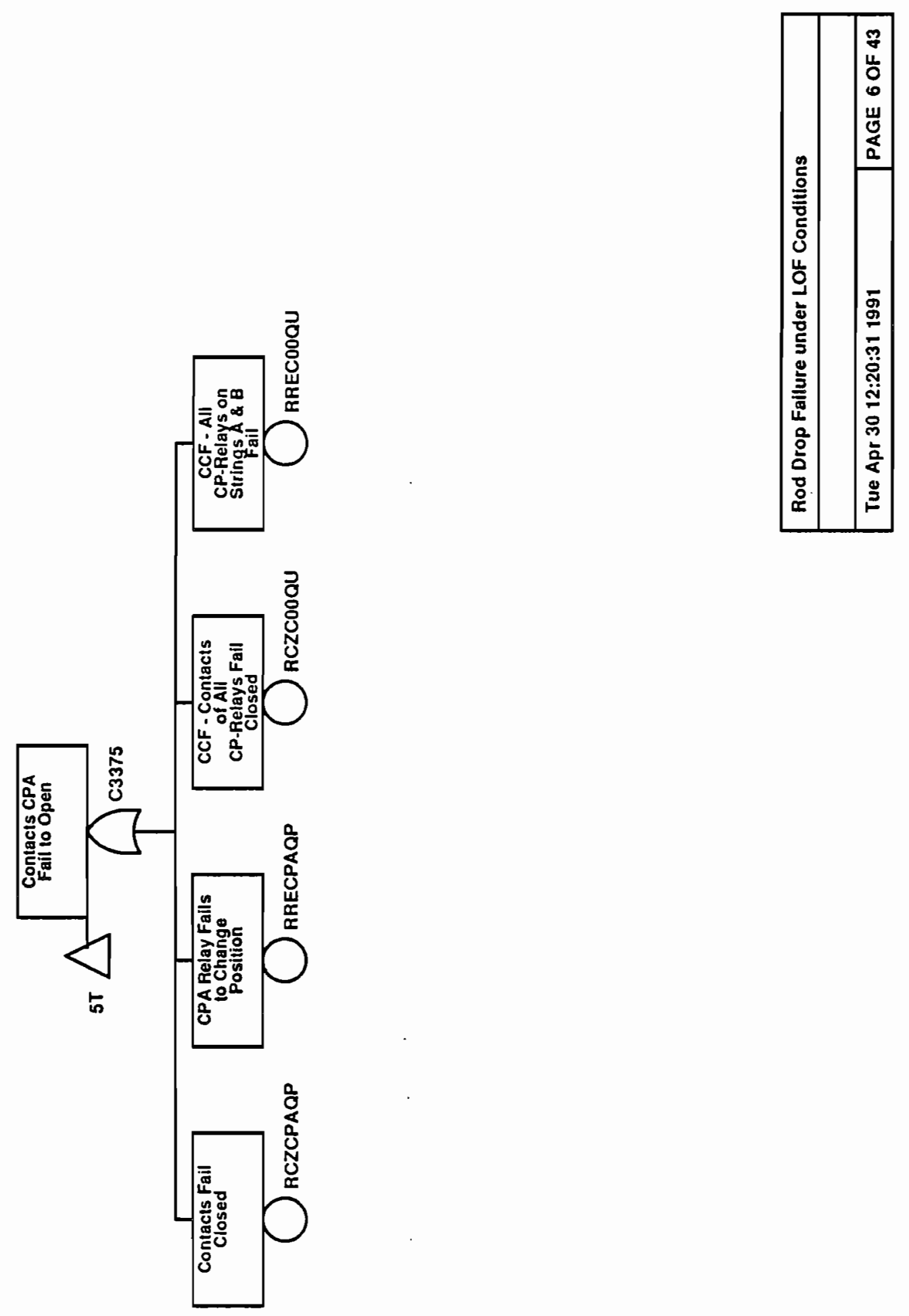




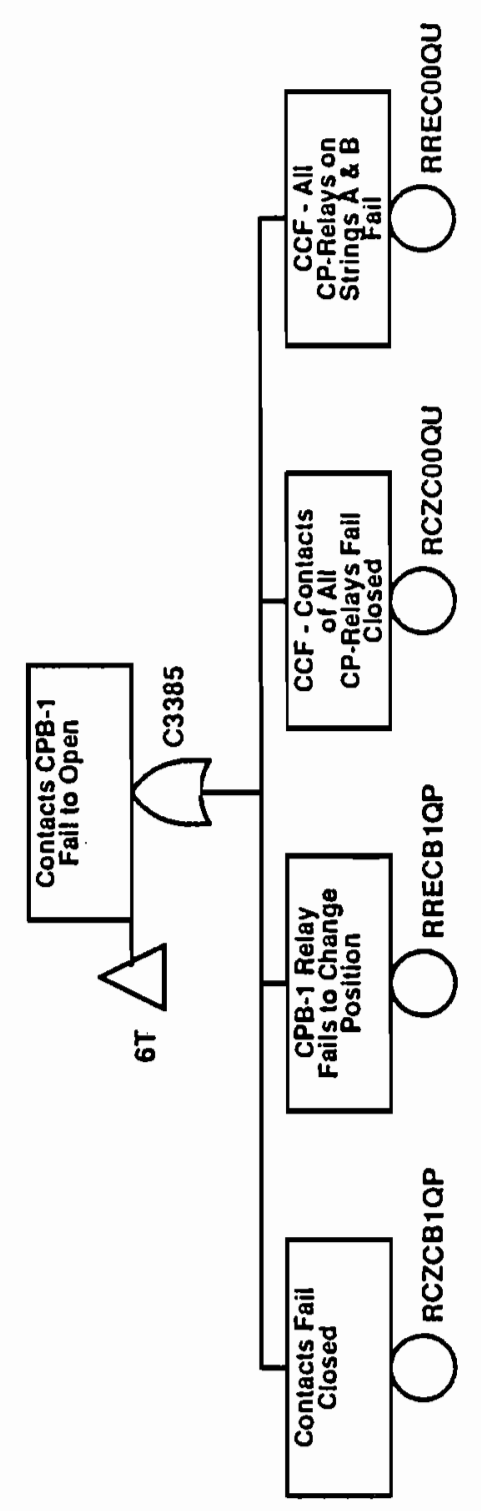




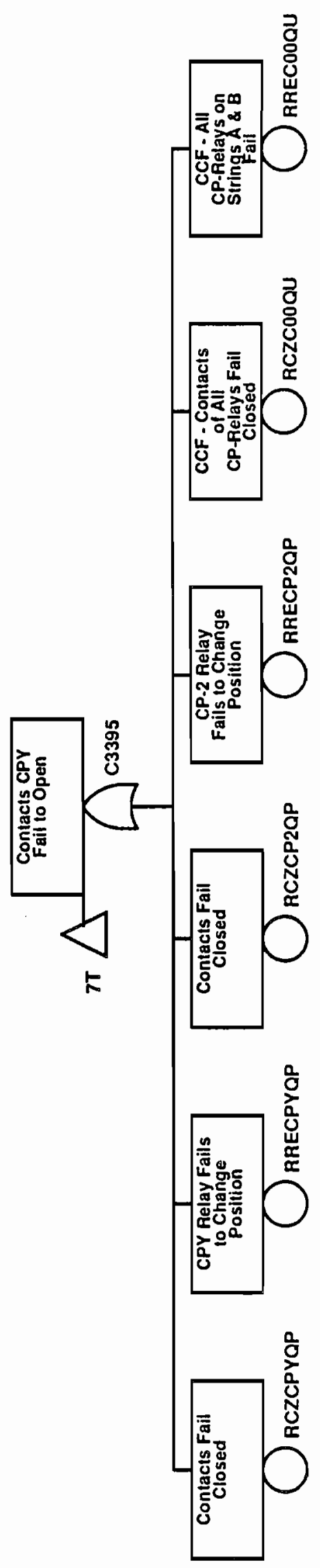

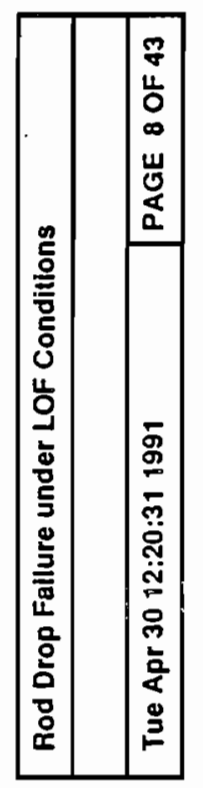




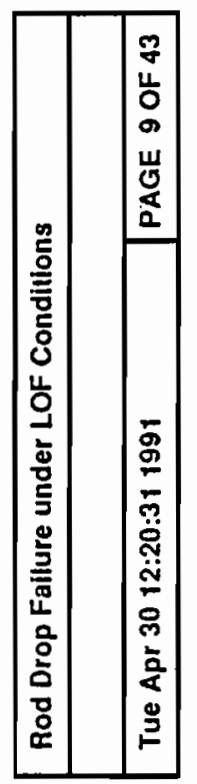

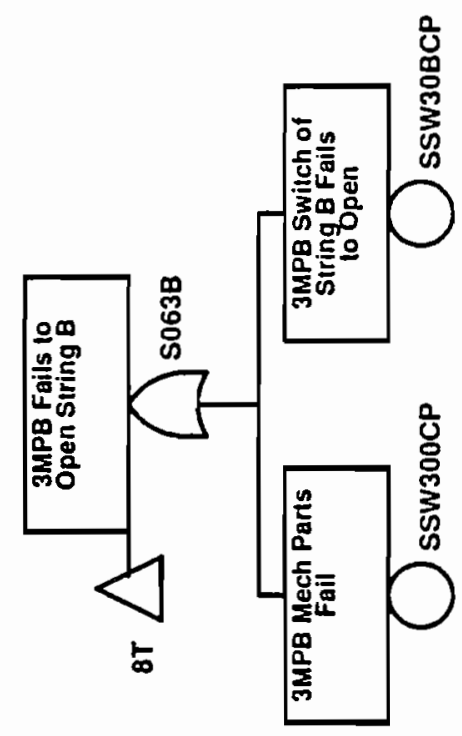

B. 2-163 


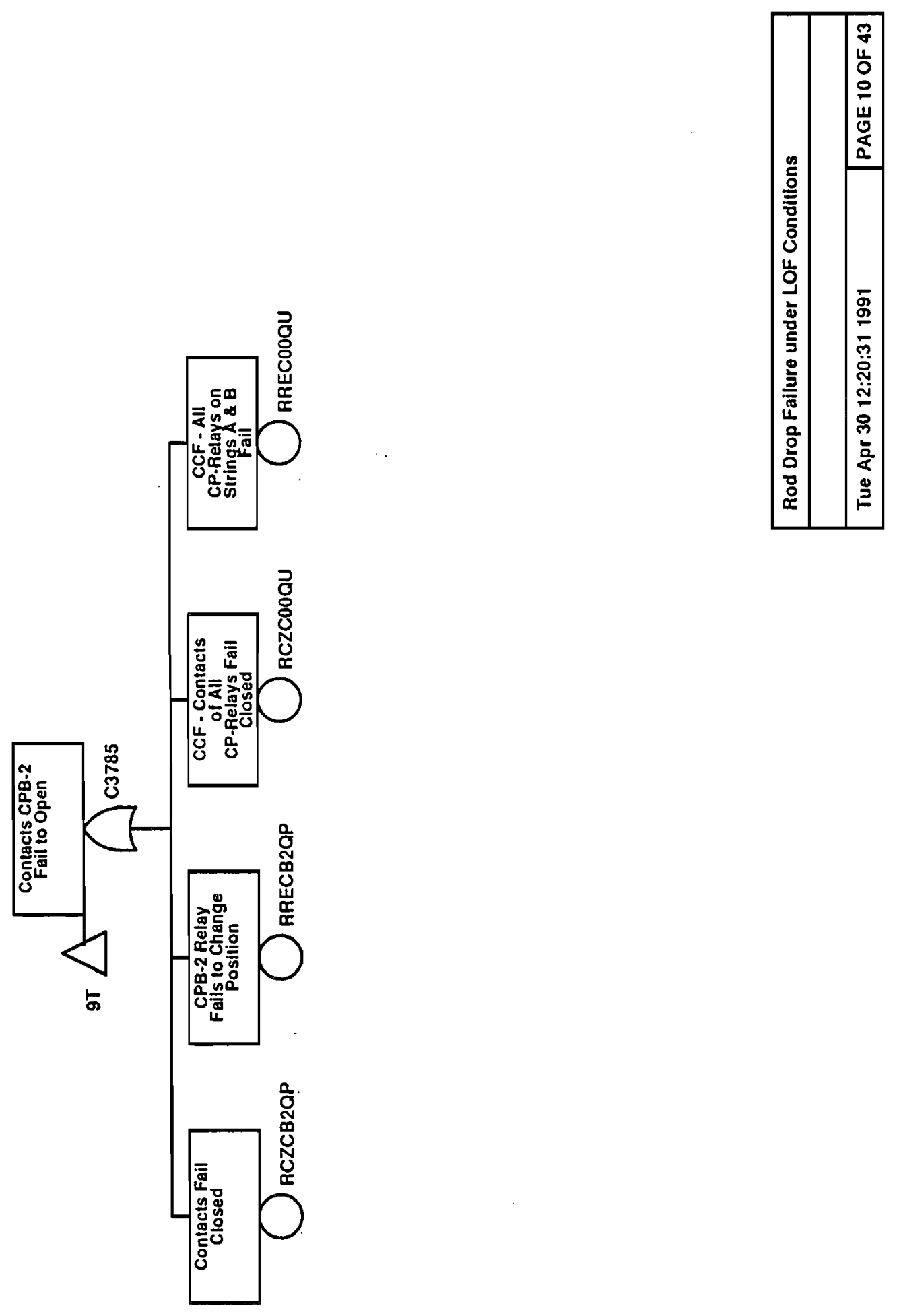

B. 2-164 


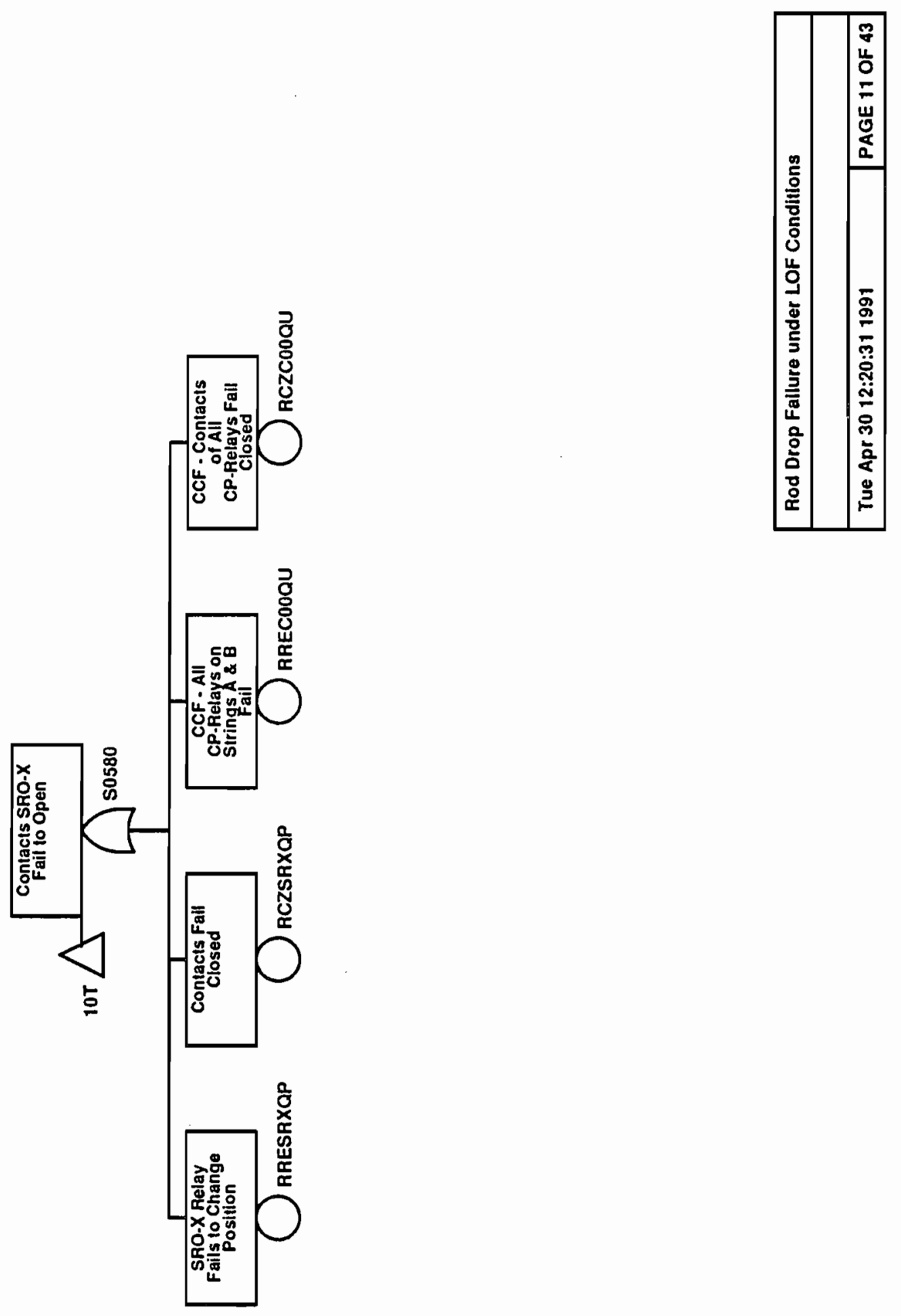

B. 2-165 

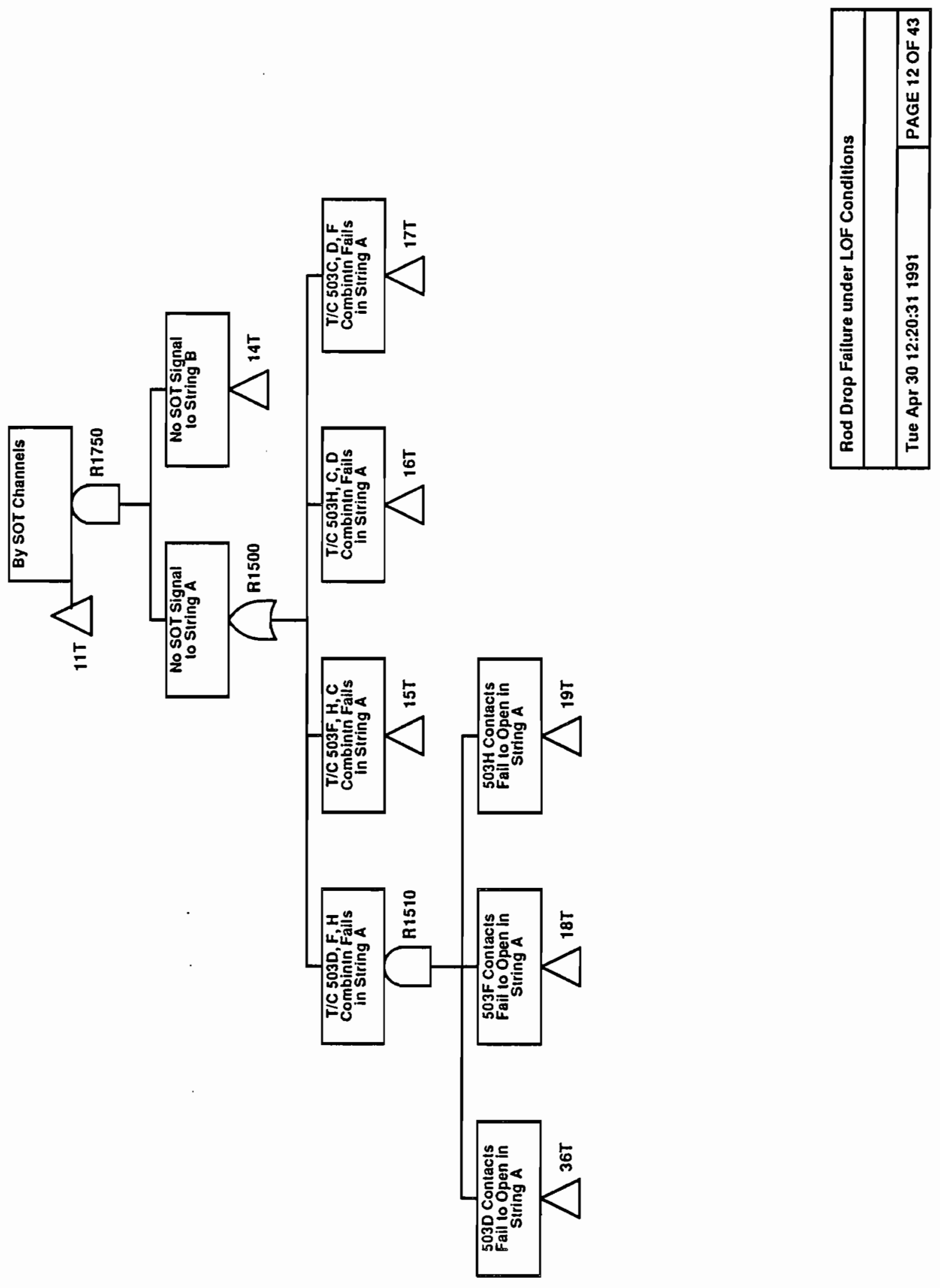

B. 2-166 


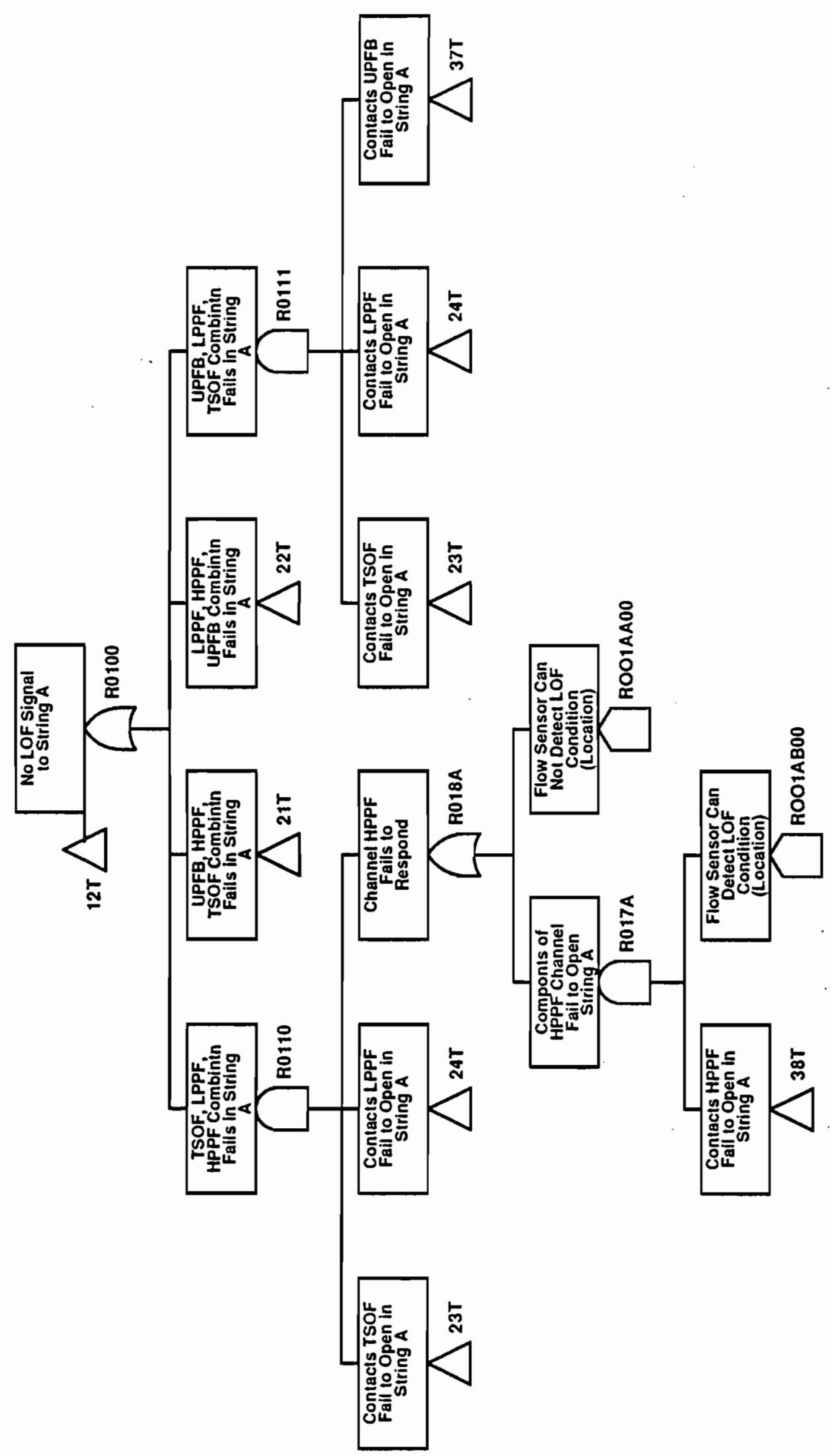

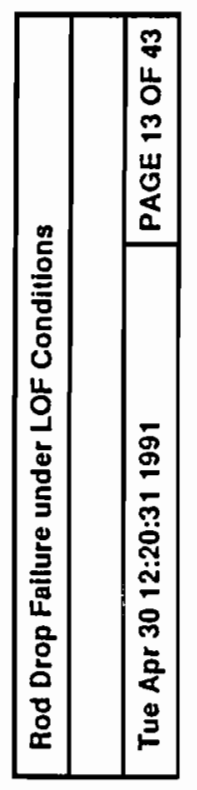




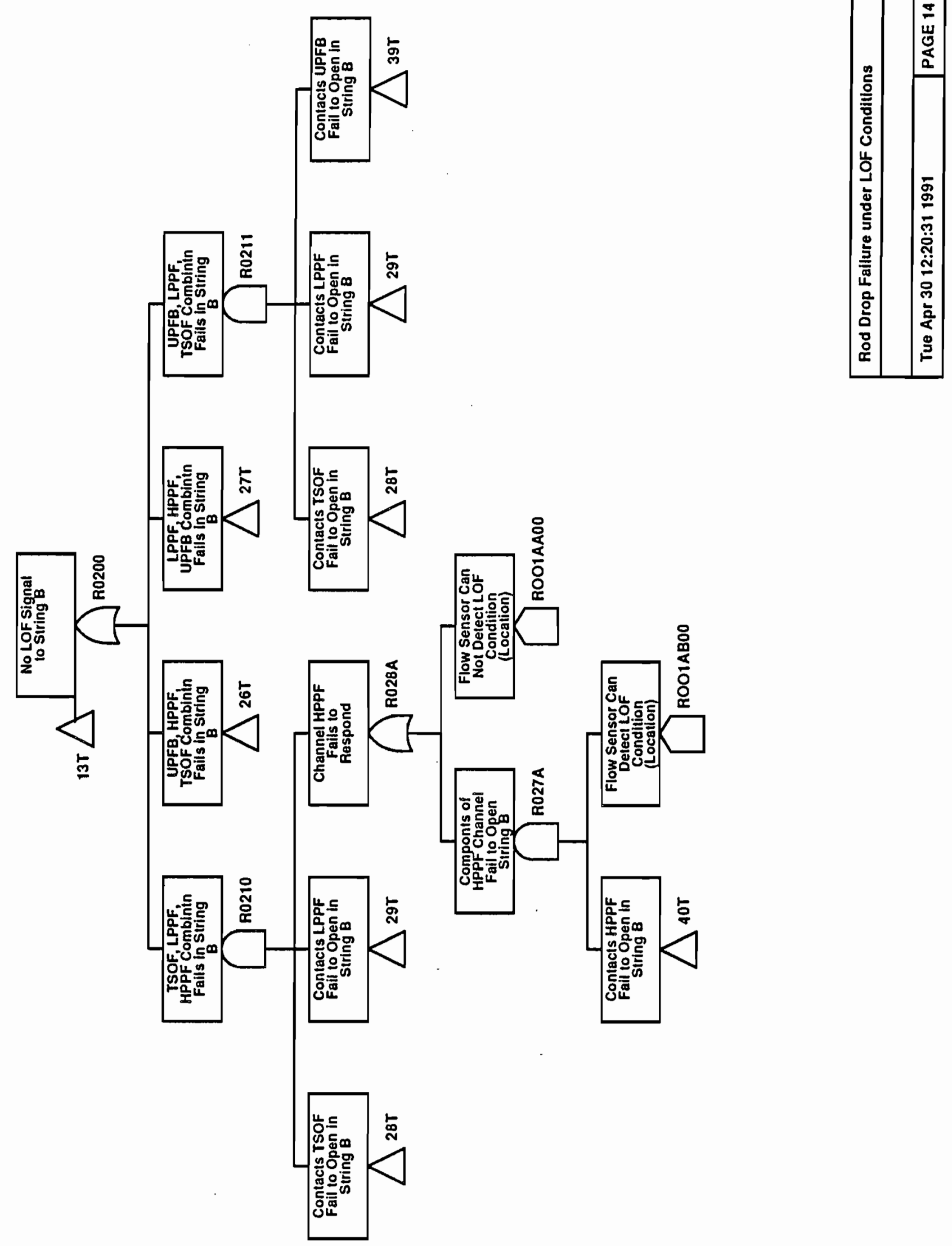




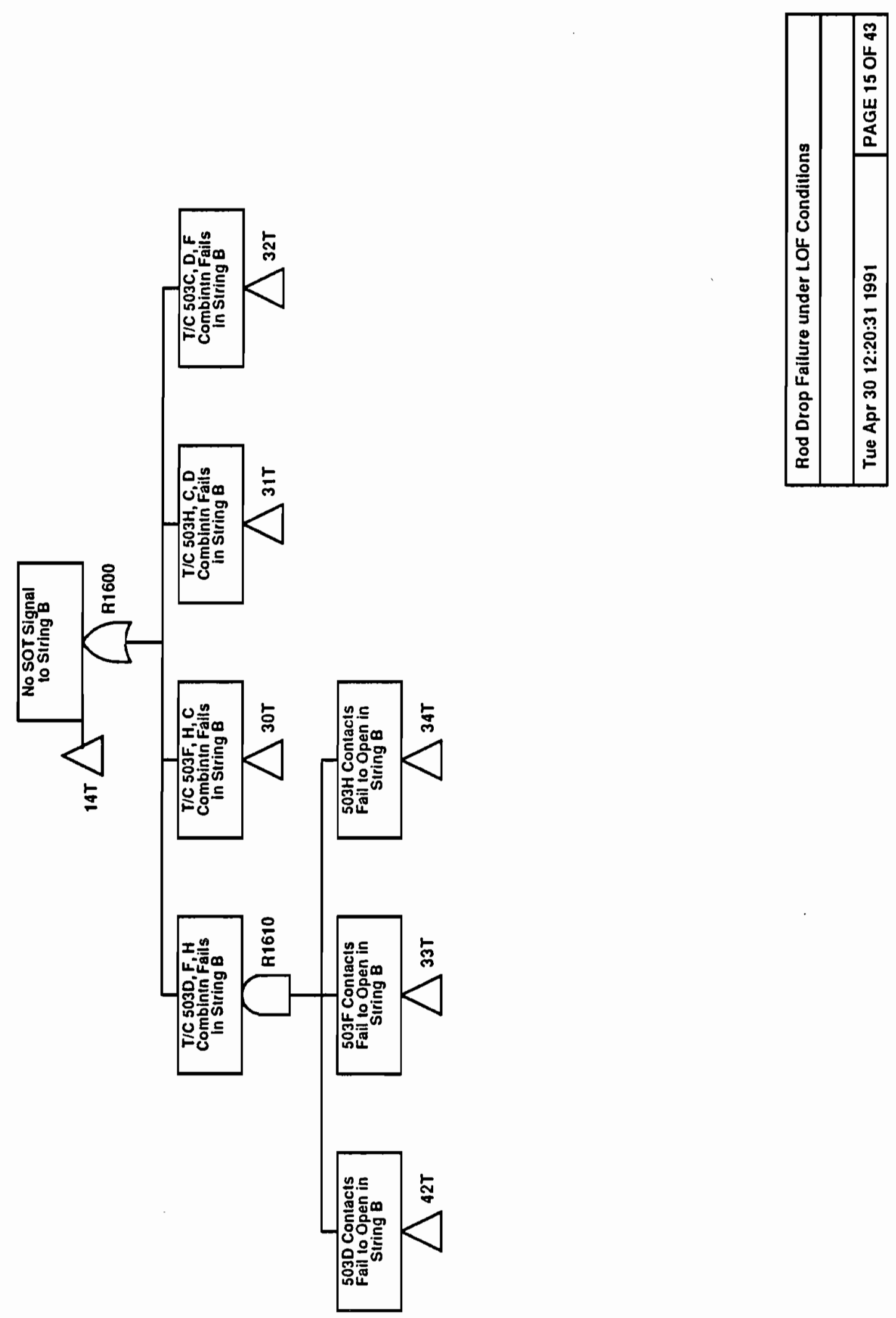

B. $2-169$ 


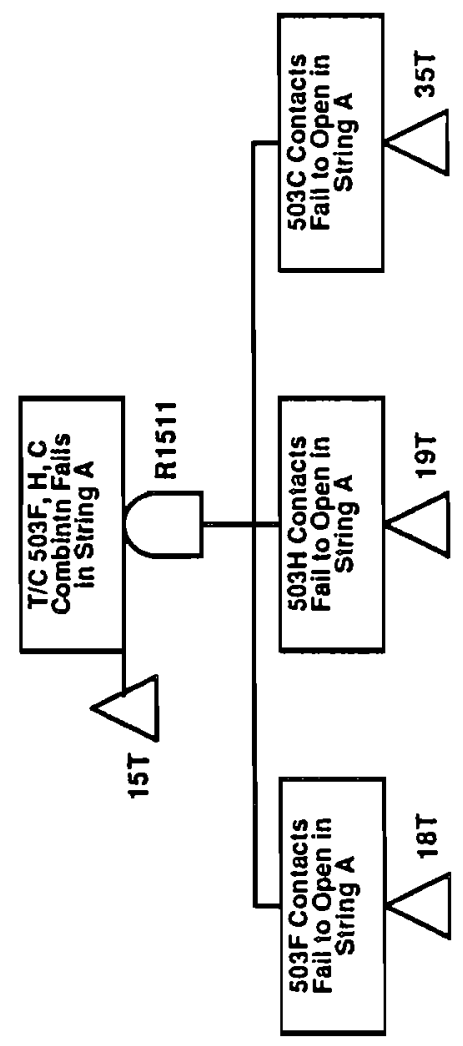

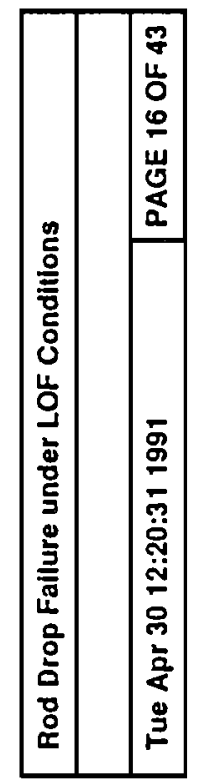

B. $2-170$ 


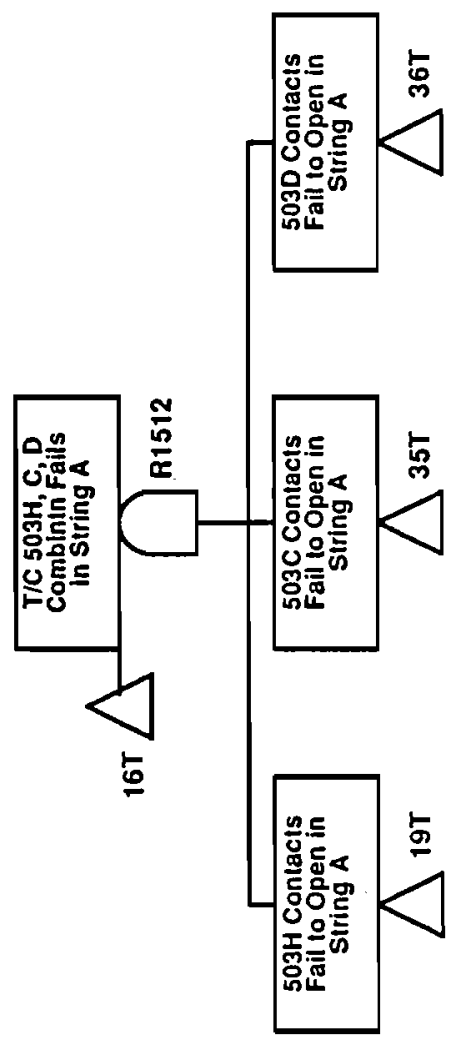

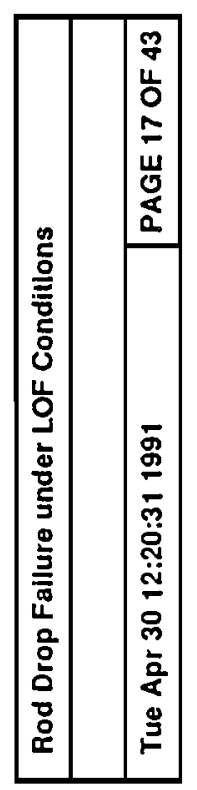




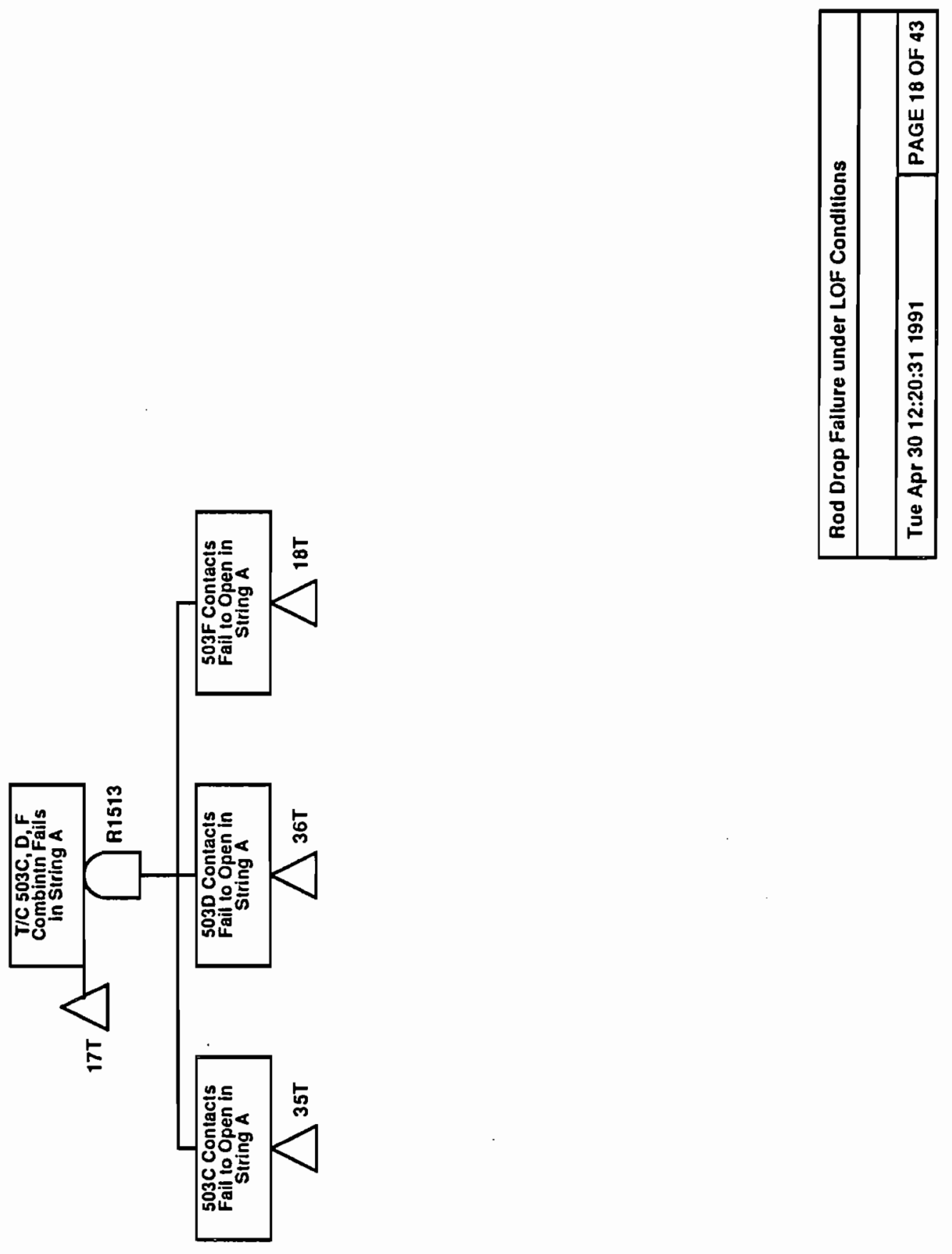



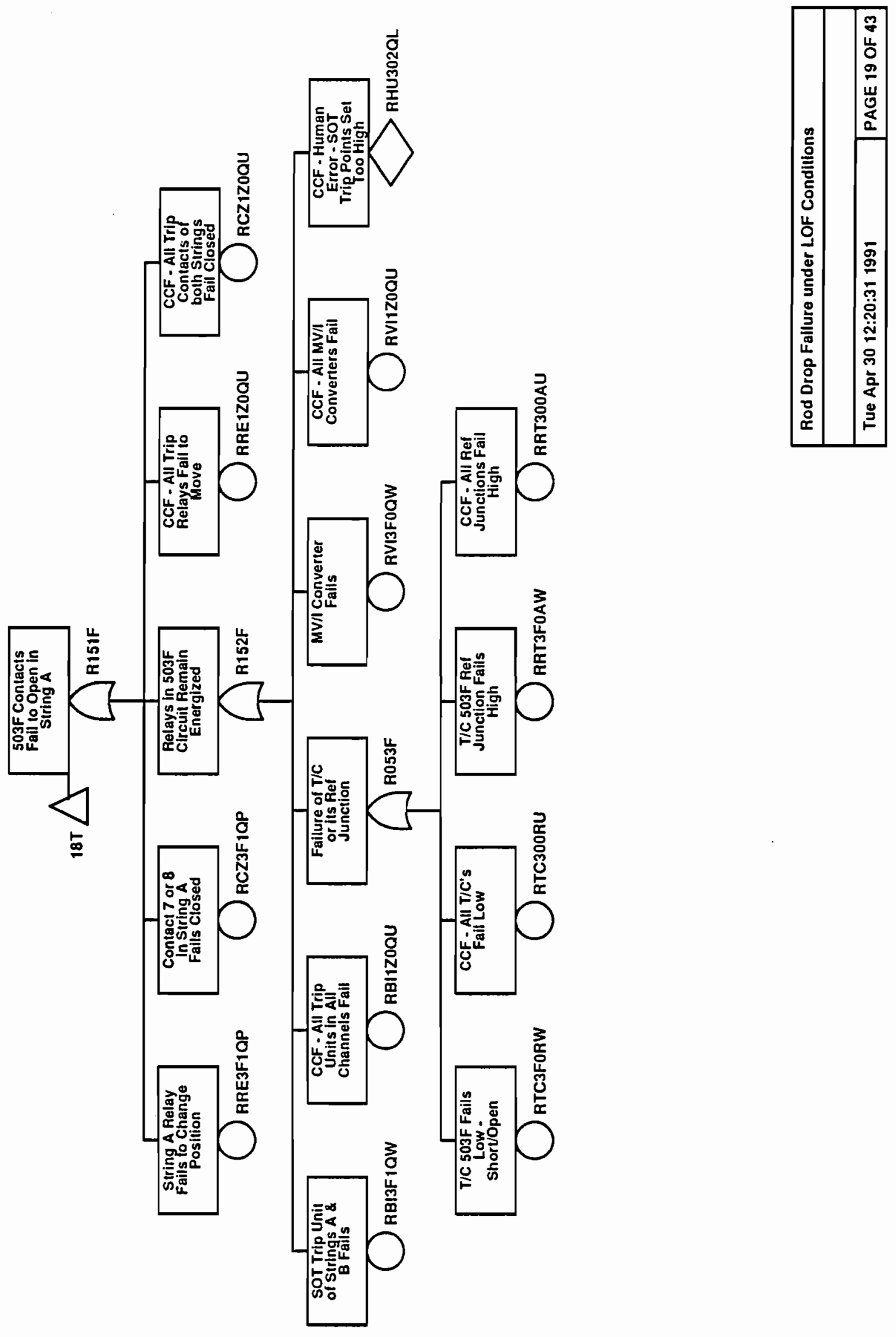

B. 2-173 

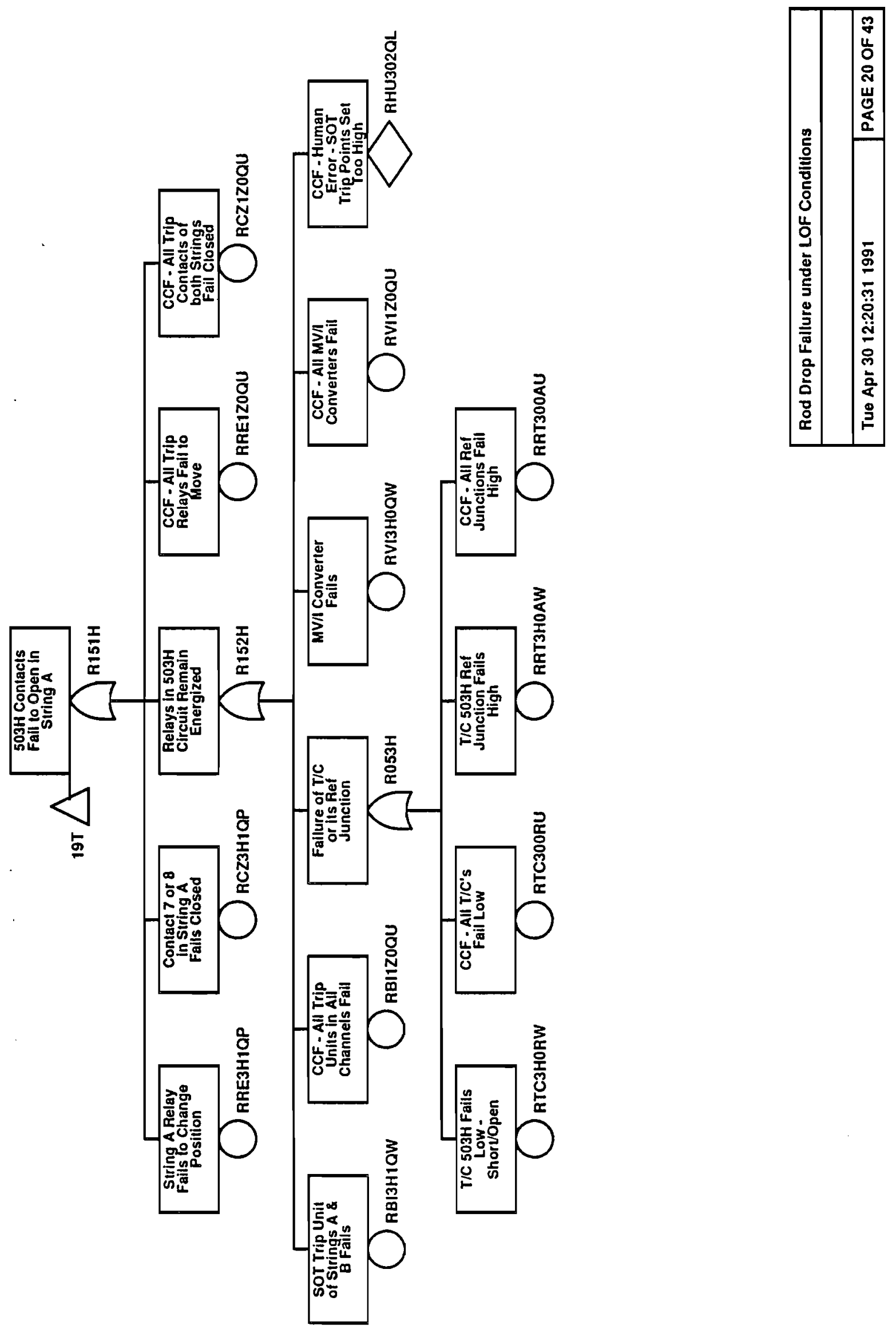

B. $2-174$ 


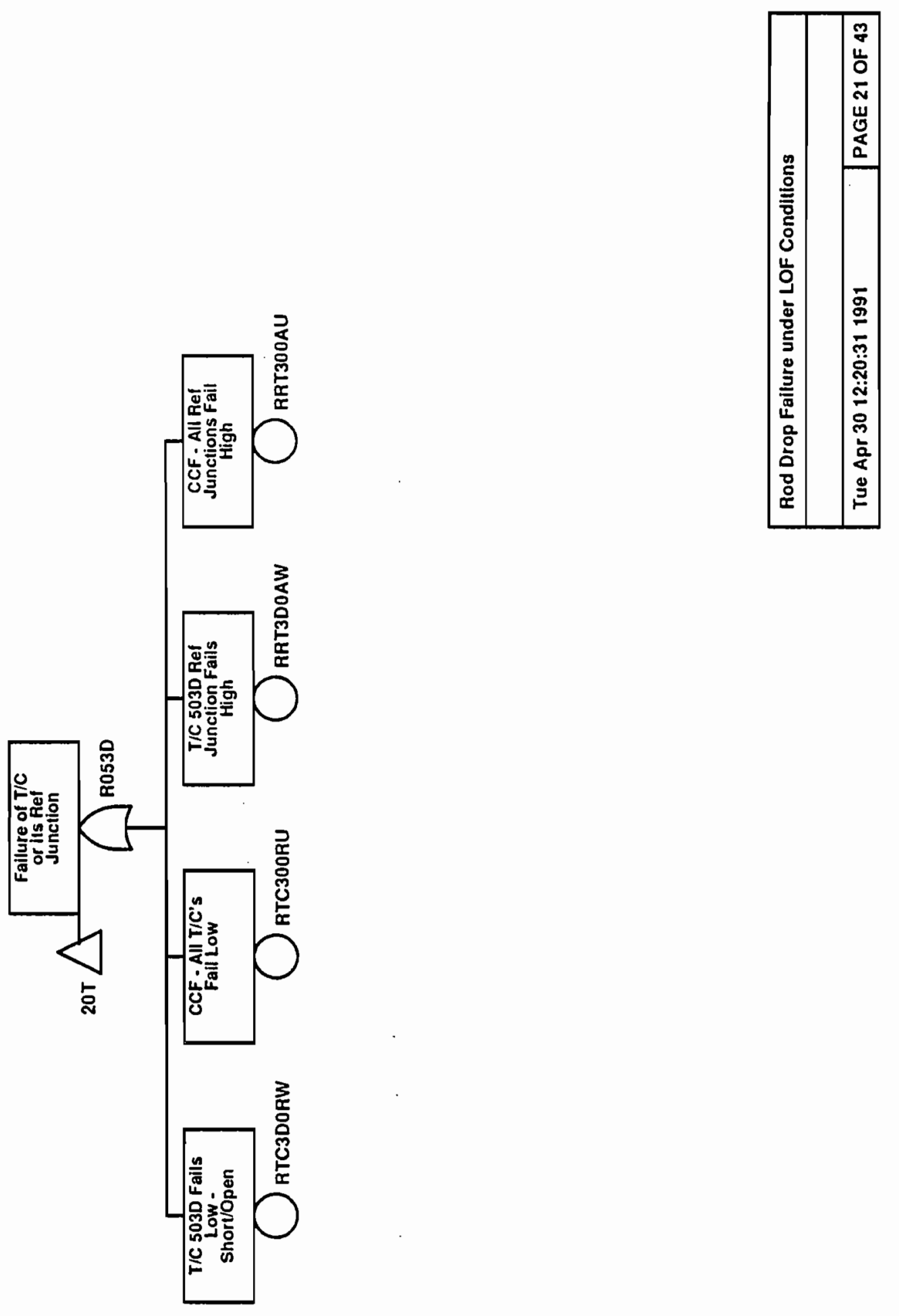




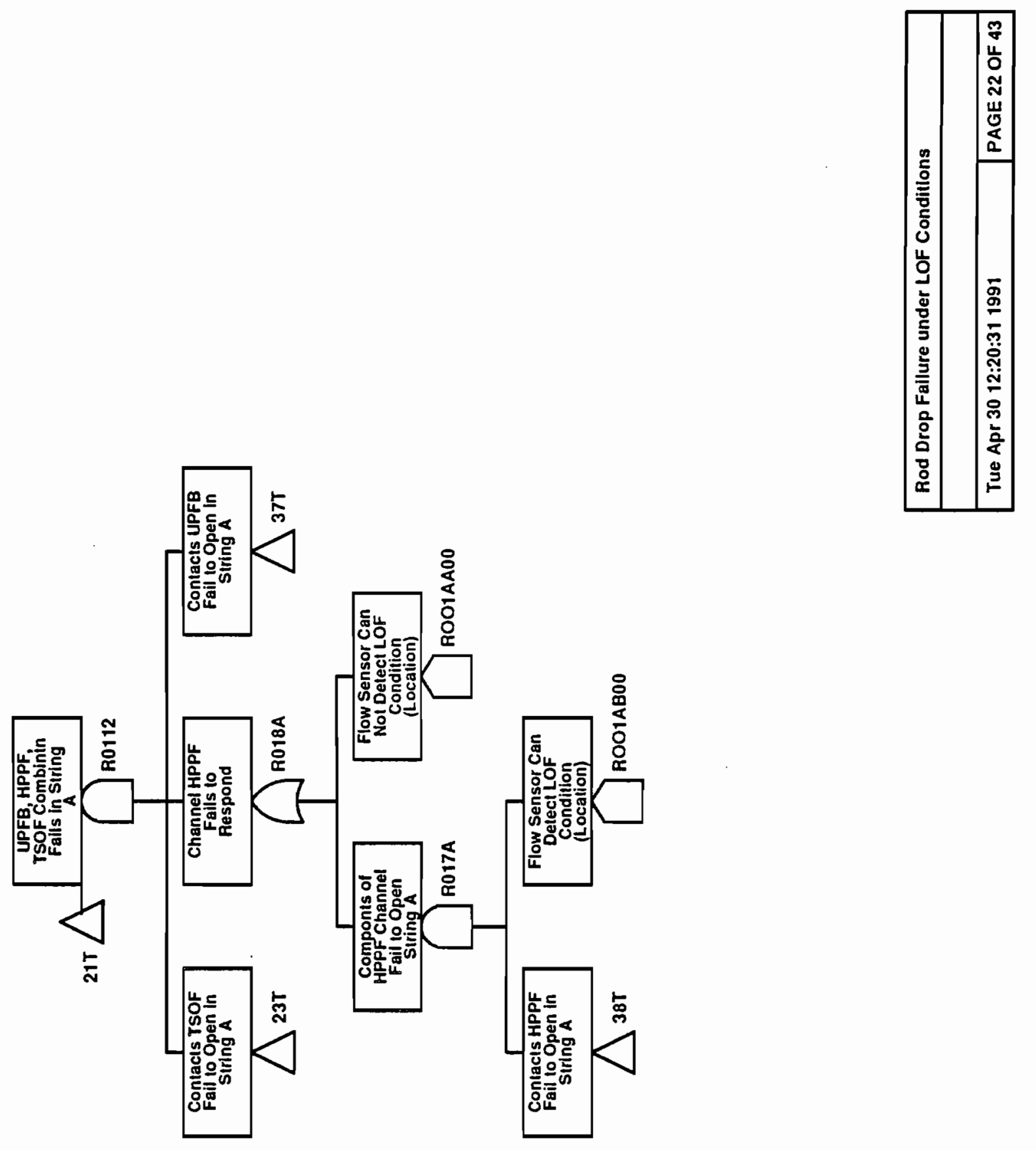




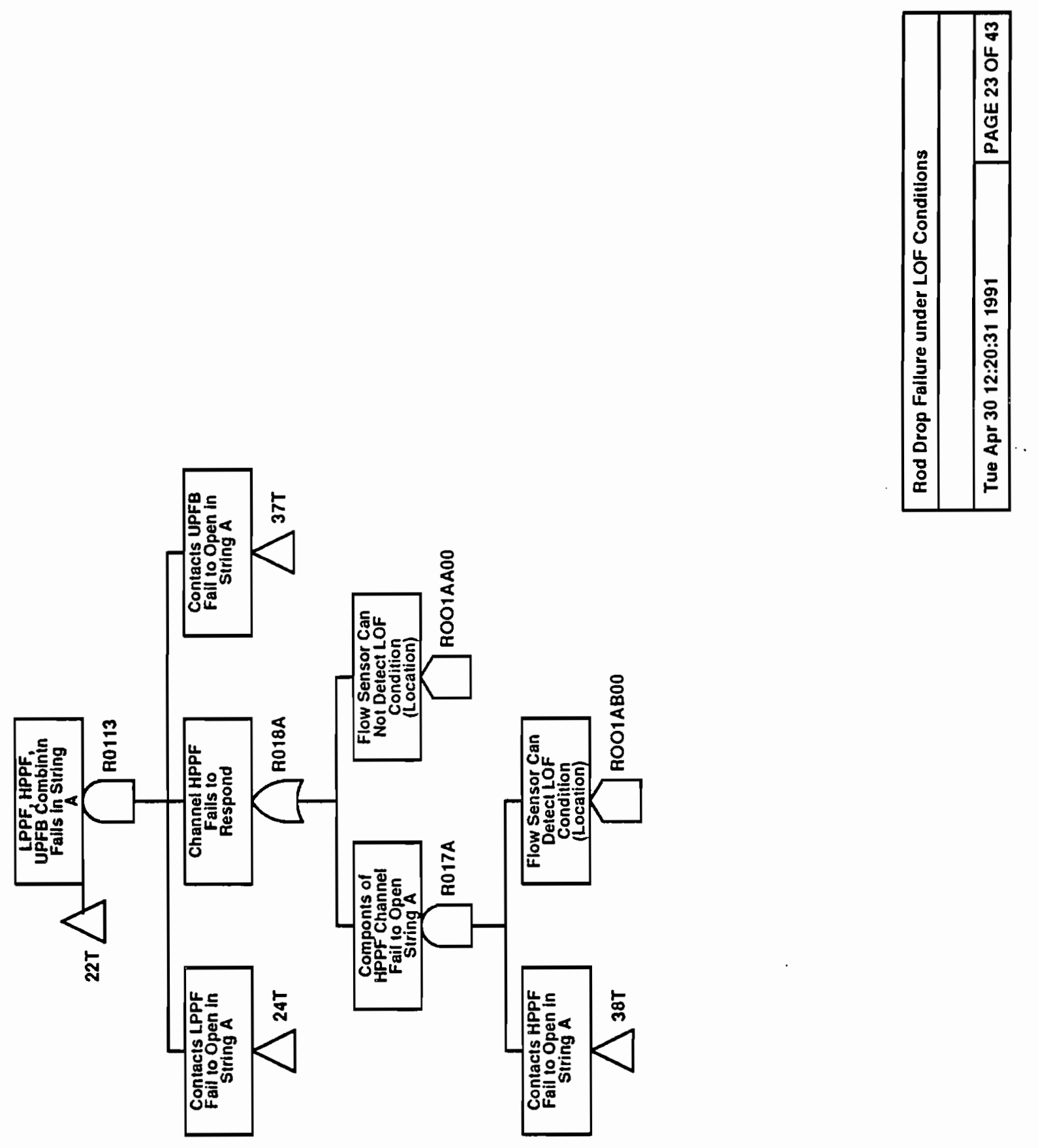



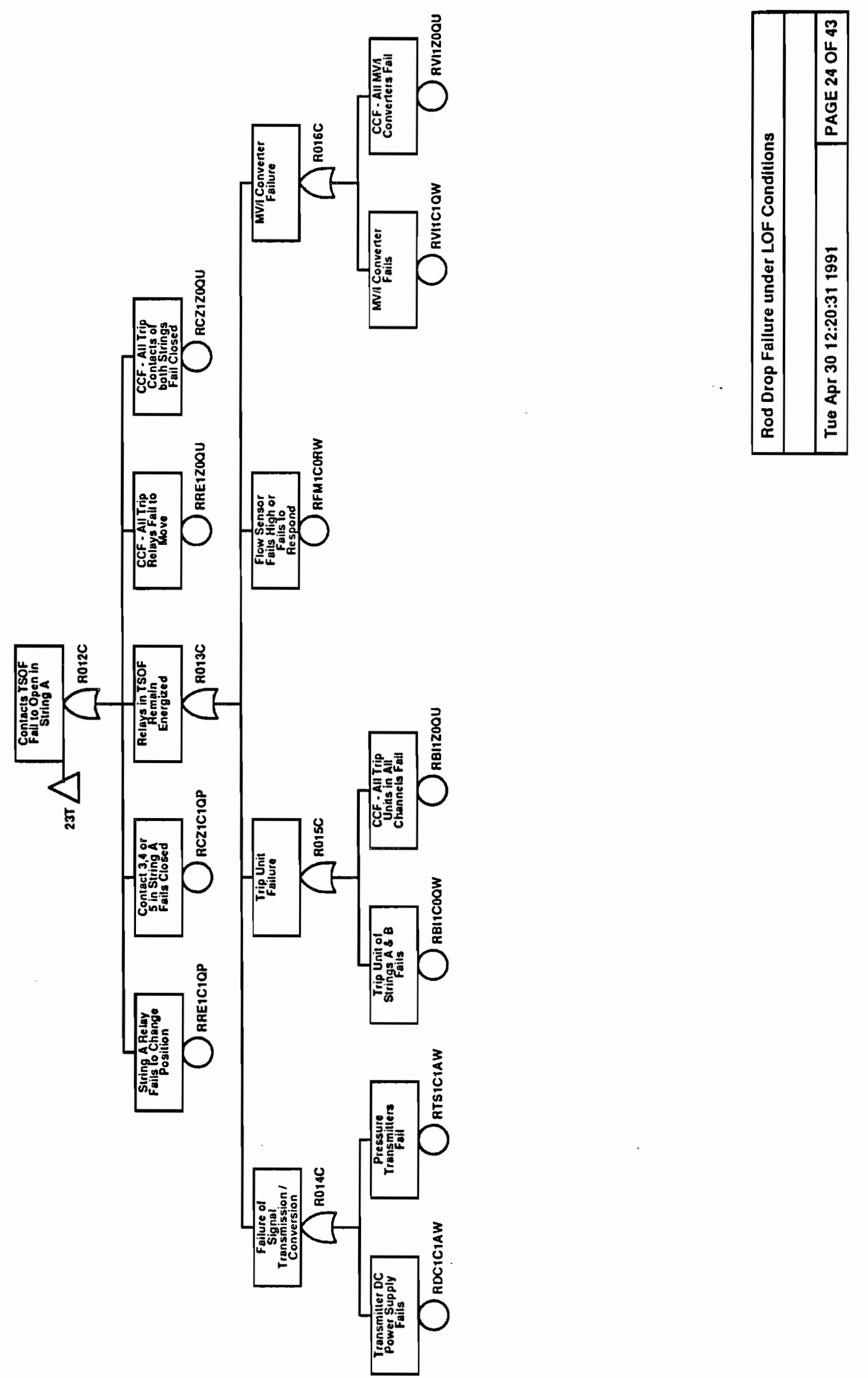

B. 2-178 


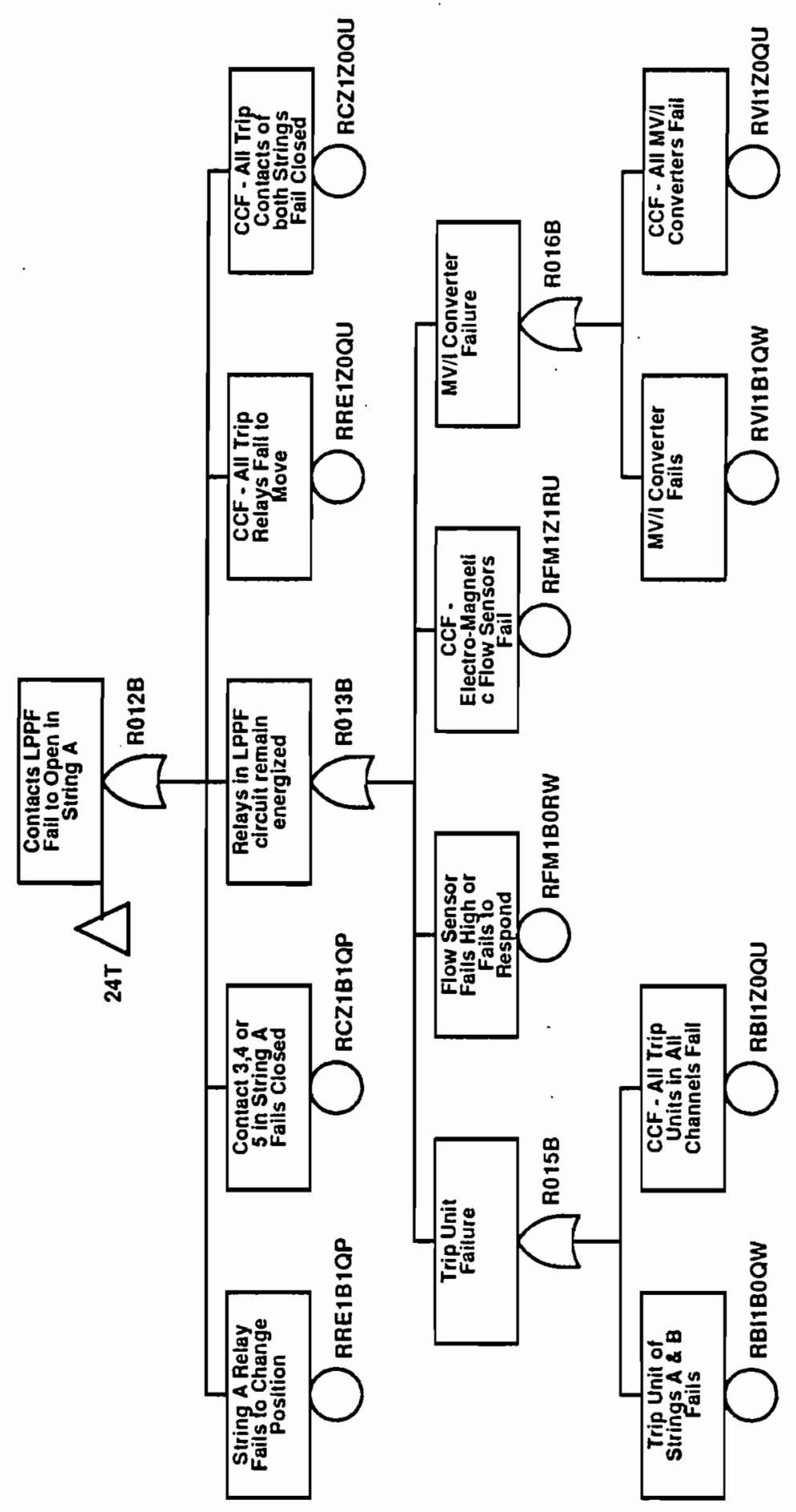



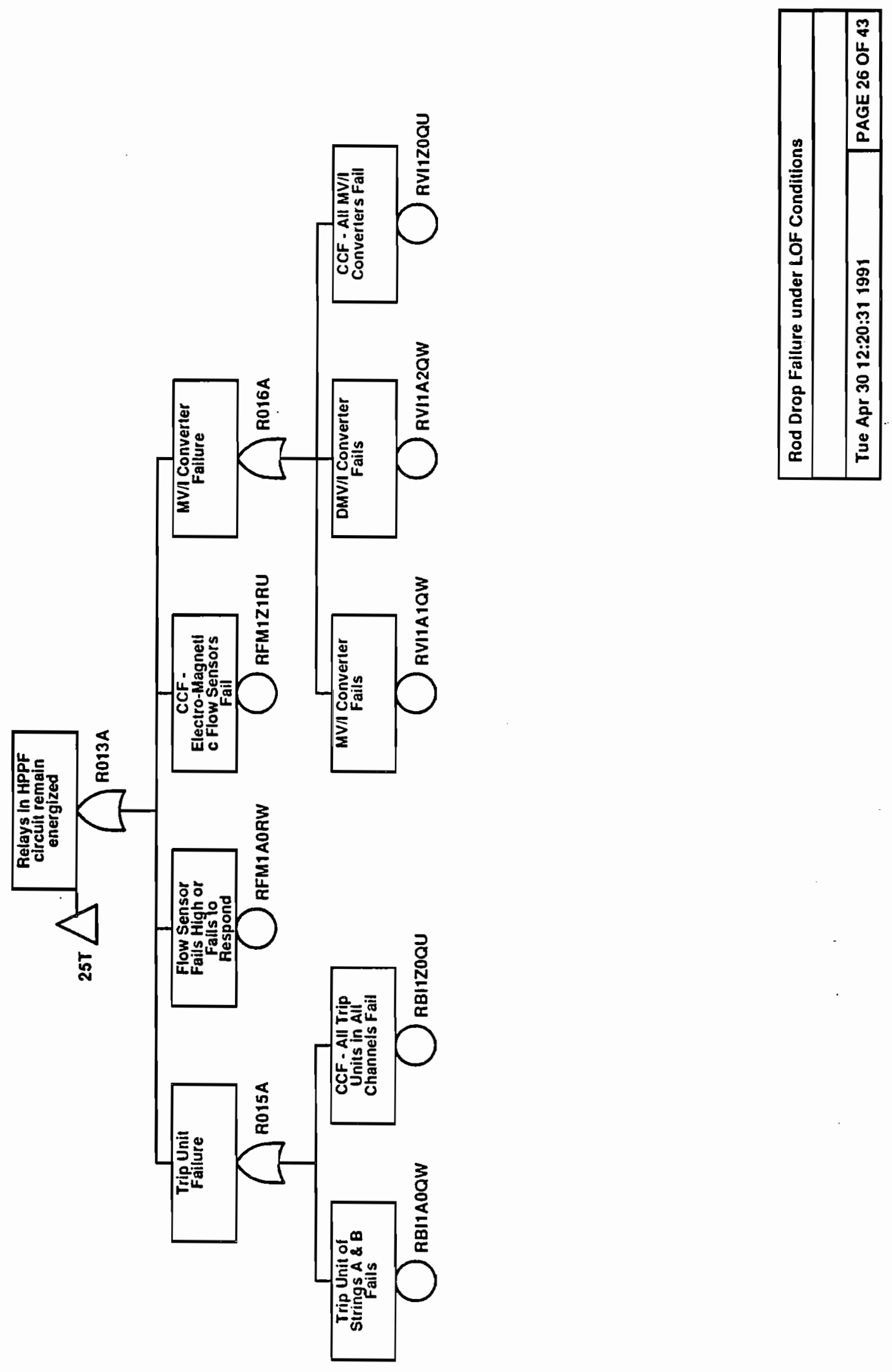


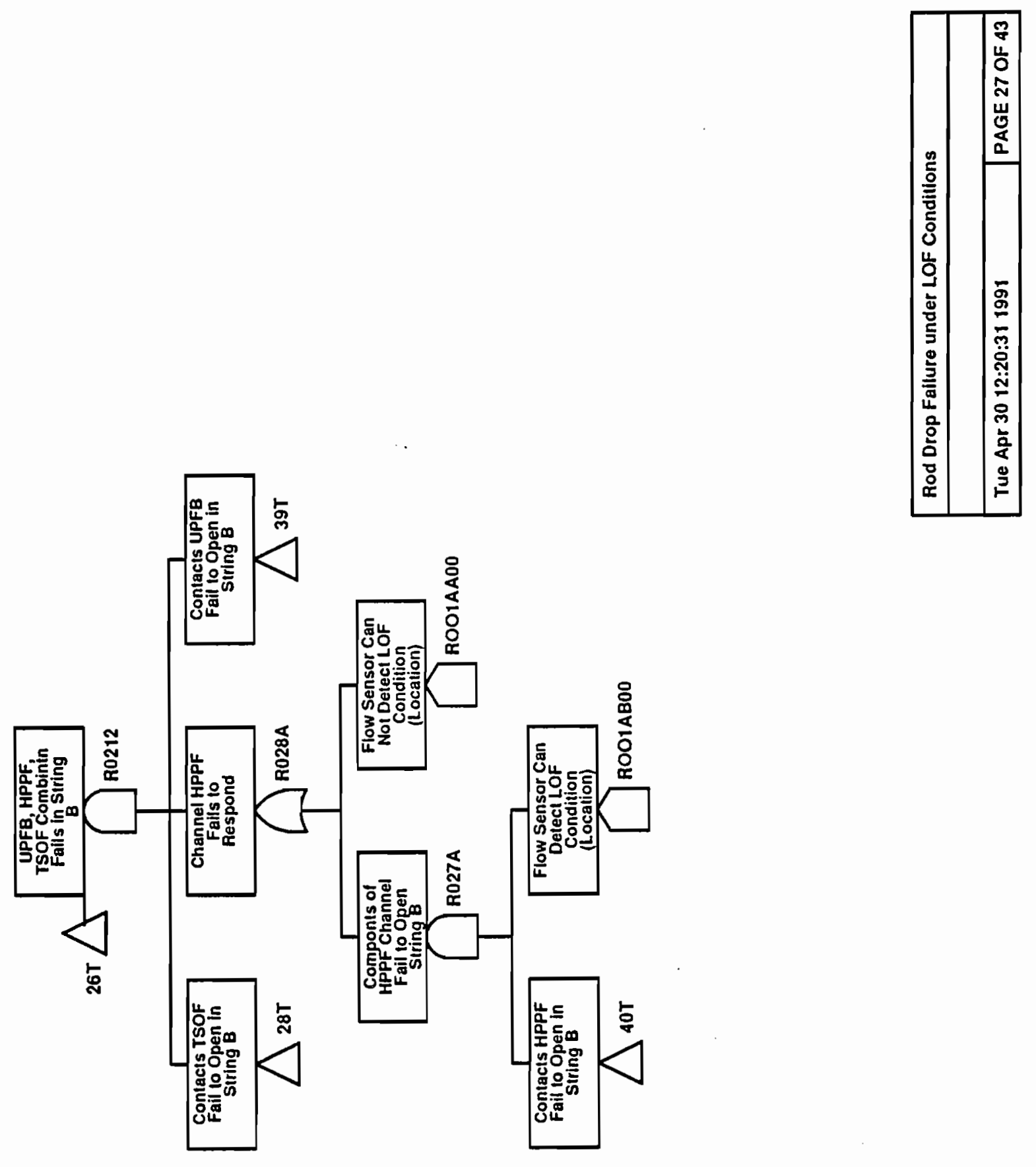




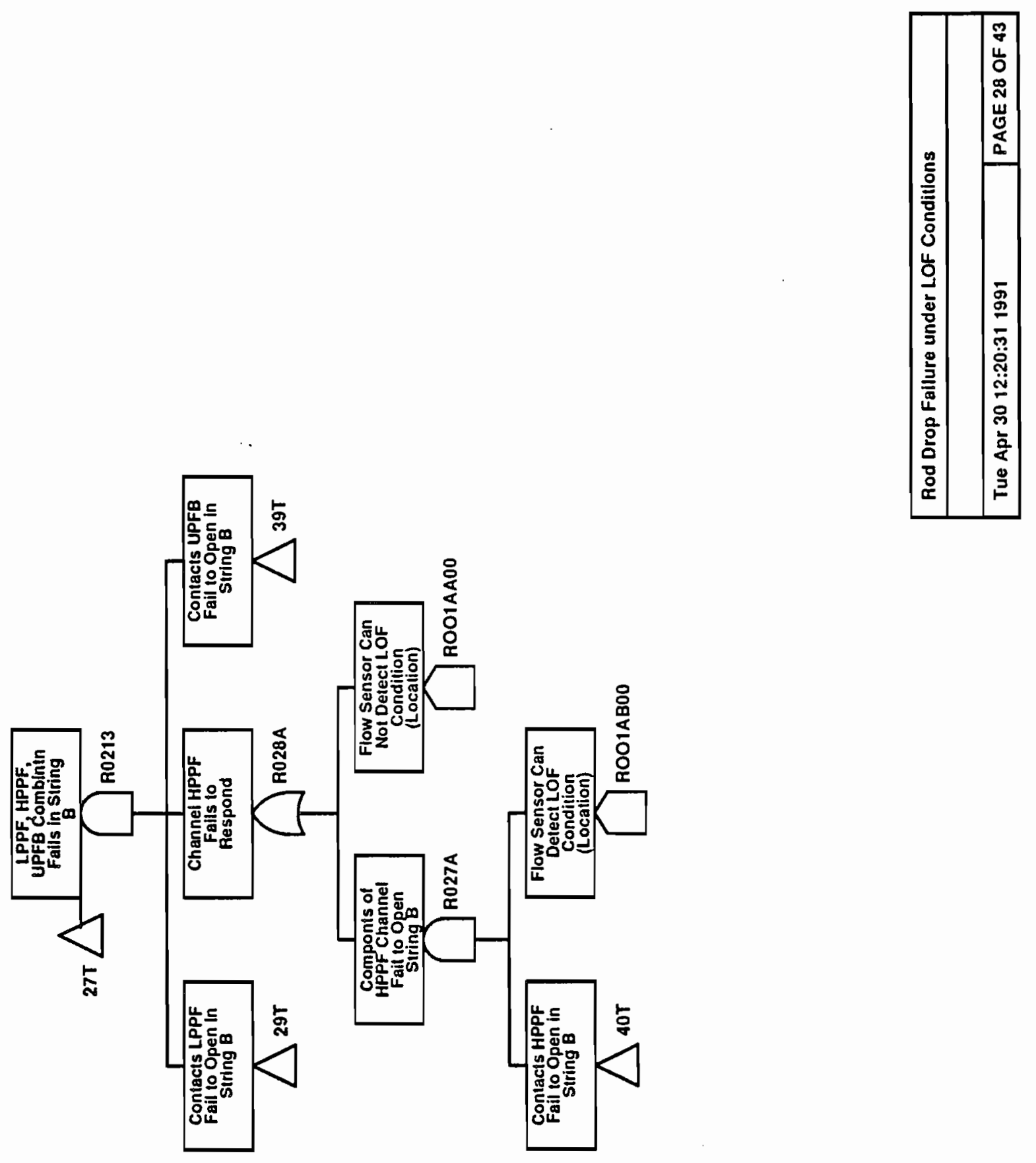



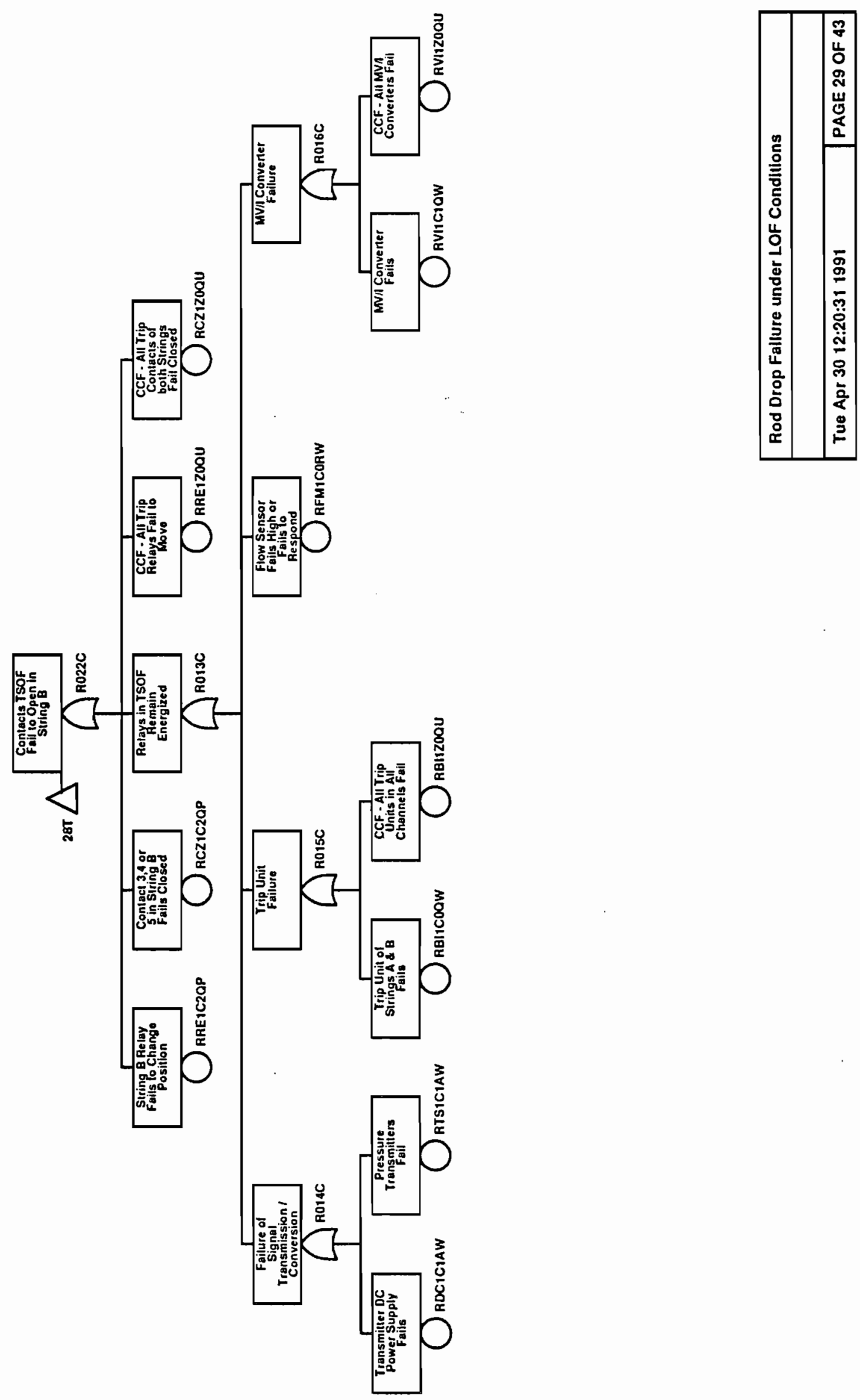

B. 2-183 


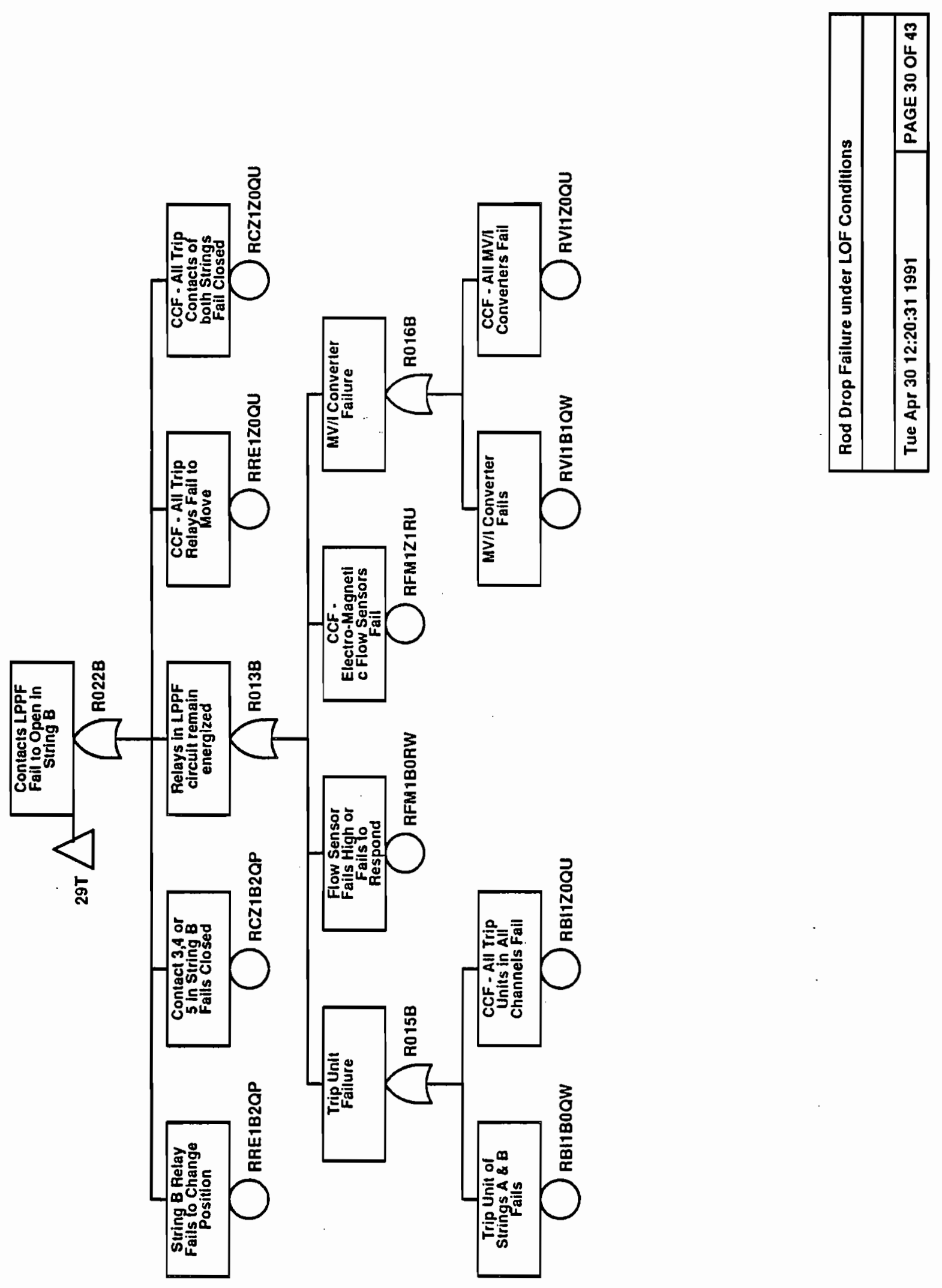




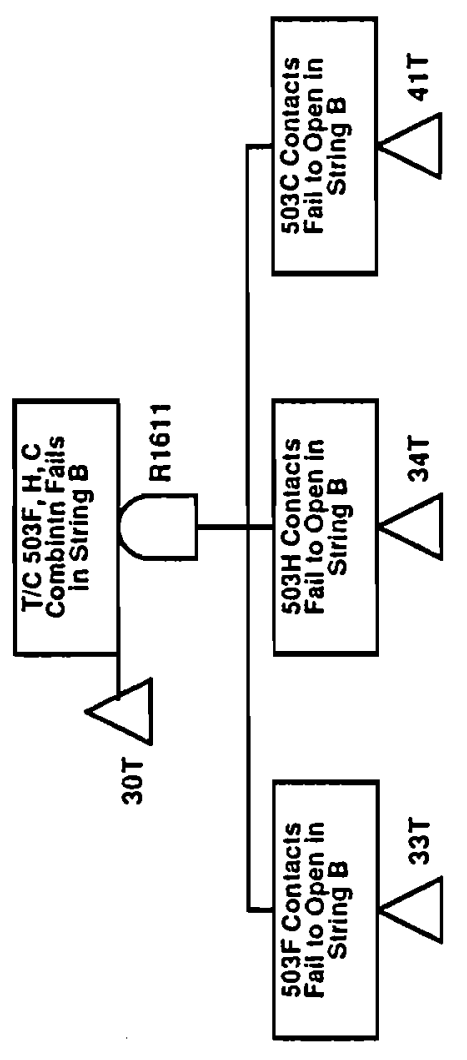

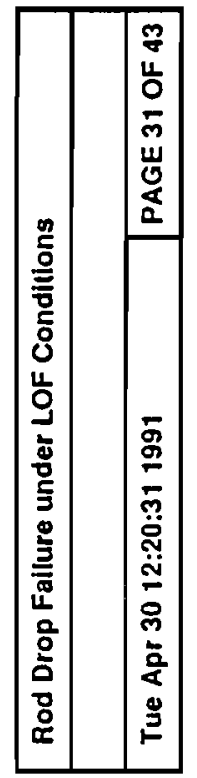




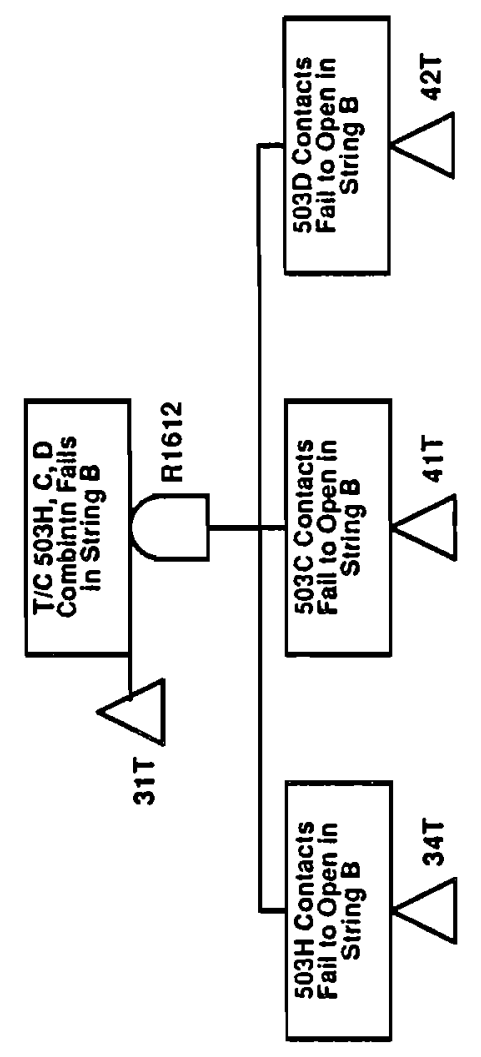

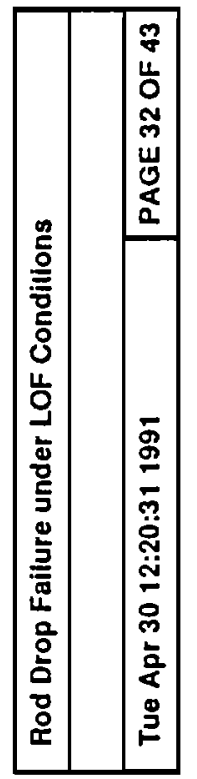




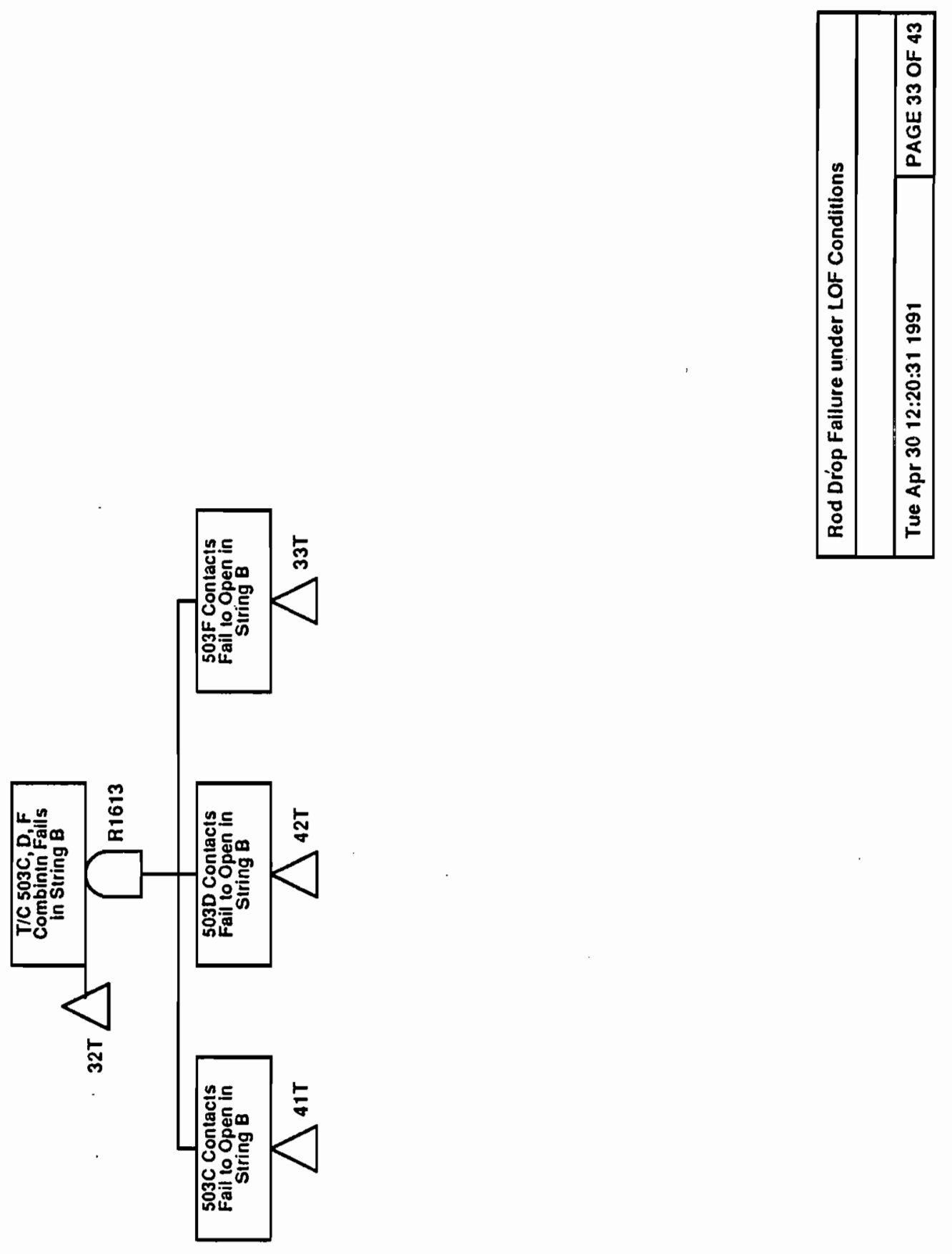



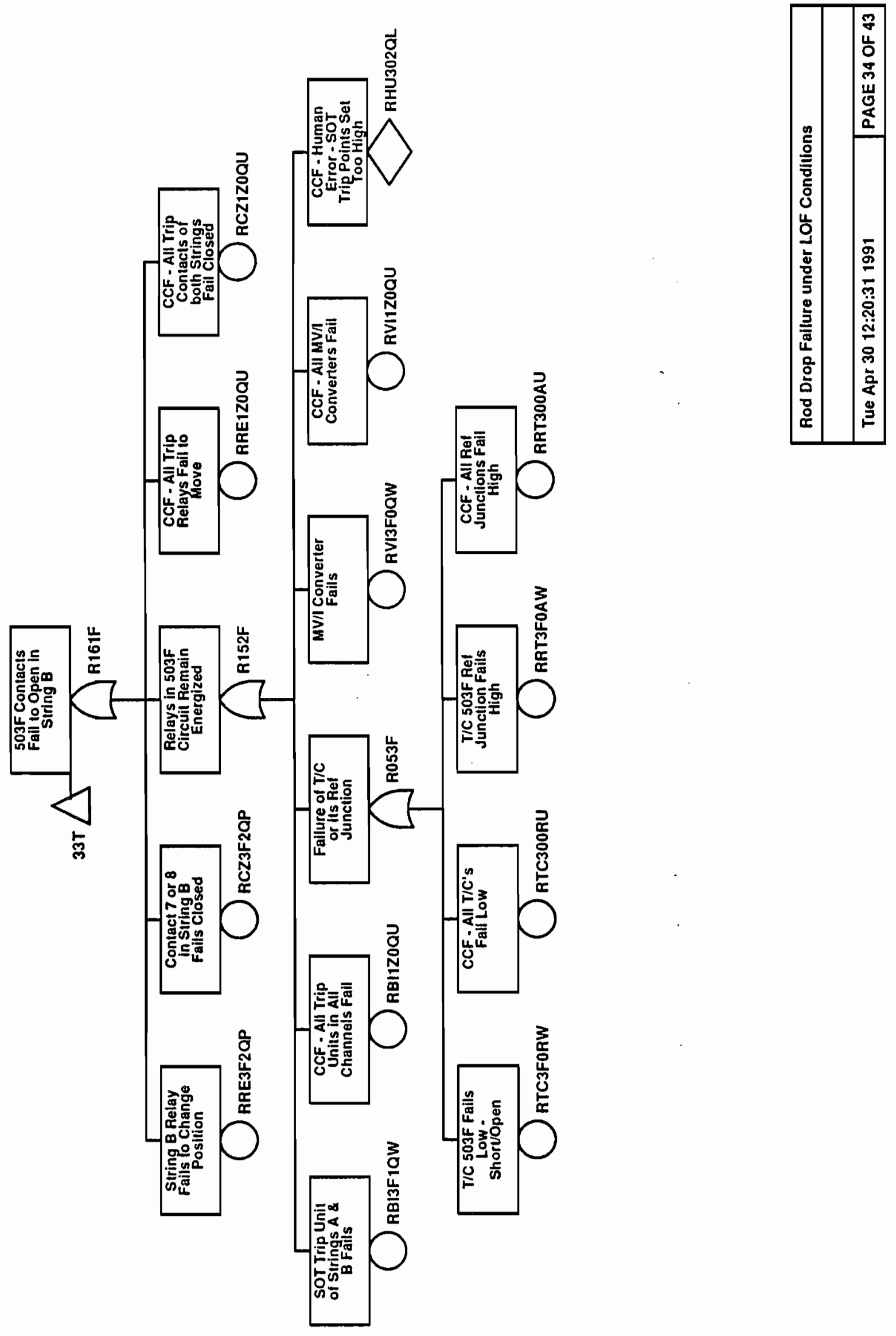

B. $2-188$ 

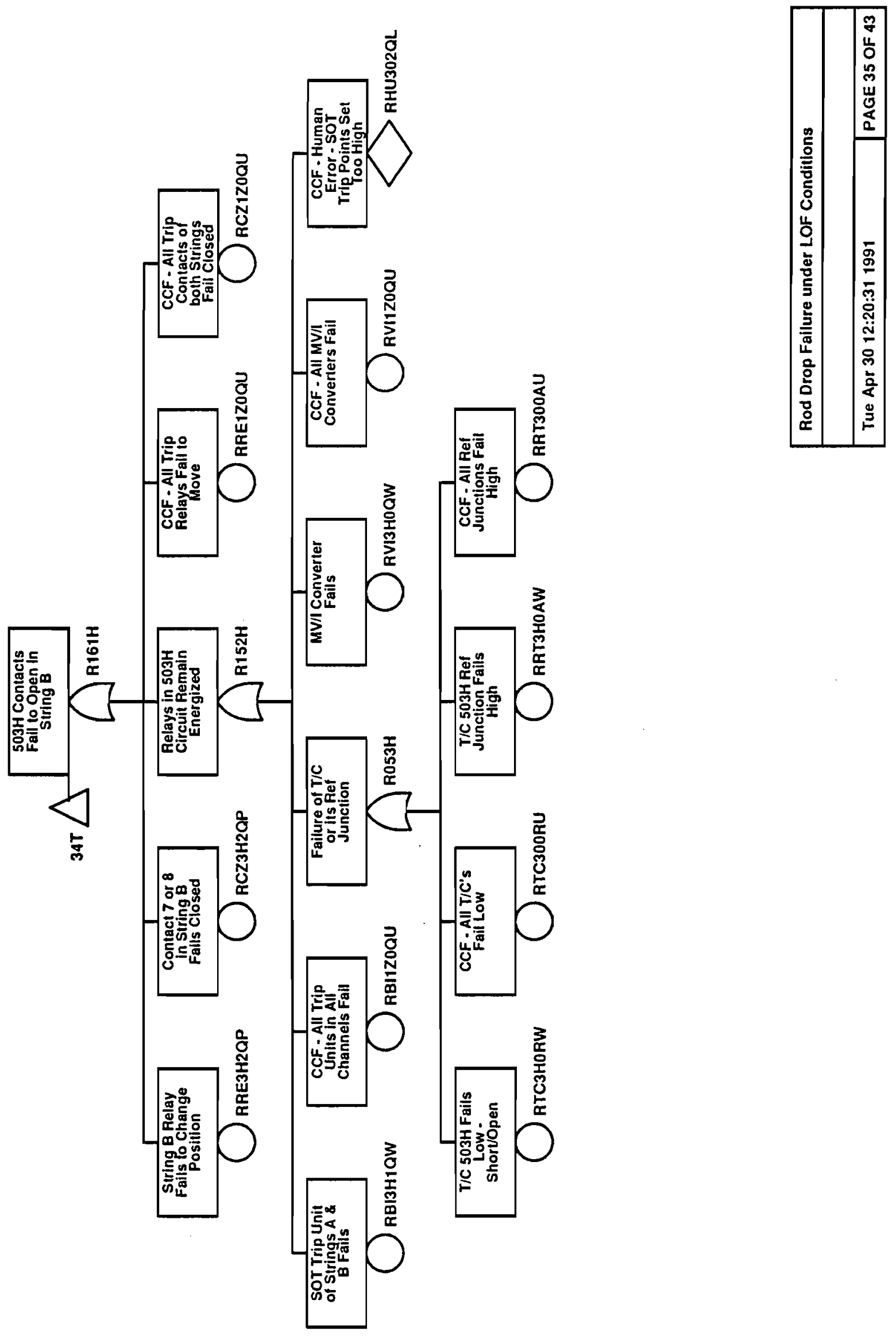

B. 2-189 


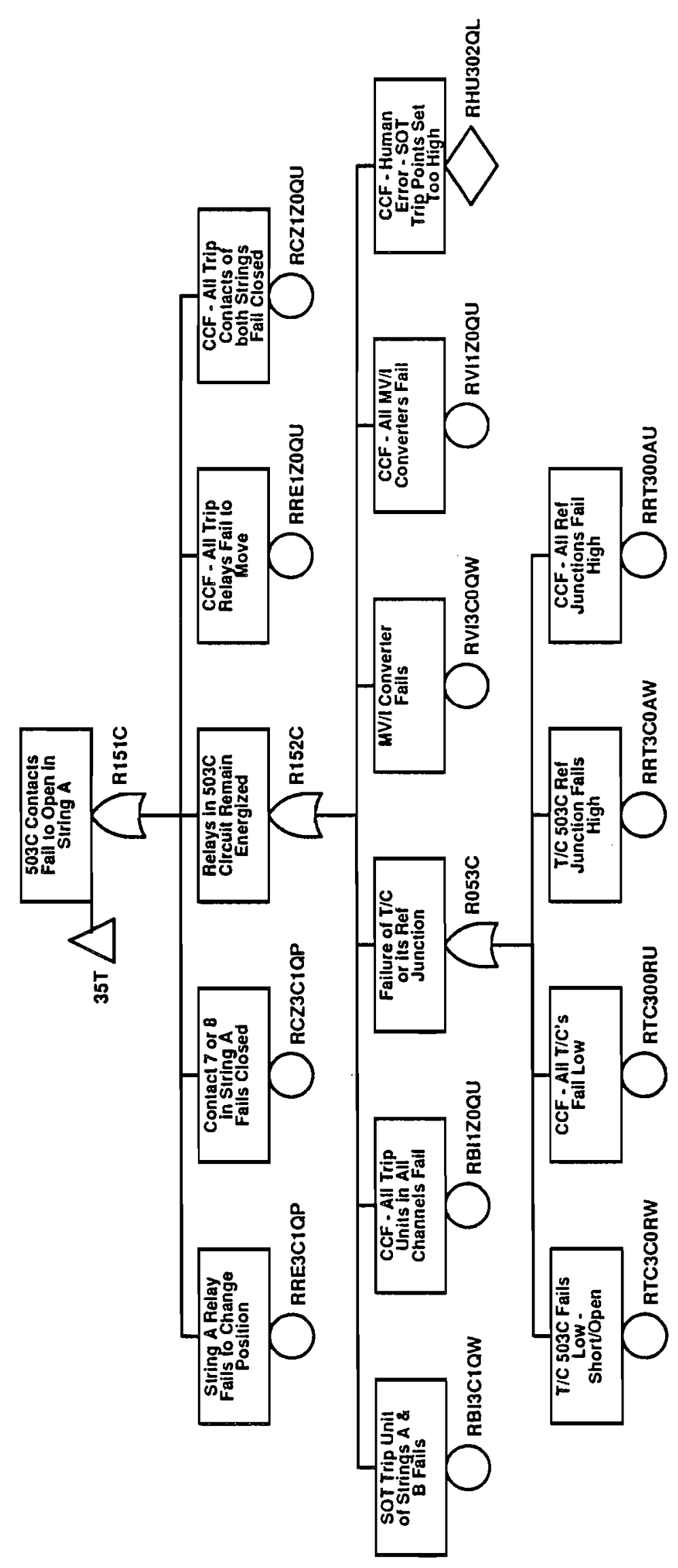

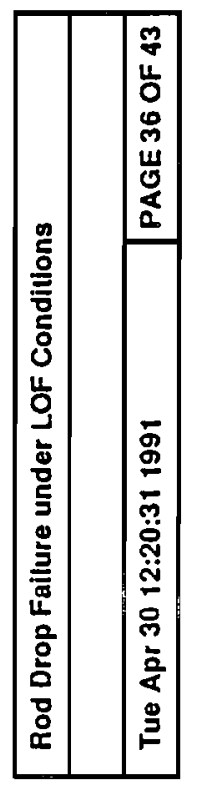




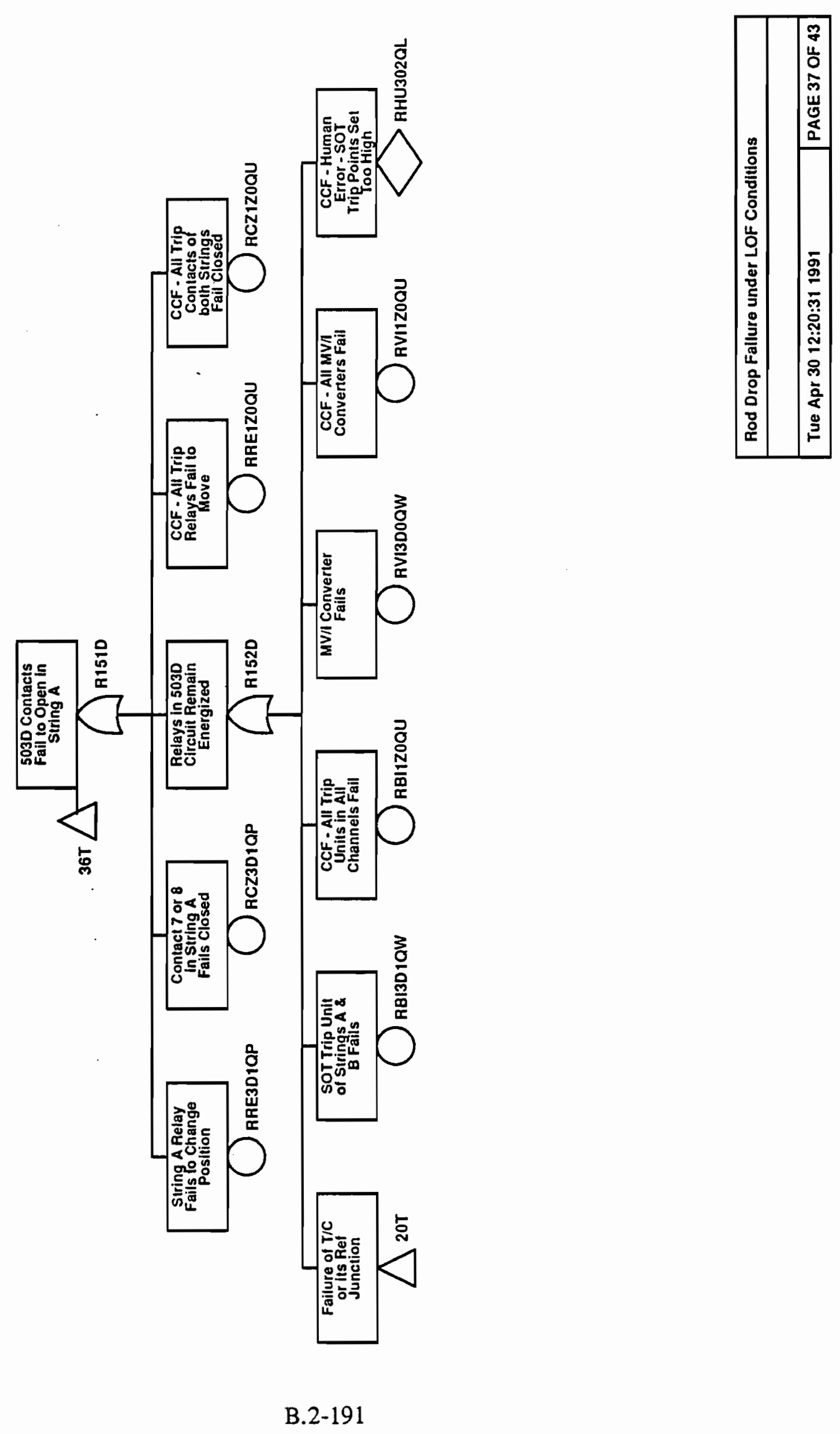

\begin{tabular}{|c|c|}
\hline$a$ & 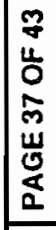 \\
\hline 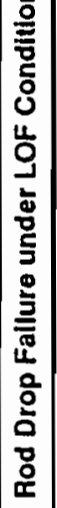 & 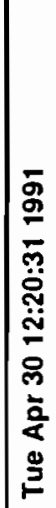 \\
\hline
\end{tabular}




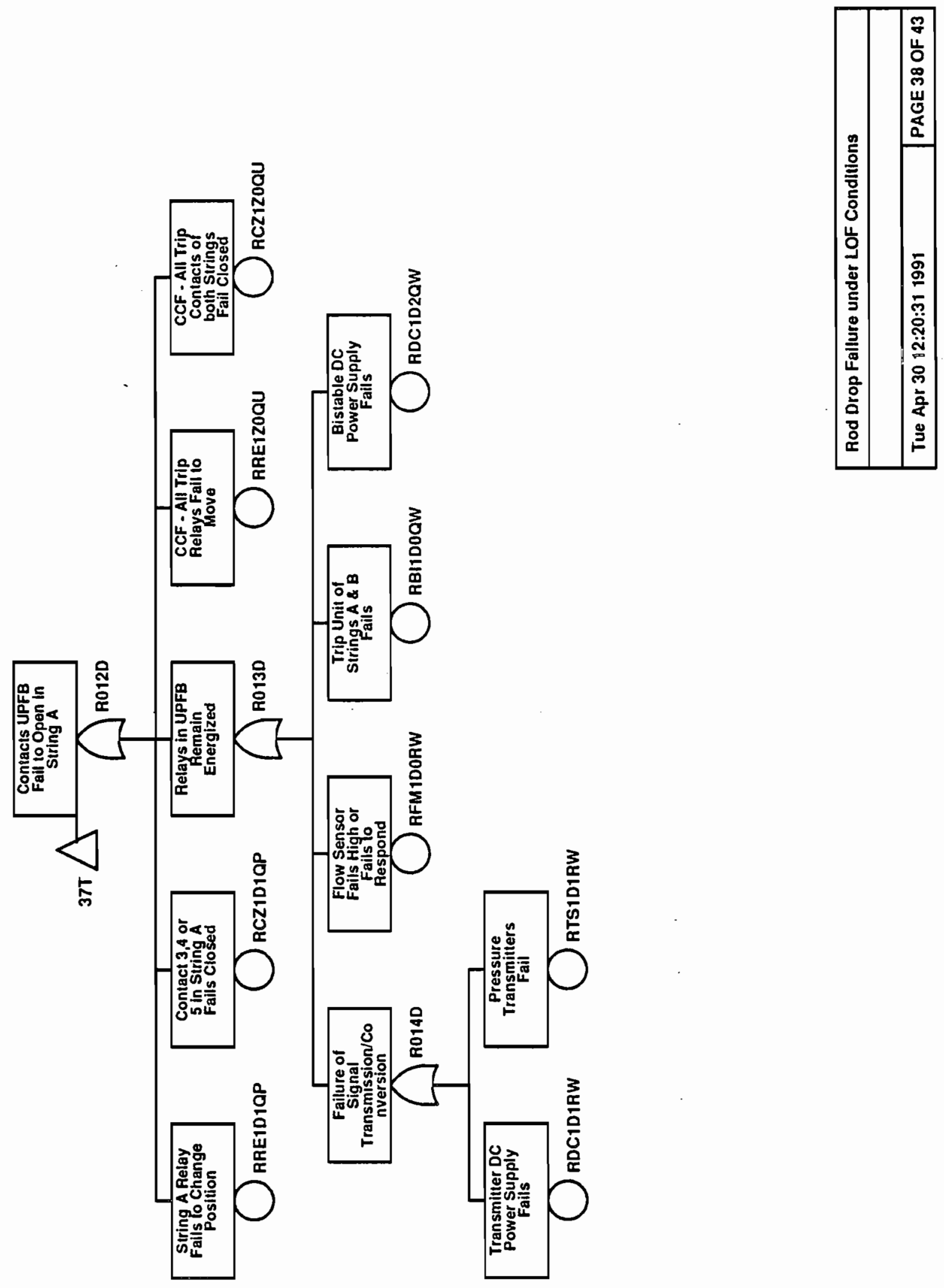

B. 2-192 


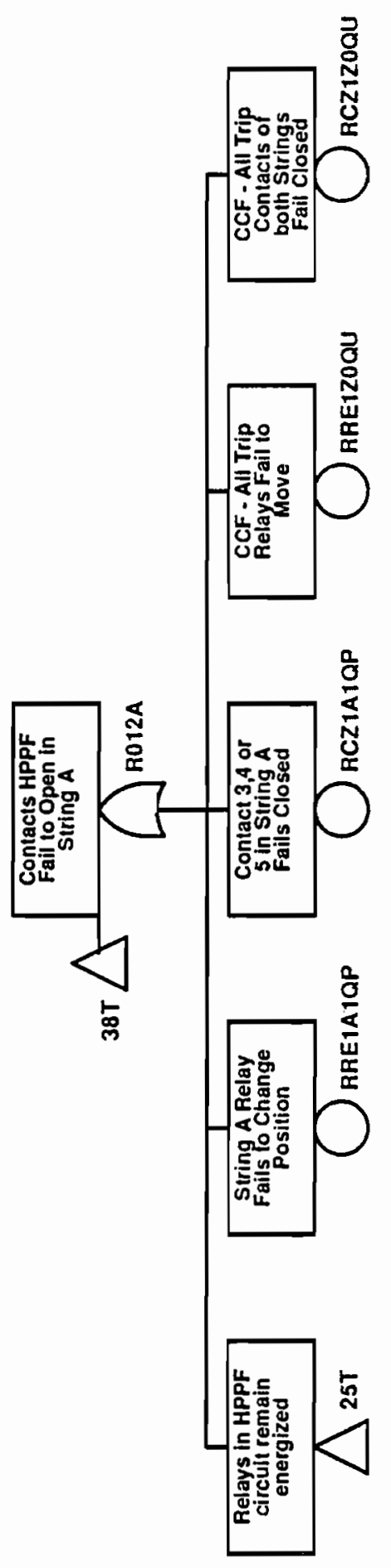

B. 2-193 


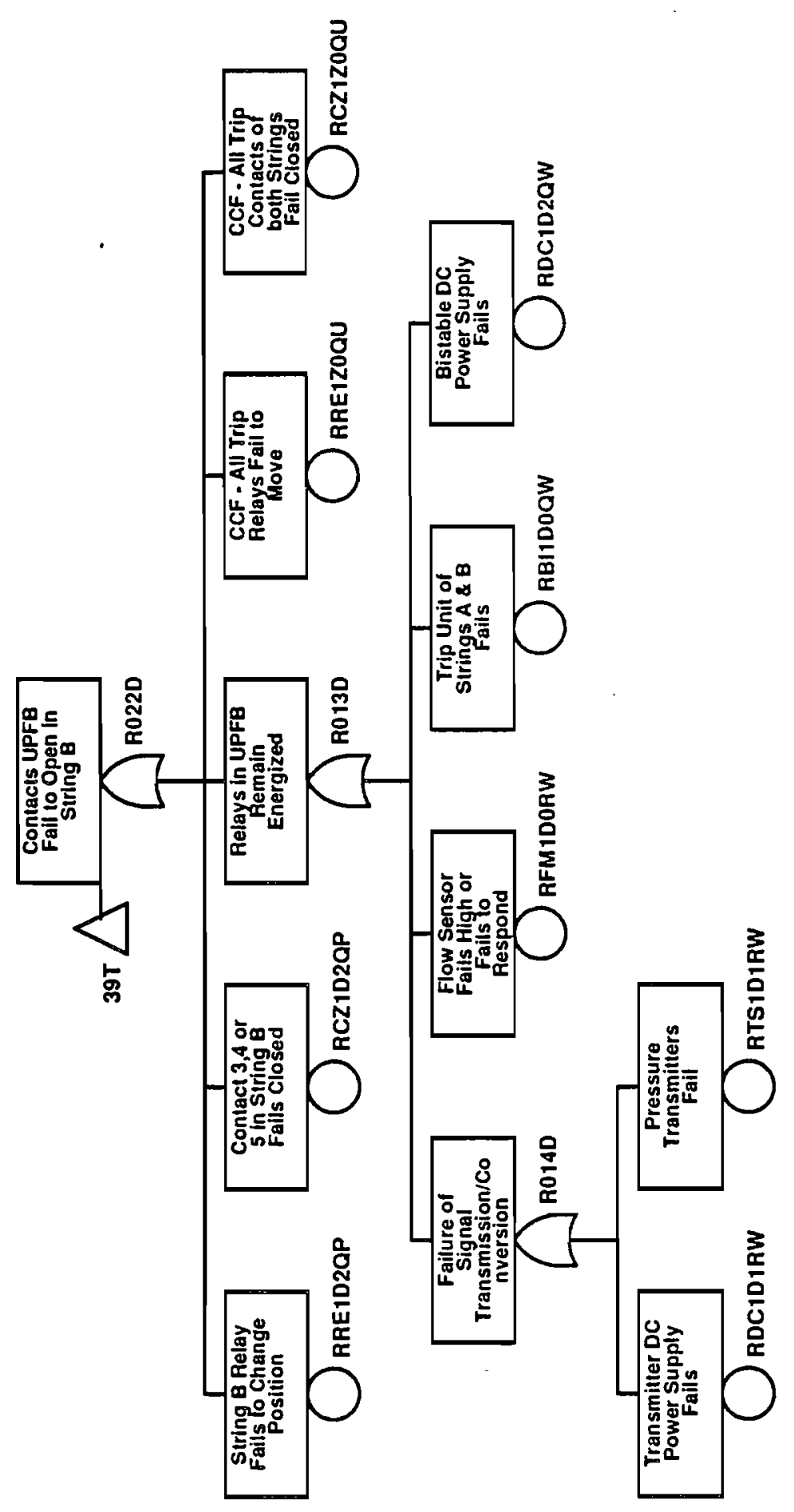




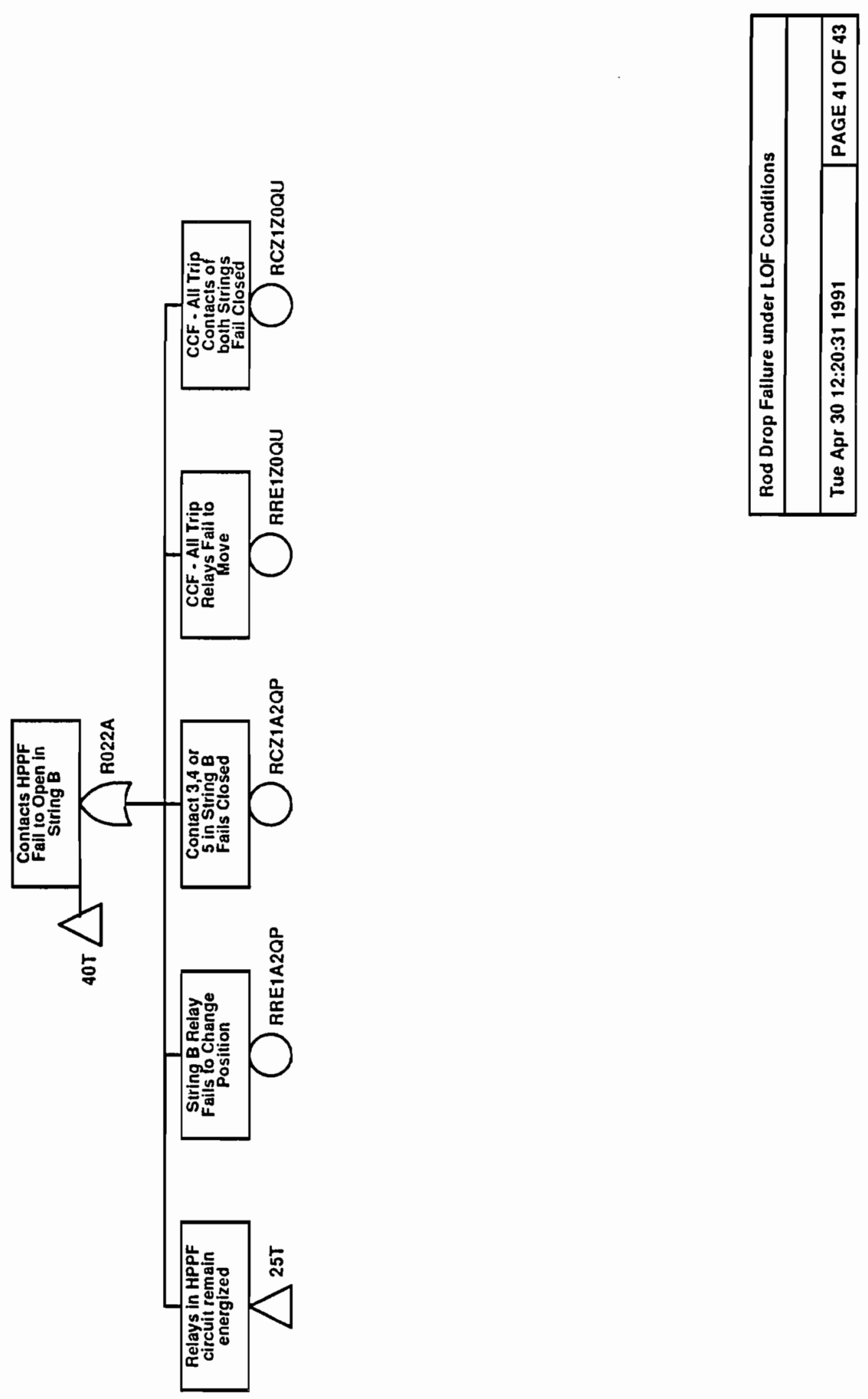



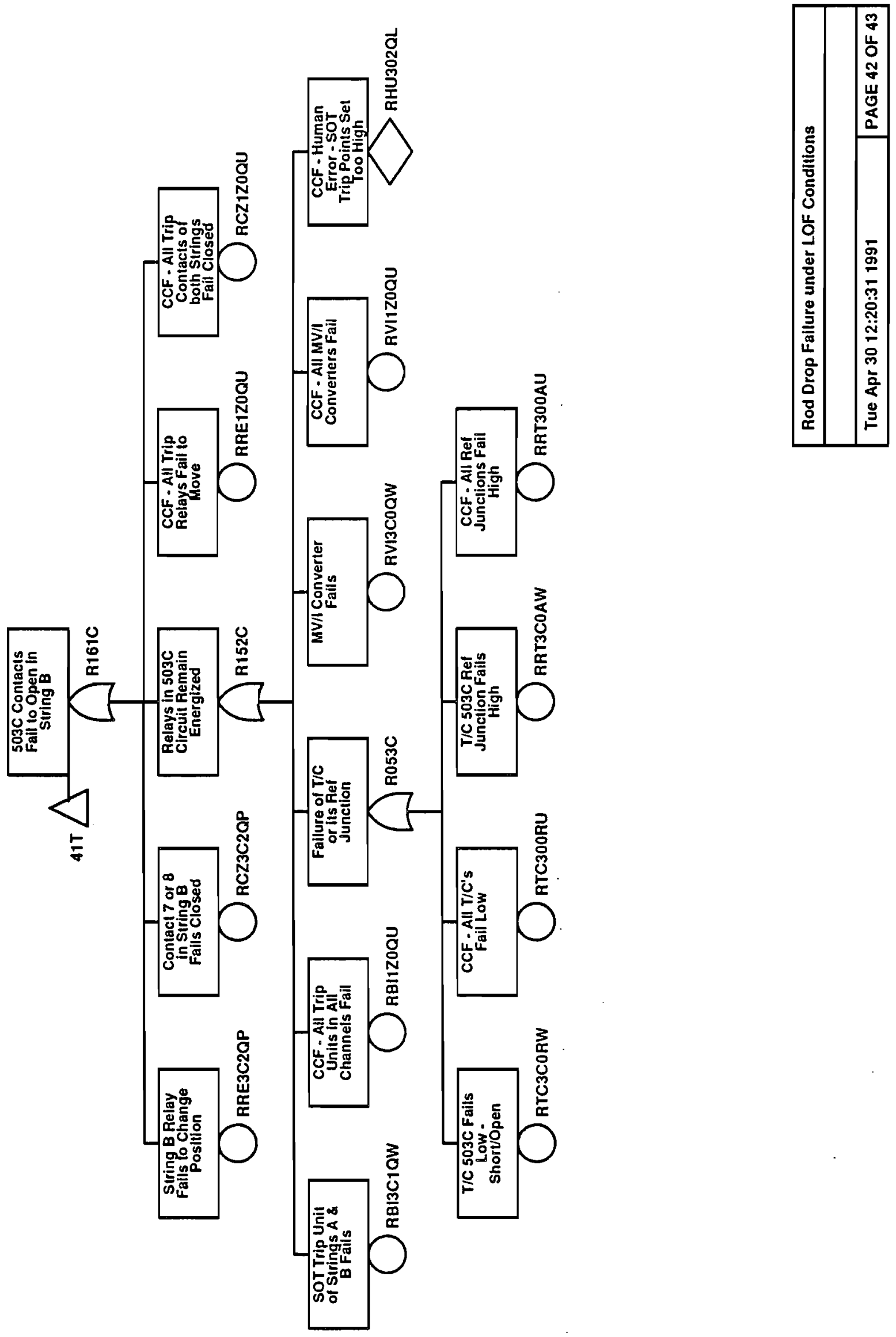


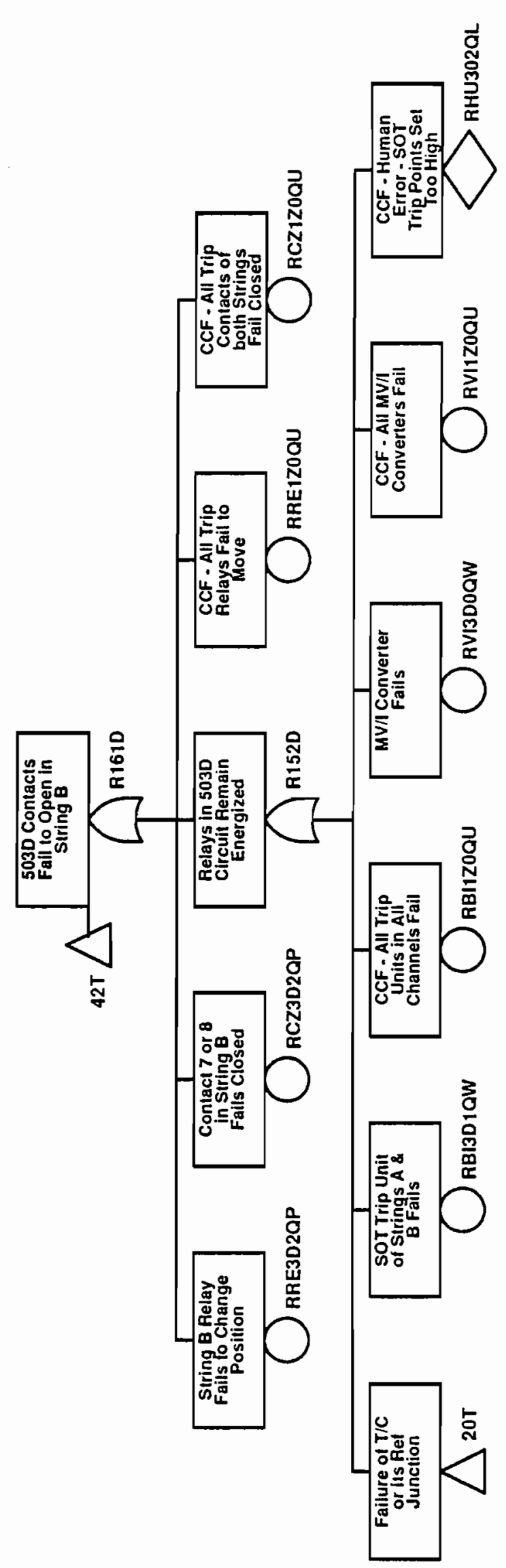

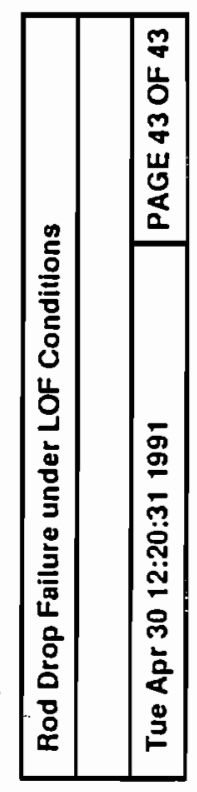

B. 2-197 


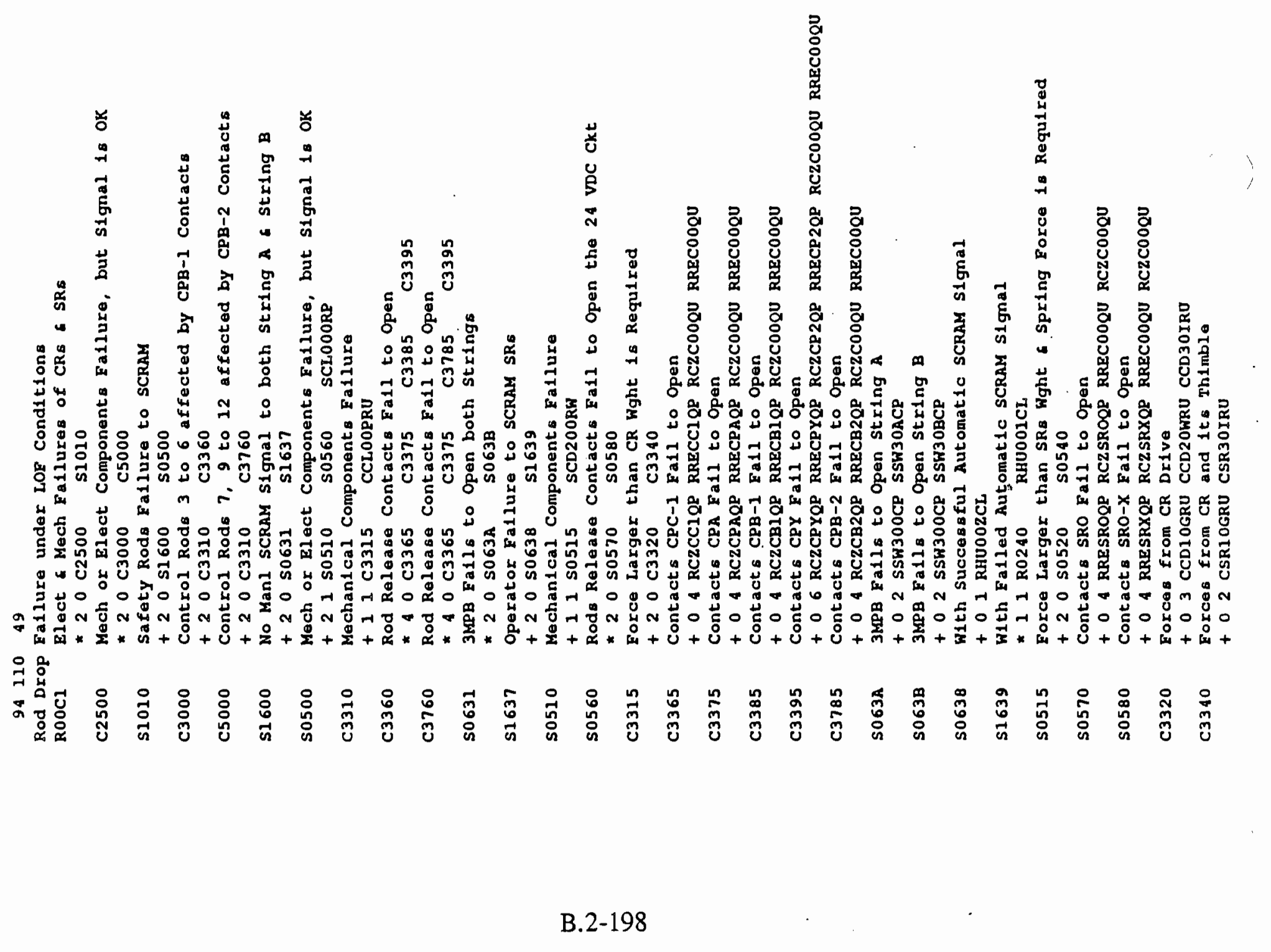




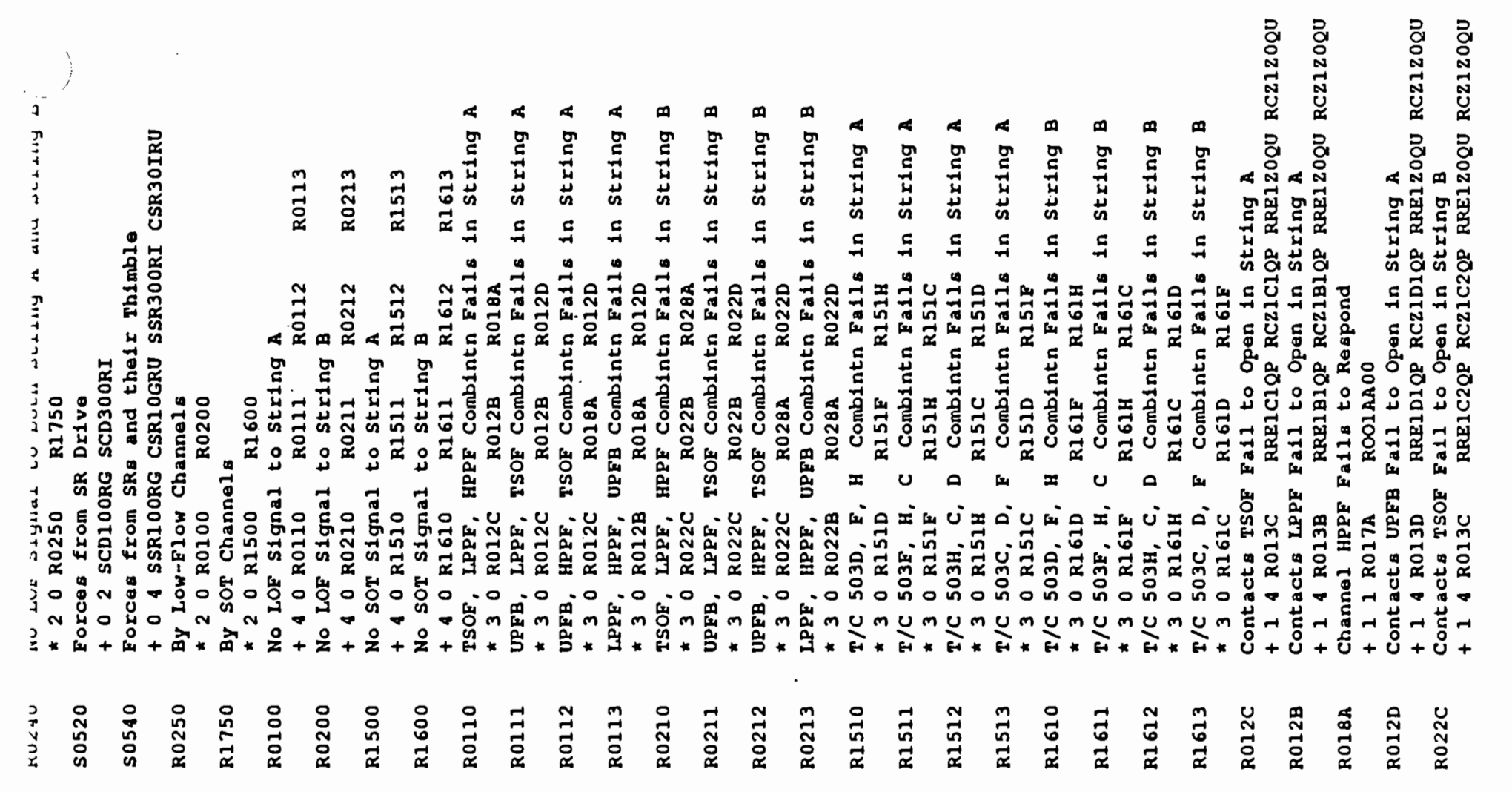




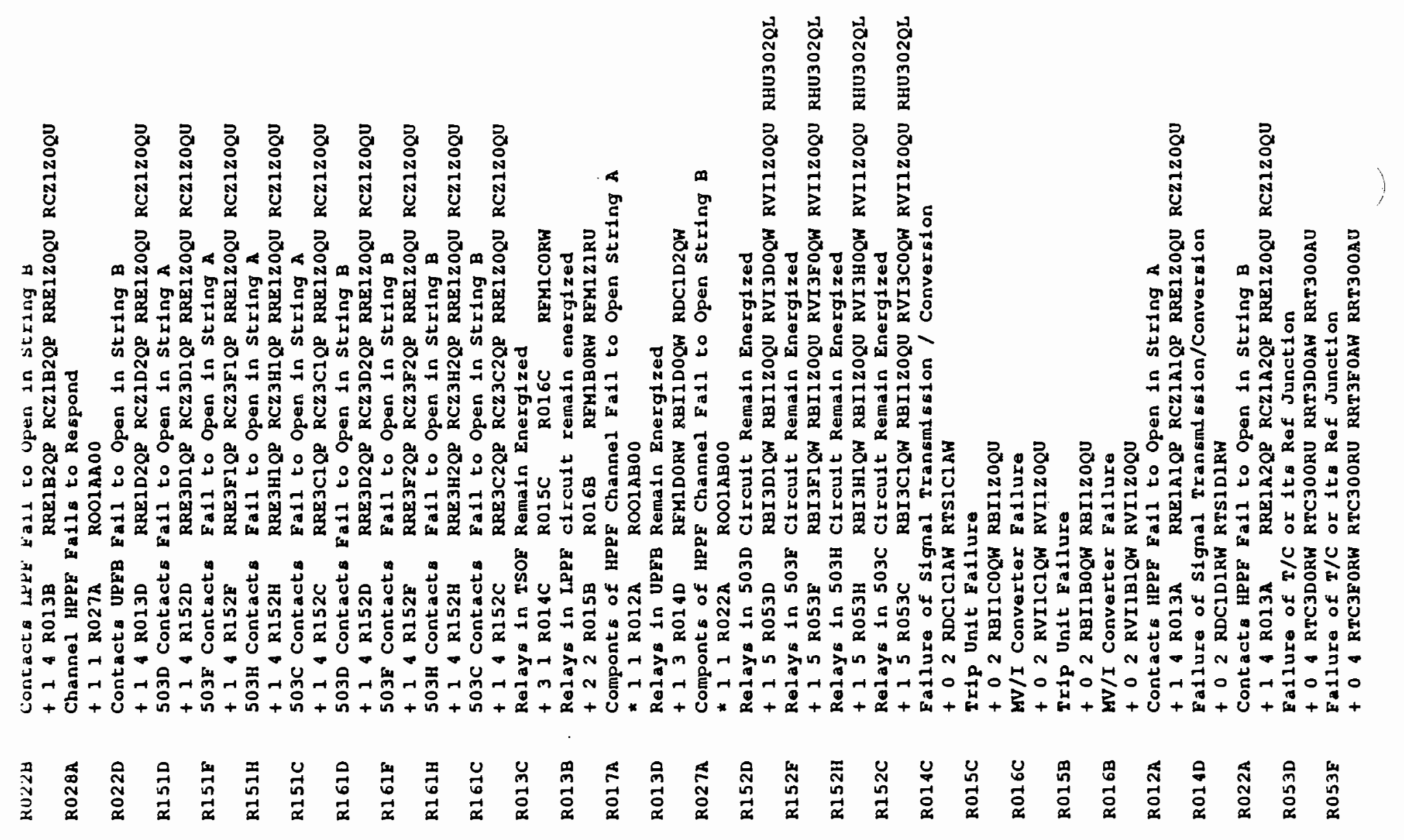




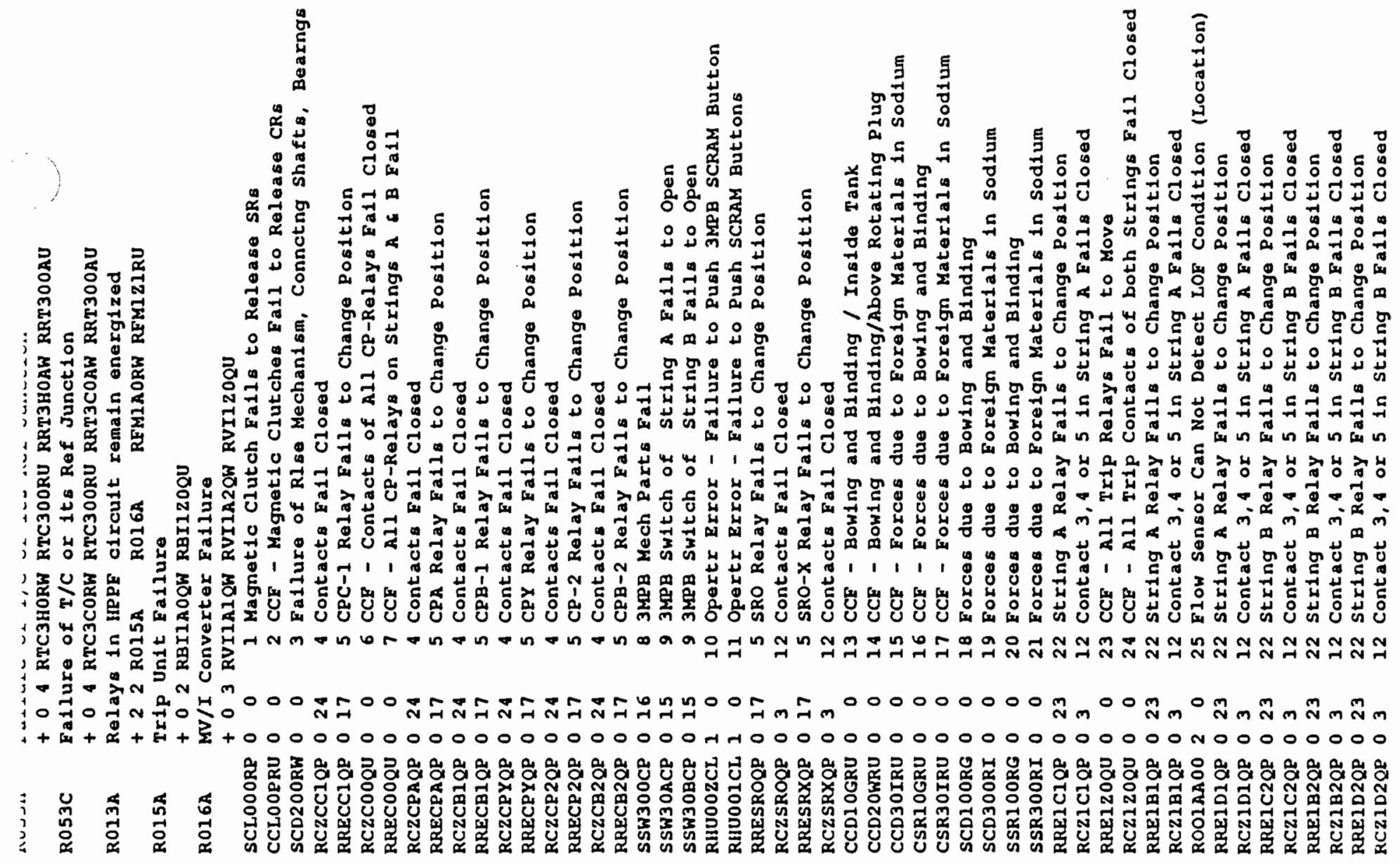




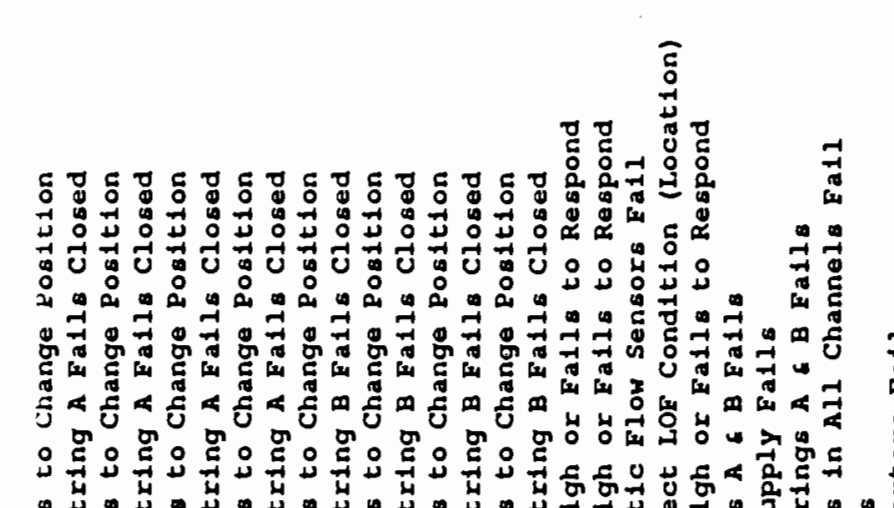

吾

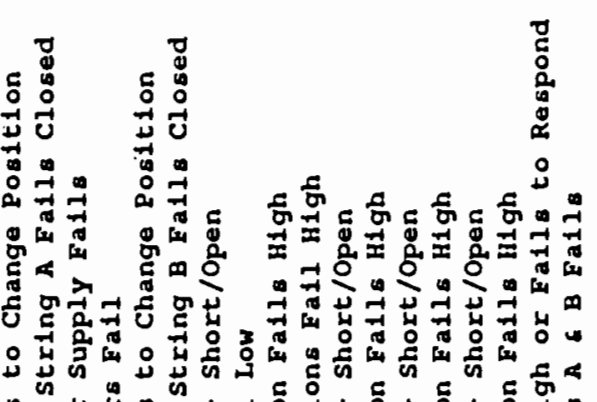

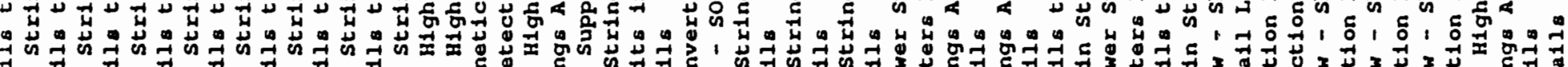

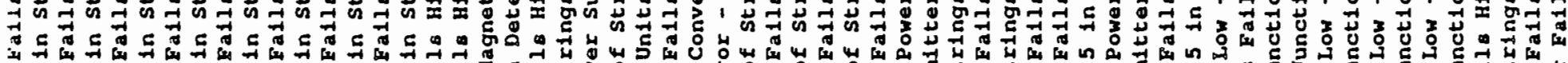

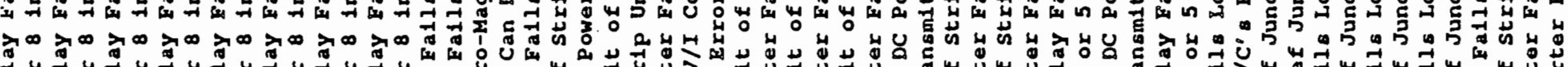

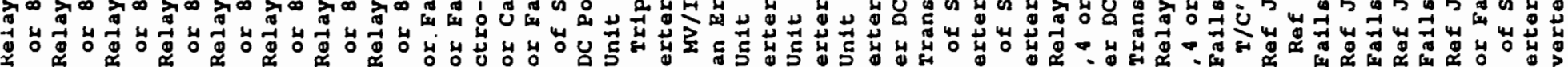
4

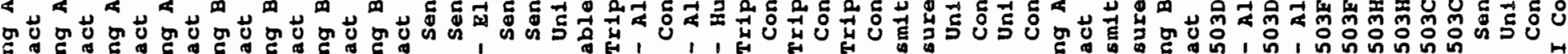

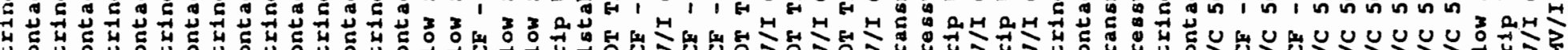

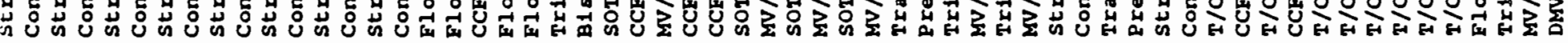

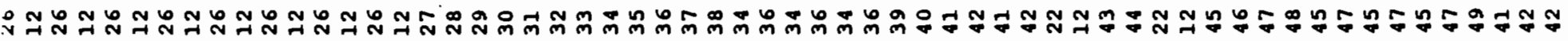

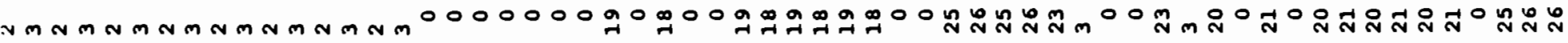
$0000000000000000000 \mathrm{~N} 0000000 \pi 00000000000000000000000000000000$

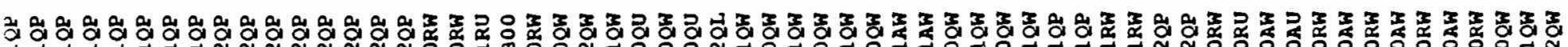

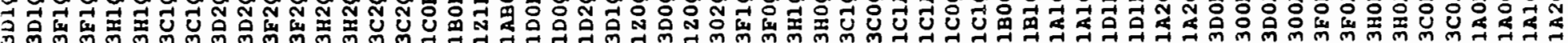

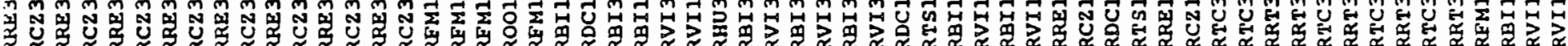




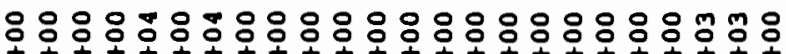

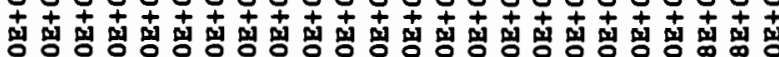

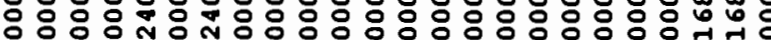
í $\dot{0} \dot{0} \dot{0} \dot{0} \dot{0} \dot{0} 0 \dot{0} \dot{0} 0 \dot{0} 0 \dot{0} 0 \dot{0} 0 \dot{0}$

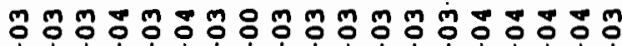

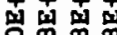

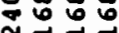

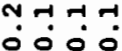

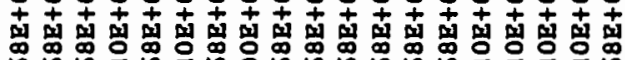

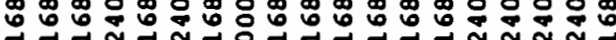

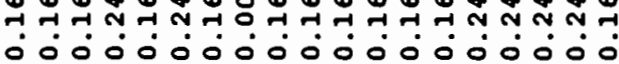

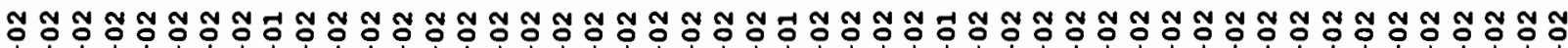

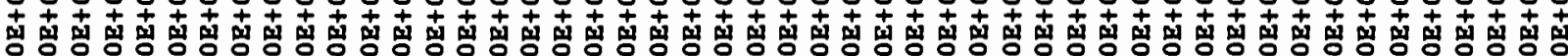

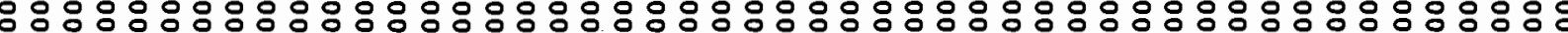

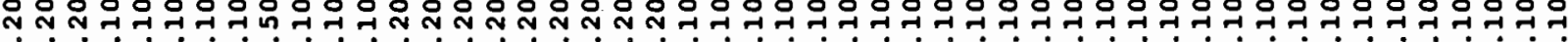

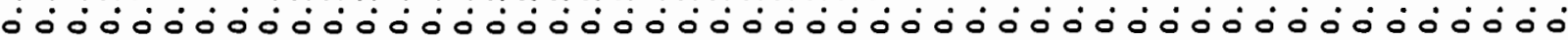

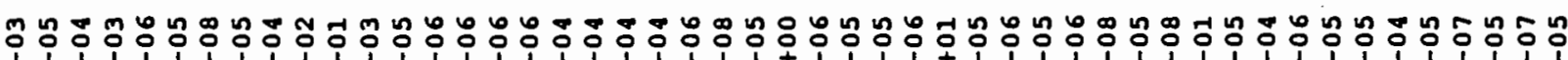

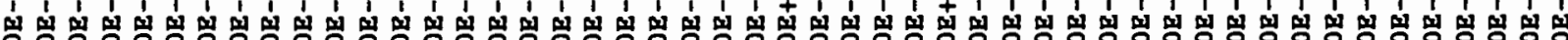

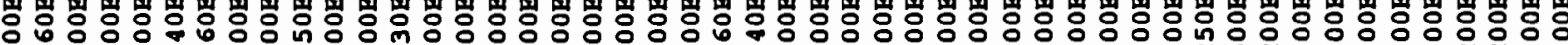

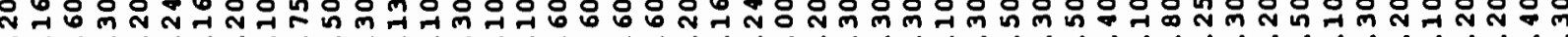

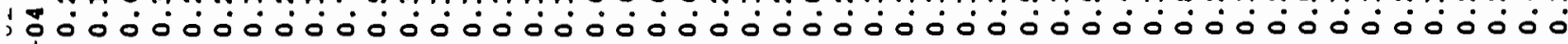
i 㠃

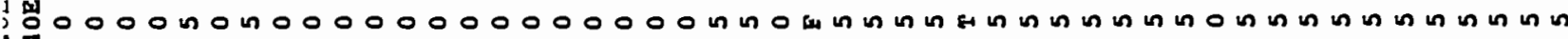

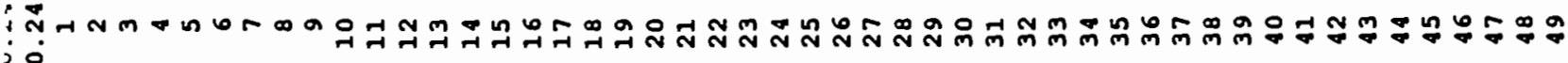



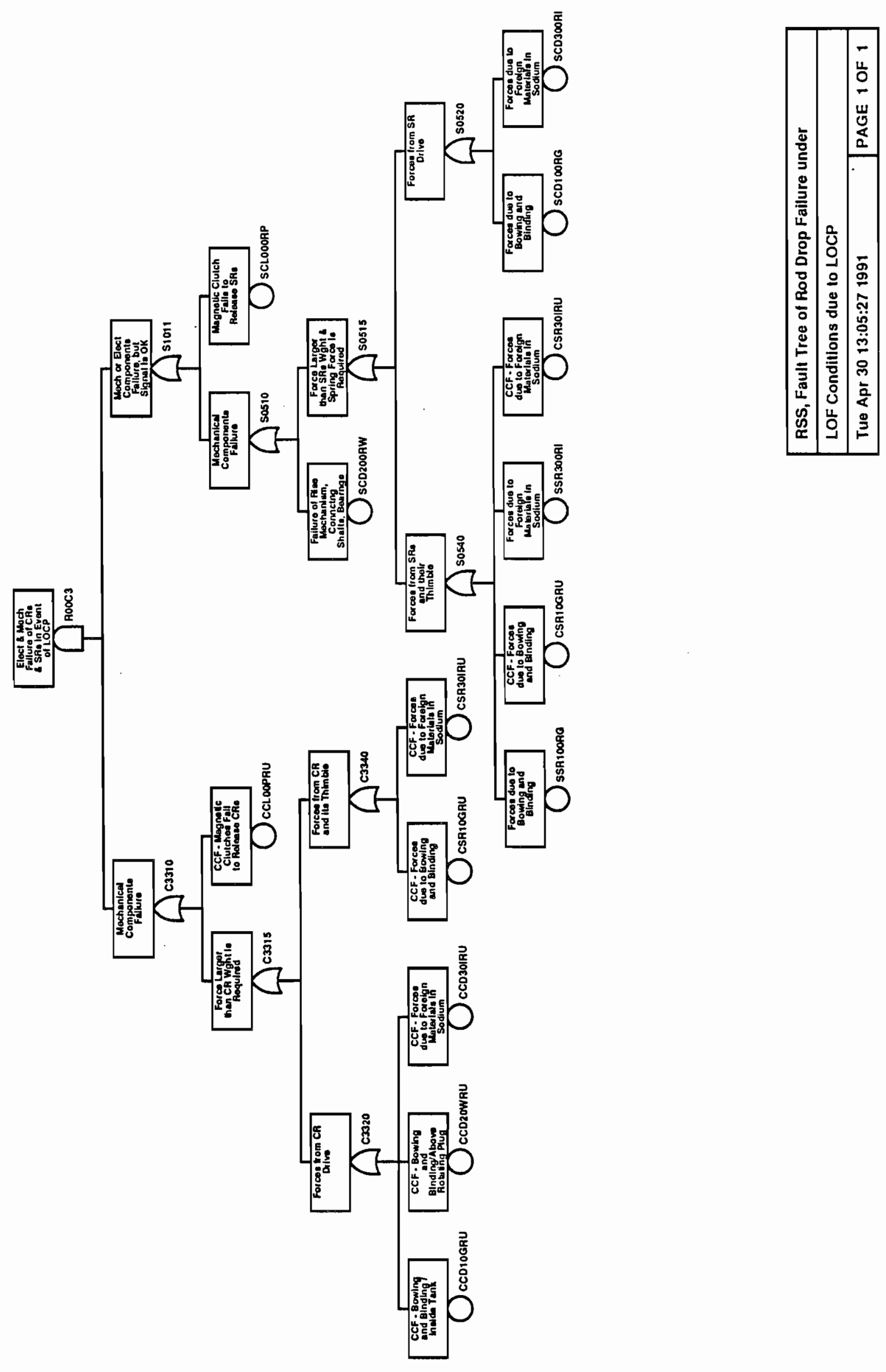

B. 2-204 


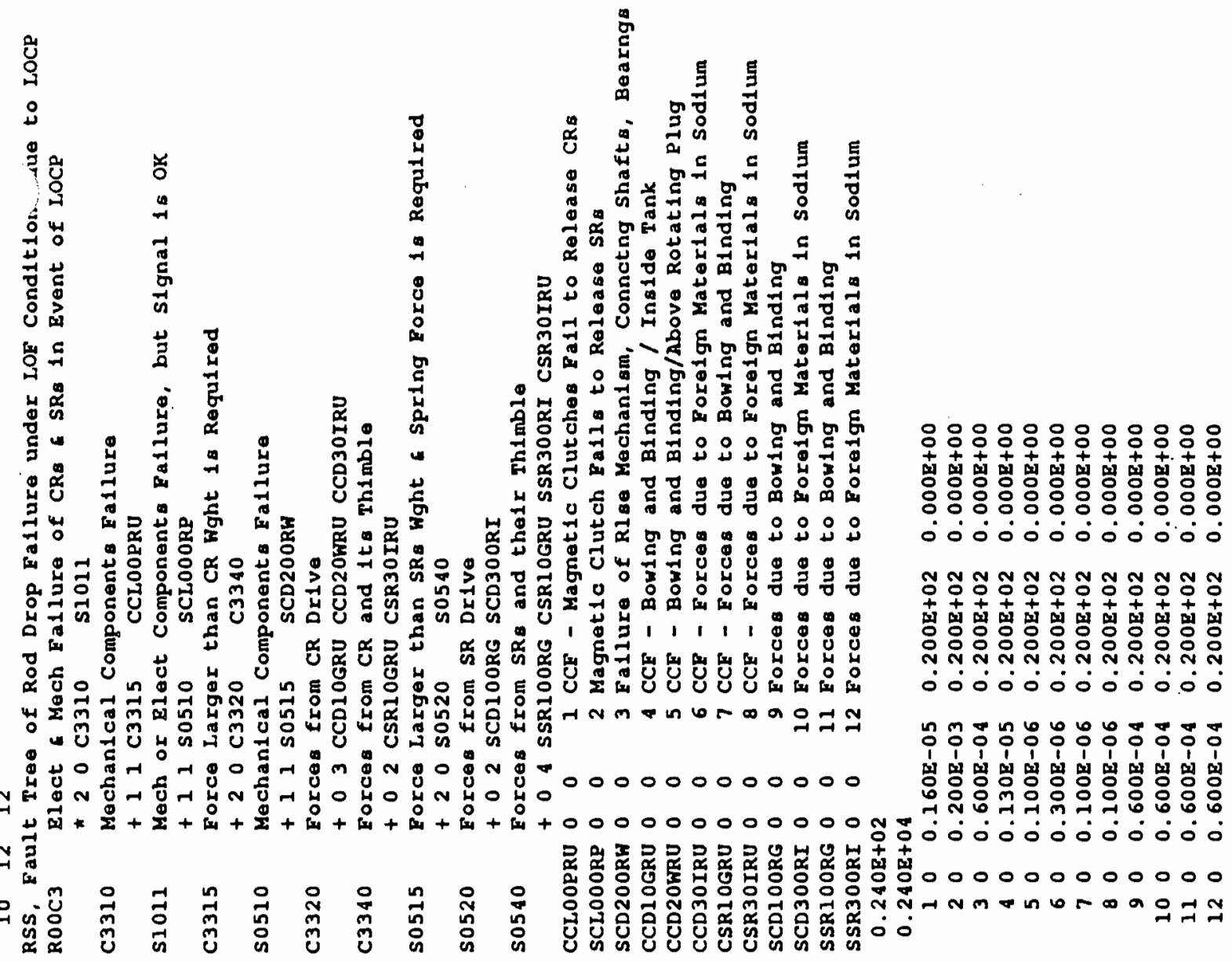




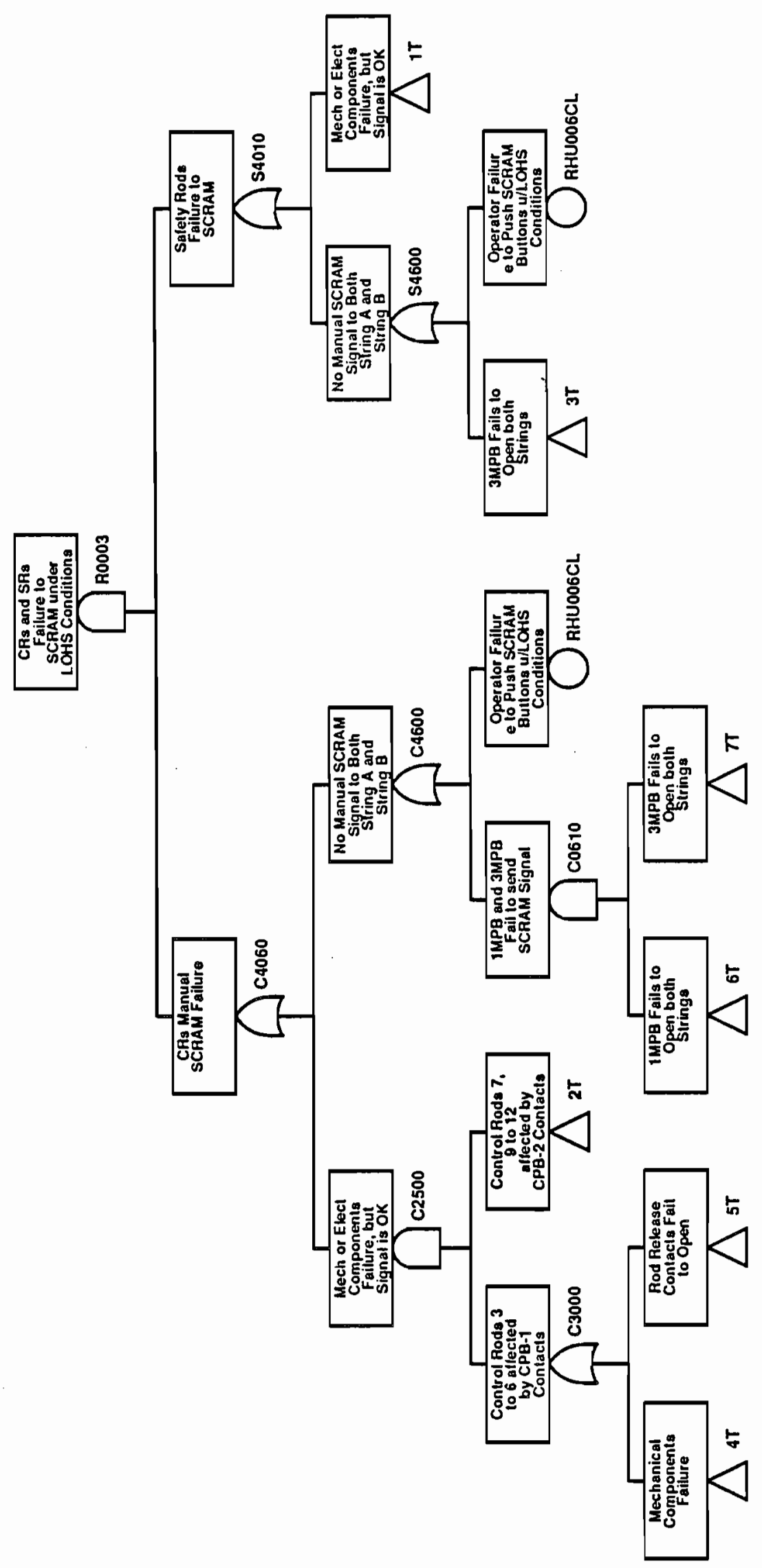

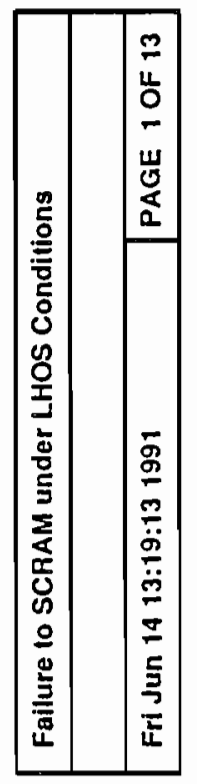



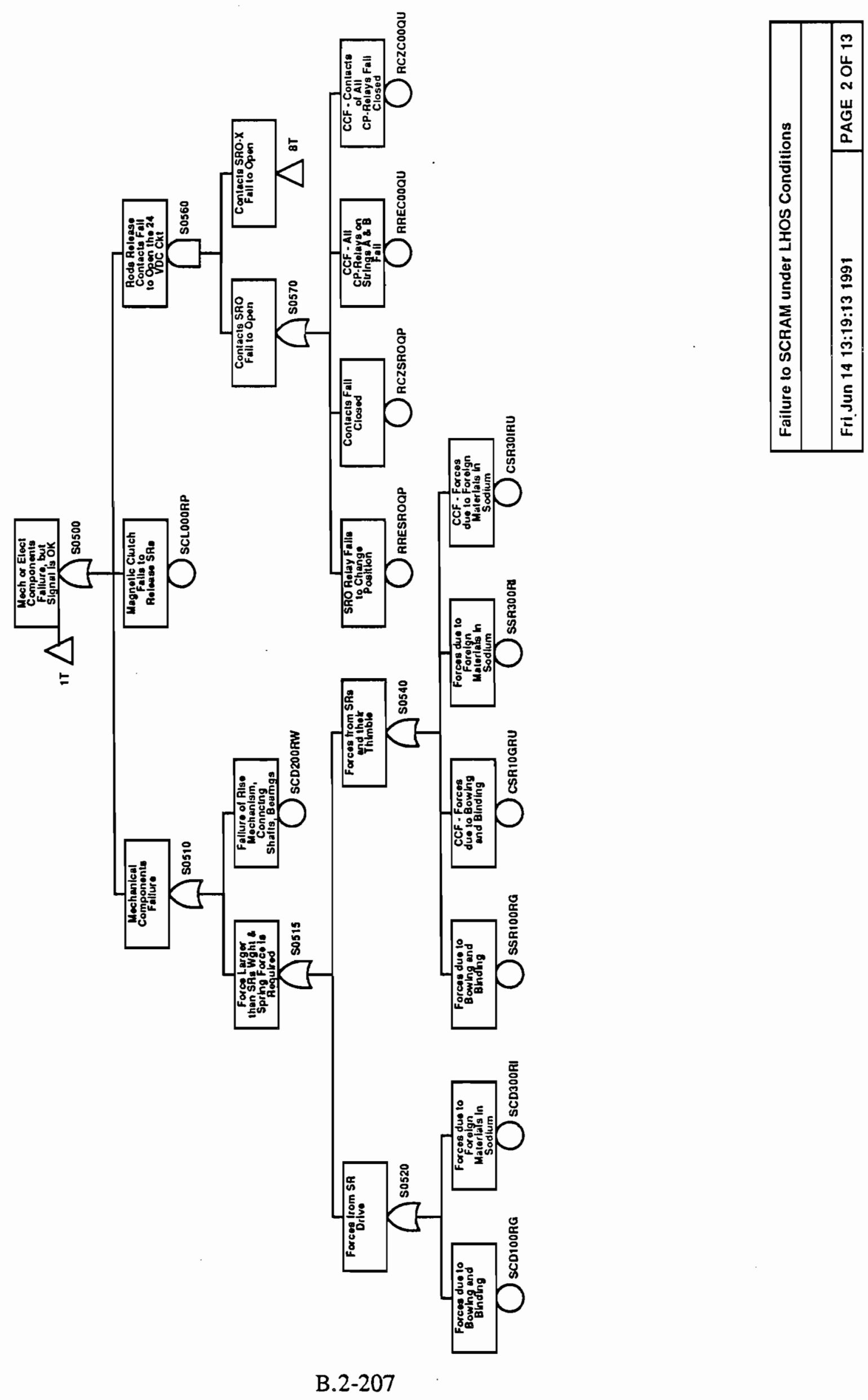


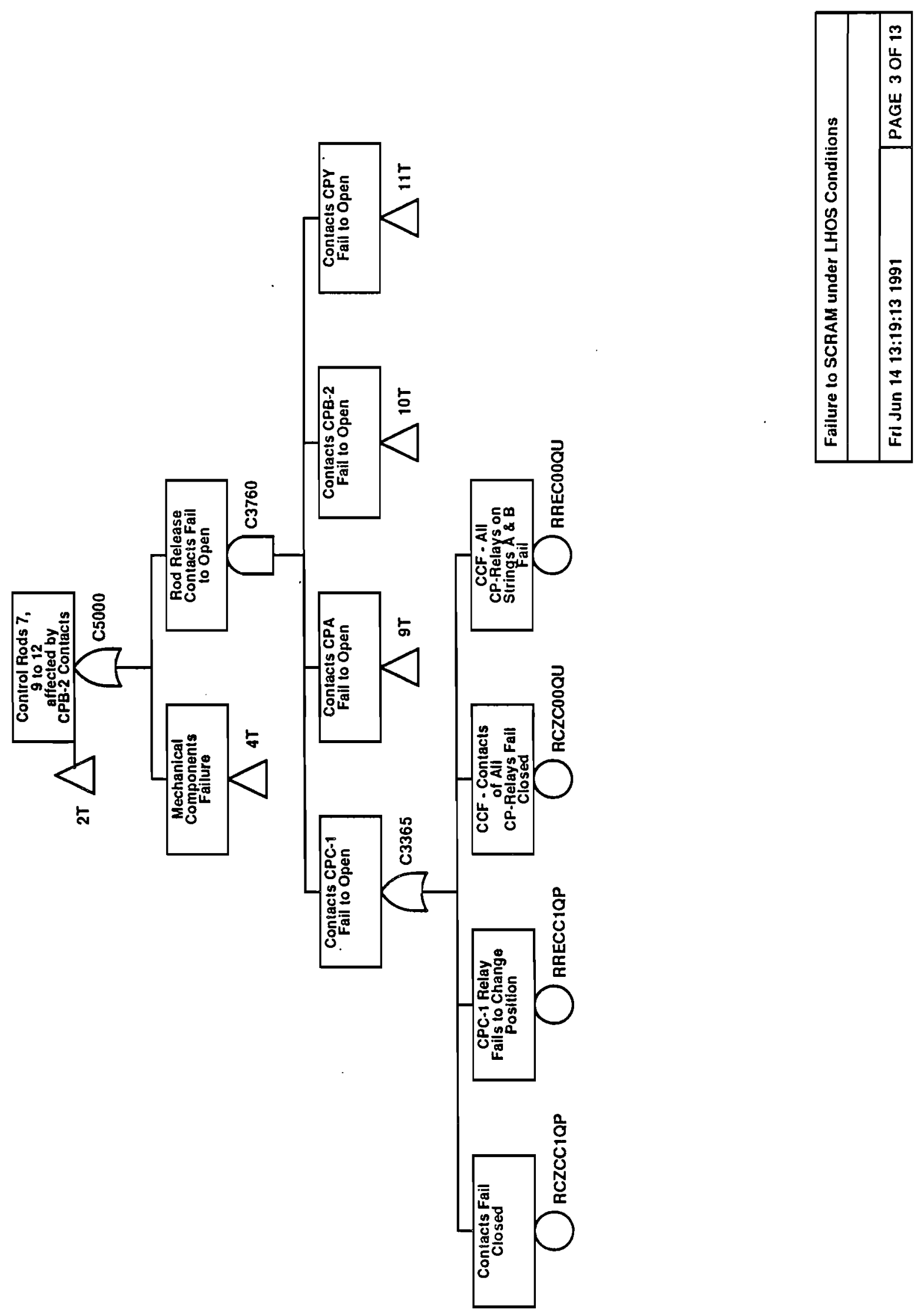




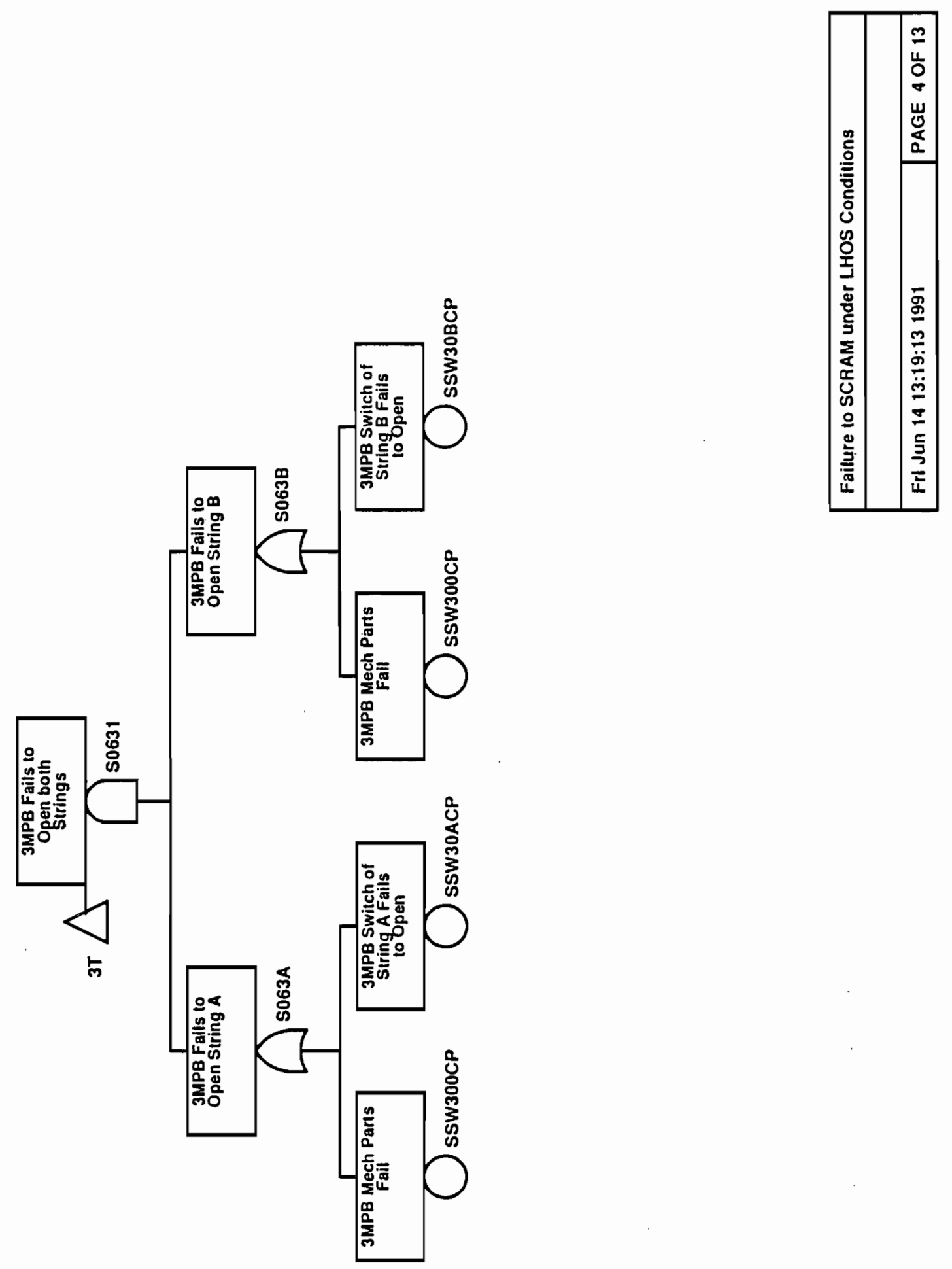




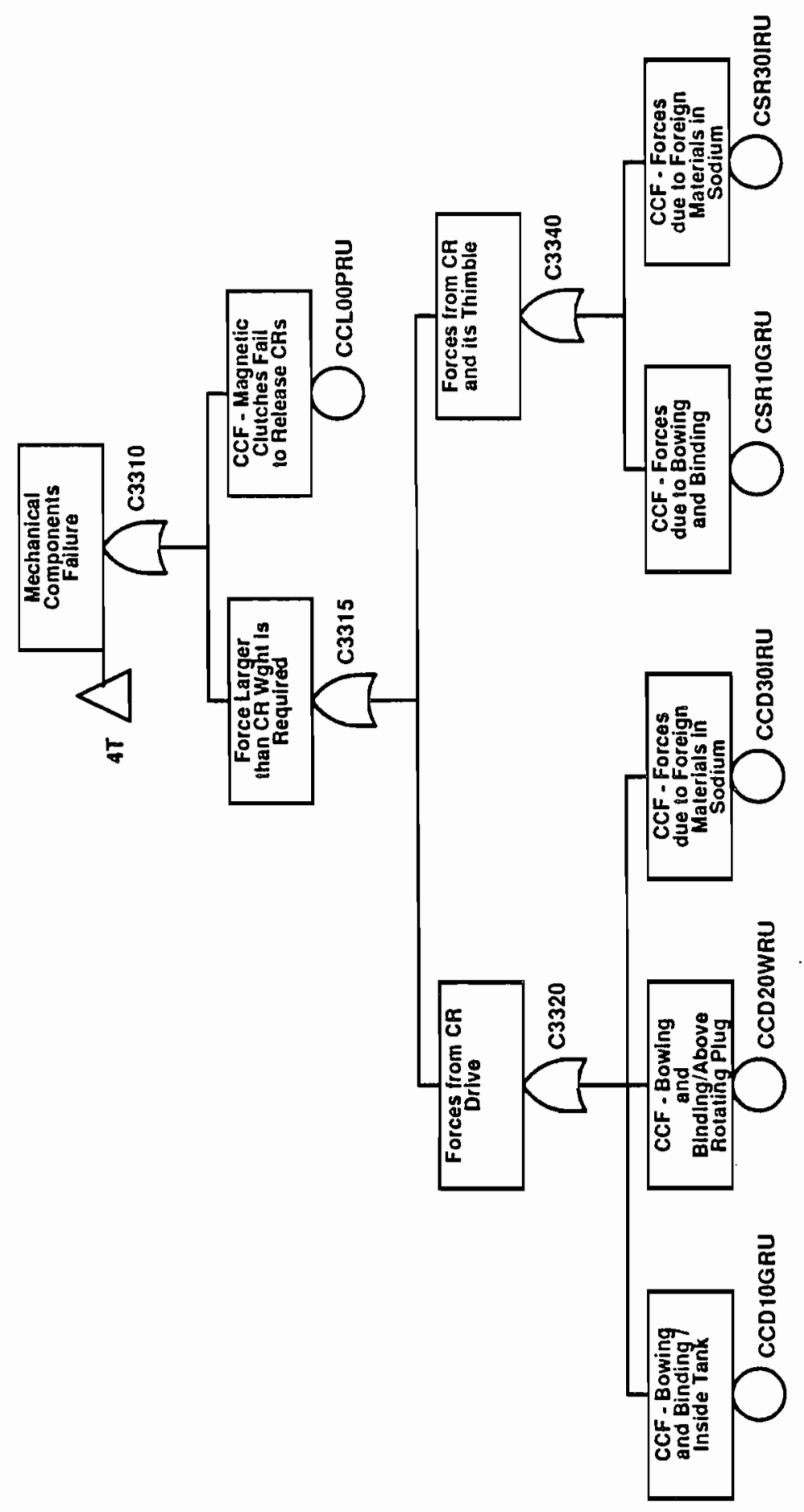



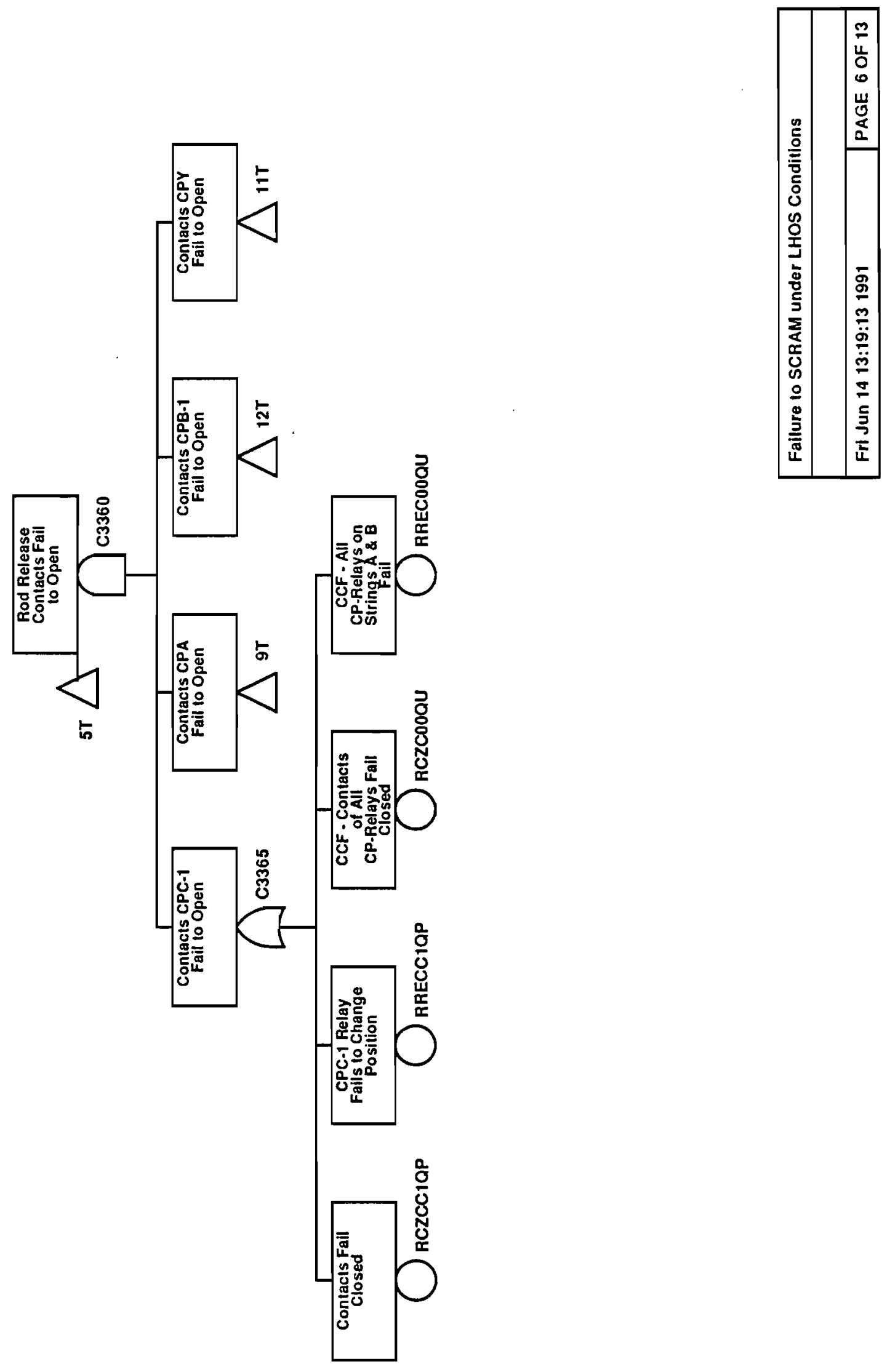

B. 2-211 


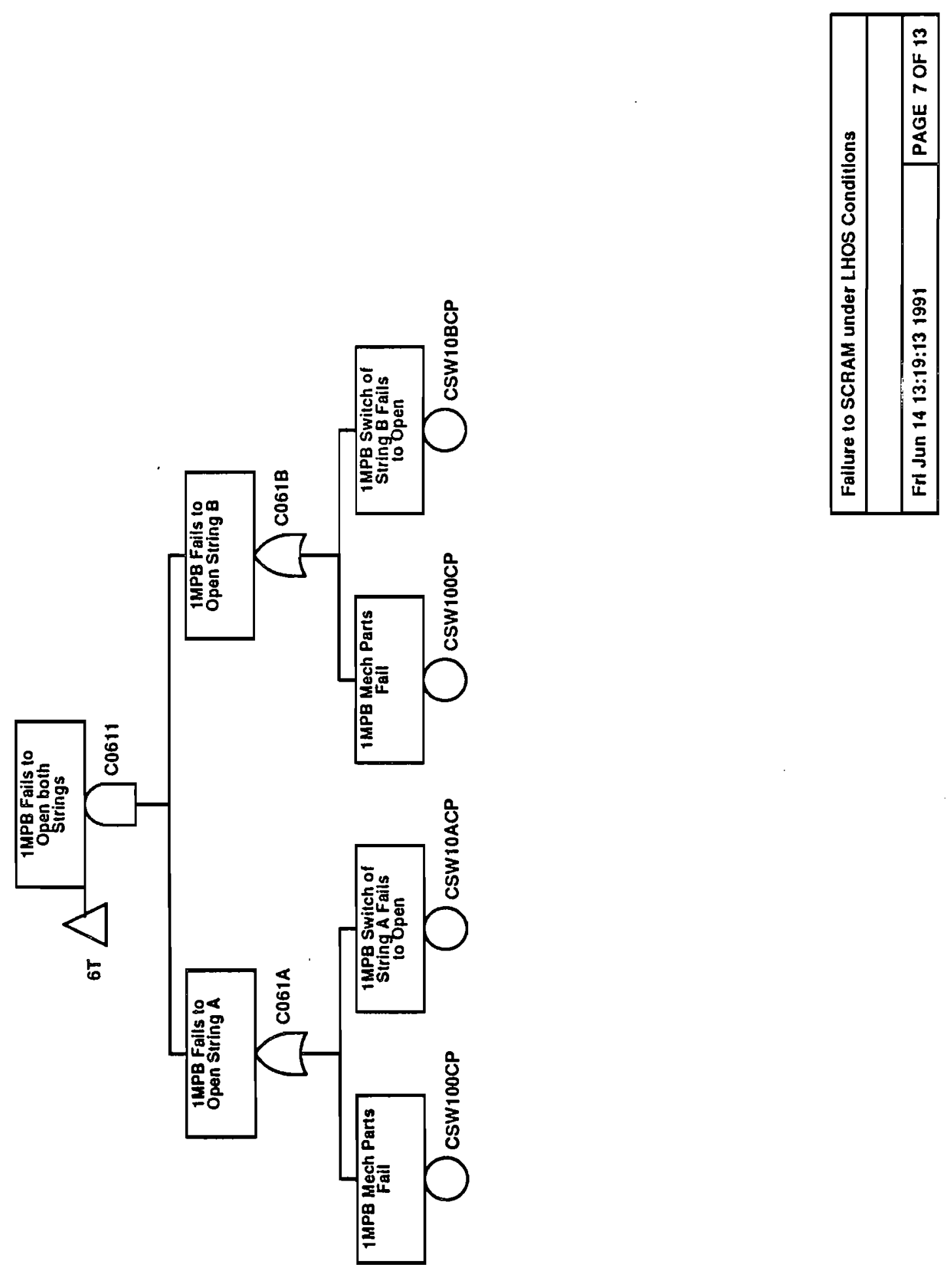




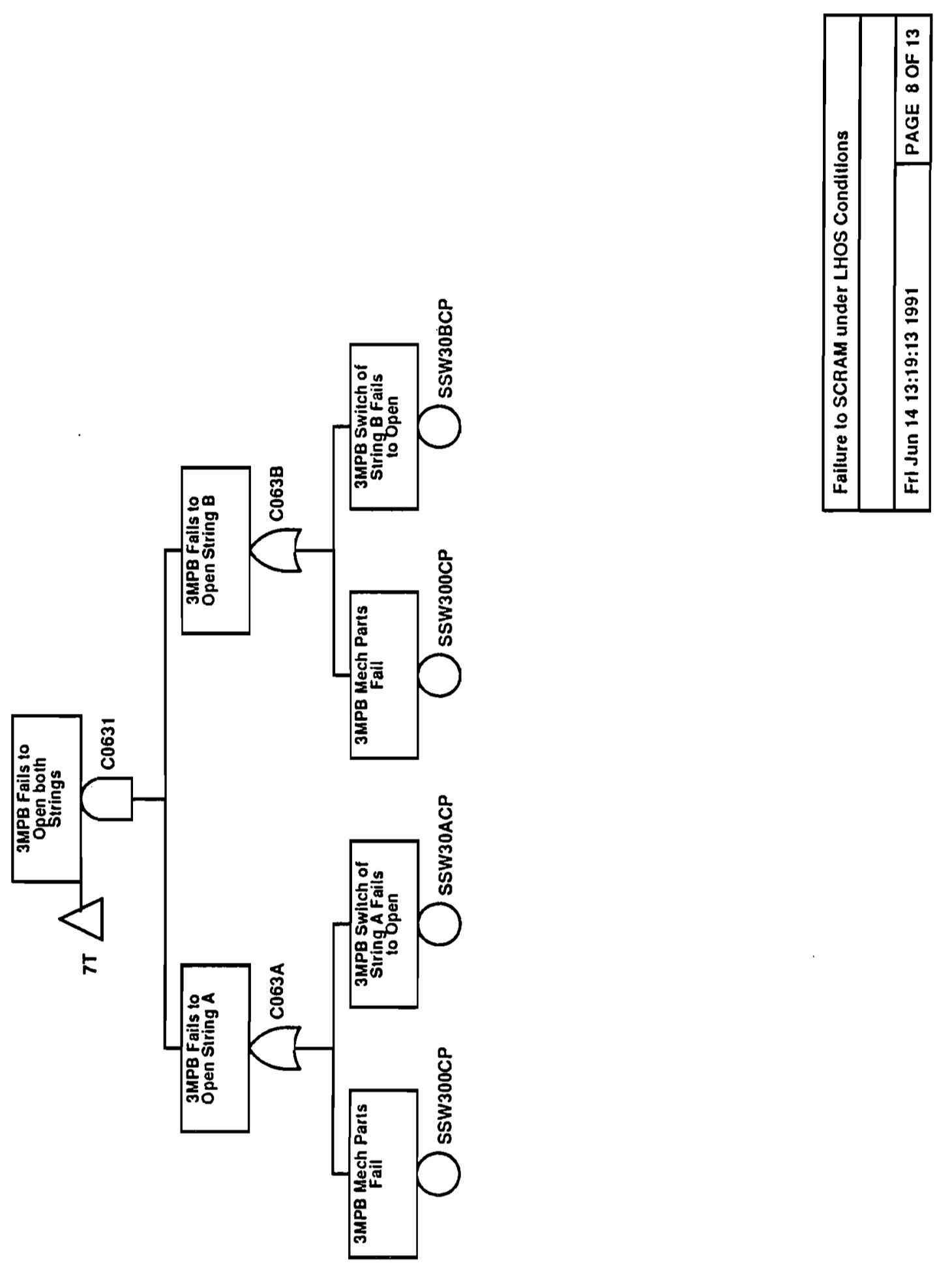




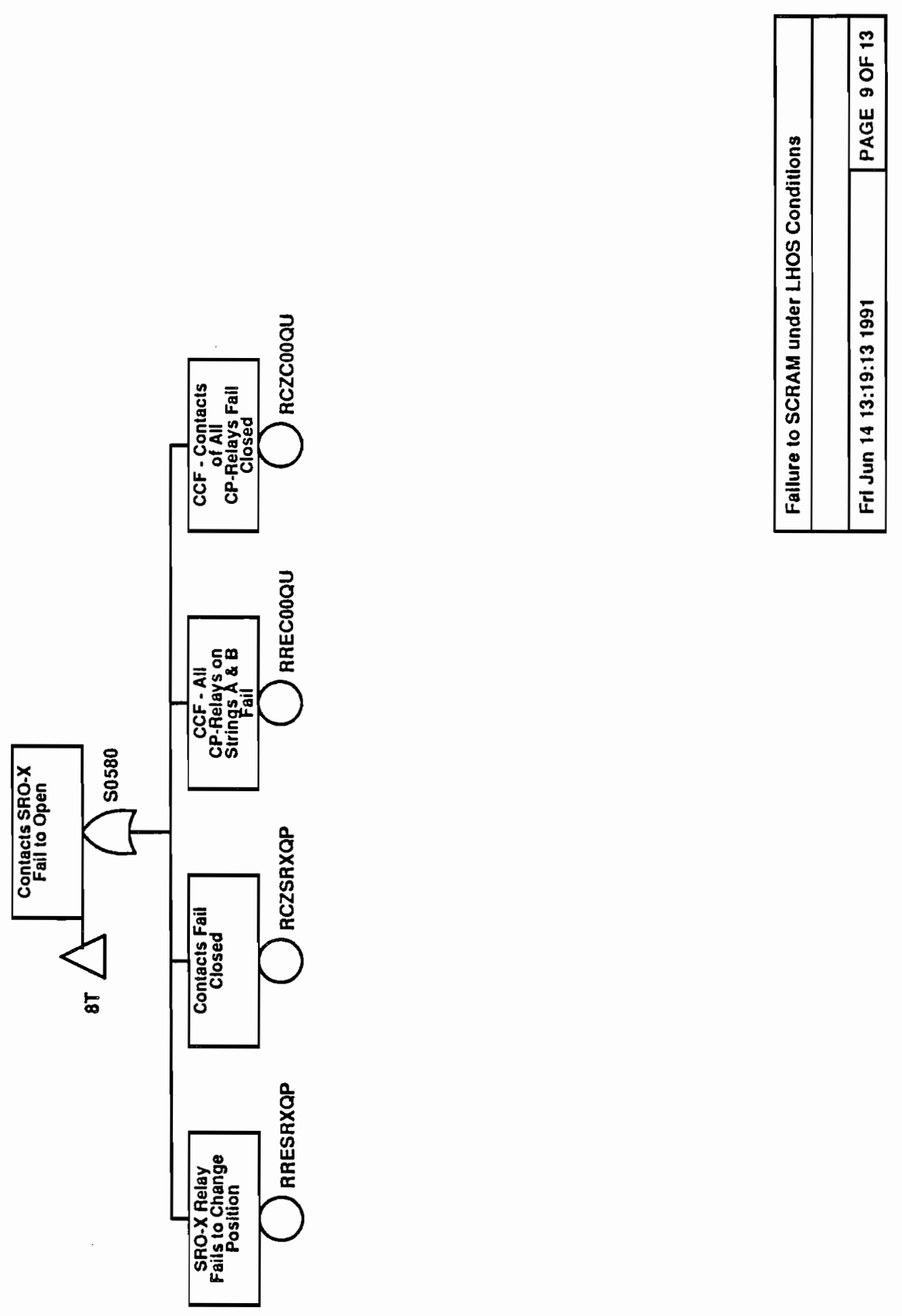

B. 2-214 


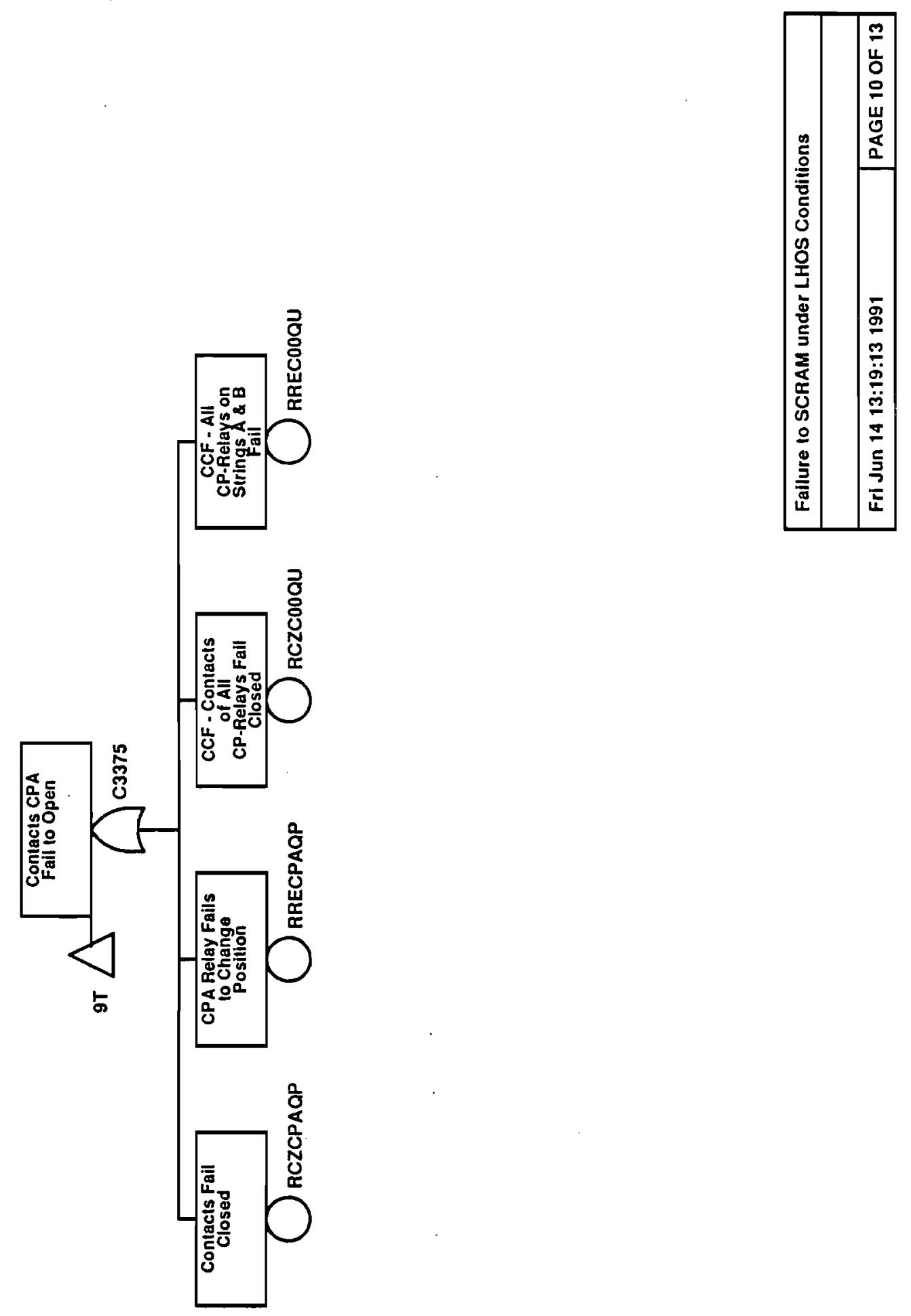




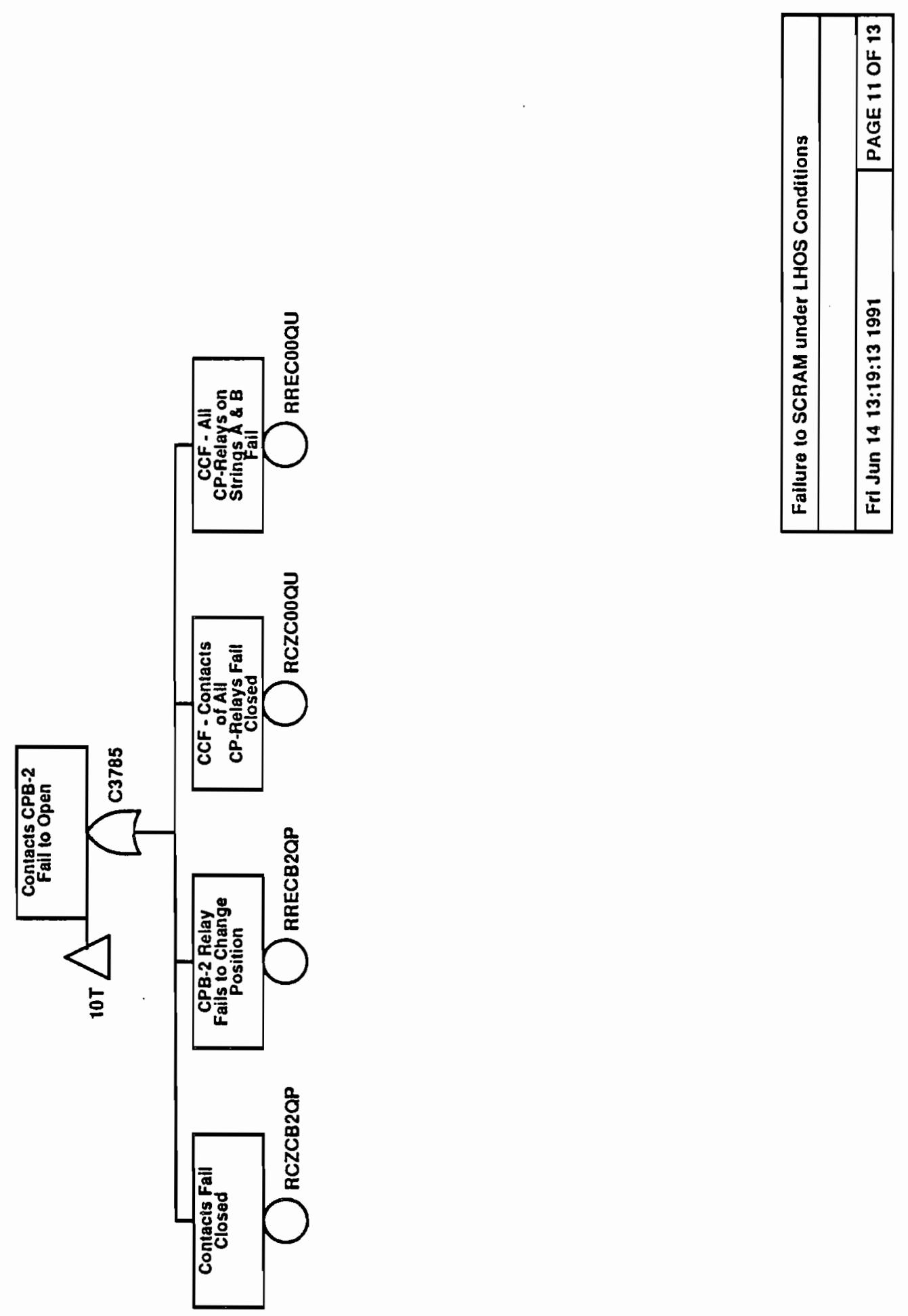

B. 2-216 


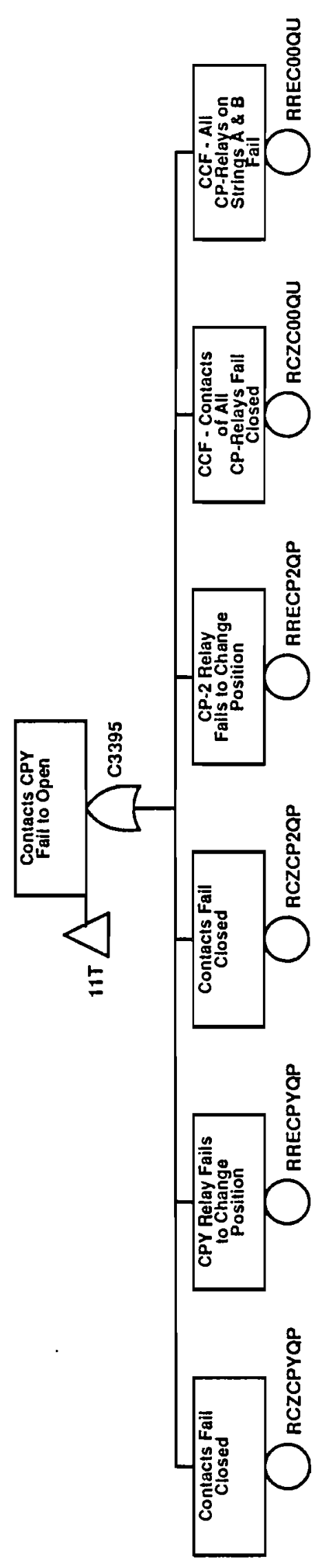

B. $2-217$ 


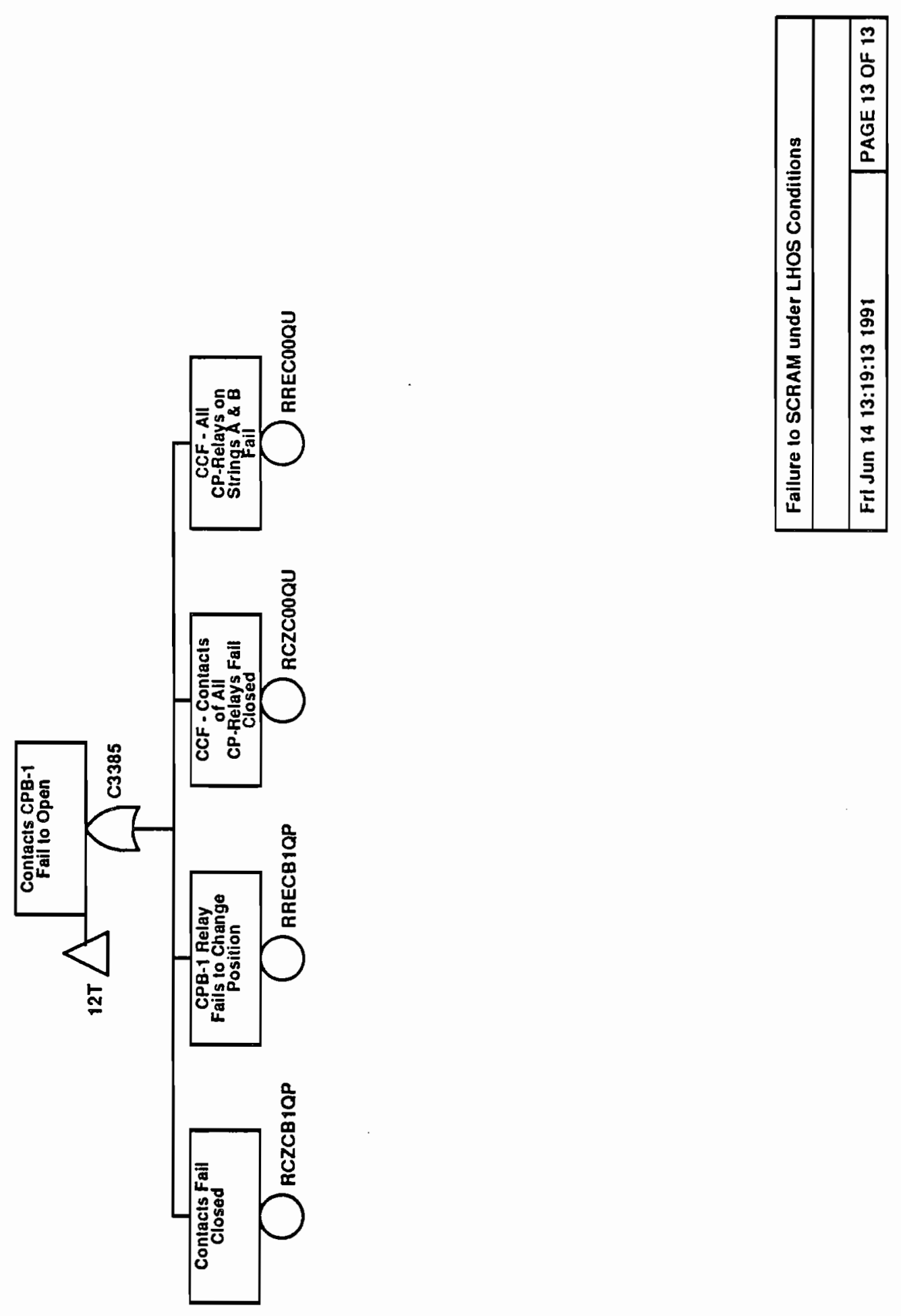




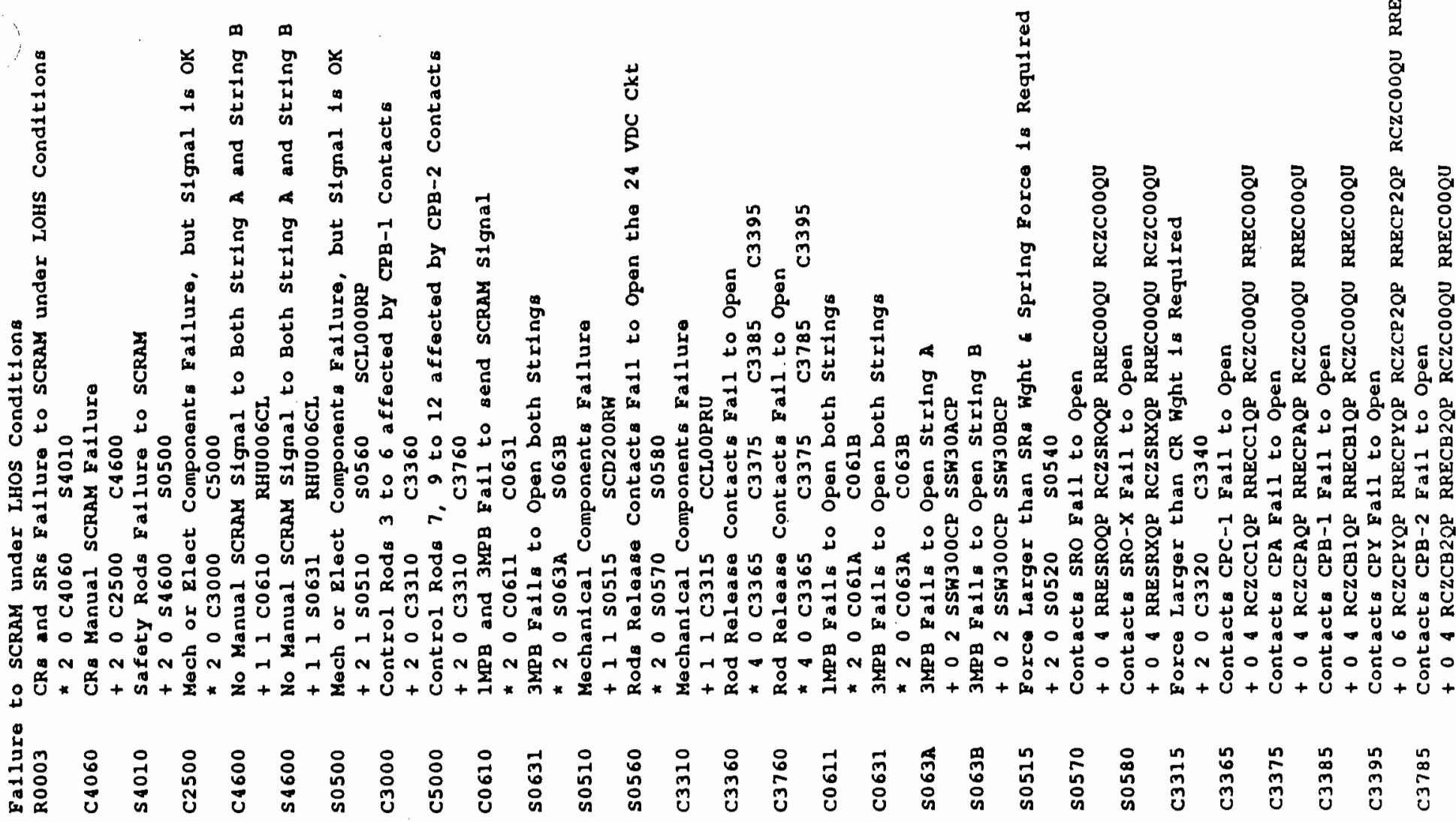




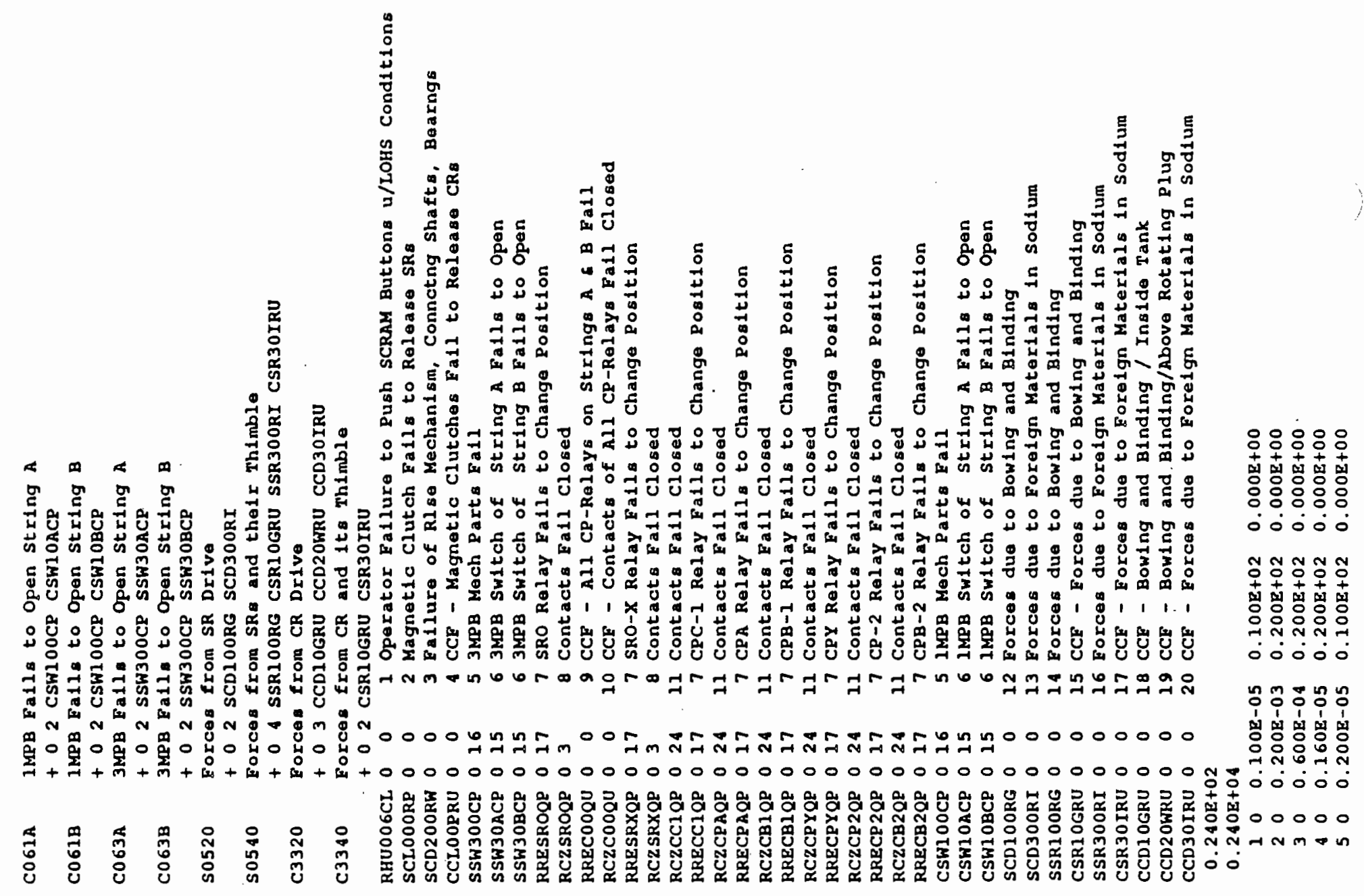




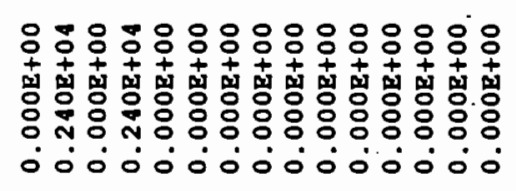

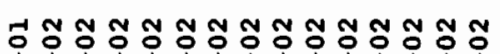

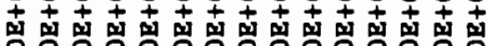

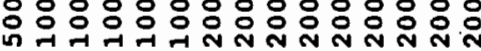
0000000000000

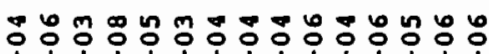

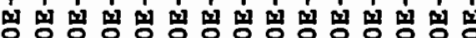

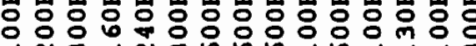

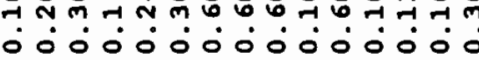
o no no 0000000000

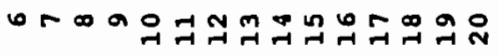




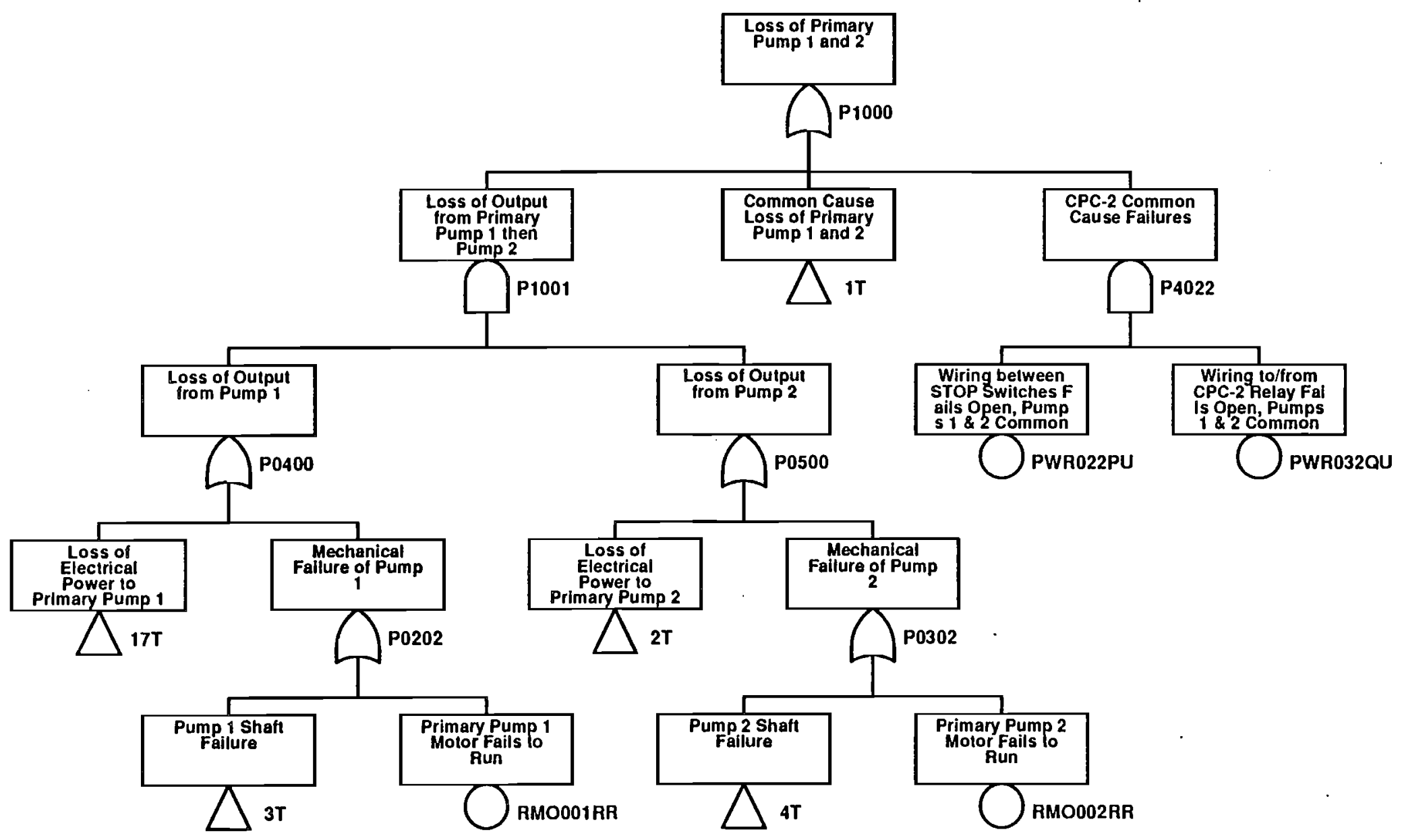

\begin{tabular}{|c|c|}
\hline \multicolumn{2}{|c|}{ Loss of Oulput Both Primary Pumps in a 1} \\
\hline hour window & \\
\hline Frl Jun 14 13:45:17 1991 & PAGE 1 OF 18 \\
\hline
\end{tabular}



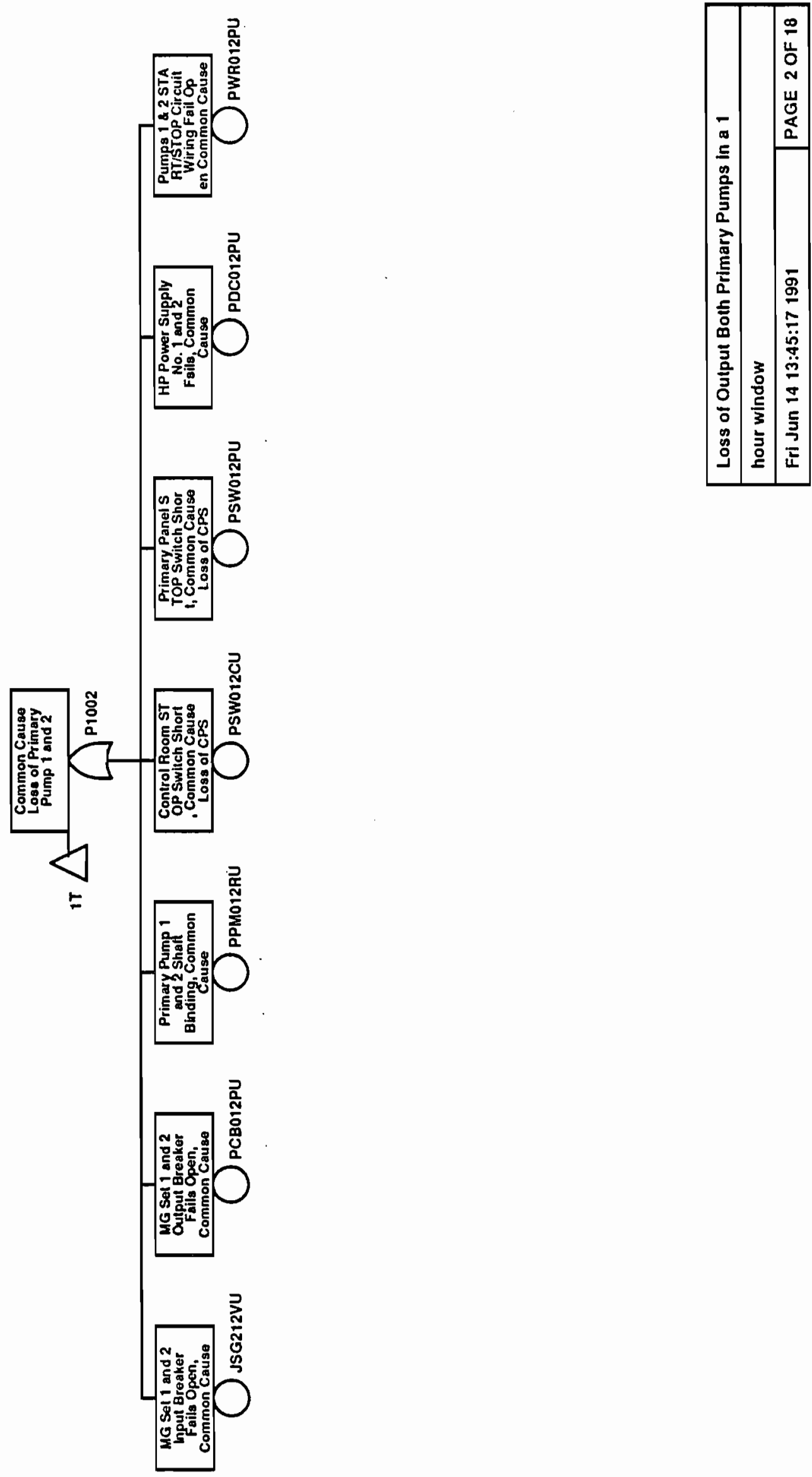

B. 2-223 


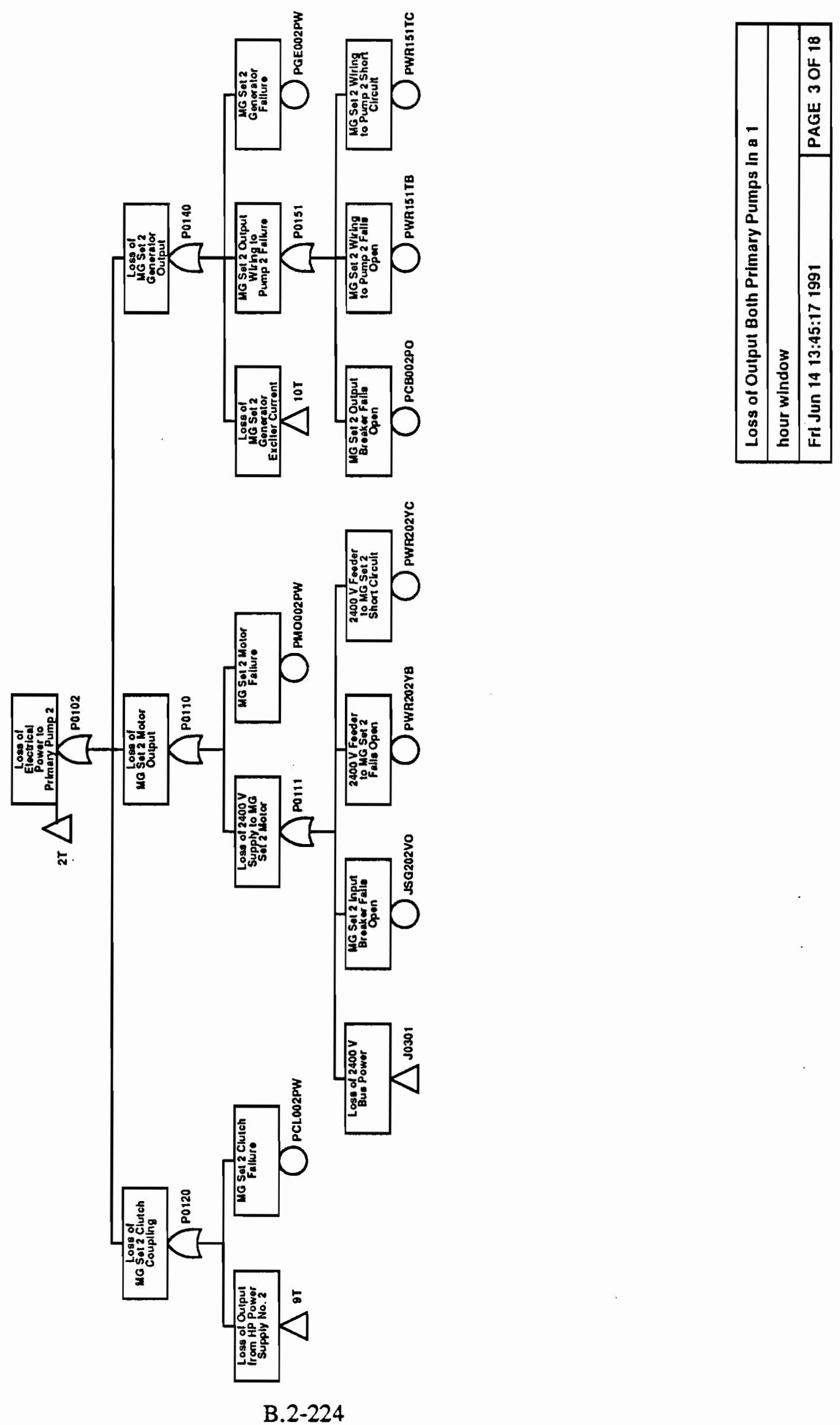



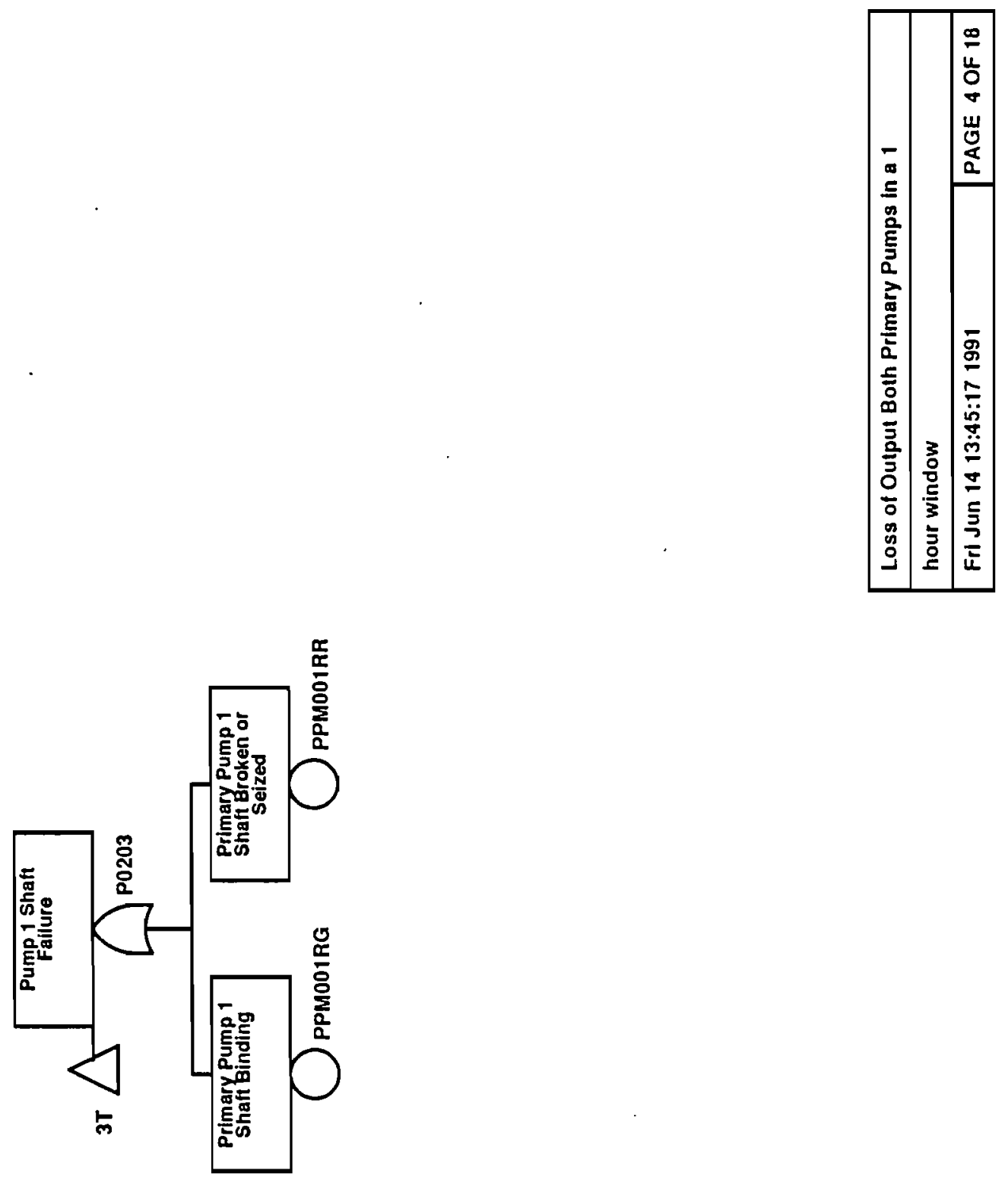


\begin{tabular}{|c|c|c|}
\hline$\overline{-}$ & & 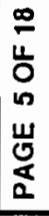 \\
\hline 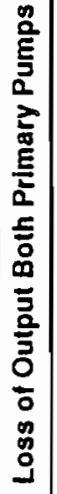 & 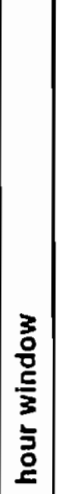 & 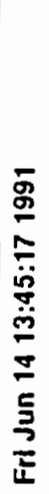 \\
\hline
\end{tabular}

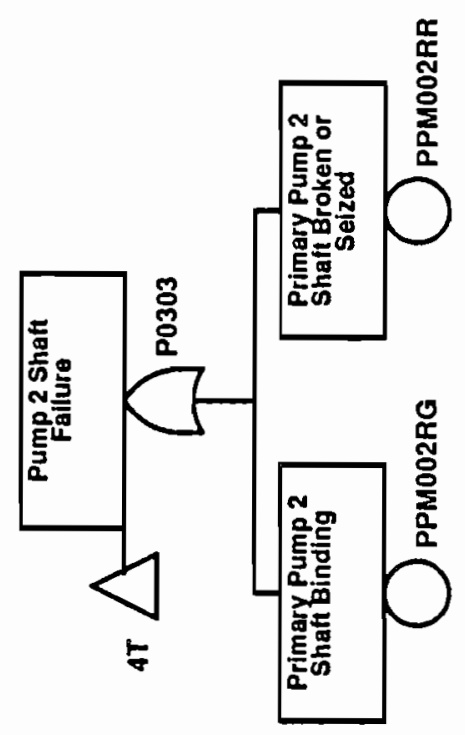




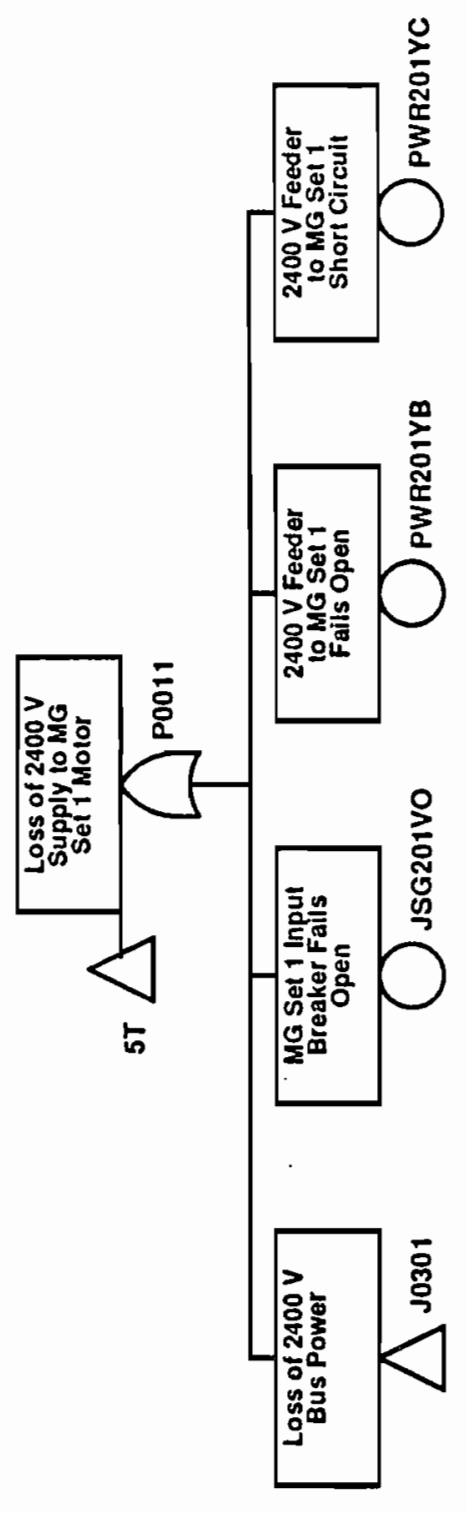

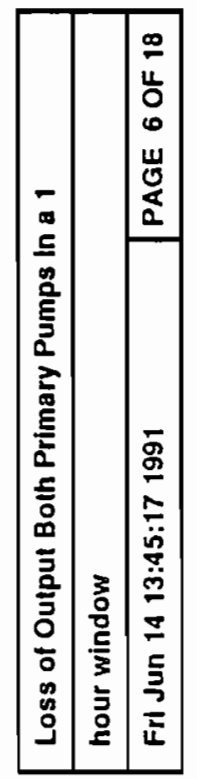




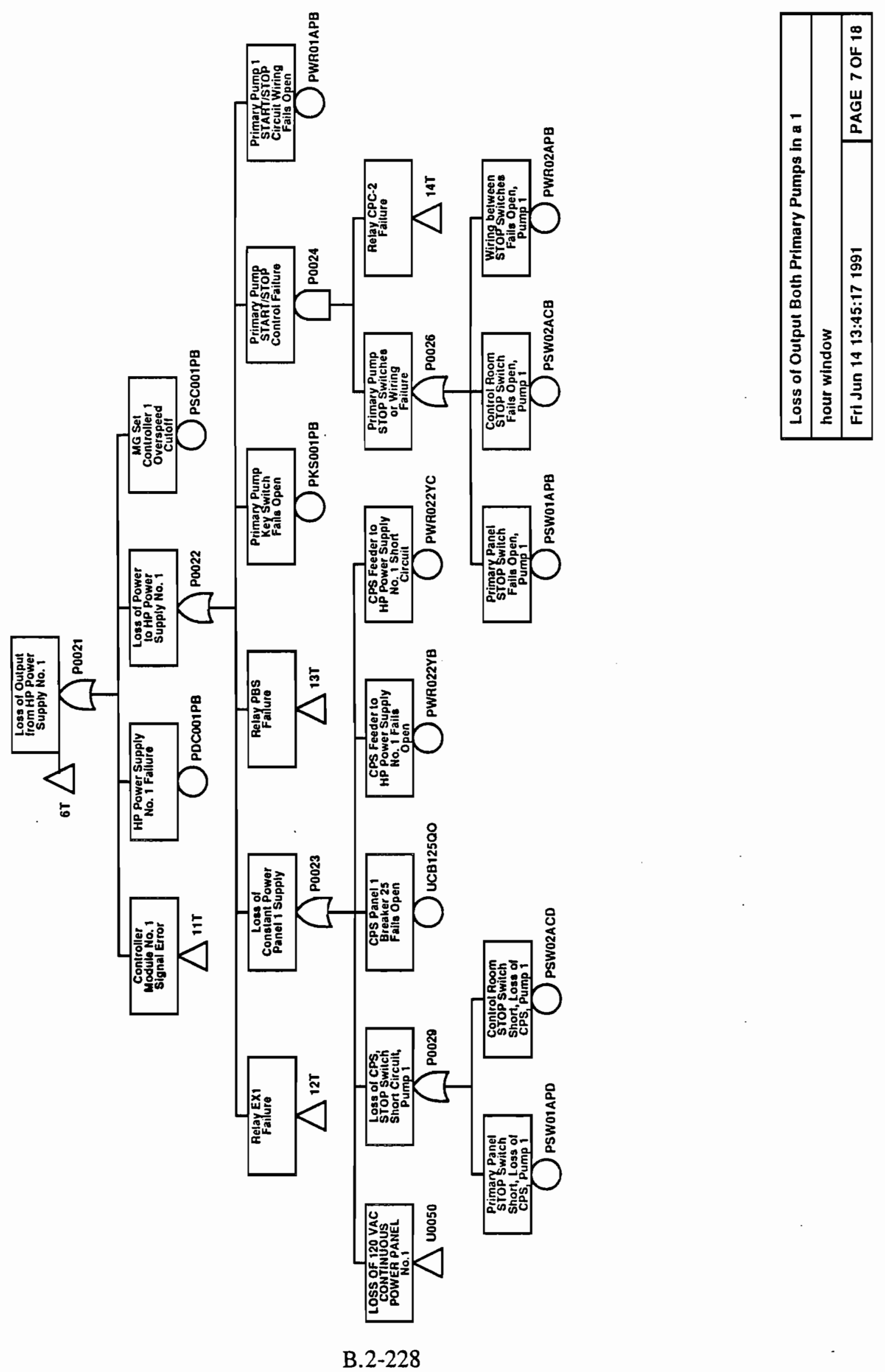



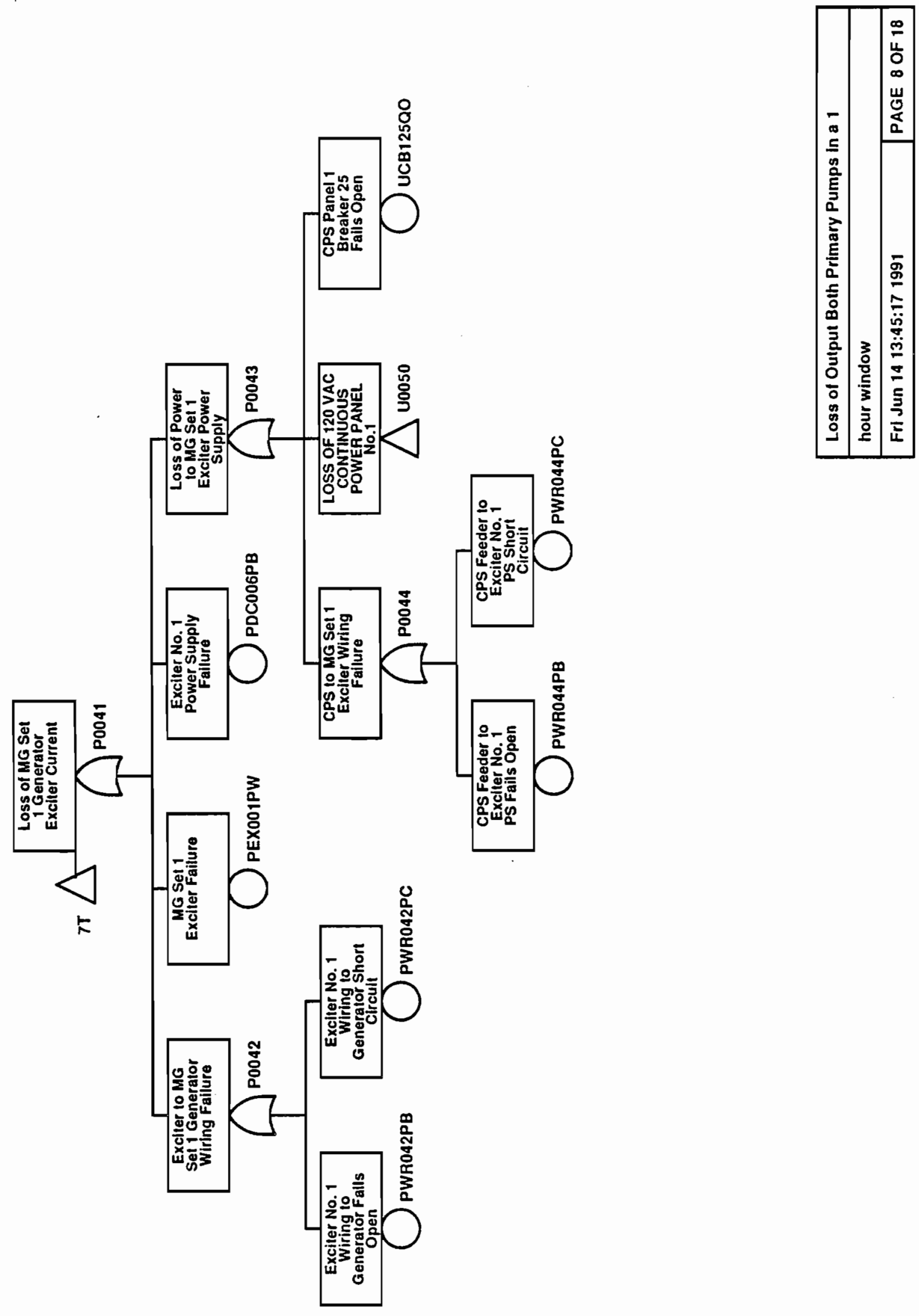


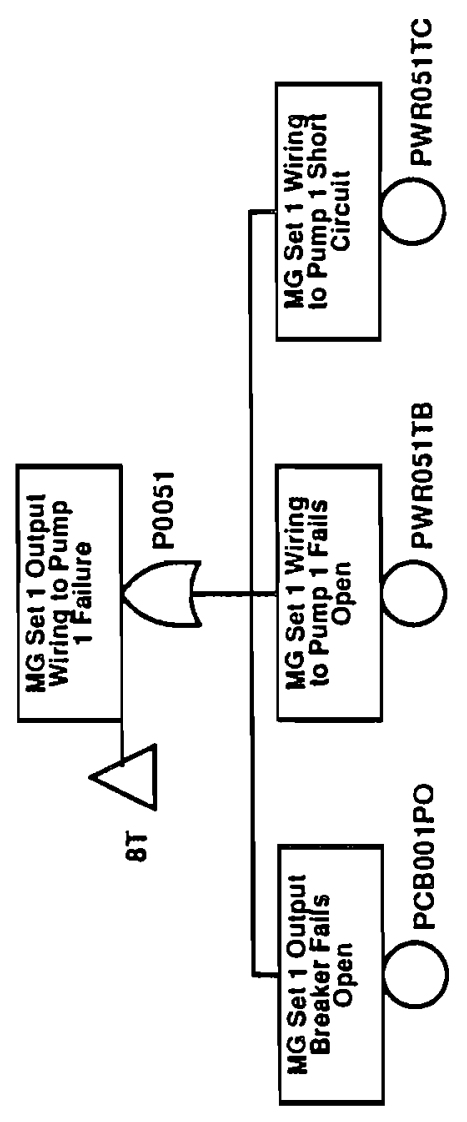

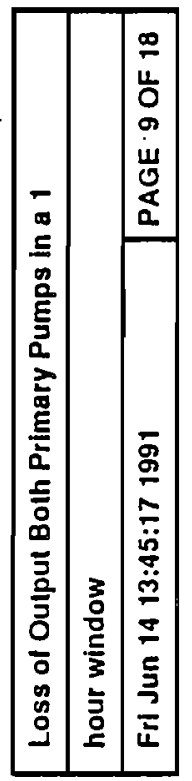



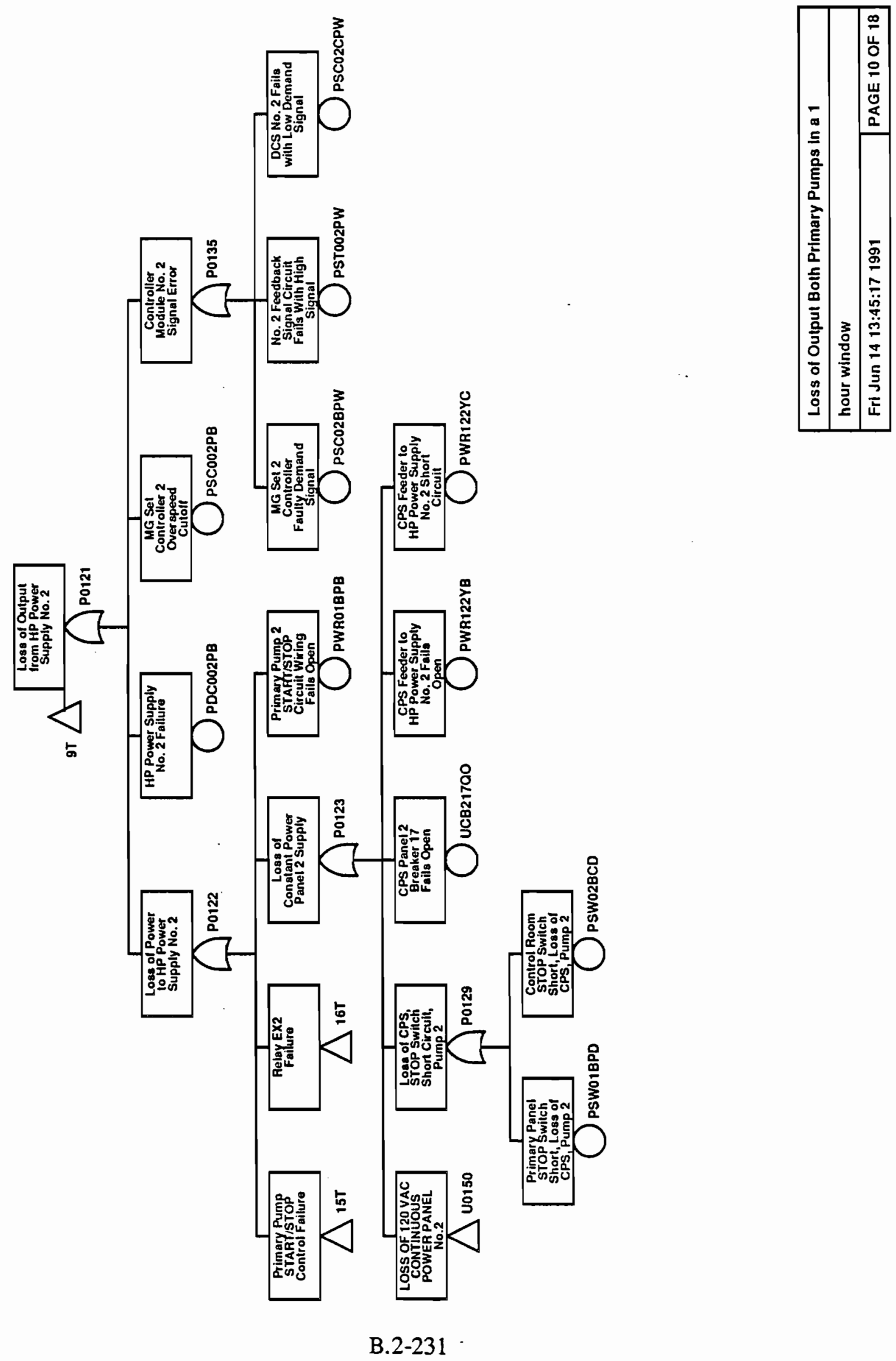


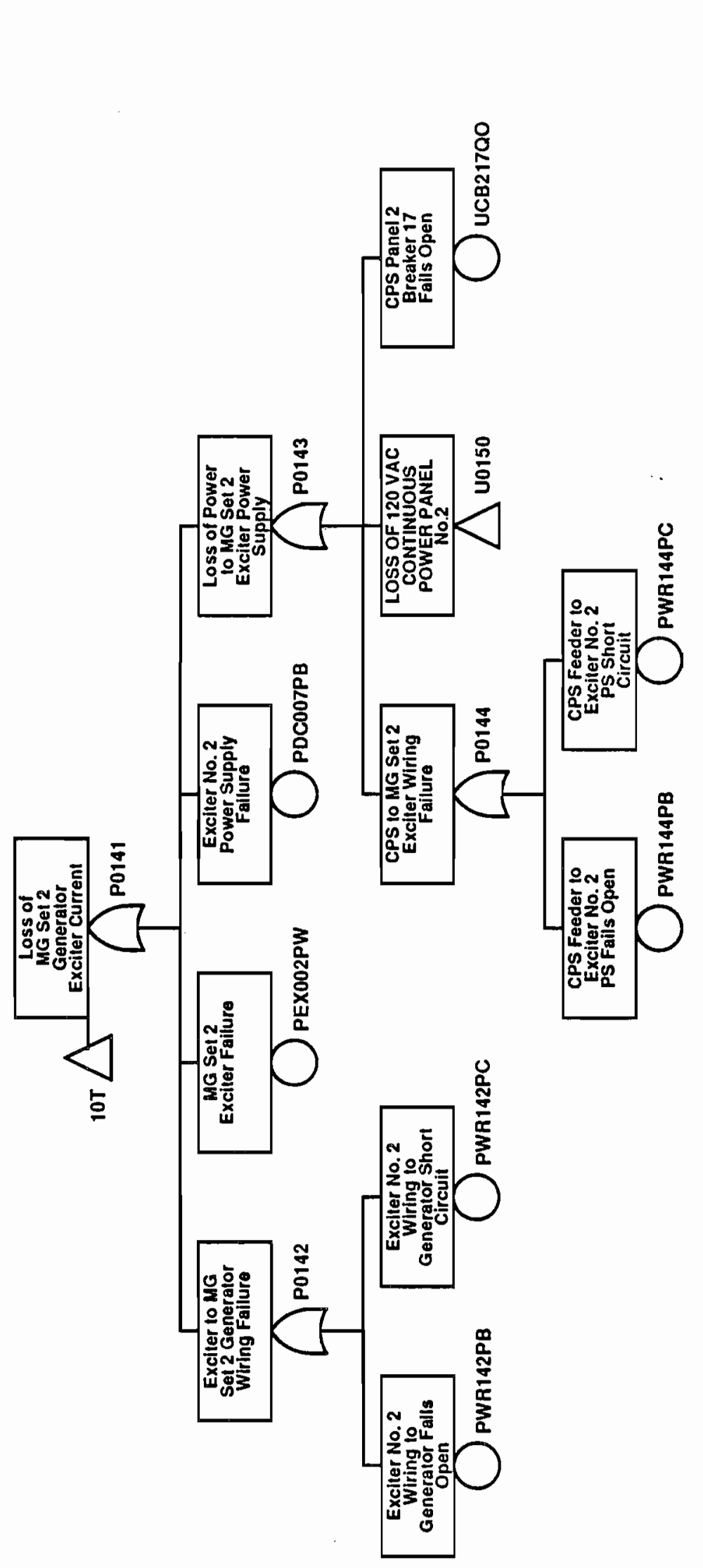

\begin{tabular}{|c|c|c|}
\hline $\bar{D}$ & & 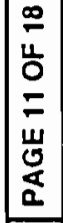 \\
\hline 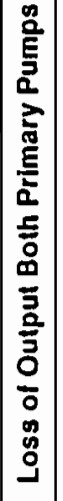 & \begin{tabular}{|c|} 
\\
\\
\\
3 \\
0 \\
0 \\
$\frac{3}{3}$ \\
3 \\
0 \\
0
\end{tabular} & 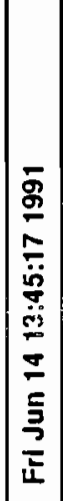 \\
\hline
\end{tabular}



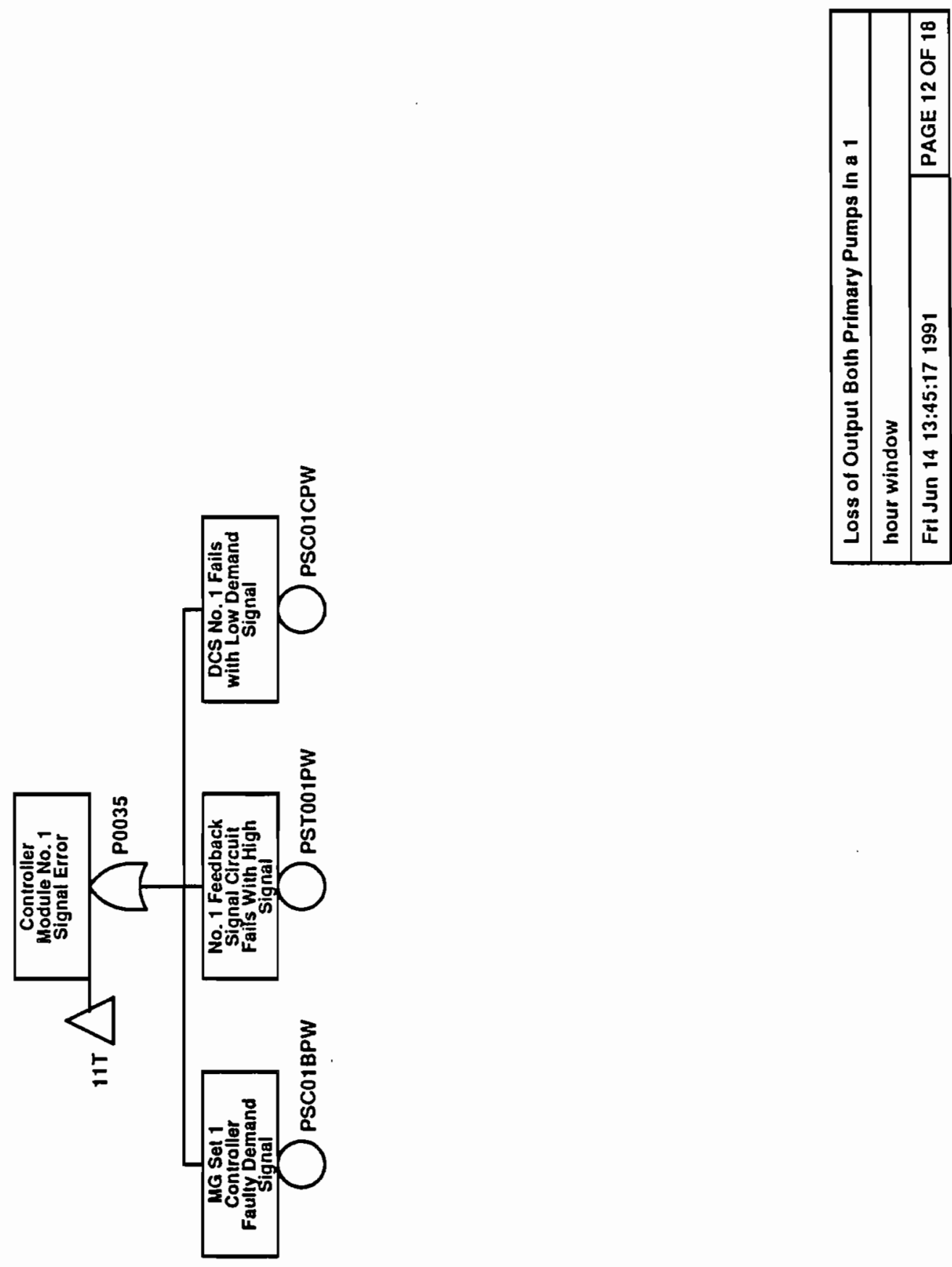


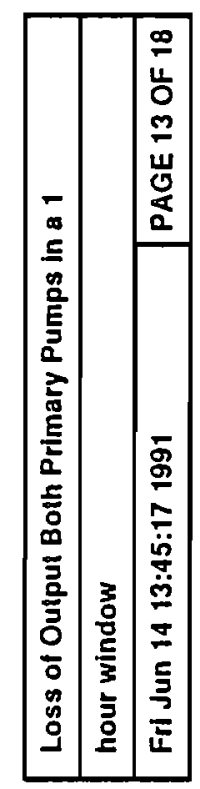

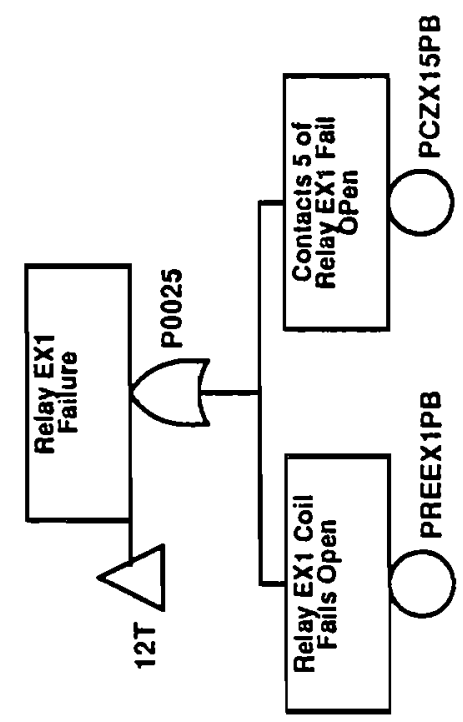




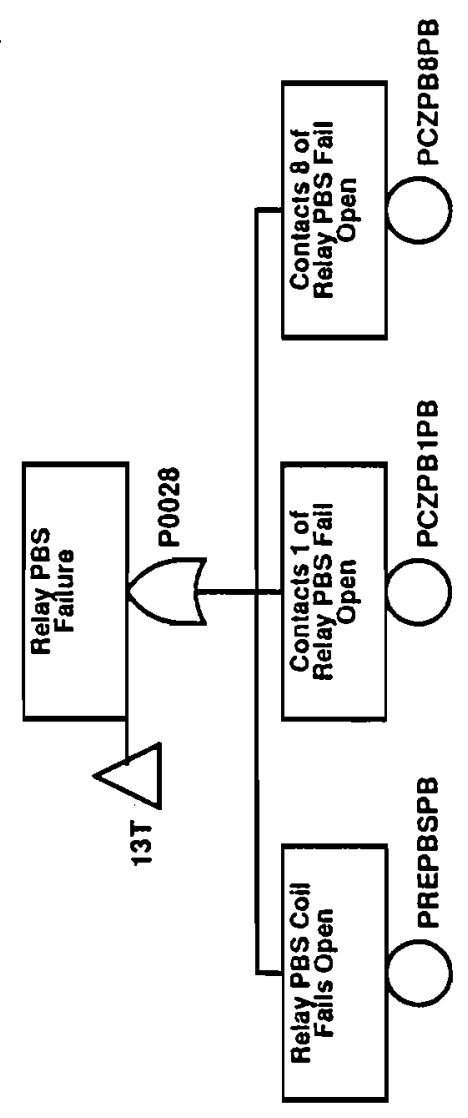

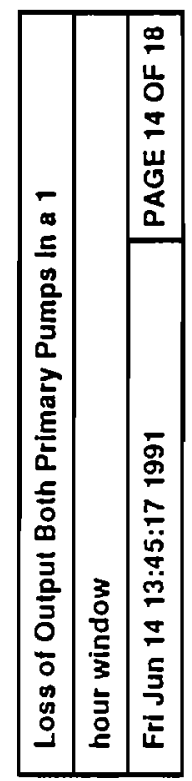




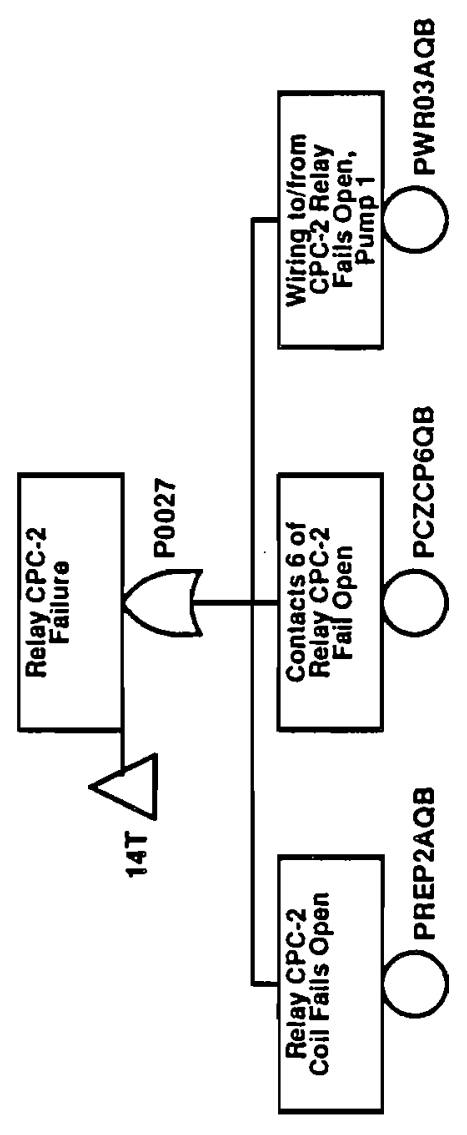




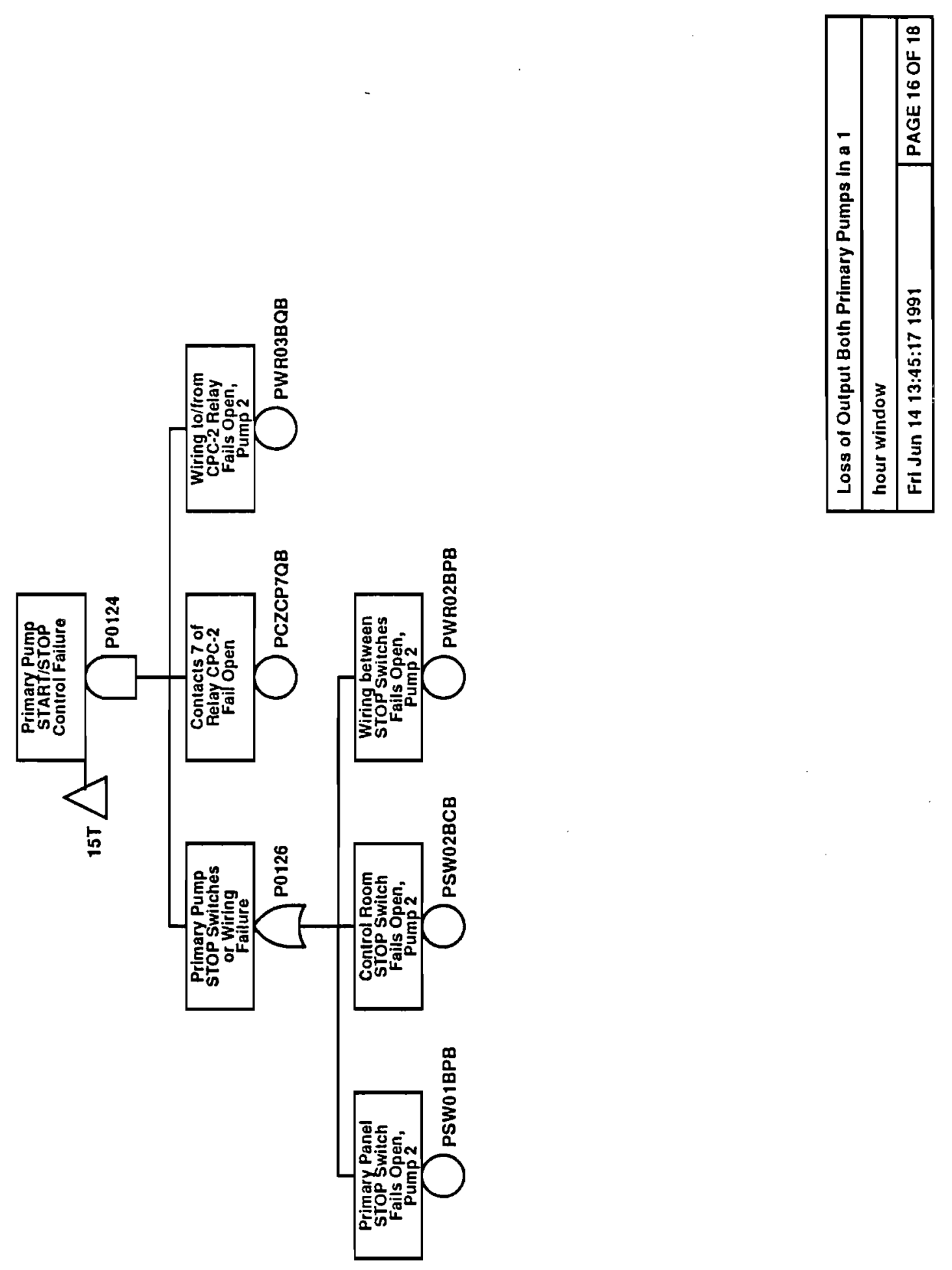




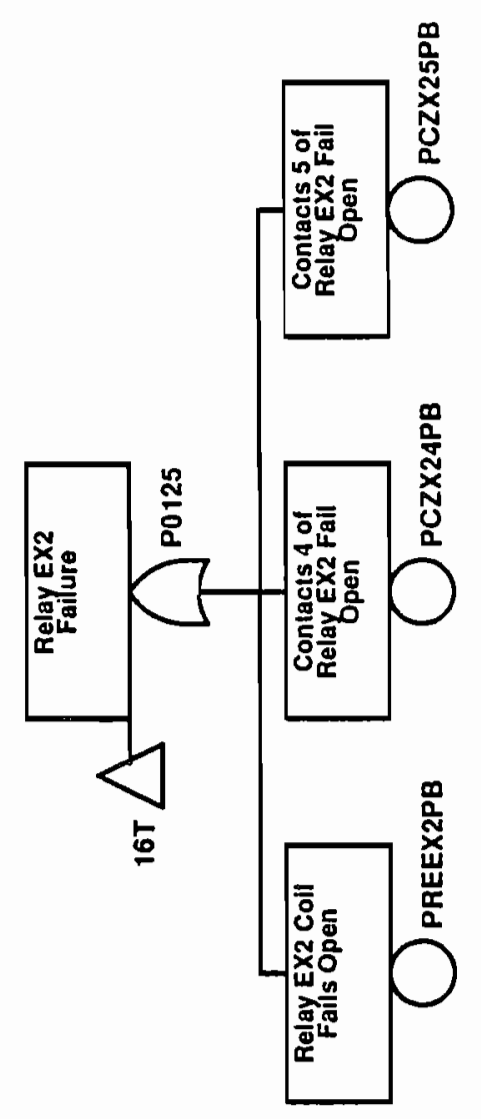

I 


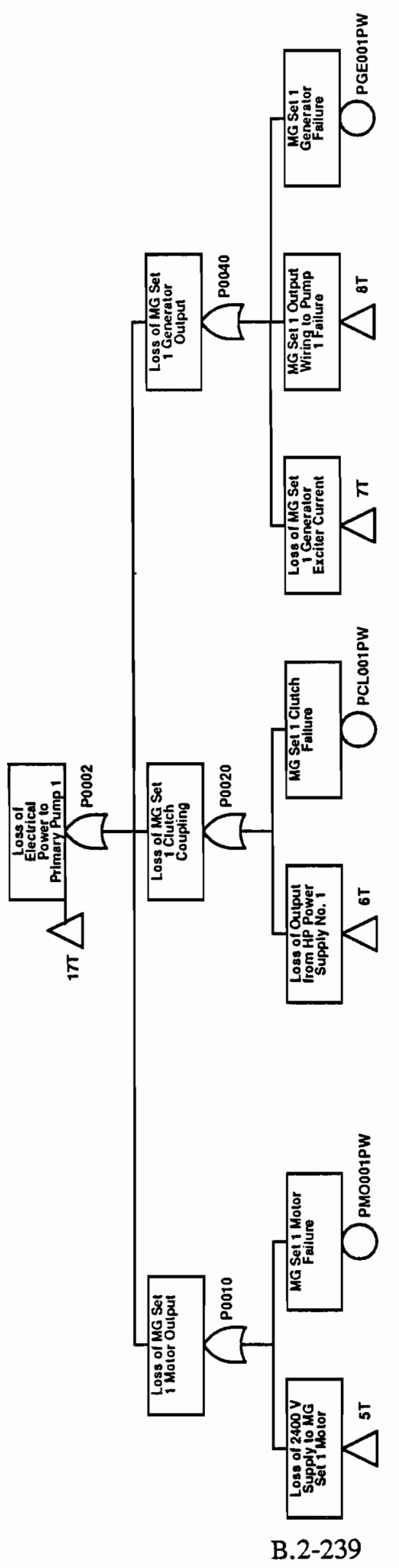




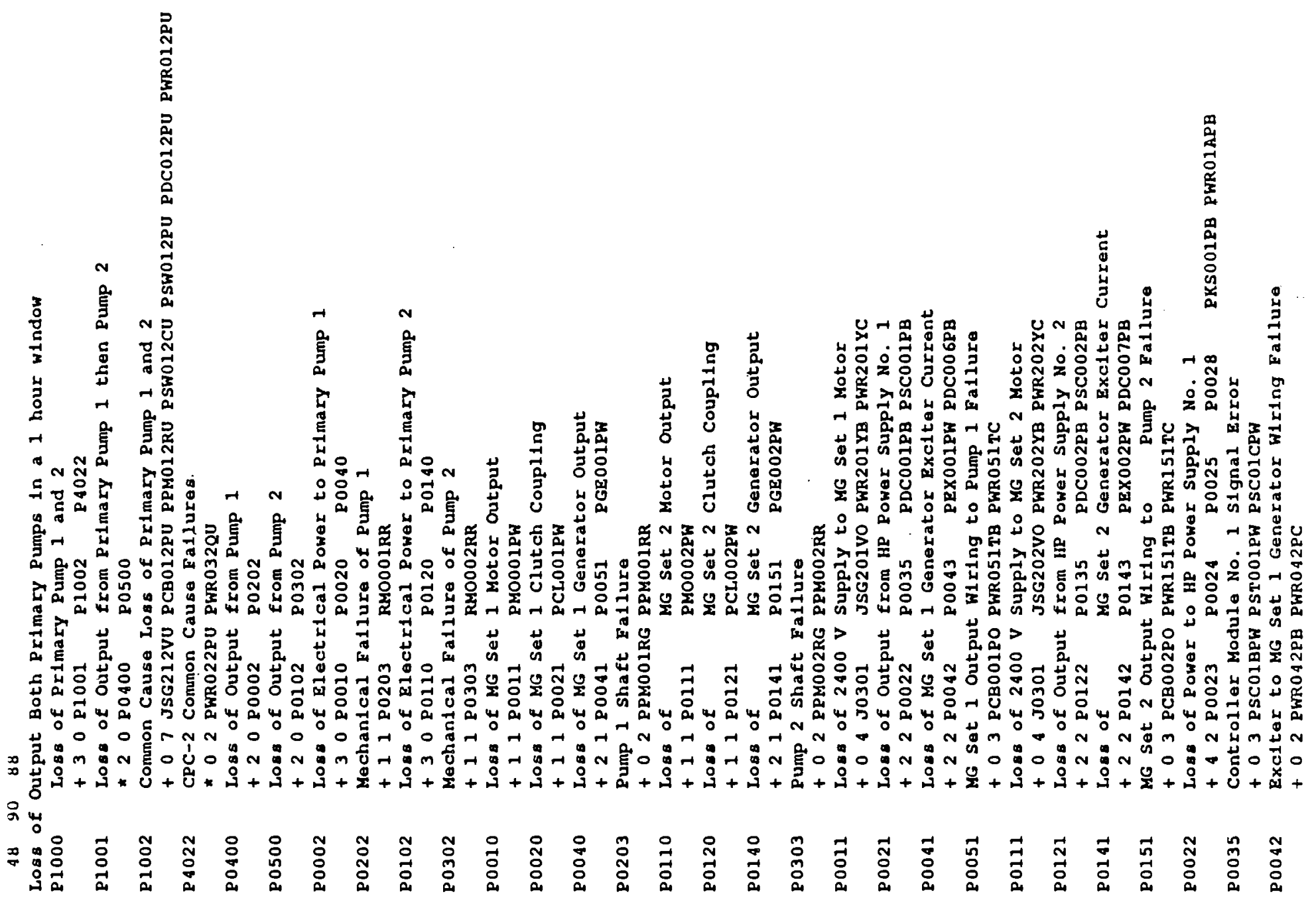




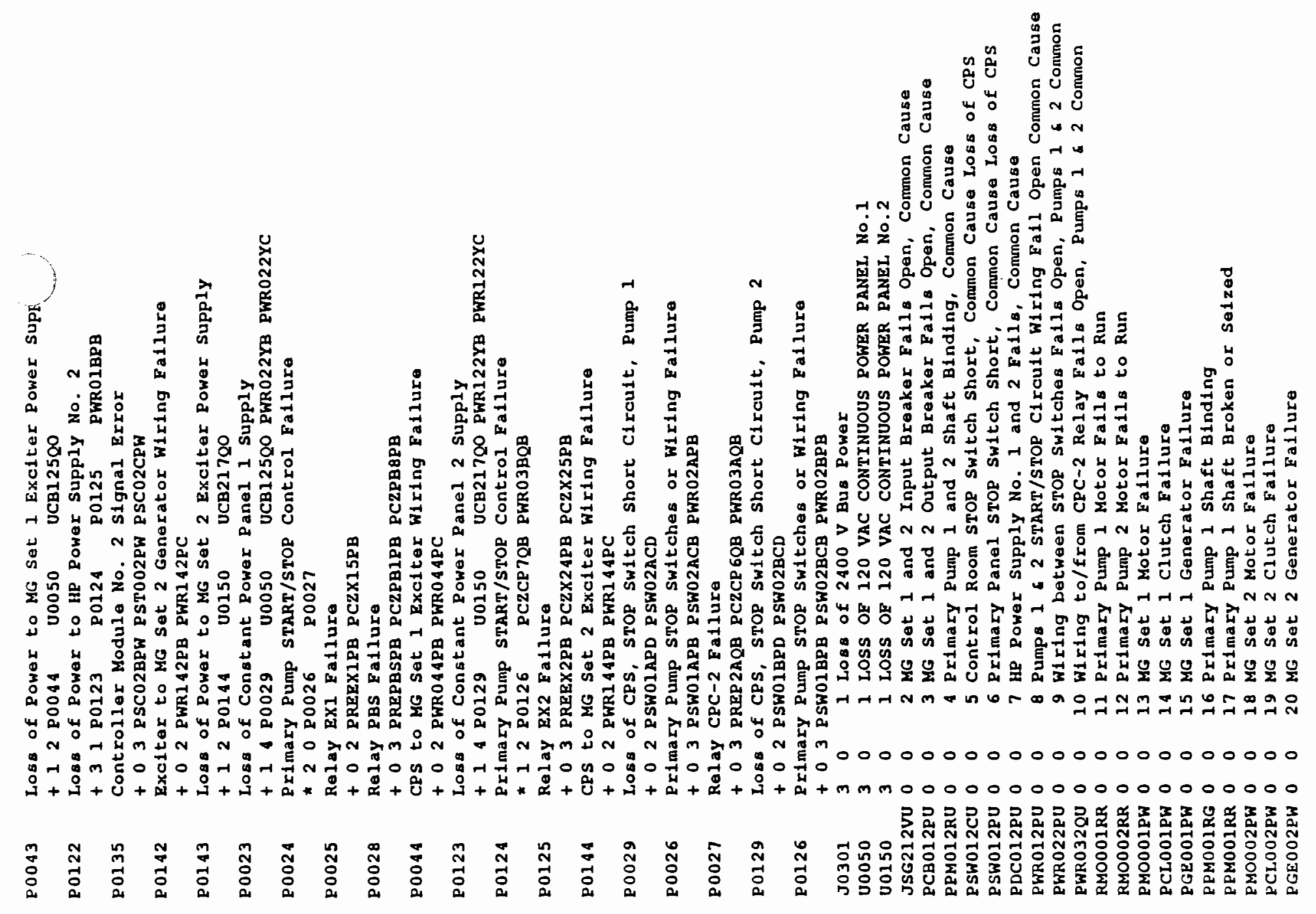




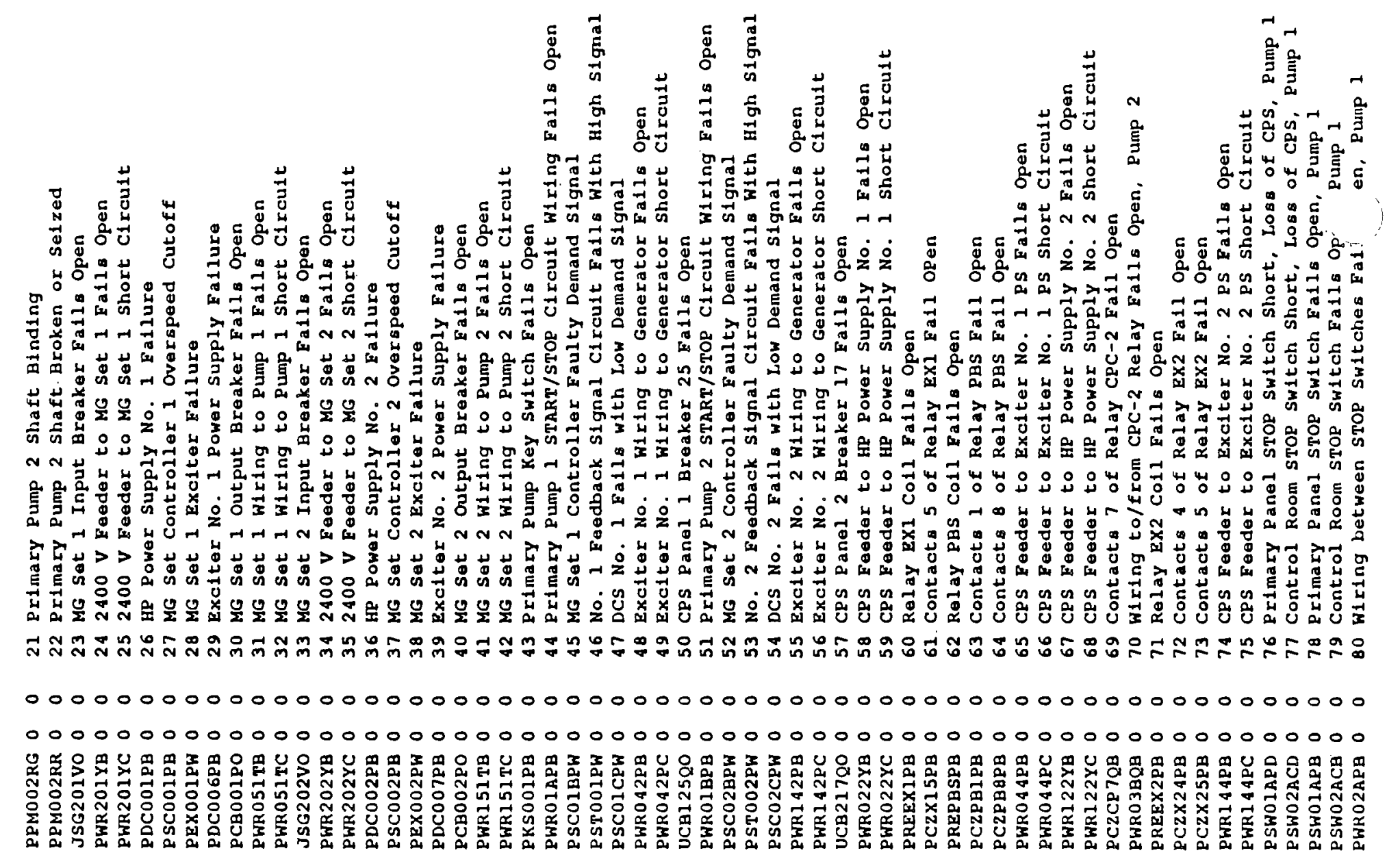




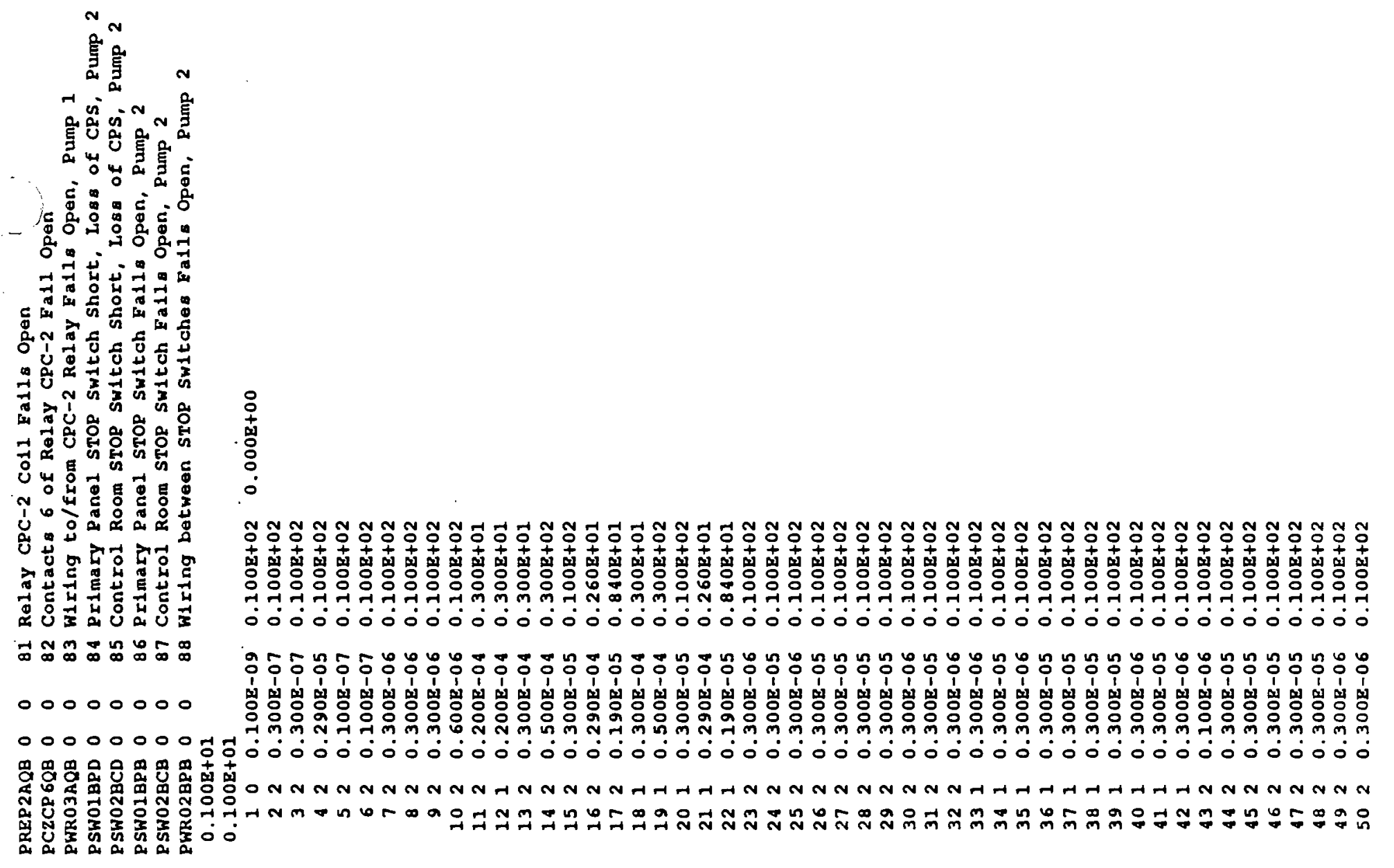




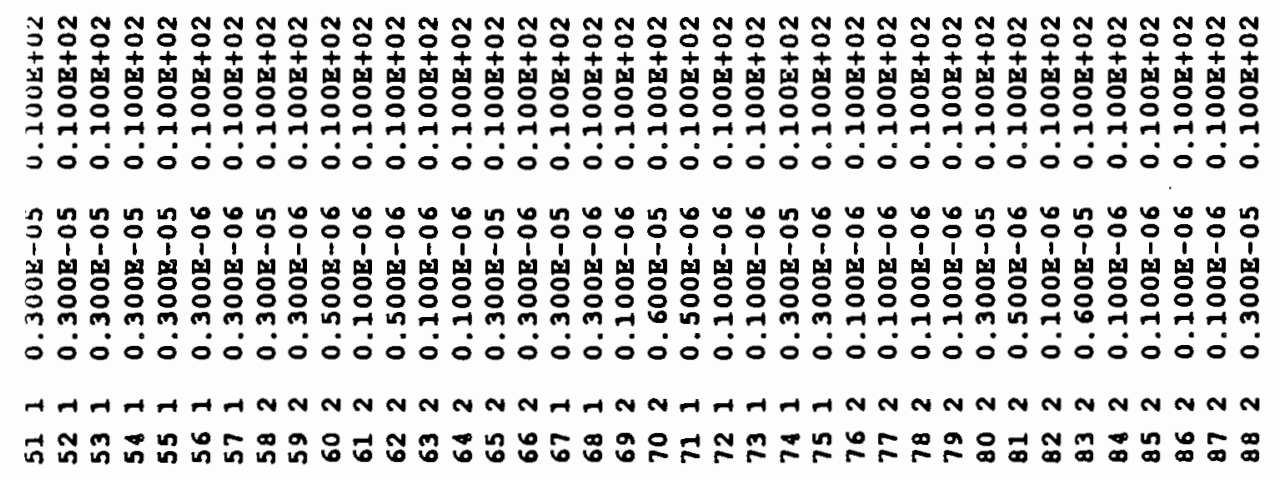




\begin{tabular}{|c|c|}
\hline & 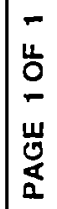 \\
\hline 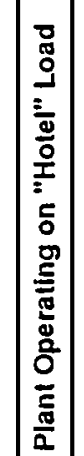 & 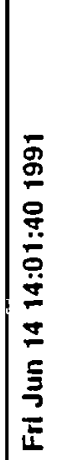 \\
\hline
\end{tabular}

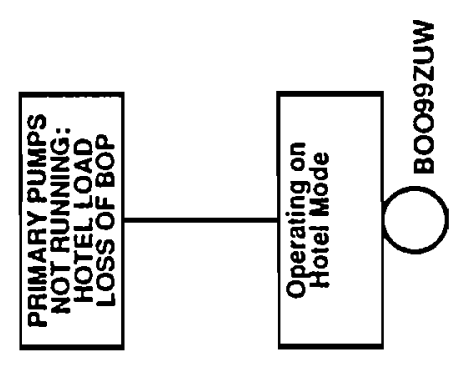




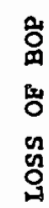

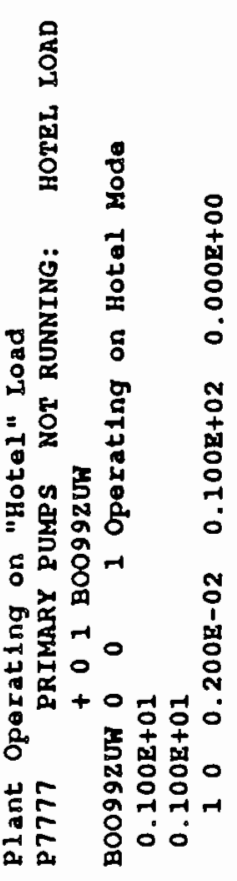

B. 2-246 

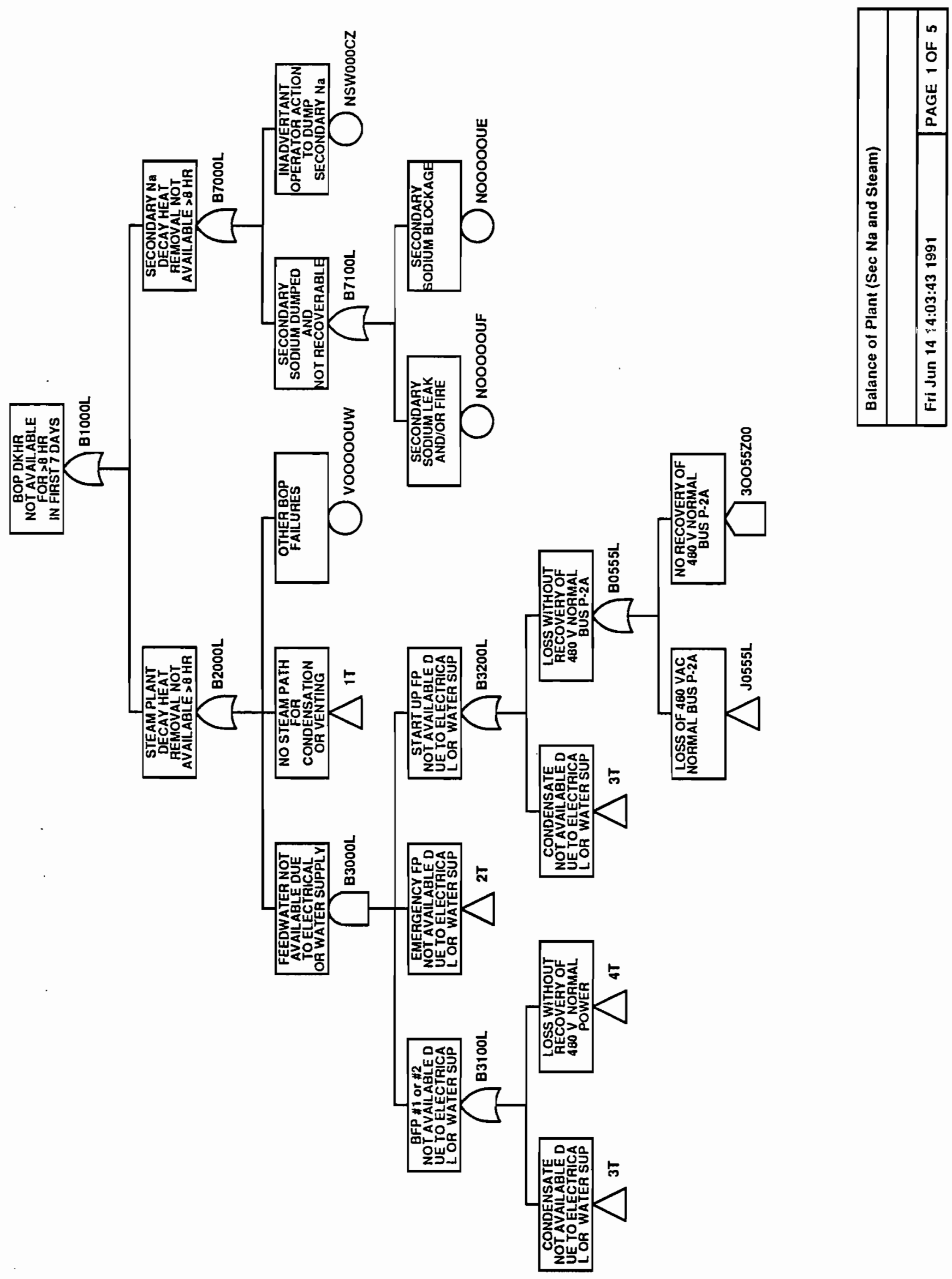


\begin{tabular}{|c|c|}
\hline & 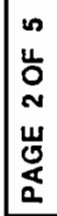 \\
\hline 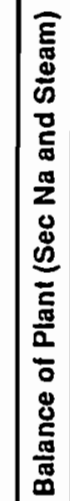 & 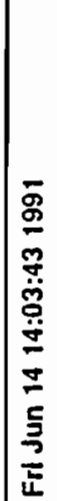 \\
\hline
\end{tabular}

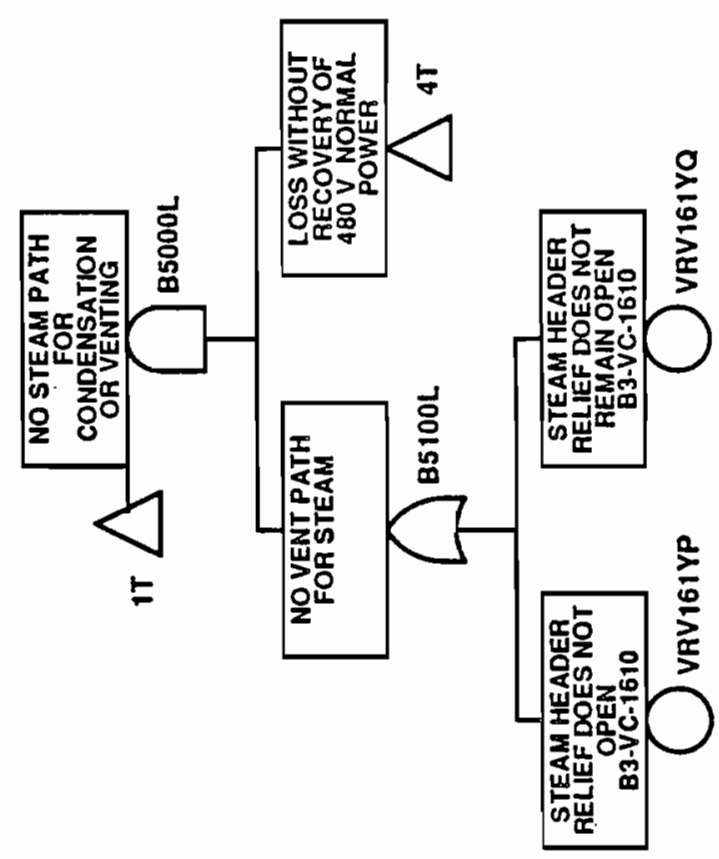




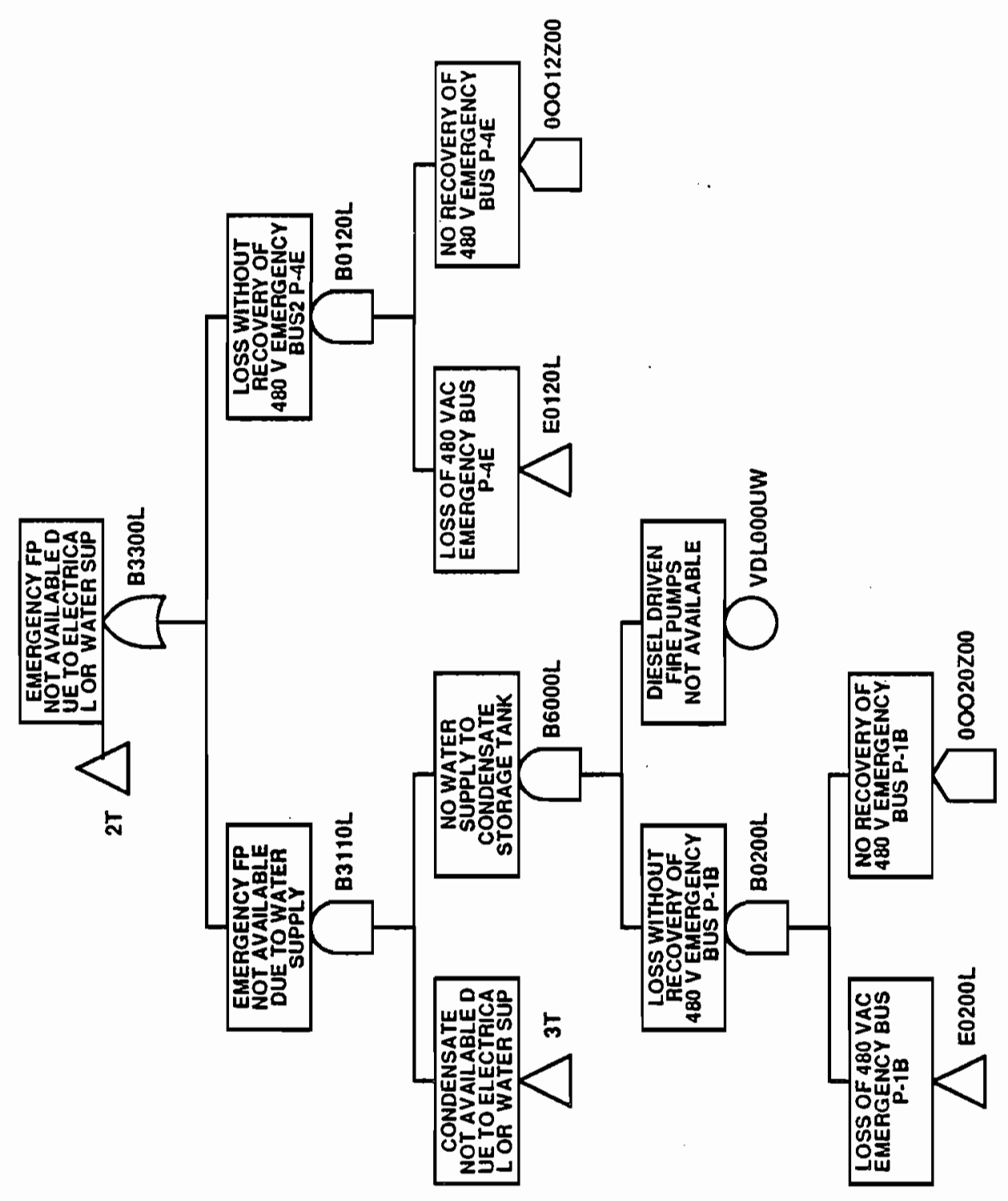

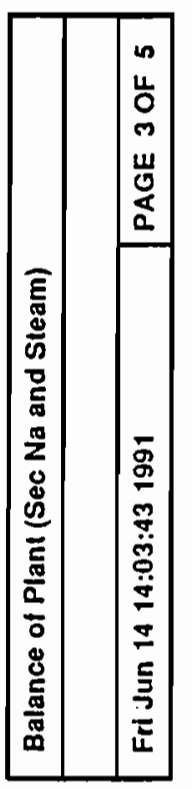




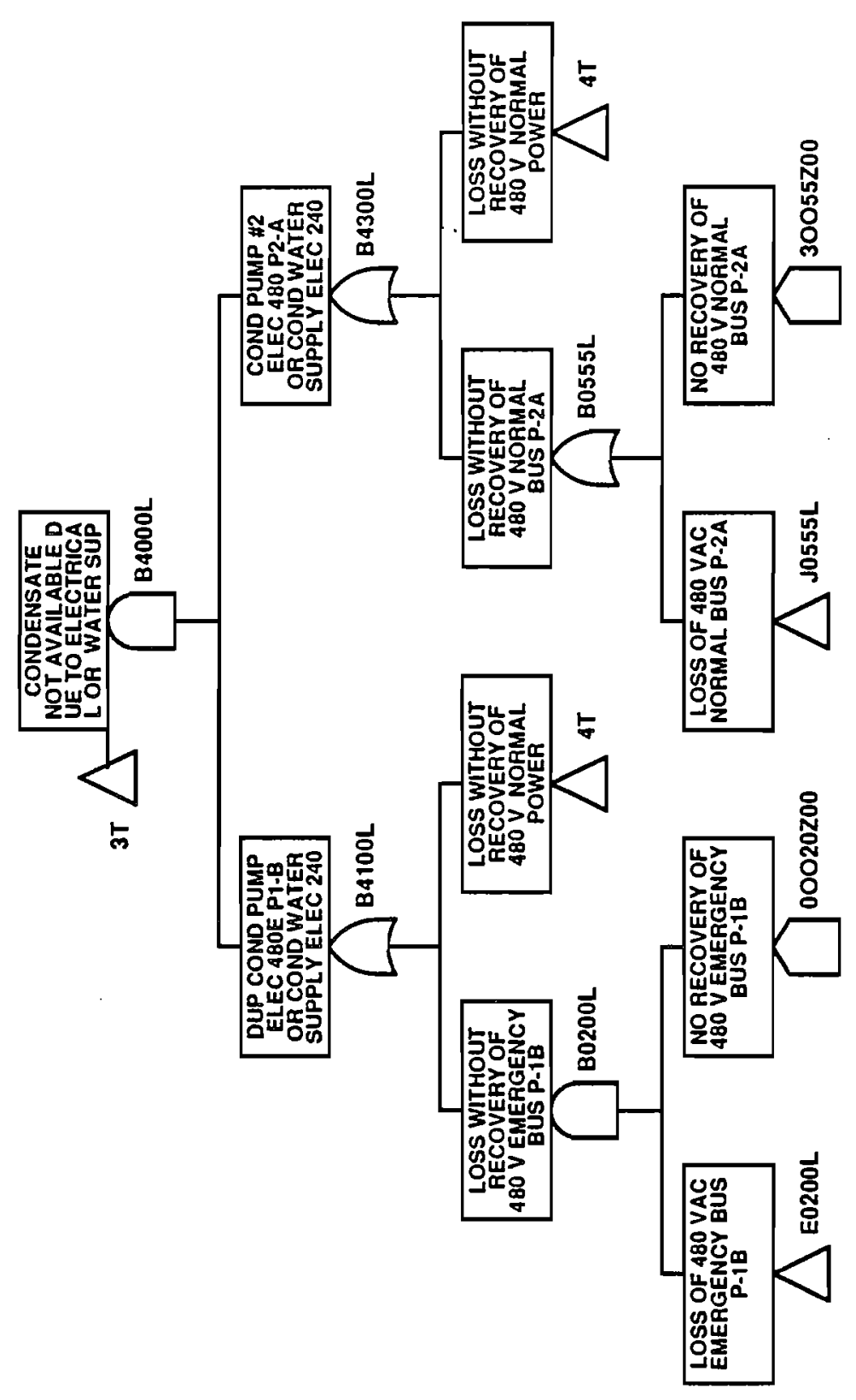

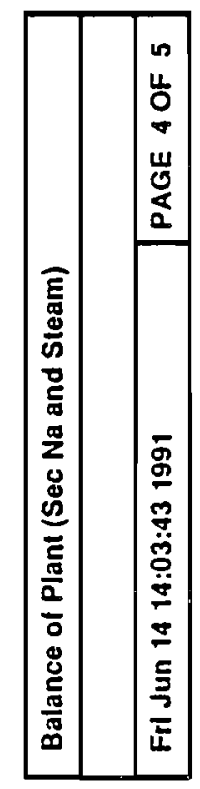

B. $2-250$ 


\begin{tabular}{|c|c|}
\hline & 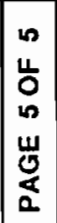 \\
\hline 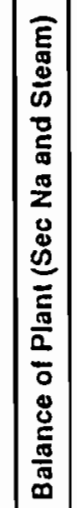 & 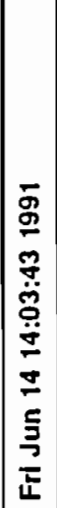 \\
\hline
\end{tabular}

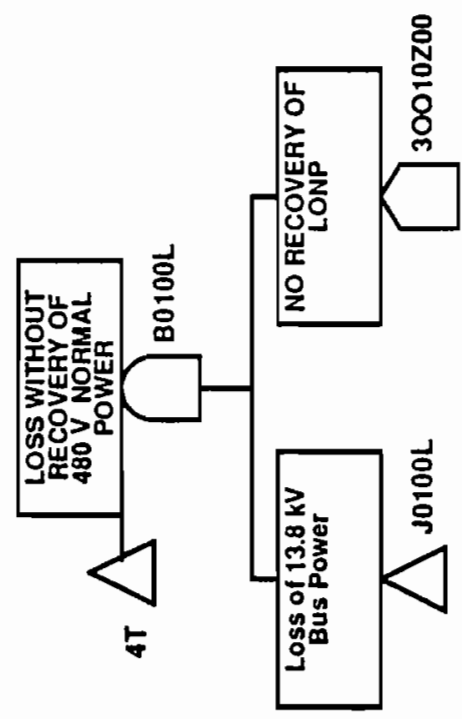

B. 2-251 


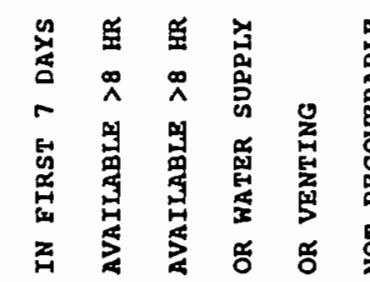

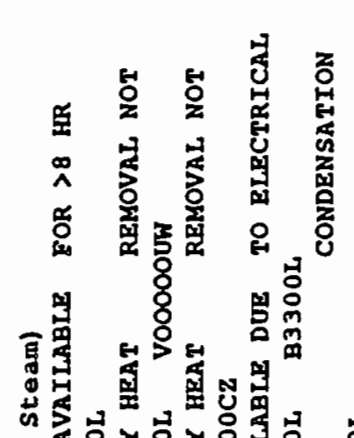

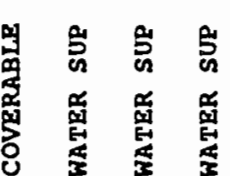

훙 훙

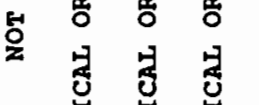

氮

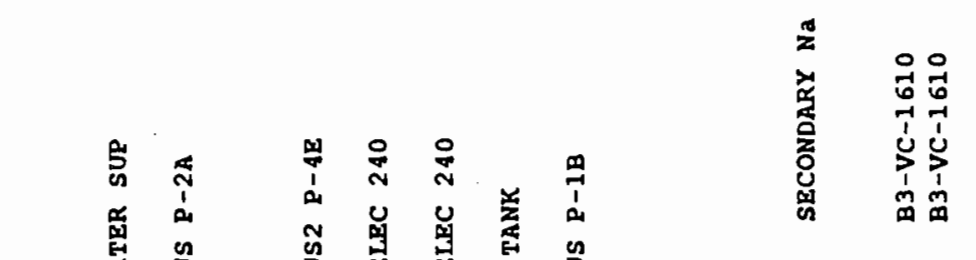

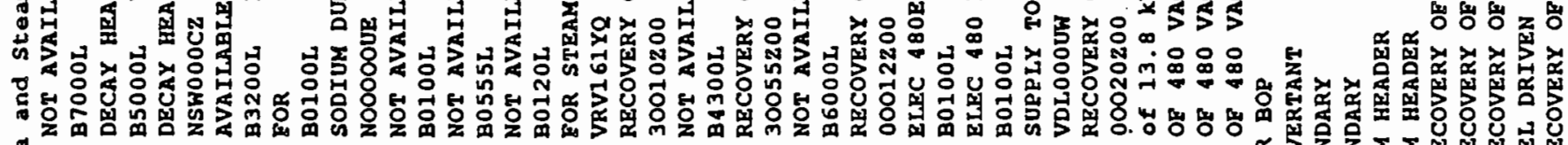
" z

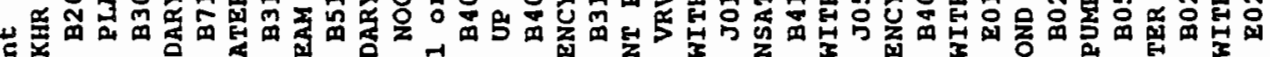

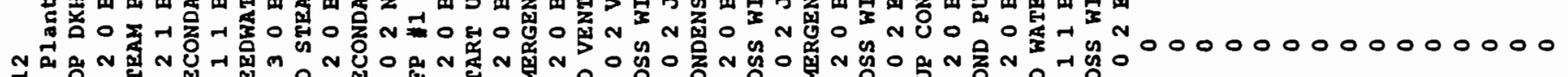
n。 આ 


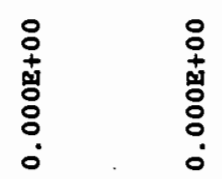

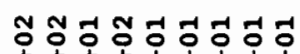

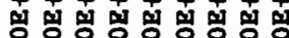

ㅇㅇㅇㅇㅇㅇㅇㅇㅇㅇㅇ

:0000.0.

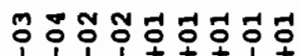

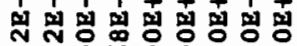

ำ

○0 00000:

O OOO OH HOF

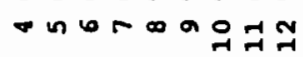

B.2-253 


\begin{tabular}{|c|c|}
\hline & $\frac{-}{\frac{1}{0}}$ \\
\hline 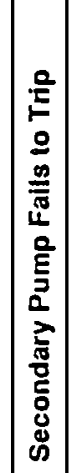 & 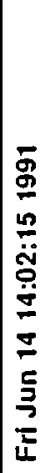 \\
\hline
\end{tabular}

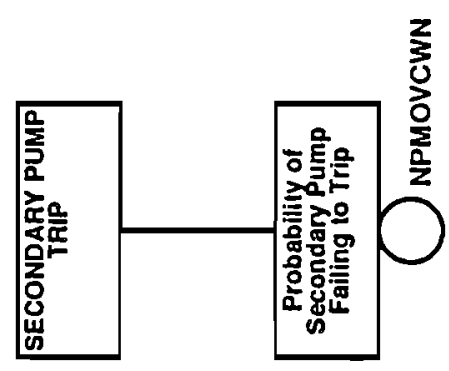




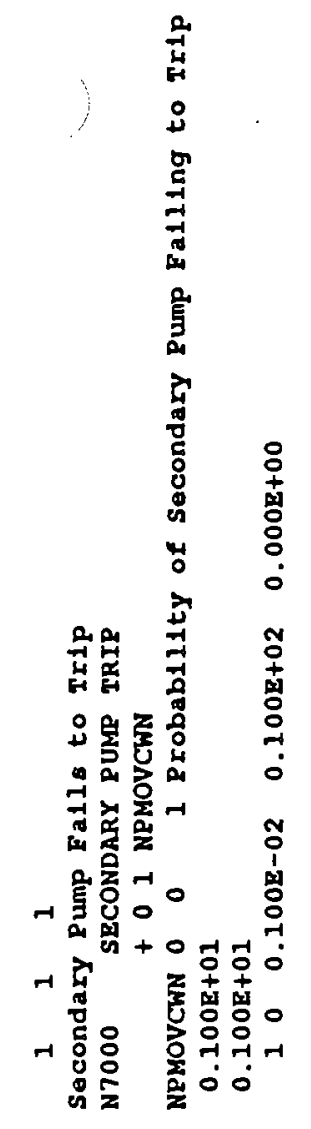

B. 2-255 


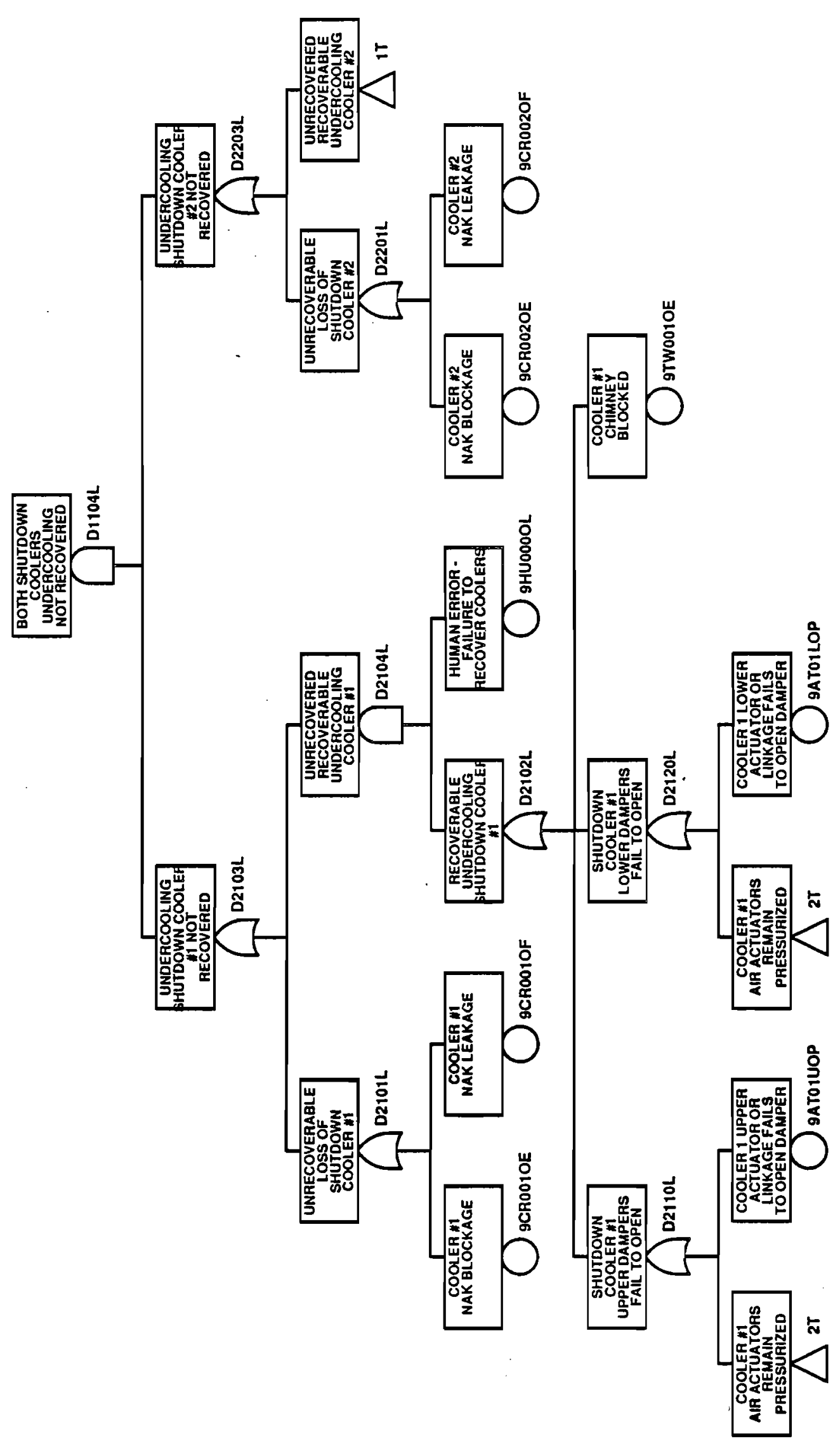

B. 2-256 


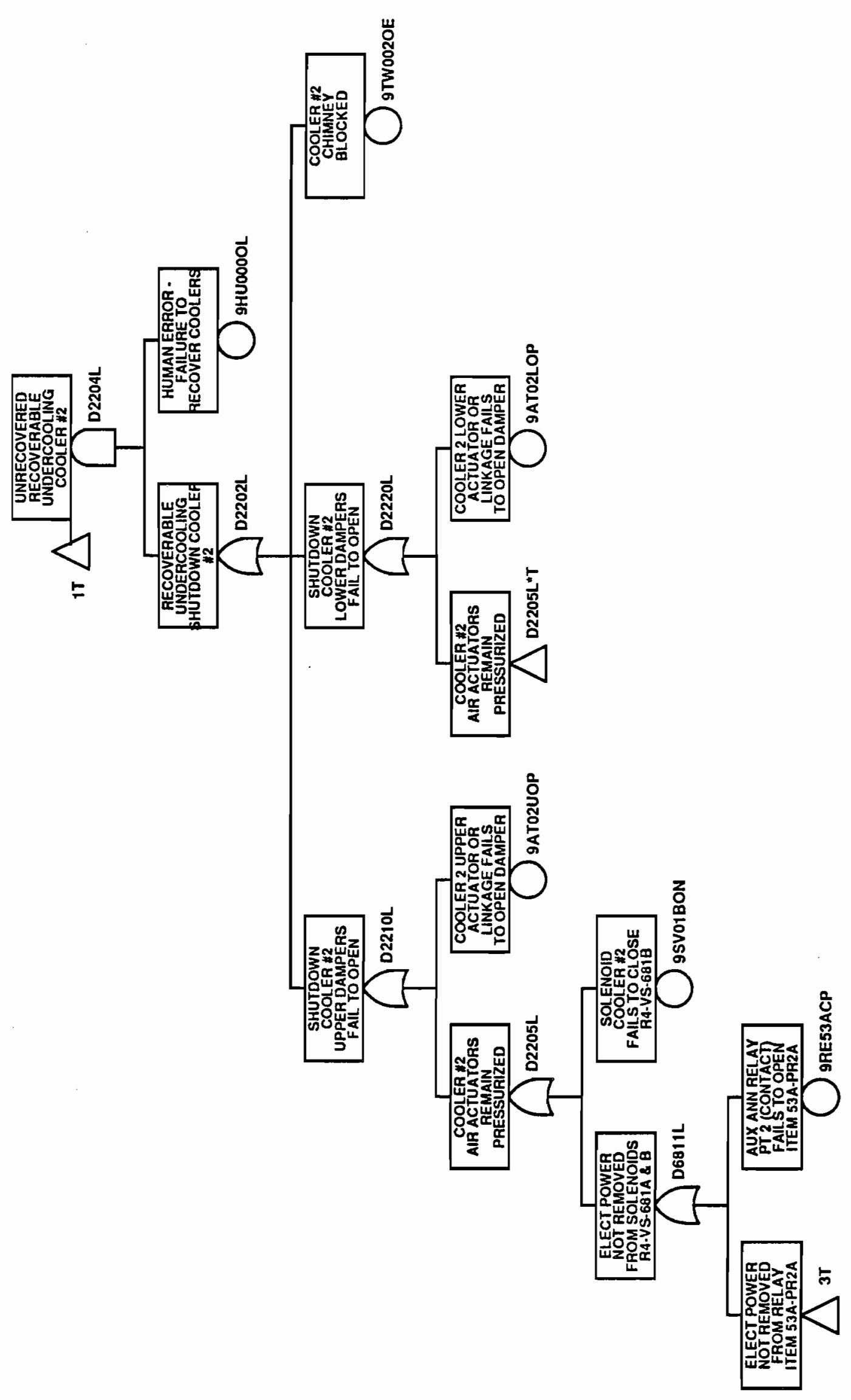




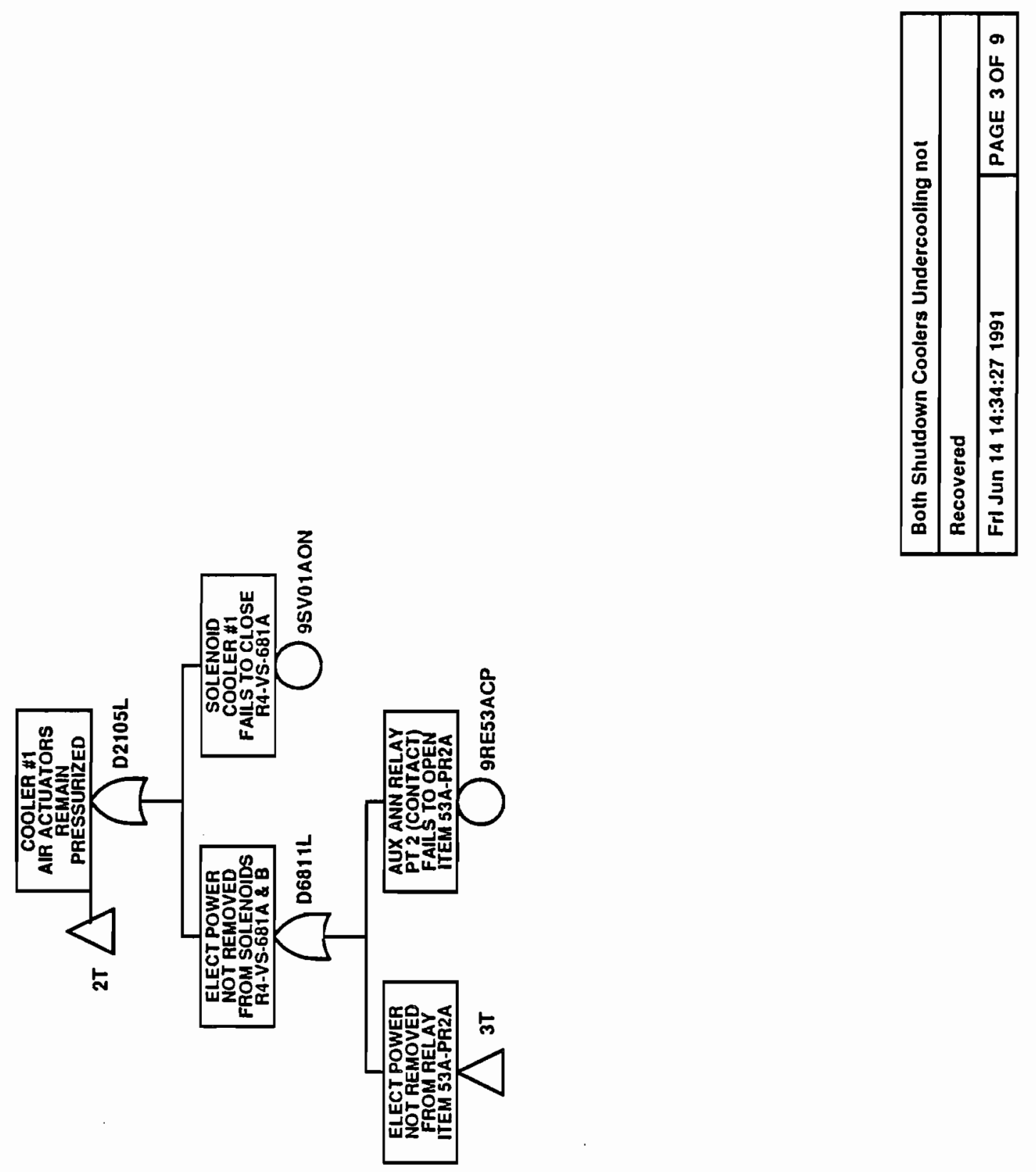

B. $2-258$ 


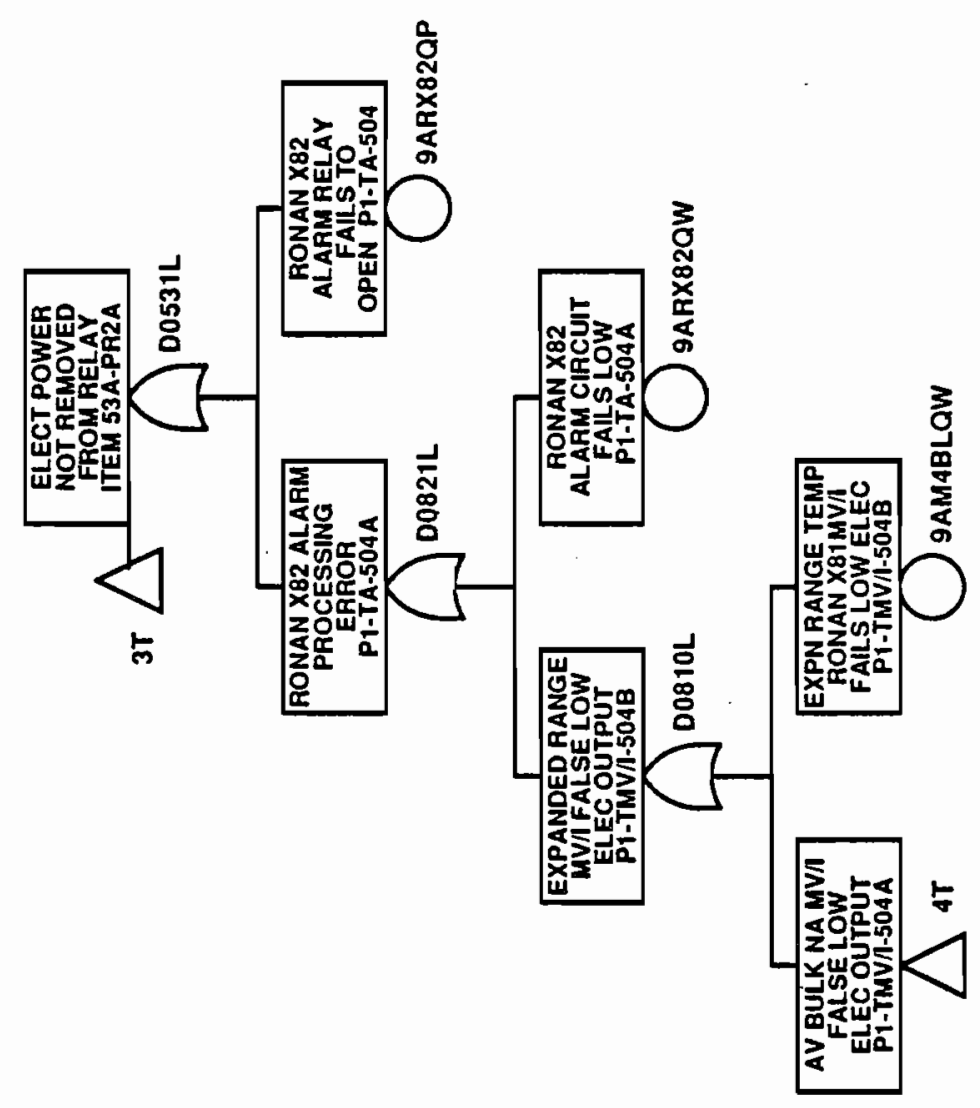

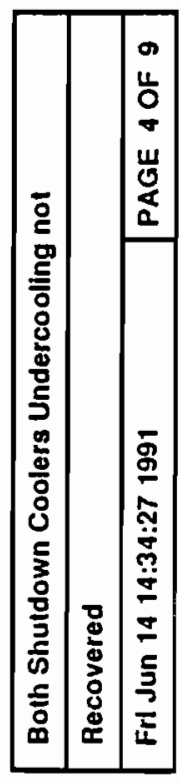




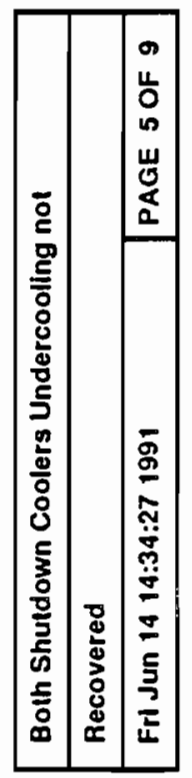

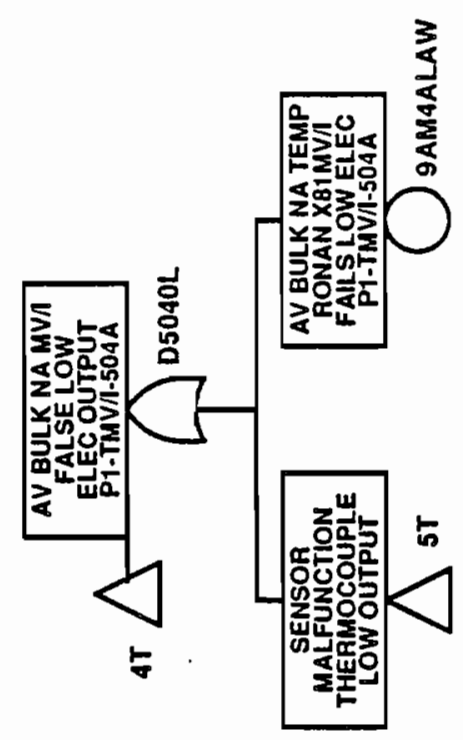




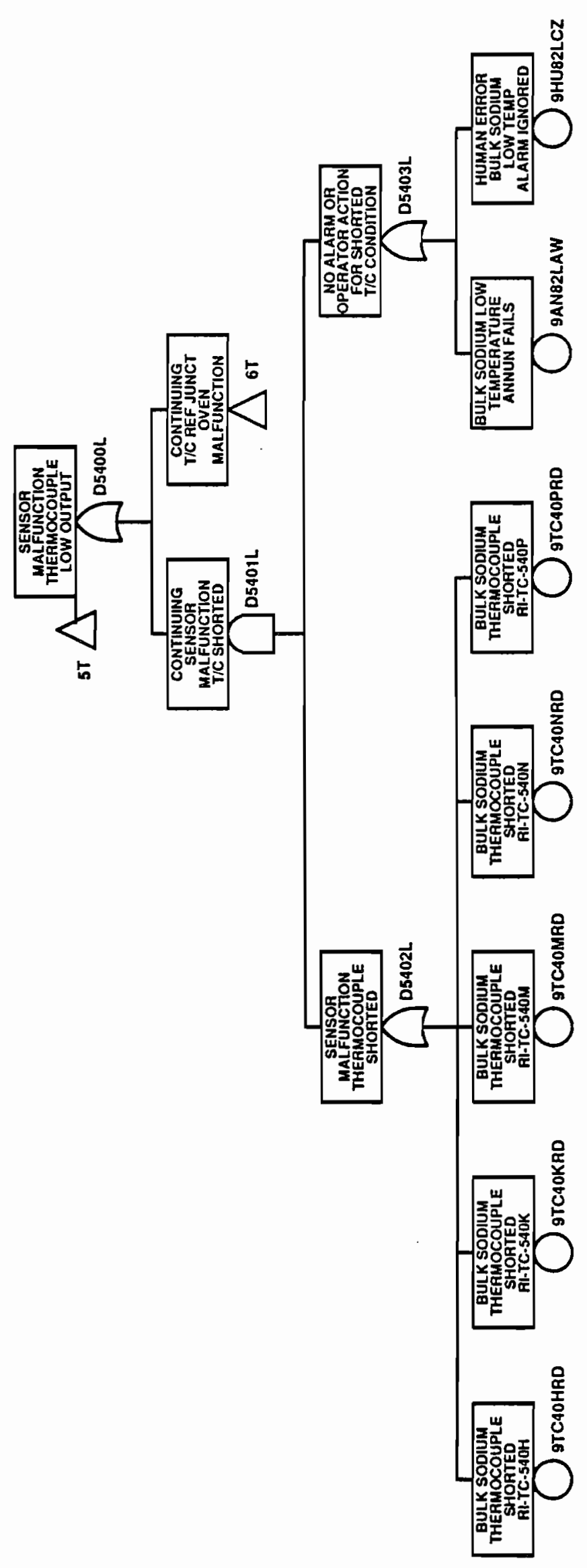

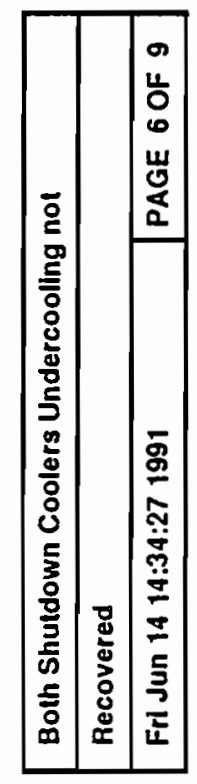



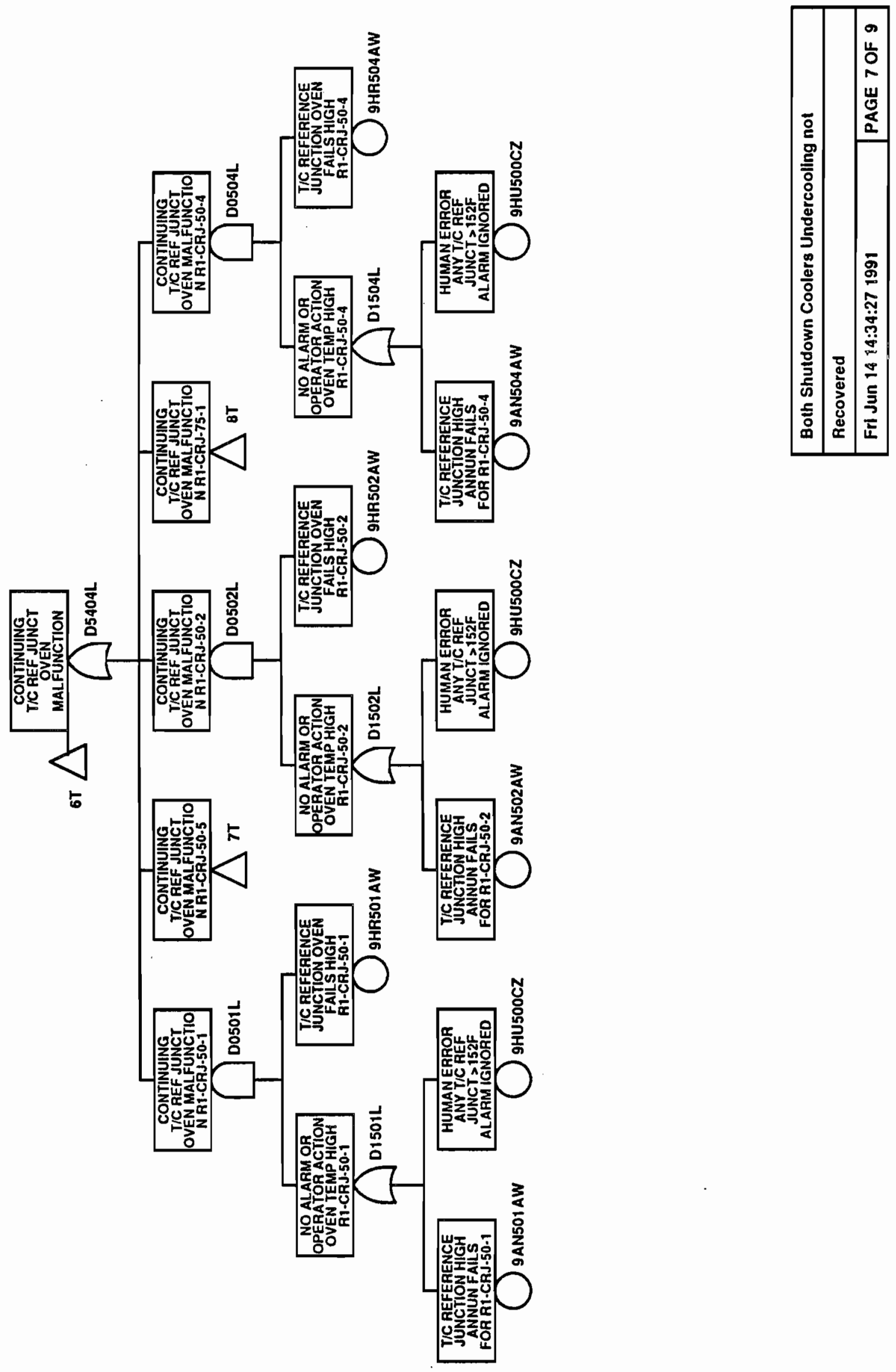


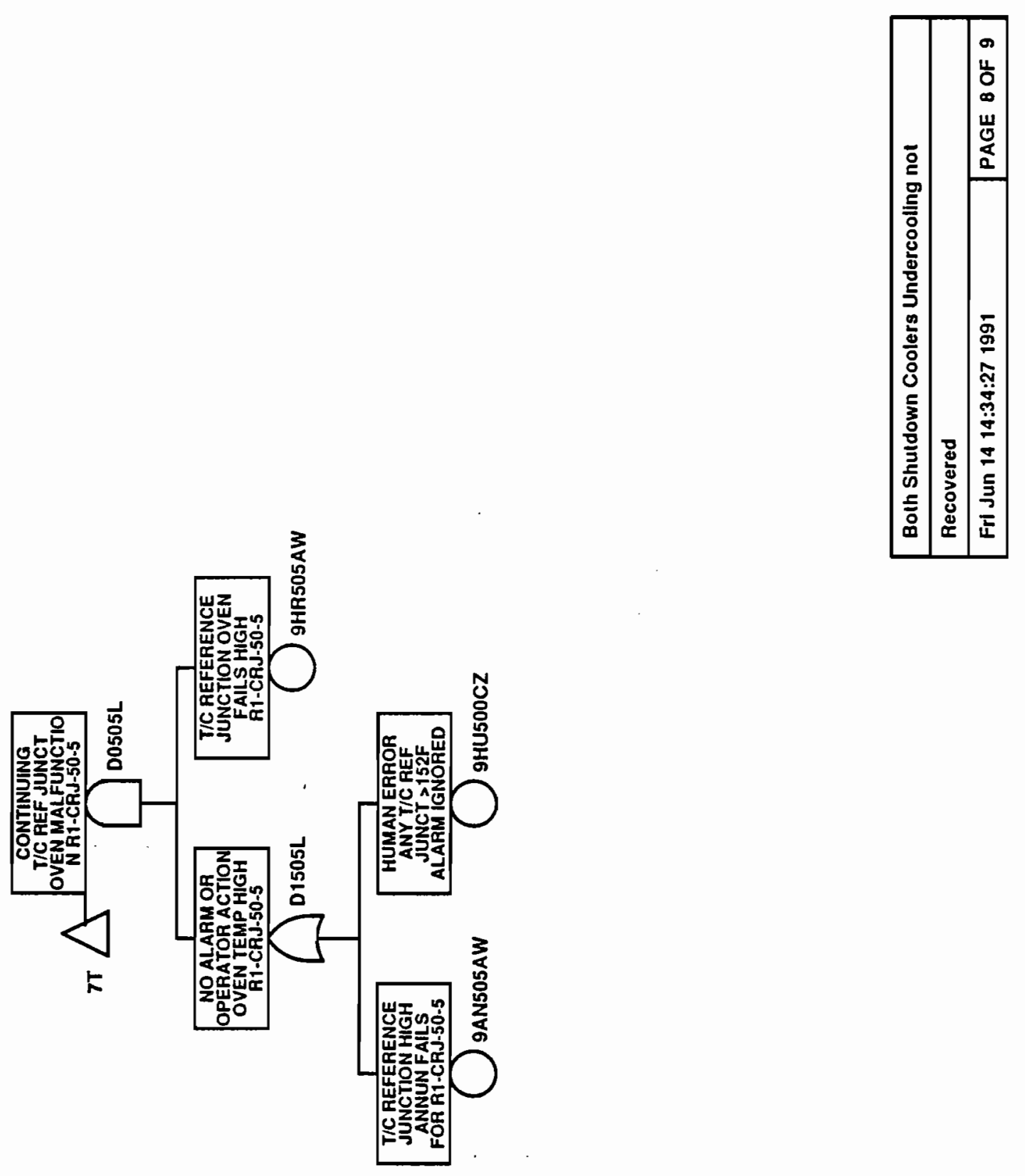




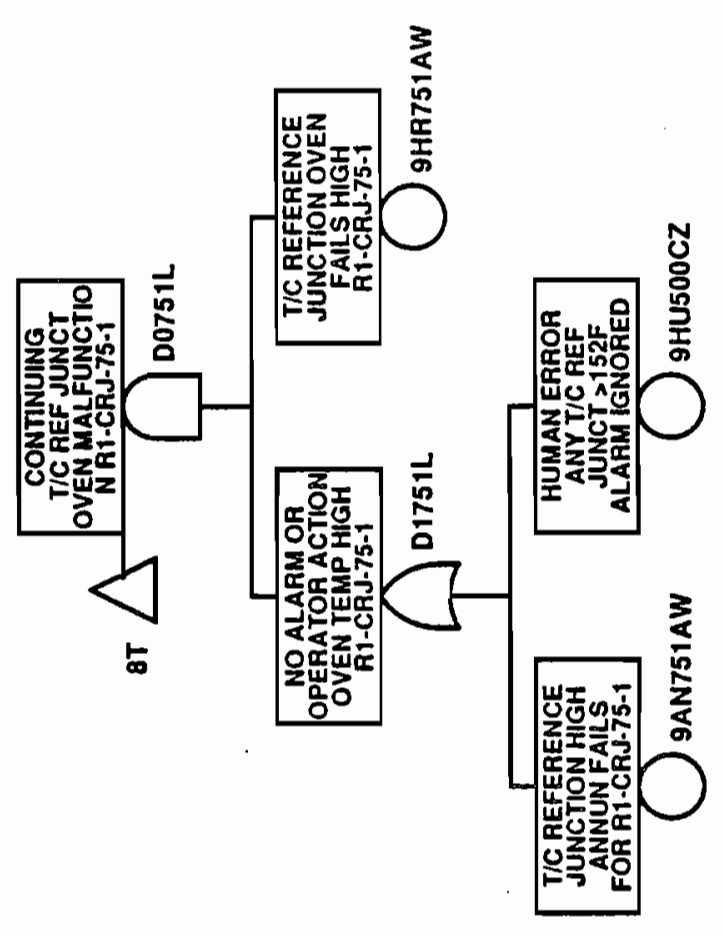

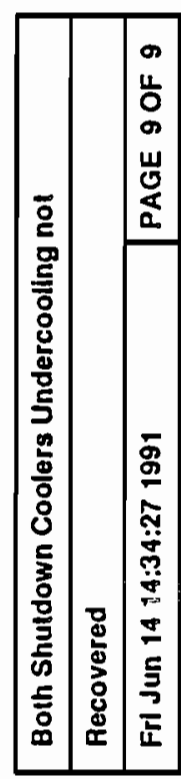




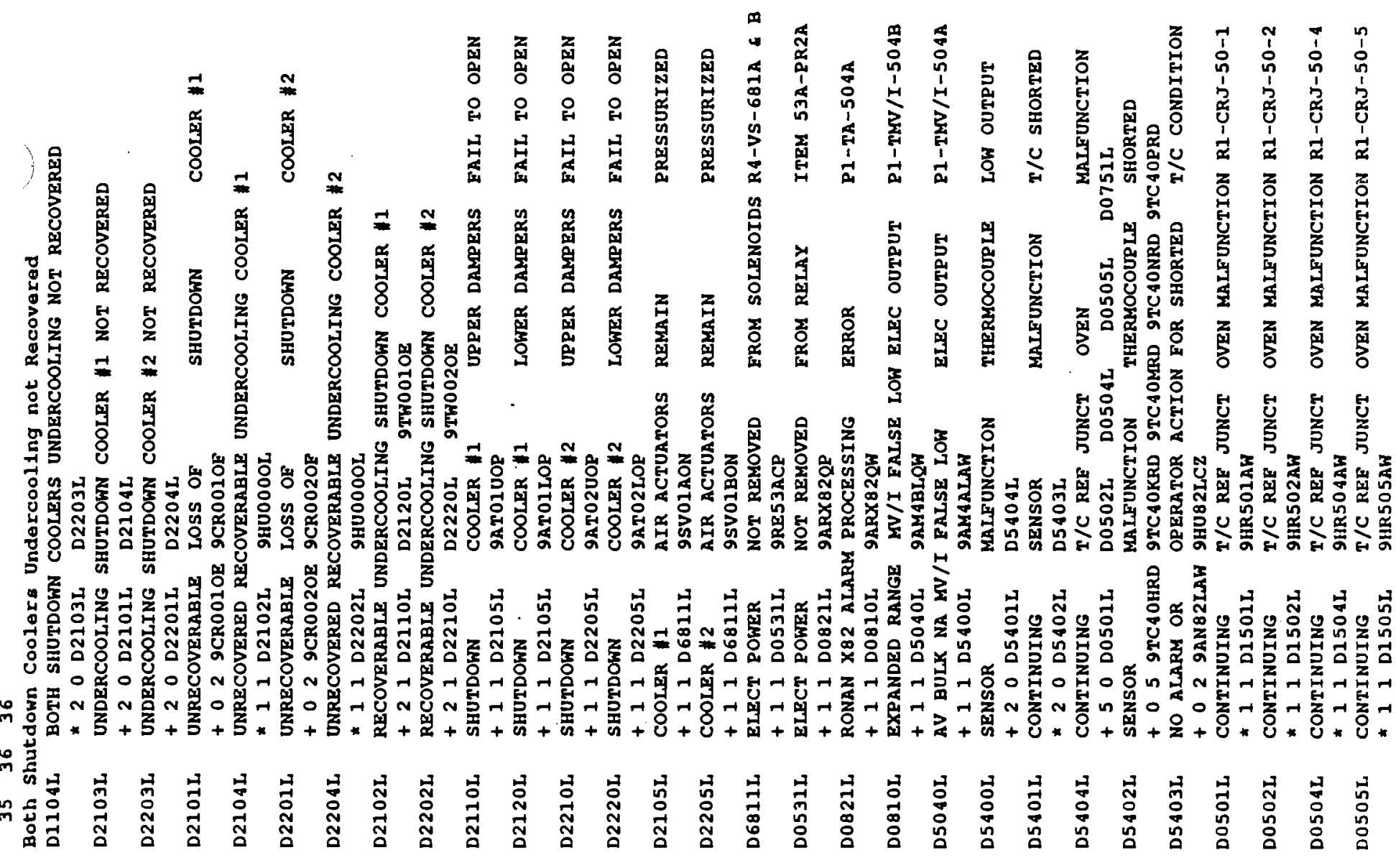




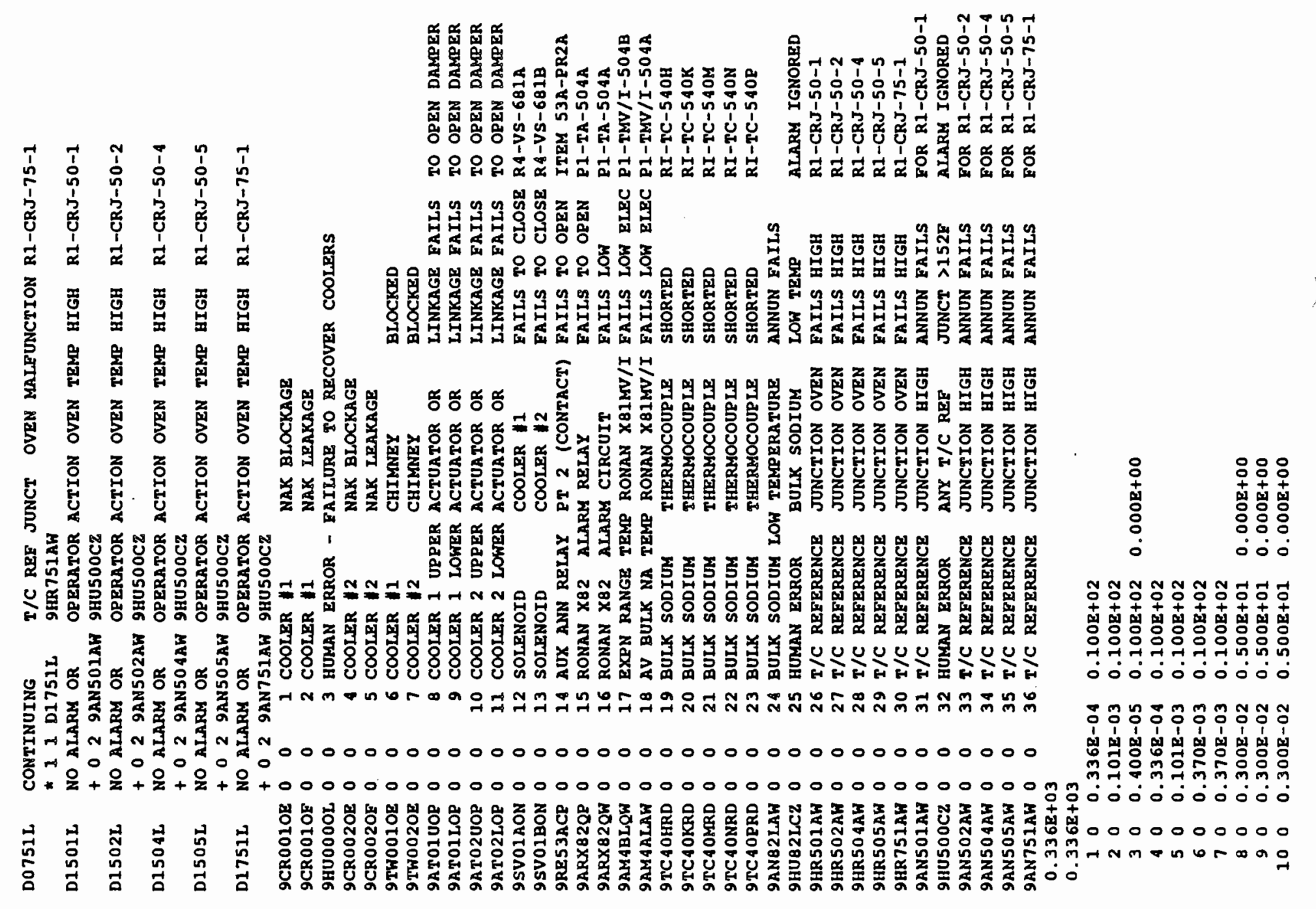



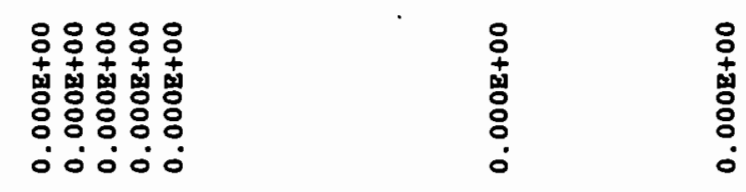

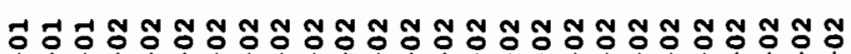

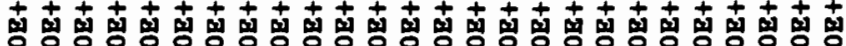

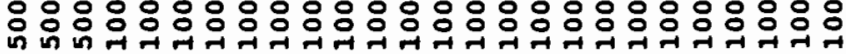

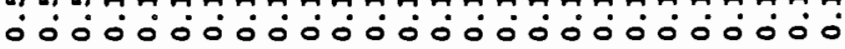

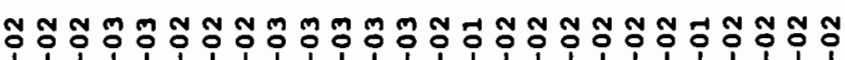

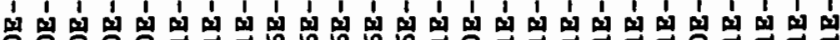

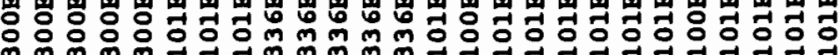
mo mo mo mo 00000000000000000000000000

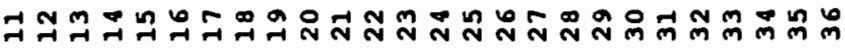




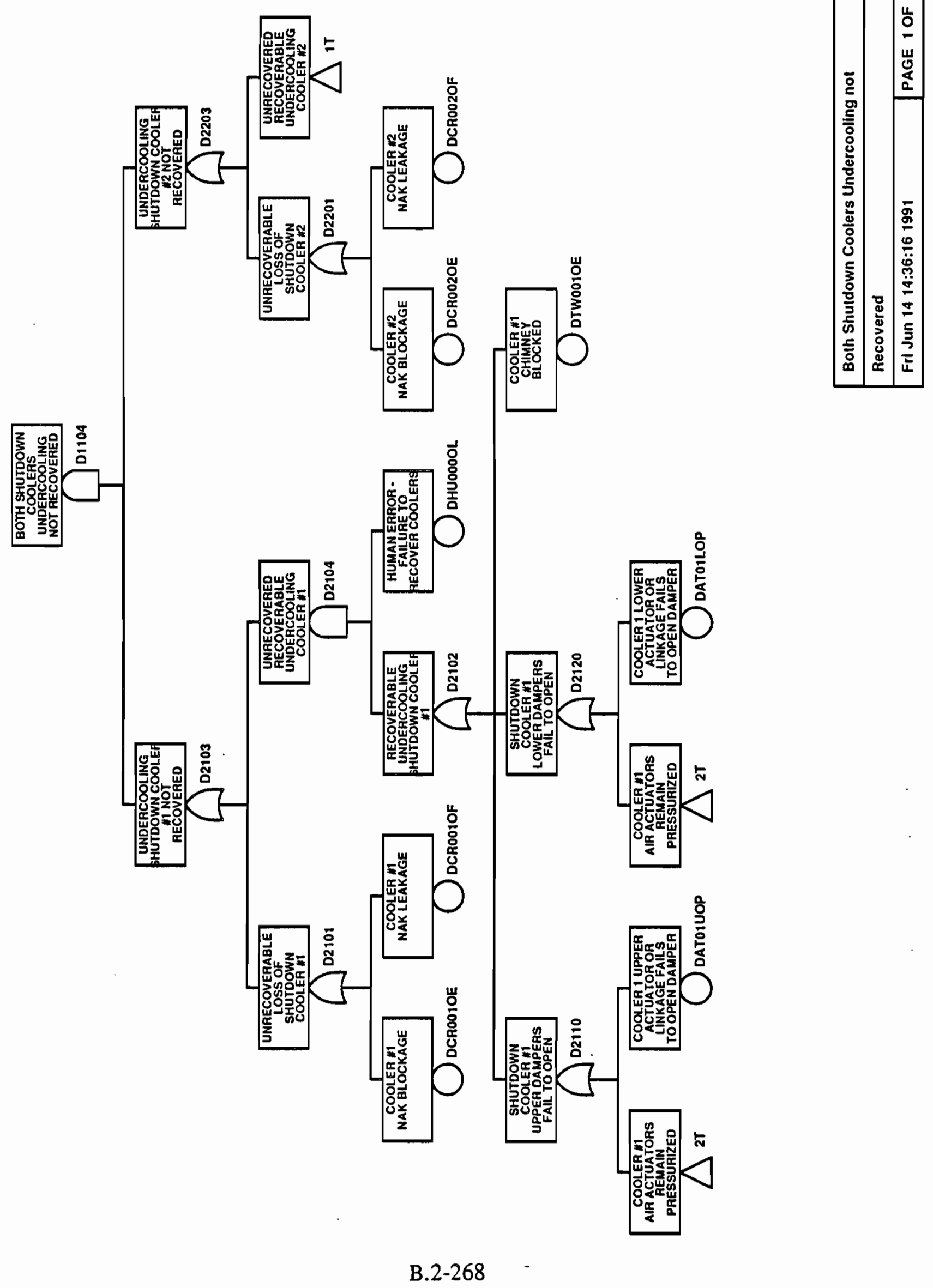




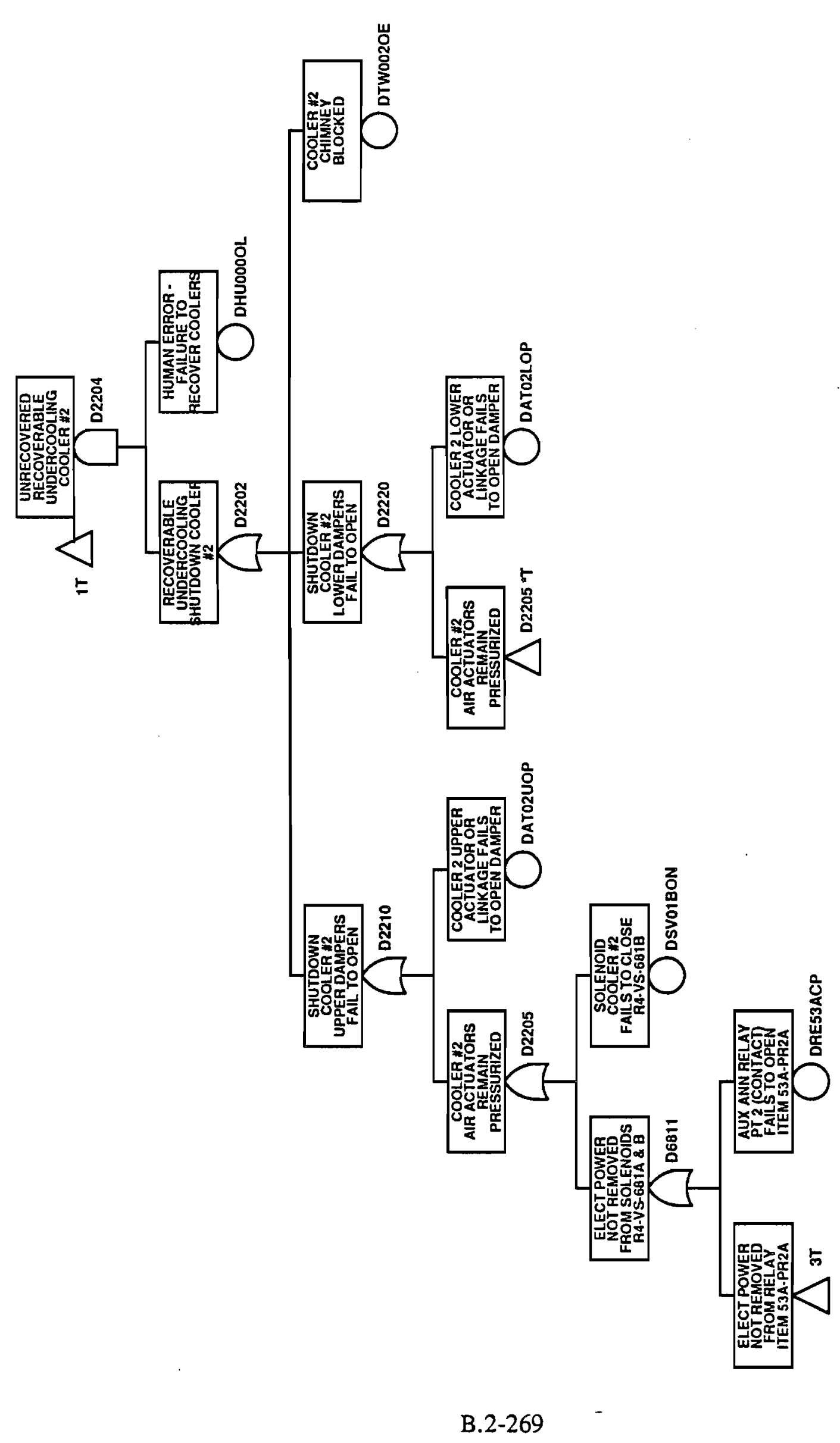



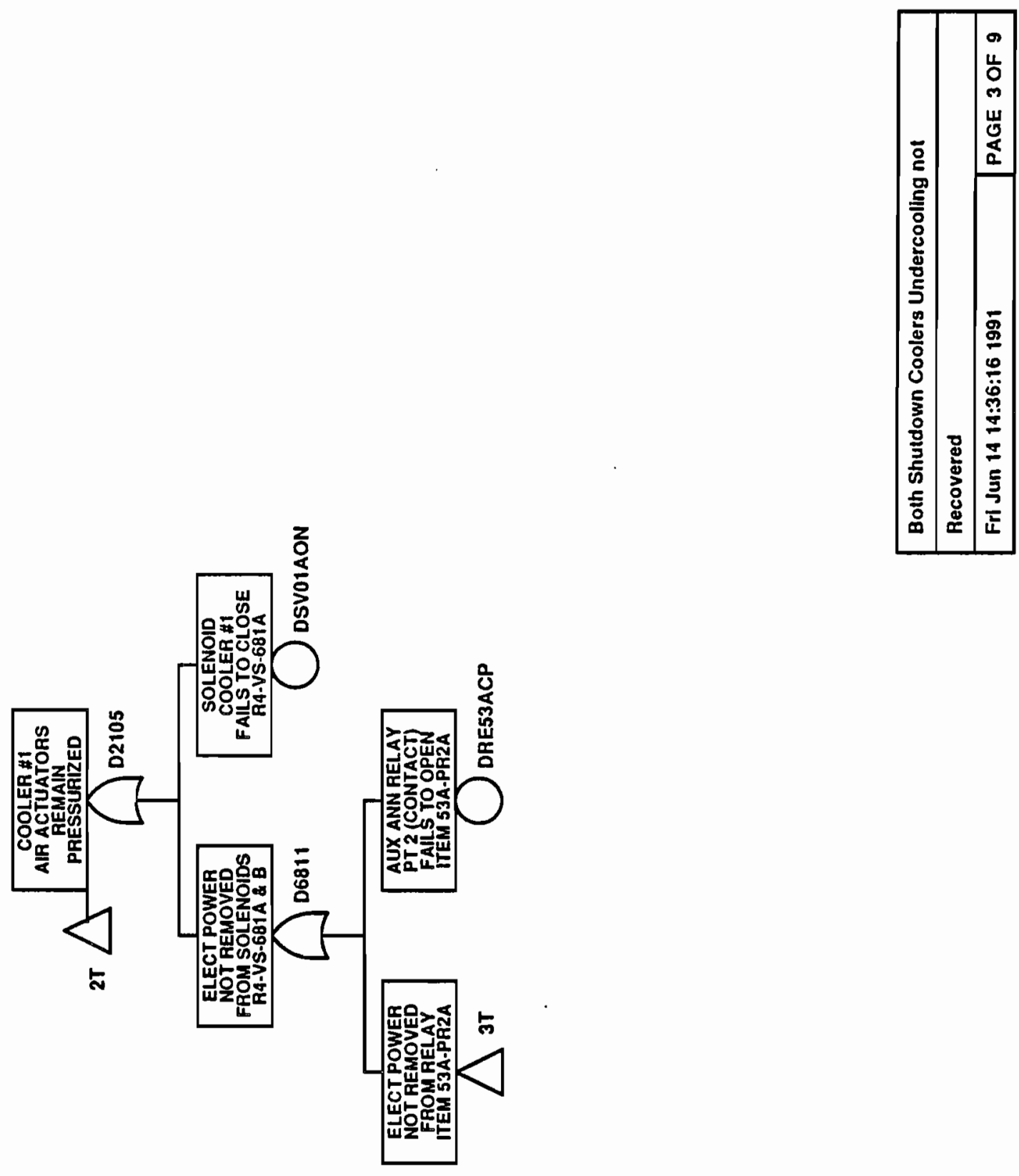


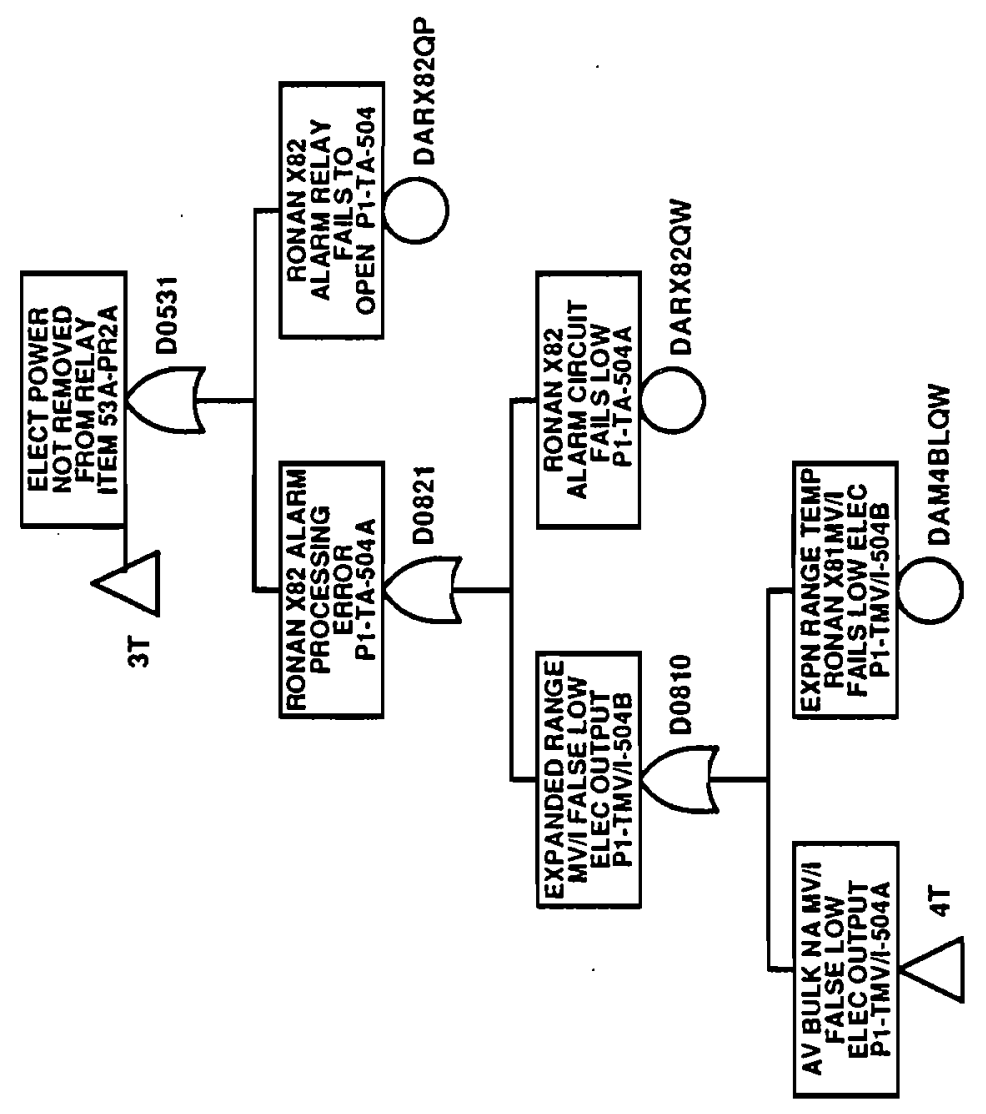

[I 


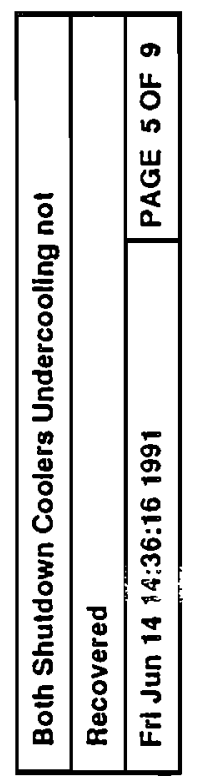

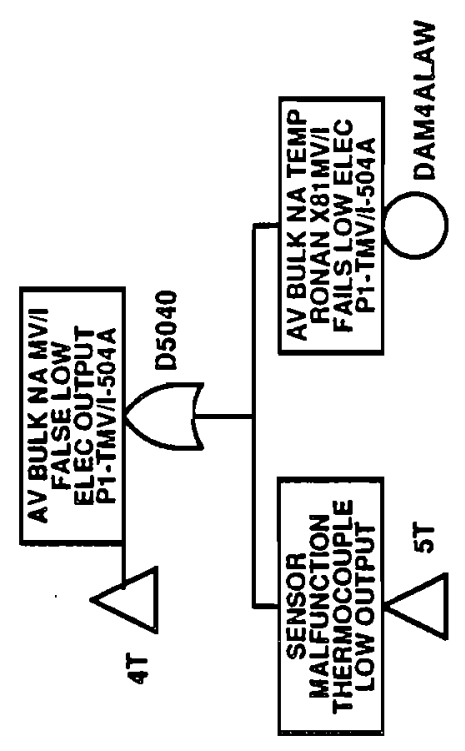

B. 2-272 


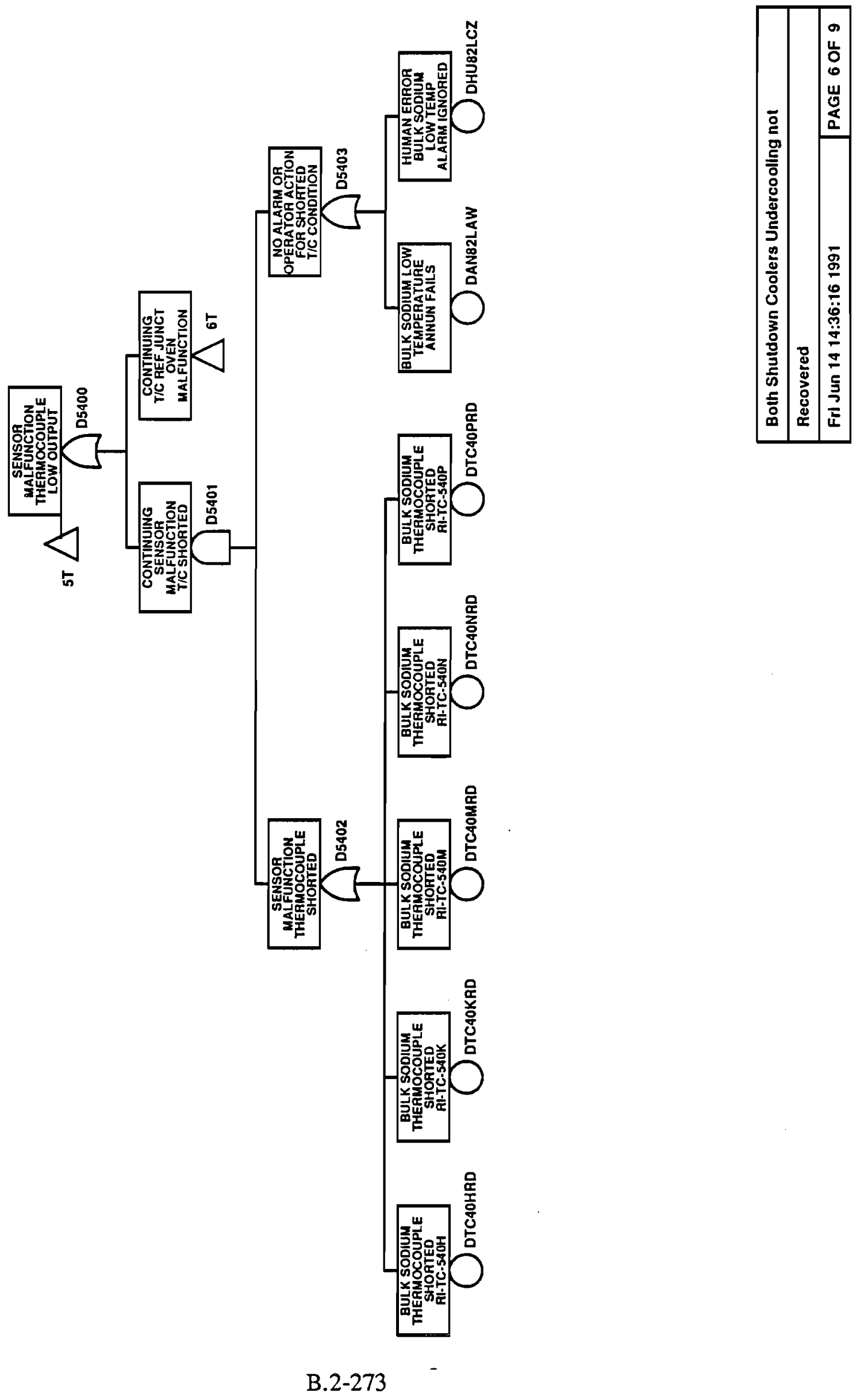



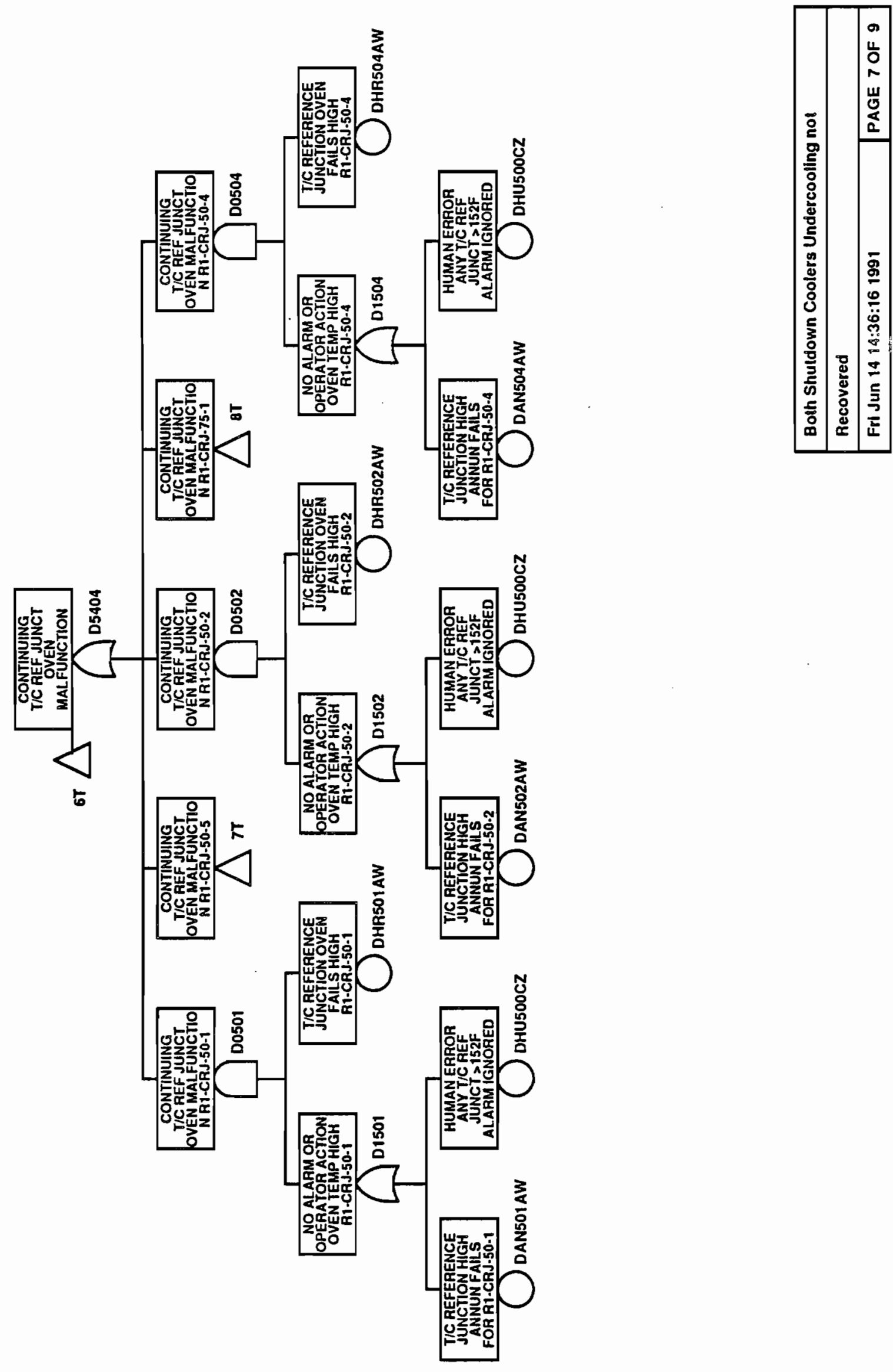

B. $2-274$ 


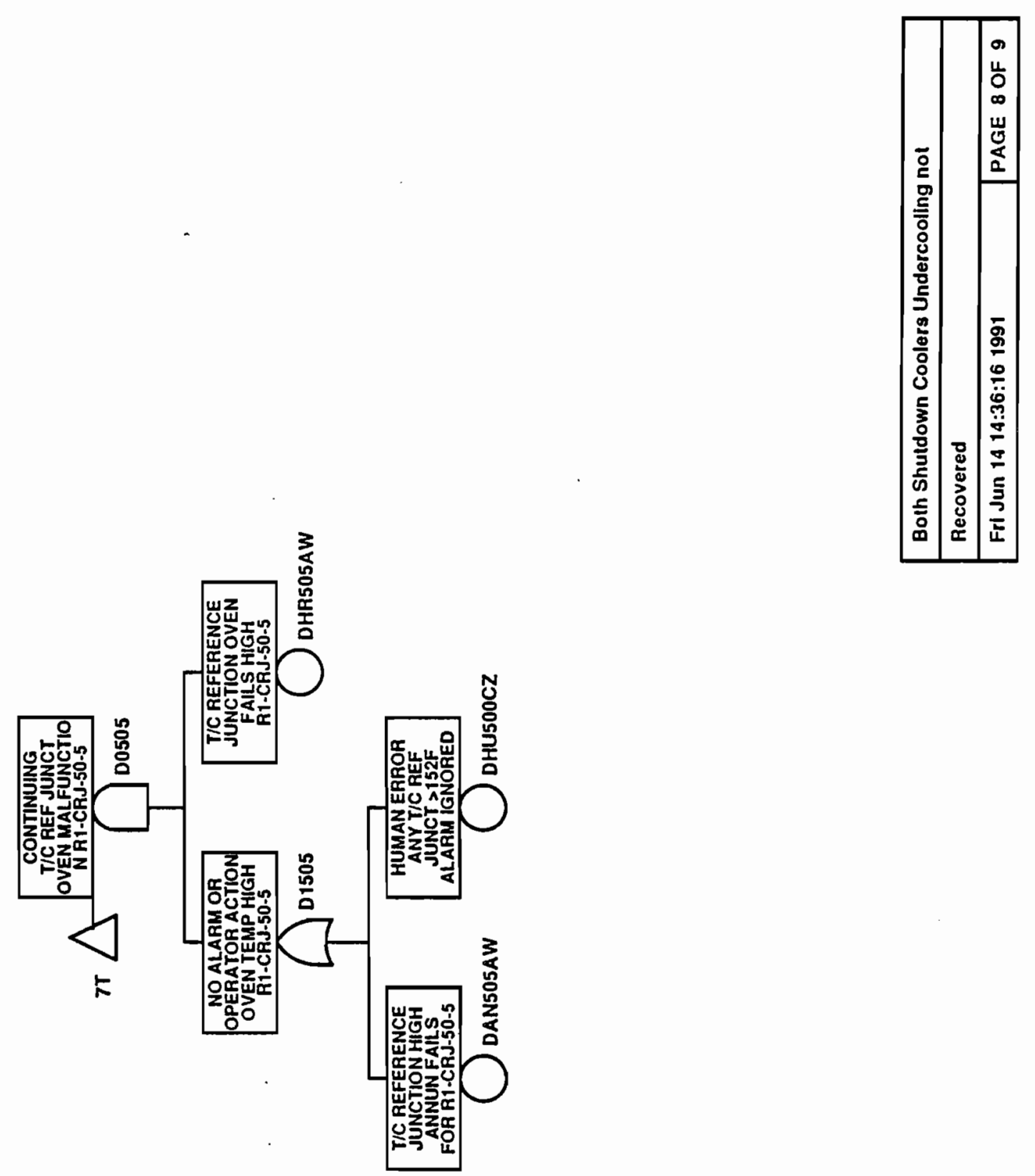




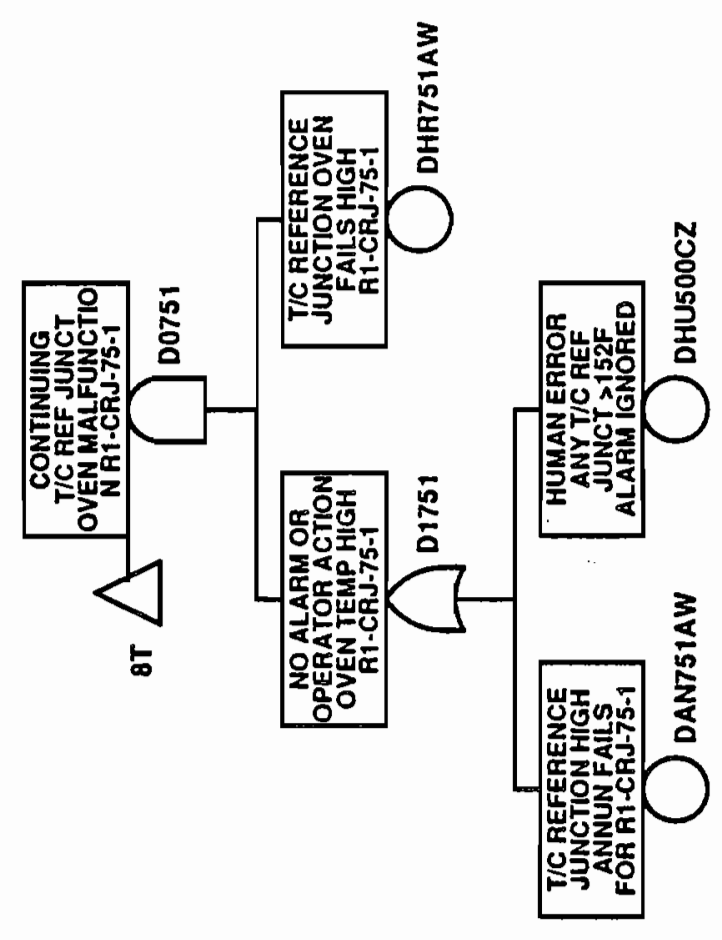

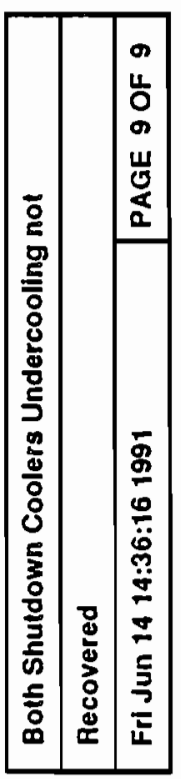




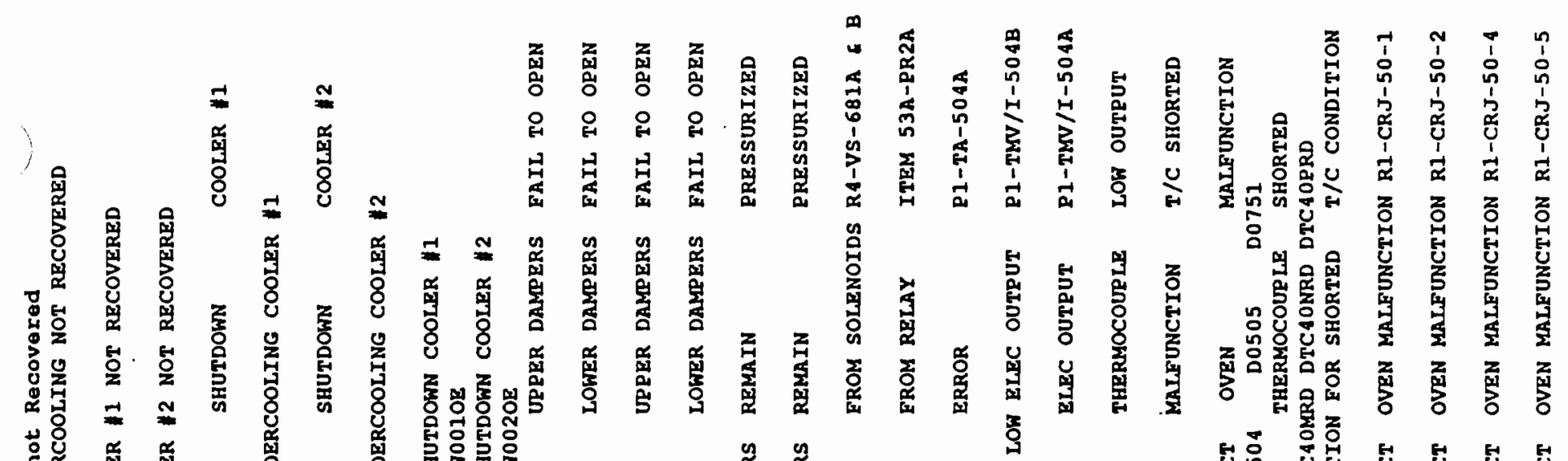

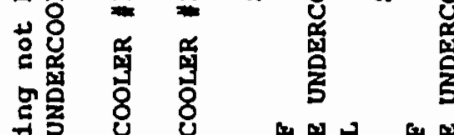

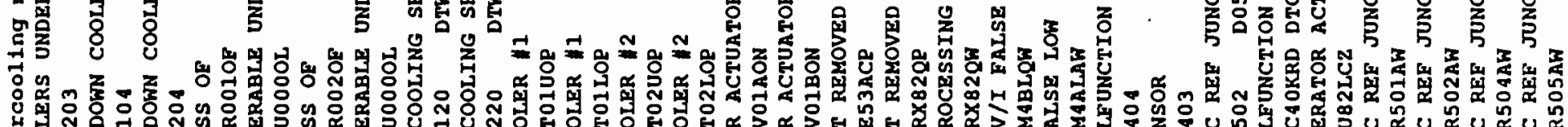

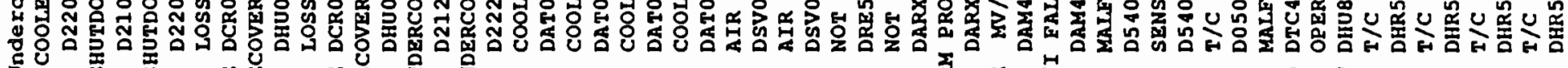

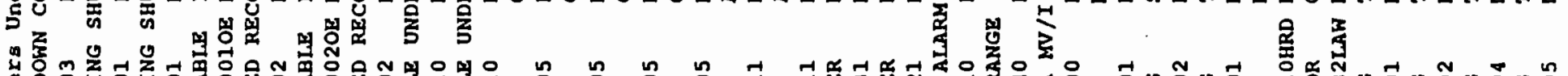

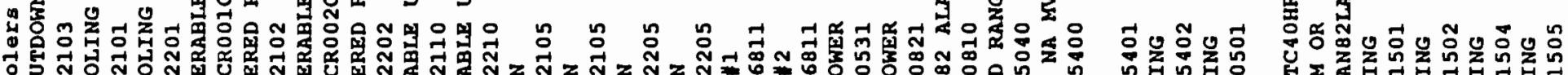

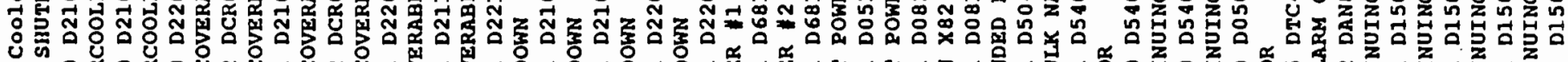

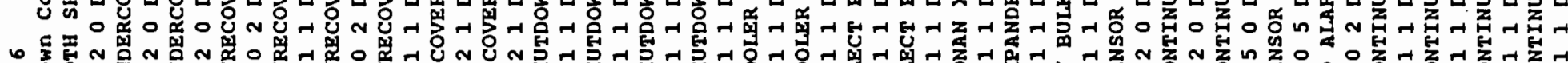

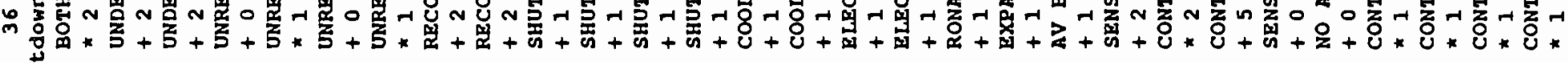
谣

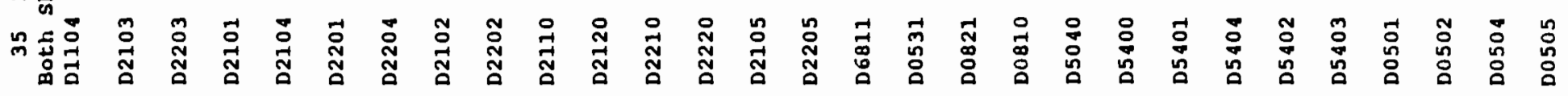




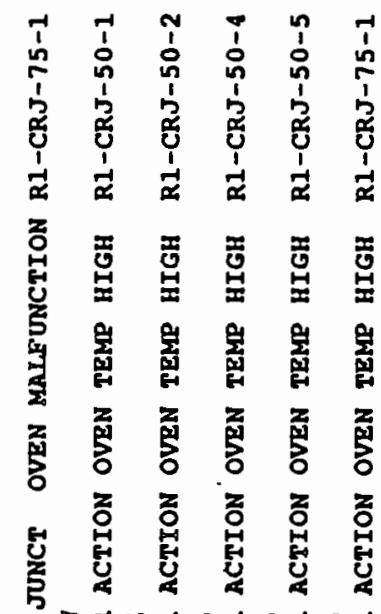

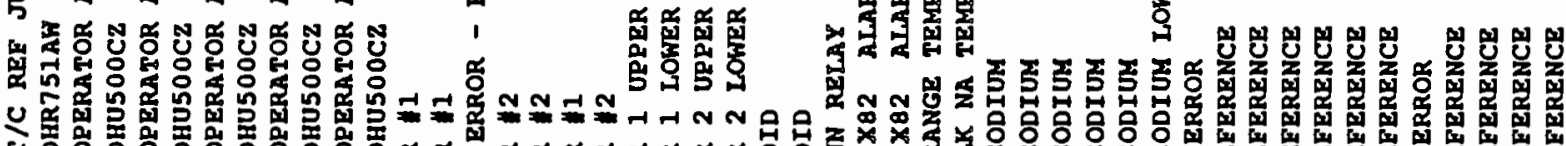

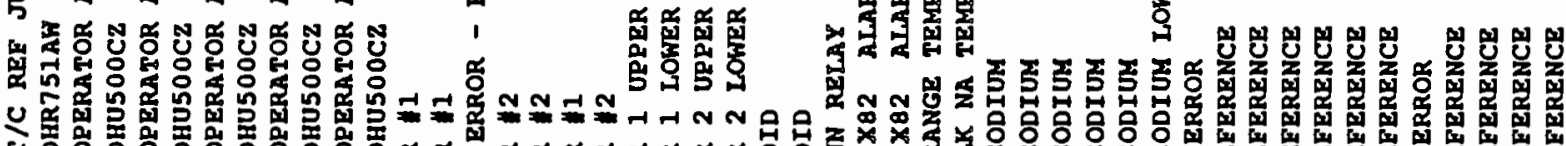

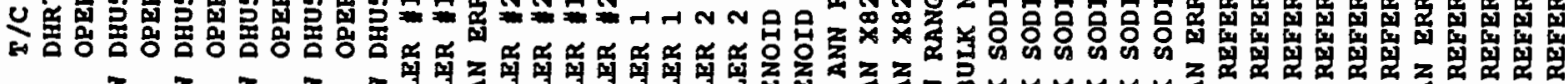
정

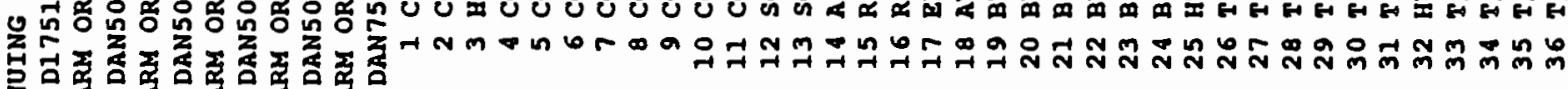
列 HE $\mathrm{U}^{-1} 0^{0}+0^{0}+0^{0}+0^{0}+0000000000000000000000000000000000000$

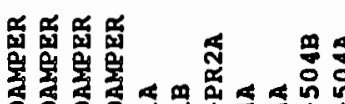

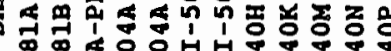

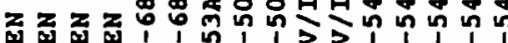

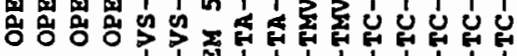

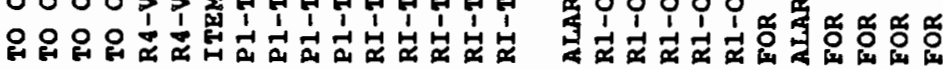

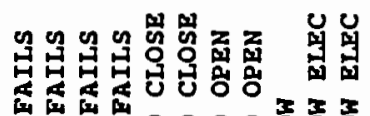

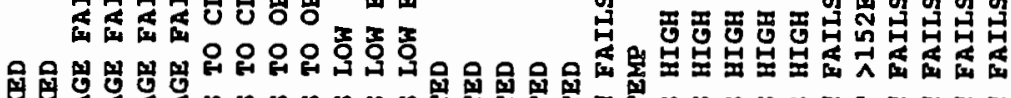

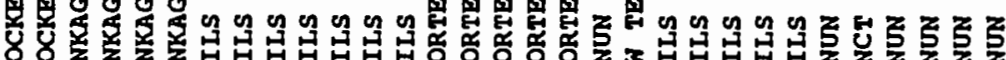

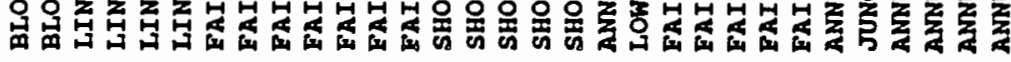

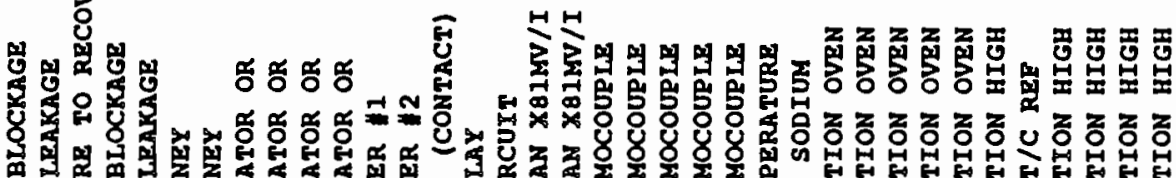

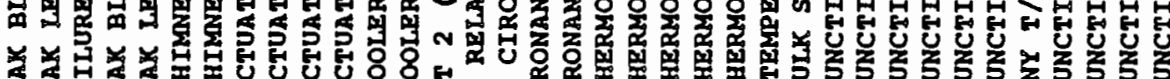

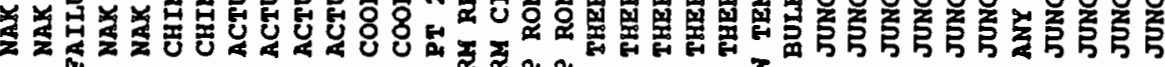
急

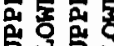

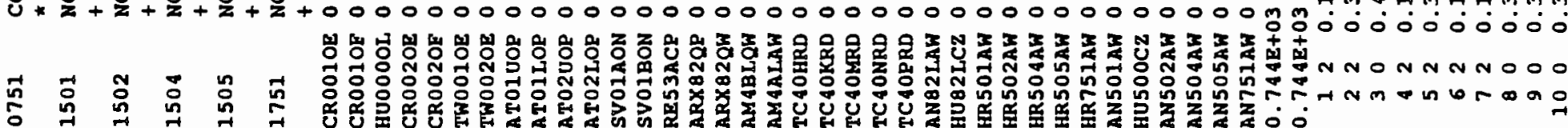




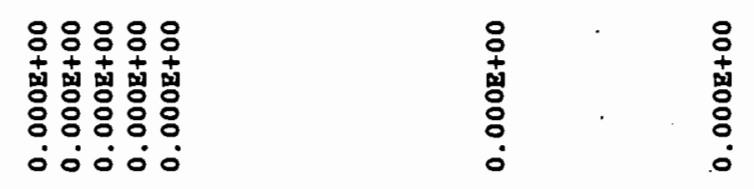

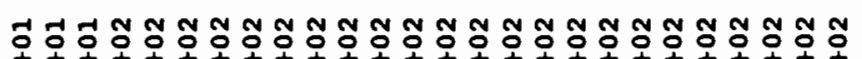

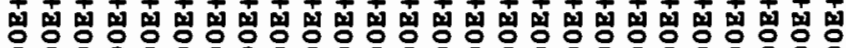

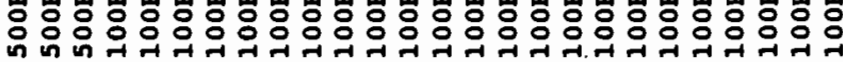

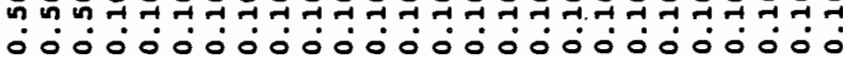

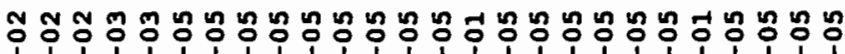

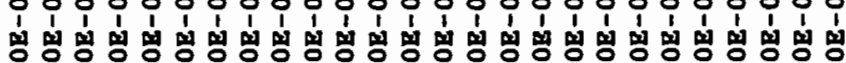

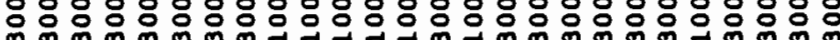

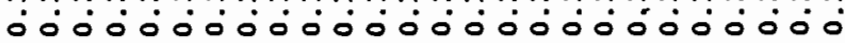

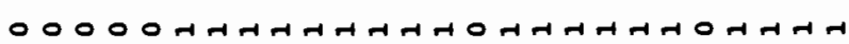

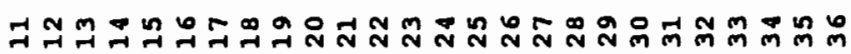




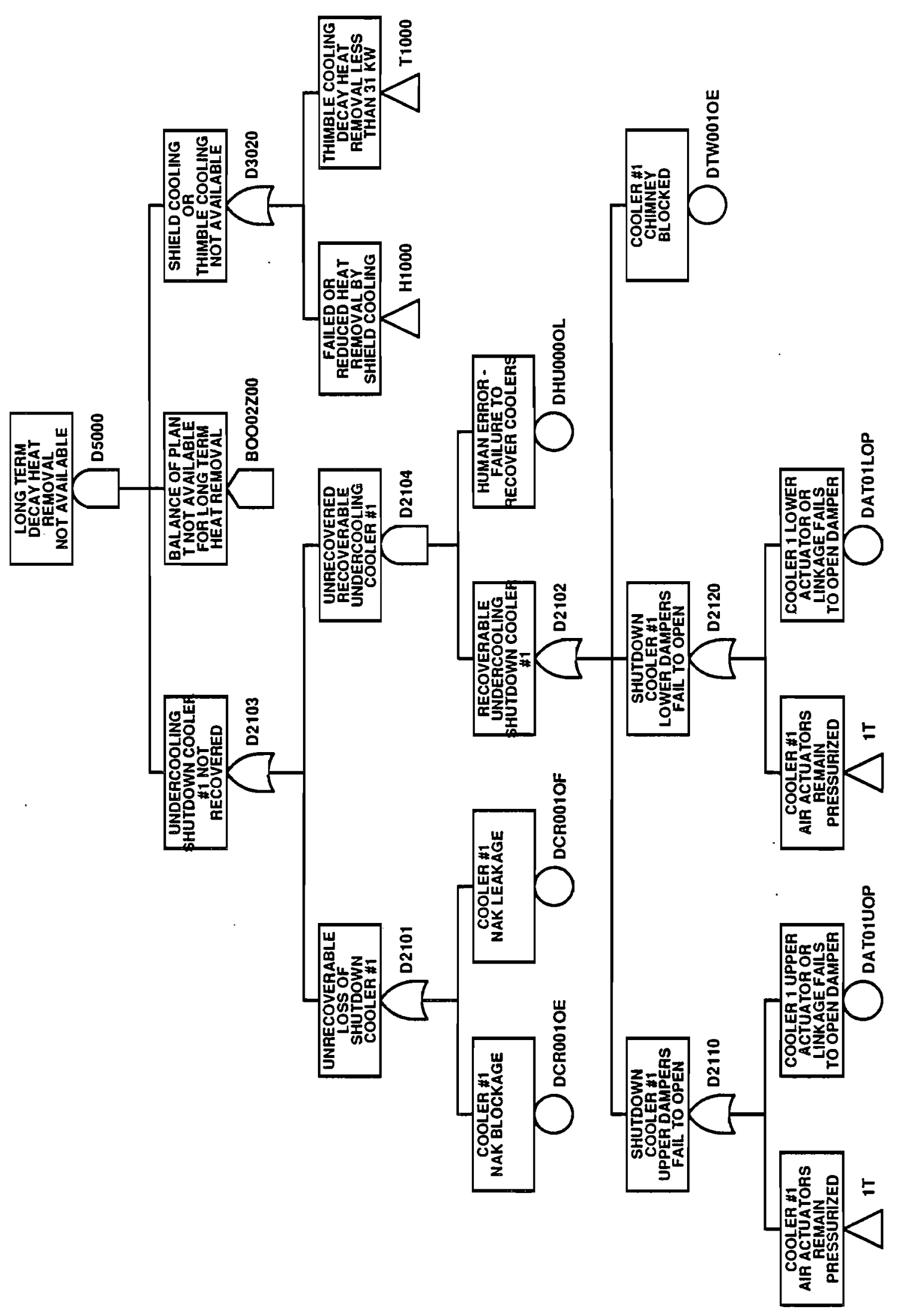




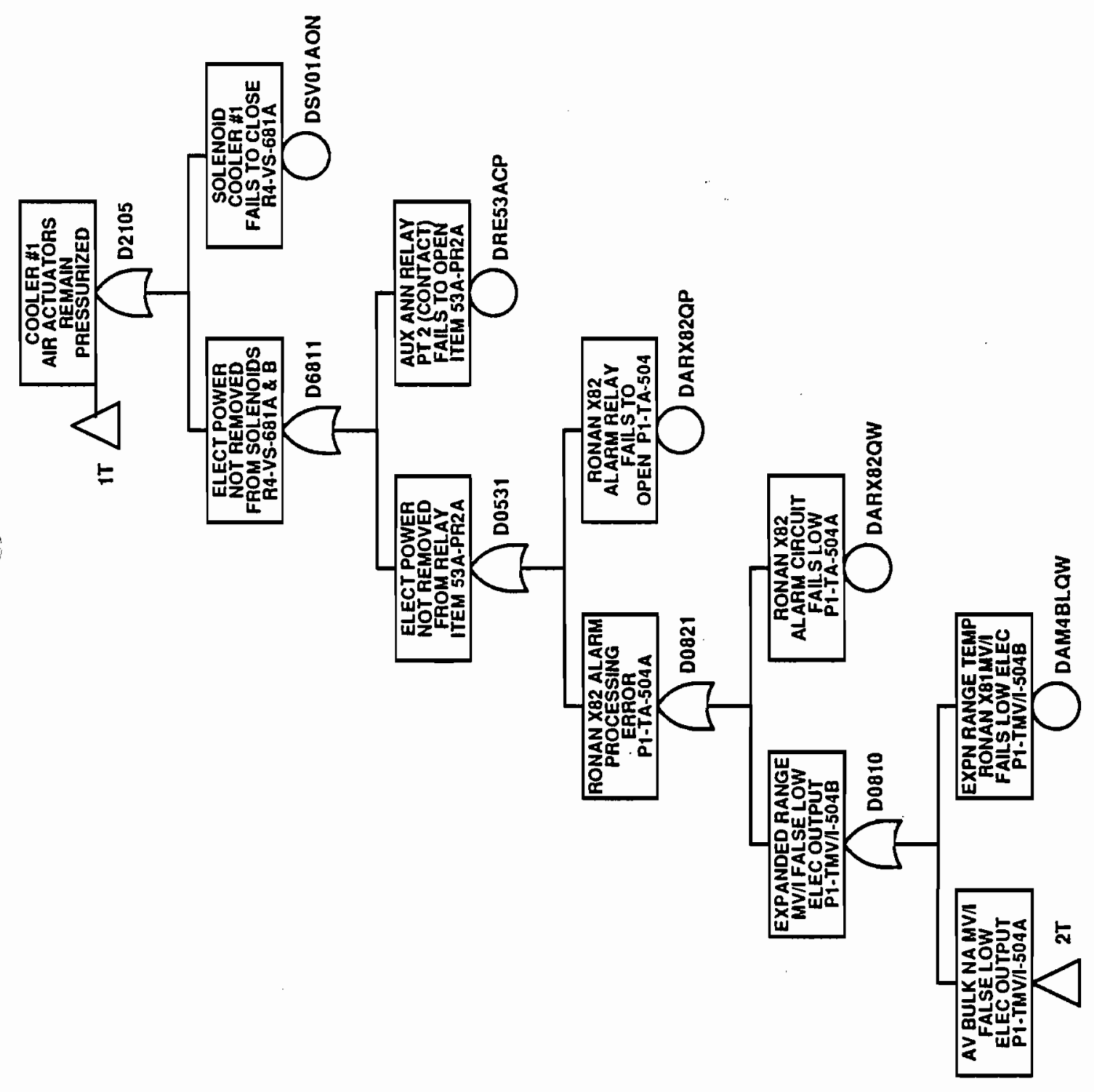




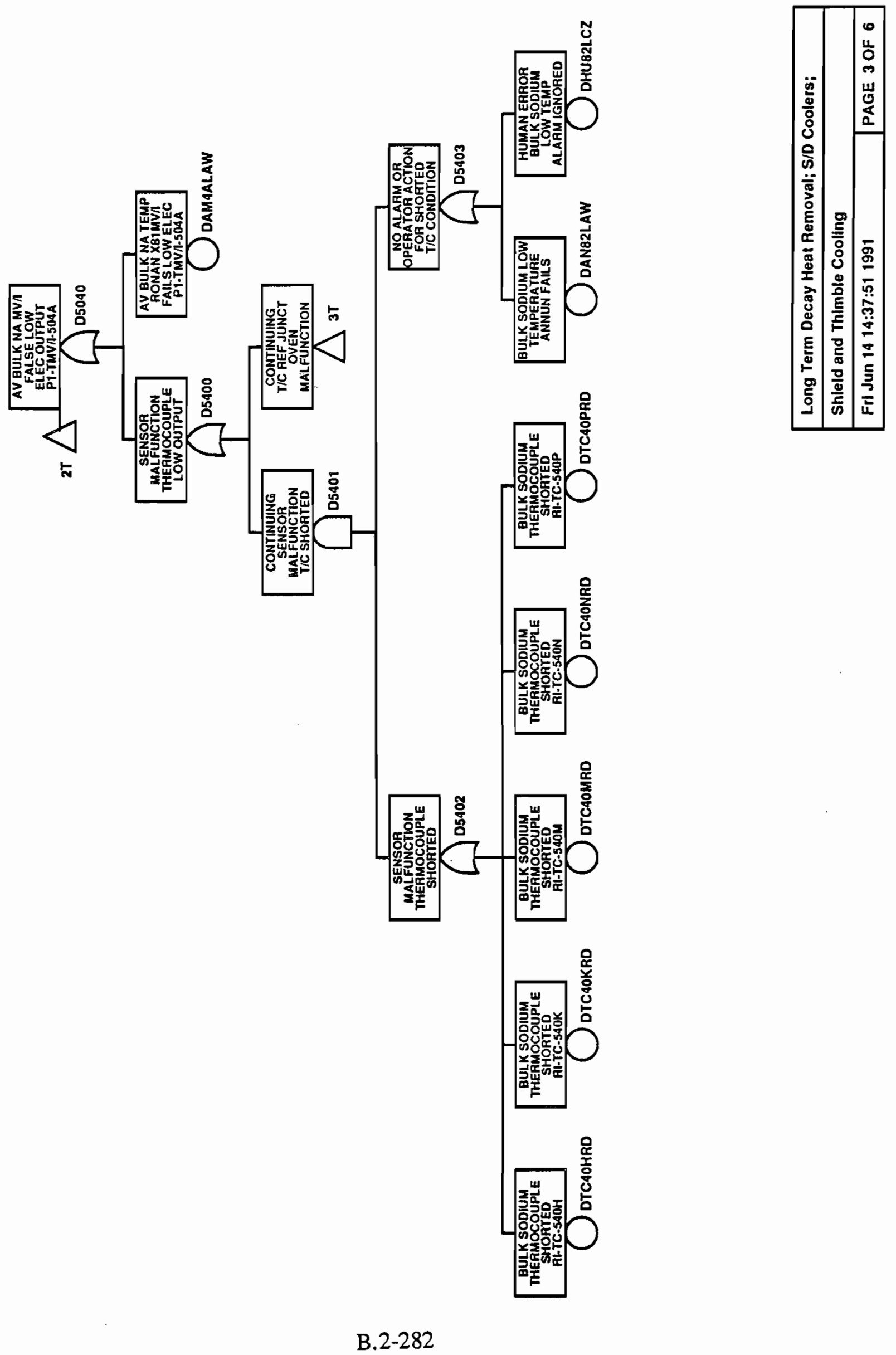



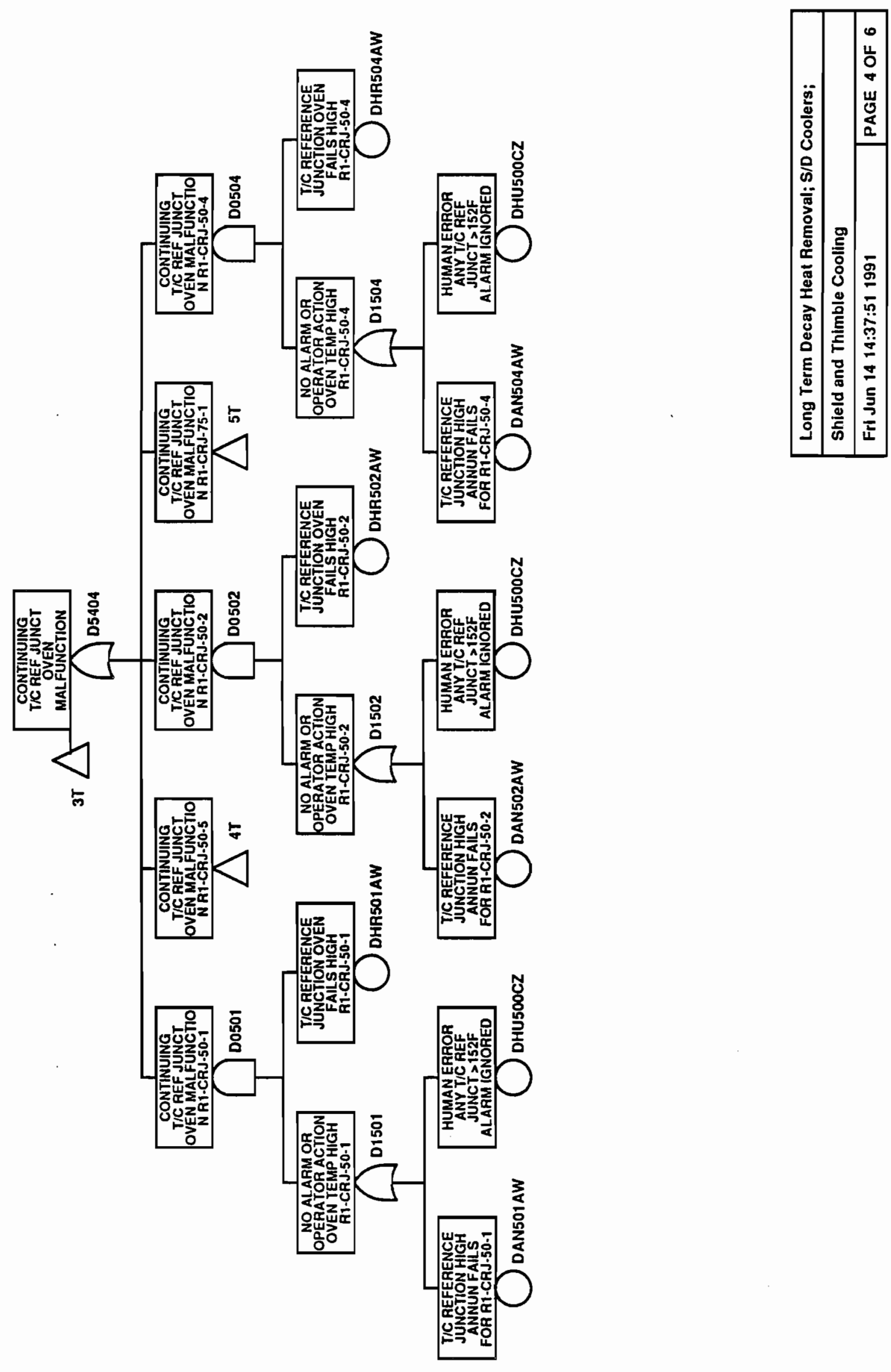


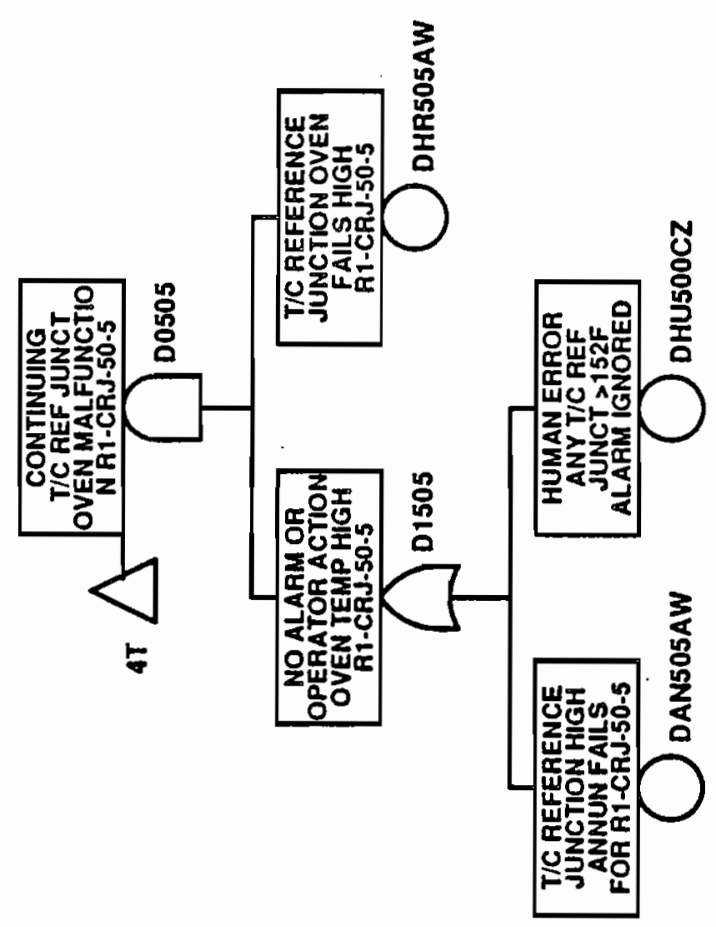




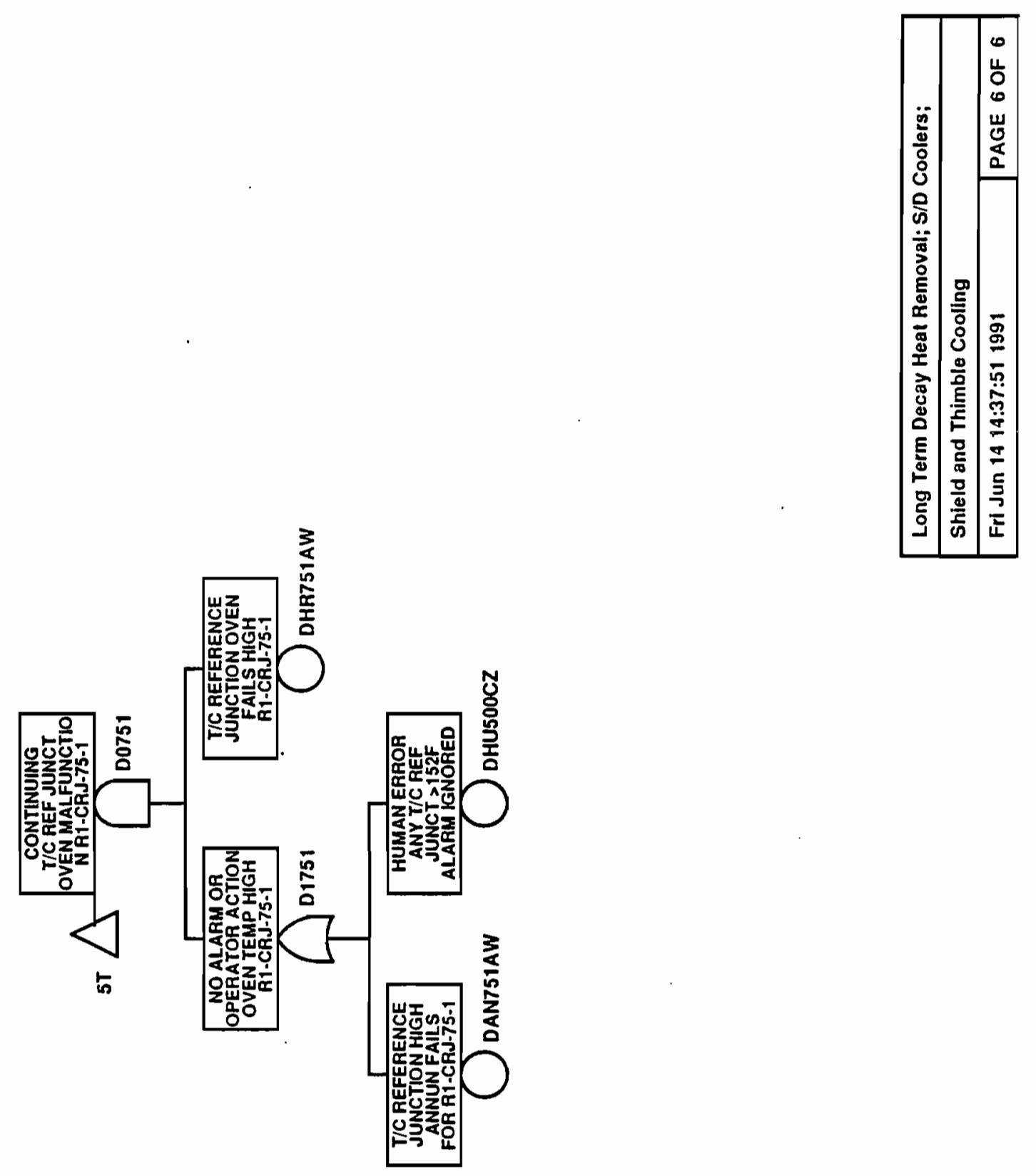




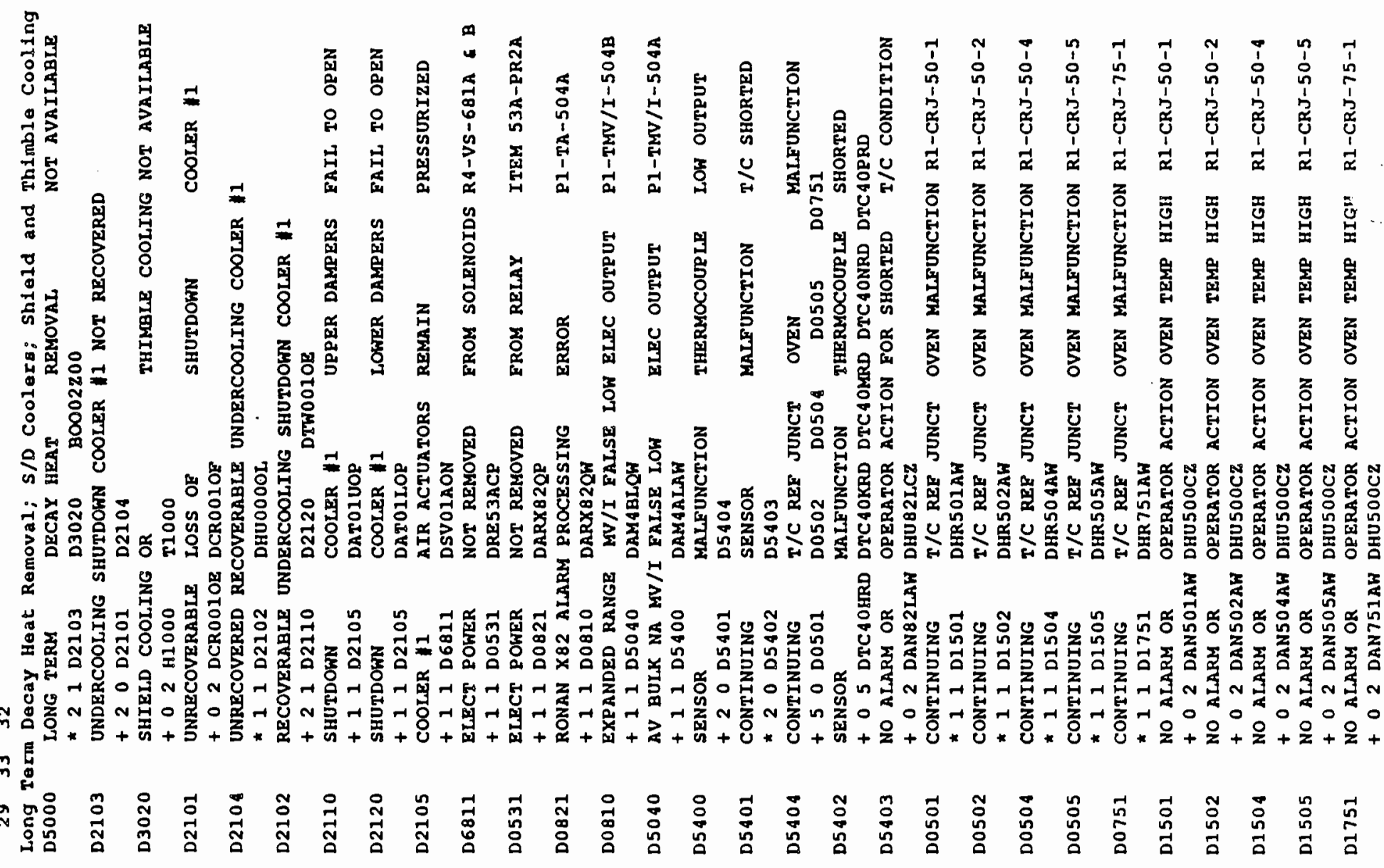




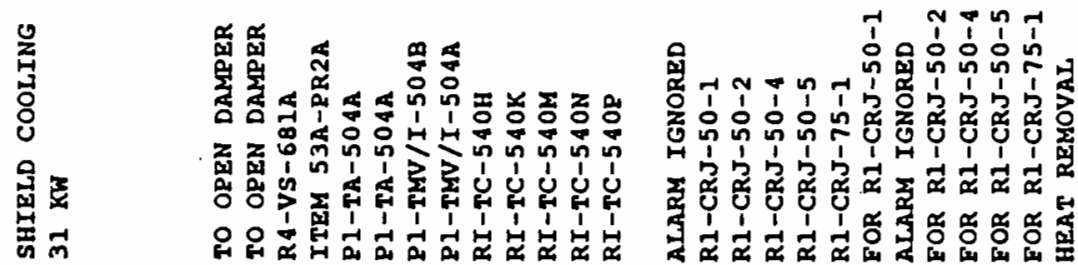

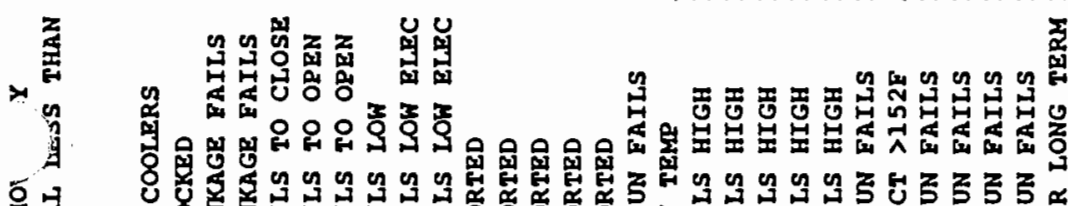

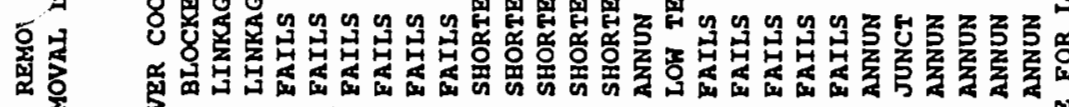

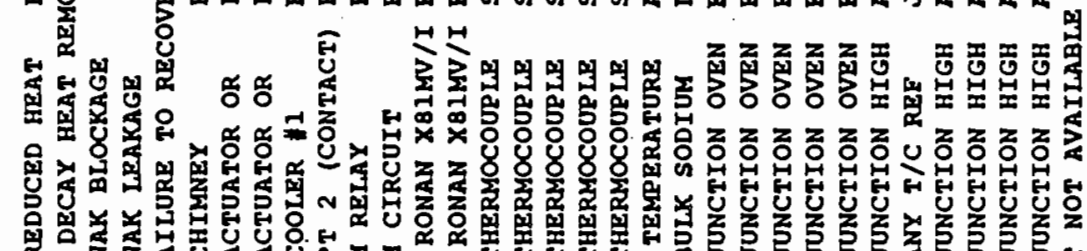

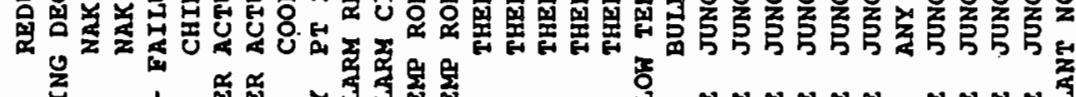

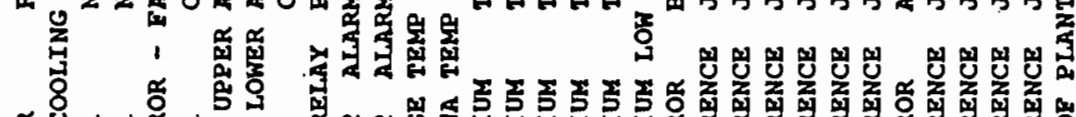

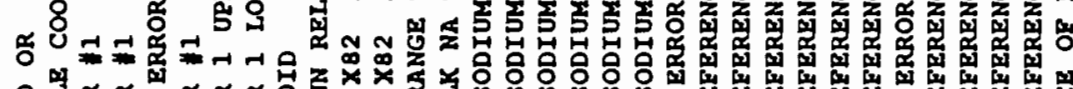

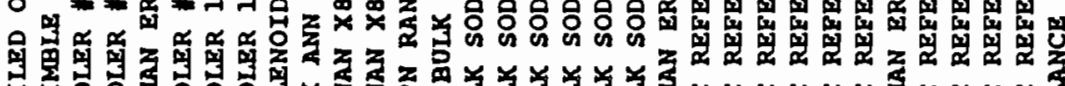

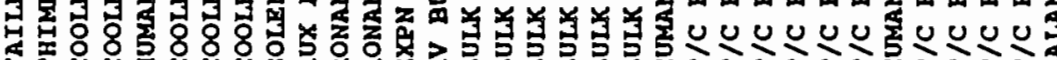
ज्ञ

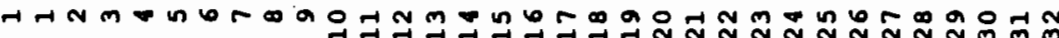
000000000000000000000000000000000 m m $000000000000000000000000000000 \mathrm{~N}$

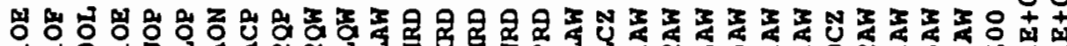
○

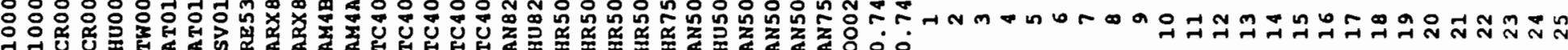

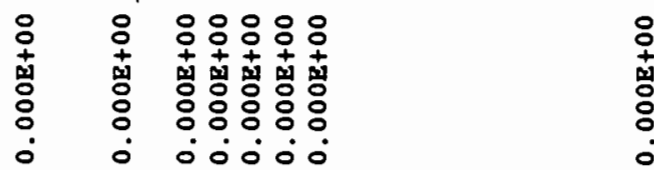

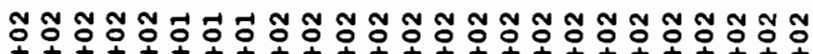

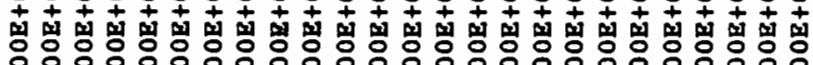

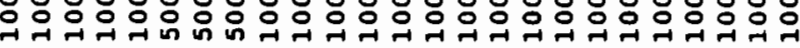

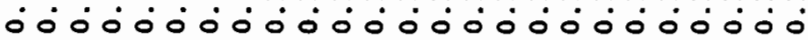

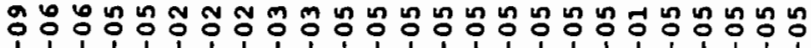

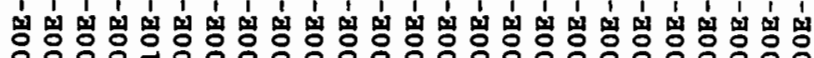

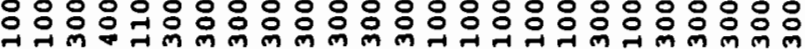
m

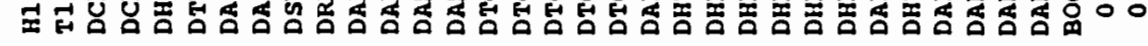




\section{8
$\vdots$
0
$\dot{0}$
0
0}

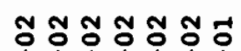

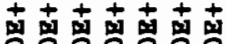

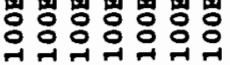

00.0.

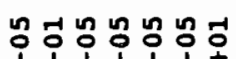

背

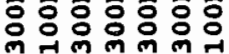

¿000000

HOAHAH

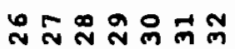



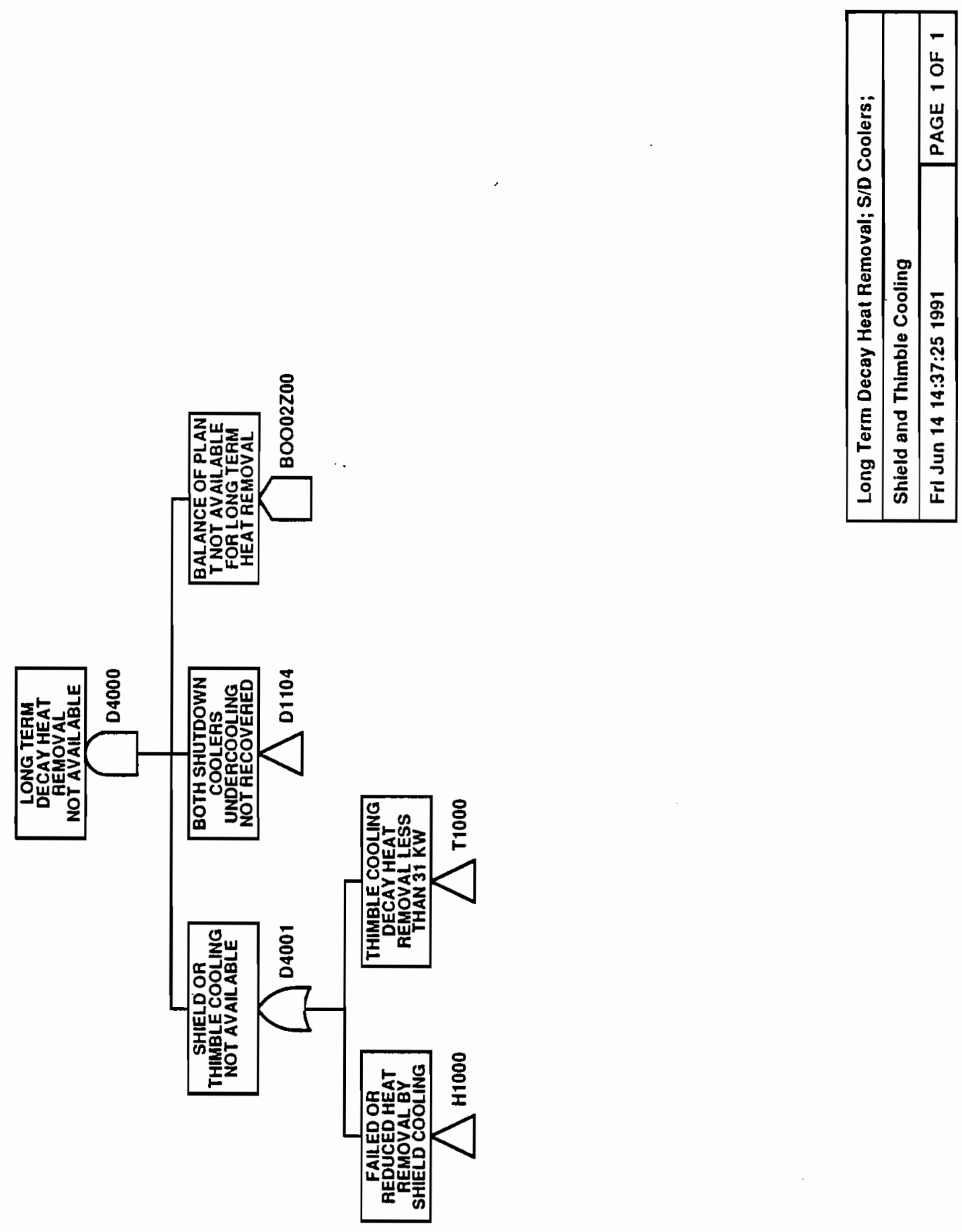

B. 2-289 


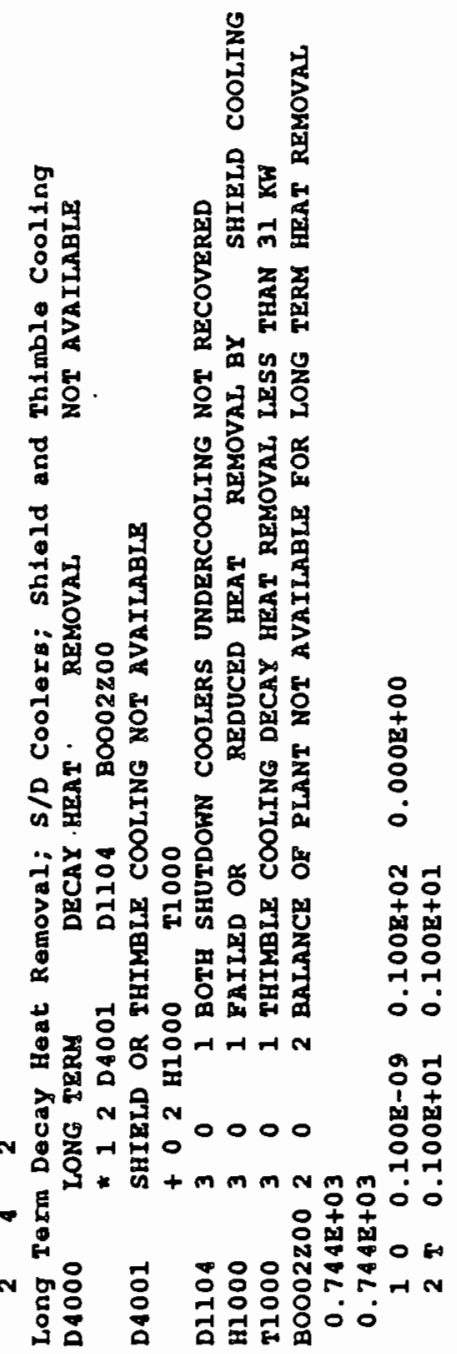




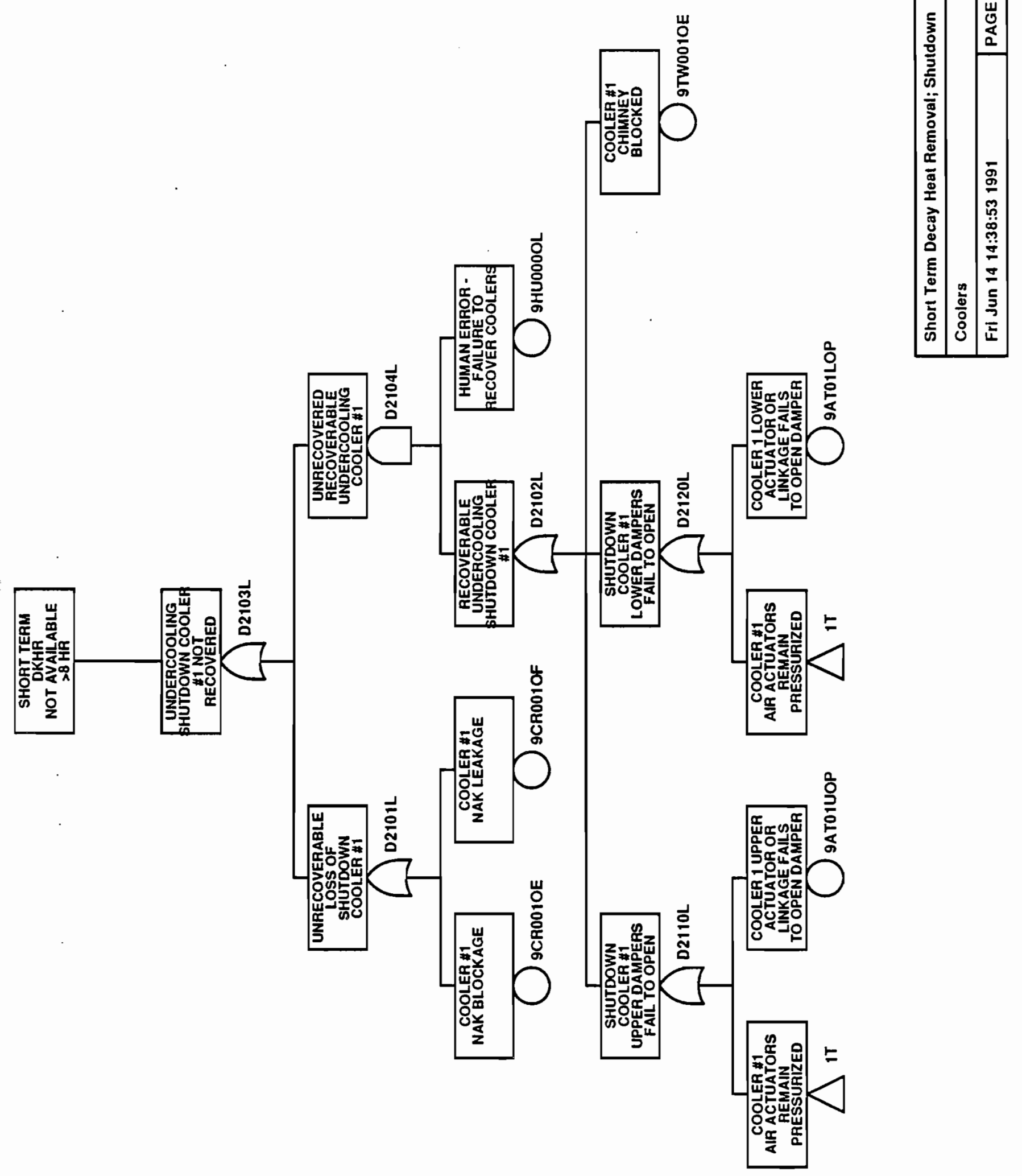




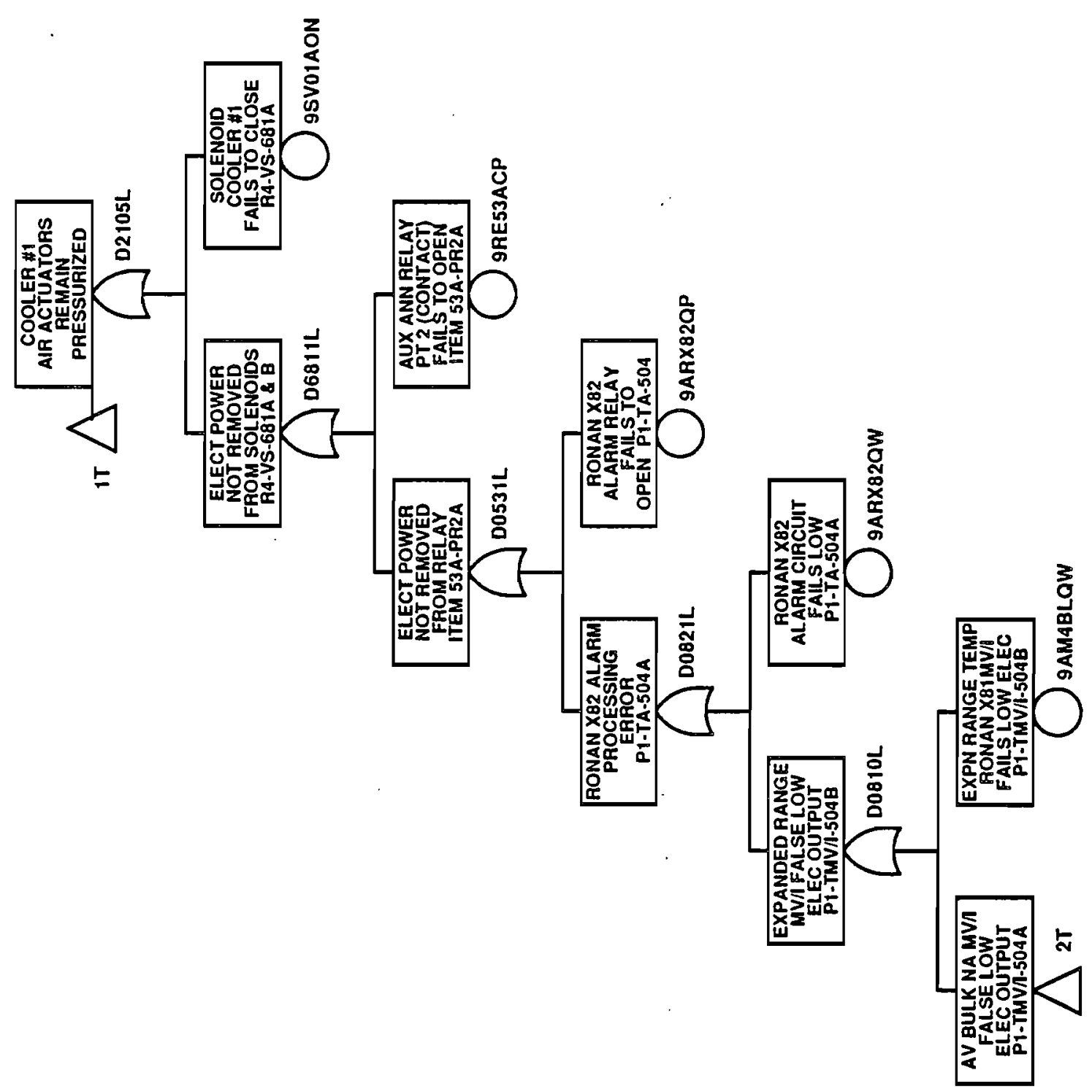



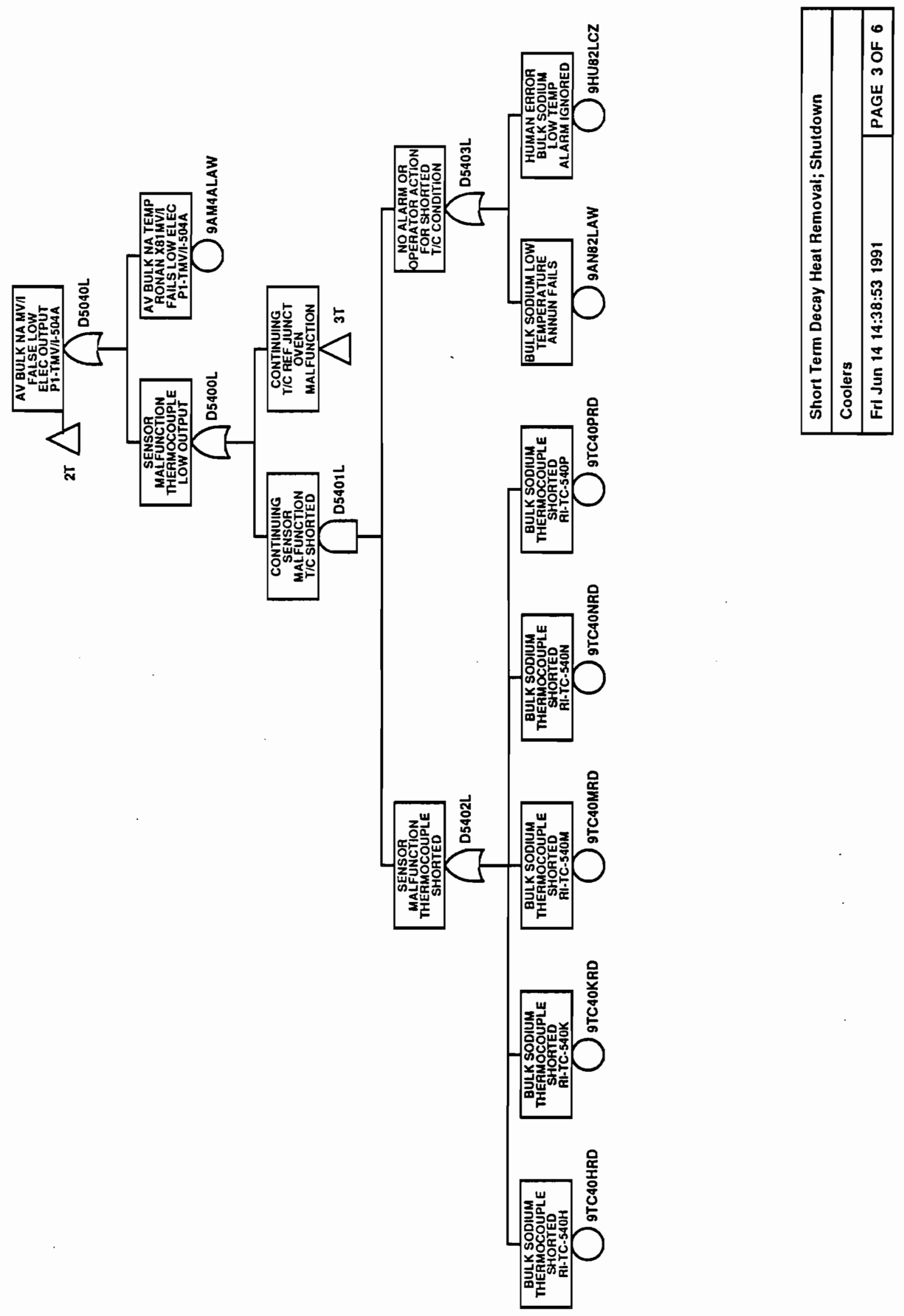

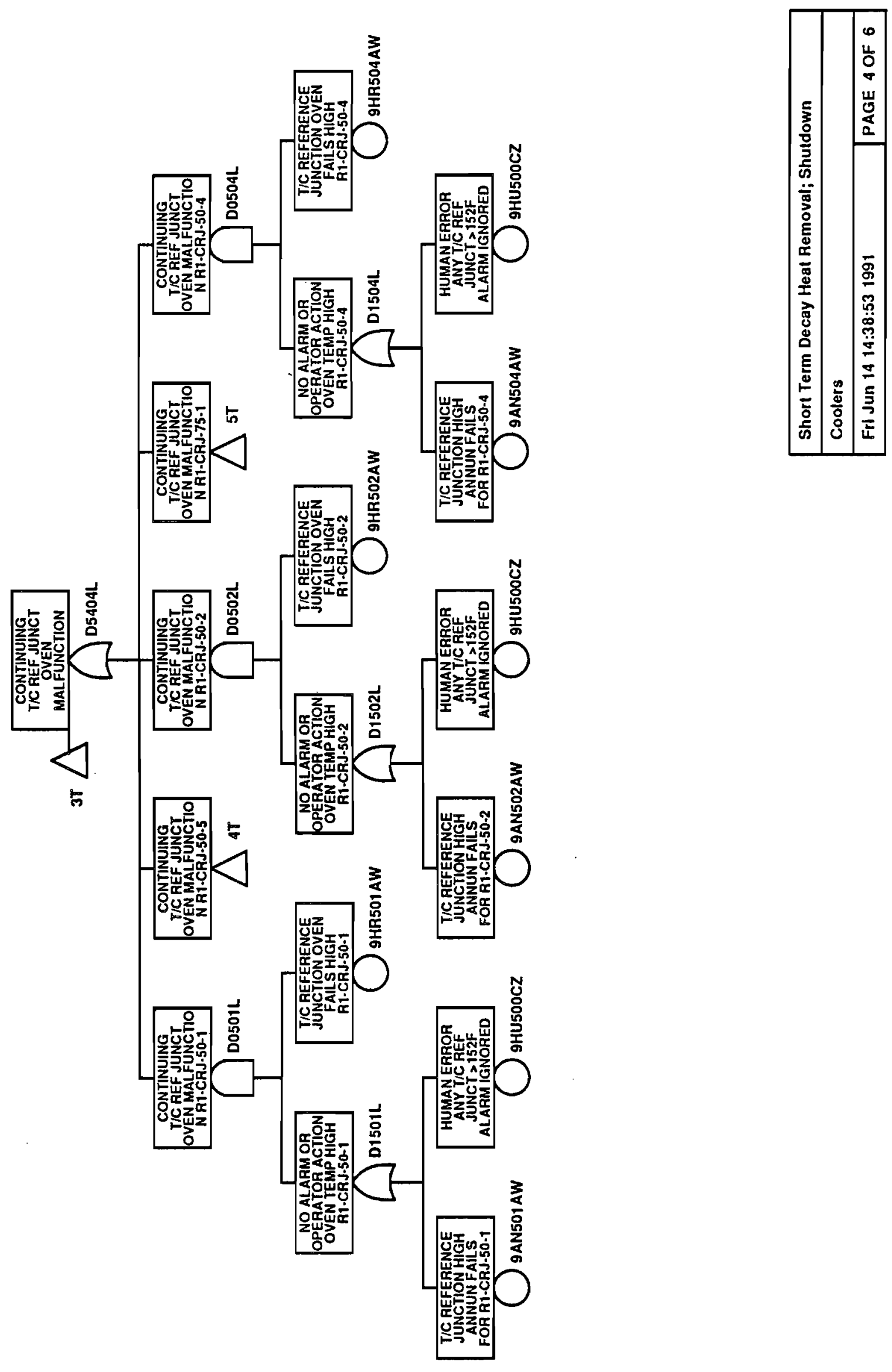


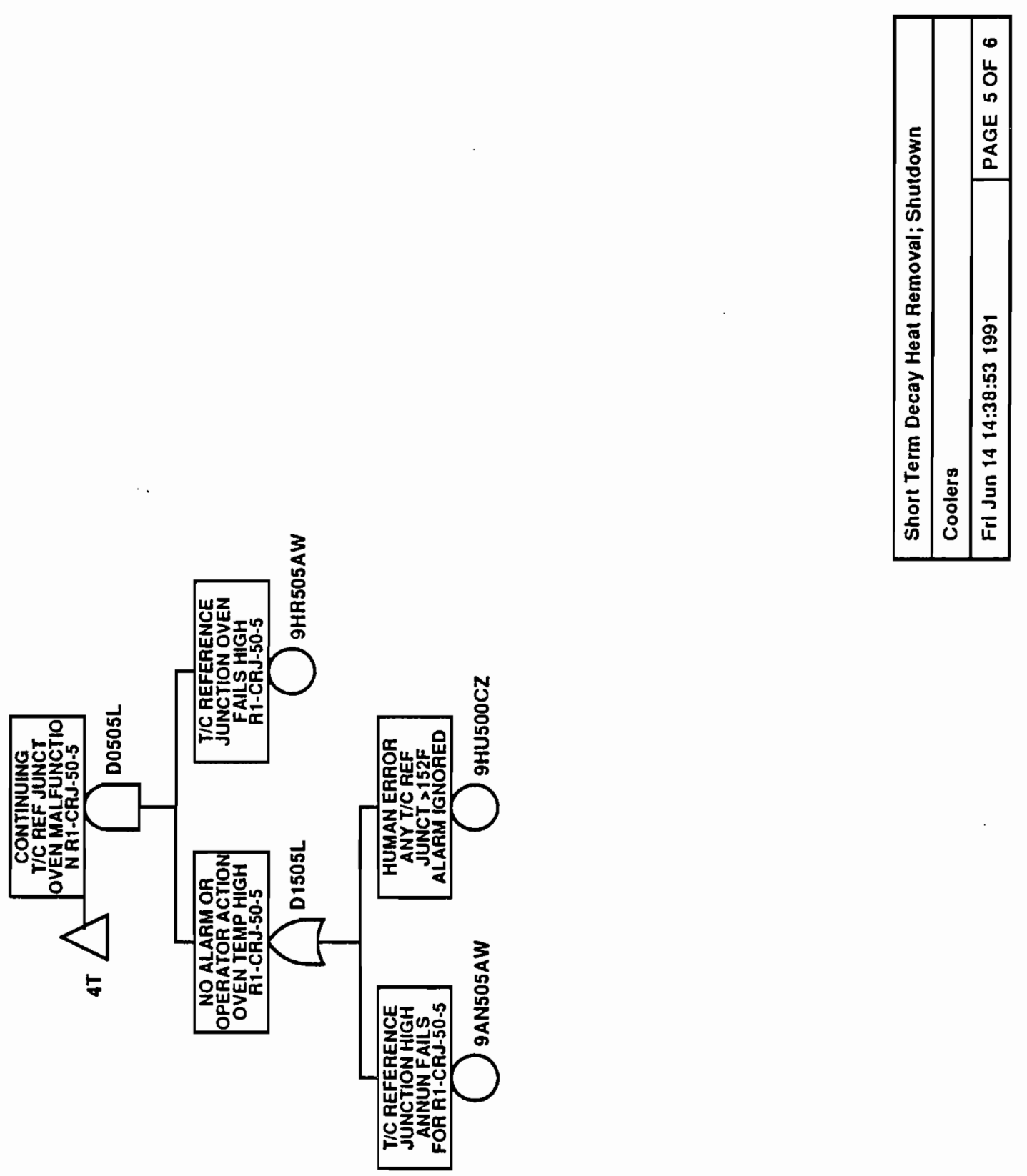



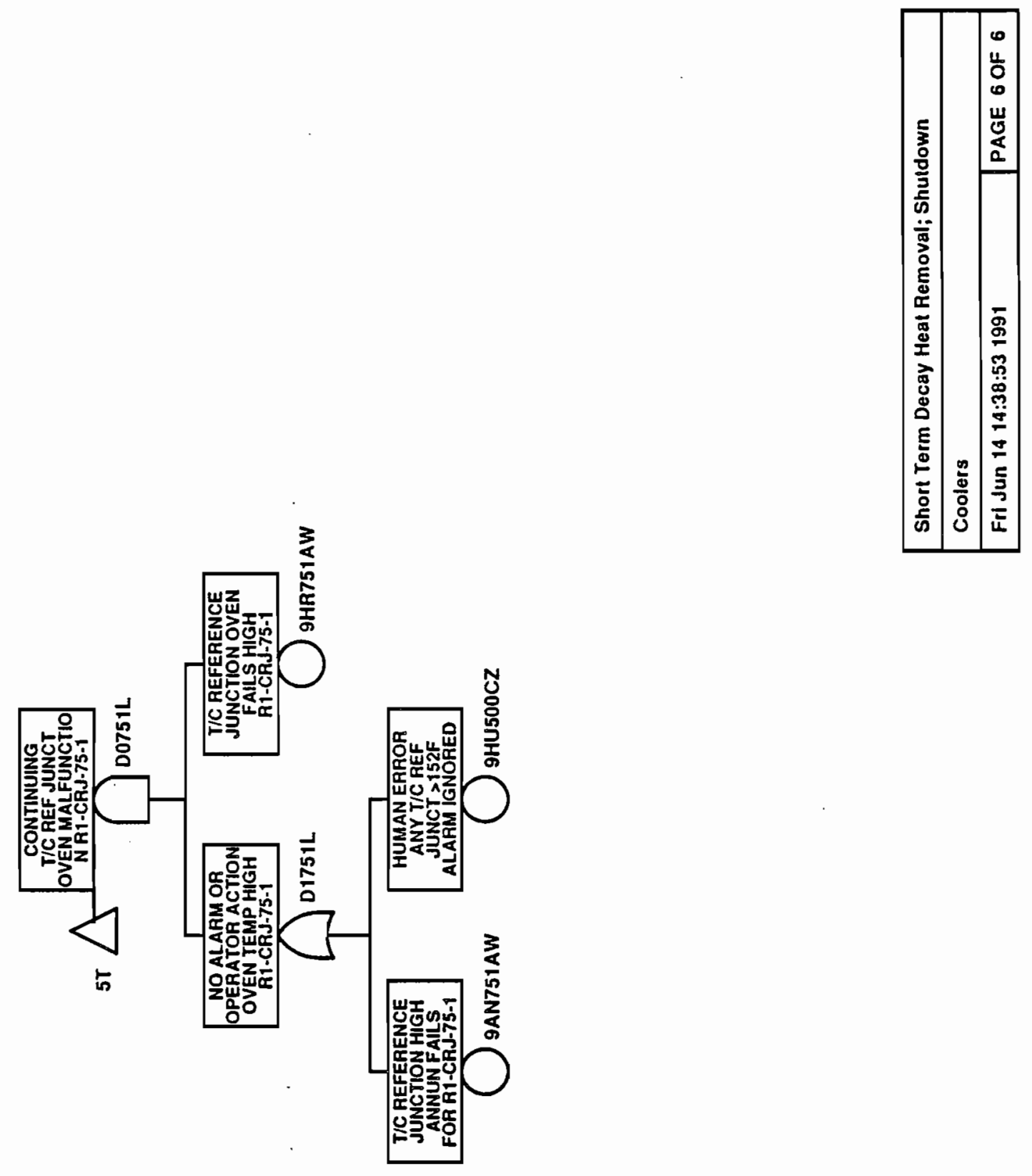


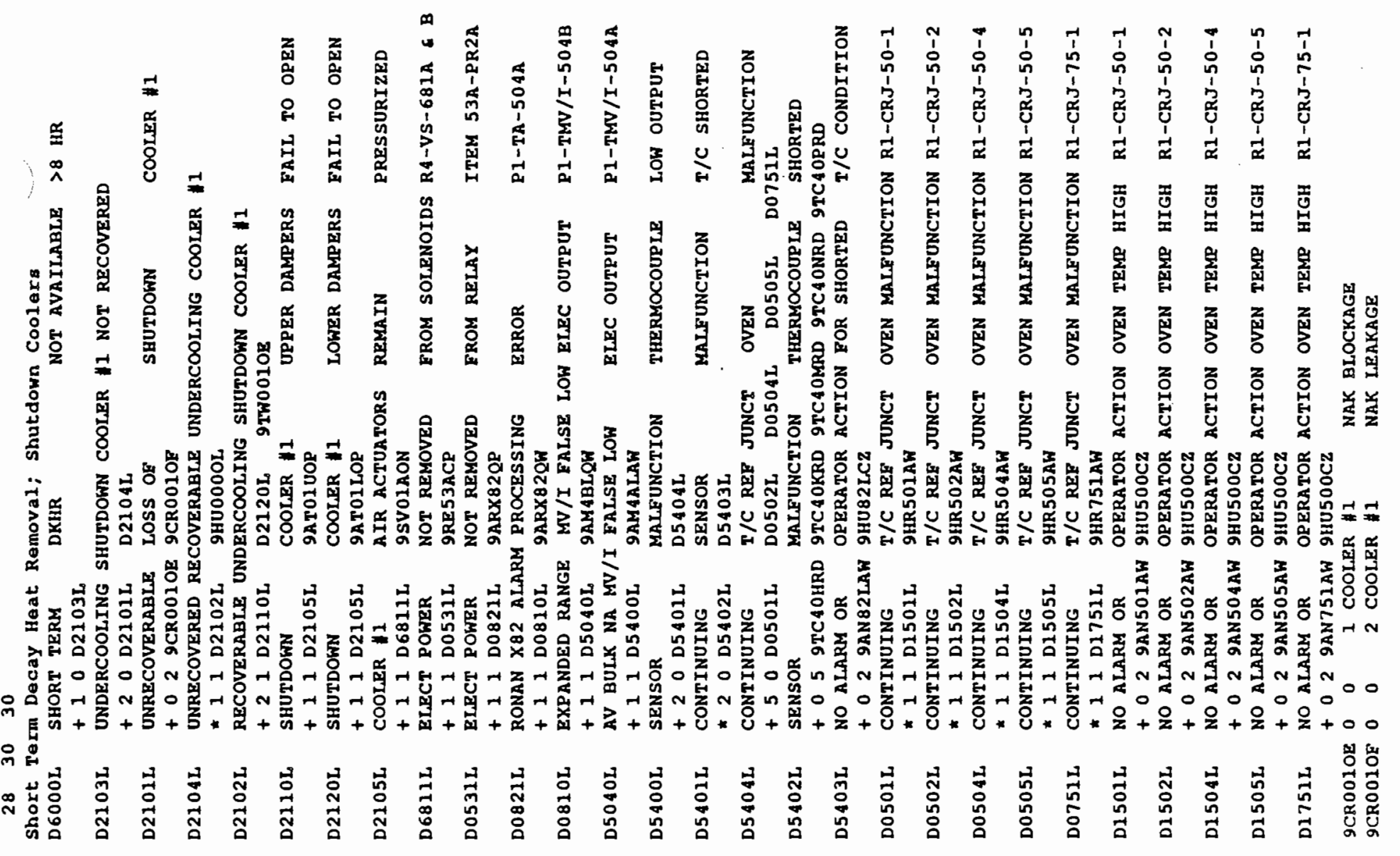




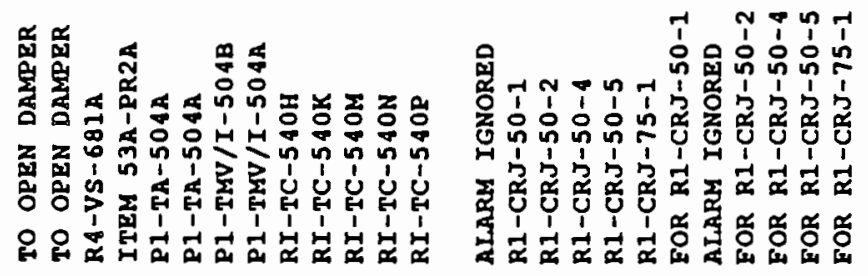

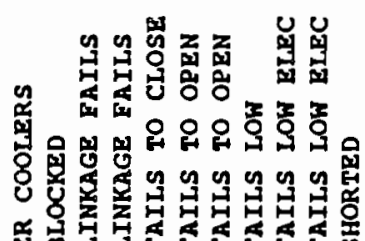

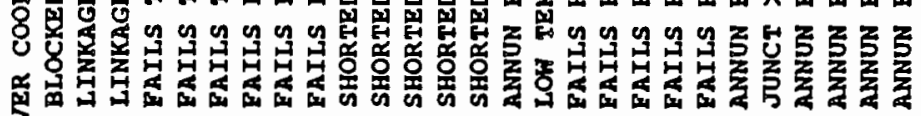

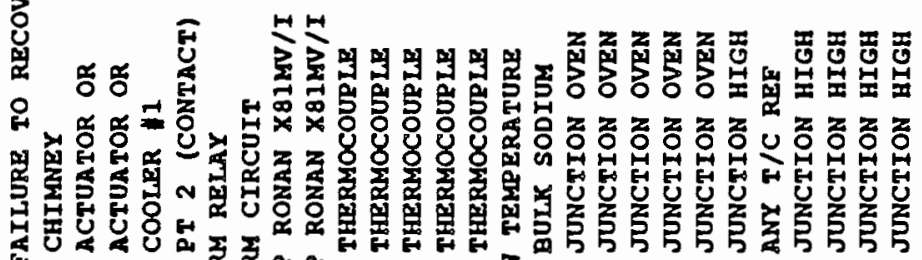

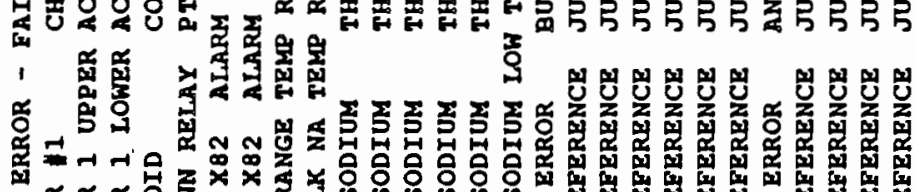

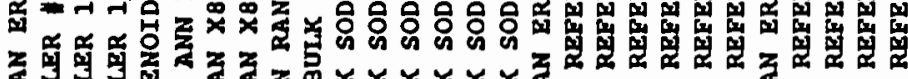

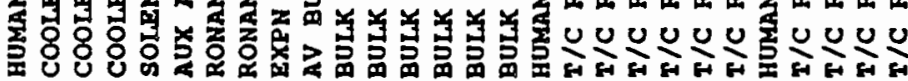

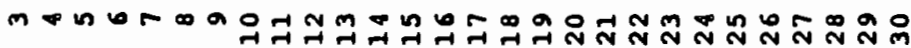
0000000000000000000000000000

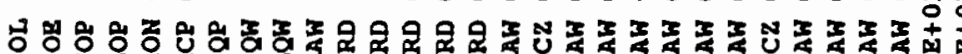

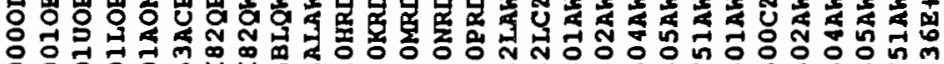

0000000000000000000000000000

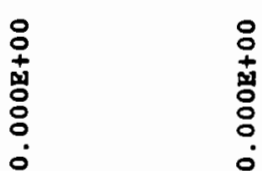

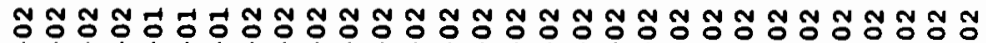

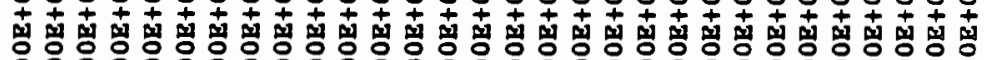

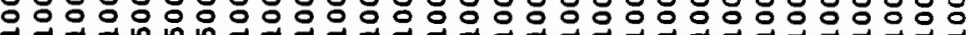

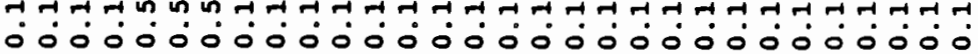

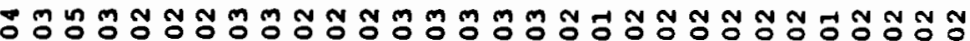
i i i i i i i i i i i i

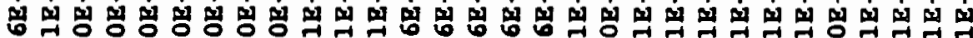

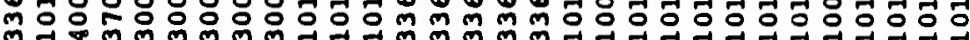

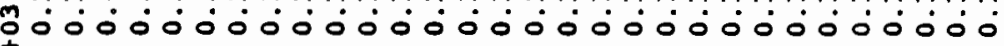
\$

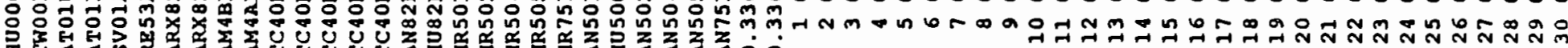

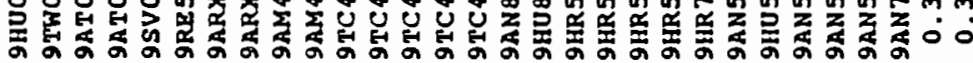




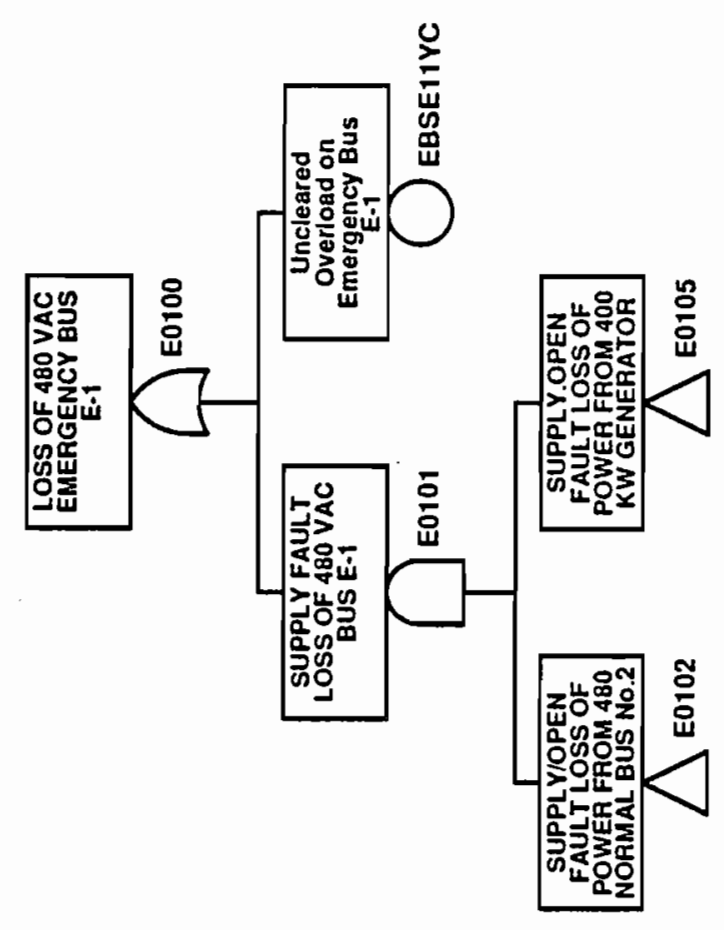

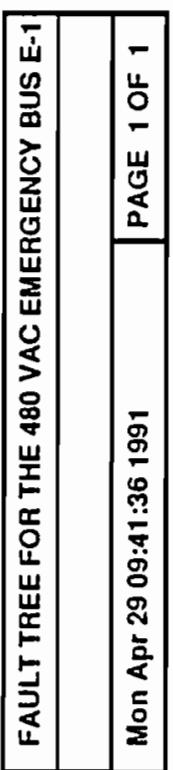




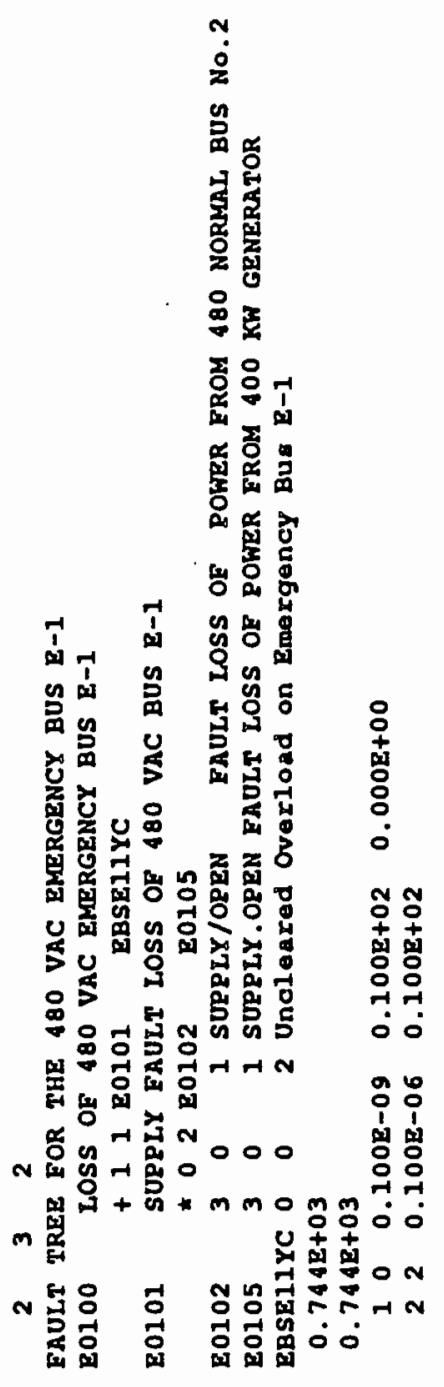

\section{B. $2-300$}




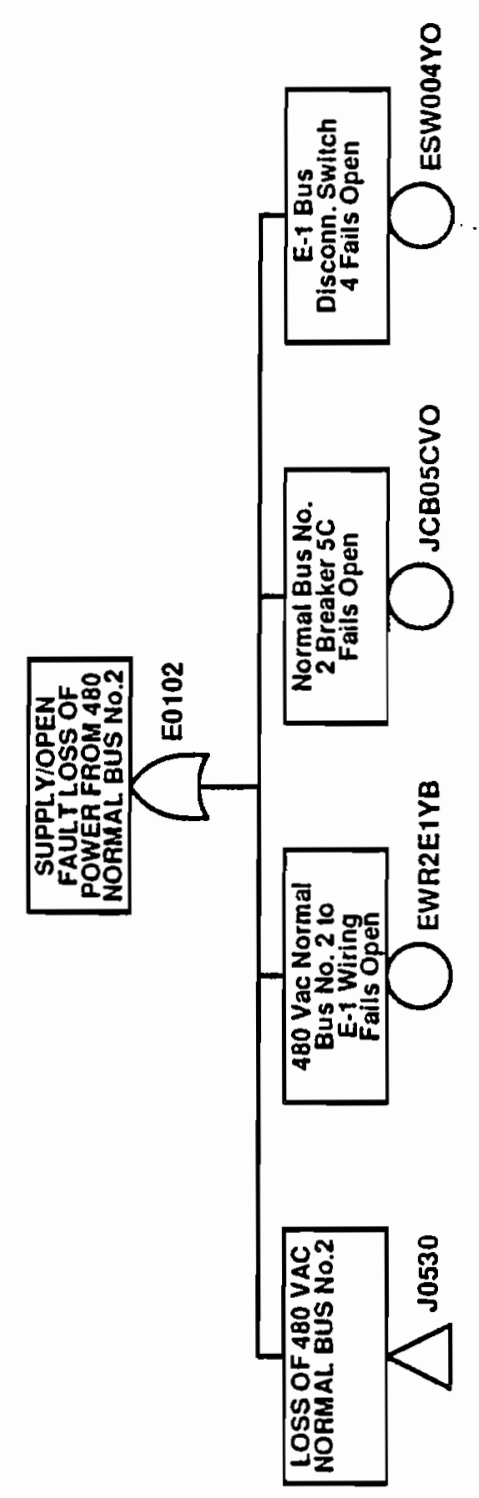

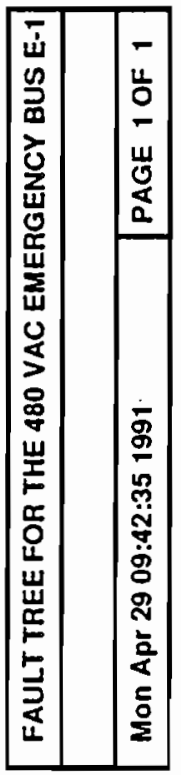

B. 2-301 


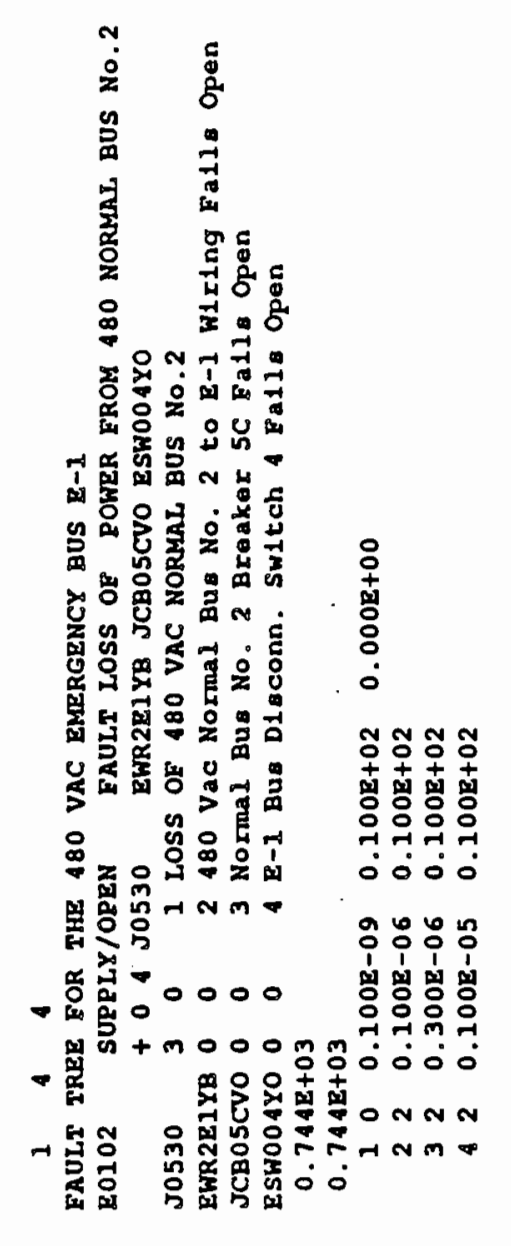

\section{B. 2-302}



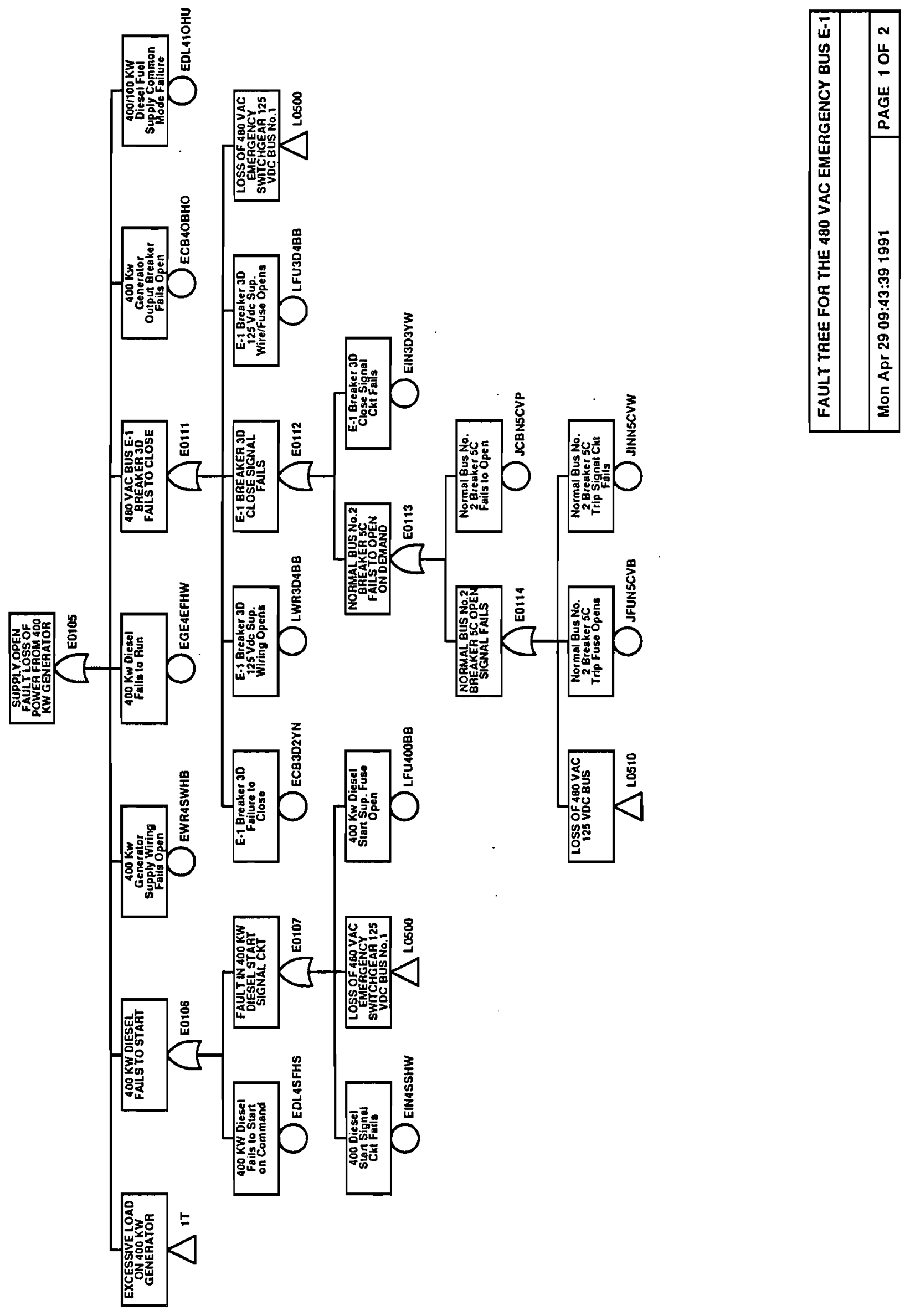

B. 2-303 


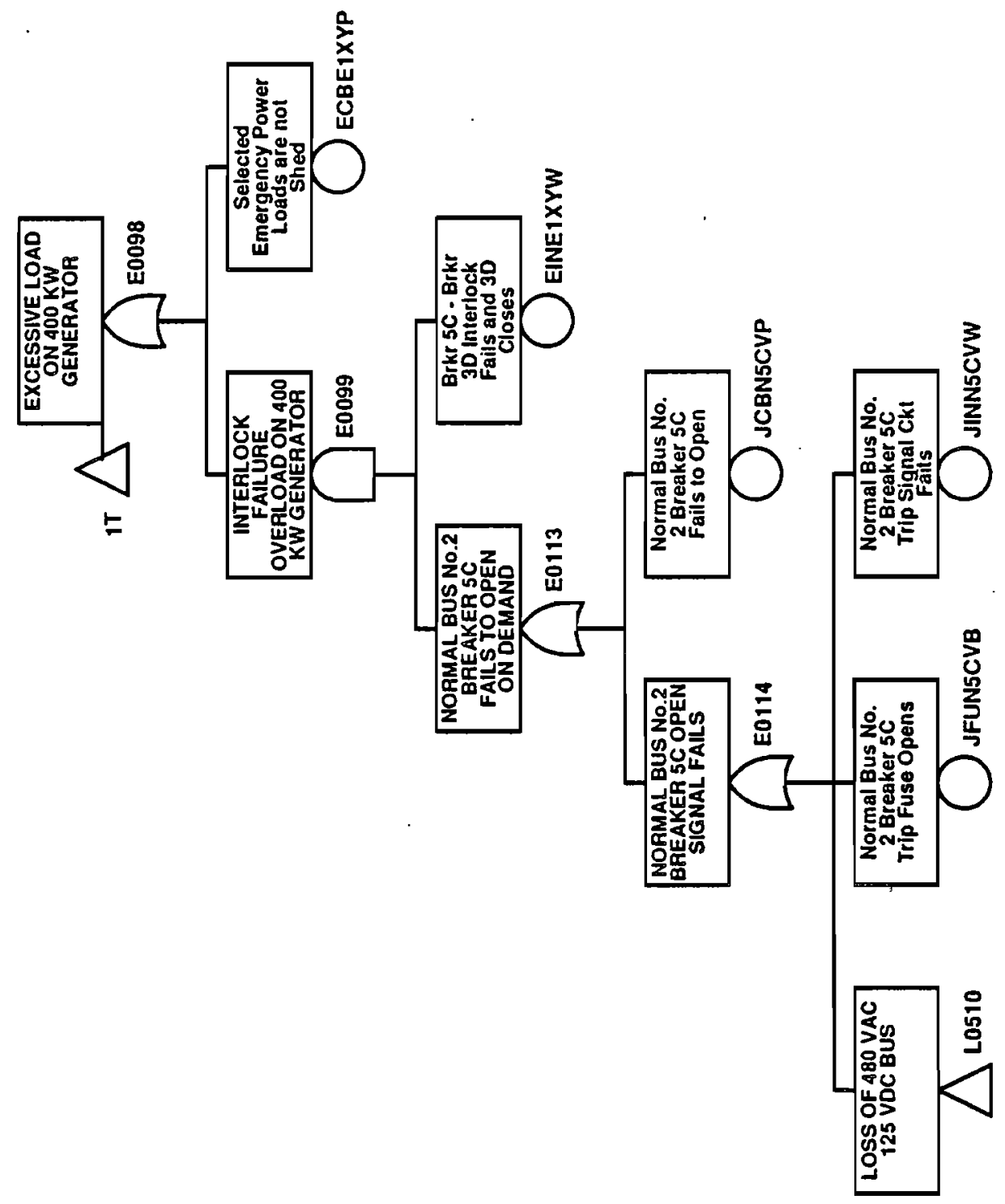




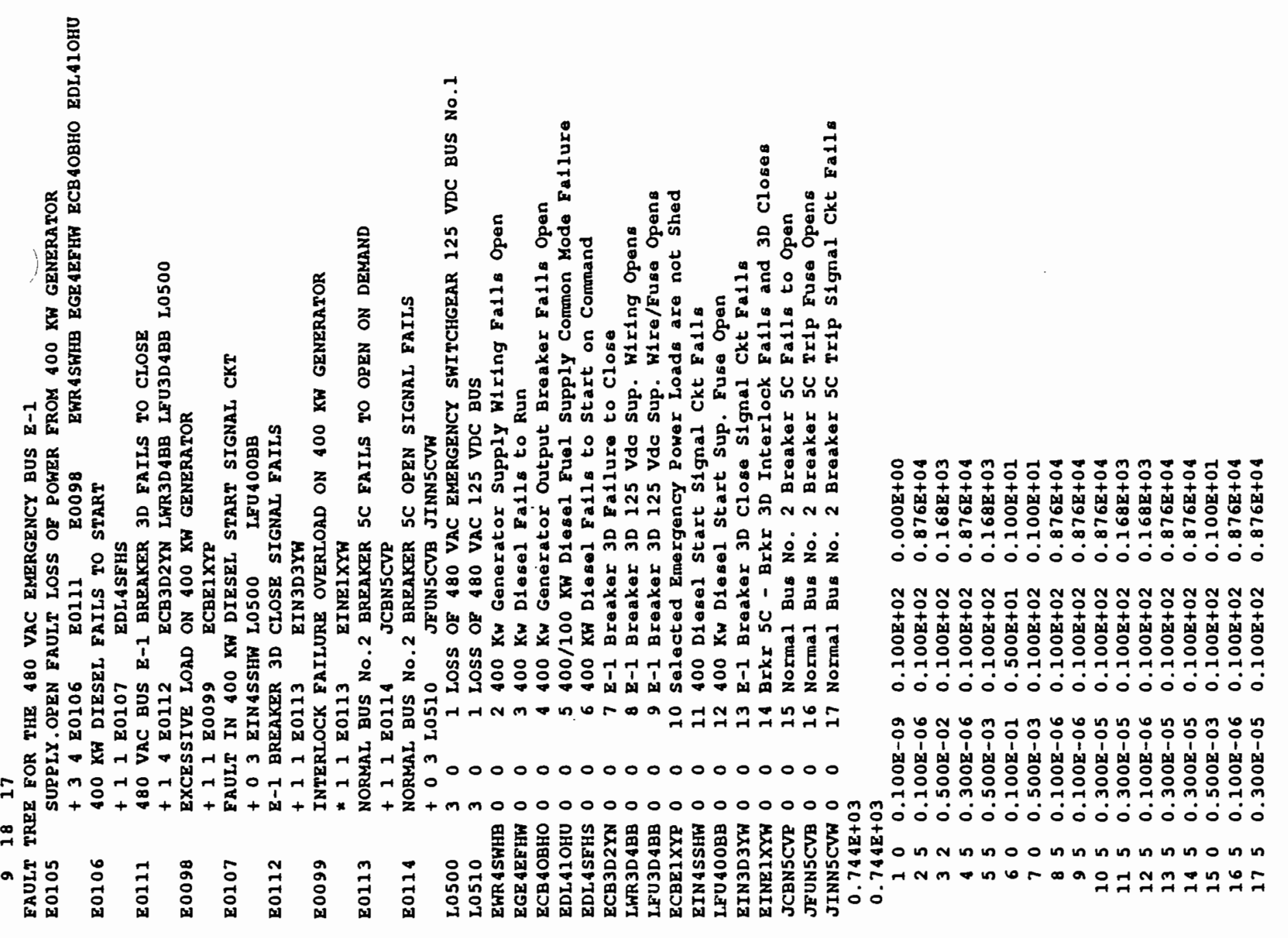



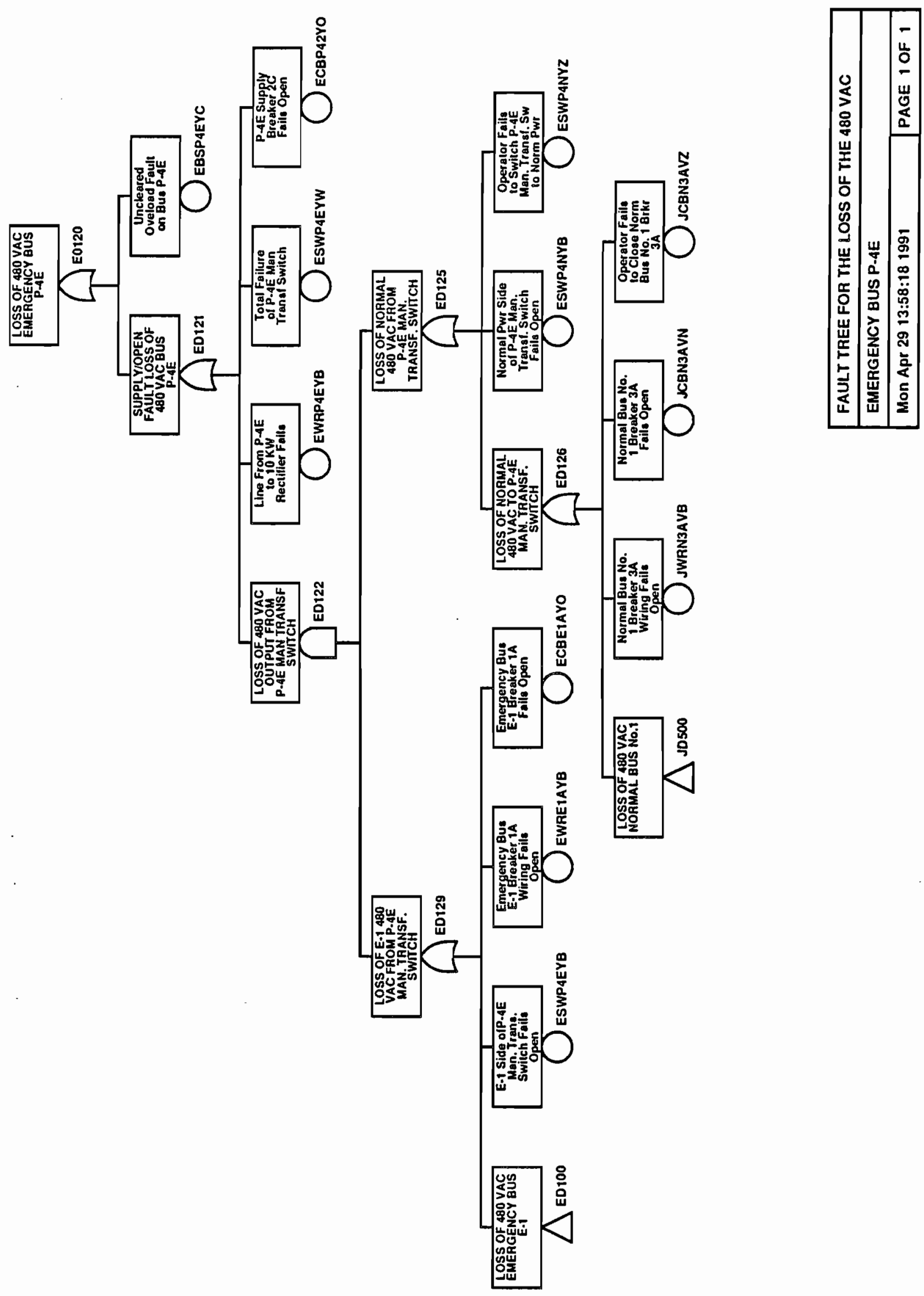


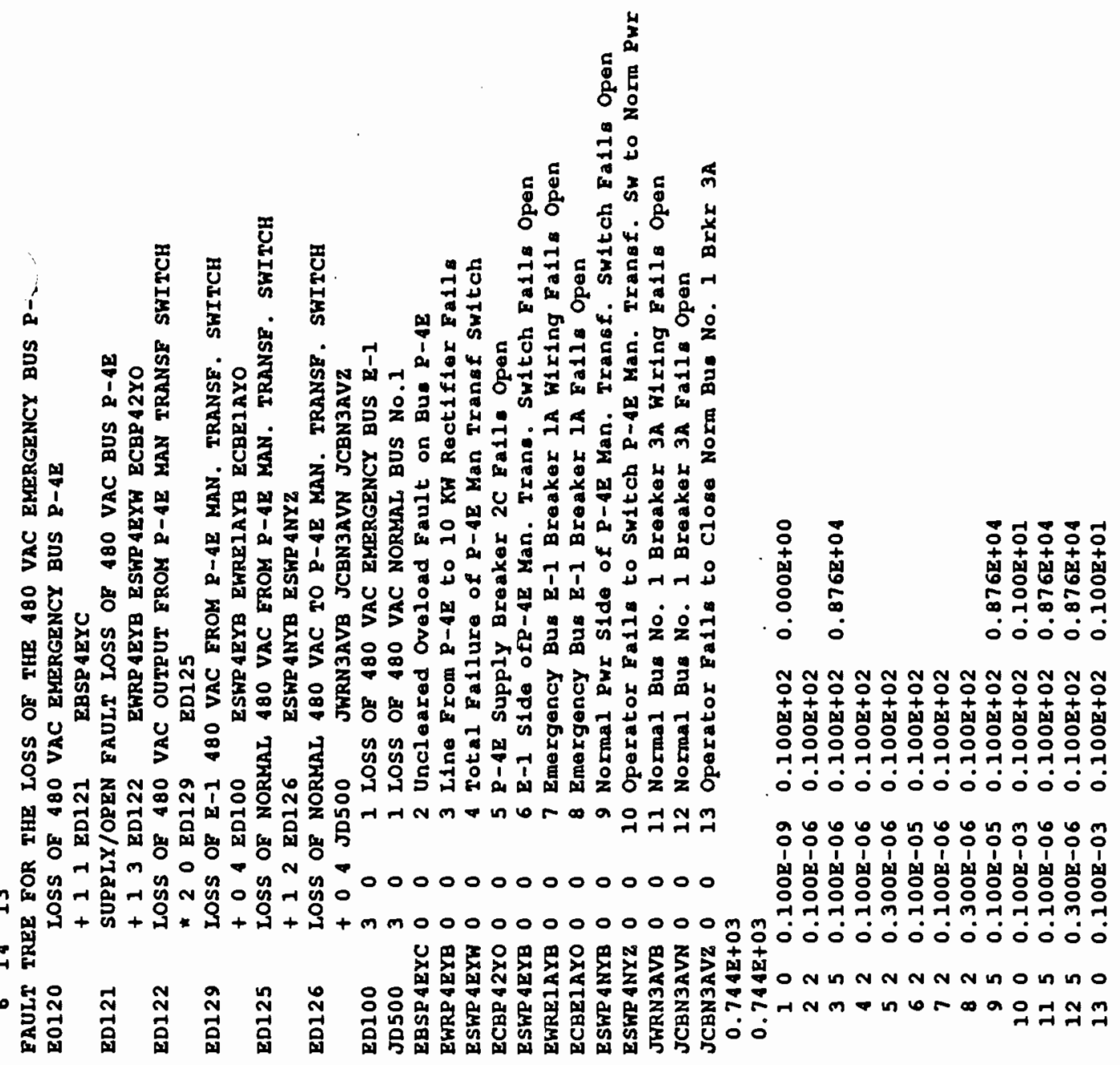



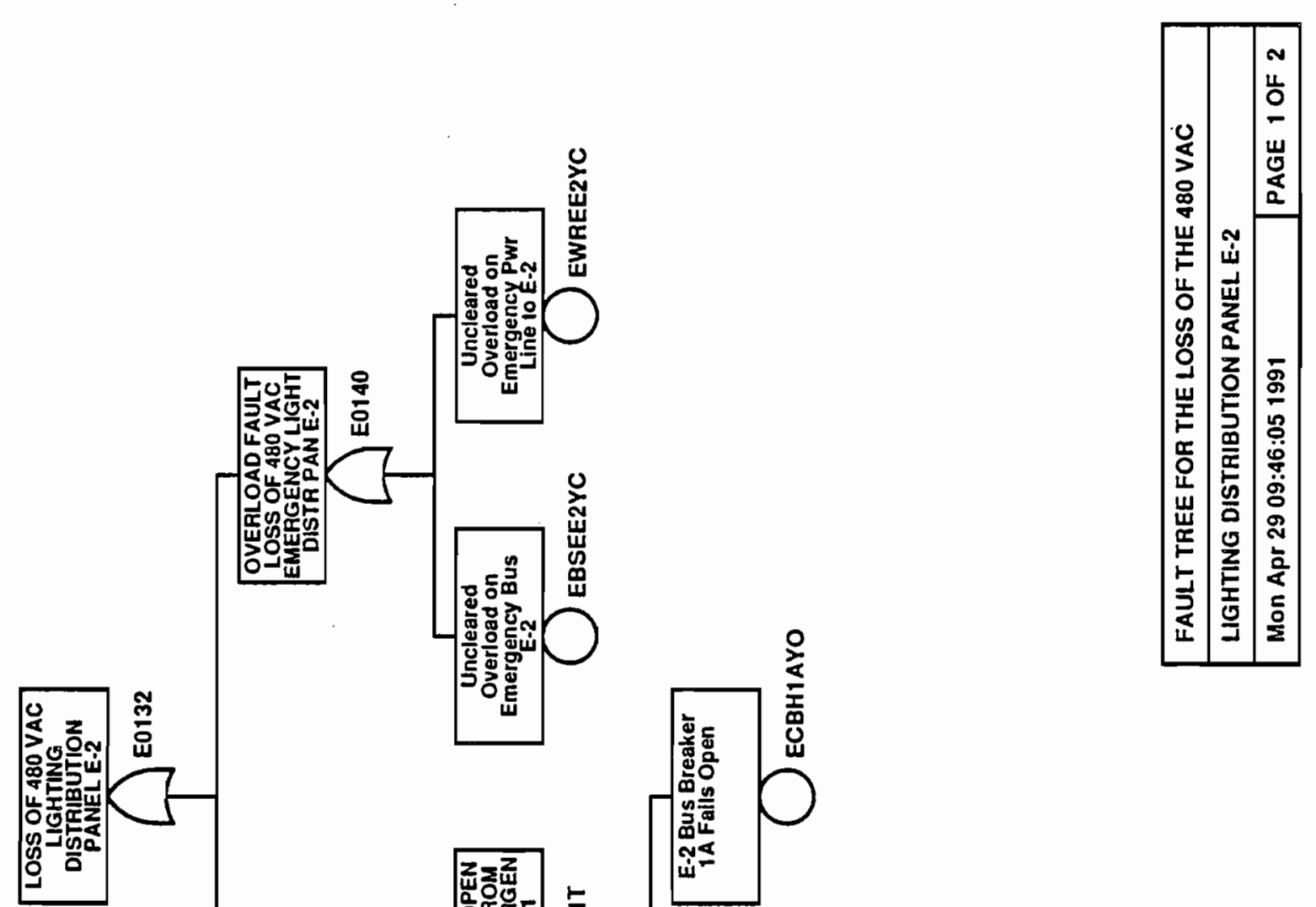


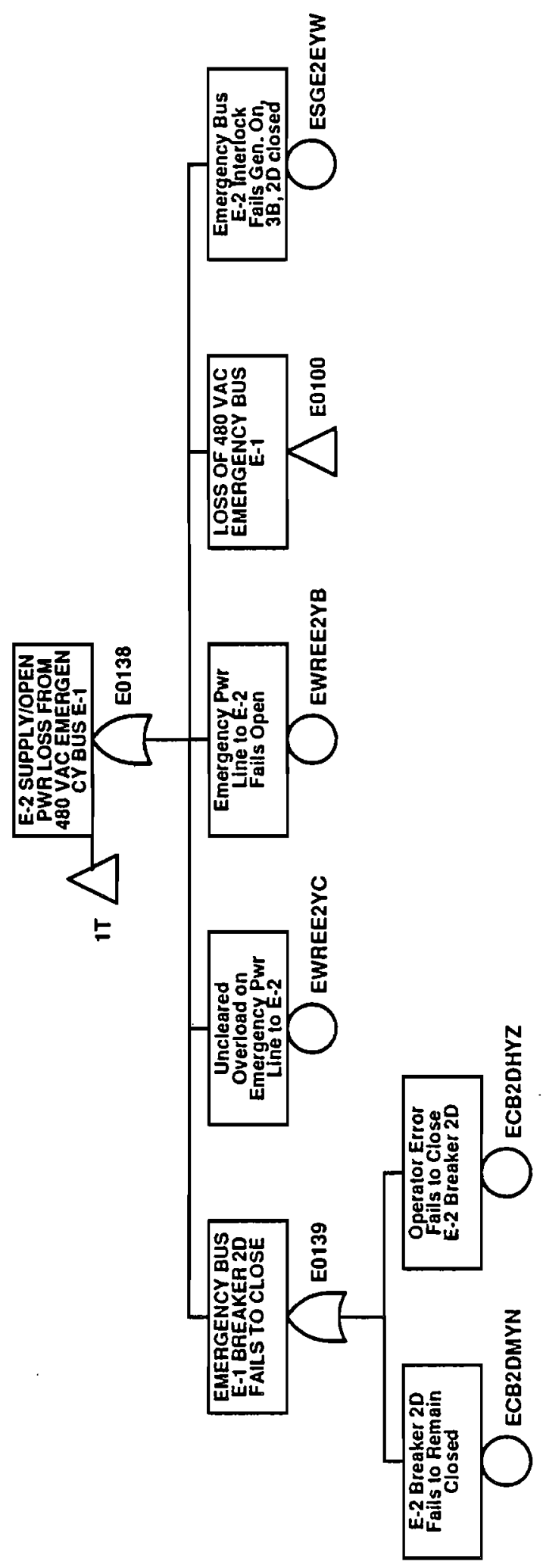

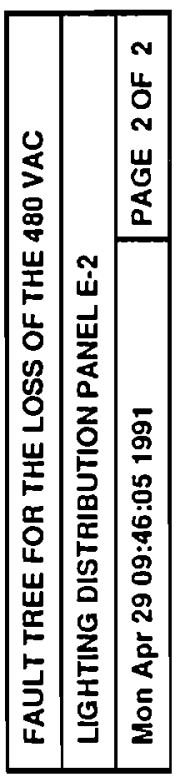

B. 2-309 


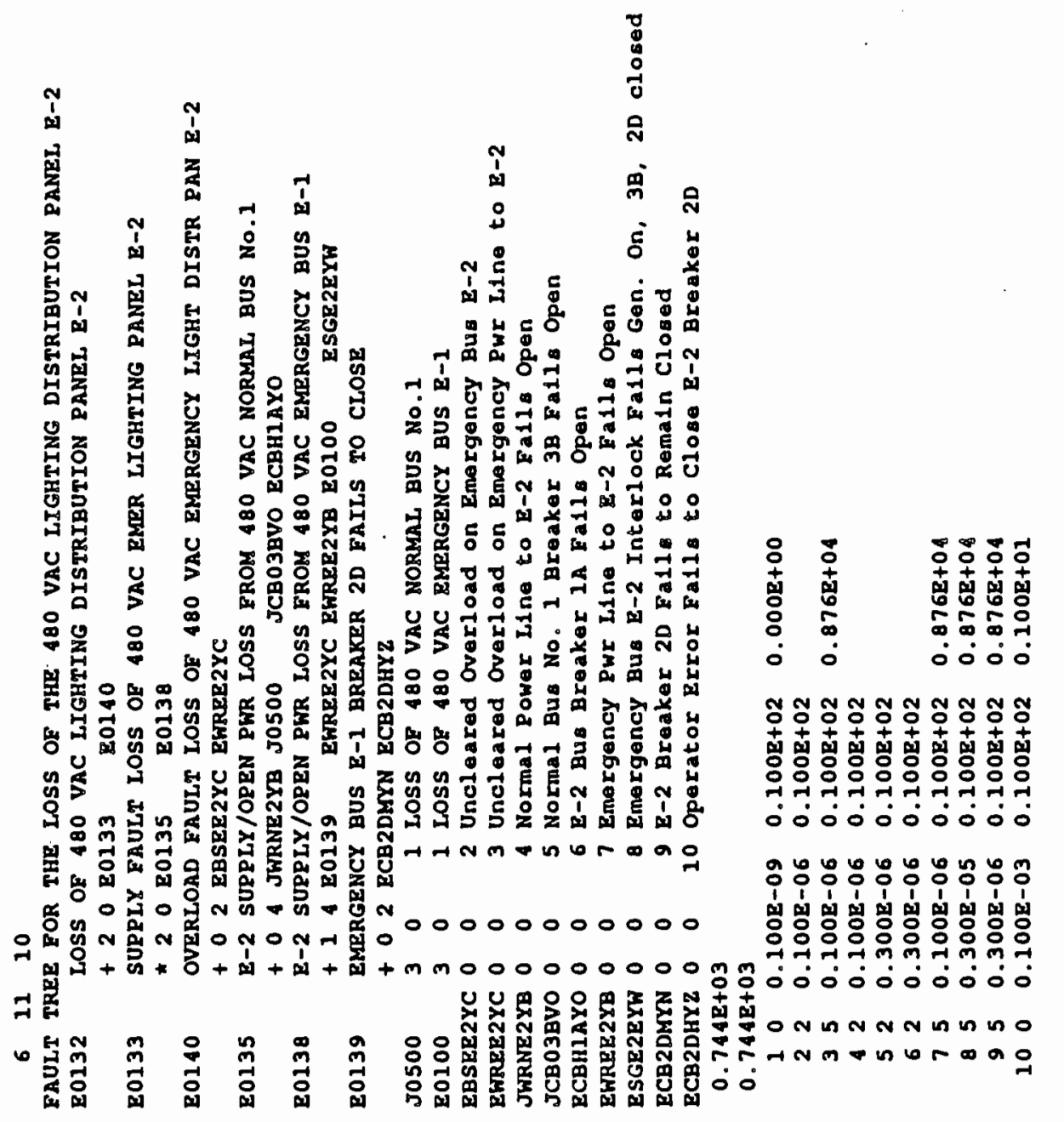




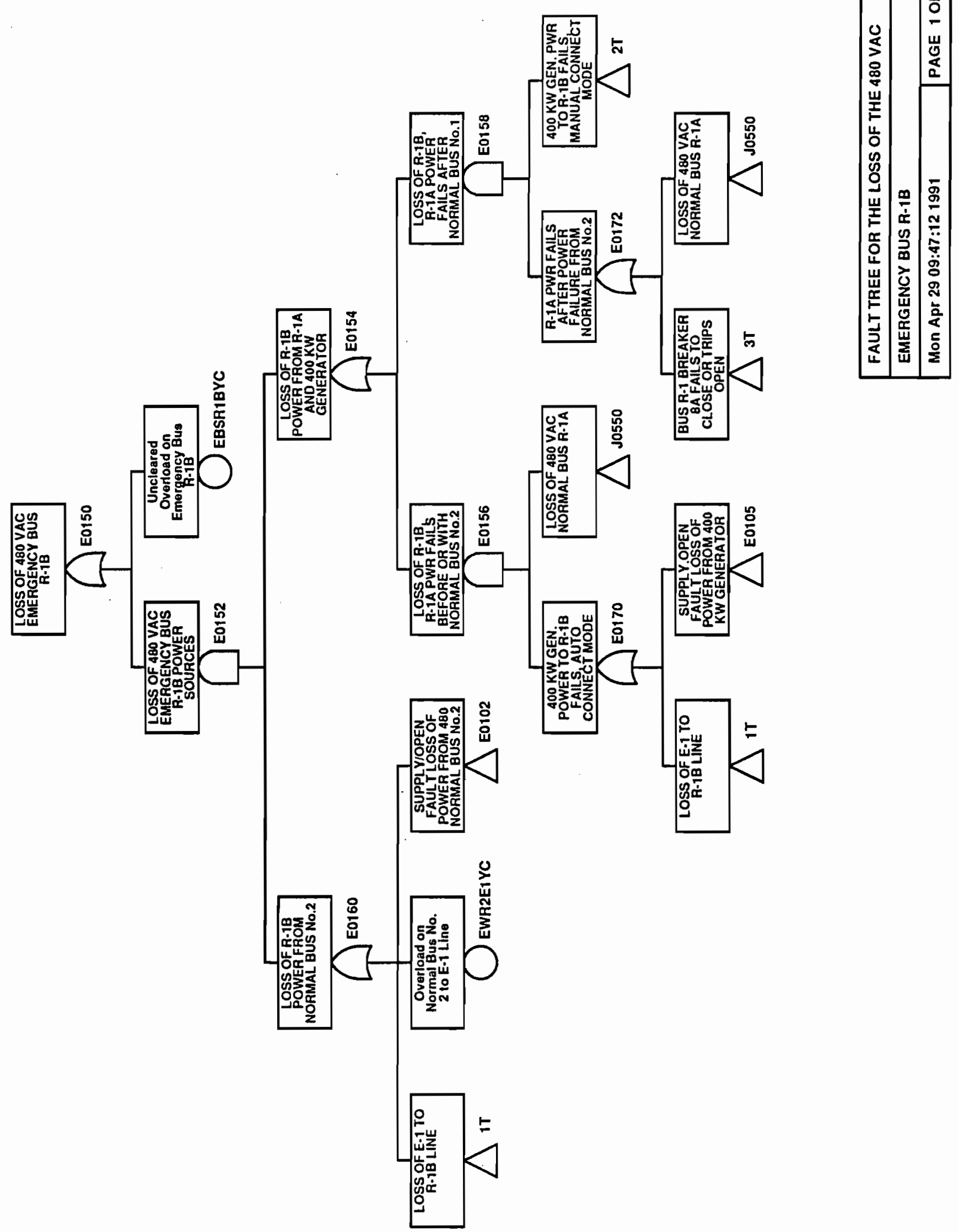

B. 2-311 


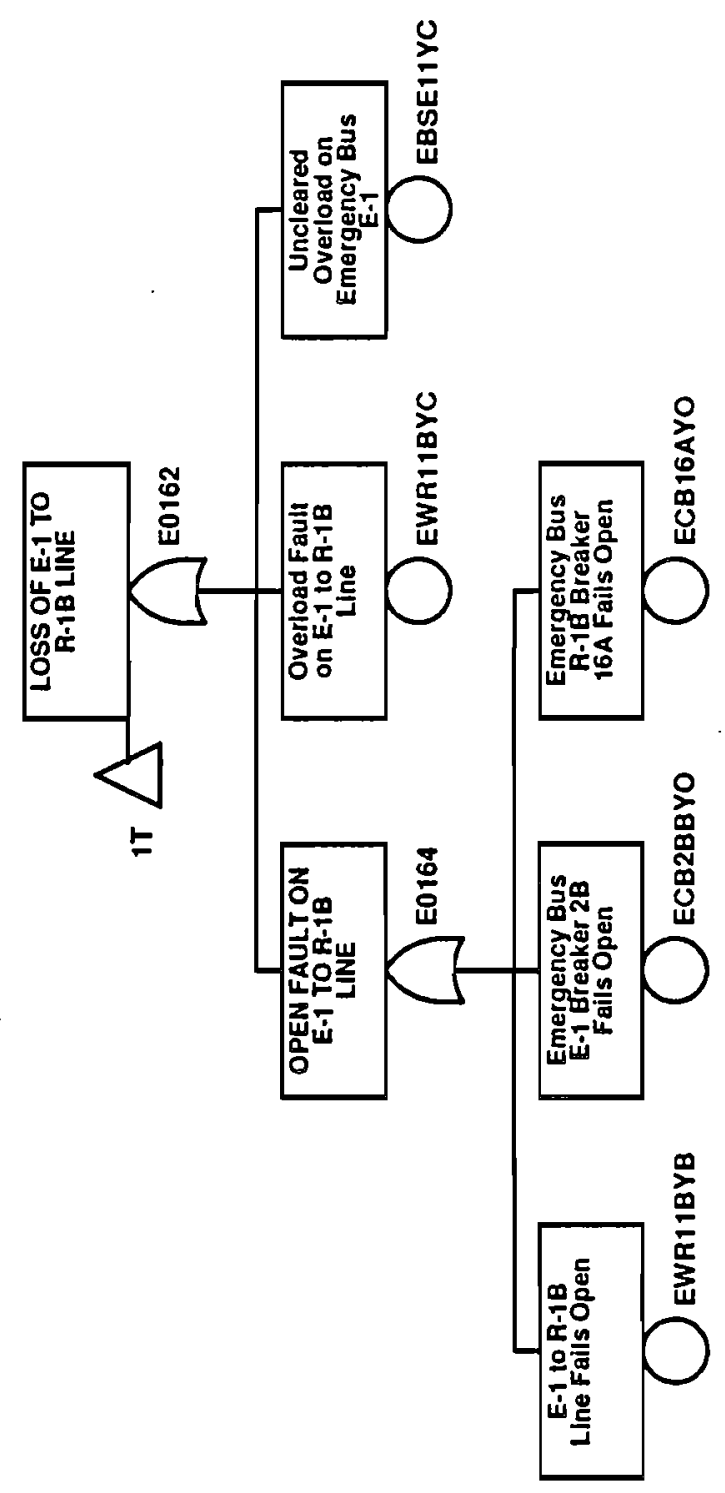

\begin{tabular}{|c|c|c|}
\hline $\begin{array}{l}0 \\
\vdots \\
\delta \\
\mathbb{E}\end{array}$ & & $\begin{array}{l}0 \\
\text { L } \\
0 \\
\text { N } \\
w \\
0 \\
\underline{a} \\
\end{array}$ \\
\hline 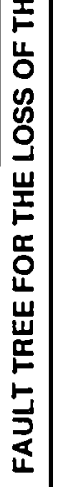 & 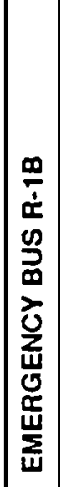 & 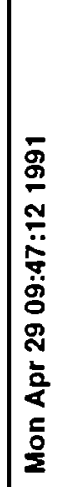 \\
\hline
\end{tabular}

B. 2-312 

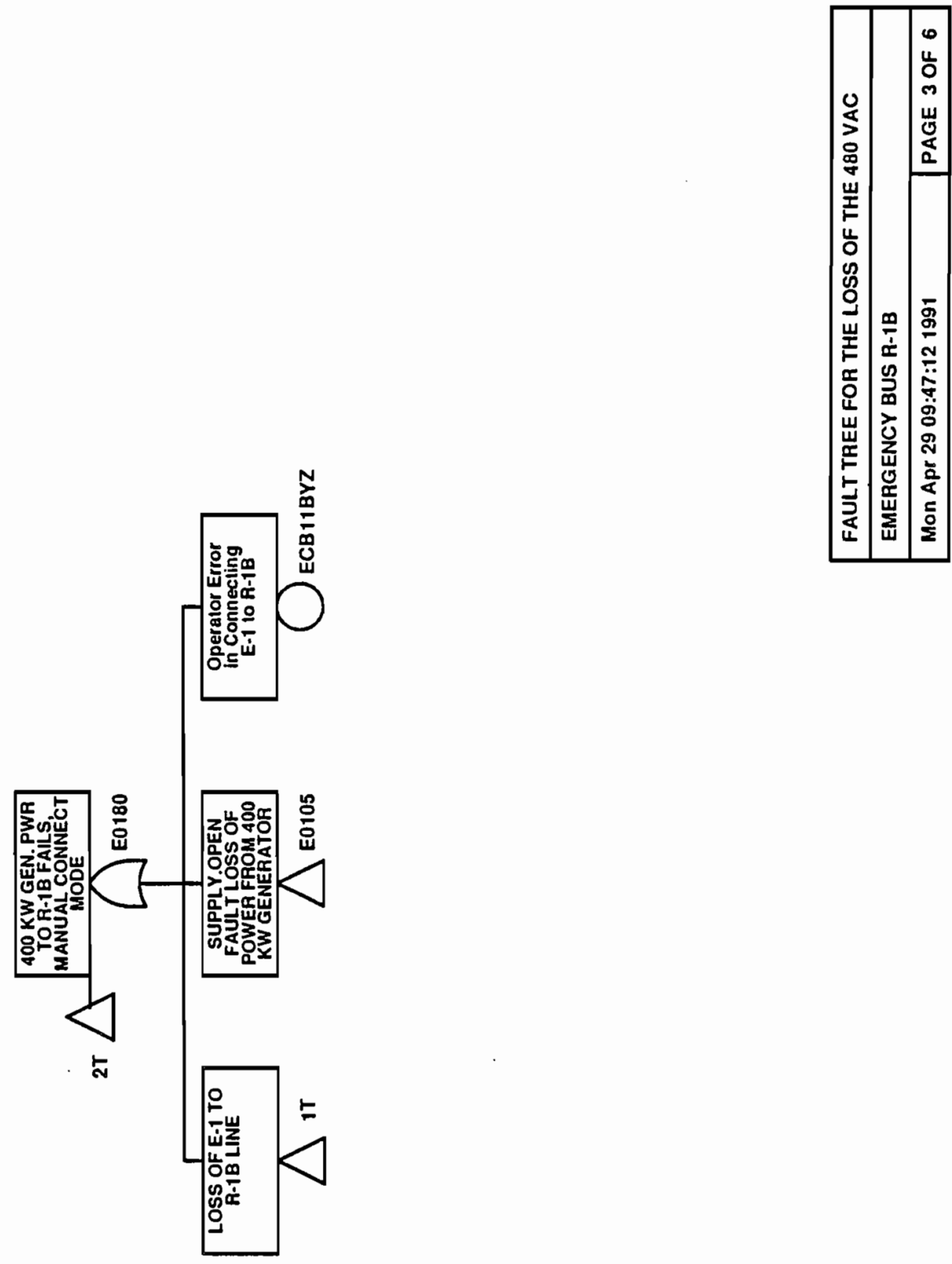

B. $2-313$ 


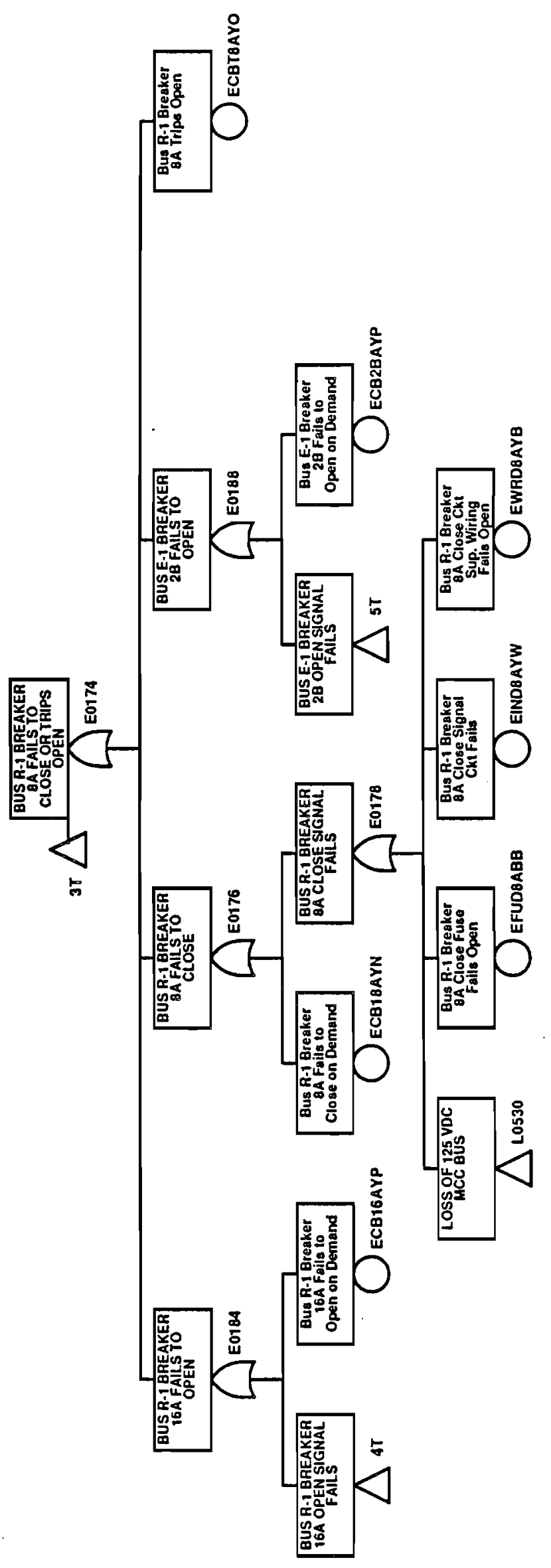

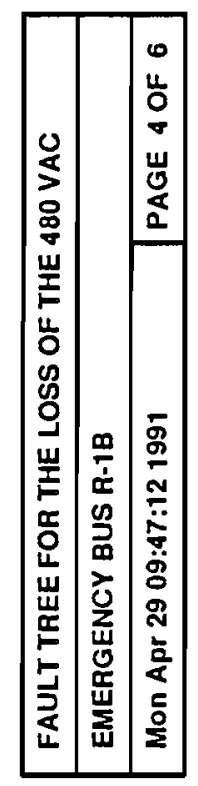




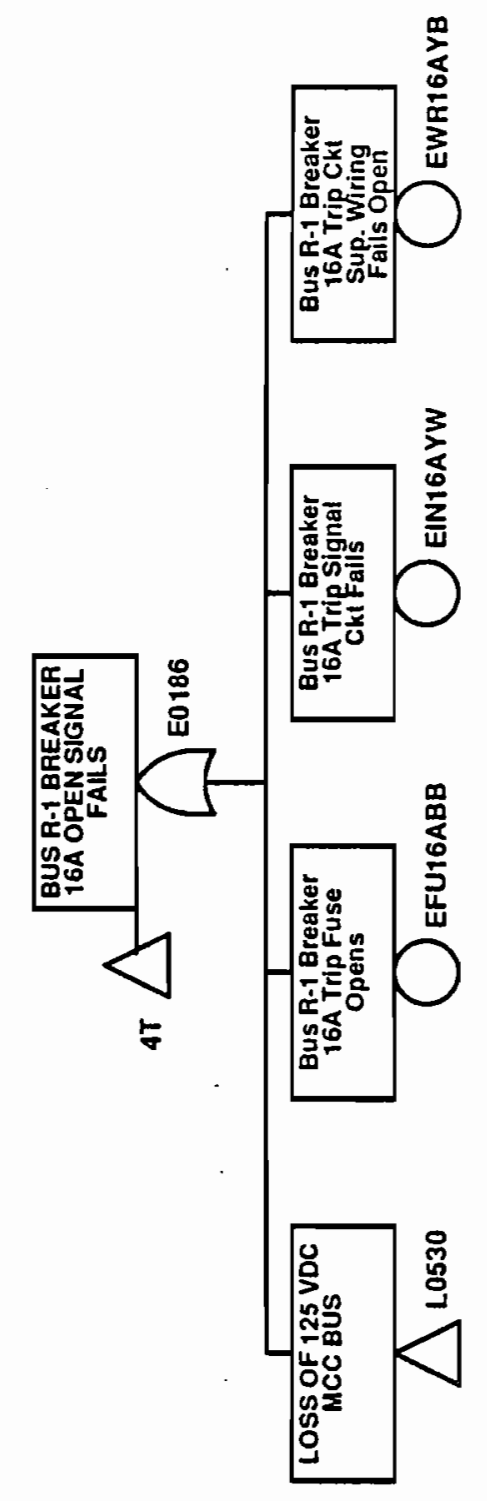

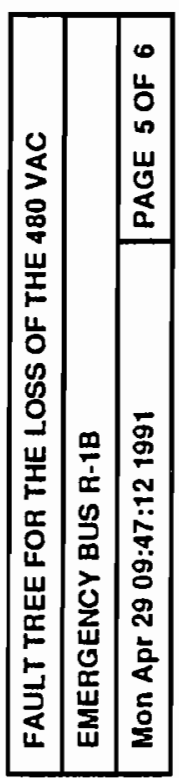




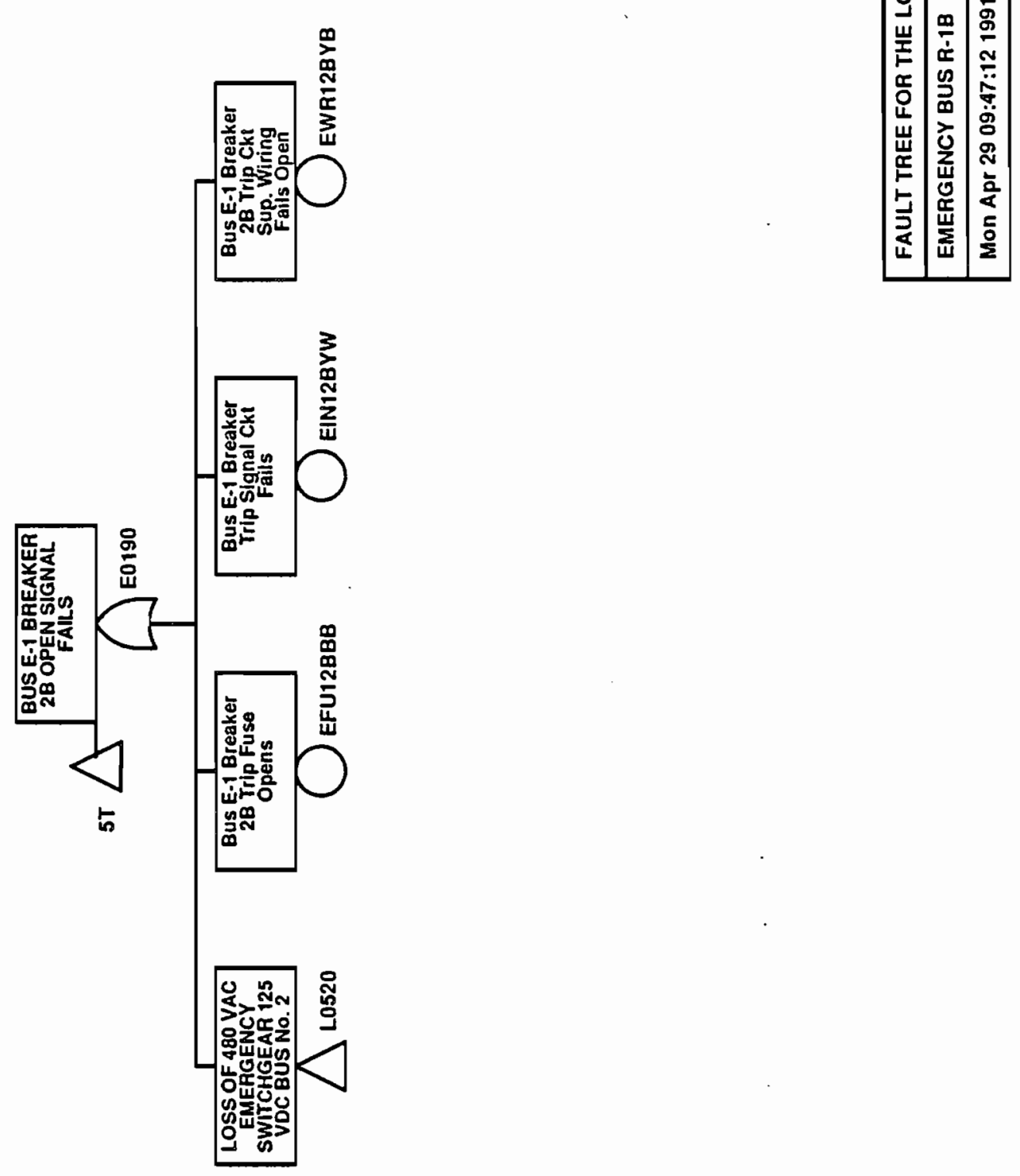

B. $2-316$ 


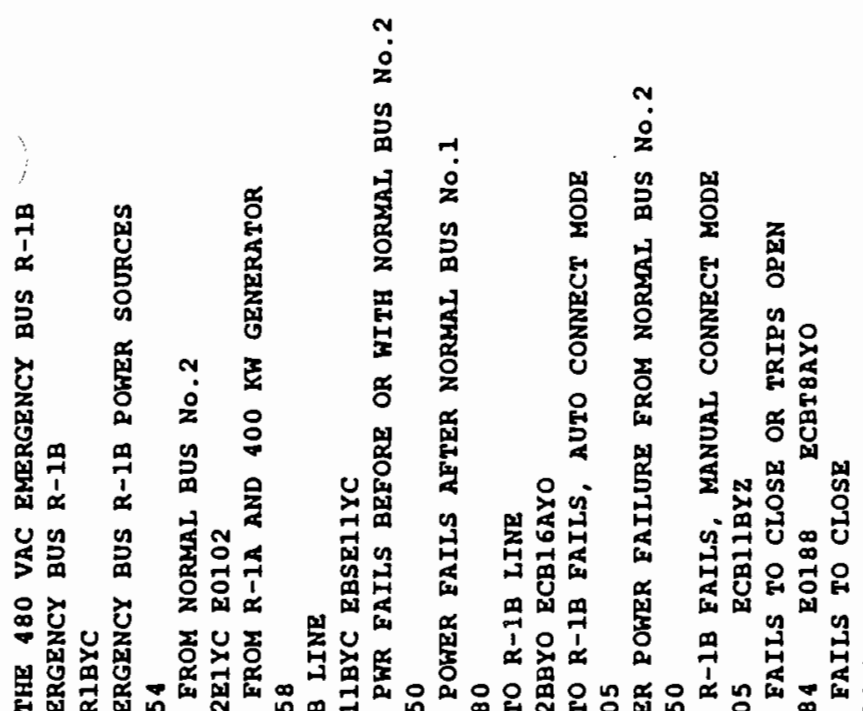

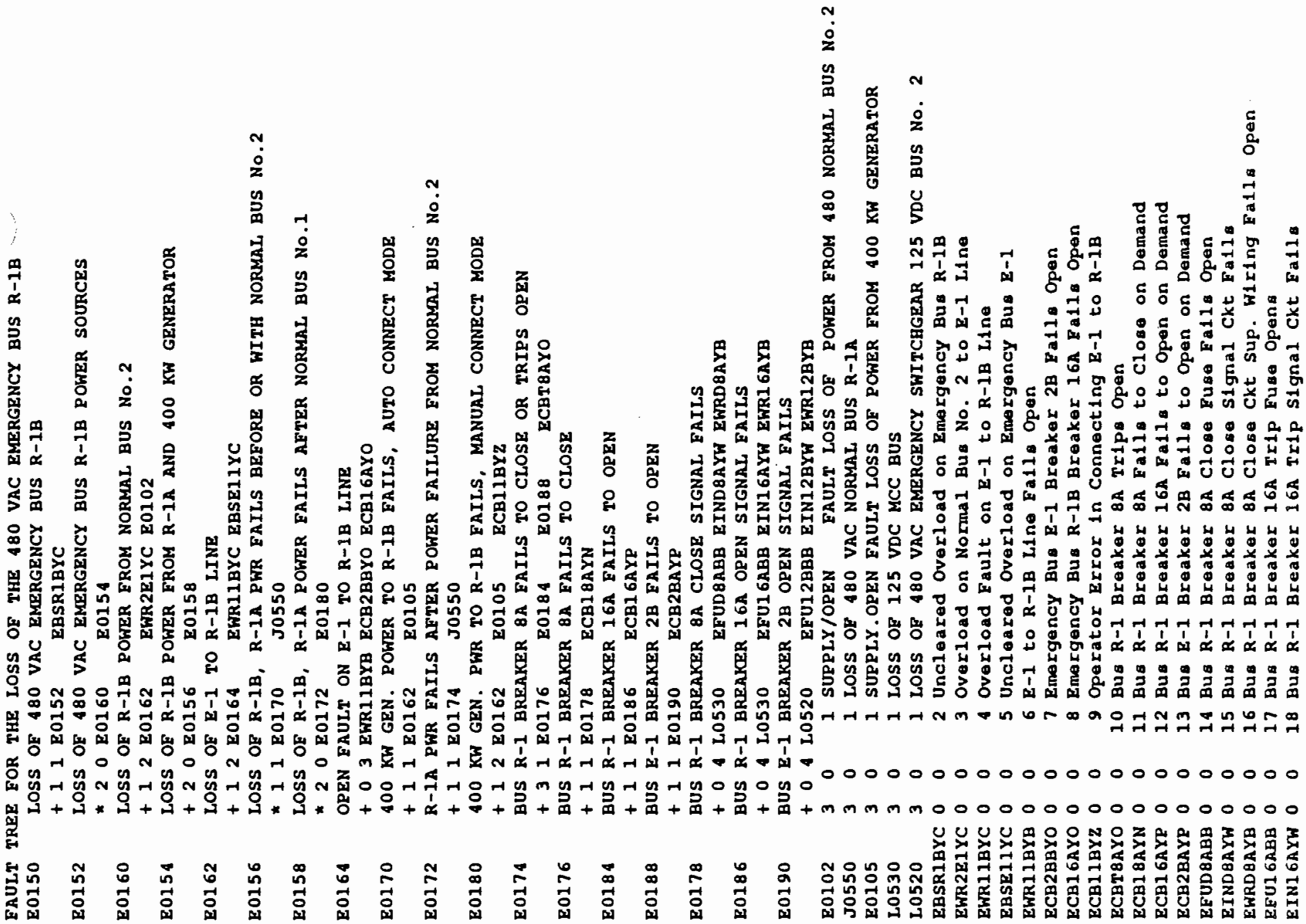




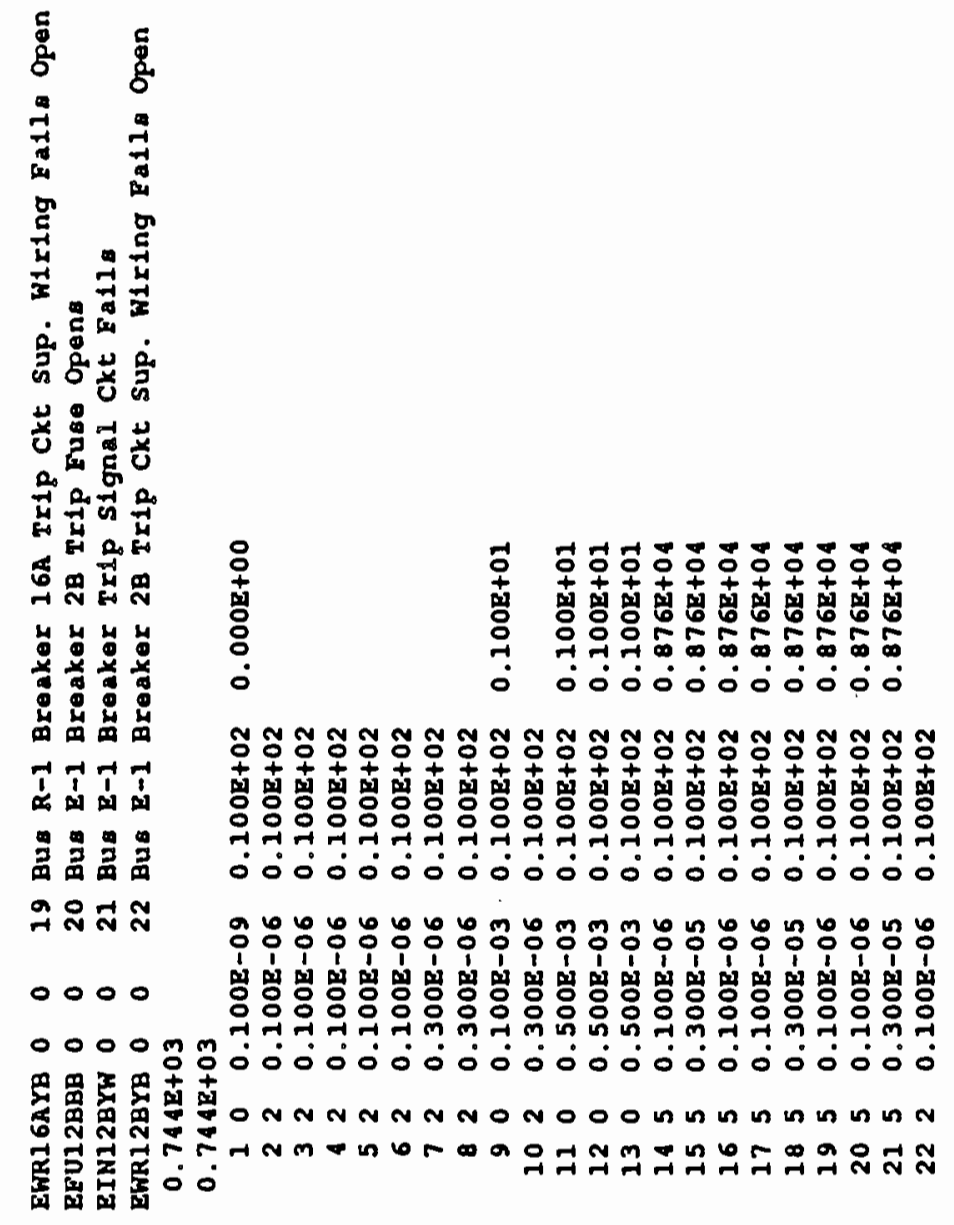




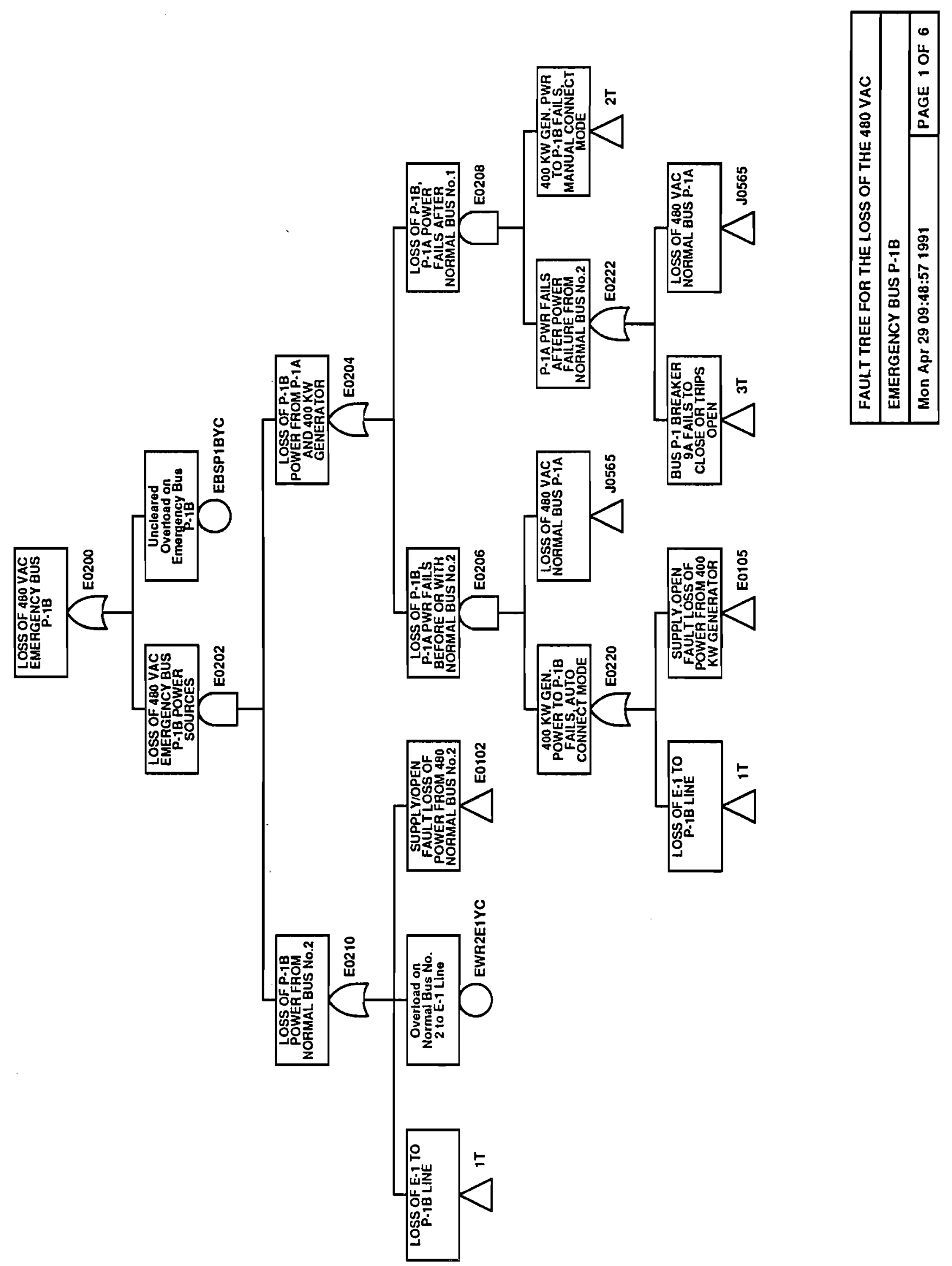

B. 2-319 


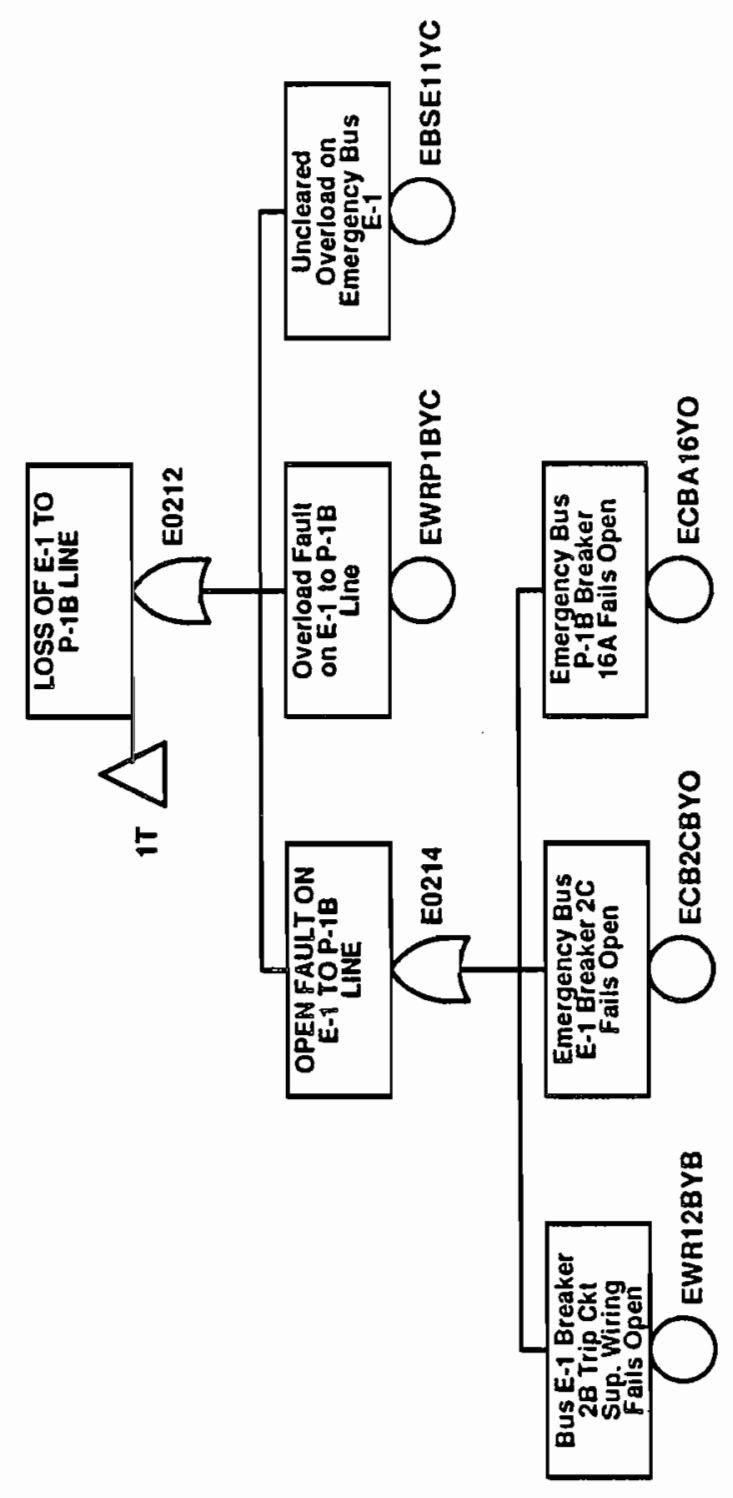

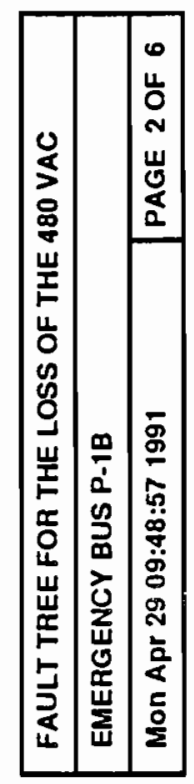

B. $2-320$ 


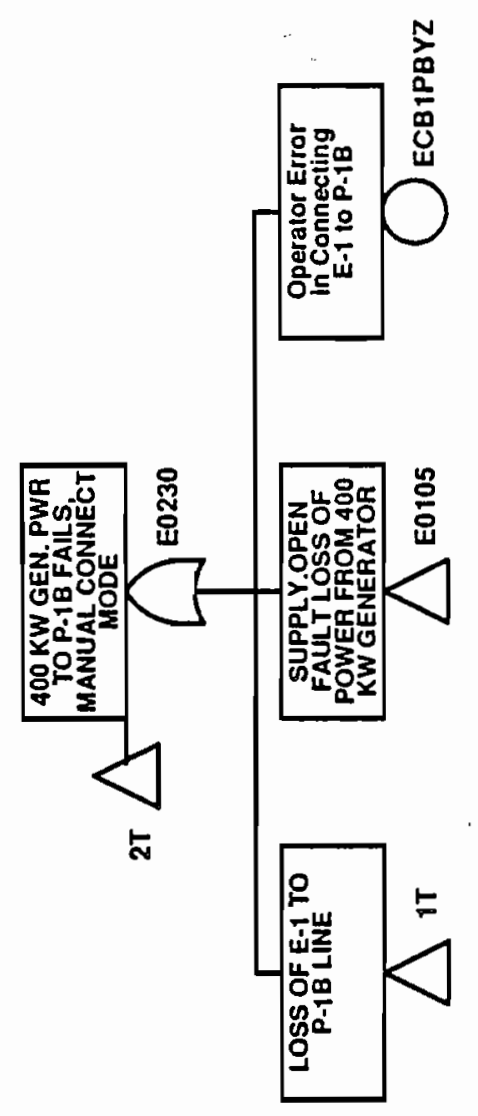

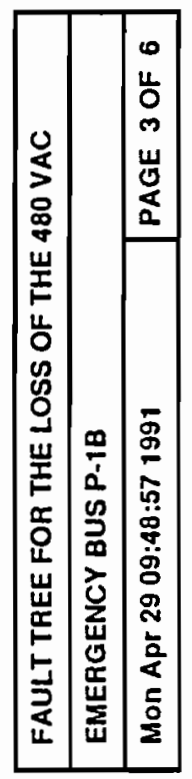



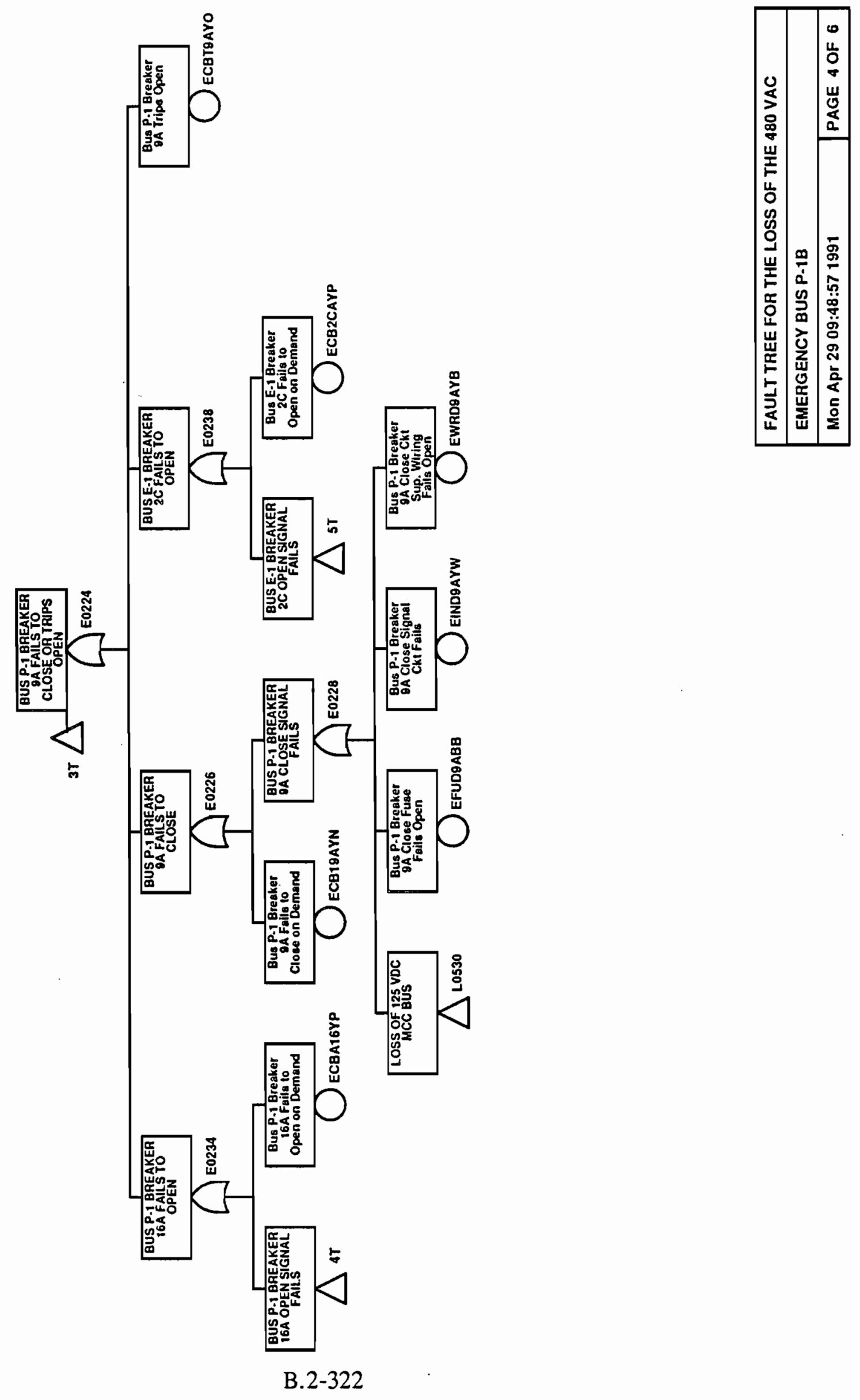


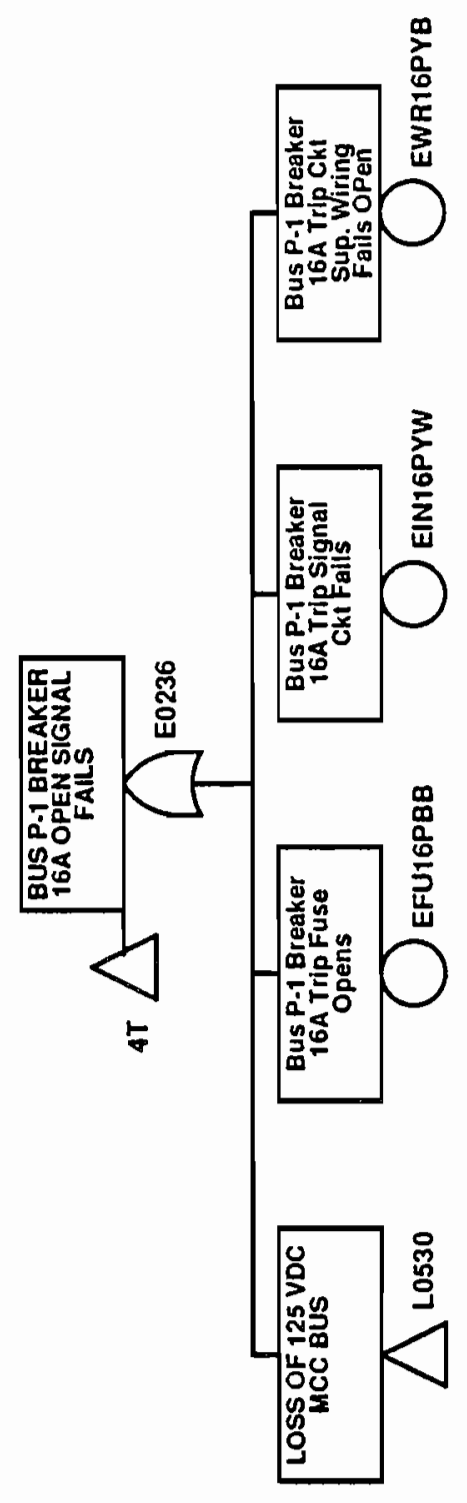

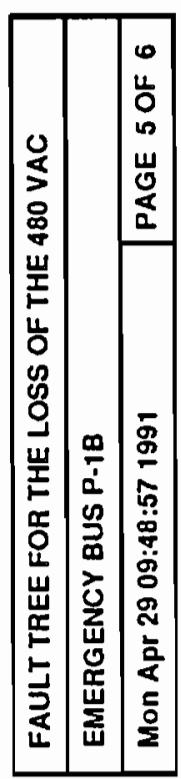




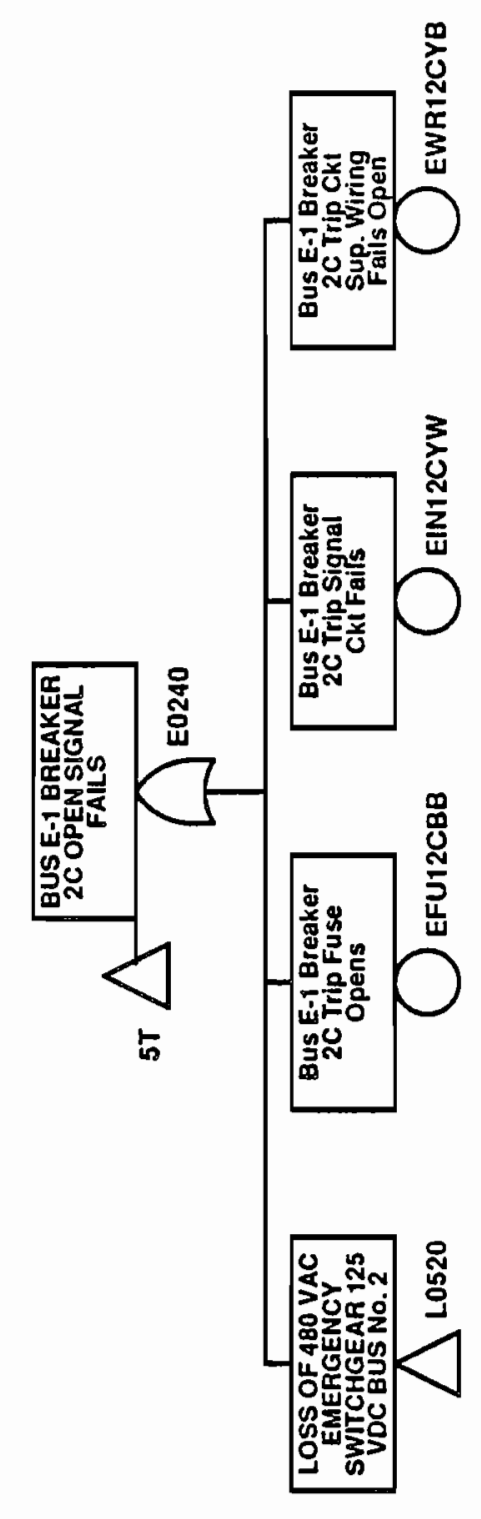

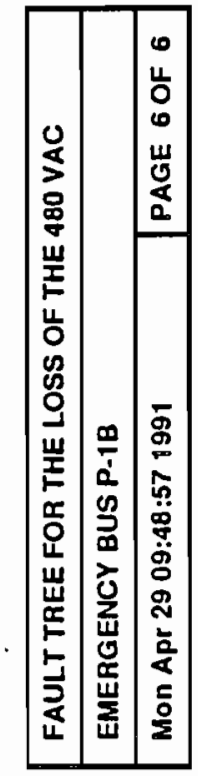




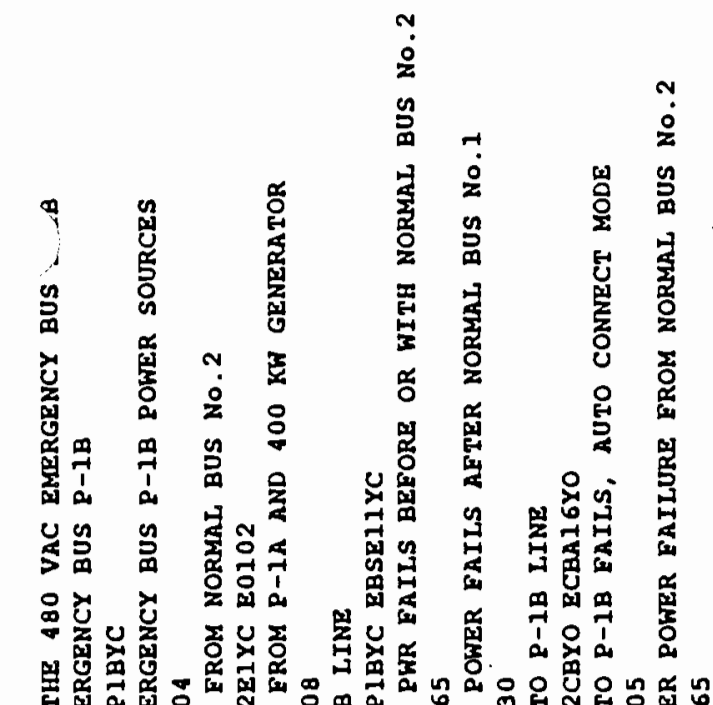

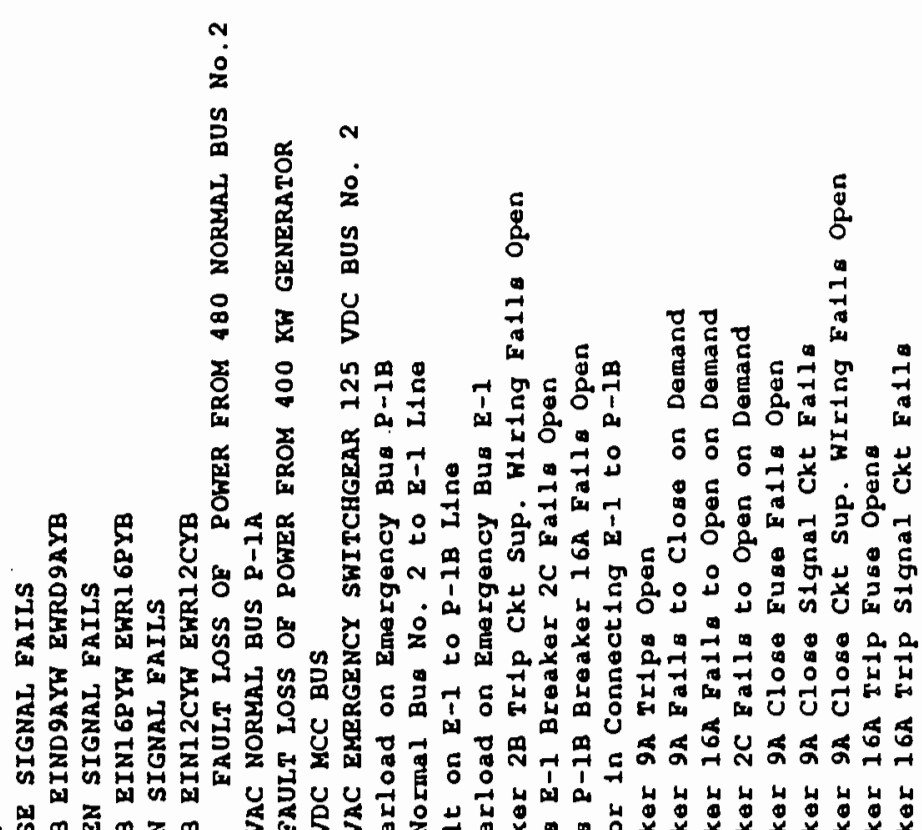

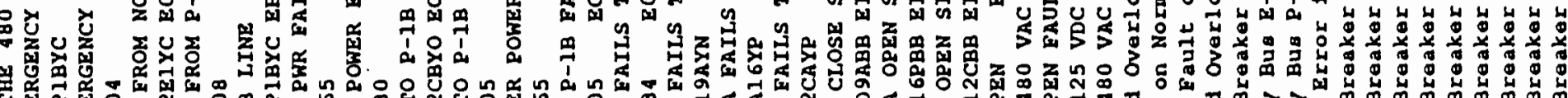

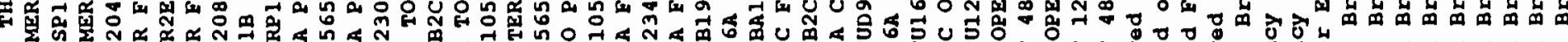

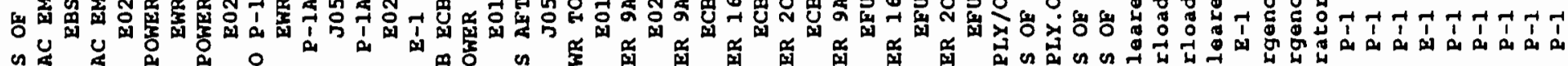

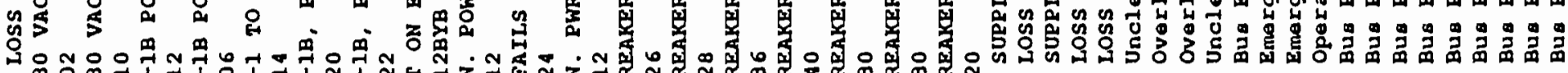

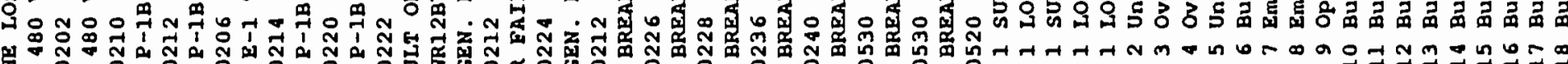
ए人

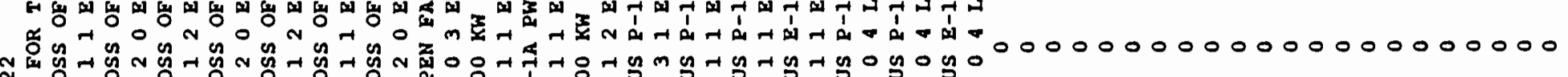

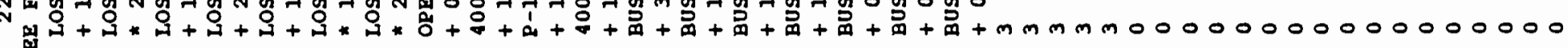

范 


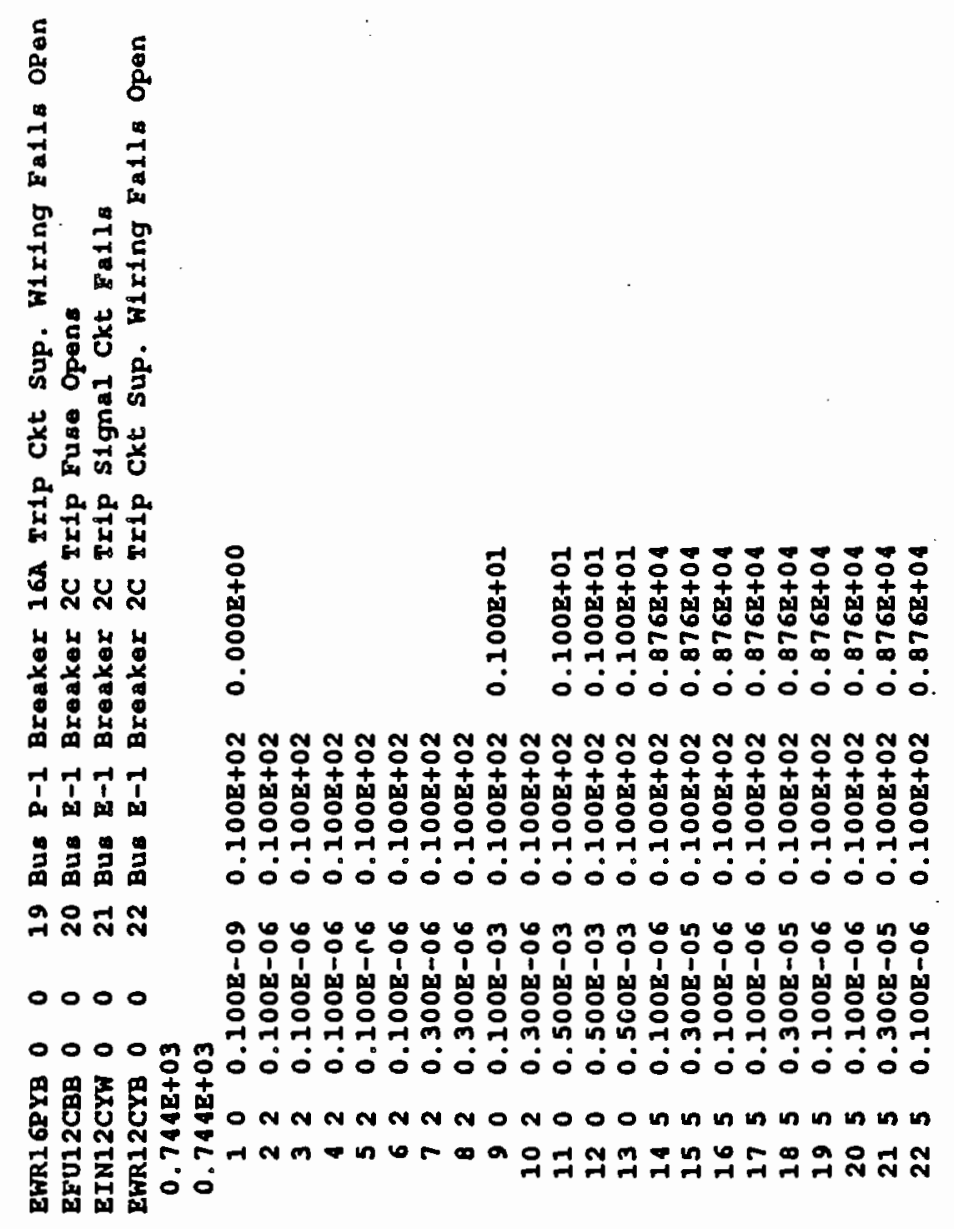




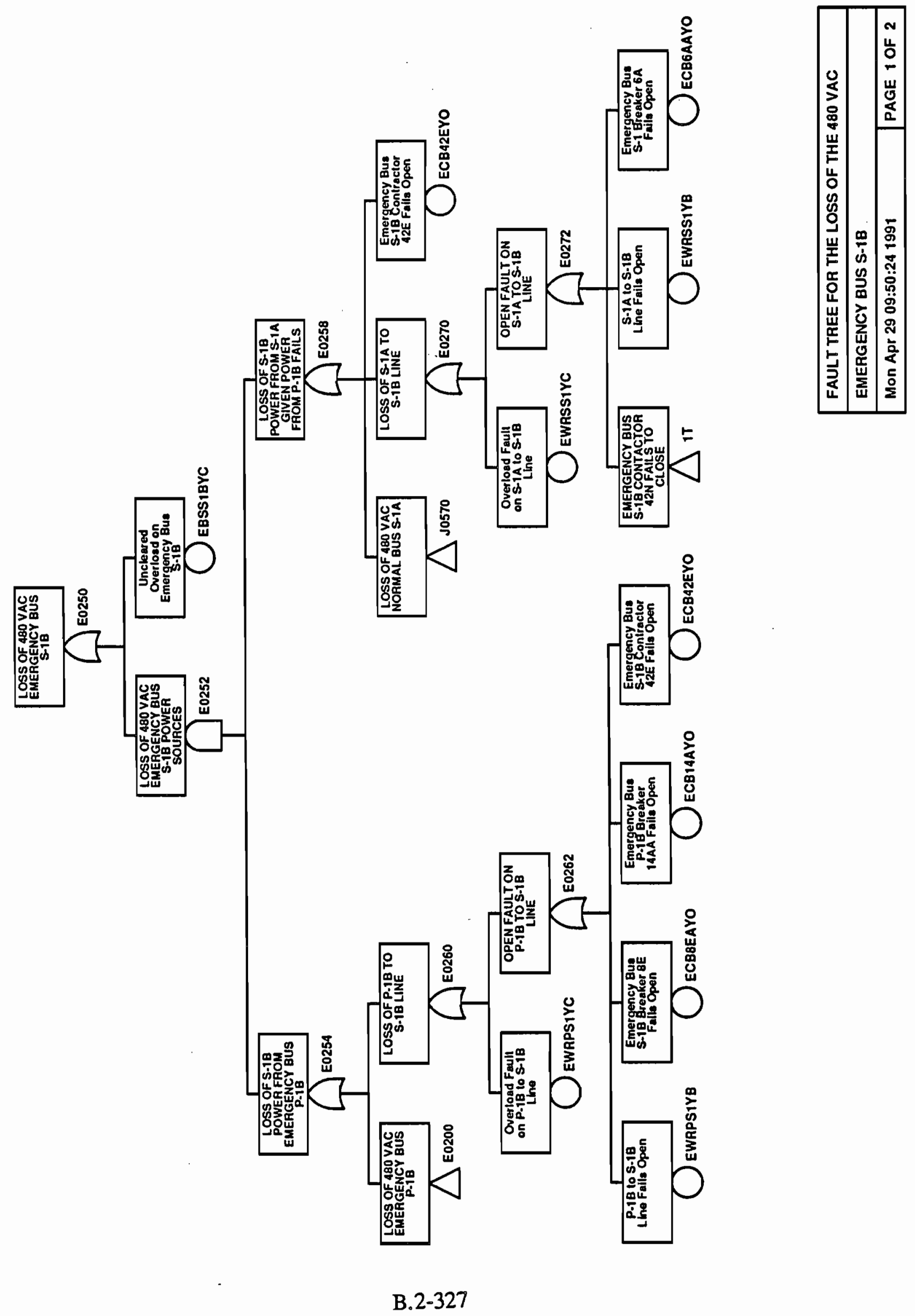




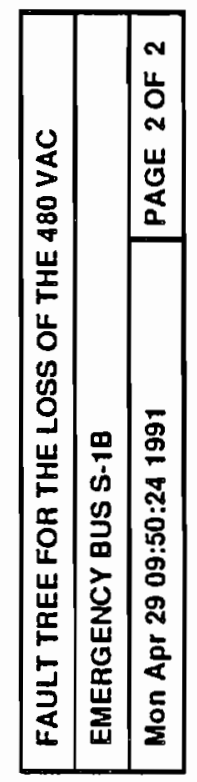

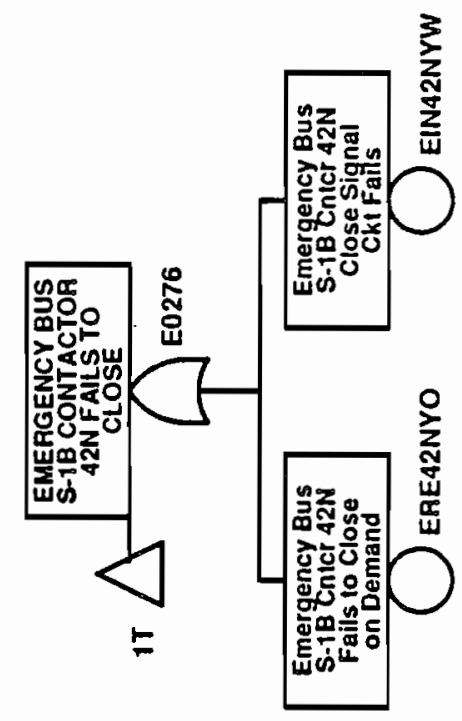

B. 2-328 


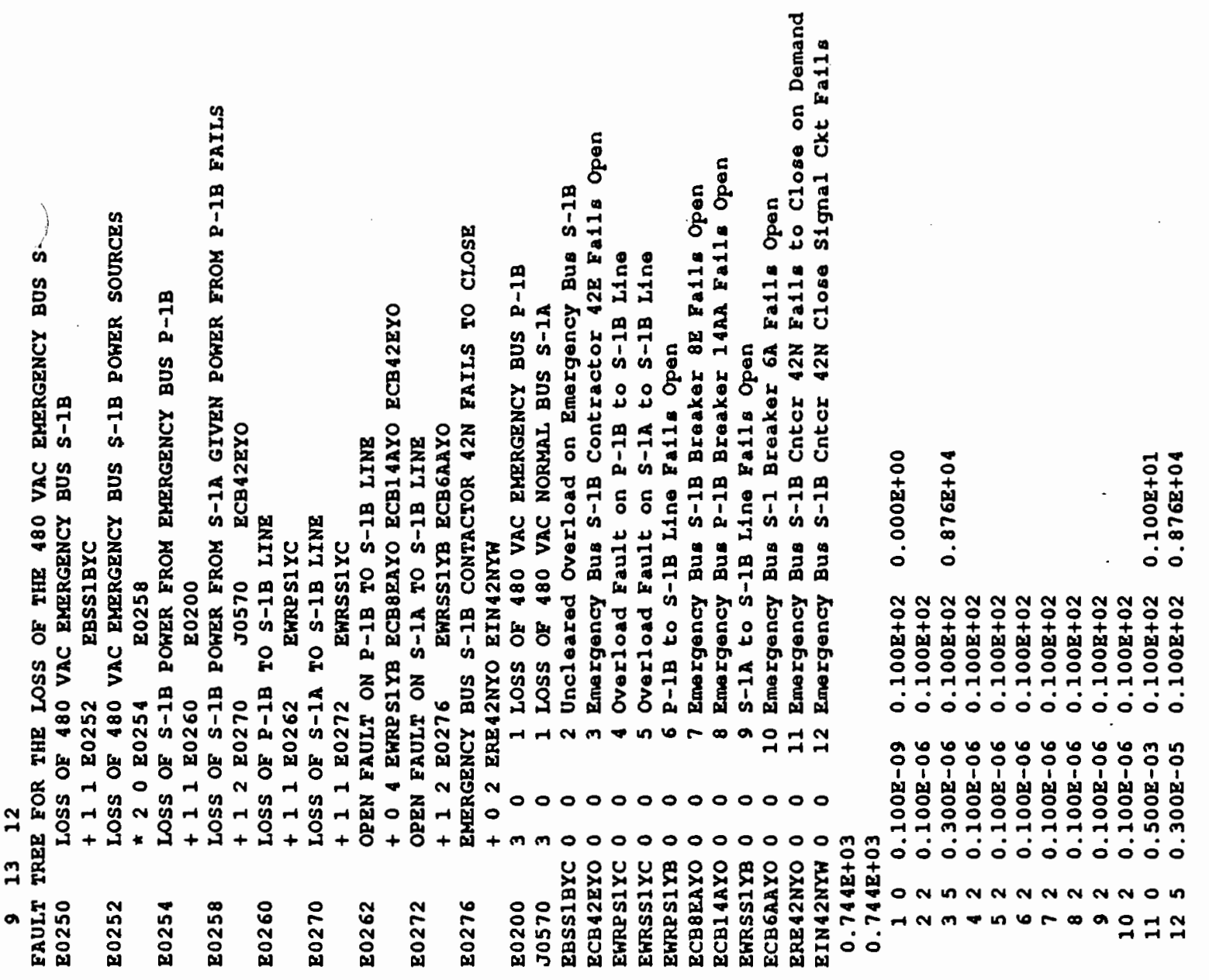

B. 2-329 


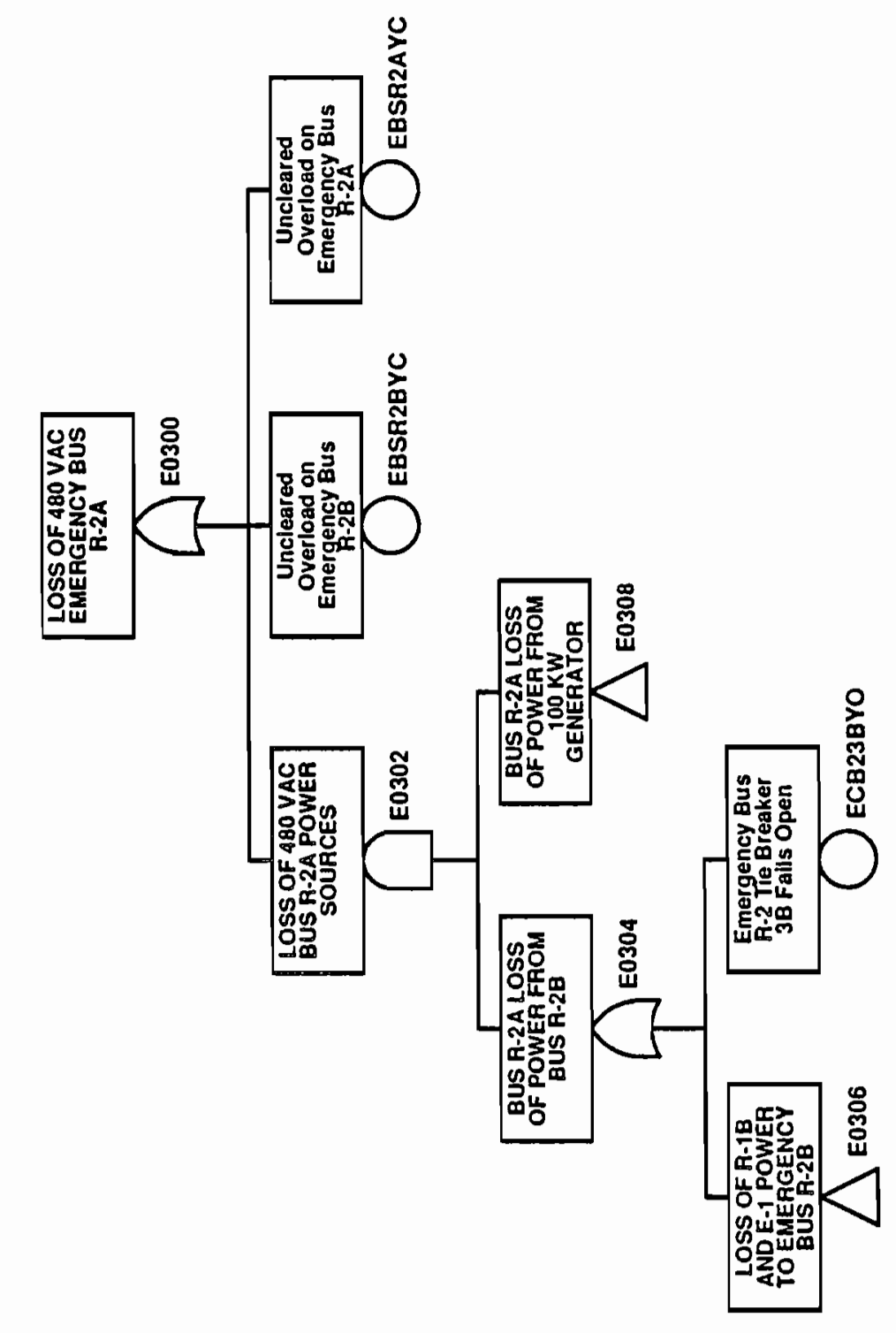

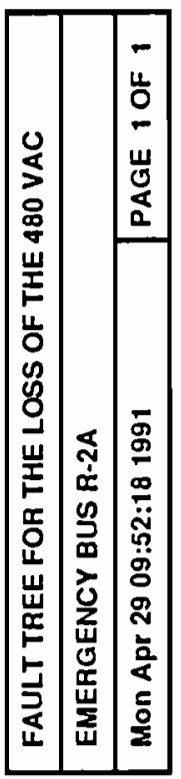




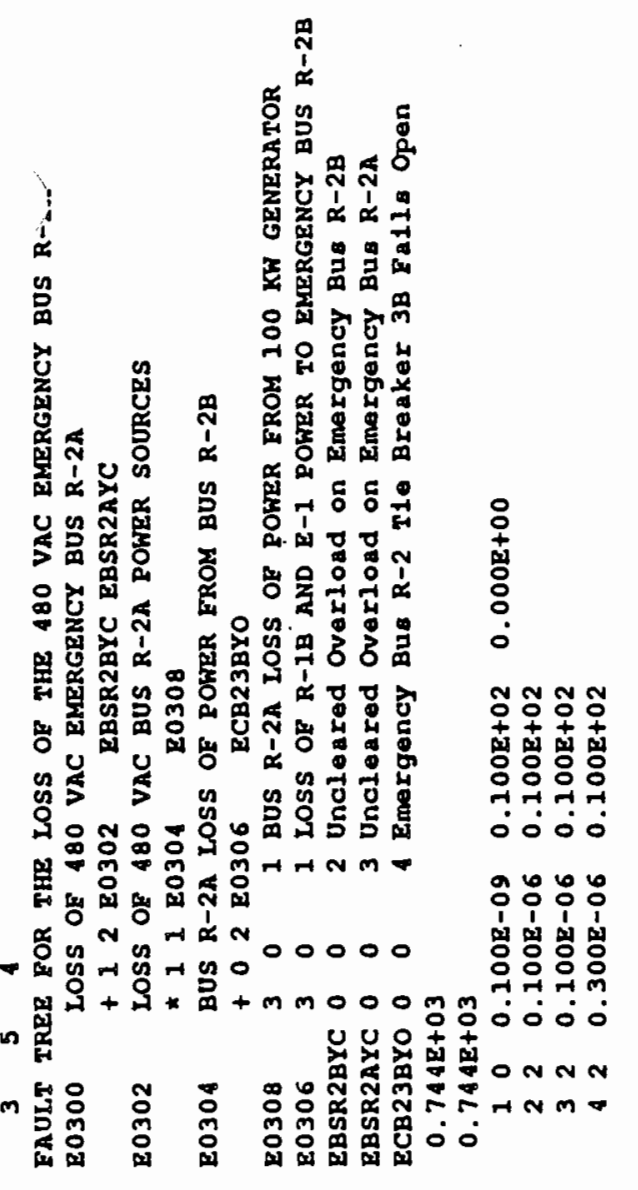

B. 2-331 


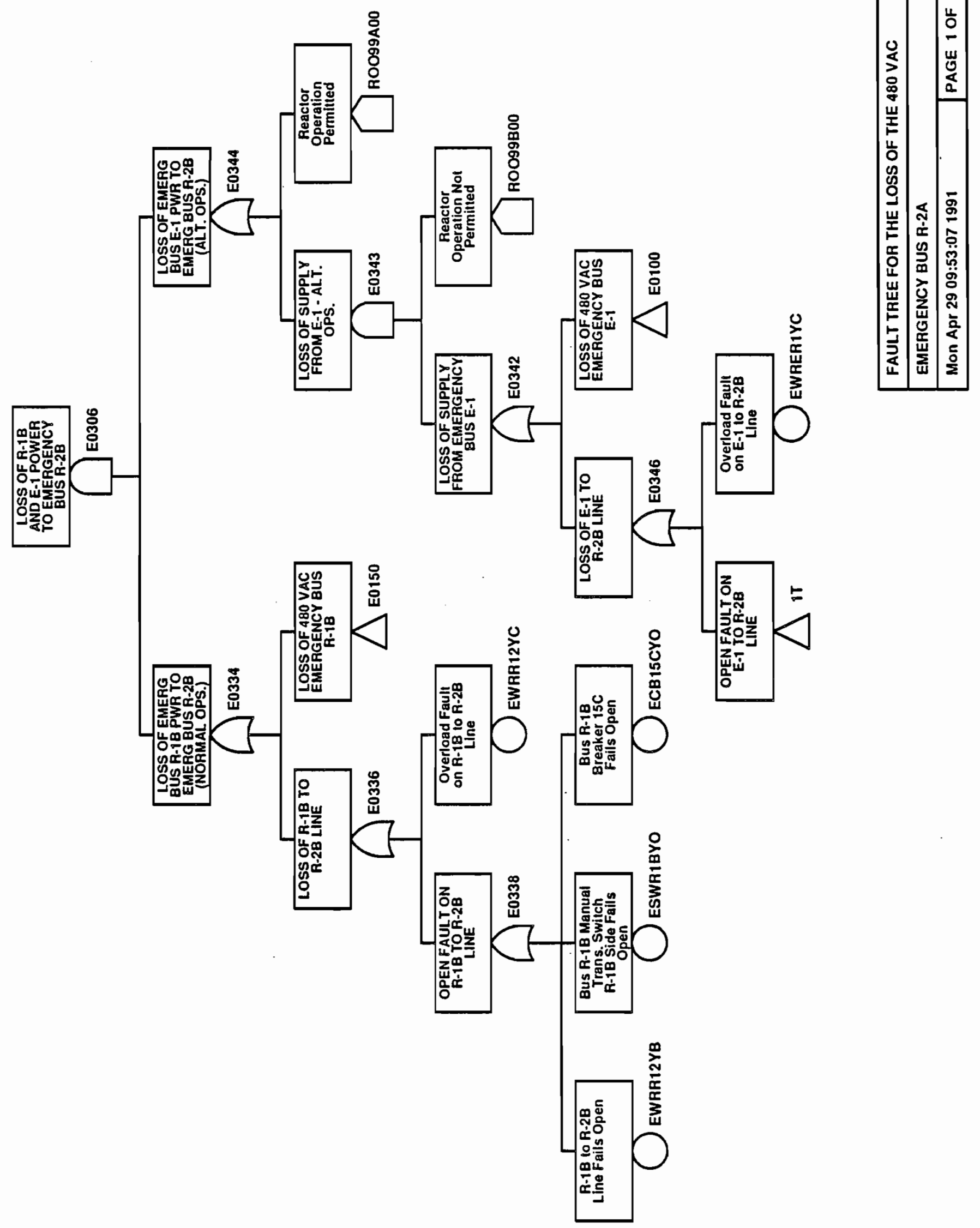




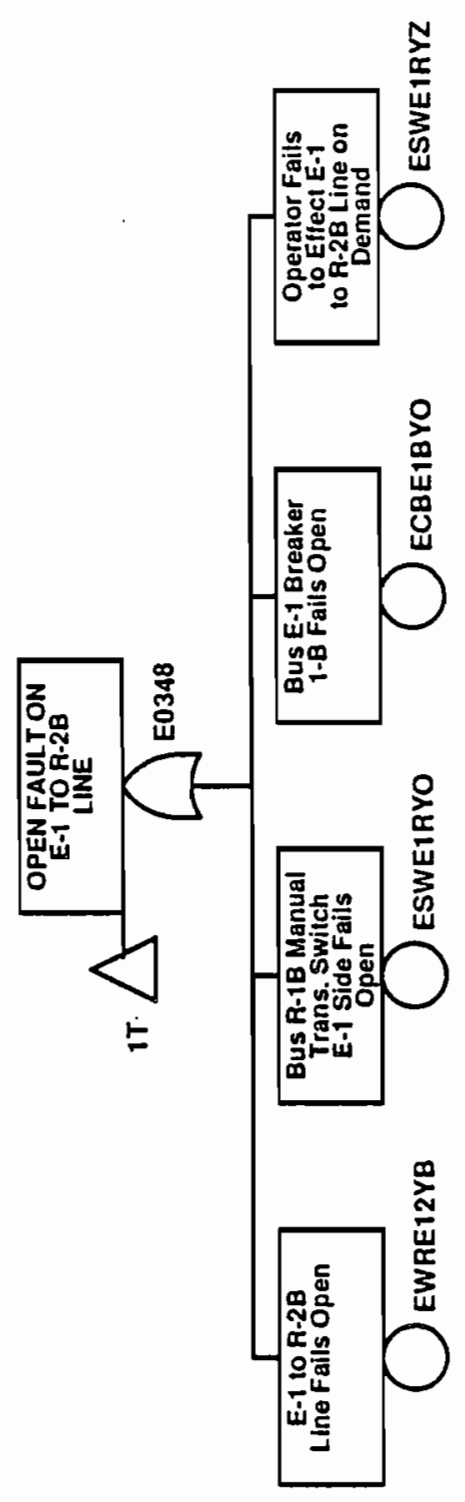

\begin{tabular}{|c|c|c|}
\hline $\begin{array}{l}0 \\
\frac{1}{3} \\
0 \\
8 \\
\square\end{array}$ & & 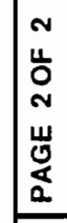 \\
\hline 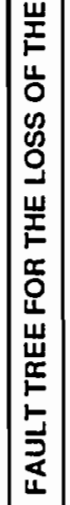 & 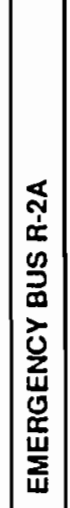 & 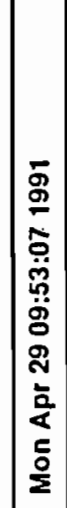 \\
\hline
\end{tabular}

\section{B. 2-333}




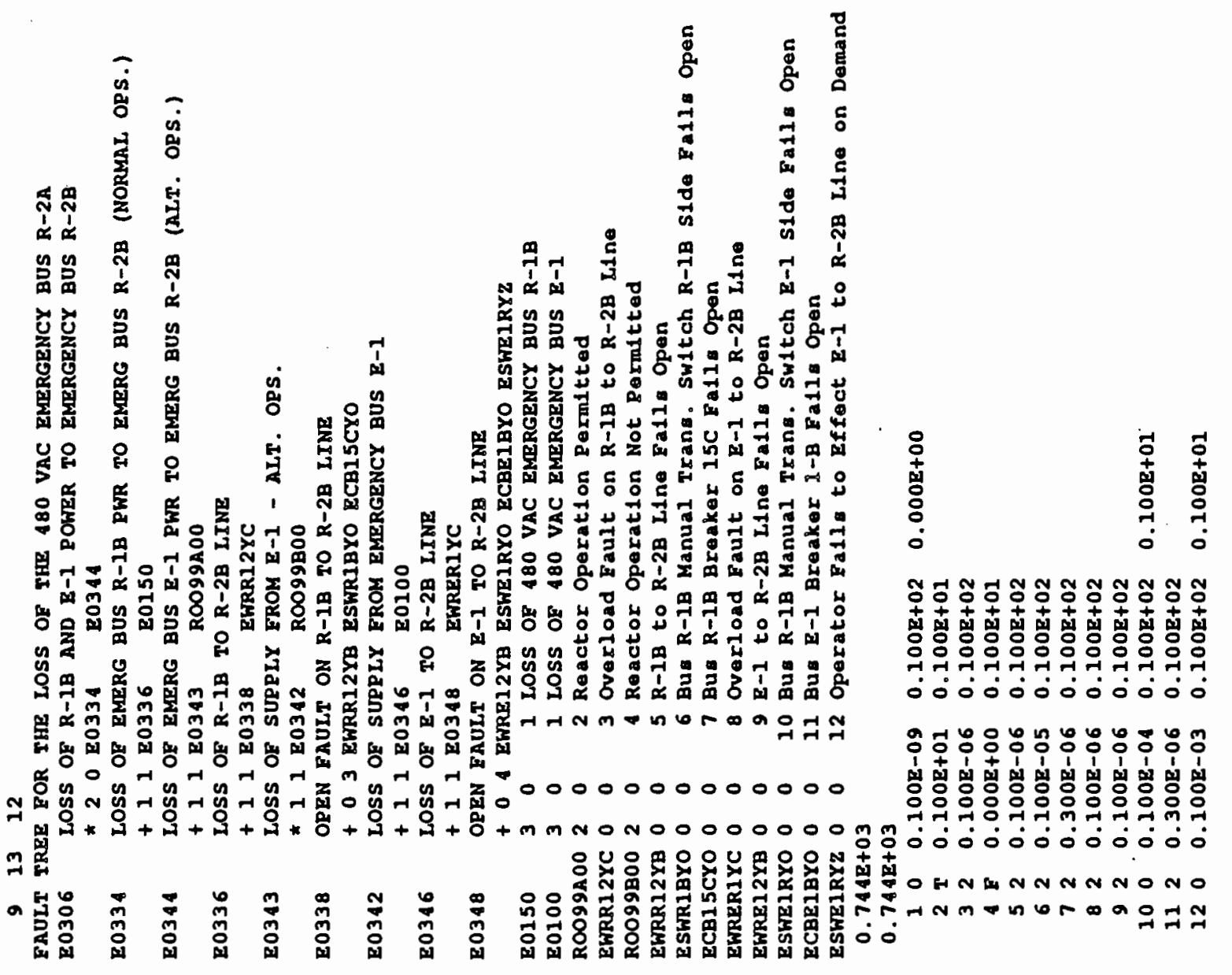



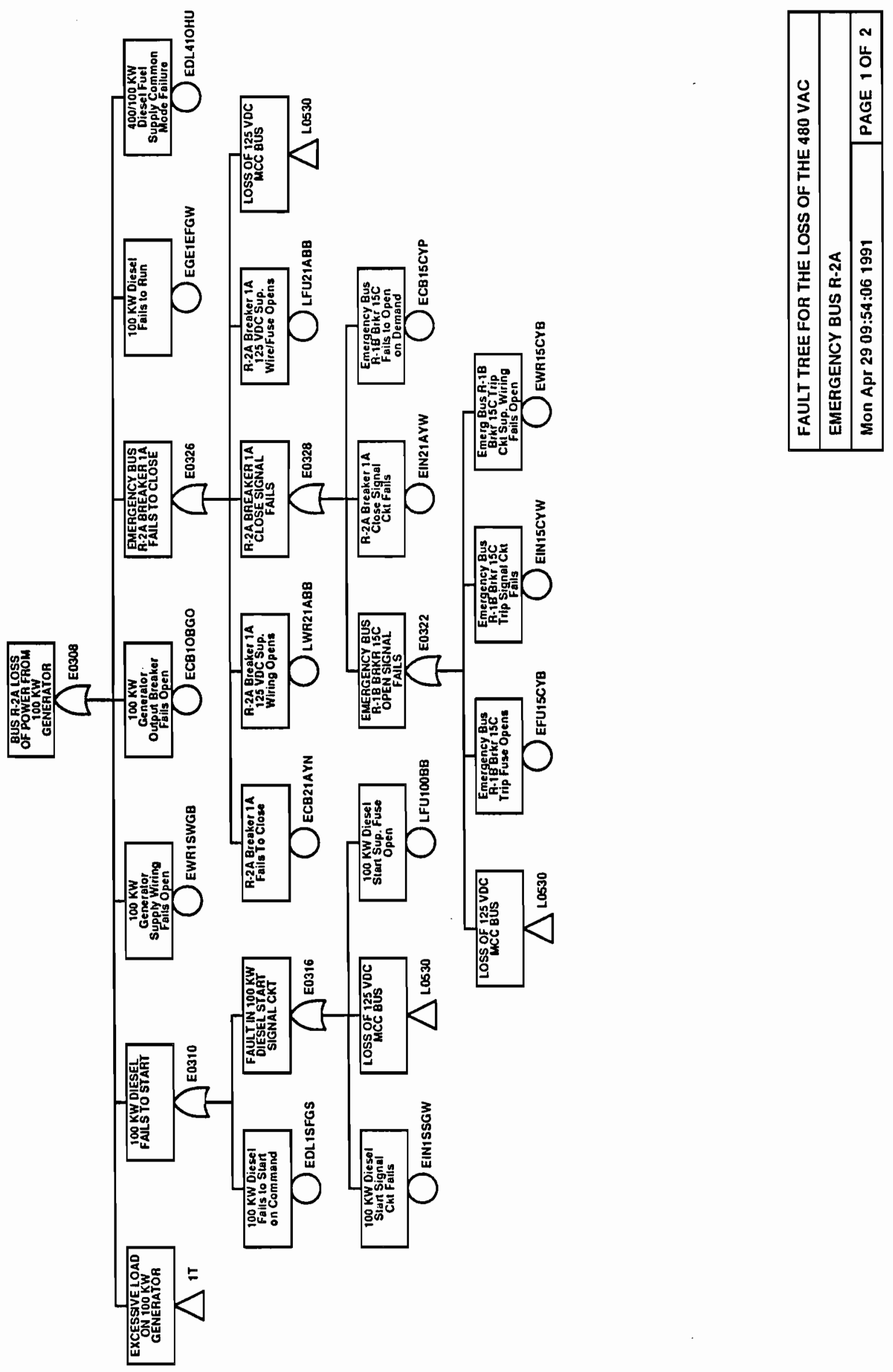

B. 2-335 

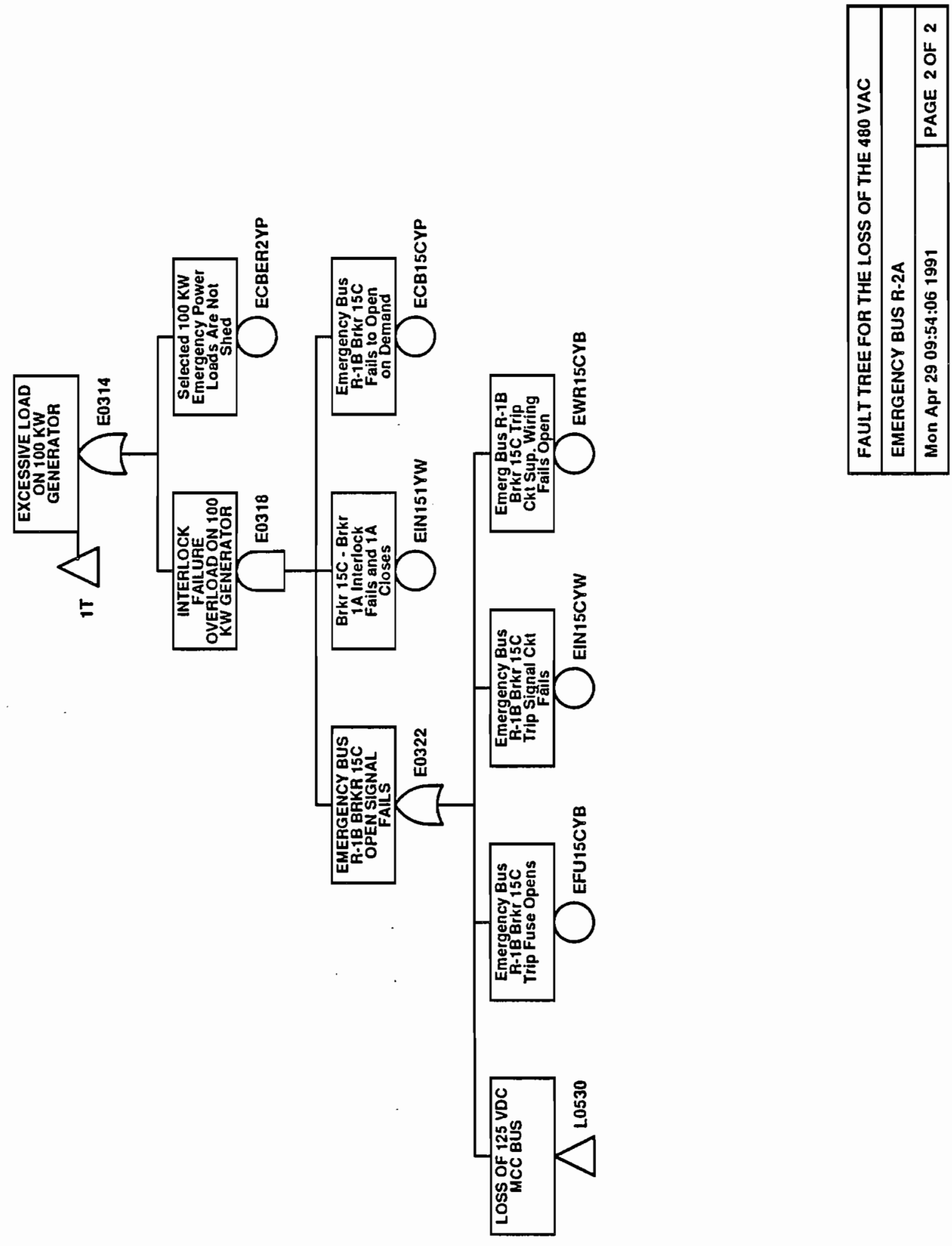


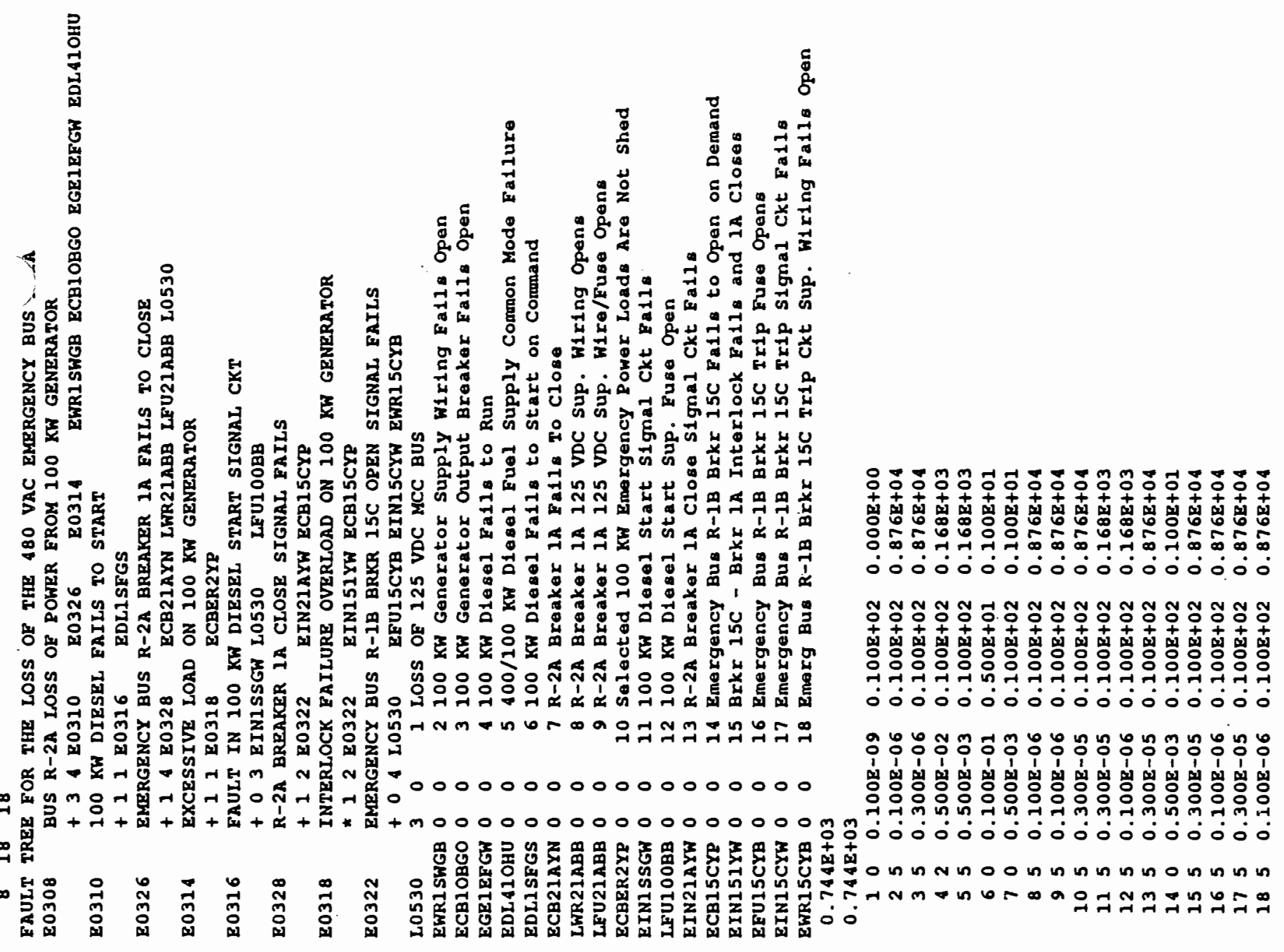




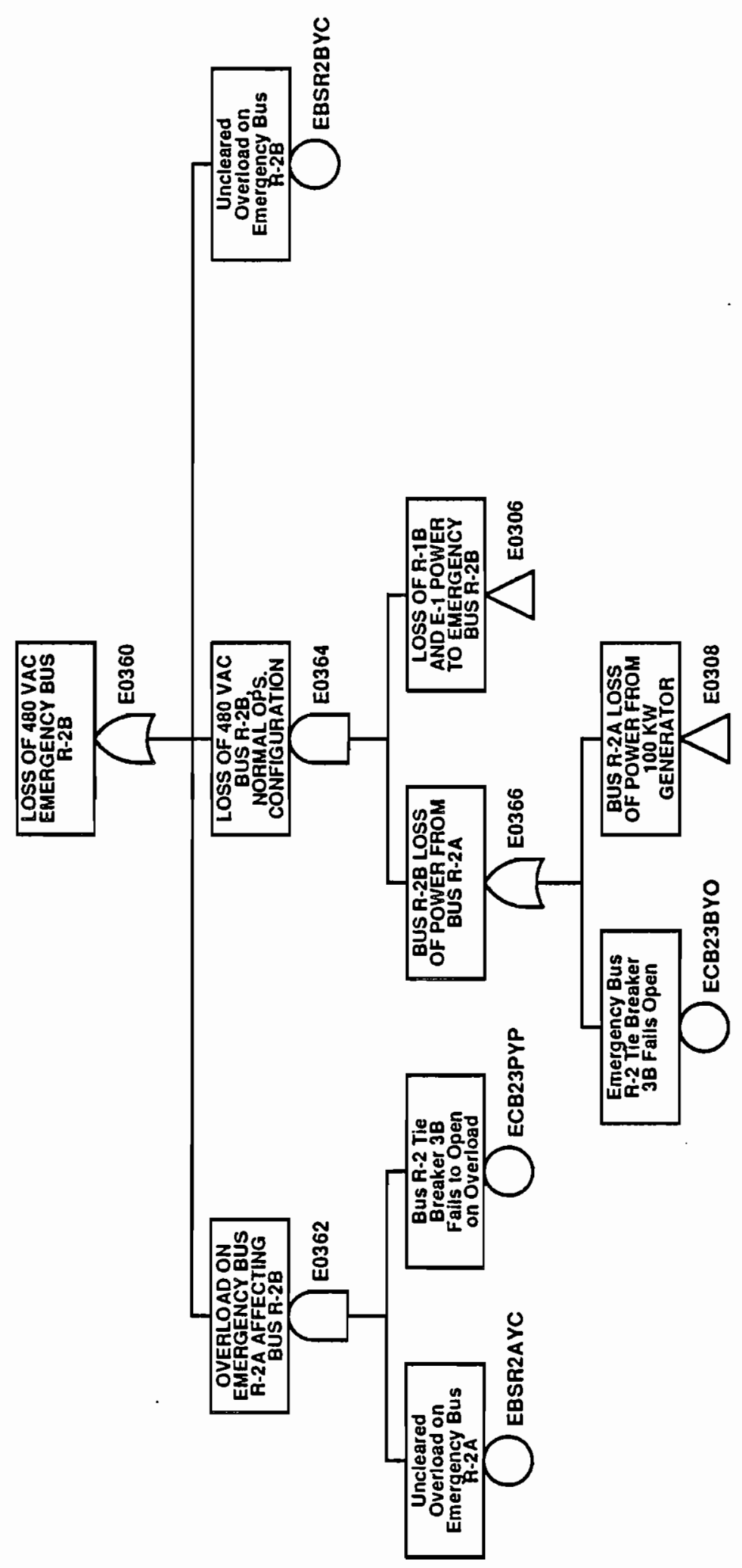

B. 2-338 


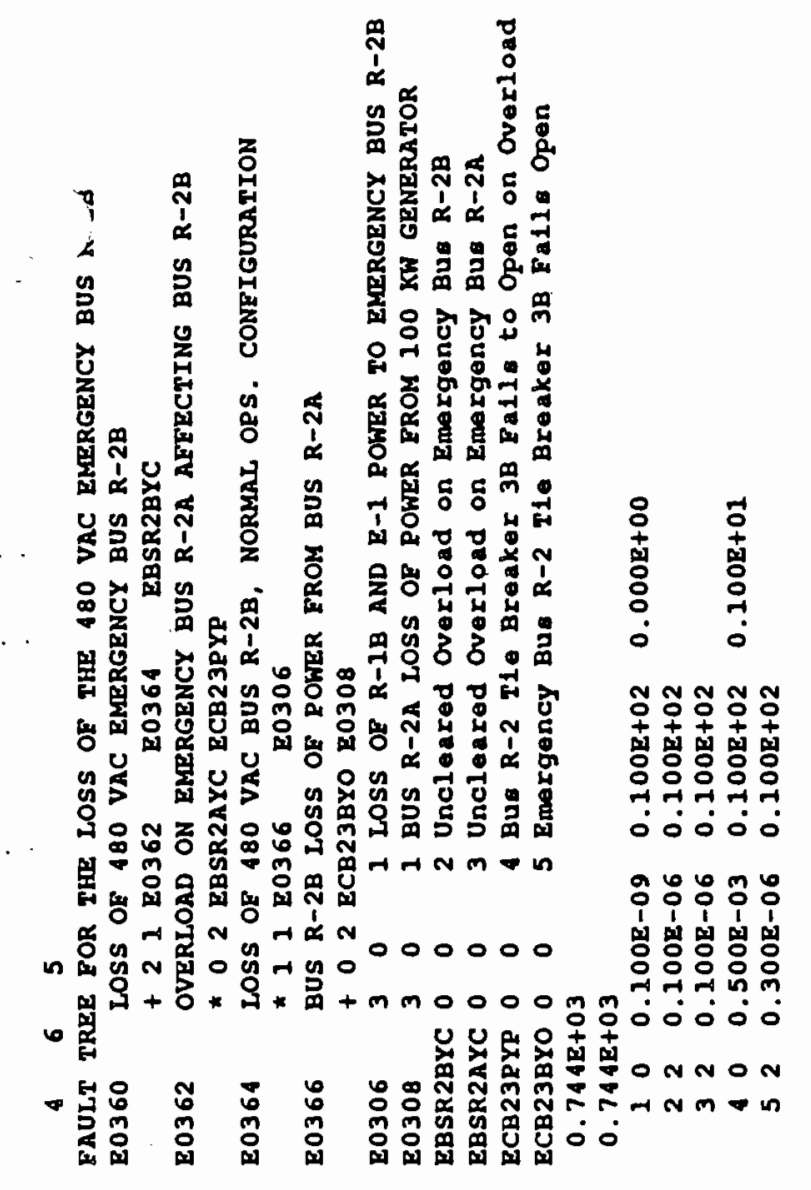




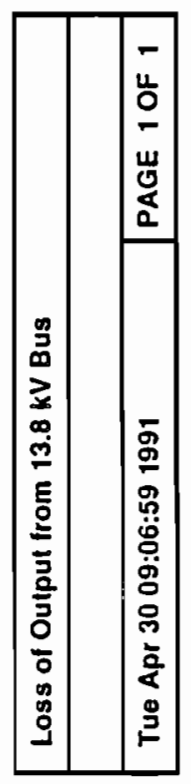

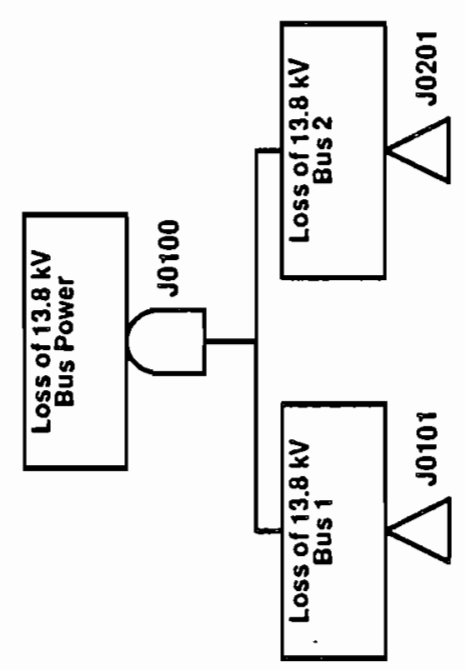




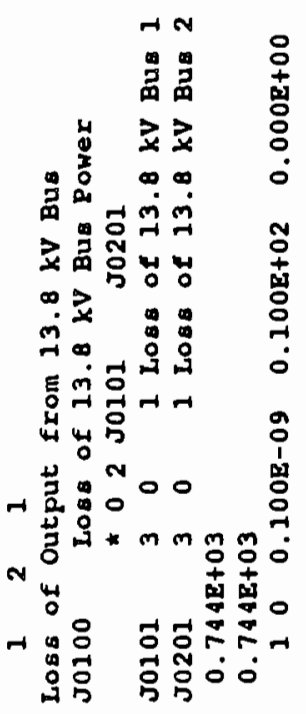

B. 2-341 


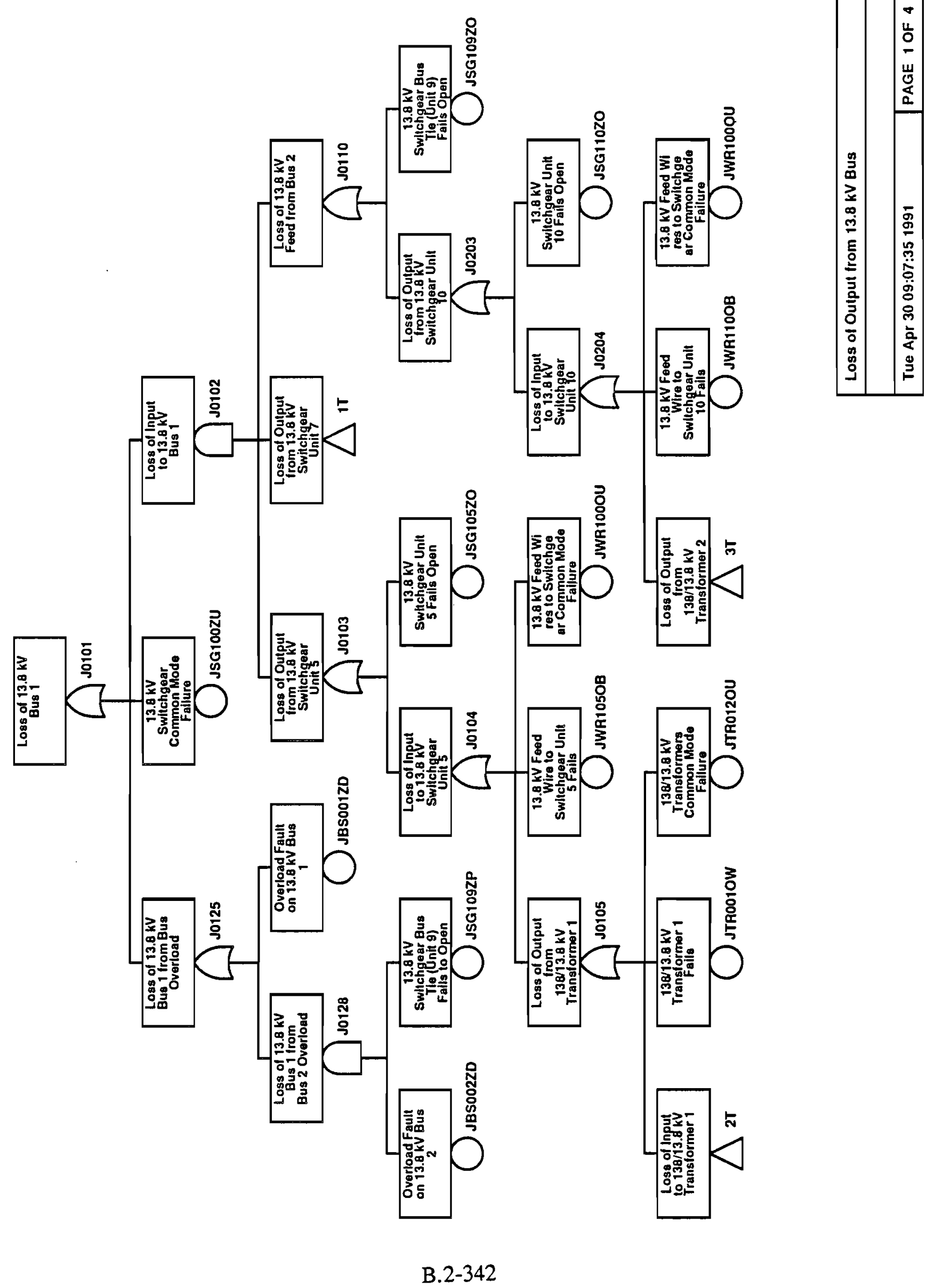




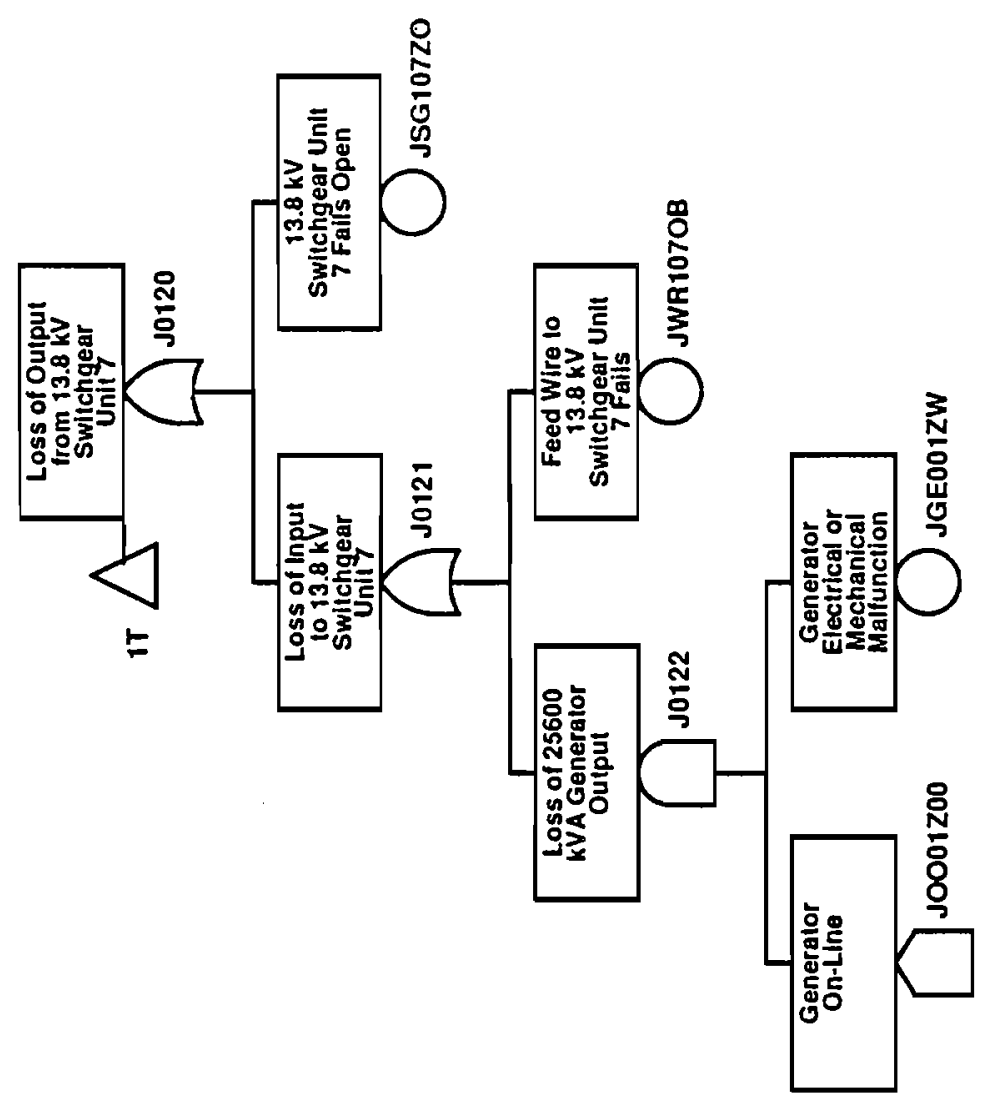

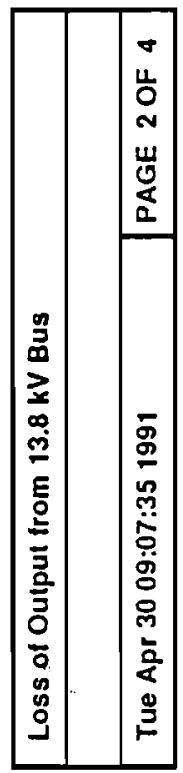




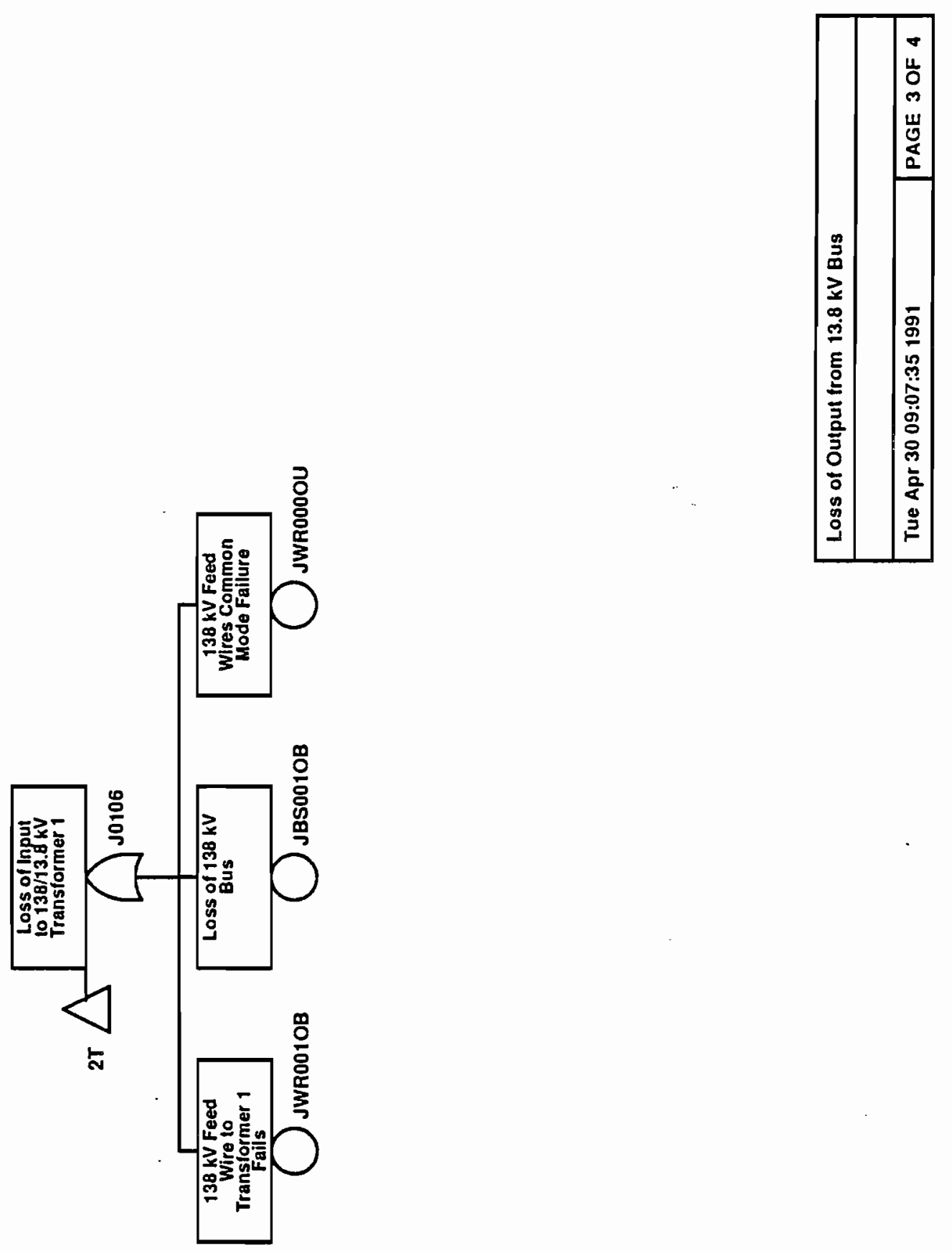




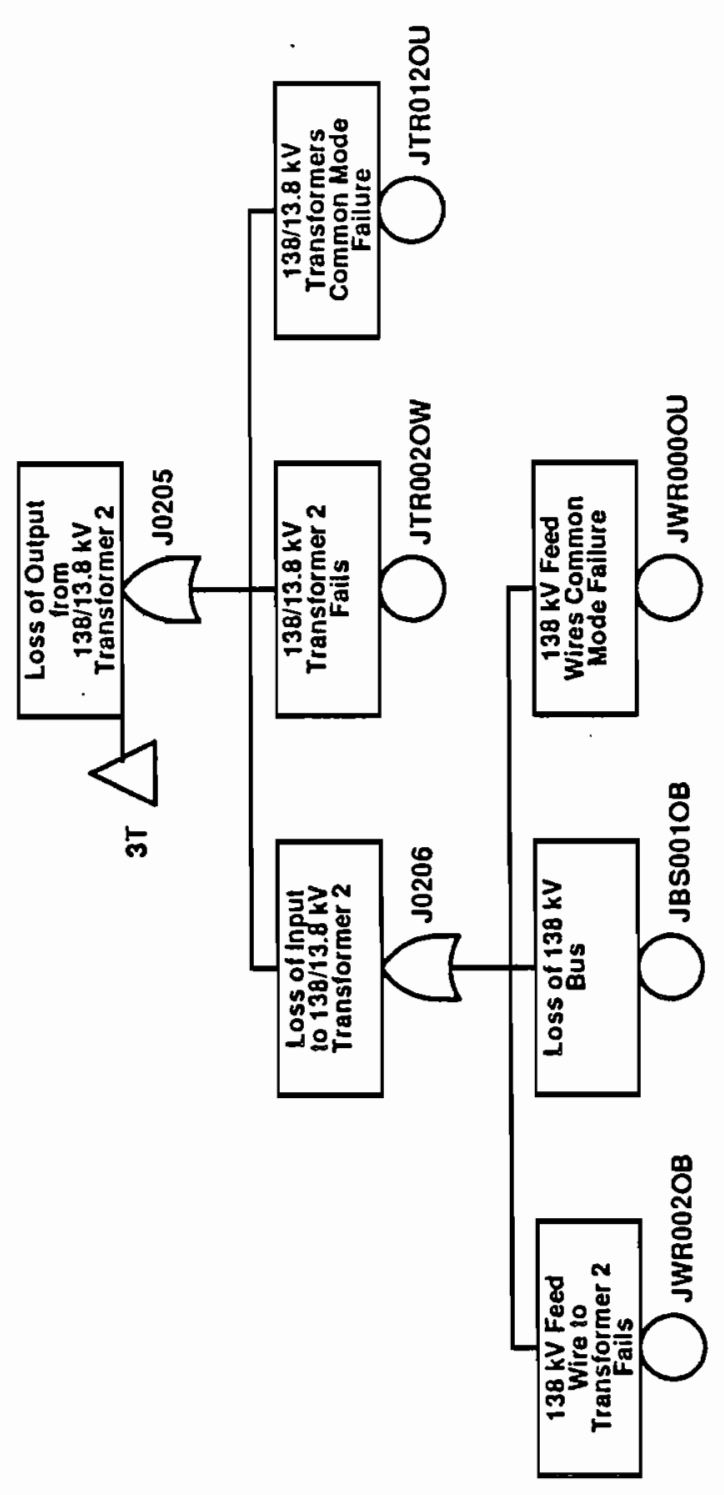

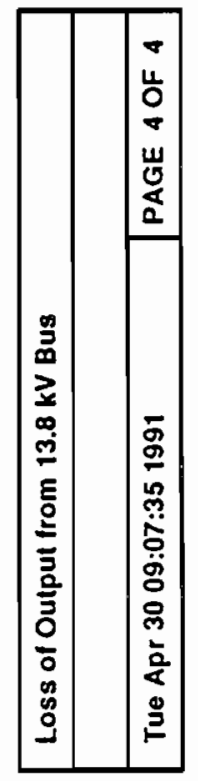




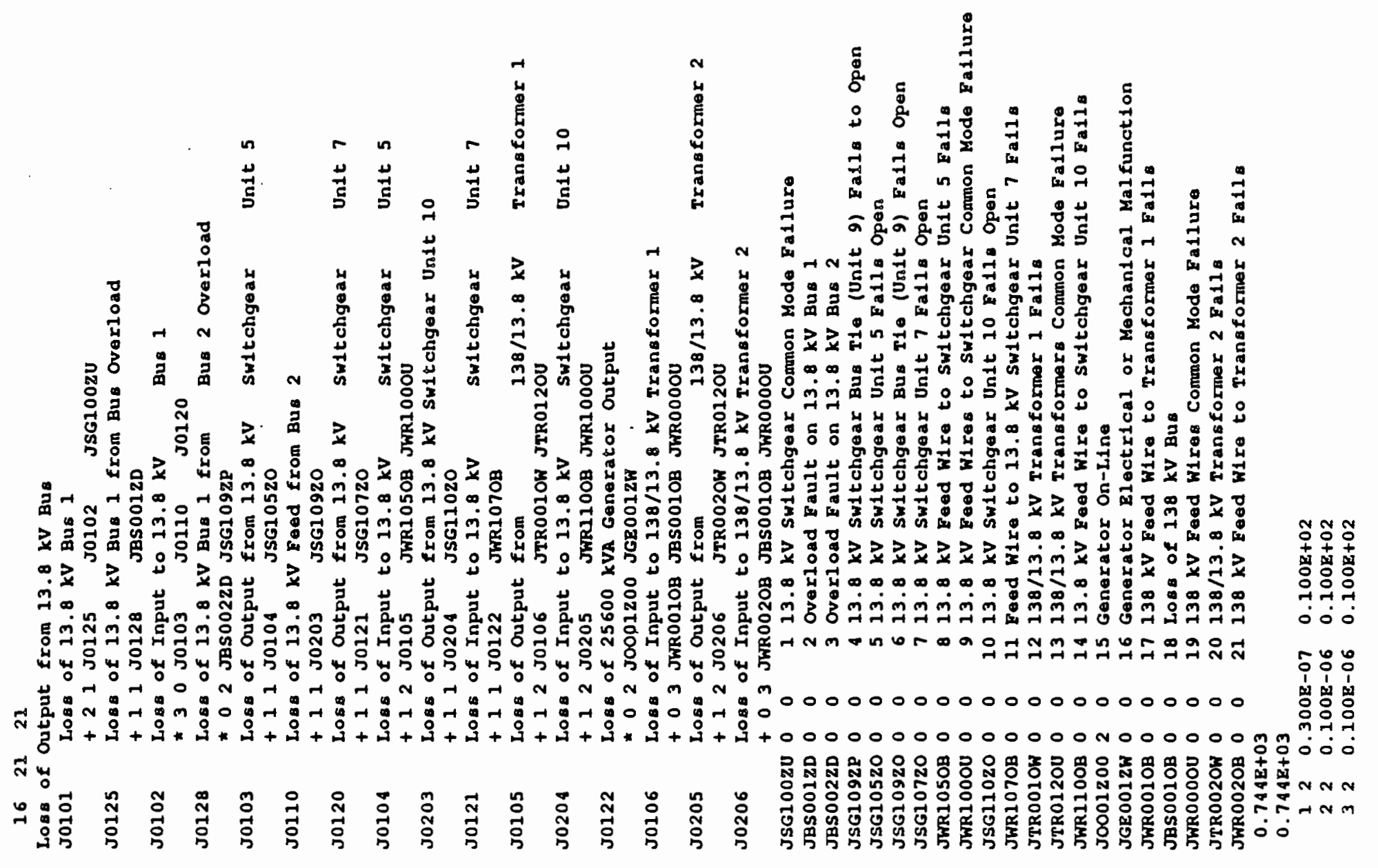

B. $2-346$ 


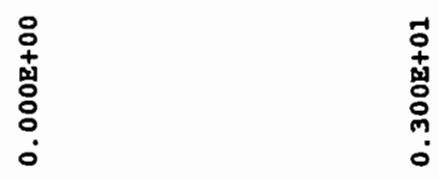

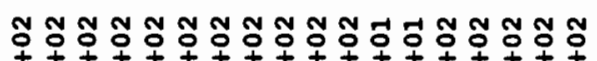

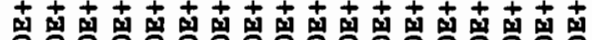

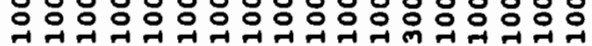

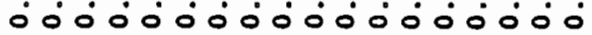

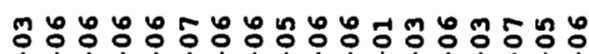

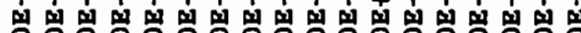
응ㅇㅇㅇㅇㅇㅇㅇㅇㅇㅇㅇㅇㅇㅇㅇㅇㅇㅇㅇㅇㅇㅇㅇㅇㅇㅇㅇㅇ йm m m

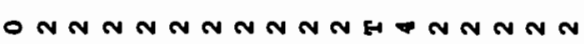
- 


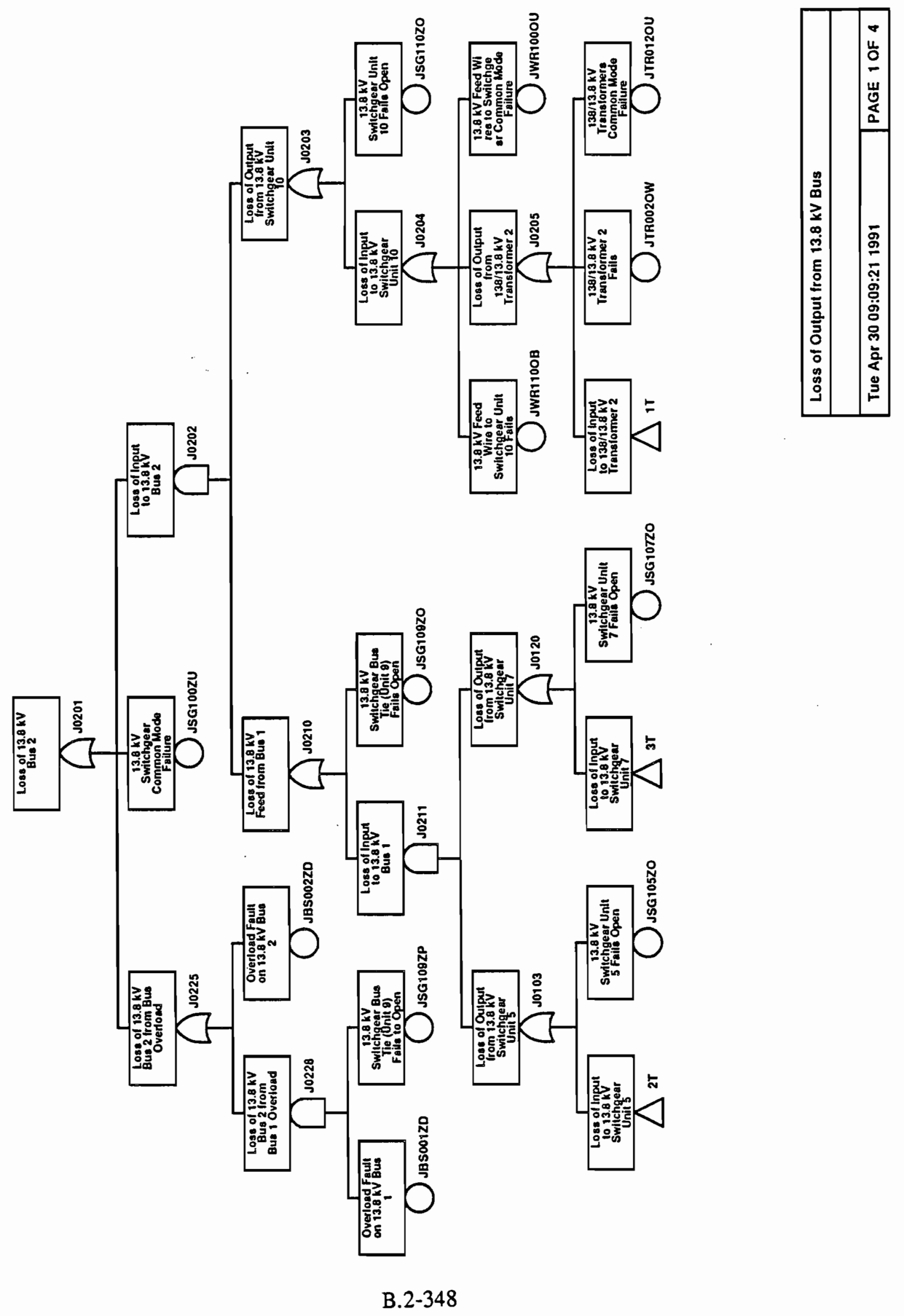




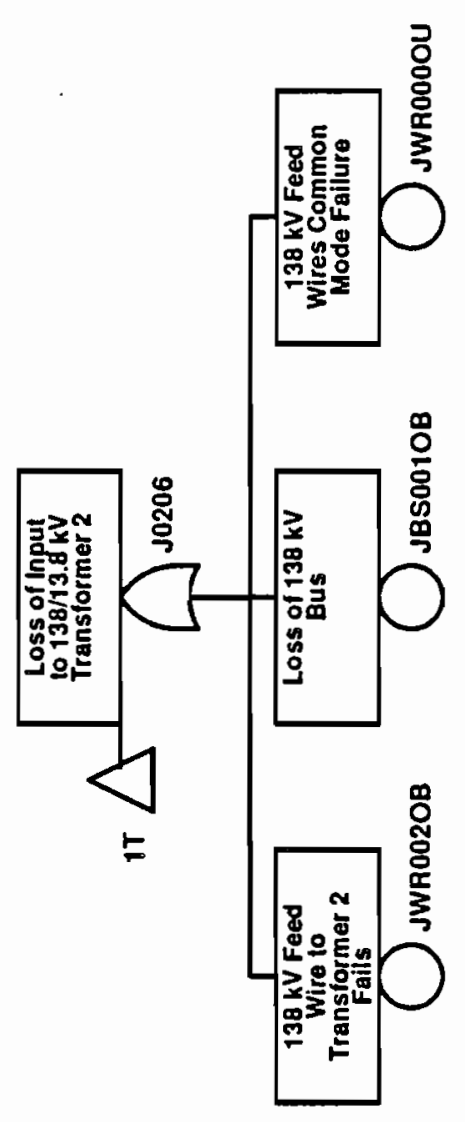



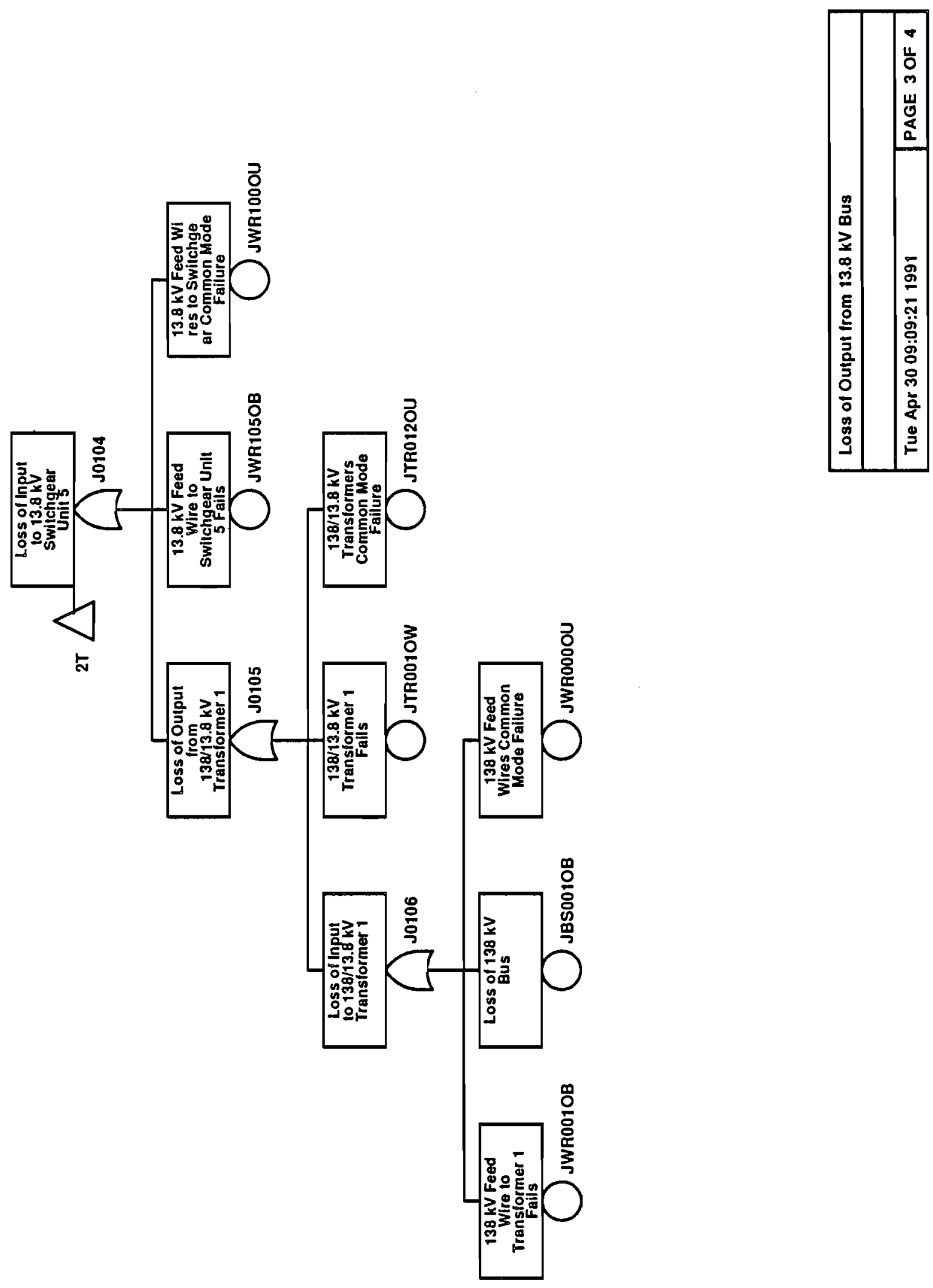

B. $2-350$ 


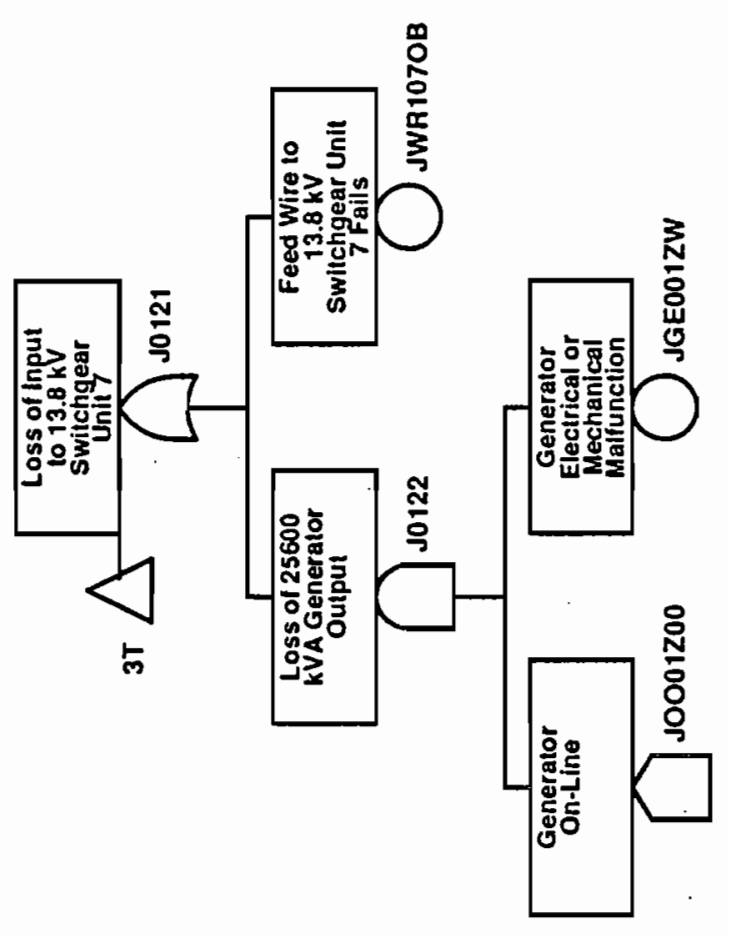

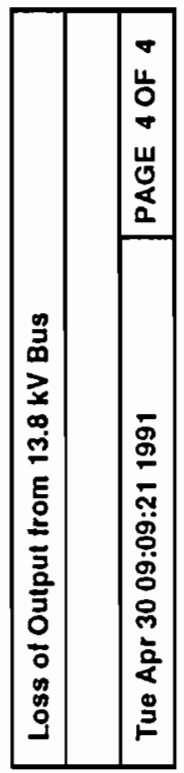




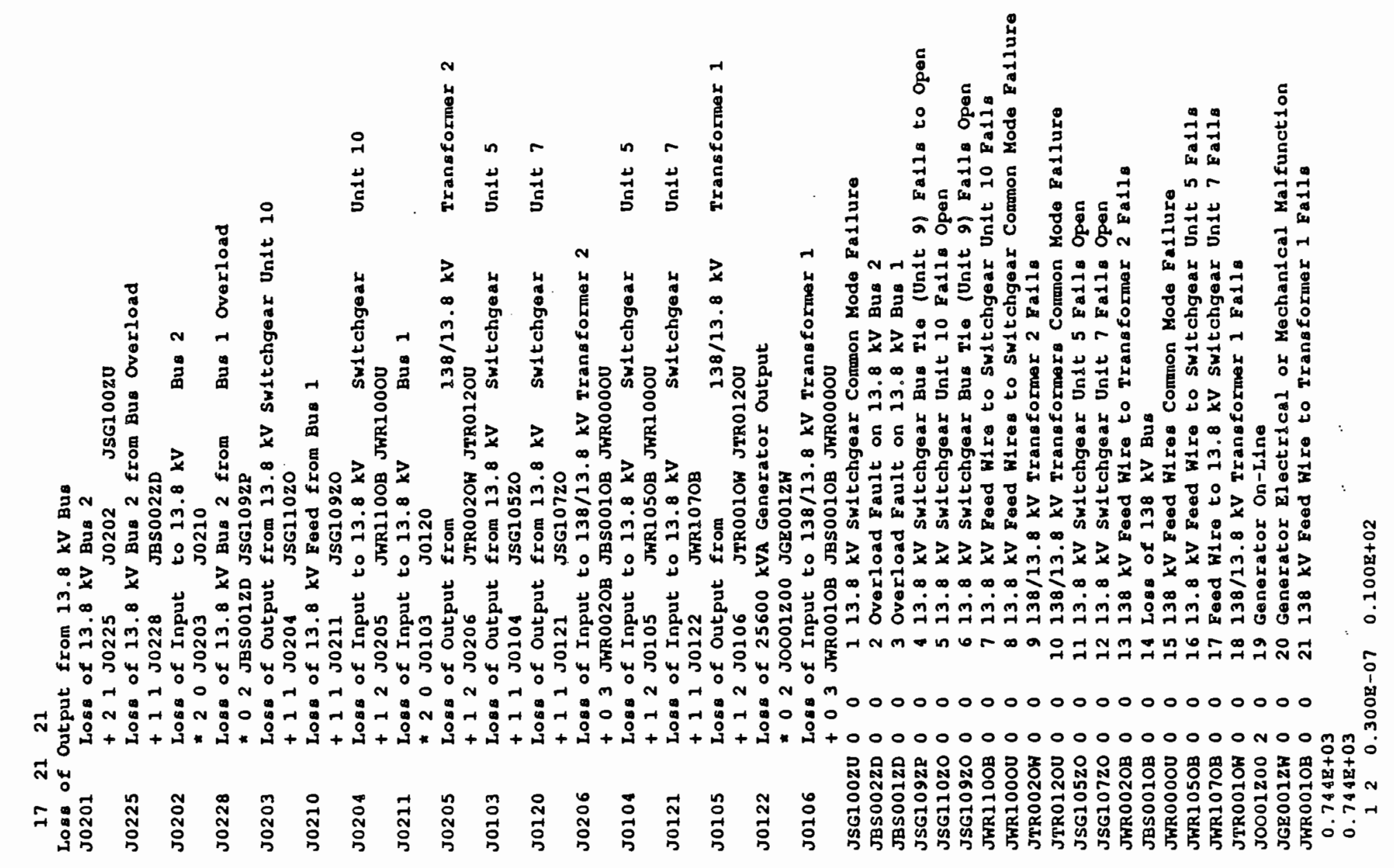



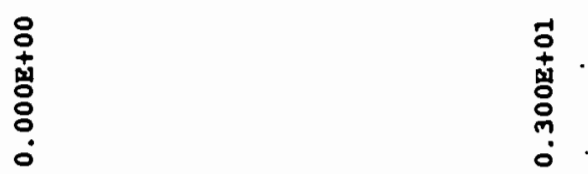

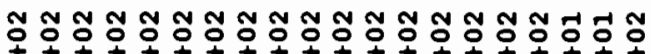

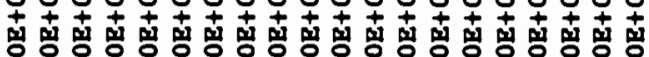

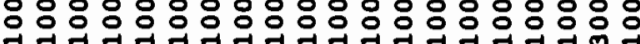

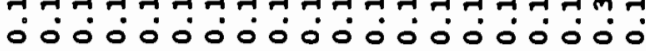

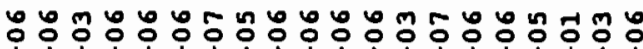
111111111111111111

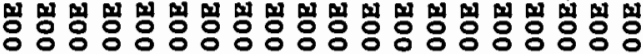

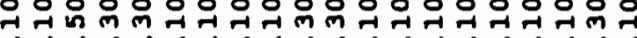

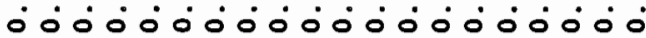
n N O N N N N N N N N N N N H N Nด แ 


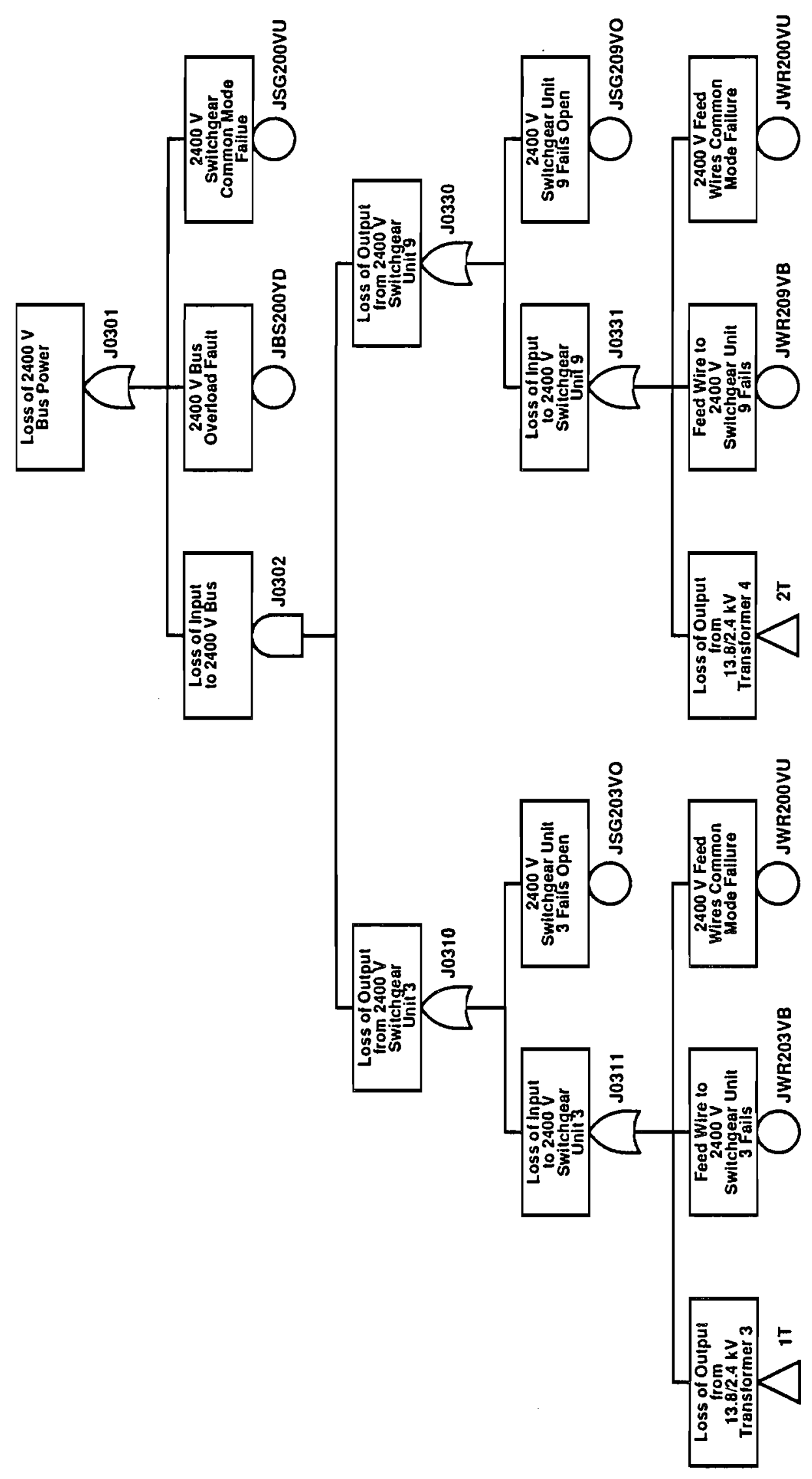




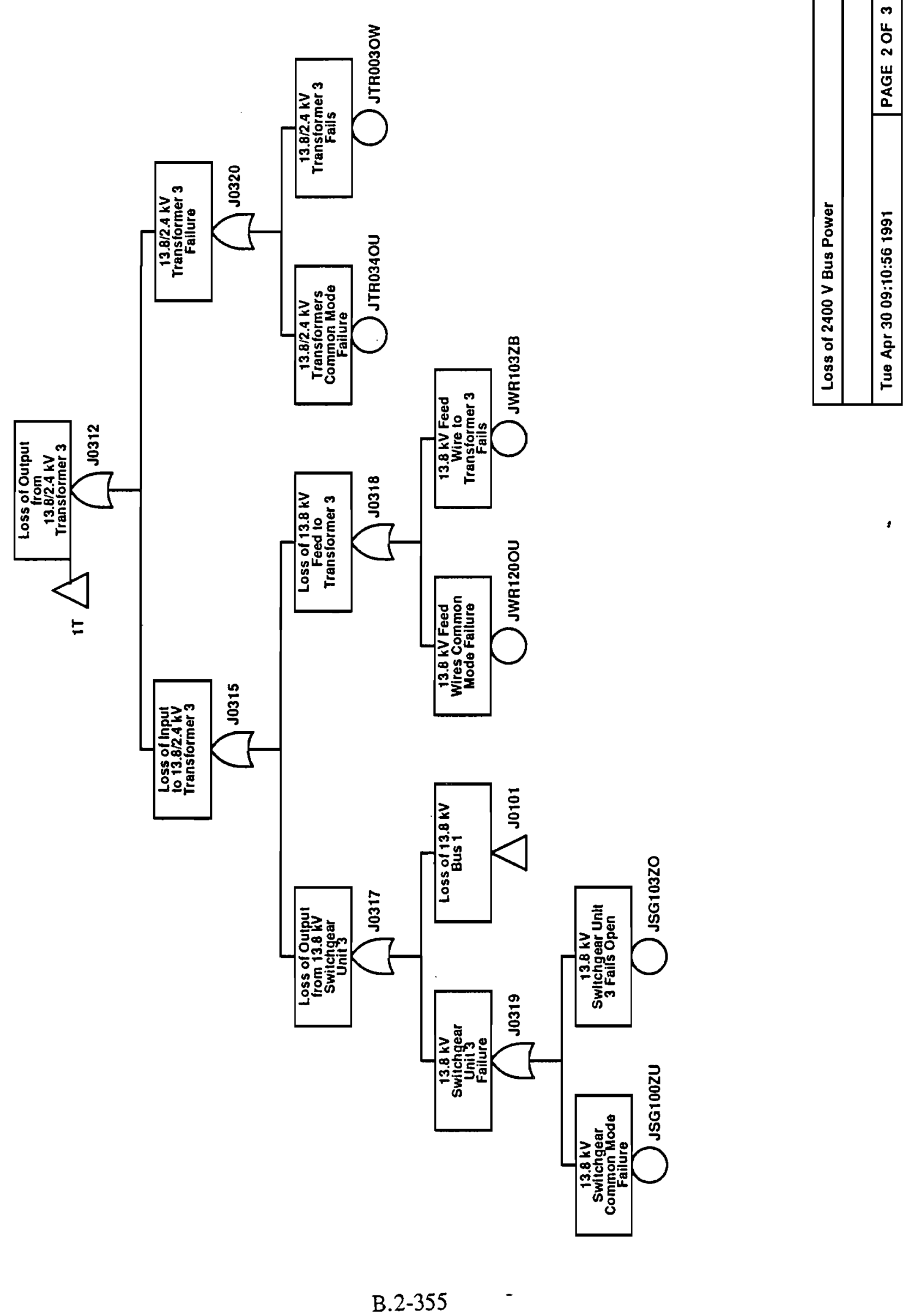




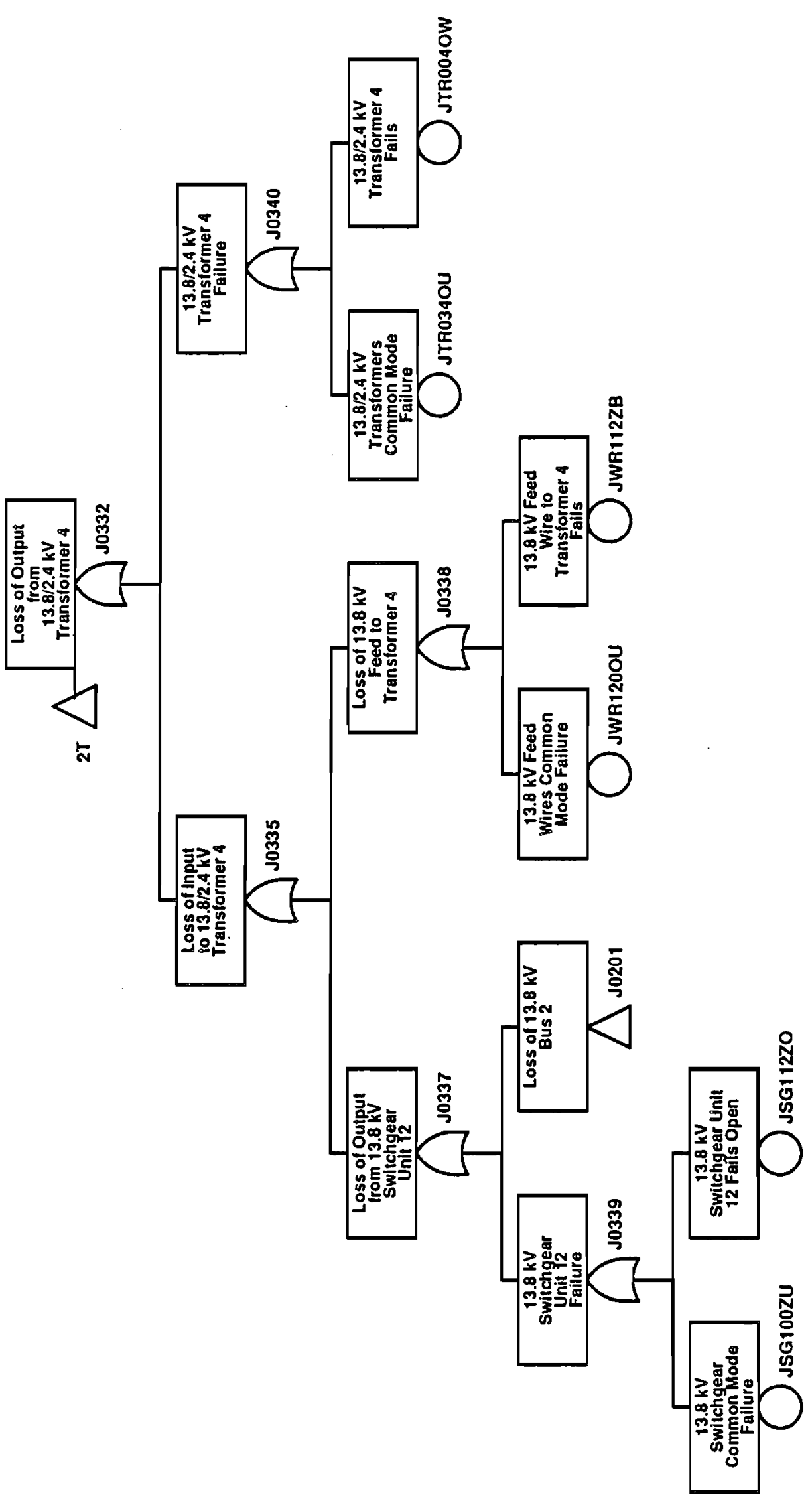




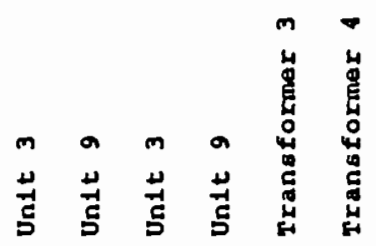

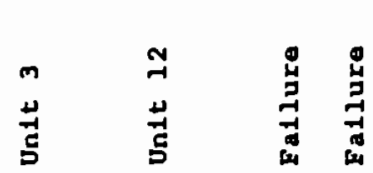

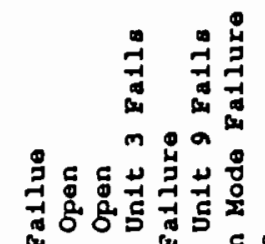

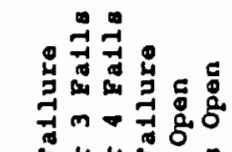

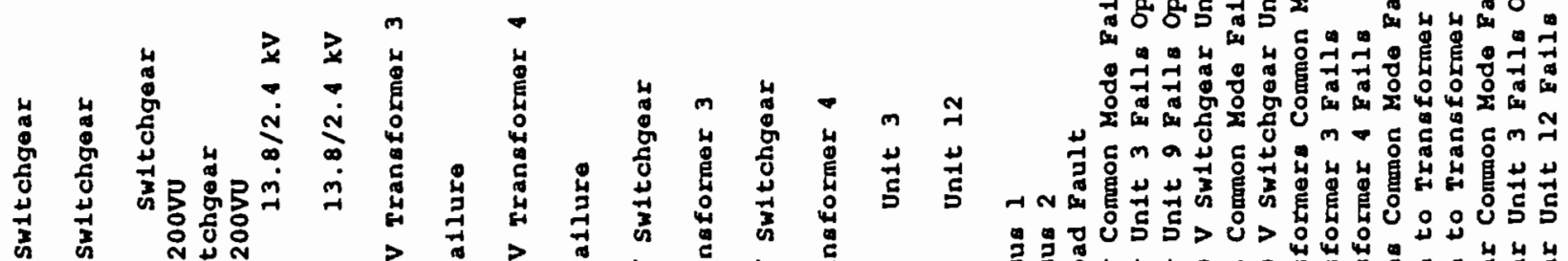

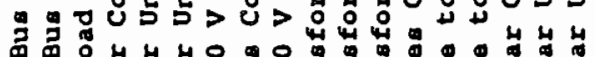

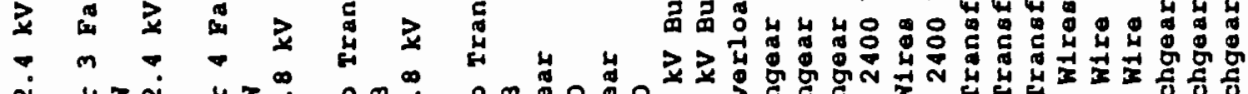
$\begin{array}{lll}\mu & 0 \\ 0 & 0\end{array}$

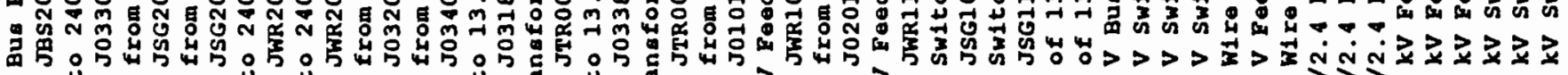
出

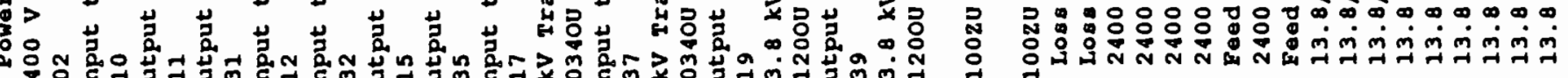

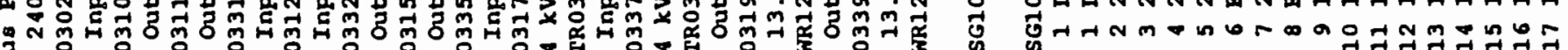

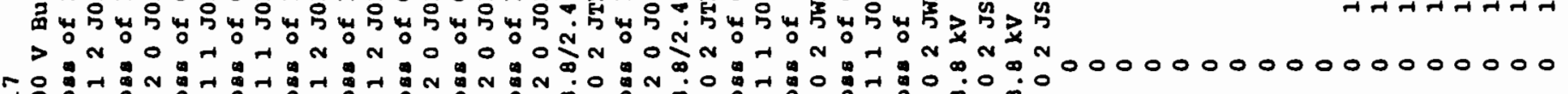

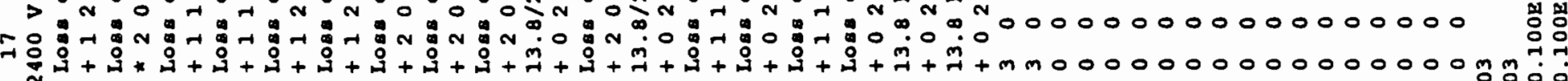

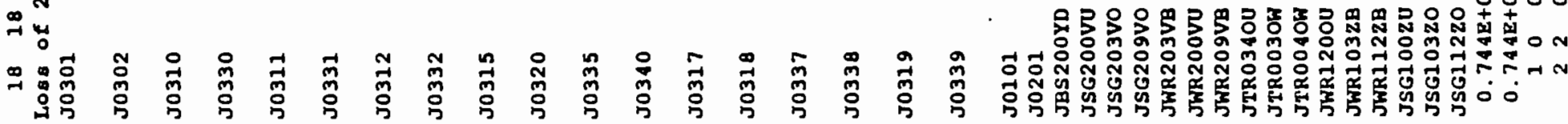




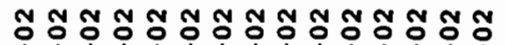

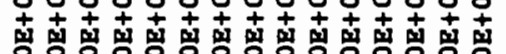

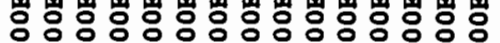

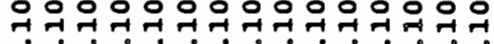
-00000000000

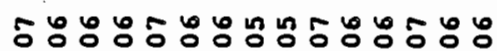

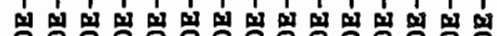

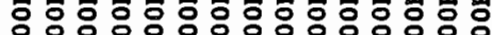

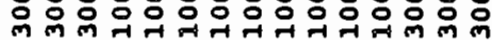
○ं $0 \dot{0} 0 \dot{0} 0 \dot{0} 0 \dot{0} 0 \dot{0}$

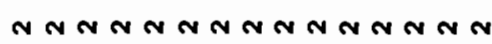
$m$ an 6 - 0 o 


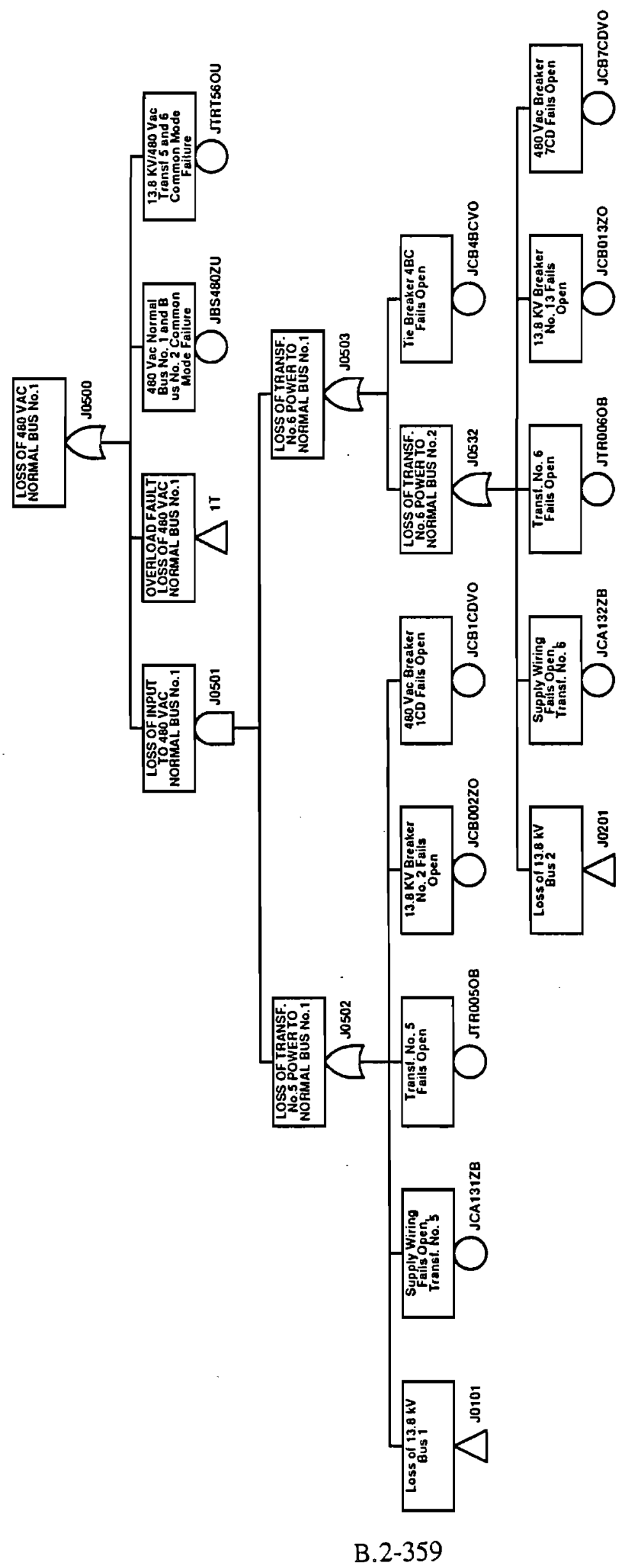

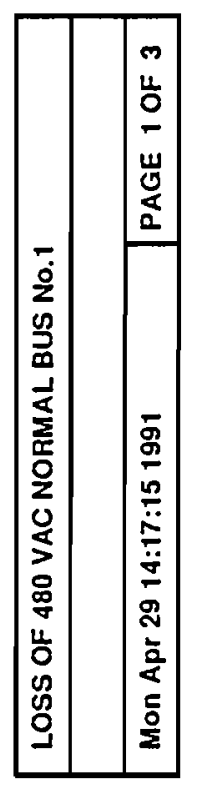




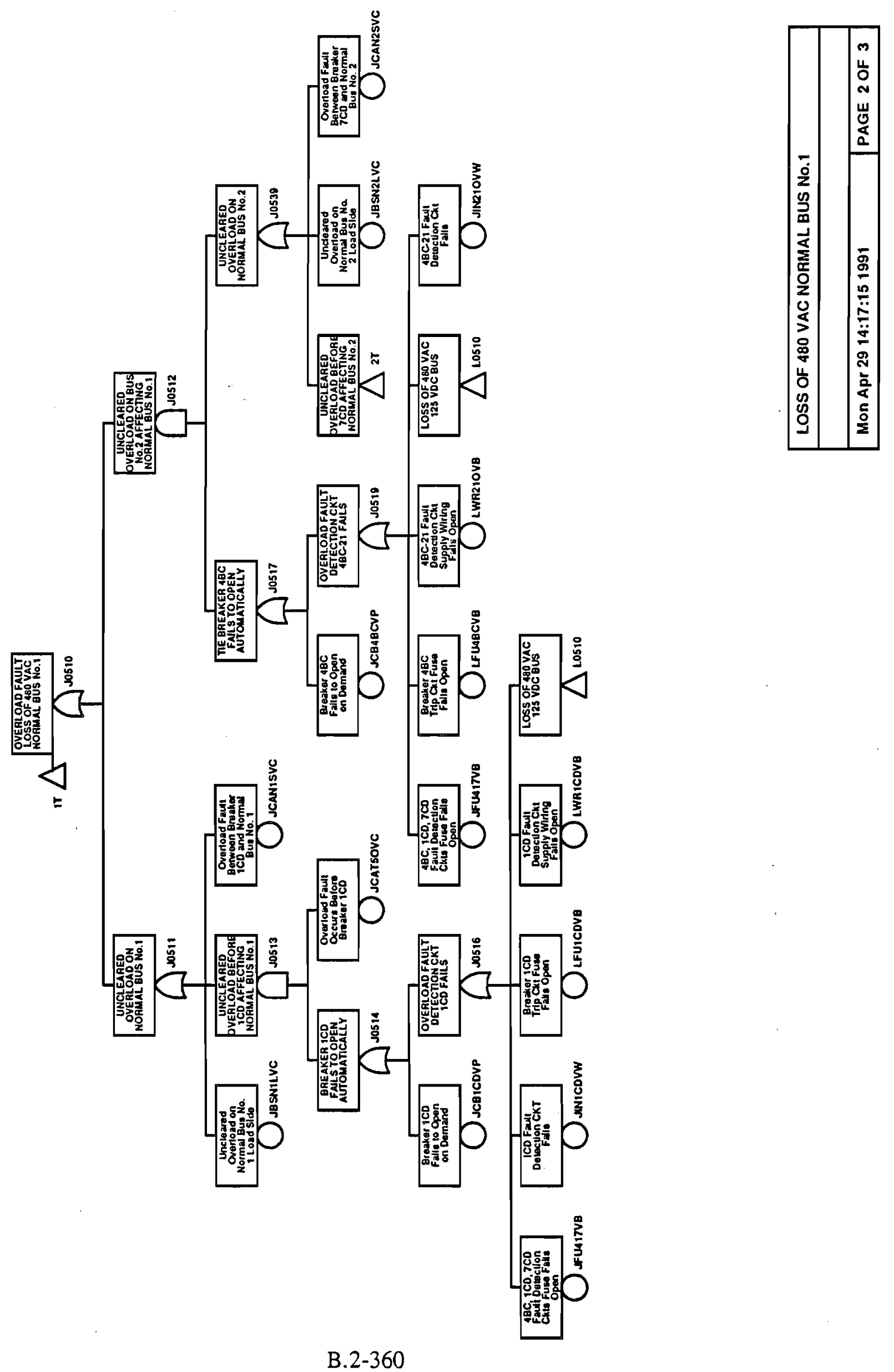




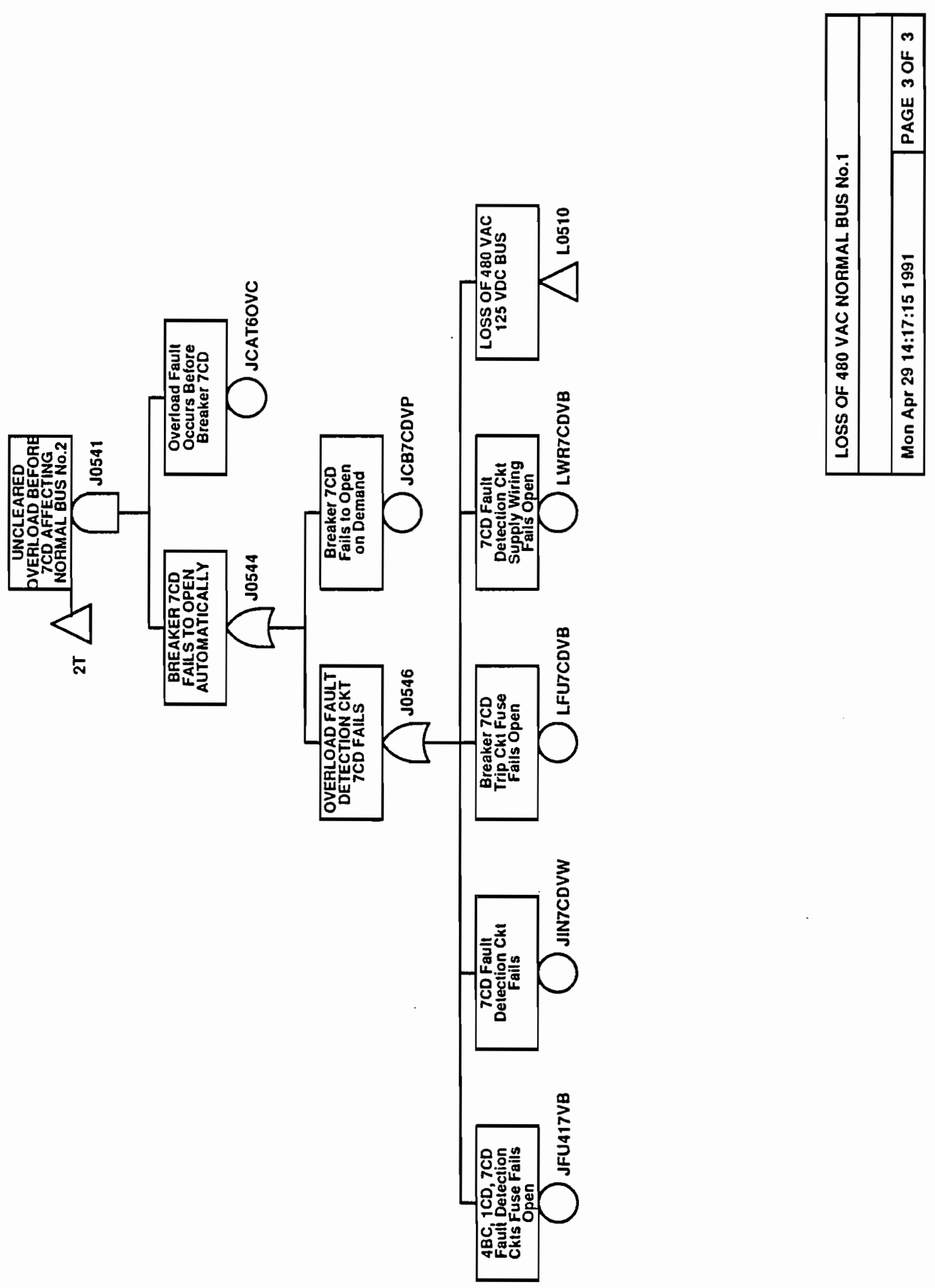




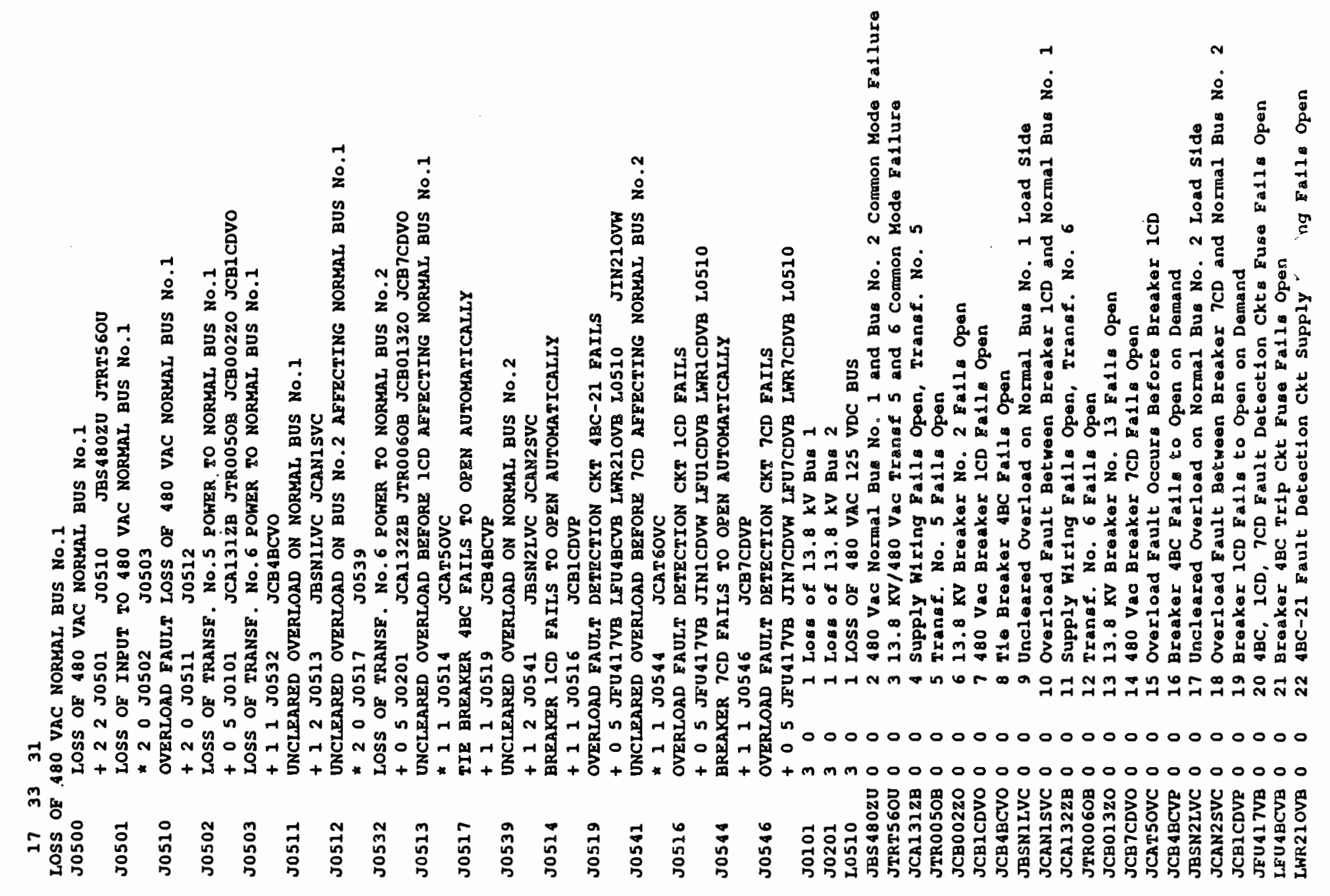




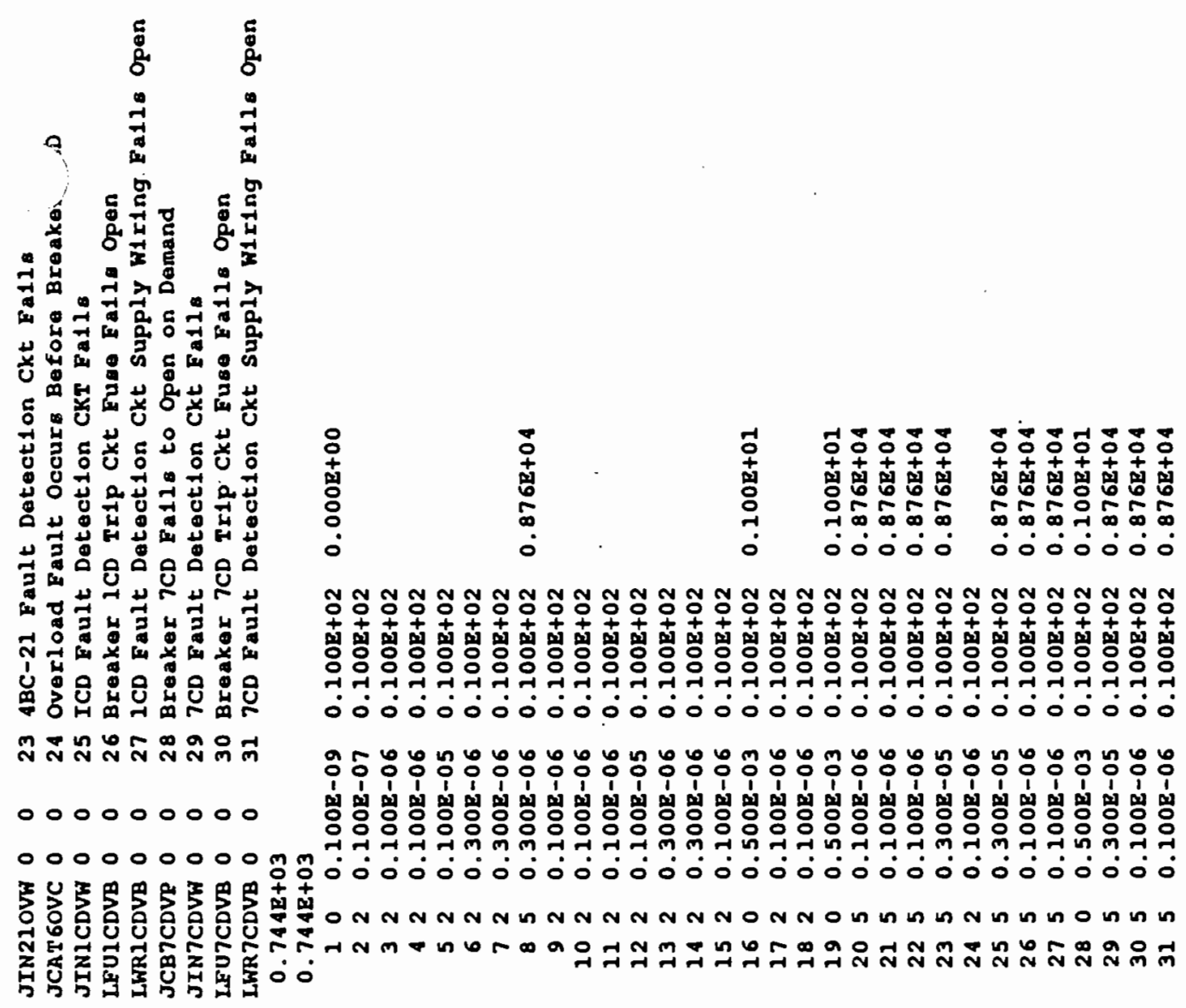




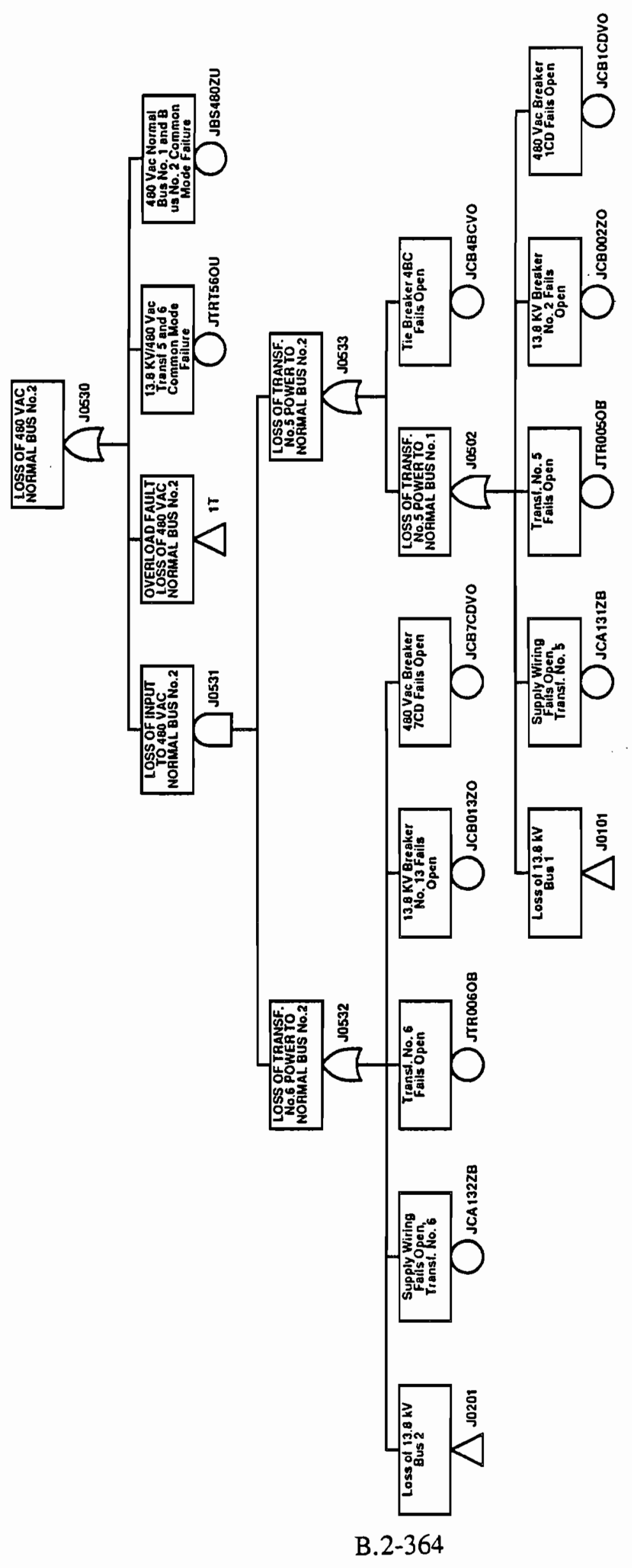




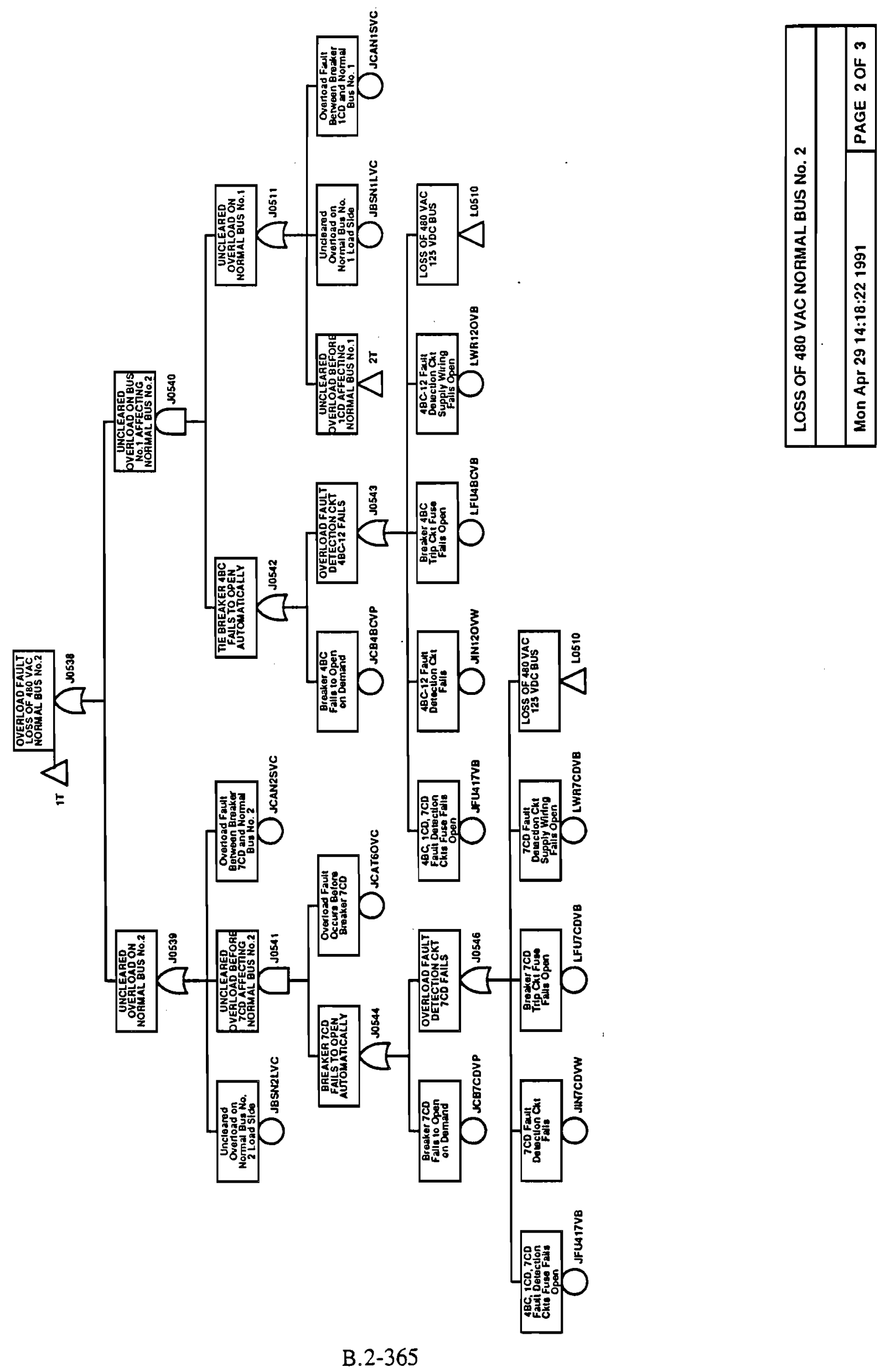



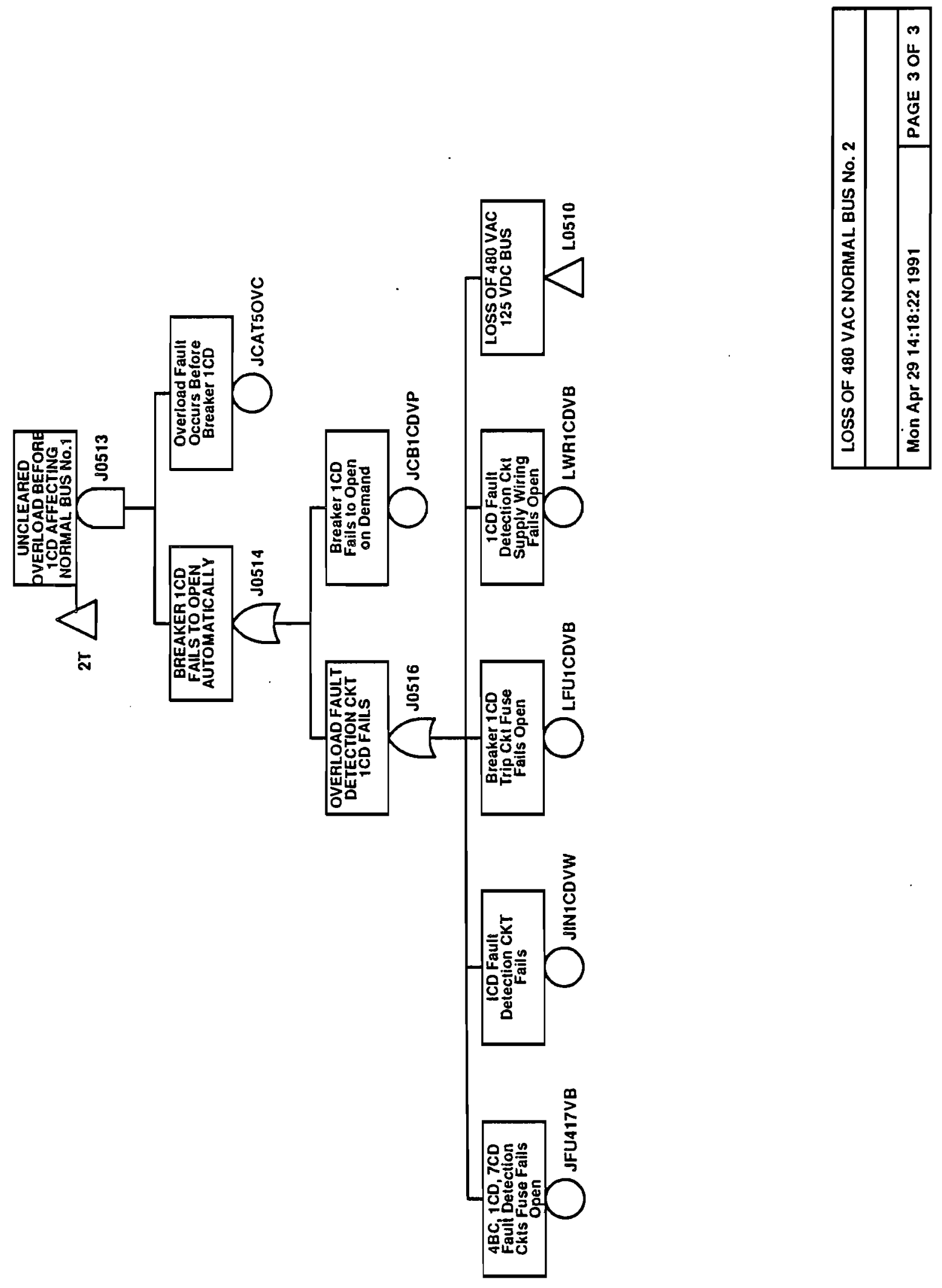

B. $2-366$ 


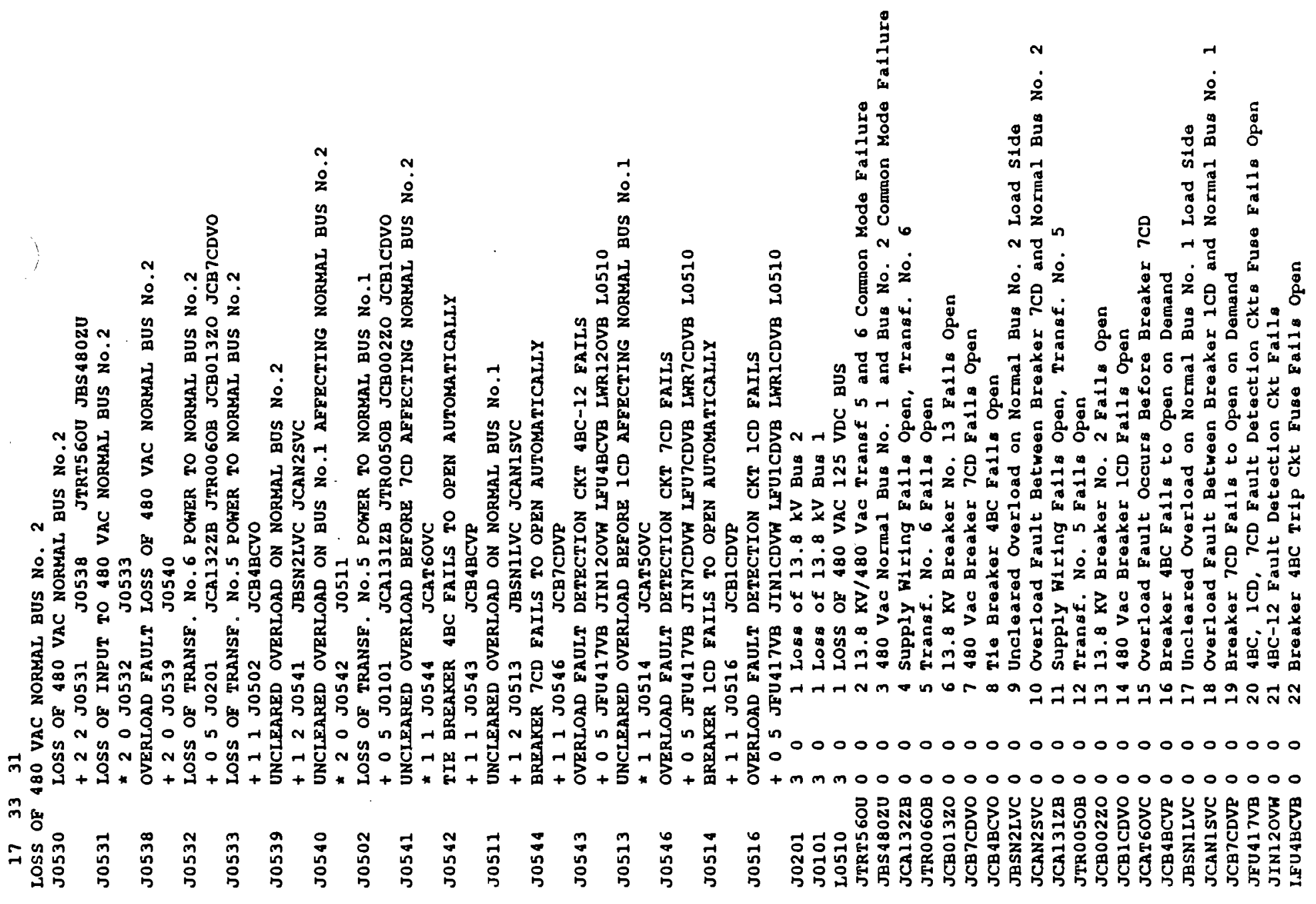




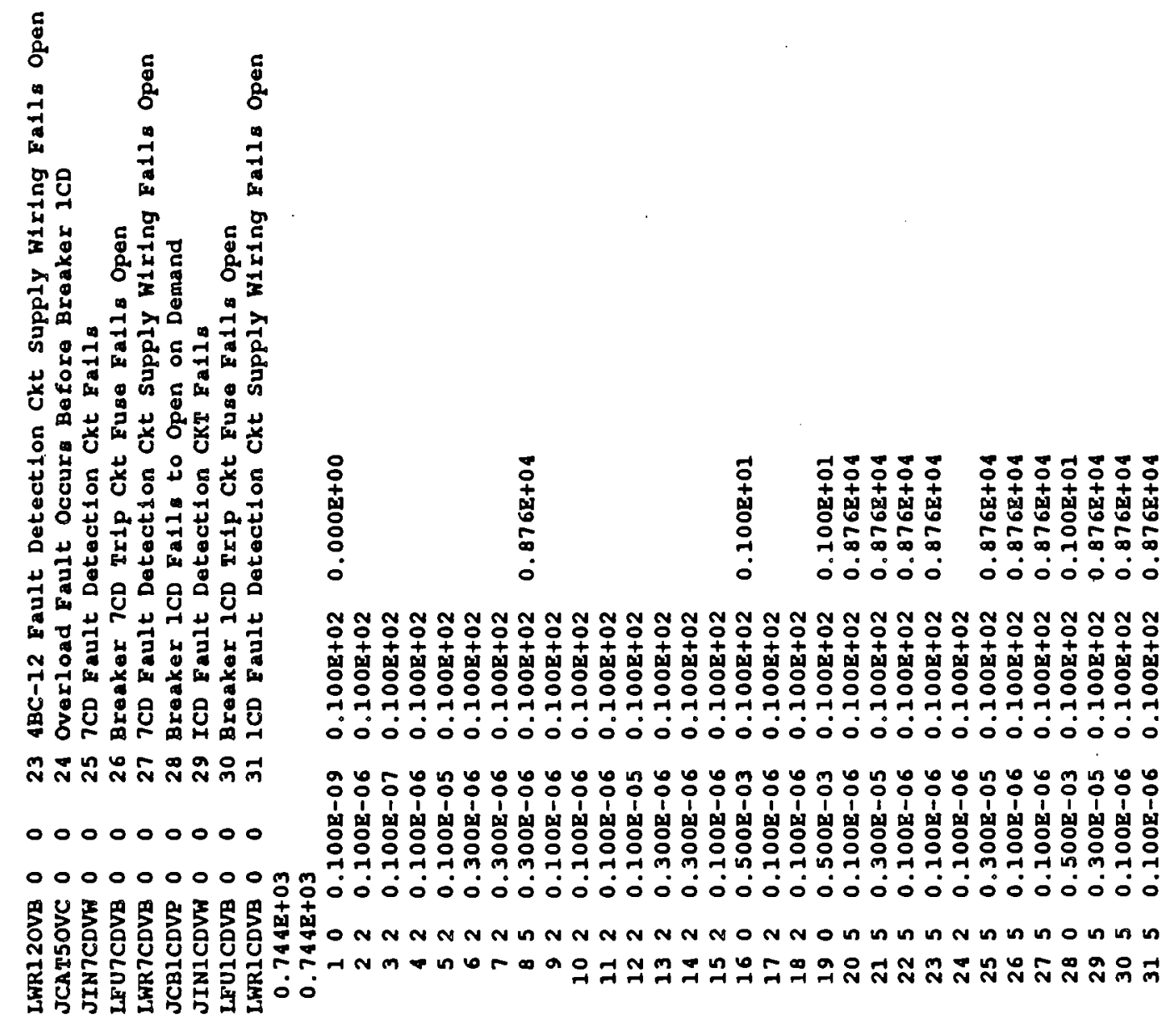




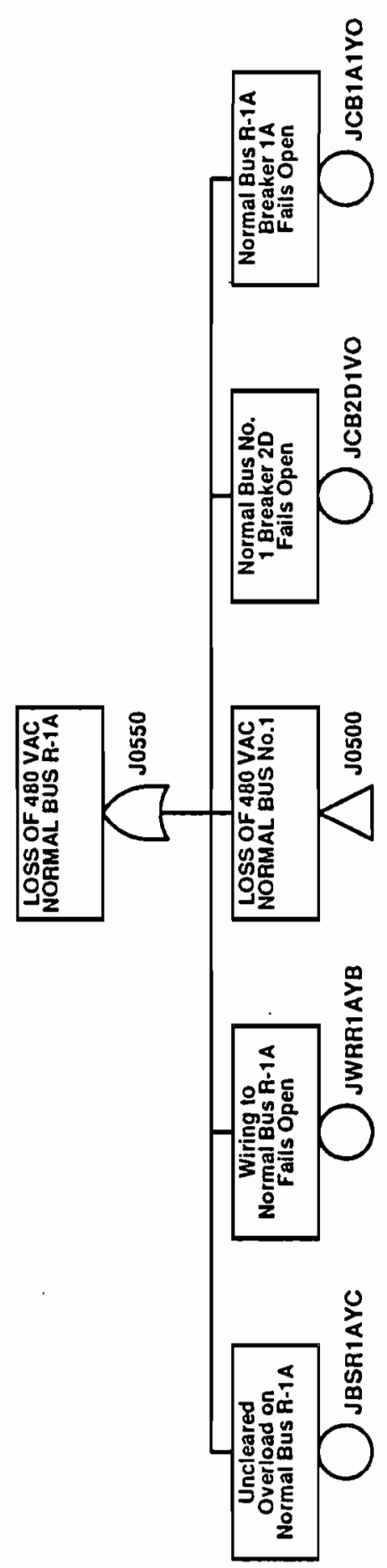

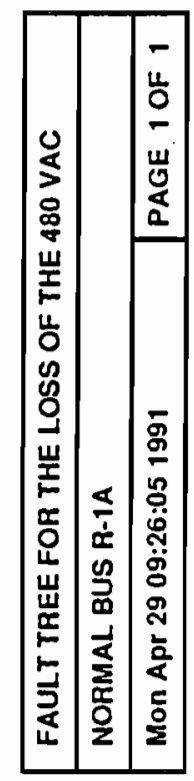




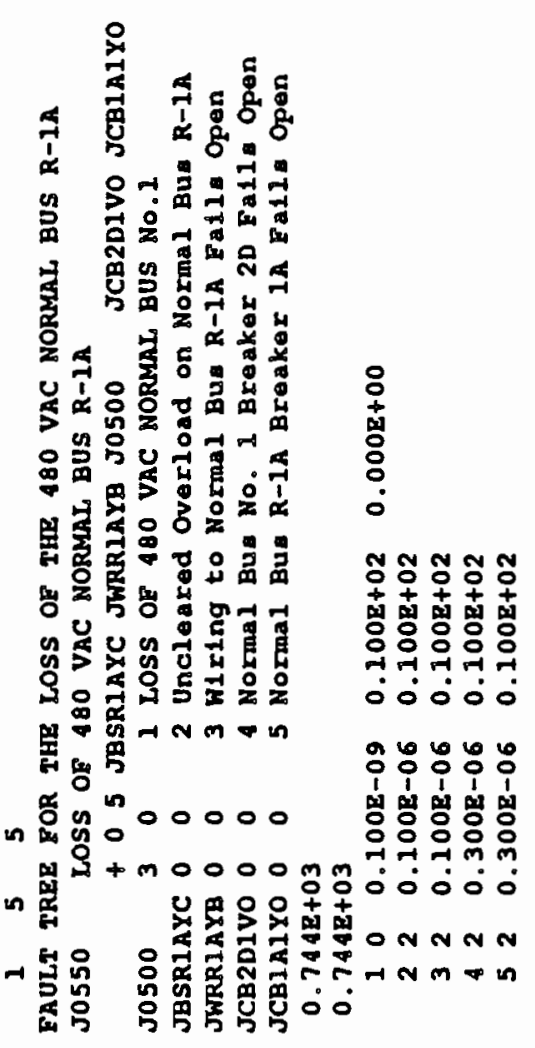




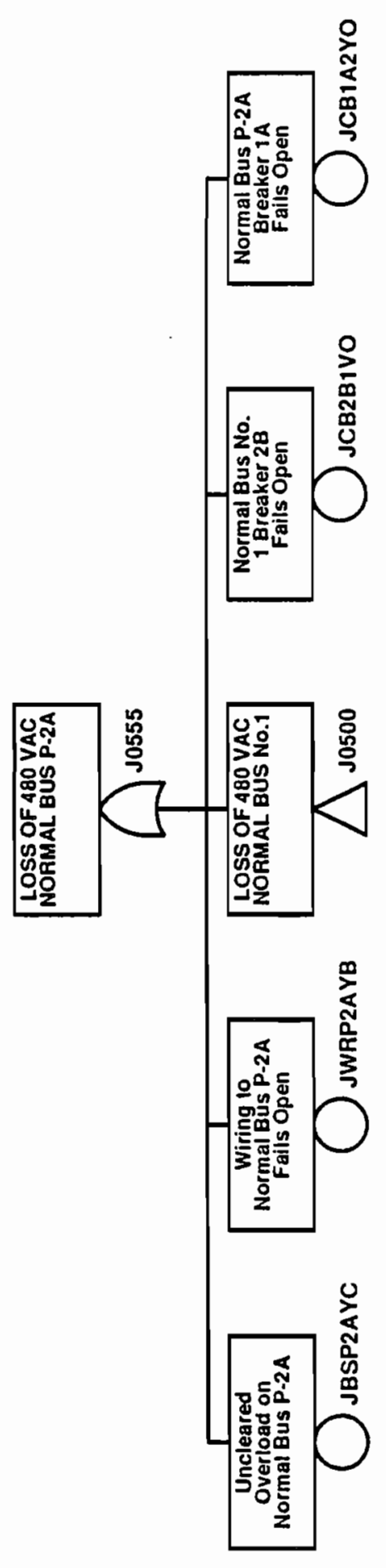

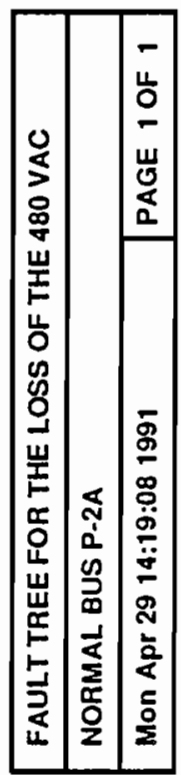

B. 2-371 


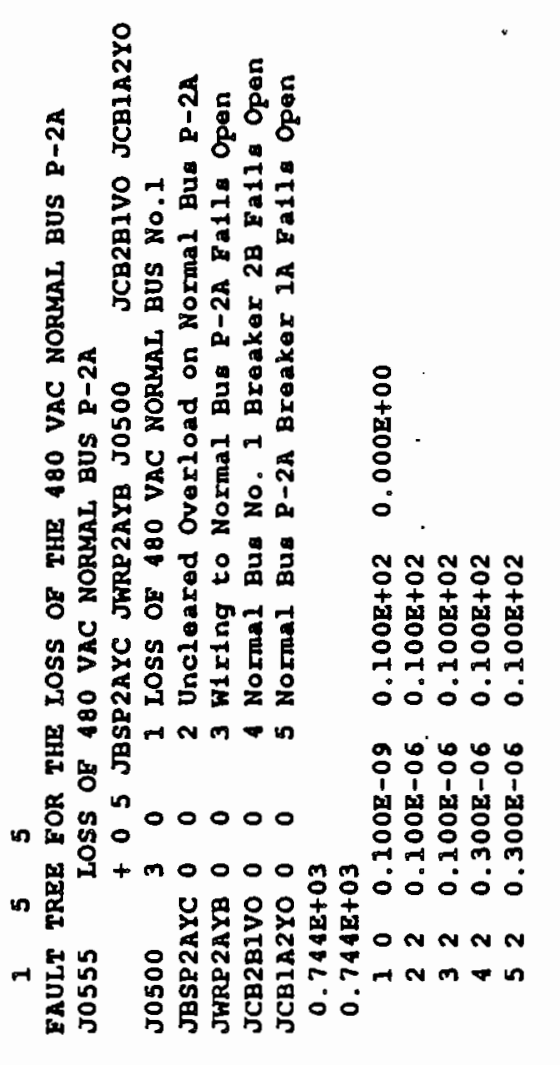



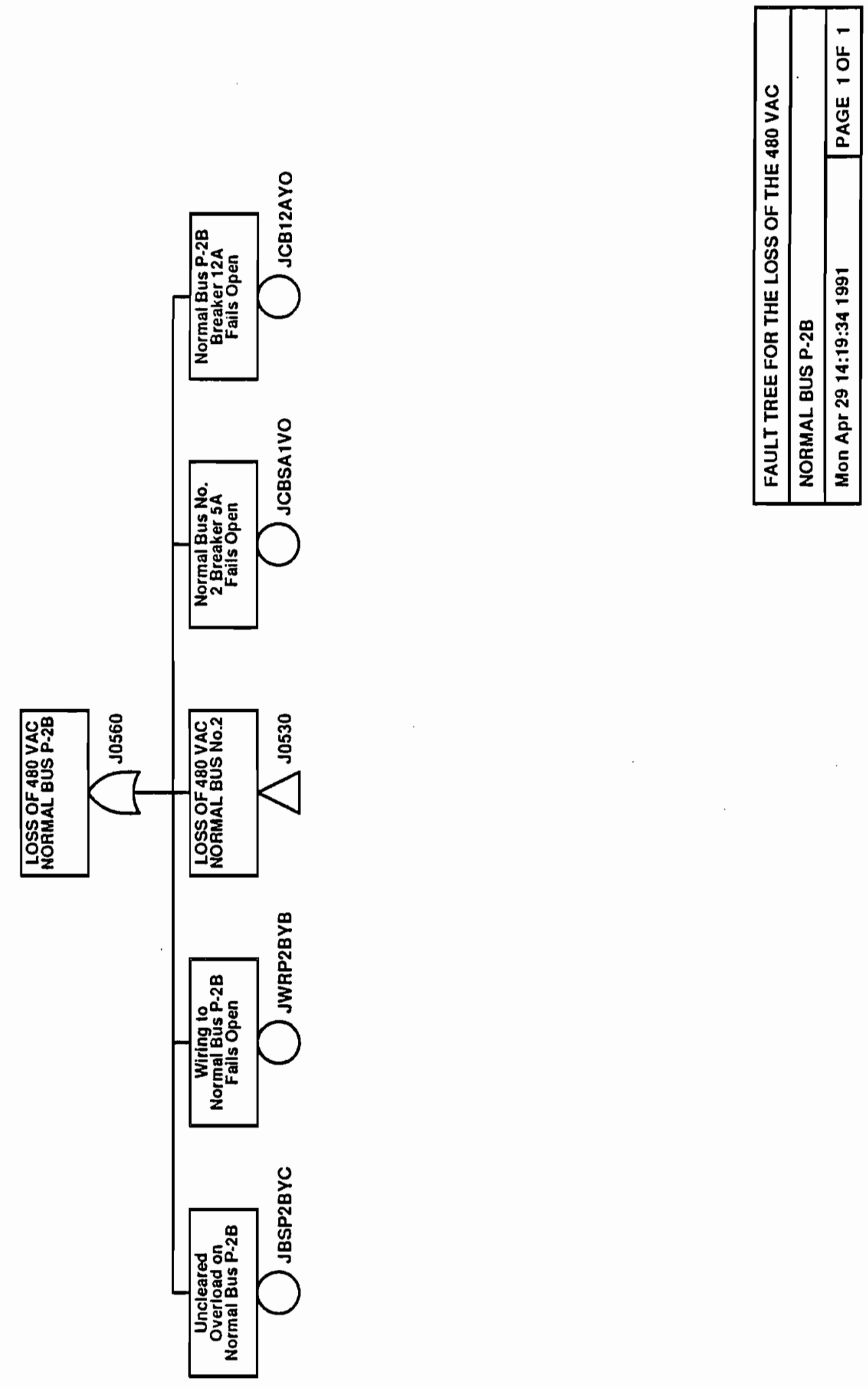

B. 2-373 


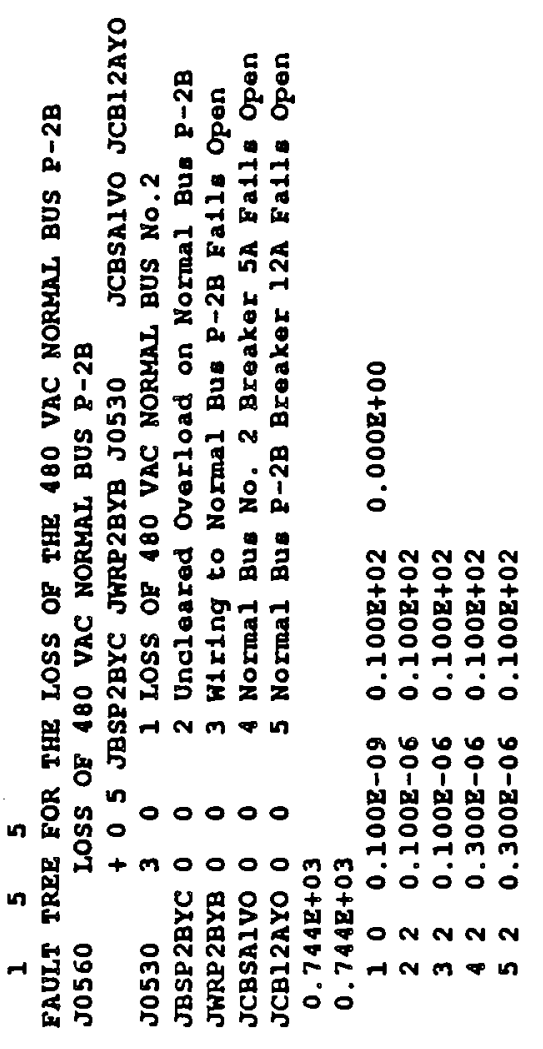



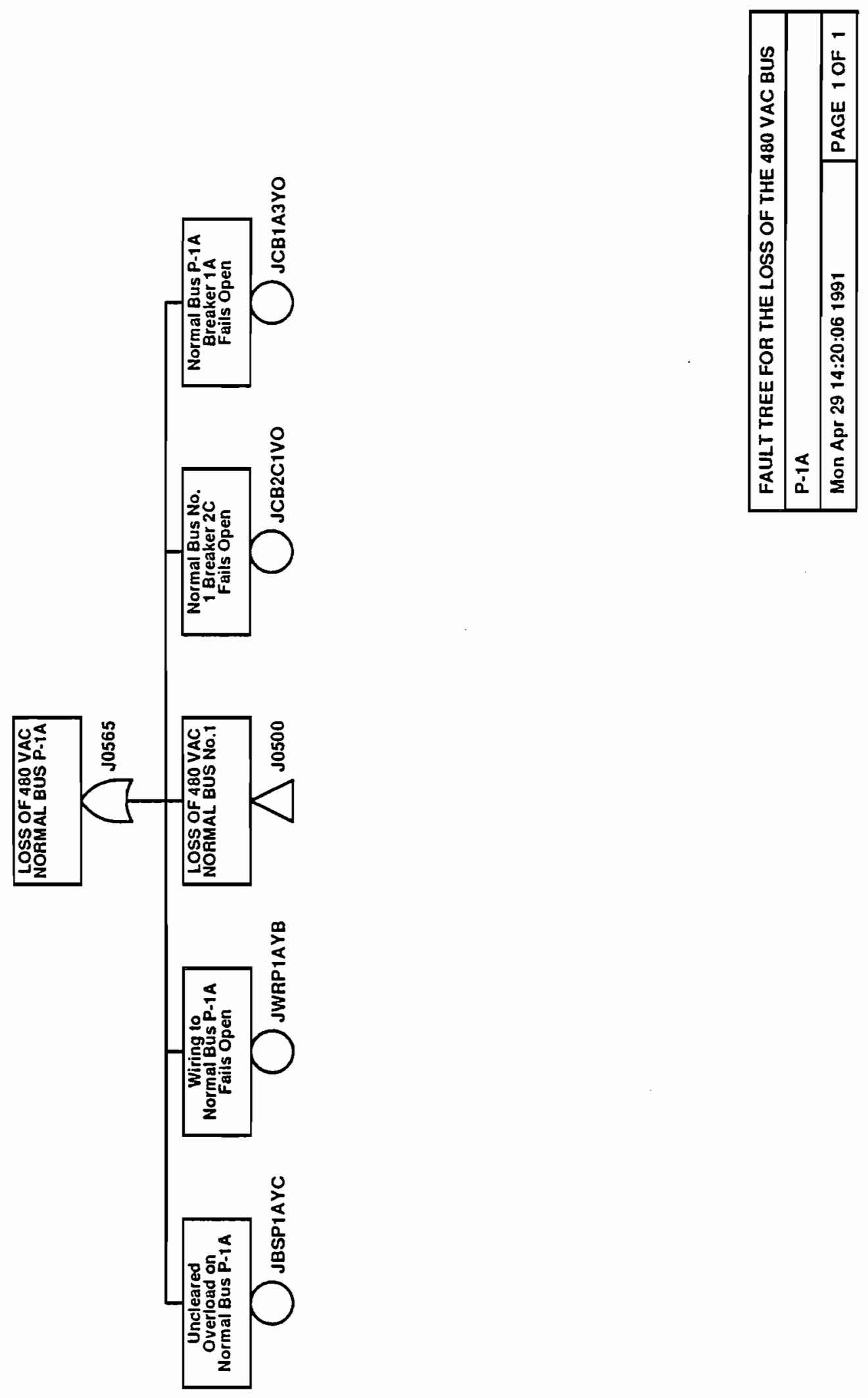

B. 2-375 


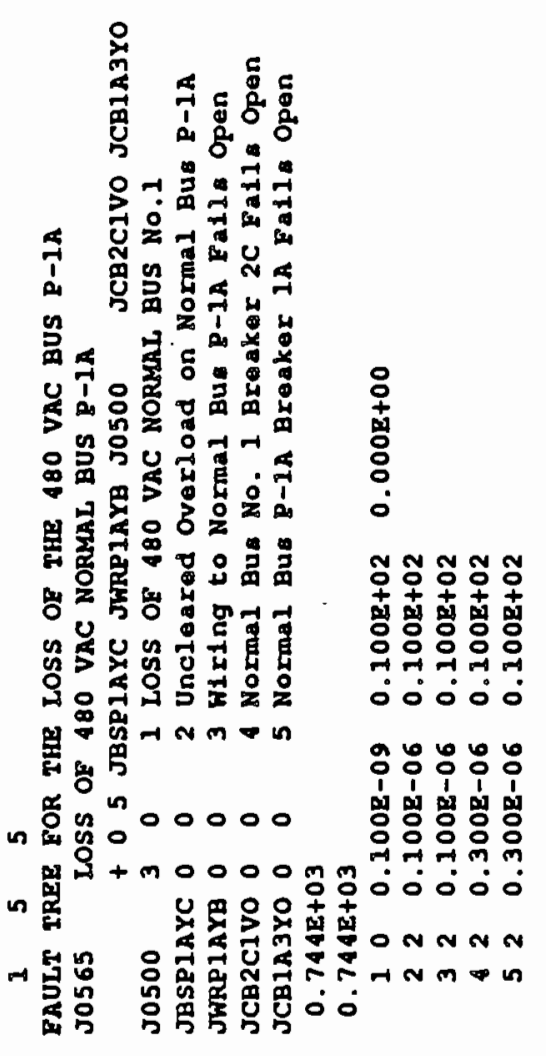



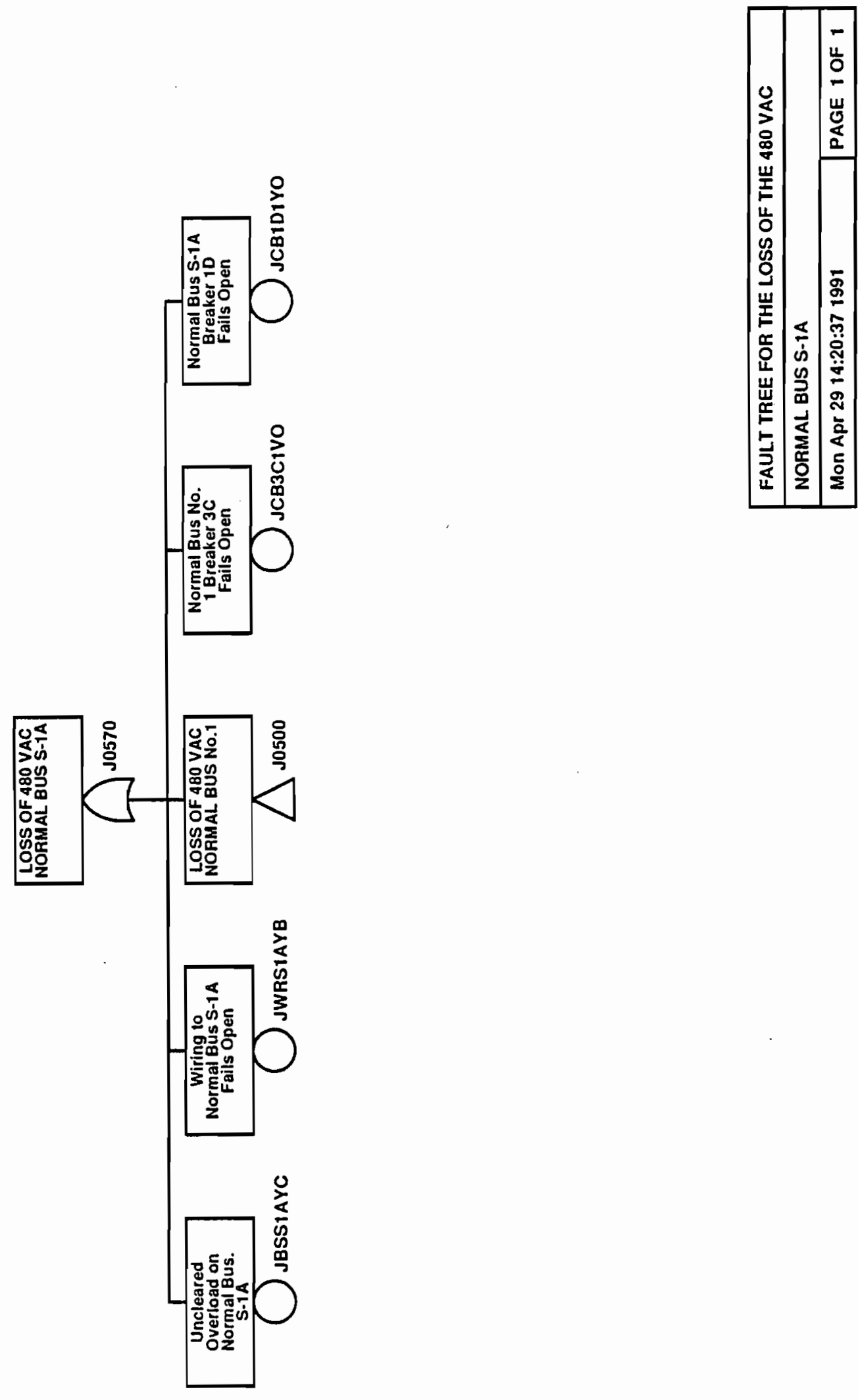


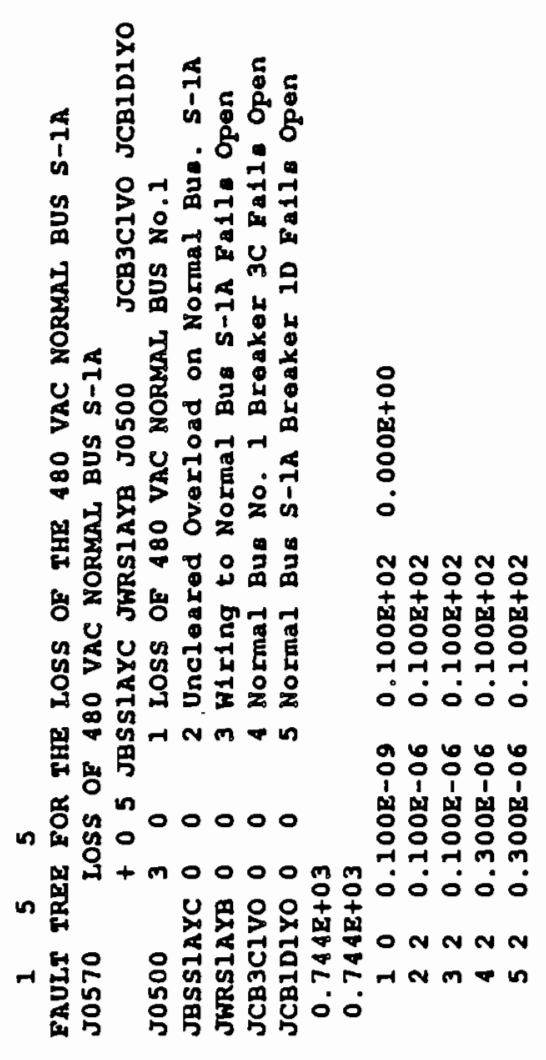



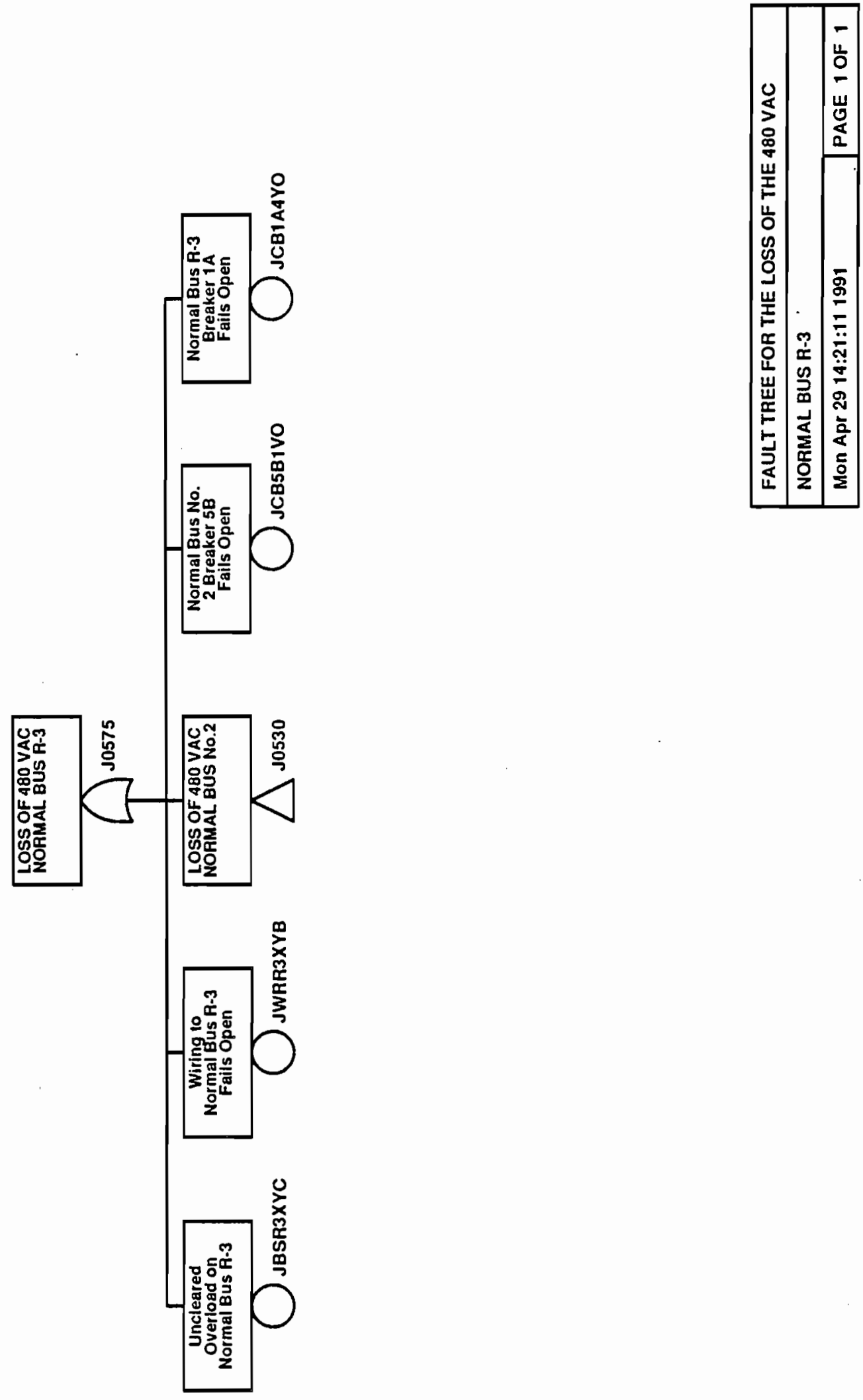


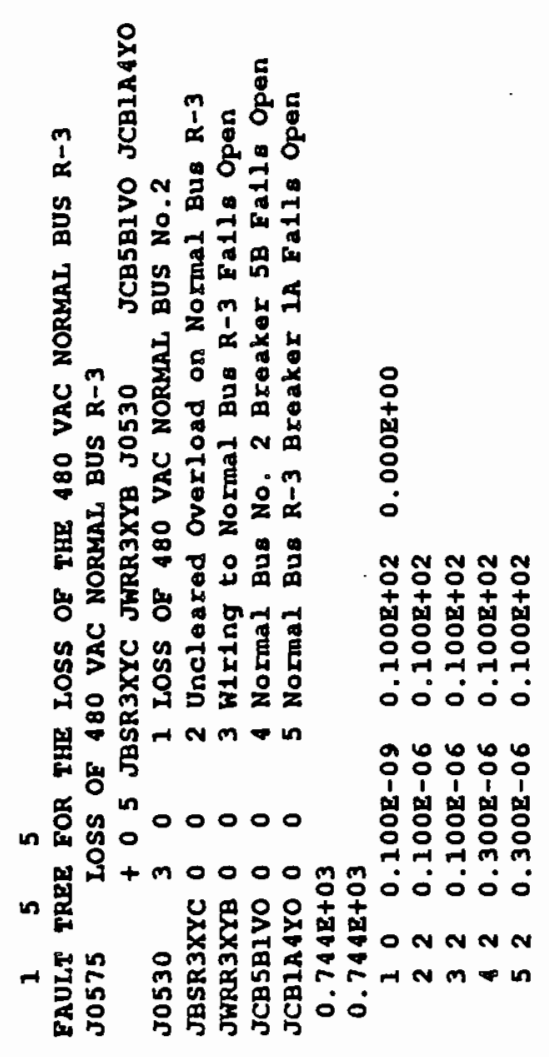



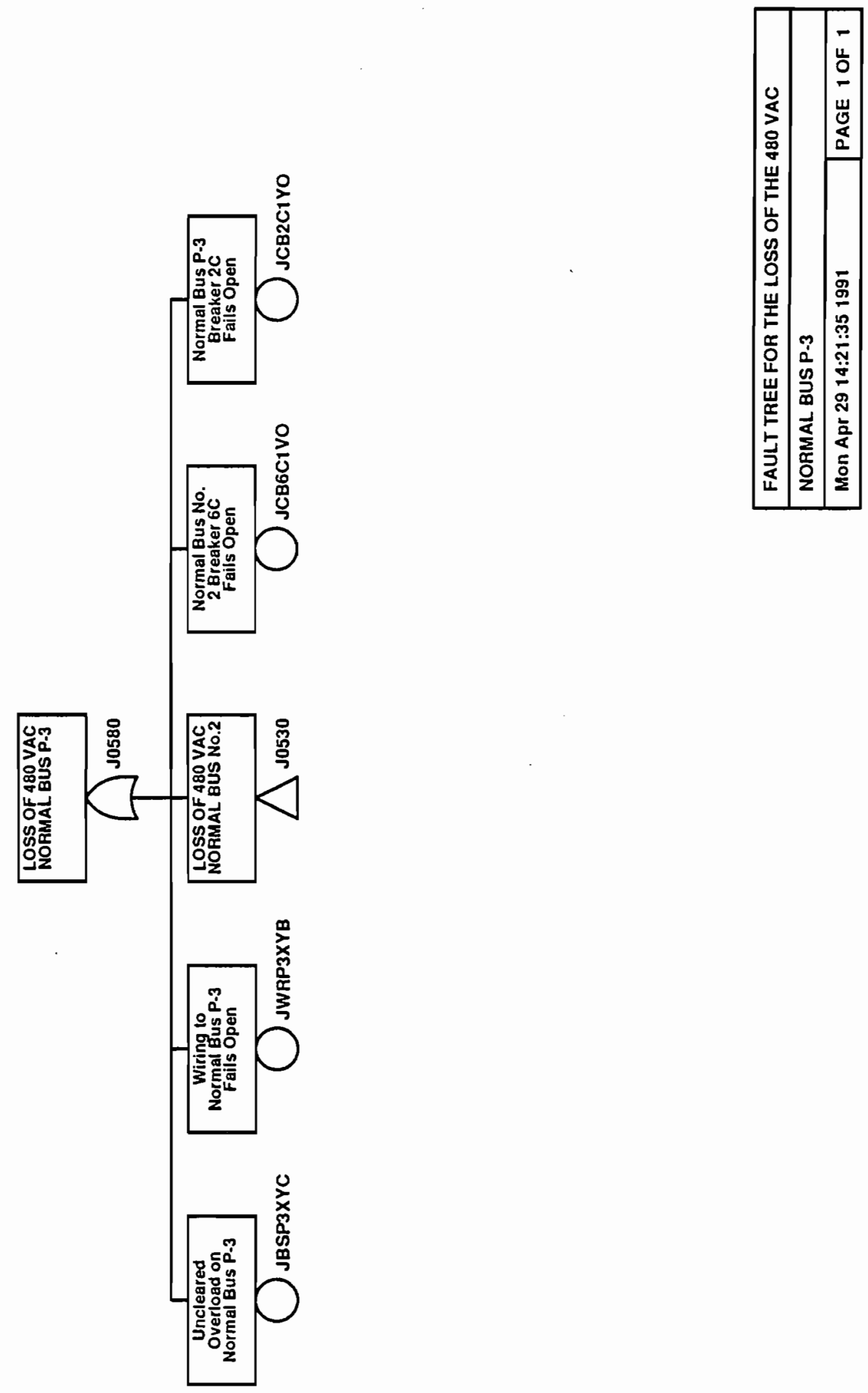


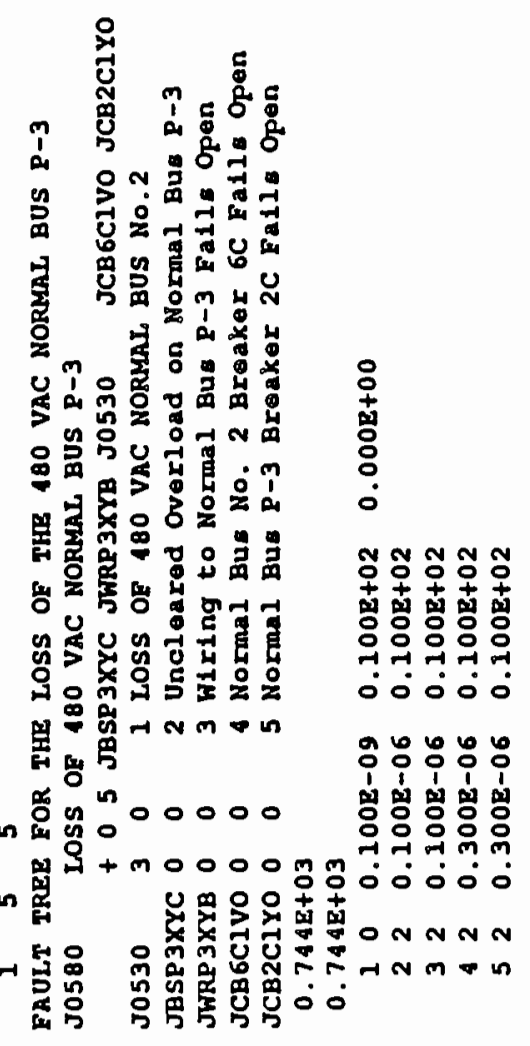




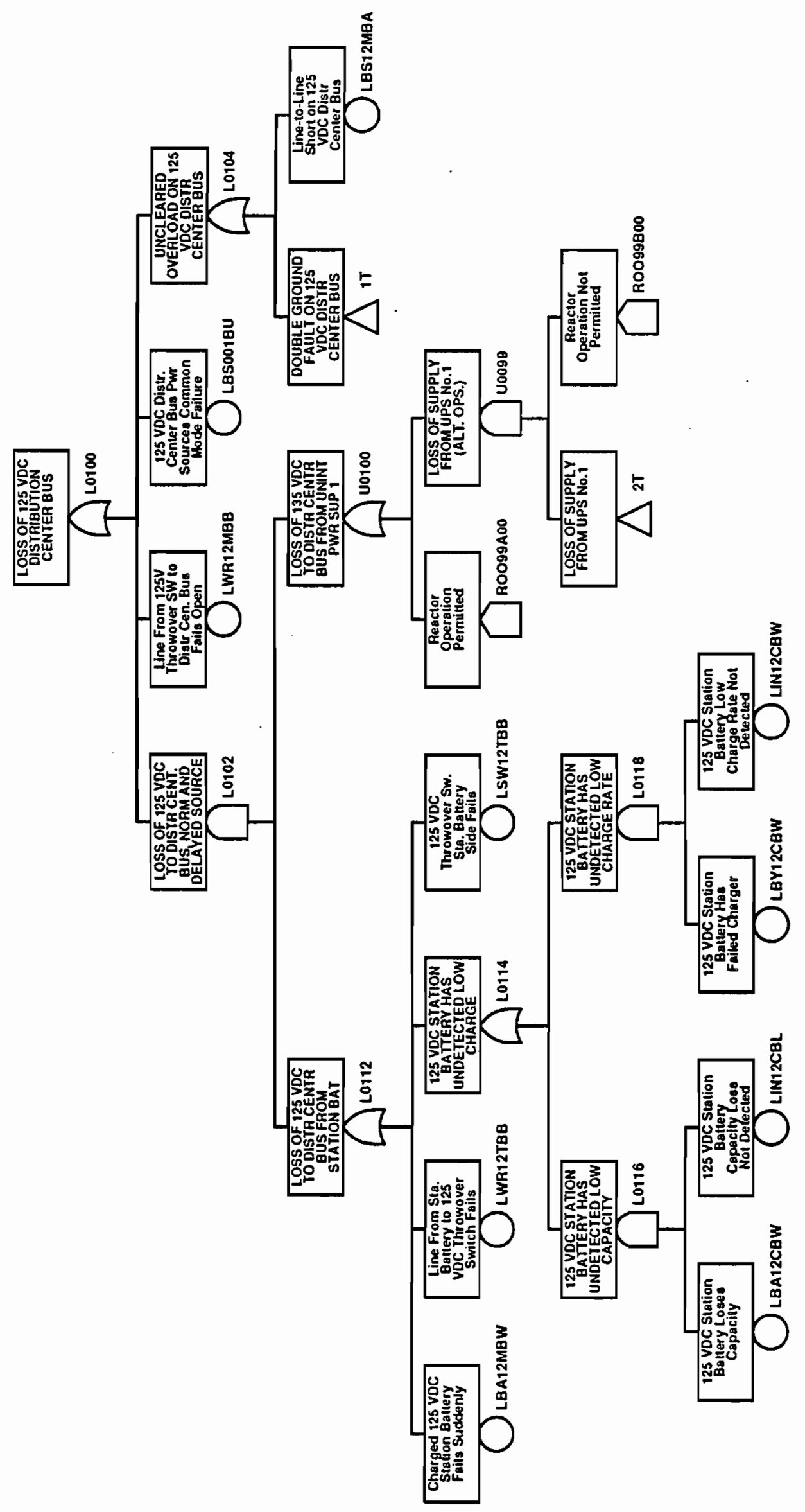

B. 2-383 


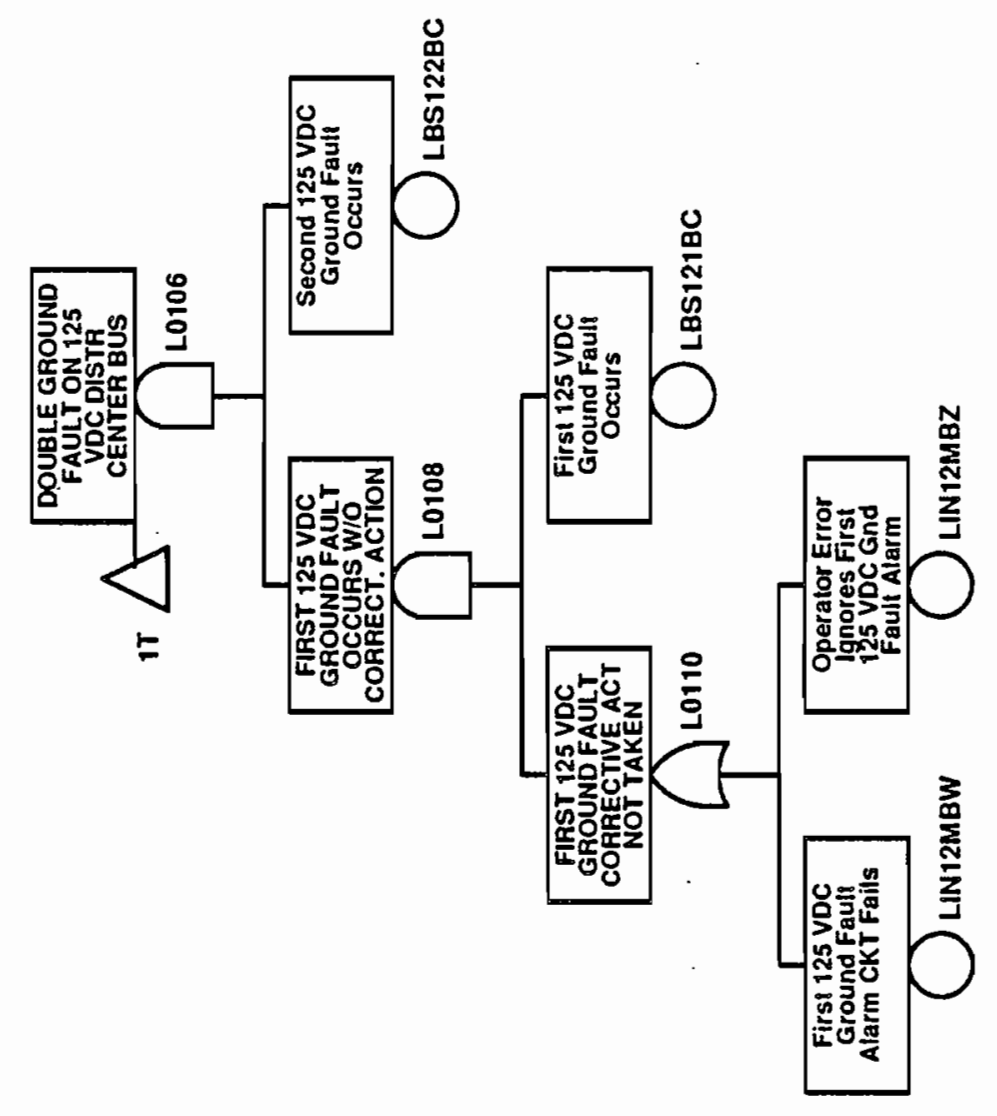

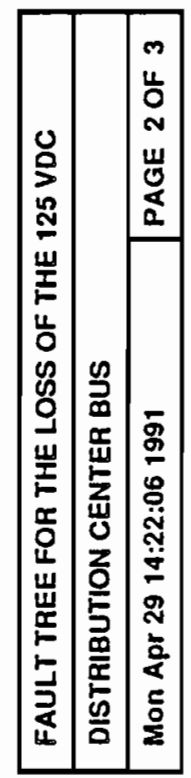



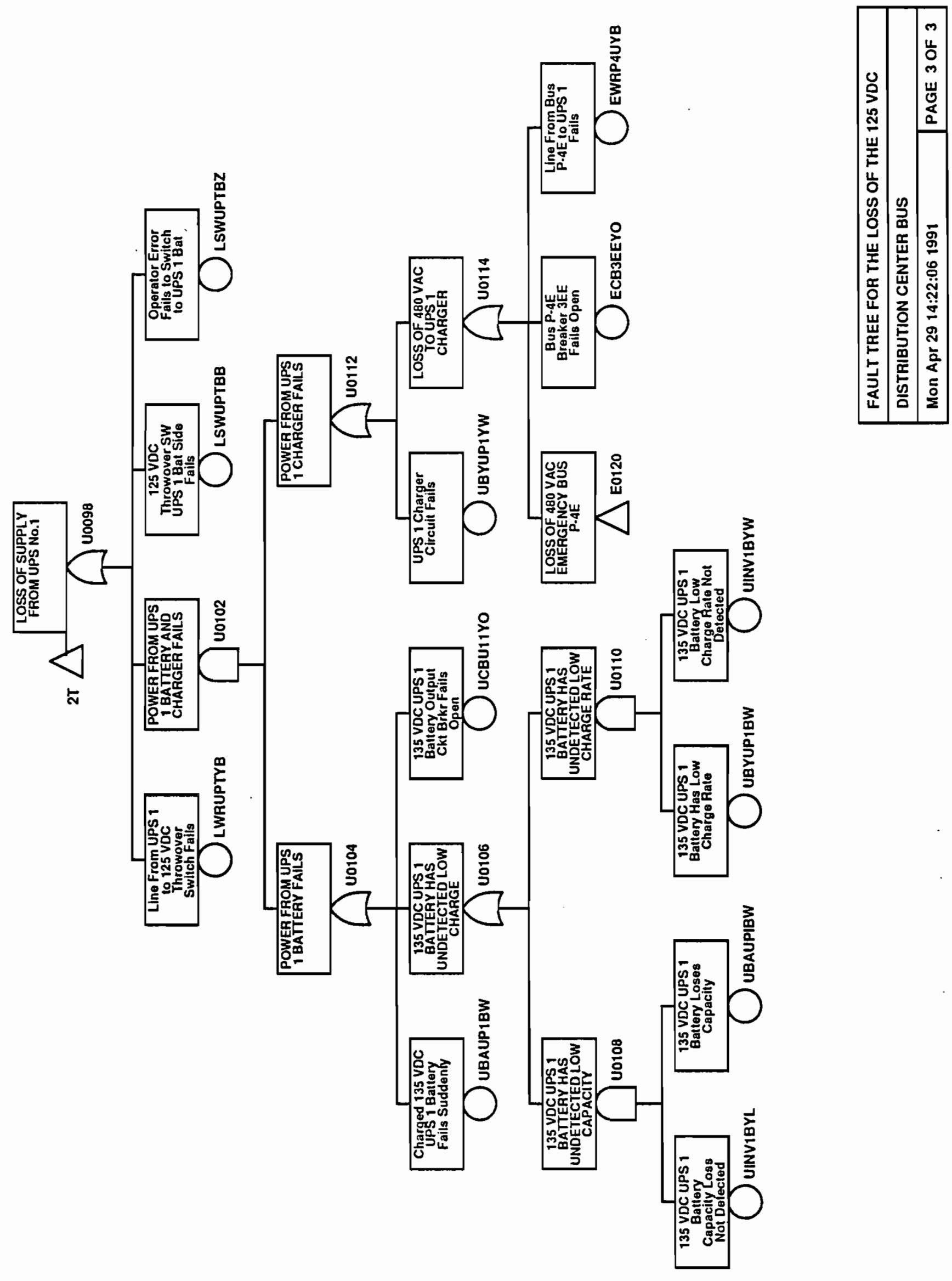

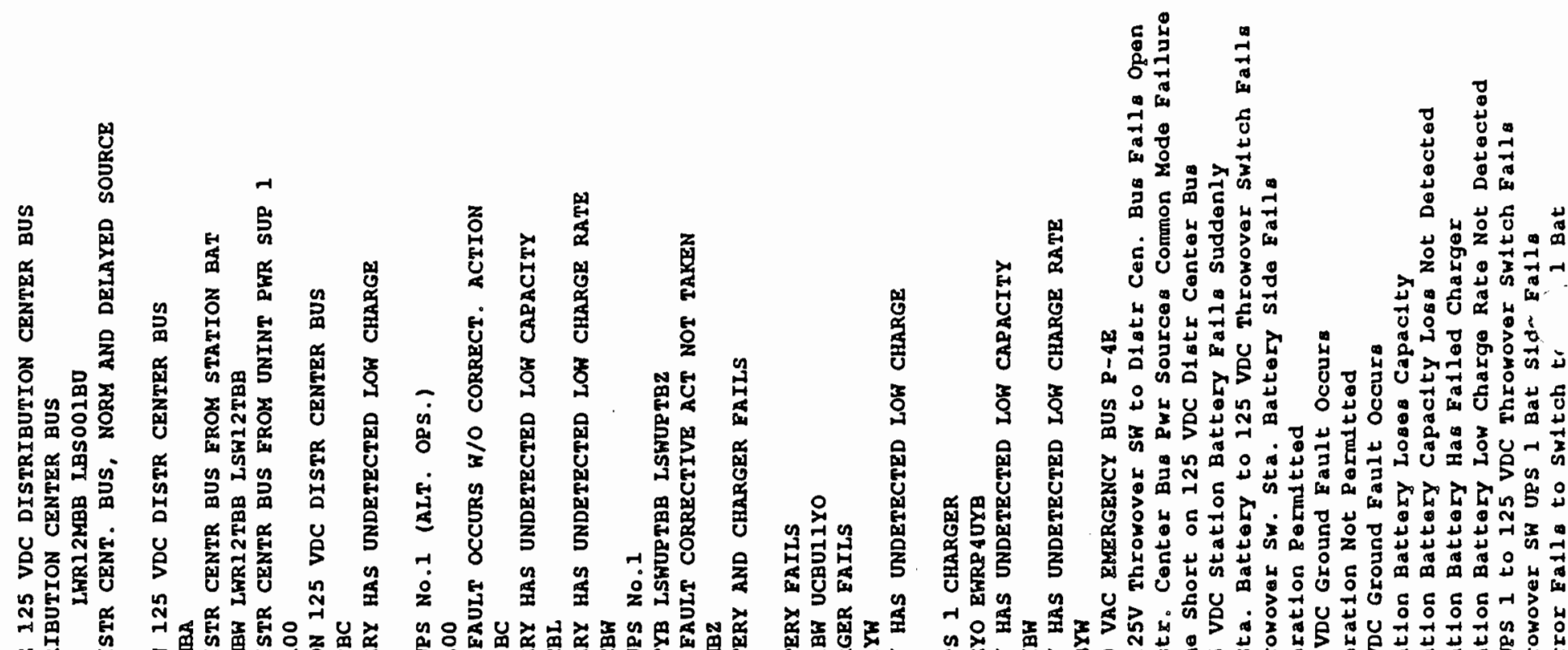

-

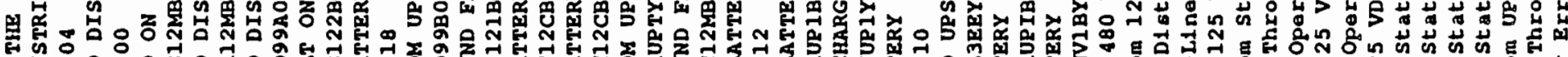

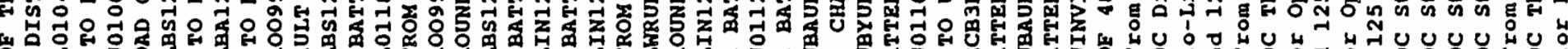

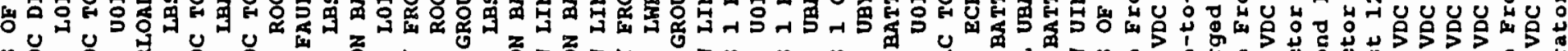
织

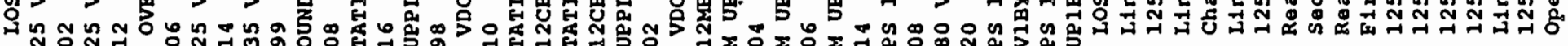

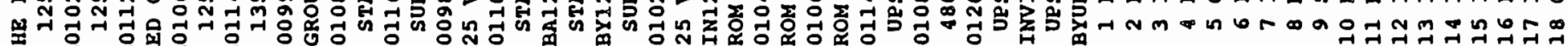

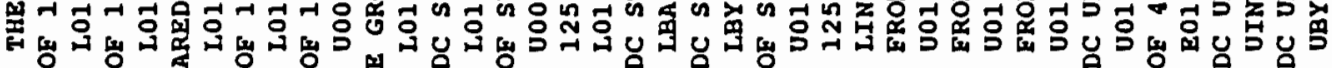

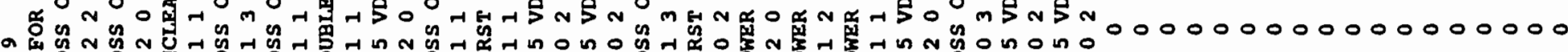

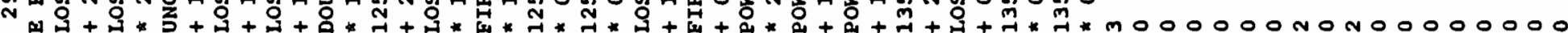
î

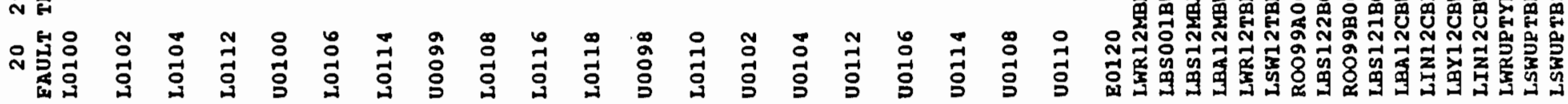




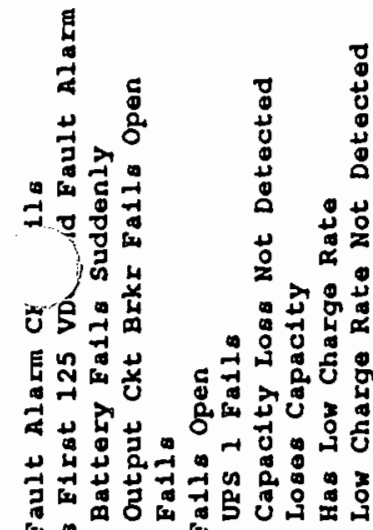

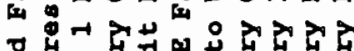

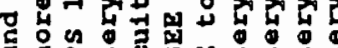

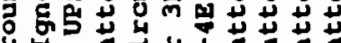

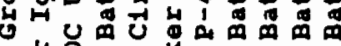
U

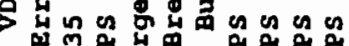
ก స广

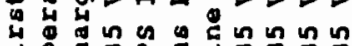

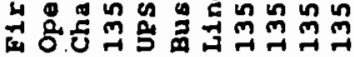

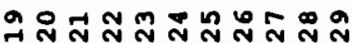

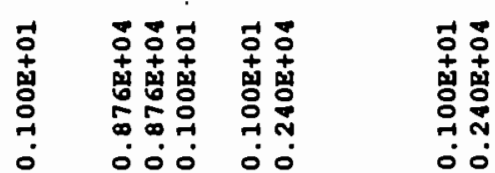

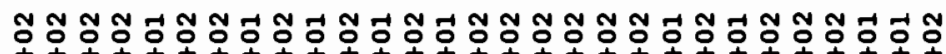

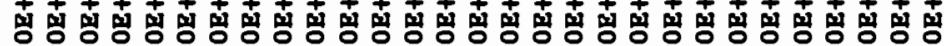

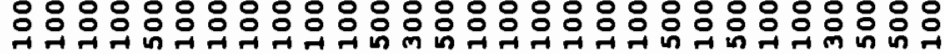

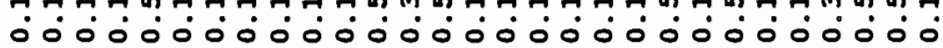

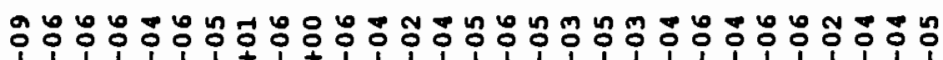

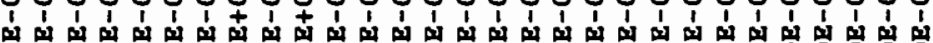

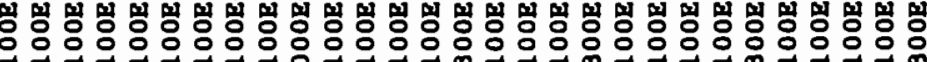

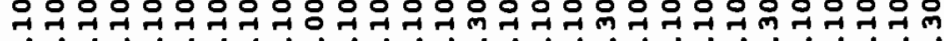
00000000000 00000000000 g g.

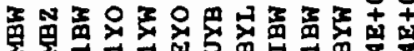

至

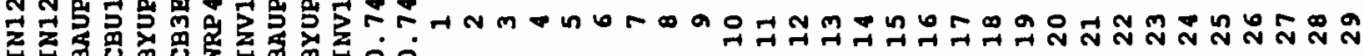

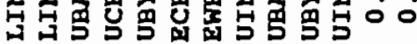



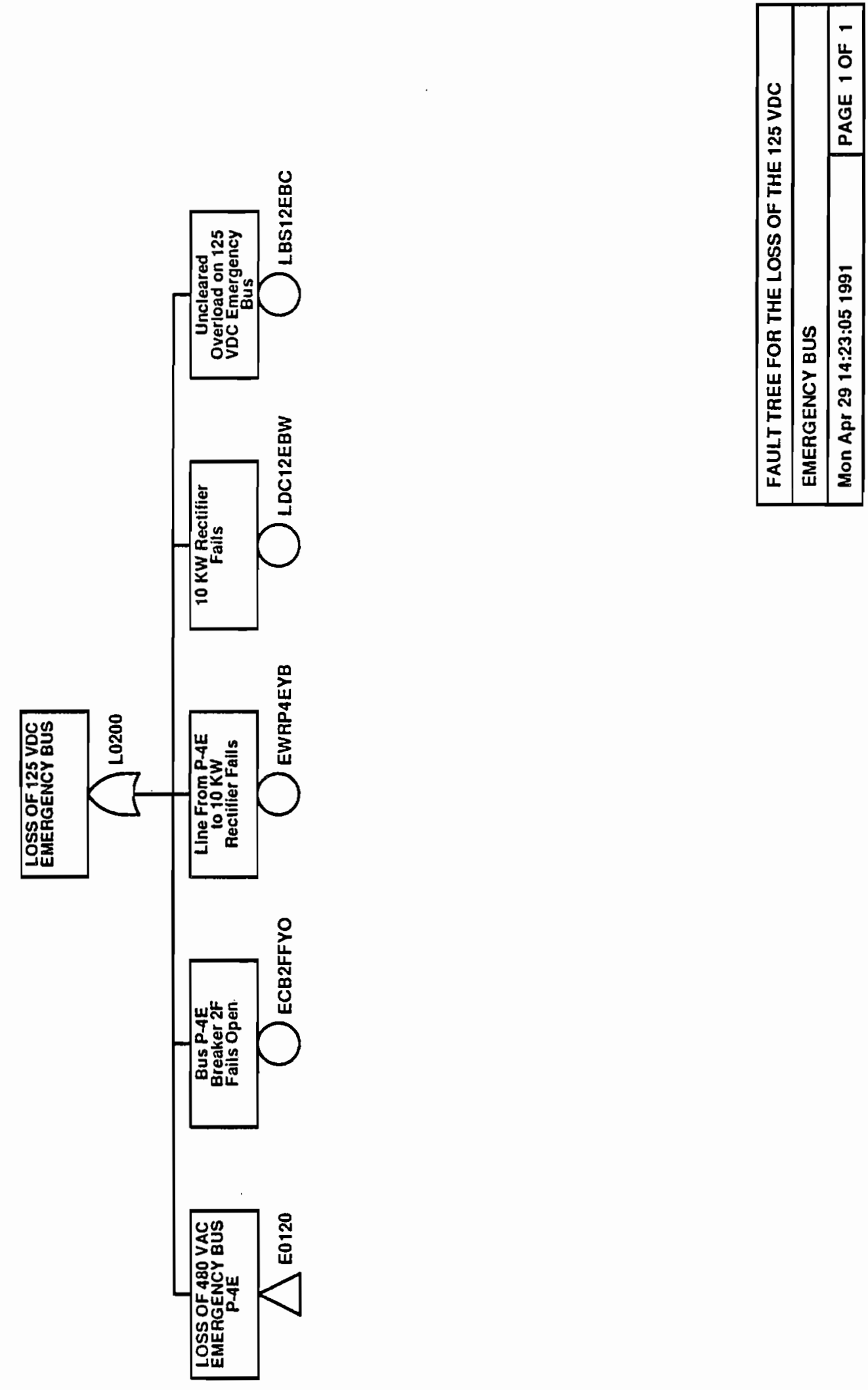


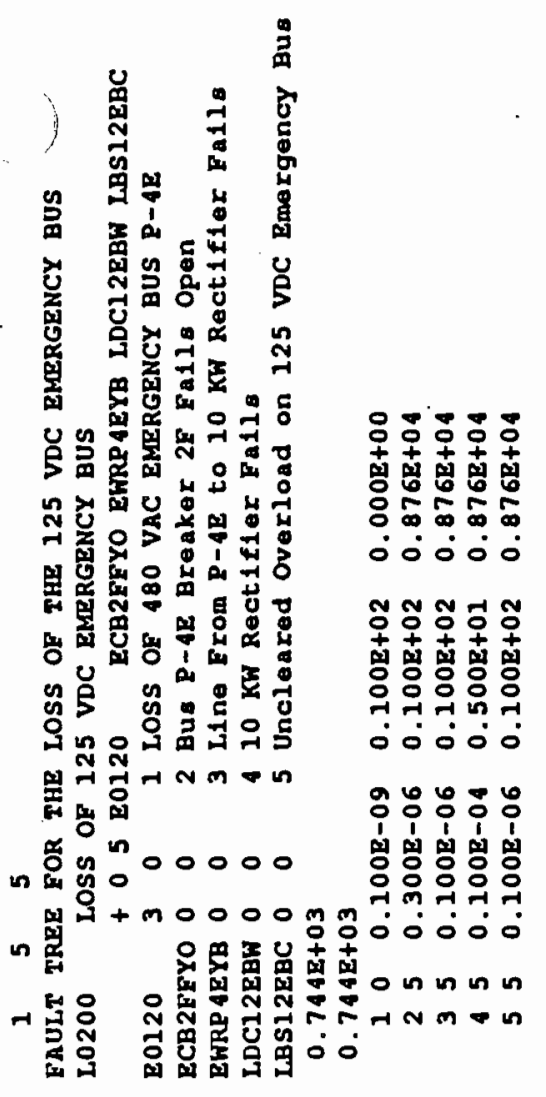

B. 2-389 


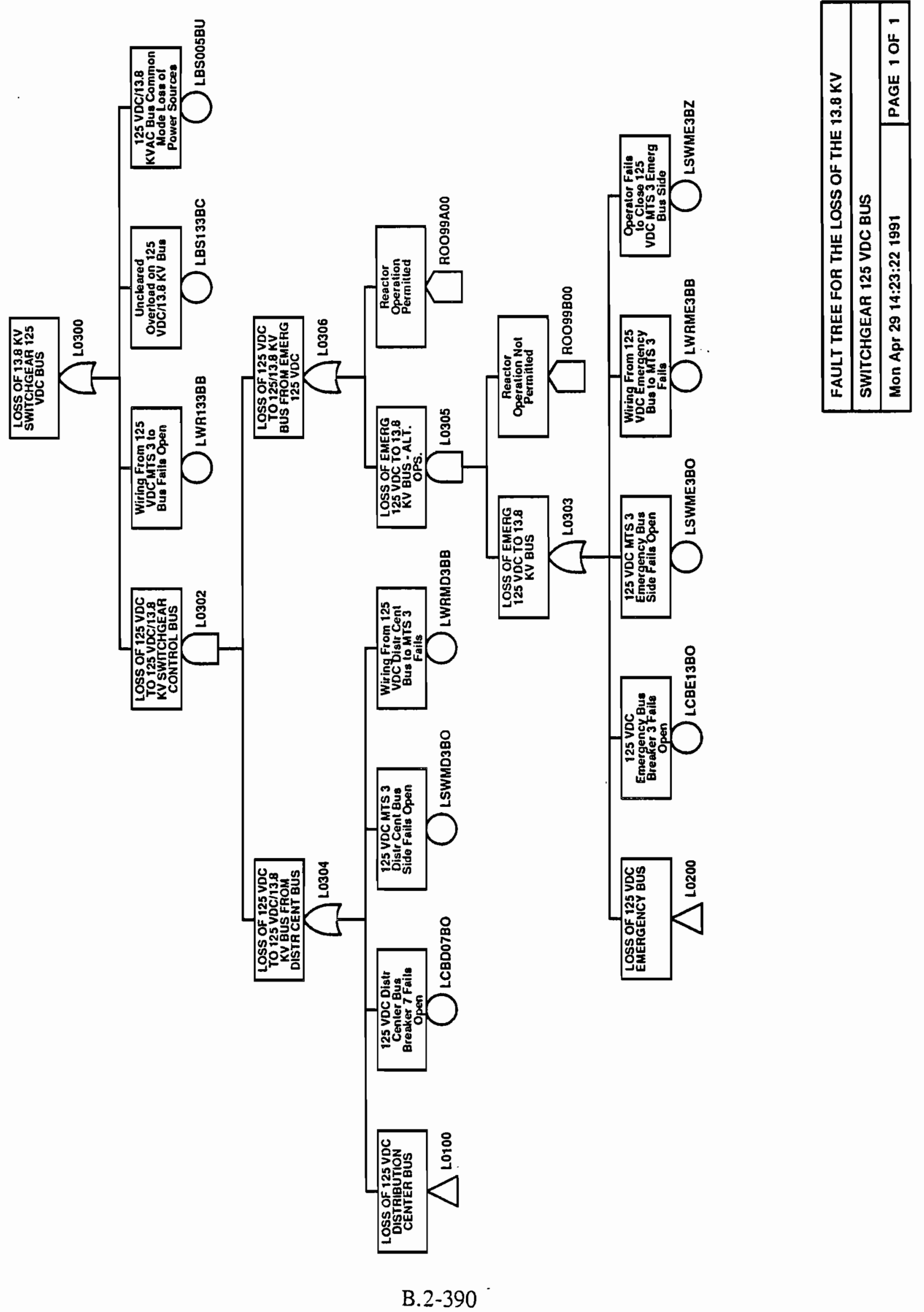




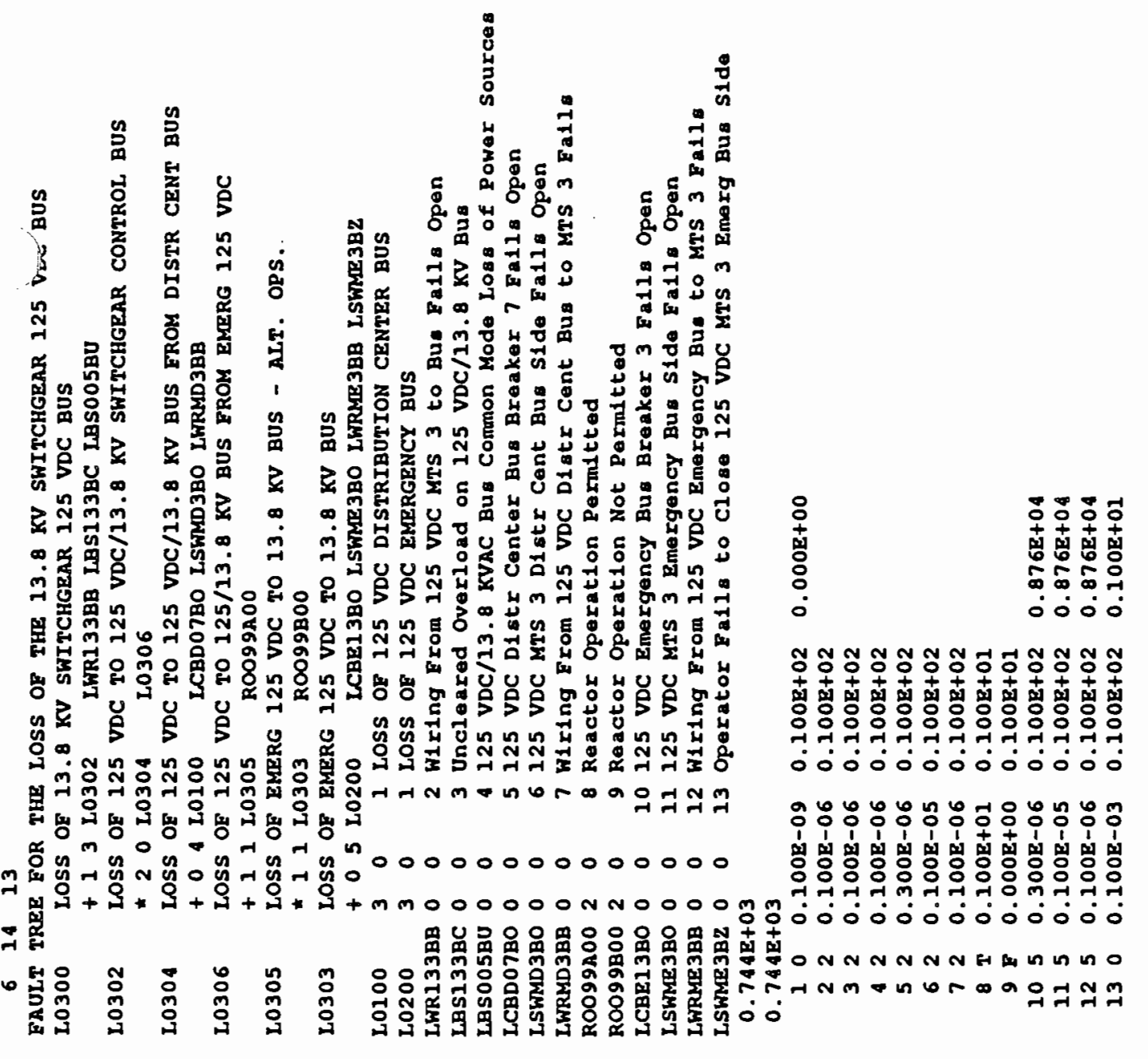




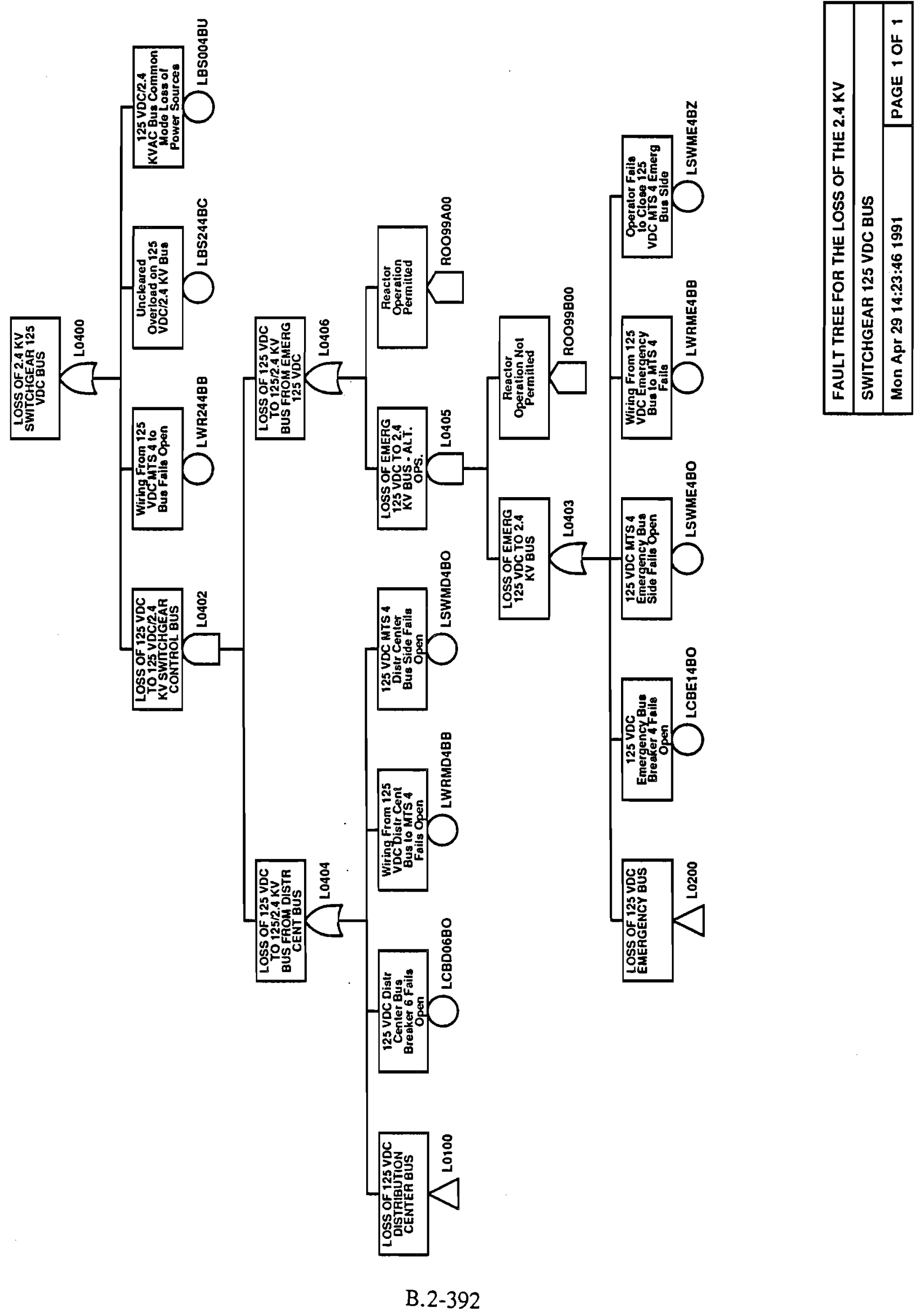




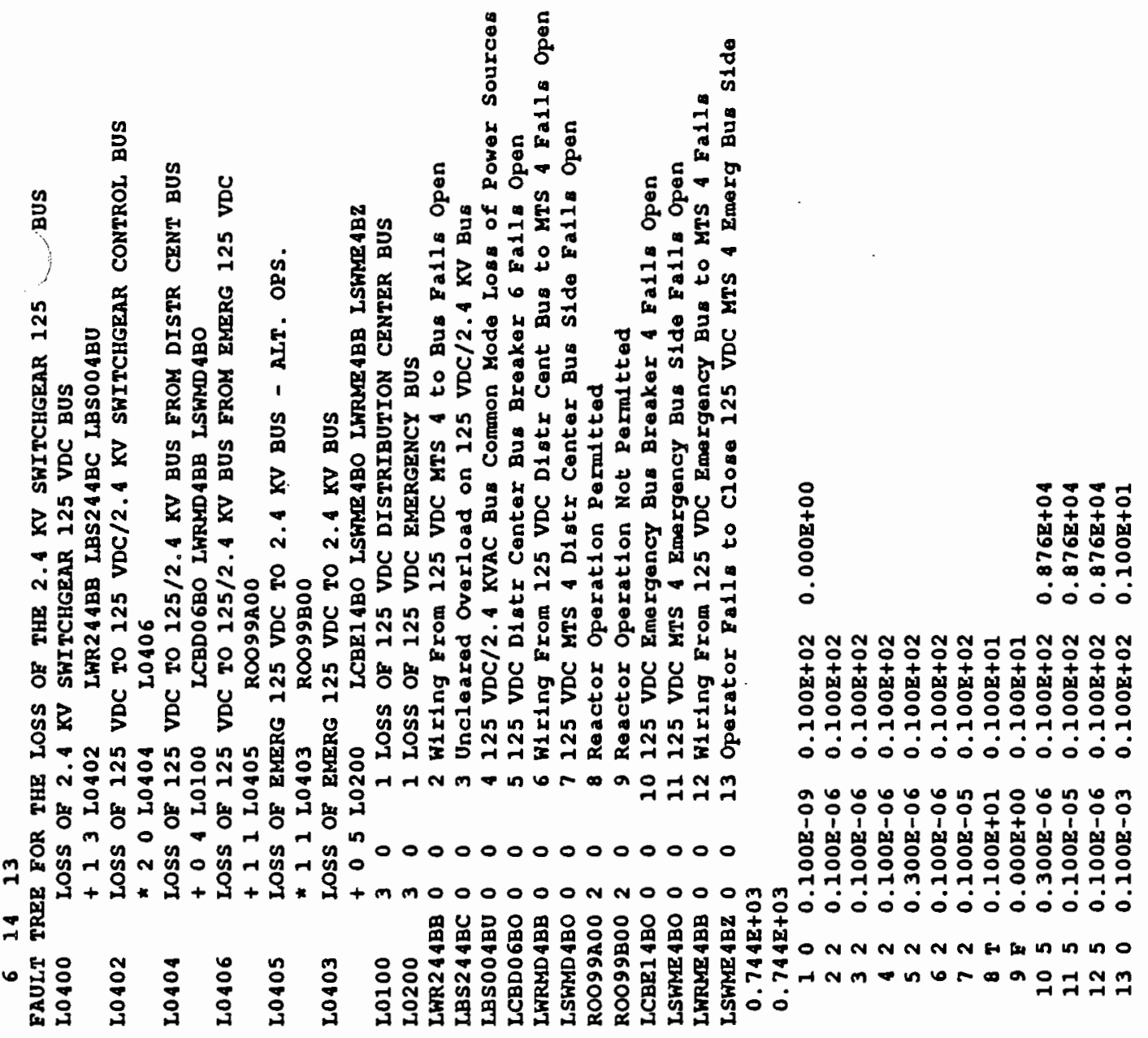




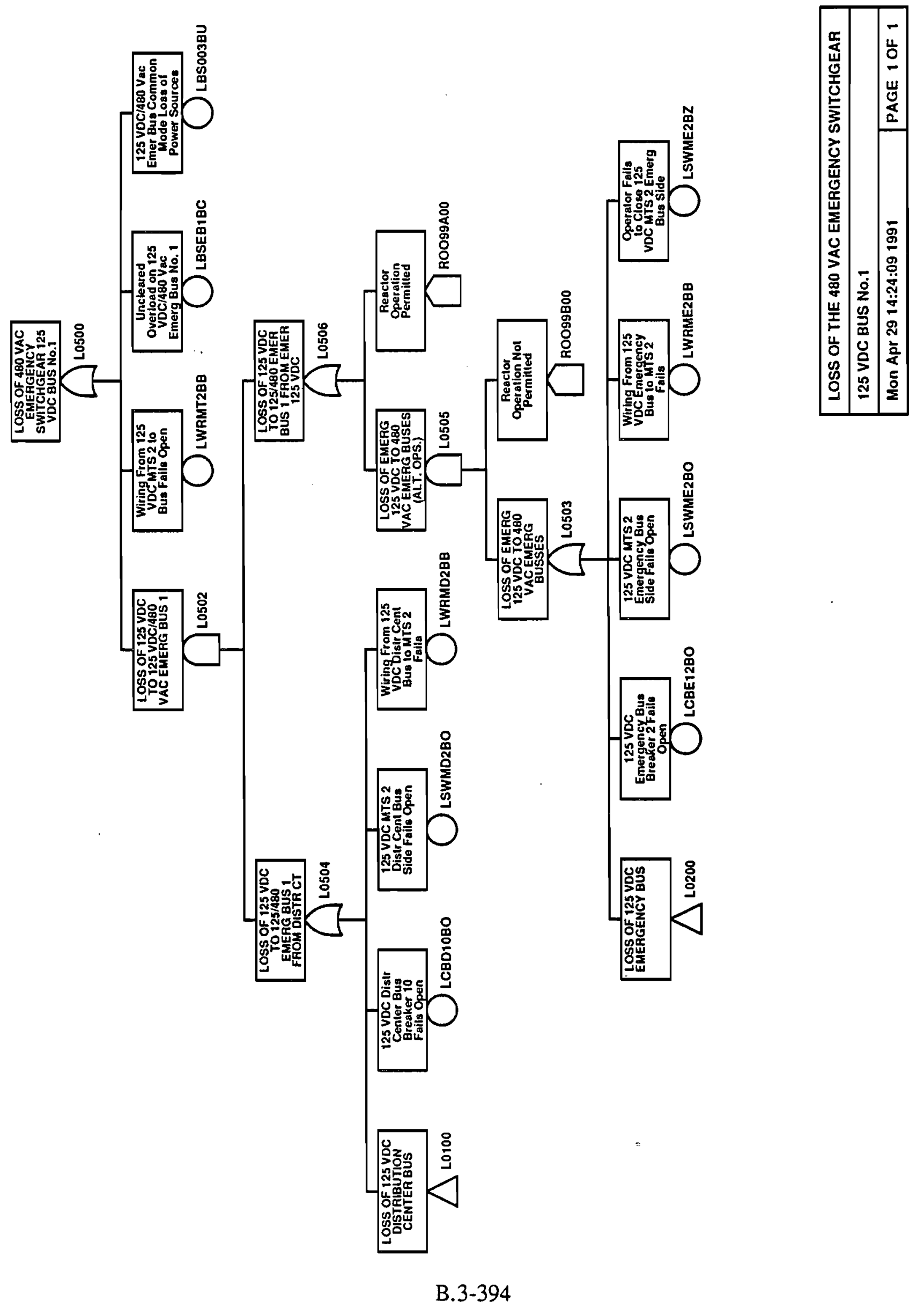




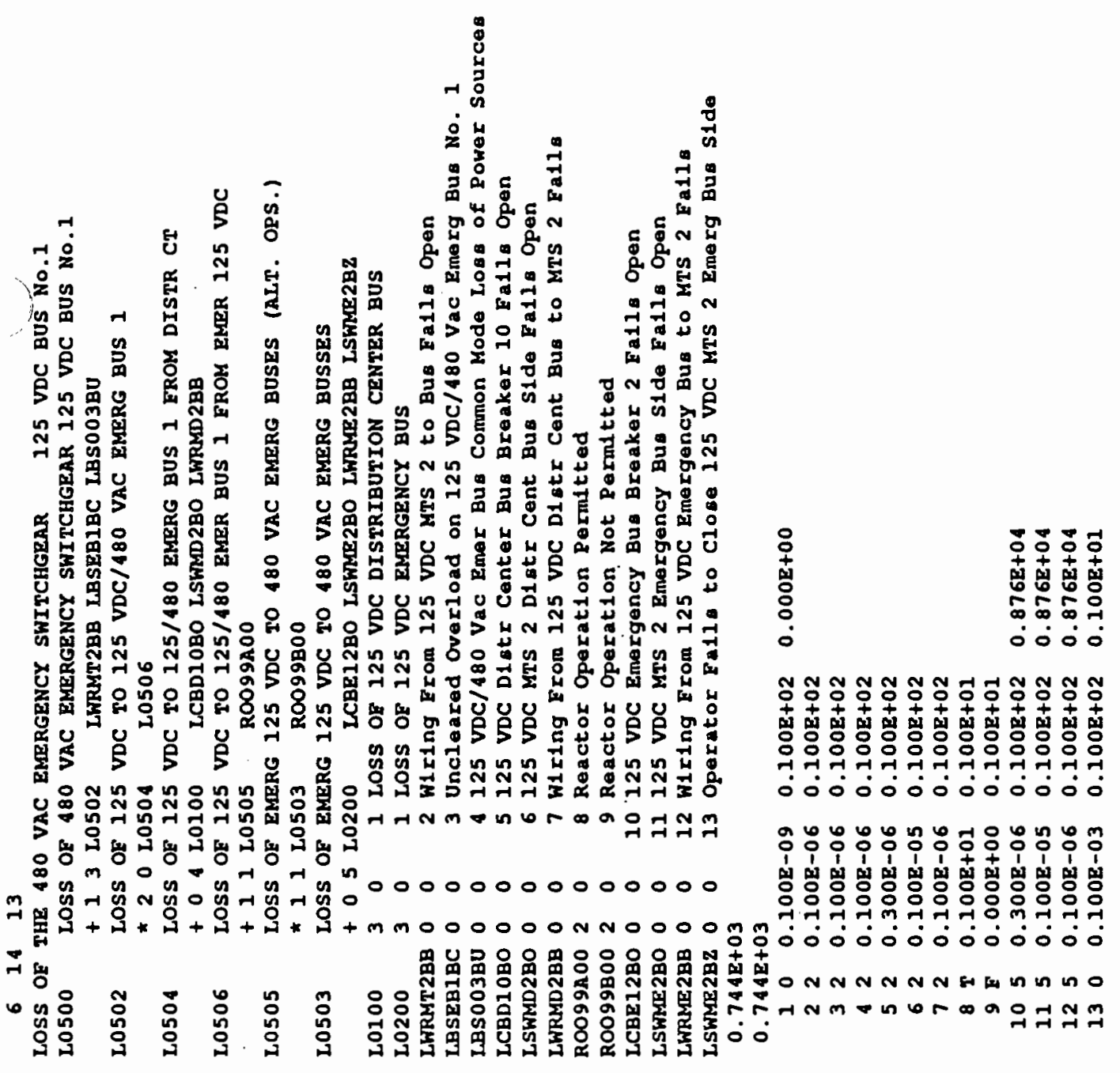




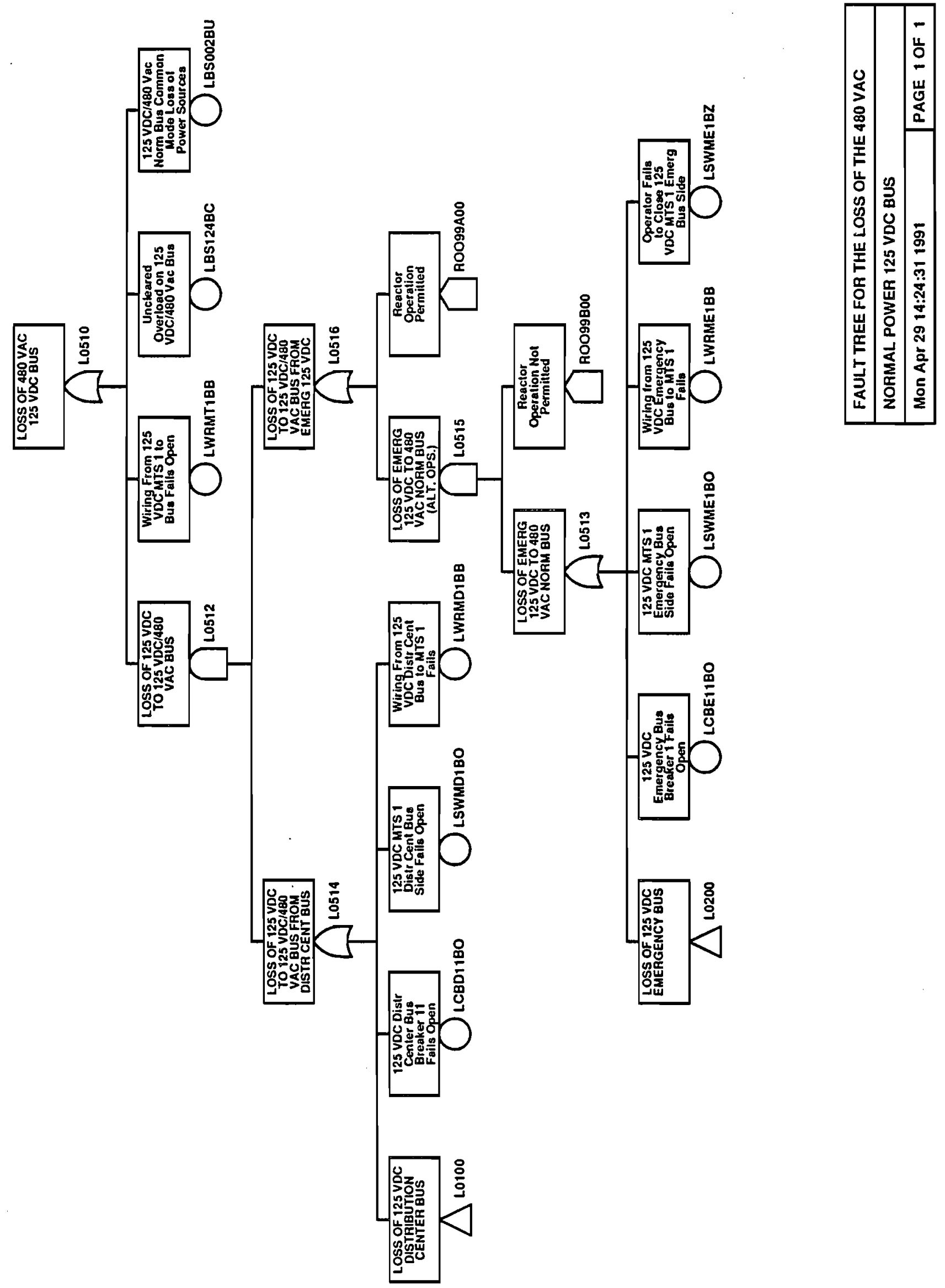

B. 2-396 


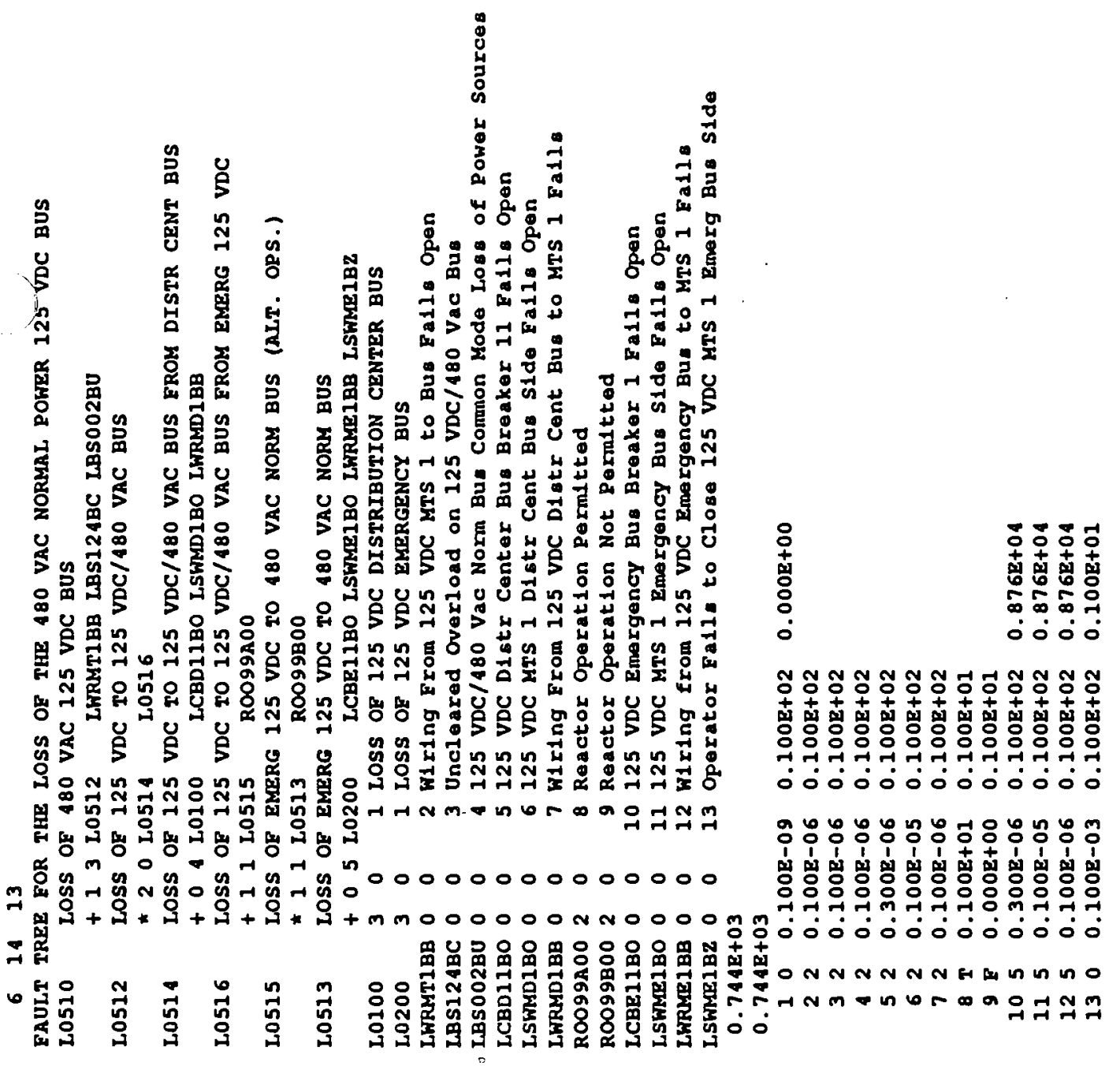




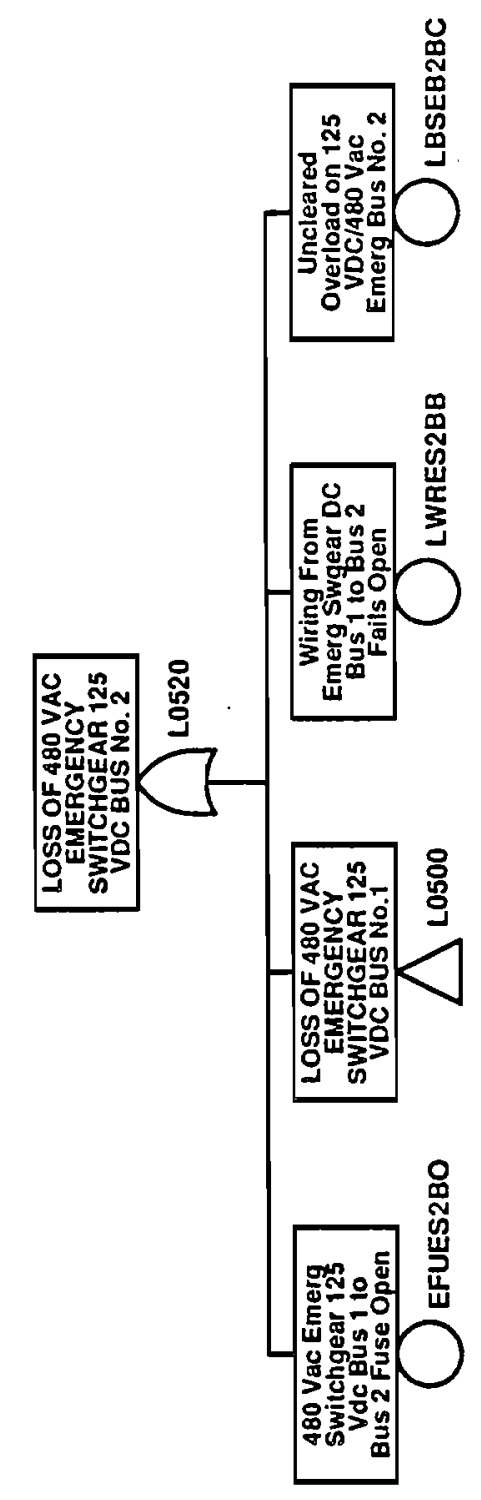

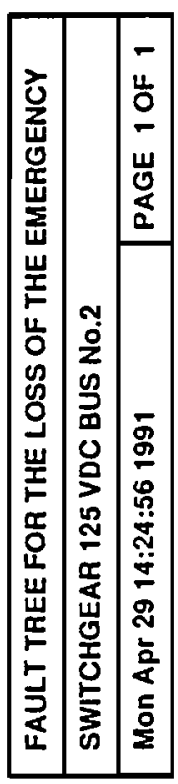




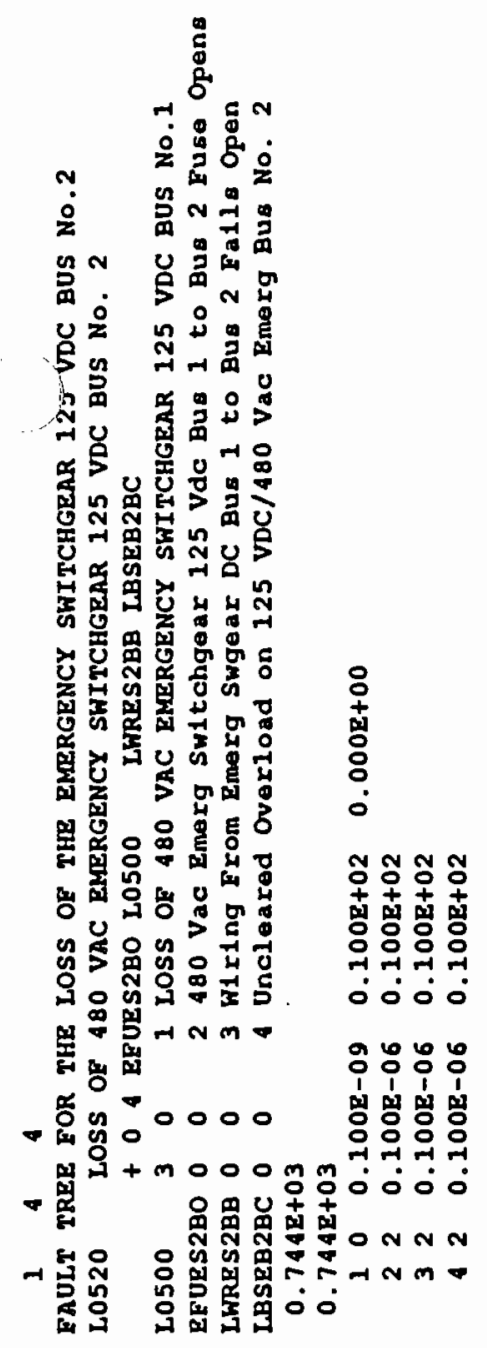

B. 2-399 


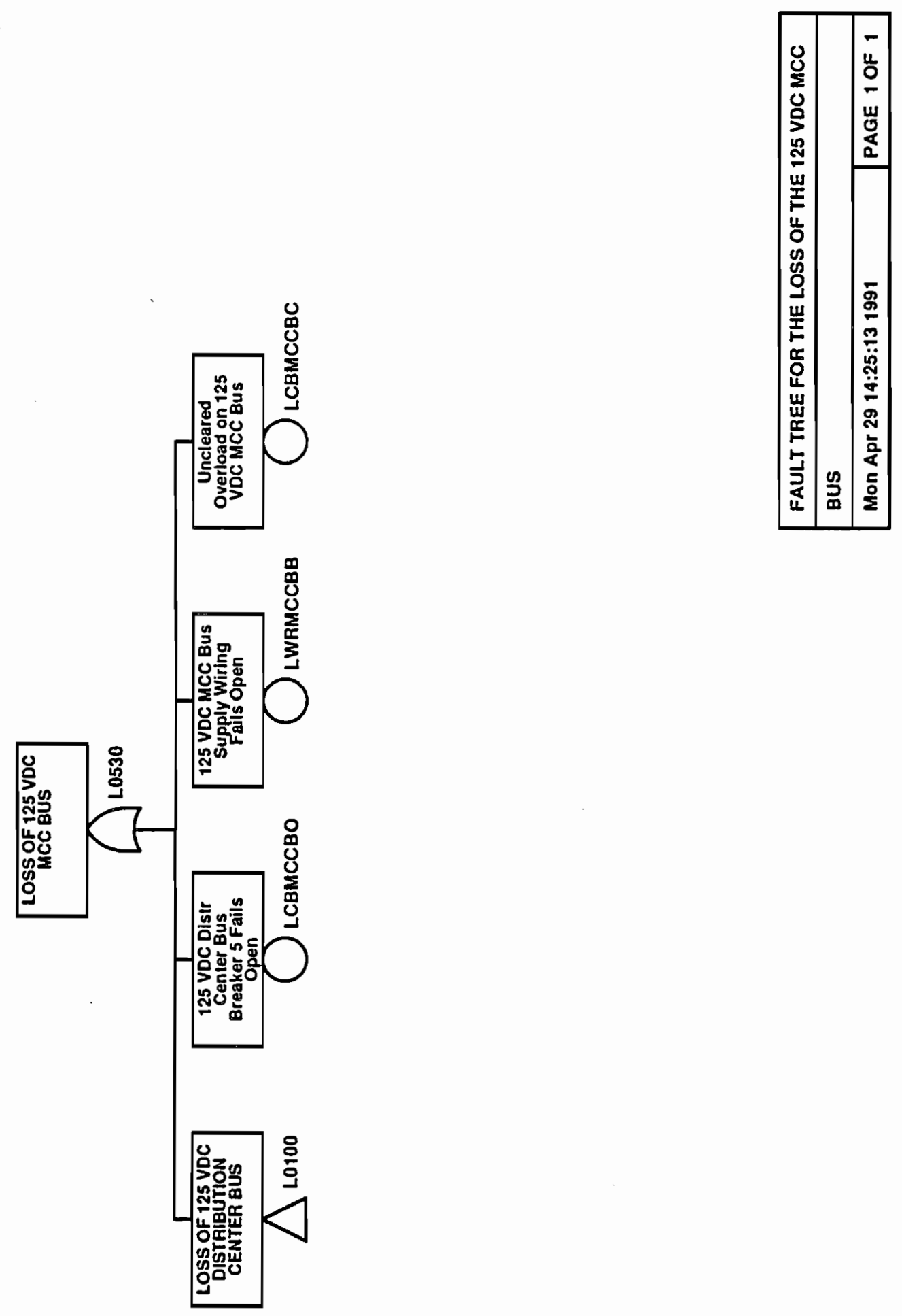




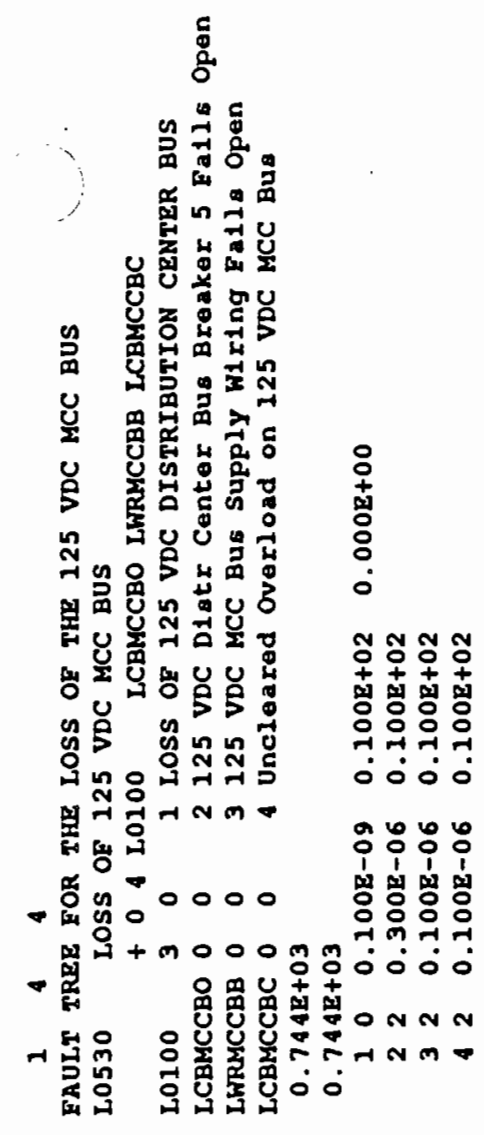

B. 2-401 


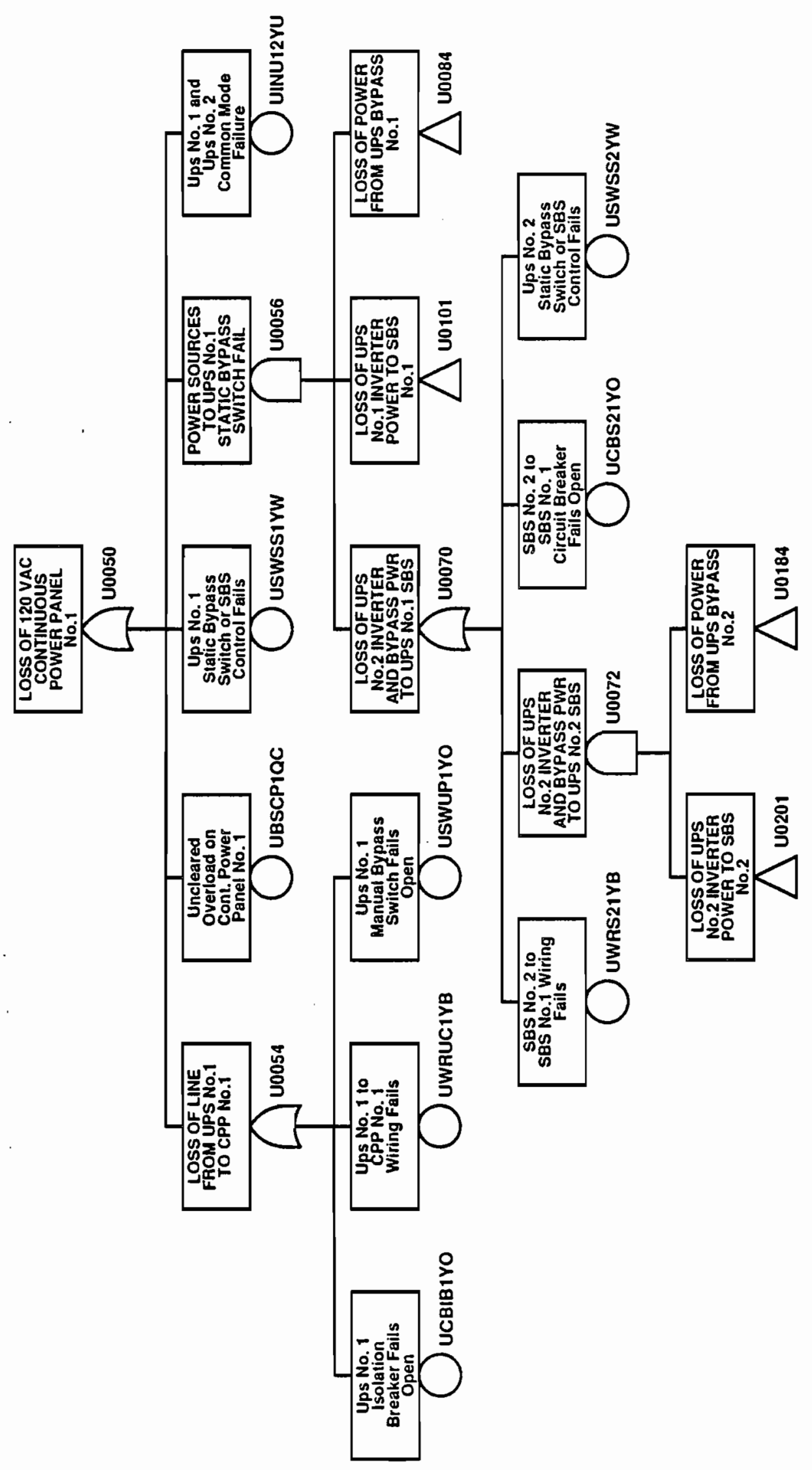

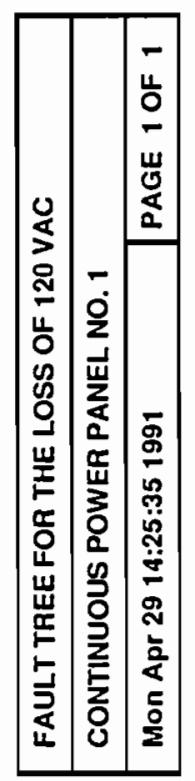




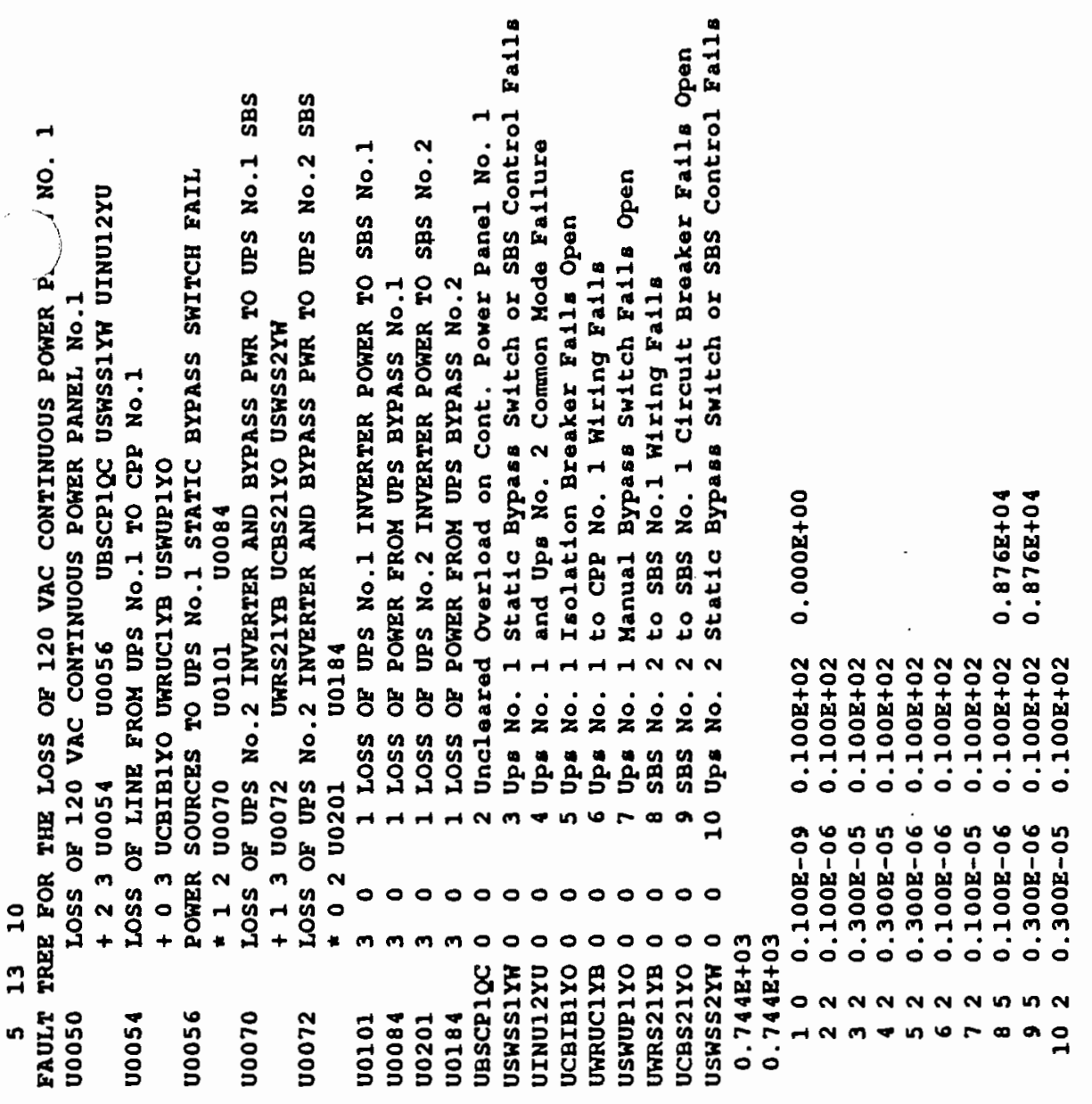




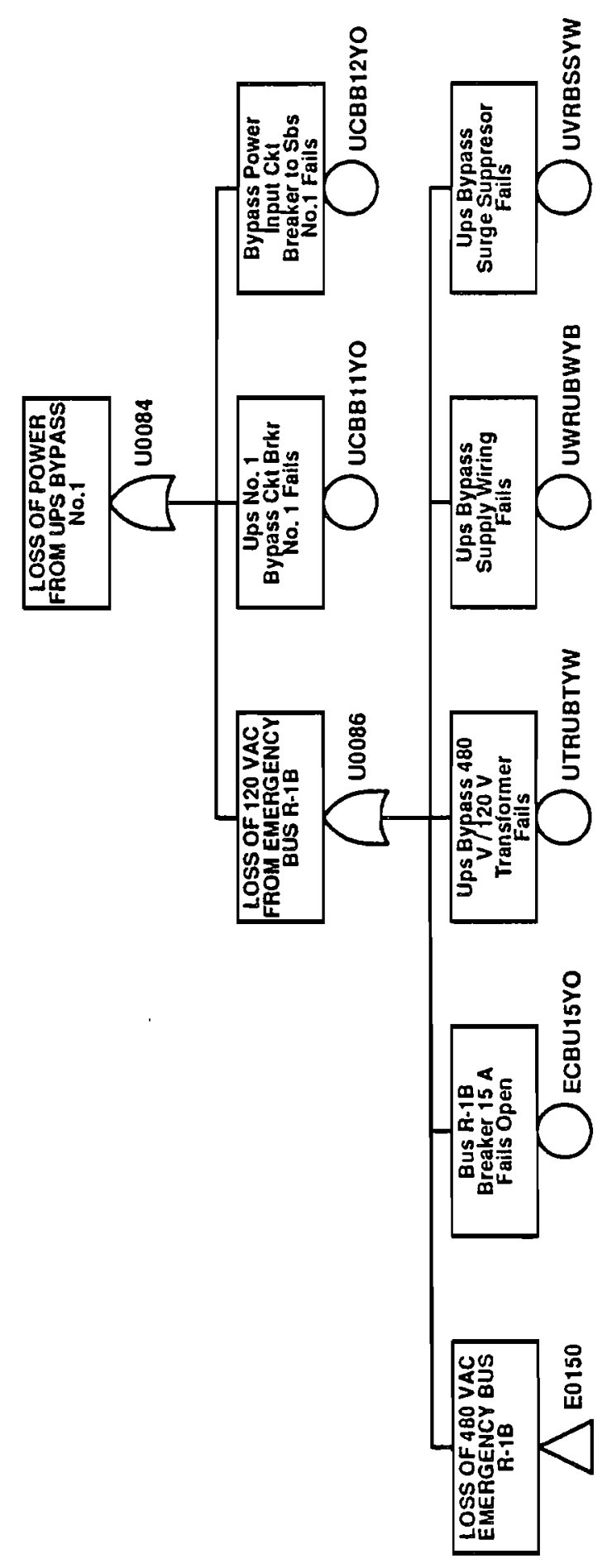

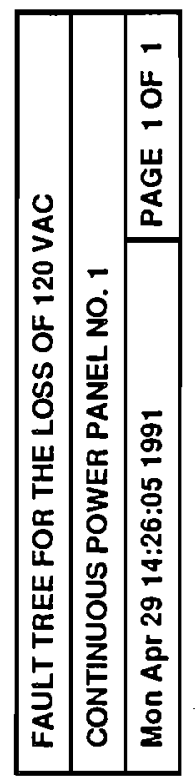




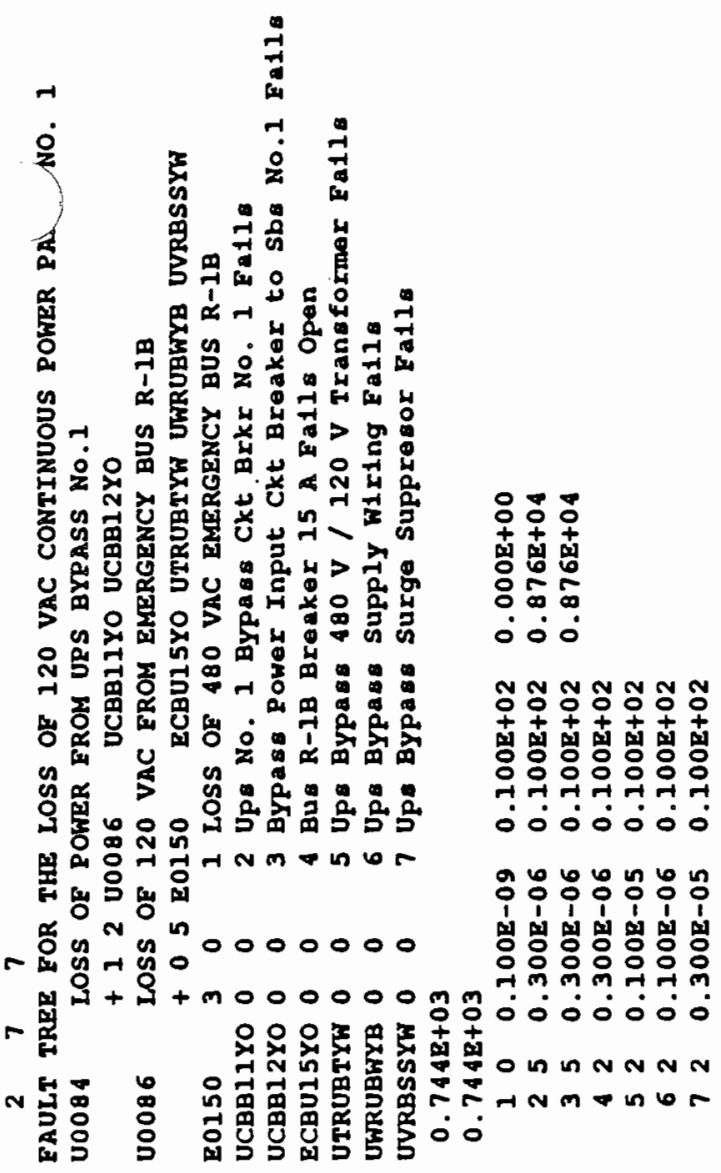

B. 2-405 


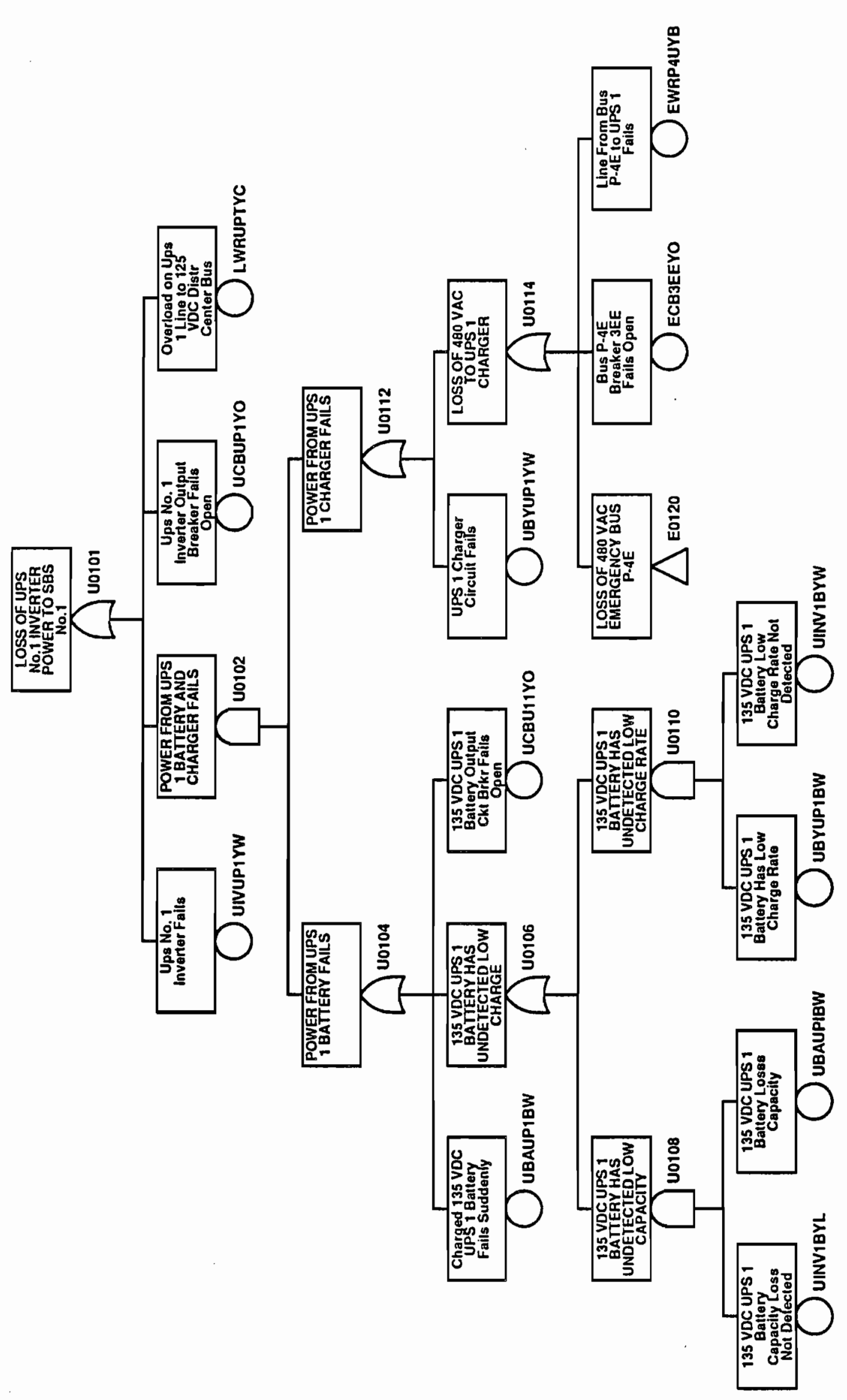




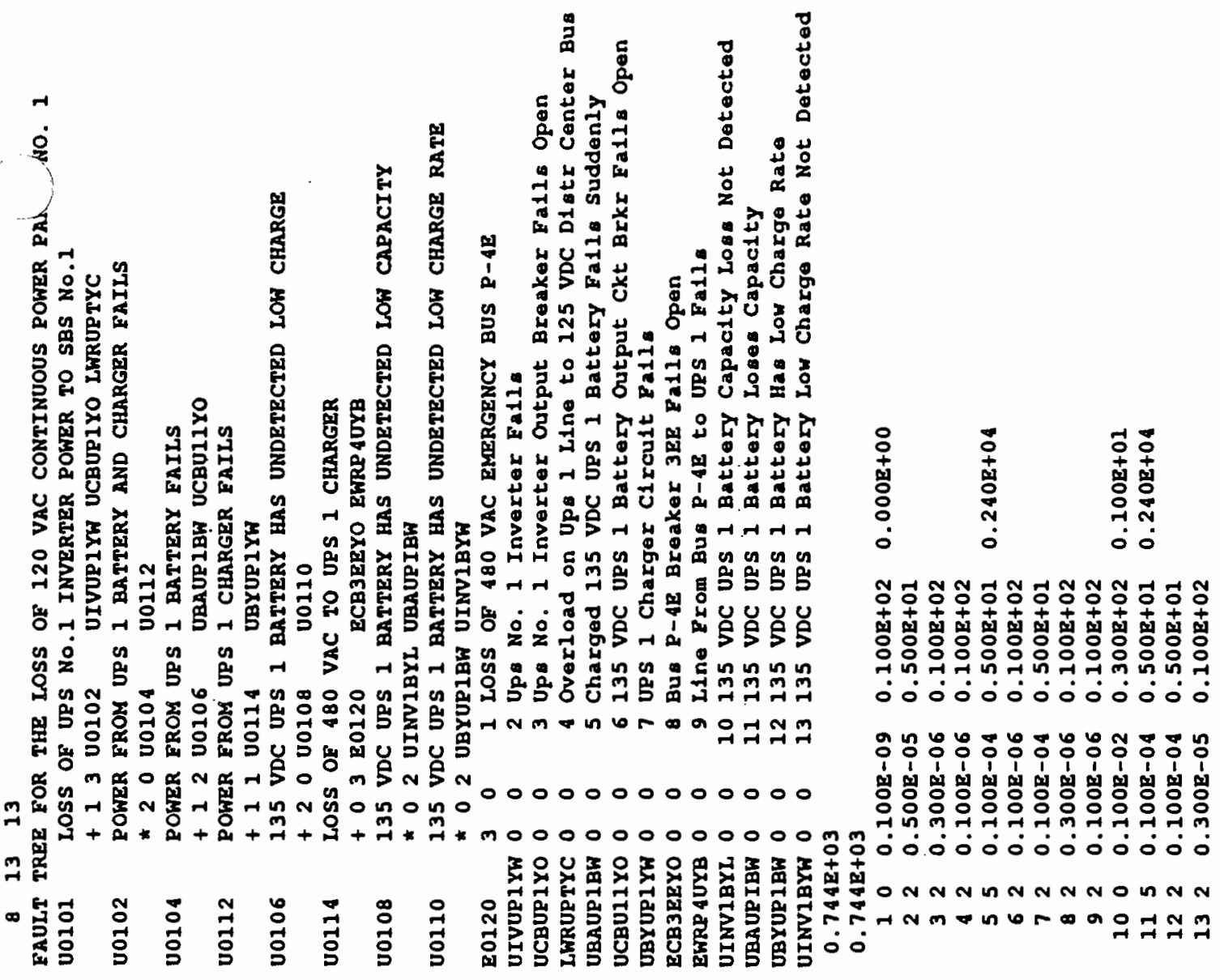




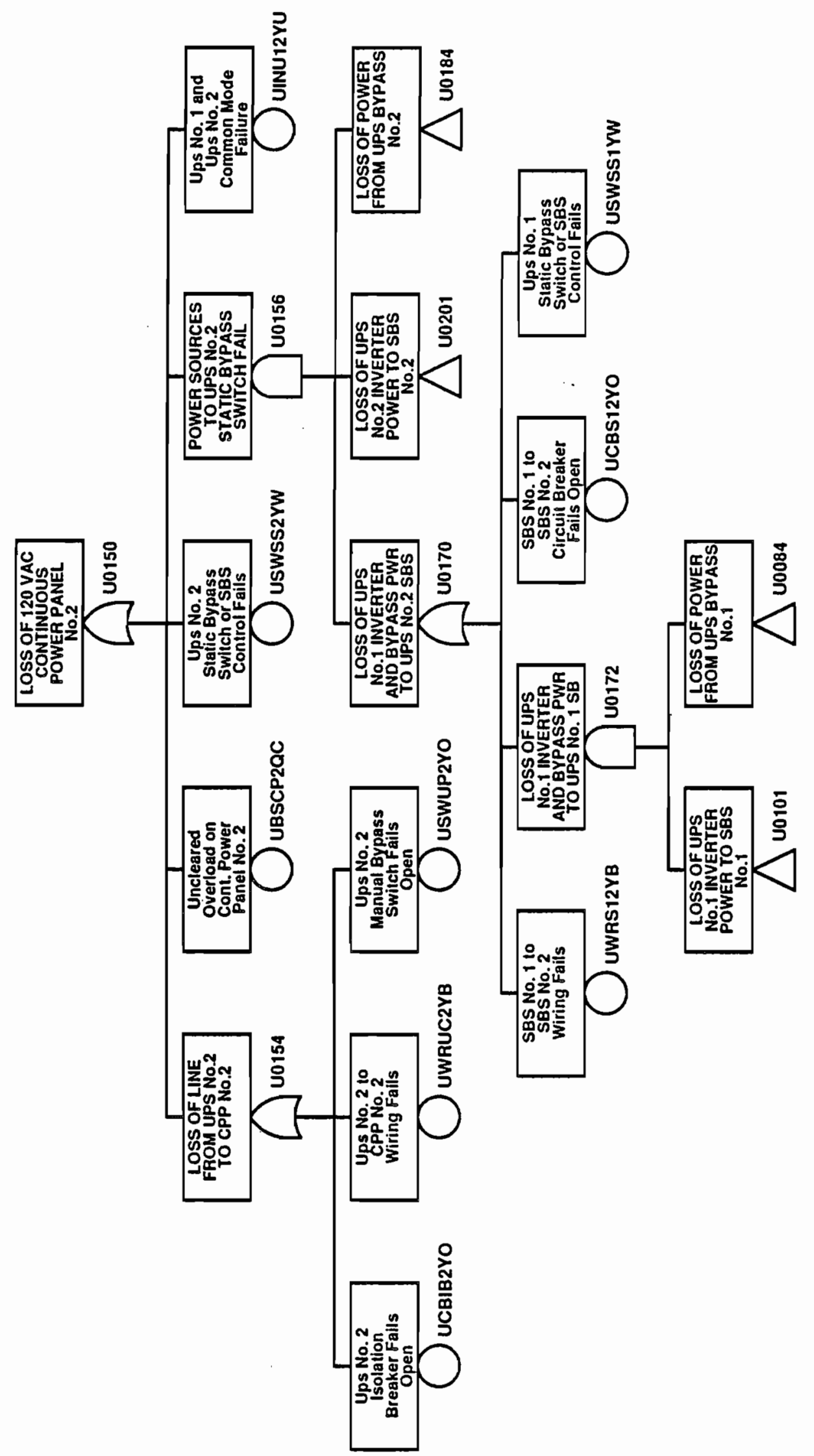

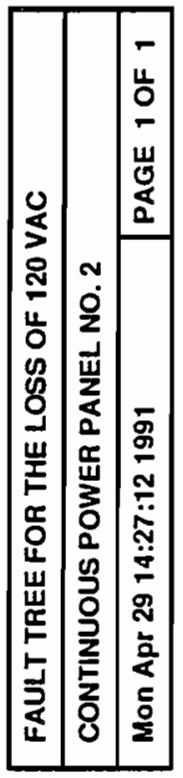




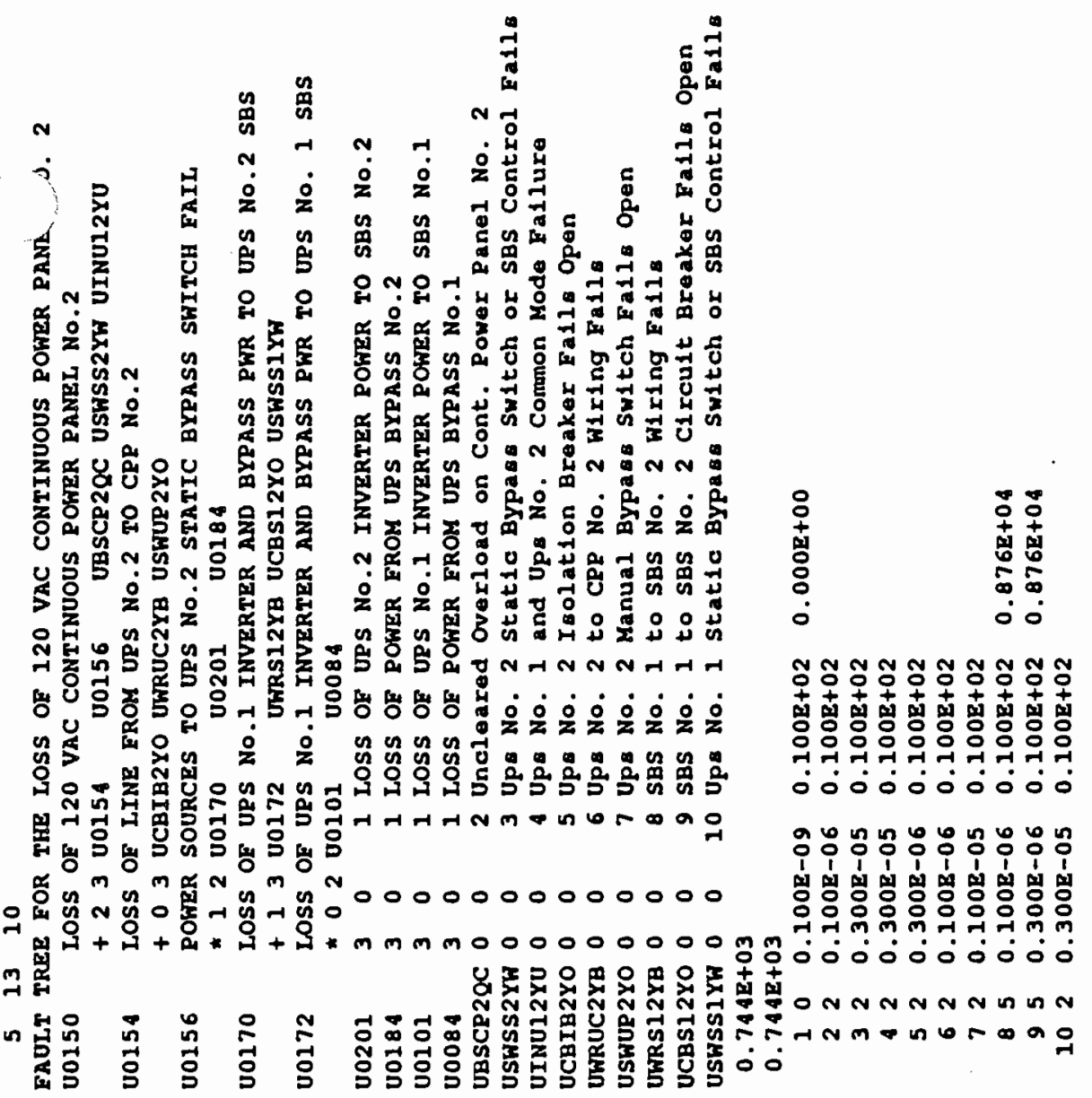



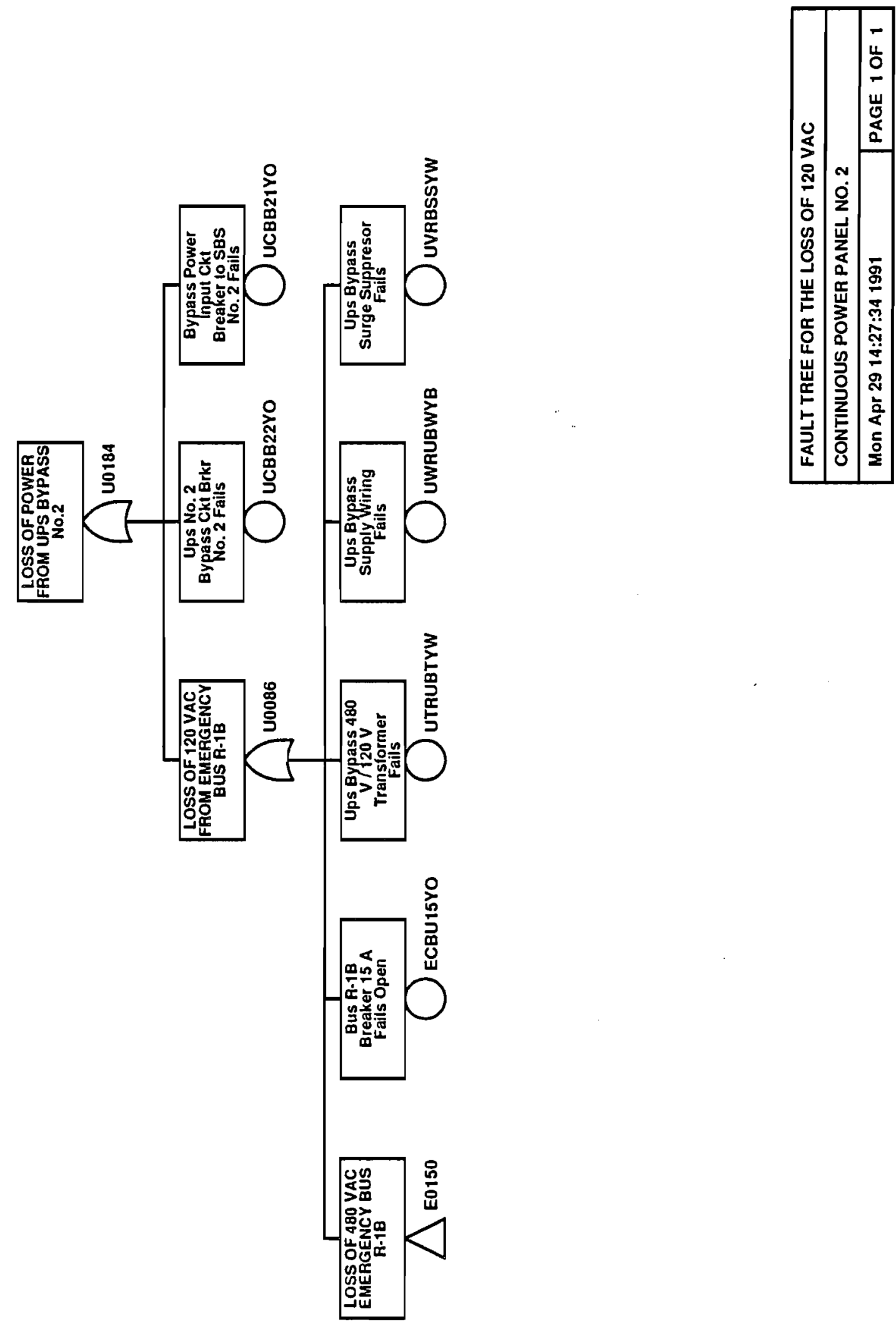


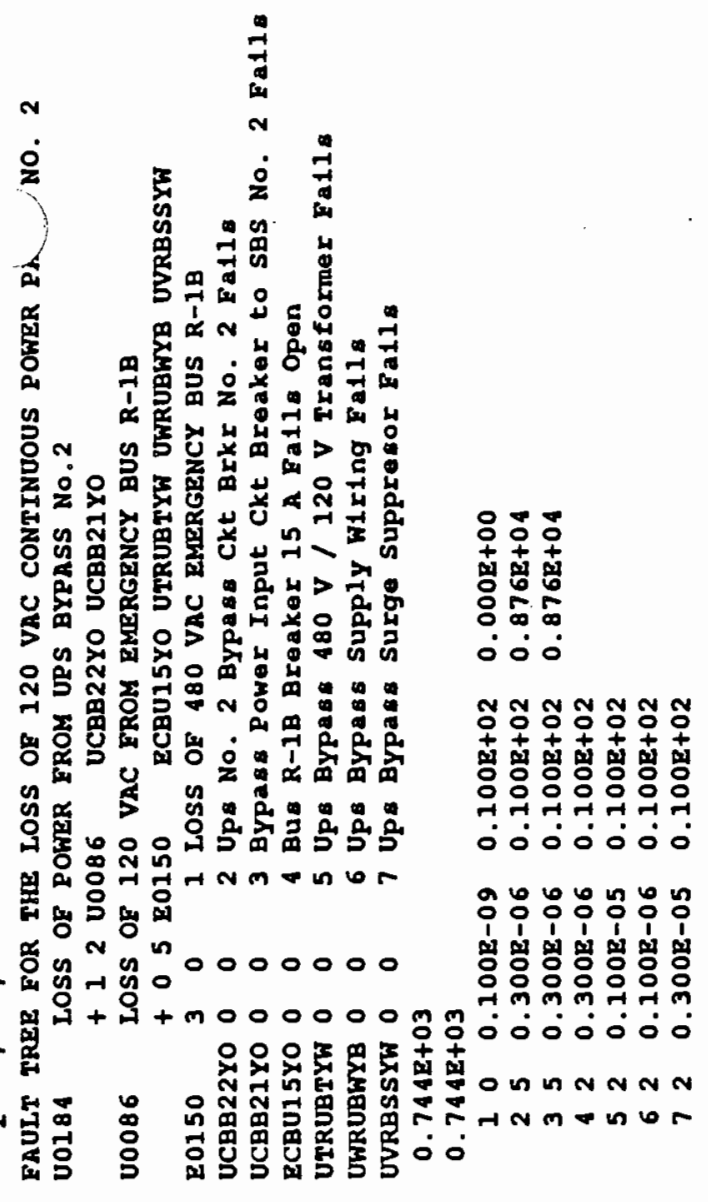




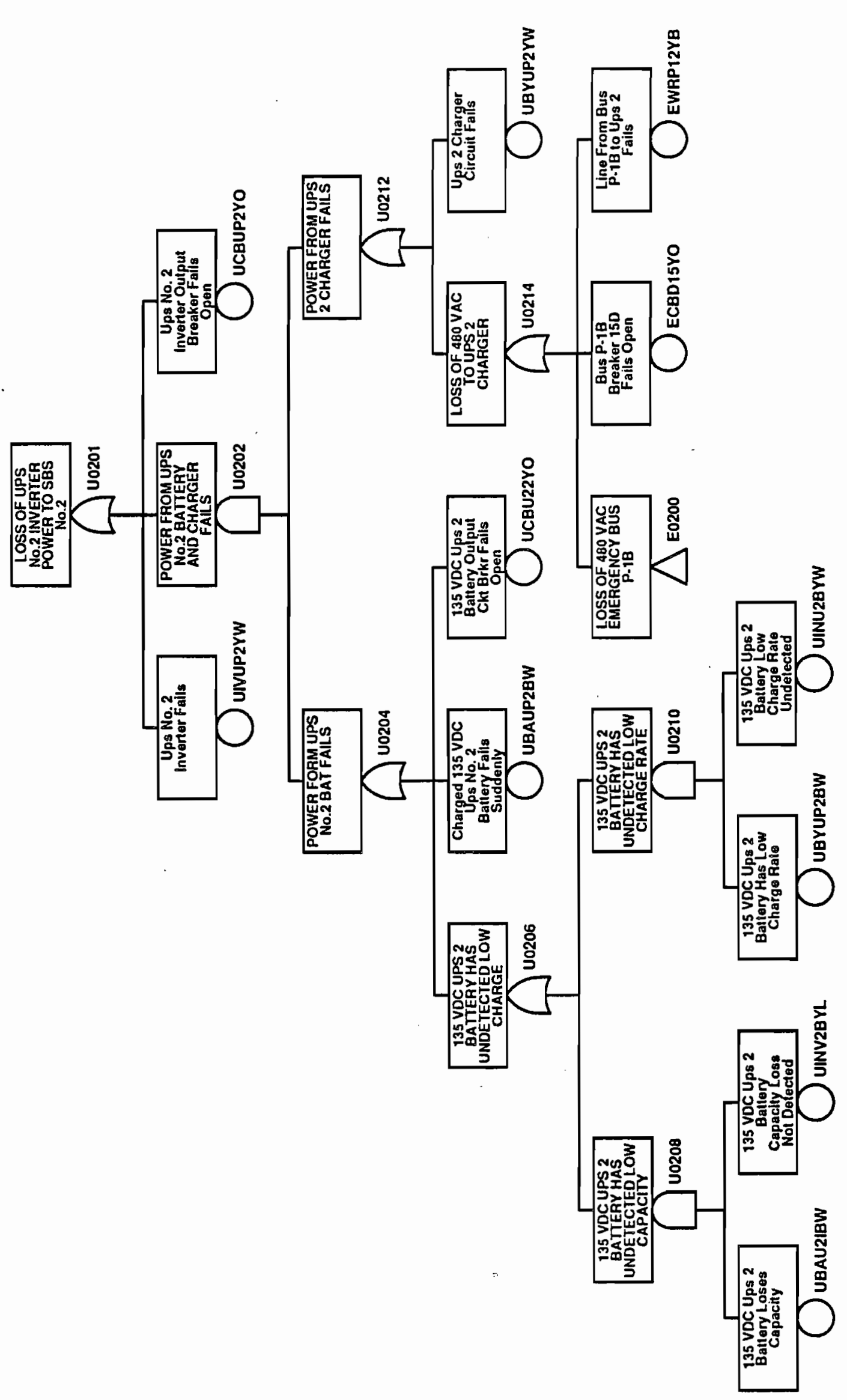




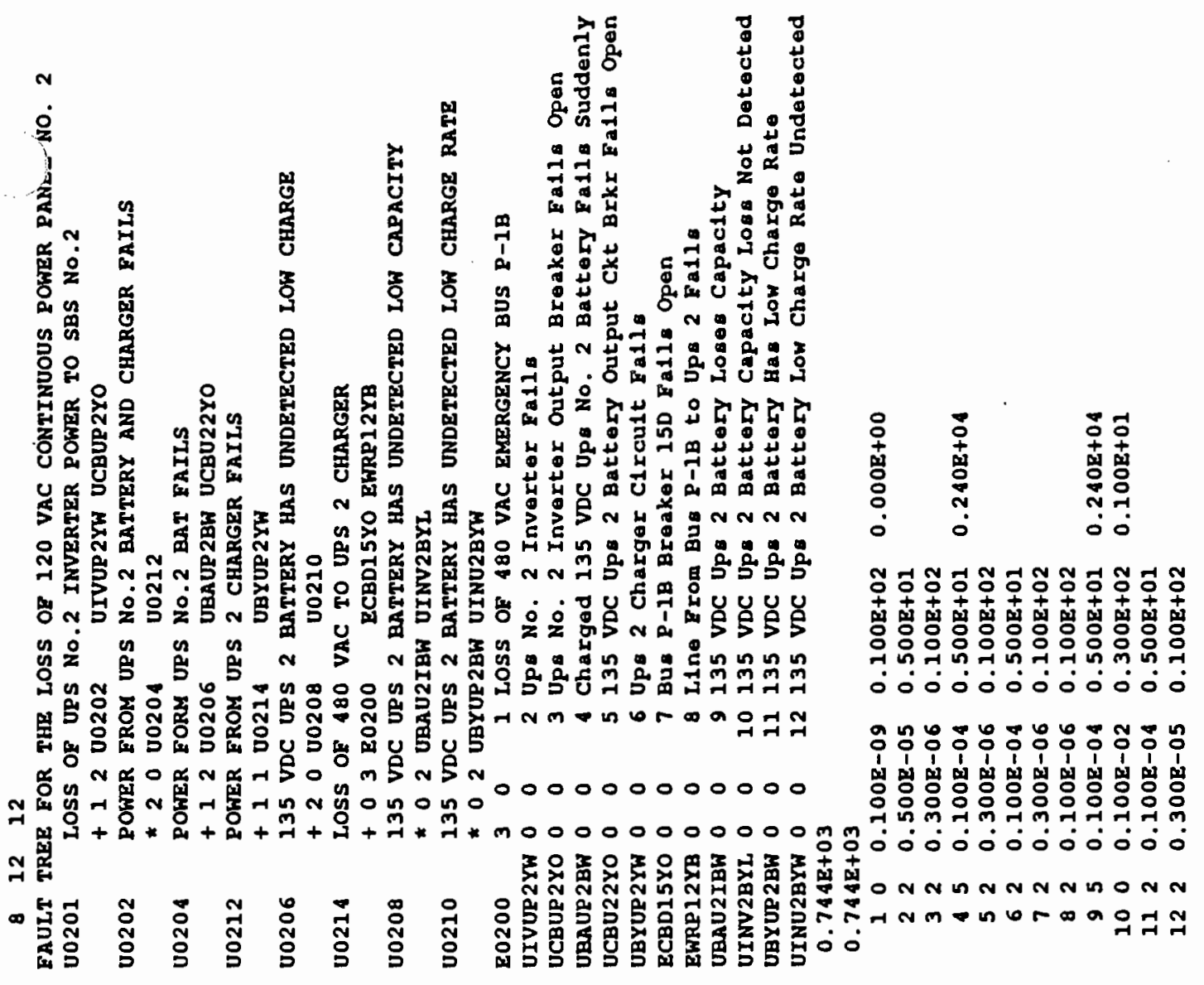




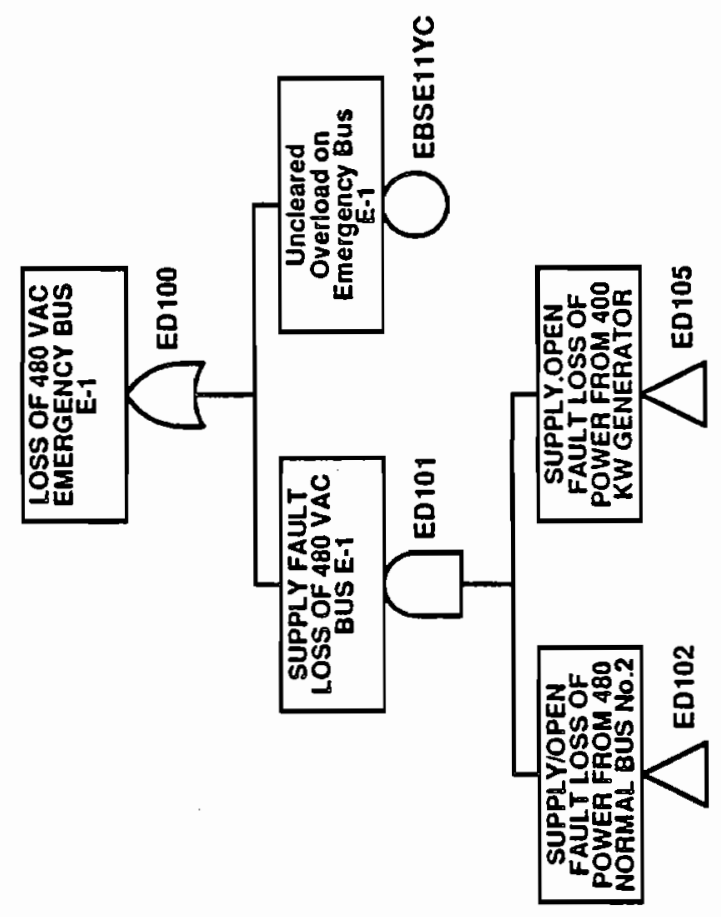

B. $2-414$ 


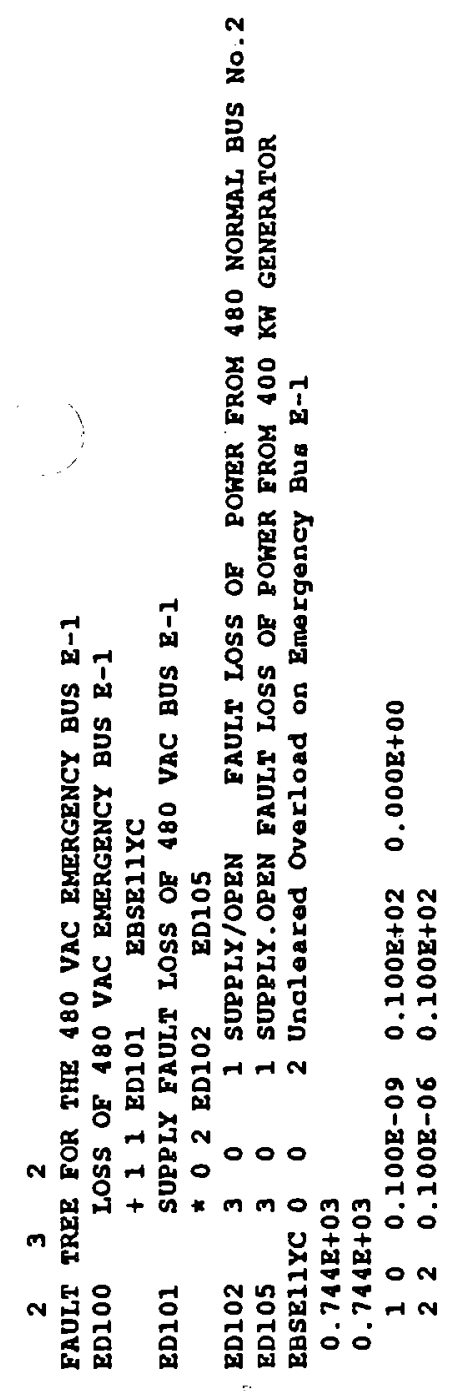

B. 2-415 


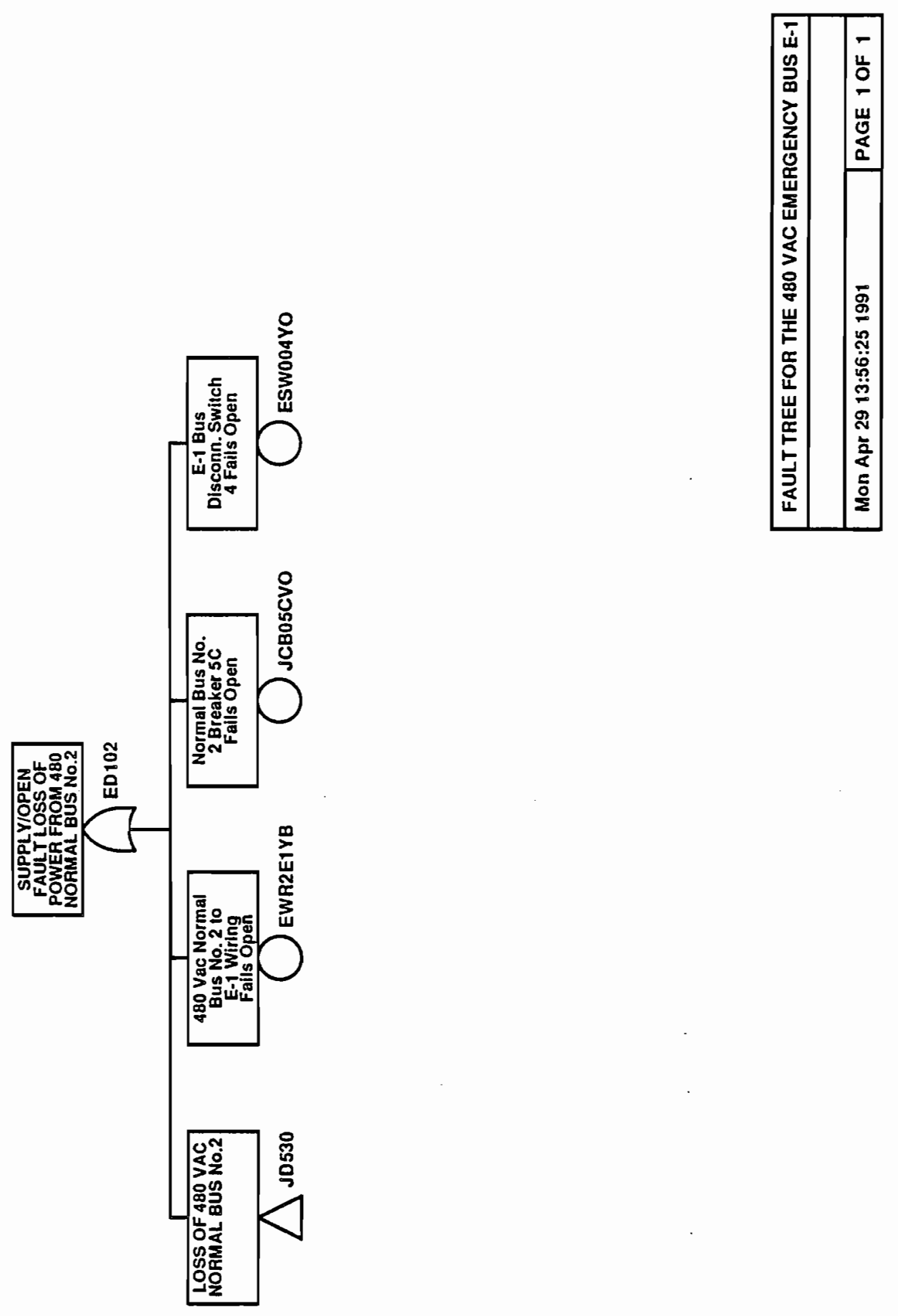

B. $2-416$ 


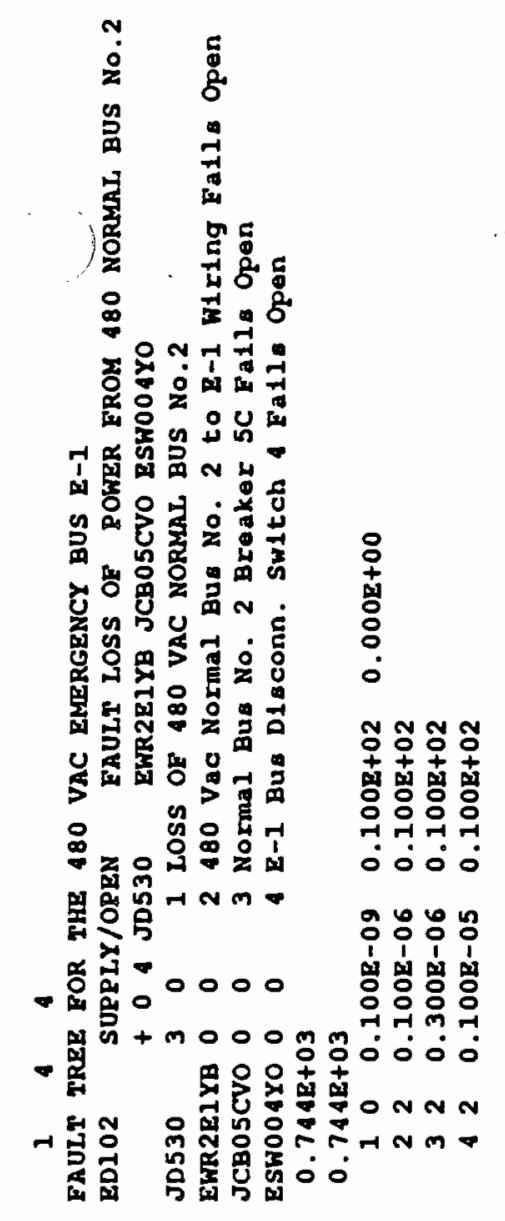




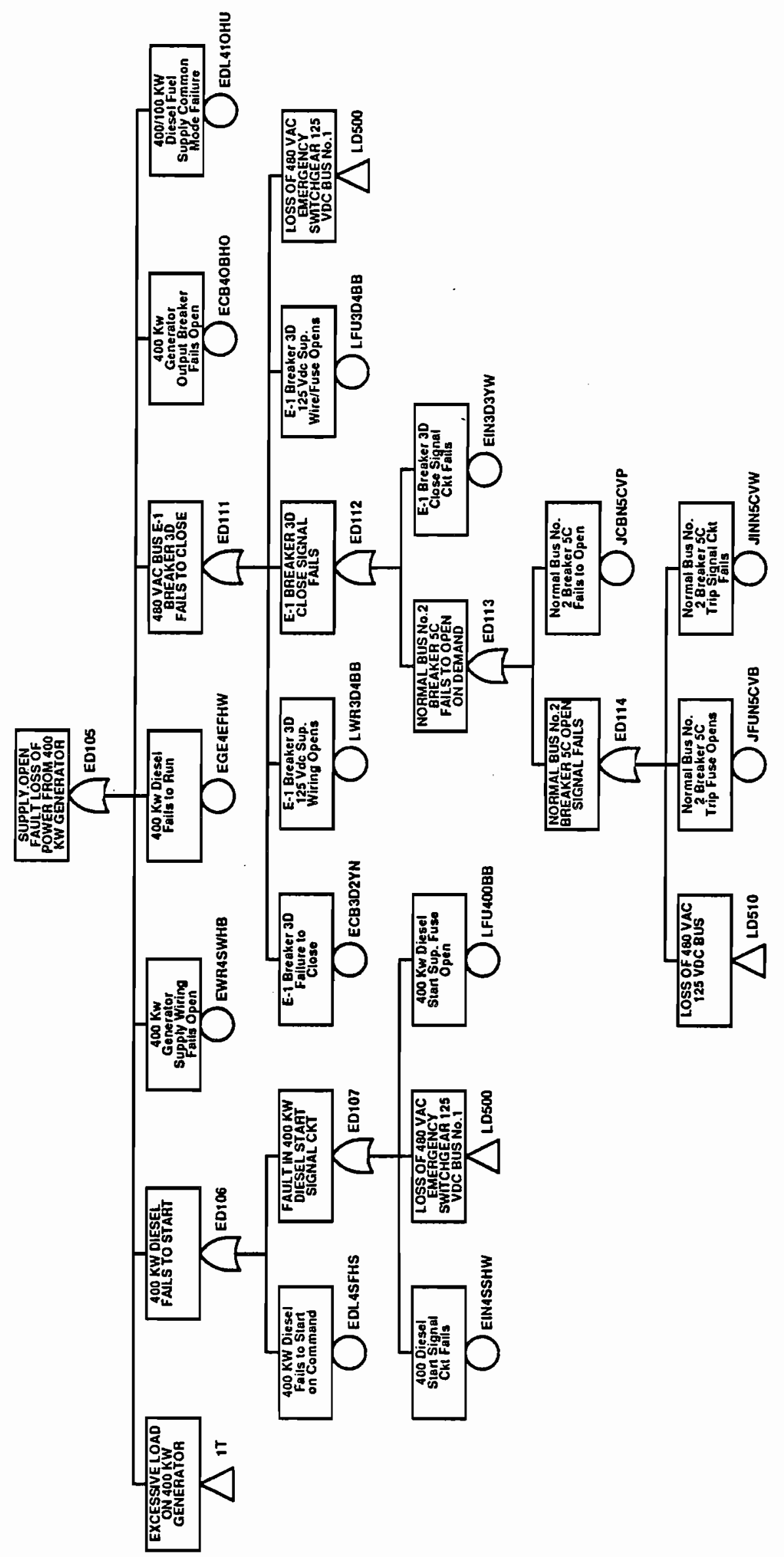

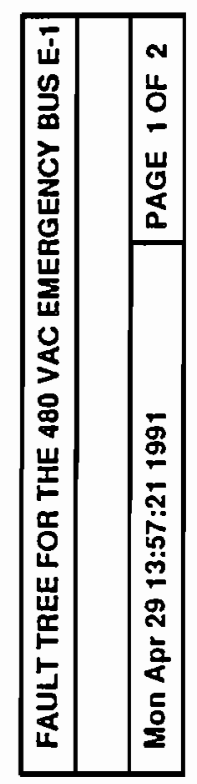




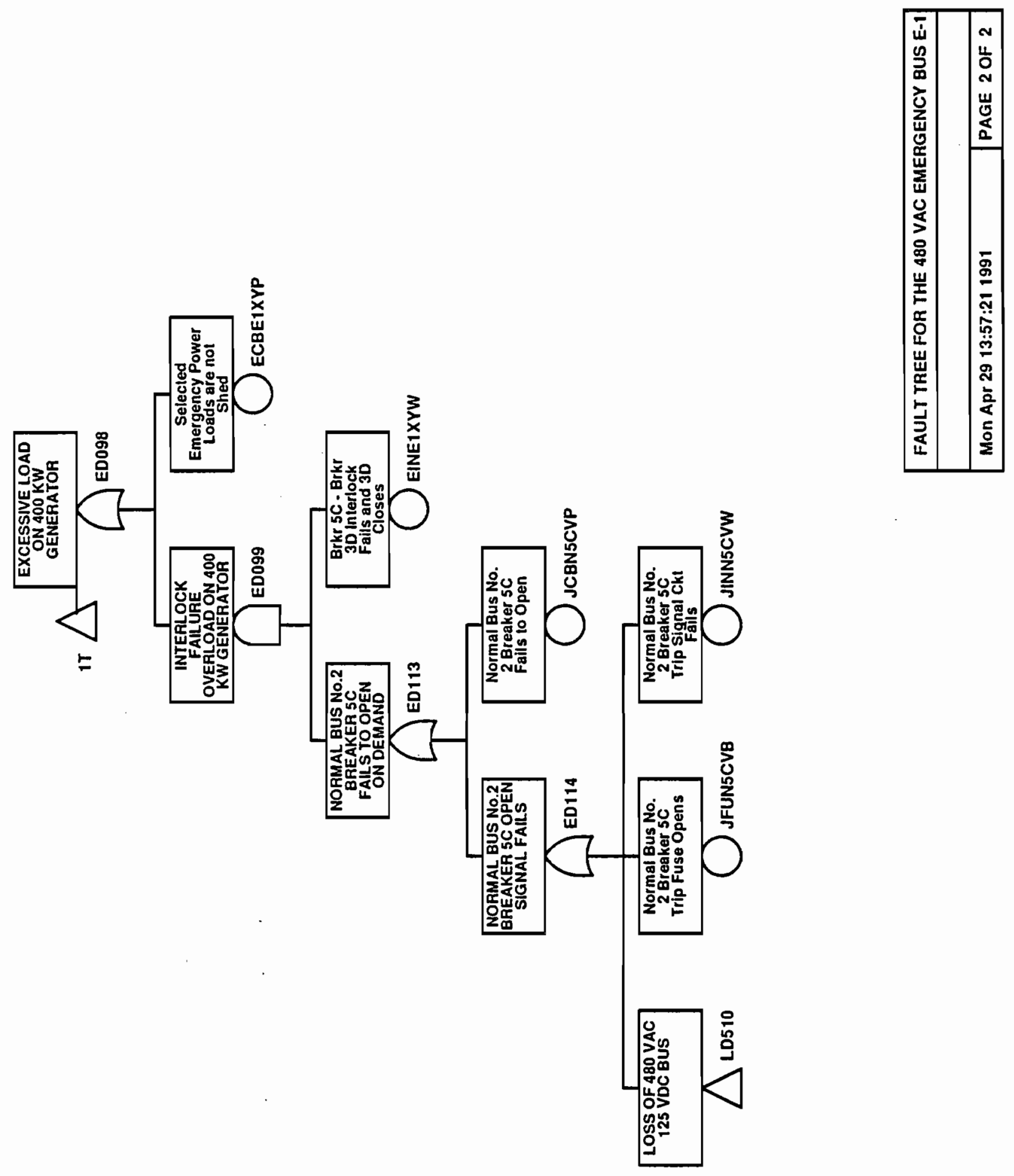




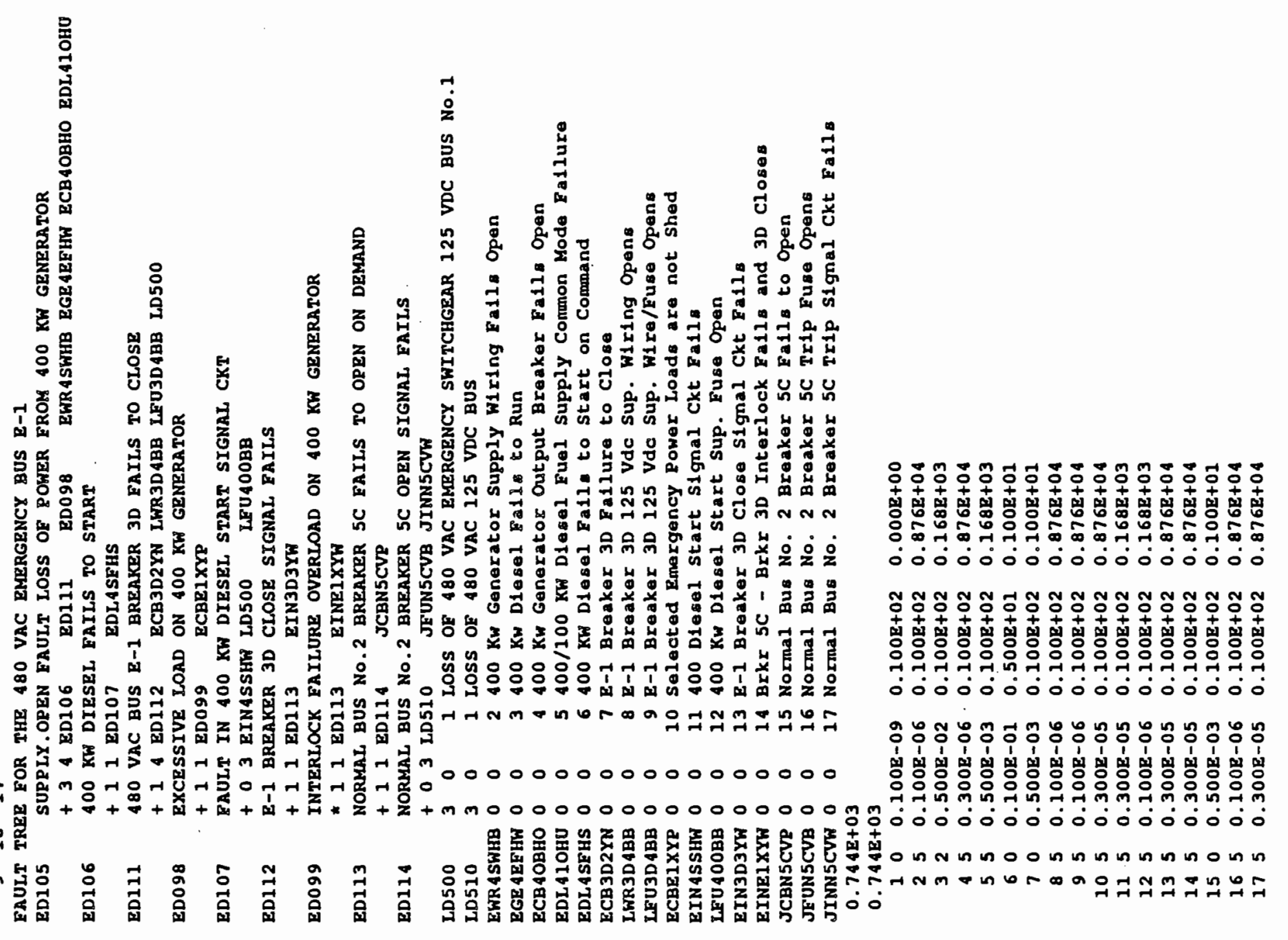




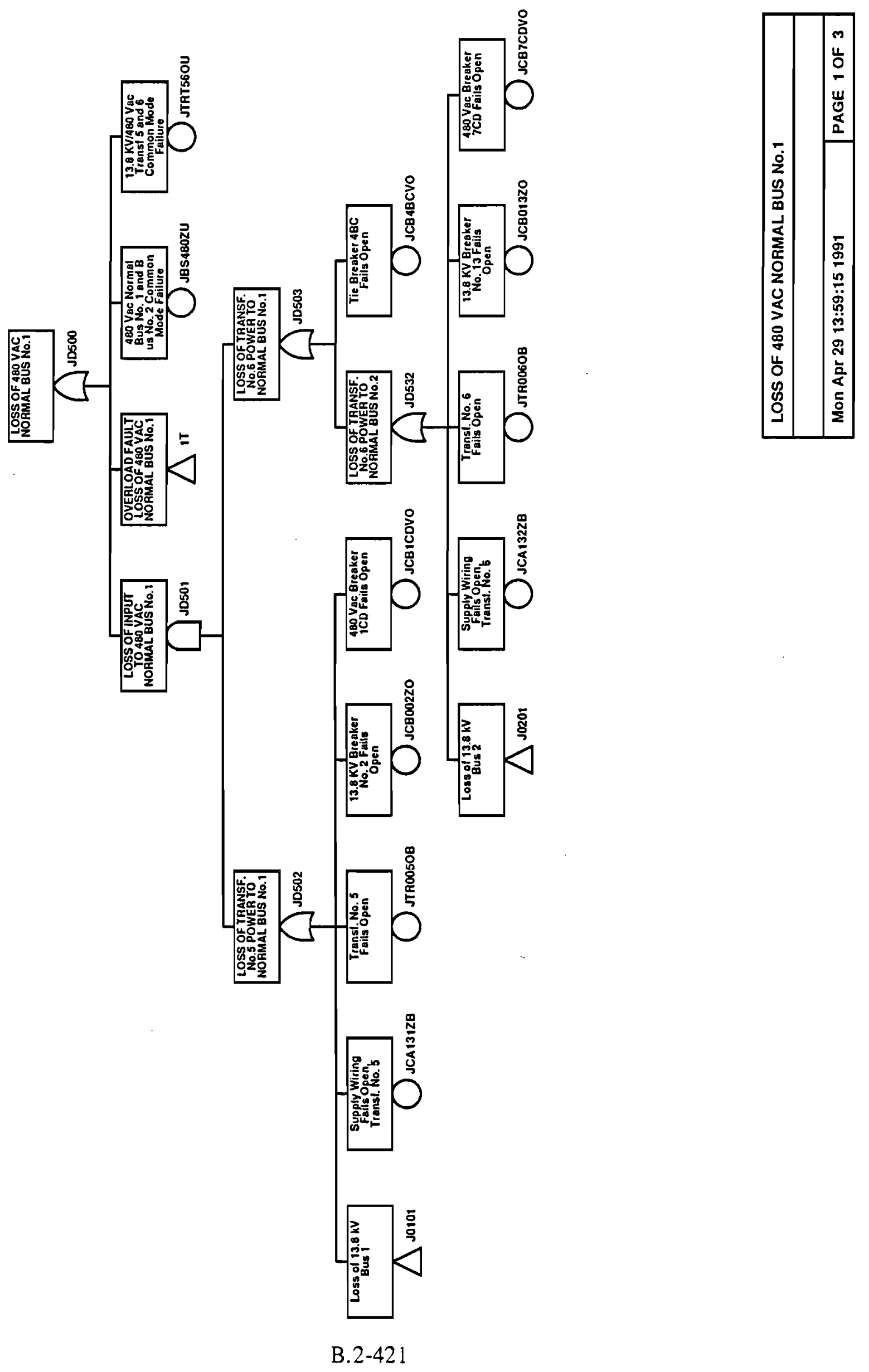




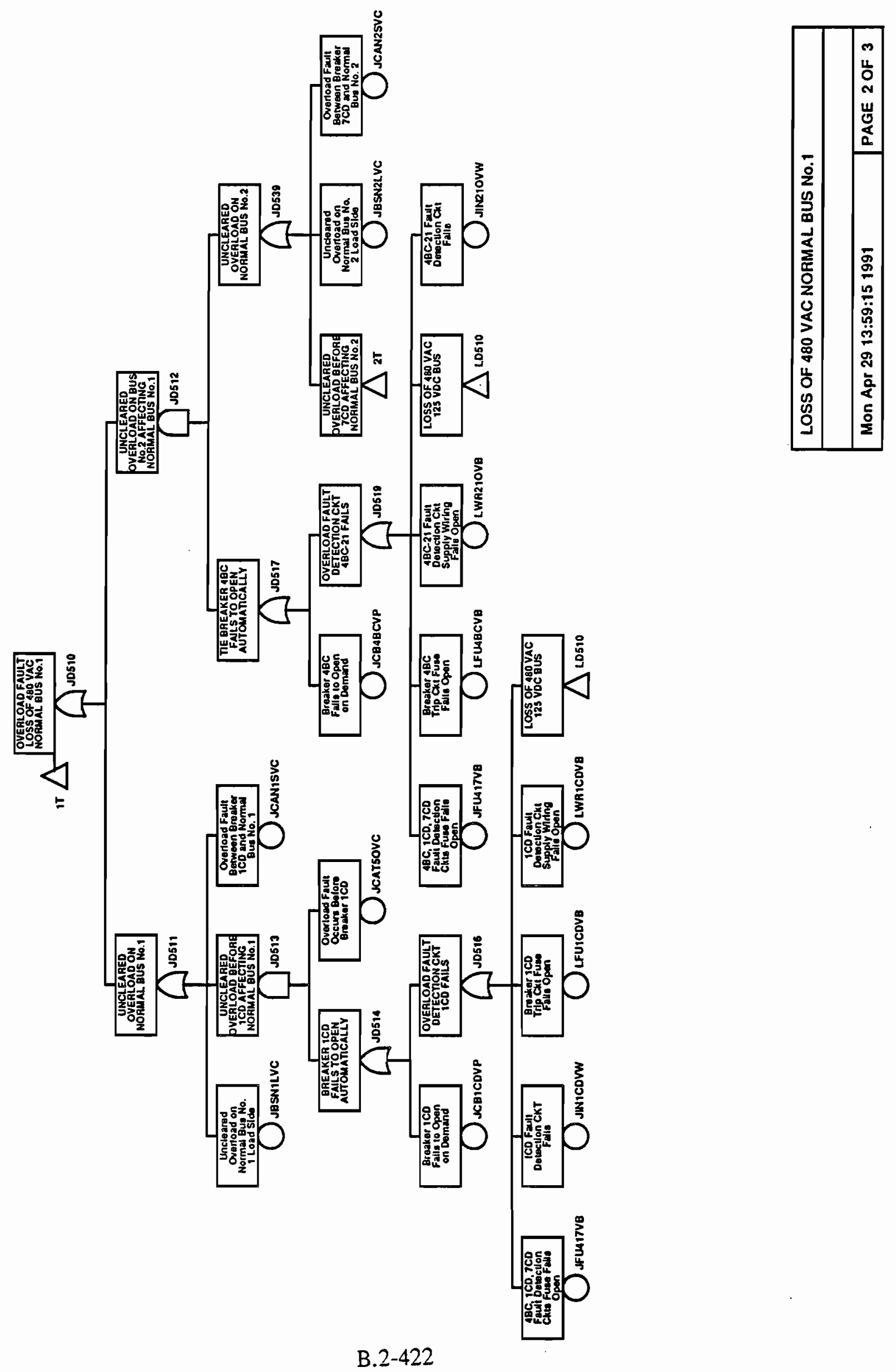




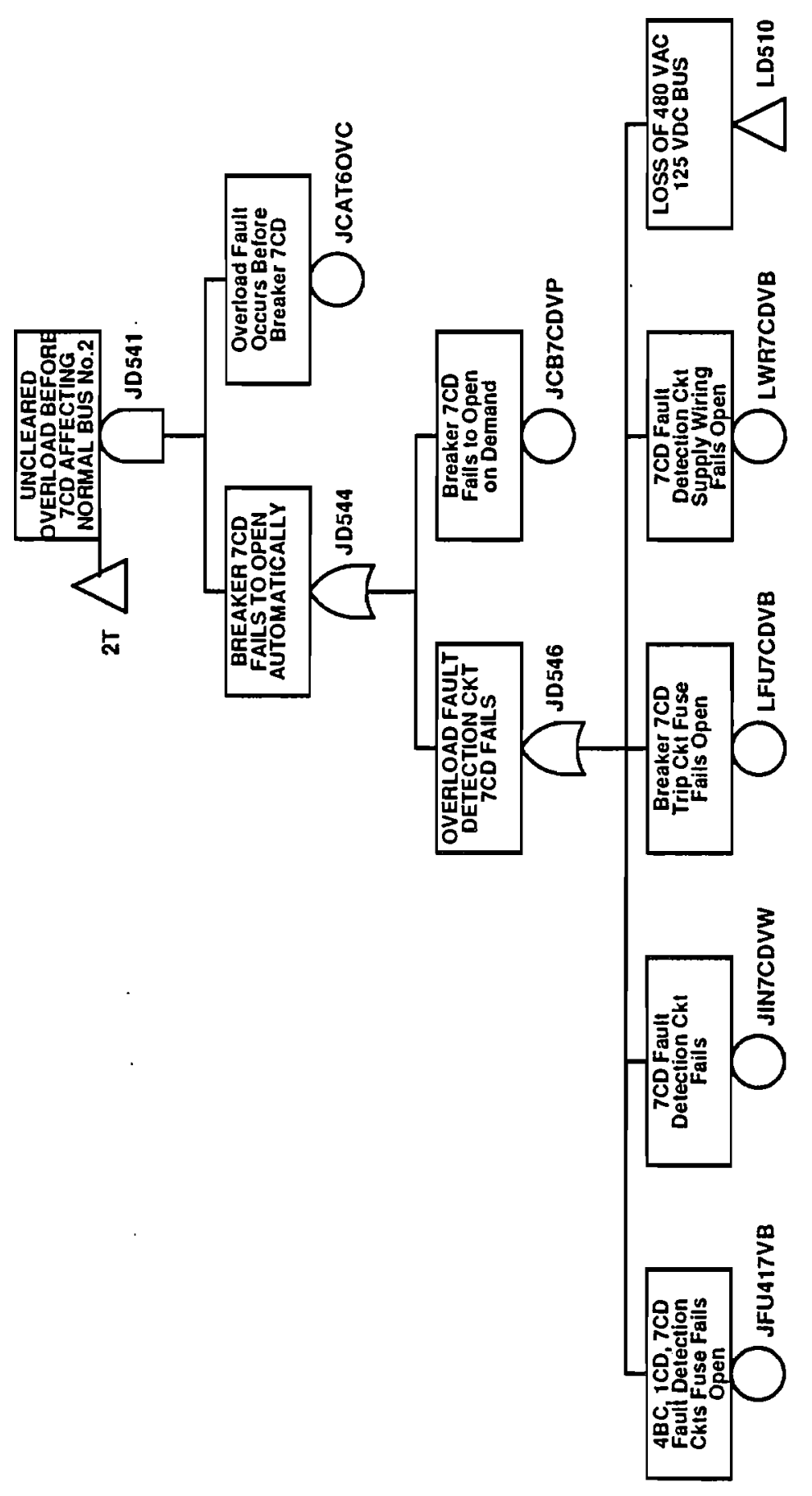

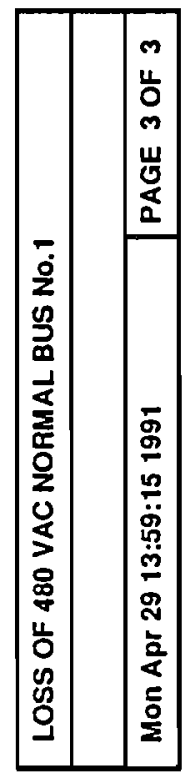




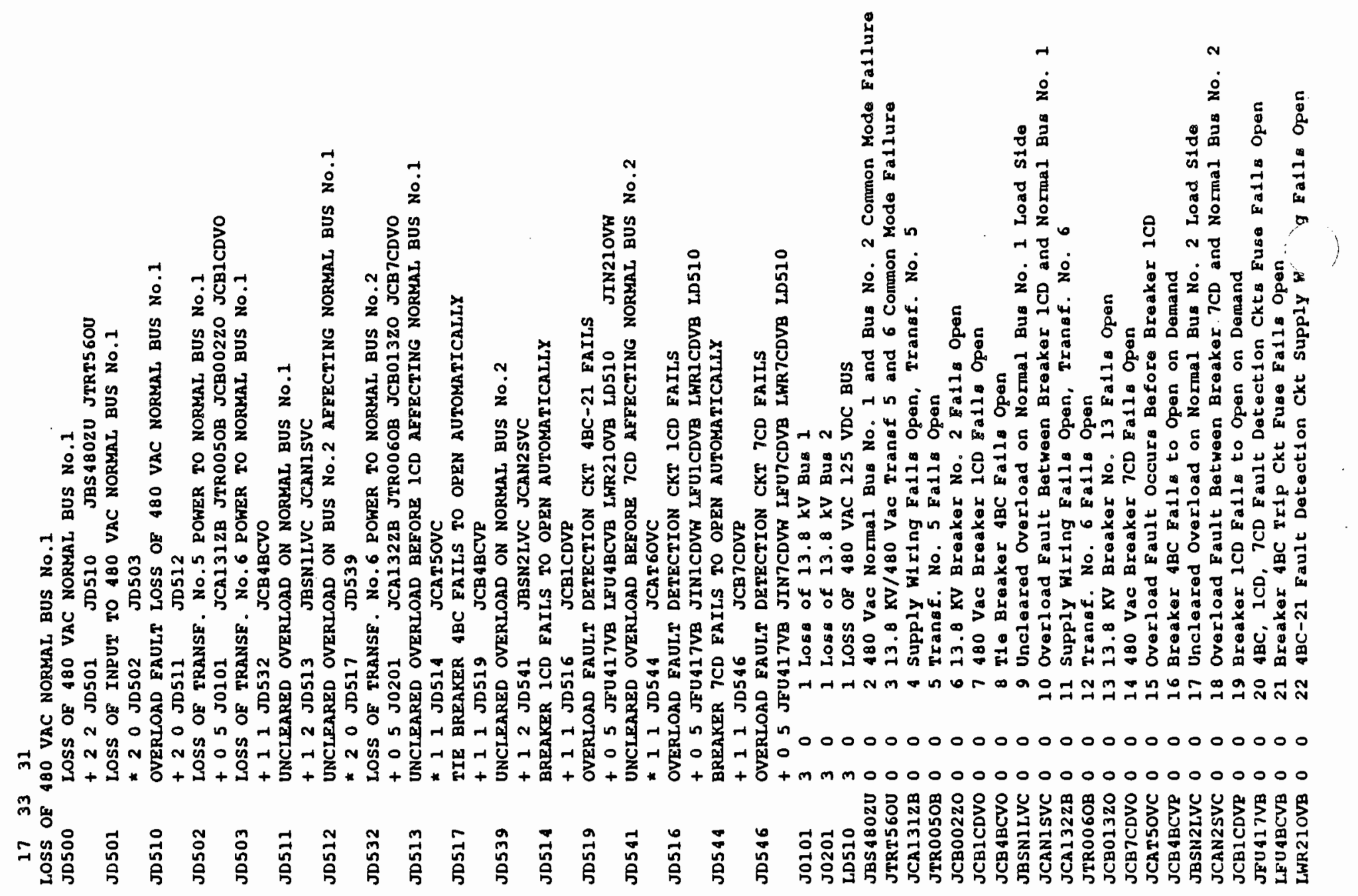



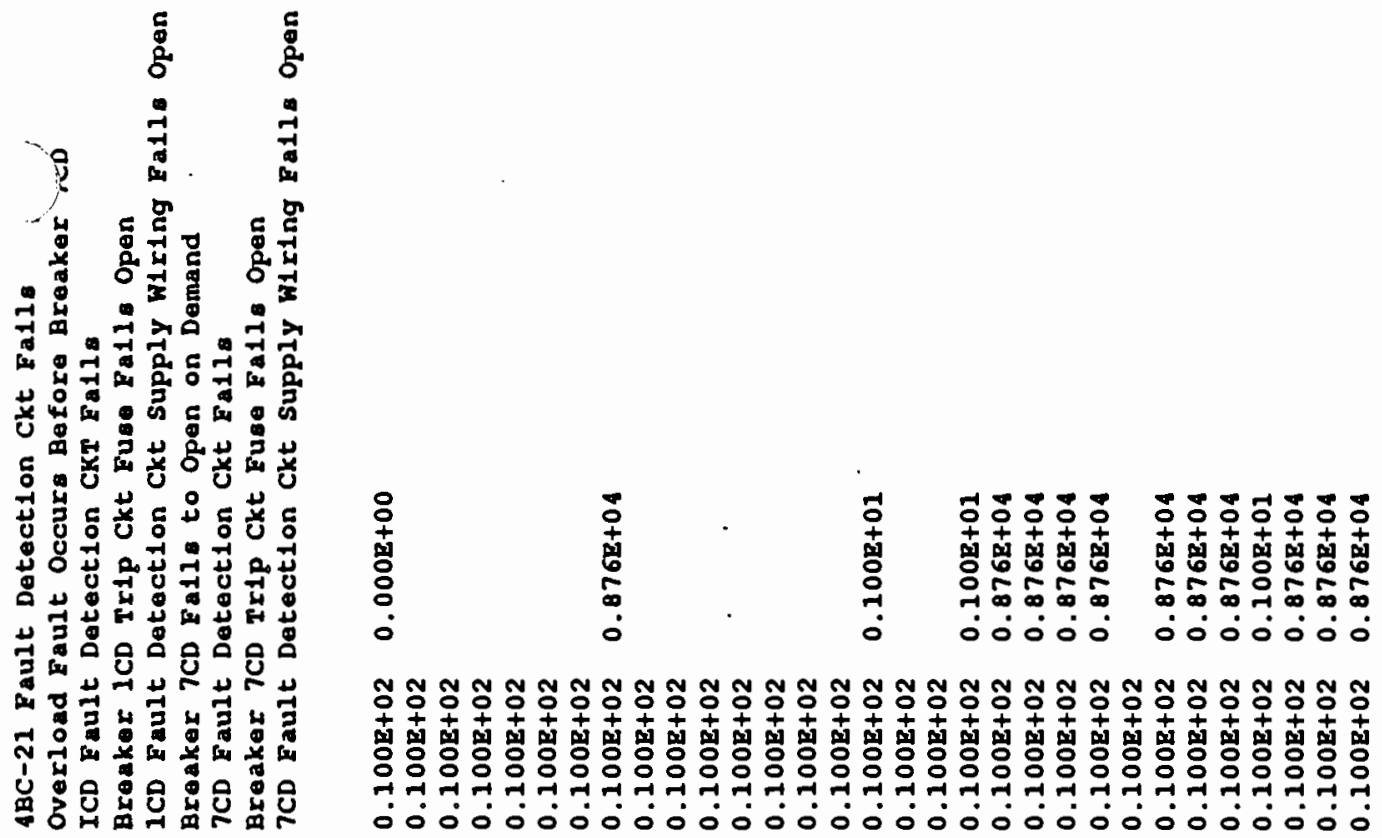

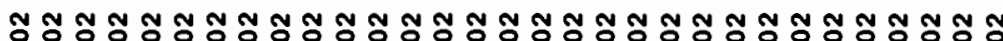

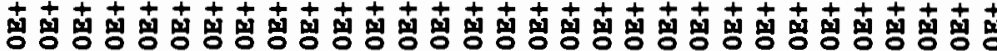

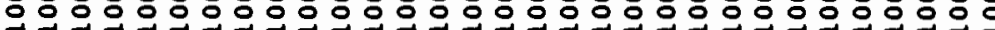
0000000000000000000000000000

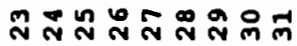

000000000 0000000000

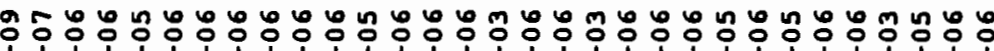

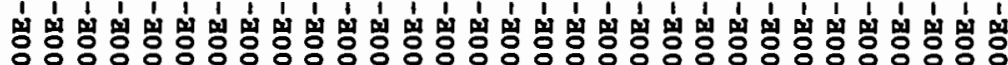

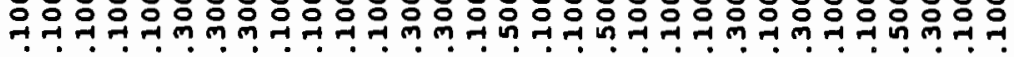
工

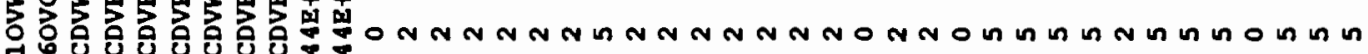

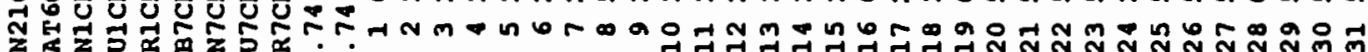

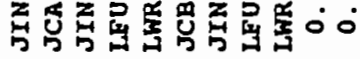




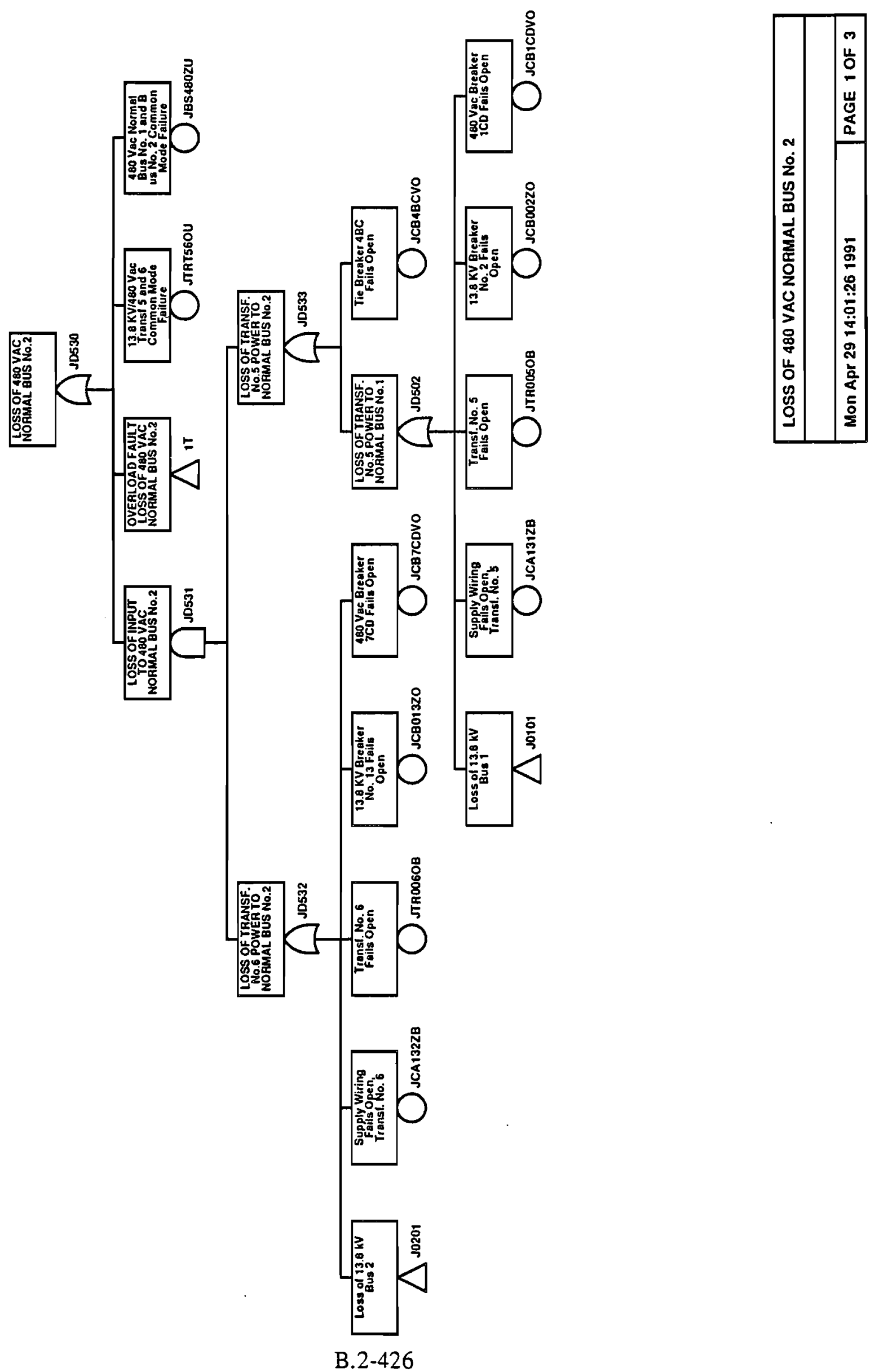




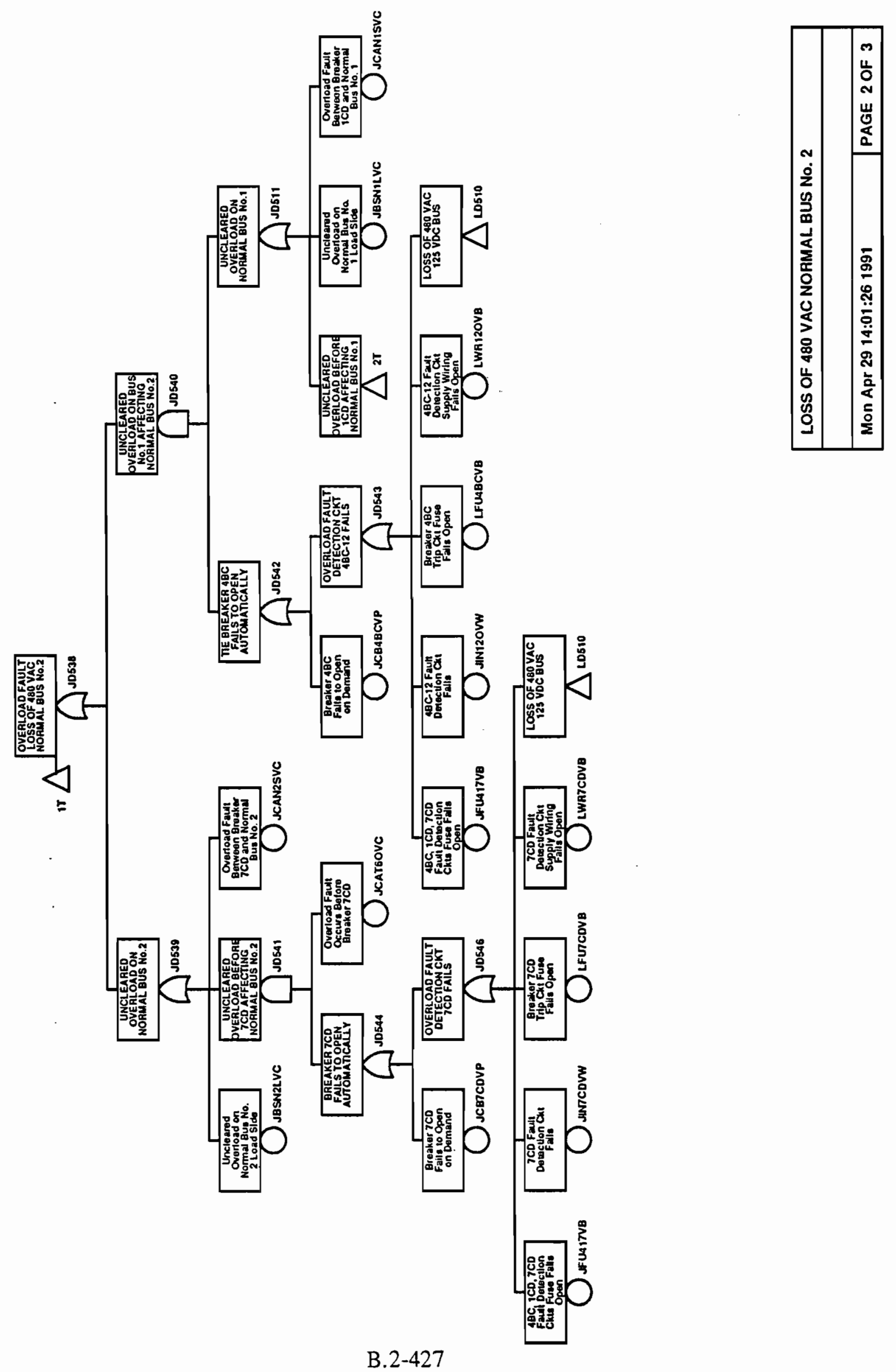




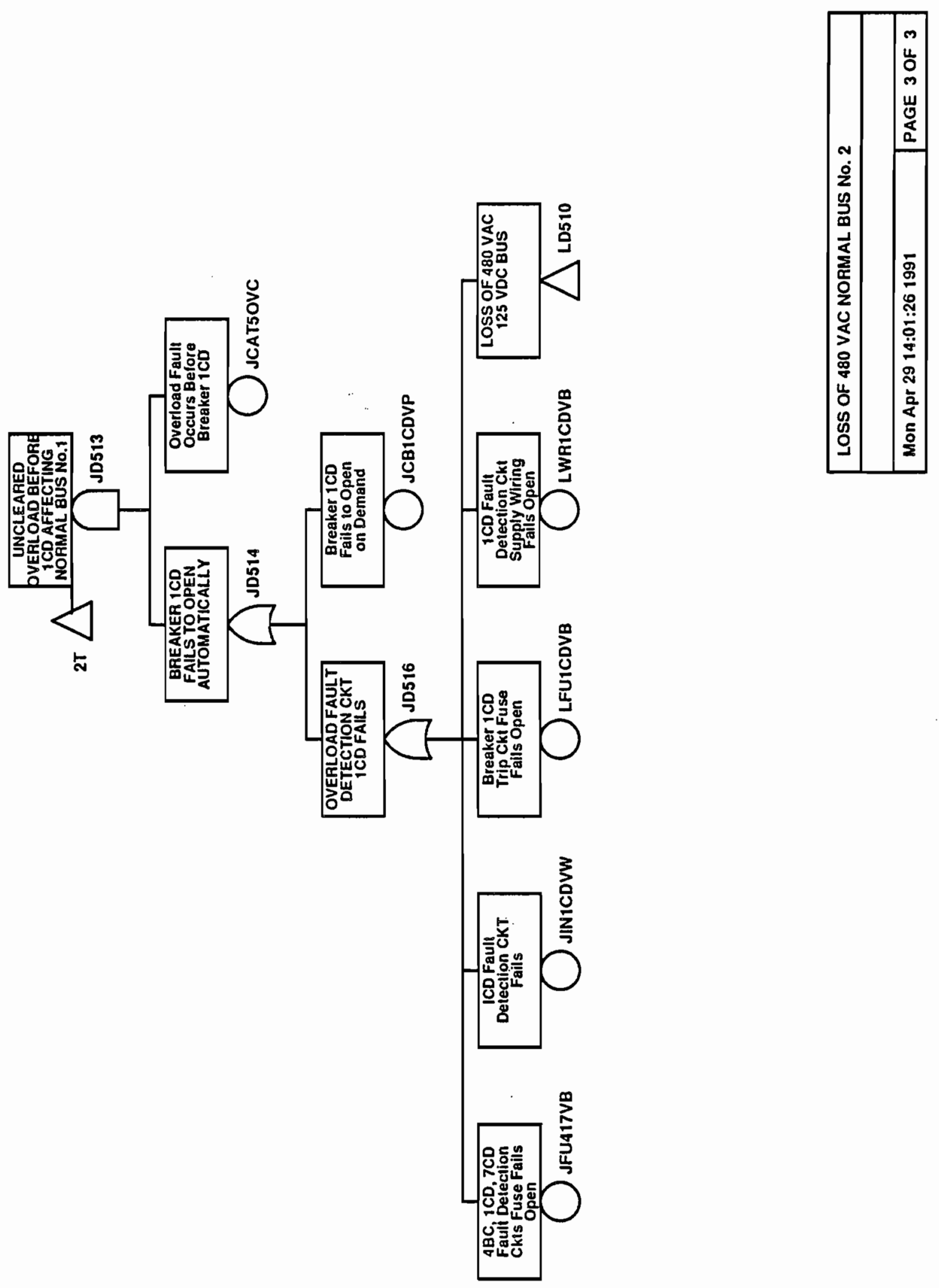




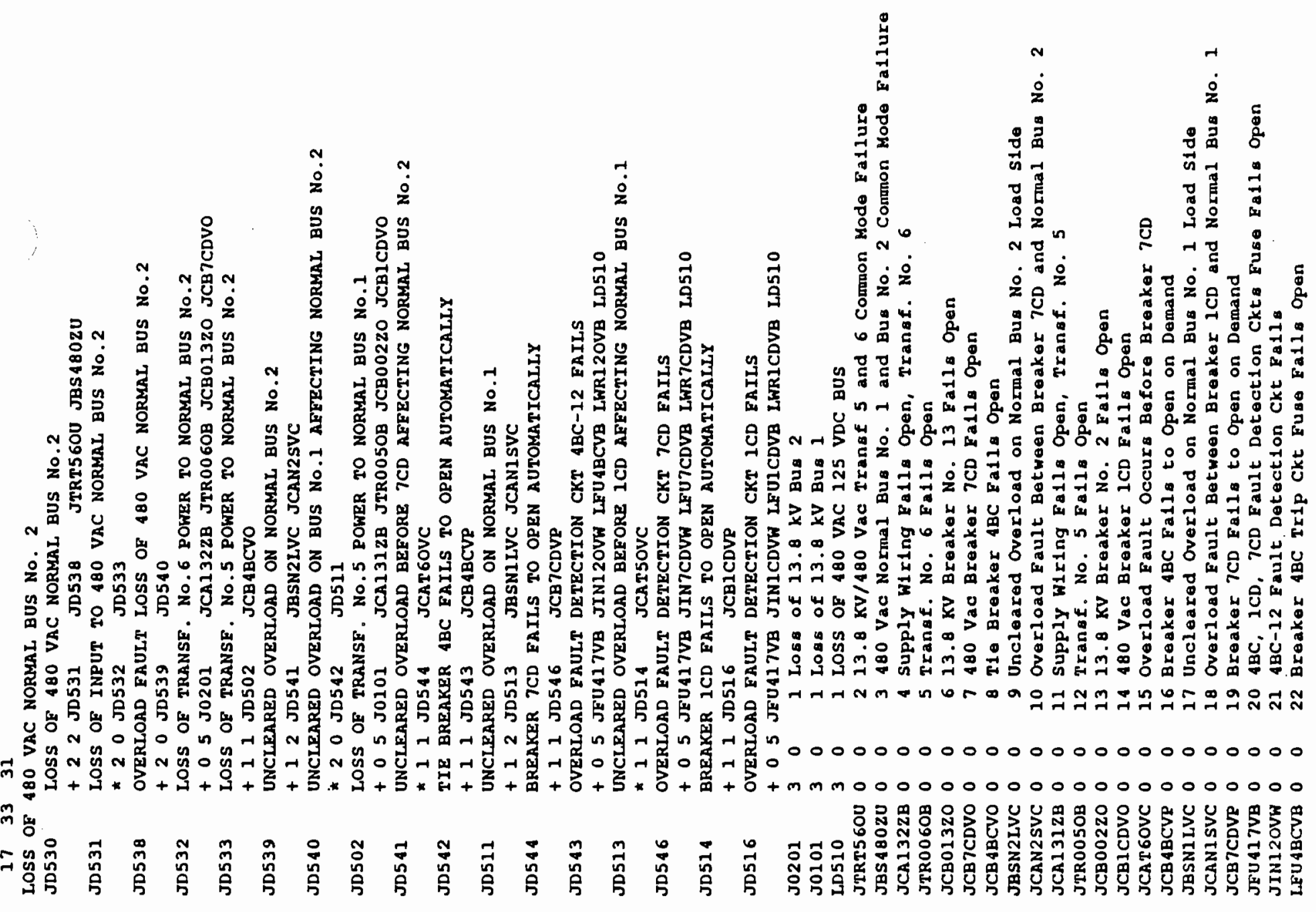



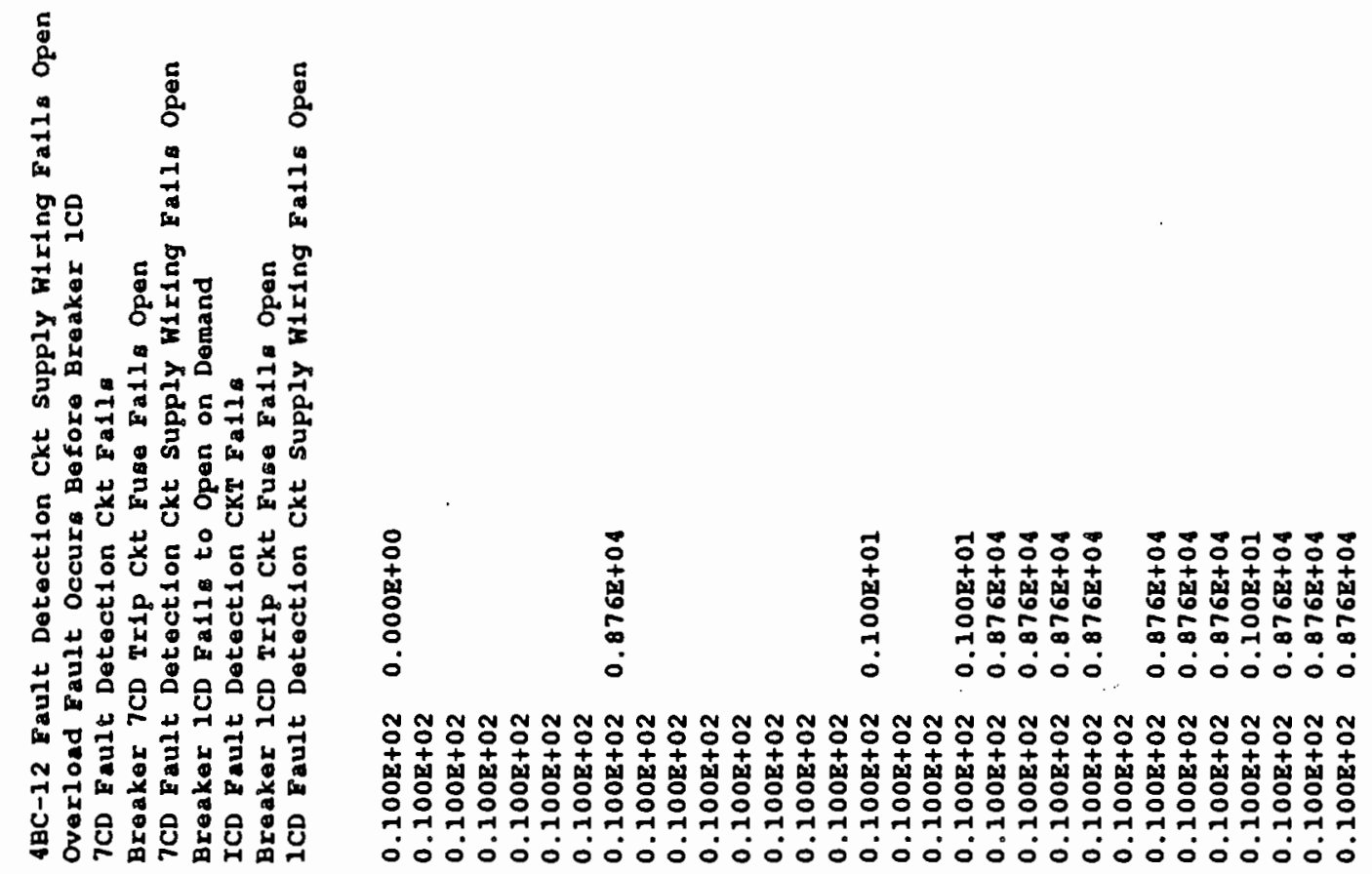

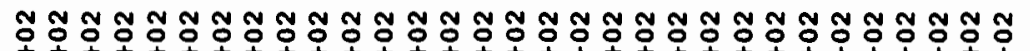

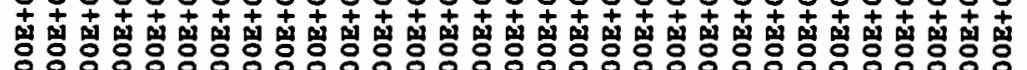

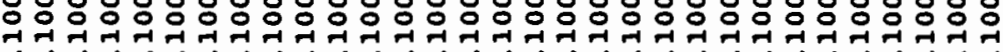

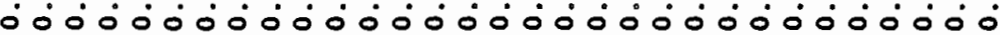

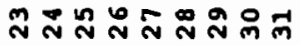

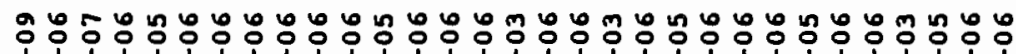

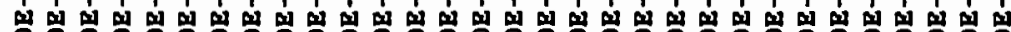

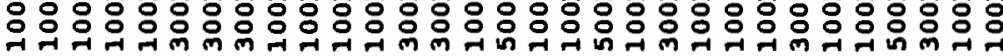
000000000

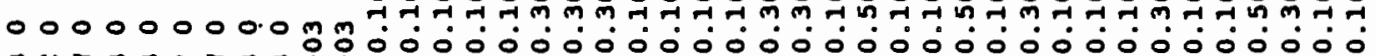

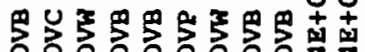

施 $\vec{E}$

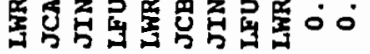




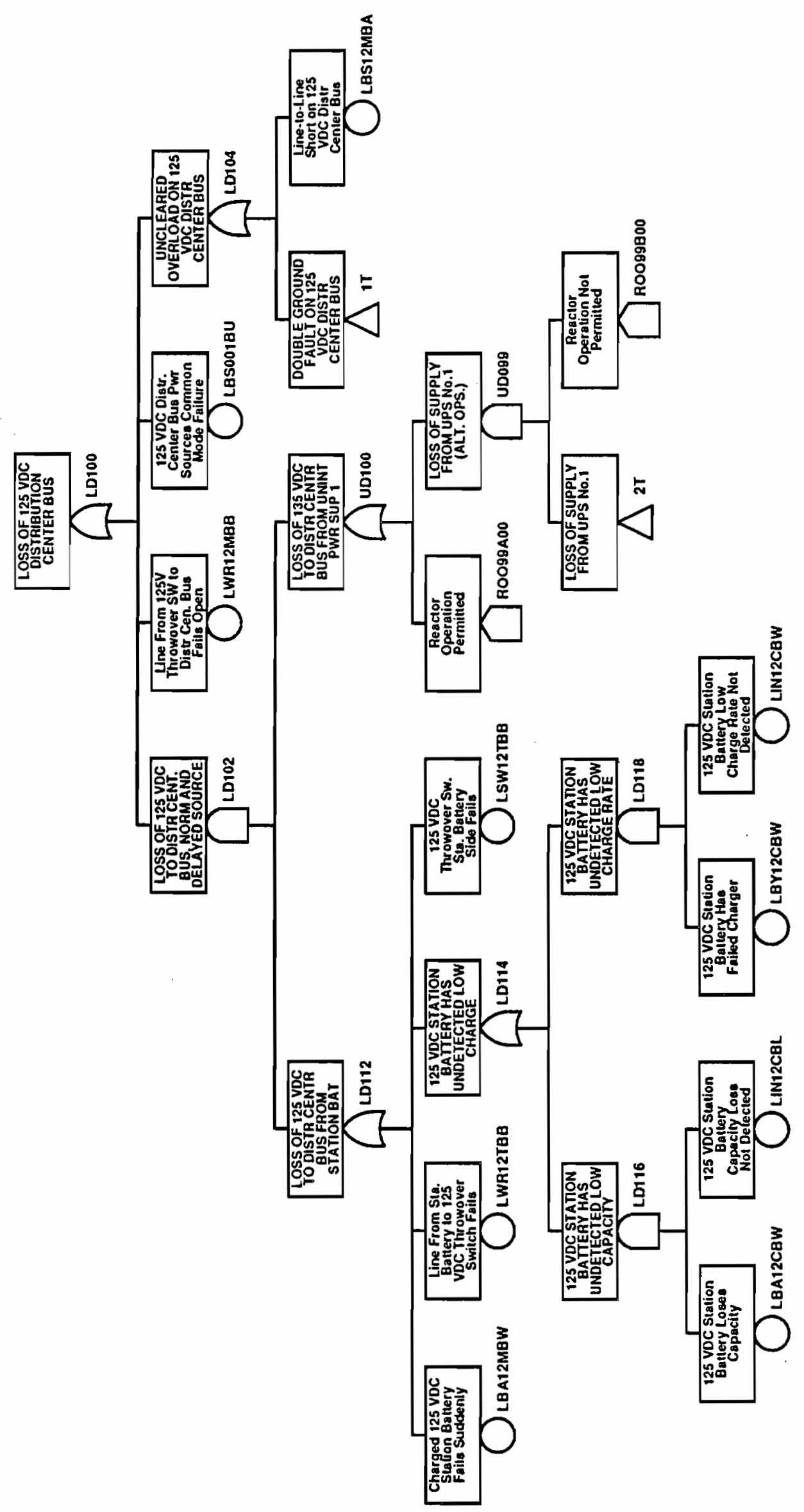

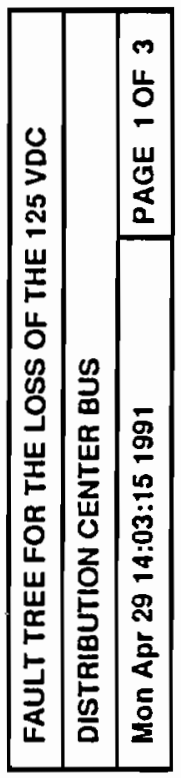



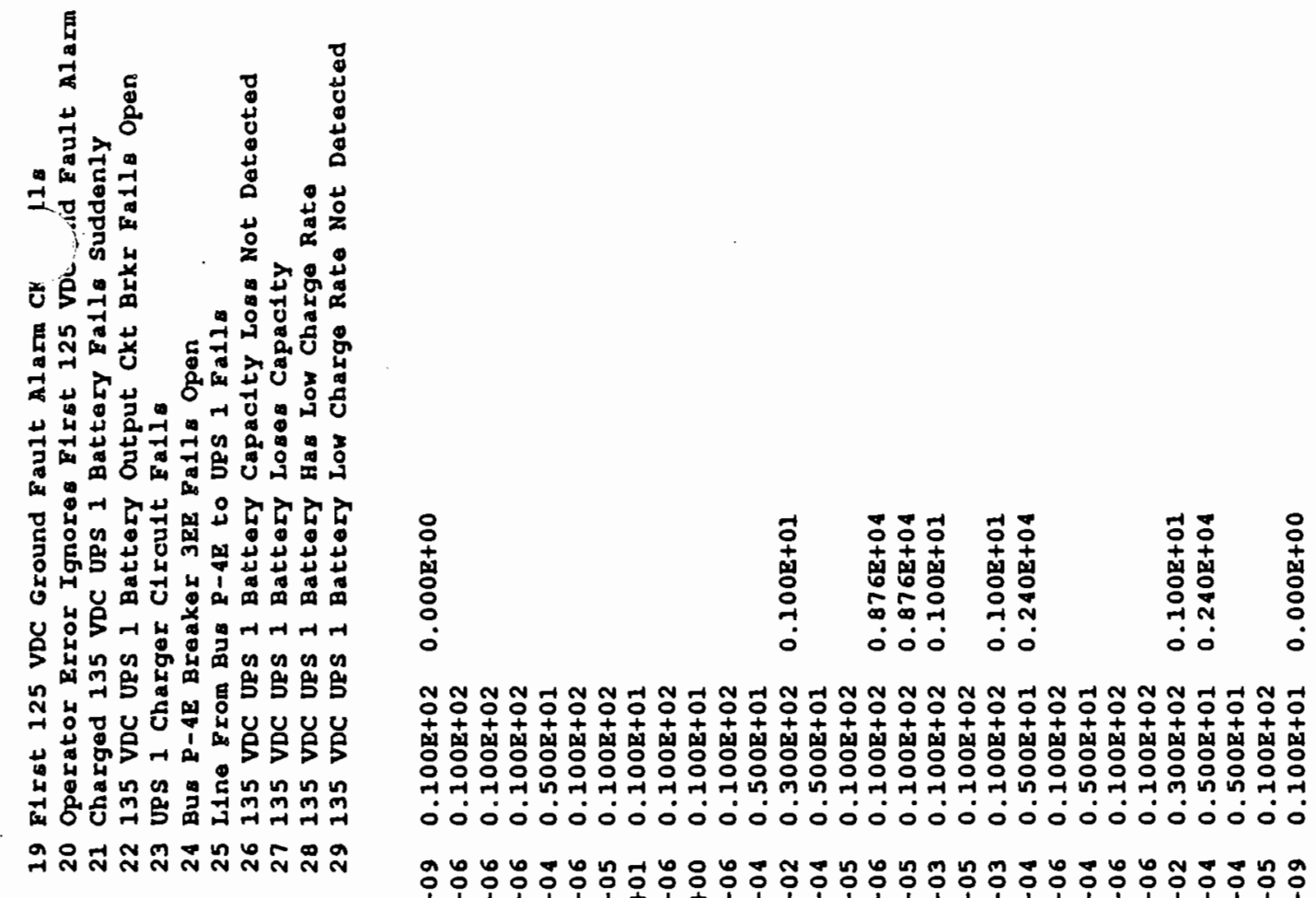

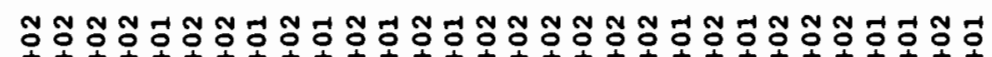

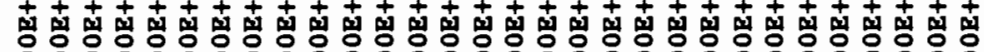

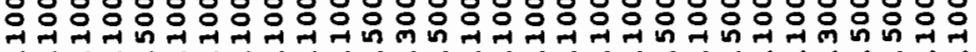

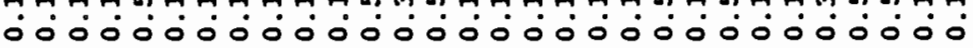

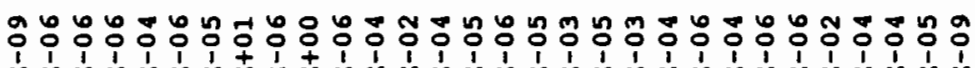

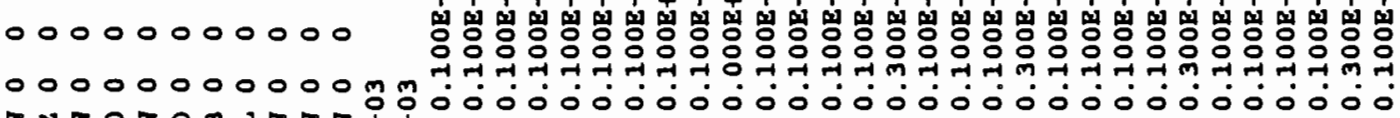
00000000000 贸

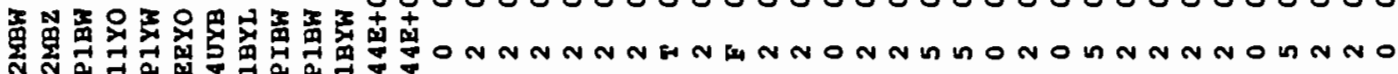

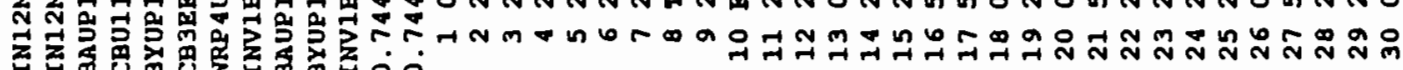

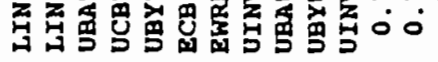




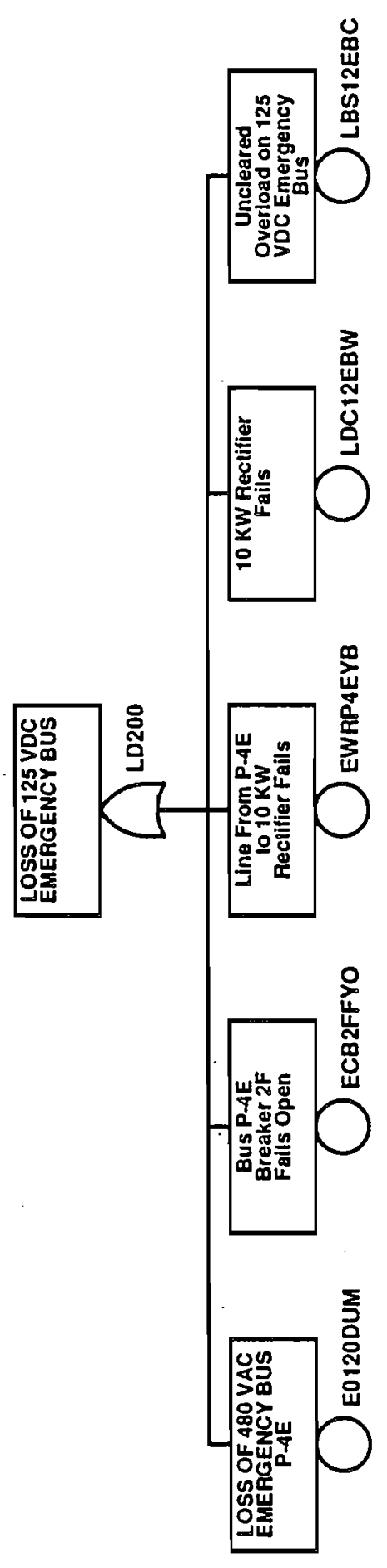

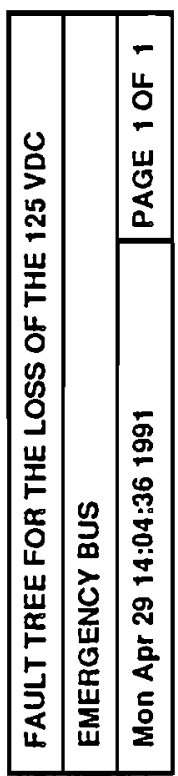




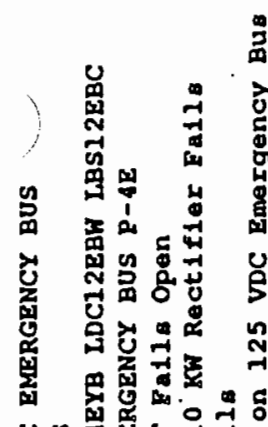

u舟的

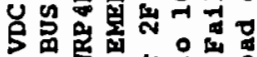

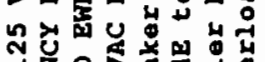

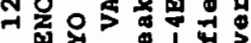

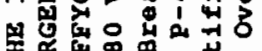

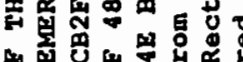

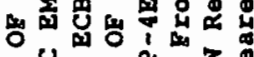

o

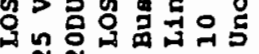

बำ

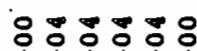

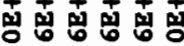

战尔尔。

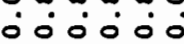

พำํำำㅇำำ

䓛南南南南声

$\circ$ 웅ㅇㅇㅇㅇ ○ं0 000

ㅇํㅇ웅훙ㅇㅇ

퐁

1 1 1 11 1

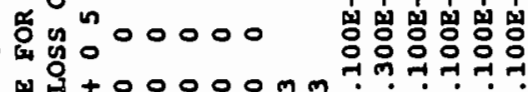

岗 + 00000 m

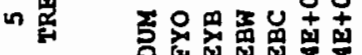

봉였

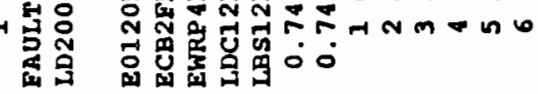




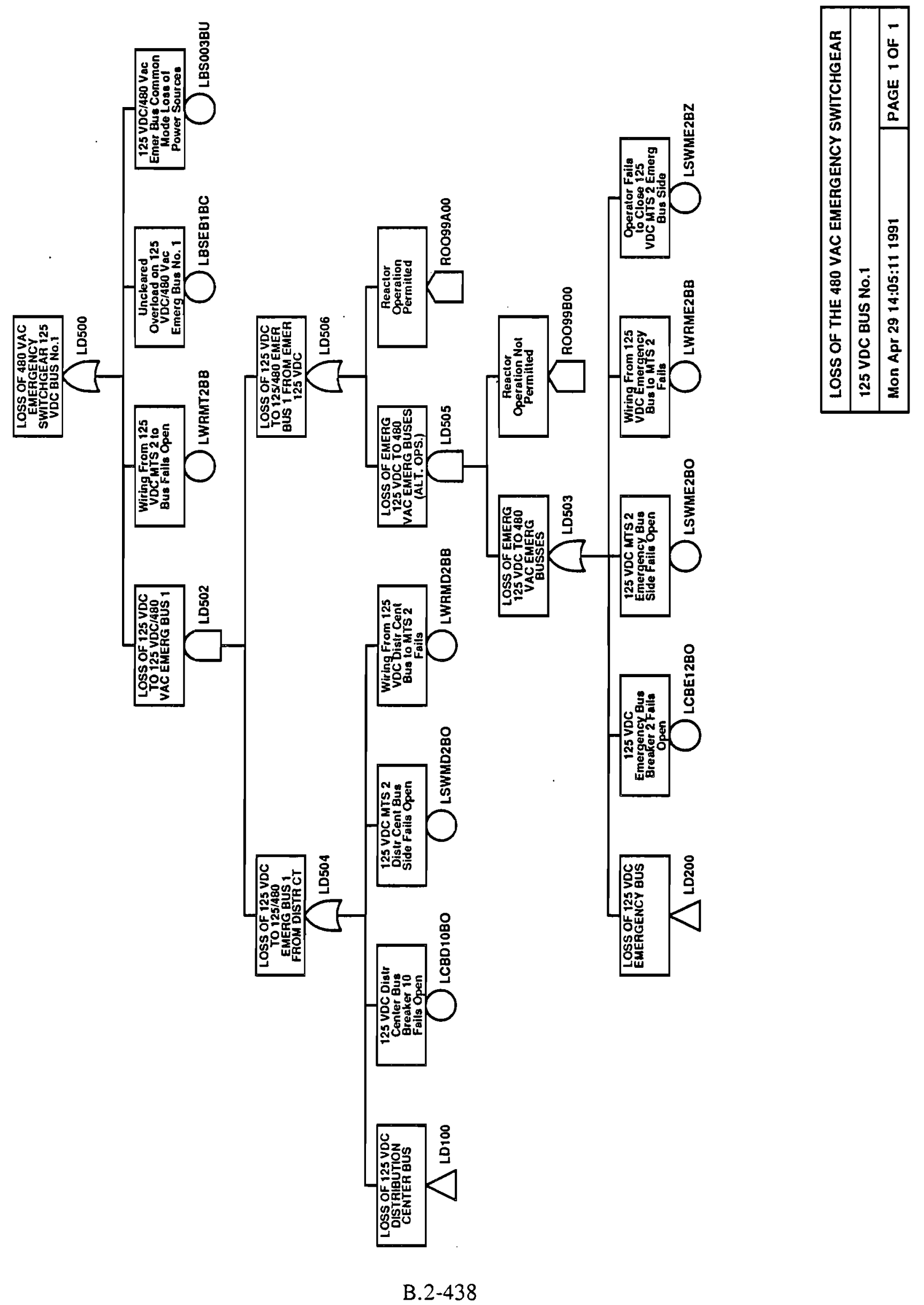




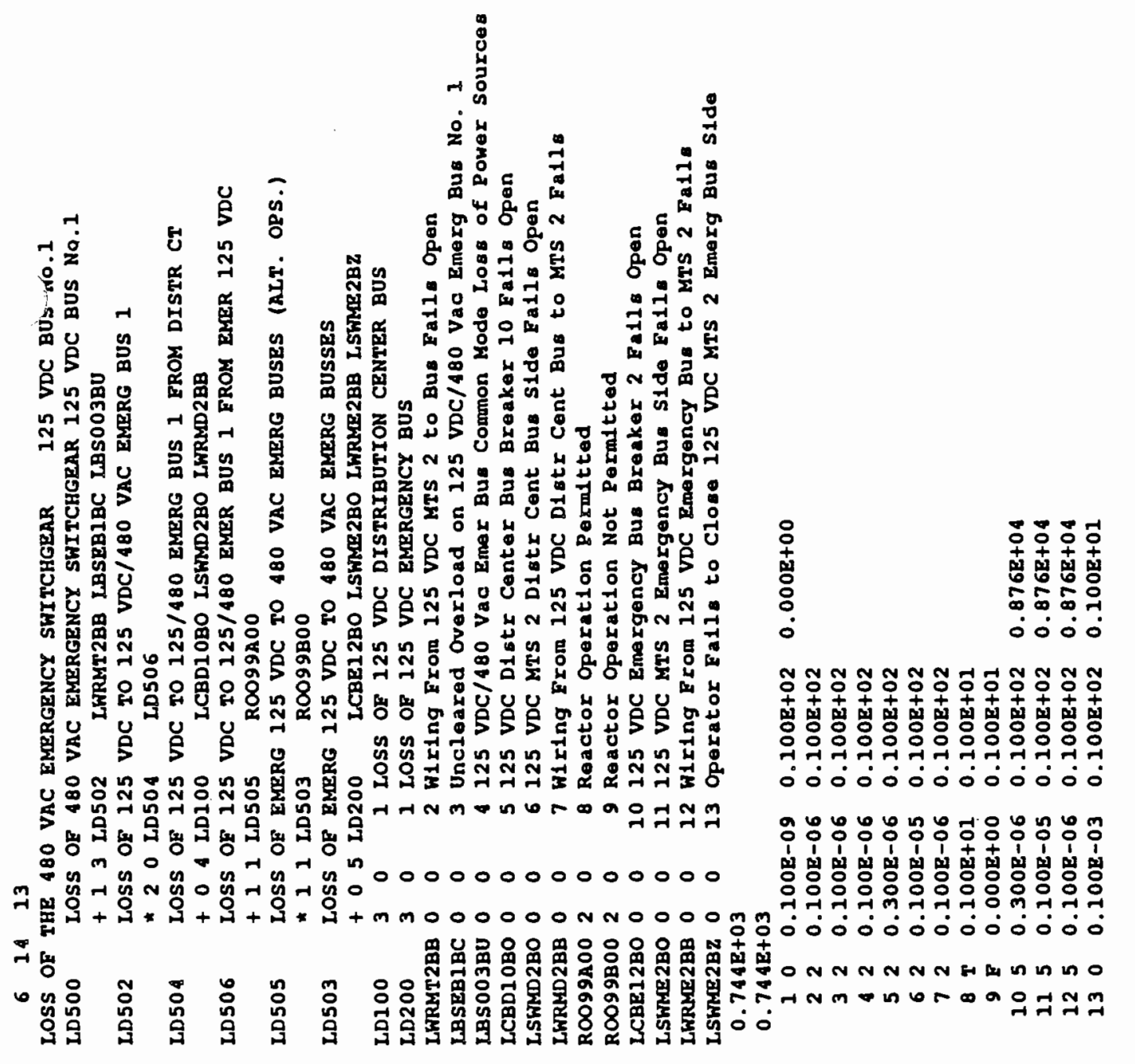

B. 2-439 


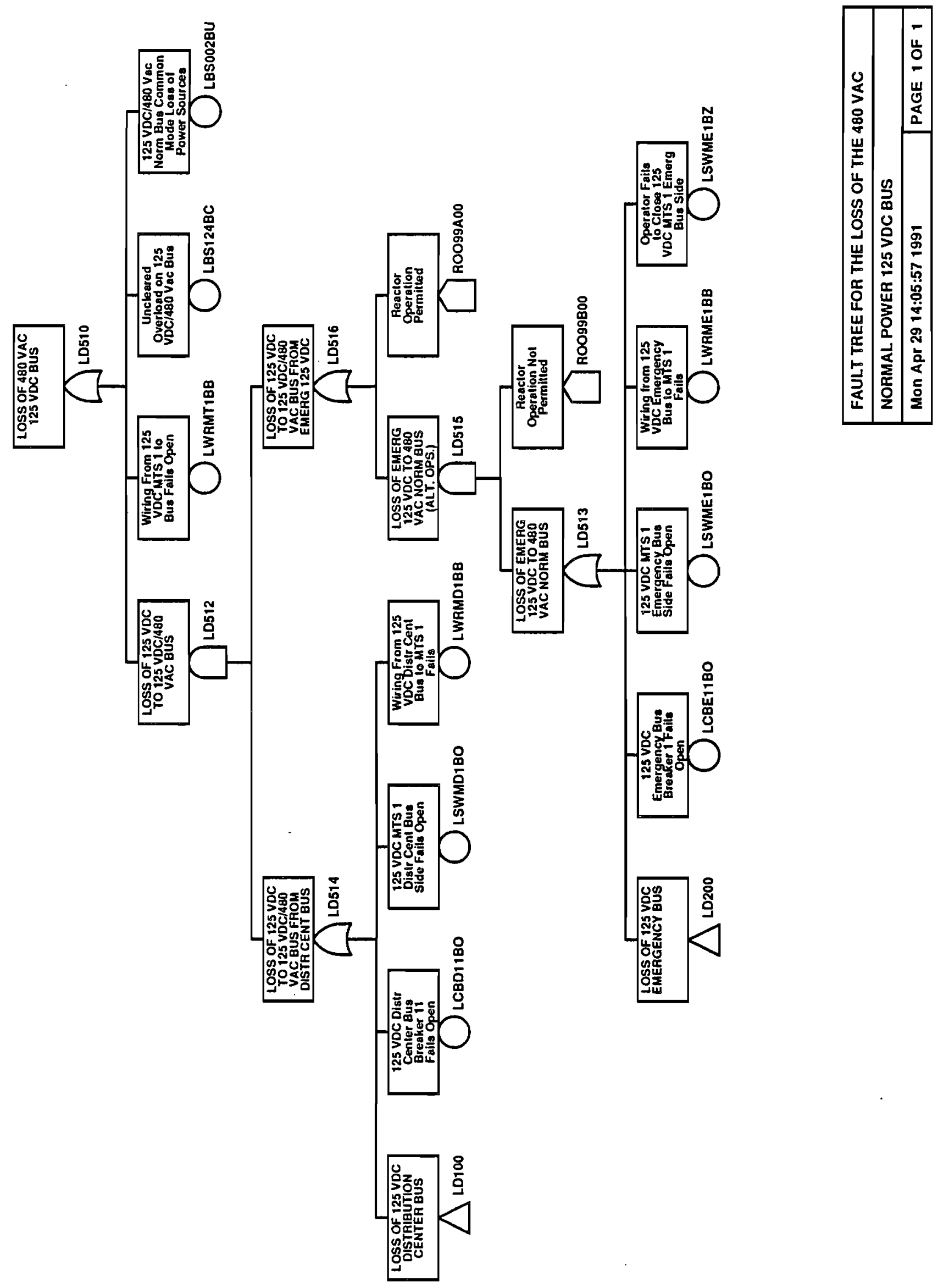




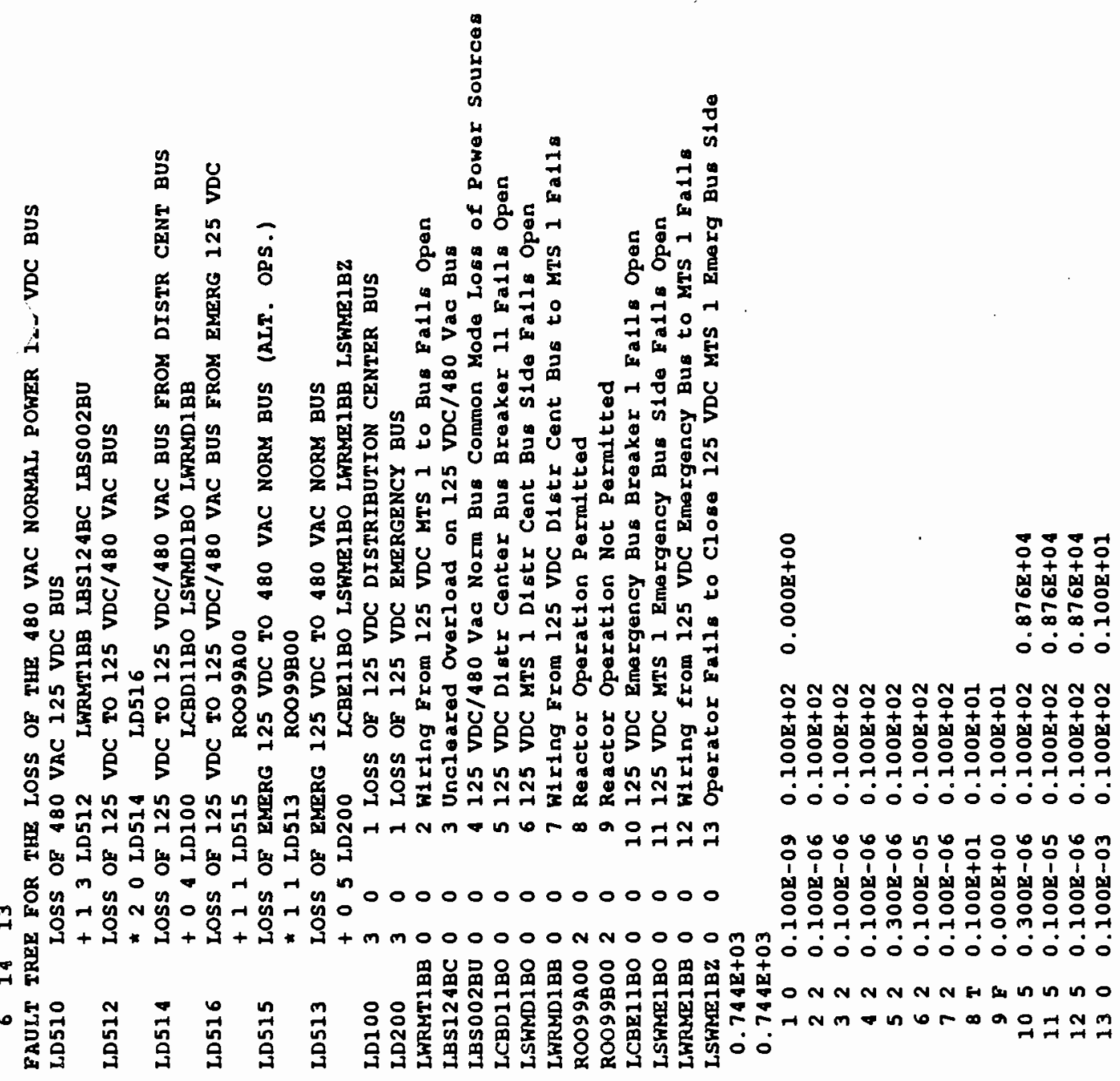




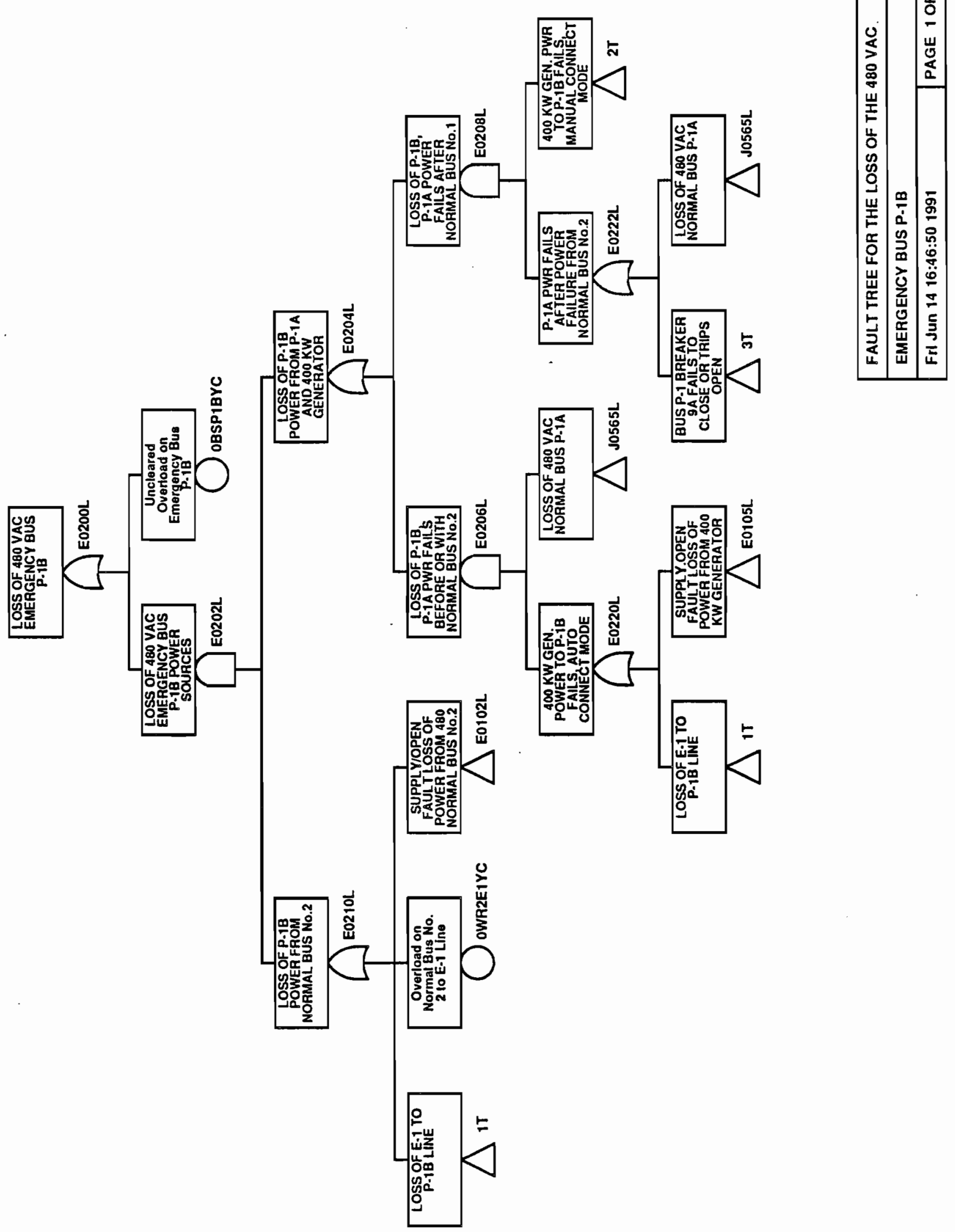

B. 2-442 

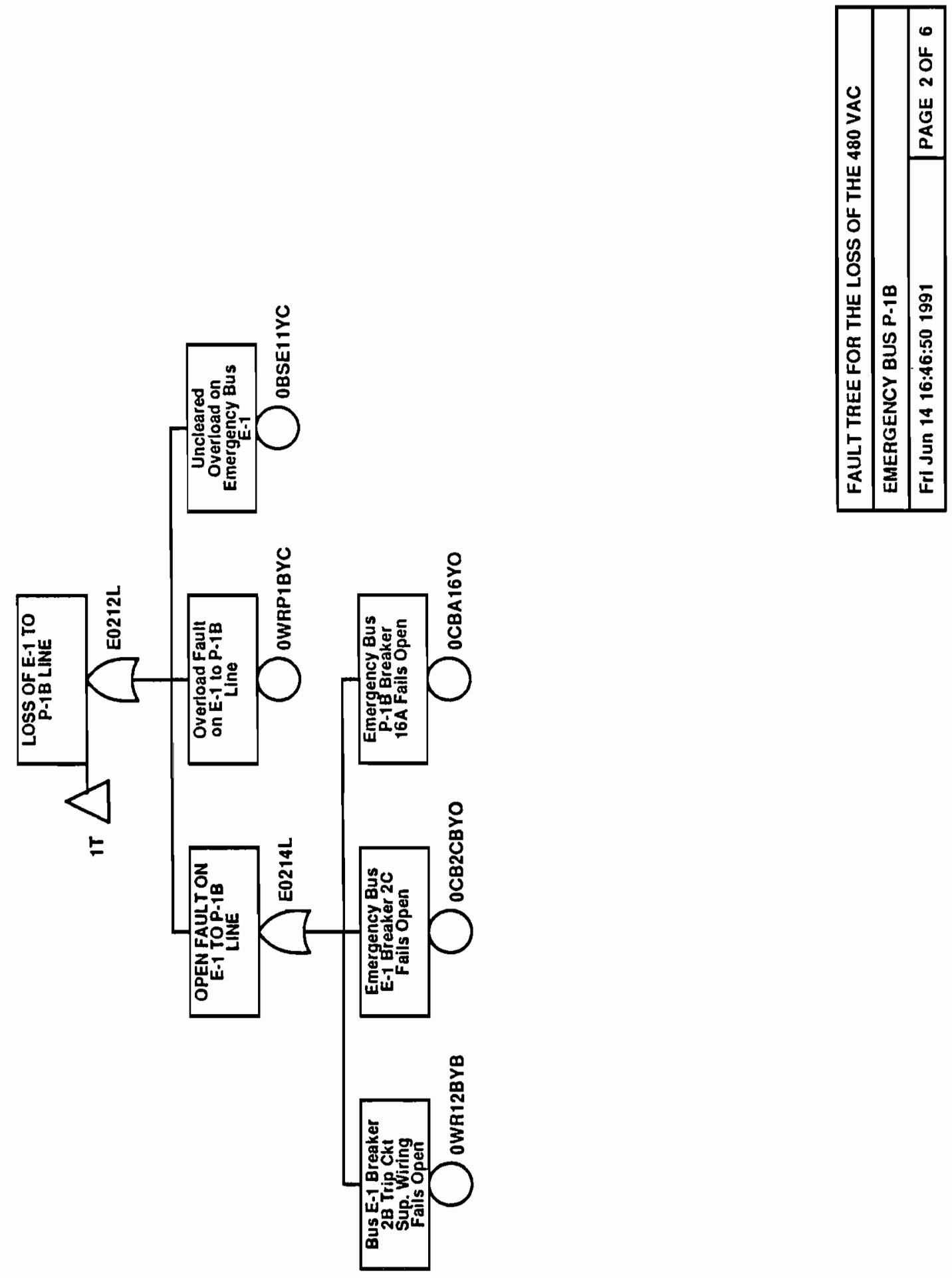

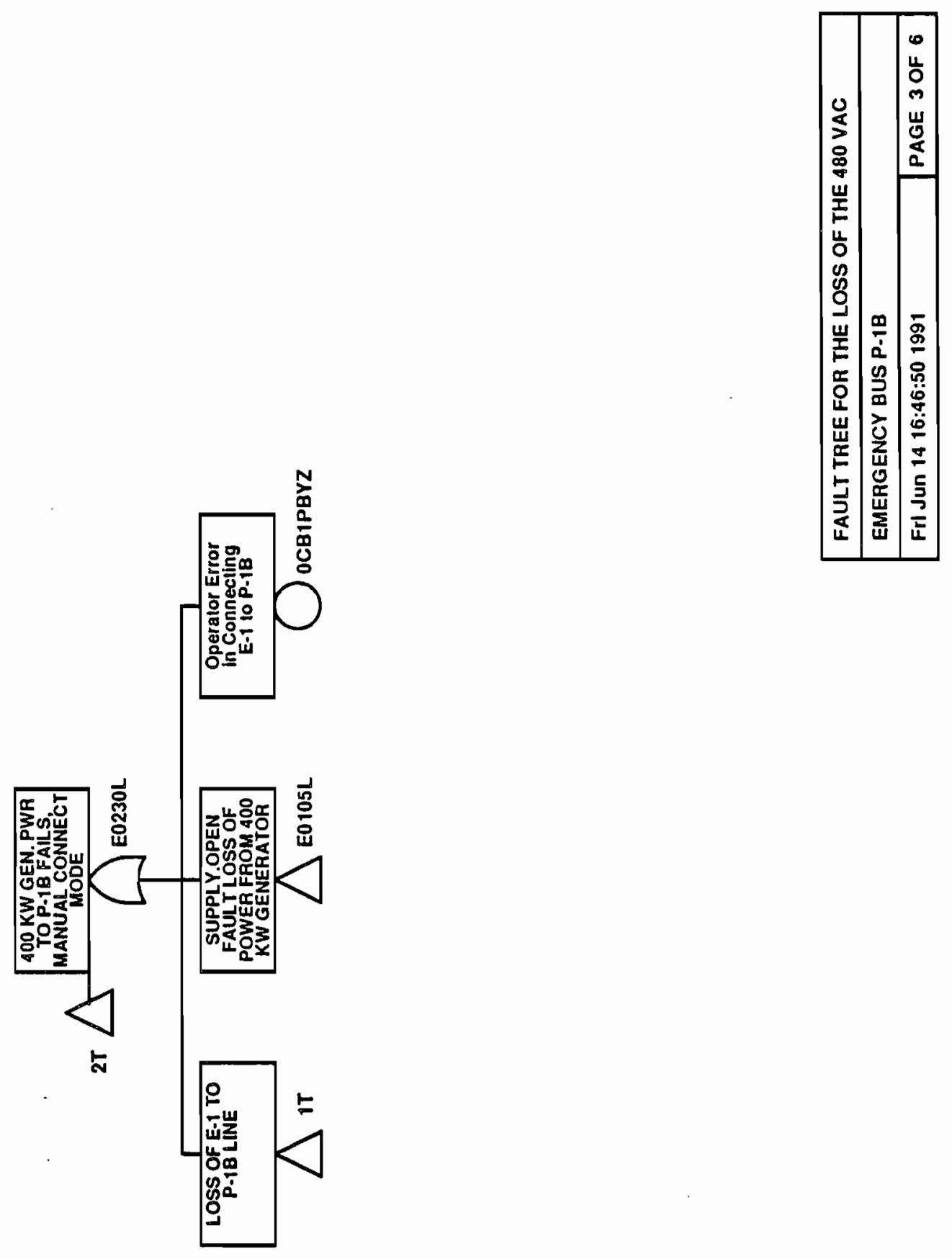

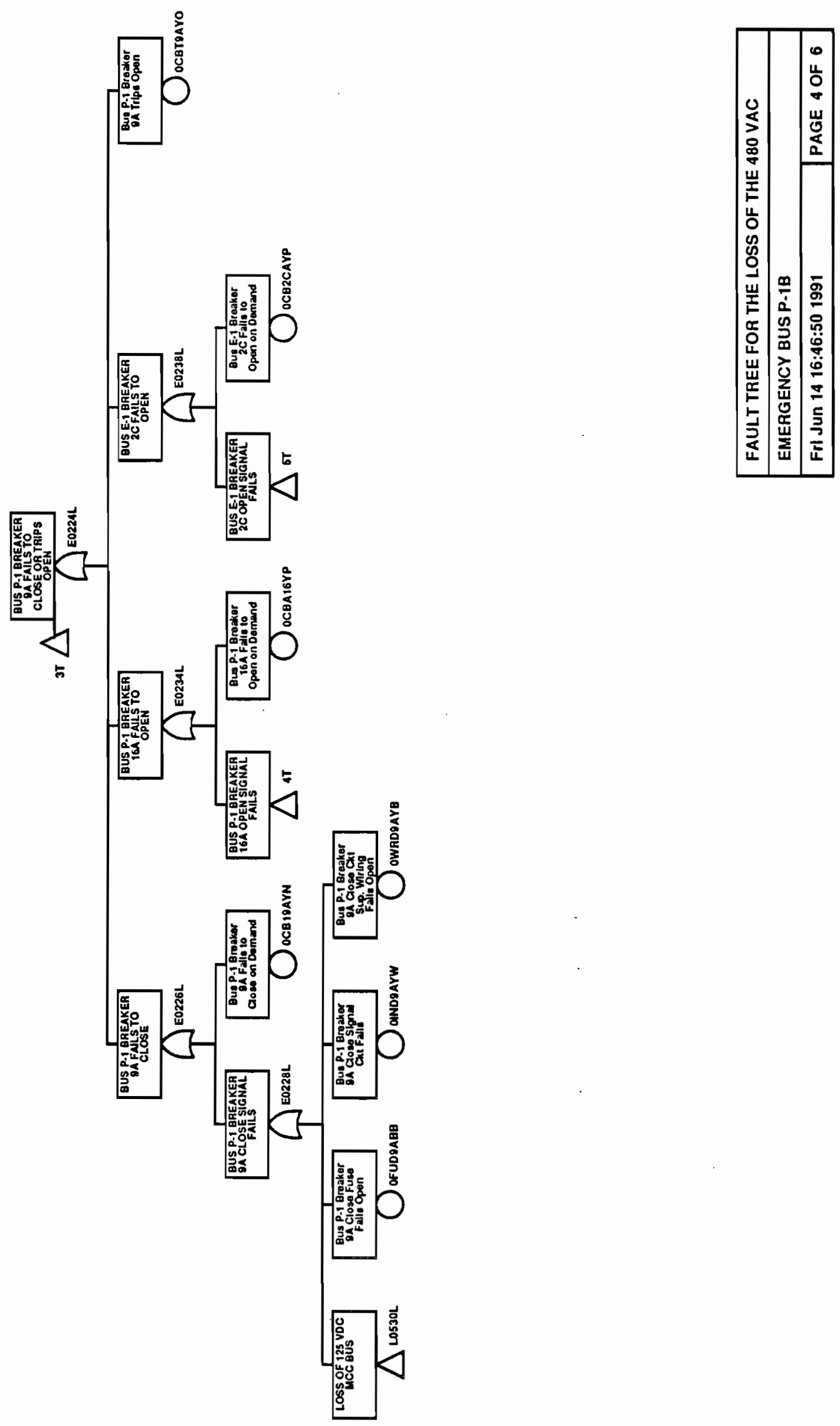

B. $2-445$ 


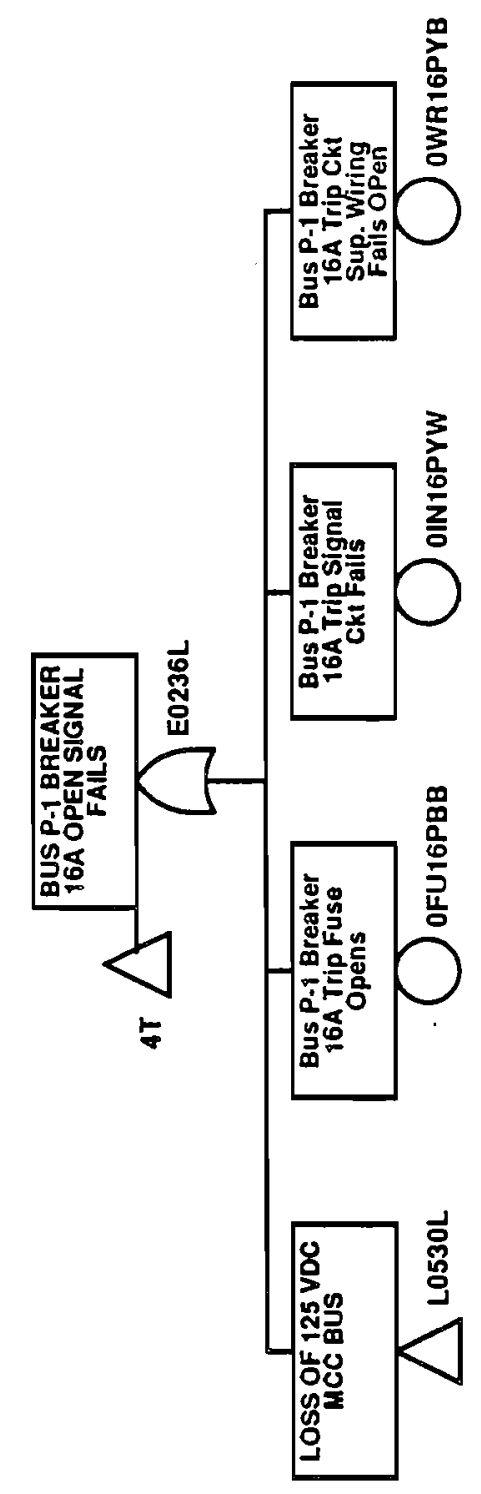

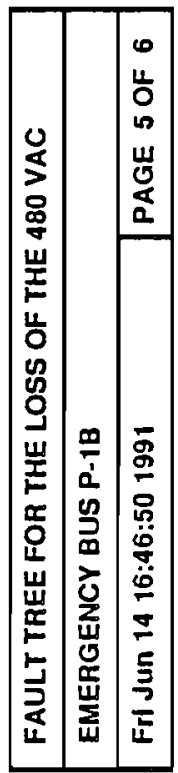



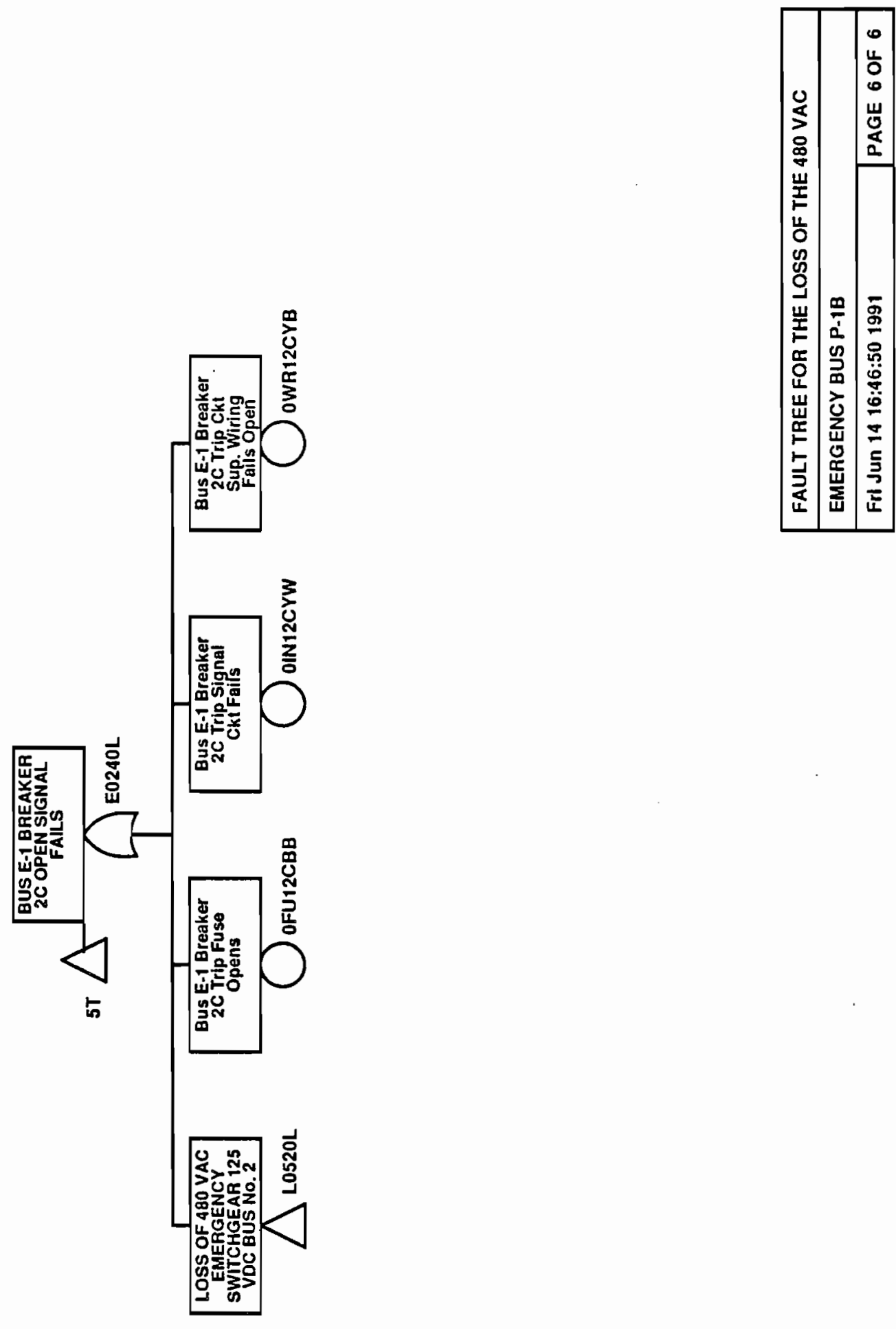


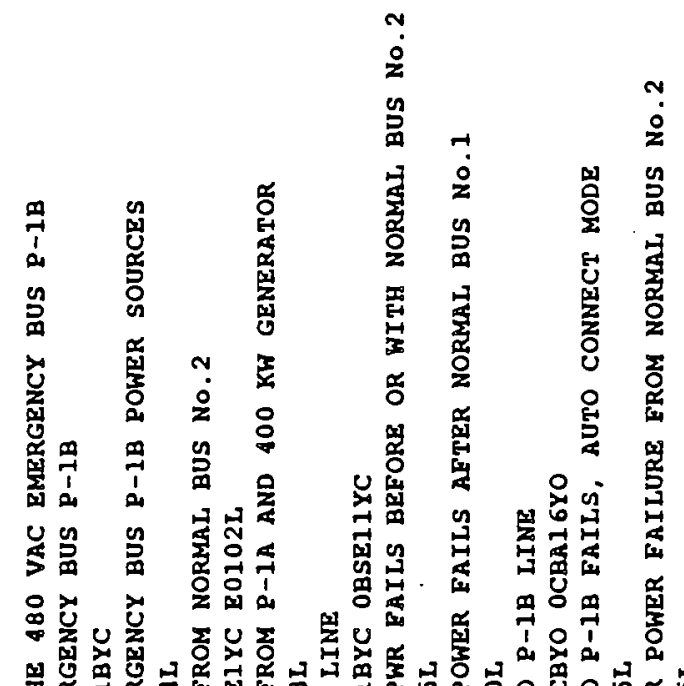

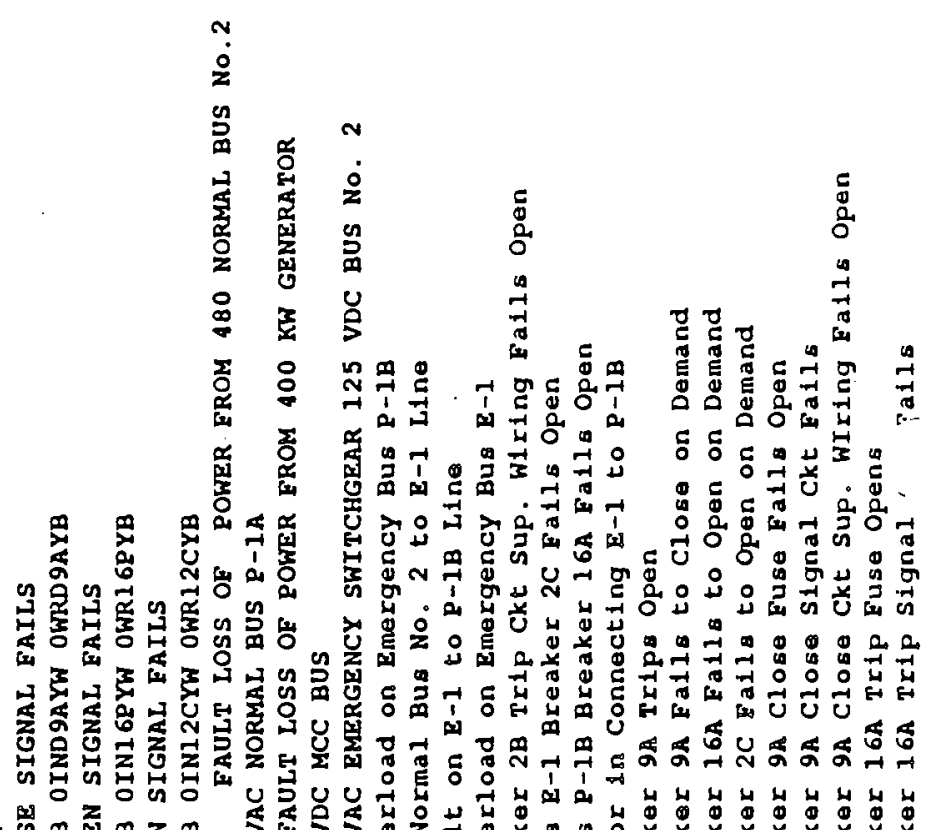

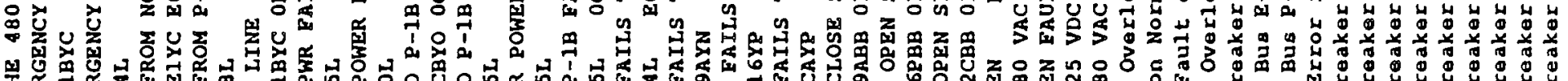

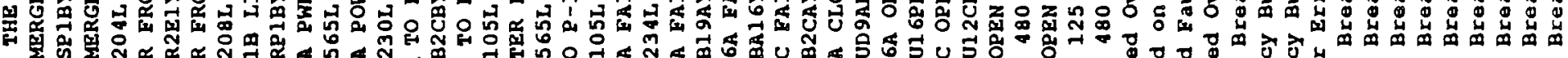

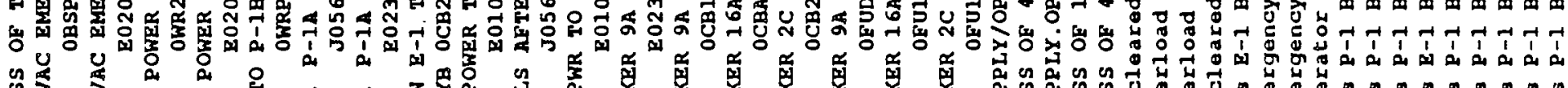

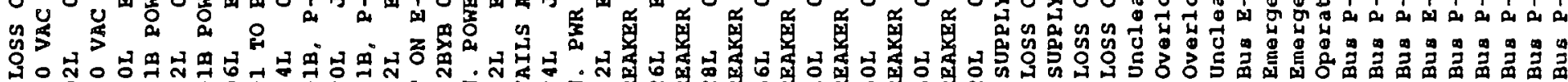

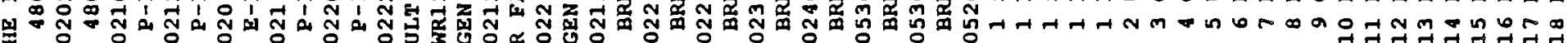

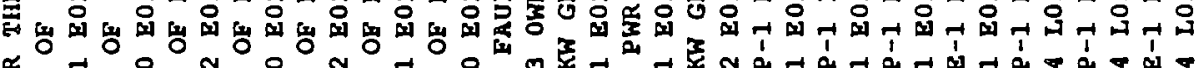
N

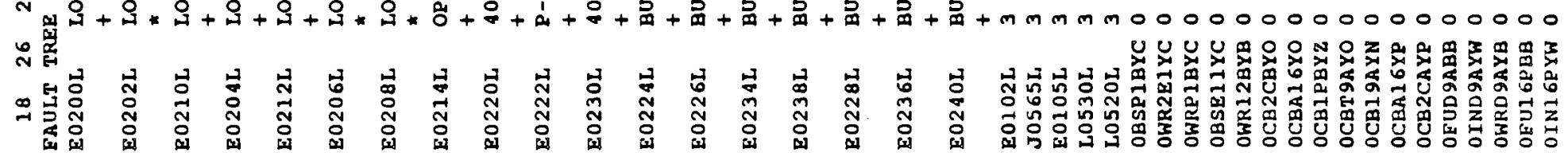




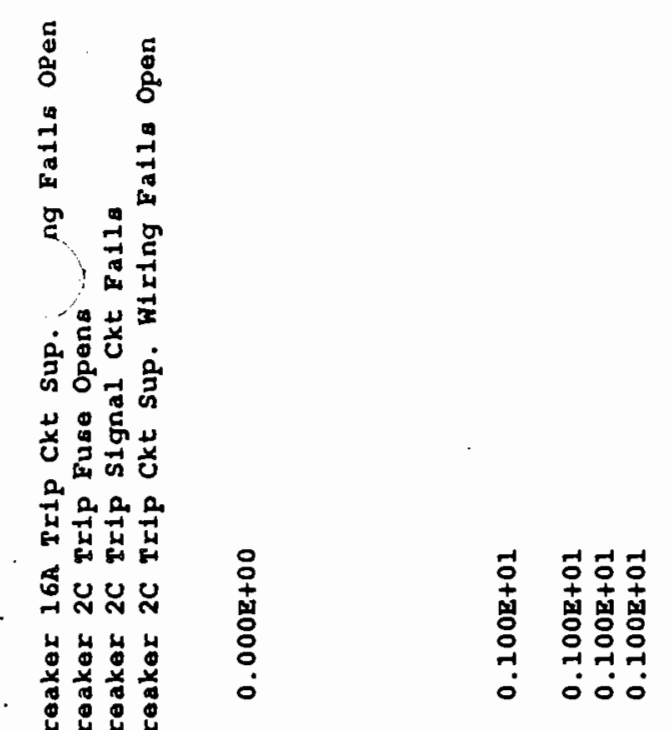

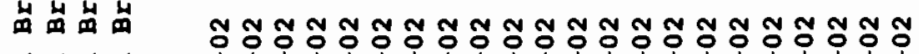

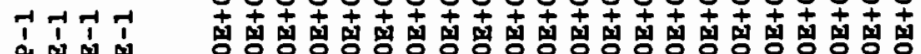

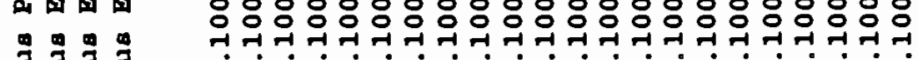

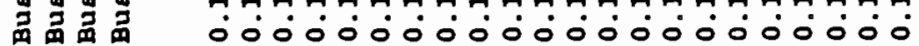
$\stackrel{-1}{\text { ก }} \underset{\text { N }}{ }$

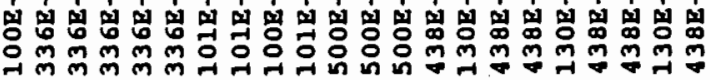
o 0 o o 0 o m

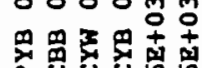

U Uु

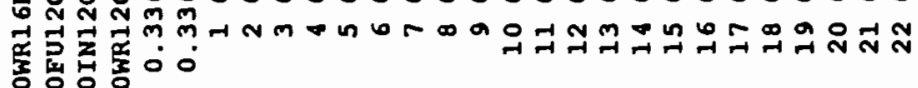




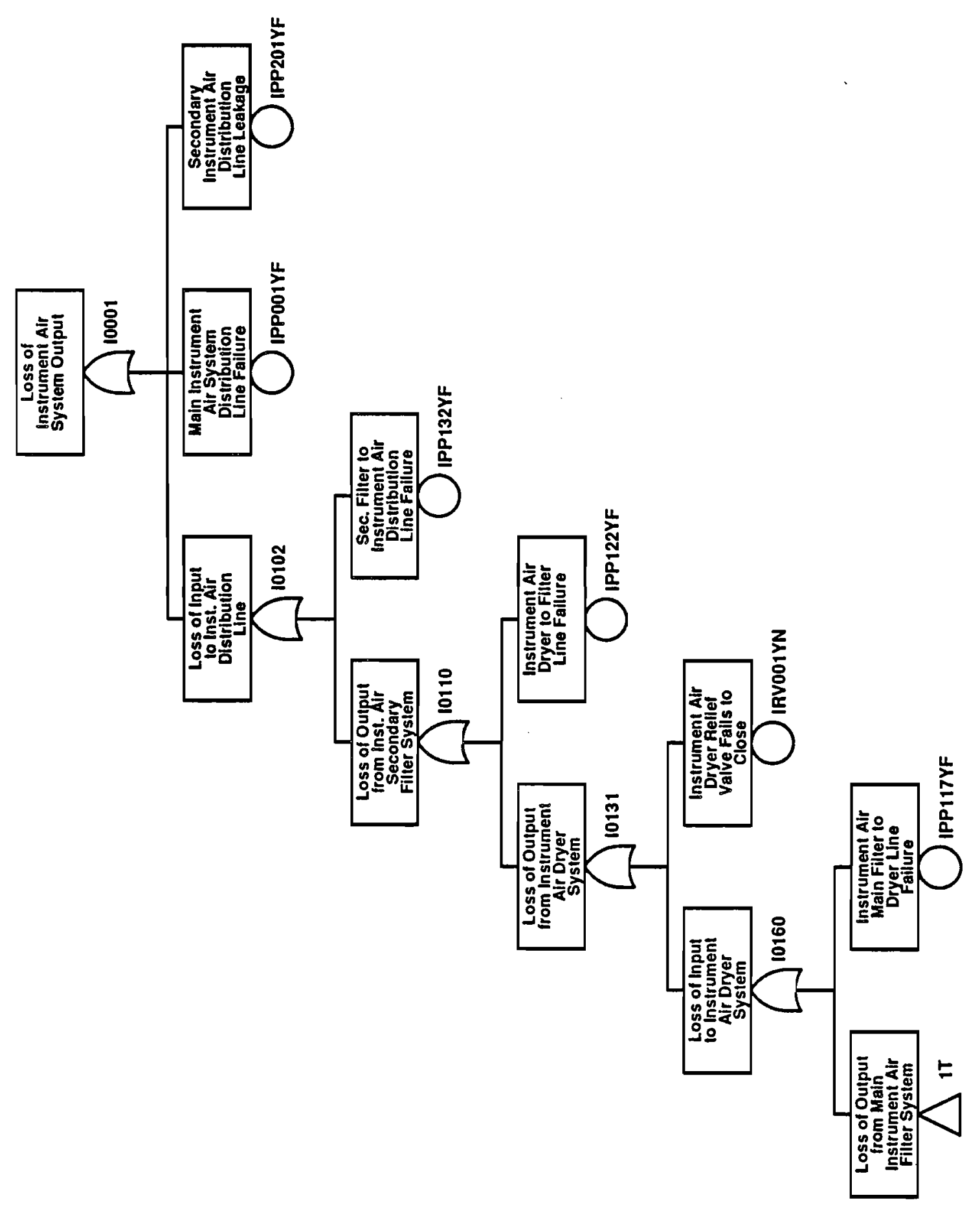



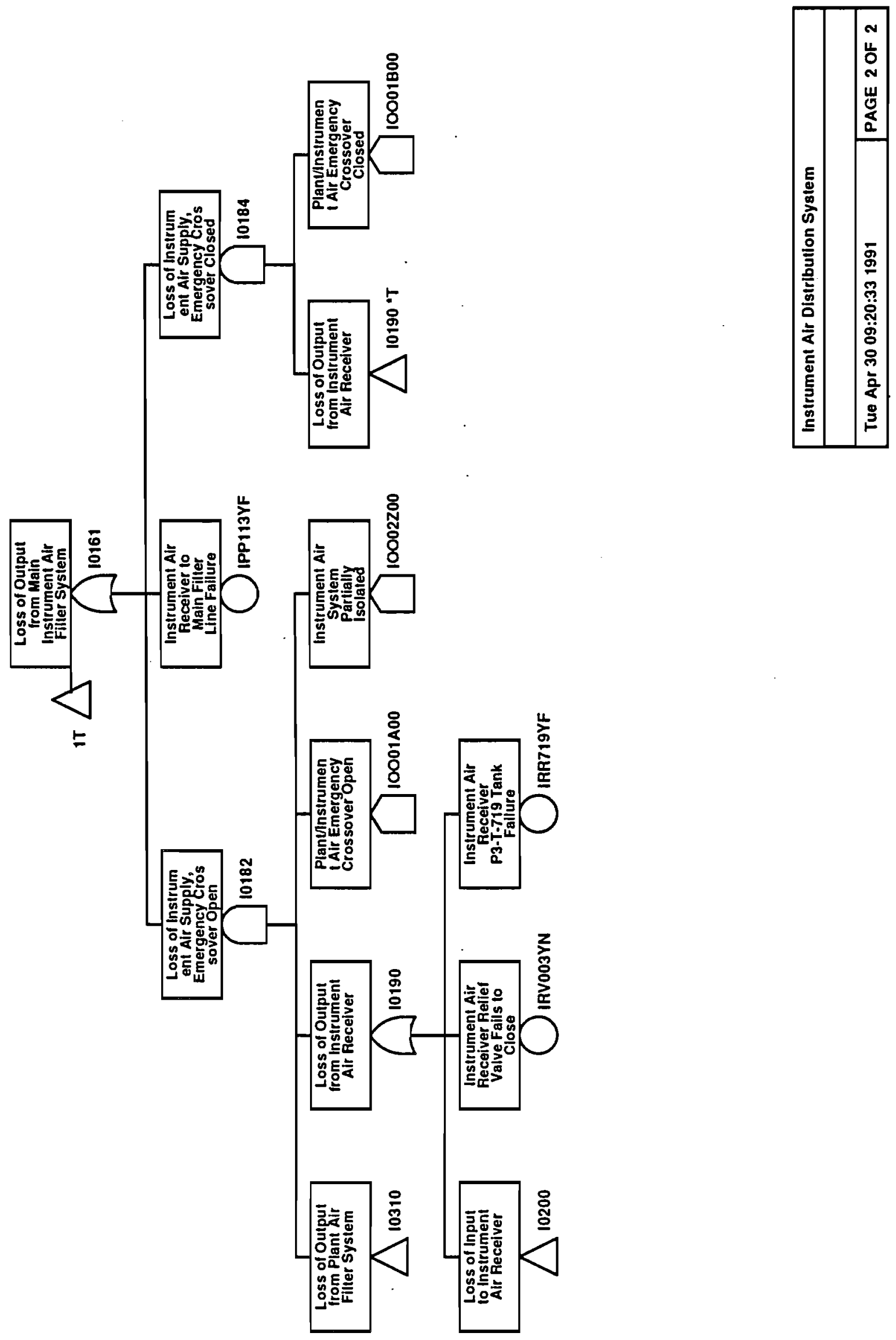

\section{B. 2-451}




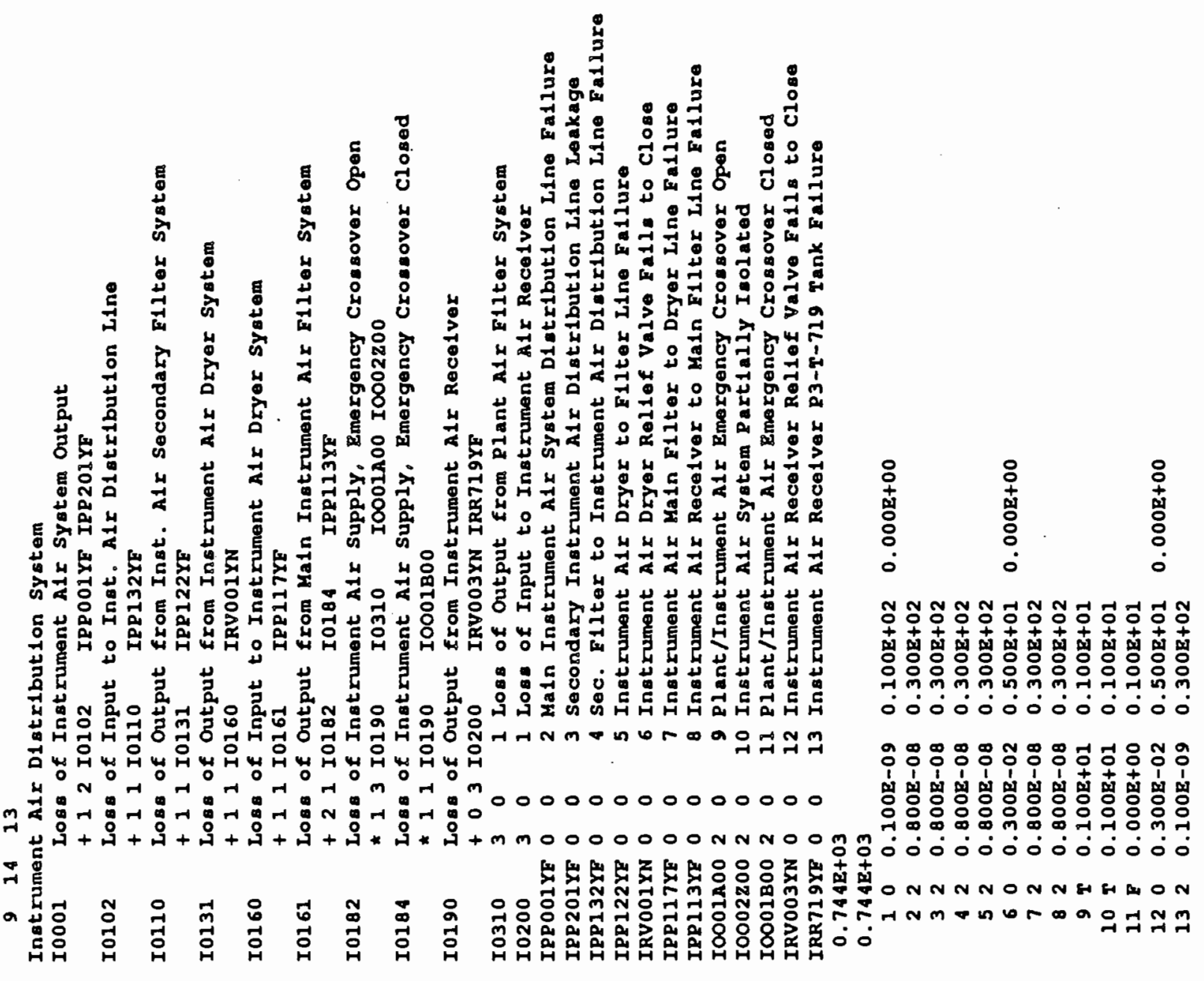

B. 2-452 


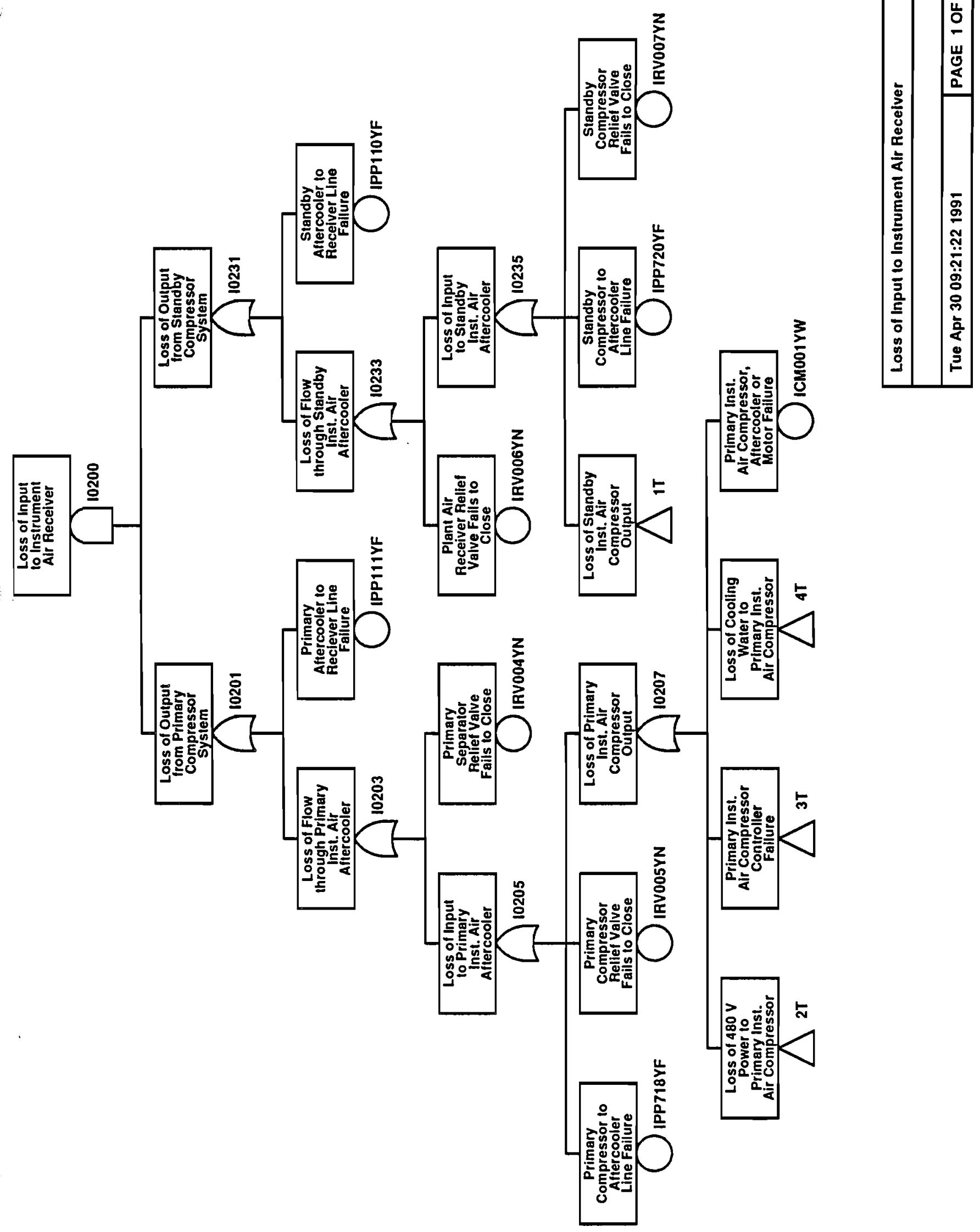




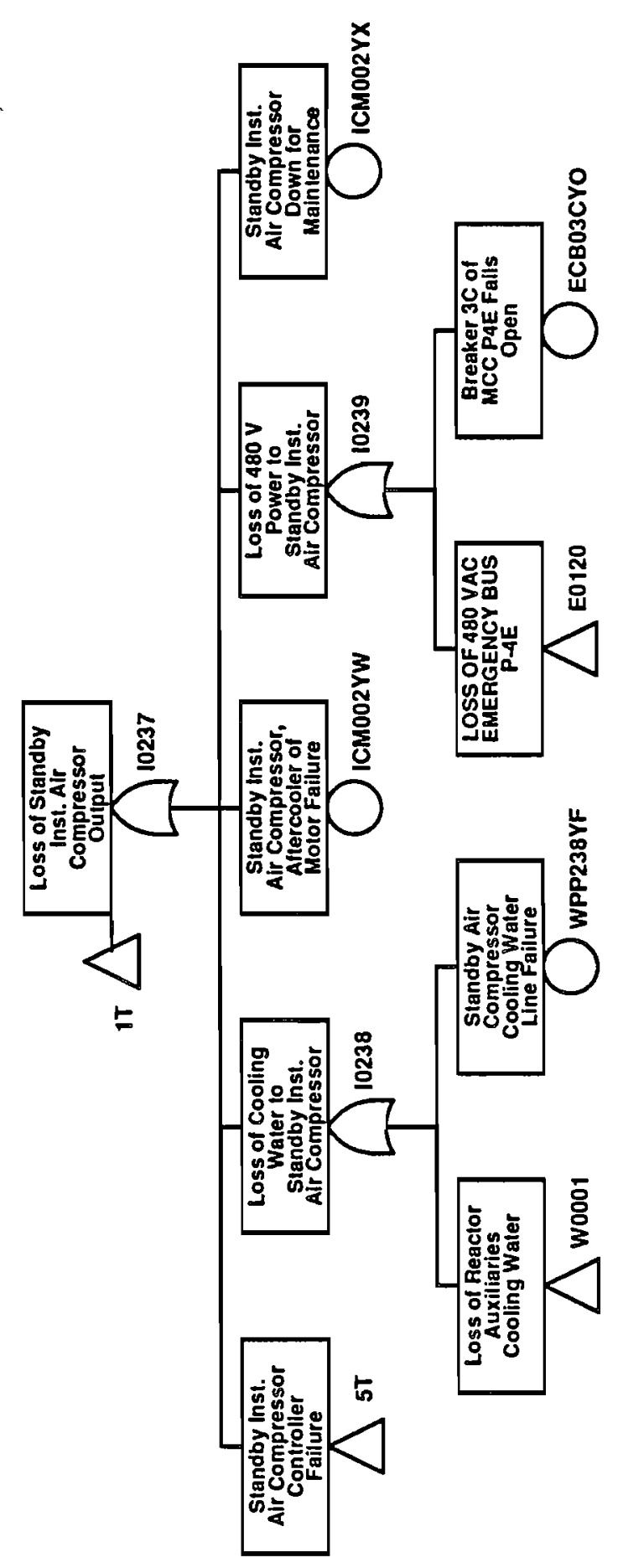

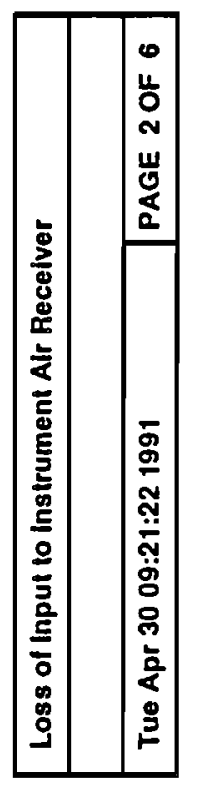



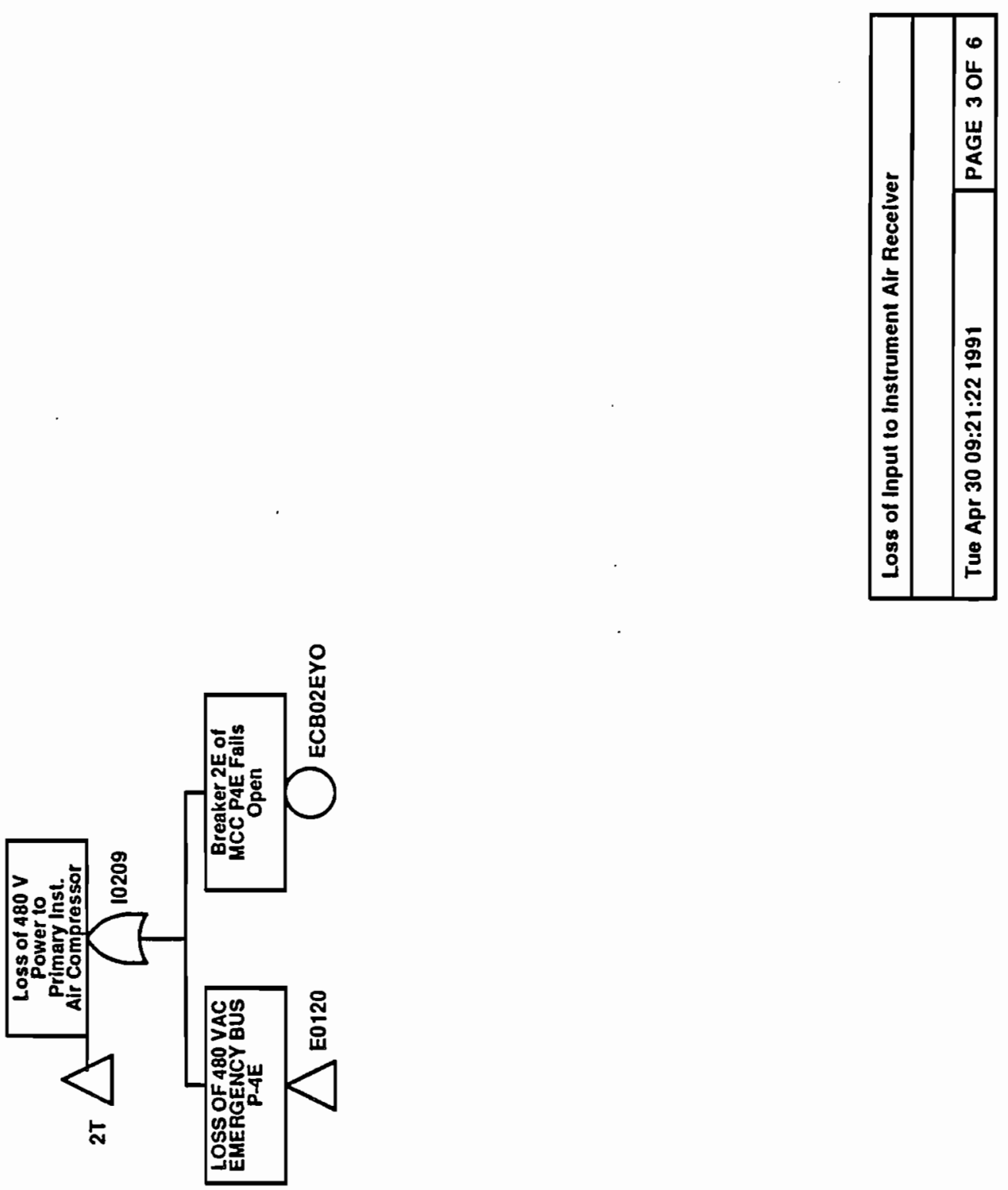


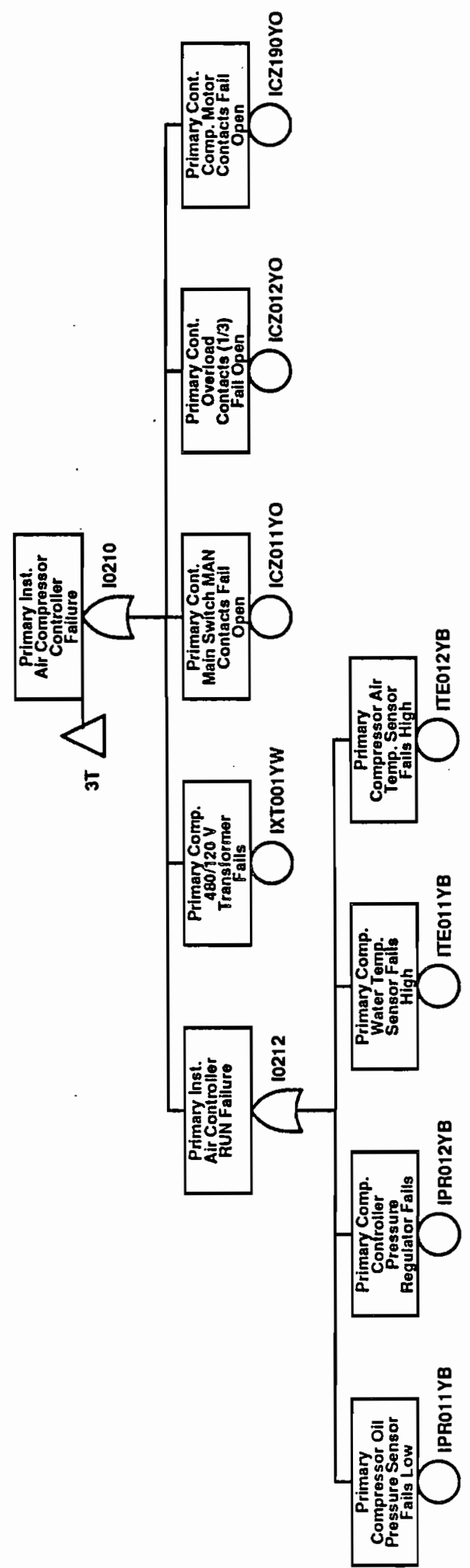

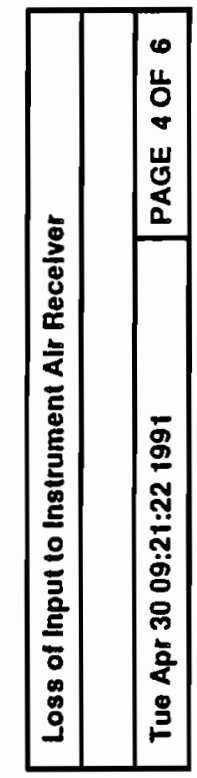

\section{B. $2-456$}




\begin{tabular}{|c|c|}
\hline$\stackrel{\circ}{0}$ & 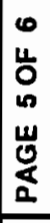 \\
\hline 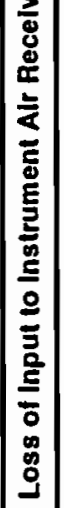 & 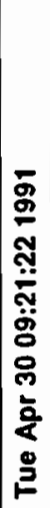 \\
\hline
\end{tabular}

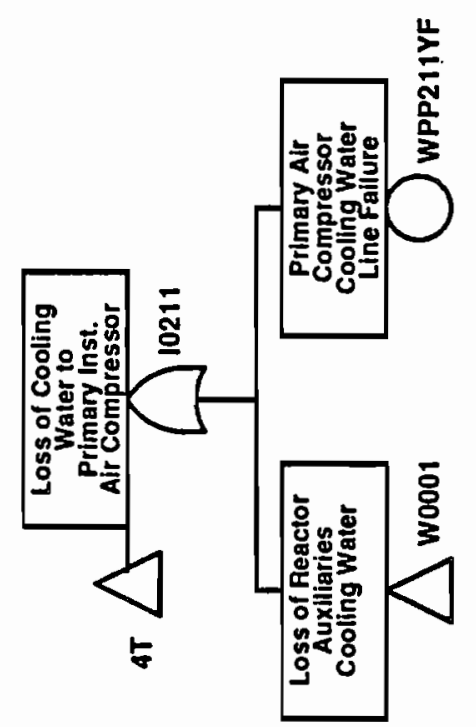




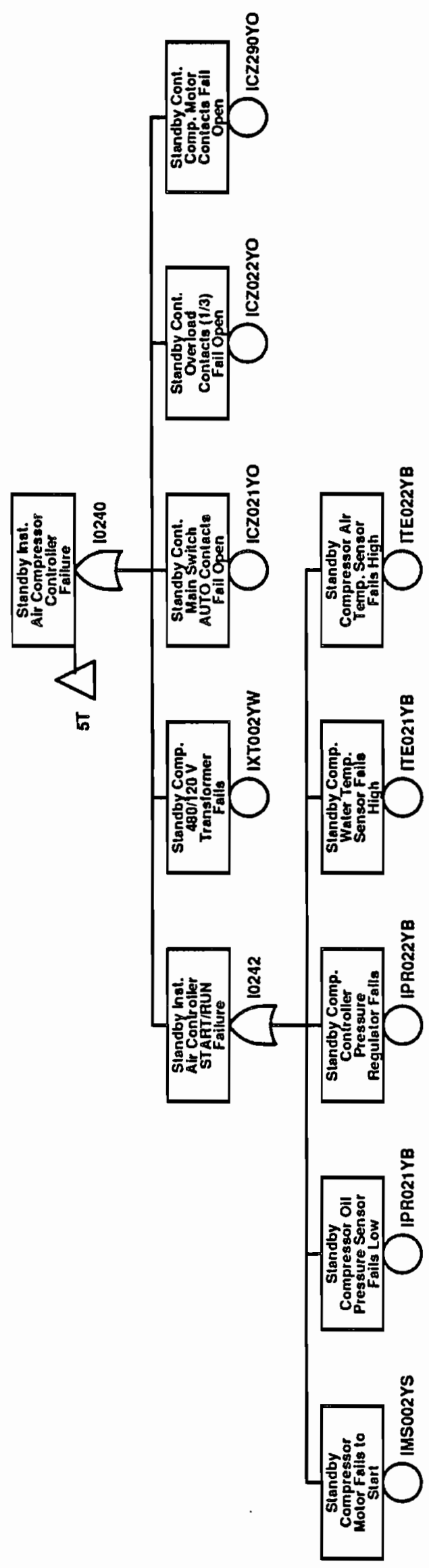

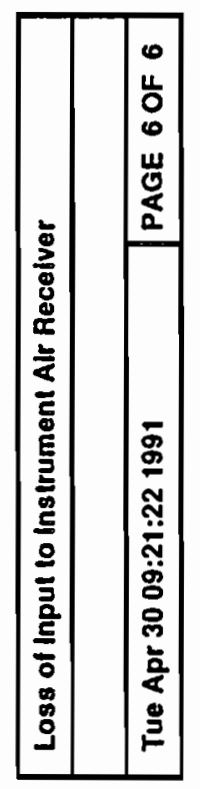

\section{B. $2-458$}




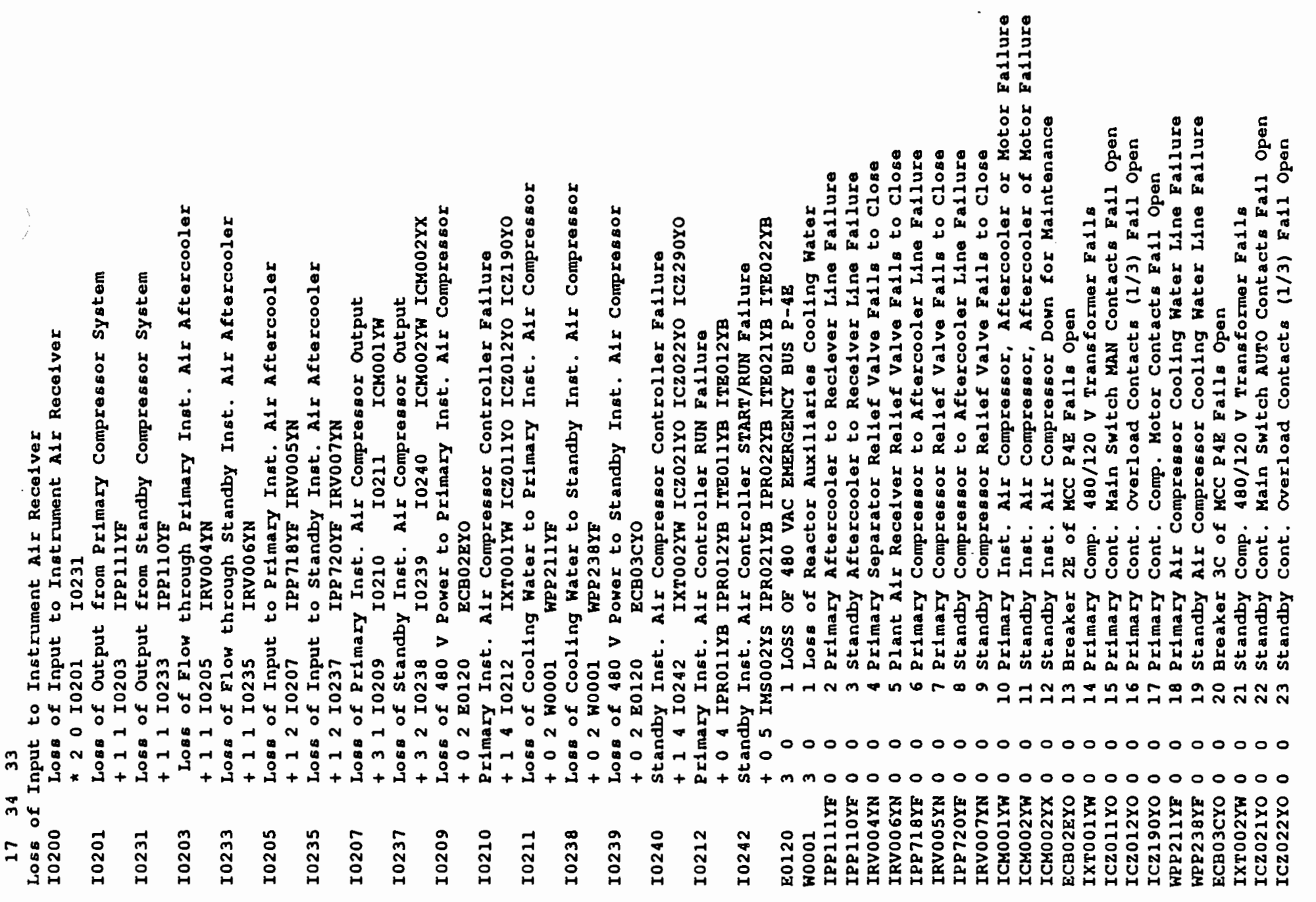



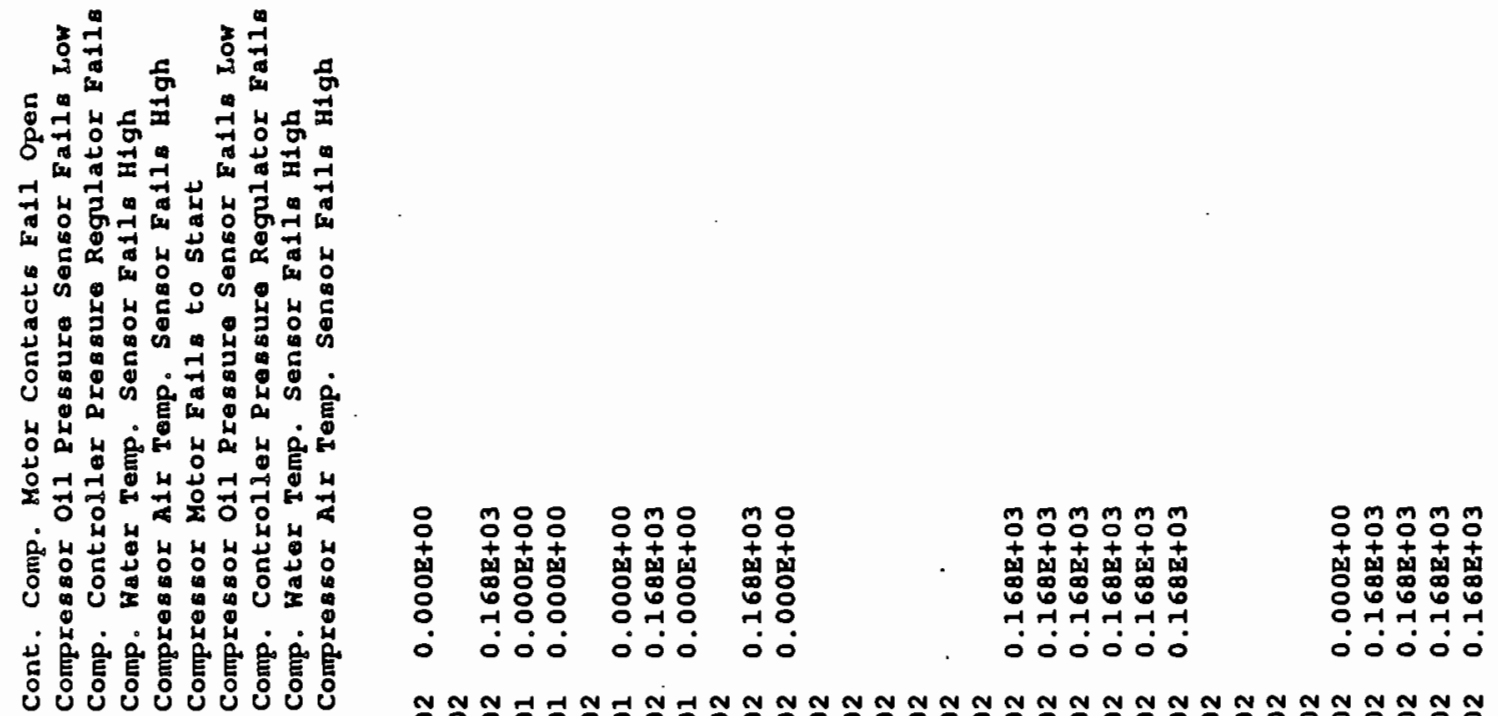
(1)

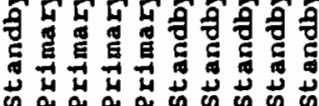

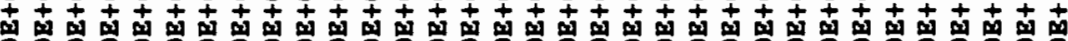

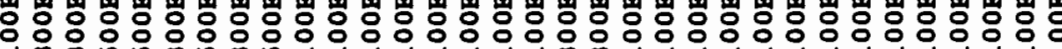

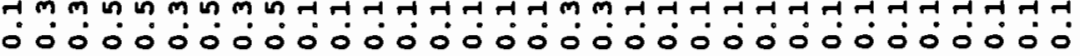

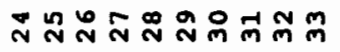

0000000000

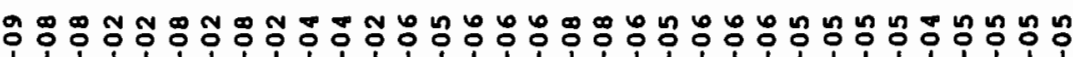

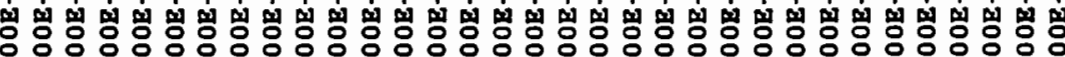

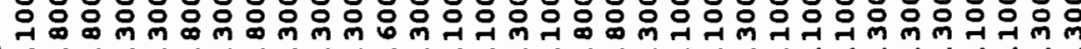

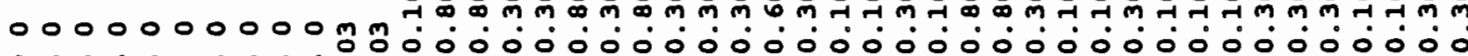

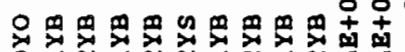

旅 N

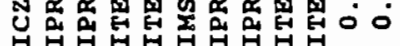




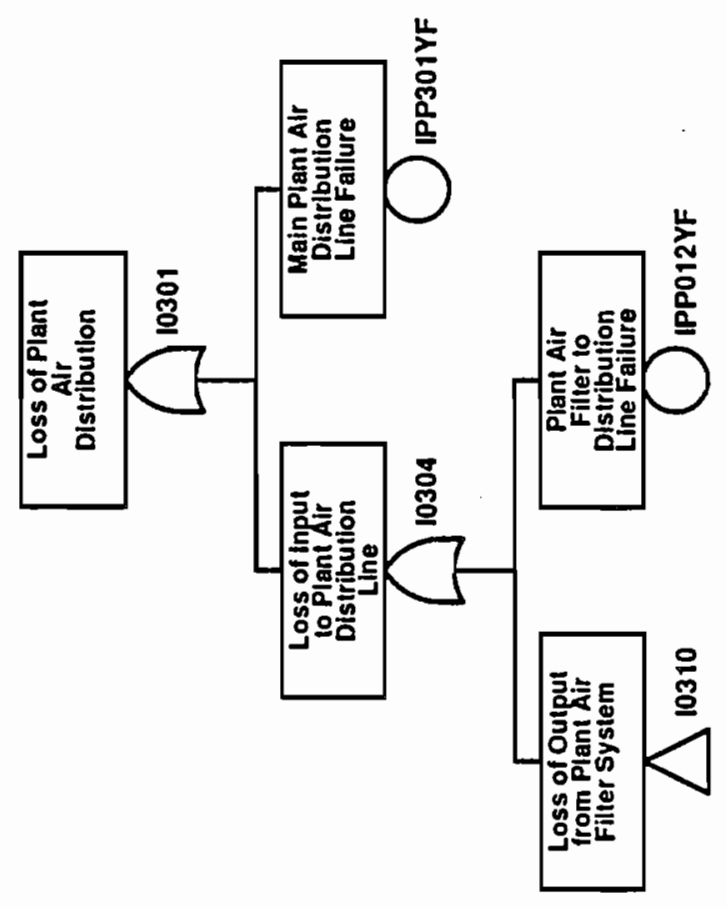




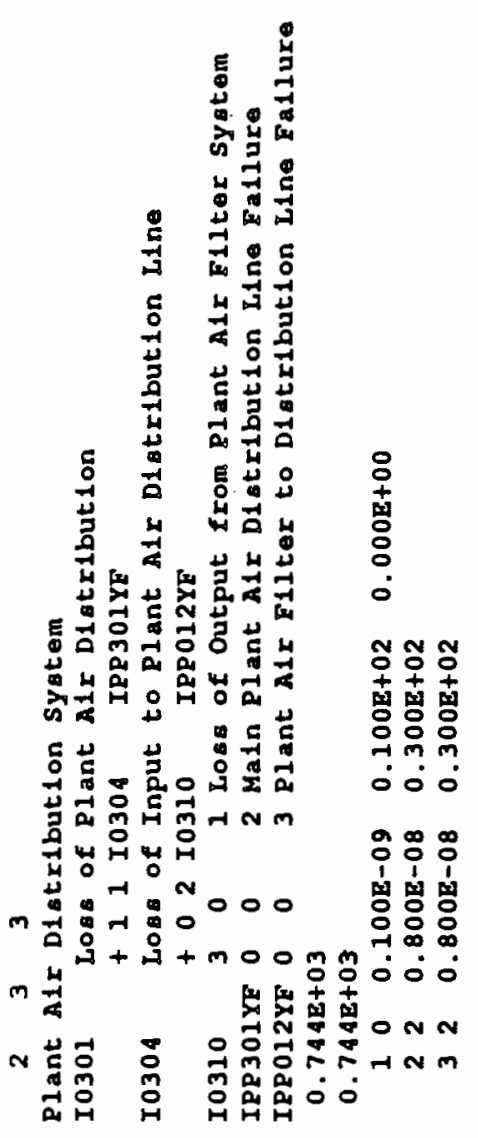

B. 2-462 


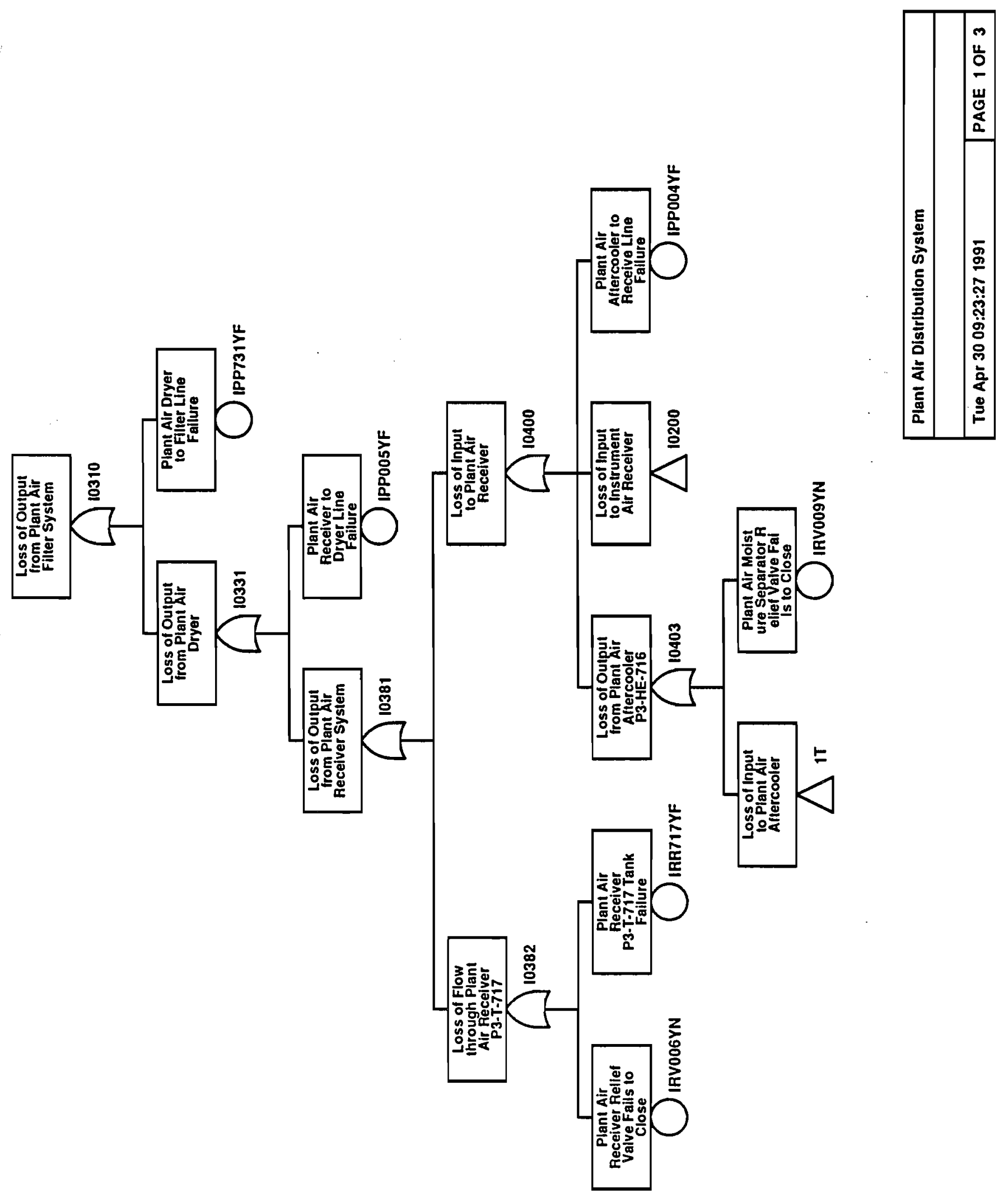



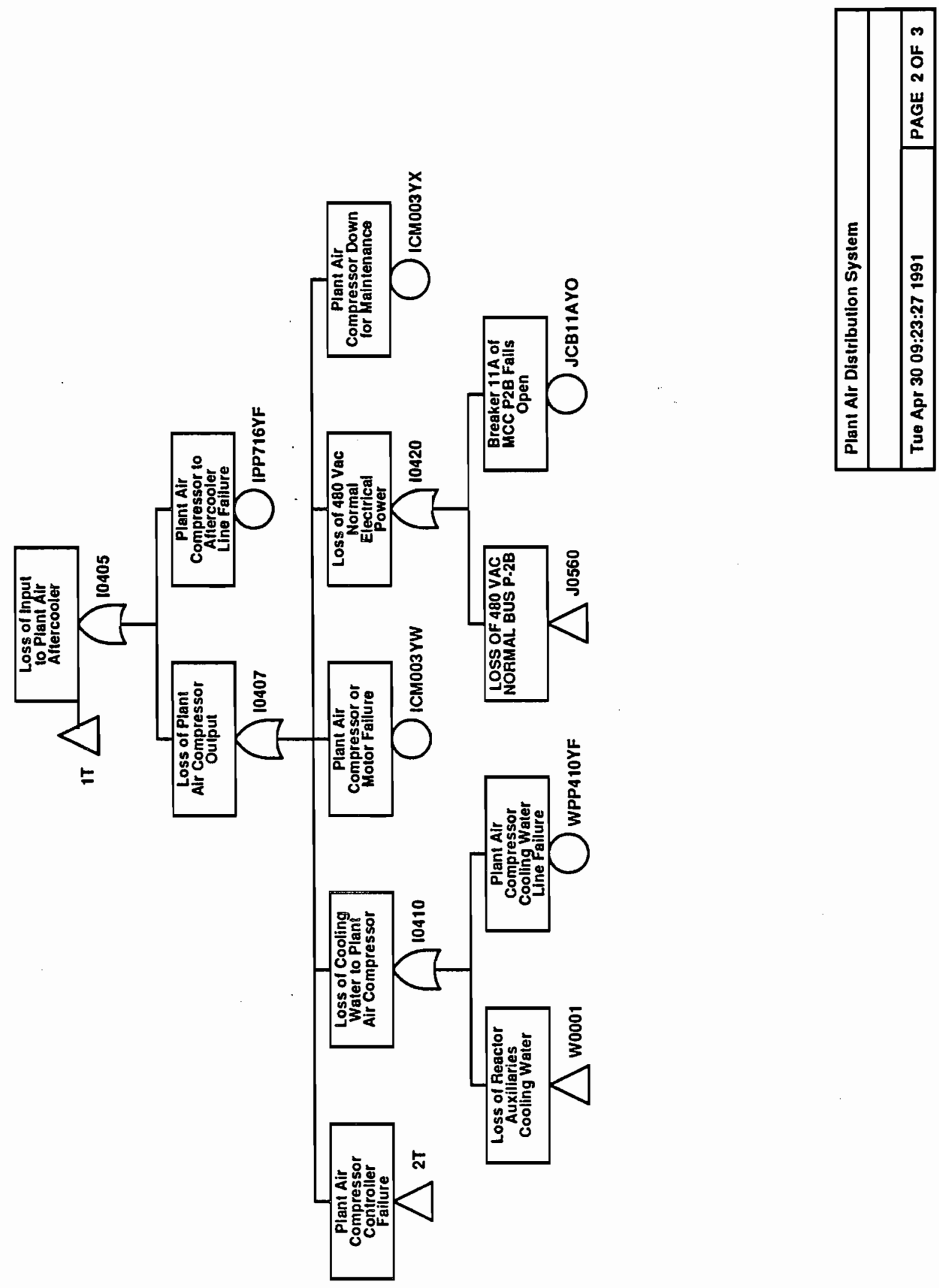

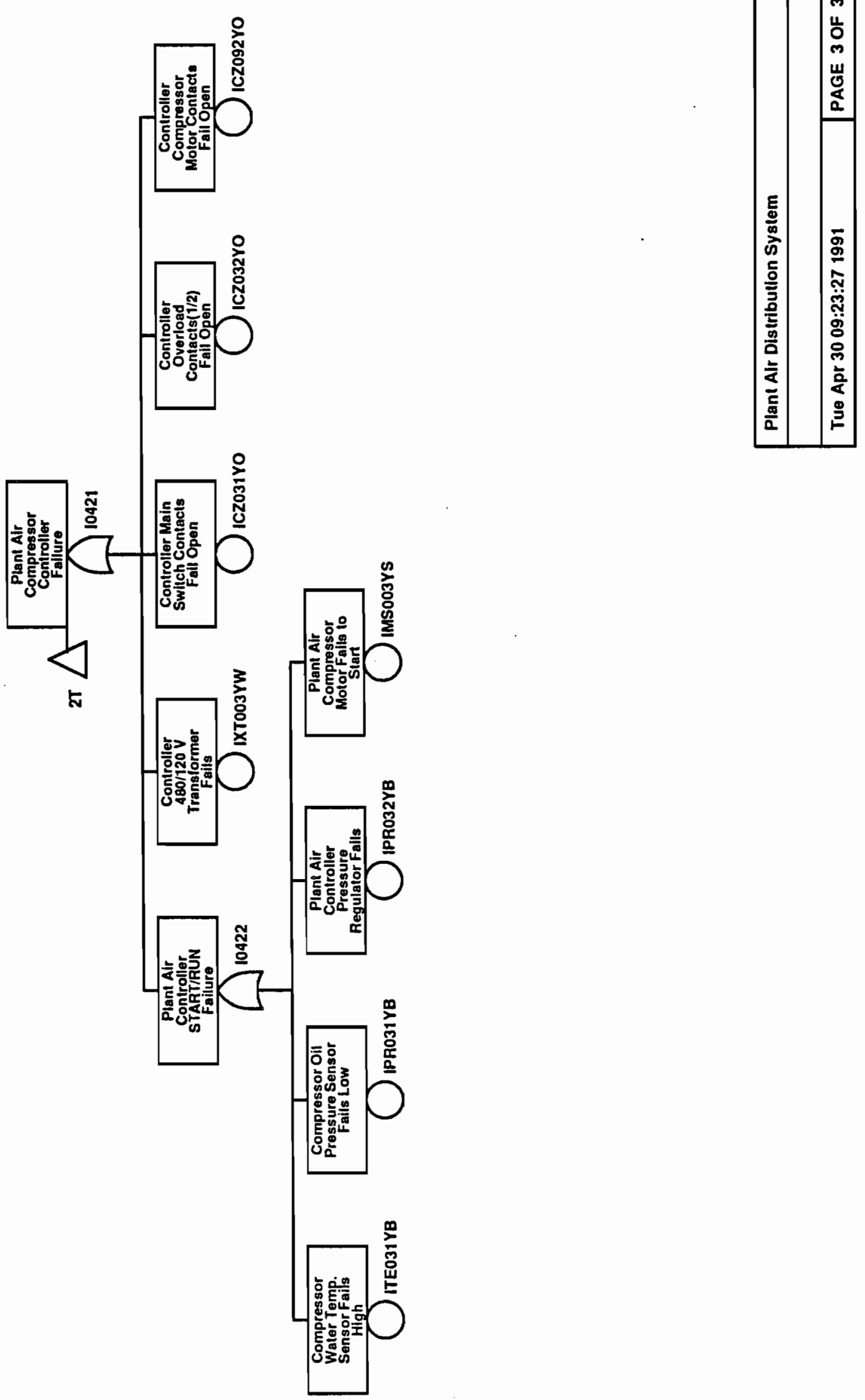

B. 2-465 


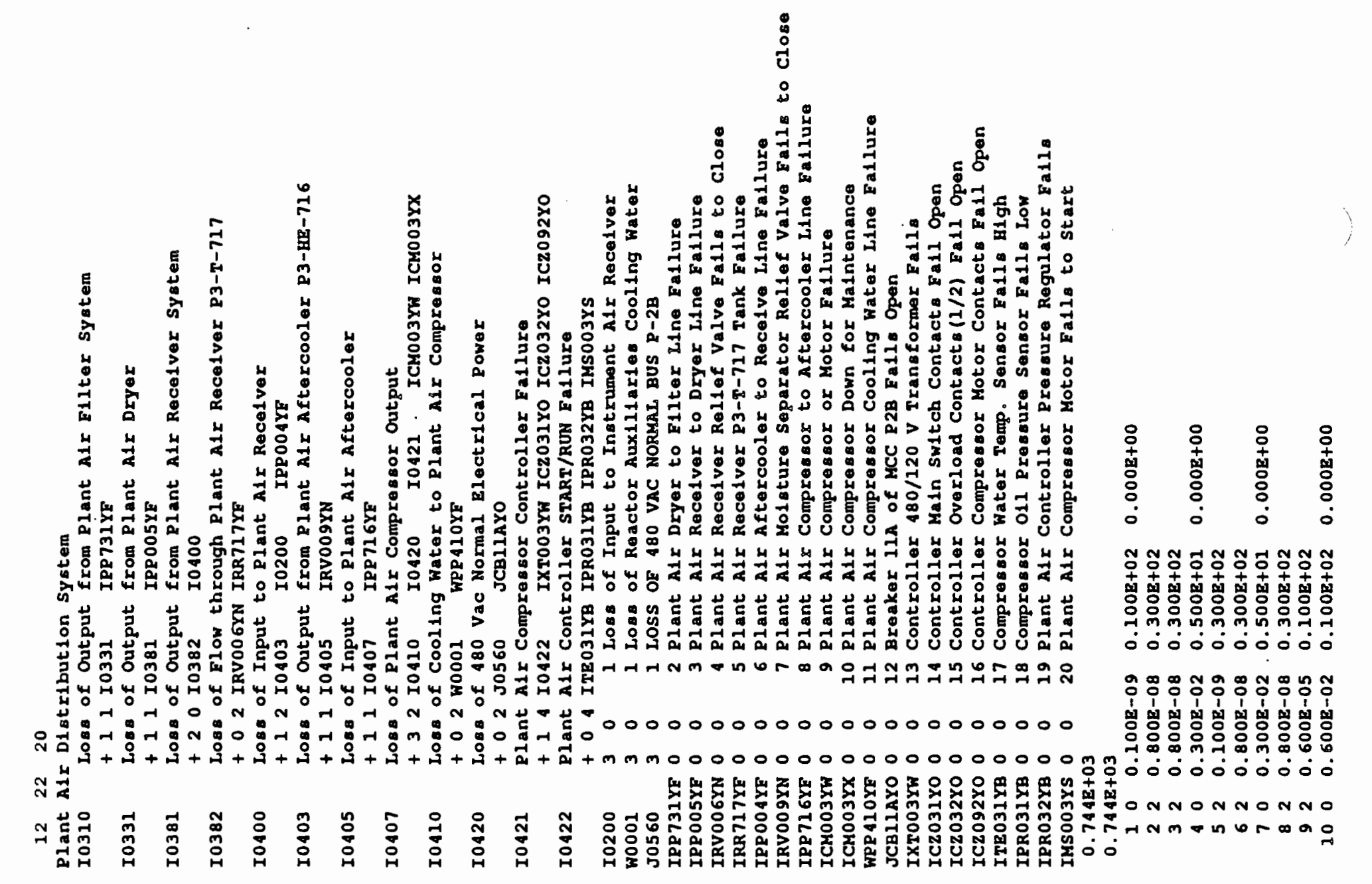




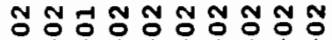

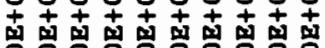

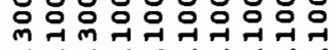

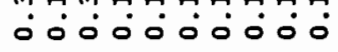

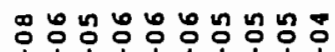

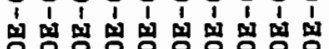

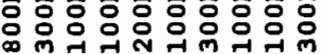
00000000

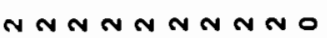

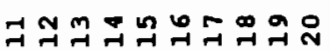




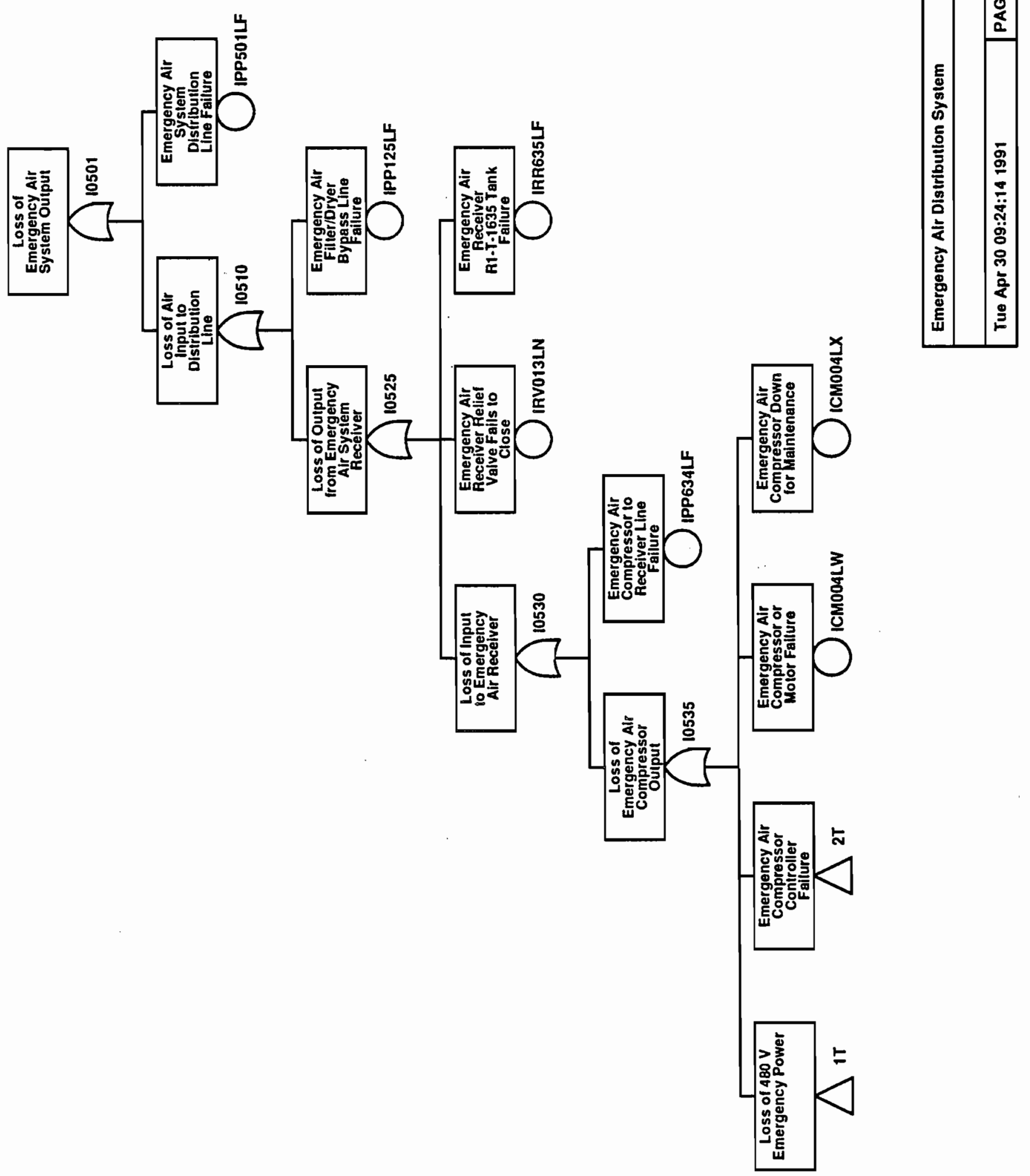



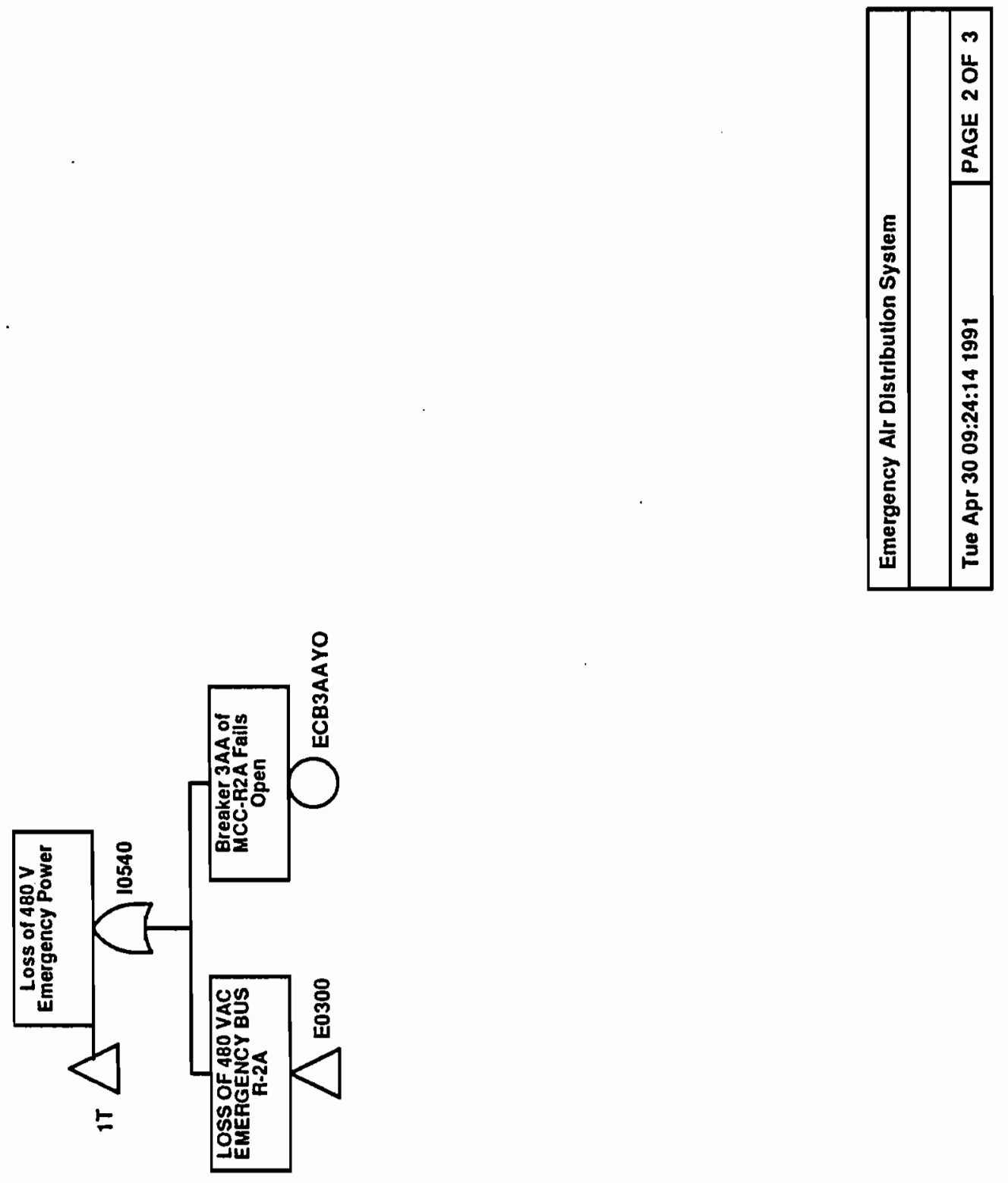


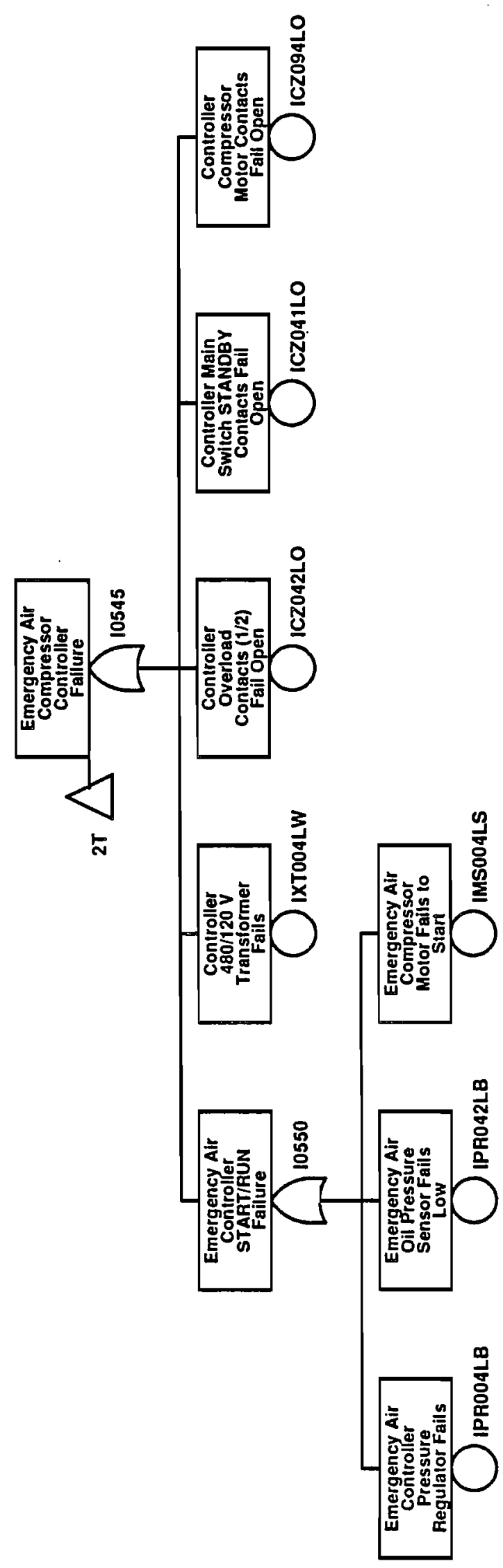

\begin{tabular}{|c|c|}
\hline & 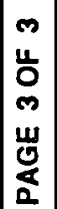 \\
\hline 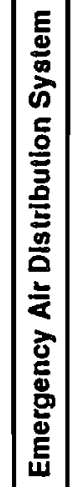 & 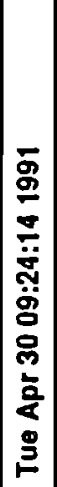 \\
\hline
\end{tabular}

B. $2-470$ 


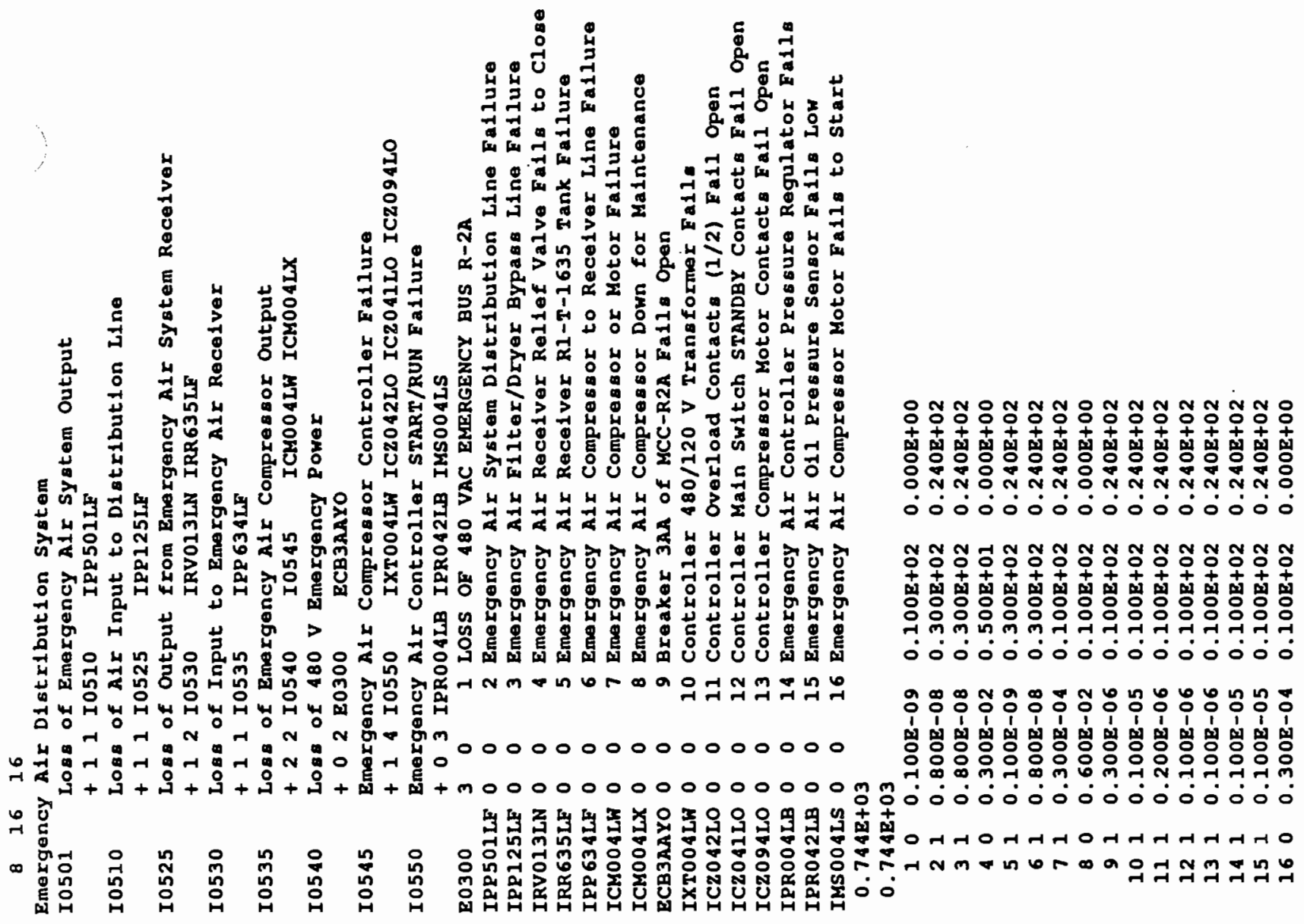



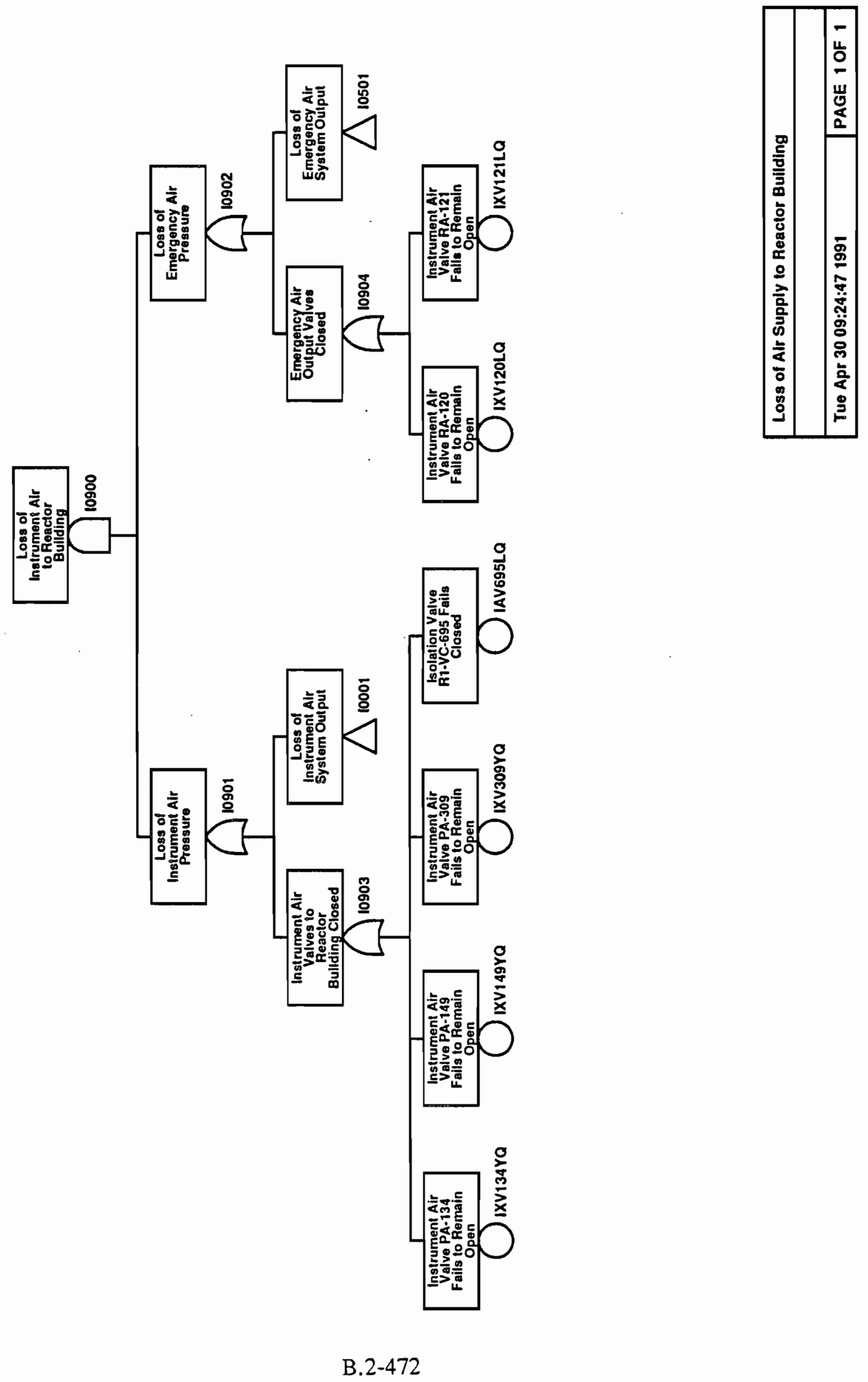


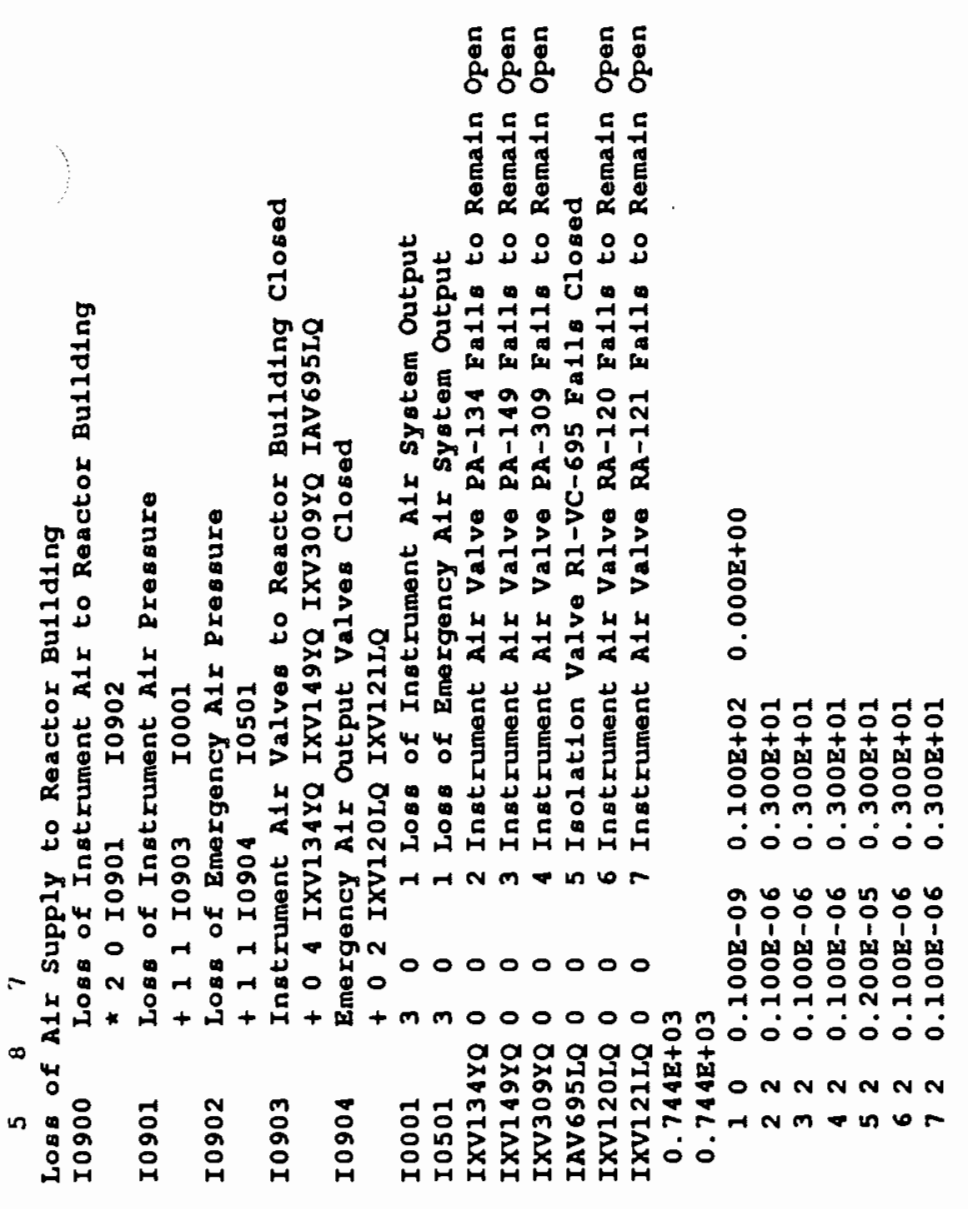

B. 2-473 


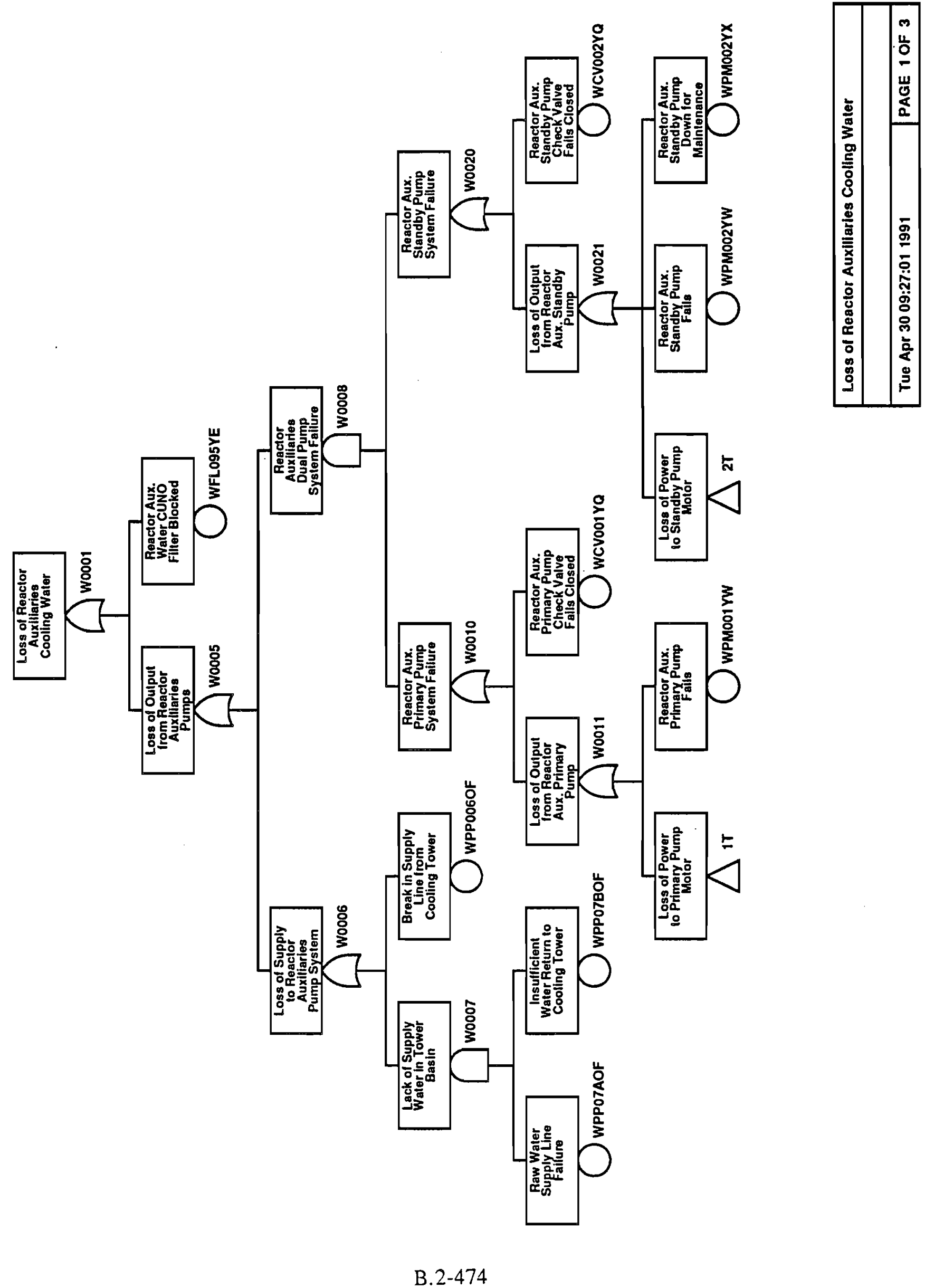



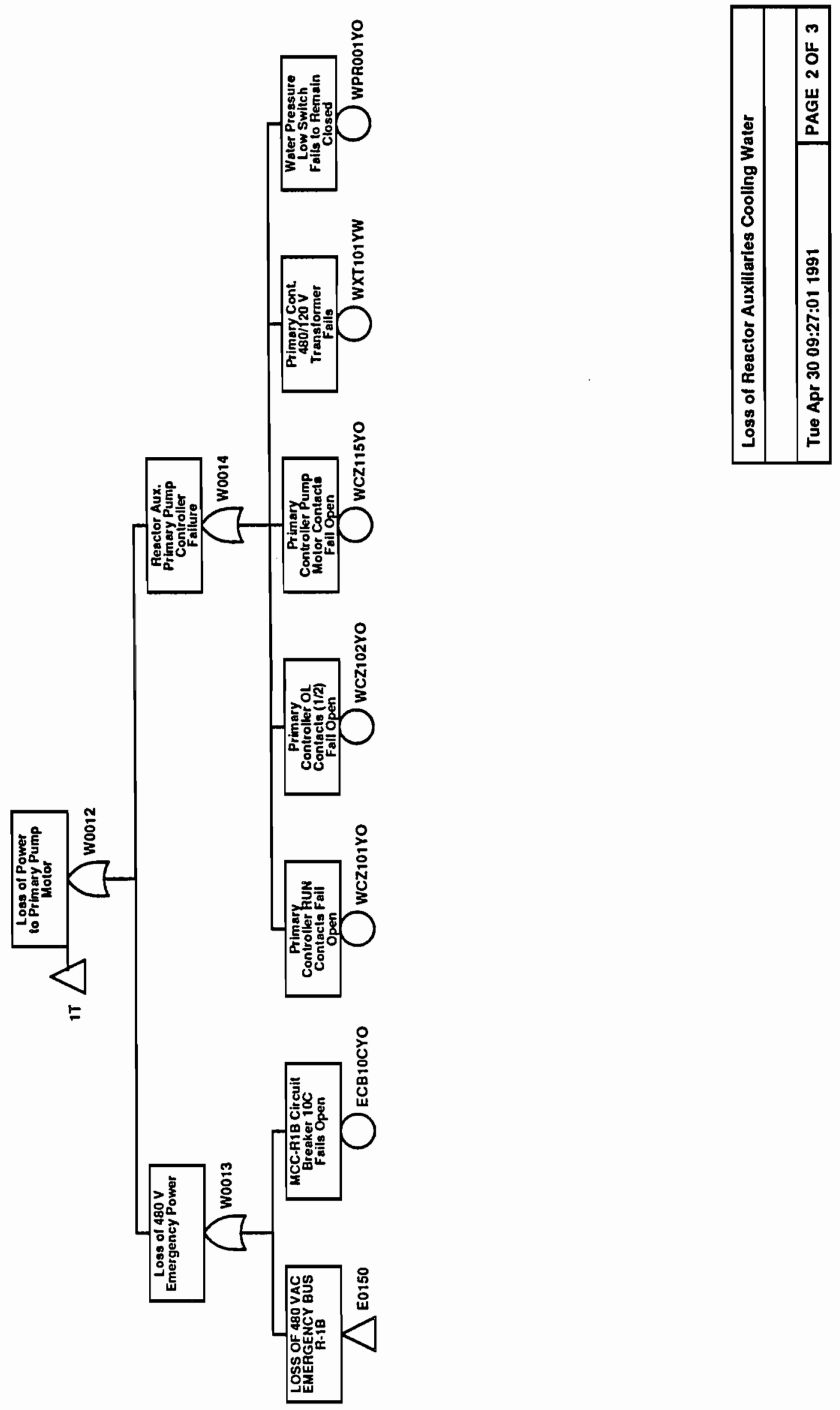

B. $2-475$ 

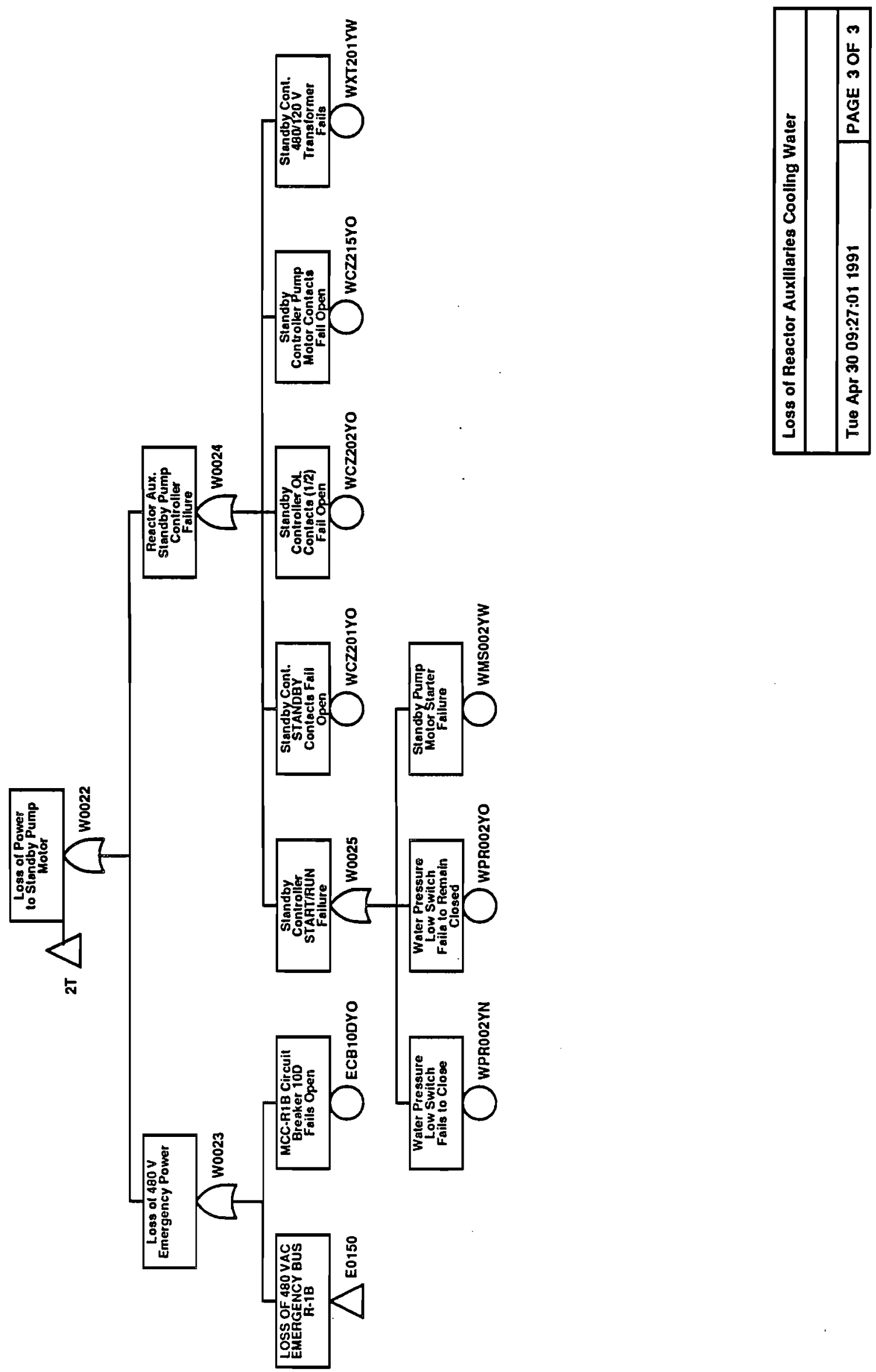

\section{B. $2-476$}




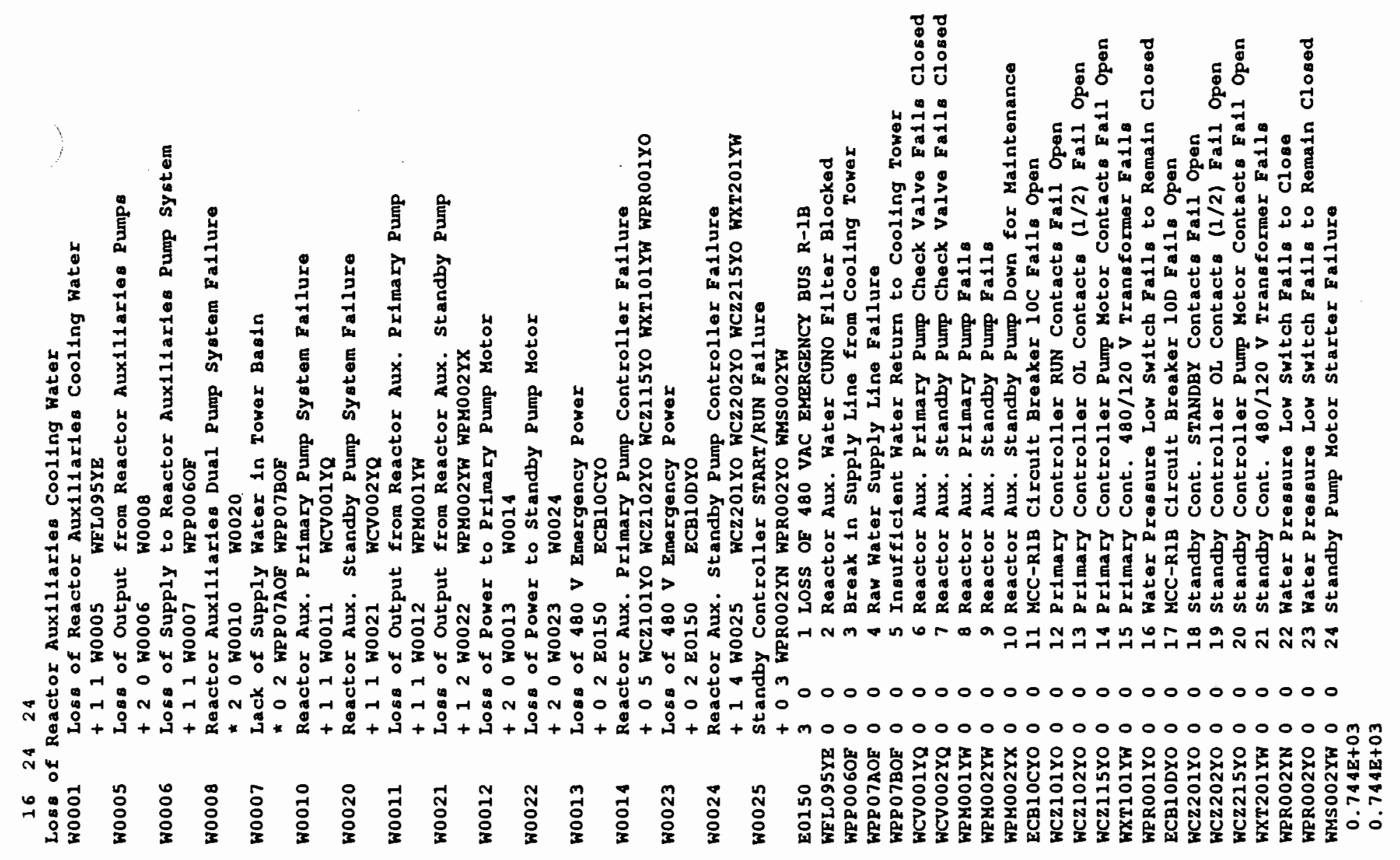




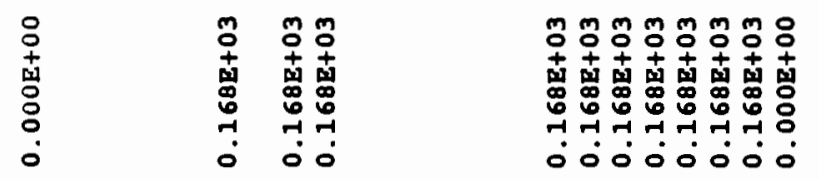

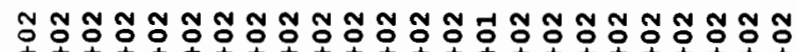

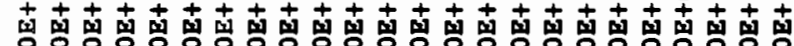
응ㅇㅇㅇㅇㅇㅇㅇㅇㅇㅇㅇㅇㅇㅇㅇㅇㅇㅇㅇㅇㅇㅇㅇㅇㅇㅇㅇㅇㅇㅇㅇㅇㅇㅇㅇㅇㅇㅇ

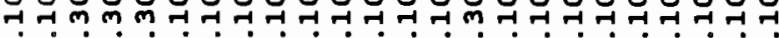
000000000000000000000000

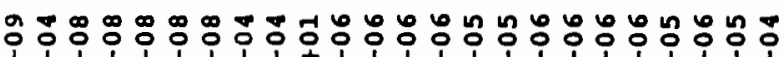

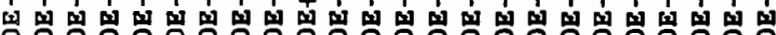
응응응ㅇㅇㅇㅇㅇㅇㅇㅇㅇㅇㅇㅇㅇㅇㅇㅇㅇㅇㅇㅇㅇㅇㅇㅇㅇㅇㅇ응

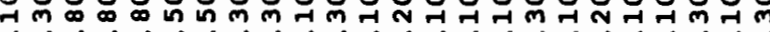

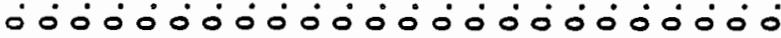

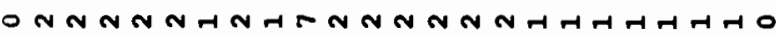

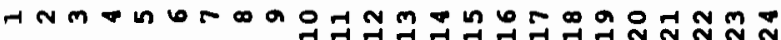




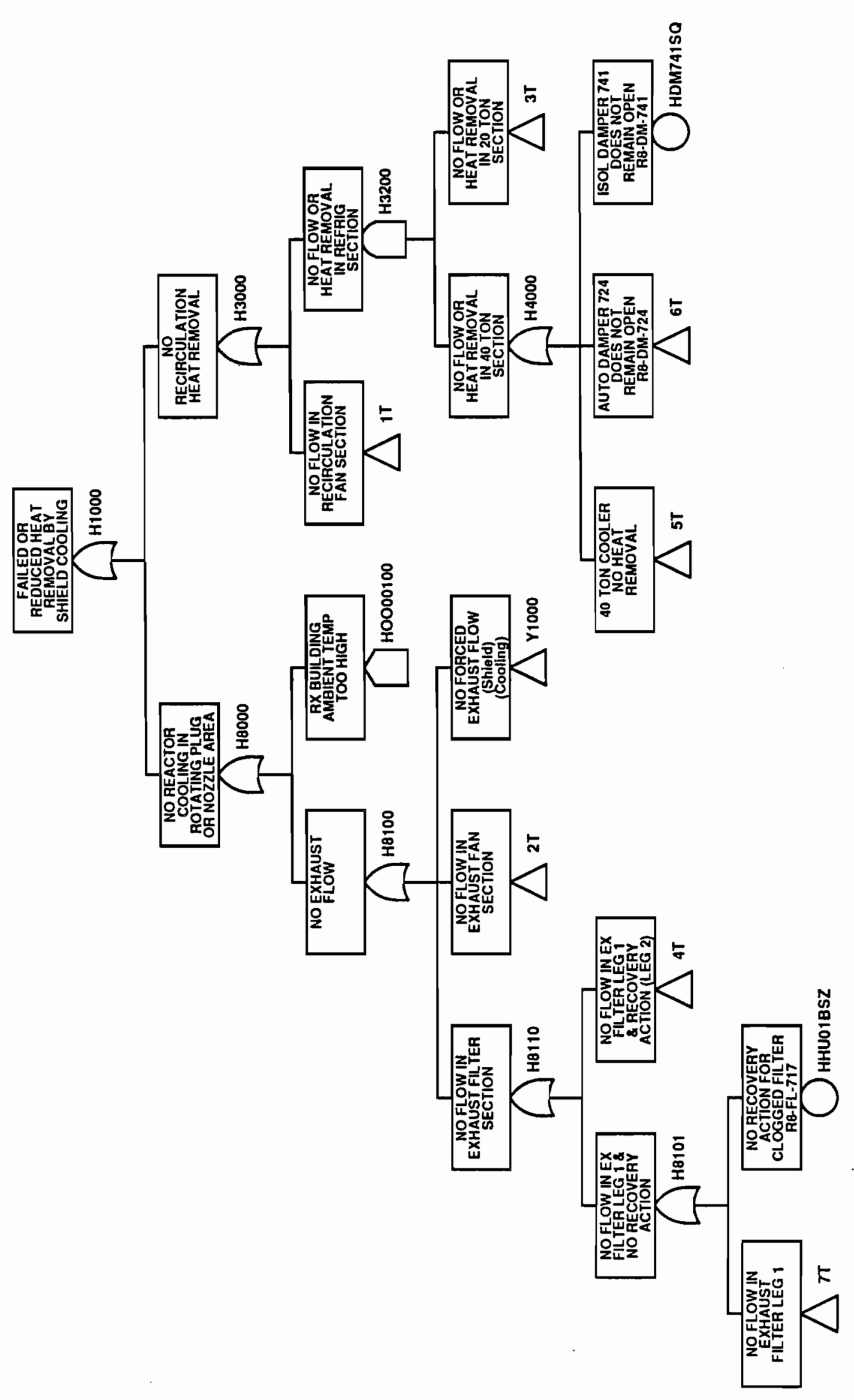




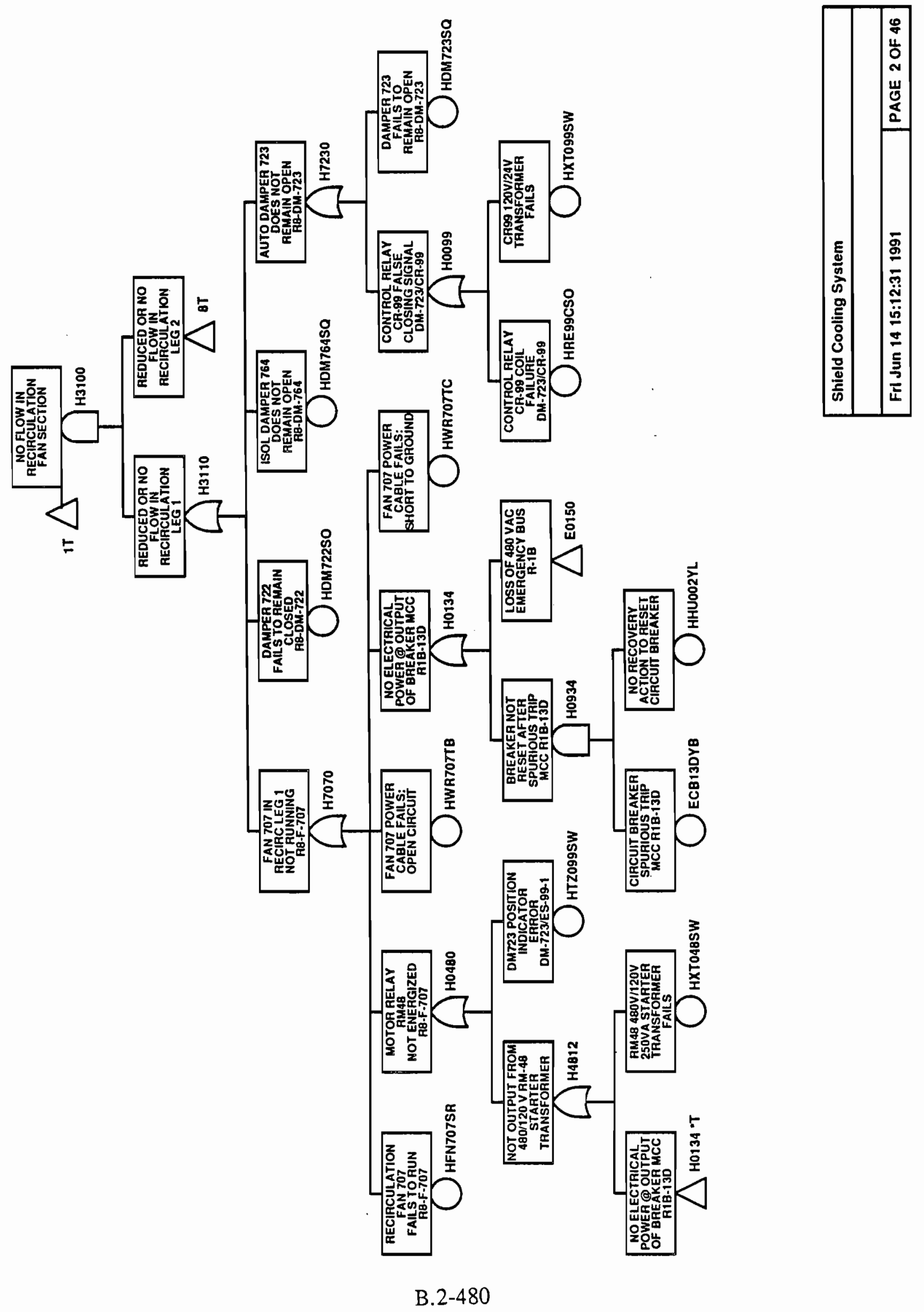



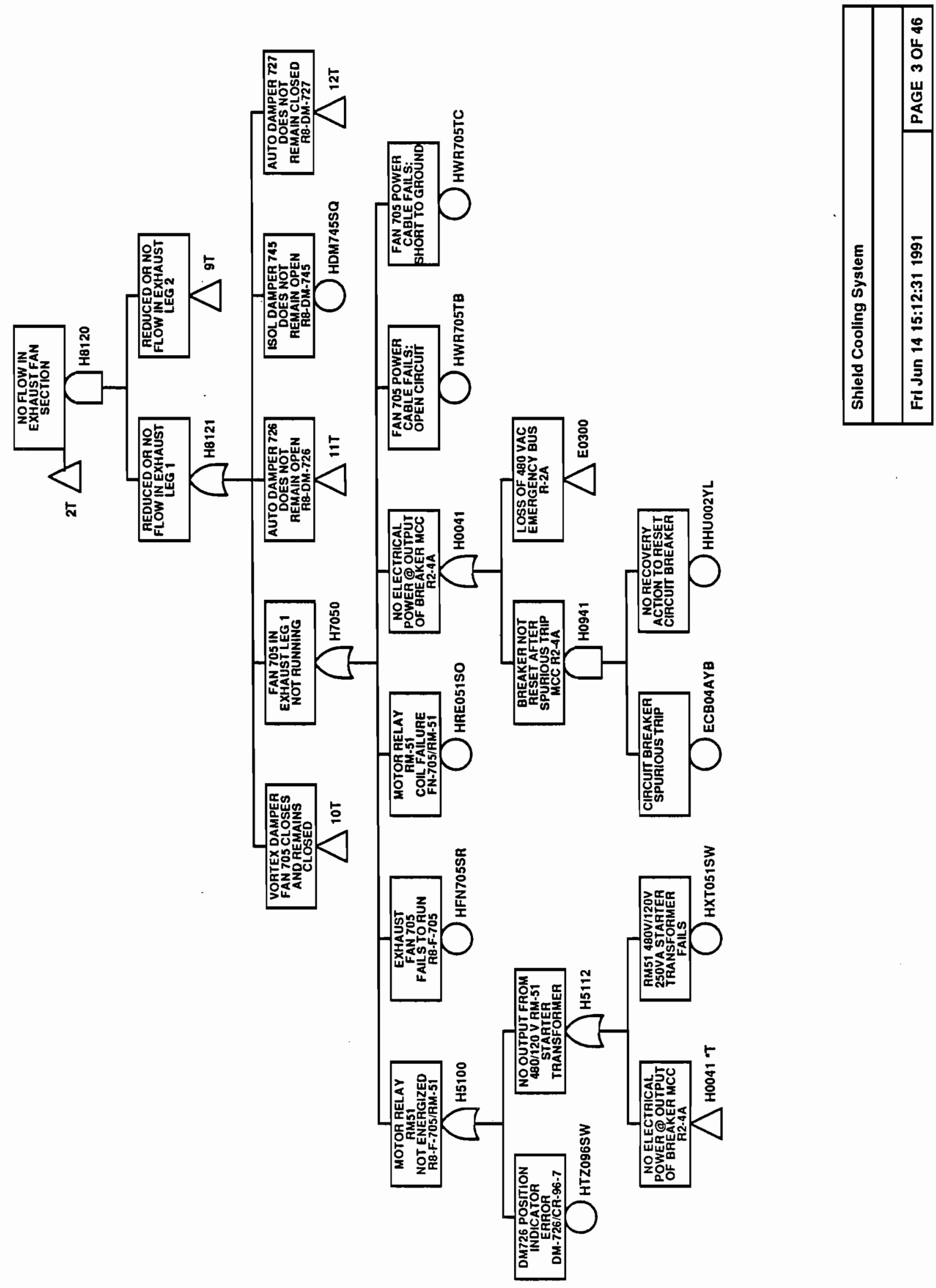

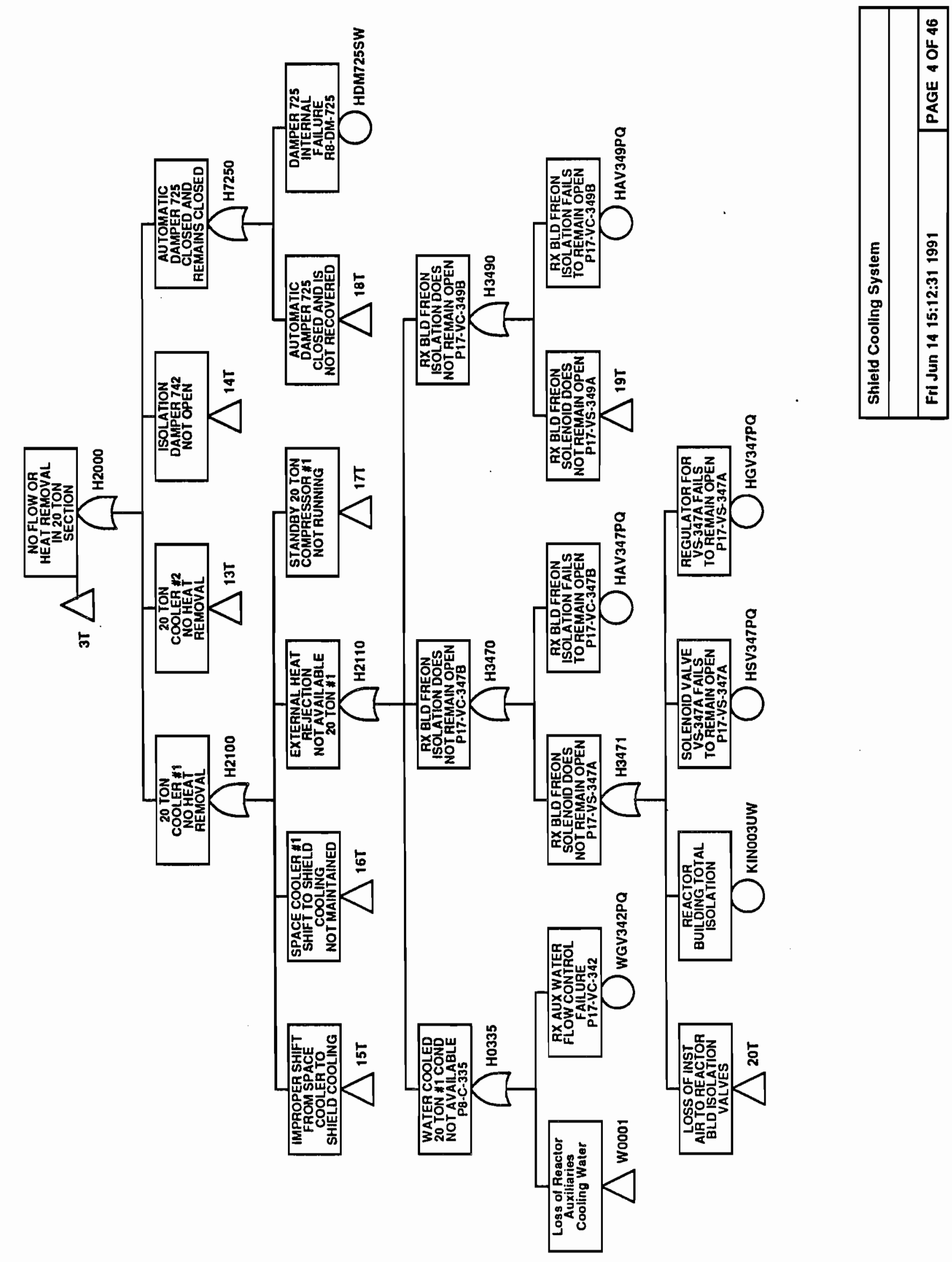


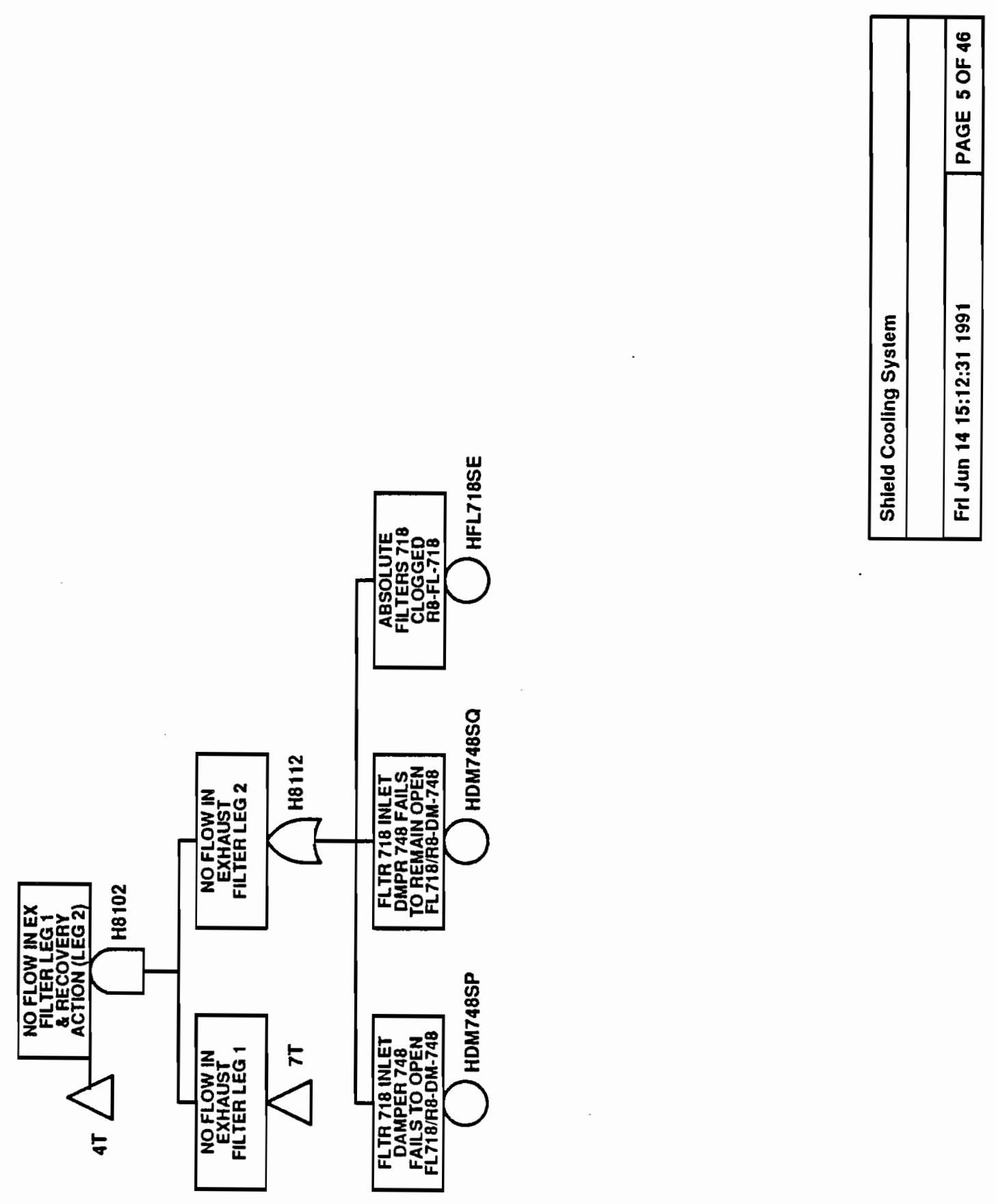



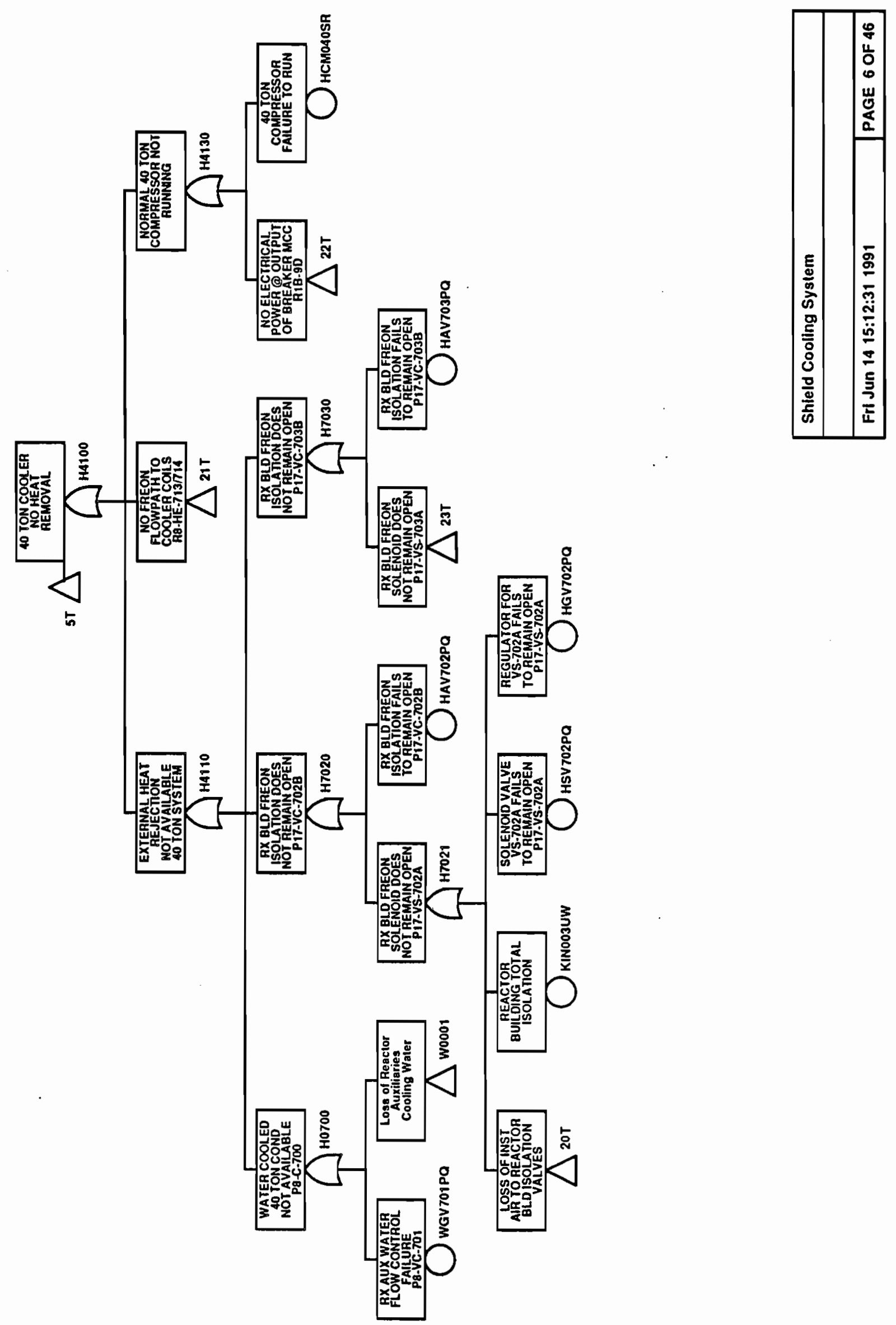

B. $2-484$ 


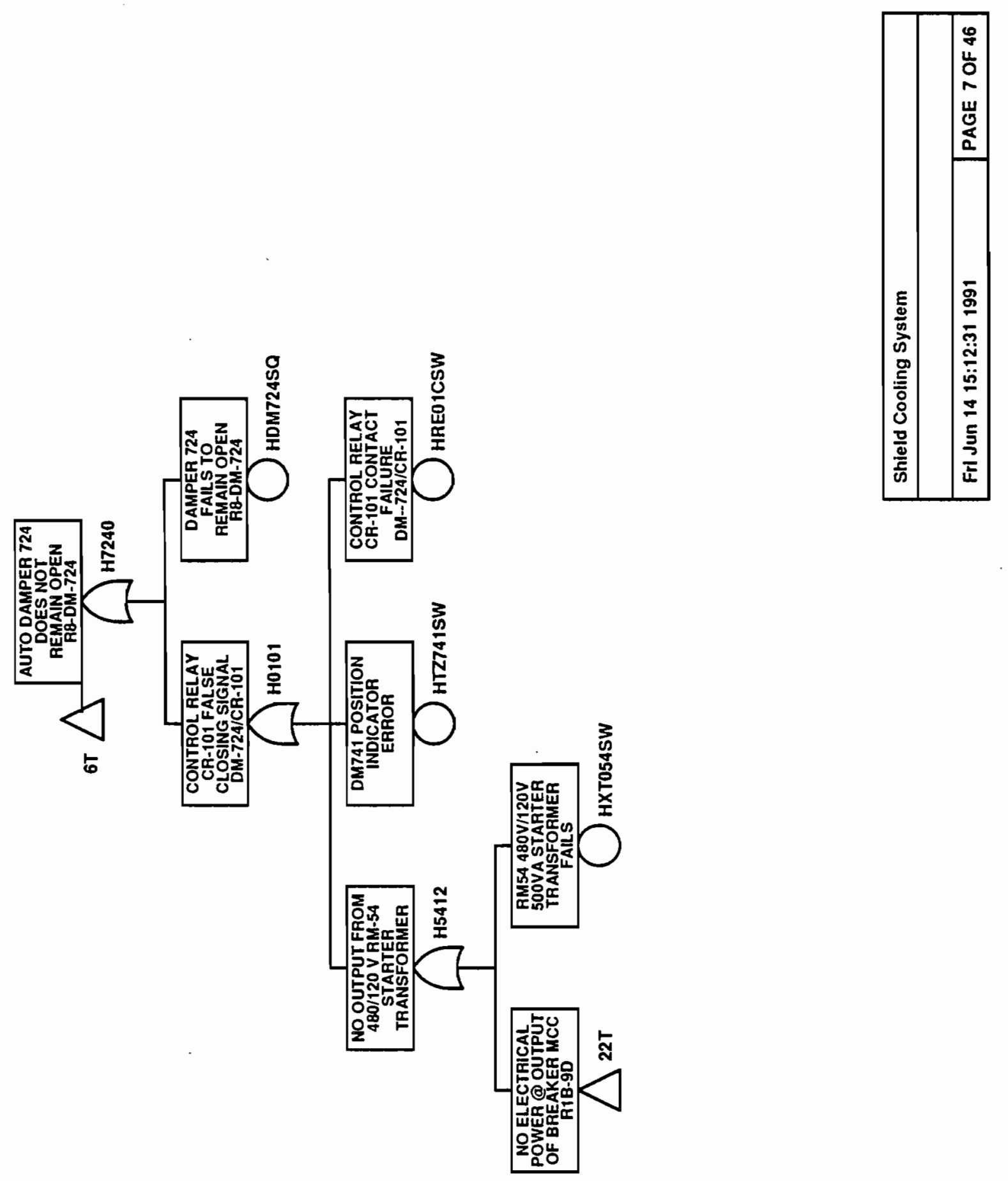




\begin{tabular}{|c|c|}
\hline & 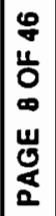 \\
\hline 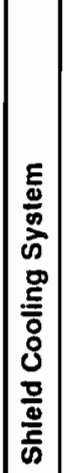 & 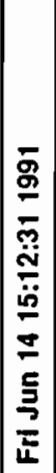 \\
\hline
\end{tabular}

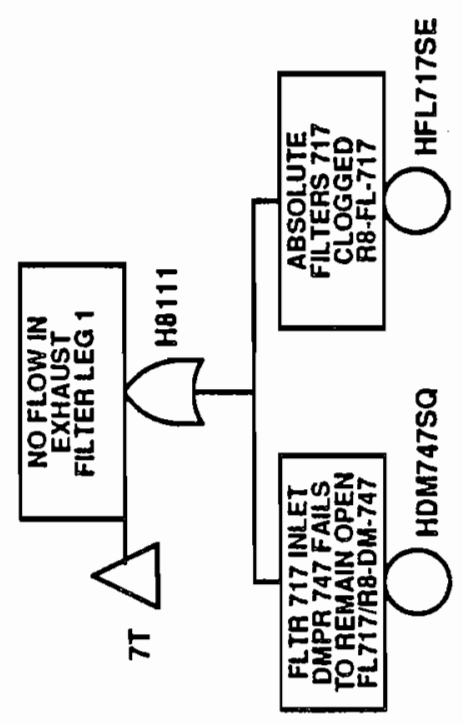

B. 3-486 


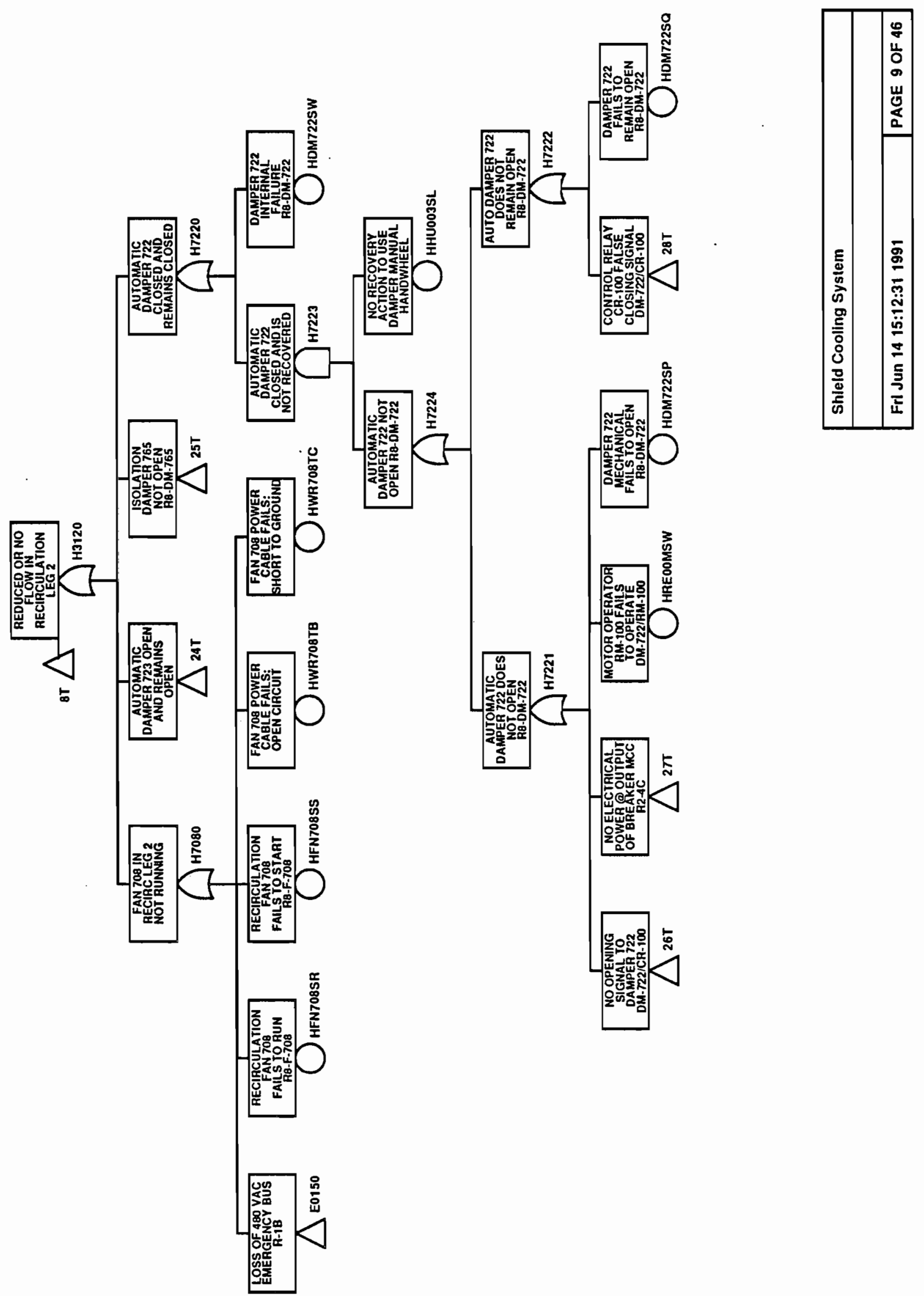



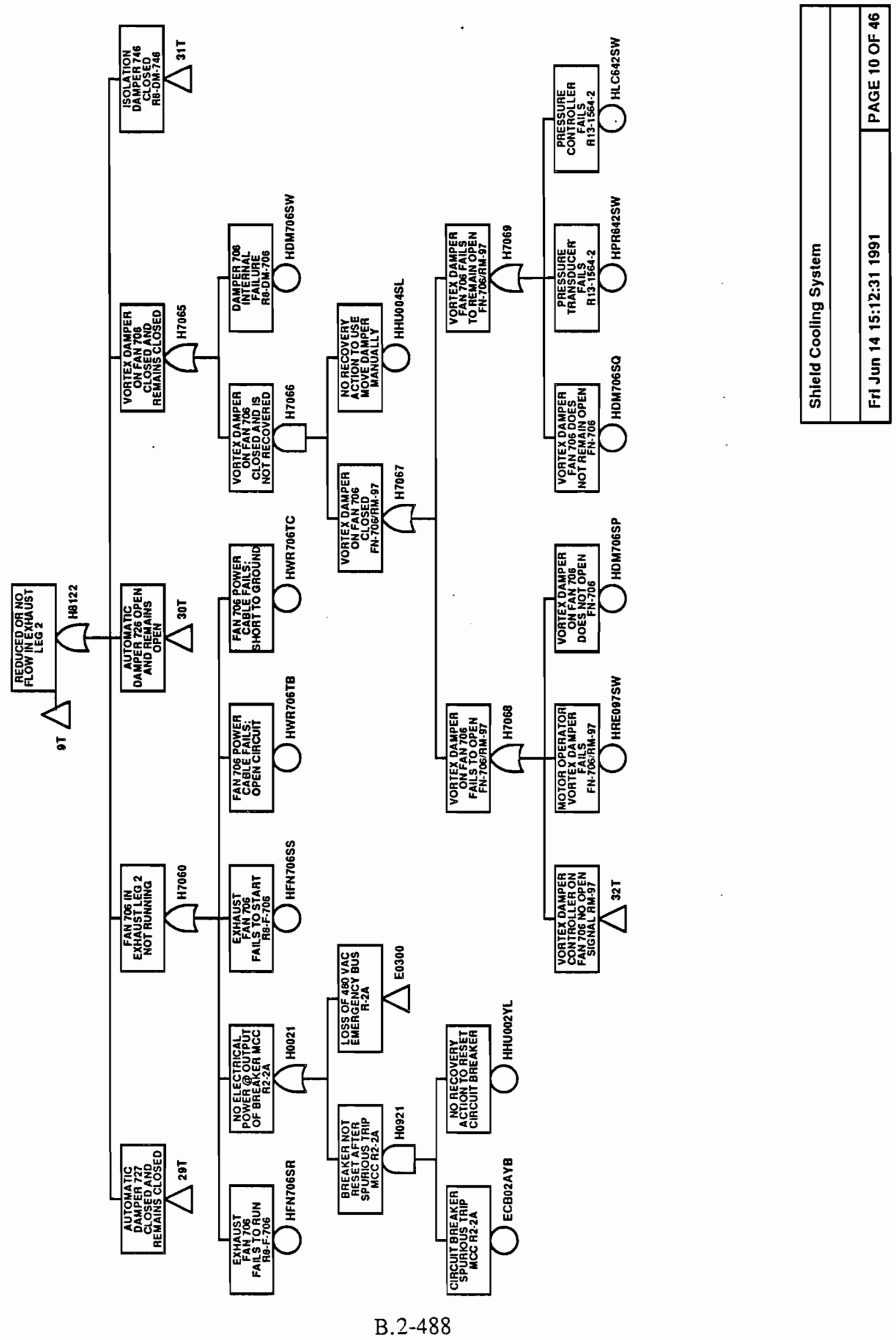


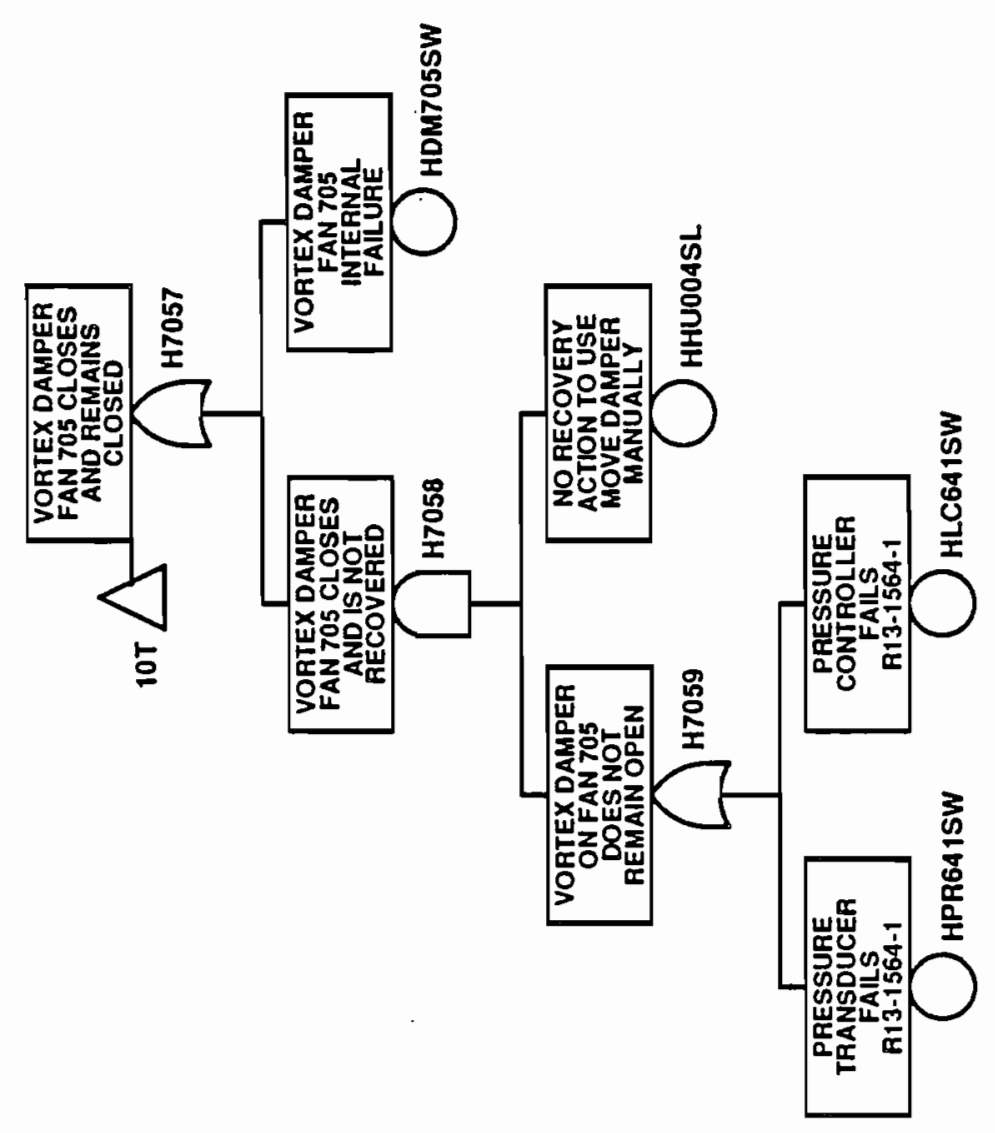

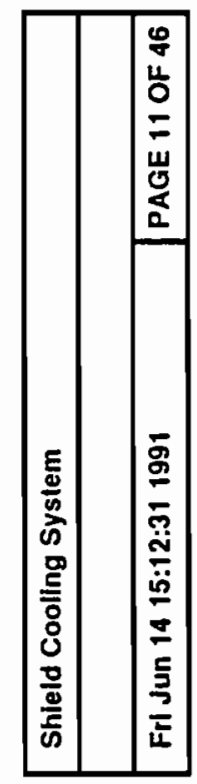




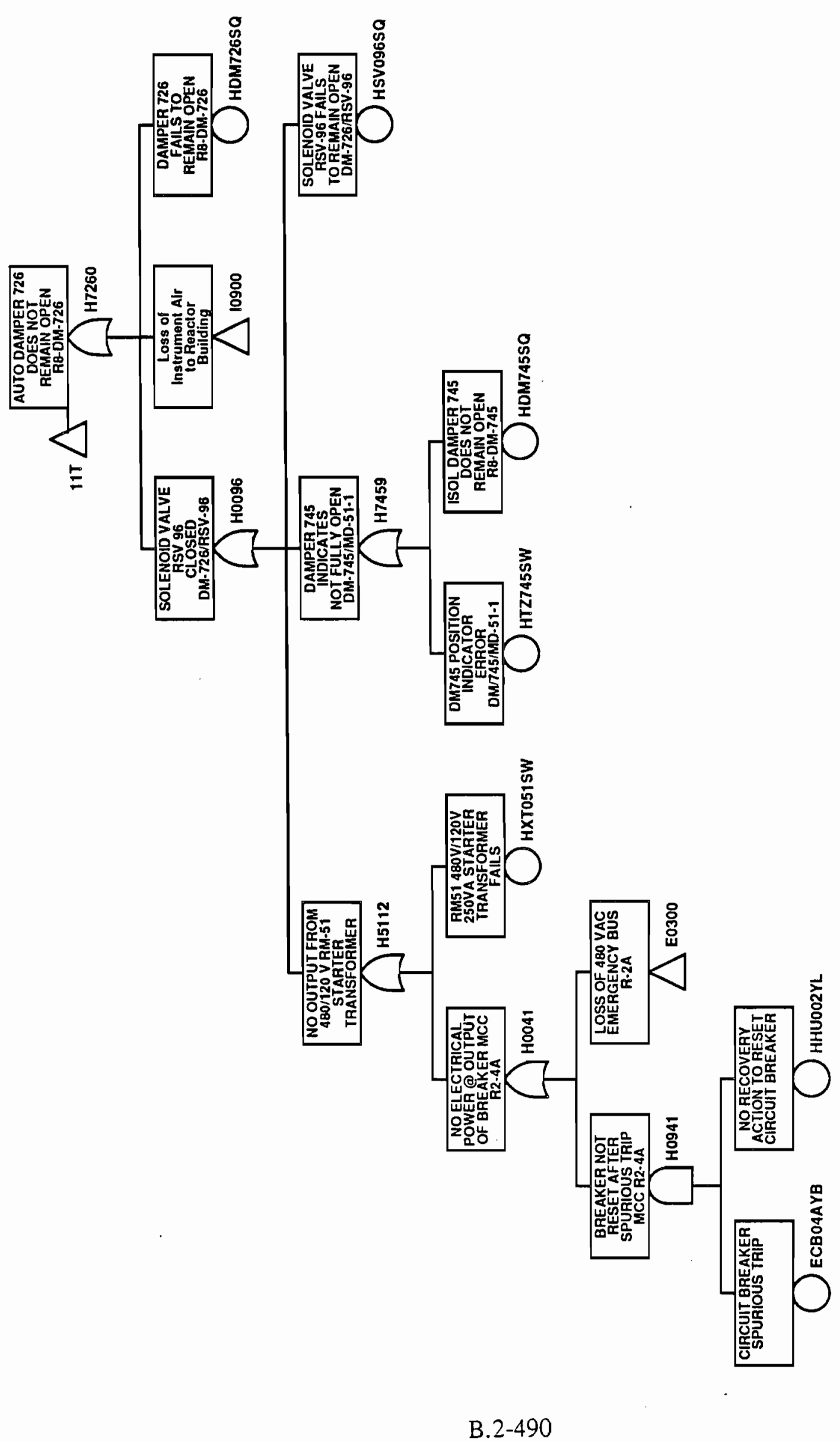

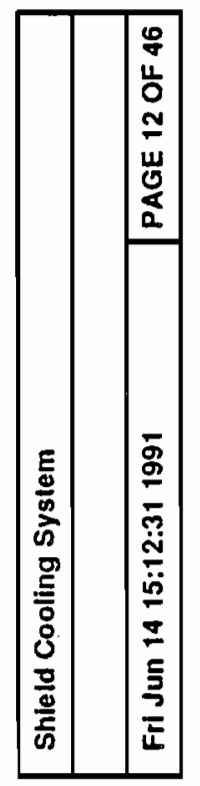




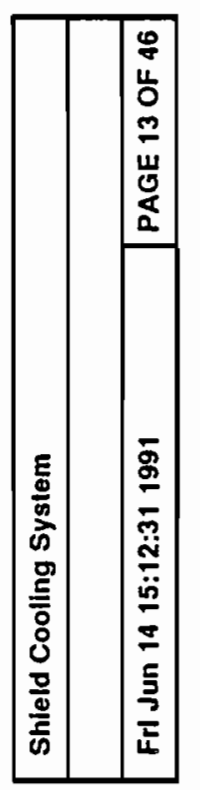

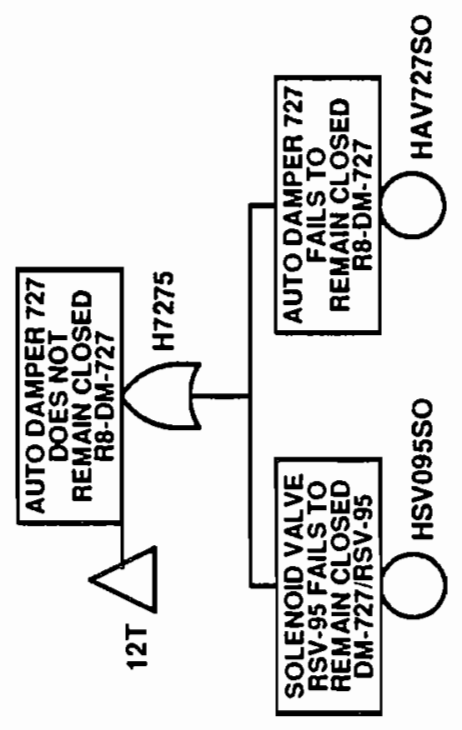



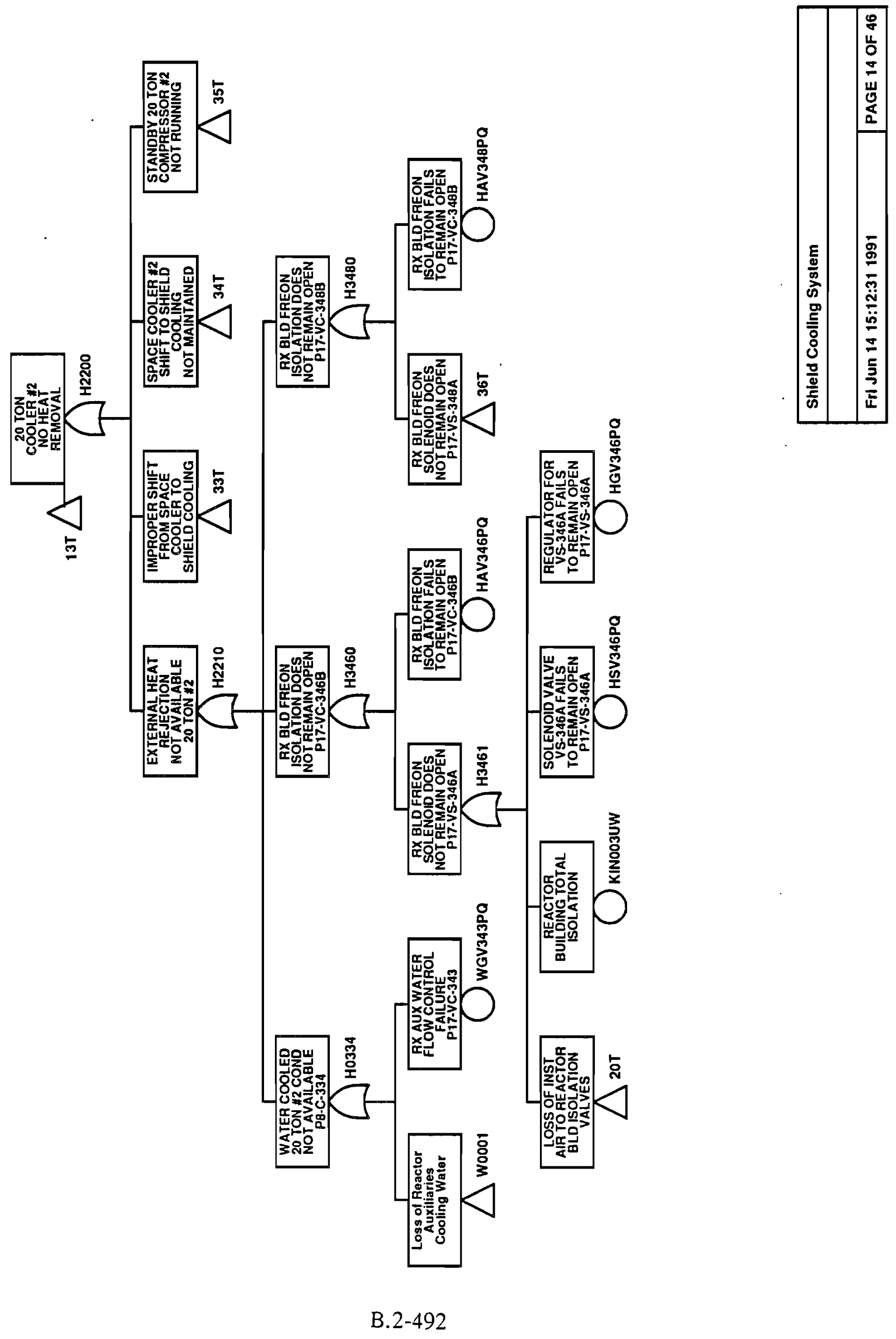


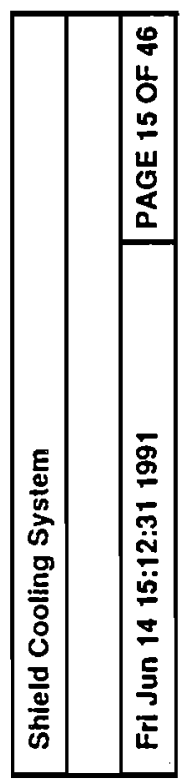

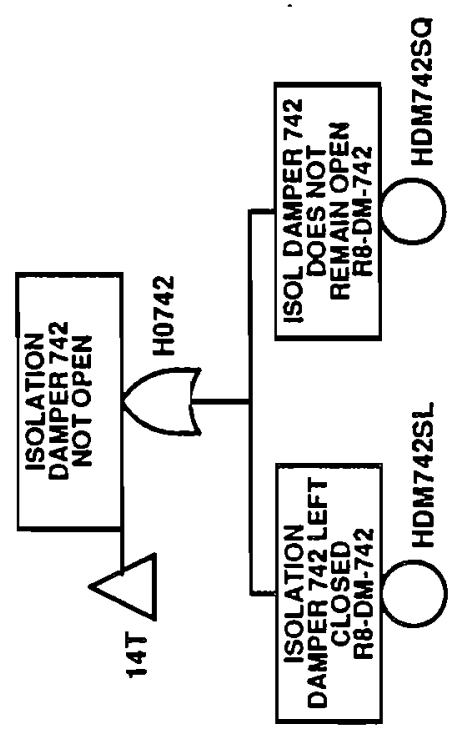



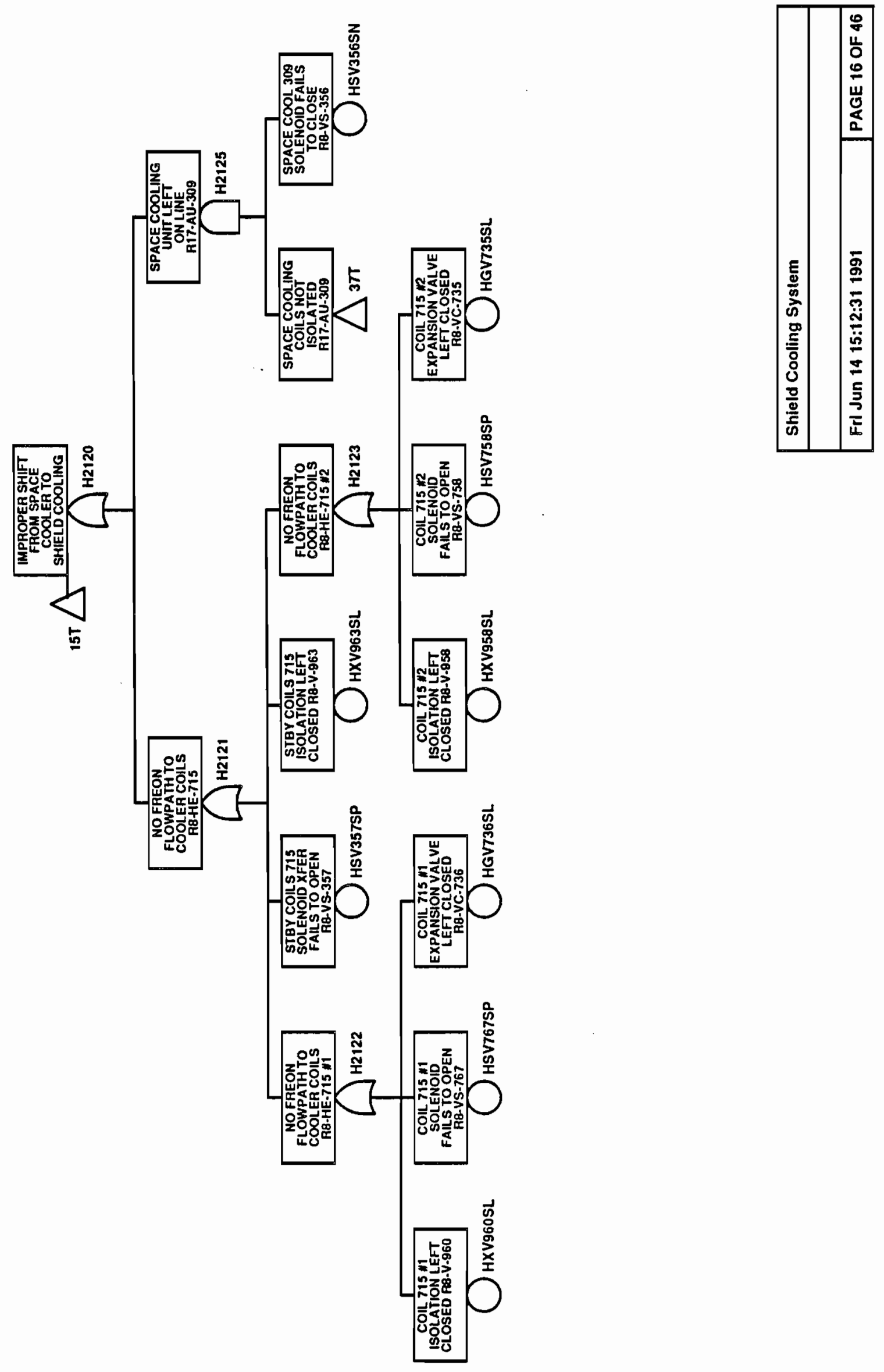


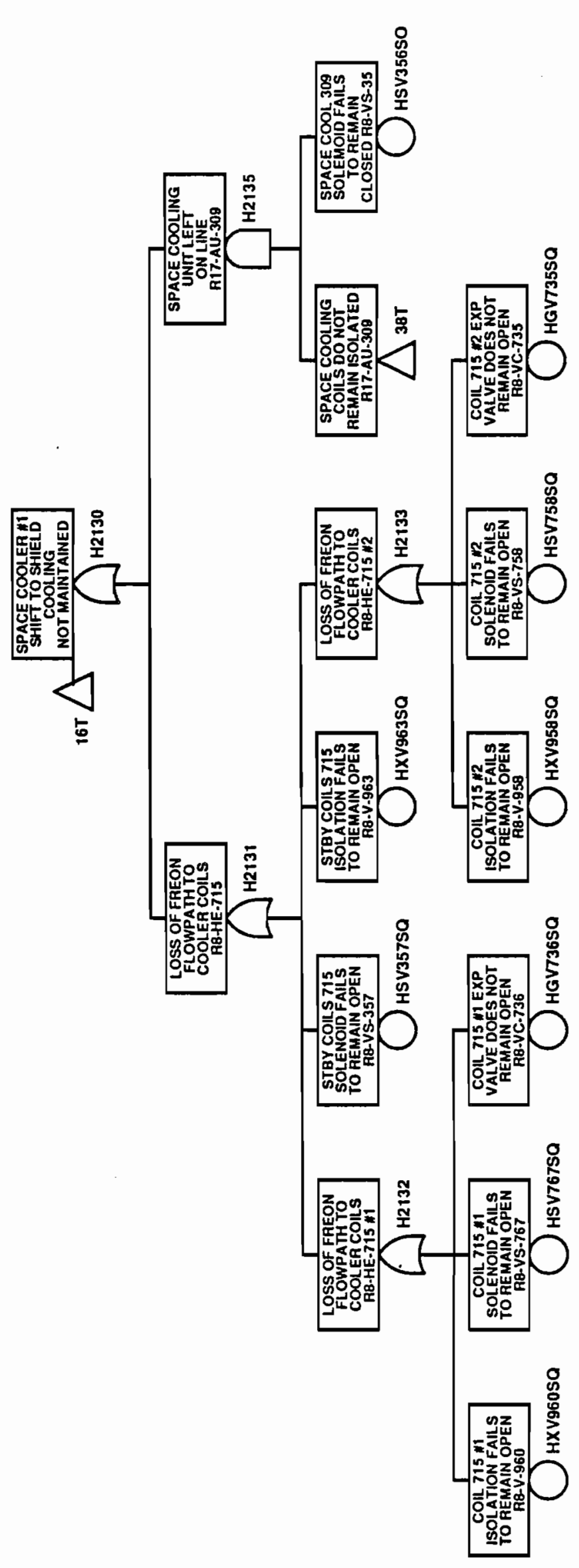

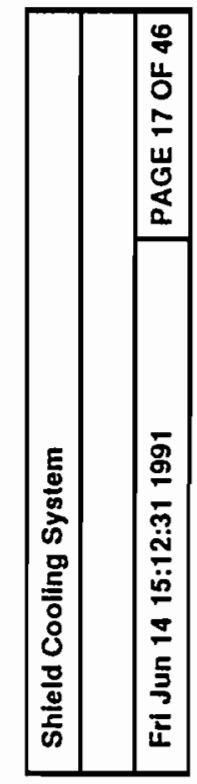




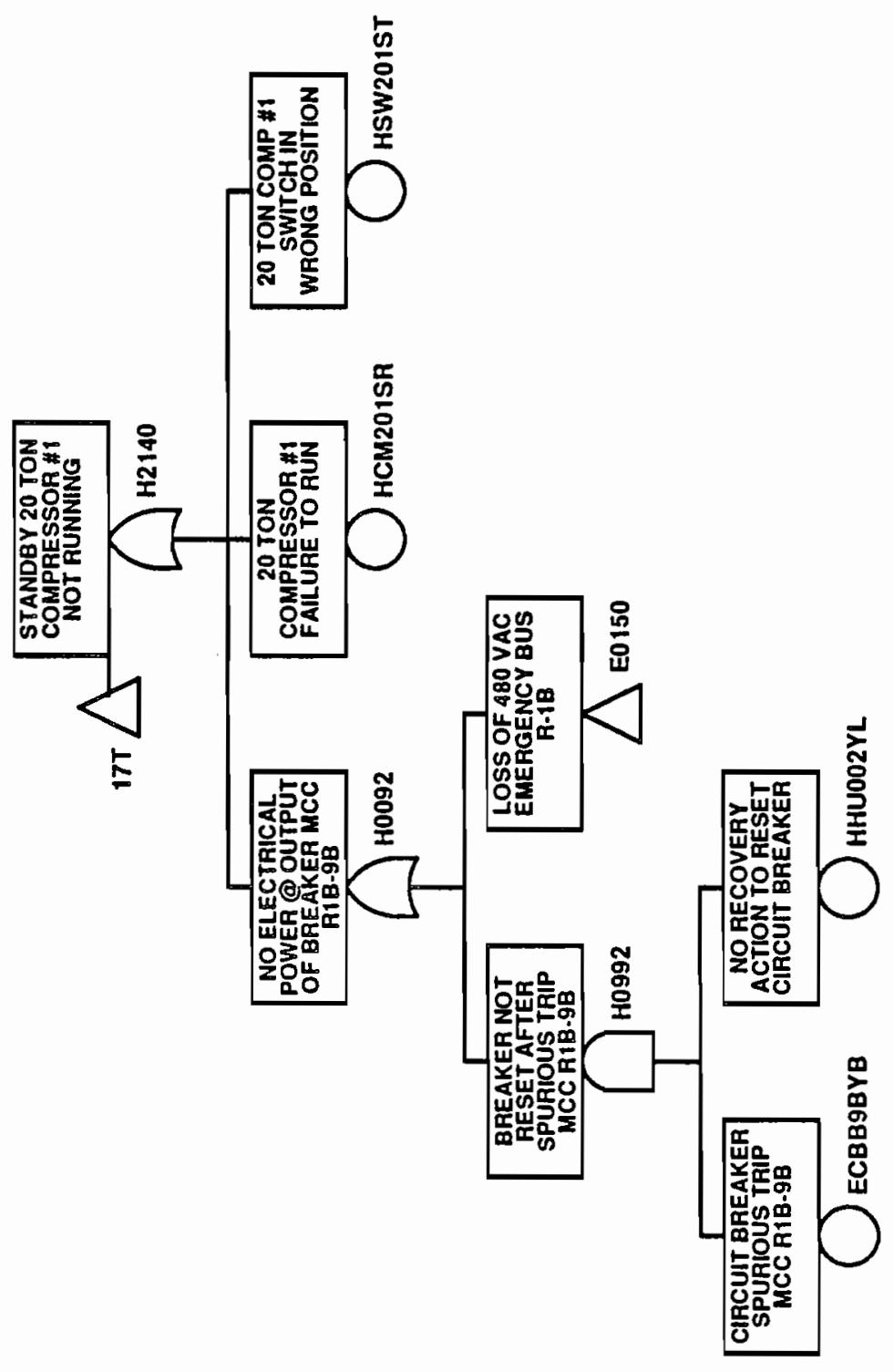




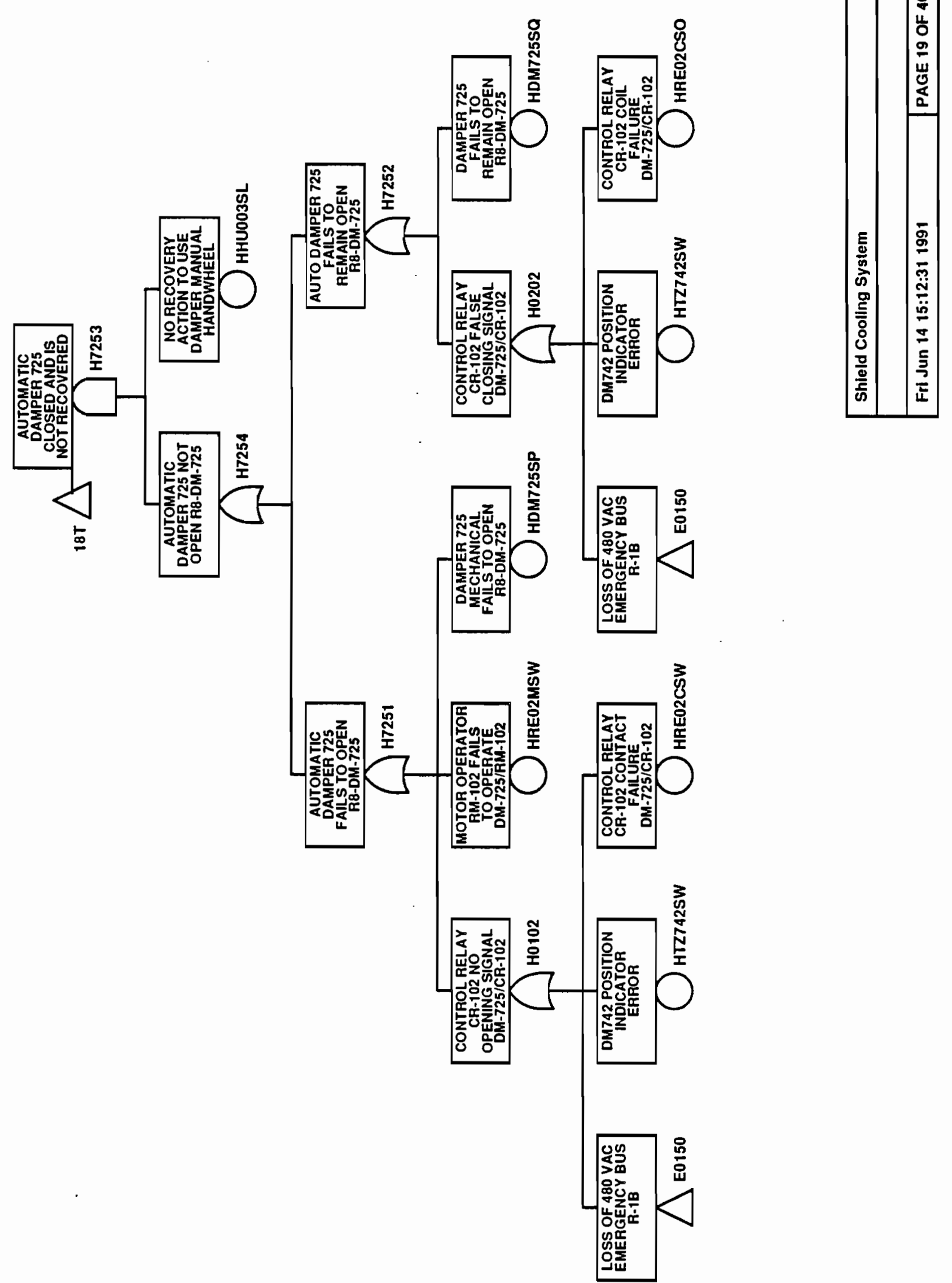




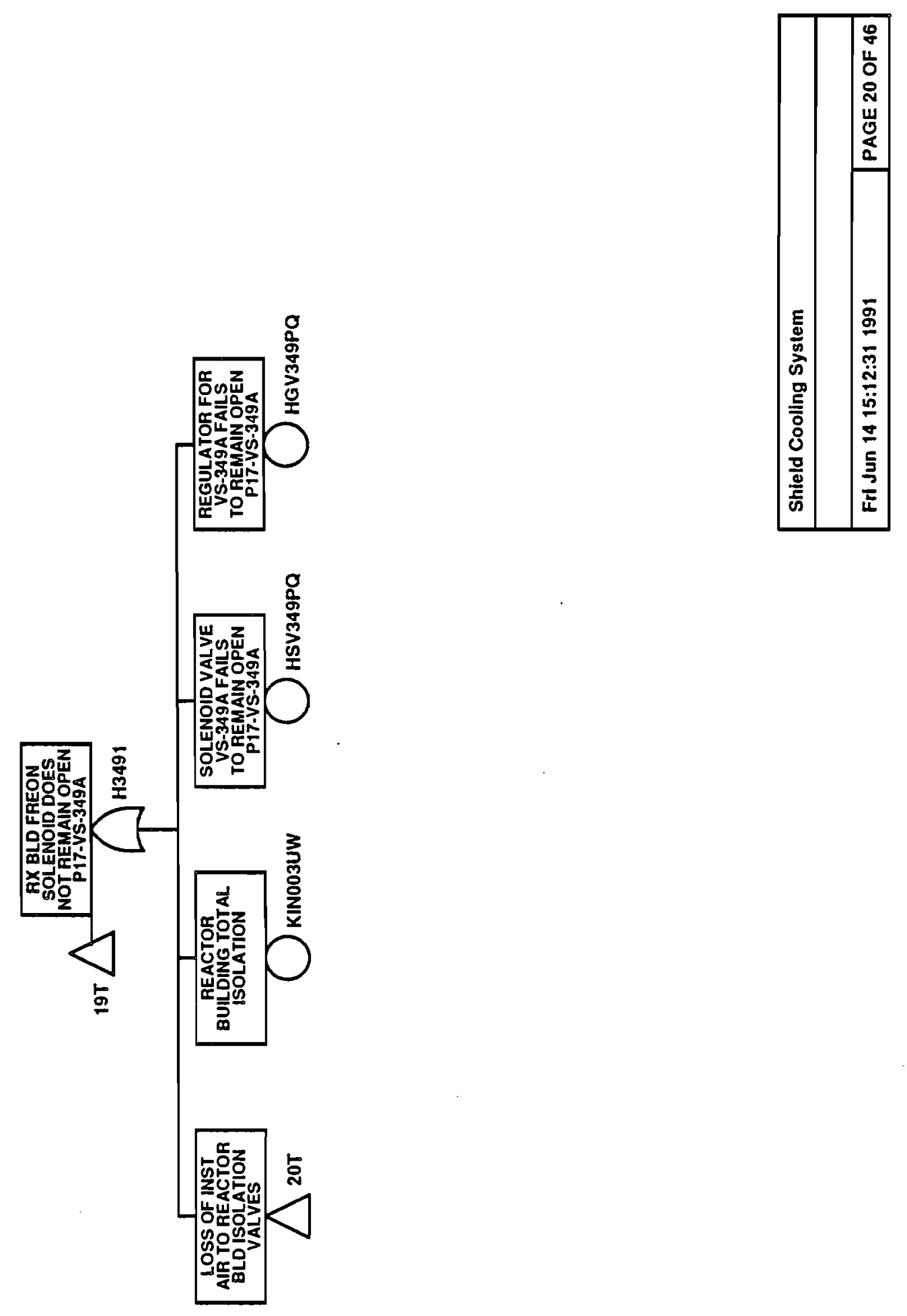




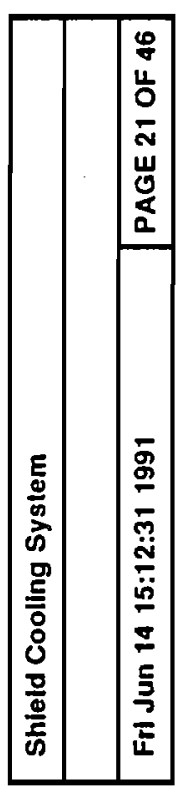

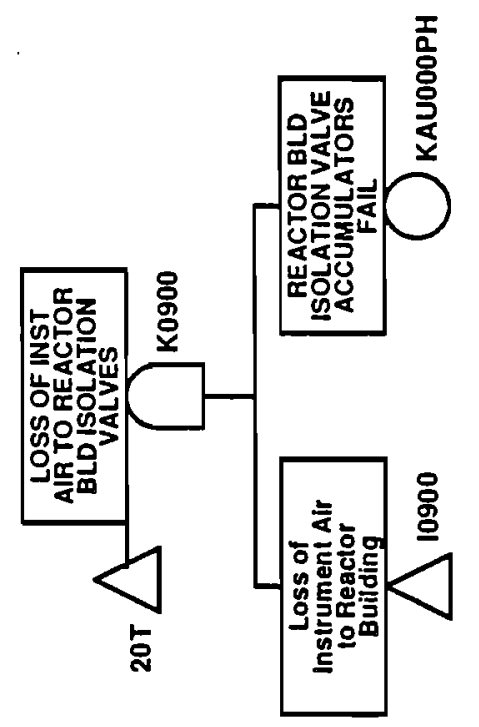

B. 2-499 

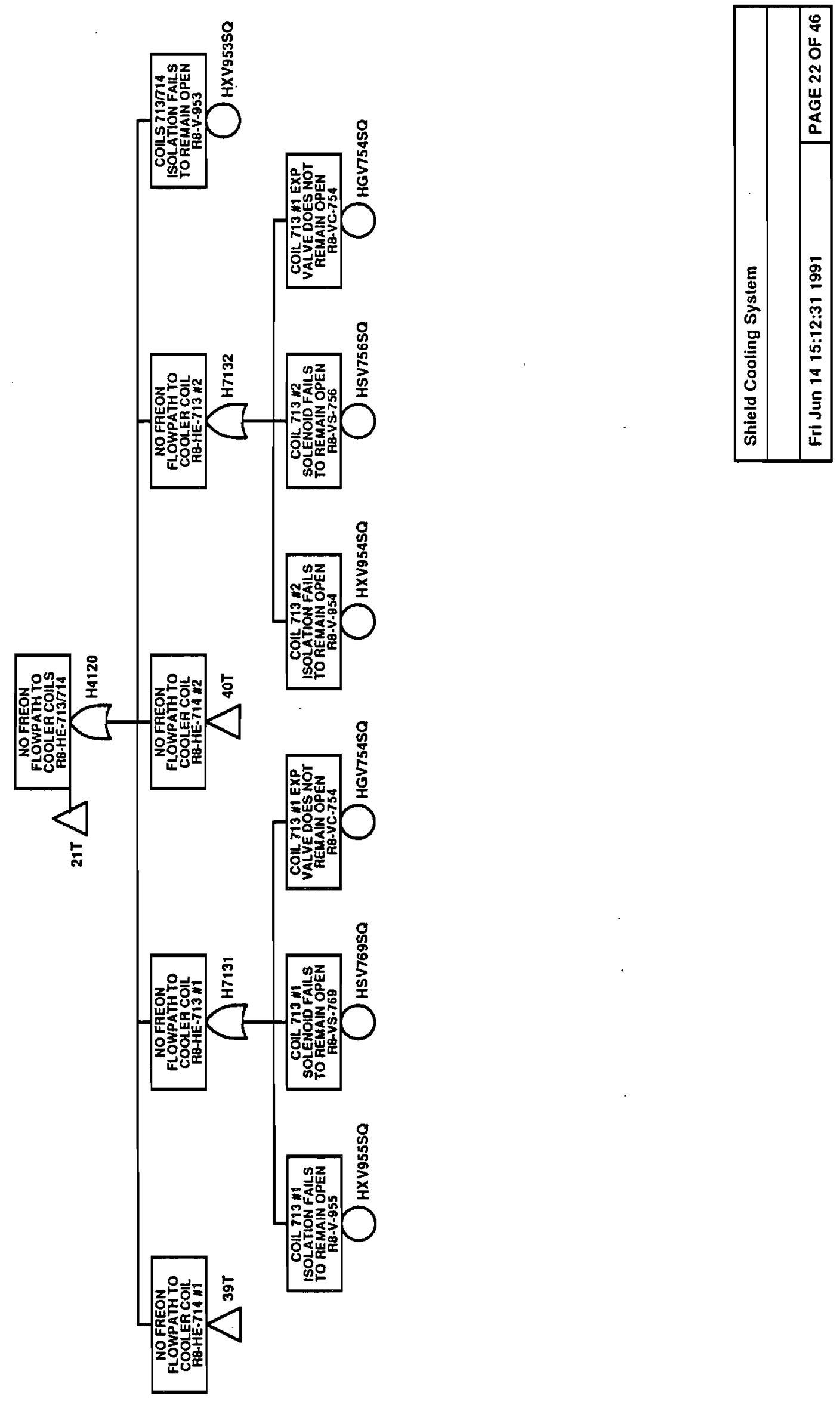

B. $2-500$ 


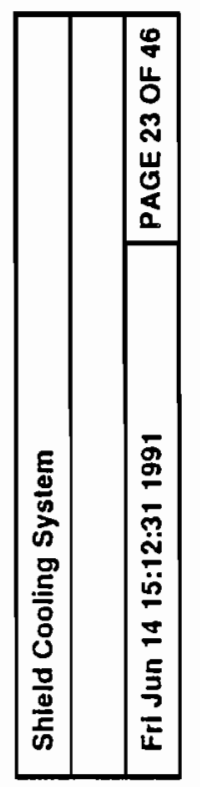

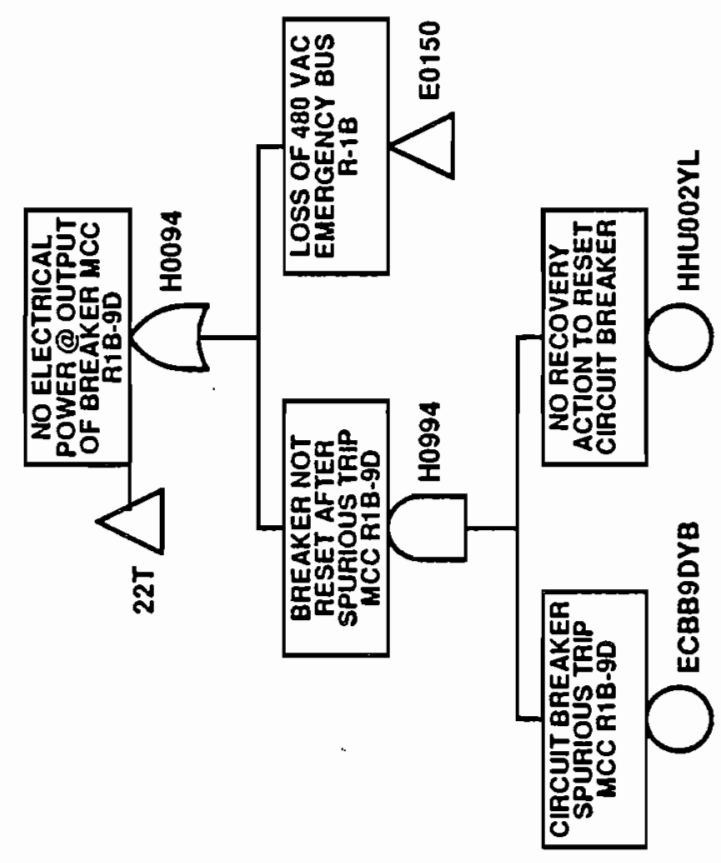




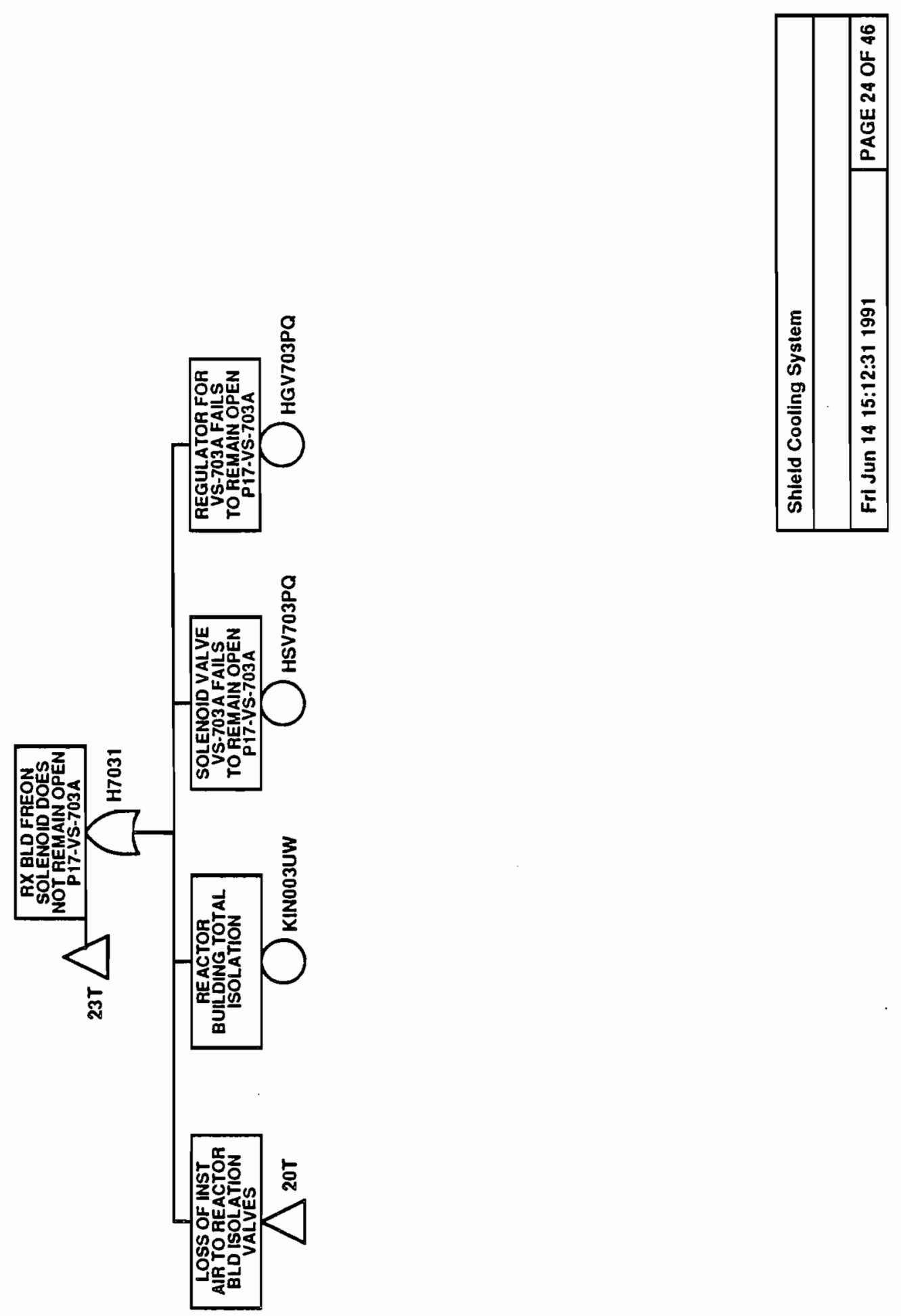

B.2-502 

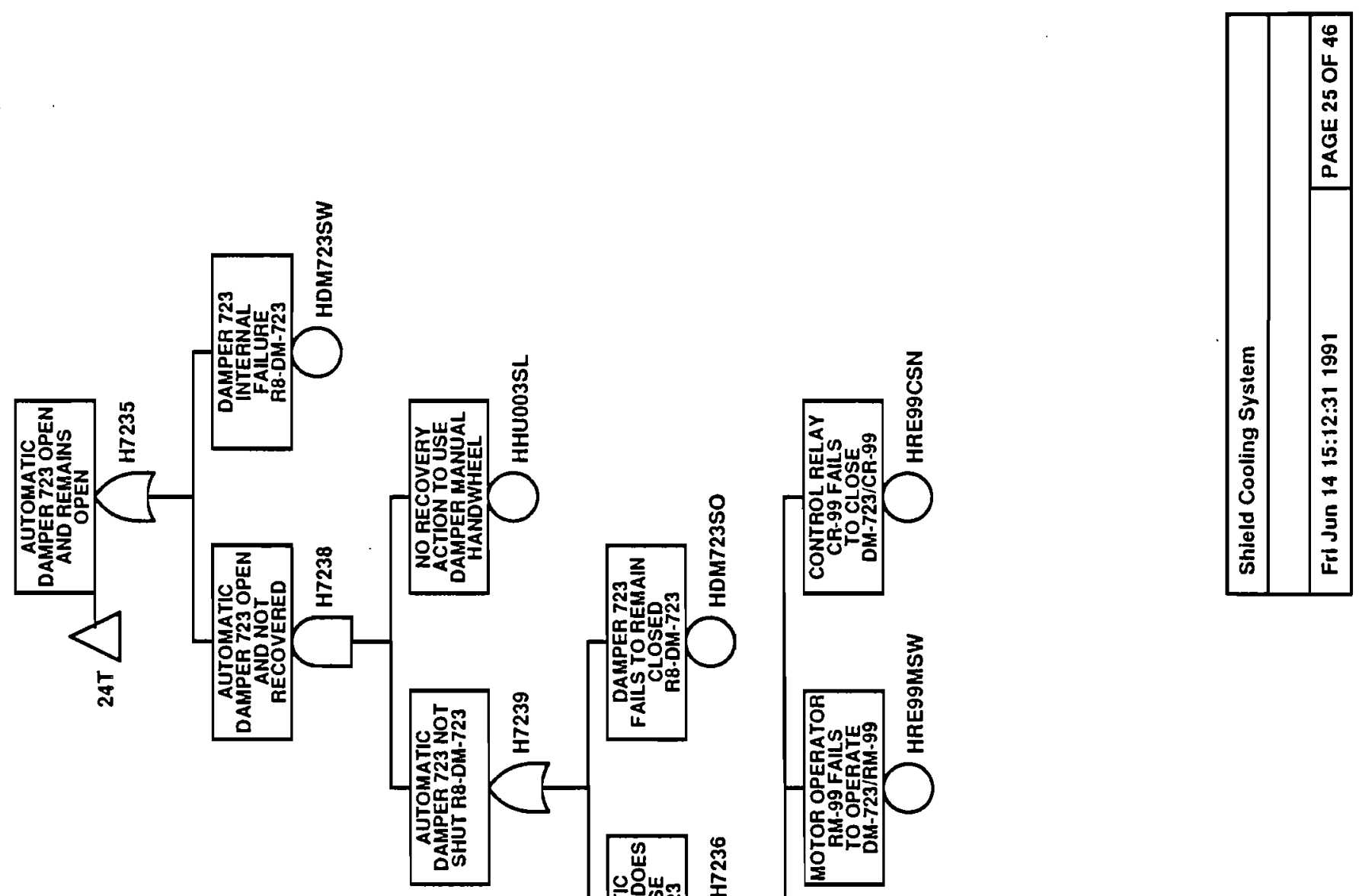


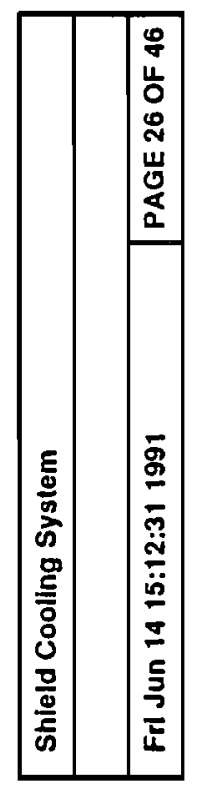

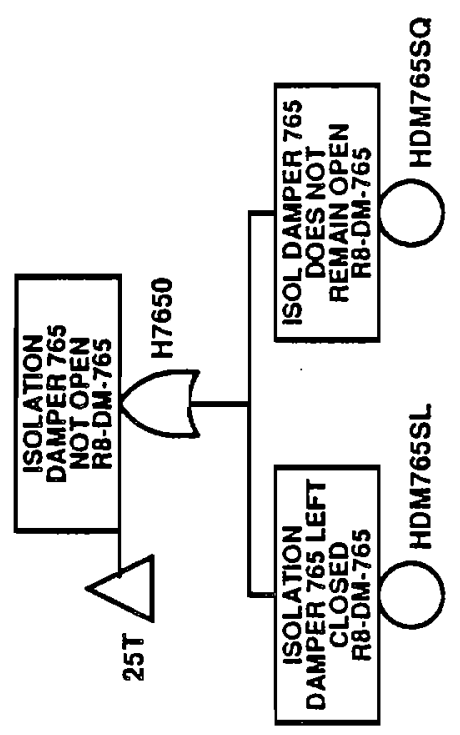

B. 2-504 


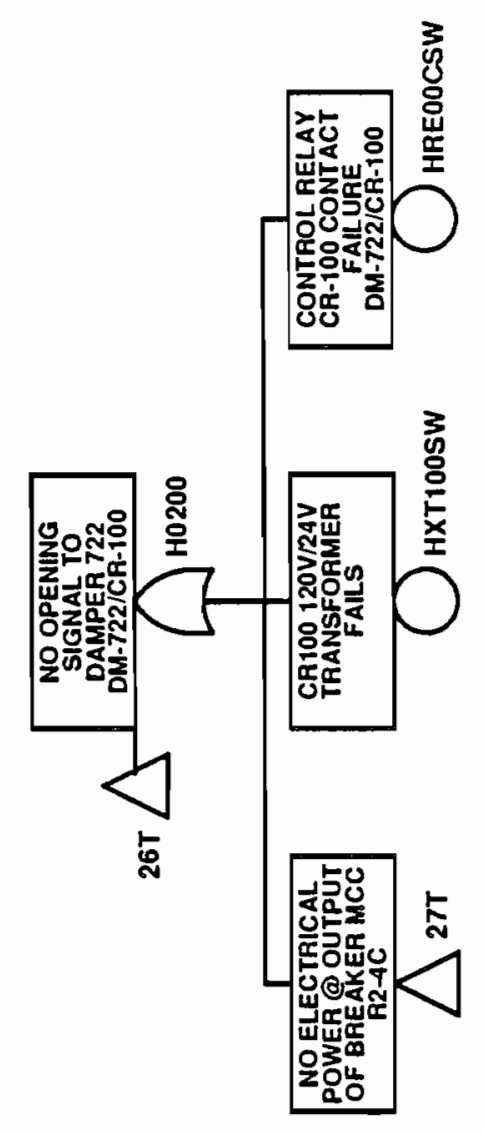

B. 2-505 


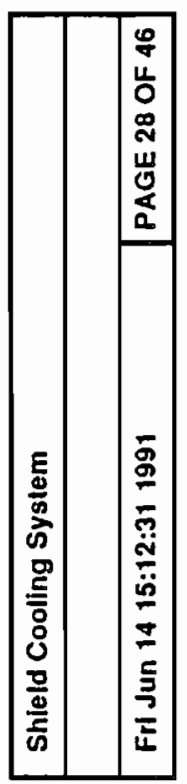

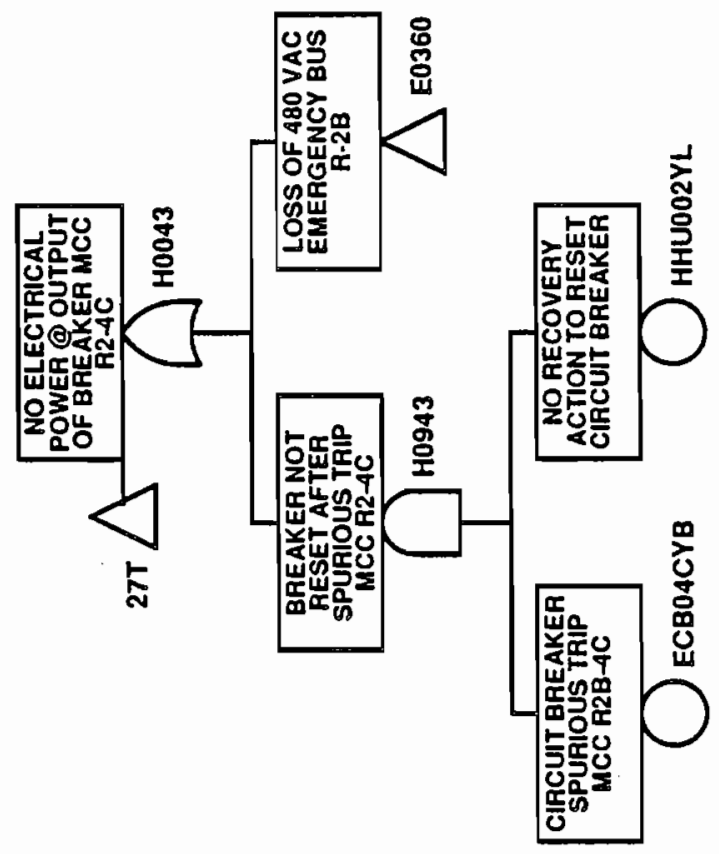

B. 2-506 


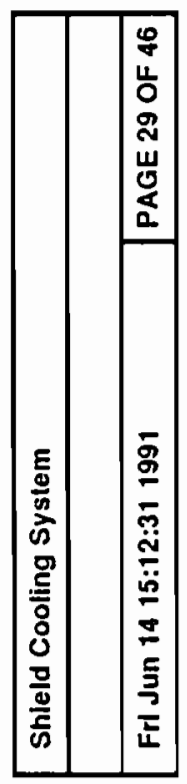

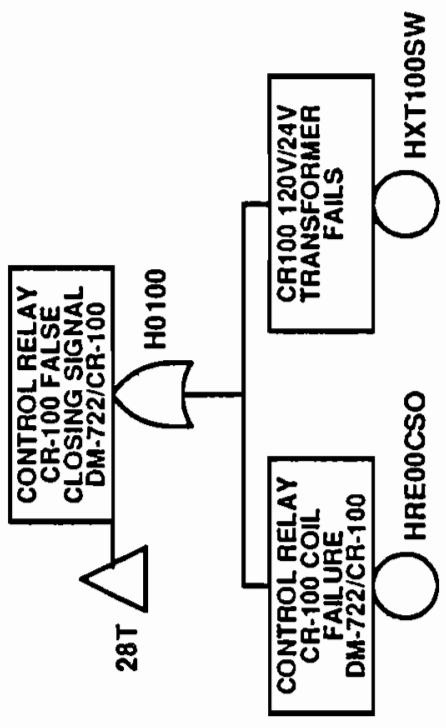




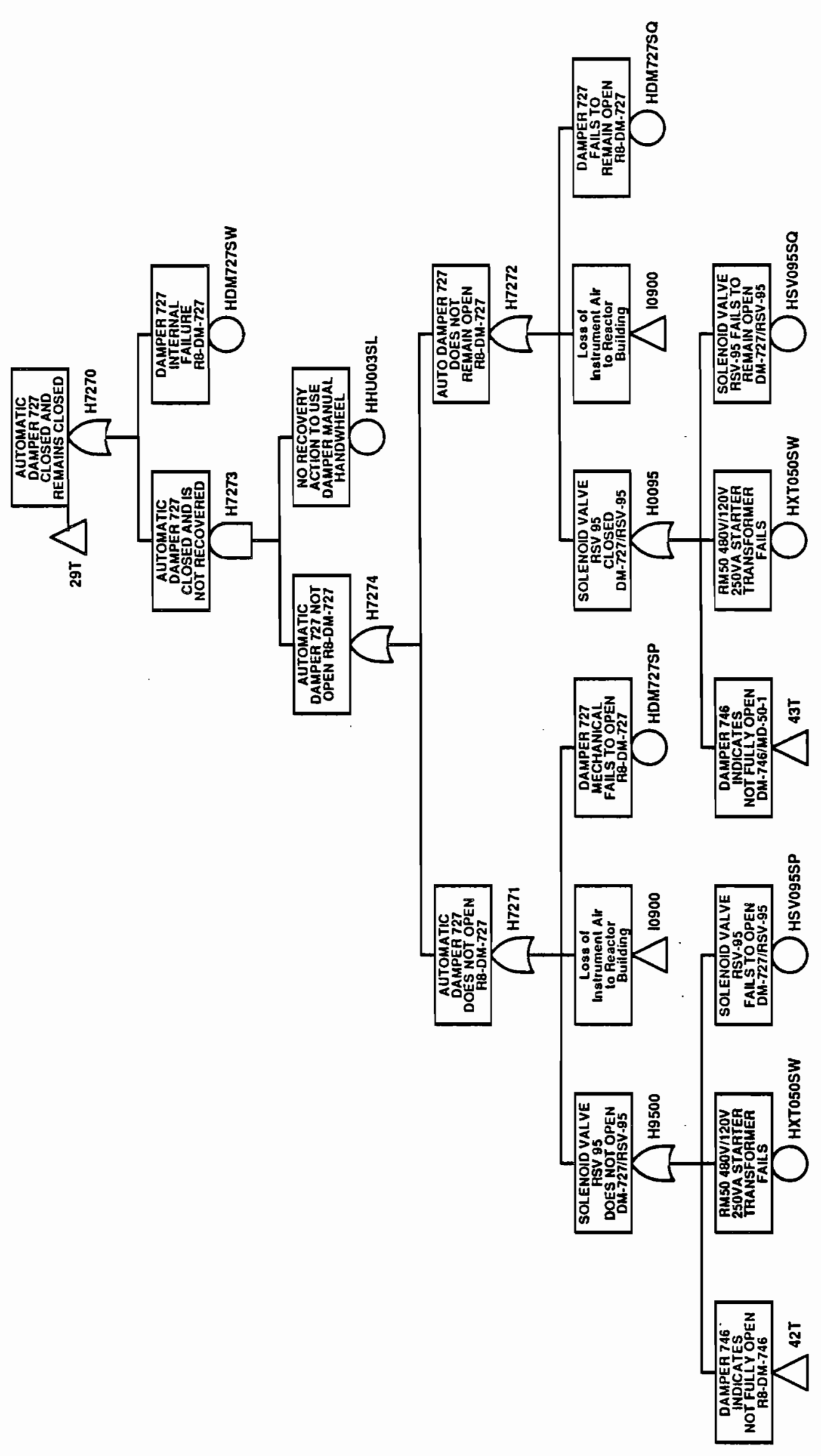

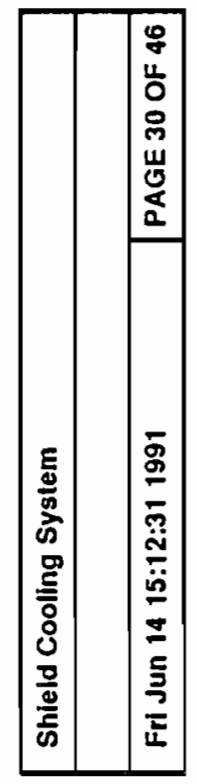




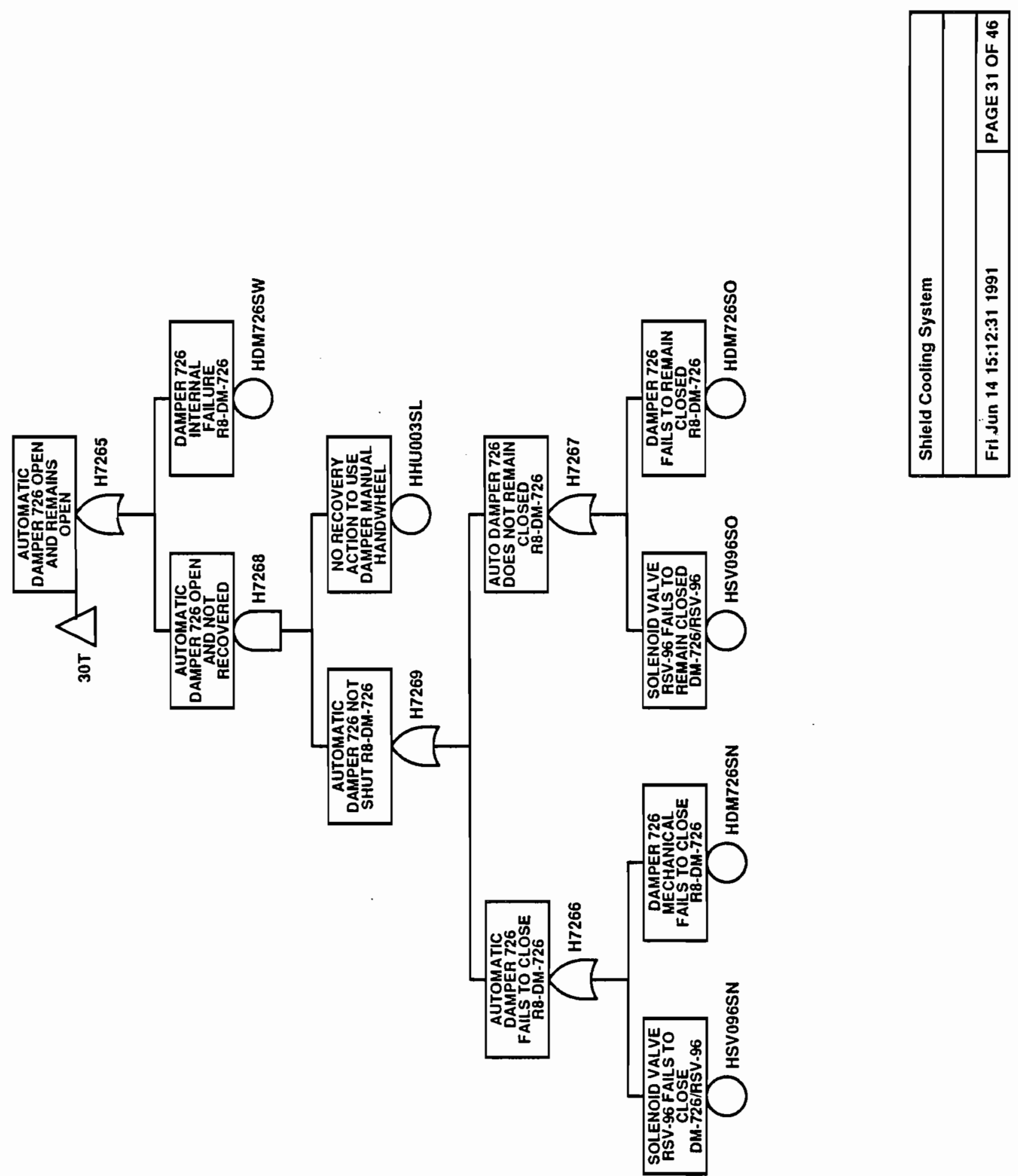




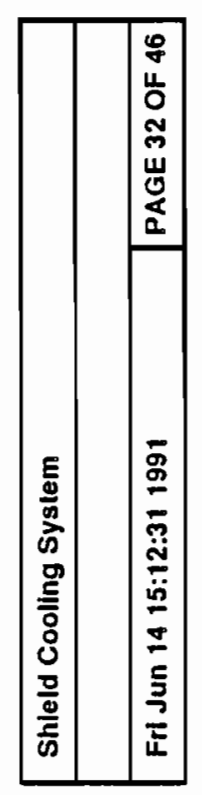

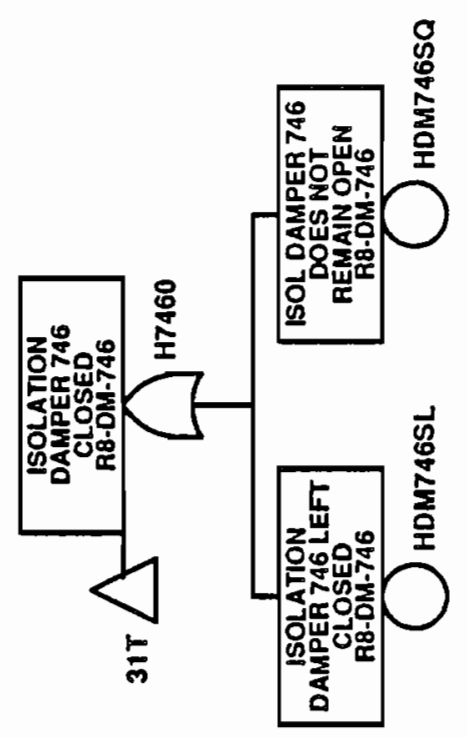




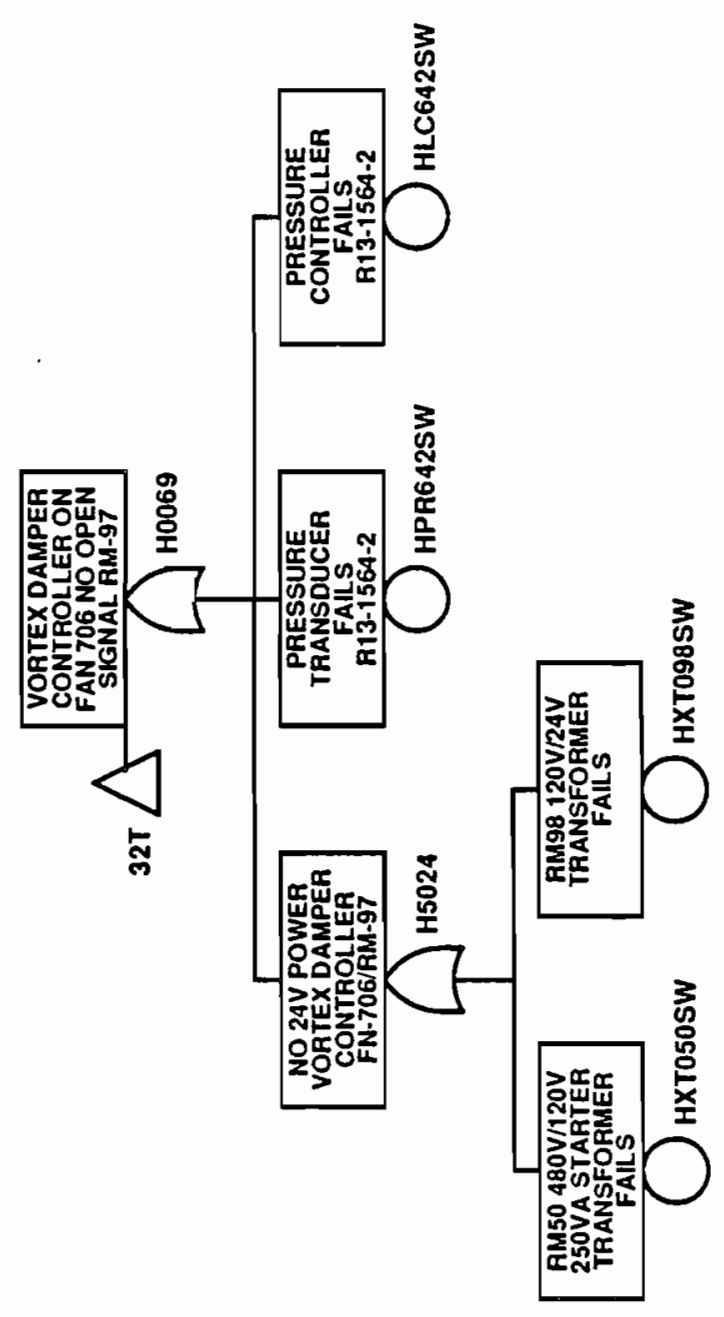

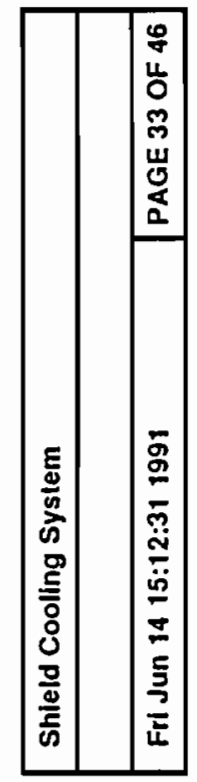



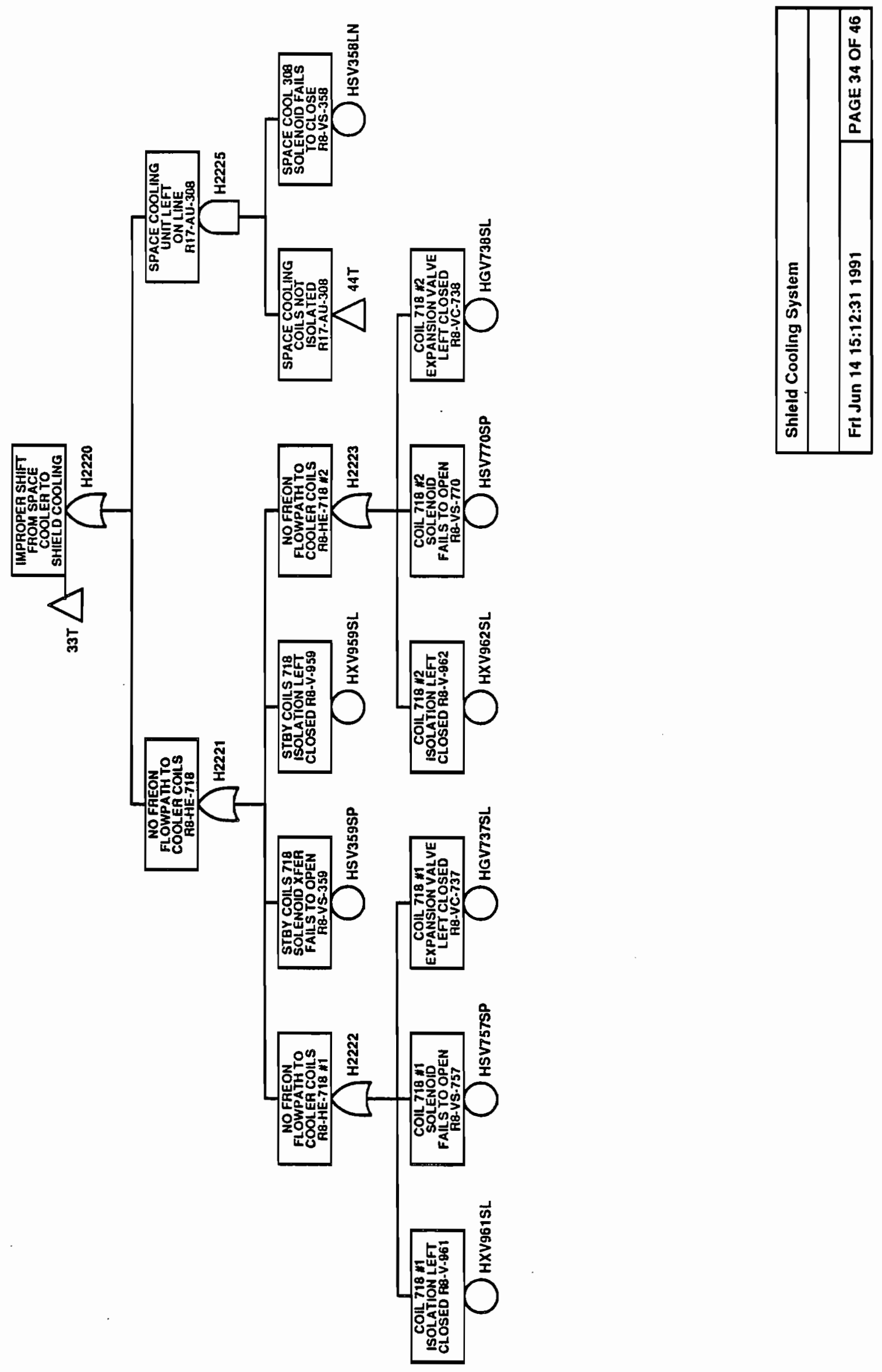


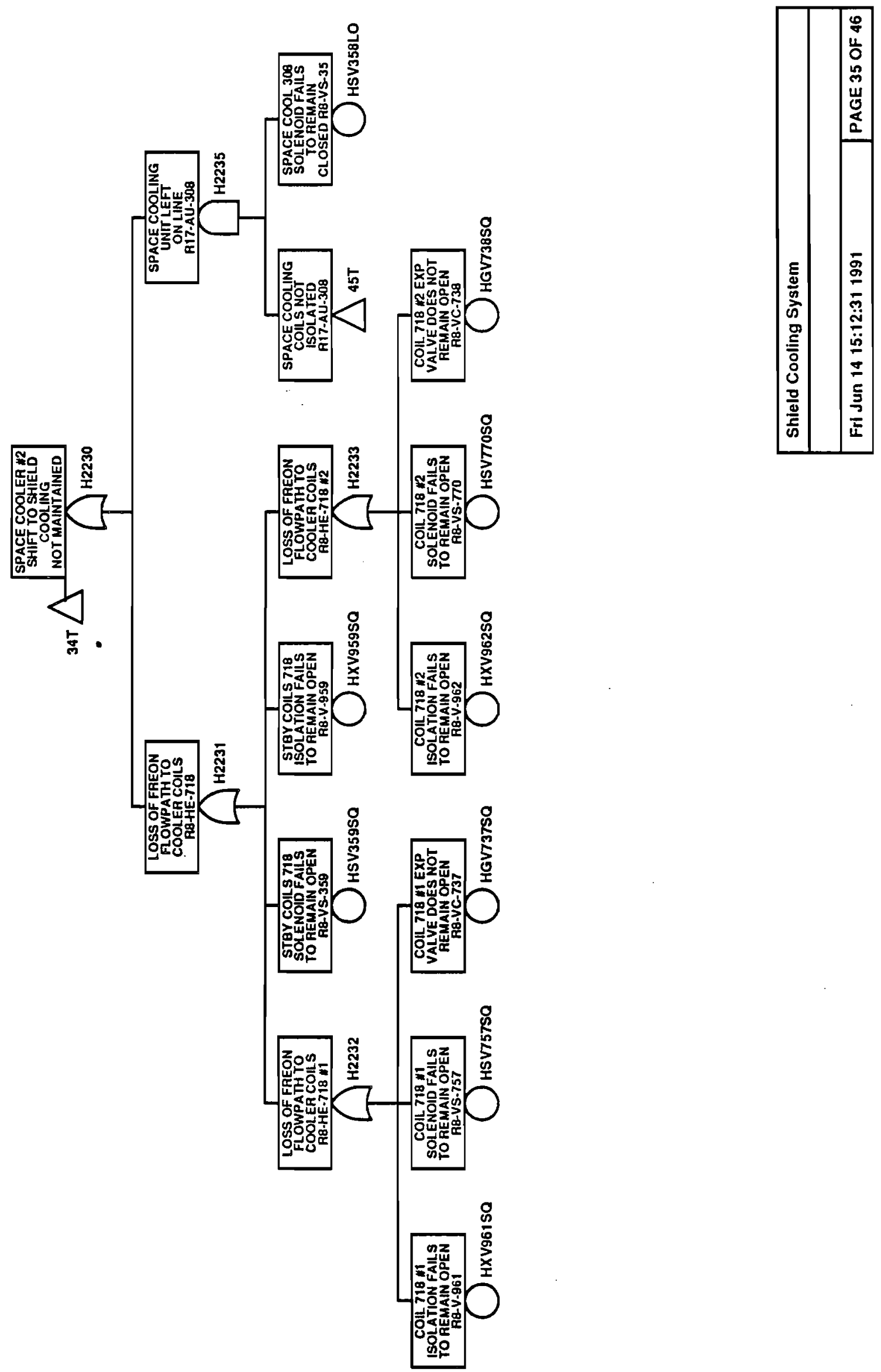

B. 2-513 


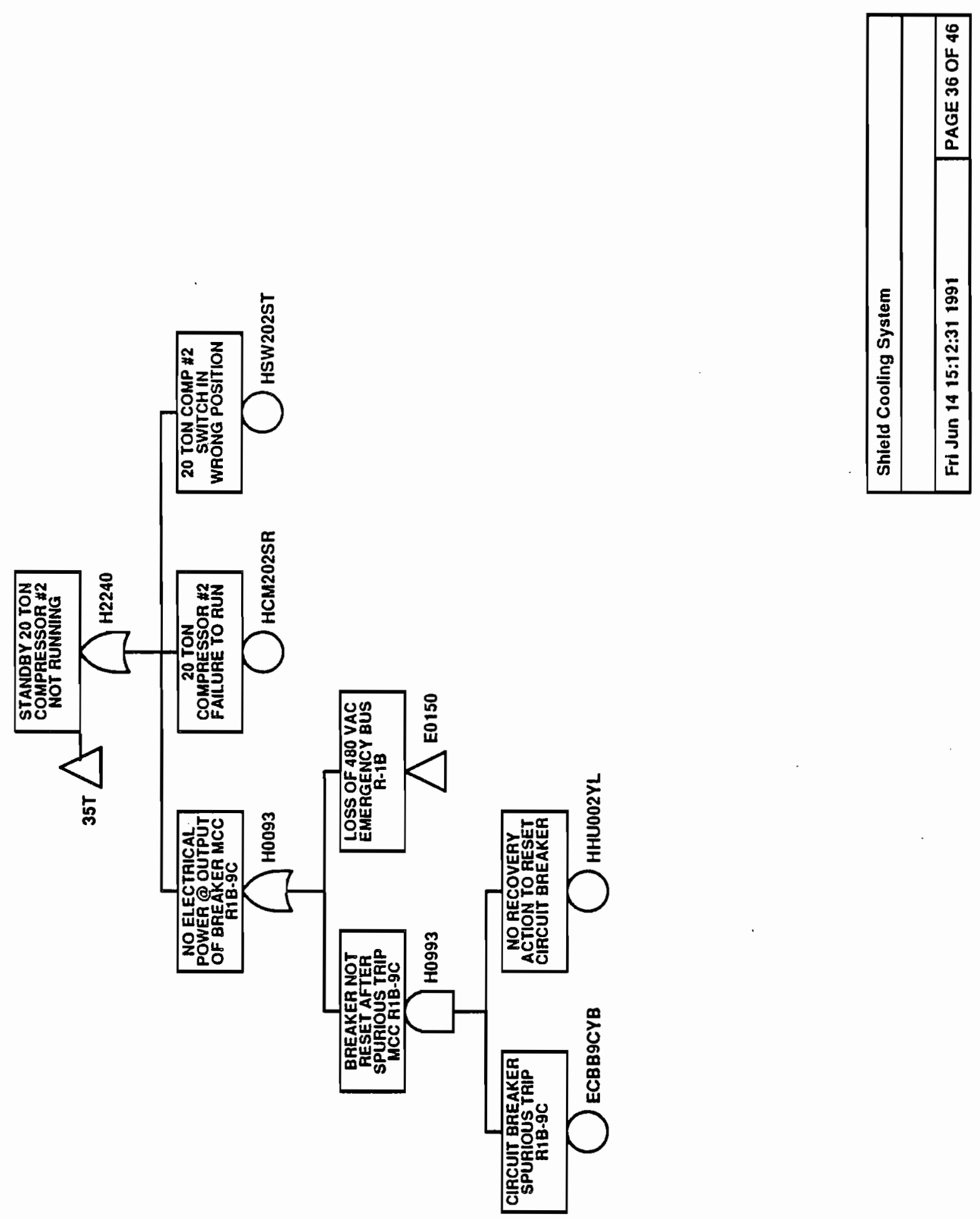




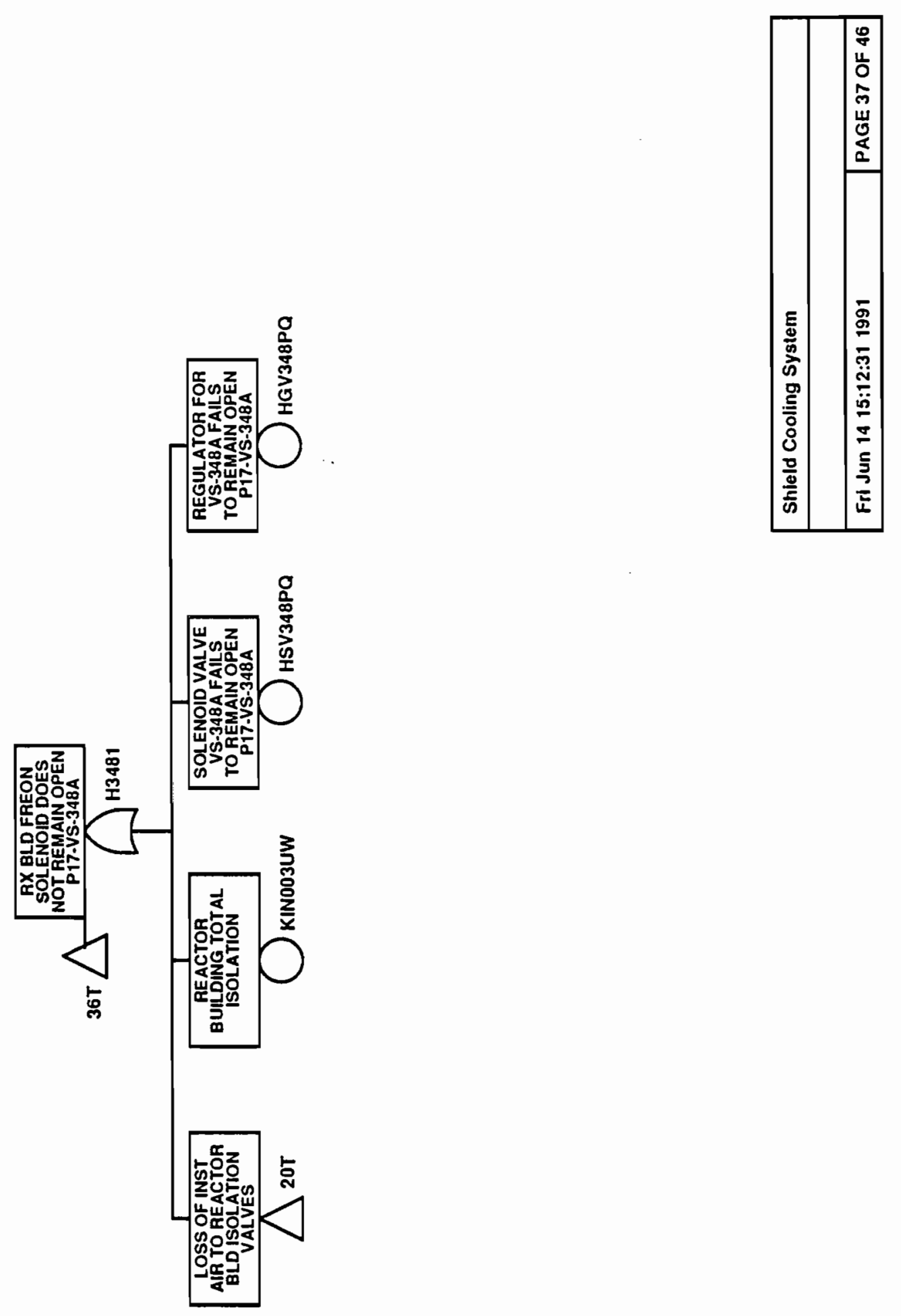




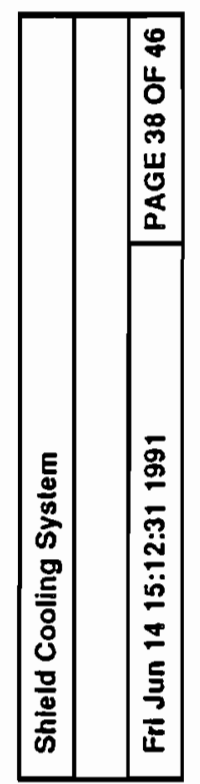

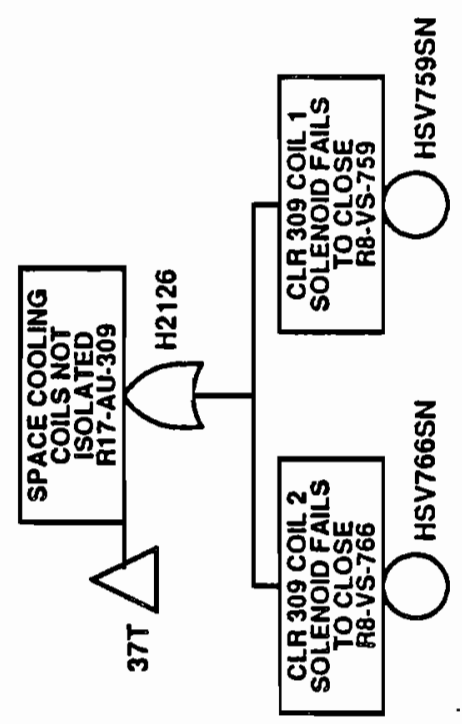




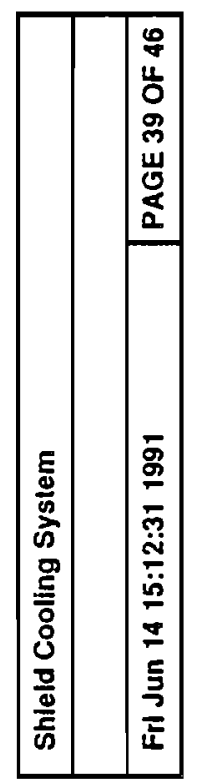

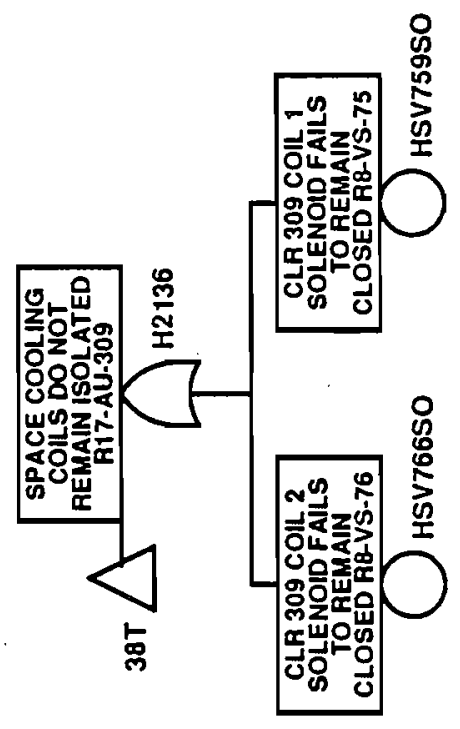




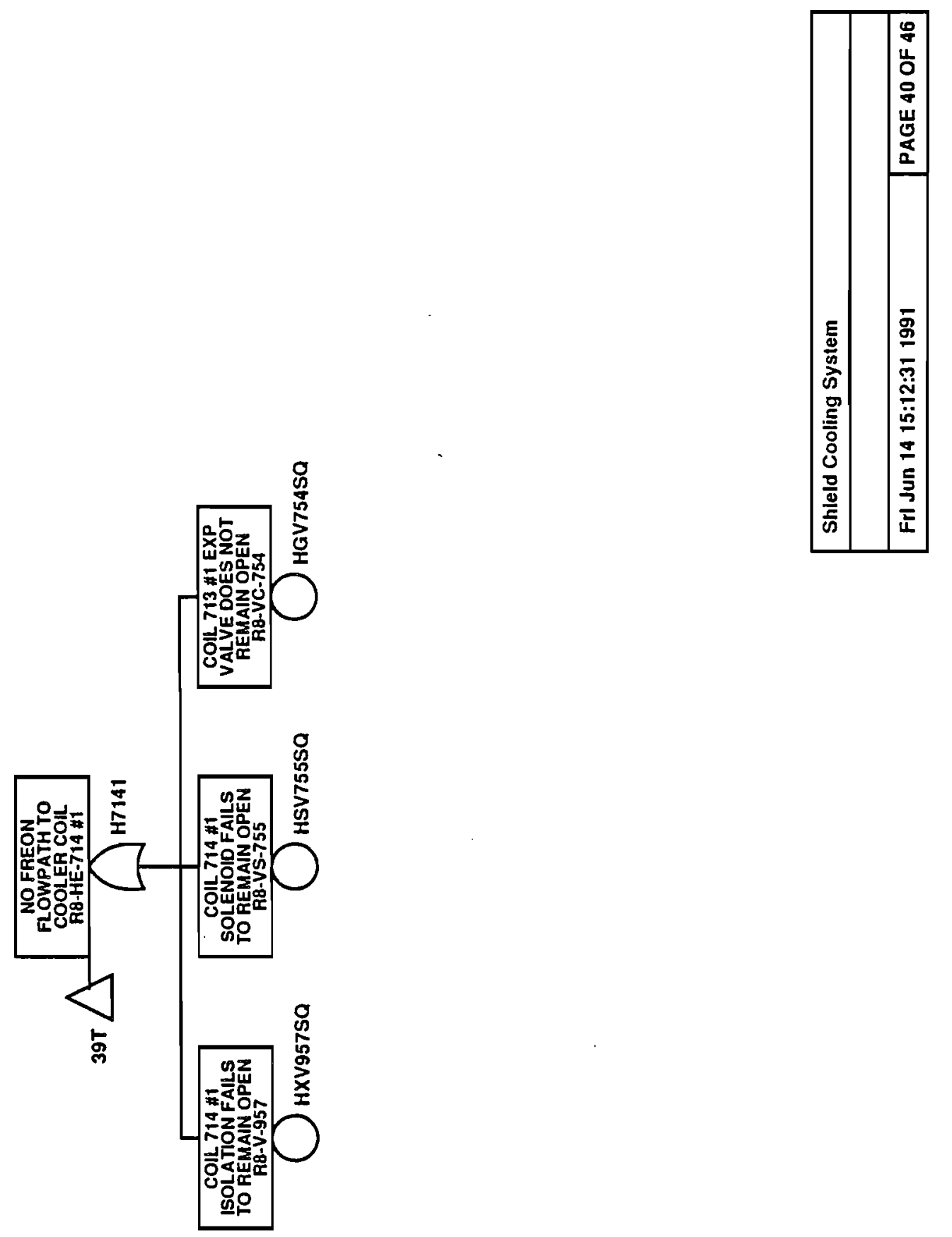

B. $2-518$ 


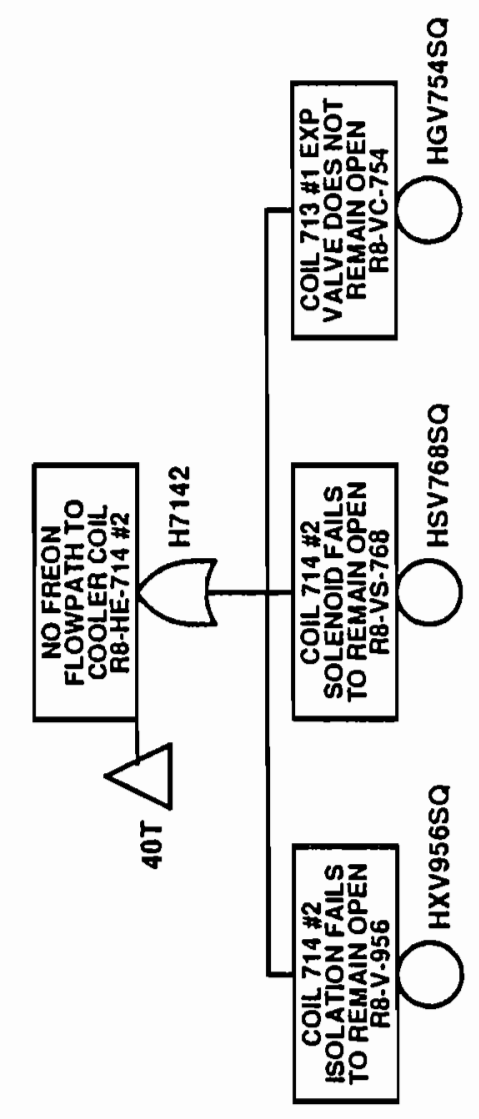

\begin{tabular}{|c|c|}
\hline & 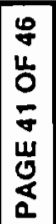 \\
\hline 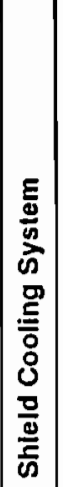 & 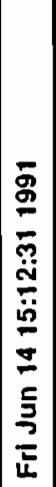 \\
\hline
\end{tabular}




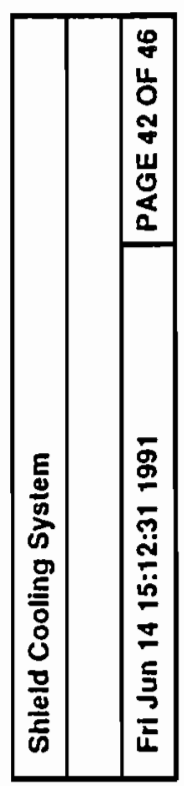

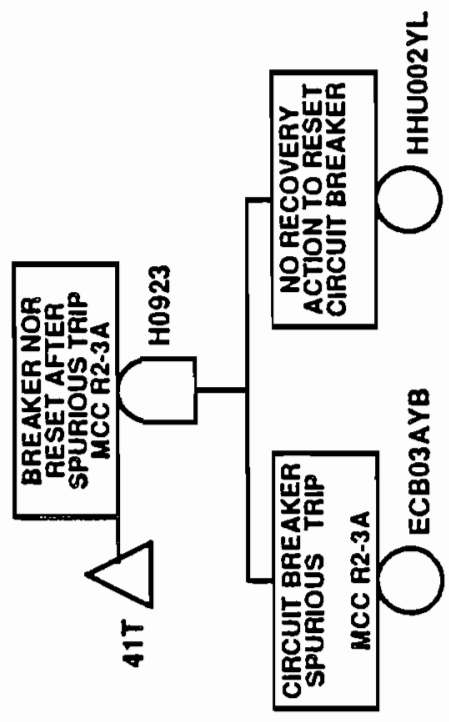

B. $2-520$ 


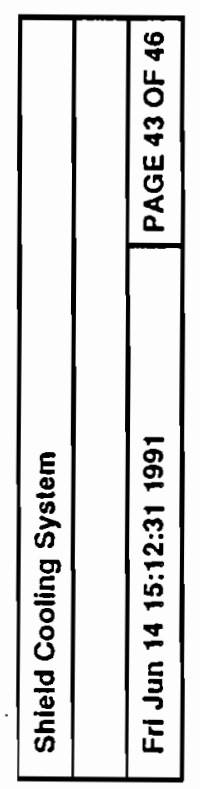

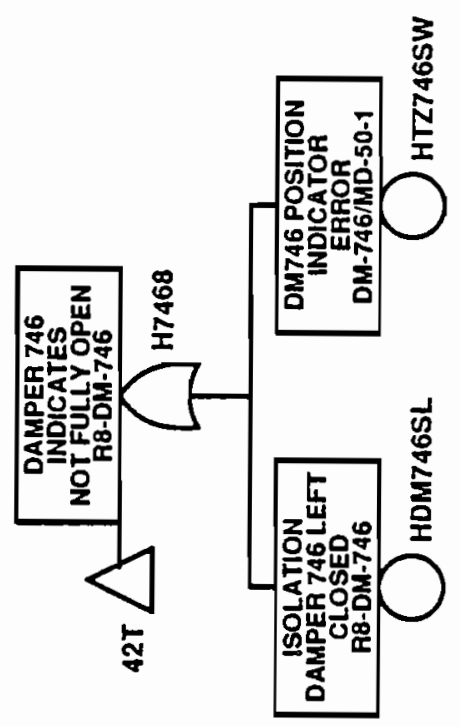

B. 2-521 


\begin{tabular}{|c|c|}
\hline & 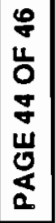 \\
\hline 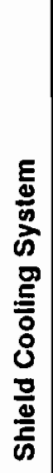 & 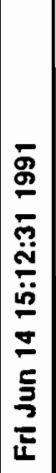 \\
\hline
\end{tabular}

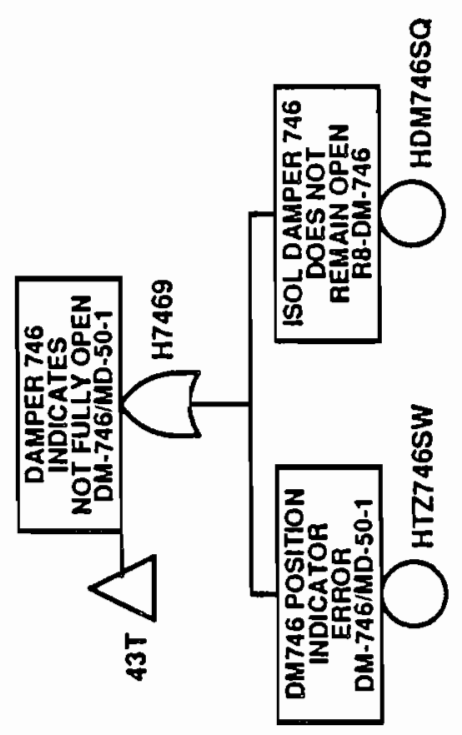

B. 2-522 


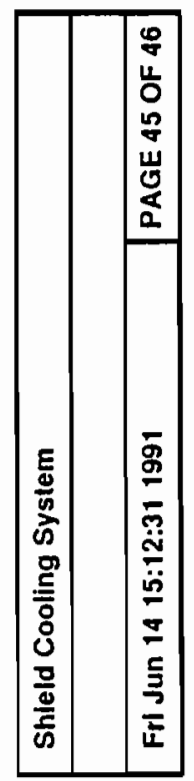

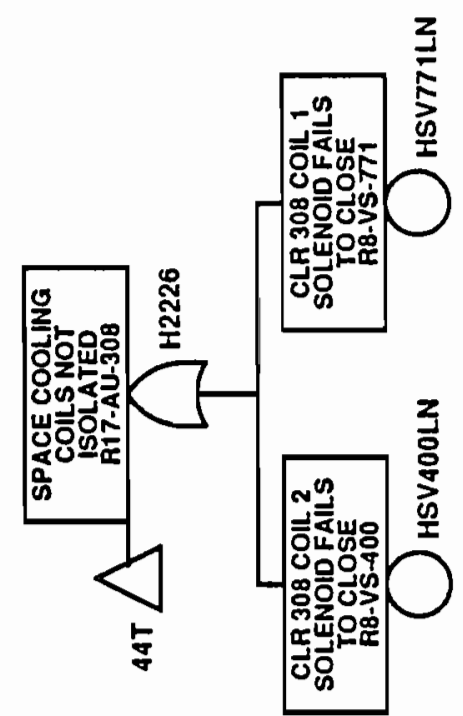

B. 2-523 


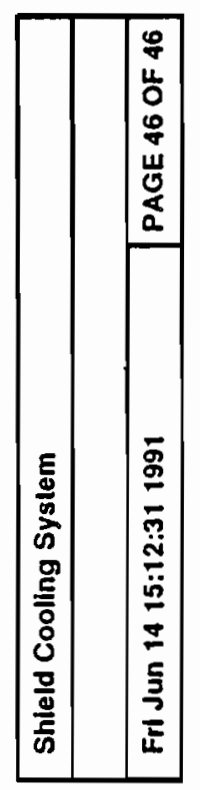

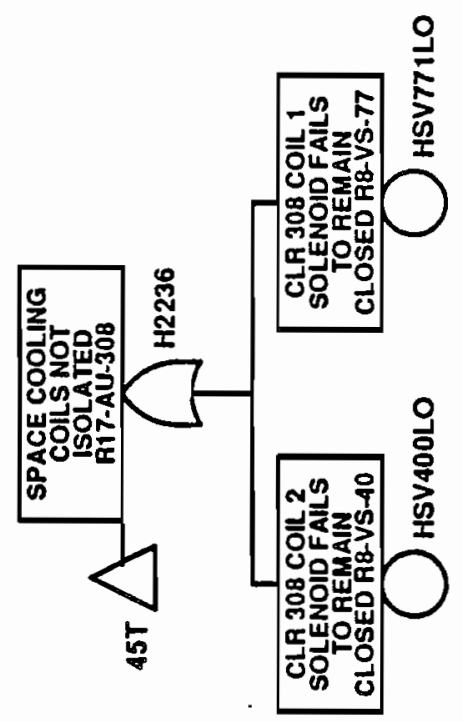




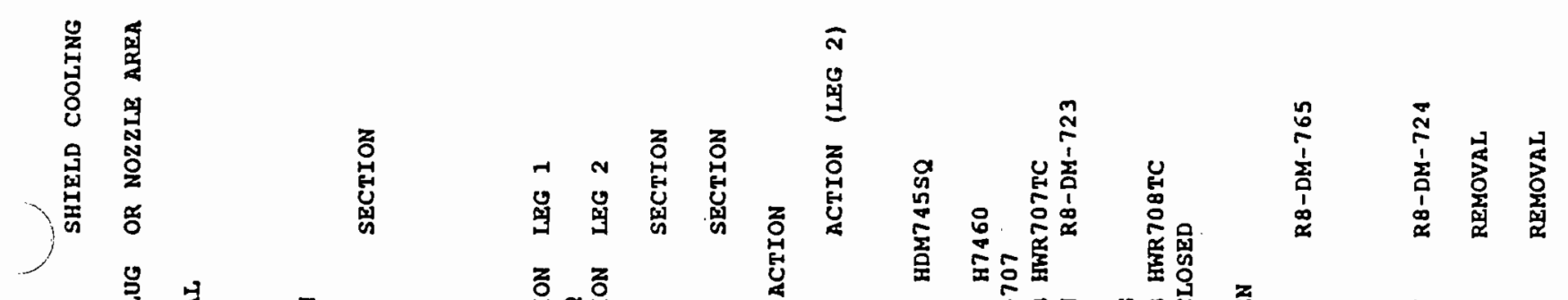

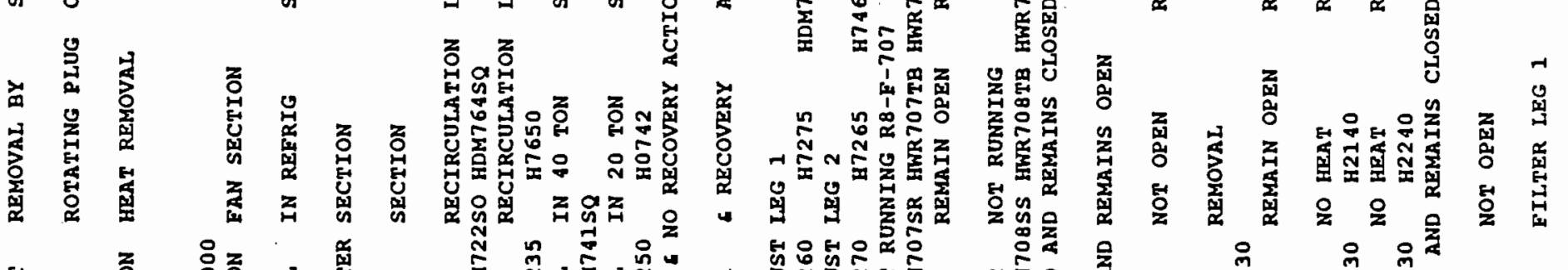

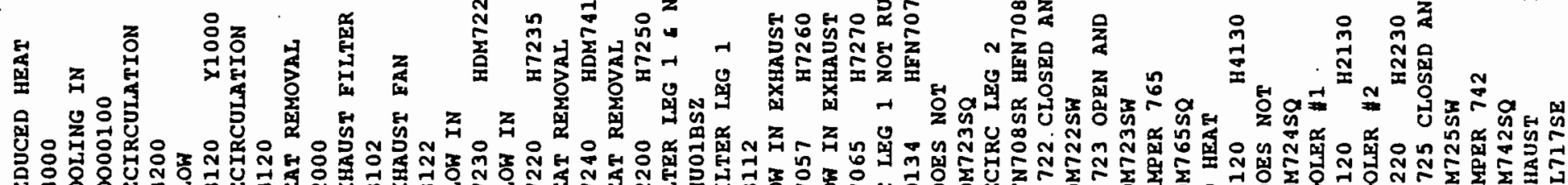

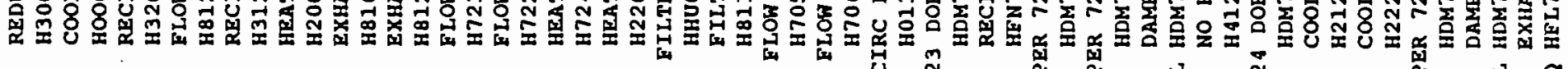

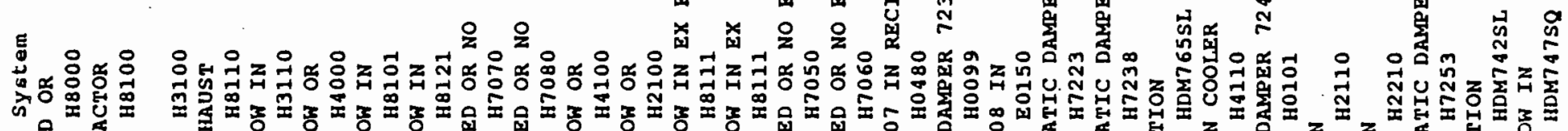

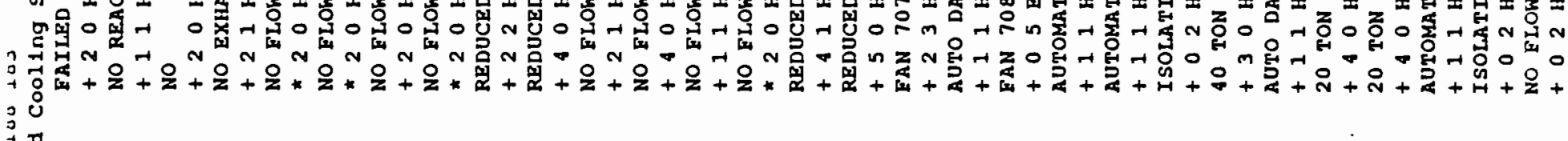

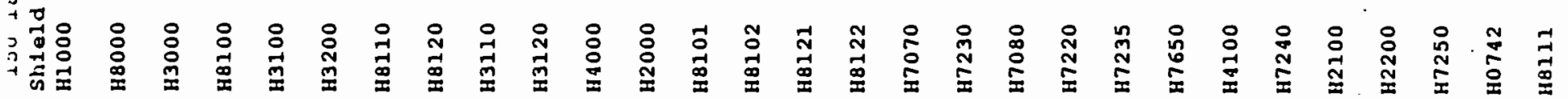




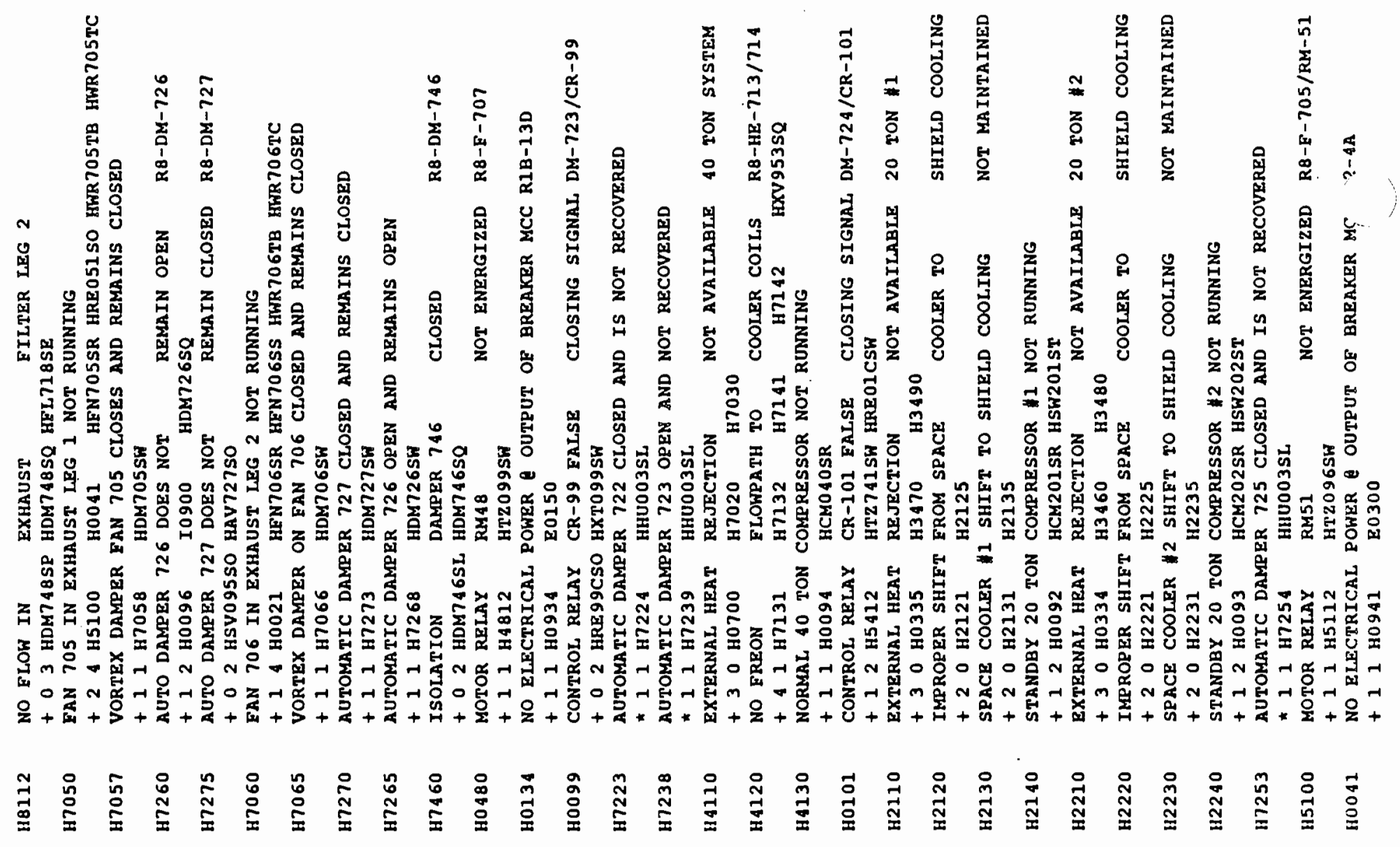




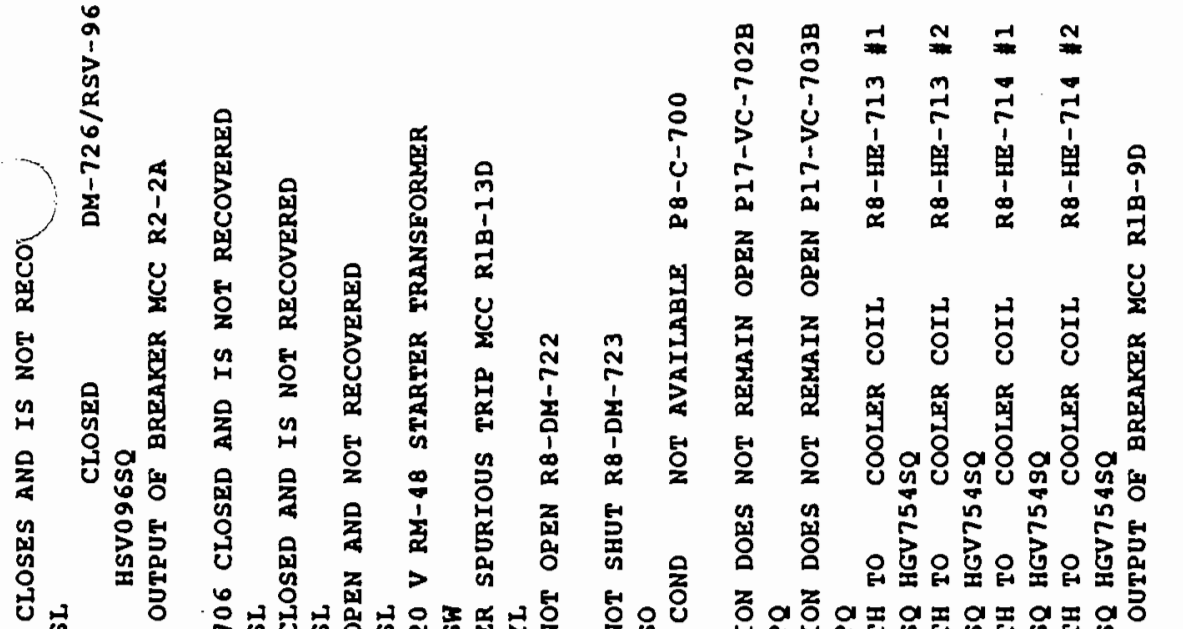

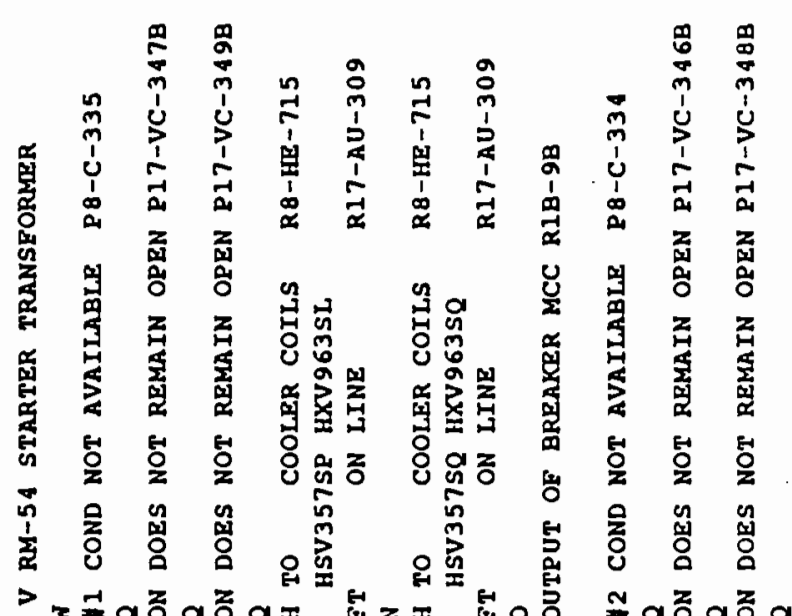
药

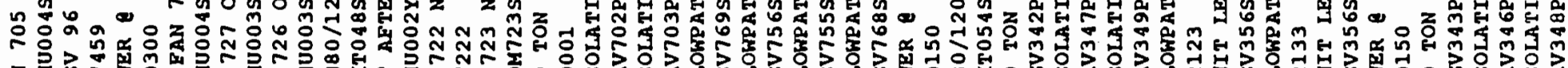

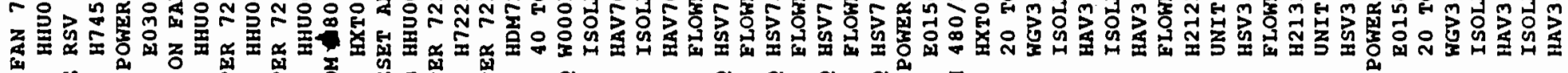

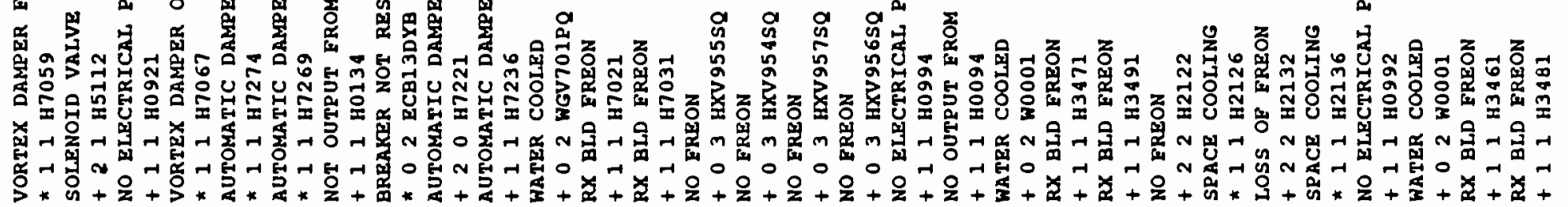

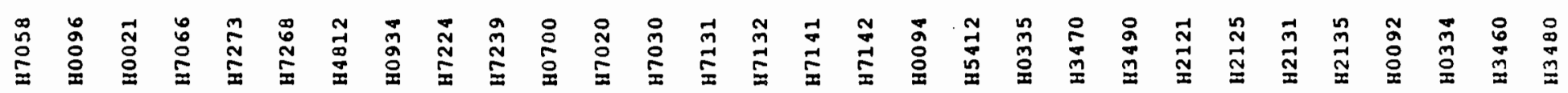




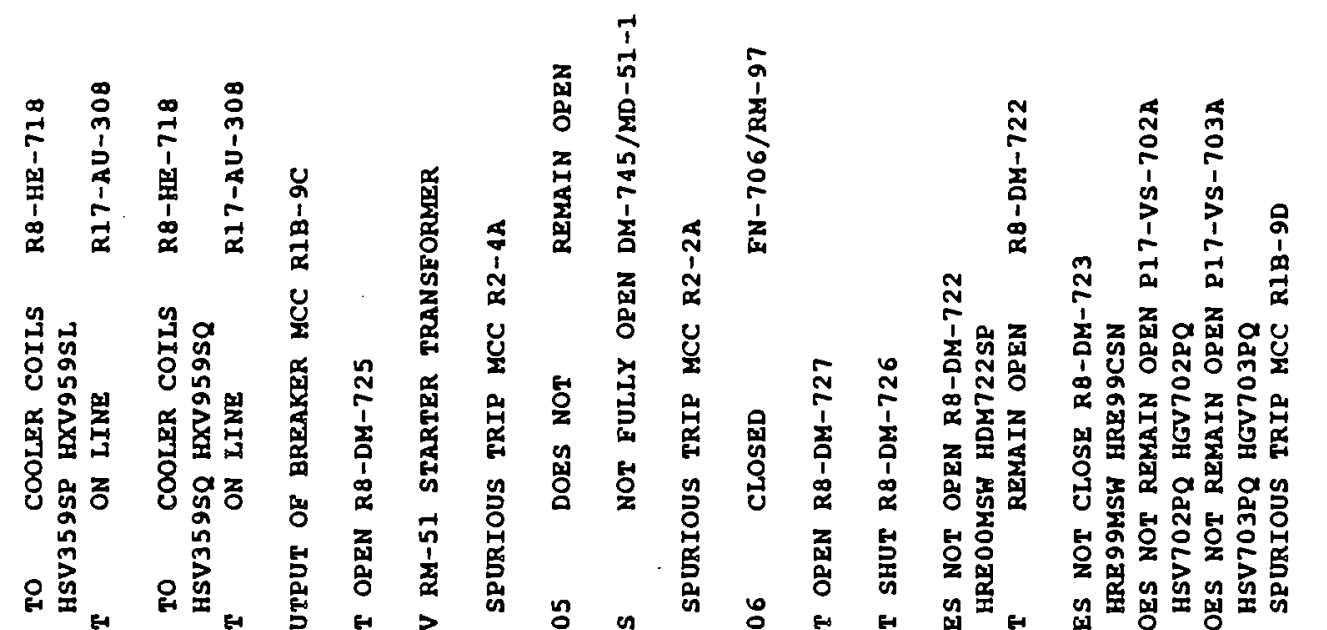

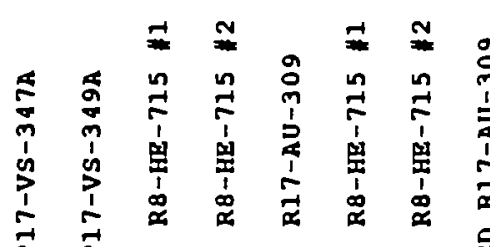

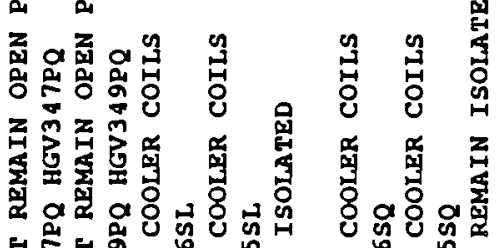
붕

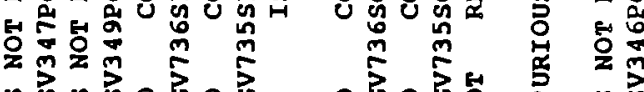

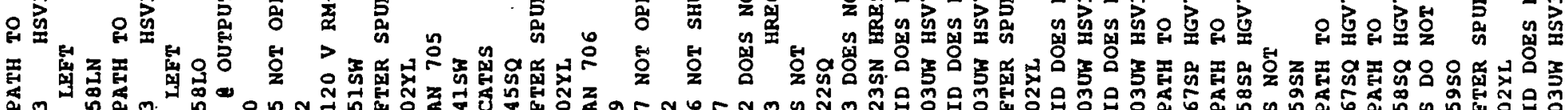

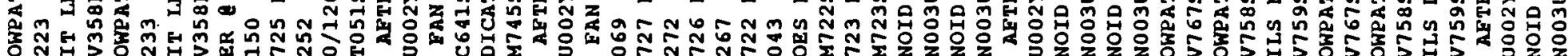

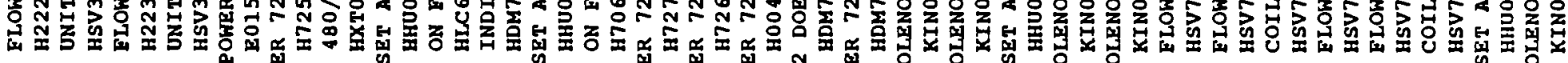

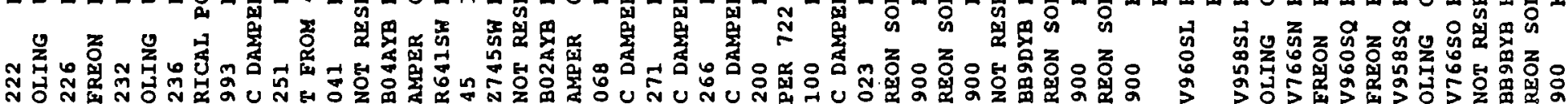
z

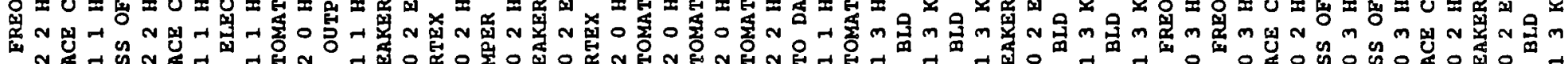

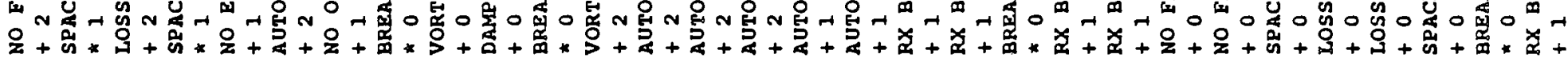

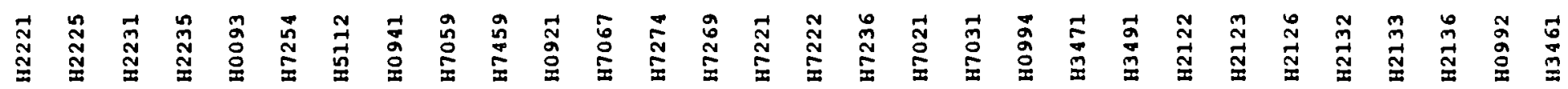




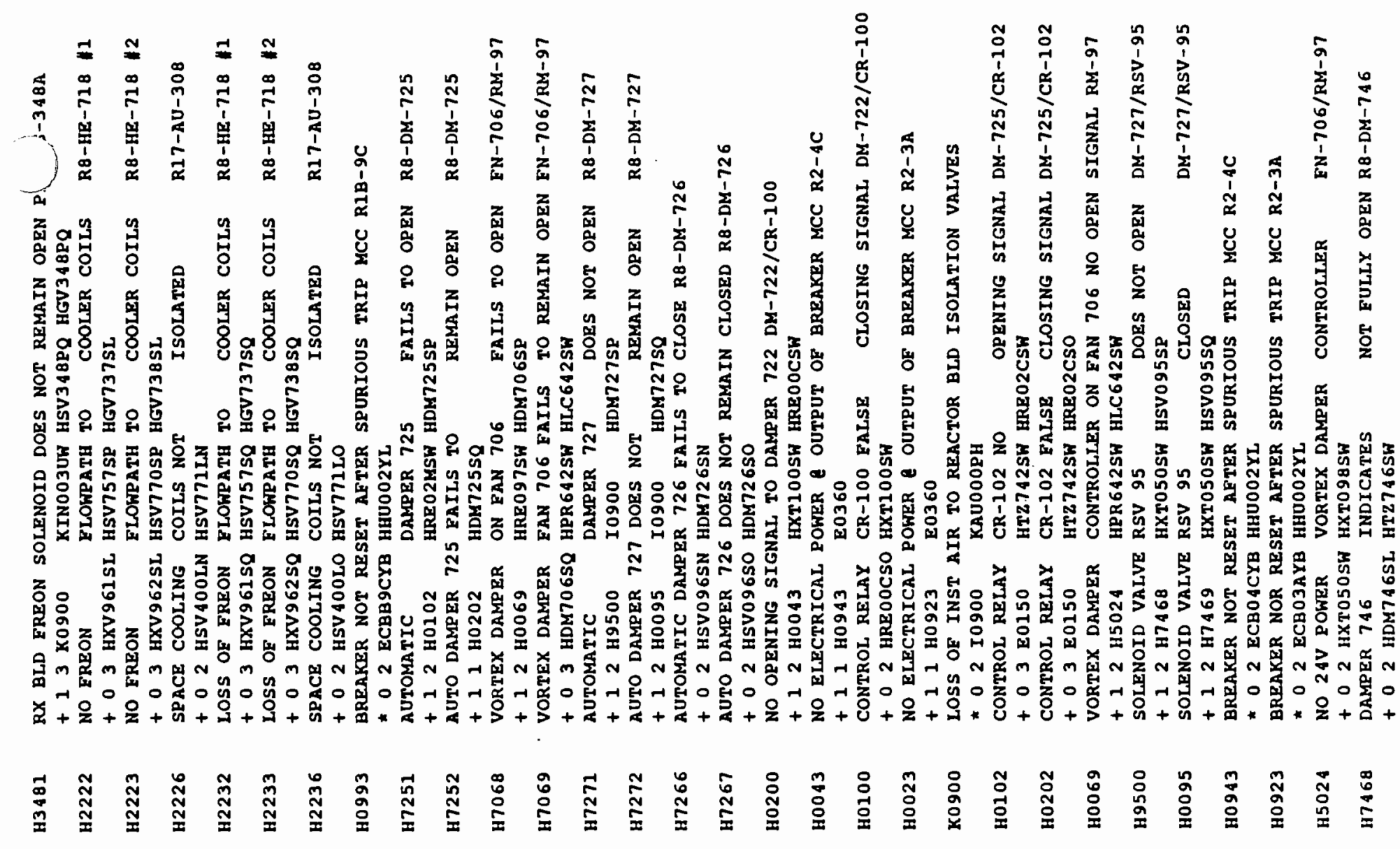




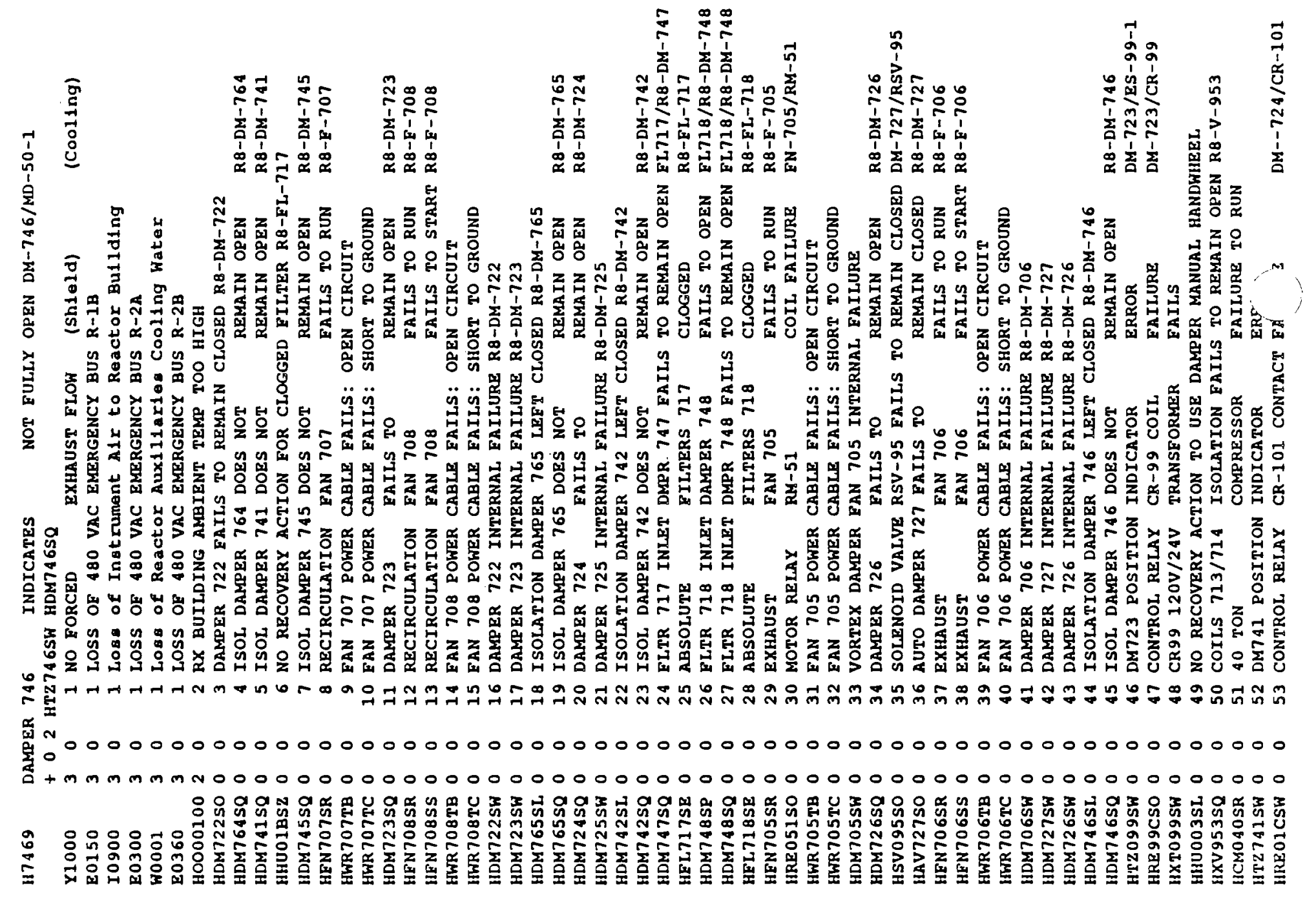




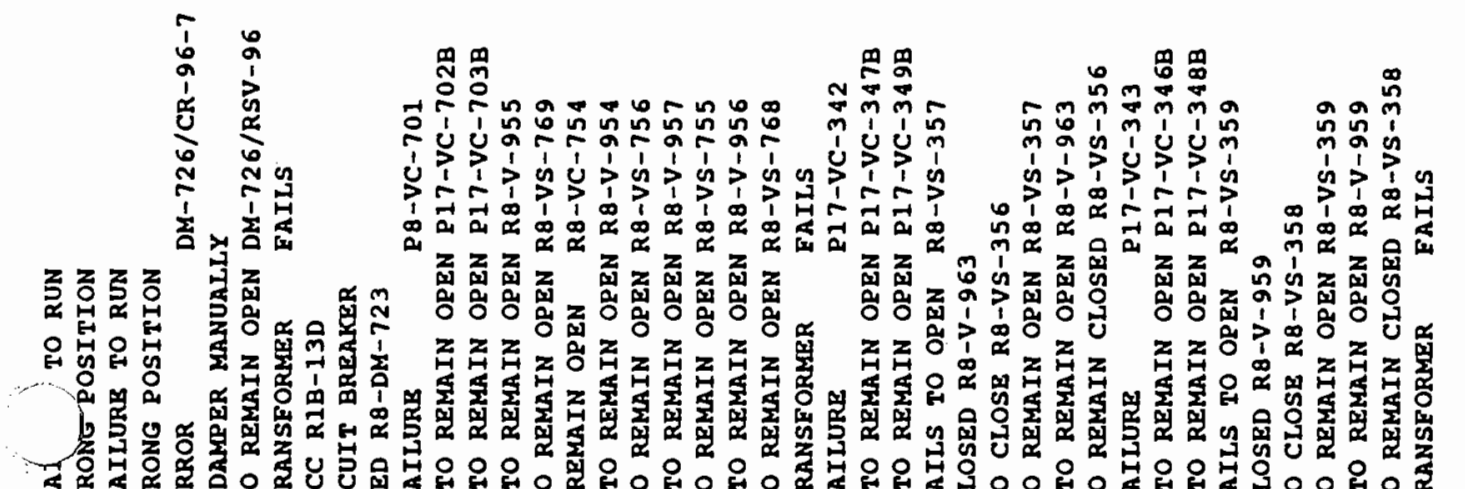

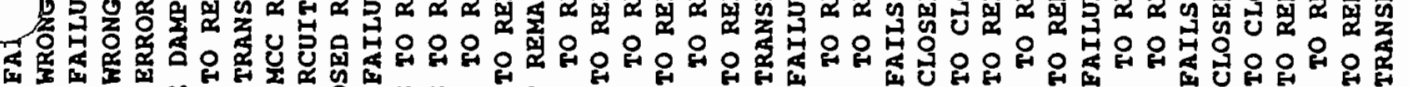

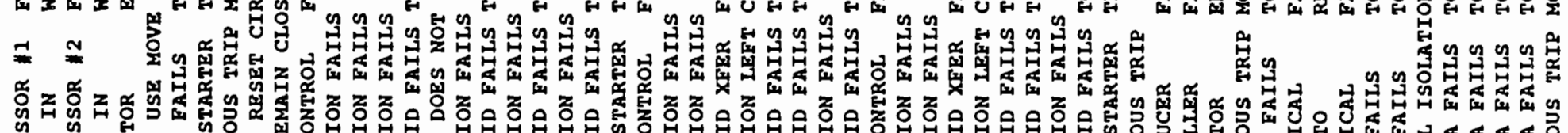

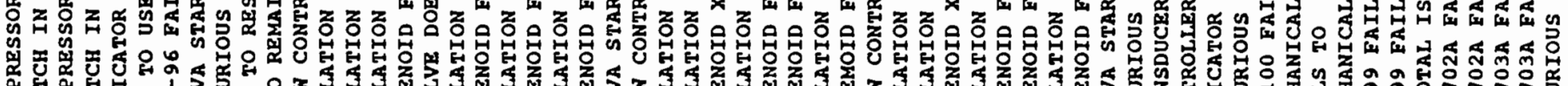

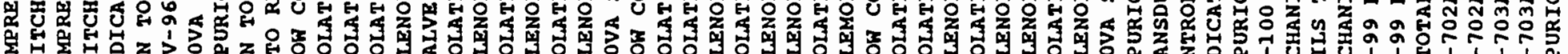

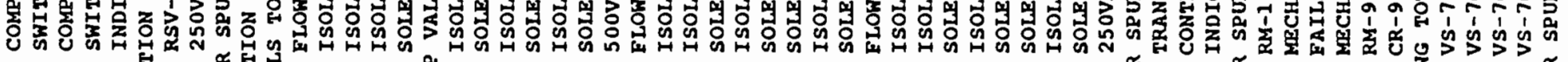

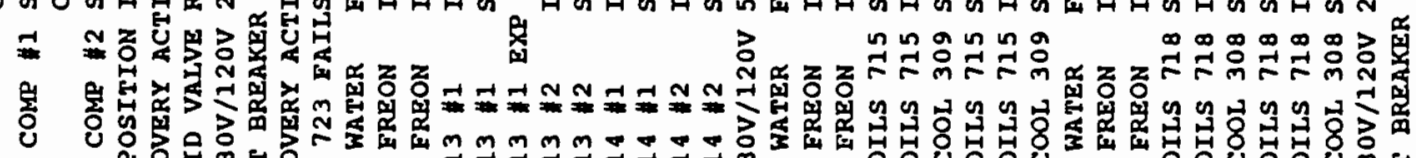

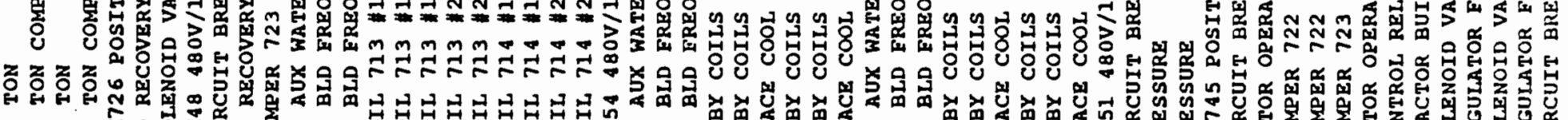

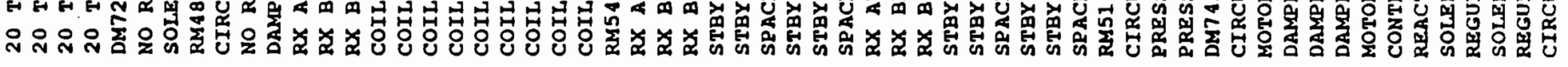

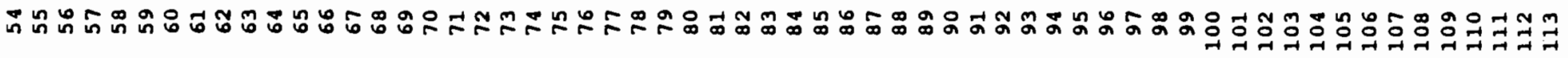

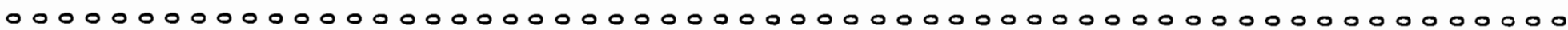

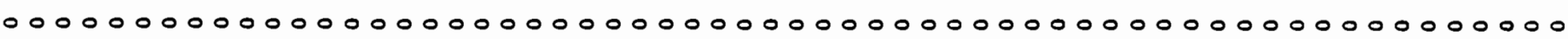

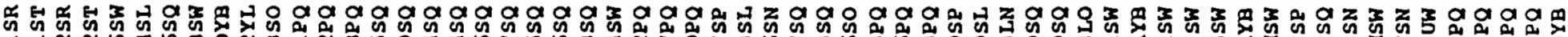

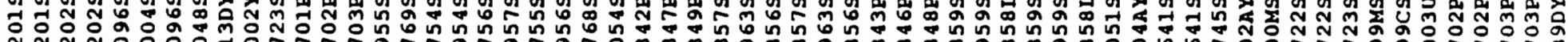

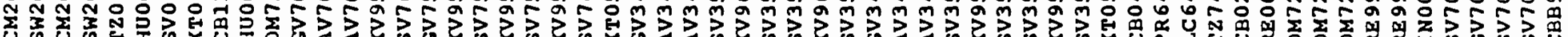

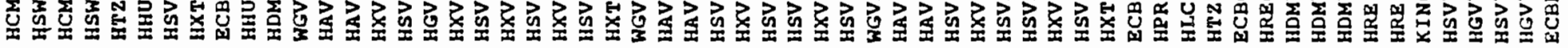




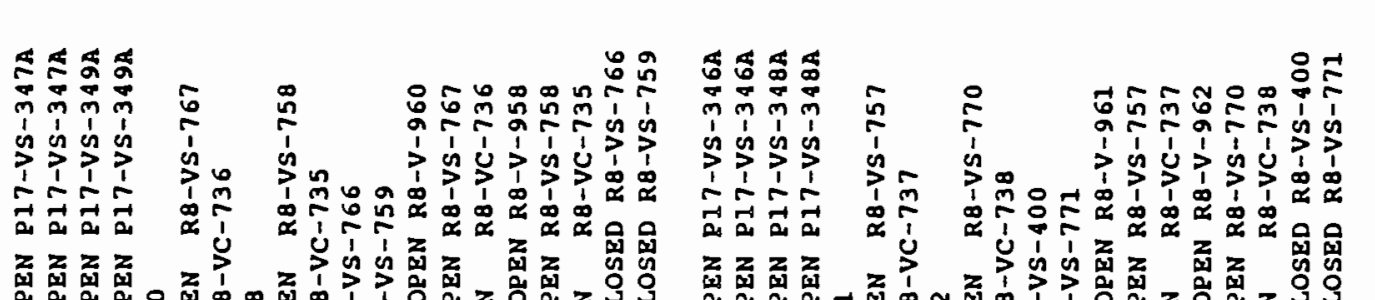

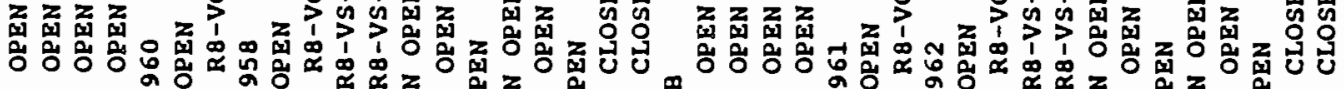

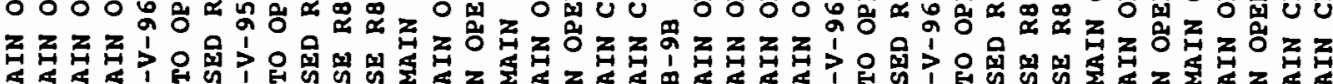

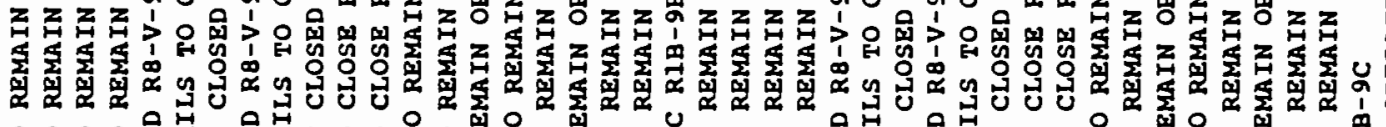

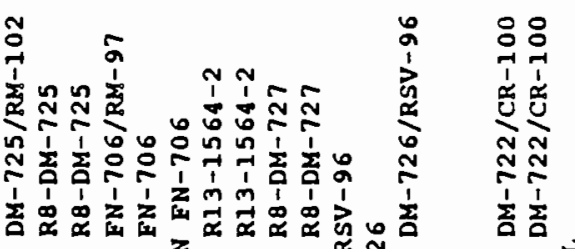

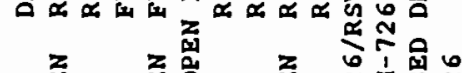

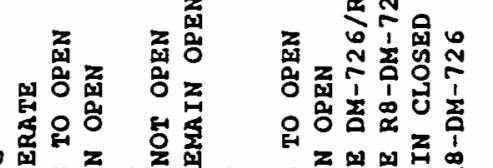

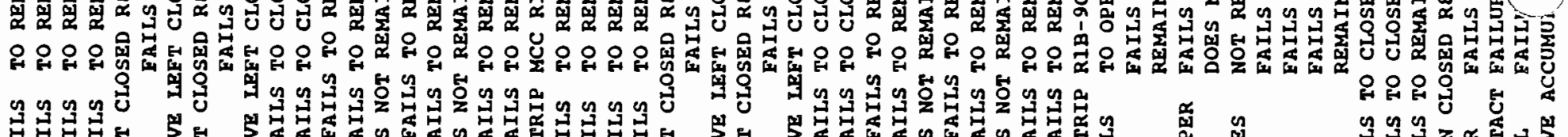

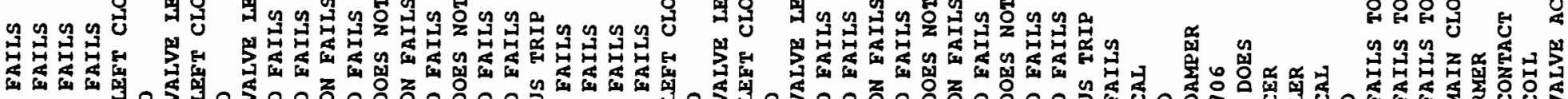

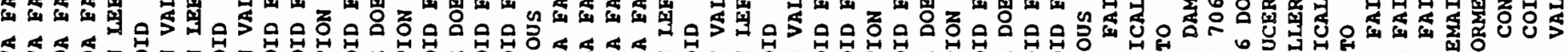

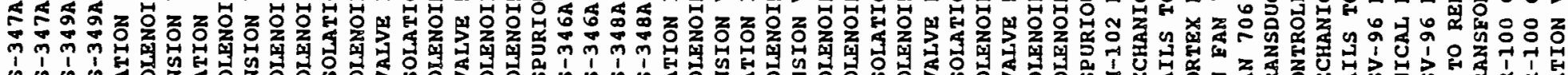

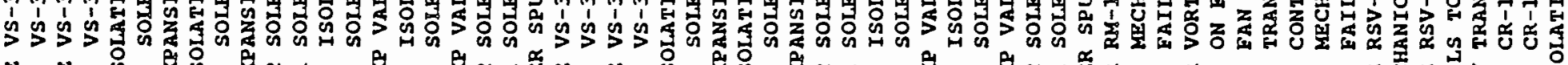
N

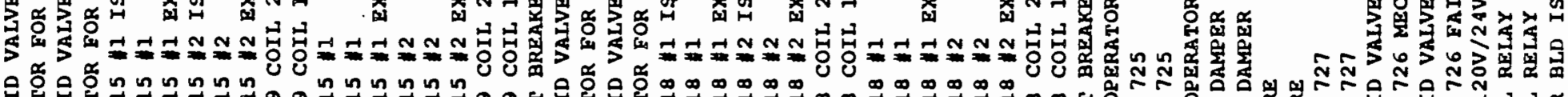

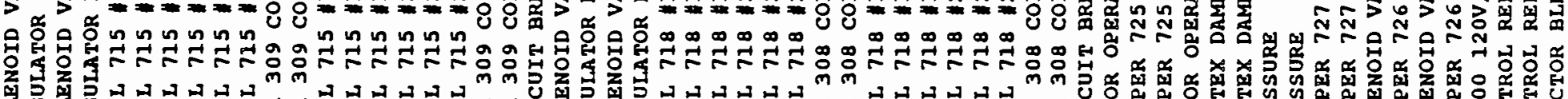

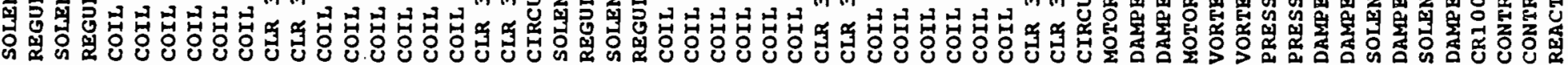

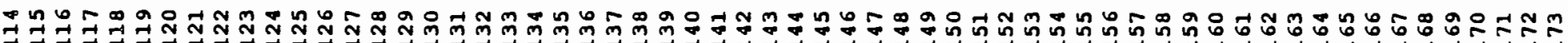
a

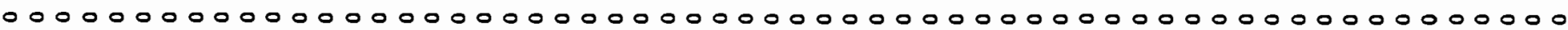

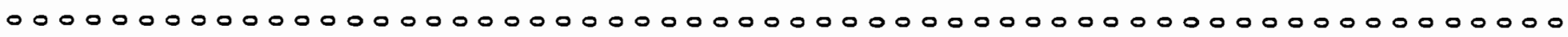

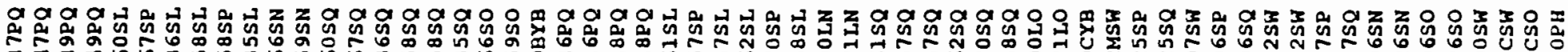

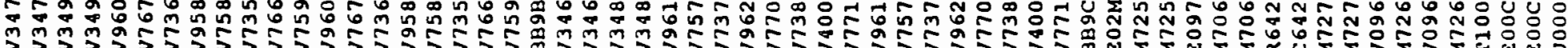

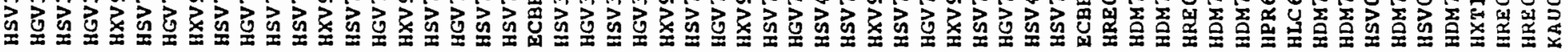



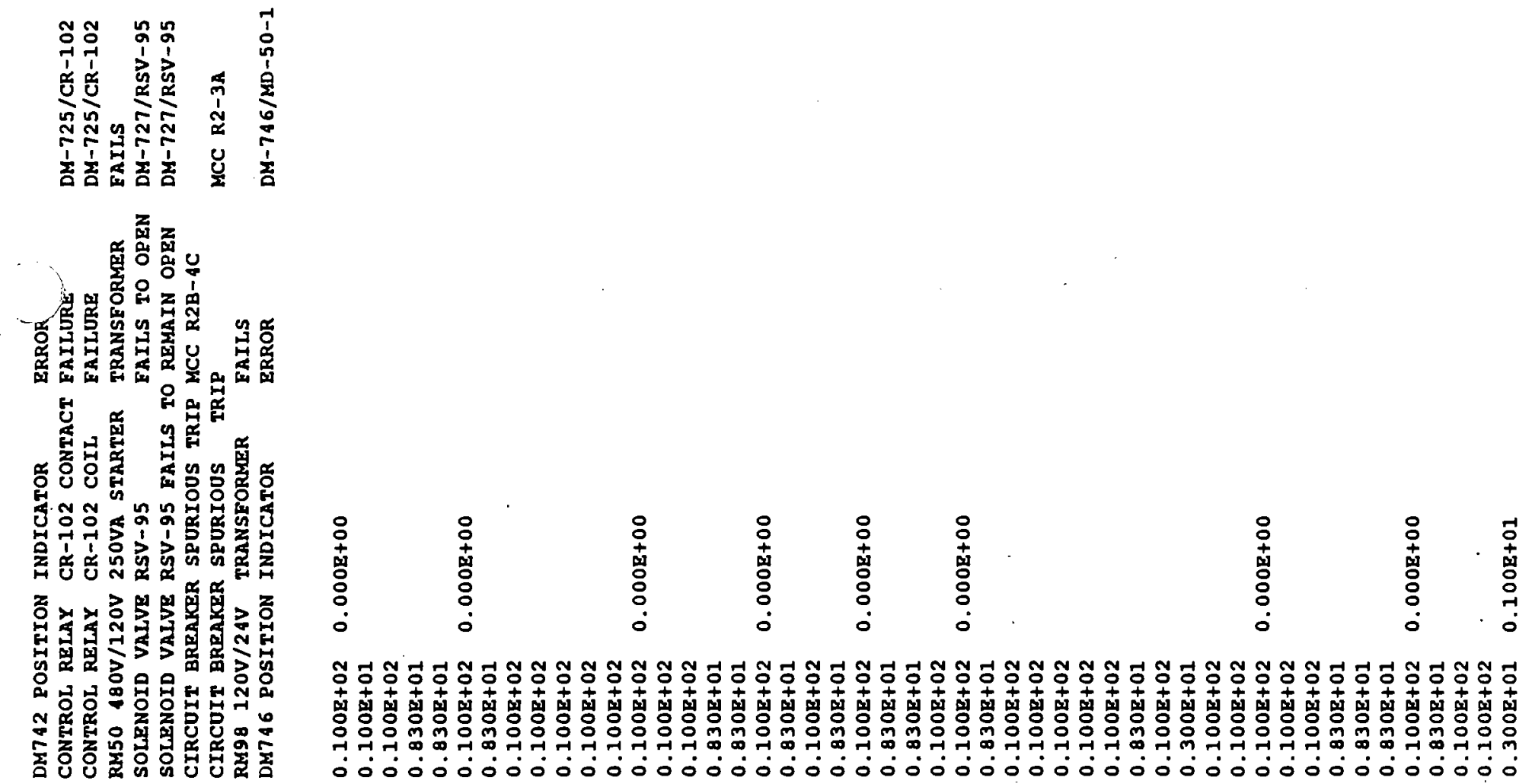

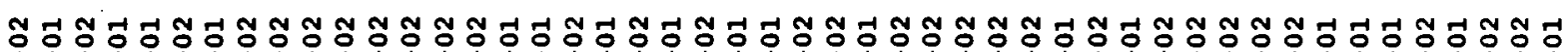

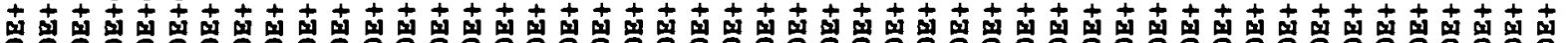

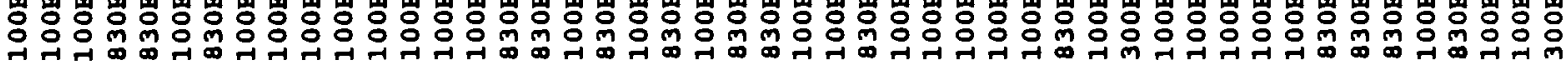

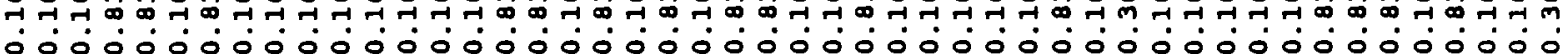

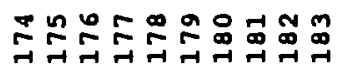

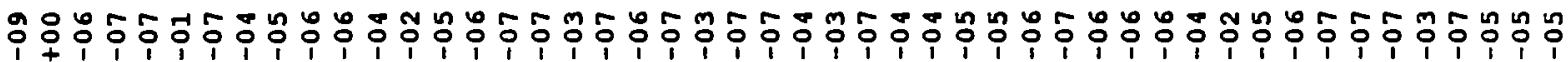

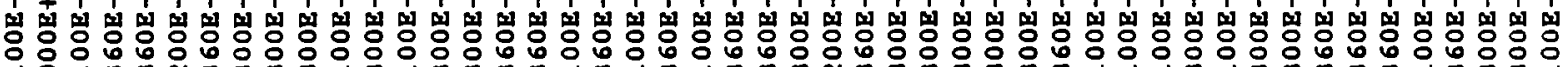

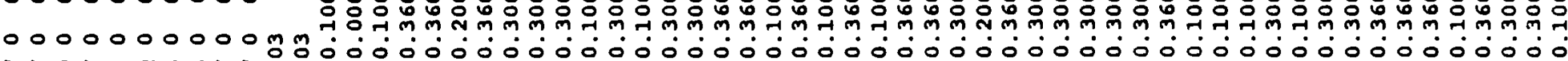
0000000000 勇

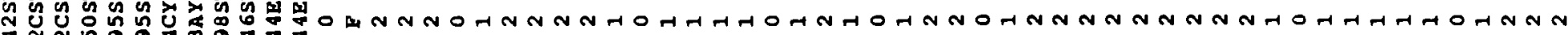
N

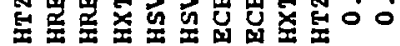




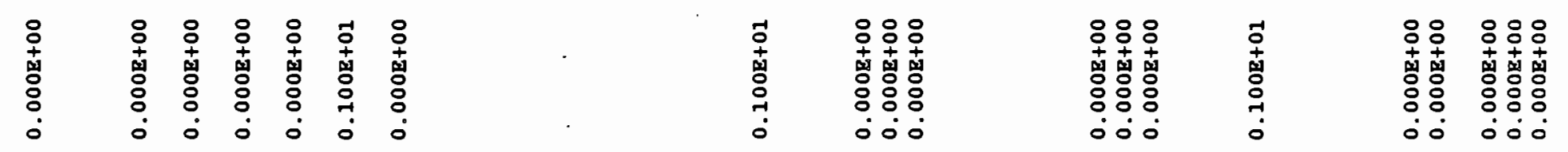

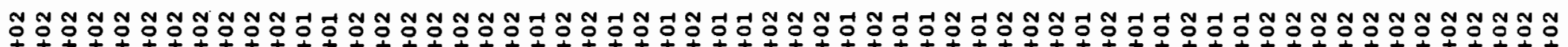

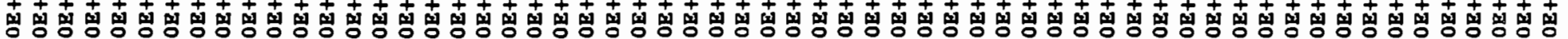

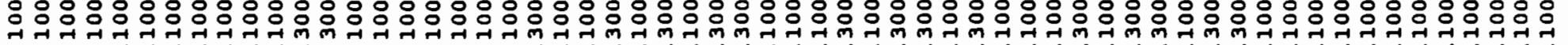

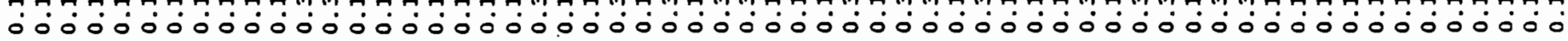

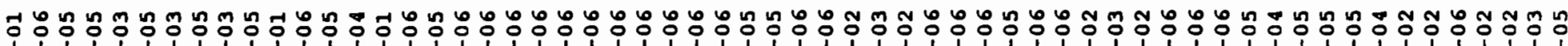

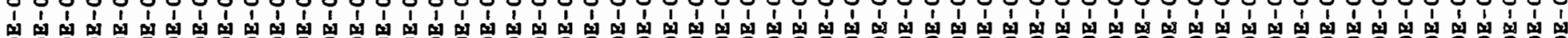

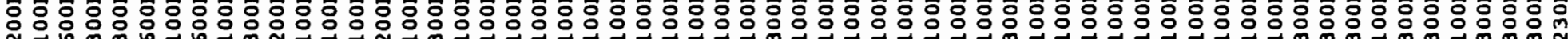

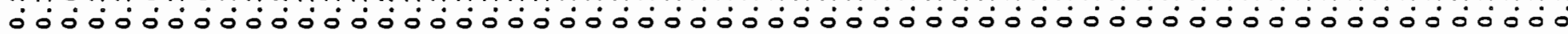

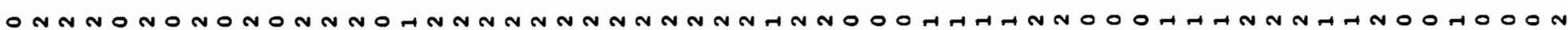

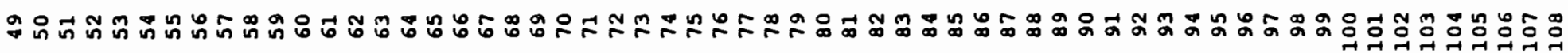



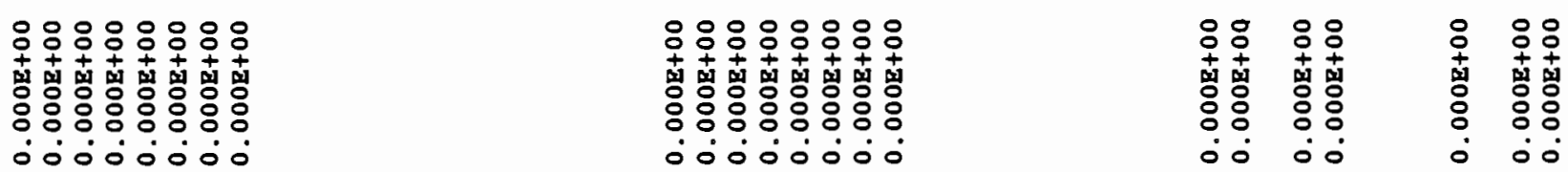

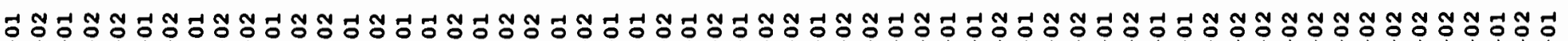

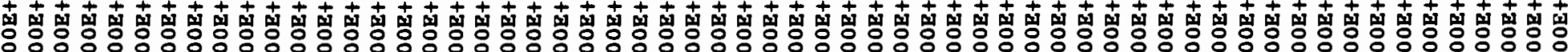

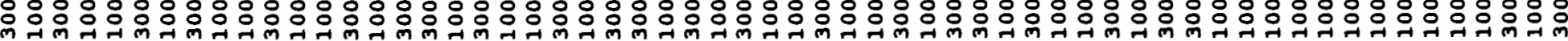

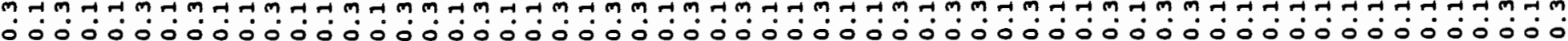

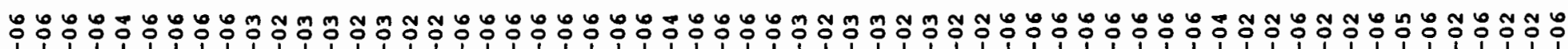

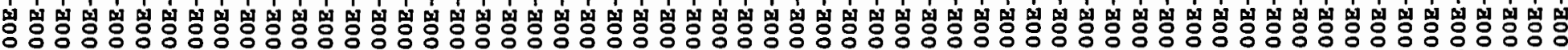

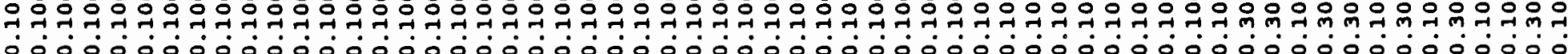

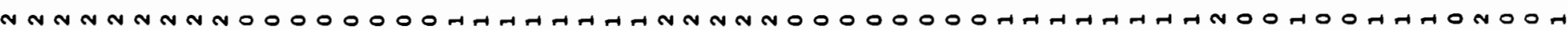

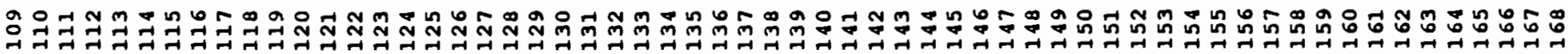




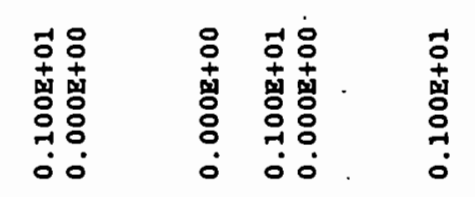

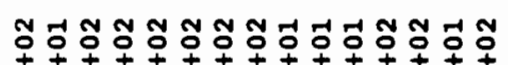

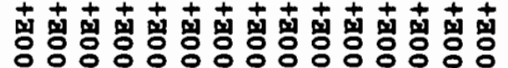

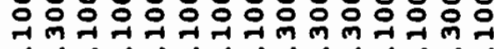
0000000000000

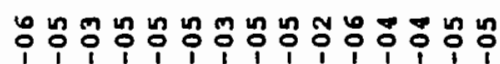

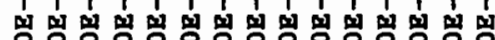

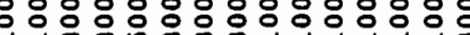

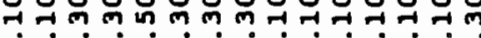
-0000000000000 hNOHAHOHNOHNNNN

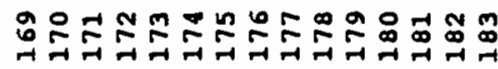




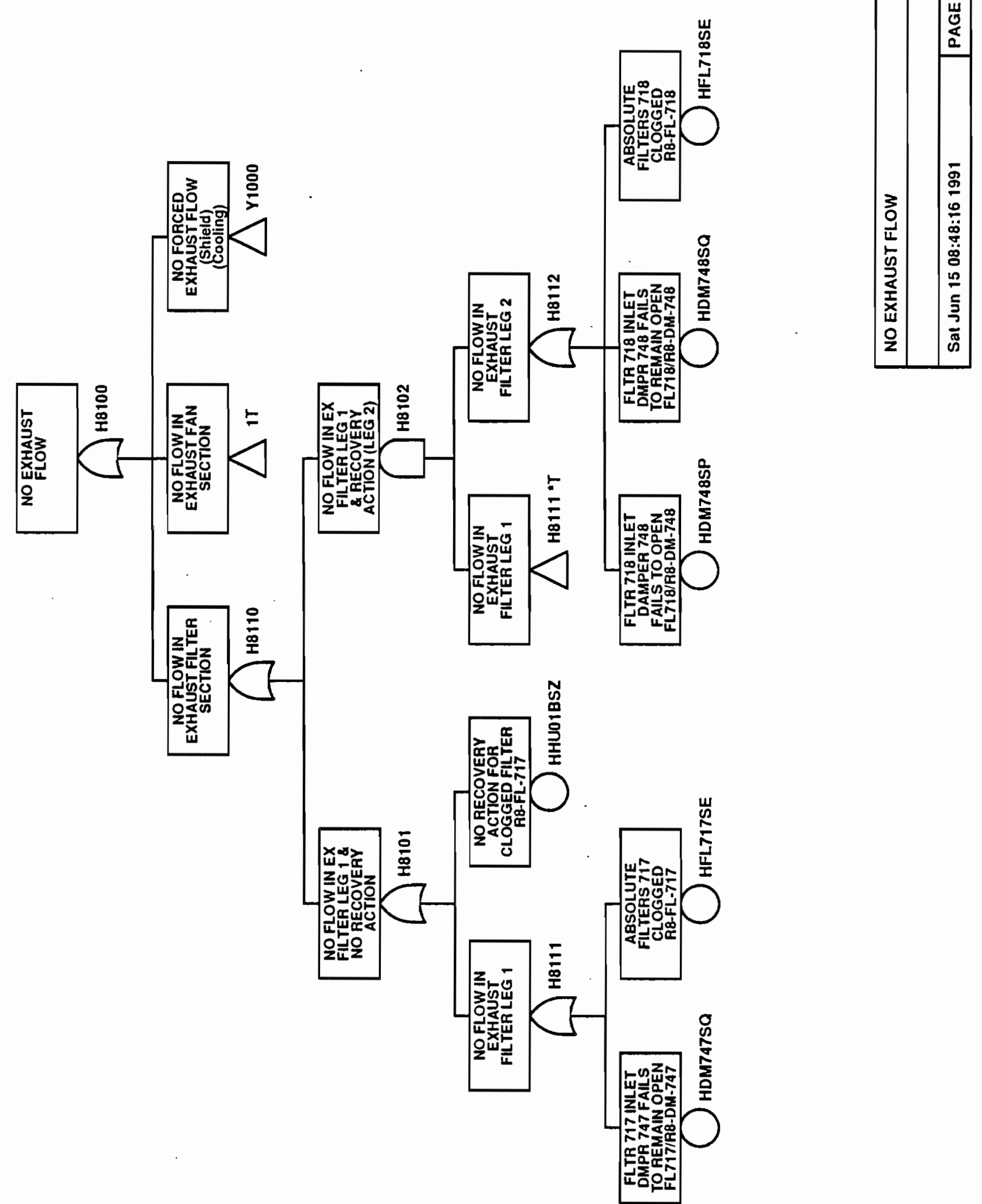




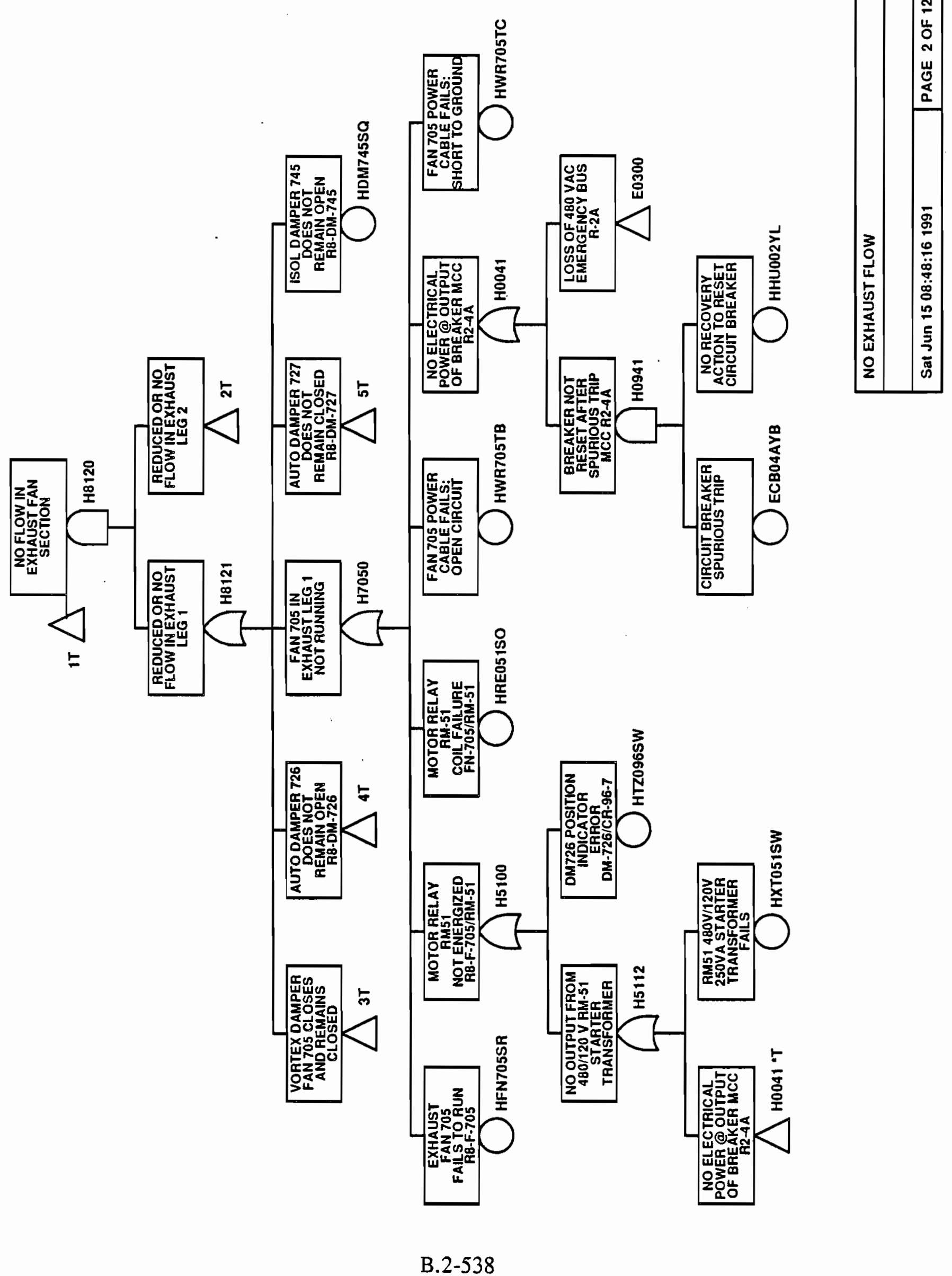




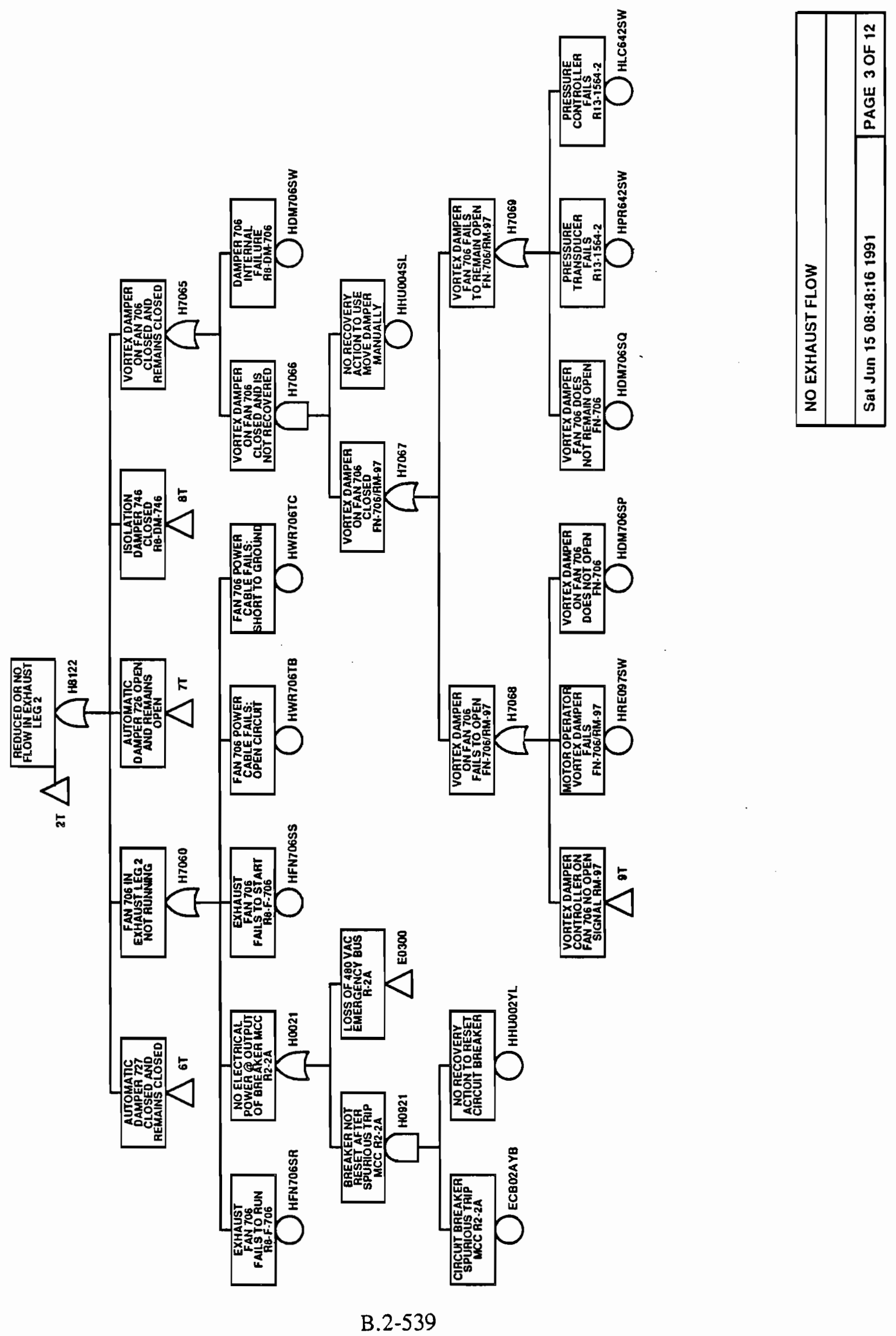




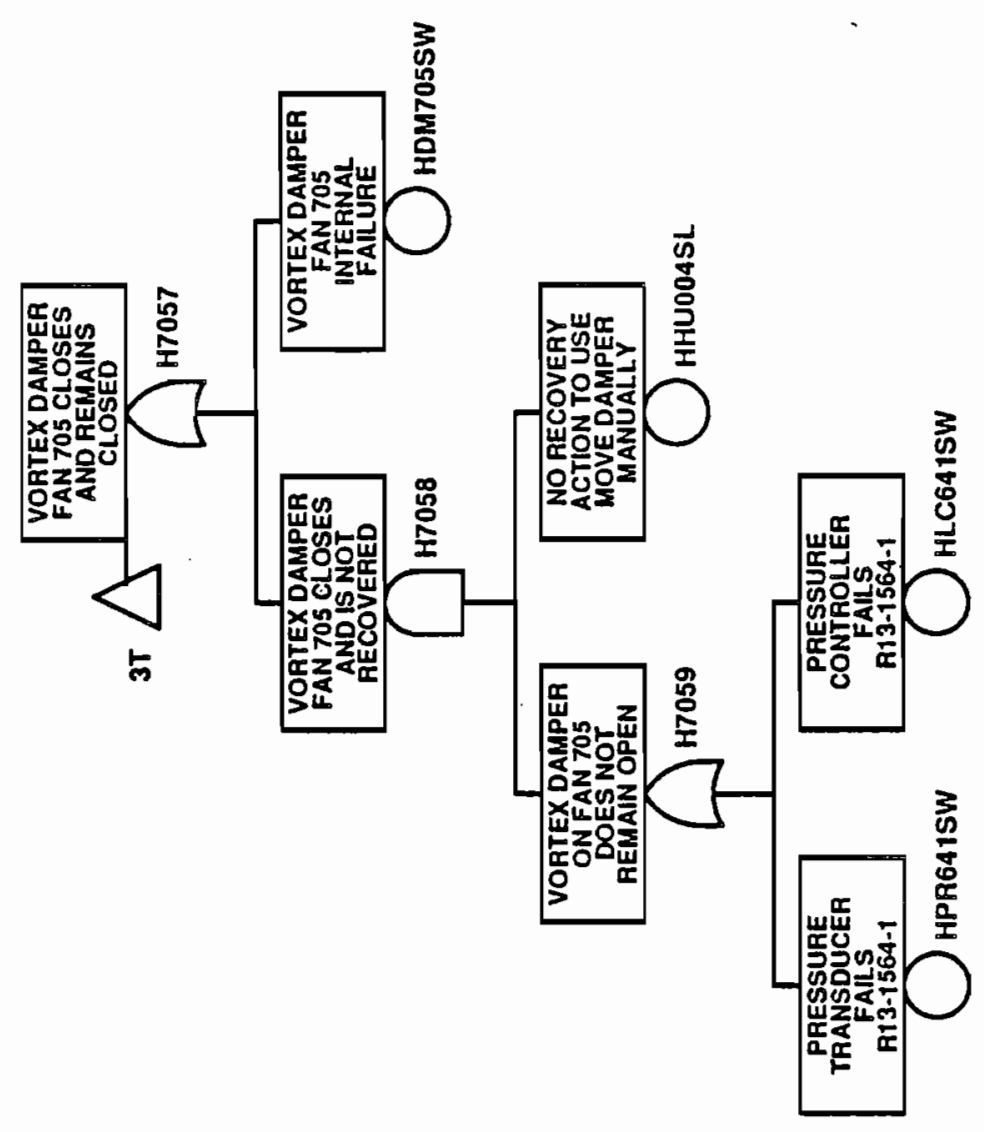

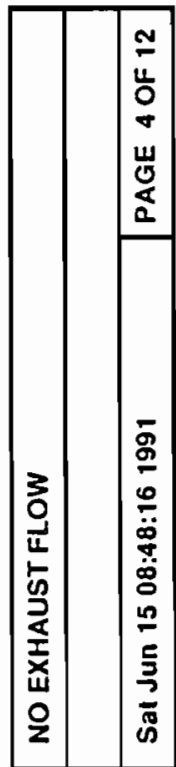




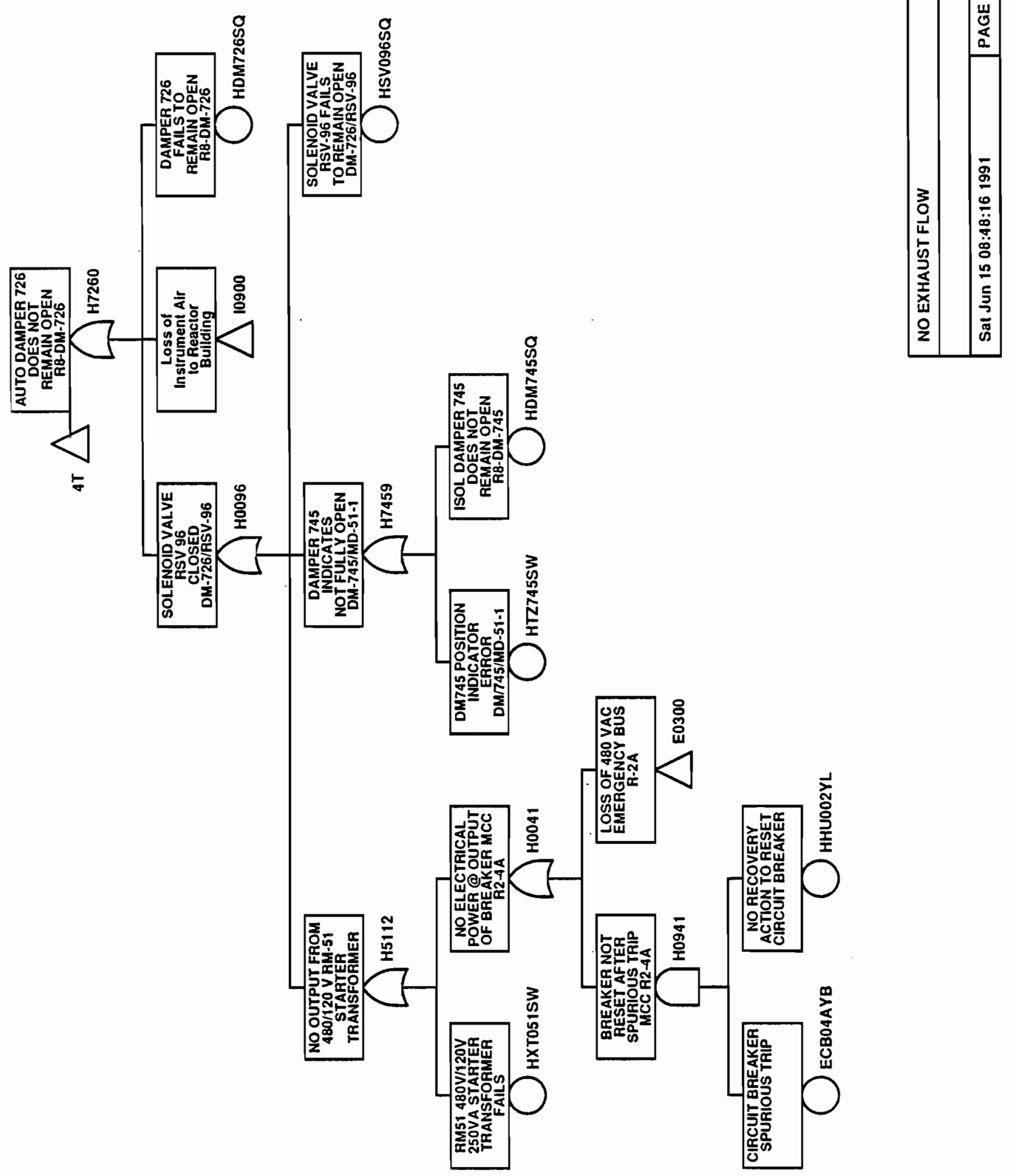




\begin{tabular}{|c|c|}
\hline & 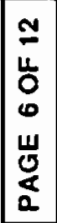 \\
\hline 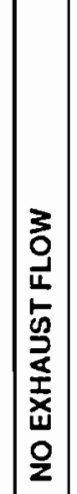 & 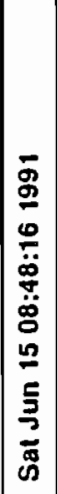 \\
\hline
\end{tabular}

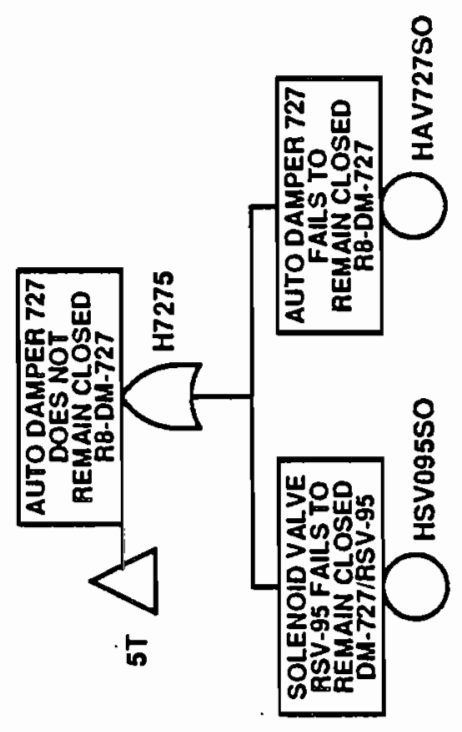




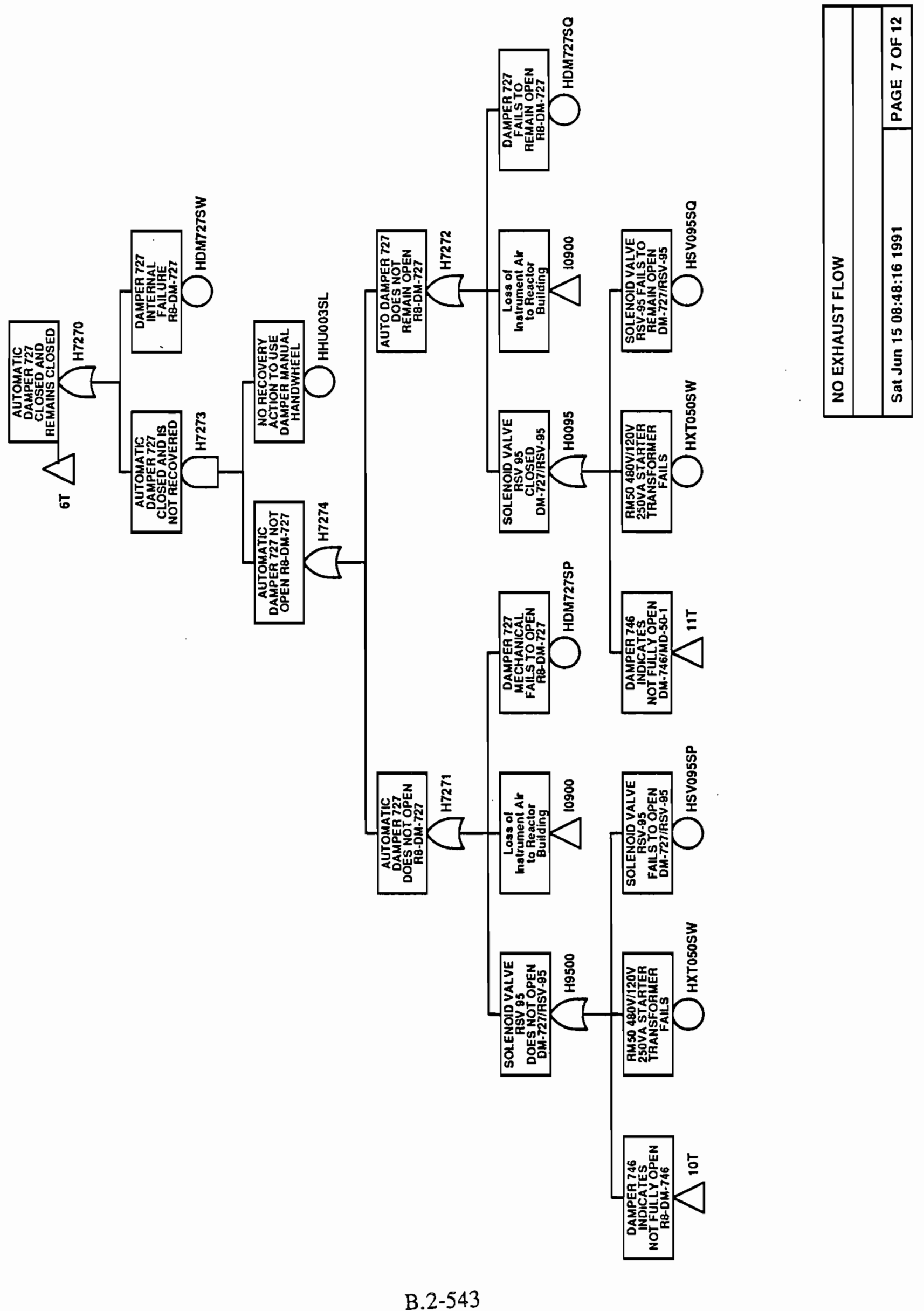




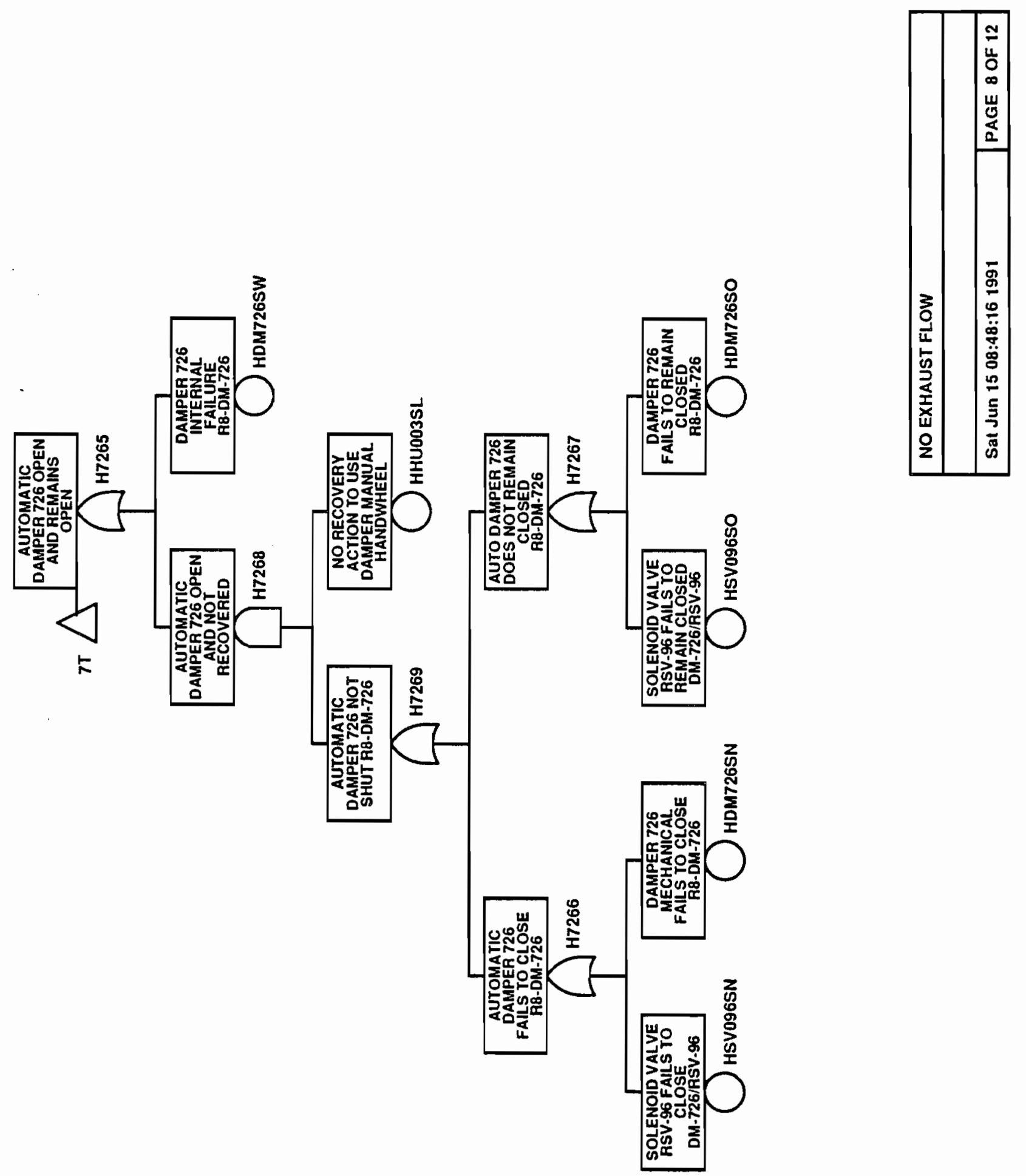




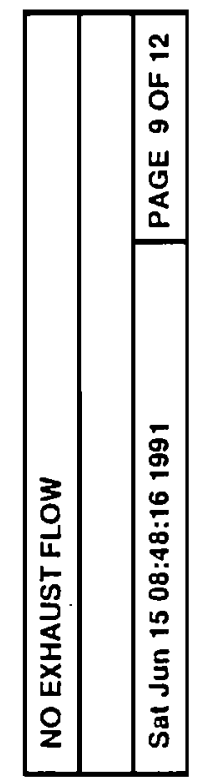

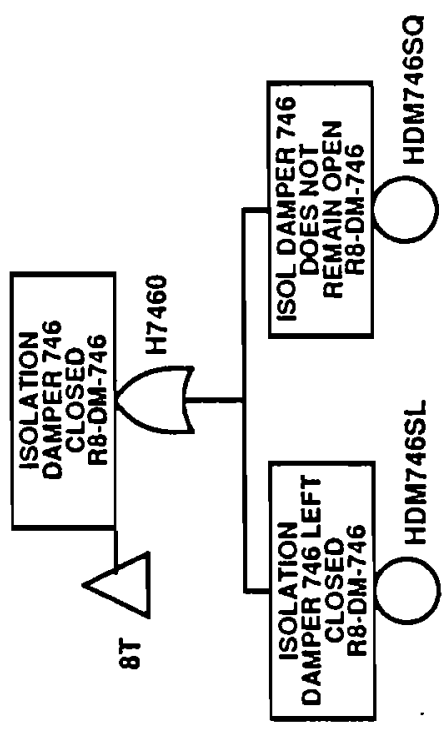




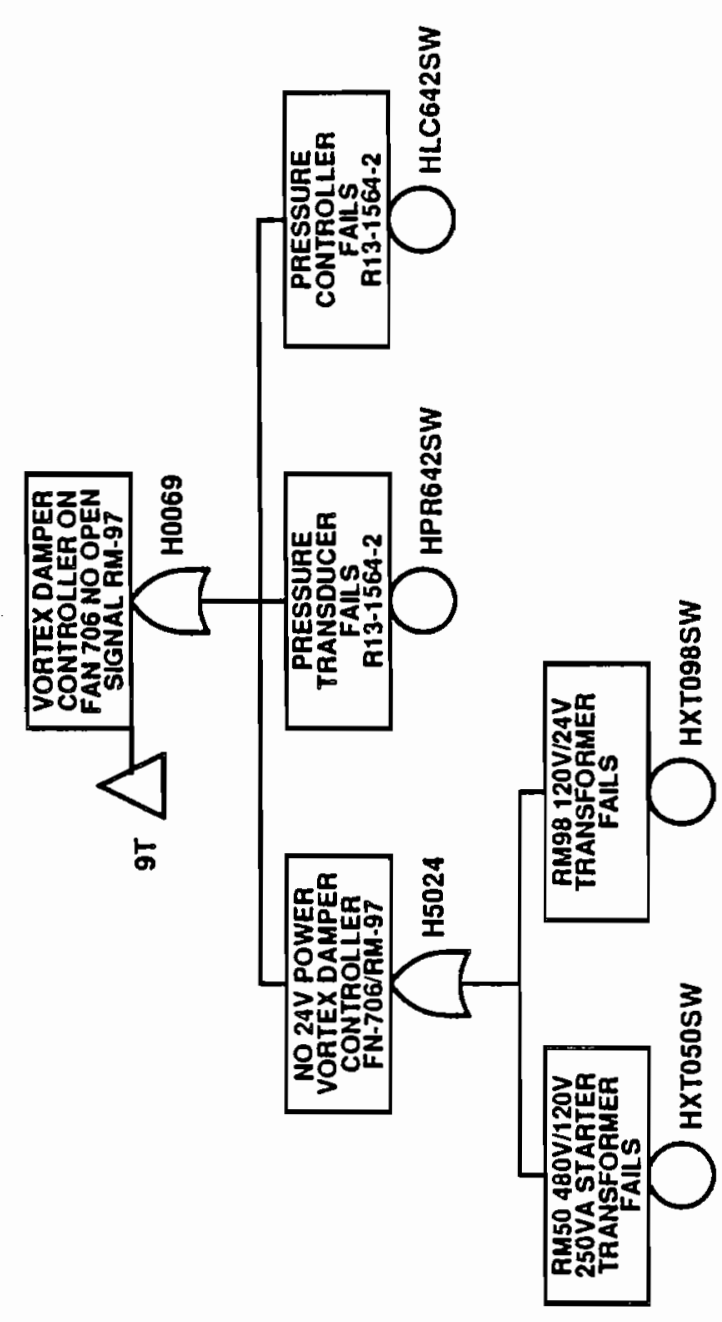

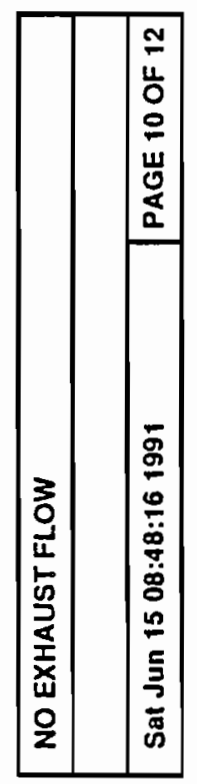




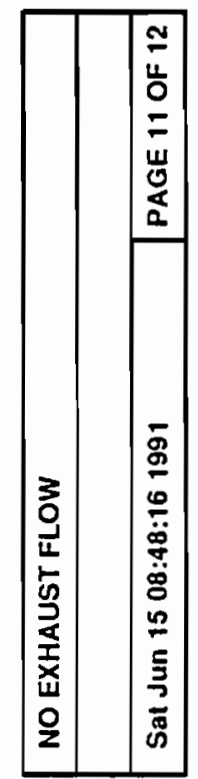

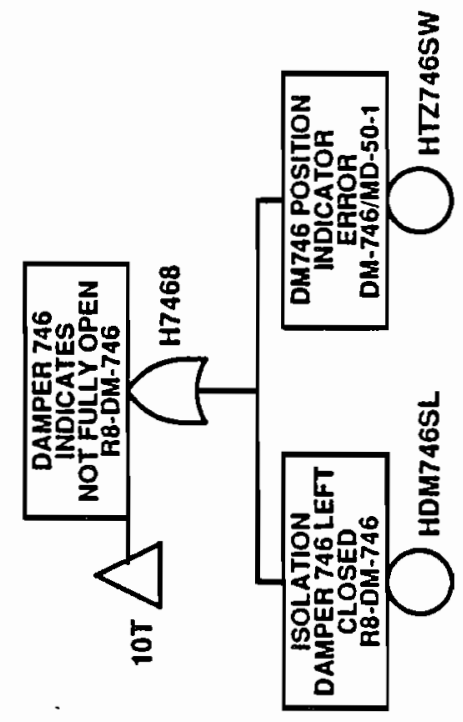




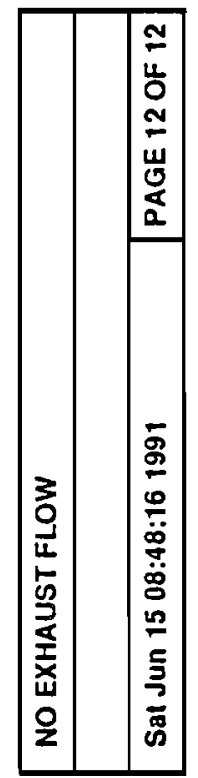

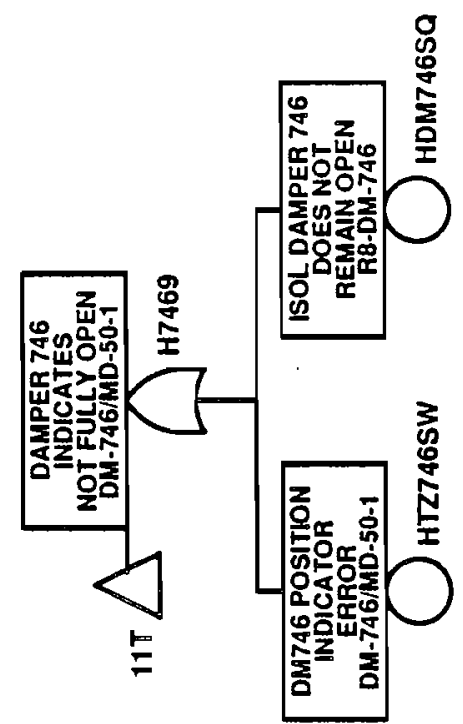




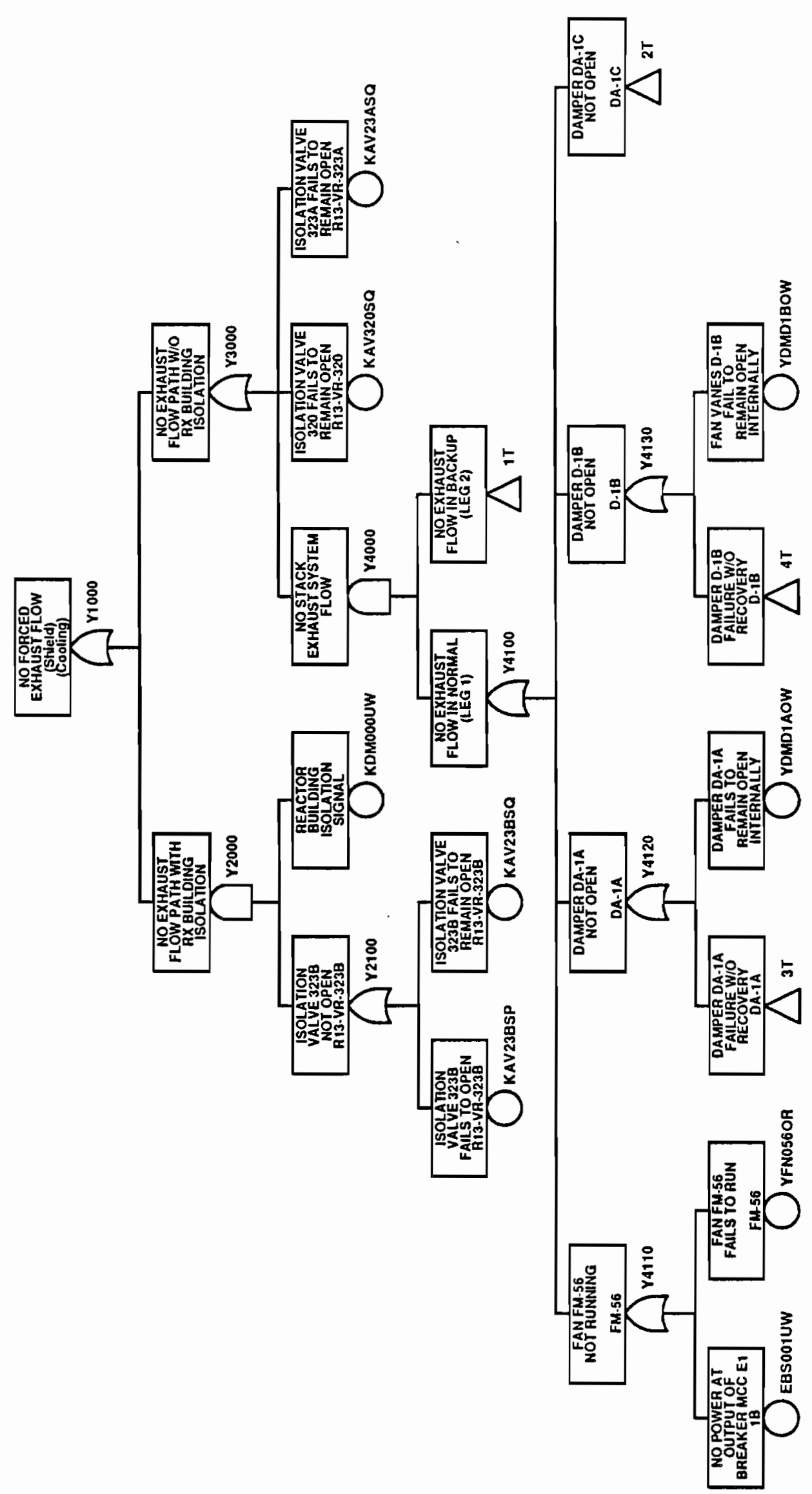

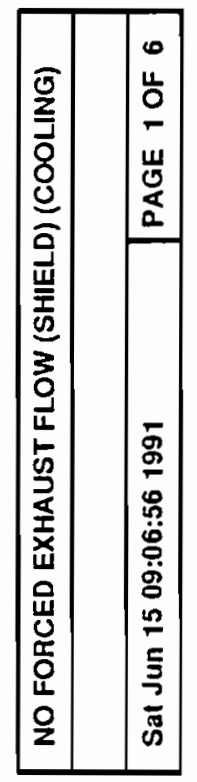

B. $2-553$ 


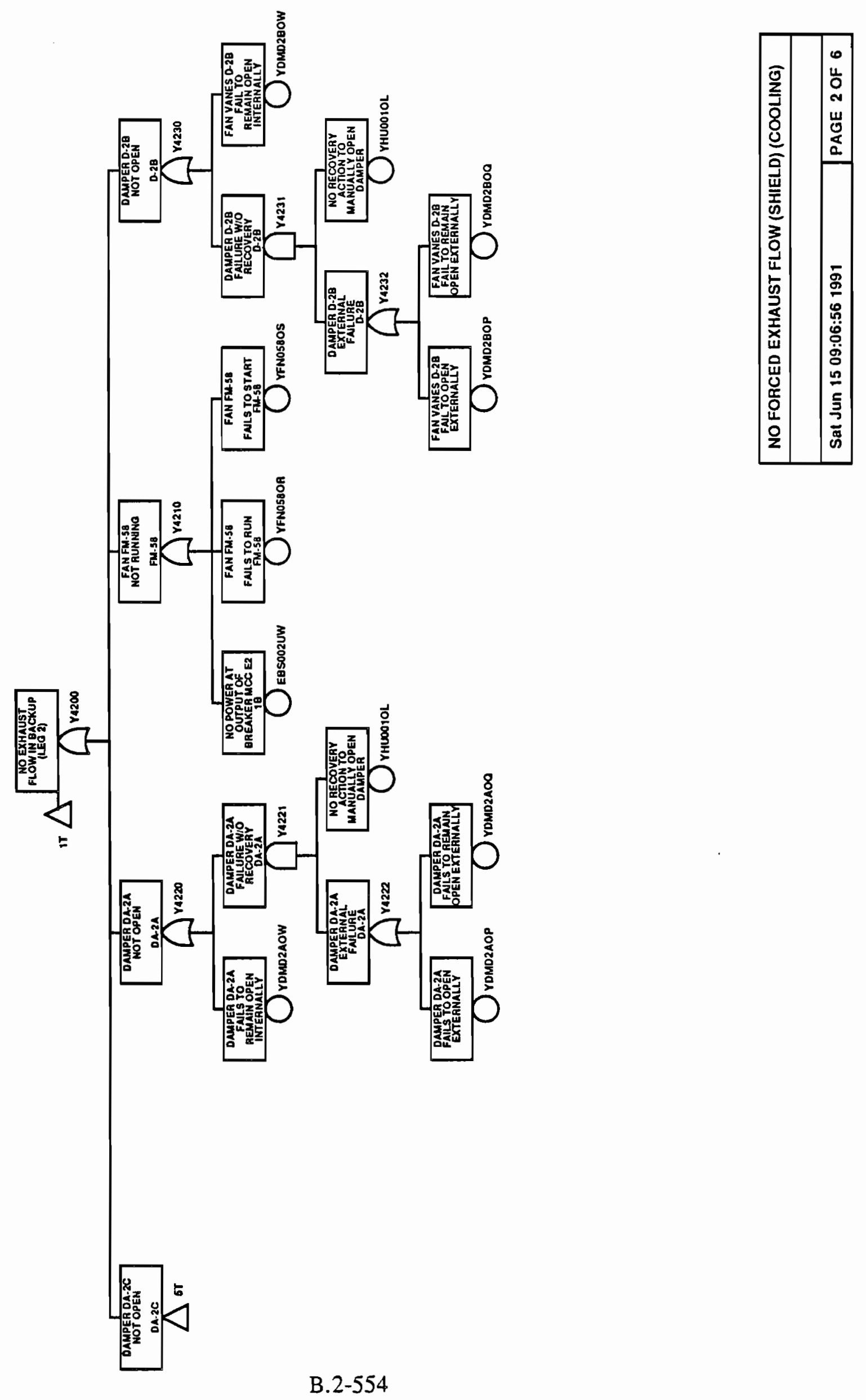




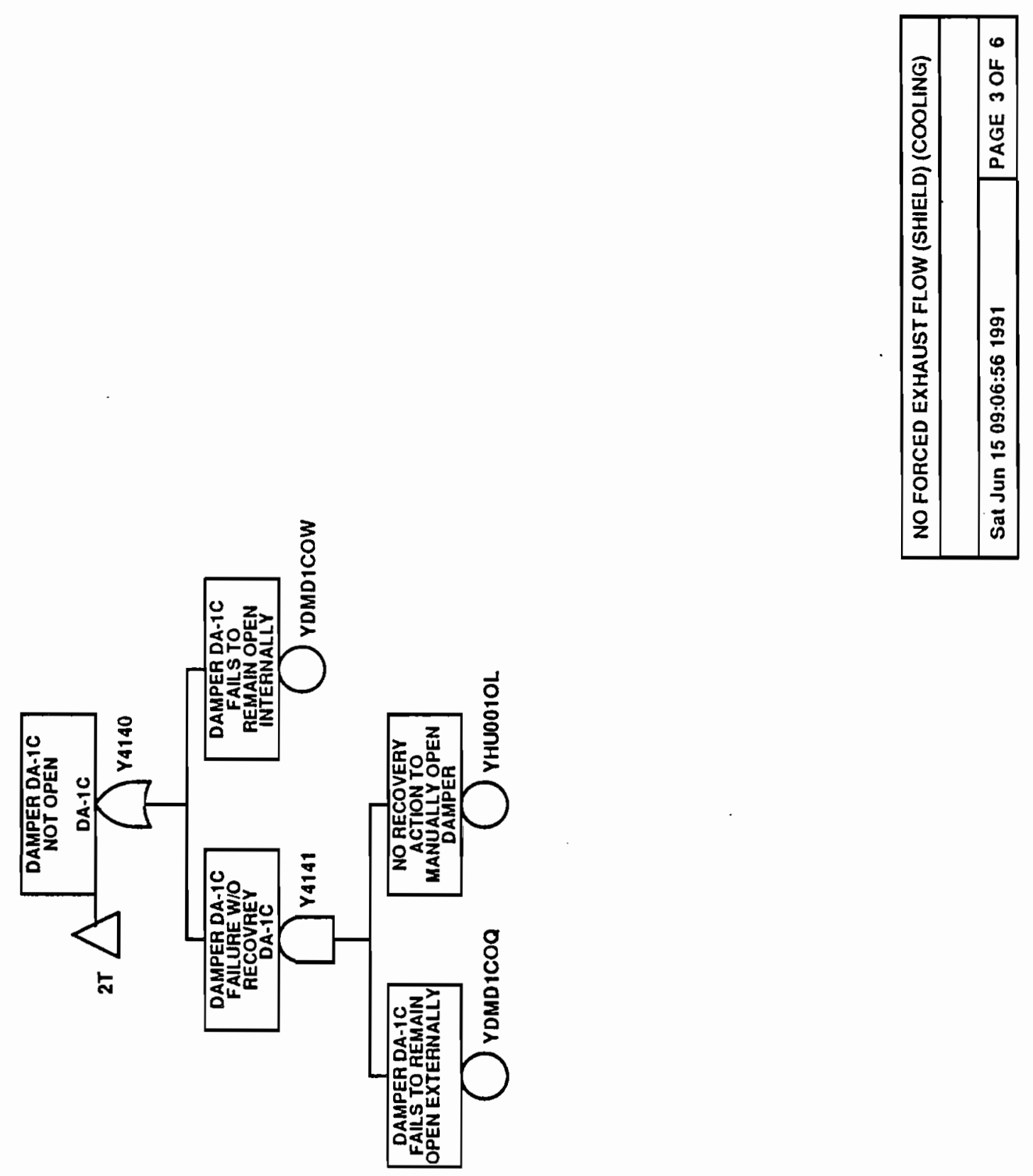

B. 2-555 


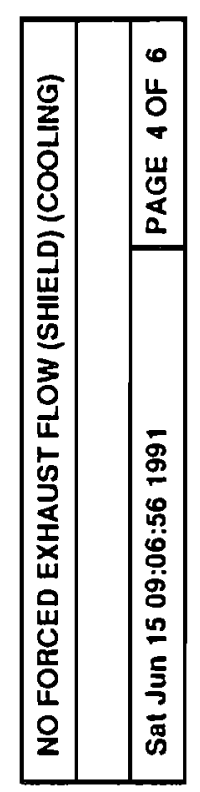

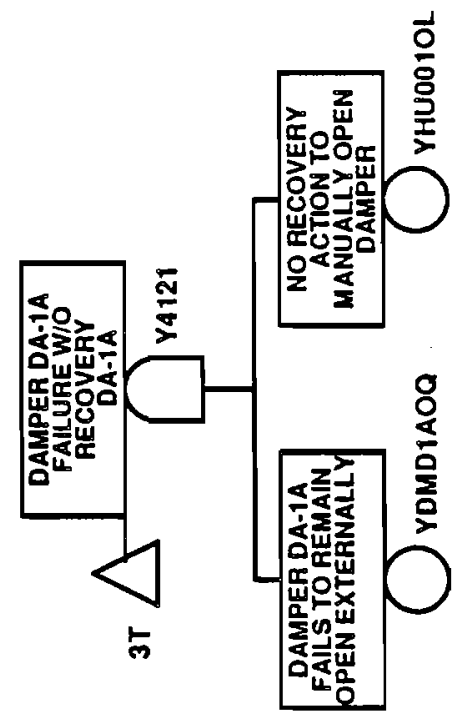




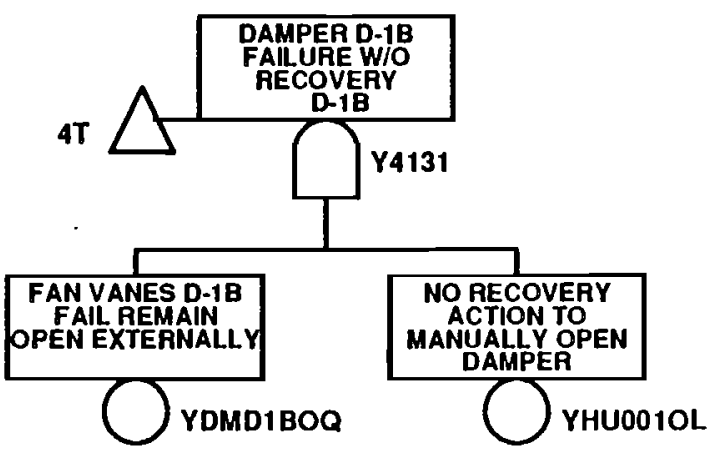

NO FORCED EXHAUST FLOW (SHIELD) (COOLING) 


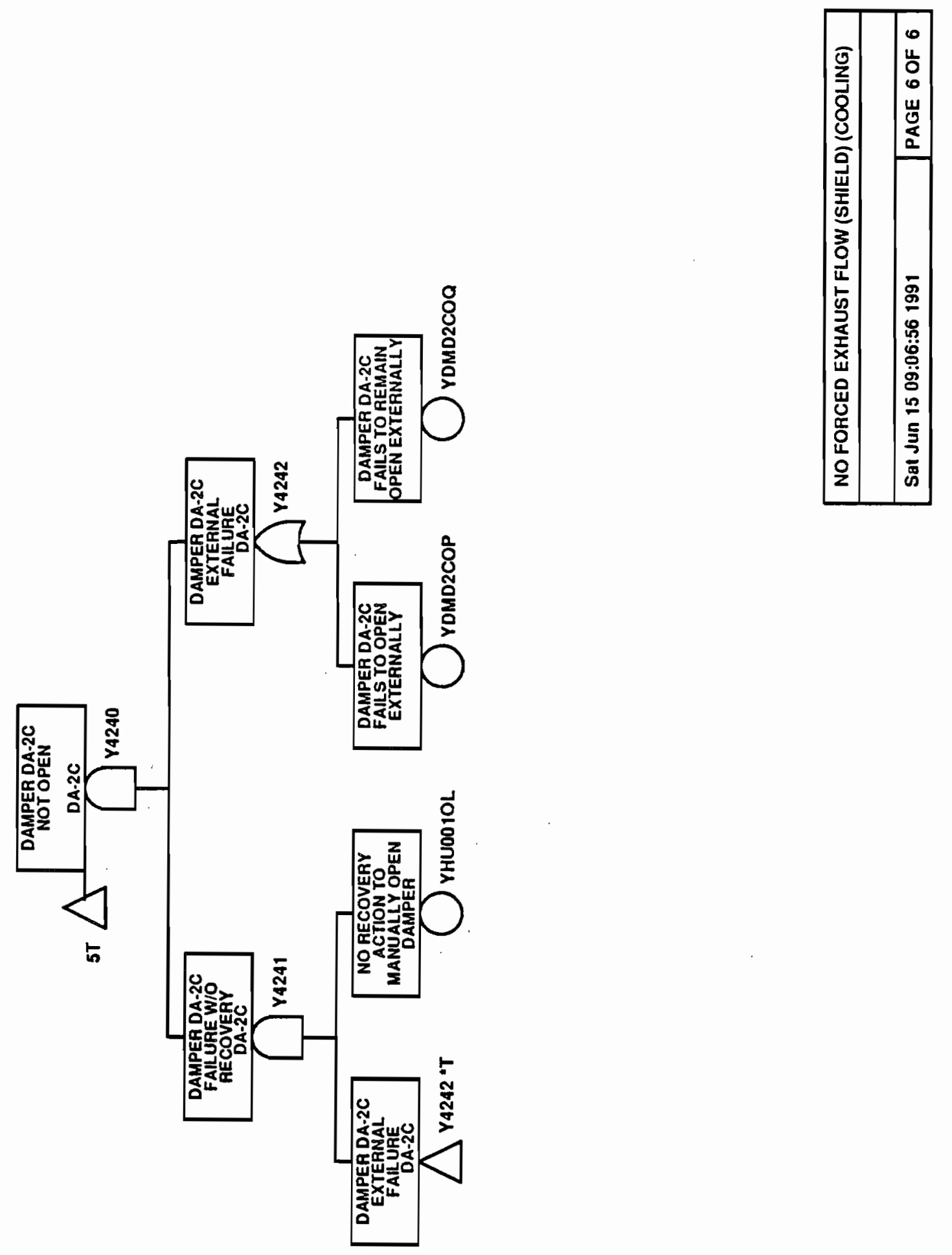


$\begin{array}{lll}24 & 25 & 25\end{array}$

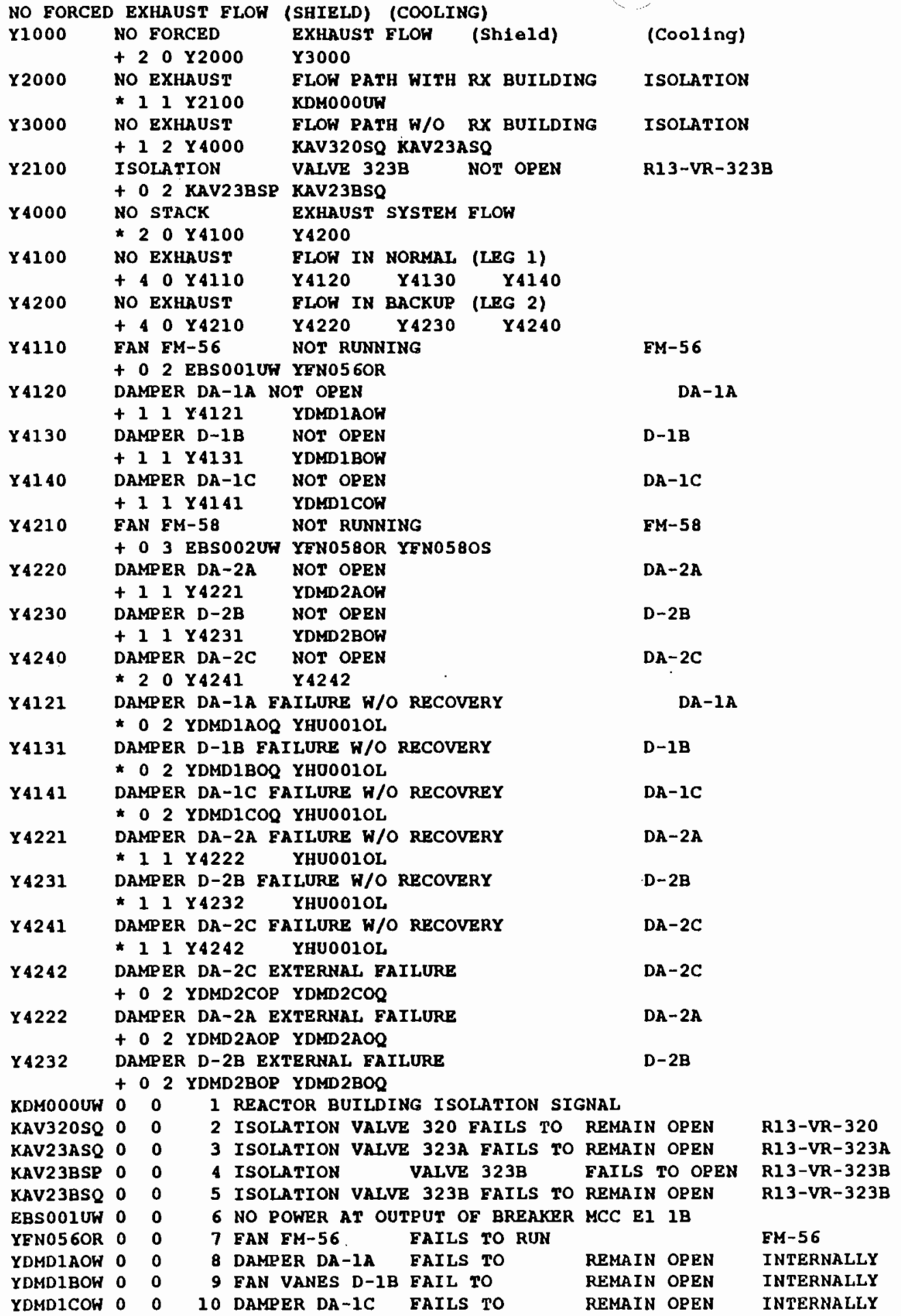


EBS002UW 0 O 11 NO POWER AT OUTPUT OF BREAKER MCC E2 1B

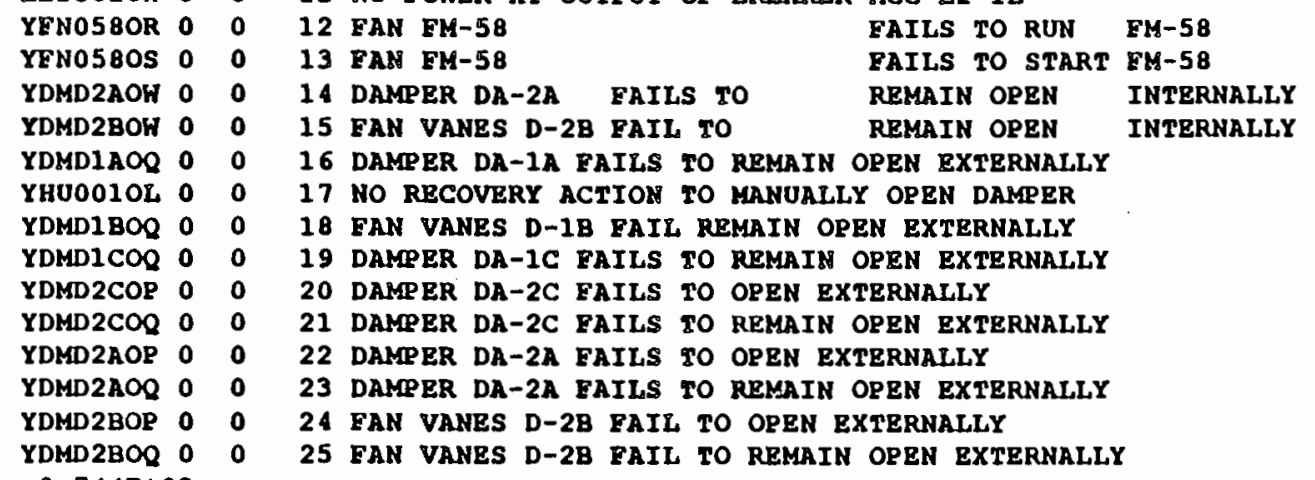

25 FAN VANES D-2B FAIL TO REMAIN OPEN EXTERNALLY

$0.744 \mathrm{E}+03$

$\begin{array}{rllll}1 & 2 & 0.100 \mathrm{E}-03 & 0.300 \mathrm{E}+02 & \\ 2 & 1 & 0.640 \mathrm{E}-07 & 0.100 \mathrm{E}+02 & \\ 3 & 2 & 0.640 \mathrm{E}-07 & 0.100 \mathrm{E}+02 & \\ 4 & 0 & 0.200 \mathrm{E}-02 & 0.100 \mathrm{E}+02 & 0.000 \mathrm{E}+00 \\ 5 & 1 & 0.100 \mathrm{E}-06 & 0.100 \mathrm{E}+02 & \\ 6 & 2 & 0.160 \mathrm{E}-05 & 0.370 \mathrm{E}+01 & \\ 7 & 2 & 0.200 \mathrm{E}-04 & 0.300 \mathrm{E}+01 & \\ 8 & 1 & 0.360 \mathrm{E}-07 & 0.830 \mathrm{E}+01 & \\ 9 & 1 & 0.360 \mathrm{E}-07 & 0.830 \mathrm{E}+01 & \\ 10 & 1 & 0.360 \mathrm{E}-07 & 0.830 \mathrm{E}+01 & \\ 11 & 1 & 0.160 \mathrm{E}-05 & 0.370 \mathrm{E}+01 & \\ 12 & 1 & 0.200 \mathrm{E}-04 & 0.300 \mathrm{E}+01 & \\ 13 & 0 & 0.300 \mathrm{E}-03 & 0.300 \mathrm{E}+01 & 0.000 \mathrm{E}+00 \\ 14 & 1 & 0.360 \mathrm{E}-07 & 0.100 \mathrm{E}+02 & \\ 15 & 1 & 0.360 \mathrm{E}-07 & 0.100 \mathrm{E}+02 & \\ 16 & 2 & 0.640 \mathrm{E}-07 & 0.100 \mathrm{E}+02 & \\ 17 & 0 & 0.100 \mathrm{E}+00 & 0.100 \mathrm{E}+02 & 0.000 \mathrm{E}+00 \\ 18 & 2 & 0.640 \mathrm{E}-07 & 0.100 \mathrm{E}+02 & \\ 19 & 2 & 0.640 \mathrm{E}-07 & 0.100 \mathrm{E}+02 & \\ 20 & 0 & 0.300 \mathrm{E}-02 & 0.100 \mathrm{E}+02 & 0.000 \mathrm{E}+00 \\ 21 & 1 & 0.640 \mathrm{E}-07 & 0.100 \mathrm{E}+02 & \\ 22 & 0 & 0.300 \mathrm{E}-02 & 0.100 \mathrm{E}+02 & 0.000 \mathrm{E}+00 \\ 23 & 1 & 0.640 \mathrm{E}-07 & 0.100 \mathrm{E}+02 & \\ 24 & 0 & 0.300 \mathrm{E}-02 & 0.100 \mathrm{E}+02 & 0.000 \mathrm{E}+00 \\ 25 & 1 & 0.640 \mathrm{E}-07 & 0.100 \mathrm{E}+02 & \end{array}$




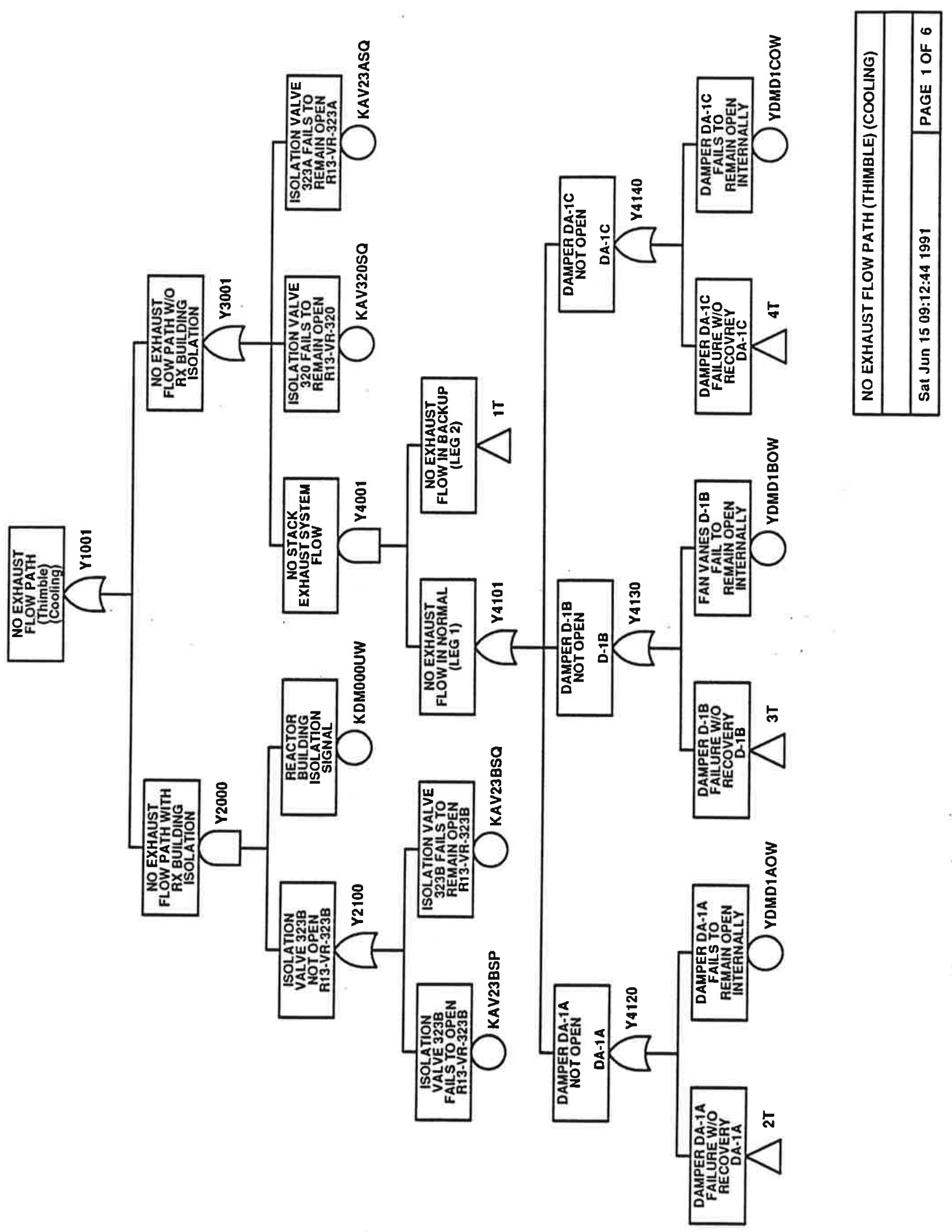




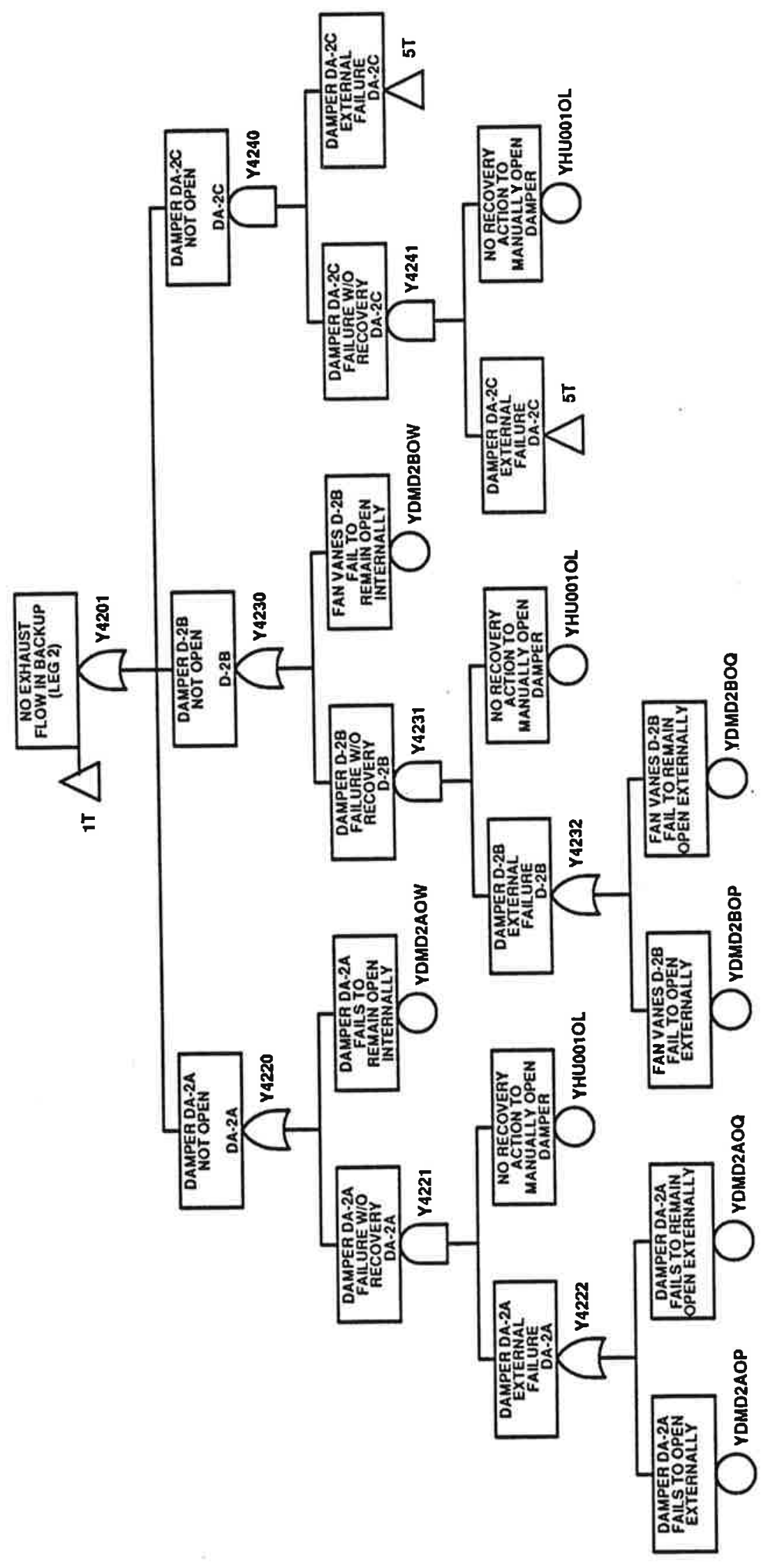

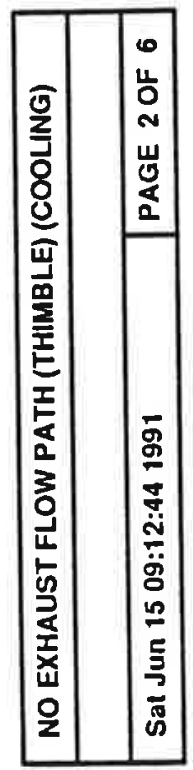




\begin{tabular}{|c|c|}
\hline 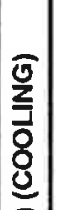 & $\begin{array}{l}0 \\
u \\
0 \\
0 \\
u \\
0 \\
0 \\
0 \\
0\end{array}$ \\
\hline 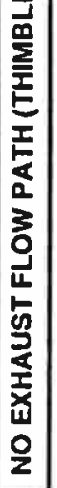 & 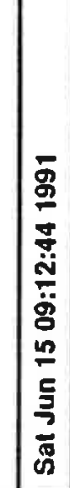 \\
\hline
\end{tabular}

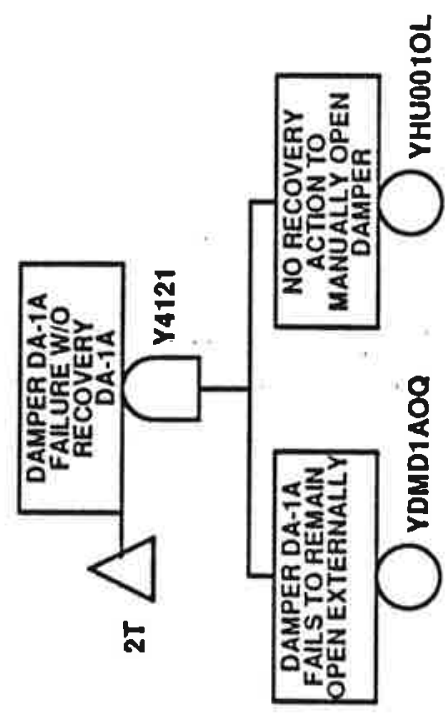




in

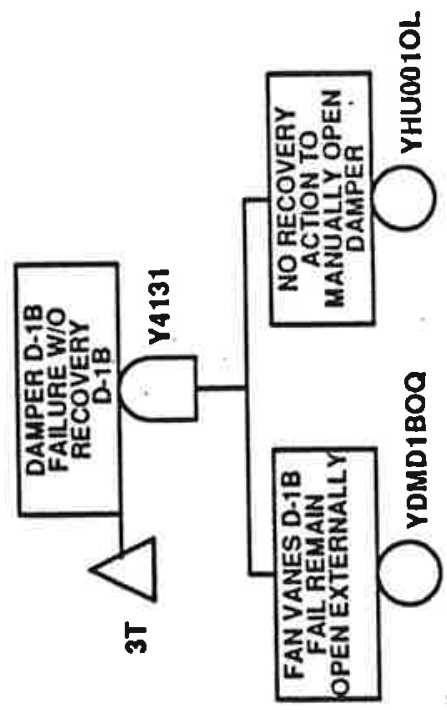

B. 2-564 


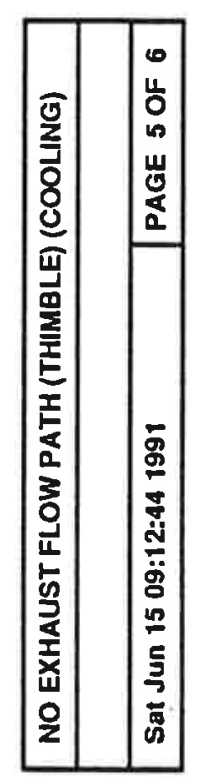

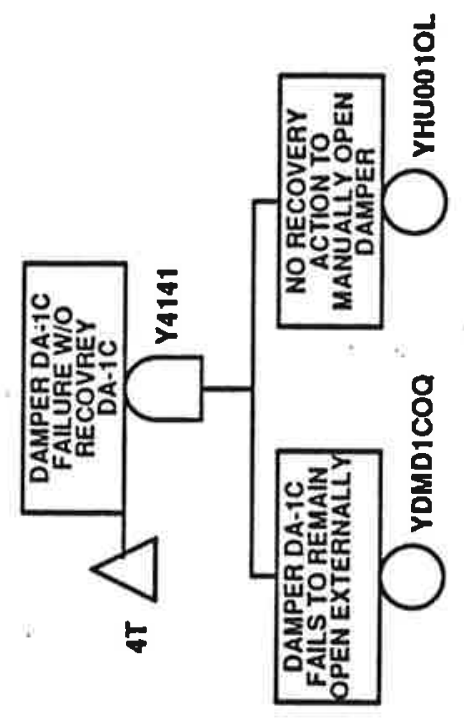




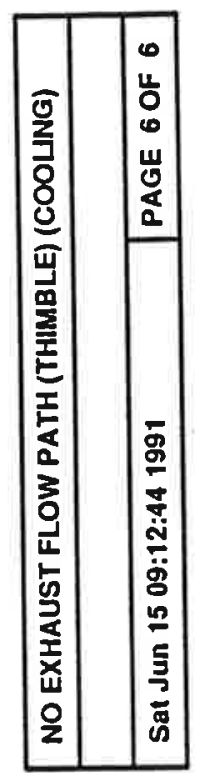

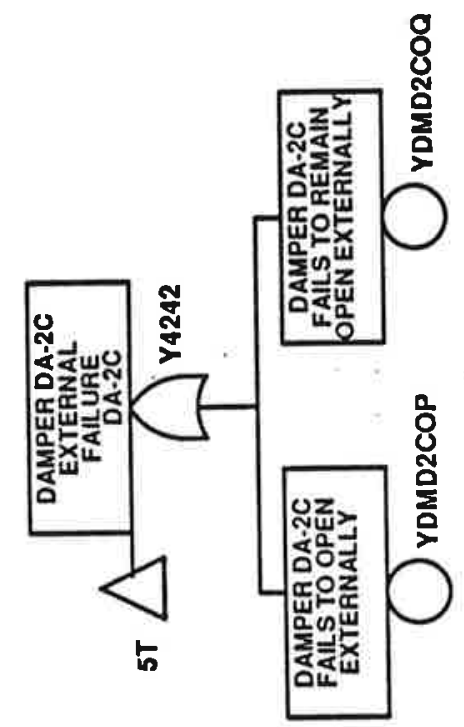

B. $2-566$ 


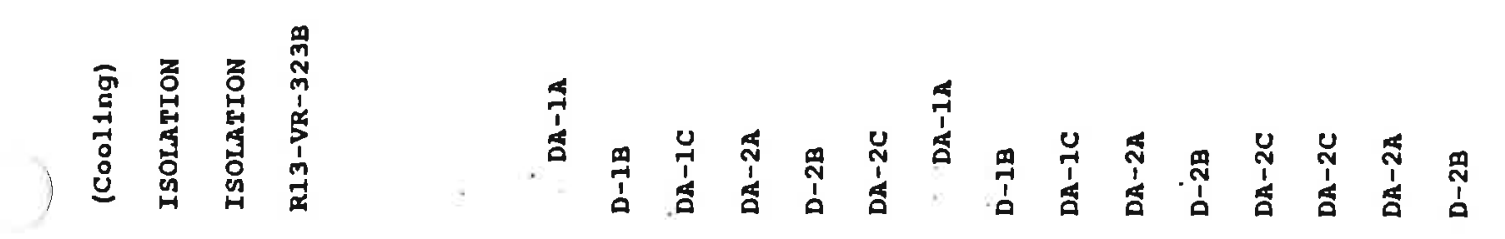

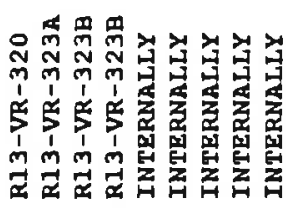

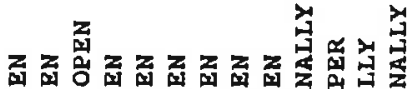

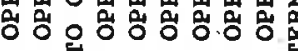

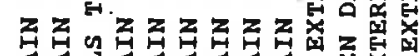

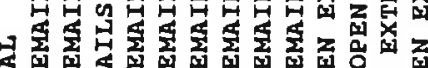

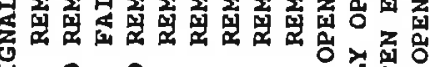
药

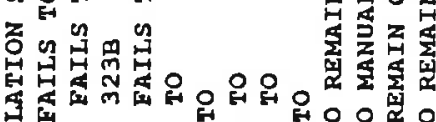

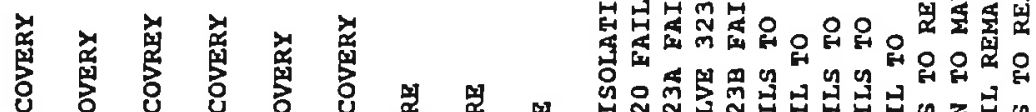

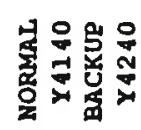

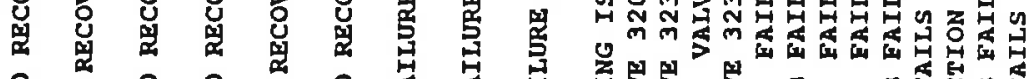

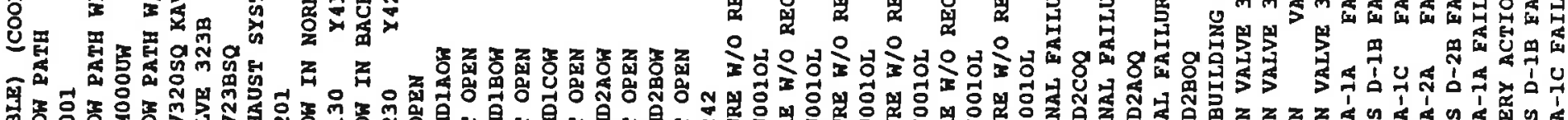

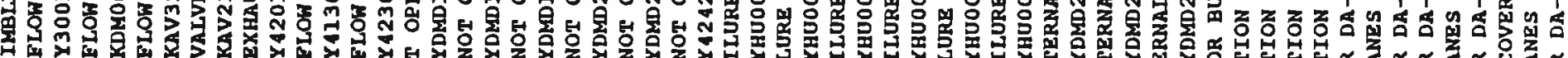

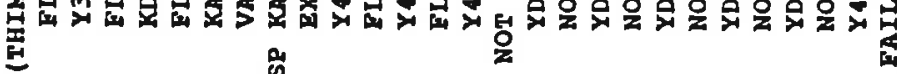
舟

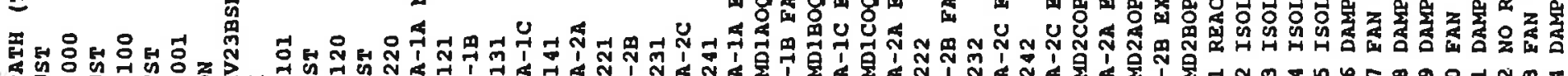

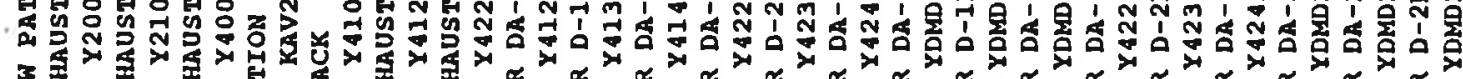

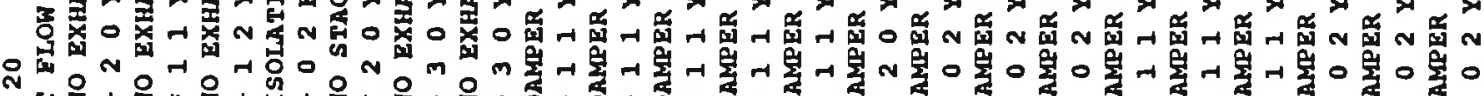
ก ละ. กับ

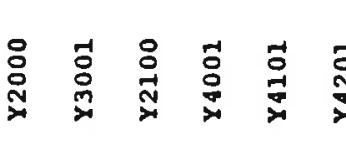




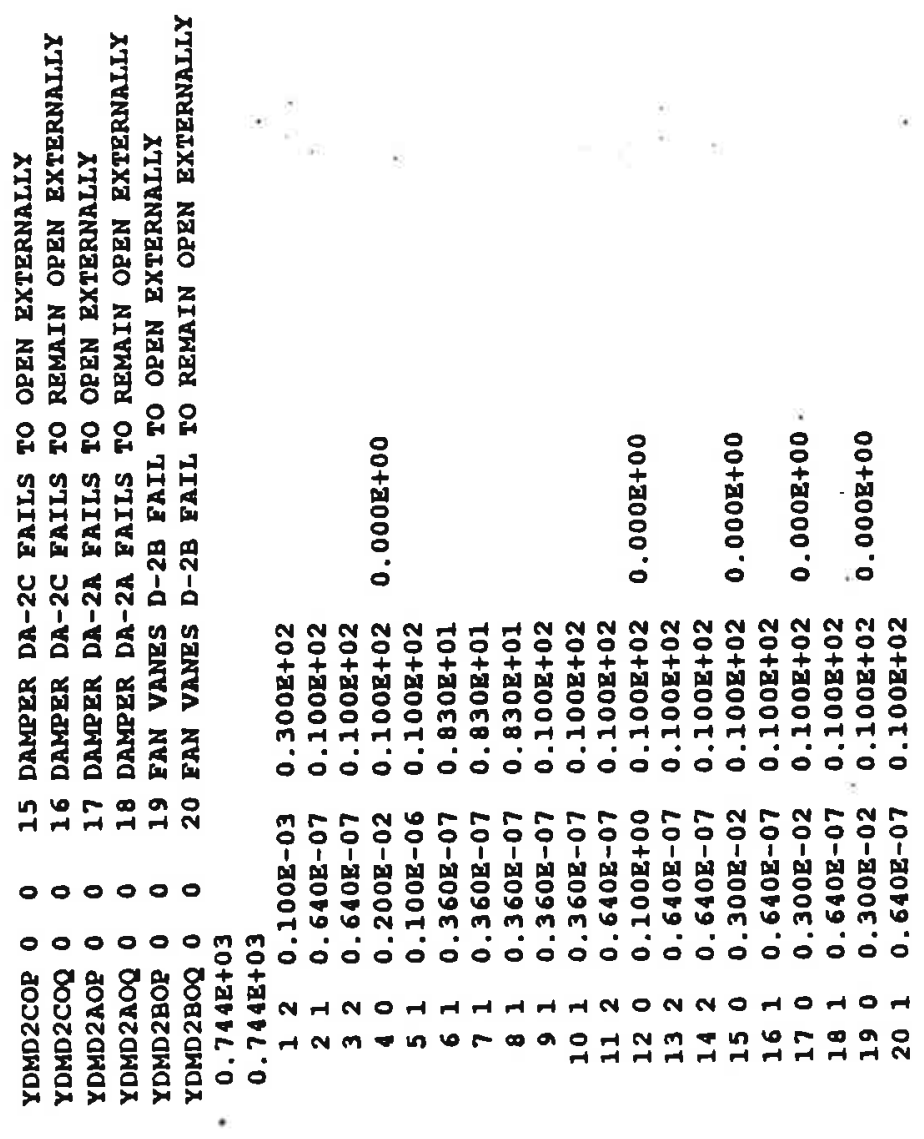




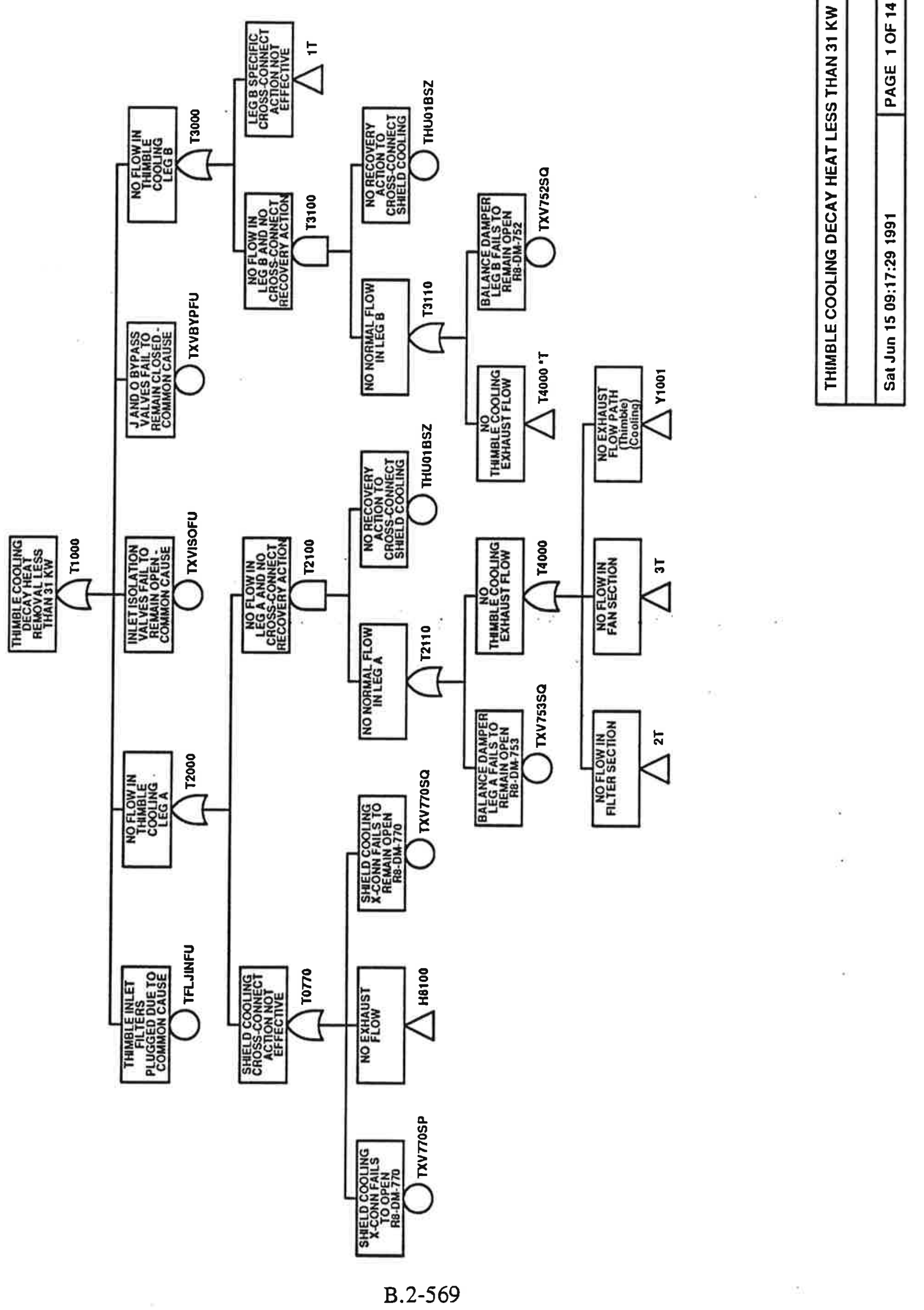




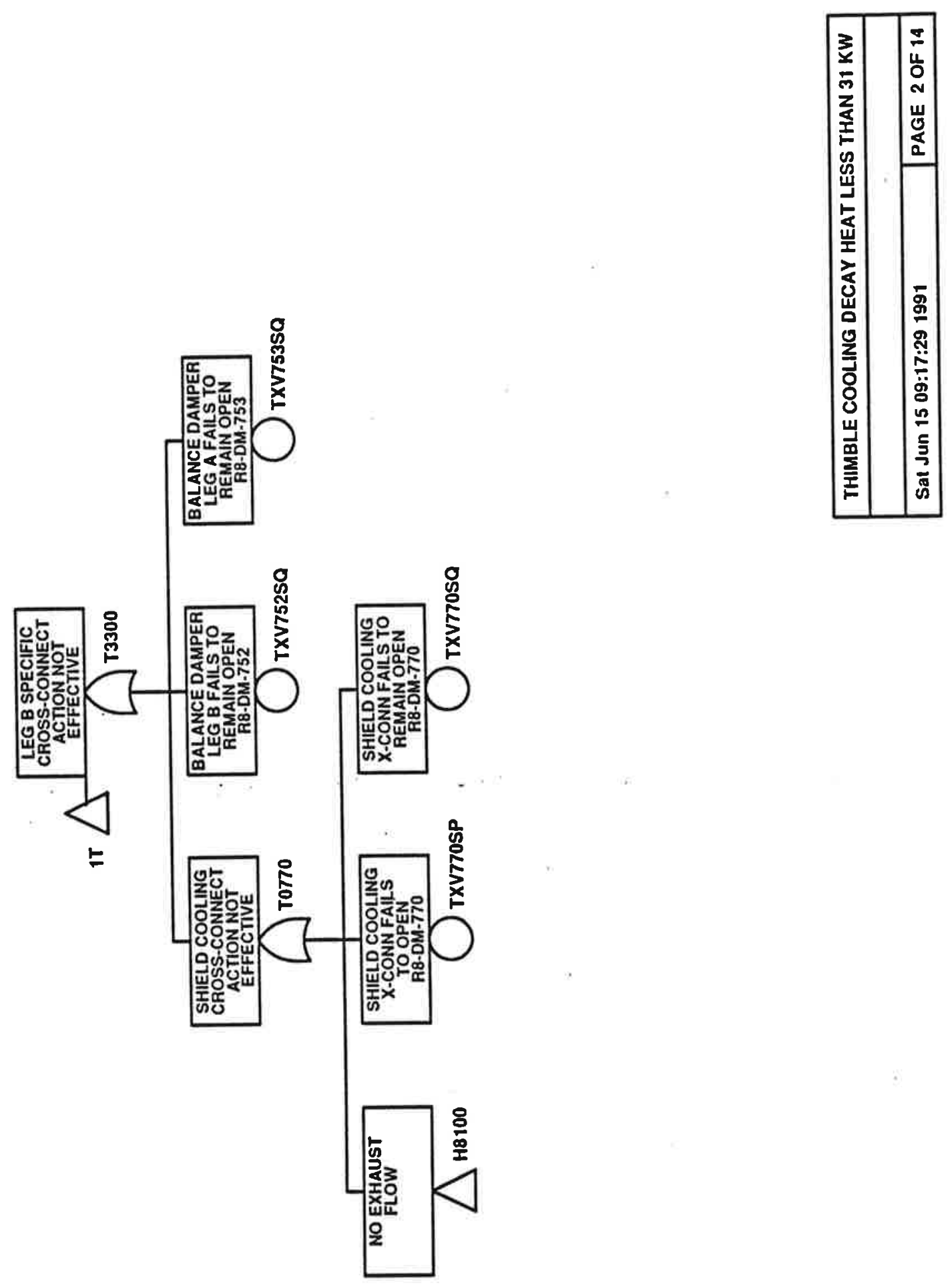

B. $2-570$ 


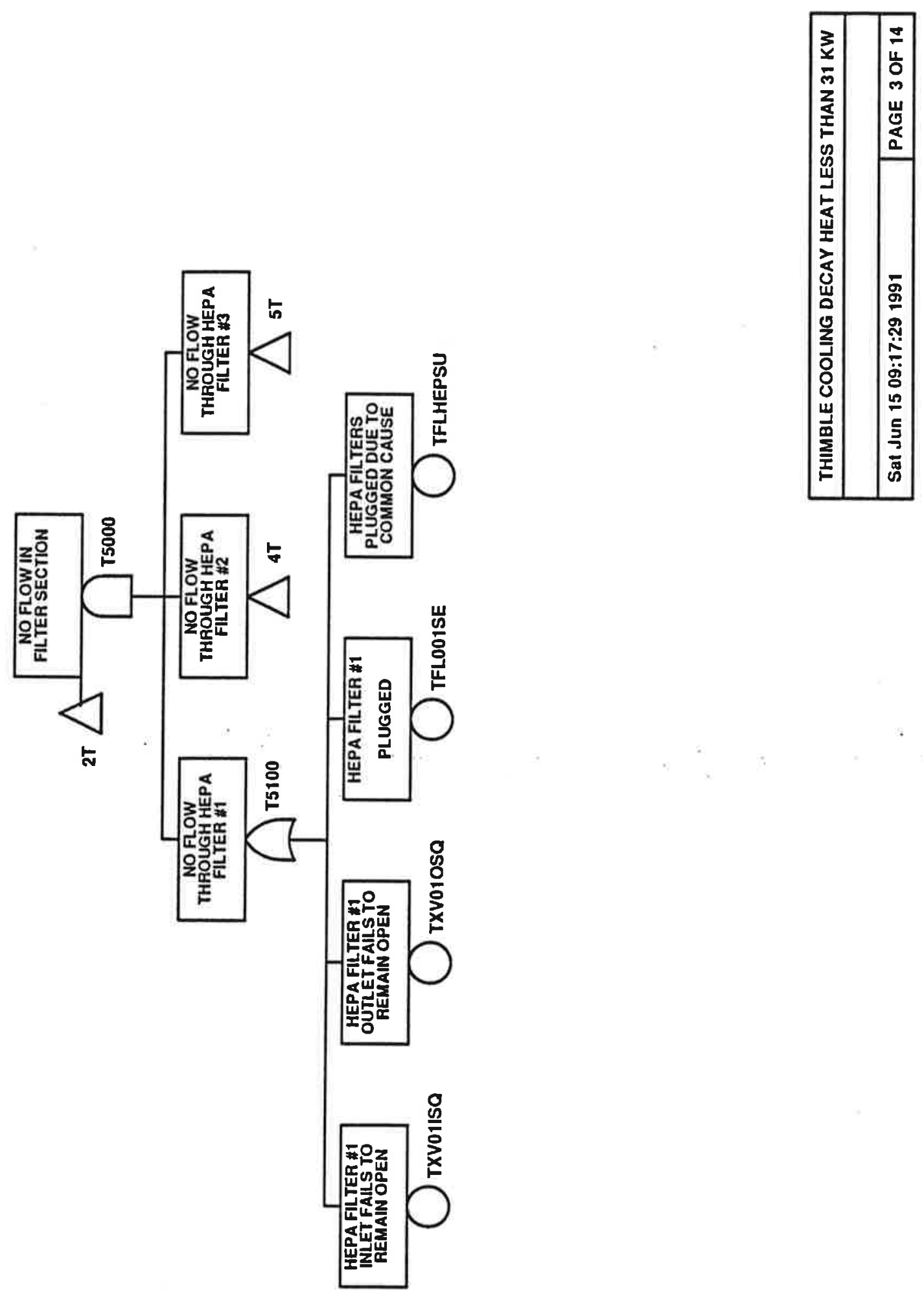

B. 2-571 


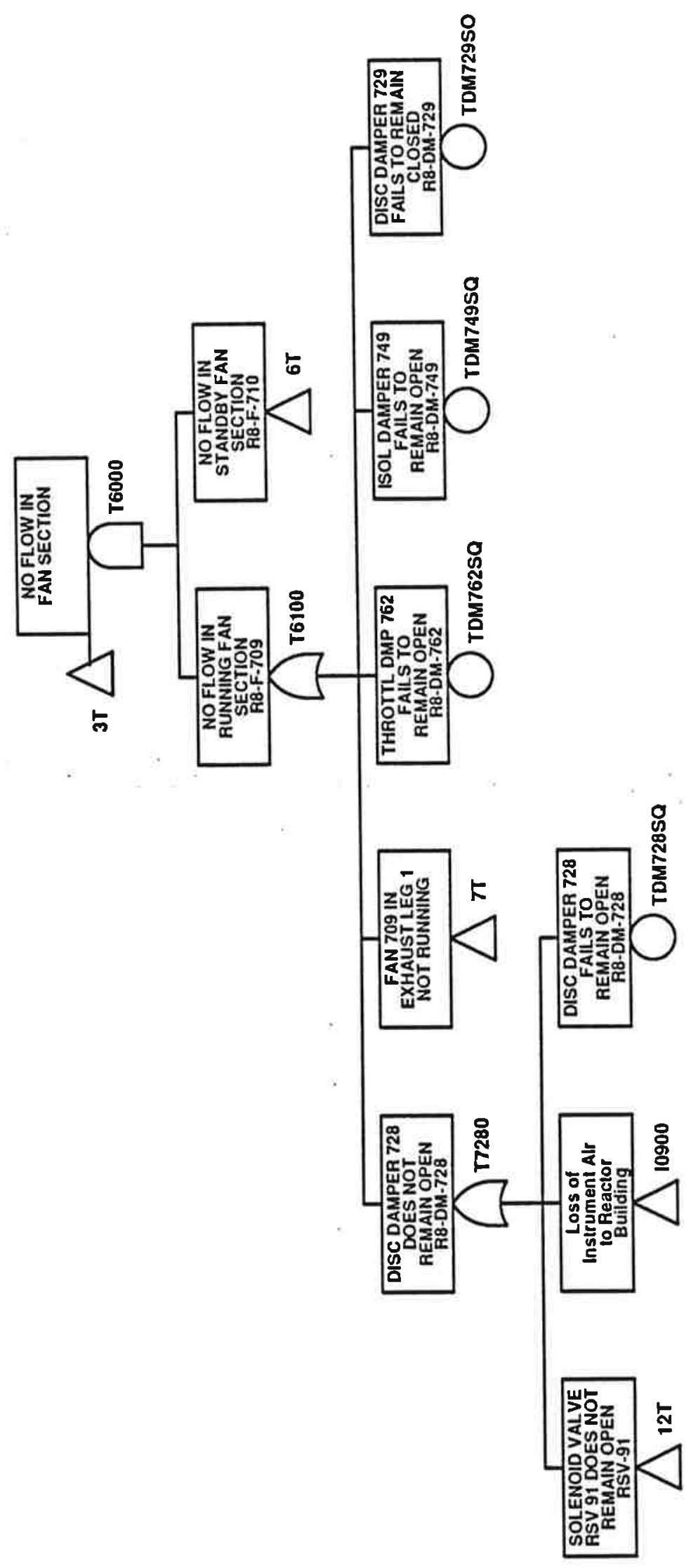

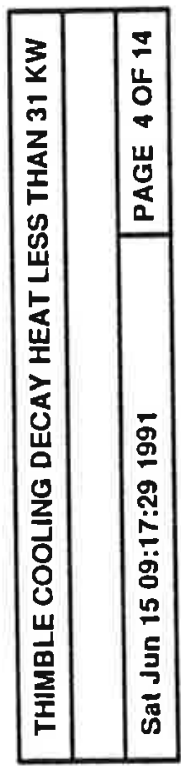




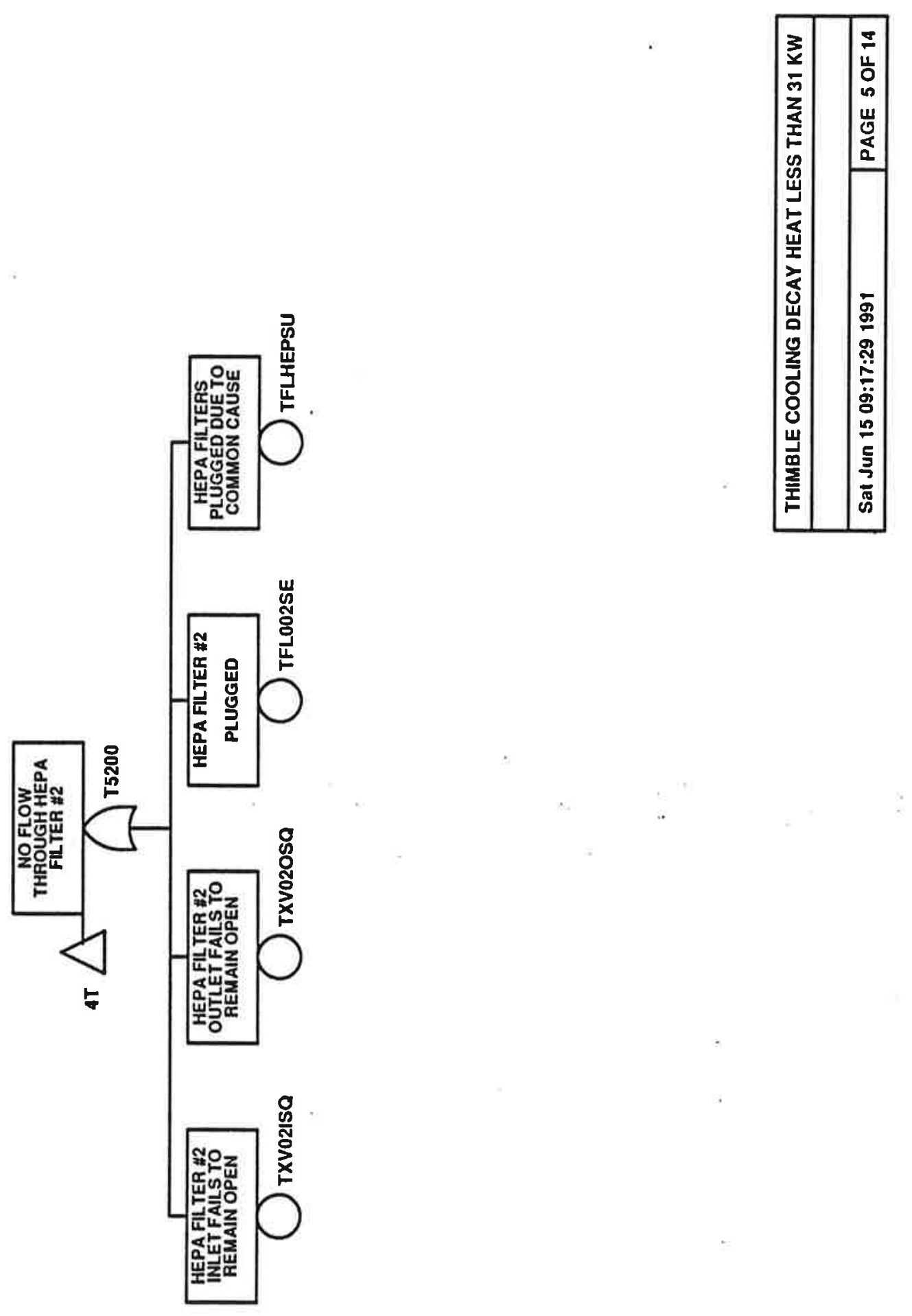




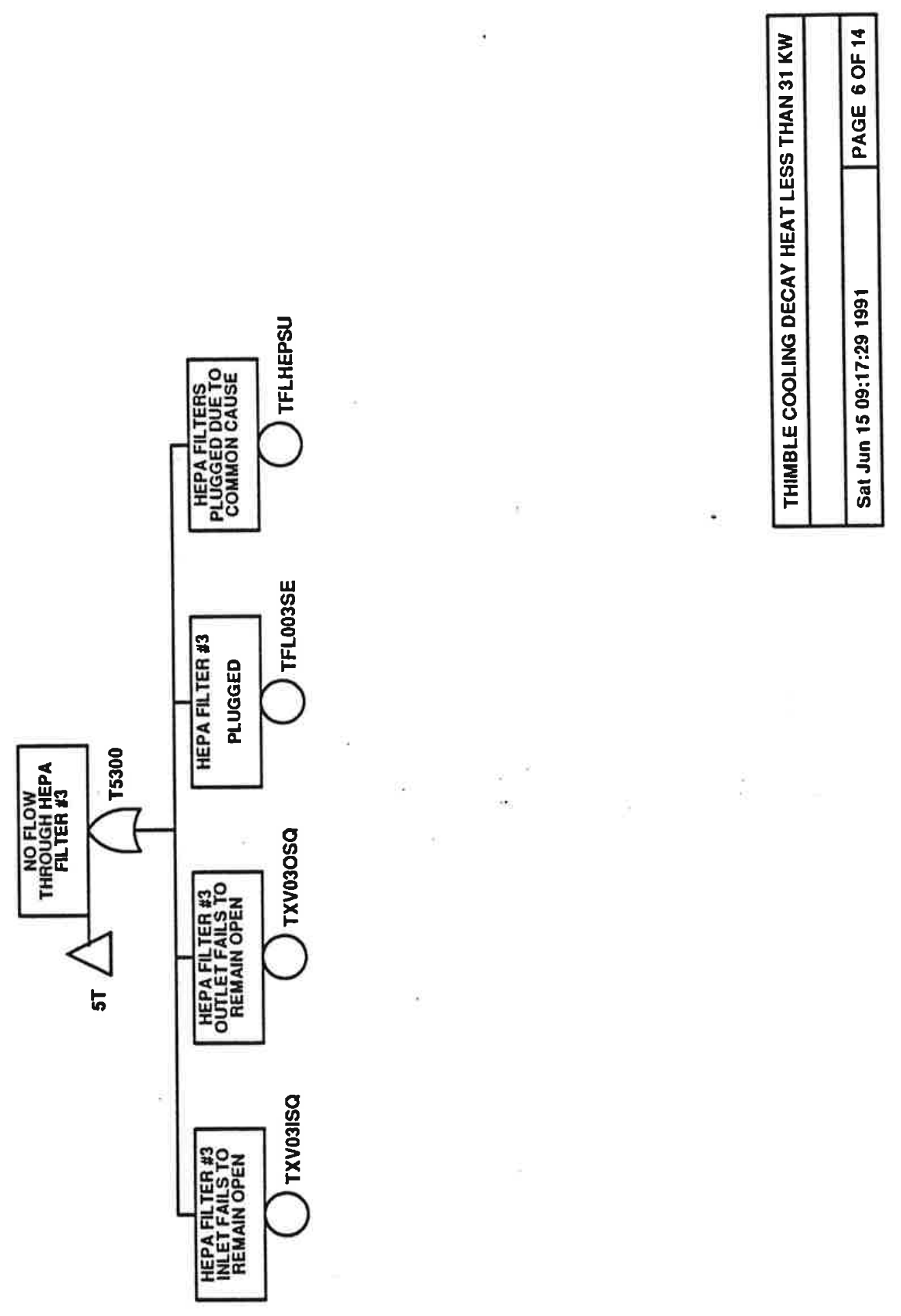




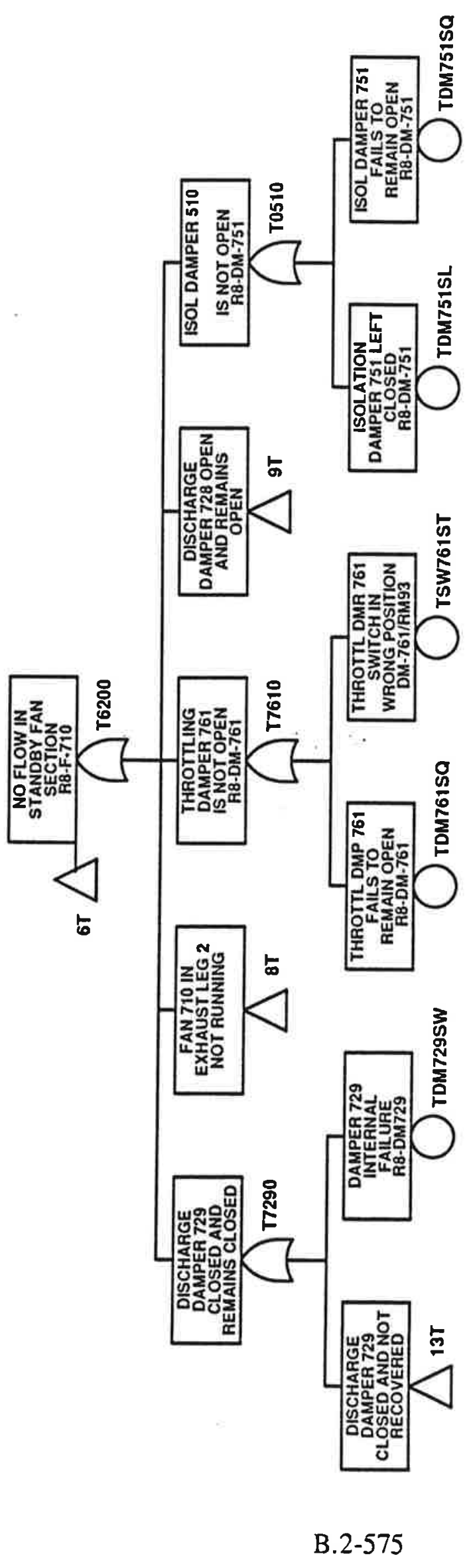

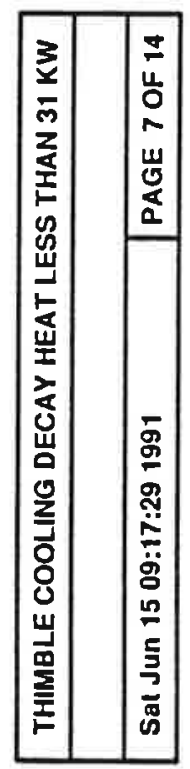



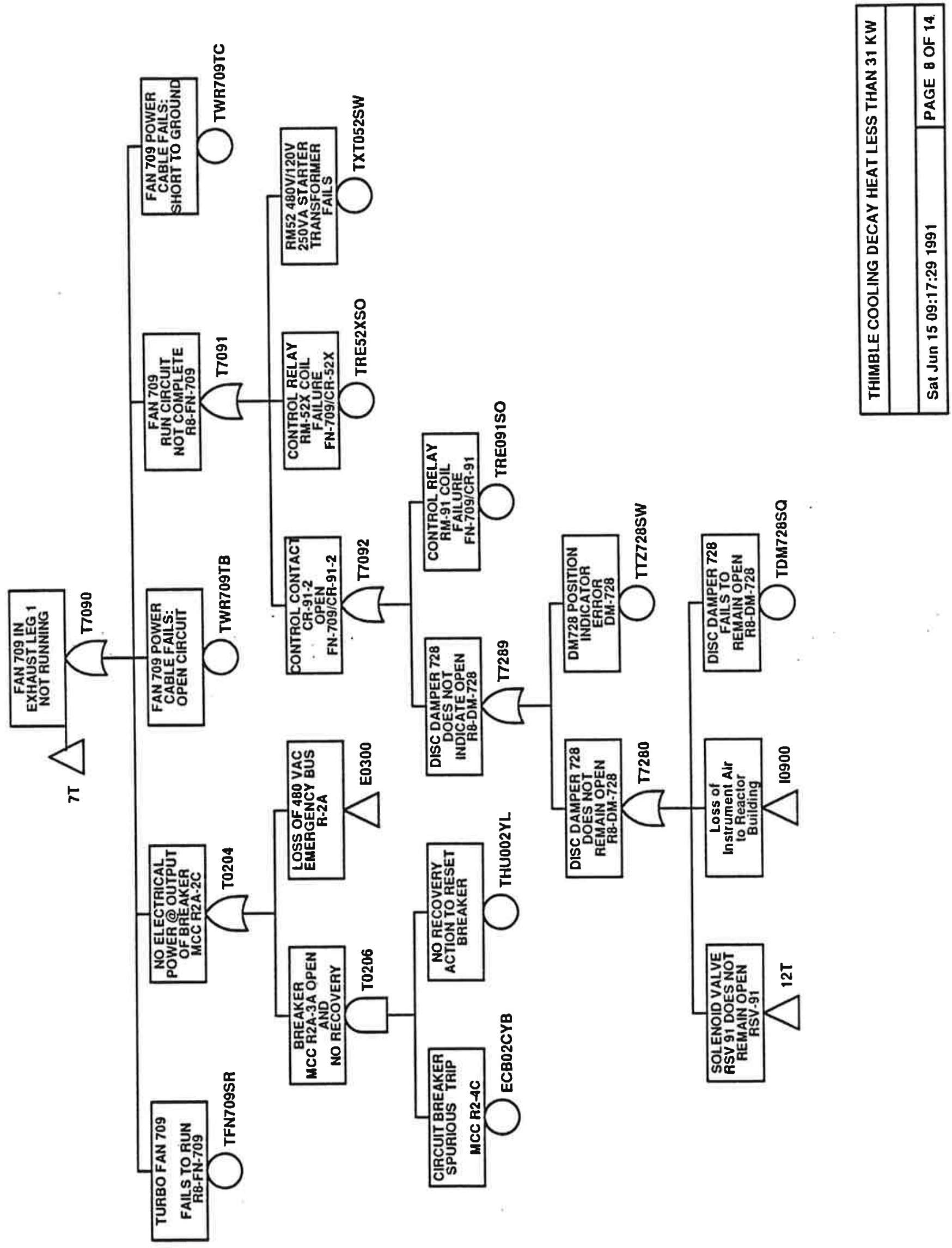

B. $2-576$ 


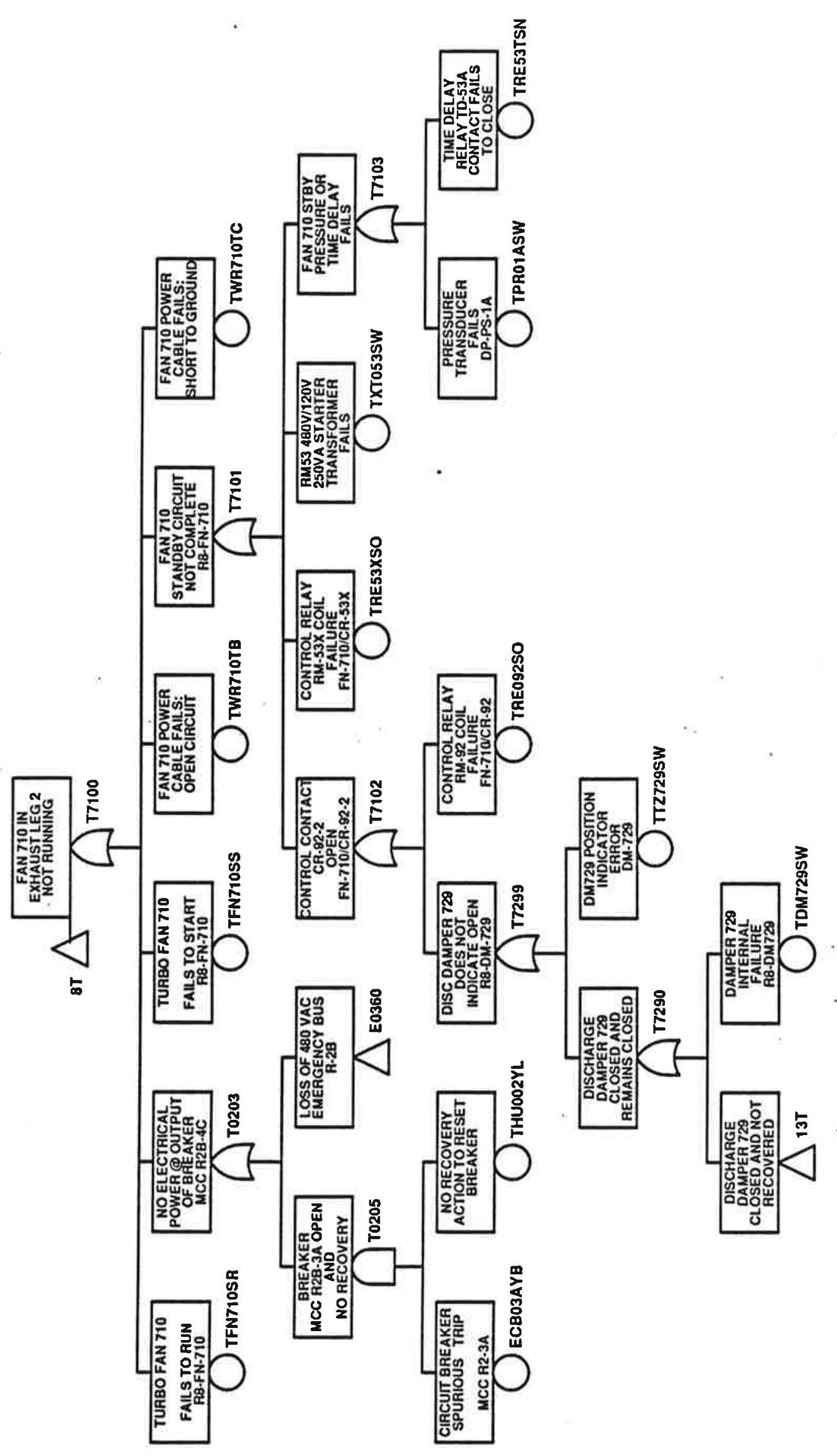

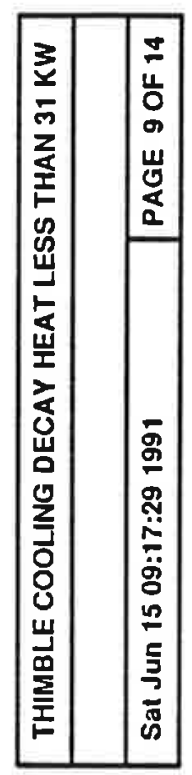

B. $2-577$ 


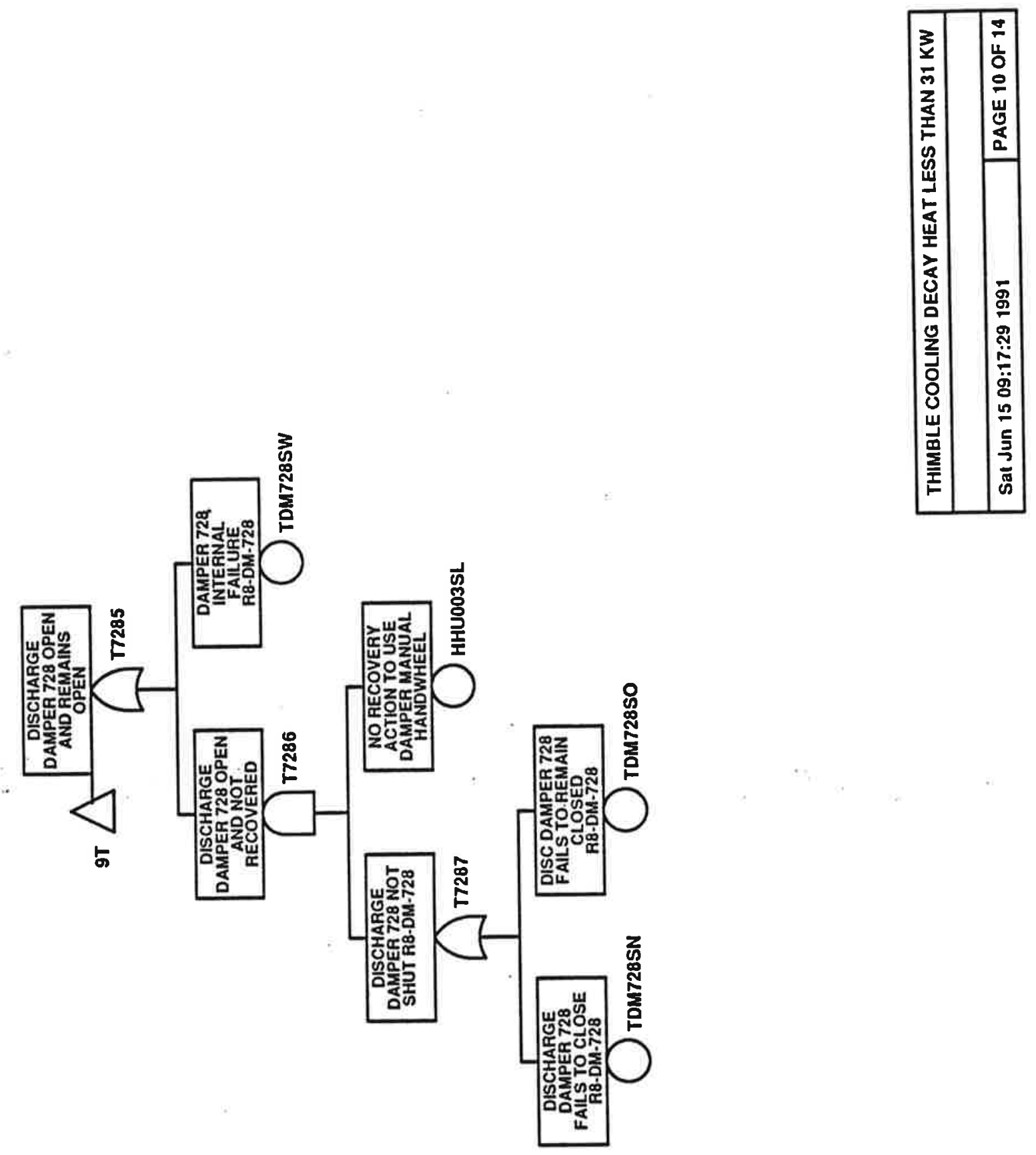




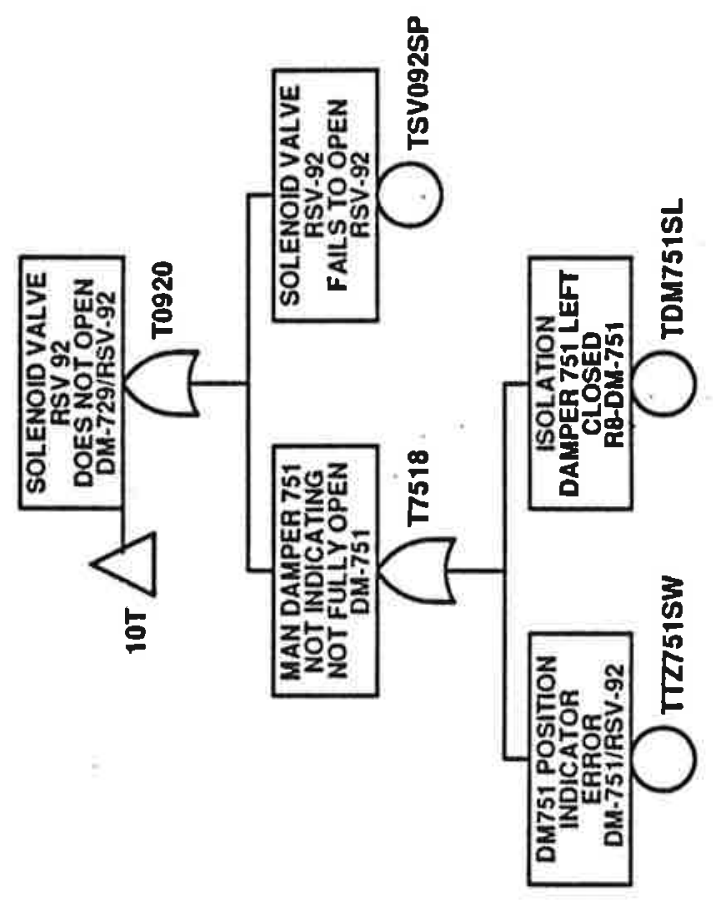

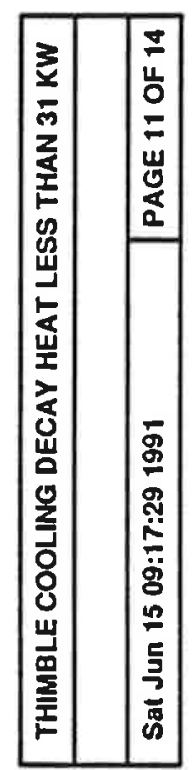




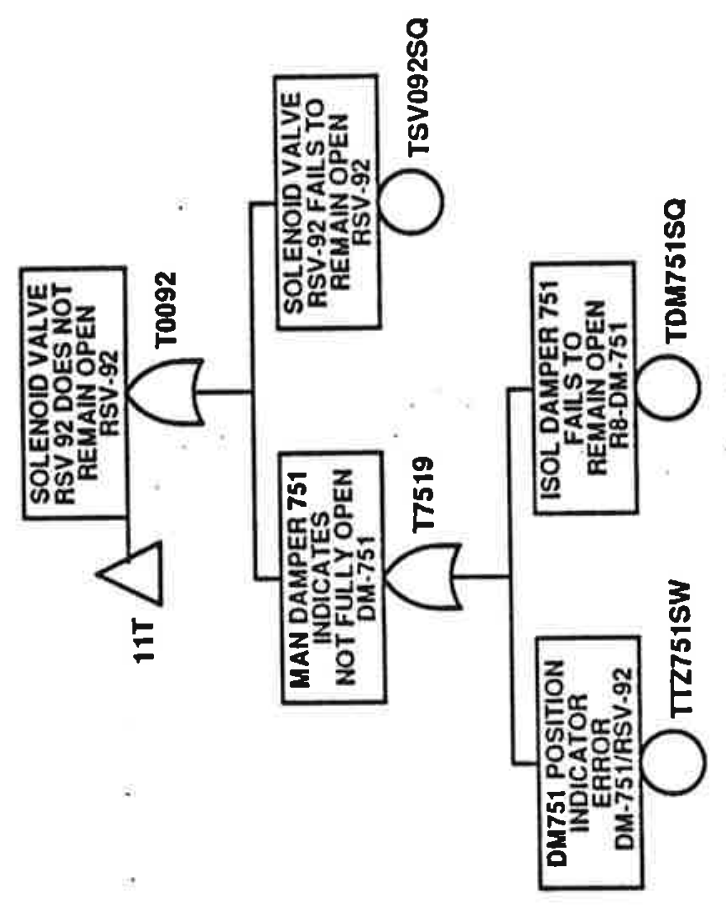

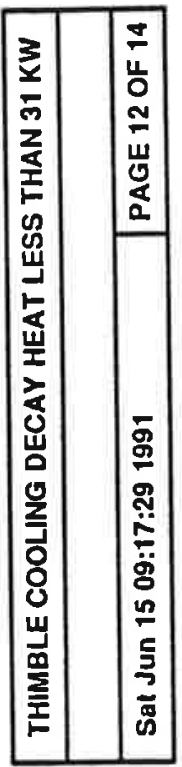




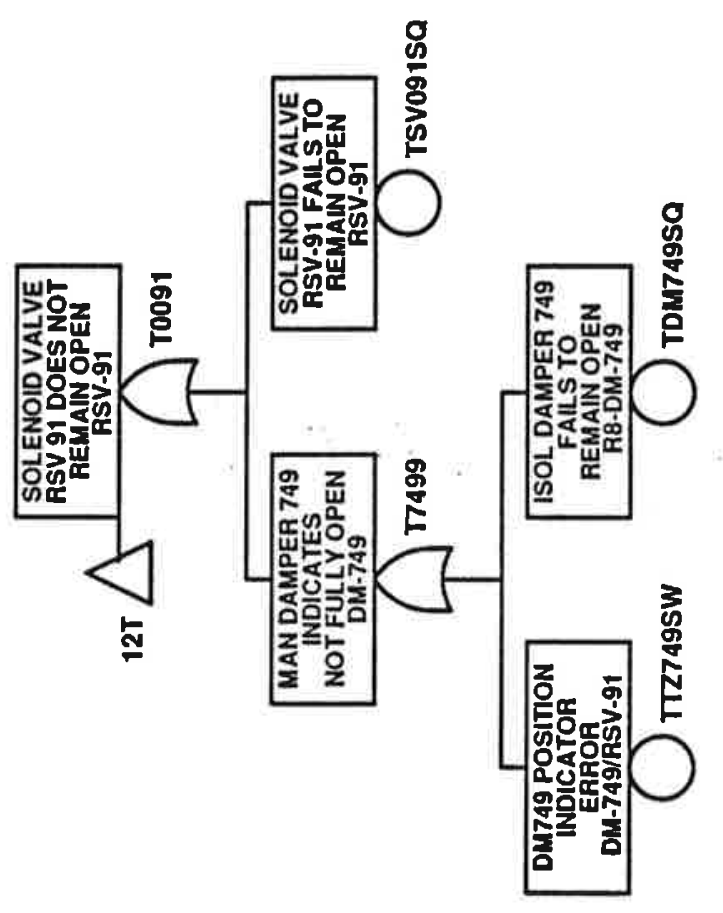

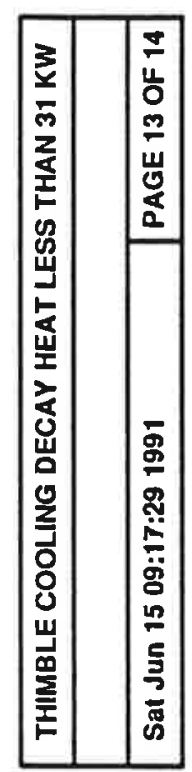




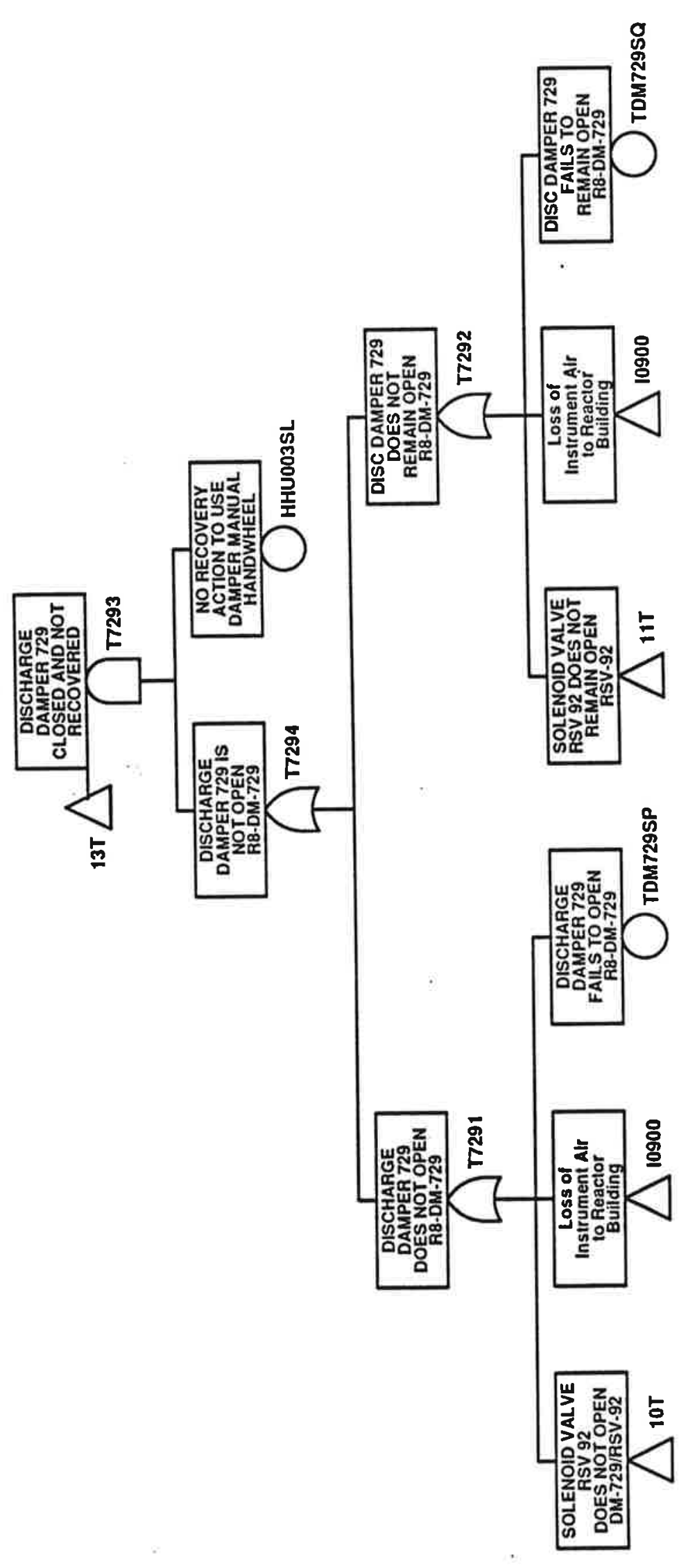

\begin{tabular}{|c|c|}
\hline $\begin{array}{l}\frac{z}{x} \\
\frac{n}{n} \\
z \\
\frac{\alpha}{5} \\
\frac{1}{n} \\
0\end{array}$ & 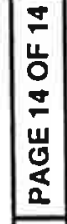 \\
\hline 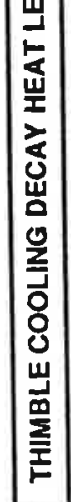 & 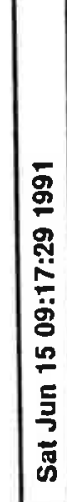 \\
\hline
\end{tabular}

\section{B. 2-582}



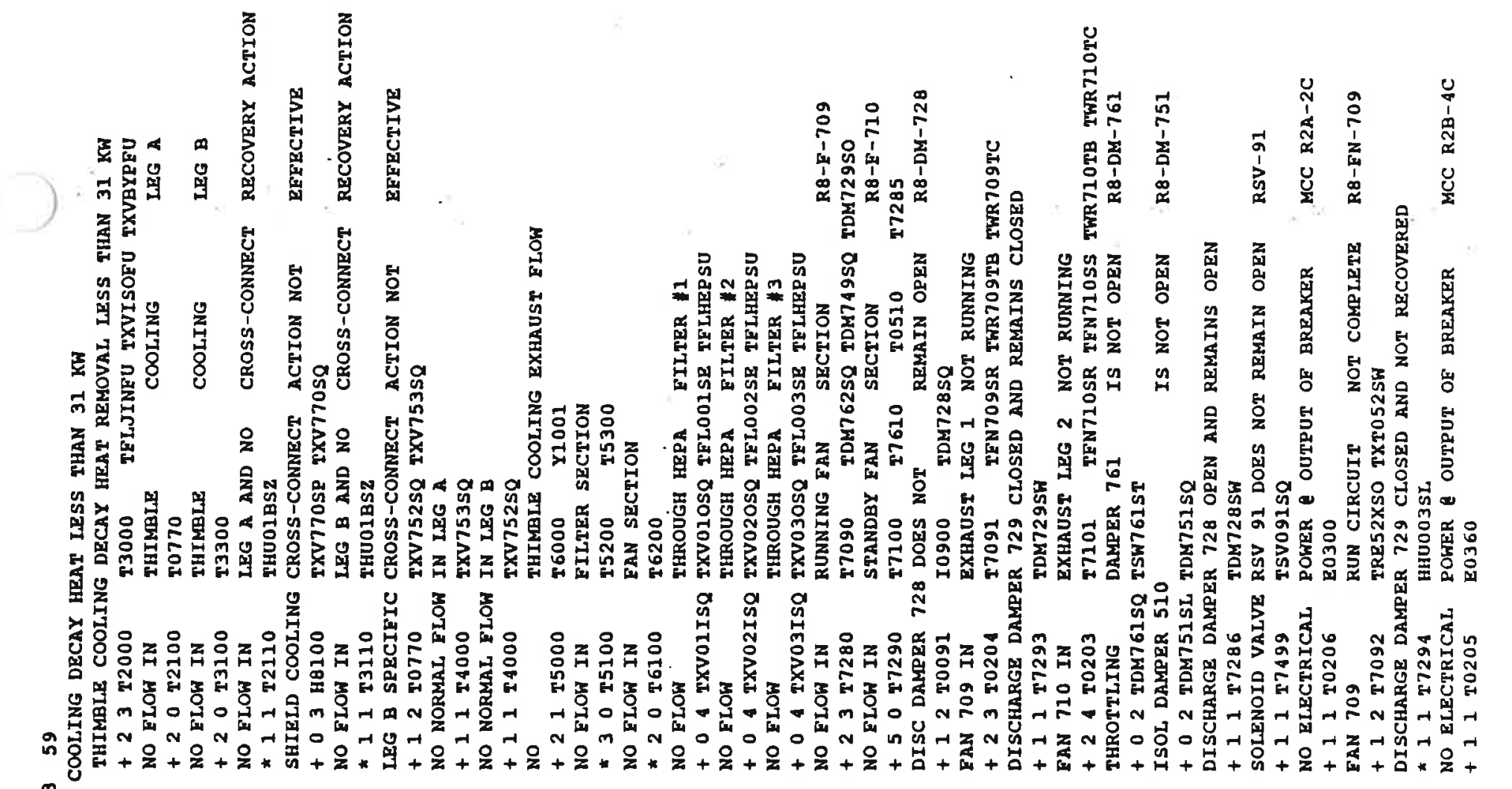

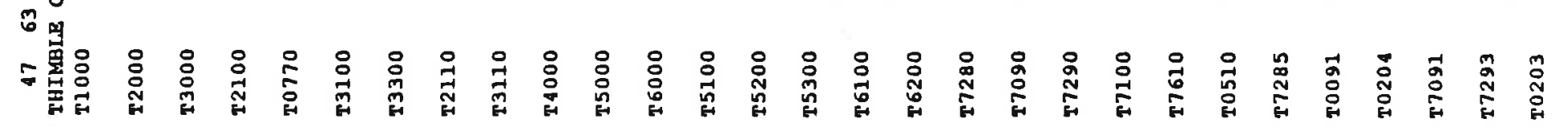




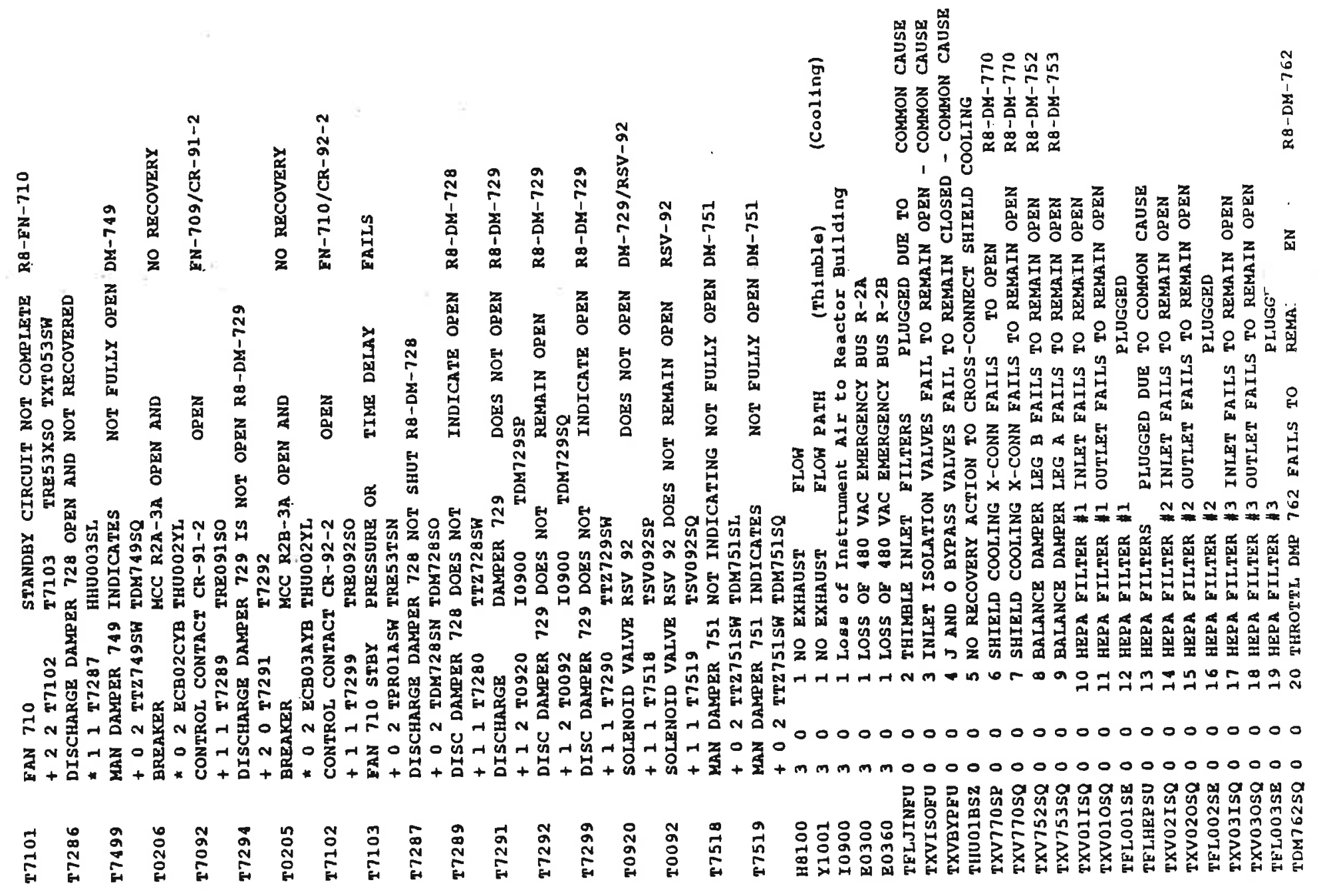




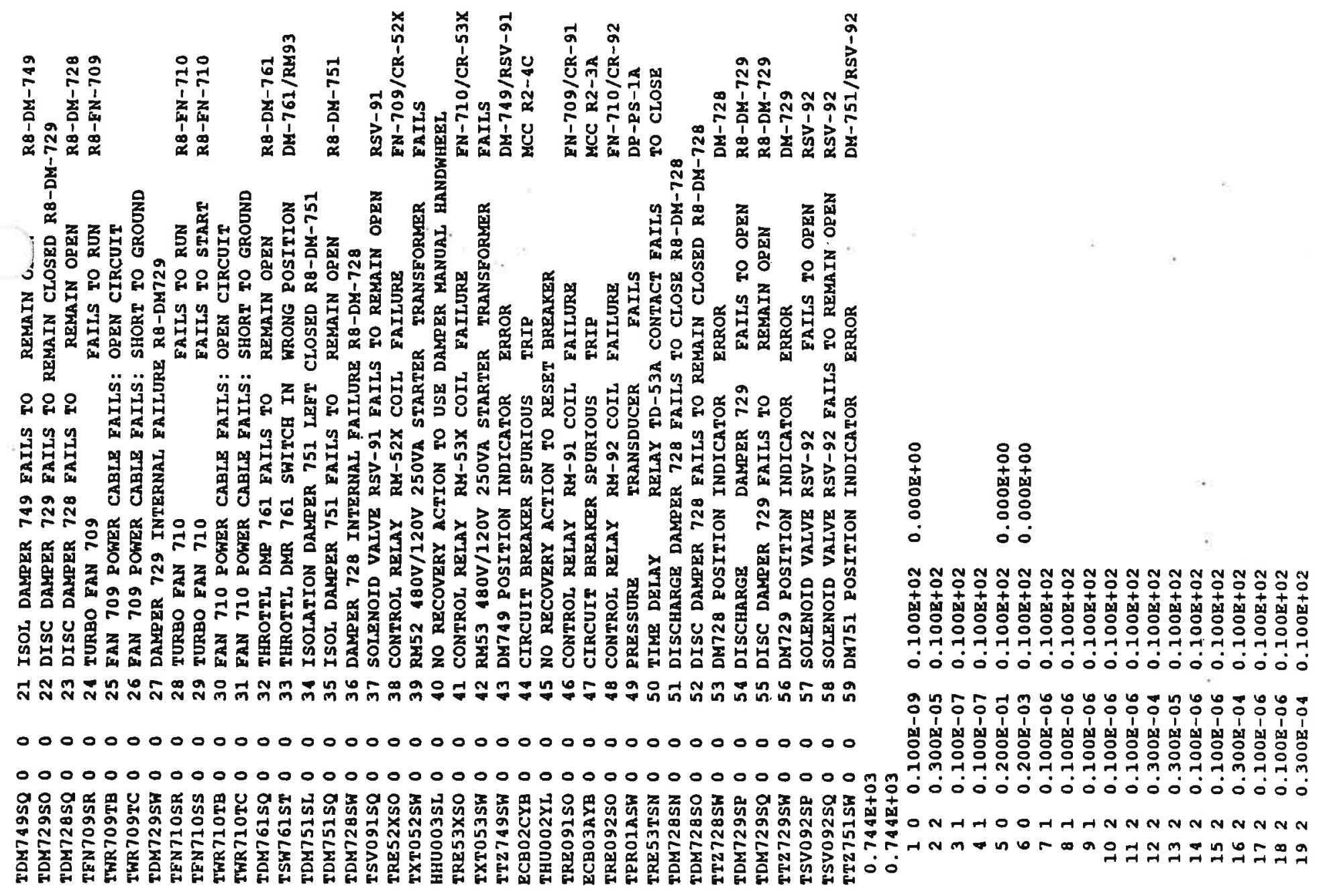




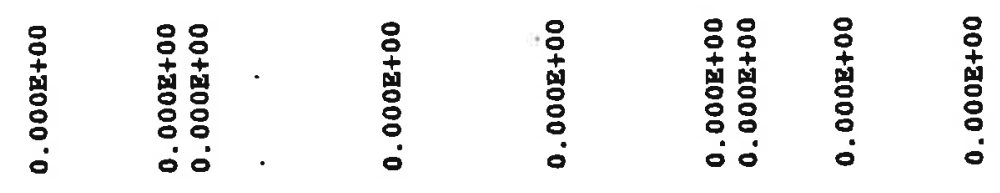

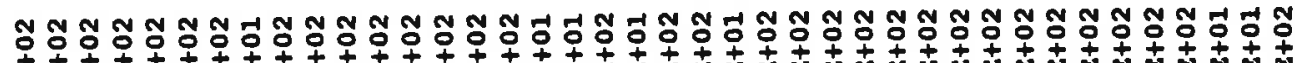

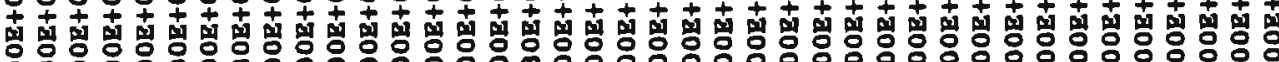

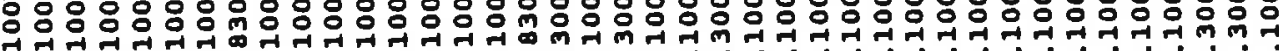

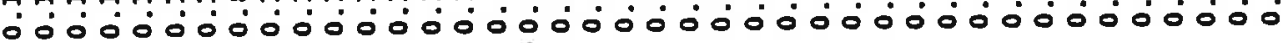

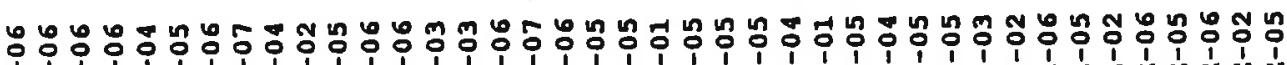

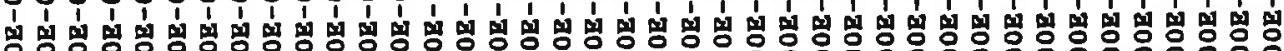

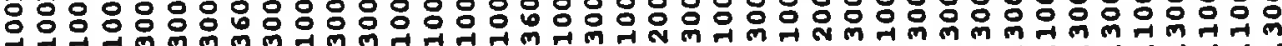

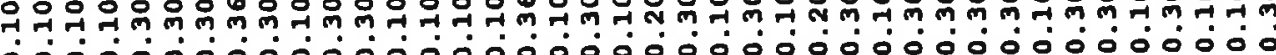
NNNNNNNHAOHAH0

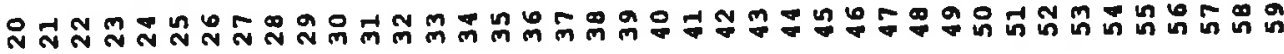




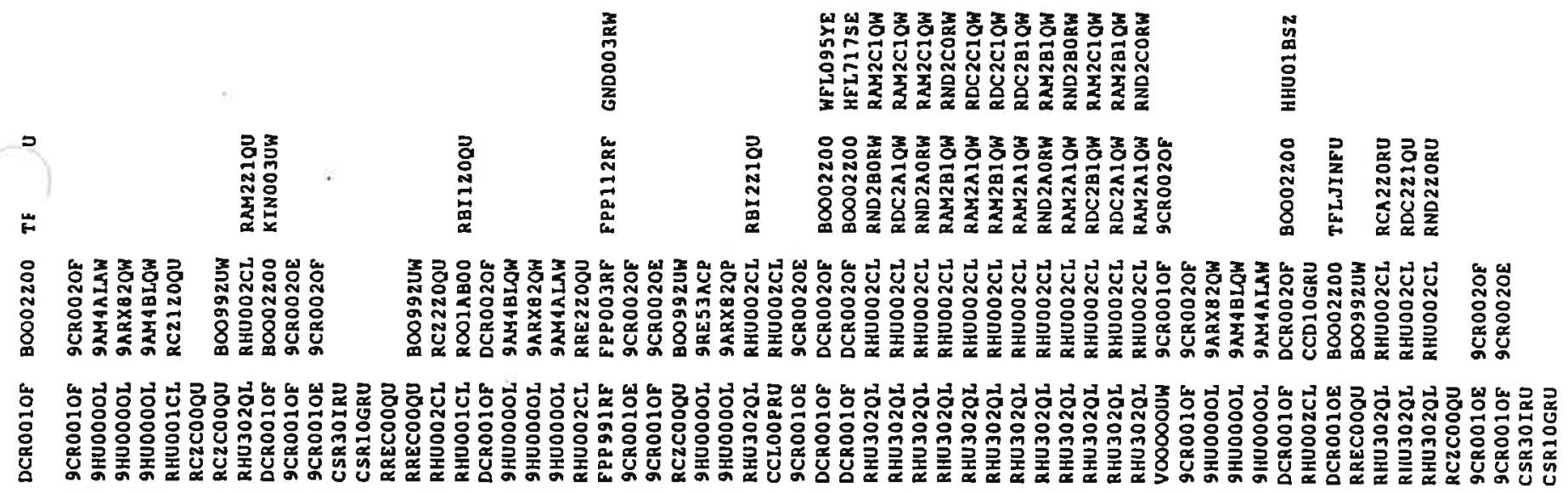

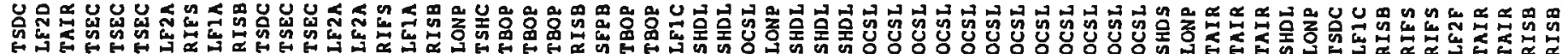

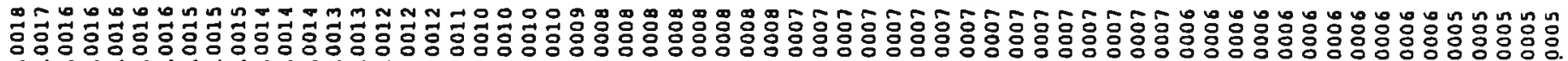

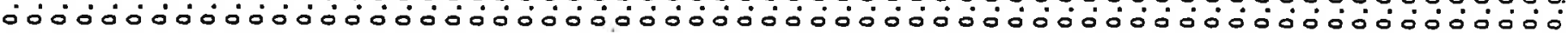

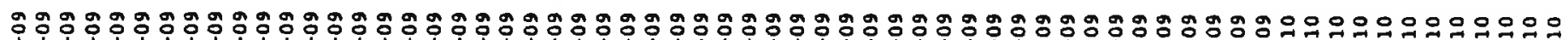

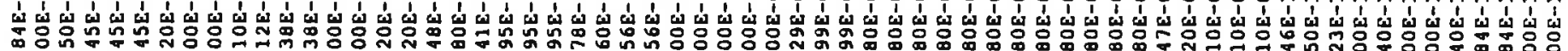

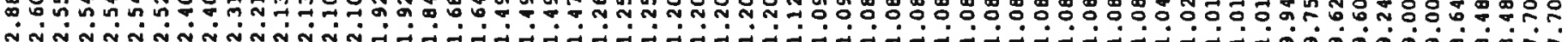

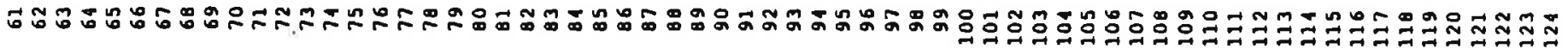

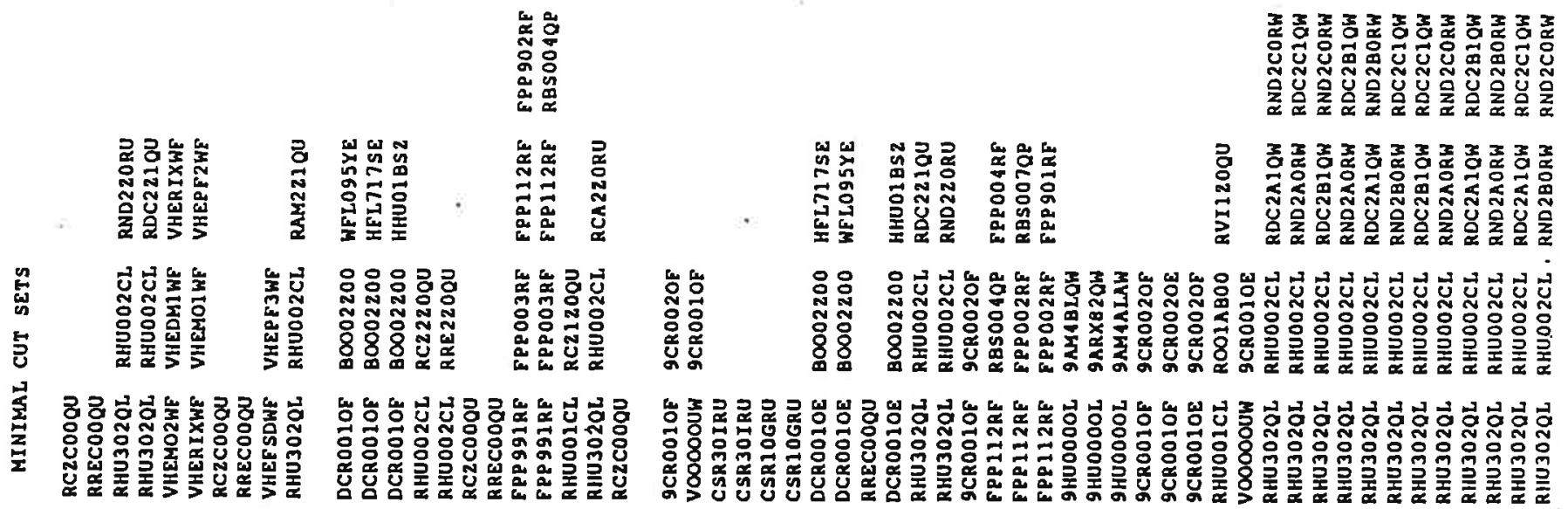




\begin{tabular}{|c|c|c|c|c|c|c|c|}
\hline 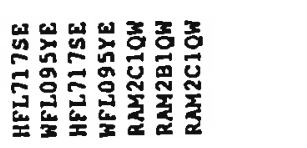 & 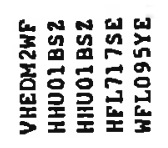 & $\begin{array}{l}\text { 岗 } \\
\text { 空 } \\
\text { 至 }\end{array}$ & 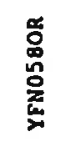 & 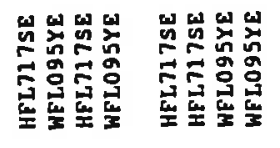 & 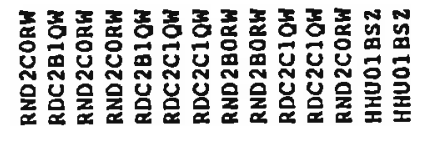 & 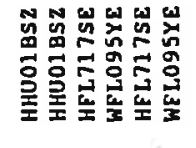 & 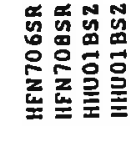 \\
\hline 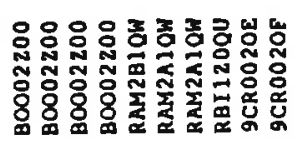 & 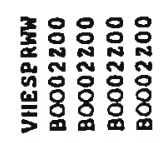 & 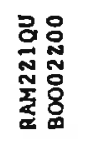 & 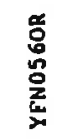 & 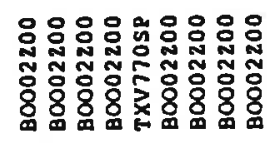 & 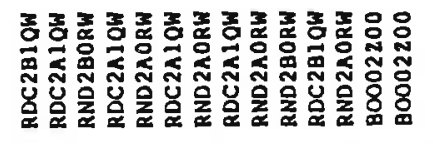 & 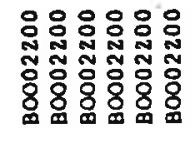 & 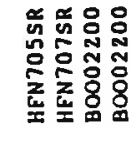 \\
\hline
\end{tabular}

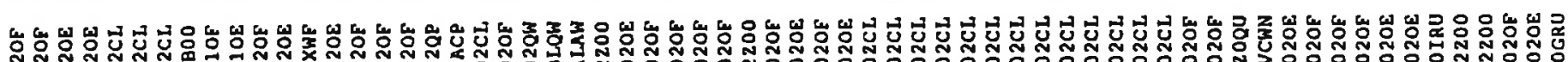

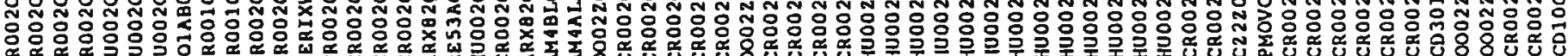

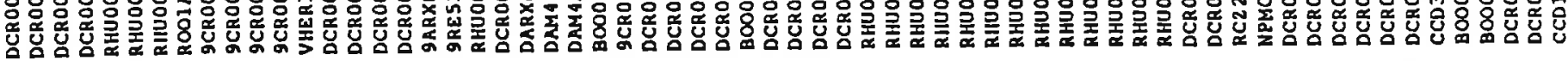

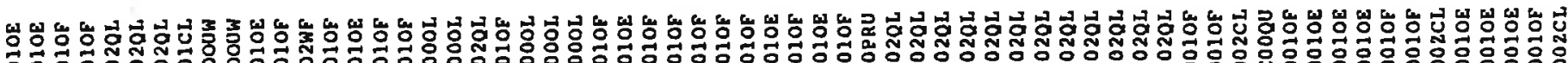

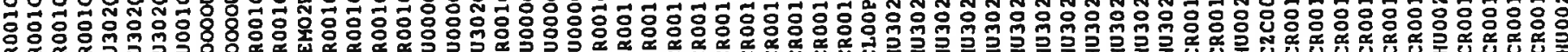

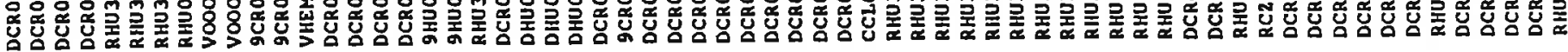

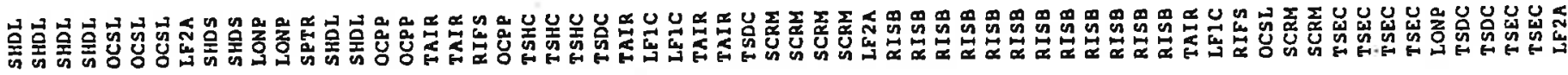

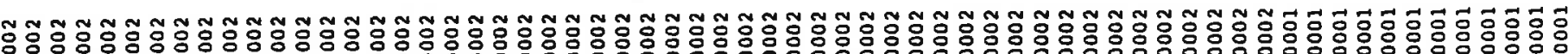

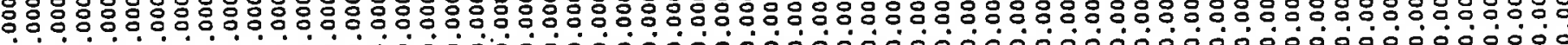

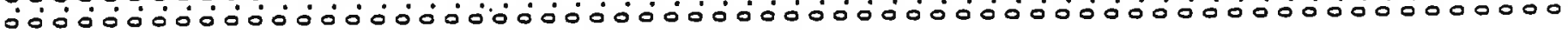

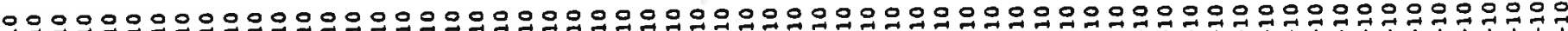

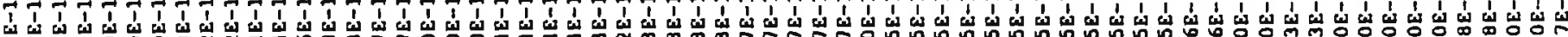

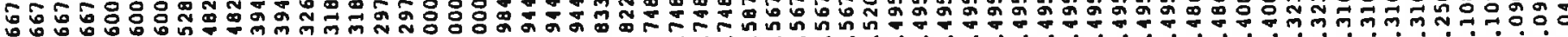

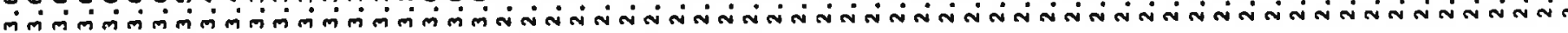

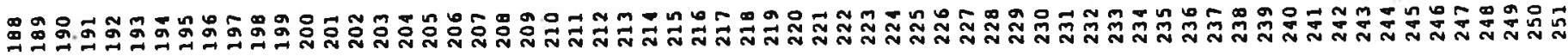

\begin{tabular}{|c|c|c|c|c|}
\hline 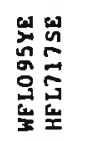 & & 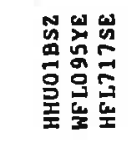 & 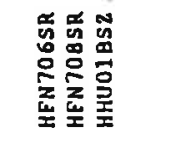 & 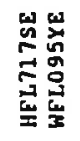 \\
\hline 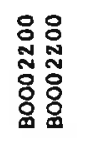 & 惡 & 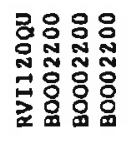 & 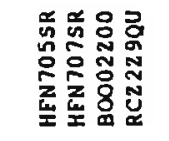 & 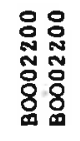 \\
\hline 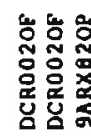 & & 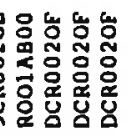 & 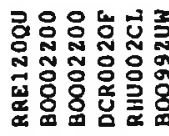 & aric \\
\hline
\end{tabular}

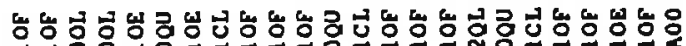

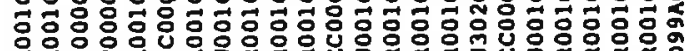

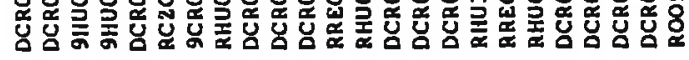

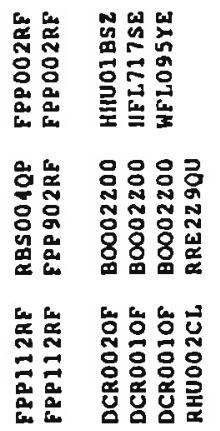

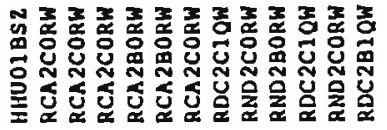

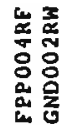

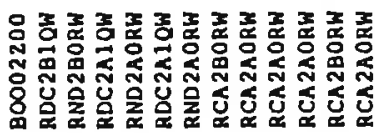

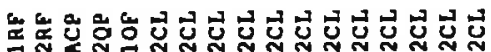

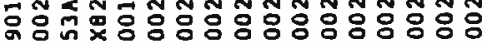

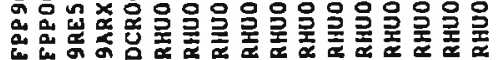

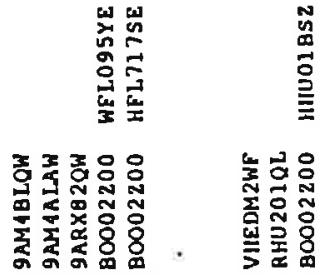

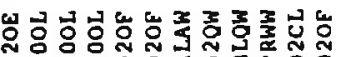

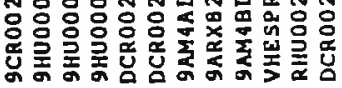
㡙

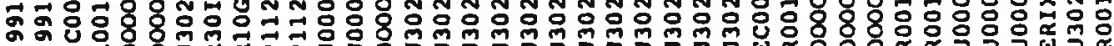

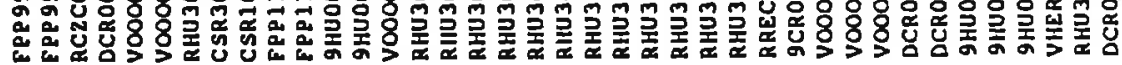

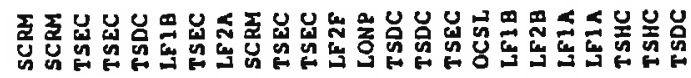

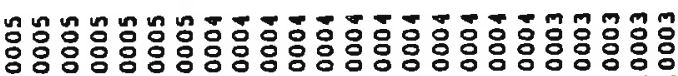

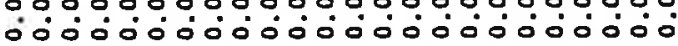

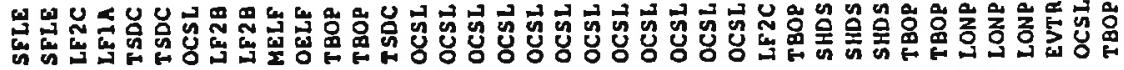

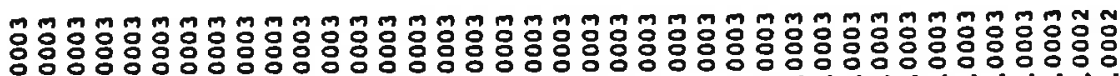

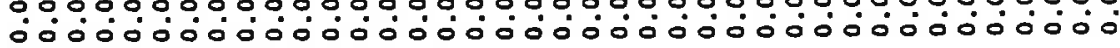

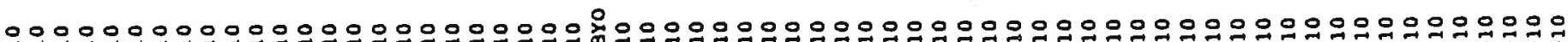

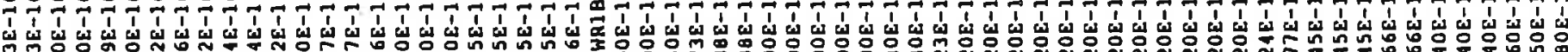

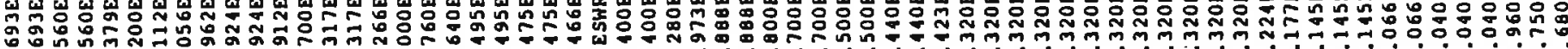

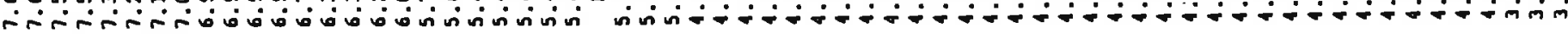

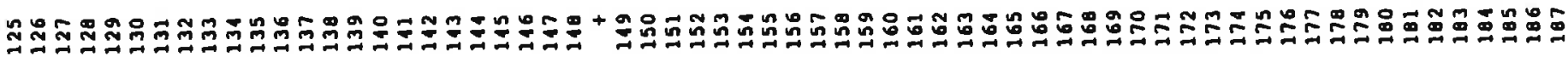




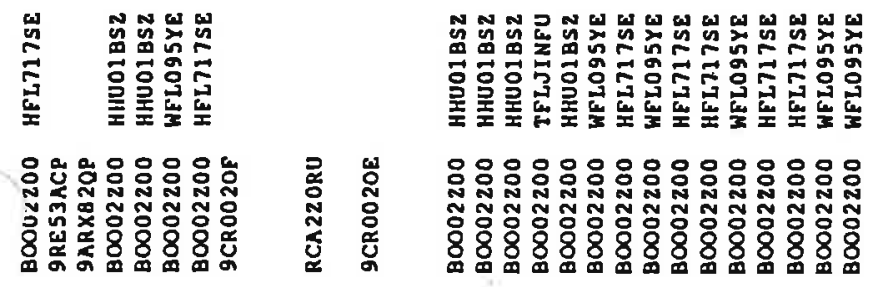

ร)

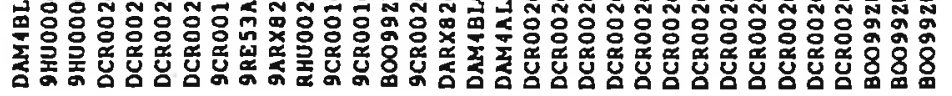

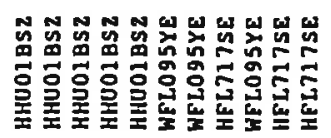

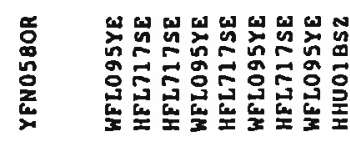

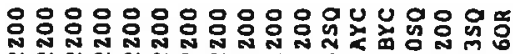

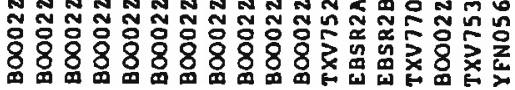

흥융유웅웅융유유

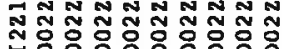

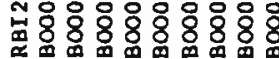

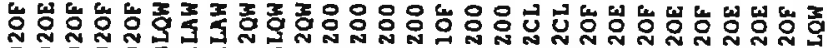

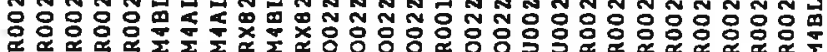

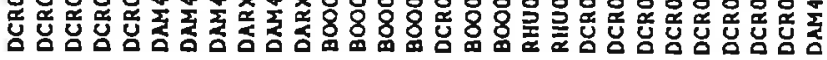

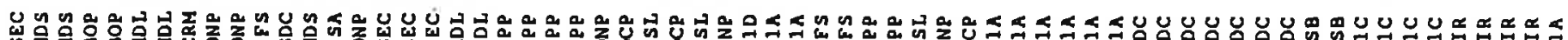

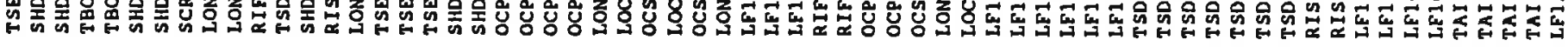

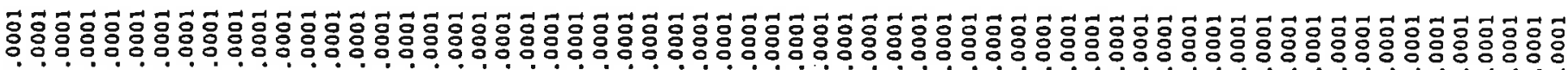

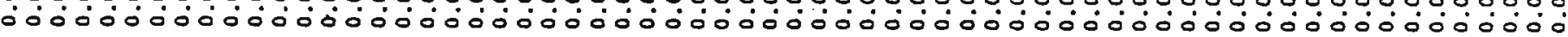

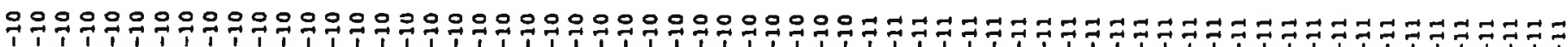

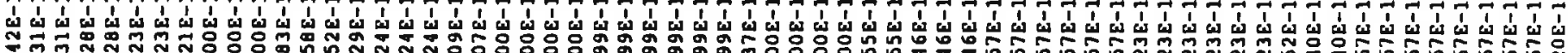

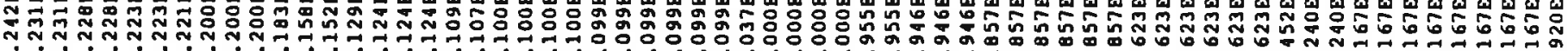

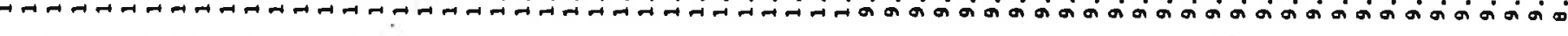

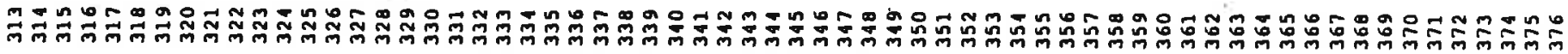

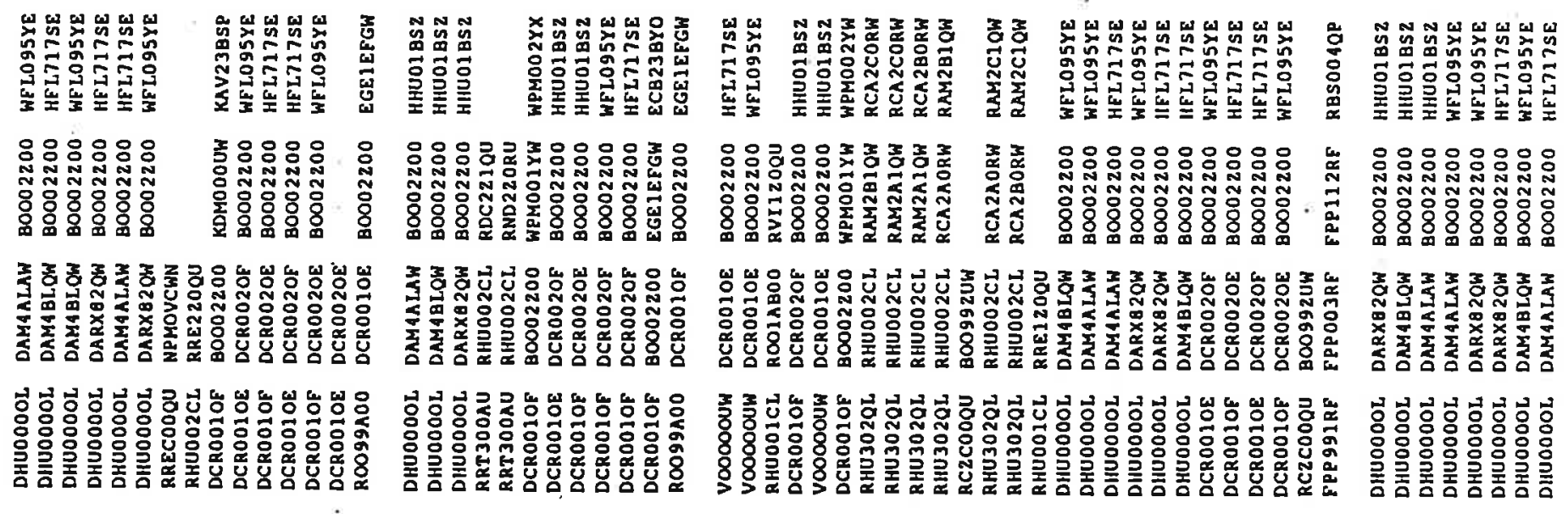

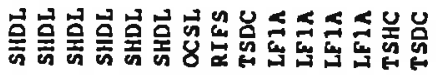

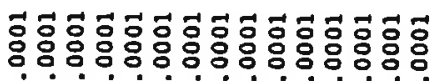

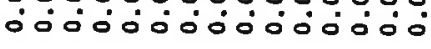

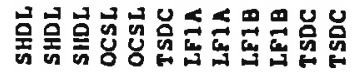

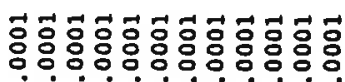

000000000000

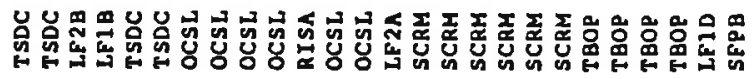

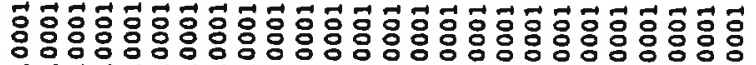

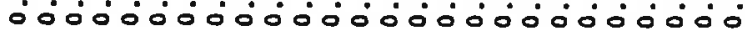

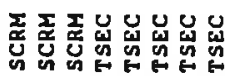

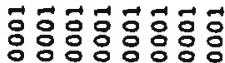

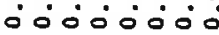

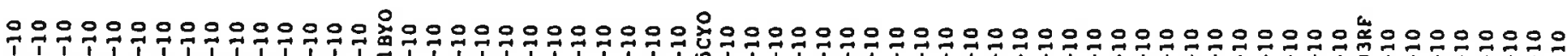

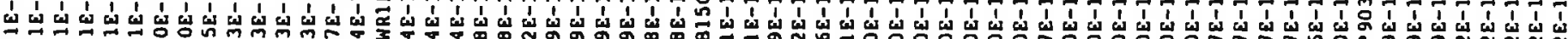

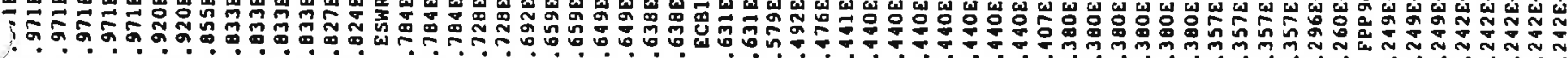

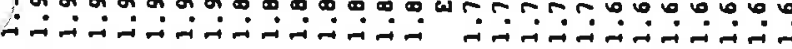

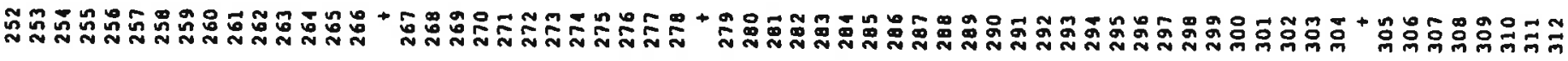




\section{嵒品 \\ 傿

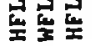

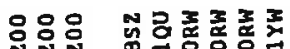

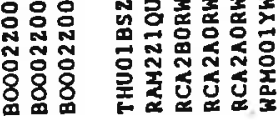

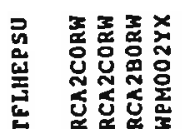

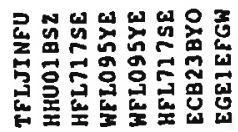

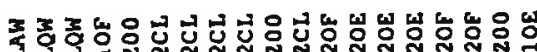

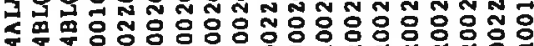

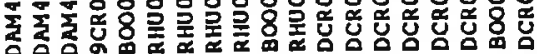

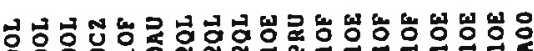

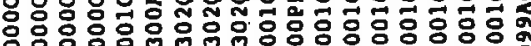

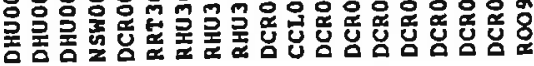

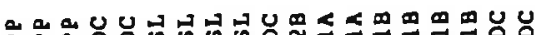

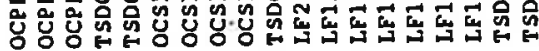
ํํำำำำ

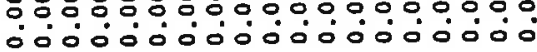

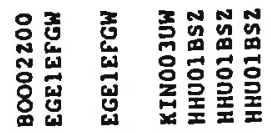

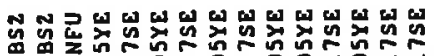
홍ํำ

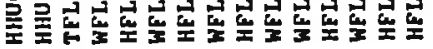

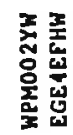

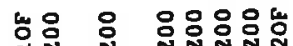

苳

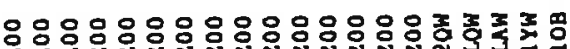

N N N N N N N N N N N N N N

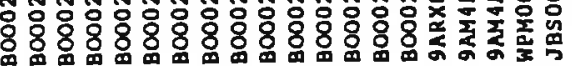

突紫

형웅

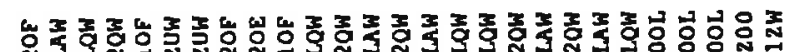

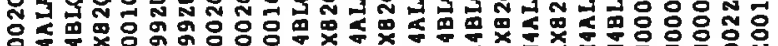

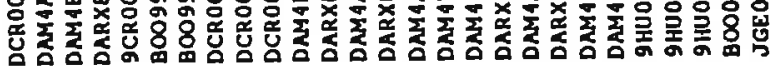

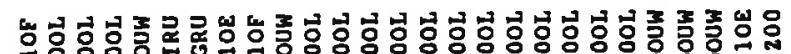

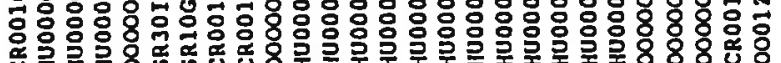

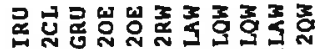



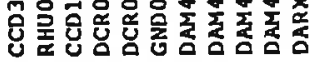

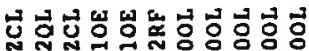

ㅇㅇㅇ영형ㅇㅇㅇㅇㅇ

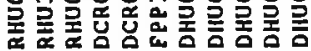

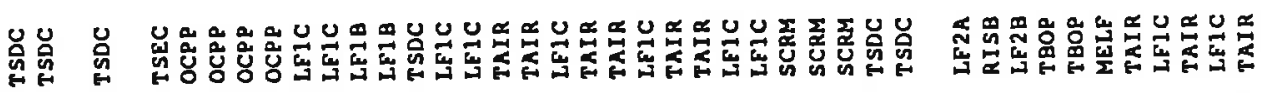

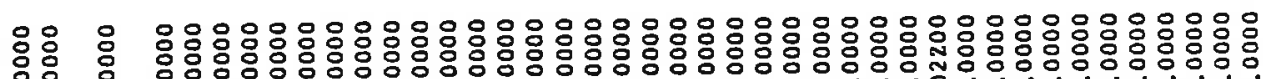

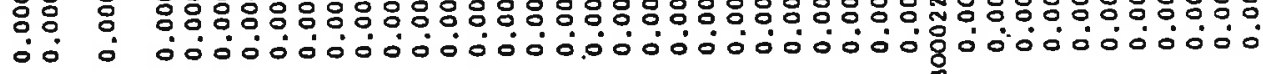

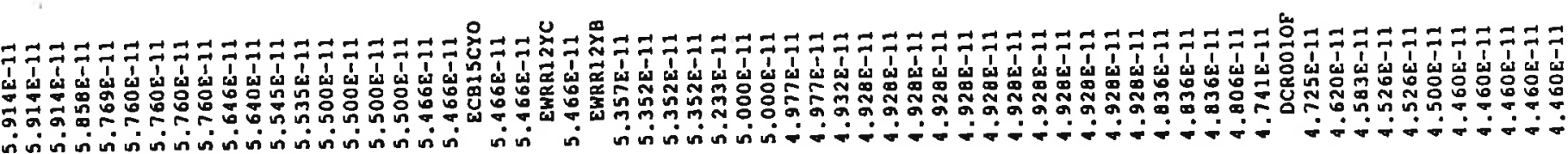

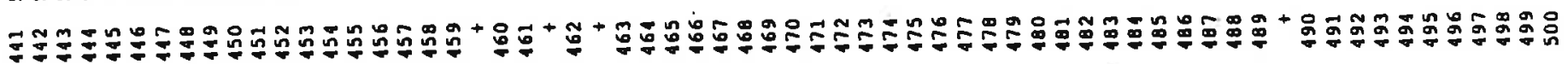

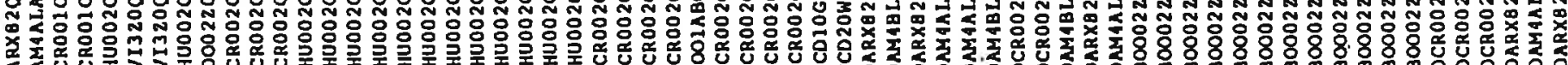

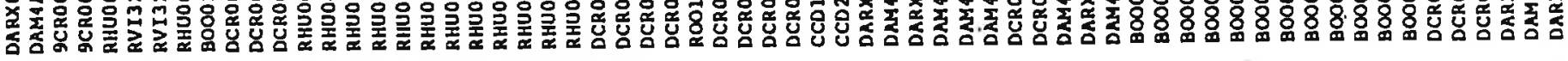

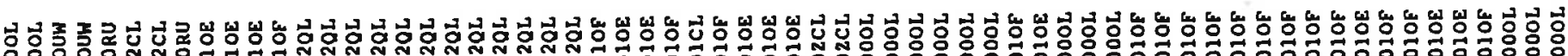

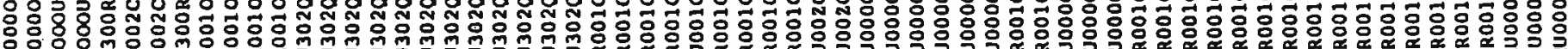

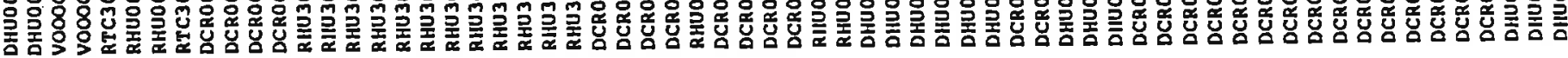

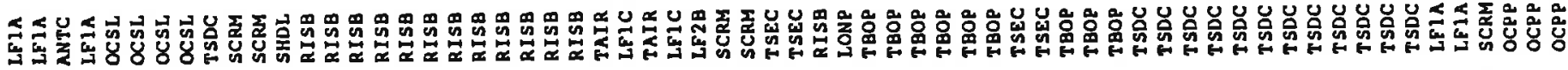

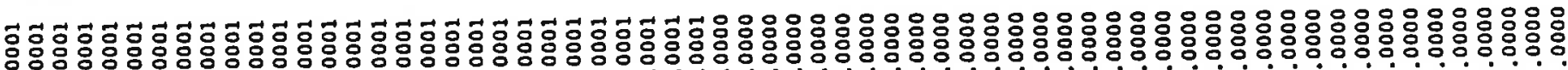

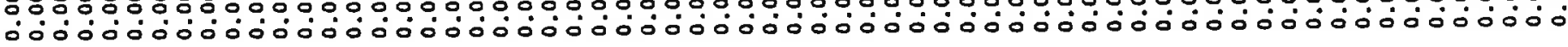

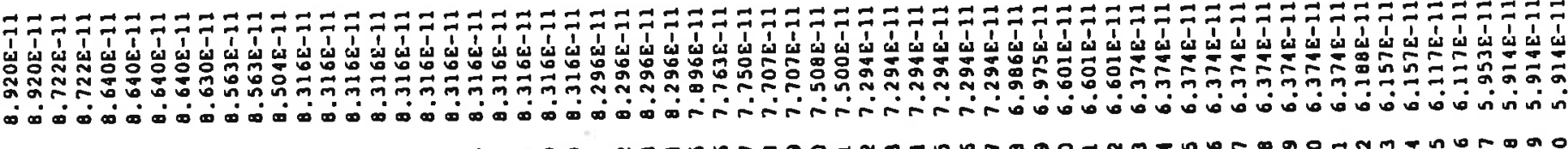

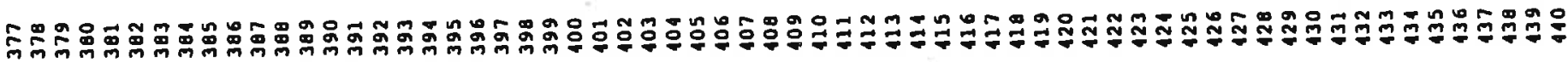




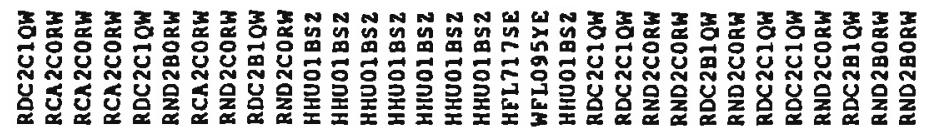

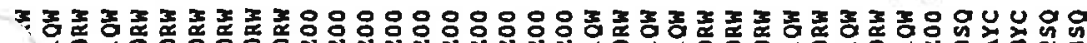

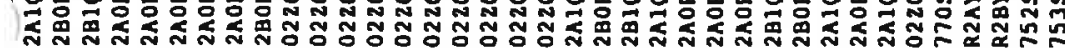

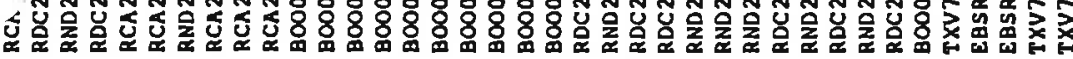

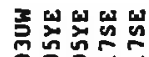

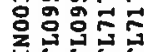

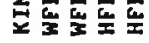

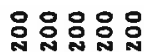

各客客囱亩

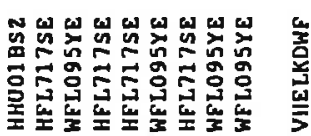

응응영웅웅응

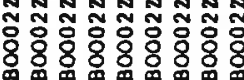

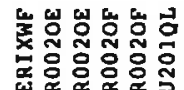

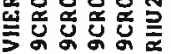

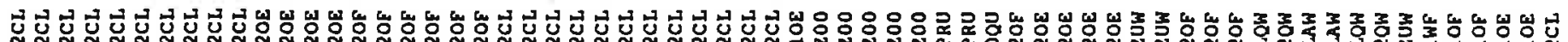

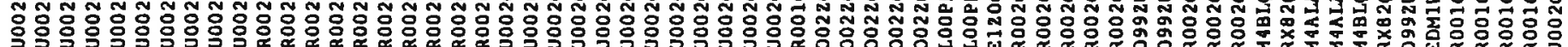

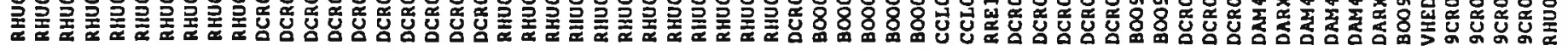

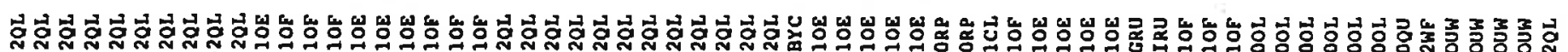

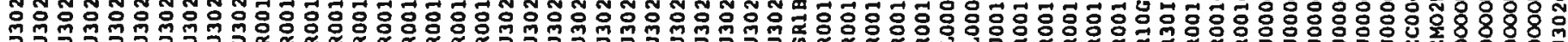

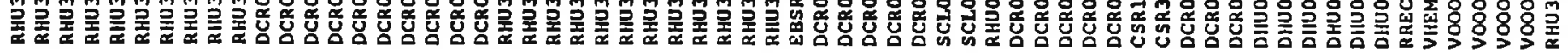

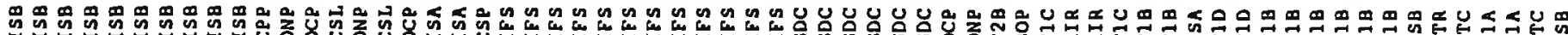

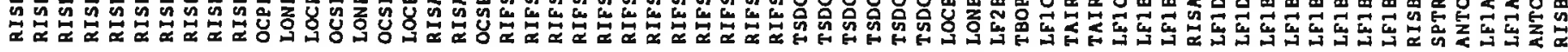

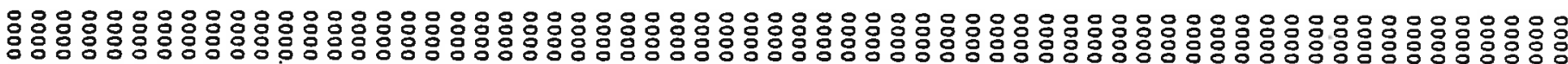

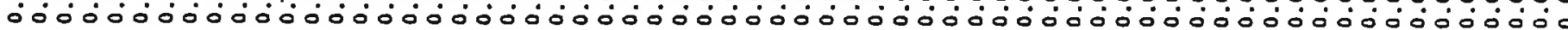

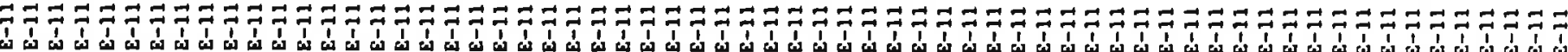

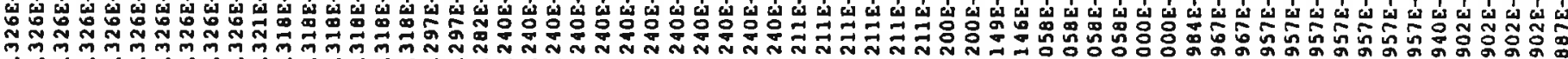

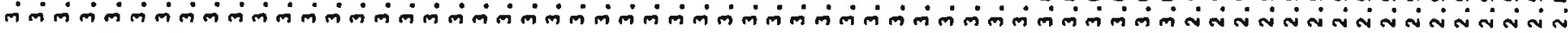

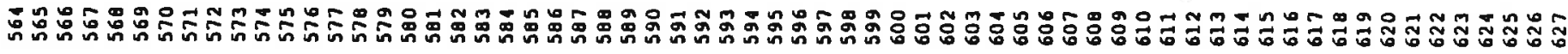

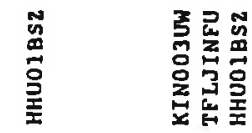

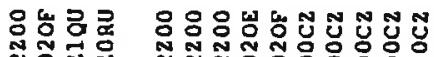

הัก

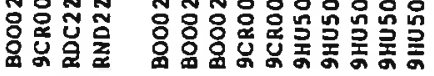

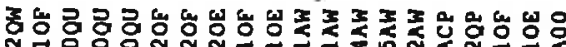

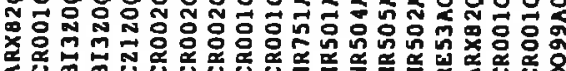

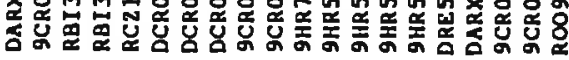

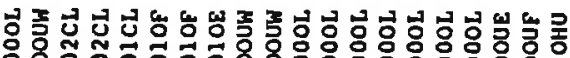

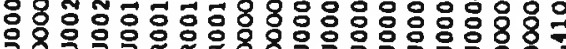

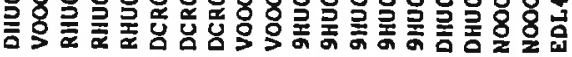

总

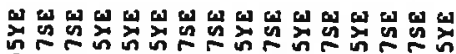

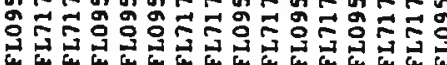

응 :

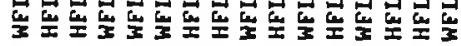

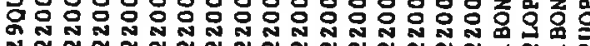

రํㅇㅇํㅇ.

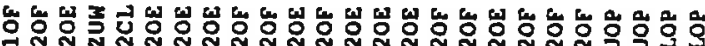

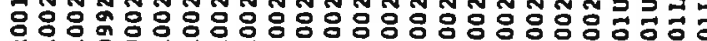

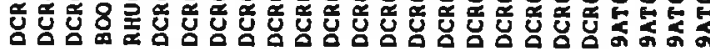

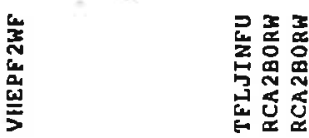

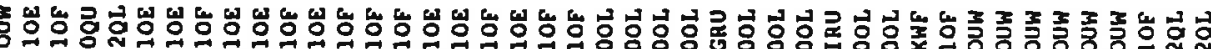

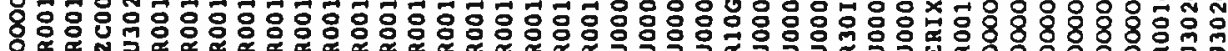

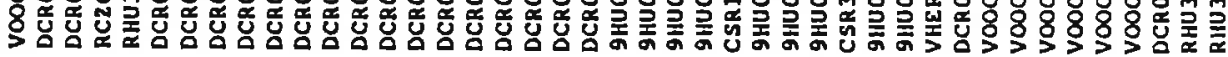

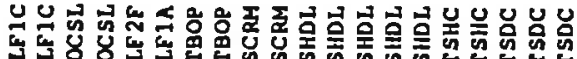
용ㅇㅇㅇㅇㅇㅇㅇㅇㅇㅇㅇㅇㅇㅇㅇㅇㅇㅇㅇㅇㅇㅇㅇ응

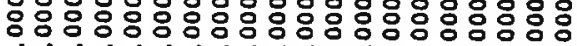

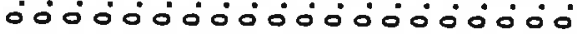

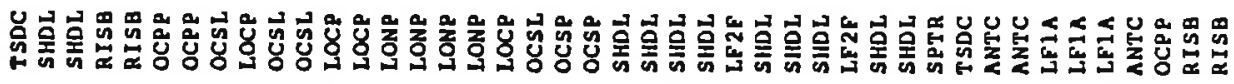

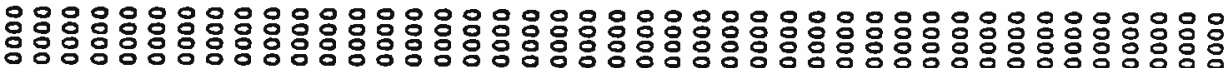

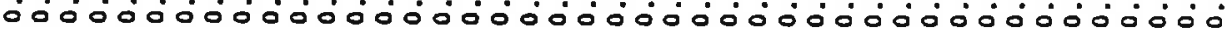

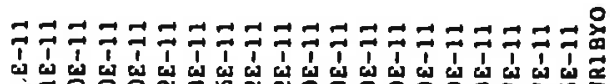

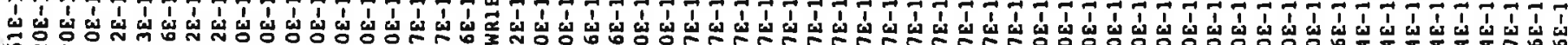
掣

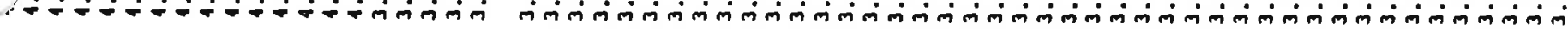

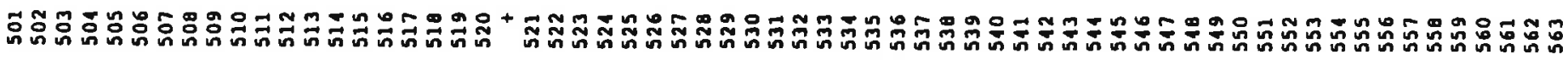




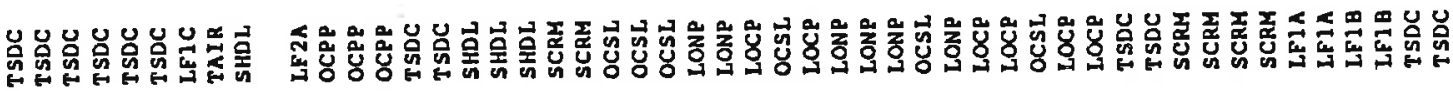

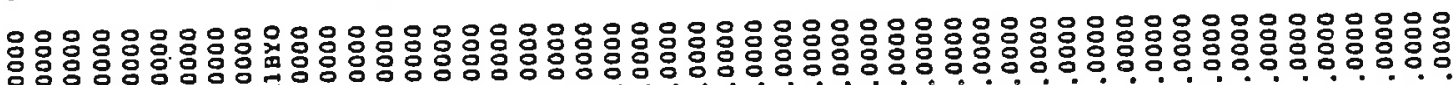

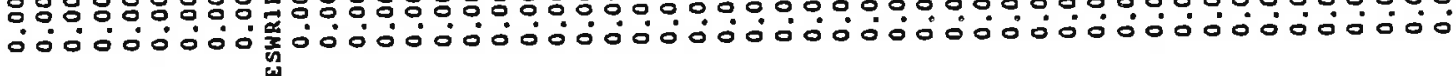

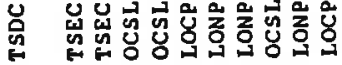

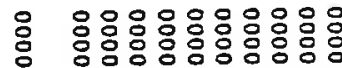 - 0000000000}

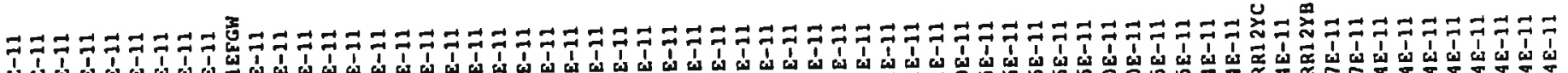

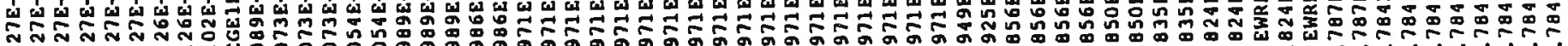
تंसं

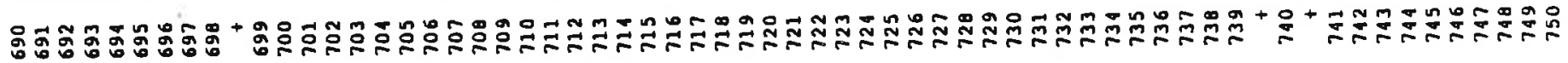

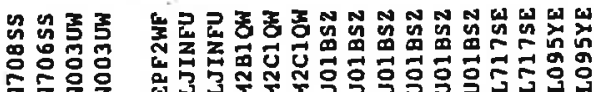

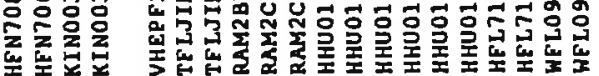

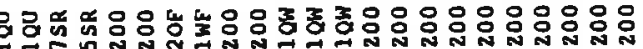

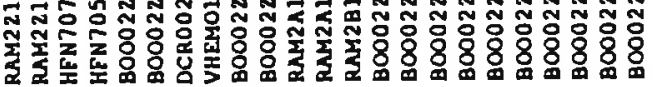

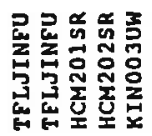

定

ֻूँ

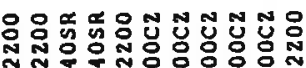

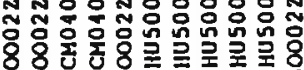

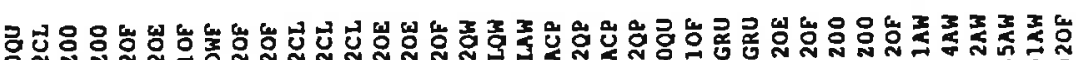

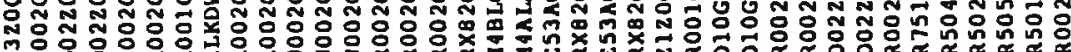

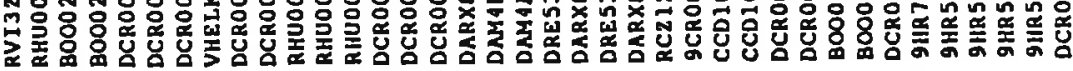

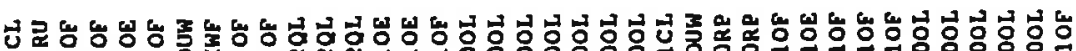

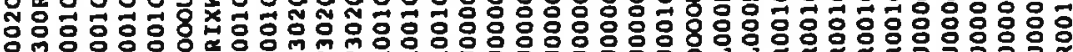

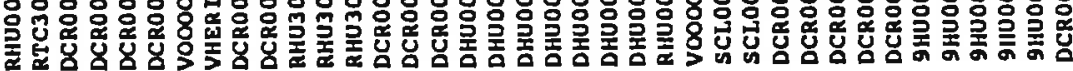

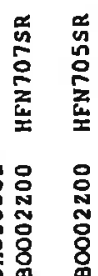

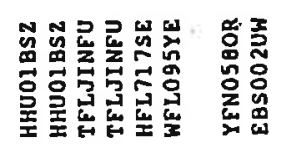

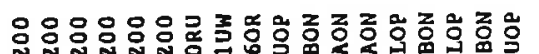

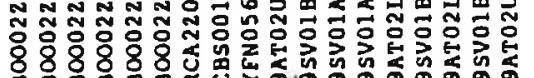

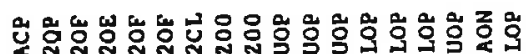

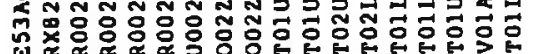

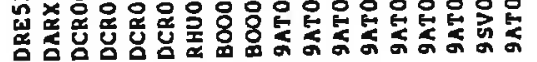

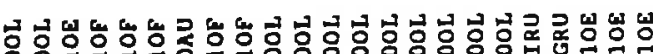

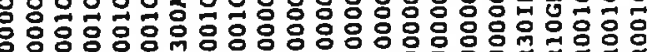

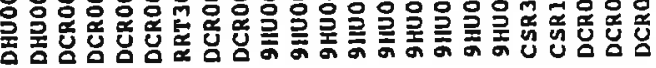

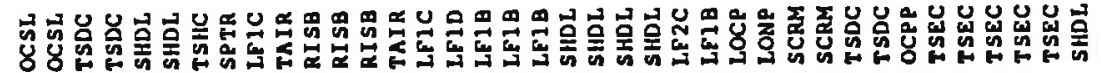

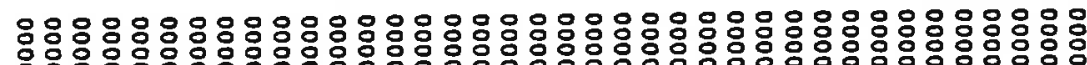

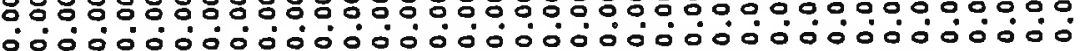

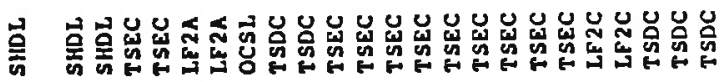

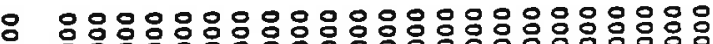

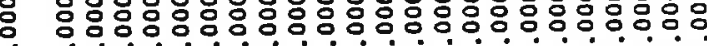
i000000000000000000000

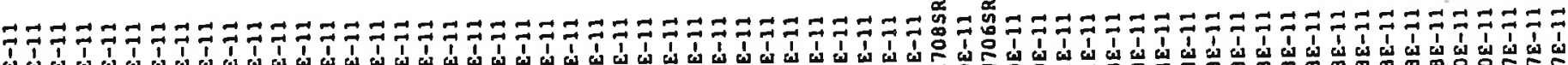

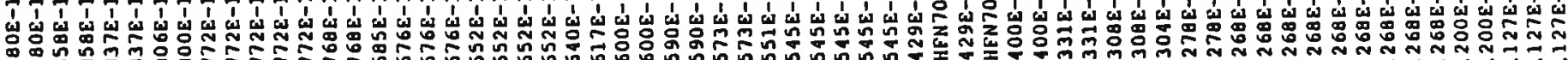
ФФ

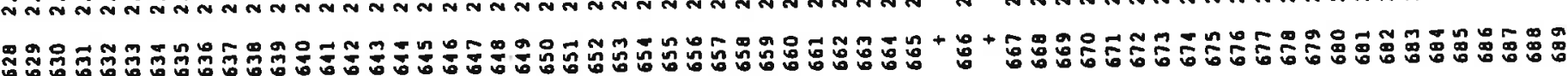




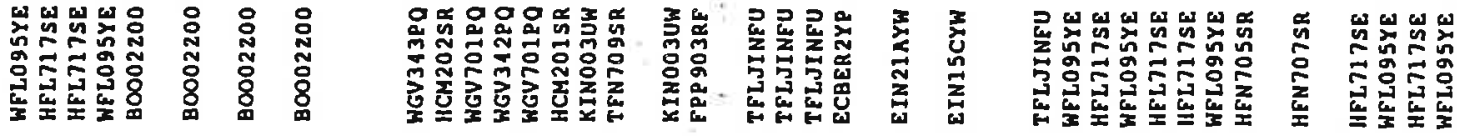 "Unumenem}

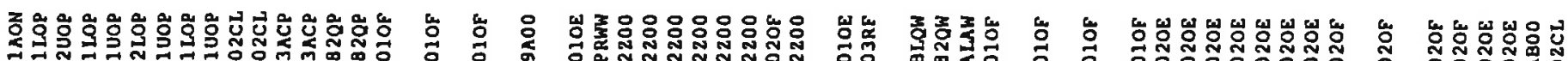
Hแแ

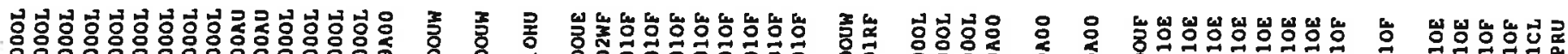

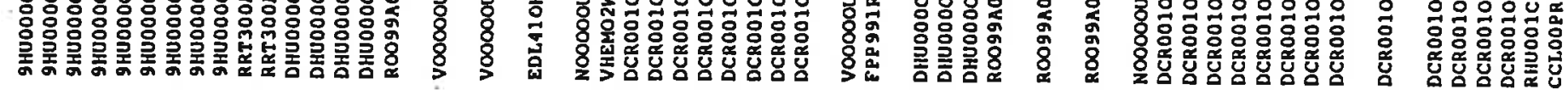

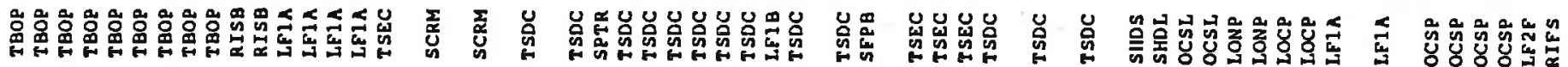

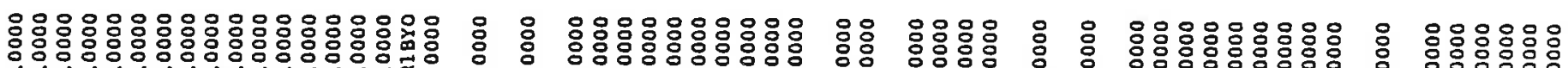

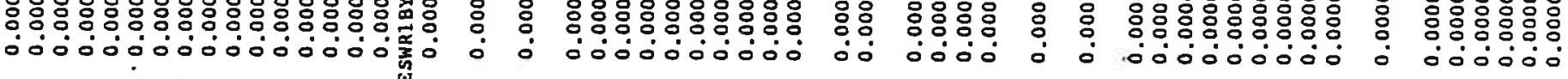

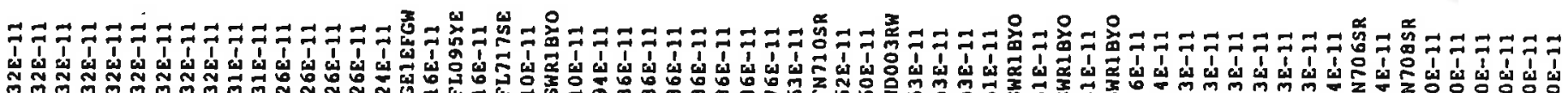

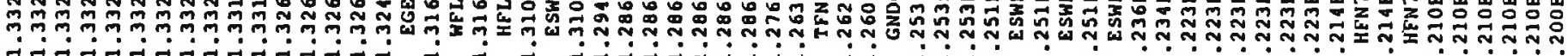

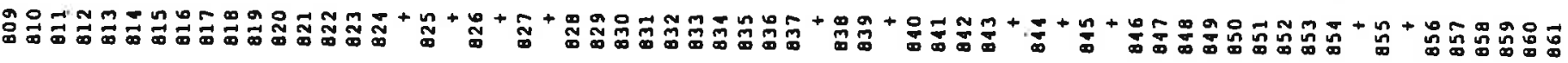

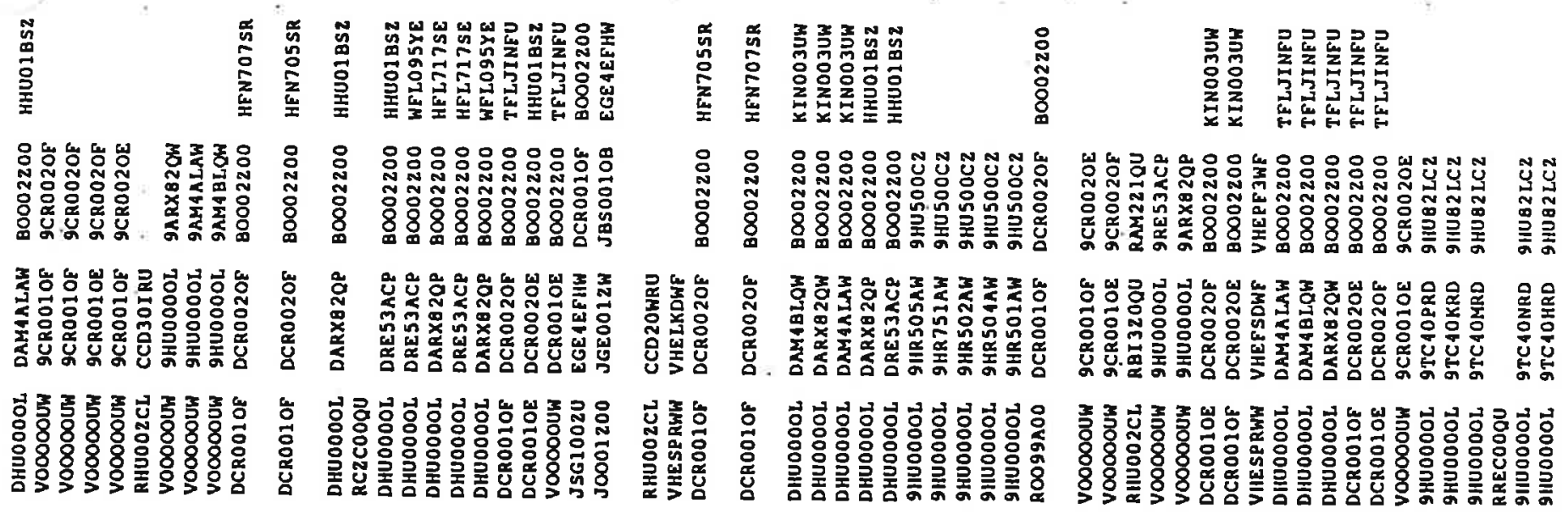

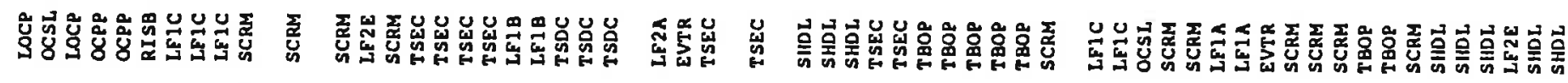

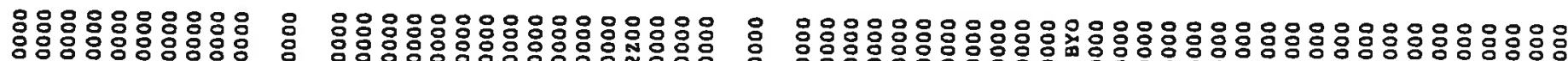

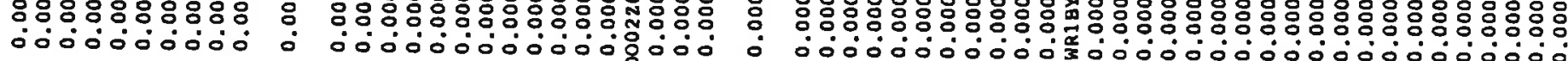

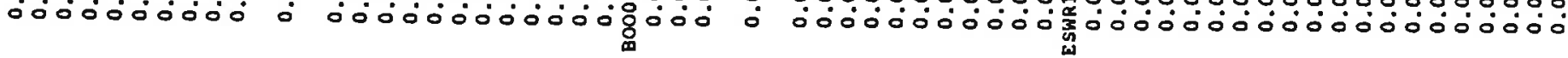

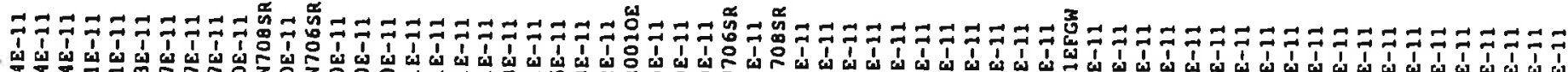

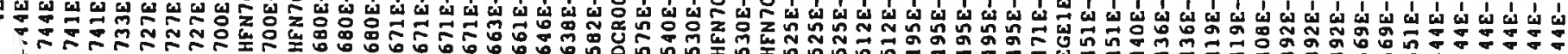
Fis

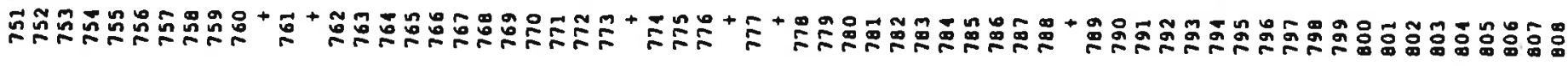




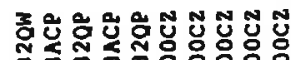

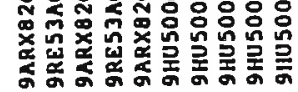

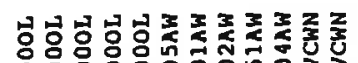

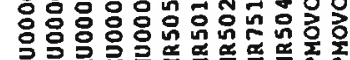

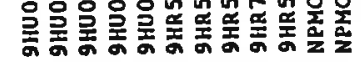

mWint!n!

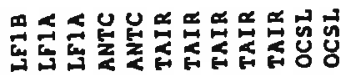

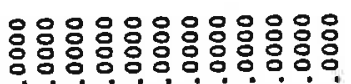

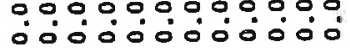

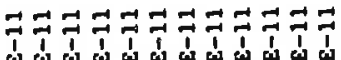

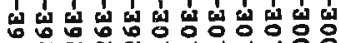

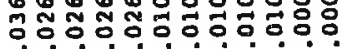

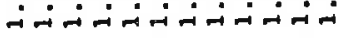

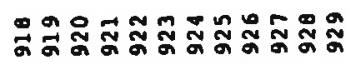

岕 点

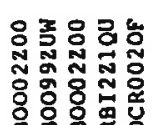

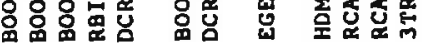

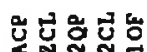

mํํㅇํㅇㅇㅠ.

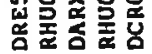

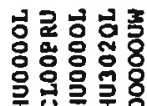

홈언옿롶

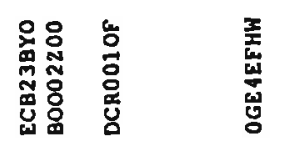

웅혀 존 뭉궁흥

일

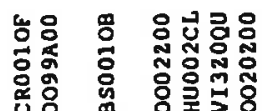

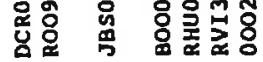

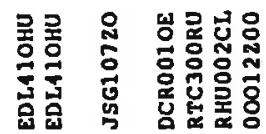

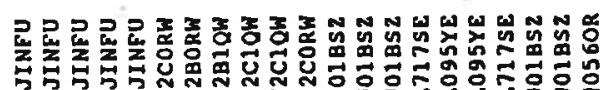

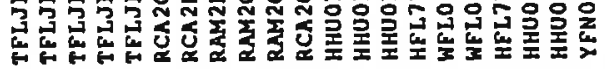

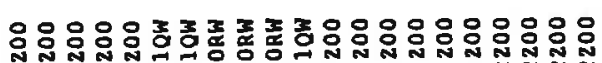

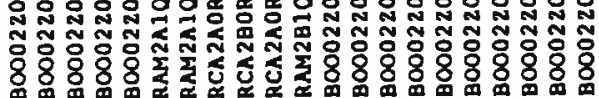

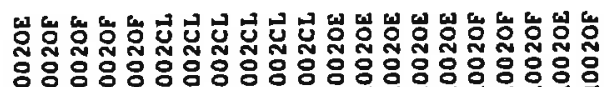

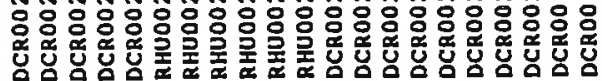

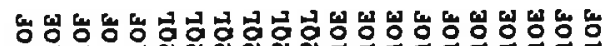

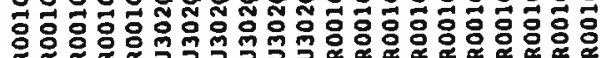

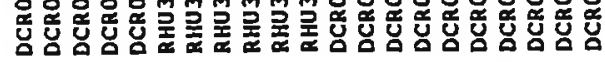

\section{离}

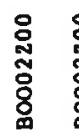

容 :

$\frac{2}{8} 8$

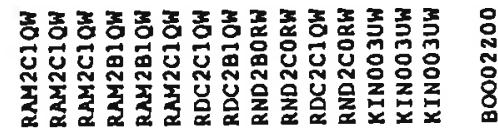

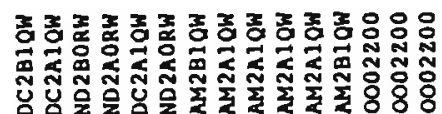

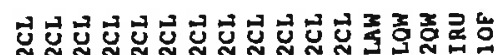

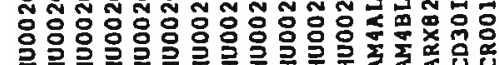

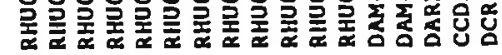

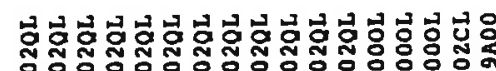

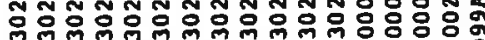

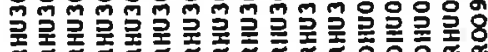

蛋旁 웅용중 กับ

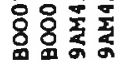

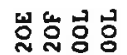
융융을옹

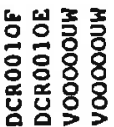

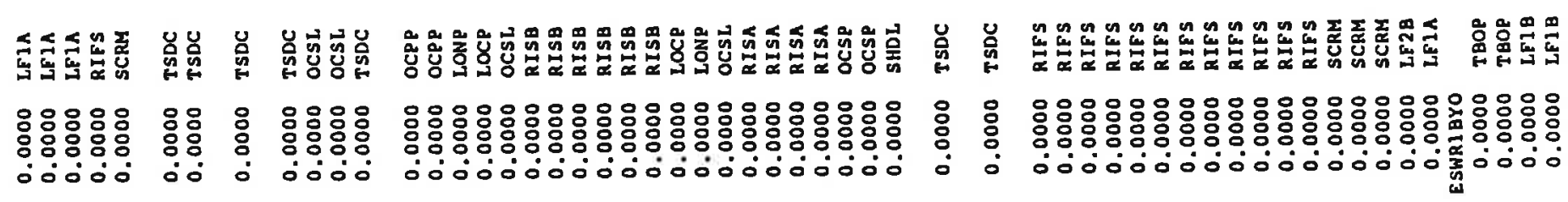

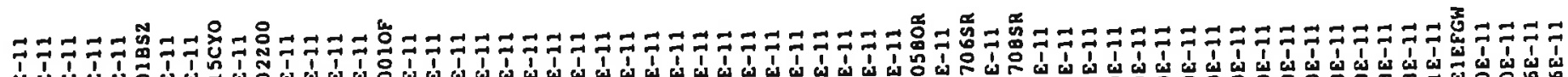

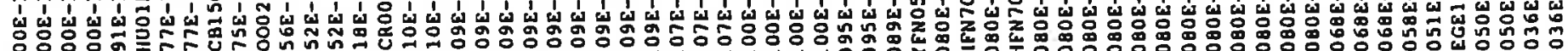

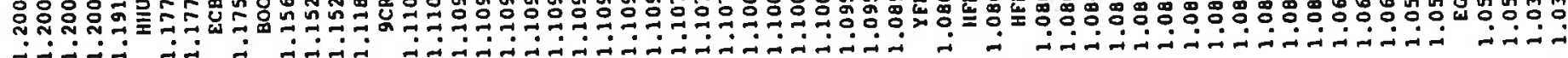

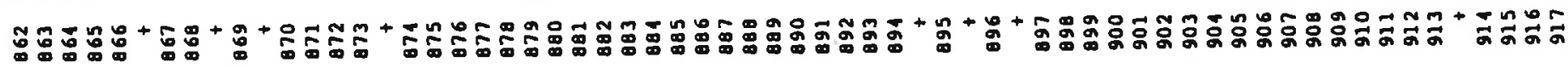




\section{Argonne}

Nuclear Science and Engineering Division

Argonne National Laboratory

9700 South Cass Avenue, Bldg. 208 Argonne, IL

60439-4842 www.anl.gov 